\title{
Utilization of a Numerical Reservoir Simulation with Water and Gas Injection for Verification of Top Down Modeling
}

\author{
Ashley Konya \\ aekonya@mix.wvu.edu
}

Follow this and additional works at: https://researchrepository.wvu.edu/etd

Part of the Other Engineering Commons

\section{Recommended Citation \\ Konya, Ashley, "Utilization of a Numerical Reservoir Simulation with Water and Gas Injection for Verification of Top Down Modeling" (2020). Graduate Theses, Dissertations, and Problem Reports. 7573. https://researchrepository.wvu.edu/etd/7573}

This Thesis is protected by copyright and/or related rights. It has been brought to you by the The Research Repository @WVU with permission from the rights-holder(s). You are free to use this Thesis in any way that is permitted by the copyright and related rights legislation that applies to your use. For other uses you must obtain permission from the rights-holder(s) directly, unless additional rights are indicated by a Creative Commons license in the record and/ or on the work itself. This Thesis has been accepted for inclusion in WVU Graduate Theses, Dissertations, and Problem Reports collection by an authorized administrator of The Research Repository @ WVU. For more information, please contact researchrepository@mail.wvu.edu. 


\title{
Utilization of a Numerical Reservoir Simulation with Water and Gas Injection for Verification of Top Down Modeling
}

\author{
Ashley Konya \\ Thesis submitted \\ to the Statler College of Engineering \\ at West Virginia University \\ in partial fulfillment of the requirements for the degree of \\ Masters in Petroleum and Natural Gas Engineering
}

Shahab Mohaghegh, Ph.D., Chair

Kashy Aminian, Ph.D.

Sam Ameri, M.S.

Mehrdad Zamirian, Ph.D.

Department of Petroleum and Natural Gas Engineering

Morgantown, West Virginia

2020

Keywords: Artificial Intelligence, Machine Learning, Numerical Reservoir Simulation, Reservoir Modeling, Data-Driven Reservoir Modeling, Top Down Modeling

Copyright 2020 Ashley Konya 


\title{
Abstract \\ Utilization of a Numerical Reservoir Simulation with Water and Gas Injection for Verification of Top Down Modeling
}

\author{
Ashley Konya
}

The primary purpose of this thesis was to confirm the capabilities of artificial intelligence and machine learning through Top Down Modeling in history matching and predicting the oil, gas, and water production rates, reservoir pressure, and water saturation, of one limb of an anticline with water and gas injection. Several other characteristics were also applied to make the model more realistic to industry standards. The second purpose of this thesis was to determine the minimum amount of training and calibration data required in order to obtain good results for this particular dataset by increasing the blind validation in one year increments with each additional model.

The aforementioned task was accomplished by first creating the described reservoir in a numerical reservoir simulator (NRS) in order to synthetically generate the dataset for the Top Down Model. Synthetic data from a NRS was desired because all of the details and values were known for the entire reservoir at all time steps. The numerical reservoir model, a unique scenario from any other reservoir built to test Top Down Modeling, had the following list of characteristics to make the model more realistic and unique from models used previously:

- One limb of an anticline with 57 production wells and 20 injection wells brought online in phases

- Water and gas injection

- Random daily and monthly shut-in dates for all production wells

- No communication between some layers

- Presence of sealed faults with no surrounding aquifer

- Water cut operating constraint and partial completion varying with time for all producers

After the NRS model was completed, the data was exported, formatted, and calculated for import into a software to begin the process of creating Top Down Models (TDMs). An iterative process to select attributes, train data-driven models (DDMs), and evaluate the training results was performed for every DDM generated. Variations of attributes selected for training were used with each DDM in an attempt to achieve the best results possible for each blind validation scenario. The length of blind validation and random partitioning of the training and calibration data changed with each model, thus making the iterative process necessary. The TDMs were built from the five DDMs and the accuracy of the predictions made were analyzed on an entire reservoir and individual well basis. If these results were determined to be unsatisfactory, the process was repeated from the selecting training attributes step.

As detailed in the results and discussion section, 7 TDMs with varying lengths of blind validation were generated and all displayed acceptable predictions. It is thus shown that AI and ML was successful in history matching and predicting entire reservoir and individual well behavior. The maximum amount of blind validation without predicting new wells was also successfully implemented and satisfactory predictions were obtained. Consequently, one years worth of data for training and calibration was sufficient to teach the TDM recompletion changes and new well behavior to in turn receive adequate predictions. 


\section{Dedication}

This thesis is dedicated to the author's family. Without their constant support, understanding, and faith, the author would not be where she is today, nor would she have obtained this degree. The author feels fortunate to have them to build her up when she is discouraged and to celebrate in her successes alongside her. 


\section{Acknowledgements}

The author would first like to thank the committee chair, professor, and research advisor Dr. Shahab Mohaghegh, for all of his support and time he has dedicated to answering a multitude of questions. His guidance and the lessons he has taught both inside and outside the classroom-are invaluable. The author would also like to thank Professor Sam Ameri, Dr. Kashy Aminian, and Dr. Mehrdad Zamirian for their recommendations throughout the entirety of the thesis and their willingness to always assist in any task.

To the author's research team and fellow graduate students, thank you for your ingenuity and comradery. The discussions that transpired were inspiring and enriching towards the learning experience at West Virginia University.

The author would like to thank Yvon Martinez, a friend and colleague, who worked closely with the author throughout the entirety of this thesis. Her positivity, ability to write codes to expedite certain processes, and friendship has made the past two years of work an enjoyable experience.

Lastly, to all of the author's friends outside of the PNGE department and the author's family, thank you for bringing diversity and unique experiences to the author's life. The fun memories that often brightened spirits after working long hours will always be cherished. 


\section{Table of Contents}

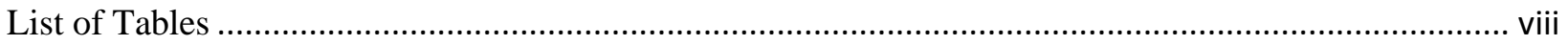

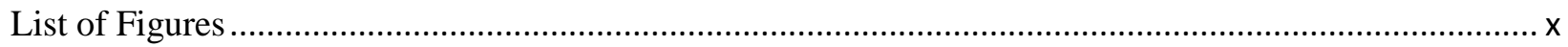

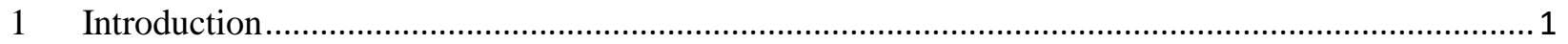

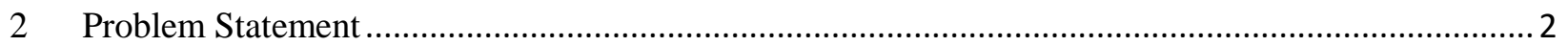

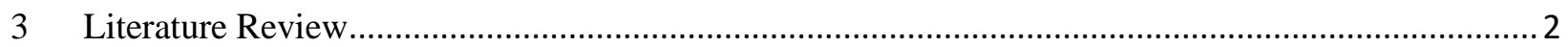

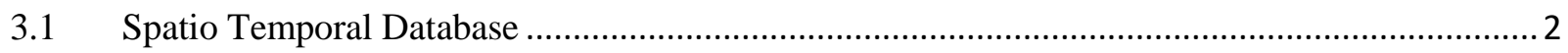

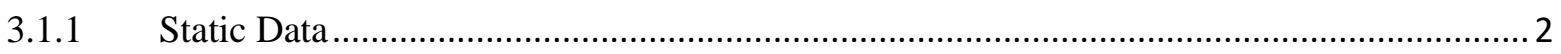

3.1.2 Blind Validation in Time and Space ….......................................................................

3.1.3 Polygon Based Properties ........................................................................................

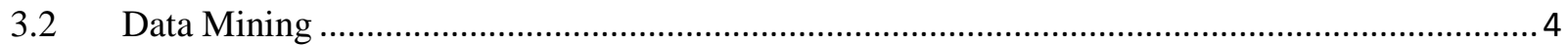

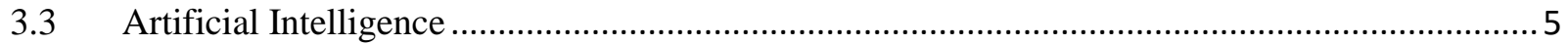

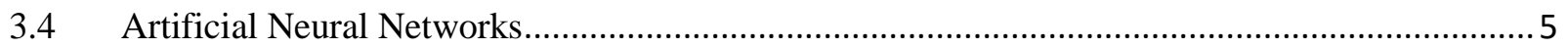

3.4.1 Supervised Learning Algorithms …..................................................................... 7

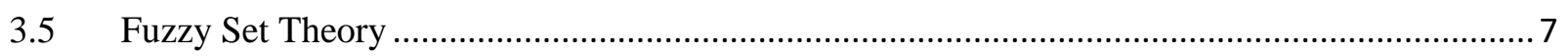

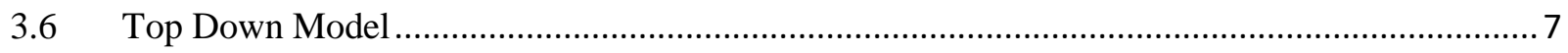

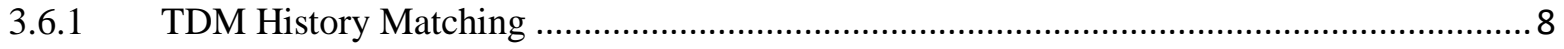

3.6.2 Post Modeling Analysis Using A Top Down Model ...................................................... 9

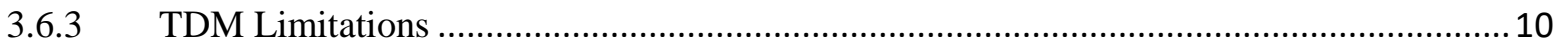

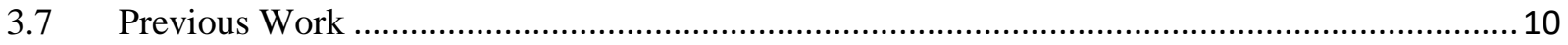

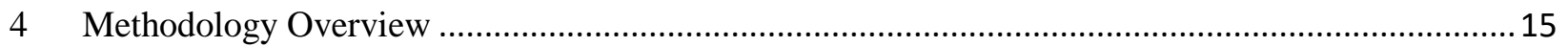

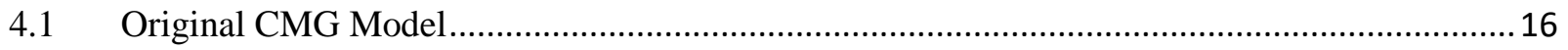

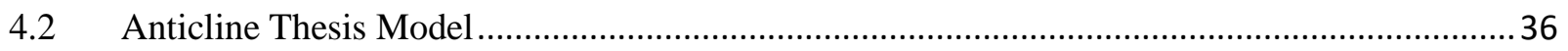

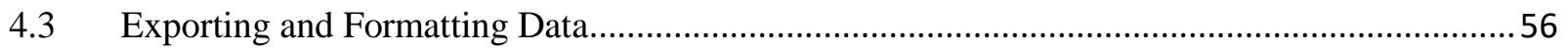

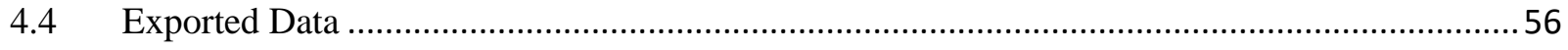

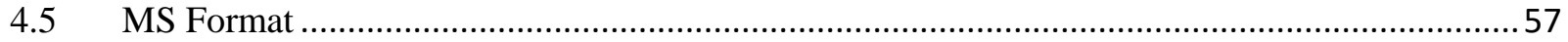

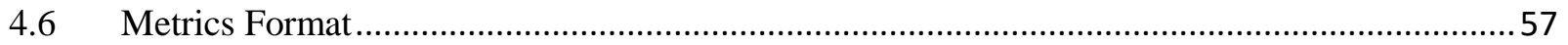

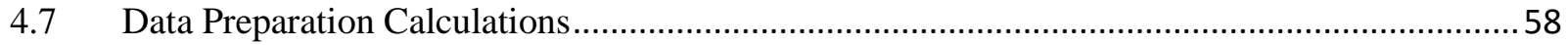

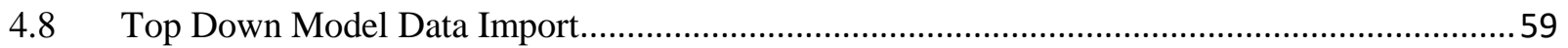

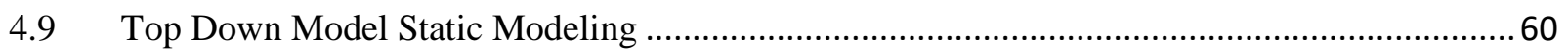

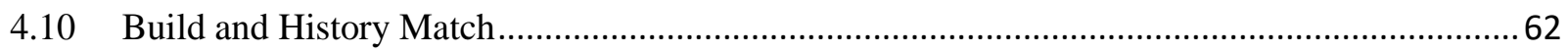

4.10.1 Random Partitioning-No Blind Validation ..............................................................6 68

4.10.2 Multi-Random Partitioning-1 Year Blind Validation .................................................... 75 
4.10.3 Multi-Random Partitioning-2 Year Blind Validation .................................................. 81

4.10.4 Multi-Random Partitioning-3 Year Blind Validation TDM ….........................................87

4.10.5 Multi-Random Partitioning- 4 Year Blind Validation ...................................................92

4.10.6 Multi-Random Partitioning- 5 Year Blind Validation ....................................................98

4.10.7 Multi-Random Partitioning- 6 Year Blind Validation .................................................. 103

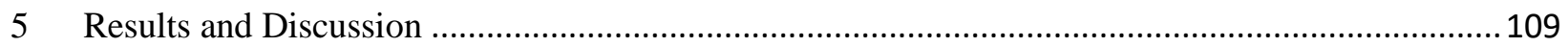

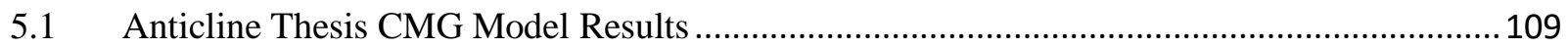

5.2 Wells Producing Until End of Simulation ...................................................................... 111

5.2.1 Largest Number of Production Days........................................................................ 112

5.2.2 Average Number of Production Days ......................................................................... 113

5.2.3 Smallest Number of Production Days ............................................................................ 114

$5.3 \quad$ Wells Not Producing at the End of Simulation................................................................. 115

5.3.1 Largest Number of Production Days............................................................................... 115

5.3.2 Smallest Number of Production Days ..................................................................... 116

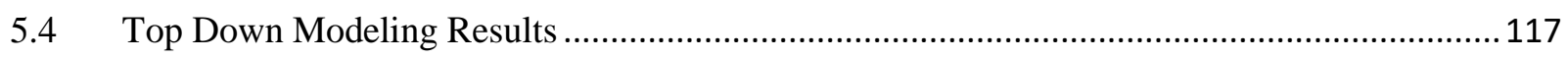

5.4.1 Random Partitioning-No Blind Validation ............................................................ 119

5.4.2 Multi-Random Partitioning-1 Year Blind Validation .................................................. 127

5.4.3 Multi-Random Partitioning-2 Year Blind Validation ................................................... 137

5.4.4 Multi-Random Partitioning- 3 Year Blind Validation ................................................... 148

5.4.5 Multi-Random Partitioning- 4 Year Blind Validation ................................................... 161

5.4.6 Multi-Random Partitioning- 5 Year Blind Validation ................................................. 172

5.4.7 Multi-Random Partitioning-6 Year Blind Validation .................................................. 184

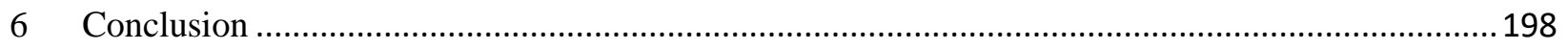

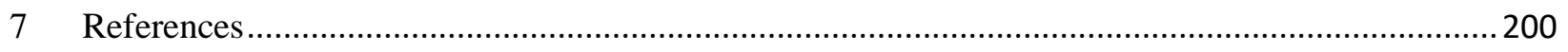

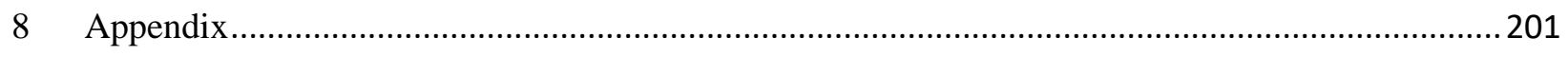

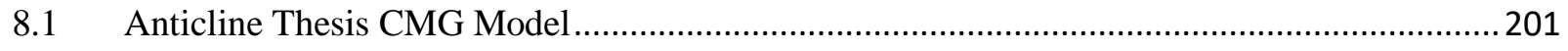

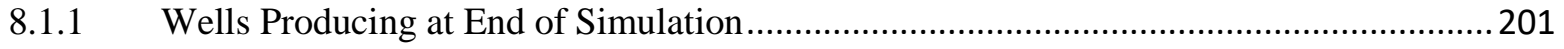

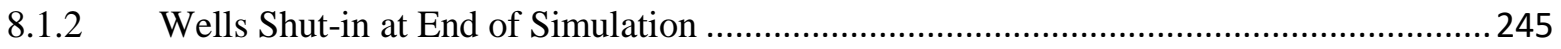

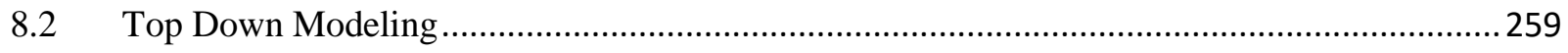

8.2.1 Random Partitioning-No Blind Validation ............................................................... 259

8.2.2 Multi-Random Partitioning- 1 Year Blind Validation ....................................................320

8.2.3 Multi-Random Partitioning- 2 Year Blind Validation ................................................... 381

8.2.4 Multi-Random Partitioning- 3 Year Blind Validation ................................................. 444 
8.2.5 Multi-Random Partitioning- 4 Year Blind Validation ..................................................509

8.2.6 Multi-Random Partitioning- 5 Year Blind Validation ...................................................577

8.2.7 Multi-Random Partitioning- 6 Year Blind Validation ..................................................641 


\section{List of Tables}

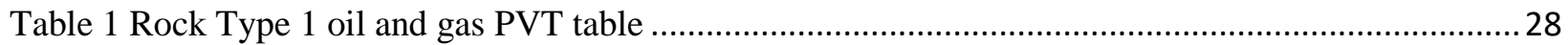

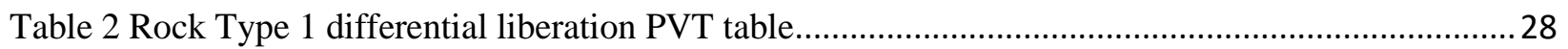

Table 3 General properties for PVT Region 1 of the Original CMG model..........................................29

Table 4 Rock Type 1's water-oil relative permeability table ..................................................................29

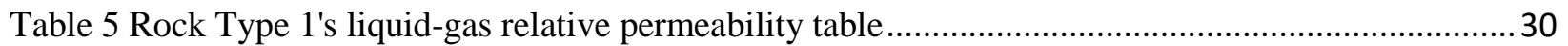

Table 6 Initial condition properties specified for the Original CMG model........................................... 31

Table 7 Phases of production well addition in the Original CMG model................................................35

Table 8 Values needed to create the grid top map file in Builder to reshape the reservoir into an anticline

Table 9 Production well completion phases for Anticline Thesis model..............................................50

Table 10 Gas injection well completions for the Anticline Thesis model ...............................................52

Table 11 Partial completion plan for all production wells in Anticline Thesis Model ..............................53

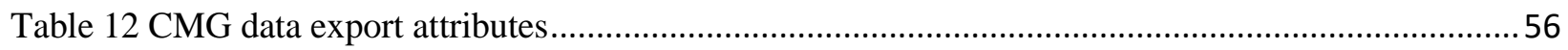

Table 13 Data-driven oil model attributes for dataset generation where oil $(\mathrm{t})$ is the output ...................62

Table 14 Data-driven gas model attributes for dataset generation where gas $(\mathrm{t})$ is the output ..................63

Table 15 Data-driven water model attributes for dataset generation where water $(\mathrm{t})$ is the output ............65

Table 16 Data-driven reservoir pressure model attributes for dataset generation where reservoir pressure

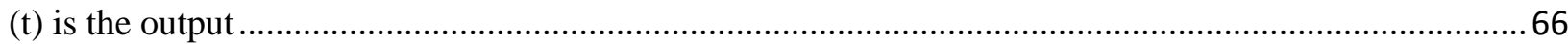

Table 17 Data-driven water saturation model attributes for dataset generation where water saturation $(t)$ is

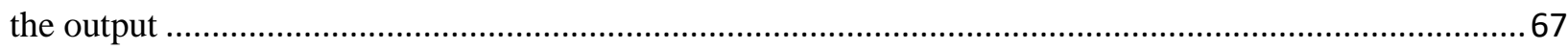

Table 18 Data-driven oil model training attributes where oil $(\mathrm{t})$ is the output........................................68

Table 19 Data-driven gas model training attributes where gas $(\mathrm{t})$ is the output .......................................69

Table 20 Data-driven water model training attributes where water $(\mathrm{t})$ is the output ..............................69

Table 21 Data-driven reservoir pressure model training attributes where reservoir pressure $(t)$ is the output

Table 22 Data-driven water saturation model training attributes where water saturation $(\mathrm{t})$ is the output.70

Table 23 Default design values for artificial neural network ............................................................... 71

Table 24 Training R Squared metrics for all data-driven models ..................................................... 71

Table 25 Data-driven oil model training attributes for Multi-Random Partitioning-1 Year Blind Validation

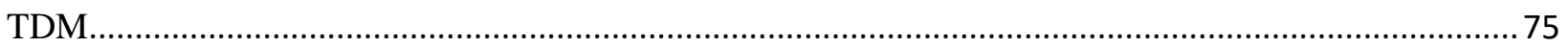

Table 26 Data-driven gas model training attributes for Multi-Random Partitioning-1 Year Blind

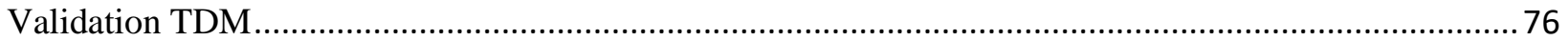

Table 27 Data-driven water model training attributes for Multi-Random Partitioning-1 Year Blind

Validation TDM................................................................................................................. 76

Table 28 Data-driven reservoir pressure training attributes for Multi-Random Partitioning-1 Year Blind

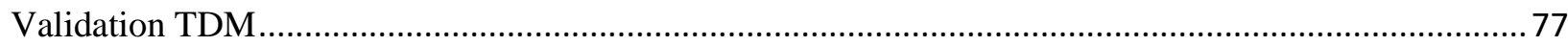

Table 29 Data-driven water saturation model training attributes for Multi-Random Partitioning-1 Year

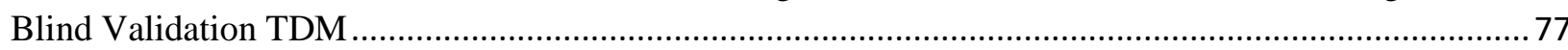

Table 30 R Squared metrics for each data-driven model in Multi-Random Partitioning-1 Year Blind

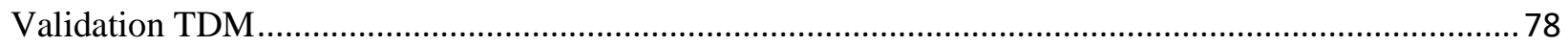

Table 31 Data-driven oil model training attributes for Multi-Random Partitioning-2 Year Blind Validation

TDM......

Table 32 Data-driven gas model training attributes for Multi-Random Partitioning-2 Year Blind Validation TDM. 
Table 33 Data-driven water model training attributes for Multi-Random Partitioning-2 Year Blind Validation TDM.

Table 34 Data-driven reservoir pressure model training attributes for Multi-Random Partitioning-2 Year

Blind Validation TDM

Table 35 Data-driven water saturation model training attributes for Multi-Random Partitioning- 2 Year

Blind Validation TDM

Table 36 R Squared metrics for all data-driven models in the Multi-Random Partitioning-2 Year Blind Validation TDM.

Table 37 Data-driven oil model training attributes for Multi-Random Partitioning- 3 Year Blind

Validation TDM.

Table 38 Data-driven gas model training attributes for Multi-Random Partitioning-3 Year Blind

Validation TDM.

Table 39 Data-driven water model training attributes for Multi-Random Partitioning-3 Year Blind

Validation TDM.

Table 40 Data-driven reservoir pressure model training attributes for Multi-Random Partitioning-3 Year

Blind Validation TDM.

Table 41 Data-driven water saturation model training attributes for Multi-Random Partitioning- 3 Year

Blind Validation TDM

Table 42 R Squared metrics for data-driven models in Multi-Random Partitioning-3 Year Blind

Validation TDM.

Table 43 Data-driven oil model training attributes for Multi-Random Partitioning-4 Year Blind Validation TDM.

Table 44 Data-driven gas model training attributes for Multi-Random Partitioning- 4 Year Blind

Validation TDM.

Table 45 Data-driven water model training for Multi-Random Partitioning-4 Year Blind Validation TDM

.93

Table 46 Data-driven reservoir pressure model training attributes for Multi-Random Partitioning- 4 Year

Blind Validation TDM

Table 47 Data-driven water saturation model training attributes for Multi-Random Partitioning- 4 Year

Blind Validation.

Table 48 R Squared metrics for all data-driven models in the Multi-Random Partitioning-4 Year Blind

Validation TDM.

Table 49 Data-driven oil model training attributes for Multi-Random Partitioning-5 Year Blind Validation TDM.

Table 50 Data-driven gas model training attributes for Multi-Random Partitioning-5 Year Blind Validation TDM.

Table 51 Data-driven water model training attributes for Multi-Random Partitioning- 5 Year Blind Validation TDM.

Table 52 Data-driven reservoir pressure model training attributes for Multi-Random Partitioning- 5 Year

Blind Validation TDM

Table 53 Data-driven water saturation model training attributes for Multi-Random Partitioning- 5 Year Blind Validation TDM 100

Table 54 R Squared metrics for all data-driven models in the Multi-Random Partitioning- 5 Year Blind Validation TDM.

Table 55 Data-driven oil model training attributes for Multi-Random Partitioning- 6 Year Blind Validation TDM. 
Table 56 Data-driven gas model training attributes for Multi-Random Partitioning- 6 Year Blind Validation TDM.

Table 57 Data-driven water model training attributes for Multi-Random Partitioning- 6 Year Blind Validation TDM. 104

Table 58 Data-driven reservoir pressure model training attributes for Multi-Random Partitioning- 6 Year

Blind Validation TDM. 105

Table 59 Data-driven water saturation model training attributes for Multi-Random Partitioning- 6 Year

Blind Validation TDM. 105

Table 60 R Squared metrics for all data-driven models in Multi-Random Partitioning- 6 Year Blind Validation TDM. 106

Table 61 Summary of all Top Down Model's individual well production prediction accuracy..... 198

\section{List of Figures}

Figure 1 Voronoi polygon grid depicting theoretical drainage area during first phase of development ...... 4 Figure 2 Diagram of an artificial neural network neuron (Mohaghegh, Data-Driven Reservoir Modeling, 2017)

Figure 3 Diagram of a simple artificial neural network (Mohaghegh, Data-Driven Reservoir Modeling, 2017) 6

Figure 4 Comparison of results from SRM and NRM after training, calibration, and verification ............11

Figure 5 Comparison of the SRM's history matching with actual outputs ............................................. 11

Figure 6 Blind validation comparison of SRM results for gas production rate vs. the realization from

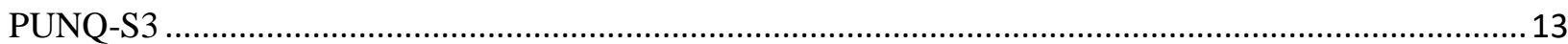

Figure 7 Well bottom hole history matching results of SRM compared against actual PUNQ-S3 values .14 Figure 8 Simulator results with SRM identified properties vs actual field values for cumulative oil production along with forecast prediction vs actual for cumulative oil production.................................15

Figure 9 Reservoir shape of Original CMG Model ............................................................................ 16

Figure 10 Side view of reservoir shape of Original CMG Model..........................................................17

Figure 11 Grid top .msh map for Layer 1 of Original CMG Model .....................................................18

Figure 12 Grid thickness for each K layer shown for J layer 1 of the Original CMG Model...................18

Figure 13 Porosity .msh map for K Layer 1 of the Original CMG Model...............................................19

Figure 14 Porosity .msh map for K Layer 2 of the Original CMG Model................................................20

Figure 15 Porosity .msh map for K Layer 3 of the Original CMG Model..............................................20

Figure 16 Porosity .msh map for K Layer 4 of the Original CMG Model...............................................21

Figure 17 Porosity .msh map for K Layer 5 of the Original CMG Model................................................21

Figure 18 Porosity .msh map for K Layer 6 of the Original CMG model ...............................................22

Figure 19 Permeability .msh map for K Layer 1 of the Original CMG model .........................................23

Figure 20 Permeability .msh map for K Layer 2 of the Original CMG model .........................................23

Figure 21 Permeability .msh map for K Layer 3 of the Original CMG model ........................................24

Figure 22 Permeability .msh map for K Layer 4 of the Original CMG model .........................................24

Figure 23 Permeability .msh map for K Layer 5 of the Original CMG model ........................................25

Figure 24 Permeability .msh map for K Layer 6 of the Original CMG model ........................................25

Figure 25 Initial water saturation .msh map for K Layers 1-6 of the Original CMG model .....................26

Figure 26 Volume modifier of 257 applied to the four sides of the reservoir in the Original CMG model27 
Figure $273 \mathrm{D}$ view of the volume modifier function on the four sides of the reservoir in the Original CMG

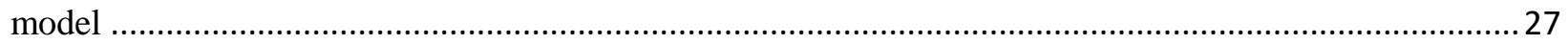

Figure 28 Default numerical controls in the Original CMG Model...................................................... 32

Figure 29 Continuation of the default numerical controls in the Original CMG model ........................... 33

Figure 30 Injector well locations in aquifer around reservoir for the Original CMG model .....................34

Figure 31 Injector-005 water injection rate and well bottom hole pressure.............................................35

Figure 32 Production well locations in reservoir portion of the Original CMG model .............................36

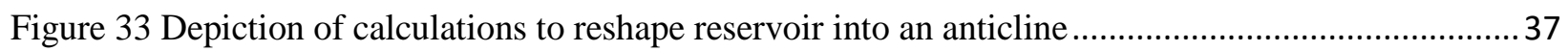

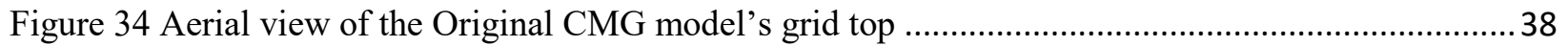

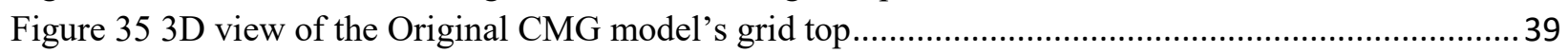

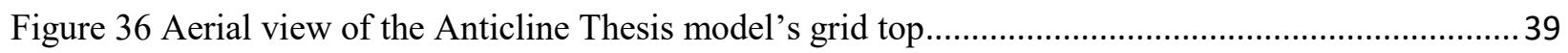

Figure 37 3D view of the Anticline Thesis model's grid top .................................................................. 40

Figure 38 Layer 1's porosity .msh file open in new CMG Builder ...................................................... 41

Figure 39 Creating a 260 x 260 Orthogonal Corner Point grid of smaller cells ...................................... 42

Figure 40 New 260 x 260 grid on top of opened Layer 1 Porosity .msh file.............................................43

Figure 41260 x 260 grid of smaller cells over the desired portion of the Layer 1 Porosity map file after

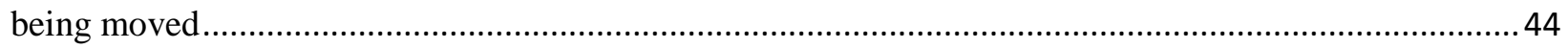

Figure 42 Shifted 260 x 260 grid directly over Layer 1 Porosity map specified in Array Properties.........45 Figure 43 Values from original map files that need replaced in the newly exported map files to increase

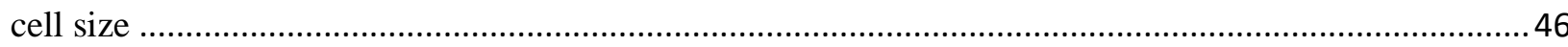

Figure 44 Water filled portions of the reservoir due to change in DWOC to $4900 \mathrm{ft}$ in Anticline Thesis

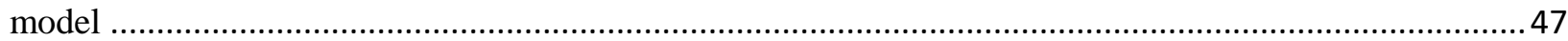

Figure 45 Gas saturation at Injector-002 for the TransK multiplier test ..................................................48

Figure 46 Aerial view of sealed fault locations in Anticline Thesis model ............................................49

Figure 47 Pressure difference caused by the two sealing faults in Anticline Thesis model .....................49

Figure 48 Legend for well locations map of each phase of production for Anticline Thesis model ..........50

Figure 49 Well locations map for each phase of production for the Anticline Thesis Model ...................51

Figure 50 Example of text file output from code used to generate random shut-in dates and alter

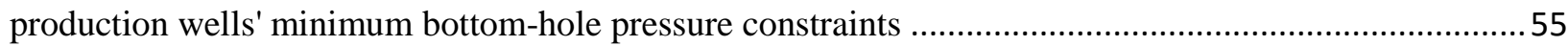

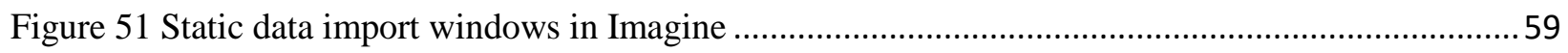

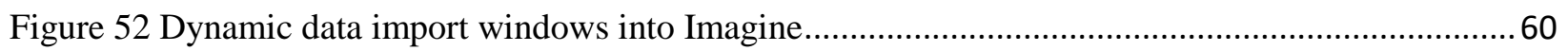

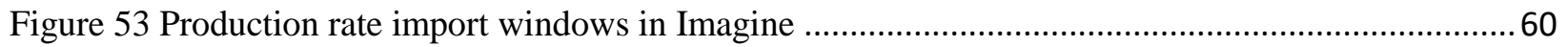

Figure 54 Cartesian grid and reservoir boundary creation in Imagine.................................................61

Figure 55 Geostatistics being calculated for spatial properties using the inverse distance weighting

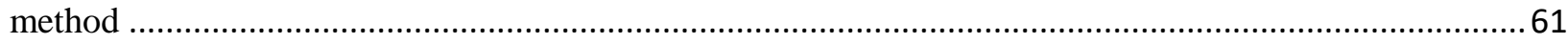

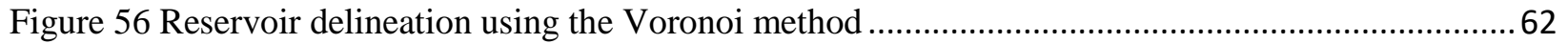

Figure 57 Data-driven oil model training results for training, calibration, and verification ........................72

Figure 58 Data-driven gas training results for training, calibration, and verification................................72

Figure 59 Data-driven water training results for training, calibration, and verification ...........................73

Figure 60 Data-driven reservoir pressure training results for training, calibration, and verification..........73

Figure 61 Data-driven water saturation training results for training, calibration, and verification ............74

Figure 62 Random partitioning-no blind validation TDM structure ................................................... 74

Figure 63 Data-driven oil model training results for Multi-Random Partitioning-1 Year Blind Validation 
Figure 64 Data-driven gas model training results for Multi-Random Partitioning-1 Year Blind Validation

Figure 65 Data-driven water model training results for Multi-Random Partitioning-1 Year Blind

Validation... 79

Figure 66 Data-driven reservoir pressure model training results for Multi-Random Partitioning-1 Year

Blind Validation.

Figure 67 Data-driven water saturation model training results for Multi-Random Partitioning-1 Year Blind Validation... .80

Figure 68 Multi-Random Partitioning-1 Year Blind Validation TDM structure ...................................... 81

Figure 69 Data-driven oil model training results for Multi-Random Partitioning- 2 Year Blind Validation

TDM.

Figure 70 Data-drive gas model training results for Multi-Random Partitioning-2 Year Blind Validation

TDM.

Figure 71 Data-driven water model training results for Multi-Random Partitioning-2 Year Blind

Validation TDM. .85

Figure 72 Data-driven reservoir pressure model training results for Multi-Random Partitioning-2 Year

Blind Validation TDM.......

Figure 73 Data-driven water saturation model training results for Multi-Random Partitioning-2 Year

Blind Validation TDM. .86

Figure 74 Structure for Multi-Random Partitioning-2 Year Blind Validation Top Down Model

Figure 75 Data-driven oil model training results for Multi-Random Partitioning-2 Year Blind Validation

TDM.

90

Figure 76 Data-driven gas model training results for Multi-Random Partitioning-3 Year Blind Validation

TDM.

Figure 77 Data-driven water model training results for Multi-Random Partitioning- 3 Year Blind

Validation TDM.

Figure 78 Data-driven reservoir pressure model training results for Multi-Random Partitioning- 3 Year

Blind Validation TDM..

Figure 79 Data-driven water saturation model training results for Multi-Random Partitioning- 3 Year Blind Validation TDM.

Figure 80 Structure for Multi-Random Partitioning- 3 Year Blind Validation Top Down Model

Figure 81 Data-driven oil model training results for Multi-Random Partitioning- 4 Year Blind Validation

TDM.

Figure 82 Data-driven gas model training results for Multi-Random Partitioning- 4 Year Blind Validation

TDM. .96

Figure 83 Data-driven water model training results for Multi-Random Partitioning-4 Year Blind

Validation TDM.

Figure 84 Data-driven reservoir pressure model training results for Multi-Random Partitioning- 4 Year

Blind Validation TDM.

Figure 85 Data-driven water saturation model training results for Multi-Random Partitioning- 4 Year

Blind Validation TDM.

Figure 86 Structure for Multi-Random Partitioning- 4 Year Blind Validation Top Down Model.....

Figure 87 Data-driven oil model training results for Multi-Random Partitioning- 5 Year Blind Validation TDM.

Figure 88 Data-driven gas model training results for Multi-Random Partitioning- 5 Year Blind Validation TDM. 101 
Figure 89 Data-driven water model training results for Multi-Random Partitioning- 5 Year Blind Validation TDM.

Figure 90 Data-driven reservoir pressure model training results for Multi-Random Partitioning- 5 Year Blind Validation TDM. 102

Figure 91 Data-driven water saturation model training results for Multi-Random Partitioning- 5 Year

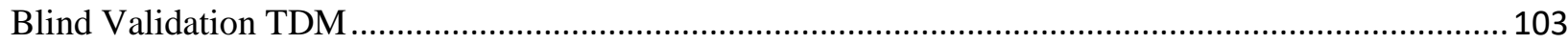

Figure 92 Structure for Multi-Random Partitioning- 5 Year Blind Validation Top Down Model ...........103 Figure 93 Data-driven oil model training results for Multi-Random Partitioning- 6 Year Blind Validation TDM. 107

Figure 94 Data-driven gas model training results for Multi-Random Partitioning- 6 Year Blind Validation TDM. 107

Figure 95 Data-driven water model training results for Multi-Random Partitioning- 6 Year Blind Validation TDM. .108

Figure 96 Data-driven reservoir pressure results for Multi-Random Partitioning- 6 Year Blind Validation TDM. 108

Figure 97 Data-driven water saturation training results for Multi-Random Partitioning- 6 Year Blind

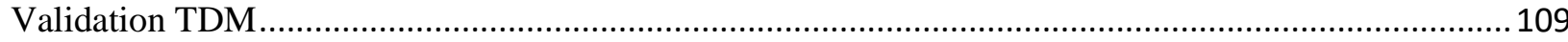

Figure 98 Structure for Multi-Random Partitioning- 6 Year Blind Validation TDM............................ 109

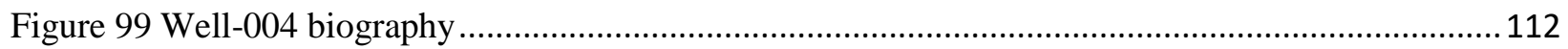

Figure 100 Well-004 saturations and pressure for every layer .......................................................112

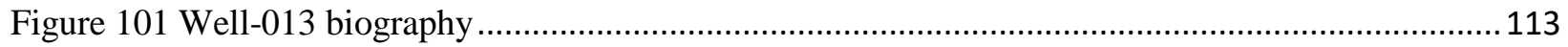

Figure 102 Well-013 saturations and pressure for every layer ........................................................113

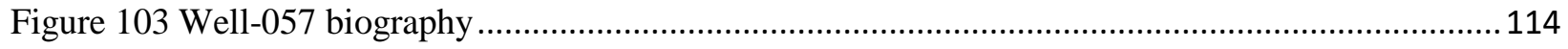

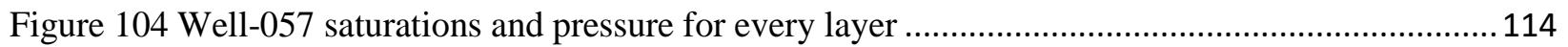

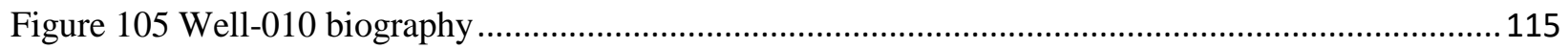

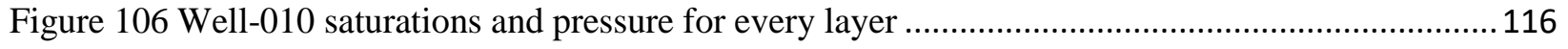

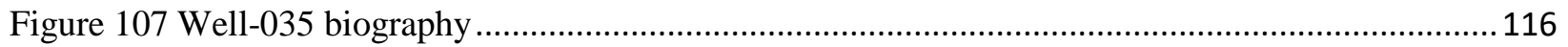

Figure 108 Well-035 saturations and pressure for every layer ........................................................117

Figure 109 Reservoir pressure heat map at January 31st, 2016 using nearest neighbor interpolation

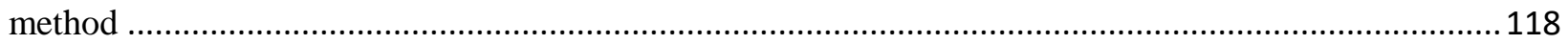

Figure 110 Reservoir pressure heat map at January 31st, 2016 using linear interpolation method..........118

Figure 111 Reservoir pressure heat map at January 31st, 2016 using cubic interpolation method ..........118

Figure 112 Entire reservoir oil results for Random Partitioning-No Blind Validation Top Down Model119 Figure 113 Entire reservoir gas results for Random Partitioning-No Blind Validation Top Down Model

Figure 114 Entire reservoir water results for Random Partitioning- No Blind Validation Top Down Model .121

Figure 115 Entire reservoir heat map for reservoir pressure at December 31st, 2015 for Random Partitioning-No Blind Validation.... .121

Figure 116 Entire reservoir heat map for reservoir pressure at June 30th, 2016 for Random Partitioning-

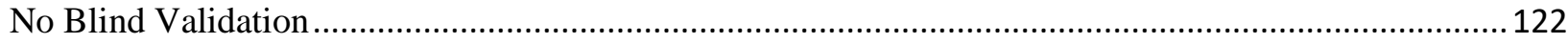

Figure 117 Entire reservoir heat map for water saturation at December 31st, 2015 for Radom Partitioning-

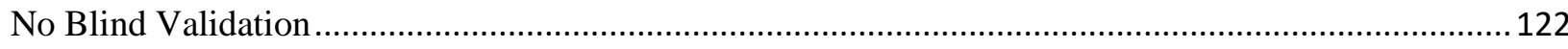
Figure 118 Entire reservoir heat map for water saturation at June 30th, 2016 for Random Partitioning- No

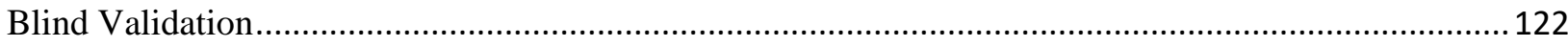


Figure 119 Well-019 oil, gas, and water rate TDM predictions vs actual simulation data for Random Partitioning-No Blind Validation TDM

Figure 120 Well-044 oil, gas, and water rate TDM predictions vs actual simulation data plots for Random Partitioning-No Blind Validation TDM 124

Figure 121 Well-010 oil, gas, and water rate TDM predictions vs actual simulation data plots for Random Partitioning-No Blind Validation TDM. 125

Figure 122 Well-047 oil, gas, and water rate TDM predictions vs actual simulation data plots for Random Partitioning-No Blind Validation TDM 126

Figure 123 Well-005 oil, gas, and water rate TDM predictions vs actual simulation data plots for Random Partitioning-No Blind Validation TDM 127

Figure 124 Entire reservoir oil results for Multi-Random Partitioning- 1 Year Blind Validation Top Down Model 128

Figure 125 Entire reservoir gas results for Multi-Random Partitioning-2 Year Blind Validation Top Down Model 128

Figure 126 Entire reservoir water results for Multi-Random Partitioning-1 Year Blind Validation Top Down Model 129

Figure 127 Entire reservoir heat map for reservoir pressure at December 31st, 2015 for 1 Year Blind Validation TDM. 130

Figure 128 Entire reservoir heat map for reservoir pressure at June 30th, 2016 for 1 Year Blind Validation TDM. 130

Figure 129 Entire reservoir heat map for water saturation at December 31st, 2015 for 1 Year Blind Validation TDM

Figure 130 Entire reservoir heat map for water saturation at June 30th, 2016 for 1 Year Blind Validation TDM 131

Figure 131 Well-015 oil, gas, and water rate TDM predictions vs actual simulation data plots for 1 Year Blind Validation TDM 132

Figure 132 Well-040 oil, gas, and water rate TDM predictions vs actual simulation data plots for 1 Year Blind Validation TDM. 133

Figure 133 Well-003 oil, gas, and water rate TDM predictions vs actual simulation data plots for 1 Year Blind Validation TDM. 134

Figure 134 Well-053 oil, gas, and water rate TDM predictions vs actual simulation data plots for 1 Year Blind Validation TDM 135

Figure 135 Well-014 oil, gas, and water rate TDM predictions vs actual simulation data plots for 1 Year Blind Validation TDM. 136

Figure 136 Well-021 oil, gas, and water rate TDM predictions vs actual simulation data plots for 1 Year Blind Validation TDM

Figure 137 Entire reservoir oil results for Multi-Random Partitioning-2 Year Blind Validation Top Down

Model 138

Figure 138 Entire reservoir gas results for Multi-Random Partitioning- 2 Year Blind Validation Top Down Model 138

Figure 139 Entire reservoir water results for Multi-Random Partitioning-2 Year Blind Validation Top Down Model 139

Figure 140 Entire reservoir heat map for reservoir pressure at December 31st, 2014 for 2 Year Blind Validation TDM 139

Figure 141 Entire reservoir heat map for reservoir pressure at June 30th, 2015 for 2 Year Blind Validation TDM. 140 
Figure 142 Entire reservoir heat map for reservoir pressure at December 31st, 2015 for 2 Year Blind Validation TDM

Figure 143 Entire reservoir heat map for reservoir pressure at June 30th, 2016 for 2 Year Blind Validation TDM. 140

Figure 144 Entire reservoir heat map for reservoir pressure at December 31st for 2 Year Blind Validation TDM. 141

Figure 145 Entire reservoir heat map for water saturation at December 31st, 2014 for 2 Year Blind Validation TDM. .141

Figure 146 Entire reservoir heat map for water saturation at June 30th, 2015 for 2 Year Blind Validation TDM. 141

Figure 147 Entire reservoir heat map for water saturation at December 31st, 2015 for 2 Year Blind Validation TDM. .142

Figure 148 Entire reservoir heat map for water saturation at June 30th, 2016 for 2 Year Blind Validation TDM. 142

Figure 149 Entire reservoir heat map for water saturation at December 31st, 2016 for 2 Year Blind Validation TDM. 142

Figure 150 Well-007 oil, gas, and water rate TDM predictions vs actual simulation data plots for 2 Year

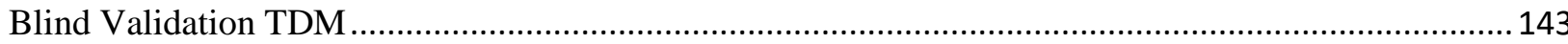
Figure 151 Well-033 oil, gas, and water rate TDM predictions vs actual simulation data plots for 2 Year

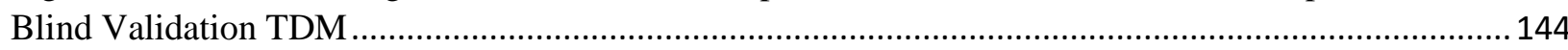
Figure 152 Well-002 oil, gas, and water rate TDM predictions vs actual simulation data plots for 2 Year

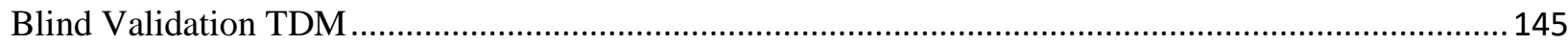
Figure 153 Well-020 oil, gas, and water rate TDM predictions vs actual simulation data plots for 2 Year

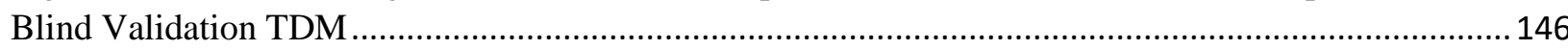
Figure 154 Well-026 oil, gas, and water rate TDM predictions vs actual simulation data plots for 2 Year Blind Validation TDM. 147 Figure 155 Well-048 oil, gas, and water rate TDM predictions vs actual simulation data plots for 2 Year

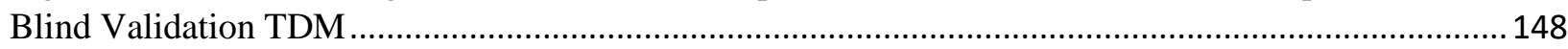
Figure 156 Entire reservoir oil results for Multi-Random Partitioning- 3 Year Blind Validation Top Down

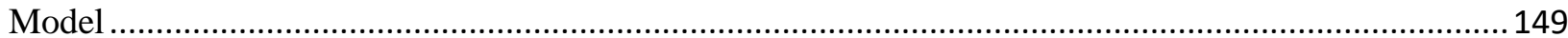

Figure 157 Entire reservoir gas results for Multi-Random Partitioning- 3 Year Blind Validation Top Down Model 149

Figure 158 Entire reservoir water results for Multi-Random Partitioning-3 Year Blind Validation Top Down Model 150 Figure 159 Entire reservoir heat map for reservoir pressure at December 31st, 2013 for 3 Year Blind Validation TDM 150 Figure 160 Entire reservoir heat map for reservoir pressure at June 30th, 2014 for 3 Year Blind Validation TDM. 151

Figure 161 Entire reservoir heat map for reservoir pressure at December 31st, 2014 for 3 Year Blind Validation TDM.

Figure 162 Entire reservoir heat map for reservoir pressure at June 30th, 2015 for 3 Year Blind Validation TDM. 151

Figure 163 Entire reservoir heat map for reservoir pressure at December 31st, 2015 for 3 Year Blind Validation TDM. 152 Figure 164 Entire reservoir heat map for reservoir pressure at June 30th, 2016 for 3 Year Blind Validation TDM. 152 
Figure 165 Entire reservoir heat map for reservoir pressure at December 31st, 2016 for 3 Year Blind Validation TDM.

Figure 166 Entire reservoir heat map for water saturation at December 31st, 2013 for 3 Year Blind Validation TDM.

Figure 167 Entire reservoir heat map for water saturation at June 30th, 2014 for 3 Year Blind Validation

TDM.

Figure 168 Entire reservoir heat map for water saturation at December 31st, 2014 for 3 Year Blind Validation TDM. 153

Figure 169 Entire reservoir heat map for water saturation at June 30th, 2015 for 3 Year Blind Validation

TDM. 154

Figure 170 Entire reservoir heat map for water saturation at December 31st, 2015 for 3 Year Blind Validation TDM. 154

Figure 171 Entire reservoir heat map for water saturation at June 30th, 2016 for 3 Year Blind Validation

TDM. 154

Figure 172 Entire reservoir heat map for water saturation at December 31st, 2016 for 3 Year Blind Validation TDM 155

Figure 173 Well-001 oil, gas, and water rate TDM predictions vs actual simulation data plots for 3 Year

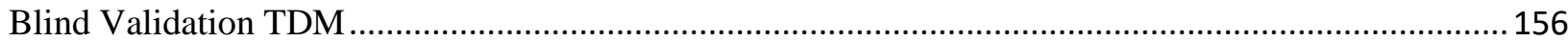
Figure 174 Well-034 oil, gas, and water rate TDM predictions vs actual simulation data plots for 3 Year

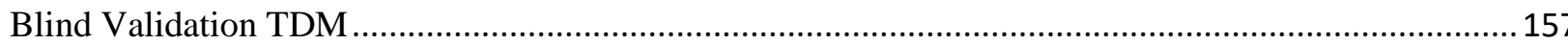
Figure 175 Well-014 oil, gas, and water rate TDM predictions vs actual simulation data plots for 3 Year Blind Validation TDM. 158 Figure 176 Well-043 oil, gas, and water rate TDM predictions vs actual simulation data plots for 3 Year Blind Validation TDM 159 Figure 177 Well-021 oil, gas, and water rate TDM predictions vs actual simulation data plots for 3 Year Blind Validation TDM. 160 Figure 178 Well-036 oil, gas, and water rate TDM predictions vs actual simulation data plots for 3 Year Blind Validation TDM. 161

Figure 179 Entire reservoir oil results for Multi-Random Partitioning- 4 Year Blind Validation Top Down Model 162

Figure 180 Entire reservoir gas results for Multi-Random Partitioning- 4 Year Blind Validation Top Down Model 162

Figure 181 Entire reservoir water results for Multi-Random Partitioning- 4 Year Blind Validation Top Down Model 163

Figure 182 Entire reservoir heat map for reservoir pressure at December 31st, 2012 for 4 Year Blind Validation TDM 163

Figure 183 Entire reservoir heat map for reservoir pressure at December 31st, 2013 for 4 Year Blind Validation TDM

Figure 184 Entire reservoir heat map for reservoir pressure at December 31st, 2014 for 4 Year Blind Validation TDM. 164

Figure 185 Entire reservoir heat map for reservoir pressure at December 31st, 2015 for 4 Year Blind Validation TDM.

Figure 186 Entire reservoir heat map for reservoir pressure at December 31st, 2016 for 4 Year Blind Validation TDM. 165

Figure 187 Entire reservoir heat map for water saturation at December 31st, 2012 for 4 Year Blind Validation TDM. 
Figure 188 Entire reservoir heat map for water saturation at December 31st, 2013 for 4 Year Blind Validation TDM

Figure 189 Entire reservoir heat map for water saturation at December 31st, 2014 for 4 Year Blind Validation TDM.

Figure 190 Entire reservoir heat map for water saturation at December 31st, 2015 for 4 Year Blind

Validation TDM.

Figure 191 Entire reservoir heat map for water saturation at December 31st, 2016 for 4 Year Blind Validation TDM.

Figure 192 Well-008 oil, gas, and water rate TDM predictions vs actual simulation data plots for 4 Year

Blind Validation TDM.

Figure 193 Well-034 oil, gas, and water rate TDM predictions vs actual simulation data plots for 4 Year

Blind Validation TDM. 168

Figure 194 Well-002 oil, gas, and water rate TDM predictions vs actual simulation data plots for 4 Year

Blind Validation TDM. 169

Figure 195 Well-038 oil, gas, and water rate TDM predictions vs actual simulation data plots for 4 Year

Blind Validation TDM.

Figure 196 Well-017 oil, gas, and water rate TDM predictions vs actual simulation data plots for 4 Year

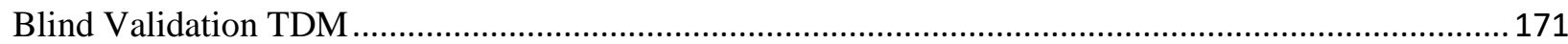

Figure 197 Well-055 oil, gas, and water rate TDM predictions vs actual simulation data plots for 4 Year

Blind Validation TDM.

Figure 198 Entire reservoir oil results for Multi-Random Partitioning- 5 Year Blind Validation Top Down

Model 173

Figure 199 Entire reservoir gas results for Multi-Random Partitioning- 5 Year Blind Validation Top

Down Model

Figure 200 Entire reservoir water results for Multi-Random Partitioning- 5 Year Blind Validation Top

Down Model

Figure 201 Entire reservoir heat map for reservoir pressure at December 31st, 2011 for 5 Year Blind Validation TDM.

Figure 202 Entire reservoir heat map for reservoir pressure at December 31st, 2012 for 5 Year Blind Validation TDM.

Figure 203 Entire reservoir heat map for reservoir pressure at December 31st, 2013 for 5 Year Blind Validation TDM.

Figure 204 Entire reservoir heat map for reservoir pressure at December 31st, 2014 for 5 Year Blind Validation TDM.

Figure 205 Entire reservoir heat map for reservoir pressure at December 31st, 2015 for 5 Year Blind Validation TDM.

Figure 206 Entire reservoir heat map for reservoir pressure at December 31st, 2016 for 5 Year Bind Validation TDM.

Figure 207 Entire reservoir heat map for water saturation at December 31st, 2011 for 5 Year Blind Validation TDM.

Figure 208 Entire reservoir heat map for water saturation at December 31st, 2012 for 5 Year Blind Validation TDM.

Figure 209 Entire reservoir heat map for water saturation at December 31st, 2013 for 5 Year Blind Validation TDM.

Figure 210 Entire reservoir heat map for water saturation at December 31st, 2014 for 5 Year Blind Validation TDM. 
Figure 211 Entire reservoir heat map for water saturation at December 31st, 2015 for 5 Year Blind Validation TDM.

Figure 212 Entire reservoir heat map for water saturation at December 31st, 2016 for 5 Year Blind Validation TDM. .178

Figure 213 Well-007 oil, gas, and water rate TDM predictions vs actual simulation data plots for 5 Year Blind Validation TDM. 179

Figure 214 Well-034 oil, gas, and water rate TDM predictions vs actual simulation data plots for 5 Year Blind Validation TDM.... 180

Figure 215 Well-010 oil, gas, and water rate TDM predictions vs actual simulation data plots for 5 Year Blind Validation TDM. 181 Figure 216 Well-020 oil, gas, and water rate TDM prediction vs actual simulation data plots for 5 Year Blind Validation TDM 182

Figure 217 Well-021 oil, gas, and water rate TDM predictions vs actual simulation data plots for 5 Year Blind Validation. 183 Figure 218 Well-057 oil, gas, and water rate TDM predictions vs actual simulation data plots for 5 Year Blind Validation TDM. 184

Figure 219 Entire reservoir oil results for Multi-Random Partitioning- 6 Year Blind Validation TDM.. 185 Figure 220 Entire reservoir gas results for Multi-Random Partitioning- 6 Year Blind Validation TDM.186 Figure 221 Entire reservoir water results for Multi-Random Partitioning- 6 Year Blind Validation TDM

Figure 222 Entire reservoir heat map for reservoir pressure at December 31st, 2010 for 6 Year Blind Validation TDM

Figure 223 Entire reservoir heat map for reservoir pressure at December 31st, 2011 for 6 Year Blind Validation TDM.

Figure 224 Entire reservoir heat map for reservoir pressure at December 31st, 2012 for 6 Year Blind Validation TDM.

Figure 225 Entire reservoir heat map for reservoir pressure at December 31st, 2013 for 6 Year Blind Validation TDM.

Figure 226 Entire reservoir heat map for reservoir pressure at December 31st, 2014 for 6 Year Blind Validation TDM.

Figure 227 Entire reservoir heat map for reservoir pressure at December 31st, 2015 for 6 Year Blind Validation TDM.

Figure 228 Entire reservoir heat map for reservoir pressure at December 31st, 2016 for 6 Year Blind Validation TDM 189

Figure 229 Entire reservoir heat map for water saturation at December 31st, 2010 for 6 Year Blind Validation TDM.

Figure 230 Entire reservoir heat map for water saturation at December 31st, 2011 for 6 Year Blind Validation TDM

Figure 231 Entire reservoir heat map for water saturation at December 31st, 2012 for 6 Year Blind Validation TDM.

Figure 232 Entire reservoir heat map for water saturation at December 31st, 2013 for 6 Year Blind Validation TDM. 190

Figure 233 Entire reservoir heat map for water saturation at December 31st, 2014 for 6 Year Blind Validation TDM. 190

Figure 234 Entire reservoir heat map for water saturation at December 31st, 2015 for 6 Year Blind Validation TDM. 
Figure 235 Entire reservoir heat map for water saturation at December 31st, 2016 for 6 Year Blind Validation TDM.

Figure 236 Well-011 oil, gas, and water rate TDM predictions vs actual simulation data plots for 6 Year Blind Validation TDM.... 192

Figure 237 Well-053 oil, gas, and water rate TDM predictions vs actual simulation data plots for 6 Year Blind Validation TDM. 193

Figure 238 Well-022 oil, gas, and water rate TDM predictions vs actual simulation data plots for 6 Year Blind Validation TDM.... 194

Figure 239 Well-056 oil, gas, and water rate TDM predictions vs actual simulation data plots for 6 Year

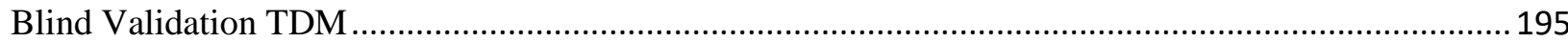
Figure 240 Well-014 oil, gas, and water TDM predictions vs actual simulation data plots for 6 Year Blind Validation TDM. 196 Figure 241 Well-046 oil, gas, and water rate TDM predictions vs actual simulation data plots for 6 Year

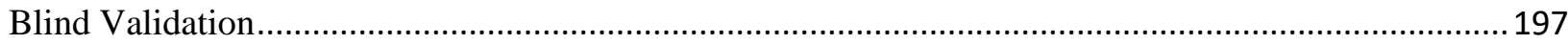

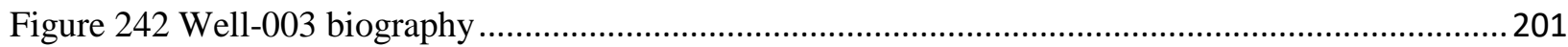

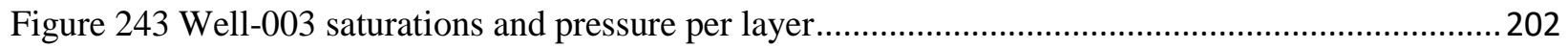

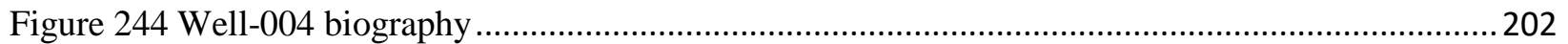

Figure 245 Well-004 saturations and pressure per layer........................................................................203

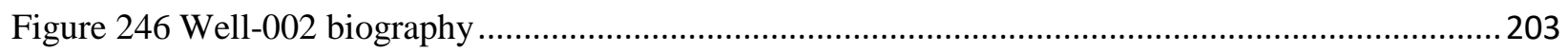

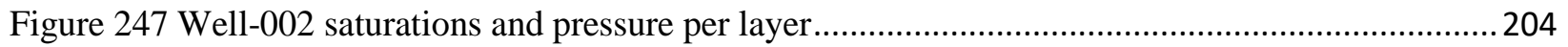

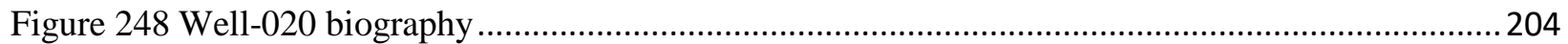

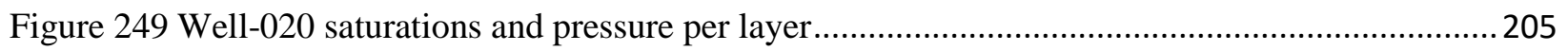

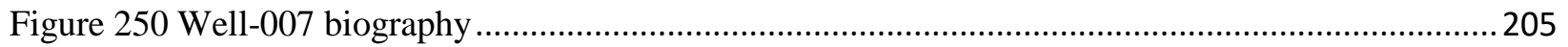

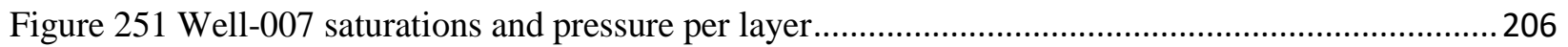

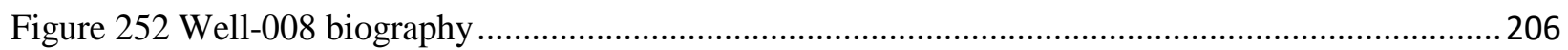

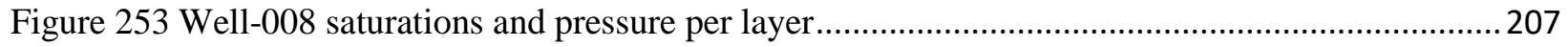

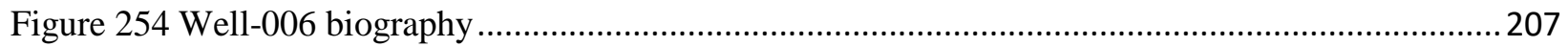

Figure 255 Well-006 saturations and pressure per layer.....................................................................208

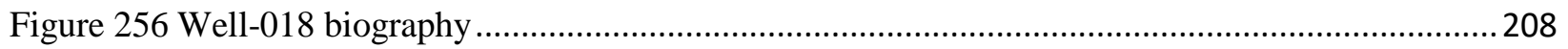

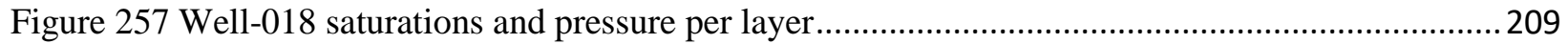

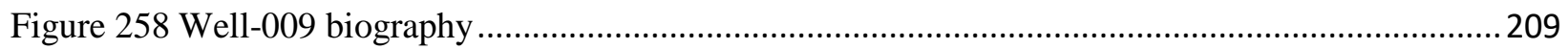

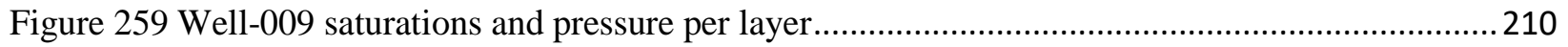

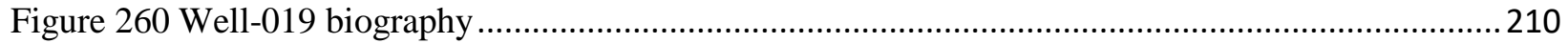

Figure 261 Well-019 saturations and pressure per layer................................................................ 211

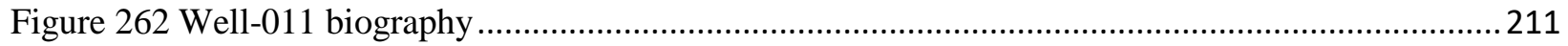

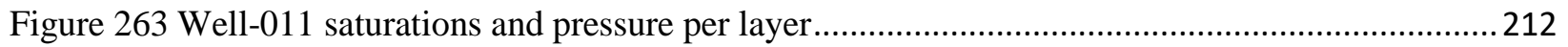

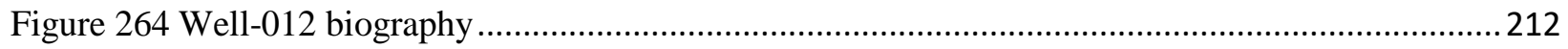

Figure 265 Well-012 saturations and pressure per layer...............................................................213

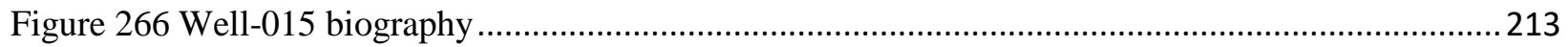

Figure 267 Well-015 saturations and pressure per layer................................................................214

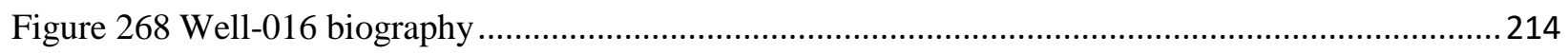

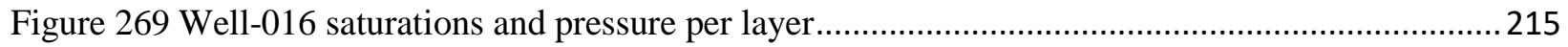

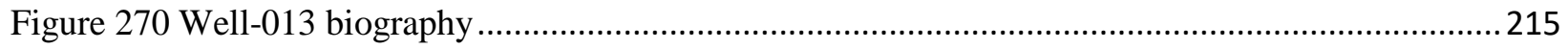

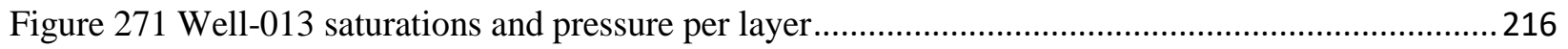

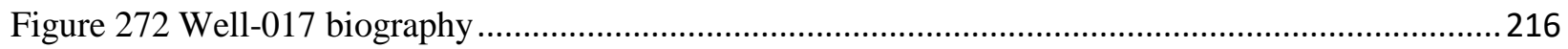




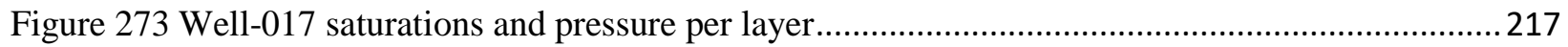

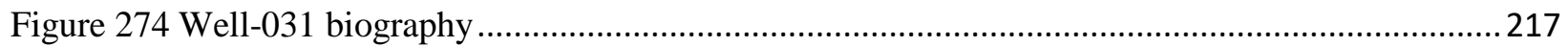

Figure 275 Well-031 saturations and pressure per layer...............................................................218

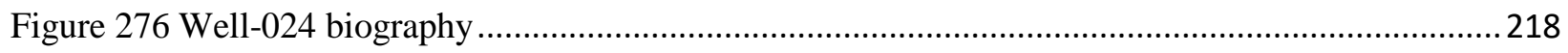

Figure 277 Well-024 saturations and pressure per layer................................................................219

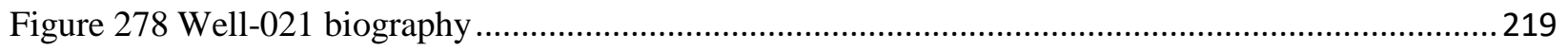

Figure 279 Well-021 saturations and pressure per layer................................................................220

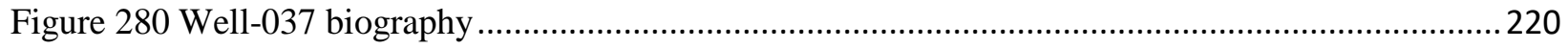

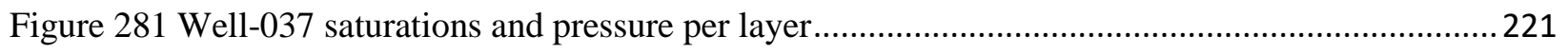

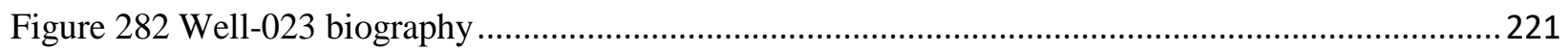

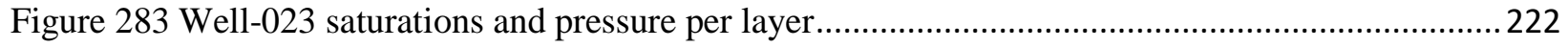

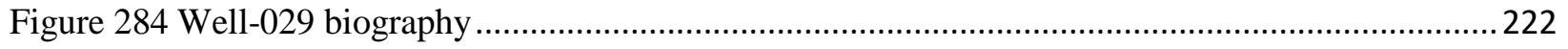

Figure 285 Well-029 saturations and pressure per layer.................................................................223

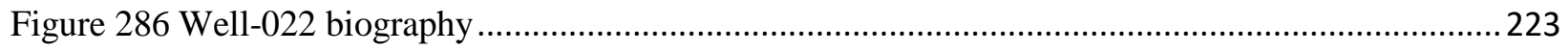

Figure 287 Well-022 saturations and pressure per layer................................................................224

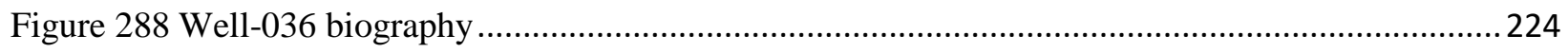

Figure 289 Well-036 saturations and pressure per layer..................................................................225

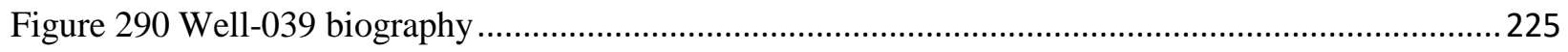

Figure 291 Well-039 saturations and pressure per layer.................................................................226

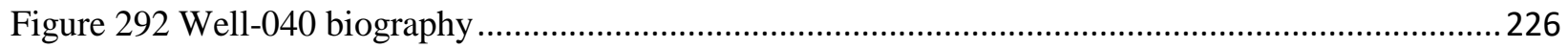

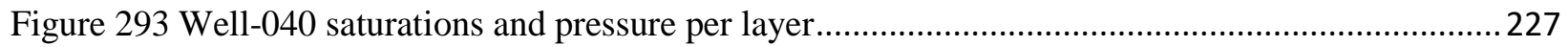

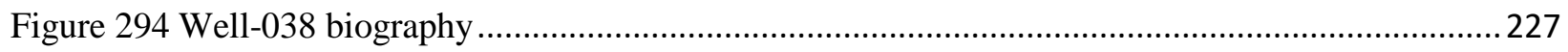

Figure 295 Well-038 saturations and pressure per layer.................................................................228

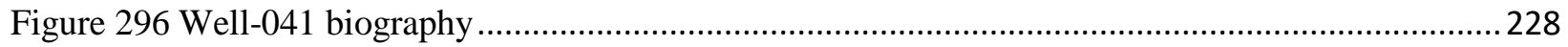

Figure 297 Well-041 saturations and pressure per layer...............................................................229

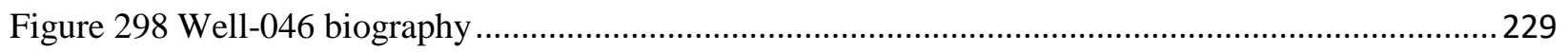

Figure 299 Well-046 saturations and pressure per layer....................................................................230

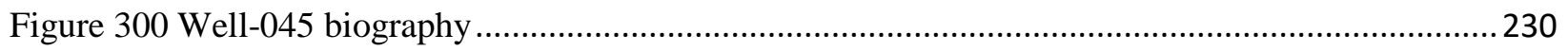

Figure $301 \mathrm{Well}-045$ saturations and pressure per layer................................................................ 231

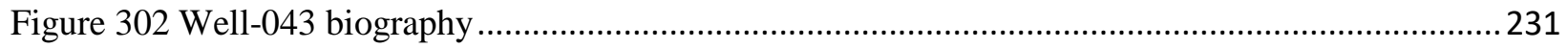

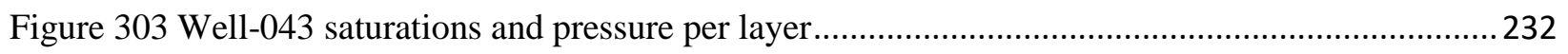

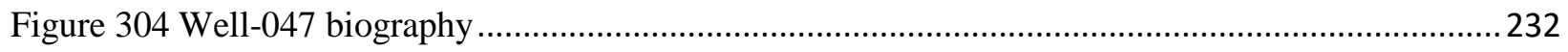

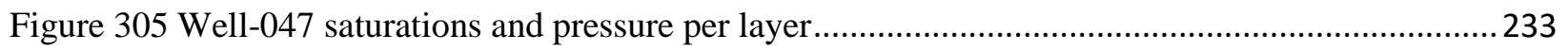

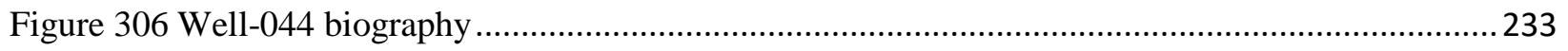

Figure 307 Well-044 saturations and pressure per layer................................................................234

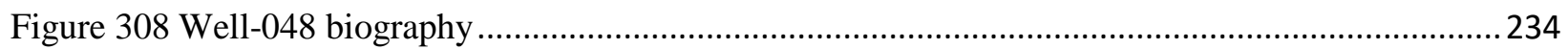

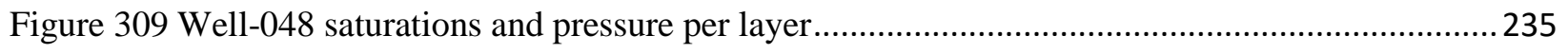

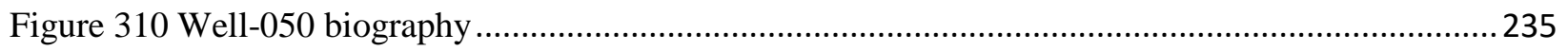

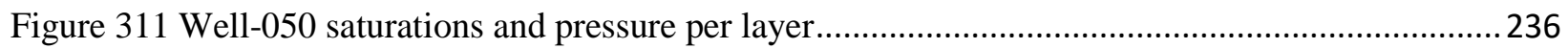

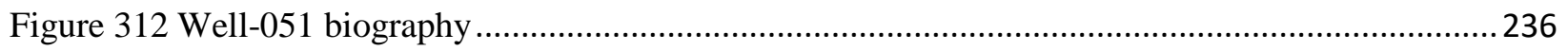

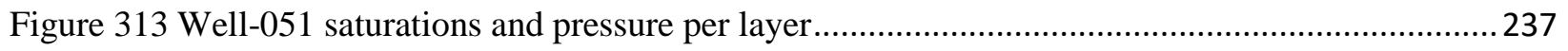

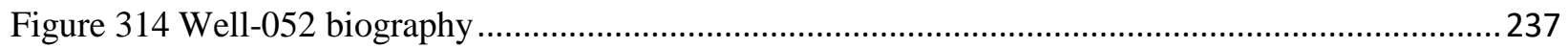

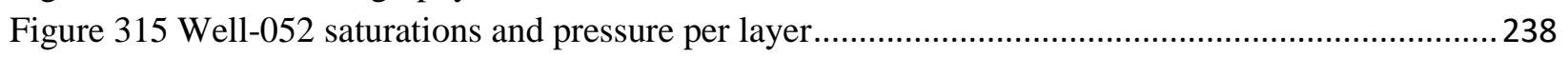

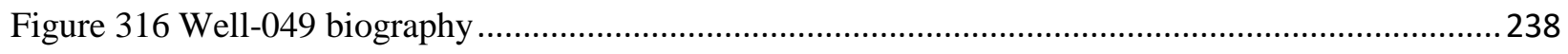




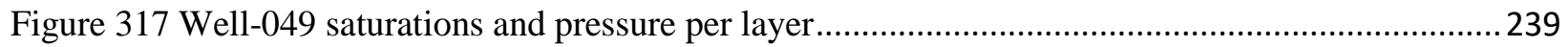

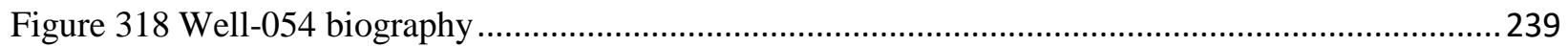

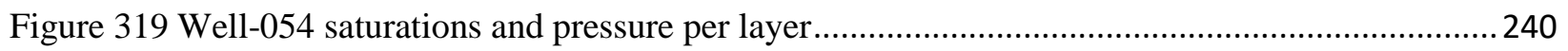

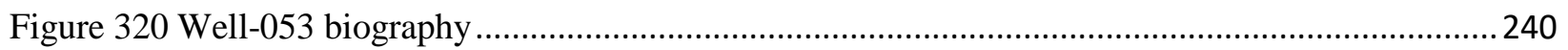

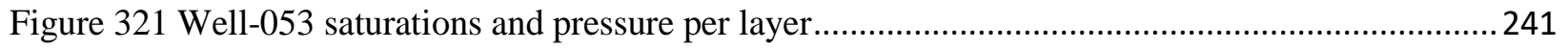

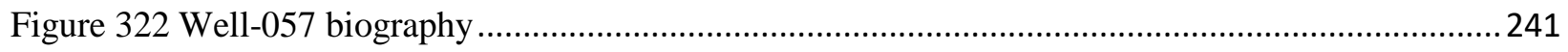

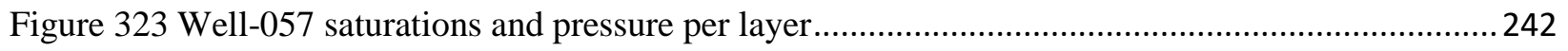

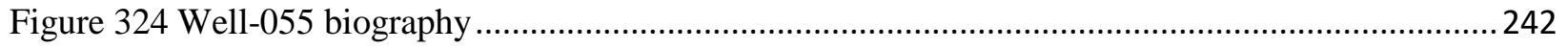

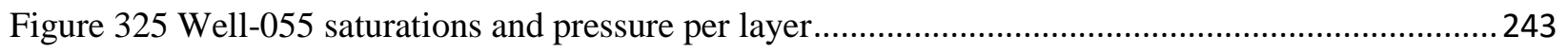

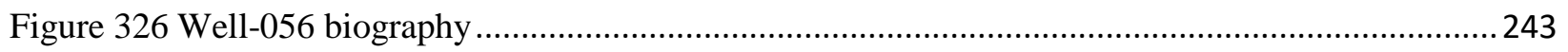

Figure 327 Well-056 saturations and pressure per layer...................................................................244

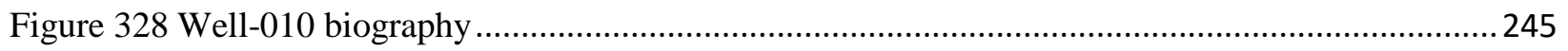

Figure 329 Well-010 saturations and pressure per layer..................................................................245

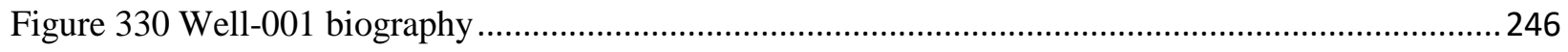

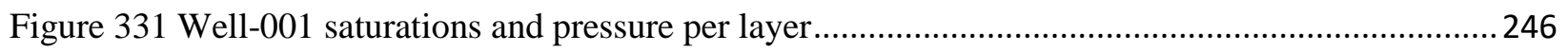

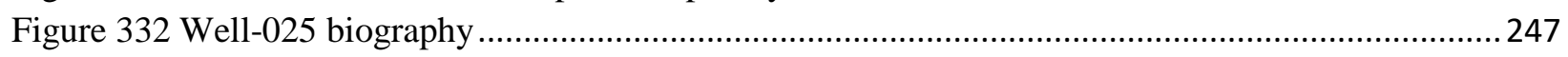

Figure 333 Well-025 saturations and pressure per layer................................................................ 247

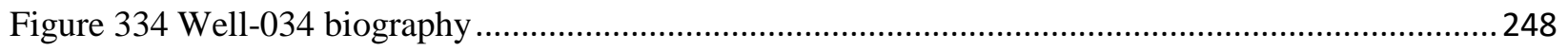

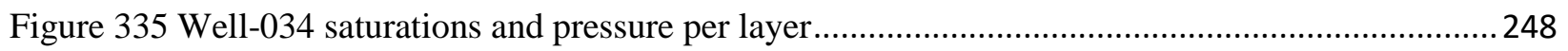

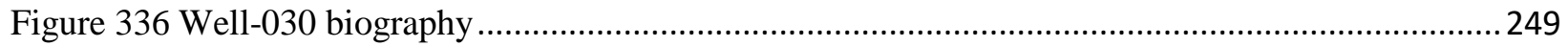

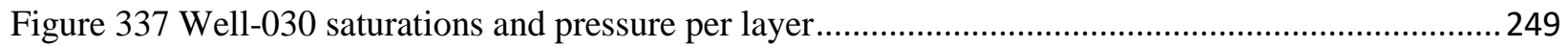

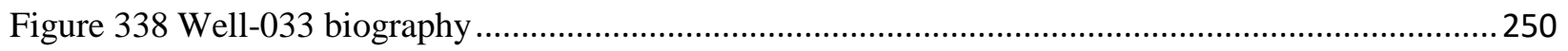

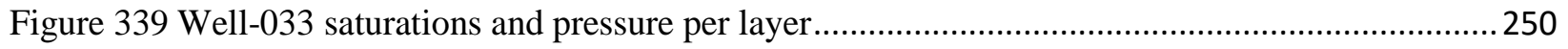

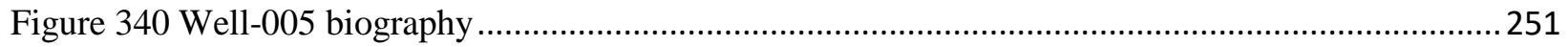

Figure 341 Well-005 saturations and pressure per layer............................................................... 251

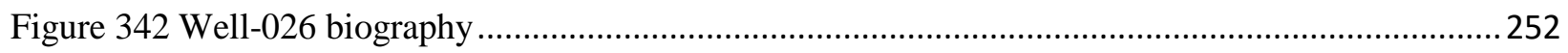

Figure 343 Well-026 saturations and pressure per layer..................................................................252

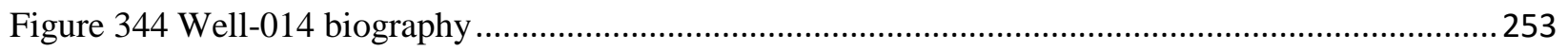

Figure 345 Well-014 saturations and pressure per layer................................................................253

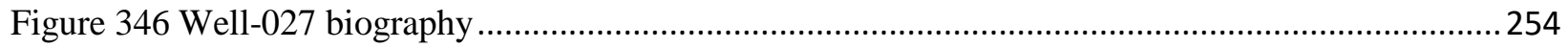

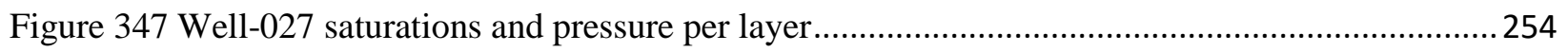

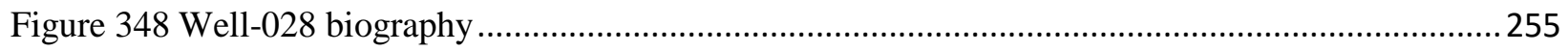

Figure 349 Well-028 saturations and pressure per layer................................................................25

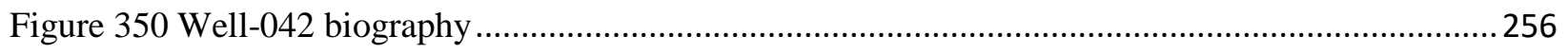

Figure 351 Well-042 saturations and pressure per layer................................................................256

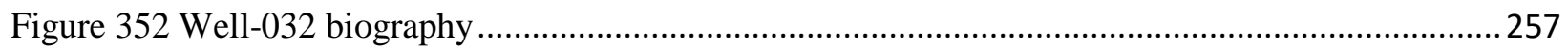

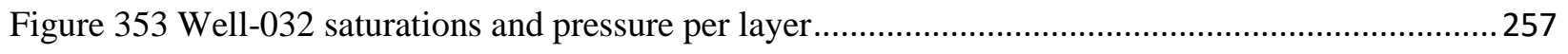

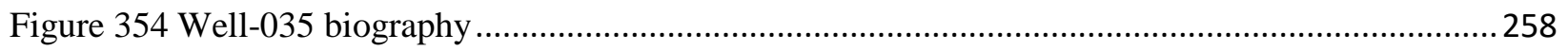

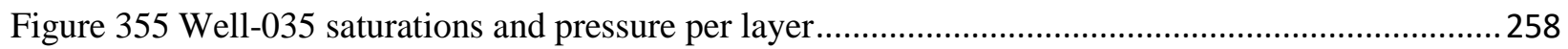

Figure 356 Well-001 oil, gas, and water rate TDM prediction vs. actual simulation data plots for No Blind

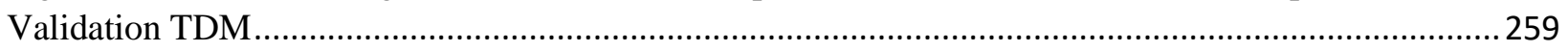

Figure 357 Well-002 oil, gas, and water TDM predictions vs. actual simulation data plots for No Blind

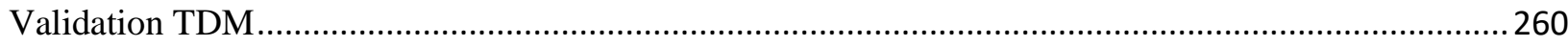


Figure 358 Well-003 oil, gas, and water rate TDM predictions vs. actual simulation data plots for No Blind Validation TDM

Figure 359 Well-004 oil, gas, and water rate TDM predictions vs actual simulation data plots for No Blind Validation TDM 262

Figure 360 Well-005 oil, gas, and water rate TDM predictions vs actual simulation data plots for No Blind Validation TDM.

Figure 361 Well-006 oil, gas, and water rate TDM predictions vs actual simulation data plots for No Blind Validation TDM. 264

Figure 362 Well-007 oil, gas, water rate TDM predictions vs actual simulation data plots for No Blind Validation TDM. .265 Figure 363 Well-008 oil, gas, and water rate TDM predictions vs actual simulation data plots for No Blind Validation TDM. 266

Figure 364 Well-009 oil, gas, and water rate TDM predictions vs actual simulation data plots for No Blind Validation TDM.

Figure 365 Well-010 oil, gas, and water rate TDM predictions vs actual simulation data plots for No Blind Validation TDM. 268

Figure 366 Well-011 oil, gas, and water rate TDM predictions vs actual simulation data plots for No Blind Validation TDM.

Figure 367 Well-012 oil, gas, and water rate TDM predictions vs actual simulation data plots for No Blind Validation TDM

Figure 368 Well-013 oil, gas, and water rate TDM predictions vs actual simulation data plots for No Blind Validation TDM.

Figure 369 Well-014 oil, gas, and water rate TDM prediction vs actual simulation data plots for No Blind Validation TDM.

Figure 370 Well-015 oil, gas, and water rate TDM predictions vs actual simulation data plots for No Blind Validation TDM....

Figure 371 Well-016 oil, gas, and water rate TDM predictions vs actual simulation data plots for No Blind Validation TDM.

Figure 372 Well-017 oil, gas, and water rate TDM predictions vs actual simulation data plots for No Blind Validation TDM.

Figure 373 Well-018 oil, gas, and water rate TDM predictions vs actual simulation data plots for No Blind Validation TDM.

Figure 374 Well-019 oil, gas, and water rate TDM prediction vs actual simulation data plots for No Blind Validation TDM.

Figure 375 Well-020 oil, gas, and water rate TDM predictions vs actual simulation data plots for No Blind Validation TDM.

Figure 376 Well-021 oil, gas, and water rate TDM predictions vs actual simulation data plots for No Blind Validation TDM.

Figure 377 Well-022 oil, gas, and water rate TDM predictions vs actual simulation data plots for No Blind Validation TDM.

Figure 378 Well-023 oil, gas, and water rate TDM predictions vs actual simulation data plots for No Blind Validation TDM

Figure 379 Well-024 oil, gas, and water rate TDM predictions vs actual simulation data plots for No Blind Validation TDM.

Figure 380 Well-025 oil, gas, and water rate TDM predictions vs actual simulation data plots for No Blind Validation TDM. 
Figure 381 Well-026 oil, gas, and water rate TDM predictions vs actual simulation data plots for No Blind Validation TDM

Figure 382 Well-027 oil, gas, and water rate TDM predictions vs actual simulation data plots for No Blind Validation TDM. 285

Figure 383 Well-028 oil, gas, and water rate TDM predictions vs actual simulation data plots for No Blind Validation TDM.

Figure 384 Well-029 oil, gas, and water rate TDM predictions vs actual simulation data plots for No Blind Validation TDM.

Figure 385 Well-030 oil, gas, and water rate TDM predictions vs actual simulation data plots for No Blind Validation TDM.

Figure 386 Well-031 oil, gas, and water rate TDM predictions vs actual simulation data plots for No Blind Validation TDM.

Figure 387 Well-032 oil, gas, and water rate TDM predictions vs actual simulation data plots for No Blind Validation TDM.

Figure 388 Well-033 oil, gas, and water rate TDM predictions vs actual simulation data plots for No Blind Validation TDM.

Figure 389 Well-034 oil, gas, and water rate TDM predictions vs actual simulation data plots for No Blind Validation TDM.

Figure 390 Well-035 oil, gas, and water rate TDM predictions vs actual simulation data plots for No Blind Validation TDM

Figure 391 Well-036 oil, gas, and water rate TDM predictions vs actual simulation data plots for No Blind Validation TDM.

Figure 392 Well-037 oil, gas, and water rate TDM predictions vs actual simulation data plots for No Blind Validation TDM.

Figure 393 Well-038 oil, gas, and water rate TDM predictions vs actual simulation data plots for No Blind Validation TDM....

Figure 394 Well-039 oil, gas, and water rate TDM predictions vs actual simulation data plots for No Blind Validation TDM.

Figure 395 Well-040 oil, gas, and water rate TDM predictions vs actual simulation data plots for No Blind Validation TDM.

Figure 396 Well-041 oil, gas, and water rate TDM predictions vs actual simulation data plots for No Blind Validation TDM.

Figure 397 Well-042 oil, gas, and water rate TDM predictions vs actual simulation data plots for No Blind Validation TDM.

Figure 398 Well-043 oil, gas, and water rate TDM predictions vs actual simulation data plots for No Blind Validation TDM. 301

Figure 399 Well-044 oil, gas, and water rate TDM predictions vs actual simulation data plots for No Blind Validation TDM.

Figure 400 Well-045 oil, gas, and water rate TDM predictions vs actual simulation data plots for No Blind Validation TDM.

Figure 401 Well-046 oil, gas, and water rate TDM predictions vs actual simulation data plots for No Blind Validation TDM.

Figure 402 Well-047 oil, gas, and water rate TDM predictions vs actual simulation data plots for No Blind Validation TDM.

Figure 403 Well-048 oil, gas, and water rate TDM predictions vs actual simulation data plots for No Blind Validation TDM. 
Figure 404 Well-049 oil. gas, and water rate TDM predictions vs actual simulation data plots for No

Blind Validation TDM

Figure 405 Well-050 oil, gas, and water rate TDM predictions vs actual simulation data plots for No Blind Validation TDM. 308

Figure 406 Well-051 oil, gas, and water rate TDM predictions vs actual simulation data plots for No Blind Validation TDM.

Figure 407 Well-052 oil, gas, and water rate TDM predictions vs actual simulation data plots for No Blind Validation TDM. 310

Figure 408 Well-053 oil, gas, and water rate TDM predictions vs actual simulation data plots for No Blind Validation TDM.

Figure 409 Well-054 oil, gas, and water rate TDM predictions vs actual simulation data plots for No Blind Validation TDM.

Figure 410 Well-055 oil, gas, and water rate TDM predictions vs actual simulation data plots for No Blind Validation TDM.

Figure 411 Well-056 oil, gas, and water rate TDM predictions vs actual simulation data plots for No Blind Validation TDM.

Figure 412 Well-057 oil, gas, and water rate TDM predictions vs actual simulation data plots for No

Blind Validation TDM.

Figure 413 Entire reservoir heat map for reservoir pressure at December 32st, 2015 for No Blind Validation TDM.

Figure 414 Entire reservoir heat map for reservoir pressure at February 29th, 2016 for No Blind Validation TDM.

Figure 415 Entire reservoir heat map for reservoir pressure at April 30th, 2016 for No Blind Validation TDM.

Figure 416 Entire reservoir heat map for reservoir pressure at June 30th, 2016 for No Blind Validation

TDM.

Figure 417 Entire reservoir heat map for reservoir pressure at August 31st, 2016 for No Blind Validation

TDM.

Figure 418 Entire reservoir heat map for reservoir pressure at October 31st, 2016 for No Blind Validation

TDM.

Figure 419 Entire reservoir heat map for reservoir pressure at December 31st, 2016 for No Blind Validation TDM.

Figure 420 Entire reservoir heat map for water saturation at December 31st, 2016 for No Blind Validation TDM. 318

Figure 421 Entire reservoir heat map for water saturation at February 29th, 2016 for No Blind Validation TDM.

Figure 422 Entire reservoir heat map for water saturation at April 30th, 2016 for No Blind Validation

TDM.

Figure 423 Entire reservoir heat map for water saturation at June 30th, 2016 for No Blind Validation TDM.

Figure 424 Entire reservoir heat map for water saturation at August 31st, 2016 for No Blind Validation TDM. 319

Figure 425 Entire reservoir heat map for water saturation for October 31st, 2016 for No Blind Validation TDM.

Figure 426 Entire reservoir heat map for water saturation at December 31st, 2016 for No Blind Validation TDM. 320 
Figure 427 Well-001 oil, gas, and water rate TDM predictions vs actual simulation data plots for 1 Year

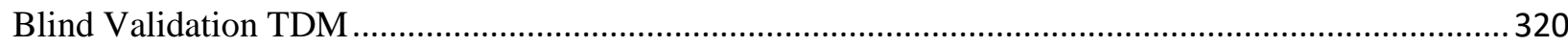

Figure 428 Well-002 oil, gas, and water rate TDM predictions vs actual simulation data plots for 1 Year

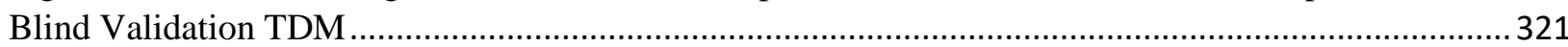

Figure 429 Well-003 oil, gas, and water rate TDM predictions vs actual simulation data plots for 1 Year

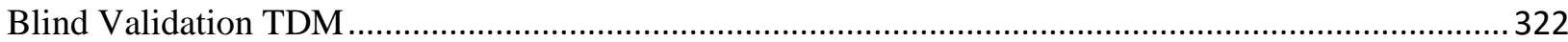
Figure 430 Well-004 oil, gas, and water rate TDM predictions vs actual simulation data plots for 1 Year

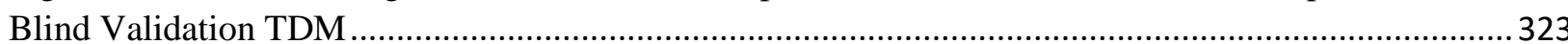
Figure 431 Well-005 oil, gas, and water rate TDM predictions vs actual simulation data plots for 1 Year

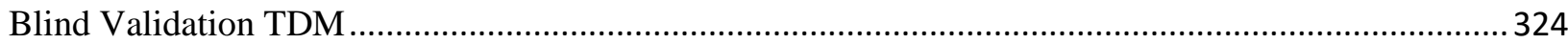
Figure 432 Well-006 oil, gas, and water rate TDM predictions vs actual simulation data plots for 1 Year

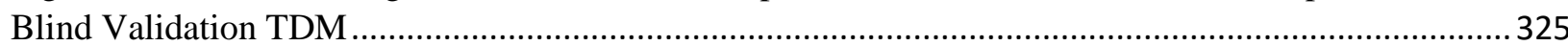
Figure 433 Well-007 oil, gas, and water rate TDM predictions vs actual simulation data for 1 Year Blind Validation TDM. 326 Figure 434 Well-008 oil, gas, and water rate TDM predictions vs actual simulation data plots for 1 Year Blind Validation TDM

Figure 435 Well-009 oil, gas, and water rate TDM predictions vs actual simulation data plots for 1 Year

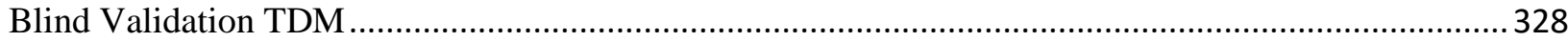
Figure 436 Well-010 oil, gas, and water rate TDM predictions vs actual simulation data plots for 1 Year

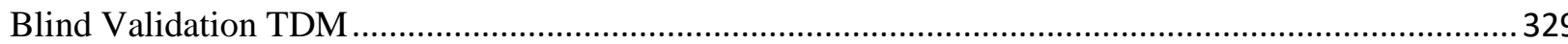
Figure 437 Well-011 oil, gas, and water rate TDM predictions vs actual simulation data plots for 1 Year Blind Validation TDM.... 330 Figure 438 Well-012 oil, gas, and water rate TDM predictions vs actual simulation data plots for 1 Year Blind Validation TDM 331 Figure 439 Well-013 oil, gas, and water rate TDM predictions vs actual simulation data plots for 1 Year Blind Validation TDM. 332 Figure 440 Well-014 oil, gas, and water rate TDM predictions vs actual simulation data plots for 1 Year Blind Validation TDM 333

Figure 441 Well-015 oil, gas, and water rate TDM predictions vs actual simulation data plots for 1 Year Blind Validation TDM. 334 Figure 442 Well-016 oil, gas, and water rate TDM predictions vs actual simulation data plots for 1 Year Blind Validation TDM 335 Figure 443 Well-017 oil, gas, and water rate TDM predictions vs actual simulation data plots for 1 Year Blind Validation TDM. 336 Figure 444 Well-018 oil, gas, and water rate TDM predictions vs actual simulation data plots for 1 Year Blind Validation TDM 337

Figure 445 Well-019 oil, gas, and water rate TDM predictions vs actual simulation data plots for 1 Year Blind Validation TDM. 338 Figure 446 Well-020 oil, gas, and water rate TDM predictions vs actual simulation data plots for 1 Year Blind Validation TDM 339 Figure 447 Well-021 oil, gas, and water rate TDM predictions vs actual simulation data plots for 1 Year Blind Validation TDM. 340 Figure 448 Well-022 oil, gas, and water rate TDM predictions vs actual simulation data plots for 1 Year Blind Validation TDM 341 Figure 449 Well-023 oil, gas, and water rate TDM predictions vs actual simulation data plots for 1 Year Blind Validation TDM. 342 
Figure 450 Well-024 oil, gas, and water rate TDM predictions vs actual simulation data plots for 1 Year

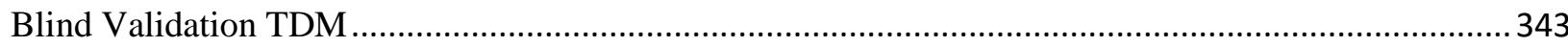

Figure 451 Well-025 oil, gas, and water rate TDM predictions vs actual simulation data plots for 1 Year

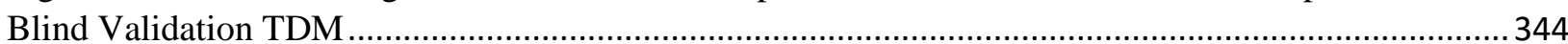

Figure 452 Well-026 oil, gas, and water rate TDM predictions vs actual simulation data plots for 1 Year

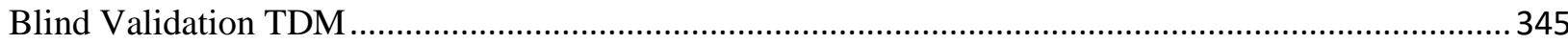
Figure 453 Well-027 oil, gas, and water rate TDM predictions vs actual simulation data plots for 1 Year

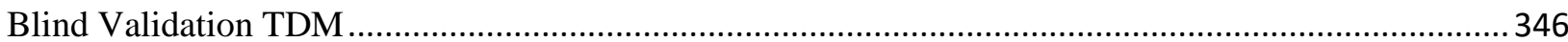
Figure 454 Well-028 oil, gas, and water rate TDM predictions vs actual simulation data plots for 1 Year

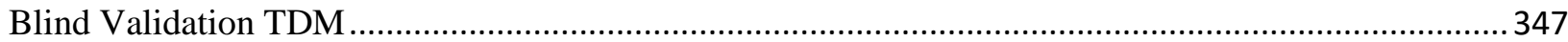
Figure 455 Well-029 oil, gas, and water rate TDM predictions vs actual simulation data plots for 1 Year

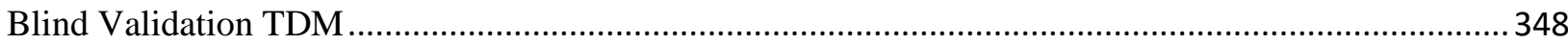
Figure 456 Well-030 oil, gas, and water rate TDM predictions vs actual simulation data plots for 1 Year

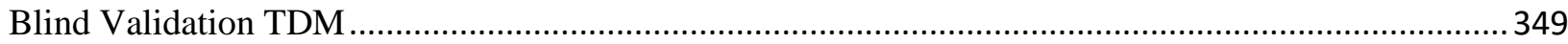
Figure 457 Well-031 oil, gas, and water rate TDM predictions vs actual simulation data plots for 1 Year

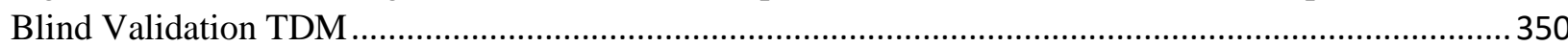
Figure 458 Well-032 oil, gas, and water rate TDM predictions vs actual simulation data plots for 1 Year

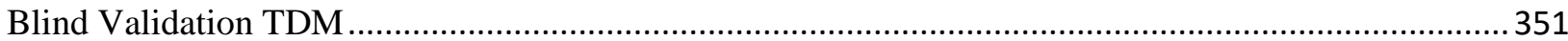
Figure 459 Well-033 oil, gas, and water rate TDM predictions vs actual simulation data plots for 1 Year

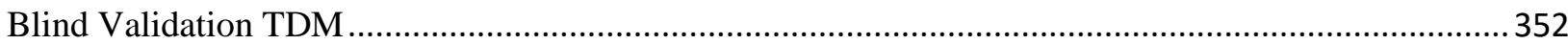
Figure 460 Well-034 oil, gas, and water rate TDM predictions vs actual simulation data plots for 1 Year

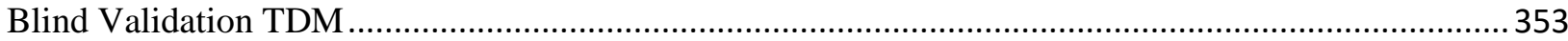
Figure 461 Well-035 oil, gas, and water rate TDM predictions vs actual simulation data plots for 1 Year

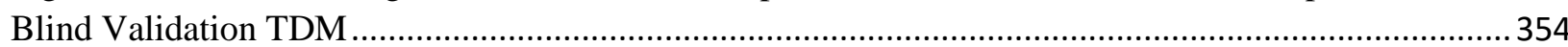
Figure 462 Well-036 oil, gas, and water rate TDM predictions vs actual simulation data plots for 1 Year

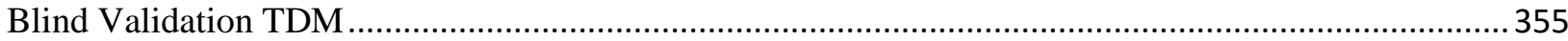
Figure 463 Well-037 oil, gas, and water rate TDM predictions vs actual simulation data plots for 1 Year

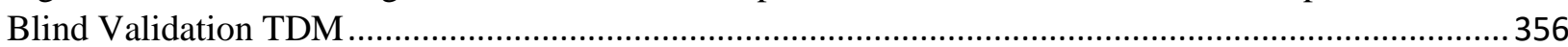
Figure 464 Well-038 oil, gas, and water rate TDM predictions vs actual simulation data plots for 1 Year Blind Validation TDM.... 357 Figure 465 Well-039 oil, gas, and water rate TDM predictions vs actual simulation data plots for 1 Year

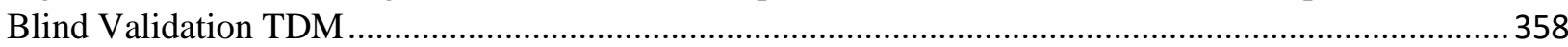
Figure 466 Well-040 oil, gas, and water rate TDM predictions vs actual simulation data plots for 1 Year Blind Validation TDM. 359 Figure 467 Well-041 oil, gas, and water rate TDM predictions vs actual simulation data plots for 1 Year

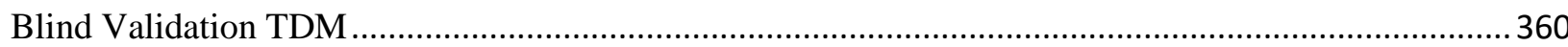
Figure 468 Well-042 oil, gas, and water rate TDM predictions vs actual simulation data plots for 1 Year Blind Validation TDM.... 361 Figure 469 Well-043 oil, gas, and water rate TDM predictions vs actual simulation data plots for 1 Year Blind Validation TDM 362 Figure 470 Well-044 oil, gas, and water rate TDM predictions vs actual simulation data plots for 1 Year Blind Validation TDM. 363 Figure 471 Well-045 oil, gas, and water rate TDM predictions vs actual simulation data plots for 1 Year Blind Validation. 364 Figure 472 Well-046 oil, gas, and water rate TDM predictions vs actual simulation data plots for 1 Year Blind Validation TDM. 365 
Figure 473 Well-047 oil, gas, and water rate TDM predictions vs actual simulation data plots for 1 Year

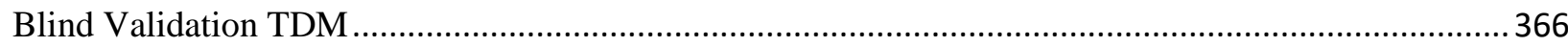

Figure 474 Well-048 oil, gas, and water rate TDM predictions vs actual simulation data plots for 1 Year

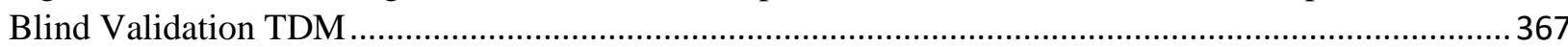

Figure 475 Well-049 oil, gas, and water rate TDM predictions vs actual simulation data plots for 1 Year

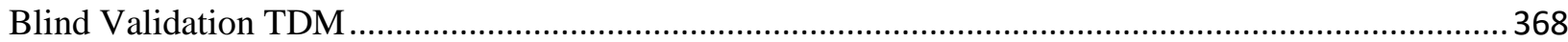

Figure 476 Well-050 oil, gas, and water rate TDM predictions vs actual simulation data plots for 1 Year

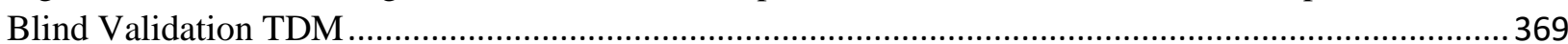

Figure 477 Well-051 oil, gas, and water rate TDM predictions vs actual simulation data plots for 1 Year

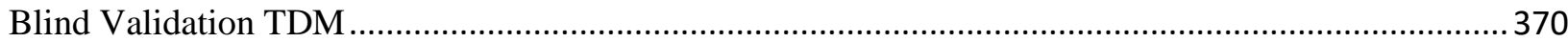

Figure 478 Well-052 oil, gas, and water rate TDM predictions vs actual simulation data plots for 1 Year

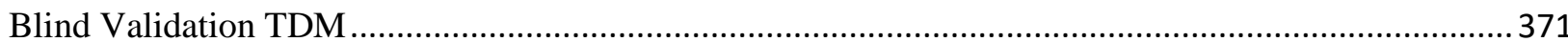

Figure 479 Well-053 oil, gas, and water rate TDM predictions vs actual simulation data plots for 1 Year

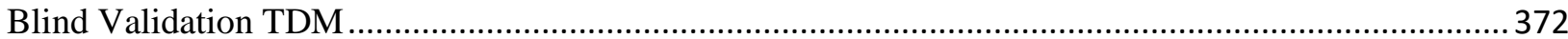
Figure 480 Well-054 oil, gas, and water rate TDM predictions vs actual simulation data plots for 1 Year

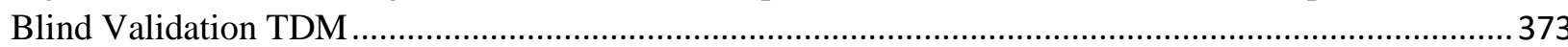
Figure 481 Well-055 oil, gas, and water rate TDM predictions vs actual simulation data plots for 1 Year

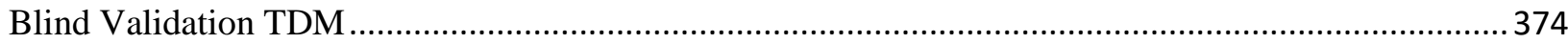
Figure 482 Well-056 oil, gas, and water rate TDM predictions vs actual simulation data plots for 1 Year

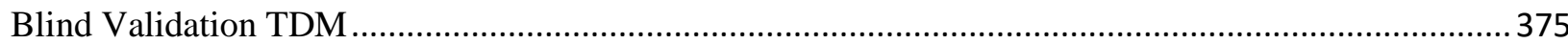
Figure 483 Well-057 oil, gas, and water rate TDM predictions vs actual simulation data plots for 1 Year

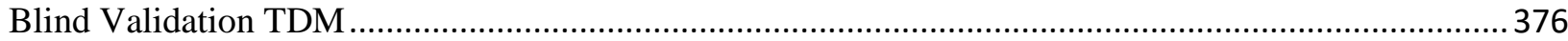
Figure 484 Entire reservoir heat map for reservoir pressure at December 31st, 2015 for 1 Year Blind Validation TDM 376

Figure 485 Entire reservoir heat map of reservoir pressure at February 29th, 2016 for 1 Year Blind Validation TDM

Figure 486 Entire reservoir heat map of reservoir pressure at April 30th, 2016 for 1 Year Blind Validation TDM

Figure 487 Entire reservoir heat map of reservoir pressure at June 30th, 2016 for 1 Year Blind Validation TDM

Figure 488 Entire reservoir heat map of reservoir pressure at August 31st, 2016 for 1 Year Blind Validation TDM

Figure 489 Entire reservoir heat map of reservoir pressure at October 31st, 2016 for 1 Year Blind Validation TDM

Figure 490 Entire reservoir heat map of reservoir pressure at December 31st, 2016 for 1 Year Blind Validation TDM 378

Figure 491 Entire reservoir heat map for water saturation at December 31st, 2015 for 1 Year Blind Validation TDM

Figure 492 Entire reservoir heat map for water saturation February 29th, 2016 for 1 Year Blind Validation TDM.

Figure 493 Entire reservoir heat map for water saturation April 30th, 2016 for 1 Year Blind Validation TDM.

Figure 494 Entire reservoir heat map for water saturation at June 30th, 2016 for 1 Year Blind Validation TDM. 380

Figure 495 Entire reservoir heat map for water saturation at August 31st, 2016 for 1 Year Blind Validation TDM 380 
Figure 496 Entire reservoir heat map for water saturation at October 31st, 2016 for 1 Year Blind Validation TDM.

Figure 497 Entire reservoir heat map for water saturation at December 31st, 2016 for 1 Year Blind Validation TDM.

Figure 498 Well-001 oil, gas, and water rate TDM predictions vs actual simulation data plots for 2 Year Blind Validation TDM.

Figure 499 Well-002 oil, gas, and water rate TDM predictions vs actual simulation data plots for 2 Year Blind Validation TDM.

Figure 500 Well-003 oil, gas, and water rate TDM predictions vs actual simulation data plots for 2 Year Blind Validation TDM.

Figure 501 Well-004 oil, gas, and water rate TDM predictions vs actual simulation data plots for 2 Year Blind Validation TDM. 384

Figure 502 Well-005 oil, gas, and water rate TDM predictions vs actual simulation data plots for 2 Year Blind Validation TDM.... 385

Figure 503 Well-006 oil, gas, and water rate TDM predictions vs actual simulation data plots for 2 Year Blind Validation TDM.... 386

Figure 504 Well-007 oil, gas, and water rate TDM predictions vs actual simulation data plots for 2 Year Blind Validation TDM.... 387

Figure 505 Well-008 oil, gas, and water rate TDM predictions vs actual simulation data plots for 2 Year Blind Validation TDM. 388

Figure 506 Well-009 oil, gas, and water rate TDM predictions vs actual simulation data plots for 2 Year Blind Validation TDM. 389

Figure 507 Well-010 oil, gas, and water rate TDM predictions vs actual simulation data plots for 2 Year Blind Validation. 390 Figure 508 Well-011 oil, gas, and water rate TDM predictions vs actual simulation data plots for 2 Year Blind Validation TDM. 391

Figure 509 Well-012 oil, gas, and water rate TDM predictions vs actual simulation data plots for 2 Year Blind Validation TDM. 392

Figure 510 Well-013 oil, gas, and water rate TDM predictions vs actual simulation data plots for 2 Year Blind Validation TDM. 393

Figure 511 Well-014 oil, gas, and water rate TDM predictions vs actual simulation data plots for 2 Year Blind Validation TDM. 394

Figure 512 Well-015 oil, gas, and water rate TDM predictions vs actual simulation data plots for 2 Year Blind Validation TDM. 395 Figure 513 Well-016 oil, gas, and water rate TDM predictions vs actual simulation data plots for 2 Year Blind Validation TDM. 396 Figure 514 Well-017 oil, gas, and water rate TDM predictions vs actual simulation data plots for 2 Year Blind Validation TDM. 397 Figure 515 Well-018 oil, gas, and water rate TDM predictions vs actual simulation data plots for 2 Year Blind Validation TDM. 398 Figure 516 Well-019 oil, gas, and water rate TDM predictions vs actual simulation data plots for 2 Year Blind Validation TDM. 399 Figure 517 Well-020 oil, gas, and water rate TDM predictions vs actual simulation data plots for 2 Year Blind Validation TDM. 400 Figure 518 Well-021 oil, gas, and water rate TDM predictions vs actual simulation data plots for 2 Year Blind Validation TDM. 401 
Figure 519 Well-022 oil, gas, and water rate TDM predictions vs actual simulation data plots for 2 Year

Blind Validation TDM.

Figure 520 Well-023 oil, gas, and water rate TDM predictions vs actual simulation data plots for 2 Year

Blind Validation TDM.... 403

Figure 521 Well-024 oil, gas, and water rate TDM predictions vs actual simulation data plots for 2 Year

Blind Validation TDM.

Figure 522 Well-025 oil, gas, and water rate TDM predictions vs actual simulation data plots for 2 Year

Blind Validation TDM. 405

Figure 523 Well-026 oil, gas, and water rate TDM predictions vs actual simulation data plots for 2 Year

Blind Validation TDM. 406

Figure 524 Well-027 oil, gas, and water rate TDM predictions vs actual simulation data plots for 2 Year

Blind Validation TDM. 407

Figure 525 Well-028 oil, gas, and water rate TDM predictions vs actual simulation data plots for 2 Year

Blind Validation TDM. 408

Figure 526 Well-029 oil, gas, and water rate TDM predictions vs actual simulation data plots for 2 Year

Blind Validation TDM. 409

Figure 527 Well-030 oil, gas, and water rate TDM predictions vs actual simulation data plots for 2 Year

Blind Validation TDM. 410

Figure 528 Well-031 oil, gas, and water rate TDM predictions vs actual simulation data plots for 2 Year

Blind Validation TDM.

Figure 529 Well-032 oil, gas, and water rate TDM predictions vs actual simulation data plots for 2 Year

Blind Validation TDM. 412

Figure 530 Well-033 oil, gas, and water rate TDM predictions vs actual simulation data plots for 2 Year

Blind Validation TDM.

Figure 531 Well-034 oil, gas, and water rate TDM predictions vs actual simulation data plots for 2 Year

Blind Validation TDM. 414

Figure 532 Well-035 oil, gas, and water rate TDM predictions vs actual simulation data plots for 2 Year

Blind Validation TDM.

Figure 533 Well-036 oil, gas, and water rate TDM predictions vs actual simulation data plots for 2 Year

Blind Validation TDM. 416

Figure 534 Well-037 oil, gas, and water rate TDM predictions vs actual simulation data plots for 2 Year

Blind Validation TDM.

Figure 535 Well-038 oil, gas, and water rate TDM predictions vs actual simulation data plots for 2 Year

Blind Validation TDM.

Figure 536 Well-039 oil, gas, and water rate TDM predictions vs actual simulation data plots for 2 Year

Blind Validation TDM.

Figure 537 Well-040 oil, gas, and water rate TDM predictions vs actual simulation data plots for 2 Year

Blind Validation TDM. . .420

Figure 538 Well-041 oil, gas, and water rate TDM predictions vs actual simulation data plots for 2 Year

Blind Validation TDM.

Figure 539 Well-042 oil, gas, and water rate TDM predictions vs actual simulation data plots for 2 Year

Blind Validation TDM. 422

Figure 540 Well-043 oil, gas, and water rate TDM predictions vs actual simulation data plots for 2 Year

Blind Validation TDM.

Figure 541 Well-044 oil, gas, and water rate TDM predictions vs actual simulation data plots for 2 Year

Blind Validation TDM. .424 
Figure 542 Well-045 oil, gas, and water rate TDM predictions vs actual simulation data plots for 2 Year

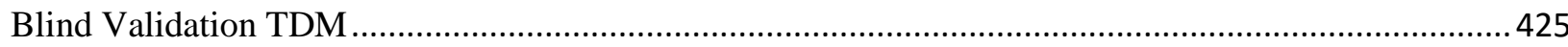

Figure 543 Well-046 oil, gas, and water rate TDM predictions vs actual simulation data plots for 2 Year

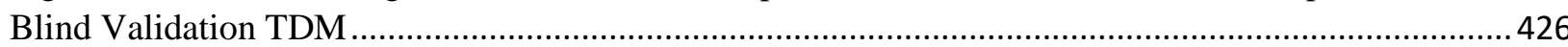

Figure 544 Well-047 oil, gas, and water rate TDM predictions vs actual simulation data plots for 2 Year

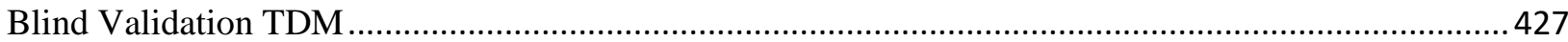

Figure 545 Well-048 oil, gas, and water rate TDM predictions vs actual simulation data plots for 2 Year

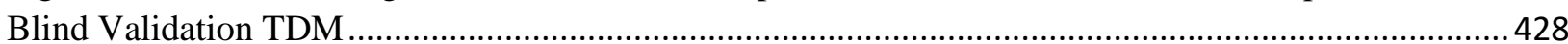

Figure 546 Well-049 oil, gas, and water rate TDM predictions vs actual simulation data plots for 2 Year

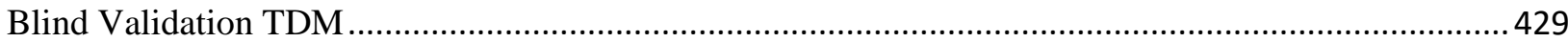

Figure 547 Well-050 oil, gas, and water rate TDM predictions vs actual simulation data plots for 2 Year

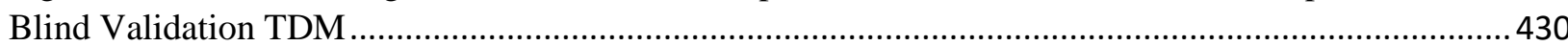

Figure 548 Well-051 oil, gas, and water rate TDM predictions vs actual simulation data plots for 2 Year

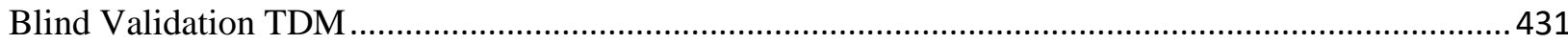

Figure 549 Well-052 oil, gas, and water rate TDM predictions vs actual simulation data plots for 2 Year

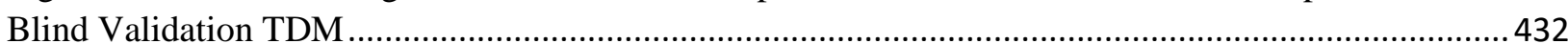

Figure 550 Well-053 oil. gas, and water rate TDM predictions vs actual simulation data plots for 2 Year

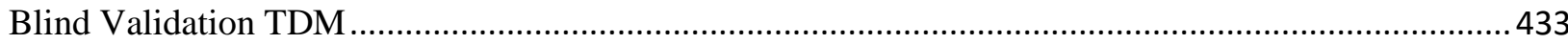

Figure 551 Well-054 oil, gas, and water rate TDM predictions vs actual simulation data plots for 2 Year

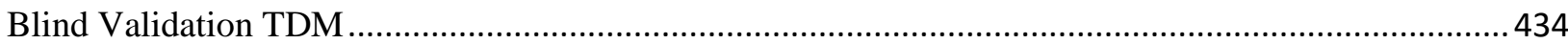

Figure 552 Well-055 oil, gas, and water rate TDM predictions vs actual simulation data plots for 2 Year

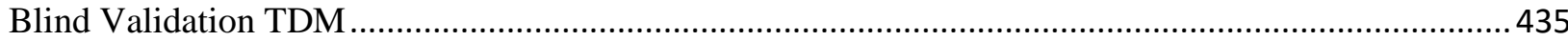

Figure 553 Well-056 oil, gas, and water rate TDM predictions vs actual simulation data plots for 2 Year

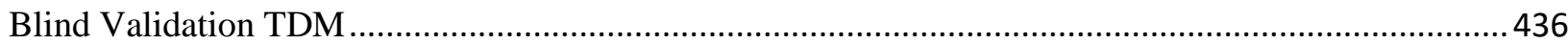

Figure 554 Well-057 oil, gas, and water rate TDM predictions vs actual simulation data plots for 2 Year

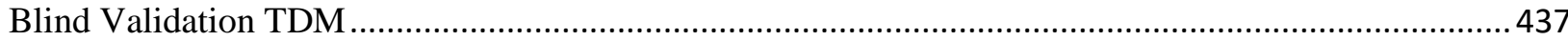

Figure 555 Entire reservoir heat map for reservoir pressure at December 31st, 2014 for 2 Year Blind

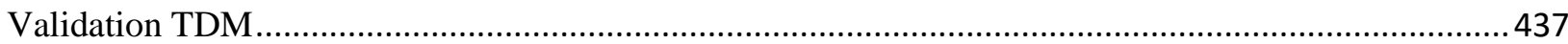

Figure 556 Entire reservoir heat map for reservoir pressure at March 31st, 2015 for 2 Year Blind

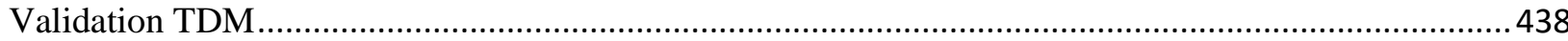

Figure 557 Entire reservoir heat map for reservoir pressure at June 30th, 2015 for 2 Year Blind Validation

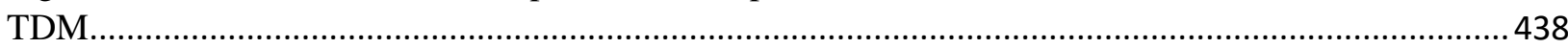

Figure 558 Entire reservoir heat map for reservoir pressure at September 30th, 2015 for 2 Year Blind

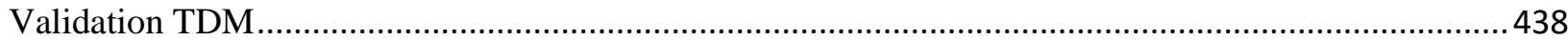

Figure 559 Entire reservoir heat map for reservoir pressure at December 31st, 2015 for 2 Year Blind

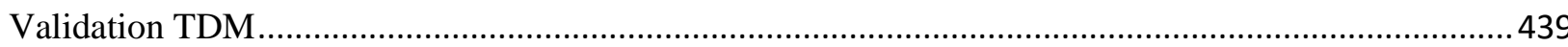

Figure 560 Entire reservoir heat map for reservoir pressure at March 31st, 2016 for 2 Year Blind

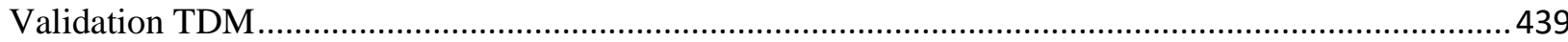

Figure 561 Entire reservoir heat map for reservoir pressure at June 30th, 2016 for 2 Year Blind Validation

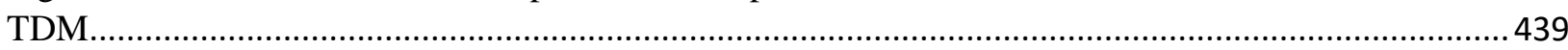

Figure 562 Entire reservoir heat map for reservoir pressure at September 30th, 2016 for 2 Year Blind

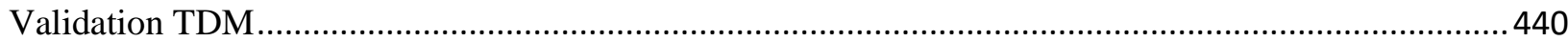

Figure 563 Entire reservoir heat map for reservoir pressure at December 31st, 2016 for 2 Year Blind

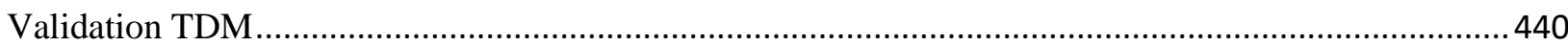

Figure 564 Entire reservoir heat map for water saturation at December 31st, 2014 for 2 Year Blind

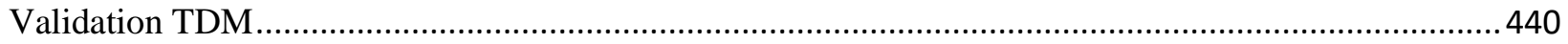


Figure 565 Entire reservoir heat map for water saturation at March 31st, 2015 for 2 Year Blind Validation

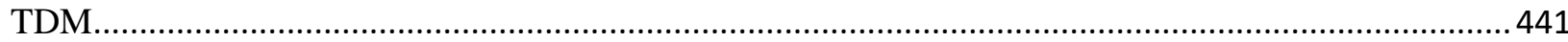

Figure 566 Entire reservoir heat map for water saturation at June 30th, 2015 for 2 Year Blind Validation

TDM. 441

Figure 567 Entire reservoir heat map for water saturation at September 30th, 2015 for 2 Year Blind Validation TDM.

Figure 568 Entire reservoir heat map for water saturation at December 31st, 2015 for 2 Year Blind Validation TDM.

Figure 569 Entire reservoir heat map for water saturation at March 31st, 2016 for 2 Year Blind Validation TDM. 442

Figure 570 Entire reservoir heat map for water saturation at June 30th, 2016 for 2 Year Blind Validation

TDM. 442

Figure 571 Entire reservoir heat map for water saturation at September 30th, 2016 for 2 Year Blind Validation TDM.

Figure 572 Entire reservoir heat map for water saturation at December 31st, 2016 for 2 Year Blind Validation TDM. .443

Figure 573 Well-001 oil, gas, and water rate TDM predictions vs actual simulation data plots for 4 Year

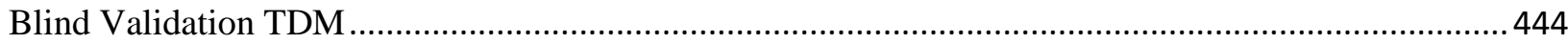
Figure 574 Well-002 oil, gas, and water rate TDM prediction vs actual simulation data plots for 3 Year Blind Validation TDM 445 Figure 575 Well-003 oil, gas, and water rate TDM predictions vs actual simulation data plots for 3 Year Blind Validation TDM. 446 Figure 576 Well-004 oil, gas, and water rate TDM prediction vs actual simulation data plots for 3 Year Blind Validation TDM. 447

Figure 577 Well-005 oil, gas, and water rate TDM prediction vs actual simulation data plots for 3 Year Blind Validation TDM. 448

Figure 578 Well-006 oil, gas, and water rate TDM prediction vs actual simulation data plots for 3 Year Blind Validation TDM. 449

Figure 579 Well-007 oil, gas, and water rate TDM predictions vs actual simulation data plots for 3 Year Blind Validation TDM. 450 Figure 580 Well-008 oil, gas, and water rate TDM predictions vs actual simulation data plots for 3 Year Blind Validation TDM. 451 Figure 581 Well-009 oil, gas, and water rate TDM predictions vs actual simulation data plots for 3 Year Blind Validation TDM. 452 Figure 582 Well-010 oil, gas, and water rate TDM predictions vs actual simulation data plots for 3 Year Blind Validation TDM 453 Figure 583 Well-011 oil, gas, and water rate TDM predictions vs actual simulation data plots for 3 Year Blind Validation TDM. 454 Figure 584 Well-012 oil, gas, and water rate TDM predictions vs actual simulation data plots for 3 Year Blind Validation TDM. 455 Figure 585 Well-013 oil, gas, and water rate TDM predictions vs actual simulation data plots for 3 Year Blind Validation TDM. 456 Figure 586 Well-014 oil, gas, and water rate TDM predictions vs actual simulation data plots for 3 Year Blind Validation TDM. 457 Figure 587 Well-015 oil, gas, and water rate TDM predictions vs actual simulation data plots for 3 Year Blind Validation TDM 458 
Figure 588 Well-016 oil, gas, and water rate TDM predictions vs actual simulation data plots for 3 Year

Blind Validation TDM.

Figure 589 Well-017 oil, gas, and water rate TDM predictions vs actual simulation data plots for 3 Year

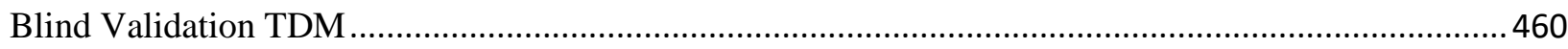

Figure 590 Well-018 oil, gas, and water rate TDM predictions vs actual simulation data plots for 3 Year

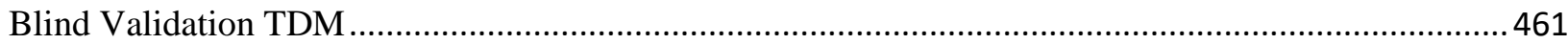

Figure 591 Well-019 oil, gas, and water rate TDM predictions vs actual simulation data plots for 3 Year

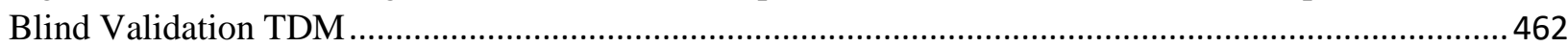

Figure 592 Well-020 oil, gas, and water rate TDM predictions vs actual simulation data plots for 3 Year

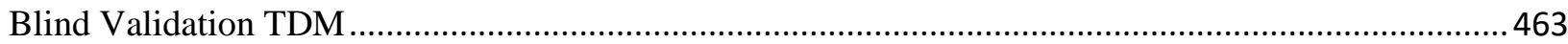

Figure 593 Well-021 oil, gas, and water rate TDM predictions vs actual simulation data plots for 3 Year

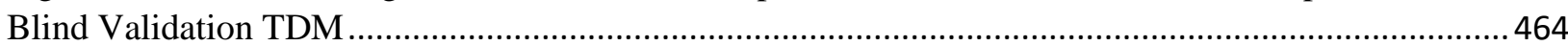

Figure 594 Well-022 oil, gas, and water rate TDM predictions vs actual simulation data plots for 3 Year

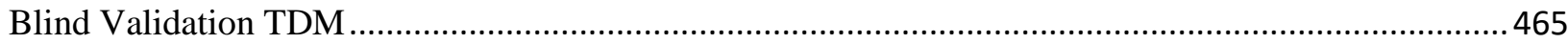

Figure 595 Well-023 oil, gas, and water rate TDM predictions vs actual simulation data plots for 3 Year

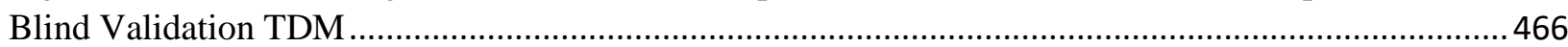

Figure 596 Well-024 oil, gas, and water rate TDM predictions vs actual simulation data plots for 3 Year

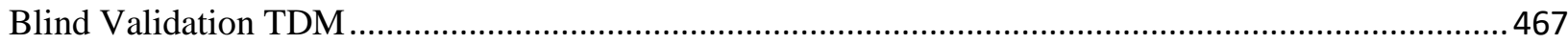

Figure 597 Well-025 oil, gas, and water rate TDM predictions vs actual simulation data plots for 3 Year

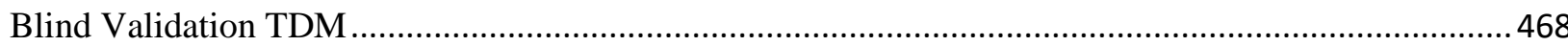

Figure 598 Well-026 oil, gas, and water rate TDM predictions vs actual simulation data plots for 3 Year

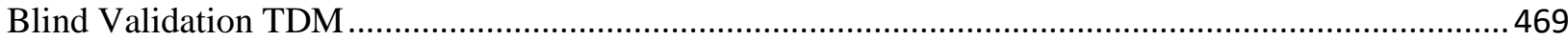

Figure 599 Well-027 oil, gas, and water rate TDM predictions vs actual simulation data plots for 3 Year

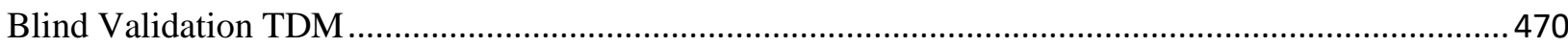

Figure 600 Well-028 oil, gas, and water rate TDM predictions vs actual simulation data plots for 3 Year

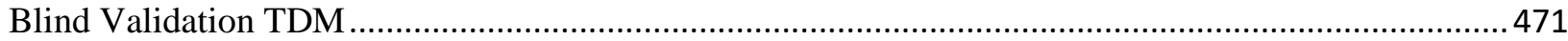

Figure 601 Well-029 oil, gas, and water rate TDM predictions vs actual simulation data plots for 3 Year

Blind Validation TDM.

Figure 602 Well-030 oil, gas, and water rate TDM predictions vs actual simulation data plots for 3 Year

Blind Validation TDM.

Figure 603 Well-031 oil, gas, and water rate TDM predictions vs actual simulation data plots for 3 Year

Blind Validation TDM.

Figure 604 Well-032 oil, gas, and water rate TDM predictions vs actual simulation data plots for 3 Year

Blind Validation TDM.

Figure 605 Well-033 oil, gas, and water rate TDM predictions vs actual simulation data plots for 3 Year

Blind Validation TDM.

Figure 606 Well-034 oil, gas, and water rate TDM predictions vs actual simulation data plots for 3 Year

Blind Validation TDM.

Figure 607 Well-035 oil, gas, ad water rate TDM predictions vs actual simulation data plots for 3 Year

Blind Validation TDM.

Figure 608 Well-036 oil, gas, and water rate TDM predictions vs actual simulation data plots for 3 Year

Blind Validation TDM.

Figure 609 Well-037 oil, gas, ad water rate TDM predictions vs actual simulation data plots for 3 Year

Blind Validation TDM..

Figure 610 Well-038 oil, gas, and water rate TDM predictions vs actual simulation data plots for 3 Year

Blind Validation TDM. 
Figure 611 Well-039 oil, gas, and water rate TDM predictions vs actual simulation data plots for 3 Year

Blind Validation TDM

Figure 612 Well-040 oil, gas, and water rate TDM predictions vs actual simulation data plots for 3 Year

Blind Validation TDM.

Figure 613 Well-041 oil, gas, and water rate TDM predictions vs actual simulation data plots for 3 Year

Blind Validation TDM.

Figure 614 Well-042 oil, gas, and water rate TDM predictions vs actual simulation data plots for 3 Year

Blind Validation TDM.

Figure 615 Well-043 oil, gas, and water rate TDM predictions vs actual simulation data plots for 3 Year

Blind Validation TDM.

Figure 616 Well-044 oil. gas, and water rate TDM predictions vs actual simulation data plots for 3 Year

Blind Validation TDM.

Figure 617 Well-045 oil, gas, and water rate TDM predictions vs actual simulation data plots for 3 Year

Blind Validation TDM.

Figure 618 Well-046 oil, gas, and water rate TDM predictions vs actual simulation data plots for 3 Year

Blind Validation TDM.

Figure 619 Well-047 oil, gas, and water rate TDM predictions vs actual simulation data plots for 3 Year

Blind Validation TDM.

Figure 620 Well-048 oil, gas, and water rate TDM predictions vs actual simulation data plots for 3 Year

Blind Validation TDM

Figure 621 Well-049 oil, gas, and water rate TDM predictions vs actual simulation data plots for 3 Year

Blind Validation TDM 492

Figure 622 Well-050 oil, gas, and water rate TDM predictions vs actual simulation data plots for 3 Year

Blind Validation TDM

Figure 623 Well-051 oil, gas, and water rate TDM predictions vs actual simulation data plots for 3 Year

Blind Validation TDM. 494

Figure 624 Well-052 oil, gas, and water rate TDM predictions vs actual simulation data plots for 3 Year

Blind Validation TDM.

Figure 625 Well-053 oil, gas, and water rate TDM predictions vs actual simulation data plots for 3 Year

Blind Validation TDM. 496

Figure 626 Well-054 oil, gas, and water rate TDM predictions vs actual simulation data plots for 3 Year

Blind Validation TDM.

Figure 627 Well-055 oil, gas, and water rate TDM predictions vs actual simulation data plots for 3 Year Blind Validation TDM.

Figure 628 Well-056 oil, gas, and water rate TDM predictions vs actual simulation data plots for 3 Year

Blind Validation TDM

Figure 629 Well-057 oil, gas, and water rate TDM predictions vs actual simulation data plots for 3 Year

Blind Validation TDM.

Figure 630 Entire reservoir heat map for reservoir pressure at December 31st, 2013 for 3 Year Blind

Validation TDM .500

Figure 631 Entire reservoir heat map for reservoir pressure at March 31st, 2014 for 3 Year Blind Validation TDM 501

Figure 632 Entire reservoir heat map for reservoir pressure at June 30th, 2014 for 3 Year Blind Validation TDM 501

Figure 633 Entire reservoir heat map for September 30th, 2014 for 3 Year Blind Validation TDM .......501 
Figure 634 Entire reservoir heat map for reservoir pressure at December 31st, 2014 for 3 Year Blind Validation TDM

Figure 635 Entire reservoir heat map for reservoir pressure at March 31st, 2015 for 3 Year Blind Validation TDM.

Figure 636 Entire reservoir heat map for reservoir pressure at June 30th, 2015 for 3 Year Blind Validation TDM. 502

Figure 637 Entire reservoir heat map for reservoir pressure at September 30th, 2015 for 3 Year Blind Validation TDM .503

Figure 638 Entire reservoir heat map for reservoir pressure at December 31st, 2015 for 3 Year Blind Validation TDM.

Figure 639 Entire reservoir heat map for reservoir pressure at March 31st, 2016 for 3 Year Blind Validation TDM. 503

Figure 640 Entire reservoir heat map for reservoir pressure at June 30th, 2016 for 3 Year Blind Validation TDM. .504

Figure 641 Entire reservoir heat map for reservoir pressure at September 30th, 2016 for 3 Year Blind Validation TDM .504

Figure 642 Entire reservoir heat map for reservoir pressure at December 31st, 2016 for 3 Year Blind Validation TDM. .504

Figure 643 Entire reservoir heat map for water saturation at December 31st, 2013 for 3 Year Blind Validation TDM. 505

Figure 644 Entire reservoir heat map for water saturation at March 31st, 2014 for 3 Year Blind Validation TDM. 505

Figure 645 Entire reservoir heat map for water saturation at June 30th, 2014 for 3 Year Blind Validation TDM. 505

Figure 646 Entire reservoir heat map for water saturation at September 30th, 2014 for 3 Year Blind Validation TDM 506

Figure 647 Entire reservoir heat map for water saturation at December 31st, 2014 for 3 Year Blind Validation TDM. 506

Figure 648 Entire reservoir heat map for water saturation at March 31st, 2015 for 3 Year Blind Validation TDM. .506

Figure 649 Entire reservoir heat map for water saturation at June 30th, 2015 for 3 Year Blind Validation TDM. 507

Figure 650 Entire reservoir heat map for water saturation at September 30th, 2015 for 3 Year Blind Validation TDM 507

Figure 651 Entire reservoir heat map for water saturation at December 31st, 2015 for 3 Year Blind Validation TDM 507

Figure 652 Entire reservoir heat map for water saturation at March 31st, 2016 for 3 Year Blind Validation TDM. 508

Figure 653 Entire reservoir heat map for water saturation at June 30th, 2016 for 3 Year Blind Validation TDM .508

Figure 654 Entire reservoir heat map for water saturation at September 30th, 2016 for 3 Year Blind Validation TDM 508

Figure 655 Entire reservoir heat map for water saturation at December 31st, 2016 for 3 Year Blind Validation TDM. 509 Figure 656 Well-001 oil, gas, and water rate TDM predictions vs actual simulation data plots for 4 Year Blind Validation TDM. 509 
Figure 657 Well-002 oil, gas, and water rate TDM predictions vs actual simulation data plots for 4 Year

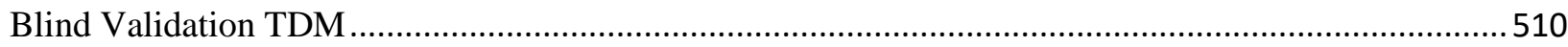

Figure 658 Well-003 oil, gas, and water rate TDM predictions vs actual simulation data plots for 4 Year

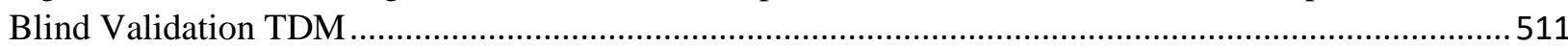

Figure 659 Well-004 oil, gas, and water rate TDM predictions vs actual simulation data plots for 4 Year

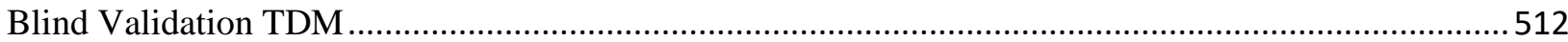
Figure 660 Well-005 oil, gas, and water rate TDM predictions vs actual simulation data plots for 4 Year

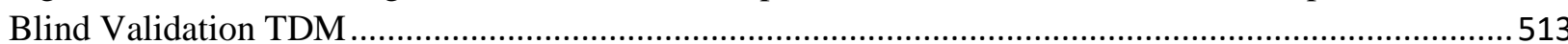
Figure 661 Well-006 oil, gas, and water rate TDM predictions vs actual simulation data plots for 4 Year

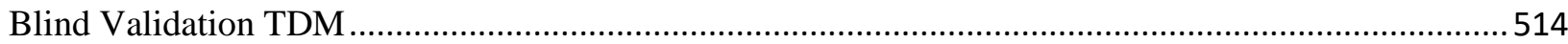
Figure 662 Well-007 oil, gas, and water rate TDM predictions vs actual simulation data plots for 4 Year

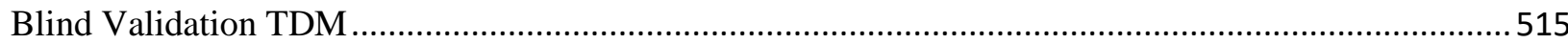
Figure 663 Well-008 oil, gas, and water rate TDM predictions vs actual simulation data plots for 4 Year

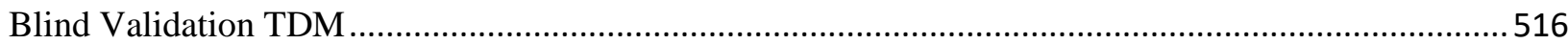
Figure 664 Well-009 oil, gas, and water rate TDM predictions vs actual simulation data plots for 4 Year

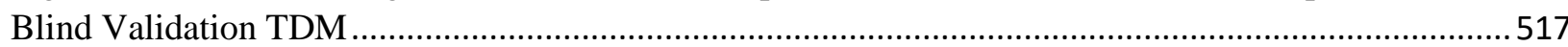
Figure 665 Well-010 oil, gas, and water rate TDM predictions vs actual simulation data plots for 4 Year

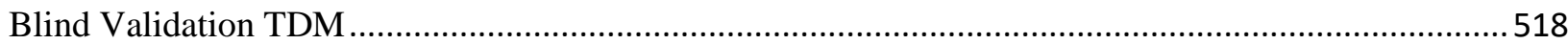
Figure 666 Well-011 oil, gas, and water rate TDM predictions vs actual simulation data plots for 4 Year

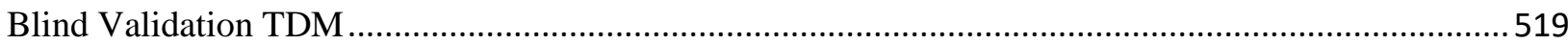
Figure 667 Well-012 oil, gas, and water rate TDM predictions vs actual simulation data plots for 4 Year

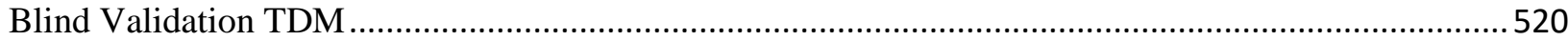
Figure 668 Well-013 oil, gas, and water rate TDM predictions vs actual simulation data plots for 4 Year

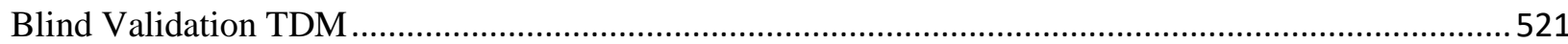
Figure 669 Well-014 oil, gas, and water rate TDM predictions vs actual simulation data plots for 4 Year

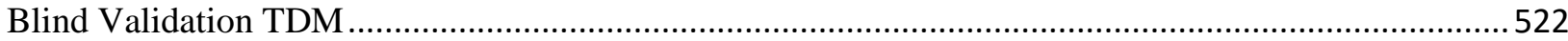
Figure 670 Well-015 oil, gas, and water rate TDM predictions vs actual simulation data plots for 4 Year

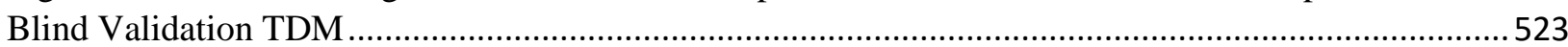
Figure 671 Well-016 oil, gas, and water rate TDM predictions vs actual simulation data plots for 4 Year

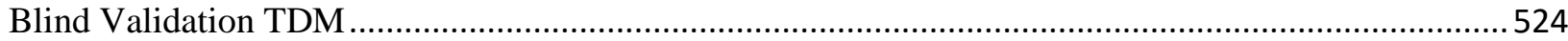
Figure 672 Well-017 oil, gas, and water rate TDM predictions vs actual simulation data plots for 4 Year

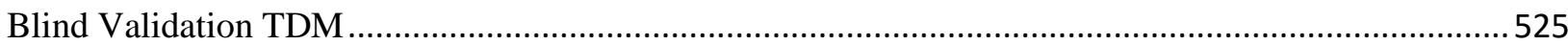
Figure 673 Well-018 oil, gas, and water rate TDM predictions vs actual simulation data plots for 4 Year Blind Validation TDM.... 526 Figure 674 Well-019 oil, gas, and water rate TDM predictions vs actual simulation data plots for 4 Year Blind Validation TDM

Figure 675 Well-020 oil, gas, and water rate TDM predictions vs actual simulation data plots for 4 Year Blind Validation TDM... 528 Figure 676 Well-021 oil, gas, and water rate TDM predictions vs actual simulation data plots for 4 Year Blind Validation TDM 529 Figure 677 Well-022 oil, gas, and water rate TDM predictions vs actual simulation data plots for 4 Year Blind Validation TDM. 530 Figure 678 Well-023 oil, gas, aand water rate TDM predictions vs actual simulation data plots for 4 Year Blind Validation TDM 531 Figure 679 Well-024 oil, gas, and water rate TDM predictions vs actual simulation data plots for 4 Year Blind Validation TDM. .532 
Figure 680 Well-025 oil, gas, and water rate TDM predictions vs actual simulation data plots for 4 Year

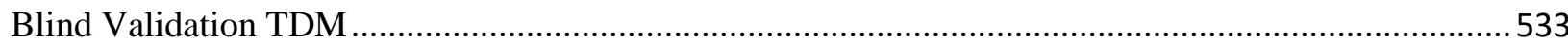

Figure 681 Well-026 oil, gas, and water rate TDM predictions vs actual simulation data plots for 4 Year

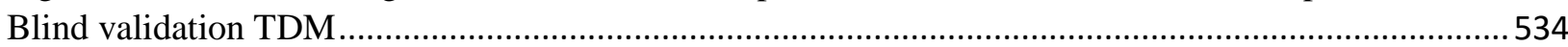

Figure 682 Well-027 oil, gas, and water rate TDM predictions vs actual simulation data plots for 4 Year

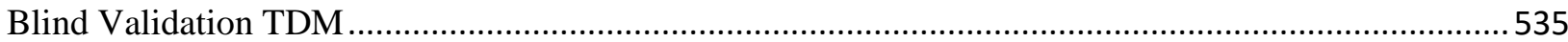
Figure 683 Well-028 oil, gas, and water rate TDM predictions vs actual simulation data plots for 4 Year

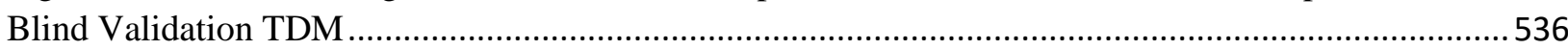
Figure 684 Well-029 oil, gas, and water rate TDM predictions vs actual simulation data plots for 4 Year

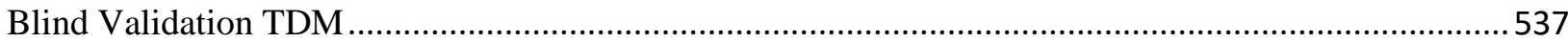
Figure 685 Well-030 oil, gas, and water rate TDM predictions vs actual simulation data plots for 4 Year

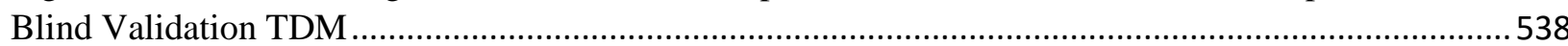
Figure 686 Well-031 oil, gas, and water rate TDM predictions vs actual simulation data plots for 4 Year

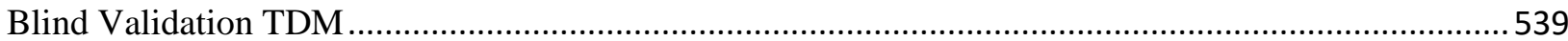
Figure 687 Well-032 oil, gas, and water rate TDM predictions vs actual simulation data plots for 4 Year

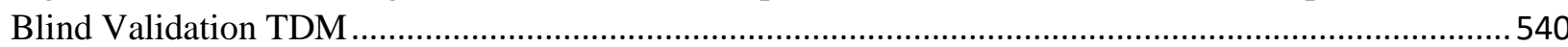
Figure 688 Well-033 oil, gas, and water rate TDM predictions vs actual simulation data plots for 4 Year

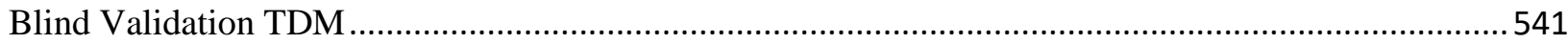
Figure 689 Well-034 oil, gas, and water rate TDM predictions vs actual simulation data plots for 4 Year

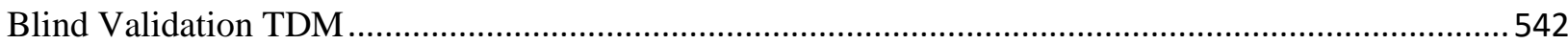
Figure 690 Well-035 oil, gas, and water rate TDM predictions vs actual simulation data plots for 4 Year

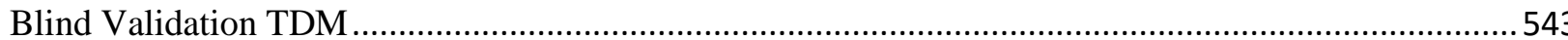
Figure 691 Well-036 oil, gas, and water rate TDM predictions vs actual simulation data plots for 4 Year

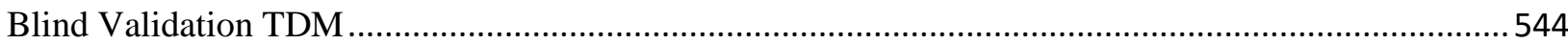
Figure 692 Well-037 oil, gas, and water rate TDM predictions vs actual simulation data plots for 4 Year

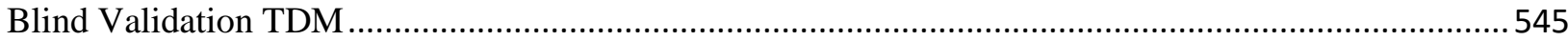
Figure 693 Well-038 oil, gas, and water rate TDM predictions vs actual simulation data plots for 4 Year

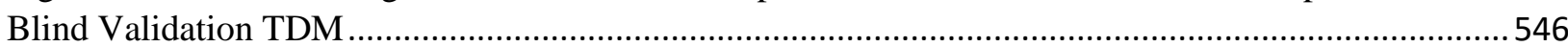
Figure 694 Well-039 oil, gas, and water rate TDM predictions vs actual simulation data plots for 4 Year Blind Validation TDM.... 547 Figure 695 Well-040 oil, gas, and water rate TDM predictions vs actual simulation data plots for 4 Year

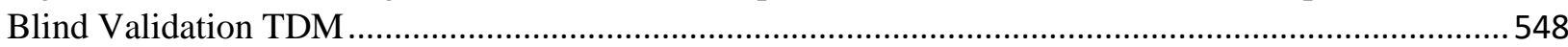
Figure 696 Well-041 oil, gas, and water rate TDM predictions vs actual simulation data plots for 4 Year Blind Validation TDM. 549 Figure 697 Well-042 oil, gas, and water rate TDM predictions vs actual simulation data plots for 4 Year

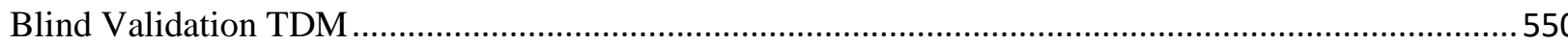
Figure 698 Well-043 oil, gas, annd water rate TDM predictions vs actual simulation data plots for 4 Year Blind Validation TDM. 551 Figure 699 Well-044 oil, gas, and water rate TDM predictions vs actual simulation data plots for 4 Year Blind Validation TDM 552 Figure 700 Well-045 oil, gas, and water rate TDM predictions vs actual simulation data plots for 4 Year Blind Validation TDM. 553 Figure 701 Well-046 oil, gas, and water rate TDM predictions vs actual simulation data plots for 4 Year Blind Validation TDM 554 Figure 702 Well-047 oil, gas, and water rate TDM predictions vs actual simulation data plots for 4 Year

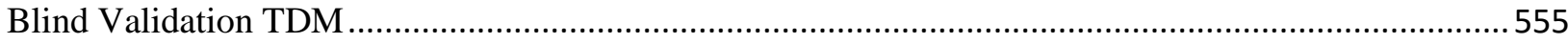


Figure 703 Well-048 oil, gas, and water rate TDM predictions vs actual simulation data plots for 4 Year

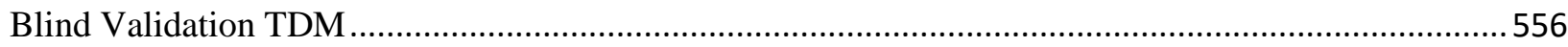

Figure 704 Well-049 oil, gas, and water rate TDM predictions vs actual simulation data plots for 4 Year

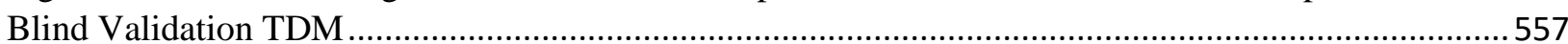

Figure 705 Well-050 oil, gas, and water rate TDM predictions vs actual simulation data plots for 4 Year

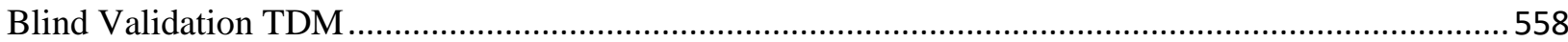

Figure 706 Well-051 oil, gas, and water rate TDM predictions vs actual simulation data plots for 4 Year

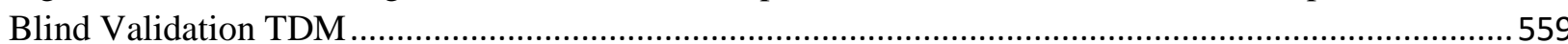

Figure 707 Well-052 oil, gas, and water rate TDM predictions vs actual simulation data plots for 4 Year

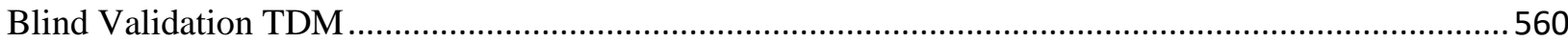

Figure 708 Well-053 oil, gas, and water rate TDM predictions vs actual simulation data plots for 4 Year

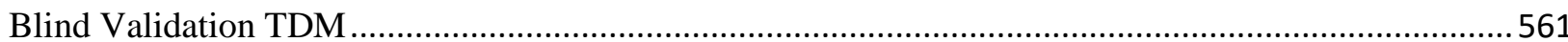
Figure 709 Well-054 oil, gas, and water rate TDM predictions vs actual simulation data plots for 4 Year

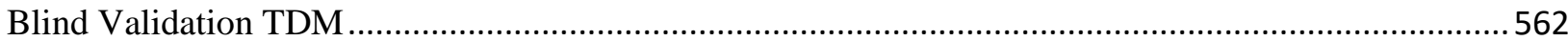
Figure 710 Well-055 oil, gas, and water rate TDM predictions vs actual simulation data plots for 4 Year

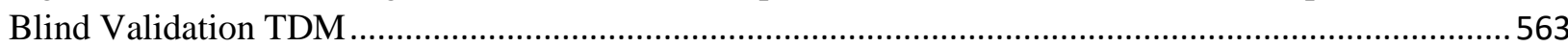
Figure 711 Well-056 oil, gas, and water rate TDM predictions vs actual simulation data plots for 4 Year

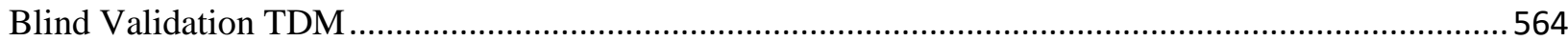
Figure 712 Well-057 oil, gas, and water rate TDM predictions vs actual simulation data plots for 4 Year

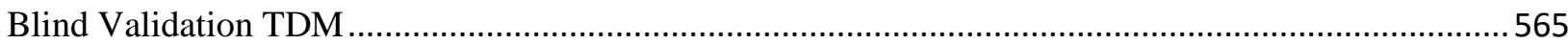
Figure 713 Entire reservoir heat map for reservoir pressure at December 31st, 2012 for 4 Year Blind

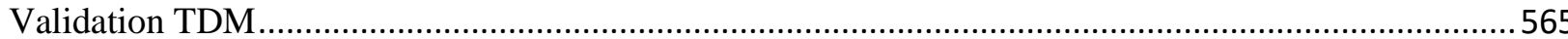

Figure 714 Entire reservoir heat map for reservoir pressure at March 31st, 2013 for 4 Year Blind

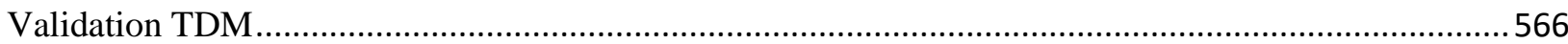
Figure 715 Entire reservoir heat map for reservoir pressure at June 30th, 2013 for 4 Year Blind Validation TDM. 566

Figure 716 Entire reservoir heat map for reservoir pressure at September 30th, 2013 for 4 Year Blind

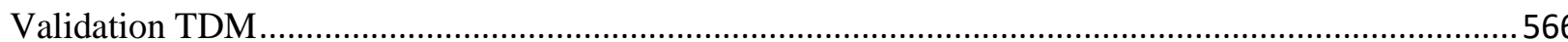

Figure 717 Entire reservoir heat map for reservoir pressure at December 31st, 2013 for 4 Year Blind Validation TDM

Figure 718 Entire reservoir heat map for reservoir pressure at March 31st, 2014 for 4 Year Blind Validation TDM

Figure 719 Entire reservoir heat map for reservoir pressure at June 30th, 2014 for 4 Year Blind Validation TDM. 567

Figure 720 Entire reservoir heat map for reservoir pressure at September 30th, 2014 for 4 Year Blind Validation TDM .568

Figure 721 Entire reservoir heat map for reservoir pressure at December 31st, 2014 for 4 Year Blind Validation TDM

Figure 722 Entire reservoir heat map for reservoir pressure at March 31st, 2015 for 4 Year Blind Validation TDM 568

Figure 723 Entire reservoir heat map for reservoir pressure at June 30th, 2015 for 4 Year Blind Validation TDM. 569

Figure 724 Entire reservoir heat map for reservoir pressure at September 30th, 2015 for 4 year Blind Validation TDM 569

Figure 725 Entire reservoir heat map for reservoir pressure at December 31st, 2015 for 4 Year Blind Validation TDM 
Figure 726 Entire reservoir heat map for reservoir pressure at March 31st, 2016 for 4 Year Blind Validation TDM

Figure 727 Entire reservoir heat map for reservoir pressure at June 30th, 2016 for 4 Year Blind Validation

TDM. .570

Figure 728 Entire reservoir heat map for reservoir pressure at September 30th, 2016 for 4 Year Blind

Validation TDM.

Figure 729 Entire reservoir heat map for reservoir pressure at December 31st, 2016 for 4 Year Blind Validation TDM.

Figure 730 Entire reservoir heat map for water saturation at December 31st, 2012 for 4 Year Blind

Validation TDM.

Figure 731 Entire reservoir heat map for water saturation at March 31st, 2013 for 4 Year Blind Validation

TDM.

Figure 732 Entire reservoir heat map for water saturation at June 30th, 2013 for 4 Year Blind Validation

TDM.

Figure 733 Entire reservoir heat map for water saturation at September 30th, 2013 for 4 Year Blind

Validation TDM.

Figure 734 Entire reservoir heat map for water saturation at December 31st, 2013 for 4 Year Blind

Validation TDM.

Figure 735 Entire reservoir heat map for water saturation at March 31st, 2014 for 4 Year Blind Validation

TDM.

Figure 736 Entire reservoir heat map for water saturation at June 30th, 2014 for 4 Year Blind Validation

TDM.

Figure 737 Entire reservoir heat map for water saturation at September 30th, 2014 for 4 Year Blind Validation TDM. 573

Figure 738 Entire reservoir heat map for water saturation at December 31st, 2014 for 4 Year Blind Validation TDM.

Figure 739 Entire reservoir heat map for water saturation at March 31st, 2015 for 4 Year Blind Validation TDM.

Figure 740 Entire reservoir heat map for water saturation at June 30th, 2015 for 4 Year Blind Validation

Figure 741 Entire reservoir heat map for water saturation at September 30th, 2015 for 4 Year Blind Validation TDM. 575

Figure 742 Entire reservoir heat map for water saturation at December 31st, 2015 for 4 Year Blind Validation TDM

Figure 743 Entire reservoir heat map for water saturation at March 31st, 2016 for 4 Year Blind Validation TDM.

Figure 744 Entire reservoir heat map for water saturation at June 30th, 2016 for 4 Year Blind Validation

TDM. 576

Figure 745 Entire reservoir heat map for water saturation at September 30th, 2016 for 4 Year Blind Validation TDM

Figure 746 Entire reservoir heat map for water saturation at December 31st, 2016 for 4 Year Blind Validation TDM

Figure 747 Well-001 oil, gas, and water rate TDM predictions vs actual simulation data plots for 5 Year Blind Validation TDM.

Figure 748 Well-002 oil, gas, and water rate TDM predictions vs actual simulation data plots for 5 Year Blind Validation TDM. 
Figure 749 Well-003 oil, gas, and water rate TDM predictions vs actual simulation data plots for 5 Year Blind Validation TDM

Figure 750 Well-004 oil, gas, and water rate TDM predictions vs actual simulation data plots for 5 Year

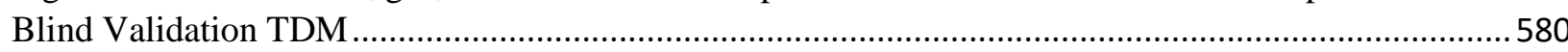

Figure 751 Well-005 oil, gas, and water rate TDM predictions vs actual simulation data plots for 5 Year

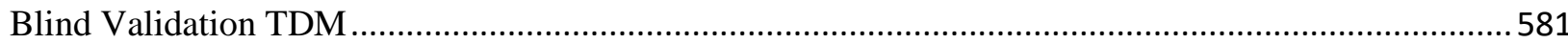

Figure 752 Well-006 oil, gas, and water rate TDM predictions vs actual simulation data plots for 5 Year

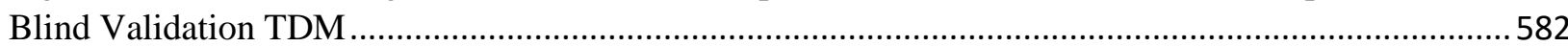

Figure 753 Well-007 oil, gas, and water rate TDM predictions vs actual simulation data plots for 5 Year

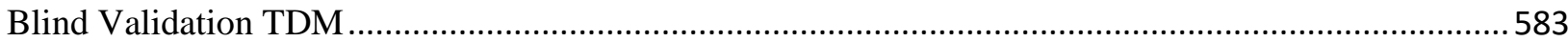
Figure 754 Well-008 oil, gas, and water rate TDM preditions vs actual simulation data plots for 5 Year

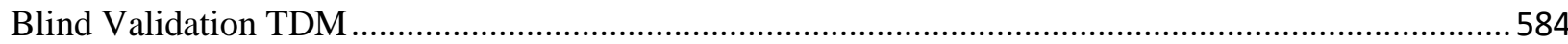
Figure 755 Well-009 oil, gas, and water rate TDM predictions vs actual simulation data plots for 5 Year

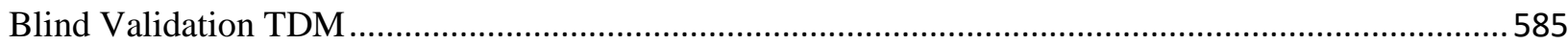
Figure 756 Well-010 oil, gas, and water rate TDM predictions vs actual simulation data plots for 5 Year

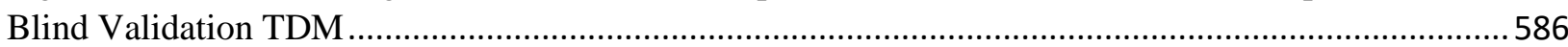
Figure 757 Well-011 oil, gas, and water rate TDM predictions vs actual simulation data plots for 5 Year

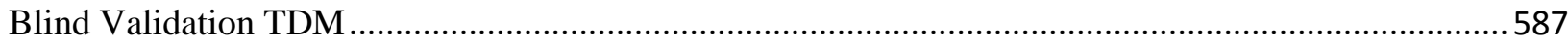
Figure 758 Well-012 oil, gas, and water rate TDM predictions vs actual simulation data plots for 5 Year

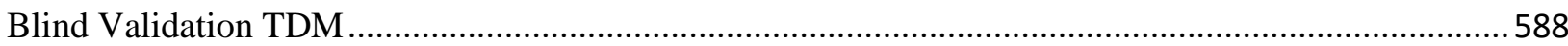
Figure 759 Well-013 oil, gas, and water rate TDM predictios vs actual simulation data plots for 5 Year

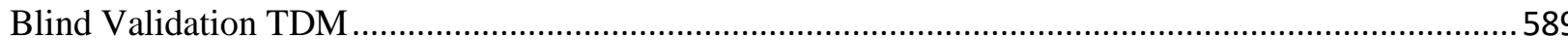
Figure 760 Well-014 oil, gas, and water rate TDM predictions vs actual simulation data plots for 5 Year

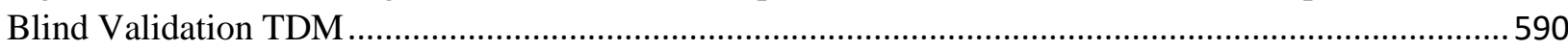
Figure 761 Well-015 oil, gas, and water rate TDM predictions vs actual simulation data plots for 5 Year

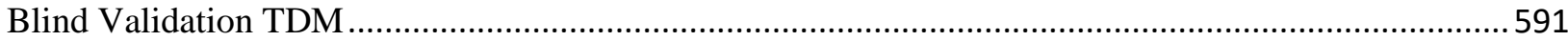
Figure 762 Well-016 oil, gas, and water rate TDM predictions vs actual simulation data plots for 5 Year

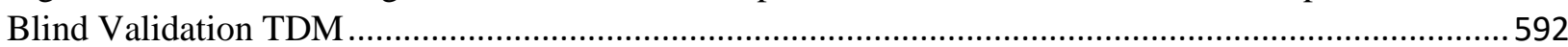
Figure 763 Well-017 oil, gas, and water rate TDM predictions vs actual simulation data plots for 5 Year

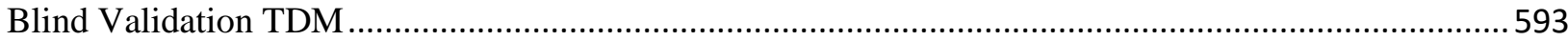
Figure 764 Well-018 oil, gas, and water rate TDM predictions vs actual simulation data plots for 5 Year

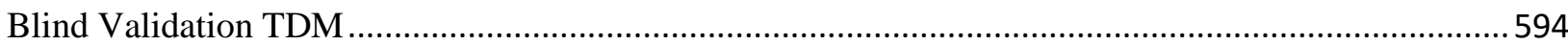
Figure 765 Well-019 oil, gas, and water rate TDM predictions vs actual simulation data plots for 5 Year Blind Validation TDM.... 595 Figure 766 Well-020 oil, gas, and water rate TDM predictions vs actual simulation data plots for 5 Year

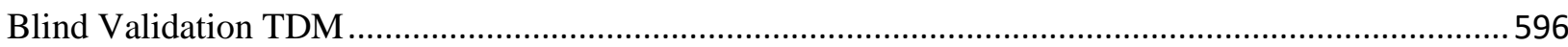
Figure 767 Well-021 oil, gas, and water rate TDM predictions vs actual simulation data plots for 5 Year

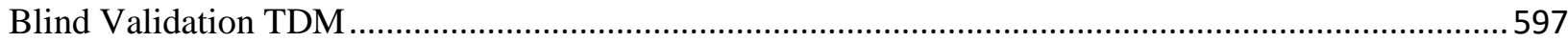
Figure 768 Well-022 oil, gas, and water rate TDM predictions vs actual simulation data plots for 5 Year

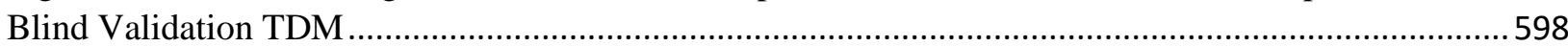
Figure 769 Well-023 oil, gas, and water rate TDM predictions vs actual simulation data plots for 5 Year

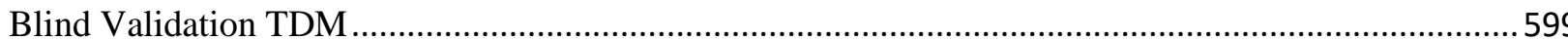
Figure 770 Well-024 oil, gas, and water rate TDM predictions vs actual simulation data plots for 5 Year Blind Validation TDM 600 Figure 771 Well-025 oil, gas, and water rate TDM predictions vs actual simulation data plots for 5 Year Blind Validation TDM. 601 
Figure 772 Well-026 oil, gas, and water rate TDM predictions vs actual simulation data plots for 5 Year

Blind Validation TDM.

Figure 773 Well-027 oil, gas, and water rate TDM predictions vs actual simulation data plots for 5 Year

Blind Validation TDM. 603

Figure 774 Well-028 oil, gas, and water rate TDM predictions vs actual simulation data plots for 5 Year

Blind Validation TDM. .604

Figure 775 Well-029 oil, gas, and water rate TDM predictions vs actual simulation data plots for 5 Year

Blind Validation TDM. 605

Figure 776 Well-030 oil, gas, and water rate TDM predictions vs actual simulation data plots for 5 Year

Blind Validation TDM. .606

Figure 777 Well-031 oil, gas, and water rate TDM predictions vs actual simulation data plots for 5 Year

Blind Validation TDM. 607

Figure 778 Well-032 oil, gas, and water rate TDM predictions vs actual simulation data plots for 5 Year Blind Validation TDM. 608

Figure 779 Well-033 oil, gas, and water rate TDM predictions vs actual simulation data plots for 5 Year

Blind Validation TDM. 609

Figure 780 Well-034 oil, gas, and water rate TDM predictions vs actual simulation data plots for 5 Year Blind Validation TDM. 610 Figure 781 Well-035 oil, gas, and water rate TDM predictions vs actual simulation data plots for 5 Year Blind Validation TDM. 611

Figure 782 Well-036 oil, gas, and water rate TDM predictions vs actual simulation data plots for 5 Year Blind Validation TDM. 612 Figure 783 Well-037 oil, gas, and water rate TDM predictions vs actual simulation data plots for 5 Year Blind Validation TDM. 613

Figure 784 Well-038 oil, gas, and water rate TDM predictions vs actual simulation data plots for 5 Year Blind Validation TDM. 614 Figure 785 Well-039 oil, gas, and water rate TDM predictions vs actual simulation data plots for 5 Year Blind Validation TDM. 615

Figure 786 Well-040 oil, gas, and water rate TDM predictions vs actual simulation data plots for 5 Year Blind Validation TDM. 616 Figure 787 Well-041 oil, gas, and water rate TDM predictions vs actual simulation data plots for 5 Year Blind Validation TDM.

Figure 788 Well-042 oil, gas, and water rate TDM predictions vs actual simulation data plots for 5 Year Blind Validation TDM. 618

Figure 789 Well-043 oil, gas, and water rate TDM predictions vs actual simulation data plots for 5 Year Blind Validation TDM. 619

Figure 790 Well-044 oil, gas, and water rate TDM predictions vs actual simulation data plots for 5 Year Blind Validation TDM. 620 Figure 791 Well-045 oil, gas, and water rate TDM predictions vs actual simulation data plots for 5 Year Blind Validation TDM. 621

Figure 792 Well-046 oil, gas, and water rate TDM predictions vs actual simulation data plots for 5 Year Blind Validation TDM. 622 Figure 793 Well-047 oil, gas, and water rate TDM predictions vs actual simulation data plots for 5 Year Blind Validation TDM. 623

Figure 794 Well-048 oil, gas, and water rate TDM predictions vs actual simulation data plots for 5 Year Blind Validation TDM. 624 
Figure 795 Well-049 oil, gas, and water rate TDM predictions vs actual simulation data plots for 5 Year

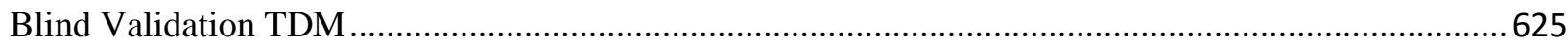

Figure 796 Well-050 oil, gas, and water rate TDM predictions vs actual simulation data plots for 5 Year

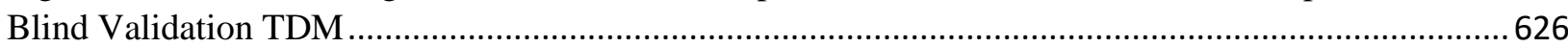

Figure 797 Well-051 oil, gas, and water rate TDM predictions vs actual simulation data plots for 5 Year

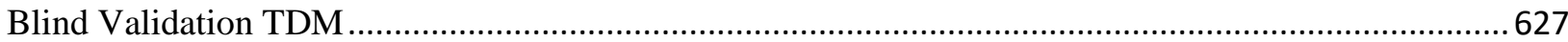

Figure 798 Well-051 oil, gas, and water rate TDM predictions vs actual simulation data plots for 5 Year

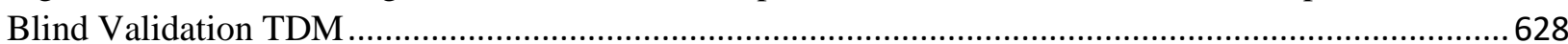

Figure 799 Well-053 oil, gas, and water rate TDM predictions vs actual simulation data plots for 5 Year

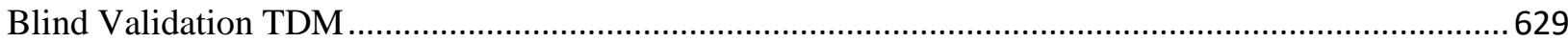
Figure 800 Well-054 oil, gas, and water rate TDM predictions vs actual simulation data plots for 5 Year

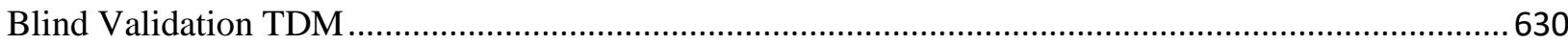
Figure 801 Well-055 oil, gas, and water rate TDM predictions vs actual simulation data plots for 5 Year

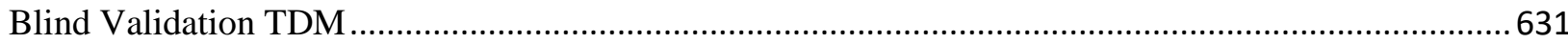
Figure 802 Well-056 oil, gas, and water rate TDM predictions vs actual simulation data plots for 5 Year

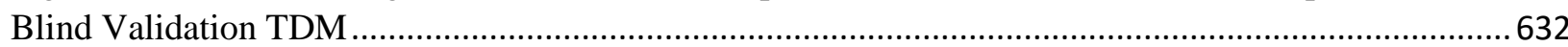
Figure 803 Well-057 oil, gas, and water rate TDM predictions vs actual simulation data plots for 5 Year

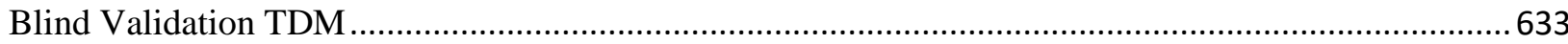
Figure 804 Entire reservoir heat map for reservoir pressure at December 31st, 2011 for 5 Year Blind Validation TDM. .633

Figure 805 Entire reservoir heat map for reservoir pressure at June 30th, 2012 for 5 Year Blind Validation TDM. 634

Figure 806 Entire reservoir heat map for reservoir pressure at December 31st, 2012 for 5 Year Blind Validation TDM .634

Figure 807 Entire reservoir heat map for reservoir pressure at June 30th, 2013 for 5 Year Blind Validation TDM. 634

Figure 808 Entire reservoir heat map for reservoir pressure at December 31st, 2013 for 5 Year Blind Validation TDM .635

Figure 809 Entire reservoir heat map for reservoir heat map at June 30th, 2014 for 5 Year Blind Validation TDM 635

Figure 810 Entire reservoir heat map for reservoir pressure at December 31st, 2014 for 5 Year Blind Validation TDM .635

Figure 811 Entire reservoir heat map for reservoir pressure at June 30th, 2015 for 5 Year Blind Validation TDM. 636

Figure 812 Entire reservoir heat map for reservoir pressure at December 31st, 2015 for 5 Year Blind Validation TDM .636

Figure 813 Entire reservoir heat map for reservoir pressure at June 30th, 2016 for 5 Year Blind Validation TDM. 636

Figure 814 Entire reservoir heat map for reservoir pressure at December 31st, 2016 for 5 Year Blind Validation TDM 637

Figure 815 Entire reservoir heat map for water saturation at December 31st, 2011 for 5 Year Blind Validation TDM

Figure 816 Entire reservoir heat map for water saturation at June 30th, 2012 for 5 Year Blind Validation TDM. 637

Figure 817 Entire reservoir heat map for water saturation at December 31st, 2012 for 5 Year Blind Validation TDM 638 
Figure 818 Entire reservoir heat map for water saturation at June 30th, 2013 for 5 Year Blind Validation TDM.

Figure 819 Entire reservoir heat map for water saturation at December 31st, 2013 for 5 Year Blind Validation TDM. 638

Figure 820 Entire reservoir heat map for water saturation at June 30th, 2014 for 5 Year Blind Validation

TDM. 639

Figure 821 Entire reservoir heat map for water saturation at December 31st, 2014 for 5 Year Blind Validation TDM. 639

Figure 822 Entire reservoir heat map for water saturation at June 30th, 2015 for 5 Year Blind Validation TDM. 639

Figure 823 Entire reservoir heat map for water saturation at December 31st, 2015 for 5 Year Blind Validation TDM. 640

Figure 824 Entire reservoir heat map for water saturation at June 30th, 2016 for 5 Year Blind Validation TDM. 640

Figure 825 Entire reservoir heat map for water saturation at December 31st, 2016 for 5 Year Blind Validation TDM .640

Figure 826 Well-001 oil, gas, and water rate TDM predictions vs actual simulation data plots for 6 Year Blind Validation TDM.

Figure 827 Well-002 oil, gas, and water rate TDM predictions vs actual simulation data plots for 6 Year Blind Validation TDM. .642

Figure 828 Well-003 oil, gas, and water rate TDM predictions vs actual simulation data plots for 6 Year Blind Validation TDM 643

Figure 829 Well-004 oil, gas, and water rate TDM predictions vs actual simulation data plots for 6 Year Blind Validation TDM. 644 Figure 830 Well-005 oil, gas, and water rate TDM predictions vs actual simulation data plots for 6 Year Blind Validation TDM. 645 Figure 831 Well-006 oil, gas, and water rate TDM predictions vs actual simulation data plots for 6 Year Blind Validation TDM. .646 Figure 832 Well-007 oil, gas, and water rate TDM predictions vs actual simulation data plots for 6 Year Blind Validation TDM. 647 Figure 833 Well-008 oil, gas, and water rate TDM predictions vs actual simulation data plots for 6 Year Blind Validation TDM. 648 Figure 834 Well-009 oil, gas, and water rate TDM predictions vs actual simulation data plots for 6 Year Blind Validation TDM

Figure 835 Well-010 oil, gas, and water rate TDM predictions vs actual simulation data plots for 6 Year Blind Validation TDM. 650

Figure 836 Well-011 oil, gas, and water rate TDM predictions vs actual simulation data plots for 6 Year Blind Validation TDM. 651 Figure 837 Well-012 oil, gas, and water rate TDM predictions vs actual simulation data plots for 6 Year Blind Validation TDM. 652 Figure 838 Well-013 oil, gas, and water rate TDM predictions vs actual simulation data plots for 6 Year Blind Validation TDM. 653 Figure 839 Well-014 oil, gas, and water rate TDM predictions vs actual simulation data plots for 6 Year Blind Validation TDM. 654 Figure 840 Well-015 oil, gas, and water rate TDM predictions vs actual simulation data plots for 6 Year Blind Validation TDM. 655 
Figure 841 Well-016 oil, gas, and water rate TDM predictions vs actual simulation data plots for 6 Year Blind Validation TDM

Figure 842 Well-017 oil, gas, and water rate TDM predictions vs actual simulation data plots for 6 Year Blind Validation TDM....

Figure 843 Well-018 oil, gas, and water rate TDM predictions vs actual simulation data plots for 6 Year Blind Validation TDM.

Figure 844 Well-019 oil, gas, and water rate TDM predictions vs actual simulation data plots for 6 Year Blind Validation TDM....

Figure 845 Well-020 oil, gas, and water rate TDM predictions vs actual simulation data plots for 6 Year Blind Validation TDM. 660

Figure 846 Well-021 oil, gas, and water rate TDM predictions vs actual simulation data plots for 6 Year

Blind Validation TDM. 661

Figure 847 Well-002 oil, gas, and water rate TDM predictions vs actual simulation data plots for 6 Year Blind Validation TDM. 662

Figure 848 Well-023 oil, gas, and water rate TDM predictions vs actual simulation data plots for 6 Year Blind Validation TDM. 663

Figure 849 Well-024 oil, gas, and water rate TDM predictions vs actual simulation data plots for 6 Year Blind Validation TDM. 664

Figure 850 Well-025 oil, gas, and water rate TDM predictions vs actual simulation data plots for 6 Year Blind Validation TDM. 665

Figure 851 Well-026 oil, gas, and water rate TDM predictions vs actual simulation data plots for 6 Year Blind Validation TDM. 666

Figure 852 Well-027 oil, gas, and water rate TDM predictions vs actual simulaiton data plots for 6 Year Blind Validation TDM.

Figure 853 Well-028 oil, gas, and water rate TDM predictions vs actual simulation data plots for 6 Year Blind Validation TDM. 668

Figure 854 Well-029 oil, gas, and water rate TDM predictions vs actual simulation data plots for 6 Year Blind Validation TDM. 669

Figure 855 Well-030 oil, gas, and water rate TDM predictions vs actual simulation data plots for 6 Year Blind Validation TDM. 670

Figure 856 Well-031 oil, gas, and water rate TDM predictions vs actual simulation data plots for 6 Year Blind Validation TDM.

Figure 857 Well-032 oil, gas, and water rate TDM predictions vs actual simulation data plots for 6 Year Blind Validation TDM.

Figure 858 Well-033 oil, gas, and water rate TDM predictions vs actual simulation data plots for 6 Year Blind Validation TDM.

Figure 859 Well-034 oil, gas, and water rate TDM predictions vs actual simulation data plots for 6 Year Blind Validation TDM.

Figure 860 Well-035 oil, gas, and water rate TDM predictions vs actual simulation data plots for 6 Year blind Validation TDM.

Figure 861 Well-036 oil, gas, and water rate TDM predictions vs actual simulation data plots for 6 Year Blind Validation TDM.

Figure 862 Well-037 oil, gas, and water rate TDM predictions vs actual simulation data plots for 6 Year Blind Validation TDM.

Figure 863 Well-038 oil, gas, and water rate TDM predictions vs actual simulation data plots for 6 Year Blind Validation TDM. 
Figure 864 Well-039 oil, gas, and water rate TDM predictions vs actual simulation data plots for 6 Year

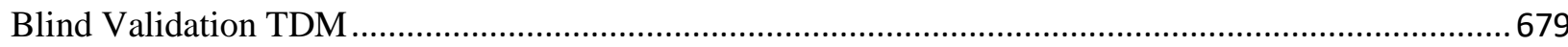

Figure 865 Well-040 oil, gas, and water rate TDM predictions vs actual simulation data plots for 6 Year

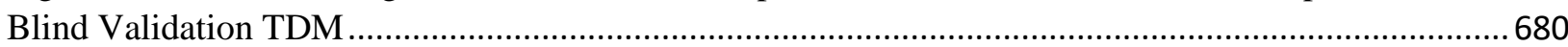

Figure 866 Well-041 oil, gas, and water rate TDM predictions vs actual simulation data plots for 6 Year

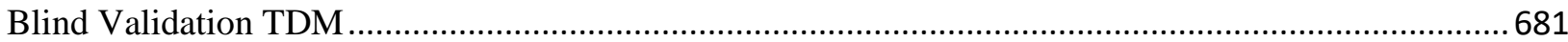

Figure 867 Well-042 oil, gas, and water rate TDM predictions vs actual simulation data plots for 6 Year

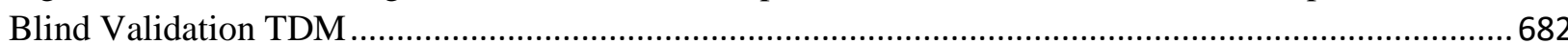

Figure 868 Well-043 oil, gas, and water rate TDM predictions vs actual simulation data plots for 6 Year

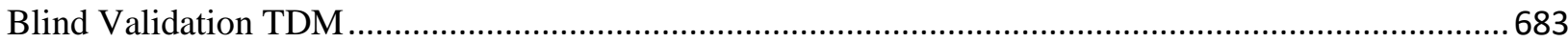

Figure 869 Well-044 oil, gas, and water rate TDM predictions vs actual simulation data plots for 6 Year

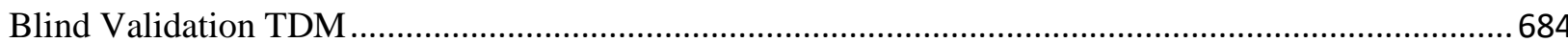
Figure 870 Well-045 oil, gas, and water rate TDM predictions vs actual simulation data plots for 6 Year

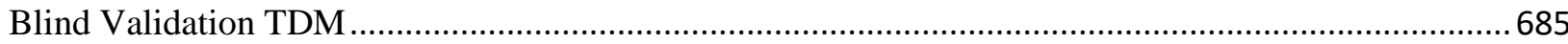
Figure 871 Well-046 oil, gas, and water rate TDM predictions vs actual simulation data plots for 6 Year

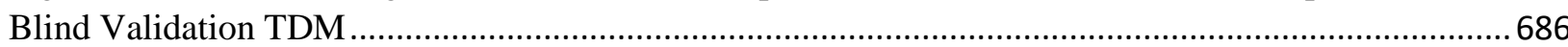
Figure 872 Well-047 oil, gas, and water rate TDM predicitons vs actual simulation data plots for 6 Year

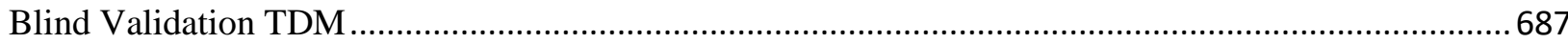
Figure 873 Well-048 oil, gas, and water rate TDM predictions vs actual simulation data plots for 6 Year

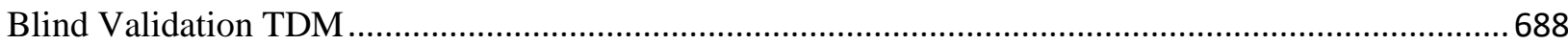
Figure 874 Well-049 oil, gas, and water rate TDM predictions vs actual simulation data plots for 6 Year Blind Validation TDM.... 689

Figure 875 Well-050 oil, gas, and water rate TDM predictions vs actual simulation data plots for 6 Year Blind Validation TDM 690 Figure 876 Well-051 oil, gas, and water rate TDM predictions vs actual simulation data plots for 6 Year Blind Validation TDM. 691

Figure 877 Well-052 oil, gas, and water rate TDM predictions vs actual simulation data plots for 6 Year Blind Validation TDM 692

Figure 878 Well-053 oil, gas, and water rate TDM predictions vs actual simulation data plots for 6 Year Blind Validation TDM. 693

Figure 879 Well-054 oil, gas, and water rate TDM predictions vs actual simulation data plots for 6 Year Blind Validation TDM 694

Figure 880 Well-055 oil, gas, and water rate TDM predictions vs actual simulation data plots for 6 Year Blind Validation TDM 695

Figure 881 Well-056 oil, gas, and water rate TDM predictions vs actual simulation data plots for 6 Year Blind Validation TDM 696

Figure 882 Well-057 oil, gas, and water rate TDM predictions vs actual simulation data plots for 6 Year Blind Validation TDM

Figure 883 Entire reservoir heat map for reservoir pressure at December 31st, 2010 for 6 Year Blind Validation TDM. 697

Figure 884 Entire reservoir heat map for reservoir pressure at June 30th, 2011 for 6 Year Blind Validation TDM. 698

Figure 885 Entire reservoir heat map for reservoir pressure at December 31st, 2011 for 6 Year Blind Validation TDM. .698 Figure 886 Entire reservoir heat map for reservoir pressure at June 30th, 2012 for 6 Year Blind Validation TDM. 698 
Figure 887 Entire reservoir heat map for reservoir pressure at December 31st, 2012 for 6 Year Blind Validation TDM

Figure 888 Entire reservoir heat map for reservoir pressure at June 30th, 2013 for 6 Year Blind Validation

TDM. 699

Figure 889 Entire reservoir heat map for reservoir pressure at December 31st, 2013 for 6 Year Blind Validation TDM.

Figure 890 Entire reservoir heat map for reservoir pressure at June 30th, 2014 for 6 Year Blind Validation

TDM. 700

Figure 891 Entire reservoir heat map for reservoir pressure at December 31st, 2014 for 6 Year Blind Validation TDM. .700

Figure 892 Entire reservoir heat map for reservoir pressure at June 30th, 2015 for 6 Year Blind Validation TDM. 700

Figure 893 Entire reservoir heat map for reservoir pressure at December 31st, 2015 for 6 Year Blind Validation TDM 701

Figure 894 Entire reservoir heat map for reservoir pressure at June 30th, 2016 for 6 Year Blind Validation TDM. 701

Figure 895 Entire reservoir heat map for reservoir pressure at December 31st, 2016 for 6 Year Blind Validation TDM. 701

Figure 896 Entire reservoir heat map for water saturation at December 31st, 2010 for 6 Year Blind Validation TDM. 702

Figure 897 Entire reservoir heat map for water saturation at June 30th, 2011 for 6 Year Blind Validation

TDM. 702

Figure 898 Entire reservoir heat map for water saturation at December 31st, 2011 for 6 Year Blind Validation TDM. 702

Figure 899 Entire reservoir heat map for water saturation at June 30th, 2012 for 6 Year Blind Validation

TDM. .703

Figure 900 Entire reservoir heat map for water saturation at December 31st, 2012 for 6 Year Blind Validation TDM. 703

Figure 901 Entire reservoir heat map for water saturation at June 30th, 2013 for 6 Year Blind Validation

TDM. 703

Figure 902 Entire reservoir heat map for water saturation at December 31st, 2013 for 6 Year Blind Validation TDM 704

Figure 903 Entire reservoir heat map for water saturation at June 30th, 2014 for 6 Year Blind Validation TDM. 704

Figure 904 Entire reservoir heat map for water saturation at December 31st, 2014 for 6 Year Blind Validation TDM 704

Figure 905 Entire reservoir heat map for water saturation at June 30th, 2015 for 6 Year Blind Validation TDM. 705

Figure 906 Entire reservoir heat map for water saturation at December 31st, 2015 for 6 Year Blind Validation TDM 705 Figure 907 Entire reservoir heat map for water saturation at June 30th, 2016 for 6 Year Blind Validation TDM. 705

Figure 908 Entire reservoir heat map for water saturation at December 31st, 2016 for 6 Year Blind Validation TDM. 


\section{Introduction}

Numerical reservoir simulation (NRM) is characterized as a "Bottom Up" strategy that first starts with a geologic model. This geologic model will provide details about the reservoir before development begins. The geologic model is created by correlating the seismic, well-log, geostatistics and mathematical models in order to determine the subsurface geology, depositional environment, and petrophysical properties. This includes, but is not limited to the number of reservoir layers, presence of sealing or nonsealing faults, permeability, porosity, etc. After the geologic model is built, the model needs to be up scaled in order to reduce the number of cells from 25-50 million for complex or large reservoirs to 2 million cells or less for the numerical reservoir model. The upscaling can be done using multiple methods including analytical (harmonic average, arithmetic average, etc. for each grid block), local flow based (single-phase flow equation simulations in every direction across a grid block), and global flow-based (pressure gradients for the entire field from certain wells to find the permeability tensor) (Society of Petroleum Engineers, 2015). Once fluid flow fundamentals have been added, the geologic model progresses into a dynamic reservoir model which is then quantified using previous production data. If the dynamic reservoir model does not match up with the historical production data (oil rate, gas/oil ratio, water/oil ratio, or reservoir pressure), it can usually be attributed to assumptions made in the geologic model or elsewhere in the NRM. These parameters can be changed in order to match the production data in a process called history matching, starting with properties that have the highest uncertainty. After history matching is complete, it is important to also perform a sensitivity analysis to determine how much the production and the simulation changes from altering specific parameters. This allows the range of expected production to be established from the worst case scenario to the best case scenario. Even for simpler conventional reservoirs, this process is long and often computationally intensive due to the number of simulation runs that need to be completed to obtain a reliable and accurate model (Intelligent Solutions Inc., n.d.) (Society of Petroleum Engineers, 2015).

While small advances to reduce the problem presented above have been made, it is still a prevalent issue today. Models with a high resolution in time and space are still not practical even with faster computers, parallel central processing units, etc.

Top Down Modeling (TDM) is a reservoir simulation model that is made entirely from field measurements and production data. When this approach is used, production, seismic, core, and well test data can provide insight into the reservoir through the use of artificial intelligence and machine learning without assumptions being made. Depending on the objective of the TDM, various algorithms and data preparation methods can be used to accomplish the desired outcome. While the minimum amount of data required for a TDM varies based on each situation, generally the more data, the better. This allows the TDM to understand a fuller range of properties that could lead to various production volumes. Because of this, TDM is not applied on new fields undergoing appraisal, because it does not have enough measured data from wells, as well as it has no production values to train the model (Mohaghegh, Shahkarami, Gholami, \& Haghighat, 2014). Consequently, a TDM is best applied to fields that at least a few years of production as an alternative modeling method or in addition to a numerical reservoir simulation model. Even though equations, such as the one for fluid flow, is not given to the Top Down Model, those equations as well as expertise in parameters that play an influential role are essential in selection of data 
used for the model. The data used in the training, calibration, and validation along with the analysis are vital to the success of the TDM (Mohaghegh, Data-Driven Reservoir Modeling, 2017).

\section{Problem Statement}

The task at hand is to confirm the capabilities of Top Down Modeling by comparing the predictions of the Top Down Models with those of a Numerical Reservoir Simulator for a reservoir with water and gas injection in one limb of an anticline. This will be done with specific reservoir characteristics to ensure its abilities to predict in a different reservoir environment with industry realizations. A multitude of TDM models will be made in an attempt to determine the minimum amount of data needed to obtain accurate predictions.

\section{Literature Review}

Data-Driven modeling is defined as "the process of applying artificial intelligence and data mining methods such as machine learning and pattern recognition in order to uncover hidden patterns in large data sets that represent fluid flow in porous media". They have several advantages such as being easy to update, improve with new data and training, and they allow for a large number of runs within a short amount of time. (Mohaghegh, Data-Driven Reservoir Modeling, 2017).

\subsection{Spatio Temporal Database}

The Spatio Temporal Database is the dataset to be used to build a data-driven reservoir model. This dataset consists of both static and dynamic data from which misleading values caused by errors are already removed. This dataset is one of the most important features of the data-driven modeling. If not properly prepared or properly quality controlled, then errors could greatly mislead and affect the model. These misleading data values do not include noise which is present in every dataset that is not generated from a numerical reservoir simulator. Quality control could include, but is not limited to ensuring that the abandonment of wells occurs on the correct date, the correct injection rate is represented in the data, completion footage is correct after recompletion of a well, etc. Once the quality control process is complete, then any changes in production, whether increases or decreases, can be explained through the provided dataset. Recompletion can cause an increase in production shown by an increase in the completion footage provided in the dynamic data and shut-ins for well maintenance can decrease production included in the number of days of production for that well, to name a few examples.

It cannot be overemphasized that each record within the spatio temporal database must be for one well only at one time step where the output is production. Within each record are all of the static, dynamic, and offset attributes that are responsible for the production amount specified as the output at one instance in time (Mohaghegh, Data-Driven Reservoir Modeling, 2017).

\subsubsection{Static Data}

Static data can be both truly static and also dynamically modified static data. Dynamically modified static data is done by taking the average with respect to the open layers. Therefore for each time step that the well is recompleted, the averaged static parameters will change. The average taken can be 
either a standard arithmetic average or a weighted average based on the thickness of the formations contributing to production. In almost all cases, the thickness of several formations is not uniform at the well location. Therefore by taking a weighted average of properties based on the open formations' thicknesses, a more accurate representation of the properties influential to an increase or decrease in production can be obtained.

\subsubsection{Blind Validation in Time and Space}

Both time and space are taken into account in a spatio temporal database. The extent of each of these is determined by how many wells there are and how long the reservoir has been producing. The resolution in time can be yearly, monthly, daily, etc. where data provided daily can be up-scaled to monthly or annually if there is enough data. The resolution in space is simply the number of wells present within the reservoir. Blind validation can be done in both time and space. If the model contains several years' worth of data, one year or more at the end of the model can be used as blind validation. After the models have been successfully trained and the TDM is built, the TDM will be deployed on the specified blind validation dataset where the TDM prediction will be compared with the actual values as a way to quality check the TDM. This can also be done in space where one well is removed entirely from the TDM. Again, once the models have been successfully trained and the TDM built, the TDM can predict the well's production based on where it is at in the reservoir and its offset producers (and offset injectors if present).

\subsubsection{Polygon Based Properties}

Two grid systems are used in a Top Down Model. One grid is a 2D Cartesian Grid where the static properties are specified at the well locations. Geostatistics such as Kriging (inverse weighting method in the case of this thesis) are used to generate the static properties for all of the grids that a well is not located in. The grid is described as being 2D because the thickness of the grids at the well's location is the actual thickness of the formation. The second grid system is known as a polygon grid. This grid maps out the drainage area for each well and also shows which Cartesian grids within a well's polygon represent the well. The polygon grid can only be created once a boundary is drawn around the existing wells to represent the approximate extent of the reservoir. As the number of wells increase as the field is developed, the drainage area, and the size of each well's Voronoi calculated polygons decreases. It is important to note that often during the beginning phases of a field's development, few wells are completed. When this happens, those well's polygons can be rather large, however it is not suggested that these wells will necessarily communicate with the boundaries or grids near the boundaries. Nevertheless, the polygon based static properties will be an arithmetic average of all the Cartesian grids within the polygon. An example where the well may not communicate with grids near the boundary is shown below. After new wells are drilled, the values for the Cartesian grids between wells are updated from the geostatistical calculations using the old and the new wells' attributes, and the theoretical drainage area of each well is updated (Mohaghegh, Data-Driven Reservoir Modeling, 2017).

The aforementioned polygon properties which are dynamically calculated based on the number of wells within a reservoir, help detail the geology and reservoir characteristics between wells. When these properties and the production history are examined, an idea of how the wells communicate and the flow path between them can be learned (Mohaghegh, Data-Driven Reservoir Modeling, 2017). 


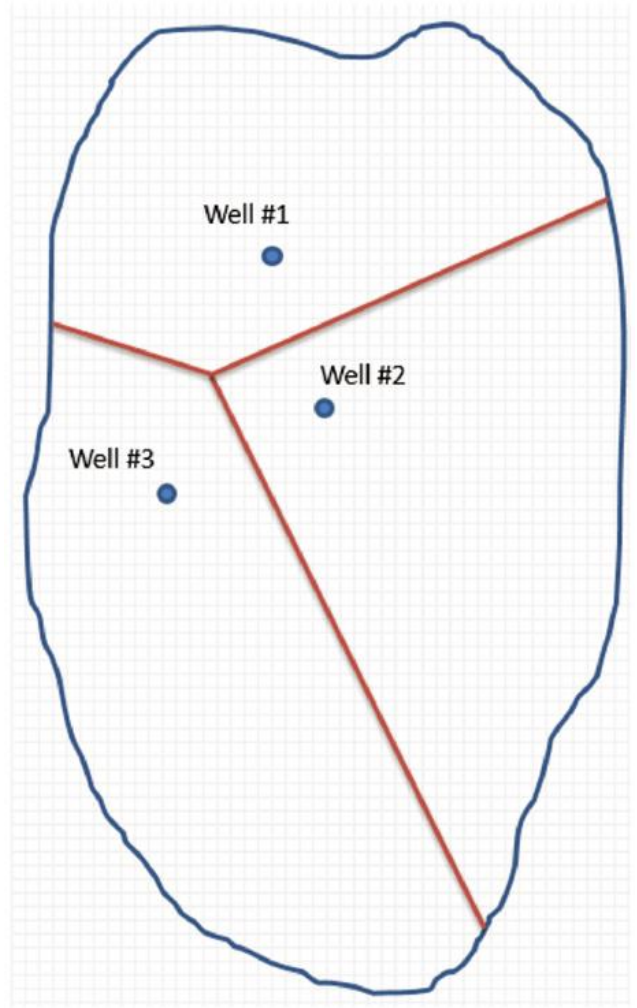

Figure 1 Voronoi polygon grid depicting theoretical drainage area during first phase of development

\subsection{Data Mining}

Data mining is the extraction of unknown and possibility useful trends from a dataset using various techniques. One technique is known as association analysis. Association analysis is the detection of attribute-value pairs that happen often in a dataset. A second method is regression analysis where models are made that detail dependent variables through the study of independent variables. Lastly, another technique is classification and prediction. Classification consists of creating models that "predict the class of objects whose class label is unknown" (Brighterion, Inc., 2017). The model can take many forms where some examples are formulas, decision trees, or if-then rules. In general, the process has the following steps (Brighterion, Inc., 2017):

1. Data cleaning and quality control

2. Data compilation and selection

3. Data formatting into appropriate forms

4. Pattern recognition and statistics to extract patterns

5. Pattern identification to determine patterns of value

6. Visualization of the patterns for users

Data mining, which is used in Top Down Modeling, also has similar limitations as is presented later in this chapter. Data mining's quality is limited to the quality of the data used. It also can only extract patterns present in the data, and therefore cannot find new patterns that have not been seen historically (Brighterion, Inc., 2017). 


\subsection{Artificial Intelligence}

Artificial Intelligence is a multitude of analytical tools at anyone trying to imitate life dispense and the biological process of learning. Some of the tools commonly used are fuzzy logic, artificial neural networks, and evolutionary programming, among a plethora of others (Mohaghegh, Data-Driven Reservoir Modeling, 2017).

\subsection{Artificial Neural Networks}

An artificial neural network is an information processing system that emulates a human's neural biology. This system consists of many pieces as well as several assumptions outlined below (Mohaghegh, Data-Driven Reservoir Modeling, 2017).

1. The processing of information occurs within components called neurons which can be compared to the nucleus of a biological neuron.

2. Connection links are the method for which signals are communicated to other neurons. Again, in a biological system, these can be associated with the axons and dendrites of neurons, or the connection of the two called a synapse.

3. Each of the aforementioned links has a value associated with it that is often multiplied with the signal being transmitted. These values often detail the level of influence of the signal and is termed as the weight of the signal.

4. A non-linear activation function (rarely, a linear activation function) calculates the output of the neuron from the summation of all the inputs. This output is then either signaled to the next set of neurons or is deemed the final output. All of the aforementioned parts are shown below in Figure 2 (Mohaghegh, Data-Driven Reservoir Modeling, 2017); (Fausset , 1994).

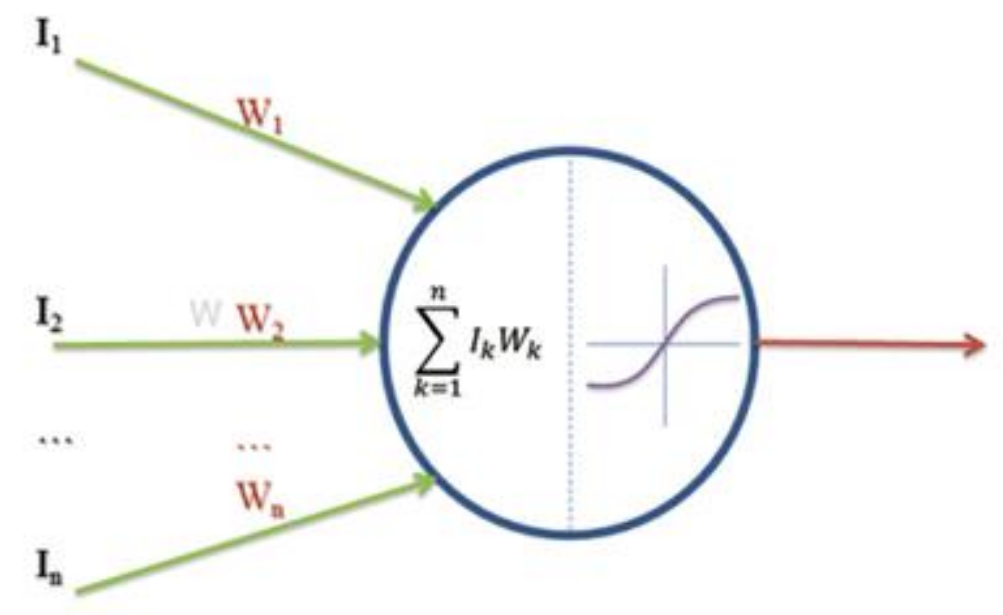

Figure 2 Diagram of an artificial neural network neuron (Mohaghegh, Data-Driven Reservoir Modeling, 2017)

A multitude of individual neurons are needed to build the artificial neural network where the structure and algorithms used are dependent on the task at hand. A neural network always has a set of input neurons, output neurons, and neurons in the middle to perform any task. The number of input and output neurons directly corresponds with the number of inputs given to the system as well as the number of outputs expected. All neurons are organized into layers, where the neurons between the input and output layers are separated into one or more layers called hidden layers. Depending on the task, an artificial neural network can be described as unsupervised or supervised. Unsupervised networks are not 
given any feedback by the trainer of the network and a majority of the algorithms are classification algorithms. A supervised network allows inputs and outputs at certain time steps to be used to train the model in order to ensure that the model produces acceptable outputs. This process can be optimized by changing algorithms and network architecture (number of hidden layers, number of hidden neurons, etc.) (Mohaghegh, Data-Driven Reservoir Modeling, 2017). An example of a simple artificial neural network is shown below.

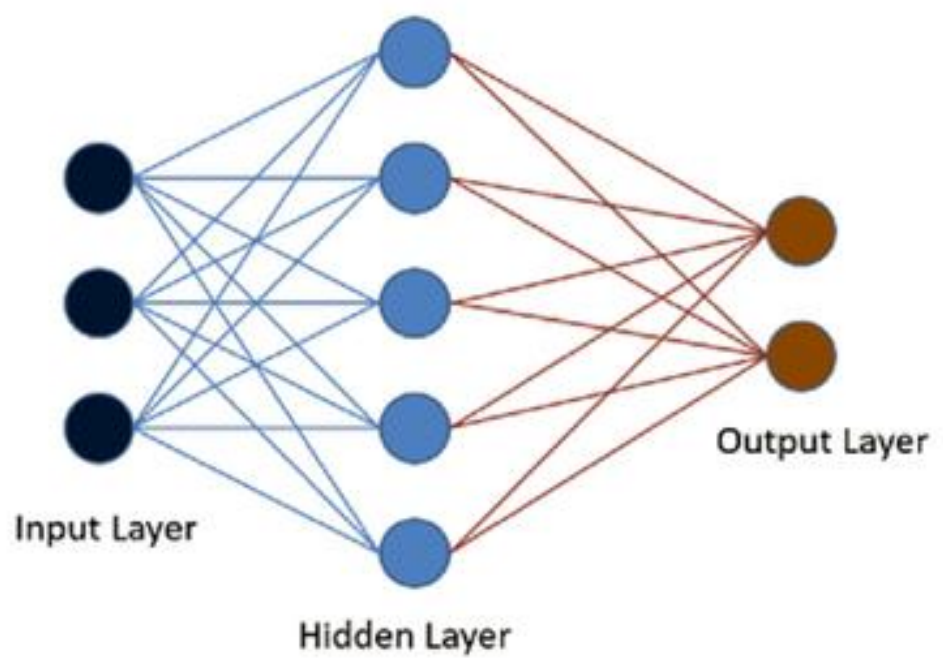

Figure 3 Diagram of a simple artificial neural network (Mohaghegh, Data-Driven Reservoir Modeling, 2017)

The most successful neural network for Top Down Modeling and the one used in this thesis is a fully connected neural network. A fully connected neural network is one where "every input neuron is connect to every hidden neuron, and every hidden neuron is connect to the output neuron" (Mohaghegh, Data-Driven Reservoir Modeling, 2017). A fully connected neural network can have any number of hidden layers, however the neural networks constructed in this work contain only one hidden layer. Each data-driven model also only has one output. This structure was chosen because it has performed best in the previous work done with data-driven reservoir modeling.

Every neuron determines the weight that should be applied to every signal it receives. A random weight is first initially assigned in the training process and is continuously adjusted as the network continues to learn. The weights multiplied by the input signals are then added together. The summation is then provided to a threshold level that determines if an output will be provided or not, which changes based on the activation function used. For this thesis, the logistic sigmoid function is used which provides a value of 0 or 1 as an output from the summation of the weights to the next layer (Mohaghegh, DataDriven Reservoir Modeling, 2017).

In order to evaluate the quality of the network's learning, the data is divided into three datasets; training, calibration, and verification. The training set is used to allow the network to learn, where the weights are adjusted as the network learns the required numbers to achieve the specified output for multiple pairs of input and output values. After each epoch in training, where an epoch is defined as all of the training data being used once to calculate the weights and the error between the predicted values and the actuals are calculated, the inputs to the calibration dataset are given to the network to allow it to predict the outputs. The predicted outputs are then compared against the provided calibration dataset outputs to deem if the network needs additional training or if it can accurately predict the calibration dataset's outputs. This process attempts to prevent the network from memorizing the dataset which would 
likely cause inaccurate predictions should new data be predicted upon. Then the training is tested once more on the verification dataset. The verification dataset is also known and referred to as within this thesis as the validation dataset. Again the inputs are provided and predicted upon, then compared against the provided verification dataset (Mohaghegh, Data-Driven Reservoir Modeling, 2017).

\subsubsection{Supervised Learning Algorithms}

A very popular algorithm used in supervised learning, and the algorithm used for the data-driven reservoir modeling in this thesis, is called a backpropagation algorithm. During the training process, the predicted value is compared against the actual output value. The error between these values is then propagated backwards throughout the network to adjust all of the weights in the network before another iteration is performed in the training. This process is done in a loop until the weights assigned reach an equilibrium with the error between the predictions and actual outputs or until the training process is stopped manually. (Mohaghegh, Data-Driven Reservoir Modeling, 2017). There are also several different backpropagation types, where Vanilla and Enhanced are both used for this thesis. The Enhanced method utilizes both the learning rate and momentum design parameters whereas the Vanilla method only uses learning rate. The momentum parameter has the ability to get the solution out of a local minima so that it can find the global minimum as well as speed up the learning process. The learning rate which is exactly as it sounds, how fast the network learns the information that is presented to it. While a very fast learning rate may seem to ideal, this could cause the global minimum to be missed and problems in convergence. Other methods provided in the software, but not used are Rprop, Quick Prop, Batch, and Weight Decay (Intelligent Solutions, Inc. , 2016).

\subsection{Fuzzy Set Theory}

There are numerous variables that affect the production of the focal well. Reservoir, completion, flow, and surface facility characteristics all play an important role as to how much oil, gas, and water are produced at every time step along with the reservoir and flowing bottom-hole pressure at all times. Not only do all of these parameters matter for the focal well, but they also matter for the wells surrounding the focal well. By including certain attributes from the wells surrounding the focal well, the interference of those wells with the focal well can be identified. Fuzzy set theory can be used to find correlations between the data to determine which parameters are most important to include in the training process as not all attributes can be used. Using too many variables in the training process has actually found to not only slow down the training process, but has also been found to provide less accurate results. While determining which variables correlate with the output, it's crucial to use reservoir engineering expertise to ensure that the correlation makes sense. The assurance of causation between the variables will help develop a quality set of inputs that will provide accurate results.

\subsection{Top Down Model}

A Top Down Model can have various structures which is dependent on the purpose of the model, the data and quantity of data available, and the reservoir being modeled. A Top Down Model however does always contain a Spatio Temporal Database, a method for feature selection such as the Key Parameter Influencer, several data-driven reservoir models deemed to be of sufficient accuracy after training, and post modeling analysis tools. After the inputs for each data-driven reservoir model are determined from the KPI and use of domain expertise, the models are trained, calibrated, and validated. 
Oil, which is often the first output and is the first output for this thesis, will include any attributes deemed important, but will not have the outputs of the other models at time t. Because oil is the first output, the other outputs will not be available at time t. Gas, another popular output and the second output of this thesis, will have oil at time t, however it will not have any other model's outputs at time t. This is due to the fact that the oil model will have finished predicting at the current time step and oil at $t$ will then be fed as an input into the gas model (Mohaghegh, Data-Driven Reservoir Modeling, 2017).

Selecting the parameters that will be used in the training of each of the data-driven models can often be a difficult task. While there are many parameters that are believed to play an influential role in production, it is not necessarily beneficial to include them all. Not only will the greater number of attributes prolong the training process, but some parameters may be unnecessary when compared with the importance of others. Because of this, the Key Parameter Influencer helps identify the variables that should be included where the lower ranking variables are deemed to be the variables overshadowed by more influential ones. There are often variables that optimize production such as injection rate or choke setting. If not already included, these optimization parameters must also be included into the model in order to identify further optimization in the future.

Top Down Models also utilize Intelligent Agents which are defined as a code that is capable of evaluating the outcome of the several data-driven reservoir models, makes a decision, and ensures that multiple constraints for field operations are satisfied during the implementation of the multiple datadriven reservoir models. This includes, but is not limited to, ensuring the sequence of the models is performed in the right order, that all minimum or maximum operating constraints are not exceeded during prediction, etc (Mohaghegh, Data-Driven Reservoir Modeling, 2017).

It is important to ensure that the fundamentals of reservoir engineering are applied to the analysis of the data and the results of a Top Down Model. One example of this is to certify that the data given to the Top Down model make sense. If there are enough records, the data makes sense, and enough data is provided to explain changes in production, then the Top Down Model should be successful. In addition, the data provided will have a range of values for each attribute. One should not expect great predictive results from values outside of the ranges used to train the model. This is due to the fact that most machine learning algorithms are not known for their extrapolative capabilities, but rather their interpolative capabilities, even if non-linear. Once a Top Down Model that can accurately predict is created, the relationship between all of the inputs can be evaluated to make sure they make sense from a geological and physical perspective. This can be achieved for each data-driven model that comprises the Top Down Model (Mohaghegh, Data-Driven Reservoir Modeling, 2017).

\subsubsection{TDM History Matching}

The history matching process for a Top Down Model is different than the history matching process of a numerical reservoir simulator. Even though in both circumstances, the measurements and reservoir characteristics are uncertain, these parameters are not changed unless a subset of the data is believed to be of better quality. During history matching of a Top Down Model, the relationships (ie the weights and connections in the artificial neural network) between the parameters are adjusted until the production data can be matched. This is different from numerical reservoir simulation history matching in that the relationships defined by equations such as Darcy's Law remain unchanged whereas the reservoir characteristics and measurements are changed until a match with the production data is found. 
There are three types of history matching for a Top Down Model: sequential, random, and mixed history matching. Sequential history matching is done when the data is organized by date and the partitioning of the datasets are therefore in sequential order. The training set will be the largest portion from the start of the simulation until the start of the calibration dataset. The verification dataset will then start on the date after the calibration dataset ends. This type of partitioning results in the calibration and verification datasets using the TDM's predictions for dynamic attributes at time steps $\mathrm{t}-1$ and $\mathrm{t}-2$ (with the exception of the first time step in the calibration dataset). For random history matching, the records in each dataset are selected at random and therefore are not in order. The dynamic inputs at $\mathrm{t}-1$ and $\mathrm{t}-2$ for the calibration and verification datasets are actual records, not predictions, due to being selected at random. Because of this, the random history matching is not considered a blind history match unlike the sequential history matching. Only when the outputs are predicted one after the other in sequential order is the history match considered blind. Mixed partitioning is when the training and calibration datasets are assigned at random and the verification dataset is the last assigned portion of the model. For example, one year at the end of the timeline can be set aside for verification whereas the rest of the dataset can be randomly assigned to training and calibration. This form of history matching allows the ability of the model to predict with its own predictions as inputs at previous time steps, to be evaluated in the verification dataset. The results obtained from the verification dataset can be an early indicator of what the forecasting results would look like and is considered blind verification, also referred to as blind validation.

After the history matching of each individual data-driven model that comprises a Top Down Model, the Top Down Model itself also needs to be validated. This is done by ensuring that the model is "internally consistent" meaning that it must be able to predict acceptable results when the inputs fed to it are also predictions it has made at a previous time steps (Mohaghegh, Data-Driven Reservoir Modeling, 2017). Similar to what was previously discussed in history matching, these predictions can be compared against the actual values for the entire Top Down Model.

\subsubsection{Post Modeling Analysis Using A Top Down Model}

Once the quality of the Top Down Model has been deemed worthy, post modeling can be done. There are several techniques of post modeling that can be done, some of which are briefly presented here.

Forecasting can be done for the data-driven model outputs, for which the length of the forecast can be specified by the modeler. Another technique is production optimization where the best operational constraints to produce the most oil can help be identified, even if there are multiple combinations of constraints to test. Examples of production optimization is finding the best combination of choke settings and artificial lift parameters. The amount of water injected into a field can also be evaluated in order to gain insight into whether too much water was injected or not enough water was injected in the past. The most beneficial water injection schedule for the future can also be forecasted for the field. Reservoir characterization, or the interaction between wells (both injection and producer wells), can also be done in the post modeling phase. This "high level map of fluid flow in a reservoir" can be used to pinpoint the location of faults and other geological features (Mohaghegh, Data-Driven Reservoir Modeling, 2017). One investigation often performed from a reservoir model is infill well location optimization. This can also be done in Top Down Modeling, where operational constraints for the proposed well can be set and the forecasted oil, water, and gas production can be examined after interference from other wells, reservoir characteristics, oil in place, and the operational conditions set are taken into account. Infill well location investigations can be done for individual locations, or multiple locations at once, depending on the objective. Type curves can be created for individual wells, sections of the field, or the entire field. The 
type curves can then show the change in production versus time as one other attribute, chosen by the modeler, is varied, thus showing the impact of that attribute. Last, but not least, uncertainty quantification can be done as part of post modeling analysis. A profile range for the output will be generated from the Monte Carlo simulation technique applied using the TDM.

\subsubsection{TDM Limitations}

There are several limitations to Top Down Modeling that will be briefly discussed. First and foremost, Top Down Modeling cannot be applied to new fields. "Approximately 20 to 30 wells and at least two years of production history is probably the minimum requirements to develop a TDM" (Mohaghegh, Data-Driven Reservoir Modeling, 2017). As previously emphasized, the data quality is extremely important to the success of a Top Down Model. If the data being used does not accurately describe the fluid flow, or is insufficient, the TDM will not be successful even if the theoretical minimum amount of data is satisfied. Top Down Models can also not be applied once large changes to the physics have been made such as switching from primary recovery to secondary recovery. A change like this would require the model to be retrained with data from the new secondary recovery method.

\subsection{Previous Work}

Artificial intelligence and machine learning (AI \& ML) have become increasing topics of interest within the oil and gas industry. While other industries have been utilizing this technology for decades, the oil and gas industry is just starting to scratch the surface of the endless possibilities. One important implementation is autonomously handling large amounts of continuous data quickly. This processed and analyzed data can then be used to do real time reservoir modeling by creating a surrogate reservoir model (SRM) that can replicate the more complex numerical reservoir model, but perform runs in fractions of the time. This application has already been tested and deemed successful for a field in the Middle East (Mohaghegh, Artificial Intelligence and Data Mining: Enabling Technology for Smart Fields, 2009). Several other applications of AI and ML within the oil and gas industry are presented below.

A surrogate reservoir model was also used to perform a history matching process for a heterogeneous reservoir created using a numerical reservoir simulator (NRM) where the objective was to perform the process more quickly than conventional methods. The NRM's data was defined as the "actual data" meaning that it is being considered as the measured production data that is typically used in the history matching process. This allows the predictions and properties from the SRM to be compared and adjusted against the NRM's data (Mohaghegh, Shahkarami, Gholami, \& Haghighat, 2014).

First a spatio-temporal database is created only using geologic realizations and measured values. The database needs to be arranged in a way that the artificial neural networks (ANN) in the SRM model can learn the connections within the dataset to reach a certain outcome or production value. Over fitting of the model, where the error for training continues to decrease, but the error in the calibration increases, can occur. This is combatted by monitoring the training and calibration process so that once the aforementioned trend is observed, the training process can be stopped. Once there is little difference between the SRM's outputs from the ANN and the output values included as the result in the database, this trained model can now be validated using a blind dataset (Mohaghegh, Shahkarami, Gholami, \& Haghighat, 2014).

After this training, calibration, and validation procedures were completed, the results from the SRM were compared with the NRM data as shown below in Figure 4. As it can be seen, not only could 
the SRM recreate the results from the NRM during the training process, but it was able to predict the performance on the blind dataset during validation. In order to introduce the uncertainty that occurs in NRM to perform history matching, ten different permeability maps were created, varying the permeability values at the well locations. Lastly, is a depiction of the history matching results for production from adjusting permeability values where a good match can be observed in Figure 5. The history matching and SRM development was accomplished much faster than conventional methods due to the pattern recognition capabilities of AI and ML (Mohaghegh, Shahkarami, Gholami, \& Haghighat, 2014).

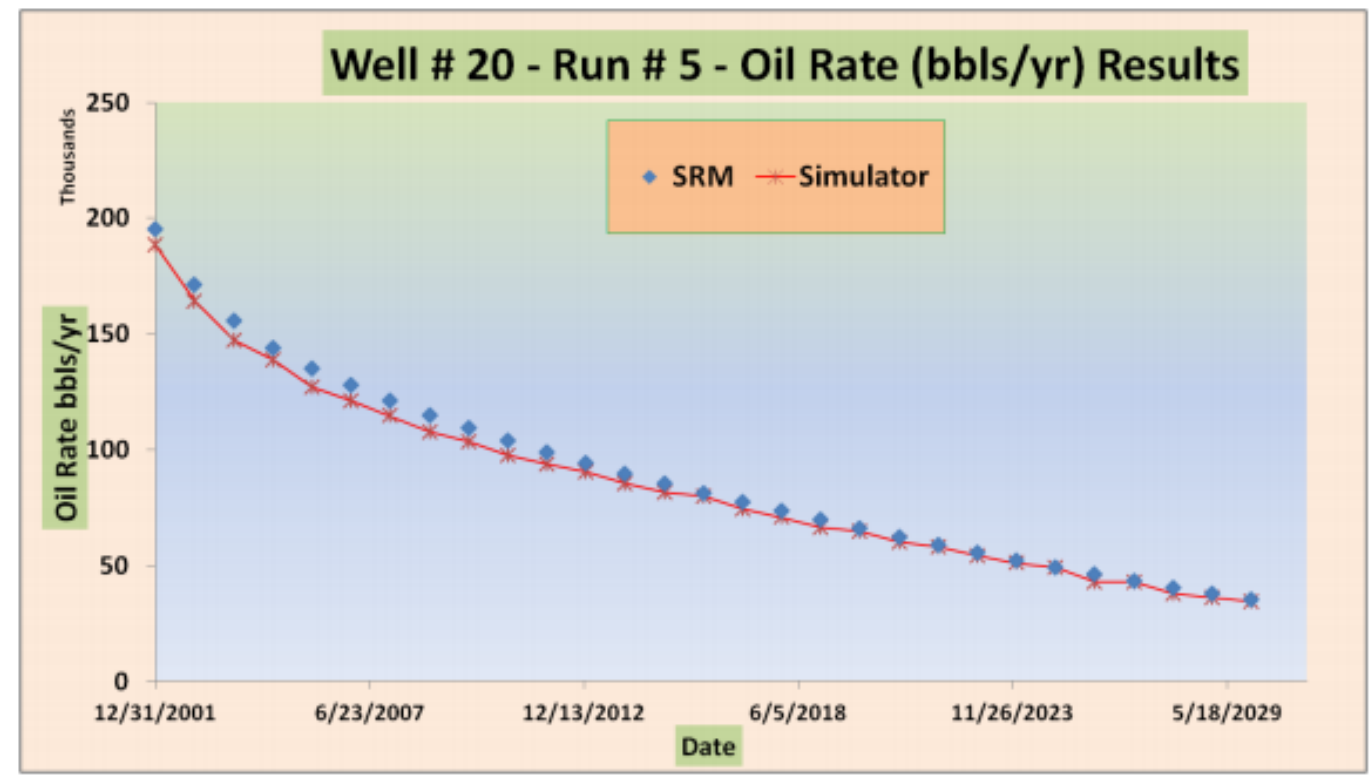

Figure 4 Comparison of results from SRM and NRM after training, calibration, and verification

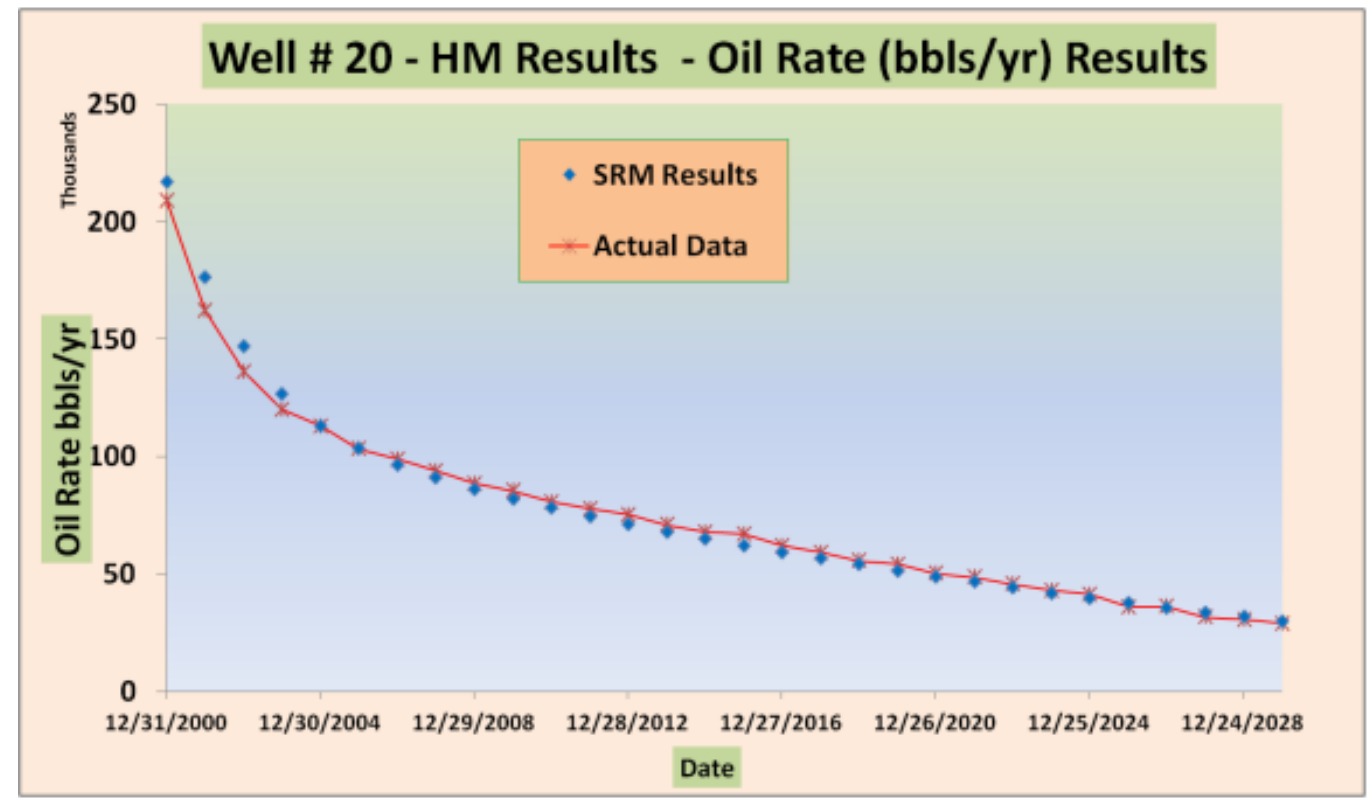

Figure 5 Comparison of the SRM's history matching with actual outputs 
Similarly to the last application of SRM for history matching, another SRM was created for history matching, however instead of an academic model, a more realistic problem was solved. The PUNQ-S3 reservoir model, often used to evaluate new methods of history matching, was used. While the last example only contained permeability as an uncertain parameter, this model's uncertain parameters are horizontal permeability, porosity, and vertical permeability. Due to the increase in complexity, a Differential Evolution (DE) algorithm and objective functions were utilized, allowing the history match process to be automated. One case from the PUNQ-S3 reservoir model was assigned to be the true case and this case's outputs were used as the "measured field data". In order to communicate the interaction and impact of multiple portions of the reservoir, both Voronoi graphs and a tier system were implemented. The tier system consisted of four tiers which each help delineate the communication within each portion of the Voronoi graph. The first tier is the well block, the second is the first row or grid blocks around the well block, the third tier are the grids around the second tier, and the fourth tier are the rest of the grids in that portion of the Voronoi graph (Shahkarami, Mohaghegh, \& Hajizadeh, 2015).

The SRM in (Mohaghegh, Shahkarami, Gholami, \& Haghighat, 2014) was tasked with matching oil production as the only output whereas the SRM within (Shahkarami, Mohaghegh, \& Hajizadeh, 2015) was tasked with matching gas production, water production, and well bottom hole pressure, all of which required separate SRM models. The static and dynamic data were prepared, including the output values at the previous two time steps as inputs for each respective SRM. All three SRMs were then trained, calibrated, and validated before combining the three networks together. A blind validation was then carried out where one entire realization of the PUNQ-S3 model was used in that the inputs were loaded into the integrated SRM and the outputs were compared with the realization's. A visualization of the comparison between the SRM results and the PUNQ-S3 actual values can be seen below in Figure 6 . While the blind validation results were not as accurate as the training results, as expected, the results were acceptable and the SRM is now ready to begin history matching (Shahkarami, Mohaghegh, \& Hajizadeh, 2015). 

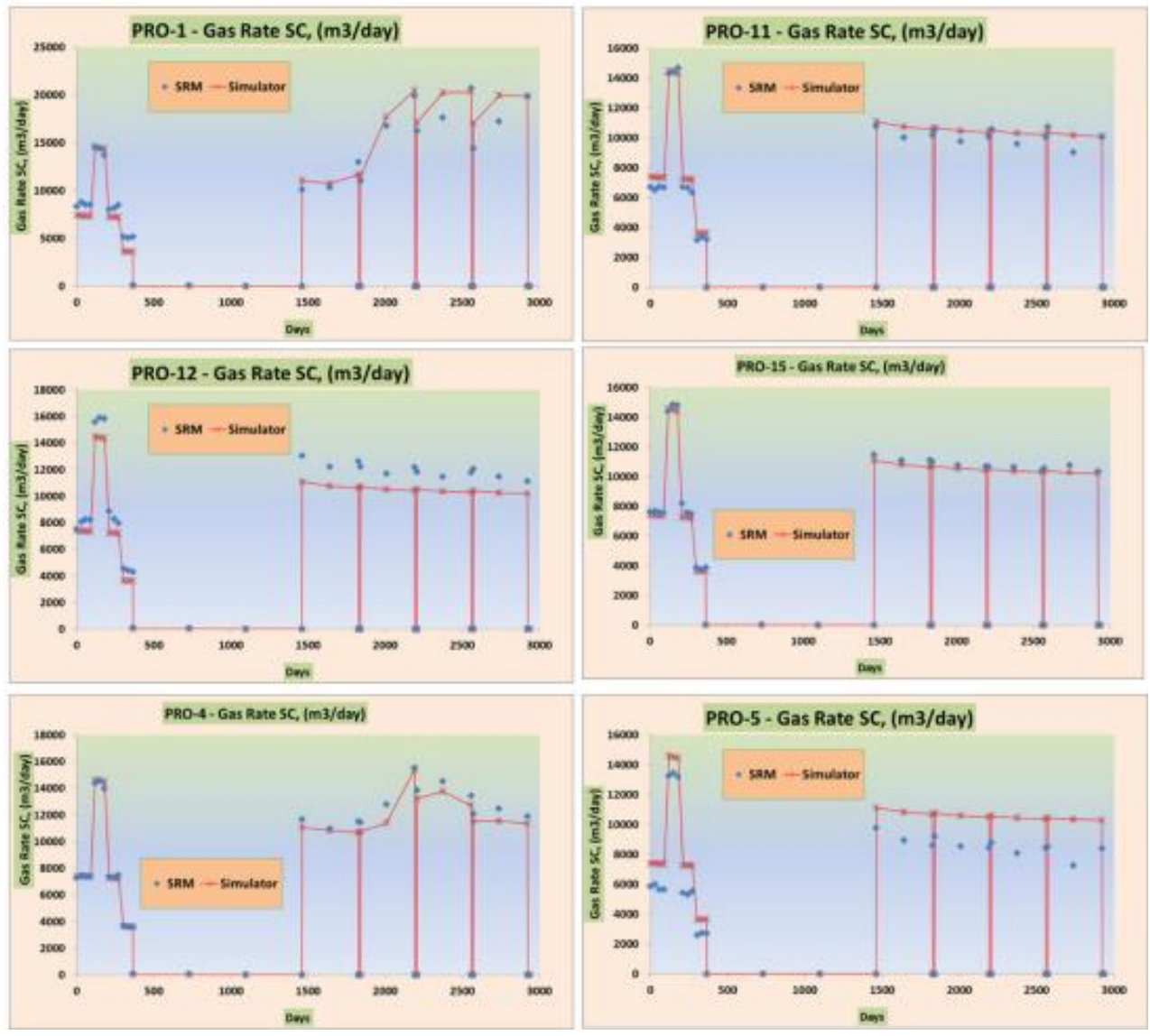

Figure 6 Blind validation comparison of SRM results for gas production rate vs. the realization from PUNQ-S3

Due to the many combinations possible that could match the actual results, the ten best combinations were determined after horizontal permeability, porosity, and vertical permeability were varied so that the outputs generated would match eight years of historical data. The match for water production was acceptable, however the match for gas production and well bottom hole pressure, as seen below in Figure 7, were exceptional. Then the best combination of inputs was inputted into the PUNQ-S3 simulator and ran. The simulator results with SRM identified properties were compared with actual field production. Next, forecasts were done for cumulative production at 16.5 years which can then be compared to the actual values. The matches from the simulator results with SRM identified properties and the actual field production were great where one such example can be seen below in Figure 8 for oil production. The forecasted values at 16.5 years were slightly overestimated for oil and gas production, but slightly underestimated for water production. While other methodologies also included automated history matching, they required numerous runs of the numerical simulator, a usually time intensive endeavor. The SRM developed in this study only required 11 runs of the simulator to perform history matching and therefore has promising potential to assist with history matching in the future (Shahkarami, Mohaghegh, \& Hajizadeh, 2015). 


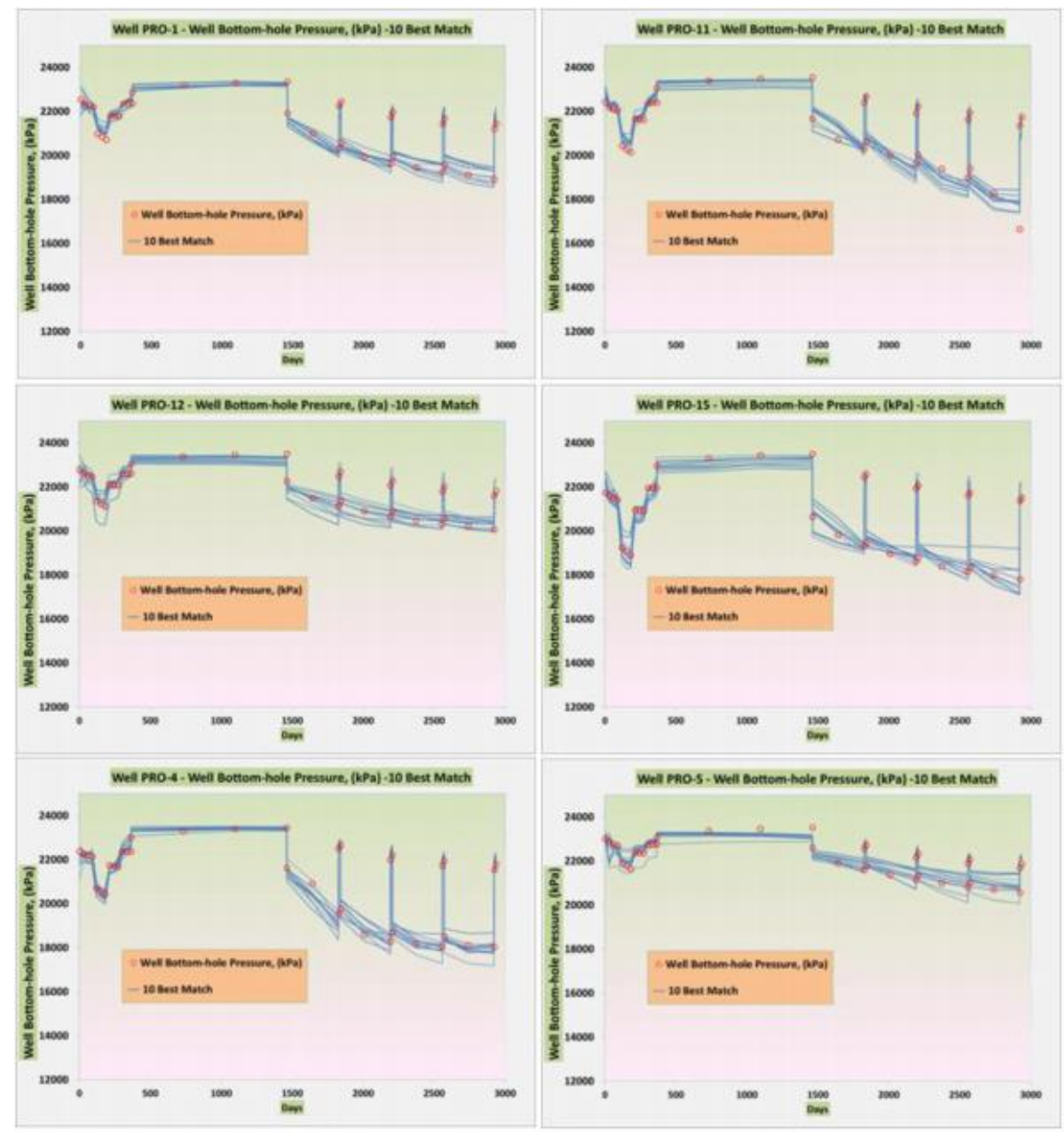

Figure 7 Well bottom hole history matching results of SRM compared against actual PUNQ-S3 values 


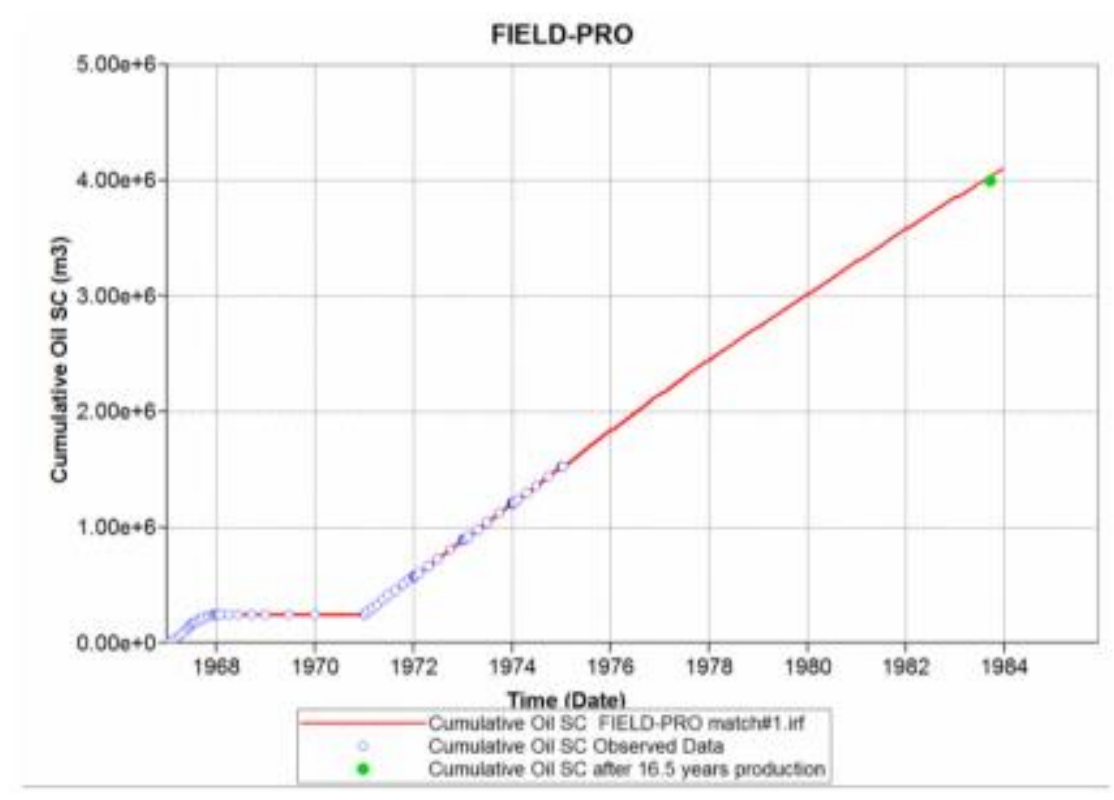

Figure 8 Simulator results with SRM identified properties vs actual field values for cumulative oil production along with forecast prediction vs actual for cumulative oil production

Synthetic data from a numerical reservoir simulation model has been previously used to confirm the capabilities of TDM, but for a different reservoir scenario and methodology in Al Hasan Mohamed Al Haifi's thesis. The reservoir was a partial dome with water injection wells and a surrounding aquifer. Twenty injection wells were brought online at the start of the simulation and continuously injected into the reservoir. Fifty-three producers were brought online in phases, however they were not recompleted in time nor did they have a water cut operational constraint. One Top Down Model was built using oil, gas, water, reservoir pressure, and water saturation data-driven models, the TDM was history matched for nineteen years. Once the history match was deemed satisfactory, the model was then used to forecast for two years immediately after the end of the history match. The forecasted results were compared with the results from the synthetic data, kept separate throughout this process. It was found that the predictions closely resembled the synthetic data and therefore the capabilities of Top Down Modeling was confirmed for this scenario (Haifi, 2019).

\section{Methodology Overview}

First a CMG model needed to be modified from the base model that was obtained from Josh Dietz, where an in depth description of the original model is provided in the next chapter. This is done by first changing several static parameters such as creating a new grid that changes the shape of the reservoir from a partial dome to one limb of an anticline. Dynamic variables were also changed, including starting both water and gas injection from the first day of production. These changes and more were performed to make the model reflect industry realizations. They are discussed in more detail in Chapter 8.

Once the above changes have been made to the CMG model, the model was tested to ensure it satisfied the above requirements and data was generated. After the final model and data set were developed, a presentation was created as documentation. Data needed for the Top-Down Model (TDM) was then created and formatted to separately input static and dynamic parameters. 
Thereafter, TDMs were developed, trained, calibrated, validated and blind tested. The amount of blind testing was increased until bad results were obtained and no efforts performed were able to achieve good results. This in turn found the "breaking point" of the models and dataset prepared which also deems the minimum amount of data required from this field to obtain good results.

\subsection{Original CMG Model}

The Original Thesis Model obtained was a 260 X 260 X 6 corner point grid. It was shaped in a partial dome as shown below Figure 9. Another viewpoint of the reservoir shape in Figure 10 illustrates that the layers of the model have varying thicknesses. These thicknesses as well as other properties were specified within the model using .msh maps outlined in more detail below. This model has a single porosity type and contains no faults nor fractures.

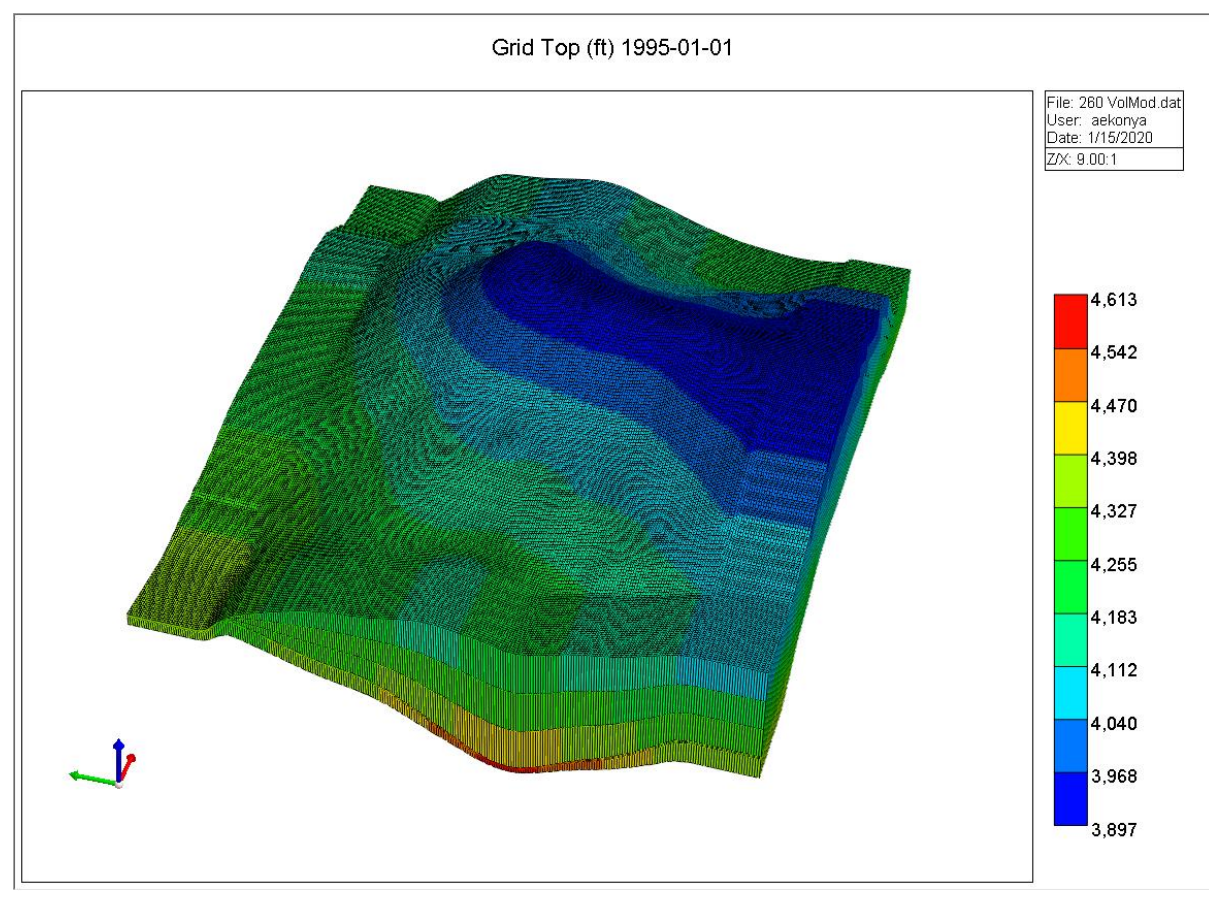

Figure 9 Reservoir shape of Original CMG Model 


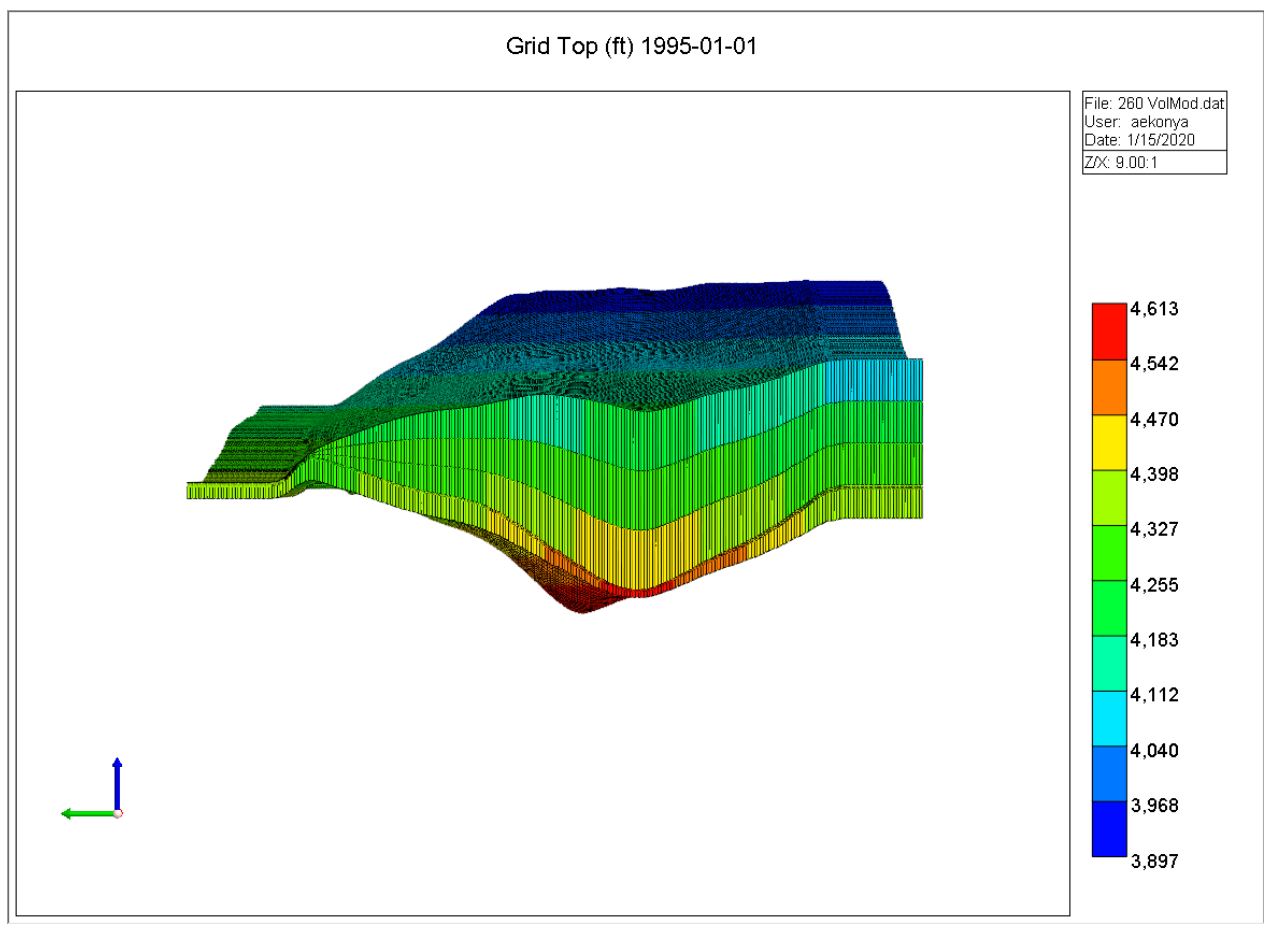

Figure 10 Side view of reservoir shape of Original CMG Model

A grid top .msh map file is specified only for Layer 1 of the model where the grid top depths of Layers 2 through 6 are calculated from the grid thickness .msh map files provided. An aerial view of the grid top .msh map for Layer 1 can be seen in Figure 11. A unique grid thickness .msh map file is specified for each layer, resulting in layers of varying grid thicknesses throughout the reservoir as seen below in Figure 12. 


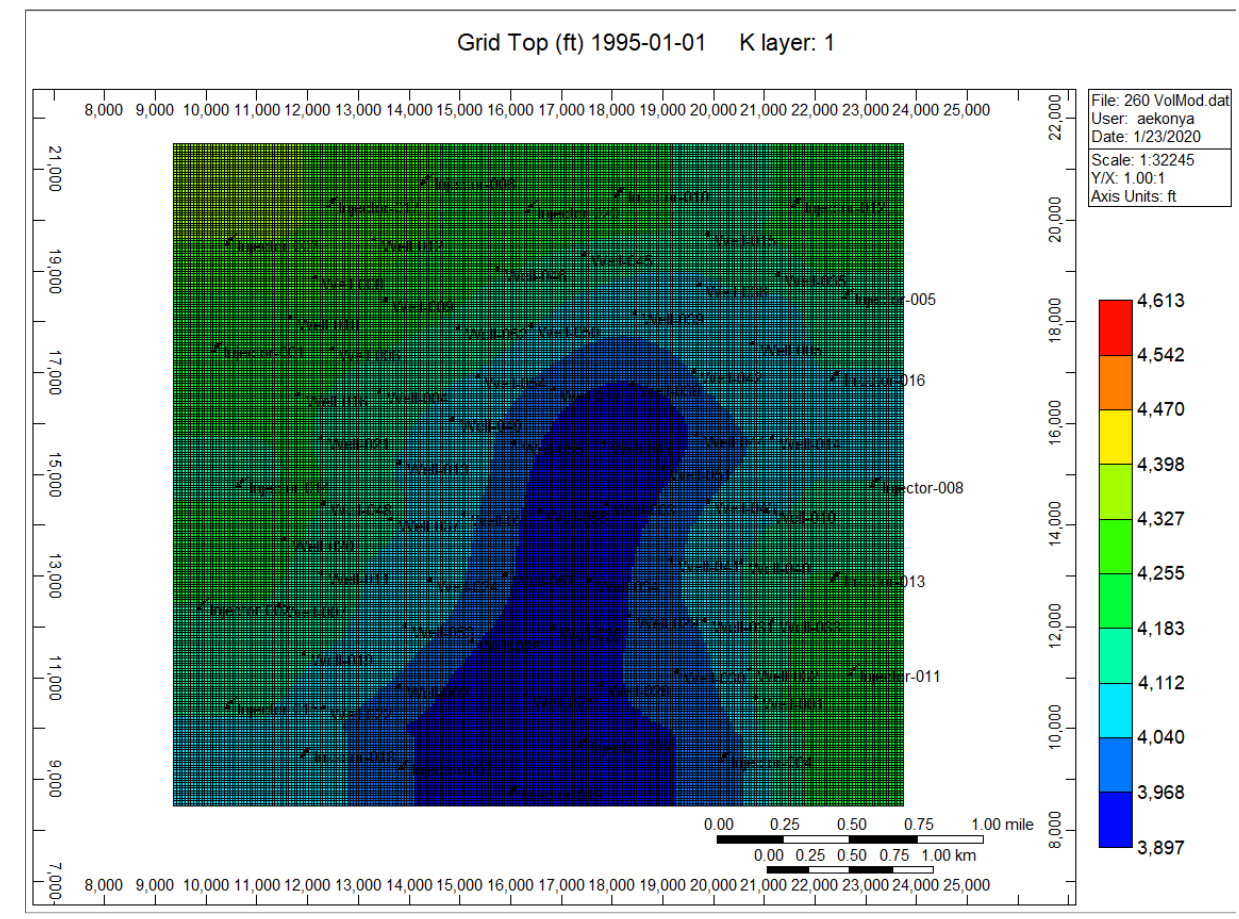

Figure 11 Grid top .msh map for Layer 1 of Original CMG Model

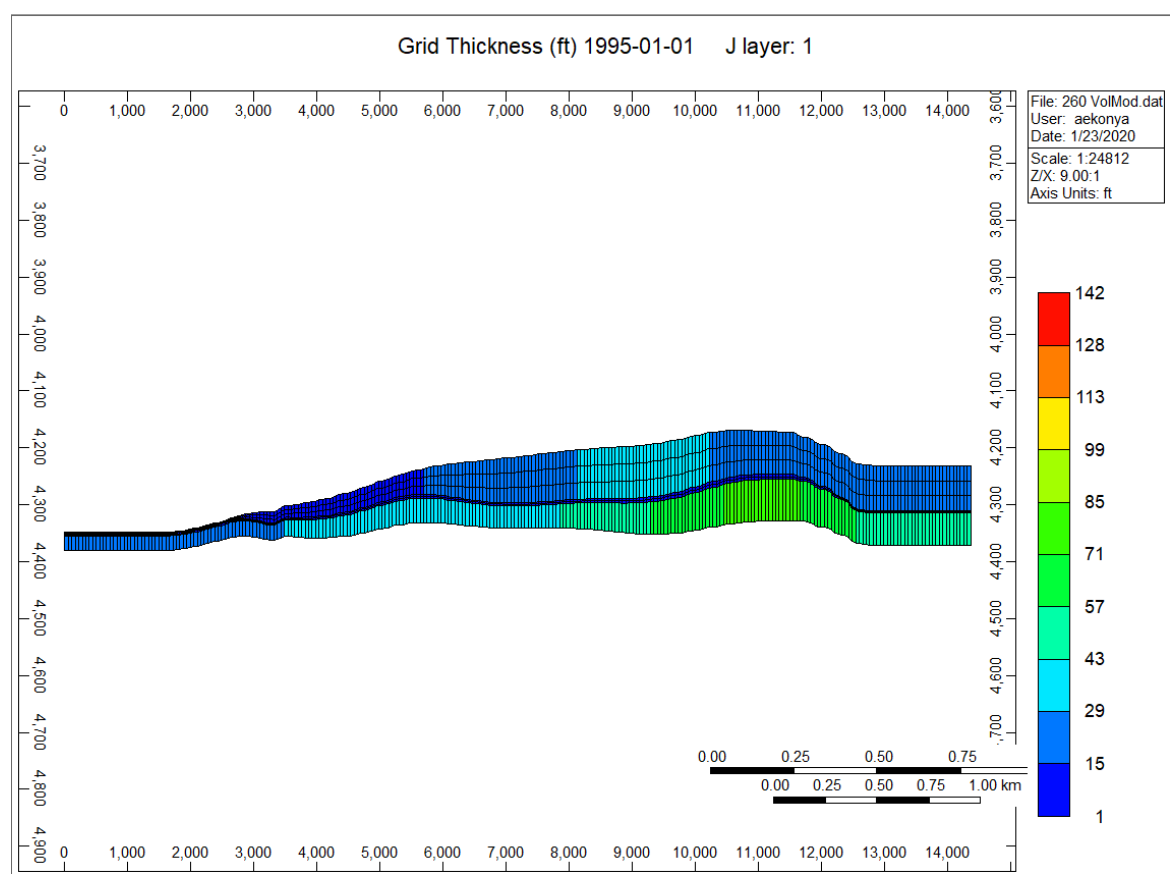

Figure 12 Grid thickness for each K layer shown for J layer 1 of the Original CMG Model

A different porosity .msh map file was also specified for all layers. The .msh porosity maps all contain a border around the center of the map where the values are constant and the center of the map's porosity values vary. This border is 31 cells in the $\mathrm{X}$ direction and 30 cells in the $\mathrm{Y}$ direction inward from the edge of the grid. The border was added to the porosity, permeability, and initial water saturation maps in order to give the model values representative of an aquifer around the reservoir. In Figure 13 below, the porosity map for the first $\mathrm{K}$ layer can be seen, where the border is easily identified and given a consistent 
porosity of 0.02 . While the border's location remains constant, its given porosity value changes with each layer. As seen in Figure 14, the border is in the same location, but the border's value is 0.04 for the entirety of Layer 2. This trend continues in that the border's porosity value increases by 0.02 as the K Layer increases yielding a porosity value of 0.12 for K Layer 6. Also depicted in Figure 13 and Figure 14, is the change in the center of the map where not only do the values vary throughout one layer's map, but the pattern of porosity values also changes from layer to layer. A depiction of each layer's porosity map is shown in Figure 13 through Figure 18.

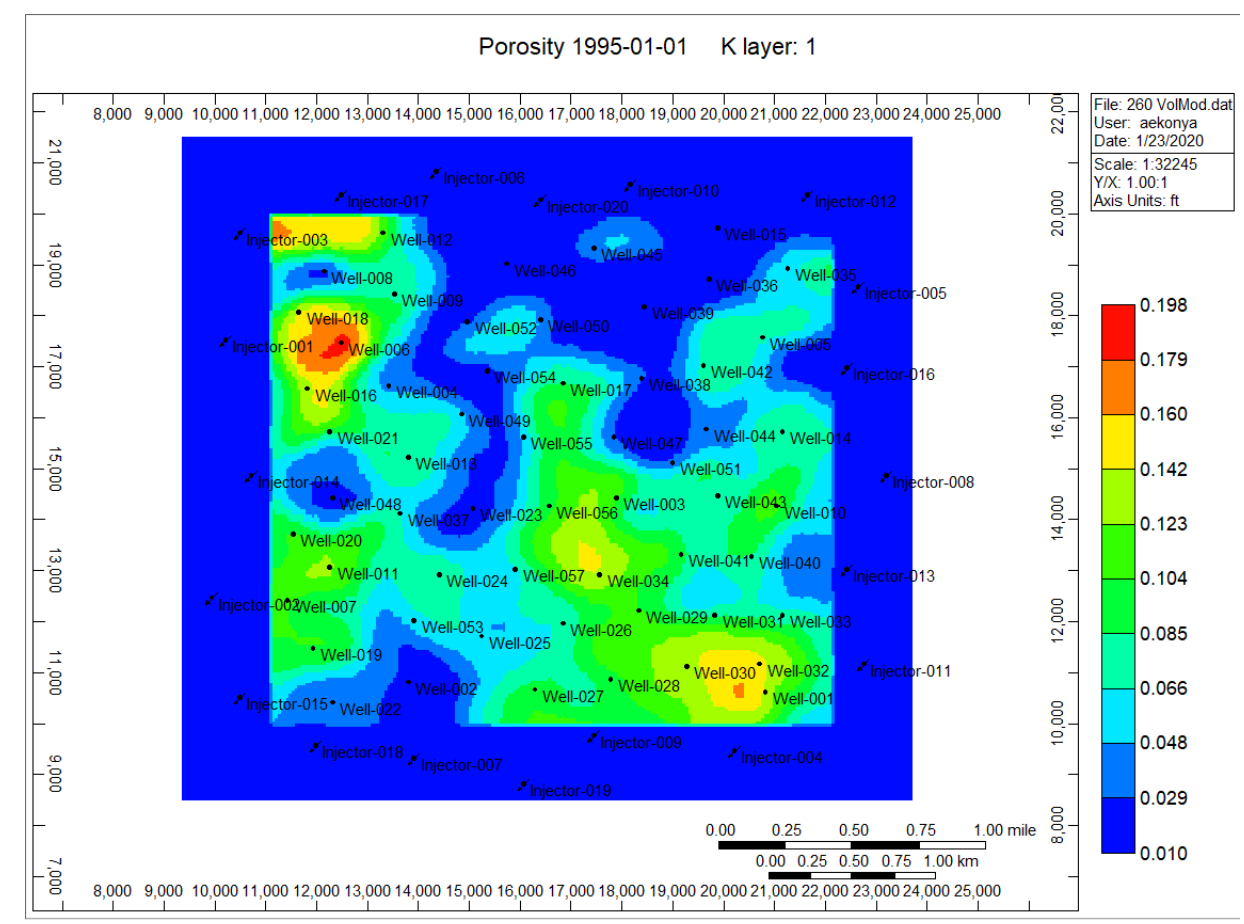

Figure 13 Porosity.msh map for K Layer 1 of the Original CMG Model 


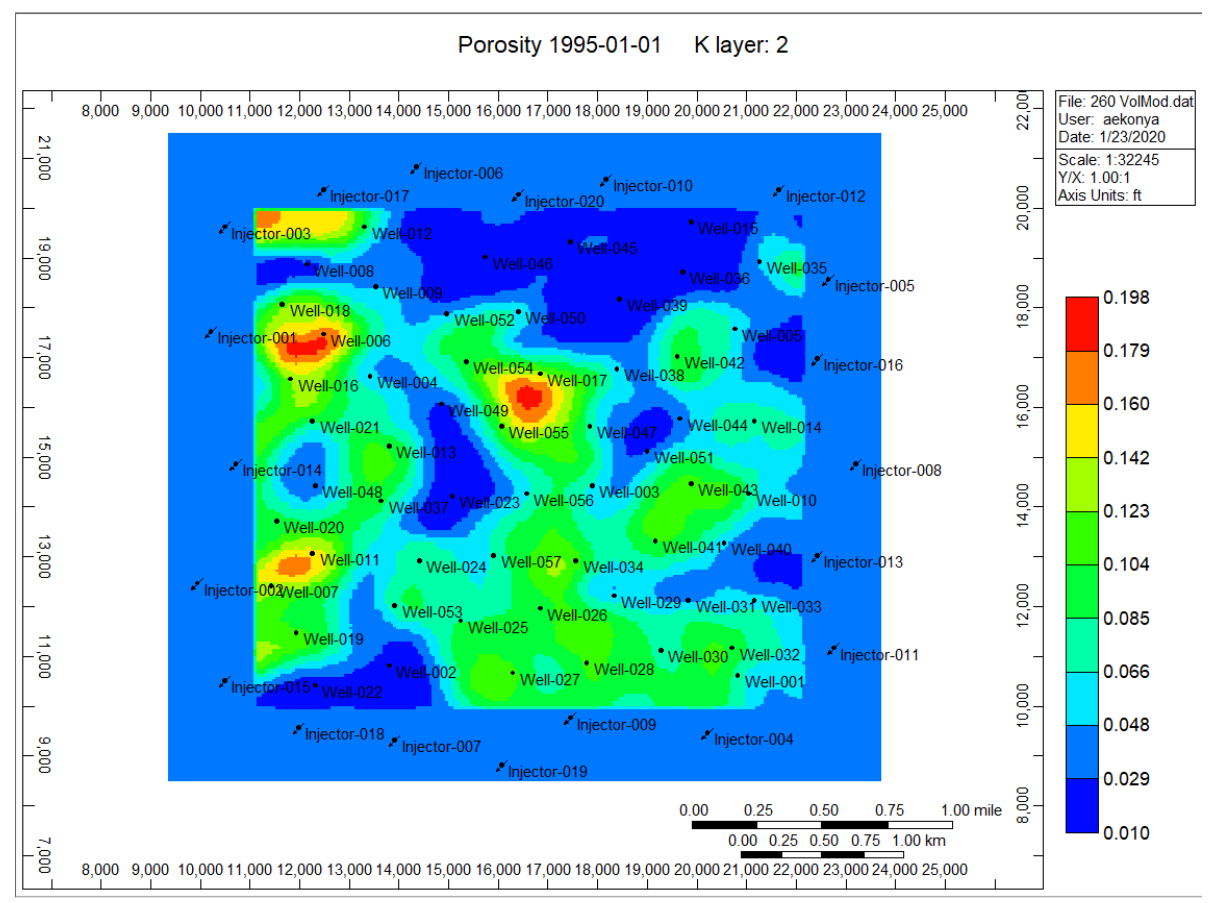

Figure 14 Porosity .msh map for K Layer 2 of the Original CMG Model

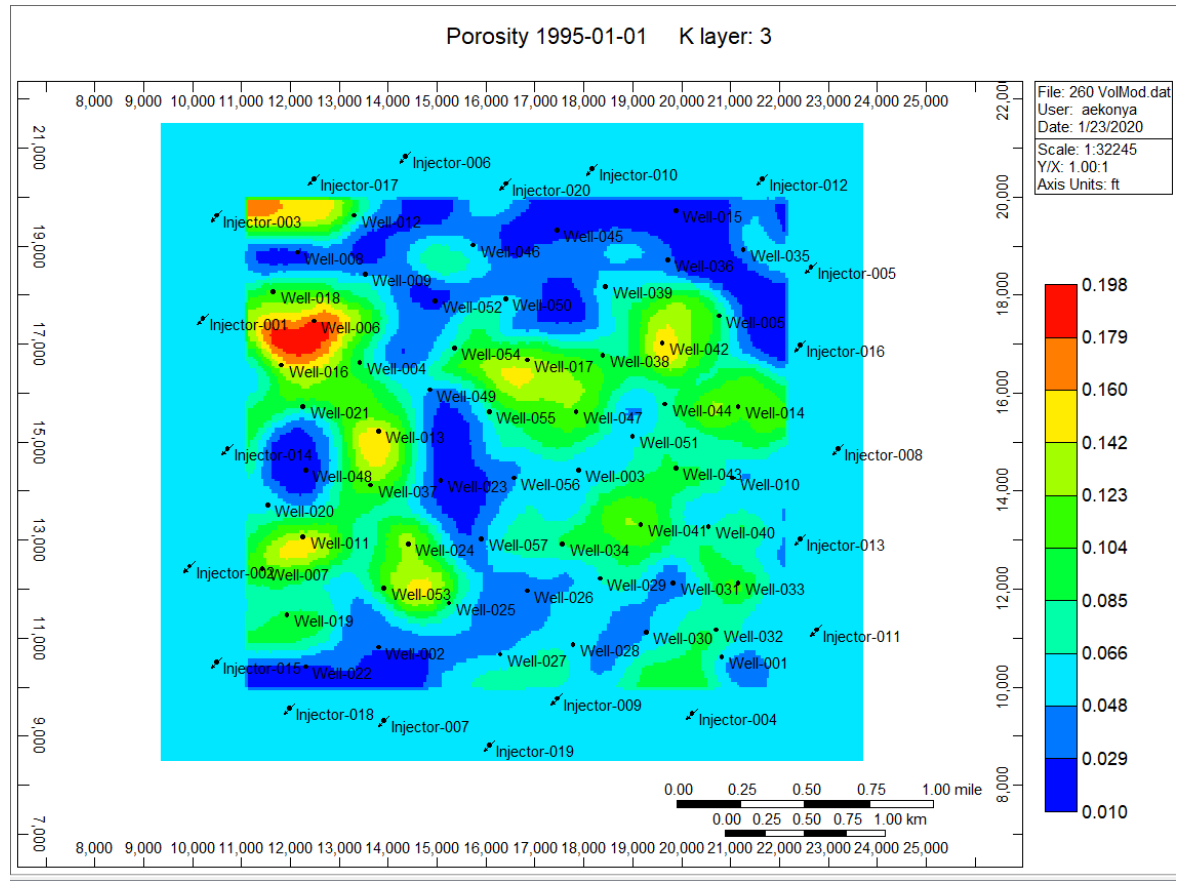

Figure 15 Porosity .msh map for K Layer 3 of the Original CMG Model 


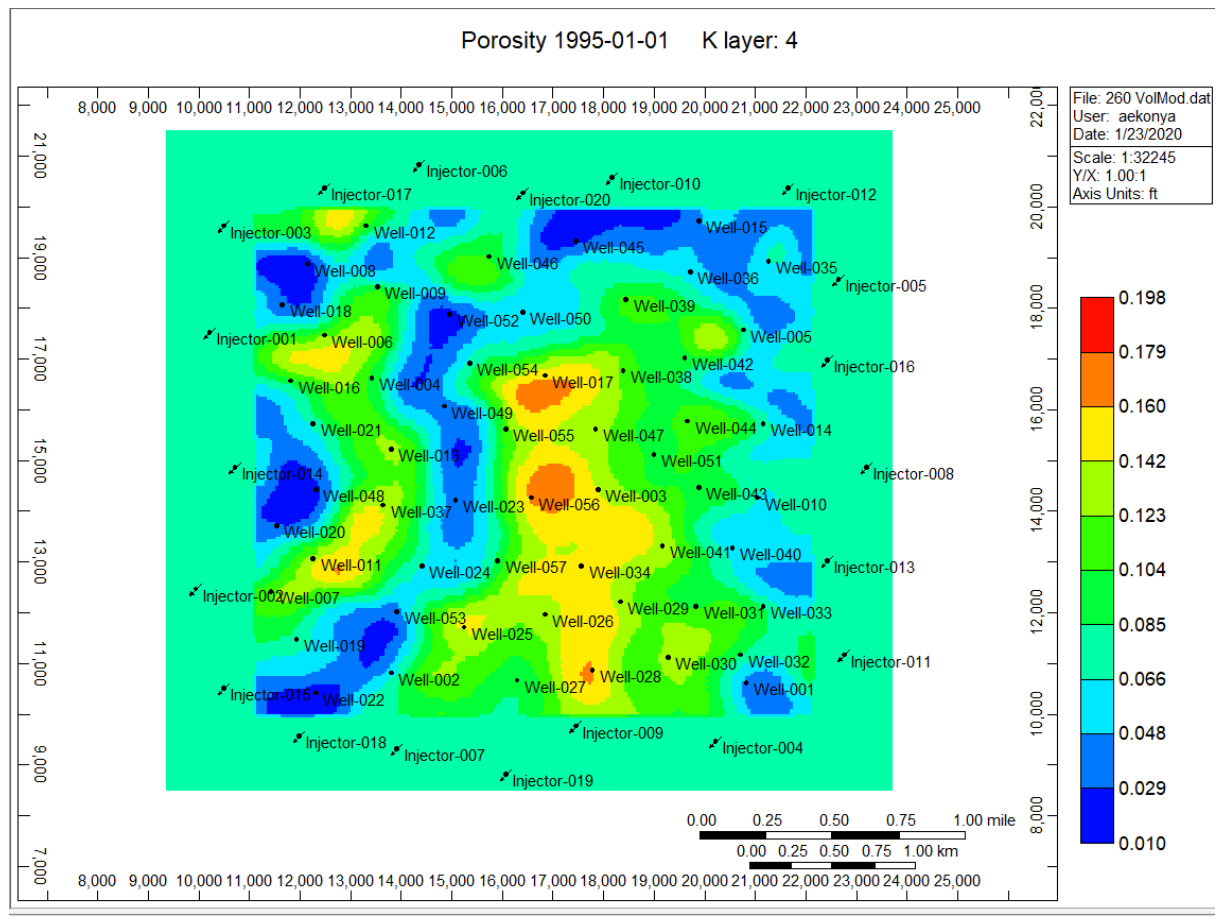

Figure 16 Porosity .msh map for K Layer 4 of the Original CMG Model

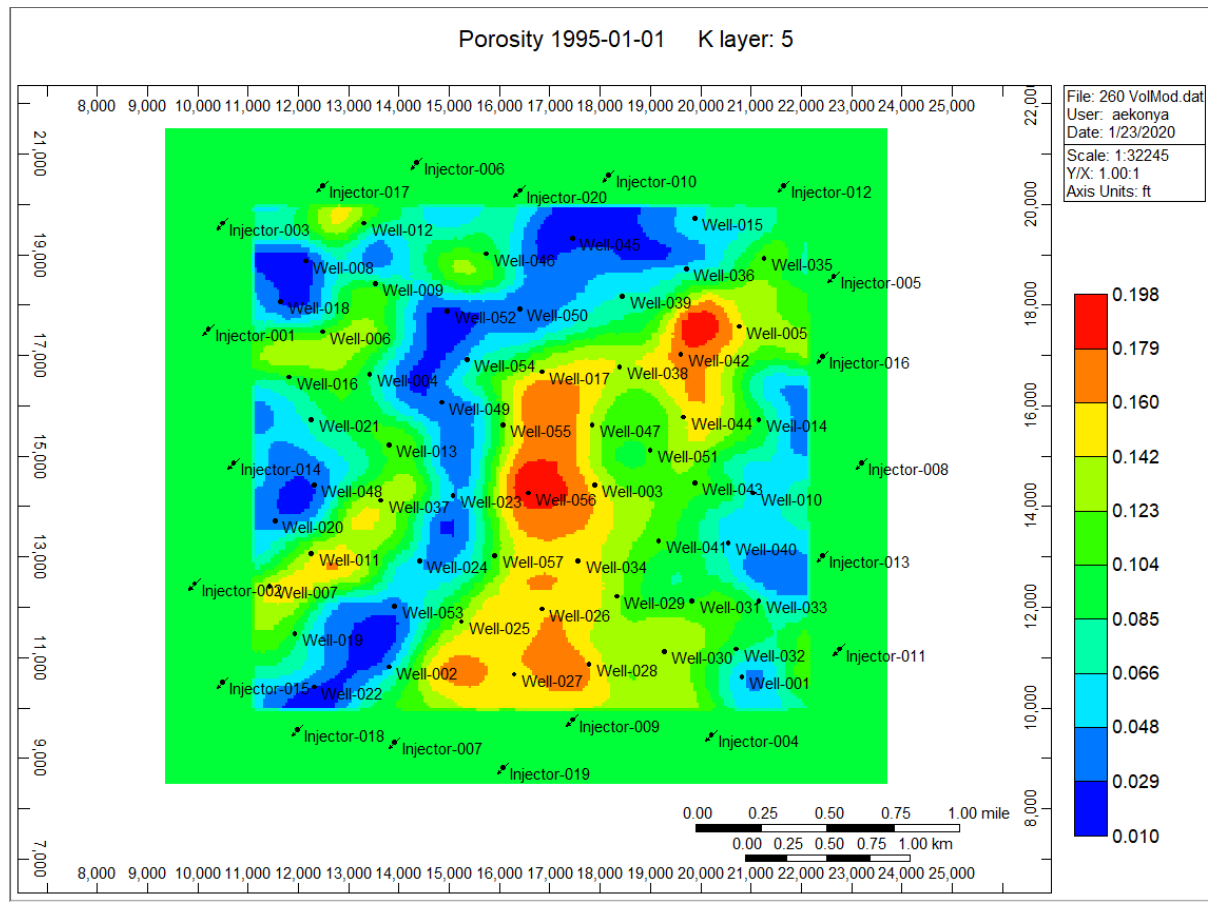

Figure 17 Porosity .msh map for K Layer 5 of the Original CMG Model 


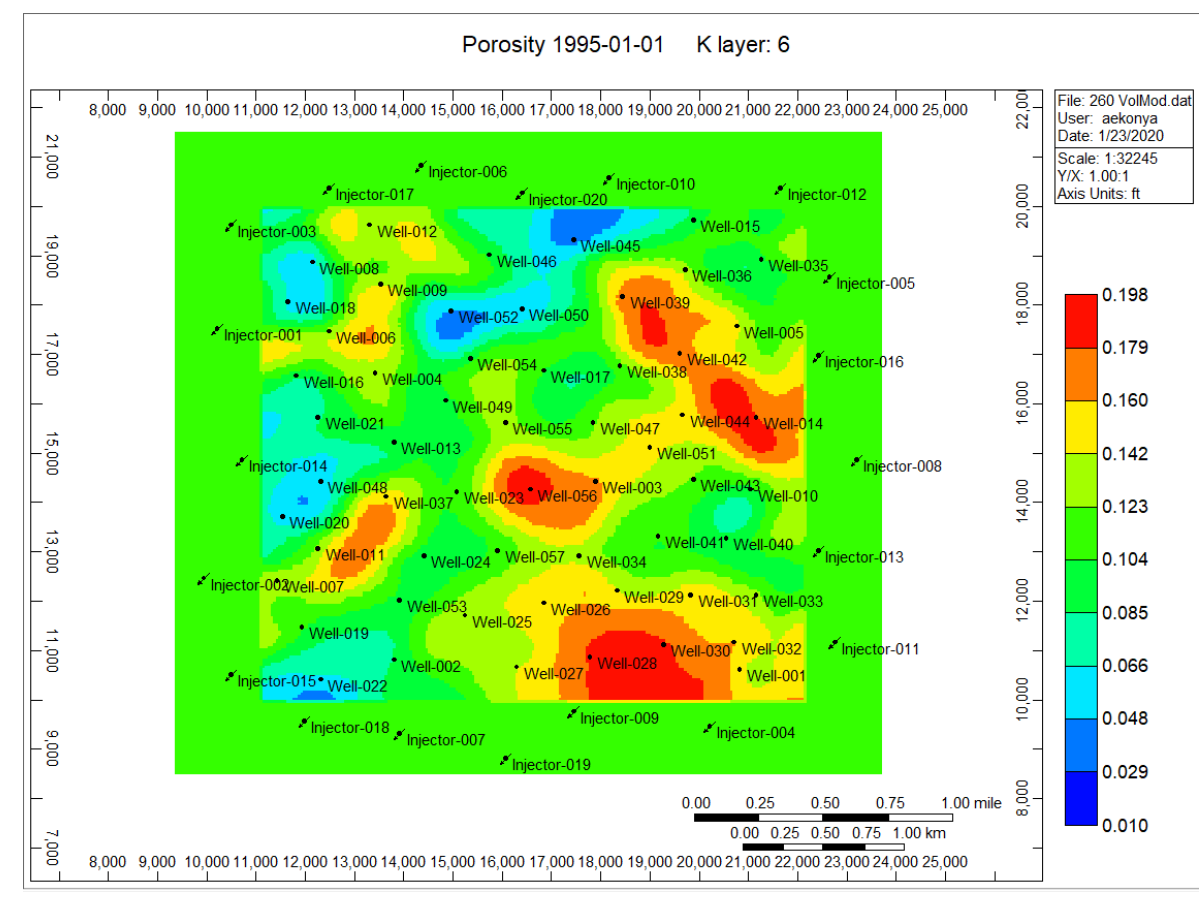

Figure 18 Porosity .msh map for K Layer 6 of the Original CMG model

Similar to porosity, permeability also has a unique permeability .msh map for each layer, all of which again have a border surrounding the center distribution of permeability values. The border has a consistent permeability value of $100 \mathrm{md}$ for all layers to simulate an aquifer. As seen in Figure 19Figure 19 , the left side of the map shows the cutoff of higher permeability values to the border's $100 \mathrm{md}$ value with only one row of cells as a gradient between the two. The .msh permeability maps were specified for Permeability I. Permeability J was set to be equal to Permeability I. Permeability K was specified to be 0.1 times Permeability I, therefore indicating that the formation is less permeable in the K direction. The 
varying distributions of permeability values for the center of the map for each layer can be seen below in Figure 19 through Figure 24.

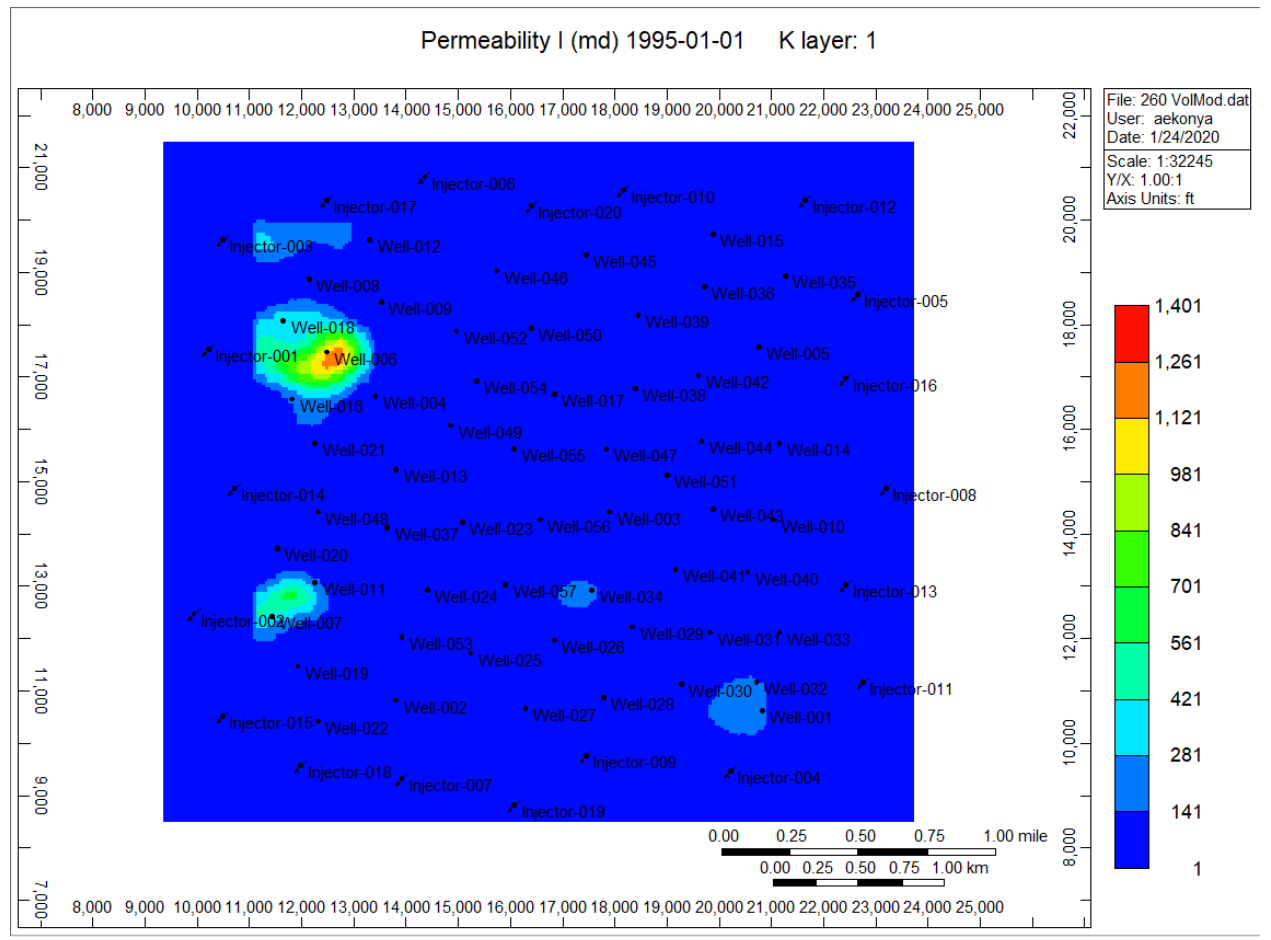

Figure 19 Permeability .msh map for K Layer 1 of the Original CMG model

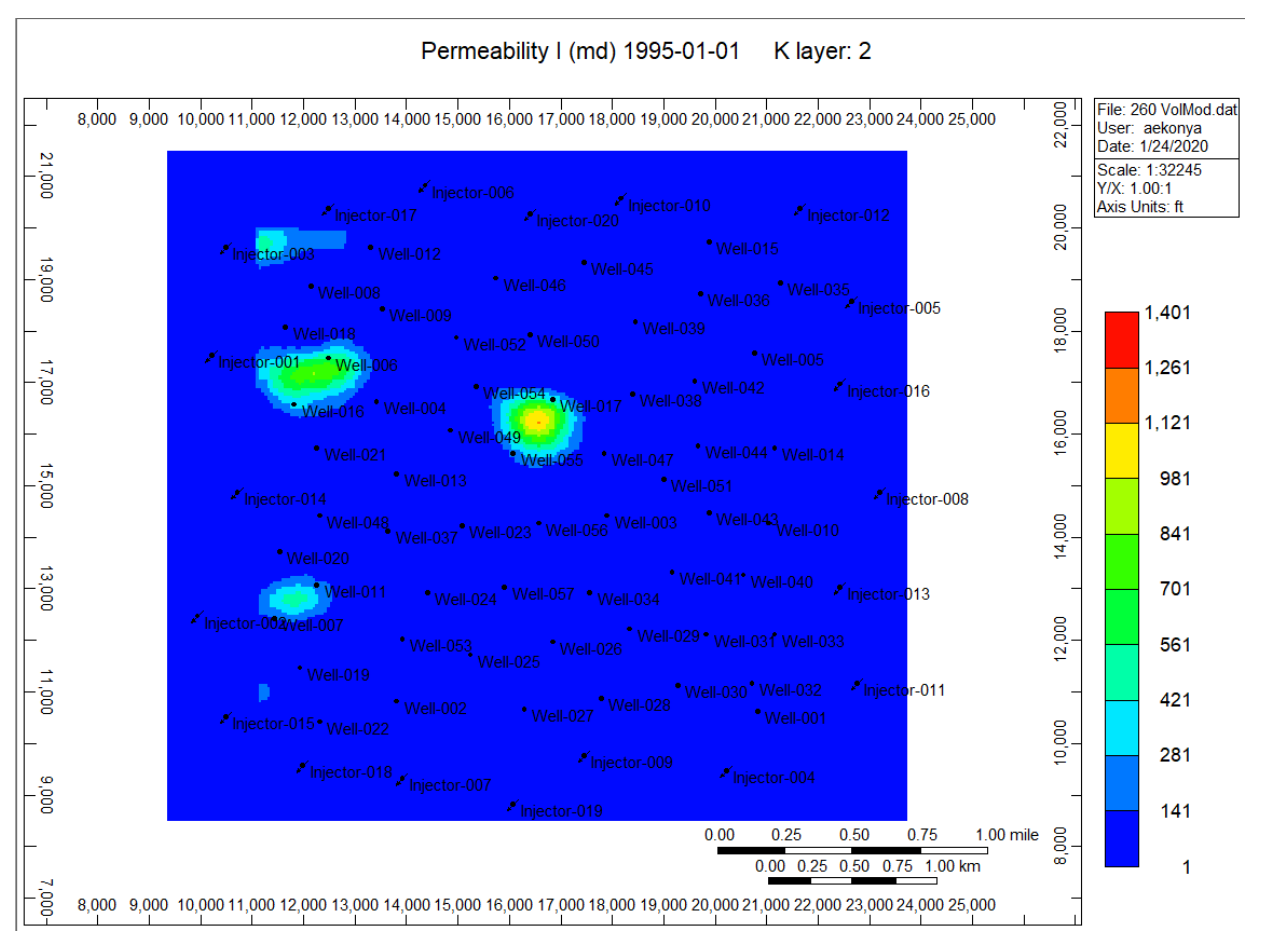

Figure 20 Permeability .msh map for K Layer 2 of the Original CMG model 


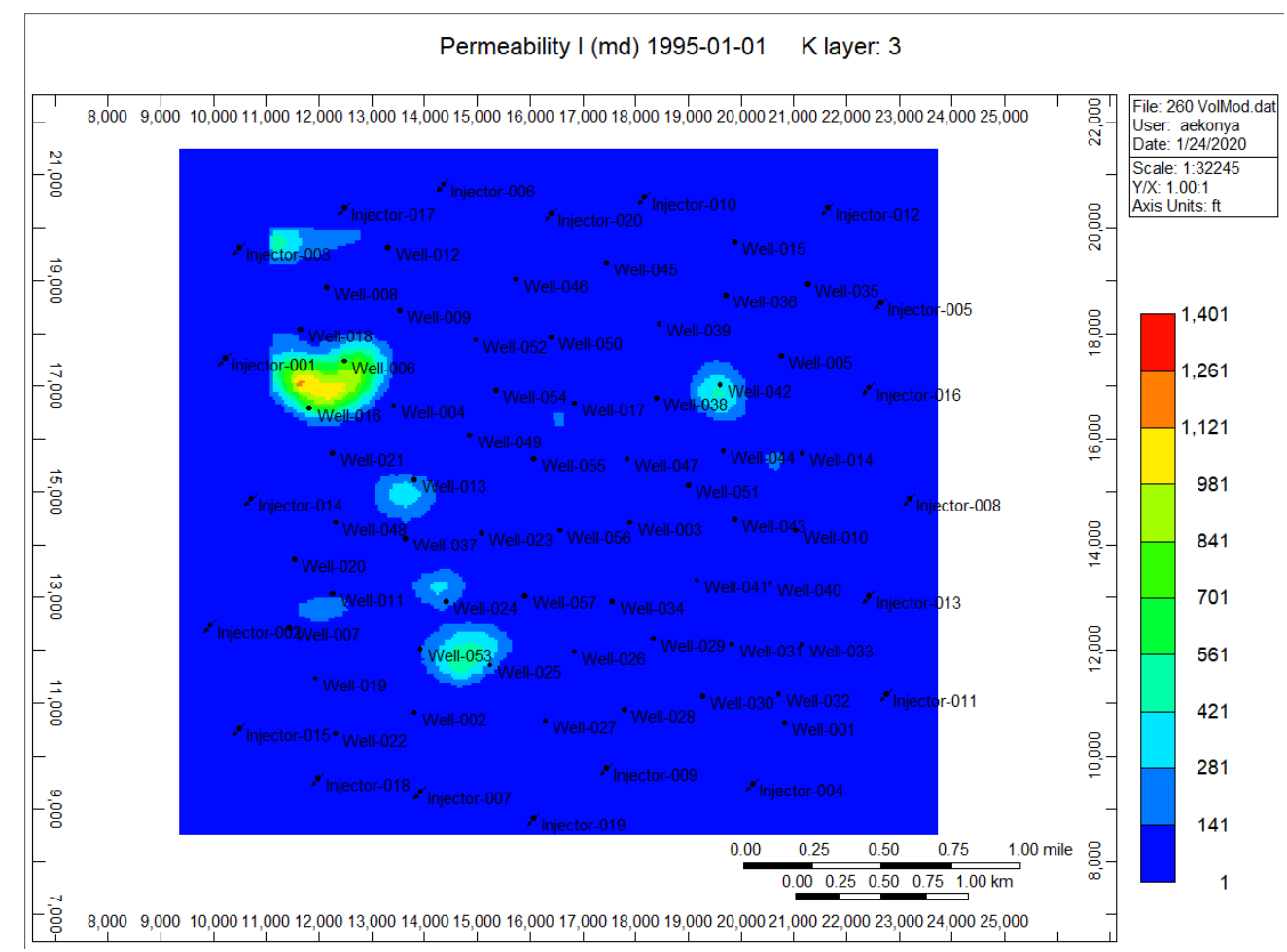

Figure 21 Permeability .msh map for K Layer 3 of the Original CMG model

Permeability I (md) 1995-01-01 K layer: 4

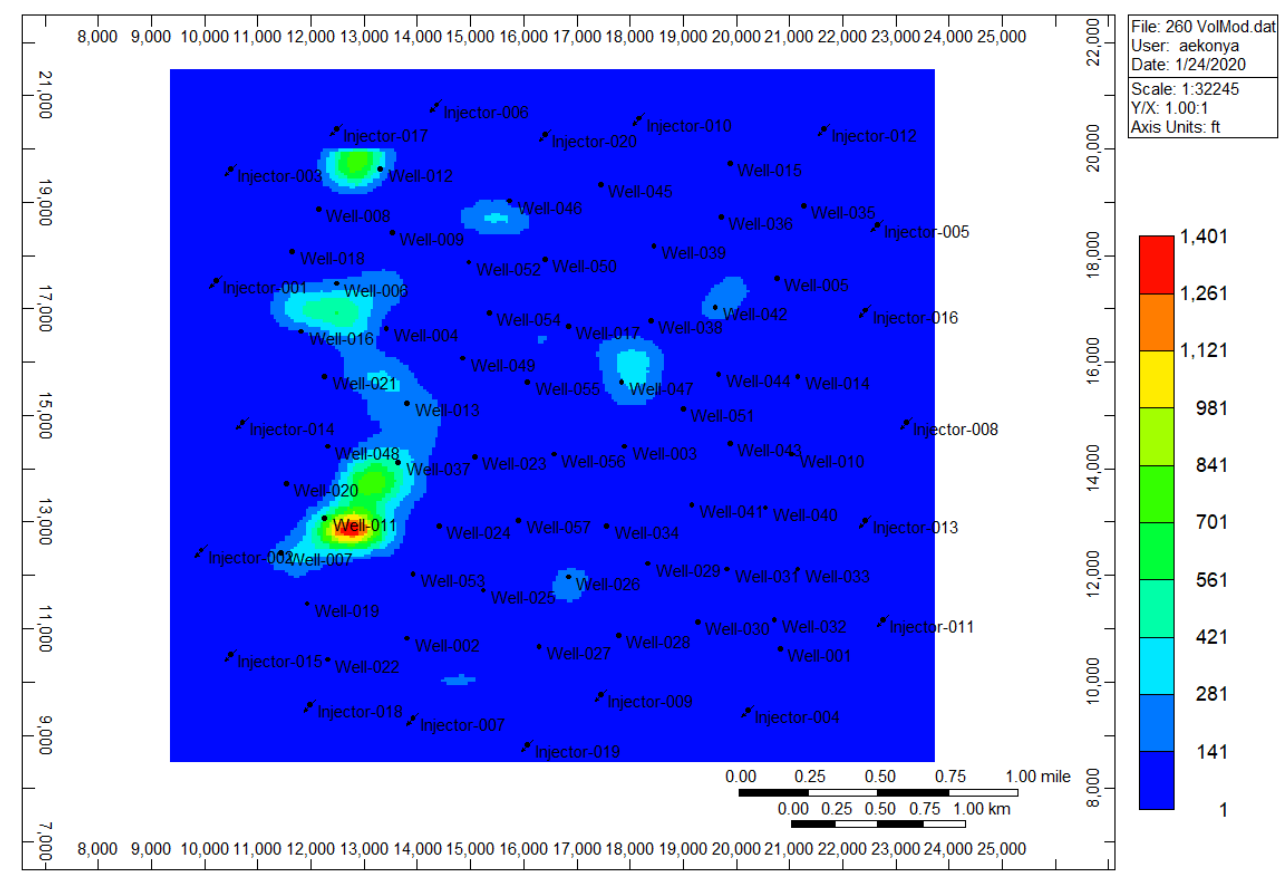

Figure 22 Permeability.msh map for K Layer 4 of the Original CMG model 


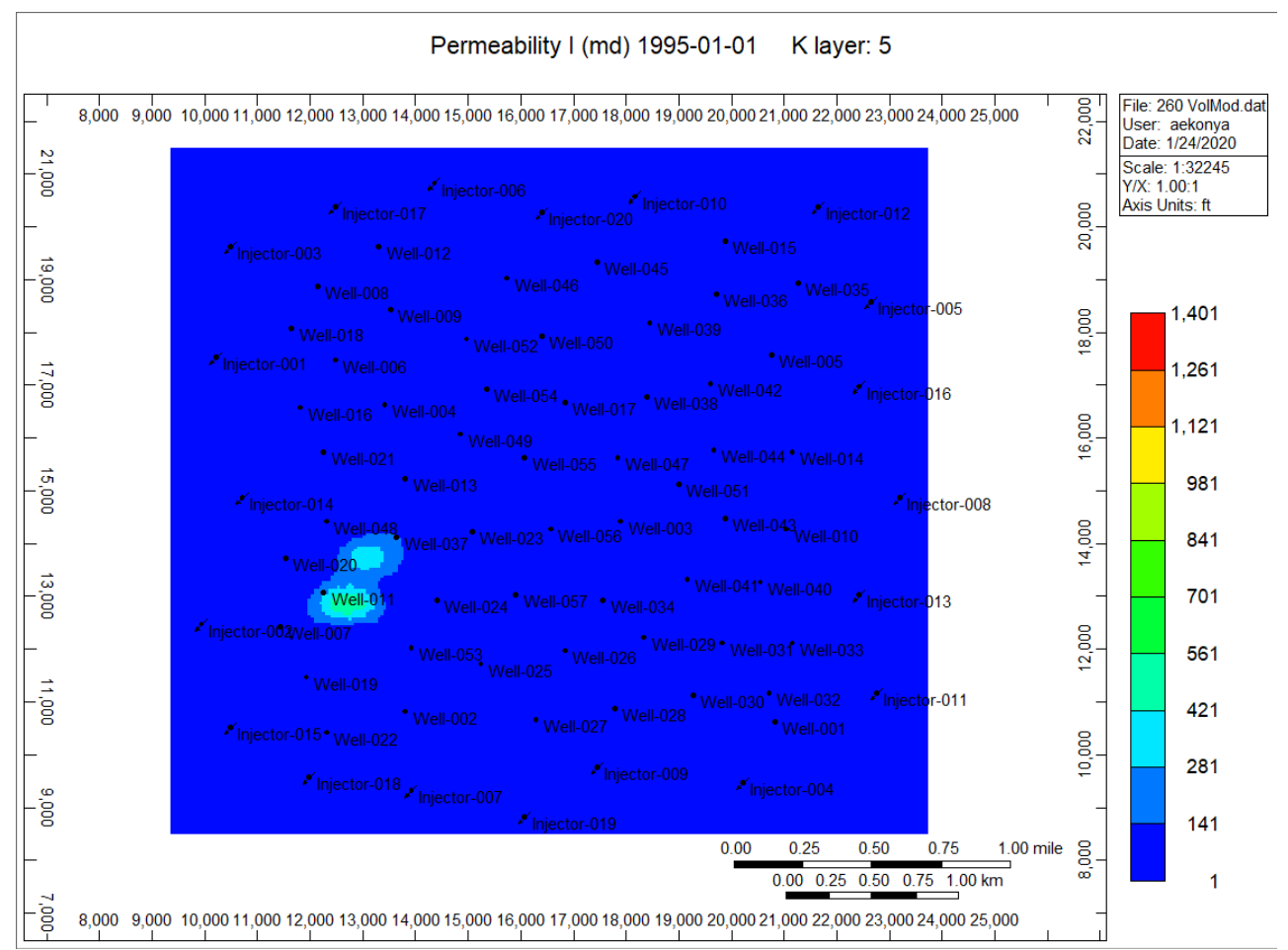

Figure 23 Permeability .msh map for K Layer 5 of the Original CMG model

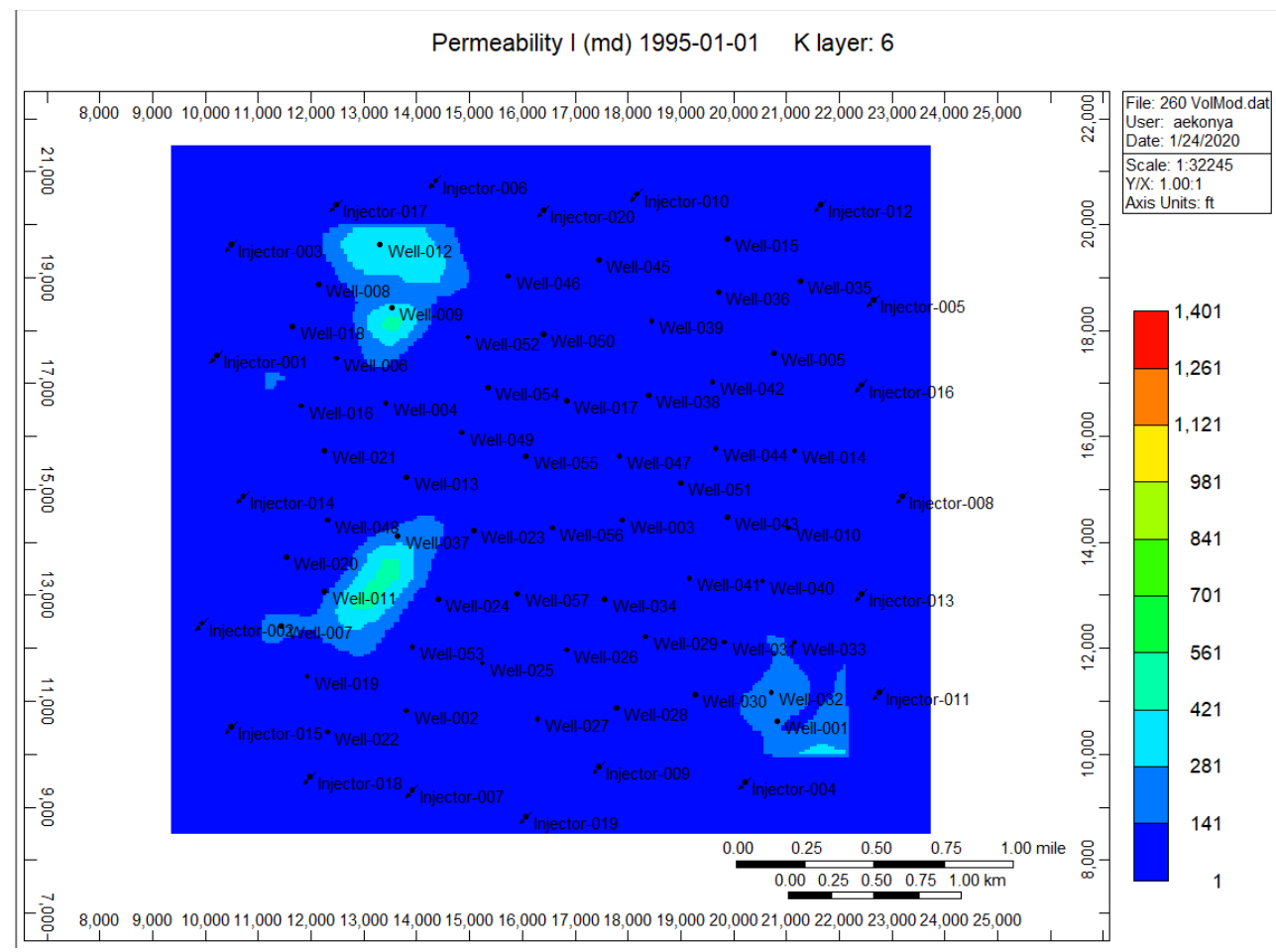

Figure 24 Permeability .msh map for K Layer 6 of the Original CMG model

The initial water saturation for the model was assigned by importing one .msh map for each layer, thus allowing all layers to have the same initial water saturation. Once again, the initial water saturation 
map has a border applied to it where the water saturation is 1.0 to simulate an aquifer and the water saturation values within the center vary. The initial water saturation map is shown in Figure 25 .

Initial Water Saturation 1995-01-01 K layer: 1

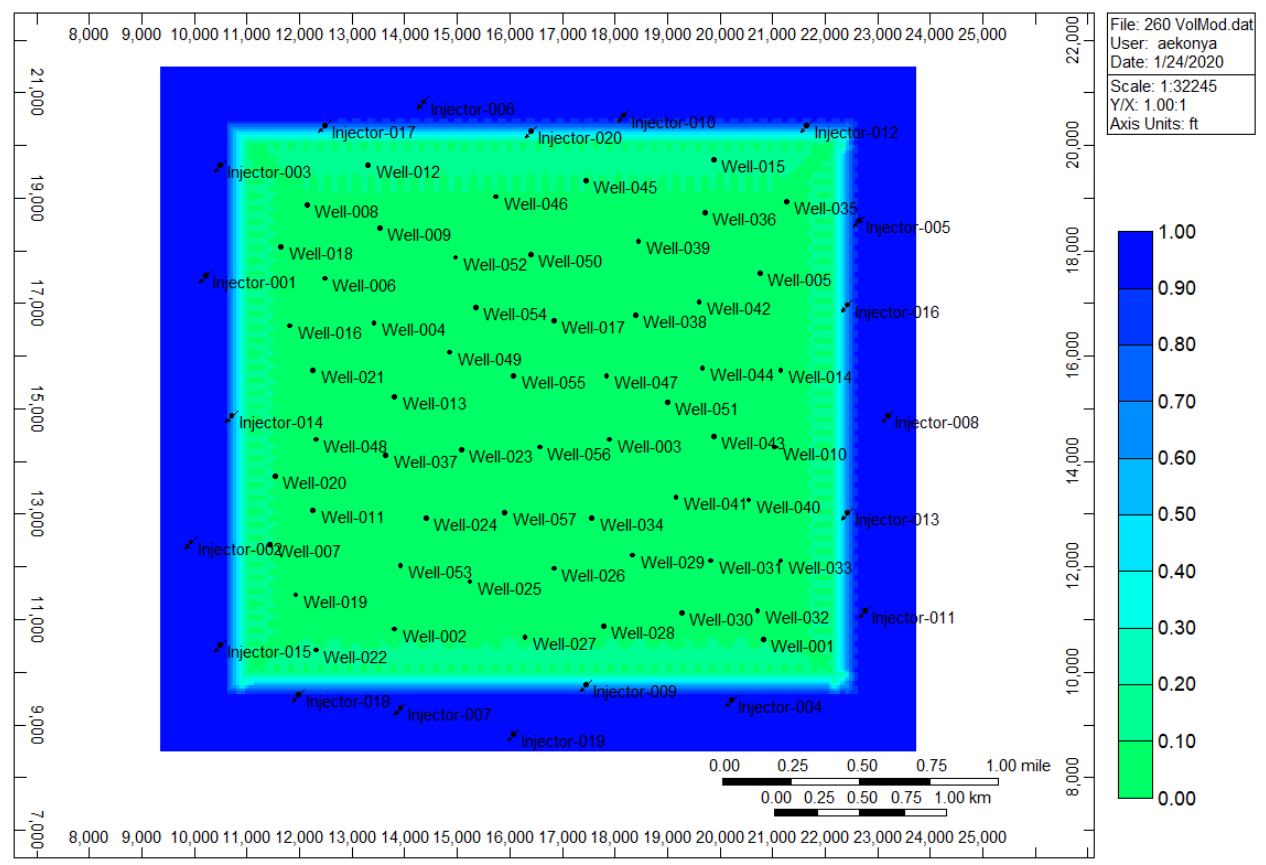

Figure 25 Initial water saturation .msh map for K Layers 1-6 of the Original CMG model

The Volume Modifier function (Volmod) is used to simulate a constant pressure boundary, an effect a surrounding aquifer would provide. This is done by multiplying the last row of grids next to each boundary on the four sides of the reservoir in all six layers by a factor of 257 . Because the multiplier is greater than 1, the last row of grids on each side can be interpreted as "associating volume external to the reservoir with a grid block" (Computer Modelling Group Ltd., 2017). In other words, the last row of cells on the four sides of the reservoir have a much larger volume than the rest of the grid because it is simulating the aquifer that extends beyond the grid. If only the border around each of the maps were used to simulate the aquifer alone, then the aquifer would be approximately $30 \mathrm{ft}$ in all directions which is not realistic. Theoretically, the grid, porosity, permeability, and initial water saturation maps could be altered to add 100+ grids on each side of the reservoir to more accurately model a realistic aquifer without the volume modifier function. This addition of hundreds of cells however, would significantly increase the run time of the model and is not an efficient solution. Therefore, in using both the border of cells to specify the aquifer's properties and the volume modifier to convey the extent of the aquifer, a realistic aquifer effect can be simulated while also reducing the run time of the simulation. Both an aerial view of the volume modifier in Layer 1 (which is the same for all layers) as well as a 3D view can be seen in Figure 26 and Figure 27 respectively. 


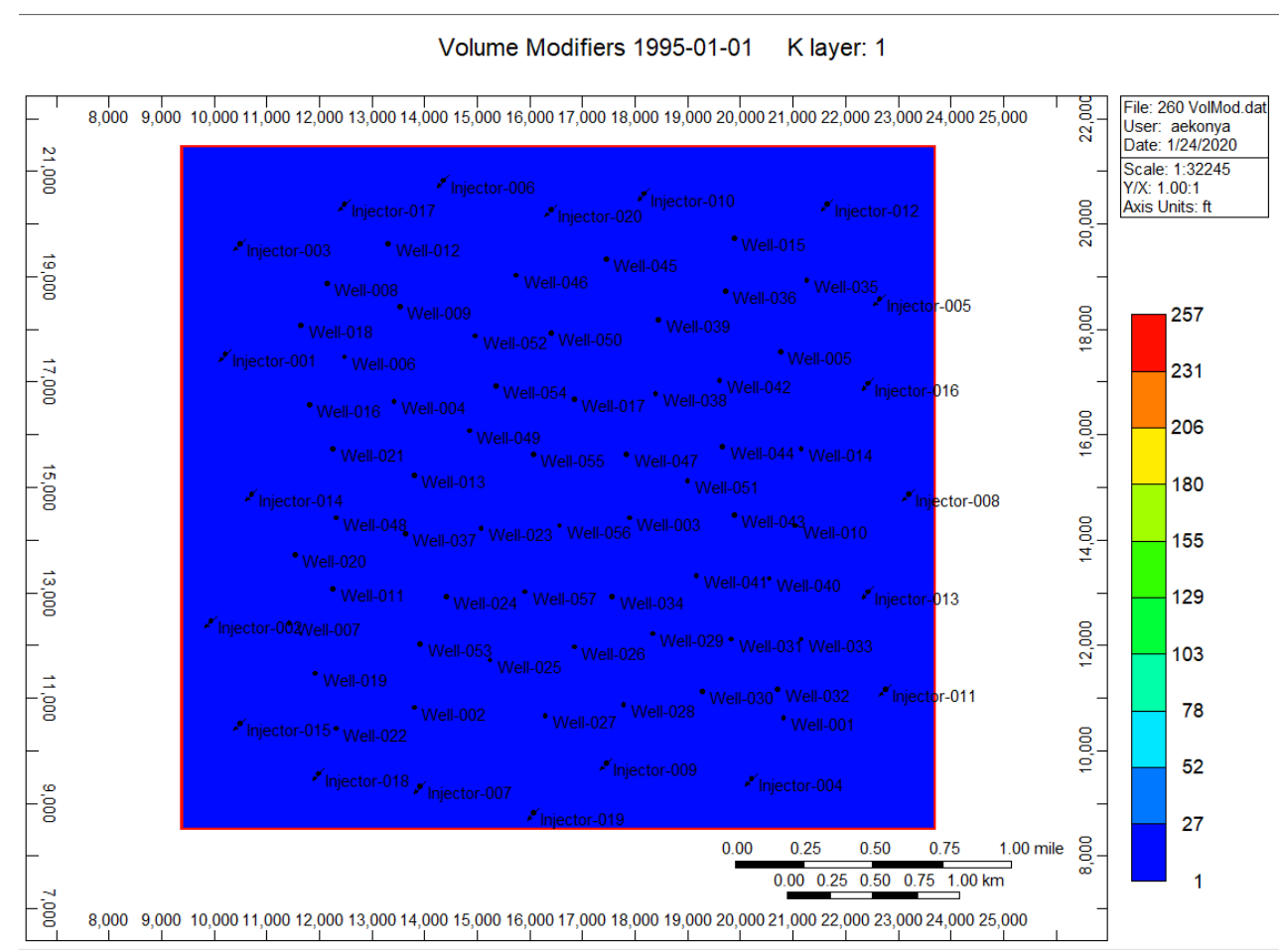

Figure 26 Volume modifier of 257 applied to the four sides of the reservoir in the Original CMG model

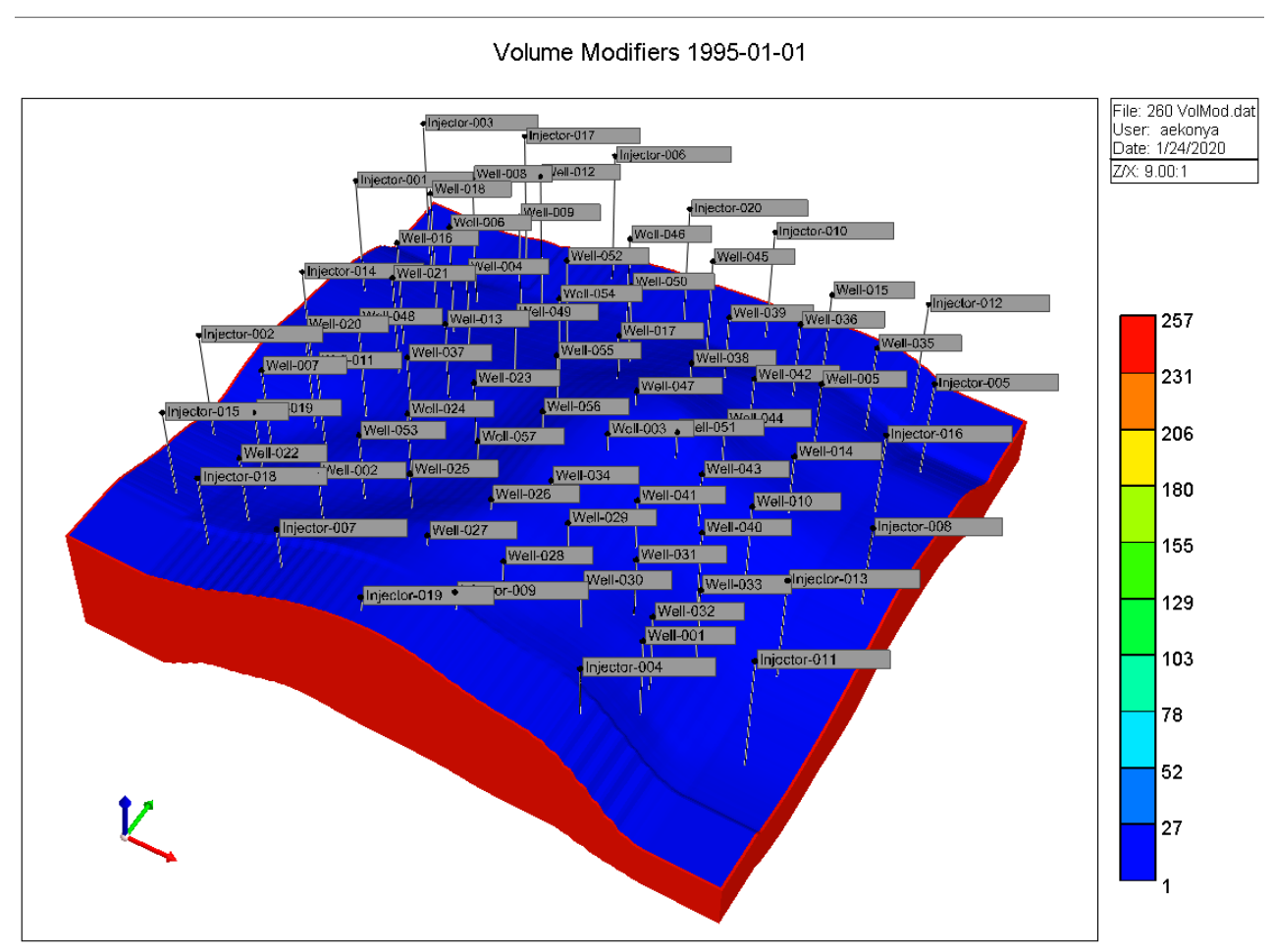

Figure $273 D$ view of the volume modifier function on the four sides of the reservoir in the Original CMG model

The rock compressibility value was assigned to be $2 \mathrm{E}-6$ 1/psi and its corresponding reference pressure was 3122 psi. The Black Oil model was chosen for the fluid model where a PVT Region was added. The oil and gas PVT table and differential liberation PVT table values are displayed below in 
Table 1 and Table 2 respectively for the added PVT Region denoted as PVT Region 1. General PVT properties such as reservoir temperature were also defined and shown in Table 3.

Table 1 Rock Type 1 oil and gas PVT table

\begin{tabular}{|l|l|l|l|l|l|l|}
\hline P (psi) & Rs (ft3/bbl) & Bo & $\begin{array}{l}\text { Eg } \\
\text { (ft3/bbl) }\end{array}$ & $\begin{array}{l}\text { Viso } \\
\text { (cp) }\end{array}$ & Visg (cp) & Co (1/psi) \\
\hline 14.696 & 5.21489 & 1.03174 & 4.97392 & 1.55343 & 0.0119415 & $3.00 \mathrm{E}-05$ \\
\hline 280.383 & 60.168 & 1.05194 & 98.176 & 1.19556 & 0.0122199 & $3.00 \mathrm{E}-05$ \\
\hline 546.07 & 127.793 & 1.07808 & 197.79 & 0.960137 & 0.0126381 & $3.00 \mathrm{E}-05$ \\
\hline 811.757 & 202.423 & 1.10829 & 303.856 & 0.805536 & 0.0131713 & $3.00 \mathrm{E}-05$ \\
\hline 1077.44 & 282.145 & 1.14188 & 415.917 & 0.697465 & 0.0138201 & $3.00 \mathrm{E}-05$ \\
\hline 1343.13 & 365.936 & 1.17846 & 532.791 & 0.617797 & 0.0145869 & $3.00 \mathrm{E}-05$ \\
\hline 1608.82 & 453.146 & 1.21774 & 652.482 & 0.55658 & 0.0154684 & $2.54 \mathrm{E}-05$ \\
\hline 1874.5 & 543.321 & 1.2595 & 772.351 & 0.507992 & 0.0164531 & $2.09 \mathrm{E}-05$ \\
\hline 2140.19 & 636.123 & 1.30358 & 889.605 & 0.468422 & 0.0175212 & $1.76 \mathrm{E}-05$ \\
\hline 2405.88 & 731.293 & 1.34984 & 1001.85 & 0.435518 & 0.0186479 & $1.51 \mathrm{E}-05$ \\
\hline 2671.57 & 828.62 & 1.39815 & 1107.44 & 0.407683 & 0.019808 & $1.32 \mathrm{E}-05$ \\
\hline 2937.25 & 927.934 & 1.44842 & 1205.53 & 0.383797 & 0.0209794 & $1.16 \mathrm{E}-05$ \\
\hline 3202.94 & 1029.09 & 1.50056 & 1295.92 & 0.363048 & 0.0221449 & $1.04 \mathrm{E}-05$ \\
\hline 3468.63 & 1131.97 & 1.55448 & 1378.83 & 0.344837 & 0.0232914 & $9.39 \mathrm{E}-06$ \\
\hline 3734.31 & 1236.46 & 1.61013 & 1454.73 & 0.328707 & 0.0244104 & $8.53 \mathrm{E}-06$ \\
\hline 4000 & 1342.48 & 1.66743 & 1524.2 & 0.314309 & 0.0254964 & $7.80 \mathrm{E}-06$ \\
\hline 4200 & 1423.25 & 1.71162 & 1572.65 & 0.304444 & 0.0262904 & $7.32 \mathrm{E}-06$ \\
\hline 4400 & 1504.8 & 1.7567 & 1618.07 & 0.295307 & 0.0270635 & $6.90 \mathrm{E}-06$ \\
\hline 4600 & 1587.11 & 1.80264 & 1660.72 & 0.286817 & 0.0278157 & $6.51 \mathrm{E}-06$ \\
\hline 4800 & 1670.15 & 1.84942 & 1700.84 & 0.278904 & 0.0285473 & $6.16 \mathrm{E}-06$ \\
\hline 5000 & 1753.9 & 1.89703 & 1738.65 & 0.271509 & 0.0292586 & $5.84 \mathrm{E}-06$ \\
\hline
\end{tabular}

Table 2 Rock Type 1 differential liberation PVT table

\begin{tabular}{|l|l|l|l|l|l|l|}
\hline $\mathbf{P}(\mathbf{p s i})$ & $\begin{array}{l}\text { Rsd } \\
\mathbf{( f t 3 / b b l )}\end{array}$ & Bod & Eg (ft3/bbl) & Viso (cp) & Visg (cp) & $\begin{array}{l}\text { Co } \\
(\mathbf{1} / \mathbf{p s i})\end{array}$ \\
\hline 14.696 & 5.21489 & 1.03174 & 4.97392 & 1.55343 & 0.0119415 & $3.00 \mathrm{E}-05$ \\
\hline 280.383 & 60.168 & 1.05194 & 98.176 & 1.19556 & 0.0122199 & $3.00 \mathrm{E}-05$ \\
\hline 546.07 & 127.793 & 1.07808 & 197.79 & 0.960137 & 0.0126381 & $3.00 \mathrm{E}-05$ \\
\hline 811.757 & 202.423 & 1.10829 & 303.856 & 0.805536 & 0.0131713 & $3.00 \mathrm{E}-05$ \\
\hline 1077.44 & 282.145 & 1.14188 & 415.917 & 0.697465 & 0.0138201 & $3.00 \mathrm{E}-05$ \\
\hline 1343.13 & 365.936 & 1.17846 & 532.791 & 0.617797 & 0.0145869 & $3.00 \mathrm{E}-05$ \\
\hline 1608.82 & 453.146 & 1.21774 & 652.482 & 0.55658 & 0.0154684 & $2.54 \mathrm{E}-05$ \\
\hline 1874.5 & 543.321 & 1.2595 & 772.351 & 0.507992 & 0.0164531 & $2.09 \mathrm{E}-05$ \\
\hline 2140.19 & 636.123 & 1.30358 & 889.605 & 0.468422 & 0.0175212 & $1.76 \mathrm{E}-05$ \\
\hline 2405.88 & 731.293 & 1.34984 & 1001.85 & 0.435518 & 0.0186479 & $1.51 \mathrm{E}-05$ \\
\hline 2671.57 & 828.62 & 1.39815 & 1107.44 & 0.407683 & 0.019808 & $1.32 \mathrm{E}-05$ \\
\hline
\end{tabular}




\begin{tabular}{|l|l|l|l|l|l|l|}
\hline 2937.25 & 927.934 & 1.44842 & 1205.53 & 0.383797 & 0.0209794 & $1.16 \mathrm{E}-05$ \\
\hline 3202.94 & 1029.09 & 1.50056 & 1295.92 & 0.363048 & 0.0221449 & $1.04 \mathrm{E}-05$ \\
\hline 3468.63 & 1131.97 & 1.55448 & 1378.83 & 0.344837 & 0.0232914 & $9.39 \mathrm{E}-06$ \\
\hline 3734.31 & 1236.46 & 1.61013 & 1454.73 & 0.328707 & 0.0244104 & $8.53 \mathrm{E}-06$ \\
\hline 4000 & 1342.48 & 1.66743 & 1524.2 & 0.314309 & 0.0254964 & $7.80 \mathrm{E}-06$ \\
\hline 4200 & 1423.25 & 1.71162 & 1572.65 & 0.304444 & 0.0262904 & $7.32 \mathrm{E}-06$ \\
\hline 4400 & 1504.8 & 1.7567 & 1618.07 & 0.295307 & 0.0270635 & $6.90 \mathrm{E}-06$ \\
\hline 4600 & 1587.11 & 1.80264 & 1660.72 & 0.286817 & 0.0278157 & $6.51 \mathrm{E}-06$ \\
\hline 4800 & 1670.15 & 1.84942 & 1700.84 & 0.278904 & 0.0285473 & $6.16 \mathrm{E}-06$ \\
\hline 5000 & 1753.9 & 1.89703 & 1738.65 & 0.271509 & 0.0292586 & $5.84 \mathrm{E}-06$ \\
\hline
\end{tabular}

Table 3 General properties for PVT Region 1 of the Original CMG model

\begin{tabular}{|l|l|}
\hline Description of General PVT Property & Value \\
\hline Reservoir Temperature (TRES) & $130 \mathrm{~F}$ \\
\hline Oil density (Stock tank oil density) & $50.863 \mathrm{lb} / \mathrm{ft} 3$ \\
\hline Gas density/gravity (Gas gravity (Air=1)) & 0.65 \\
\hline Water phase density (DENSITY WATER) & $62.0104 \mathrm{lb} / \mathrm{ft} 3$ \\
\hline Water Formation Volume Factor (BWI) & 1.01332 \\
\hline Water Compressibility (CW) & $3.14934 \mathrm{E}-61 / \mathrm{psi}$ \\
\hline Reference Pressure for Water FVF (REFPW) & $14.969 \mathrm{psi}$ \\
\hline Water Viscosity (VWI) & $0.567336 \mathrm{cp}$ \\
\hline Pressure dependence of water viscosity (CVW) & $0.0 \mathrm{cp} / \mathrm{psi}$ \\
\hline
\end{tabular}

A rock type was added and named Rock Type 1 in order to input the relative permeability data. The method for evaluating 2-phase KRO was chosen to be Stone's Second Model, SWSG. Rock Type 1 is water wet and its water-oil relative permeability table and its liquid-gas relative permeability table are shown below in Error! Reference source not found. and Table 5. When specifying the water-oil relative permeability values, the option "include capillary pressure (drainage curve if using hysteresis)" is not selected. For the liquid-gas relative permeability table input, the liquid-gas $\mathrm{Kr}$ table dependency is selected to be gas saturation and the option "include capillary pressure (drainage curve if using hysteresis)" is selected.

Table 4 Rock Type 1's water-oil relative permeability table

\begin{tabular}{|l|l|l|}
\hline Sw & krw & krow \\
\hline 0.1 & 0 & 1 \\
\hline 0.15 & 0.00332 & 0.782512 \\
\hline 0.2 & 0.013281 & 0.602047 \\
\hline 0.25 & 0.029883 & 0.454285 \\
\hline 0.3 & 0.053125 & 0.335145 \\
\hline 0.35 & 0.083008 & 0.240789 \\
\hline
\end{tabular}




\begin{tabular}{|l|l|l|}
\hline 0.4 & 0.119531 & 0.167627 \\
\hline 0.45 & 0.162695 & 0.112322 \\
\hline 0.5 & 0.2125 & 0.071794 \\
\hline 0.55 & 0.268945 & 0.043223 \\
\hline 0.6 & 0.332031 & 0.024061 \\
\hline 0.65 & 0.401758 & 0.012035 \\
\hline 0.7 & 0.478125 & 0.005154 \\
\hline 0.75 & 0.561133 & 0.001727 \\
\hline 0.8 & 0.650781 & 0.00037 \\
\hline 0.85 & 0.74707 & $2.66 \mathrm{E}-05$ \\
\hline 0.9 & 0.85 & 0 \\
\hline & & \\
\hline
\end{tabular}

Table 5 Rock Type 1's liquid-gas relative permeability table

\begin{tabular}{|l|l|l|l|}
\hline Sg & krg & krog & Pcog (psi) \\
\hline 0 & 0 & 1 & 0 \\
\hline 0.025 & $4.77 \mathrm{E}-07$ & 0.90773 & 1.8232 \\
\hline 0.05 & $1.53 \mathrm{E}-05$ & 0.818488 & 2.6295 \\
\hline 0.075 & 0.000116 & 0.732378 & 3.2862 \\
\hline 0.1 & 0.000488 & 0.649519 & 3.8746 \\
\hline 0.125 & 0.00149 & 0.570045 & 4.4263 \\
\hline 0.15 & 0.003708 & 0.494106 & 4.958 \\
\hline 0.175 & 0.008014 & 0.421875 & 5.4801 \\
\hline 0.2 & 0.015625 & 0.353553 & 6 \\
\hline 0.225 & 0.028157 & 0.289379 & 6.5235 \\
\hline 0.25 & 0.047684 & 0.22964 & 7.0553 \\
\hline 0.275 & 0.076795 & 0.174693 & 7.6 \\
\hline 0.3 & 0.118652 & 0.125 & 8.1616 \\
\hline 0.325 & 0.177046 & 0.08119 & 8.7445 \\
\hline 0.35 & 0.256454 & 0.044194 & 9.353 \\
\hline
\end{tabular}




\begin{tabular}{|l|l|l|l|}
\hline 0.375 & 0.362098 & 0.015625 & 9.992 \\
\hline 0.4 & 0.5 & 0 & 10.6667 \\
\hline
\end{tabular}

Under the initial conditions tab, the "water, oil" option was used for the "perform gravitycapillary equilibrium of a reservoir initially containing" characteristic. The properties outlined in Table 6 were inputted under the initial conditions tab along with selecting the "add phase pressure correction to ensure that the reservoir is initially in gravitation equilibrium" option for the phase pressure correction specification.

Table 6 Initial condition properties specified for the Original CMG model

\begin{tabular}{|l|l|}
\hline Property & Value \\
\hline Reference Pressure (REFPRES) & $3122 \mathrm{psi}$ \\
\hline Reference Depth (REFDEPTH) & $4300 \mathrm{ft}$ \\
\hline Water-Oil Contact Depth (DWOC) & $4500 \mathrm{ft}$ \\
\hline Constant Bubble Point Pressure (PB) & $1500 \mathrm{psi}$ \\
\hline
\end{tabular}

All default values were kept in the numerical tab as displayed in the figures below. 


\begin{tabular}{|c|c|c|}
\hline Keyword Description & Default Value & Dataset Value \\
\hline \multicolumn{3}{|l|}{ Timestep Control Keywords } \\
\hline \multicolumn{3}{|l|}{ Maximum Number of Timesteps (MAXSTEPS) } \\
\hline Maximum Simulation Time (MAXTIME) & 8036 & \\
\hline Maximum Simulation Date (MAXDATE) & 2017-01-01 & \\
\hline Maximum Time Step Size (DTMAX) & 365 day & \\
\hline Minimum Time Step Size (DTMIN) & 0.001 day & \\
\hline First Time Step Size after Well Change (DTWELL) & 1 day & \\
\hline \multicolumn{3}{|l|}{ Maximum CPU Seconds (MAXCPU) } \\
\hline \multicolumn{3}{|l|}{ Maximum Elapsed Time in Seconds (MAXCLK) } \\
\hline \multicolumn{3}{|l|}{ Normal Variation per Time Step (NORM) } \\
\hline Pressure (PRESS) & $435.113 p s i$ & \\
\hline Saturation (SATUR) & 0.1 & \\
\hline Bubble Point Pressure (PBUB) & $435.113 \mathrm{psi}$ & \\
\hline Dew Point Pressure (PDW) & $1450.38 \mathrm{psi}$ & \\
\hline \multicolumn{3}{|l|}{ Maximum Changes per Time Step (MAXCHANGE) } \\
\hline Pressure (PRESS) & $870.227 \mathrm{psi}$ & \\
\hline Saturation (SATUR) & 0.2 & \\
\hline Bubble Point Pressure (PBUB) & $870.227 \mathrm{psi}$ & \\
\hline Dew Point Pressure (PDW) & $2900.75 \mathrm{psi}$ & \\
\hline \multicolumn{3}{|l|}{ Solution Method Keywords } \\
\hline \multicolumn{3}{|l|}{ Relaxation Option (RELAX) } \\
\hline Over-elaxation in Accumulation (ACC) & ON & $\boldsymbol{v}$ \\
\hline Under-relaxation for Gas Saturation Disappearance (GAS) & OFF & $\mathbf{v}$ \\
\hline Maximum Newton Iterations (NEWTONCYC) & 10 & \\
\hline Maximum Time Step Cuts (NCUTS) & 4 & \\
\hline Adaptive Implicit Method (AIM) & Stability Switching And Threshold Criterion & $\mathbf{r}$ \\
\hline Frequency of Checking for Backward Switching (BACK) & & \\
\hline Threshold Pressure (THRESH) & 0.25 & \\
\hline Threshold Saturation or Polymer Concentration (THRESH) & 0.25 & \\
\hline Set Well Blocks and Neighbours to Implicit (AIMWELL) & Well blocks & $\mathbf{T}$ \\
\hline Convergence Tolerance (CONVERGE) & Residual & Residual \\
\hline Maximum Scaled Residual - All Equations (MAXRES) & 0.1 & \\
\hline Maximum Scaled Residual - Oil Equation (MAXRES OIL) & 0.1 & \\
\hline Maximum Scaled Residual - Water Equation (MAXRES WATER) & 0.1 & \\
\hline Maximum Scaled Residual - Seawater Equation (MAXRES SEAWATER) & 0.0005 & \\
\hline Maximum Scaled Residual - Gas Equation (MAXRES GAS) & 0.1 & \\
\hline Maximum Scaled Residual - Solvent Equation (MAXRES SOLVENT) & 0.1 & \\
\hline Maximum Scaled Residual - Polymer Equation (MAXRES POLYMER) & 0.0005 & \\
\hline Maximum Scaled Residual - Lightoil Equation (MAXRES LIGHTOIL) & 0.1 & \\
\hline Maximum Scaled Residual - Other Equation (MAXRES OTHER) & 0.1 & \\
\hline Average of All (Phase or Component) Equations (TOTRES) & 0.001 & \\
\hline Well Equation (WELLRES) & 0.001 & \\
\hline
\end{tabular}

Figure 28 Default numerical controls in the Original CMG Model 


\begin{tabular}{|c|c|c|}
\hline Keyword Description & Default Value & Dataset Value \\
\hline Adaptive Implicit Method (AlM) & Stability Switching And Threshold Criterion & 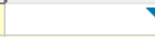 \\
\hline \multicolumn{3}{|l|}{ Frequency of Checking for Backward Switching (BACK) } \\
\hline Threshold Pressure (THRESH) & 0.25 & \\
\hline Threshold Saturation or Polymer Concentration (THRESH) & 0.25 & \\
\hline Set Well Blocks and Neighbours to Implicit (AIMWELL) & Well blocks & r \\
\hline Convergence Tolerance (CONVERGE) & Residual & Residual \\
\hline Maximum Scaled Residual - All Equations (MAXRES) & 0.1 & \\
\hline Maximum Scaled Residual - Oil Equation (MAXRES OIL) & 0.1 & \\
\hline Maximum Scaled Residual - Water Equation (MAXRES WATER) & 0.1 & \\
\hline Maximum Scaled Residual - Seawater Equation (MAXRES SEAWATER) & 0.0005 & \\
\hline Maximum Scaled Residual - Gas Equation (MAXRES GAS) & 0.1 & \\
\hline Maximum Scaled Residual - Solvent Equation (MAXRES SOLVENT) & 0.1 & \\
\hline Maximum Scaled Residual - Polymer Equation (MAXRES POLYMER) & 0.0005 & \\
\hline Maximum Scaled Residual - Lightoil Equation (MAXRES LIGHTOIL) & 0.1 & \\
\hline Maximum Scaled Residual - Other Equation (MAXRES OTHER) & 0.1 & \\
\hline Average of All (Phase or Component) Equations (TOTRES) & 0.001 & \\
\hline Well Equation (WELLRES) & 0.001 & \\
\hline Residual Multiplier Equation for Fracture Blocks (FRACRES) & 1 & \\
\hline Well Bottom Hole Pressure Tolerance (WELLBHP) & 10 & \\
\hline Polymer Concentration Tolerance (POLY) & 0.005 & \\
\hline Light Oil Volume Fraction Tolerance (API) & 0.005 & \\
\hline \multicolumn{3}{|l|}{ Linear Solver Keywords } \\
\hline Linear Solver Precision (PRECC) & 0.0001 & \\
\hline Linear Solver Ordering (SORDER) & REDBLACK & T \\
\hline Linear Solver Factorization (SDEGREE) & 1 & Tran \\
\hline Linear Solver Pivot Stabilization (PIVOT) & OFF & r \\
\hline Linear Solver Iterations (ITERMAX) & 40 & \\
\hline Linear Solver Orthogonalizations (NORTH) & 30 & \\
\hline Linear Solver Tolerance (RELTOL) & 0.1 & \\
\hline Linear Solver Failure Behavior (SOLFAIL) & CONTINUE & r \\
\hline \multicolumn{3}{|l|}{ Parallel Processing Keywords } \\
\hline Target number of domains (JACDOMS) & 2 & \\
\hline Target number of planes per Jacobian domain (DPLANES) & 4 & \\
\hline Number of threads to be used (PNTHRDS) & 1 & \\
\hline AIMSOL/PARASOL Switch (SOLVER) & AIMSOL & T \\
\hline \multicolumn{3}{|l|}{ Number of scaling and GMRES vector operation classes (PNPROSL) } \\
\hline \multicolumn{3}{|l|}{ Factorization degree within PARASOL classes (PDEGAA) } \\
\hline \multicolumn{3}{|l|}{ Factorization degree between PARASOL classes (PDEGAB) } \\
\hline \multicolumn{3}{|l|}{ Red-Black ordering check for PARASOL (CHECKRB) } \\
\hline \multicolumn{3}{|l|}{ Dynamic Grid Keywords } \\
\hline \multicolumn{3}{|l|}{ Distance from wells the protected region of non-amalgamation extends areally (DYNAGRID-WLN-A) } \\
\hline Distance from wells the protected region of non-amalgamation extends vertically (DYNAGRID-WLN-V) & & \\
\hline
\end{tabular}

Figure 29 Continuation of the default numerical controls in the Original CMG model

The Original CMG model contained 20 injection wells and 57 producer wells. All of the injection wells were added to the model at the start of simulation at 1-1-1995 and specified as "Injector

MOBWEIGHT" type wells. By selecting this type of well, the total mobility of the injector's grids are taken into account when calculating the flow rate. The equations by which this is done are shown below (Eghonghon, 2015).

$$
\begin{gathered}
q=\text { Well Index } * \text { Phase Mobility } *\left(P_{\text {block }}-P_{\text {block }}\right) \\
\text { Phase Mobility } \left.=k_{i} / \mu_{i} \text { where } i=\text { moving fluid (oil, water, gas, etc. }\right) \\
\text { Well Index }=\frac{2 * \pi * h * \sqrt{k_{x} k_{y}}}{\ln \left({ }^{r_{o}} / r_{w}\right)+s} \text { where } s=\text { skin factor }
\end{gathered}
$$

Injection wells were placed within the aquifer portion of the grid, or in other words, where the border is shown in the porosity, permeability, and initial water saturation maps. The injection wells encompassed the reservoir on all four sides and injected water into the aquifer to help provide pressure 
support to the producer wells. A visual with only the injectors displayed in the aquifer portion of the reservoir on the initial water saturation map for K Layer 1 is shown below in Figure 30. They were allowed to operate at a maximum bottom hole pressure of 4000 psi for the entirety of the simulation which ends at 1-1-2017. By only specifying a maximum bottom hole pressure of 4000psi, the injector will inject as much water as possible until it reaches the 4000 psi constraint and continue to inject as much water as possible to stay at that pressure as seen below in Figure 31. The injection wells injected water into all six layers.

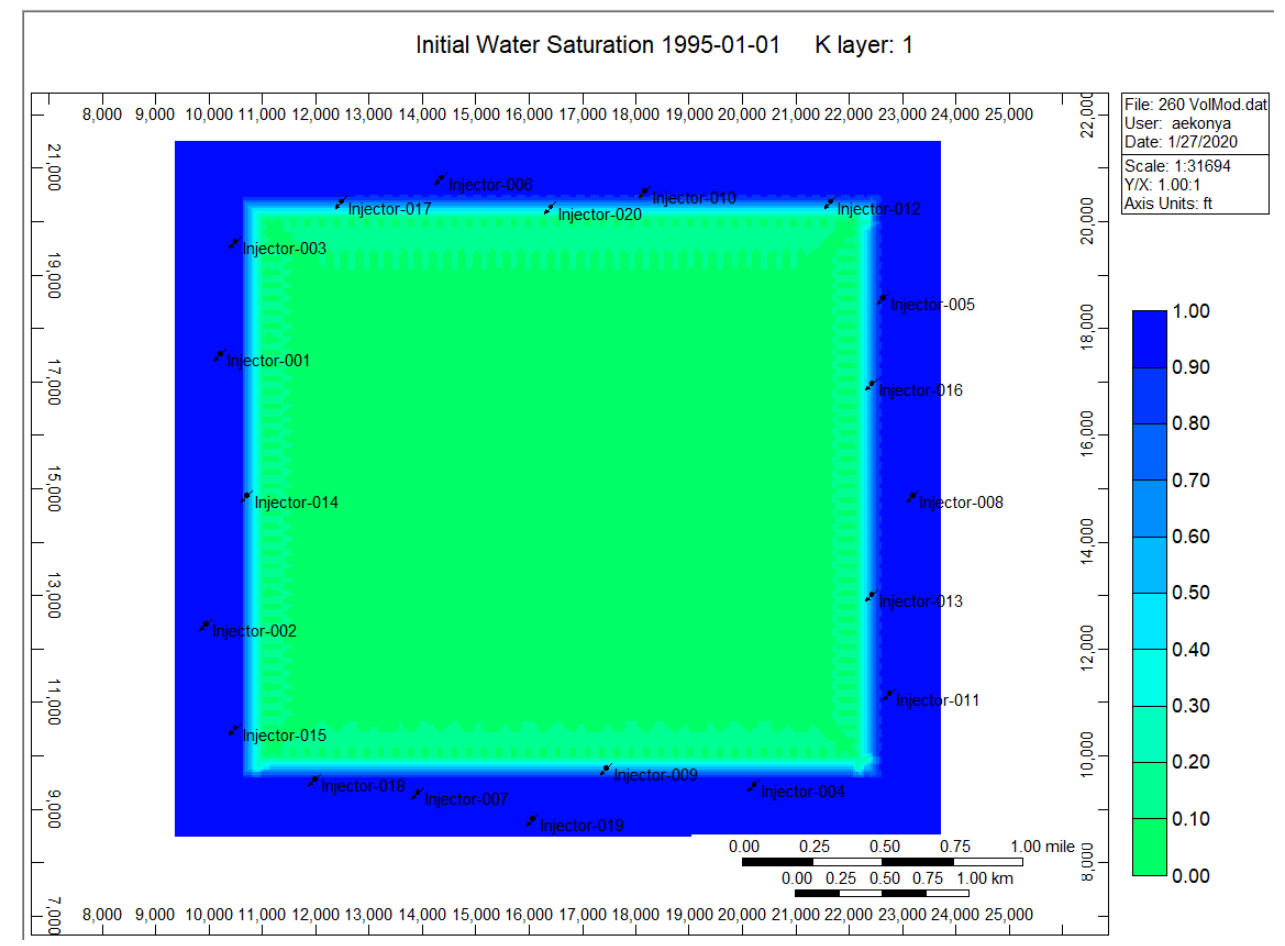

Figure 30 Injector well locations in aquifer around reservoir for the Original CMG model 


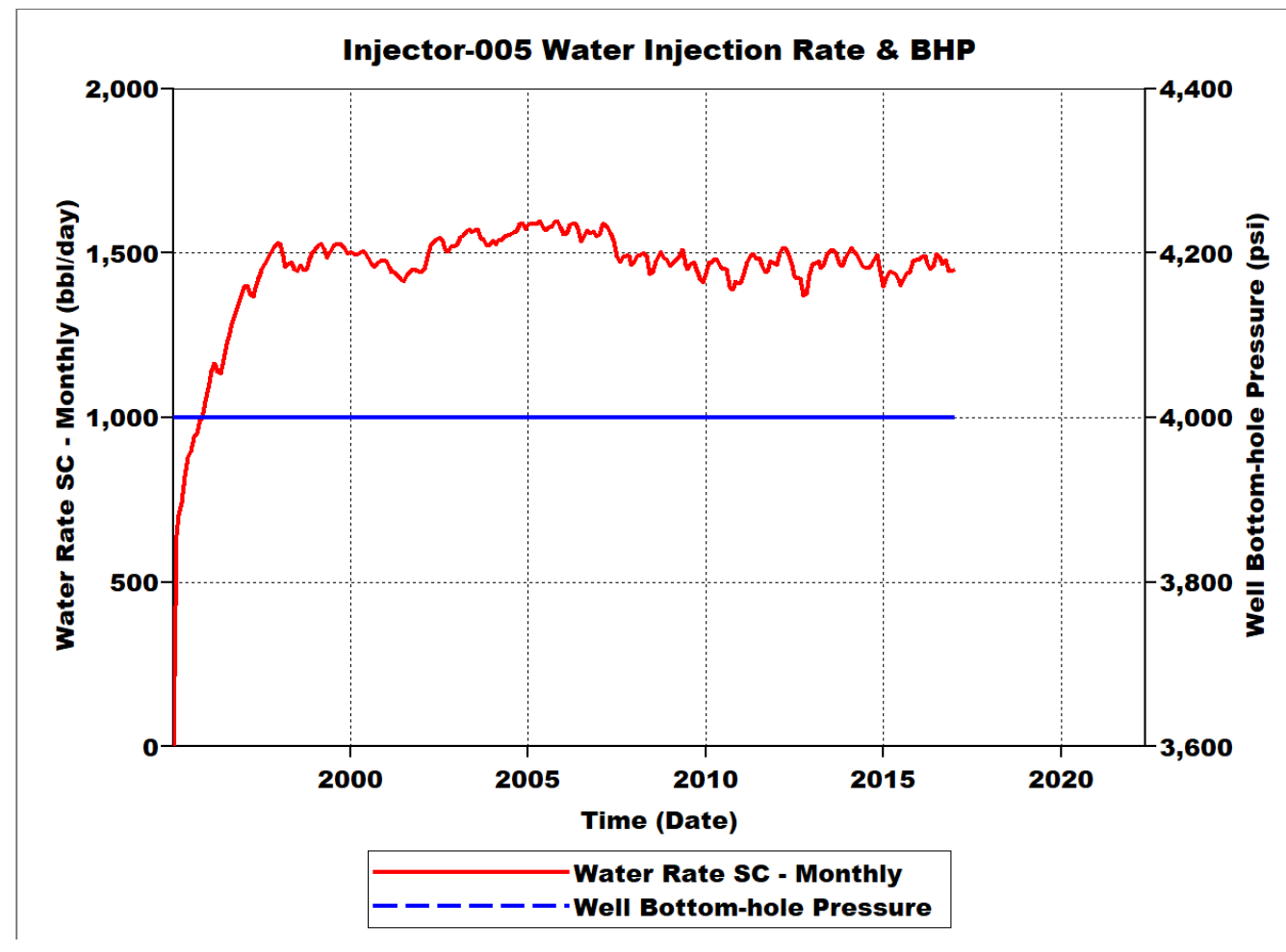

Figure 31 Injector-005 water injection rate and well bottom hole pressure

All 57 producers were also added to the model at the start of the simulation, 1-1-1995. Producer wells 6 through 57 were shut in at 1-1-1995 after being defined and then opened according to various phases of production. These phases of production are outlined in Table 7.

Table 7 Phases of production well addition in the Original CMG model

\begin{tabular}{|c|c|c|}
\hline Wells & Phase of Production & Date Phase Begins \\
\hline $1-5$ & Phase 1 & $1-1-1995$ \\
\hline $6-20$ & Phase 2 & $1-1-1998$ \\
\hline $21-37$ & Phase 3 & $1-1-2002$ \\
\hline $38-42$ & Phase 4 Part 1 & $1-1-2007$ \\
\hline $43-47$ & Phase 4 Part 2 & $1-1-2008$ \\
\hline $48-52$ & Phase 4 Part 3 & $1-1-2009$ \\
\hline $53-57$ & Phase 4 Part 4 & $1-1-2010$ \\
\hline
\end{tabular}

Wells 1 through 5 were defined and operated with a minimum bottom hole pressure constraint of approximately 1700 psi and produced from all layers. Wells 6 through 57 were first defined as being operated at a minimum bottom hole pressure of $28 \mathrm{psi}$, however when the wells were brought online in their respective phases, their minimum bottom hole pressure constraint was modified to be a value around 1700 psi. Wells 6 through 57 also produced from all six layers. The well locations are shown in Figure 32 where it can be seen that all production wells are within the reservoir portion of the reservoir. 


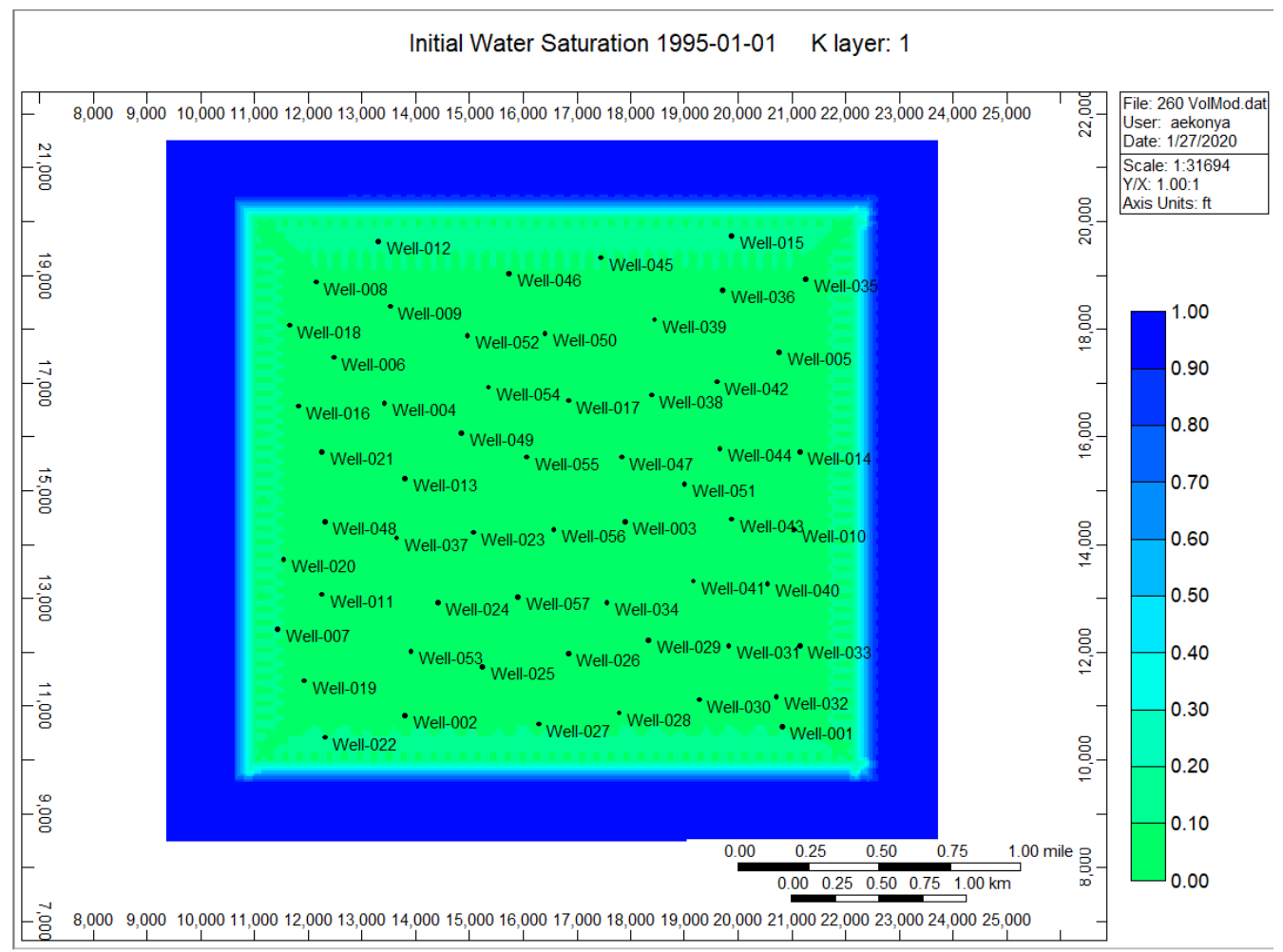

Figure 32 Production well locations in reservoir portion of the Original CMG model

Every month after a well is brought online, the minimum bottom hole pressure constraint is adjusted to be a value between 1660 psi and 1800 psi. This in turn changes how much oil is produced and the well bottom hole pressure value for the well as the production wells will produce as much as is required to reach the minimum bottom hole pressure constraint. Also, by changing this constraint a more realistic pressure response is achieved from each well because real wells in industry do not have a constant pressure profile for the entire life of the well.

\subsection{Anticline Thesis Model}

The 250x250 grid version of the Original CMG model (also referred to as the base model) was obtained and the modification process was started, however upon trying to validate the dataset and run it for one time step, fatal errors were encountered. Troubleshooting was done to determine a solution to this error, however one was not easily found. Upon consultation with the creator of the Original CMG model, it was found that the error had been received before, for which the recommendation was to recreate the changes on a newer updated Original CMG model. Therefore this model was obtained from the creator where the grid was now 260x260. This version of the model will be referred to as the Original CMG model and is the model presented in detail in the Original CMG model section above.

The alterations were recreated on the 260x260 version of the Original CMG model and the comparison between the Original CMG model and the Anticline Thesis model (the current CMG model), including depictions, will be shown below. First, the grid top was changed from a partial dome to one limb of an anticline. For simplicity, the reservoir will be referred to as an anticline throughout this thesis even though it is only the upward sloping half of an anticline, or the upward sloping limb of an anticline. In order to do so, the maximum grid top value, minimum grid top value, maximum grid centroid $\mathrm{x}$, 
minimum grid centroid $\mathrm{x}$, maximum grid centroid $\mathrm{y}$, and minimum grid centroid $\mathrm{y}$ were determined. The difference between the max and min of the grid top value and grid centroid $\mathrm{x}$ were calculated. The change in the $\mathrm{x}$ direction and grid top value for each step was calculated by dividing each respective difference by 9. Nine was established to be the number of steps needed to allow the grid top to have a realistic shape without its surface being too smooth or too blocky. From the upper left corner of the reservoir, the grid centroid $\mathrm{x}$ increased by its calculated step whereas the grid top value decreased by its calculated step size for a constant grid centroid y value. The inverse relationship is performed for the grid centroid $\mathrm{x}$ and grid top value for the opposite side of the reservoir, starting at the bottom right corner, again for a constant grid centroid y value. The above operation is illustrated in Figure 33. The data value groups (grid centroid $\mathrm{x}$, grid centroid $\mathrm{y}$, and grid top value) were numbered 1-20 and are called "observation numbers" as seen in Table 8. The highlighted rows in Table 8 are the four corners of the reservoir which corresponds with the $(\mathrm{x}, \mathrm{y})$ values shown in Figure 33.

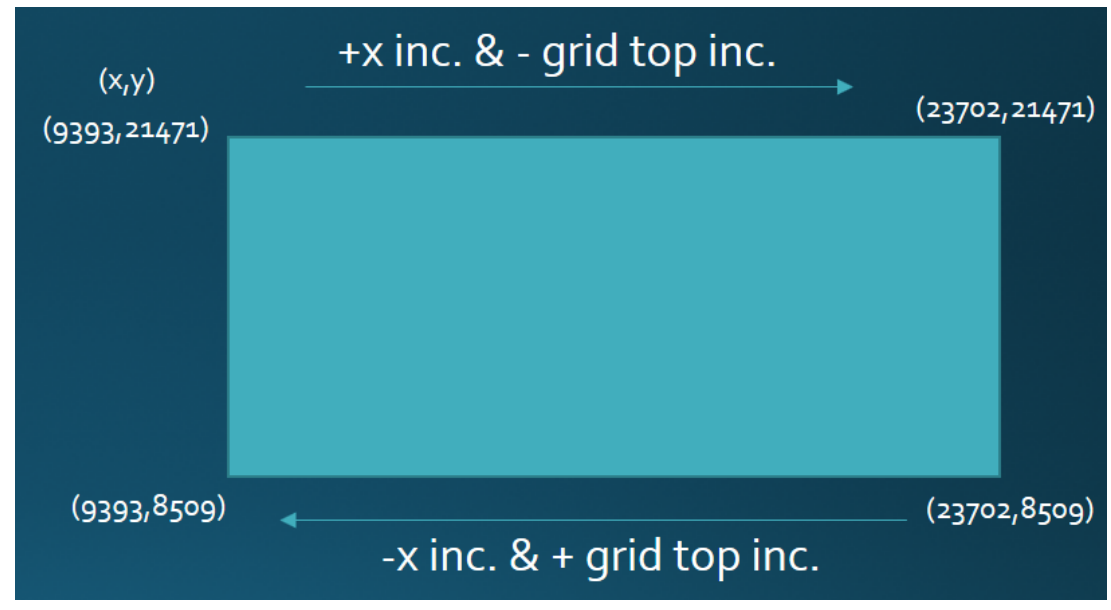

Figure 33 Depiction of calculations to reshape reservoir into an anticline

Table 8 Values needed to create the grid top map file in Builder to reshape the reservoir into an anticline

\begin{tabular}{|r|r|l|l|}
\hline $\begin{array}{l}\text { Observation } \\
\text { Numbers }\end{array}$ & X & Y & $\begin{array}{l}\text { Grid Top } \\
\text { Value }\end{array}$ \\
\hline 1 & 9393 & 21471 & 4613.00 \\
\hline 2 & 10982.89 & 21471 & 4533.44 \\
\hline 3 & 12572.78 & 21471 & 4453.89 \\
\hline 4 & 14162.67 & 21471 & 4374.33 \\
\hline 5 & 15752.56 & 21471 & 4294.78 \\
\hline 6 & 17342.44 & 21471 & 4215.22 \\
\hline 7 & 18932.33 & 21471 & 4135.67 \\
\hline 8 & 20522.22 & 21471 & 4056.11 \\
\hline 9 & 22112.11 & 21471 & 3976.56 \\
\hline 10 & 23702 & 21471 & 3897.00 \\
\hline 11 & 23702 & 8509 & 3897.00 \\
\hline 12 & 22112.11 & 8509 & 3976.56 \\
\hline & & & \\
\hline
\end{tabular}




\begin{tabular}{|r|r|r|r|}
\hline 13 & 20522.22 & 8509 & 4056.11 \\
\hline 14 & 18932.33 & 8509 & 4135.67 \\
\hline 15 & 17342.44 & 8509 & 4215.22 \\
\hline 16 & 15752.56 & 8509 & 4294.78 \\
\hline 17 & 14162.67 & 8509 & 4374.33 \\
\hline 18 & 12572.78 & 8509 & 4453.89 \\
\hline 19 & 10982.89 & 8509 & 4533.44 \\
\hline 20 & 9393 & 8509 & 4613.00 \\
\hline
\end{tabular}

Next, the Create a Map File option within Builder was used where the observation numbers, $\mathrm{x}$ locations (grid centroid x values), y locations (grid centroid y values), and grid top values shown in Table 8 were copied in and the file was saved. The saved .msh file was then assigned to Layer 1's Grid Top in Array Properties within Builder. The grid tops for Layers 2-6 are determined using the Grid Top from Layer 1 and the thickness maps inputted into the model for each layer. A snapshot of the aerial view and $3 \mathrm{D}$ view of the grid top from the Original CMG model is shown below in Figure 34 and Figure 35. The reshaped grid top in the Anticline Thesis model is shown in Figure 36 and Figure 37.

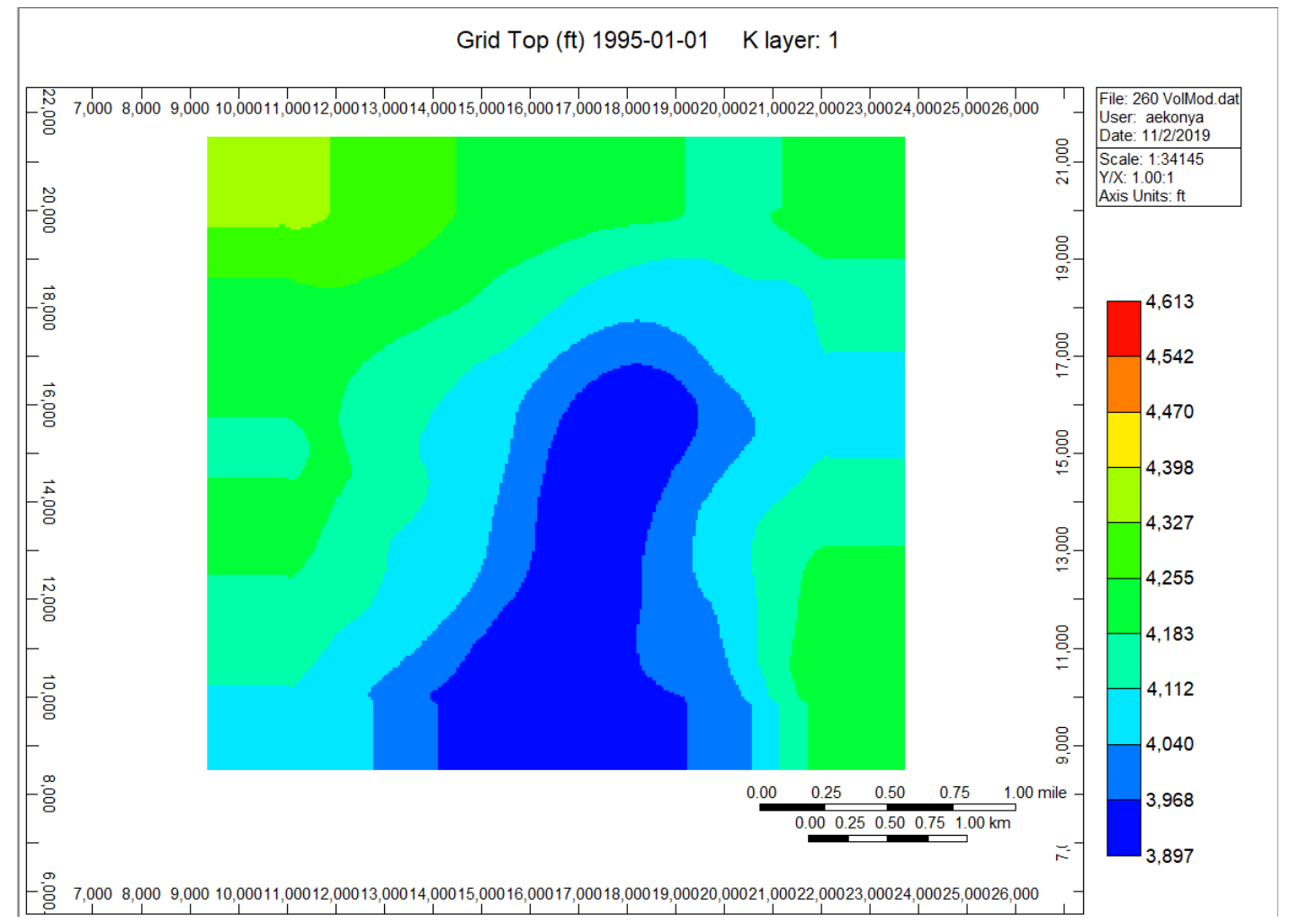

Figure 34 Aerial view of the Original CMG model's grid top 


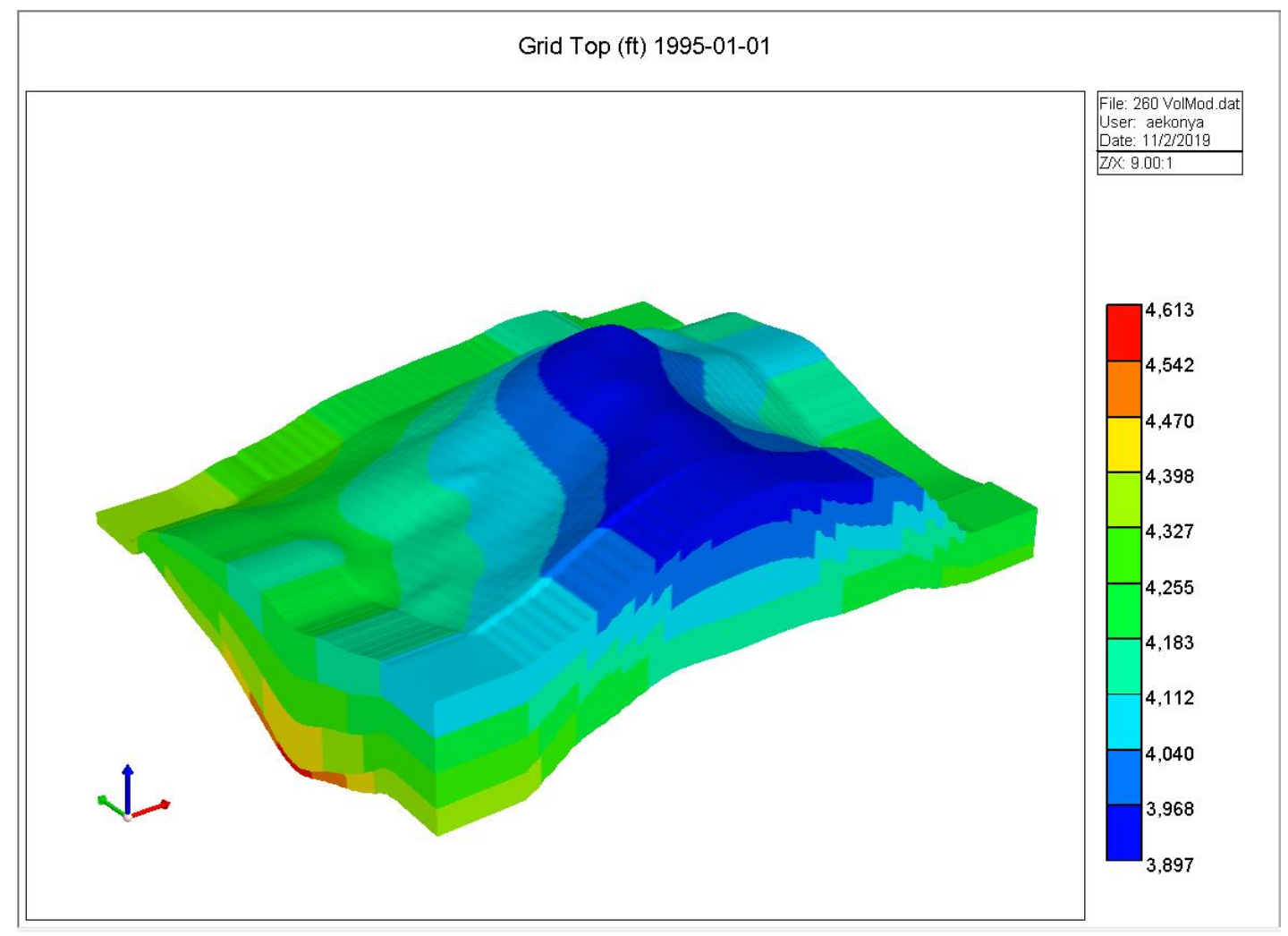

Figure $353 D$ view of the Original CMG model's grid top

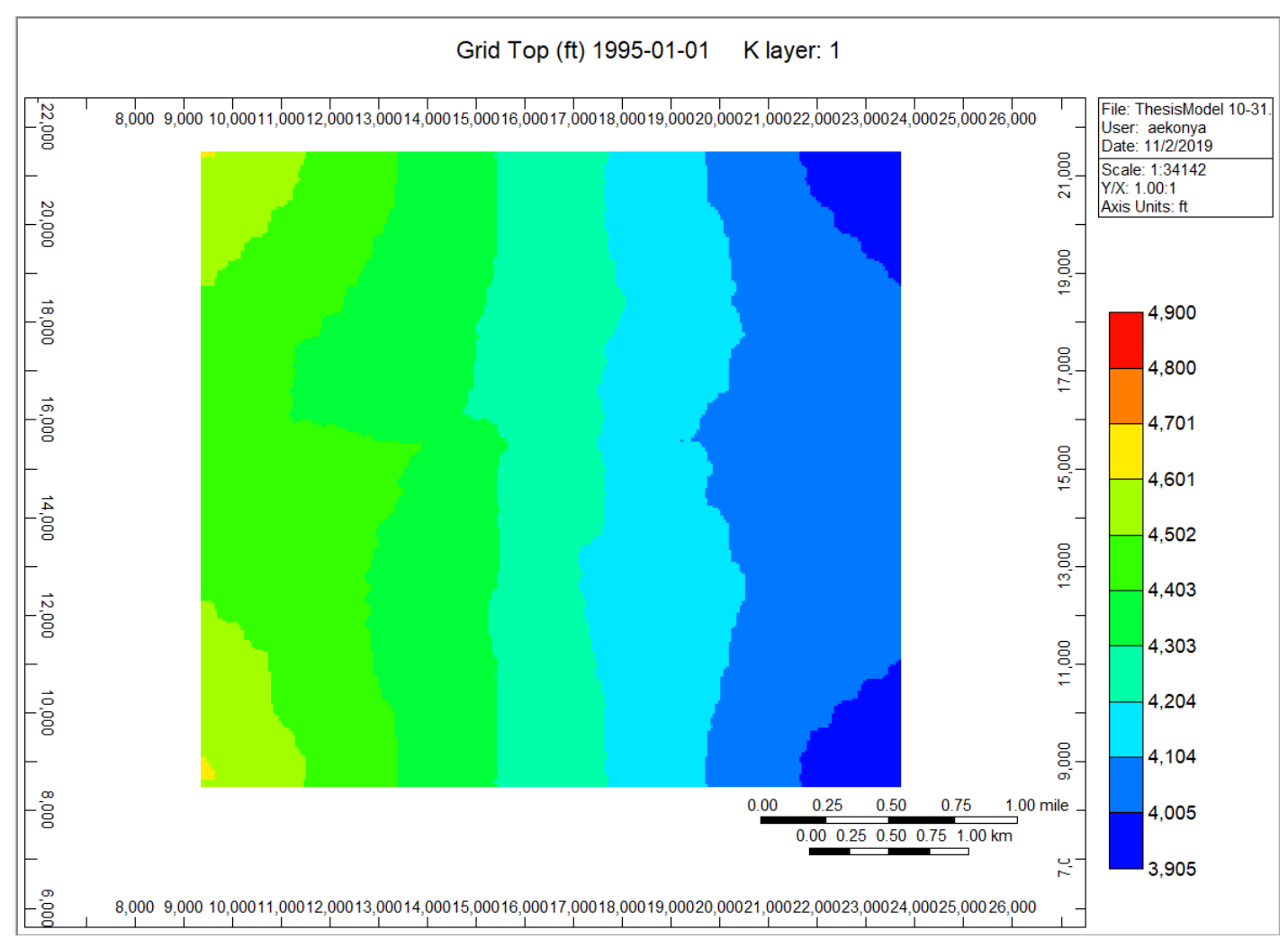

Figure 36 Aerial view of the Anticline Thesis model's grid top 


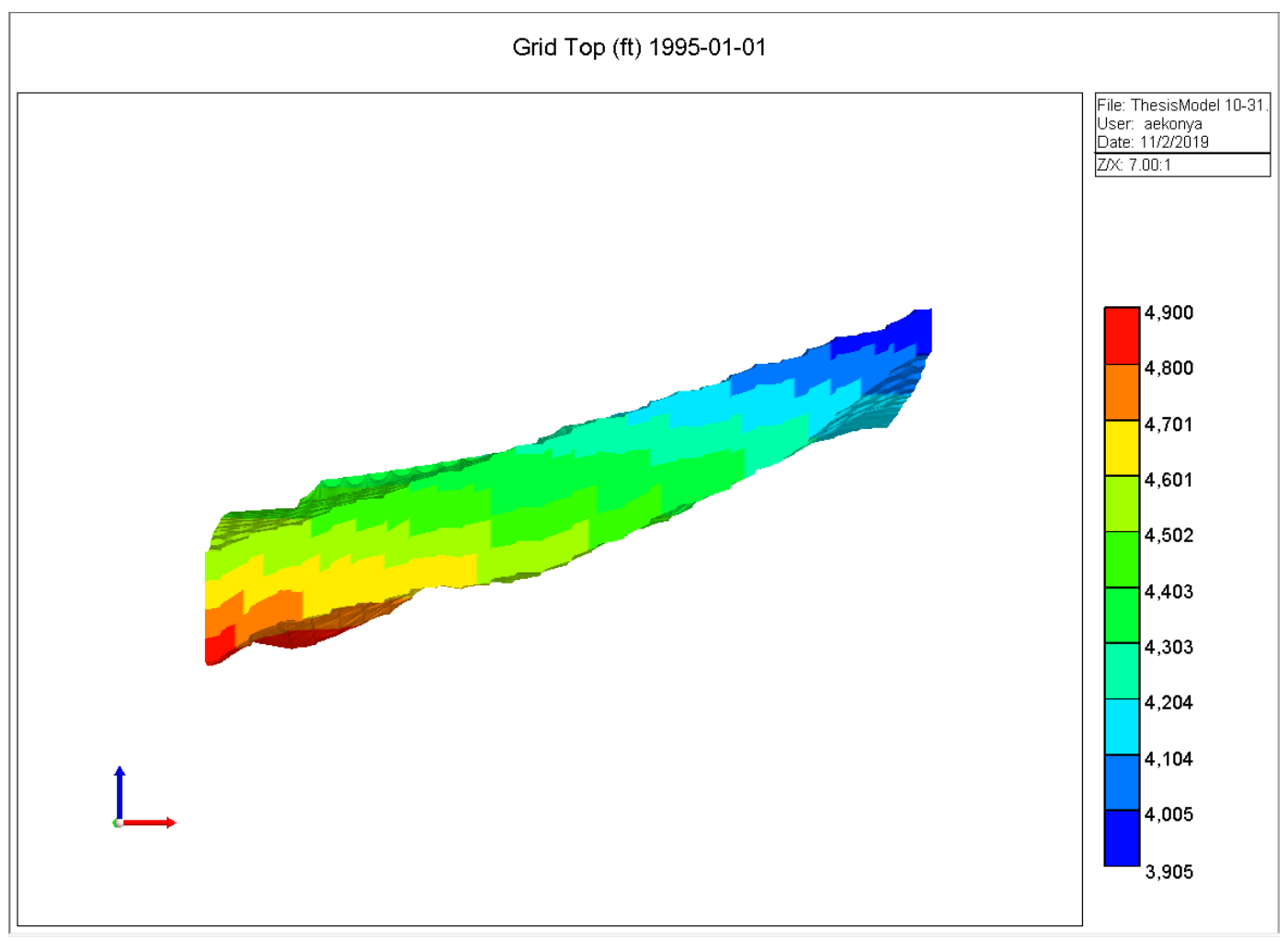

Figure 37 3D view of the Anticline Thesis model's grid top

The Original CMG model also had a volume modifier applied to four sides of the reservoir to simulate an aquifer and apply a constant pressure boundary. The volume modifier was removed from the Anticline Thesis model completely as a constant pressure boundary does not apply. Depictions of the volume modifier in the Original CMG model can be seen above in Figure 26 and Figure 27.

The porosity, permeability, and initial water saturation maps also needed to be edited in order to remove the effect of a surrounding aquifer. The aforementioned maps contained a border around the maps with specified values that represented an aquifer. The following steps were taken to alter the original maps. First a new CMG Builder window was opened, and the IMEX Simulator and Field units option were selected. The Porosity map for Layer 1 was opened by going to File and Open Map File as shown in Figure 38. 


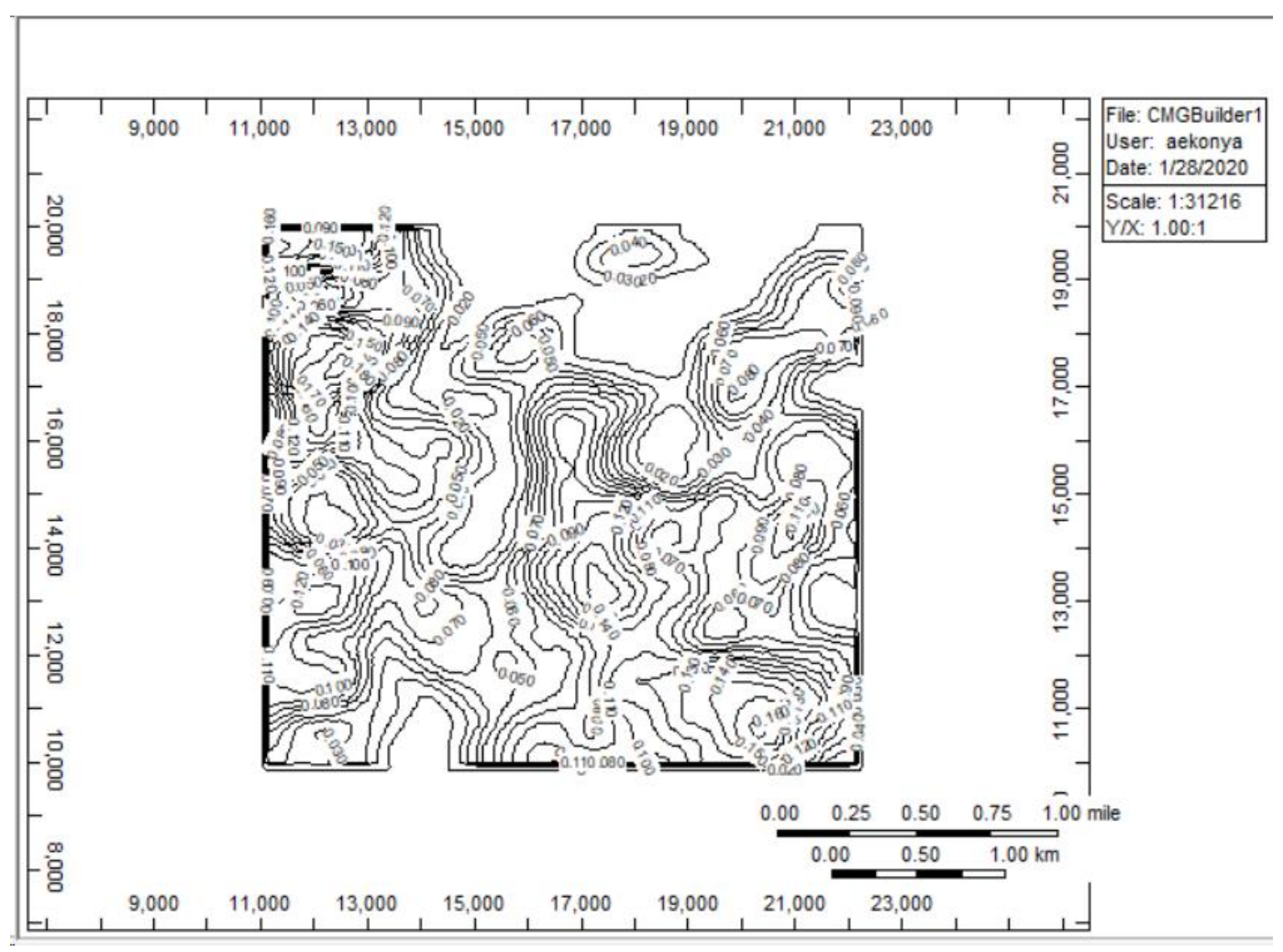

Figure 38 Layer 1's porosity.msh file open in new CMG Builder

Next, a 260 x 260 grid of smaller cell sizes were overlain on the opened map file. The desired cell size was calculated first by multiplying 200 by the cell size in the $\mathrm{x}$ direction (55.25) and 200 by the cell size in the $\mathrm{y}$ direction (50.05). By doing this multiplication, the length of the map in the $\mathrm{x}$ and $\mathrm{y}$ direction for only the reservoir portion is found. This is due to 30 cells on the left, right, above, and below (from an aerial perspective) of the reservoir being values representative of an aquifer which equals 60 cells total in the $\mathrm{x}$ and $\mathrm{y}$ direction. The equations are shown below.

$$
\begin{aligned}
& 200 * 55.25=11050 \text { length in } x \text { direction } \\
& 200 * 50.05=10010 \text { length } \text { in } y \text { direction }
\end{aligned}
$$

Because the desired reservoir portion is only $200 \times 200$ of normal sized cells, a 260 × 260 grid overlaying the same area would require smaller cell sizes. These smaller cell sizes to overlay a $260 \times 260$ grid over the same length in the $\mathrm{x}$ and $\mathrm{y}$ direction are calculated using the equations below.

$$
\begin{aligned}
& \frac{11050}{260}=42.5 \text { each cell will be } 42.5 \text { feet in the } x \text { direction } \\
& \frac{10010}{260}=38.5 \text { each cell will be } 38.5 \mathrm{ft} \text { in the } y \text { direction }
\end{aligned}
$$

A 260 x 260 grid of smaller cells is overlain on the opened Layer 1 Porosity .msh file by using the Create an Orthogonal Corner Point Grid Option. The number of grid blocks in the I, J, and K directions is specified to be 260, 260, and 6 respectively. The block widths in the I and J directions are the values calculated in the previous step. All of the grid specifications needed are shown in Figure 39. After the grid is added, Figure 40 shows the display of the newly added grid on top of the opened .msh file. 


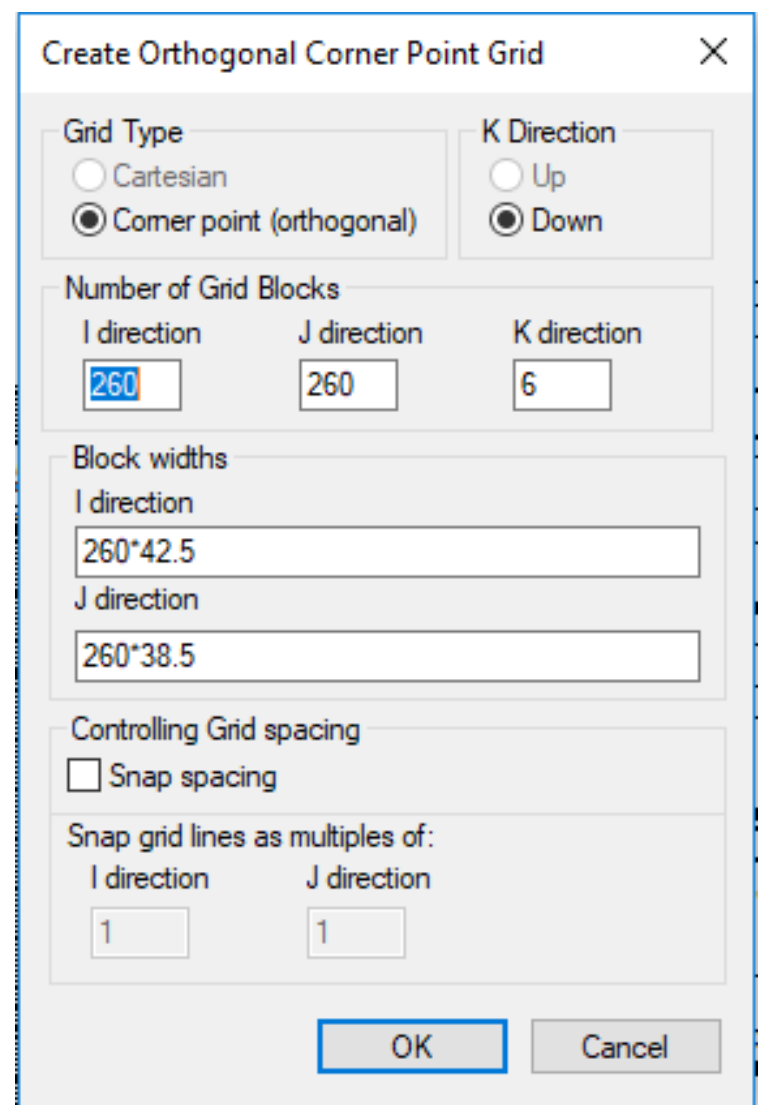

Figure 39 Creating a $260 \times 260$ Orthogonal Corner Point grid of smaller cells 


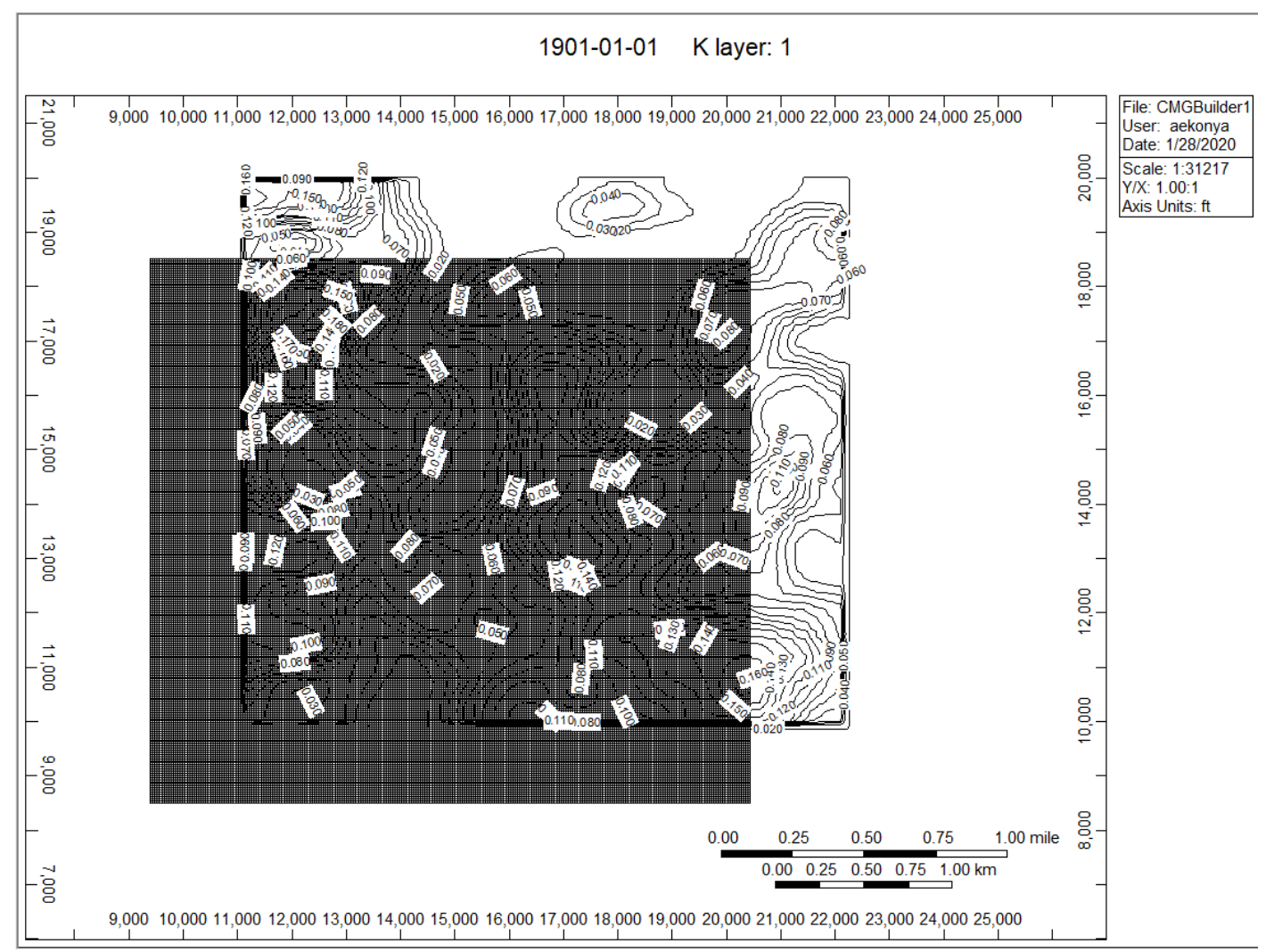

Figure 40 New $260 \times 260$ grid on top of opened Layer 1 Porosity .msh file

Now that the $260 \times 260$ grid was added, it needs to be moved to be directly over the reservoir portion of the opened Layer 1 Porosity .msh file. This is done by first selecting the Edit Grid option then holding down the SHIFT key and dragging the grid with the computer mouse until it lines up with the .msh file as shown below. 


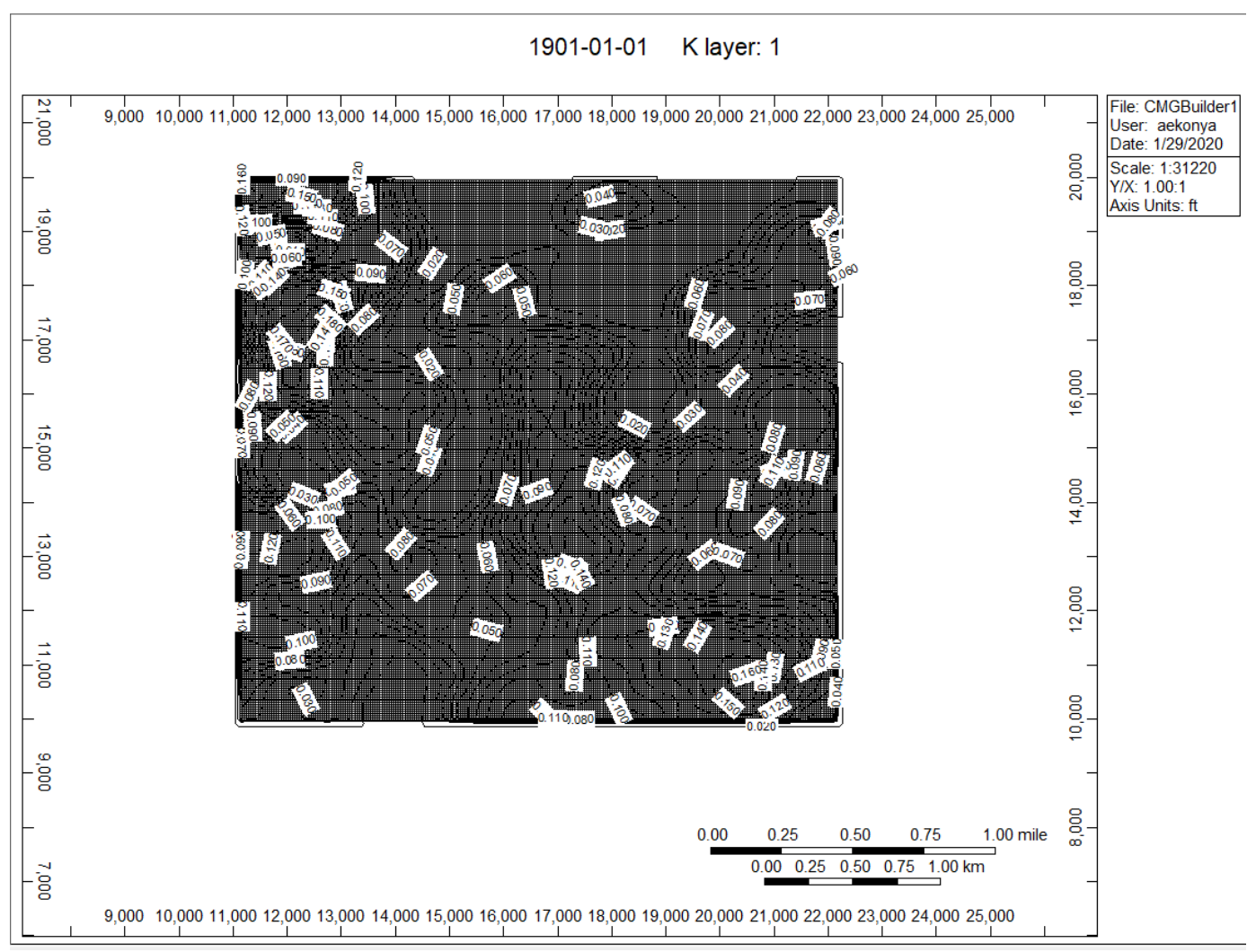

Figure $41260 \times 260$ grid of smaller cells over the desired portion of the Layer 1 Porosity map file after being moved

All of the permeability, porosity, and grid thickness (to keep files that will be used uniform) were specified under Array Properties for each layer. Once the maps were specified, the grid was directly over the desired portions of the newly specified maps as shown below. The porosity, permeability, and grid thickness maps were then exported in a .msh format. This effectively exported only the reservoir portion of the properties with a $260 \times 260$ grid of smaller cells and the aquifer properties in the surrounding border have been cropped out. 


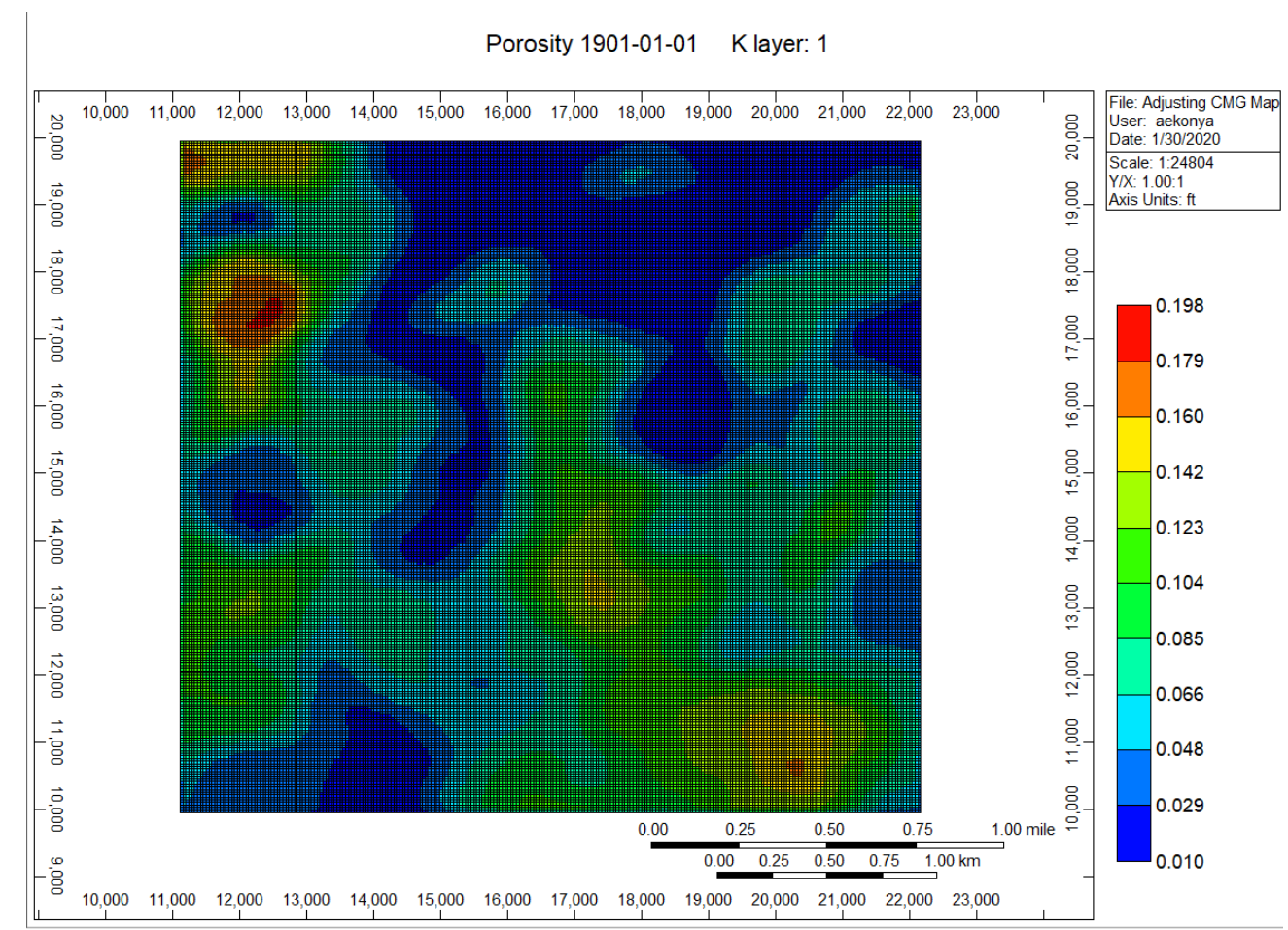

Figure 42 Shifted $260 \times 260$ grid directly over Layer 1 Porosity map specified in Array Properties

To keep the wells in the same locations, the cells within the smaller cell sized 260 x 260 grid need to be enlarged to their original size before the alteration process began, thus expanding the reservoir section to cover the original reservoir and border sections. This enlargement of the cells is done by simply copying and pasting certain values from the original map files to the recently exported map files. The values that need to be adjusted are the xoffset, y offset, xmin, ymin, xmax, ymax, DI, and DJ values shown in Figure 43. 


\begin{tabular}{|c|c|c|c|c|c|c|}
\hline RESUI & IS XOFFSET & 9400.79 & & & & \\
\hline RESUI & IS YOFFSET & 21529 & & & & \\
\hline RESUI & IS KDIR DOWN & & & & & \\
\hline ORIG & N TOPLEFT & & & & & \\
\hline XMIN & 27.625 & & & & & \\
\hline YMIN & 25.025 & & & & & \\
\hline XMAX & 14337.4 & & & & & \\
\hline YMAX & 12988 & & & & & \\
\hline ORDE: & NEXTCOLUMN & & & & & \\
\hline DI & 55.25 & 55.25 & 55.25 & 55.25 & & \\
\hline & 55.25 & 55.25 & 55.25 & 55.25 & 55.25 & 55.25 \\
\hline & 55.25 & 55.25 & 55.25 & 55.25 & 55.25 & 55.25 \\
\hline & 55.25 & 55.25 & 55.25 & 55.25 & 55.25 & 55.25 \\
\hline & 55.25 & 55.25 & 55.25 & 55.25 & 55.25 & 55.25 \\
\hline & 55.25 & 55.25 & 55.25 & 55.25 & 55.25 & 55.25 \\
\hline & 55.25 & 55.25 & 55.25 & 55.25 & 55.25 & 55.25 \\
\hline & 55.25 & 55.25 & 55.25 & 55.25 & 55.25 & 55.25 \\
\hline & 55.25 & 55.25 & 55.25 & 55.25 & 55.25 & 55.25 \\
\hline & 55.25 & 55.25 & 55.25 & 55.25 & 55.25 & 55.25 \\
\hline & 55.25 & 55.25 & 55.25 & 55.25 & 55.25 & 55.25 \\
\hline & 55.25 & 55.25 & 55.25 & 55.25 & 55.25 & 55.25 \\
\hline & 55.25 & 55.25 & 55.25 & 55.25 & 55.25 & 55.25 \\
\hline & 55.25 & 55.25 & 55.25 & 55.25 & 55.25 & 55.25 \\
\hline & 55.25 & 55.25 & 55.25 & 55.25 & 55.25 & 55.25 \\
\hline & 55.25 & 55.25 & 55.25 & 55.25 & 55.25 & 55.25 \\
\hline & 55.25 & 55.25 & 55.25 & 55.25 & 55.25 & 55.25 \\
\hline & 55.25 & 55.25 & 55.25 & 55.25 & 55.25 & 55.25 \\
\hline & 55.25 & 55.25 & 55.25 & 55.25 & 55.25 & 55.25 \\
\hline & 55.25 & 55.25 & 55.25 & 55.25 & 55.25 & 55.25 \\
\hline & 55.25 & 55.25 & 55.25 & 55.25 & 55.25 & 55.25 \\
\hline & 55.25 & 55.25 & 55.25 & 55.25 & 55.25 & 55.25 \\
\hline & 55.25 & 55.25 & 55.25 & 55.25 & 55.25 & 55.25 \\
\hline & 55.25 & 55.25 & 55.25 & 55.25 & 55.25 & 55.25 \\
\hline & 55.25 & 55.25 & 55.25 & 55.25 & 55.25 & 55.25 \\
\hline & 55.25 & 55.25 & 55.25 & 55.25 & 55.25 & 55.25 \\
\hline & 55.25 & 55.25 & 55.25 & 55.25 & 55.25 & 55.25 \\
\hline & 55.25 & 55.25 & 55.25 & 55.25 & 55.25 & 55.25 \\
\hline & 55.25 & 55.25 & 55.25 & 55.25 & 55.25 & 55.25 \\
\hline & 55.25 & 55.25 & 55.25 & 55.25 & 55.25 & 55.25 \\
\hline & 55.25 & 55.25 & 55.25 & 55.25 & 55.25 & 55.25 \\
\hline & 55.25 & 55.25 & 55.25 & 55.25 & 55.25 & 55.25 \\
\hline & 55.25 & 55.25 & 55.25 & 55.25 & 55.25 & 55.25 \\
\hline & 55.25 & 55.25 & 55.25 & 55.25 & 55.25 & 55.25 \\
\hline & 55.25 & 55.25 & 55.25 & 55.25 & 55.25 & 55.25 \\
\hline & 55.25 & 55.25 & 55.25 & 55.25 & 55.25 & 55.25 \\
\hline & 55.25 & 55.25 & 55.25 & 55.25 & 55.25 & 55.25 \\
\hline & 55.25 & 55.25 & 55.25 & 55.25 & 55.25 & 55.25 \\
\hline & 55.25 & 55.25 & 55.25 & 55.25 & 55.25 & 55.25 \\
\hline & 55.25 & 55.25 & 55.25 & 55.25 & 55.25 & 55.25 \\
\hline & 55.25 & 55.25 & 55.25 & 55.25 & 55.25 & 55.25 \\
\hline & 55.25 & 55.25 & 55.25 & 55.25 & 55.25 & 55.25 \\
\hline & 55.25 & 55.25 & 55.25 & 55.25 & 55.25 & 55.25 \\
\hline & 55.25 & 55.25 & 55.25 & & & \\
\hline $\mathrm{DJ}$ & 50.05 & 50.05 & 50.05 & 50.05 & & \\
\hline & 50.05 & 50.05 & 50.05 & 50.05 & 50.05 & 50.05 \\
\hline & 50.05 & 50.05 & 50.05 & 50.05 & 50.05 & 50.05 \\
\hline & 50.05 & 50.05 & 50.05 & 50.05 & 50.05 & 50.05 \\
\hline & 50.05 & 50.05 & 50.05 & 50.05 & 50.05 & 50.05 \\
\hline
\end{tabular}

Figure 43 Values from original map files that need replaced in the newly exported map files to increase cell size 
Once the values have been updated, the map files have successfully been adjusted to remove the border of aquifer properties from the Original CMG model. The adjusted porosity, permeability, and grid thickness map files were then all specified in Array Properties in the Anticline Thesis model.

Instead of using the initial water saturation map, a constant initial water saturation was assigned due to the limited variation of the initial saturation map. The initial saturation map varied from 0.01 to 0.05 throughout the reservoir portion and therefore a value of 0.05 was assigned to be the initial water saturation for the entire grid and for all layers.

The depth of the water oil contact point (DWOC) was original set to 4500ft in the Original CMG model when the reservoir was shaped as a partial dome. However after reshaping the reservoir, the specified DWOC would have effectively made almost half of the anticline be filled with water and no oil. Because of this and the desire to produce oil from all layers and from most of the reservoir, the DWOC was moved to $4900 \mathrm{ft}$. At the depth of $4900 \mathrm{ft}$, only a few small portions of the reservoir, at the deepest portions, would be filled with water. The water filled portions of the reservoir are shown by arrows below in Figure 44.

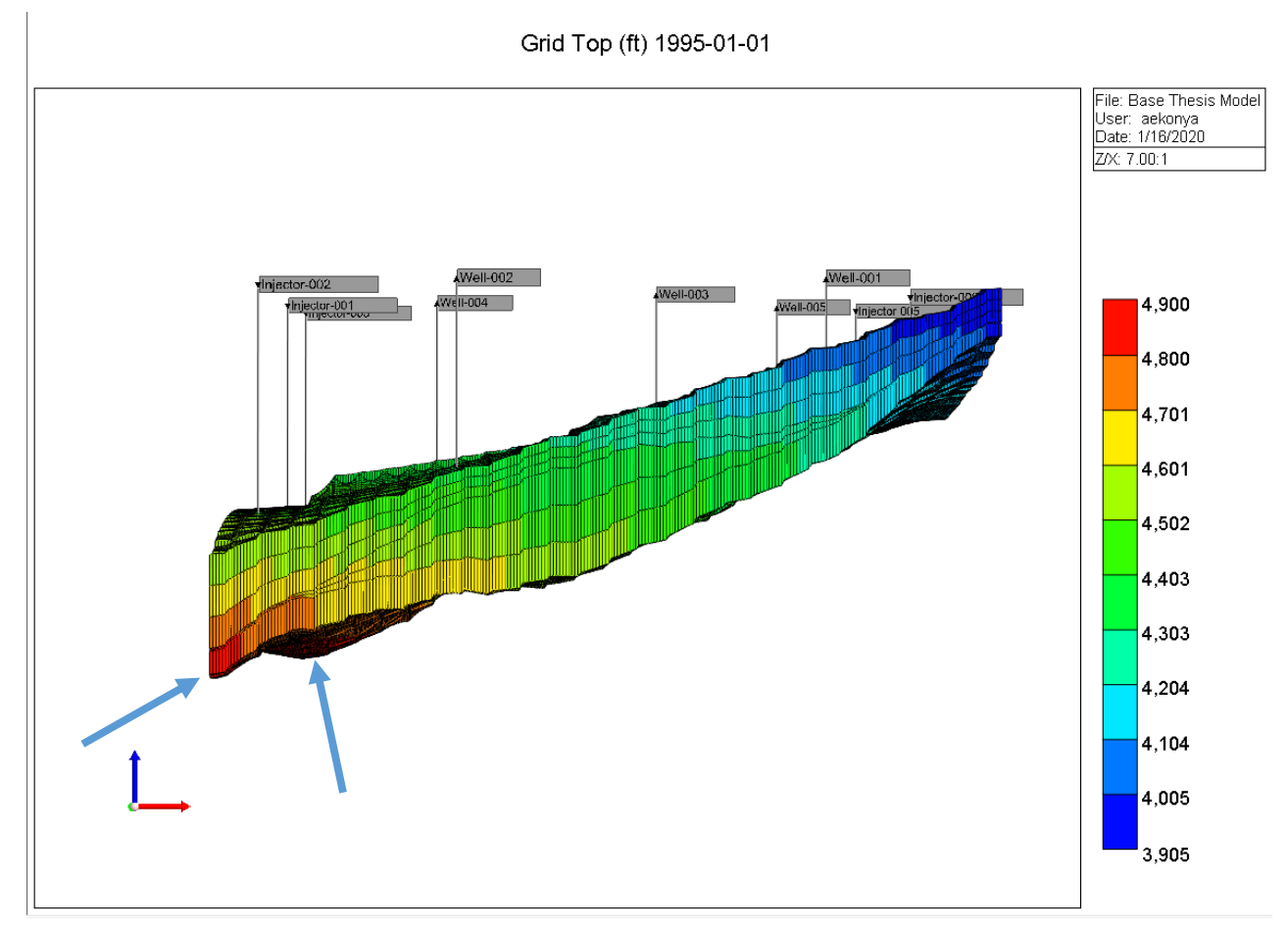

Figure 44 Water filled portions of the reservoir due to change in DWOC to $4900 \mathrm{ft}$ in Anticline Thesis model

Non-vertical communication was established for each geological layer by using the Transmissibility K Multiplier function (TransK) within Builder. There are three geological layers where Layers 1 and 2, Layers 3 and 4, and Layers 5 and 6 make up each respective geological layer. TransK was used instead of allowing the permeability in the $\mathrm{K}$ direction to equal 0 because if the permeability in the $\mathrm{K}$ direction is equal to 0 then the layer cannot communicate with the layer above it nor the layer below it. Due to the fact that there are two simulation layers per geological layer, communication does need to occur between certain layers. TransK was set to 0 in Layers 2 and 4 due to the definition of the TransK function's application provided in the IMEX manual. The manual states "If flow between a pair of blocks is considered, and they both lie on the same grid, then it is the block with the lowest $\mathrm{K}$ index for a $\mathrm{K}$ direction pair, that supplies the multiplier... that is, a directional multiplier applies to a block's interface 
with its neighbor with the higher index in that direction" (Computer Modeling Group LTD., 2017). Therefore by setting TransK equal to 0 in Layer 2, Layer 2 has no communication with Layer 3 (which has a higher index), but does have communication with Layer 1, as intended. This application was tested with a simplified model that allowed gas injection into Layers 2, 5, and 6 in order to see which layers the gas would propagate into. As seen in Figure 45, even though gas is not injected into Layer 1, it has a higher gas saturation value because it is allowed to communicate with Layer 2, which is currently injecting. Communication was successfully limited between Layers 2 and 3 and Layers 4 and 5 as desired, proving that the TransK multiplier was effective.

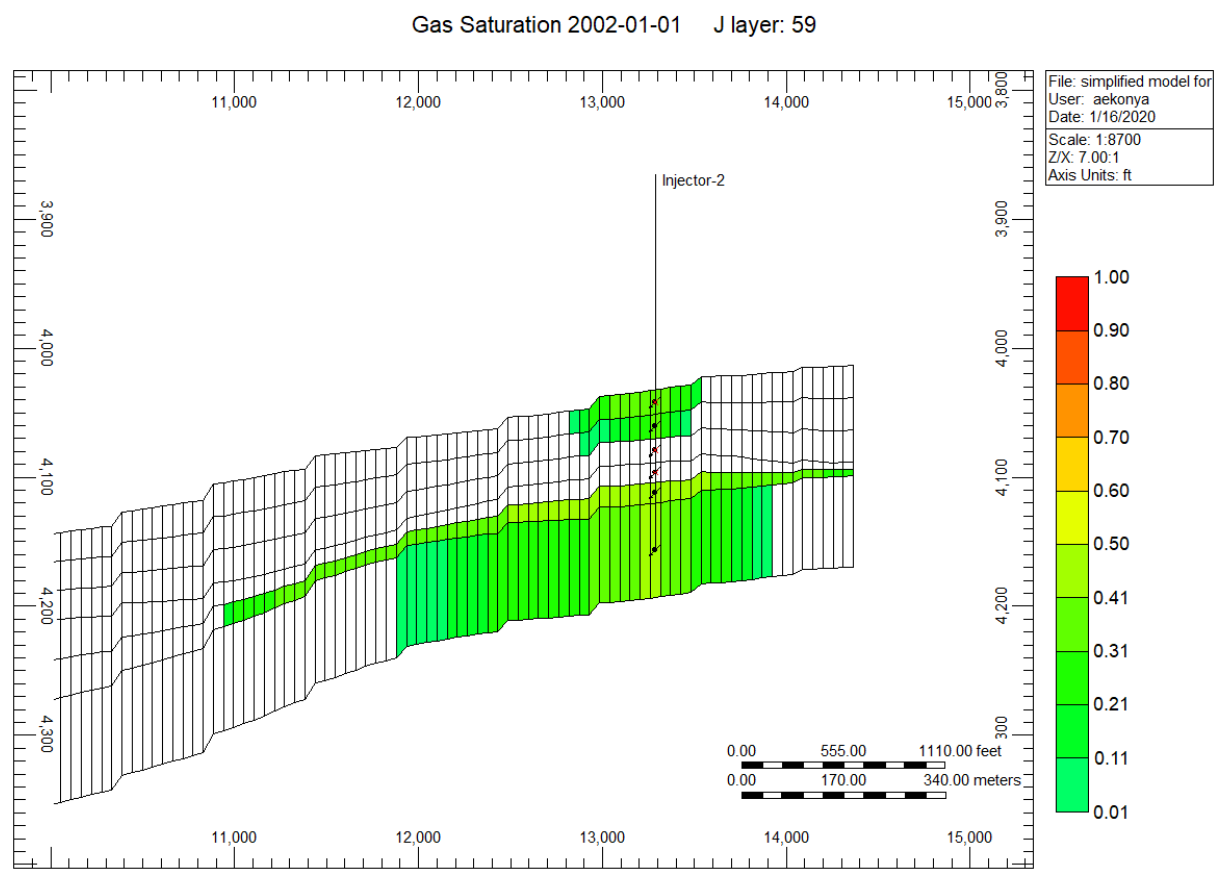

Figure 45 Gas saturation at Injector-002 for the TransK multiplier test

Sealed faults were also added to the Anticline Thesis model by creating a new map file. The "Add points with mouse clicks" option was used to select the desired locations of the two faults to be added to the model. One fault was placed on the lower right side of the reservoir (from an aerial perspective) directly between several wells and injectors. Another fault was placed in the middle of the reservoir where one end was near Injector-002. The location of the faults respective to the wells can be seen from an aerial perspective below. Once the locations were selected and added to each respective fault, the .msh file was saved. The "Set Transmissibility Multiplier across Faults" option was then used to set the multiplier to 0 for both faults. In doing this, the faults became sealing faults in which a pressure difference could be seen the fault, as desired and shown in Figure 47. 


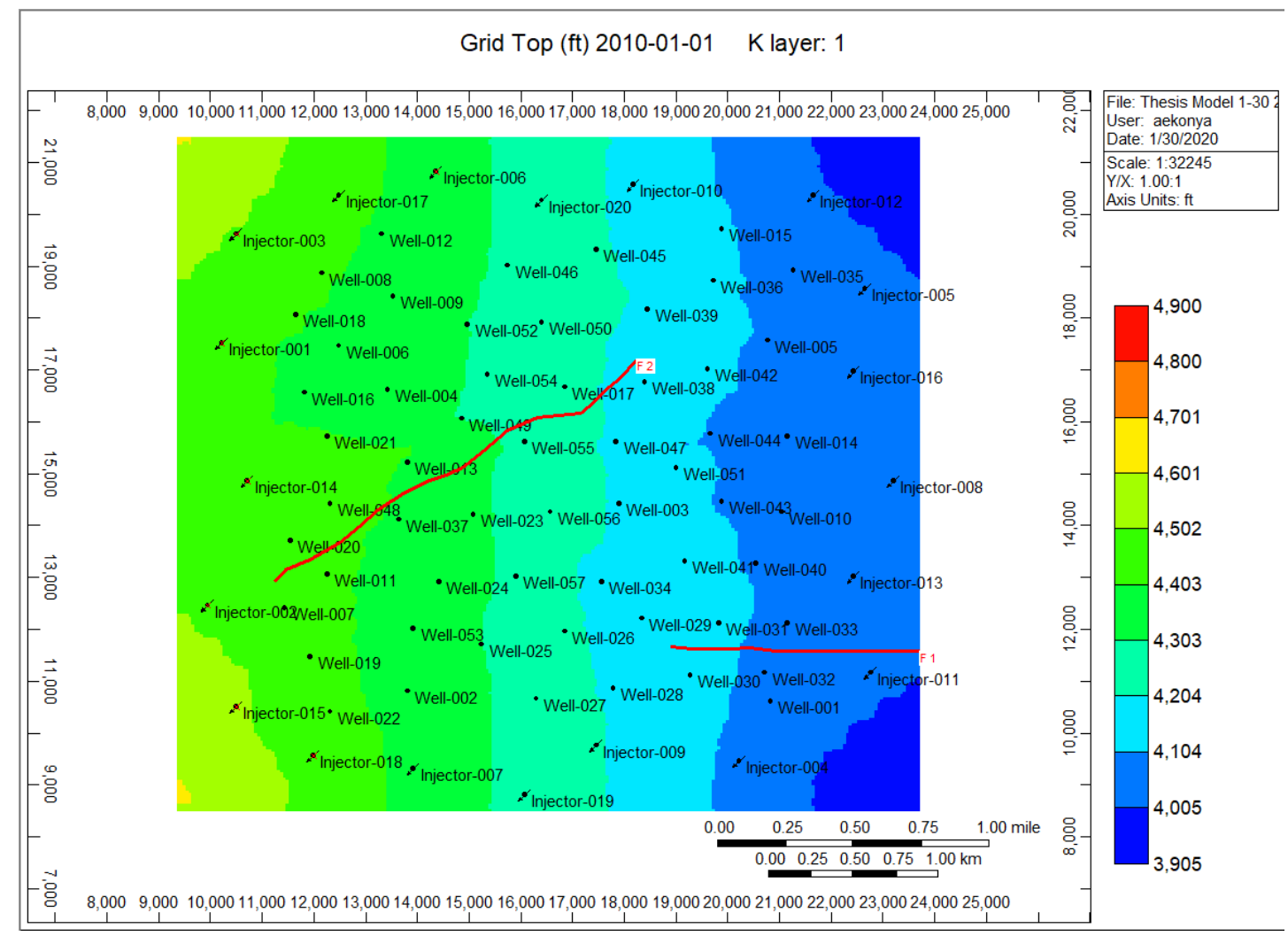

Figure 46 Aerial view of sealed fault locations in Anticline Thesis model

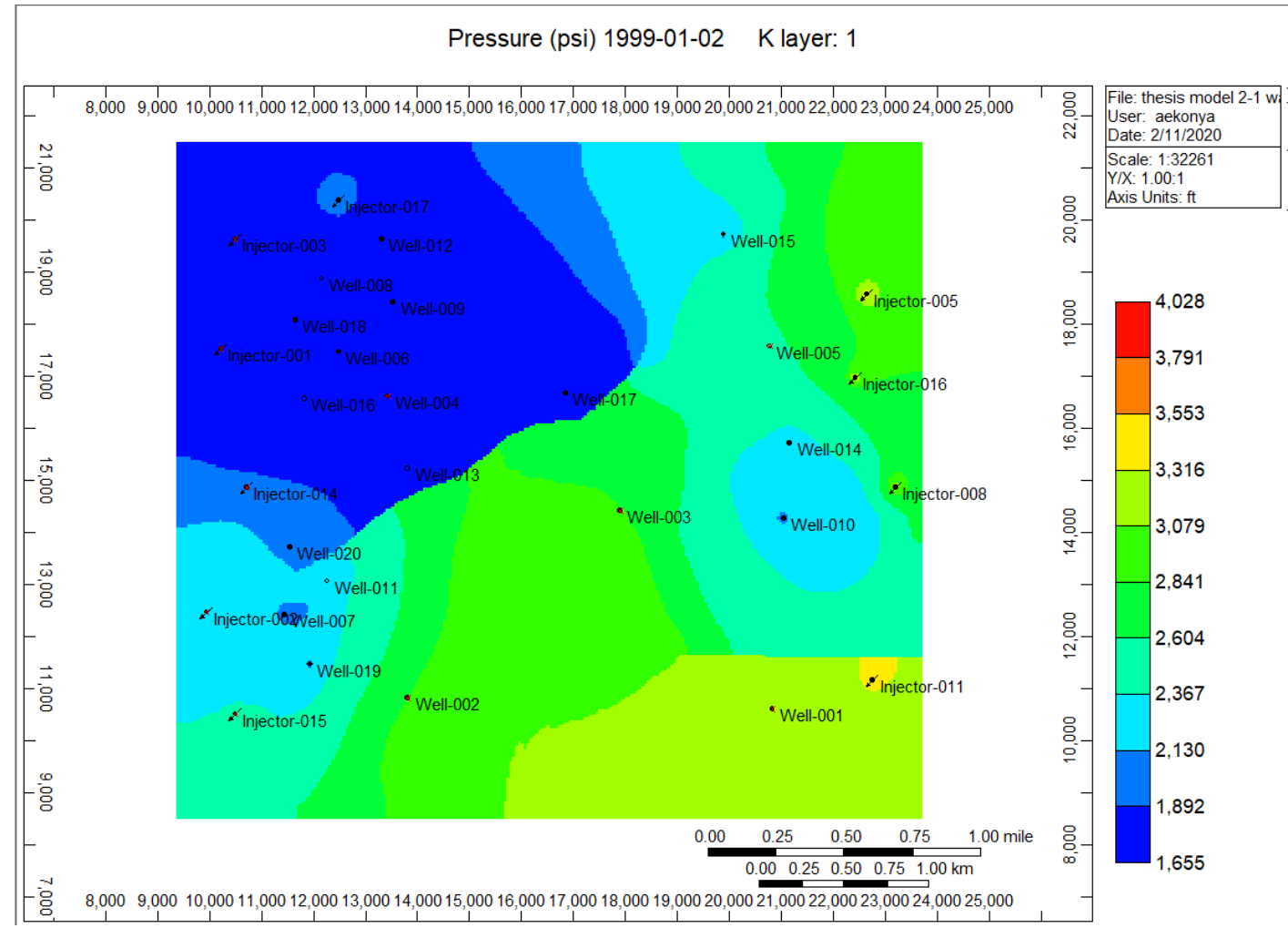

Figure 47 Pressure difference caused by the two sealing faults in Anticline Thesis model 
The production wells were completed in four phases of production where the fourth phase was split into four sub-phases, allowing the gradual increase of reservoir development up to 57 wells. In Table 9 below are the various phases, the year the phase began, and the number of production and injection wells that were added during that phase. Once the wells were added to the reservoir, the wells were allowed to produce, unlike the Original CMG model. For simplicity, the production wells were drilled in chronological order to allow completion throughout the entirety of the reservoir. A depiction of the various well locations according to each phase and type of well is shown in Figure 49 with its corresponding legend in Figure 48.

Table 9 Production well completion phases for Anticline Thesis model

\begin{tabular}{|c|c|c|c|}
\hline Phase & Year Wells Were Added & $\begin{array}{c}\text { Number of Production } \\
\text { Wells Added }\end{array}$ & $\begin{array}{c}\text { Number of } \\
\text { Injection Wells } \\
\text { Added }\end{array}$ \\
\hline 1 & 1995 & 5 & 5 \\
\hline 2 & 1998 & 15 & 5 \\
\hline 3 & 2002 & 17 & 5 \\
\hline 4 Part 1 & 2007 & 5 & 2 \\
\hline 4 Part 2 & 2008 & 5 & 1 \\
\hline 4 Part 3 & 2009 & 5 & 1 \\
\hline 4 Part 4 & 2010 & 5 & 1 \\
\hline
\end{tabular}

\begin{tabular}{|ll|}
\hline Color & \multicolumn{1}{c|}{ Shape } \\
Green- Oil Producer & $\triangle$ Phase 1 \\
Orange- Gas Injector & Phase 2 \\
Blue-Water Injector & Phase 3 \\
& $\square$ Phase 4 Part 1 \\
& Phase 4 Part 2 \\
& $\bigcirc$ Phase 4 Part 3 \\
& $\bigcirc$ Phase 4 Part 4 \\
\hline
\end{tabular}

Figure 48 Legend for well locations map of each phase of production for Anticline Thesis model 


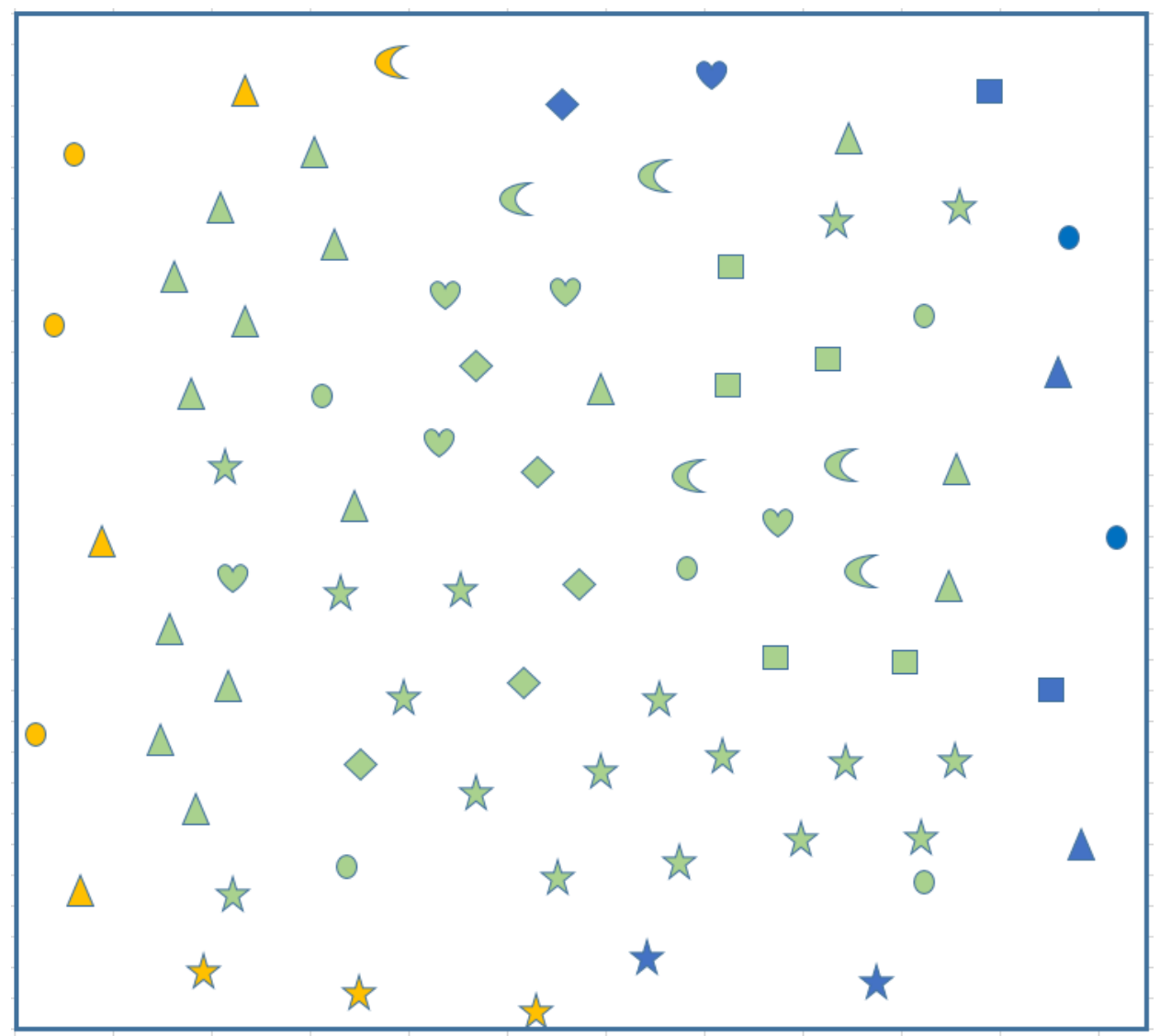

Figure 49 Well locations map for each phase of production for the Anticline Thesis Model

In the Original CMG model obtained, all of the injection wells were drilled and completed on the first day of production, January $1^{\text {st }}, 1995$. Water was injected in all injection wells. In the Anticline Thesis model, the injection wells were completed in phases, similar to the production wells. The injection wells began injection based on their proximity to the production wells that were currently producing. Both gas and water injection wells were specified where all of the injectors on the left side of the reservoir or down dip, were gas injection and all of the injectors on the right side of the reservoir and up dip, were water injection. There are 10 water and gas injection wells each. The location of the gas and water injection wells can be seen in orange and blue with shapes denoting their respective phases in Figure 49 above.

Upon running the model after specifying the gas injection wells, the model would fail and a nonconvergence error would be received. After several tests, this error was due to the constraints applied to the injector wells. In the Original CMG model, the only constraint applied to the wells was a maximum bottom-hole pressure of 4,000 psi. While half of the wells were switched to gas injection in the Anticline Thesis model, the original injection constraint of a maximum bottom-hole pressure of 4,000 psi was left intact. This effectively allowed the injectors to inject as much gas as was needed until it reached and maintained a bottom-hole pressure of 4,000 psi. For the gas injectors to reach that pressure and maintain it, incredibly large volumes of gas were injected into the reservoir and resulted in the model failing to converge on saturation and pressure calculations for the areas near the gas injectors. The non-convergence error was solved by setting a primary constraint of a maximum of 20,000,000 $\mathrm{ft}^{3} /$ day STG surface gas injection rate followed by a secondary constraint of a maximum bottom-hole pressure of 4,000 psi. Because the convergence error was solely due to the gas injection, the only injection constraint on the water injectors continues to be a maximum bottom-hole pressure of 4,000 psi. 
Water injection wells were specified to inject into all layers, however the gas injection wells were completed in different layers. This was done in an attempt to even out the gas injected into each layer as some layers are easier to inject into. The table below outlines which layers each injector injects into.

Table 10 Gas injection well completions for the Anticline Thesis model

\begin{tabular}{|l|l|}
$\begin{array}{l}\text { Injector } \\
\text { Name }\end{array}$ & $\begin{array}{l}\text { Layers } \\
\text { Completed }\end{array}$ \\
\hline Injector-001 & Layer 2 \\
\hline Injector-002 & Layer 2 \\
\hline Injector-003 & Layers 3-6 \\
\hline Injector-006 & Layers 4-6 \\
\hline Injector-007 & All Layers \\
\hline Injector-014 & Layers 3-6 \\
\hline Injector-015 & Layer 2 \\
\hline Injector-017 & All Layers \\
\hline Injector-018 & Layers 3-6 \\
\hline Injector-019 & All Layers \\
\hline
\end{tabular}

Production wells also underwent a partial completion strategy where recompletion at a later time was utilized to replicate real world reservoir management methods. Table 11 below shows the layers completed at the start of each phase as well as the year each well was opened. The producing layers at each time step were chosen based on the well's location within the reservoir and its proximity to an injection well. Because breakthrough from an injection well can greatly affect production within a well, wells that were closest to injectors were completed and produced from all layers in an attempt to produce before breakthrough occurred. 
Table 11 Partial completion plan for all production wells in Anticline Thesis Model

\begin{tabular}{|c|c|c|c|c|c|c|c|c|}
\hline $\begin{array}{c}\text { Well } \\
\text { Name }\end{array}$ & $\begin{array}{l}\text { Year } \\
\text { Open }\end{array}$ & $\begin{array}{l}\text { Producing } \\
\text { Layer-P1 }\end{array}$ & $\begin{array}{l}\text { Producing } \\
\text { Layer-P2 }\end{array}$ & $\begin{array}{c}\text { Producing } \\
\text { Layer-P3 }\end{array}$ & $\begin{array}{c}\text { Producing } \\
\text { Layer-P4 } \\
\text { A } \\
\end{array}$ & $\begin{array}{c}\text { Producing } \\
\text { Layer-P4 } \\
\text { B } \\
\end{array}$ & $\begin{array}{c}\text { Producing } \\
\text { Layer-P4 } \\
\text { C } \\
\end{array}$ & $\begin{array}{c}\text { Producing } \\
\text { Layer-P4 } \\
\text { D } \\
\end{array}$ \\
\hline Well-01 & 1995 & 5,6 & 5,6 & $3,4,5,6$ & $3,4,5,6$ & $1,2,3,4,5,6$ & $1,2,3,4,5,6$ & $1,2,3,4,5,6$ \\
\hline Well-02 & 1995 & 5,6 & 5,6 & $3,4,5,6$ & $3,4,5,6$ & $1,2,3,4,5,6$ & $1,2,3,4,5,6$ & $1,2,3,4,5,6$ \\
\hline Well-03 & 1995 & 5,6 & 5,6 & $3,4,5,6$ & $3,4,5,6$ & $1,2,3,4,5,6$ & $1,2,3,4,5,6$ & $1,2,3,4,5,6$ \\
\hline Well-04 & 1995 & 5,6 & 5,6 & $3,4,5,6$ & $3,4,5,6$ & $1,2,3,4,5,6$ & $1,2,3,4,5,6$ & $1,2,3,4,5,6$ \\
\hline Well-05 & 1995 & 5,6 & 5,6 & $3,4,5,6$ & $3,4,5,6$ & $1,2,3,4,5,6$ & $1,2,3,4,5,6$ & $1,2,3,4,5,6$ \\
\hline Well-06 & 1998 & & All Layers & All Layers & All Layers & All Layers & All Layers & All Layers \\
\hline Well-07 & 1998 & & All Layers & All Layers & All Layers & All Layers & All Layers & All Layers \\
\hline Well-08 & 1998 & & All Layers & All Layers & All Layers & All Layers & All Layers & All Layers \\
\hline Well-09 & 1998 & & All Layers & All Layers & All Layers & All Layers & All Layers & All Layers \\
\hline Well-10 & 1998 & & All Layers & All Layers & All Layers & All Layers & All Layers & All Layers \\
\hline Well-11 & 1998 & & All Layers & All Layers & All Layers & All Layers & All Layers & All Layers \\
\hline Well-12 & 1998 & & All Layers & All Layers & All Layers & All Layers & All Layers & All Layers \\
\hline Well-13 & 1998 & & All Layers & All Layers & All Layers & All Layers & All Layers & All Layers \\
\hline Well-14 & 1998 & & All Layers & All Layers & All Layers & All Layers & All Layers & All Layers \\
\hline Well-15 & 1998 & & All Layers & All Layers & All Layers & All Layers & All Layers & All Layers \\
\hline Well-16 & 1998 & & All Layers & All Layers & All Layers & All Layers & All Layers & All Layers \\
\hline Well-17 & 1998 & & All Layers & All Layers & All Layers & All Layers & All Layers & All Layers \\
\hline Well-18 & 1998 & & All Layers & All Layers & All Layers & All Layers & All Layers & All Layers \\
\hline Well-19 & 1998 & & All Layers & All Layers & All Layers & All Layers & All Layers & All Layers \\
\hline Well-20 & 1998 & & All Layers & All Layers & All Layers & All Layers & All Layers & All Layers \\
\hline Well-21 & 2002 & & & $4,5,6$ & $4,5,6$ & $2,3,4,5,6$ & $2,3,4,5,6$ & $1,2,3,4,5,6$ \\
\hline Well-22 & 2002 & & & $4,5,6$ & $4,5,6$ & $2,3,4,5,6$ & $2,3,4,5,6$ & $1,2,3,4,5,6$ \\
\hline Well-23 & 2002 & & & $4,5,6$ & $4,5,6$ & $2,3,4,5,6$ & $2,3,4,5,6$ & $1,2,3,4,5,6$ \\
\hline Well-24 & 2002 & & & $4,5,6$ & $4,5,6$ & $2,3,4,5,6$ & $2,3,4,5,6$ & $1,2,3,4,5,6$ \\
\hline Well-25 & 2002 & & & $4,5,6$ & $4,5,6$ & $2,3,4,5,6$ & $2,3,4,5,6$ & $1,2,3,4,5,6$ \\
\hline Well-26 & 2002 & & & $4,5,6$ & $4,5,6$ & $2,3,4,5,6$ & $2,3,4,5,6$ & $1,2,3,4,5,6$ \\
\hline Well-27 & 2002 & & & $4,5,6$ & $4,5,6$ & $2,3,4,5,6$ & $2,3,4,5,6$ & $1,2,3,4,5,6$ \\
\hline Well-28 & 2002 & & & $4,5,6$ & $4,5,6$ & $2,3,4,5,6$ & $2,3,4,5,6$ & $1,2,3,4,5,6$ \\
\hline Well-29 & 2002 & & & $4,5,6$ & $4,5,6$ & $2,3,4,5,6$ & $2,3,4,5,6$ & $1,2,3,4,5,6$ \\
\hline Well-30 & 2002 & & & $4,5,6$ & $4,5,6$ & $2,3,4,5,6$ & $2,3,4,5,6$ & $1,2,3,4,5,6$ \\
\hline Well-31 & 2002 & & & $4,5,6$ & $4,5,6$ & $2,3,4,5,6$ & $2,3,4,5,6$ & $1,2,3,4,5,6$ \\
\hline Well-32 & 2002 & & & $4,5,6$ & $4,5,6$ & $2,3,4,5,6$ & $2,3,4,5,6$ & $1,2,3,4,5,6$ \\
\hline Well-33 & 2002 & & & $4,5,6$ & $4,5,6$ & $2,3,4,5,6$ & $2,3,4,5,6$ & $1,2,3,4,5,6$ \\
\hline Well-34 & 2002 & & & $4,5,6$ & $4,5,6$ & $2,3,4,5,6$ & $2,3,4,5,6$ & $1,2,3,4,5,6$ \\
\hline Well-35 & 2002 & & & $4,5,6$ & $4,5,6$ & $2,3,4,5,6$ & $2,3,4,5,6$ & $1,2,3,4,5,6$ \\
\hline Well-36 & 2002 & & & $4,5,6$ & $4,5,6$ & $2,3,4,5,6$ & $2,3,4,5,6$ & $1,2,3,4,5,6$ \\
\hline Well-37 & 2002 & & & $4,5,6$ & $4,5,6$ & $2,3,4,5,6$ & $2,3,4,5,6$ & $1,2,3,4,5,6$ \\
\hline
\end{tabular}




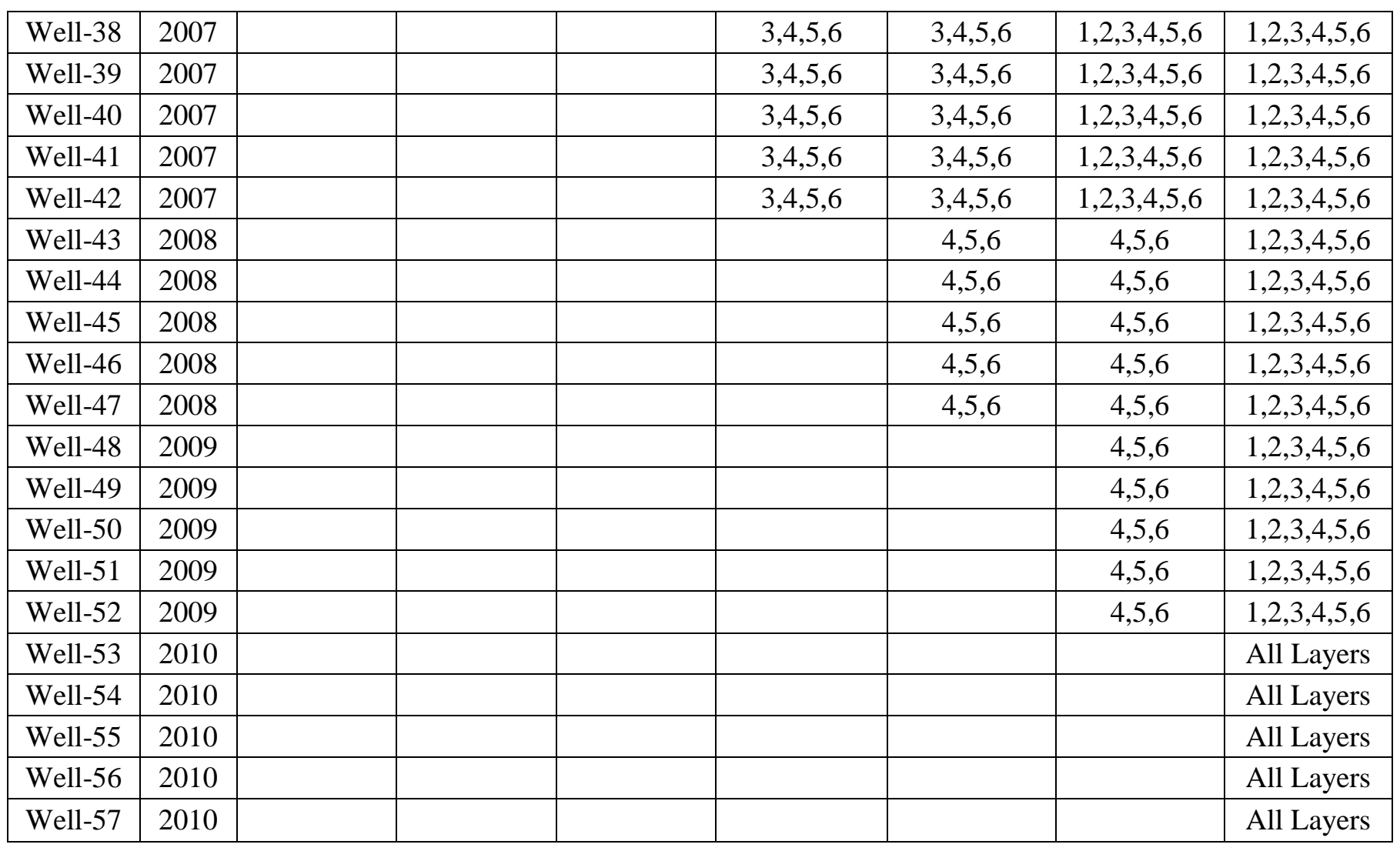

Production wells were also given random shut-in dates to simulate workover events that occur in industry. These shut-in dates could range from one whole month, several months in a row, to daily shutins. A code was utilized to randomly generate these dates by first assigning a random bottom-hole pressure value to every well for every day that the well is online after it has been added in its respective phase. The pressure value assigned is randomly generated within a specified range and is used to continuously adjust the minimum bottom-hole pressure constraint for the production wells. After extensive analysis was done on the reservoir pressure and production behavior with gas and water injection, the pressure range of 1600 psi to 1750 psi was chosen. This range was deemed most efficient because it would allow black oil to be produced as it is above the bubble point pressure of 1500 psi, but allow the minimum bottom-hole pressure constraint to be low enough that wells that have difficulty receiving pressure support from the injectors could still produce a moderate amount of oil. Once these pressure values were created and assigned to dates for each well, twenty percent of the total number of days of production were shut-in in 30 day increments. These 30 day shut-in increments could occur sporadically throughout the life of a well or several could happen consecutively, thus simulating various types of workovers performed in the field. After these monthly shut-ins were added, five percent of the remaining producing dates for all the wells were shut-in on a daily basis.

Wells were brought back online from the shut-in dates while simultaneously adjusting the production well's minimum bottom-hole pressure to one of the random values selected. While the well is producing, the well's minimum bottom-hole pressure constraint is adjusted daily in order to vary the pressure response for the well. This creates a realistic pressure effect for the production wells because if not applied, the production wells will produce any amount of oil until it reaches and maintains the minimum bottom-hole pressure constraint, therefore keeping a constant pressure throughout the life of the 
well which is unrealistic. The pressure response therefore varies slightly within the 1600 to 1750 range, no spikes from 1600 directly to 1750 or dramatic spikes of any value, are observed. The code was written in a way that the shut-in dates and the altering of the minimum bottom-hole pressure constraints were written to a text file in the format required for CMG. Therefore, after the text file of the desired shut-in dates and altering commands was obtained, the contents could be copied and pasted directly into the .DAT file. An example of a portion of the text file is shown in Figure 50.

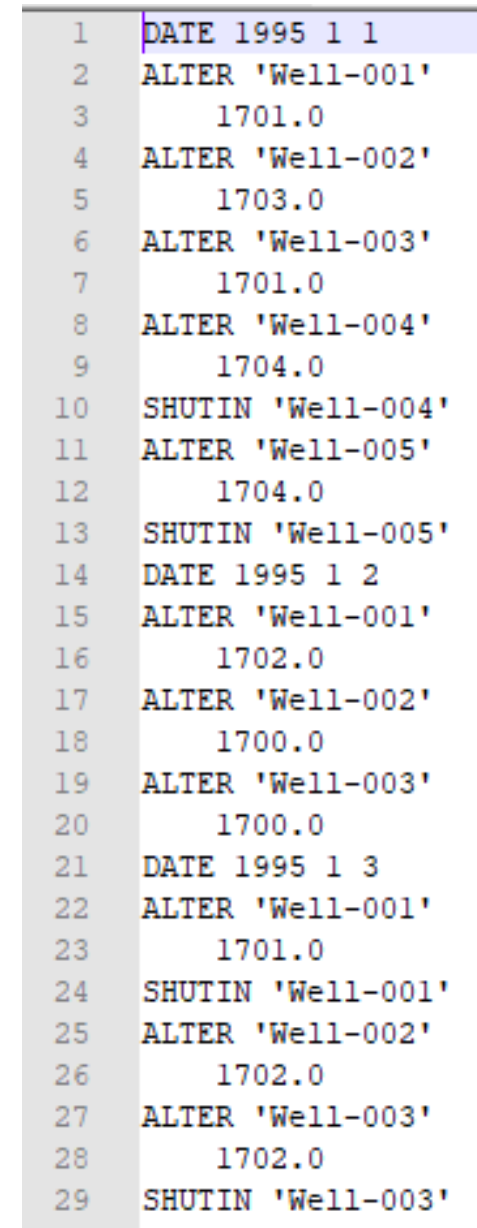

Figure 50 Example of text file output from code used to generate random shut-in dates and alter production wells' minimum bottom-hole pressure constraints

Along with the minimum bottom-hole pressure constraint as the primary production well operational constraint, a secondary monitoring constraint was assigned to all production wells. A monitoring water cut fraction value of 0.9 was assigned as shut-in repeat where if a well was found to violate that constraint, the time step would be repeated with that well shut-in. This prevents the large volume of water being produced that day if it violated the water cut, and all of the calculations were repeated. In using a water-cut constraint, an industry standard of reducing the produced water was applied which also allowed the injected water to propagate farther and provide pressure support more efficiently. 


\subsection{Exporting and Formatting Data}

Multiple codes were written within Python by Yvon Martinez in order to streamline the data formatting process for Imagine. The data was formatted into several different formats which are outlined below.

\subsection{Exported Data}

Due to the limited capability of CMG to write SPECIAL OUTSRF commands, two identical runs with the exception of different SPECIAL OUTSRF commands were performed. This allowed the same values for production, etc. to be obtained, however the values for the eight cells surrounding the well center and the well center grid block were obtained for all six layers for all 77 wells (producers and injectors) for oil saturation, gas saturation, water saturation, and pressure. Data was exported from CMG Results on a well basis, layer basis, and the special history basis on a monthly time increment. Data exported on a layer basis and well basis only contained values for when the well was open. Data on a layer basis, even though it is formatted for the well center, also did not provide saturation values. The special history data however is available for all dates within the simulation and must be specified by specific block addresses, one by one. Because of the aforementioned reasons, in order to have pressure values at all time steps and to have saturation values, exported data on a monthly special history basis is essential. A table including the attributes in each export is shown below.

Table 12 CMG data export attributes

\begin{tabular}{|l|l|l|}
\hline Monthly Well Based & Monthly Layer Based & Monthly Special History \\
\hline Cumulative Gas SC (ft3) & Gas Rate SC-Monthly (ft3/day) & Gas Saturation \\
\hline Cumulative Oil SC (ft3) & Oil Rate SC-Monthly (bbl/day) & Oil Saturation \\
\hline Cumulative Water (ft3) & Water Rate SC-Monthly (bbl/day) & Water Saturation \\
\hline Gas Oil Ratio (ft3/bbl) & Well Block Pressure (psi) & Pressure \\
\hline Gas Rate SC-Monthly (ft3/day) & Well Pressure (psi) & \\
\hline Oil Rate SC-Monthly (bbl/day) & & \\
\hline Water Cut-\% & & \\
\hline Water Rate SC-Monthly (bbl/day) & & \\
\hline Well Bottom-hole Pressure (psi) & & \\
\hline
\end{tabular}

The monthly well based export, contains the aforementioned attributes for every well, including injectors, in separate tabs. Within each well's tab is the value for the attribute for every month of the simulation, from January $1^{\text {st }}, 1995$ to January $1^{\text {st }}, 2017$ which is a total of 265 months. The monthly layer based export had a separate tab for every layer, therefore each of the 77 wells had six tabs each. Within each of these tabs were the aforementioned attributes for all of the 265 months. Due to how the models had to be run for the special history, the monthly special history was exported by attribute for producers and injectors respectively. Therefore there was one excel file for the producers' gas saturation and one excel file for the injector's gas saturation values. These two excel sheets were manually combined into one excel sheet for all of the gas saturation values for all of the wells. This process was done for all of the attributes, thus yielding four excel sheets total. Within each attributes' excel sheet is one tab that consists of the gas saturation, etc. values for the 4,158 grid blocks for the 265 months.

The property maps for grid bottom, grid paydepth, grid thickness, porosity, permeability in I, J, and $\mathrm{K}$, grid top, and initial water saturation were exported in XYZ format for every layer including the 
well locations in each file. This was later used to collect and format the static data for use in Imagine and for record keeping purposes.

\subsection{MS Format}

MS format is the format used for in house record keeping. This format contains attribute properties in columns for each producer and injectors' rows. For the static data, there is one row for each well where the following attributes are specified for each of the six layers for that well: grid bottom, grid top, grid paydepth, grid thickness, initial water saturation, permeability I, permeability J, permeability K, and porosity. Also specified for every well is its $\mathrm{x}$ and y location, top, TVD (true vertical depth), and its well type. Static as dynamic data is also created in MS format due to the weighted average of permeability and porosity changing when wells are recompleted and layers are added. One excel sheet contains the information for one attribute of the following four; porosity, permeability I, permeability J, and permeability K. Each column within the sheet contains the weighted average with respect to grid thickness of the attribute for a well where each row details the value at that monthly time step. As wells are recompleted or brought online, the values at that time step for that specific well will change where the blank cells represent wells that are not online yet.

The special history data was also processed for the MS format for all 77 wells. Only one excel sheet and one tab is used for all of the information where each column is a different attribute per layer. The attributes included are oil saturation, water saturation, gas saturation, and grid block pressure (also known as reservoir pressure) as well as the average for all of the layers for the aforementioned properties. Each row includes the data for one well at one monthly time step. Then the next row is for the same well and the next time step until all of the monthly time steps are reported and the next well begins.

The last subset of data within the MS format was dynamic data. Gas, oil, and water saturations and grid block pressure were also reported in a dynamic format, however instead of including all of the layers' values, only the average for every well's six layers is reported. The columns denote the well name and the rows are the monthly time steps. Each saturation is reported in its own excel sheet. Well pressure or flowing bottom-hole pressure (FBHP) has the same format as the previously described grid block pressure in its own excel document. Finally, a dynamic sheet containing the well pressure (FBHP), oil rate, gas rate, water rate, and well block pressure (Reservoir Pressure) for all wells at all monthly time steps is created. These values are reported for all six layers as well as an average where the time steps are the rows and the properties are the columns.

\subsection{Metrics Format}

The Metrics format was used specifically for import into Imagine. While the static data for the MS format and the Metric format are formatted same, averages needed to be calculated for import into Imagine. The required values were gathered and averaged from the MS format static data file and one value was produced for each well for each parameter. On the other hand, the dynamic data is formatted differently. Instead of the monthly dates organized by rows, the monthly dates are the columns. Each row in the Metrics format is therefore the well name, and only one property can be represented in each excel sheet. The following properties are formatted for import into Imagine: completion footage, flowing bottom-hole pressure, reservoir pressure, oil saturation, gas saturation, water saturation, injected water rate, injected gas rate, produced oil rate, produced gas rate, and produced water rate. Due to the recompletion of wells with time, porosity, permeability I, permeability $\mathrm{J}$, and permeability $\mathrm{K}$ are also 
organized in a dynamic format. This was done to account for the difference in the weighted average when a new layer is added because porosity and permeability are specified for every layer. Therefore, the porosity and permeabilities also had a dynamic format even though they are typically thought of as static attributes.

\subsection{Data Preparation Calculations}

Imagine does not accept data per layer, it is only compatible with data imported per well. Because of this, several calculations needed to be performed in order to provide realistic values per well when a majority of the data exported from CMG was provided for each well and for each layer. The equations used, beginning with the static data, are explained in detail below.

The static data used for import into Imagine contained the well name, well type, $\mathrm{x}$ and $\mathrm{y}$ locations, porosity as a percentage, permeability I, permeability J, permeability $\mathrm{K}$, initial water saturation as a percentage, top, TVD, and pay thickness for every well. Pay thickness was calculated by taking the summation of all six layers' grid thickness. Porosity was calculated by using the weighted average equation below where the "L" represents Layer. Top was assigned the value of Grid Top from Layer 1 for all wells. TVD was deemed to be the Grid Bottom from Layer 6 for all wells. The equation to calculate the weighted average of all three permeabilities, very similar to porosity, is also shown here. Because initial water saturation was set to be 0.05 for the entire grid, $5 \%$ was set for every well.

$$
\begin{gathered}
\text { Equation 1 Porosity weighted average equation } \\
\varphi_{\text {well }}=\frac{\sum h_{L 1} * \varphi_{L 1}+h_{L 2} * \varphi_{L 2}+h_{L 3} * \varphi_{L 3}+h_{L 4} * \varphi_{L 4}+h_{L 5} * \varphi_{L 5}+h_{L 6} * \varphi_{L 6}}{\sum h_{1}+h_{2}+h_{3}+h_{4}+h_{5}+h_{6}} * 100 \\
\text { Equation 2 Permeability I, J, and K weighted average equation } \\
k_{\text {well }}=\frac{\sum h_{L 1} * k_{L 1}+h_{L 2} * k_{L 2}+h_{L 3} * k_{L 3}+h_{L 4} * k_{L 4}+h_{L 5} * k_{L 5}+h_{L 6} * k_{L 6}}{\sum h_{L 1}+h_{L 2}+h_{L 3}+h_{L 4}+h_{L 5}+h_{L 6}}
\end{gathered}
$$

The static as dynamic data, created for porosity and permeability, used the same equations as above, but took into account the partial completion applied to the wells. A partial completion excel file was made where the dates that each individual layer was opened and closed was recorded, whether it was due to assigned recompletion of the well or due to the water cut constraint being reached and the well shut-in. Whenever the well was not online or shut-in after reaching water cut, the value for porosity and permeability were calculated using the weighted average of all layers. In doing this, the possibility of teaching the neural network that a porosity and permeability value of zero can occur, is avoided. Whenever the well was open, the weighted average of only the open layers, denoted as Open Layers Permeability or Open Layers Porosity, was found in order to more accurately teach the neural network that different magnitudes of porosity and permeability can aid in achieving varying amounts of production.

Once the water, gas, and oil rates exported from $\mathrm{CMG}$ per well were formatted into the Metrics format for import into Imagine, the data for injector and producers were separated into two excel files. The rates exported for the injectors are the amounts injected whereas the rates from the producers were produced. These two properties need to exported separately. The partial completion excel file is again used to create the completion footage with respect to time file. The pay thickness is added for only the open layers and if the well is closed, the well is given a 0 value. Flowing bottom-hole pressure (FBHP) is computed using the weighted average equation below and the "Well Pressure" values from CMG. 
Flowing bottom-hole pressure is weighted with only the grid thickness of each formation because FBHP is a function of operational constraints controlled by the operator. When the well is producing, only the open layers are used in the equation, when the well is shut-in, the FBHP is equal to the reservoir pressure at that time for that well. The two pressure values are set equal to each other because the pressure difference causes production, thus giving the neural network the opportunity to learn the consequence of having a pressure differential versus not having one.

Equation 3 Flowing bottom-hole pressure weighted average equation

$$
F B H P_{\text {well }}=\frac{\sum P_{L 1} * h_{L 1}+P_{L 2} * h_{L 2}+P_{L 3} * h_{L 3}+P_{L 4} * h_{L 4}+P_{L 5} * h_{L 5}+P_{L 6} * h_{L 6}}{\sum h_{1}+h_{2}+h_{3}+h_{4}+h_{5}+h_{6}}
$$

Reservoir pressure also utilizes a weighted average equation, but with respect to kh, permeability I multiplied by grid thickness. Reservoir pressure is the response of the reservoir and is directly dependent on the fluid flow through the rock. Consequently, permeability (which also takes into account porosity) and grid thickness must be used. While the well is shut-in the weighted average as shown below is computed for all reservoir layers, while the well is producing, only the open layers are accounted for in the equation.

Equation 4 Reservoir pressure weighted average equation

$=\frac{P r_{\text {well }}}{\sum P_{L 1} * h_{L 1} * k_{L 1}+P_{L 2} * h_{L 2} * k_{L 2}+P_{L 3} * h_{L 3} * k_{L 3}+P_{L 4} * h_{L 4} * k_{L 4}+P_{L 5} * h_{L 5} * k_{L 5}+P_{L 6} * h_{L 6} * k_{L 6}}$

Oil, gas, and water saturations are computed in the same exact manner as reservoir pressure on account of also being directly dependent on the fluid flow through the rock and the ability of that fluid to be displaced. All of the saturation values were given as percentages, therefore each value was multiplied by 100 .

\subsection{Top Down Model Data Import}

Three different types of data were imported into the Imagine software: static, static as dynamic, and dynamic data. The figures below shows both the window used to select and load the desired files, as well as the window used to select the attributes being imported from the drop down options. An example window for static data, dynamic data, and production rate data are included here.

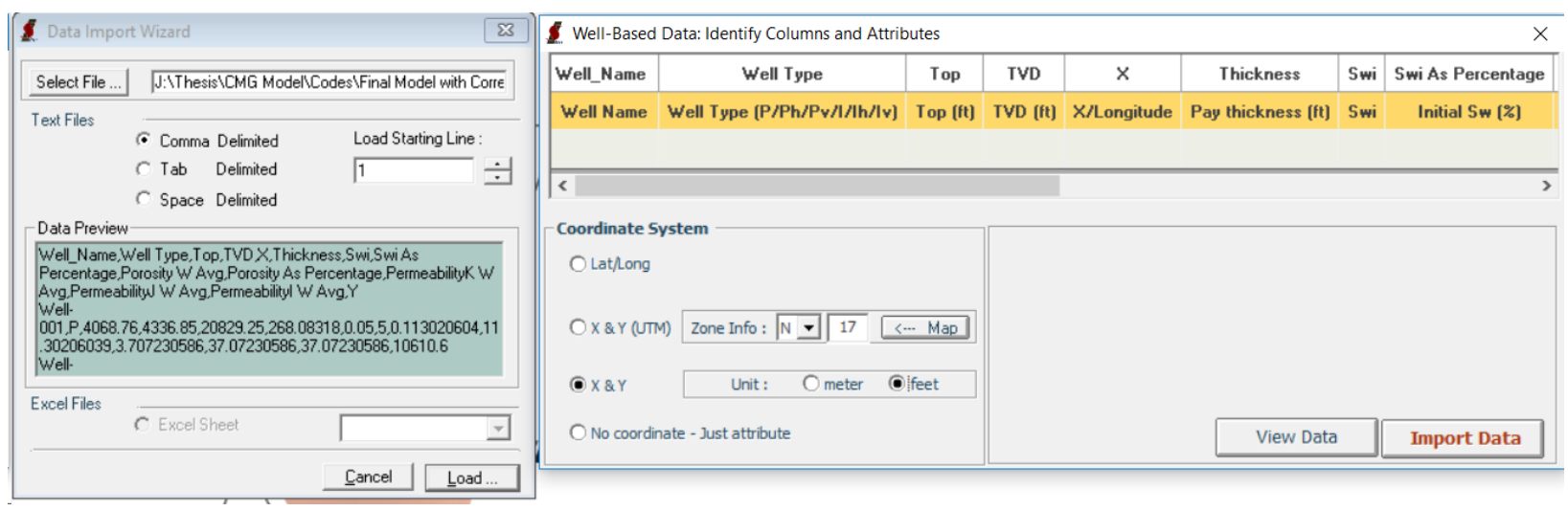

Figure 51 Static data import windows in Imagine 


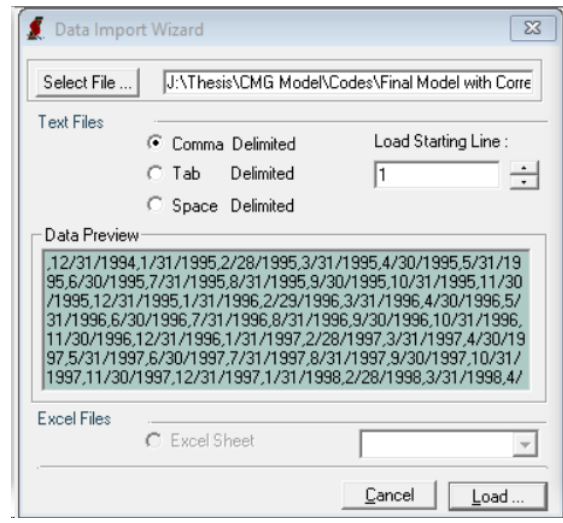

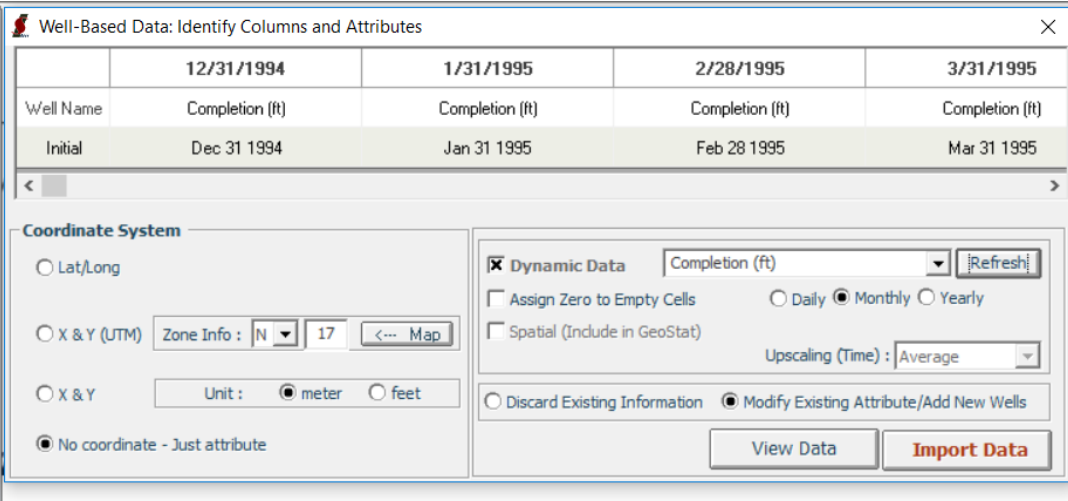

Figure 52 Dynamic data import windows into Imagine

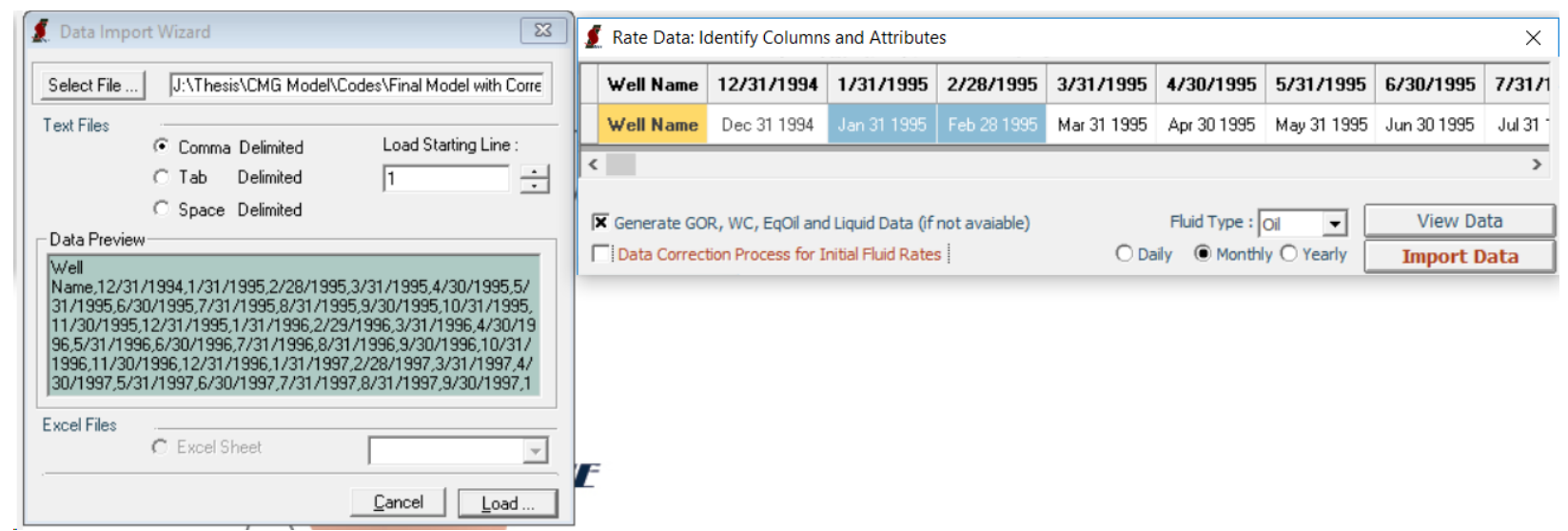

Figure 53 Production rate import windows in Imagine

\subsection{Top Down Model Static Modeling}

After all of the data has been imported, the static modeling for the Top Down Model must be completed. This consists of first created a Cartesian grid for which the size of each grid can be specified in order to ensure that two wells are not within the same grid block. The grid block size used for all of these TDMs is 5 acres. Next, the reservoir boundary needs to be drawn around the wells. The boundary does not need to be exact, but should be as similar as possible to the actual shape of the reservoir. The boundary is saved before starting to calculate the geostatistics. The final boundary and grid is shown below. 


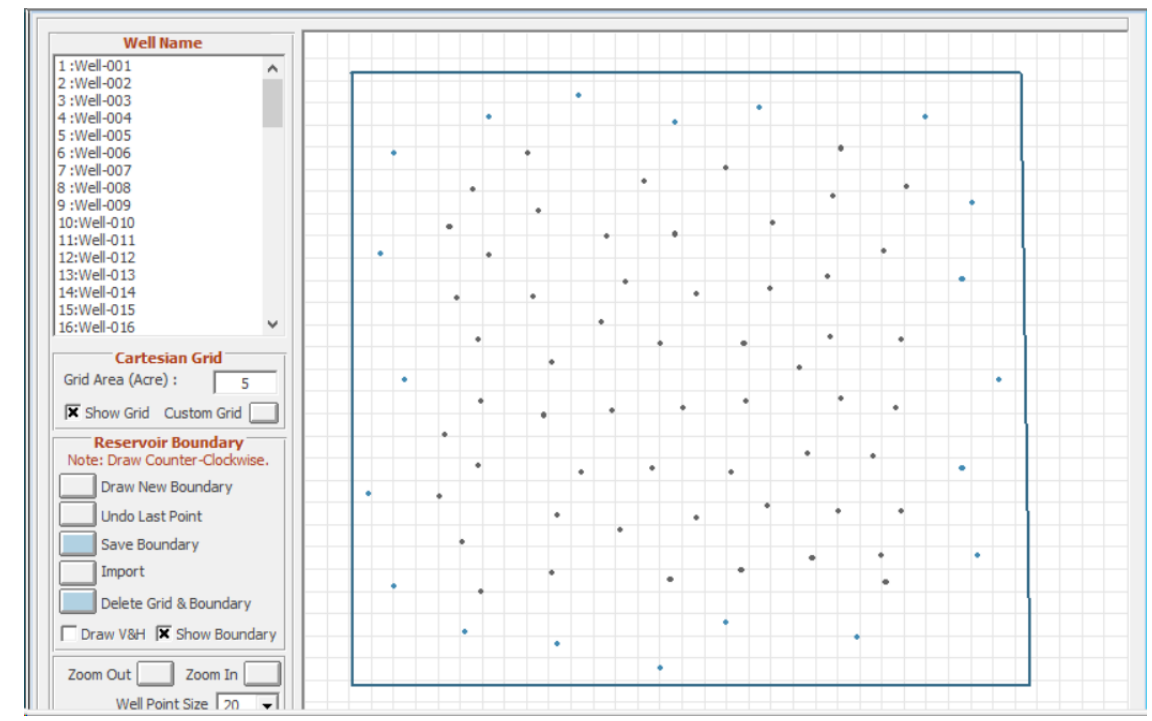

Figure 54 Cartesian grid and reservoir boundary creation in Imagine

The geostatistics are calculated for all attributes listed as being spatial properties, or in other words being a reservoir specific property. Spatial properties include permeability, porosity, water saturation, oil saturation, gas saturation, etc. There are several methods that can be used, however the inverse weighting method is used to calculate all geostatistics for every TDM. The window used to calculate geostatistics is shown below.

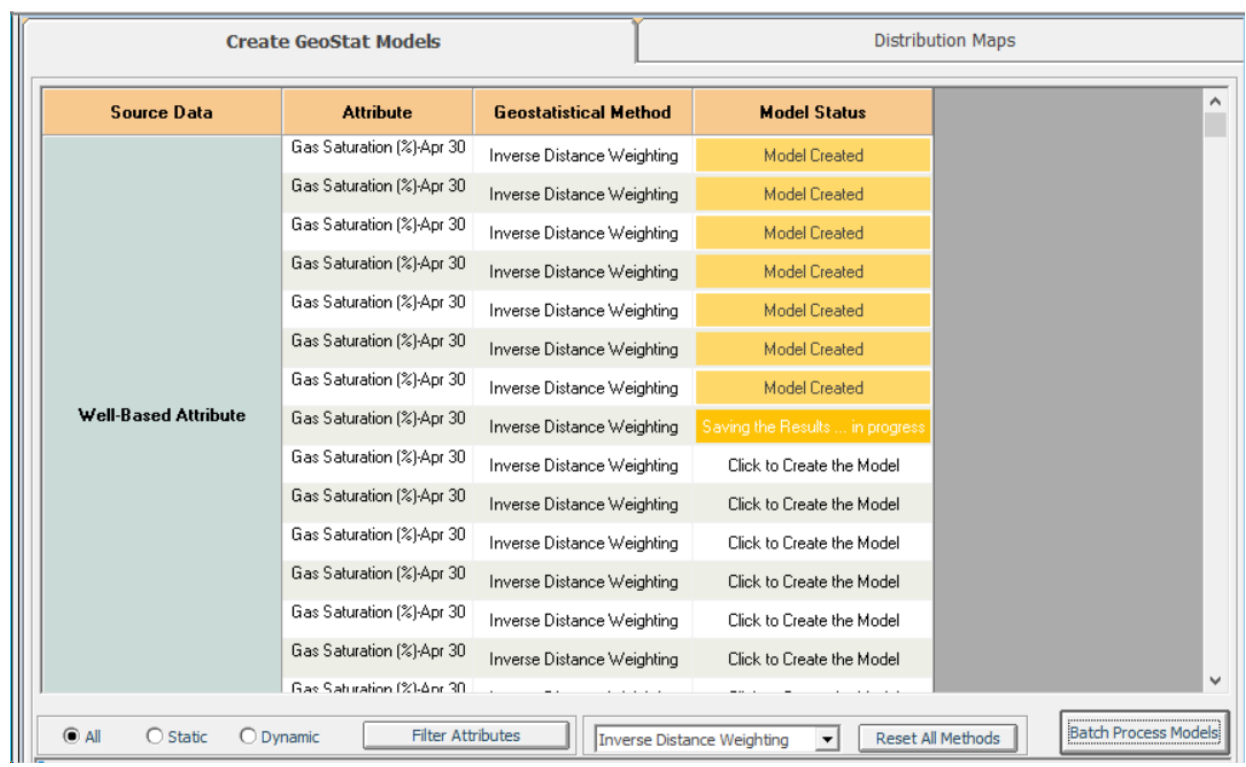

Figure 55 Geostatistics being calculated for spatial properties using the inverse distance weighting method

Next in the static modeling process is reservoir delineation. Reservoir delineation consists of dividing the reservoir into drainage area blocks for each well taking into consideration the boundary drawn in the previous step. Two methods can be used, the Voronoi method and the Clustering method. For all of the TDMs, the Voronoi method was used to perform reservoir delineation. The completed reservoir delineation is shown in Figure 56. The last step of the static modeling is the volumetric calculations. 


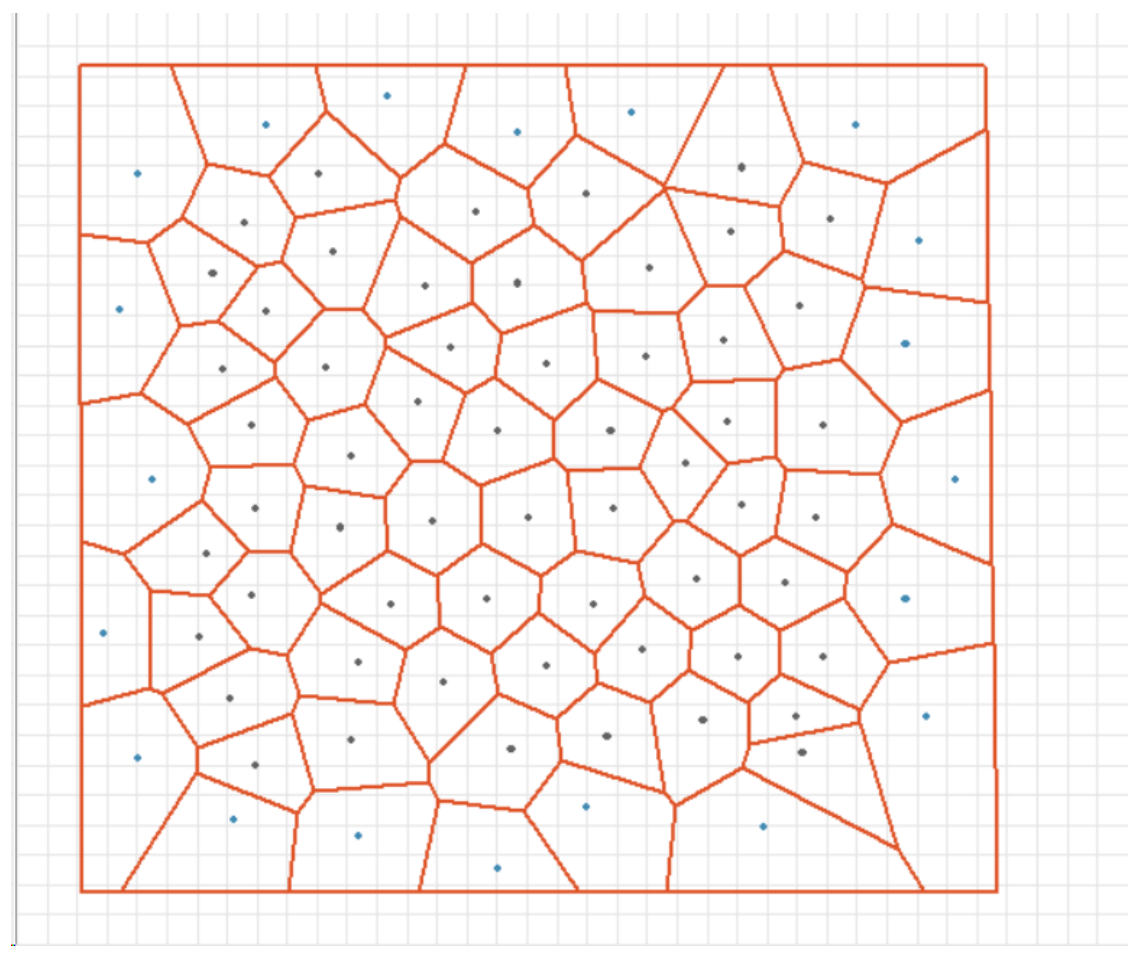

Figure 56 Reservoir delineation using the Voronoi method

\subsection{Build and History Match}

Each data driven model that is used to comprise the Top Down Model which are oil, gas, water, reservoir pressure, and water saturation were created. Every model used two offset producers and two offset injectors on a monthly time basis. The development and deployment dates for all models were set from December $31^{\text {st }}, 1994$ to December $31^{\text {st }}, 2016$. Once these characteristics have been specified, the data-driven model is added and the attributes that will be included to generate the data-driven model's dataset were be selected. The attributes selected to create the model's dataset do not have to be the exact attributes that will be used to train the models. Additional variables can be selected in case a model needs to be retrained with new variables. This in turn eliminates the waiting process of creating a new dataset to include the variables that wish to be tested. A table of the parameters selected to create each model: oil, gas, water, reservoir pressure, and water saturation are shown below. For the offset producer and offset injector, the listed attributes are included for both the first and second offset producers and injectors when the dataset is created. These tables are the same for every oil model created etc. regardless of the amount of blind validation.

Table 13 Data-driven oil model attributes for dataset generation where oil (t) is the output

\begin{tabular}{|l|l|l|}
\hline Focal & Offet Producer & Offset Injector \\
\hline X \&Y & X \&Y & X \&Y \\
\hline Drainage Area & Drainage Area & Drainage Area \\
\hline Pay Thickness & Distance t, t-1, t-2 & Distance t, t-1, t-2 \\
\hline Porh (1-Sw) & Pay Thickness & Pay Thickness \\
\hline Permeability & Permeability & Permeability \\
\hline
\end{tabular}




\begin{tabular}{|c|c|c|}
\hline Porosity & Porh (1-Sw) & Porosity \\
\hline Top & Porosity & Porh (1-Sw) \\
\hline TVD & Top & Top \\
\hline Completion $\mathrm{t}, \mathrm{t}-1, \mathrm{t}-2$ & TVD & TVD \\
\hline FBHP $t, t-1, t-2$ & Completion $t, t-1, t-2$ & Completion $\mathrm{t}, \mathrm{t}-1, \mathrm{t}-2$ \\
\hline Gas Saturation t-1,t-2 & FBHP t, t-1, t-2 & FBHP t, t-1, t-2 \\
\hline Oil Saturation t-1,t-2 & Gas Saturation t-1,t-2 & Gas Saturation t-1,t-2 \\
\hline $\begin{array}{l}\text { Open Layers Permeability t, t-1, } \\
\text { t-2 }\end{array}$ & Oil Saturation t-1,t-2 & Injected Gas Rate $t, t-1, t-2$ \\
\hline Open Layers Porosity t, t-1, t-2 & $\begin{array}{l}\text { Open Layers Permeability t, t-1, } \\
\text { t-2 }\end{array}$ & Injected Water Rate $t, t-1, t-2$ \\
\hline Reservoir Pressure t-1, t-2 & Open Layers Porosity t, t-1, t-2 & Oil Saturation t-1,t-2 \\
\hline Water Saturation t-1, t-2 & Reservoir Pressure t-1, t-2 & $\begin{array}{l}\text { Open Layers Permeability t, } t-1 \text {, } \\
\text { t-2 }\end{array}$ \\
\hline Pay Thickness Poly Ave & Water Saturation t-1, t-2 & Open Layers Porosity t, t-1, t-2 \\
\hline Permeability Poly Ave & Pay Thickness Poly Ave & Reservoir Pressure t-1, t-2 \\
\hline Porh (1-Sw) Poly Ave & Permeability Poly Ave & Water Saturation t-1, t-2 \\
\hline Porosity Poly Ave & Porh (1-Sw) Poly Ave & Pay Thickness Poly Ave \\
\hline q1 Monthly-Oil-Poly Ave & Porosity Poly Ave & Permeability Poly Ave \\
\hline Gas Saturation Poly Ave t-1, t-2 & q1 Monthly-Oil-Poly Ave & Porh (1-Sw) Poly Ave \\
\hline Oil Saturation Poly Ave t-1, t-2 & Gas Saturation Poly Ave t-1, t-2 & Porosity Poly Ave \\
\hline $\begin{array}{l}\text { Open Layers Permeability Poly } \\
\text { Ave } t, t-1, t-2\end{array}$ & Oil Saturation Poly Ave t-1, t-2 & Gas Saturation Poly Ave t-1, t-2 \\
\hline $\begin{array}{l}\text { Open Layers Porosity Poly Ave t, } \\
t-1, t-2\end{array}$ & $\begin{array}{l}\text { Open Layers Permeability Poly } \\
\text { Ave t, } \mathrm{t}-1, \mathrm{t}-2\end{array}$ & Oil Saturation Poly Ave t-1, t-2 \\
\hline $\begin{array}{l}\text { Reservoir Pressure Poly Ave t-1, } \\
\text { t-2 }\end{array}$ & $\begin{array}{l}\text { Open Layers Porosity Poly Ave t, } \\
\text { t-1, t-2 }\end{array}$ & $\begin{array}{l}\text { Open Layers Permeability Poly } \\
\text { Ave } t, t-1, t-2\end{array}$ \\
\hline $\begin{array}{l}\text { Water Saturation Poly Ave t-1, t- } \\
2\end{array}$ & $\begin{array}{l}\text { Reservoir Pressure Poly Ave t-1, } \\
\text { t-2 }\end{array}$ & $\begin{array}{l}\text { Open Layers Porosity Poly Ave t, } \\
t-1, t-2\end{array}$ \\
\hline EQ-Oil t-1, t-2 & \begin{tabular}{|l} 
Water Saturation Poly Ave t-1, $t-$ \\
2
\end{tabular} & $\begin{array}{l}\text { Reservoir Pressure Poly Ave t-1, } \\
\text { t-2 }\end{array}$ \\
\hline$q$ gas $t-1, t-2$ & EQ-Oil t-1, t-2 & $\begin{array}{l}\text { Water Saturation Poly Ave t-1, t- } \\
2\end{array}$ \\
\hline GOR $\mathrm{t}-1, \mathrm{t}-2$ & $q$ gas $\mathrm{t}-1, \mathrm{t}-2$ & \\
\hline Liquid t-1, t-2 & GOR t-1, t-2 & \\
\hline$q$ oil $t-1, t-2$ & Liquid t-1, t-2 & \\
\hline$q$ water $\mathrm{t}-1, \mathrm{t}-2$ & q oil t-1, t-2 & \\
\hline \multirow[t]{2}{*}{ WC t-1, t-2 } & $q$ water $t-1, t-2$ & \\
\hline & WC t-1, t-2 & \\
\hline
\end{tabular}

Table 14 Data-driven gas model attributes for dataset generation where gas $(t)$ is the output

\begin{tabular}{|l|l|l|}
\hline Focal & Offset Producer & Offset Injector \\
\hline X \&Y & X \&Y & X \&Y \\
\hline Drainage Area & Drainage Area & Drainage Area \\
\hline
\end{tabular}




\begin{tabular}{|c|c|c|}
\hline Pay Thickness & Distance $t, t-1, t-2$ & Distance $t, t-1, t-2$ \\
\hline Porh (1-Sw) & Pay Thickness & Pay Thickness \\
\hline Permeability & Permeability & Permeability \\
\hline Porosity & Porh (1-Sw) & Porosity \\
\hline Top & Porosity & Porh (1-Sw) \\
\hline TVD & Top & Top \\
\hline Completion $t, t-1, t-2$ & TVD & TVD \\
\hline FBHP $t, t-1, t-2$ & Completion $\mathrm{t}, \mathrm{t}-1, \mathrm{t}-2$ & Completion $\mathrm{t}, \mathrm{t}-1, \mathrm{t}-2$ \\
\hline Gas Saturation t-1,t-2 & FBHP $\mathrm{t}, \mathrm{t}-1, \mathrm{t}-2$ & FBHP $t, t-1, t-2$ \\
\hline Oil Saturation t-1,t-2 & Gas Saturation t-1,t-2 & Gas Saturation t-1,t-2 \\
\hline $\begin{array}{l}\text { Open Layers Permeability t, t-1, } \\
\text { t-2 }\end{array}$ & Oil Saturation t-1,t-2 & Injected Gas Rate $t, t-1, t-2$ \\
\hline Open Layers Porosity t, t-1, t-2 & $\begin{array}{l}\text { Open Layers Permeability t, t-1, } \\
\text { t-2 }\end{array}$ & Injected Water Rate $t, t-1, t-2$ \\
\hline Reservoir Pressure t-1, t-2 & Open Layers Porosity t, t-1, t-2 & Oil Saturation t-1,t-2 \\
\hline Water Saturation t-1, t-2 & Reservoir Pressure t-1, t-2 & $\begin{array}{l}\text { Open Layers Permeability t, t-1, } \\
\text { t-2 }\end{array}$ \\
\hline Pay Thickness Poly Ave & Water Saturation t-1, t-2 & Open Layers Porosity t, t-1, t-2 \\
\hline Permeability Poly Ave & Pay Thickness Poly Ave & Reservoir Pressure t-1, t-2 \\
\hline Porh (1-Sw) Poly Ave & Permeability Poly Ave & Water Saturation t-1, t-2 \\
\hline Porosity Poly Ave & Porh (1-Sw) Poly Ave & Pay Thickness Poly Ave \\
\hline q1 Monthly-Gas-Poly Ave & Porosity Poly Ave & Permeability Poly Ave \\
\hline q1 Monthly-GOR-Poly Ave & q1 Monthly-Gas-Poly Ave & Porh (1-Sw) Poly Ave \\
\hline Gas Saturation Poly Ave t-1, t-2 & q1 Monthly-GOR-Poly Ave & Porosity Poly Ave \\
\hline Oil Saturation Poly Ave t-1, t-2 & Gas Saturation Poly Ave t-1, t-2 & Gas Saturation Poly Ave t-1, t-2 \\
\hline $\begin{array}{l}\text { Open Layers Permeability Poly } \\
\text { Ave } t, t-1, t-2\end{array}$ & Oil Saturation Poly Ave t-1, t-2 & Oil Saturation Poly Ave t-1, t-2 \\
\hline $\begin{array}{l}\text { Open Layers Porosity Poly Ave t, } \\
\text { t-1, t-2 }\end{array}$ & $\begin{array}{l}\text { Open Layers Permeability Poly } \\
\text { Ave t, } t-1, t-2\end{array}$ & $\begin{array}{l}\text { Open Layers Permeability Poly } \\
\text { Ave } t, t-1, t-2\end{array}$ \\
\hline $\begin{array}{l}\text { Reservoir Pressure Poly Ave t-1, } \\
\text { t-2 }\end{array}$ & $\begin{array}{l}\text { Open Layers Porosity Poly Ave t, } \\
\text { t-1, t-2 }\end{array}$ & $\begin{array}{l}\text { Open Layers Porosity Poly Ave t, } \\
t-1, t-2\end{array}$ \\
\hline $\begin{array}{l}\text { Water Saturation Poly Ave t-1, t- } \\
2\end{array}$ & $\begin{array}{l}\text { Reservoir Pressure Poly Ave t-1, } \\
\text { t-2 }\end{array}$ & $\begin{array}{l}\text { Reservoir Pressure Poly Ave t-1, } \\
\text { t-2 }\end{array}$ \\
\hline EQ-Oil t-1, t-2 & $\begin{array}{l}\text { Water Saturation Poly Ave t-1, } t- \\
2\end{array}$ & $\begin{array}{l}\text { Water Saturation Poly Ave t-1, t- } \\
2\end{array}$ \\
\hline$q$ gas $\mathrm{t}-1, \mathrm{t}-2$ & EQ-Oil t-1, t-2 & \\
\hline GOR $\mathrm{t}-1, \mathrm{t}-2$ & $q$ gas $\mathrm{t}-1, \mathrm{t}-2$ & \\
\hline Liquid $t-1, t-2$ & GOR $\mathrm{t}-1, \mathrm{t}-2$ & \\
\hline$q$ oil $t, t-1, t-2$ & Liquid t-1, t-2 & \\
\hline$q$ water $t-1, t-2$ & q oil t, t-1, t-2 & \\
\hline \multirow[t]{2}{*}{ WC t-1, t-2 } & $q$ water $t-1, t-2$ & \\
\hline & WC t-1, t-2 & \\
\hline
\end{tabular}


Table 15 Data-driven water model attributes for dataset generation where water $(t)$ is the output

\begin{tabular}{|c|c|c|}
\hline Focal & Offset Producer & Offset Injector \\
\hline $\mathbf{X} \& Y$ & $\mathbf{X} \& \mathbf{Y}$ & $\mathbf{X} \& \mathbf{Y}$ \\
\hline Drainage Area & Drainage Area & Drainage Area \\
\hline Pay Thickness & Distance $t, t-1, t-2$ & Distance $t, t-1, t-2$ \\
\hline Porh (1-Sw) & Pay Thickness & Pay Thickness \\
\hline Permeability & Permeability & Permeability \\
\hline Porosity & Porh (1-Sw) & Porosity \\
\hline Top & Porosity & Porh (1-Sw) \\
\hline TVD & Top & Top \\
\hline Completion $\mathrm{t}, \mathrm{t}-1, \mathrm{t}-2$ & TVD & TVD \\
\hline FBHP $t, t-1, t-2$ & Completion $\mathbf{t}, \mathbf{t}-\mathbf{1}, \mathrm{t}-\mathbf{2}$ & Completion $\mathrm{t}, \mathrm{t}-1, \mathrm{t}-2$ \\
\hline Gas Saturation t-1,t-2 & FBHP $\mathrm{t}, \mathrm{t}-1, \mathrm{t}-2$ & FBHP $\mathrm{t}, \mathrm{t}-1, \mathrm{t}-2$ \\
\hline Oil Saturation t-1,t-2 & Gas Saturation t-1,t-2 & Gas Saturation t-1,t-2 \\
\hline $\begin{array}{l}\text { Open Layers Permeability t, t-1, } \\
\text { t-2 }\end{array}$ & Oil Saturation t-1,t-2 & Injected Gas Rate $t, t-1, t-2$ \\
\hline Open Layers Porosity $t, t-1, t-2$ & $\begin{array}{l}\text { Open Layers Permeability } t, t-1, \\
\text { t-2 }\end{array}$ & Injected Water Rate $t, t-1, t-2$ \\
\hline Reservoir Pressure t-1, t-2 & Open Layers Porosity t, $t-1, t-2$ & Oil Saturation t-1,t-2 \\
\hline Water Saturation t-1, t-2 & Reservoir Pressure t-1, t-2 & $\begin{array}{l}\text { Open Layers Permeability } t, t-1 \text {, } \\
t-2\end{array}$ \\
\hline Pay Thickness Poly Ave & Water Saturation t-1, t-2 & Open Layers Porosity $t, t-1, t-2$ \\
\hline Permeability Poly Ave & Pay Thickness Poly Ave & Reservoir Pressure t-1, t-2 \\
\hline Porh (1-Sw) Poly Ave & Permeability Poly Ave & Water Saturation t-1, t-2 \\
\hline Porosity Poly Ave & Porh (1-Sw) Poly Ave & Pay Thickness Poly Ave \\
\hline q1 Monthly-Water-Poly Ave & Porosity Poly Ave & Permeability Poly Ave \\
\hline q1 Monthly-WC-Poly Ave & q1 Monthly-Water-Poly Ave & Porh (1-Sw) Poly Ave \\
\hline Gas Saturation Poly Ave t-1, t-2 & q1 Monthly-WC-Poly Ave & Porosity Poly Ave \\
\hline Oil Saturation Poly Ave t-1, t-2 & Gas Saturation Poly Ave t-1, t-2 & Gas Saturation Poly Ave t-1, t-2 \\
\hline $\begin{array}{l}\text { Open Layers Permeability Poly } \\
\text { Ave } t, \mathrm{t}-1, \mathrm{t}-2\end{array}$ & Oil Saturation Poly Ave t-1, t-2 & Oil Saturation Poly Ave t-1, t-2 \\
\hline $\begin{array}{l}\text { Open Layers Porosity Poly Ave t, } \\
t-1, t-2\end{array}$ & $\begin{array}{l}\text { Open Layers Permeability Poly } \\
\text { Ave t, } t-1, t-2\end{array}$ & $\begin{array}{l}\text { Open Layers Permeability Poly } \\
\text { Ave t, } t-1, t-2\end{array}$ \\
\hline $\begin{array}{l}\text { Reservoir Pressure Poly Ave t-1, } \\
\text { t-2 }\end{array}$ & $\begin{array}{l}\text { Open Layers Porosity Poly Ave } t \text {, } \\
t-1, t-2\end{array}$ & $\begin{array}{l}\text { Open Layers Porosity Poly Ave } t \text {, } \\
t-1, t-2\end{array}$ \\
\hline $\begin{array}{l}\text { Water Saturation Poly Ave t-1, t- } \\
2\end{array}$ & $\begin{array}{l}\text { Reservoir Pressure Poly Ave t-1, } \\
\text { t-2 }\end{array}$ & $\begin{array}{l}\text { Reservoir Pressure Poly Ave t-1, } \\
\text { t-2 }\end{array}$ \\
\hline EQ-Oil t, t-1, t-2 & \begin{tabular}{|l}
$\begin{array}{l}\text { Water Saturation Poly Ave t-1, t- } \\
2\end{array}$ \\
\end{tabular} & $\begin{array}{l}\text { Water Saturation Poly Ave t-1, t- } \\
2\end{array}$ \\
\hline$q$ gas $t, t-1, t-2$ & EQ-Oil t, t-1, t-2 & \\
\hline GOR $t, t-1, t-2$ & $q$ gas $t, t-1, t-2$ & \\
\hline Liquid $t-1, t-2$ & GOR $\mathrm{t}, \mathrm{t}-1, \mathrm{t}-2$ & \\
\hline
\end{tabular}




\begin{tabular}{|l|l|l|}
\hline q oil $t, t-1, t-2$ & Liquid $t-1, t-2$ & \\
\hline q water $t-1, t-2$ & q oil $t, t-1, t-2$ & \\
\hline WC $t-1, t-2$ & q water $t-1, t-2$ & \\
\hline & WC $t-1, t-2$ & \\
\hline
\end{tabular}

Table 16 Data-driven reservoir pressure model attributes for dataset generation where reservoir pressure $(t)$ is the output

\begin{tabular}{|c|c|c|}
\hline Focal & Offset Producer & Offset Injector \\
\hline $\mathbf{X} \& \mathbf{Y}$ & $\mathbf{X} \& \mathbf{Y}$ & $\mathbf{X} \& \mathbf{Y}$ \\
\hline Drainage Area & Drainage Area & Drainage Area \\
\hline Pay Thickness & Distance $t, t-1, t-2$ & Distance $t, t-1, t-2$ \\
\hline Porh (1-Sw) & Pay Thickness & Pay Thickness \\
\hline Permeability & Permeability & Permeability \\
\hline Porosity & \begin{tabular}{|l|} 
Porh (1-Sw) \\
\end{tabular} & Porosity \\
\hline Top & Porosity & Porh (1-Sw) \\
\hline TVD & Top & Top \\
\hline Completion $t, t-1, t-2$ & TVD & TVD \\
\hline FBHP $t, t-1, t-2$ & Completion $\mathrm{t}, \mathrm{t}-1, \mathrm{t}-2$ & Completion $\mathrm{t}, \mathrm{t}-1, \mathrm{t}-2$ \\
\hline Gas Saturation t-1,t-2 & FBHP t, t-1, t-2 & FBHP t, t-1, t-2 \\
\hline Oil Saturation t-1,t-2 & Gas Saturation t-1,t-2 & Gas Saturation t-1,t-2 \\
\hline $\begin{array}{l}\text { Open Layers Permeability t, } t-1 \text {, } \\
t-2\end{array}$ & Oil Saturation t-1,t-2 & Injected Gas Rate $t, t-1, t-2$ \\
\hline Open Layers Porosity t, t-1, t-2 & $\begin{array}{l}\text { Open Layers Permeability t, t-1, } \\
\text { t-2 }\end{array}$ & Injected Water Rate t, t-1, t-2 \\
\hline Reservoir Pressure t-1, t-2 & Open Layers Porosity t, t-1, t-2 & Oil Saturation t-1,t-2 \\
\hline Water Saturation t-1, t-2 & Reservoir Pressure t-1, t-2 & $\begin{array}{l}\text { Open Layers Permeability t, t-1, } \\
\text { t-2 }\end{array}$ \\
\hline Pay Thickness Poly Ave & Water Saturation t-1, t-2 & Open Layers Porosity t, t-1, t-2 \\
\hline Permeability Poly Ave & Pay Thickness Poly Ave & Reservoir Pressure t-1, t-2 \\
\hline Porh (1-Sw) Poly Ave & Permeability Poly Ave & Water Saturation t-1, t-2 \\
\hline Porosity Poly Ave & Porh (1-Sw) Poly Ave & Pay Thickness Poly Ave \\
\hline Gas Saturation Poly Ave t-1, t-2 & Porosity Poly Ave & Permeability Poly Ave \\
\hline Oil Saturation Poly Ave t-1, t-2 & Gas Saturation Poly Ave t-1, t-2 & Porh (1-Sw) Poly Ave \\
\hline $\begin{array}{l}\text { Open Layers Permeability Poly } \\
\text { Ave } t, t-1, t-2\end{array}$ & Oil Saturation Poly Ave t-1, t-2 & Porosity Poly Ave \\
\hline $\begin{array}{l}\text { Open Layers Porosity Poly Ave t, } \\
\text { t-1,t-2 }\end{array}$ & $\begin{array}{l}\text { Open Layers Permeability Poly } \\
\text { Ave t, } t-1, t-2\end{array}$ & Gas Saturation Poly Ave t-1, t-2 \\
\hline $\begin{array}{l}\text { Reservoir Pressure Poly Ave t-1, } \\
\text { t-2 }\end{array}$ & $\begin{array}{l}\text { Open Layers Porosity Poly Ave t, } \\
t-1, t-2\end{array}$ & Oil Saturation Poly Ave t-1, t-2 \\
\hline $\begin{array}{l}\text { Water Saturation Poly Ave t-1, t- } \\
2\end{array}$ & $\begin{array}{l}\text { Reservoir Pressure Poly Ave t-1, } \\
\text { t-2 }\end{array}$ & $\begin{array}{l}\text { Open Layers Permeability Poly } \\
\text { Ave } t, t-1, t-2\end{array}$ \\
\hline EQ-Oil t, t-1, t-2 & $\begin{array}{l}\text { Water Saturation Poly Ave t-1, t- } \\
2\end{array}$ & $\begin{array}{l}\text { Open Layers Porosity Poly Ave t, } \\
\text { t-1,t-2 }\end{array}$ \\
\hline$q$ gas $t, t-1, t-2$ & EQ-Oil t, t-1, t-2 & $\begin{array}{l}\text { Reservoir Pressure Poly Ave t-1, } \\
\text { t-2 }\end{array}$ \\
\hline
\end{tabular}




\begin{tabular}{|l|l|l|}
\hline GOR $t, t-1, t-2$ & q gas $t, t-1, t-2$ & Water Saturation Poly Ave t-1, $t-$ \\
\hline Liquid $t, t-1, t-2$ & GOR $t, t-1, t-2$ & \\
\hline q oil $t, t-1, t-2$ & Liquid $t, t-1, t-2$ & \\
\hline q water $t, t-1, t-2$ & q oil $t, t-1, t-2$ & \\
\hline WC $t, t-1, t-2$ & q water $t, t-1, t-2$ & \\
\hline & WC $t, t-1, t-2$ & \\
\hline
\end{tabular}

Table 17 Data-driven water saturation model attributes for dataset generation where water saturation (t) is the output

\begin{tabular}{|c|c|c|}
\hline Focal & Offset Producer & Offset Injector \\
\hline $\mathbf{X} \& \mathbf{Y}$ & $\mathbf{X} \& \mathbf{Y}$ & $\mathbf{X} \& \mathbf{Y}$ \\
\hline Drainage Area & Drainage Area & Drainage Area \\
\hline Pay Thickness & Distance $t, t-1, t-2$ & Distance $t, t-1, t-2$ \\
\hline Porh (1-Sw) & Pay Thickness & Pay Thickness \\
\hline Permeability & Permeability & Permeability \\
\hline Porosity & Porh (1-Sw) & Porosity \\
\hline Top & Porosity & Porh (1-Sw) \\
\hline TVD & Top & Top \\
\hline Completion $\mathrm{t}, \mathrm{t}-1, \mathrm{t}-2$ & TVD & TVD \\
\hline FBHP $t, t-1, t-2$ & Completion $t, t-1, t-2$ & Completion $\mathrm{t}, \mathrm{t}-1, \mathrm{t}-2$ \\
\hline Gas Saturation t-1,t-2 & FBHP $t, t-1, t-2$ & FBHP $\mathrm{t}, \mathrm{t}-1, \mathrm{t}-2$ \\
\hline Oil Saturation t-1,t-2 & Gas Saturation t-1,t-2 & Gas Saturation t-1,t-2 \\
\hline $\begin{array}{l}\text { Open Layers Permeability t, t-1, } \\
\text { t-2 }\end{array}$ & Oil Saturation t-1,t-2 & Injected Gas Rate t, t-1, t-2 \\
\hline Open Layers Porosity t, t-1, t-2 & $\begin{array}{l}\text { Open Layers Permeability t, t-1, } \\
\text { t-2 }\end{array}$ & Injected Water Rate t, t-1, t-2 \\
\hline Reservoir Pressure t, t-1, t-2 & Open Layers Porosity t, t-1, t-2 & Oil Saturation t-1,t-2 \\
\hline Water Saturation t-1, t-2 & Reservoir Pressure t, t-1, t-2 & $\begin{array}{l}\text { Open Layers Permeability t, t-1, } \\
\text { t-2 }\end{array}$ \\
\hline Pay Thickness Poly Ave & Water Saturation t-1, t-2 & Open Layers Porosity t, t-1, t-2 \\
\hline Permeability Poly Ave & Pay Thickness Poly Ave & Reservoir Pressure t, t-1, t-2 \\
\hline Porh (1-Sw) Poly Ave & Permeability Poly Ave & Water Saturation t-1, t-2 \\
\hline Porosity Poly Ave & Porh (1-Sw) Poly Ave & Pay Thickness Poly Ave \\
\hline q1 Monthly-Water-Poly Ave & Porosity Poly Ave & Permeability Poly Ave \\
\hline q1 Monthly-WC-Poly Ave & q1 Monthly-Water-Poly Ave & Porh (1-Sw) Poly Ave \\
\hline Gas Saturation Poly Ave t-1, t-2 & q1 Monthly-WC-Poly Ave & Porosity Poly Ave \\
\hline Oil Saturation Poly Ave t-1, t-2 & Gas Saturation Poly Ave t-1, t-2 & Gas Saturation Poly Ave t-1, t-2 \\
\hline $\begin{array}{l}\text { Open Layers Permeability Poly } \\
\text { Ave } t, t-1, t-2\end{array}$ & Oil Saturation Poly Ave t-1, t-2 & Oil Saturation Poly Ave t-1, t-2 \\
\hline $\begin{array}{l}\text { Open Layers Porosity Poly Ave t, } \\
\text { t-1,t-2 }\end{array}$ & $\begin{array}{l}\text { Open Layers Permeability Poly } \\
\text { Ave } t, t-1, t-2\end{array}$ & $\begin{array}{l}\text { Open Layers Permeability Poly } \\
\text { Ave } t, t-1, t-2\end{array}$ \\
\hline $\begin{array}{l}\text { Reservoir Pressure Poly Ave t, t- } \\
1, t-2\end{array}$ & $\begin{array}{l}\text { Open Layers Porosity Poly Ave t, } \\
t-1, t-2\end{array}$ & $\begin{array}{l}\text { Open Layers Porosity Poly Ave } t \text {, } \\
t-1, t-2\end{array}$ \\
\hline
\end{tabular}




\begin{tabular}{|c|c|c|}
\hline $\begin{array}{l}\text { Water Saturation Poly Ave t-1, t- } \\
2\end{array}$ & $\begin{array}{l}\text { Reservoir Pressure Poly Ave t, t- } \\
\text { 1, t-2 }\end{array}$ & $\begin{array}{l}\text { Reservoir Pressure Poly Ave t, } t- \\
1, t-2\end{array}$ \\
\hline EQ-Oil t, t-1, t-2 & $\begin{array}{l}\text { Water Saturation Poly Ave t-1, t- } \\
2\end{array}$ & $\begin{array}{l}\text { Water Saturation Poly Ave t-1, t- } \\
2\end{array}$ \\
\hline$q$ gas $t, t-1, t-2$ & EQ-Oil t, t-1, t-2 & \\
\hline GOR t, t-1, t-2 & $q$ gas $t, t-1, t-2$ & \\
\hline Liquid t, t-1, t-2 & GOR $t, t-1, t-2$ & \\
\hline$q$ oil $t, t-1, t-2$ & Liquid t, t-1, t-2 & \\
\hline$q$ water $t, t-1, t-2$ & $q$ oil $t, t-1, t-2$ & \\
\hline \multirow[t]{2}{*}{ WC t, t-1, t-2 } & $q$ water $t, t-1, t-2$ & \\
\hline & WC t, t-1, t-2 & \\
\hline
\end{tabular}

Once the dataset is generated for each data-driven model, the dataset can be cleaned. First all of the values where oil $(\mathrm{t})=0$, for all data-driven models, was removed from the dataset. This was done because an oil production of 0 indicates a random shut-in event, as it was created that way in the numerical reservoir simulation. By excluding the time step where oil is equal to 0 , the possibility of confusing the network is reduced. Next, any values that were empty were assigned a 0 value in order for all parameters to have a value. Finally the records were sorted by wells, then sorted by date. This allows for proper blind validation to be assigned in the future and was also done in this case to be uniform. Now the desired variables can be selected for training. Each data-driven model for every Top Down Model had slightly different input variables along with partitioning, which are outlined separately below and grouped by the Top Down Model.

\subsubsection{Random Partitioning-No Blind Validation}

While there is validation within this model, the validation is not blind. This is due to the values being randomly dispersed throughout all of the data. The tables included below show the variables selected to train each data-driven model in the following order: oil, gas, water, reservoir pressure, and water saturation. The variables in the offset producers and offset injectors columns are selected for both the first and second offset producer and injector to train the model. Domain expertise and iterative process until good training results were obtained were used to determine the final training attributes selected for these data-driven models and all data-drive models presented hereafter.

Table 18 Data-driven oil model training attributes where oil $(t)$ is the output

\begin{tabular}{|l|l|l|}
\hline Focal & Offset Producers & Offset Injectors \\
\hline Time & Distance t & Distance t \\
\hline Completion t & Completion t & Completion t \\
\hline FBHP t & FBHP t & FBHP t \\
\hline Oil Saturation t-1 & FBHP t-1 & Injected Gas t \\
\hline Oil Saturation t-2 & Oil Saturation t-1 & Injected Gas t-1 \\
\hline Open Layers Permeability t & Oil Saturation t-2 & Injected Water t \\
\hline Open Layers Porosity t & q oil t-1 & Open Layers Permeability t \\
\hline Open Layers Porosity t-1 & & Open Layers Porosity t \\
\hline
\end{tabular}




\begin{tabular}{|l|l|l|}
\hline Reservoir Pressure t-1 & & \\
\hline Oil Saturation t-1 Poly Ave & & \\
\hline Open Layers Permeability Poly Ave t & \\
\hline q oil t-1 & & \\
\hline q oil t-2 & & \\
\hline q liquid t-1 & & \\
\hline q gas t-1 & & \\
\hline
\end{tabular}

Table 19 Data-driven gas model training attributes where gas $(t)$ is the output

\begin{tabular}{|l|l|l|}
\hline Focal & Offset Producers & Offset Injectors \\
\hline Time & Distance t & Distance t \\
\hline Top & Top & Completion t \\
\hline TVD & TVD & FBHP t \\
\hline Completion t & Completion t & Gas Saturation t-1 \\
\hline FBHP t & FBHP t & Injected Gas t \\
\hline FBHP t-1 & FBHP t-1 & Injected Gas t-1 \\
\hline Gas Saturation t-1 & Gas Saturation t-1 & Injected Gas t-2 \\
\hline Gas Saturation t-2 & Gas Saturation t-2 & Open Layers Permeability t \\
\hline Open Layers Permeability t & Reservoir Pressure t-1 & \\
\hline Open Layers Porosity t & q gas t-1 & \\
\hline Reservoir Pressure t-1 & q gas t-2 & \\
\hline q gas t-1 & & \\
\hline q gas t-2 & & \\
\hline q oil t & & \\
\hline
\end{tabular}

Table 20 Data-driven water model training attributes where water $(t)$ is the output

\begin{tabular}{|l|l|l|}
\hline Focal & Offset Producers & Offset Injectors \\
\hline Time & Distance t & Distance t \\
\hline Top & Top & FBHP t \\
\hline TVD & TVD & Injected Water Rate t \\
\hline Completion & Completion t & Injected Water Rate t-1 \\
\hline FBHP t & FBHP t & Injected Water Rate t-2 \\
\hline FBHP t-1 & FBHP t-1 & Open Layers Permeability t \\
\hline Reservoir Pressure t-1 & Open Layers Permeability t & Water Saturation t-1 \\
\hline Open Layers Permeability t & Reservoir Pressure t-1 & \\
\hline Open Layers Porosity t & Water Saturation t-1 & \\
\hline Water Saturation t-1 & Water Saturation t-2 & \\
\hline Water Saturation t-2 & q water t-1 & \\
\hline q water t-1 & q water t-2 & \\
\hline q water t-2 & & \\
\hline
\end{tabular}




\begin{tabular}{|l|l|l|}
\hline q gas t & & \\
\hline q oil t & & \\
\hline
\end{tabular}

Table 21 Data-driven reservoir pressure model training attributes where reservoir pressure $(t)$ is the output

\begin{tabular}{|l|l|l|}
\hline Focal & Offset Producers & Offset Injectors \\
\hline Time & Distance t & Distance t \\
\hline Top & Top & FBHP t \\
\hline TVD & TVD & FBHP t-1 \\
\hline Completion t & Completion t & FBHP t-2 \\
\hline FBHP t & FBHP t & Injected Gas Rate t \\
\hline FBHP t-1 & FBHP t-1 & Injected Water Rate t \\
\hline FBHP t-2 & FBHP t-2 & Reservoir Pressure t-1 \\
\hline Open Layers Permeability t & Reservoir Pressure t-1 & Reservoir Pressure t-2 \\
\hline Open Layers Porosity t & Reservoir Pressure t-2 & \\
\hline Reservoir Pressure t-1 & & \\
\hline Reservoir Pressure t-2 & & \\
\hline q oil t & & \\
\hline q gas t & & \\
\hline q water t & & \\
\hline
\end{tabular}

Table 22 Data-driven water saturation model training attributes where water saturation $(t)$ is the output

\begin{tabular}{|l|l|l|}
\hline Focal & Offset Producers & Offset Injectors \\
\hline Time & Distance t & Distance t \\
\hline Pay Thickness & Completion t & Gas Saturation t-1 \\
\hline Top & Top & Oil Saturation t-1 \\
\hline TVD & TVD & Injected Water Rate t \\
\hline Completion t & Gas Saturation t-1 & Injected Water Rate t-1 \\
\hline FBHP t & Oil Saturation t-1 & Injected Water Rate t-2 \\
\hline Gas Saturation t-1 & Water Saturation t-1 & Water Saturation t-1 \\
\hline Oil Saturation t-1 & Water Saturation t-2 & Water Saturation t-2 \\
\hline $\begin{array}{l}\text { Open Layers Permeability } \\
\text { t }\end{array}$ & & \\
\hline Open Layers Porosity t & & \\
\hline Reservoir Pressure t & & \\
\hline Water Saturation t-1 & & \\
\hline Water Saturation t-2 & & \\
\hline q water t & & \\
\hline
\end{tabular}

Once these attributes were selected, Random Selection was used for the data partitioning. Three percentages were entered in order to randomly assign the data values into the three subgroups: training, 
calibration, and verification. The training dataset included $80 \%$ of the 6811 total number of records, calibration had $10 \%$, and verification had $10 \%$. The default values for learning rate, momentum, etc. were used because while these values could fine tune the model they should not make or break the model. These defaults are included in a table below. The training was then started and continued until good results were obtained. This often included trialing various combinations of input parameters and ensuring that certain data points were included in training in order to ensure the model learned the behavior correctly. For example, sometimes the first month of a well being brought online in a new phase was randomly assigned to the calibration or verification dataset. This point was included in the training dataset instead to allow the model to learn about the new well, especially if it was one of the wells that produced more or less than the majority of the other wells.

Table 23 Default design values for artificial neural network

\begin{tabular}{|l|l|}
\hline \multicolumn{2}{|c|}{ Input Layer } \\
\hline Momentum & 0.1 \\
\hline Learning Rate & 0.01 \\
\hline Weight Decay & 0.2 \\
\hline & Output Layer \\
\hline Momentum & 0.1 \\
\hline Learning Rate & 0.01 \\
\hline Weight Decay & 0.2 \\
\hline Activation Function & Logistic \\
\hline Save Type & Save Best Calibration Set \\
\hline
\end{tabular}

The training results for oil, gas, water, reservoir pressure, and water saturation are shown below. Each data-driven model's results are shown for training, calibration, and verification datasets, or in other words all partition cases, combined into one plot. One of the evaluation metrics of the training progress is the R Squared value. The combined training, calibration, and verification R Squared value for each of the models shown below are included in the next table. While a high R Squared value was desired to show the correlation between the actual outputs and the predicted outputs, the models were deemed sufficient after deploying the Top Down Model and looking at those results. This was done because even though oil's R Squared was 89\%, it still provided good results after the Top Down Model was deployed. With this said, the R Squared metric can be one early indication of the accuracy of each model, but accuracy should always be verified with the results gathered after deployment. The aforementioned logic was used for all seven Top Down Models to be presented.

Table 24 Training $R$ Squared metrics for all data-driven models

\begin{tabular}{|l|l|}
\hline \multicolumn{2}{|l|}{$\begin{array}{l}\text { All Partition Cases R } \\
\text { Squared }\end{array}$} \\
\hline Oil & $89.00 \%$ \\
\hline Gas & $98.70 \%$ \\
\hline Water & $96.60 \%$ \\
\hline Reservoir Pressure & $98.00 \%$ \\
\hline Water Saturation & $99.70 \%$ \\
\hline
\end{tabular}




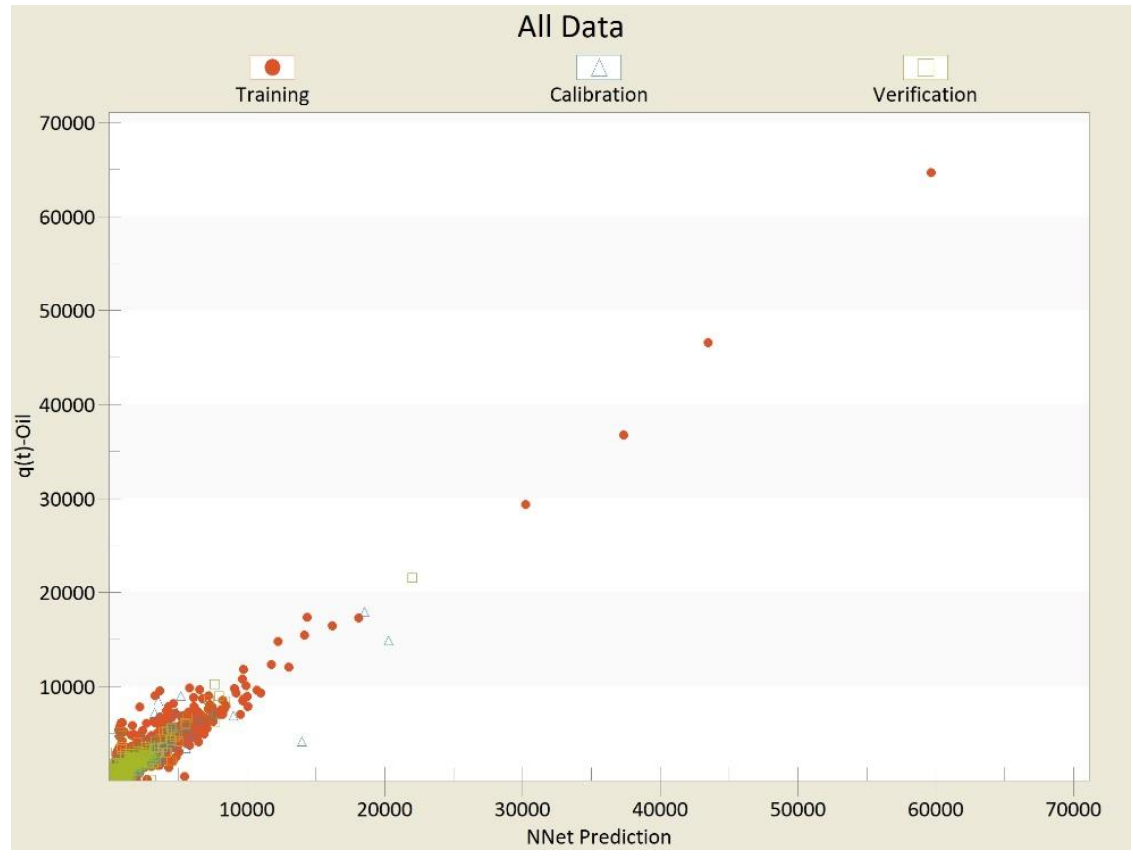

Figure 57 Data-driven oil model training results for training, calibration, and verification

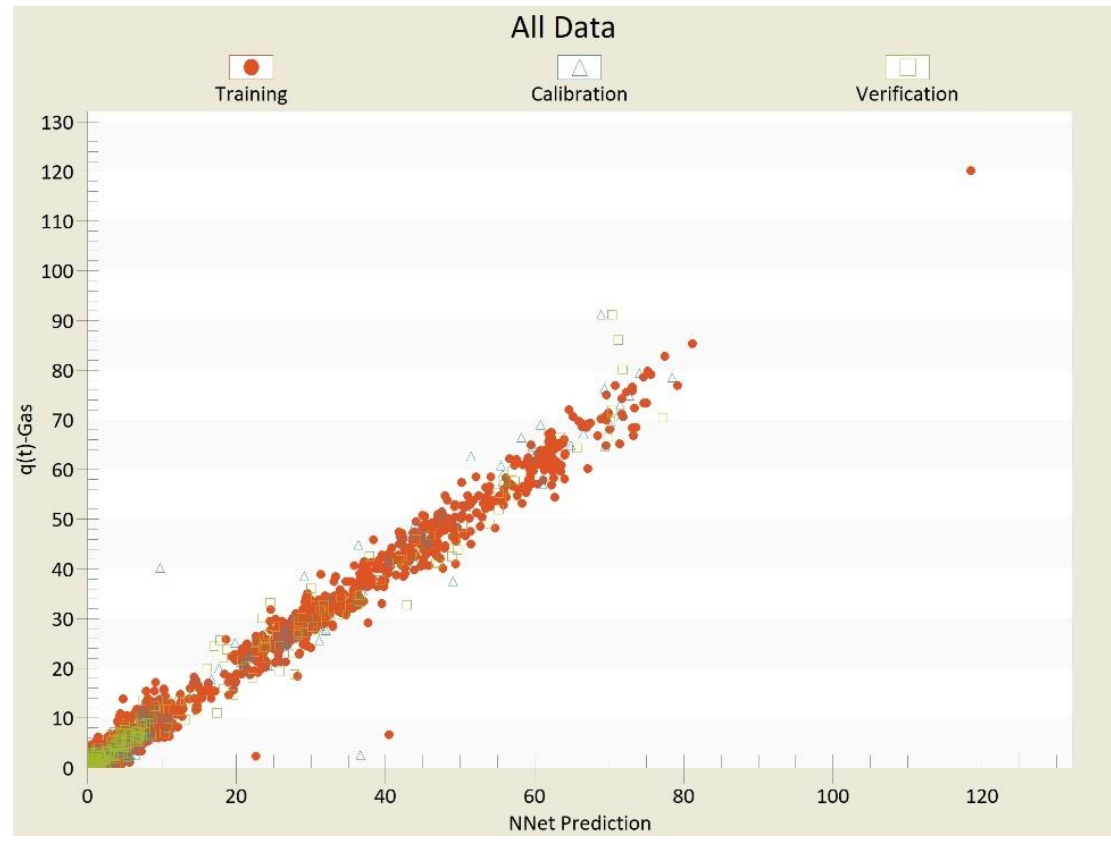

Figure 58 Data-driven gas training results for training, calibration, and verification 


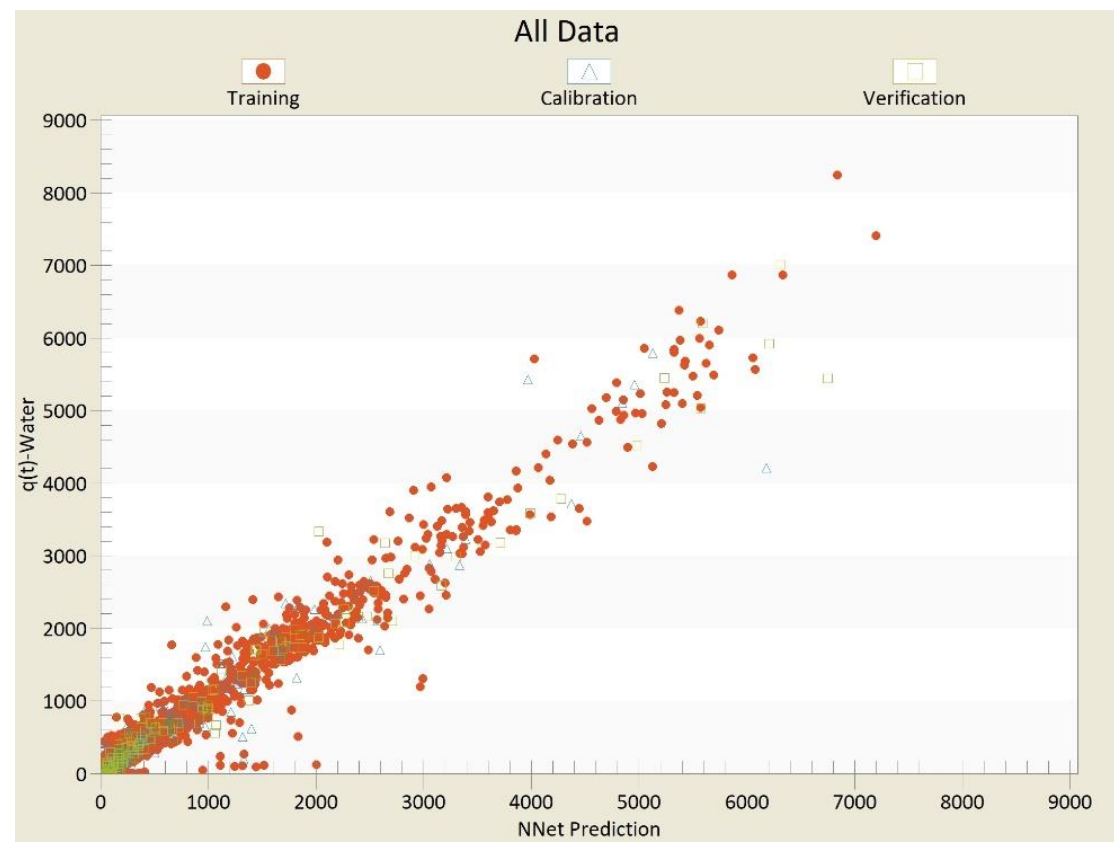

Figure 59 Data-driven water training results for training, calibration, and verification

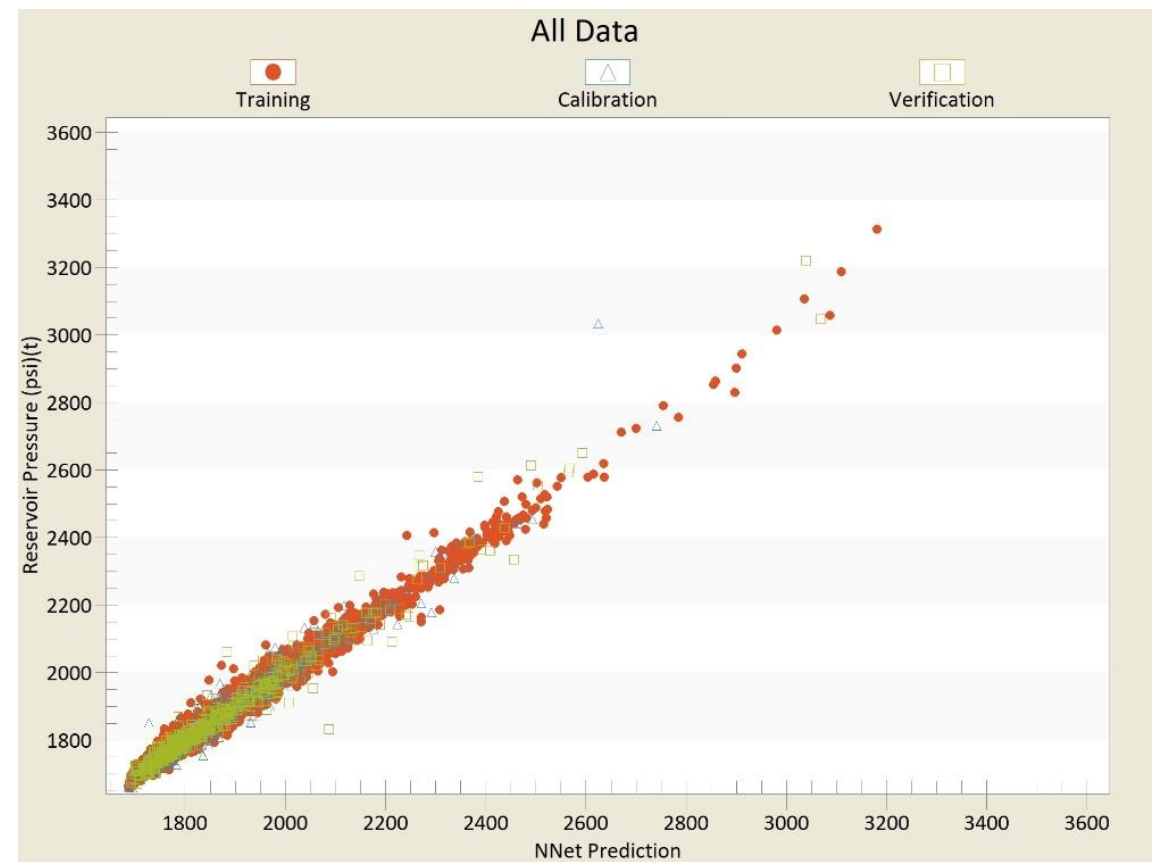

Figure 60 Data-driven reservoir pressure training results for training, calibration, and verification 


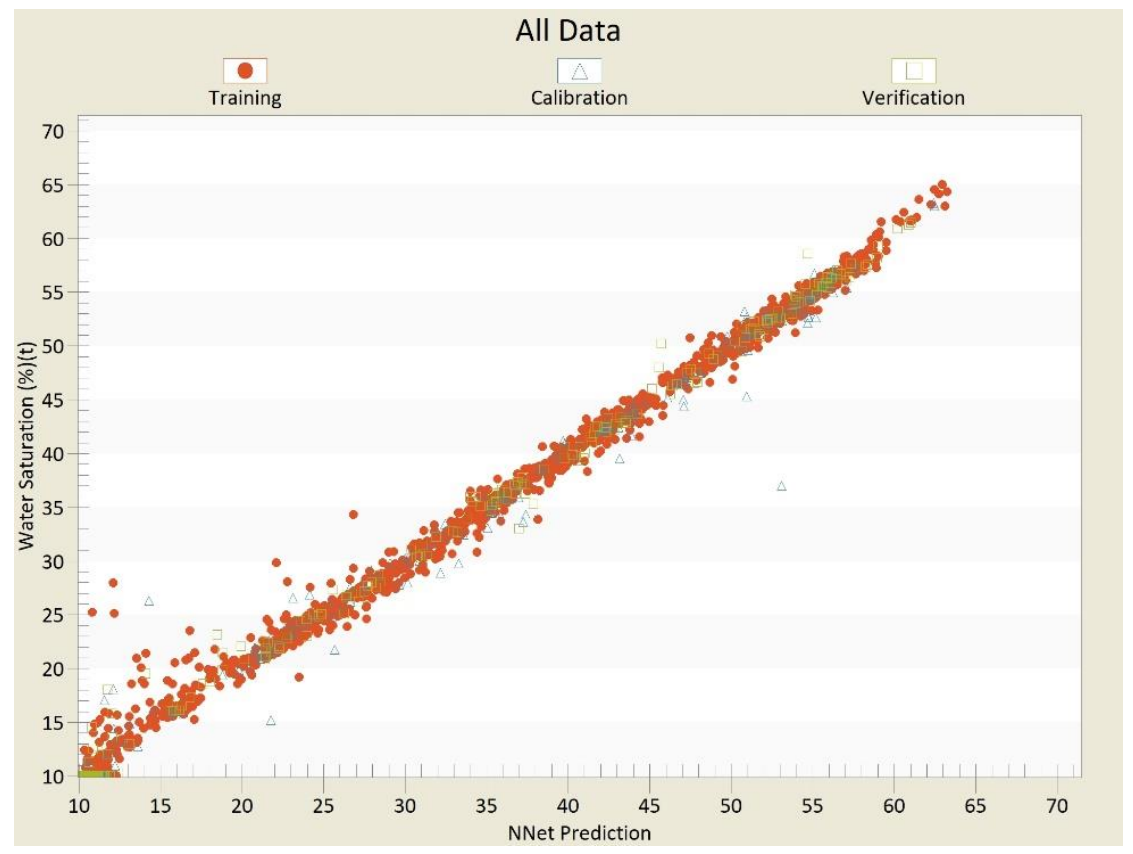

Figure 61 Data-driven water saturation training results for training, calibration, and verification

To create the Top Down Model, the last output (water saturation) was selected and the TDM was named and created. Next the second to last output (reservoir pressure) was chosen and inserted into level 2. It was ensured that reservoir pressure at time $t$ was an input into the water saturation training process, otherwise the TDM construction would not work. Then water was selected and inserted into level 3, ensuring that water $t$ time $t$ was an input into the reservoir pressure training process. Next gas inserted into level 4 , ensuring that gas at time $t$ was an input into the water training process. Finally, oil was inserted into level 5, making sure that oil at time $\mathrm{t}$ was an input into the gas training process.

\begin{tabular}{|c|c|c|c|c|c|c|c|c|c|c|c|c|c|c|}
\hline \multicolumn{3}{|c|}{ LEVEL 5} & \multicolumn{3}{|c|}{ LEVEL 4} & \multicolumn{3}{|c|}{ LEVEL 3} & \multicolumn{3}{|c|}{ LEVEL 2} & \multicolumn{3}{|c|}{ LEVEL 1} \\
\hline Linked Inputs & Detail & Output & Linked Inputs & Detail & Output & Linked Inputs & Detail & Output & Linked Inputs & Detail & Output & Linked Inputs & Detail & Outpi \\
\hline & 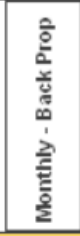 & $\begin{array}{l}\overline{0} \\
\frac{1}{E} \\
\frac{1}{5} \\
\end{array}$ & $\begin{array}{l}\overline{\overline{0}} \\
\text { ثิ }\end{array}$ & 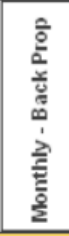 & 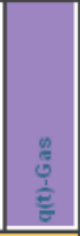 & 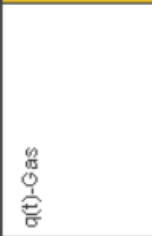 & 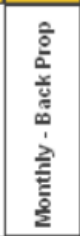 & $\begin{array}{l}\frac{d}{0} \\
\frac{1}{E} \\
\frac{1}{5} \\
\end{array}$ & 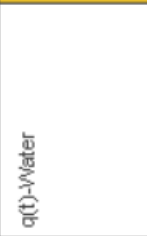 & 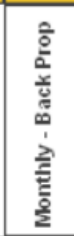 & 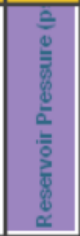 & 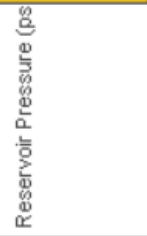 & 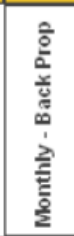 & 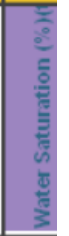 \\
\hline \multicolumn{3}{|c|}{ Oil No Blind-3 } & \multicolumn{3}{|c|}{ Gas } & \multicolumn{3}{|c|}{ Water } & \multicolumn{3}{|c|}{ Reservoir Pressure } & \multicolumn{3}{|c|}{ Water Saturation-2 } \\
\hline
\end{tabular}

Figure 62 Random partitioning-no blind validation TDM structure

Now that the Top Down Model has been built, the model can be deployed. The TDM Deployment window was loaded where the recently created TDM is selected. The desired dates of deployment are verified to be correct, along with the input parameters. The selected model is deployed for which results are shown in the next chapter. After the deployment results were deemed to be of quality, or in other words the Top Down Model's predictions were accurate, the results from each level of the Top Down Model were exported in .csv format. The entire reservoir oil, gas, and water plots with cumulative and rate production and number of active wells were also exported. 
The exported oil, water, gas, reservoir pressure, and water saturation tables were read by a python program that then plotted the predictions versus the actual data. This code written by Yvon Martinez, greatly expedited the graphing process for each individual well. The code graphed actual vs predicted data for every well for oil, water, gas, reservoir pressure, and water saturation. A few examples of these plots per well are shown in the results section, however the rest are included in each subsection of Appendix 8.2. These exported results are also used in another code that creates a side by side comparison of two heat maps, one for the actual data and one for the predicted data. These are created for the entire reservoir water saturation and reservoir pressure for specified dates. A third and final code is then used to gather the plots for each well together into one image for easy formatting.

\subsubsection{Multi-Random Partitioning-1 Year Blind Validation}

A different form of partitioning is used with this dataset in order to assign the last year of the simulation to validation. In doing so, all of the validation data used except for the first two values, will be using the models predicted values for parameters at time steps t- 1 and $\mathrm{t}-2$. This is one of the best ways to test the validity of the model and deem if it is accurate enough to function using its own predictions and also gives an early indication of how it will perform during forecasting. The tables shown here list the attributes used for each data-driven model during the training process which are slightly different than the attributes used in the random partitioning- no blind case previously presented. The attributes were adjusted in order to obtain better training and deployment results with the different partitioning used. As previously stated, the offset producer and offset injector variables listed are used for both the first and second closest offset producers and injectors.

Table 25 Data-driven oil model training attributes for Multi-Random Partitioning-1 Year Blind Validation TDM

\begin{tabular}{|l|l|l|}
\hline Focal & Offset Producer & Offset Injector \\
\hline Time & Distance t & Distance t \\
\hline Drainage Area & Completion t & Completion t \\
\hline Completion t & FBHP t & FBHP t \\
\hline FBHP t & FBHP t-1 & Injected Gas Rate t \\
\hline FBHP t-1 & Oil Saturation t-1 & Injected Gas Rate t-1 \\
\hline Oil Saturation t-1 & Oil Saturation t-2 & Injected Water Rate t \\
\hline Oil Saturation t-2 & Reservoir Pressure t-1 & Injected Water Rate t-1 \\
\hline Open Layers Permeability t & q oil t-1 & Open Layers Permeability t \\
\hline Open Layers Permeability t-1 & q oil t-2 & \\
\hline Open Layers Permeability t-2 & $\begin{array}{l}\text { Open Layers } \\
\text { Permeability t }\end{array}$ & \\
\hline Open Layers Porosity t & & \\
\hline Reservoir Pressure t-1 & & \\
\hline q oil t-1 & & \\
\hline q oil t-2 & & \\
\hline WC t-1 & & \\
\hline q gas t-1 & & \\
\hline
\end{tabular}


Table 26 Data-driven gas model training attributes for Multi-Random Partitioning-1 Year Blind Validation TDM

\begin{tabular}{|l|l|l|}
\hline Focal & Offset Producer & Offset Injector \\
\hline Time & Distance $\mathbf{~}$ & Distance $\mathbf{~}$ \\
\hline Top & Top & Completion t \\
\hline TVD & TVD & FBHP t \\
\hline Completion t & Completion t & Gas Saturation t-1 \\
\hline FBHP t & FBHP t & Injected Gas Rate t \\
\hline FBHP t-1 & FBHP t-1 & Injected Gas Rate t-1 \\
\hline Gas Saturation t-1 & Gas Saturation t-1 & Injected Gas Rate t-2 \\
\hline Gas Saturation t-2 & Gas Saturation t-2 & Open Layers Permeability t \\
\hline Open Layers Permeability t & $\begin{array}{l}\text { Reservoir Pressure } \\
\text { t-1 }\end{array}$ \\
\hline Open Layers Porosity t & q gas t-1 & \\
\hline Reservoir Pressure t-1 & q gas t-2 & \\
\hline q gas t-1 & & \\
\hline q gas t-2 & & \\
\hline q oil t & & \\
\hline
\end{tabular}

Table 27 Data-driven water model training attributes for Multi-Random Partitioning-1 Year Blind Validation TDM

\begin{tabular}{|l|l|l|}
\hline Focal & Offset Producer & Offset Injector \\
\hline Time & Distance t & Distance t \\
\hline Top & Top & FBHP t \\
\hline TVD & TVD & Injected Water Rate t \\
\hline Completion t & Completion t & Injected Water Rate t-1 \\
\hline FBHP t & FBHP t & Injected Water Rate t-2 \\
\hline FBHP t-1 & FBHP t-1 & Water Saturation t-1 \\
\hline Reservoir Pressure t-1 & $\begin{array}{l}\text { Open Layers Permeability } \\
\text { t }\end{array}$ & $\begin{array}{l}\text { Open Layers Permeability } \\
\text { t }\end{array}$ \\
\hline $\begin{array}{l}\text { Open Layers Permeability } \\
\text { t }\end{array}$ & Reservoir Pressure t-1 & \\
\hline Open Layers Porosity t & Water Saturation t-1 & \\
\hline Water Saturation t-1 & Water Saturation t-2 & \\
\hline Water Saturation t-2 & q water t-1 & \\
\hline q water t-1 & q water t-2 & \\
\hline q water t-2 & & \\
\hline q gas t & & \\
\hline q oil t & & \\
\hline
\end{tabular}


Table 28 Data-driven reservoir pressure training attributes for Multi-Random Partitioning-1 Year Blind Validation TDM

\begin{tabular}{|l|l|l|}
\hline Focal & Offset Producer & Offset Injector \\
\hline Time & Distance t & Distance t \\
\hline Top & Top & FBHP t \\
\hline TVD & TVD & FBHP t-1 \\
\hline Completion t & Completion t & FBHP t-2 \\
\hline FBHP t & FBHP t & $\begin{array}{l}\text { Reservoir Pressure t- } \\
\text { 1 }\end{array}$ \\
\hline FBHP t-1 & FBHP t-1 & $\begin{array}{l}\text { Reservoir Pressure t- } \\
\text { F }\end{array}$ \\
\hline FBHP t-2 & FBHP t-2 & Injected Gas Rate t \\
\hline $\begin{array}{l}\text { Open Layers Permeability } \\
\text { t }\end{array}$ & Reservoir Pressure t-1 & $\begin{array}{l}\text { Injected Water Rate } \\
\text { t }\end{array}$ \\
\hline Open Layers Porosity t & Reservoir Pressure t-2 & \\
\hline Reservoir Pressure t-1 & & \\
\hline Reservoir Pressure t-2 & & \\
\hline q oil t & & \\
\hline q gas t & & \\
\hline q water t & & \\
\hline
\end{tabular}

Table 29 Data-driven water saturation model training attributes for Multi-Random Partitioning-1 Year Blind Validation TDM

\begin{tabular}{|l|l|l|}
\hline Focal & Offset Producer & Offset Injector \\
\hline Time & Distance t & Distance t \\
\hline Top & Completion t & Gas Saturation t-1 \\
\hline TVD & Top & Oil Saturation t-1 \\
\hline Completion t & TVD & Injected Water Rate t \\
\hline FBHP t & Gas Saturation t-1 & $\begin{array}{l}\text { Injected Water Rate t- } \\
\mathbf{1}\end{array}$ \\
\hline Gas Saturation t-1 & Oil Saturation t-1 & $\begin{array}{l}\text { Injected Water Rate t- } \\
\mathbf{2}\end{array}$ \\
\hline Oil Saturation t-1 & Water Saturation t-1 & Water Saturation t-1 \\
\hline $\begin{array}{l}\text { Open Layers Permeability } \\
\text { t }\end{array}$ & Water Saturation t-2 & Water Saturation t-2 \\
\hline Open Layers Porosity t & & \\
\hline Reservoir Pressure t & & \\
\hline Water Saturation t-1 & & \\
\hline Water Saturation t-2 & & \\
\hline q water t & & \\
\hline
\end{tabular}

Multi-Random Partitioning was used to assign the last year of the model to blind validation. The rest of the data was split between training and calibration, $80 \%$ and $20 \%$ respectively. The training dataset 
had 5090 records, calibration had 1272 records, and verification had 449 records. Once again, the default neural network values shown in Table 23 are used for these data-driven models along with the data-driven models that will presented in the following sections. The R Squared metric for each data-driven model is shown below for all partition cases (training, calibration, and blind validation), one indication of the correlation between actual outputs and predicted outputs. The training results for all the Multi-Random Partitioning- 1 Year Blind Validation data driven models are combined into one plot showing all partition cases below.

Table 30 R Squared metrics for each data-driven model in Multi-Random Partitioning-1 Year Blind Validation TDM

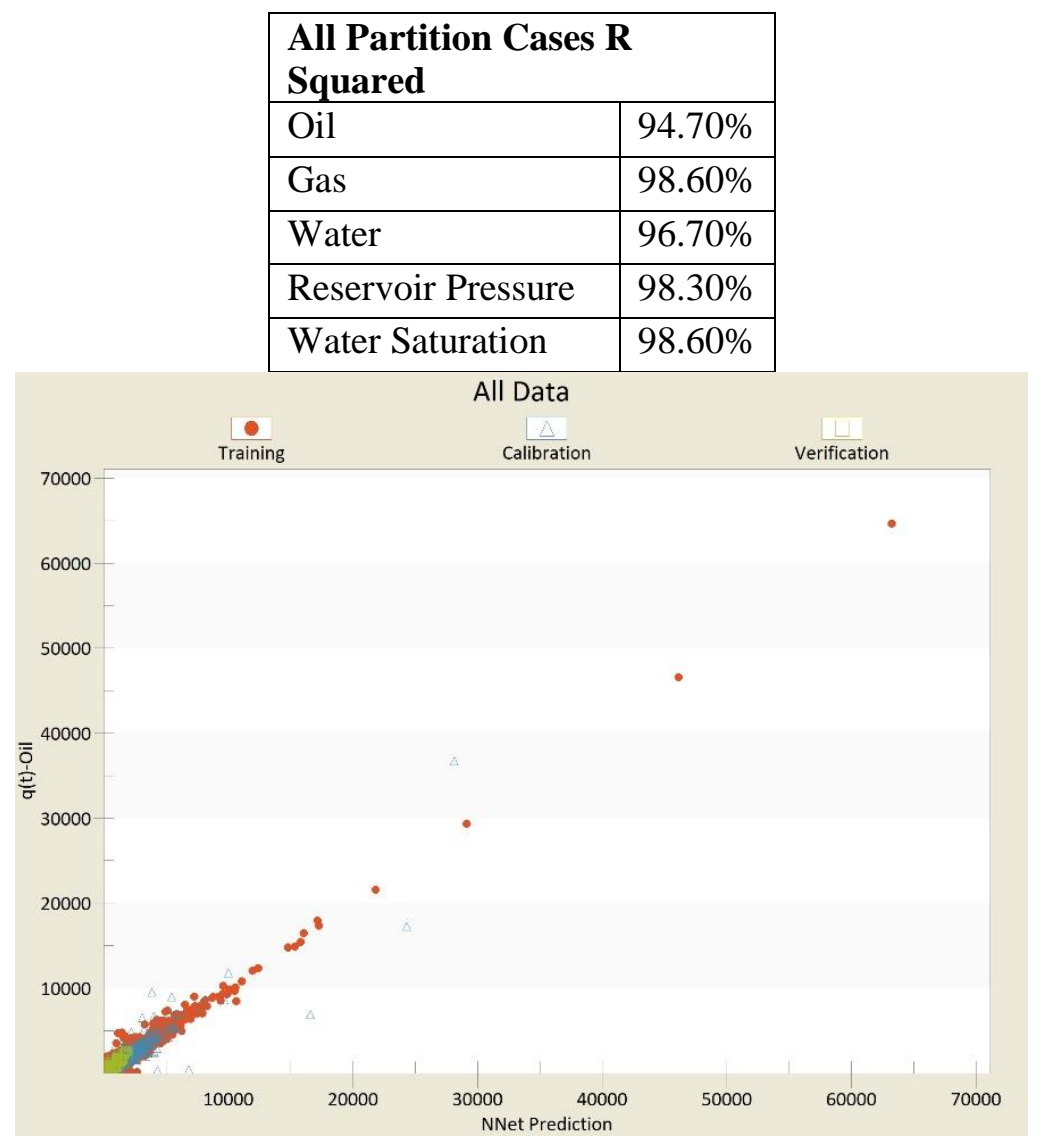

Figure 63 Data-driven oil model training results for Multi-Random Partitioning-1 Year Blind Validation 


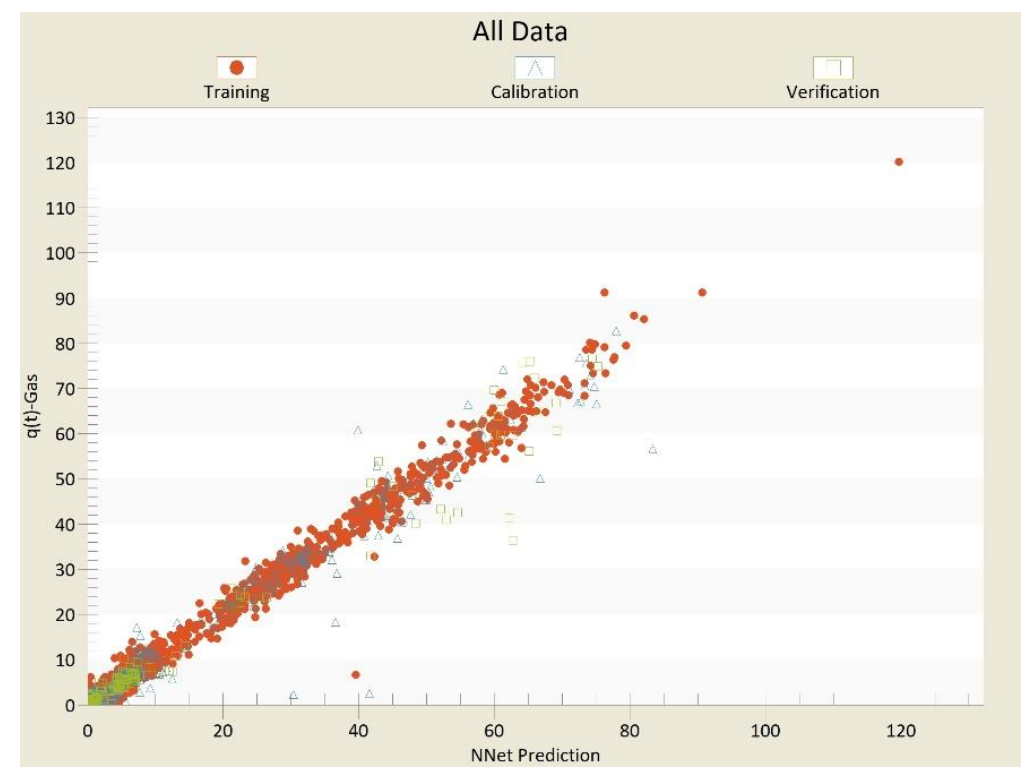

Figure 64 Data-driven gas model training results for Multi-Random Partitioning-1 Year Blind Validation

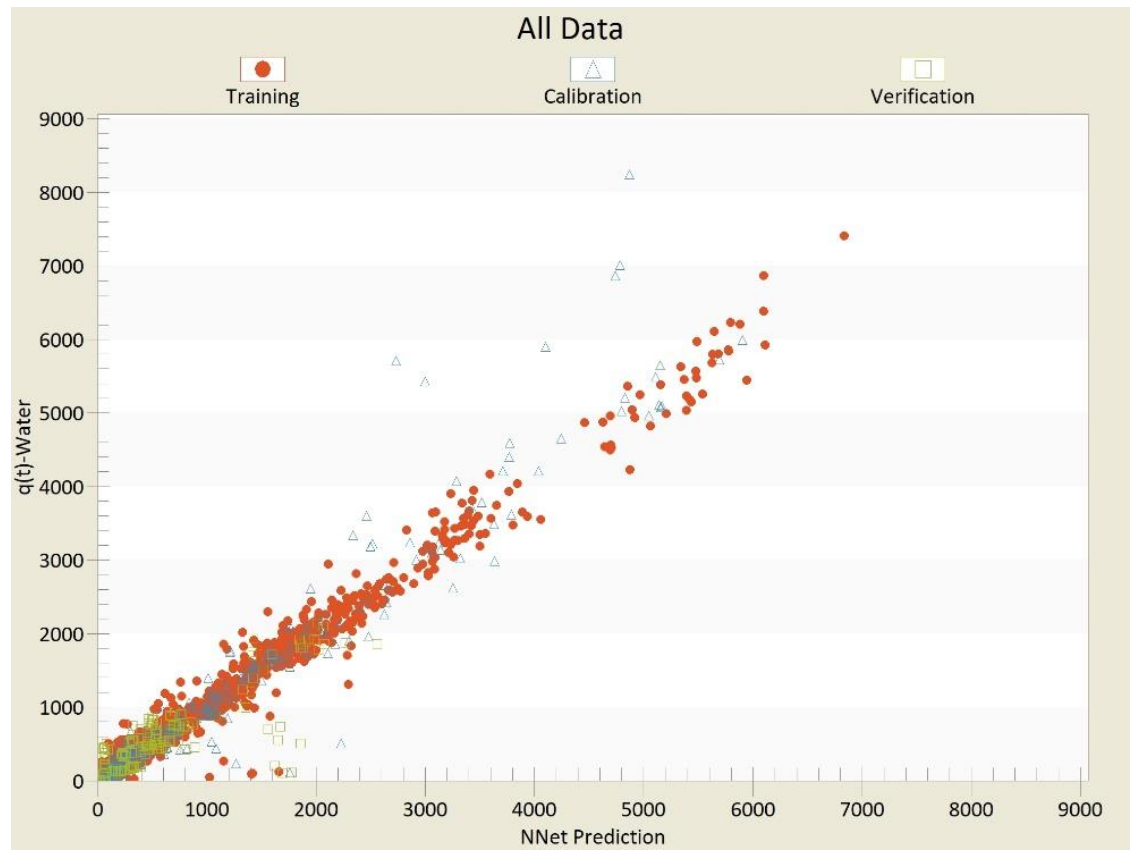

Figure 65 Data-driven water model training results for Multi-Random Partitioning-1 Year Blind Validation 


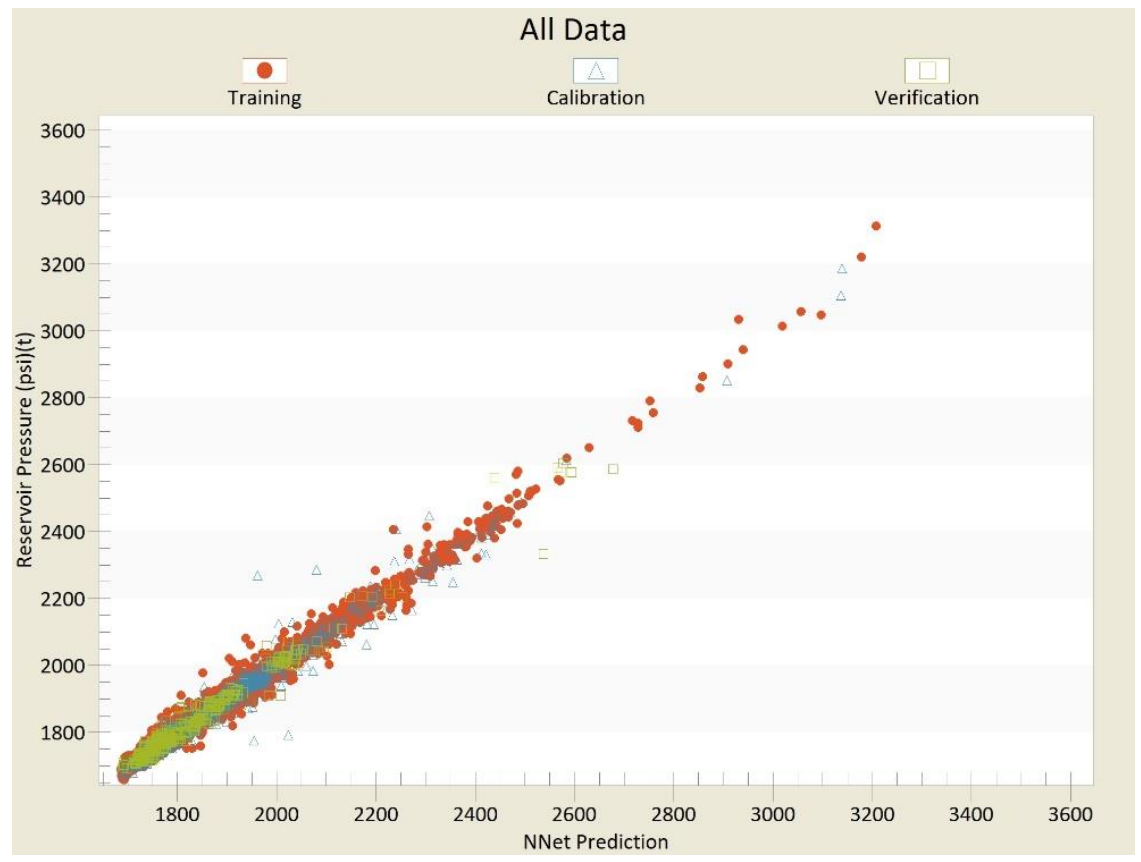

Figure 66 Data-driven reservoir pressure model training results for Multi-Random Partitioning-1 Year Blind Validation

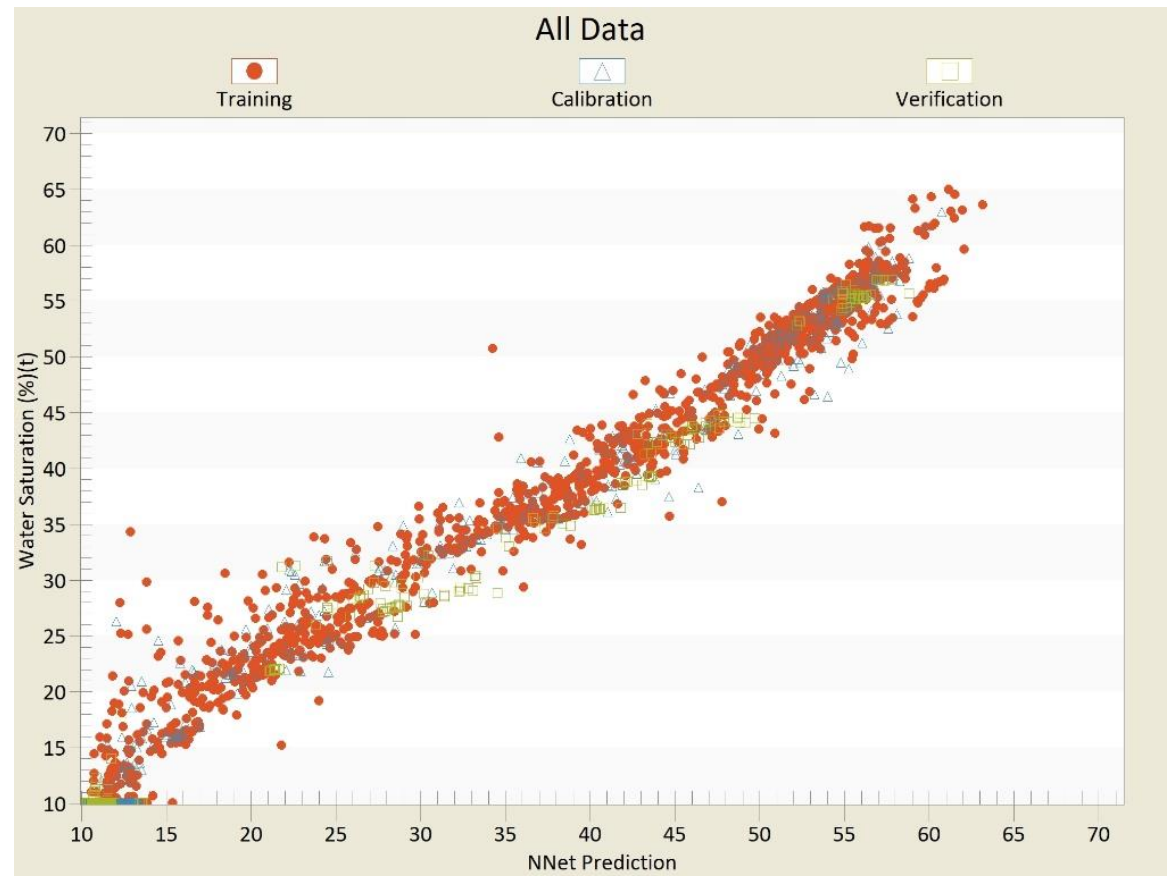

Figure 67 Data-driven water saturation model training results for Multi-Random Partitioning-1 Year Blind Validation

The Top Down Model was constructed in a similar fashion to the Random Partitioning-No Blind Validation case previously explained. The structure is shown next, where the output from one model can be seen as an input into the next model. The results from the TDM deployment are discussed in the results and discussion chapter. 


\begin{tabular}{|c|c|c|c|c|c|c|c|c|c|c|c|c|c|c|}
\hline \multicolumn{3}{|c|}{ LEVEL 5} & \multicolumn{3}{|c|}{ LEVEL 4} & \multicolumn{3}{|c|}{ LEVEL 3} & \multicolumn{3}{|c|}{ LEVEL 2} & \multicolumn{3}{|c|}{ LEVEL 1} \\
\hline \multirow[t]{2}{*}{ Linked Inputs } & Detail & Output & Linked Inputs & Detail & Output & Linked Inputs & Detail & Output & Linked Inputs & Detail & Output & Linked Inputs & Detail & Output \\
\hline & 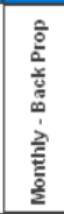 & $\begin{array}{l}\overline{0} \\
\frac{1}{1} \\
\frac{1}{5} \\
\end{array}$ & 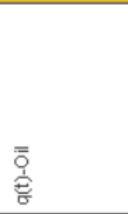 & 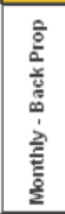 & 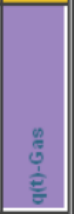 & 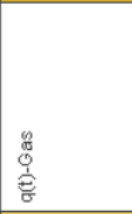 & 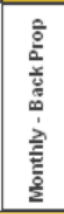 & $\begin{array}{l}\frac{\mathrm{g}}{\pi} \\
\frac{\mathrm{y}}{3} \\
\frac{1}{\mathrm{v}} \\
\end{array}$ & 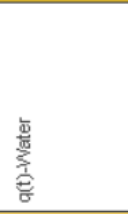 & 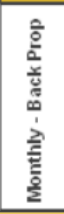 & 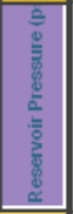 & 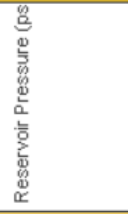 & 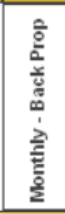 & 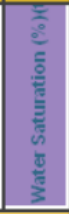 \\
\hline \multicolumn{3}{|c|}{ Oil } & \multicolumn{3}{|c|}{ Gas-1Yr } & \multicolumn{3}{|c|}{ Water-1Yr } & \multicolumn{3}{|c|}{ Reservoir Pressure-1Yr } & \multicolumn{3}{|c|}{ Water Saturation 2-1Y } \\
\hline
\end{tabular}

Figure 68 Multi-Random Partitioning-1 Year Blind Validation TDM structure

The same exporting process as discussed in Section 6.10.1 was done for the results in the MultiRandom Partitioning-1 Year Blind Validation Top Down Model. The three codes were used to help visualize the results and group the plots together. These plots are shown in this TDM's respective results section and Appendix 8.2.2.1.

\subsubsection{Multi-Random Partitioning-2 Year Blind Validation}

The same type of partitioning as the 1 Year Blind Validation case was also used for the 2 Year Blind Validation case. The only difference was instead of having blind validation from December $31^{\text {st }}, 2015$ to December $31^{\text {st }}$, 2016, blind validation was assigned to be from December $31^{\text {st }}, 2014$ to December $31^{\text {st }}$, 2016. Also, because the training and calibration records are assigned randomly, the records used are not the same between the oil, gas, water, reservoir pressure and water saturation data-driven models within a Top Down Model nor are they the same between Top Down Models. The training dataset had 4762 records, calibration had 1191 records, and the blind validation had 858 records. The variables used in each training are shown below.

Table 31 Data-driven oil model training attributes for Multi-Random Partitioning-2 Year Blind Validation TDM

\begin{tabular}{|l|l|l|}
\hline Focal & Offset Producer & Offset Injector \\
\hline Time & Distance t & Distance t \\
\hline Drainage Area & Completion t & Completion t \\
\hline Completion t & FBHP t & FBHP t \\
\hline FBHP t & FBHP t-1 & Injected Gas Rate t \\
\hline FBHP t-1 & Oil Saturation t-1 & Injected Gas Rate t-1 \\
\hline Oil Saturation t-1 & Oil Saturation t-2 & Injected Water Rate t \\
\hline Oil Saturation t-2 & $\begin{array}{l}\text { Open Layers Permeability } \\
\text { t }\end{array}$ & Injected Water Rate t-1 \\
\hline Open Layers Permeability t & Reservoir Pressure t-1 & $\begin{array}{l}\text { Open Layers Permeability } \\
\text { t }\end{array}$ \\
\hline $\begin{array}{l}\text { Open Layers Permeability t- } \\
\text { 1 }\end{array}$ & q oil t-1 & \\
\hline $\begin{array}{l}\text { Open Layers Permeability t- } \\
\mathbf{2}\end{array}$ & q oil t-2 & \\
\hline Open Layers Porosity t & & \\
\hline Reservoir Pressure t-1 & & \\
\hline
\end{tabular}




\begin{tabular}{|l|l|l|}
\hline q oil t-1 & & \\
\hline q oil t-2 & & \\
\hline WC t-1 & & \\
\hline q gas t-1 & & \\
\hline
\end{tabular}

Table 32 Data-driven gas model training attributes for Multi-Random Partitioning-2 Year Blind Validation TDM

\begin{tabular}{|l|l|l|}
\hline Focal & Offset Producer & Offset Injector \\
\hline Time & Distance t & Distance t \\
\hline Top & Top & Completion t \\
\hline TVD & TVD & FBHP t \\
\hline Completion t & Completion t & Gas Saturation t-1 \\
\hline FBHP t & FBHP t & Injected Gas Rate t \\
\hline FBHP t-1 & FBHP t-1 & Injected Gas Rate t-1 \\
\hline Gas Saturation t-1 & Gas Saturation t-1 & Injected Gas Rate t-2 \\
\hline Gas Saturation t-2 & Gas Saturation t-2 & $\begin{array}{l}\text { Open Layers Permeability } \\
\text { t }\end{array}$ \\
\hline $\begin{array}{l}\text { Open Layers Permeability } \\
\text { t }\end{array}$ & Reservoir Pressure t-1 & \\
\hline Open Layers Porosity t & q gas t-1 & \\
\hline Reservoir Pressure t-1 & q gas t-2 & \\
\hline q gas t-1 & & \\
\hline q gas t-2 & & \\
\hline q oil t & & \\
\hline
\end{tabular}

Table 33 Data-driven water model training attributes for Multi-Random Partitioning-2 Year Blind Validation TDM

\begin{tabular}{|l|l|l|}
\hline Focal & Offset Producer & Offset Injector \\
\hline Time & Distance t & Distance t \\
\hline Completion t & Completion t & FBHP t \\
\hline FBHP t & FBHP t & Injected Water Rate t \\
\hline FBHP t-1 & FBHP t-1 & Injected Water Rate t-1 \\
\hline Reservoir Pressure t-1 & $\begin{array}{l}\text { Open Layers Permeability } \\
\text { t }\end{array}$ & Injected Water Rate t-2 \\
\hline Open Layers Permeability t & Reservoir Pressure t-1 & Water Saturation t-1 \\
\hline Water Saturation t-1 & Water Saturation t-1 & $\begin{array}{l}\text { Open Layers Permeability } \\
\text { t }\end{array}$ \\
\hline Water Saturation t-2 & Water Saturation t-2 & \\
\hline q water t-1 & q water t-1 & \\
\hline q water t-2 & q water t-2 & \\
\hline q gas t & & \\
\hline q oil t & & \\
\hline
\end{tabular}




\begin{tabular}{|l|l|l|}
\hline Water Saturation Poly Ave t-1 & & \\
\hline $\begin{array}{l}\text { Open Layers Permeability Poly Ave } \\
\text { t }\end{array}$ & & \\
\hline Reservoir Pressure Poly Ave t-1 & & \\
\hline
\end{tabular}

Table 34 Data-driven reservoir pressure model training attributes for Multi-Random Partitioning-2 Year Blind Validation TDM

\begin{tabular}{|l|l|l|}
\hline Focal & Offset Producer & Offset Injector \\
\hline Time & Distance t & Distance t \\
\hline TVD & TVD & FBHP t \\
\hline Completion t & Completion t & FBHP t-1 \\
\hline FBHP t & FBHP t & FBHP t-2 \\
\hline FBHP t-1 & FBHP t-1 & $\begin{array}{l}\text { Reservoir Pressure t- } \\
\text { 1 }\end{array}$ \\
\hline FBHP t-2 & FBHP t-2 & $\begin{array}{l}\text { Reservoir Pressure t- } \\
2\end{array}$ \\
\hline $\begin{array}{l}\text { Open Layers } \\
\text { Permeability t }\end{array}$ & Reservoir Pressure t-1 & Injected Gas Rate t \\
\hline Open Layers Porosity t & Reservoir Pressure t-2 & $\begin{array}{l}\text { Injected Water Rate } \\
\text { t }\end{array}$ \\
\hline Reservoir Pressure t-1 & & \\
\hline Reservoir Pressure t-2 & & \\
\hline q oil t & & \\
\hline q gas t & & \\
\hline q water t & & \\
\hline
\end{tabular}

Table 35 Data-driven water saturation model training attributes for Multi-Random Partitioning- 2 Year Blind Validation TDM

\begin{tabular}{|l|l|l|}
\hline Focal & Offset Producer & Offset Injector \\
\hline Time & Distance t & Distance t \\
\hline Top & Completion t & Gas Saturation t-1 \\
\hline TVD & Top & Oil Saturation t-1 \\
\hline Completion t & TVD & Injected Water Rate t \\
\hline FBHP t & Gas Saturation t-1 & $\begin{array}{l}\text { Injected Water Rate t- } \\
\mathbf{1}\end{array}$ \\
\hline Gas Saturation t-1 & Oil Saturation t-1 & $\begin{array}{l}\text { Injected Water Rate t- } \\
\mathbf{2}\end{array}$ \\
\hline Oil Saturation t-1 & $\begin{array}{l}\text { Water Saturation t- } \\
\mathbf{1}\end{array}$ & Water Saturation t-1 \\
\hline $\begin{array}{l}\text { Open Layers Permeability } \\
\text { t }\end{array}$ & $\begin{array}{l}\text { Water Saturation t- } \\
\mathbf{2}\end{array}$ & Water Saturation t-2 \\
\hline Open Layers Porosity t & & \\
\hline Reservoir Pressure t & & \\
\hline Water Saturation t-1 & & \\
\hline Water Saturation t-2 & & \\
\hline
\end{tabular}


Next, the plots containing the training, calibration, and blind validation actual values versus predicted values will be shown along with a table of the $\mathrm{R}$ Squared evaluation metrics for all of the data driven models in the Multi-Random Partitioning-2 Year Blind Validation TDM.

Table 36 R Squared metrics for all data-driven models in the Multi-Random Partitioning-2 Year Blind Validation TDM

\begin{tabular}{|l|r|}
\hline \multicolumn{2}{|c|}{ All Partition Cases R Squared } \\
\hline Oil & $93.30 \%$ \\
\hline Gas & $98.70 \%$ \\
\hline Water & $97.70 \%$ \\
\hline Reservoir Pressure & $97.90 \%$ \\
\hline Water Saturation & $99.50 \%$ \\
\hline
\end{tabular}

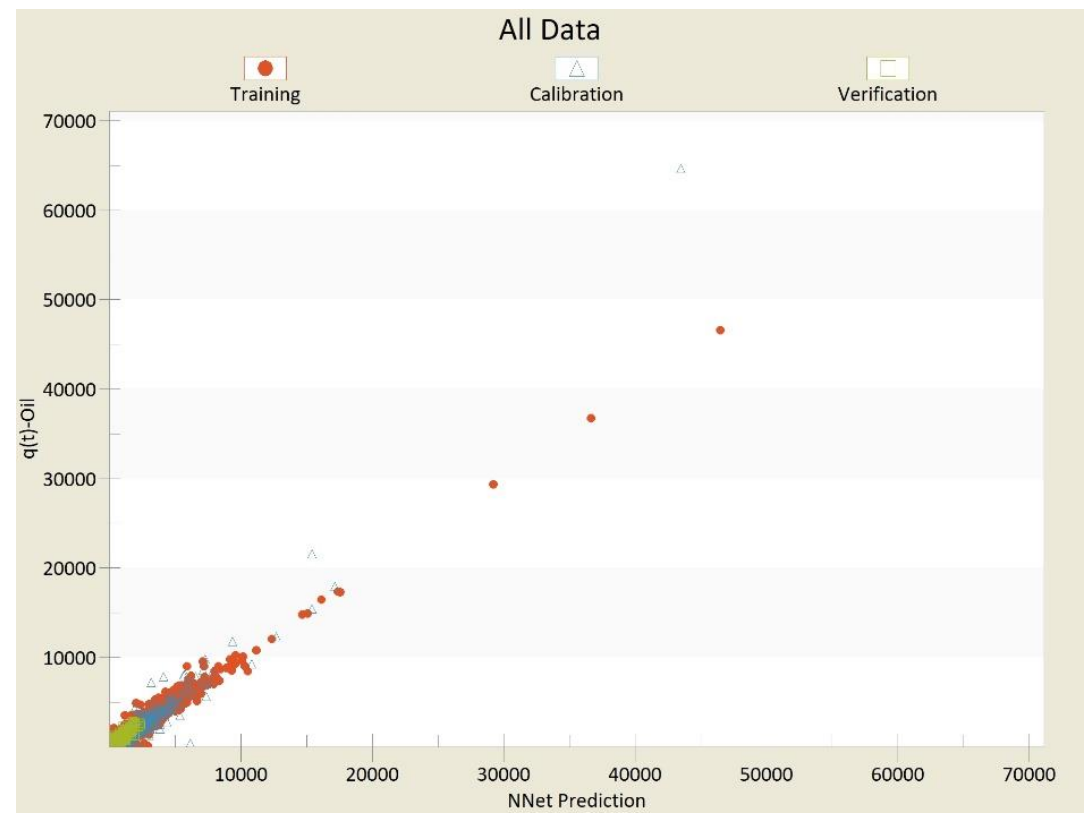

Figure 69 Data-driven oil model training results for Multi-Random Partitioning- 2 Year Blind Validation TDM 


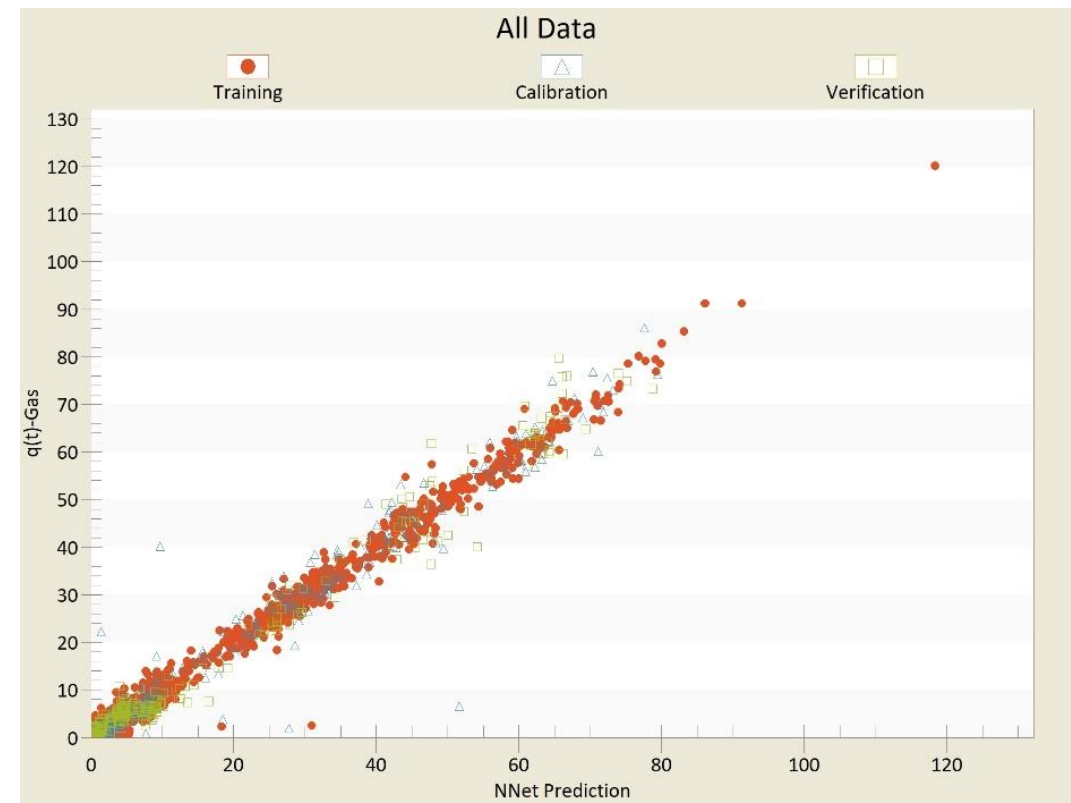

Figure 70 Data-drive gas model training results for Multi-Random Partitioning-2 Year Blind Validation TDM

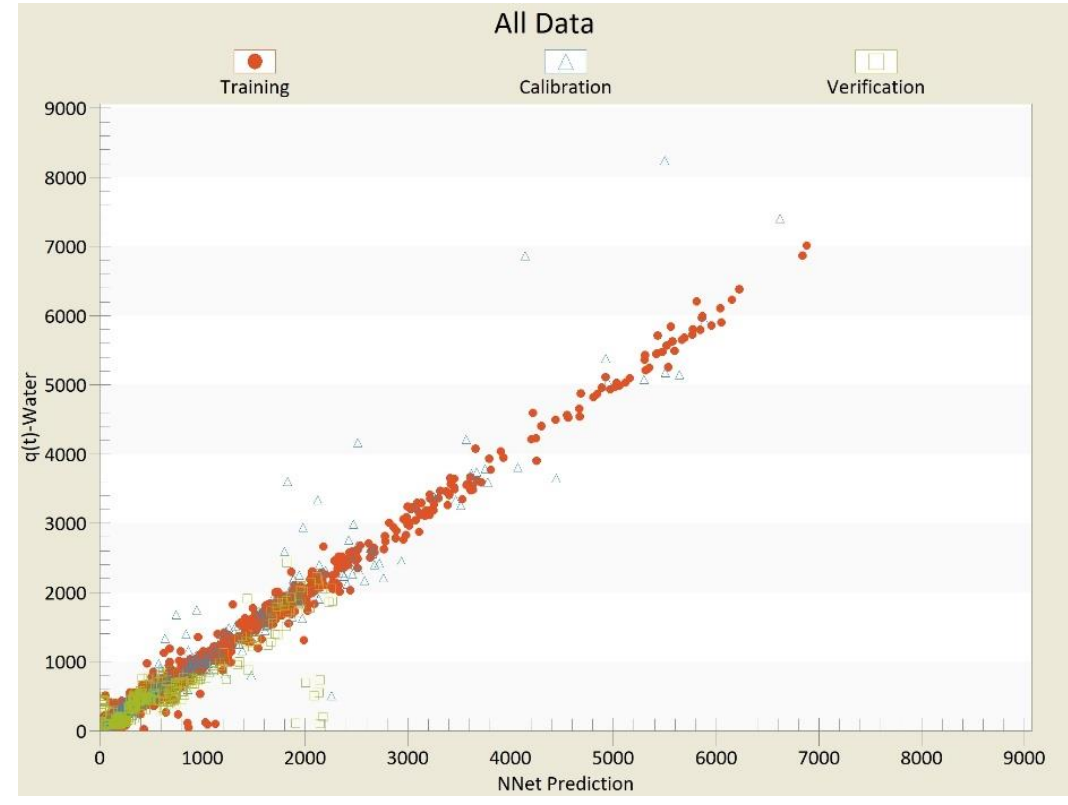

Figure 71 Data-driven water model training results for Multi-Random Partitioning-2 Year Blind Validation TDM 


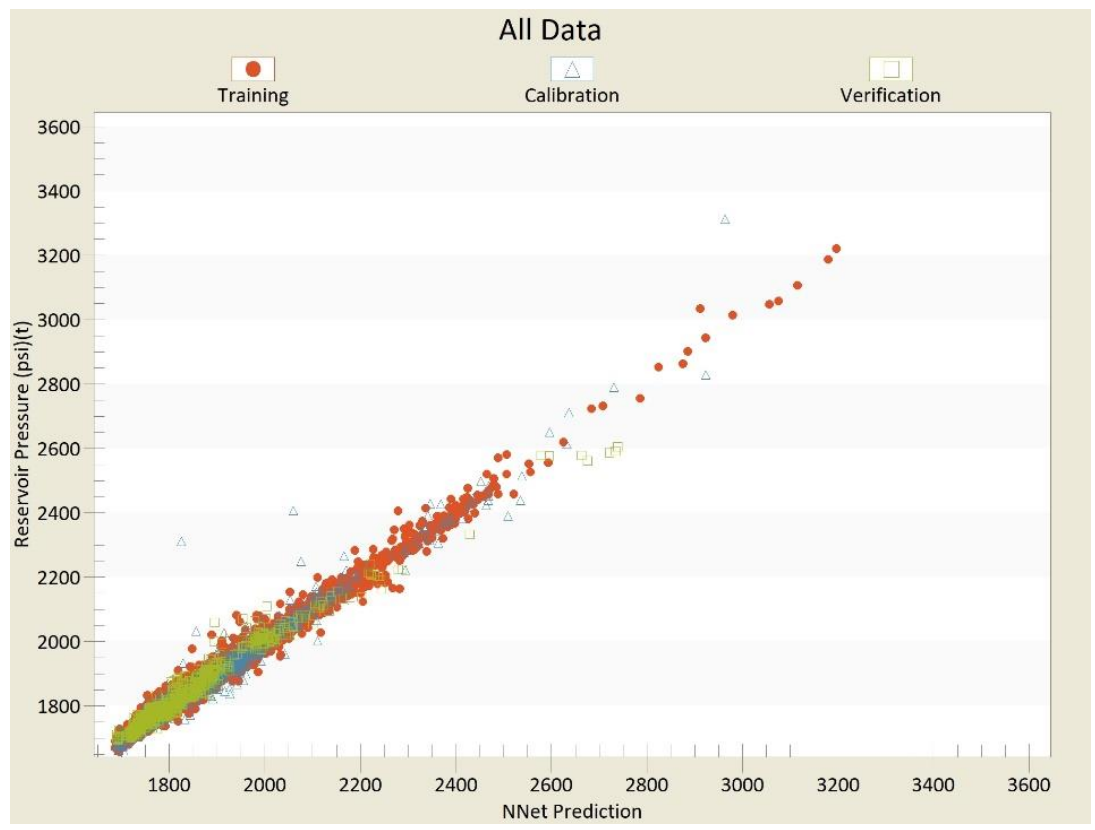

Figure 72 Data-driven reservoir pressure model training results for Multi-Random Partitioning-2 Year Blind Validation TDM

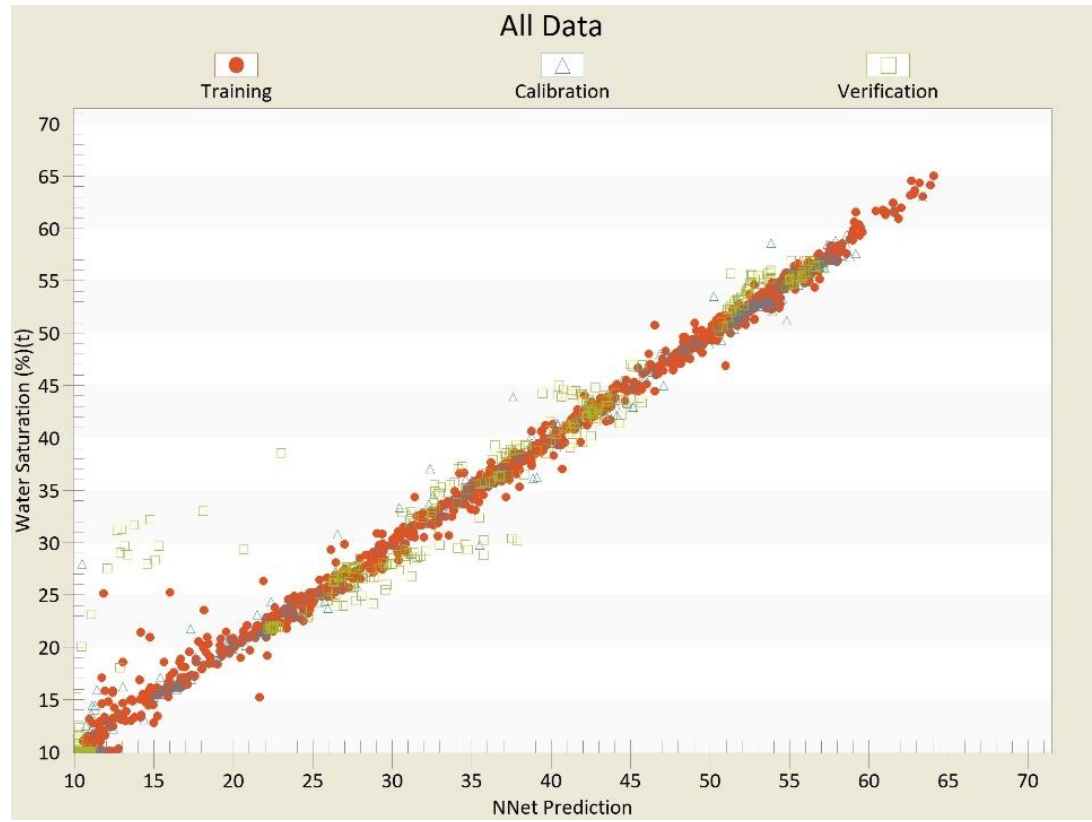

Figure 73 Data-driven water saturation model training results for Multi-Random Partitioning-2 Year Blind Validation TDM

After these results were obtained, the Top Down Model was built, where the structure is displayed here. The model was deployed, the results exported, and the plots generated using the python codes. The results are shown in the results and discussion chapter. 


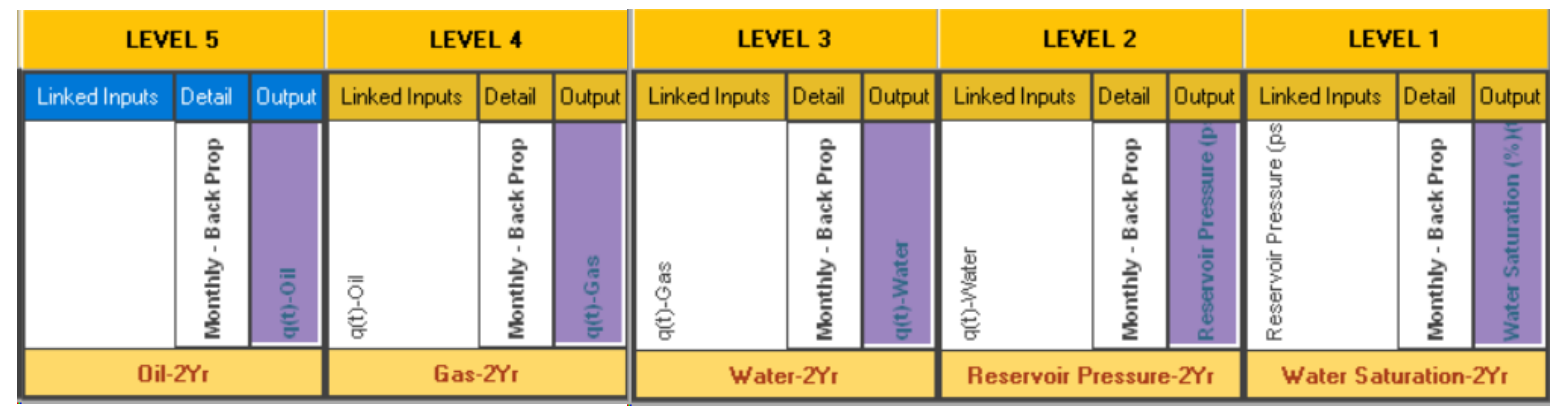

Figure 74 Structure for Multi-Random Partitioning-2 Year Blind Validation Top Down Model

\subsubsection{Multi-Random Partitioning-3 Year Blind Validation TDM}

Multi-Random Partitioning was used for the 3 Year Blind Validation case where the blind validation started on December $31^{\text {st }}, 2013$. The training dataset had 4422 records, calibration had 1105 , and verification had 1284 records each. The training attributes used to train the oil, gas, water, reservoir pressure, and water saturation models are included below.

Table 37 Data-driven oil model training attributes for Multi-Random Partitioning- 3 Year Blind Validation TDM

\begin{tabular}{|l|l|l|}
\hline Focal & Offset Producer & Offset Injector \\
\hline Time & Distance t & Distance t \\
\hline Drainage Area & Completion t & Completion t \\
\hline Completion t & FBHP t & FBHP t \\
\hline FBHP t & FBHP t-1 & Injected Gas Rate t \\
\hline FBHP t-1 & Oil Saturation t-1 & Injected Water Rate t \\
\hline Oil Saturation t-1 & Oil Saturation t-2 & Open Layers Permeability t \\
\hline Oil Saturation t-2 & Reservoir Pressure t-1 & \\
\hline Open Layers Permeability t & q oil t-1 & \\
\hline Open Layers Permeability t-1 & q oil t-2 & \\
\hline Open Layers Permeability t-2 & Open Layers Permeability t & \\
\hline Open Layers Porosity t & & \\
\hline Reservoir Pressure t-1 & & \\
\hline Oil Saturation Poly Ave t-1 & & \\
\hline Open Layers Permeability Poly Ave t & & \\
\hline Open Layers Porosity Poly Ave t & & \\
\hline q oil t-1 & & \\
\hline q oil t-2 & & \\
\hline q WC t-1 & & \\
\hline q gas t-1 & & \\
\hline
\end{tabular}

Table 38 Data-driven gas model training attributes for Multi-Random Partitioning-3 Year Blind Validation TDM

\begin{tabular}{|l|l|l|}
\hline Focal & Offset Producer & Offset Injector \\
\hline Time & Distance t & Distance t \\
\hline
\end{tabular}




\begin{tabular}{|l|l|l|}
\hline Top & Top & Completion t \\
\hline TVD & TVD & FBHP t \\
\hline Completion t & Completion t & Gas Saturation t-1 \\
\hline FBHP t & FBHP t & Injected Gas Rate t \\
\hline FBHP t-1 & FBHP t-1 & Injected Gas Rate t-1 \\
\hline Gas Saturation t-1 & Gas Saturation t-1 & Injected Gas Rate t-2 \\
\hline Gas Saturation t-2 & Gas Saturation t-2 & Open Layers Permeability t \\
\hline Open Layers Permeability t & Reservoir Pressure t-1 & \\
\hline Open Layers Porosity t & q gas t-1 & \\
\hline Reservoir Pressure t-1 & q gas t-2 & \\
\hline q gas t-1 & & \\
\hline q gas t-2 & & \\
\hline q oil t & & \\
\hline
\end{tabular}

Table 39 Data-driven water model training attributes for Multi-Random Partitioning-3 Year Blind Validation TDM

\begin{tabular}{|l|l|l|}
\hline Focal & Offset Producer & Offset Injector \\
\hline Time & Distance t & Distance t \\
\hline Completion t & Completion t & FBHP t \\
\hline FBHP t & FBHP t & Injected Water Rate t \\
\hline FBHP t-1 & FBHP t-1 & Injected Water Rate t-1 \\
\hline Reservoir Pressure t-1 & Open Layers Permeability t & Injected Water Rate t-2 \\
\hline Open Layers Permeability t & Reservoir Pressure t-1 & Water Saturation t-1 \\
\hline Open Layers Porosity t & Water Saturation t-1 & Open Layers Permeability t \\
\hline Water Saturation t-1 & Water Saturation t-2 & \\
\hline Water Saturation t-2 & q water t-1 & \\
\hline q water t-1 & q water t-2 & \\
\hline q water t-2 & & \\
\hline q gas t & & \\
\hline q oil t & & \\
\hline Water Saturation t-1 Poly Ave & & \\
\hline Open Layers Permeability t Poly Ave & & \\
\hline Reservoir Pressure t-1 Poly Ave & & \\
\hline
\end{tabular}

Table 40 Data-driven reservoir pressure model training attributes for Multi-Random Partitioning-3 Year Blind Validation TDM

\begin{tabular}{|l|l|l|}
\hline Focal & Offset Producer & Offset Injector \\
\hline Time & Distance $\mathbf{t}$ & Distance $\mathbf{t}$ \\
\hline TVD & TVD & FBHP $\mathbf{~}$ \\
\hline Completion $\mathbf{t}$ & Completion $\mathbf{t}$ & FBHP $\mathbf{t}-\mathbf{1}$ \\
\hline FBHP $\mathbf{t}$ & FBHP $\mathbf{t}$ & FBHP $\mathbf{t}-\mathbf{2}$ \\
\hline
\end{tabular}




\begin{tabular}{|l|l|l|}
\hline FBHP t-1 & FBHP t-1 & Reservoir Pressure t-1 \\
\hline FBHP t-2 & FBHP t-2 & Reservoir Pressure t-2 \\
\hline Open Layers Permeability t & Reservoir Pressure t-1 & Injected Gas Rate t \\
\hline Open Layers Porosity t & Reservoir Pressure t-2 & Injected Water Rate t \\
\hline Reservoir Pressure t-1 & & \\
\hline Reservoir Pressure t-2 & & \\
\hline q oil t & & \\
\hline q gas t & & \\
\hline q water t & & \\
\hline
\end{tabular}

Table 41 Data-driven water saturation model training attributes for Multi-Random Partitioning- 3 Year Blind Validation TDM

\begin{tabular}{|l|l|l|}
\hline Focal & Offset Producer & Offset Injector \\
\hline Time & Distance t & Distance t \\
\hline Top & Top & Gas Saturation t-1 \\
\hline TVD & TVD & Injected Water Rate t \\
\hline Completion t & Completion t & Injected Water Rate t-1 \\
\hline FBHP t & Gas Saturation t-1 & Injected Water Rate t-2 \\
\hline Gas Saturation t-1 & Oil Saturation t-1 & Oil Saturation t-1 \\
\hline Oil Saturation t-1 & Water Saturation t-1 & Water Saturation t-1 \\
\hline Open Layers Permeability t & Water Saturation t-2 & Water Saturation t-2 \\
\hline Open Layers Porosity t & & \\
\hline Reservoir Pressure t & & \\
\hline Water Saturation t-1 & & \\
\hline Water Saturation t-2 & & \\
\hline q water t & & \\
\hline
\end{tabular}

The training results for all partition cases (training, calibration, and validation) are shown below for all five of the data-driven models along with the R Squared evaluation metric for each of the models used to comprise the Multi-Random Partitioning- 3 Year Blind Validation TDM.

Table 42 R Squared metrics for data-driven models in Multi-Random Partitioning-3 Year Blind Validation TDM

\begin{tabular}{|l|c|}
\hline \multicolumn{2}{|c|}{ All Partition Cases R Squared } \\
\hline Oil & $95.24 \%$ \\
\hline Gas & $98.36 \%$ \\
\hline Water & $96.63 \%$ \\
\hline Reservoir Pressure & $98.00 \%$ \\
\hline Water Saturation & $99.25 \%$ \\
\hline & \\
\hline
\end{tabular}




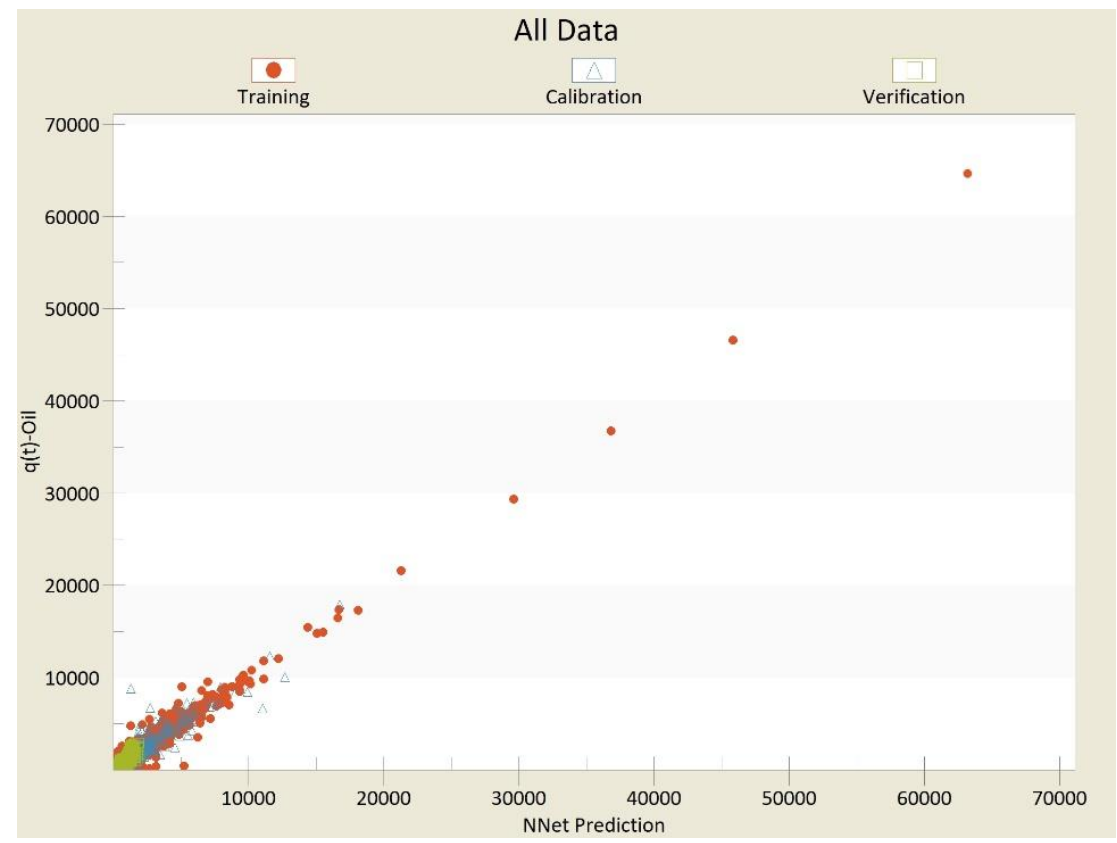

Figure 75 Data-driven oil model training results for Multi-Random Partitioning-2 Year Blind Validation TDM

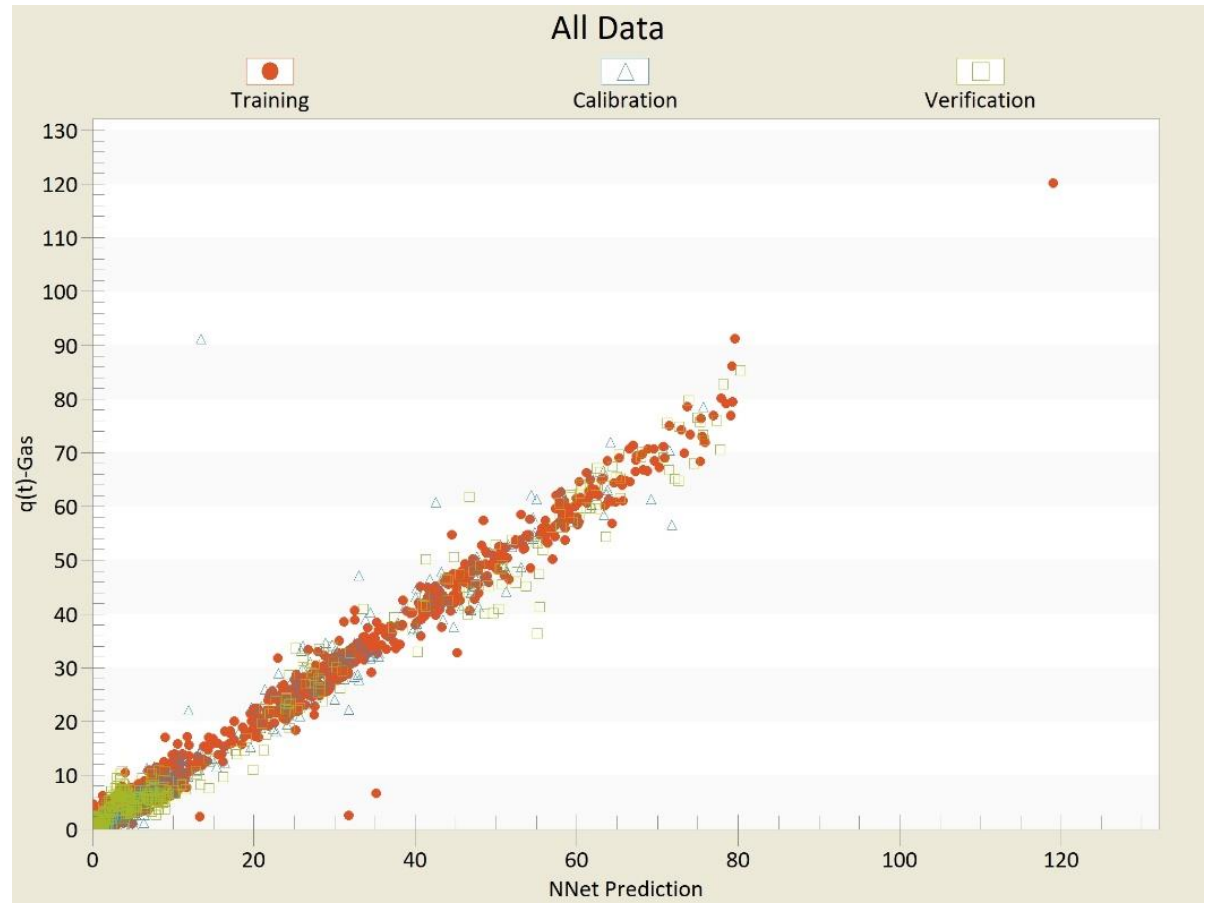

Figure 76 Data-driven gas model training results for Multi-Random Partitioning-3 Year Blind Validation TDM 


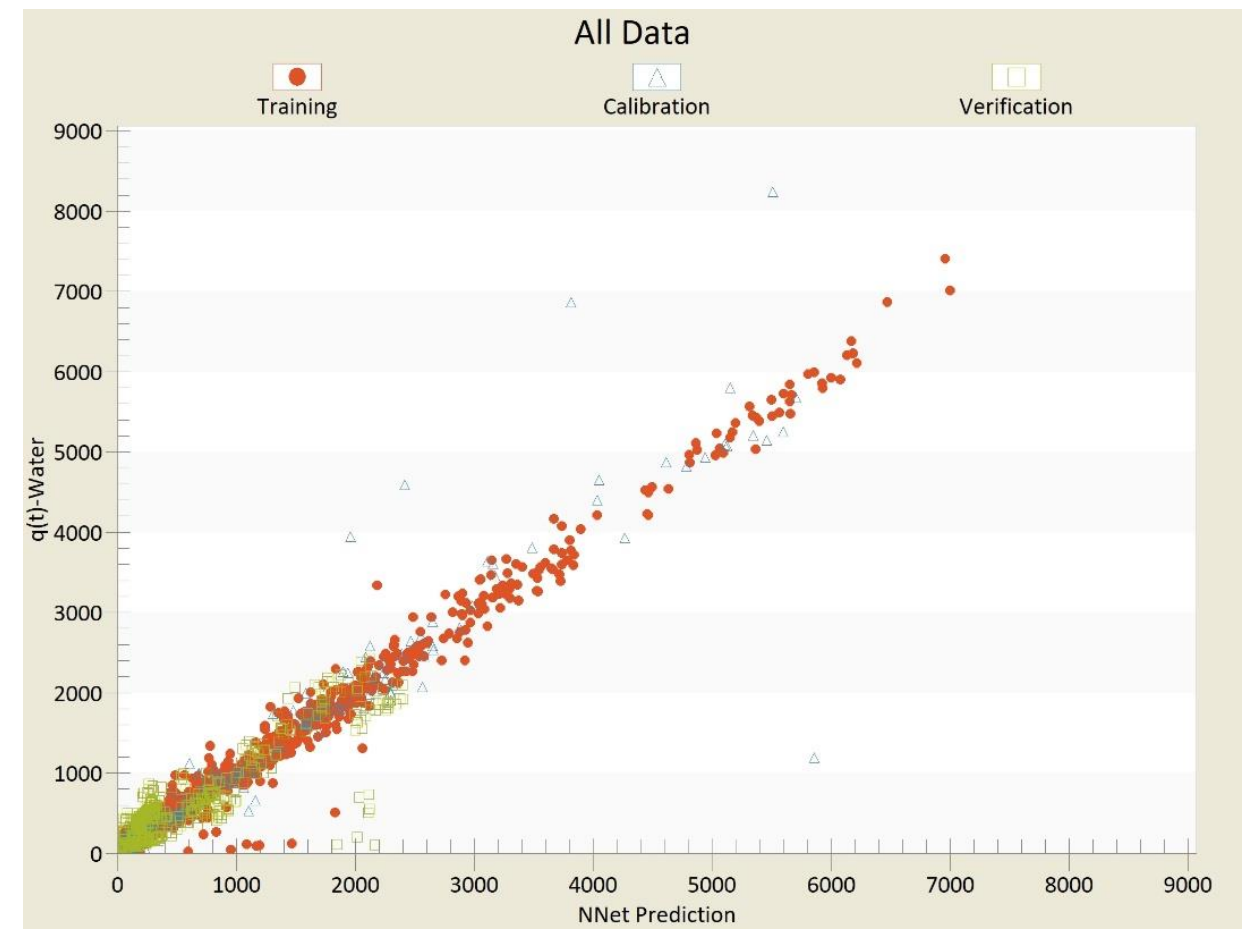

Figure 77 Data-driven water model training results for Multi-Random Partitioning- 3 Year Blind Validation TDM

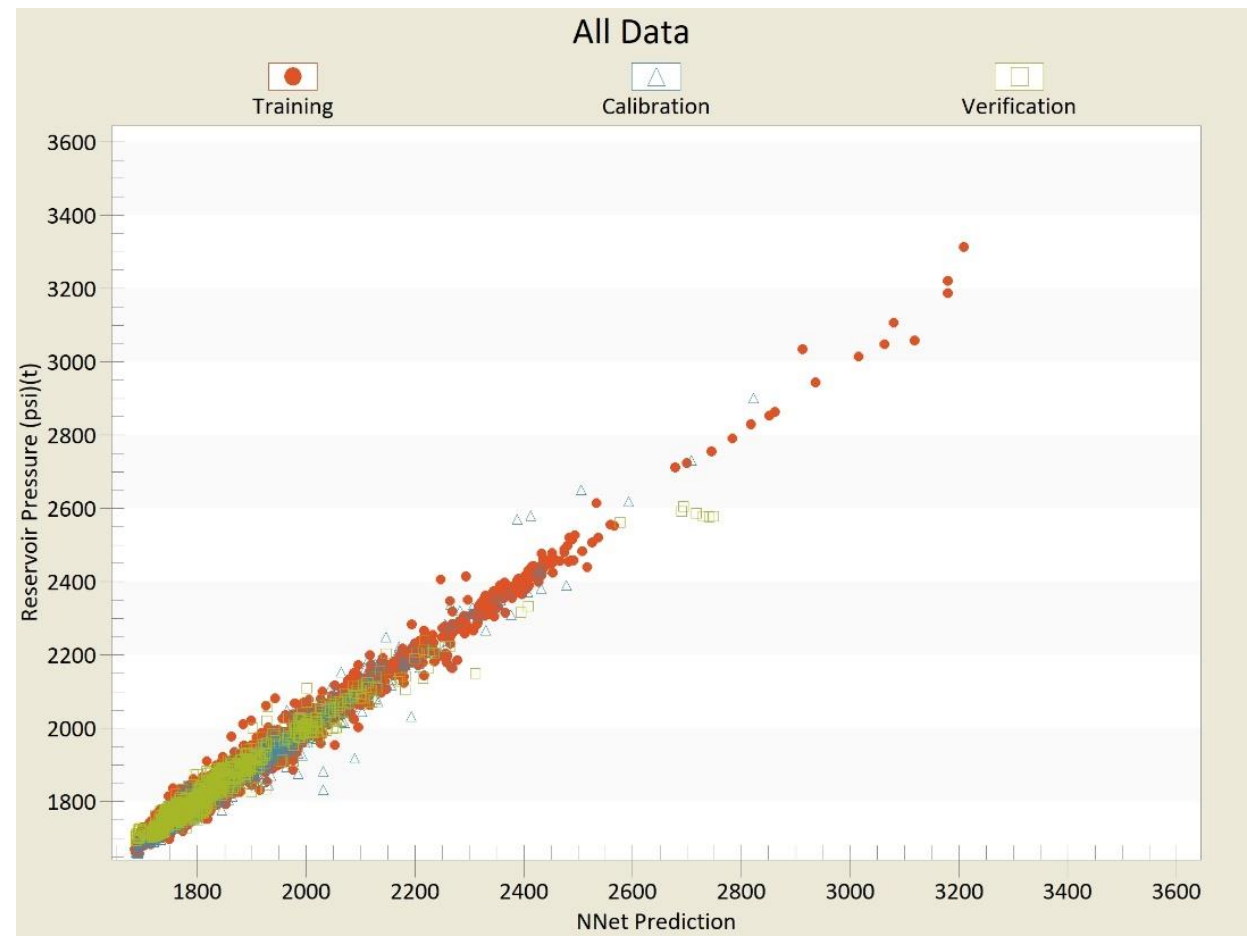

Figure 78 Data-driven reservoir pressure model training results for Multi-Random Partitioning- 3 Year Blind Validation TDM 


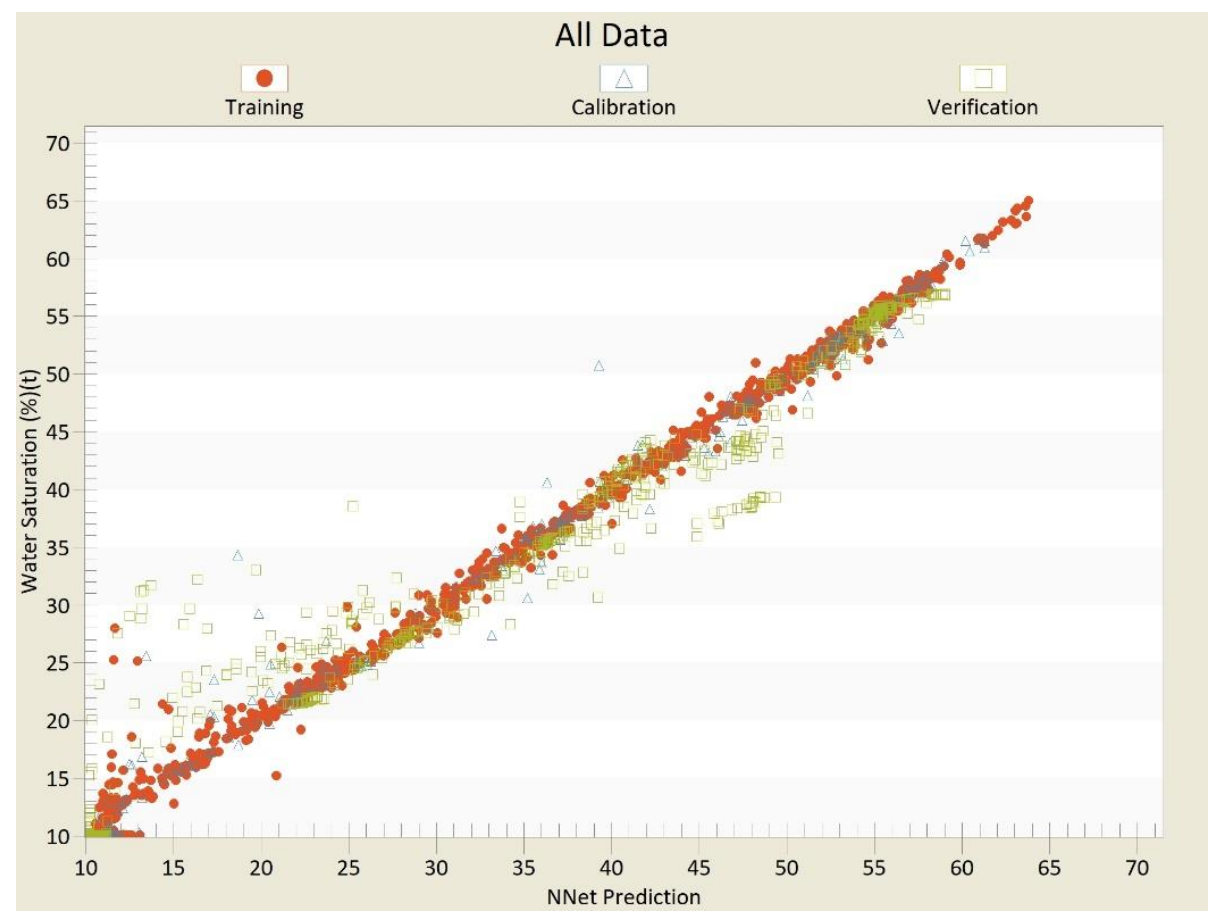

Figure 79 Data-driven water saturation model training results for Multi-Random Partitioning- 3 Year Blind Validation TDM

Once these models were successfully trained, the Top Down Model displayed below was built. The TDM was then deployed, results were exported, plots generated, and the wells were classified into groups.

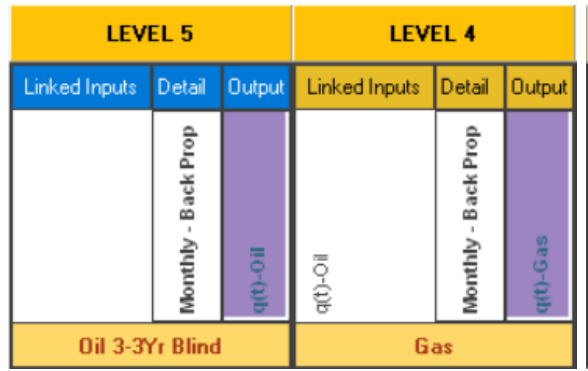

\begin{tabular}{|c|c|c|c|c|c|c|c|c|}
\hline \multicolumn{3}{|c|}{ LEVEL 3} & \multicolumn{3}{|c|}{ LEVEL 2} & \multicolumn{3}{|c|}{ LEVEL 1} \\
\hline Linked Inputs & Detail & Output & Linked Inputs & Detail & Output & Linked Inputs & Detail & Output \\
\hline 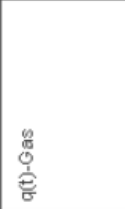 & 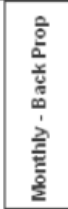 & $\begin{array}{l}\frac{d}{2} \\
\frac{1}{2} \\
\frac{1}{5} \\
\end{array}$ & 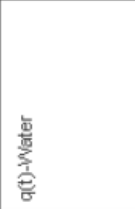 & 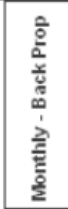 & 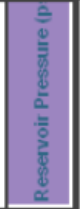 & 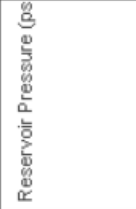 & 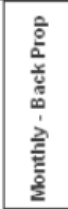 & 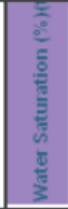 \\
\hline \multicolumn{3}{|c|}{ Water } & \multicolumn{3}{|c|}{ Beservoir Pressure-3Yr Blind } & \multicolumn{3}{|c|}{ Water Saturation-3Yr Blind } \\
\hline
\end{tabular}

Figure 80 Structure for Multi-Random Partitioning- 3 Year Blind Validation Top Down Model

\subsubsection{Multi-Random Partitioning- 4 Year Blind Validation}

Again, Multi-Random Partitioning was utilized for the 4 Year Blind Validation scenario. Blind validation started on December 31 $1^{\text {st }}, 2012$ and continued until the last date of December $31^{\text {st }}, 2016$. Because of this, verification had 1725 records, calibration had 1017 records, and training had 4069 records. Shown below are the training attributes used in each data-driven model for all five models.

Table 43 Data-driven oil model training attributes for Multi-Random Partitioning-4 Year Blind Validation TDM

\begin{tabular}{|l|l|l|}
\hline Focal & Offset Producer & Offset Injector \\
\hline Time & Distance t & Distance t \\
\hline Drainage Area & Completion t & Completion t \\
\hline
\end{tabular}




\begin{tabular}{|l|l|l|}
\hline Completion t & FBHP t & FBHP t \\
\hline FBHP t & FBHP t-1 & Injected Gas Rate t \\
\hline FBHP t-1 & Oil Saturation t-1 & Injected Water Rate t \\
\hline Oil Saturation t-1 & Oil Saturation t-2 & Open Layers Permeability t \\
\hline Oil Saturation t-2 & Reservoir Pressure t-1 & \\
\hline Open Layers Permeability t & q oil t-1 & \\
\hline Open Layers Permeability t-1 & q oil t-2 & \\
\hline Open Layers Permeability t-2 & Open Layers Permeability t & \\
\hline Open Layers Porosity t & & \\
\hline Reservoir Pressure t-1 & & \\
\hline Oil Saturation Poly Ave t-1 & & \\
\hline $\begin{array}{l}\text { Open Layers Permeability Poly } \\
\text { Ave t }\end{array}$ & & \\
\hline Open Layers Porosity Poly Ave & & \\
\hline q oil t-1 & & \\
\hline q oil t-2 & & \\
\hline WC t-1 & & \\
\hline q gas t-1 & & \\
\hline
\end{tabular}

Table 44 Data-driven gas model training attributes for Multi-Random Partitioning- 4 Year Blind Validation TDM

\begin{tabular}{|l|l|l|}
\hline Focal & Offset Producer & Offset Injector \\
\hline Time & Distance t & Distance t \\
\hline Top & Top & Completion t \\
\hline TVD & TVD & FBHP t \\
\hline Completion t & Completion t & Gas Saturation t-1 \\
\hline FBHP t & FBHP t & Injected Gas Rate t \\
\hline FBHP t-1 & FBHP t-1 & Injected Gas Rate t-1 \\
\hline Gas Saturation t-1 & Gas Saturation t-1 & Injected Gas Rate t-2 \\
\hline Gas Saturation t-2 & Gas Saturation t-2 & Open Layers Permeability t \\
\hline Open Layers Permeability t & Reservoir Pressure t-1 \\
\hline Open Layers Porosity t & q gas t-1 & \\
\hline Reservoir Pressure t-1 & q gas t-2 & \\
\hline q gas t-1 & \multicolumn{2}{|}{} \\
\hline q gas t-2 & \multicolumn{2}{|l}{} \\
\hline q oil t & \multicolumn{2}{|l}{} \\
\hline
\end{tabular}

Table 45 Data-driven water model training for Multi-Random Partitioning-4 Year Blind Validation TDM

\begin{tabular}{|l|l|l|}
\hline Focal & Offset Producer & Offset Injector \\
\hline Time & Distance $\mathbf{t}$ & Distance $\mathbf{t}$ \\
\hline
\end{tabular}




\begin{tabular}{|l|l|l|}
\hline Completion t & Completion t & FBHP t \\
\hline FBHP t & FBHP t & Injected Water Rate t \\
\hline FBHP t-1 & FBHP t-1 & Injected Water Rate t-1 \\
\hline Reservoir Pressure t-1 & Open Layers Permeability t & Injected Water Rate t-2 \\
\hline Open Layers Permeability t & Reservoir Pressure t-1 & Water Saturation t-1 \\
\hline Open Layers Porosity t & Water Saturation t-1 & Open Layers Permeability t \\
\hline Water Saturation t-1 & Water Saturation t-2 & \\
\hline Water Saturation t-2 & q water t-1 & \\
\hline q water t-1 & q water t-2 & \\
\hline q water t-2 & & \\
\hline q gas t & & \\
\hline q oil t & & \\
\hline Water Saturation t-1 Poly Ave & & \\
\hline $\begin{array}{l}\text { Open Layers Permeability t Poly } \\
\text { Ave }\end{array}$ & & \\
\hline Reservoir Pressure t-1 Poly Ave & & \\
\hline
\end{tabular}

Table 46 Data-driven reservoir pressure model training attributes for Multi-Random Partitioning- 4 Year Blind Validation TDM

\begin{tabular}{|l|l|l|}
\hline Focal & Offset Producer & Offset Injector \\
\hline Time & Distance t & Distance t \\
\hline TVD & TVD & FBHP t \\
\hline FBHP t & Completion t & FBHP t-1 \\
\hline FBHP t-1 & FBHP t & FBHP t-2 \\
\hline FBHP t-2 & FBHP t-1 & Reservoir Pressure t-1 \\
\hline Open Layers Permeability t & Reservoir Pressure t-1 & Injected Gas Rate t \\
\hline Open Layers Porosity t & Reservoir Pressure t-2 & Injected Water Rate t \\
\hline Reservoir Pressure t-1 & & \\
\hline Reservoir Pressure t-2 & & \\
\hline q oil t & & \\
\hline q gas t & & \\
\hline q water t & & \\
\hline & & \\
\hline
\end{tabular}

Table 47 Data-driven water saturation model training attributes for Multi-Random Partitioning- 4 Year Blind Validation

\begin{tabular}{|l|l|l|}
\hline Focal & Offset Producer & Offset Injector \\
\hline Time & Distance $\mathbf{t}$ & Distance t \\
\hline Top & Completion t & Gas Saturation t-1 \\
\hline TVD & Top & Oil Saturation t-1 \\
\hline
\end{tabular}




\begin{tabular}{|l|l|l|}
\hline Completion t & TVD & Injected Water Rate t \\
\hline FBHP t & Gas Saturation t-1 & Injected Water Rate t-1 \\
\hline Gas Saturation t-1 & Oil Saturation t-1 & Injected Water Rate t-2 \\
\hline Oil Saturation t-1 & Water Saturation t-1 & Water Saturation t-1 \\
\hline Open Layers Permeability t & Water Saturation t-2 & Water Saturation t-2 \\
\hline Open Layers Porosity t & & \\
\hline Reservoir Pressure t & & \\
\hline Water Saturation t-1 & & \\
\hline Water Saturation t-2 & & \\
\hline q water t & & \\
\hline
\end{tabular}

The aforementioned attributes were used to train each respective model where the training results for all five models are shown below. The R Squared for all partition cases is also shown for each datadriven model used to build the Multi-Random Partitioning- 4 Year Blind Validation TDM.

Table 48 R Squared metrics for all data-driven models in the Multi-Random Partitioning-4 Year Blind Validation TDM

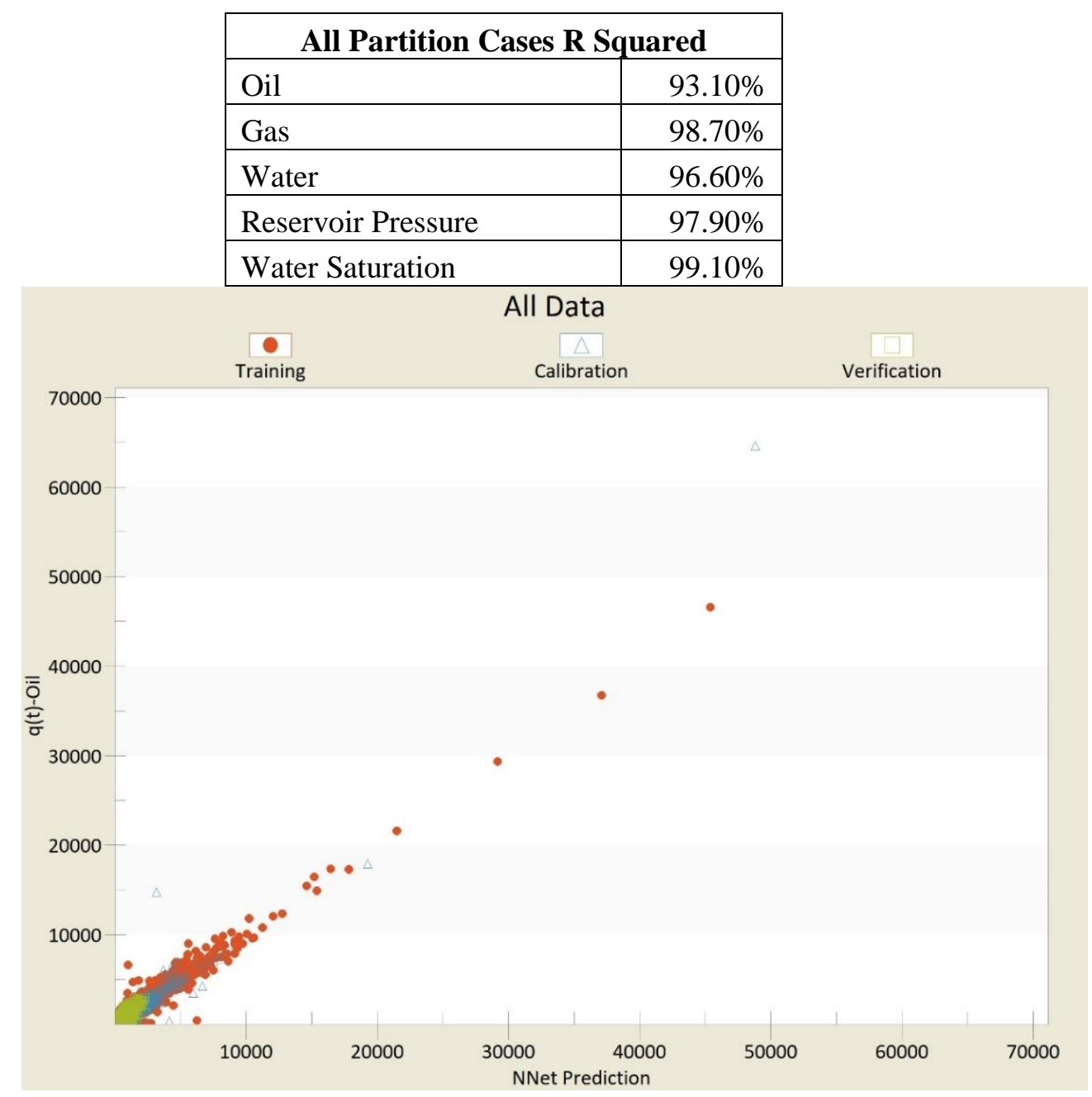

Figure 81 Data-driven oil model training results for Multi-Random Partitioning- 4 Year Blind Validation TDM 


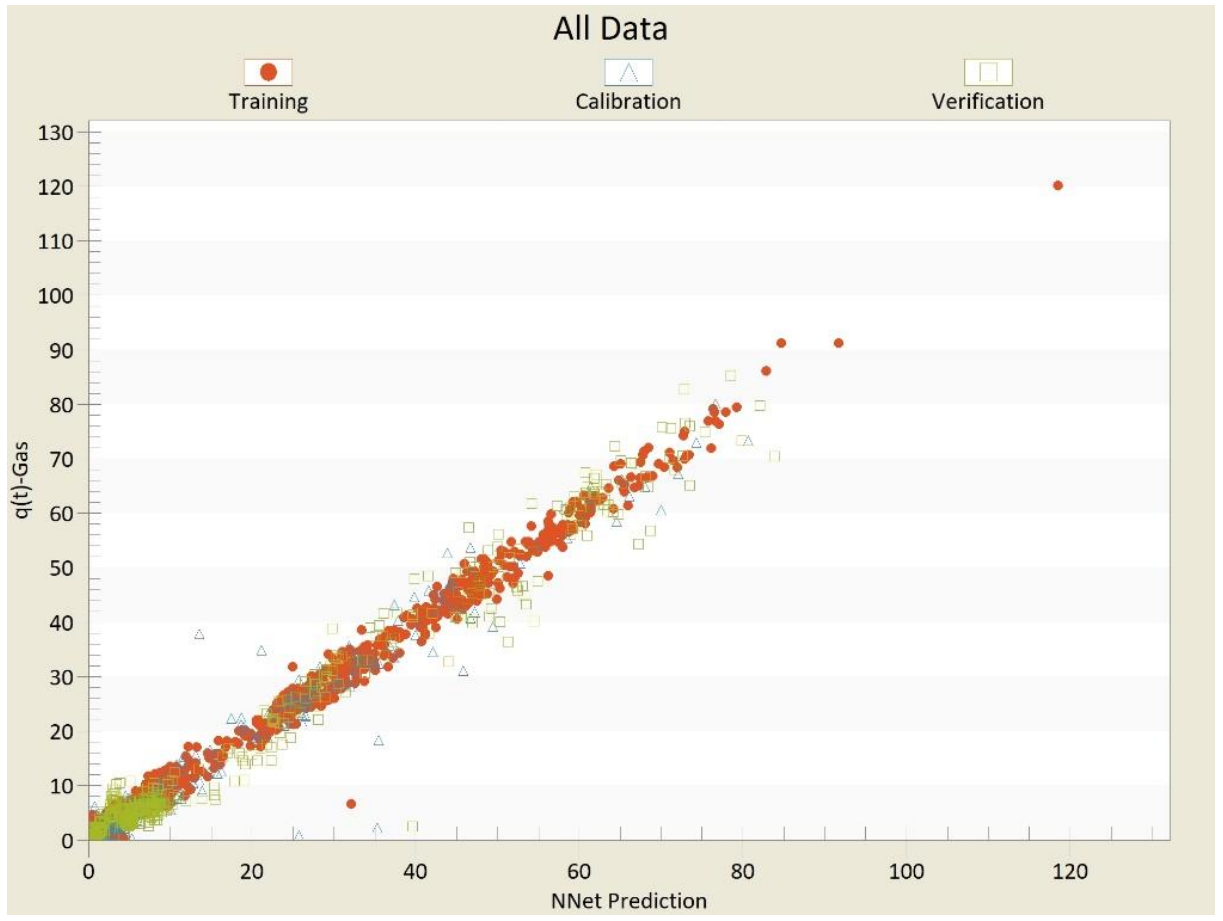

Figure 82 Data-driven gas model training results for Multi-Random Partitioning- 4 Year Blind Validation TDM

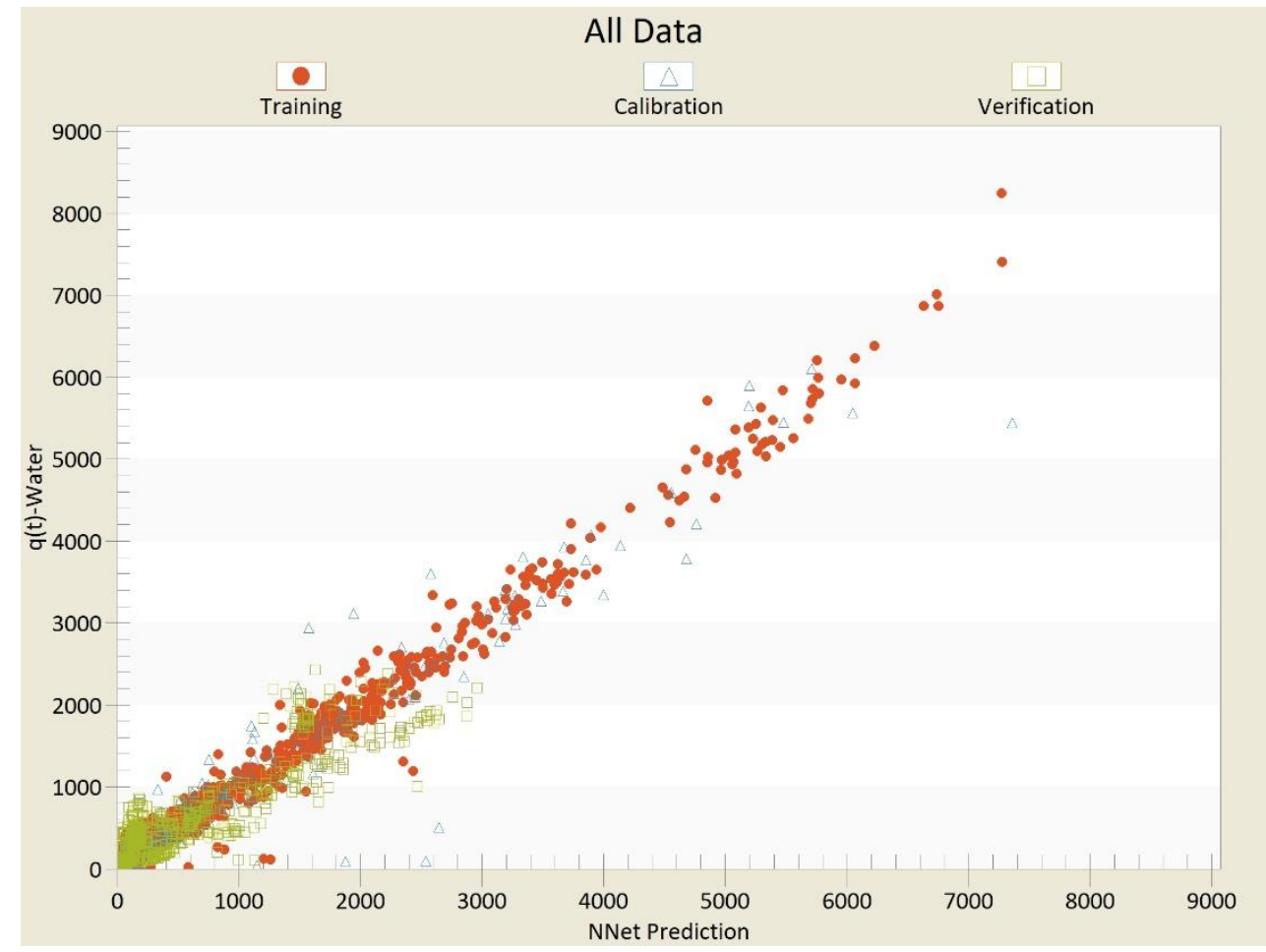

Figure 83 Data-driven water model training results for Multi-Random Partitioning-4 Year Blind Validation TDM 


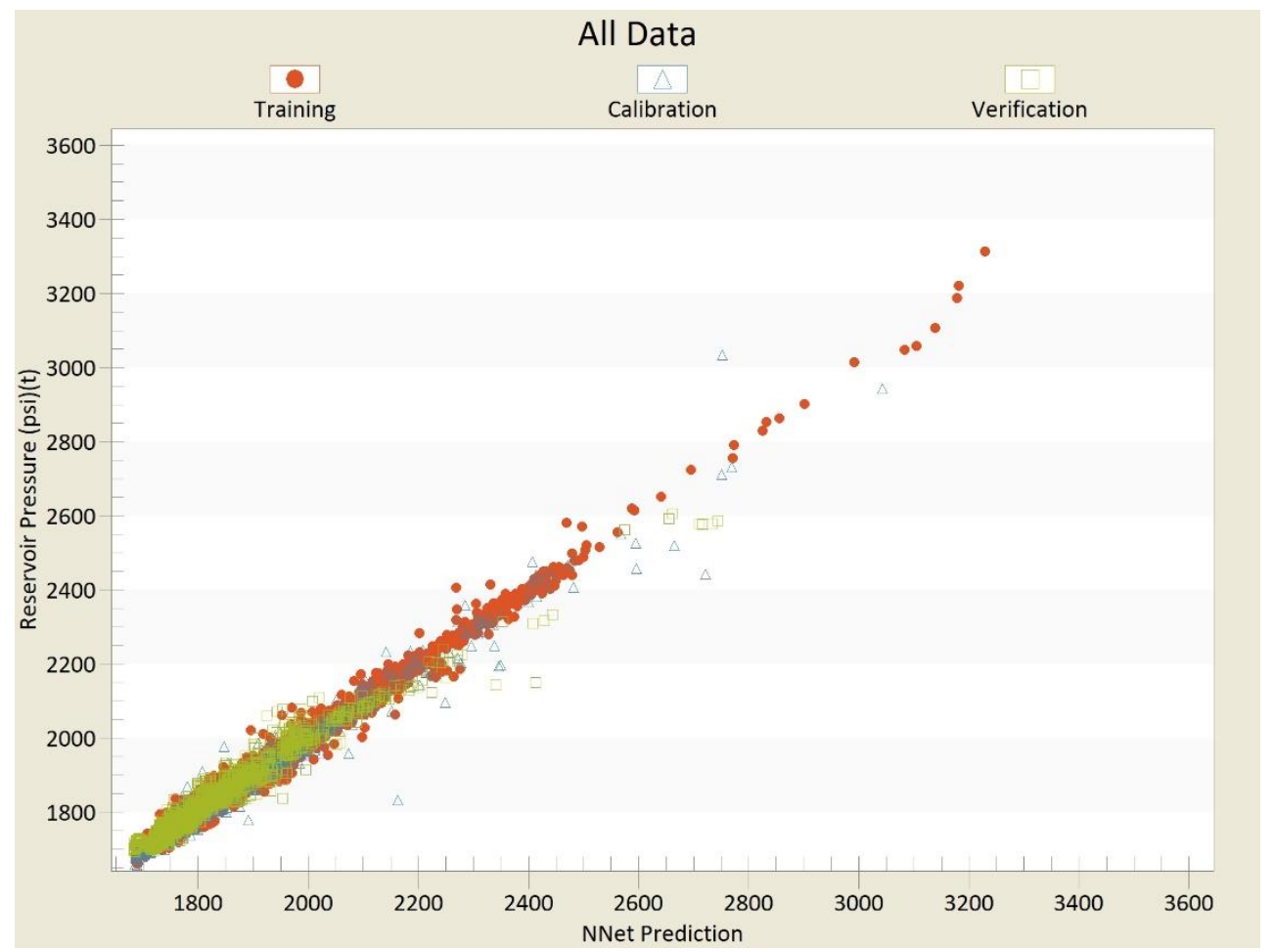

Figure 84 Data-driven reservoir pressure model training results for Multi-Random Partitioning- 4 Year Blind Validation TDM

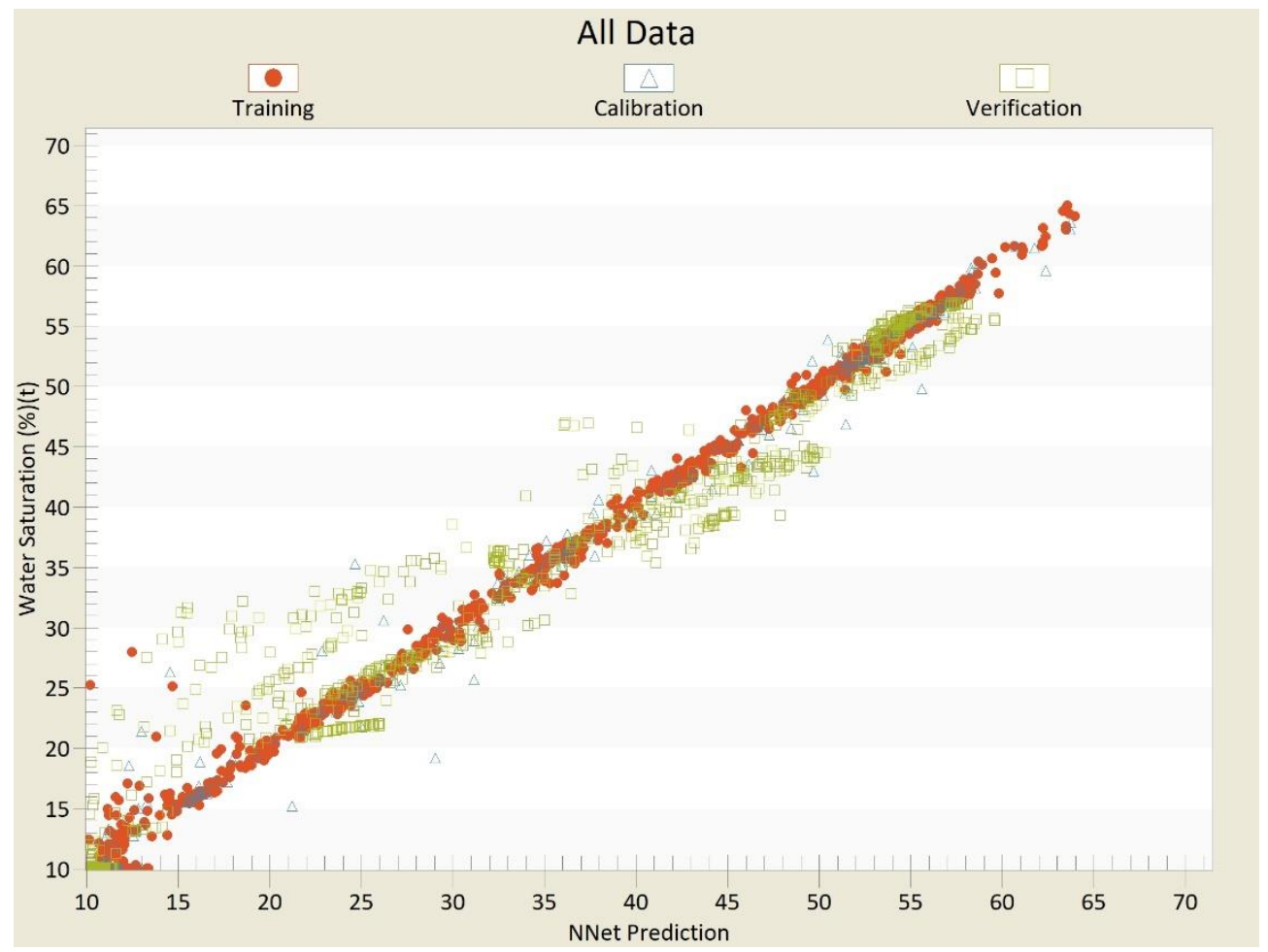

Figure 85 Data-driven water saturation model training results for Multi-Random Partitioning- 4 Year Blind Validation TDM

The Top Down Model was created after the training was completed as shown below. The same procedure for export and graph generation was followed as the previous Top Down Models. 


\begin{tabular}{|c|c|c|c|c|c|c|c|c|c|c|c|c|c|c|}
\hline \multicolumn{3}{|c|}{ LEVEL 5} & \multicolumn{3}{|c|}{ LEVEL 4} & \multicolumn{3}{|c|}{ LEVEL 3} & \multicolumn{3}{|c|}{ LEVEL 2} & \multicolumn{3}{|c|}{ LEVEL 1} \\
\hline Linked Inputs & Detail & Output & Linked Inputs & Detail & Output & Linked Inputs & Detail & Output & Linked Inputs & Detail & Output & Linked Inputs & Detail & Output \\
\hline & 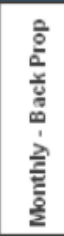 & $\begin{array}{l}\overline{0} \\
\frac{1}{1} \\
\frac{1}{5}\end{array}$ & 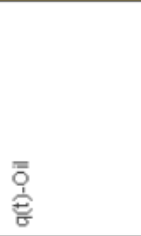 & 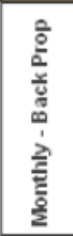 & 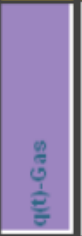 & 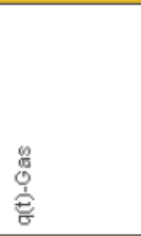 & 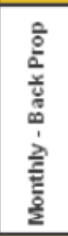 & $\begin{array}{l}\frac{d}{3} \\
\frac{2}{3} \\
\frac{1}{y} \\
\end{array}$ & 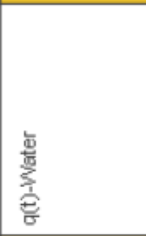 & 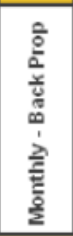 & 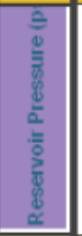 & 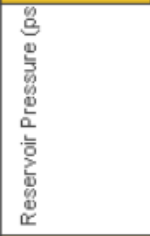 & 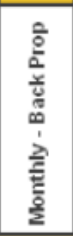 & 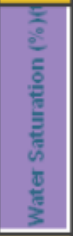 \\
\hline \multicolumn{3}{|c|}{ Oil 2-4 Y, Blind } & \multicolumn{3}{|c|}{ Gas-4Yr Blind } & \multicolumn{3}{|c|}{ Water-4Y, Blind } & \multicolumn{3}{|c|}{ Beservoir Pressure-4Yr Blint } & \multicolumn{3}{|c|}{ Water Saturation-4Yr Blind } \\
\hline
\end{tabular}

Figure 86 Structure for Multi-Random Partitioning- 4 Year Blind Validation Top Down Model

\subsubsection{Multi-Random Partitioning- 5 Year Blind Validation}

The 5 Year Blind Validation case started validation on December $31^{\text {st }}, 2011$ and continued until December $31^{\text {st }}$, 2016. The training, calibration, and verification datasets had 3701, 925, and 2185 records respectively. The attributes used in the training of each of the five models are shown in the tables below.

Table 49 Data-driven oil model training attributes for Multi-Random Partitioning-5 Year Blind Validation TDM

\begin{tabular}{|l|l|l|}
\hline Focal & Offset Producer & Offset Injector \\
\hline Time & Distance t & Distance t \\
\hline Drainage Area & Completion t & Completion t \\
\hline Completion t & FBHP t & FBHP t \\
\hline FBHP t & FBHP t-1 & Injected Gas Rate t \\
\hline FBHP t-1 & Oil Saturation t-1 & Injected Water Rate t \\
\hline Oil Saturation t-1 & Oil Saturation t-2 & Open Layers Permeability t \\
\hline Oil Saturation t-2 & Reservoir Pressure t-1 & \\
\hline Open Layers Permeability t & q oil t-1 & \\
\hline Open Layers Permeability t-1 & q oil t-2 & \\
\hline Open Layers Permeability t-2 & Open Layers Permeability t & \\
\hline Open Layers Porosity t & & \\
\hline Reservoir Pressure t-1 & & \\
\hline Oil Saturation t-1 Poly Ave & & \\
\hline Open Layers Permeability t Poly Ave & \\
\hline Open Layers Porosity t Poly Ave & & \\
\hline q oil t-1 & & \\
\hline q oil t-2 & & \\
\hline WC t-1 & & \\
\hline q gas t-1 & & \\
\hline
\end{tabular}

Table 50 Data-driven gas model training attributes for Multi-Random Partitioning-5 Year Blind Validation TDM

\begin{tabular}{|l|l|l|}
\hline Focal & Offset Producer & Offset Injector \\
\hline Time & Distance t & Distance t \\
\hline
\end{tabular}




\begin{tabular}{|l|l|l|}
\hline Top & Top & Completion t \\
\hline TVD & TVD & FBHP t \\
\hline Completion t & Completion t & Gas Saturation t-1 \\
\hline FBHP t & FBHP t 1P & Injected Gas Rate t \\
\hline FBHP t-1 & FBHP t-1 1P & Injected Gas Rate t-1 \\
\hline Gas Saturation t-1 & Gas Saturation t-1 & Injected Gas Rate t-2 \\
\hline Gas Saturation t-2 & Gas Saturation t-2 & Open Layers Permeability t \\
\hline Open Layers Permeability t & Reservoir Pressure t-1 & \\
\hline Open Layers Porosity t & q gas t-1 & \\
\hline Reservoir Pressure t-1 & q gas t-2 & \\
\hline q gas t-1 & & \\
\hline q gas t-2 & & \\
\hline q oil t & & \\
\hline
\end{tabular}

Table 51 Data-driven water model training attributes for Multi-Random Partitioning- 5 Year Blind Validation TDM

\begin{tabular}{|l|l|l|}
\hline Focal & Offset Producer & Offset Injector \\
\hline Time & Distance t & Distance t \\
\hline Completion t & Completion t & FBHP t \\
\hline FBHP t & FBHP t & Injected Water Rate t \\
\hline FBHP t-1 & FBHP t-1 & Injected Water Rate t-1 \\
\hline Reservoir Pressure t-1 & Open Layers Permeability t & Injected Water Rate t-2 \\
\hline Open Layers Permeability t & Reservoir Pressure t-1 & Water Saturation t-1 \\
\hline Open Layers Porosity t & Water Saturation t-1 & Open Layers Permeability t \\
\hline Water Saturation t-1 & Water Saturation t-2 & \\
\hline Water Saturation t-2 & q water t-1 & \\
\hline q water t-1 & q water t-2 & \\
\hline q water t-2 & & \\
\hline q gas t & & \\
\hline q oil t & & \\
\hline Water Saturation t-1 Poly Ave & & \\
\hline $\begin{array}{l}\text { Open Layers Permeability t Poly } \\
\text { Ave }\end{array}$ & & \\
\hline Reservoir Pressure t-1 Poly Ave & & \\
\hline
\end{tabular}

Table 52 Data-driven reservoir pressure model training attributes for Multi-Random Partitioning- 5 Year Blind Validation TDM

\begin{tabular}{|l|l|l|}
\hline Focal & Offset Producer & Offset Injector \\
\hline Time & Distance $\mathbf{t}$ & Distance $\mathbf{t}$ \\
\hline TVD & TVD & FBHP t \\
\hline Completion $\mathbf{t}$ & Completion $\mathbf{t}$ & FBHP t-1 \\
\hline
\end{tabular}




\begin{tabular}{|l|l|l|}
\hline FBHP t & FBHP t & FBHP t-2 \\
\hline FBHP t-1 & FBHP t-1 & Reservoir Pressure t-1 \\
\hline FBHP t-2 & FBHP t-2 & Reservoir Pressure t-2 \\
\hline Open Layers Permeability t & Reservoir Pressure t-1 & Injected Gas Rate t \\
\hline Open Layers Porosity t & Reservoir Pressure t-2 & Injected Water Rate t \\
\hline Reservoir Pressure t-1 & & \\
\hline Reservoir Pressure t-2 & & \\
\hline q oil t & & \\
\hline q gas t & & \\
\hline q water $\mathbf{t}$ & & \\
\hline
\end{tabular}

Table 53 Data-driven water saturation model training attributes for Multi-Random Partitioning- 5 Year Blind Validation TDM

\begin{tabular}{|l|l|l|}
\hline Focal & Offset Producer & Offset Injector \\
\hline Time & Distance t & Distance t \\
\hline TVD & Completion t & Gas Saturation t-1 \\
\hline Completion t & TVD & Oil Saturation t-1 \\
\hline FBHP t & Gas Saturation t-1 & Injected Water Rate t \\
\hline Gas Saturation t-1 & Oil Saturation t-1 & Injected Water Rate t-1 \\
\hline Oil Saturation t-1 & Water Saturation t-1 & Injected Water Rate t-2 \\
\hline Open Layers Permeability t & Water Saturation t-2 & Water Saturation t-1 \\
\hline Open Layers Porosity t & & Water Saturation t-2 \\
\hline Reservoir Pressure t & & \\
\hline Water Saturation t-1 & & \\
\hline Water Saturation t-2 & & \\
\hline q water t & & \\
\hline
\end{tabular}

The parameters shown above were used to train each individual model. The R Squared metric and training results for all partition cases for all five models are shown below for the 5 Year Blind Validation TDM.

Table 54 R Squared metrics for all data-driven models in the Multi-Random Partitioning- 5 Year Blind Validation TDM

\begin{tabular}{|l|r|}
\hline R Squared for All Partition Cases \\
\hline Oil & $88.30 \%$ \\
\hline Gas & $98.40 \%$ \\
\hline Water & $93.50 \%$ \\
\hline Reservoir Pressure & $96.60 \%$ \\
\hline Water Saturation & $97.20 \%$ \\
\hline
\end{tabular}




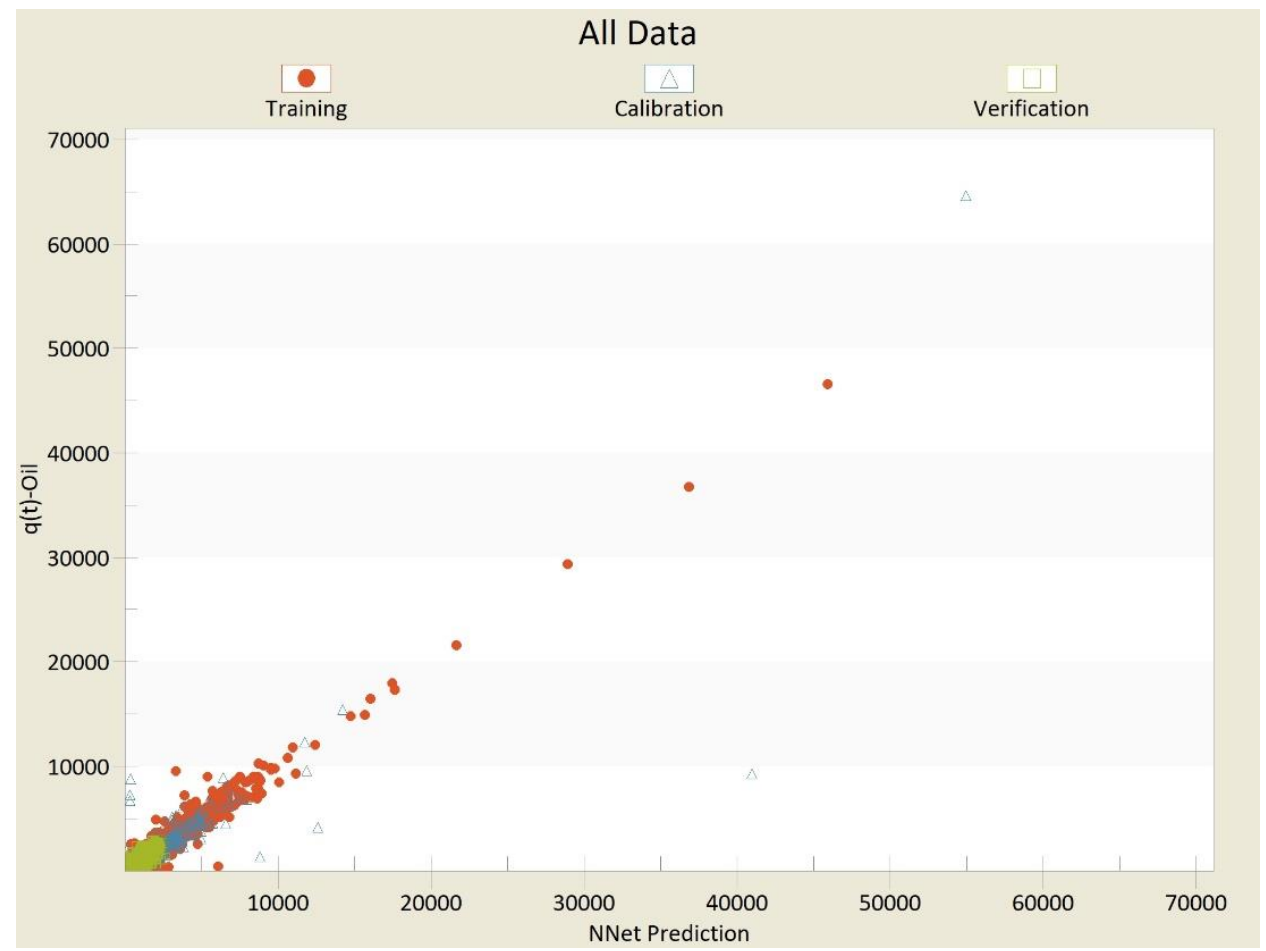

Figure 87 Data-driven oil model training results for Multi-Random Partitioning- 5 Year Blind Validation TDM

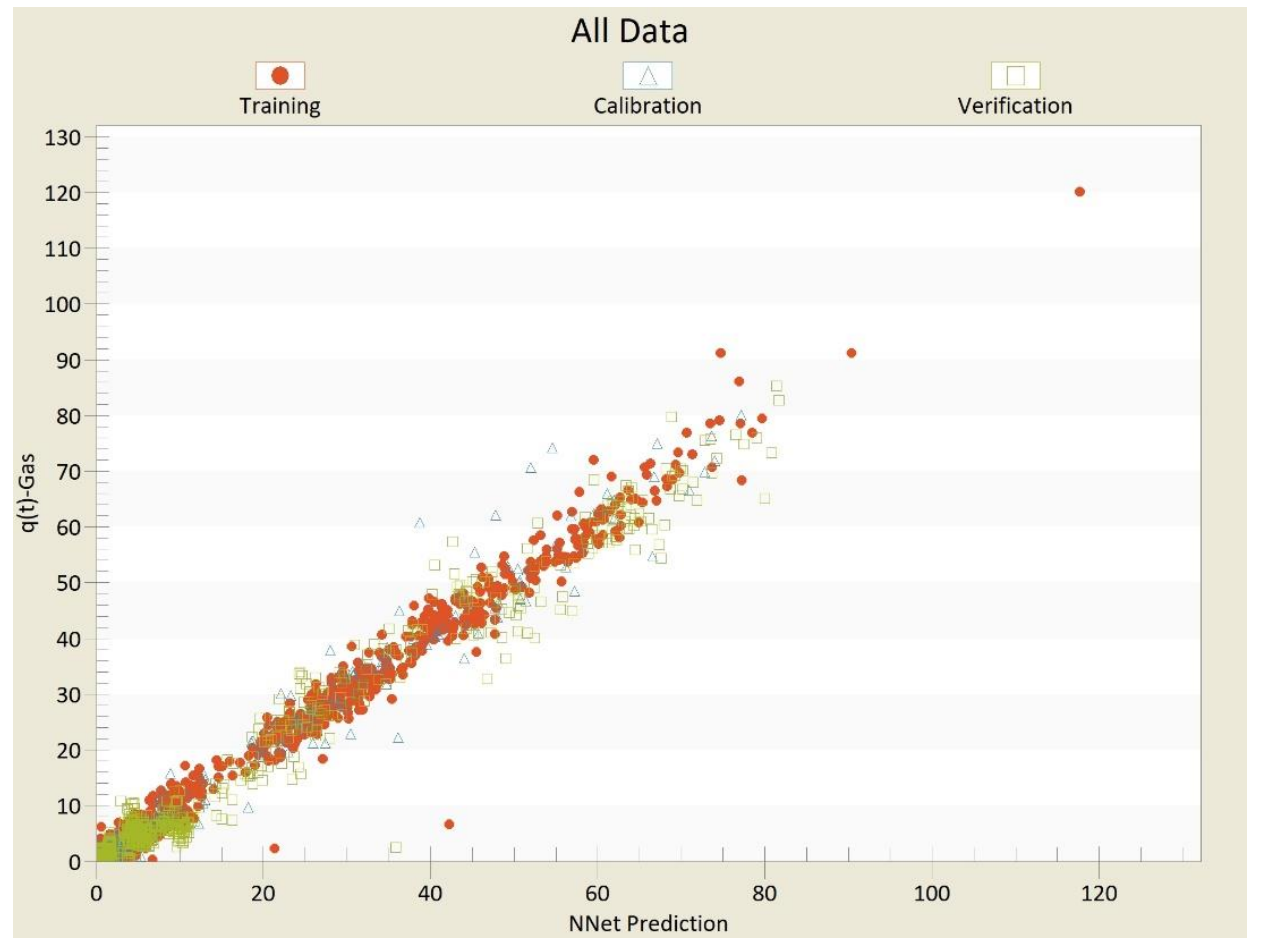

Figure 88 Data-driven gas model training results for Multi-Random Partitioning- 5 Year Blind Validation TDM 


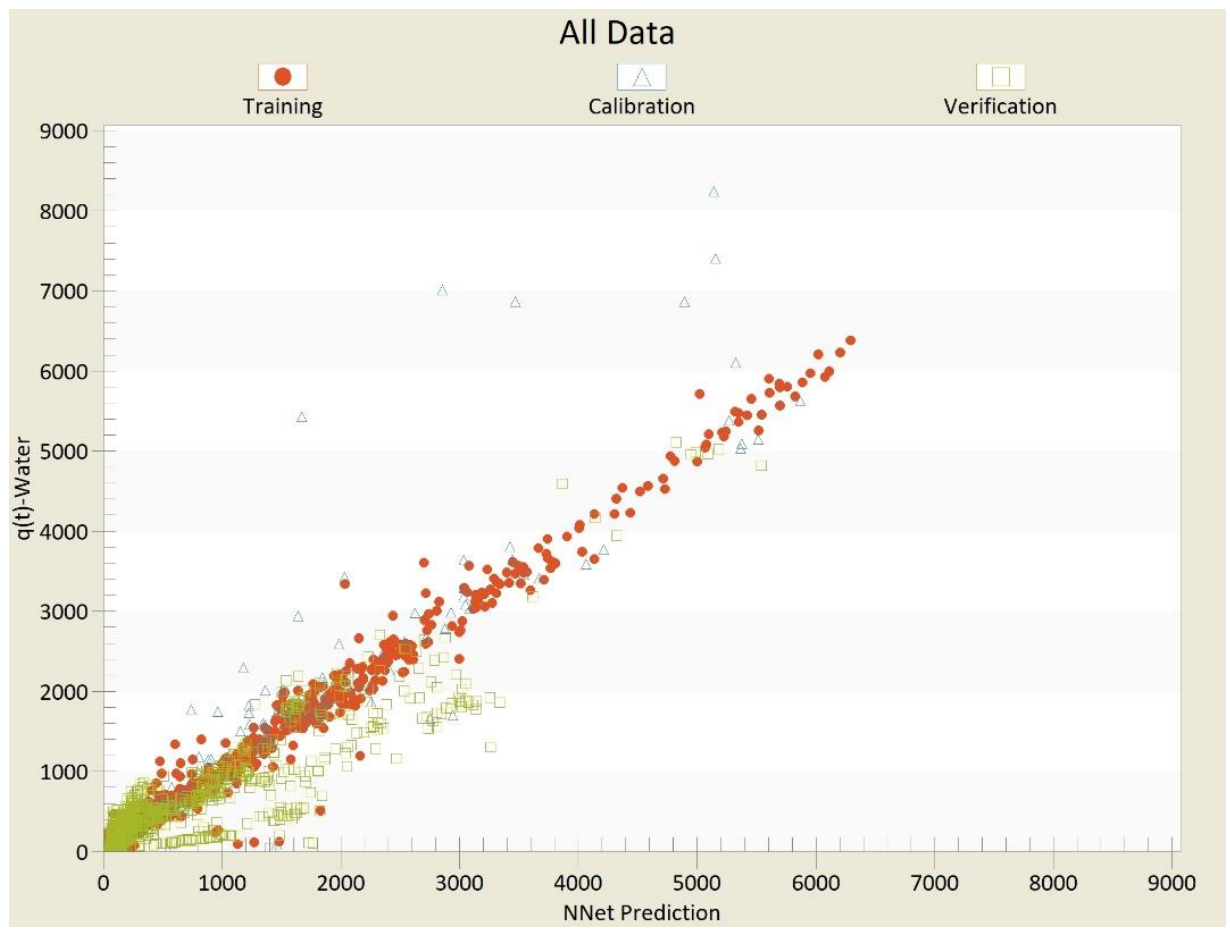

Figure 89 Data-driven water model training results for Multi-Random Partitioning- 5 Year Blind Validation TDM

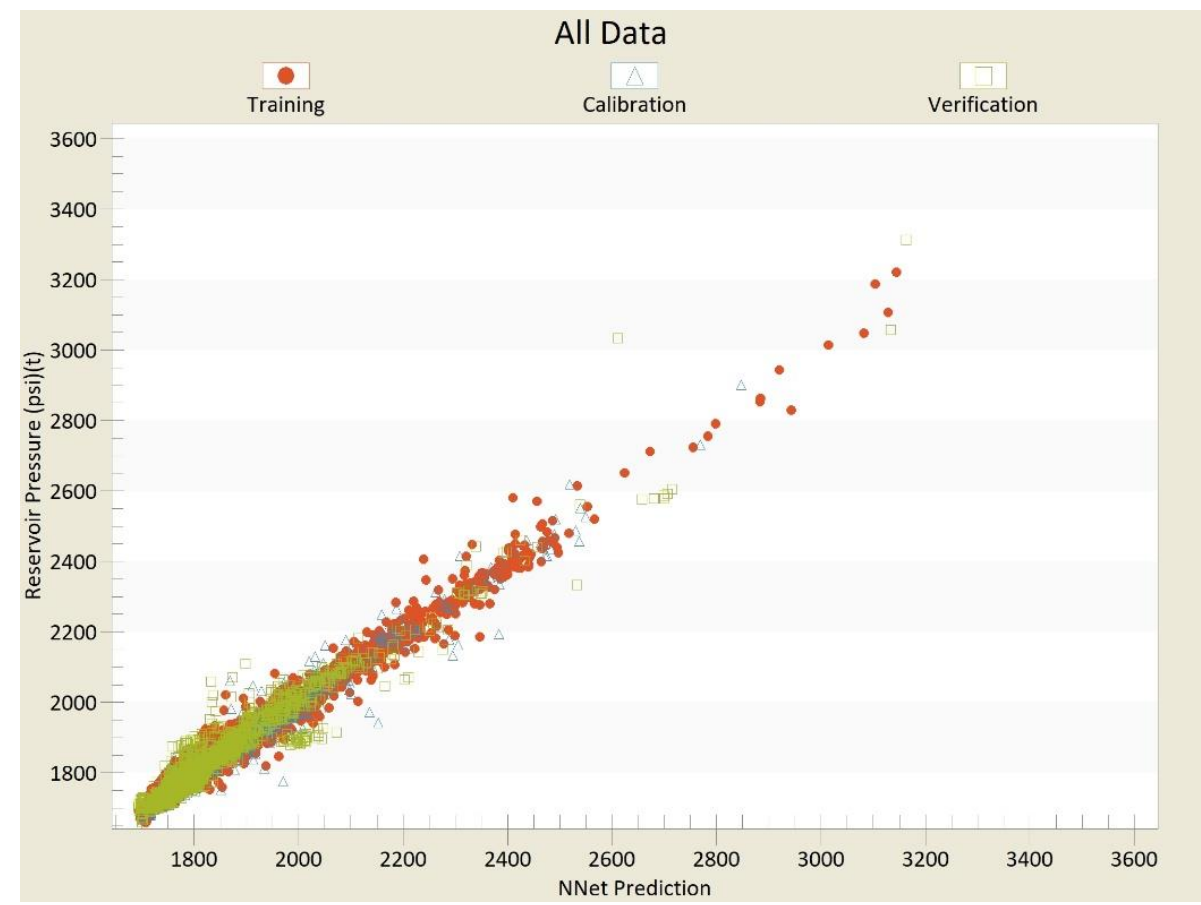

Figure 90 Data-driven reservoir pressure model training results for Multi-Random Partitioning- 5 Year Blind Validation TDM 


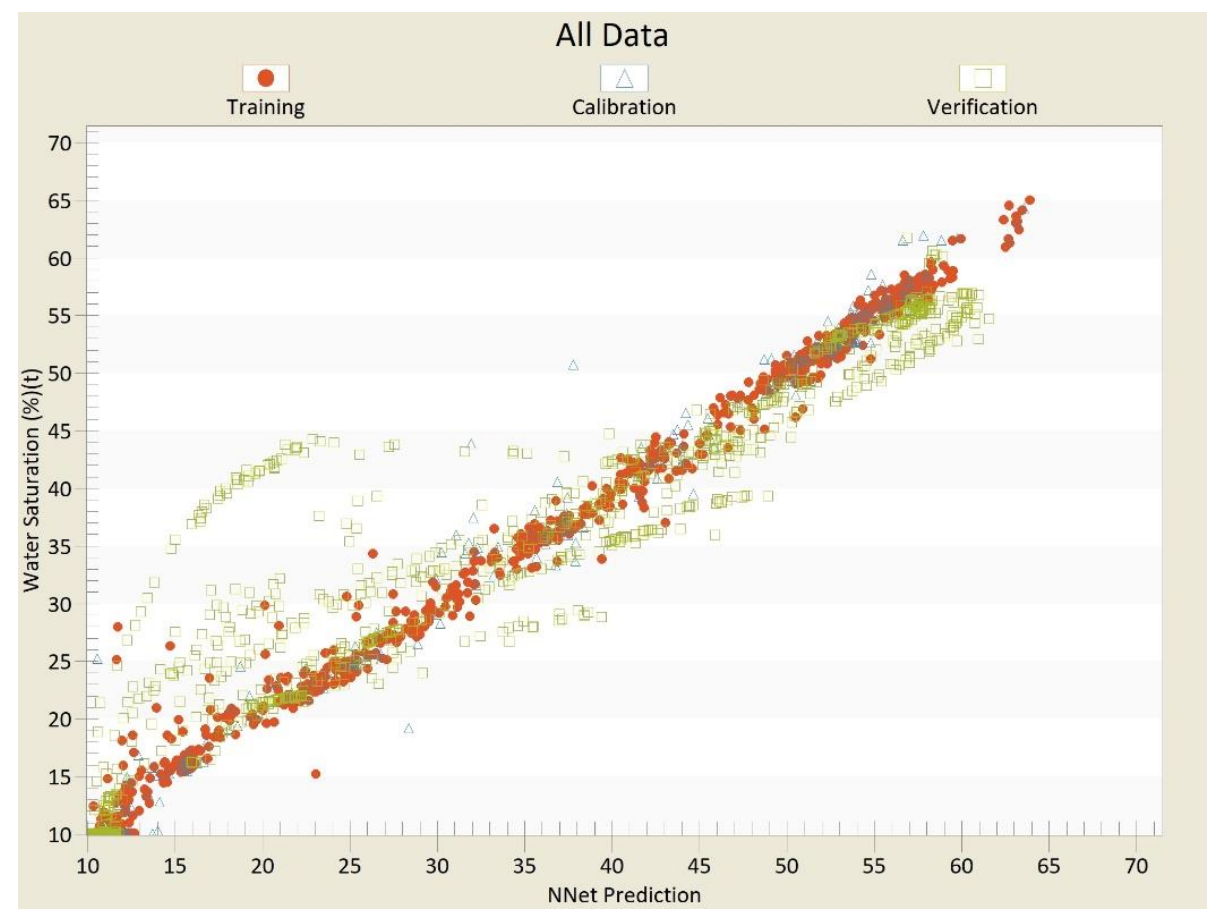

Figure 91 Data-driven water saturation model training results for Multi-Random Partitioning- 5 Year Blind Validation TDM

The 5 Year Blind Validation Top Down Model was created after the training results previously shown were obtained. The same procedure for export and graph generation was followed as the previous Top Down Models and the structure of the TDM is displayed below.

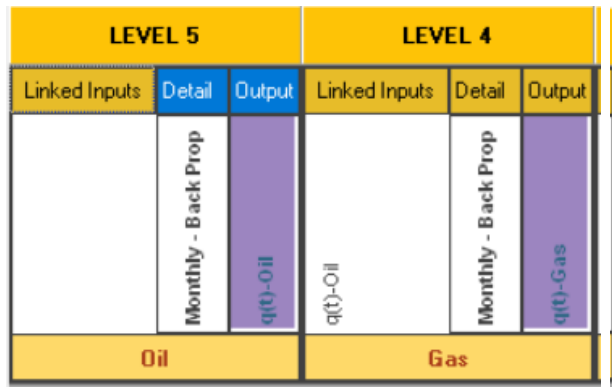

\begin{tabular}{|c|c|c|c|c|c|c|c|c|}
\hline \multicolumn{3}{|c|}{ LEVEL 3} & \multicolumn{3}{|c|}{ LEVEL 2} & \multicolumn{3}{|c|}{ LEVEL 1} \\
\hline Linked Inputs & Detail & Output & Linked Inputs & Detail & Output & Linked Inputs & Detail & Output \\
\hline 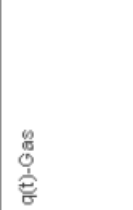 & 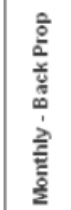 & $\begin{array}{l}\frac{\mathrm{y}}{\mathrm{y}} \\
\frac{1}{\frac{1}{2}}\end{array}$ & $\begin{array}{l}\text { 点 } \\
\text { 竞 } \\
\text { 悹 }\end{array}$ & 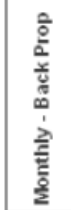 & 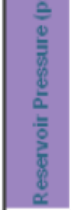 & 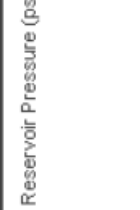 & 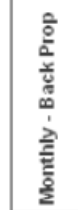 & $\frac{\pi}{3}$ \\
\hline & \multicolumn{3}{|c|}{ Reservoir Pressure } & \multicolumn{3}{|c|}{ Water Saturation } \\
\hline
\end{tabular}

Figure 92 Structure for Multi-Random Partitioning- 5 Year Blind Validation Top Down Model

\subsubsection{Multi-Random Partitioning- 6 Year Blind Validation}

The last Top Down Model created was the Multi-Random Partitioning- 6 Year Blind Validation case. The blind validation started on December $31^{\text {st }}, 2010$ and continued until December $31^{\text {st }}, 2016$. The verification dataset had 2651 records, calibration had 832 records, and the training dataset had 3328 records. Each model's training attributes are reported below. The attributes used are shown for the focal well, offset producer, and offset injector. Once again, because two offset producers and injectors were used, the listed offset producer attributes were selected for both the first offset producer and the second offset producer. The same process was followed for the first offset injector and the second offset injector, the same as previous models.

Table 55 Data-driven oil model training attributes for Multi-Random Partitioning- 6 Year Blind Validation TDM

\section{Focal}




\begin{tabular}{|l|l|l|}
\hline Time & Distance t & Distance t \\
\hline Drainage Area & Completion t & Completion t \\
\hline Completion t & FBHP t & FBHP t \\
\hline FBHP t & FBHP t-1 & Injected Gas Rate t \\
\hline FBHP t-1 & Oil Saturation t-1 & Injected Water Rate t \\
\hline Oil Saturation t-1 & Oil Saturation t-2 & Open Layers Permeability t \\
\hline Open Layers Permeability t & Reservoir Pressure t-1 & \\
\hline Open Layers Permeability t-1 & q oil t-1 & \\
\hline Open Layers Permeability t-2 & q oil t-2 & \\
\hline Open Layers Porosity t & Open Layers Permeability t & \\
\hline Reservoir Pressure t-1 & & \\
\hline Oil Saturation t-1 Poly Ave & & \\
\hline Open Layers Permeability t Poly Ave & & \\
\hline Open Layers Porosity t Poly Ave & & \\
\hline q oil t-1 & & \\
\hline q oil t-2 & & \\
\hline WC t-1 & & \\
\hline q gas t-1 & & \\
\hline
\end{tabular}

Table 56 Data-driven gas model training attributes for Multi-Random Partitioning- 6 Year Blind Validation TDM

\begin{tabular}{|l|l|l|}
\hline Focal & Offset Producer & Offset Injector \\
\hline Time & Distance t & Distance t \\
\hline Top & Top & Completion t \\
\hline TVD & TVD & FBHP t \\
\hline Completion t & Completion t & Gas Saturation t-1 \\
\hline FBHP t & FBHP t & Injected Gas Rate t \\
\hline FBHP t-1 & FBHP t-1 & Injected Gas Rate t-1 \\
\hline Gas Saturation t-1 & Gas Saturation t-1 & Injected Gas Rate t-2 \\
\hline Gas Saturation t-2 & Gas Saturation t-2 & Open Layers Permeability t \\
\hline Open Layers Permeability t & Reservoir Pressure t-1 \\
\hline Open Layers Porosity t & q gas t-1 & \\
\hline Reservoir Pressure t-1 & q gas t-2 & \\
\hline q gas t-1 & & \\
\hline q gas t-2 & & \\
\hline q oil t & \multicolumn{2}{|l}{} \\
\hline
\end{tabular}

Table 57 Data-driven water model training attributes for Multi-Random Partitioning- 6 Year Blind Validation TDM

\begin{tabular}{|l|l|l|}
\hline Focal & Offset Producer & Offset Injector \\
\hline
\end{tabular}




\begin{tabular}{|l|l|l|}
\hline Time & Distance t & Distance t \\
\hline Completion t & Completion t & FBHP t \\
\hline FBHP t & FBHP t & Injected Water Rate t \\
\hline FBHP t-1 & FBHP t-1 & Injected Water Rate t-1 \\
\hline Reservoir Pressure t-1 & Open Layers Permeability t & Injected Water Rate t-2 \\
\hline Open Layers Permeability t & Reservoir Pressure t-1 & Water Saturation t-1 \\
\hline Open Layers Porosity t & Water Saturation t-1 & Open Layers Permeability t \\
\hline Water Saturation t-1 & Water Saturation t-2 & \\
\hline Water Saturation t-2 & q water t-1 & \\
\hline q water t-1 & q water t-2 & \\
\hline q water t-2 & & \\
\hline q gas t & & \\
\hline q oil t & & \\
\hline Water Saturation t-1 Poly Ave & & \\
\hline Open Layers Permeability t Poly Ave & & \\
\hline Reservoir Pressure t-1 Poly Ave & & \\
\hline
\end{tabular}

Table 58 Data-driven reservoir pressure model training attributes for Multi-Random Partitioning- 6 Year Blind Validation TDM

\begin{tabular}{|l|l|l|}
\hline Focal & Offset Producer & Offset Injector \\
\hline Time & Distance t & Distance t \\
\hline TVD & TVD & FBHP t \\
\hline Completion t & Completion t & FBHP t-1 \\
\hline FBHP t & FBHP t & FBHP t-2 \\
\hline FBHP t-1 & FBHP t-1 & Reservoir Pressure t-1 \\
\hline FBHP t-2 & FBHP t-2 & Reservoir Pressure t-2 \\
\hline Open Layers Permeability t & Reservoir Pressure t-1 & Injected Gas Rate t \\
\hline Open Layers Porosity t & Reservoir Pressure t-2 & Injected Water Rate t \\
\hline Reservoir Pressure t-1 & & \\
\hline Reservoir Pressure t-2 & & \\
\hline q oil t & & \\
\hline q gas t & & \\
\hline q water t & & \\
\hline
\end{tabular}

Table 59 Data-driven water saturation model training attributes for Multi-Random Partitioning- 6 Year Blind Validation TDM

\begin{tabular}{|l|l|l|}
\hline Focal & Offset Producer & Offset Injector \\
\hline Time & Distance $\mathbf{t}$ & Distance t \\
\hline Top & Completion $\mathbf{t}$ & Gas Saturation t-1 \\
\hline TVD & Top & Oil Saturation t-1 \\
\hline Completion t & TVD & Injected Water Rate t \\
\hline
\end{tabular}




\begin{tabular}{|l|l|l|}
\hline FBHP t & Gas Saturation t-1 & Injected Water Rate t-1 \\
\hline Gas Saturation t-1 & Oil Saturation t-1 & Injected Water Rate t-2 \\
\hline Oil Saturation t-1 & Water Saturation t-1 & Water Saturation t-1 \\
\hline Open Layers Permeability t & Water Saturation t-2 & Water Saturation t-2 \\
\hline Open Layers Porosity t & Water Saturation t-1 Poly Ave & Water Saturation t-1 Poly Ave \\
\hline Reservoir Pressure t & & \\
\hline Water Saturation t-1 & & \\
\hline Water Saturation t-2 & & \\
\hline q water t & & \\
\hline Water Saturation t-1 Poly Ave & & \\
\hline
\end{tabular}

Once each model was sufficiently trained, the training results were examined. The R Squared evaluation metric and the training results for all partitioning cases for the oil, gas, water, reservoir pressure, and water saturation models are included below.

Table 60 R Squared metrics for all data-driven models in Multi-Random Partitioning- 6 Year Blind Validation TDM

\begin{tabular}{|l|r|}
\hline \multicolumn{2}{|l|}{ R Squared for All Partition Cases } \\
\hline Oil & $92.90 \%$ \\
\hline Gas & $98.10 \%$ \\
\hline Water & $92.80 \%$ \\
\hline Reservoir Pressure & $97.30 \%$ \\
\hline Water Saturation & $97.90 \%$ \\
\hline
\end{tabular}




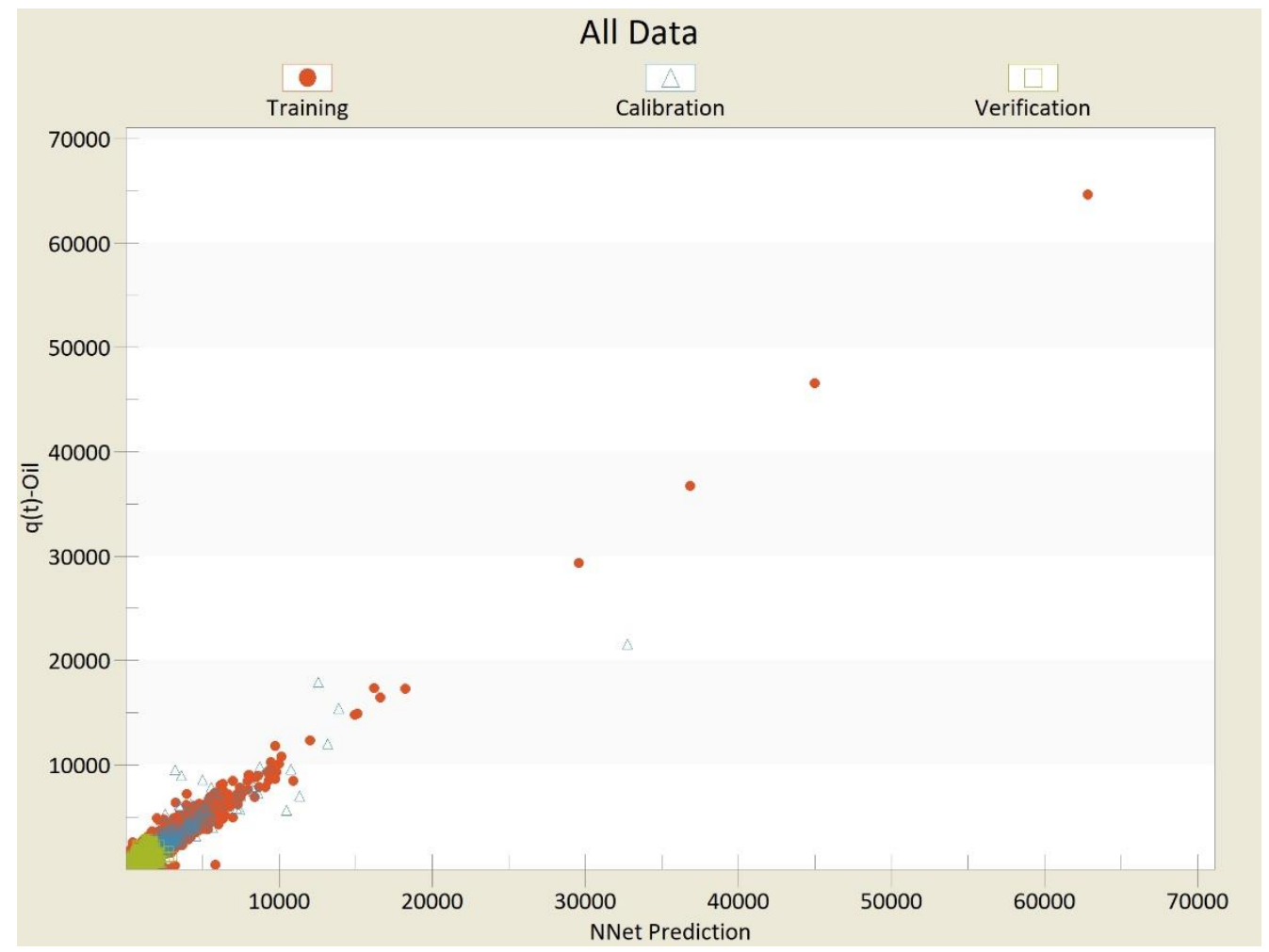

Figure 93 Data-driven oil model training results for Multi-Random Partitioning- 6 Year Blind Validation TDM

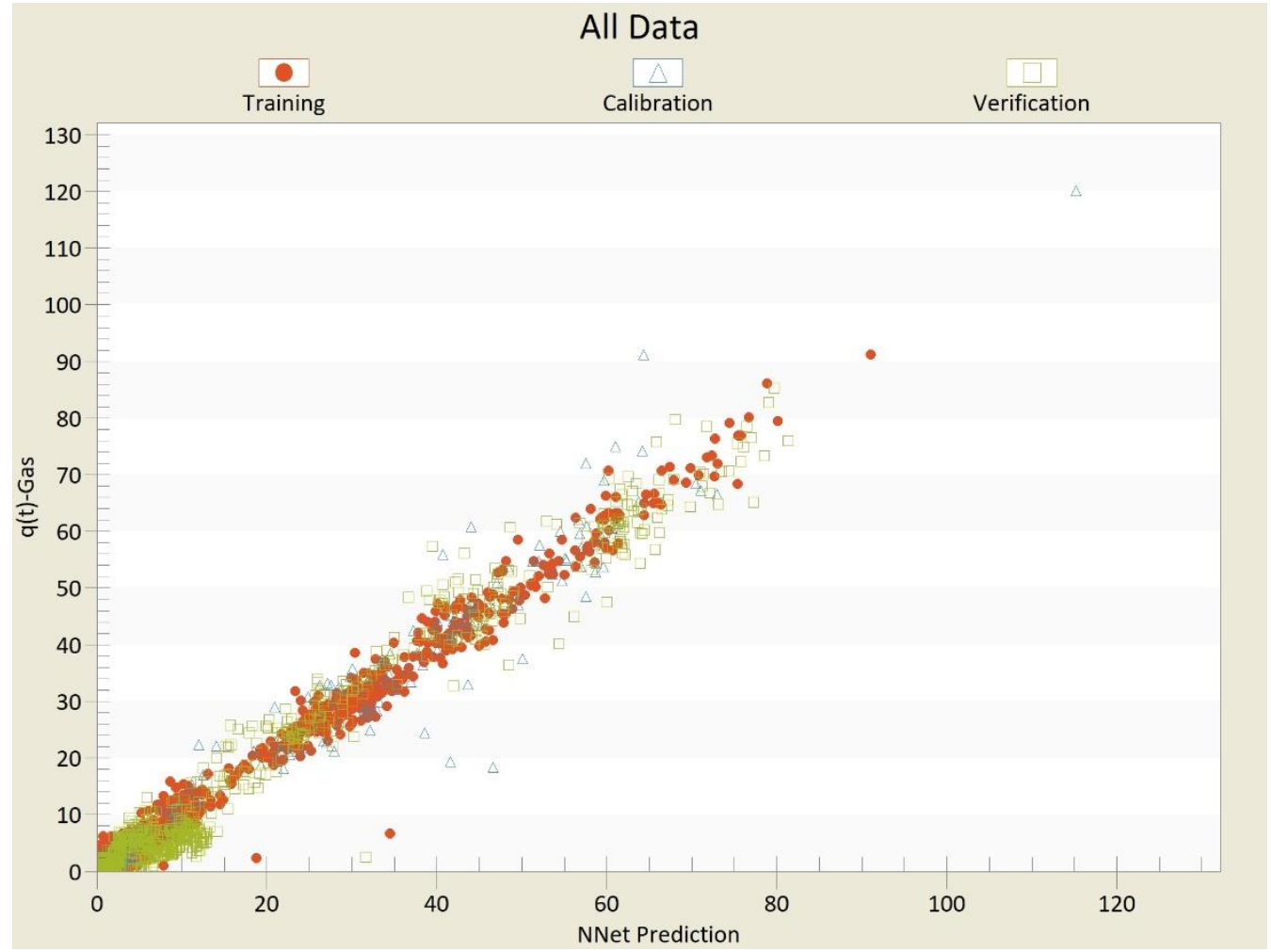

Figure 94 Data-driven gas model training results for Multi-Random Partitioning- 6 Year Blind Validation TDM 


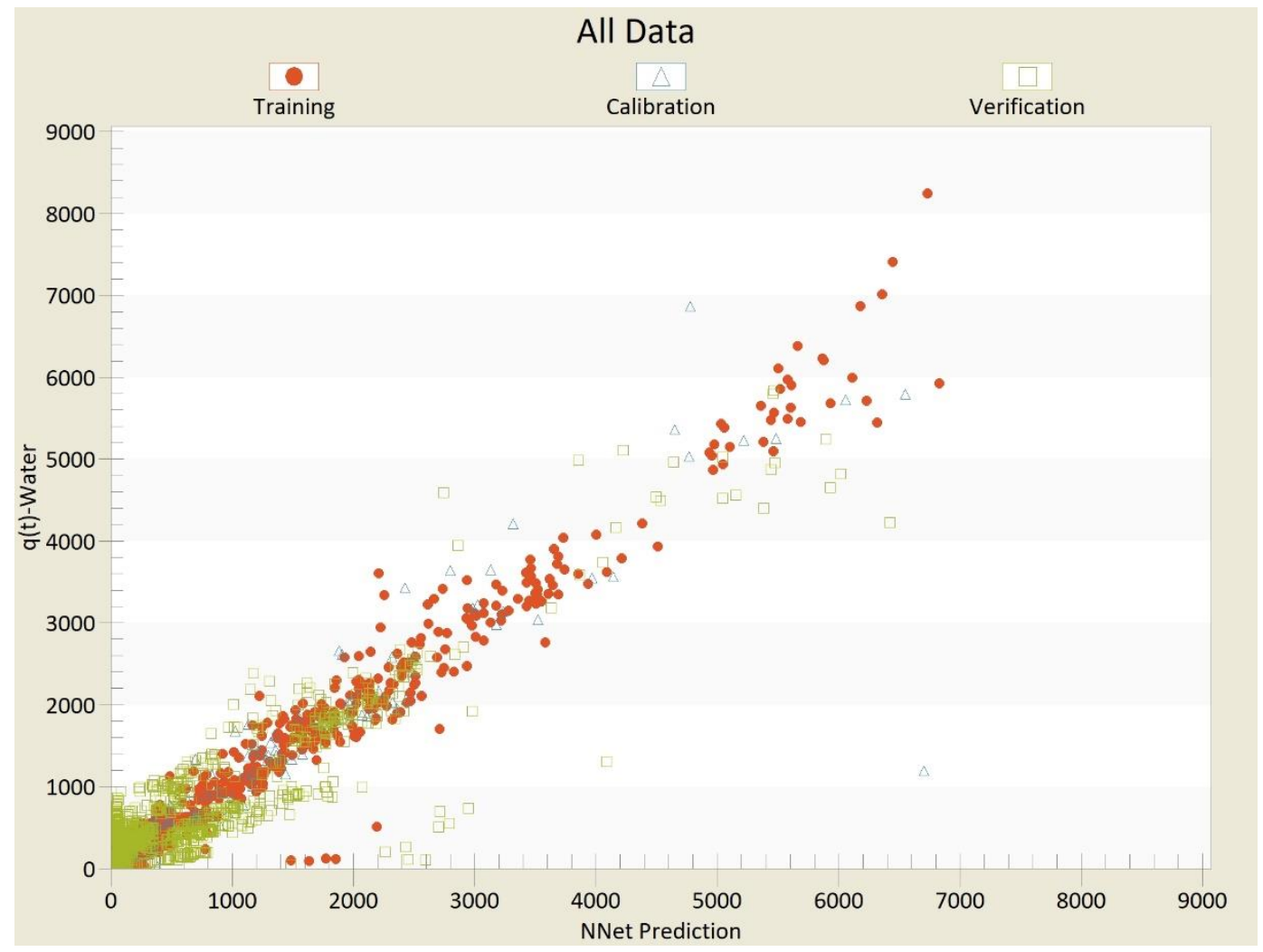

Figure 95 Data-driven water model training results for Multi-Random Partitioning- 6 Year Blind Validation TDM

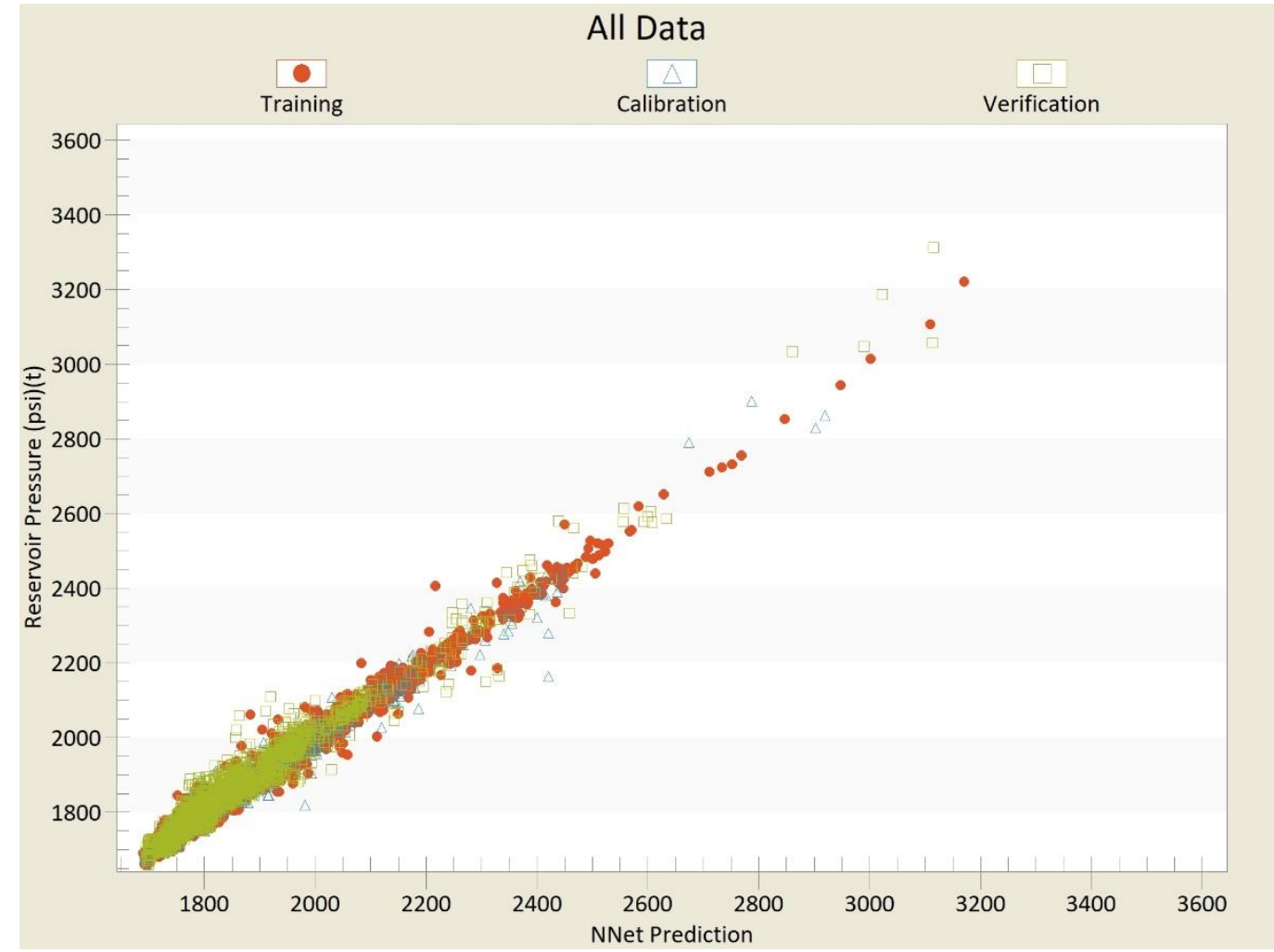

Figure 96 Data-driven reservoir pressure results for Multi-Random Partitioning- 6 Year Blind Validation TDM 


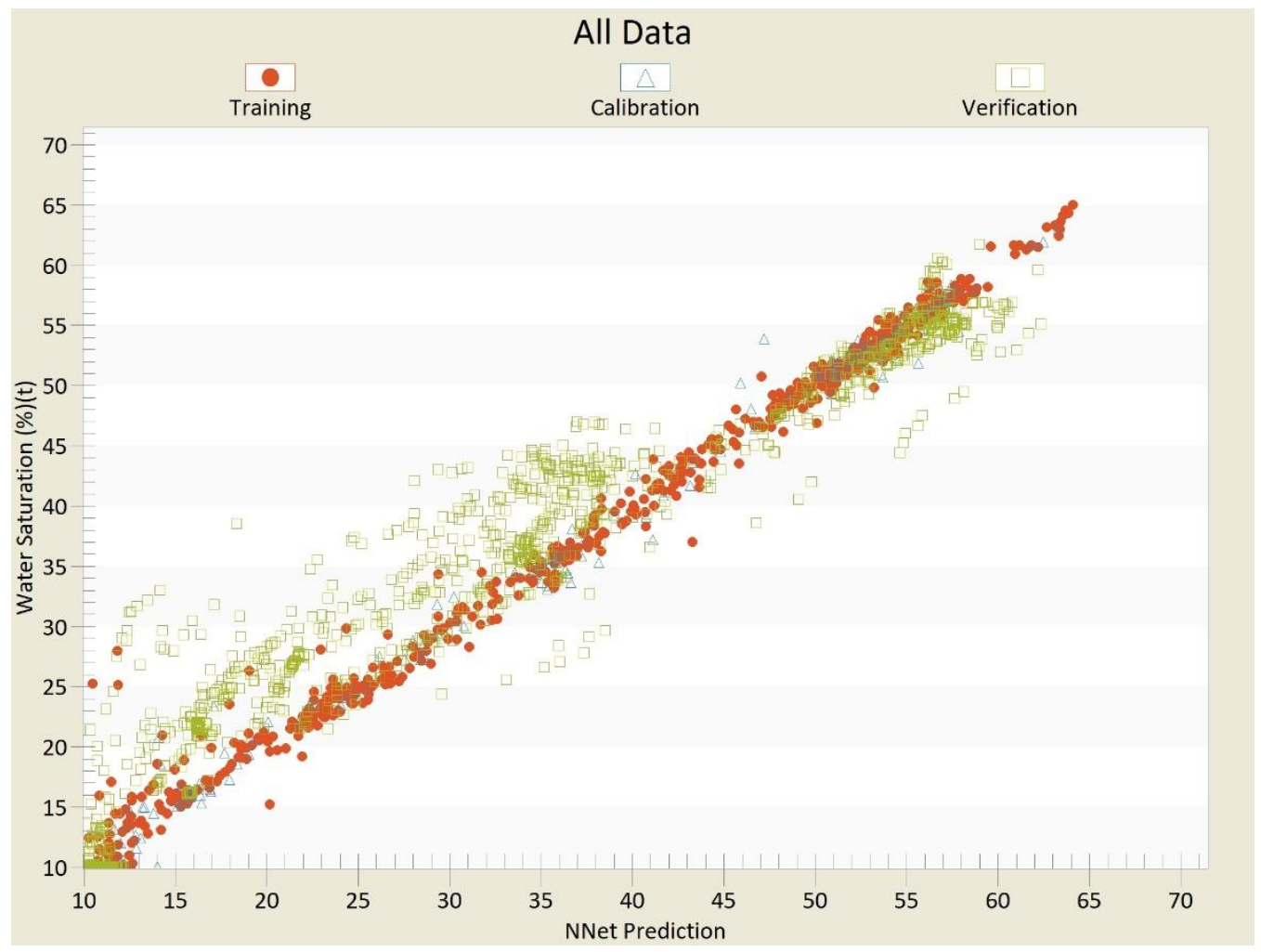

Figure 97 Data-driven water saturation training results for Multi-Random Partitioning- 6 Year Blind Validation TDM

Using the above trained models, the Top Down Model was built. The export and graph creation methodology explained for the previous models was also performed for this Top Down Model. The structure of the TDM is shown here.

\begin{tabular}{|c|c|c|c|c|c|c|c|c|c|c|c|c|c|c|}
\hline \multicolumn{3}{|c|}{ LEVEL 5} & \multicolumn{3}{|c|}{ LEVEL 4} & \multicolumn{3}{|c|}{ LEVEL 3} & \multicolumn{3}{|c|}{ LEVEL 2} & \multicolumn{3}{|c|}{ LEVEL 1} \\
\hline Linked Inputs & Detal & Output & Linked Inputs & Detail & Output & Linked Inputs & Detail & Output & Linked Inputs & Detail & Output & Linked Inputs & Detail & Outpu \\
\hline & 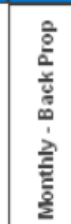 & $\begin{array}{l}\overline{0} \\
\overline{\frac{1}{5}}\end{array}$ & 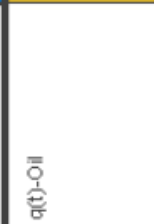 & 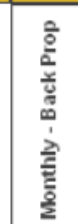 & $\begin{array}{l}\frac{0}{5} \\
\frac{5}{5} \\
\frac{1}{5}\end{array}$ & 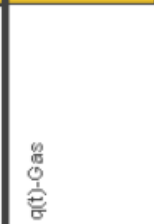 & 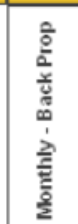 & $\frac{y_{0}}{\frac{2}{2}}$ & $\begin{array}{l}\text { 要 } \\
\text { 竞 } \\
\text { 悹 }\end{array}$ & 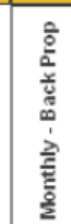 & & 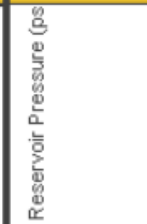 & 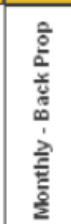 & 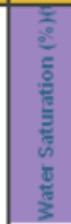 \\
\hline \multicolumn{3}{|c|}{ Oil } & \multicolumn{3}{|c|}{ Gas } & \multicolumn{3}{|c|}{ Water-6 Yr Blind } & \multicolumn{3}{|c|}{ leservoir Pressure-6 Yr Blin } & \multicolumn{3}{|c|}{ Water Saturation-6 Yr Blind } \\
\hline
\end{tabular}

Figure 98 Structure for Multi-Random Partitioning- 6 Year Blind Validation TDM

\section{Results and Discussion}

\subsection{Anticline Thesis CMG Model Results}

Once all of these changes were made and a successful run was completed, the results were examined. The wells, when open, were deemed to produce the minimum of $30 \mathrm{bbls}$ per day for the majority of their lifetimes. Realistic pressure responses were achieved and random shut-ins to simulate workover events were executed. The wells that were shut-in due to violating the water cut allowance and the dates for which shut-in occurred were recorded. It was determined that an acceptable number of wells were shut-in from water-cut and as a result, the current injection rates did not need further modification. While additional well drilling, recompletion, longer simulation time, or other constraints could have also 
been executed, those were not within the desired mission of this thesis. The desired mission of this thesis was to create and gather synthetic data from a numerical reservoir simulator that simulated industry practices on the upward sloping half of an anticline reservoir with water and gas injection and then use that data to create a data-driven reservoir model using artificial intelligence and machine learning (AI \& ML). After this data-driven model is created, the capabilities of AI \& ML are to be tested to see if it can learn how the various attributes correlate to the oil, gas, and water produced, and how much data is required to be successful. The objective was not to focus on perfecting a CMG model and fully complete the reservoir presented in the most economical way possible as is often the goal in real life practices where appraisal, completion, and facility costs are of great concern.

For the data-driven reservoir modeling purposes outlined above, all of the producers were categorized into two groups: producing at the end of the simulation and not producing at the end of the simulation. Wells not producing at the end of the simulation were wells defined as shut-in before 1-12017 due to violating the water cut constraint, which was a total of 14 wells. As a result of the small number of wells within this group, it was subdivided into only two subgroups: largest number of production days and shortest number of production days. Wells producing at the end of simulation yielded the minimum amount of oil per day for a majority of dates from when they were brought online until 1-1-2017, a total of 43 wells. This was also subdivided into three groups: largest number of production days, average number of production days, and shortest number of production days.

Wells were categorized in this manner and not the volume of production as a result of how the predictions will be made within Imagine. Blind validation in time will be used where one, two, etc. years will be separated from the end of simulation. Then the remaining dataset will be trained, calibrated, and verified on whereas the separated data will be used for blind deployment. In other words, the separated data will not be seen during training, calibration, and verification and only once all of the models have finished that process and a TDM has been built, will the separated data be used to judge the quality of the model and the accuracy of the predictions. Wells that are missing the last year, etc. cannot be used in the blind validation process because while predictions could technically be made, there is no real synthetic production data to compare it with.

The number of days a well produced was calculated from data exported from CMG. The oil, gas, and water production was exported for all wells on a daily basis. For all 57 producers, a column was added where an if statement returned a 1 if the oil production value was zero for that day, otherwise it returned a 0 value. A summation of this column then determined the number of days that the well did not produce oil, whether it was from a random shut-in or from exceeding the allowed water cut limit. Next, the total number of days between when the well was brought online and the end date of the simulation was calculated for each phase of production. The number of total days a well is online minus the number of days that the well's oil production was equal to zero was calculated and deemed the number of active production days for that well. The wells within each category, producing at the end of the simulation and not producing at the end of the simulation, were sorted based on the total number of active production days and thus separated into their respective subgroups.

Well biographies were made for all wells which included the focal well's oil, gas, and water production, the minimum production limit, the water cut percentage limit, the focal well's water cut and well bottom-hole pressure, the two closest offset injectors' injection rates, and the two closest offset producers' oil production rates on one graph. Completion in time, the location of the focal well and offset wells on a map, production statistics about that well, saturation values for all layers, and pressure values for all layers are also shown with each well biography. Included below are examples from each of the subgroups mentioned in the paragraph above. 


\subsection{Wells Producing Until End of Simulation}

An example for the largest, average, and smallest number of active production days is included below. In the legend shown on the right are the various symbols and colors representing the multiple curves. It is important to note that for some wells, the two closest offset injectors were both gas injectors with the same specified rate of 20,000,000 $\mathrm{ft}^{3} /$ day, thus making them difficult to distinguish in some cases when the maximum bottom-hole pressure for the injectors were not reached, which is why different symbols are implemented. For Well-057 below, one offset injector is a gas injector depicted by an orange symbol and line and one is a water injector depicted by a simple dashed blue line. Curves begin to show within these plots when the well is brought online. Therefore, injector curves may be seen at different time steps than the focal well's curves.

On the small map shown below the legend are five dots. The red dot shows the location of the focal well, the two green dots are the offset producers, and the two blue dots are the offset injectors. The colors of the dots have no indication of fluid injected, etc. as they do indicate in the legend and the larger graph of production rates. A bar graph is included that shows the layers completed for each well. The grid thickness for each layer is included in the legend for that graph. The completion graph is underneath the production rates graph because they share the same timeline. Therefore the date that completion changes are made can be read from the $\mathrm{x}$ axis of the production rates graph. For example, Well-004 below completes layers 1 and 2 on January $1^{\text {st }}, 2008$ and after this date, all layers are completed until the end of the simulation.

Because Well-057 is closest to both a water and gas injector, all three saturations change for each layer, which is not the case for many wells. For Well-004 below, the two closest offset injectors are gas injectors and therefore a change in the gas saturation can be seen in all layers, however a noticeable change is not seen in the water saturation. When a well is not near a water injector, its water saturation is expected to remain relatively constant due to the low initial water saturation presented in this reservoir. A similar trend can be send about gas saturation, where if a well is not near a gas injector, then its gas saturation is expected to stay relatively constant due to no free gas being present in the reservoir. Free gas would have the potential to occur if the reservoir pressure were to fall below the bubble point, however it does not in this simulation. Also seen within this plot is the field cumulative oil production as well as the well's oil, gas, and water cumulative production. 


\subsubsection{Largest Number of Production Days}

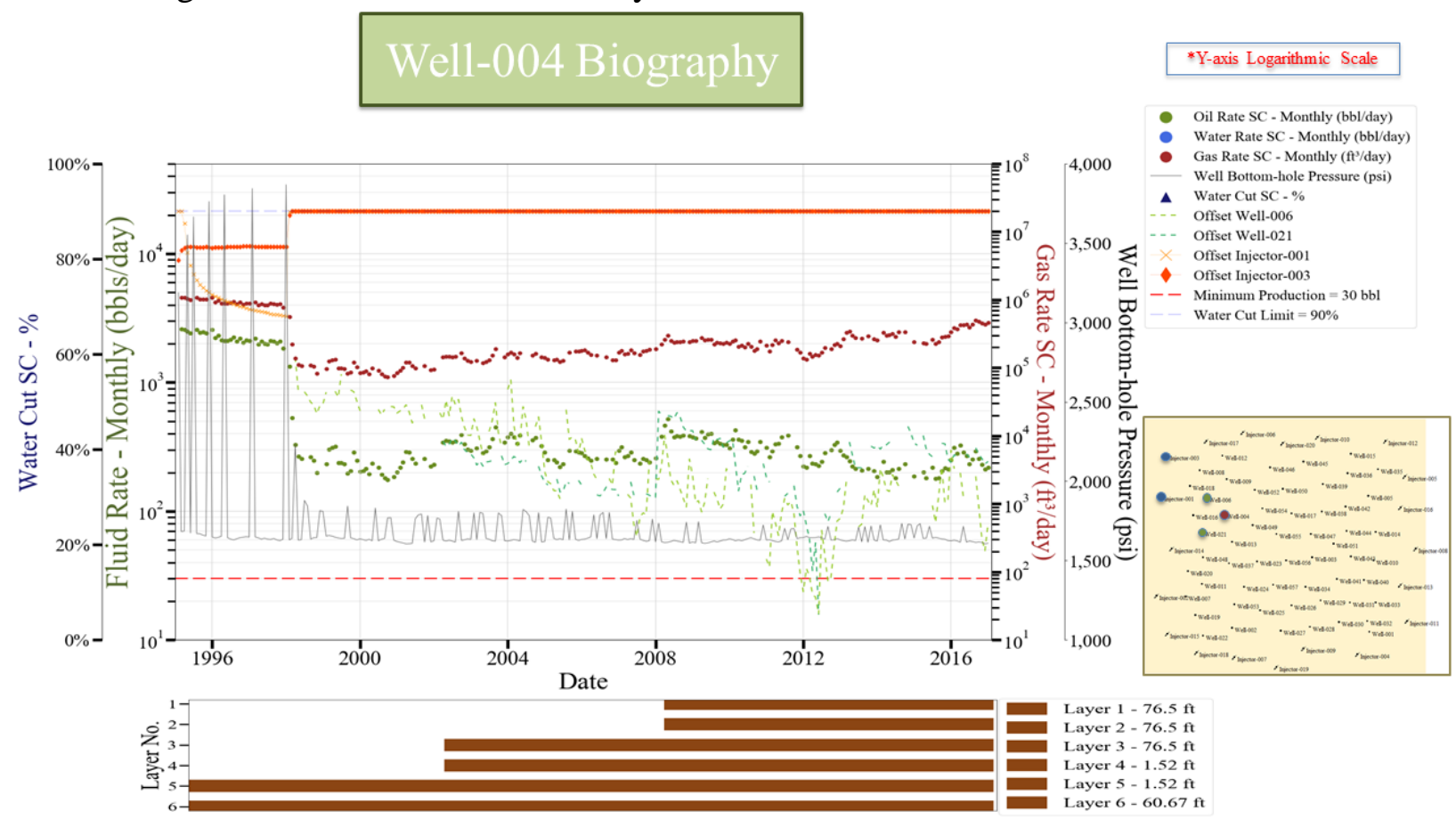

Figure 99 Well-004 biography
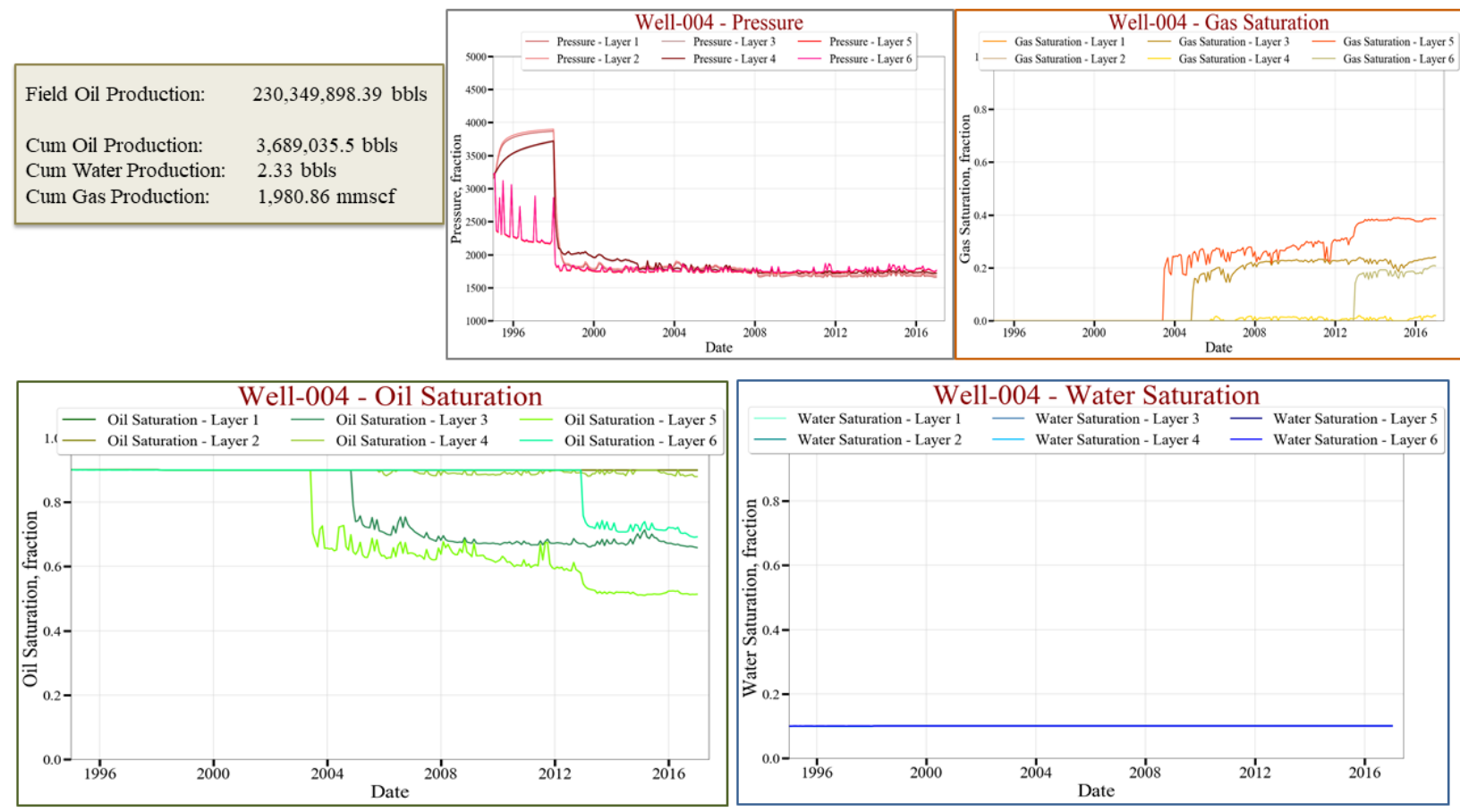

Figure 100 Well-004 saturations and pressure for every layer 


\subsubsection{Average Number of Production Days}

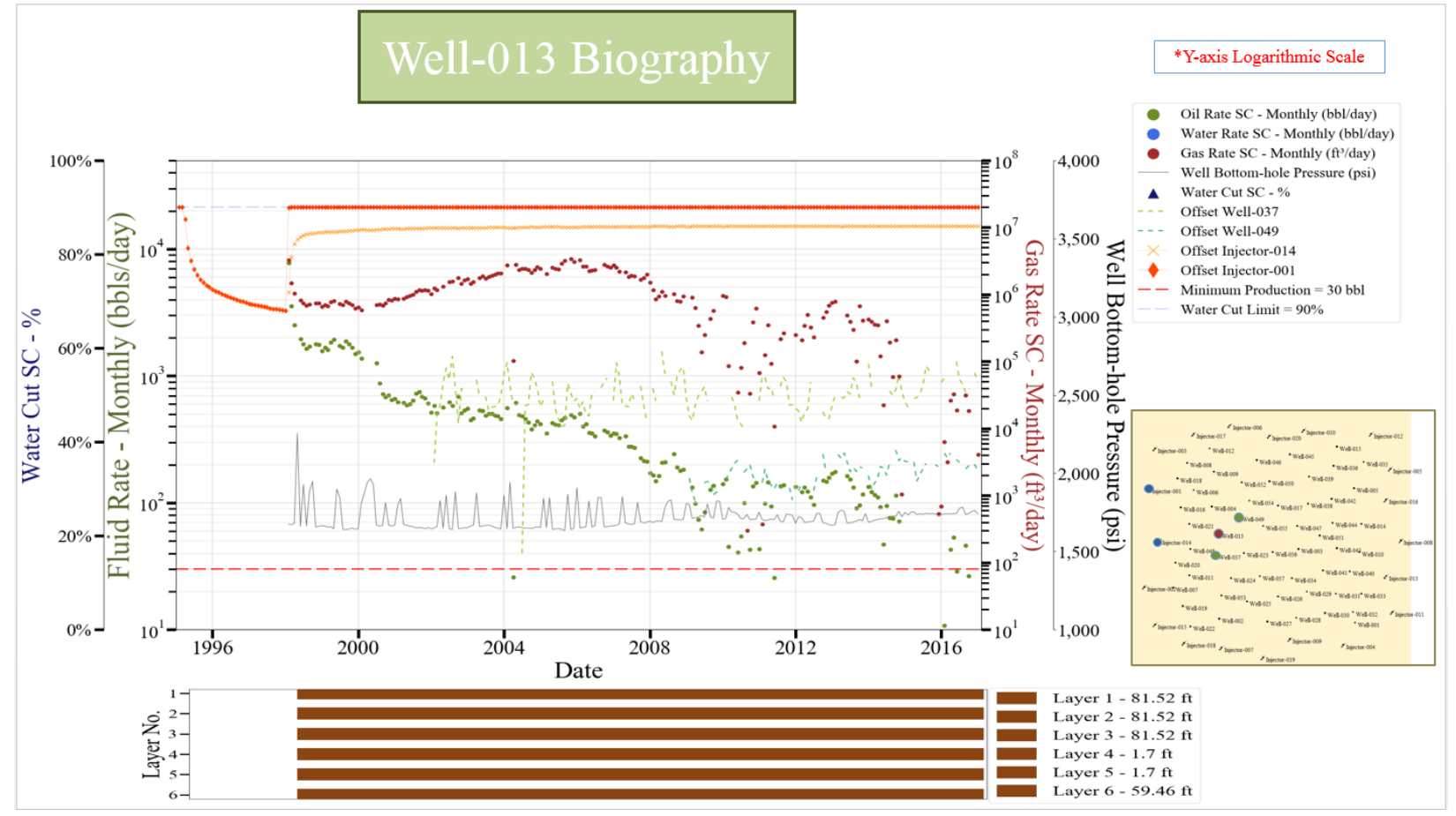

Figure 101 Well-013 biography
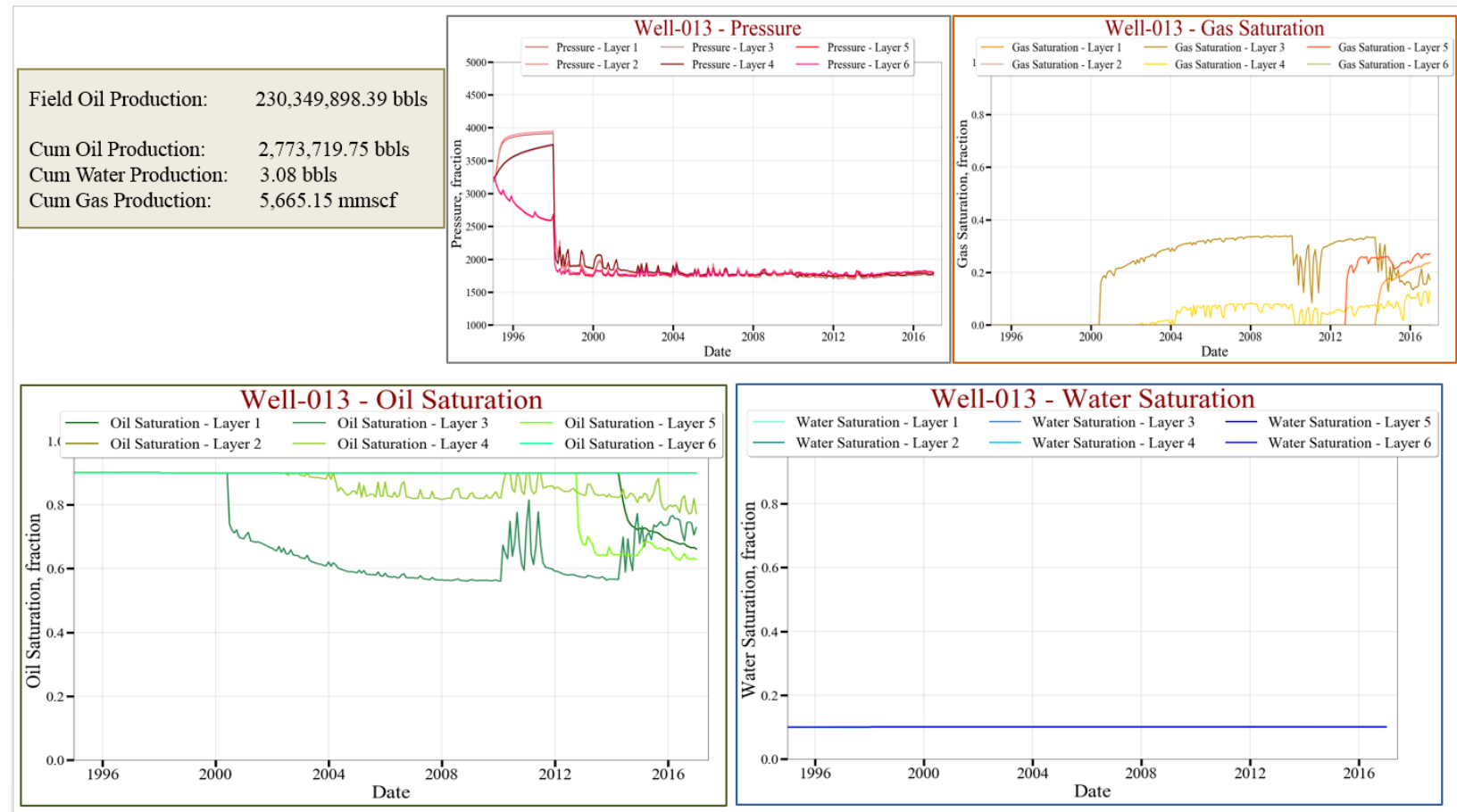

Figure 102 Well-013 saturations and pressure for every layer 


\subsubsection{Smallest Number of Production Days}

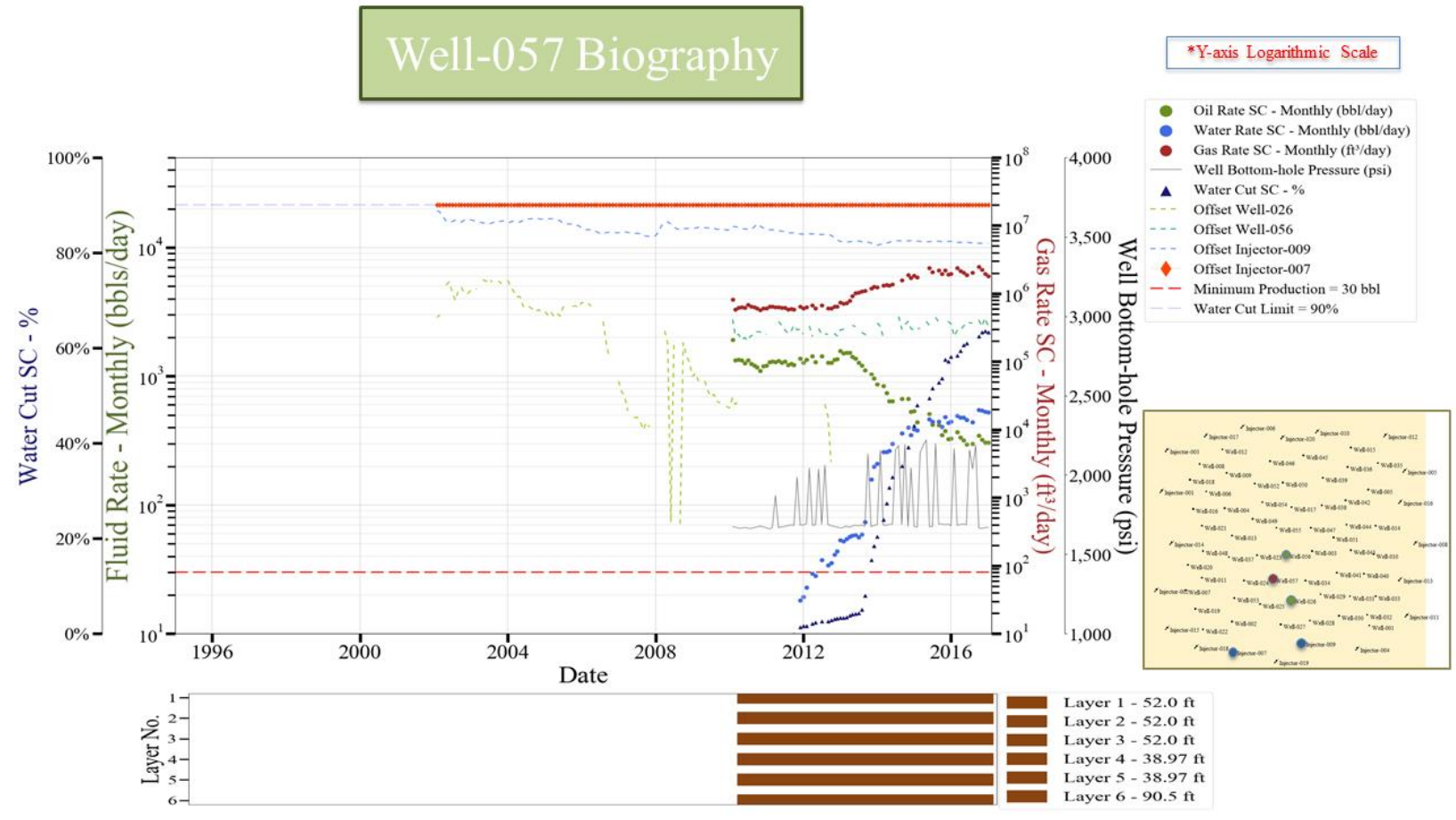

Figure 103 Well-057 biography
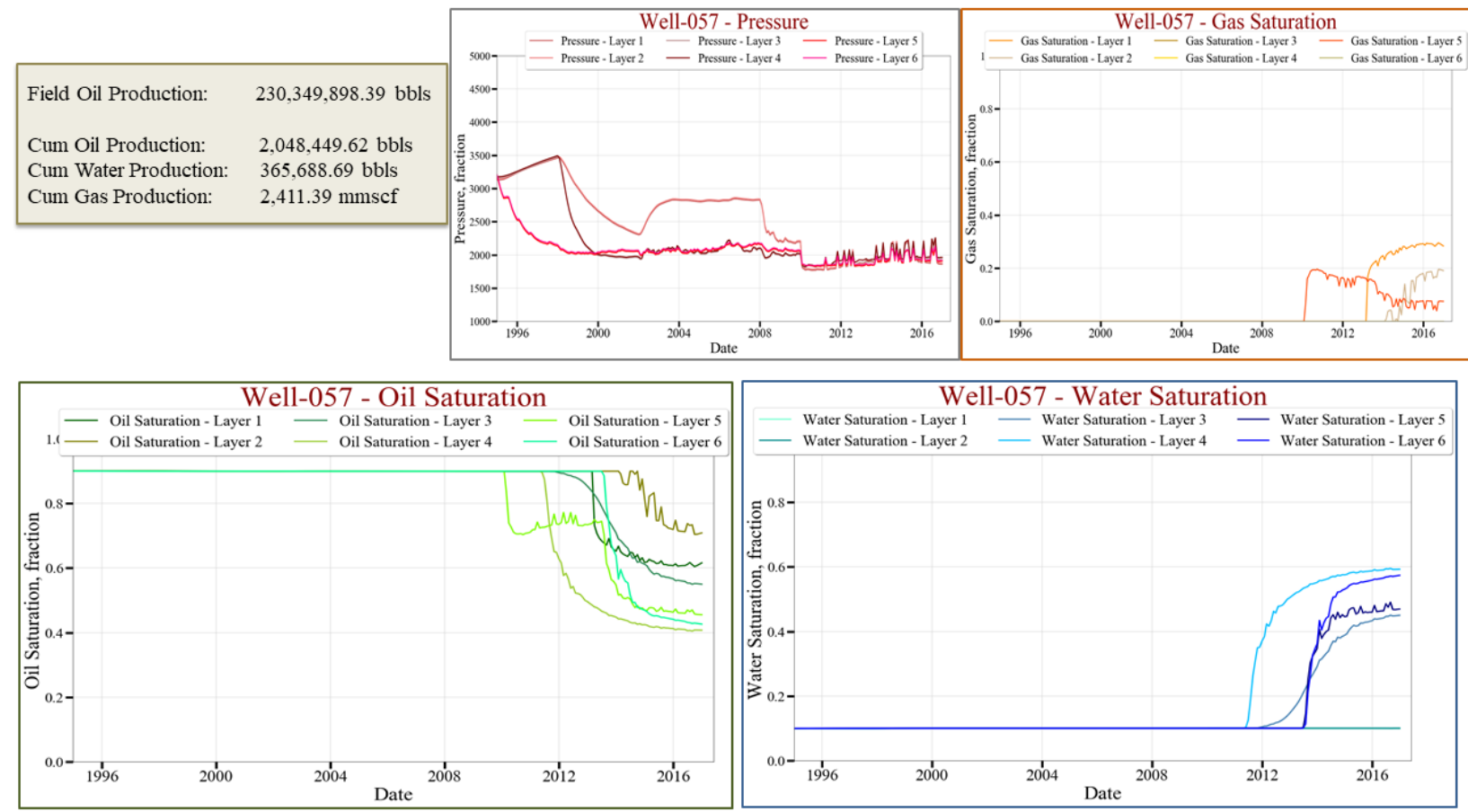

Figure 104 Well-057 saturations and pressure for every layer 


\subsection{Wells Not Producing at the End of Simulation}

The statements made about the graphs above, apply for the examples shown below. The steady rise of water cut, represented by blue triangles, can be seen in the example below. It is important to note that when the water cut triangles near the water cut limit line, a constant blue dashed line at $90 \%$, the oil, gas, and water production for the focal well terminate due to shut-in. Well-035 produces for a very short amount of time before being shut-in due to water cut. This is caused by Well-035's proximity to the sealing fault on the right side of the reservoir and its proximity to the water injectors. Because Well-035 is one of the closest wells to the water injectors, it becomes quickly water flooded. In fact, Well-035 is water flooded so quickly, that it is shut-in before it can be re-completed as planned in the partial completion plan, thus producing out of only layers 4, 5, and 6 . While other wells do experience the high water cut, Well-035 is the only well to have been affected so drastically out of all the wells within the reservoir. It is the only well that after being put online, immediately exceeds the water cut, shuts in for a period of time, and reopens for a few months of production before being shut-in permanently. Out of 57 wells, only two wells do not fulfill their planned partial completion, Well-035 and Well-028. Well-035 shuts in before it completes layers 1, 2 and 3 whereas Well-028 shuts in before it can complete layer 1.

\subsubsection{Largest Number of Production Days}

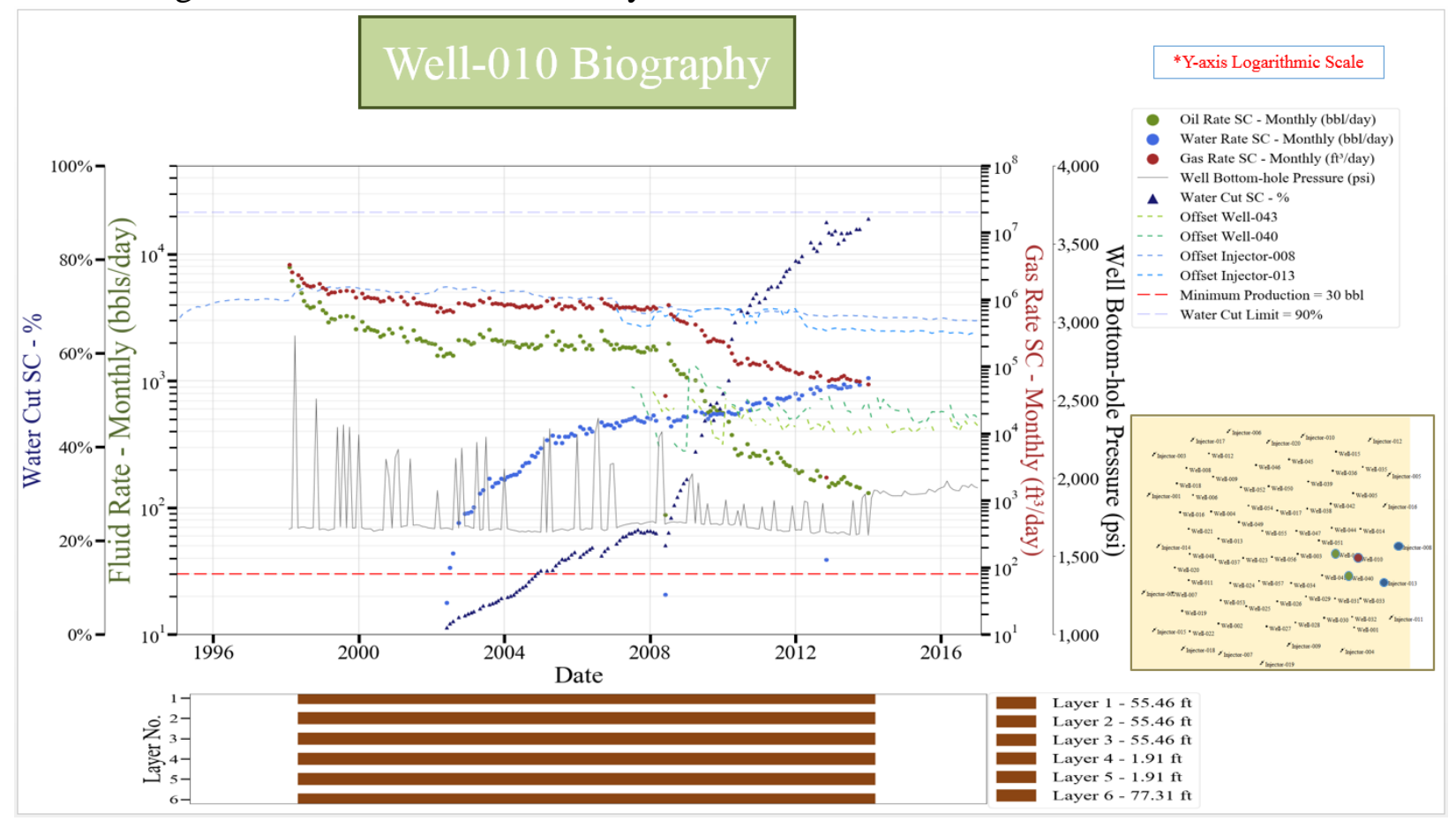

Figure 105 Well-010 biography 

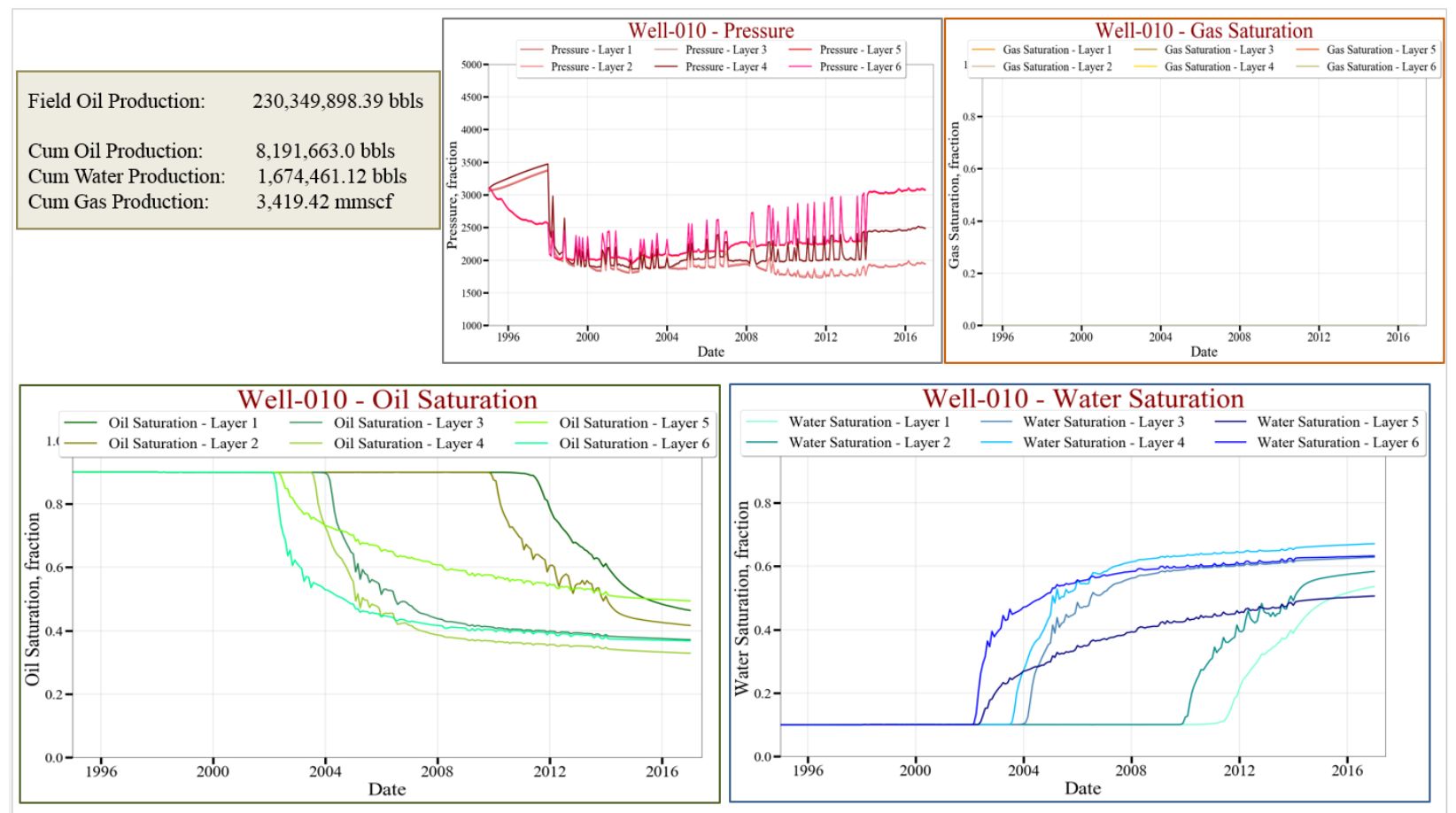

Figure 106 Well-010 saturations and pressure for every layer

\subsubsection{Smallest Number of Production Days}

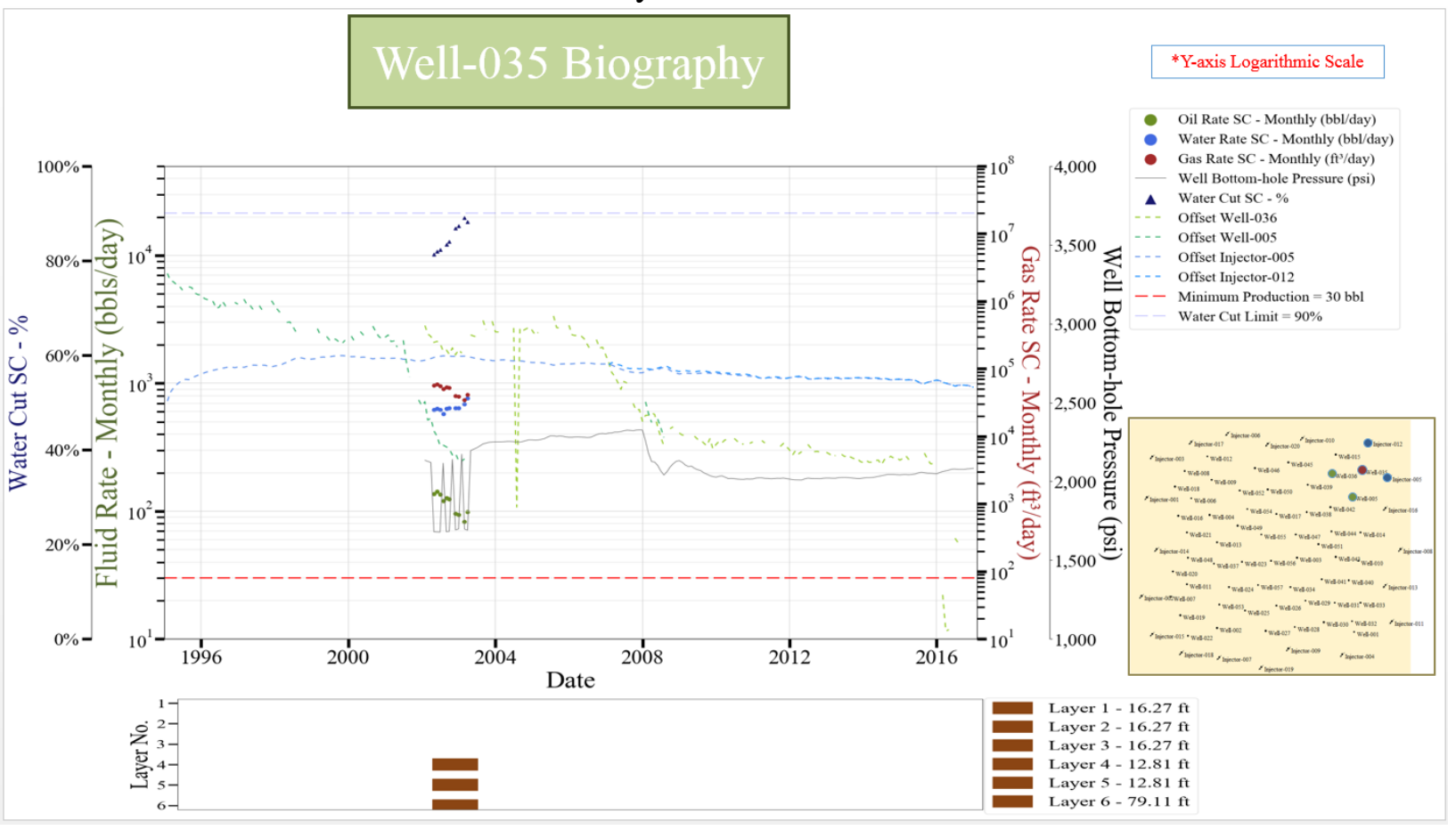

Figure 107 Well-035 biography 

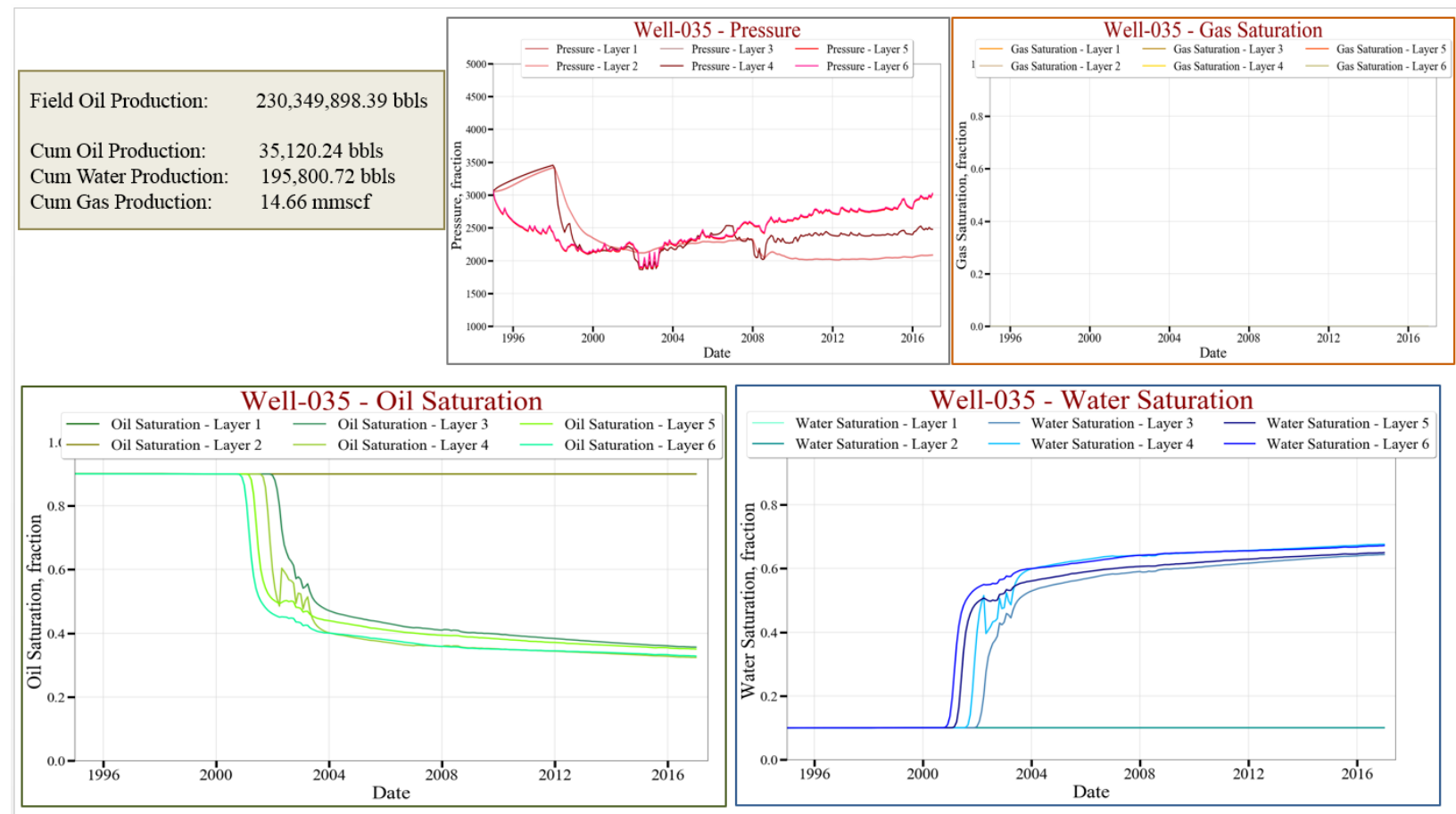

Figure 108 Well-035 saturations and pressure for every layer

\subsection{Top Down Modeling Results}

The actual data exported from the numerical reservoir simulator is compared with the predicted data from each Top Down Model below in their own subsections. The comparison is represented in three ways. The first depictions shown will be the entire reservoir plot showing cumulative production, production rate, and number of active wells. The cumulative production and production rate is shown for both the predicted and actual data for oil, gas, and water. Second, the heat maps for reservoir pressure and water saturation for the entire reservoir are included. Heat maps were generated using a code developed by Yvon Martinez. The heat maps were made for each month within a range of specified dates that vary for each Top Down Model. The values of water saturation and reservoir pressure are reported at each well location, and in order to fill in the rest of the map, an interpolation method needed to be used. The cubic interpolation method was used because it provided the smoothest and closest results when compared with linear and nearest neighbor methods. Examples at the same time step for reservoir pressure are shown below to compare the three interpolation methods. 
Reservoir Pressure (psi) at 2016-01-31
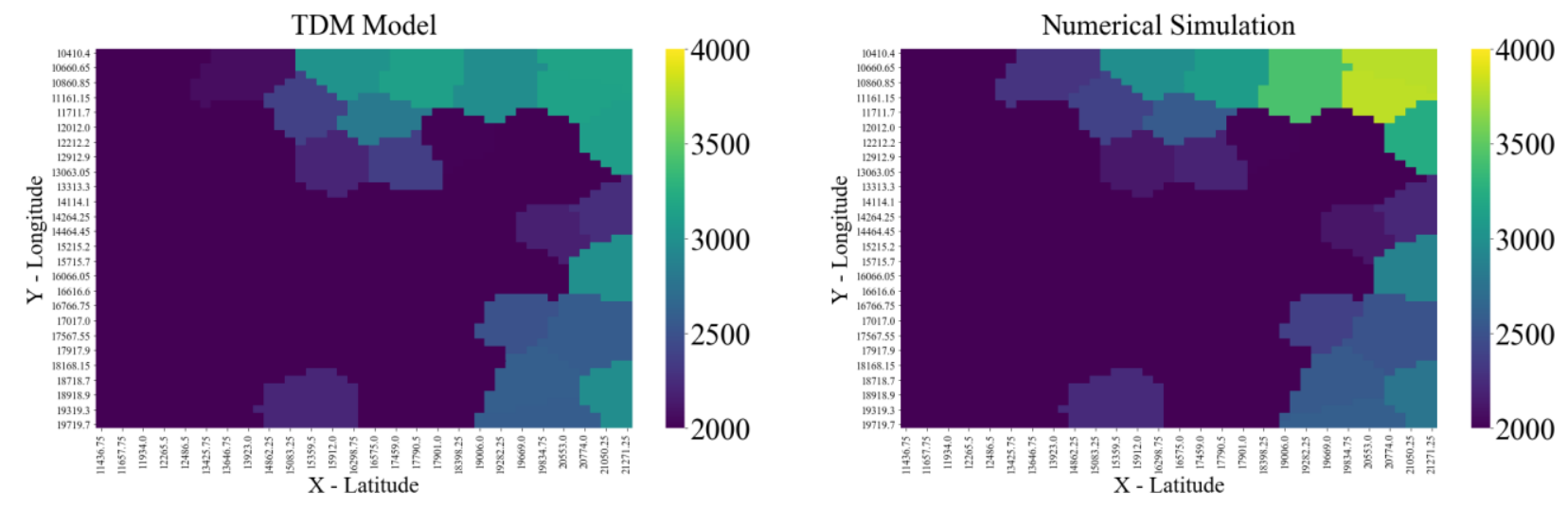

Figure 109 Reservoir pressure heat map at January 31st, 2016 using nearest neighbor interpolation method

Reservoir Pressure (psi) at 2016-01-31
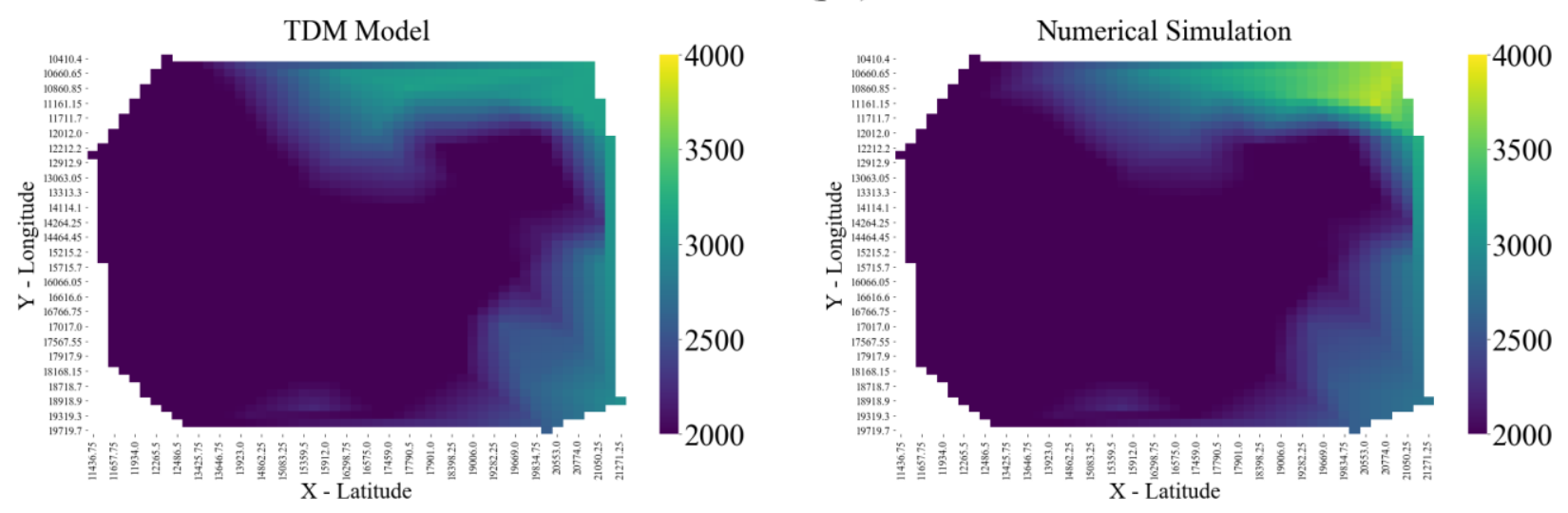

Figure 110 Reservoir pressure heat map at January 31st, 2016 using linear interpolation method

Reservoir Pressure (psi) at 2016-01-31
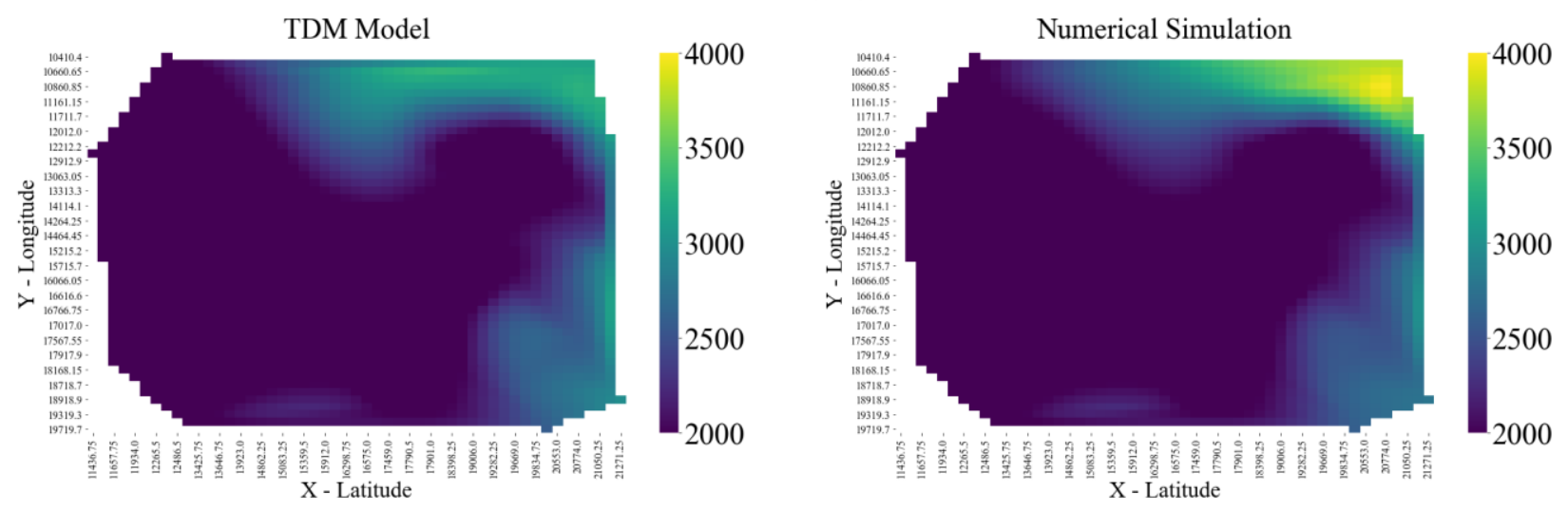

Figure 111 Reservoir pressure heat map at January 31st, 2016 using cubic interpolation method

Lastly, the third plots shown for each Top Down Model are the individual well rate plots which show both the TDM predictions and the actual simulation data for individual wells. The individual well plots were classified into three groups based on how well the predictions fit the actual data: poor, average, 
and good. Two examples are shown for each classification for each model in the results whereas all of the individual well production plots are provided in the respective Top Down Model section in Appendix 8.2.

\subsubsection{Random Partitioning-No Blind Validation}

First, the results for the entire reservoir can be seen for oil, gas, and water. The darker green cumulative curve is the actual field data, also referred to as simulator data whereas the lighter green cumulative curve is the predicted TDM curve. The predicted cumulative production is very close to the actual cumulative production, just slightly above. The predicted rate is also very close to the actual field rate with only a slight discrepancy seen in the very early life of the well where only 5 wells are producing.

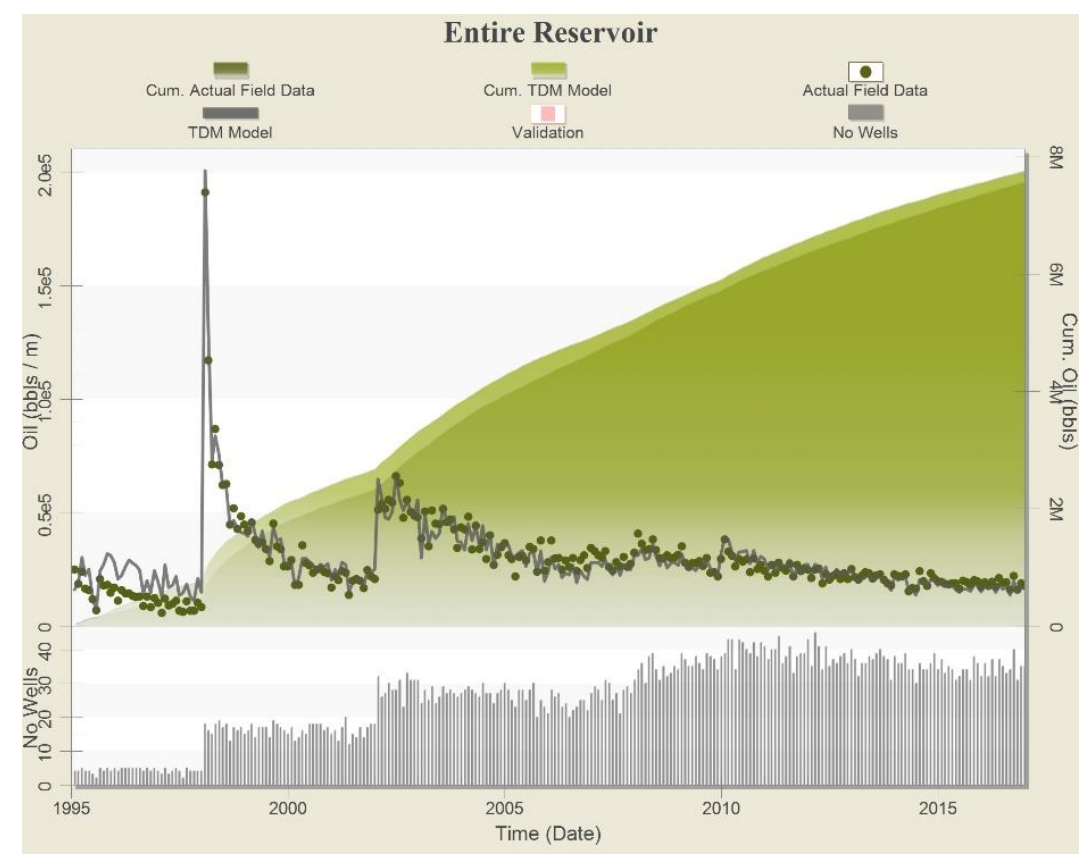

Figure 112 Entire reservoir oil results for Random Partitioning-No Blind Validation Top Down Model

Similar to the oil results, the gas predicted cumulative production is in light green, however the actual cumulative production is in orange. This color scheme is the same for the production rate data. Despite the fluctuating behavior in the gas rate data, the predicted curve matches the pattern rather well even though it does not match the exact value for some of the data points. This fluctuation is due to the maximum bottom hole pressure operating condition on the injectors as well as the random shut-in schedule for the producers. The wells that are nearest to the gas injectors are expected to produce the most gas and when they are randomly shut-in, especially for whole months at a time, then opened again, the amount of gas produced is expected to fluctuate. The cumulative prediction is extremely close to the actual cumulative production as seen in Figure 113. 


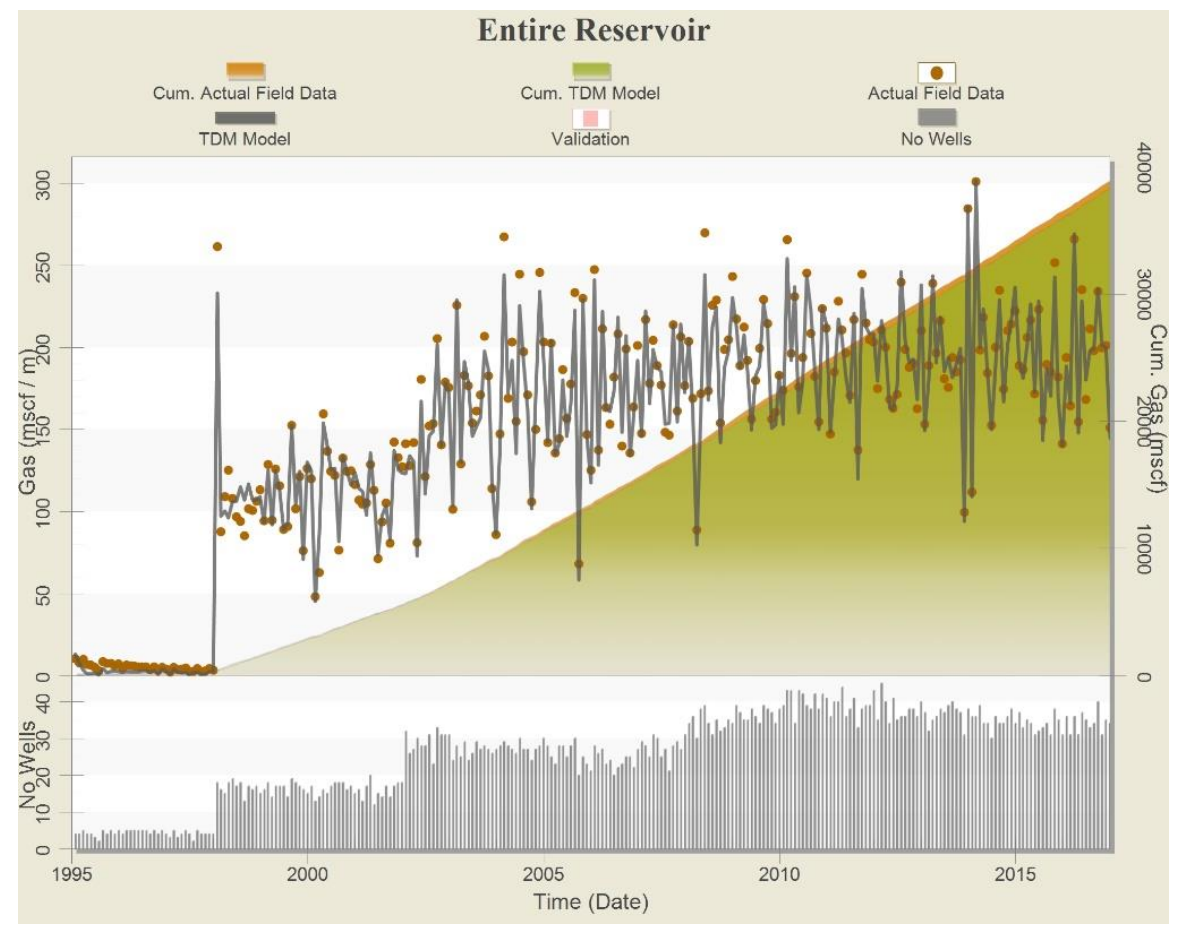

Figure 113 Entire reservoir gas results for Random Partitioning-No Blind Validation Top Down Model

For the entire reservoir water results, the actual simulator data is demonstrated in blue and the predicted results are in green. The Top Down Model anticipates the actual results with good accuracy. The rises and falls in the water production curve can be attributed to wells being shut-in due to violating the water cut operating constraint, and wells nearing the water cut constraint, but not yet violating it. The water cut constraint was set to $90 \%$, where if violated, the time step was repeated in order to perform calculations again with the well shut-in. The repetition avoids the large production of water at the time step where water was over $90 \%$ of total production for that well. 


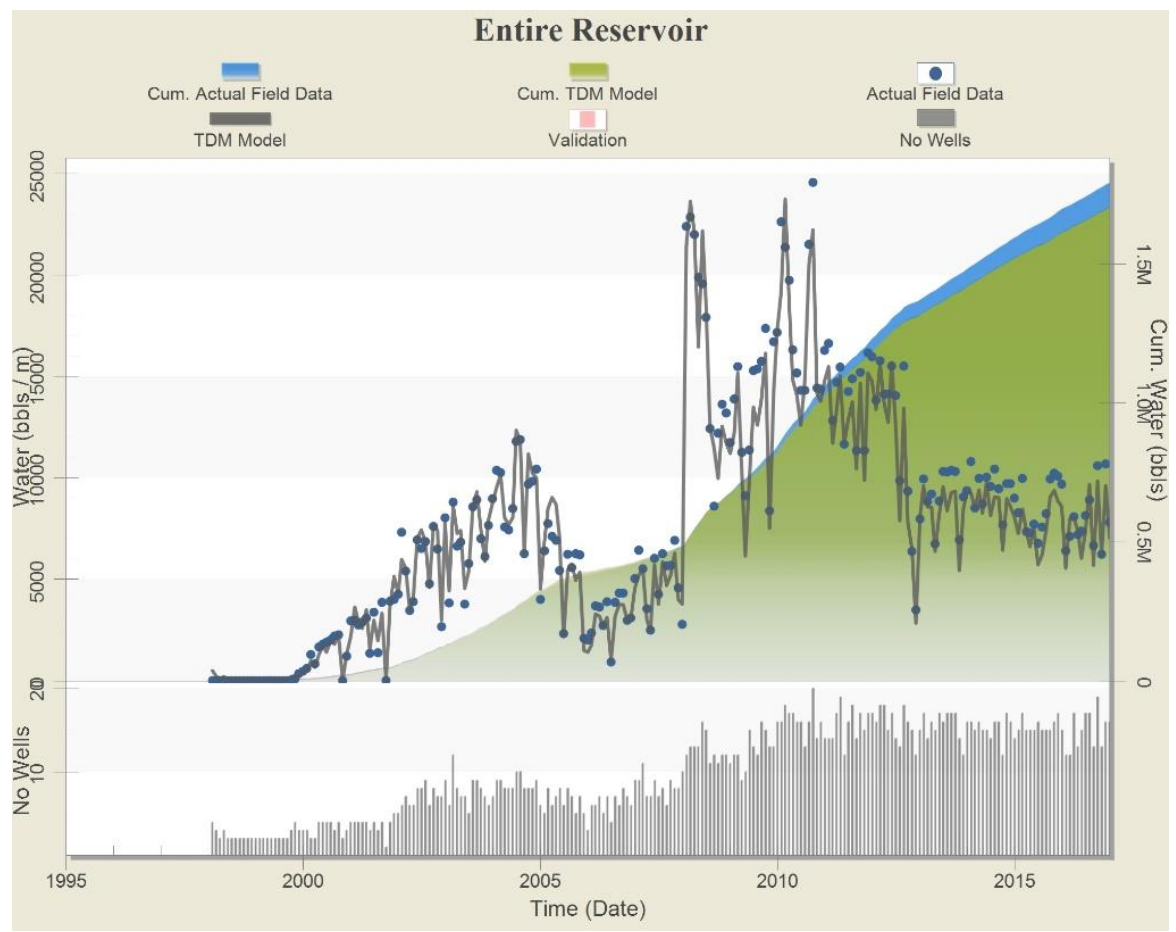

Figure 114 Entire reservoir water results for Random Partitioning- No Blind Validation Top Down Model

Heat maps were created to better represent entire reservoir results for water saturation and reservoir pressure. The heat map is shown at January $31^{\text {st }}, 2016$ and June $31^{\text {st }}, 2016$ for reservoir pressure and water saturation below. These two dates were chosen because they are in the last year of the simulation where the training, calibration, and validation is randomly placed throughout the entire dataset. As it can be seen in the comparisons below, the TDM predictions match the numerical simulation data with good accuracy. An error plot is also shown with a scale from 0 to $30 \%$ where a dark red represents an error of 30 or higher percent. Additional heat maps for both reservoir pressure and water saturation can be seen in Appendix 8.2.1.2 for every two months during the last year of simulation.

Reservoir Pressure (psi) at 2015-12-31
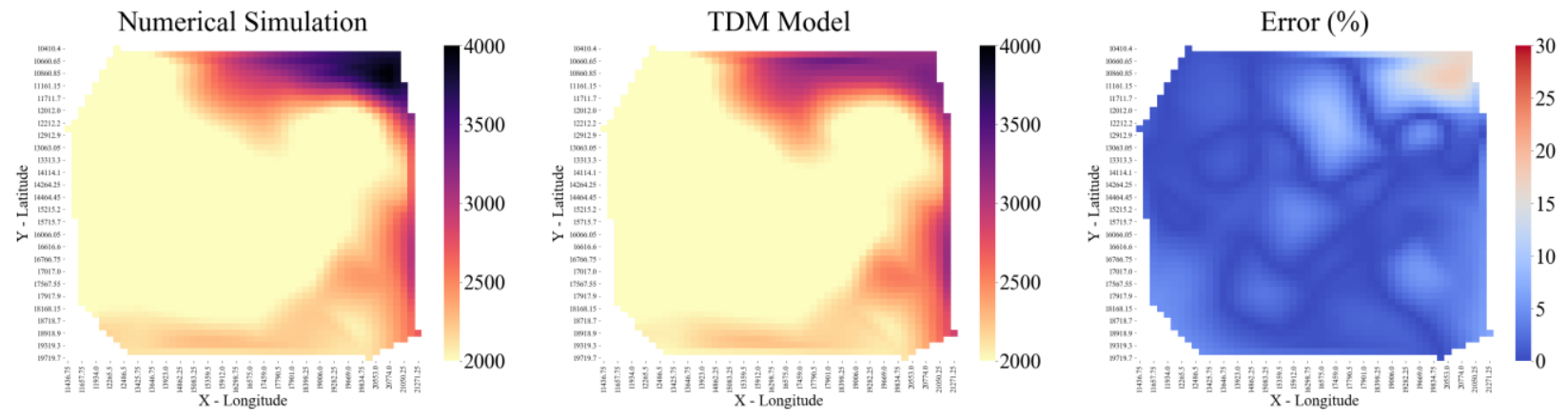

Figure 115 Entire reservoir heat map for reservoir pressure at December 31st, 2015 for Random Partitioning-No Blind Validation 
Reservoir Pressure (psi) at 2016-06-30
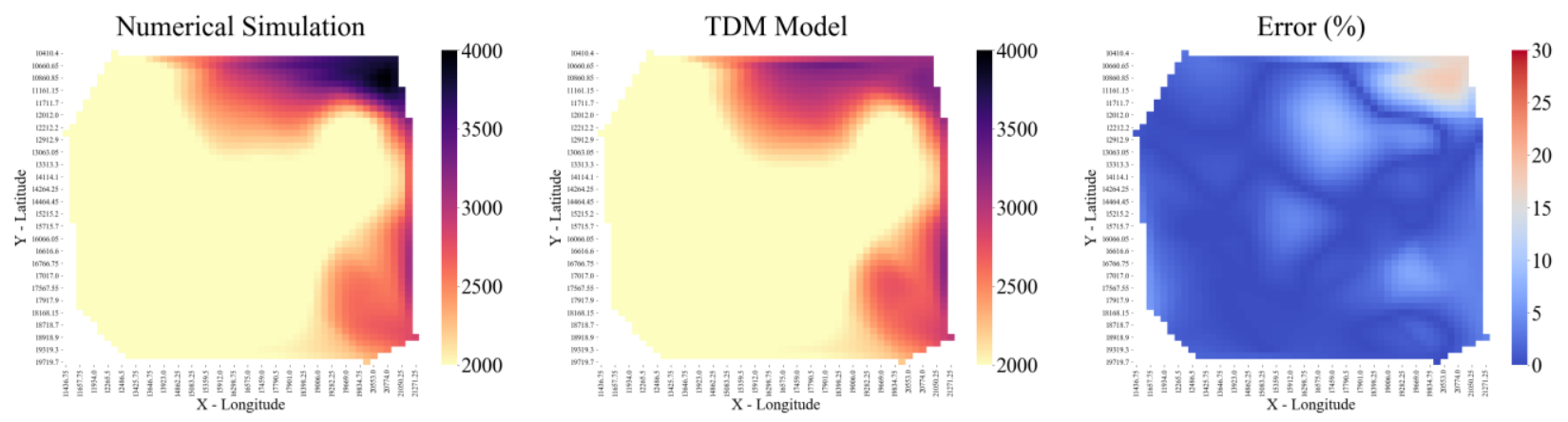

Figure 116 Entire reservoir heat map for reservoir pressure at June 30th, 2016 for Random Partitioning-No Blind Validation

Water Saturation $(\%)$ at $2015-12-31$
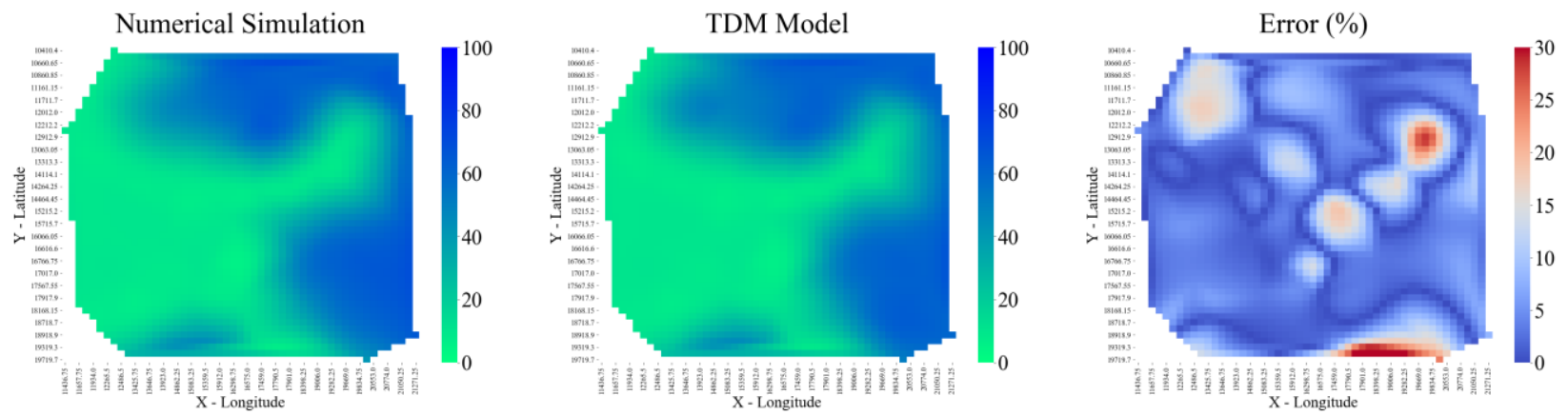

Figure 117 Entire reservoir heat map for water saturation at December 31st, 2015 for Radom Partitioning-No Blind Validation Water Saturation (\%) at 2016-06-30
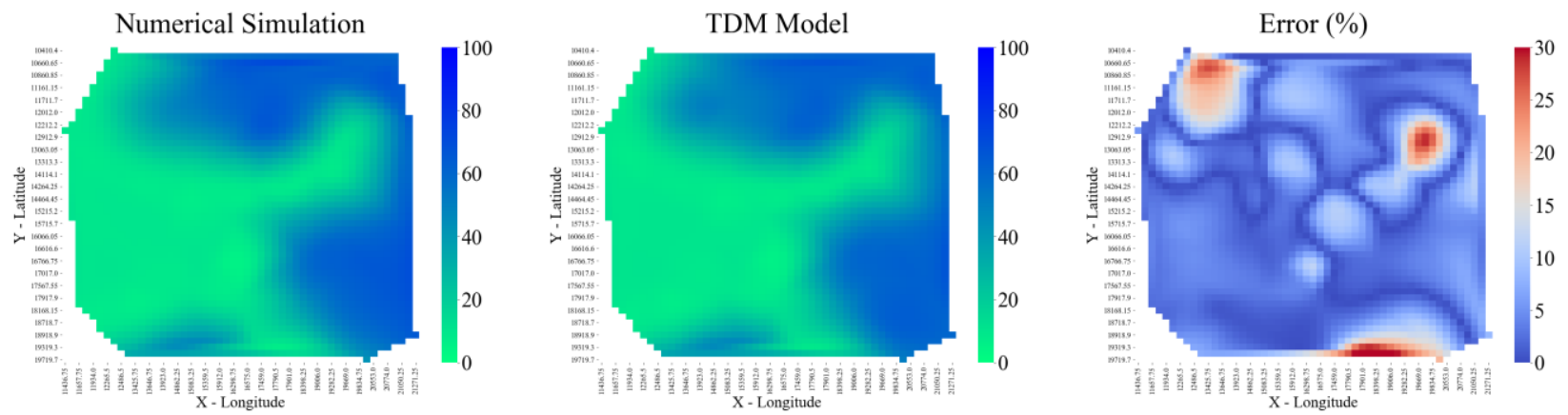

Figure 118 Entire reservoir heat map for water saturation at June 30th, 2016 for Random Partitioning- No Blind Validation

Several example wells are shown next where their individual TDM predictions are compared with the actual simulation data for oil, gas, and water rate. The scaling was chosen after the maximum was found for each well. From there, the maximum chosen for the individual well graphs was the value that a majority of wells had in common. It is important to note that a few wells were outliers, meaning that their maximum production rate for oil, gas, and/or water was higher than the majority of other wells. These outliers therefore are graphed on a different scale in order to see the data. These two sets of maximums were used for each Top Down Model created, as the actual simulation data does not change between cases. 
Two examples are shown for each classification: poor, average, and good. As can be seen in the good example plots, Well-019 and Well-044, the prediction matches the simulation data rather well. Well-019's water production is minimal due to its location in the reservoir. Well-019 is much closer to the gas injectors than the water injectors, therefore it will have a larger production of gas as seen. Conversely, Well-044 is much closer to water injectors than gas injectors, therefore its water production is higher than its gas production. Thirty-seven wells were deemed to have a good fit which is about $65 \%$ of the total number of wells.
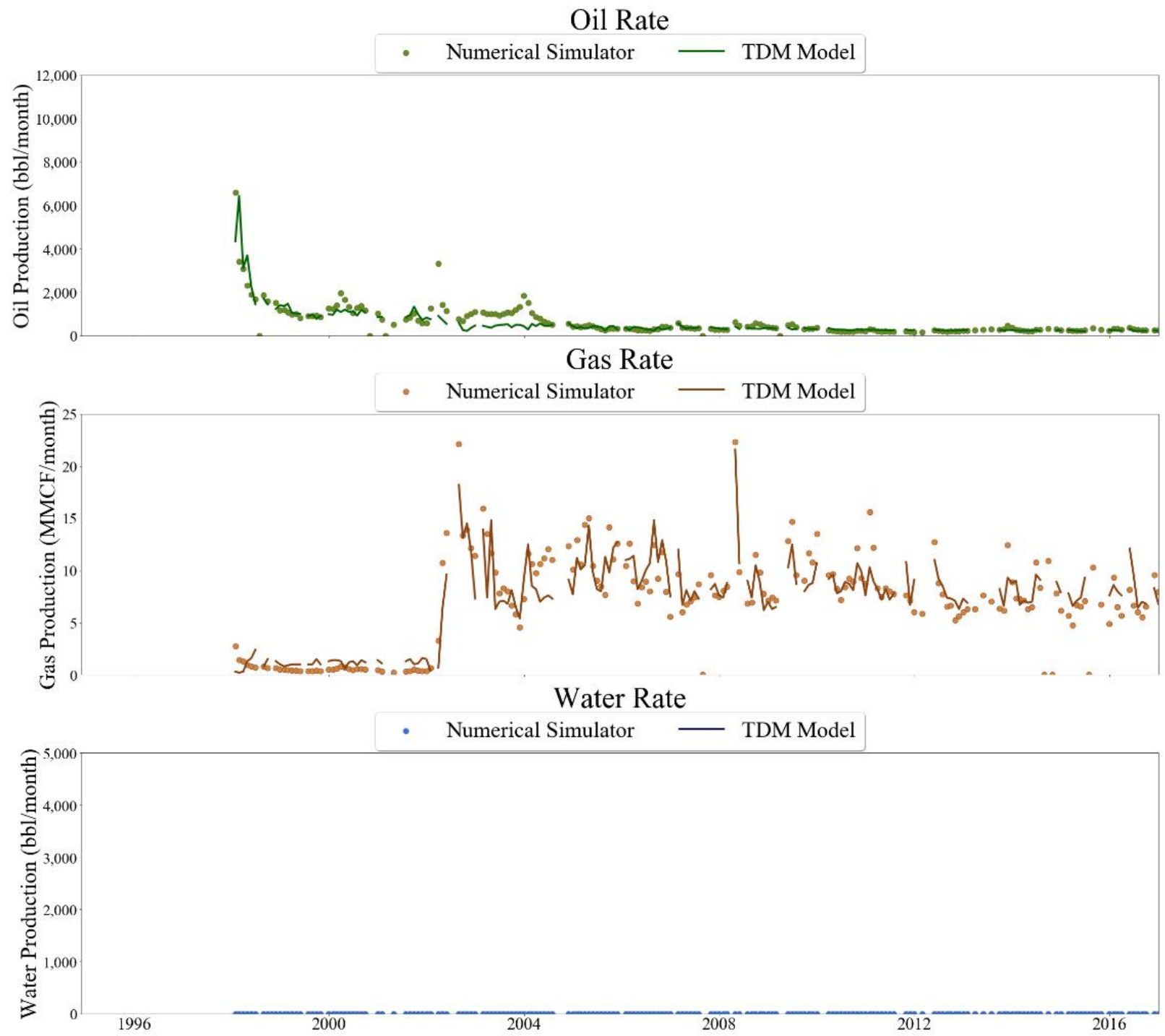

Figure 119 Well-019 oil, gas, and water rate TDM predictions vs actual simulation data for Random Partitioning-No Blind Validation TDM 


\section{Oil Rate}
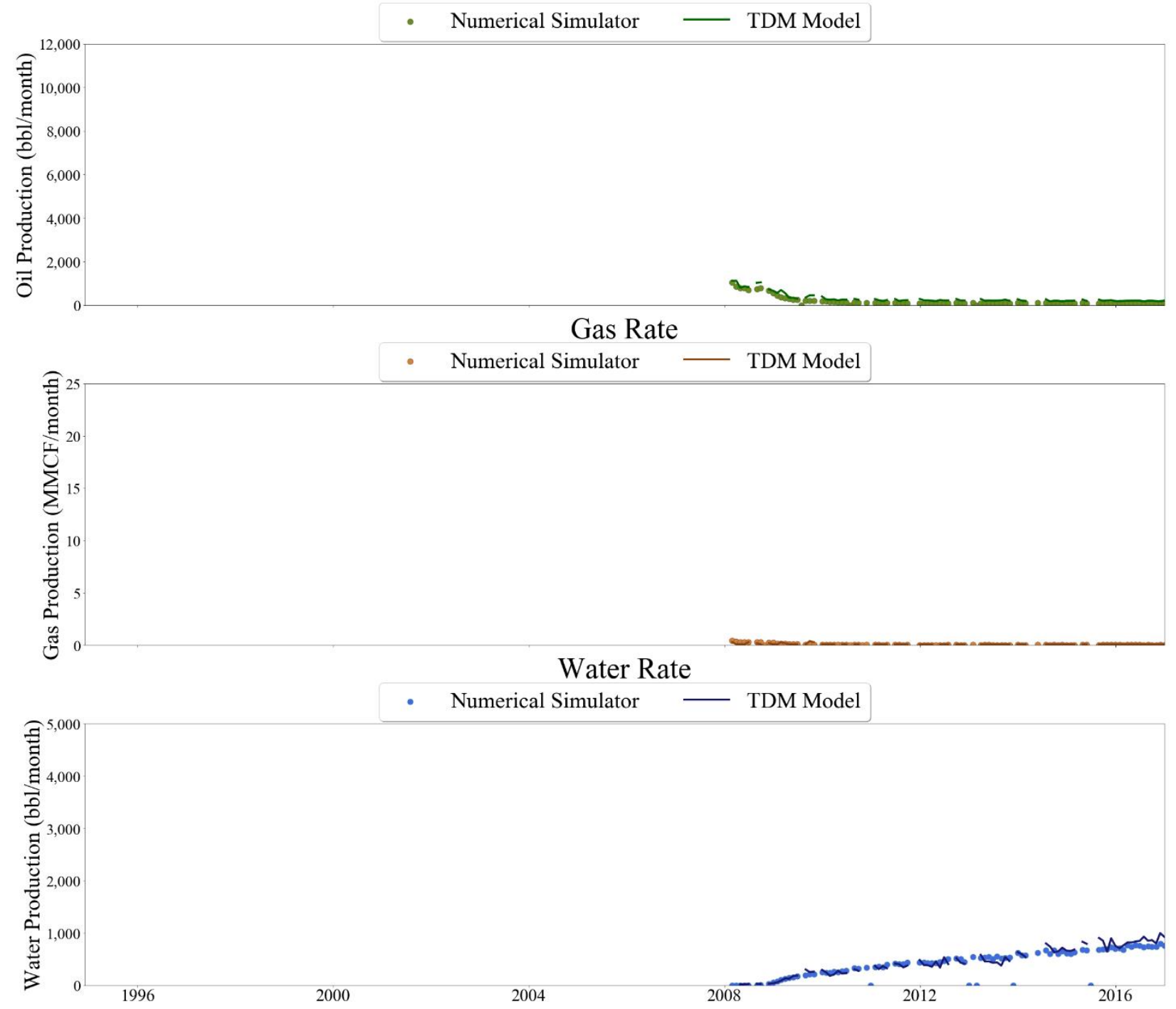

Figure 120 Well-044 oil, gas, and water rate TDM predictions vs actual simulation data plots for Random Partitioning-No Blind Validation TDM

Well-010 was categorized as being an average well because while it follows the trend of the actual data, the value of the predictions is slightly off in the oil and gas plots. Similar behavior can be seen for Well-047 where in the oil plot, the predictions are slightly above or below the actual values. Nineteen wells were characterized as having an average accuracy when comparing the TDM predictions vs the actual data which is about $33 \%$ of the total number of wells. 


\section{Oil Rate}

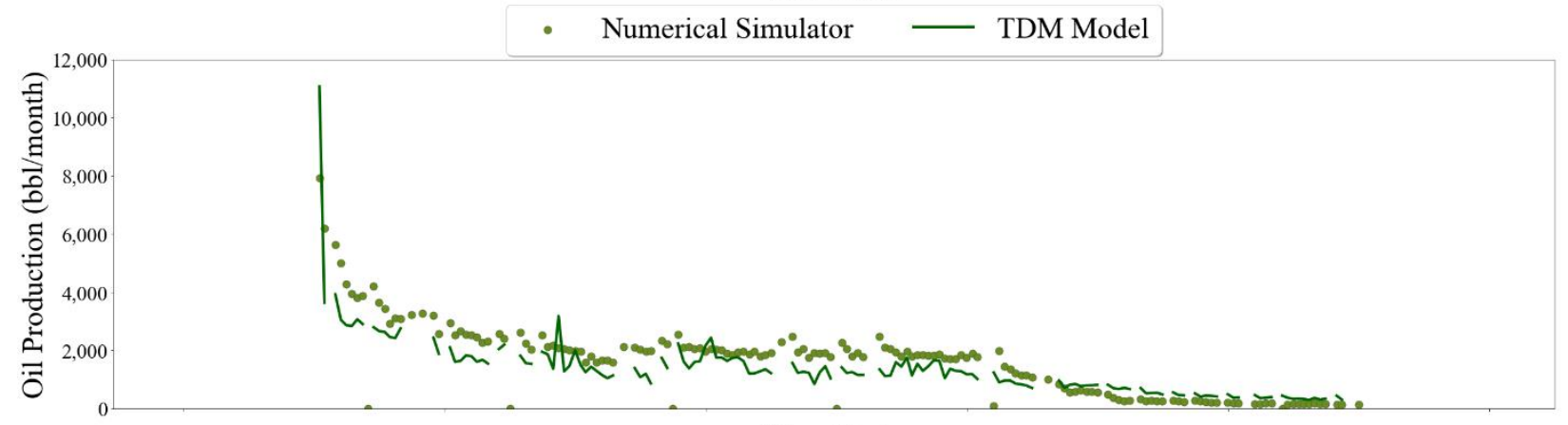

Gas Rate
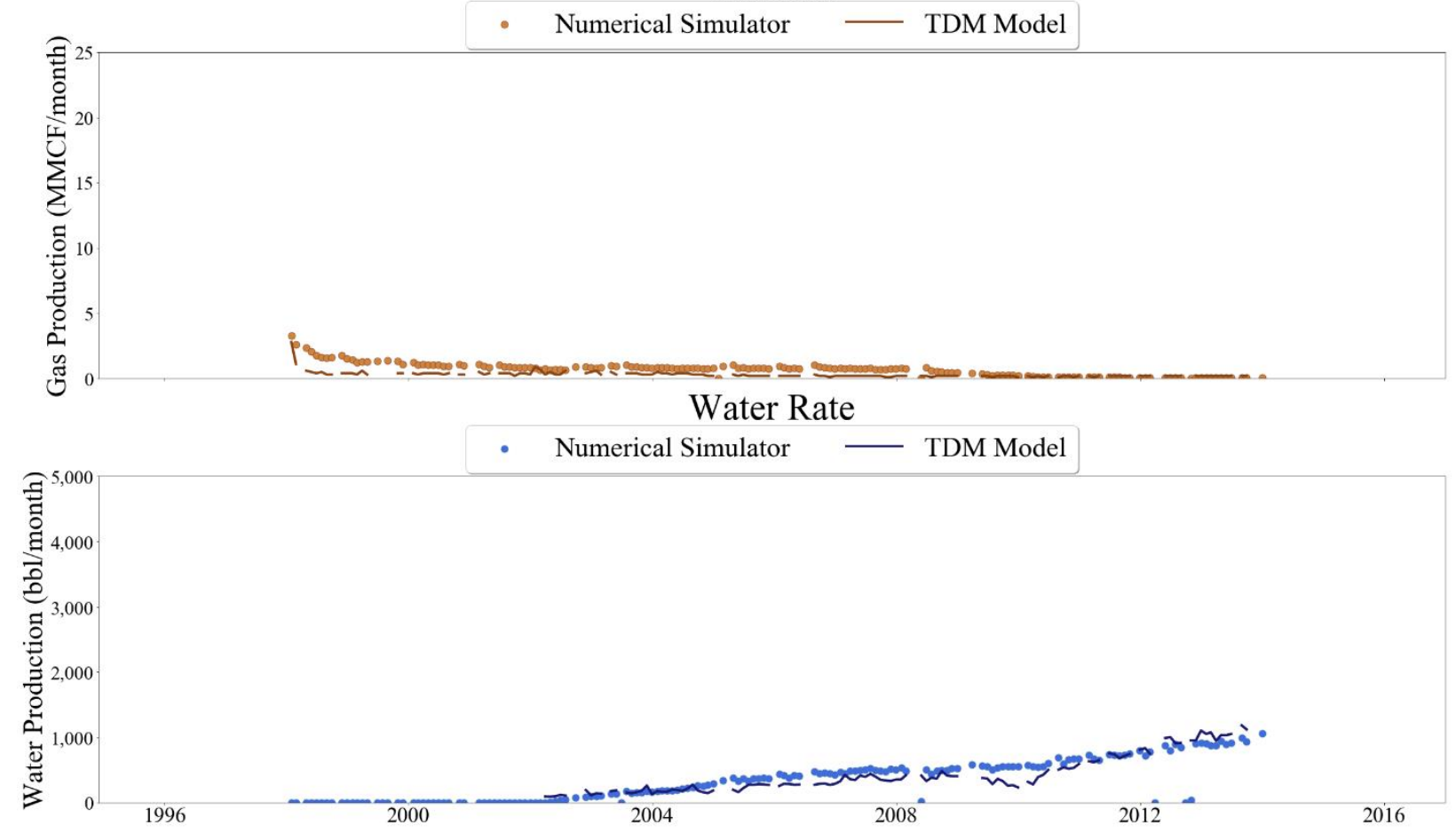

Figure 121 Well-010 oil, gas, and water rate TDM predictions vs actual simulation data plots for Random Partitioning-No Blind Validation TDM 


\section{Oil Rate}

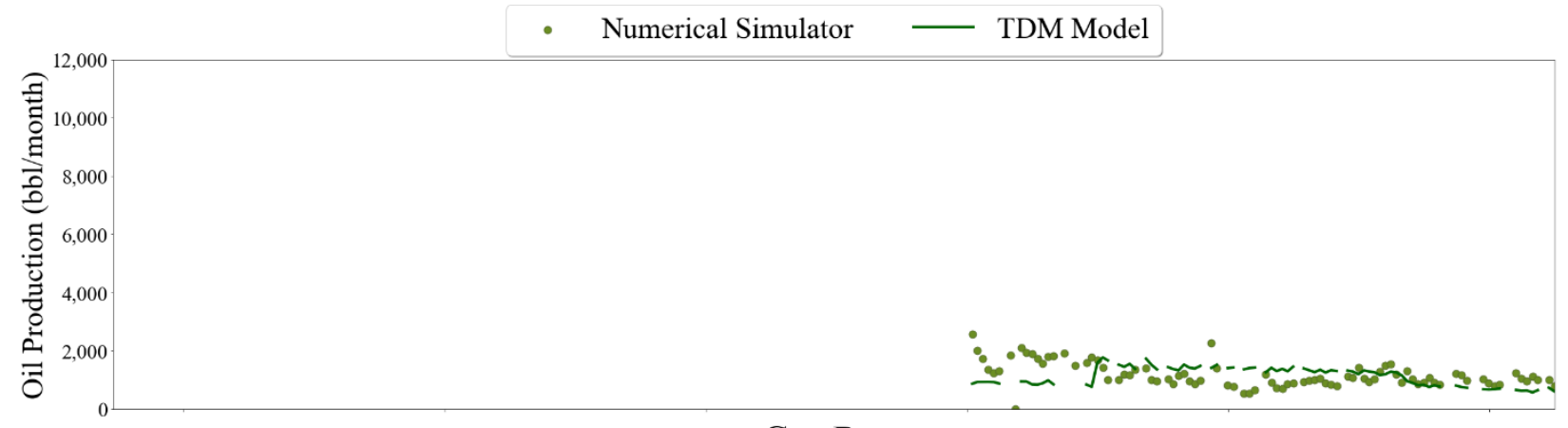

Gas Rate
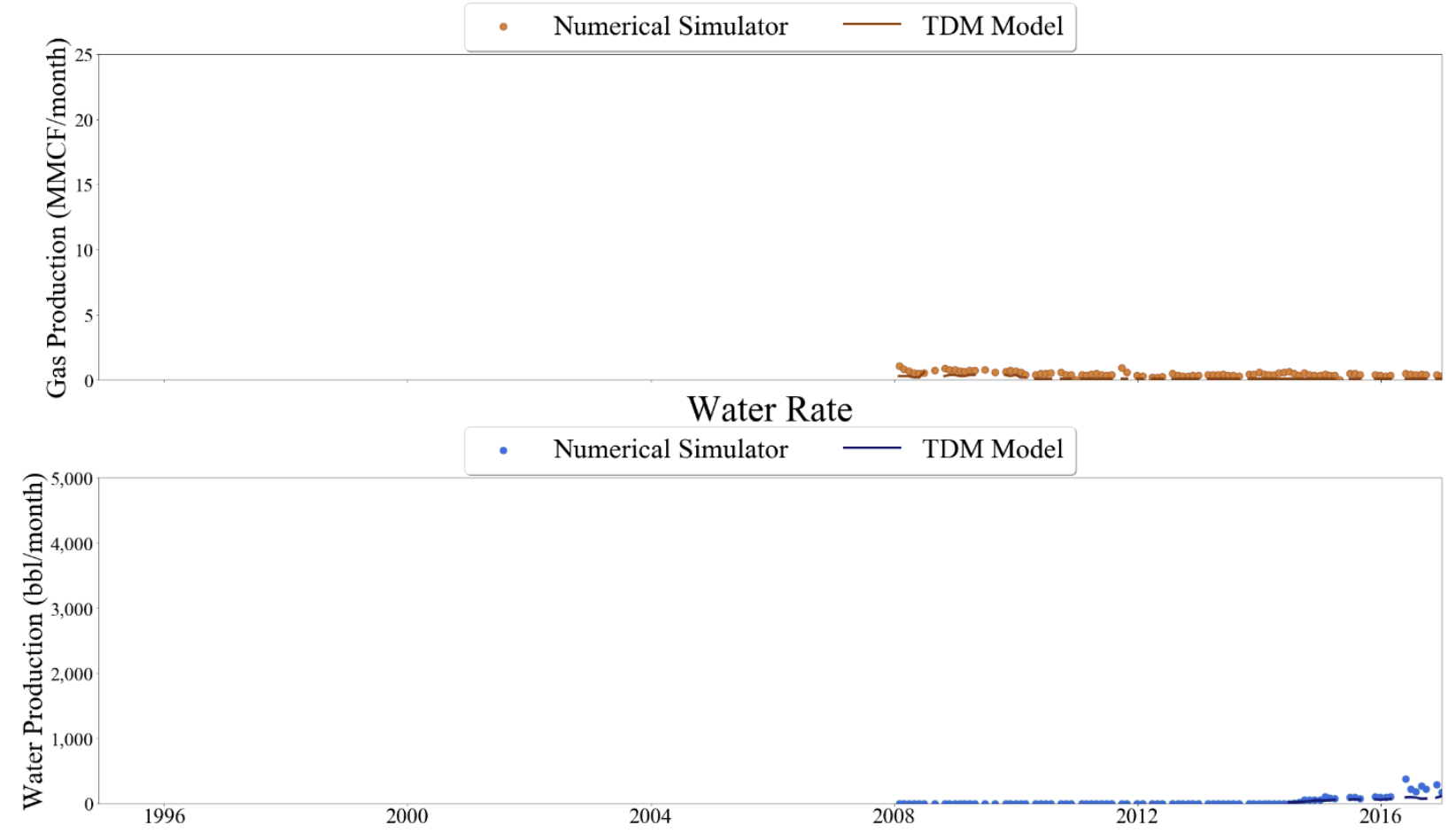

Figure 122 Well-047 oil, gas, and water rate TDM predictions vs actual simulation data plots for Random Partitioning-No Blind Validation TDM

Well-005 shown below, was deemed the only well to obtain truly poor results as clearly seen in the oil plot. While the overall decreasing trend is matched for half of the present timeline, the beginning portion of predicted results is too far from the actual results to be considered an average fit. This well was deemed poor solely for the oil plot because as seen, the water and gas production predictions were of acceptable accuracy. Because only one well had this behavior, only $1.7 \%$ of the total number of wells had a poor fit. All individual well production profiles can be found in the Appendix 8.2.1.1 in order of well number. 


\section{Oil Rate}
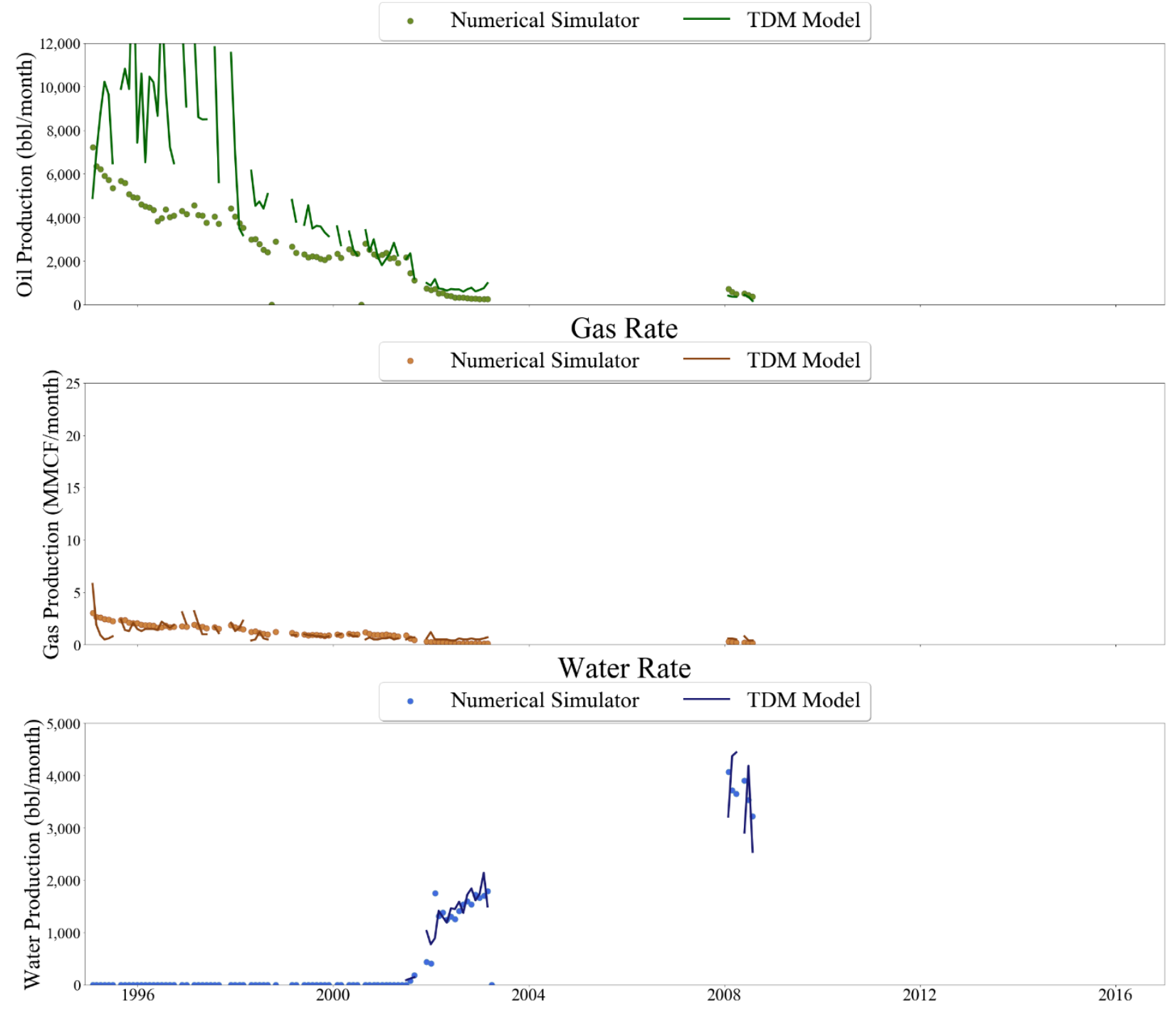

Figure 123 Well-005 oil, gas, and water rate TDM predictions vs actual simulation data plots for Random Partitioning-No Blind Validation TDM

Because an accurate model was able to be obtained with no blind validation with respect to the percentage of wells with good, poor, and average fits, the entire reservoir oil, gas, and water plot accuracies, and lastly, the reservoir pressure and water saturation heat maps; another TDM was built. The next Top Down Model was made with one year of blind validation which requires the use of MultiRandom Partitioning.

\subsubsection{Multi-Random Partitioning-1 Year Blind Validation}

The entire reservoir results are shown below for oil, gas, and water. The color scheme detailed in the previous section remains the same. The red line on the graph depicts where the training and calibration stops and where the one year of blind validation begins at December $31^{\text {st }}, 2015$. The accuracy of the Top Down Model is apparent in the plots, where the TDM prediction for cumulative water production slightly underestimates the actual cumulative water production. The explanations for the behavior of the actual 
data provided in the No Blind Validation case holds true for all Top Down Models presented in the results as the actual data from the numerical reservoir simulator does not change.

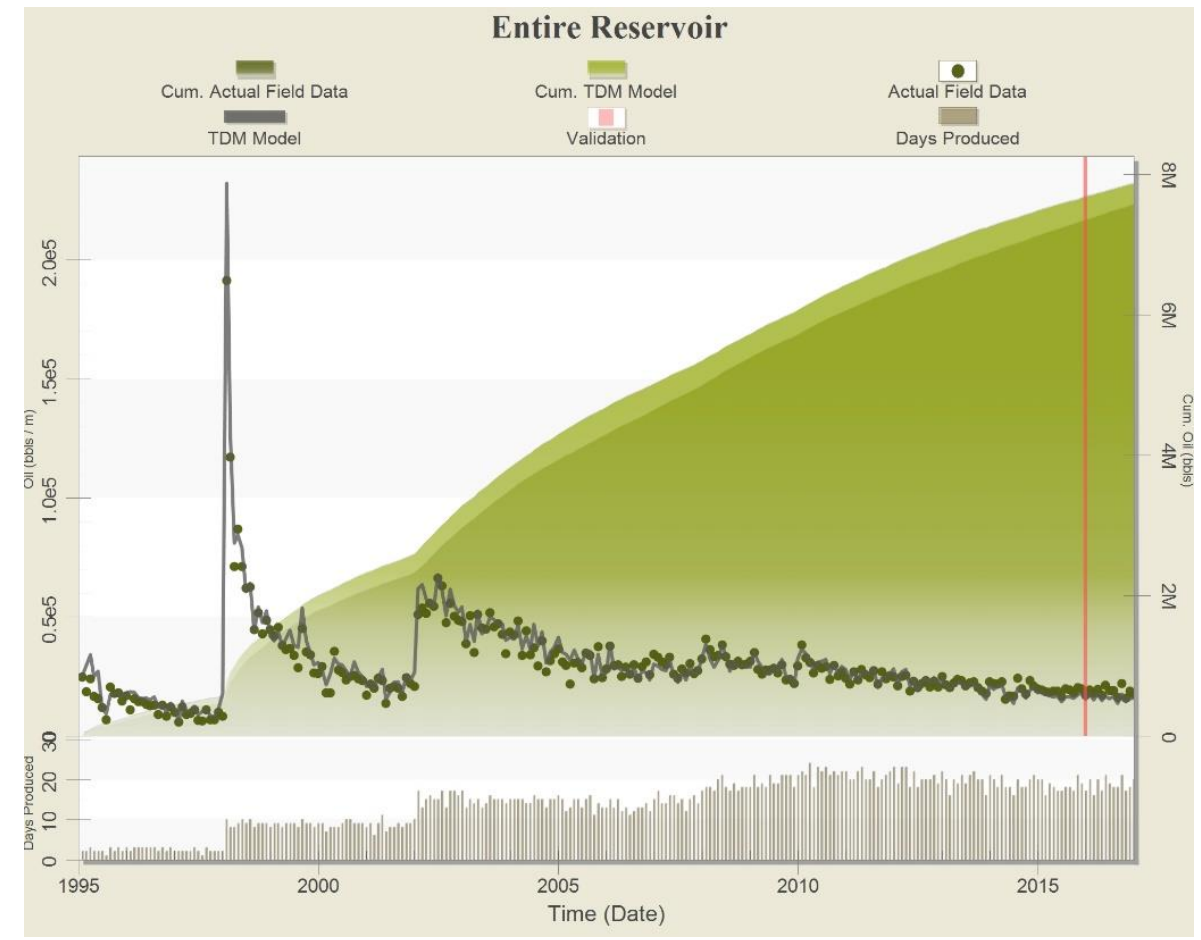

Figure 124 Entire reservoir oil results for Multi-Random Partitioning- 1 Year Blind Validation Top Down Model

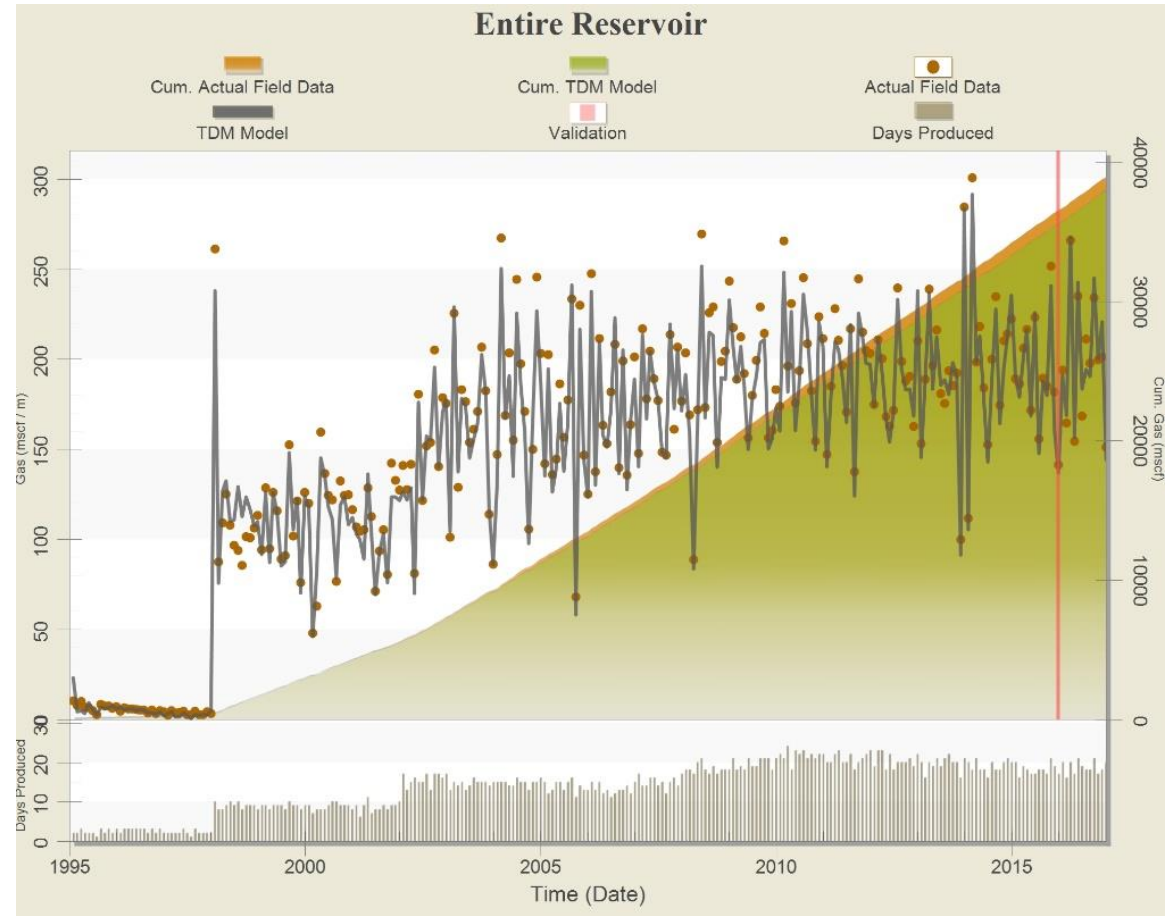

Figure 125 Entire reservoir gas results for Multi-Random Partitioning-2 Year Blind Validation Top Down Model 


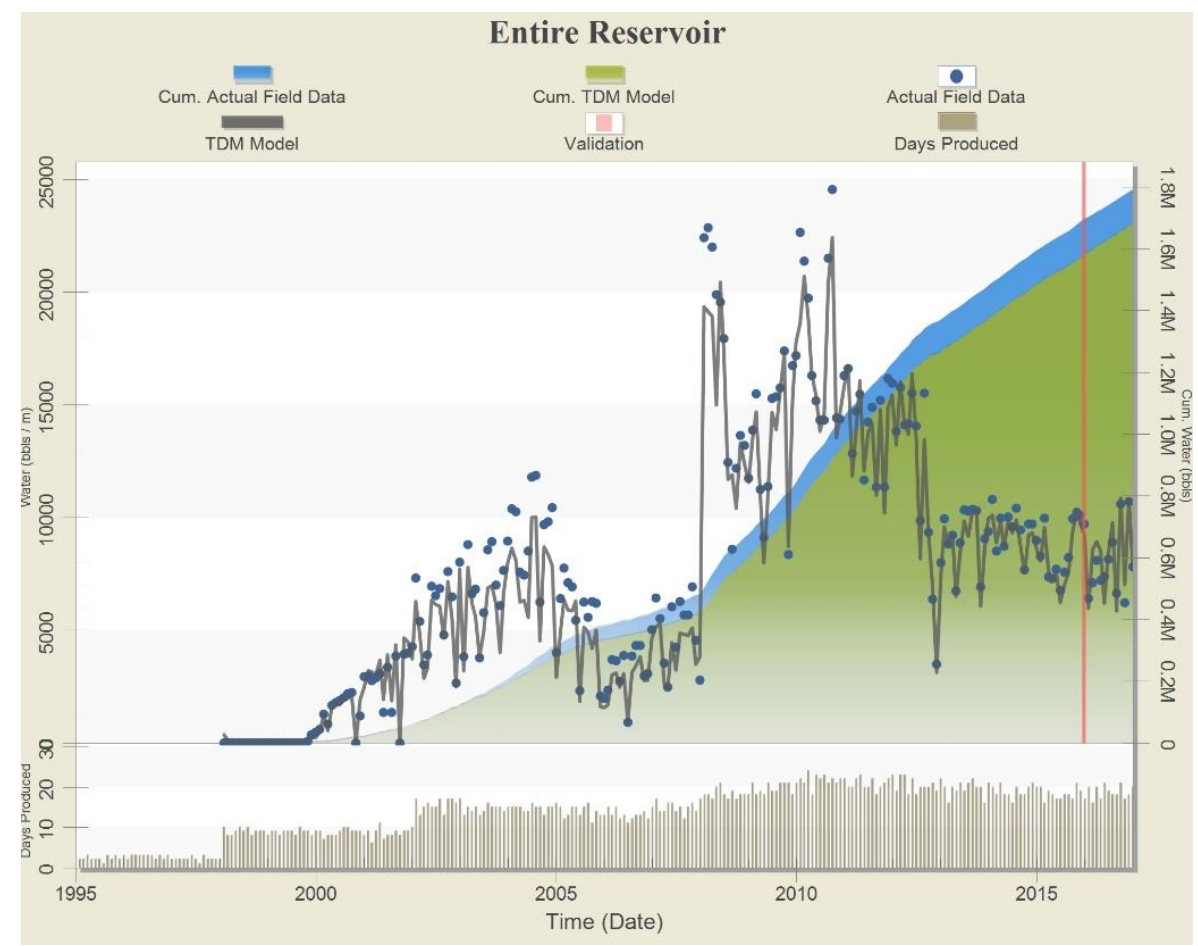

Figure 126 Entire reservoir water results for Multi-Random Partitioning-1 Year Blind Validation Top Down Model

The entire reservoir heat maps are shown for water saturation and reservoir pressure at two dates, one at the start of the one year blind validation, and one mid-way through the blind validation. The error between the numerical simulation and the Top Down Model is shown on the third plot where dark red is again showing an error of $30+\%$. While there is very little error on the reservoir pressure plots, there is some error within the water saturation plots. The error is believed to be in a few data points that are then propagated to be larger due to the interpolation between points to fill in the heat map. The error could also be partially attributed to the method used to fill in values for the well when no prediction value was calculated. The water saturation was not predicted within the software at times when the well was shut-in for the entire month and therefore a feed forward technique was used to fill in the empty predictions using the previously predicted values. When comparing these predicted values with the simulation values, especially in instances when wells were shut-in for two or more consecutive months, this could be a likely source for some of the error generated. This is one area of improvement suggested for future investigation that holds true for the remaining Top Down Models presented in this thesis. The error in the water saturation maps could also be due to the length of the well's life, thus affecting the quantity of data able to be learned from before that time step, in addition to the well's location in the reservoir. With all of this said, the water saturation heat maps were still found to be acceptable. More heat maps for both attributes are included in Appendix 8.2.2.2 in two month intervals for the 1 year of blind validation. 
Reservoir Pressure (psi) at 2015-12-31
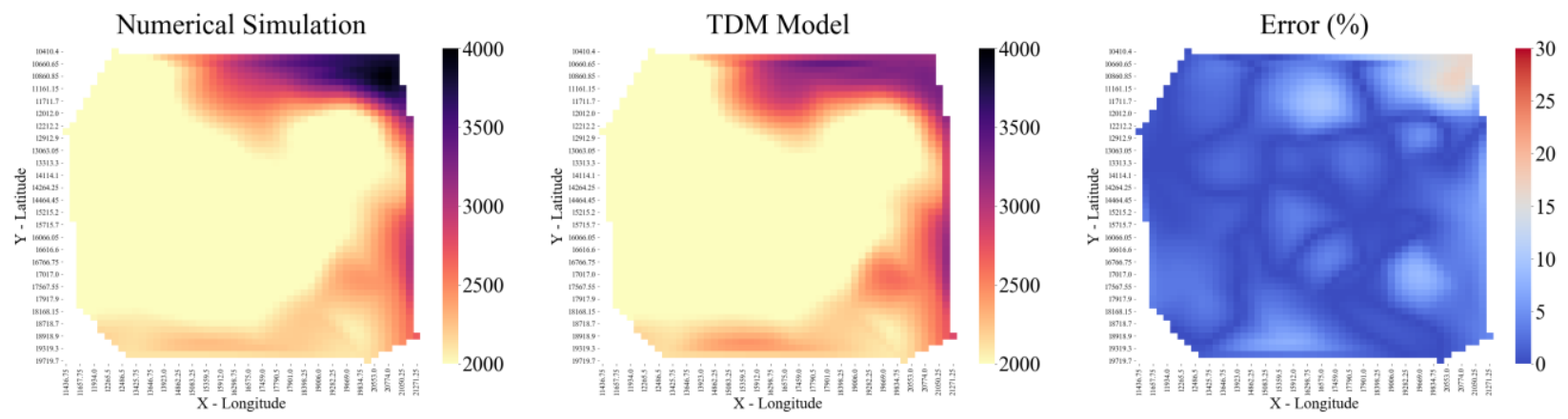

Figure 127 Entire reservoir heat map for reservoir pressure at December 31st, 2015 for 1 Year Blind Validation TDM

Reservoir Pressure (psi) at 2016-06-30
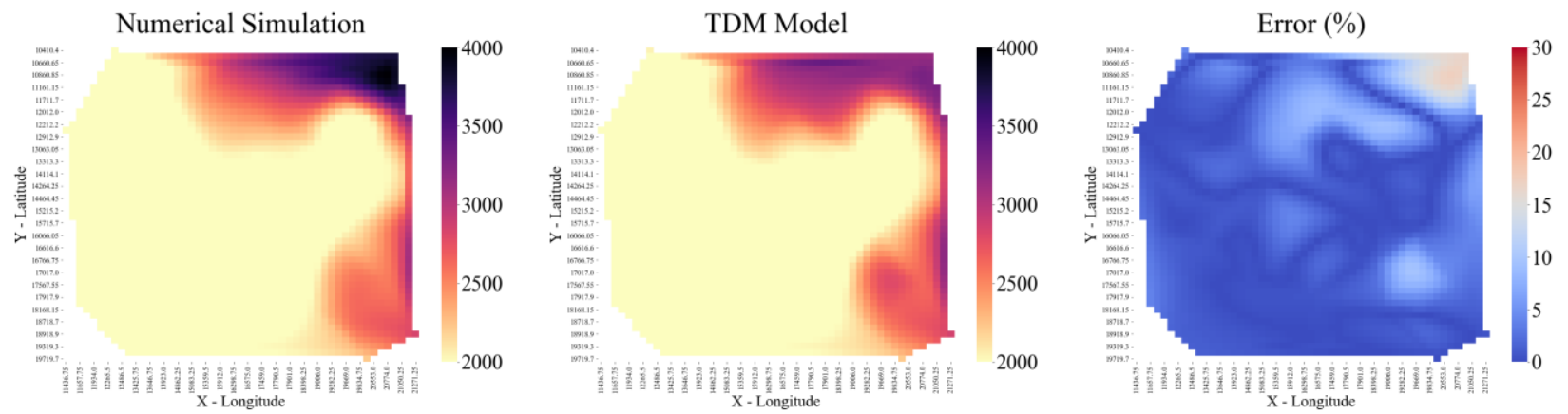

Figure 128 Entire reservoir heat map for reservoir pressure at June 30th, 2016 for 1 Year Blind Validation TDM

\section{Water Saturation (\%) at 2015-12-31}
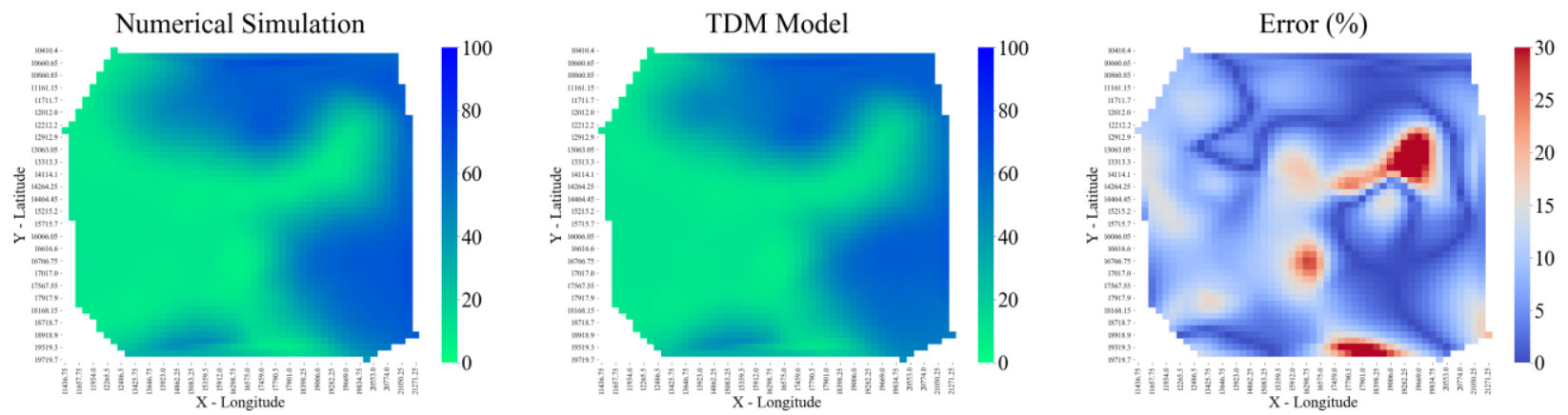

Figure 129 Entire reservoir heat map for water saturation at December 31st, 2015 for 1 Year Blind Validation TDM 
Water Saturation (\%) at 2016-06-30
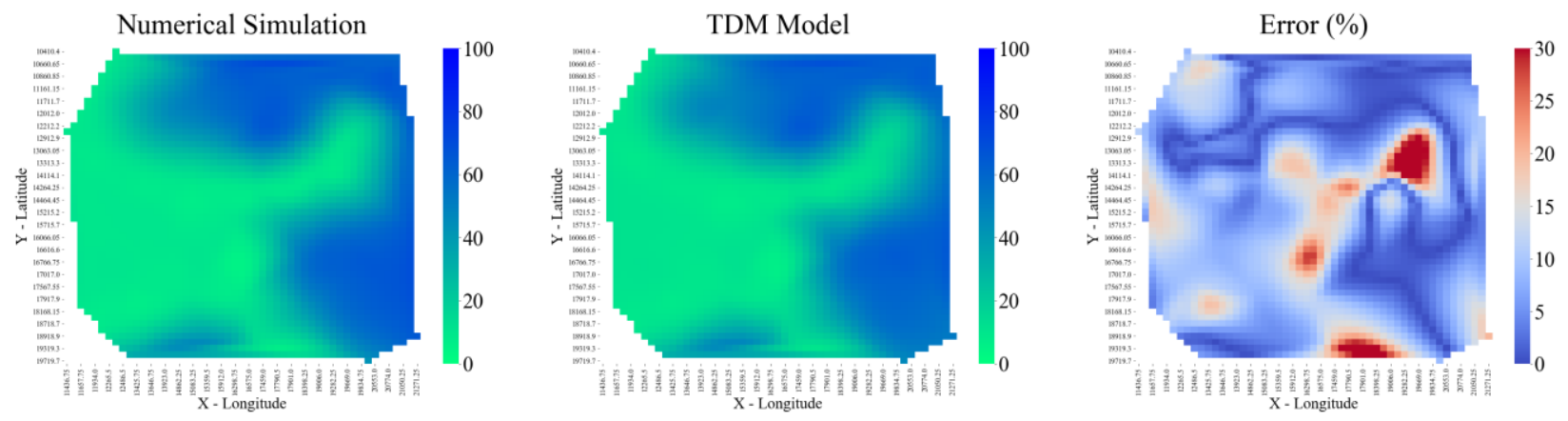

Figure 130 Entire reservoir heat map for water saturation at June 30th, 2016 for 1 Year Blind Validation TDM

Two examples are again displayed for good, average, and poor fits, in that order. The red line, similar to the entire reservoir production plots, show where the one year of blind validation begins. Well015 and Well-040 are two examples of wells with good predictions. Thirty-four wells were deemed to have good predictions which is $60 \%$ of the wells. 

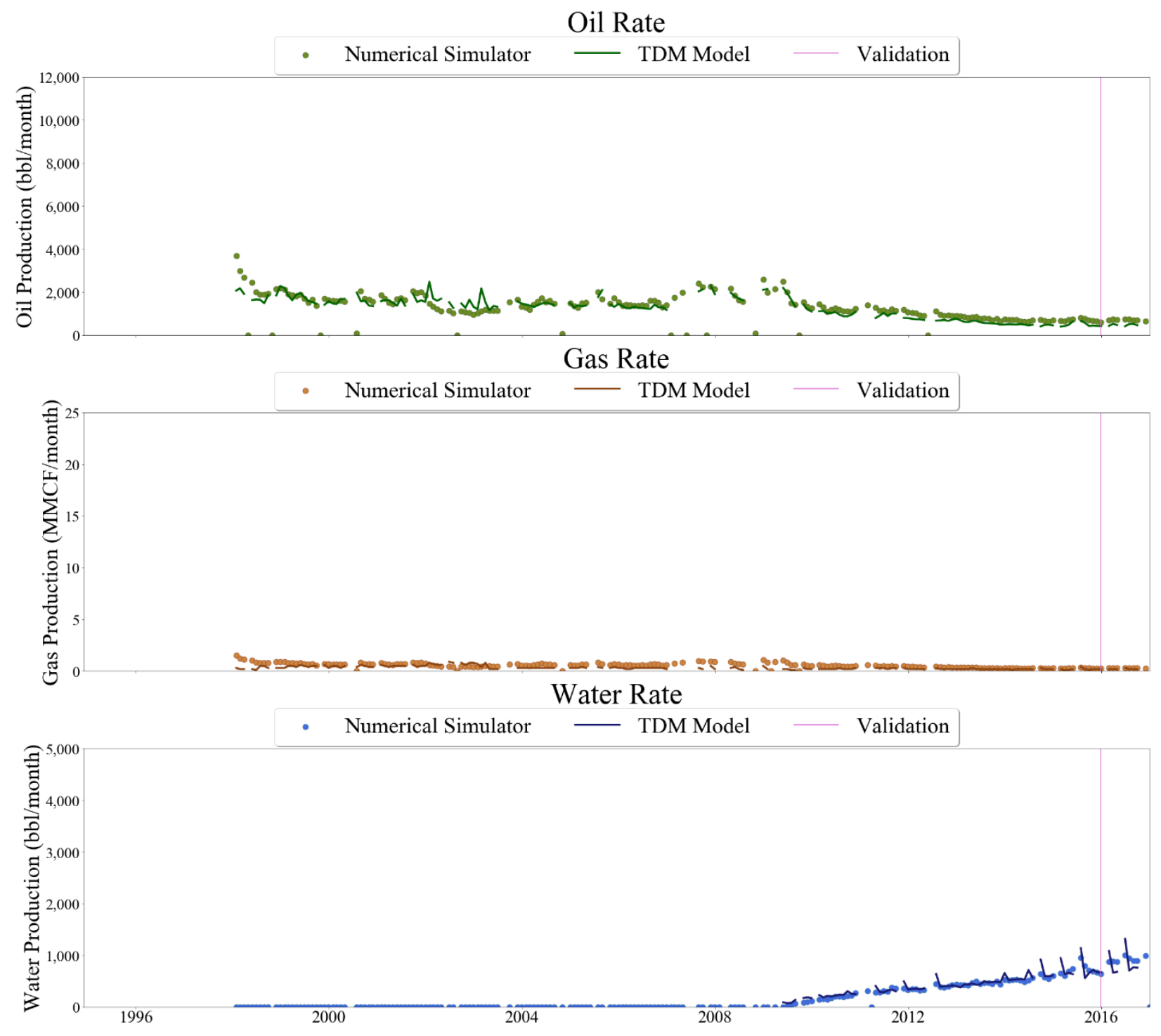

Figure 131 Well-015 oil, gas, and water rate TDM predictions vs actual simulation data plots for 1 Year Blind Validation TDM 


\section{Oil Rate}
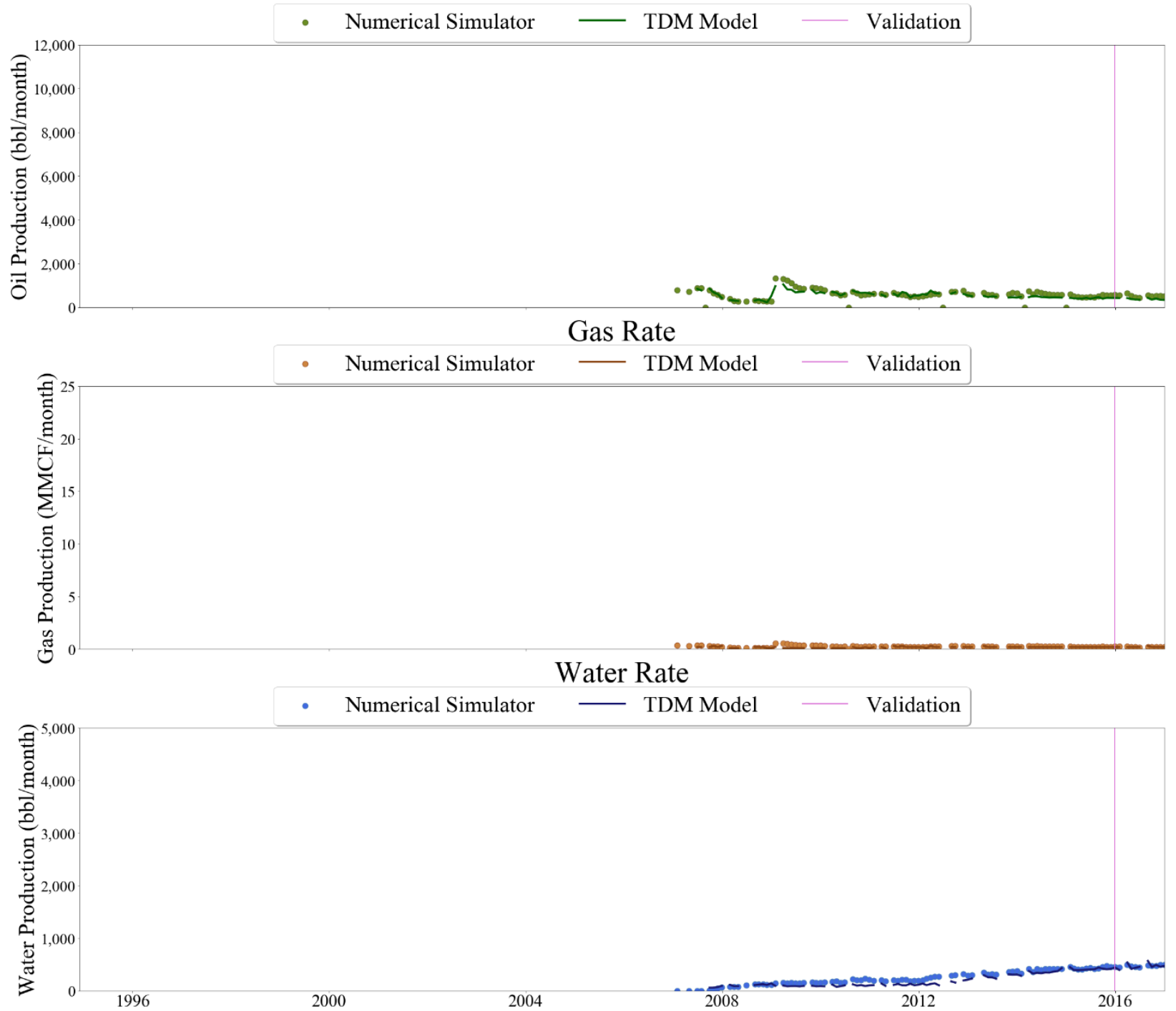

Figure 132 Well-040 oil, gas, and water rate TDM predictions vs actual simulation data plots for 1 Year Blind Validation TDM

Well-003 and Well-053 are two examples showing average accuracy when comparing the predictions with the actual data. For Well-003, the predictions are slightly above or below the actual values in the oil and gas plots where little to no water is produced, making that prediction easy to get. Well-053 was determined to be average due to the slight difference in the water plot. Overall, nineteen wells had an average accuracy which was about $33 \%$ of all the wells. 

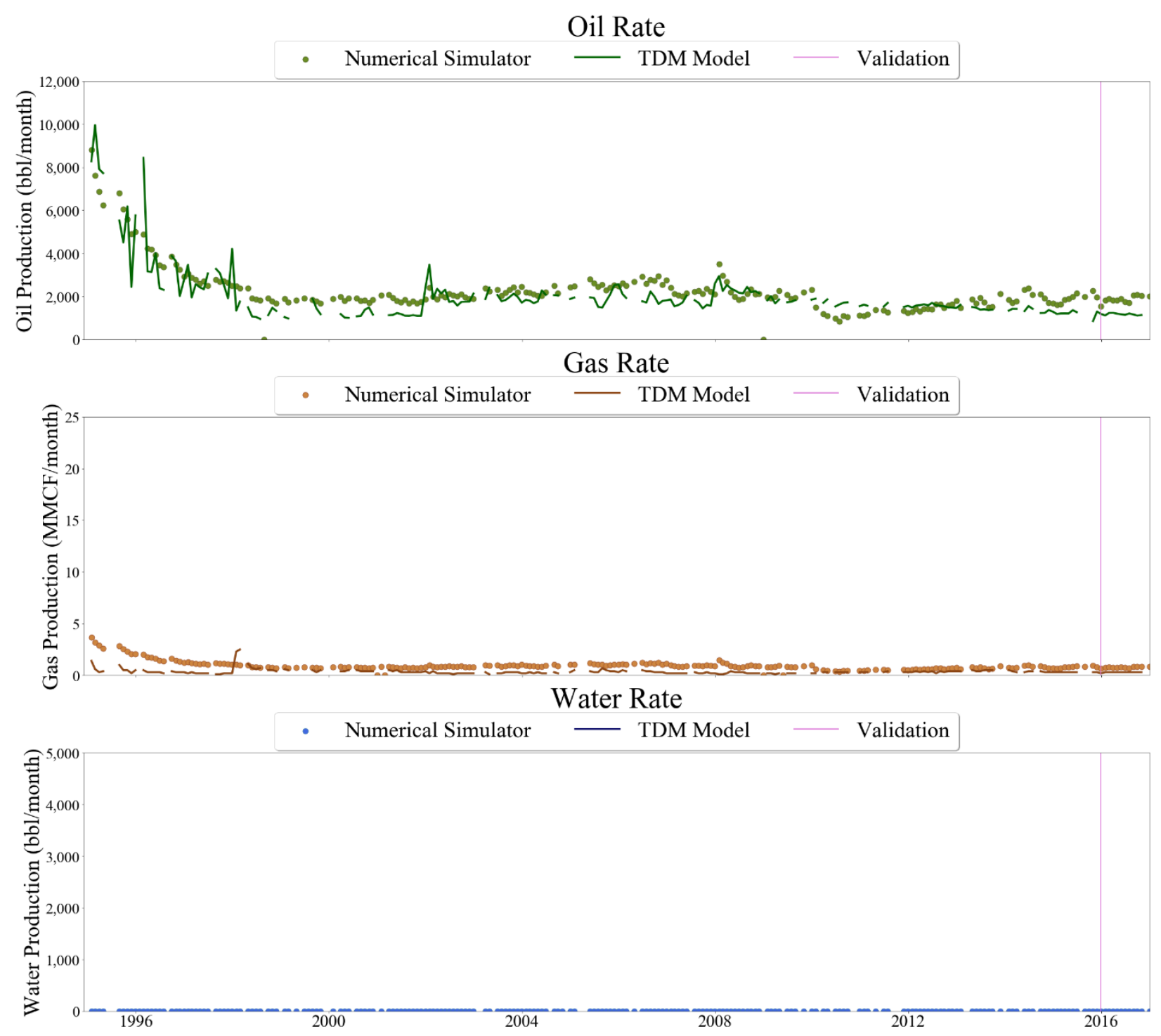

Figure 133 Well-003 oil, gas, and water rate TDM predictions vs actual simulation data plots for 1 Year Blind Validation TDM 


\section{Oil Rate}
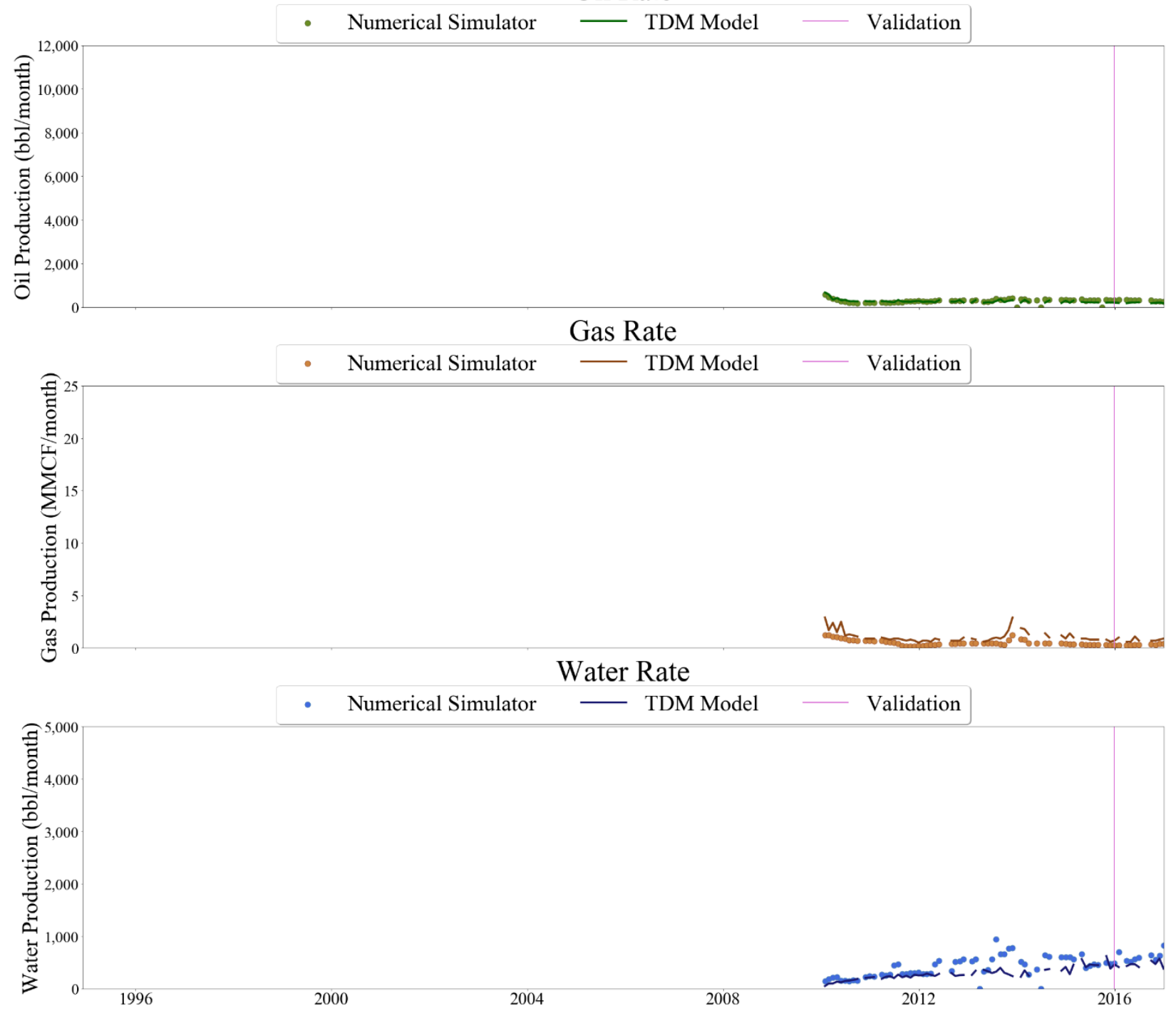

Figure 134 Well-053 oil, gas, and water rate TDM predictions vs actual simulation data plots for 1 Year Blind Validation TDM

Only four wells or 7\% of the wells had a poor fit as seen below for Well-014 and Well-021.

While the oil plot for Well-014 is accurate, the beginning part of the gas and the latter portion of the water plots are not close in value even though the general trend of the data is followed. For Well-021, the gas plot trend is not followed by the prediction. The predicted values are rather constant where a downward then upward trend is seen in the actual data. All of the individual well production plots can be found in Appendix 8.2.2.1, sorted by well number. 


\section{Oil Rate}

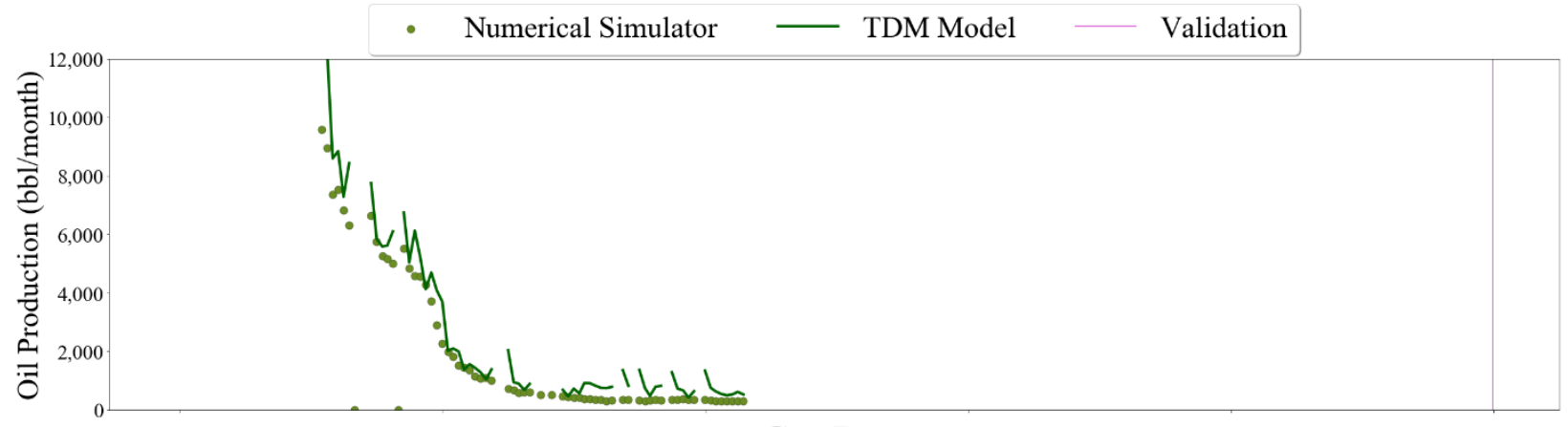

Gas Rate
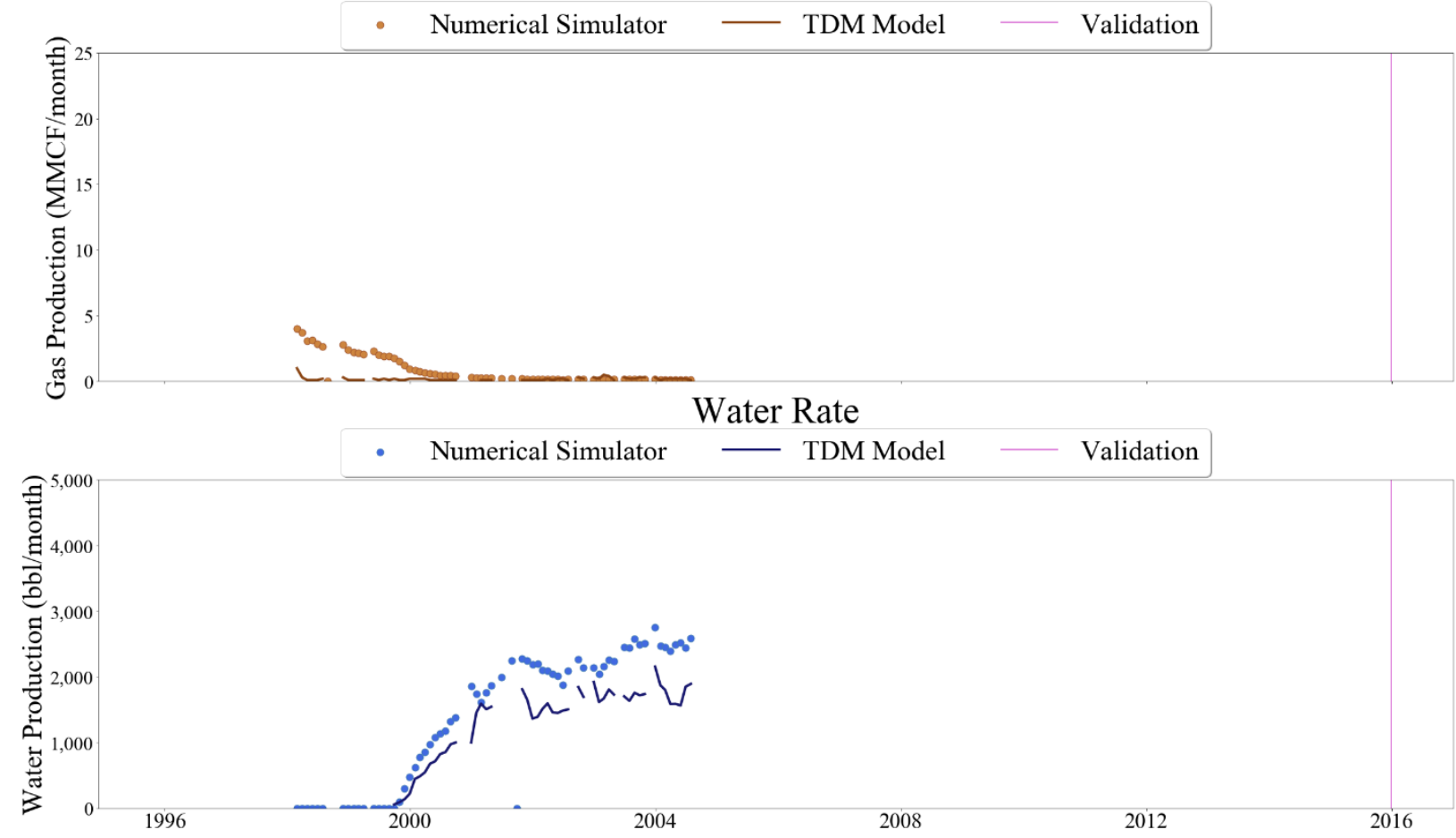

Figure 135 Well-014 oil, gas, and water rate TDM predictions vs actual simulation data plots for 1 Year Blind Validation TDM 

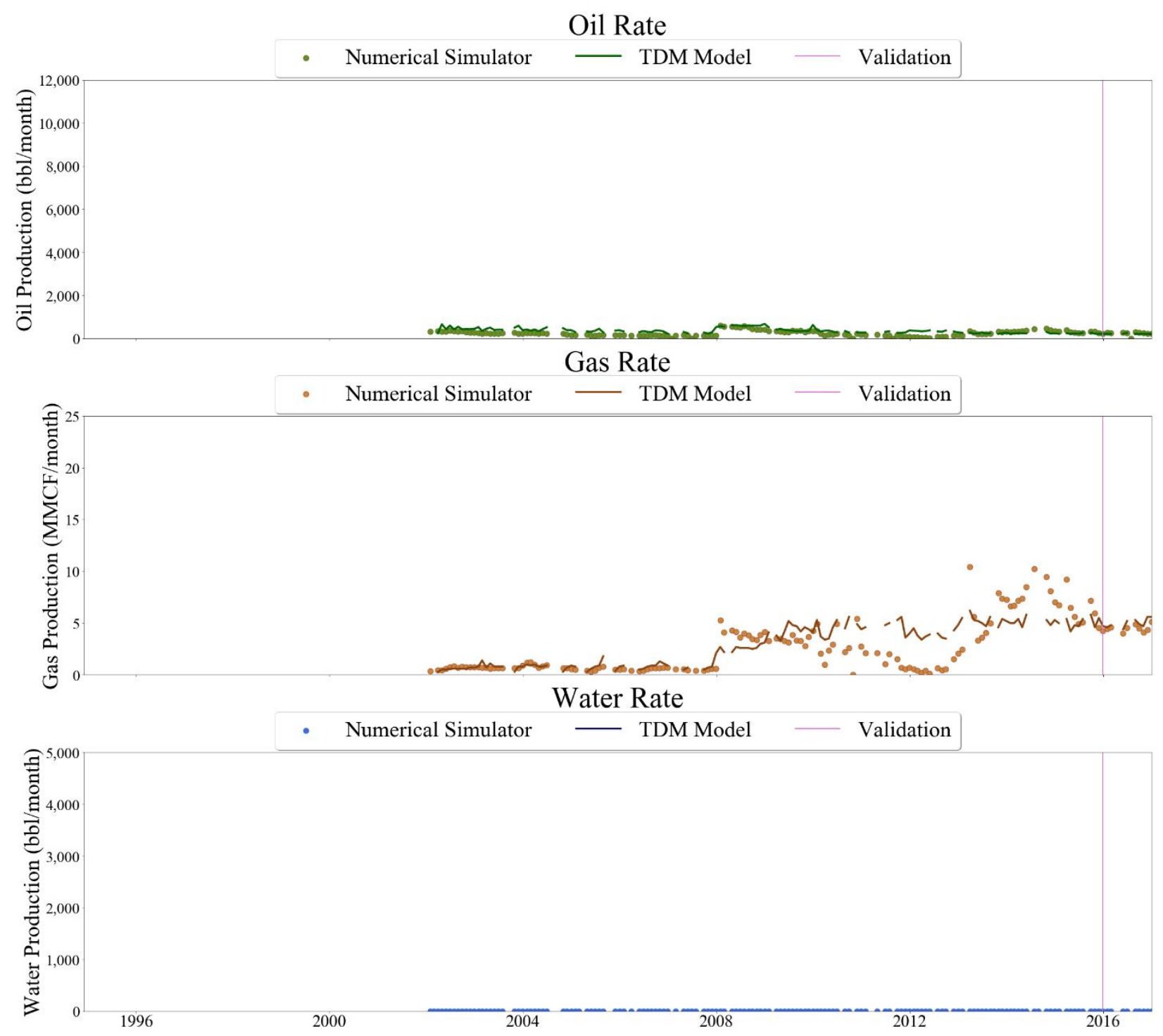

Figure 136 Well-021 oil, gas, and water rate TDM predictions vs actual simulation data plots for 1 Year Blind Validation TDM

The above results for the Multi-Random Partitioning- 1 Year Blind Validation were deemed of sufficient quality. Therefore another Top Down Model with two years of blind validation was created in order to determine if good results could also be achieved through that model.

\subsubsection{Multi-Random Partitioning-2 Year Blind Validation}

The oil, gas, and water results for the entire reservoir in the Multi-Random Partitioning- 2 Year Blind Validation Top Down Model are in Figure 137Figure 137, Figure 138, and Figure 139 respectively. The accurate match of the predictions vs the simulation data can be seen in all three plots. Again, the cumulative water prediction slightly under predicts the actual cumulative water production. The red line at December 31 $1^{\text {st }}, 2014$ shows the start of the two years of blind validation until December $31^{\text {st }}, 2016$. 


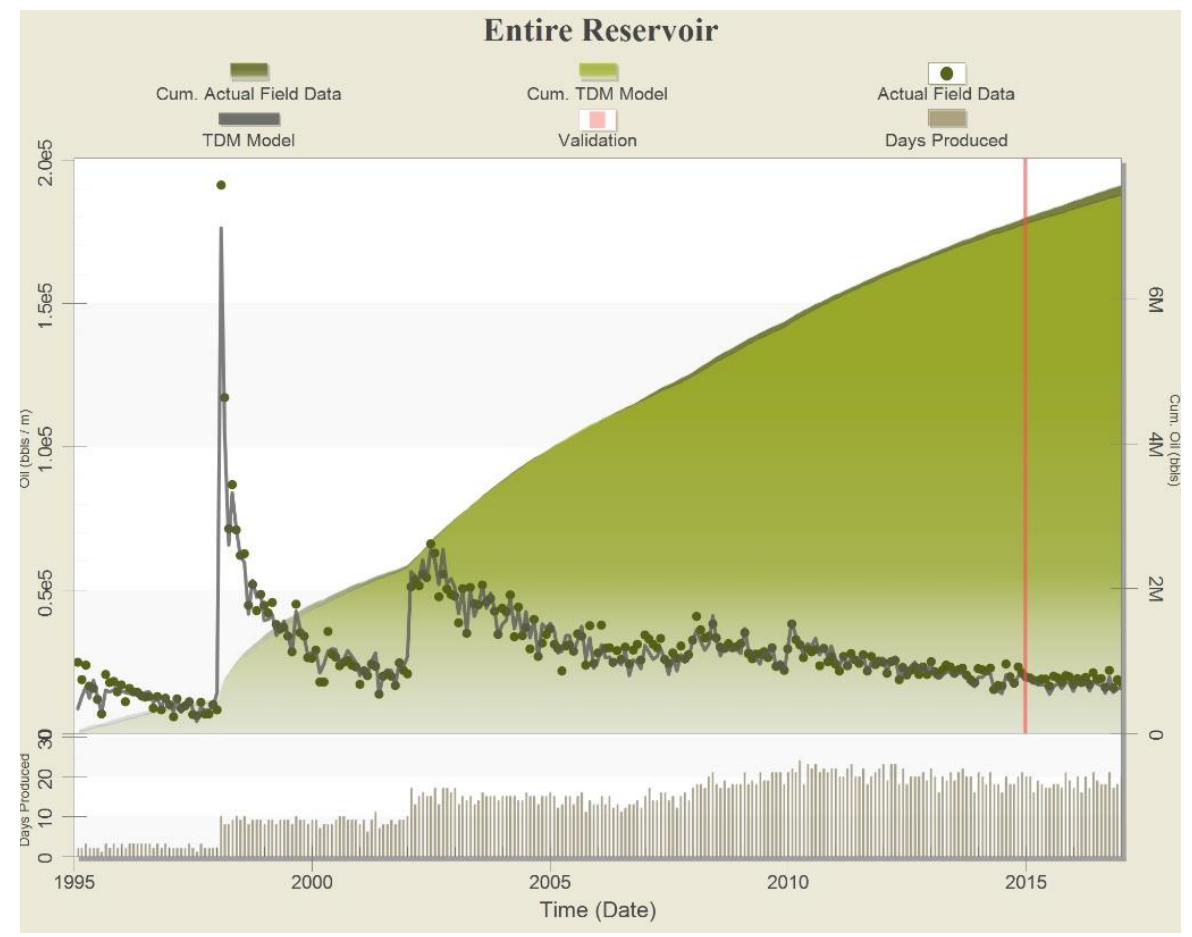

Figure 137 Entire reservoir oil results for Multi-Random Partitioning-2 Year Blind Validation Top Down Model

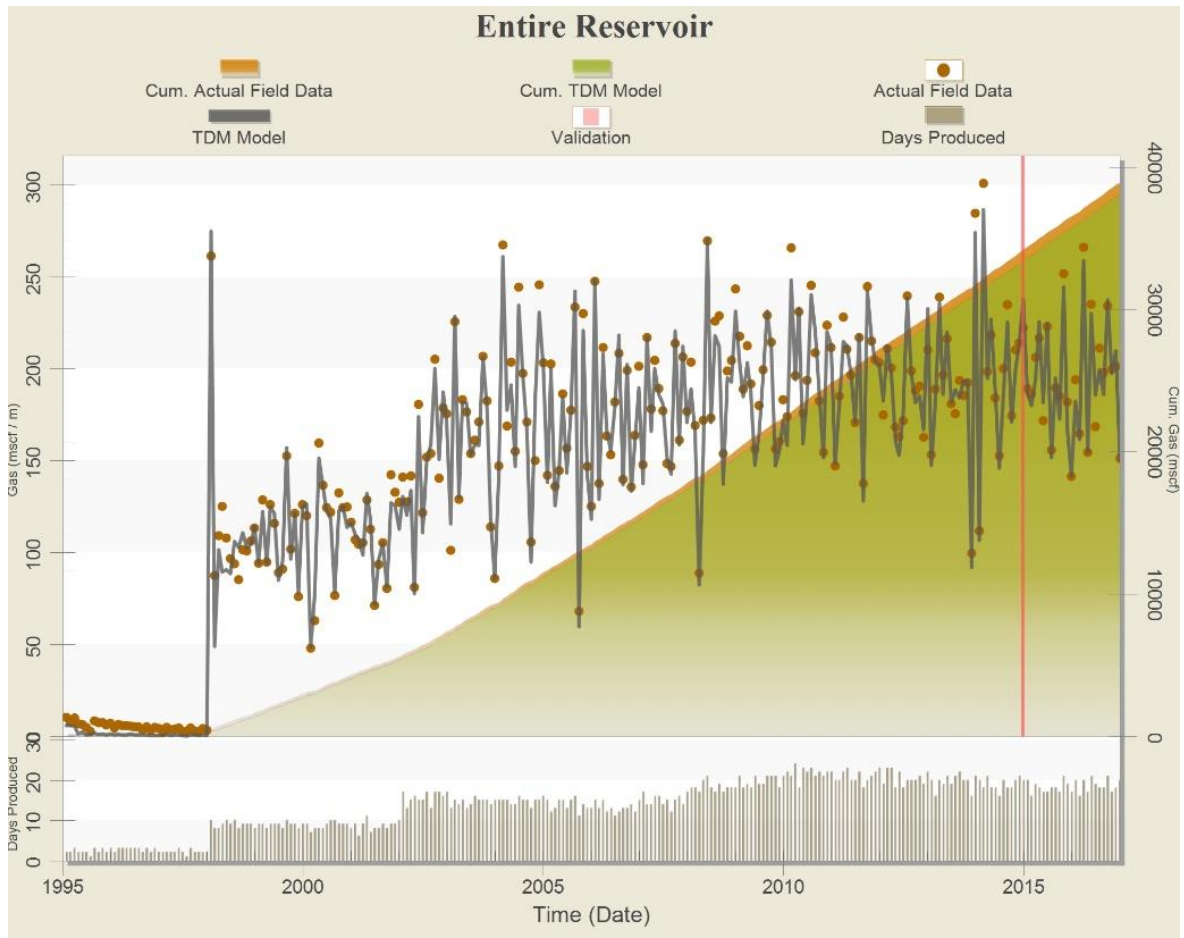

Figure 138 Entire reservoir gas results for Multi-Random Partitioning- 2 Year Blind Validation Top Down Model 


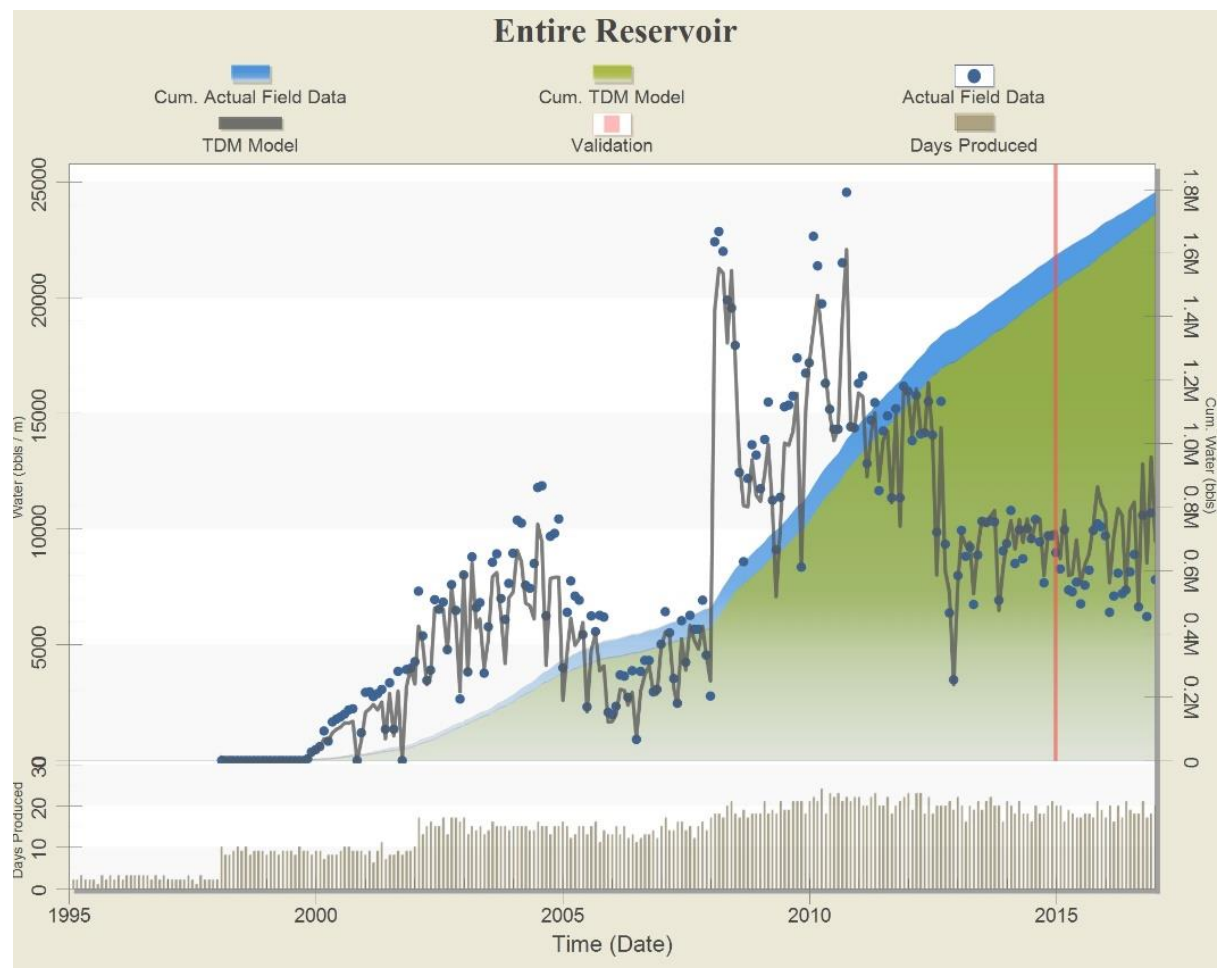

Figure 139 Entire reservoir water results for Multi-Random Partitioning-2 Year Blind Validation Top Down Model

The heat maps for water saturation and reservoir pressure are shown below for the 2 Year Blind Validation TDM. The plots are shown in 6 month increments from the start of the blind validation at December $31^{\text {st }}, 2014$ to December $31^{\text {st }}, 2016$. Additional heat maps are shown in the Appendix 8.2.3.2 in 3 month increments for both water saturation and reservoir pressure. The previous explanation for the water saturation error can also be applied here.

Reservoir Pressure (psi) at 2014-12-31
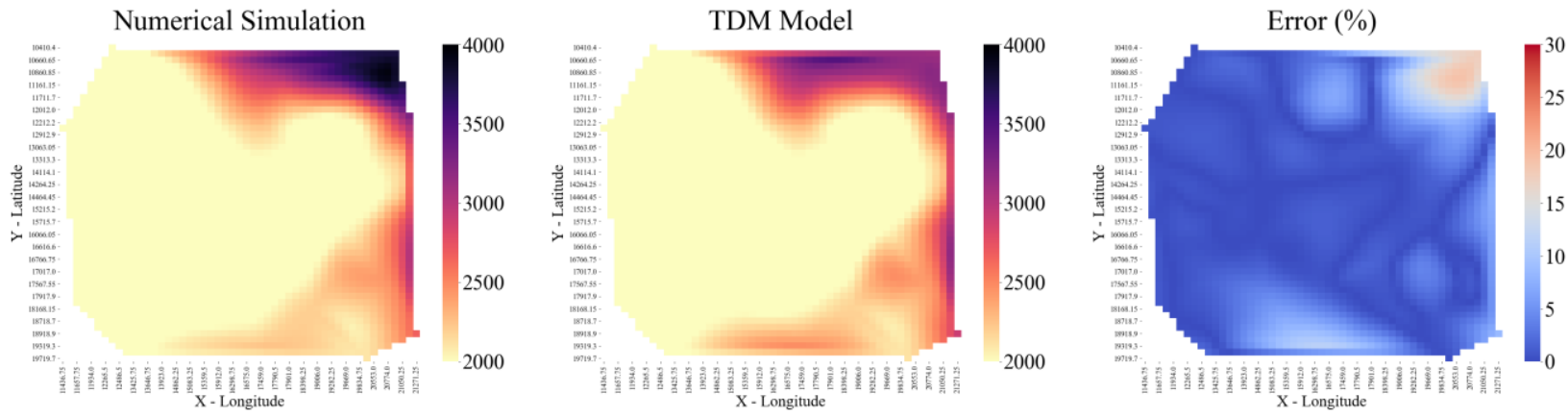

Figure 140 Entire reservoir heat map for reservoir pressure at December 31st, 2014 for 2 Year Blind Validation TDM 
Reservoir Pressure (psi) at 2015-06-30
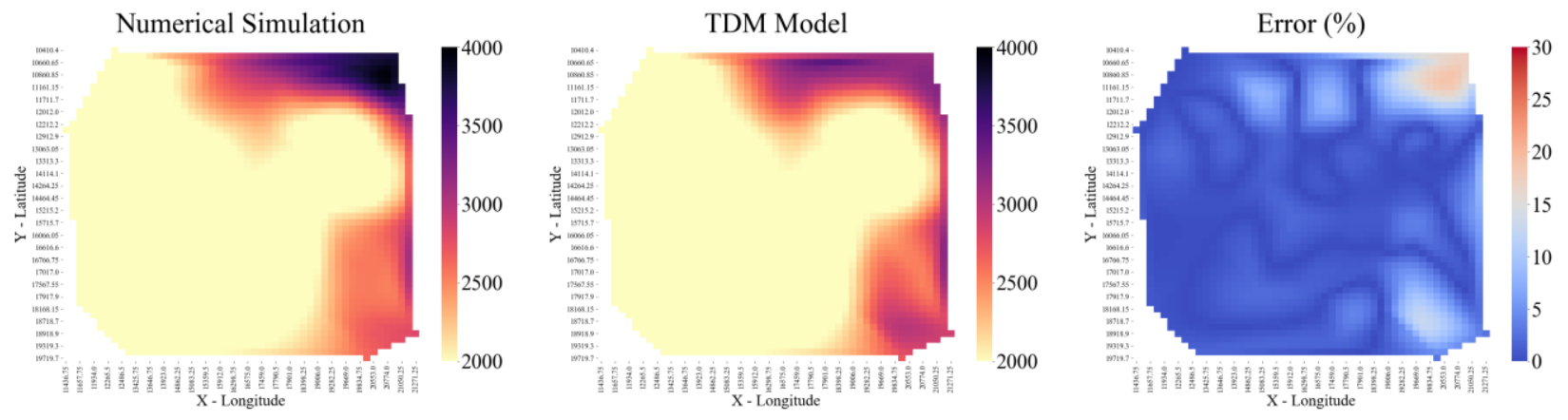

Figure 141 Entire reservoir heat map for reservoir pressure at June 30th, 2015 for 2 Year Blind Validation TDM

Reservoir Pressure (psi) at 2015-12-31
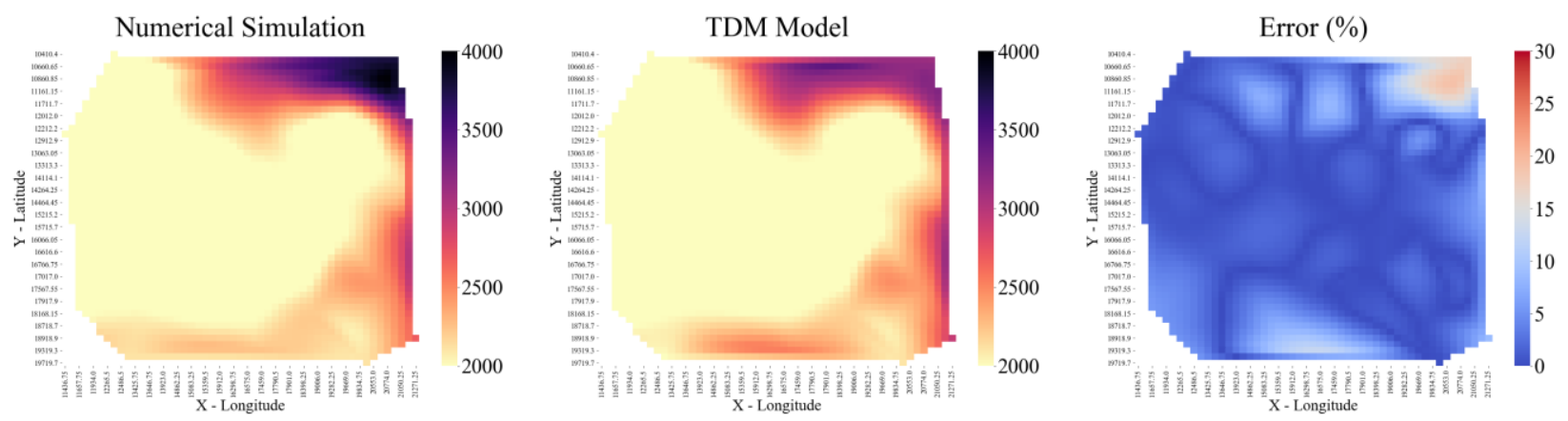

Figure 142 Entire reservoir heat map for reservoir pressure at December 31st, 2015 for 2 Year Blind Validation TDM

Reservoir Pressure (psi) at 2016-06-30
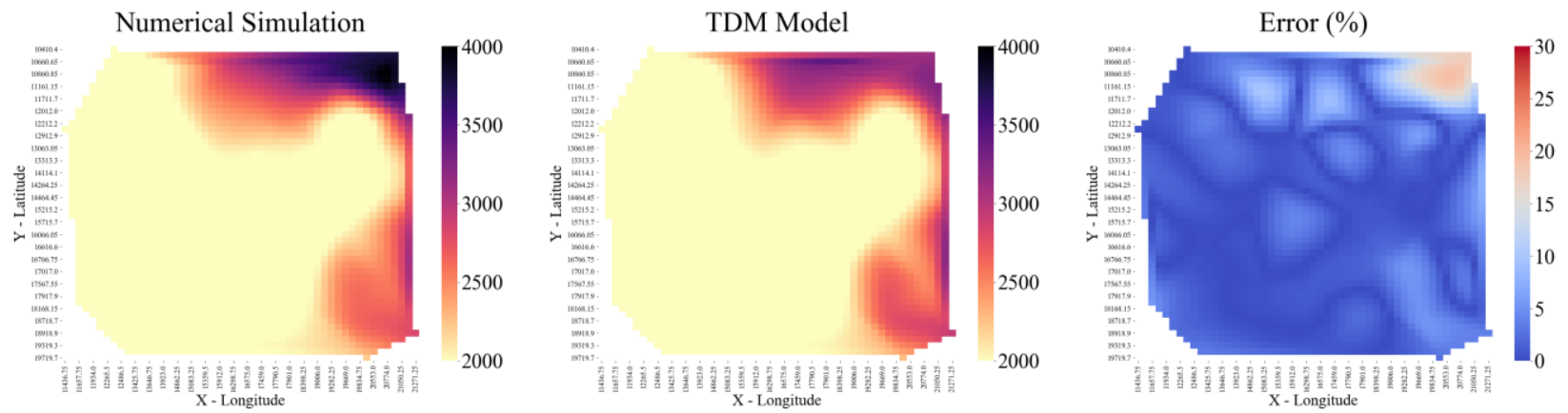

Figure 143 Entire reservoir heat map for reservoir pressure at June 30th, 2016 for 2 Year Blind Validation TDM 
Reservoir Pressure (psi) at 2016-12-31
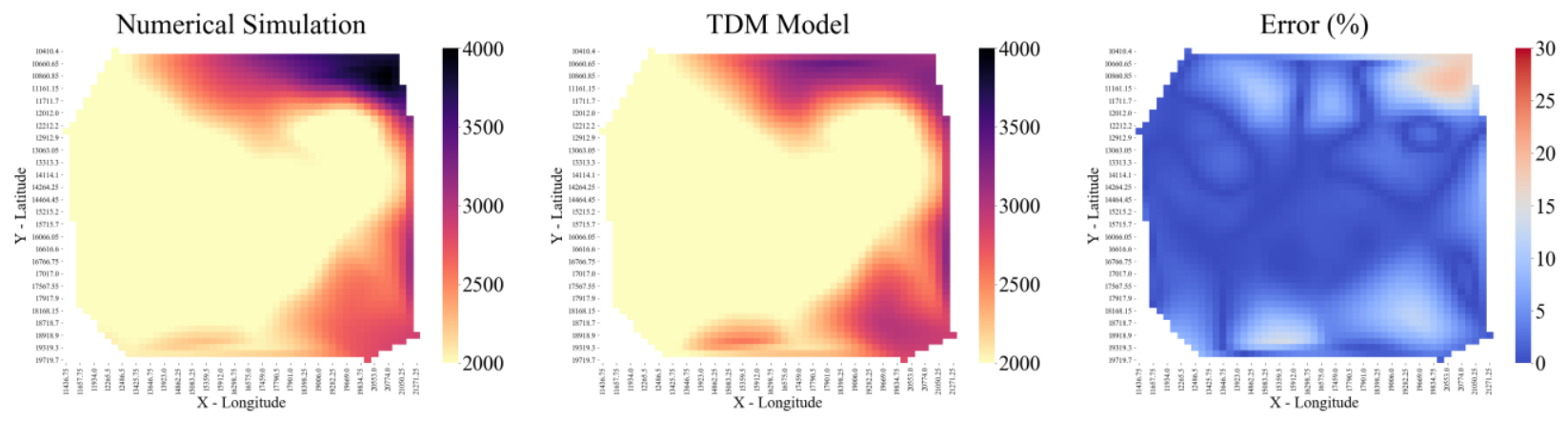

Figure 144 Entire reservoir heat map for reservoir pressure at December 31st for 2 Year Blind Validation TDM

Water Saturation (\%) at 2014-12-31
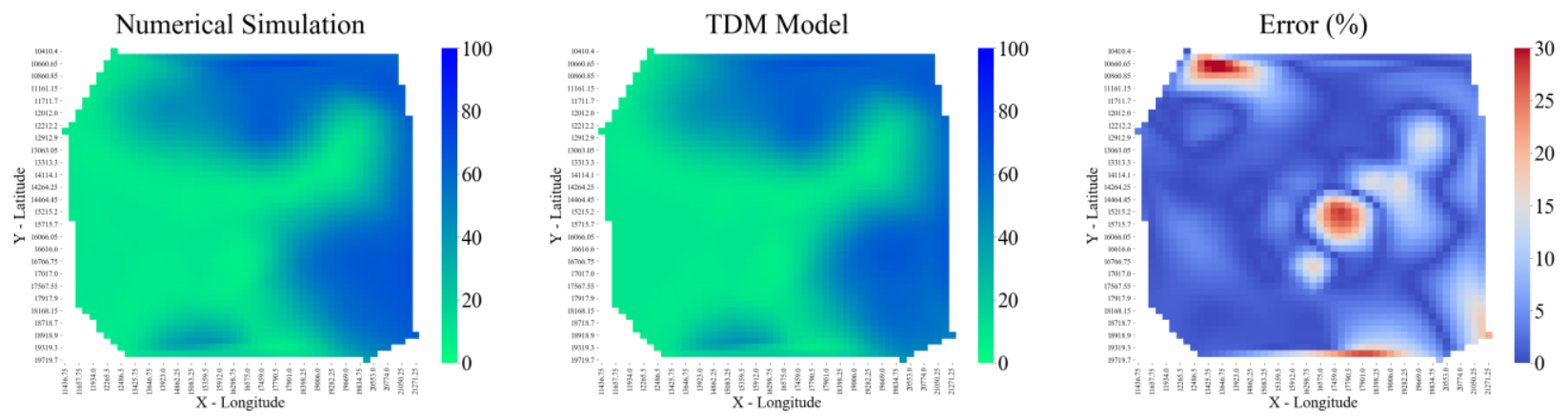

Figure 145 Entire reservoir heat map for water saturation at December 31st, 2014 for 2 Year Blind Validation TDM

Water Saturation (\%) at 2015-06-30
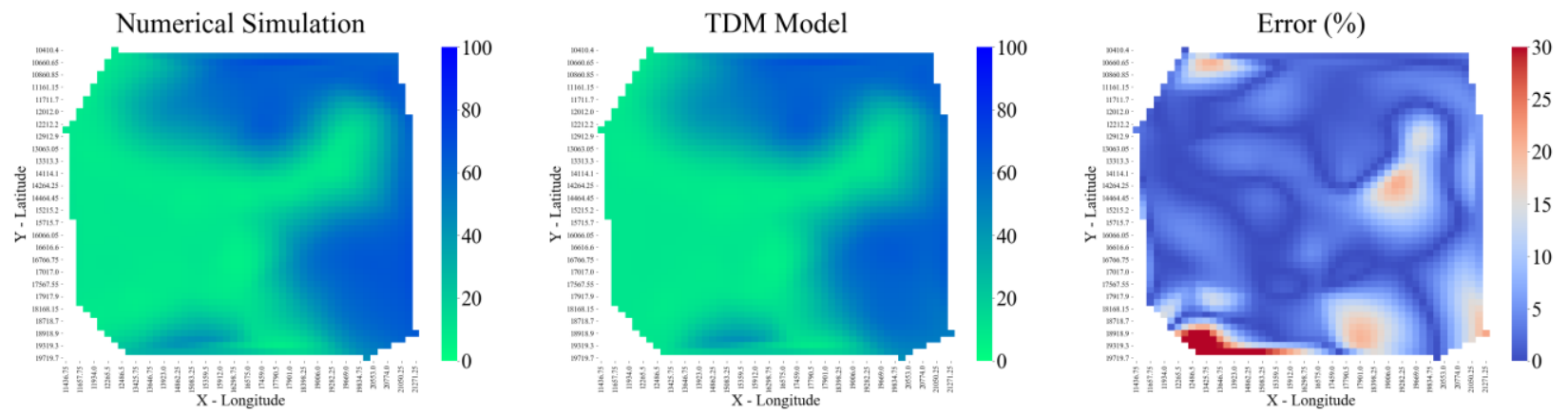

Figure 146 Entire reservoir heat map for water saturation at June 30th, 2015 for 2 Year Blind Validation TDM 
Water Saturation (\%) at 2015-12-31
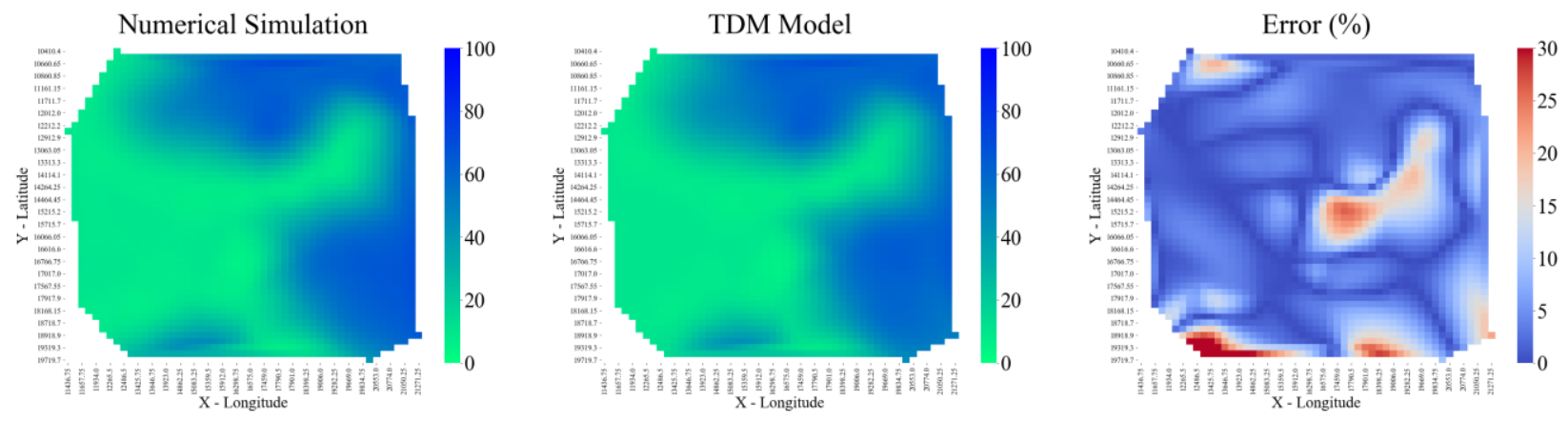

Figure 147 Entire reservoir heat map for water saturation at December 31st, 2015 for 2 Year Blind Validation TDM

Water Saturation (\%) at 2016-06-30
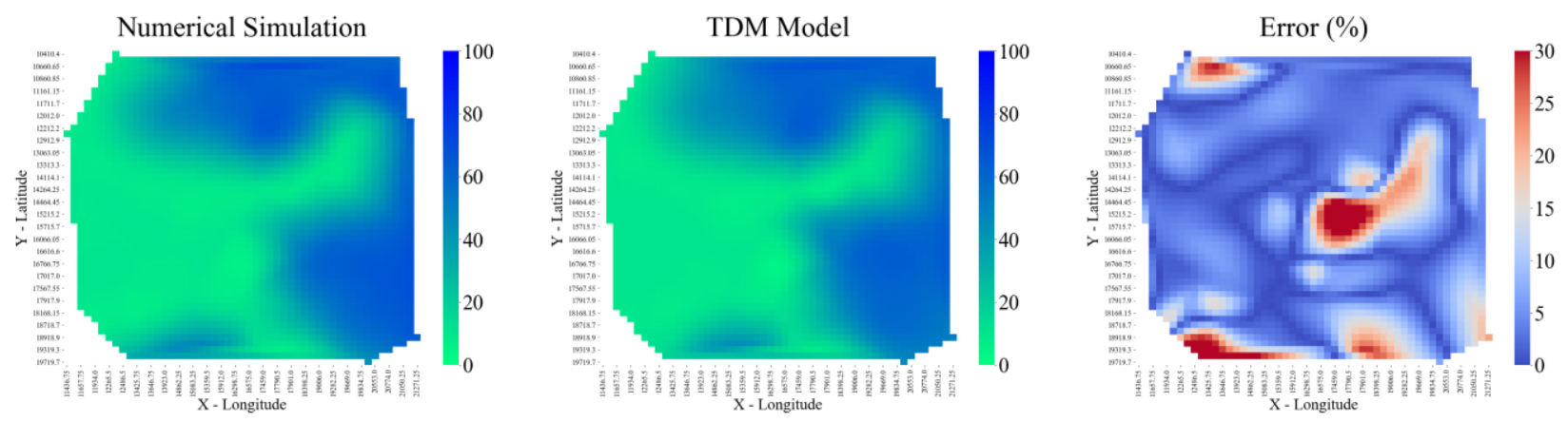

Figure 148 Entire reservoir heat map for water saturation at June 30th, 2016 for 2 Year Blind Validation TDM

Water Saturation (\%) at 2016-12-31
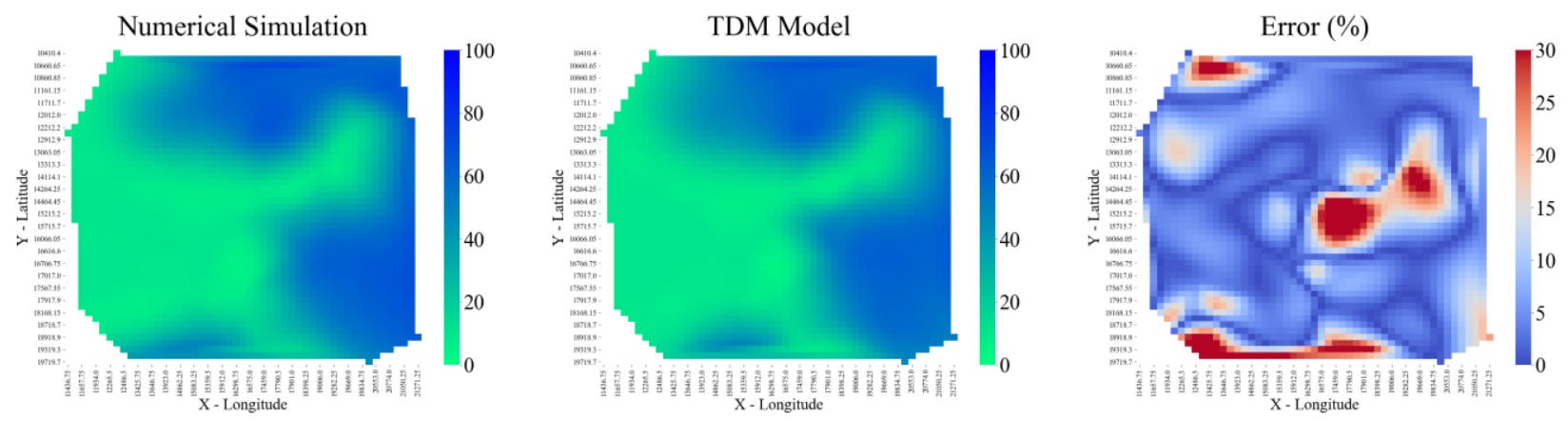

Figure 149 Entire reservoir heat map for water saturation at December 31st, 2016 for 2 Year Blind Validation TDM

Finally, the individual well production plot examples are shown below with the red line drawn on December $31^{\text {st }}$, 2014, the beginning of the two year blind validation. Well-007 and Well-033 show good accuracy among two of the 37 wells assigned to that category. Consequently, $65 \%$ of wells were considered to be of good quality. 


\section{Oil Rate}

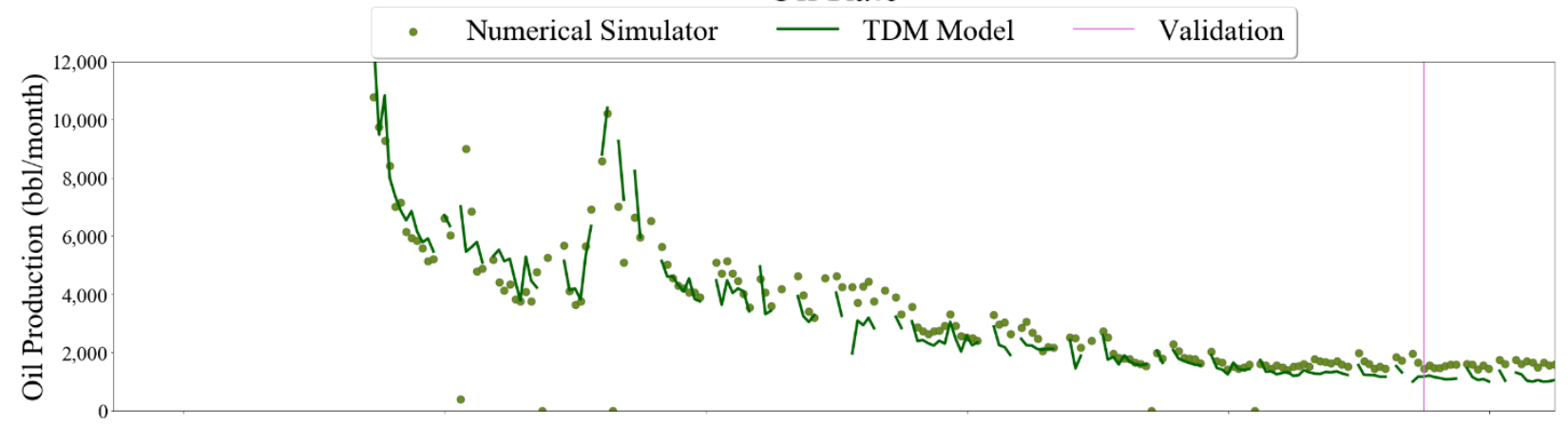

Gas Rate

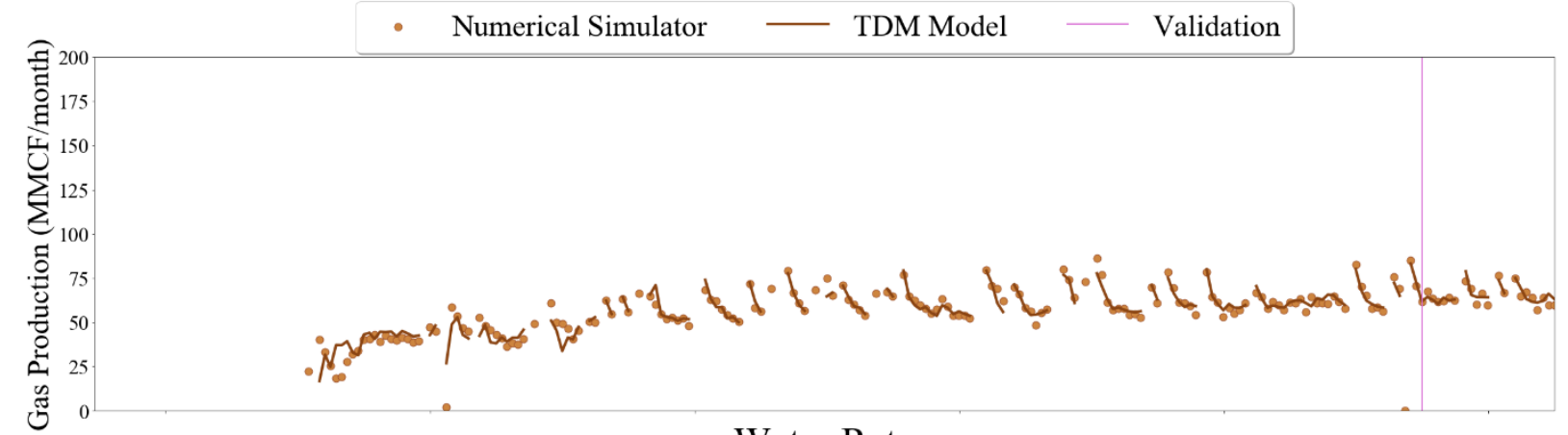

Water Rate

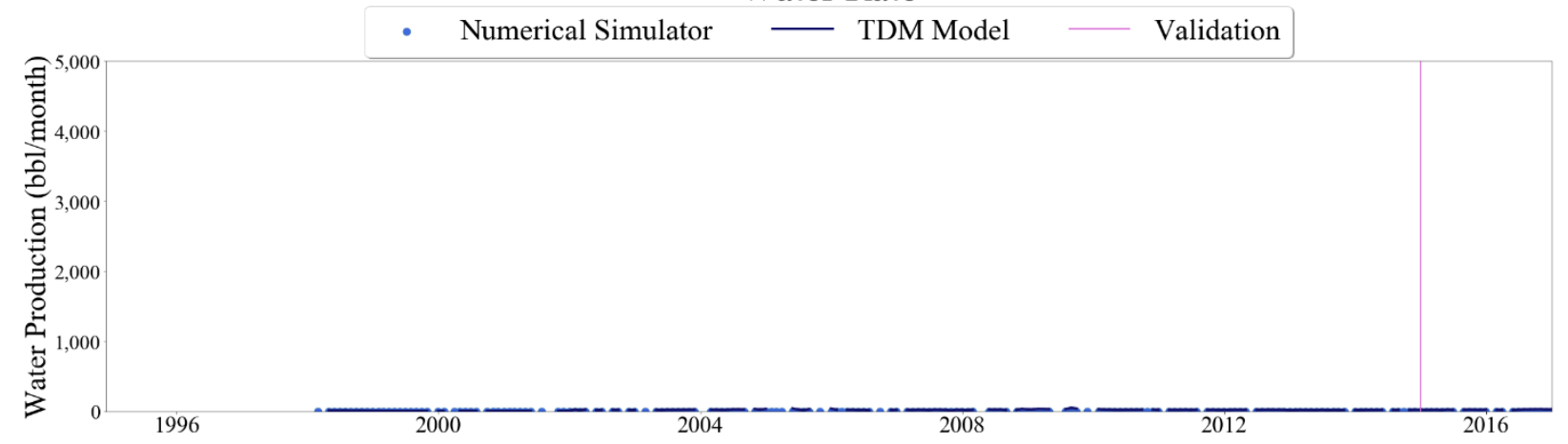

Figure 150 Well-007 oil, gas, and water rate TDM predictions vs actual simulation data plots for 2 Year Blind Validation TDM 


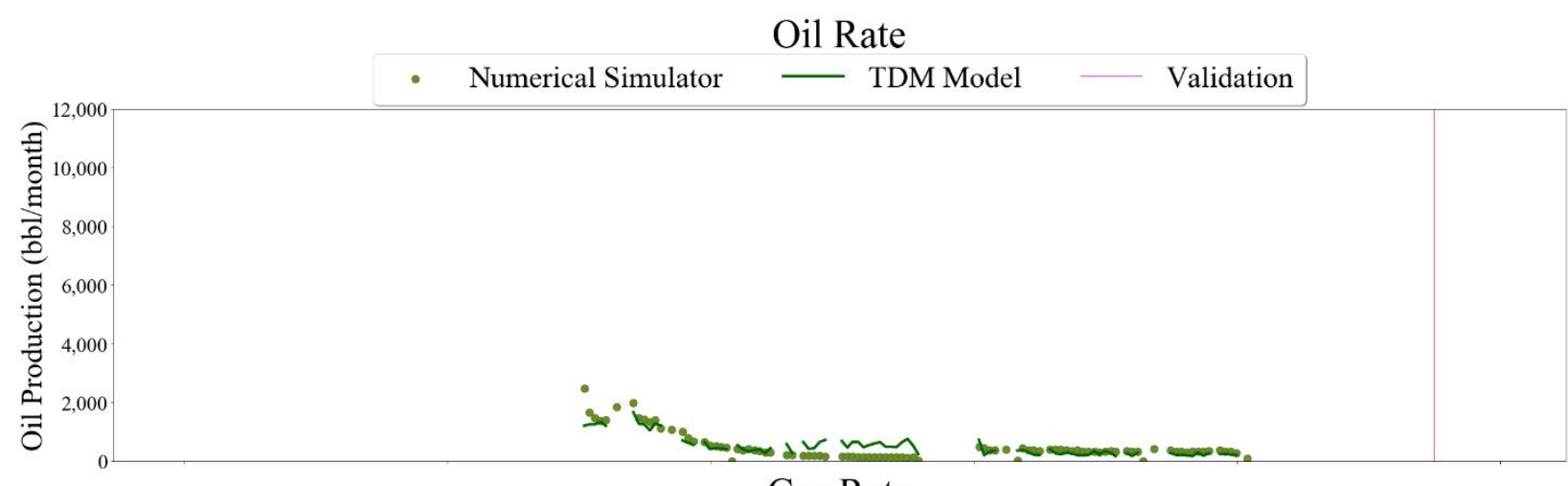

Gas Rate
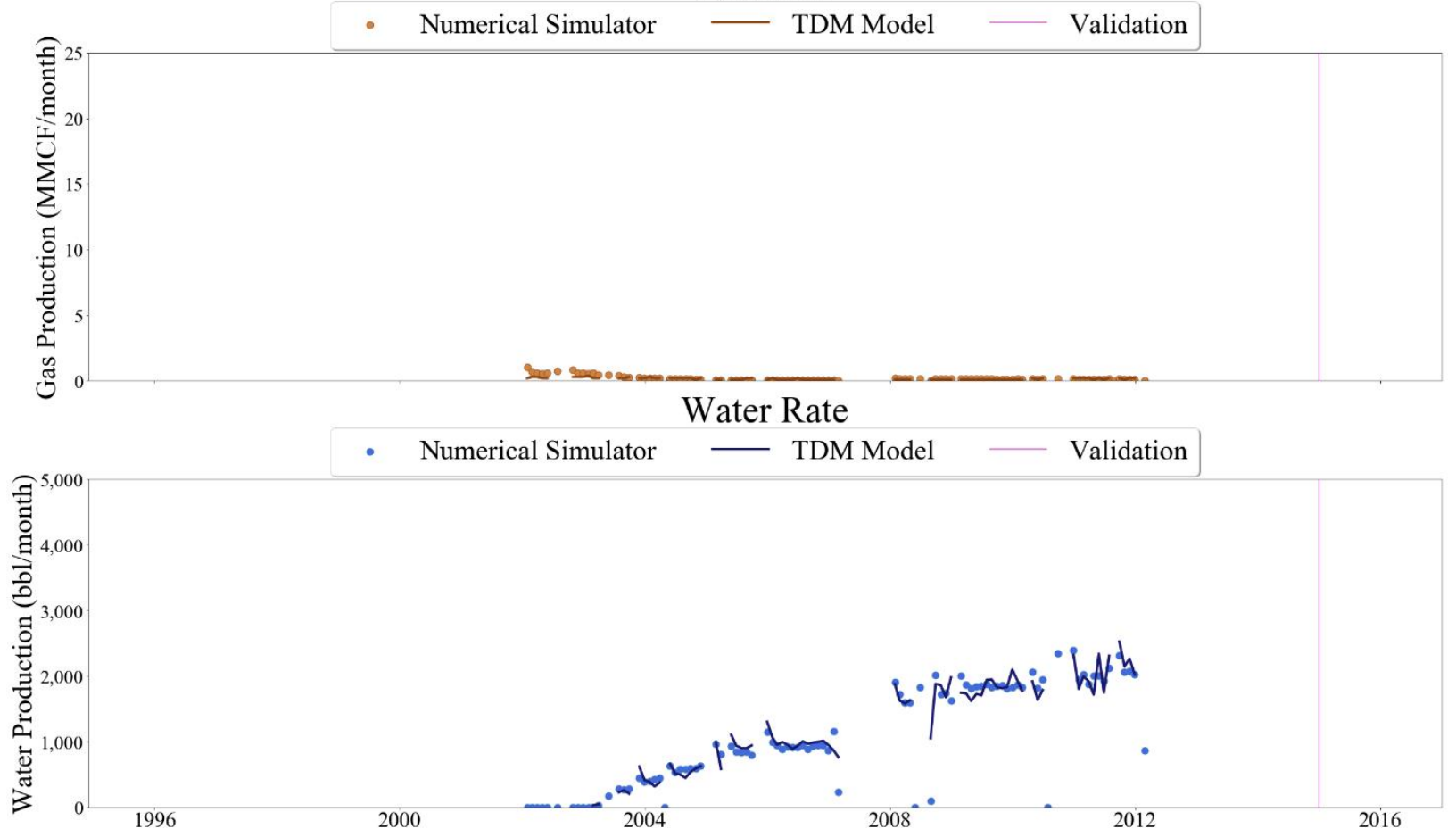

Figure 151 Well-033 oil, gas, and water rate TDM predictions vs actual simulation data plots for 2 Year Blind Validation TDM

Twenty-eight percent of the wells were considered to have average accuracy like Well-002 and Well-020. Well-002's predictions matched the actual data in the gas and water plots, however in the oil plot, the predictions were slightly over and under estimated even though it followed the general trend of the data. For Well-020, the predictions somewhat overestimated the actual data in the oil plot, but more or less overlaid the gas plot. 

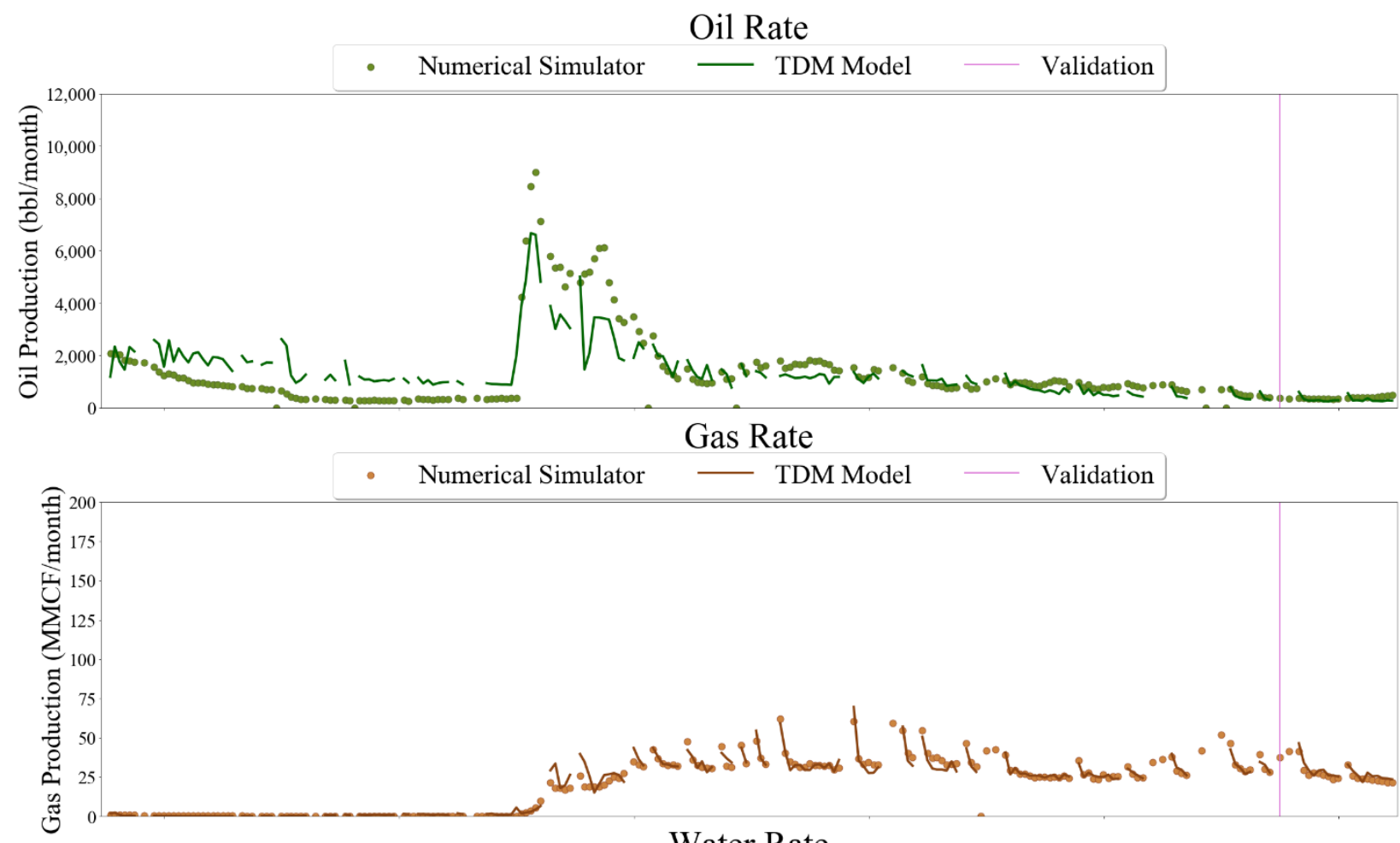

Water Rate

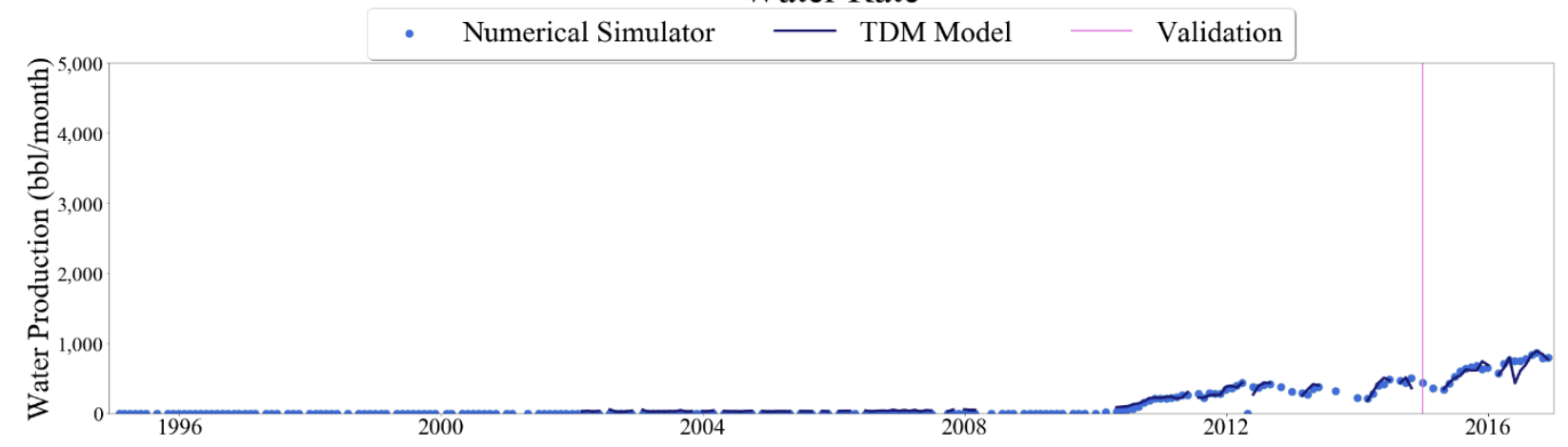

Figure 152 Well-002 oil, gas, and water rate TDM predictions vs actual simulation data plots for 2 Year Blind Validation TDM 

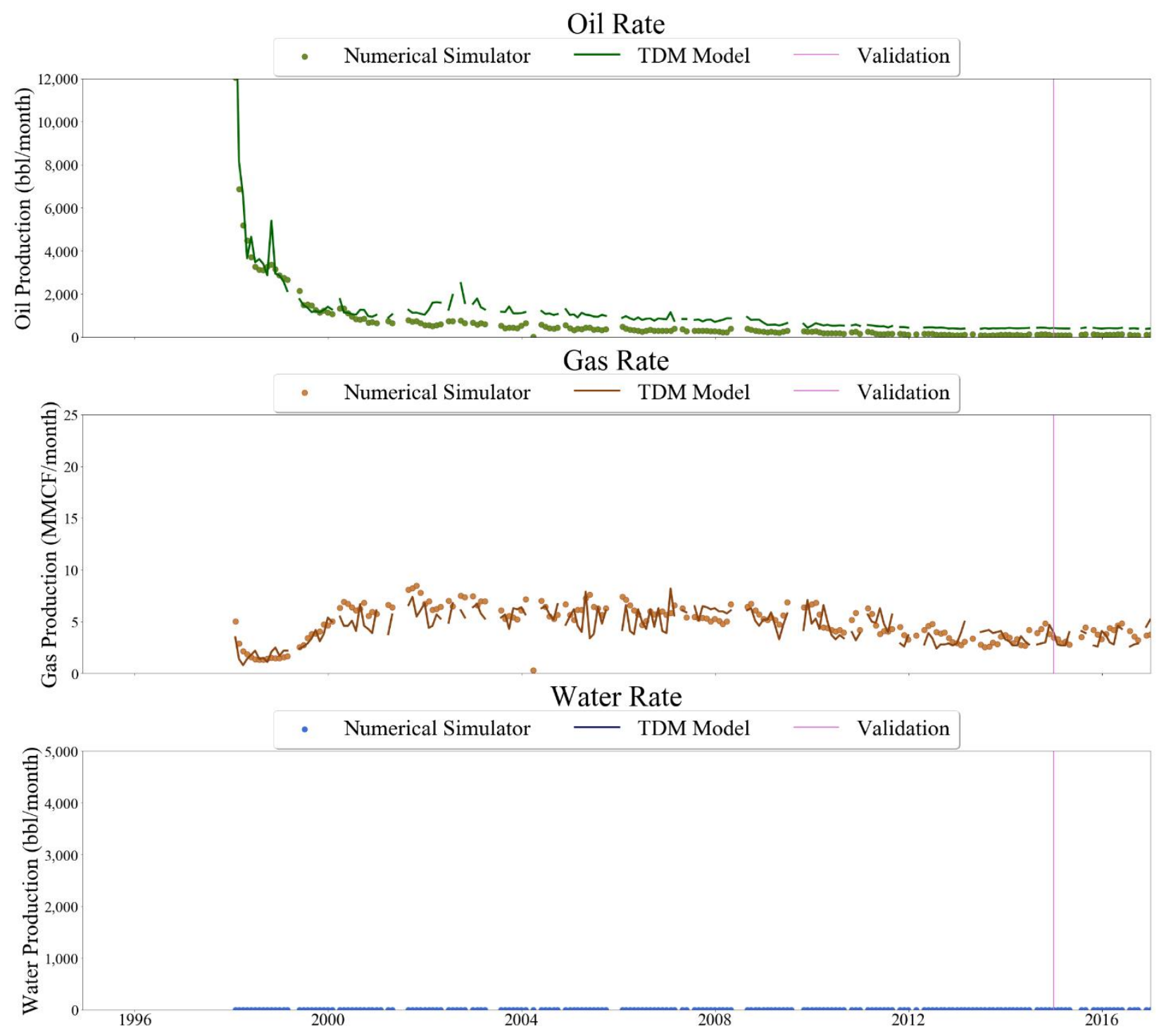

Figure 153 Well-020 oil, gas, and water rate TDM predictions vs actual simulation data plots for 2 Year Blind Validation TDM

Four wells, or $7 \%$ of the total wells, were thought to be of poor quality. Wells 26 and 48 are examples of the poor quality. Well-026's water prediction largely underestimated actual production whereas Well-048's gas prediction mainly overestimated actual production. In Appendix 8.2.3.1, the individual well production profiles can be found for all wells for the 2 Year Blind Validation case. 


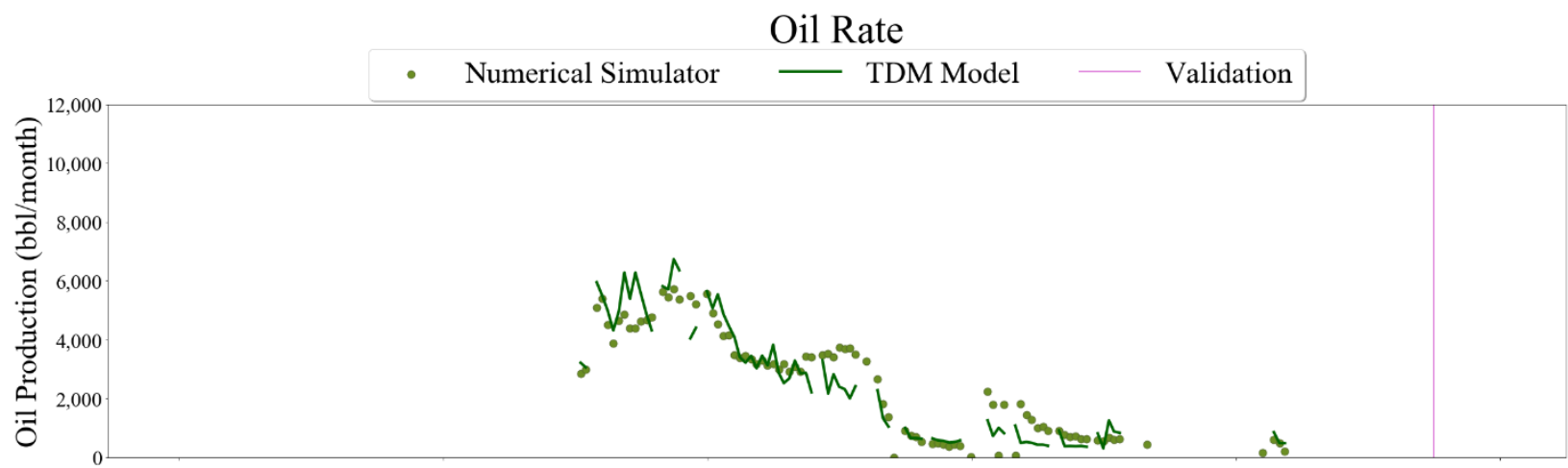

\section{Gas Rate}
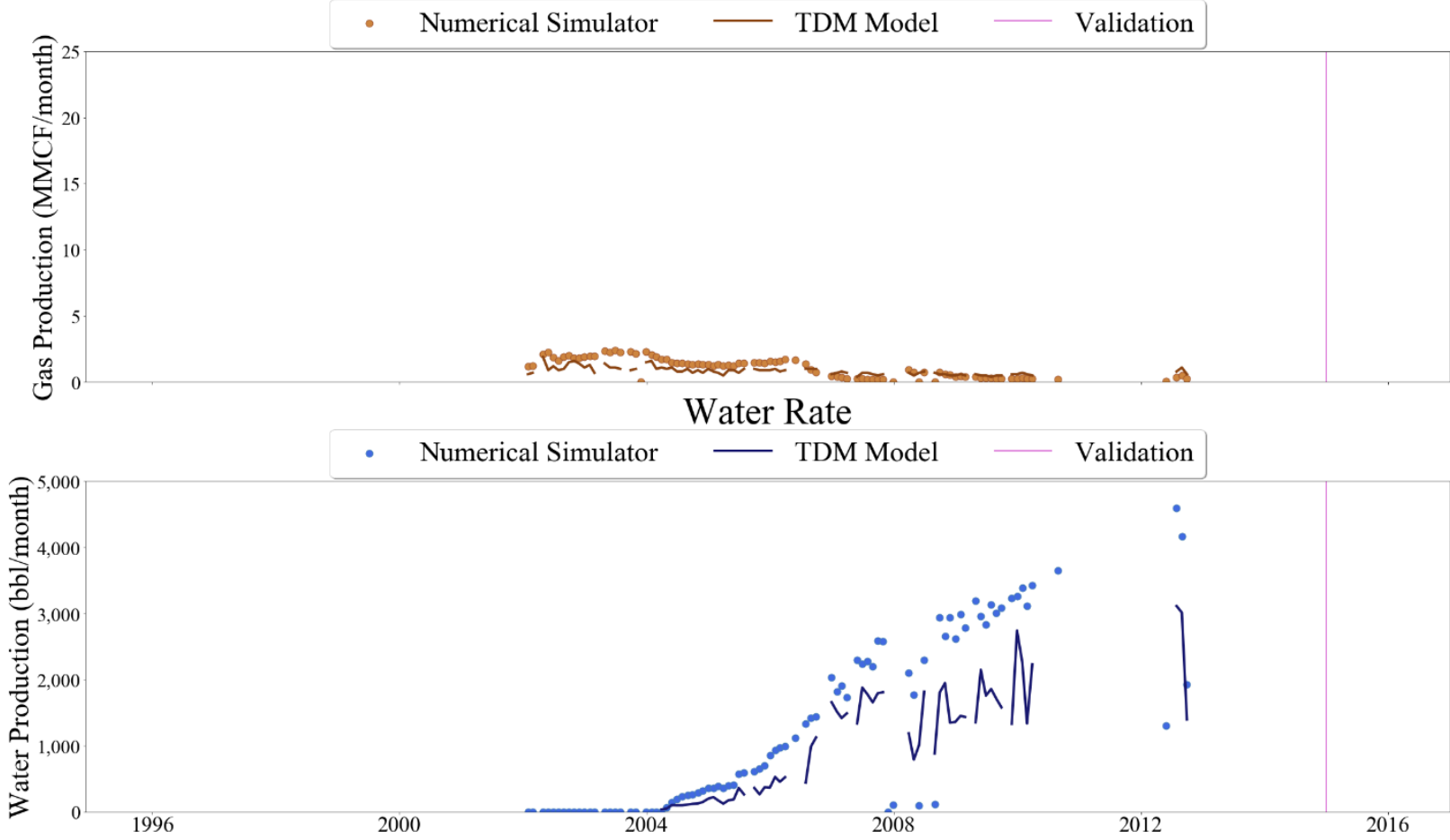

Figure 154 Well-026 oil, gas, and water rate TDM predictions vs actual simulation data plots for 2 Year Blind Validation TDM 


\section{Oil Rate}
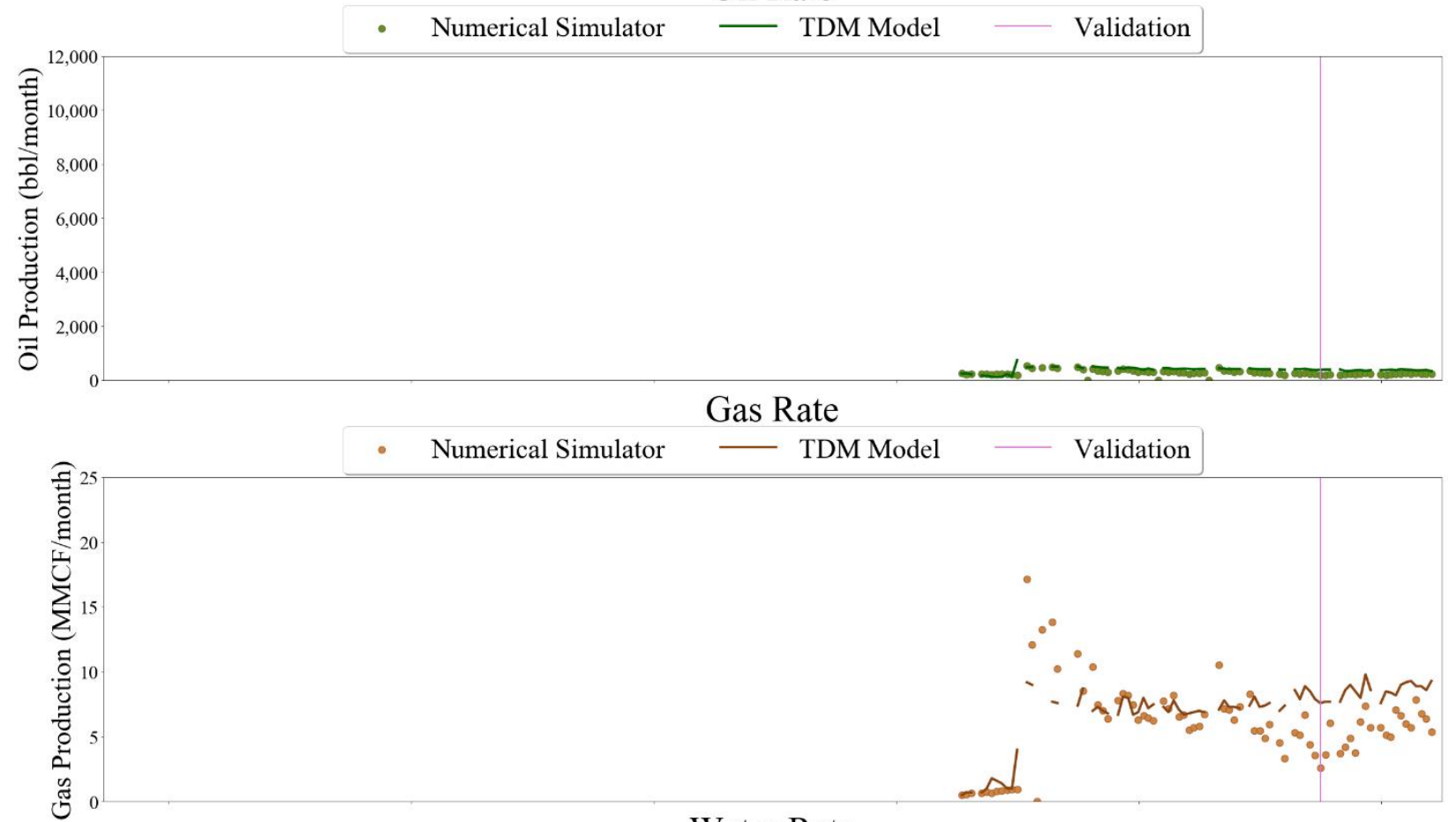

Water Rate

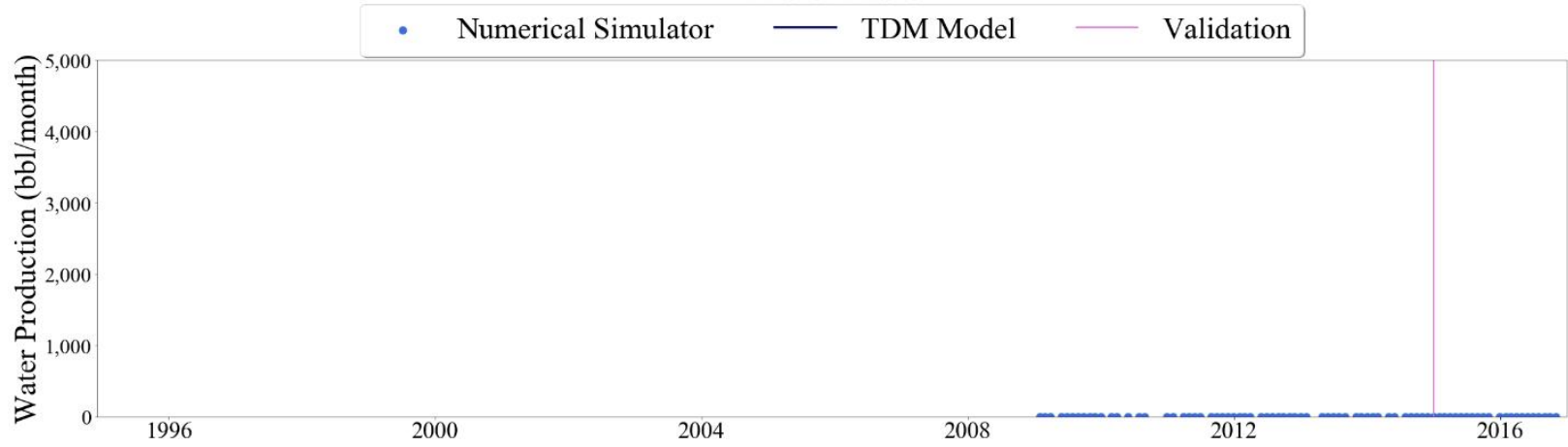

Figure 155 Well-048 oil, gas, and water rate TDM predictions vs actual simulation data plots for 2 Year Blind Validation TDM

Because all of the aforementioned plots were deemed of sufficient accuracy, an additional year was added to the blind validation to see if acceptable results could also be obtained with that model. Presented next will be the results from the Multi-Random Partitioning- 3 Year Blind Validation model.

\subsubsection{Multi-Random Partitioning- 3 Year Blind Validation}

The 3 Year Blind Validation entire reservoir oil, gas, and water results are shown below. The blind validation begins on December 31 $1^{\text {st }}, 2013$ and continues until December $31^{\text {st }}$, 2016. An exceptional match can be seen in all three plots. 


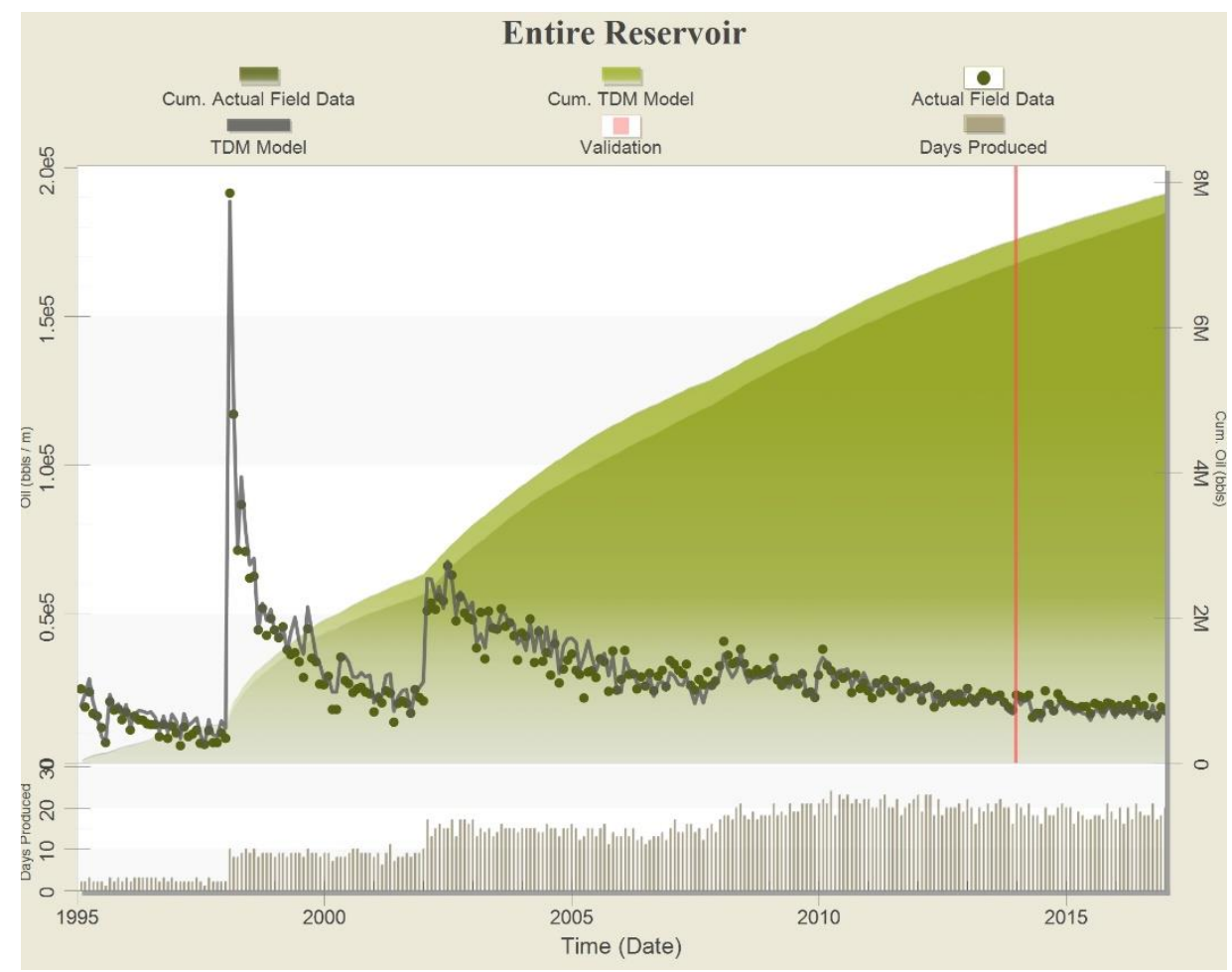

Figure 156 Entire reservoir oil results for Multi-Random Partitioning- 3 Year Blind Validation Top Down Model

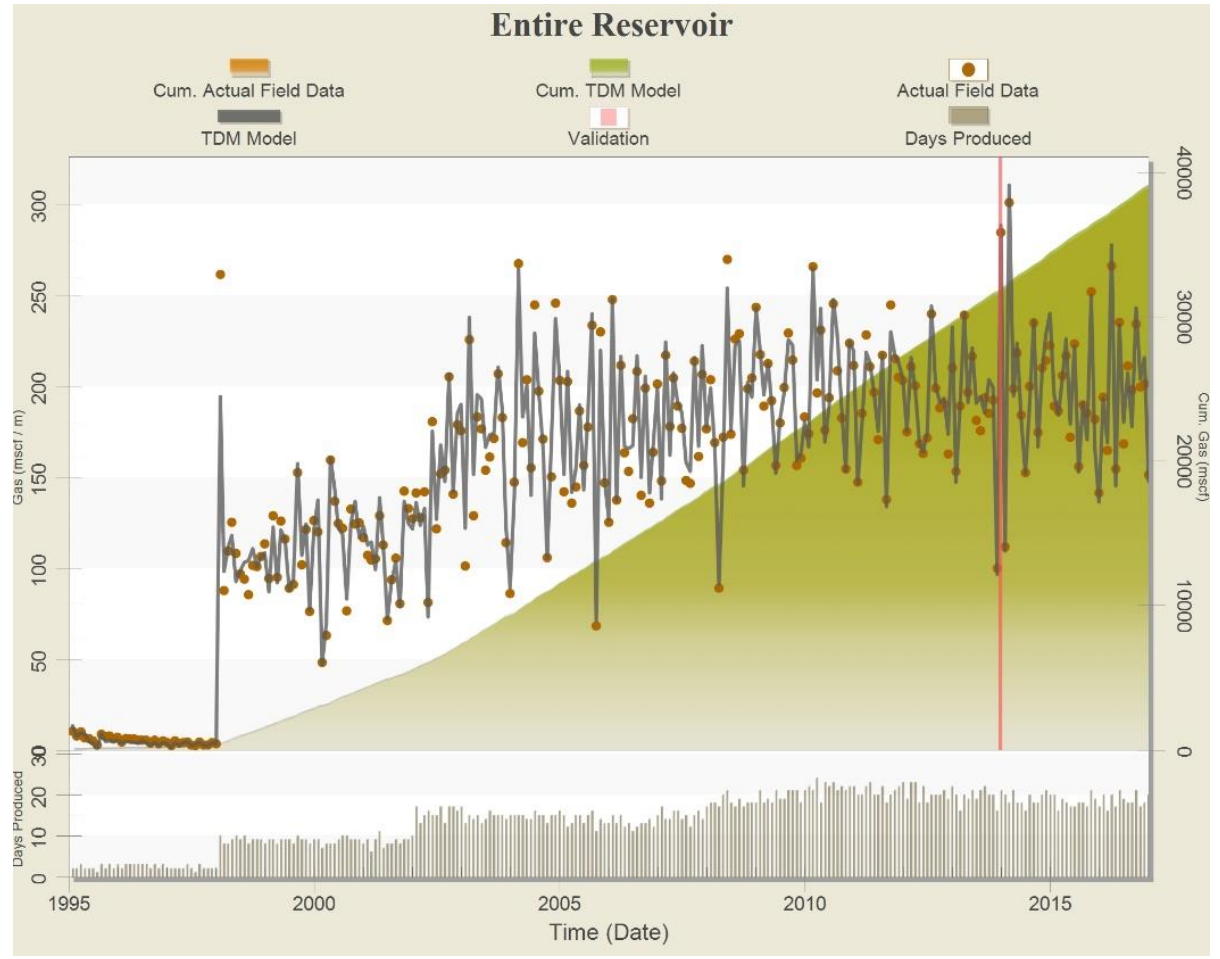

Figure 157 Entire reservoir gas results for Multi-Random Partitioning- 3 Year Blind Validation Top Down Model 


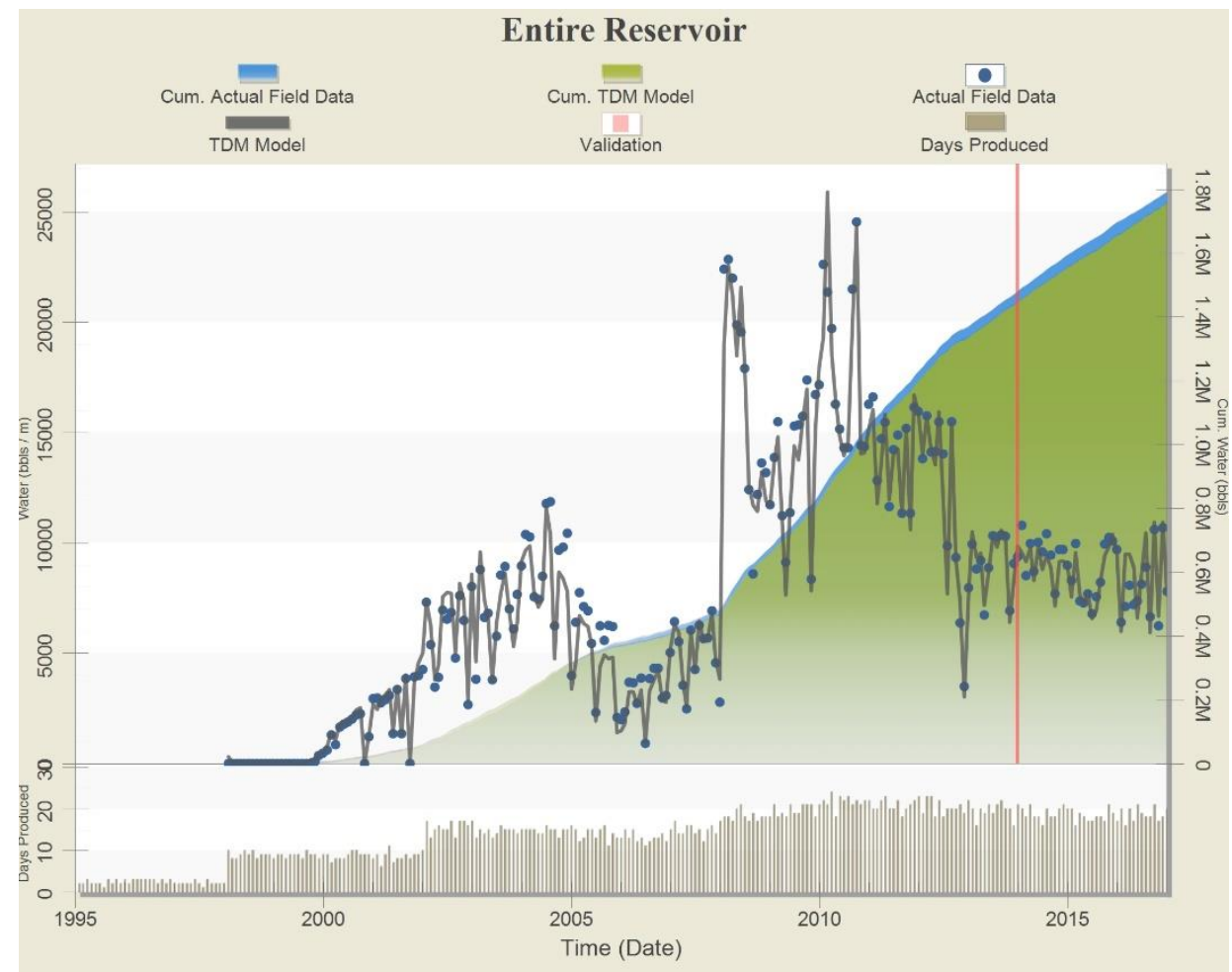

Figure 158 Entire reservoir water results for Multi-Random Partitioning-3 Year Blind Validation Top Down Model

Reservoir pressure and water saturation heat maps are included in six month intervals from the start of the blind validation, December $31^{\text {st }}, 2013$, to the end of simulation, December $31^{\text {st }}, 2016$ below. Additional heat maps can be found in the Appendix 8.2.4.2 in 3 month intervals in the 3 Year Blind Validation section.

Reservoir Pressure (psi) at 2013-12-31
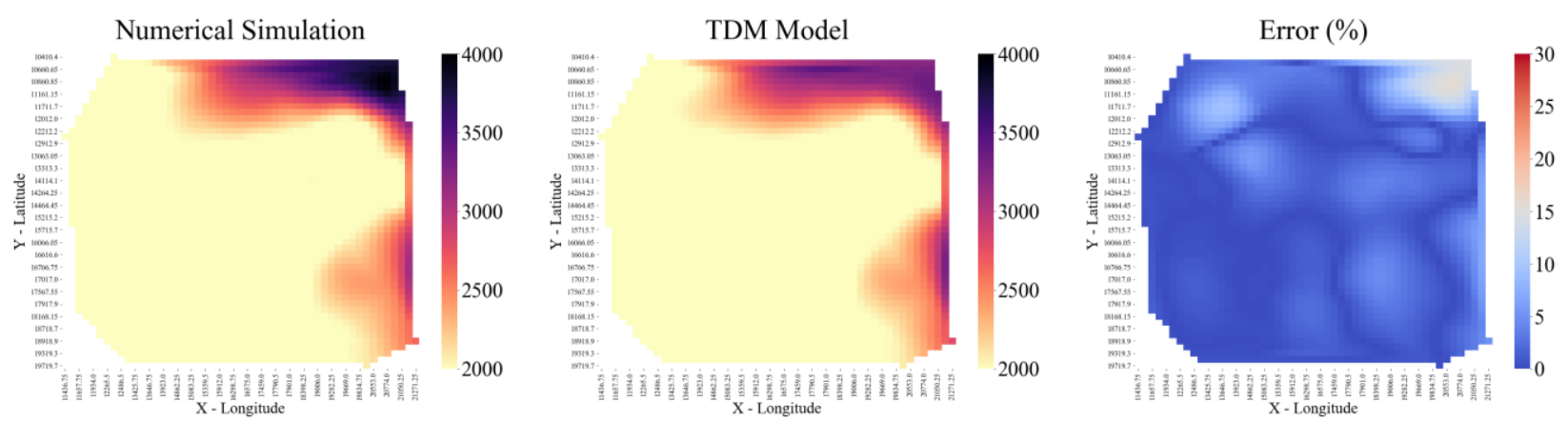

Figure 159 Entire reservoir heat map for reservoir pressure at December 31 st, 2013 for 3 Year Blind Validation TDM 
Reservoir Pressure (psi) at 2014-06-30
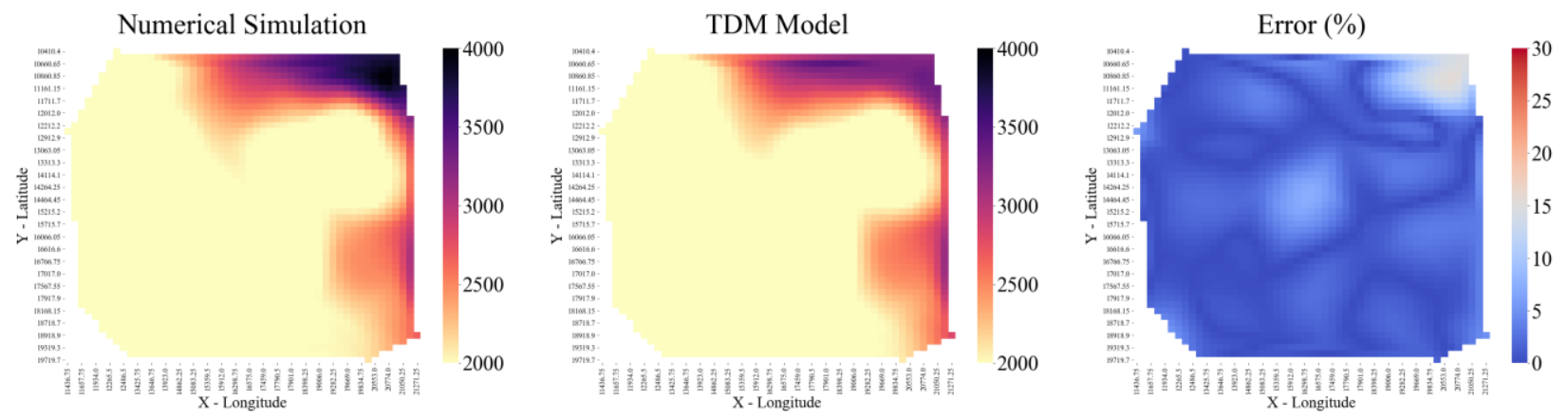

Figure 160 Entire reservoir heat map for reservoir pressure at June 30th, 2014 for 3 Year Blind Validation TDM

Reservoir Pressure (psi) at 2014-12-31
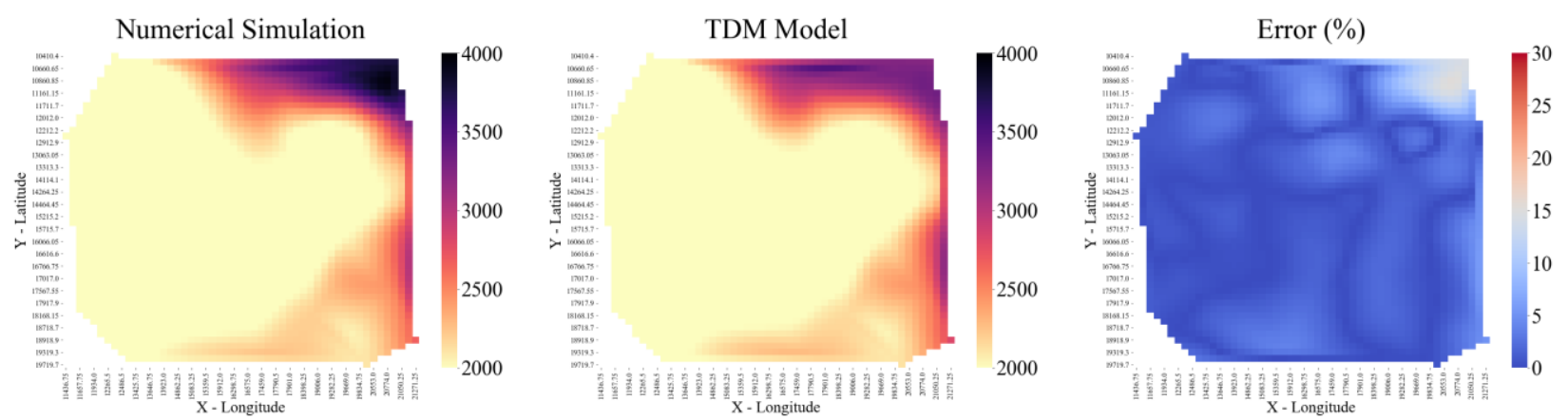

Figure 161 Entire reservoir heat map for reservoir pressure at December 31st, 2014 for 3 Year Blind Validation TDM

Reservoir Pressure (psi) at 2015-06-30
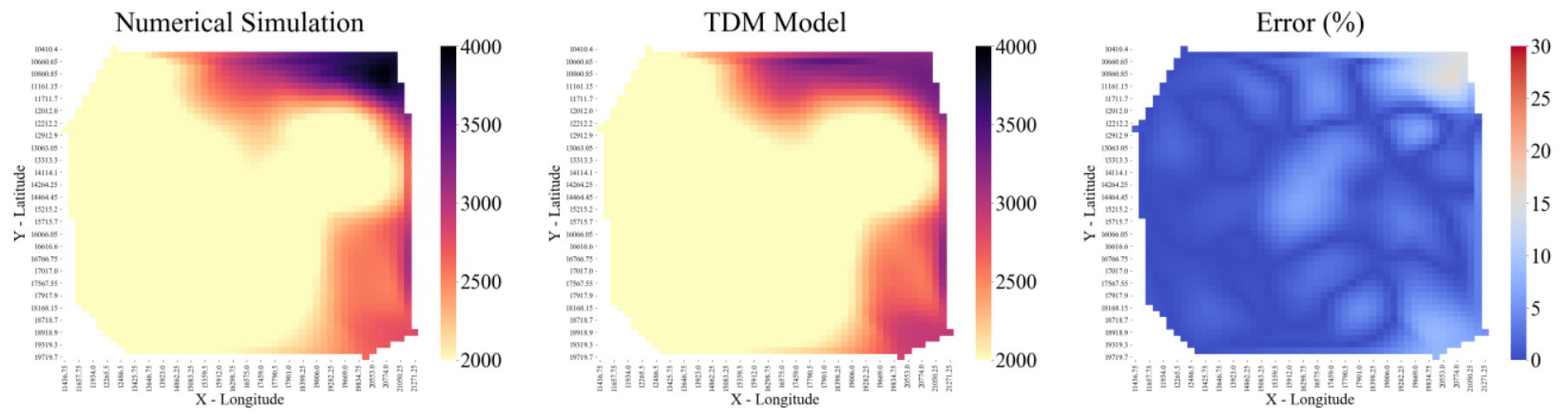

Figure 162 Entire reservoir heat map for reservoir pressure at June 30th, 2015 for 3 Year Blind Validation TDM 
Reservoir Pressure (psi) at 2015-12-31
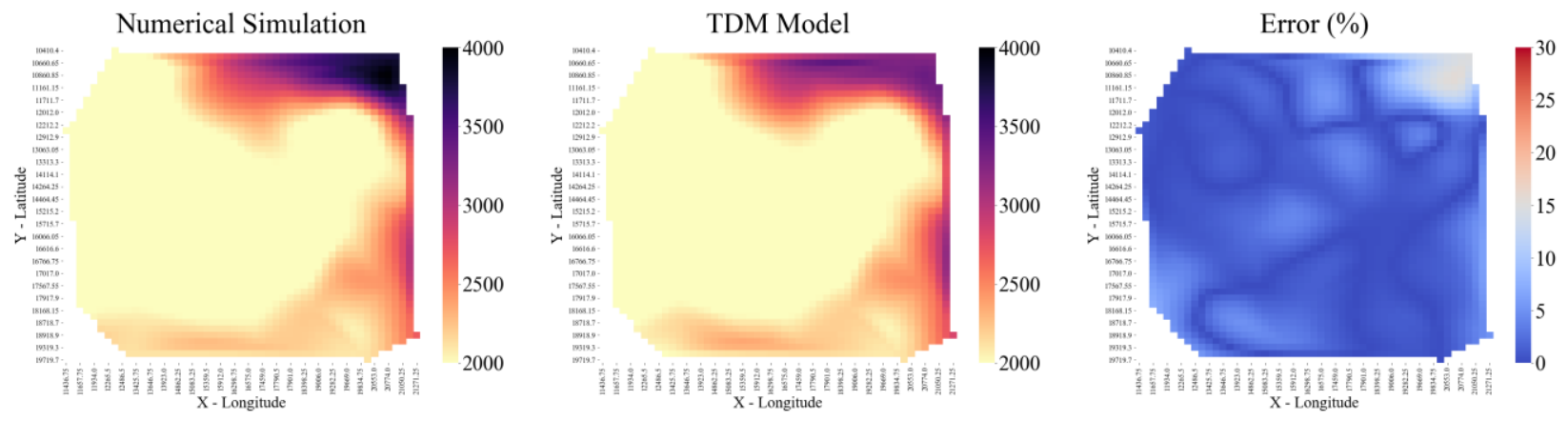

Figure 163 Entire reservoir heat map for reservoir pressure at December 31st, 2015 for 3 Year Blind Validation TDM

\section{Reservoir Pressure (psi) at 2016-06-30}
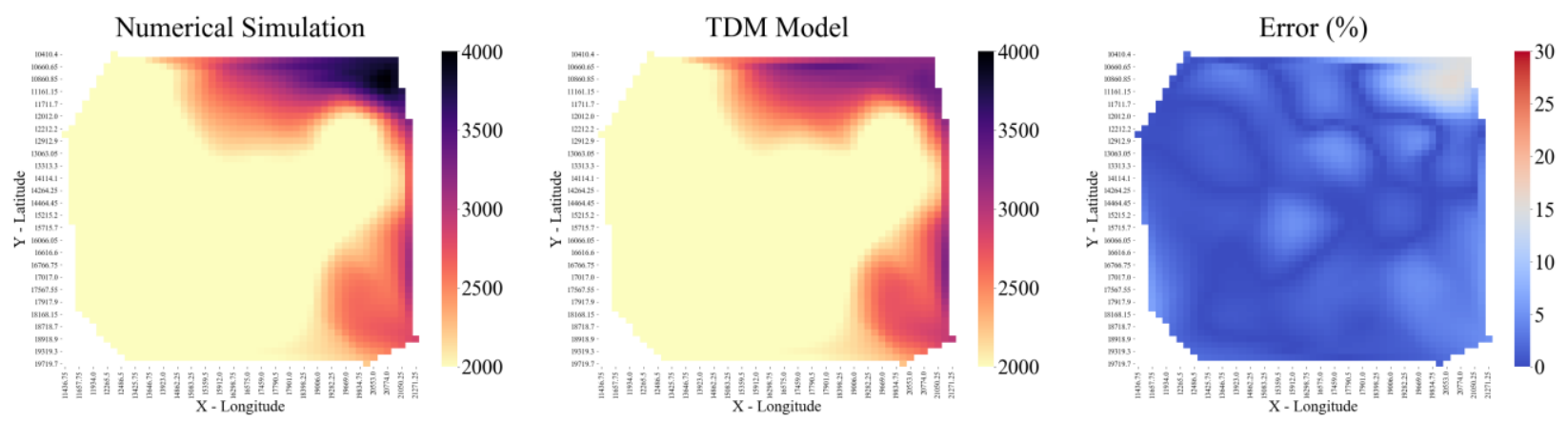

Figure 164 Entire reservoir heat map for reservoir pressure at June 30th, 2016 for 3 Year Blind Validation TDM

Reservoir Pressure (psi) at 2016-12-31
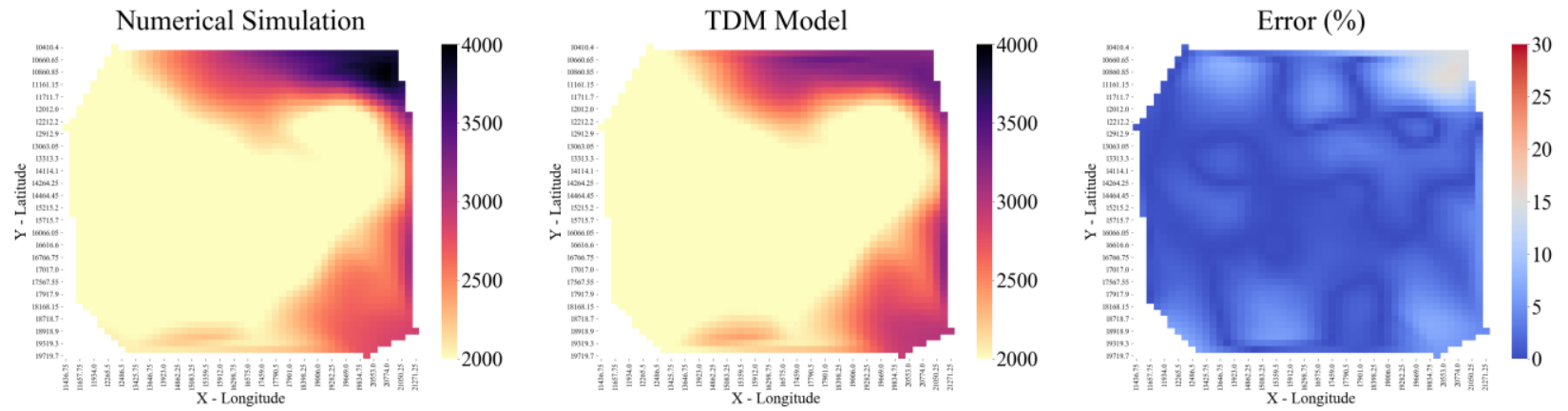

Figure 165 Entire reservoir heat map for reservoir pressure at December 31st, 2016 for 3 Year Blind Validation TDM 
Water Saturation (\%) at 2013-12-31
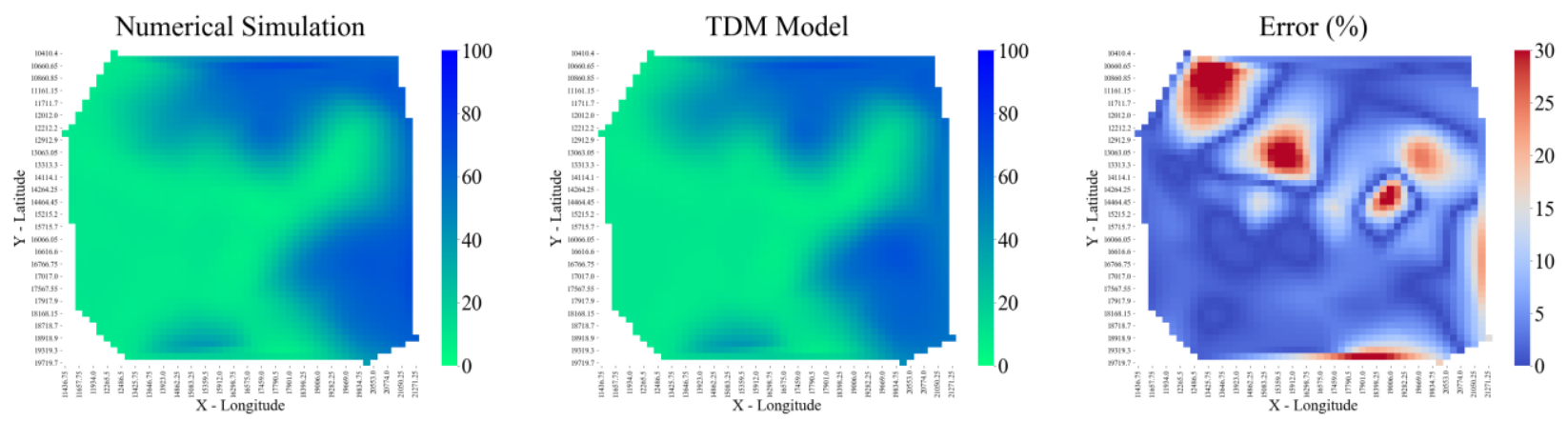

Figure 166 Entire reservoir heat map for water saturation at December 31st, 2013 for 3 Year Blind Validation TDM

Water Saturation (\%) at 2014-06-30
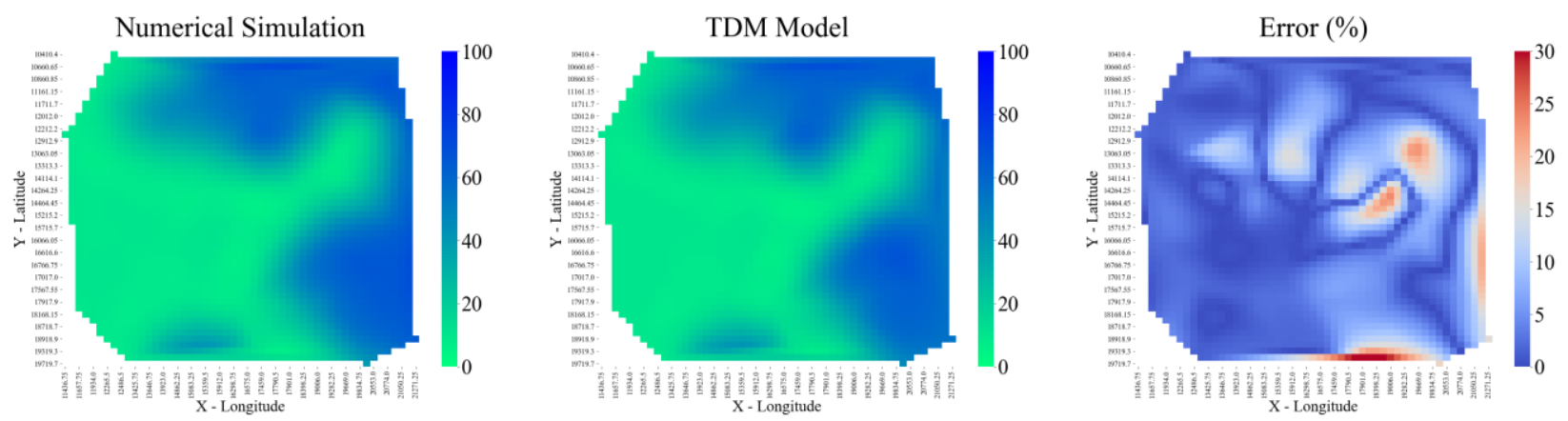

Figure 167 Entire reservoir heat map for water saturation at June 30th, 2014 for 3 Year Blind Validation TDM

Water Saturation (\%) at 2014-12-31
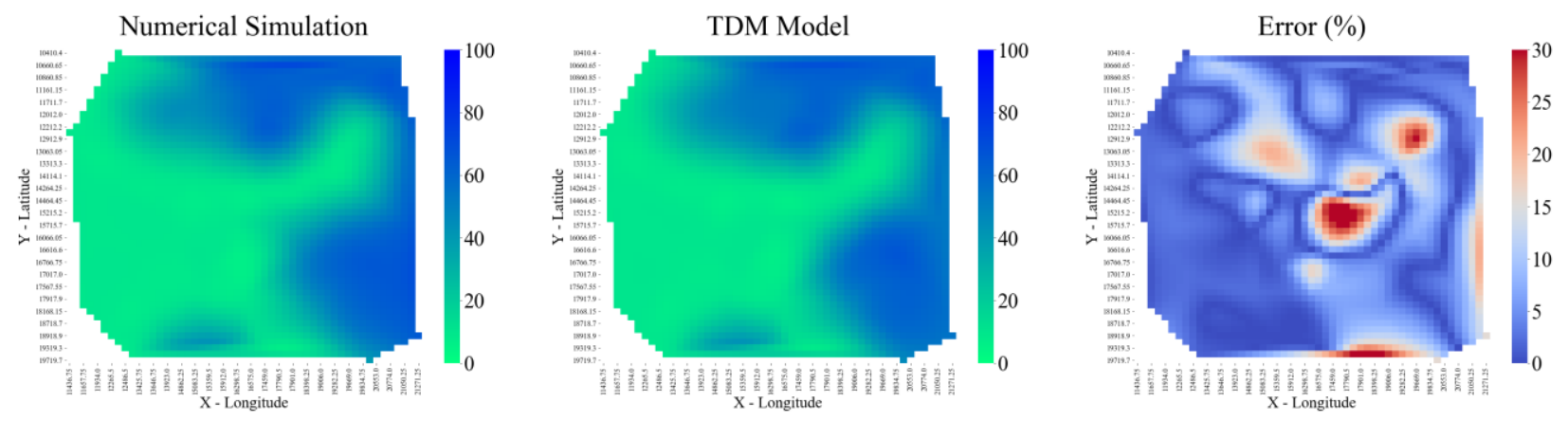

Figure 168 Entire reservoir heat map for water saturation at December 31st, 2014 for 3 Year Blind Validation TDM 
Water Saturation (\%) at 2015-06-30
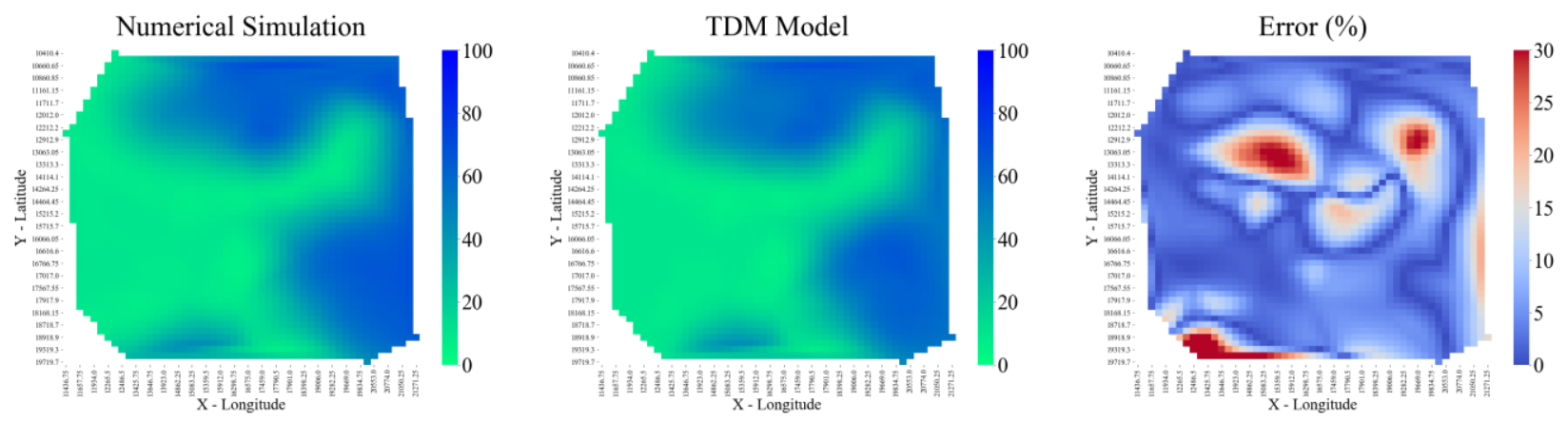

Figure 169 Entire reservoir heat map for water saturation at June 30th, 2015 for 3 Year Blind Validation TDM

Water Saturation (\%) at 2015-12-31
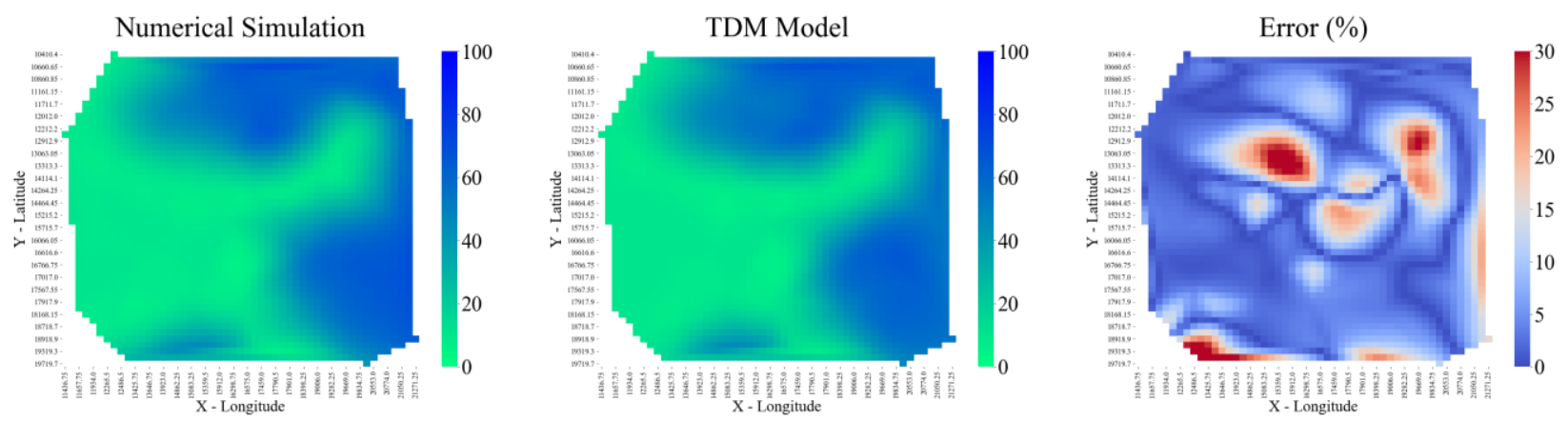

Figure 170 Entire reservoir heat map for water saturation at December 31st, 2015 for 3 Year Blind Validation TDM

Water Saturation (\%) at 2016-06-30
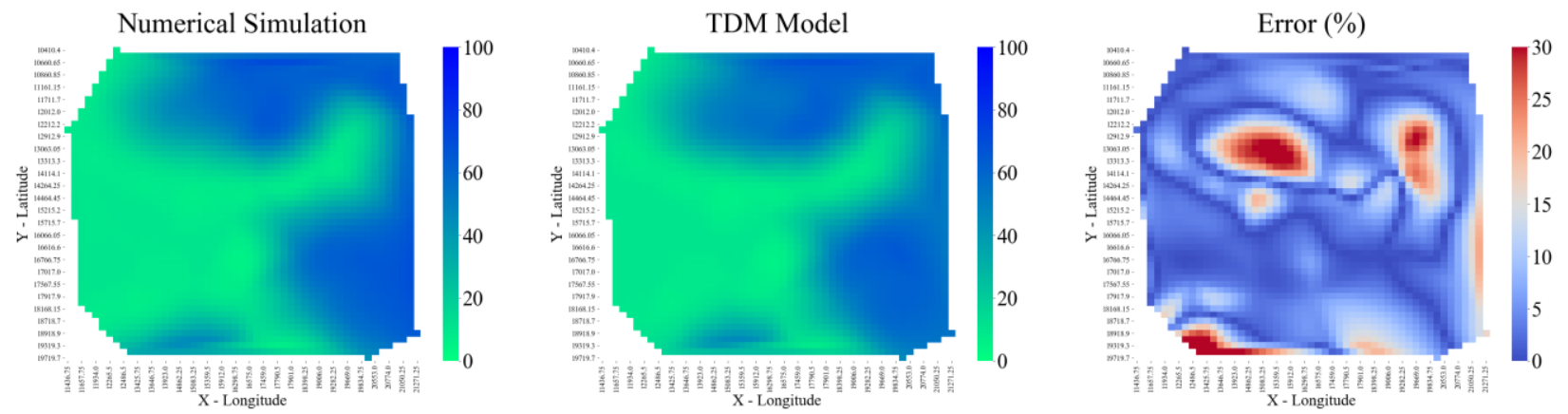

Figure 171 Entire reservoir heat map for water saturation at June 30th, 2016 for 3 Year Blind Validation TDM 


\section{Water Saturation (\%) at 2016-12-31}
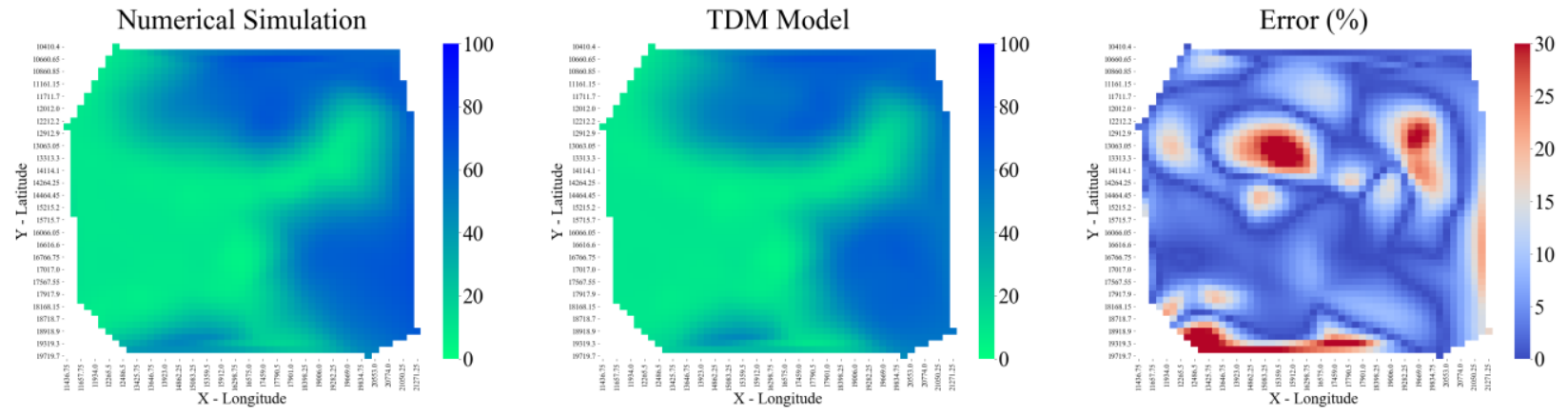

Figure 172 Entire reservoir heat map for water saturation at December 31st, 2016 for 3 Year Blind Validation TDM

Well-001 and Well-034 are two examples of wells that have good predictions. Thirty-nine out of the fifty-seven wells were considered to have a good fit, which is $68 \%$ of the wells. Again, the redline shows the beginning of blind validation at December $31^{\text {st }}, 2013$. 


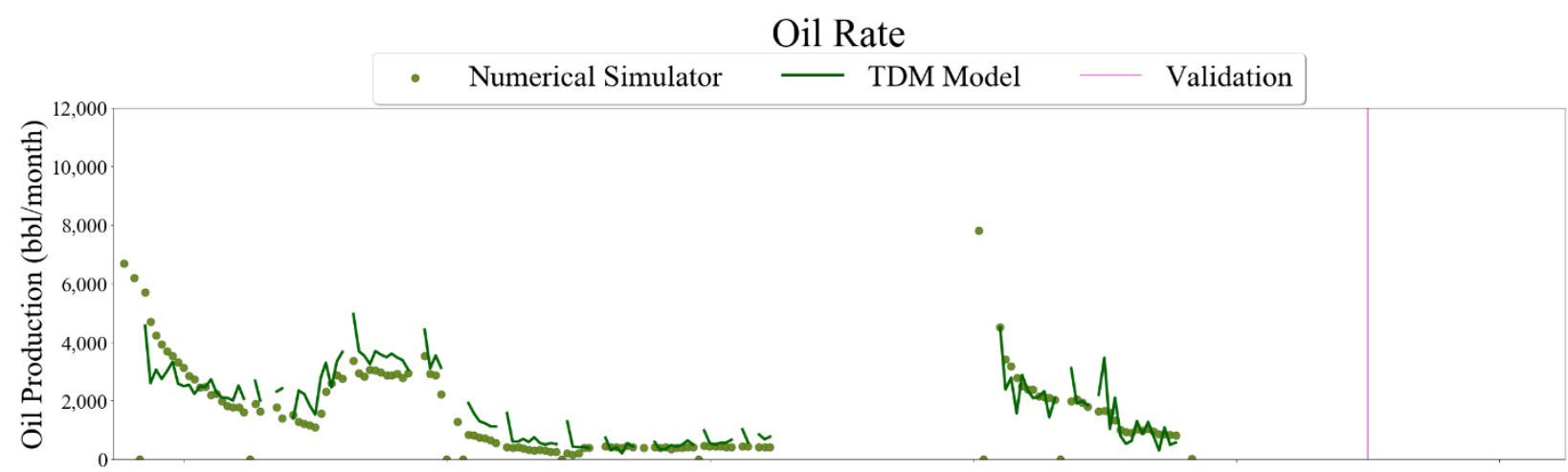

\section{Gas Rate}

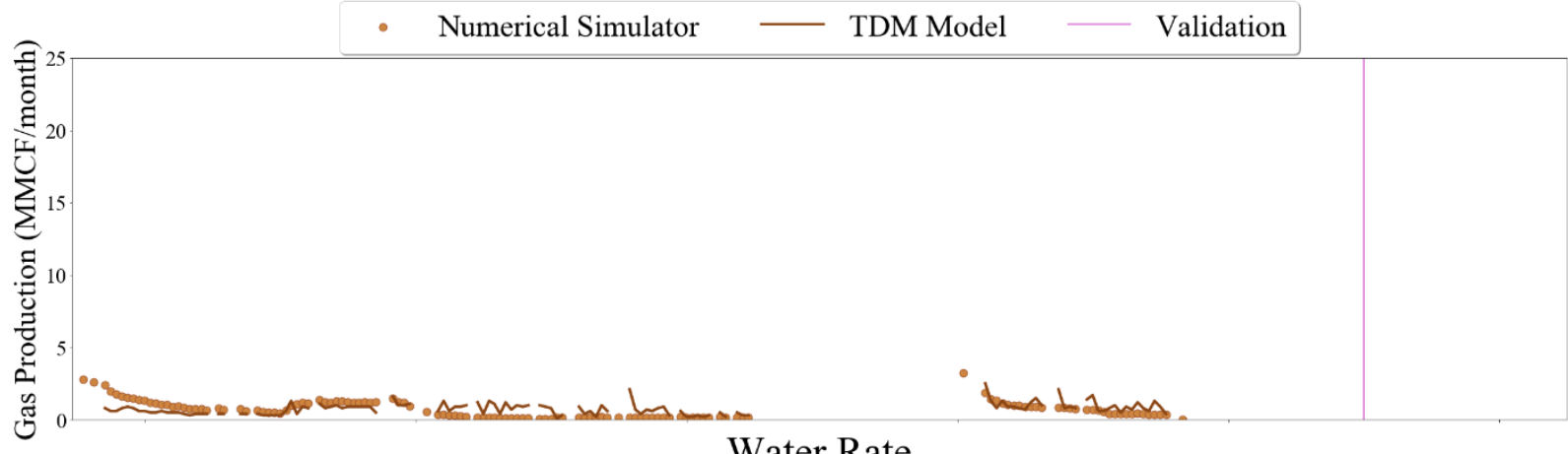

Water Rate

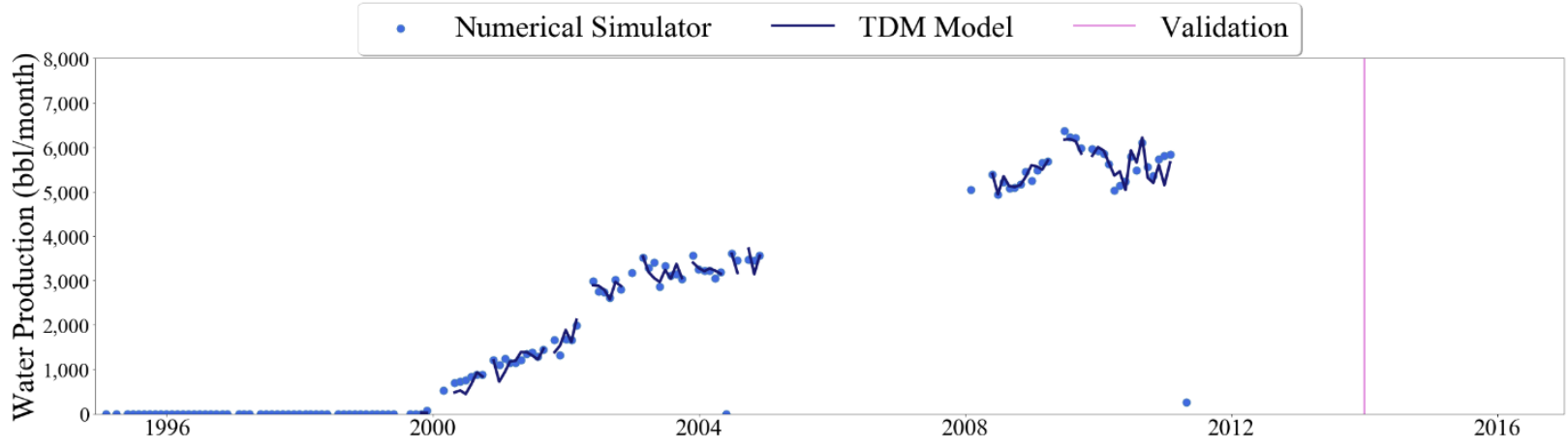

Figure 173 Well-001 oil, gas, and water rate TDM predictions vs actual simulation data plots for 3 Year Blind Validation TDM 

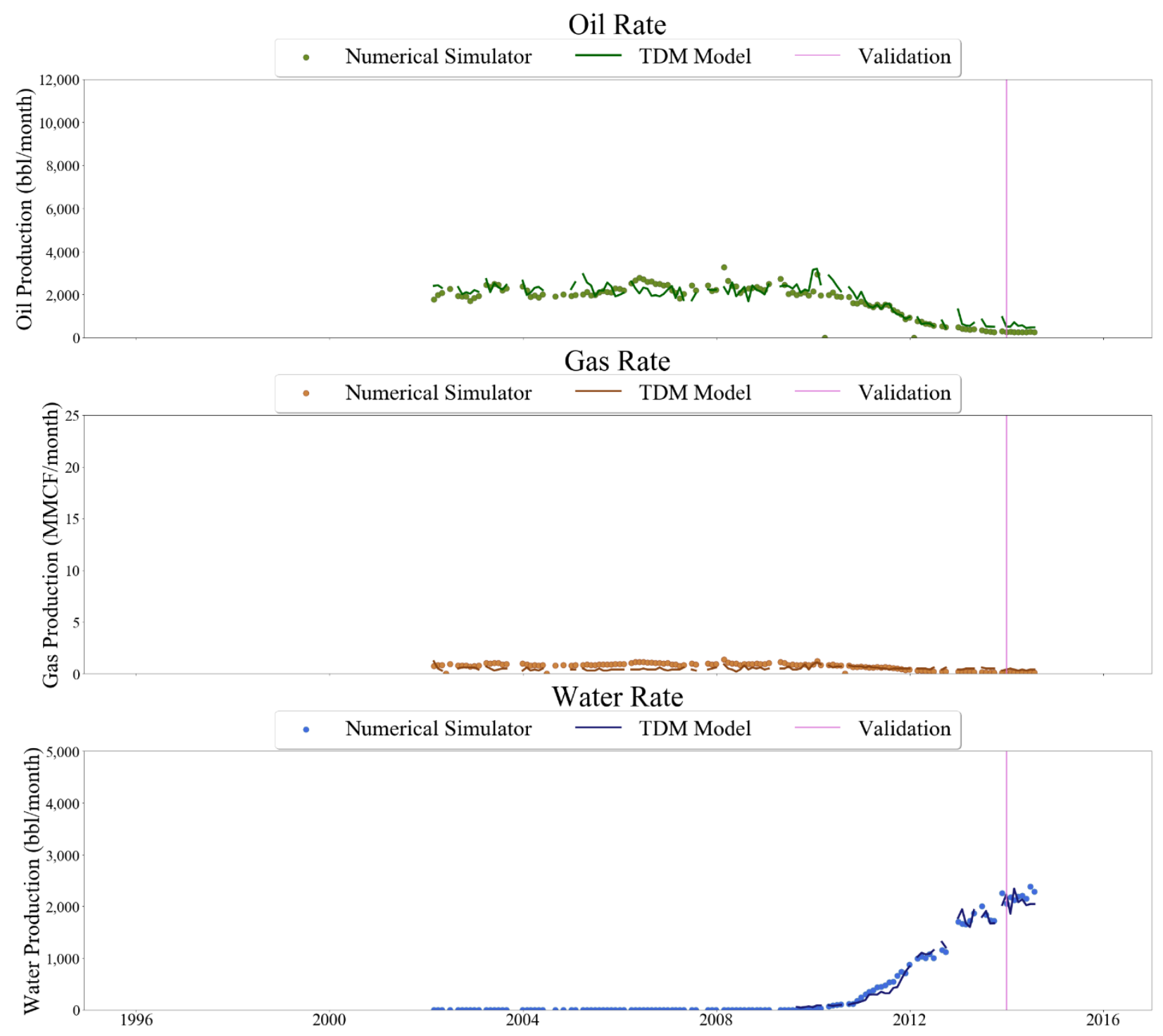

Figure 174 Well-034 oil, gas, and water rate TDM predictions vs actual simulation data plots for 3 Year Blind Validation TDM

Two examples of average fits are also included below, Well-014 and Well-043. Well-014 was decided to be average due to the slight difference in prediction versus actual data in the gas and water plots. Well-043's prediction slightly underestimated the actual data in the water plot and was therefore deemed to be of average accuracy. Fifteen wells had average accuracy which is $26 \%$ of wells. 


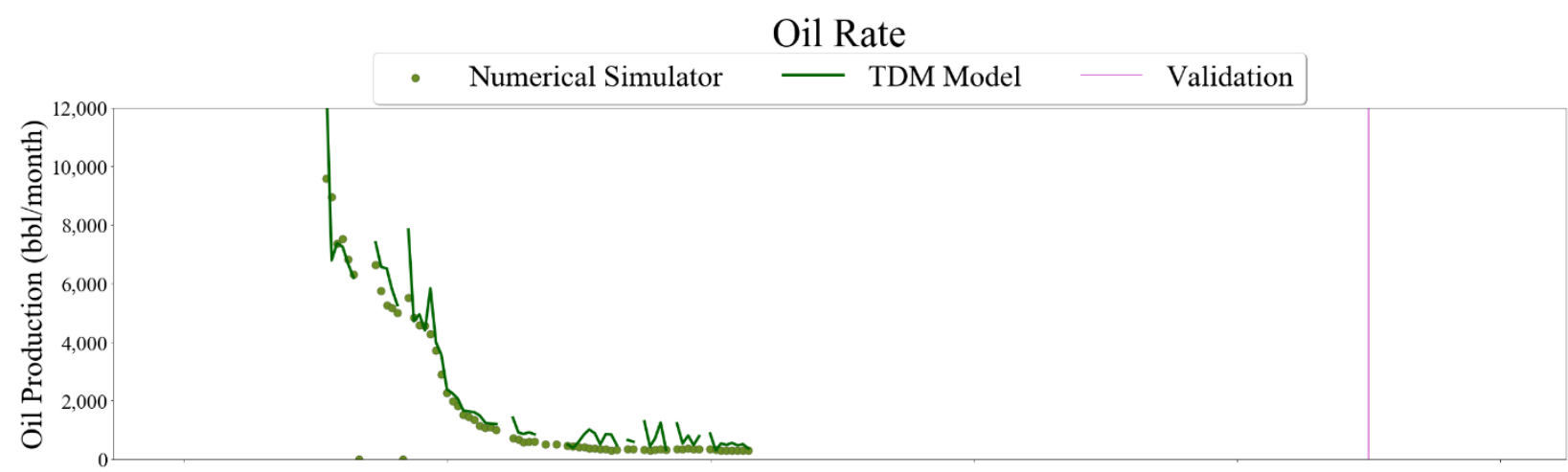

\section{Gas Rate}

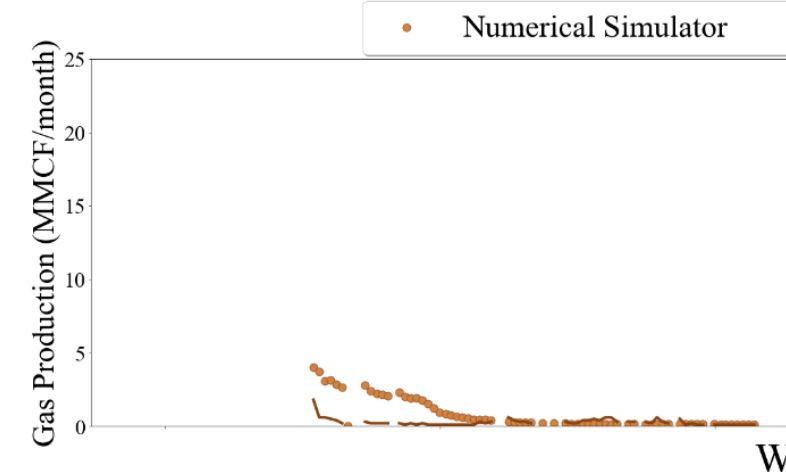

TDM Model —_ Validation

Water Rate

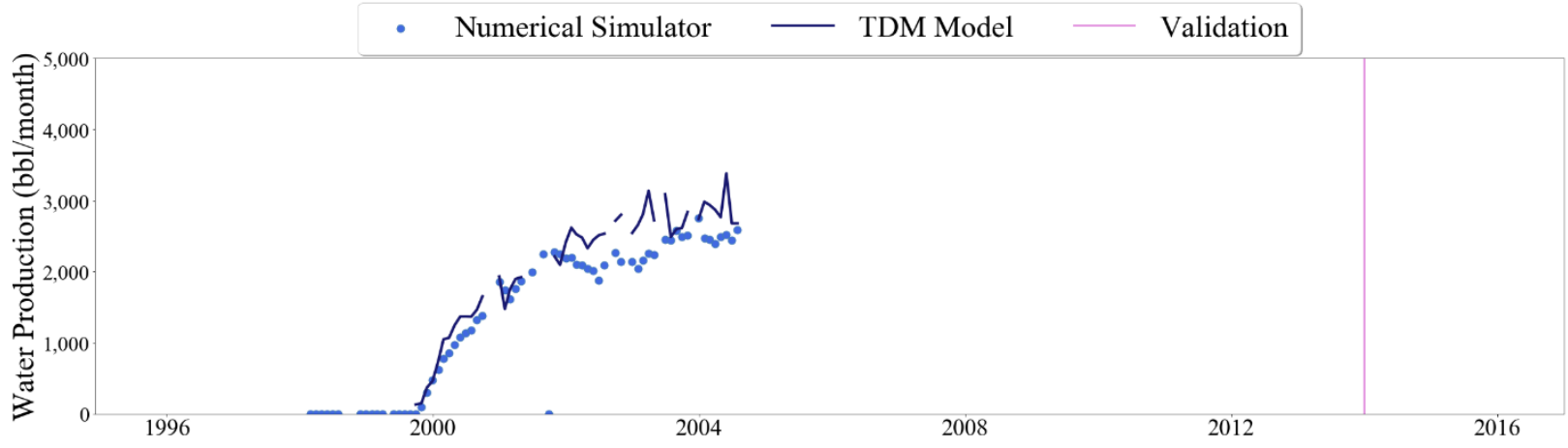

Figure 175 Well-014 oil, gas, and water rate TDM predictions vs actual simulation data plots for 3 Year Blind Validation TDM 


\section{Oil Rate}
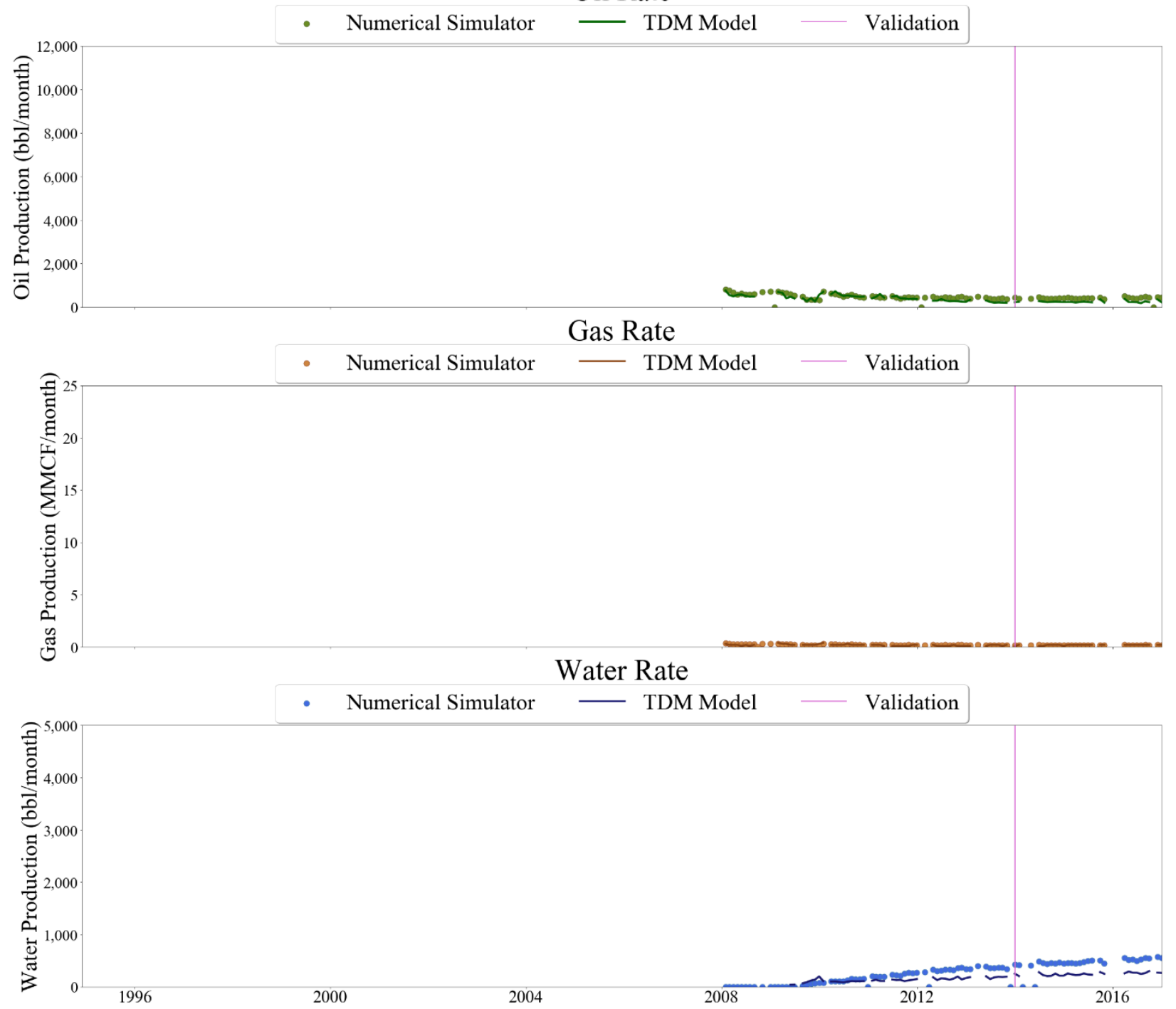

Figure 176 Well-043 oil, gas, and water rate TDM predictions vs actual simulation data plots for 3 Year Blind Validation TDM

Only three wells were believed to have poor accuracy, Well-016, Well-021, and Well-036, totaling 5\% of wells. The latter two of the three are included here. Well-021 had a poor fit due to the behavior seen in the gas plot where it failed to fully predict the ride in gas production at the beginning of blind validation. Well-036 had a poor fit due to it over predicting the beginning portions of the oil and gas plots. The individual well production plots can be examined in Appendix 8.2.4.1, in order by well number. 

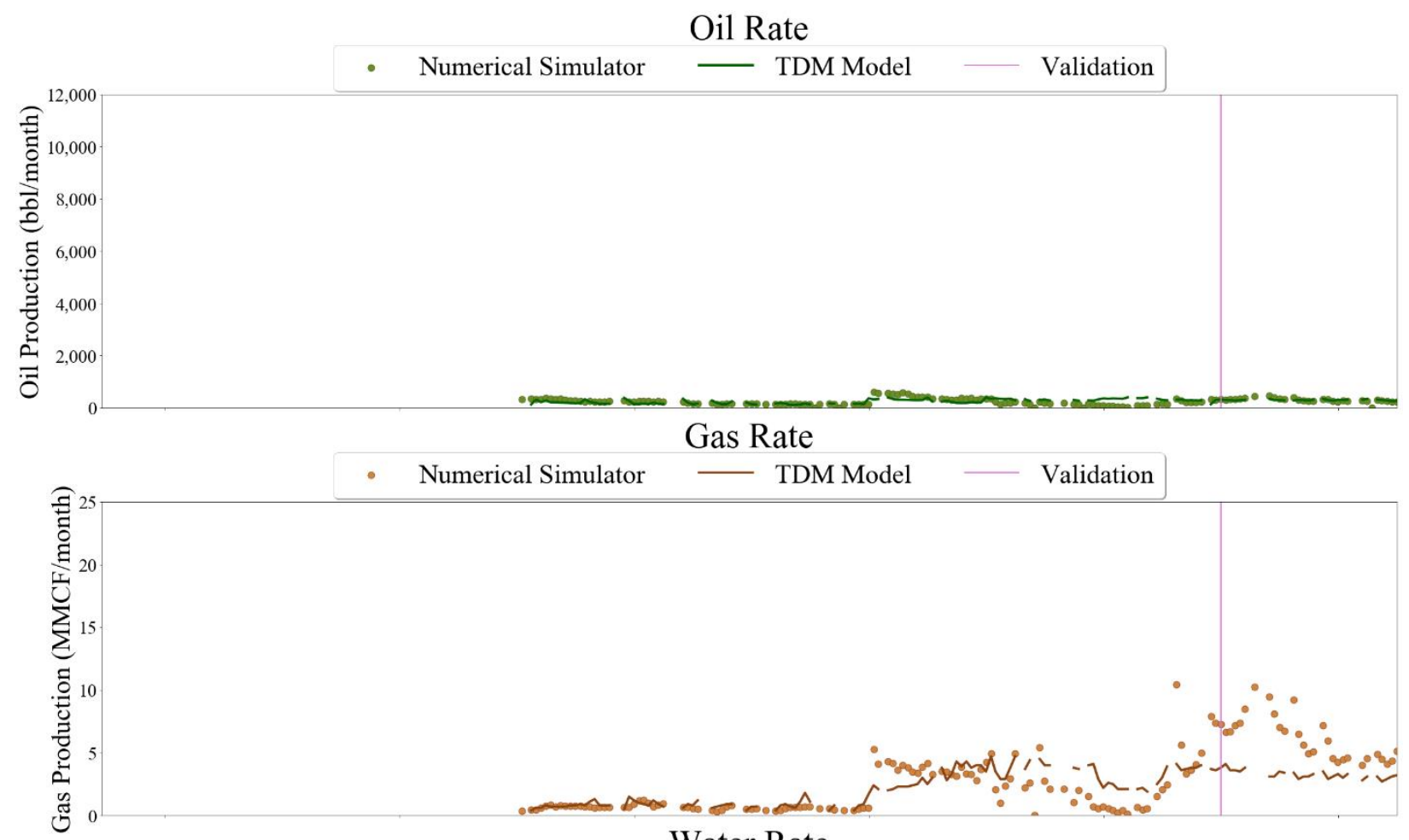

Water Rate

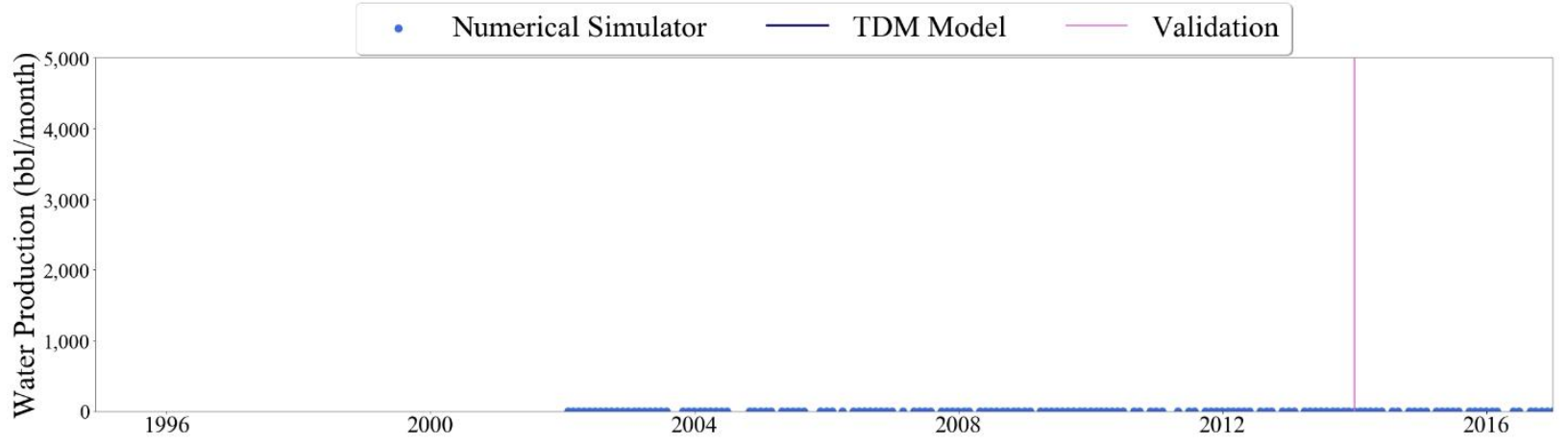

Figure 177 Well-021 oil, gas, and water rate TDM predictions vs actual simulation data plots for 3 Year Blind Validation TDM 

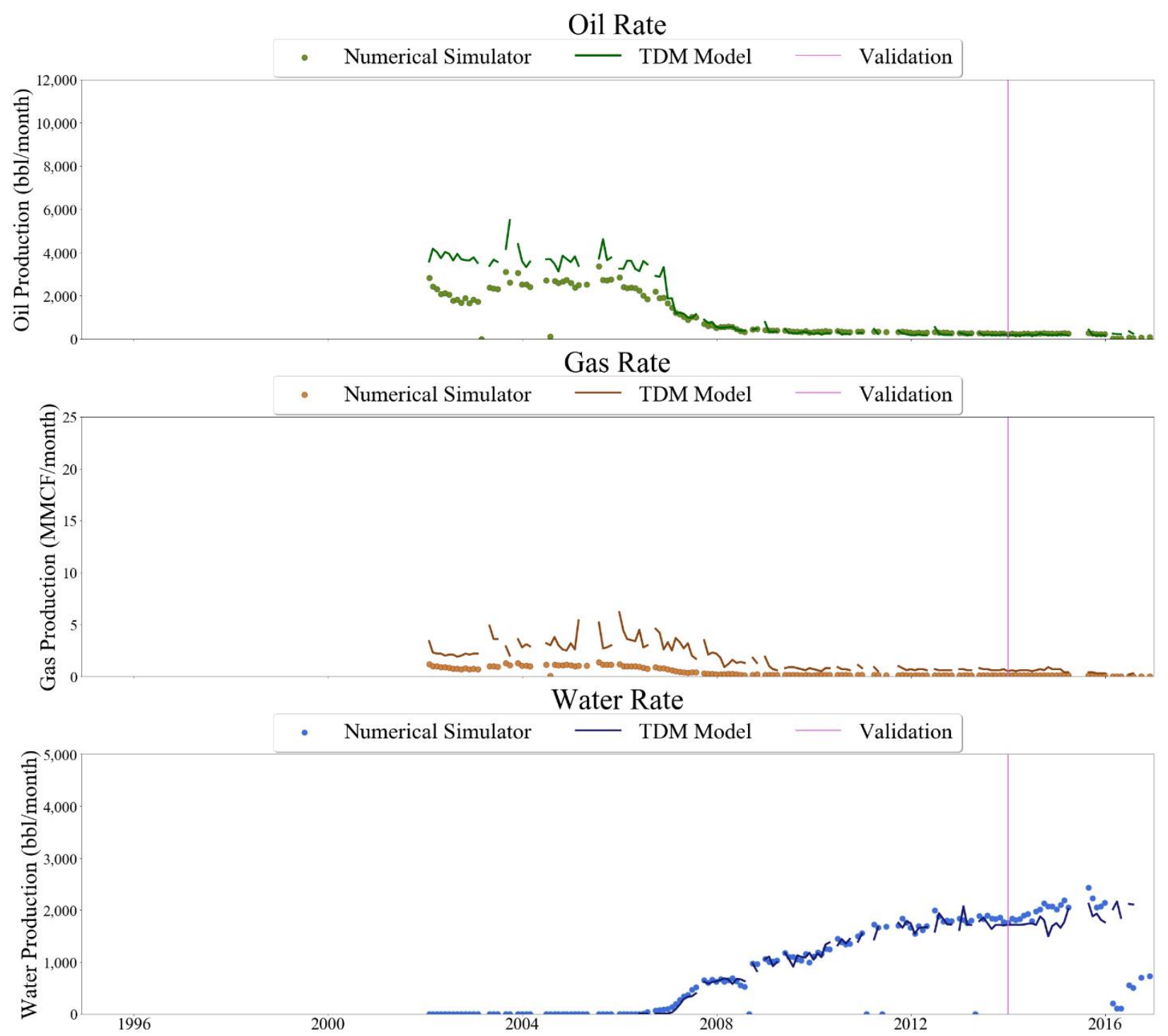

Figure 178 Well-036 oil, gas, and water rate TDM predictions vs actual simulation data plots for 3 Year Blind Validation TDM

The results outlined above were adequate and therefore another Top Down Model was made with an additional year of blind validation. The results for Multi-Random Partitioning-4 Year Blind Validation are explained next.

\subsubsection{Multi-Random Partitioning- 4 Year Blind Validation}

Entire reservoir oil, gas, and water results for the 4 Year Blind Validation case is shown below. The red line indicating the start of blind validation is at December $31^{\text {st }}, 2012$. The three plots show a great match between the prediction data and the actual simulation data besides a slightly lower prediction in cumulative water production than actual. 


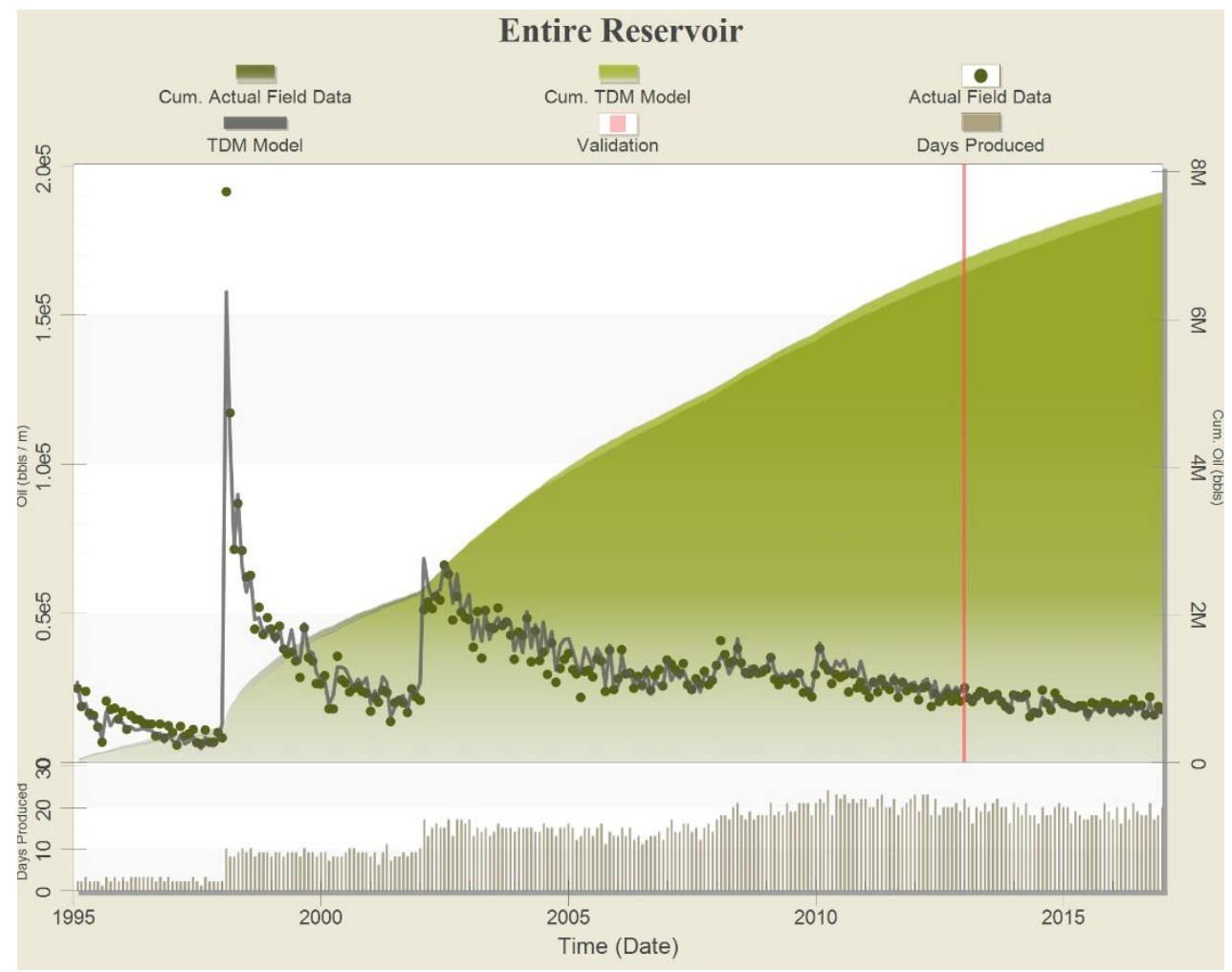

Figure 179 Entire reservoir oil results for Multi-Random Partitioning- 4 Year Blind Validation Top Down Model

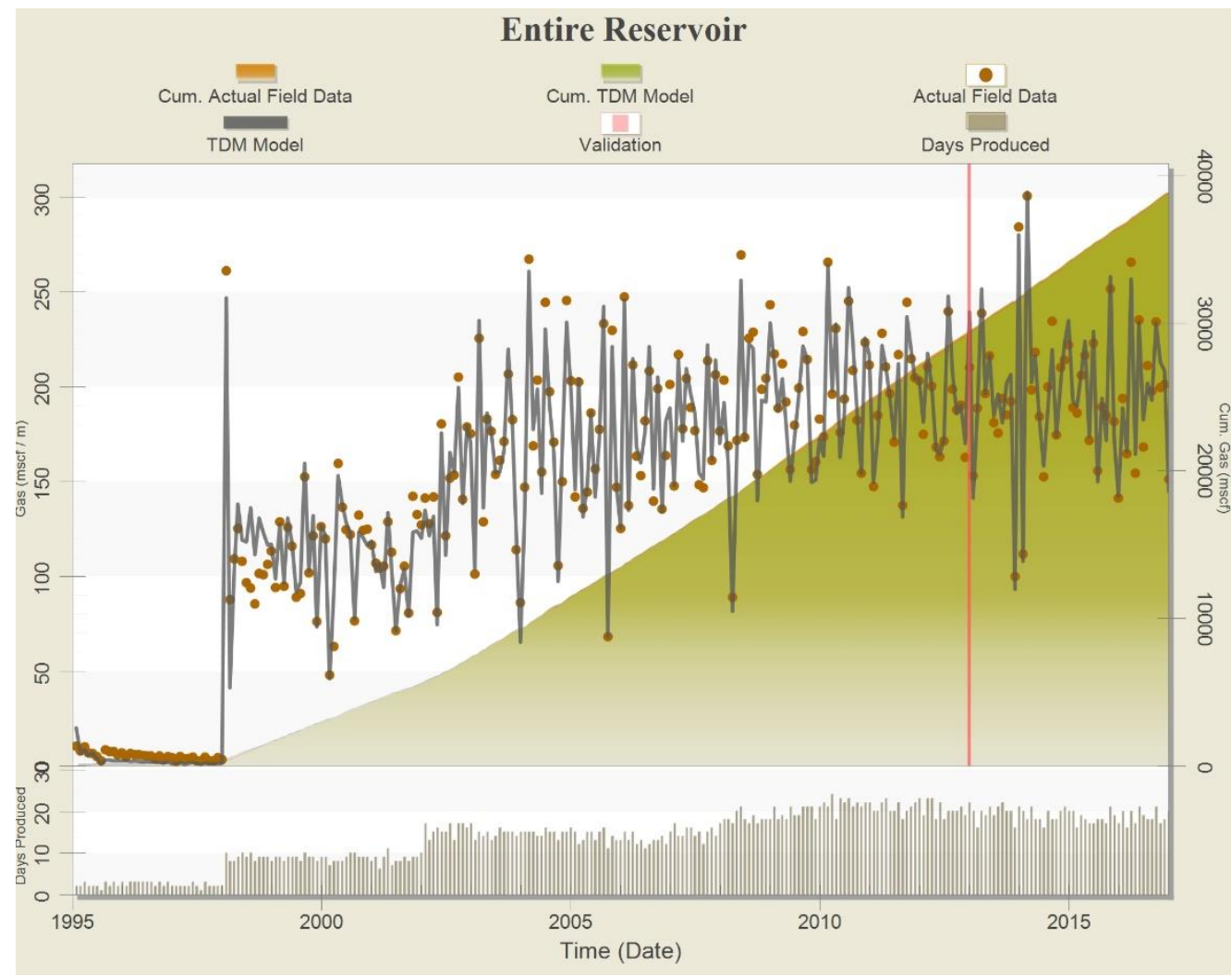

Figure 180 Entire reservoir gas results for Multi-Random Partitioning- 4 Year Blind Validation Top Down Model 


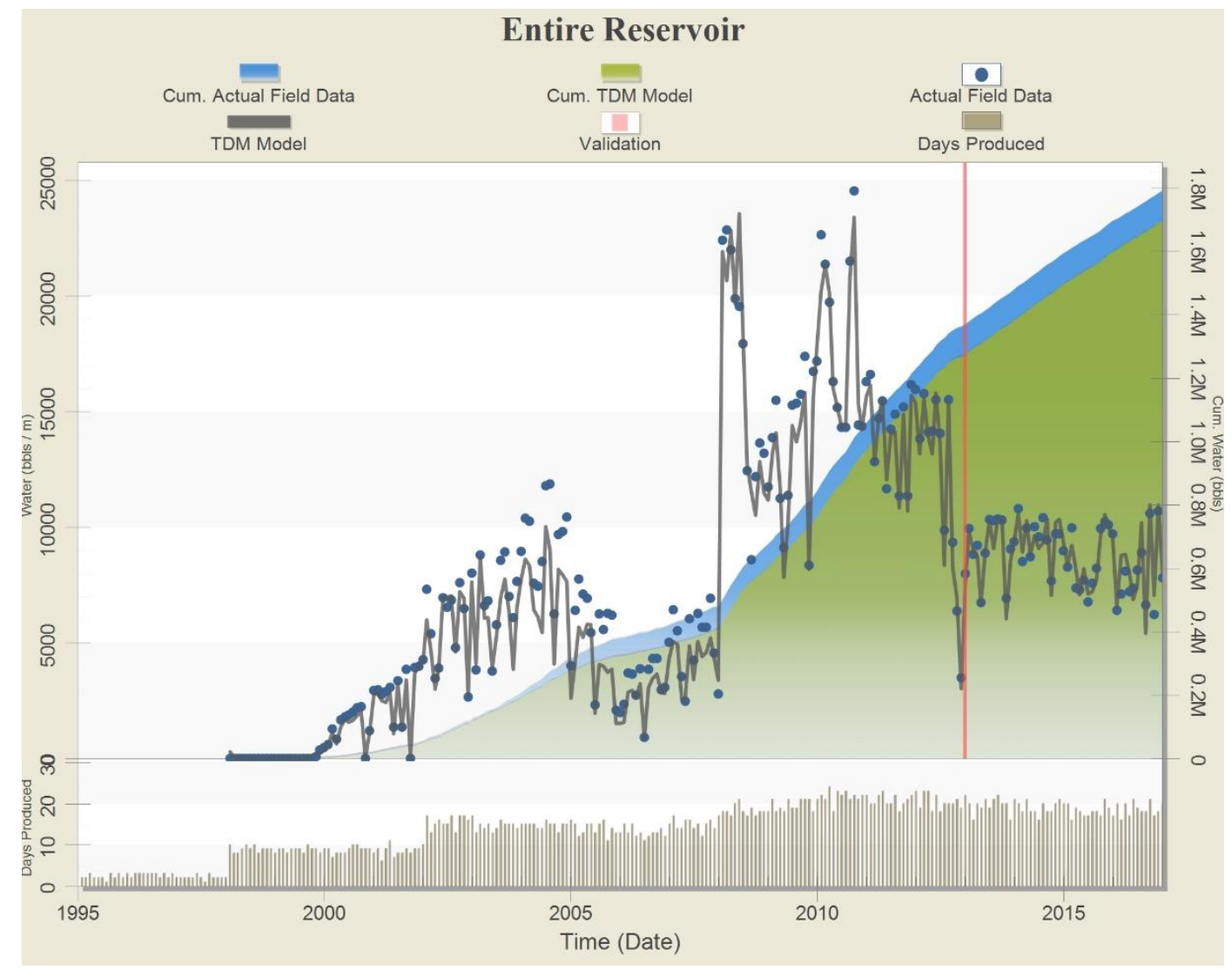

Figure 181 Entire reservoir water results for Multi-Random Partitioning- 4 Year Blind Validation Top Down Model

Heat maps for the entire reservoir for water saturation and reservoir pressure are included below in one year intervals from the start of the blind validation at December 31st, 2012. As previously stated, the error is shown in the third plot where the dark red color represents error of $30 \%$ or more. More heat maps are included in Appendix 8.2.5.2, in 3 month intervals for the entirety of the blind validation.

Reservoir Pressure (psi) at 2012-12-31
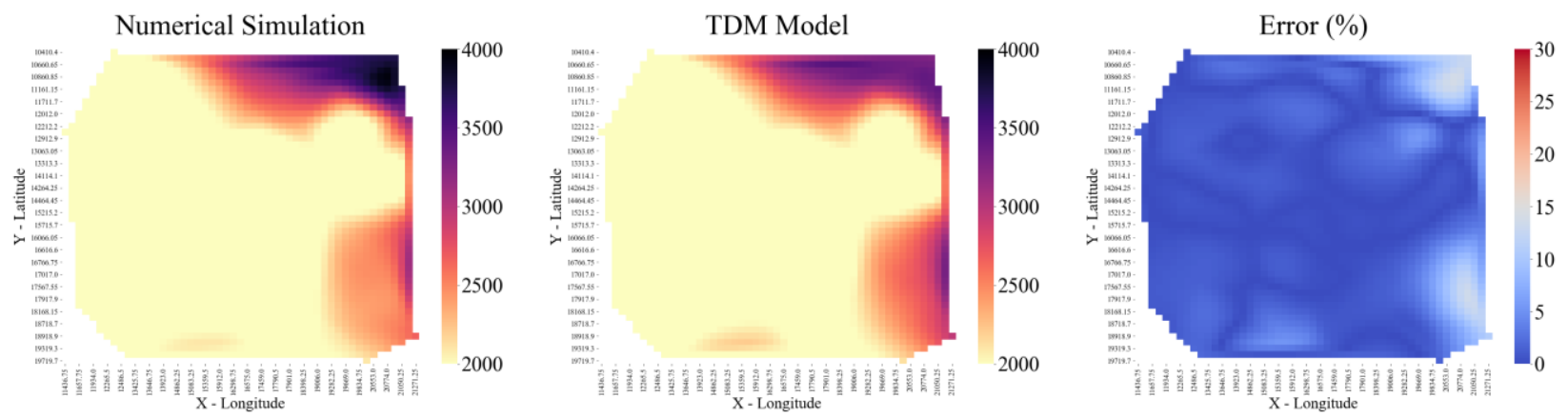

Figure 182 Entire reservoir heat map for reservoir pressure at December 31st, 2012 for 4 Year Blind Validation TDM 
Reservoir Pressure (psi) at 2013-12-31
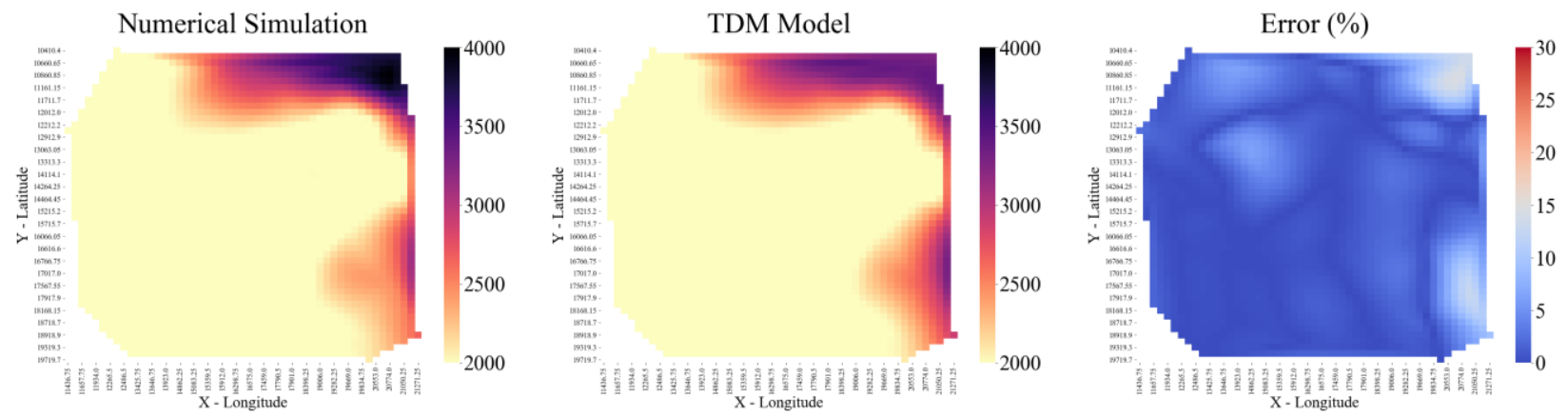

Figure 183 Entire reservoir heat map for reservoir pressure at December 31st, 2013 for 4 Year Blind Validation TDM

Reservoir Pressure (psi) at 2014-12-31
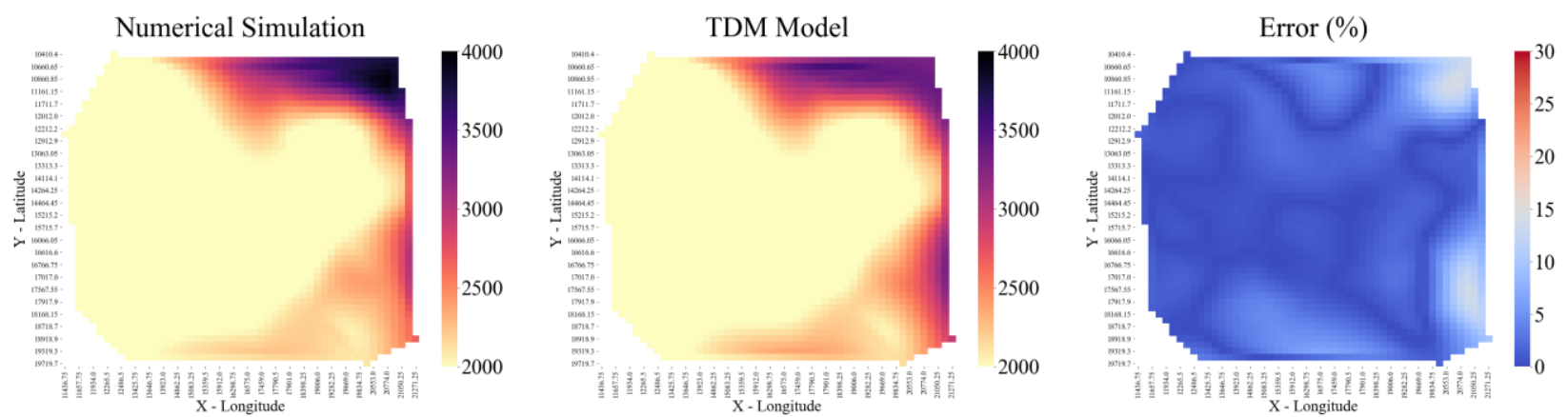

Figure 184 Entire reservoir heat map for reservoir pressure at December 31st, 2014 for 4 Year Blind Validation TDM

Reservoir Pressure (psi) at 2015-12-31
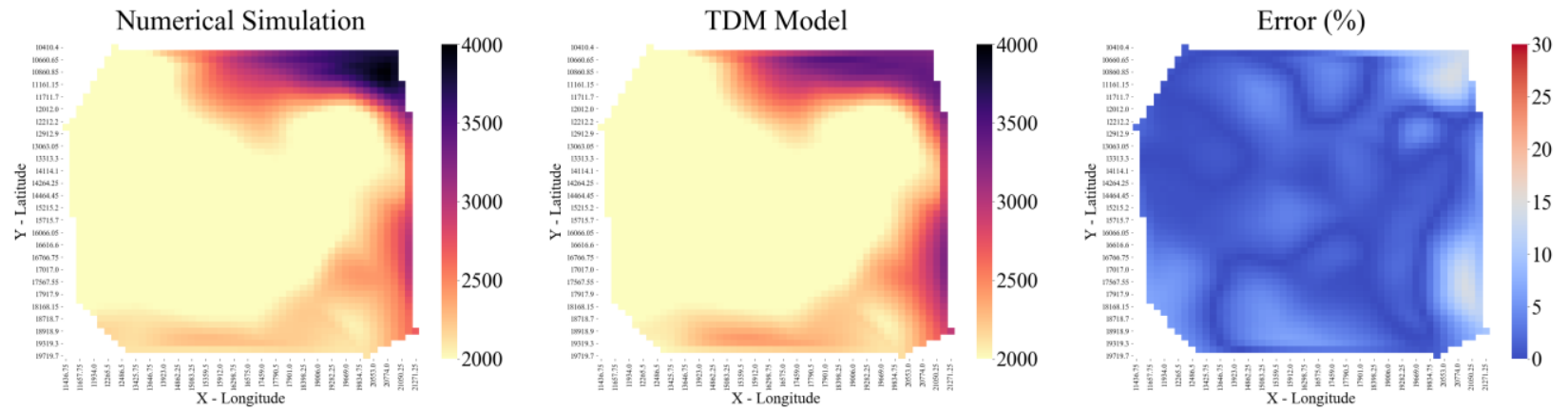

Figure 185 Entire reservoir heat map for reservoir pressure at December 31st, 2015 for 4 Year Blind Validation TDM 
Reservoir Pressure (psi) at 2016-12-31
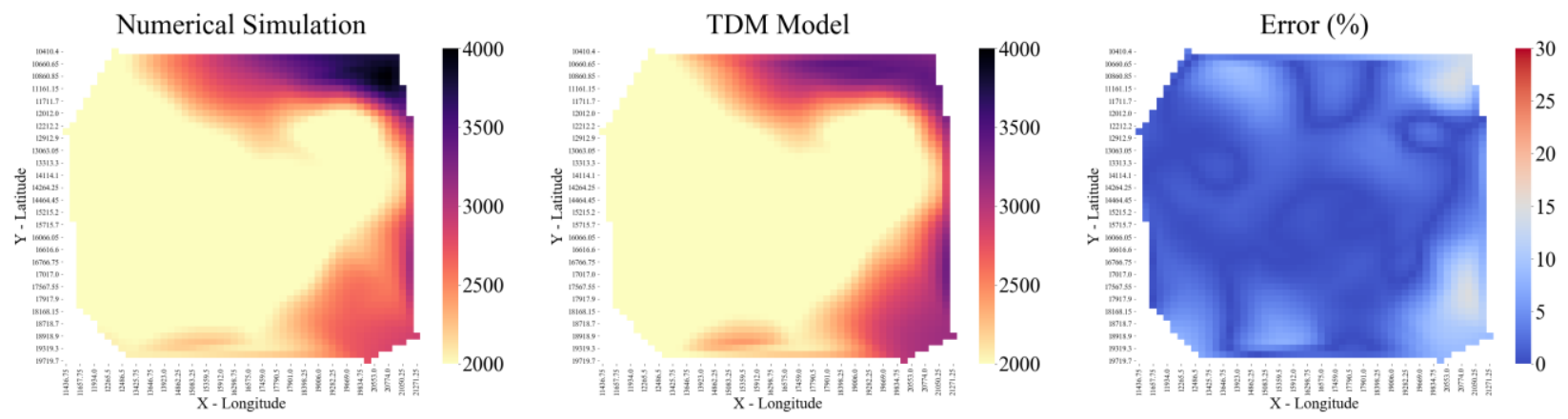

Figure 186 Entire reservoir heat map for reservoir pressure at December 31st, 2016 for 4 Year Blind Validation TDM

Water Saturation (\%) at 2012-12-31
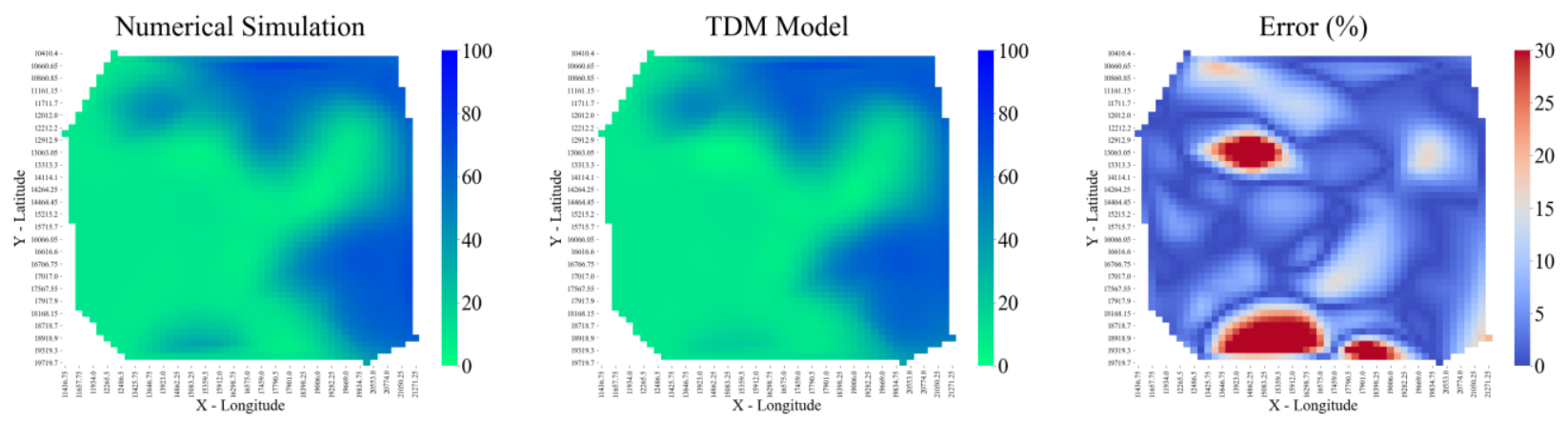

Figure 187 Entire reservoir heat map for water saturation at December 31st, 2012 for 4 Year Blind Validation TDM

Water Saturation (\%) at 2013-12-31
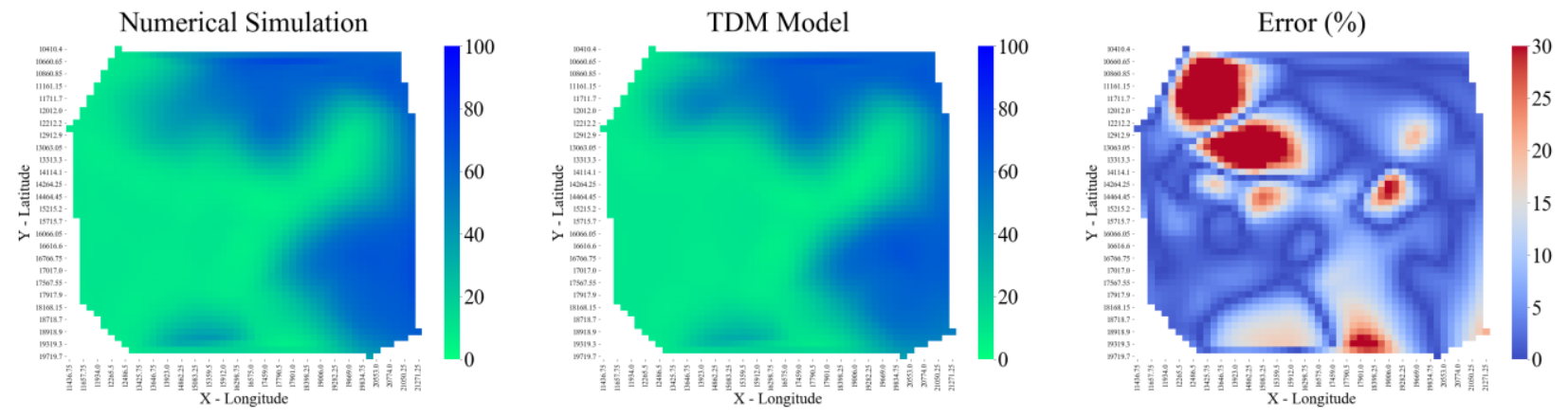

Figure 188 Entire reservoir heat map for water saturation at December 31st, 2013 for 4 Year Blind Validation TDM 
Water Saturation (\%) at 2014-12-31
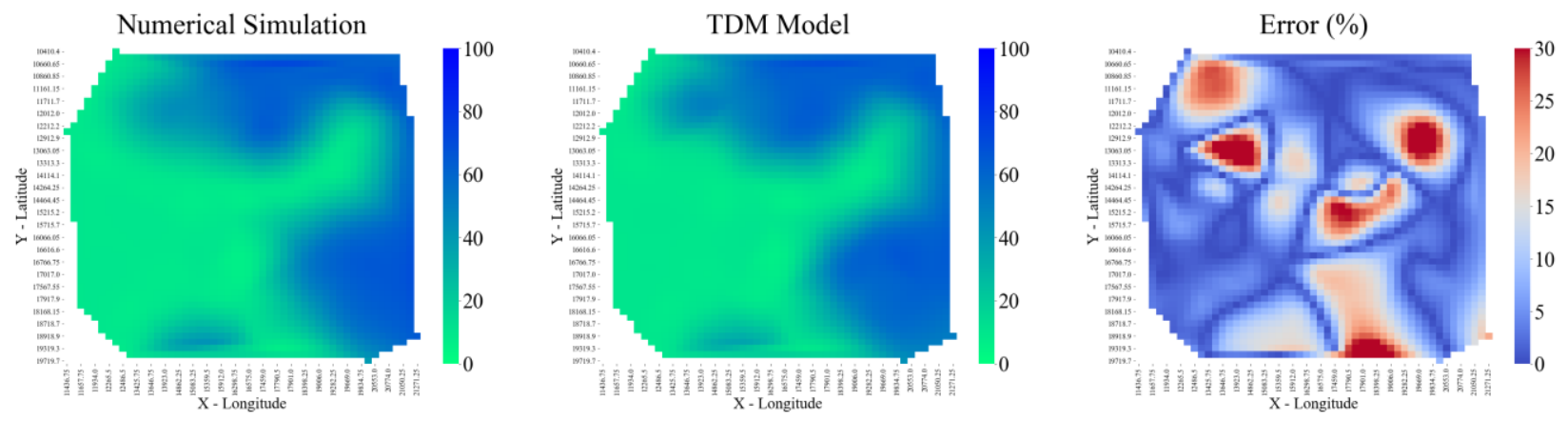

Figure 189 Entire reservoir heat map for water saturation at December 31st, 2014 for 4 Year Blind Validation TDM

Water Saturation (\%) at 2015-12-31
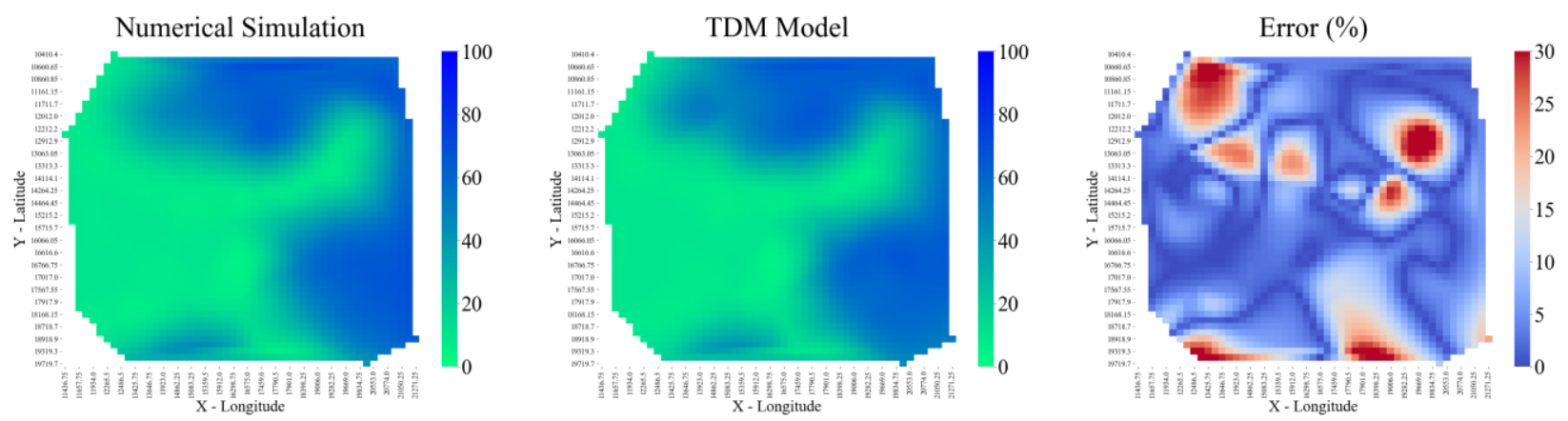

Figure 190 Entire reservoir heat map for water saturation at December 31st, 2015 for 4 Year Blind Validation TDM

Water Saturation (\%) at 2016-12-31
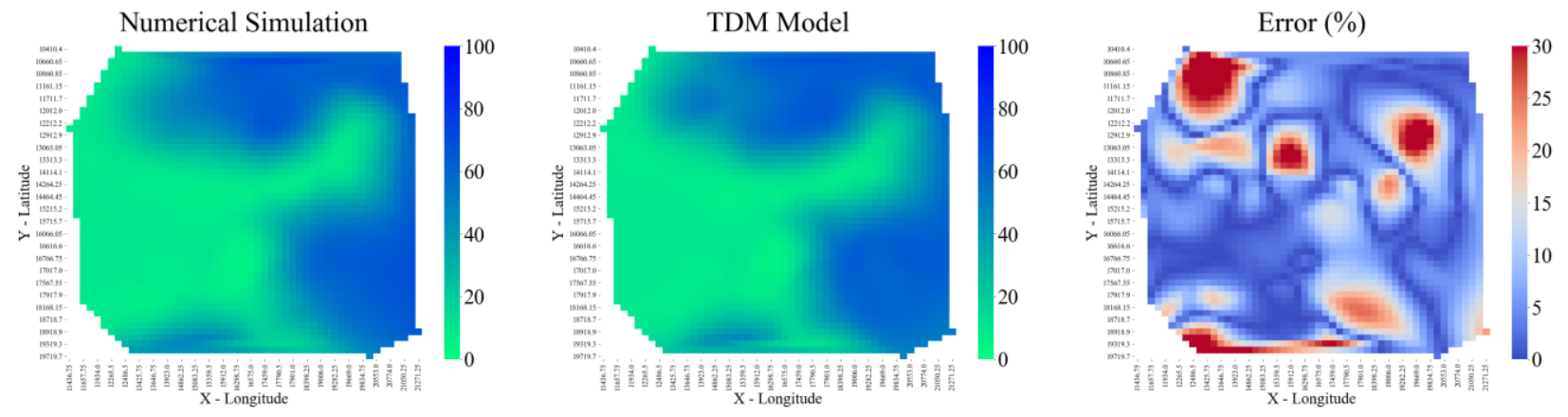

Figure 191 Entire reservoir heat map for water saturation at December 31st, 2016 for 4 Year Blind Validation TDM

Well-008 and Well-034 are displayed below as examples of wells with good predictions. These two wells along with an additional 32 wells believed to have good accuracy comprise about $60 \%$ of the 57 wells. The red line on the plots below is drawn on December $31^{\text {st }}, 2012$. 


\section{Oil Rate}
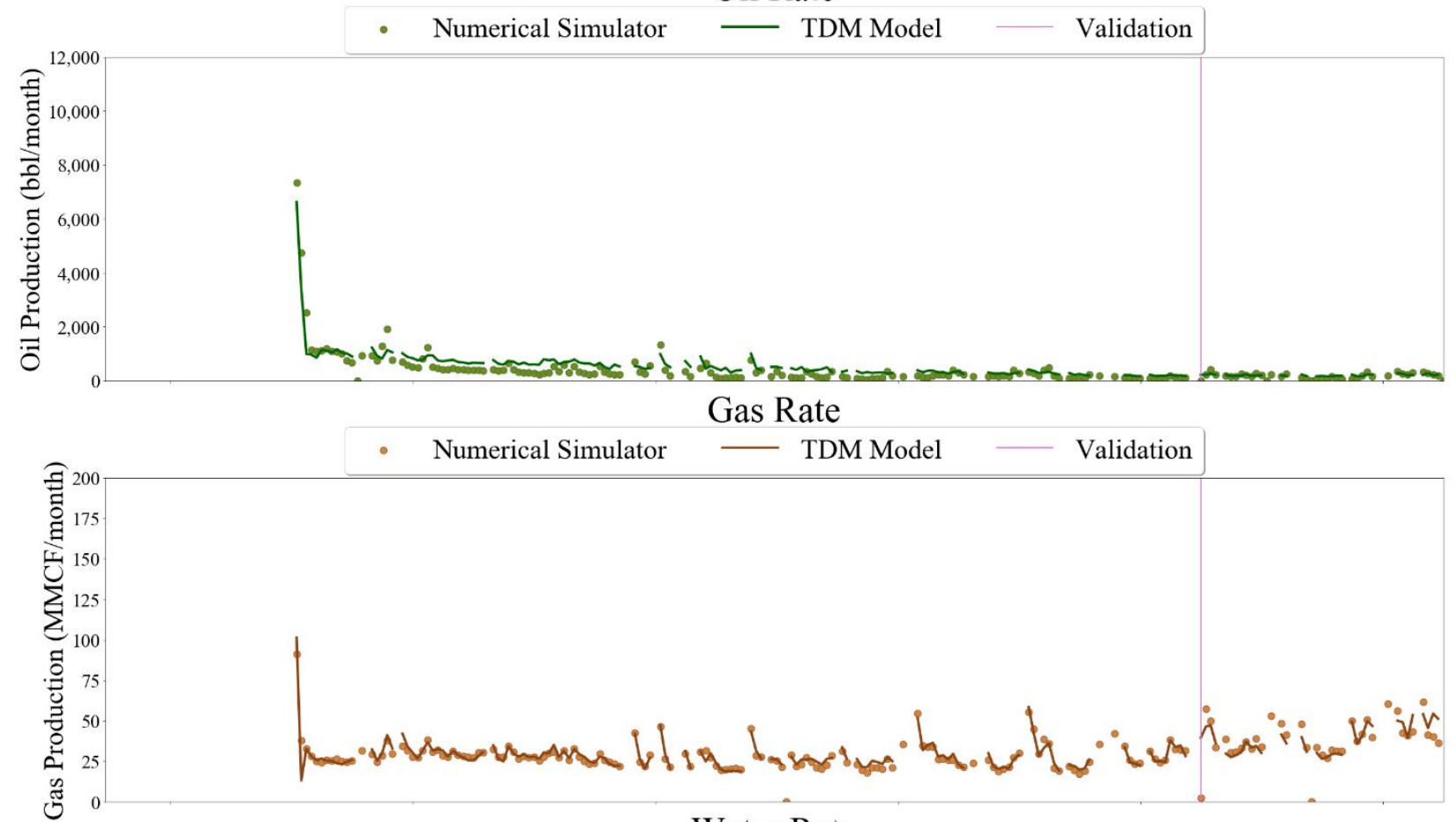

\section{Water Rate}

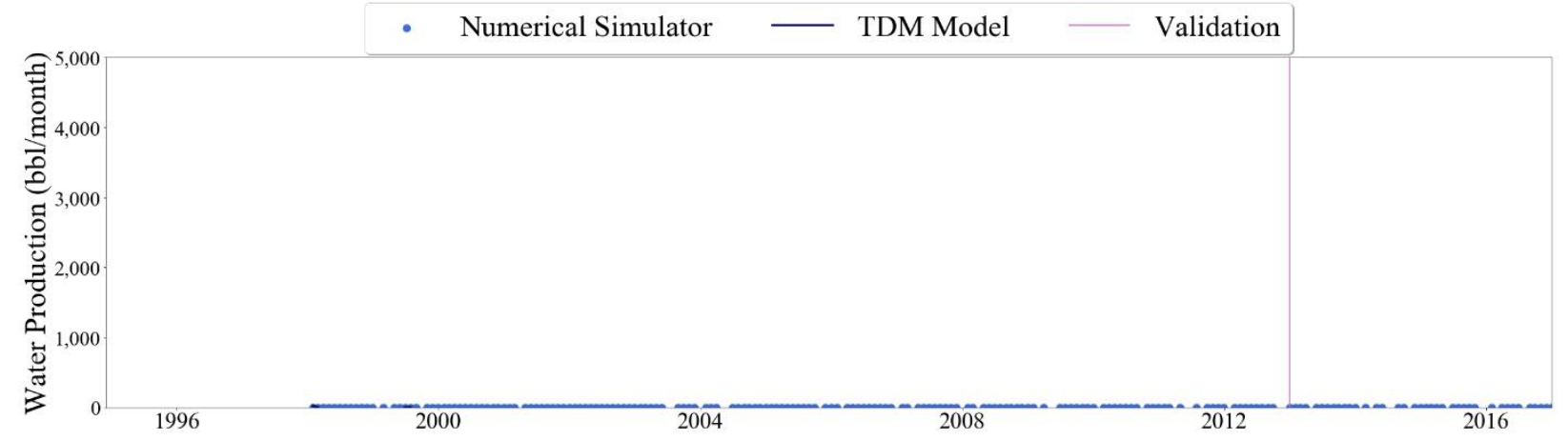

Figure 192 Well-008 oil, gas, and water rate TDM predictions vs actual simulation data plots for 4 Year Blind Validation TDM 

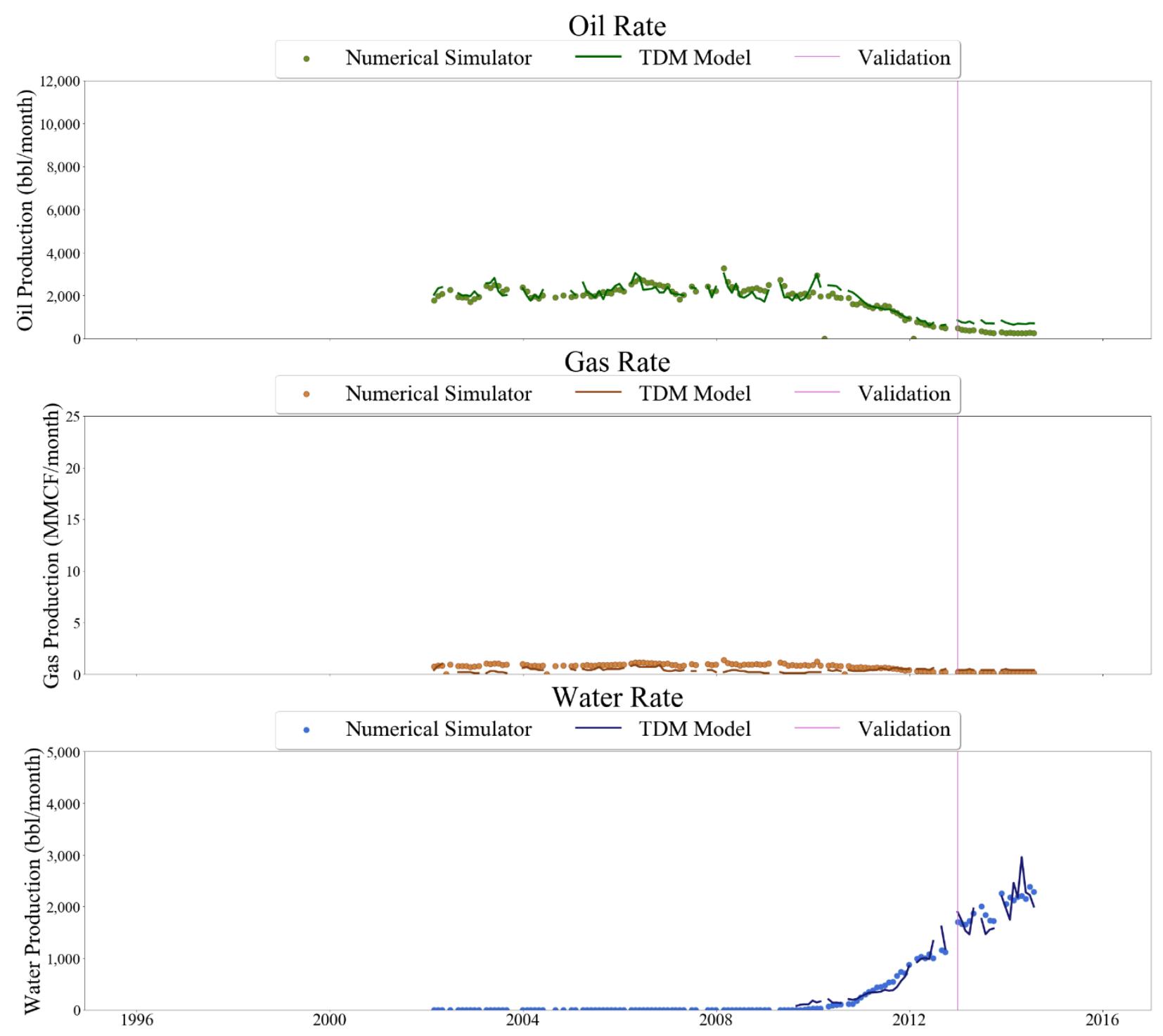

Figure 193 Well-034 oil, gas, and water rate TDM predictions vs actual simulation data plots for 4 Year Blind Validation TDM

Well-002 below was placed in the average category due to the difference between predictions and actual data in a small middle portion of the oil plot and a latter part of the water plot. Well-038 on the other hand was average due to its oil prediction at the beginning of the data for that well in addition to the latter portion of the water graph. Twenty-six percent or fifteen wells in total were placed in the average category. 


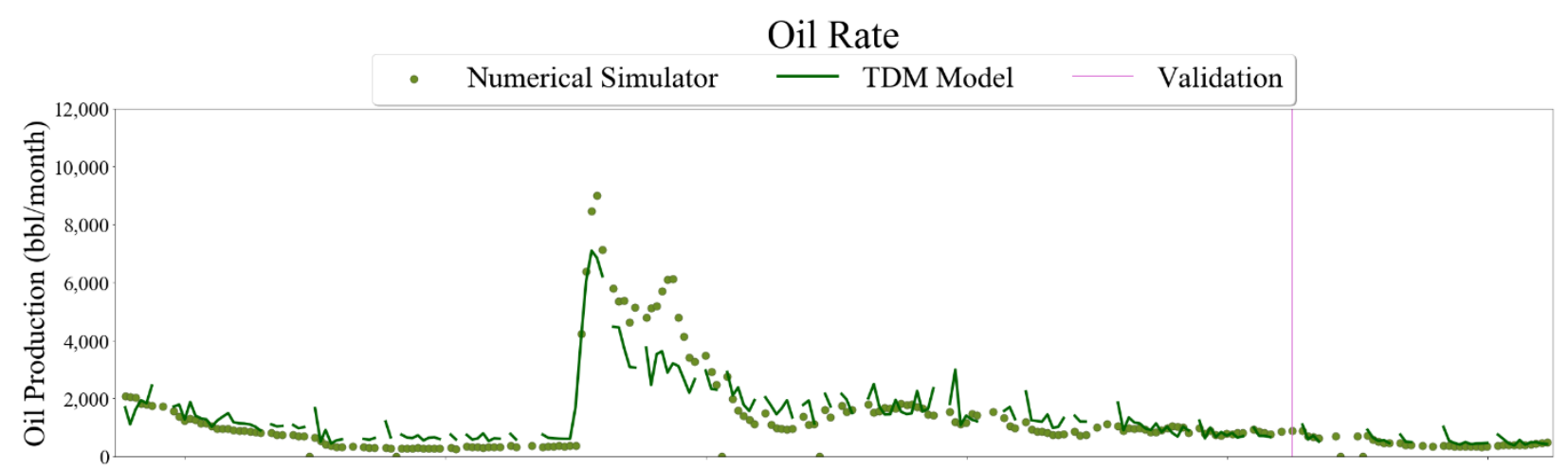

Gas Rate

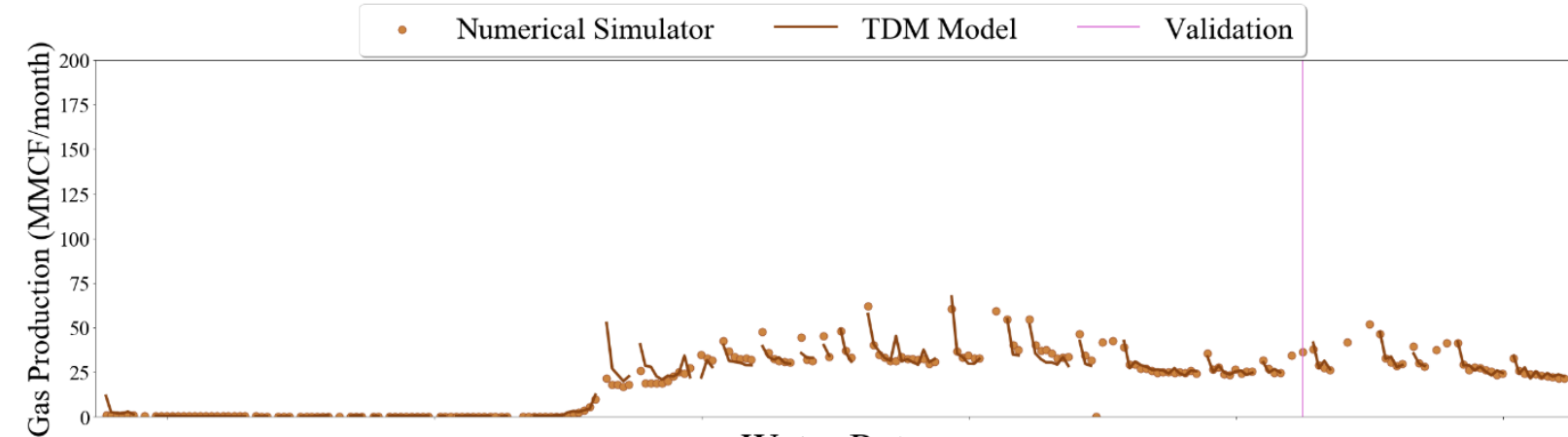

Water Rate

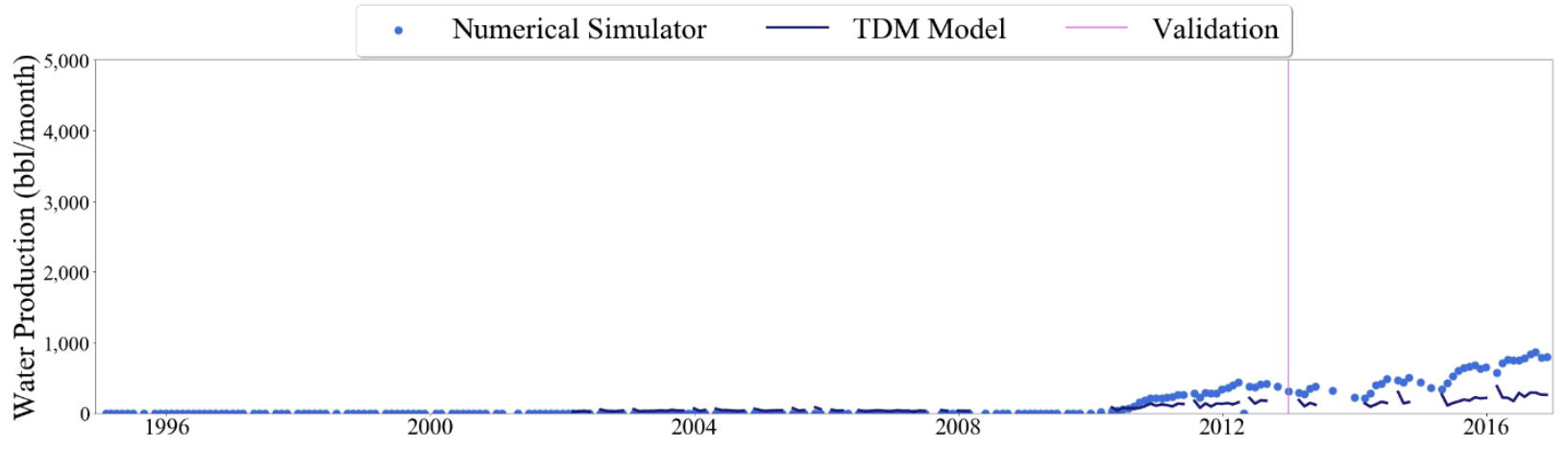

Figure 194 Well-002 oil, gas, and water rate TDM predictions vs actual simulation data plots for 4 Year Blind Validation TDM 


\section{Oil Rate}

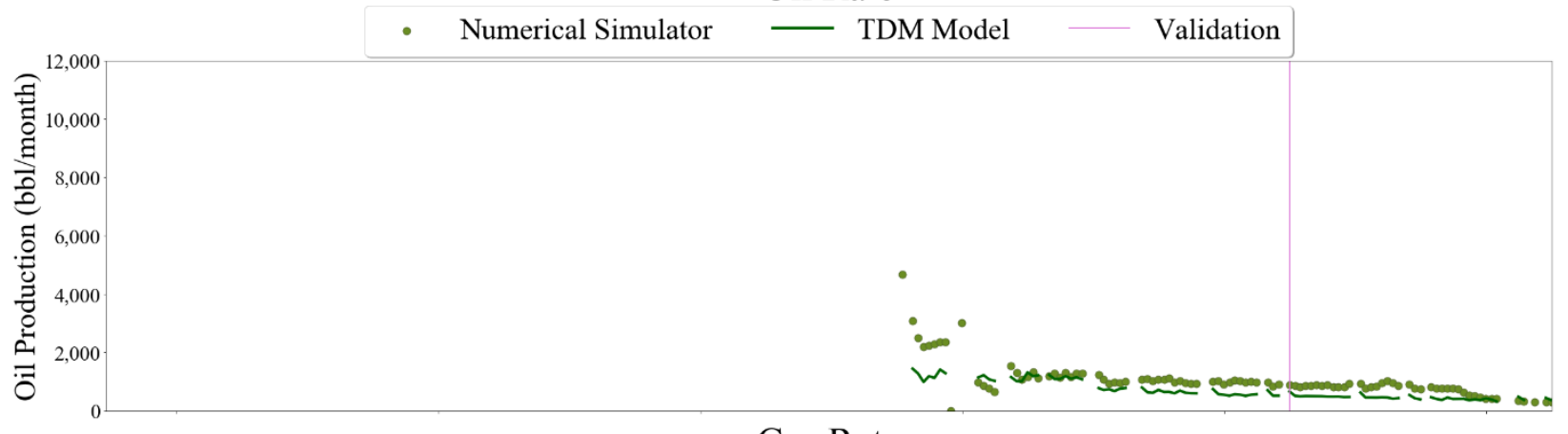

\section{Gas Rate}
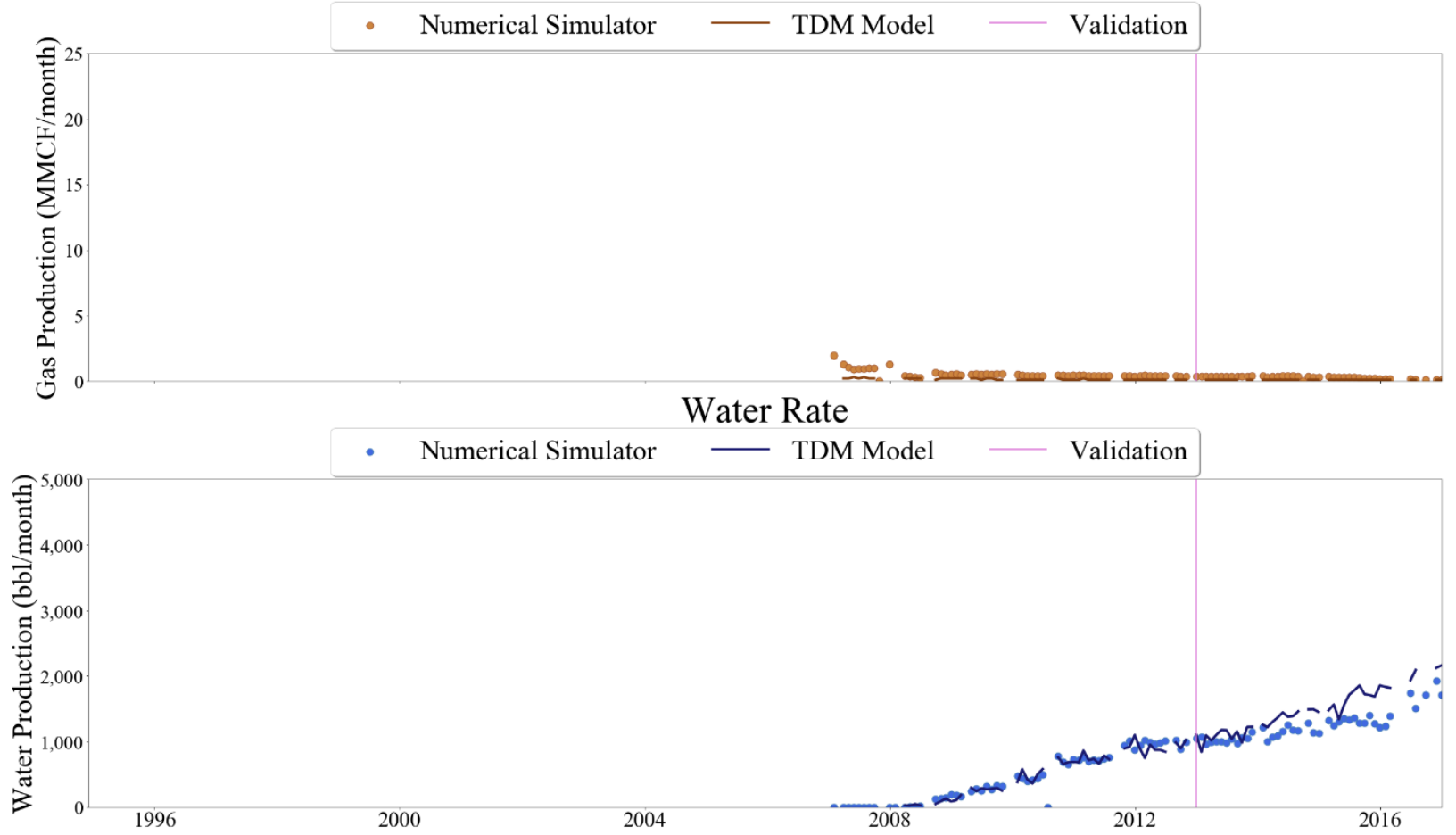

Figure 195 Well-038 oil, gas, and water rate TDM predictions vs actual simulation data plots for 4 Year Blind Validation TDM

Seven wells were thought to be of poor quality in the 4 Year Blind Validation scenario, about $12 \%$. Well-017 was thought to have a poor fit due to the over prediction in the latter portion of the oil model. Similarly, Well-055 had a poor fit for the same reason as Well-017. The individual well production plots are included in Appendix 8.2.5.2. 

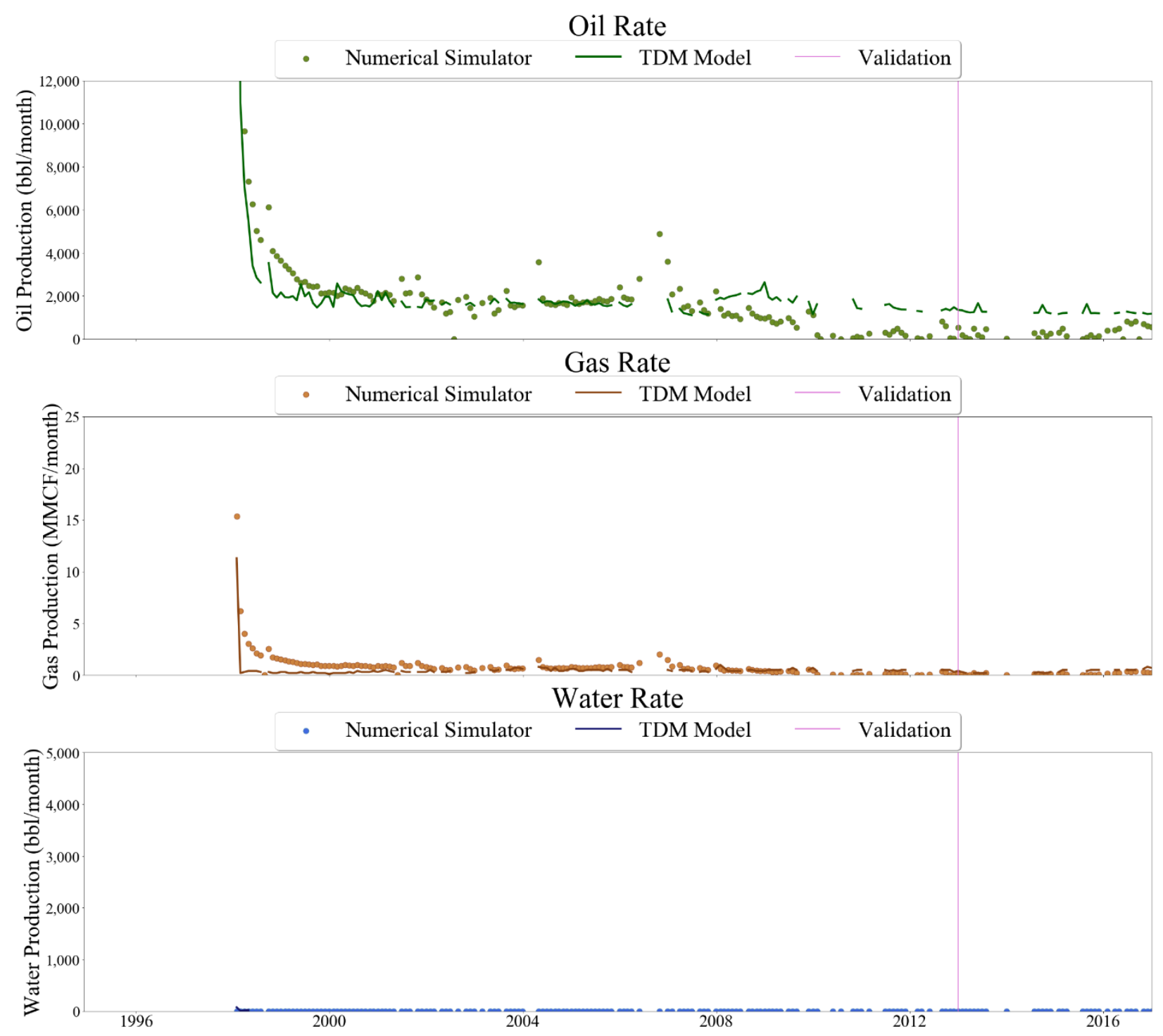

Figure 196 Well-017 oil, gas, and water rate TDM predictions vs actual simulation data plots for 4 Year Blind Validation TDM 


\section{Oil Rate}

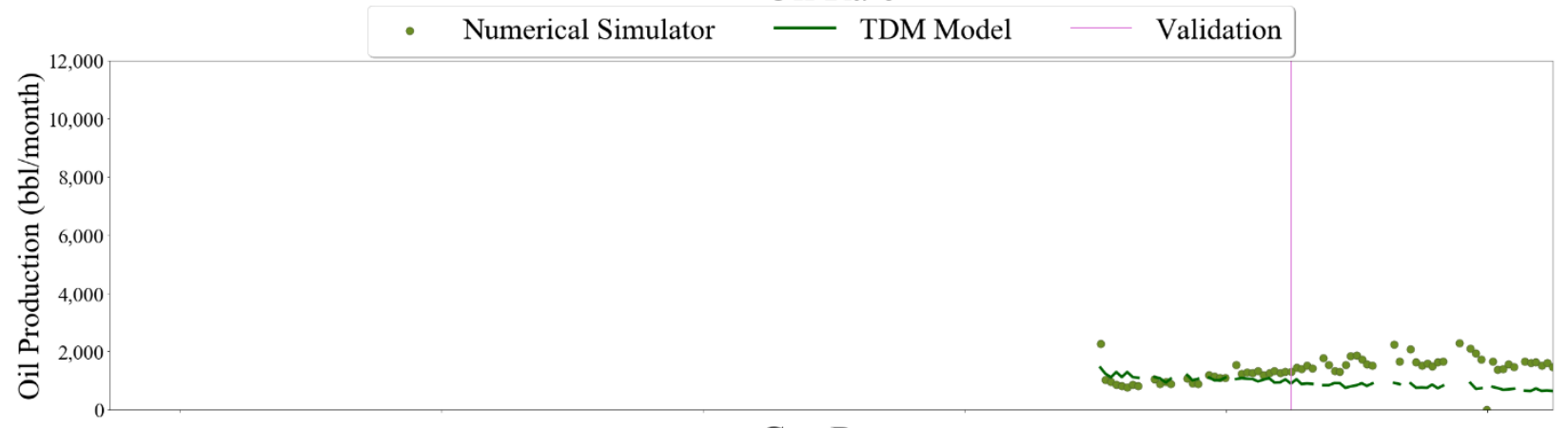

Gas Rate
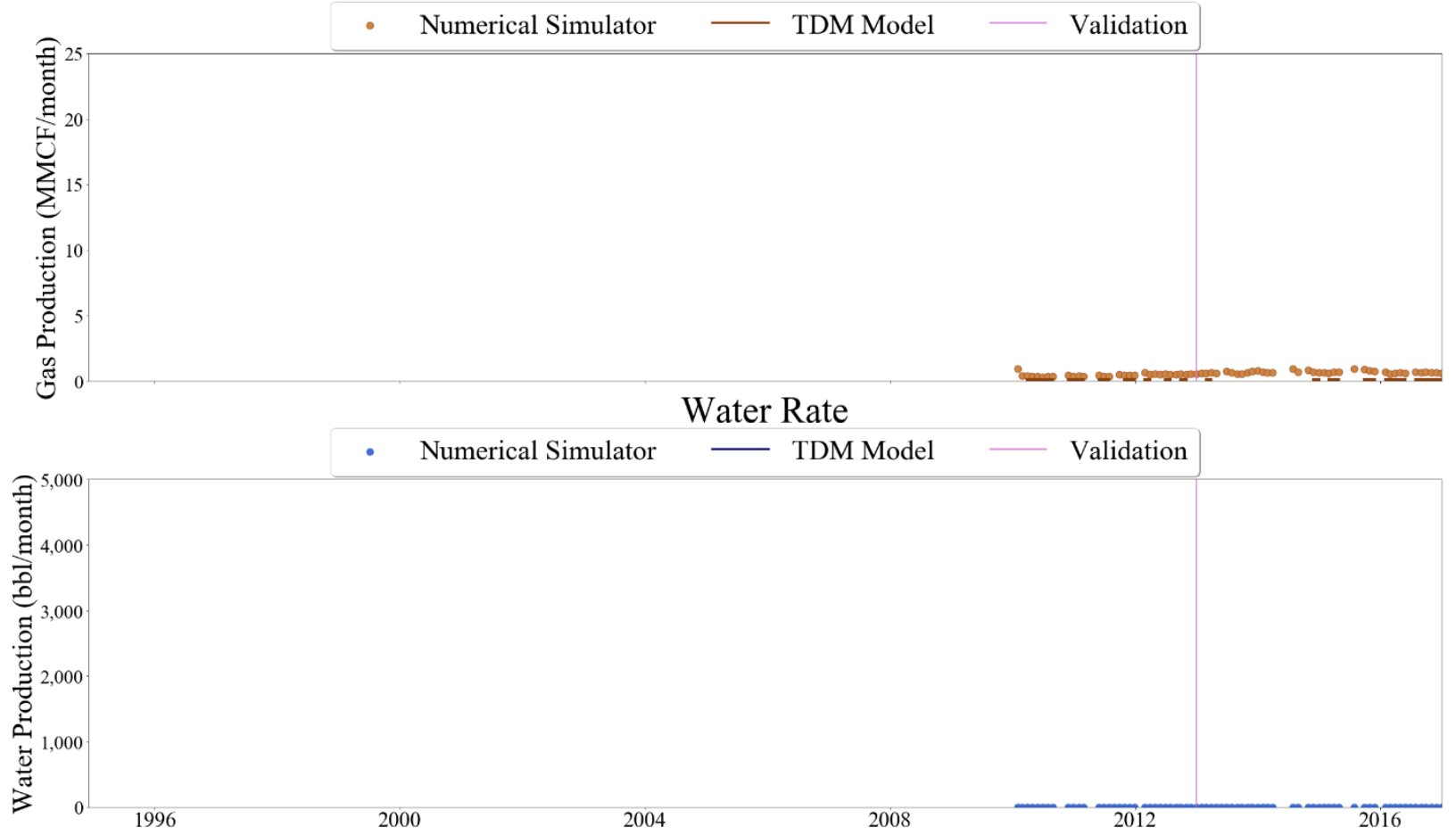

Figure 197 Well-055 oil, gas, and water rate TDM predictions vs actual simulation data plots for 4 Year Blind Validation TDM

All plots and results shown for the 4 Year Blind Validation case were adequate. Another Top Down Model was created with another year of blind validation and called Multi-Random Partitioning- 5 Year Blind Validation. This model was again developed to see if good results could also be obtained with less data assigned to training and calibration, and more data assigned to blind validation to test the model. Those results are presented next.

\subsubsection{Multi-Random Partitioning- 5 Year Blind Validation}

The 5 Year Blind Validation case entire reservoir oil, gas, and water results are shown below. The red line appears at December $31^{\text {st }}, 2011$ where the blind validation begins. The accuracy of the predictions is evident in the plots below where only the water prediction is slightly off at the end of the simulation. 


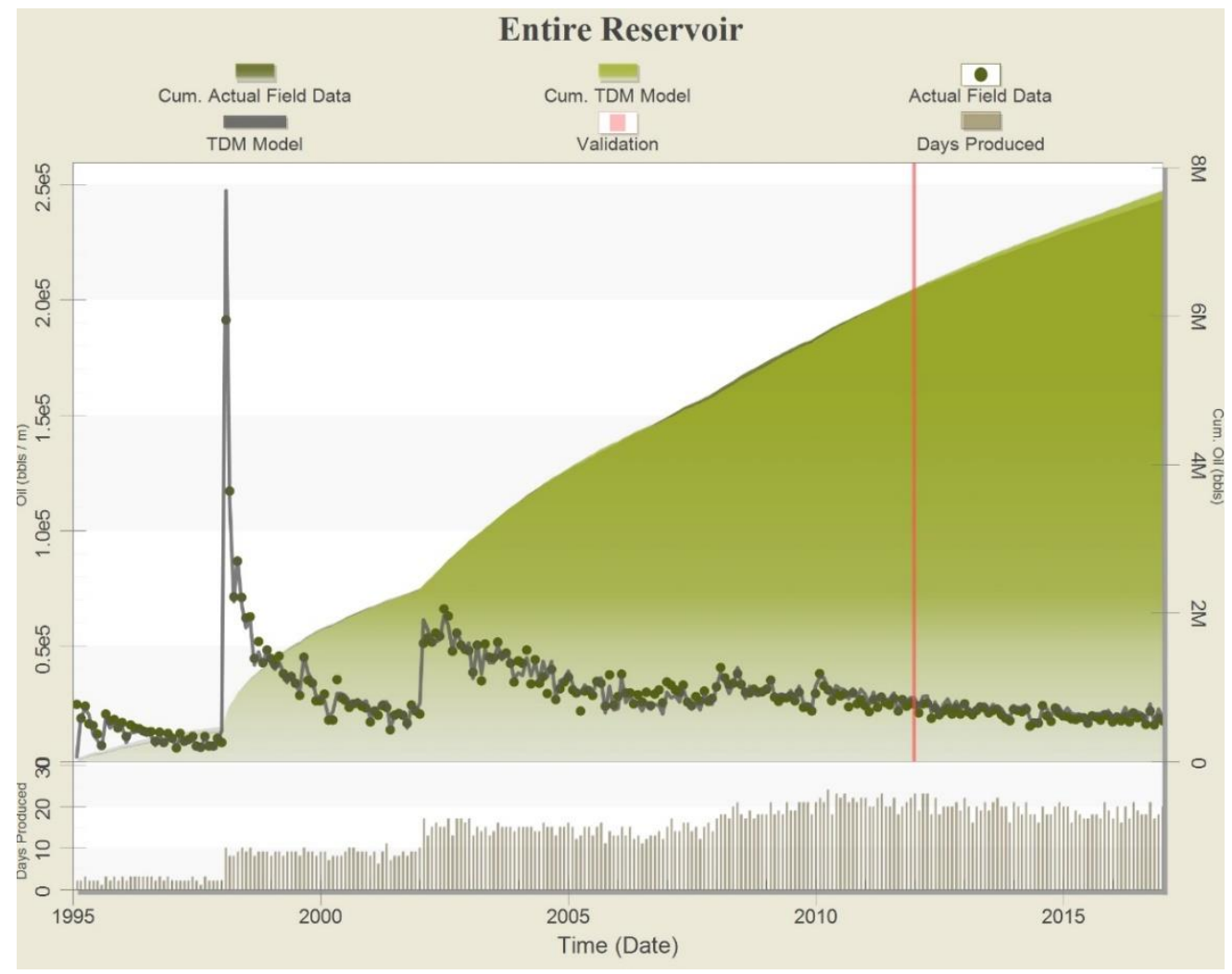

Figure 198 Entire reservoir oil results for Multi-Random Partitioning- 5 Year Blind Validation Top Down Model

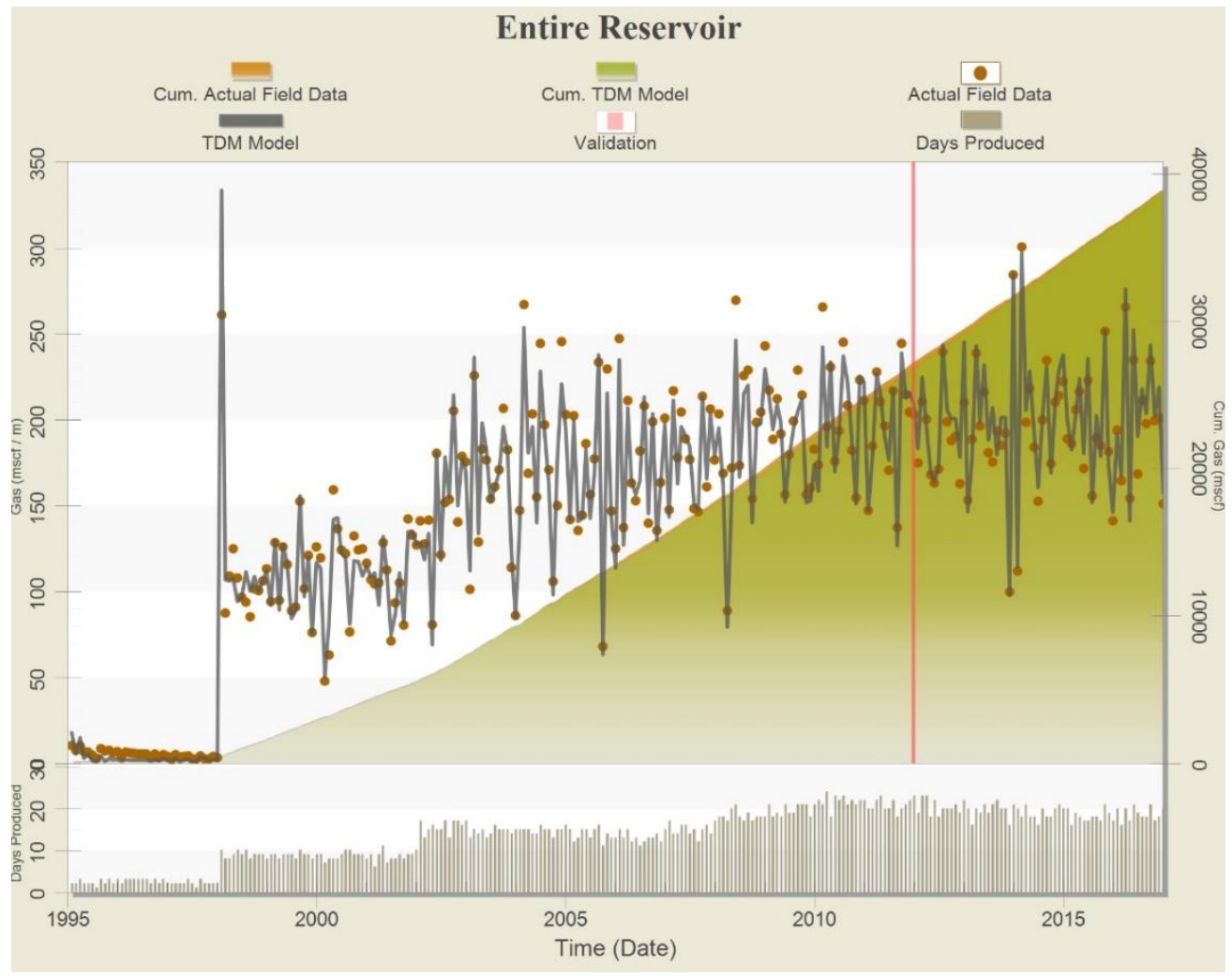

Figure 199 Entire reservoir gas results for Multi-Random Partitioning- 5 Year Blind Validation Top Down Model 


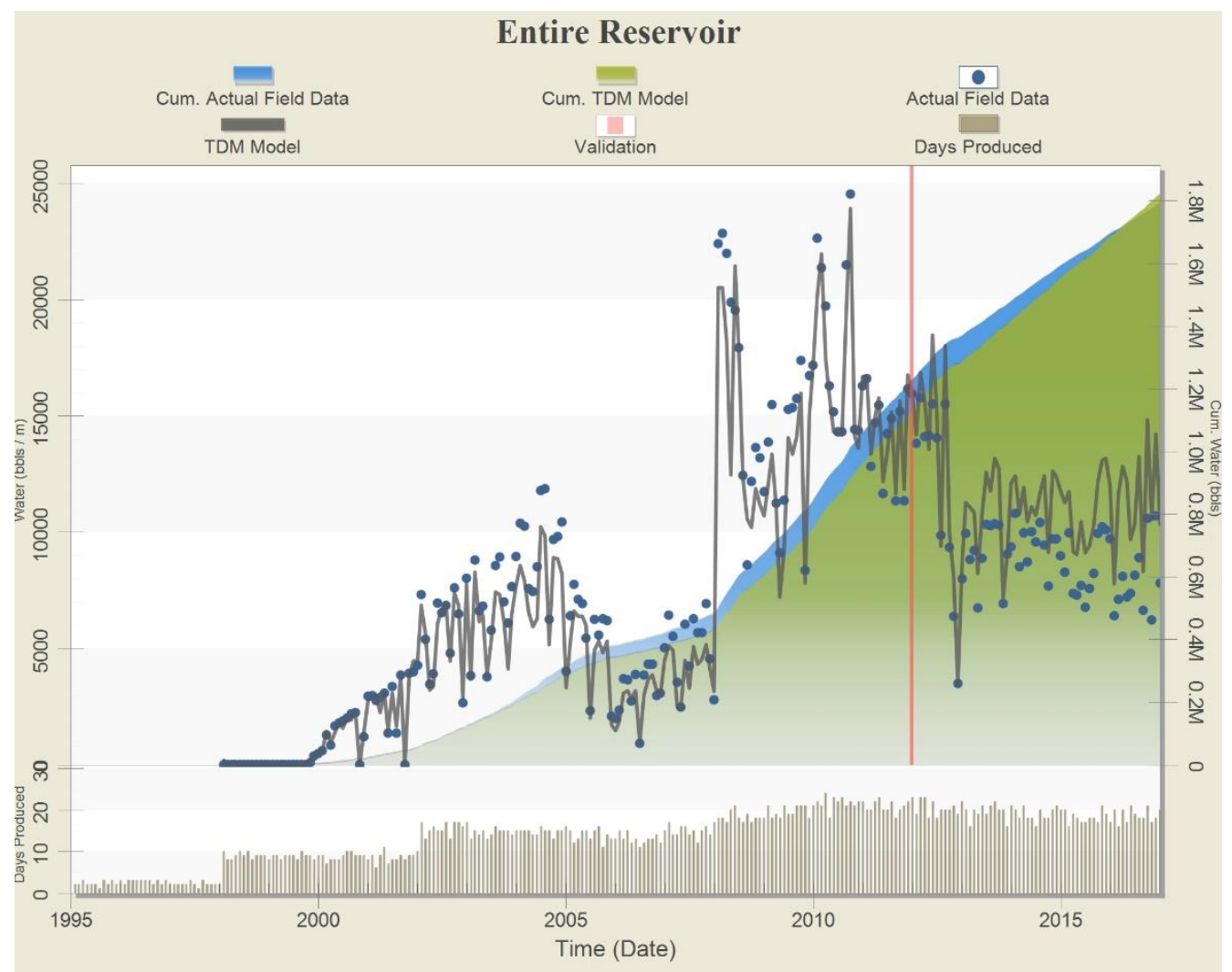

Figure 200 Entire reservoir water results for Multi-Random Partitioning- 5 Year Blind Validation Top Down Model

Next, the entire reservoir heat maps are provided for reservoir pressure and water saturation in one year intervals from the start of the blind validation. In the same manner as the previous plots, the first plot is the TDM predictions, the next are the actual values, and the last plot is the error heat map. Additional heat maps are given in Appendix 8.2.6.2, in six month intervals for reservoir pressure and water saturation for the entirety of the blind validation.

Reservoir Pressure (psi) at 2011-12-31
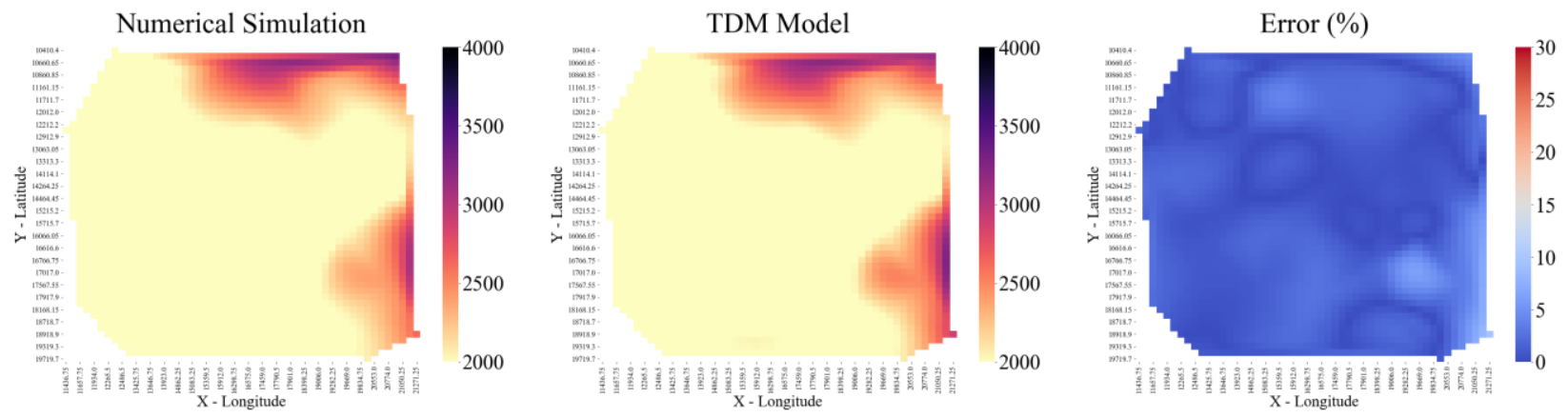

Figure 201 Entire reservoir heat map for reservoir pressure at December 31st, 2011 for 5 Year Blind Validation TDM 
Reservoir Pressure (psi) at 2012-12-31
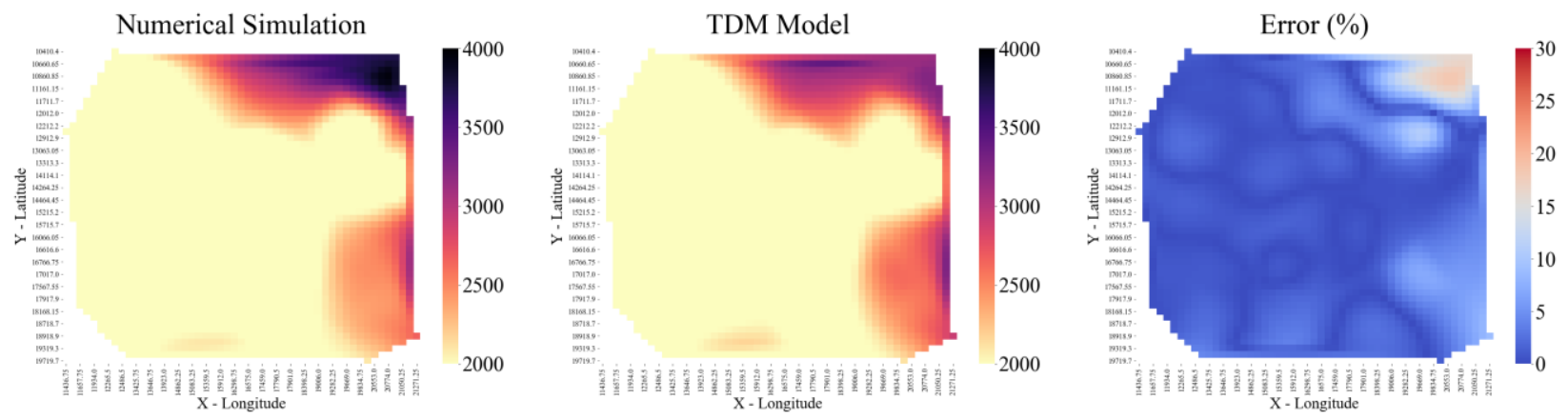

Figure 202 Entire reservoir heat map for reservoir pressure at December 31st, 2012 for 5 Year Blind Validation TDM

Reservoir Pressure (psi) at 2013-12-31
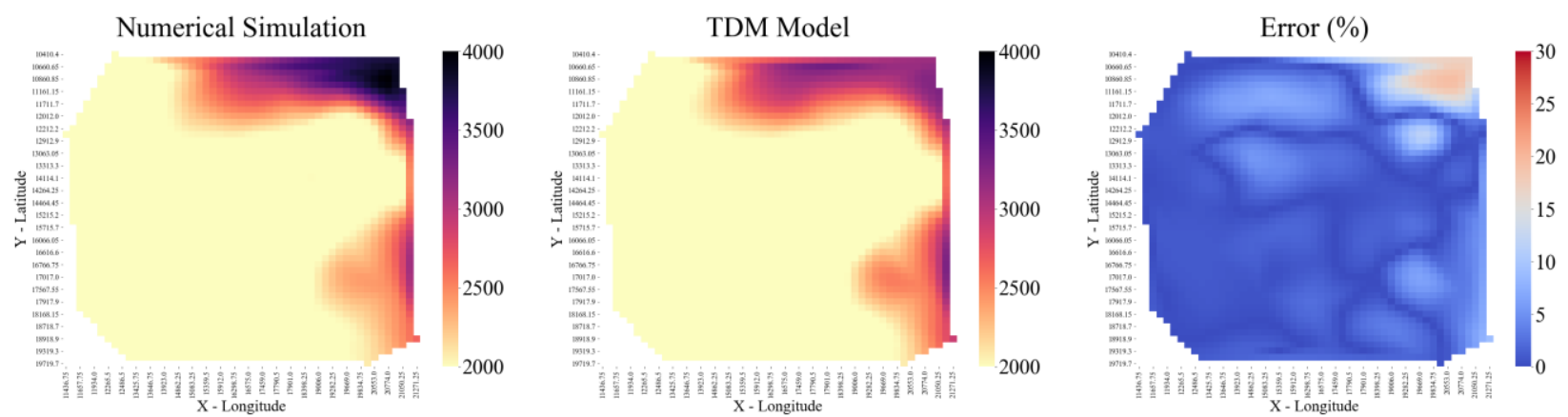

Figure 203 Entire reservoir heat map for reservoir pressure at December 31st, 2013 for 5 Year Blind Validation TDM

Reservoir Pressure (psi) at 2014-12-31
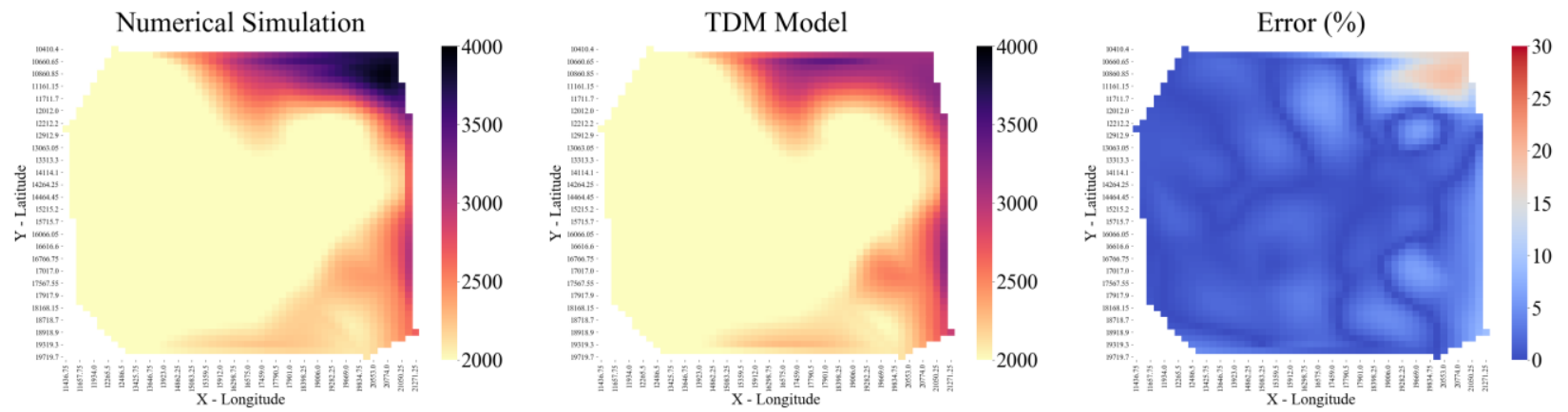

Figure 204 Entire reservoir heat map for reservoir pressure at December 31st, 2014 for 5 Year Blind Validation TDM 
Reservoir Pressure (psi) at 2015-12-31
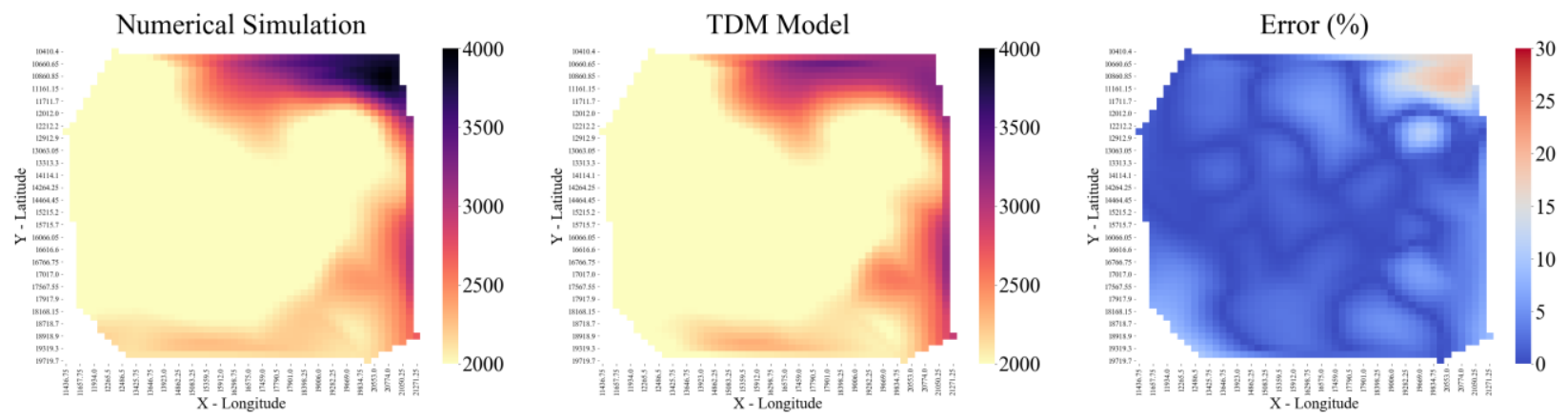

Figure 205 Entire reservoir heat map for reservoir pressure at December 31st, 2015 for 5 Year Blind Validation TDM

Reservoir Pressure (psi) at 2016-12-31
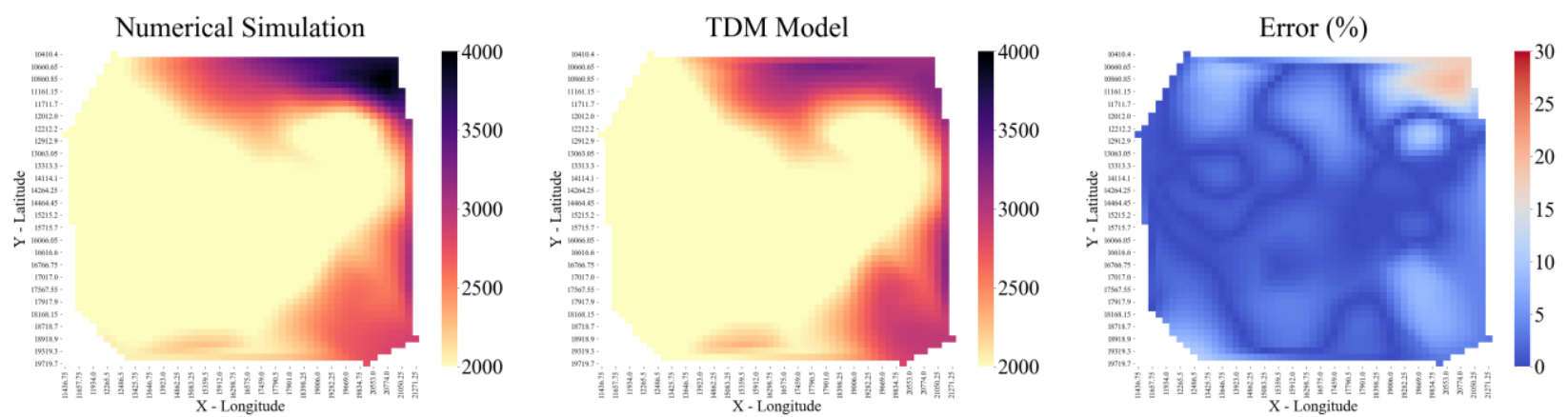

Figure 206 Entire reservoir heat map for reservoir pressure at December 31st, 2016 for 5 Year Bind Validation TDM

Water Saturation (\%) at 2011-12-31
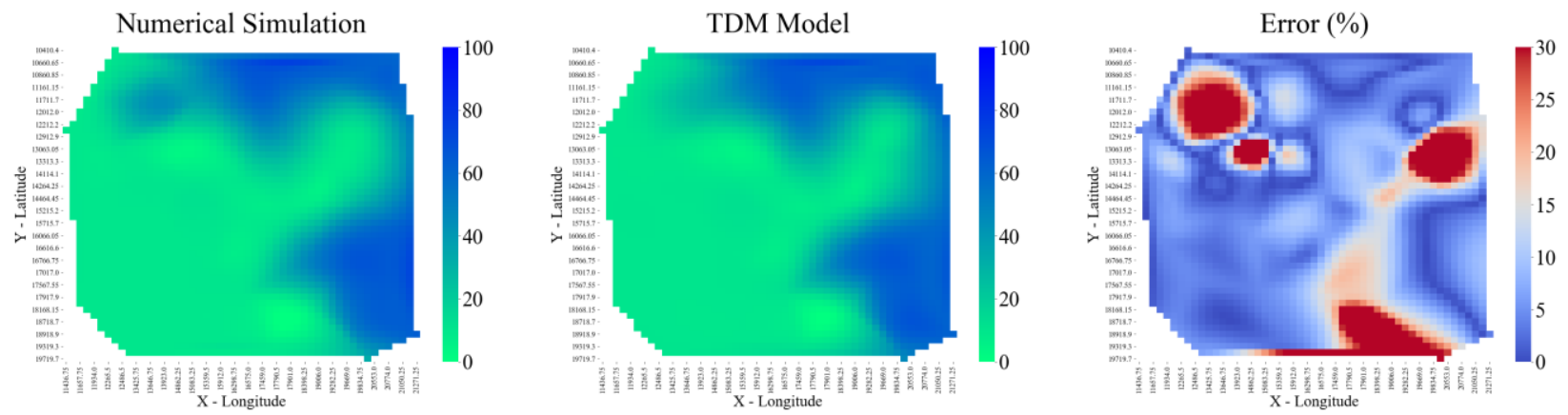

Figure 207 Entire reservoir heat map for water saturation at December 31st, 2011 for 5 Year Blind Validation TDM 
Water Saturation (\%) at 2012-12-31
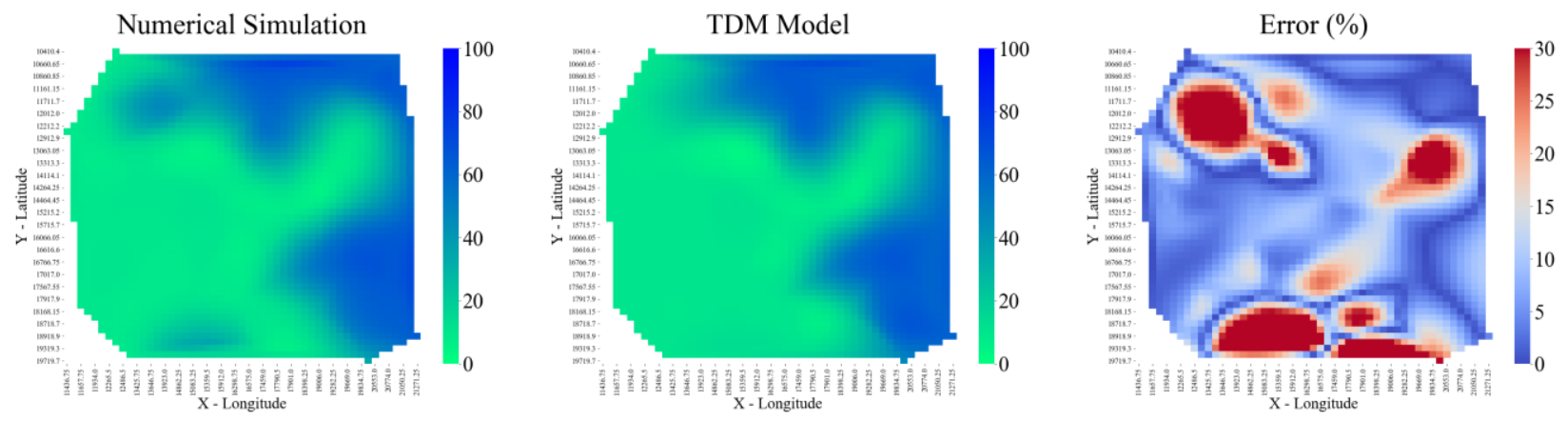

Figure 208 Entire reservoir heat map for water saturation at December 31st, 2012 for 5 Year Blind Validation TDM

Water Saturation (\%) at 2013-12-31
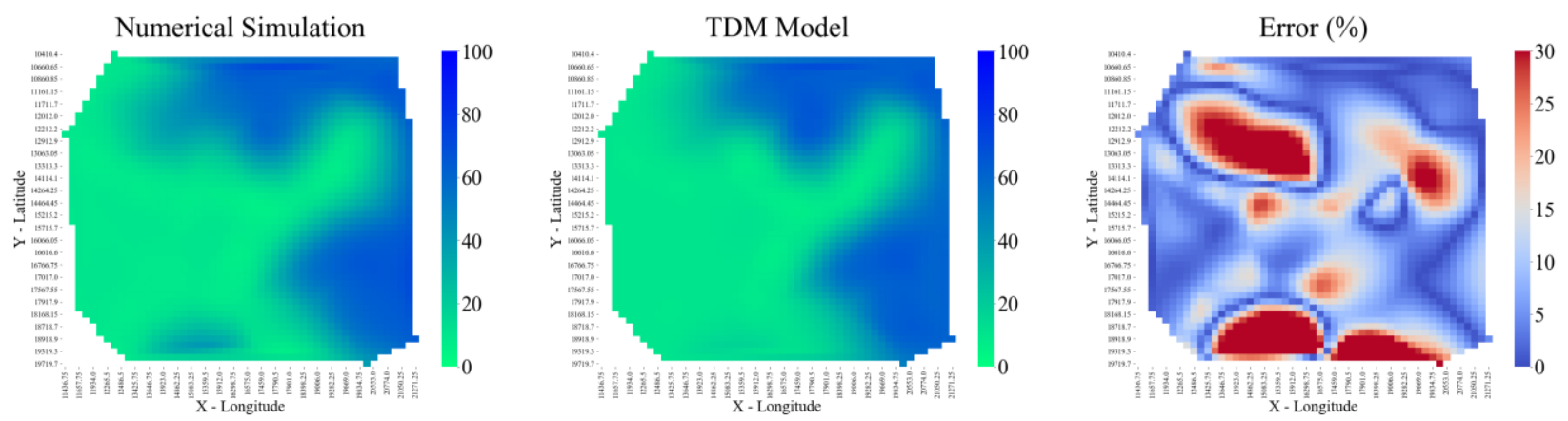

Figure 209 Entire reservoir heat map for water saturation at December 31st, 2013 for 5 Year Blind Validation TDM

Water Saturation (\%) at 2014-12-31
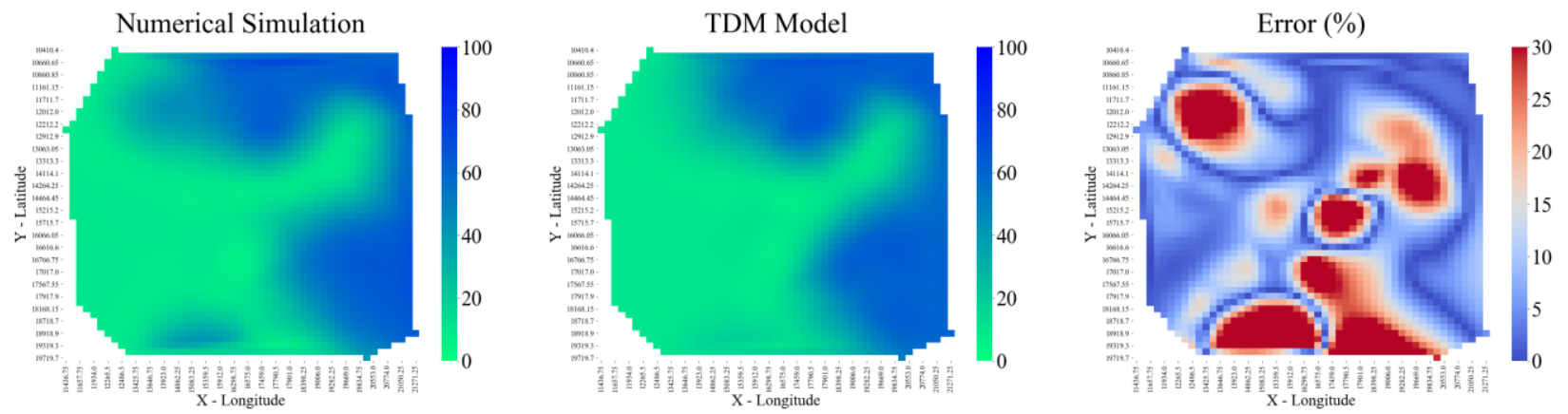

Figure 210 Entire reservoir heat map for water saturation at December 31st, 2014 for 5 Year Blind Validation TDM 
Water Saturation (\%) at 2015-12-31
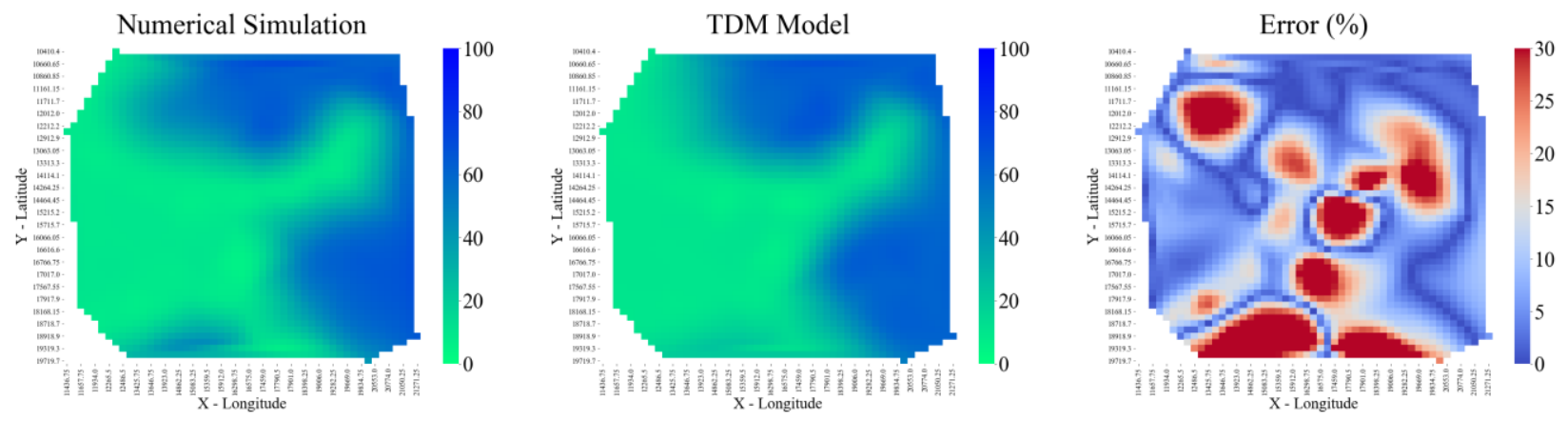

Figure 211 Entire reservoir heat map for water saturation at December 31st, 2015 for 5 Year Blind Validation TDM

Water Saturation (\%) at 2016-12-31
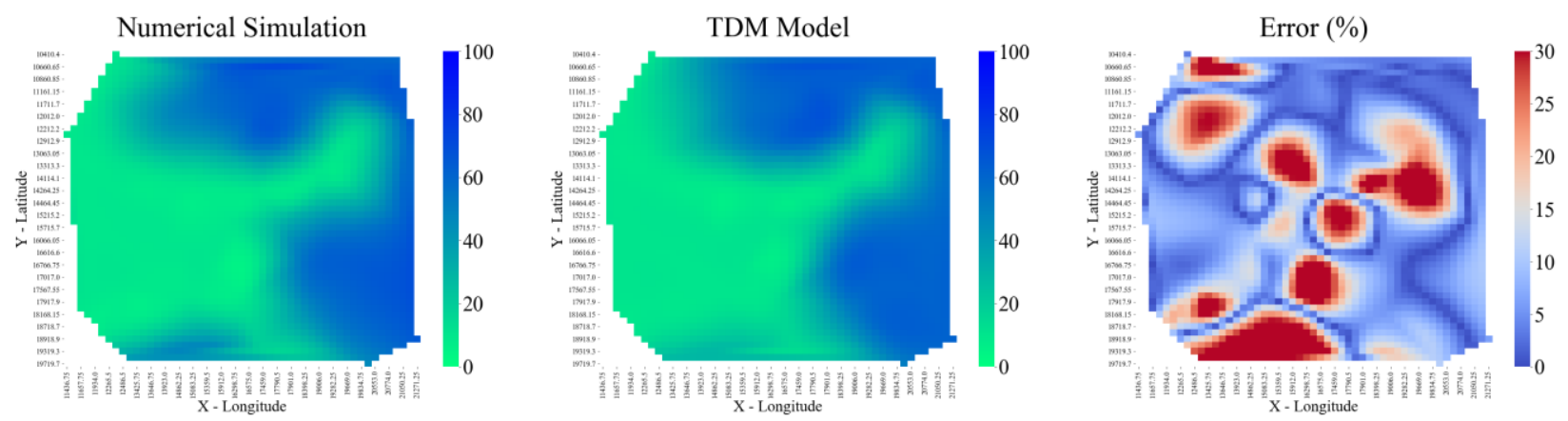

Figure 212 Entire reservoir heat map for water saturation at December 31st, 2016 for 5 Year Blind Validation TDM

The individual well production plots were characterized into three groups: good, average, and poor, just like the previous models. Two examples of wells with good accuracy are Well-007 and Well034. The red line is placed at December $31^{\text {st }}, 2011$ for the beginning of blind validation in each well. Thirty-seven wells were characterized as having good prediction accuracy, which is about $65 \%$ of the wells. As previously mentioned, the location of the well plays a very important role into which fluids are produced. If the well is close to a gas injector, gas will be produced, but no water will be produced. The inverse is also true. Only if the well is close to both a water and gas injector will the well produce both fluids. 

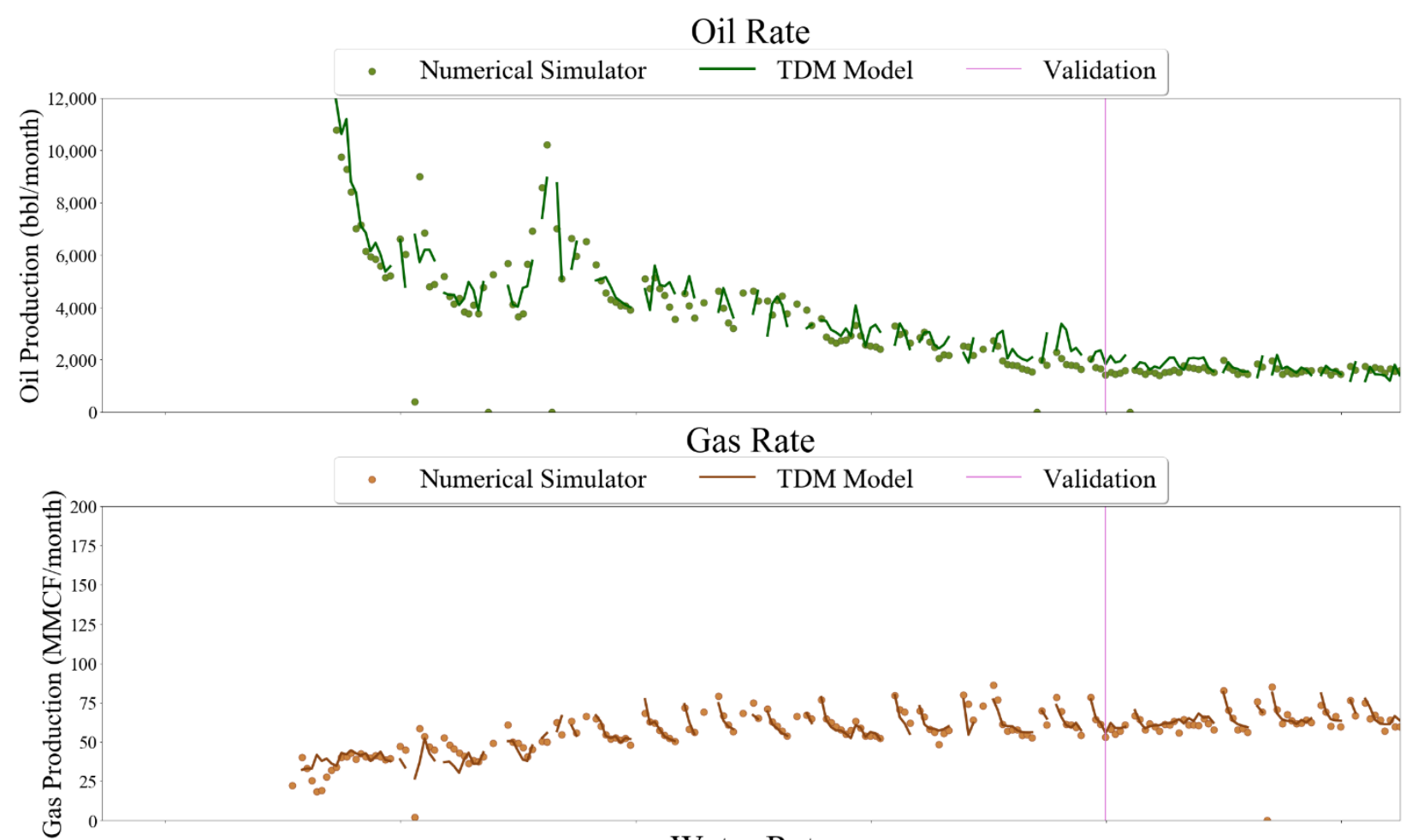

Water Rate

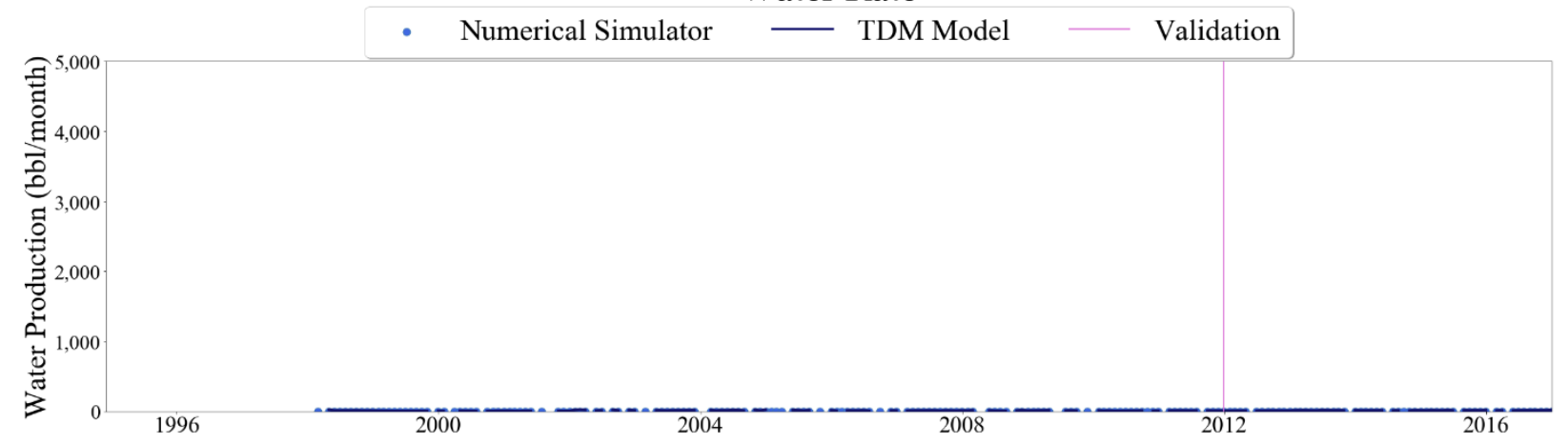

Figure 213 Well-007 oil, gas, and water rate TDM predictions vs actual simulation data plots for 5 Year Blind Validation TDM 

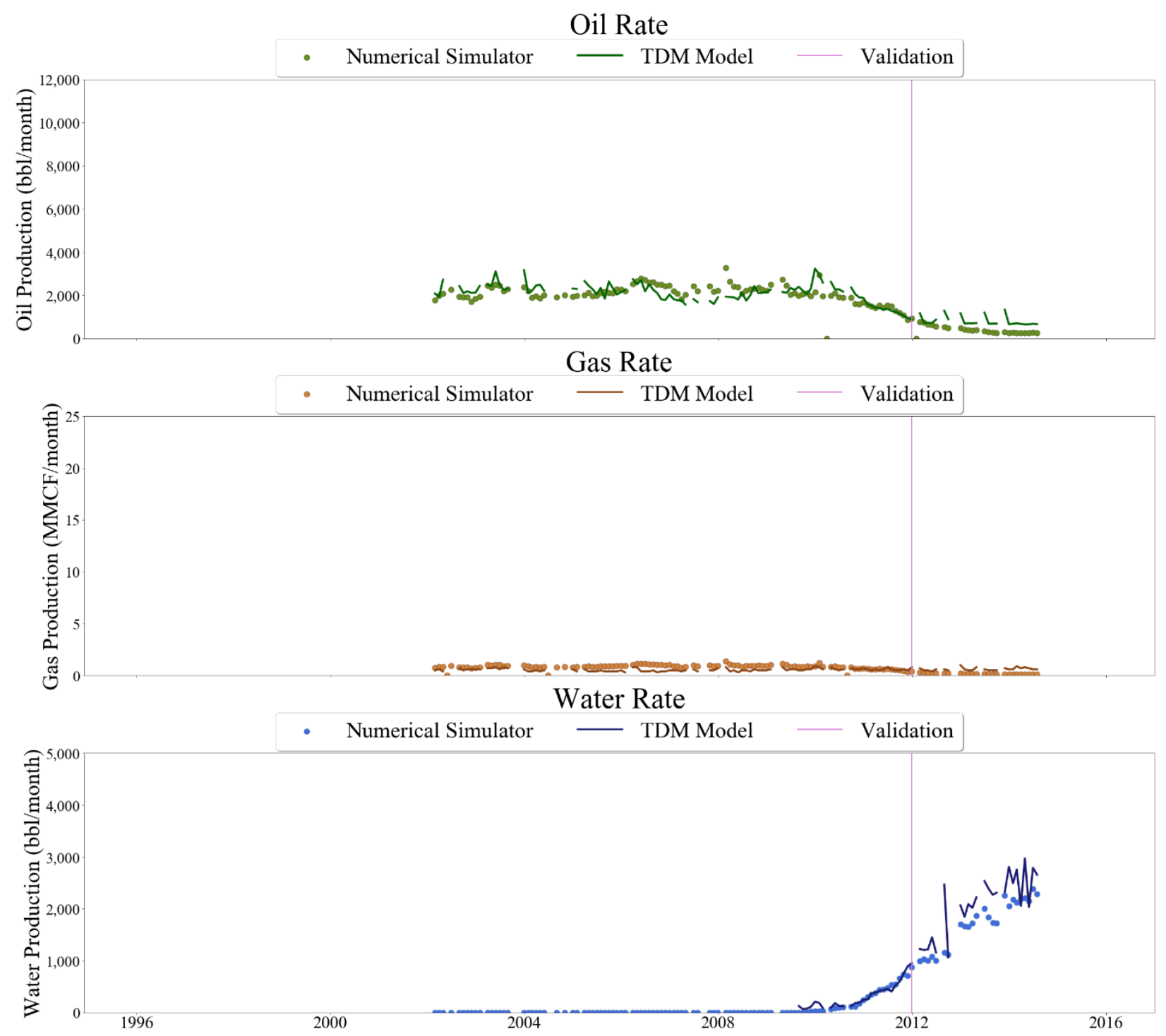

Figure 214 Well-034 oil, gas, and water rate TDM predictions vs actual simulation data plots for 5 Year Blind Validation TDM

Twelve wells, or $21 \%$ of wells, were believed to have average predictions. Well-010 was deemed average due to slightly under predicting at times in the oil plot as well as slightly over predicting the last portion of the water plot before the well was shut-in due to violating the water cut constraint. While Well020's predictions were close to the actual values, the predictions did not quite match for part of the oil and gas plots. 


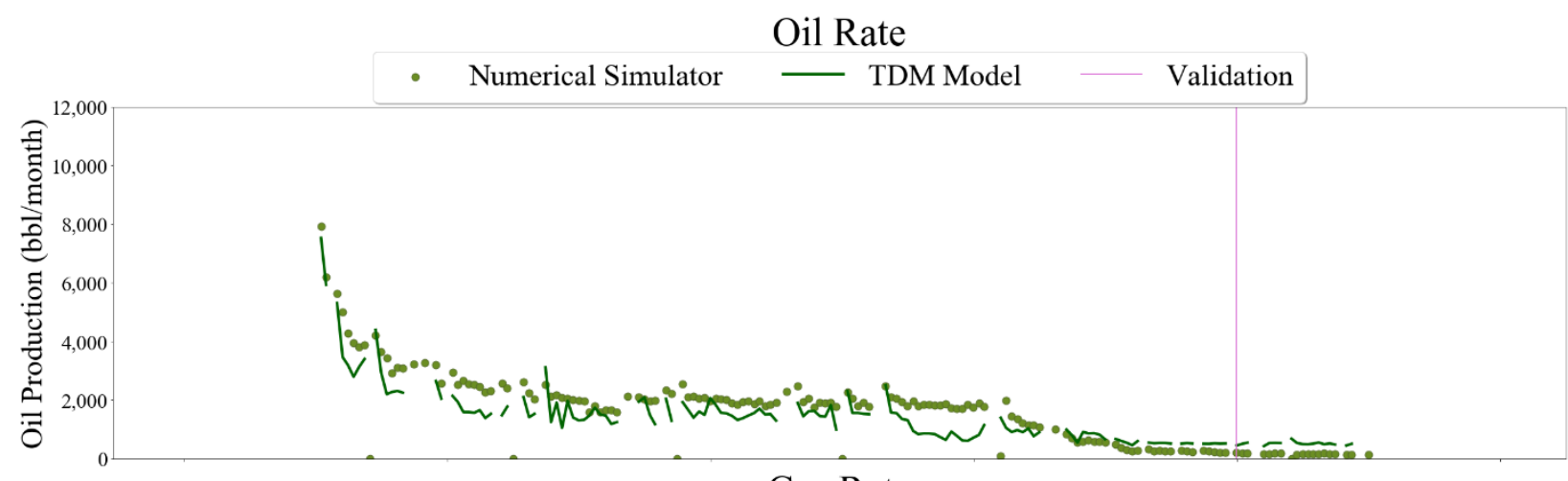

\section{Gas Rate}
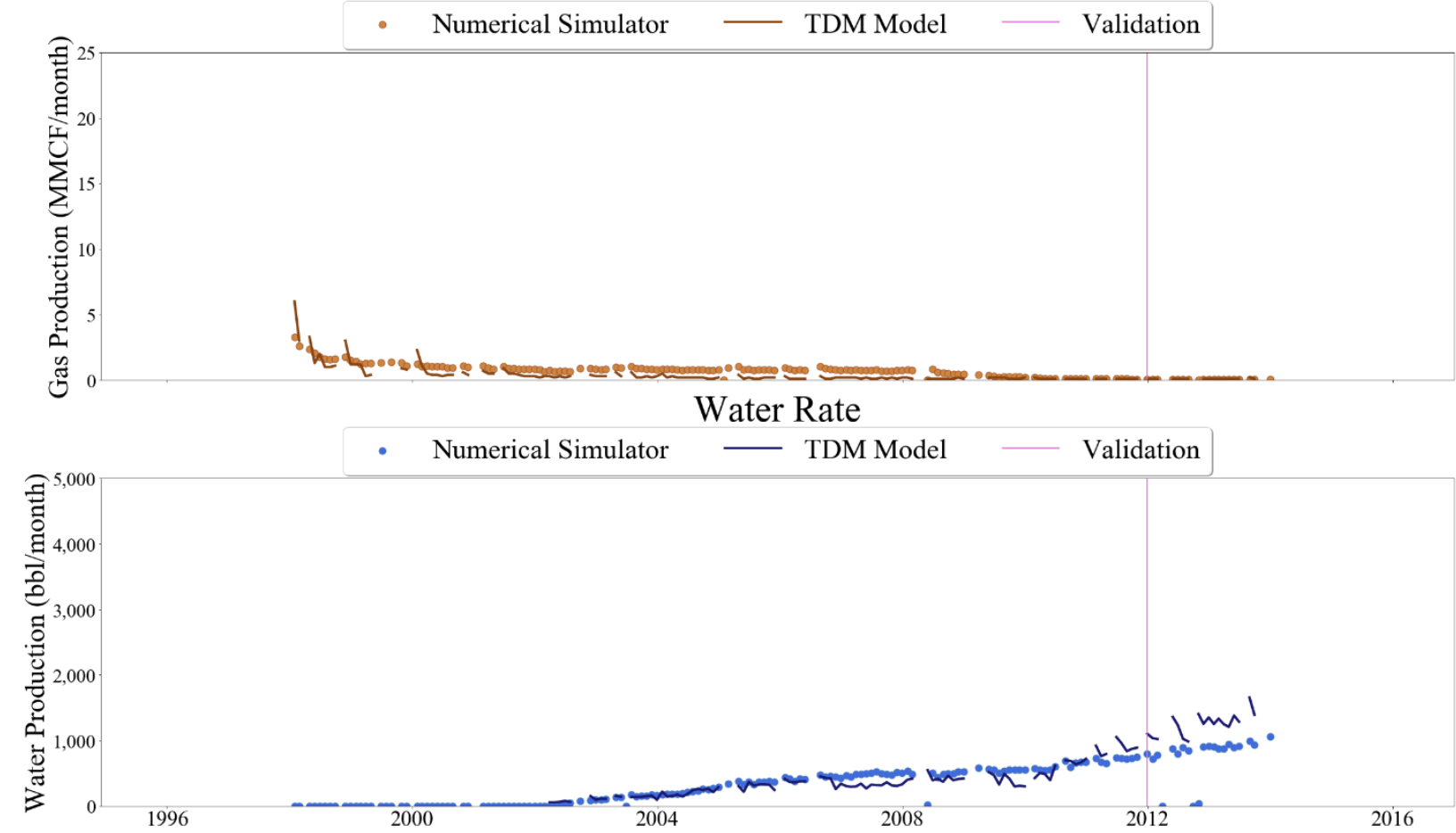

Figure 215 Well-010 oil, gas, and water rate TDM predictions vs actual simulation data plots for 5 Year Blind Validation TDM 


\section{Oil Rate}
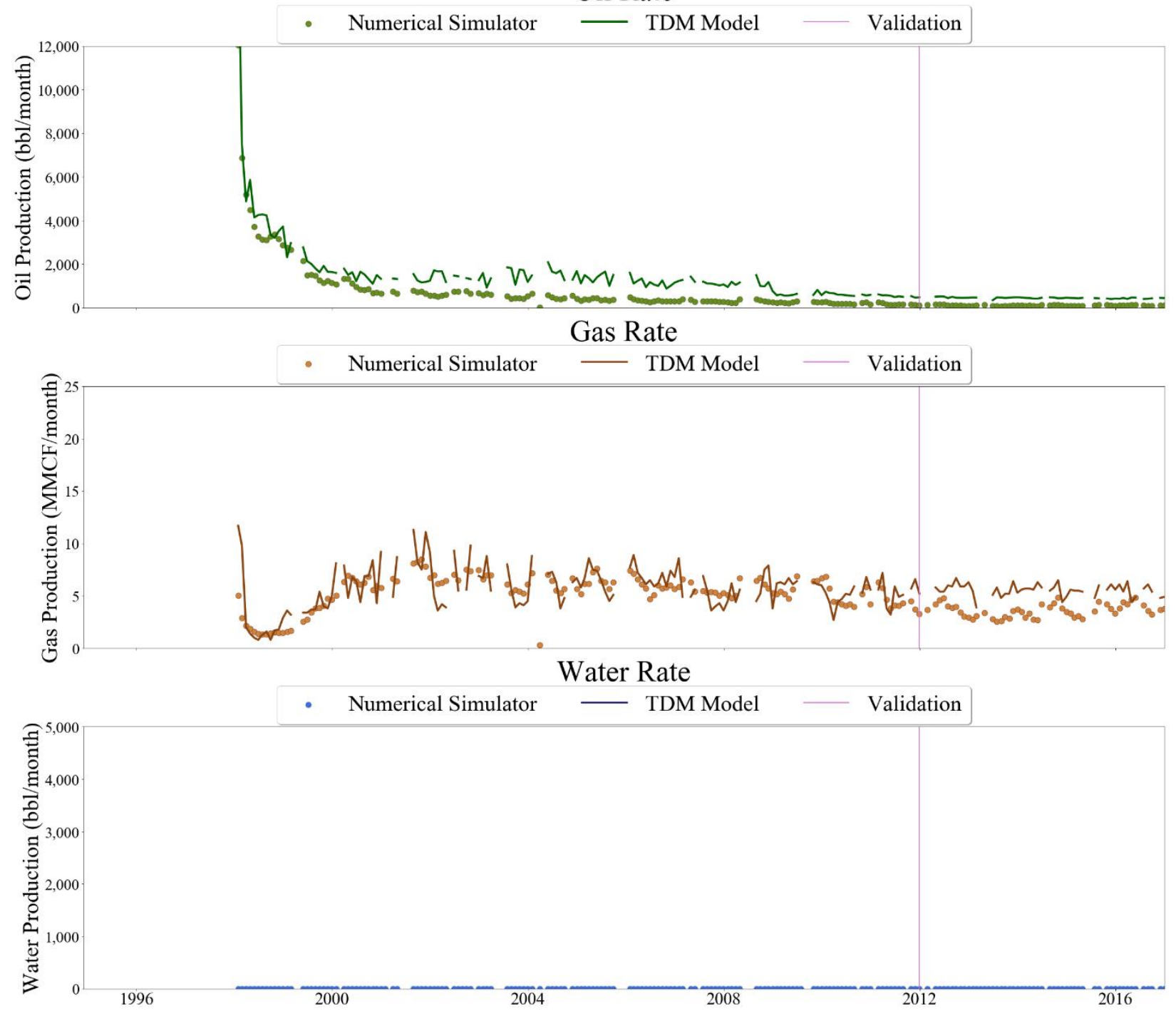

Figure 216 Well-020 oil, gas, and water rate TDM prediction vs actual simulation data plots for 5 Year Blind Validation TDM

The last category, the poor wells, had 7 wells or about $12 \%$ of the total wells. One example is Well-021 where the TDM failed to follow the trend of the actual data in the gas plot only for the second half of the well's life. Well-057 on the other hand greatly over predicted the water production the last few years of the well's life while at the same time slightly under predicting the gas production. All of the individual well production plots can be found in Appendix 8.2.6.1, for the 5 Year Blind Validation case. 

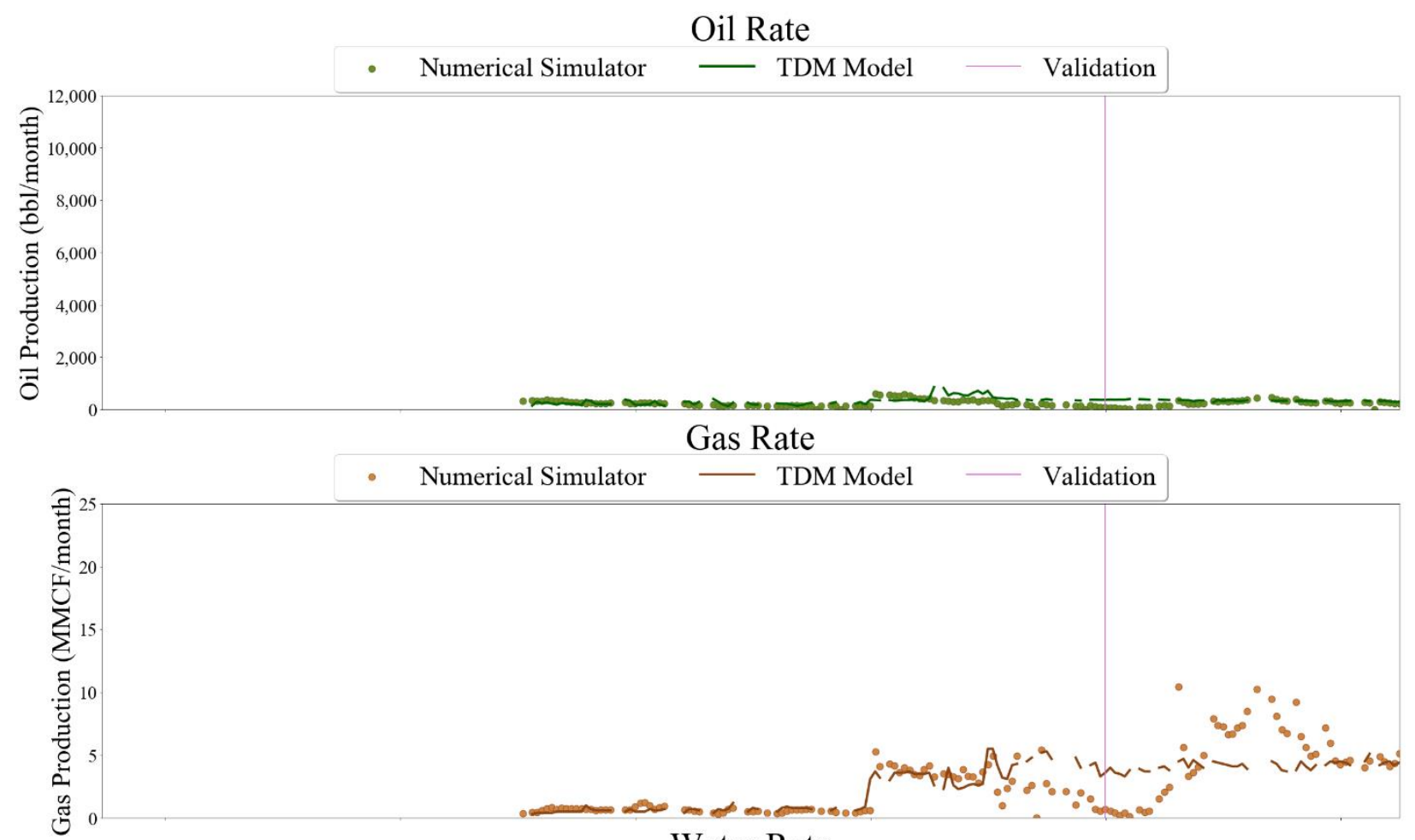

Water Rate

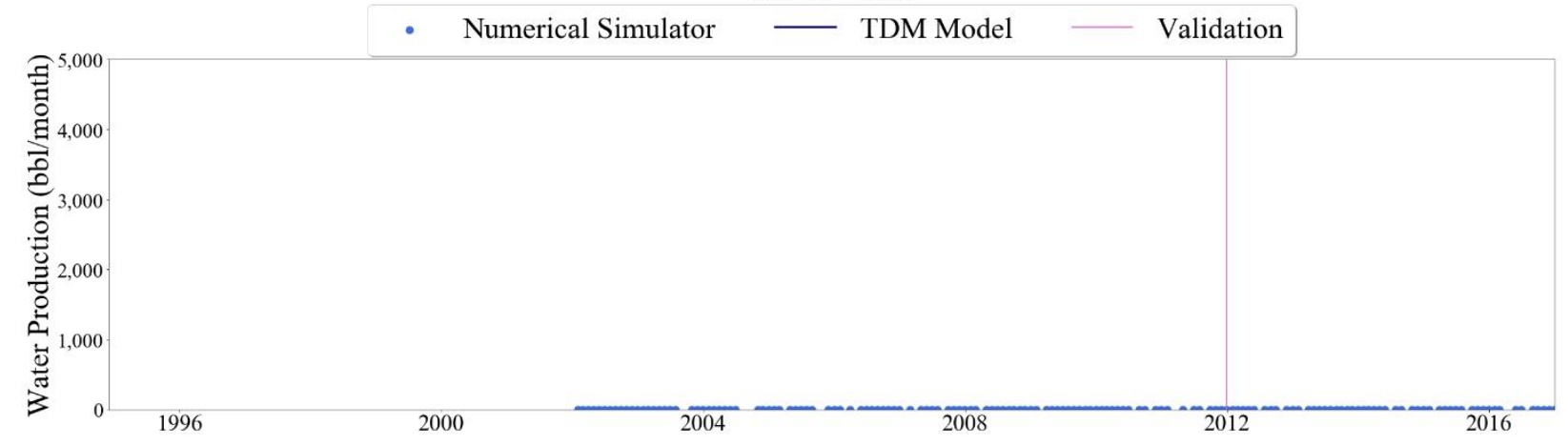

Figure 217 Well-021 oil, gas, and water rate TDM predictions vs actual simulation data plots for 5 Year Blind Validation 


\section{Oil Rate}
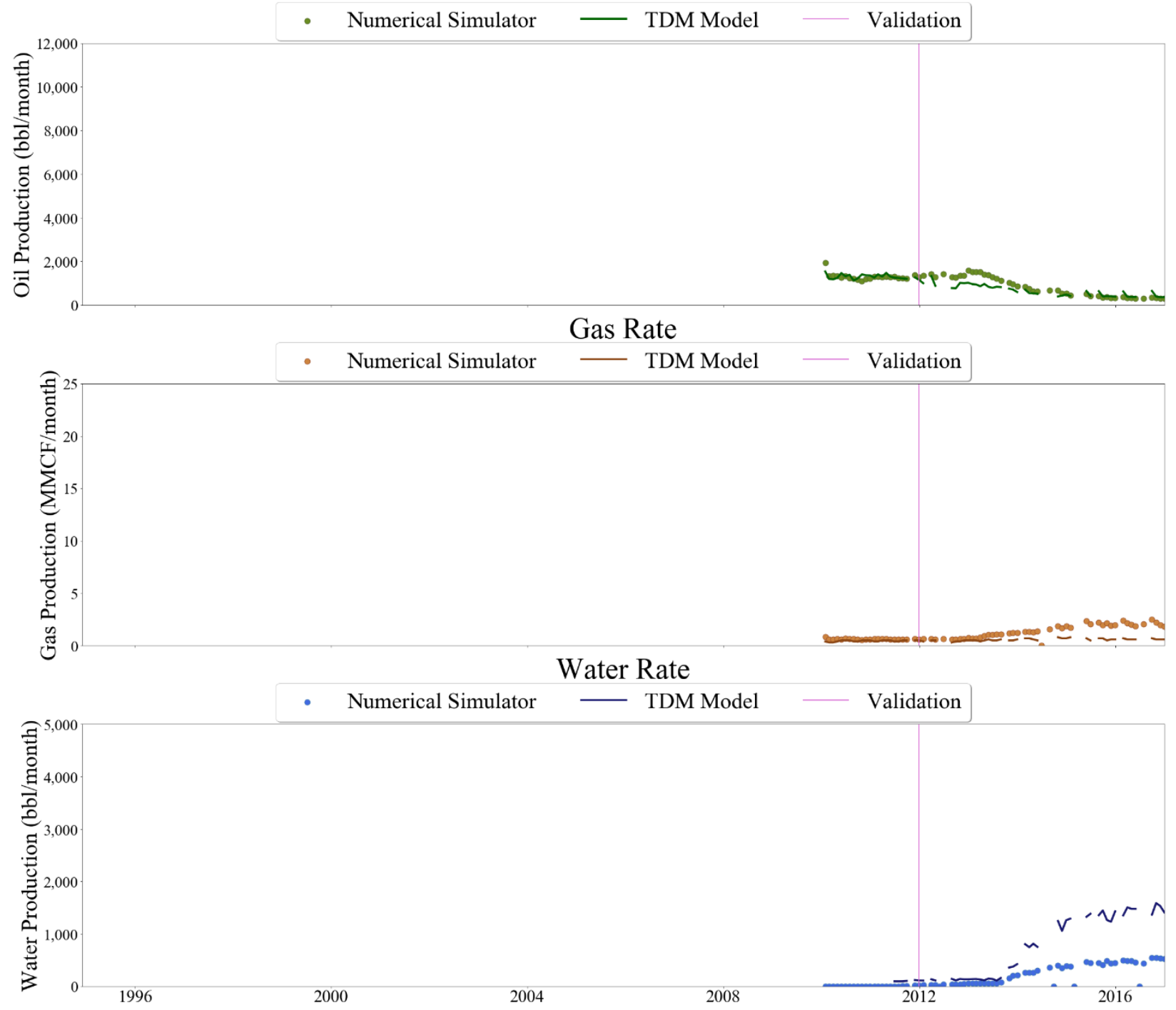

Figure 218 Well-057 oil, gas, and water rate TDM predictions vs actual simulation data plots for 5 Year Blind Validation TDM

The above results, with the majority of wells having a good fit, favorable entire reservoir results, and acceptable entire reservoir heat maps were deemed sufficient. Because of this one more model, the last TDM model, was developed with another year of blind validation. This model was called the MultiRandom Partitioning- 6 Year Blind Validation case. The additional important of this model is explained in the next results section.

\subsubsection{Multi-Random Partitioning-6 Year Blind Validation}

The last model created had 6 years of blind validation beginning on December $31^{\text {st }}, 2010$. This model has extra importance due to the last possible year of blind validation being added without predicting a phase of production. In other words, five new wells are brought online for the first time at December $31^{\text {st }}$, 2009 and are dispersed throughout the reservoir. Therefore the 6 Year Blind Validation case is predicting those five wells after only learning from a years' worth of their data. Similarly, the 6 Year Blind Validation case is predicting another five wells that are brought online at December $31^{\text {st }}, 2008$ with only 
two years worth of data. In addition, multiple wells are recompleted as of December $31^{\text {st }}, 2009$ for which production behavior will change due to additional completion footage. Again, the TDM will only have one years worth of data to learn the change in behavior in the wells that are recompleted.

First the entire reservoir oil, gas, and water results are shown below with the red line at December $31^{\text {st }}, 2010$, the start of the blind validation. Predictions with little error can be seen in all three plots where only a slight difference can be seen between the cumulative water production and its prediction.

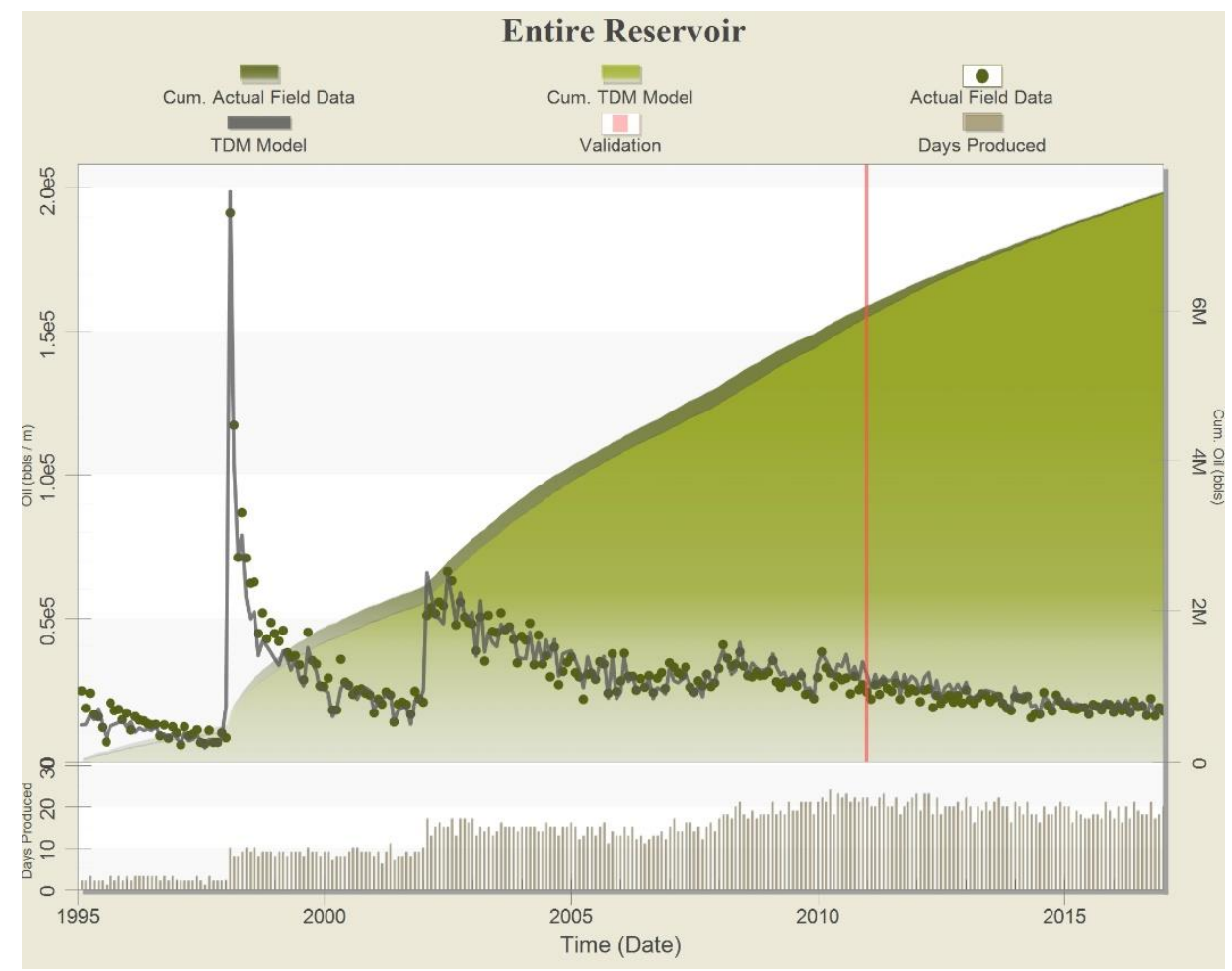

Figure 219 Entire reservoir oil results for Multi-Random Partitioning- 6 Year Blind Validation TDM 


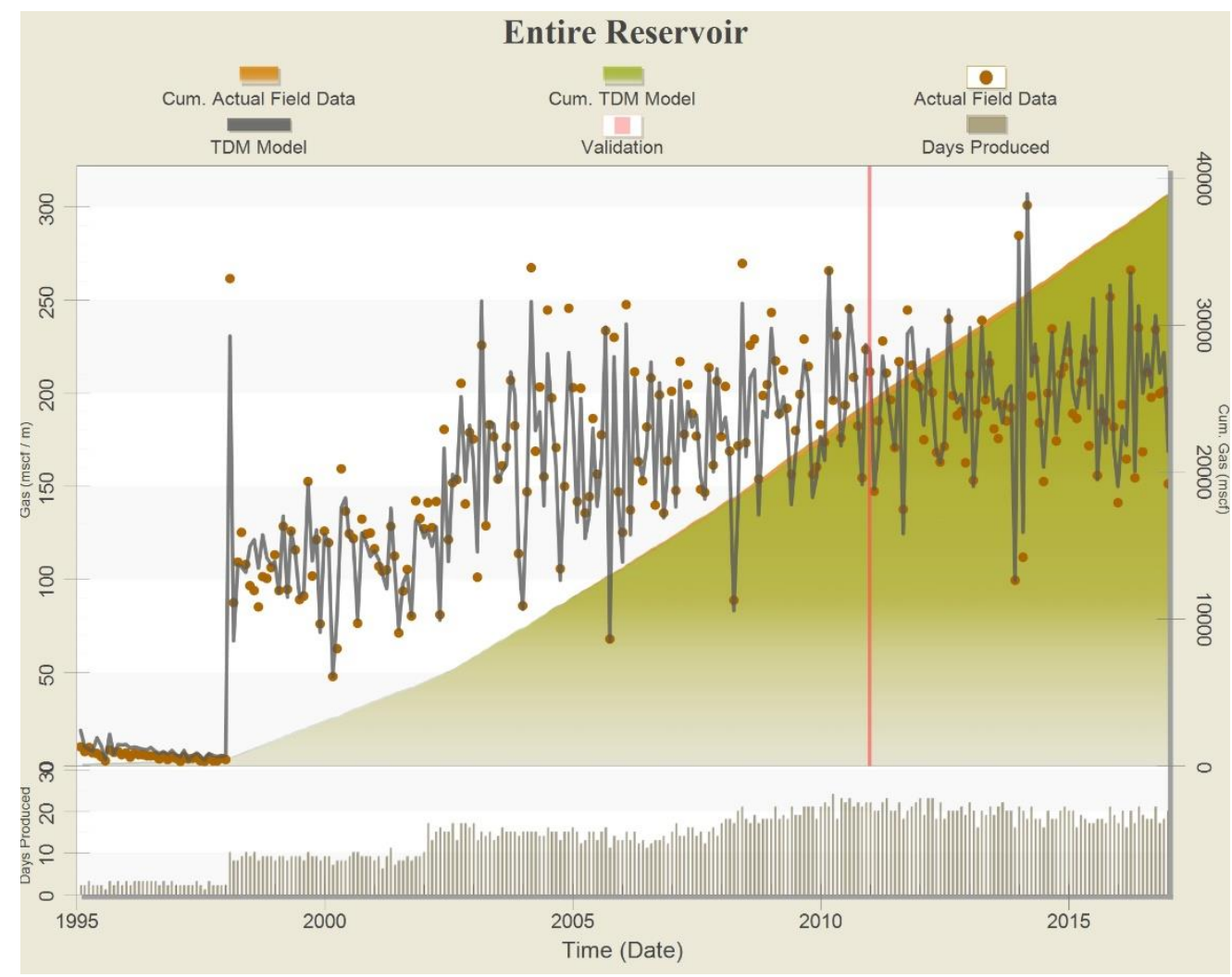

Figure 220 Entire reservoir gas results for Multi-Random Partitioning- 6 Year Blind Validation TDM

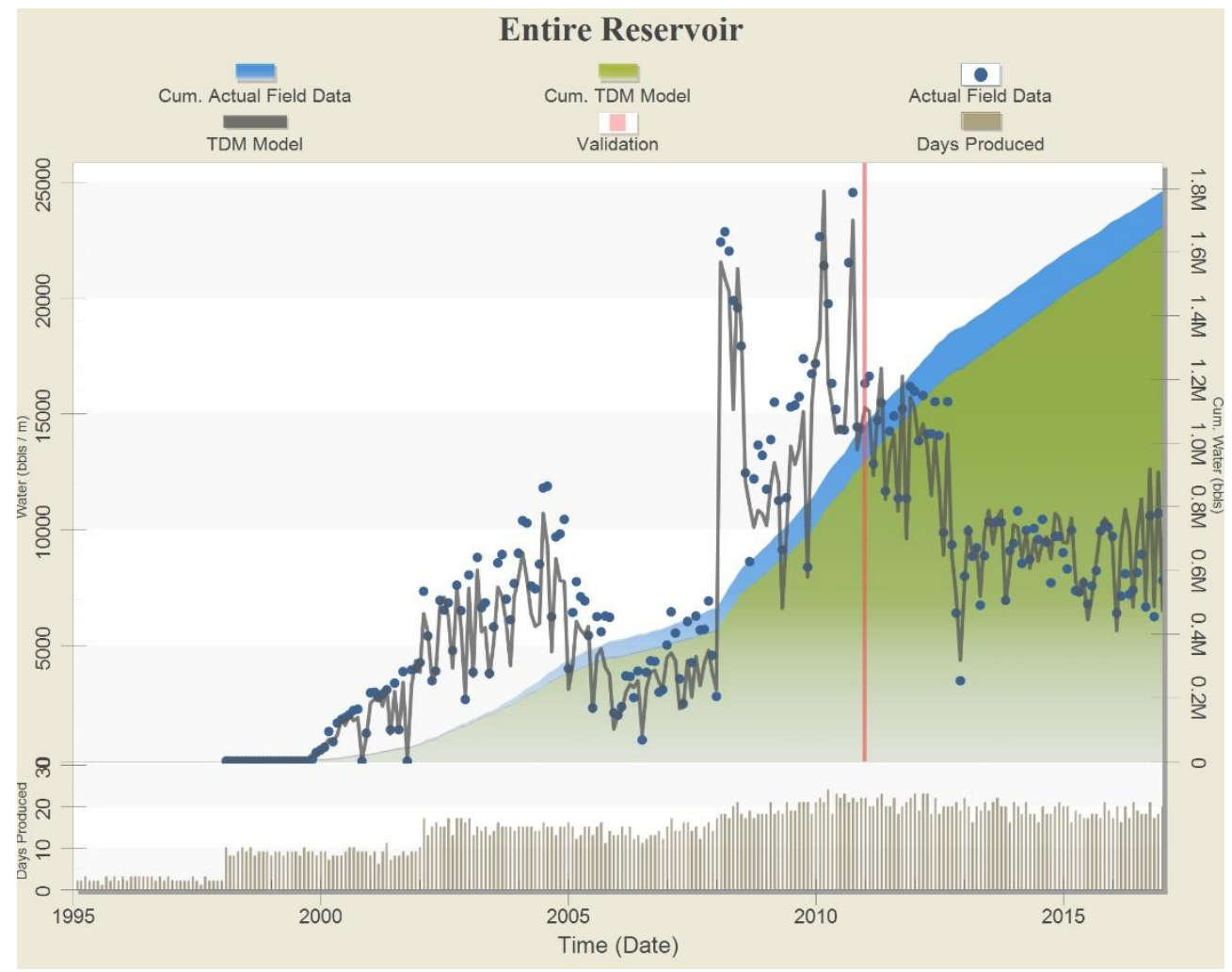

Figure 221 Entire reservoir water results for Multi-Random Partitioning- 6 Year Blind Validation TDM 
Entire reservoir heat maps for reservoir pressure then water saturation are shown next in one year intervals, starting at December $31^{\text {st }}, 2010$. More heat maps for both attributes are shown in Appendix 8.2.7.2, in six month intervals for the entirety of the blind validation. Little to no error can be seen in the reservoir pressure plots where some error can be seen in the water saturation plots. The error is again believed to be due to the length of the well's life, the well's location in the reservoir, and the interpolation technique used when no value was predicted during shut-ins. However even with this error, the water saturation heat maps were still found to be acceptable.

Reservoir Pressure (psi) at 2010-12-31
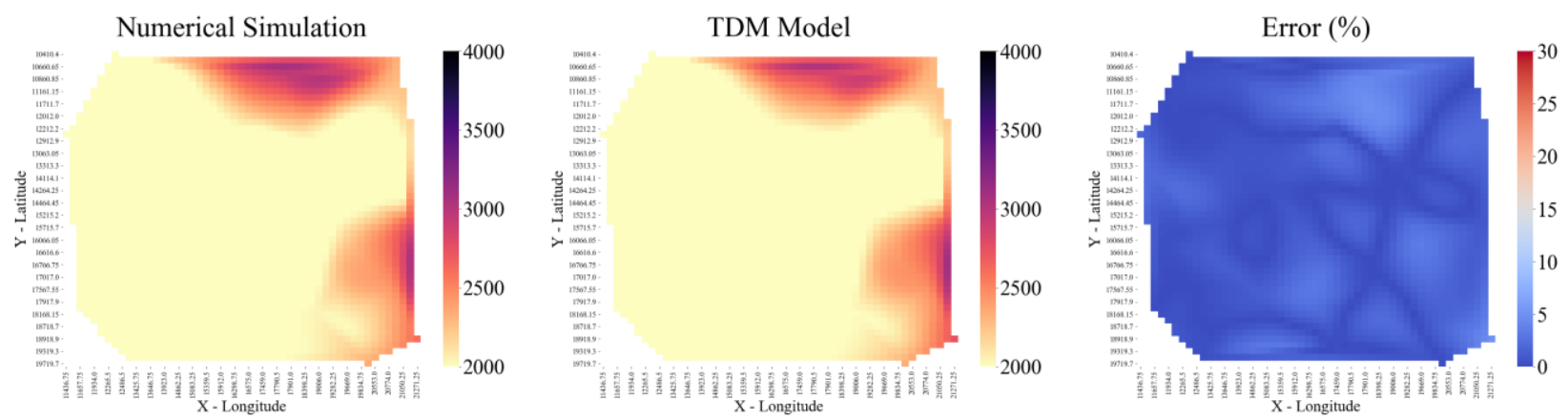

Figure 222 Entire reservoir heat map for reservoir pressure at December 31st, 2010 for 6 Year Blind Validation TDM

Reservoir Pressure (psi) at 2011-12-31
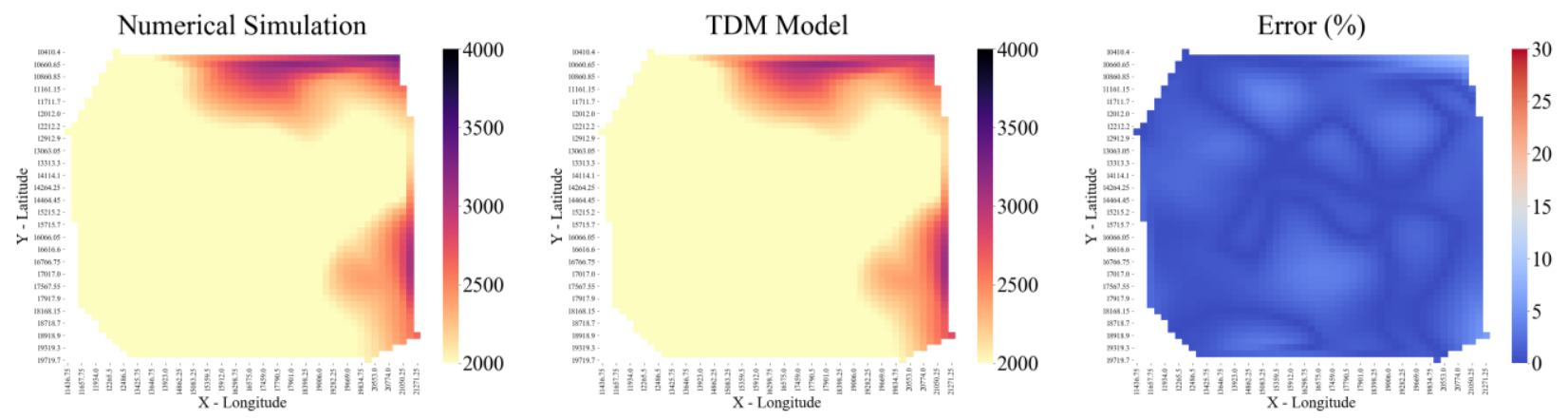

Figure 223 Entire reservoir heat map for reservoir pressure at December 31st, 2011 for 6 Year Blind Validation TDM

Reservoir Pressure (psi) at 2012-12-31
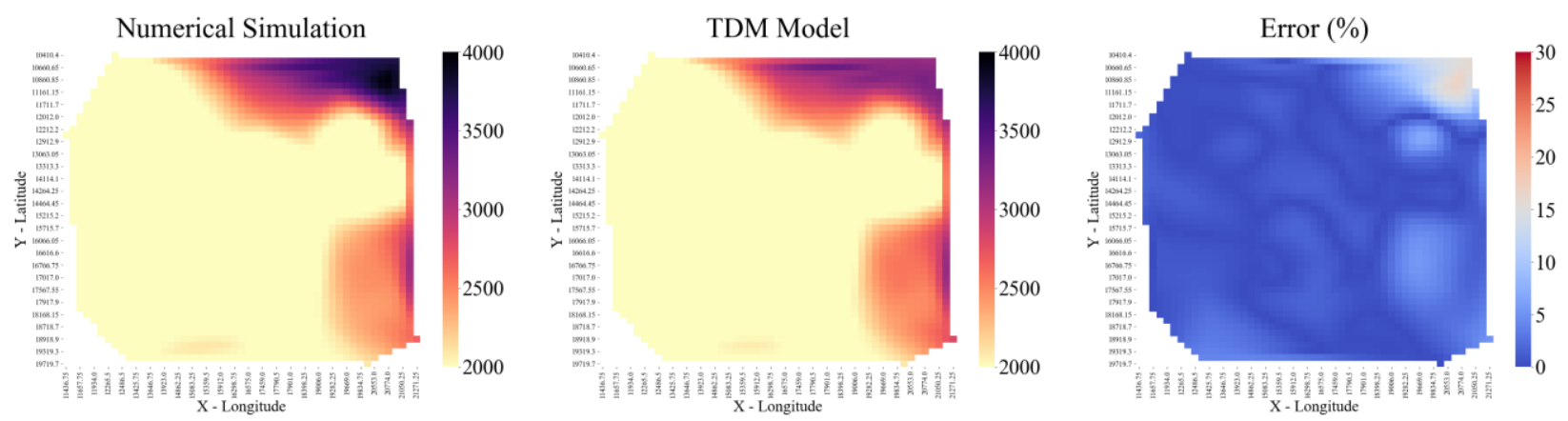

Figure 224 Entire reservoir heat map for reservoir pressure at December 31st, 2012 for 6 Year Blind Validation TDM 
Reservoir Pressure (psi) at 2013-12-31
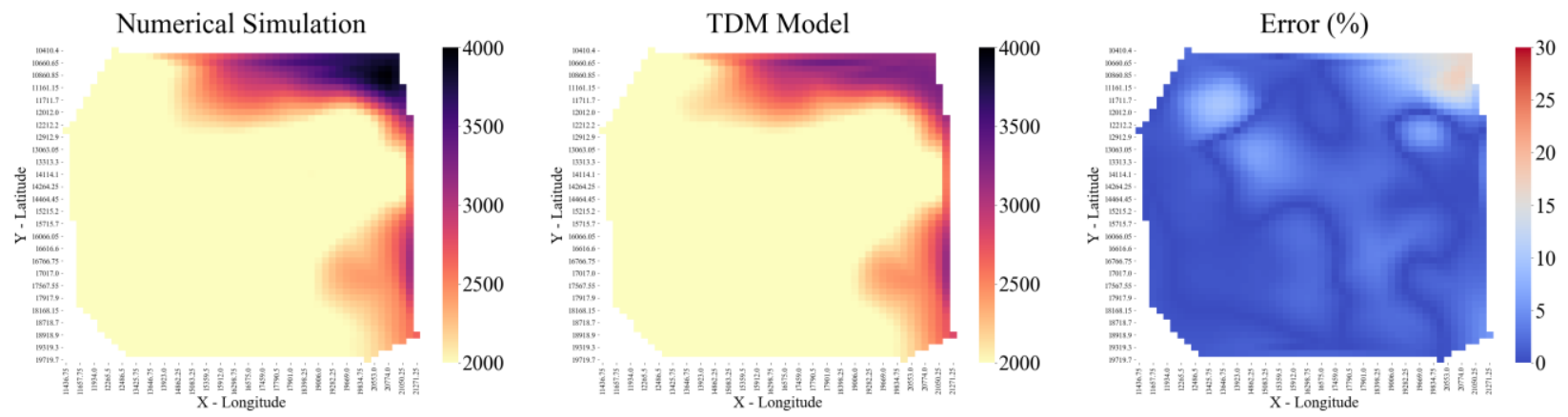

Figure 225 Entire reservoir heat map for reservoir pressure at December 31st, 2013 for 6 Year Blind Validation TDM

Reservoir Pressure (psi) at 2014-12-31
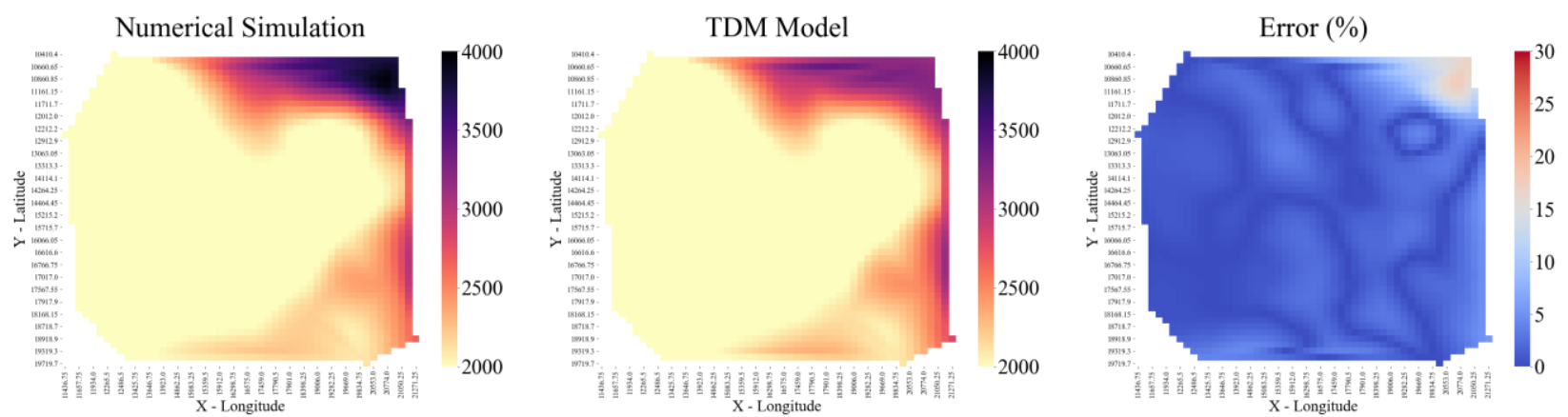

Figure 226 Entire reservoir heat map for reservoir pressure at December 31st, 2014 for 6 Year Blind Validation TDM

Reservoir Pressure (psi) at 2015-12-31
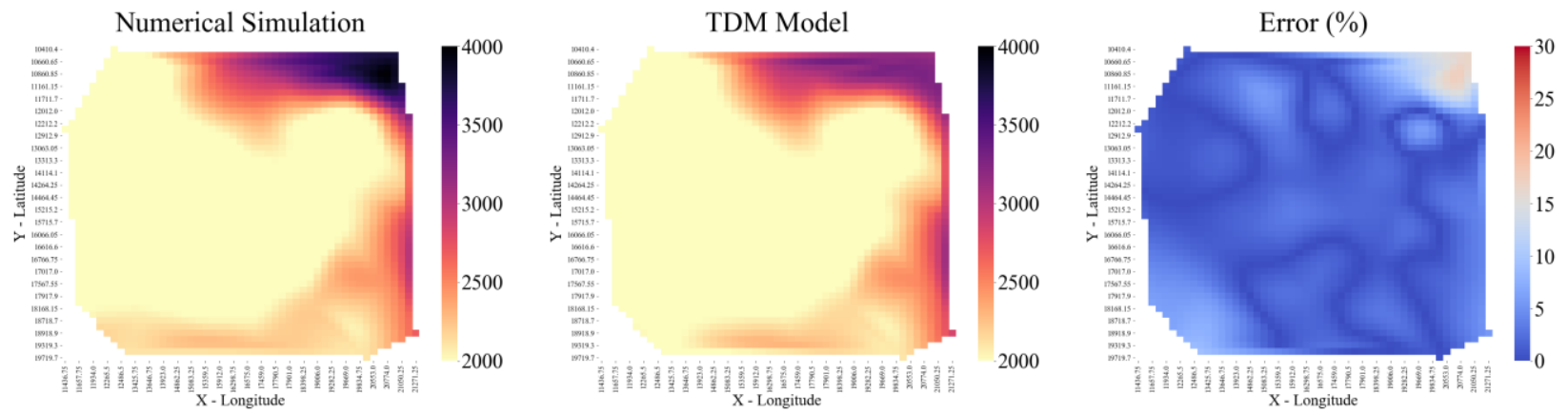

Figure 227 Entire reservoir heat map for reservoir pressure at December 31st, 2015 for 6 Year Blind Validation TDM 
Reservoir Pressure (psi) at 2016-12-31
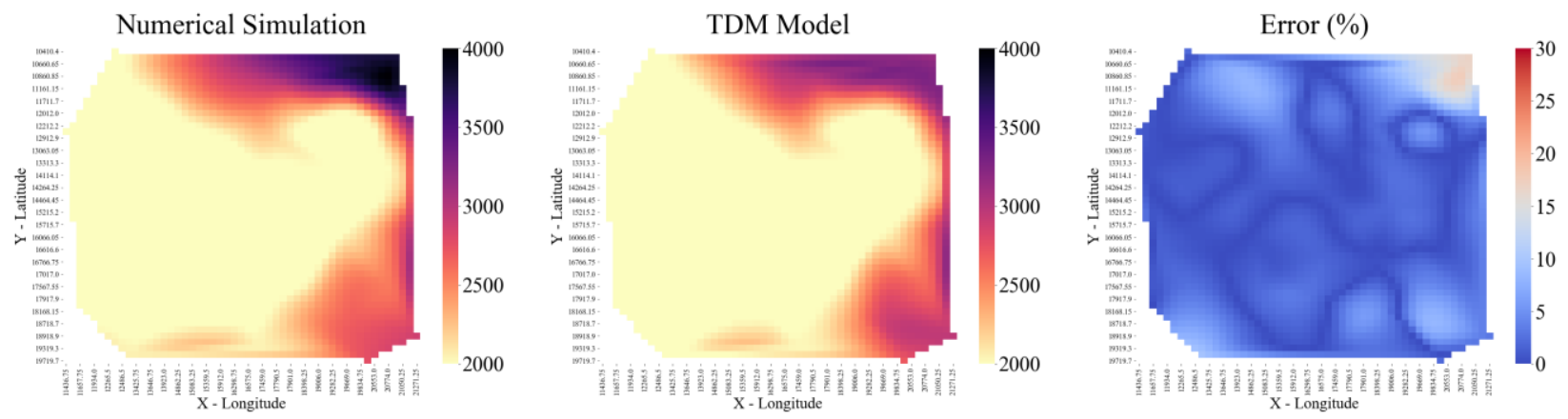

Figure 228 Entire reservoir heat map for reservoir pressure at December 31st, 2016 for 6 Year Blind Validation TDM

Water Saturation (\%) at 2010-12-31
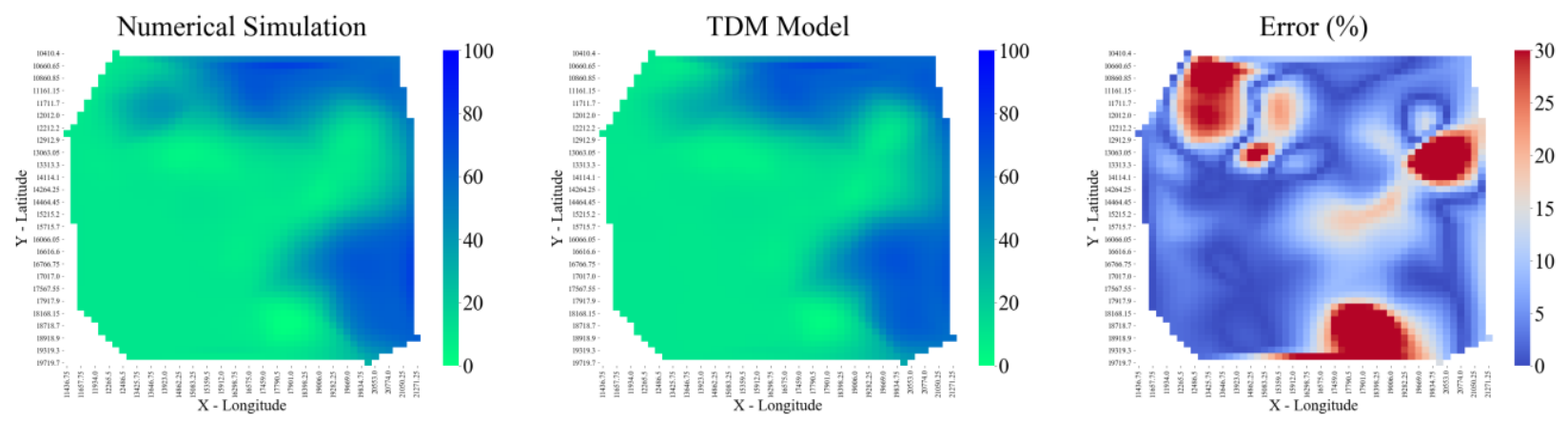

Figure 229 Entire reservoir heat map for water saturation at December 31st, 2010 for 6 Year Blind Validation TDM

Water Saturation (\%) at 2011-12-31
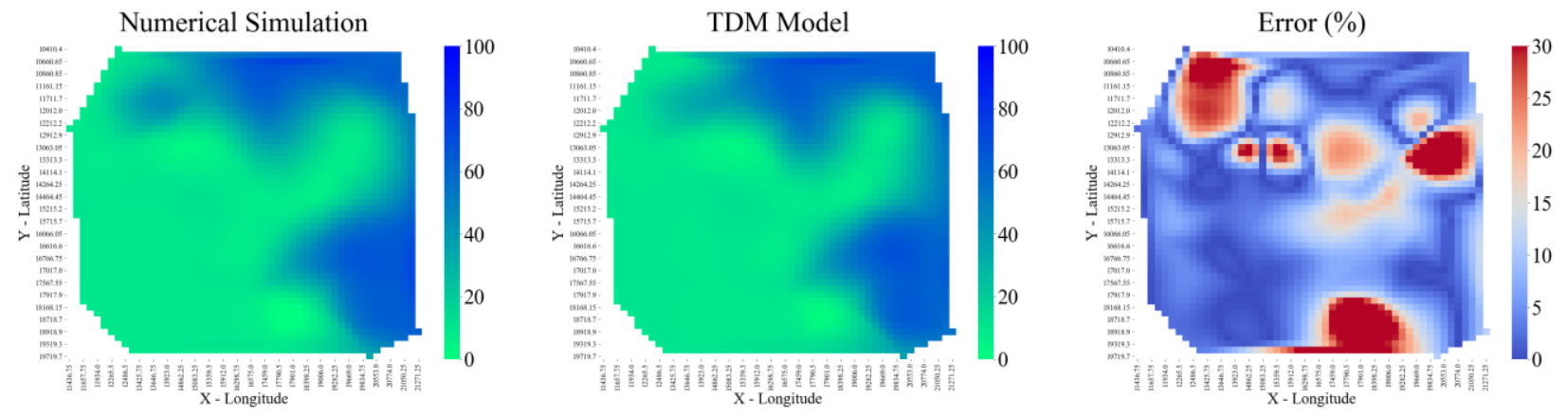

Figure 230 Entire reservoir heat map for water saturation at December 31st, 2011 for 6 Year Blind Validation TDM 
Water Saturation (\%) at 2012-12-31
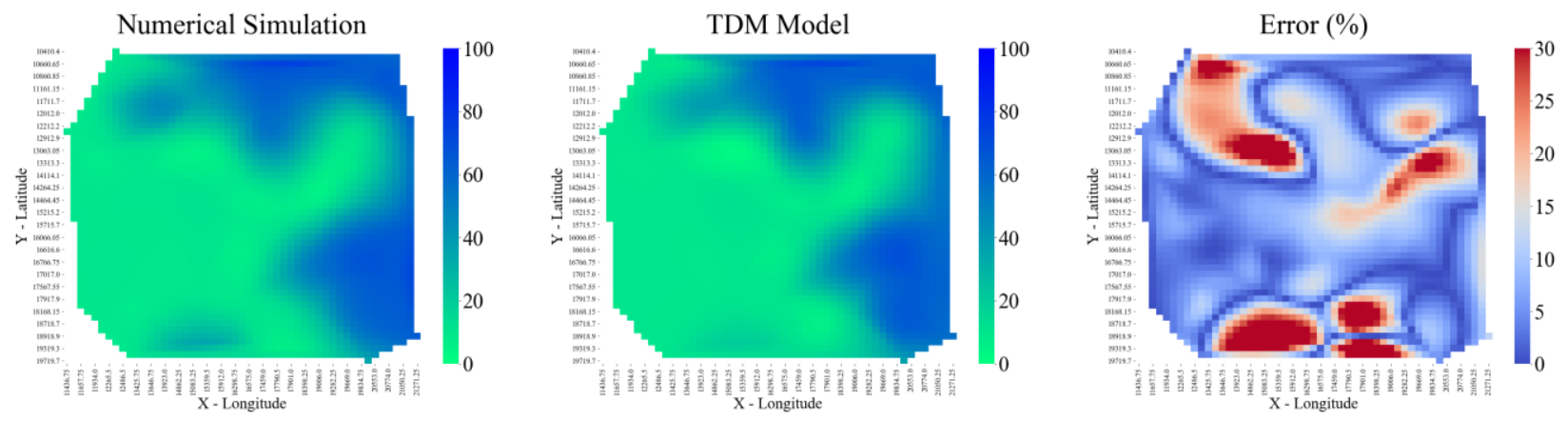

Figure 231 Entire reservoir heat map for water saturation at December 31st, 2012 for 6 Year Blind Validation TDM

Water Saturation (\%) at 2013-12-31
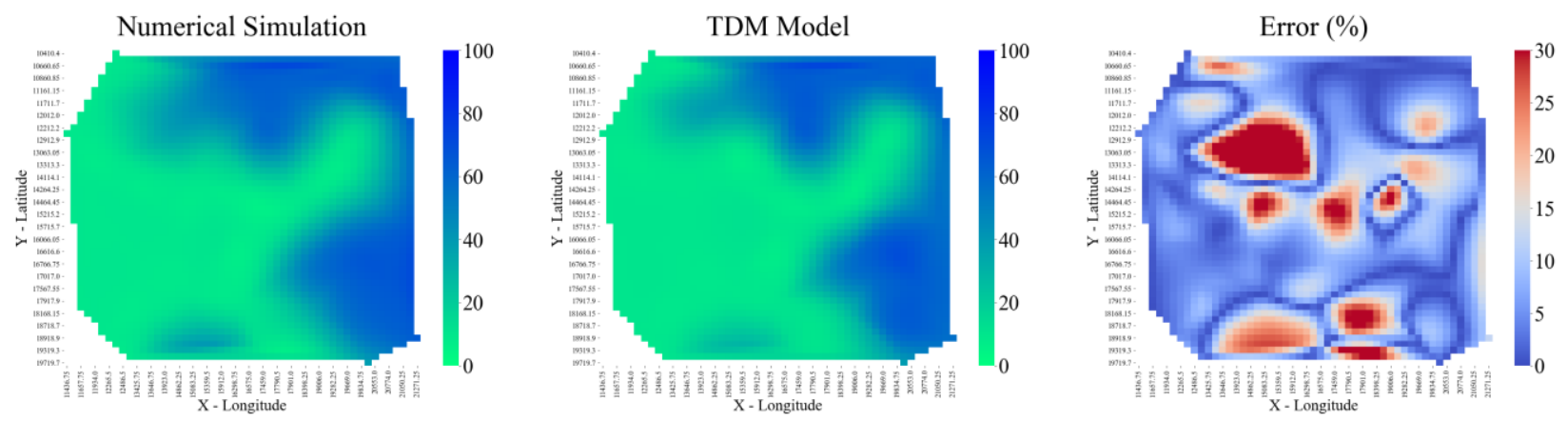

Figure 232 Entire reservoir heat map for water saturation at December 31st, 2013 for 6 Year Blind Validation TDM

Water Saturation (\%) at 2014-12-31
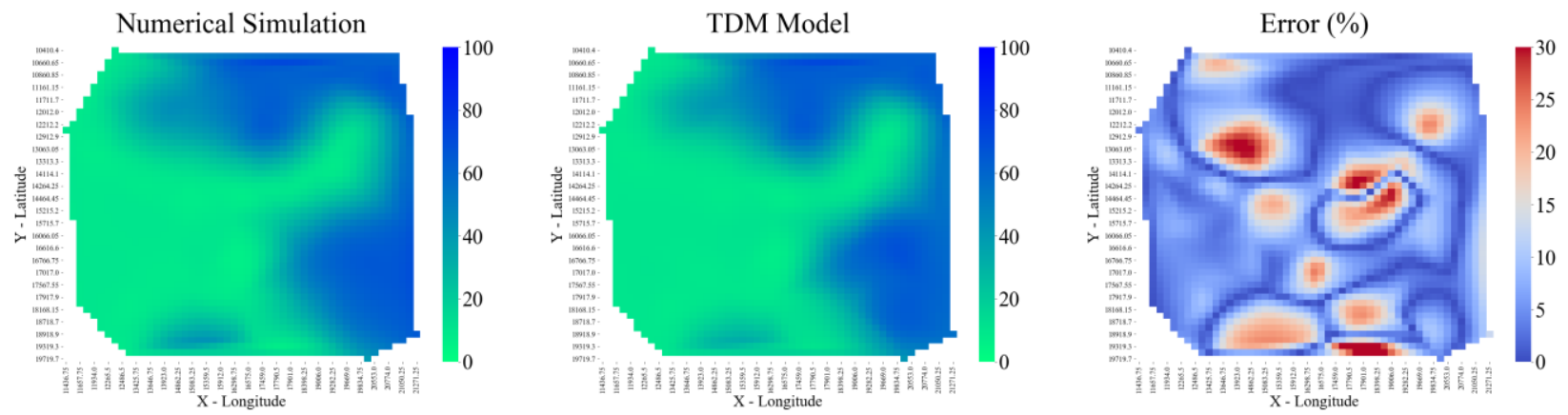

Figure 233 Entire reservoir heat map for water saturation at December 31st, 2014 for 6 Year Blind Validation TDM 
Water Saturation (\%) at 2015-12-31
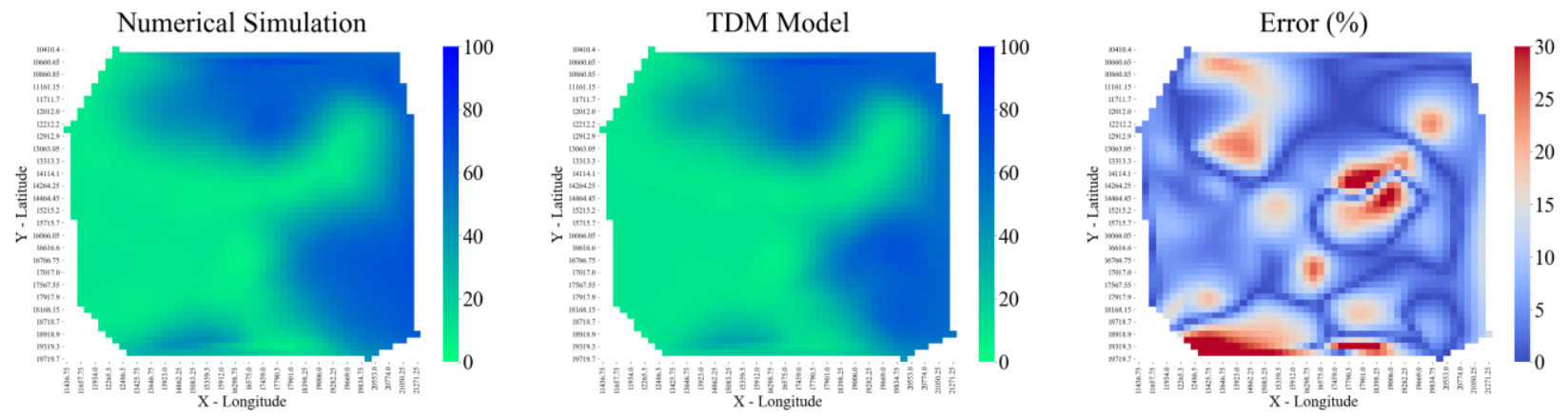

Figure 234 Entire reservoir heat map for water saturation at December 31st, 2015 for 6 Year Blind Validation TDM

Water Saturation (\%) at 2016-12-31
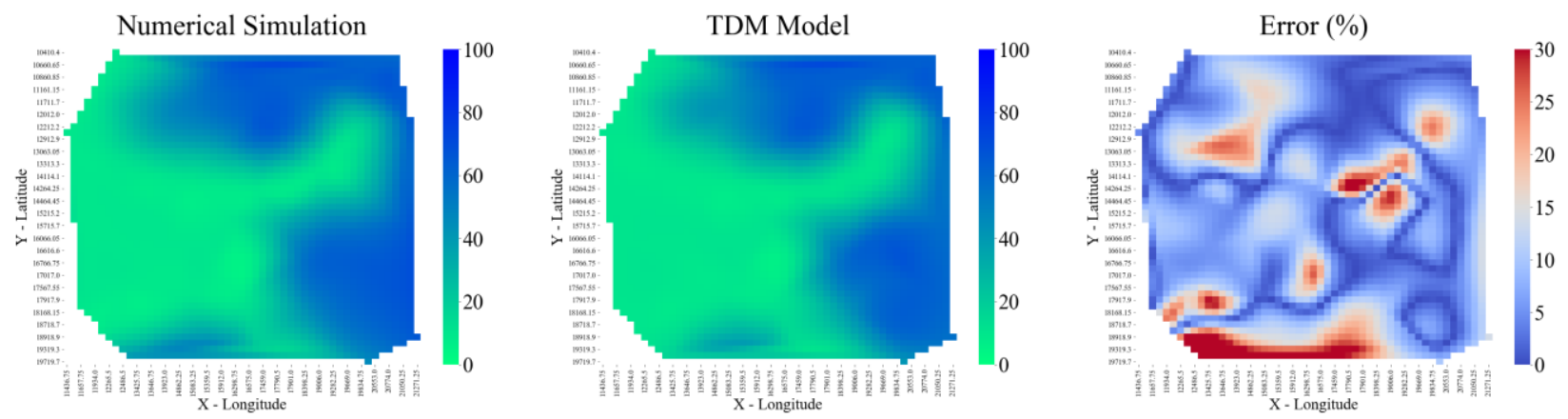

Figure 235 Entire reservoir heat map for water saturation at December 31st, 2016 for 6 Year Blind Validation TDM

All individual well plots were analyzed and categorized based on how well the predictions matched the actual data, the same procedure for the previous models. In the same manner as the entire reservoir production plots, the red line is drawn at December 31 $1^{\text {st }}, 2010$ to depict the start of blind validation. The good accuracy is clear in the example plots below for Well-011 and Well-053 below, despite the limited data for the TDM to learn from for Well-053. Well-053 was brought online at December $31^{\text {st }}, 2009$, and is in fact one of the wells that the TDM could only learn the behavior of the well from one years worth of data. Thirty-one wells were decided to be of good quality, $54 \%$ of the total wells. 


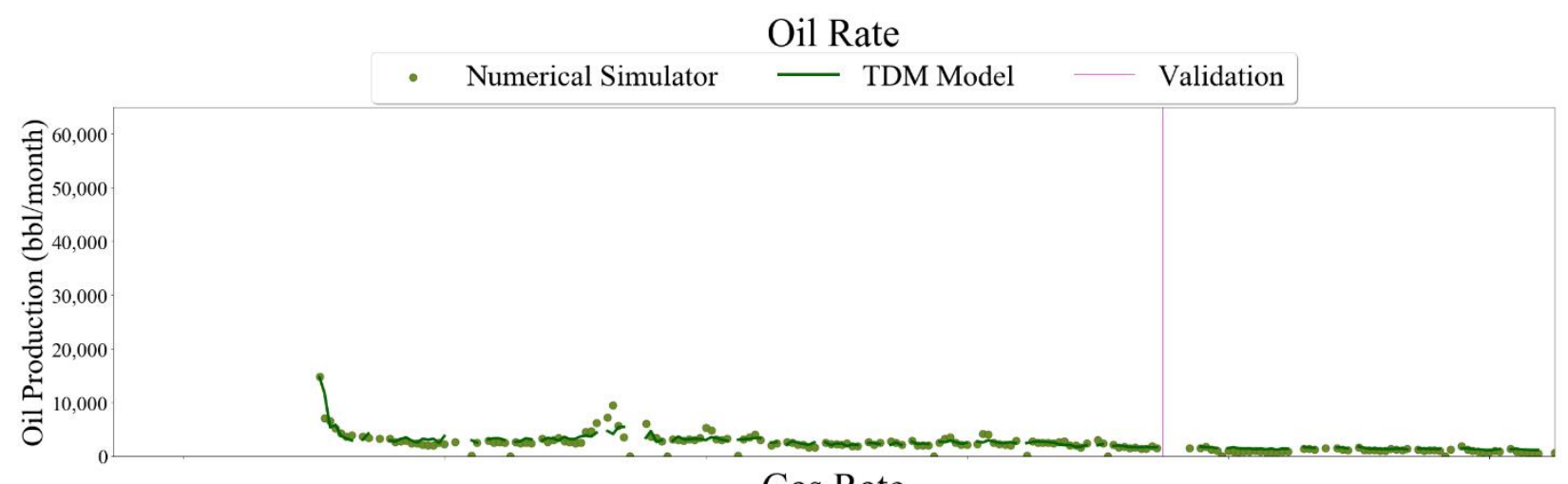

Gas Rate

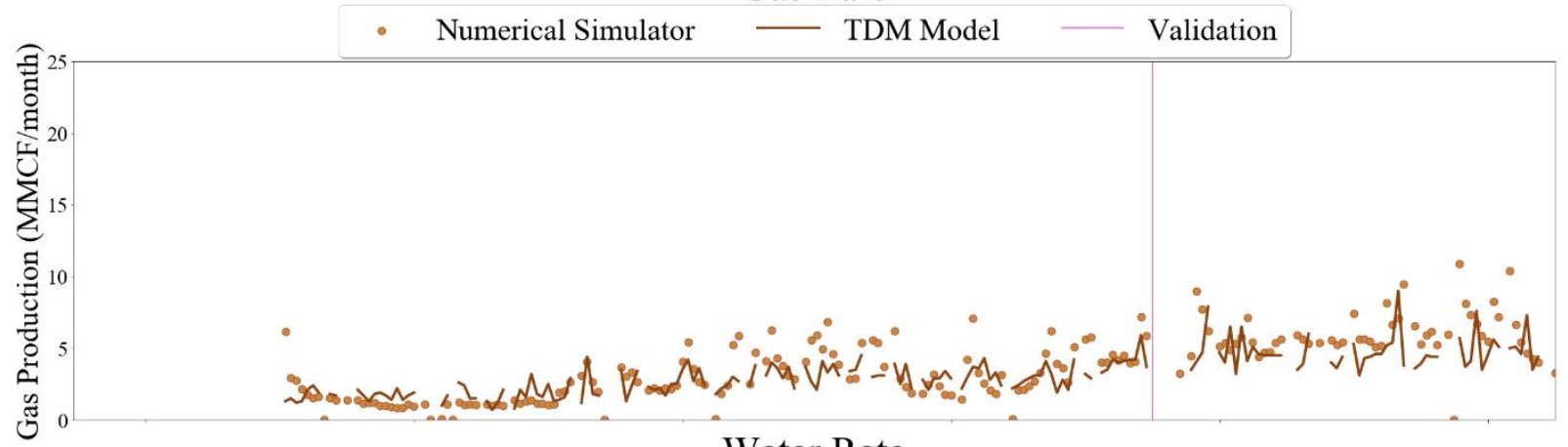

Water Rate

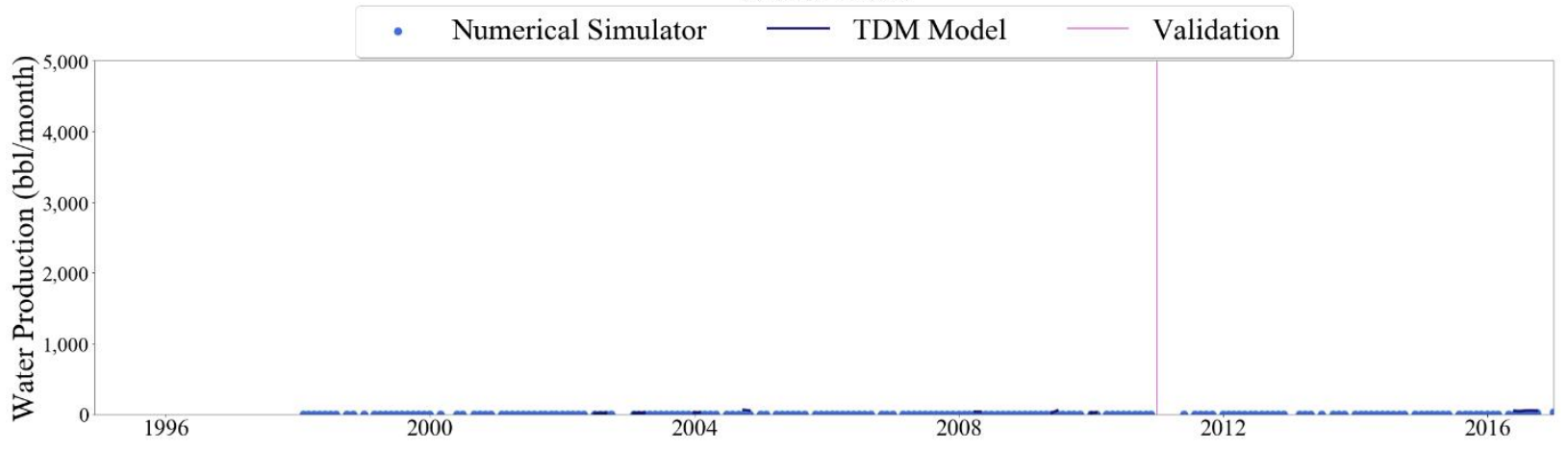

Figure 236 Well-011 oil, gas, and water rate TDM predictions vs actual simulation data plots for 6 Year Blind Validation TDM 


\section{Oil Rate}
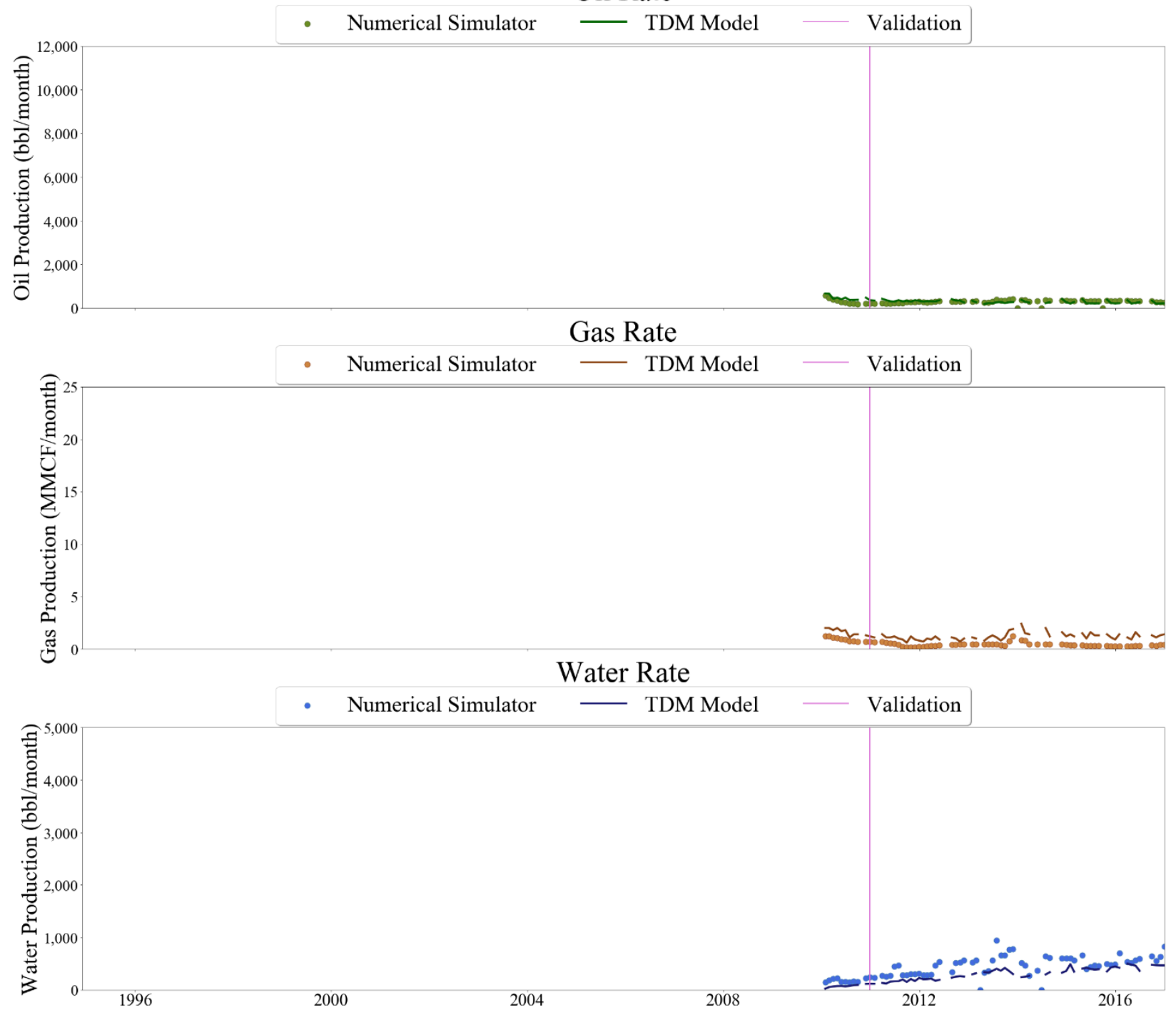

Figure 237 Well-053 oil, gas, and water rate TDM predictions vs actual simulation data plots for 6 Year Blind Validation TDM

Seventeen wells, or about $30 \%$ of the total wells, were categorized as having average accuracy. Well-022 was said to have an average fit due solely to the gas plot seen below. While the prediction values do not differ largely from the actual values, a better fit to the trend of the data, especially for the last year, is desired. Well-056 is another well within this category that was placed there because of its predictions in the oil plot. Well-056 is one of the wells that the TDM only had a years worth of data to learn from, and while it learned the overall trend, its predicted rate is slightly lower than the actual. 

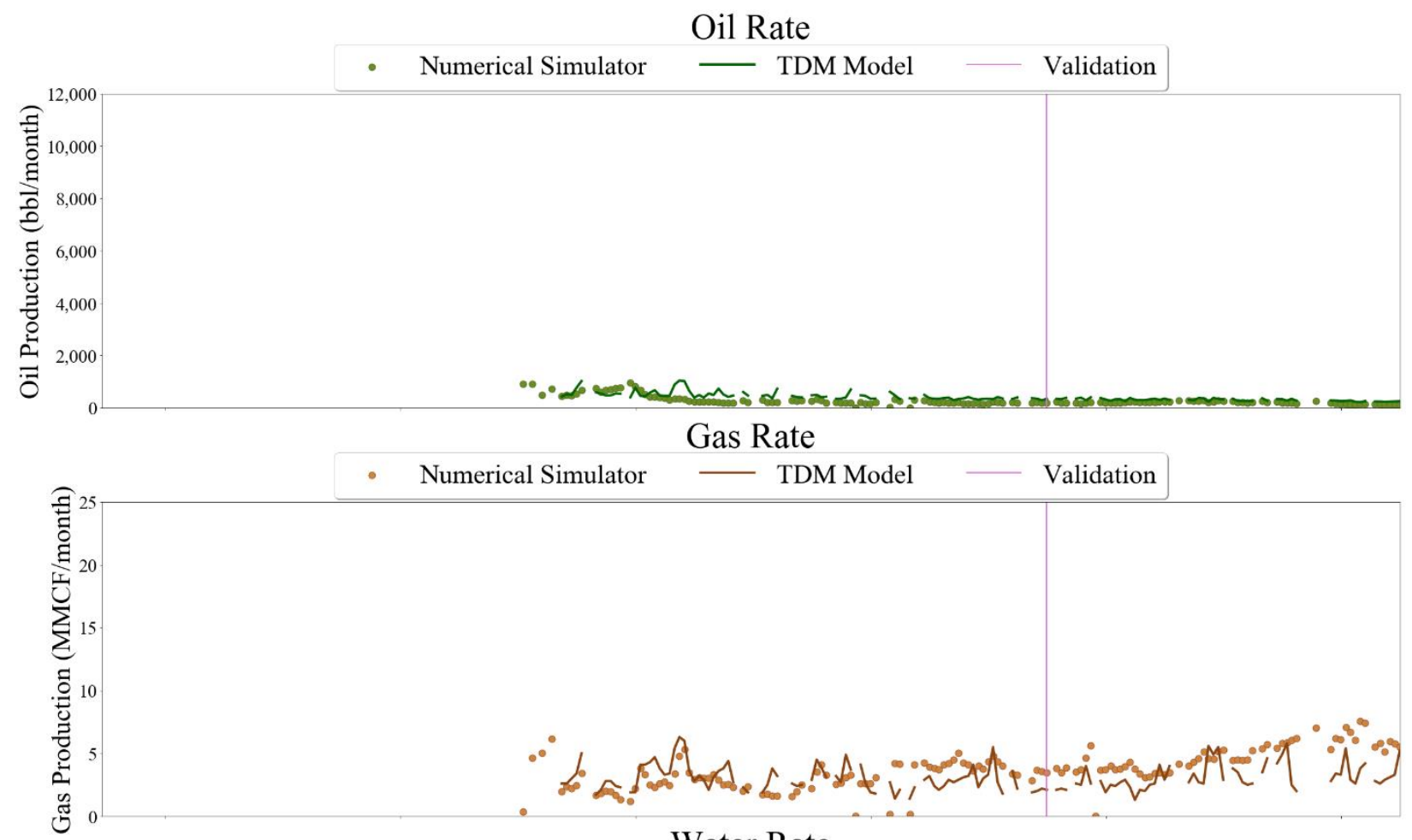

\section{Water Rate}

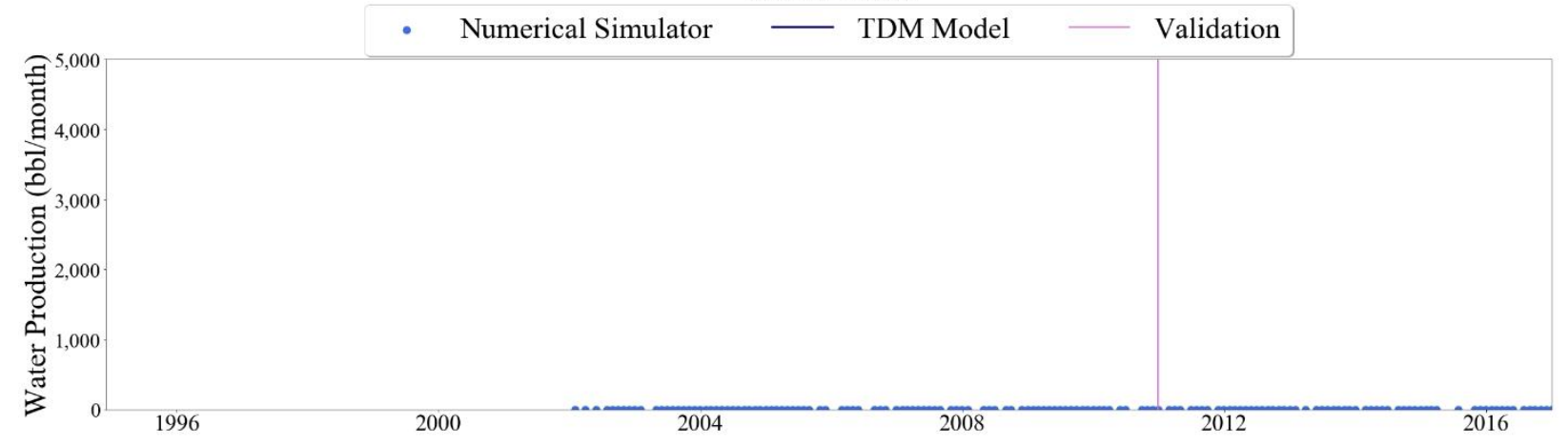

Figure 238 Well-022 oil, gas, and water rate TDM predictions vs actual simulation data plots for 6 Year Blind Validation TDM 


\section{Oil Rate}

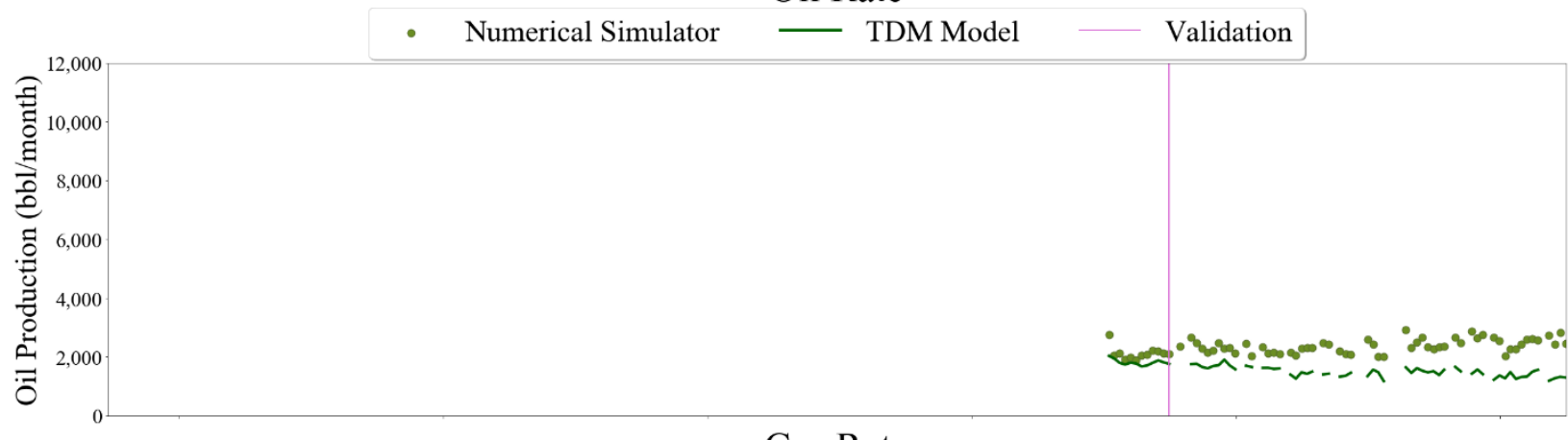

\section{Gas Rate}
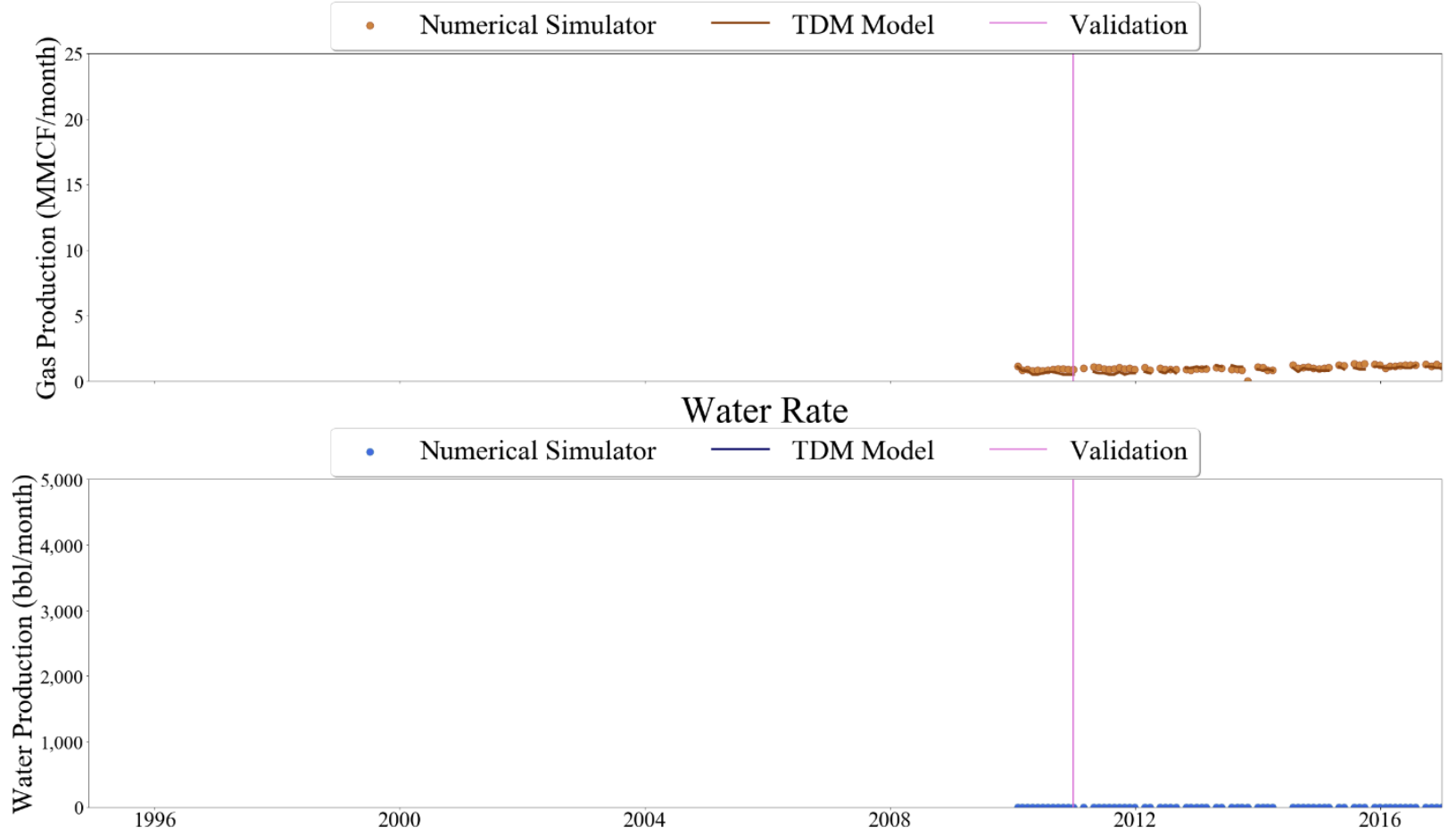

Figure 239 Well-056 oil, gas, and water rate TDM predictions vs actual simulation data plots for 6 Year Blind Validation TDM

Well-014 is one example of a well with poor overall prediction quality. The quality is attributed to the beginning of the gas plot and the end of the water plot's contrast in prediction and actual data. This difference also cannot be attributed to the length of blind validation, as the contrast occurs well before the blind validation begins. Well-046 is a second example, where the poor prediction is seen in the water plot starting about two years after blind validation begins. The TDM predicted a larger increase in water production than the actual slight increase seen in the data for the last four years of simulation. About $16 \%$ or 9 out of 57 wells were categorized as having a poor fit. All of the 6 Year Blind Validation TDM's individual well production plots can be seen in Appendix 8.2.7.1. 


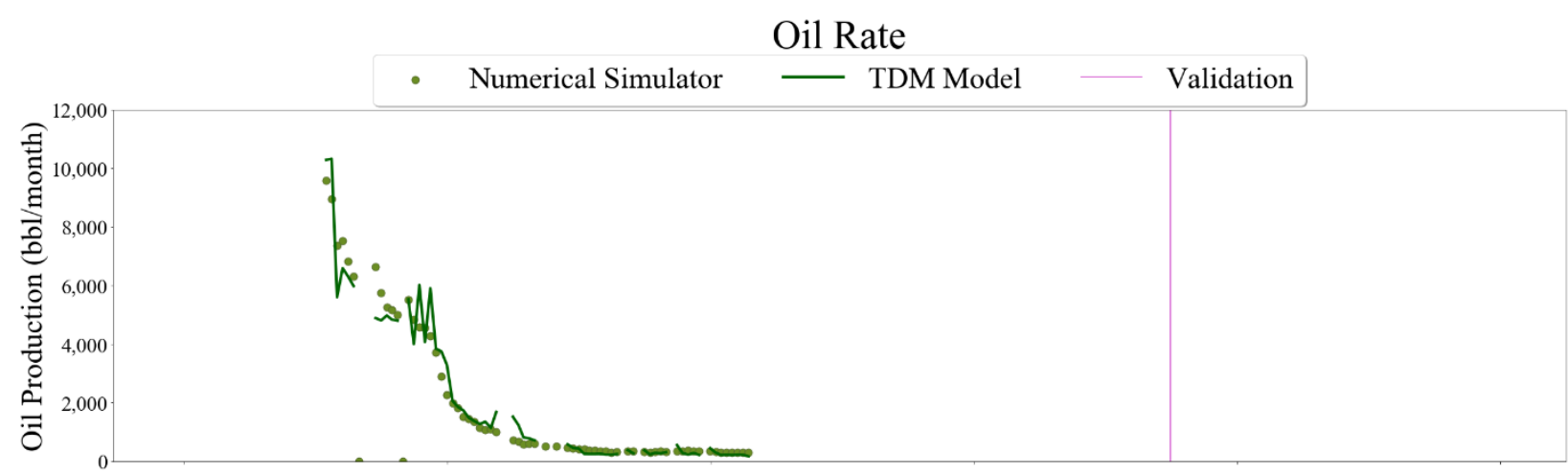

\section{Gas Rate}

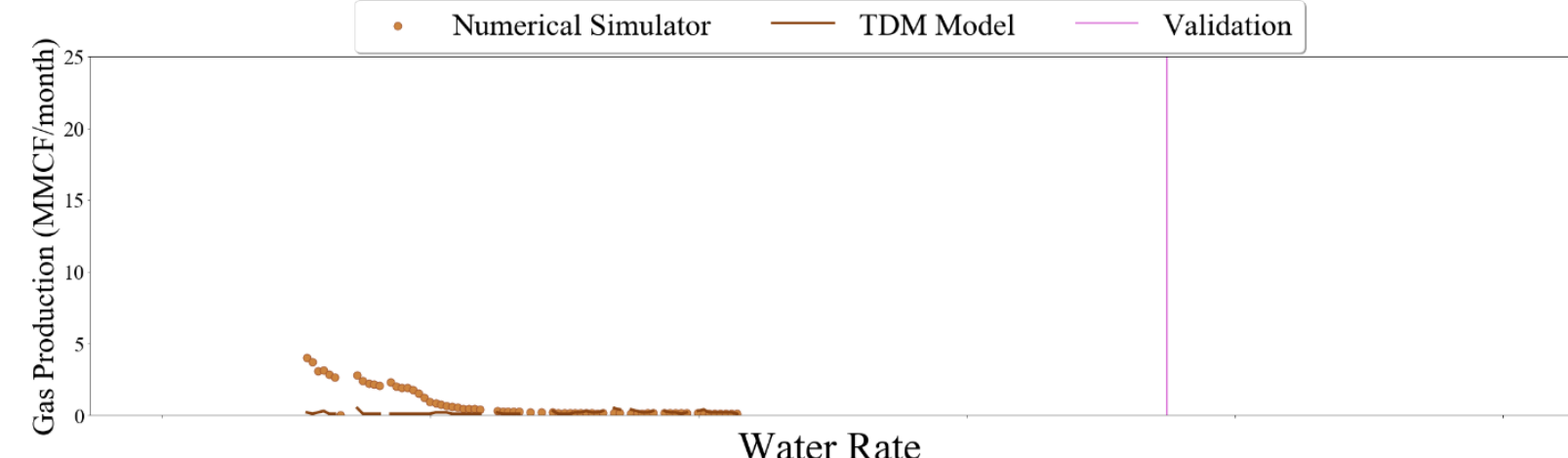

Water Rate

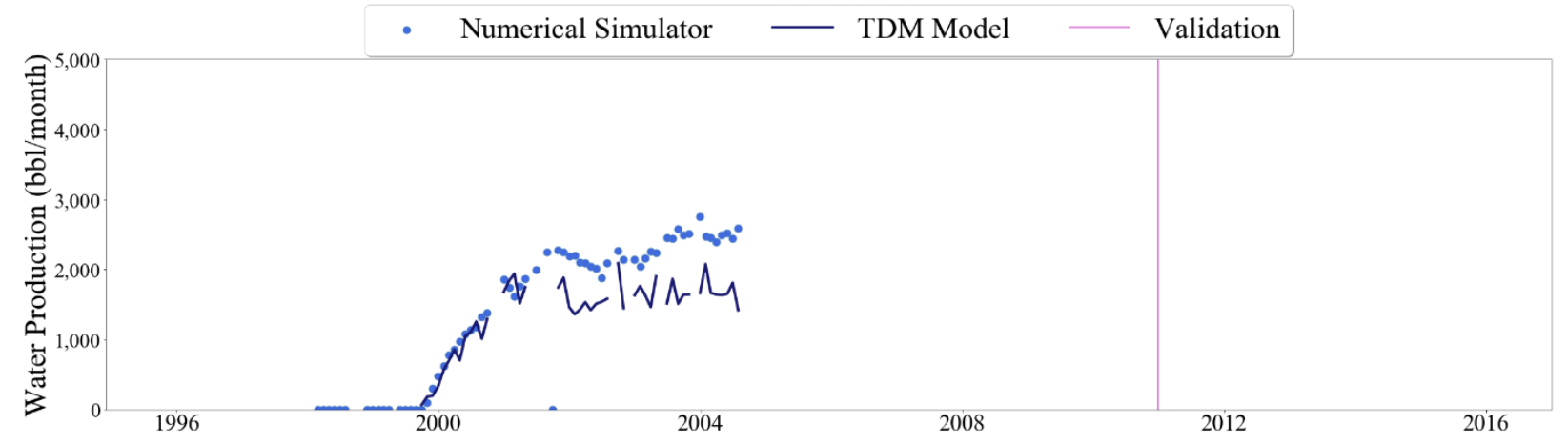

Figure 240 Well-014 oil, gas, and water TDM predictions vs actual simulation data plots for 6 Year Blind Validation TDM 


\section{Oil Rate}
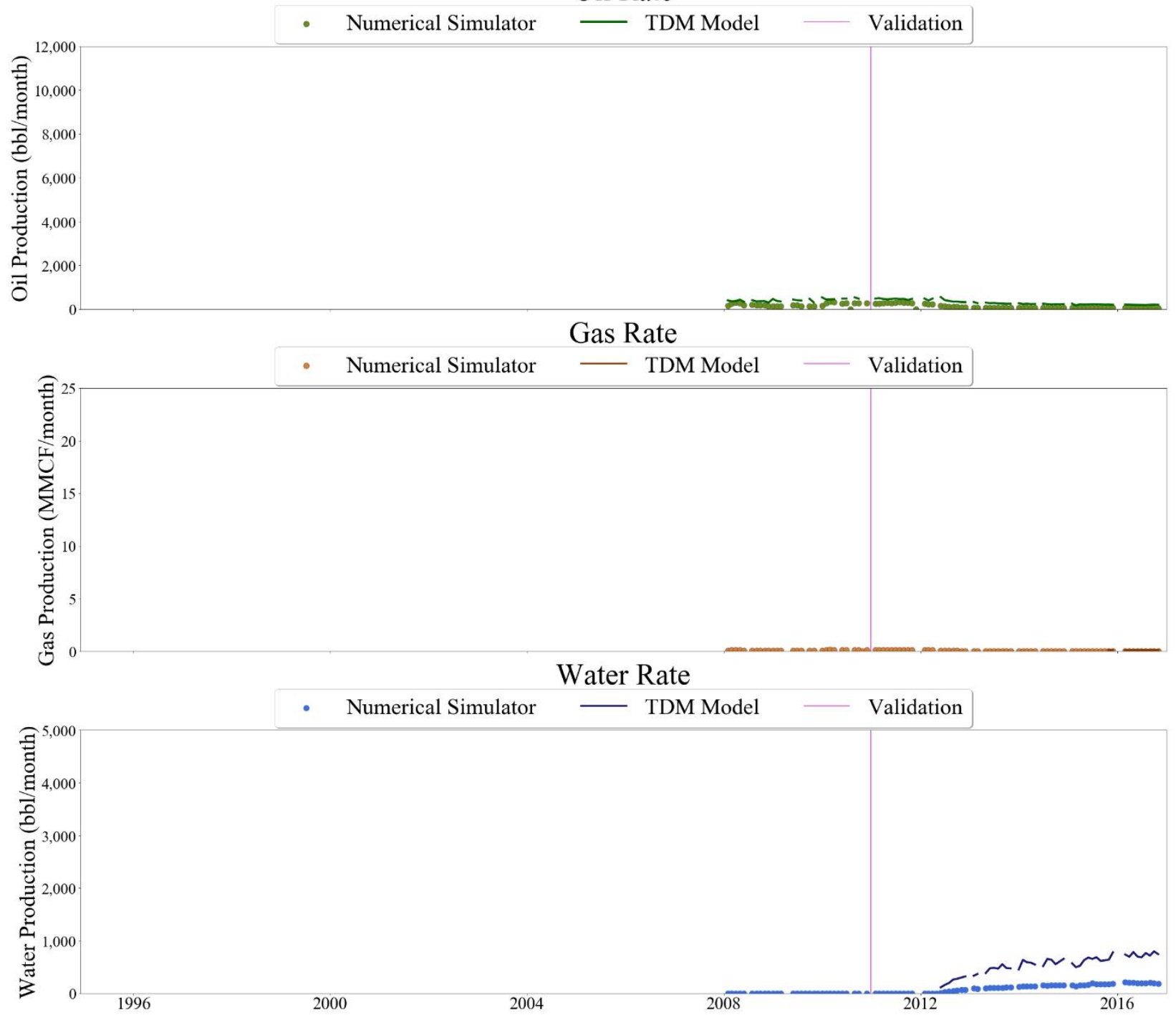

Figure 241 Well-046 oil, gas, and water rate TDM predictions vs actual simulation data plots for 6 Year Blind Validation

Overall, the Multi-Random Partitioning- 6 Year Blind Validation TDM had less wells categorized as good accuracy and more wells categorized as poor than another other model generated, and understandably so. It is important to note that the difference in accuracy between the 6 Year Blind Validation case and the remaining models was not significant, in fact when considering all of the results (entire reservoir production plots, heat maps, and individual plots) the model is still considered an adequately accurate model. The capabilities of artificial intelligence and machine learning has been proven throughout these several models, but especially with this last model due to the TDM's ability to provide acceptable or even good results with less than one year of learning changed behavior (from recompletion of a well) or the behavior of an entirely new well.

Another TDM model was not built with an additional year of blind validation because the maximum amount of blind validation without predicting a new phase of production was already tested with the 6 Year Blind Validation TDM case. Developing a Top Down Model with 7 years of blind validation beginning in December $31^{\text {st }}$, 2009, and subsequently predicting five wells production, reservoir 
pressure, and water saturation, after only learning from those wells' offsets that were brought online earlier, is believed to be an excellent topic for future discovery.

\section{Conclusion}

First, a numerical reservoir simulator was used to create a more realistic model where everything was known about the model. The model was analyzed and the characteristics previously presented were ensured to be satisfied. Then each well from the NRM was examined and categorized into two main categories: wells producing at the end of simulation and wells not producing at the end of simulation. Fourteen wells were shut-in before the end of the simulation due to violating the operating water cut constraint. These wells were then divided once more into wells with the longest and shortest well lives within the not producing at the end of the simulation group. There were 43 wells producing at the end of the simulation, of which they were subdivided into three categories: longest, average, and shortest well lives. Wells were grouped into these categories to gain a better understanding of which individual wells would be tested with the increase in blind validation. Well biographies were created as a visual summary for every well.

Next, artificial intelligence and machine learning was used to develop 7 Top Down Models where conclusions were drawn from each model. The results for each model are succinctly outlined in the table below. Each model is first identified by the name of the model as referred to in this thesis, the number of years of blind validation, as well as when the blind validation started. For each model, the number of good, average, and poor well predictions when compared with the simulation data is reported along with the percentage of the total 57 wells that category represents. It is important to note that all 7 TDMs showed good accuracy in the entire reservoir oil, gas, and water production plots.

Table 61 Summary of all Top Down Model's individual well production prediction accuracy

\begin{tabular}{|c|c|c|c|c|c|c|c|c|}
\hline $\begin{array}{c}\text { Model } \\
\text { Name }\end{array}$ & $\begin{array}{c}\text { Years of } \\
\text { Blind } \\
\text { Validation }\end{array}$ & $\begin{array}{c}\text { Start Date } \\
\text { of Blind } \\
\text { Validation }\end{array}$ & $\begin{array}{c}\text { \# of Good } \\
\text { Well } \\
\text { Predictions }\end{array}$ & $\begin{array}{c}\text { \# of } \\
\text { Average } \\
\text { Well } \\
\text { Predictions }\end{array}$ & $\begin{array}{c}\text { \# of Poor } \\
\text { Well } \\
\text { Predictions }\end{array}$ & $\begin{array}{c}\text { \% of } \\
\text { Good }\end{array}$ & $\begin{array}{c}\text { \% of } \\
\text { Average }\end{array}$ & $\begin{array}{c}\text { \% of } \\
\text { Poor }\end{array}$ \\
\hline $\begin{array}{c}\text { Random } \\
\text { Partitioning- } \\
\text { No Blind } \\
\text { Validation }\end{array}$ & 0 & -- & 37 & 19 & 1 & $64.91 \%$ & $33.33 \%$ & $1.75 \%$ \\
\hline $\begin{array}{c}\text { Multi- } \\
\text { Random } \\
\text { Partitioning- } \\
\begin{array}{c}\text { Y Year } \\
\text { Blind } \\
\text { Validation }\end{array}\end{array}$ & 1 & $12 / 31 / 2015$ & 34 & 19 & 4 & $59.65 \%$ & $33.33 \%$ & $7.02 \%$ \\
\hline $\begin{array}{c}\text { Multi- } \\
\text { Random } \\
\text { Partitioning- } \\
\begin{array}{c}2 \text { Year } \\
\text { Blind } \\
\text { Validation }\end{array}\end{array}$ & 2 & $12 / 31 / 2014$ & 37 & 16 & 4 & $64.91 \%$ & $28.07 \%$ & $7.02 \%$ \\
\hline $\begin{array}{c}\text { Multi- } \\
\text { Random } \\
\text { Partitioning- } \\
3 \text { Year }\end{array}$ & 3 & $12 / 31 / 2013$ & 39 & 15 & 3 & $68.42 \%$ & $26.32 \%$ & $5.26 \%$ \\
\hline
\end{tabular}




\begin{tabular}{|c|c|c|c|c|c|c|c|c|}
\hline $\begin{array}{c}\text { Blind } \\
\text { Validation }\end{array}$ & & & & & & & & \\
\hline $\begin{array}{c}\text { Multi- } \\
\text { Random } \\
\text { Partitioning- } \\
4 \text { Year } \\
\text { Blind } \\
\text { Validation }\end{array}$ & 4 & $12 / 31 / 2012$ & 34 & 15 & 7 & $59.65 \%$ & $26.32 \%$ & $12.28 \%$ \\
\hline $\begin{array}{c}\text { Multi- } \\
\text { Random } \\
\text { Partitioning- } \\
5 \text { Year } \\
\text { Blind } \\
\text { Validation }\end{array}$ & 5 & $12 / 31 / 2011$ & 37 & 12 & 7 & $64.91 \%$ & $21.05 \%$ & $12.28 \%$ \\
\hline $\begin{array}{c}\text { Multi- } \\
\text { Random } \\
\text { Partitioning- } \\
6 \text { Year } \\
\text { Blind } \\
\text { Validation }\end{array}$ & 6 & $12 / 31 / 2010$ & 31 & 17 & 9 & $54.39 \%$ & $29.82 \%$ & $15.79 \%$ \\
\hline
\end{tabular}

The entire reservoir heat maps developed for water saturation and reservoir pressure provided important insight. Reservoir pressure showed little to no error in the heat map, proving that the Top Down Model is not only capable of predicting production rates, but also reservoir pressure. Water saturation however did show more error in similar areas for all models where the amount of error varied with each time step. The error could be attributed to one or a combination of the following reasons: the feed forward interpolation method used for time steps where the water saturation was not predicted in the Top Down Model, the length of the well's life, and the location of the well in the reservoir. One possible solution to reduce the error in the water saturation heat maps is to fill in the empty prediction values during shut-in dates by interpolating the water saturation from the surrounding wells instead of the feed forward technique.

It is important to note that the Numerical Reservoir Model was not history matched because there was no real industry data to history match it against, the data from the NRM model was treated as the truth for the TDM model. The TDM model however was history matched to the NRM exported data, in a fraction of the time that conventional history matching of NRMs typically take. While each run of the NRM model used in this thesis on average took 19 hours, each run of the TDM model created only took on average 3-4 hours to execute. Due to the reduced run time, and the reduced number of runs required by the automated history matching process within the TDM, the TDM was deemed much more efficient in the history matching process than a typical NRM model, thus making TDM a useful complement to NRM models for industry applications.

In conclusion, the results examined for each Top Down Model was found to be sufficient with an acceptable amount of error. The capabilities of using artificial intelligence and machine learning to teach the fluid flow through porous media in each Top Down Model in the specified reservoir was successful. Each Top Down Model was able to history match and predict the oil, gas, and water production, reservoir pressure, and water saturation for a full field reservoir using solely measured data with no equations or 
assumptions. The minimum amount of data in order to obtain good results was also determined by the incremental one year increase in blind validation with each model. One year was deemed to be the minimum amount of data required for this dataset to obtain acceptable predictions.

While another year of blind validation could have been added to the 6 Year Blind Validation TDM to further test the abilities of this technology, the purpose of this thesis was not to test whether the Top Down Model could predict a new phase of production wells brought online along with new recompletions of wells without any data after the start of the new wells or data after recompletion. The author believes that further investigation into this topic would be of great value.

\section{References}

Brighterion, Inc. (2017, January 20). A closer look at Al: data mining. Retrieved from Brighterion Mastercard: https://brighterion.com/data-mining/

Computer Modeling Group LTD. (2017). Transmissibility Multipliers (Optional). Retrieved from IMEX User Guide:

file://C:/Program\%20Files\%20(x86)/CMG/Manuals/2017.10/IMEX/Default.htm\#Resources/Ima ges/WelcomeToIMEXHelp.htm\%3FTocPath\%3D 1

Computer Modelling Group Ltd. (2017). Pore Volume Modifiers (Optional). Retrieved from IMEX 2017.10 User Guide:

file://C:/Program\%20Files\%20(x86)/CMG/Manuals/2017.10/IMEX/Default.htm\#WordDocumen ts/Reservoir\%20Description/VOLMOD_PoreVolumeModifiers.htm?Highlight=volume\%20modifi er

Eberhart, R. C., Simpson, P. K., \& Dobbins, R. W. (1996). Computational Intelligence PC Tools. Boston, Massachusetts: Academic Press Professional.

Eghonghon, O. (2015). Feasibility Study Through Numerical Simulation of Waterflooding and Gas Injection for a Carbonate Oil Fleld. State College: The Pennsylvania State University.

Fausset, L. V. (1994). Fundaamentals of Neural Networks: Architectures, Algorithms, and Applications. Upper Saddle River, New Jersey: Prentice Hall.

Haifi, A. H. (2019). Confirmation of Data-Driven Reservoir Modeling Using Numerical Reservoir Simulation. Morgantown, West Virginia : West Virginia University.

Intelligent Solutions Inc. (n.d.). Top-Down, Intelligent Reservoir Models . Retrieved from Intelligent Solutions Inc.

Intelligent Solutions, Inc. . (2016). Imagine-APM User Manual. Morgantown, West Virginia.

Mohaghegh, S. (2009). Artificial Intelligence and Data Mining: Enabling Technology for Smart Fields. The Way Ahead, 14-16.

Mohaghegh, S. (2017). Data-Driven Reservoir Modeling. Richardson: Society of Petroleum Engineers.

Mohaghegh, S., Shahkarami, A., Gholami, V., \& Haghighat, S. (2014). Artificial Intelligence (Al) Assisted History Matching. SPE, 1-26. 
Pore Volume Modifiers (Optional). (n.d.). Retrieved from IMEX Black Oil \& Unconventional Simulator.

Ross, T. J. (1995). Fuzzy Logic With Engineering Applications. New York City: McGraw-Hill.

Shahkarami, A., Mohaghegh, S., \& Hajizadeh, Y. (2015). Assisted History Matching Using Pattern Recognition Technology. SPE, 1-23.

Society of Petroleum Engineers. (2015, June 3). Reservoir Simulator Applications. Retrieved from PetroWiki: https://petrowiki.org/Reservoir_simulation_applications

\section{Appendix}

\subsection{Anticline Thesis CMG Model}

8.1.1 Wells Producing at End of Simulation

\subsubsection{Largest Number of Production Days}

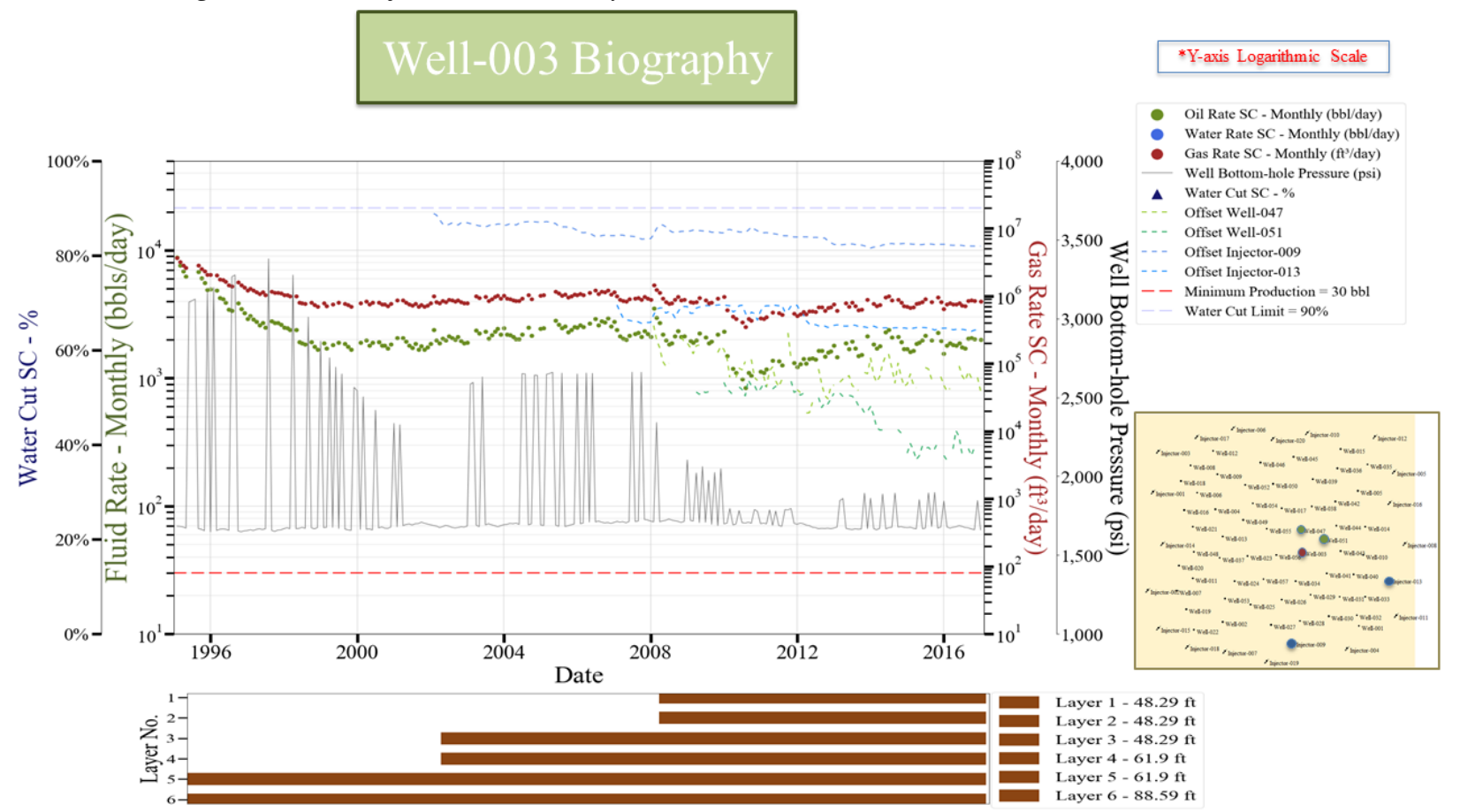

Figure 242 Well-003 biography 

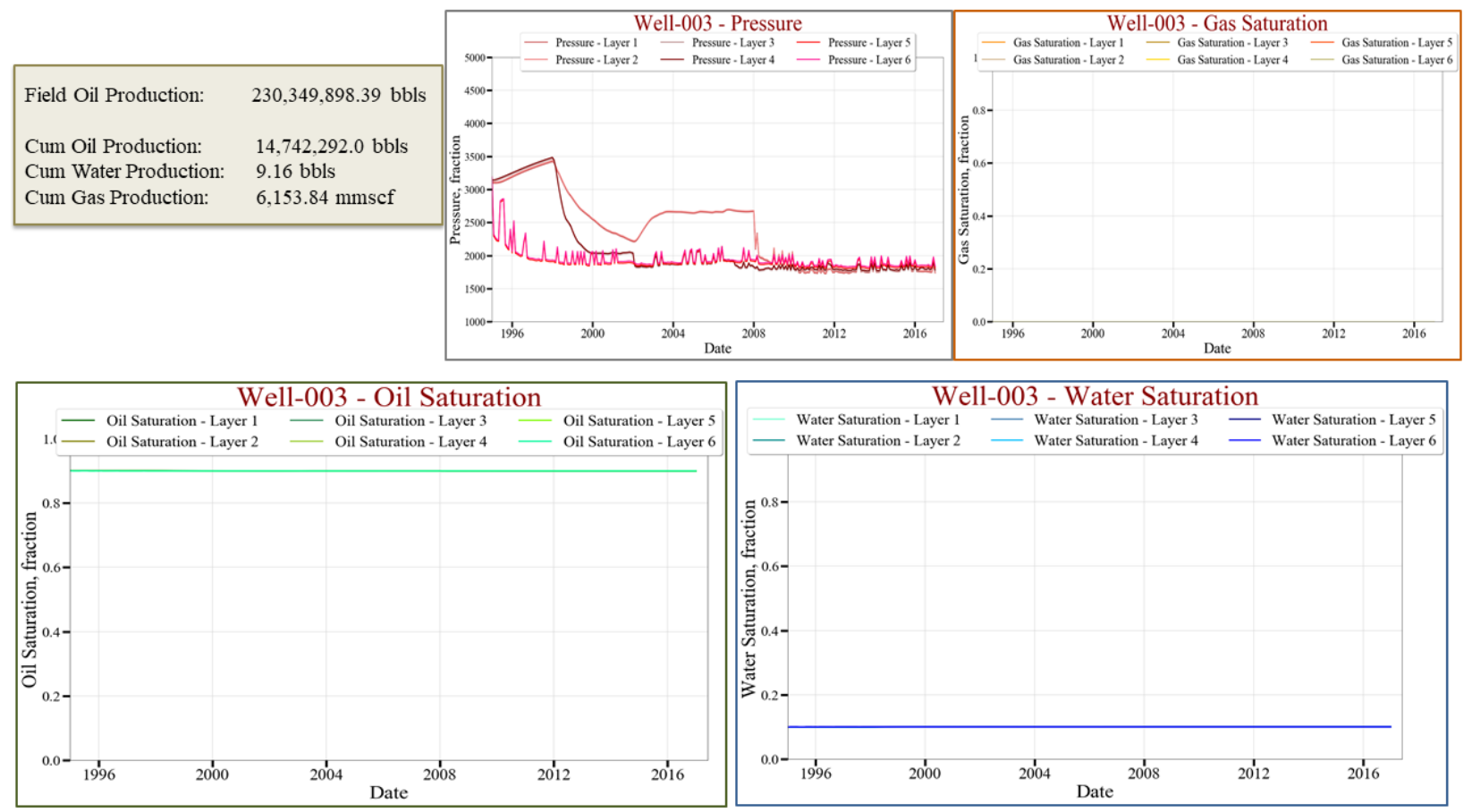

Figure 243 Well-003 saturations and pressure per layer

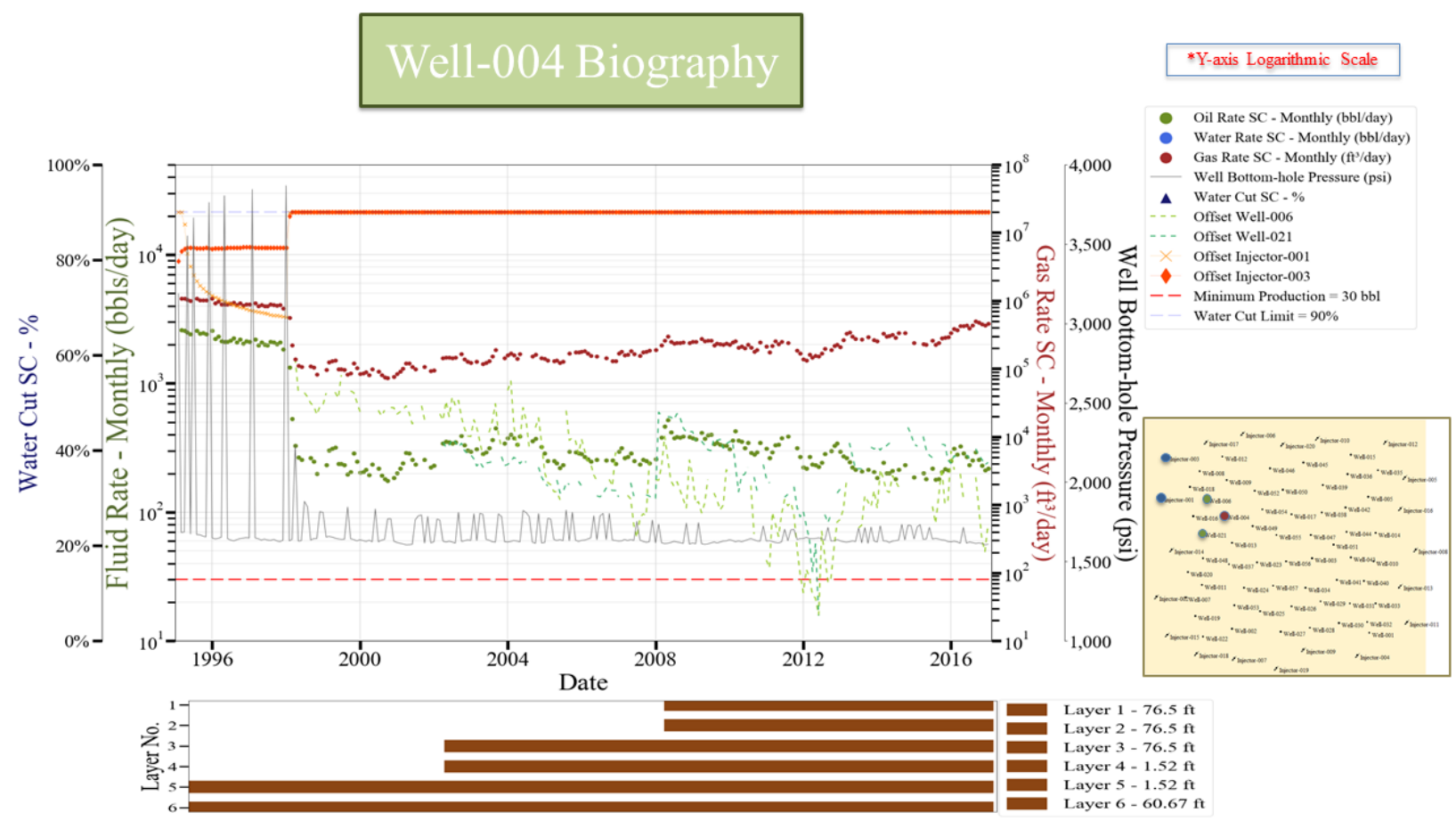

Figure 244 Well-004 biography 

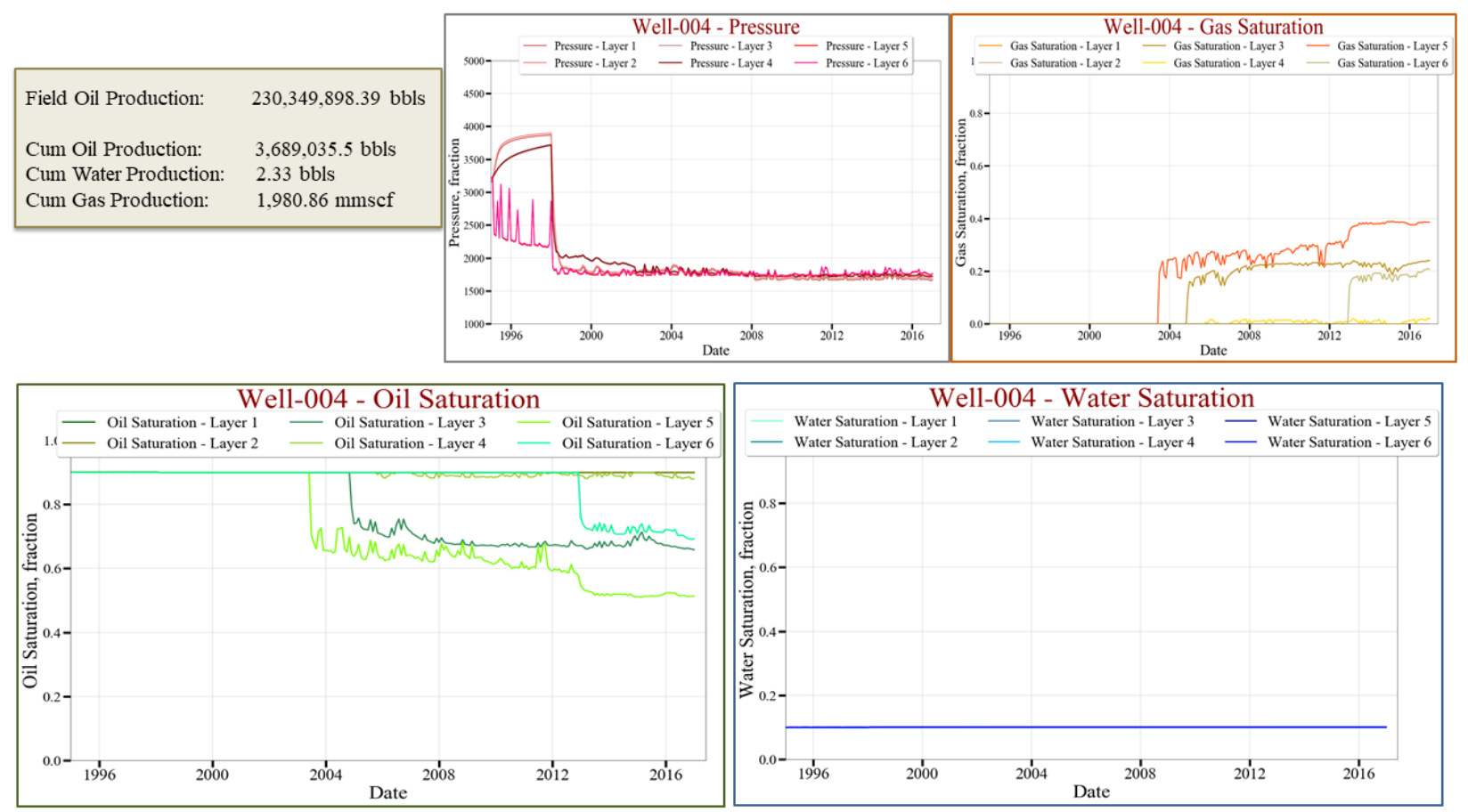

Figure 245 Well-004 saturations and pressure per layer

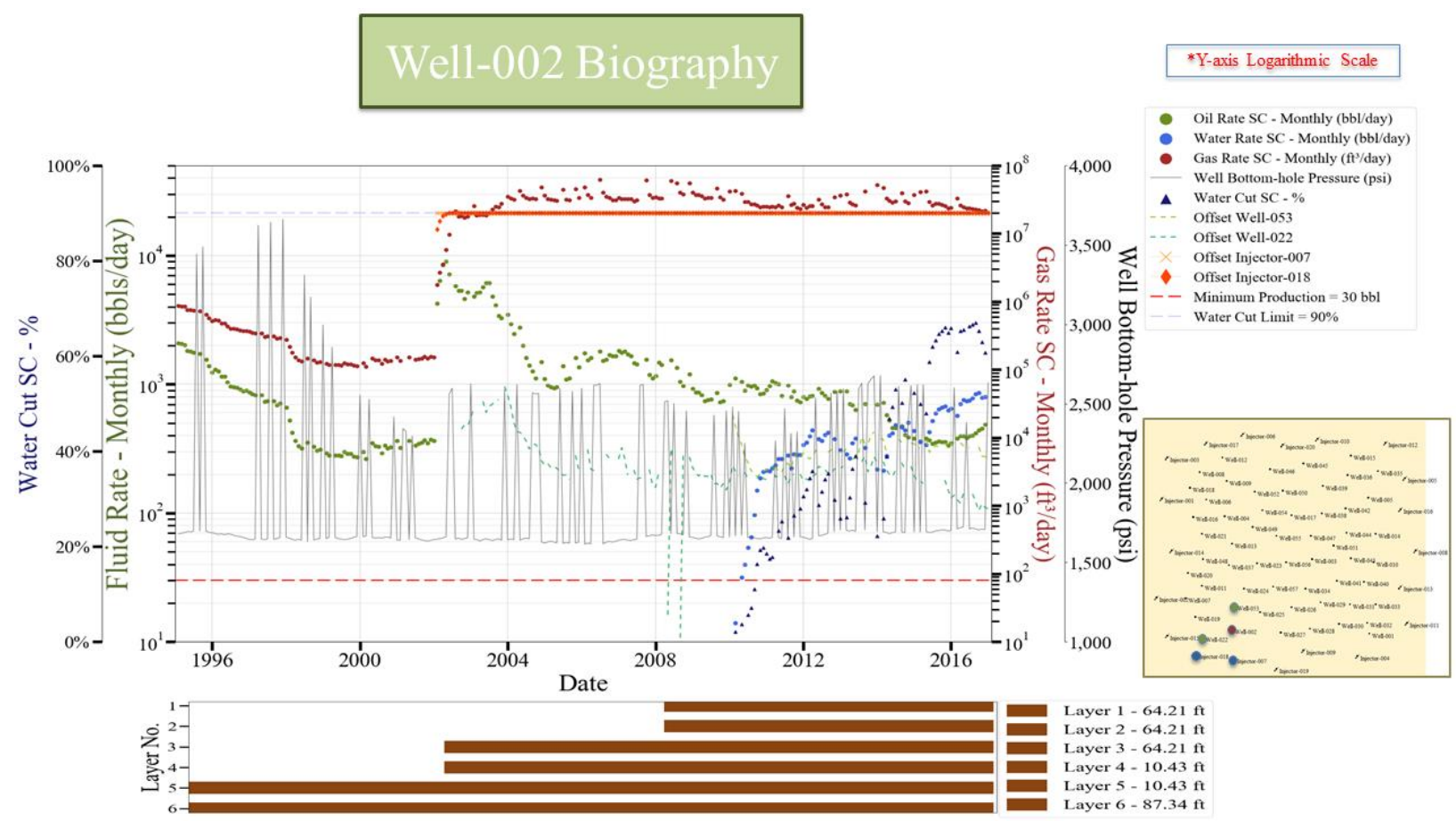

Figure 246 Well-002 biography 

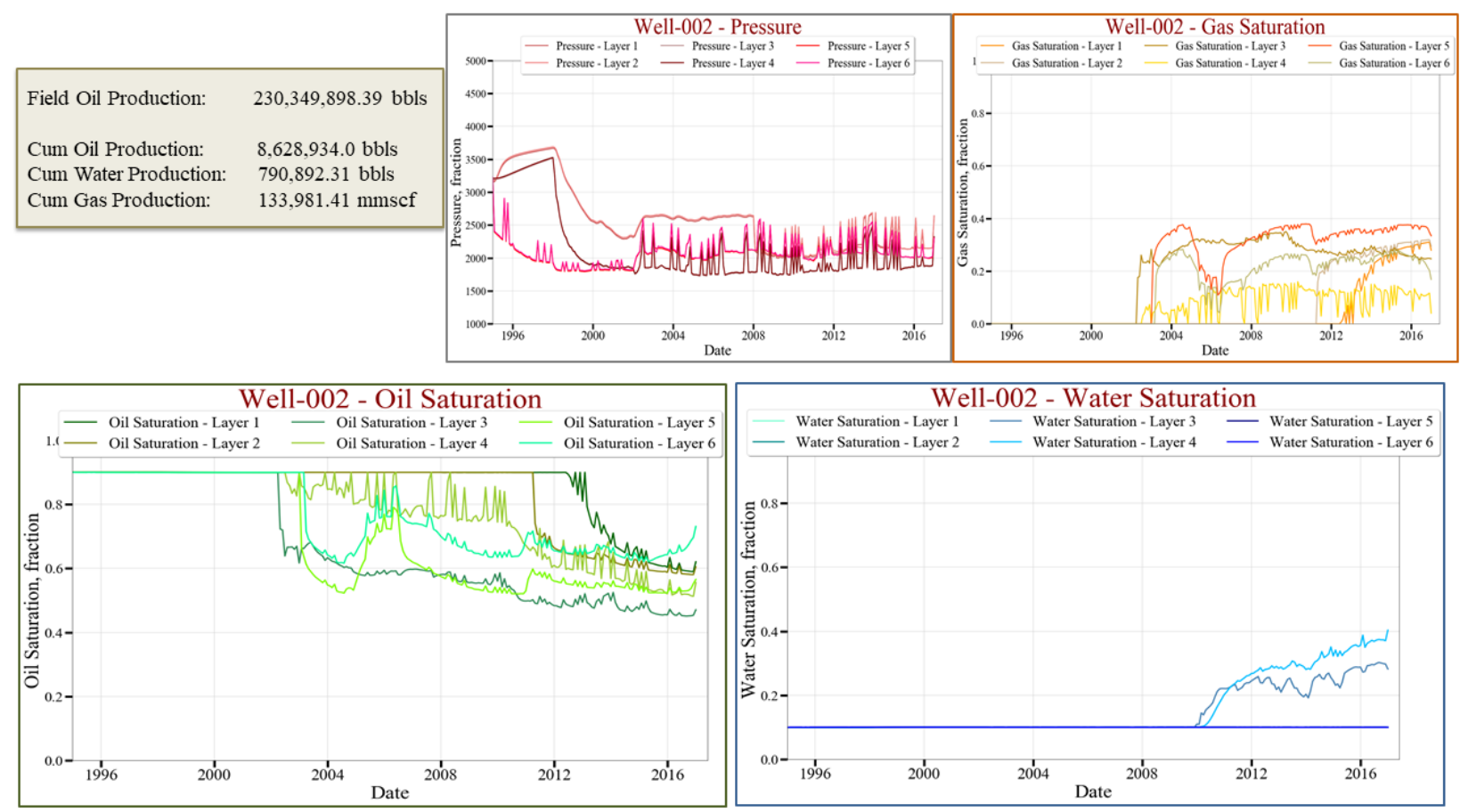

Figure 247 Well-002 saturations and pressure per layer

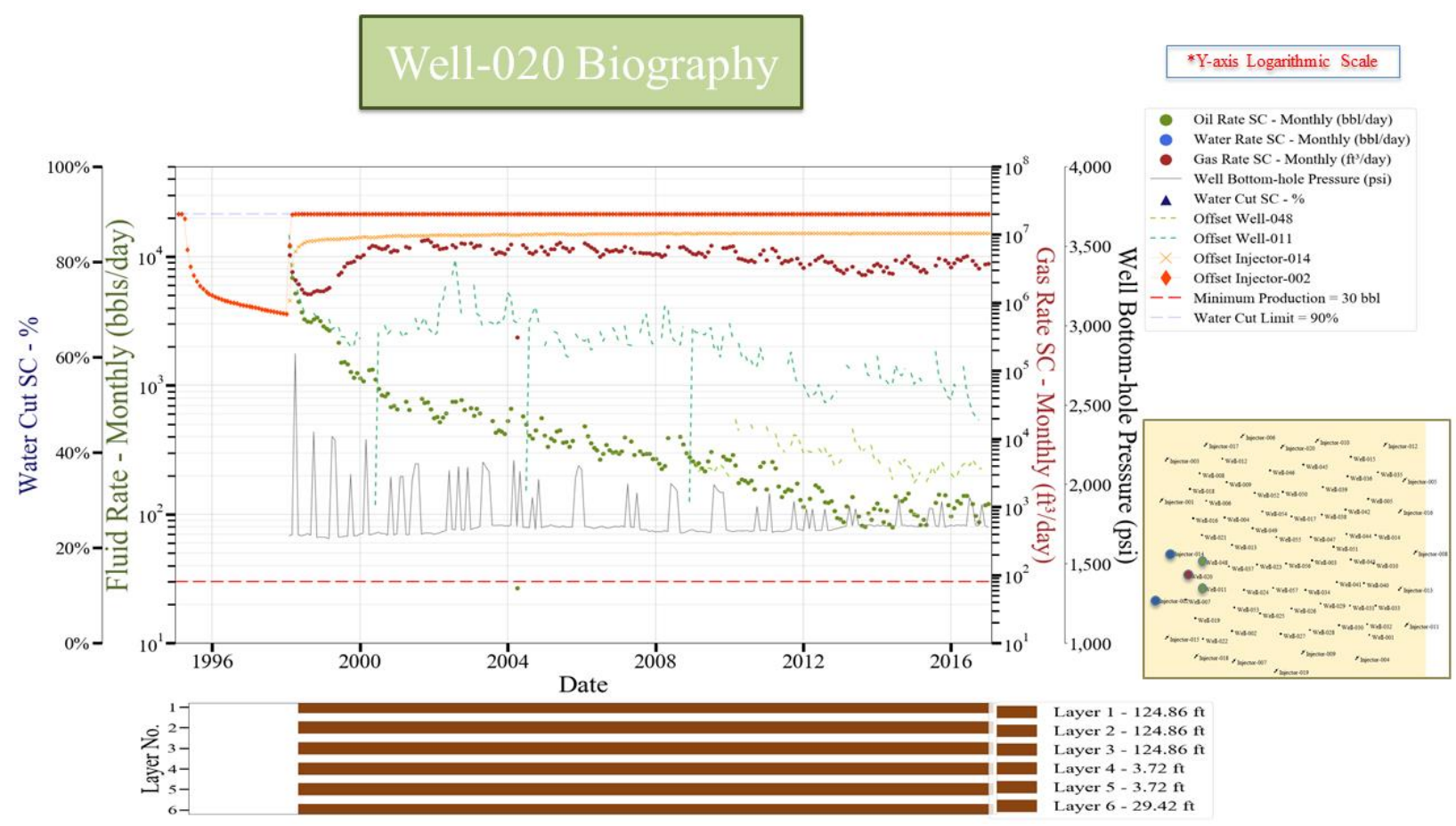

Figure 248 Well-020 biography 

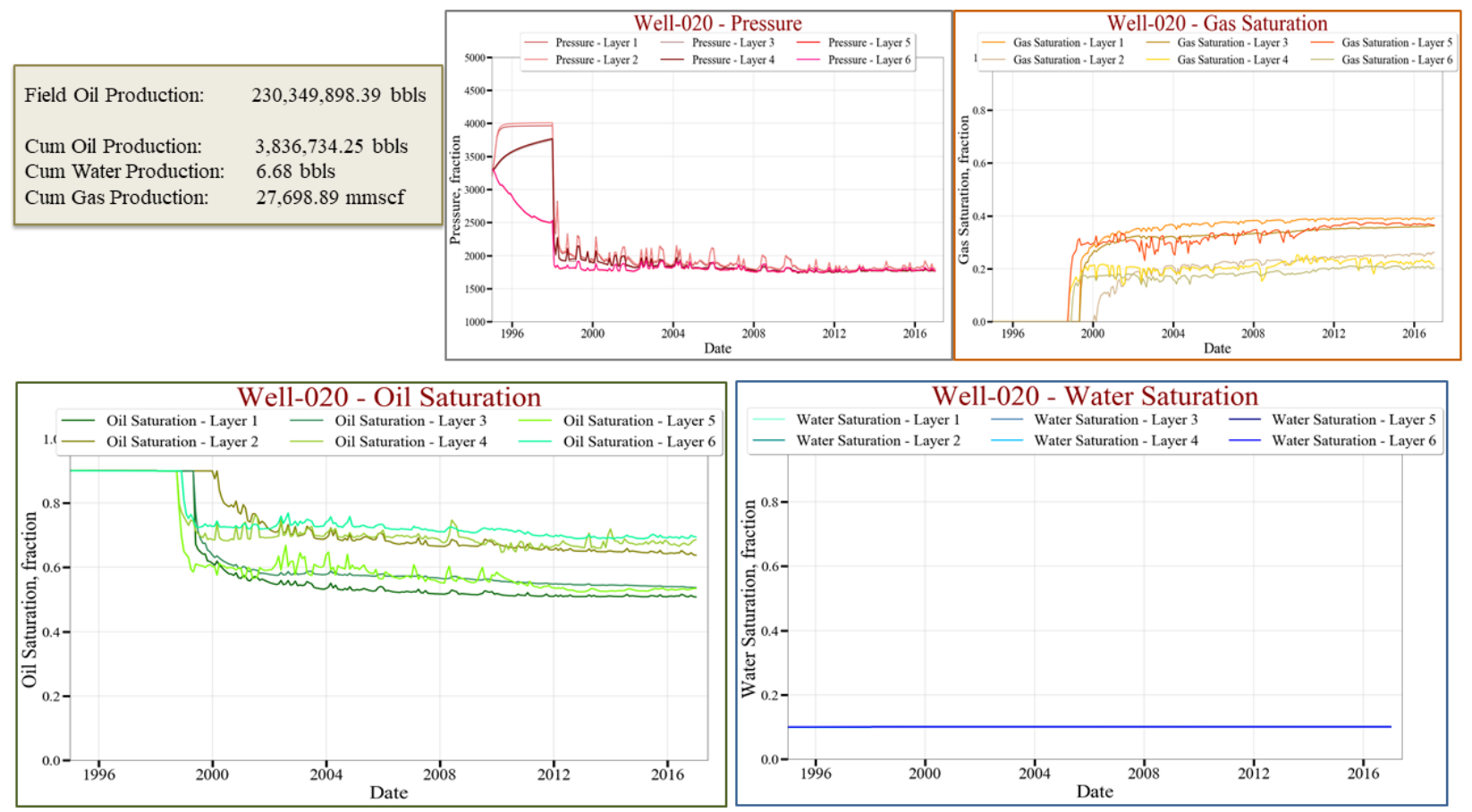

Figure 249 Well-020 saturations and pressure per layer

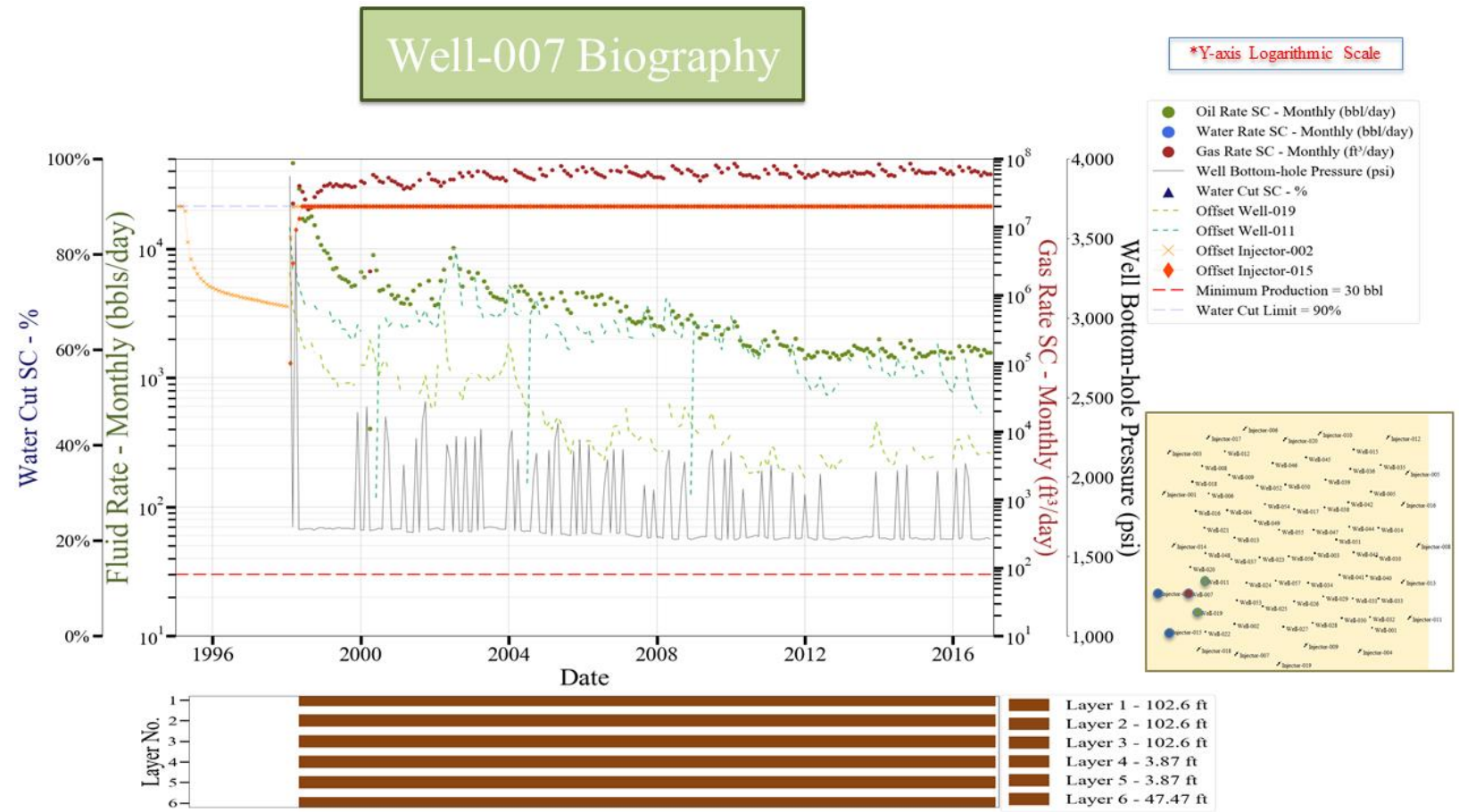

Figure 250 Well-007 biography 

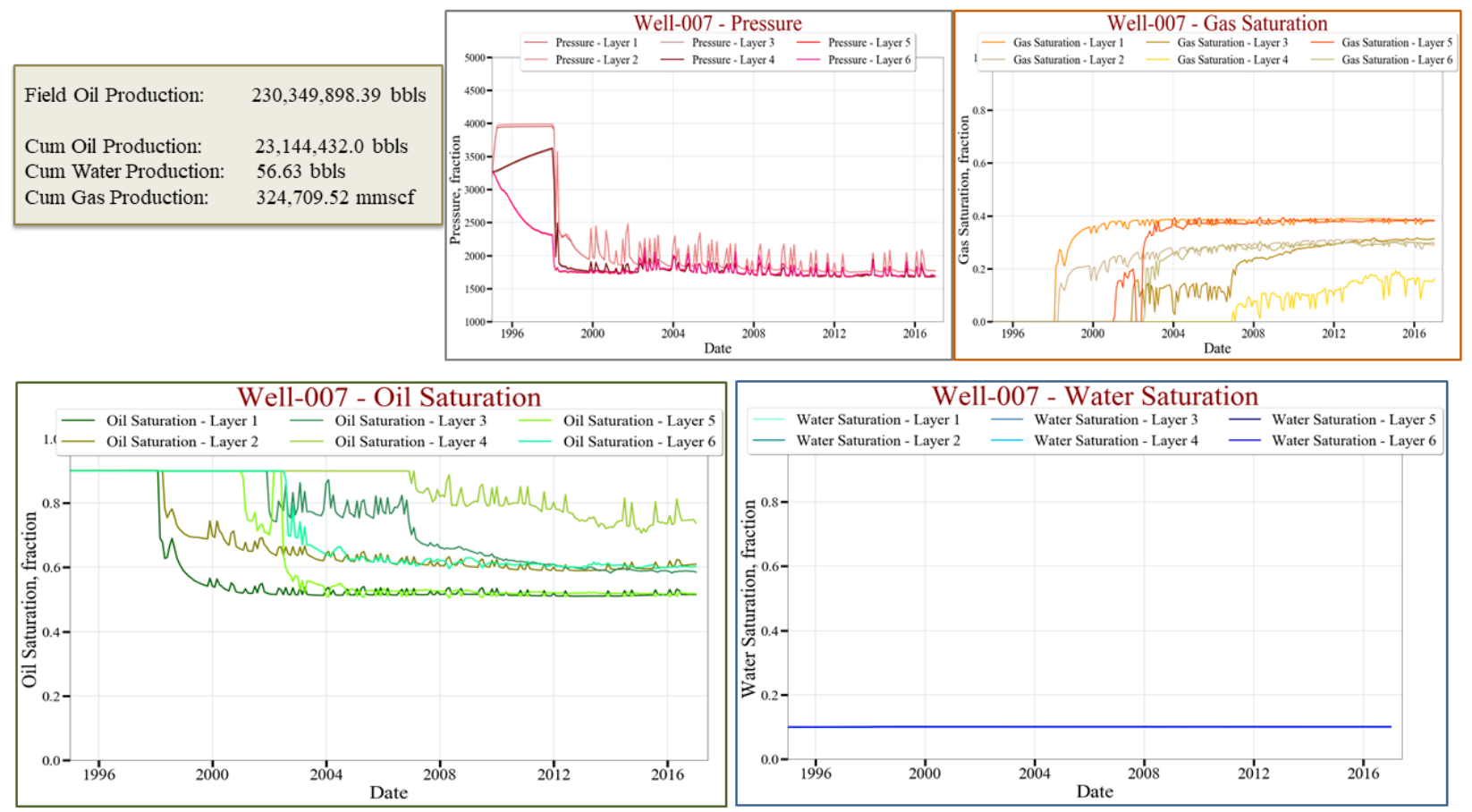

Figure 251 Well-007 saturations and pressure per layer

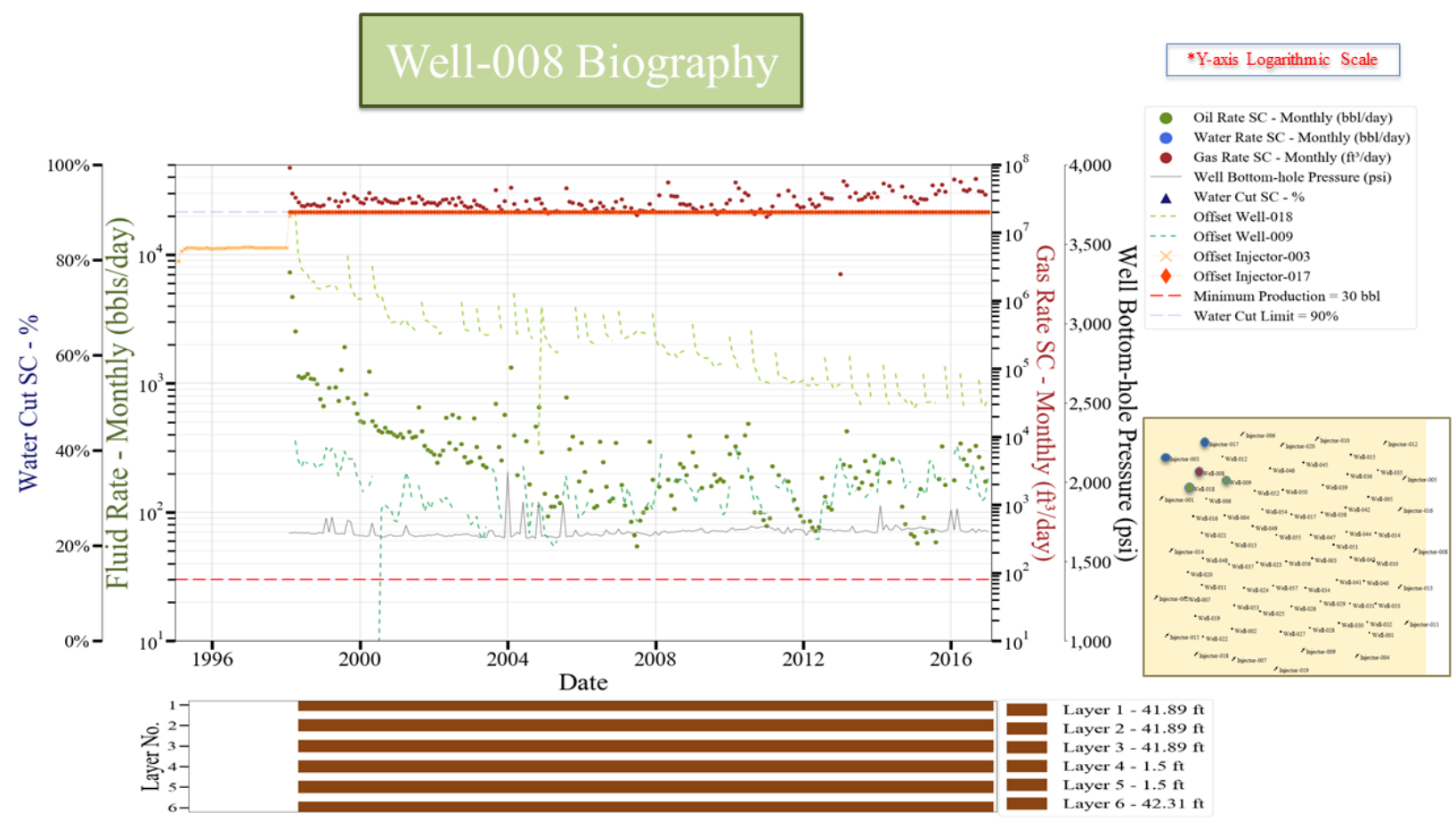

Figure 252 Well-008 biography 

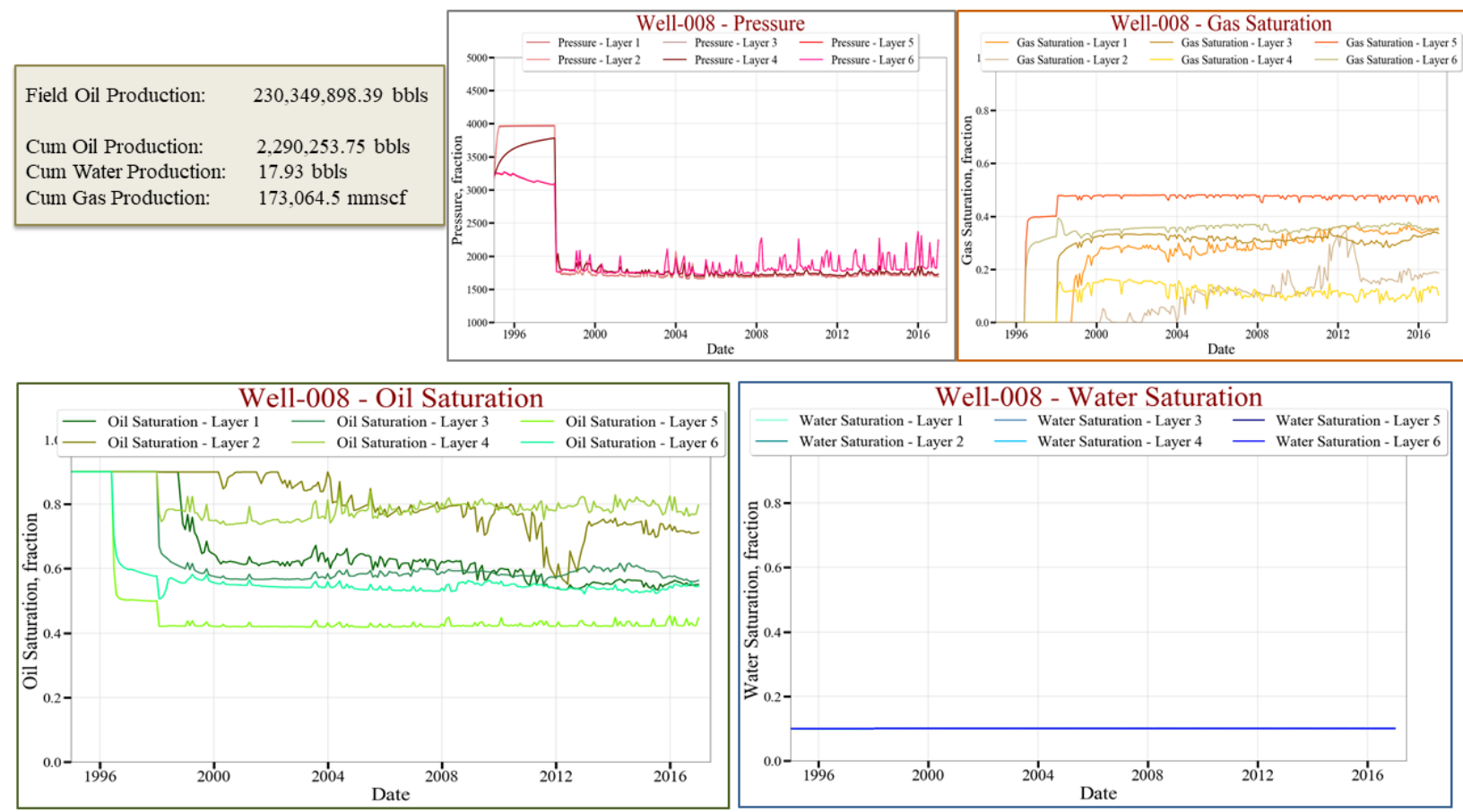

Figure 253 Well-008 saturations and pressure per layer

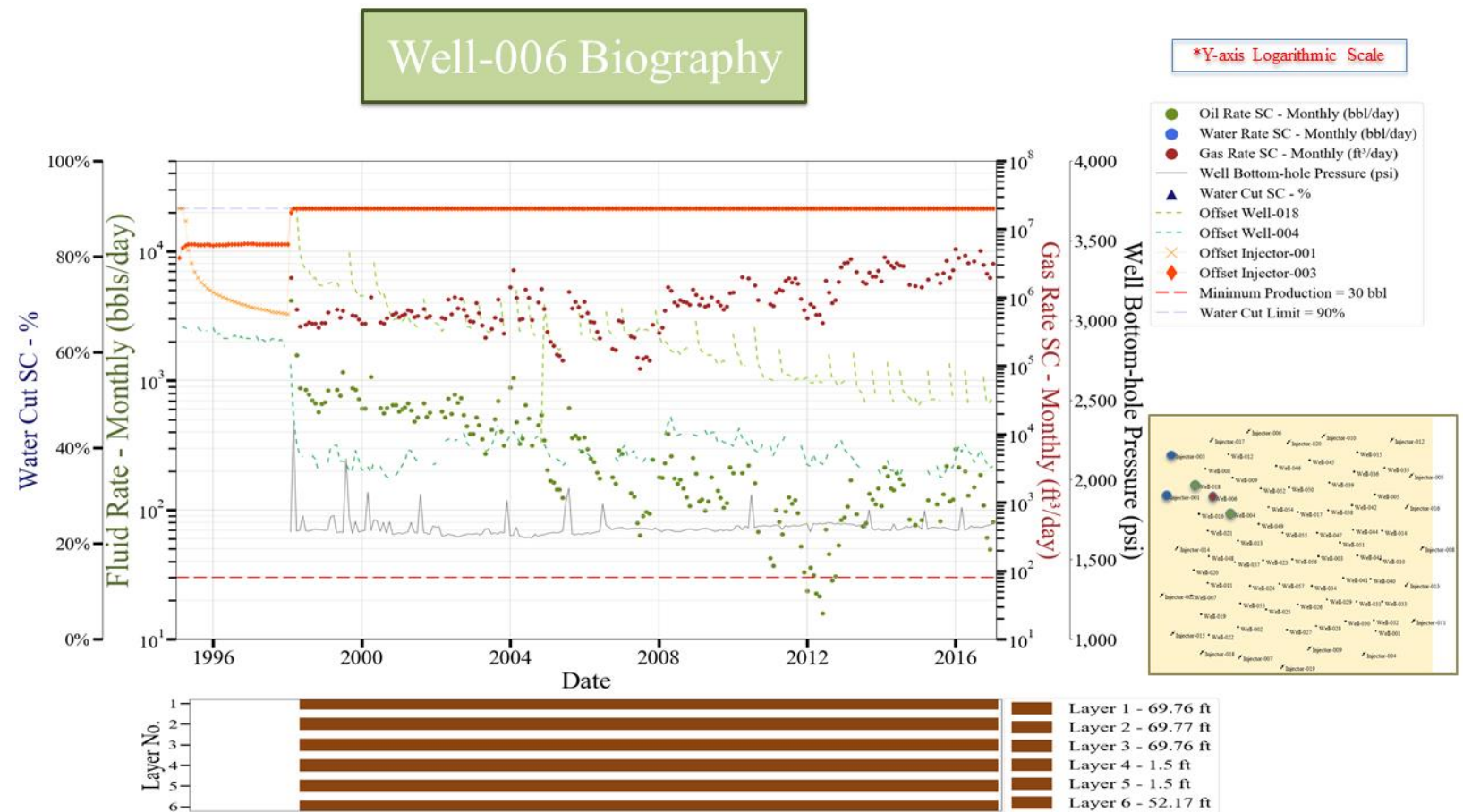

Figure 254 Well-006 biography 

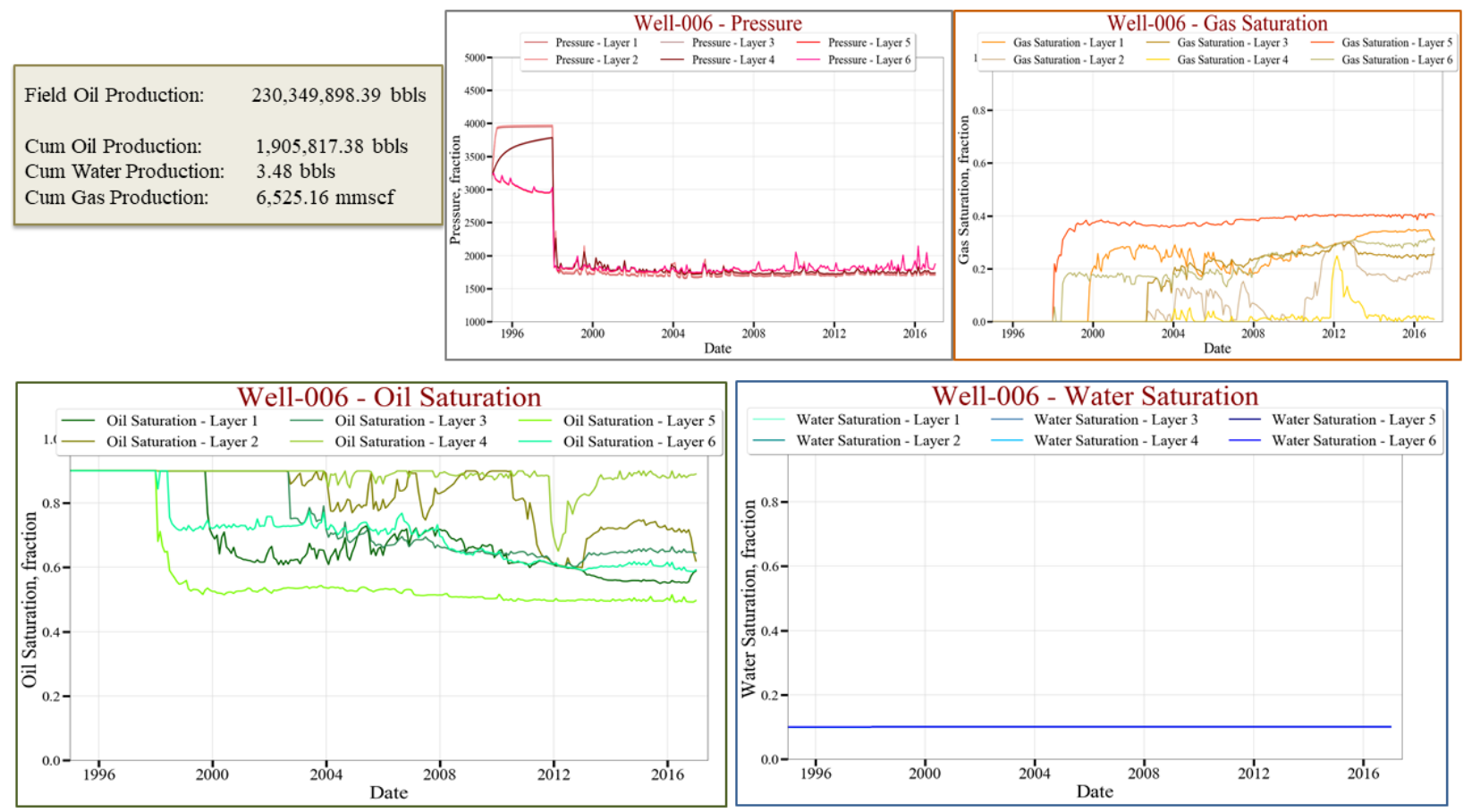

Figure 255 Well-006 saturations and pressure per layer

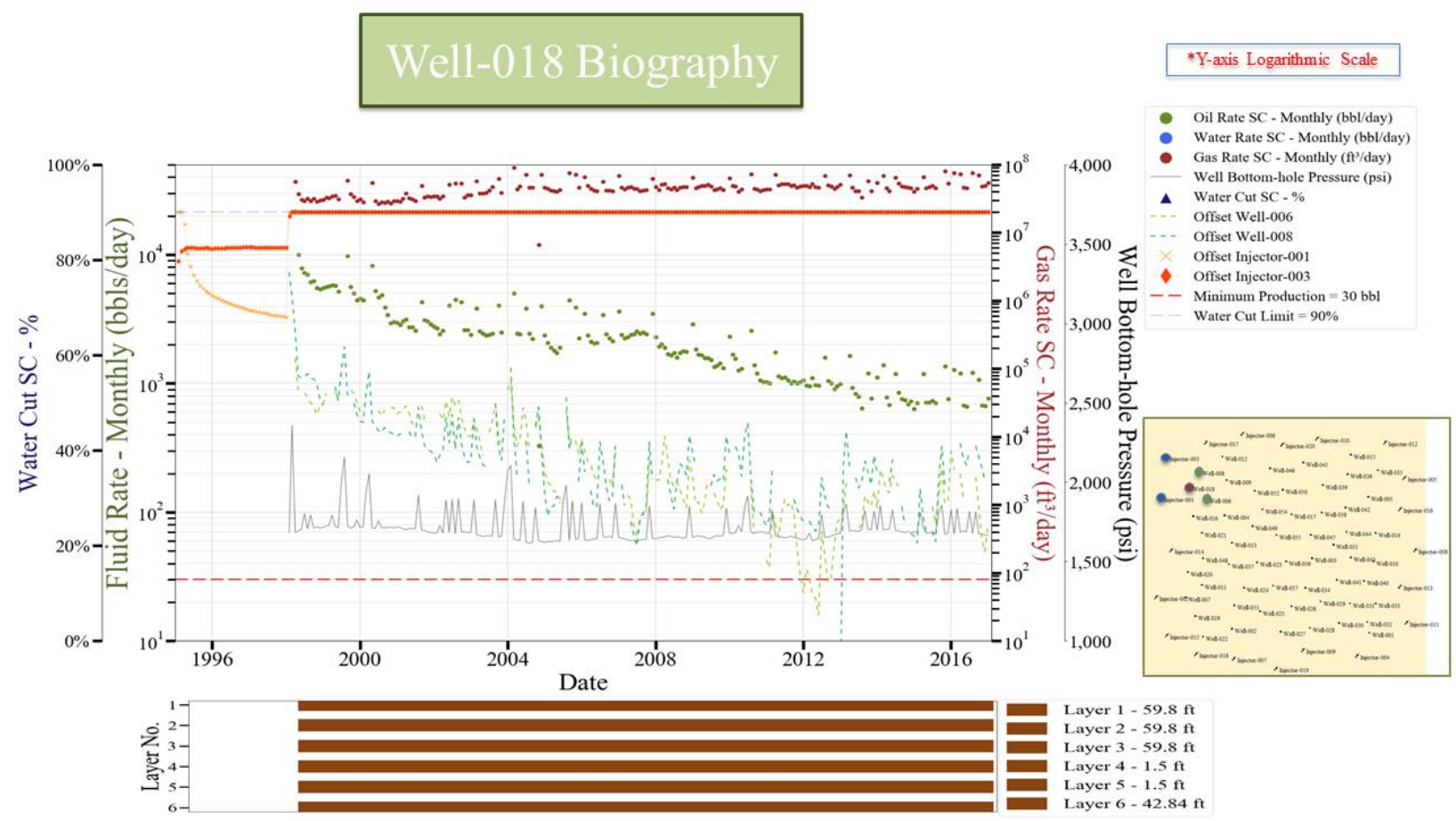

Figure 256 Well-018 biography 

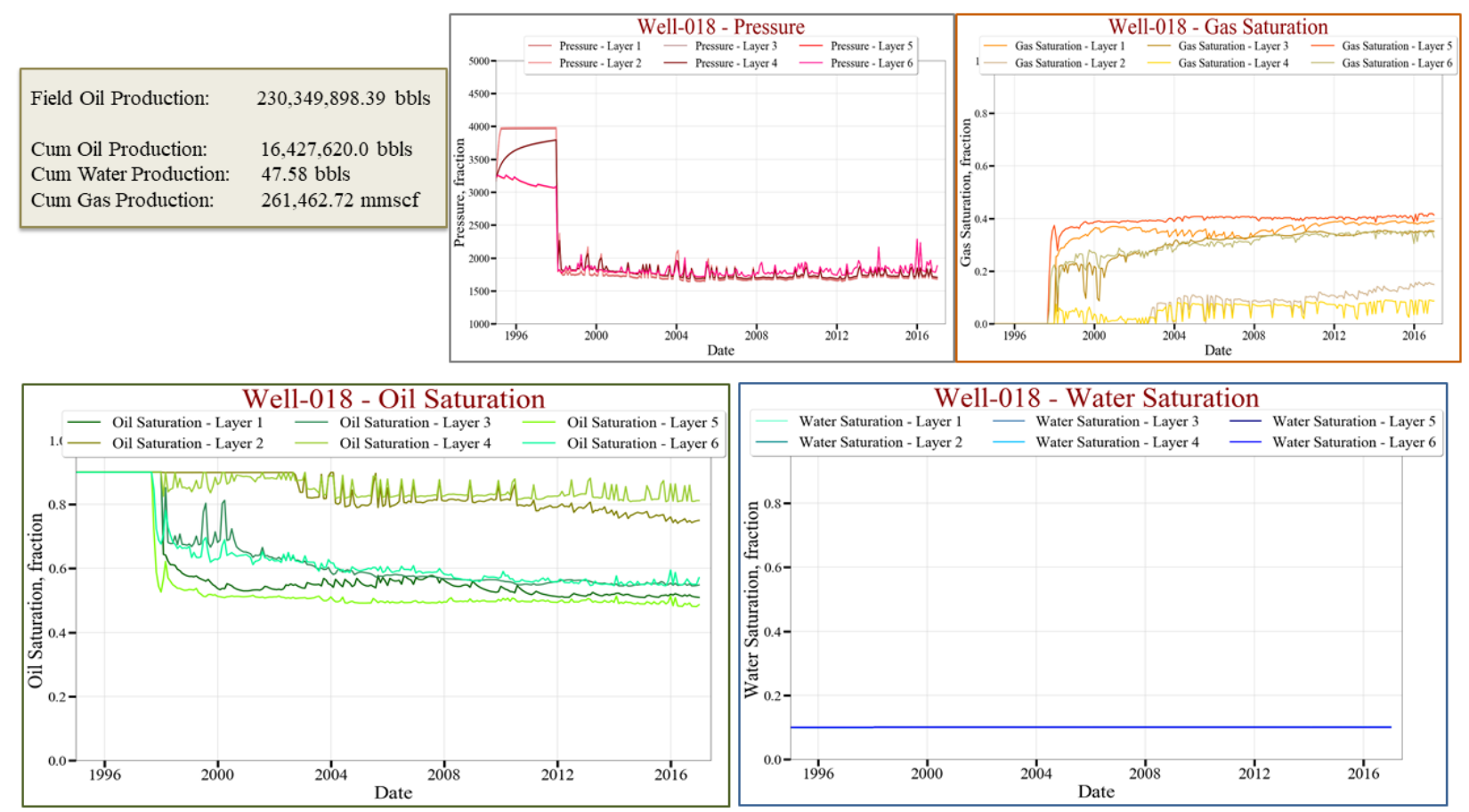

Figure 257 Well-018 saturations and pressure per layer

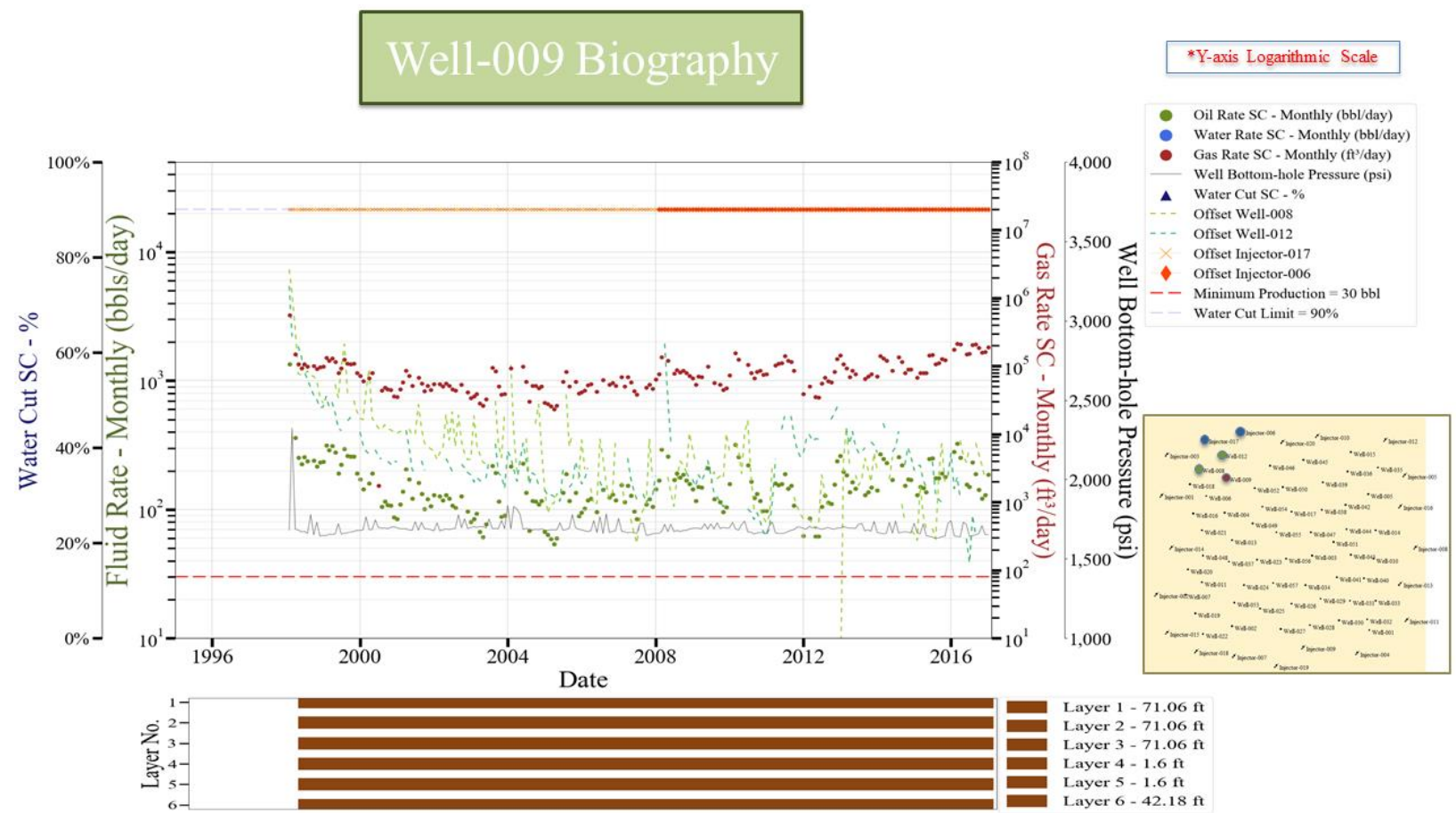

Figure 258 Well-009 biography 

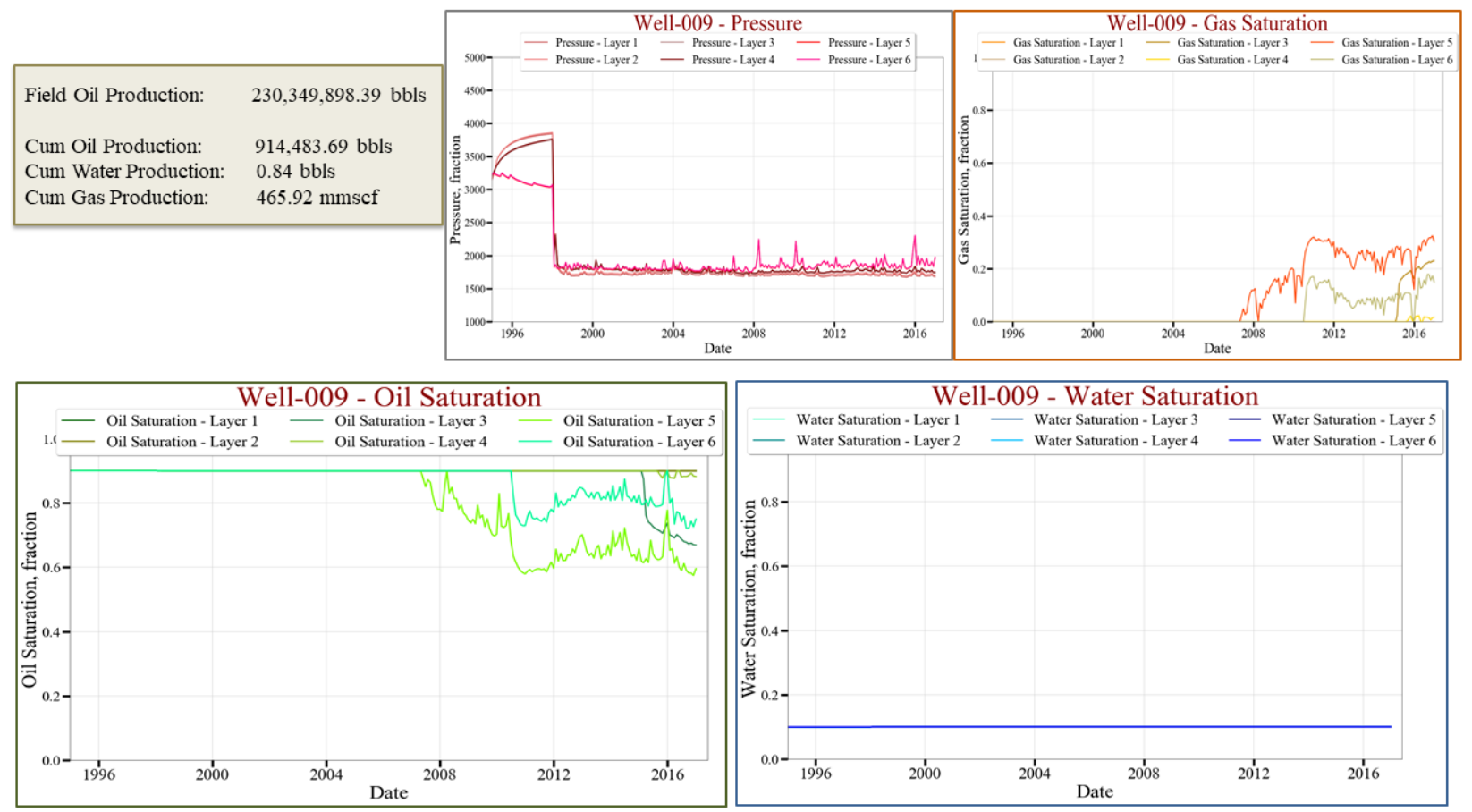

Figure 259 Well-009 saturations and pressure per layer

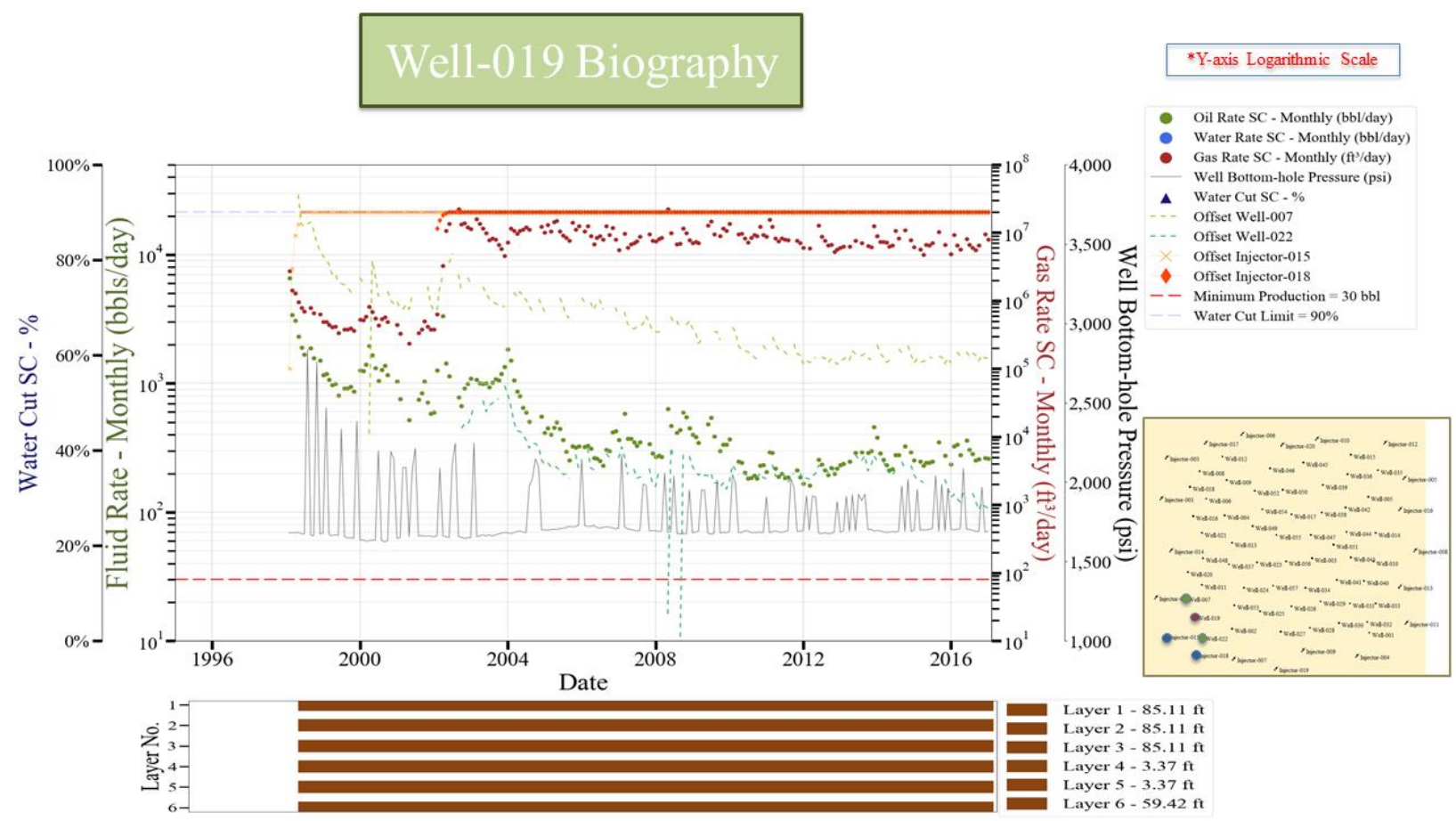

Figure 260 Well-019 biography 

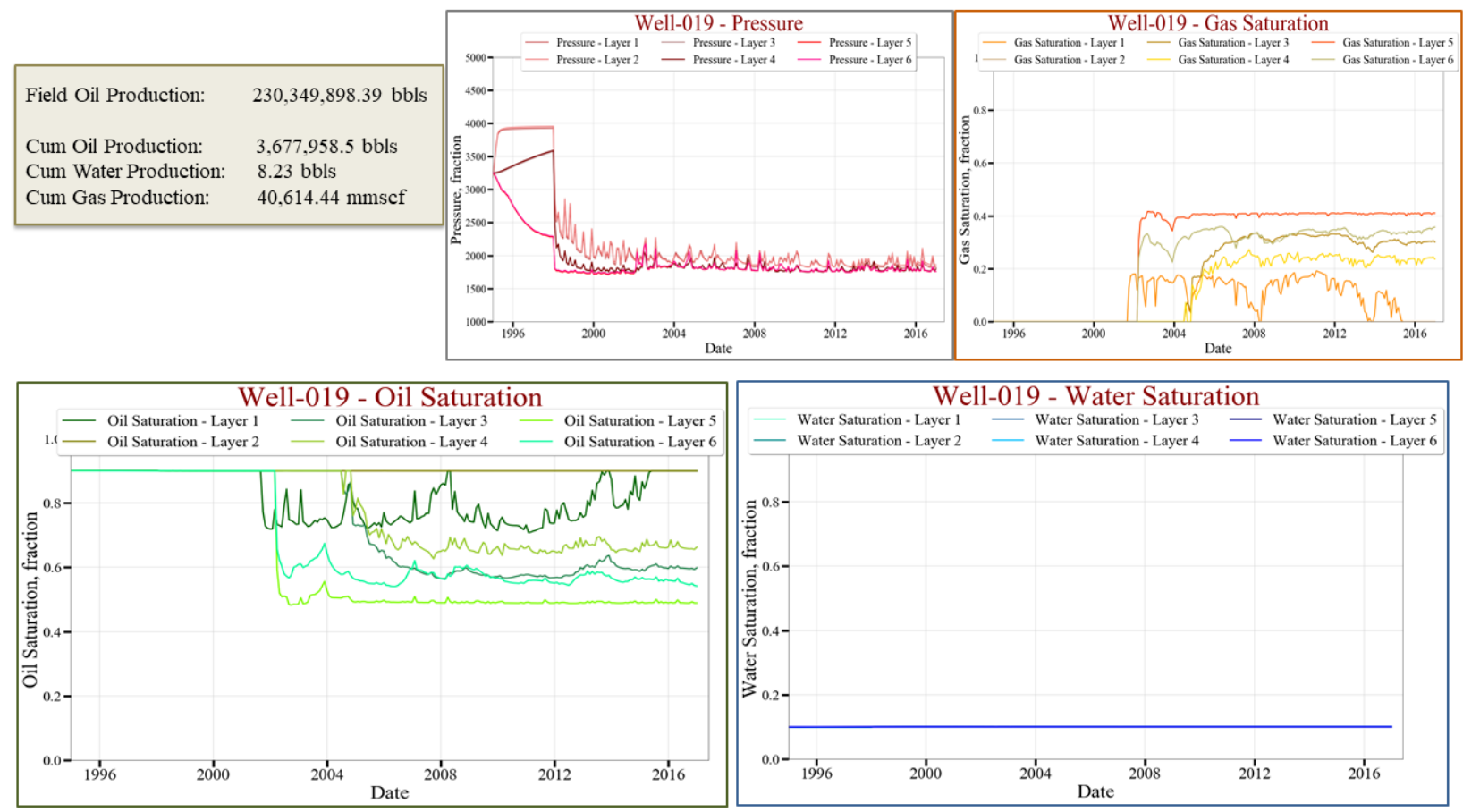

Figure 261 Well-019 saturations and pressure per layer

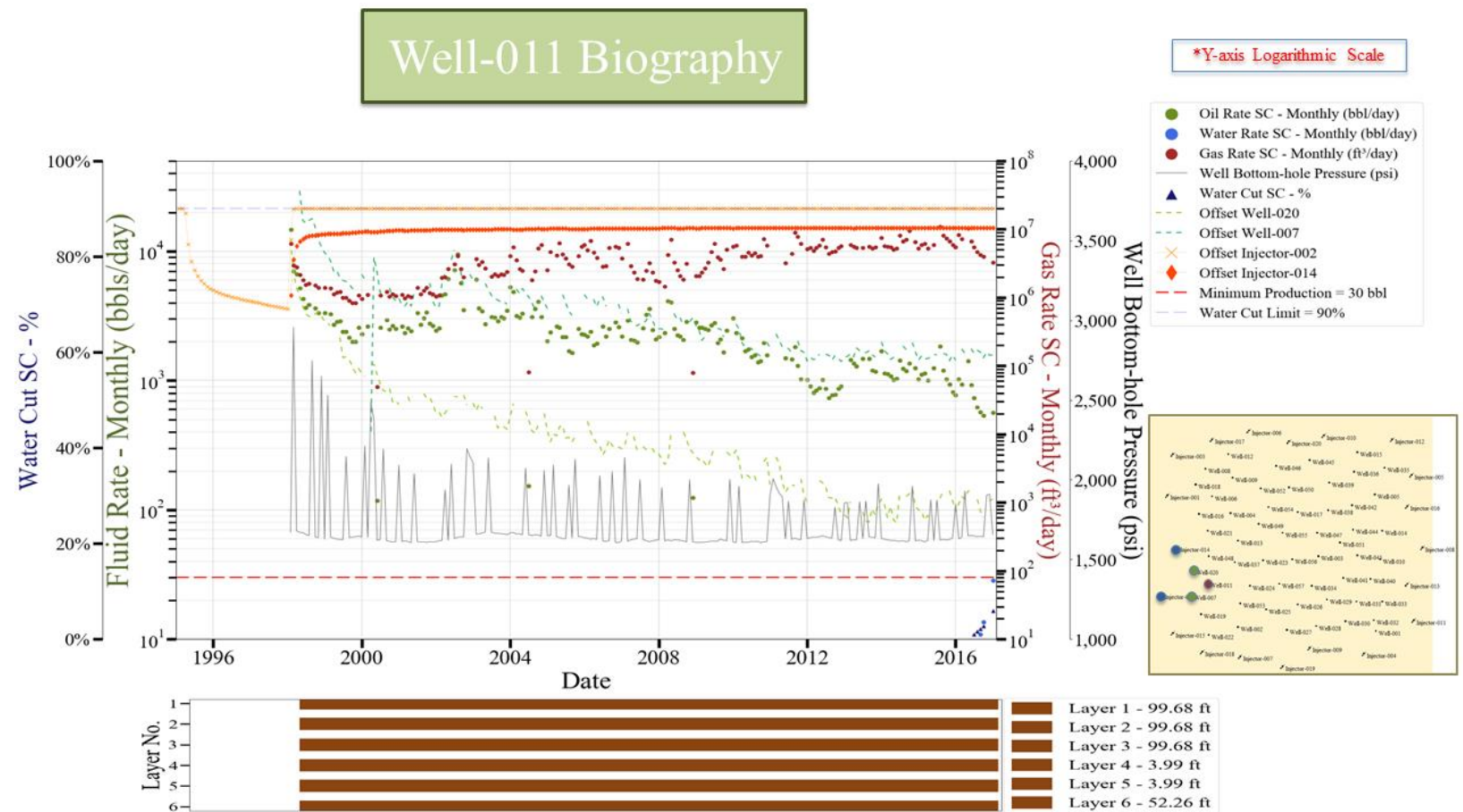

Figure 262 Well-011 biography 

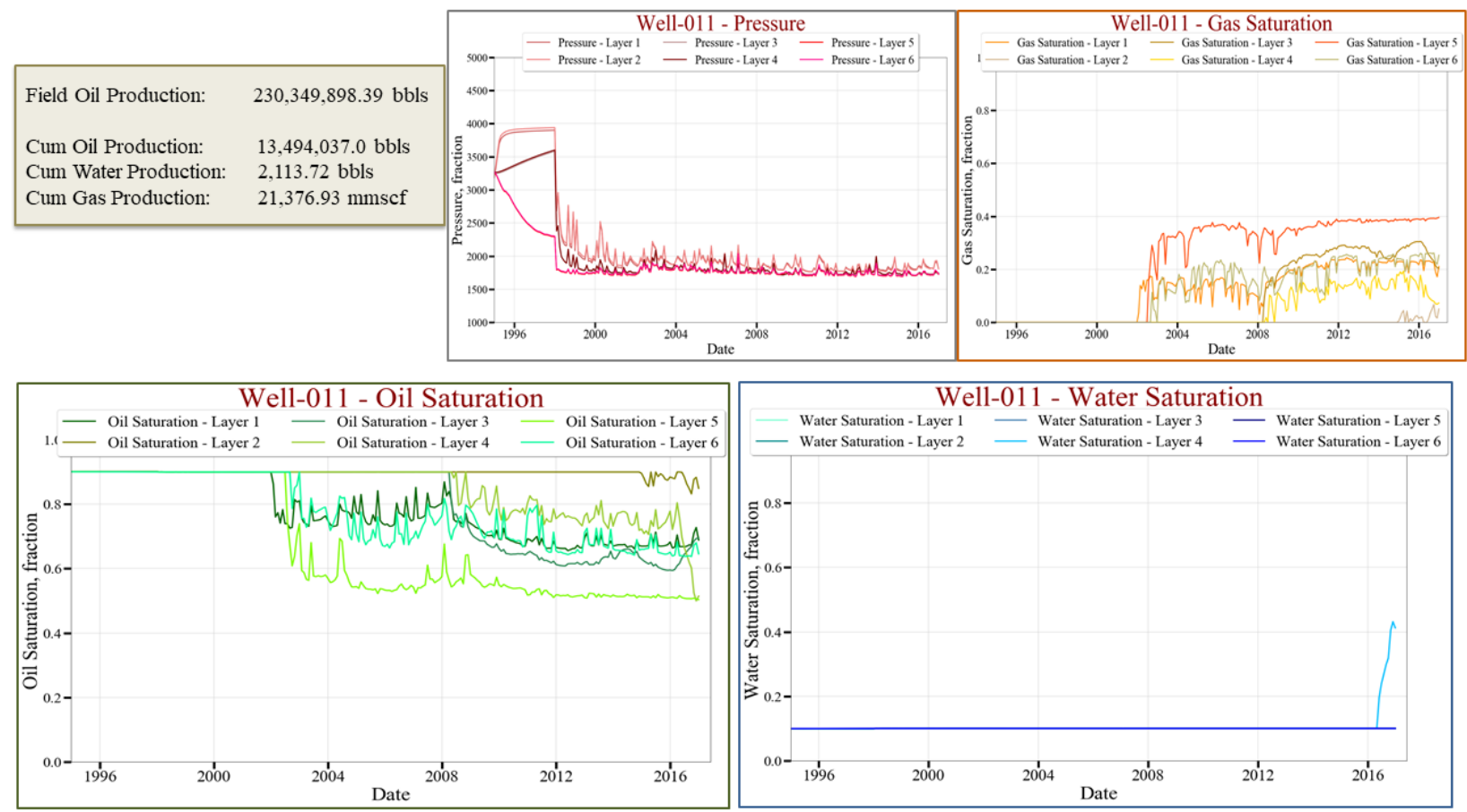

Figure 263 Well-011 saturations and pressure per layer

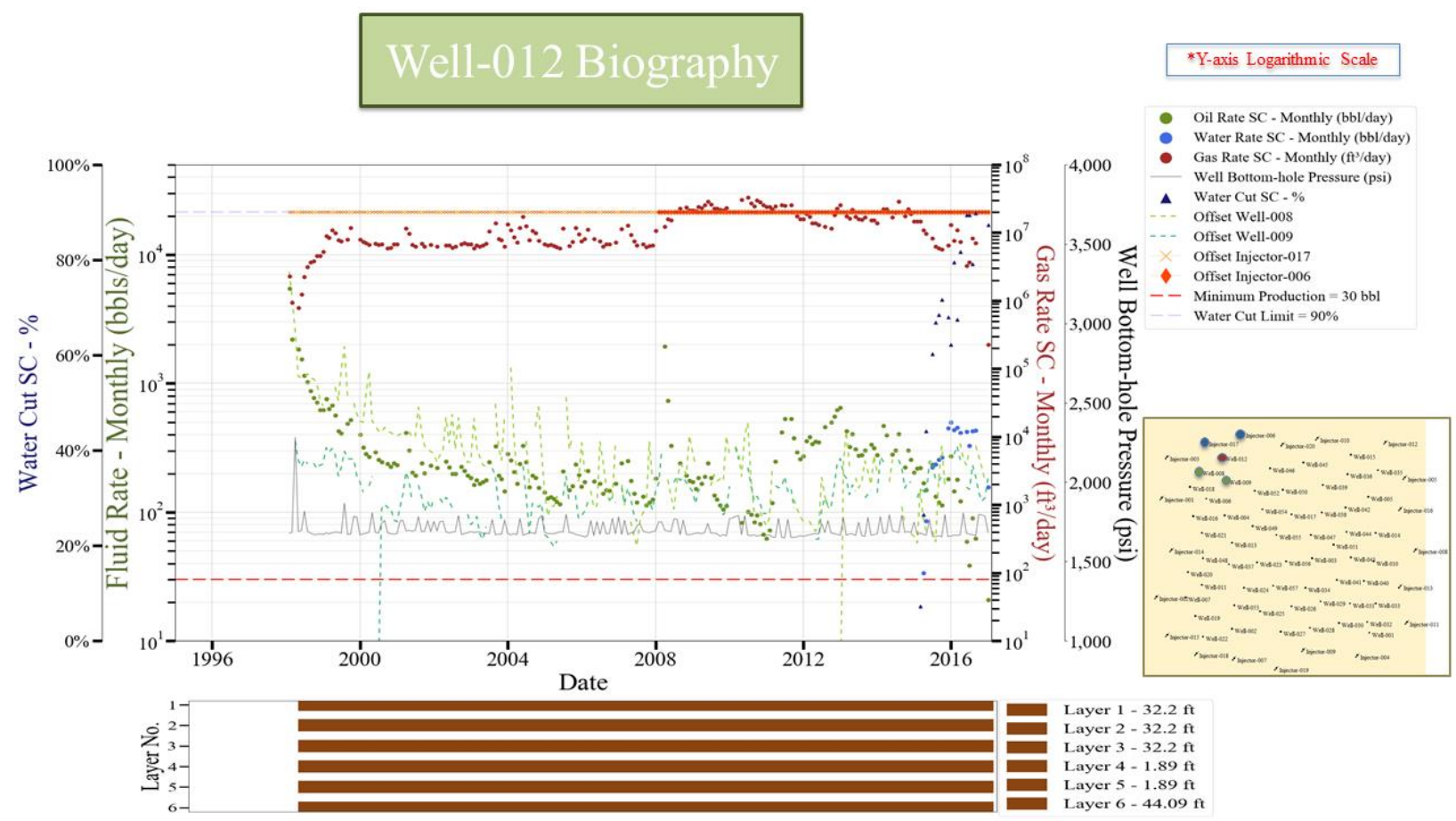

Figure 264 Well-012 biography 

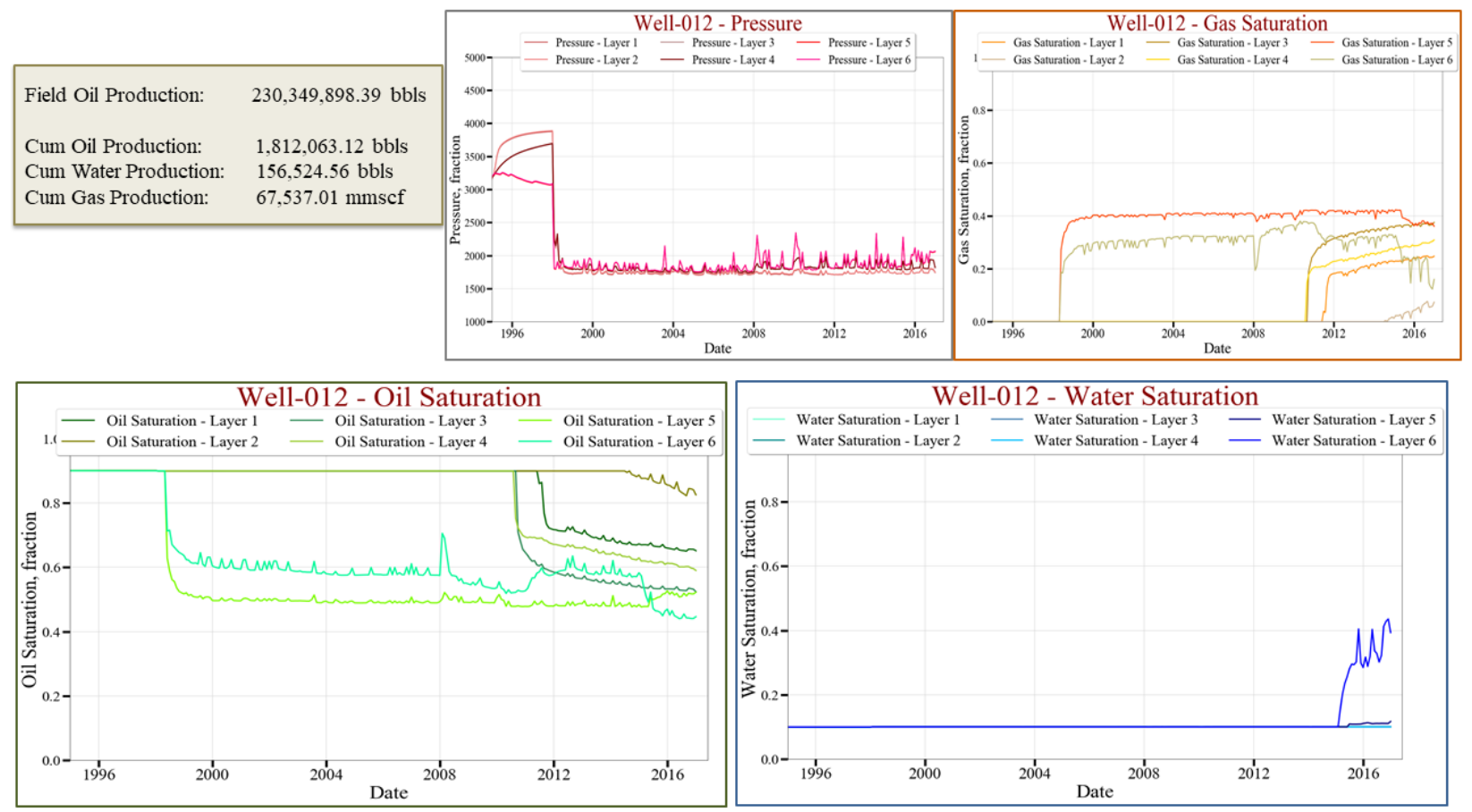

Figure 265 Well-012 saturations and pressure per layer

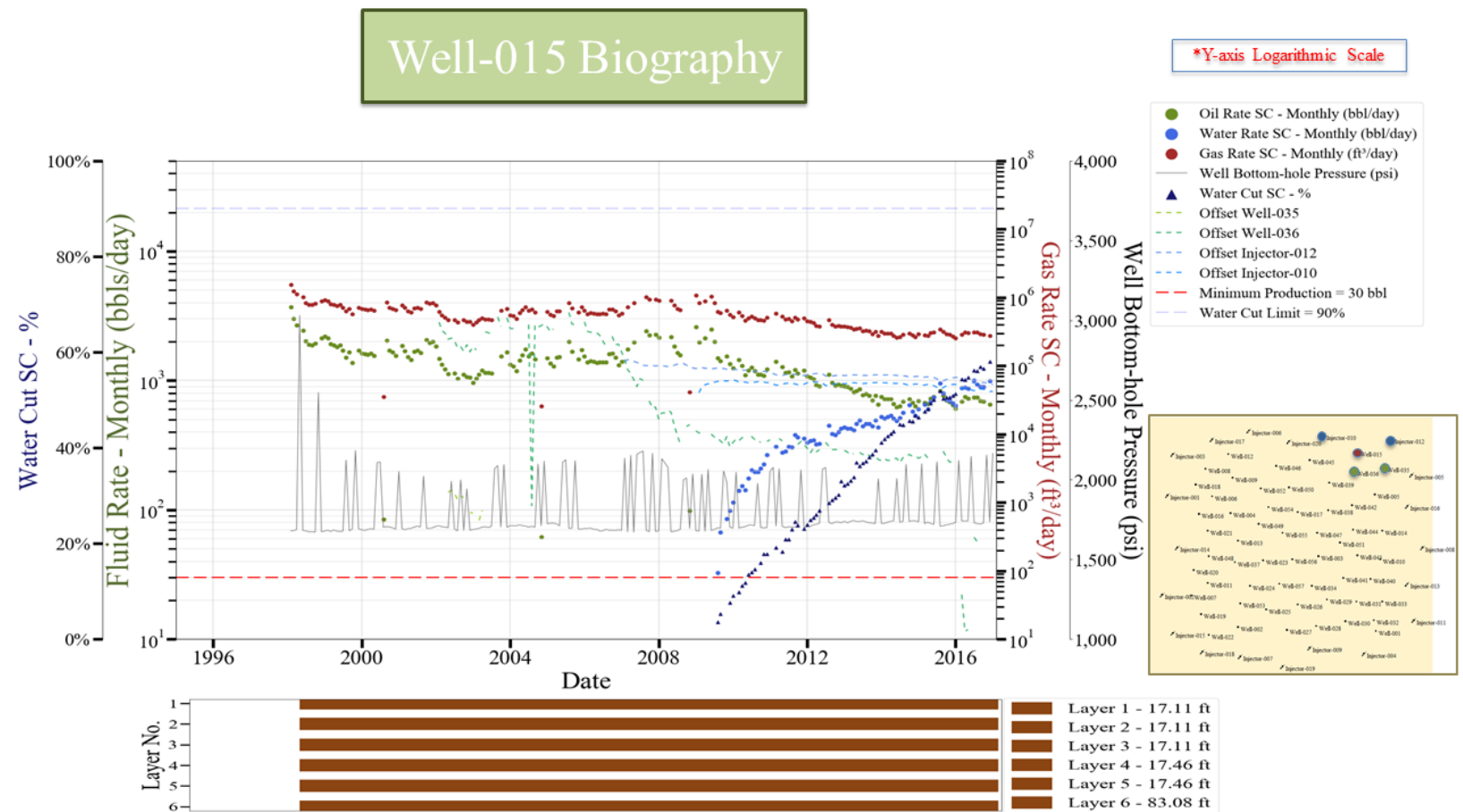

Figure 266 Well-015 biography 

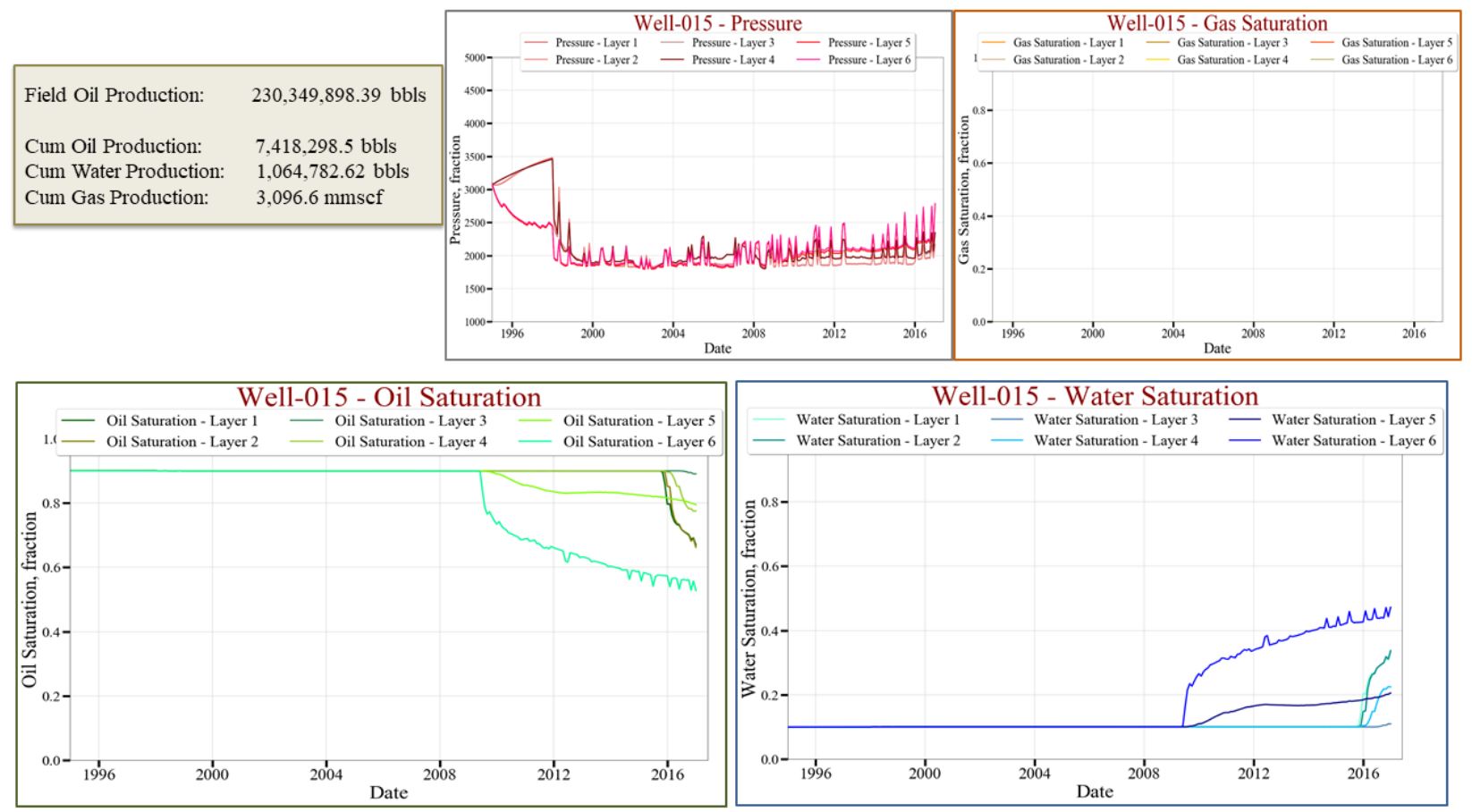

Figure 267 Well-015 saturations and pressure per layer

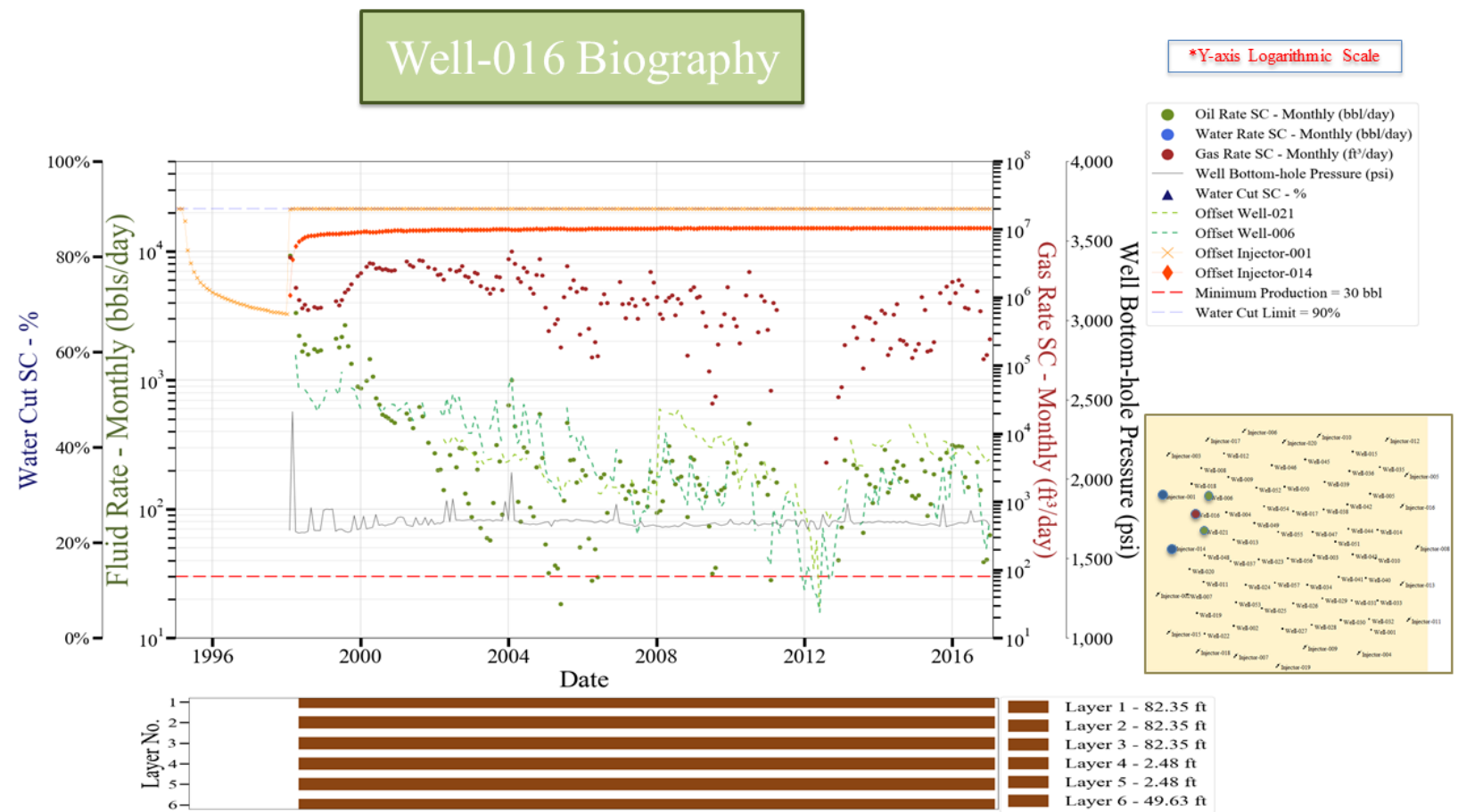

Figure 268 Well-016 biography 

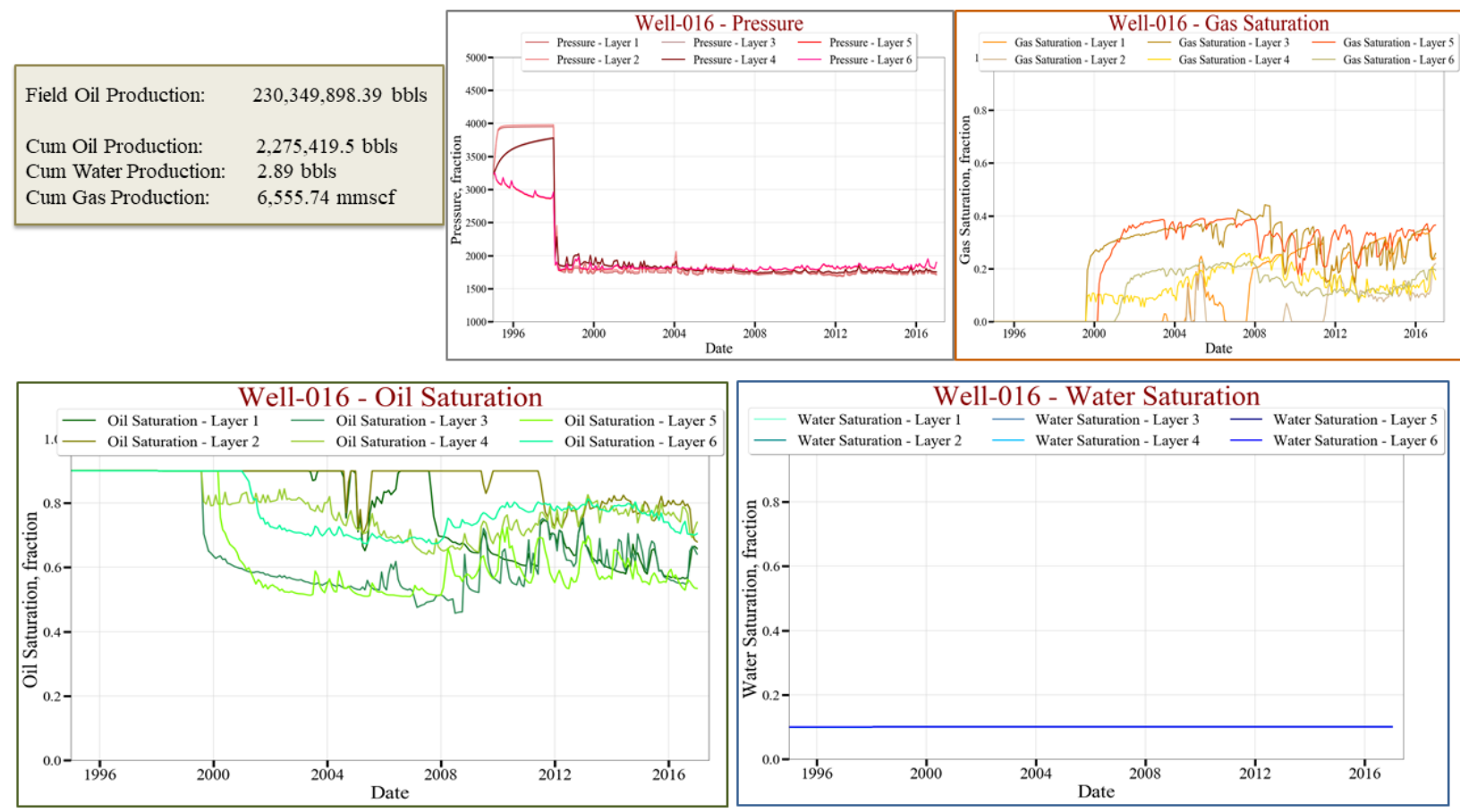

Figure 269 Well-016 saturations and pressure per layer

\subsubsection{Average Number of Production Days}

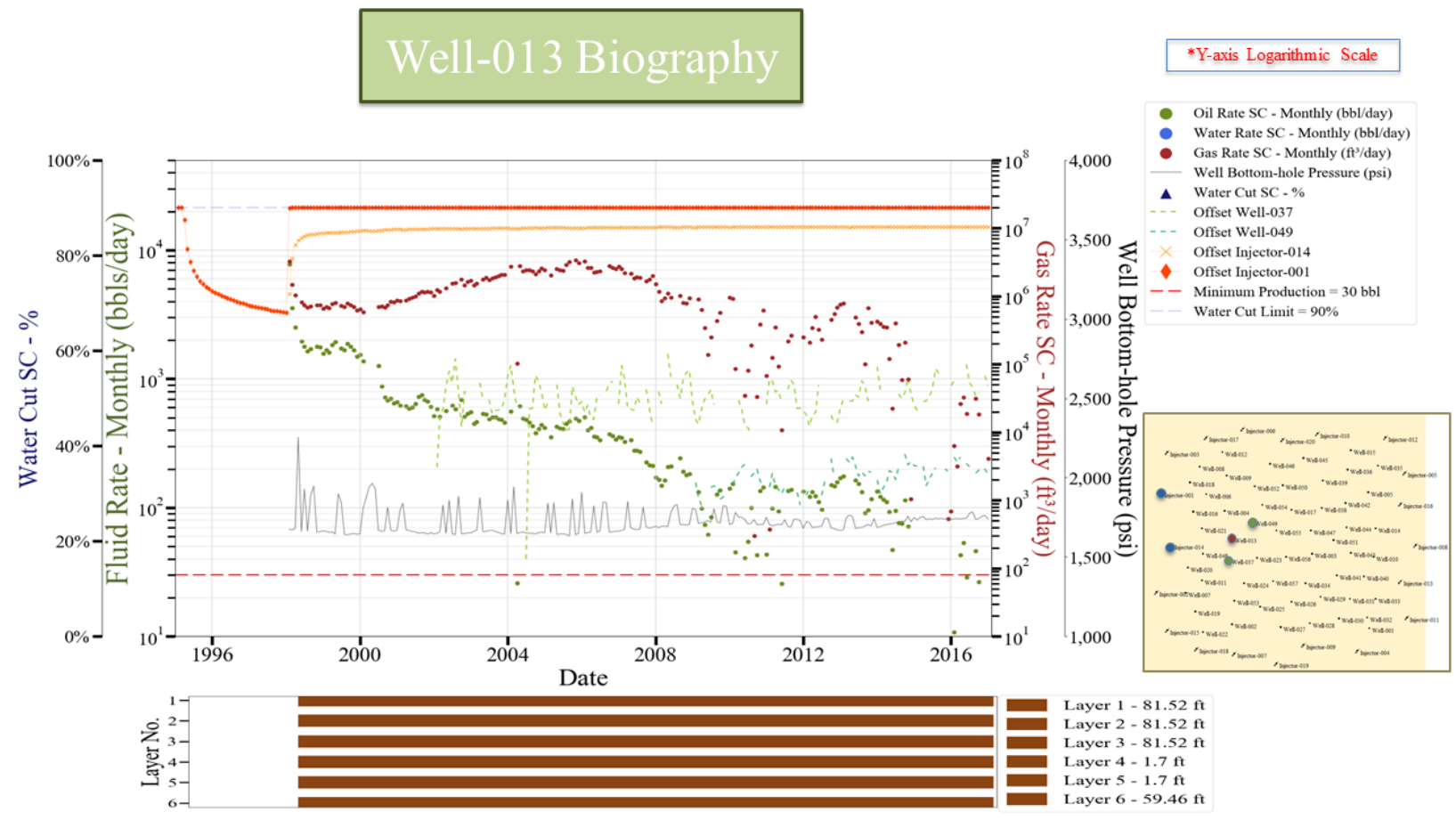

Figure 270 Well-013 biography 

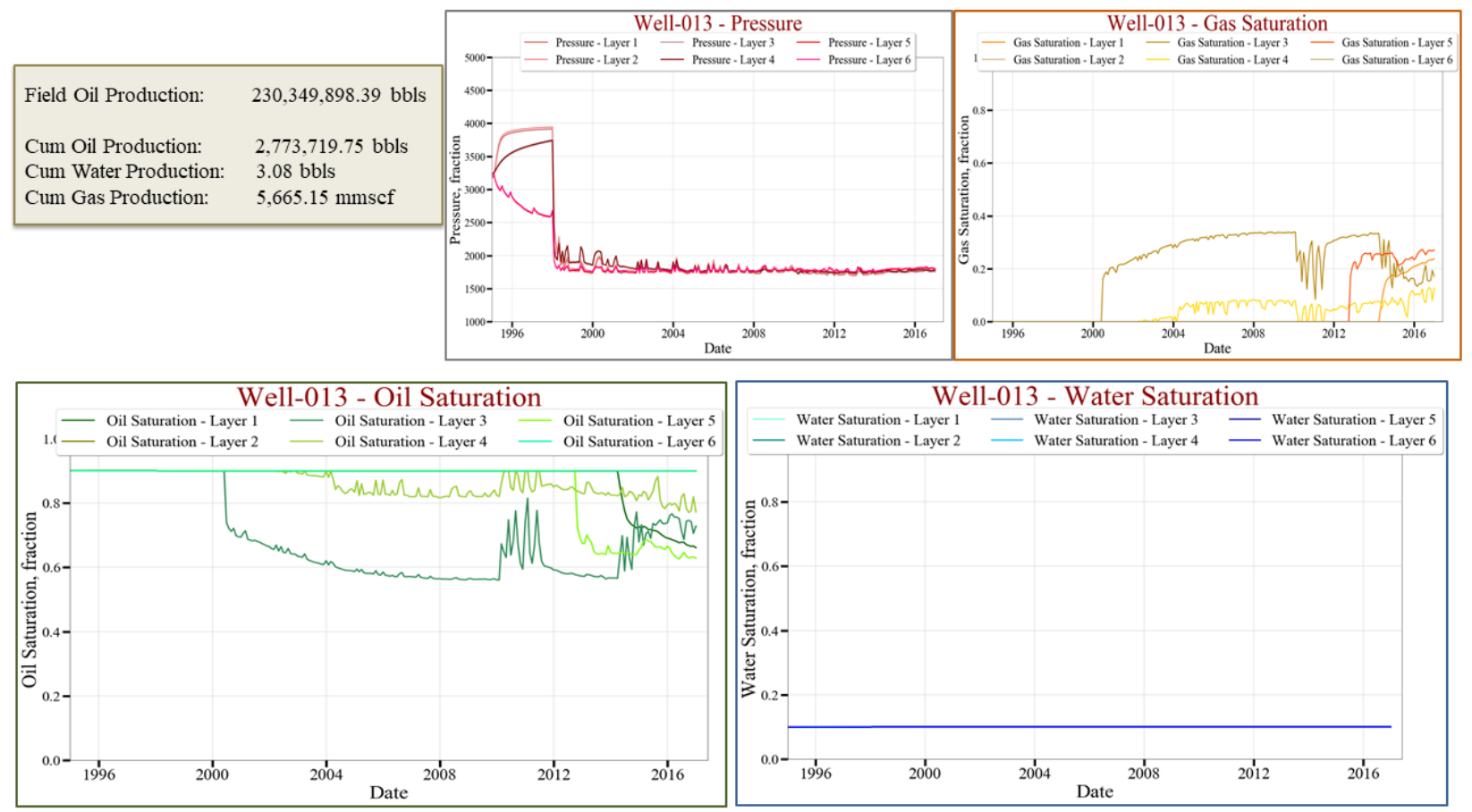

Figure 271 Well-013 saturations and pressure per layer

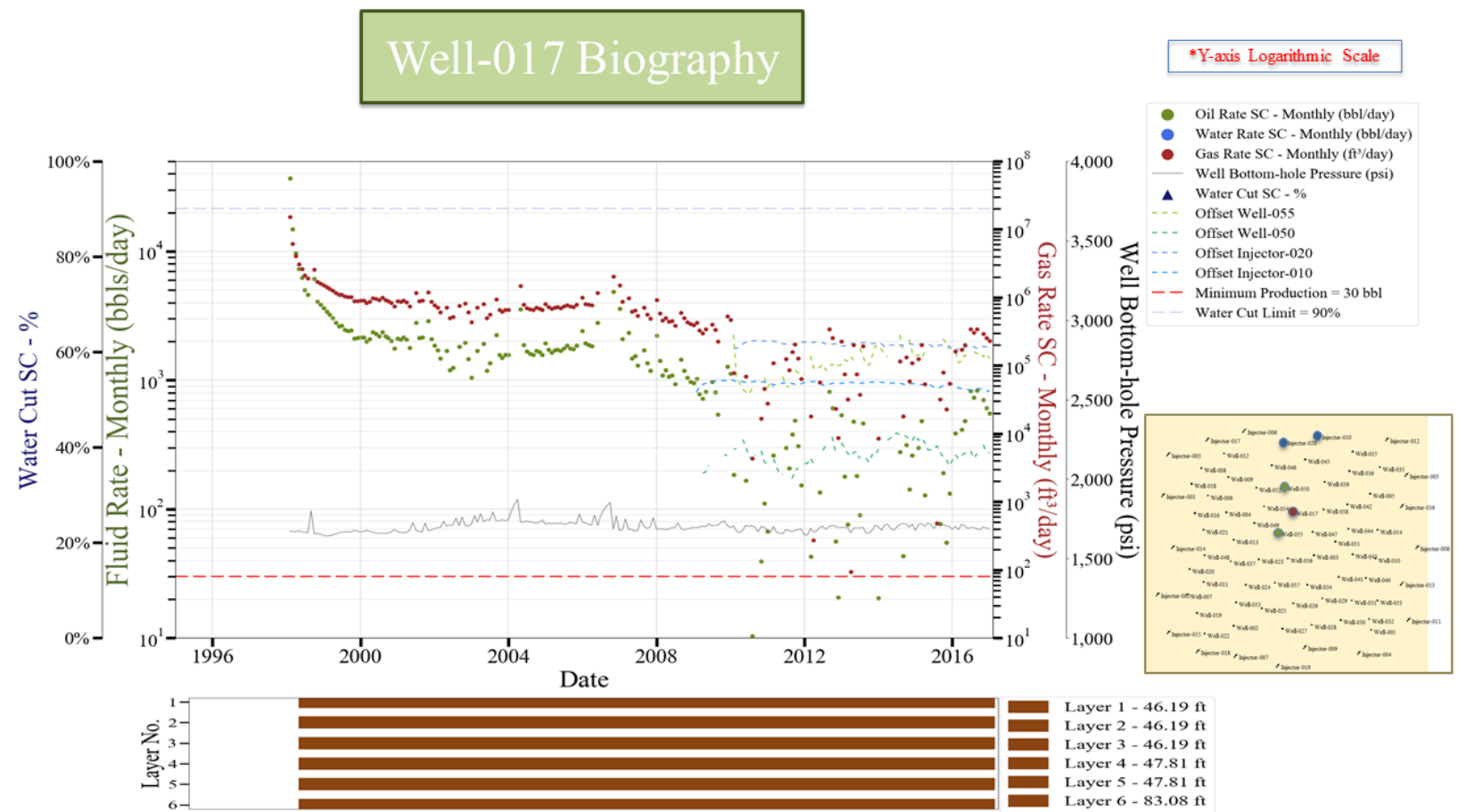

Figure 272 Well-017 biography 

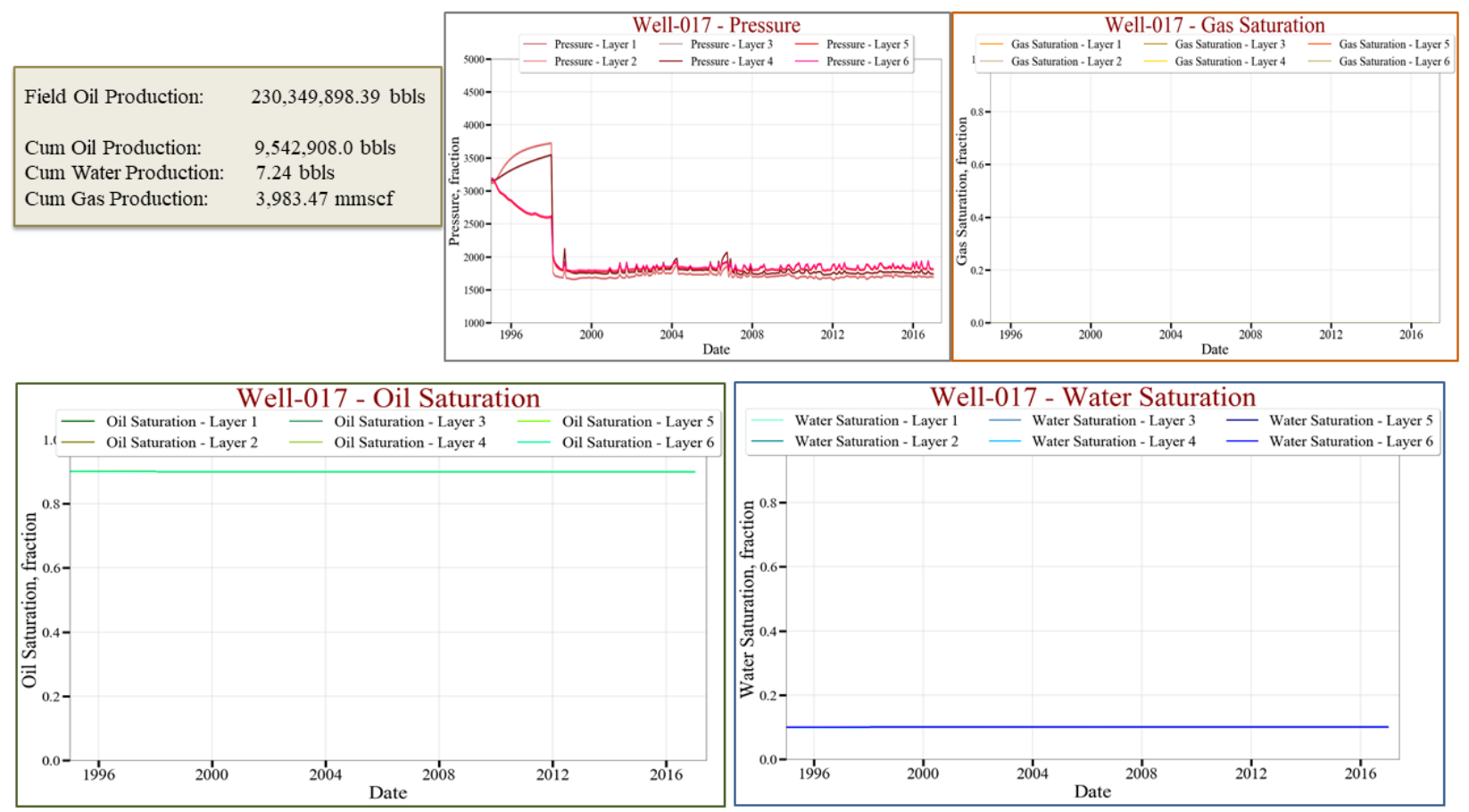

Figure 273 Well-017 saturations and pressure per layer

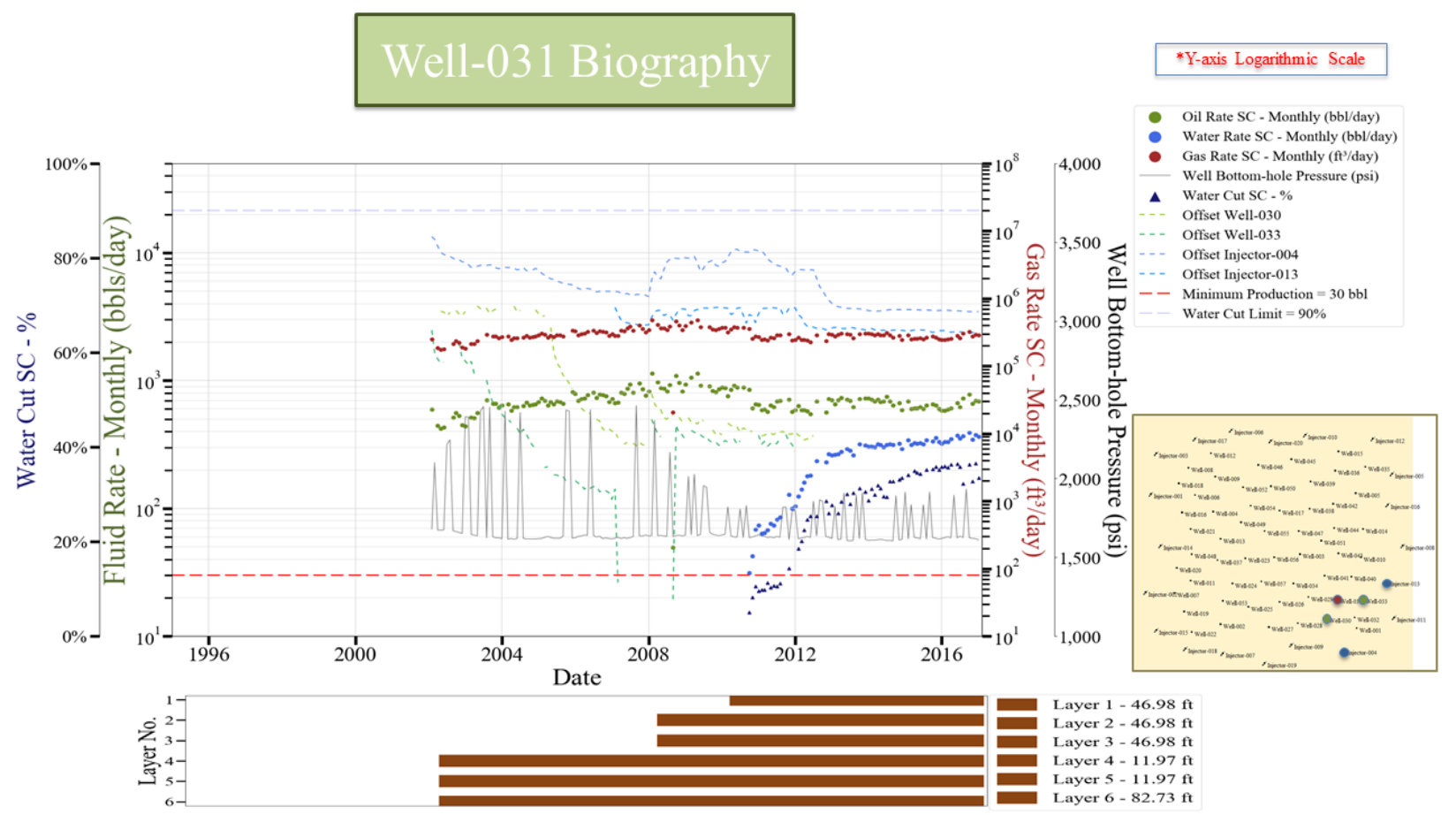

Figure 274 Well-031 biography 

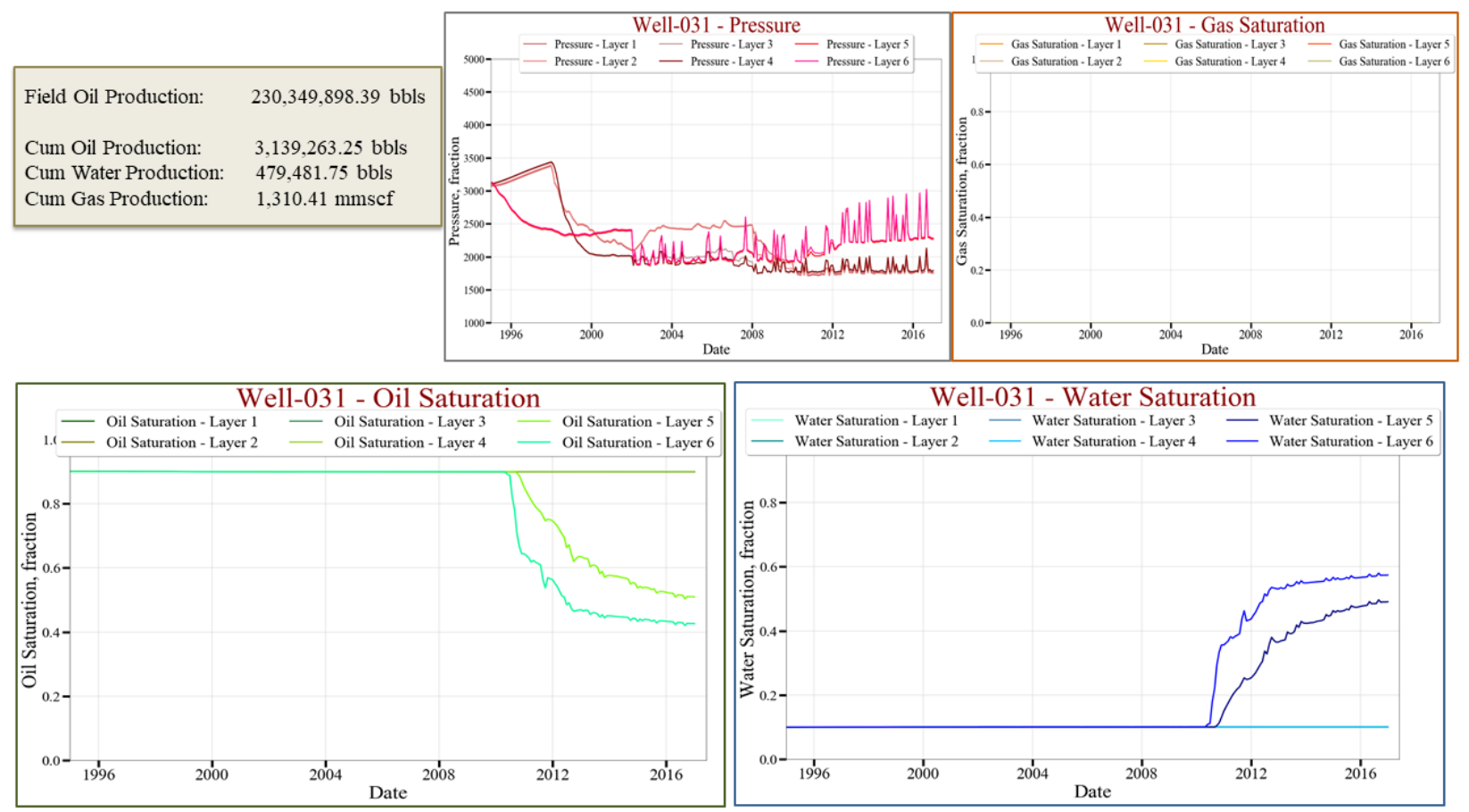

Figure 275 Well-031 saturations and pressure per layer

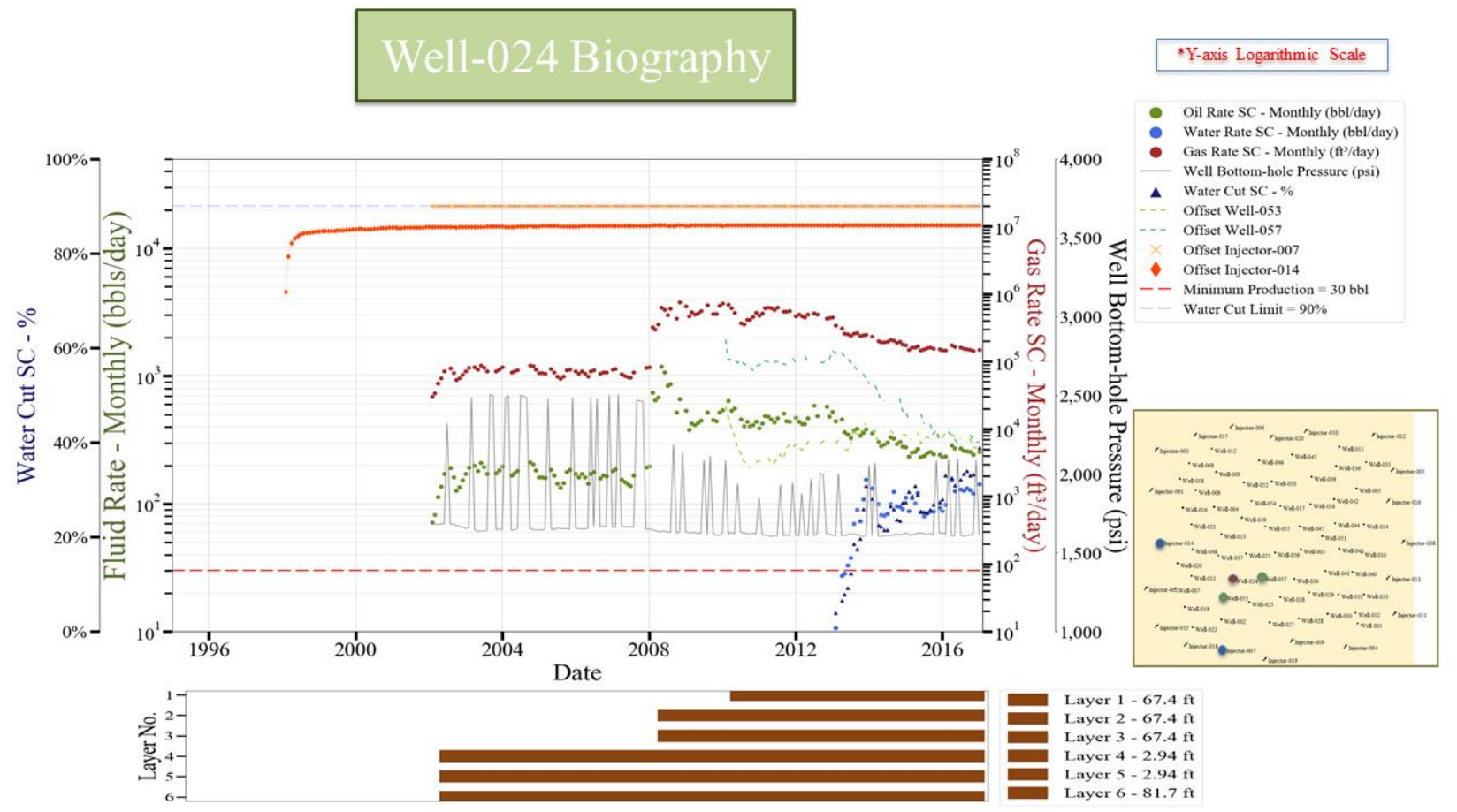

Figure 276 Well-024 biography 

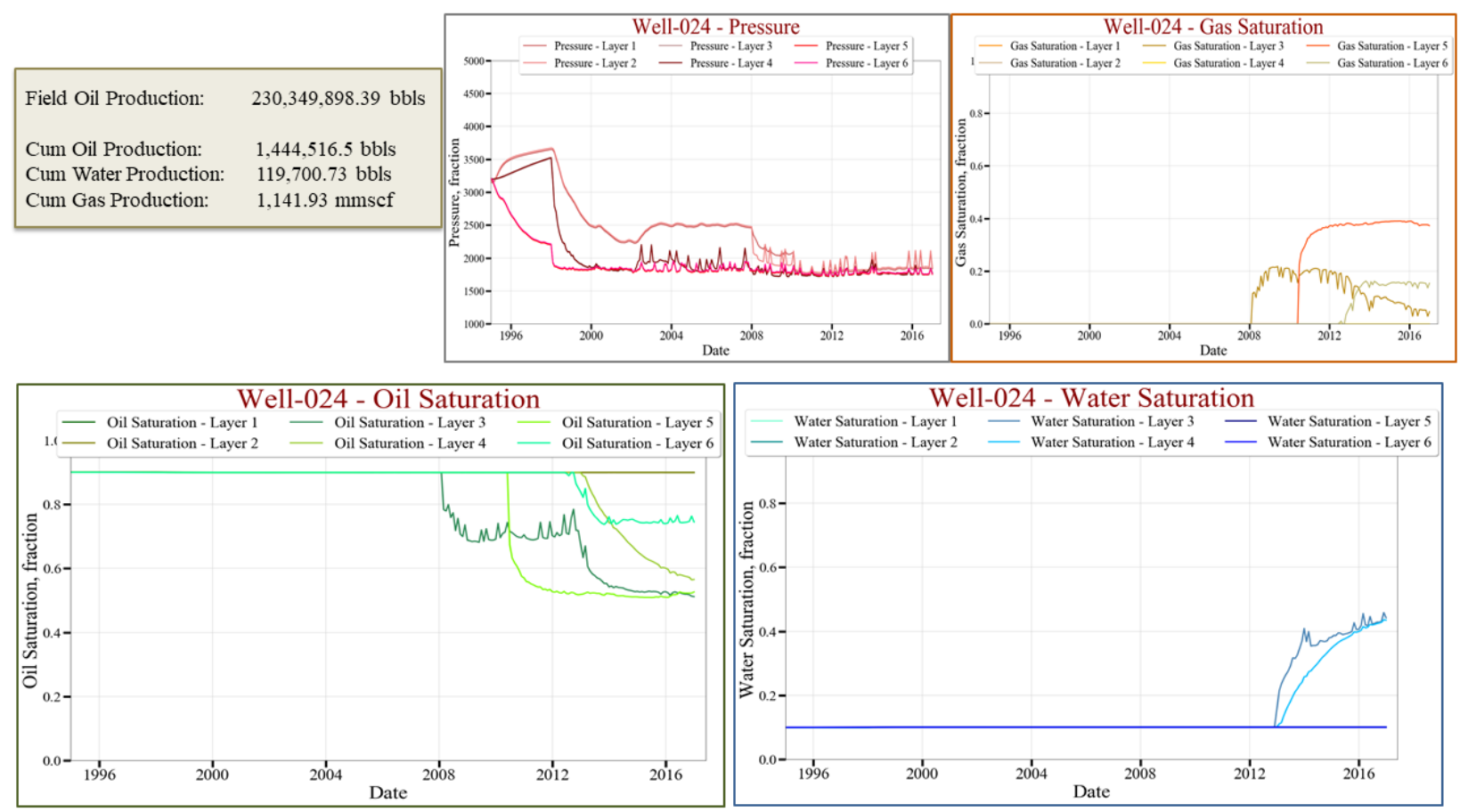

Figure 277 Well-024 saturations and pressure per layer

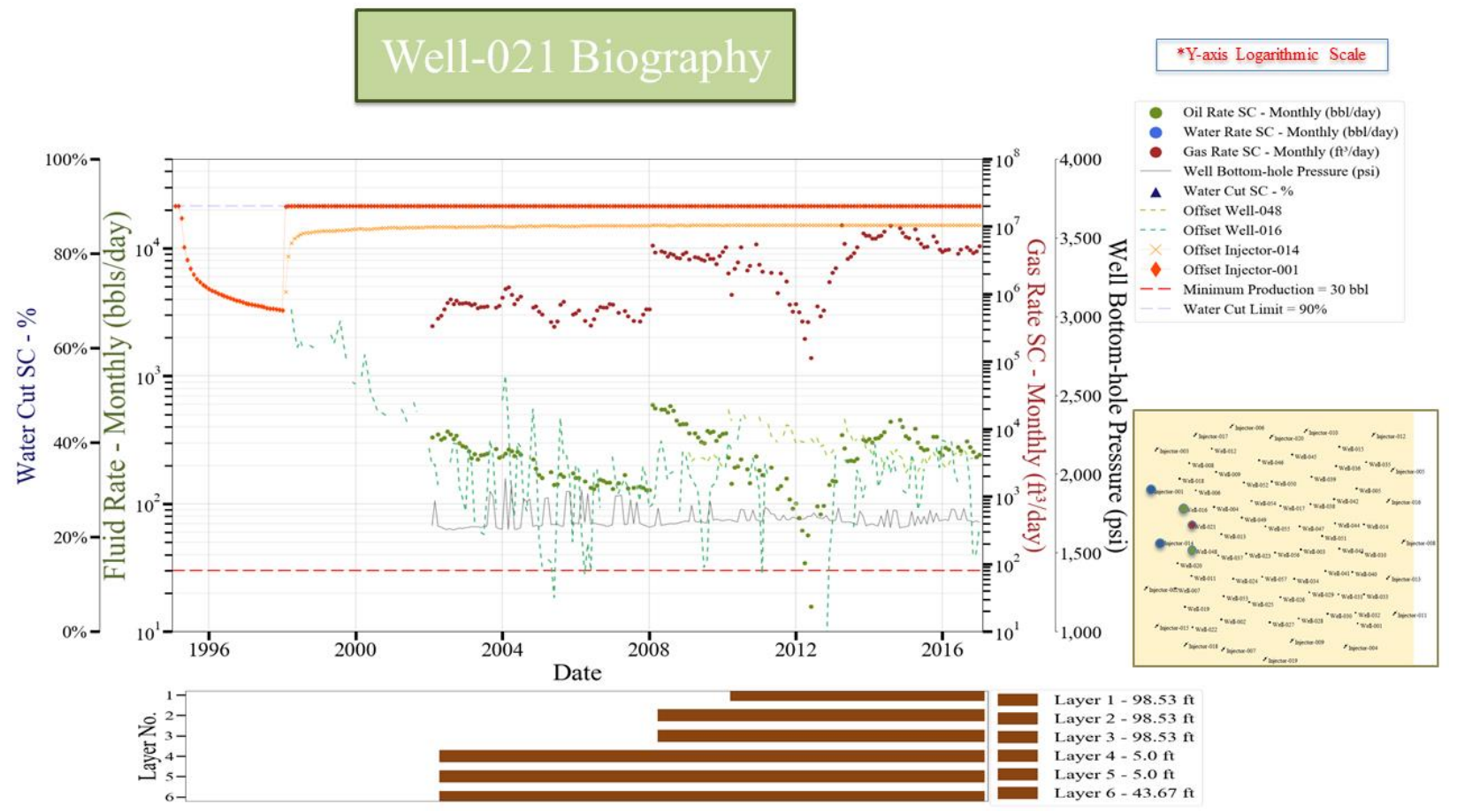

Figure 278 Well-021 biography 

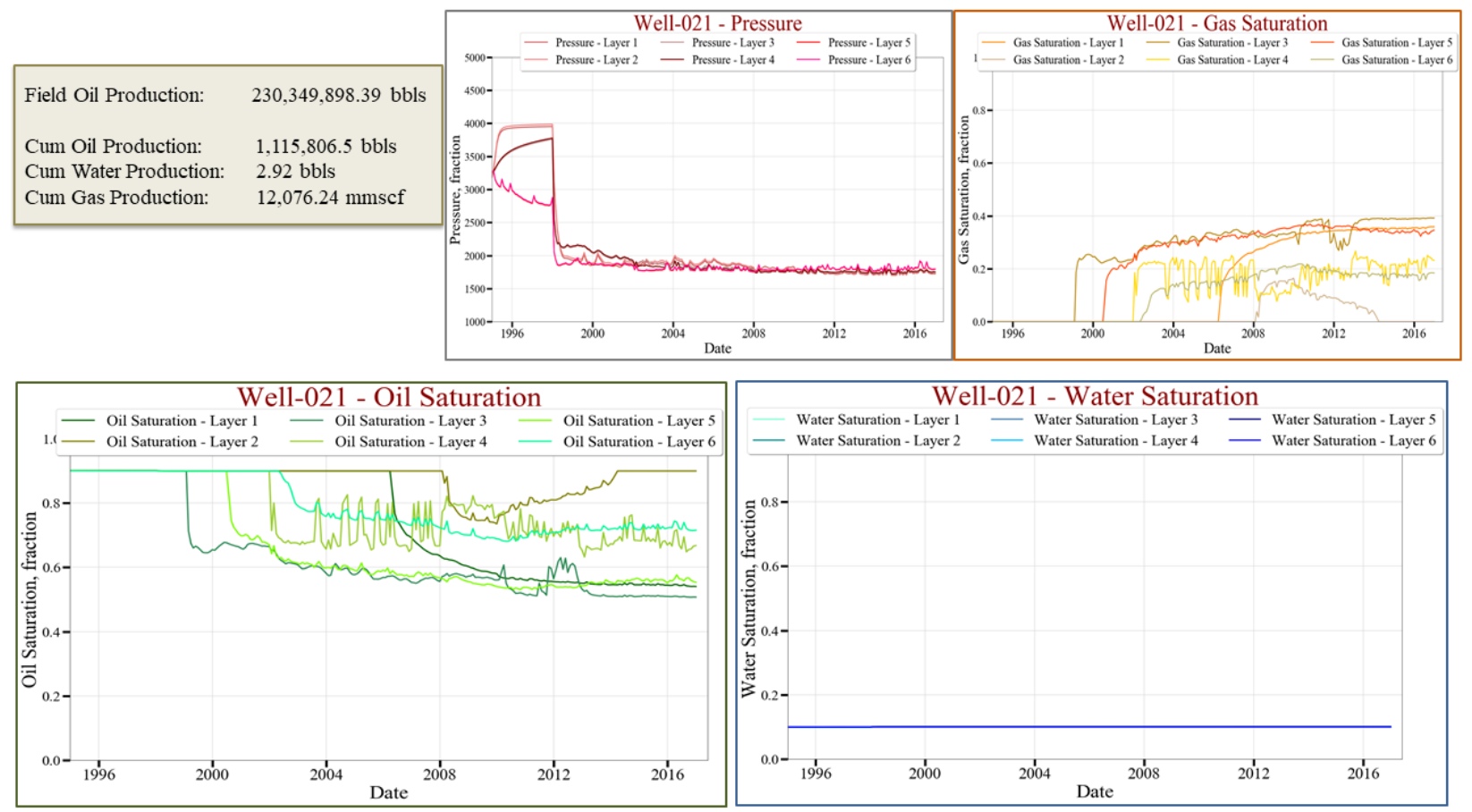

Figure 279 Well-021 saturations and pressure per layer

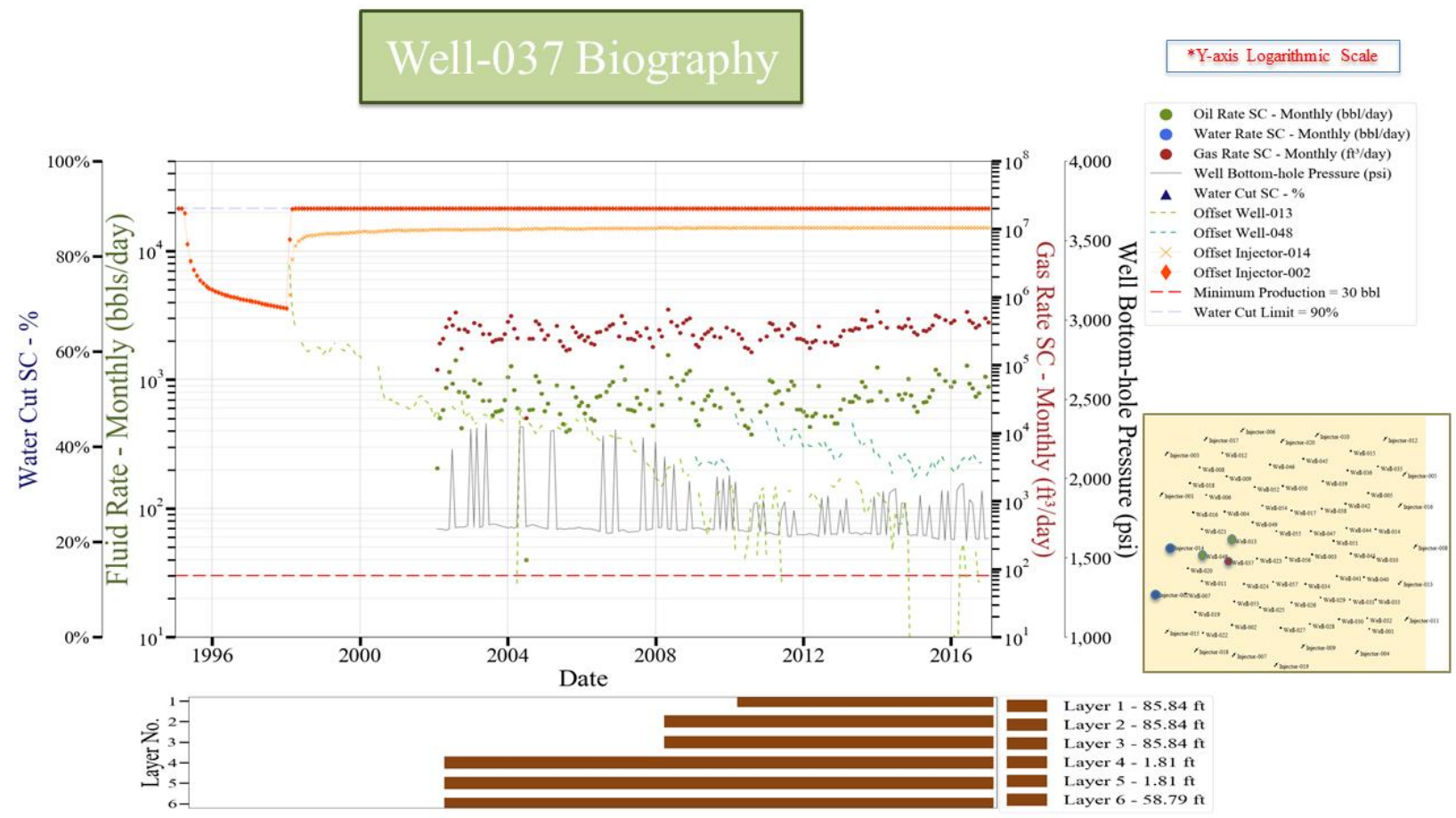

Figure 280 Well-037 biography 

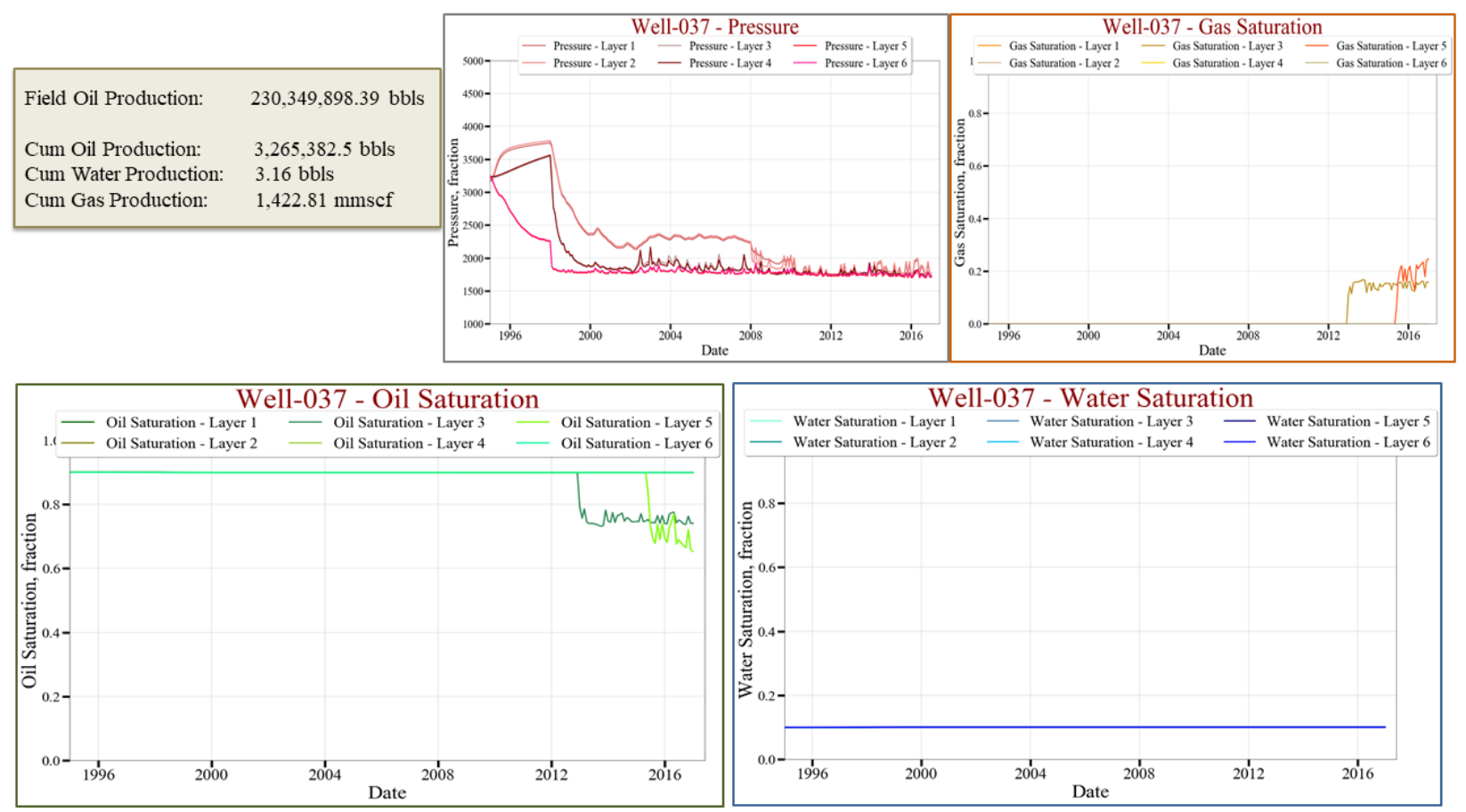

Figure 281 Well-037 saturations and pressure per layer

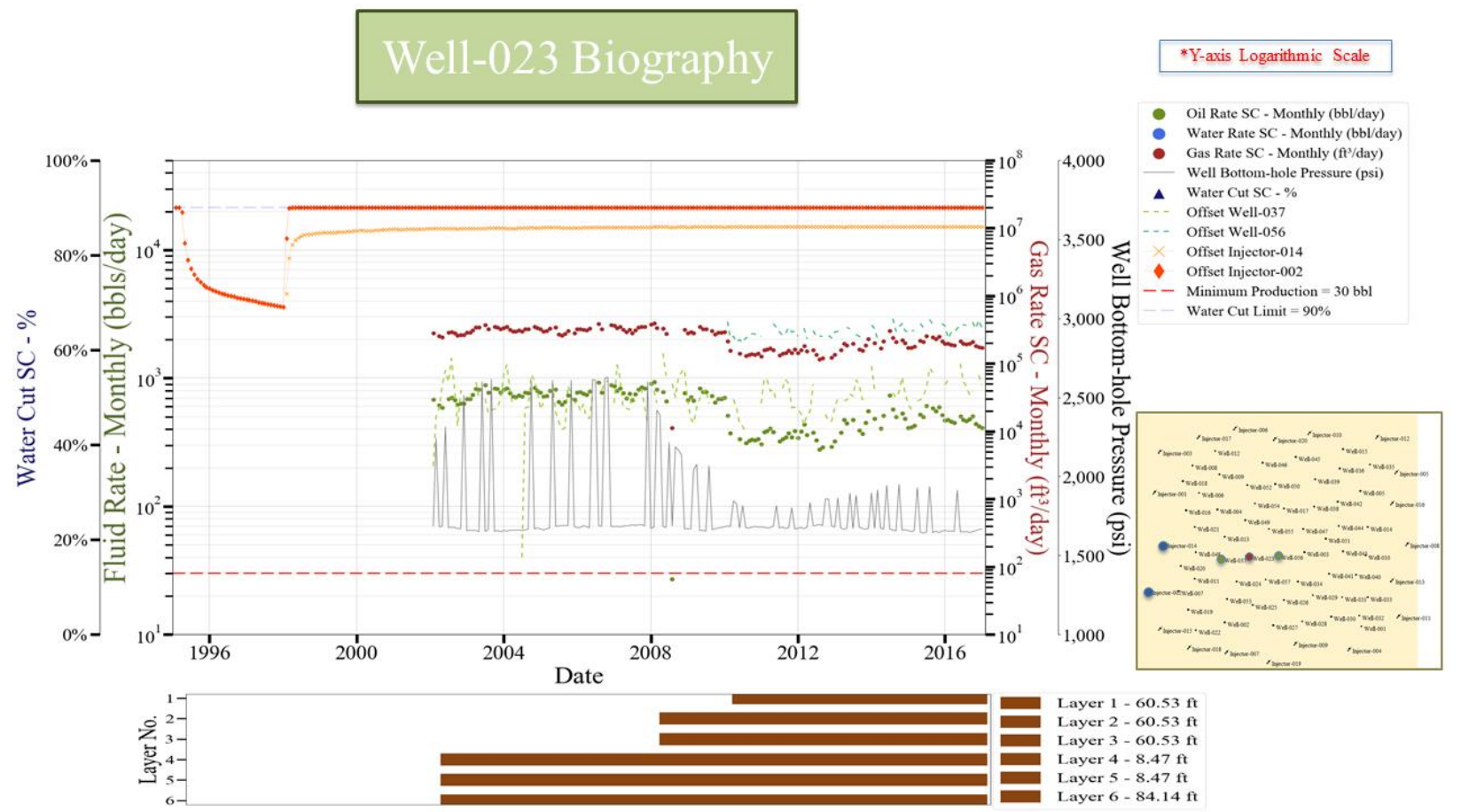

Figure 282 Well-023 biography 

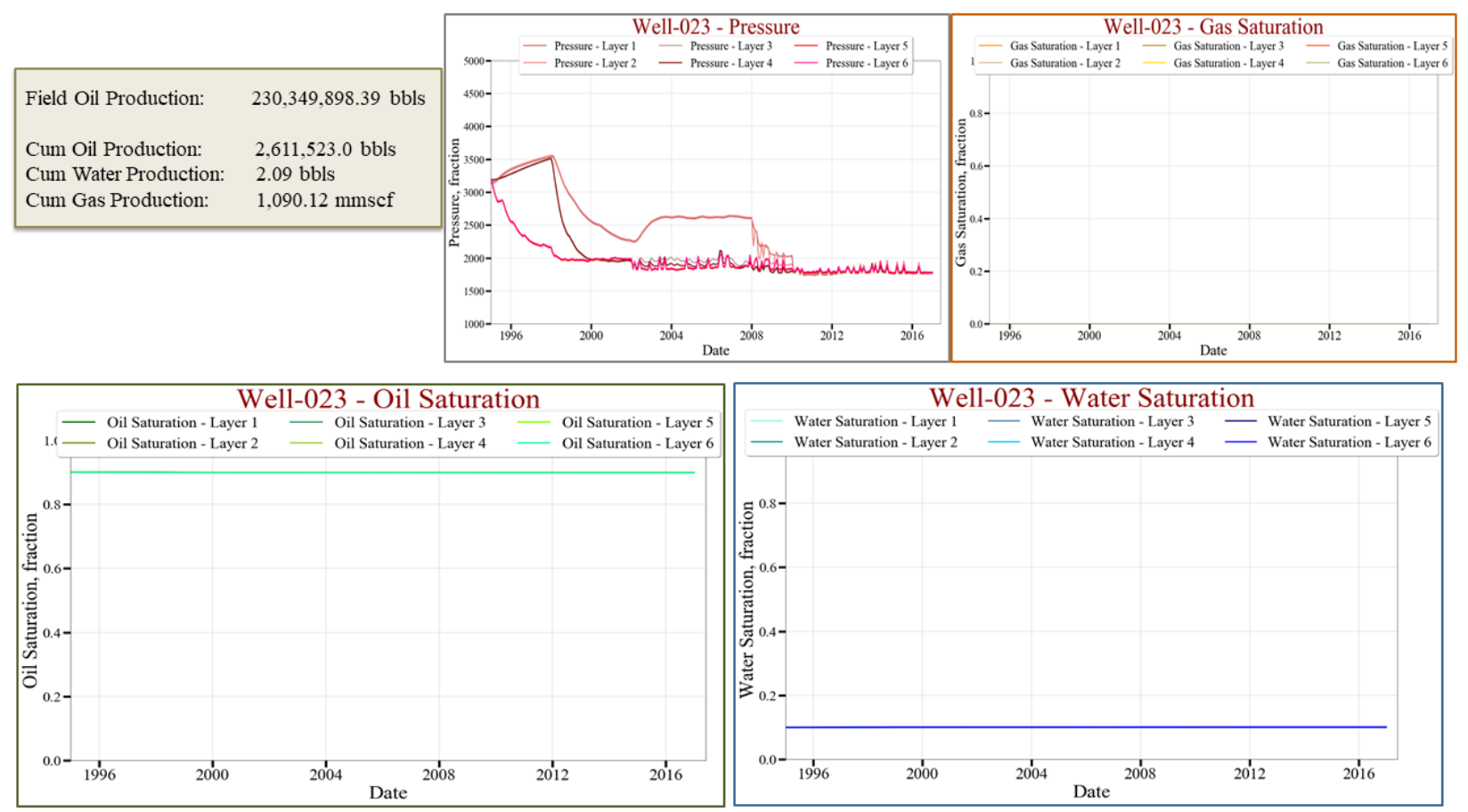

Figure 283 Well-023 saturations and pressure per layer

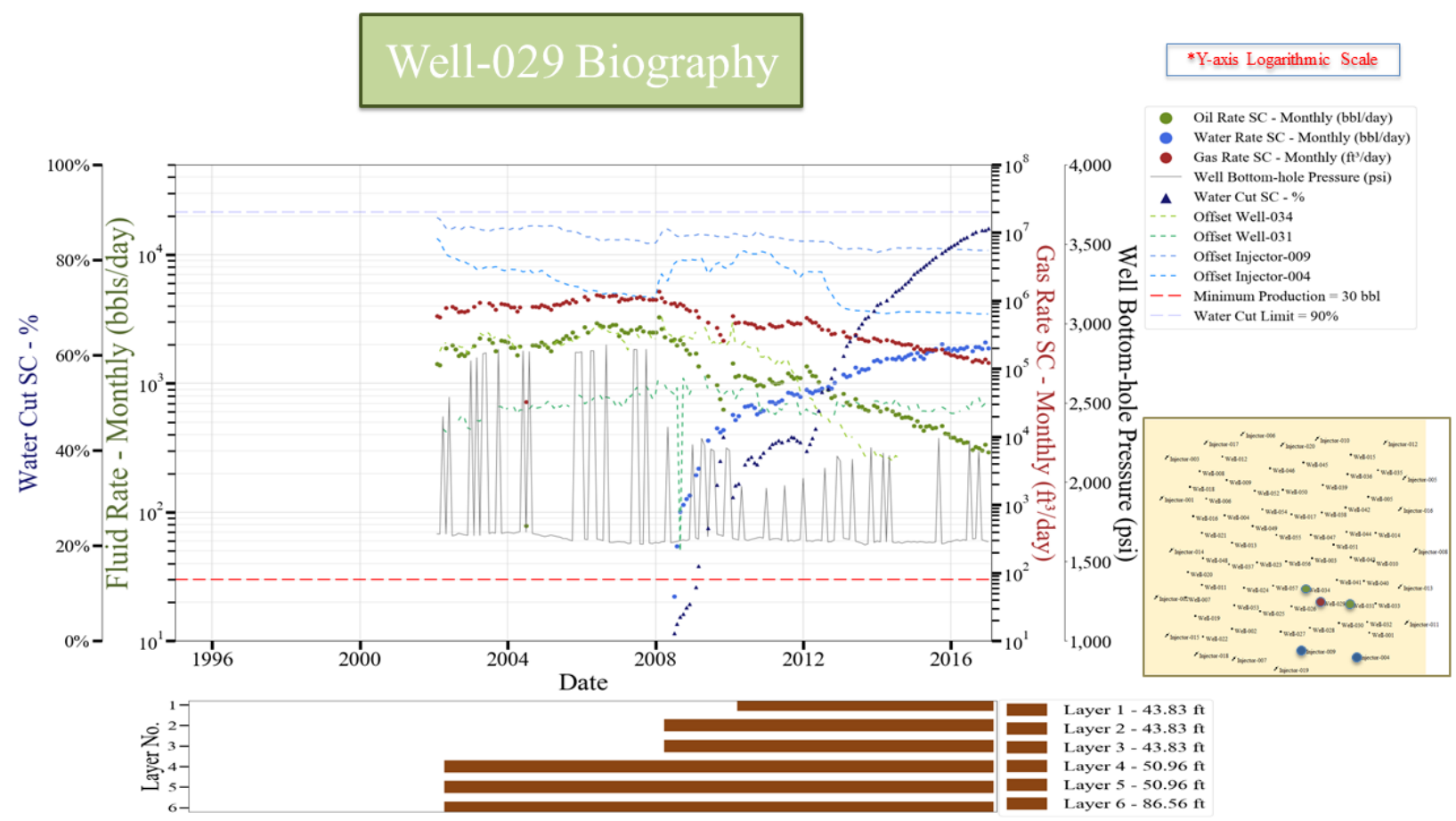

Figure 284 Well-029 biography 

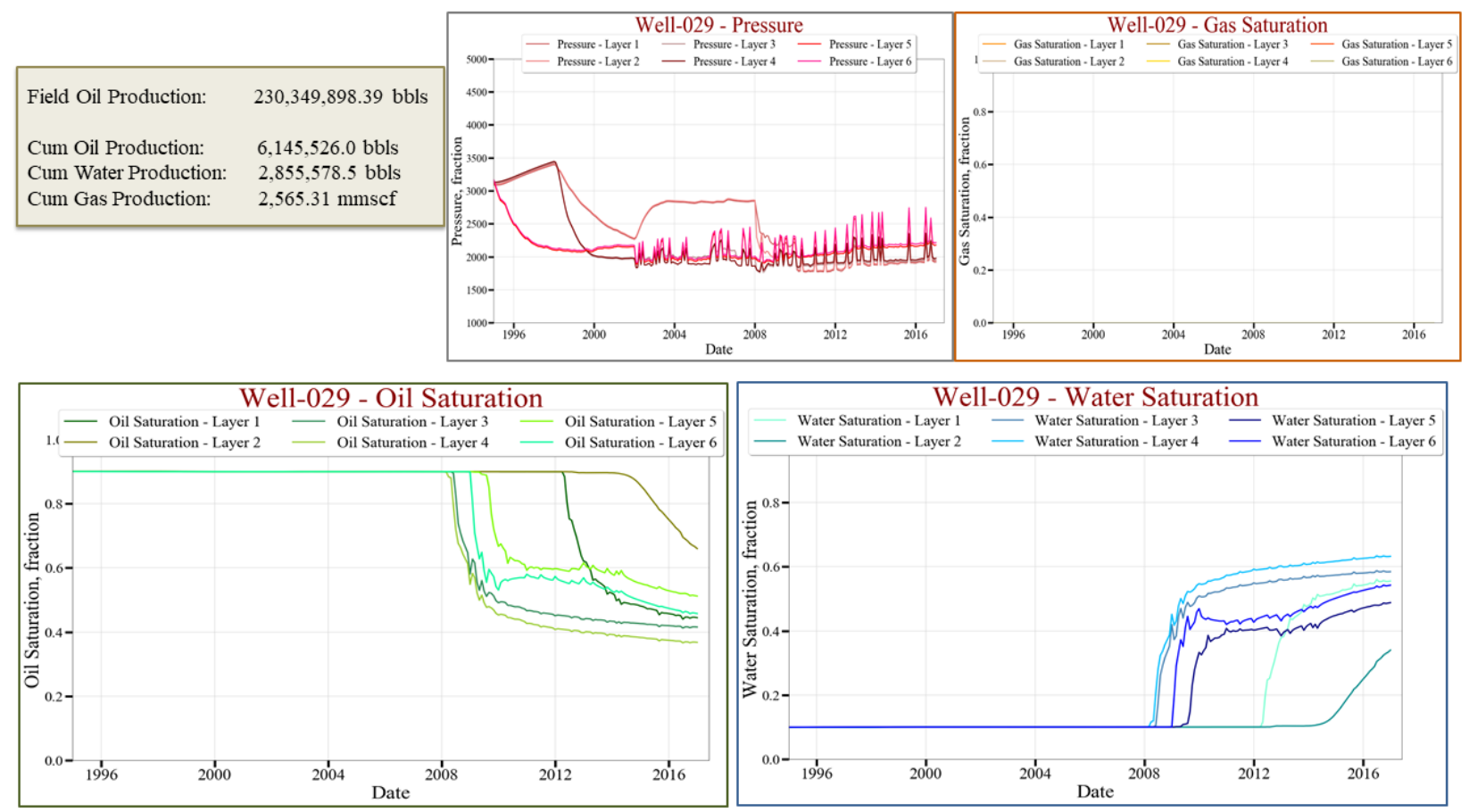

Figure 285 Well-029 saturations and pressure per layer

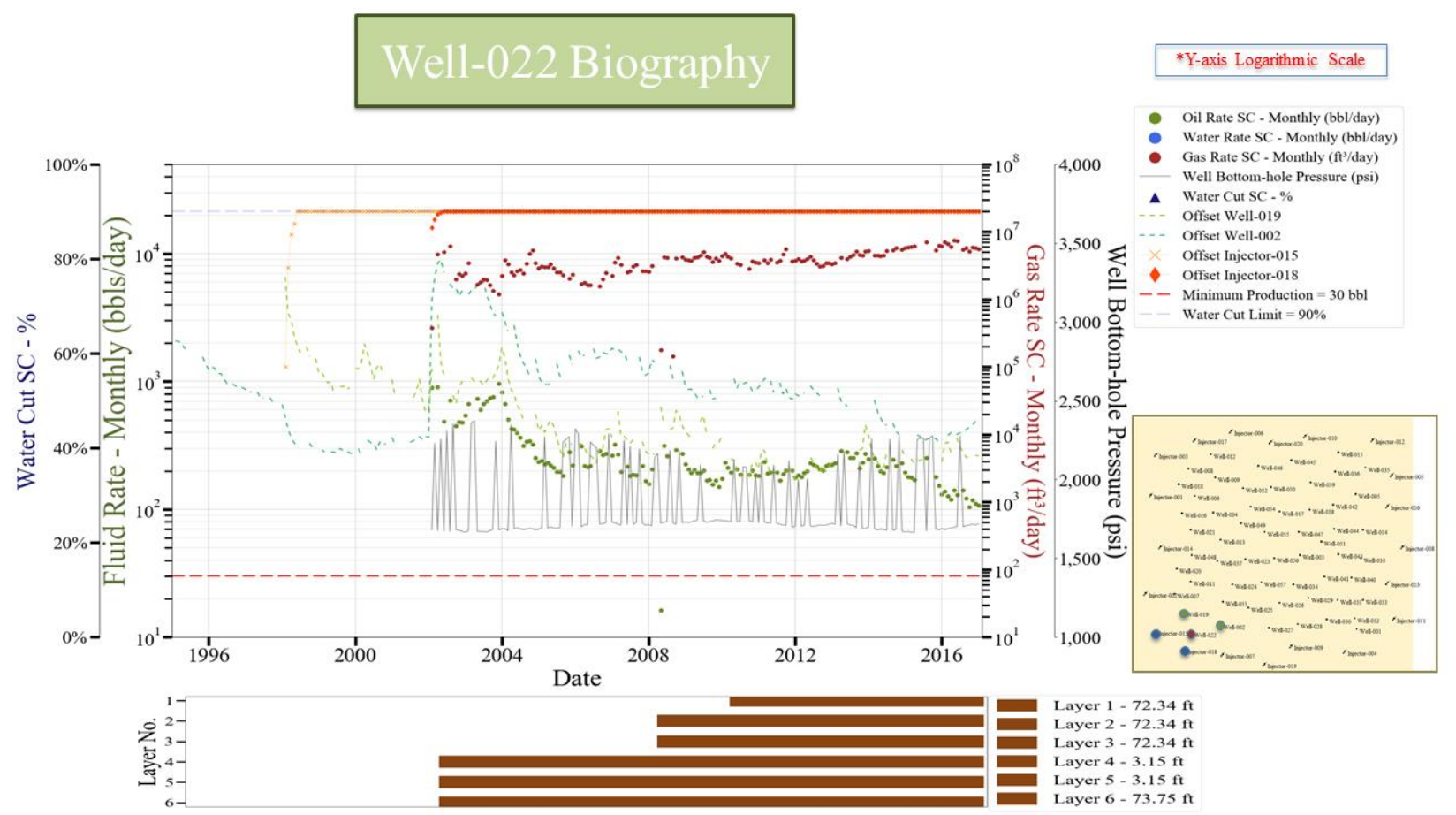

Figure 286 Well-022 biography 

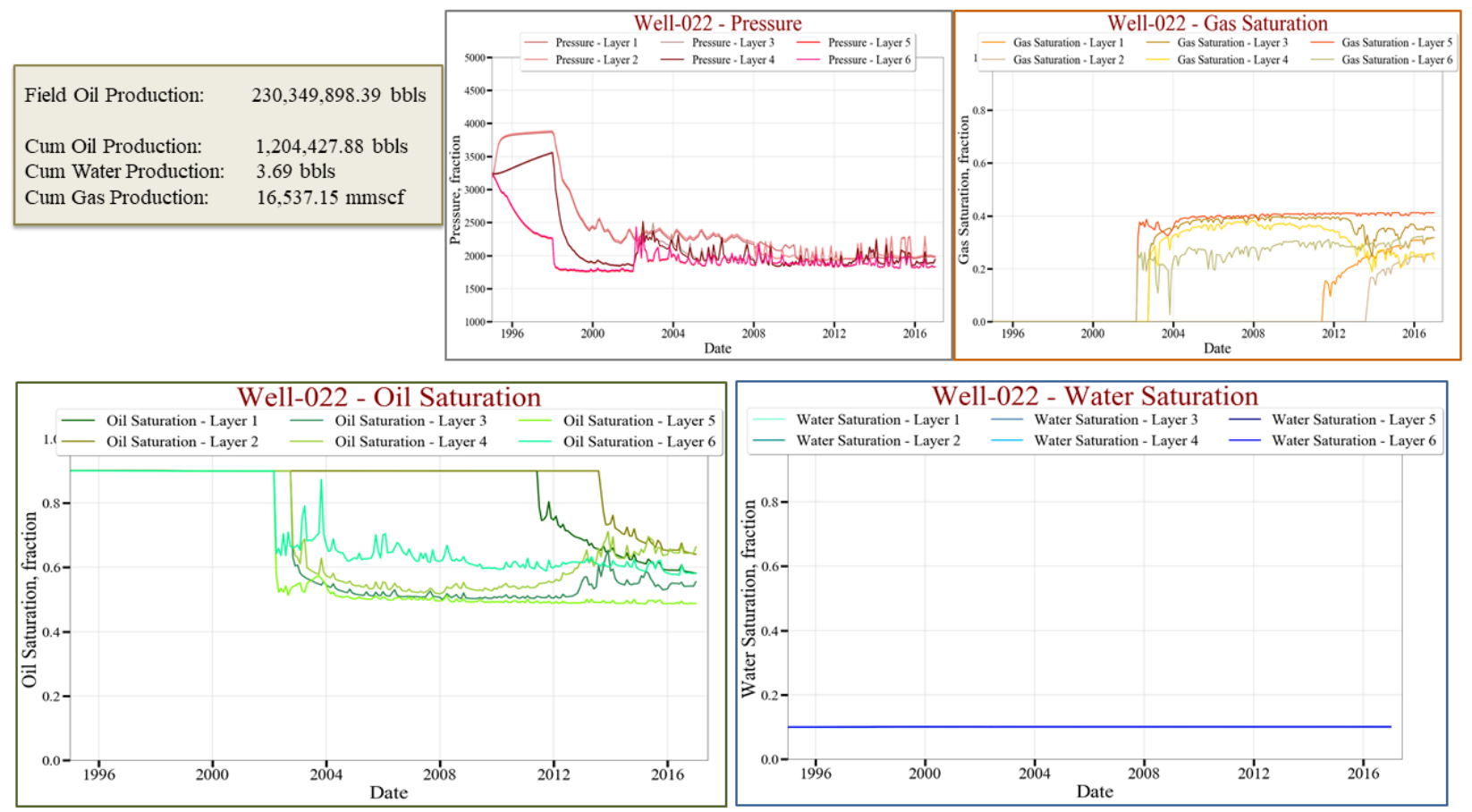

Figure 287 Well-022 saturations and pressure per layer

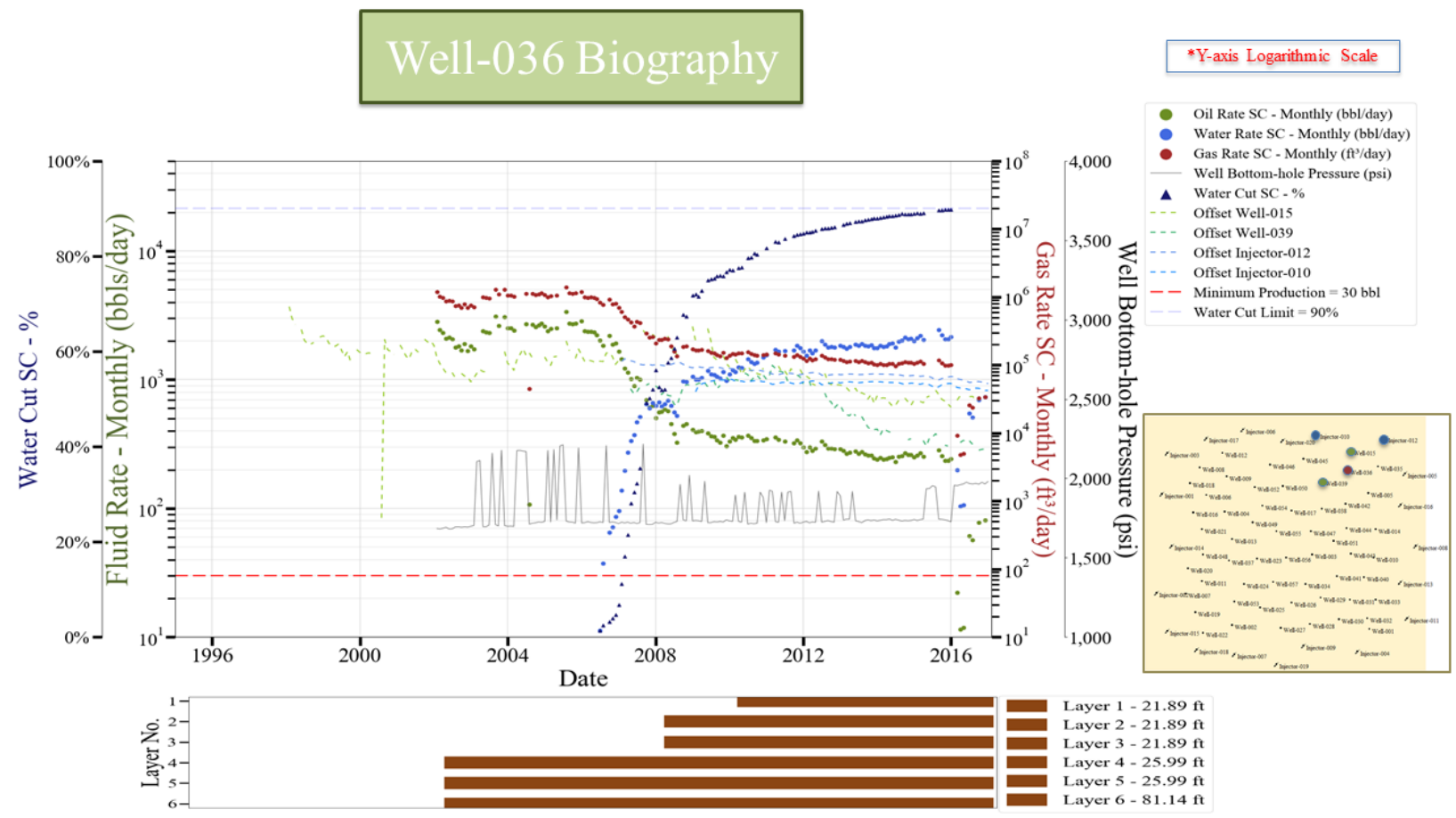

Figure 288 Well-036 biography 

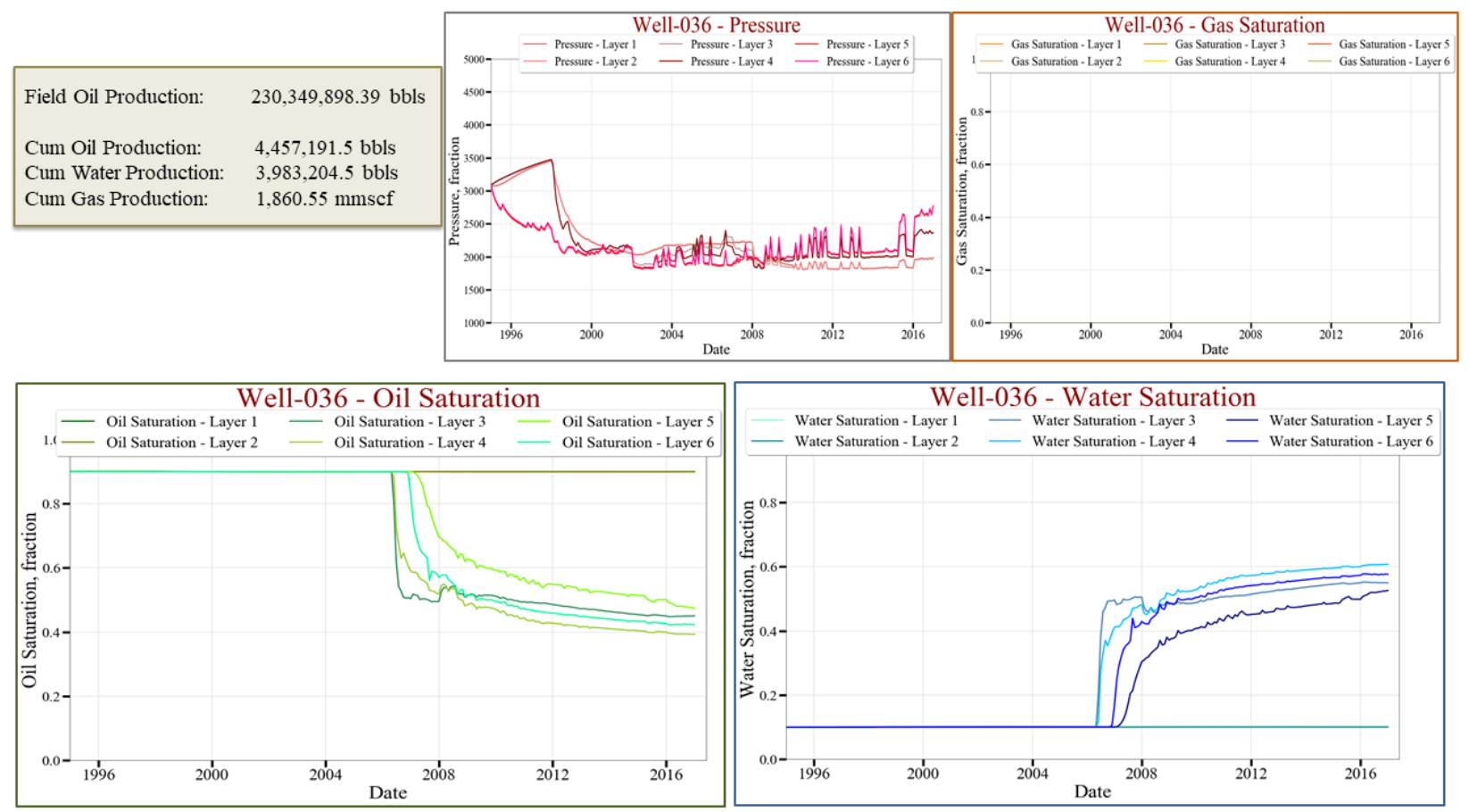

Figure 289 Well-036 saturations and pressure per layer

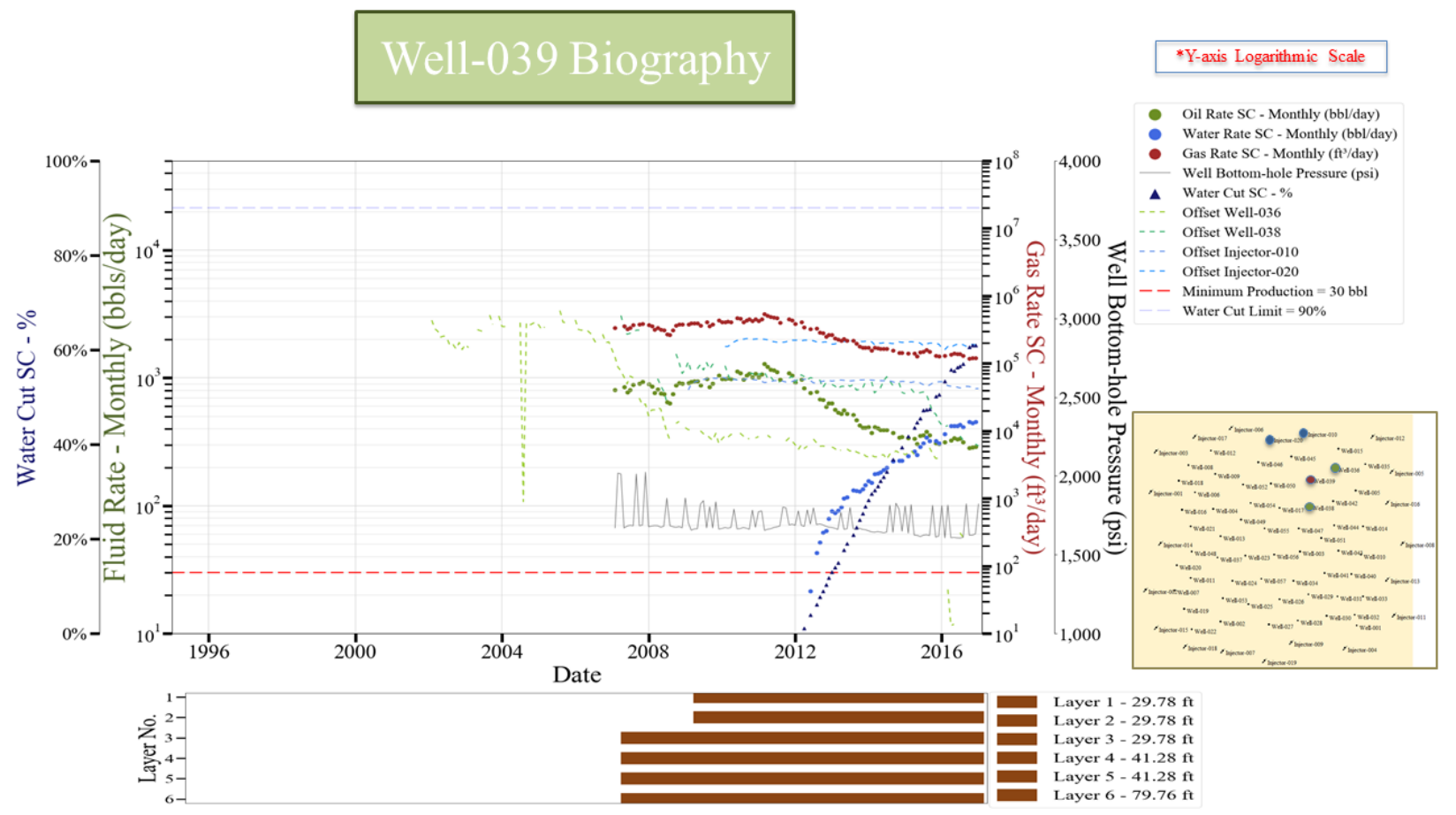

Figure 290 Well-039 biography 

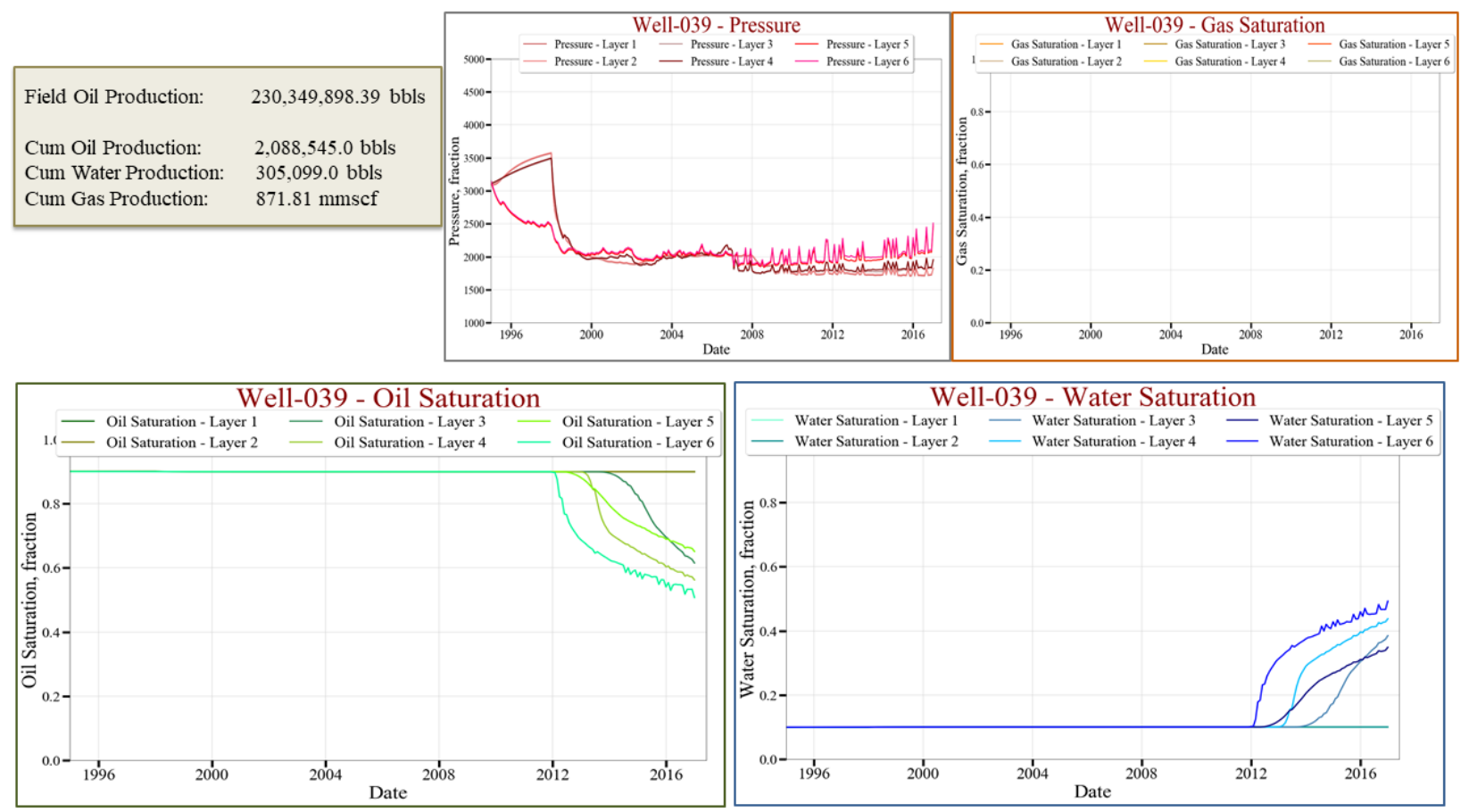

Figure 291 Well-039 saturations and pressure per layer

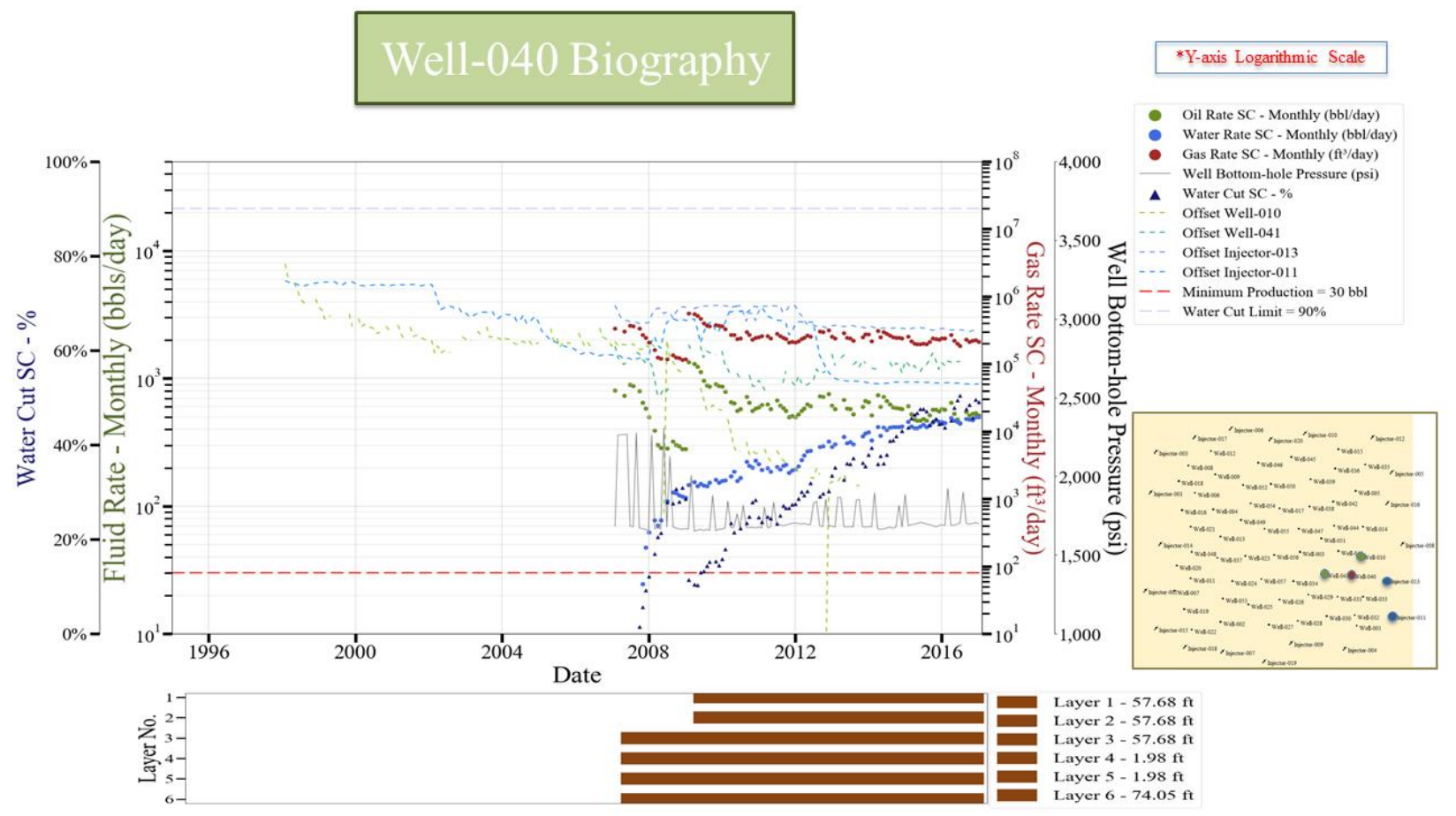

Figure 292 Well-040 biography 

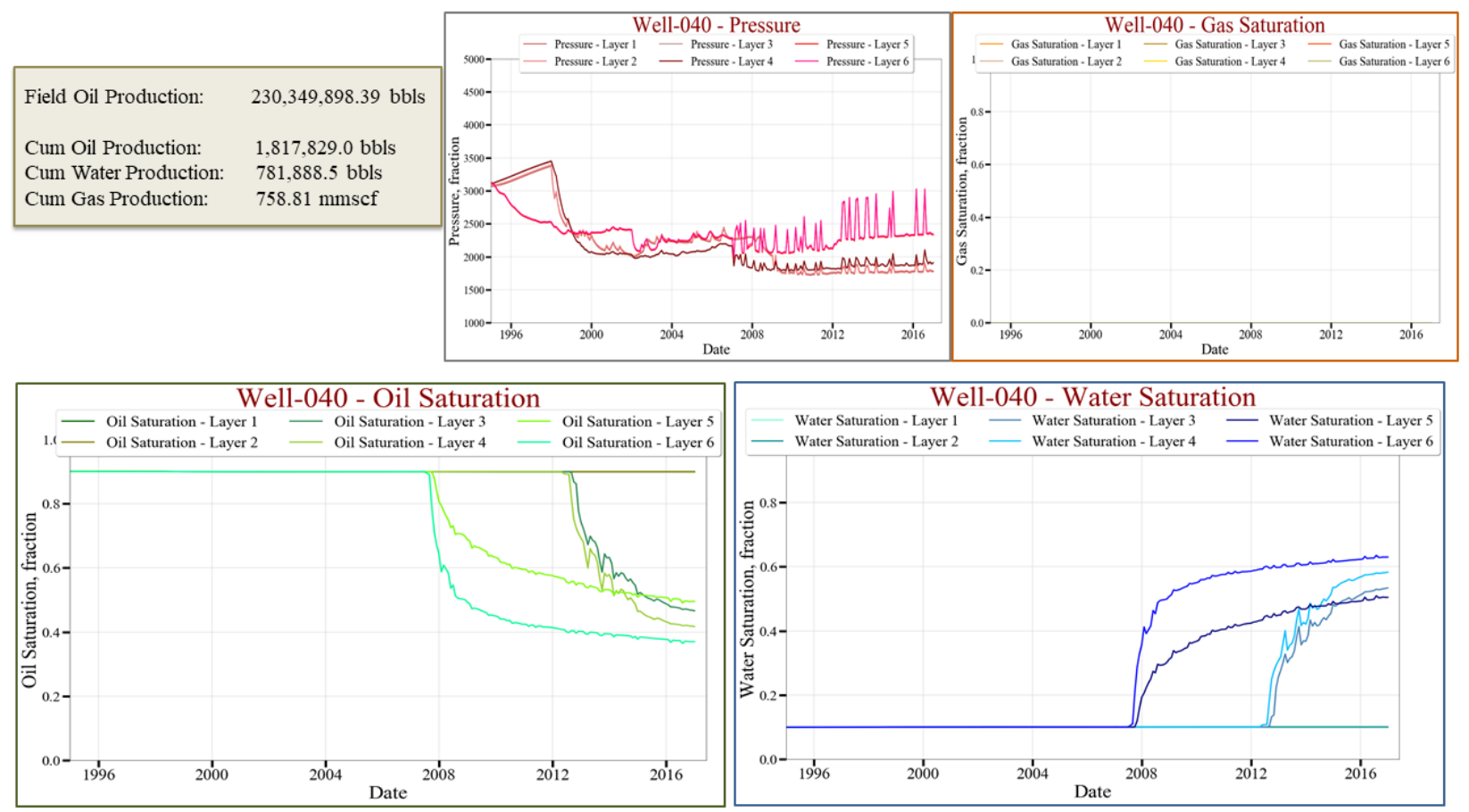

Figure 293 Well-040 saturations and pressure per layer

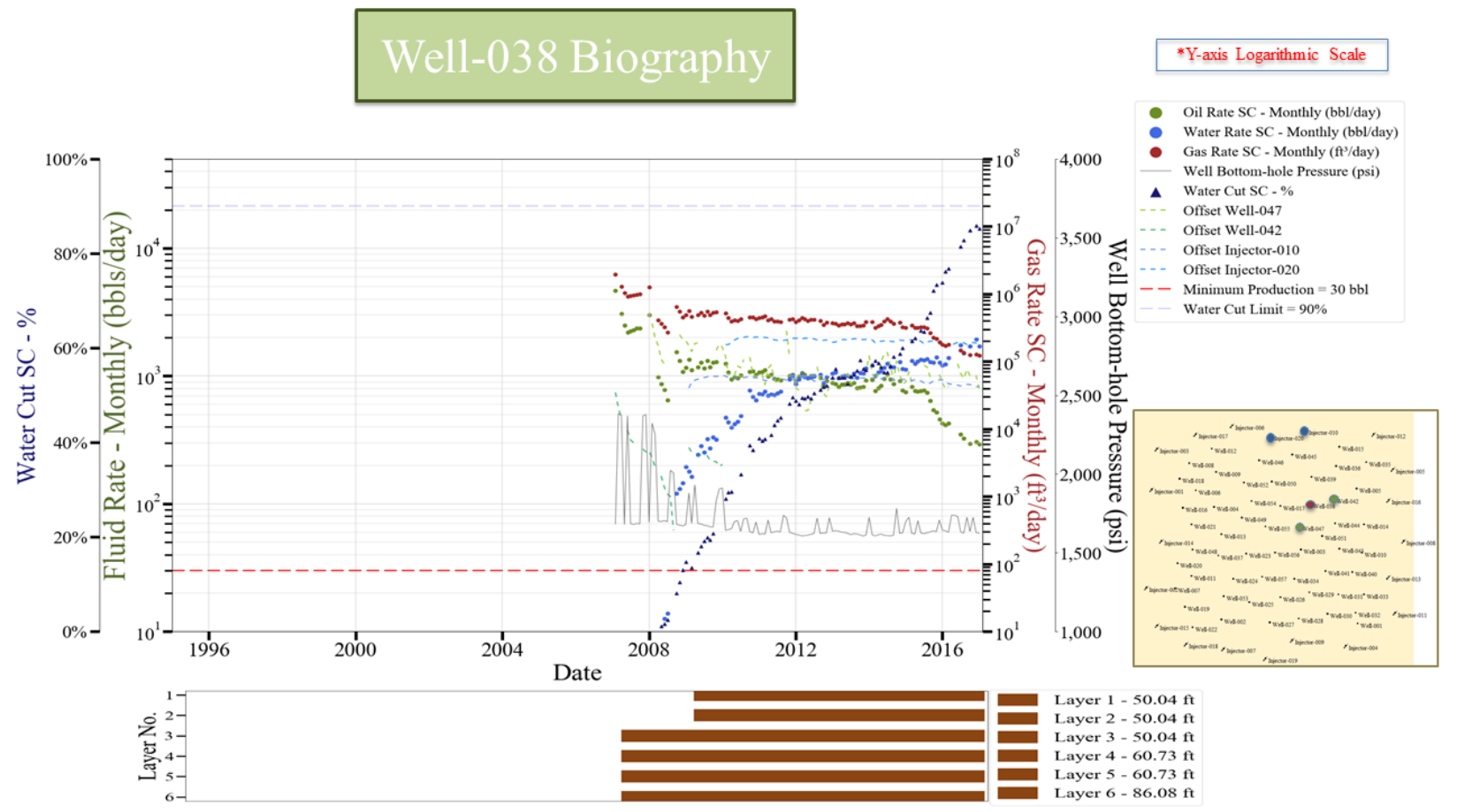

Figure 294 Well-038 biography 

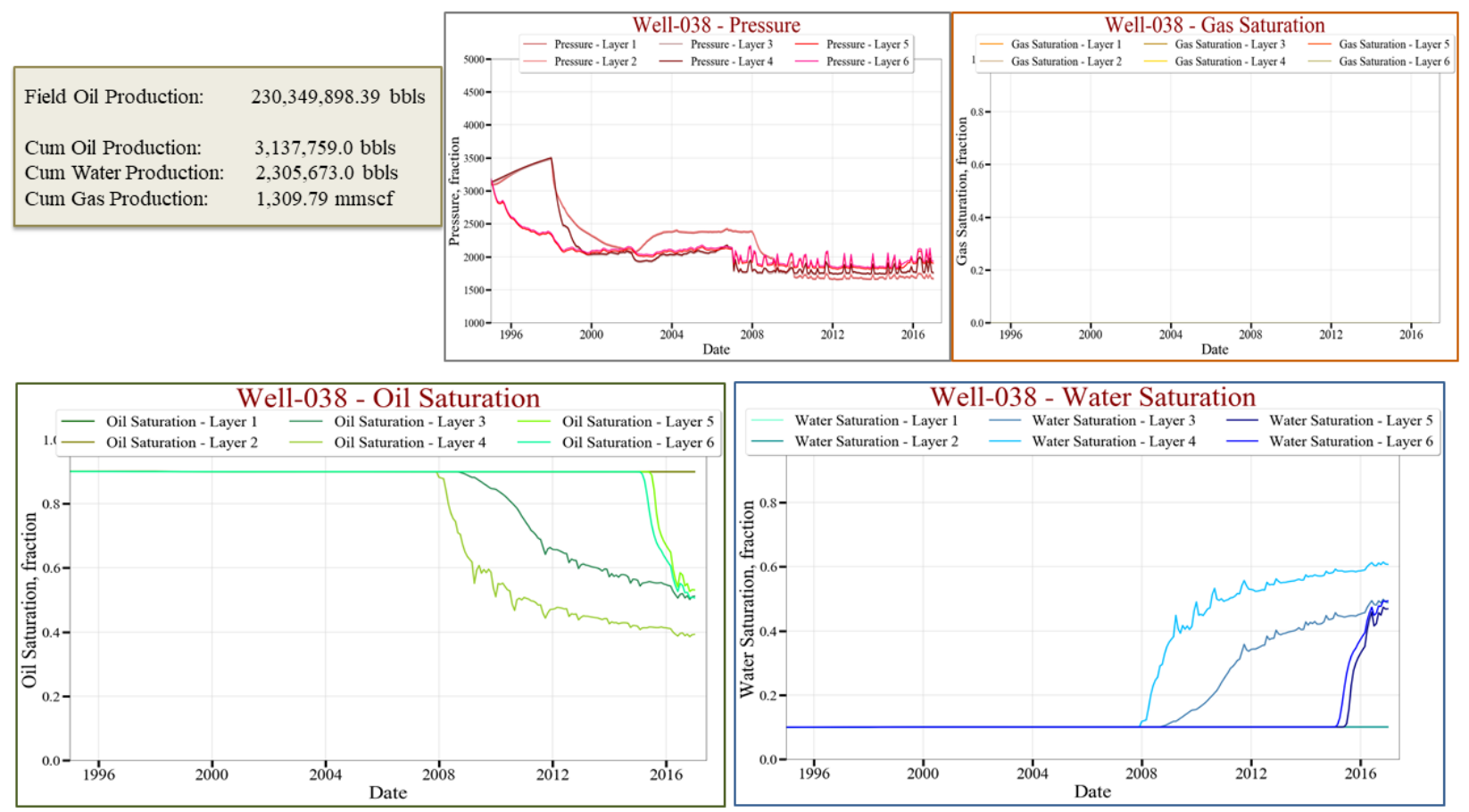

Figure 295 Well-038 saturations and pressure per layer

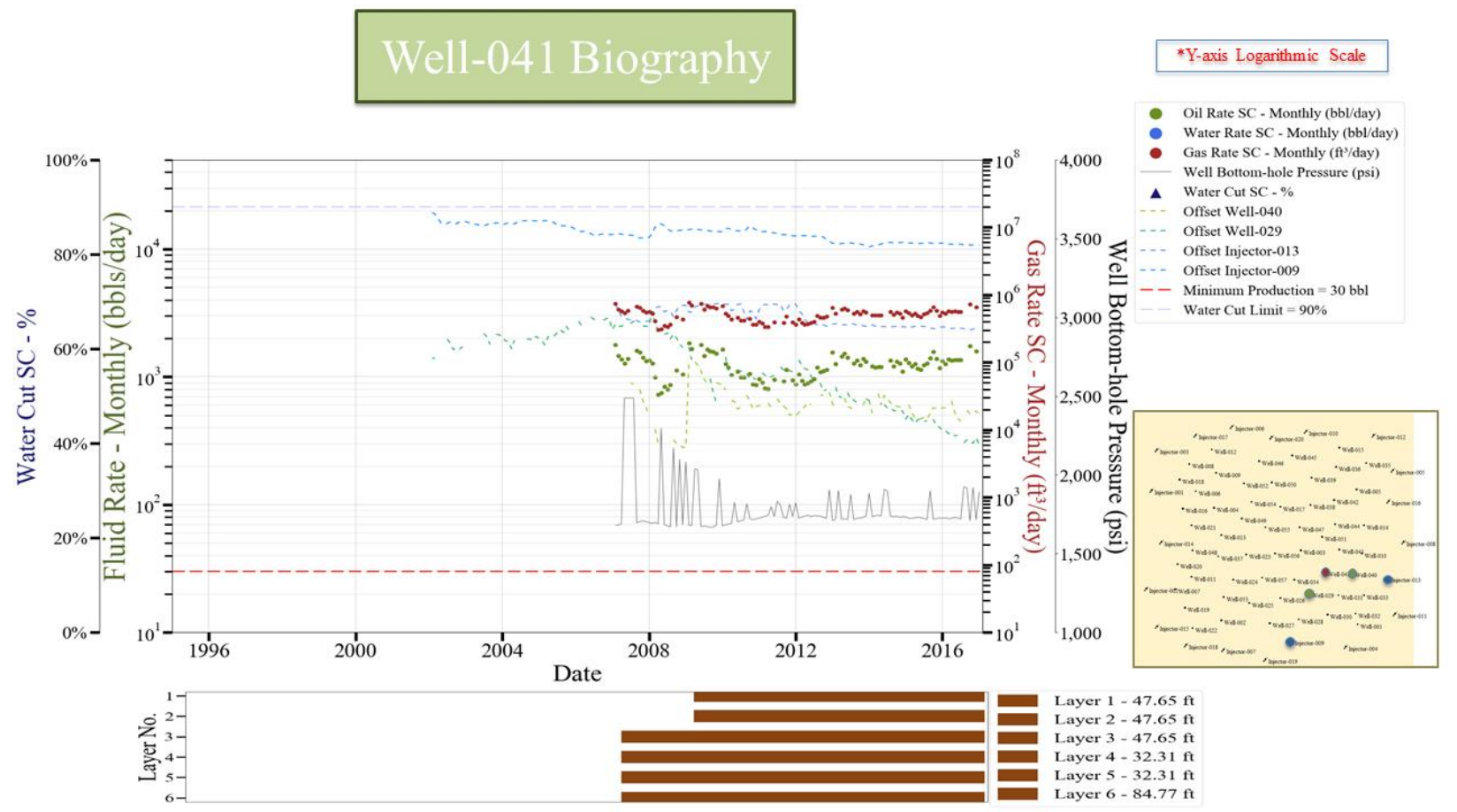

Figure 296 Well-041 biography 

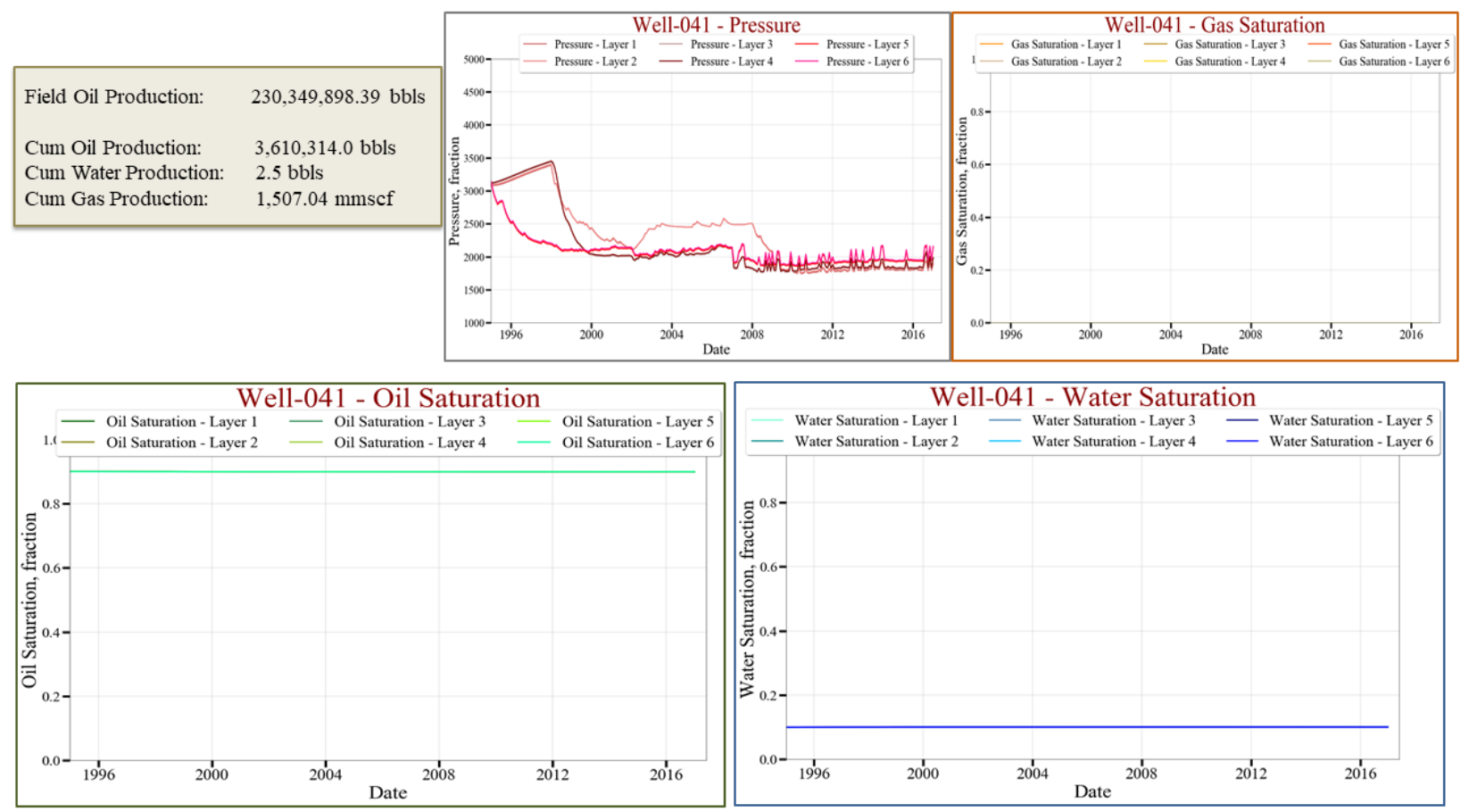

Figure 297 Well-041 saturations and pressure per layer

\subsubsection{Smallest Number of Production Days}

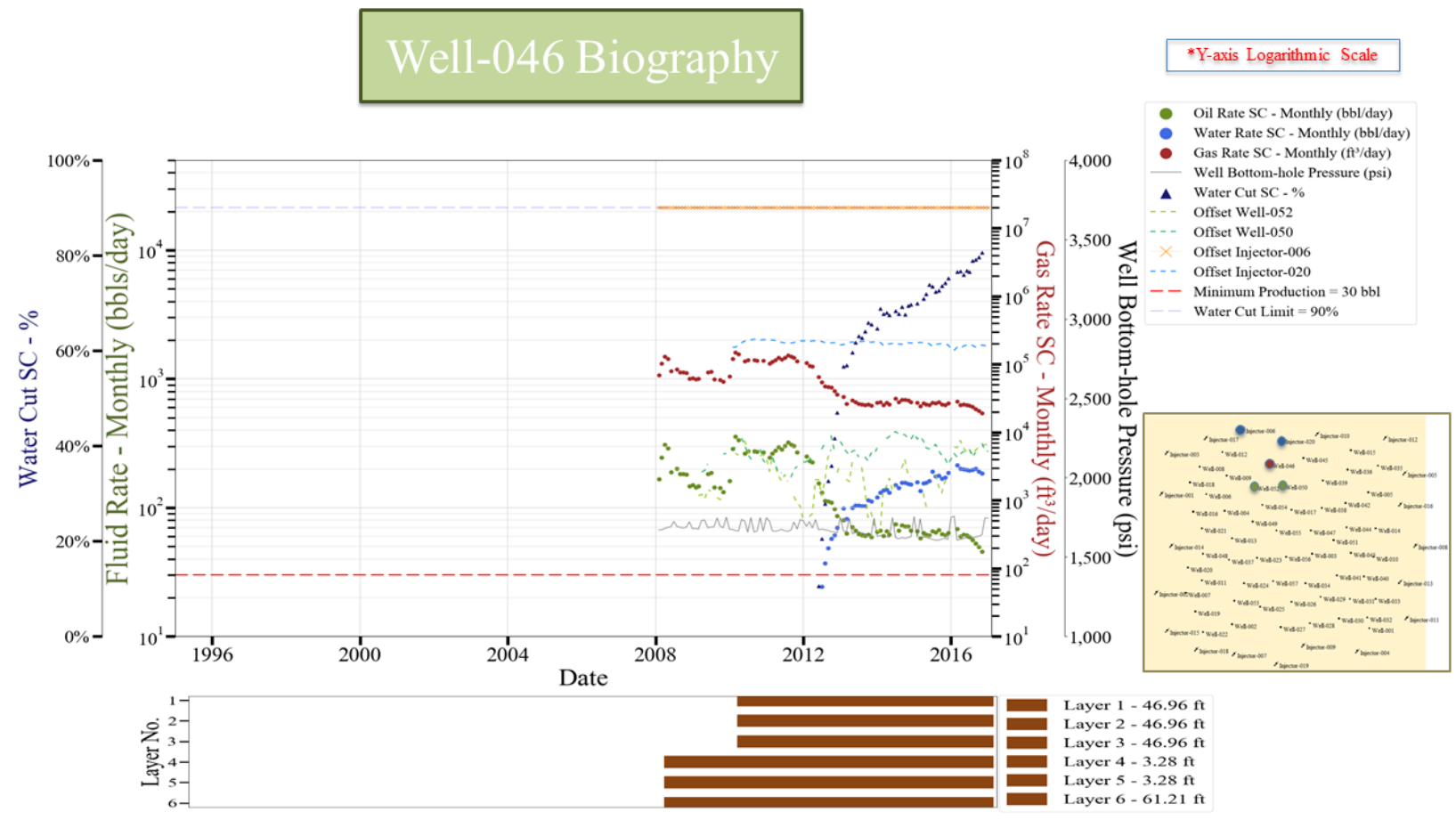

Figure 298 Well-046 biography 

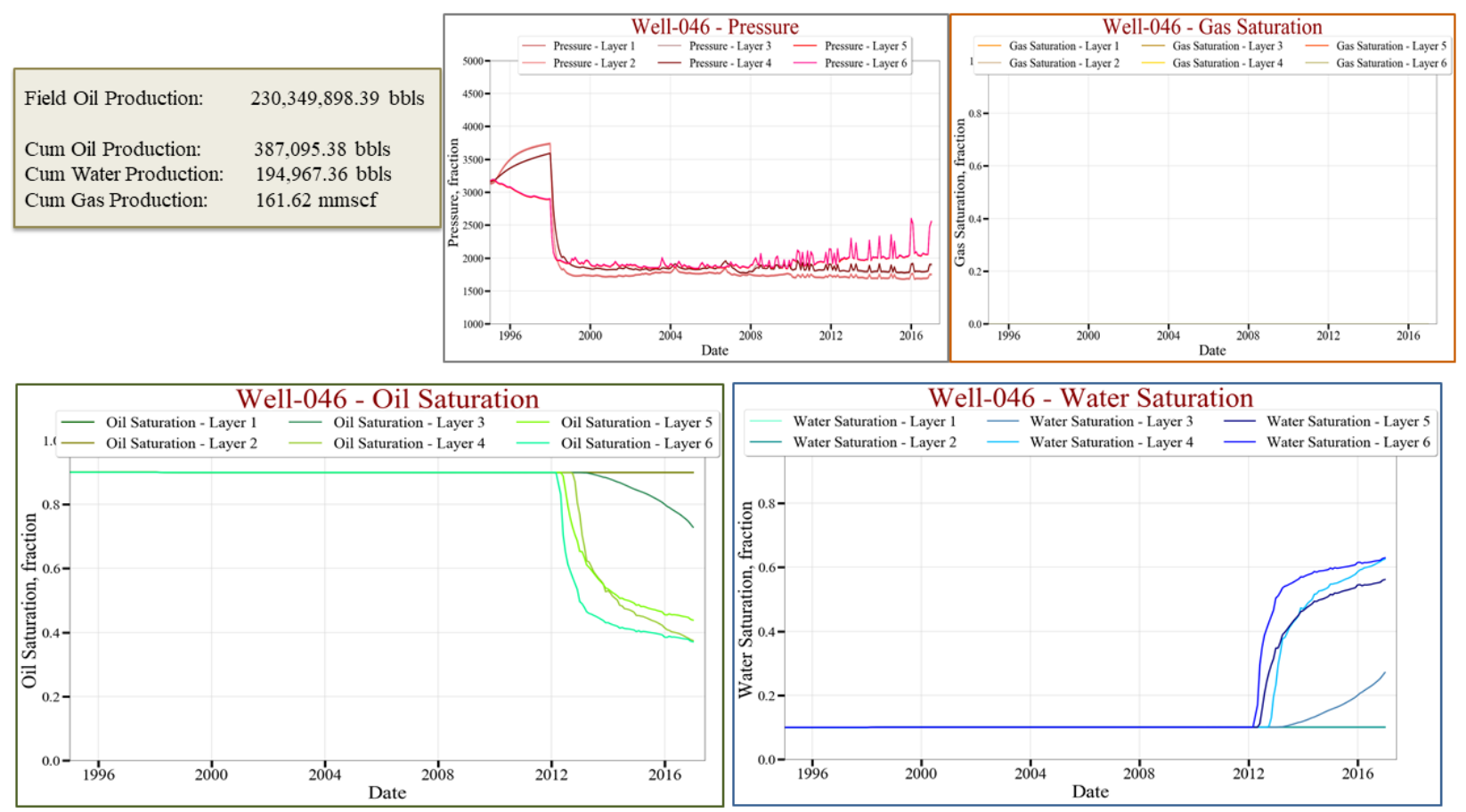

Figure 299 Well-046 saturations and pressure per layer

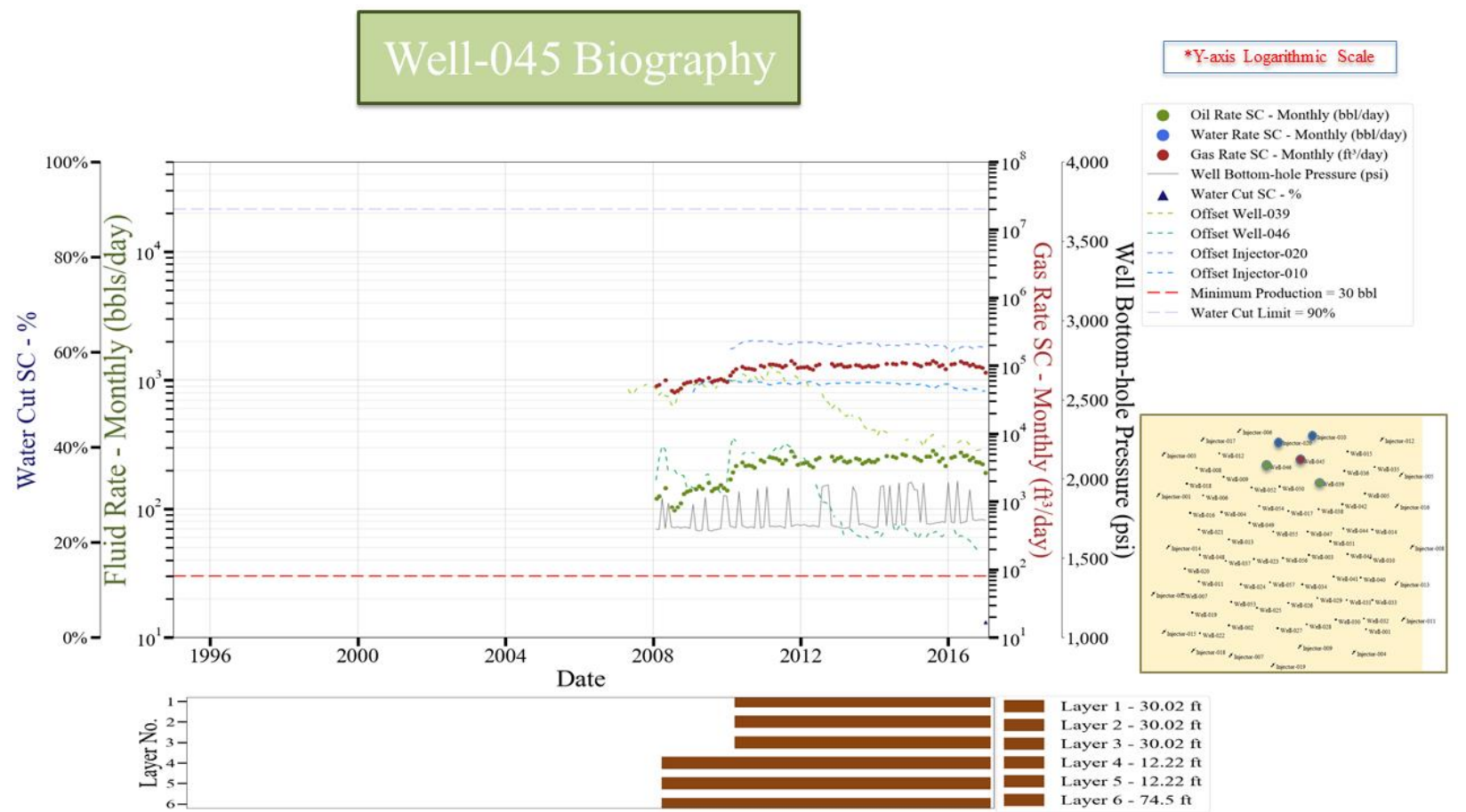

Figure 300 Well-045 biography 

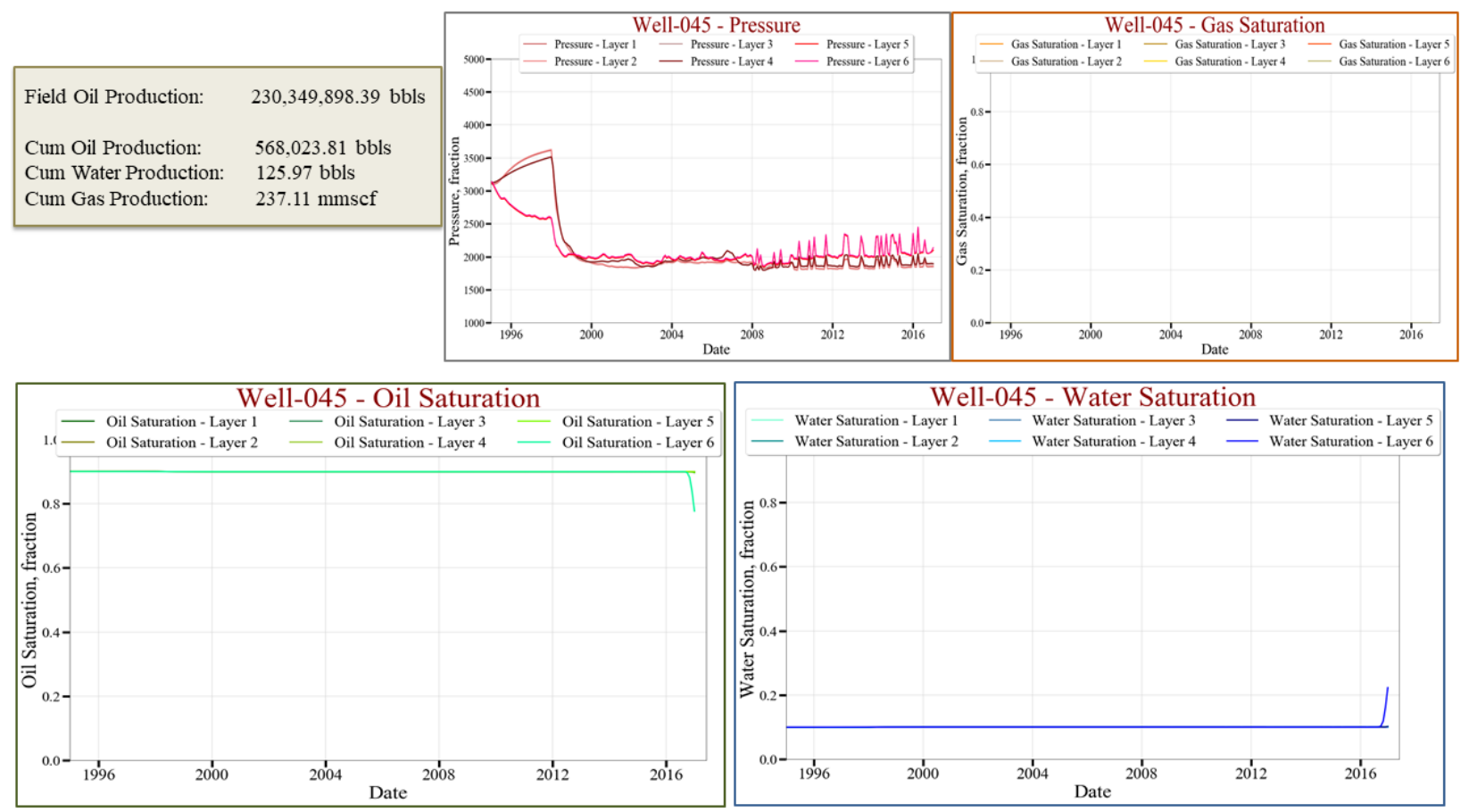

Figure 301 Well-045 saturations and pressure per layer

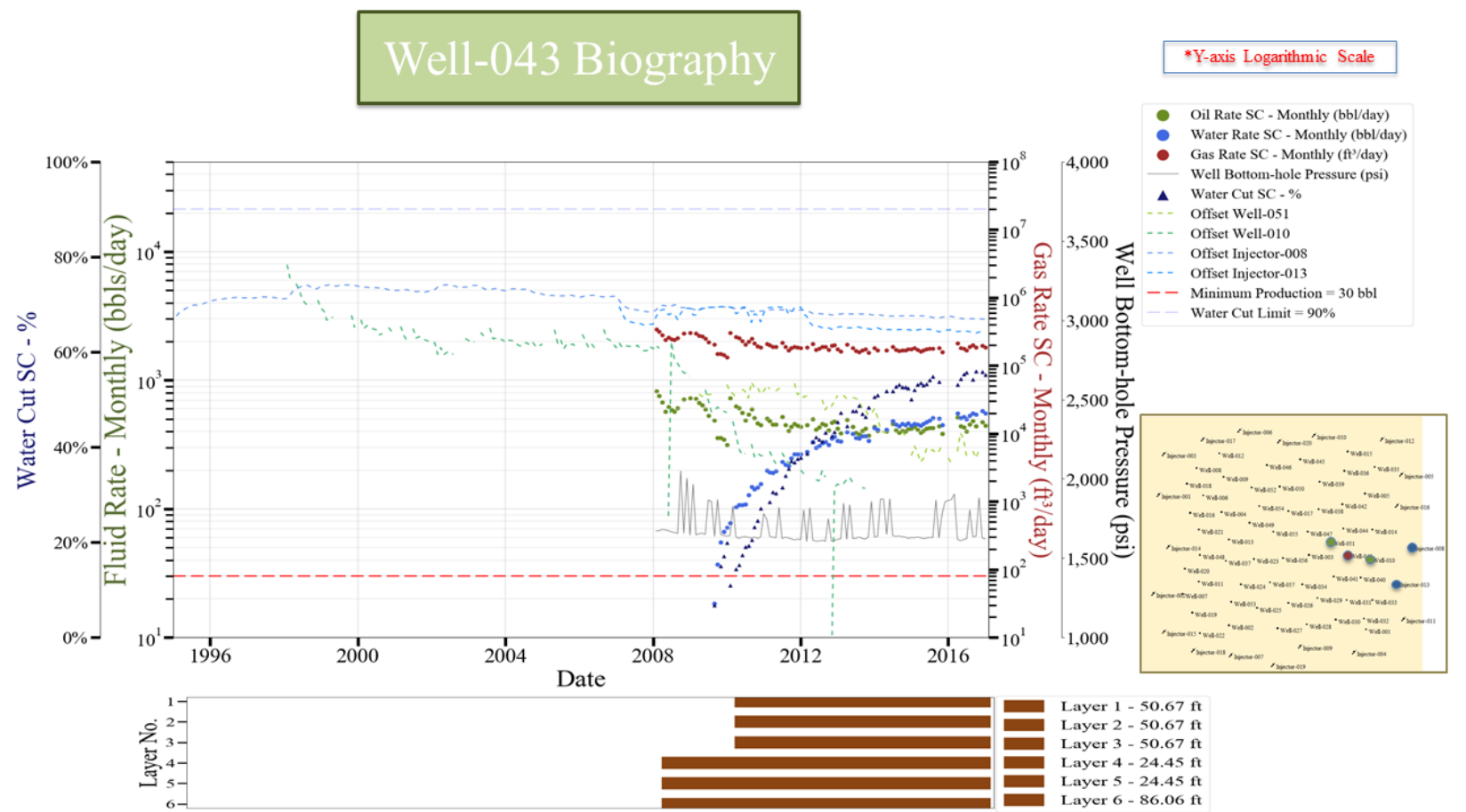

Figure 302 Well-043 biography 

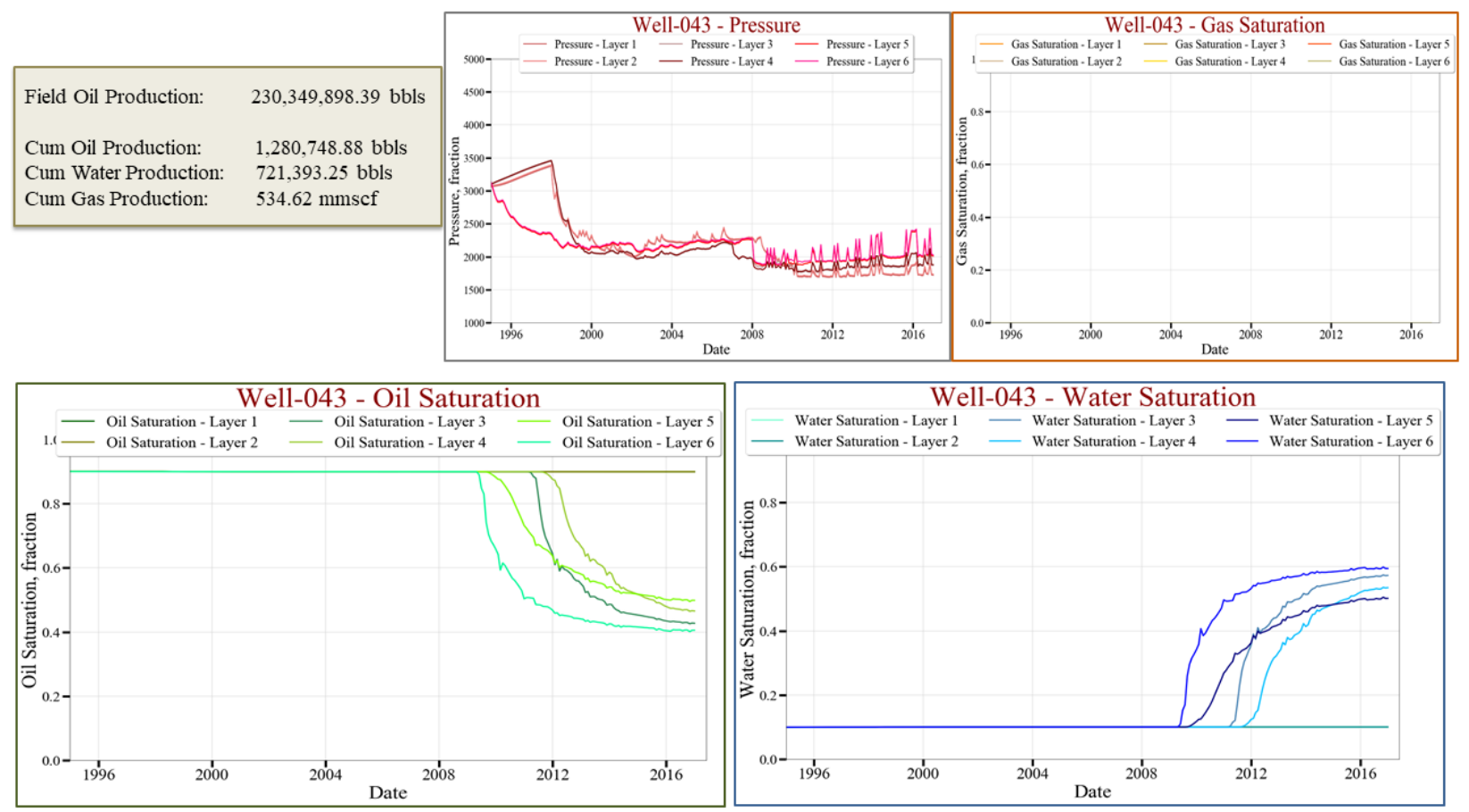

Figure 303 Well-043 saturations and pressure per layer

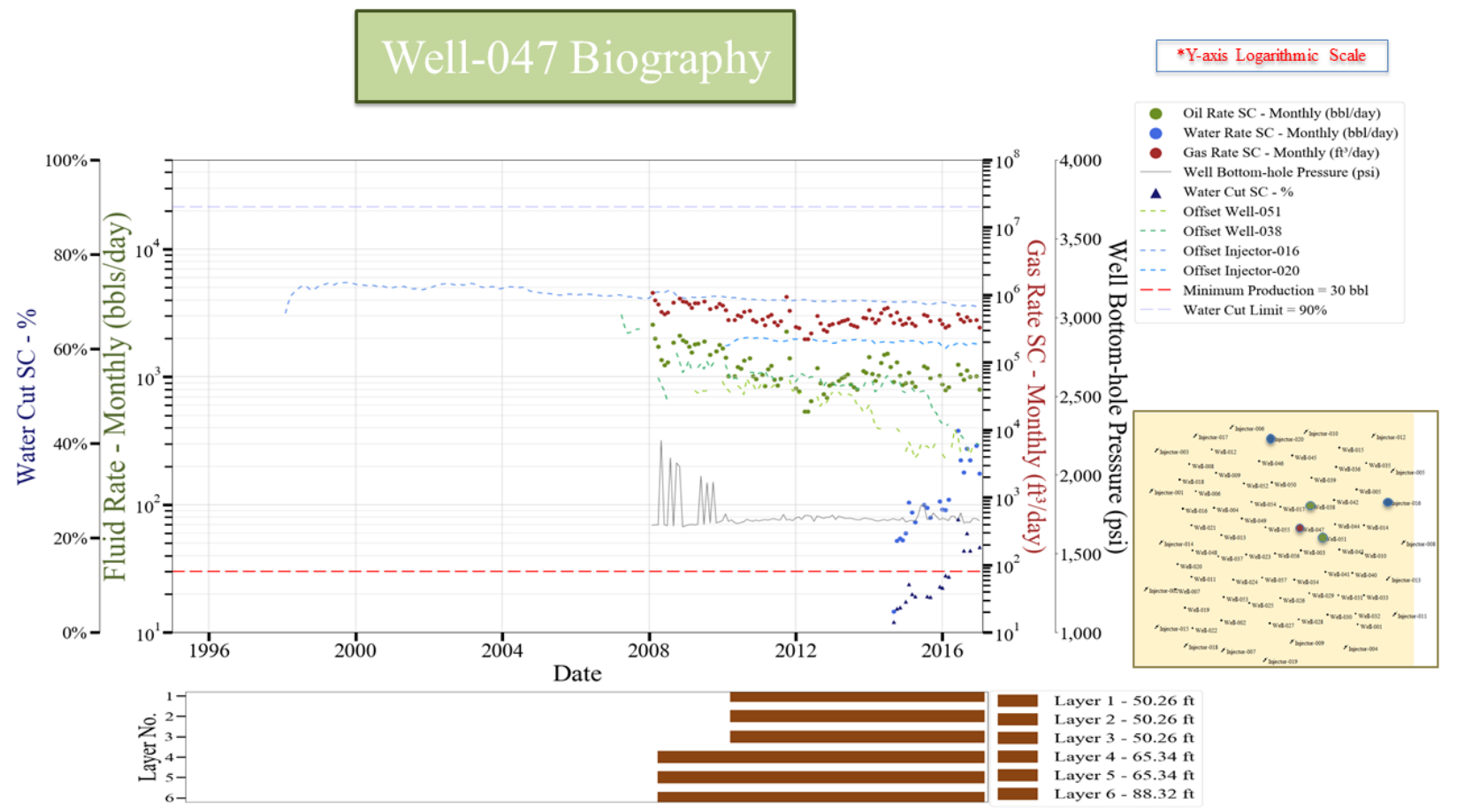

Figure 304 Well-047 biography 

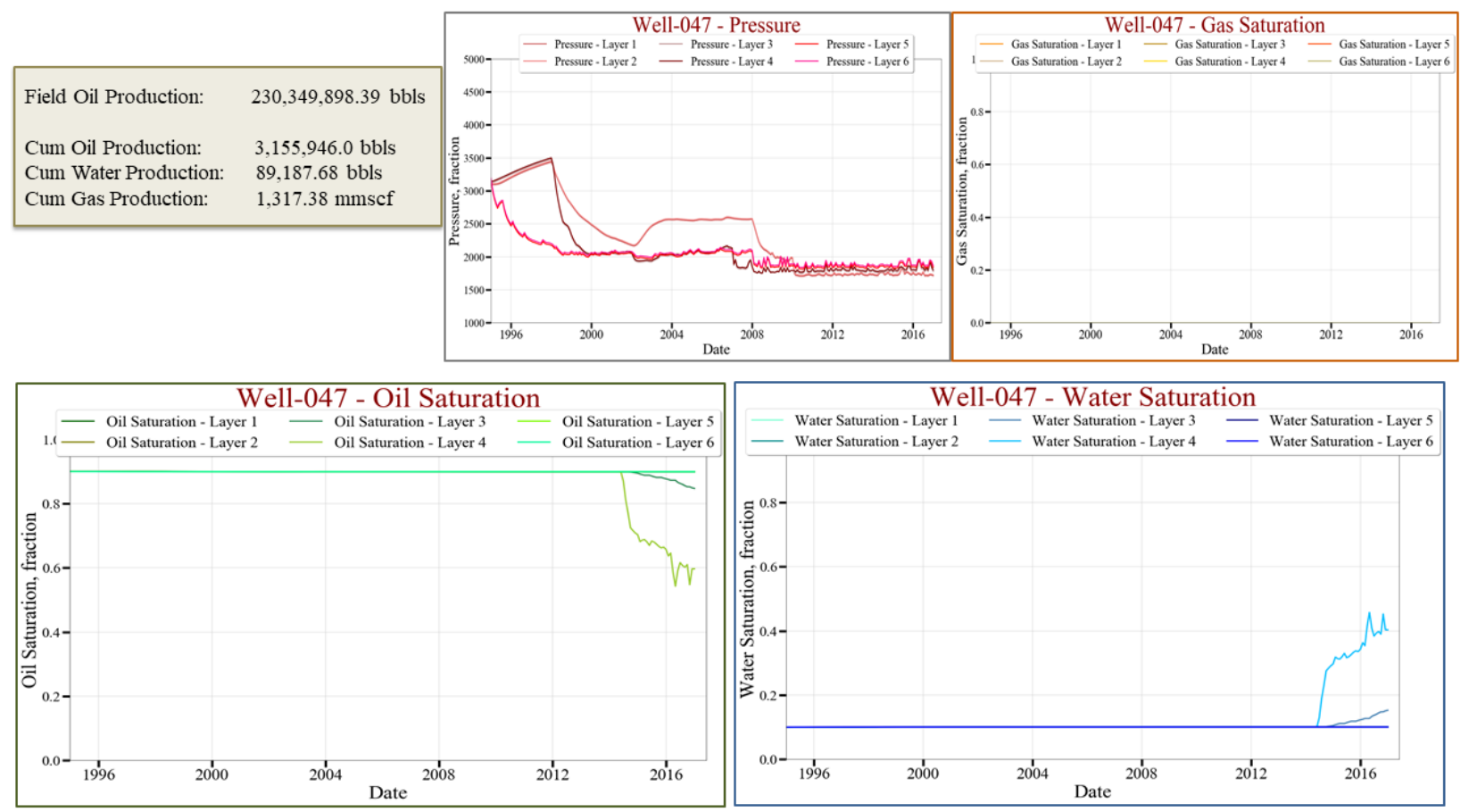

Figure 305 Well-047 saturations and pressure per layer

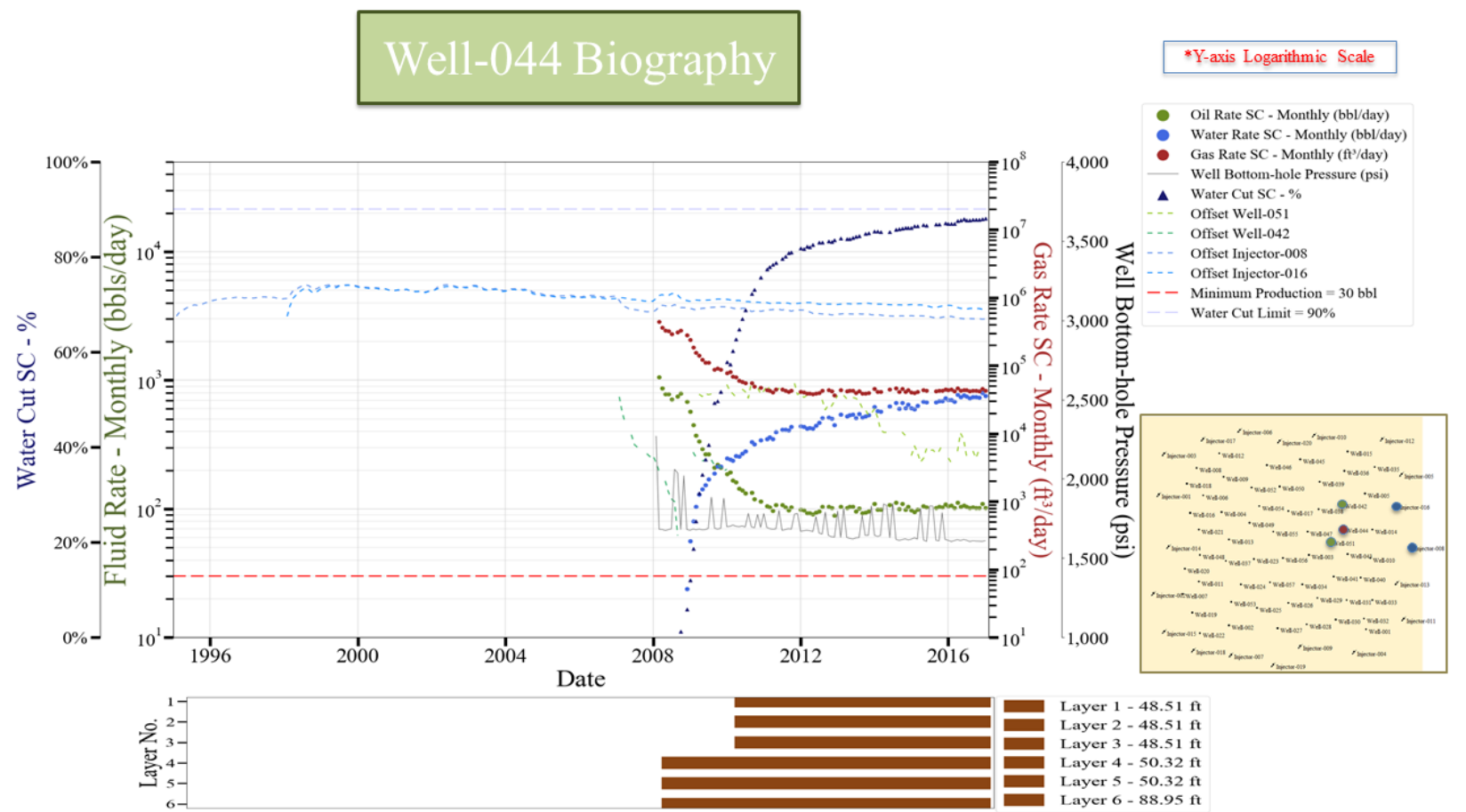

Figure 306 Well-044 biography 

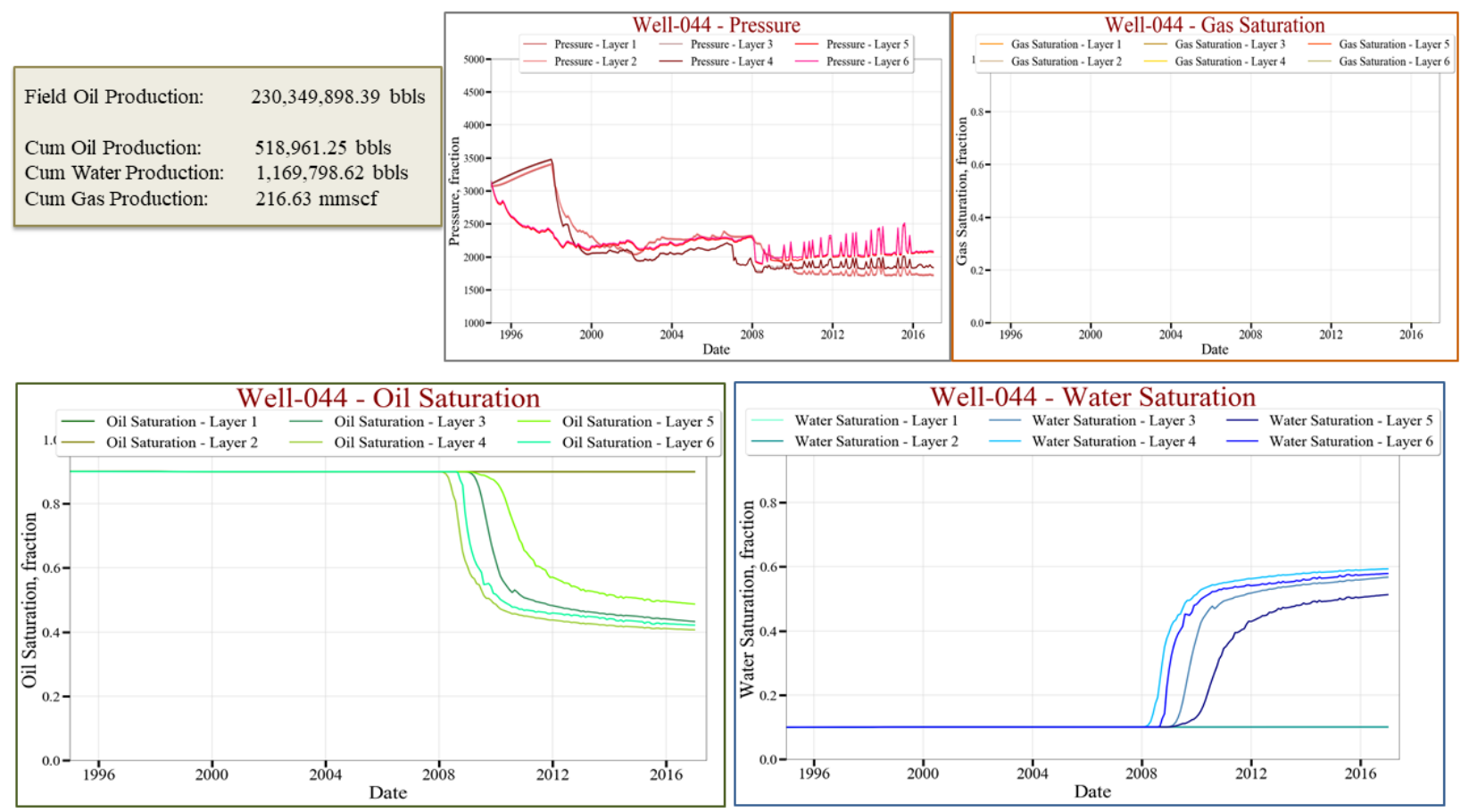

Figure 307 Well-044 saturations and pressure per layer

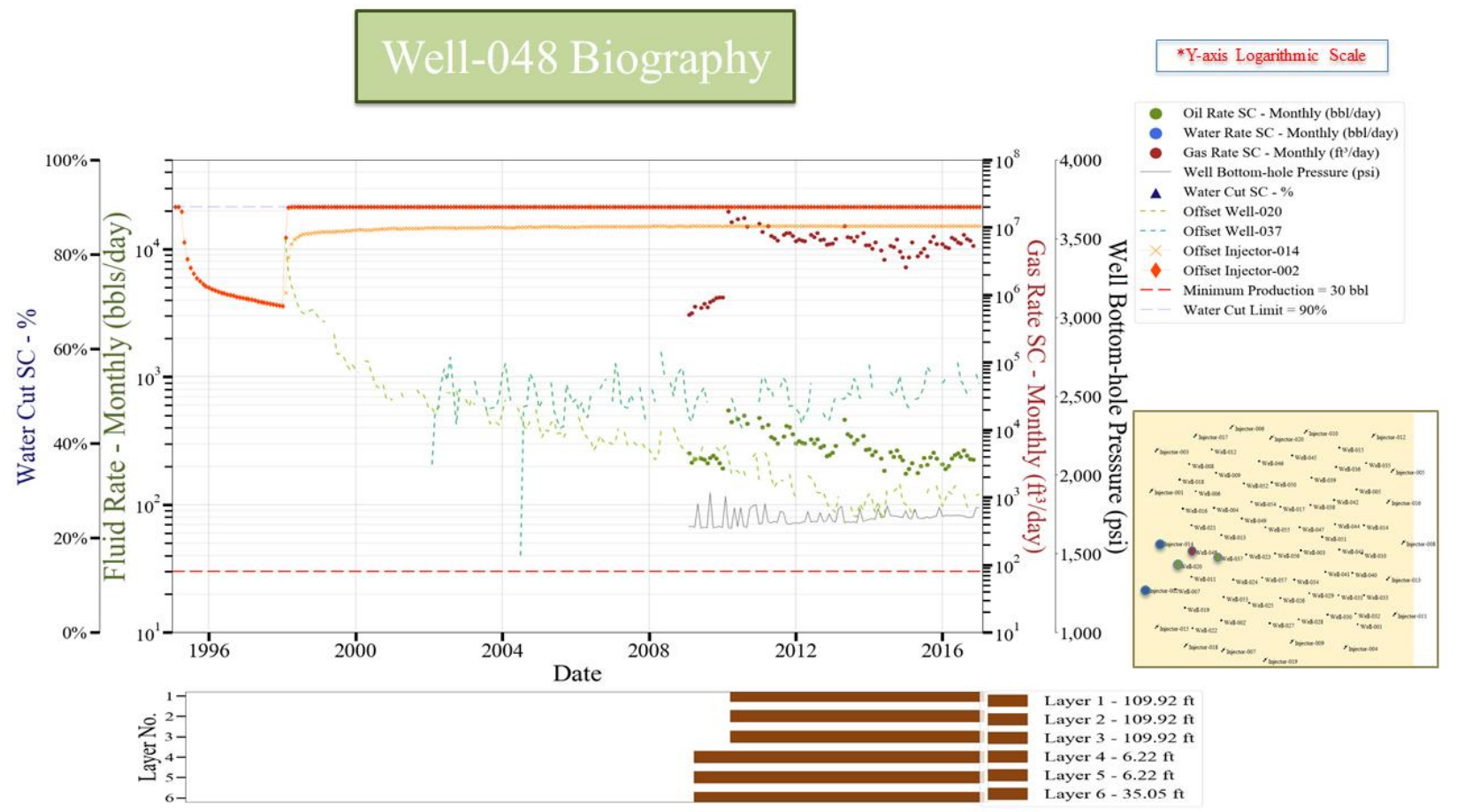

Figure 308 Well-048 biography 

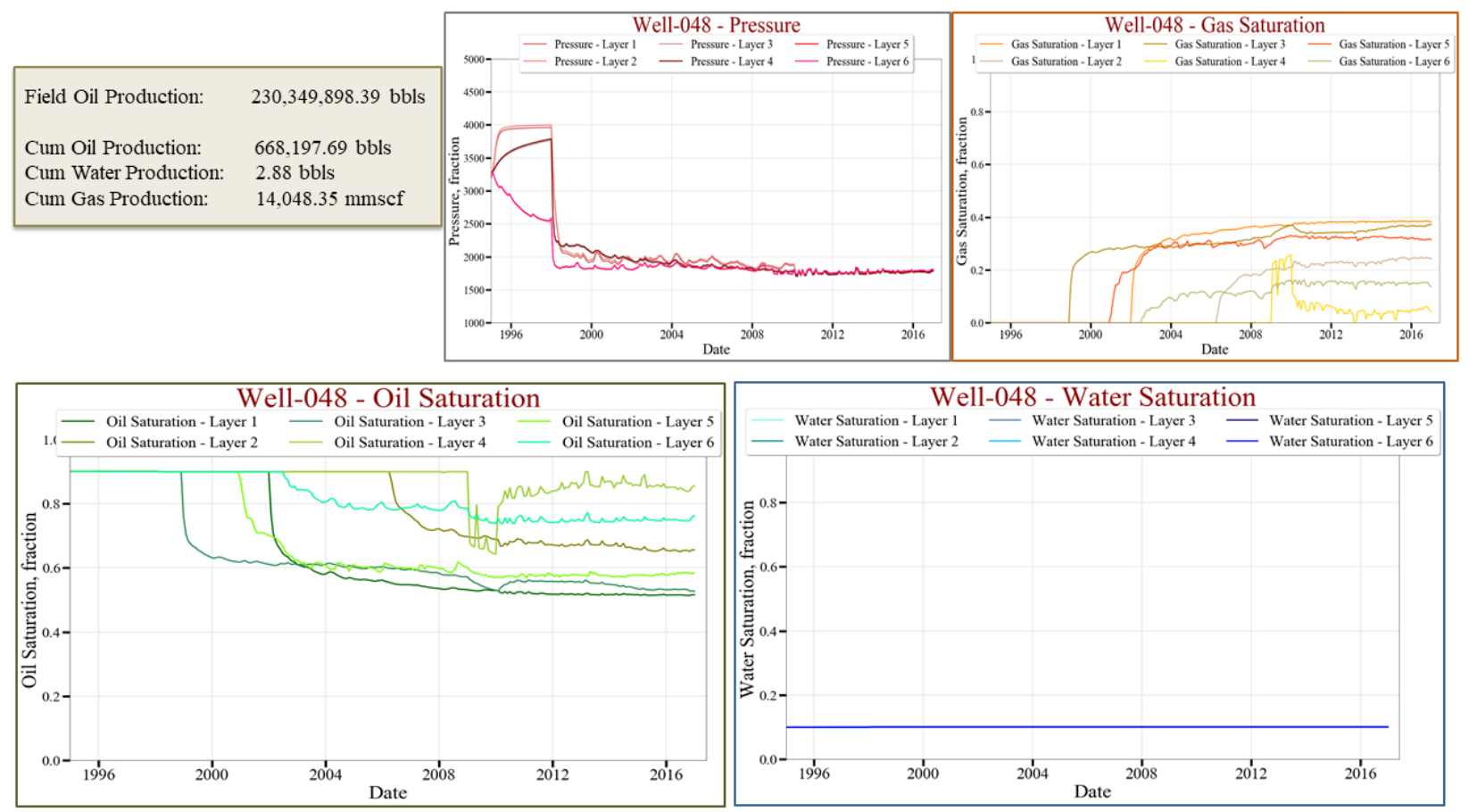

Figure 309 Well-048 saturations and pressure per layer

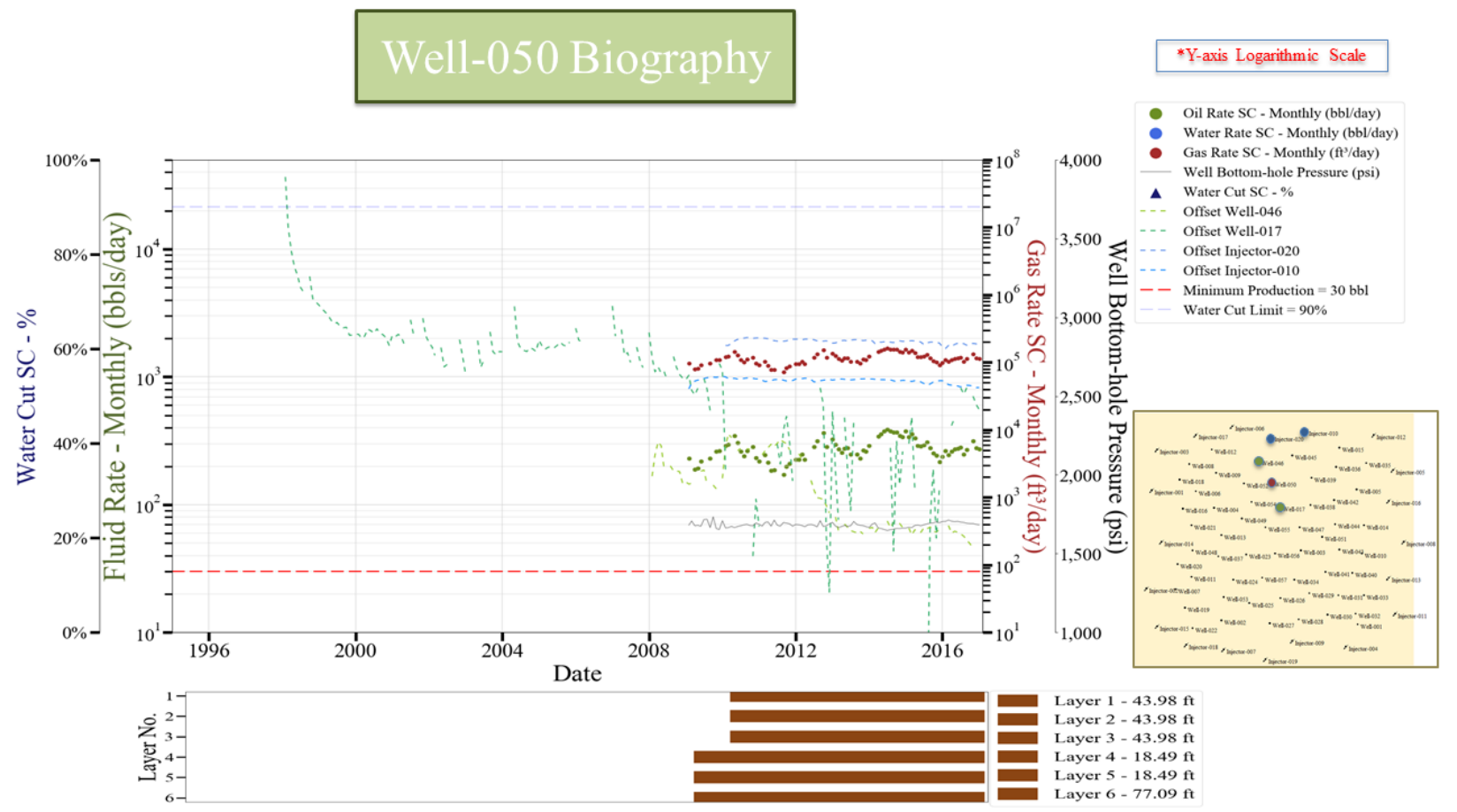

Figure 310 Well-050 biography 

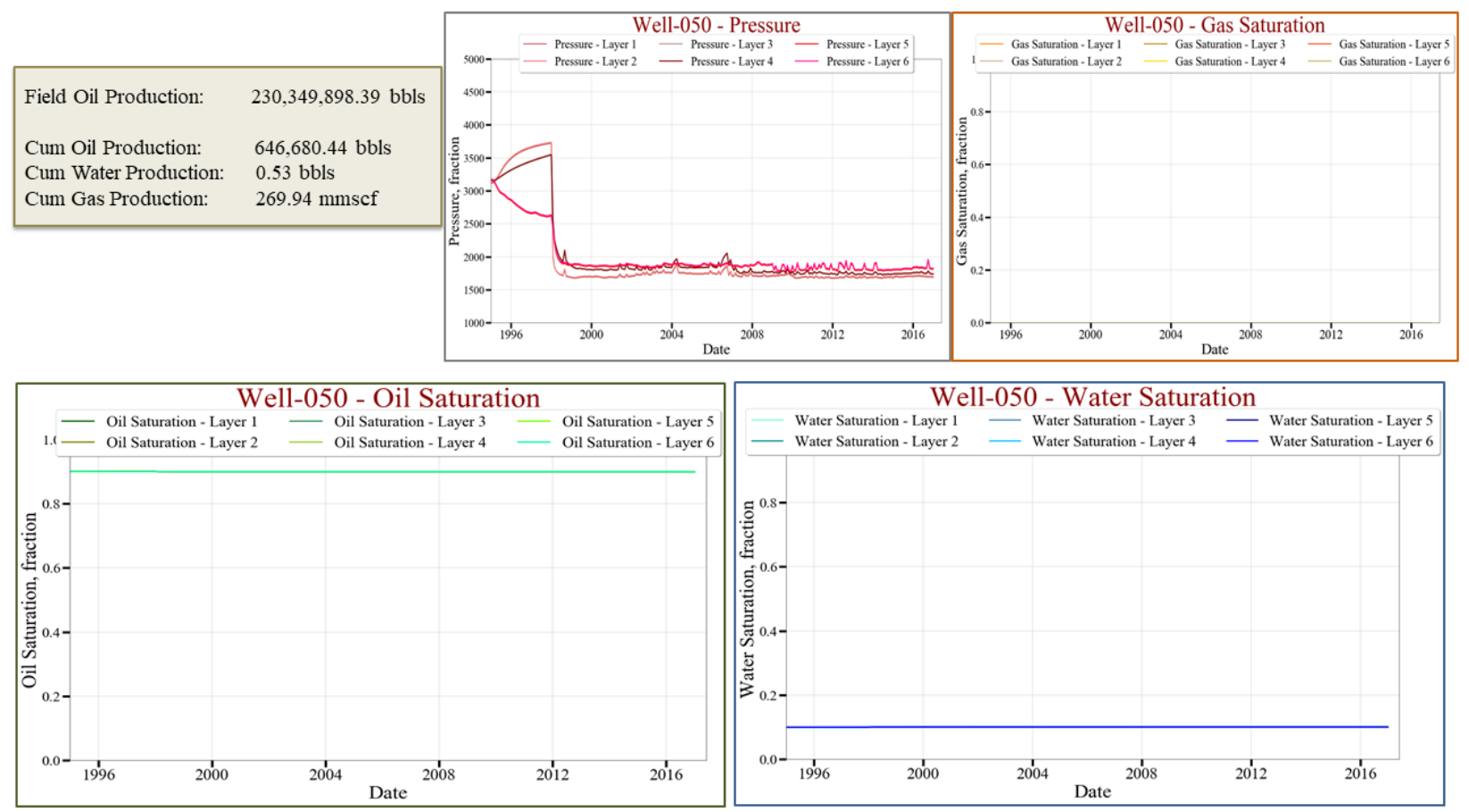

Figure 311 Well-050 saturations and pressure per layer

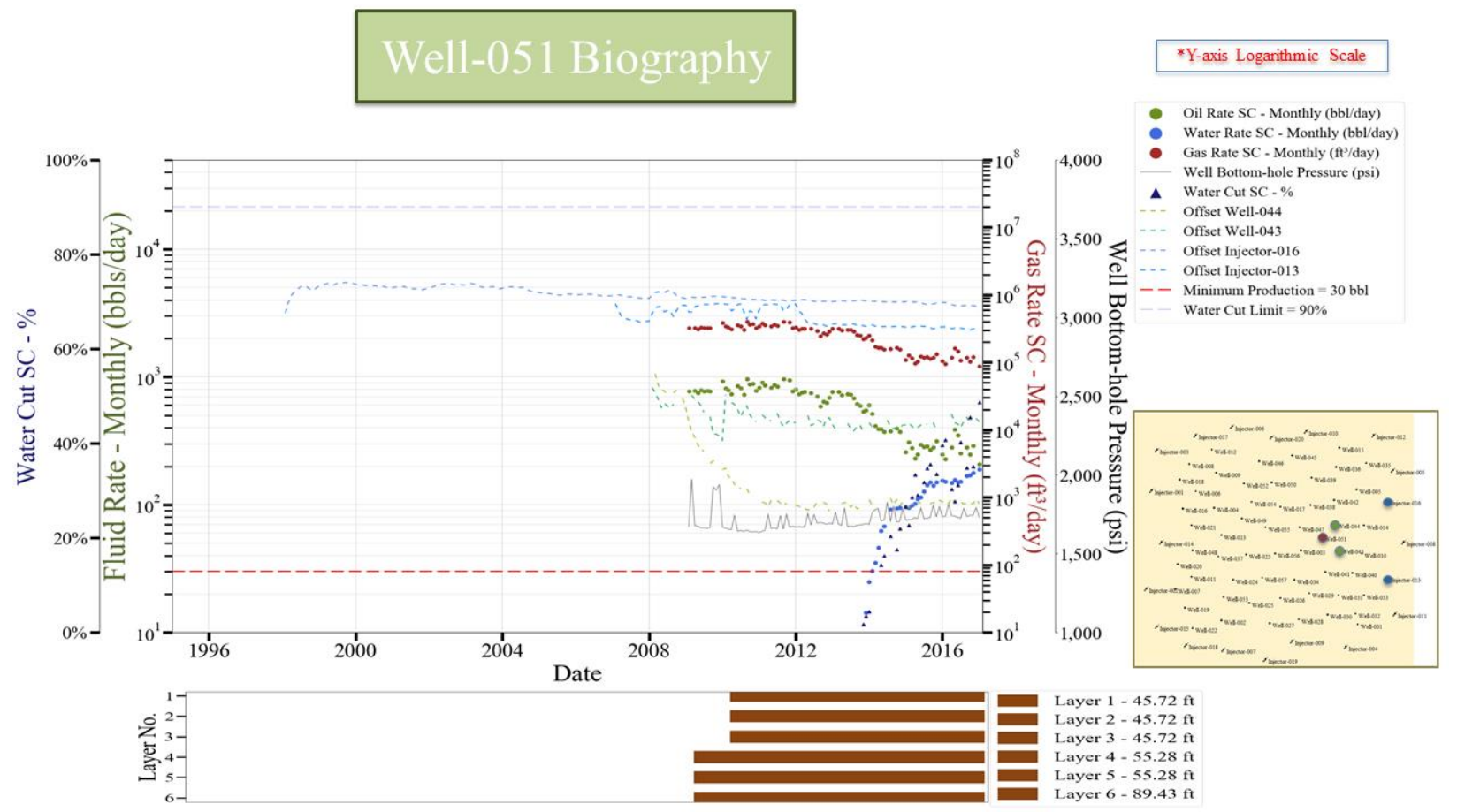

Figure 312 Well-051 biography 

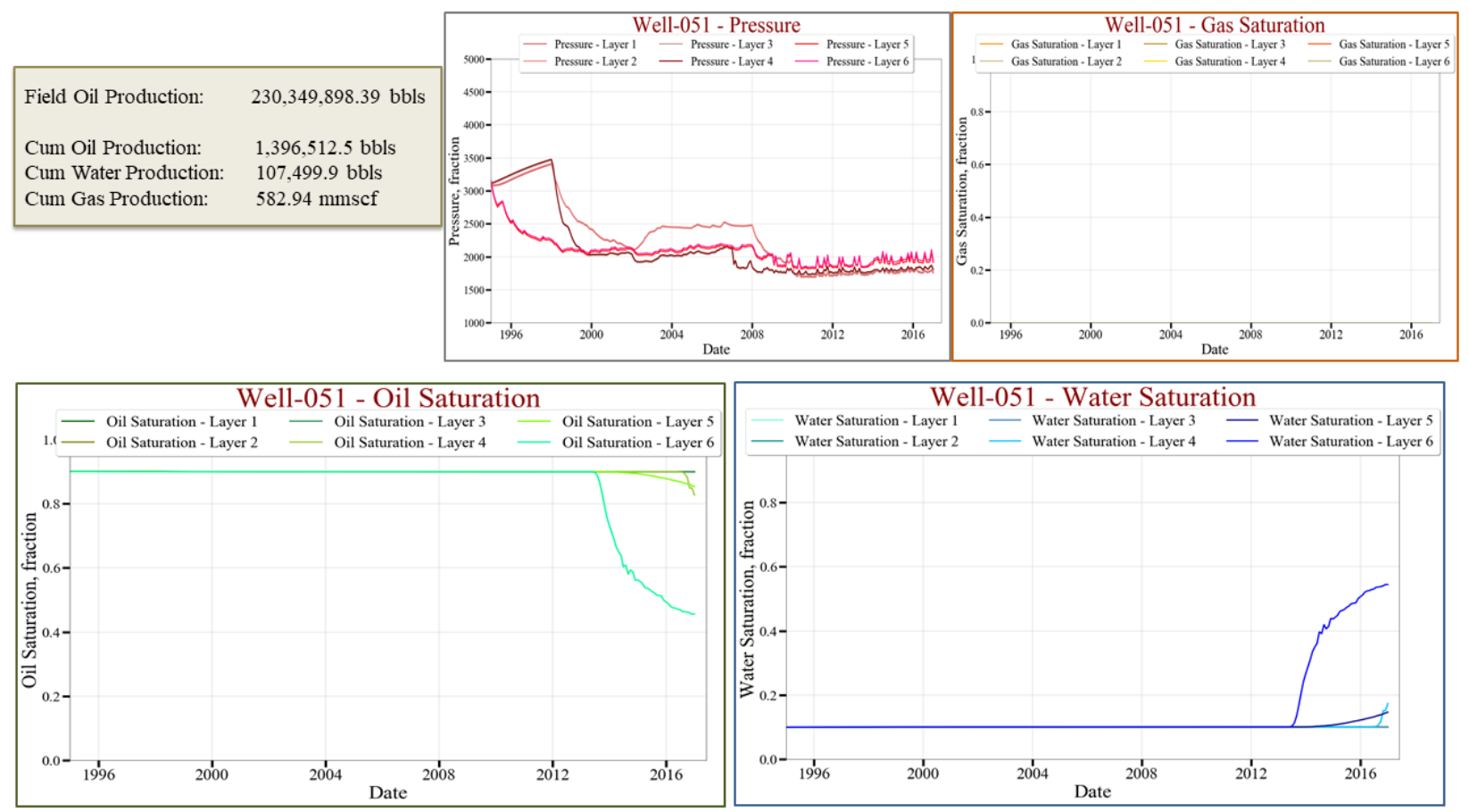

Figure 313 Well-051 saturations and pressure per layer

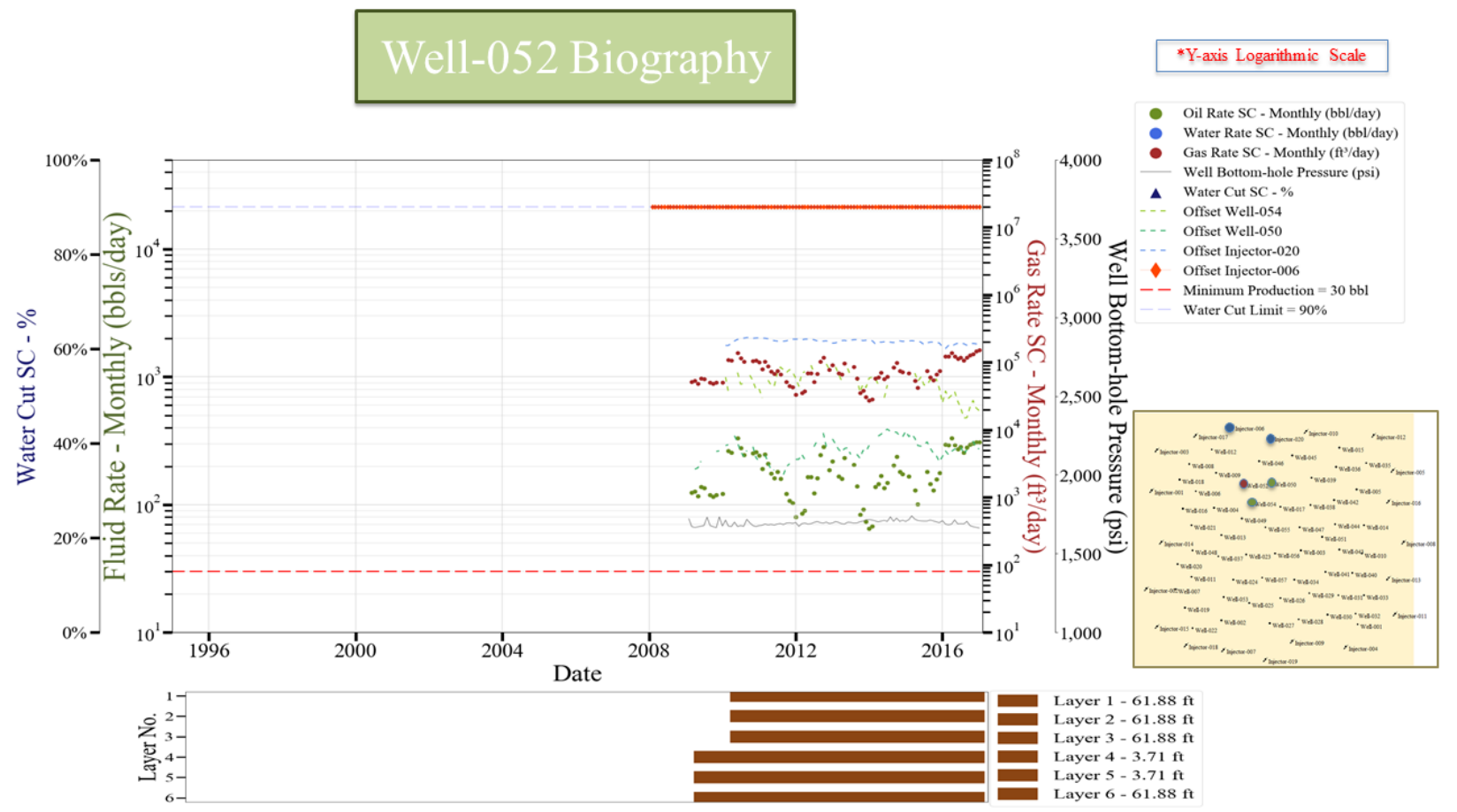

Figure 314 Well-052 biography 

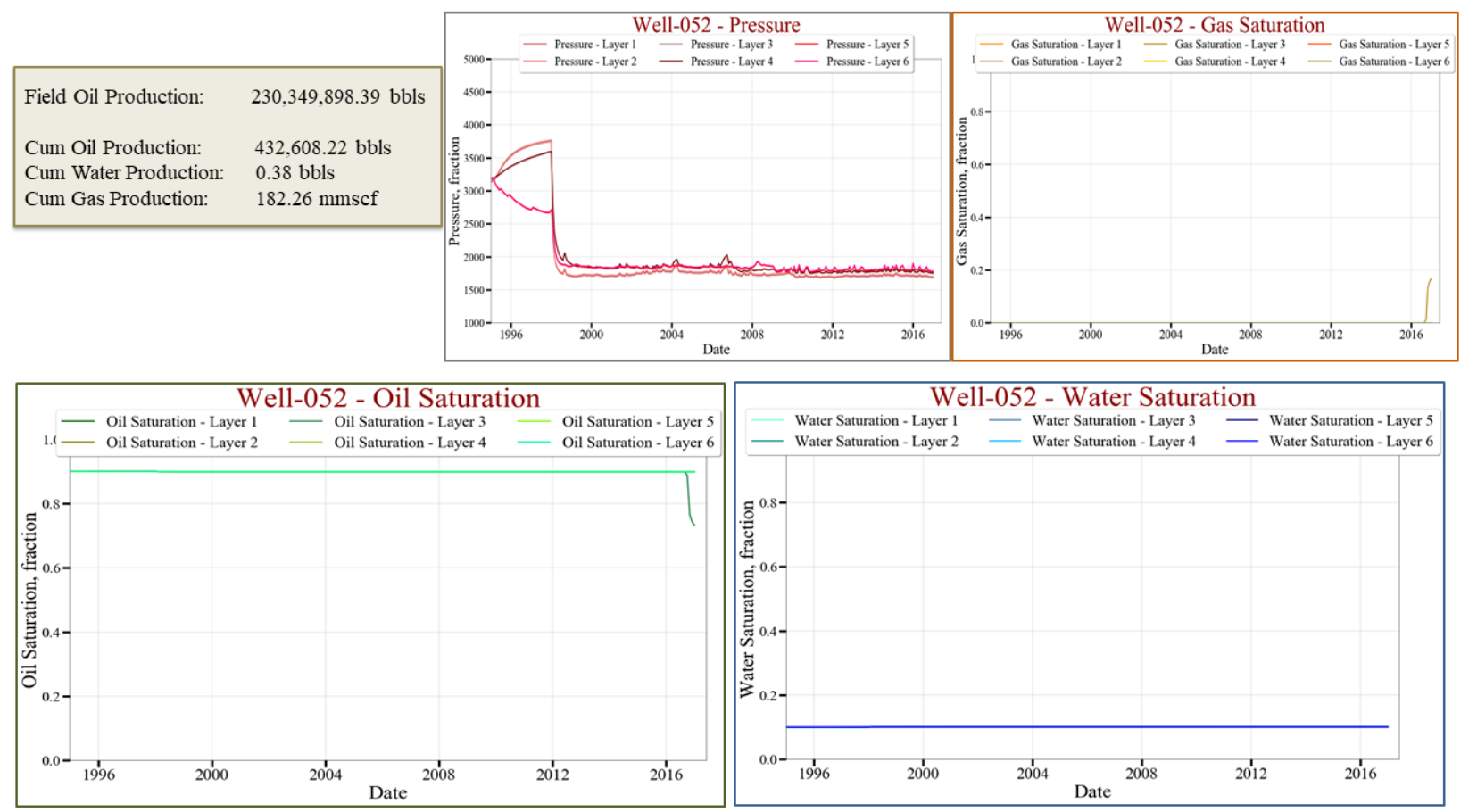

Figure 315 Well-052 saturations and pressure per layer

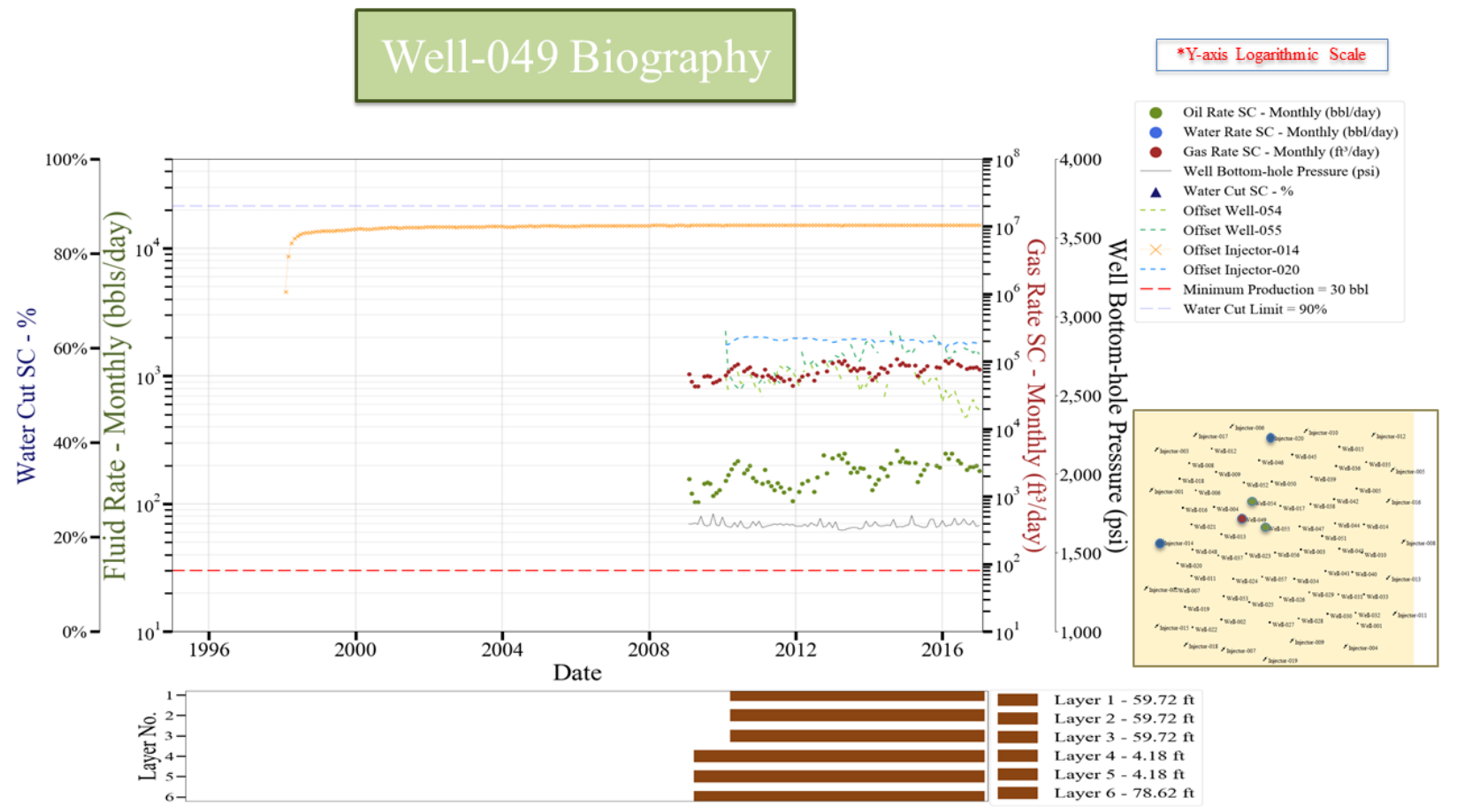

Figure 316 Well-049 biography 

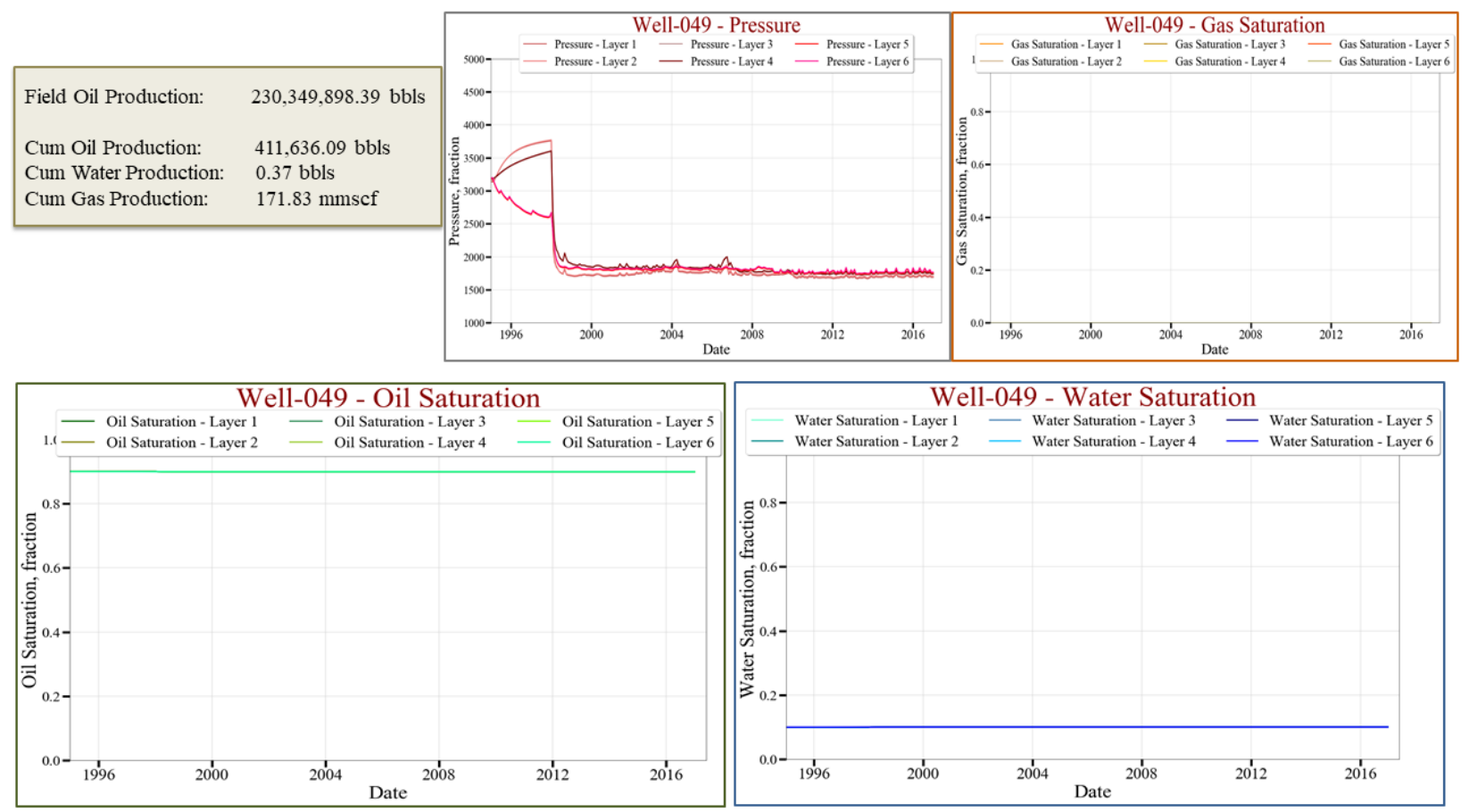

Figure 317 Well-049 saturations and pressure per layer

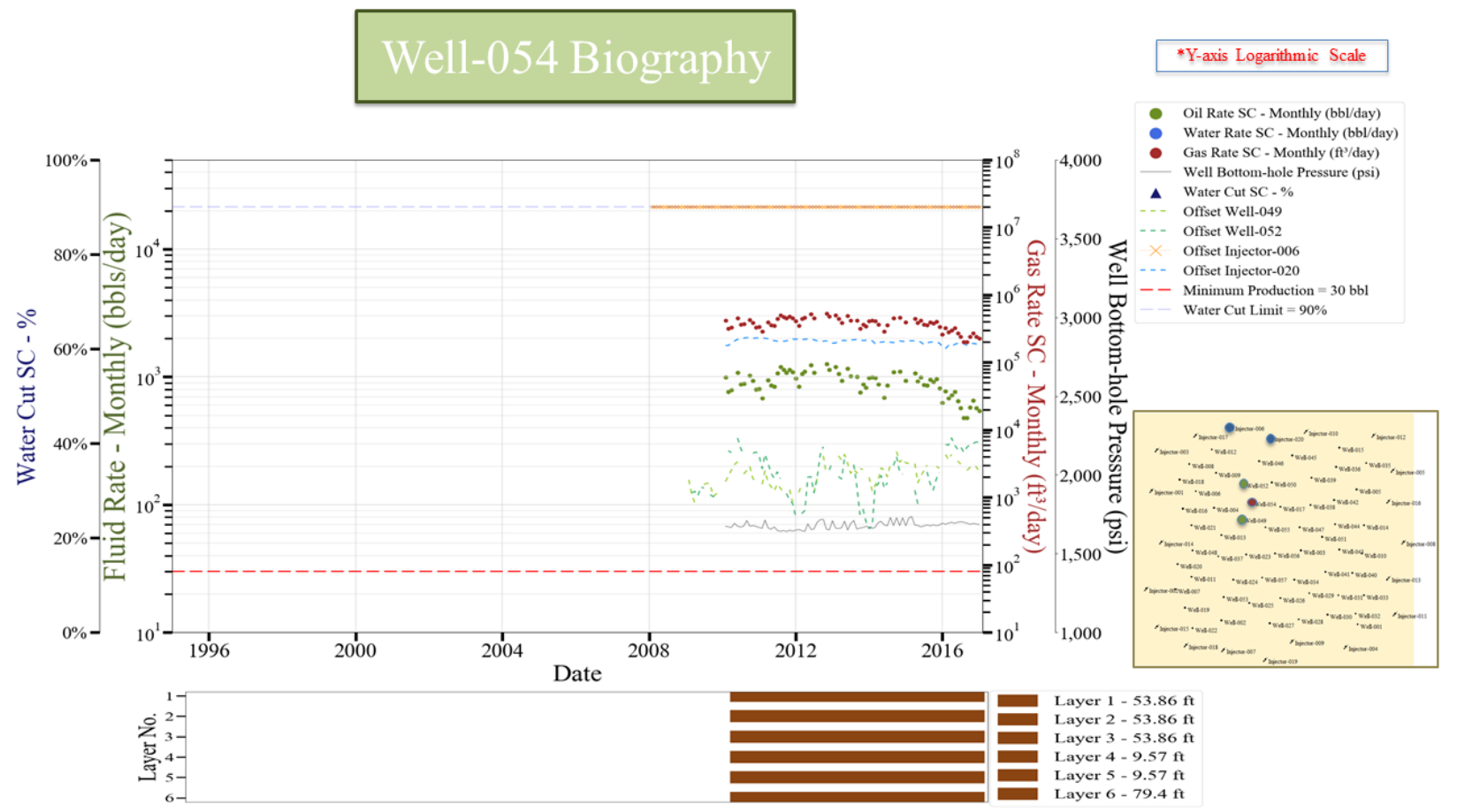

Figure 318 Well-054 biography 

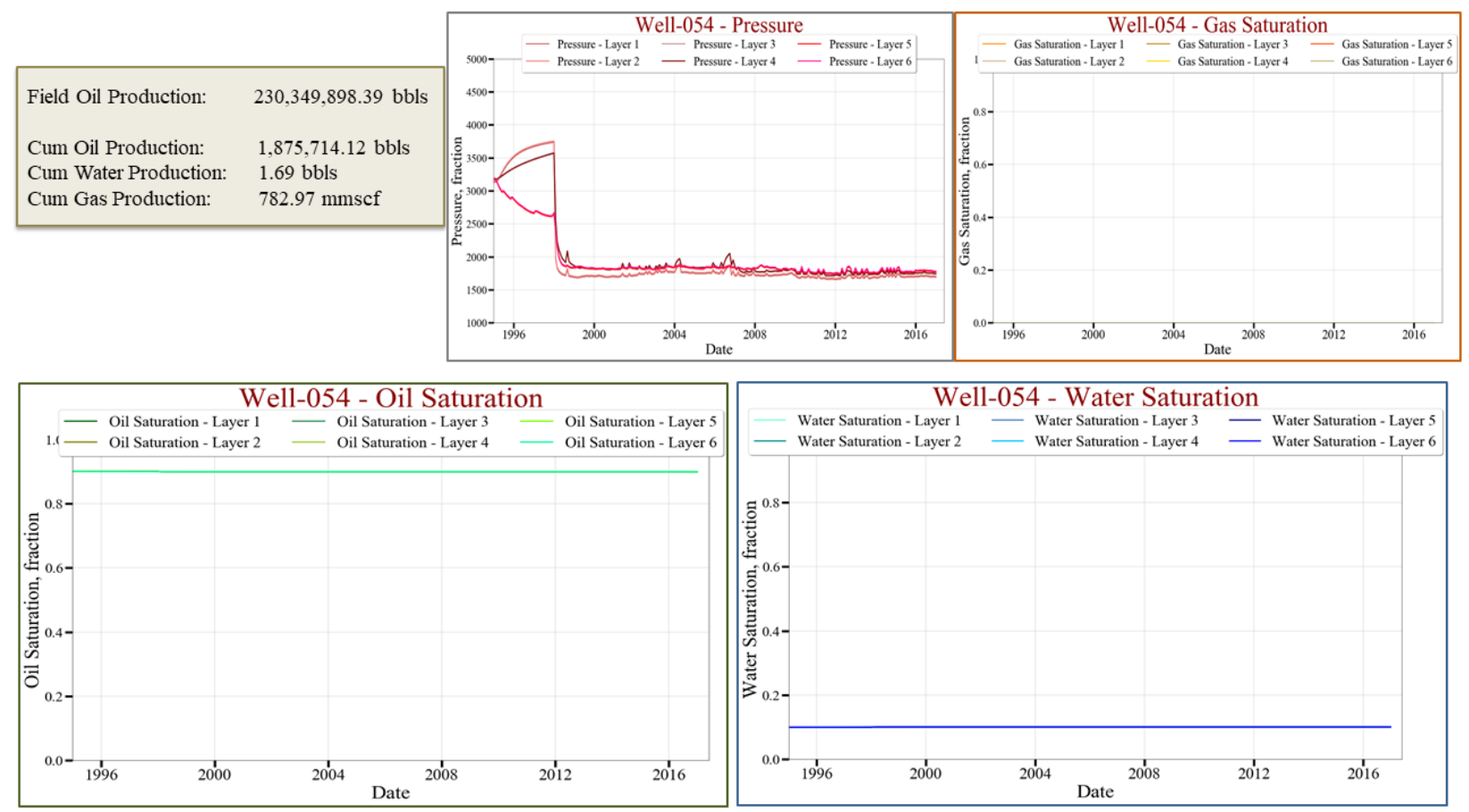

Figure 319 Well-054 saturations and pressure per layer

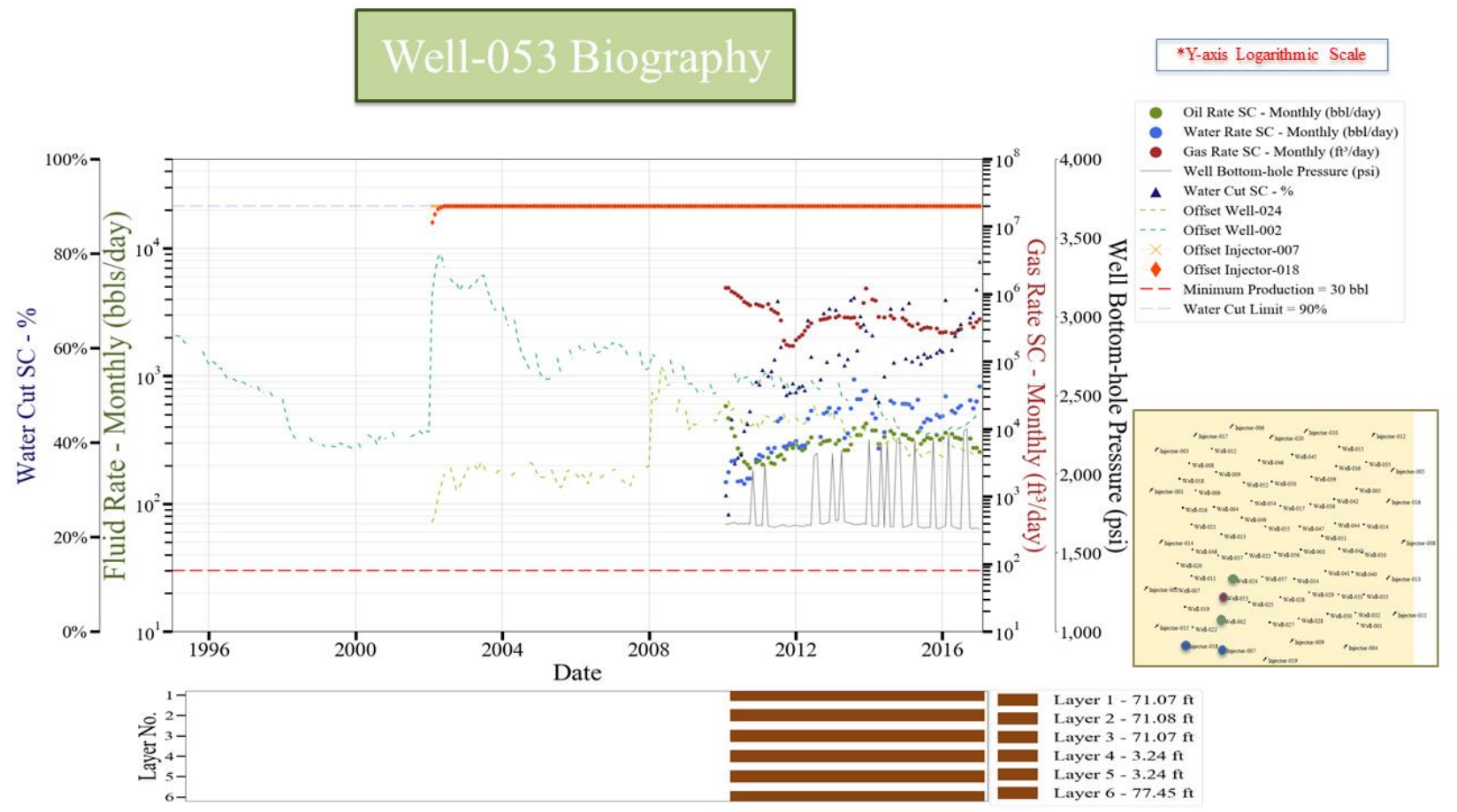

Figure 320 Well-053 biography 

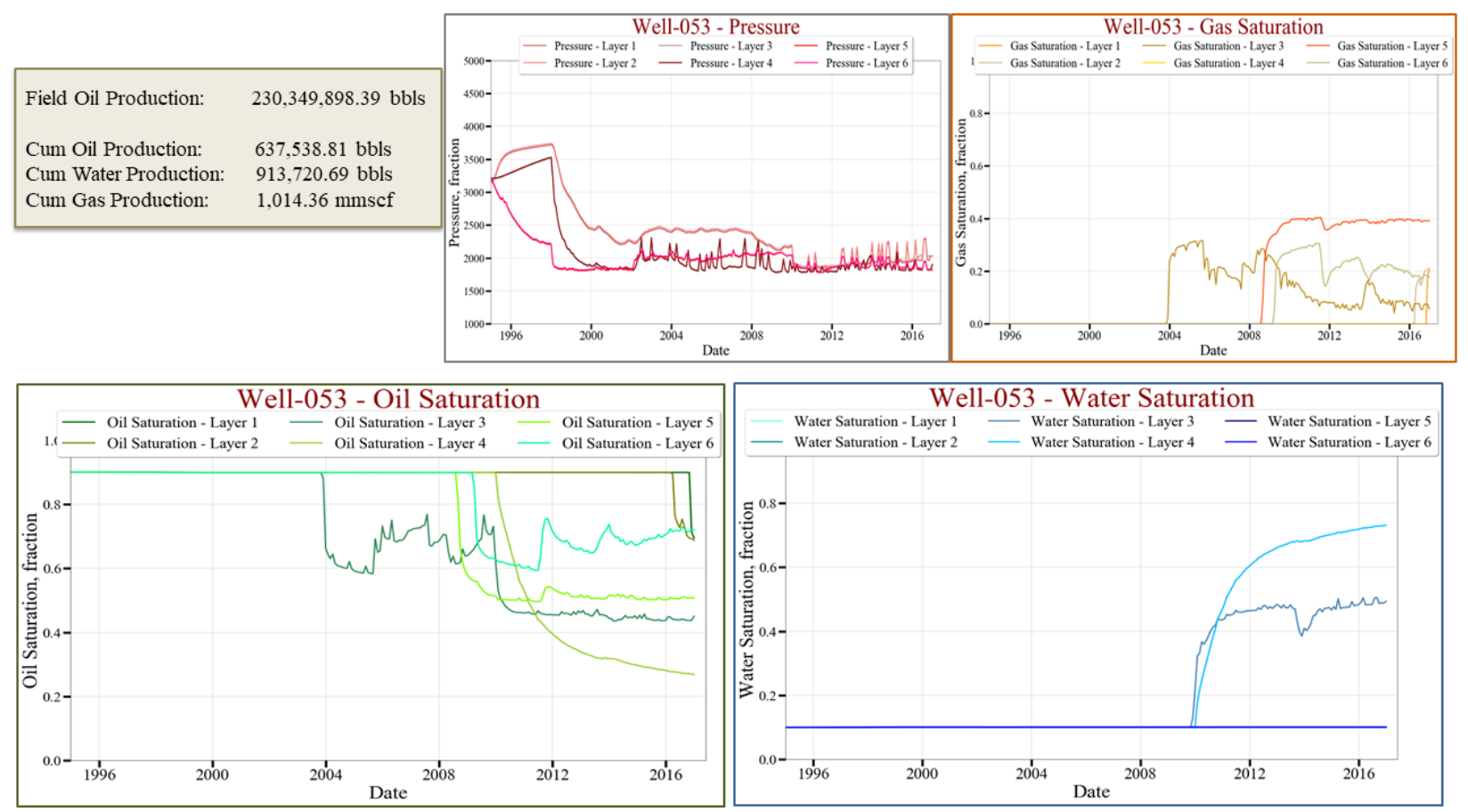

Figure 321 Well-053 saturations and pressure per layer

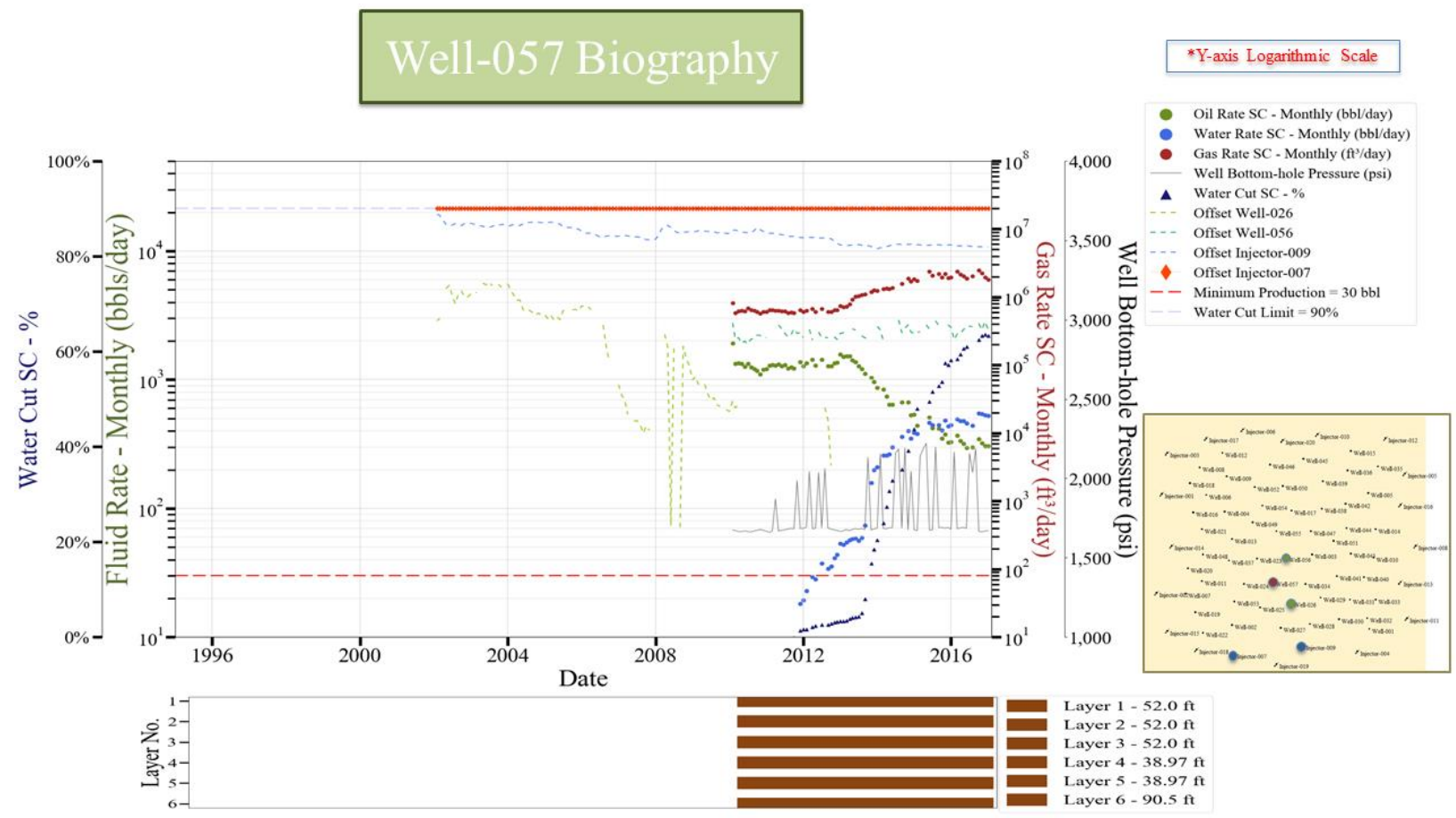

Figure 322 Well-057 biography 

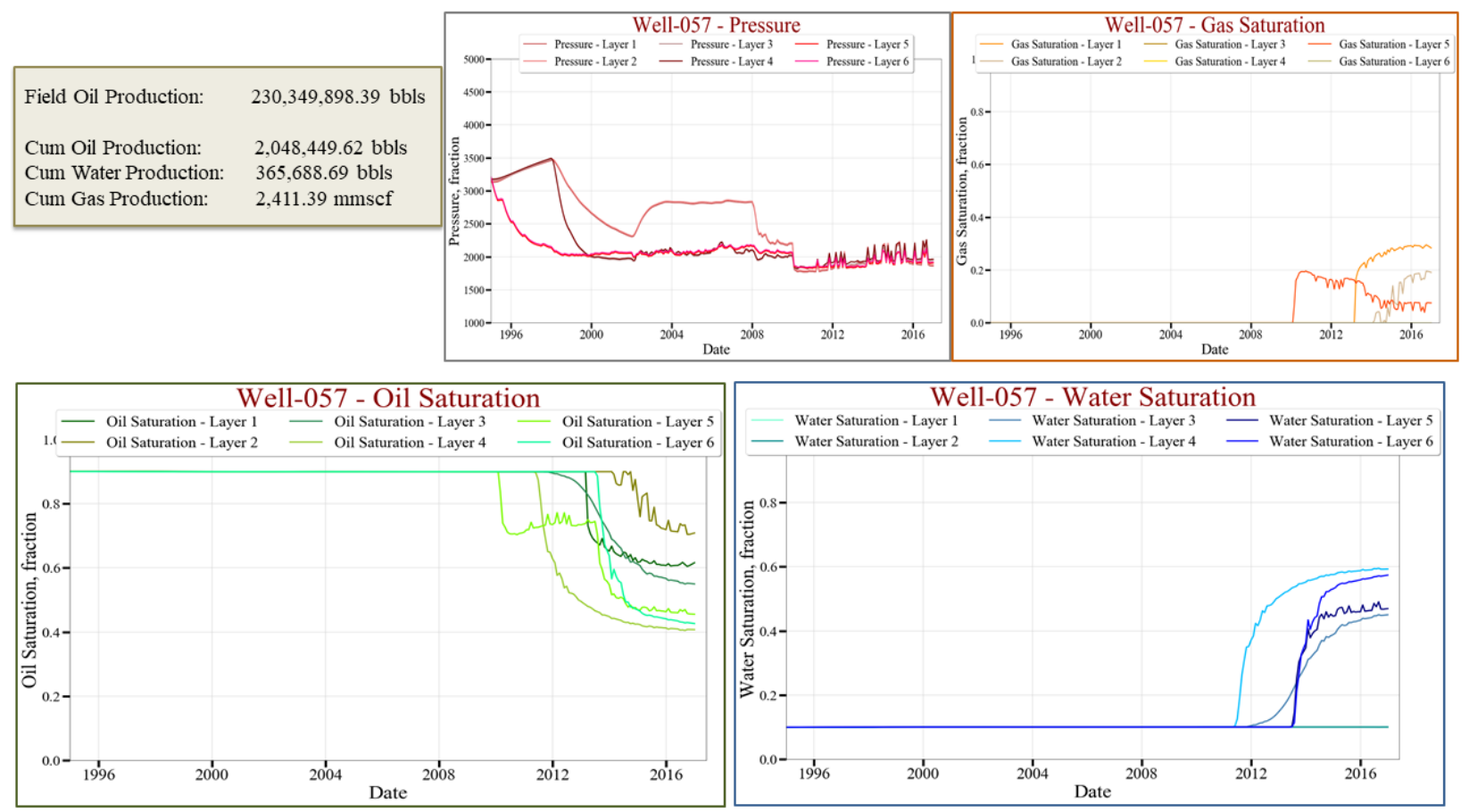

Figure 323 Well-057 saturations and pressure per layer

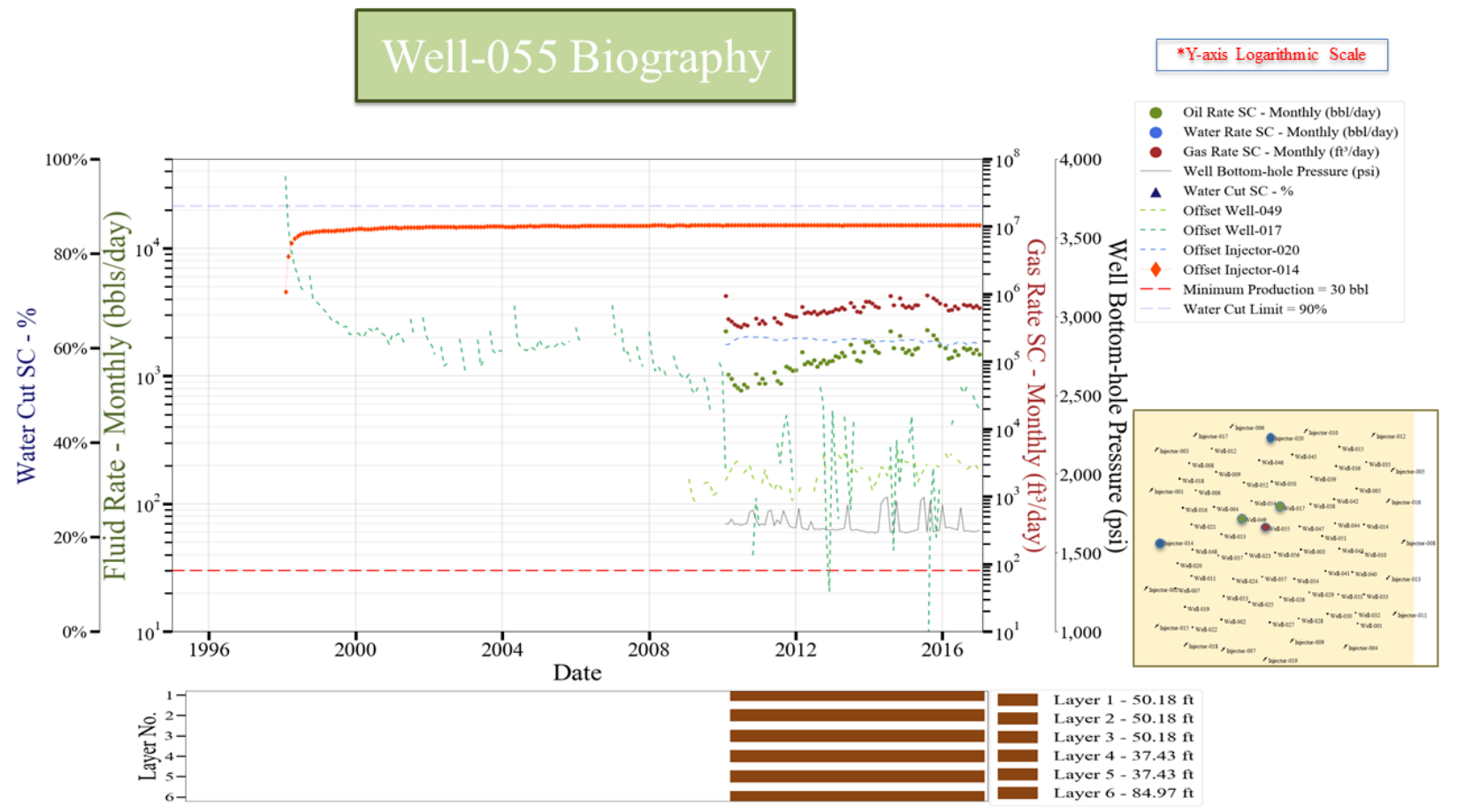

Figure 324 Well-055 biography 

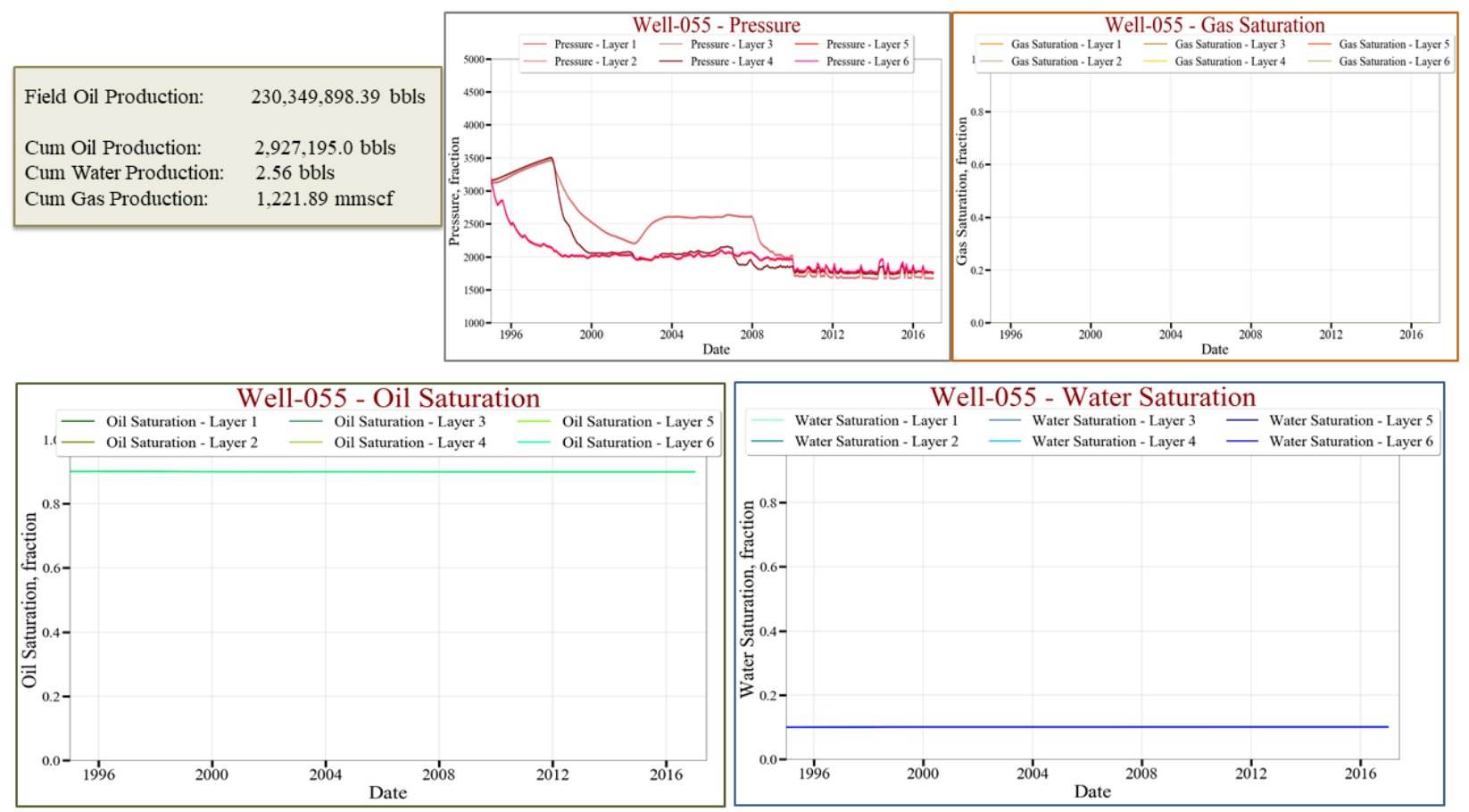

Figure 325 Well-055 saturations and pressure per layer

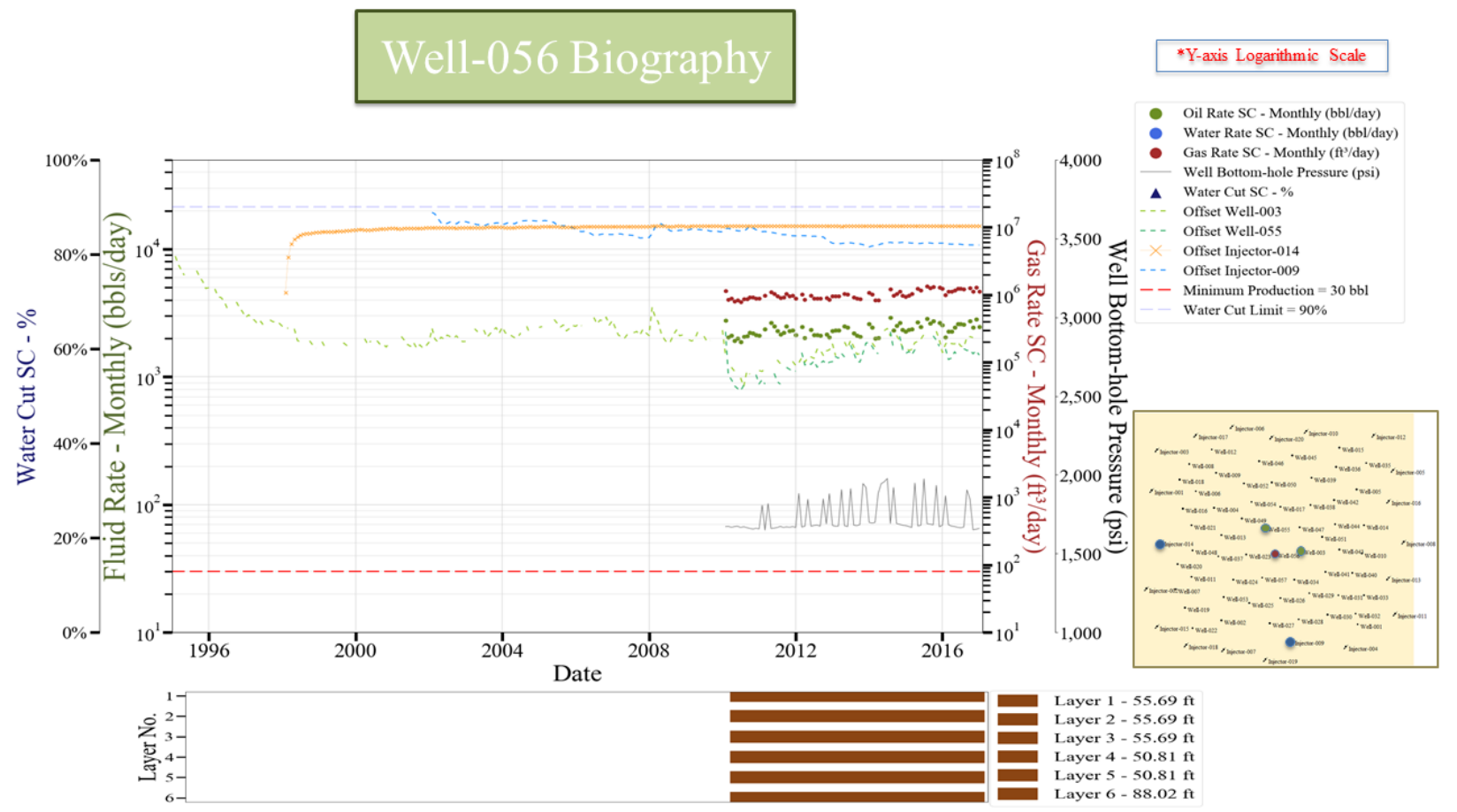

Figure 326 Well-056 biography 

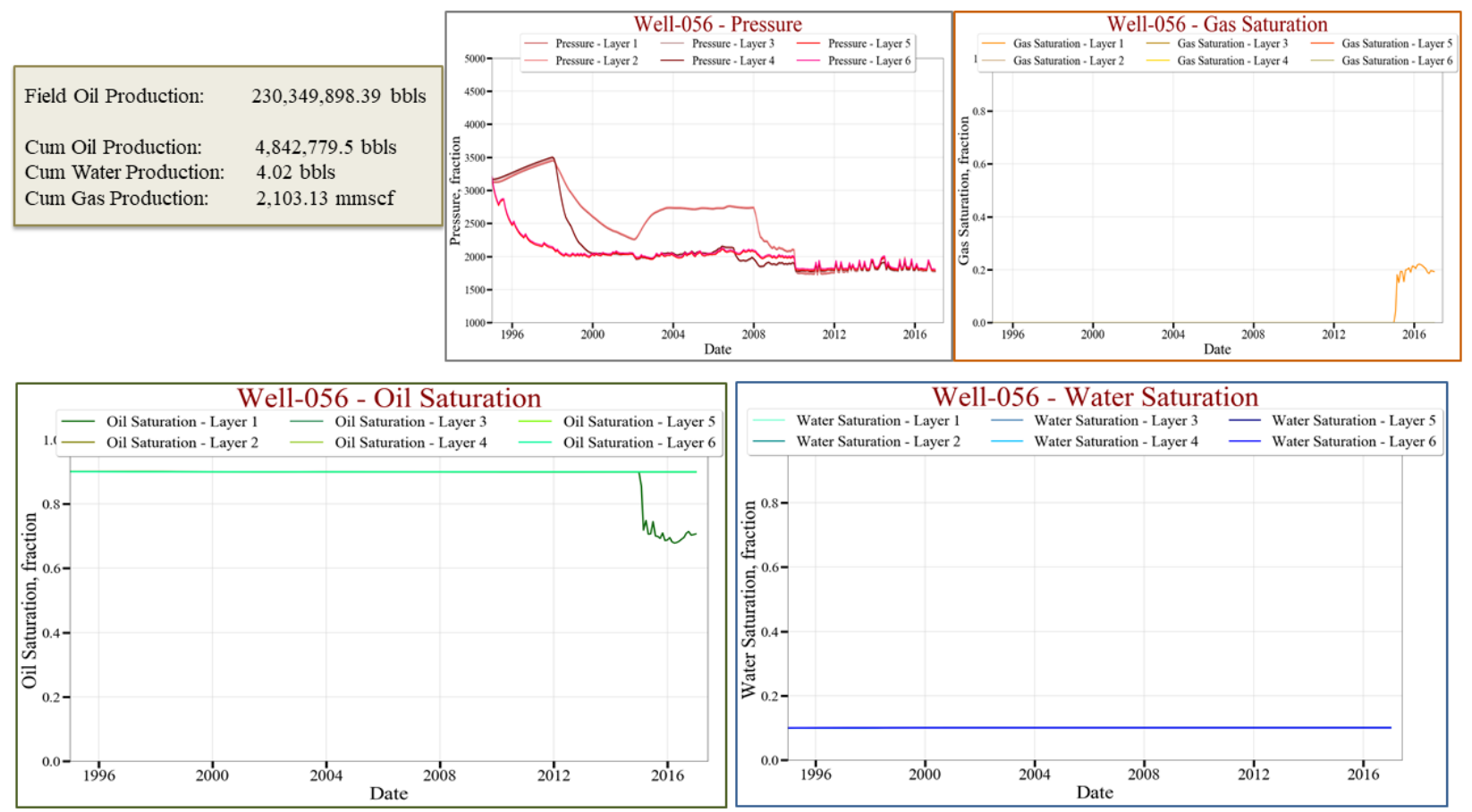

Figure 327 Well-056 saturations and pressure per layer 


\subsubsection{Wells Shut-in at End of Simulation}

\subsubsection{Largest Number of Production Days}

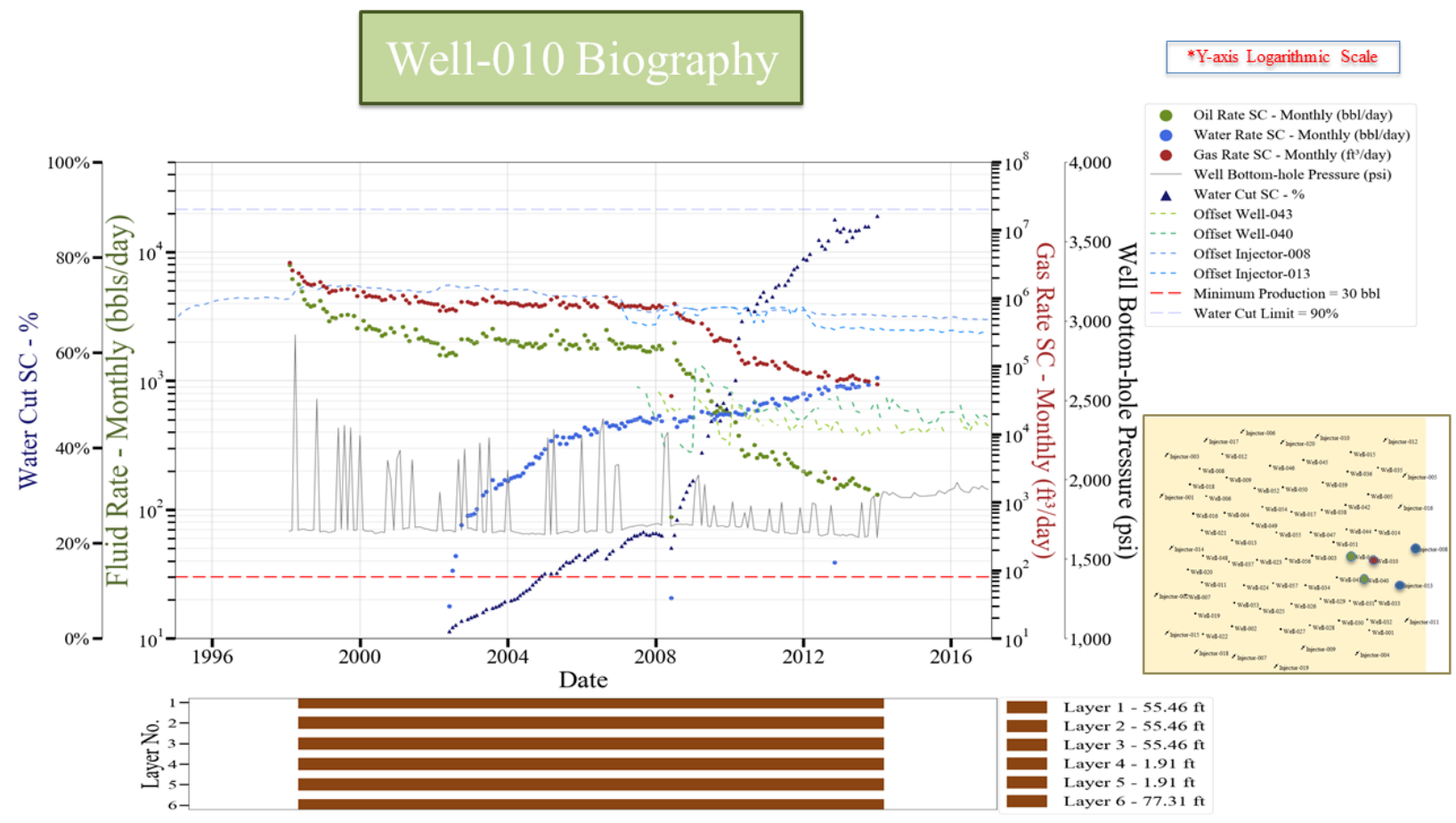

Figure 328 Well-010 biography
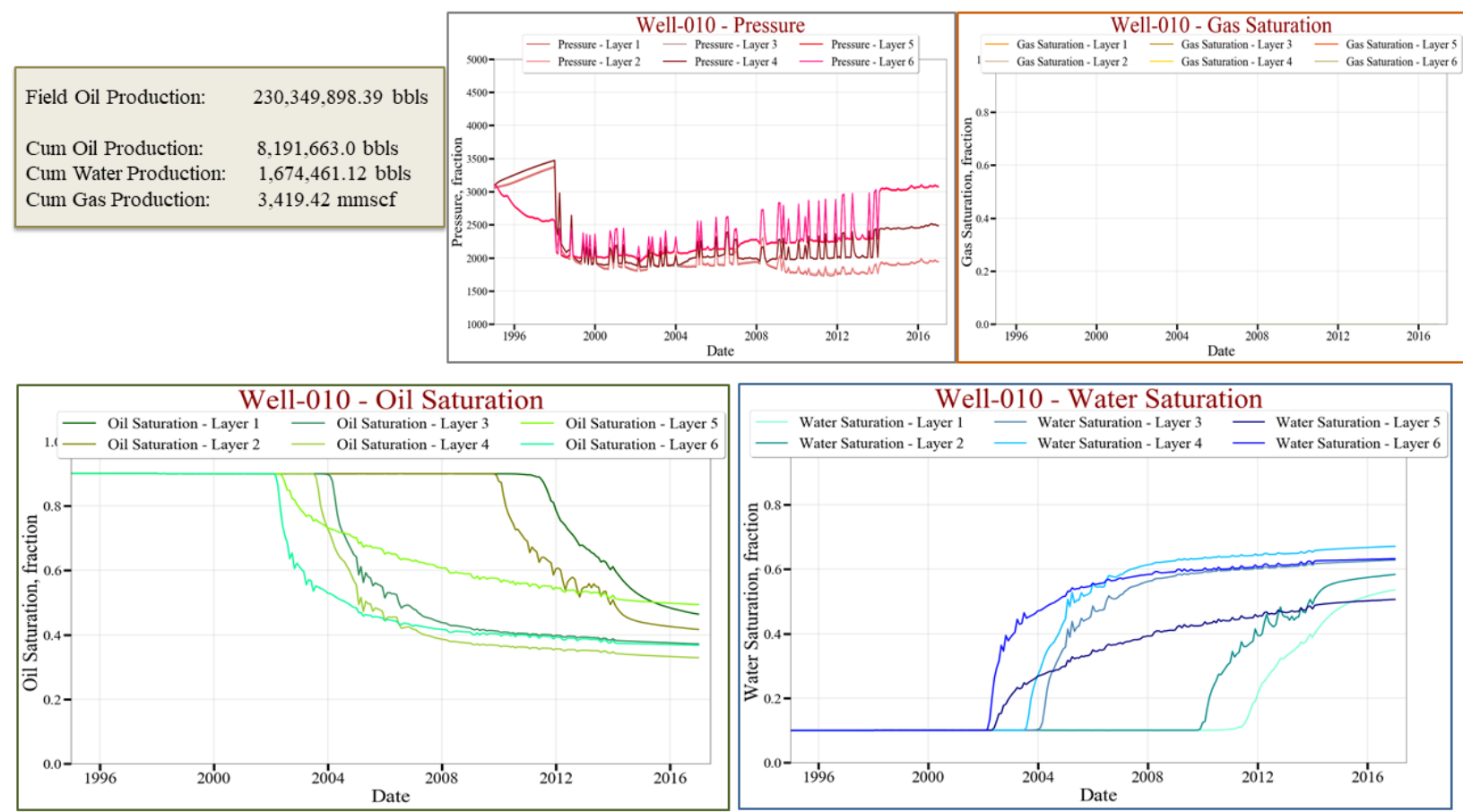

Figure 329 Well-010 saturations and pressure per layer 


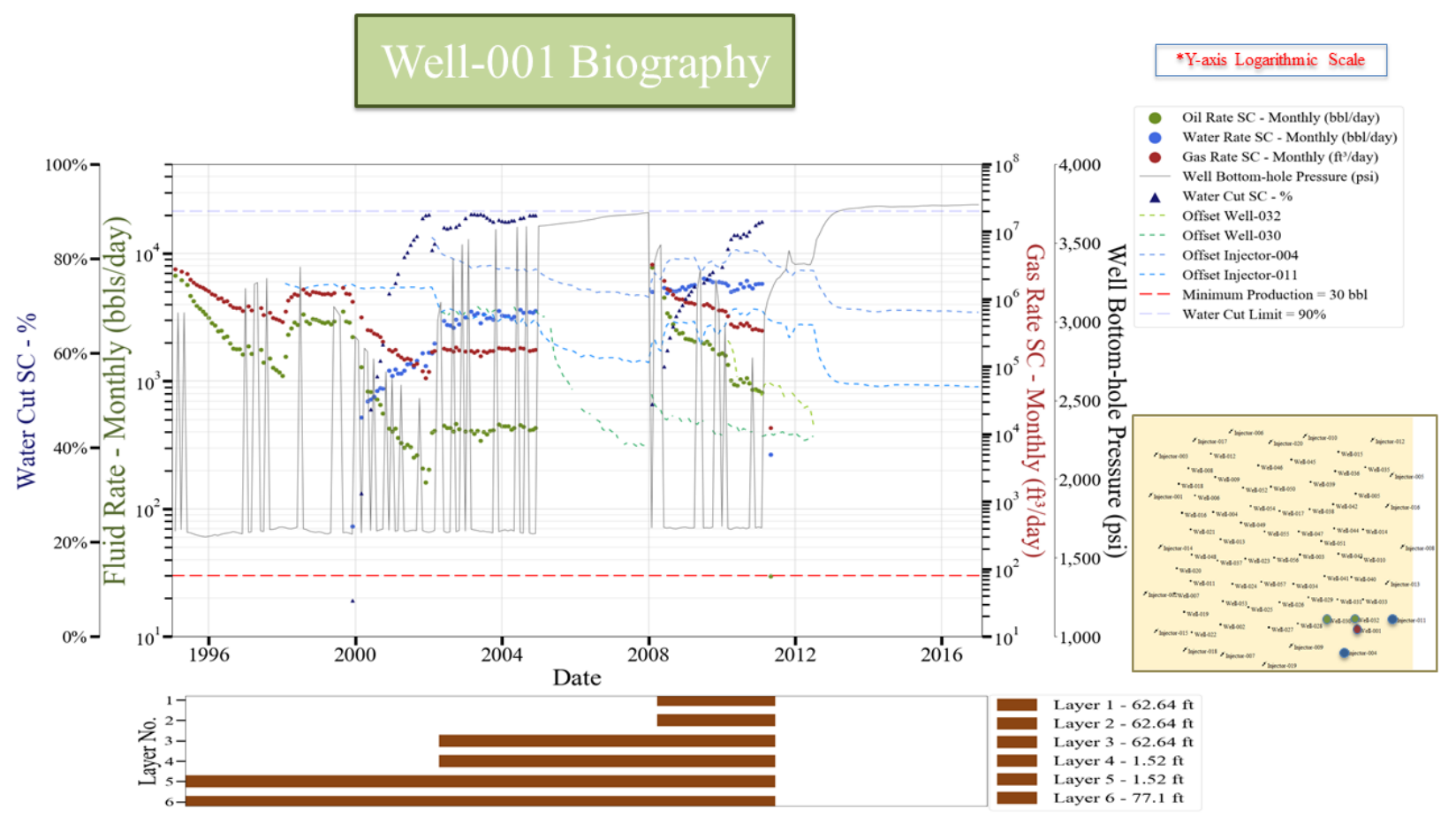

Figure 330 Well-001 biography

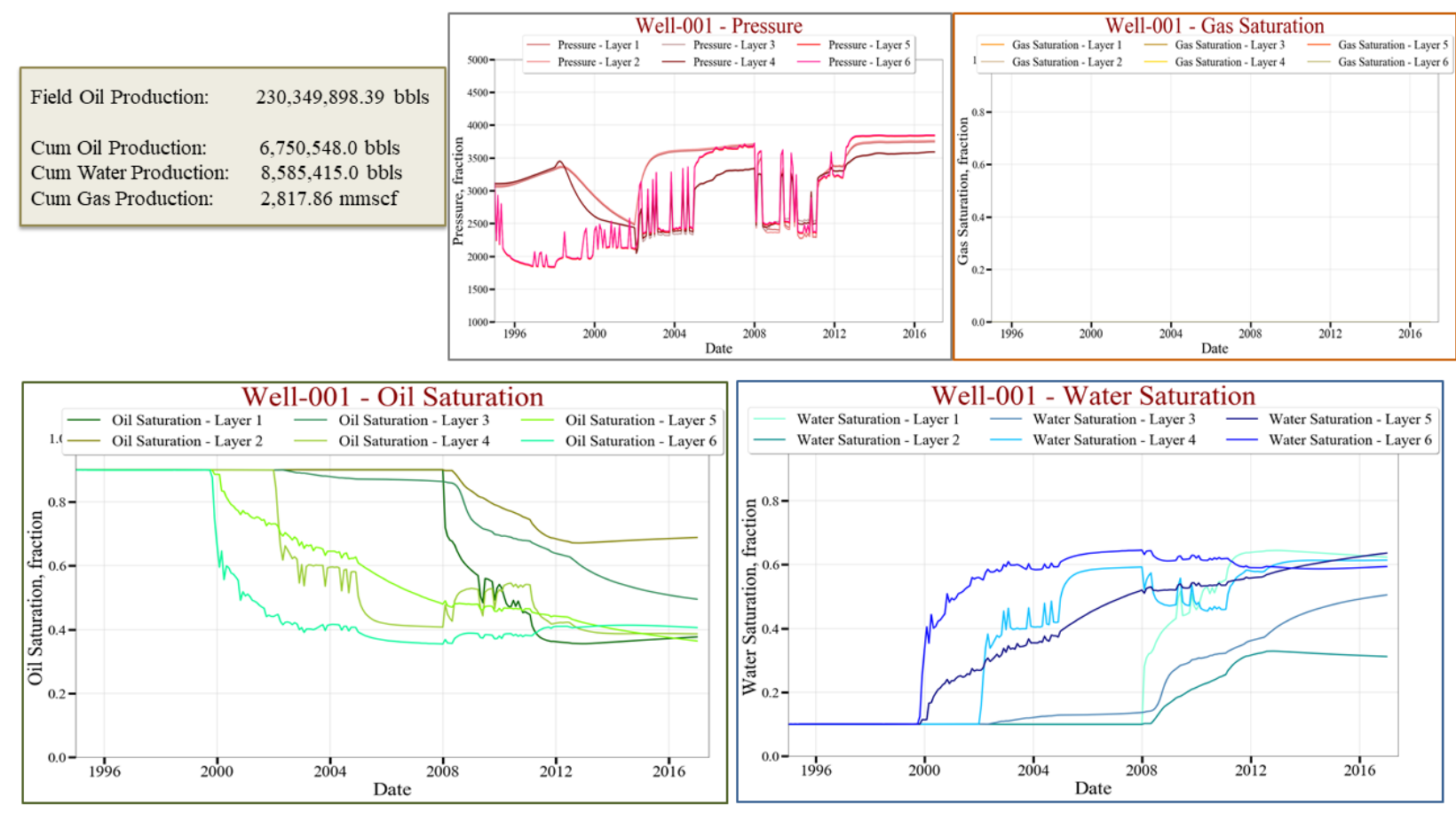

Figure 331 Well-001 saturations and pressure per layer 


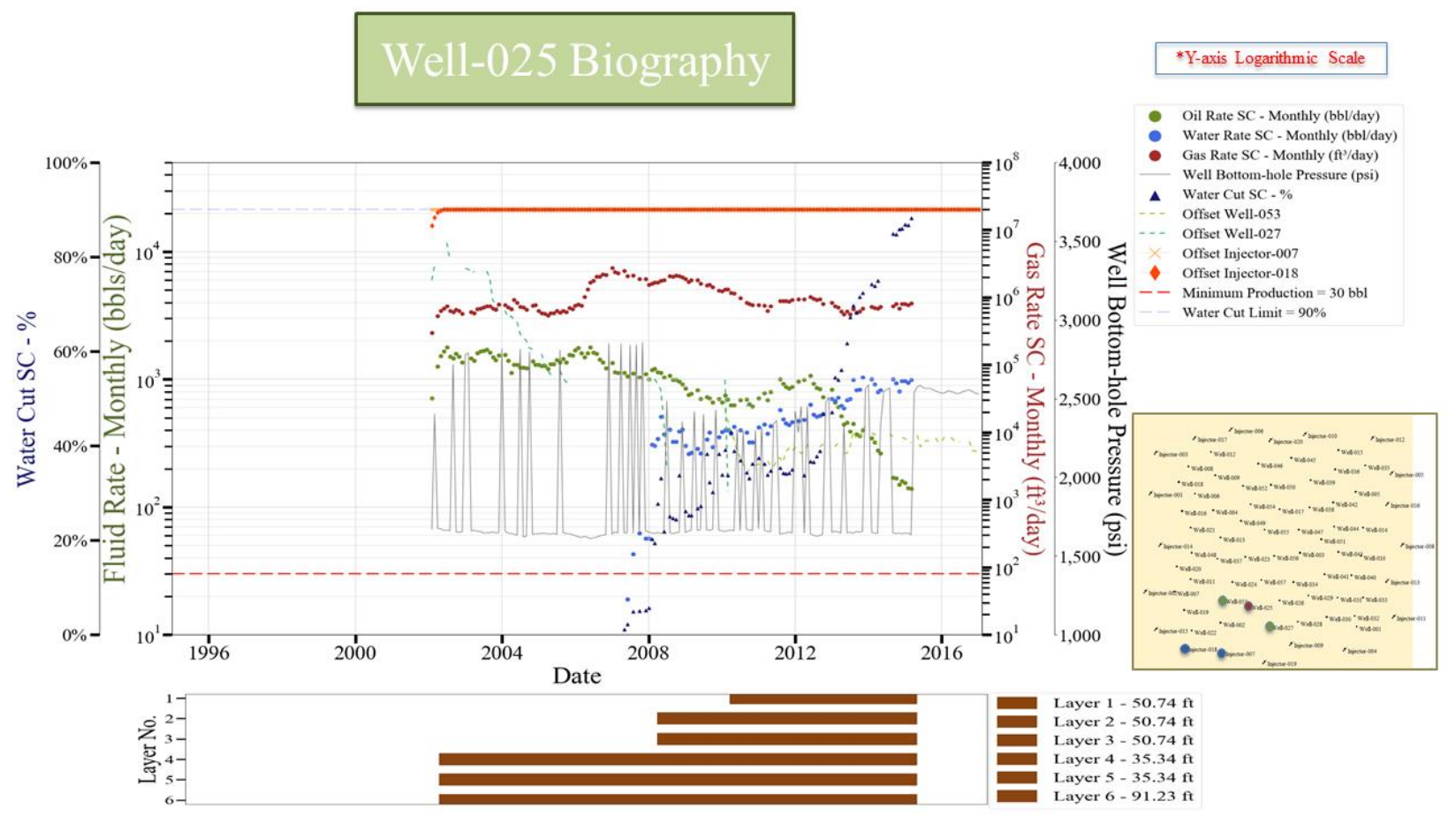

Figure 332 Well-025 biography
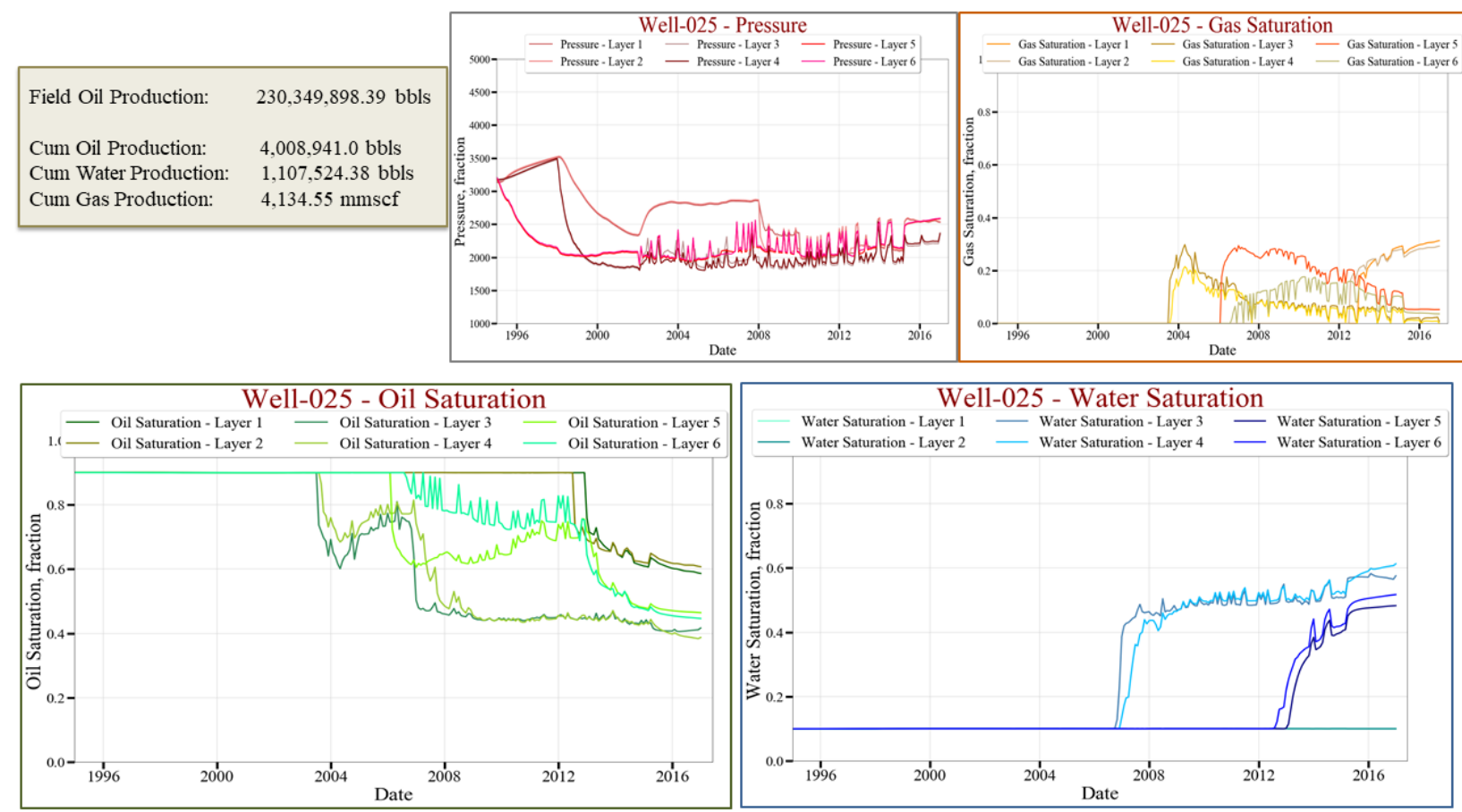

Figure 333 Well-025 saturations and pressure per layer 


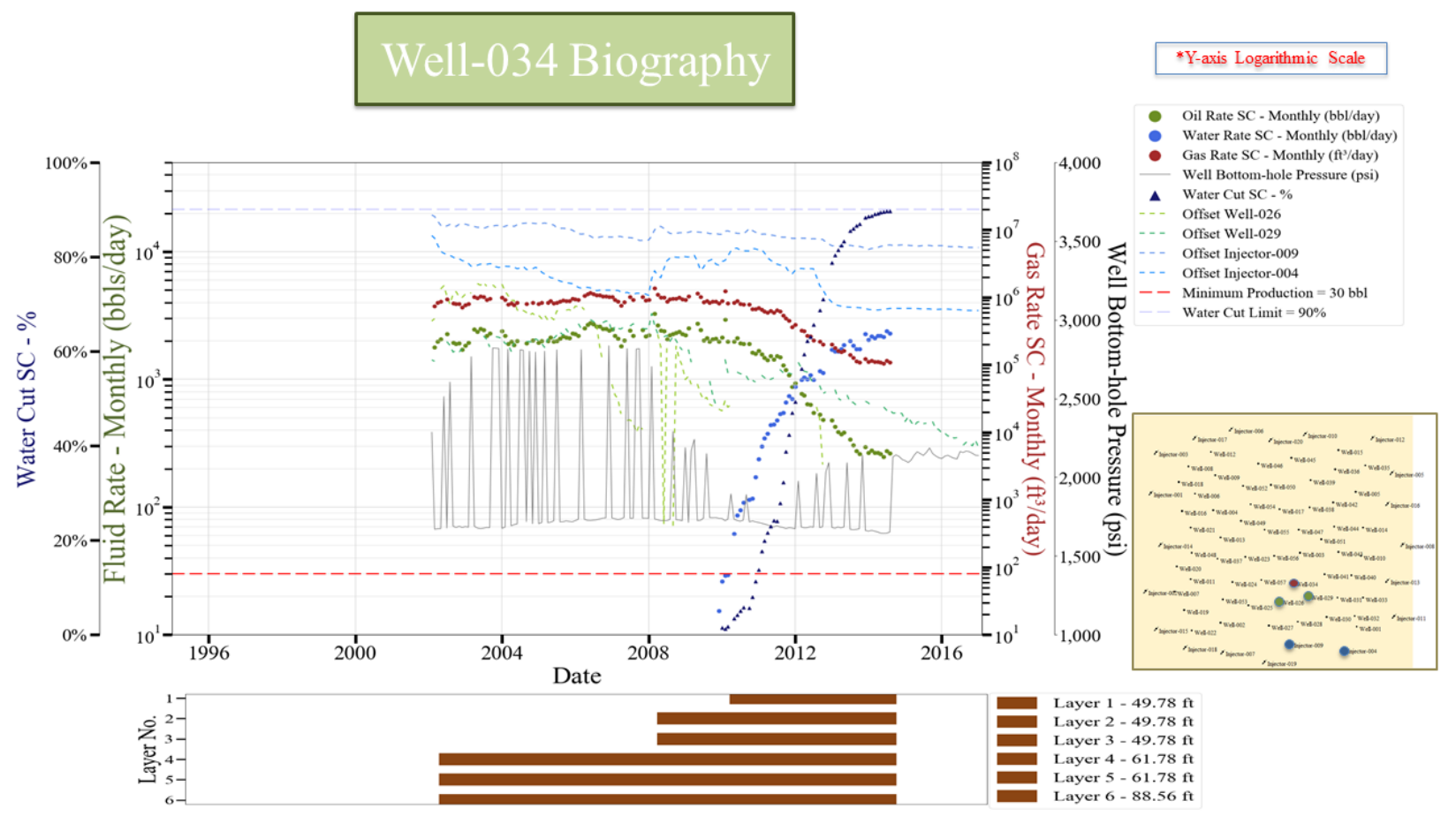

Figure 334 Well-034 biography
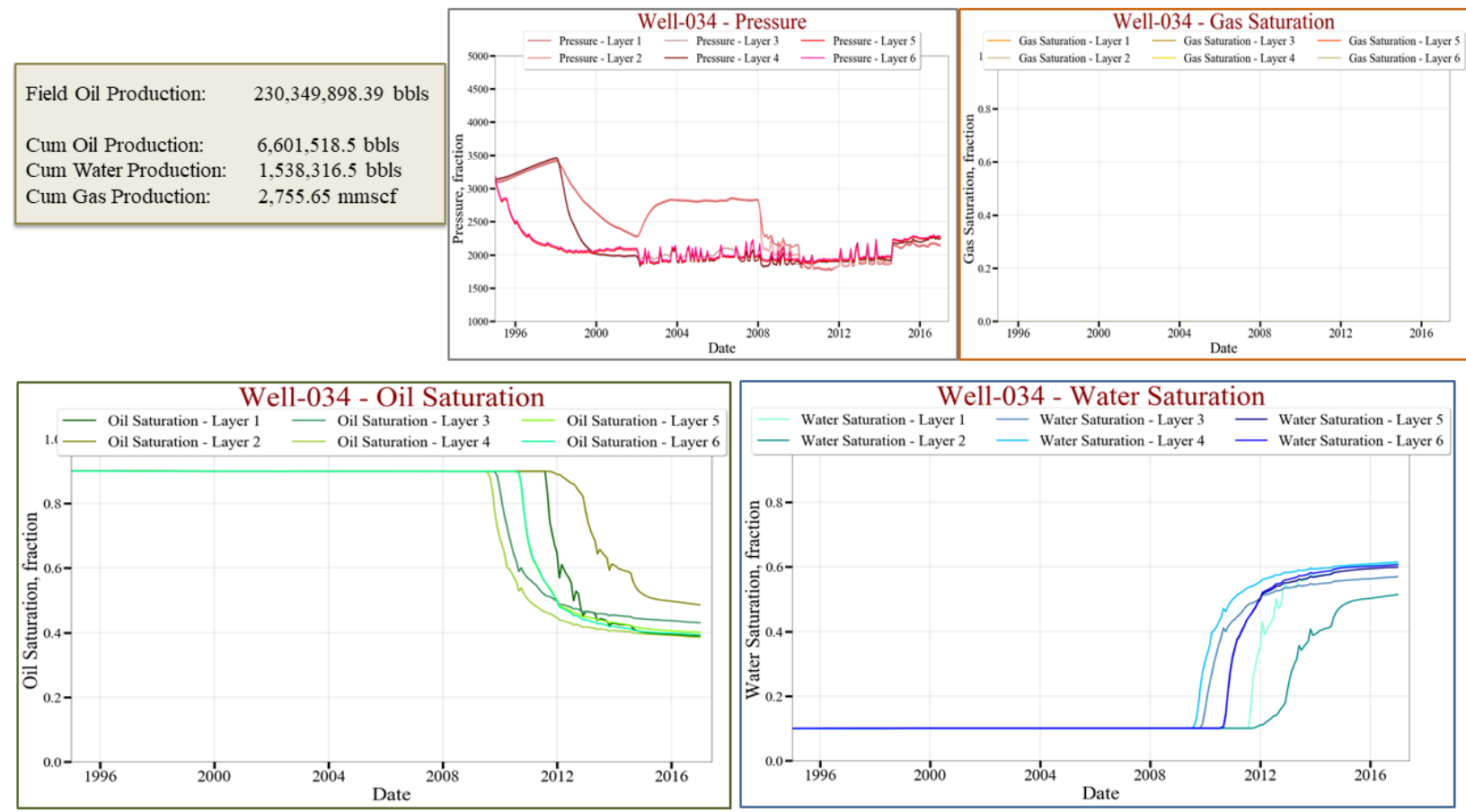

Figure 335 Well-034 saturations and pressure per layer 


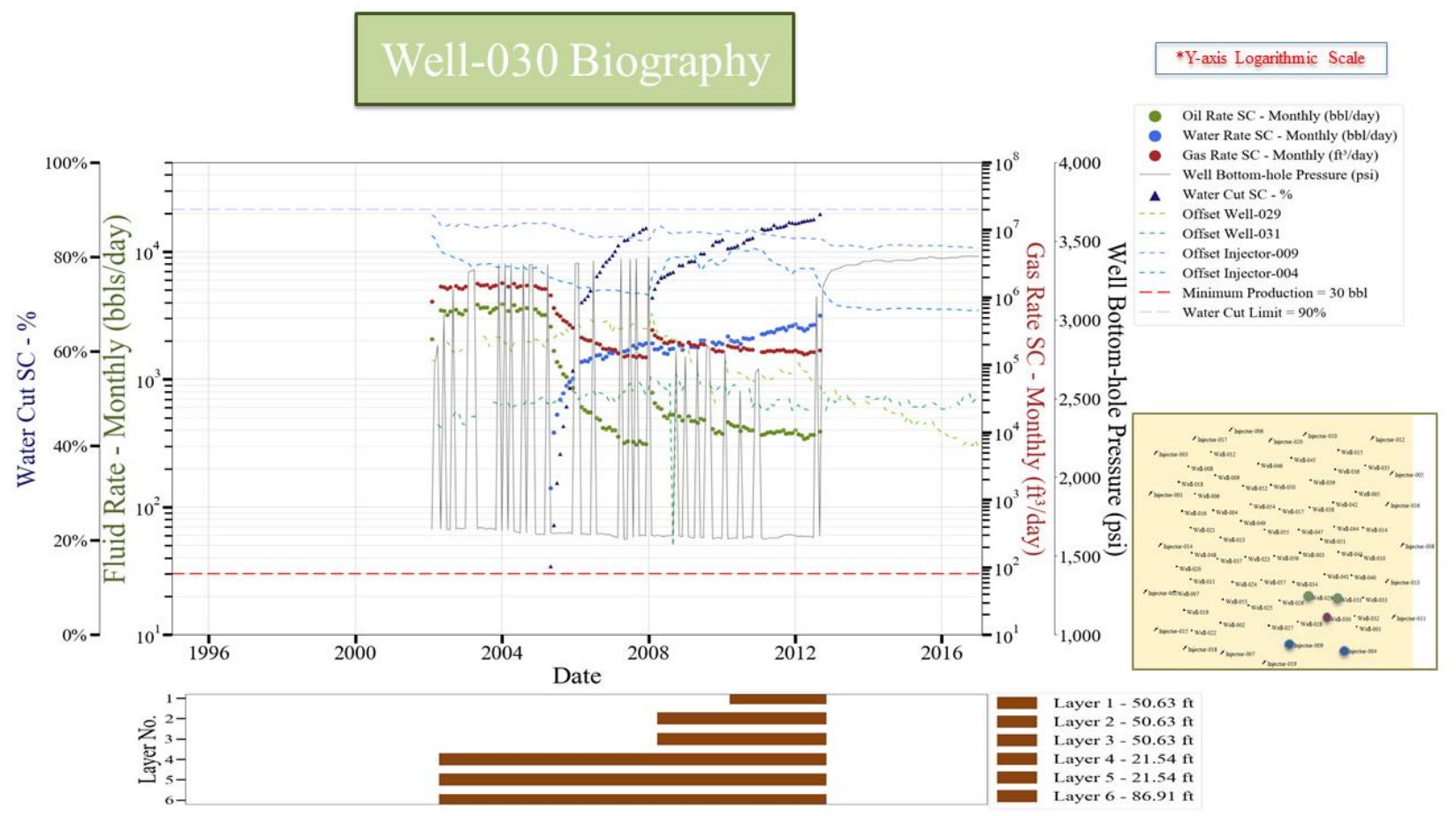

Figure 336 Well-030 biography
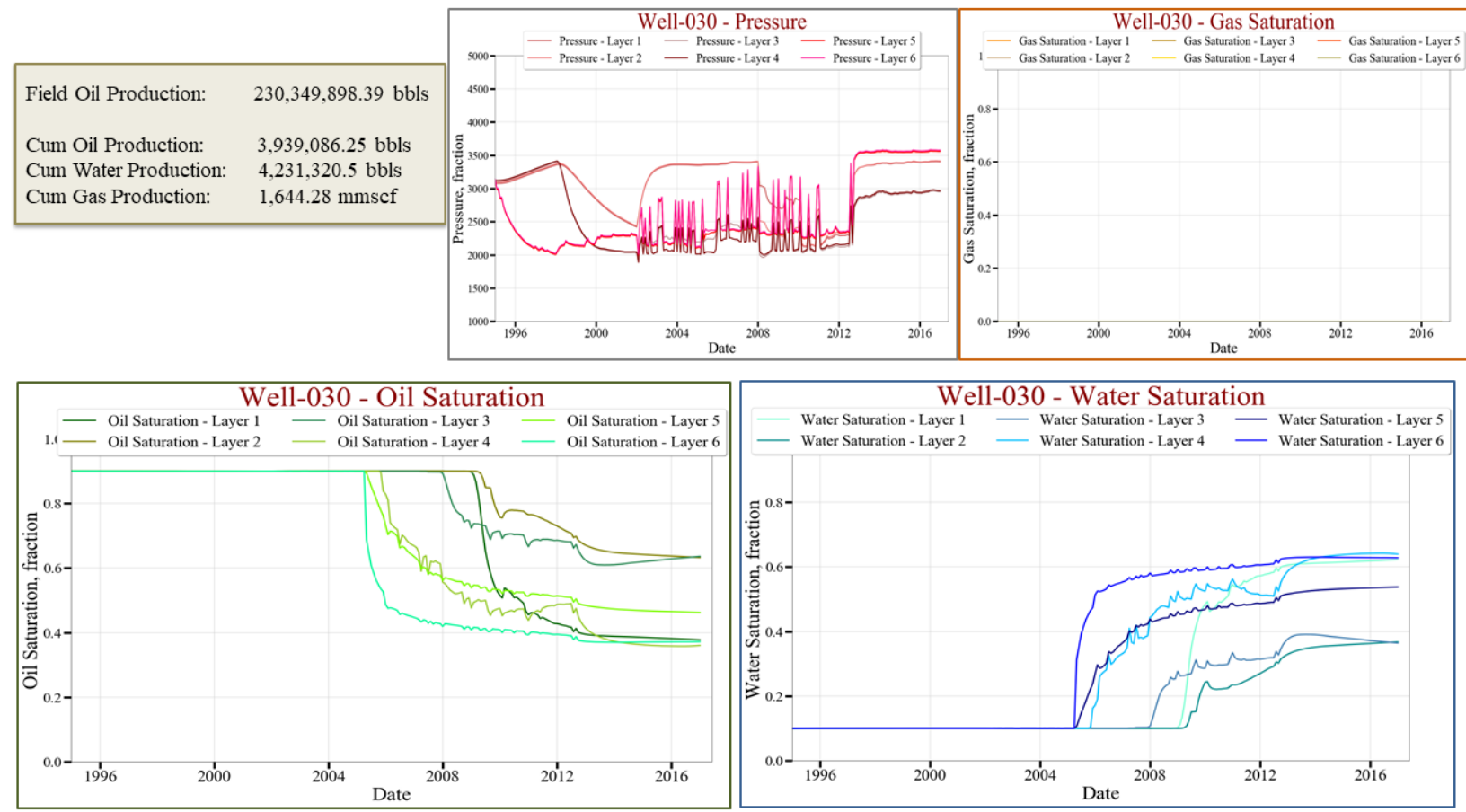

Figure 337 Well-030 saturations and pressure per layer 


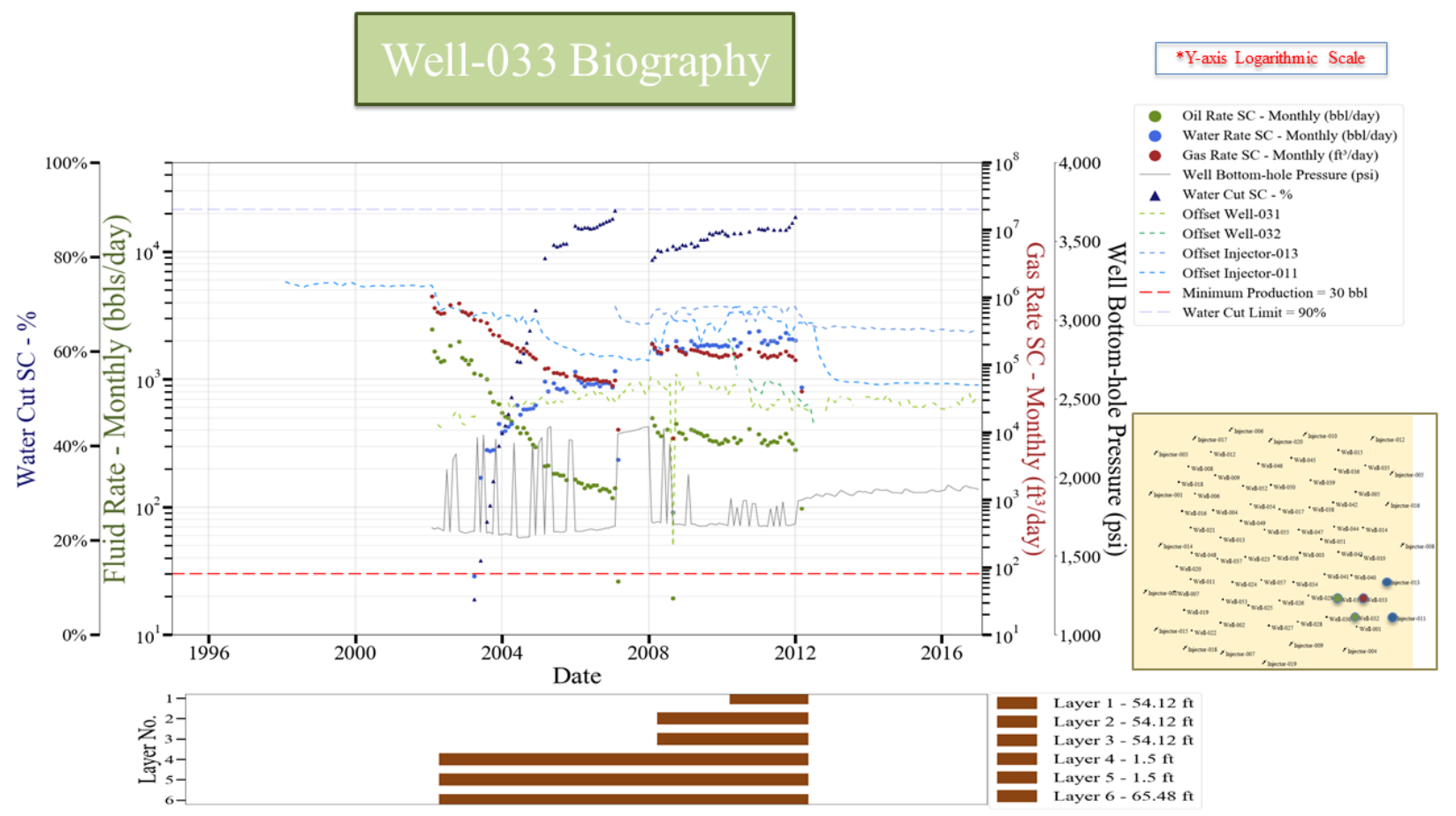

Figure 338 Well-033 biography
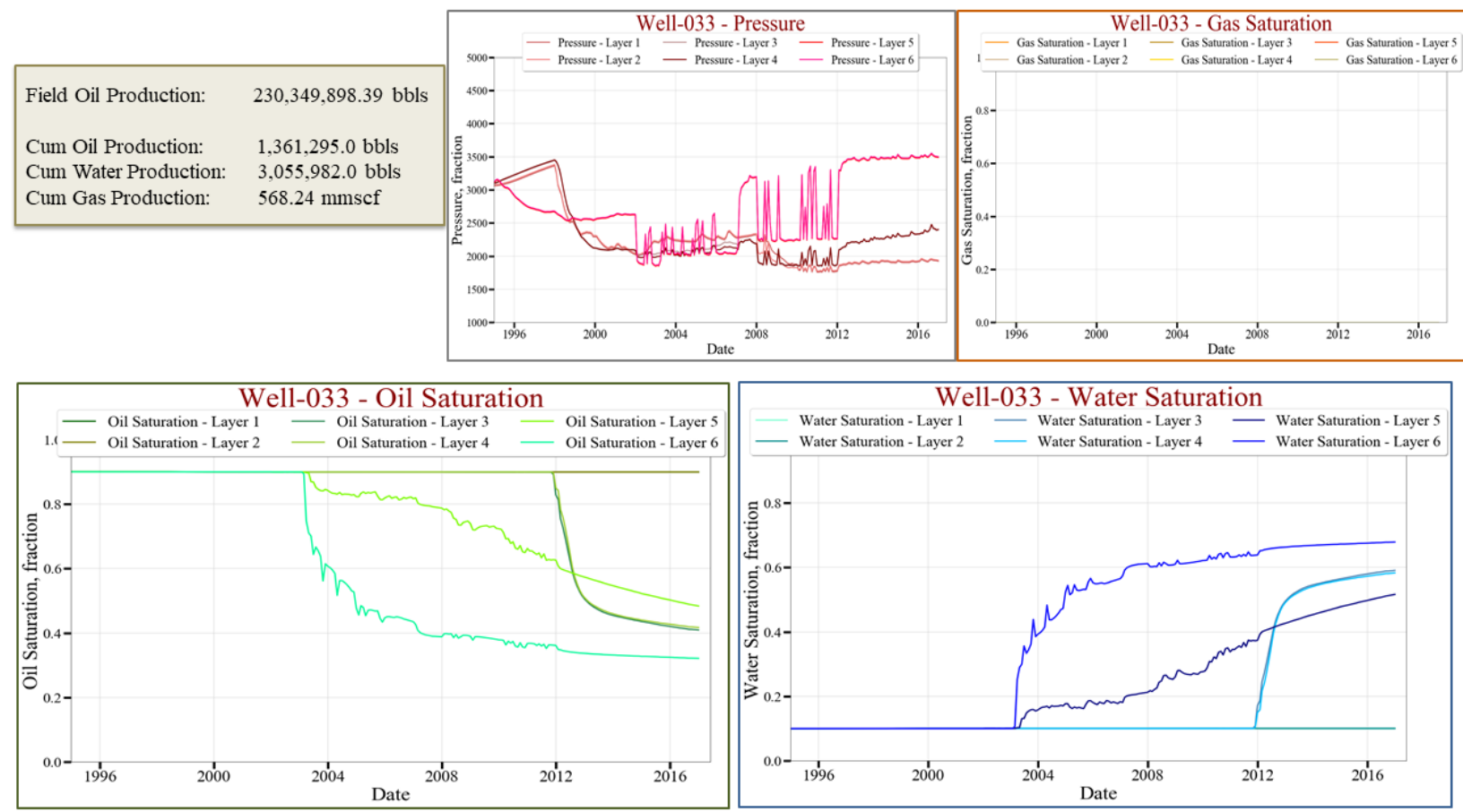

Figure 339 Well-033 saturations and pressure per layer 


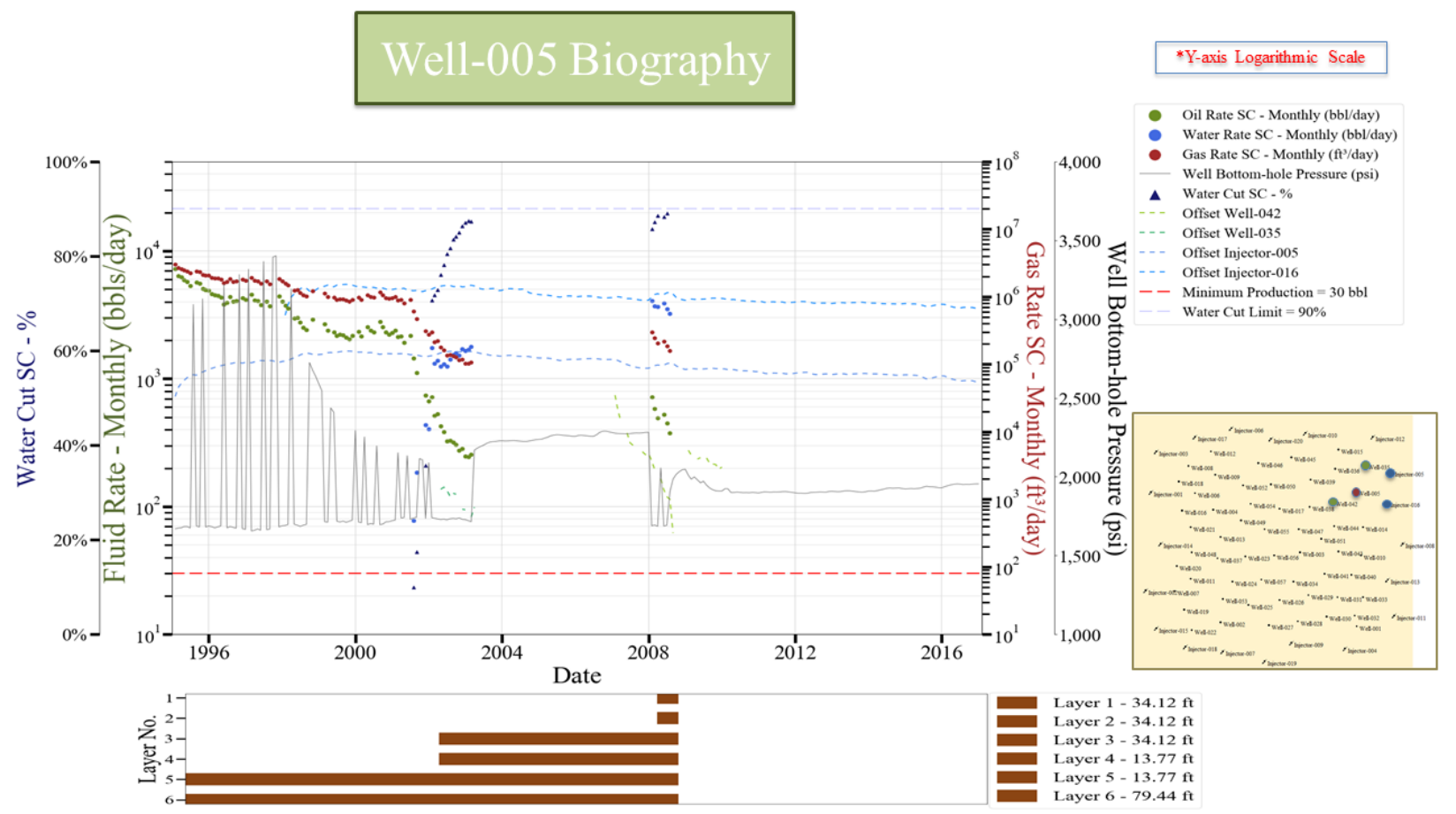

Figure 340 Well-005 biography

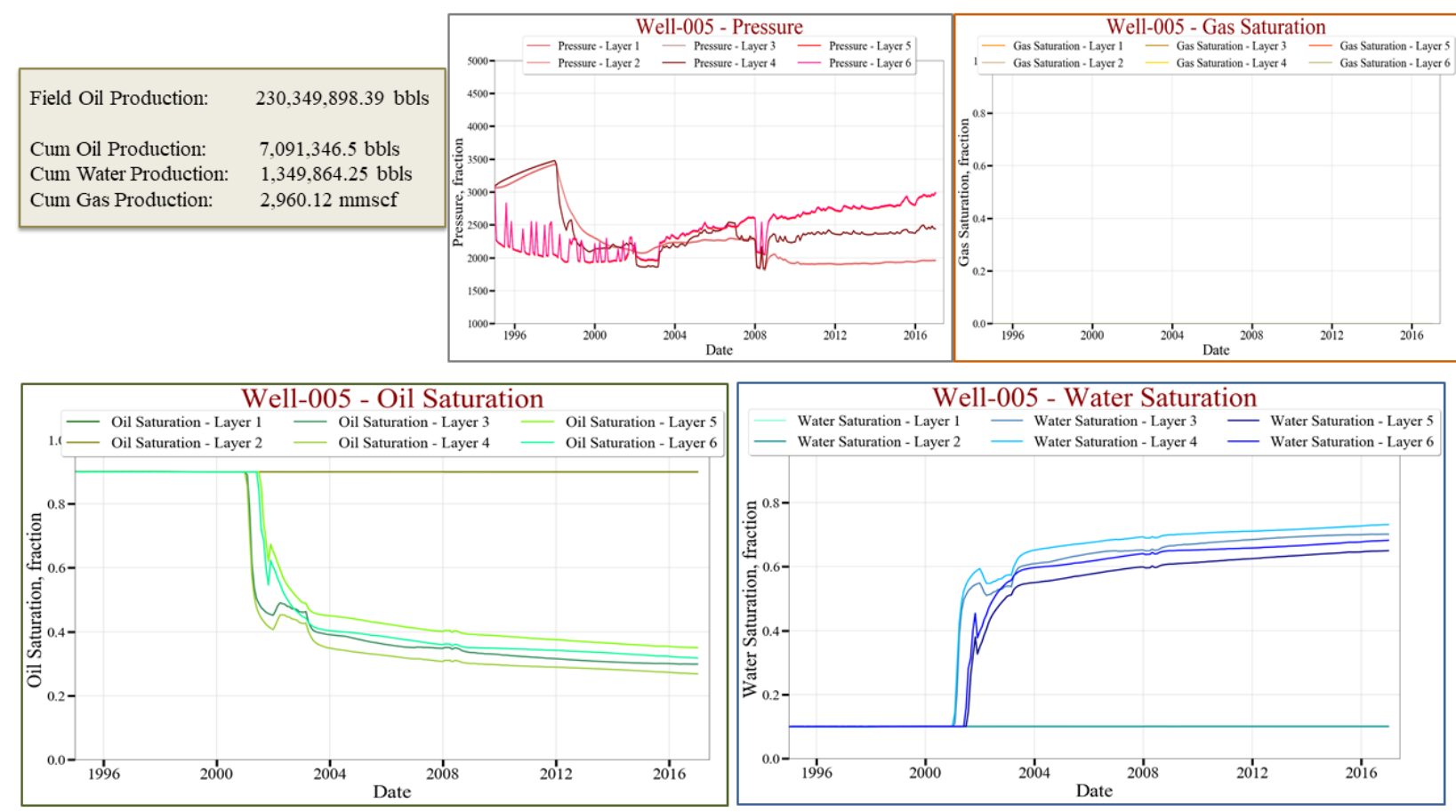

Figure 341 Well-005 saturations and pressure per layer 


\subsubsection{Lowest Number of Production Days}

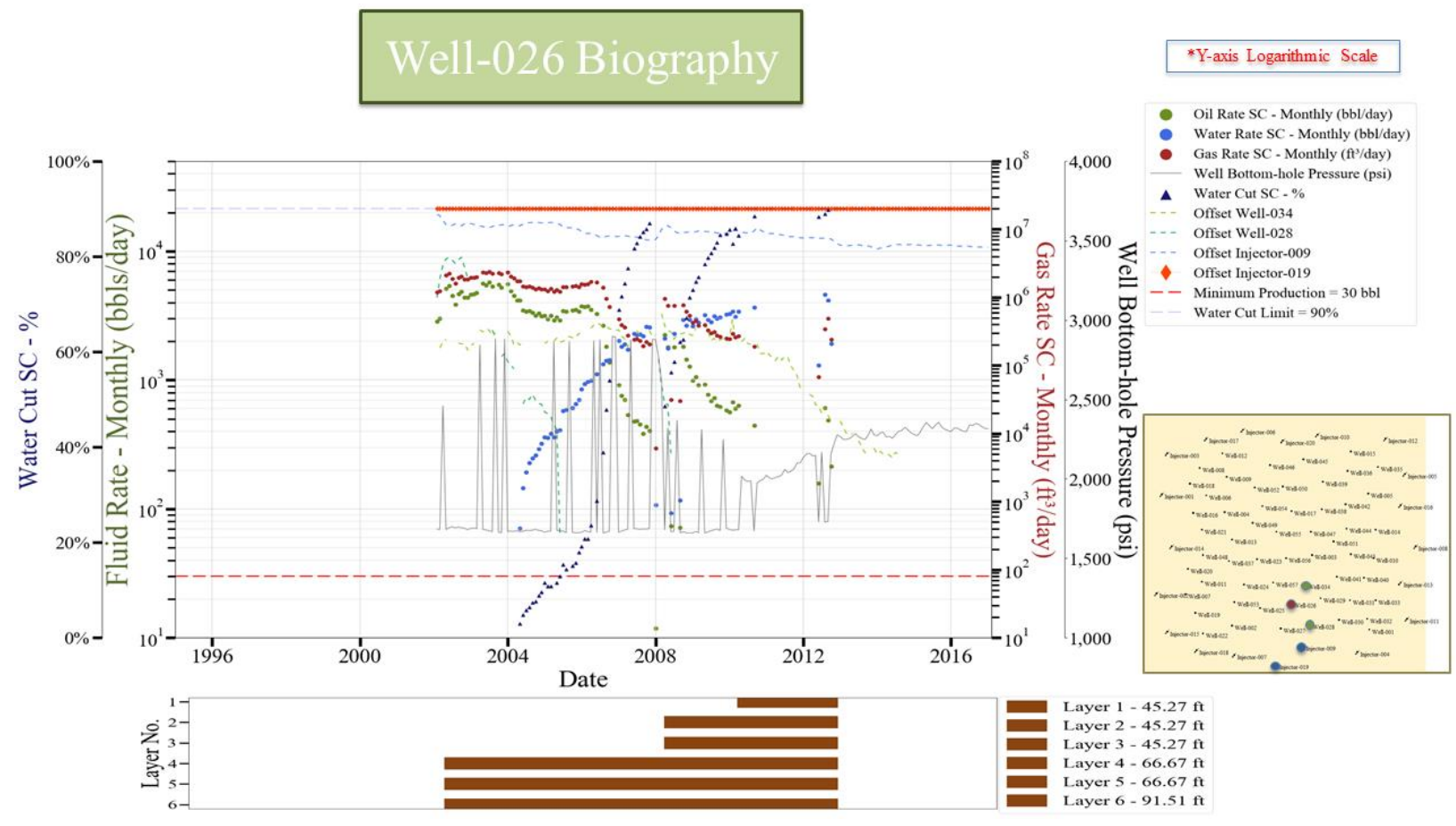

Figure 342 Well-026 biography
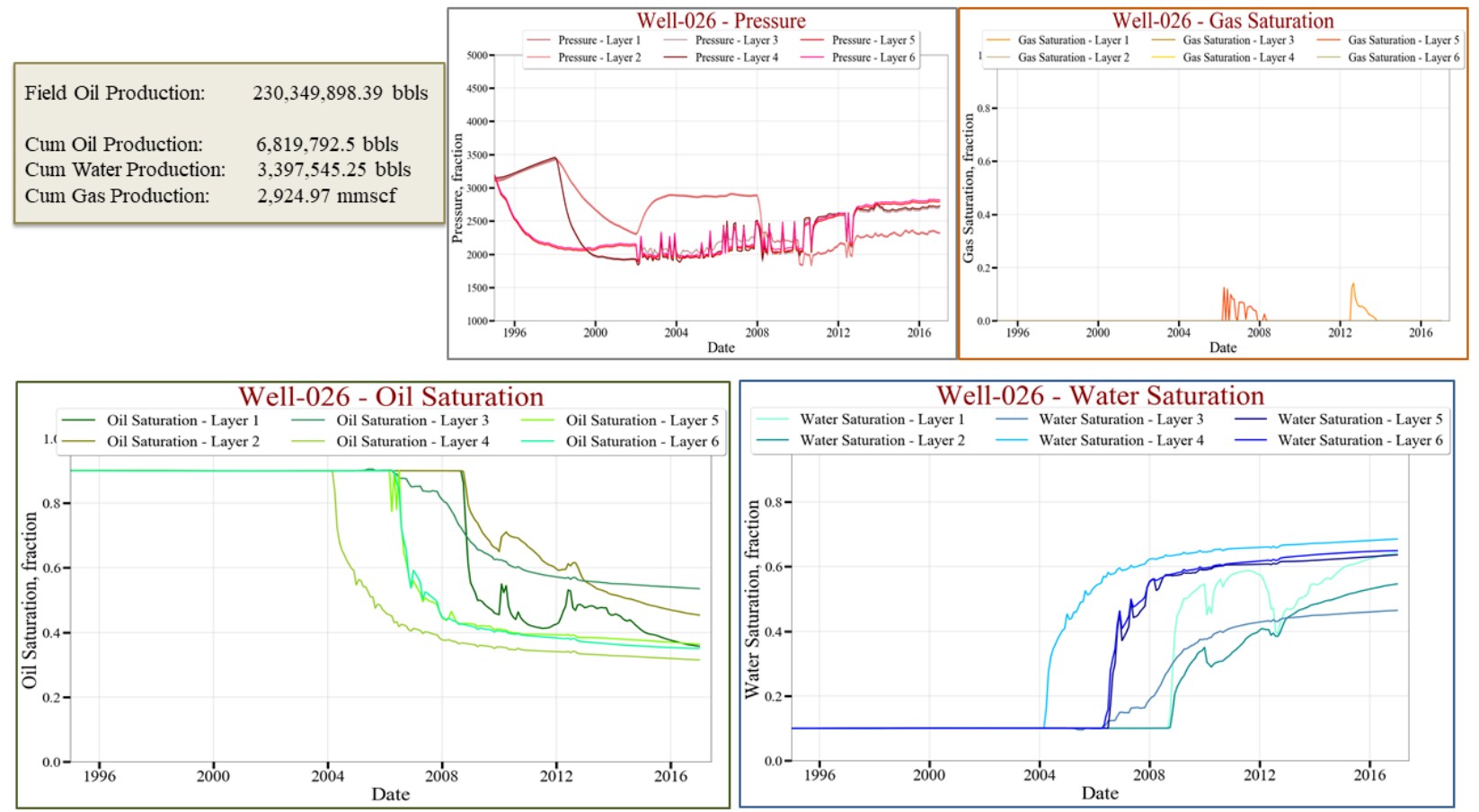

Figure 343 Well-026 saturations and pressure per layer 


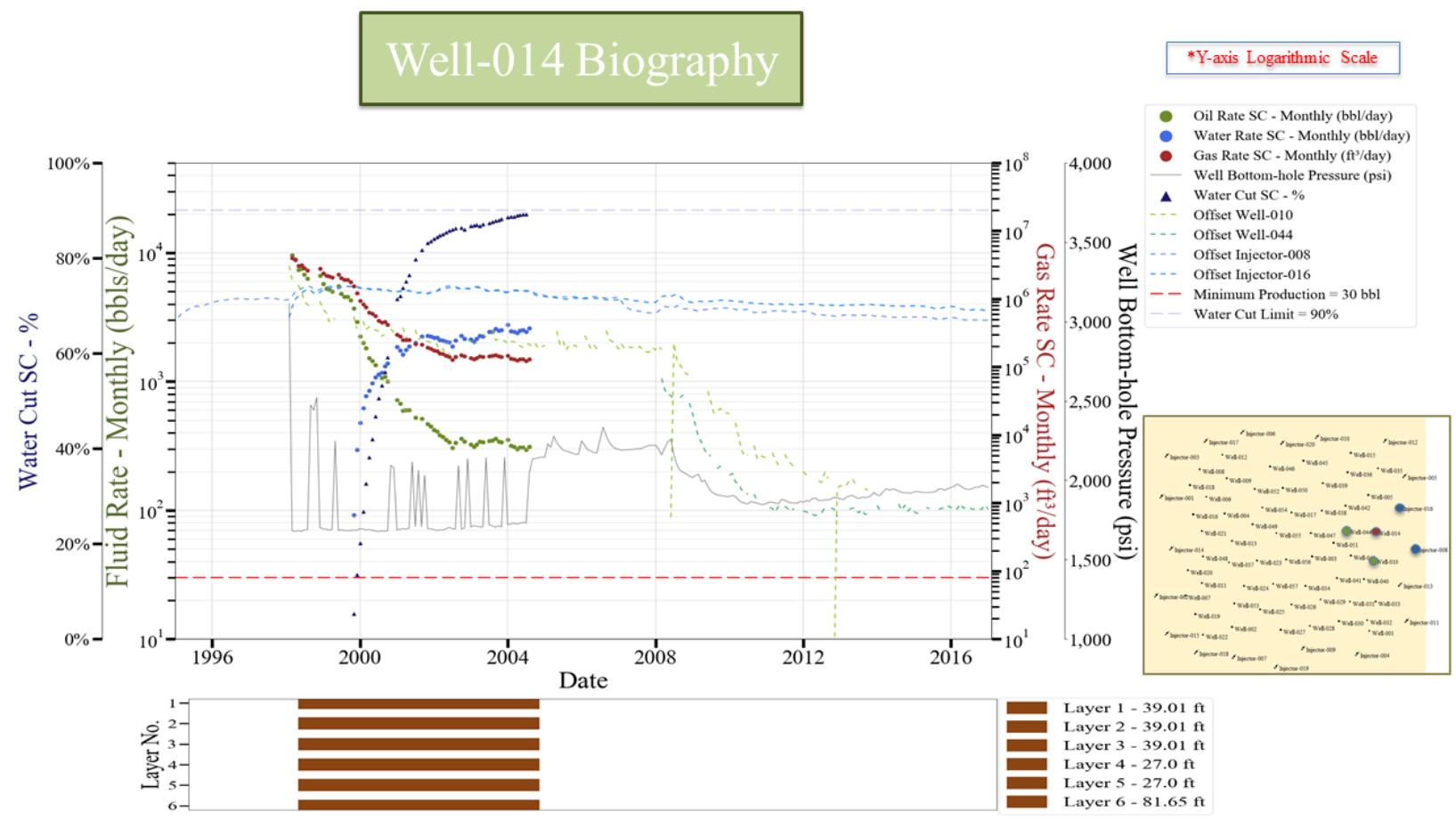

Figure 344 Well-014 biography
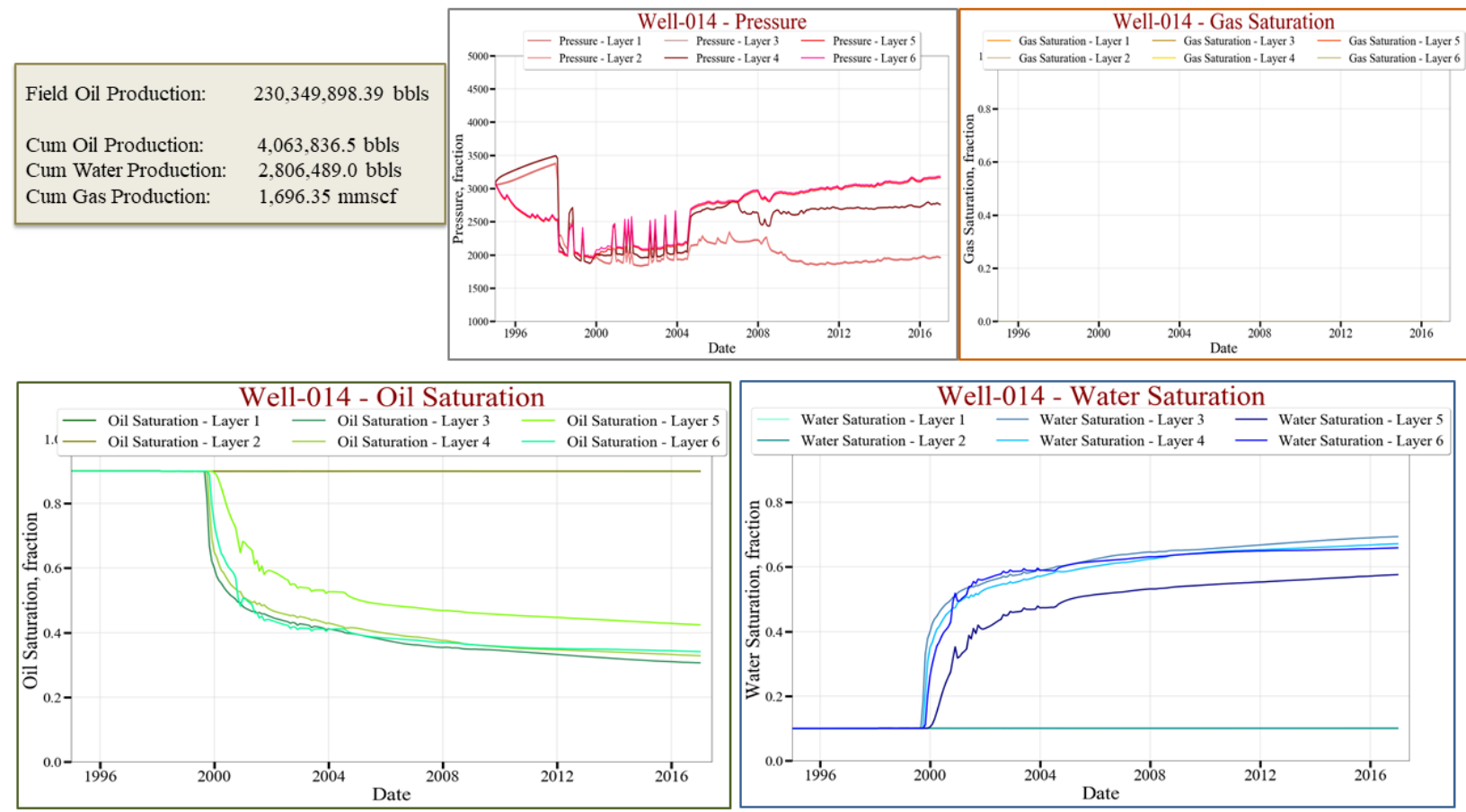

Figure 345 Well-014 saturations and pressure per layer 


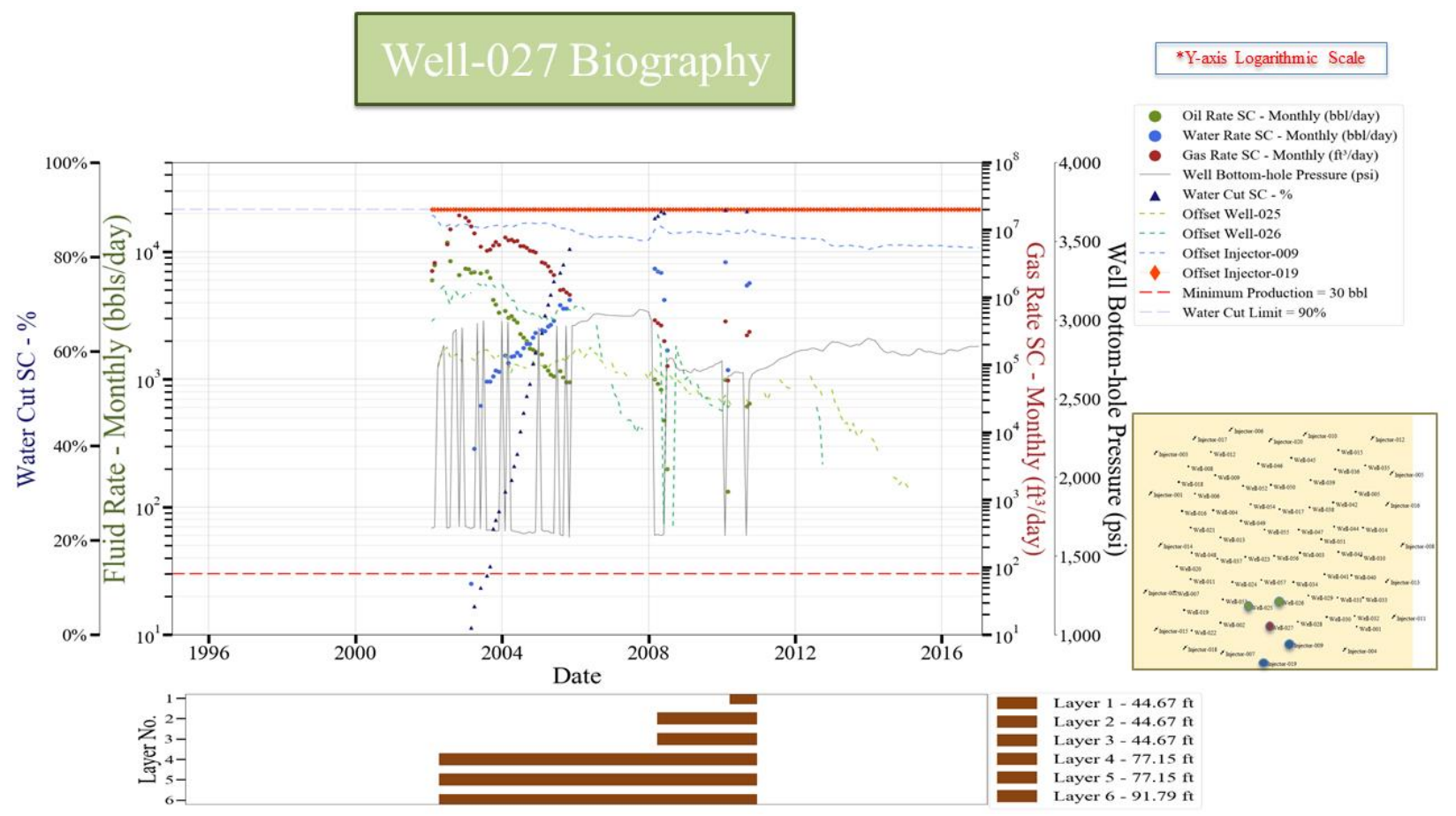

Figure 346 Well-027 biography
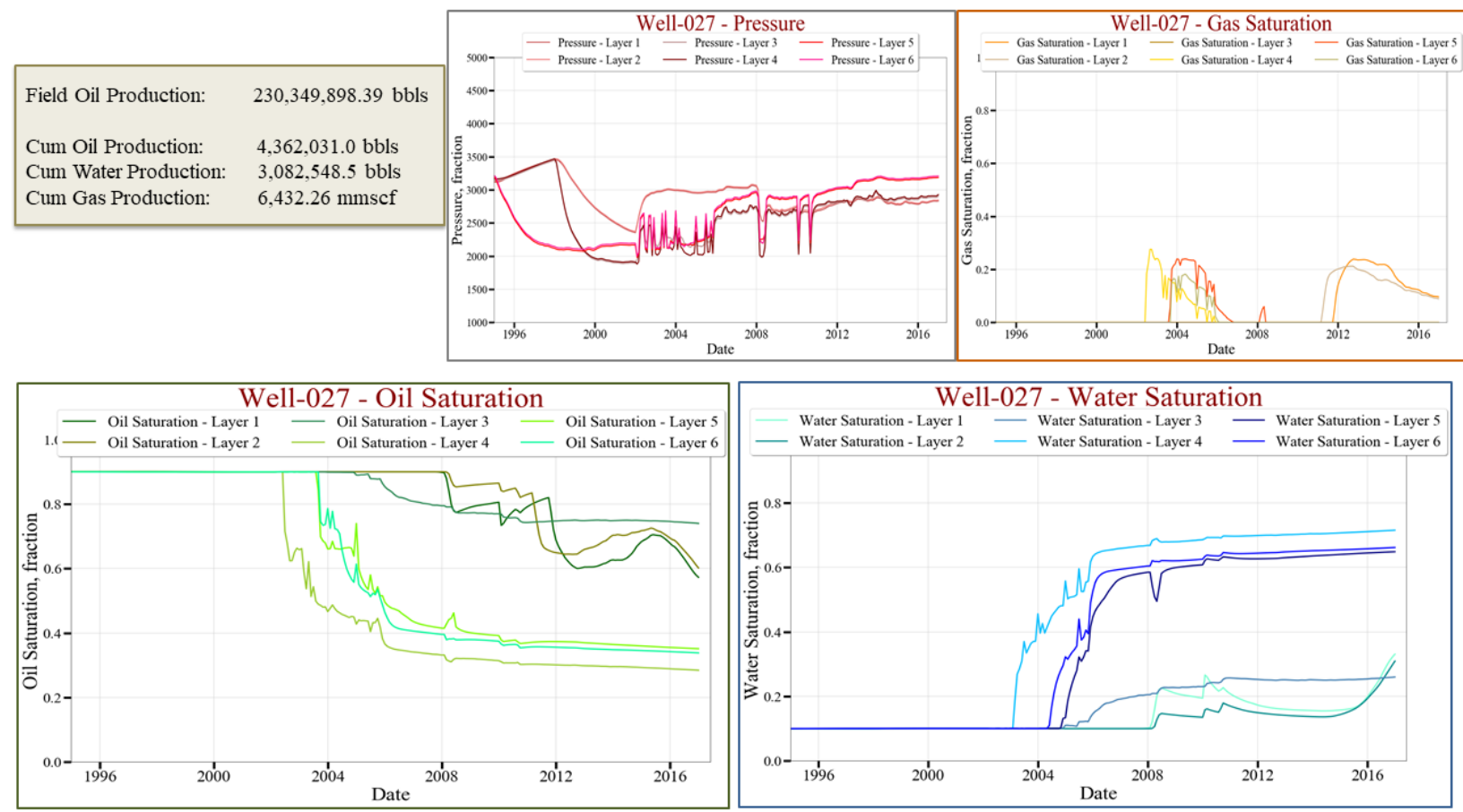

Figure 347 Well-027 saturations and pressure per layer 


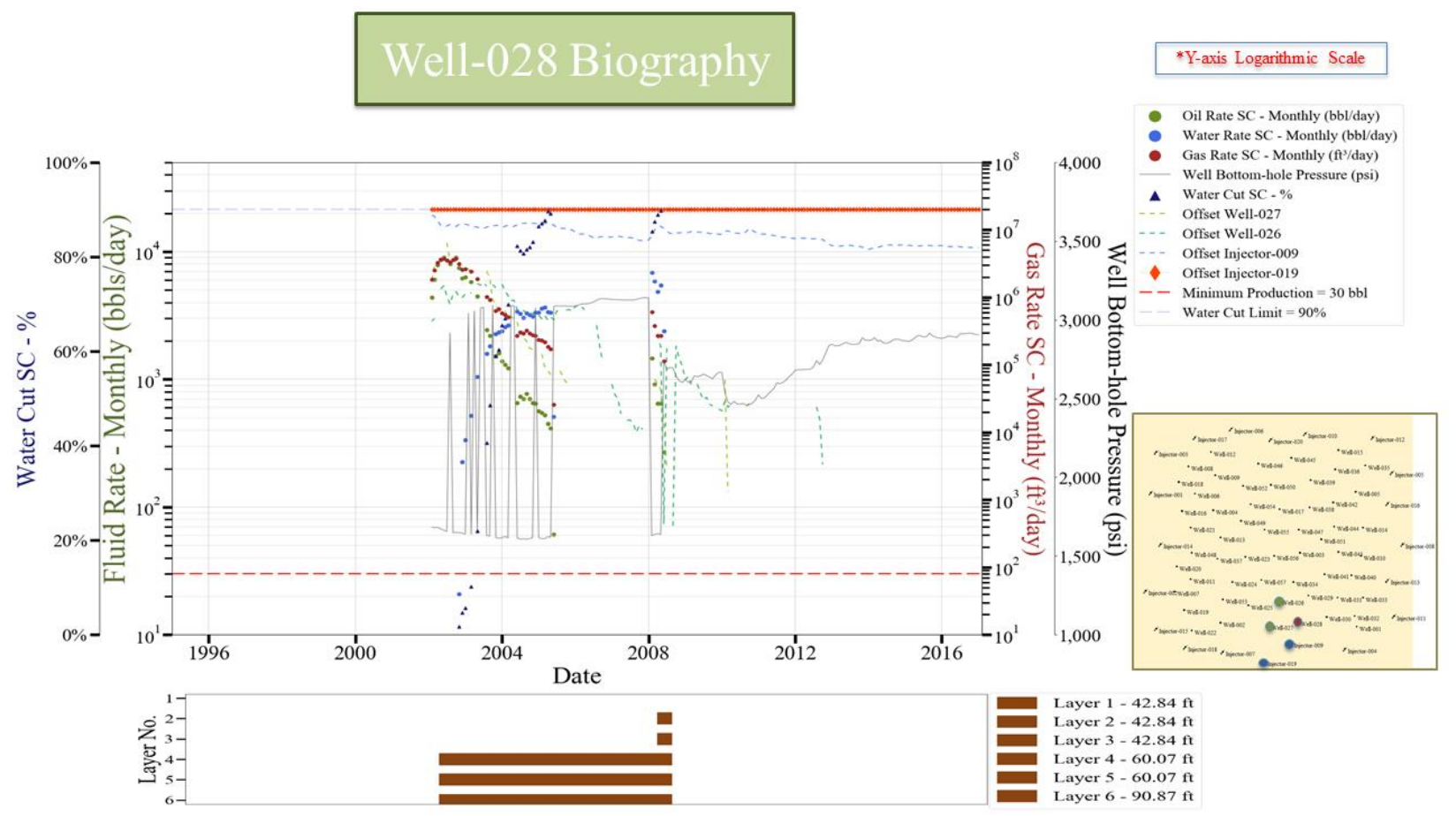

Figure 348 Well-028 biography
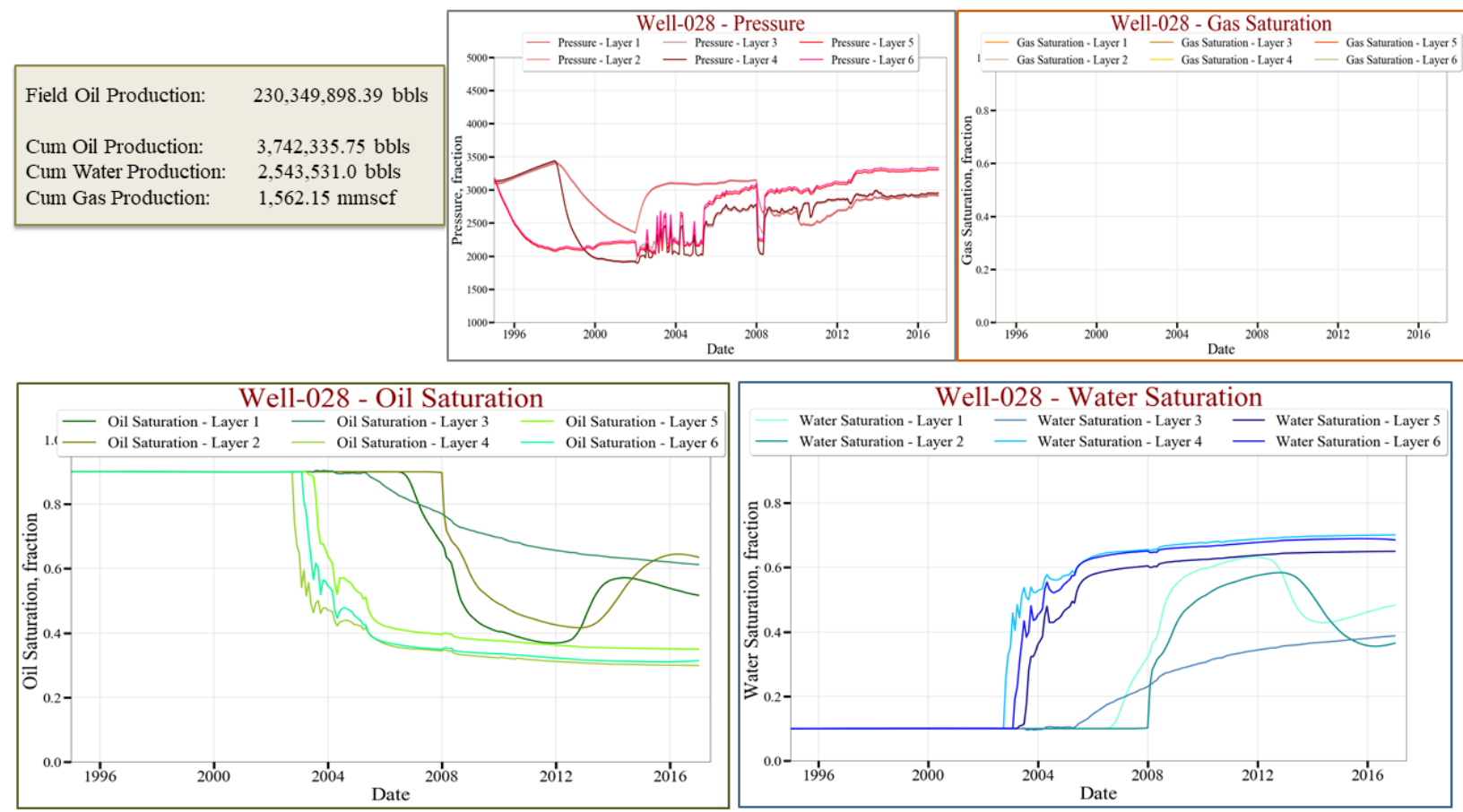

Figure 349 Well-028 saturations and pressure per layer 


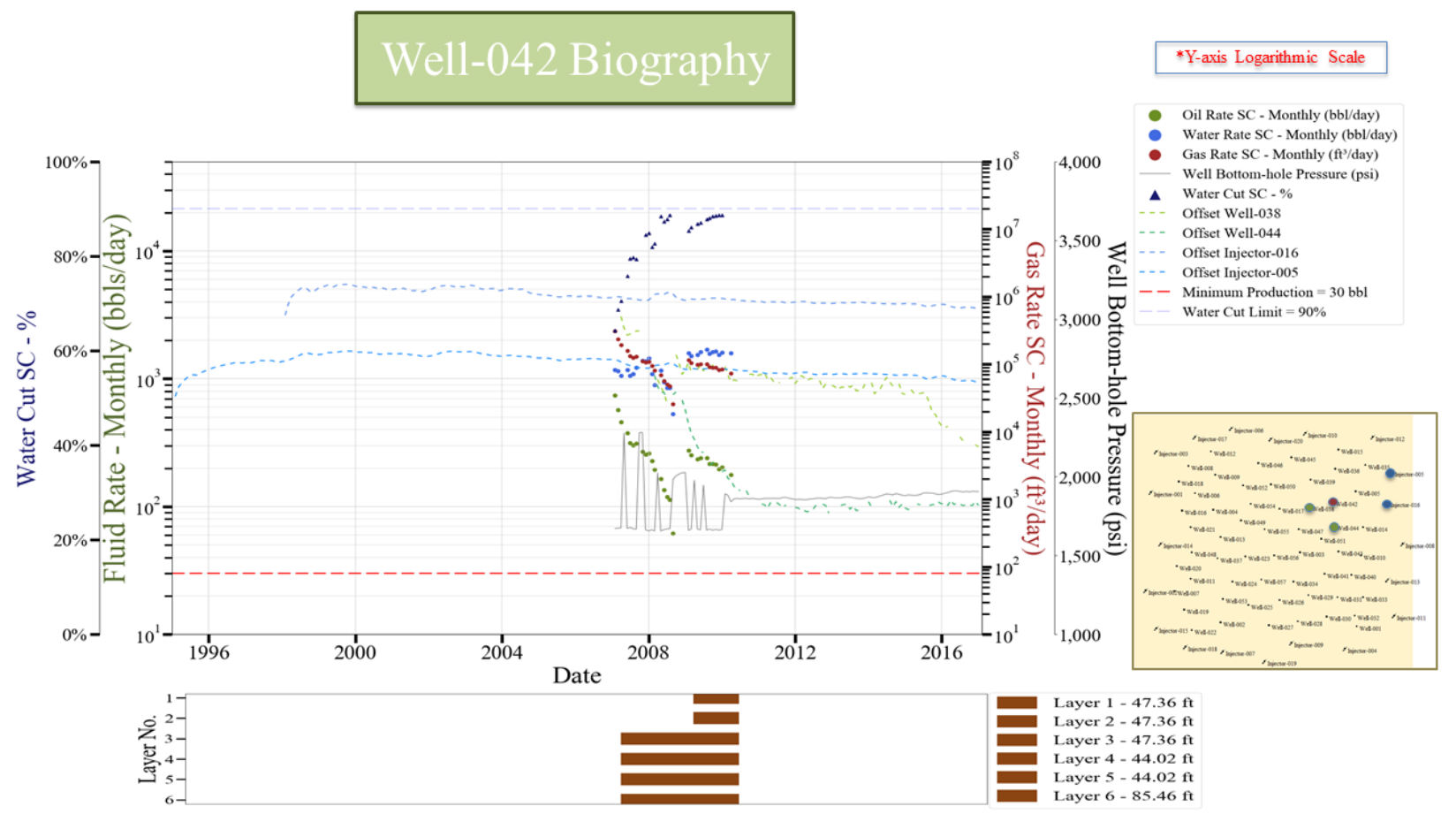

Figure 350 Well-042 biography
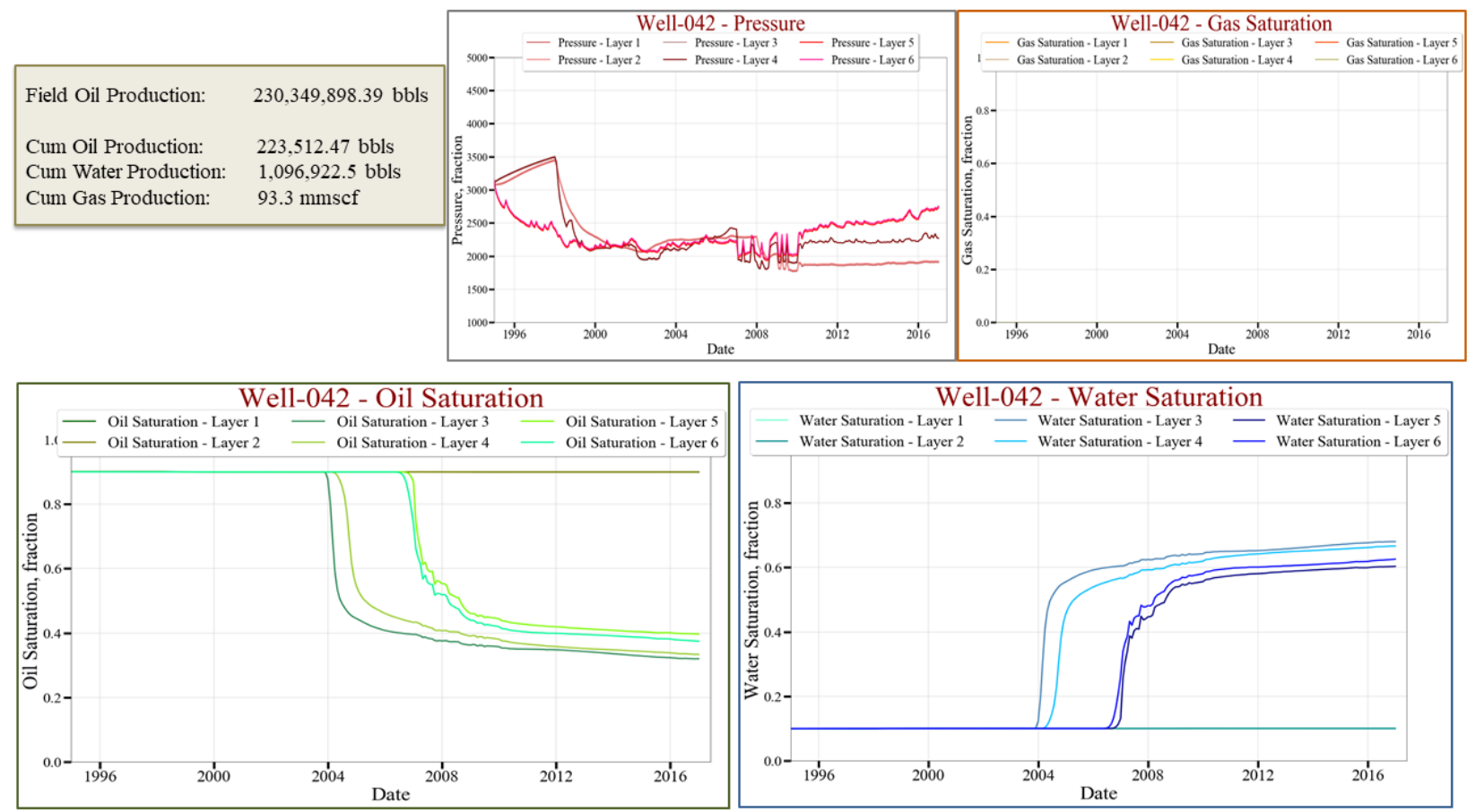

Figure 351 Well-042 saturations and pressure per layer 


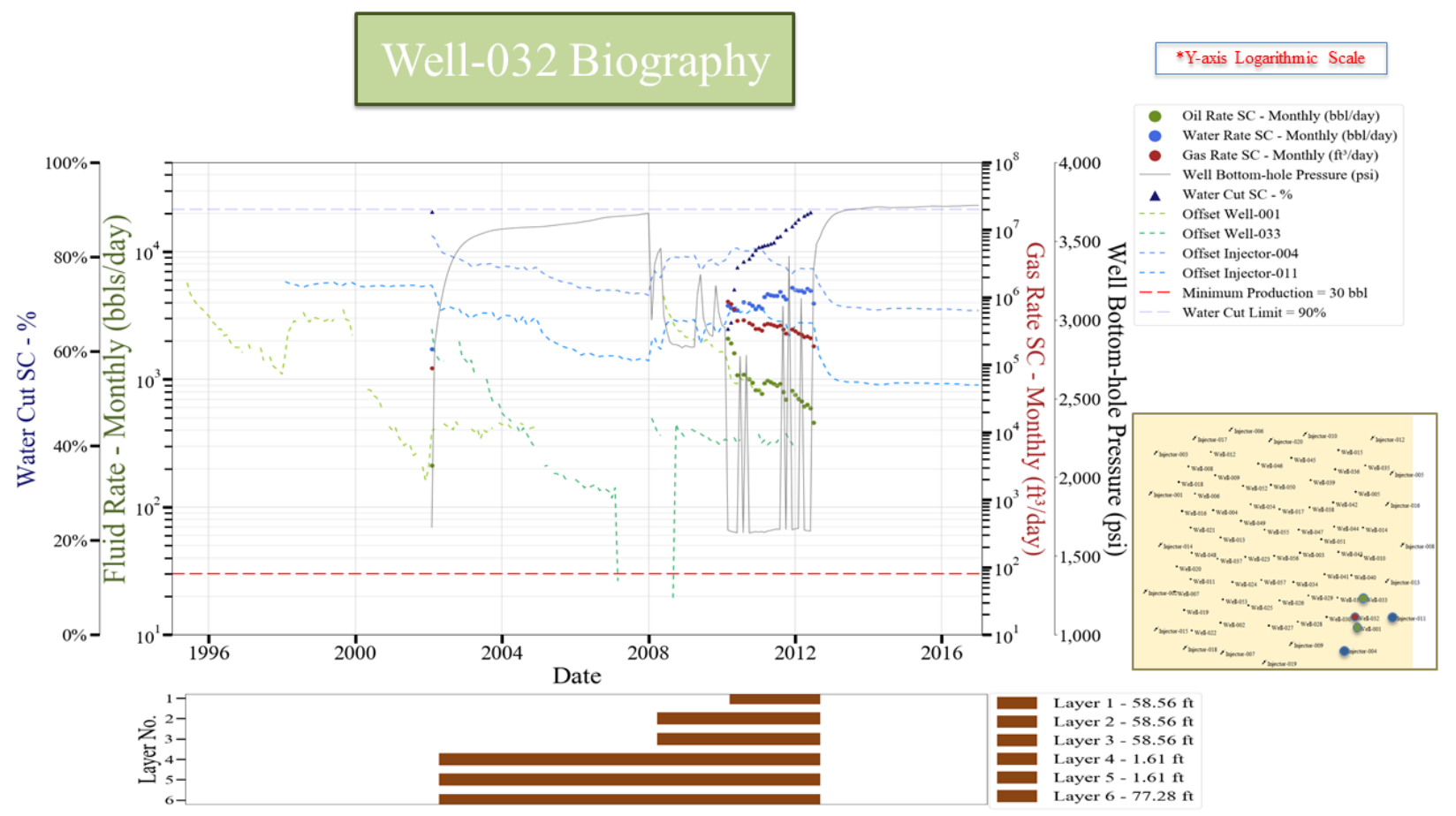

Figure 352 Well-032 biography
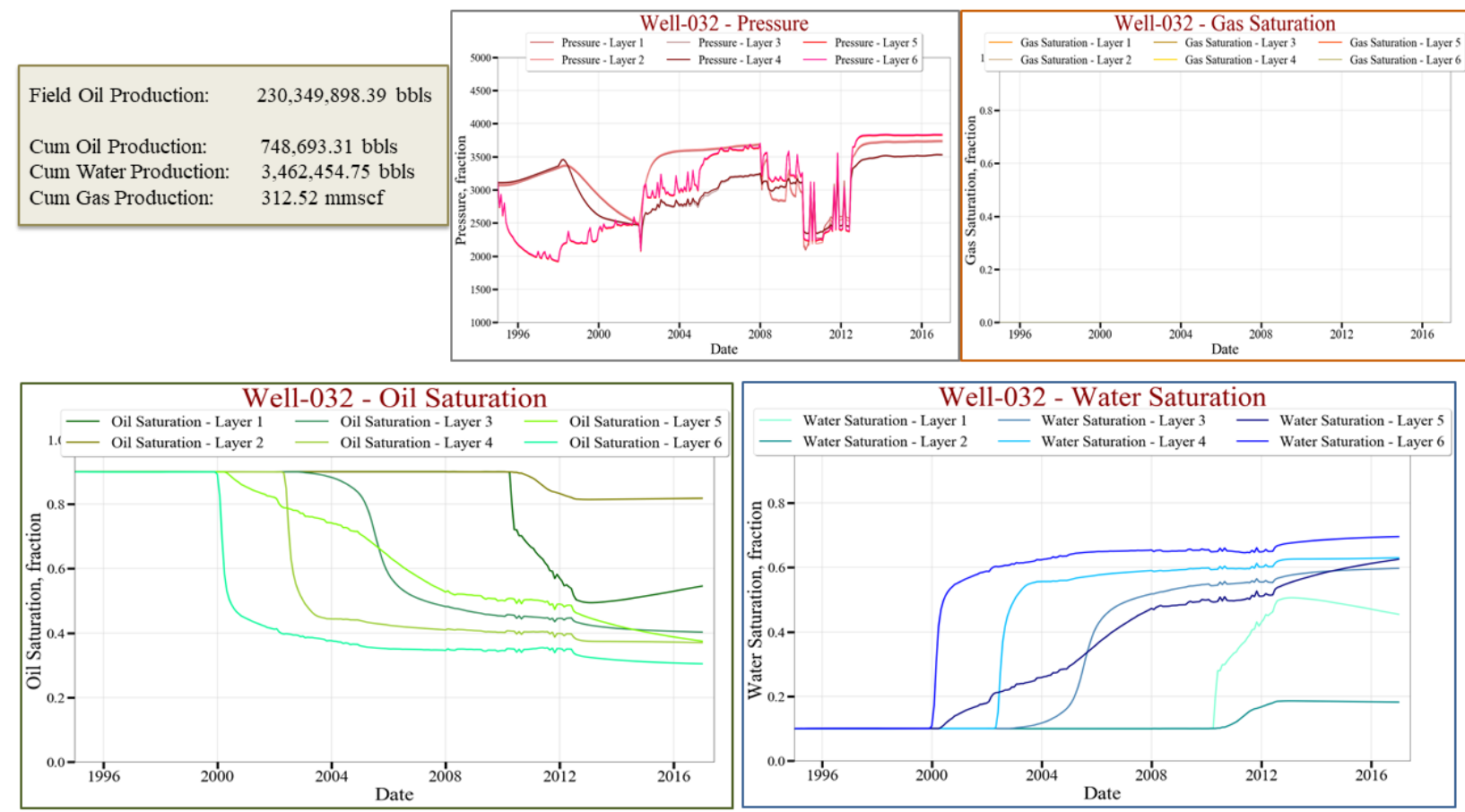

Figure 353 Well-032 saturations and pressure per layer 


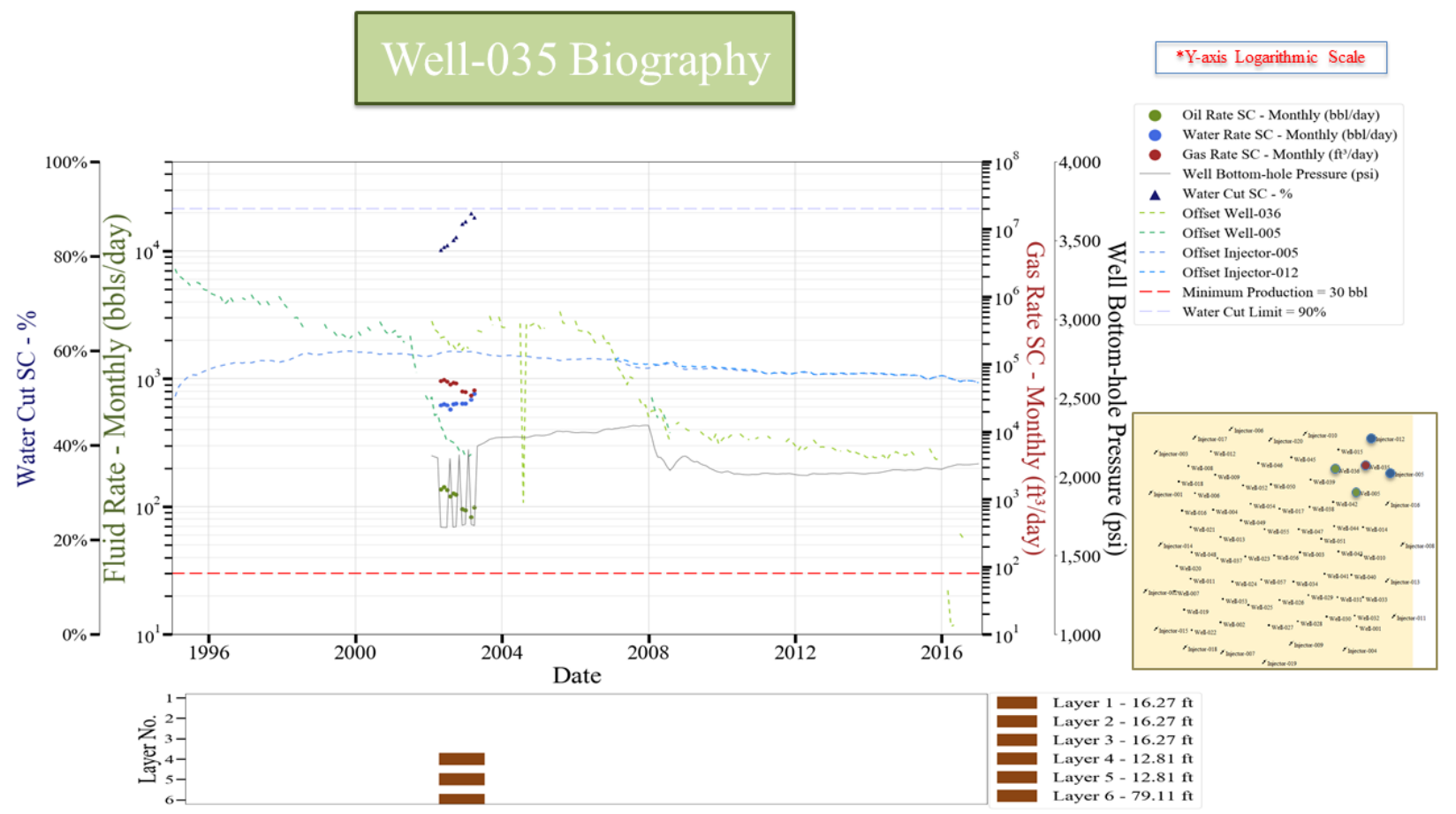

Figure 354 Well-035 biography
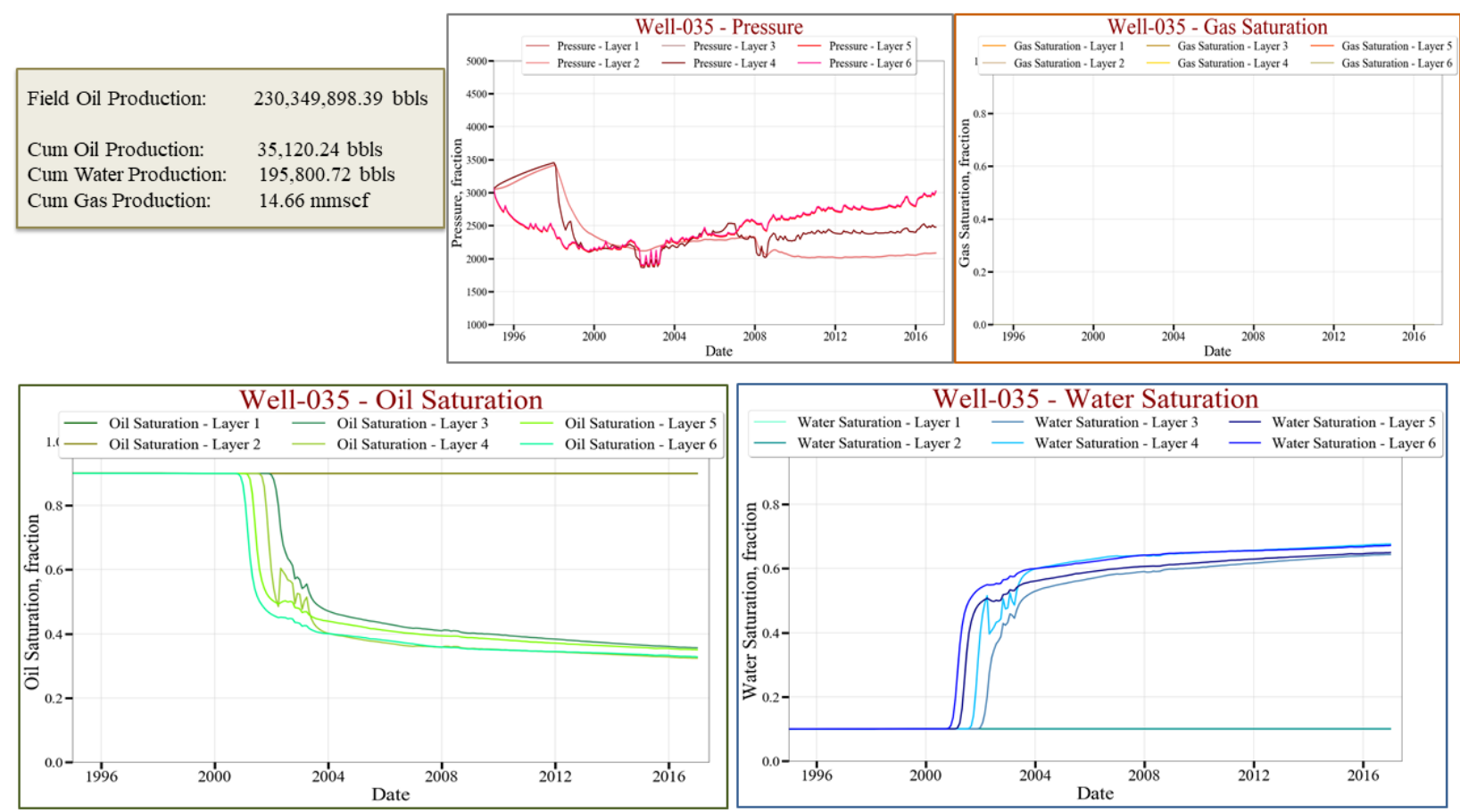

Figure 355 Well-035 saturations and pressure per layer 


\subsection{Top Down Modeling}

\subsubsection{Random Partitioning-No Blind Validation}

\subsubsection{Individual Well Production Plots}

\section{Oil Rate}

- Numerical Simulator

TDM Model

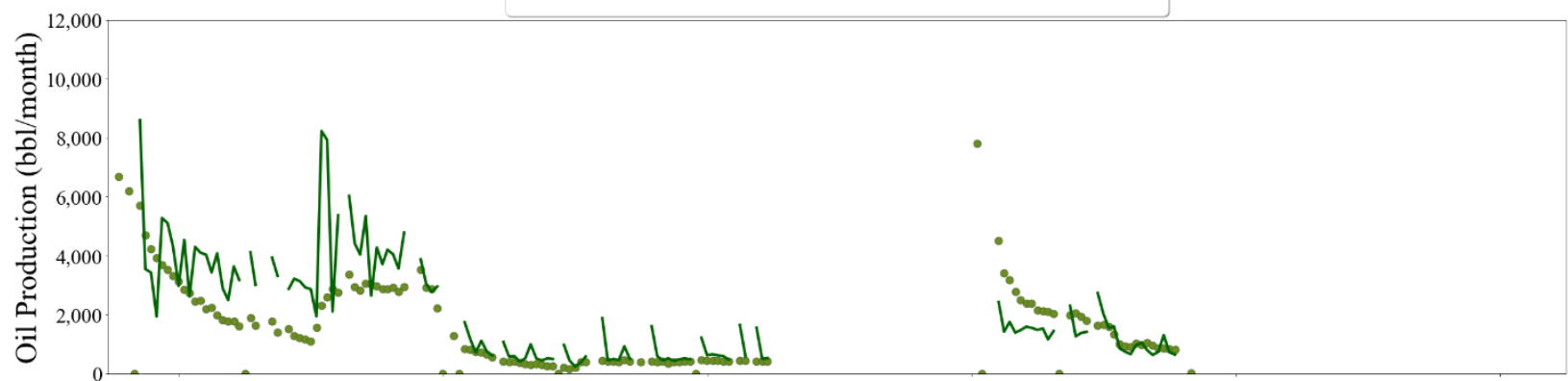

Gas Rate
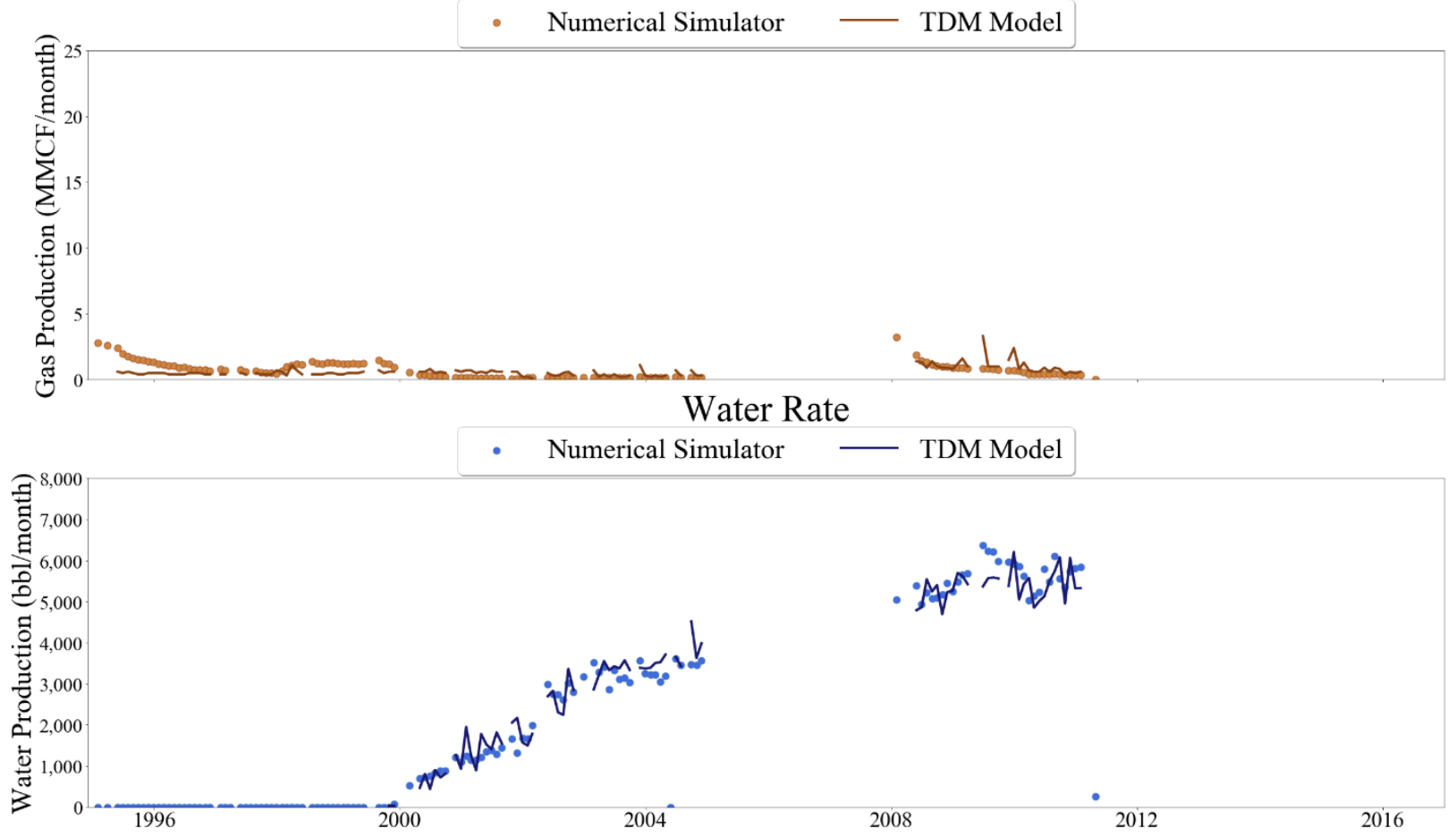

Figure 356 Well-001 oil, gas, and water rate TDM prediction vs. actual simulation data plots for No Blind Validation TDM 


\section{Oil Rate}
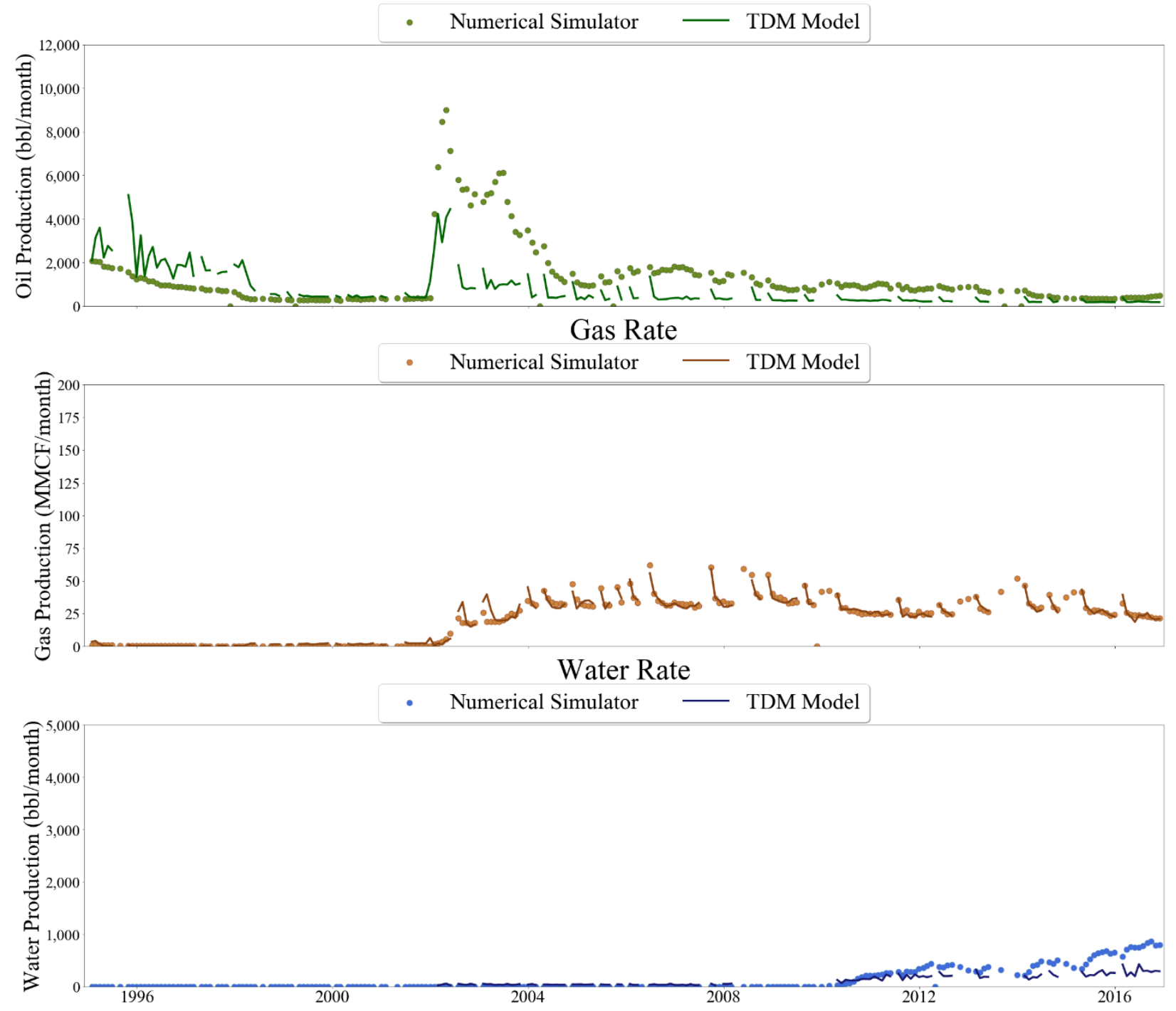

Figure 357 Well-002 oil, gas, and water TDM predictions vs. actual simulation data plots for No Blind Validation TDM 


\section{Oil Rate}

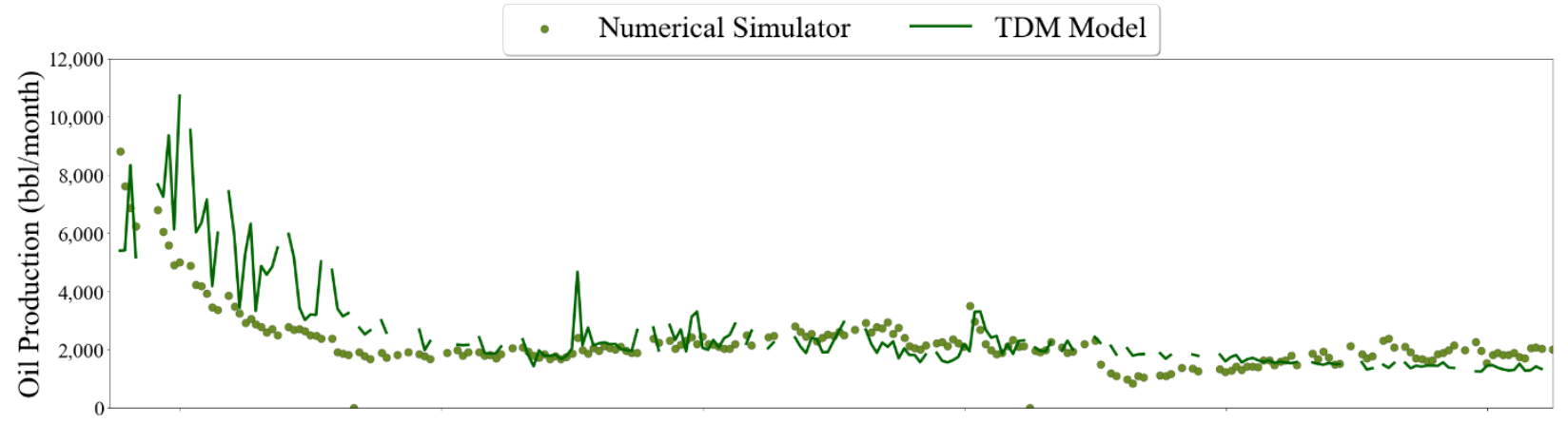

\section{Gas Rate}
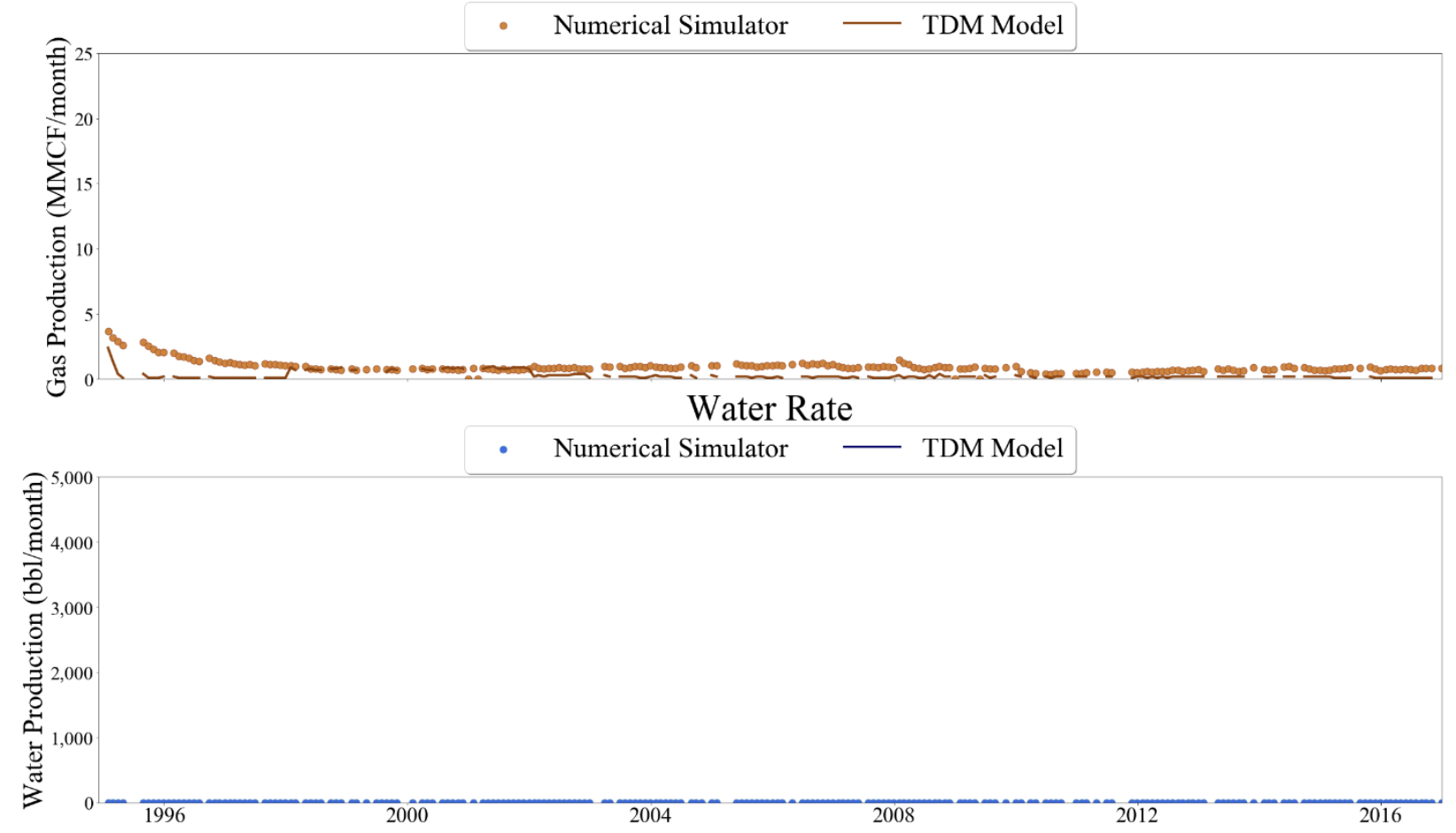

Figure 358 Well-003 oil, gas, and water rate TDM predictions vs. actual simulation data plots for No Blind Validation TDM 


\section{Oil Rate}

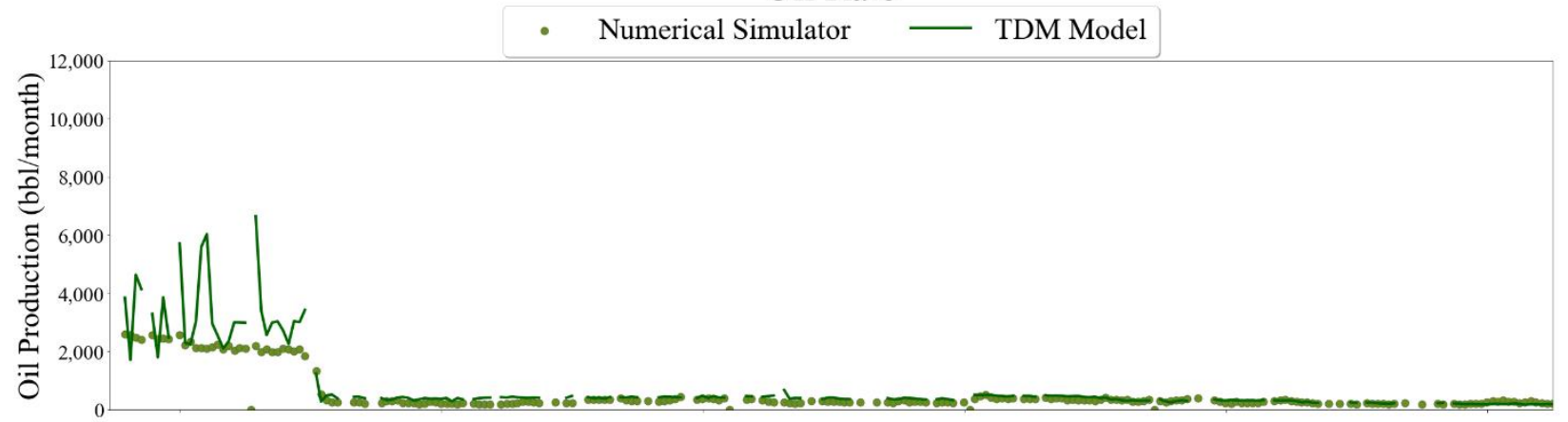

Gas Rate
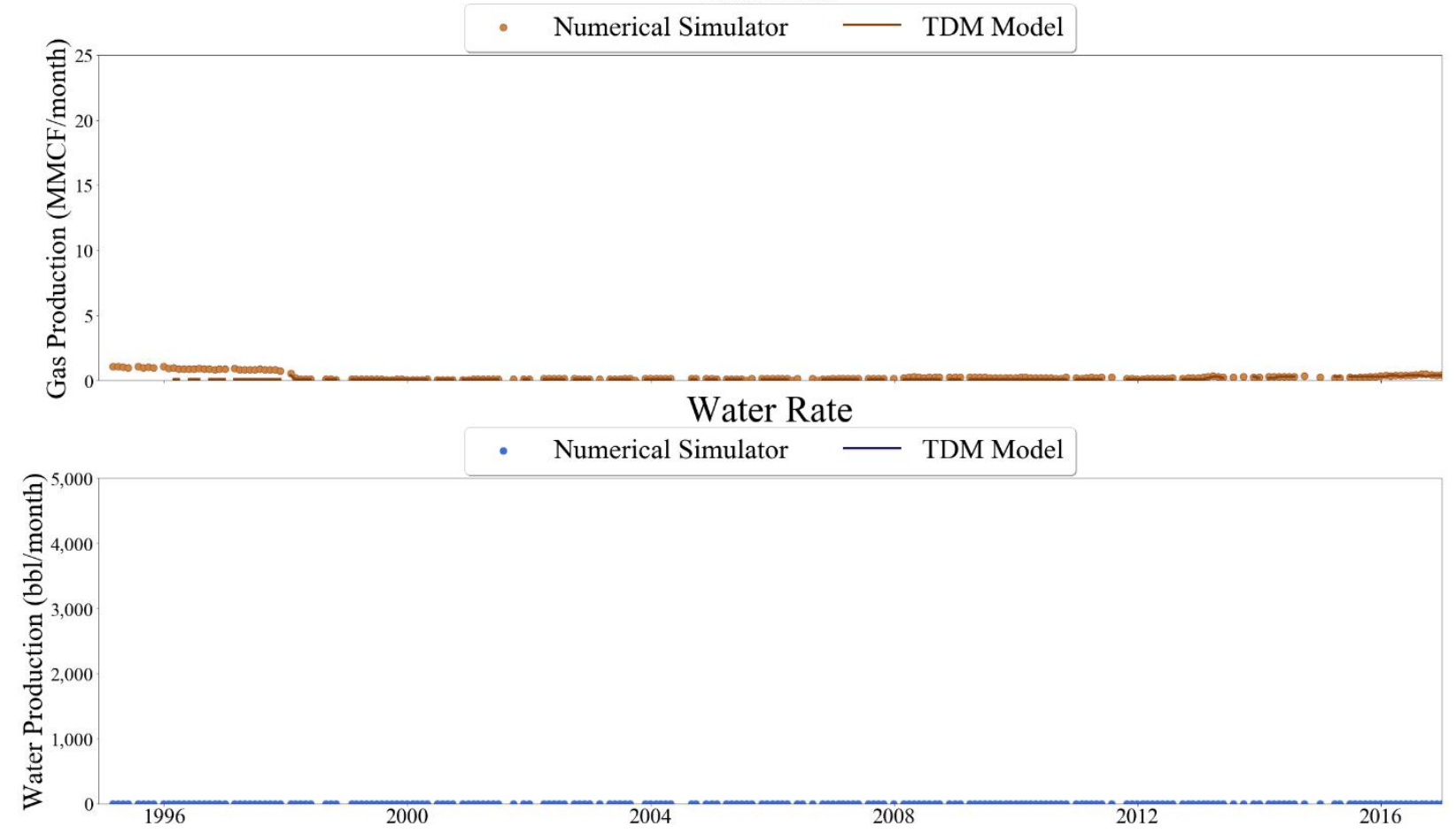

Figure 359 Well-004 oil, gas, and water rate TDM predictions vs actual simulation data plots for No Blind Validation TDM 


\section{Oil Rate}

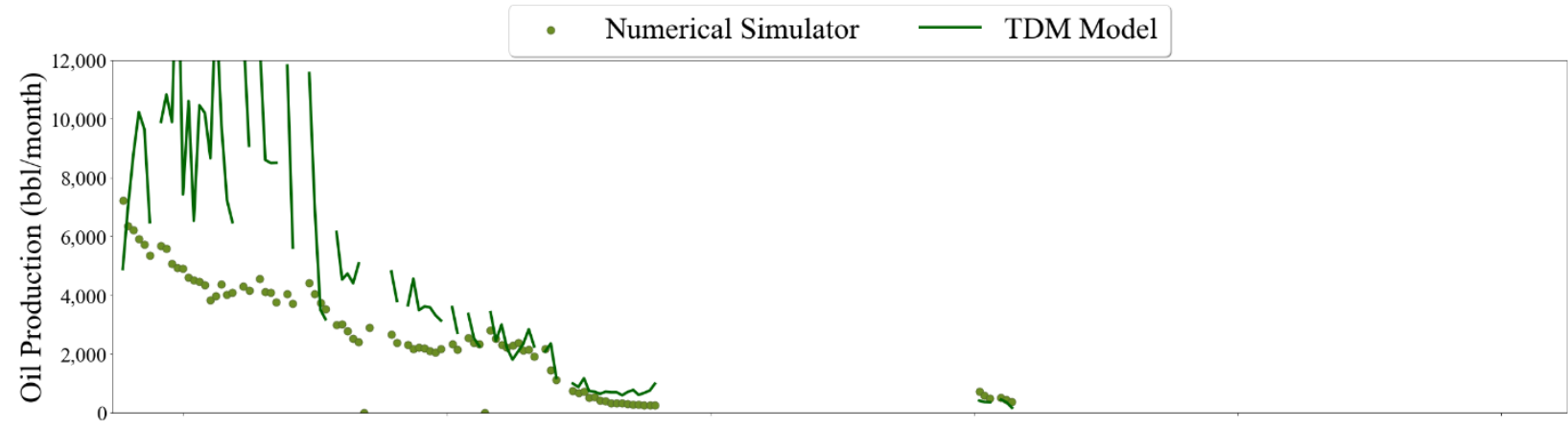

\section{Gas Rate}
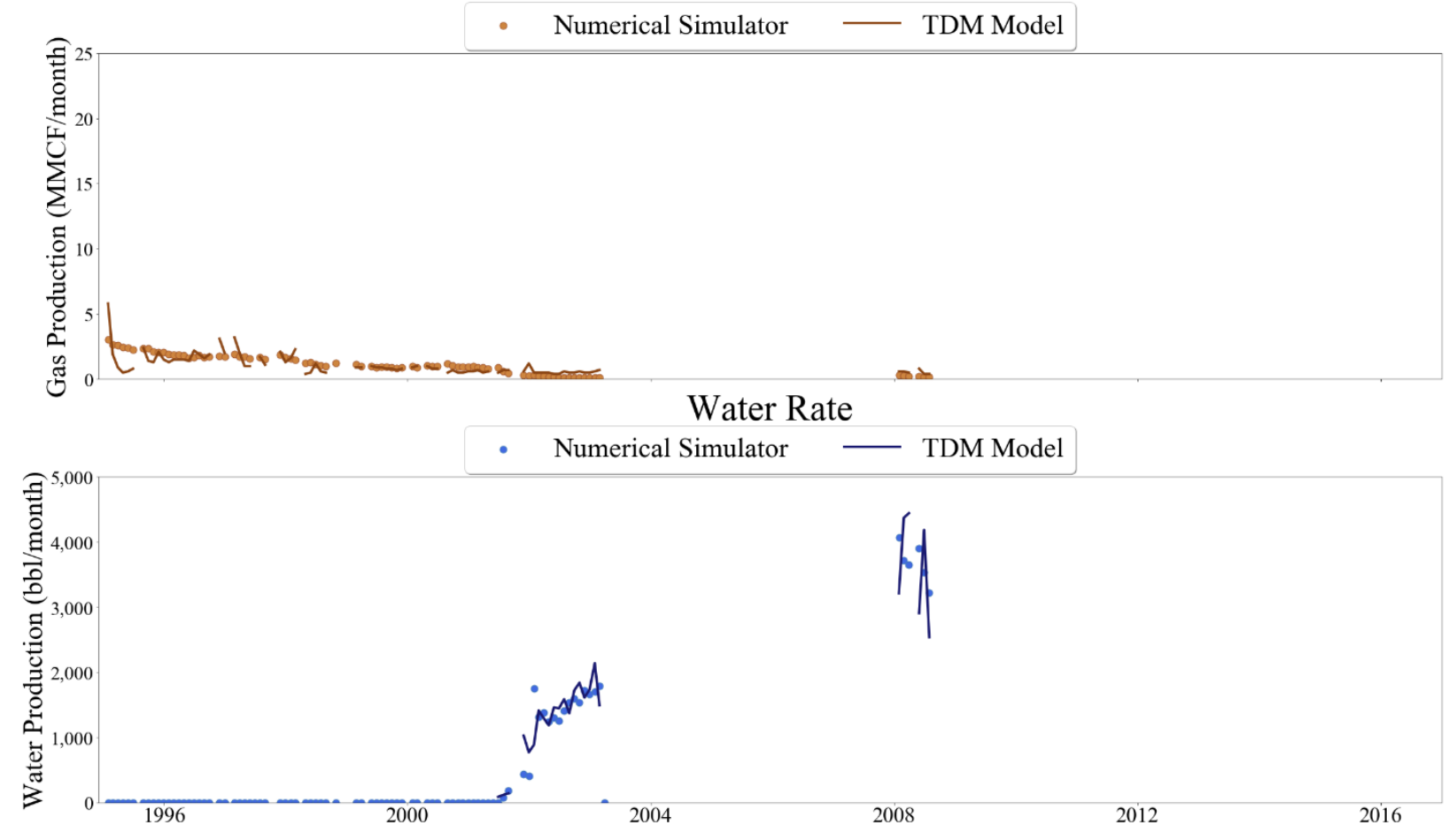

Figure 360 Well-005 oil, gas, and water rate TDM predictions vs actual simulation data plots for No Blind Validation TDM 


\section{Oil Rate}
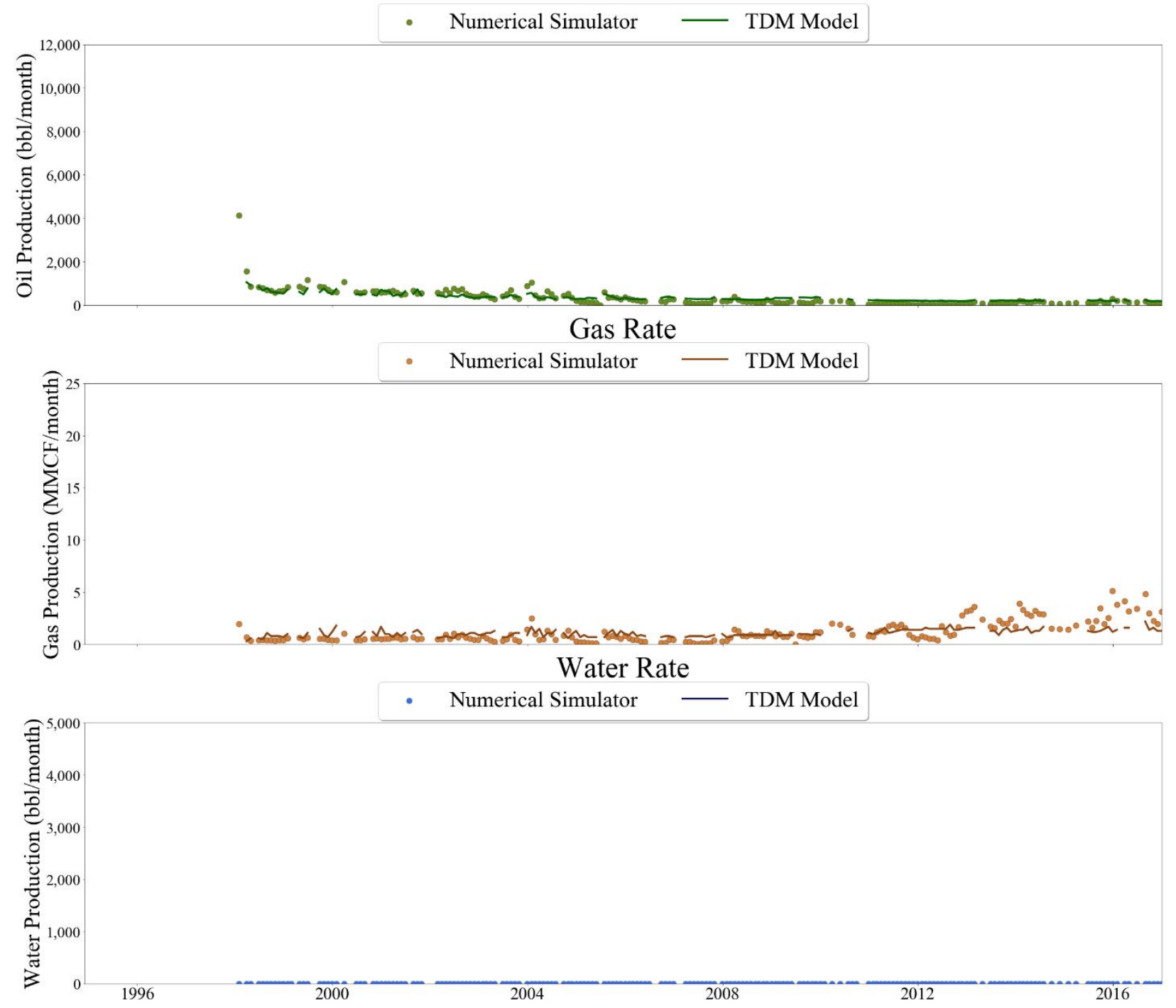

Figure 361 Well-006 oil, gas, and water rate TDM predictions vs actual simulation data plots for No Blind Validation TDM 


\section{Oil Rate}

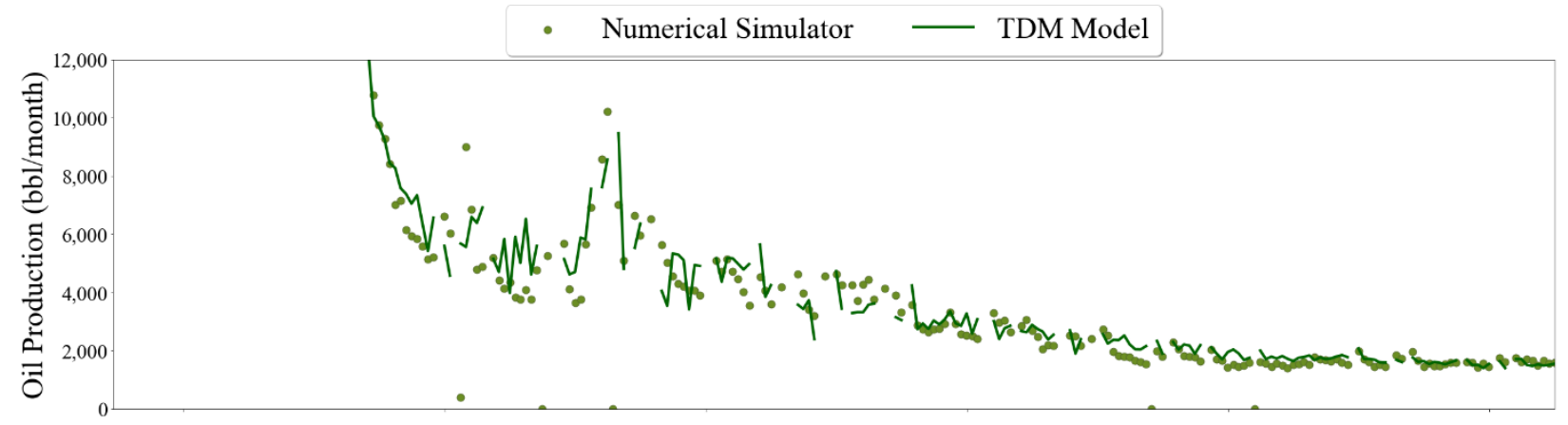

\section{Gas Rate}
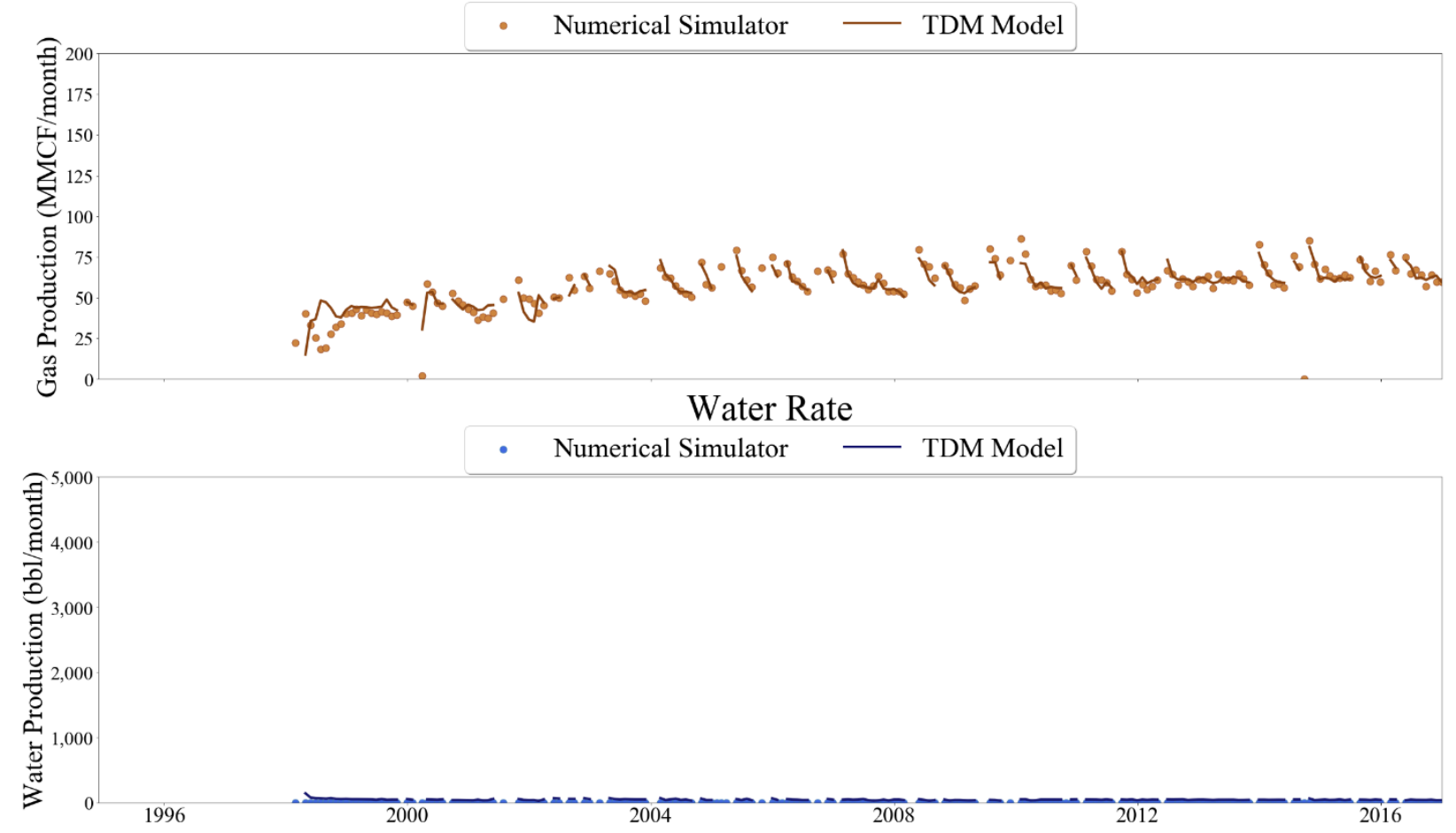

Figure 362 Well-007 oil, gas, water rate TDM predictions vs actual simulation data plots for No Blind Validation TDM 


\section{Oil Rate}
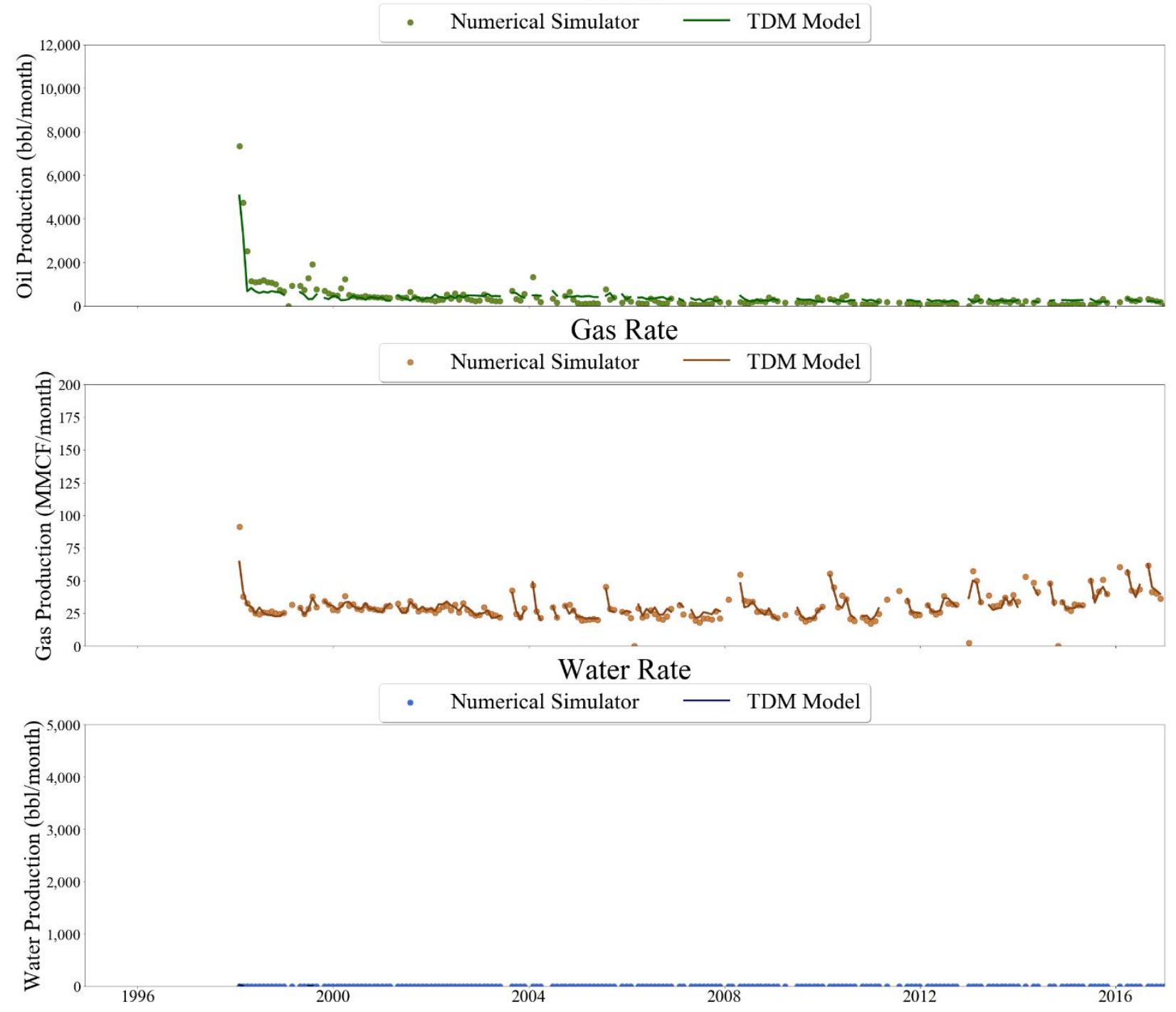

Figure 363 Well-008 oil, gas, and water rate TDM predictions vs actual simulation data plots for No Blind Validation TDM 


\section{Oil Rate}
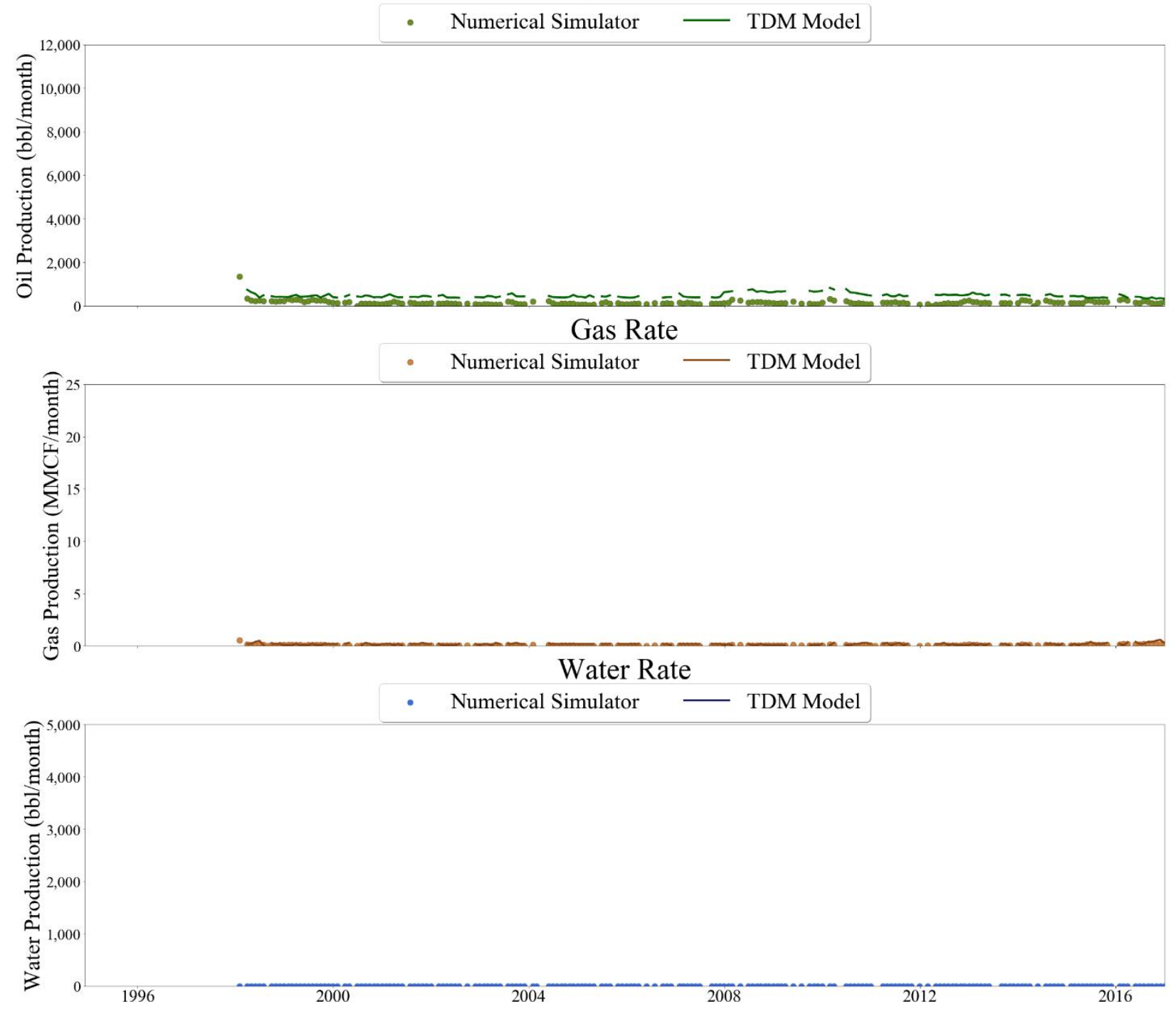

Figure 364 Well-009 oil, gas, and water rate TDM predictions vs actual simulation data plots for No Blind Validation TDM 


\section{Oil Rate}

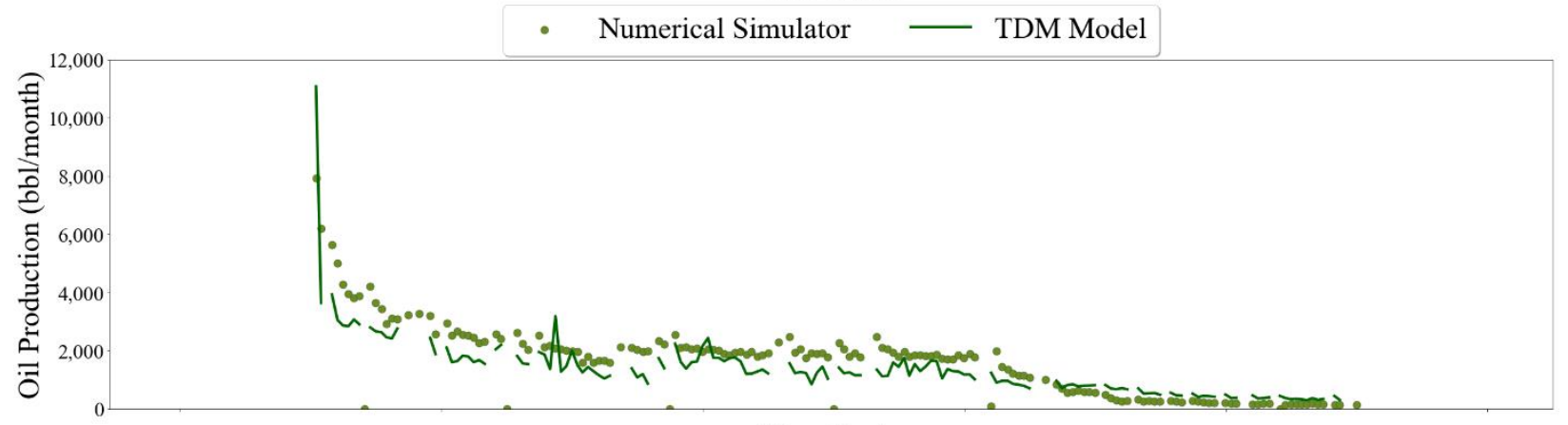

Gas Rate
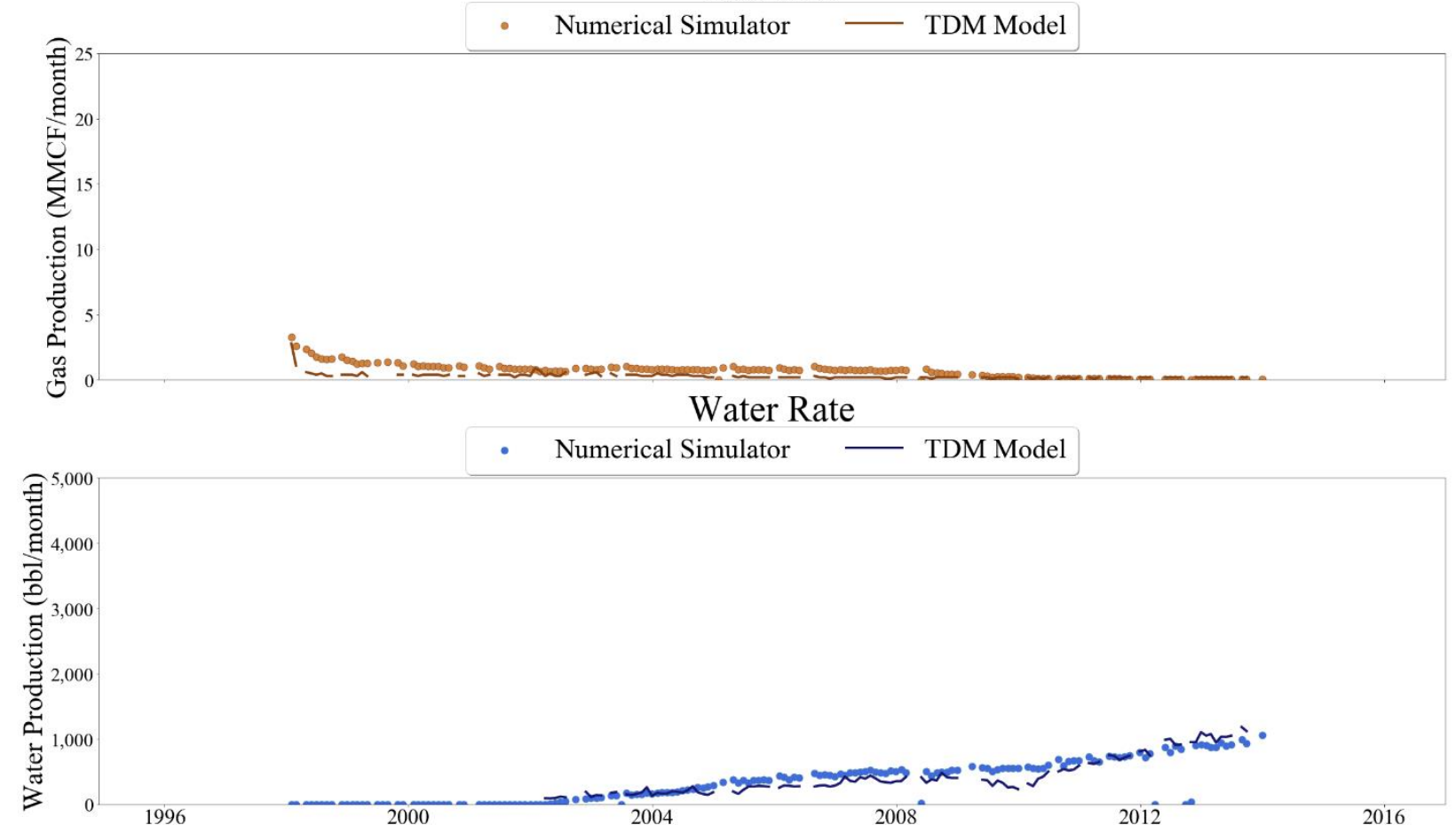

Figure 365 Well-010 oil, gas, and water rate TDM predictions vs actual simulation data plots for No Blind Validation TDM 


\section{Oil Rate}

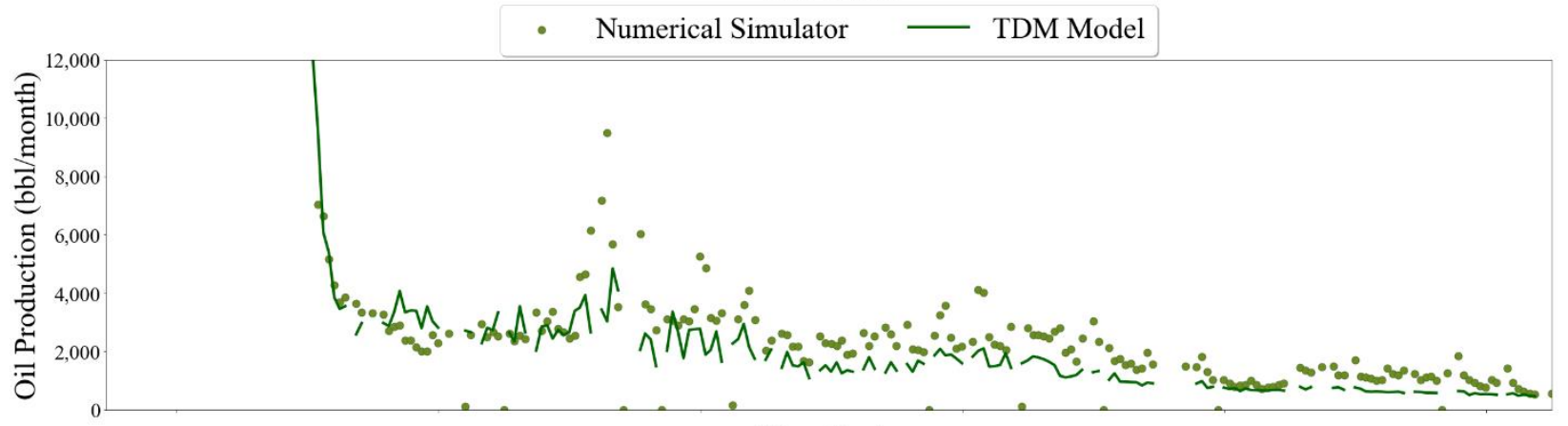

Gas Rate
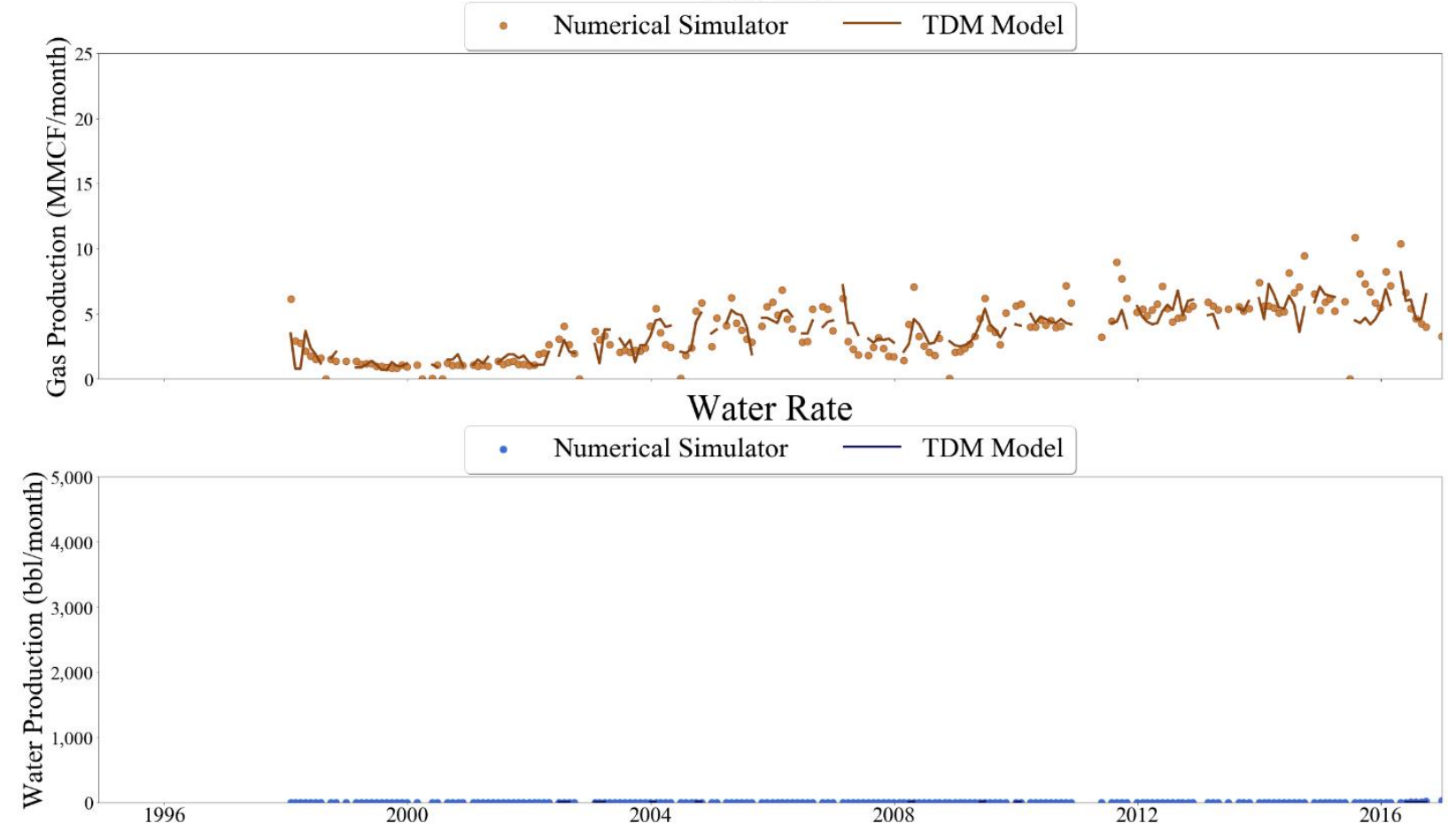

Figure 366 Well-011 oil, gas, and water rate TDM predictions vs actual simulation data plots for No Blind Validation TDM 


\section{Oil Rate}
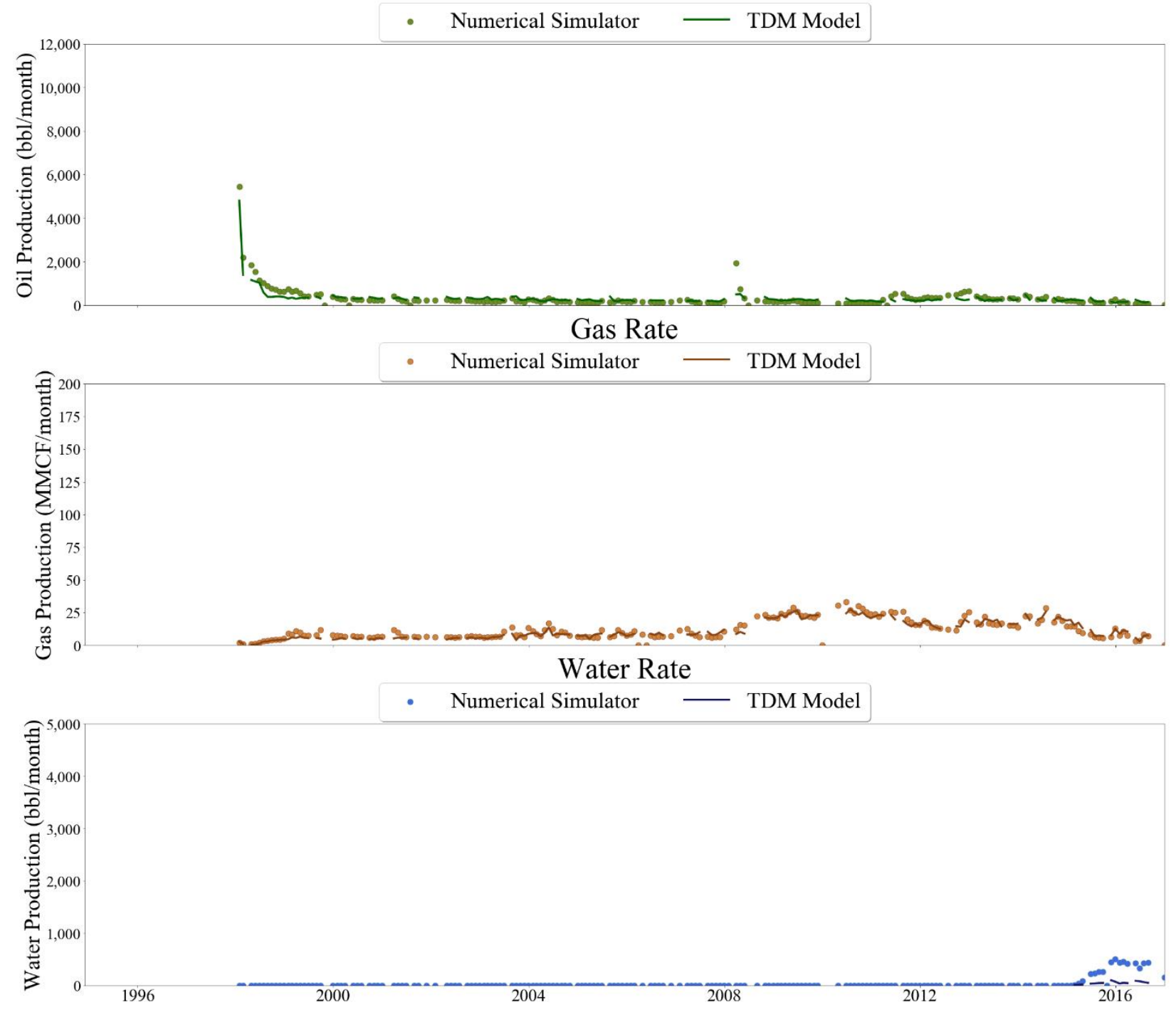

Figure 367 Well-012 oil, gas, and water rate TDM predictions vs actual simulation data plots for No Blind Validation TDM 


\section{Oil Rate}
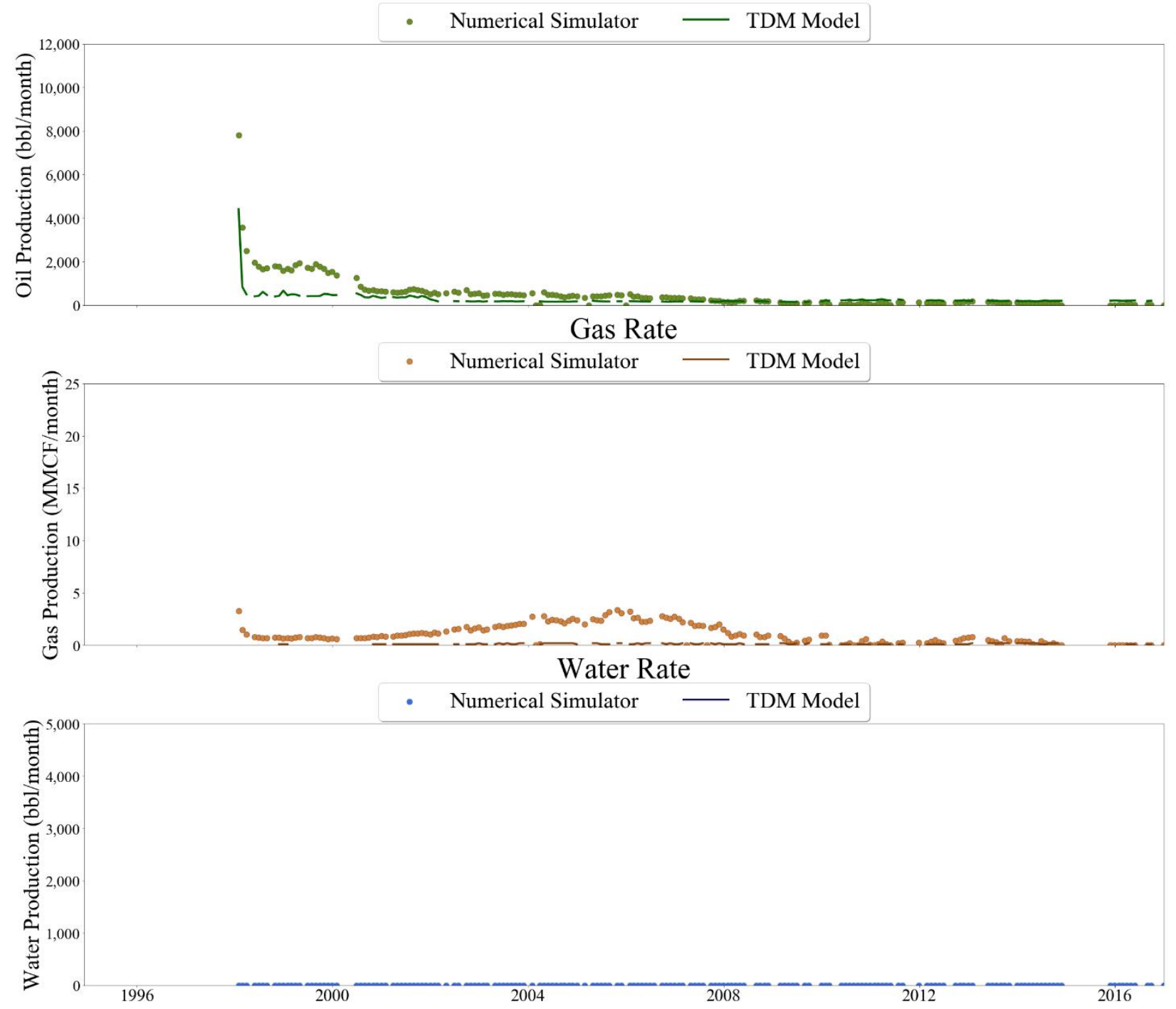

Figure 368 Well-013 oil, gas, and water rate TDM predictions vs actual simulation data plots for No Blind Validation TDM 


\section{Oil Rate}

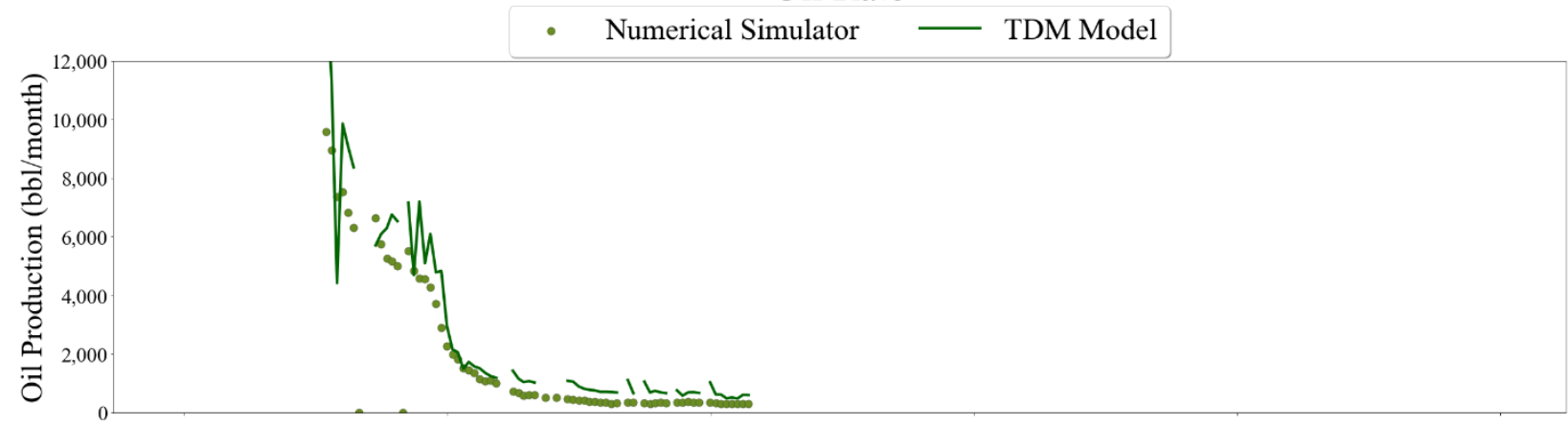

Gas Rate
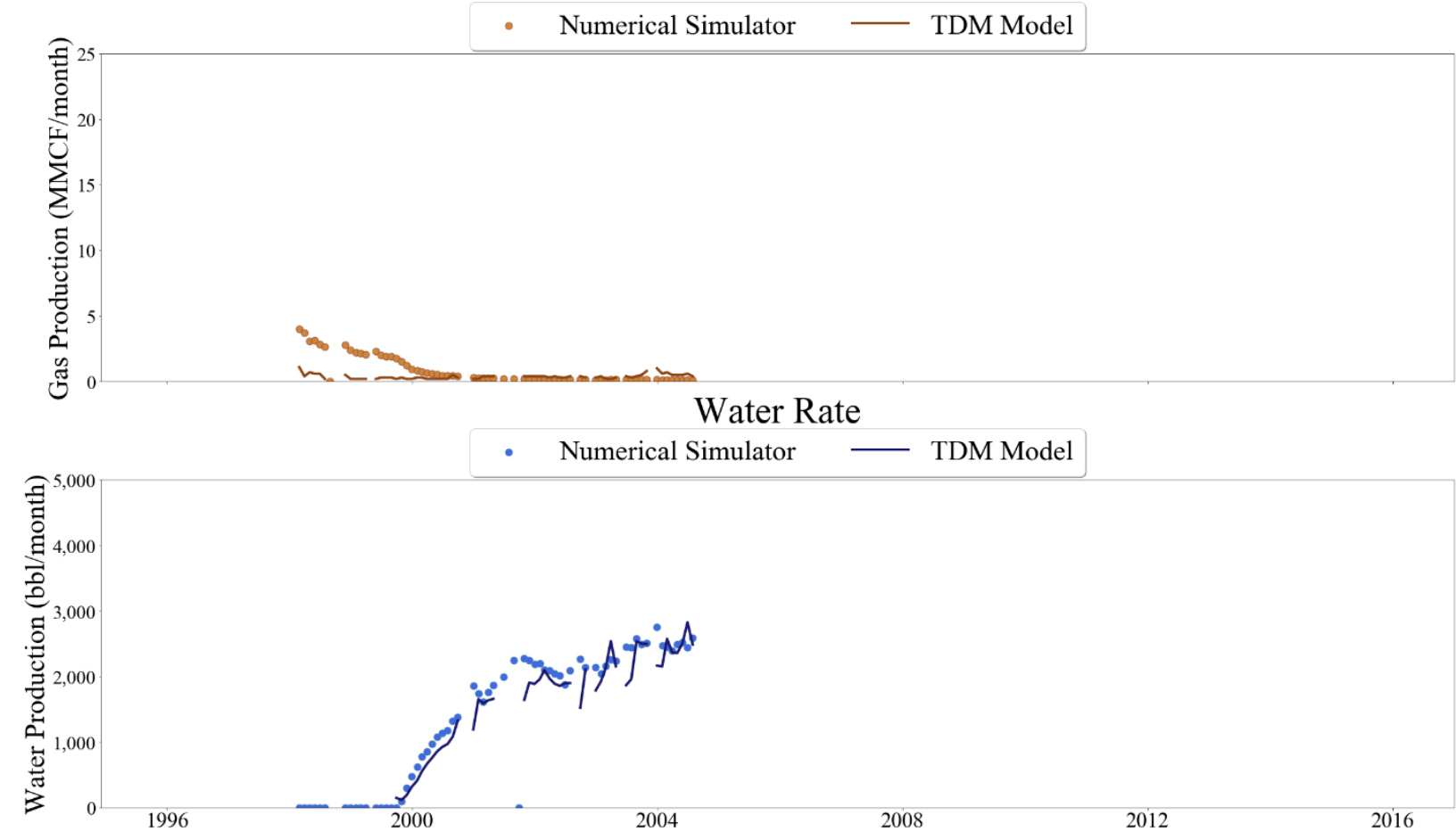

Figure 369 Well-014 oil, gas, and water rate TDM prediction vs actual simulation data plots for No Blind Validation TDM 


\section{Oil Rate}

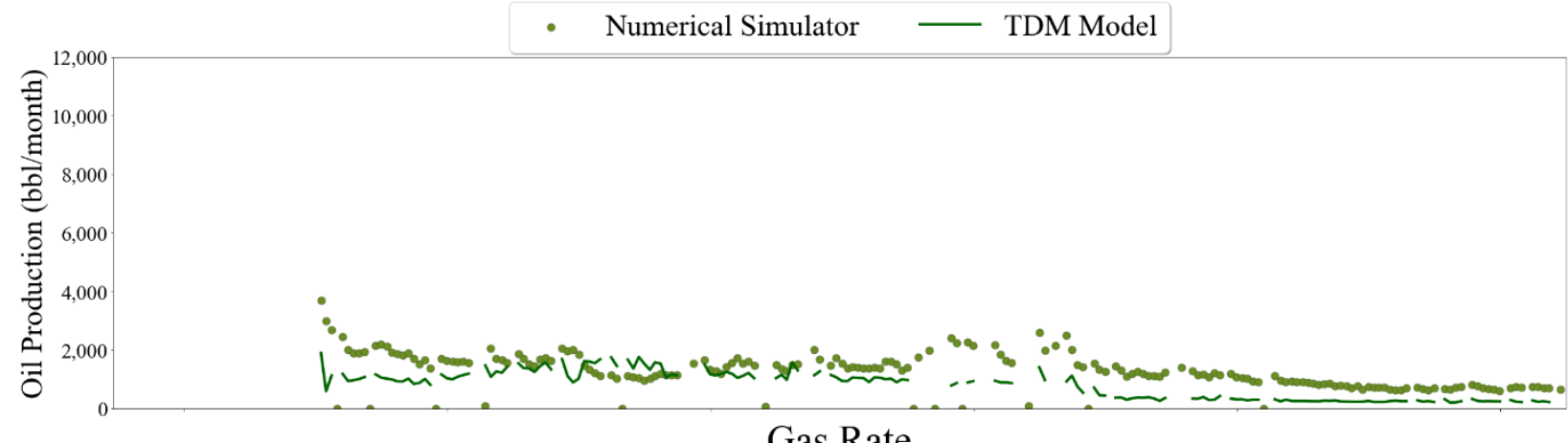

Gas Rate
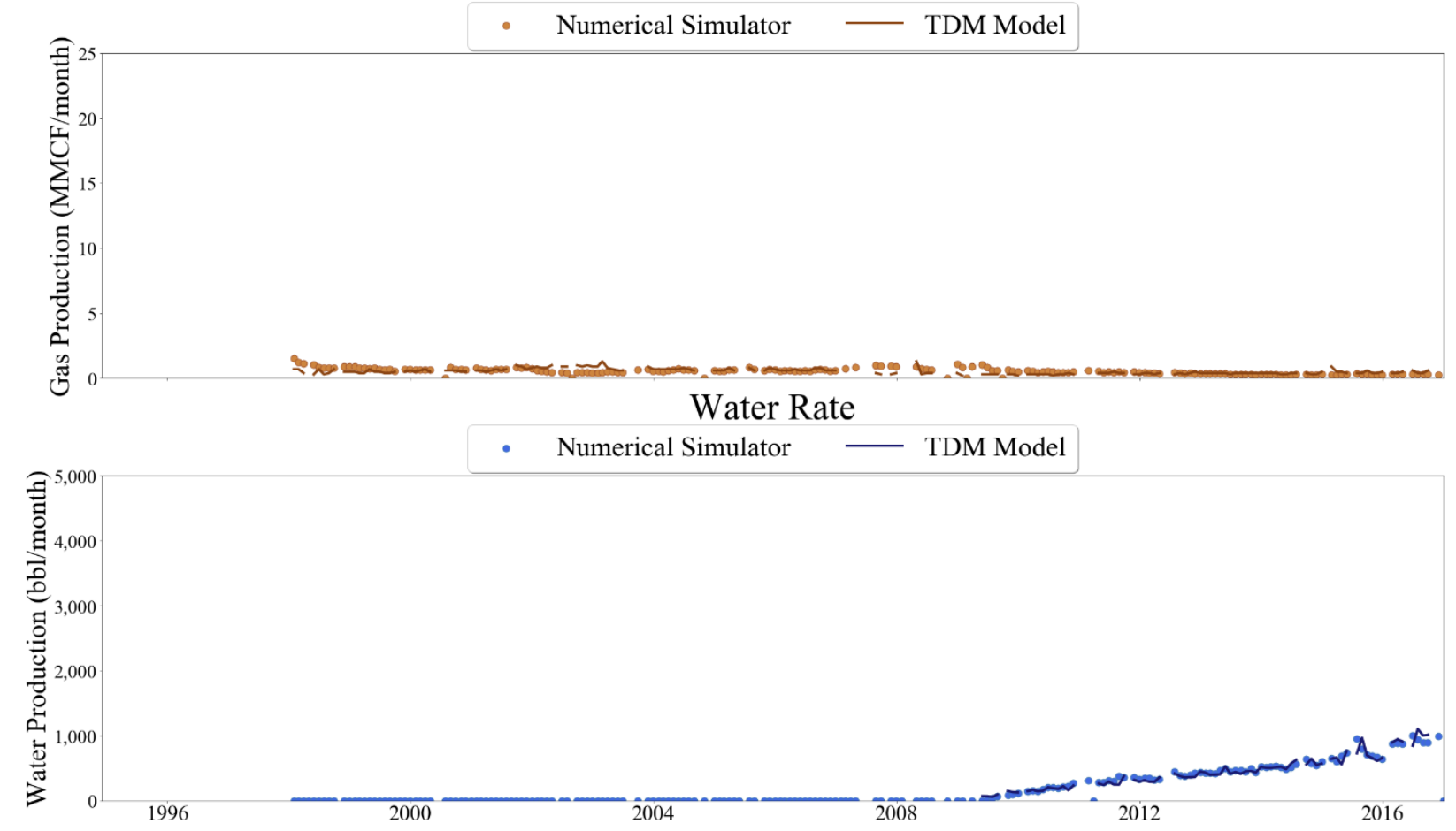

Figure 370 Well-015 oil, gas, and water rate TDM predictions vs actual simulation data plots for No Blind Validation TDM 


\section{Oil Rate}
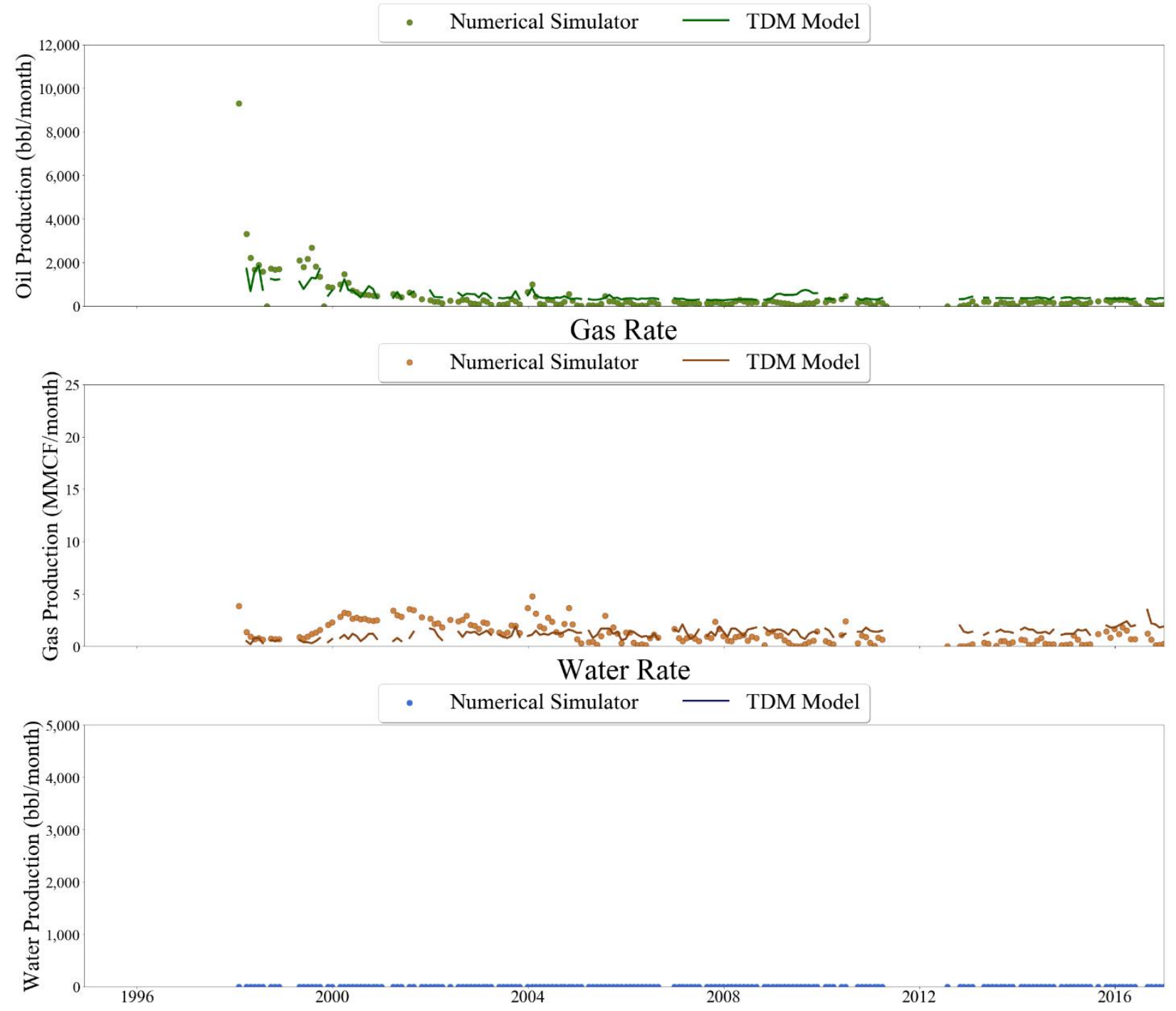

Figure 371 Well-016 oil, gas, and water rate TDM predictions vs actual simulation data plots for No Blind Validation TDM 


\section{Oil Rate}
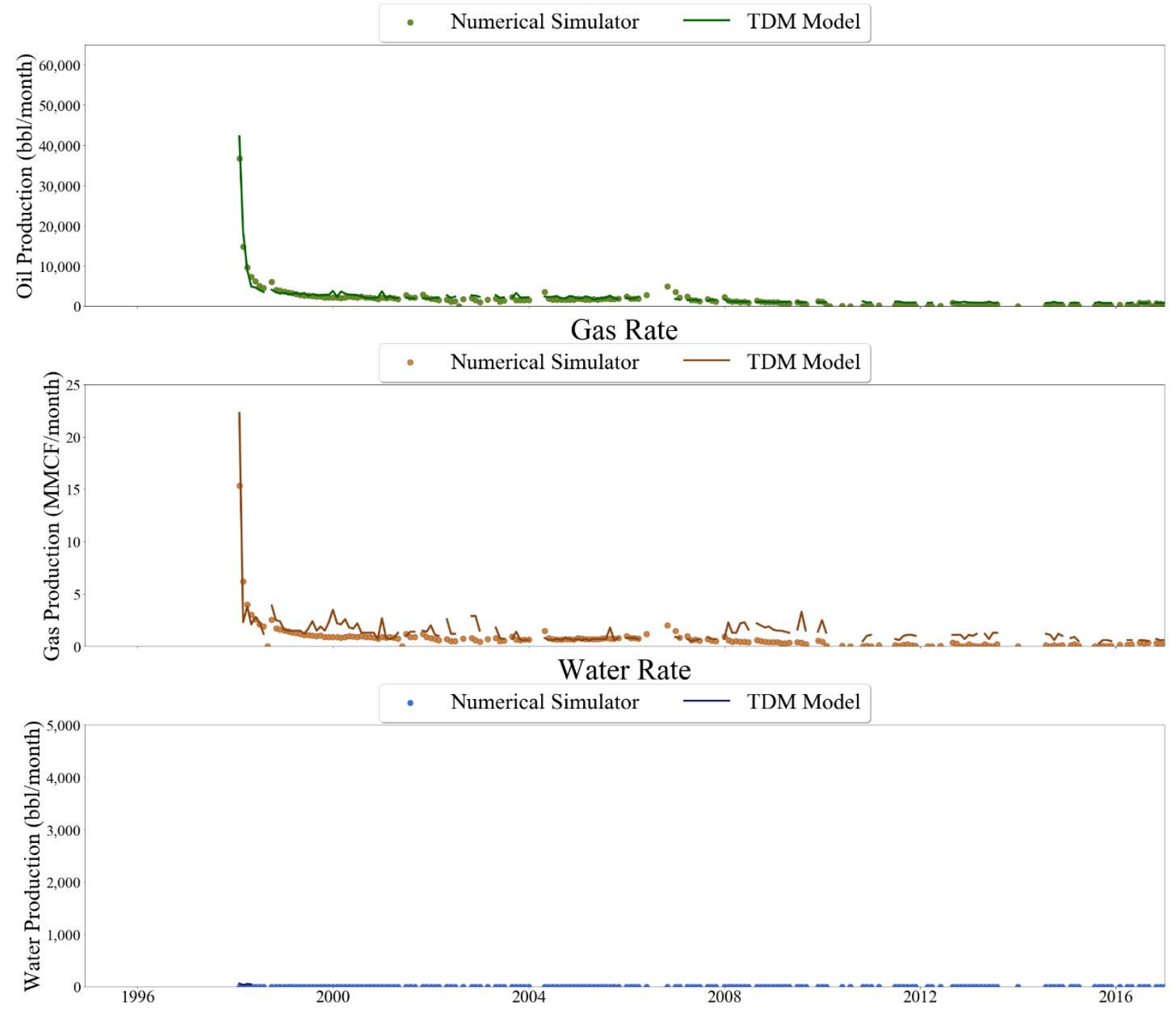

Figure 372 Well-017 oil, gas, and water rate TDM predictions vs actual simulation data plots for No Blind Validation TDM 


\section{Oil Rate}

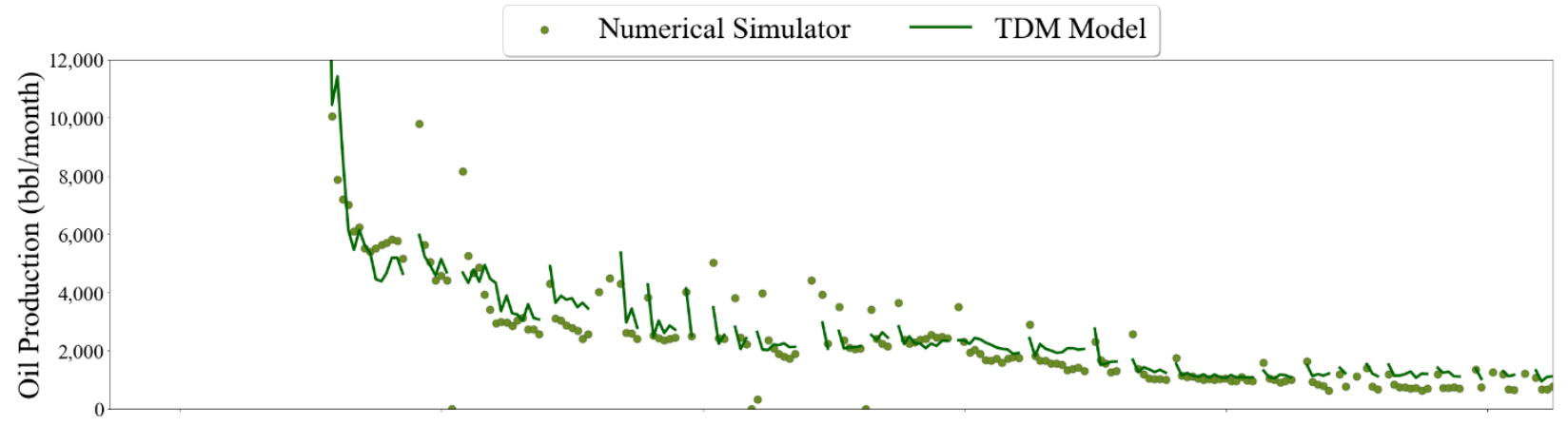

Gas Rate
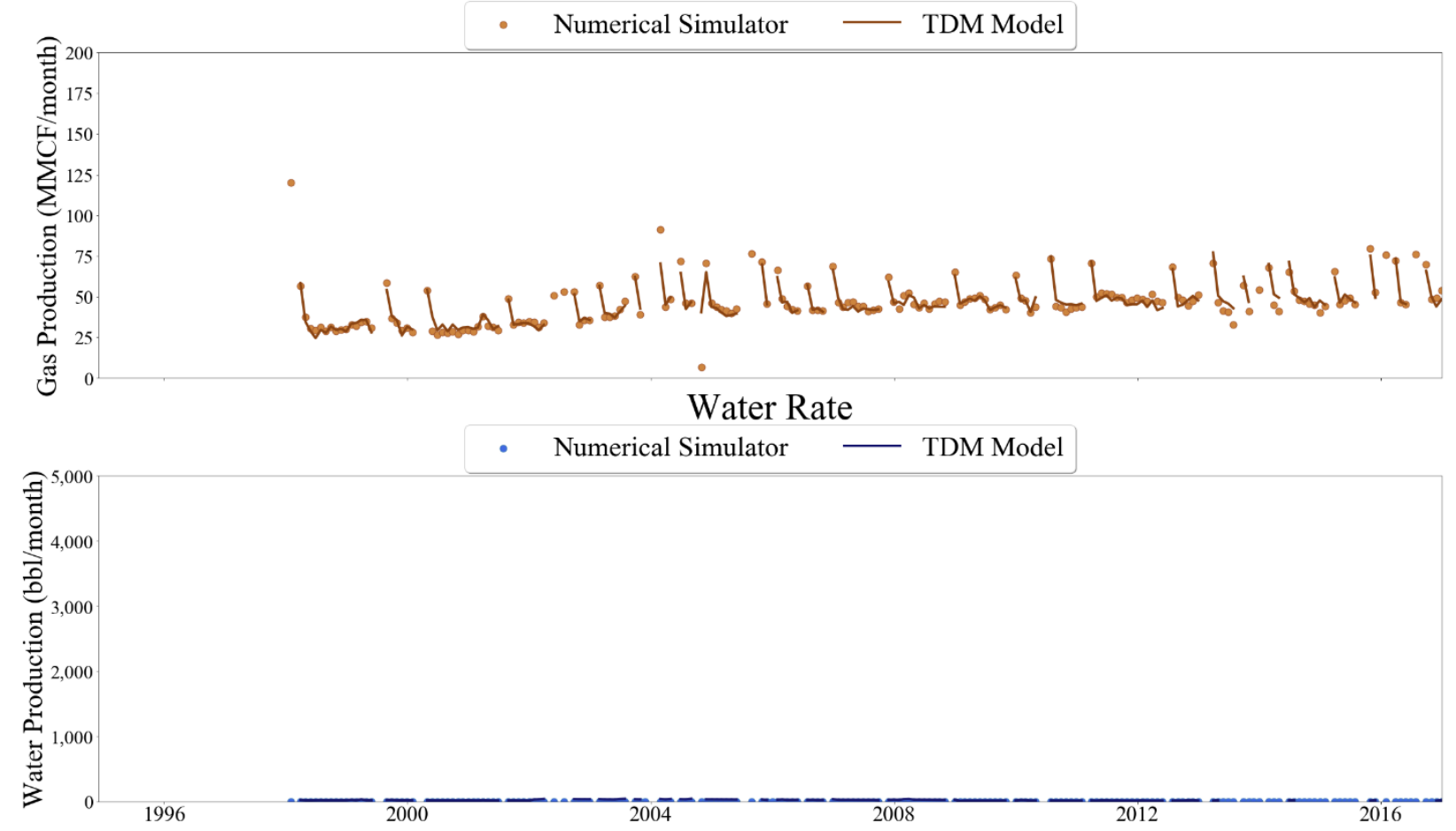

Figure 373 Well-018 oil, gas, and water rate TDM predictions vs actual simulation data plots for No Blind Validation TDM 


\section{Oil Rate}
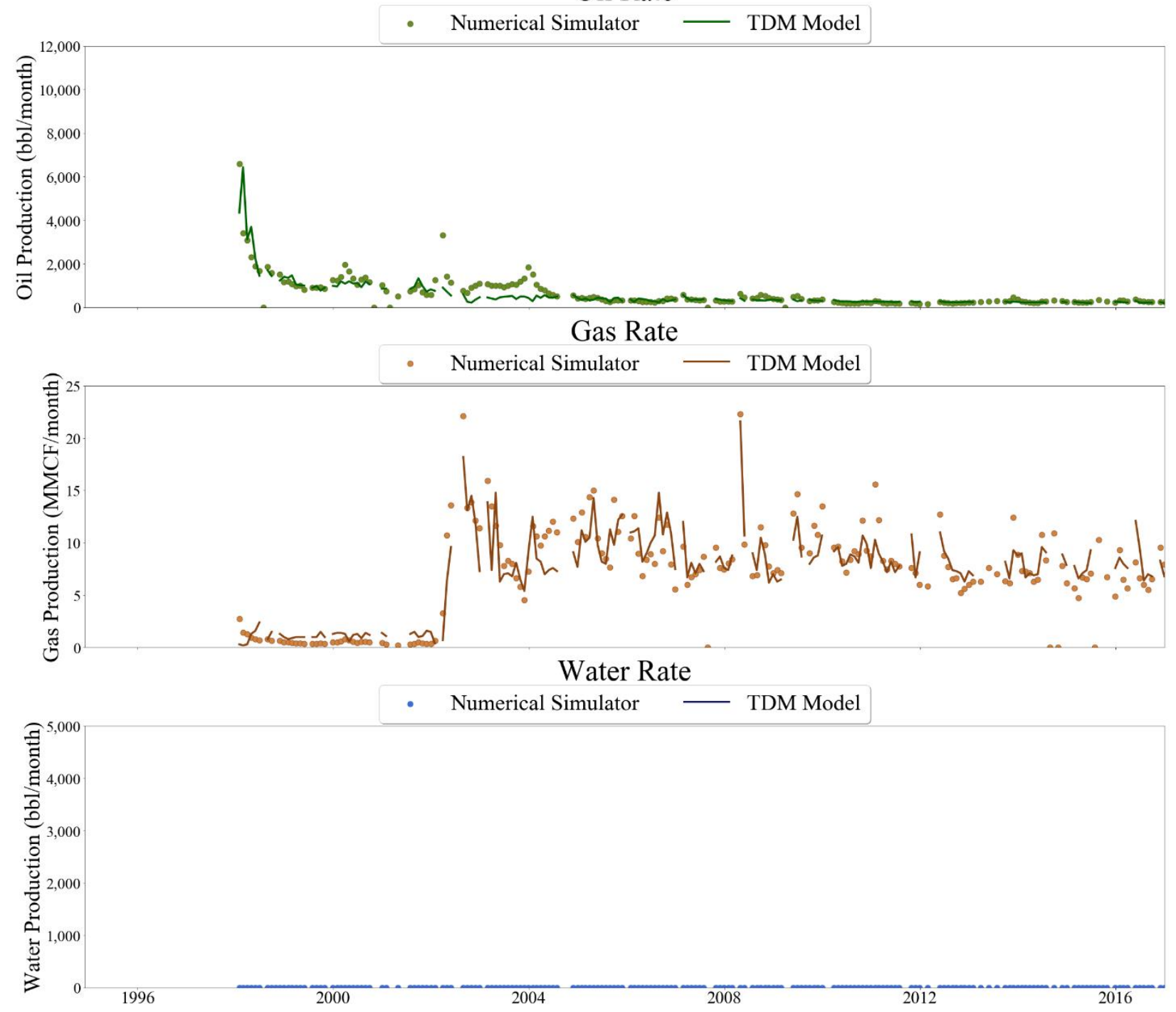

Figure 374 Well-019 oil, gas, and water rate TDM prediction vs actual simulation data plots for No Blind Validation TDM 


\section{Oil Rate}
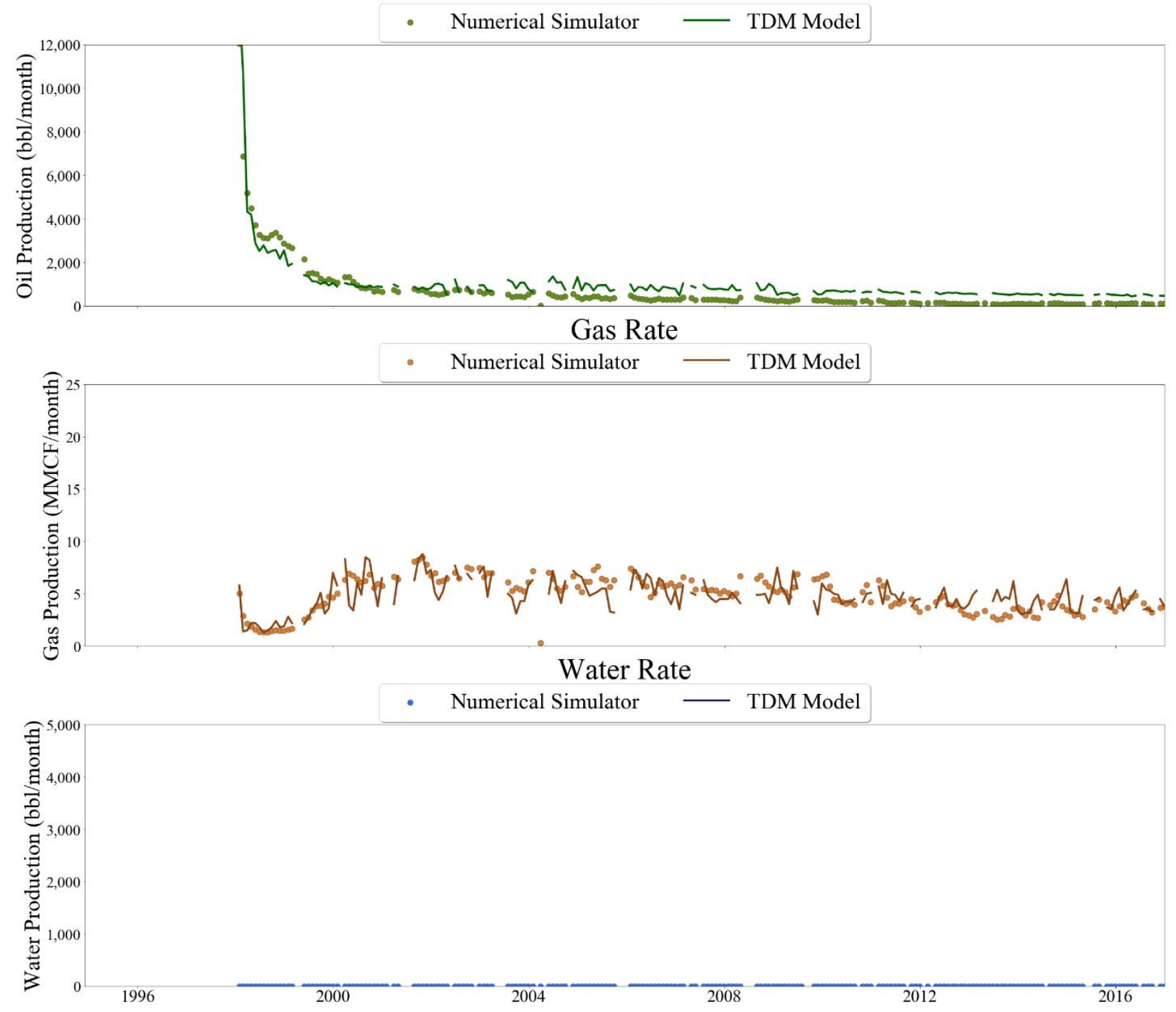

Figure 375 Well-020 oil, gas, and water rate TDM predictions vs actual simulation data plots for No Blind Validation TDM 


\section{Oil Rate}
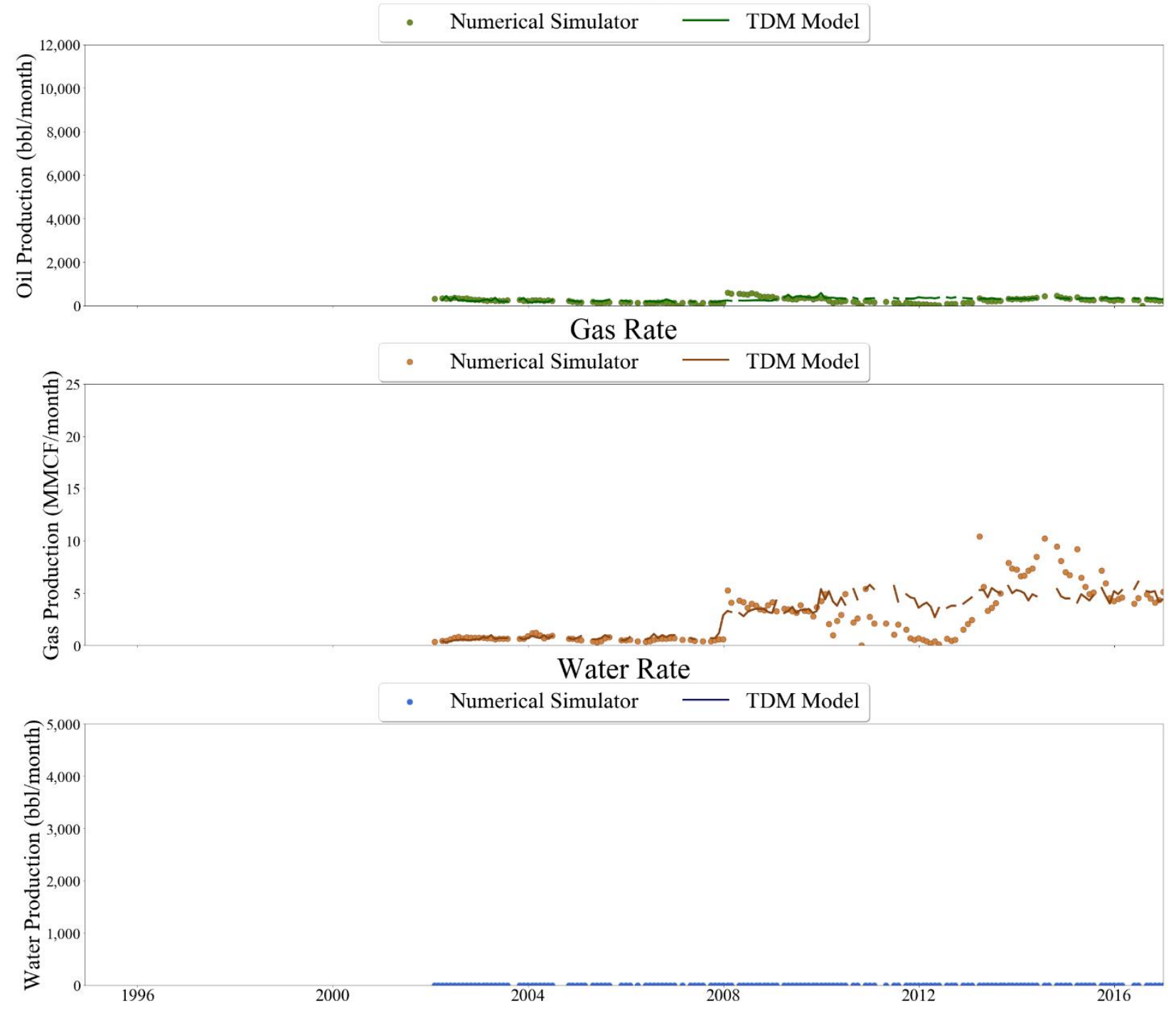

Figure 376 Well-021 oil, gas, and water rate TDM predictions vs actual simulation data plots for No Blind Validation TDM 


\section{Oil Rate}
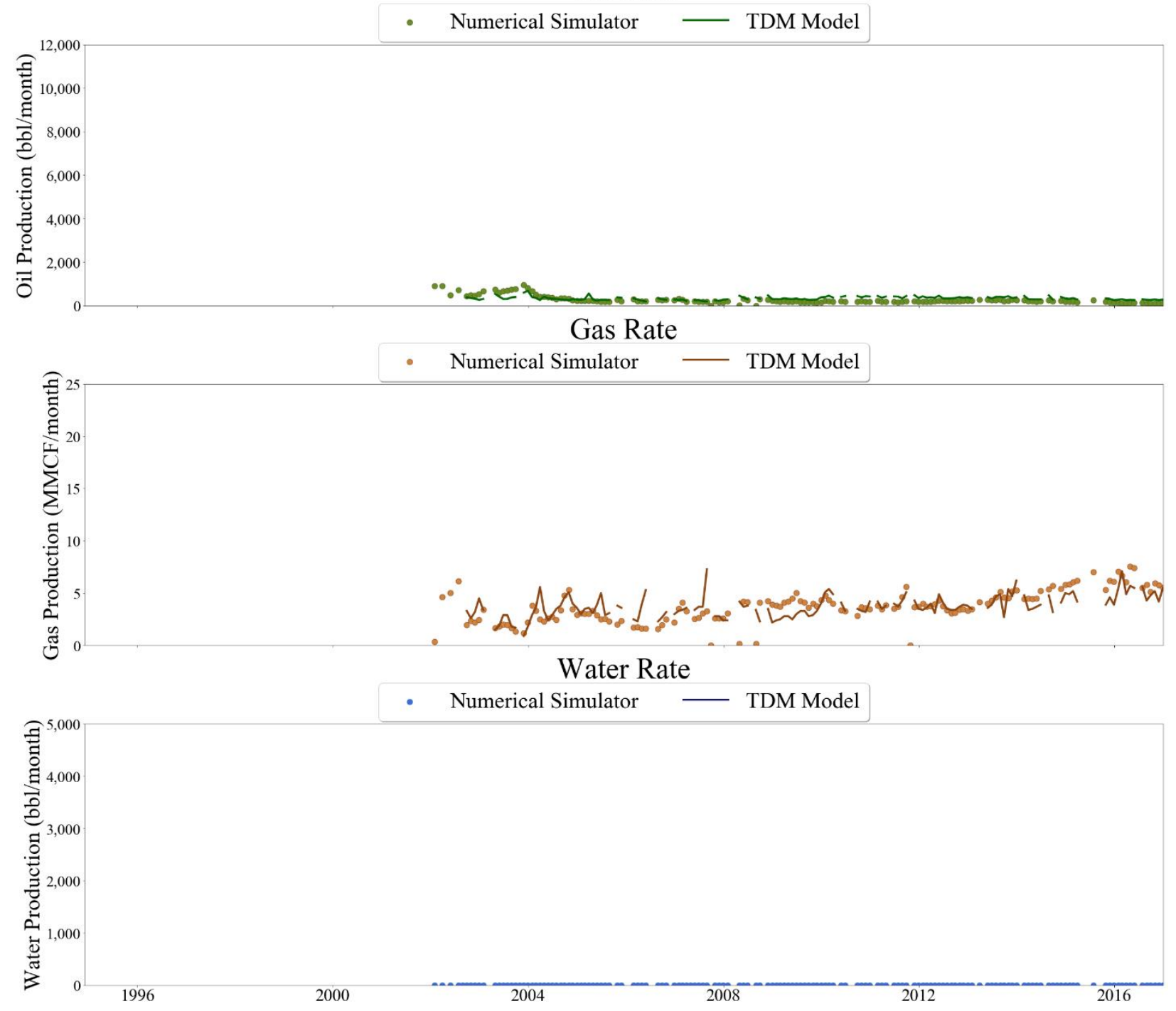

Figure 377 Well-022 oil, gas, and water rate TDM predictions vs actual simulation data plots for No Blind Validation TDM 


\section{Oil Rate}
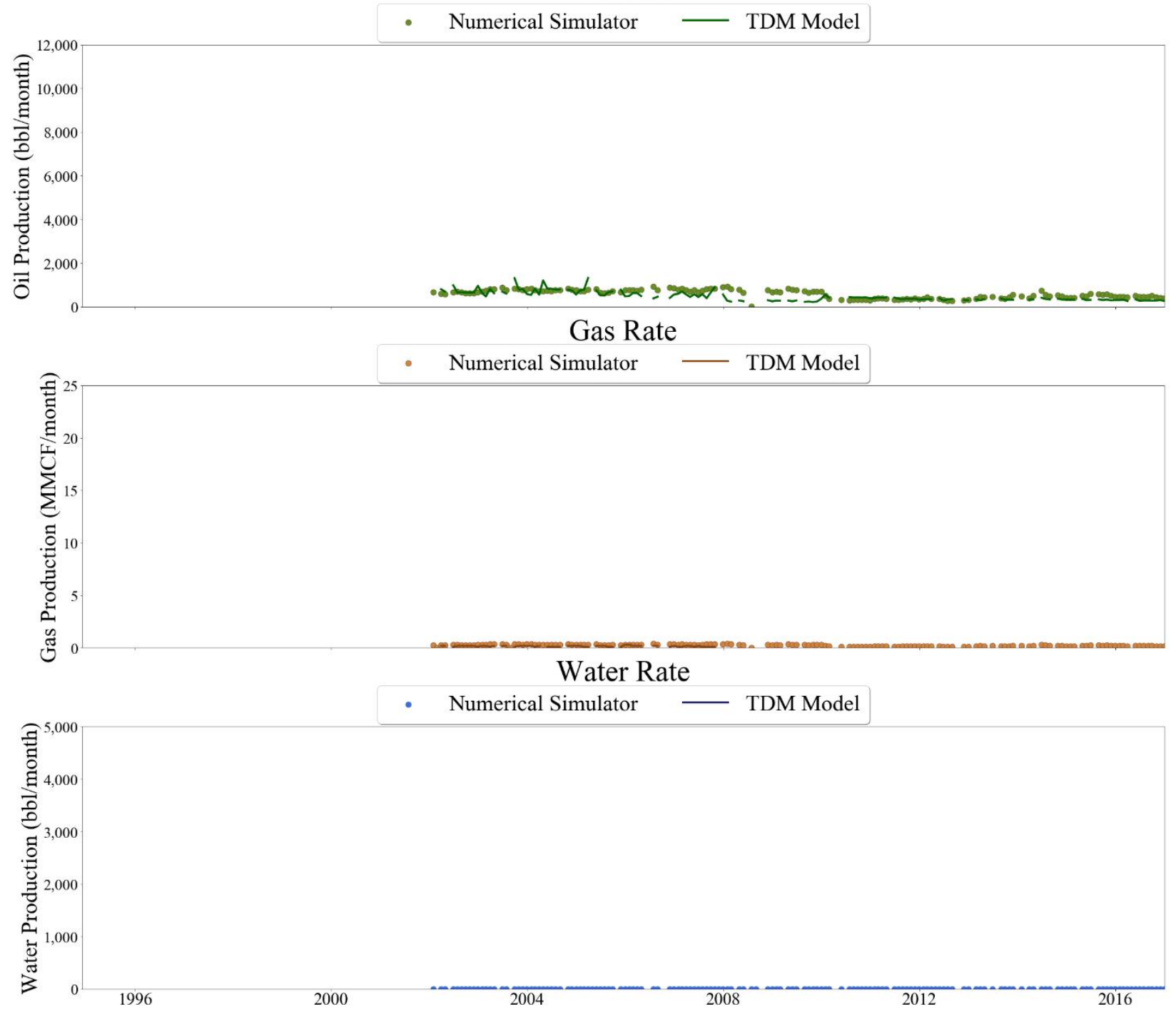

Figure 378 Well-023 oil, gas, and water rate TDM predictions vs actual simulation data plots for No Blind Validation TDM 


\section{Oil Rate}

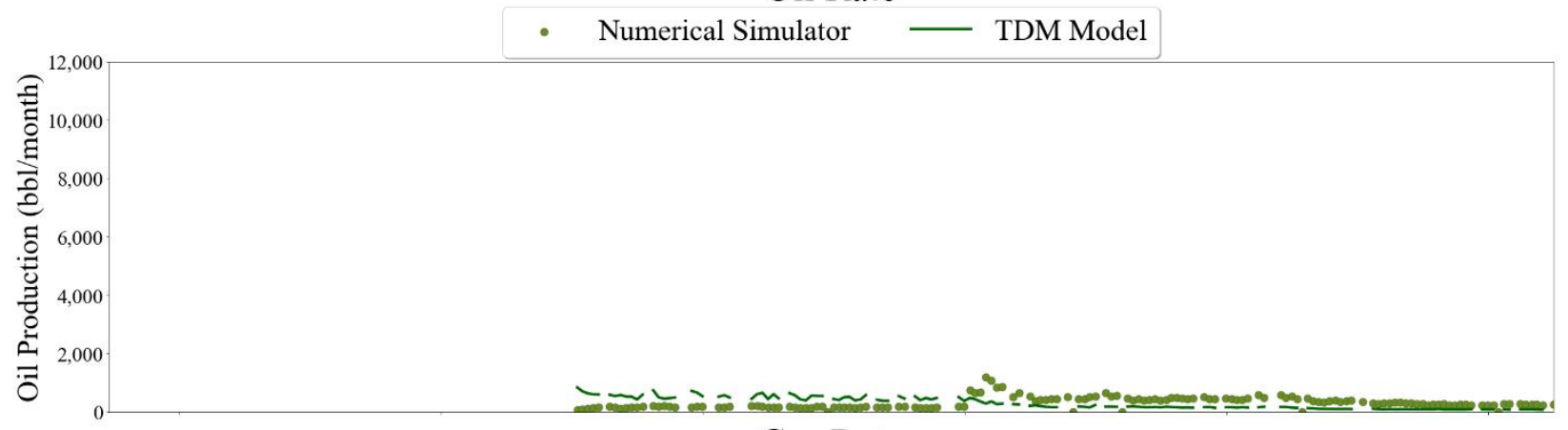

Gas Rate
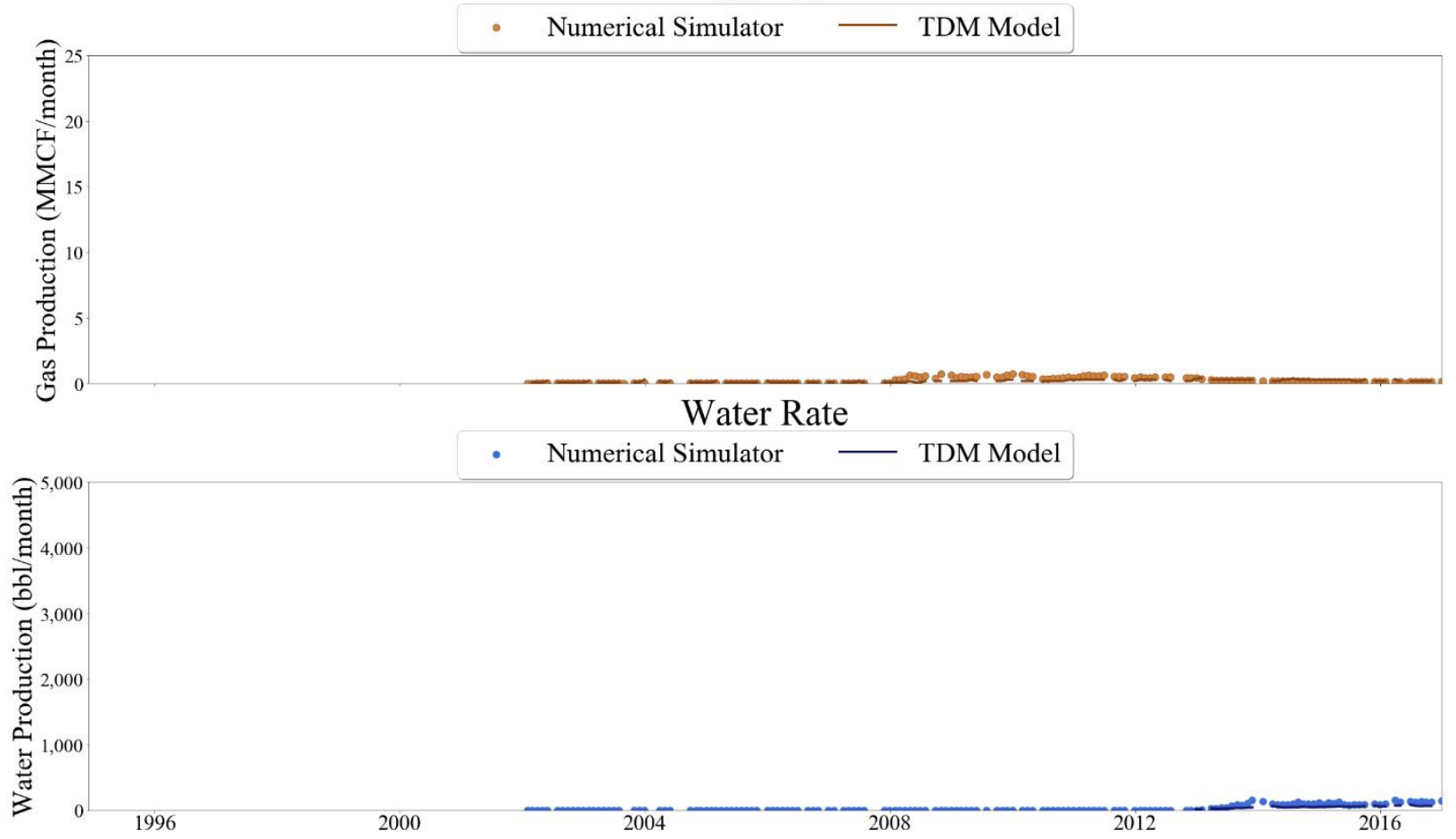

Figure 379 Well-024 oil, gas, and water rate TDM predictions vs actual simulation data plots for No Blind Validation TDM 


\section{Oil Rate}

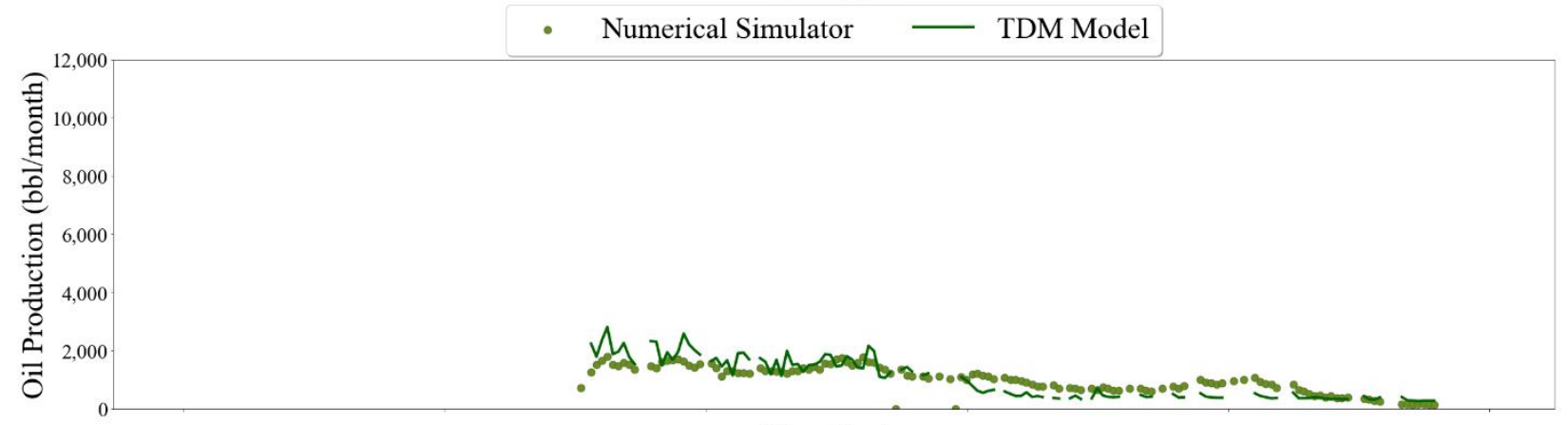

Gas Rate
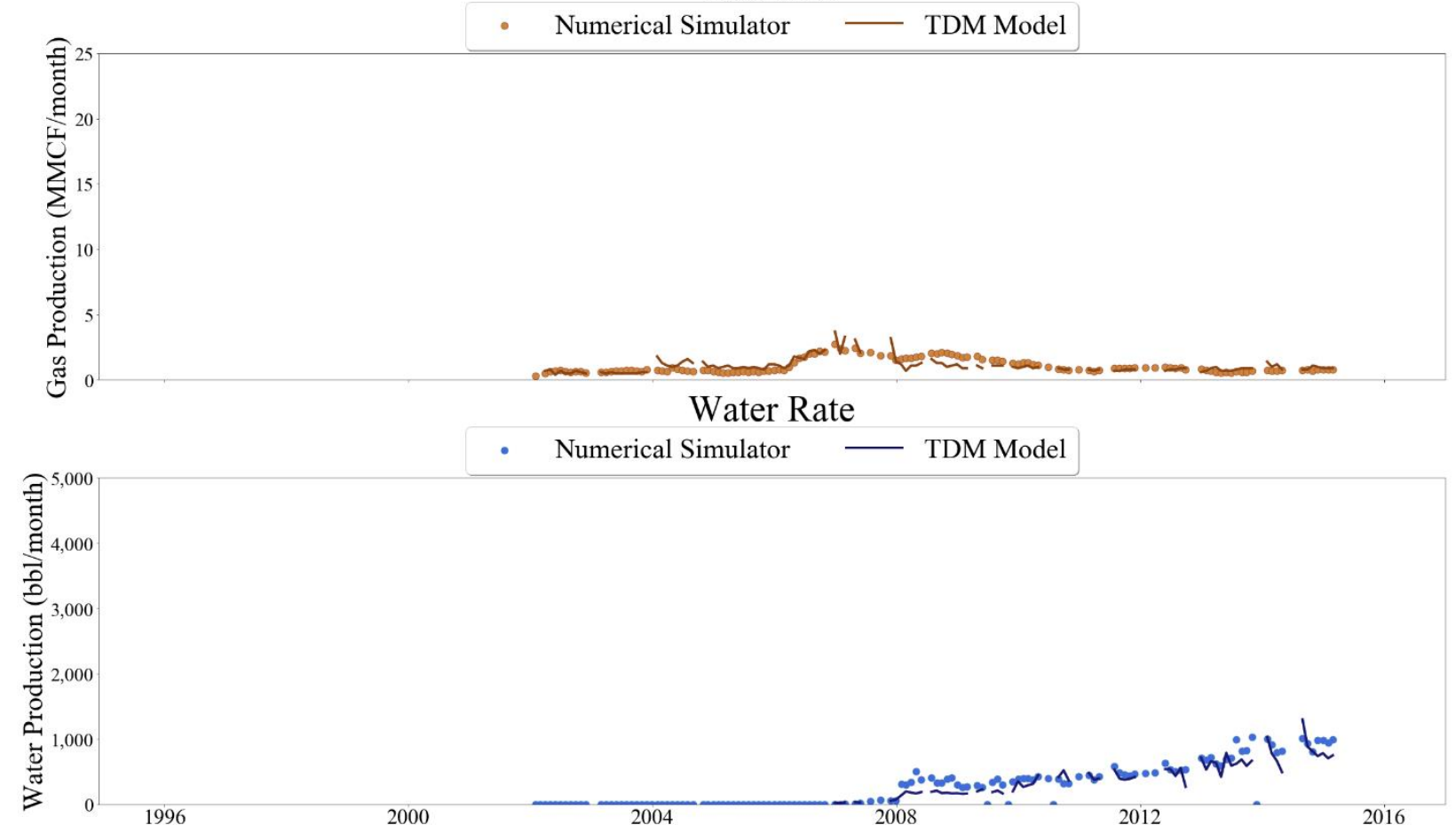

Figure 380 Well-025 oil, gas, and water rate TDM predictions vs actual simulation data plots for No Blind Validation TDM 


\section{Oil Rate}

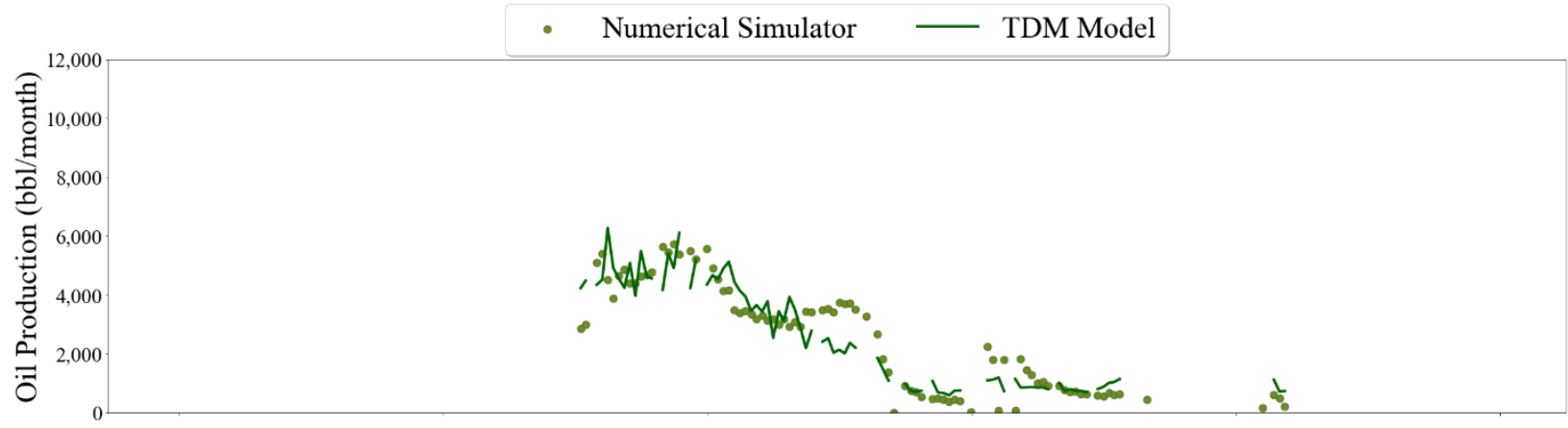

\section{Gas Rate}
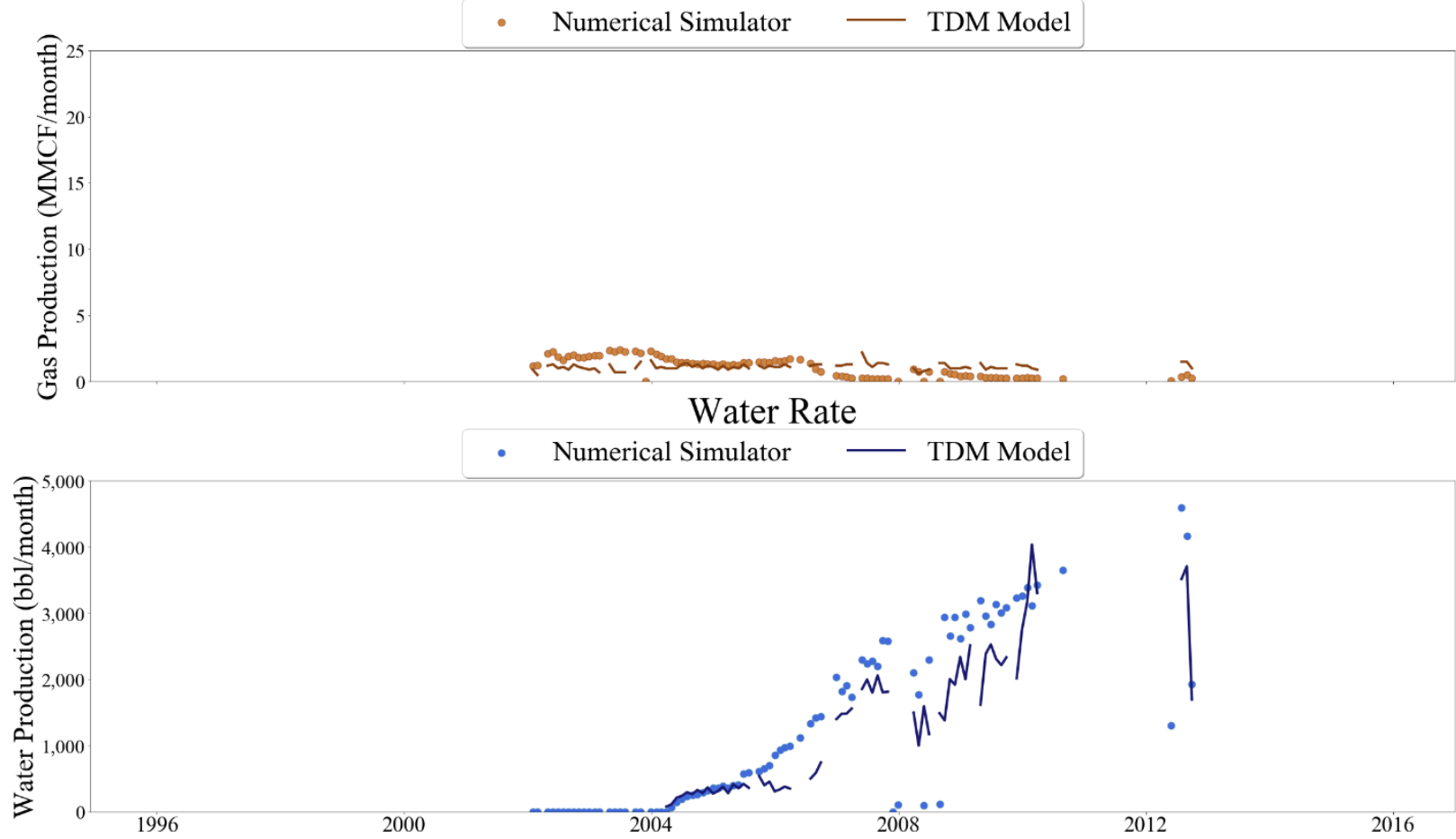

Figure 381 Well-026 oil, gas, and water rate TDM predictions vs actual simulation data plots for No Blind Validation TDM 


\section{Oil Rate}

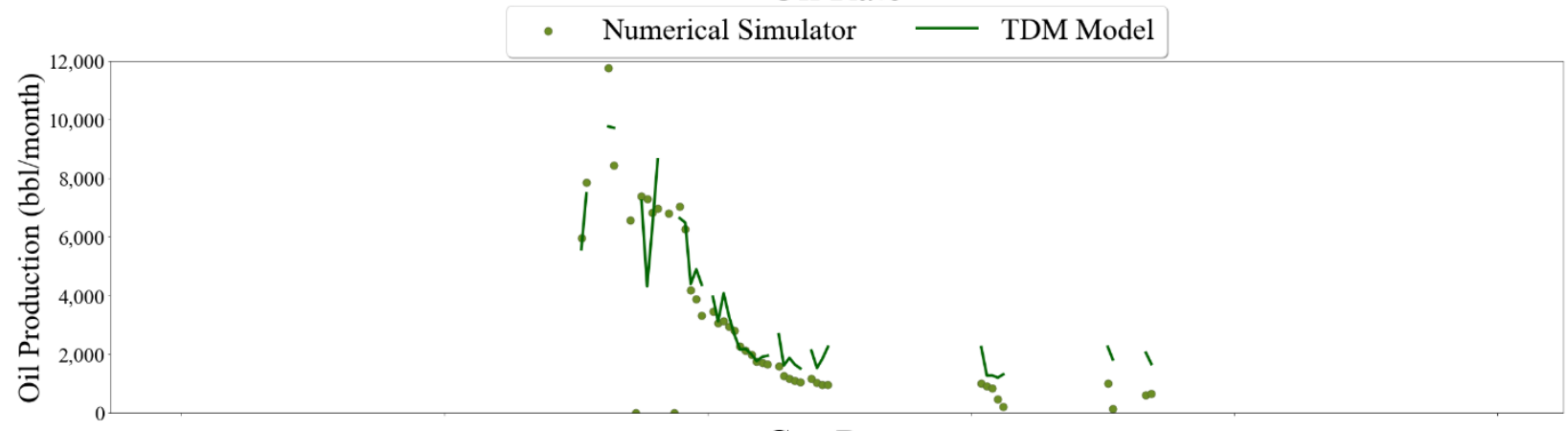

Gas Rate
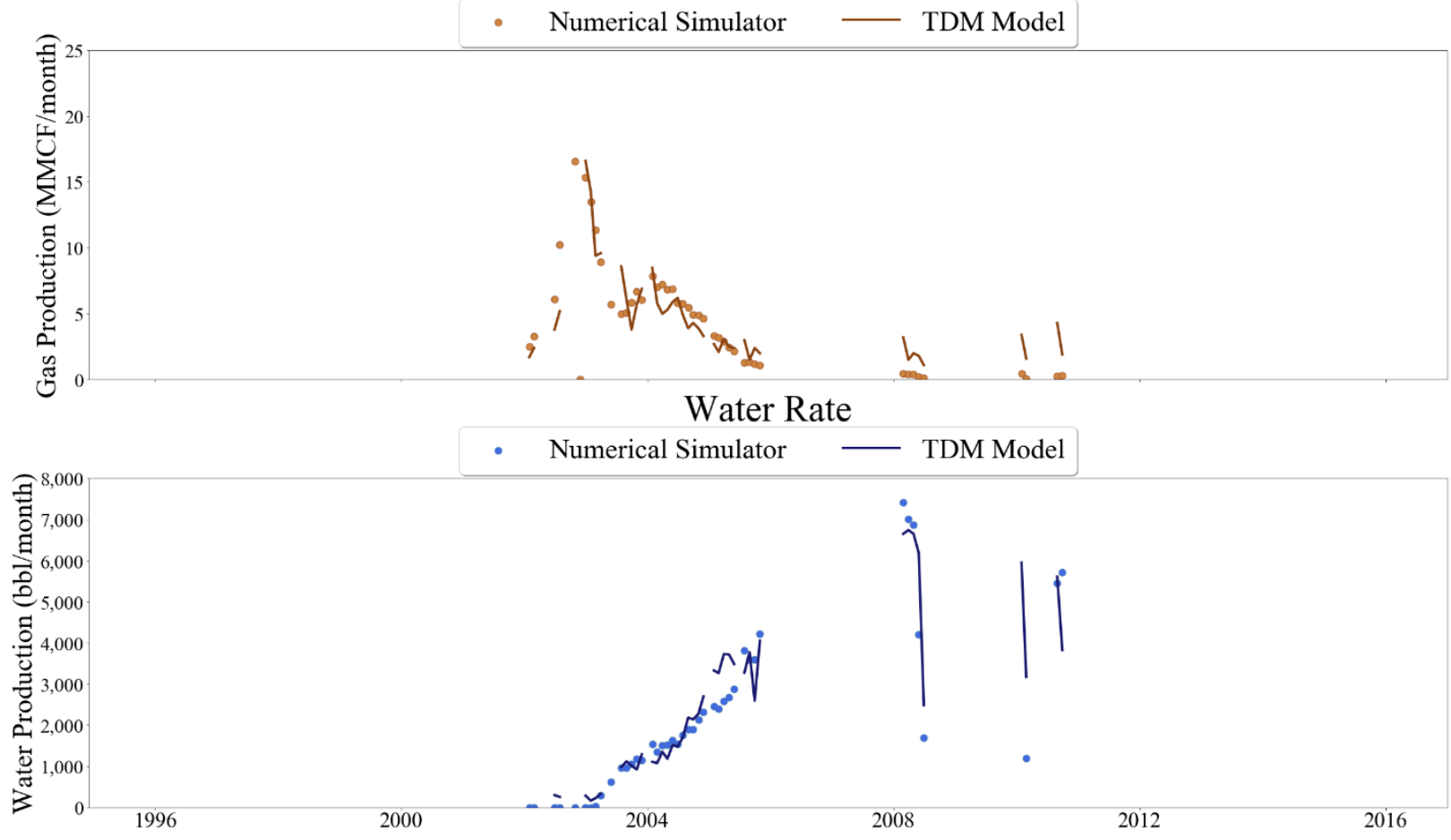

Figure 382 Well-027 oil, gas, and water rate TDM predictions vs actual simulation data plots for No Blind Validation TDM 


\section{Oil Rate}

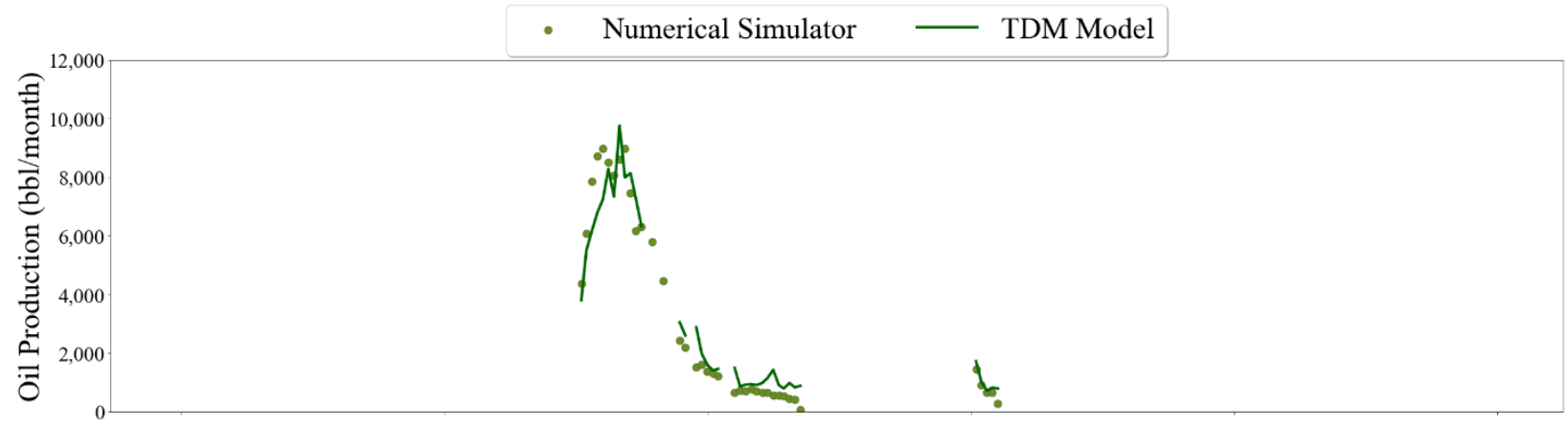

Gas Rate
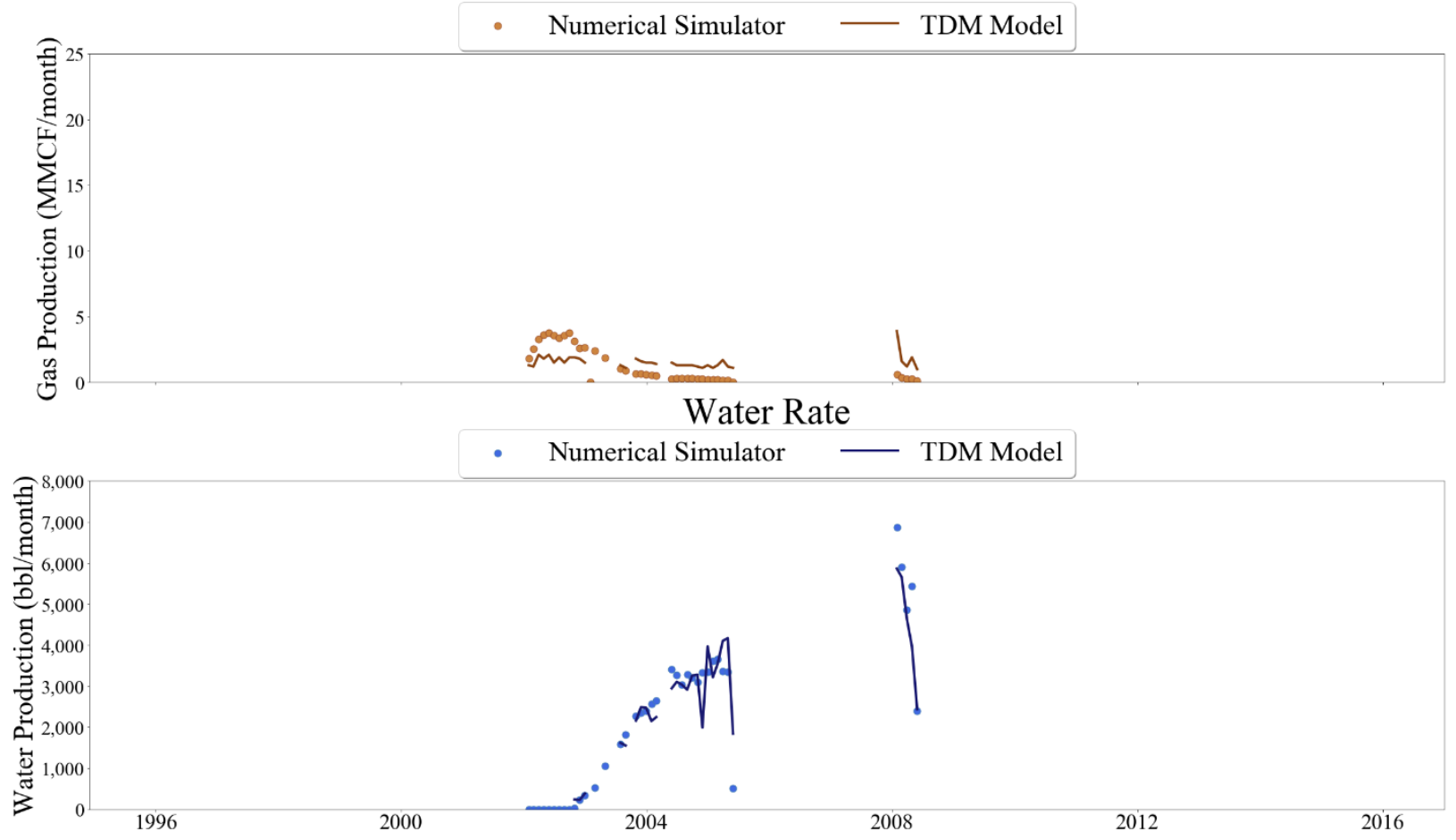

Figure 383 Well-028 oil, gas, and water rate TDM predictions vs actual simulation data plots for No Blind Validation TDM 


\section{Oil Rate}
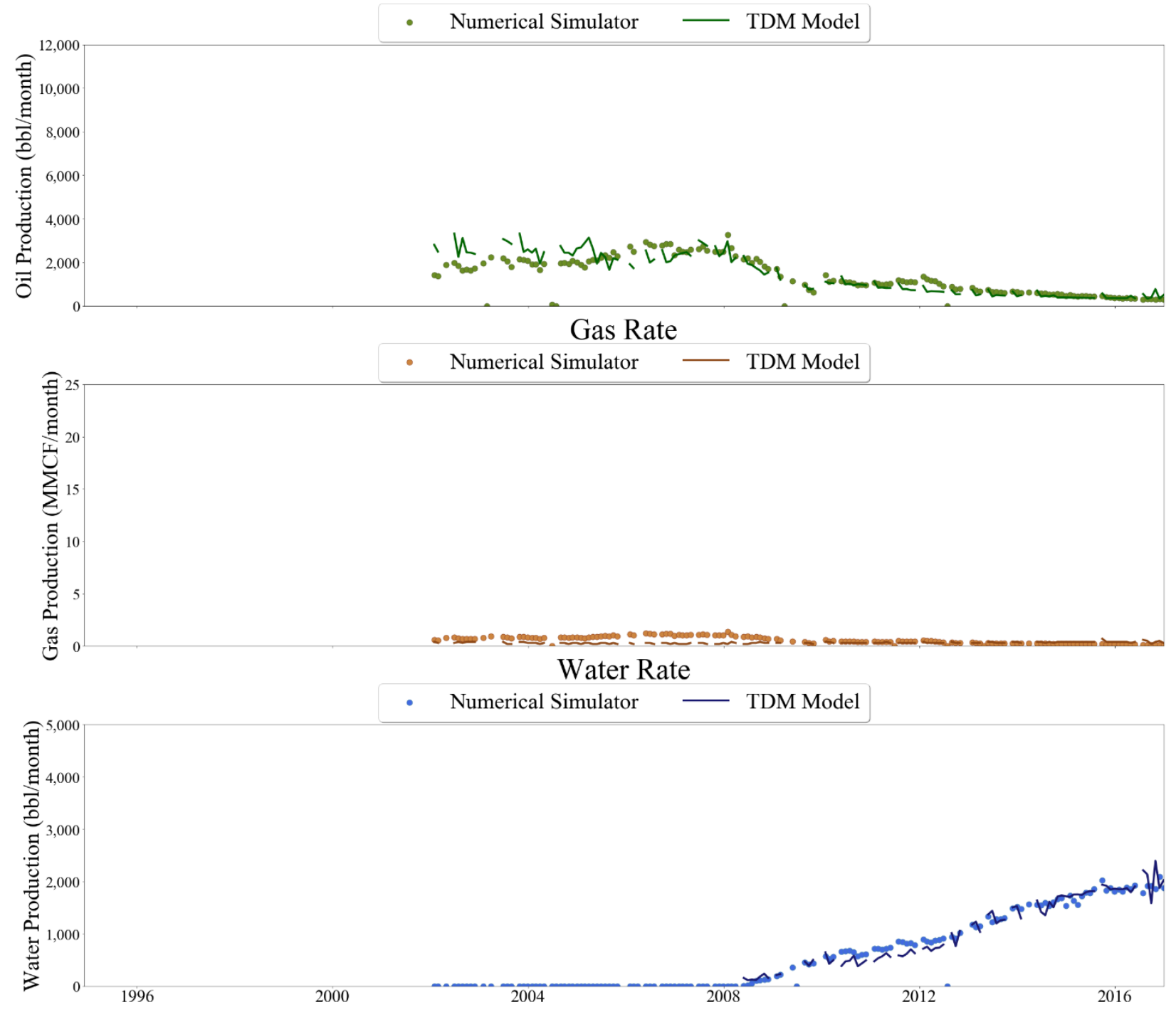

Figure 384 Well-029 oil, gas, and water rate TDM predictions vs actual simulation data plots for No Blind Validation TDM 


\section{Oil Rate}

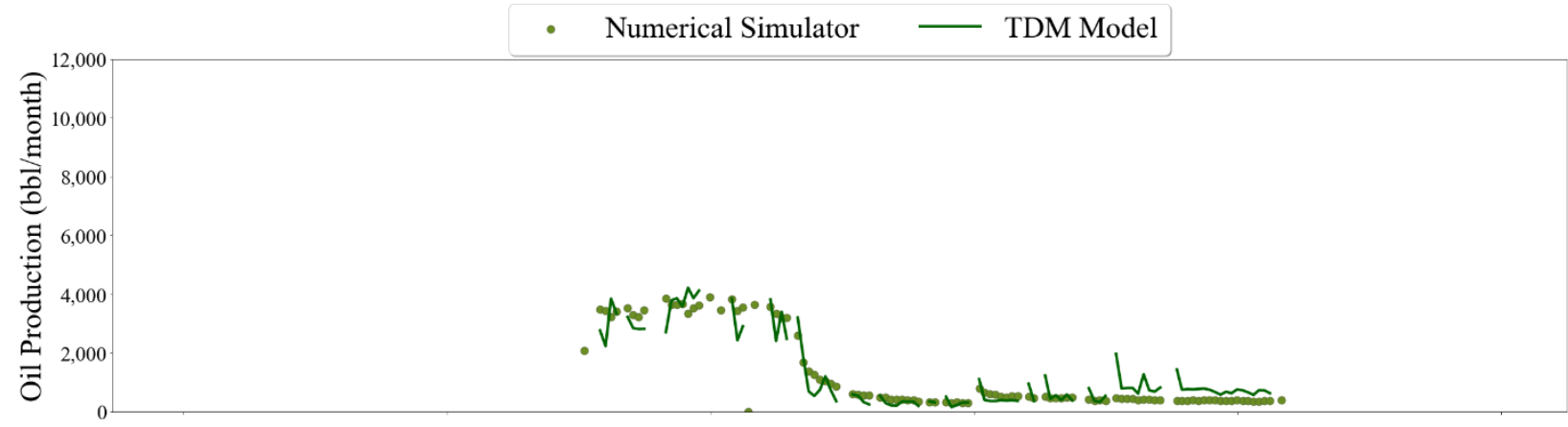

Gas Rate
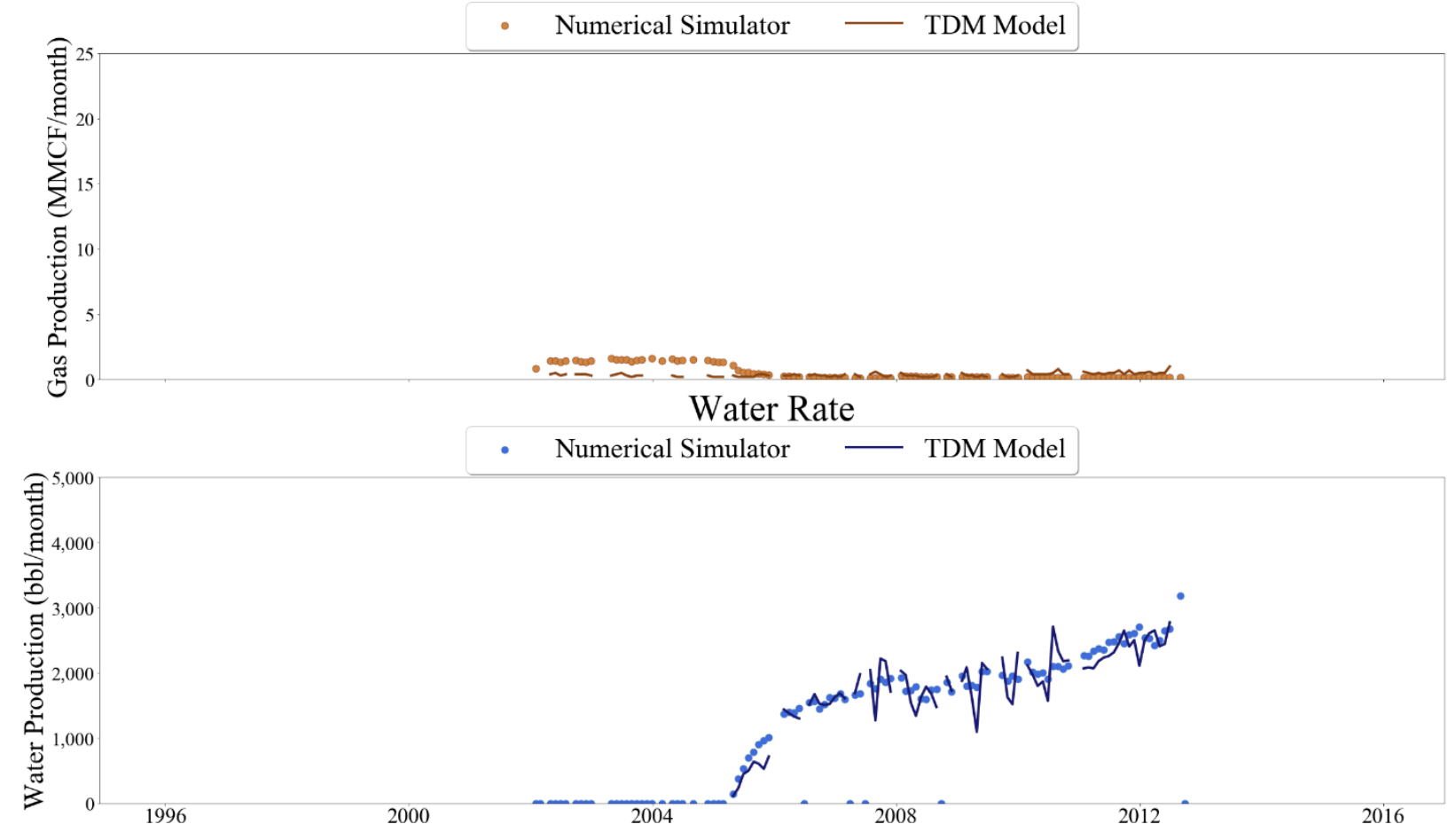

Figure 385 Well-030 oil, gas, and water rate TDM predictions vs actual simulation data plots for No Blind Validation TDM 


\section{Oil Rate}
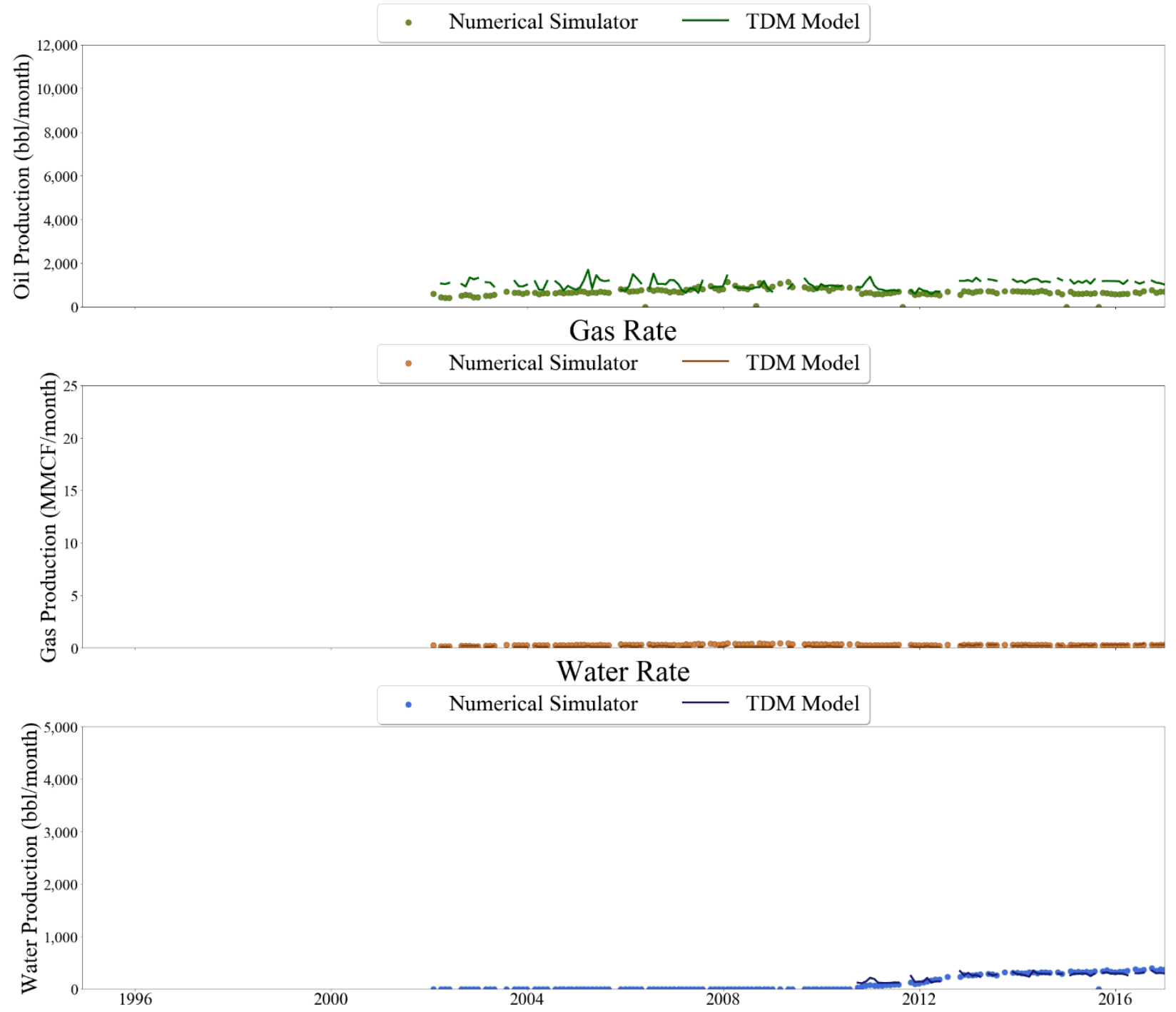

Figure 386 Well-031 oil, gas, and water rate TDM predictions vs actual simulation data plots for No Blind Validation TDM 


\section{Oil Rate}

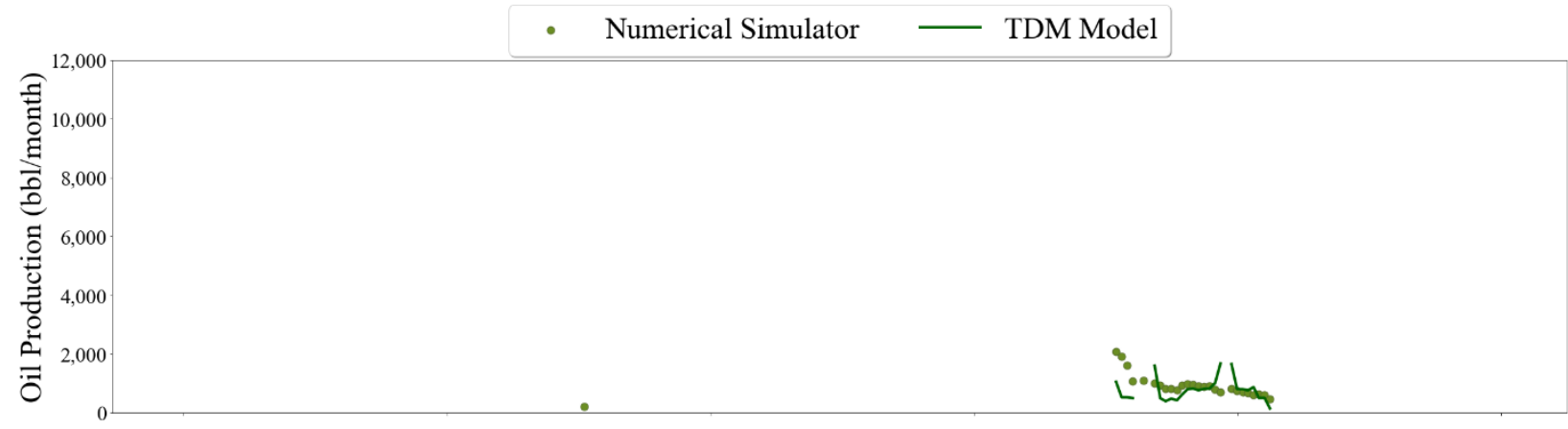

Gas Rate
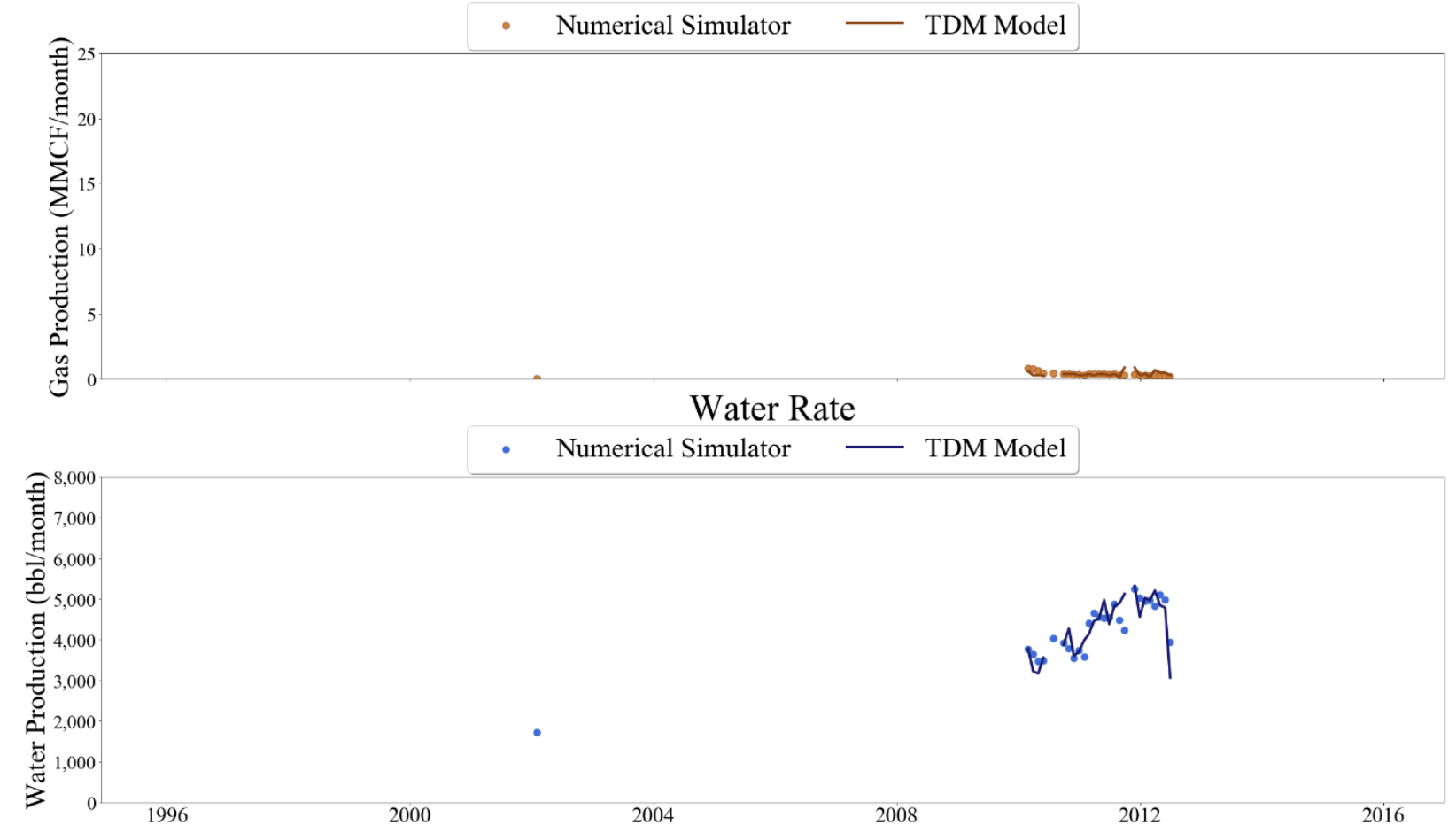

Figure 387 Well-032 oil, gas, and water rate TDM predictions vs actual simulation data plots for No Blind Validation TDM 


\section{Oil Rate}

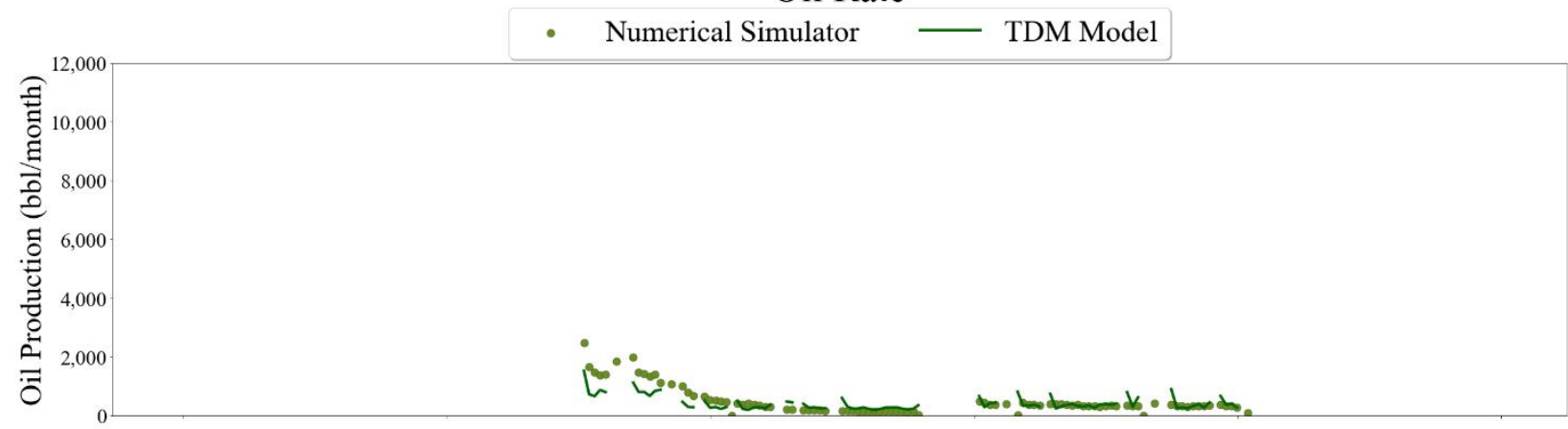

Gas Rate
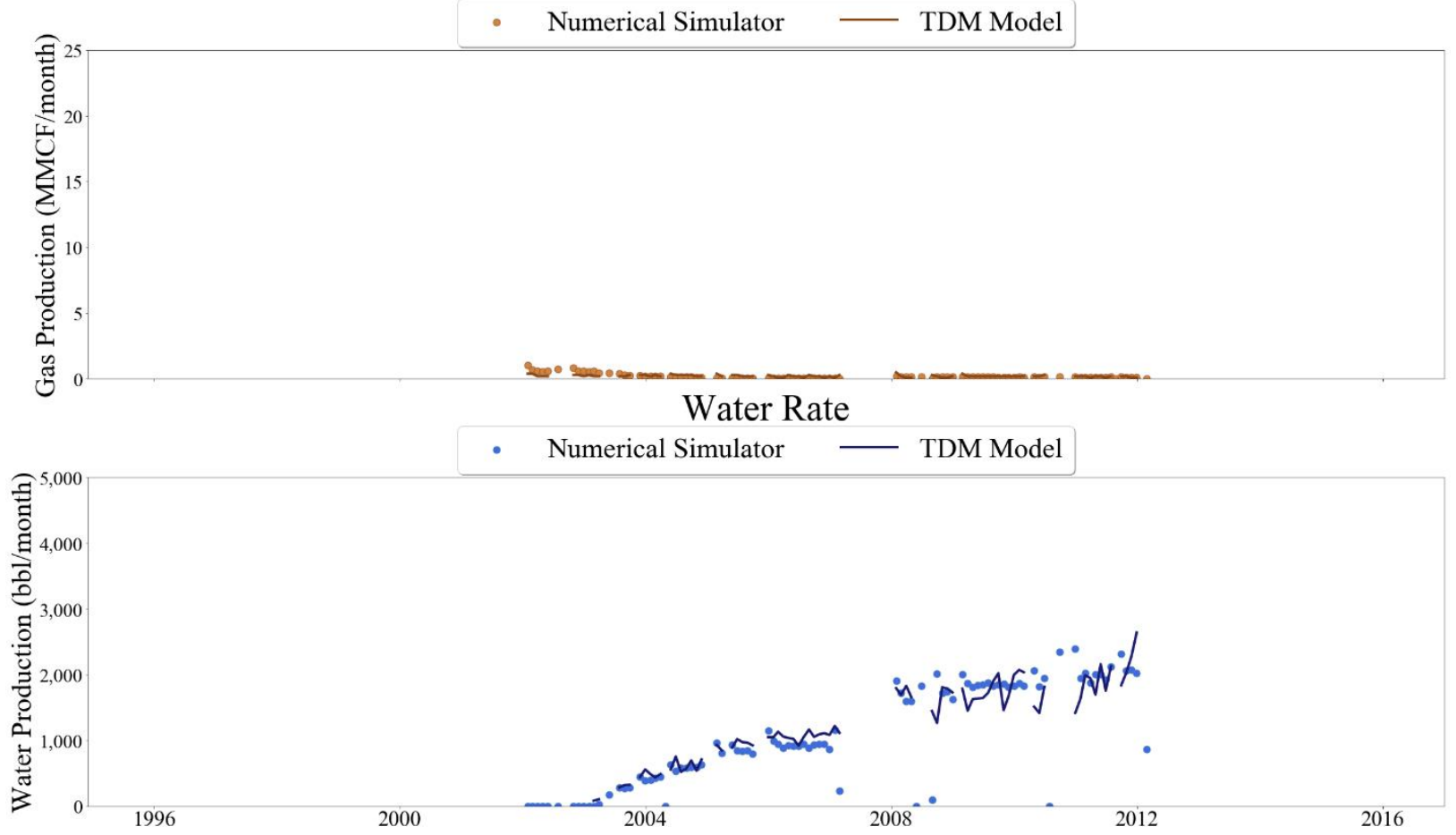

Figure 388 Well-033 oil, gas, and water rate TDM predictions vs actual simulation data plots for No Blind Validation TDM 


\section{Oil Rate}

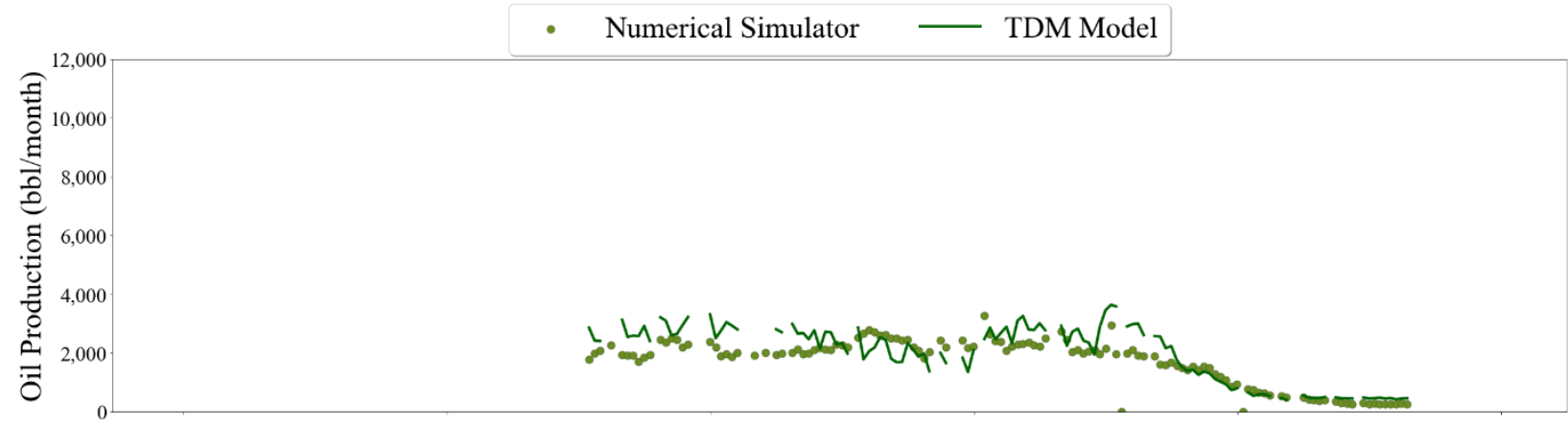

\section{Gas Rate}
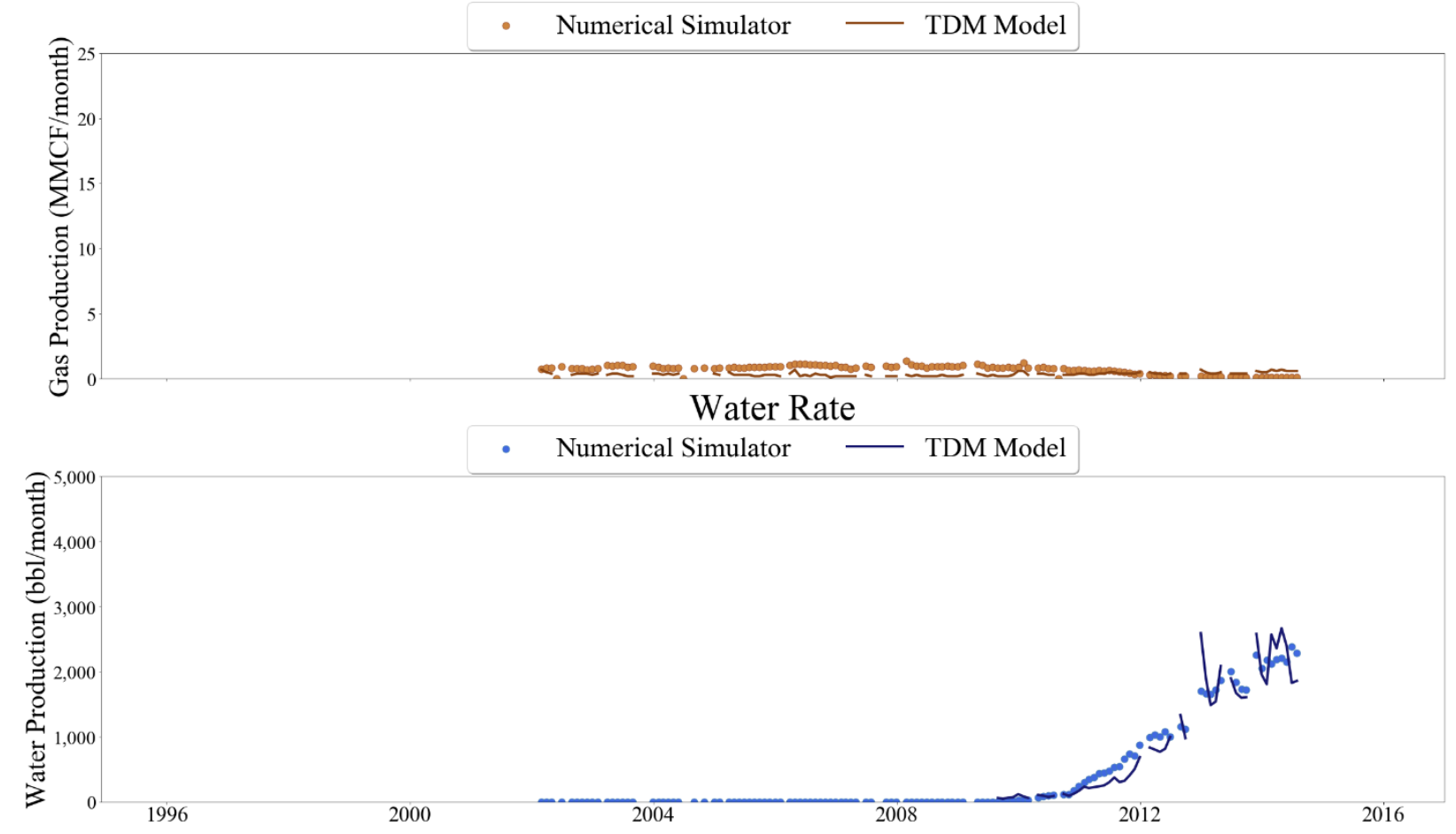

Figure 389 Well-034 oil, gas, and water rate TDM predictions vs actual simulation data plots for No Blind Validation TDM 


\section{Oil Rate}

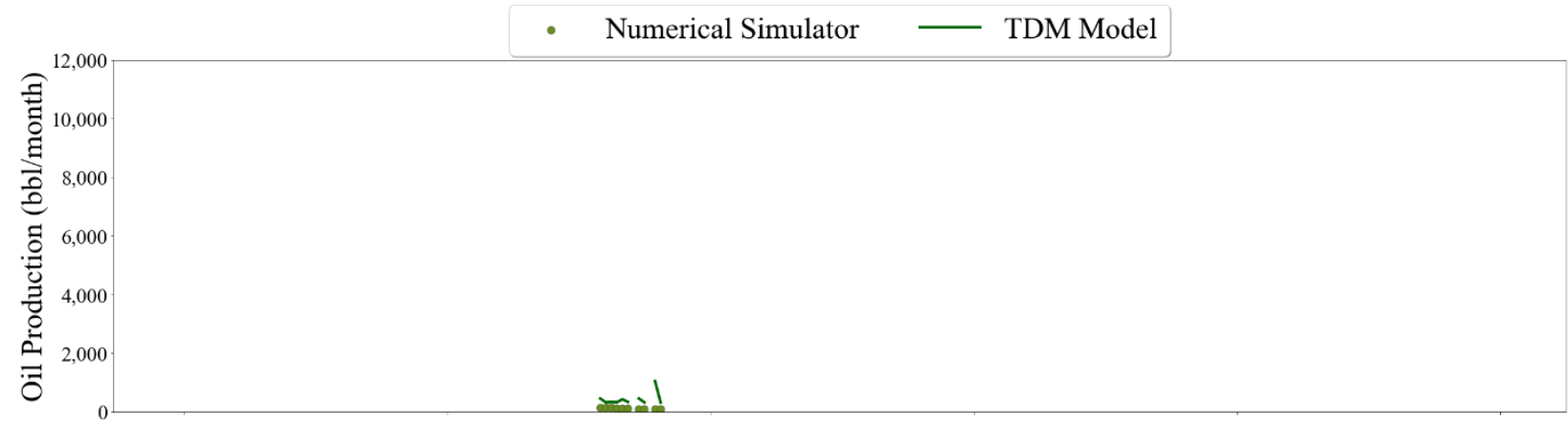

\section{Gas Rate}

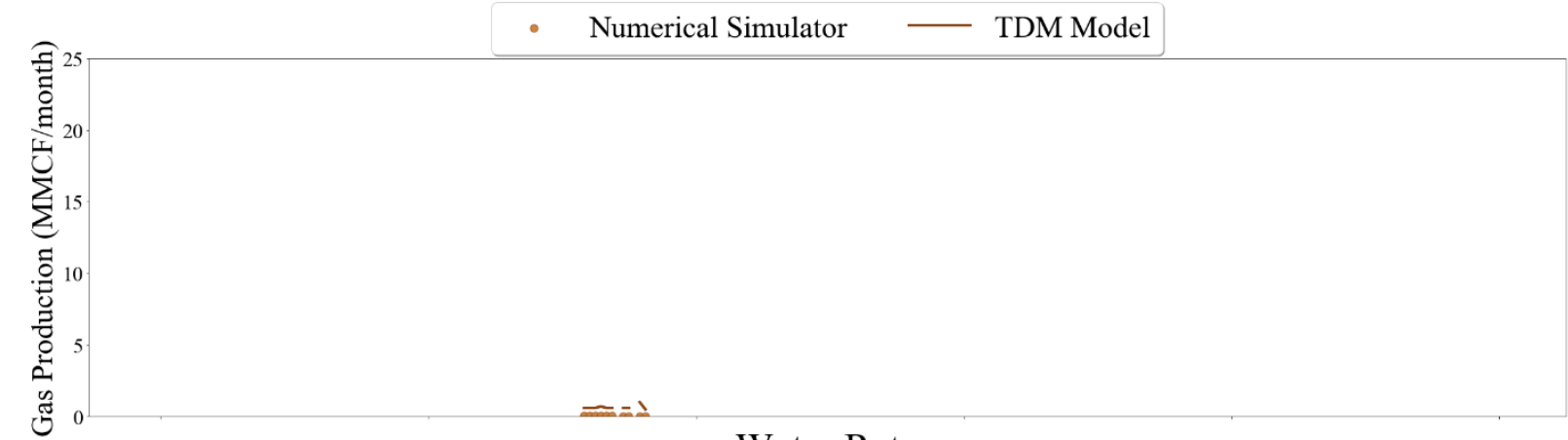

Water Rate

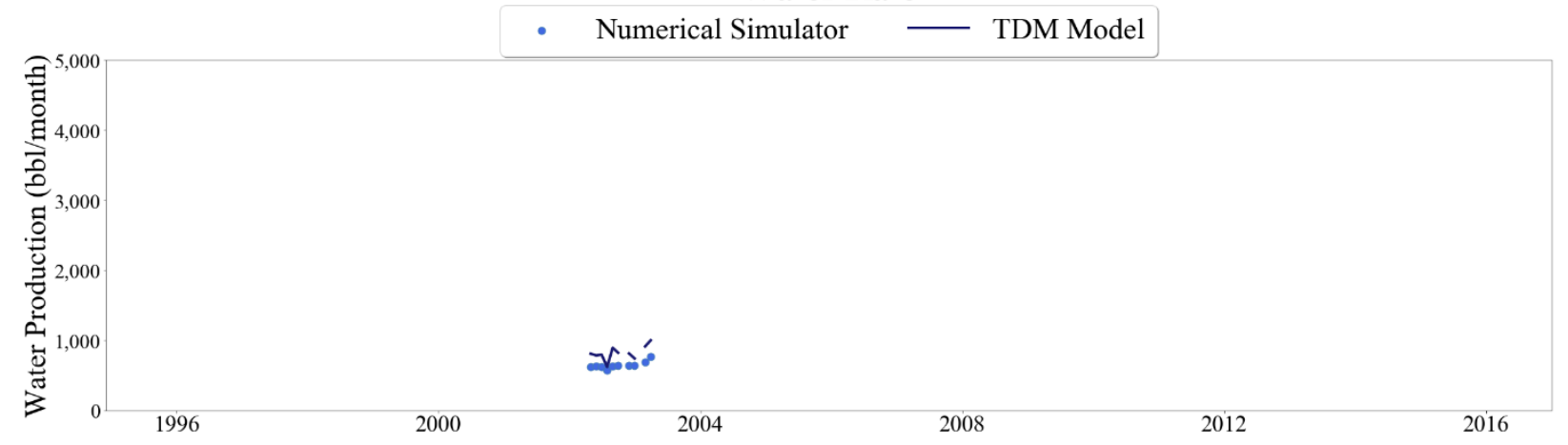

Figure 390 Well-035 oil, gas, and water rate TDM predictions vs actual simulation data plots for No Blind Validation TDM 


\section{Oil Rate}
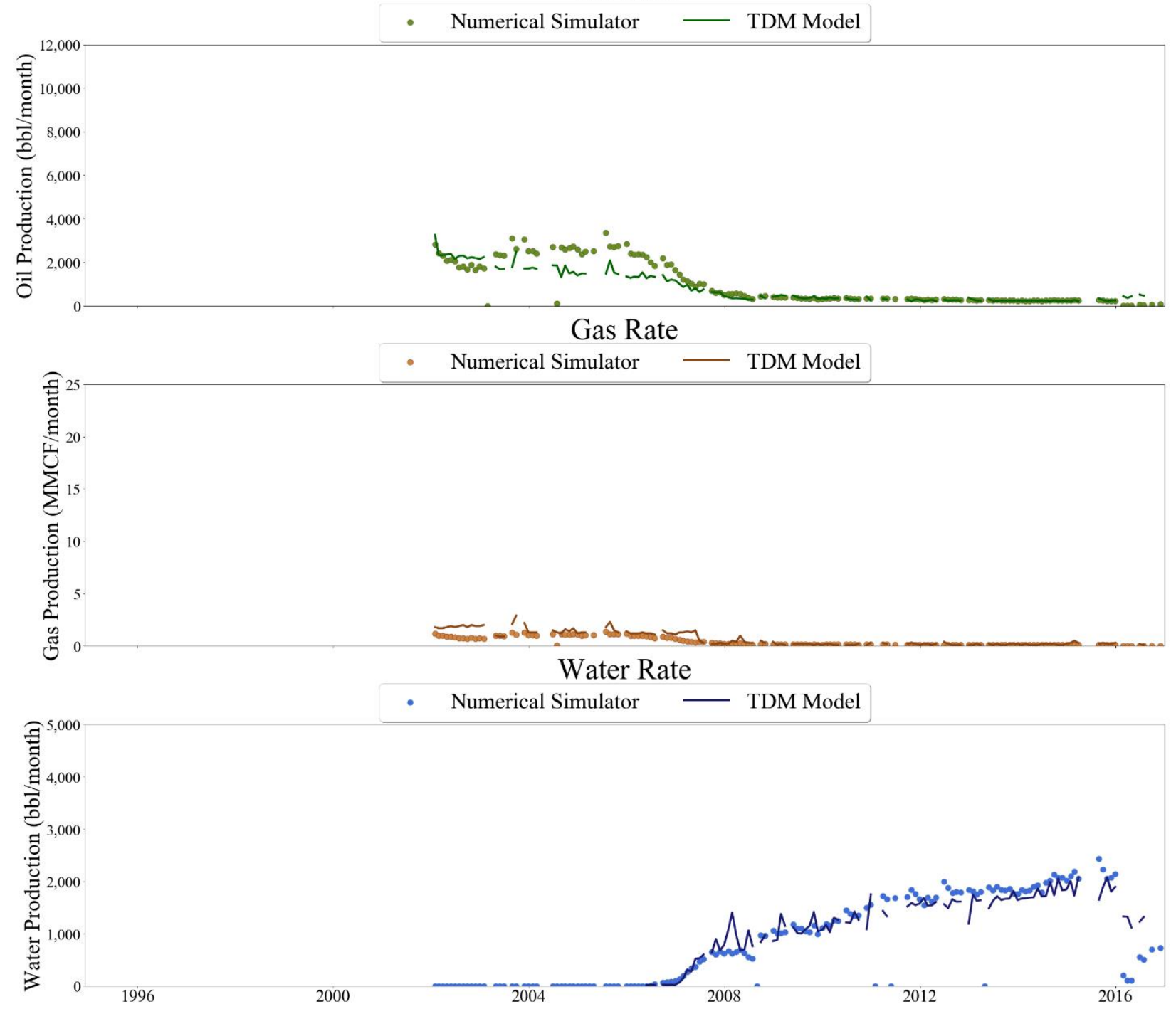

Figure 391 Well-036 oil, gas, and water rate TDM predictions vs actual simulation data plots for No Blind Validation TDM 


\section{Oil Rate}
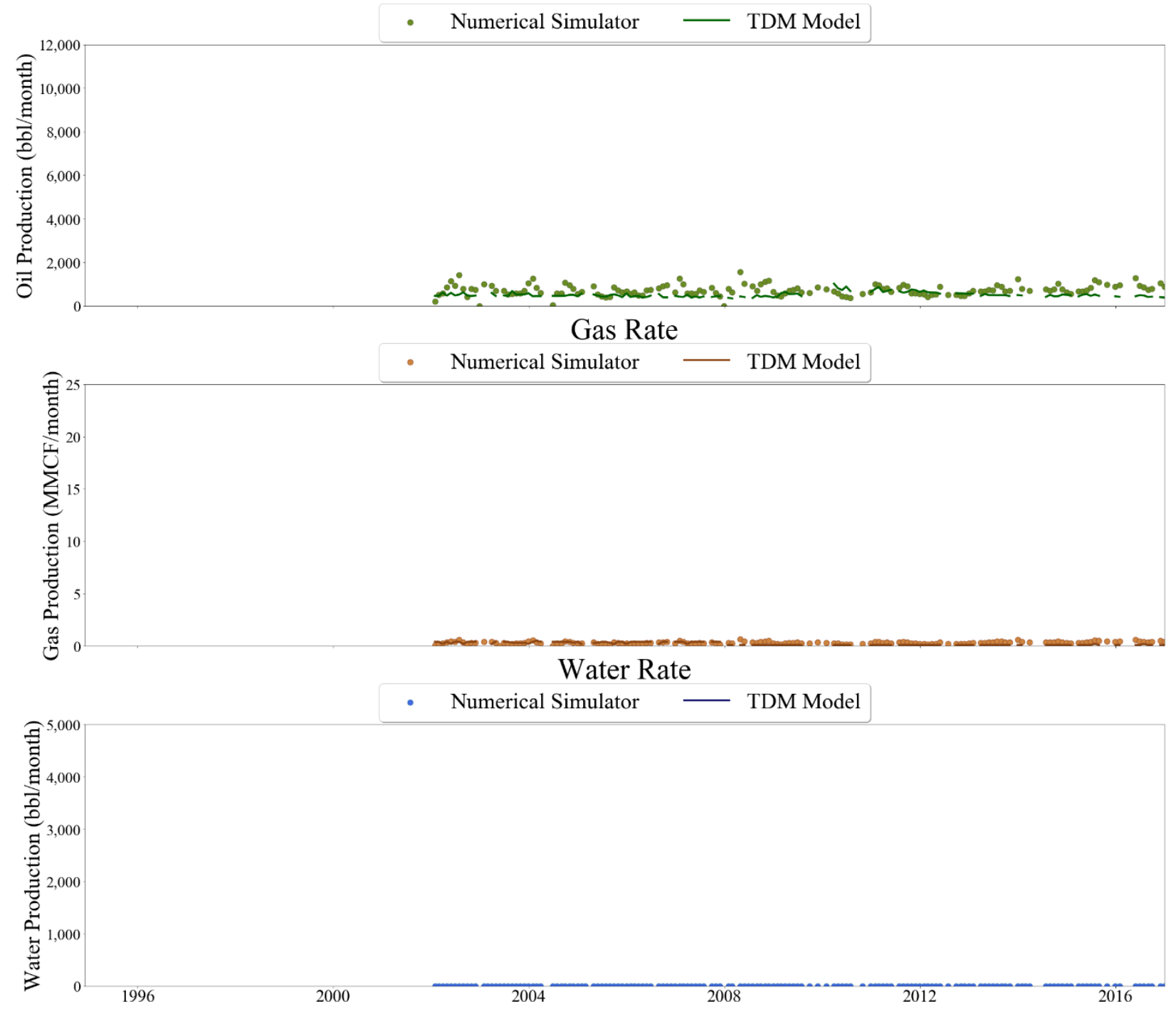

Figure 392 Well-037 oil, gas, and water rate TDM predictions vs actual simulation data plots for No Blind Validation TDM 


\section{Oil Rate}
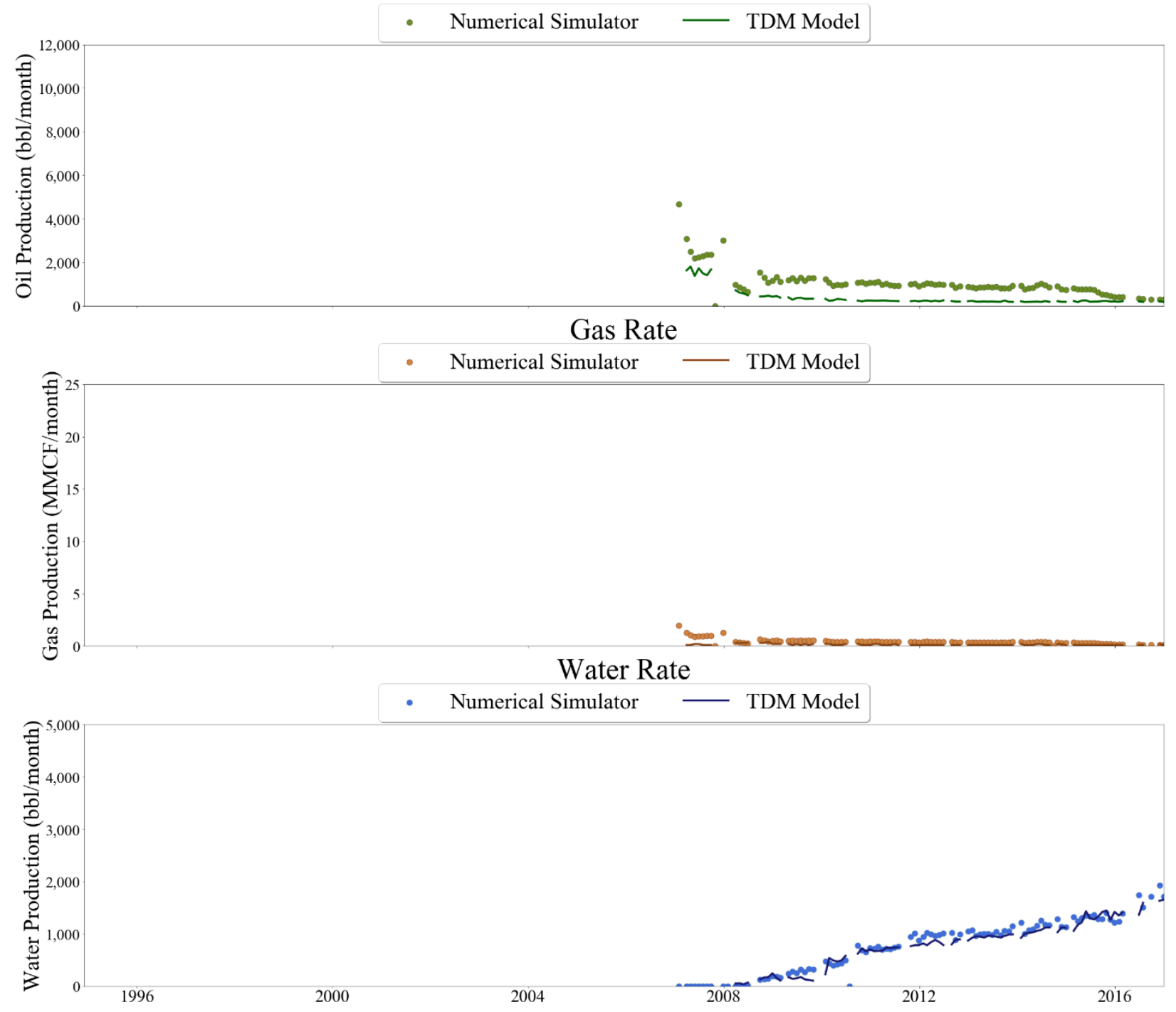

Figure 393 Well-038 oil, gas, and water rate TDM predictions vs actual simulation data plots for No Blind Validation TDM 


\section{Oil Rate}

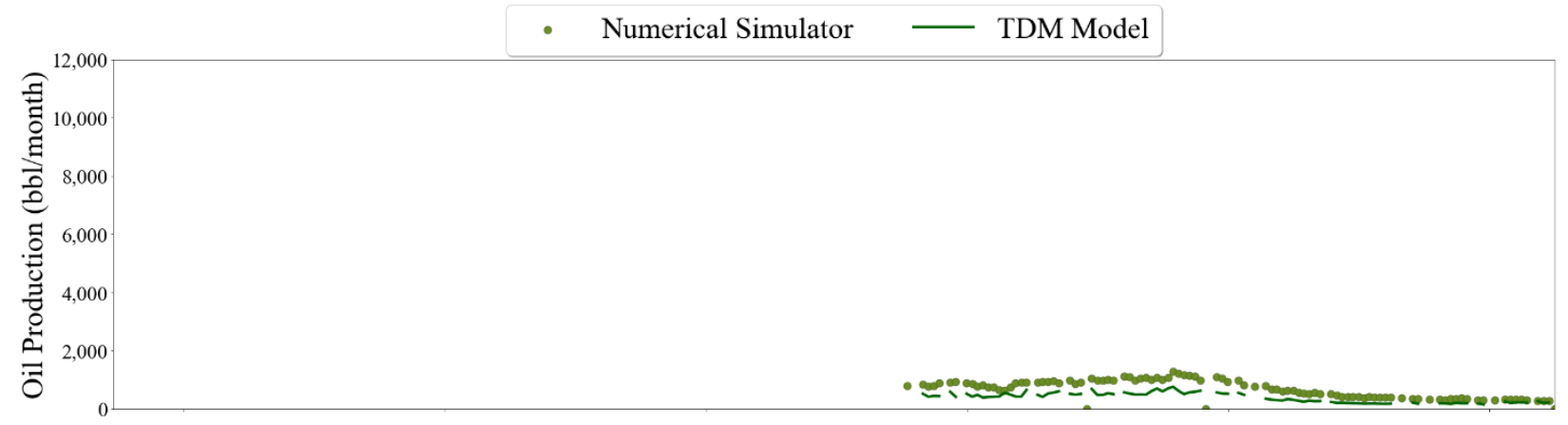

\section{Gas Rate}
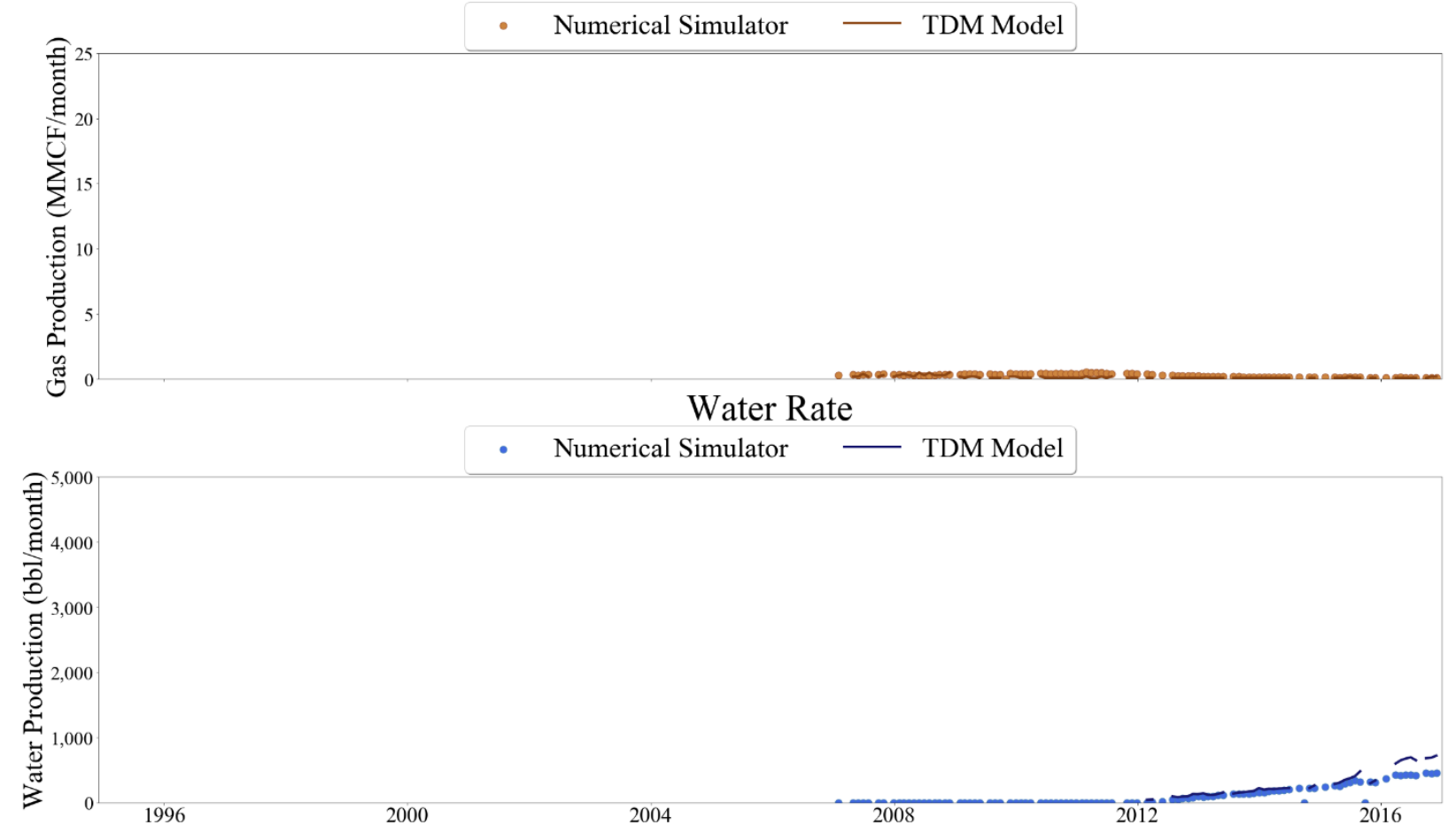

Figure 394 Well-039 oil, gas, and water rate TDM predictions vs actual simulation data plots for No Blind Validation TDM 


\section{Oil Rate}

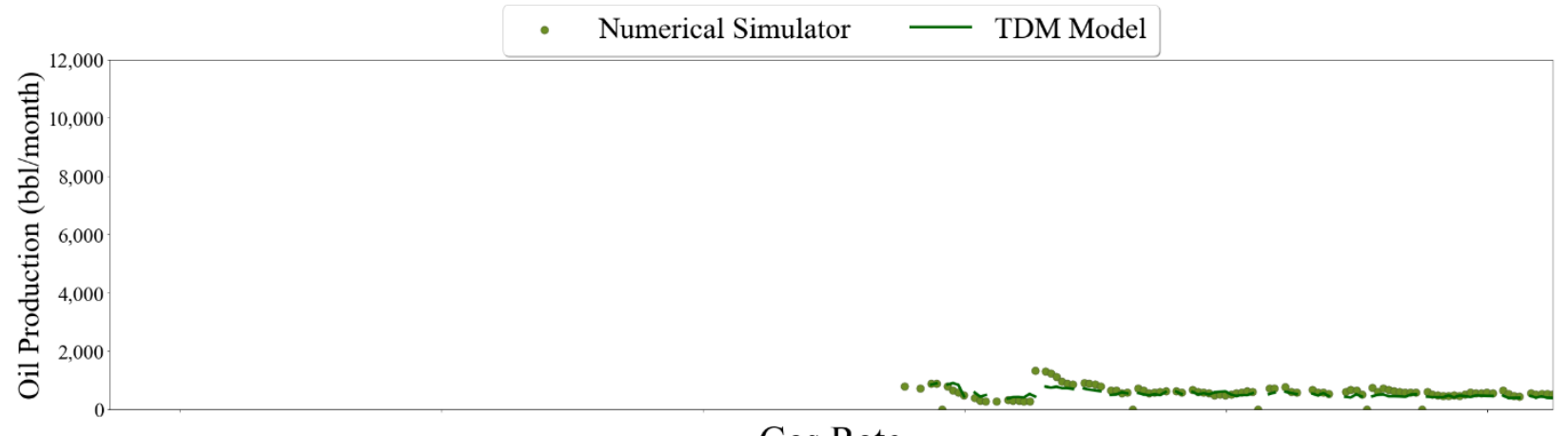

Gas Rate
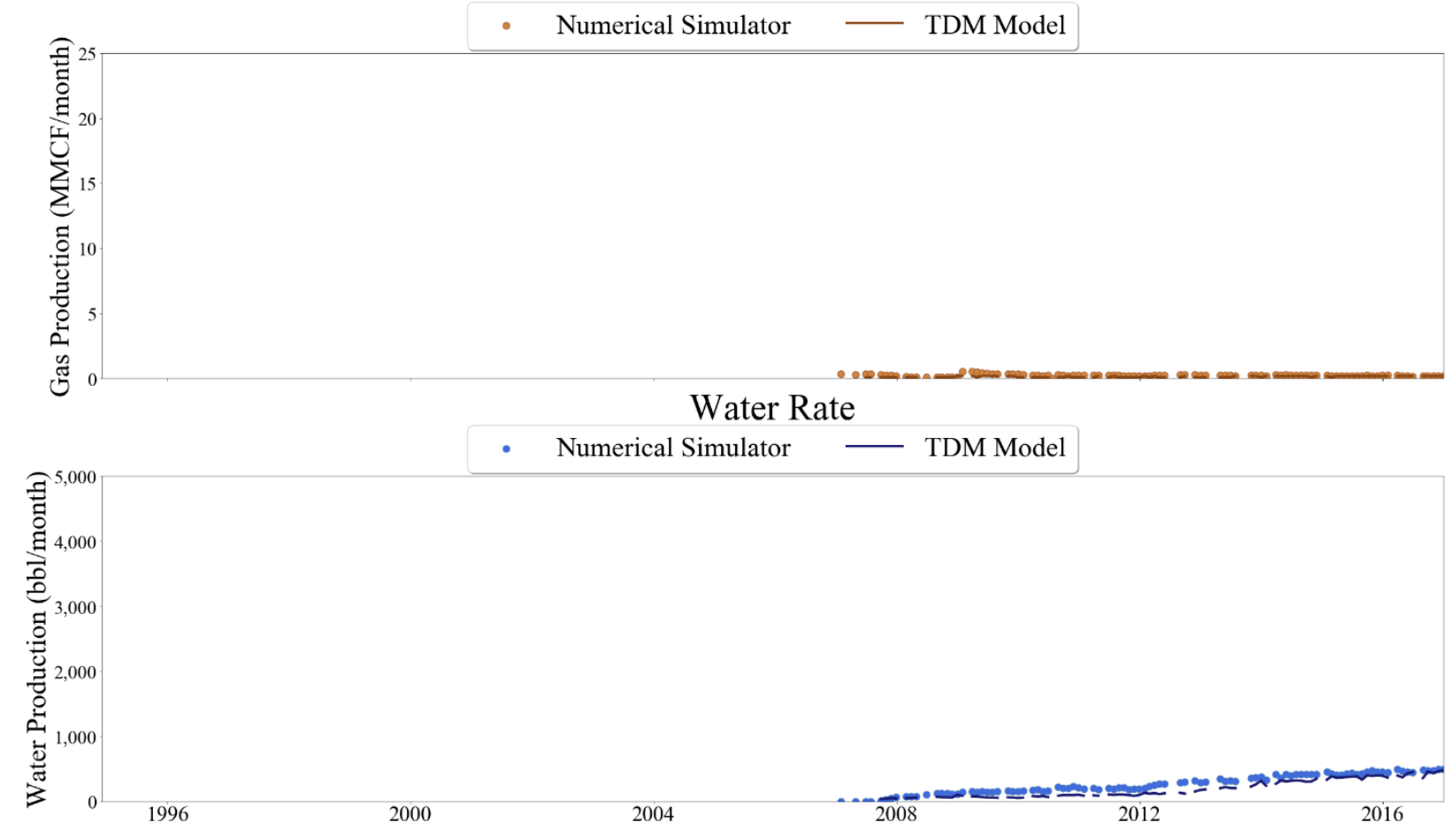

Figure 395 Well-040 oil, gas, and water rate TDM predictions vs actual simulation data plots for No Blind Validation TDM 


\section{Oil Rate}

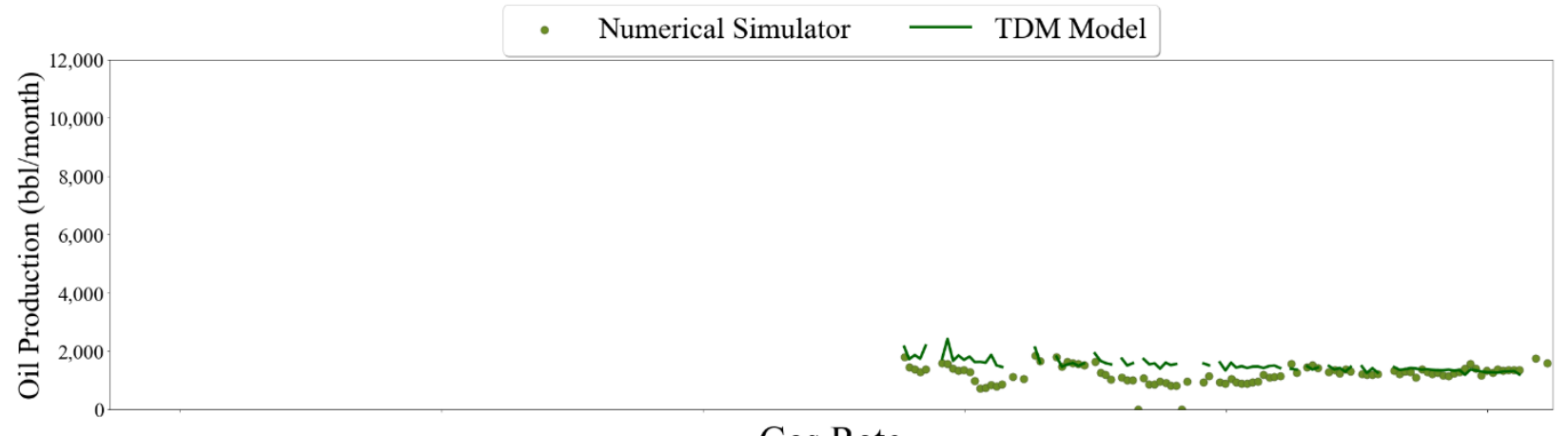

Gas Rate
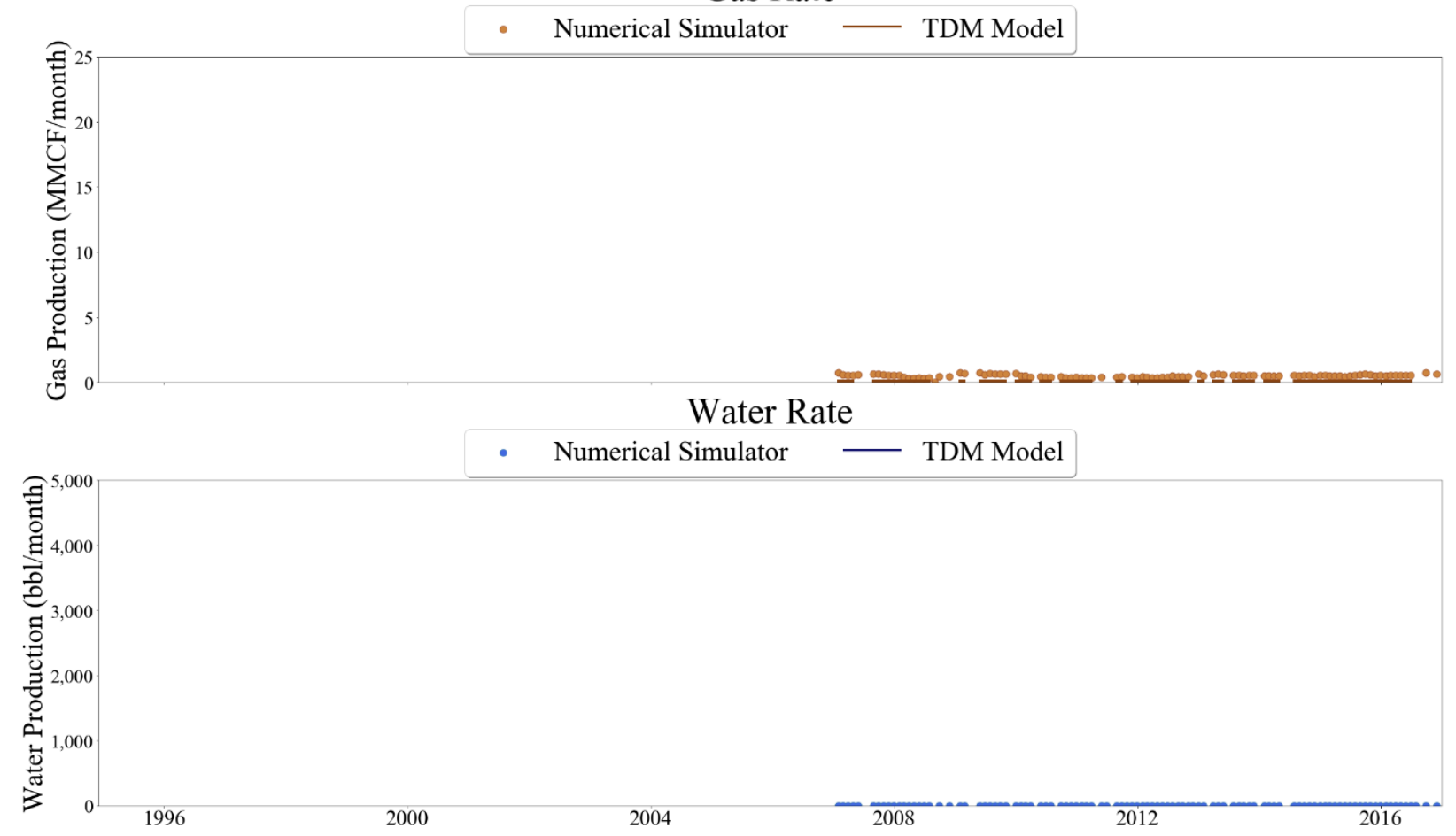

Figure 396 Well-041 oil, gas, and water rate TDM predictions vs actual simulation data plots for No Blind Validation TDM 


\section{Oil Rate}

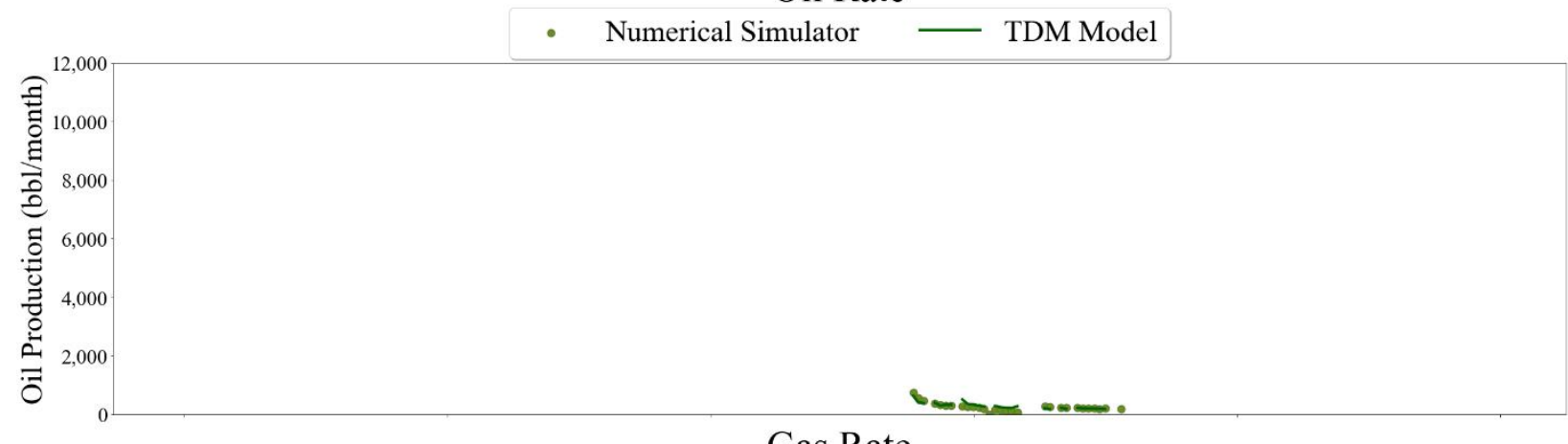

Gas Rate
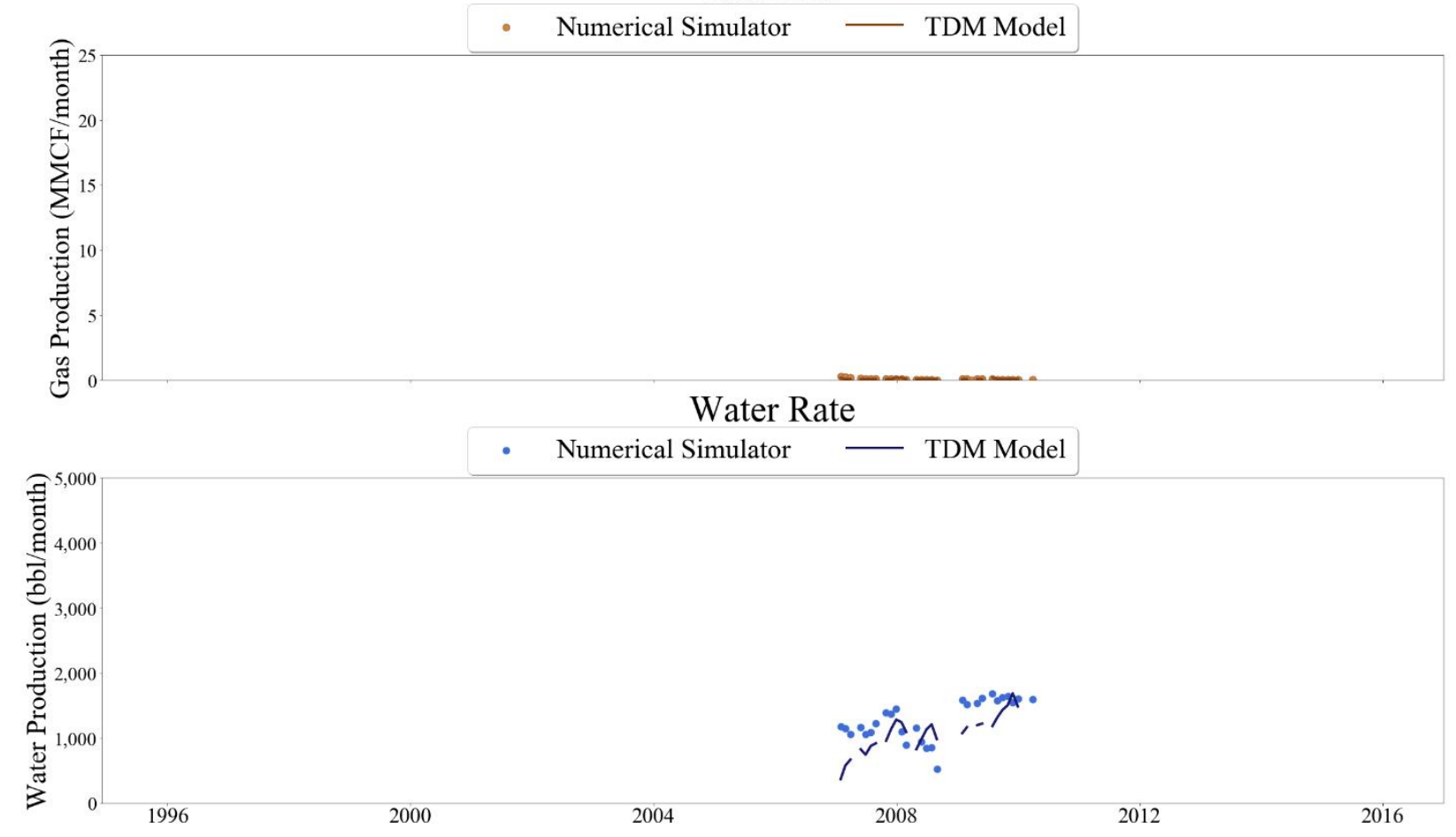

Figure 397 Well-042 oil, gas, and water rate TDM predictions vs actual simulation data plots for No Blind Validation TDM 


\section{Oil Rate}

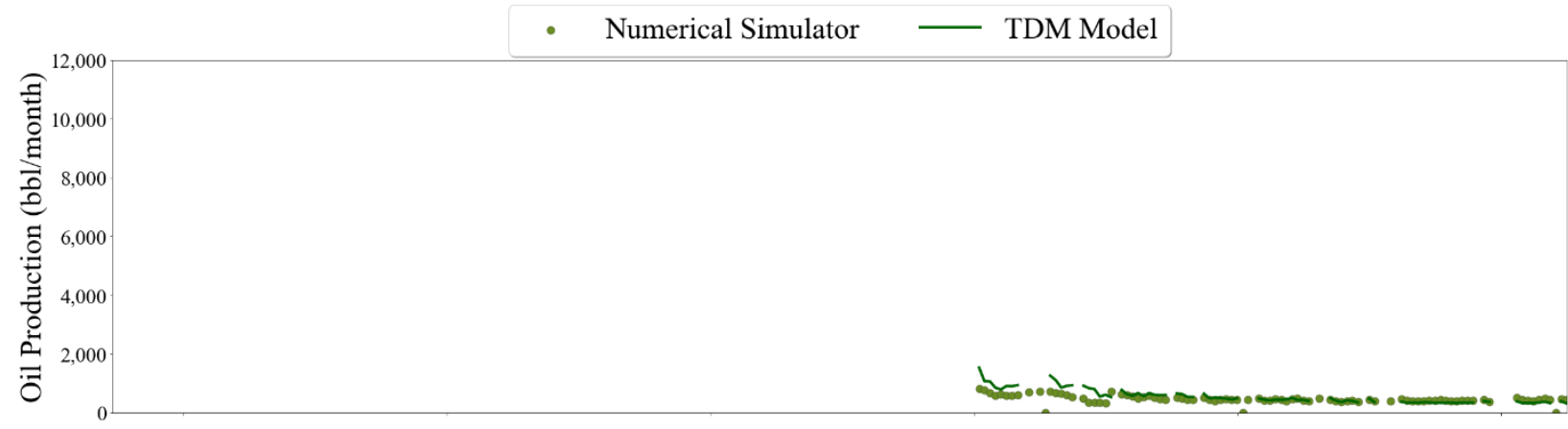

Gas Rate
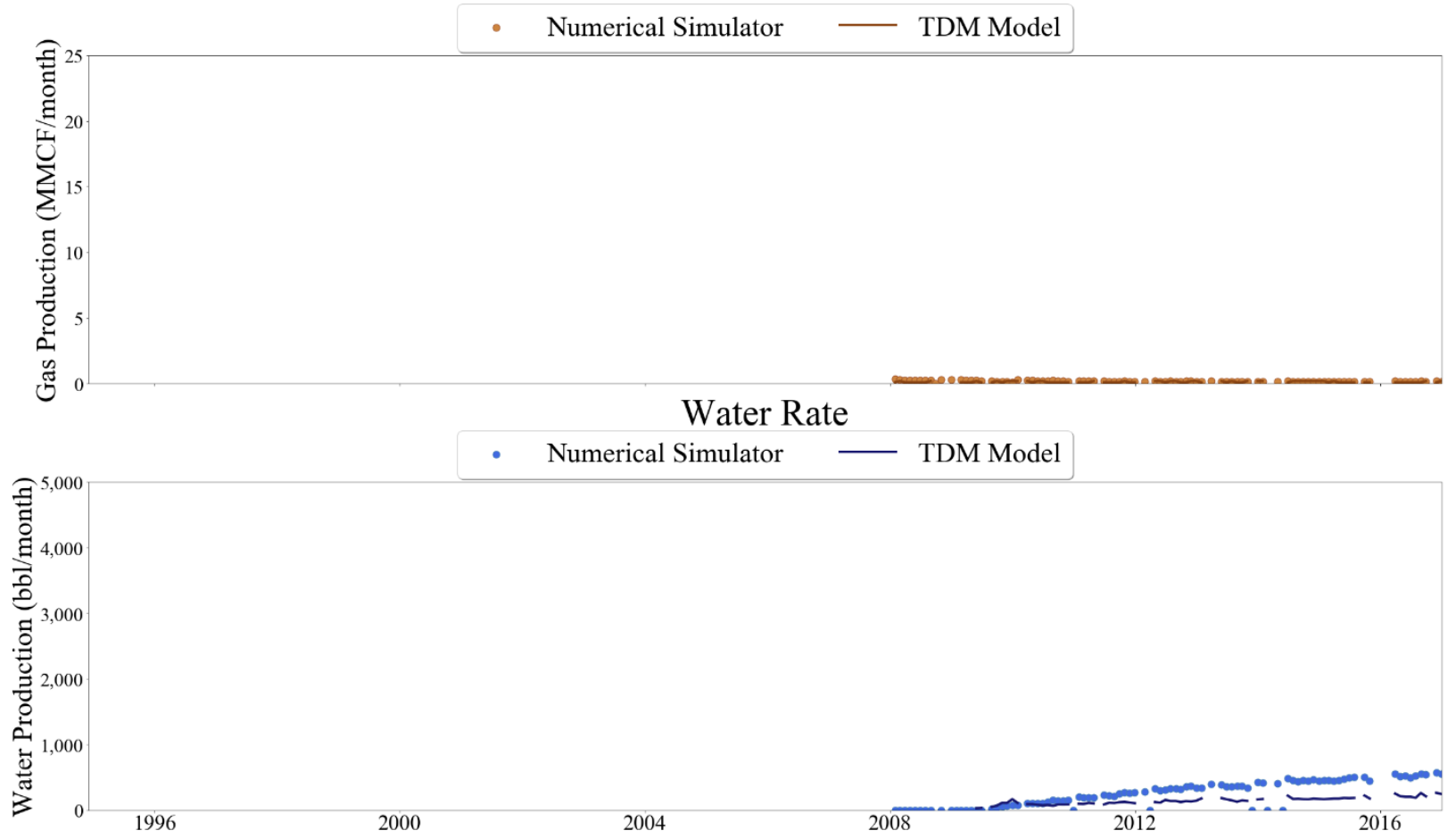

Figure 398 Well-043 oil, gas, and water rate TDM predictions vs actual simulation data plots for No Blind Validation TDM 


\section{Oil Rate}
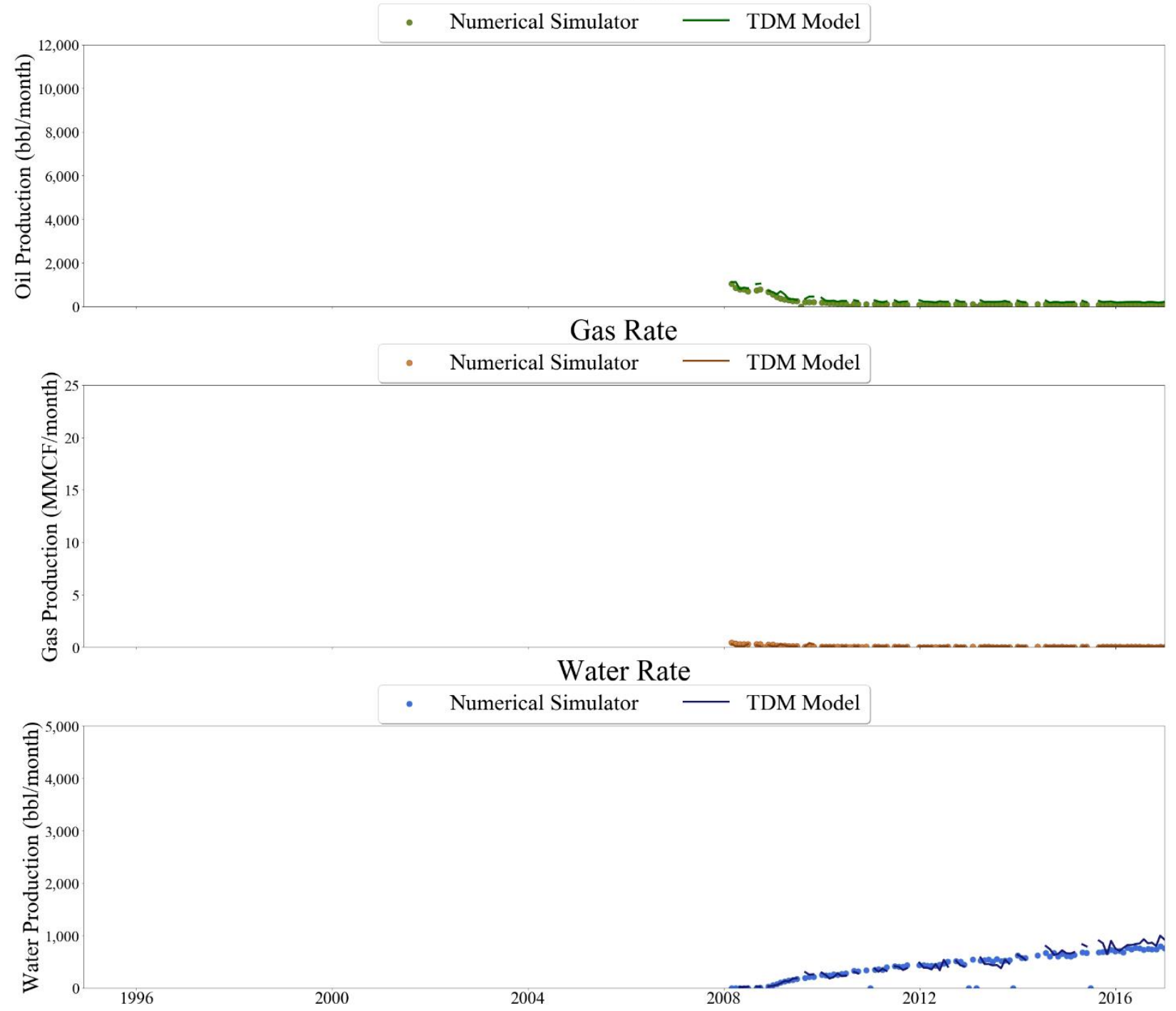

Figure 399 Well-044 oil, gas, and water rate TDM predictions vs actual simulation data plots for No Blind Validation TDM 


\section{Oil Rate}
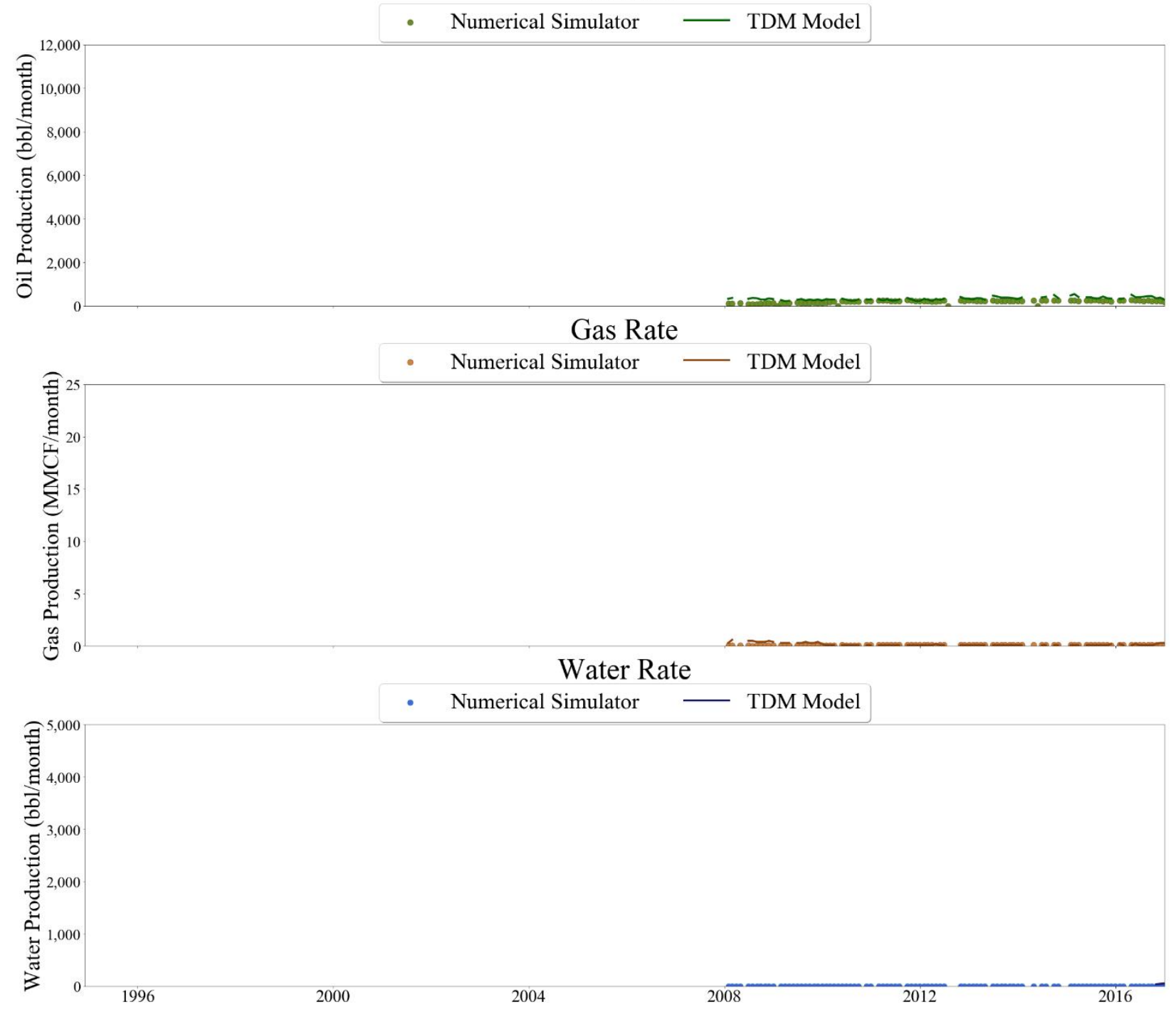

Figure 400 Well-045 oil, gas, and water rate TDM predictions vs actual simulation data plots for No Blind Validation TDM 


\section{Oil Rate}
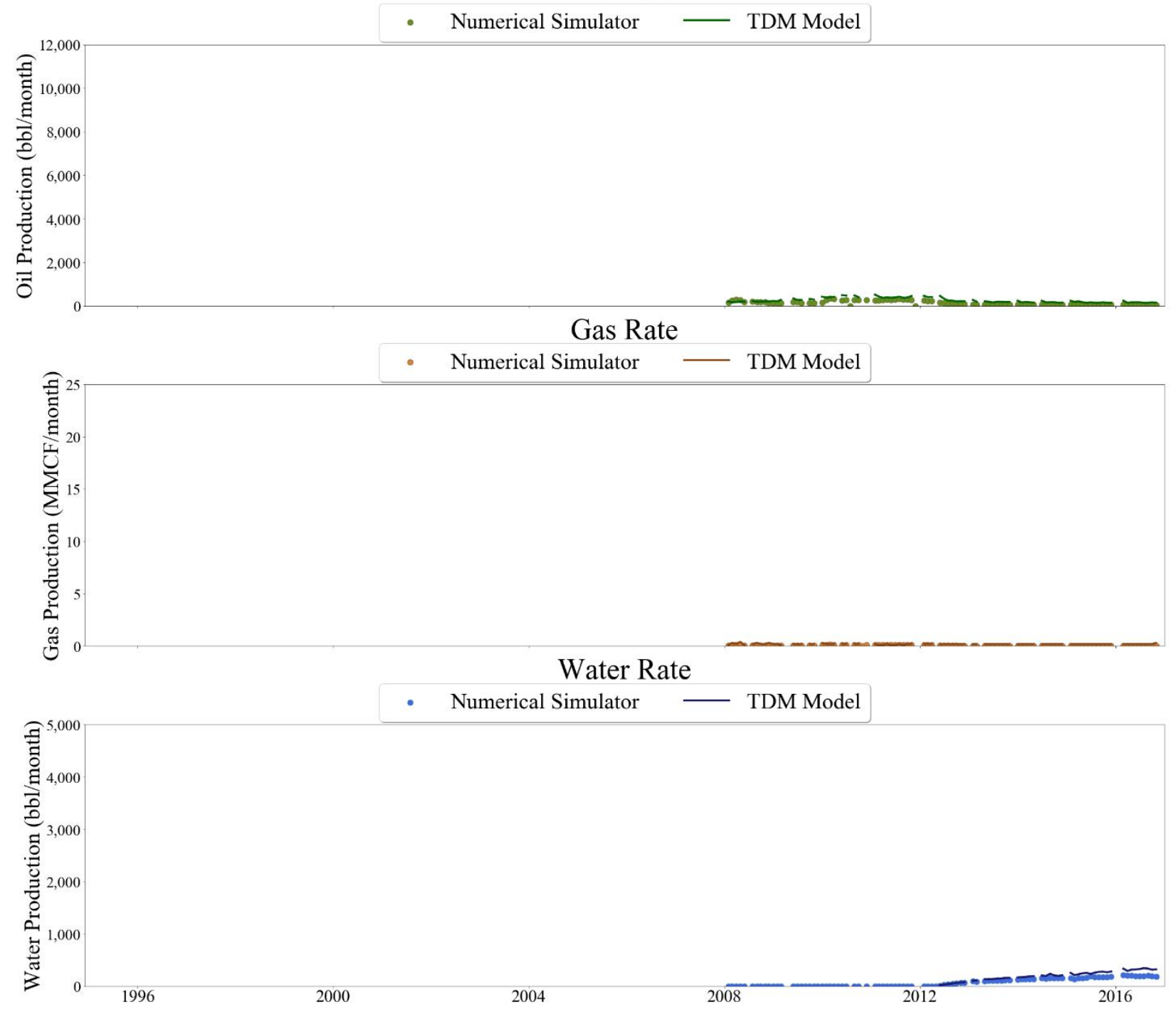

Figure 401 Well-046 oil, gas, and water rate TDM predictions vs actual simulation data plots for No Blind Validation TDM 


\section{Oil Rate}

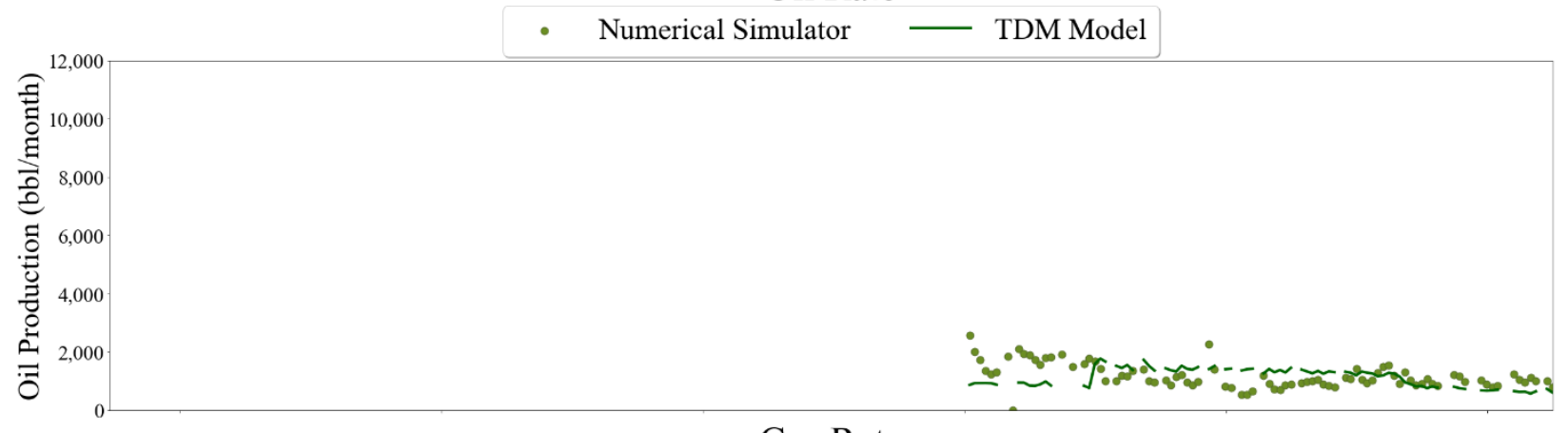

\section{Gas Rate}
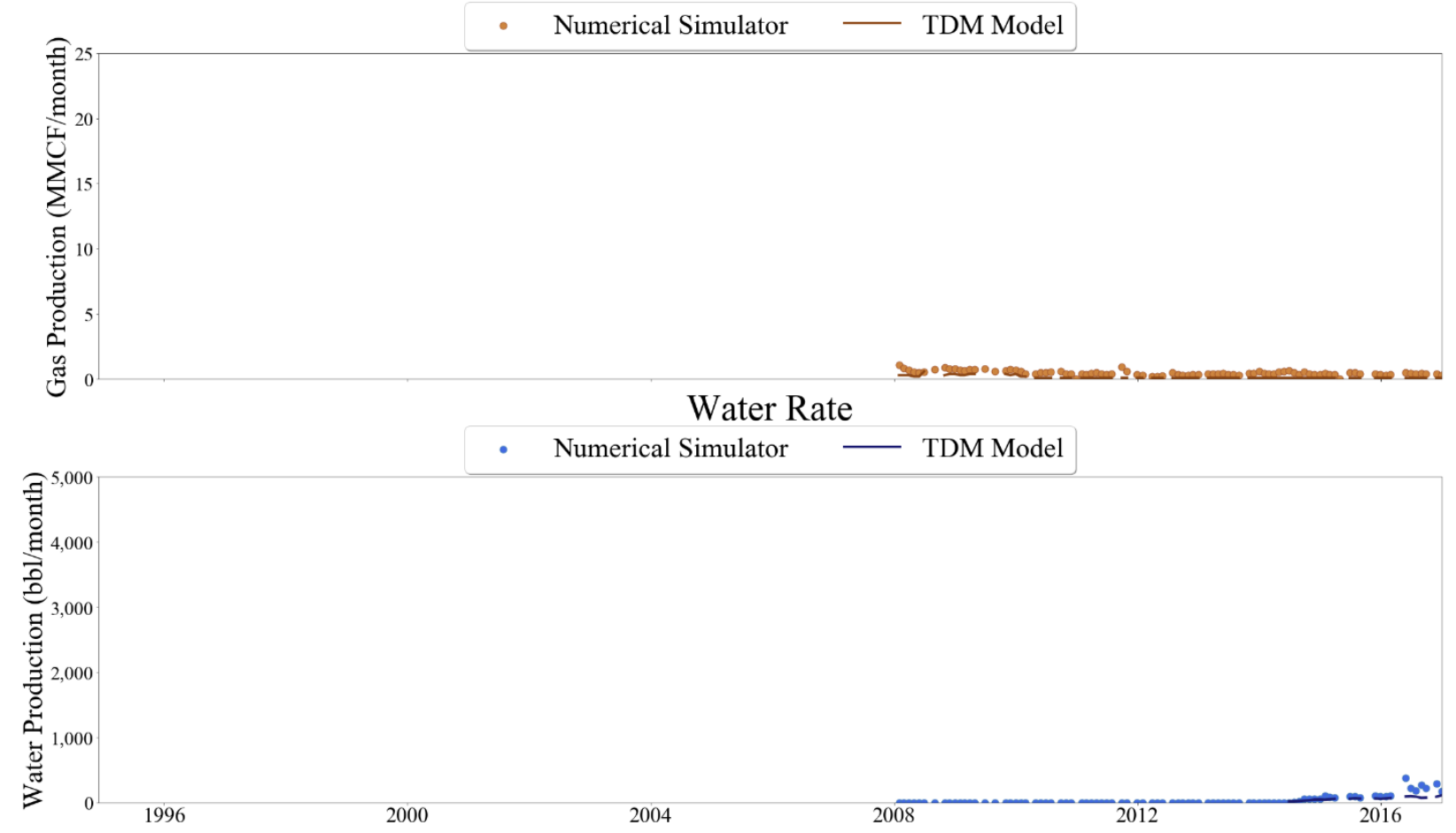

Figure 402 Well-047 oil, gas, and water rate TDM predictions vs actual simulation data plots for No Blind Validation TDM 


\section{Oil Rate}

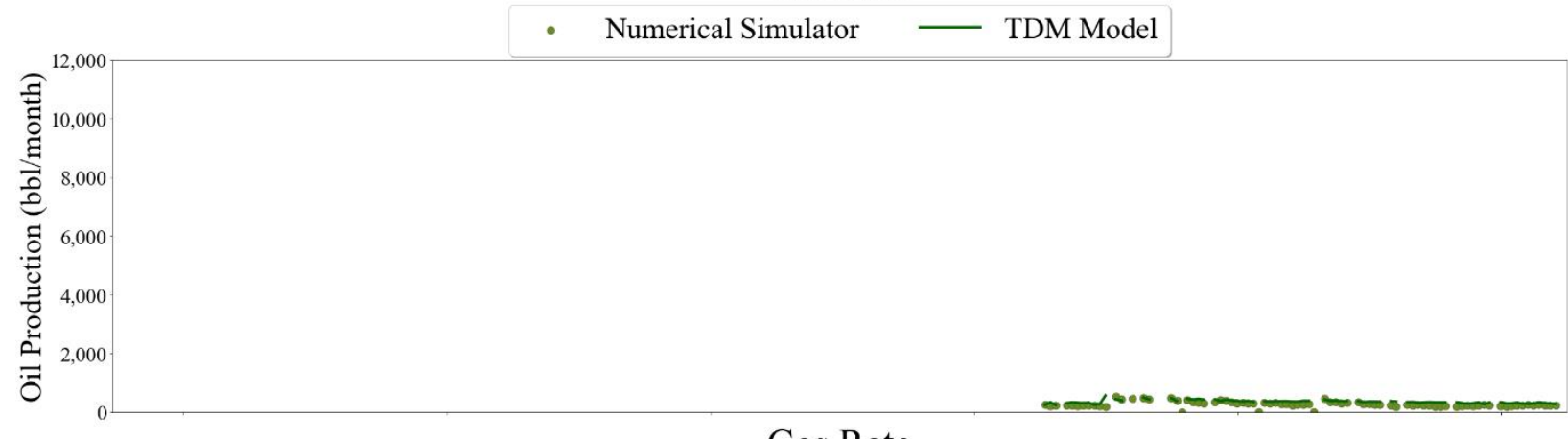

\section{Gas Rate}

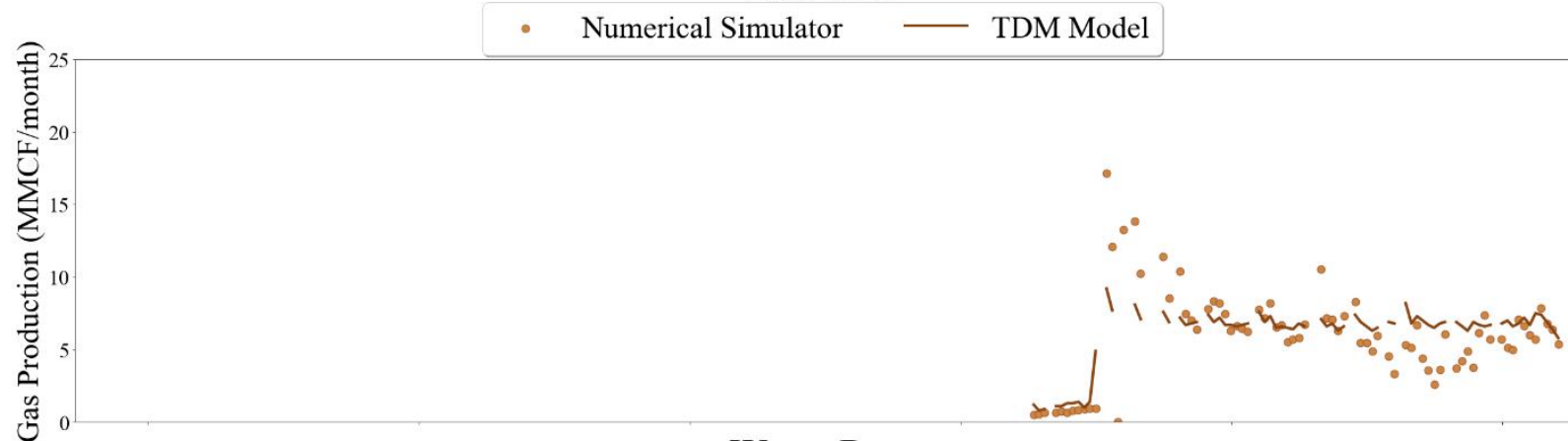

Water Rate

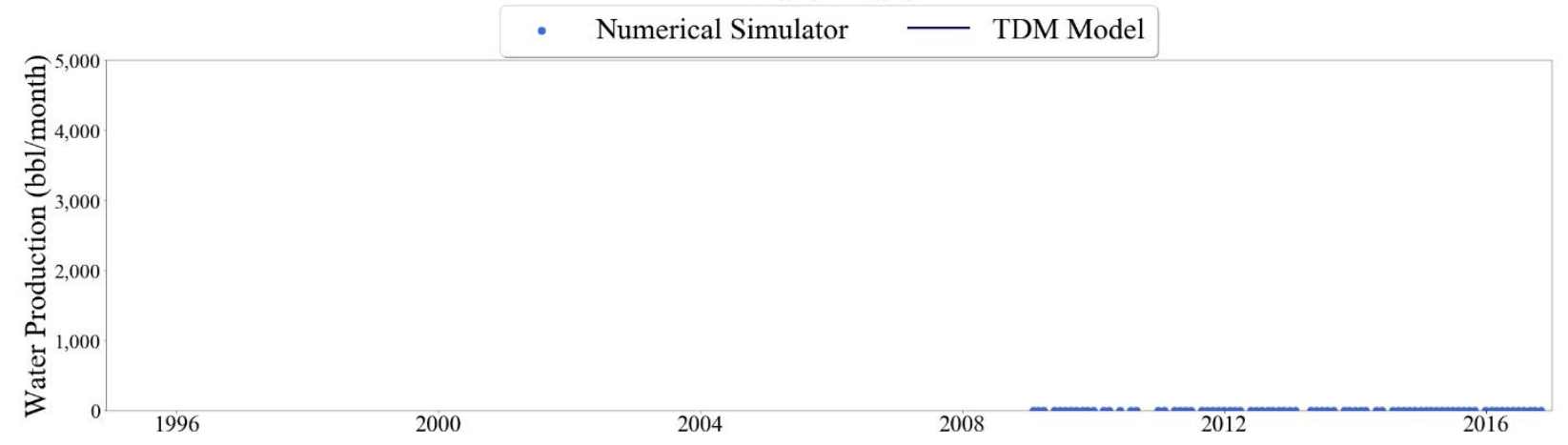

Figure 403 Well-048 oil, gas, and water rate TDM predictions vs actual simulation data plots for No Blind Validation TDM 


\section{Oil Rate}
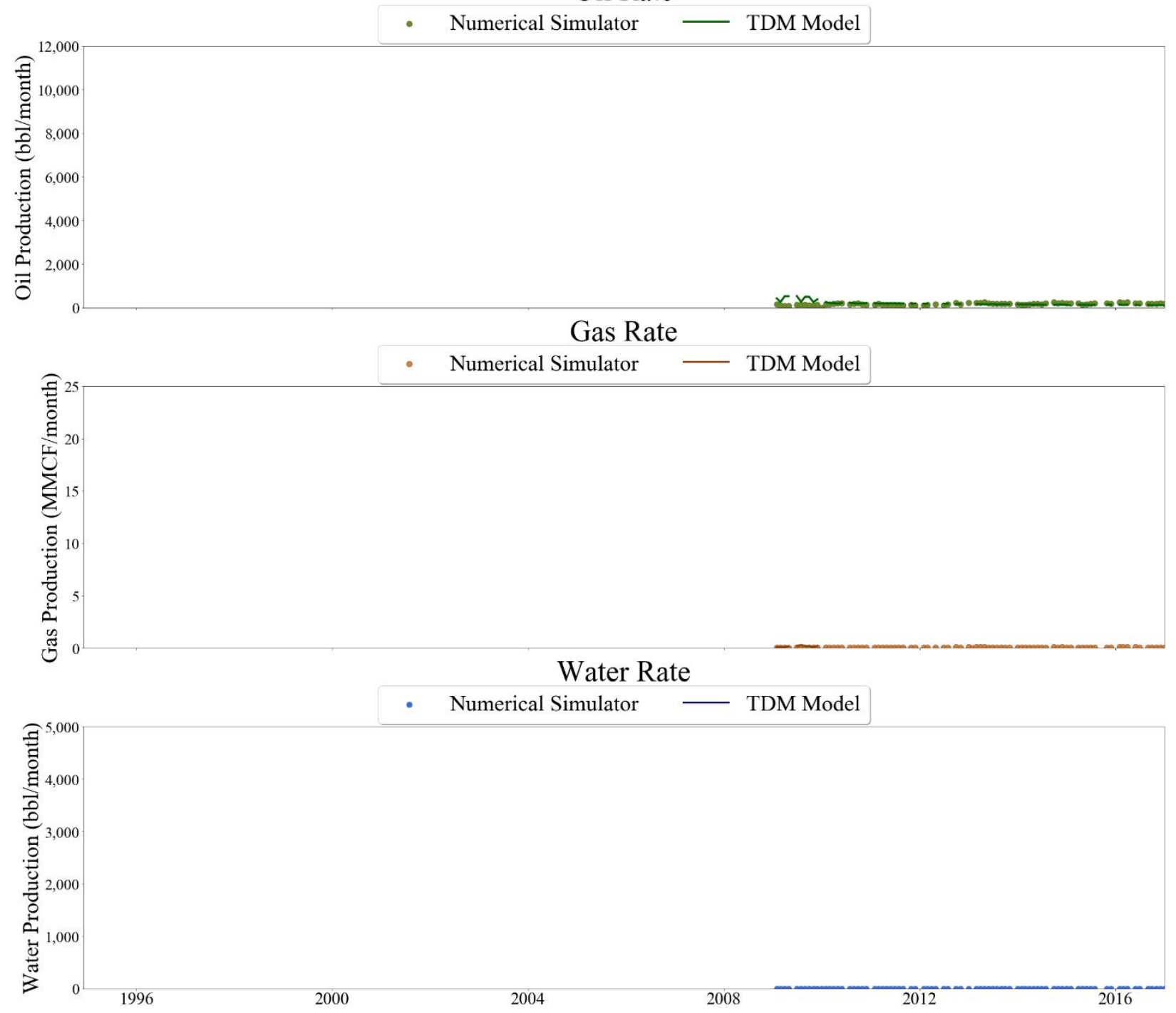

Figure 404 Well-049 oil. gas, and water rate TDM predictions vs actual simulation data plots for No Blind Validation TDM 


\section{Oil Rate}
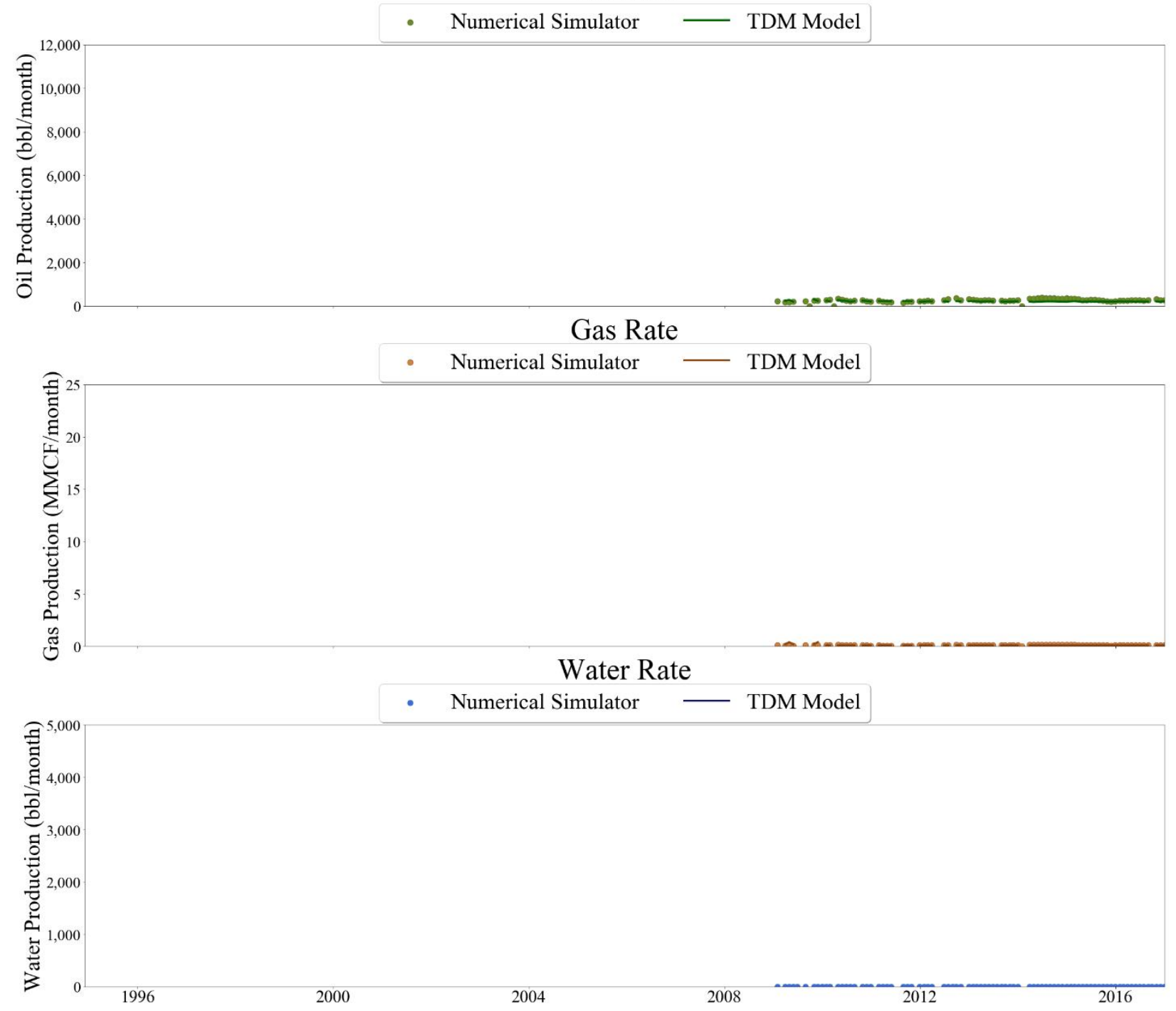

Figure 405 Well-050 oil, gas, and water rate TDM predictions vs actual simulation data plots for No Blind Validation TDM 


\section{Oil Rate}

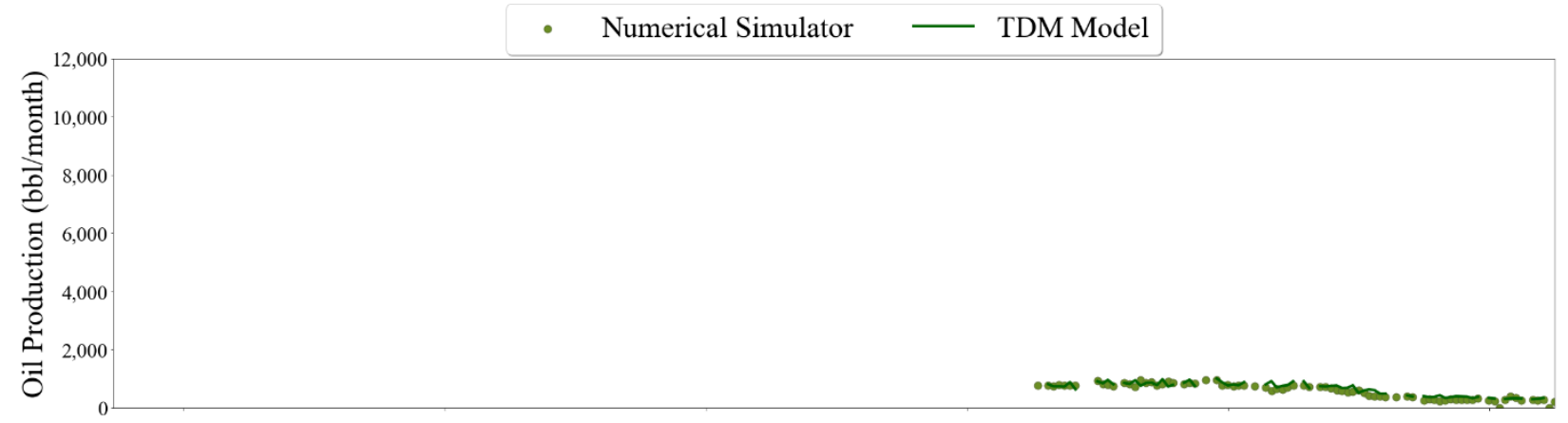

\section{Gas Rate}
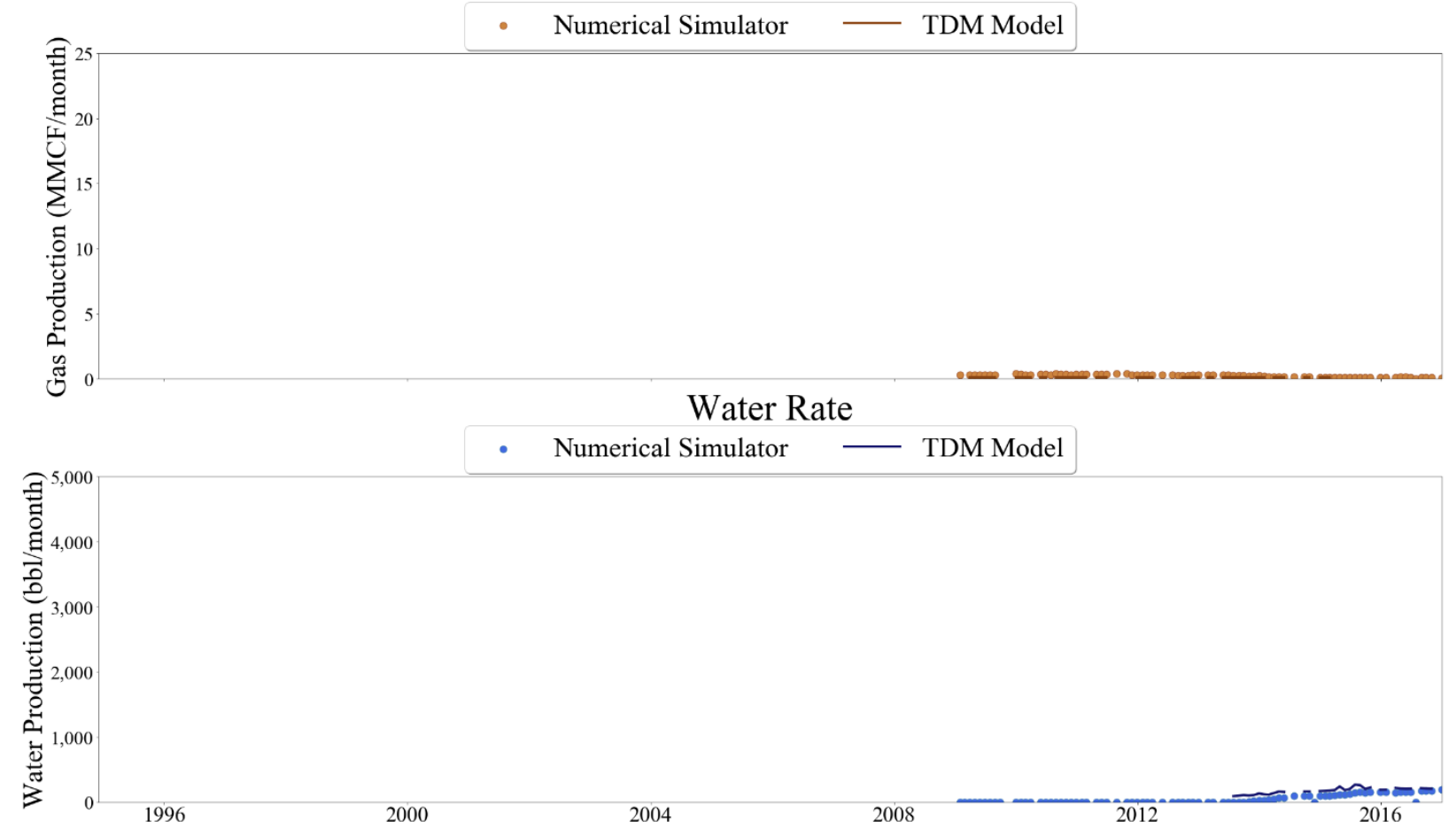

Figure 406 Well-051 oil, gas, and water rate TDM predictions vs actual simulation data plots for No Blind Validation TDM 


\section{Oil Rate}

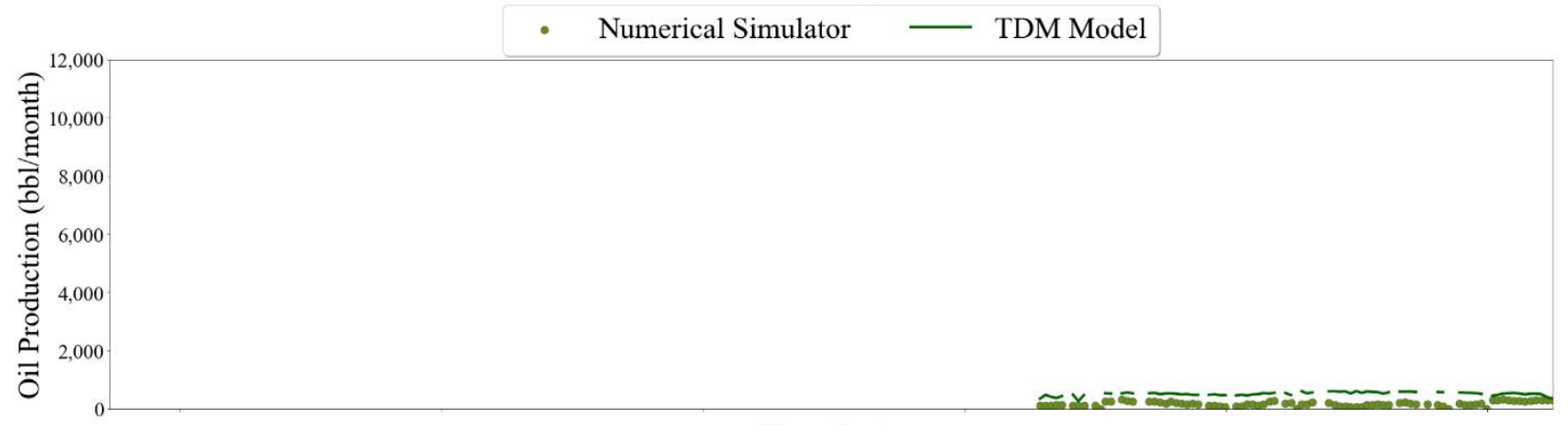

Gas Rate
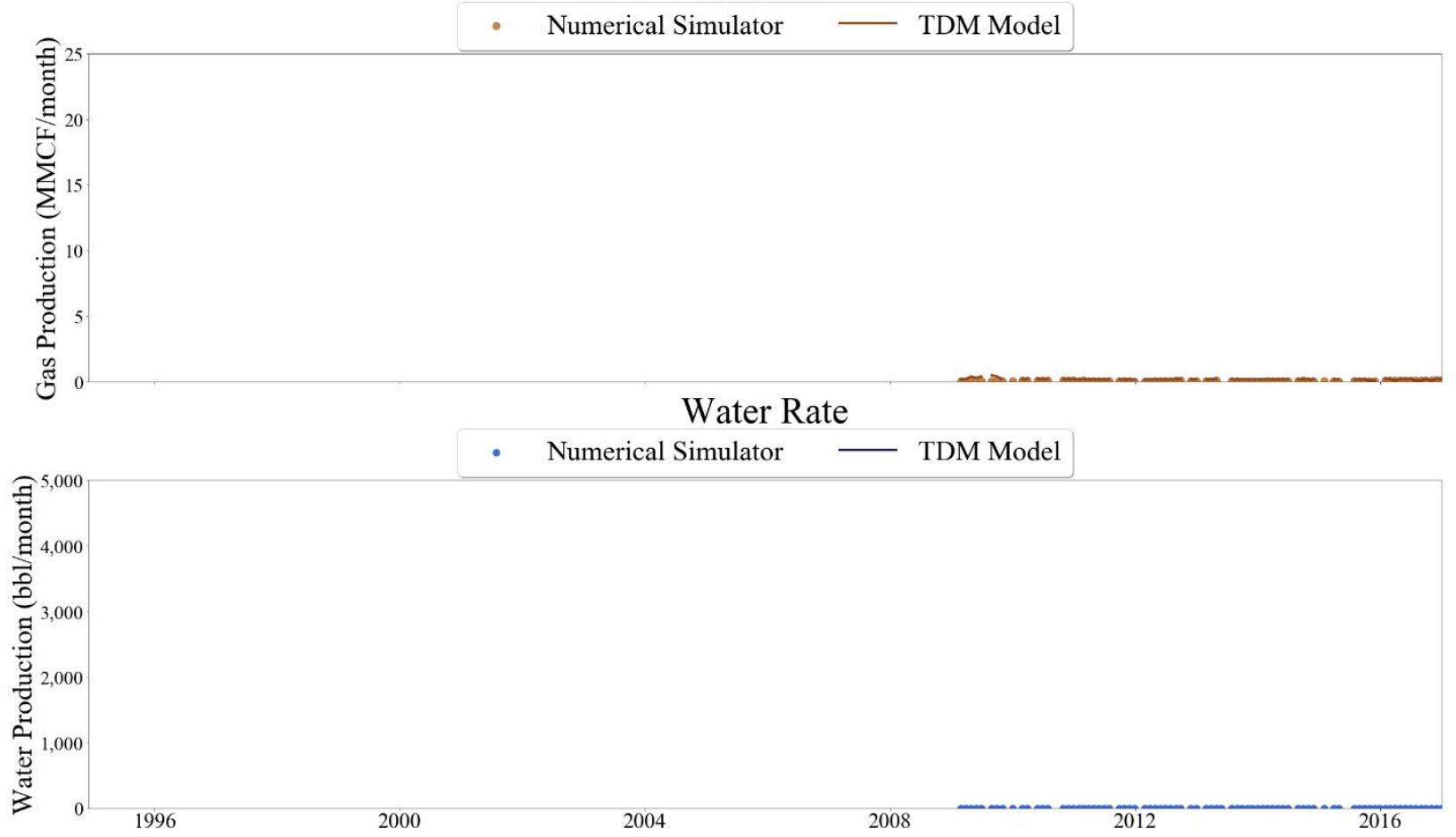

Figure 407 Well-052 oil, gas, and water rate TDM predictions vs actual simulation data plots for No Blind Validation TDM 


\section{Oil Rate}
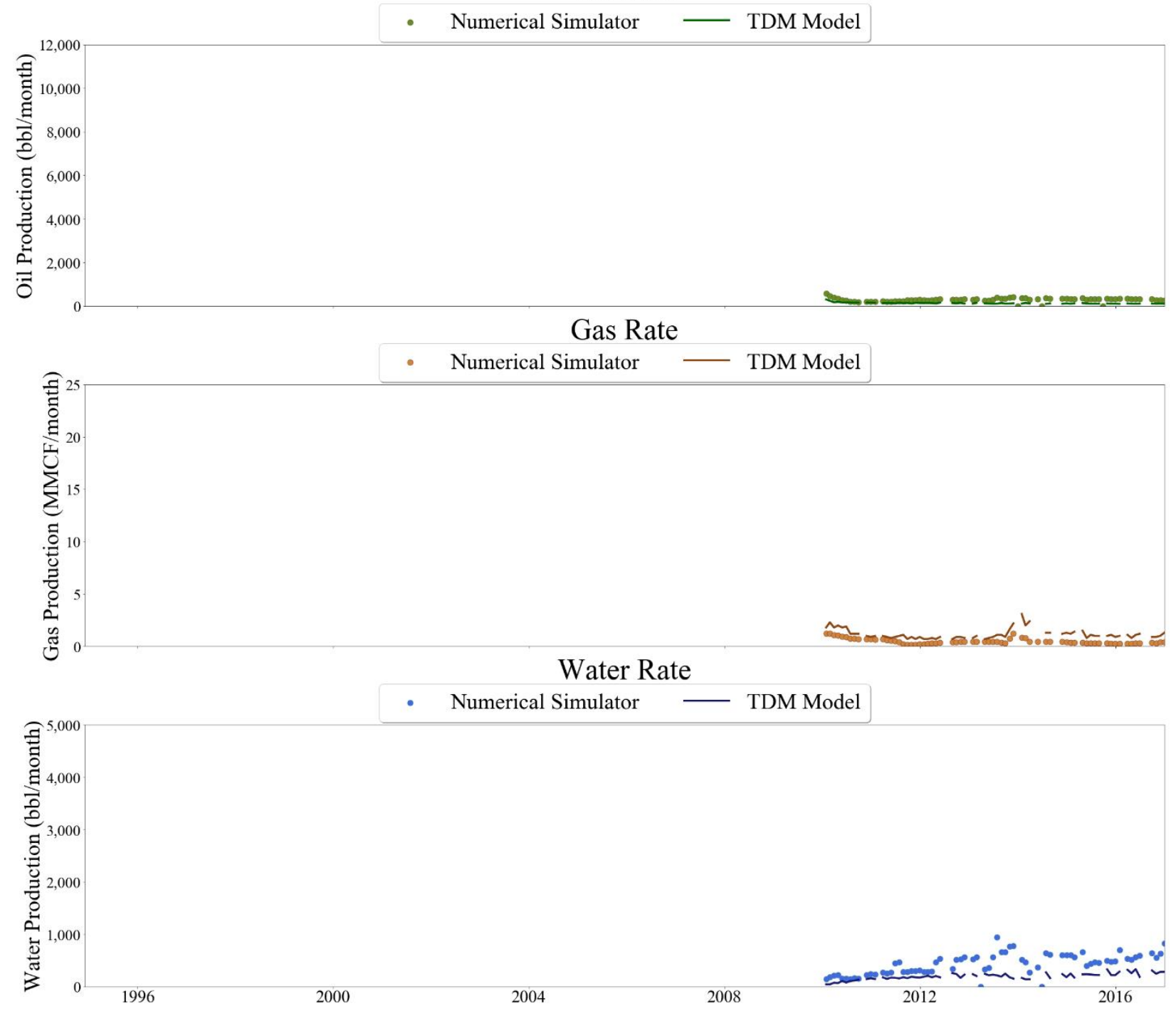

Figure 408 Well-053 oil, gas, and water rate TDM predictions vs actual simulation data plots for No Blind Validation TDM 


\section{Oil Rate}

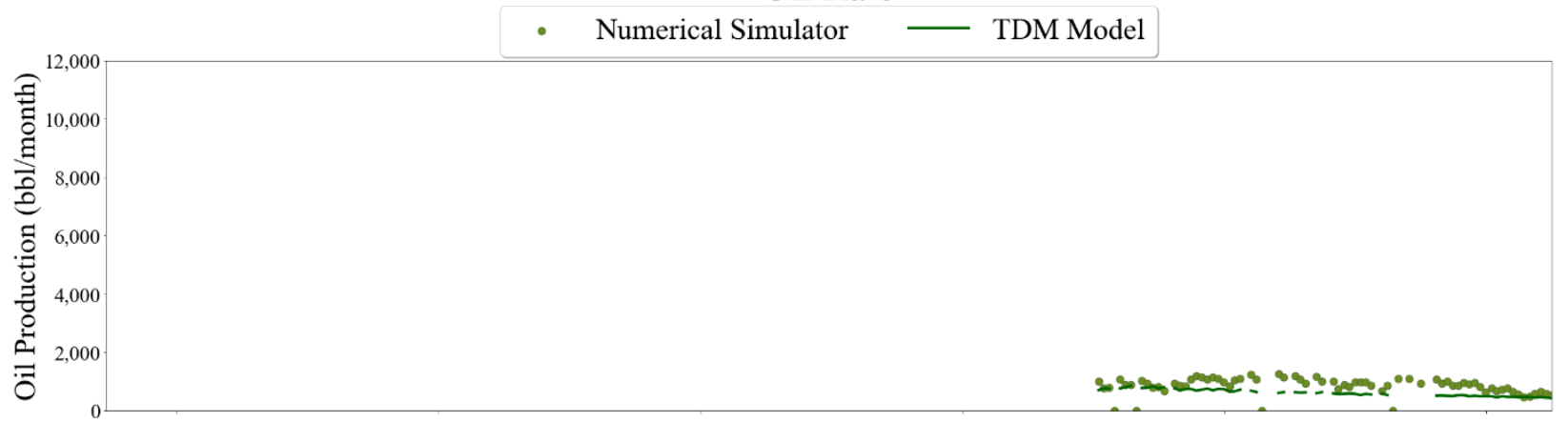

\section{Gas Rate}
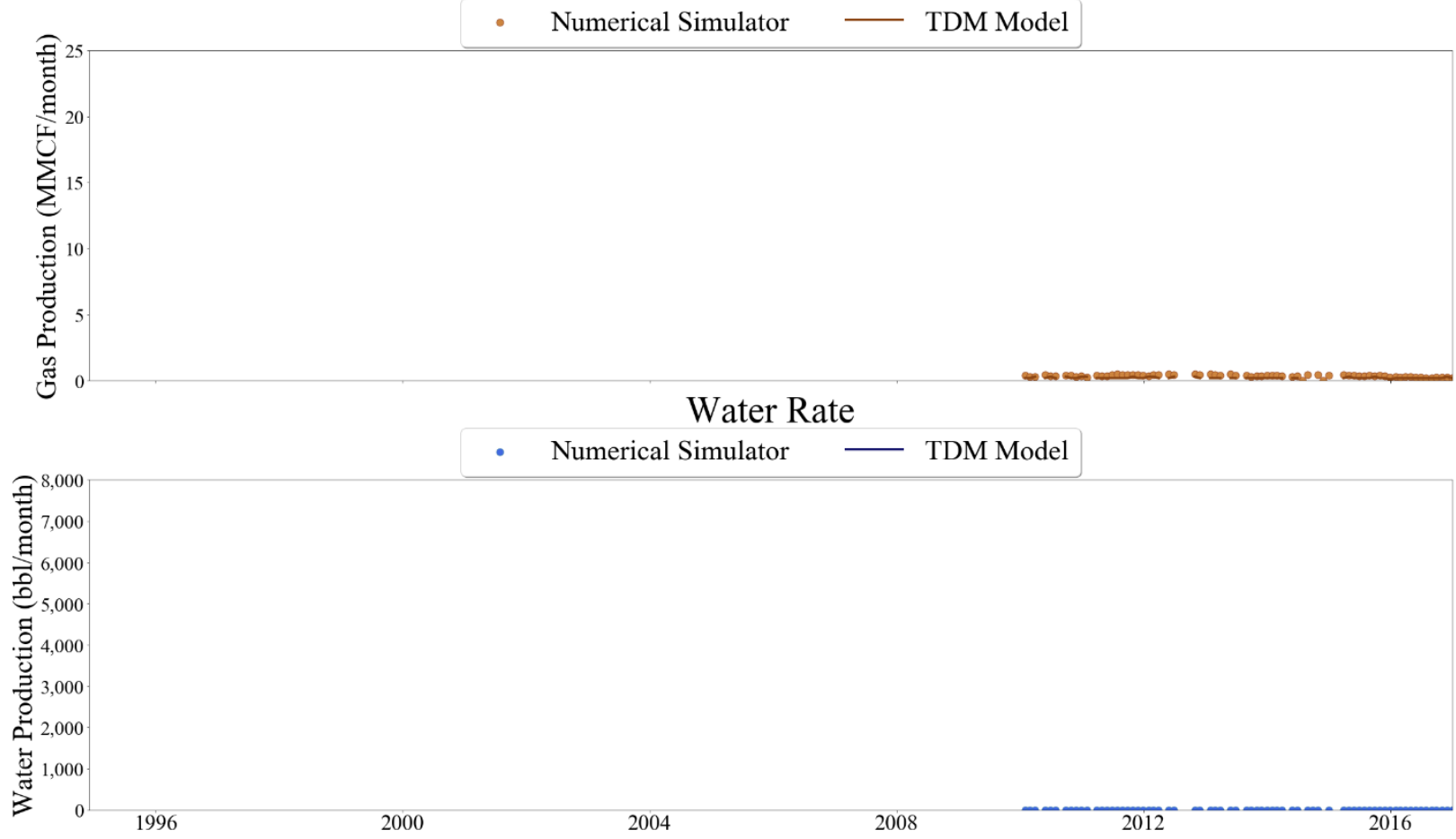

Figure 409 Well-054 oil, gas, and water rate TDM predictions vs actual simulation data plots for No Blind Validation TDM 


\section{Oil Rate}

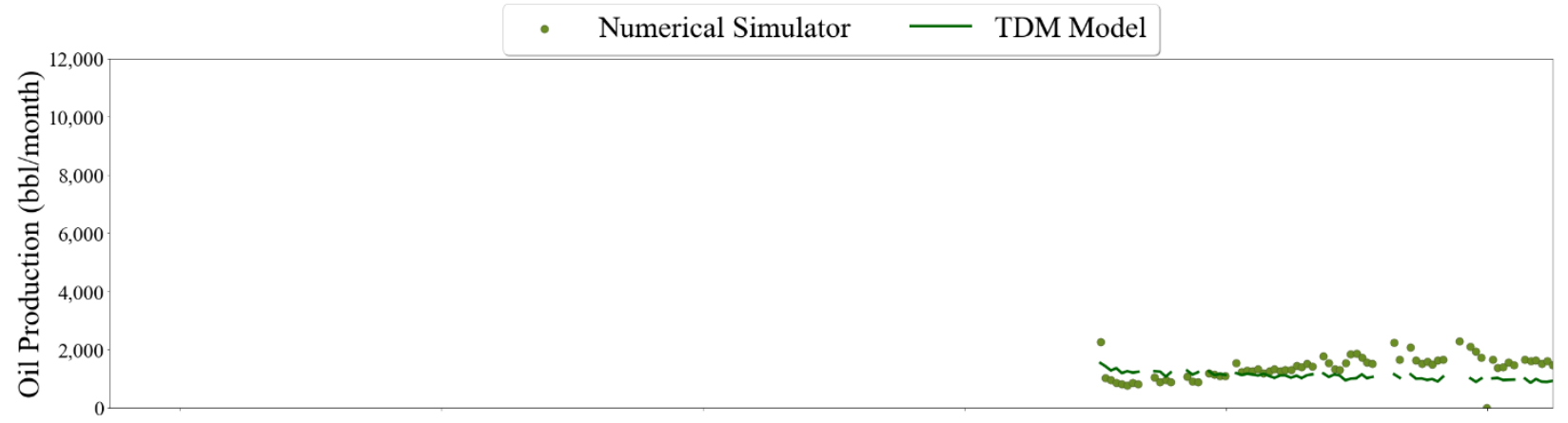

\section{Gas Rate}
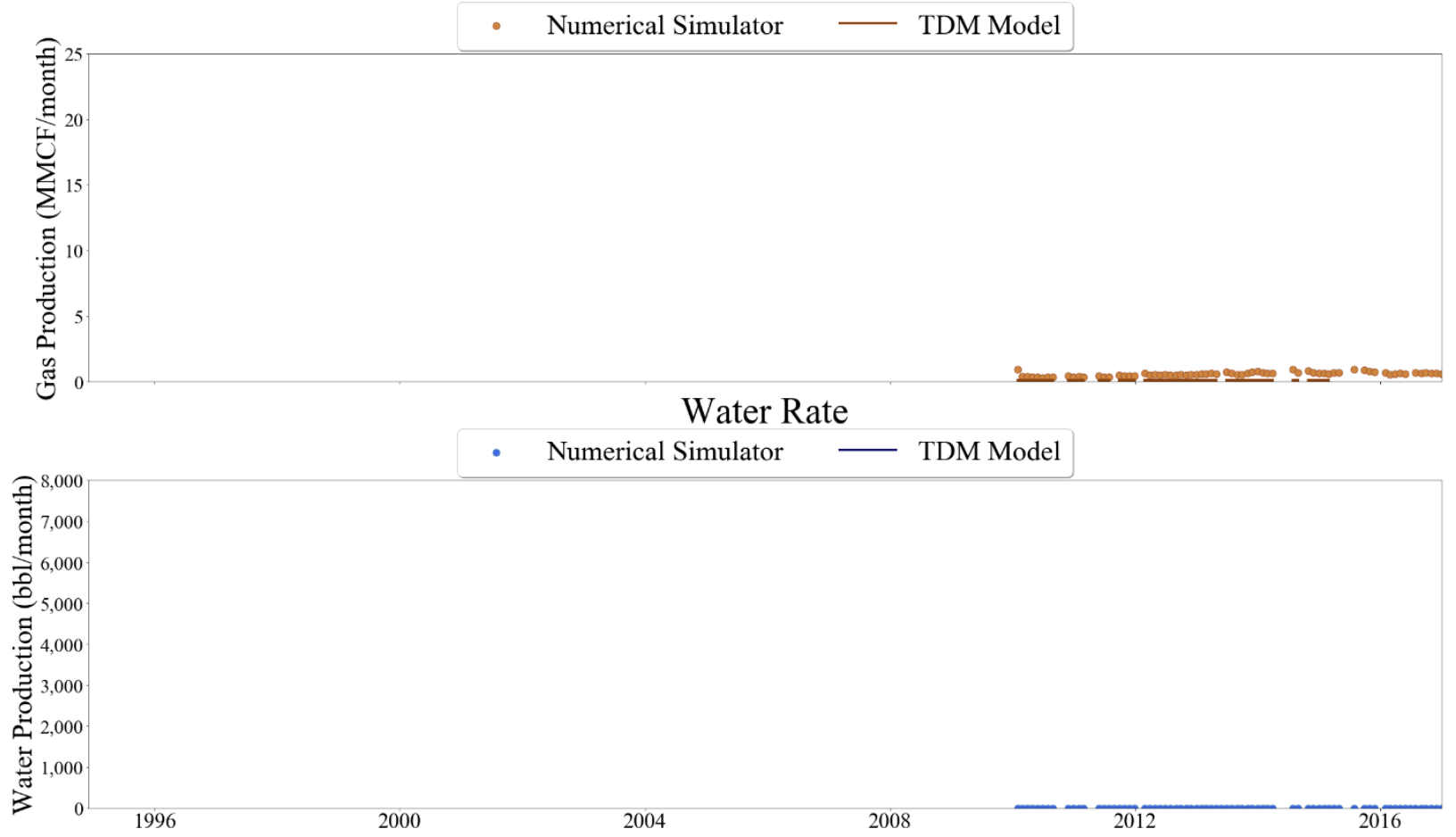

Figure 410 Well-055 oil, gas, and water rate TDM predictions vs actual simulation data plots for No Blind Validation TDM 


\section{Oil Rate}

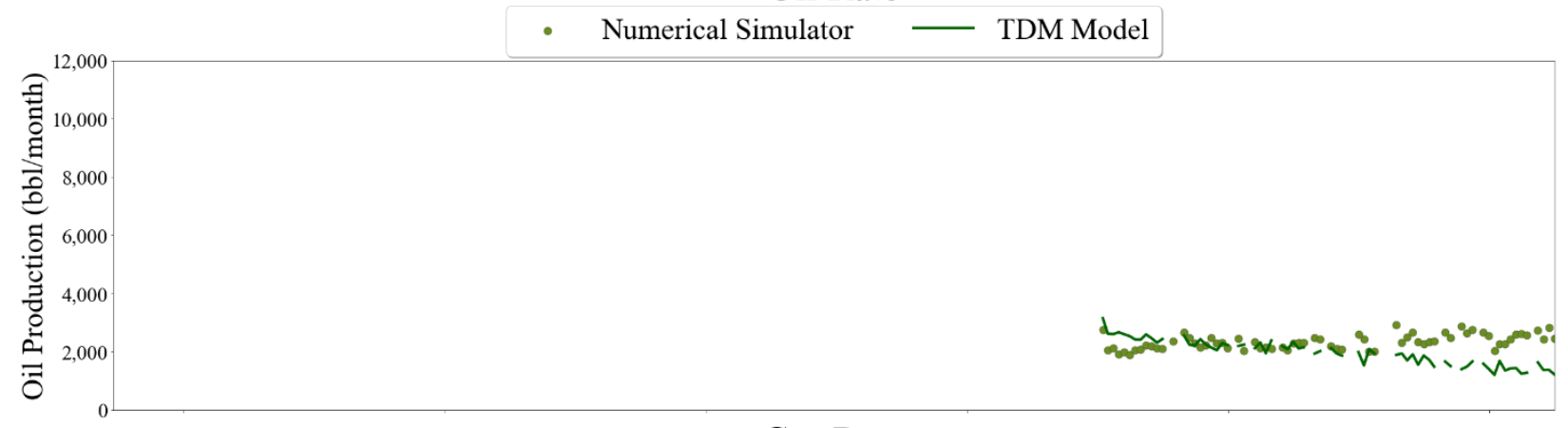

\section{Gas Rate}
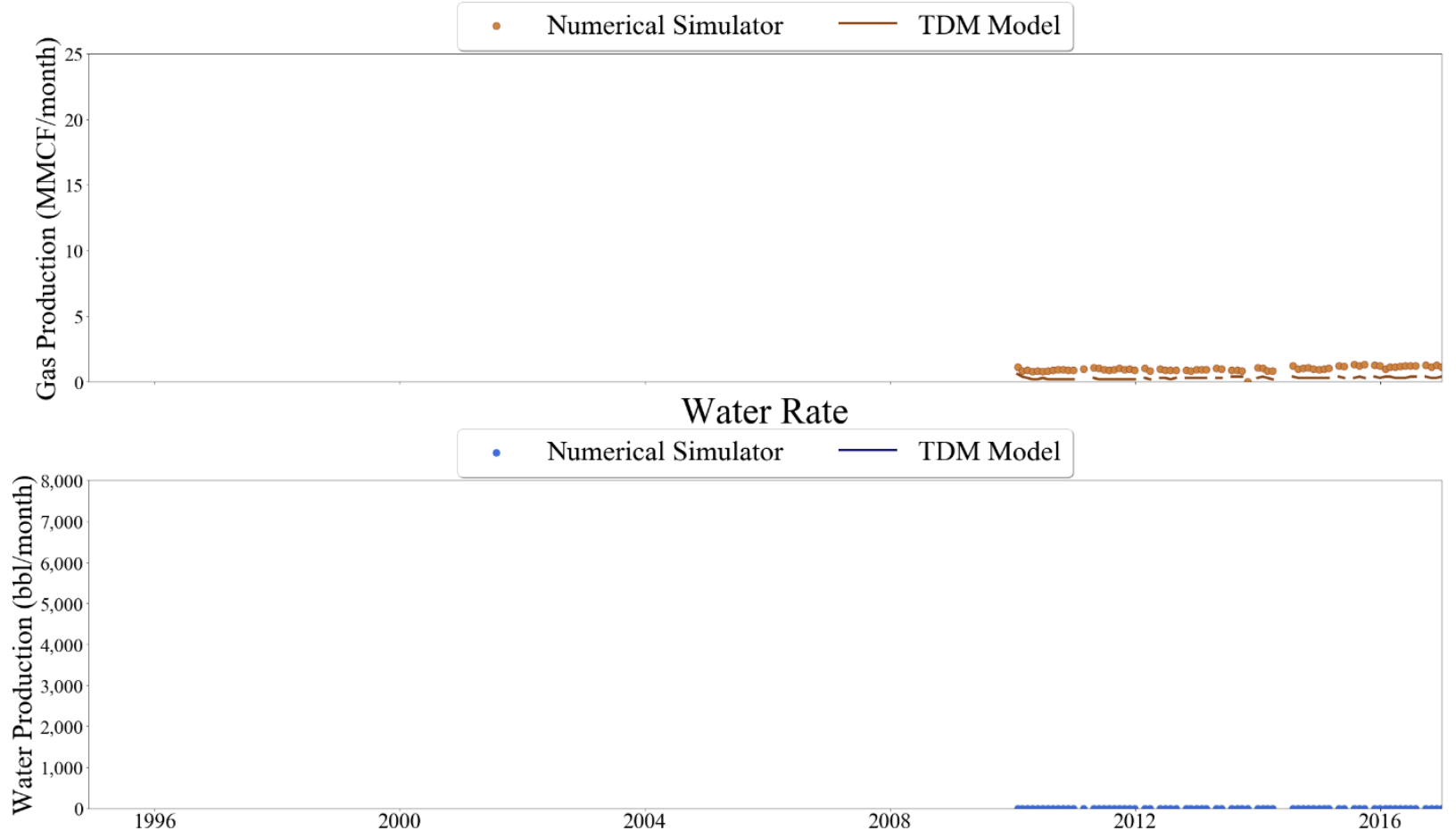

Figure 411 Well-056 oil, gas, and water rate TDM predictions vs actual simulation data plots for No Blind Validation TDM 


\section{Oil Rate}

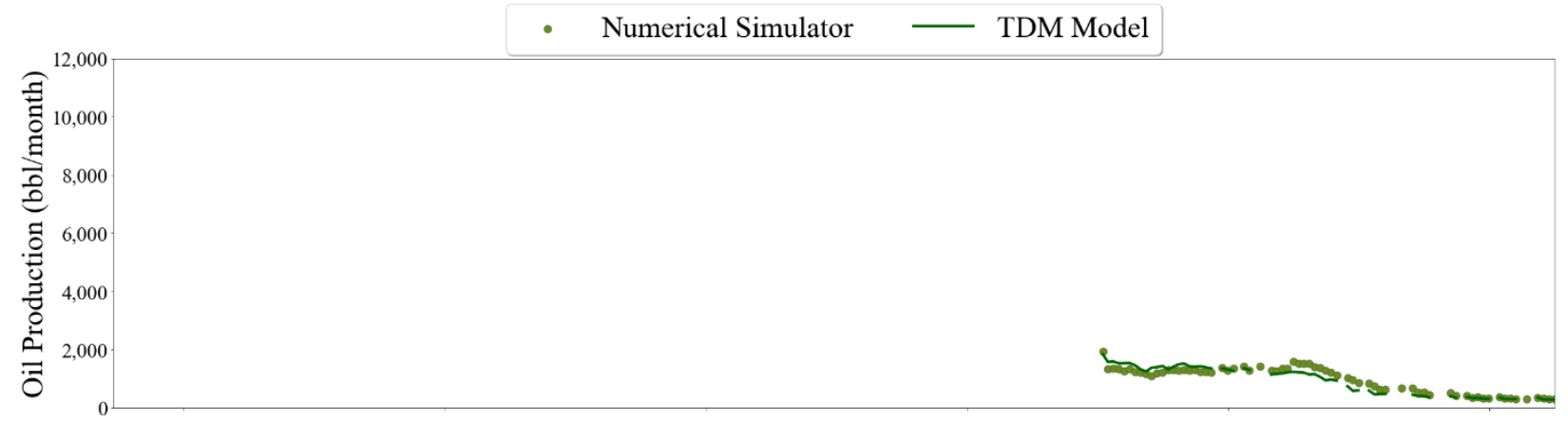

\section{Gas Rate}
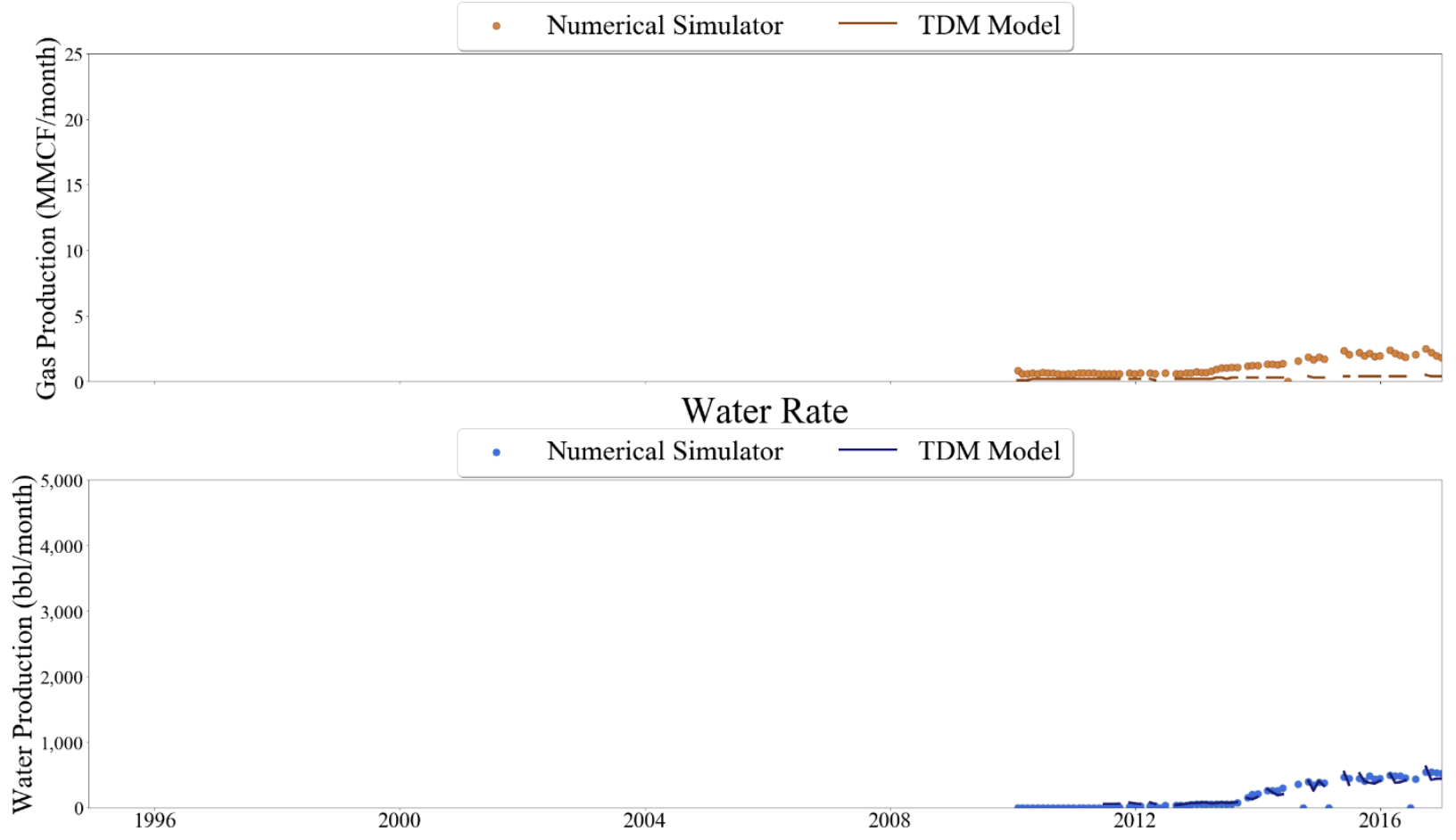

Figure 412 Well-057 oil, gas, and water rate TDM predictions vs actual simulation data plots for No Blind Validation TDM

\subsubsection{Heat Maps}

Reservoir Pressure (psi) at 2015-12-31
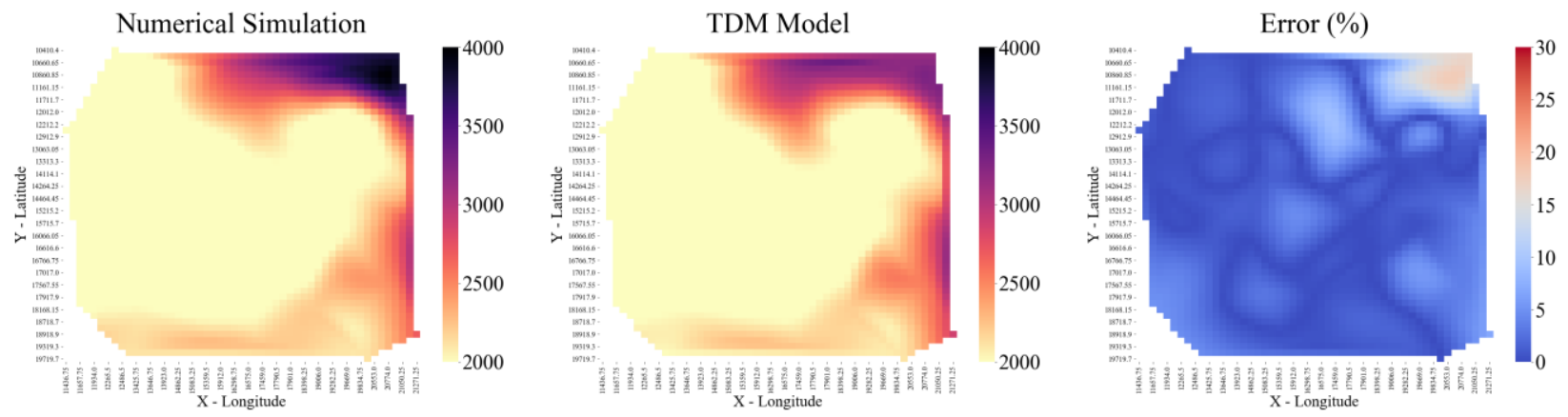

Figure 413 Entire reservoir heat map for reservoir pressure at December 32st, 2015 for No Blind Validation TDM 
Reservoir Pressure (psi) at 2016-02-29
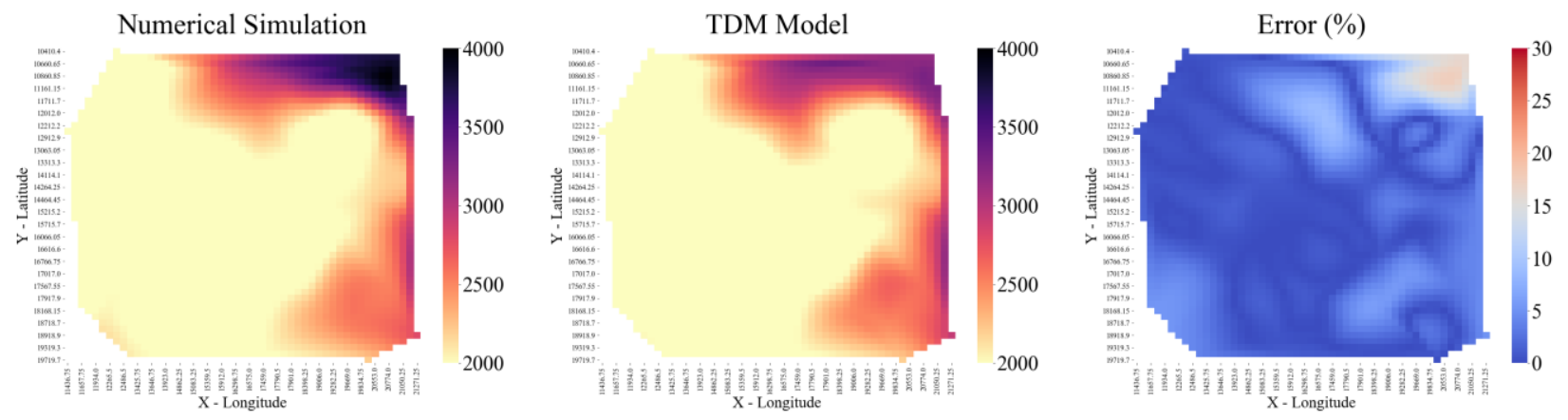

Figure 414 Entire reservoir heat map for reservoir pressure at February 29th, 2016 for No Blind Validation TDM

Reservoir Pressure (psi) at 2016-04-30
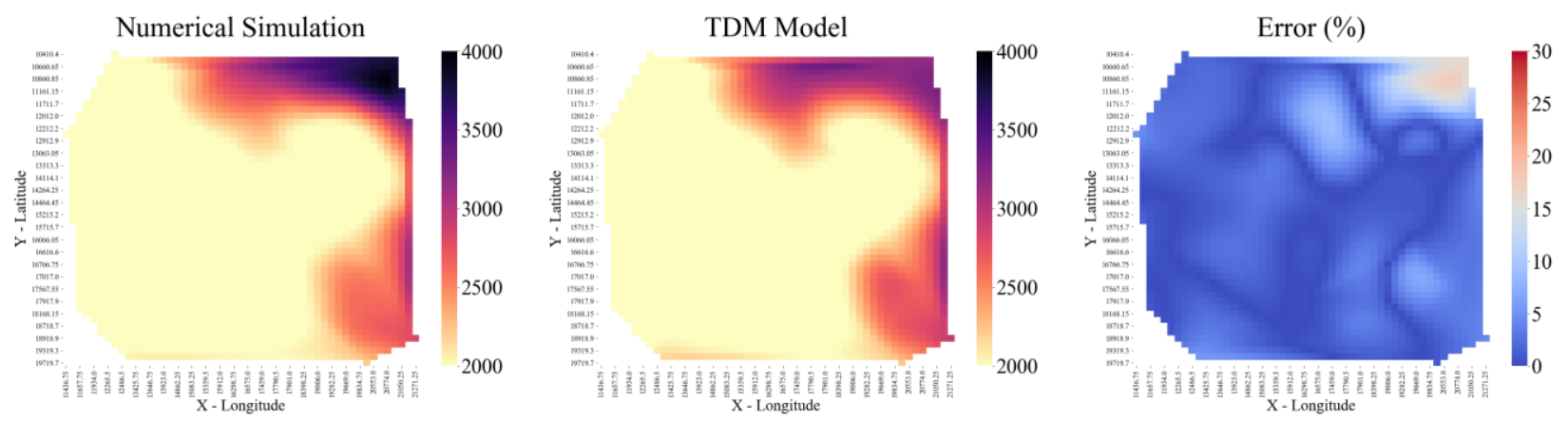

Figure 415 Entire reservoir heat map for reservoir pressure at April 30th, 2016 for No Blind Validation TDM

Reservoir Pressure (psi) at 2016-06-30
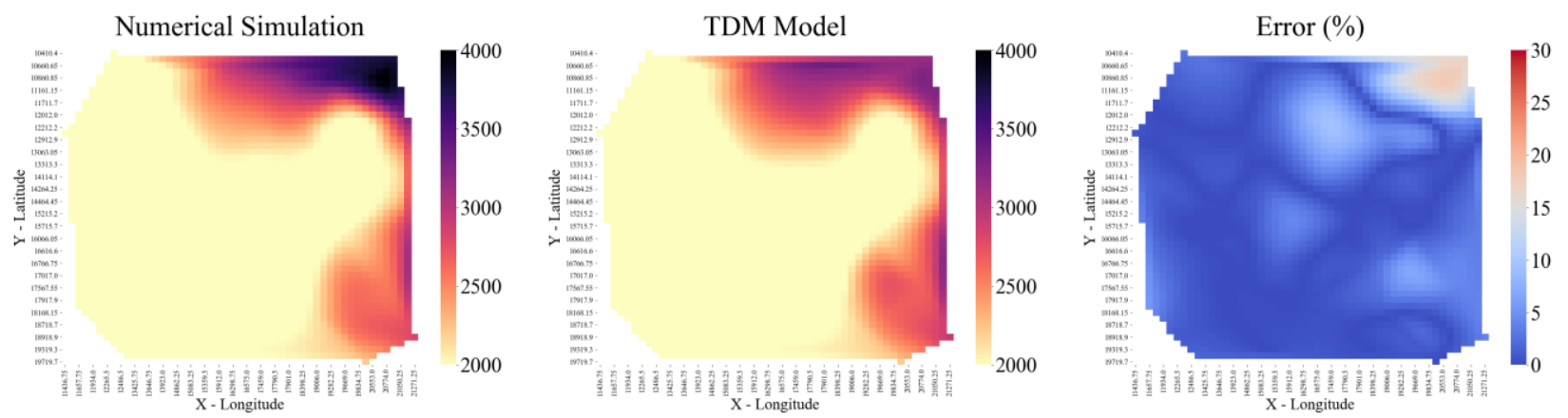

Figure 416 Entire reservoir heat map for reservoir pressure at June 30th, 2016 for No Blind Validation TDM 
Reservoir Pressure (psi) at 2016-08-31
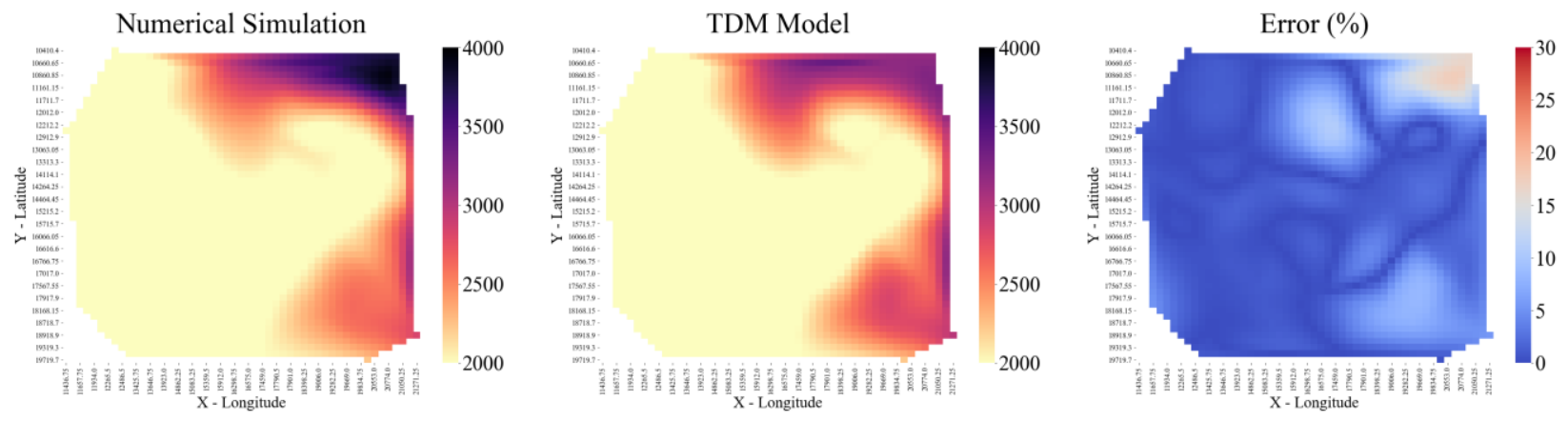

Figure 417 Entire reservoir heat map for reservoir pressure at August 31st, 2016 for No Blind Validation TDM

Reservoir Pressure (psi) at 2016-10-31
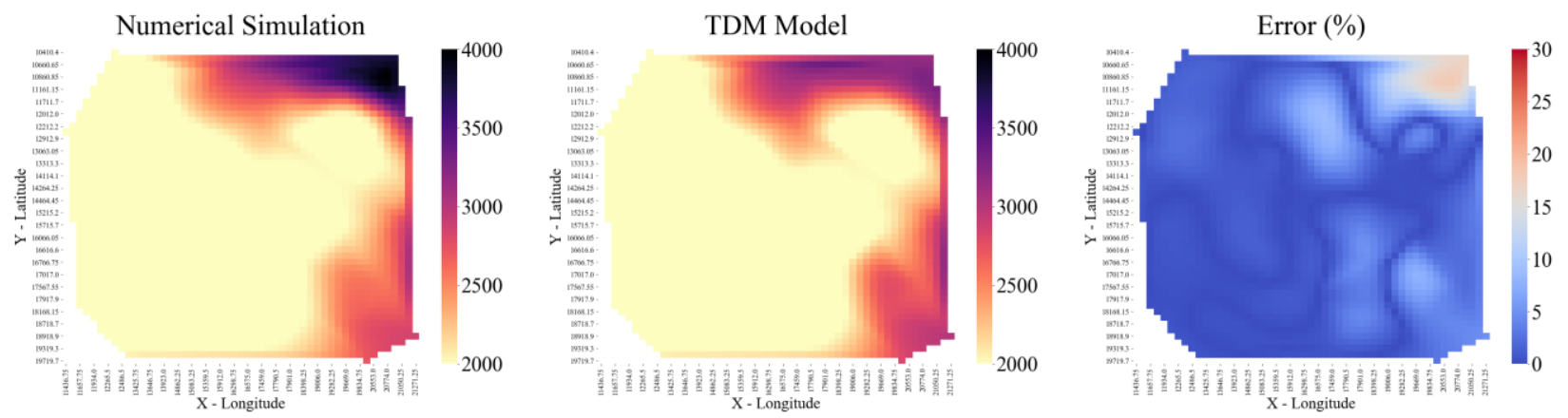

Figure 418 Entire reservoir heat map for reservoir pressure at October 31st, 2016 for No Blind Validation TDM

Reservoir Pressure (psi) at 2016-12-31
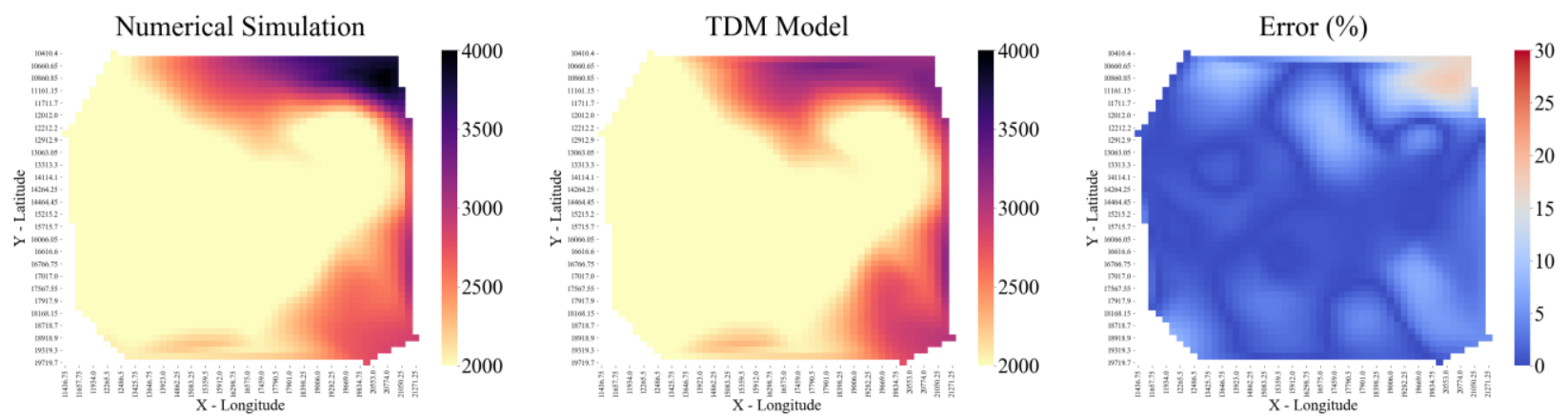

Figure 419 Entire reservoir heat map for reservoir pressure at December 31st, 2016 for No Blind Validation TDM 
Water Saturation (\%) at 2015-12-31
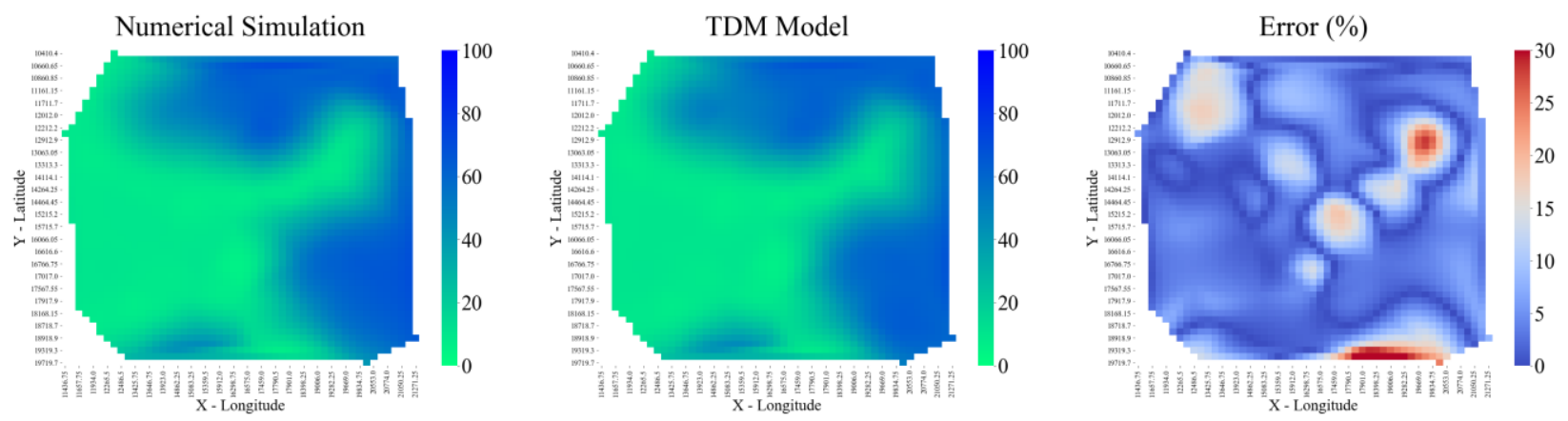

Figure 420 Entire reservoir heat map for water saturation at December 31st, 2016 for No Blind Validation TDM

Water Saturation $(\%)$ at 2016-02-29
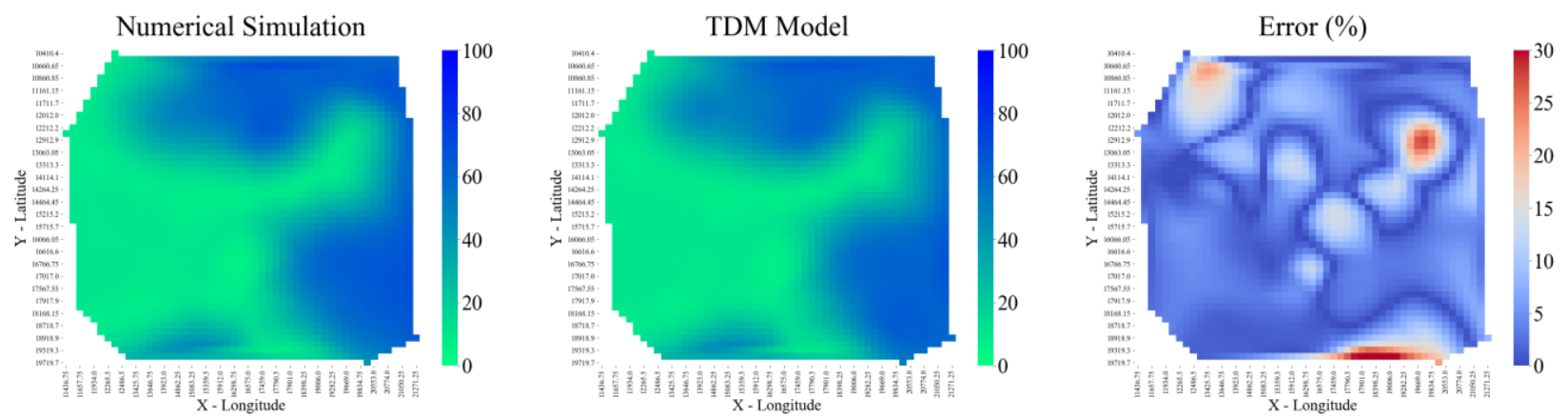

Figure 421 Entire reservoir heat map for water saturation at February 29th, 2016 for No Blind Validation TDM

\section{Water Saturation (\%) at 2016-04-30}
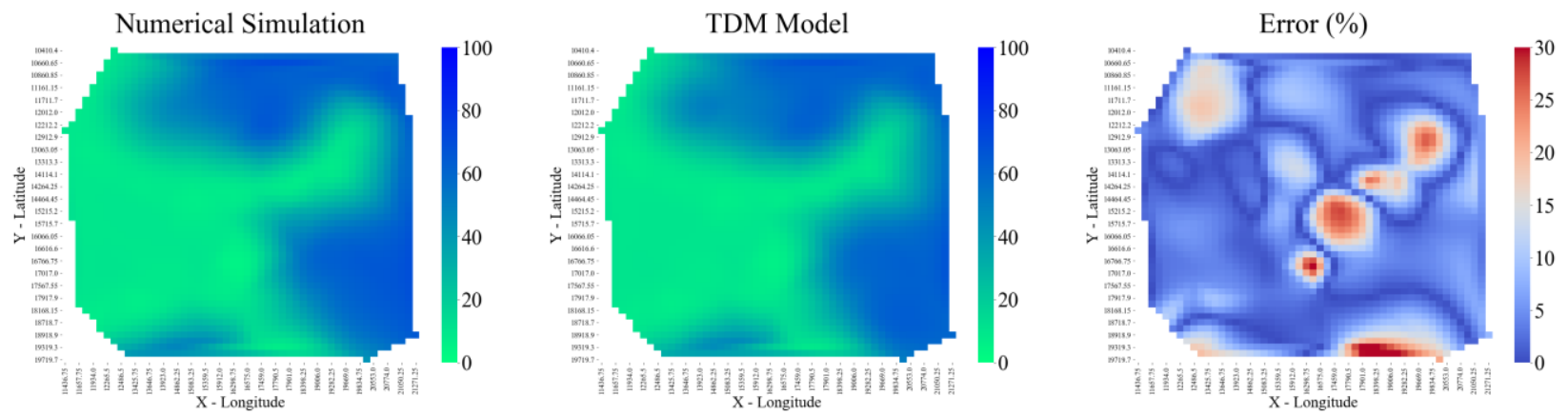

Figure 422 Entire reservoir heat map for water saturation at April 30th, 2016 for No Blind Validation TDM 
Water Saturation (\%) at 2016-06-30
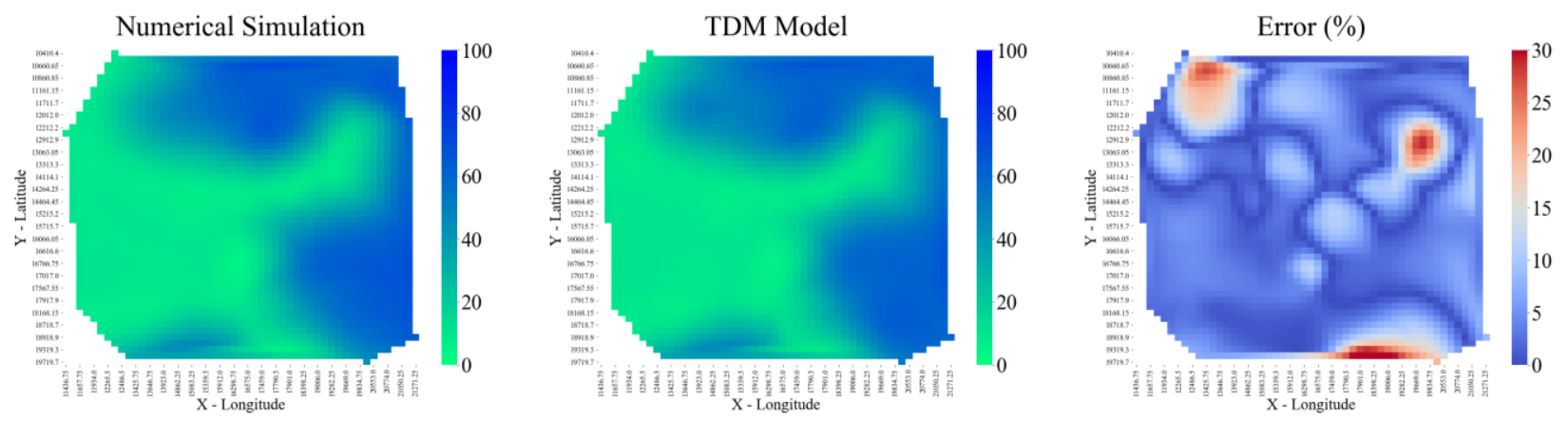

Figure 423 Entire reservoir heat map for water saturation at June 30th, 2016 for No Blind Validation TDM

Water Saturation (\%) at 2016-08-31
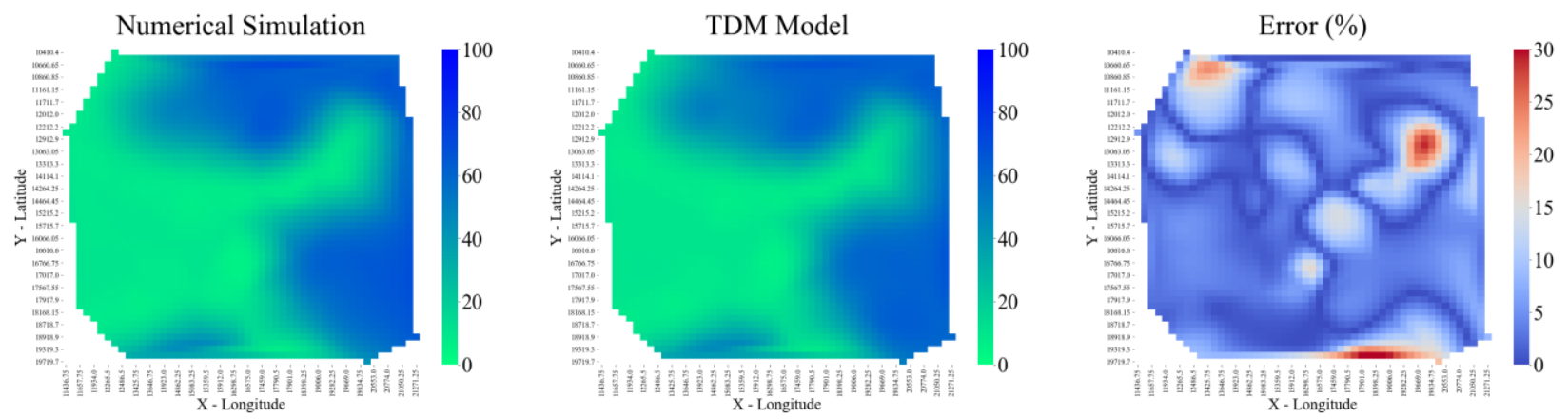

Figure 424 Entire reservoir heat map for water saturation at August 31st, 2016 for No Blind Validation TDM

\section{Water Saturation (\%) at 2016-10-31}
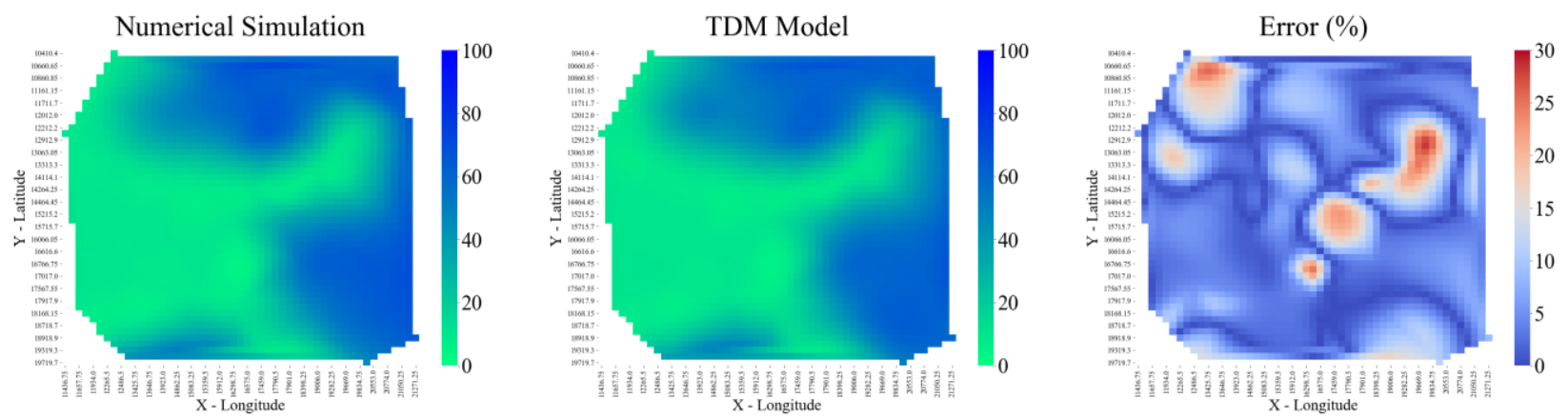

Figure 425 Entire reservoir heat map for water saturation for October 31st, 2016 for No Blind Validation TDM 
Water Saturation (\%) at 2016-12-31
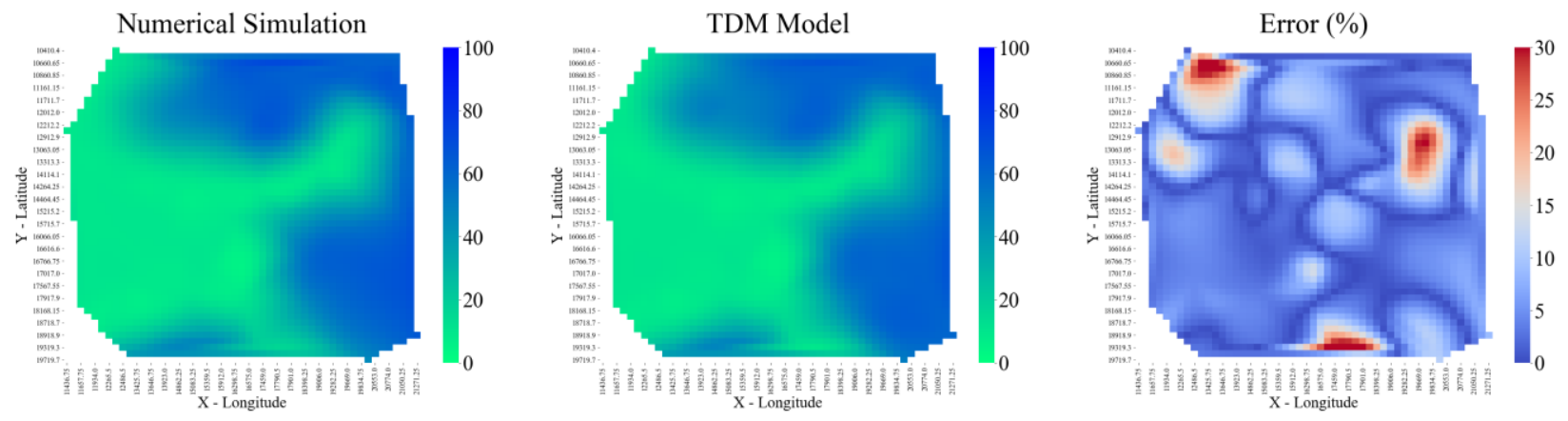

Figure 426 Entire reservoir heat map for water saturation at December 31st, 2016 for No Blind Validation TDM

\subsubsection{Multi-Random Partitioning- 1 Year Blind Validation}

\subsubsection{Individual Well Production Plots}

\section{Oil Rate}

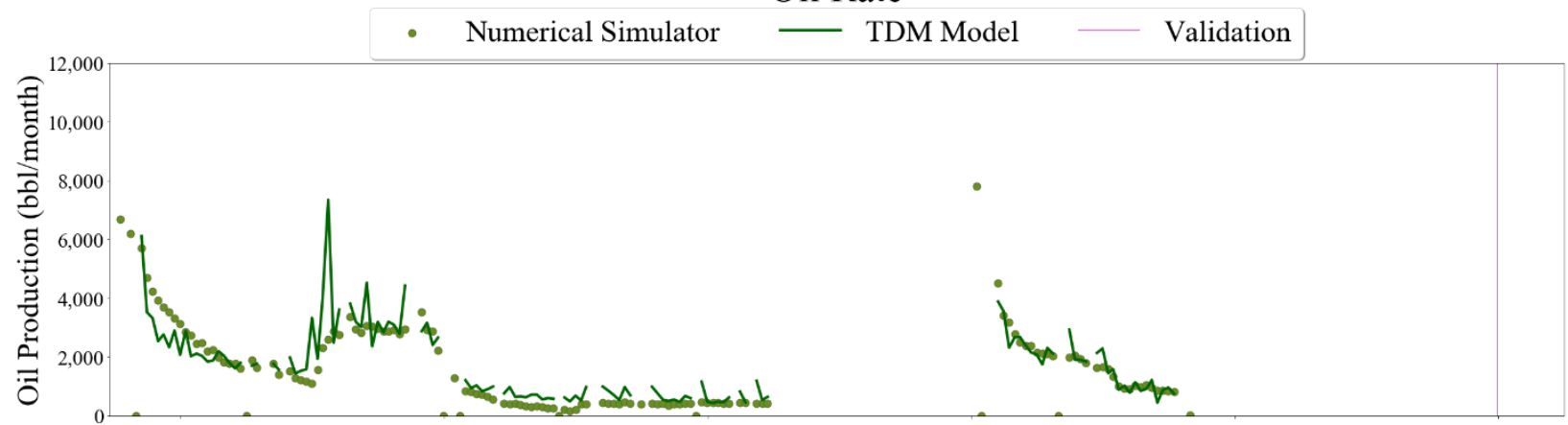

Gas Rate

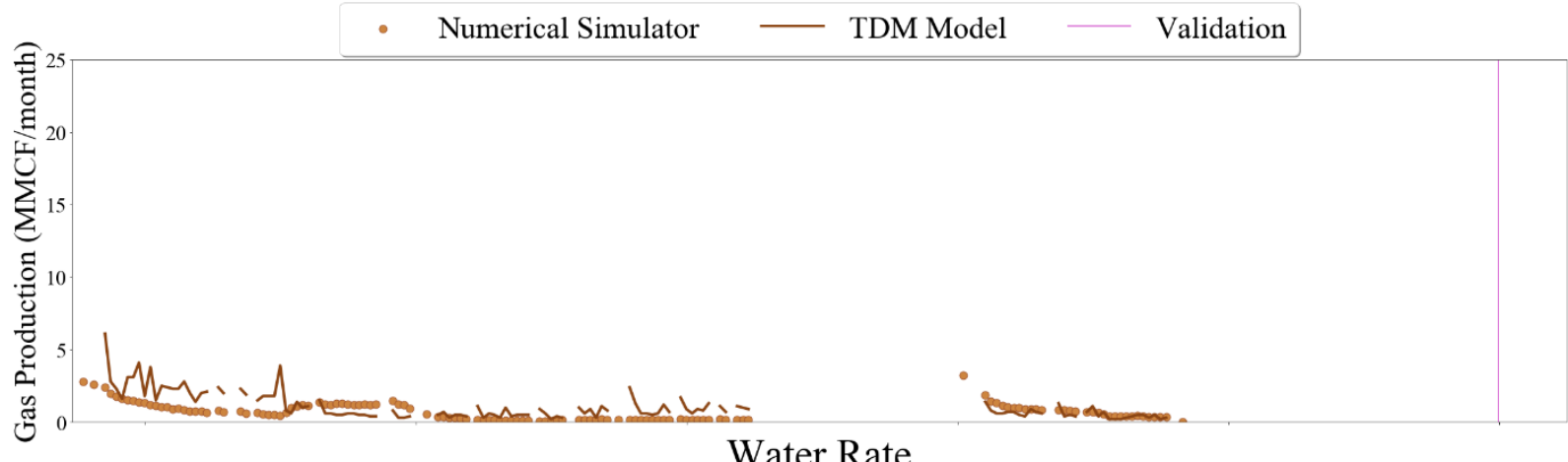

Water Rate

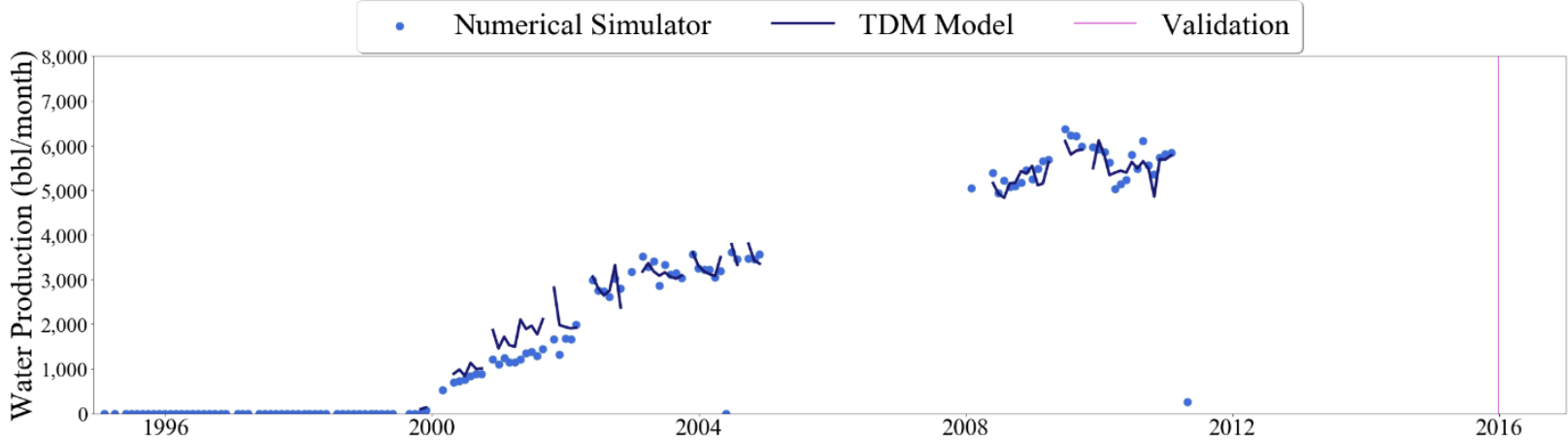

Figure 427 Well-001 oil, gas, and water rate TDM predictions vs actual simulation data plots for 1 Year Blind Validation TDM 

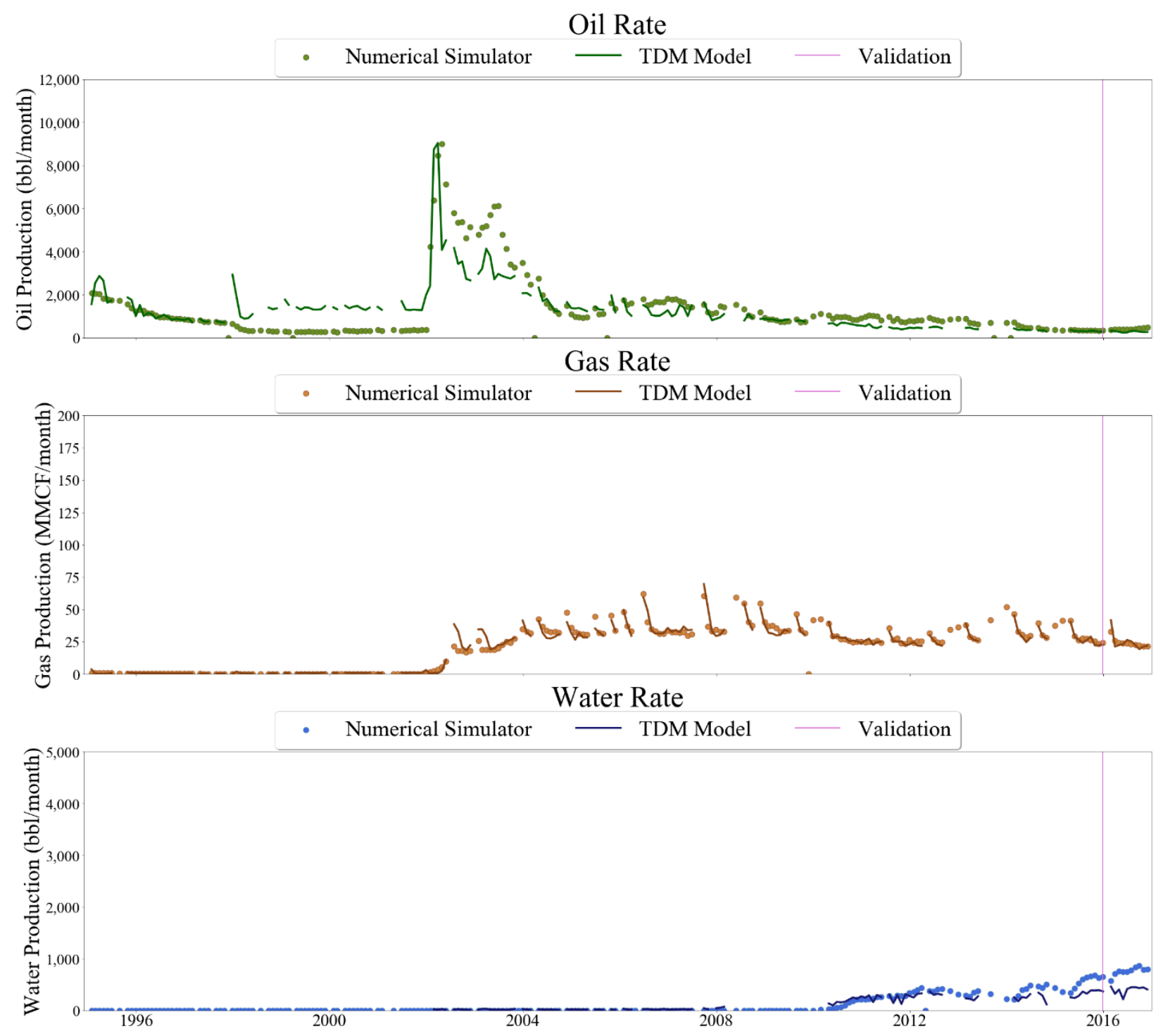

Figure 428 Well-002 oil, gas, and water rate TDM predictions vs actual simulation data plots for 1 Year Blind Validation TDM 


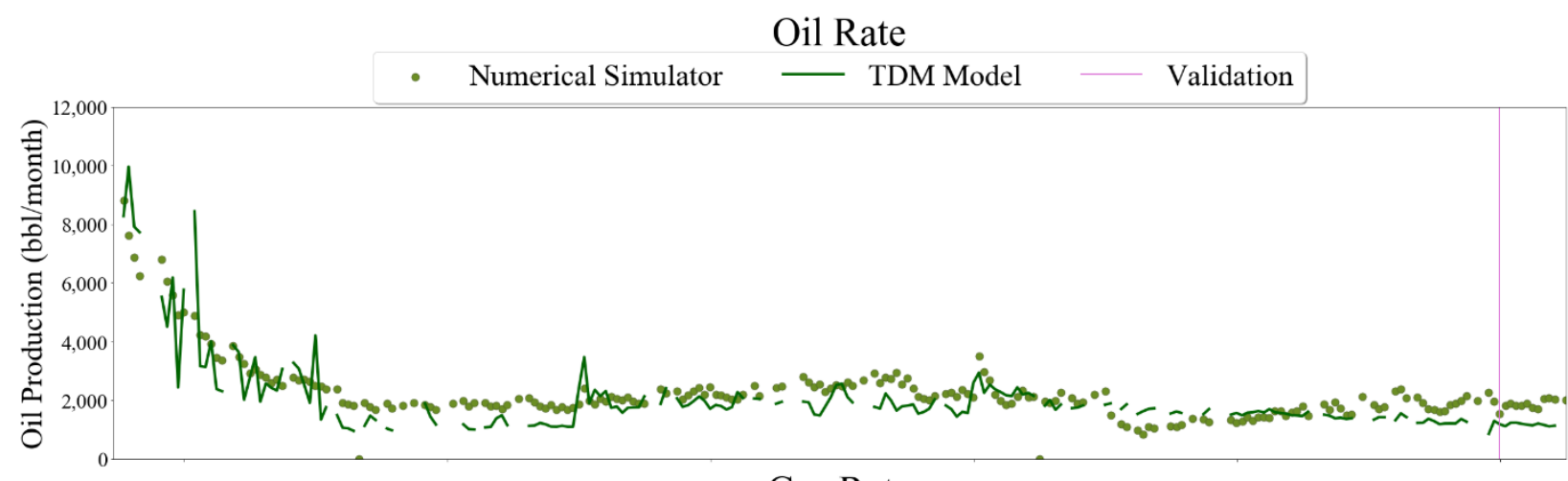

\section{Gas Rate}
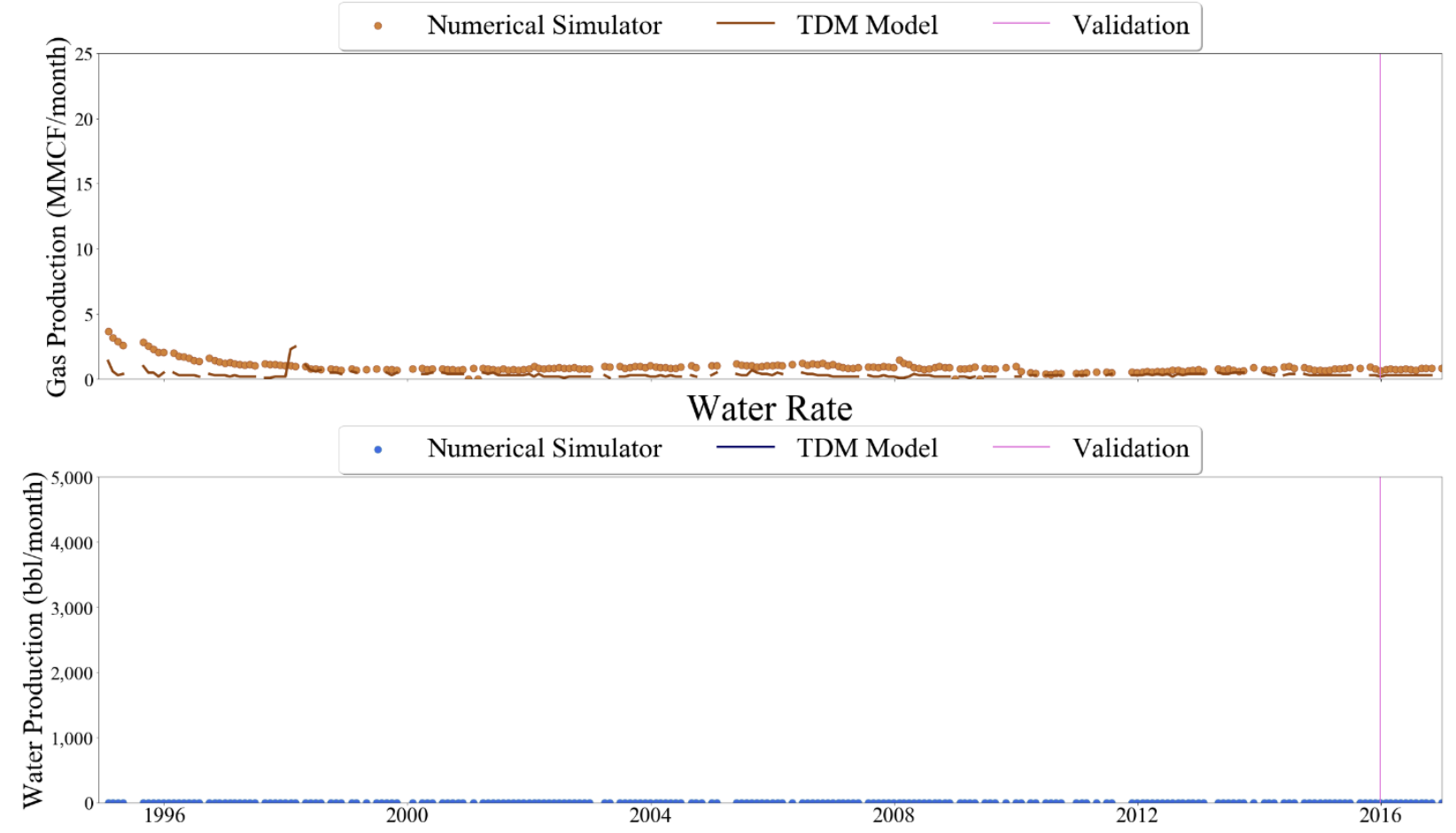

Figure 429 Well-003 oil, gas, and water rate TDM predictions vs actual simulation data plots for 1 Year Blind Validation TDM 


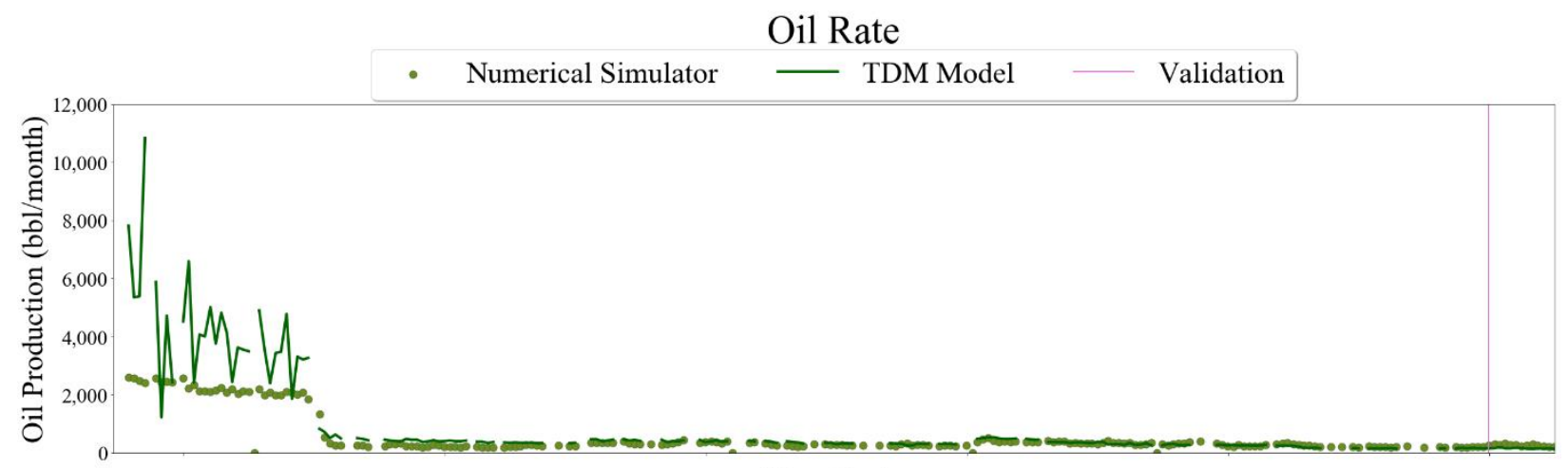

Gas Rate
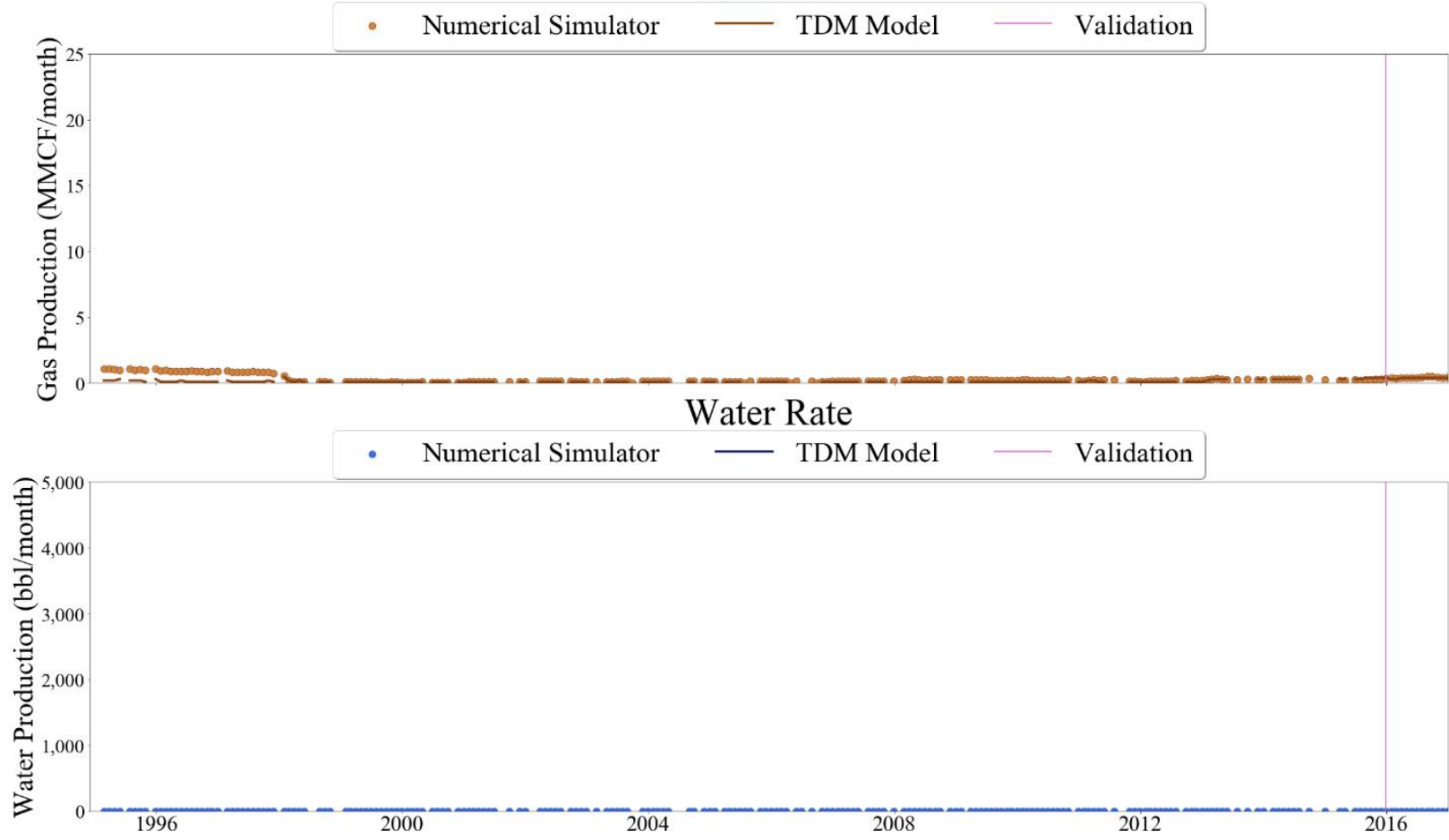

Figure 430 Well-004 oil, gas, and water rate TDM predictions vs actual simulation data plots for 1 Year Blind Validation TDM 


\section{Oil Rate}

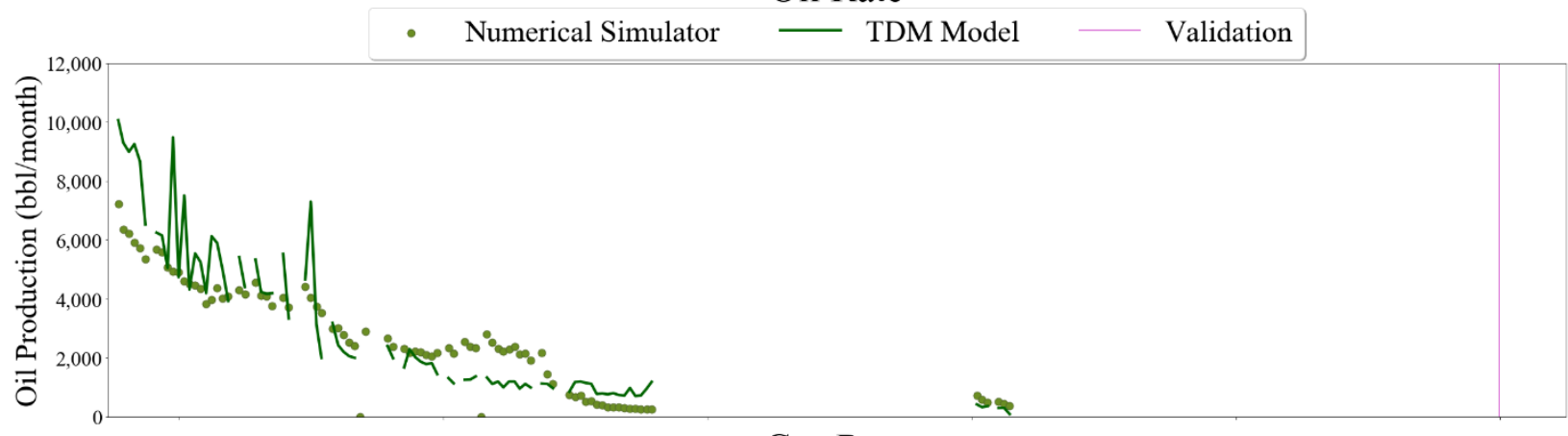

Gas Rate
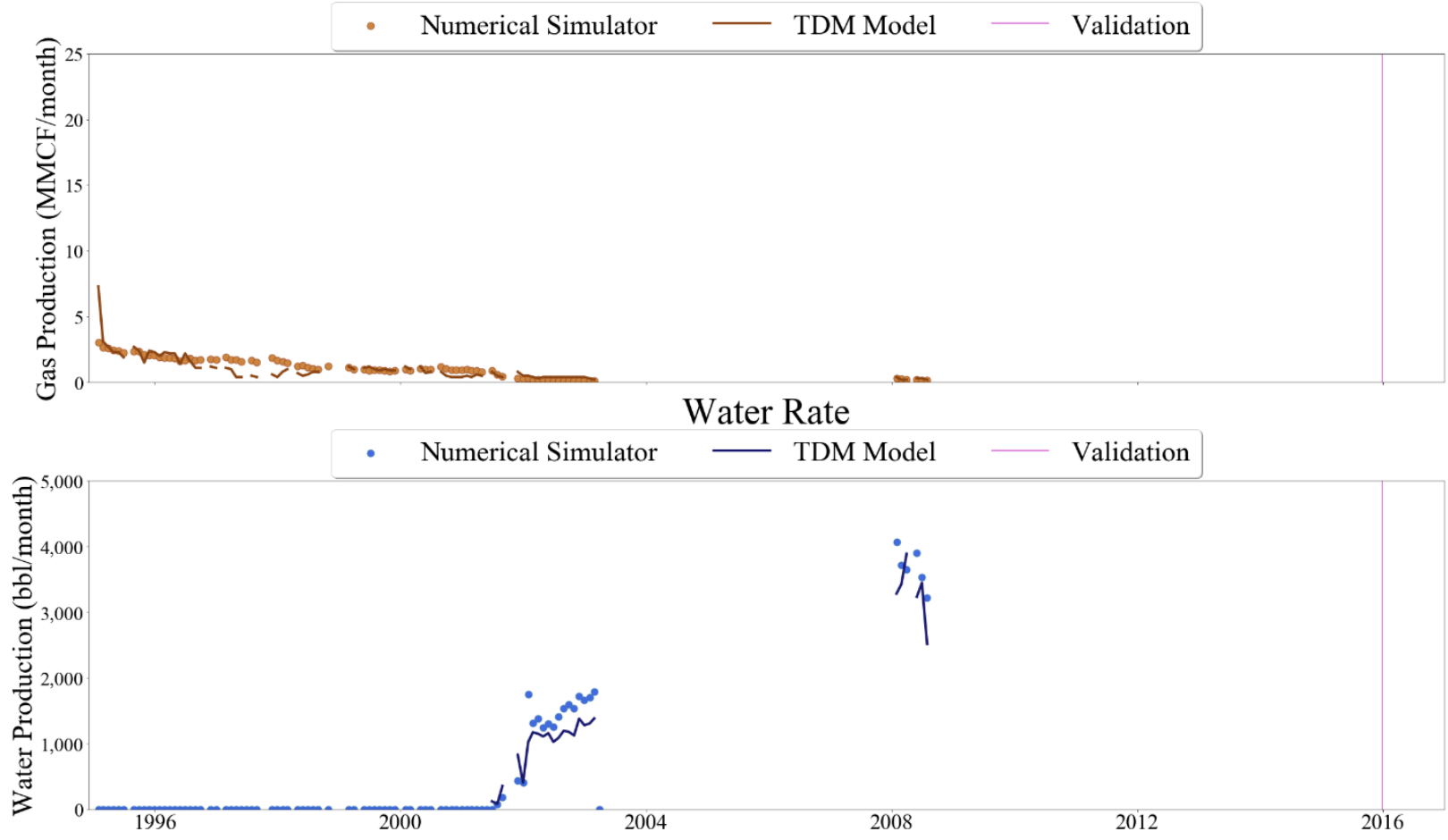

Figure 431 Well-005 oil, gas, and water rate TDM predictions vs actual simulation data plots for 1 Year Blind Validation TDM 


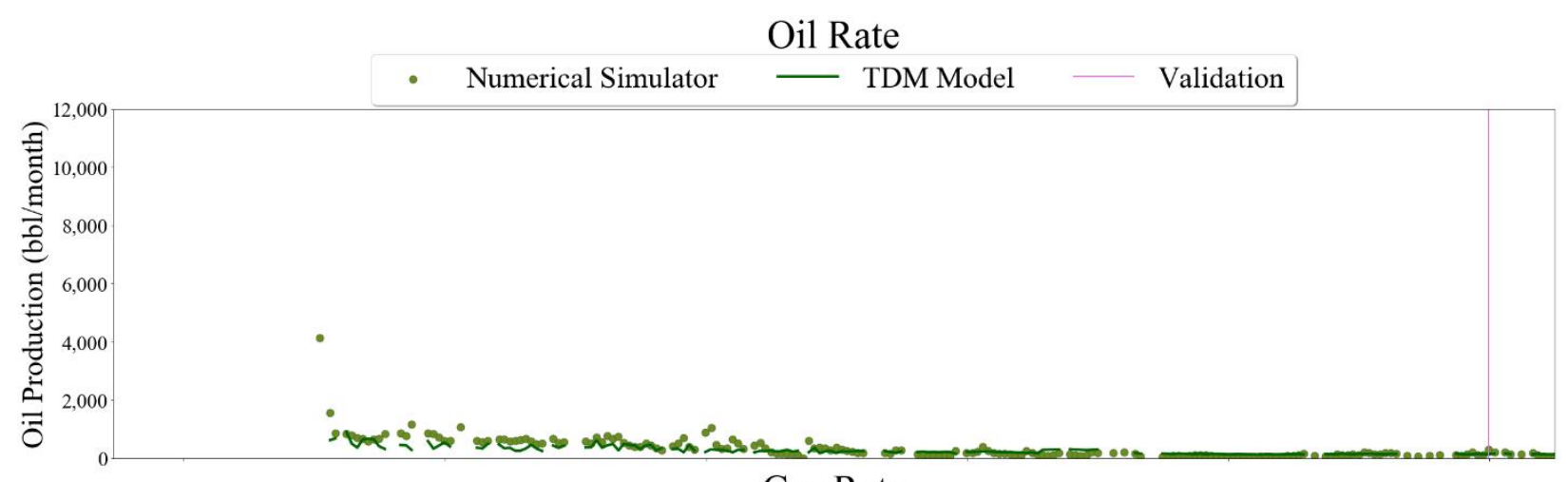

Gas Rate
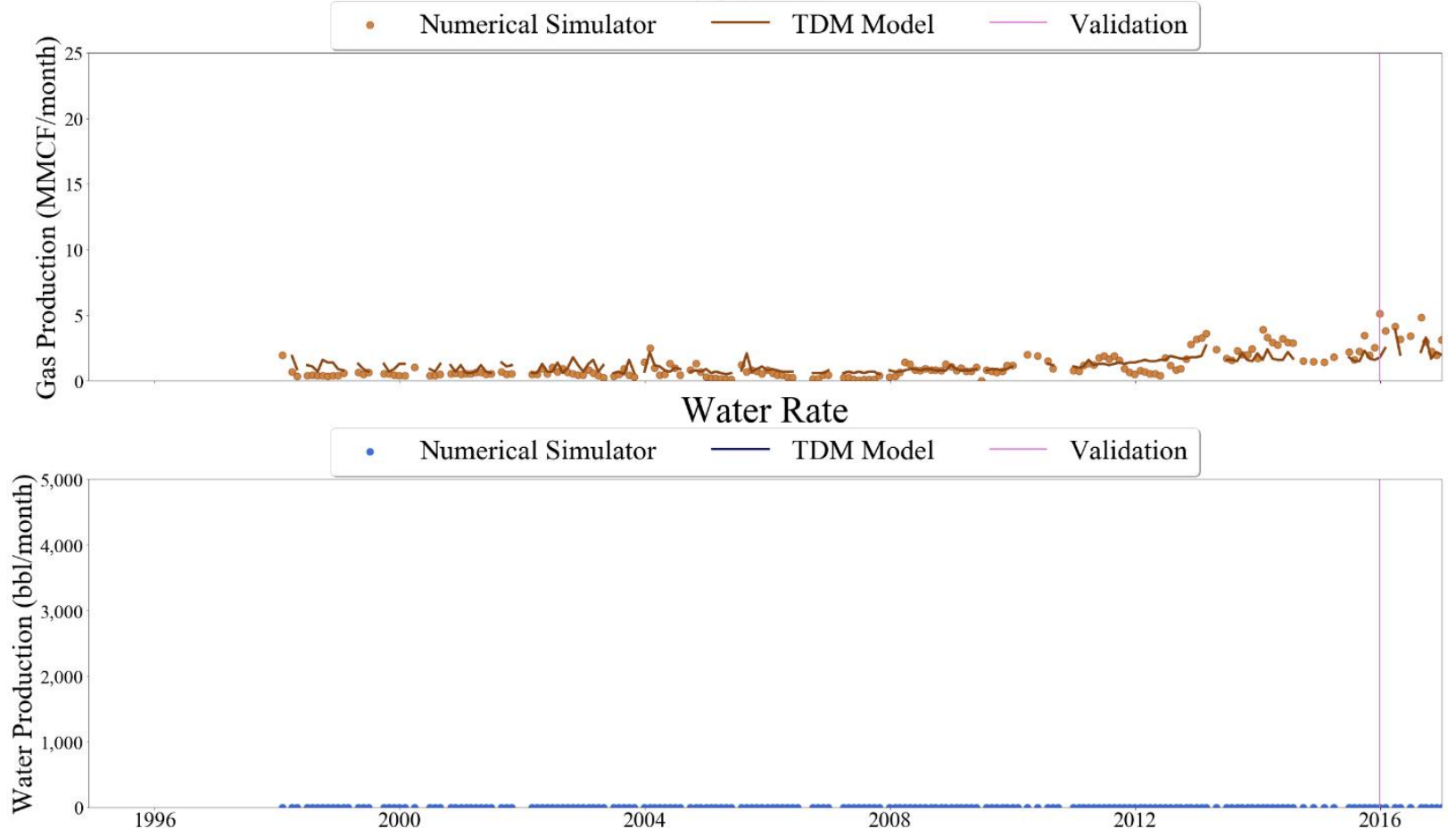

Figure 432 Well-006 oil, gas, and water rate TDM predictions vs actual simulation data plots for 1 Year Blind Validation TDM 


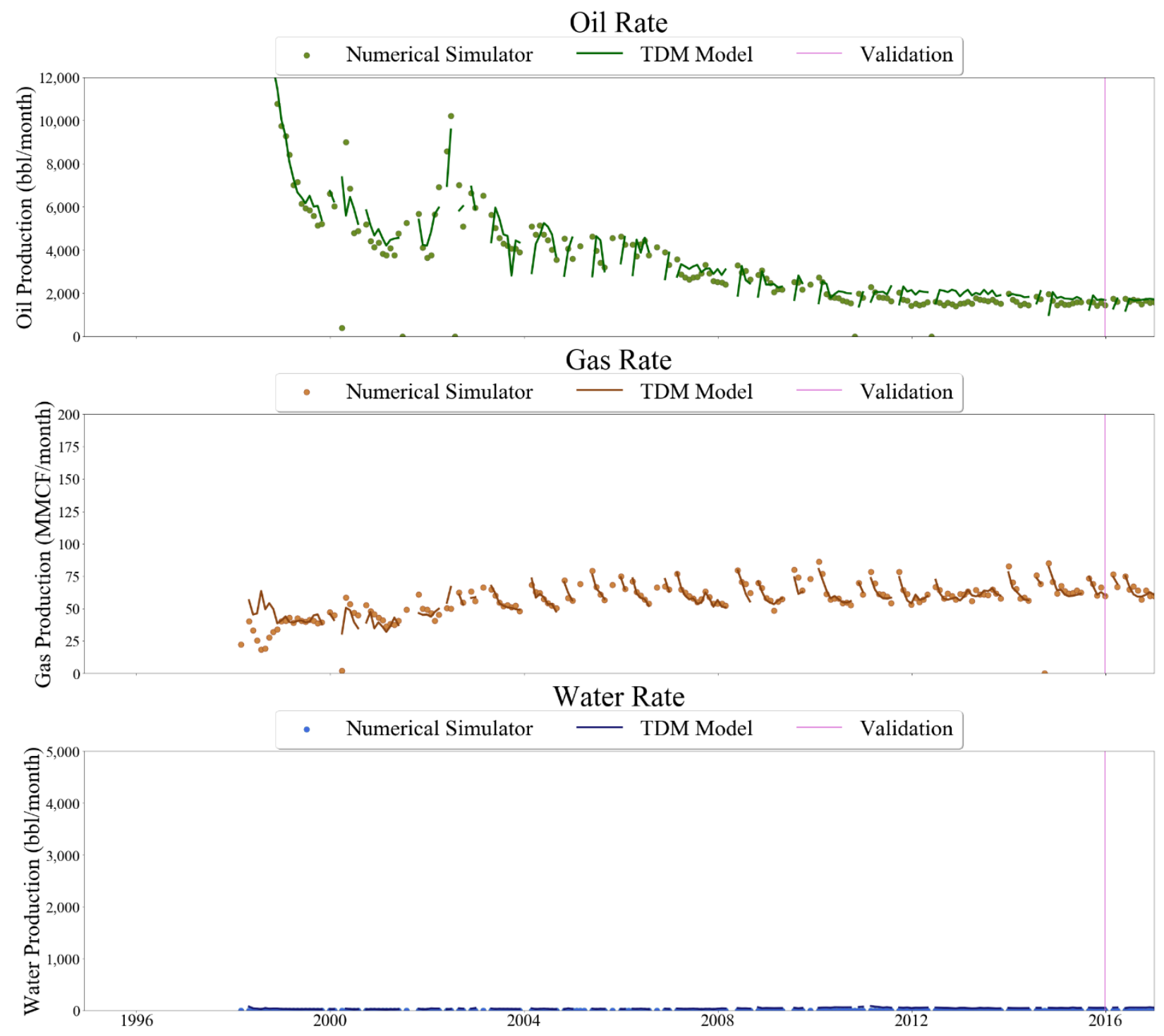

Figure 433 Well-007 oil, gas, and water rate TDM predictions vs actual simulation data for 1 Year Blind Validation TDM 

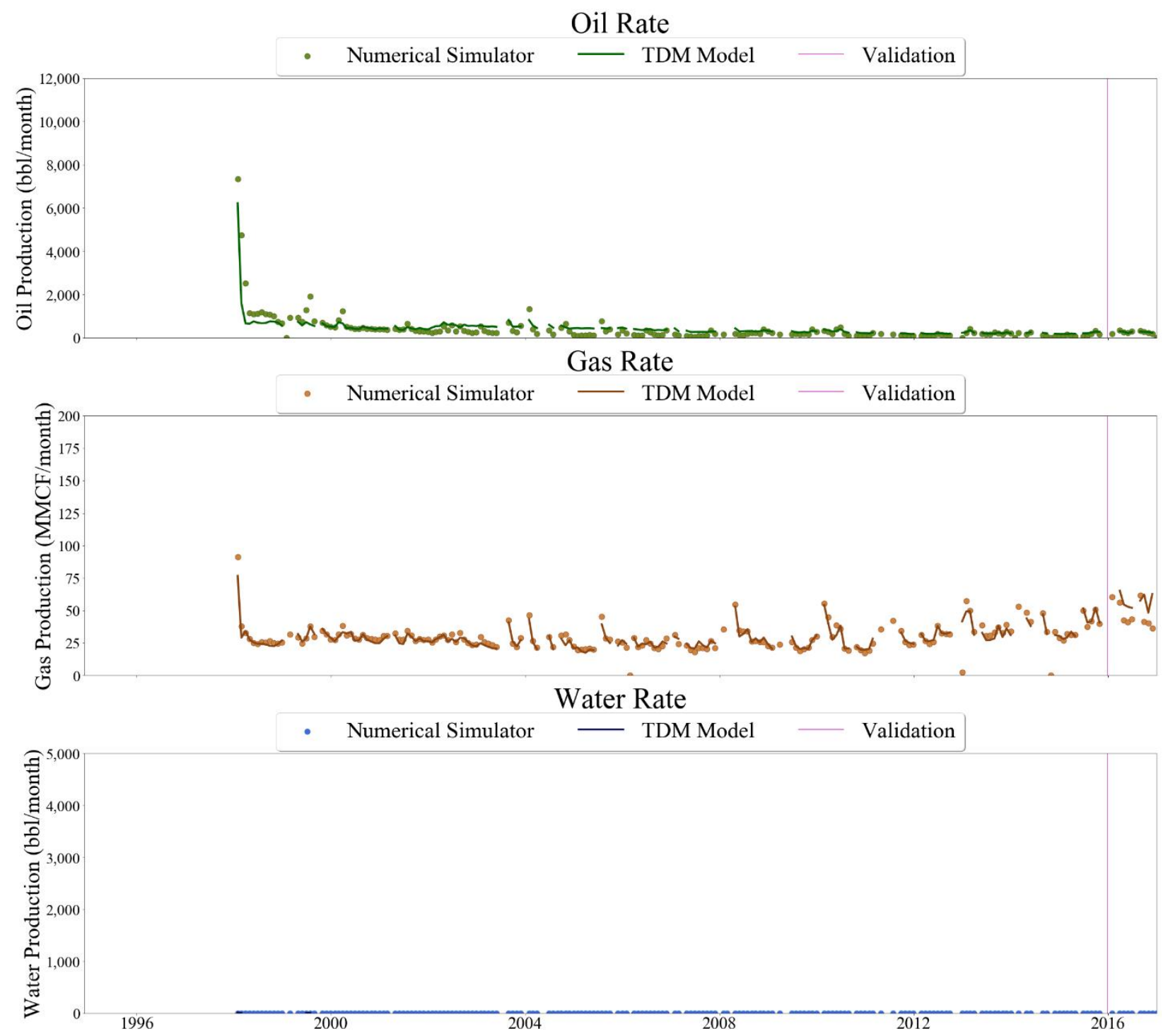

Figure 434 Well-008 oil, gas, and water rate TDM predictions vs actual simulation data plots for 1 Year Blind Validation TDM 

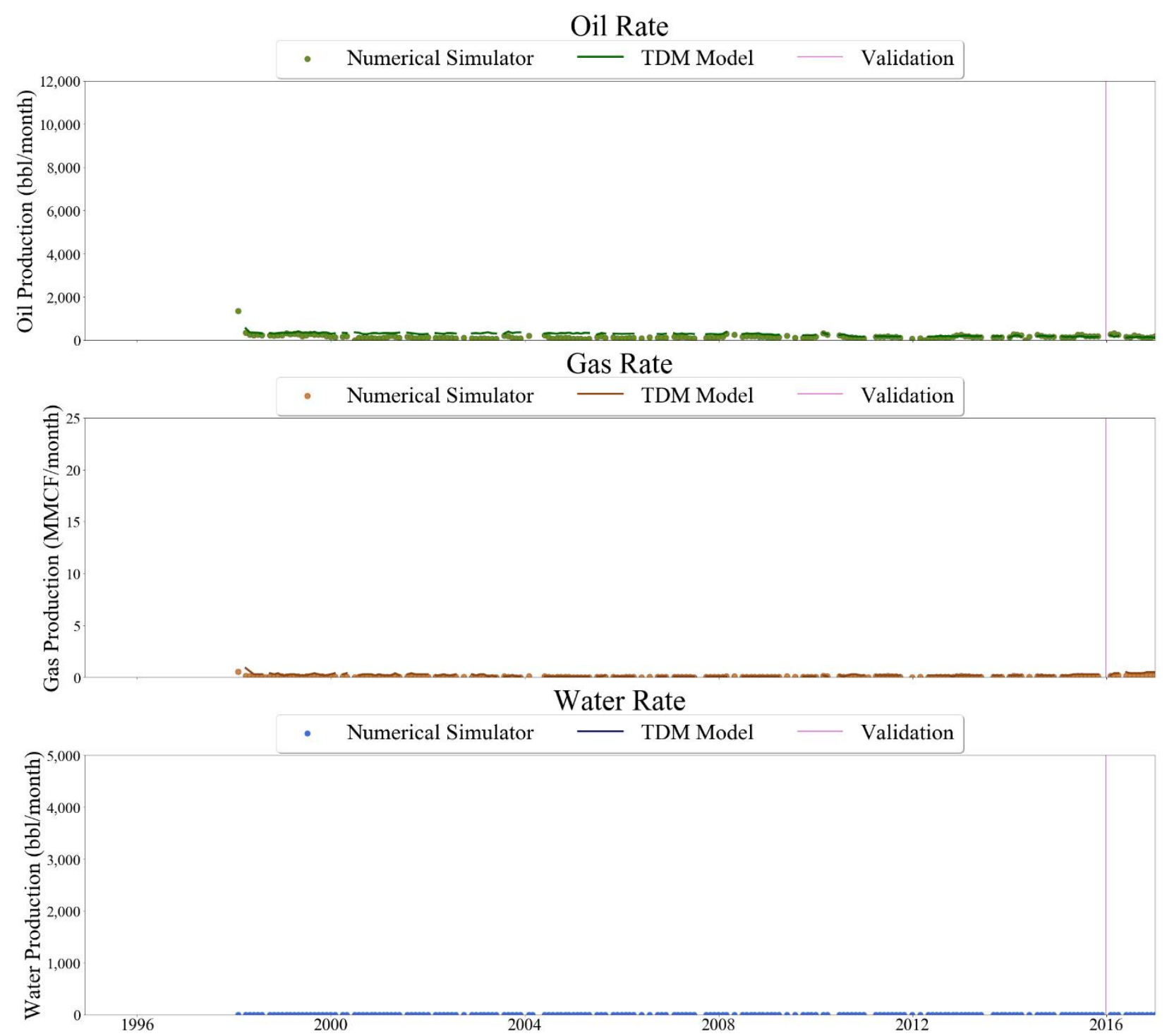

Figure 435 Well-009 oil, gas, and water rate TDM predictions vs actual simulation data plots for 1 Year Blind Validation TDM 

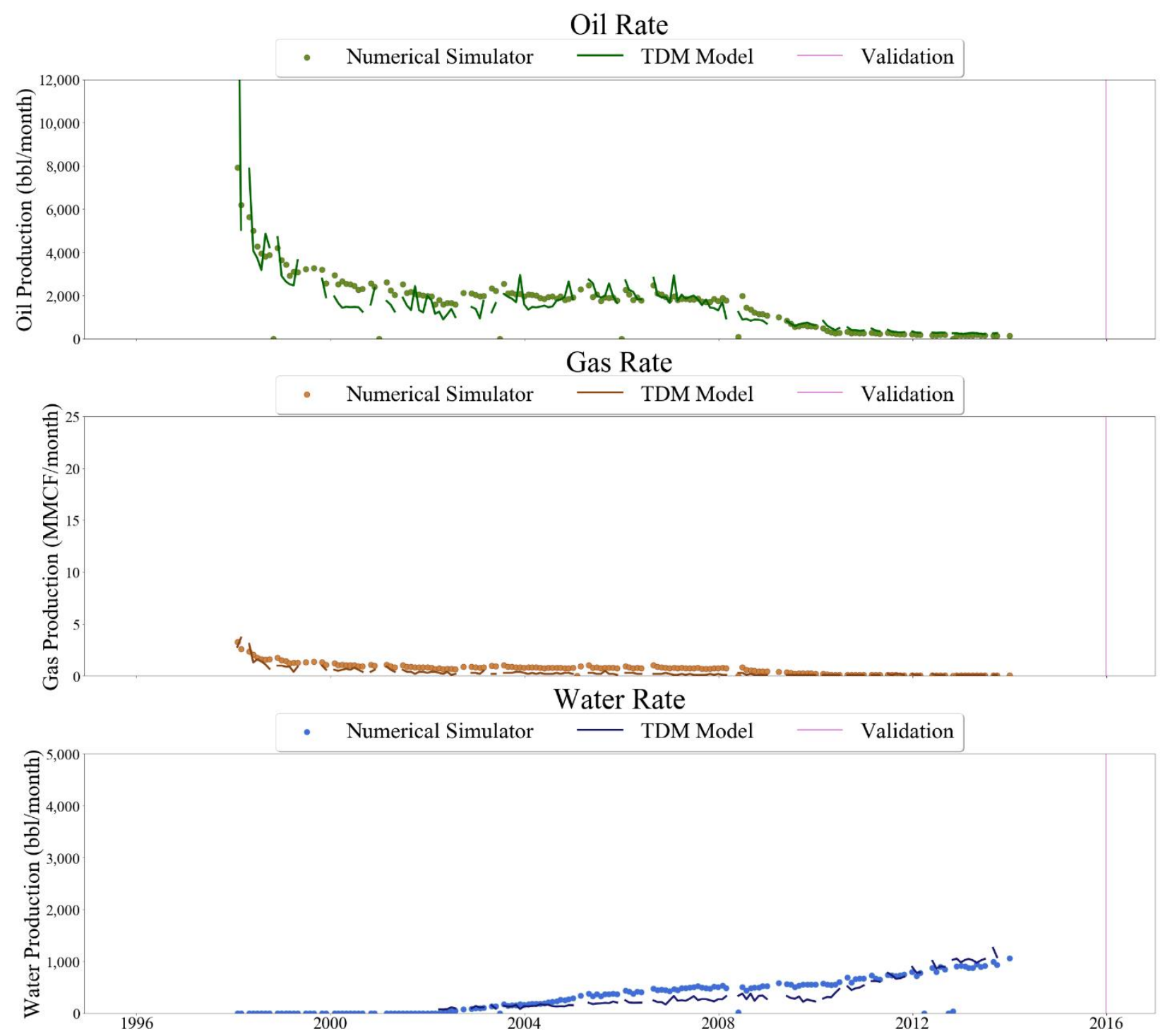

Figure 436 Well-010 oil, gas, and water rate TDM predictions vs actual simulation data plots for 1 Year Blind Validation TDM 

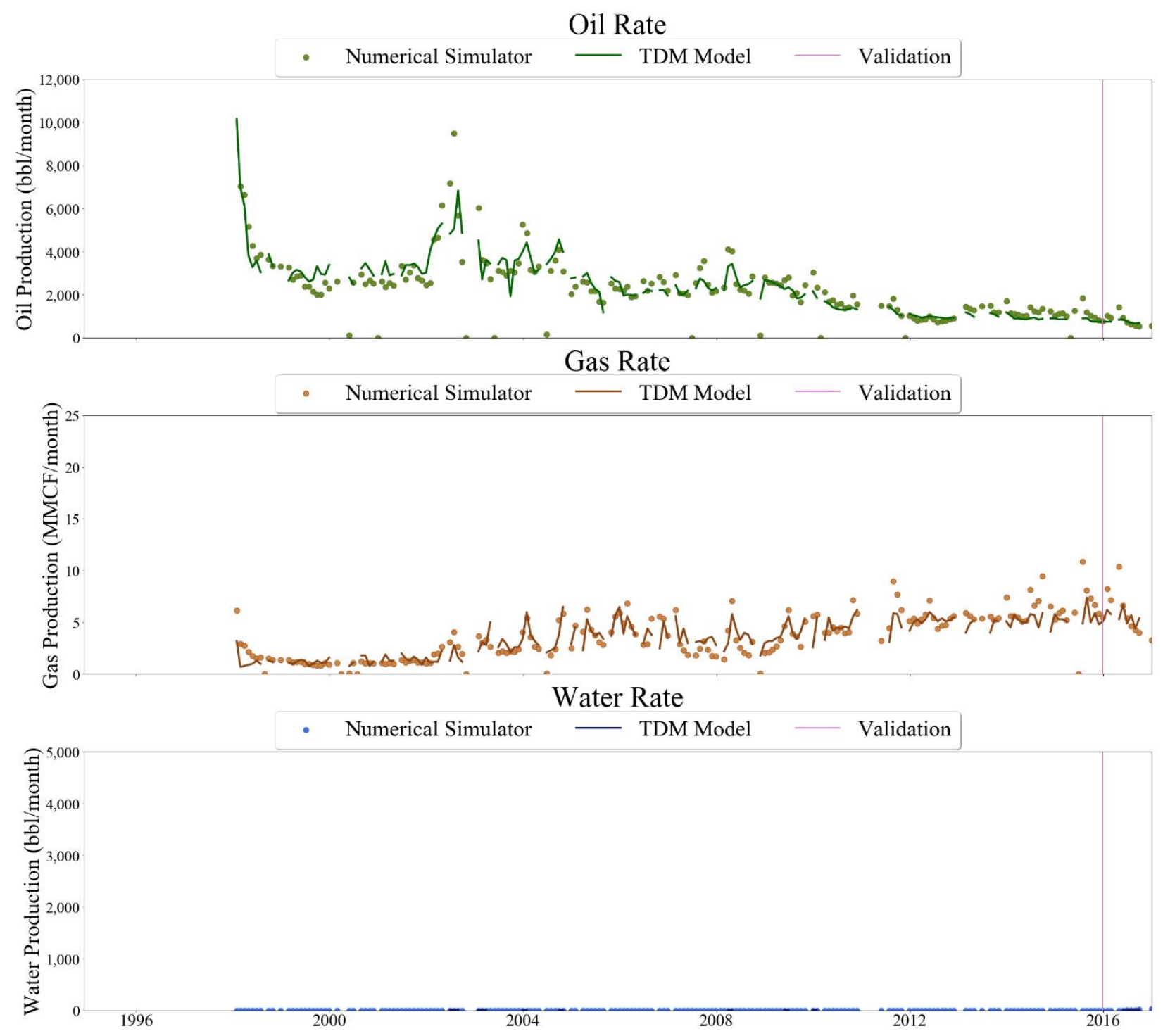

Figure 437 Well-011 oil, gas, and water rate TDM predictions vs actual simulation data plots for 1 Year Blind Validation TDM 

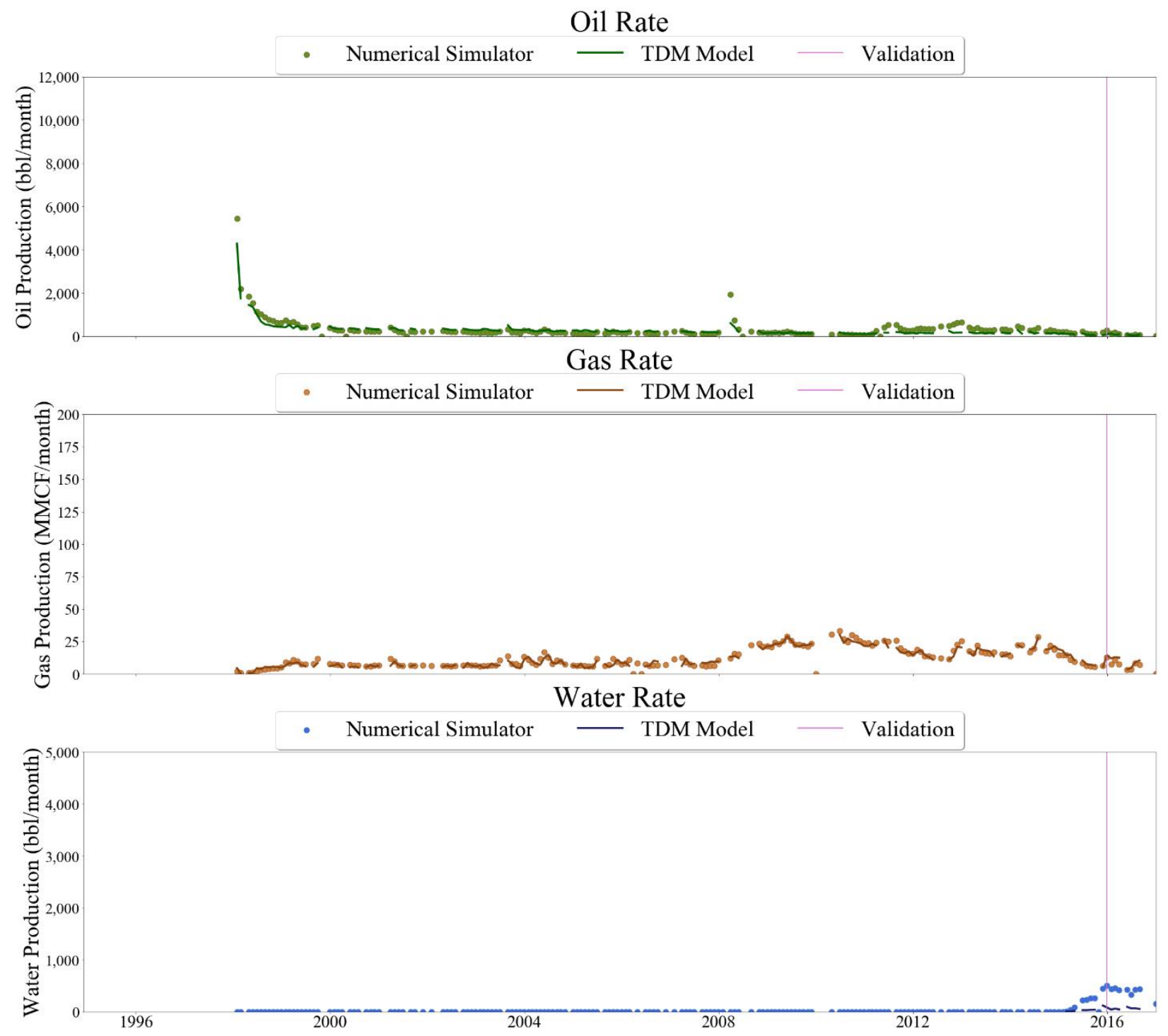

Figure 438 Well-012 oil, gas, and water rate TDM predictions vs actual simulation data plots for 1 Year Blind Validation TDM 

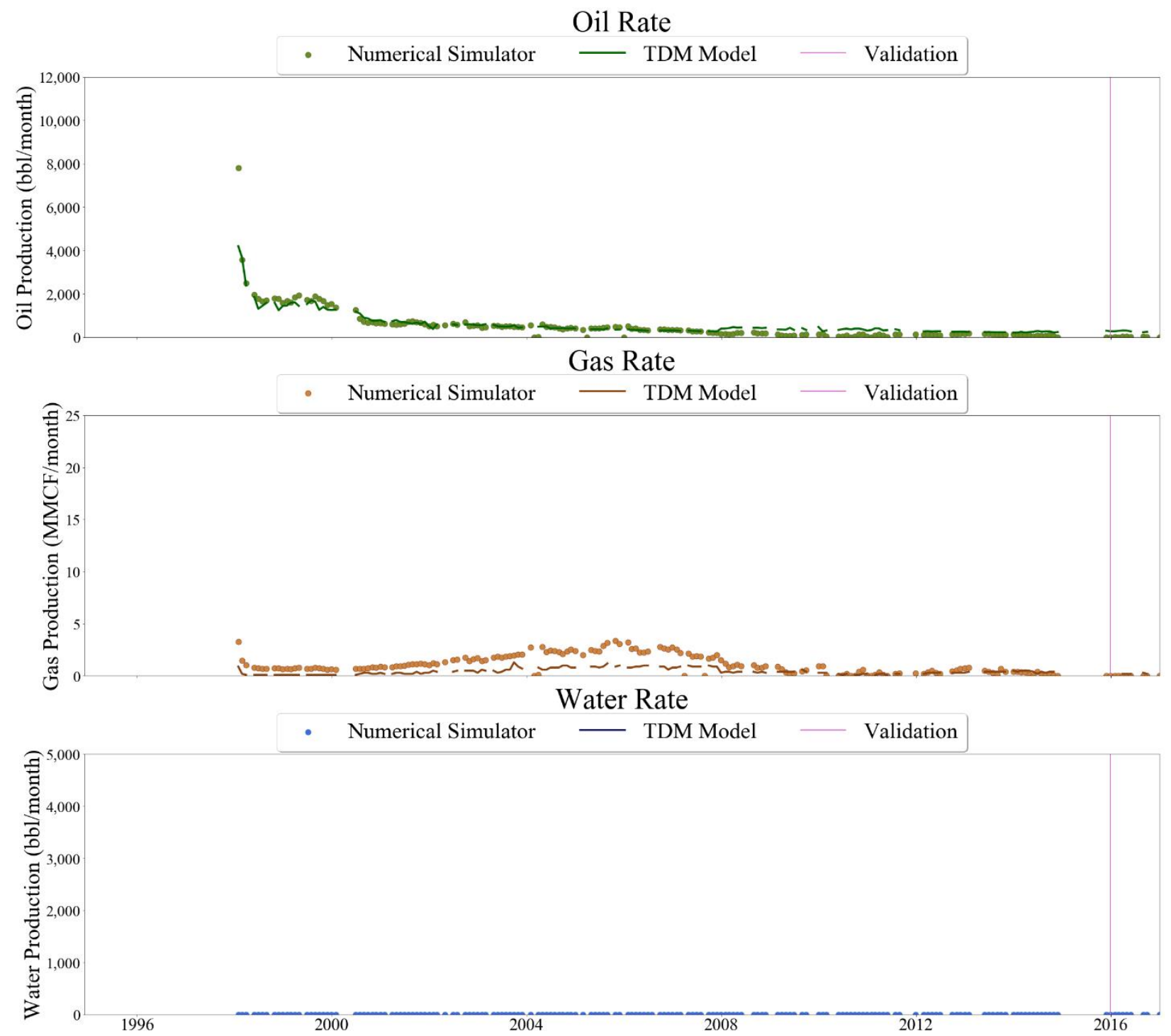

Figure 439 Well-013 oil, gas, and water rate TDM predictions vs actual simulation data plots for 1 Year Blind Validation TDM 


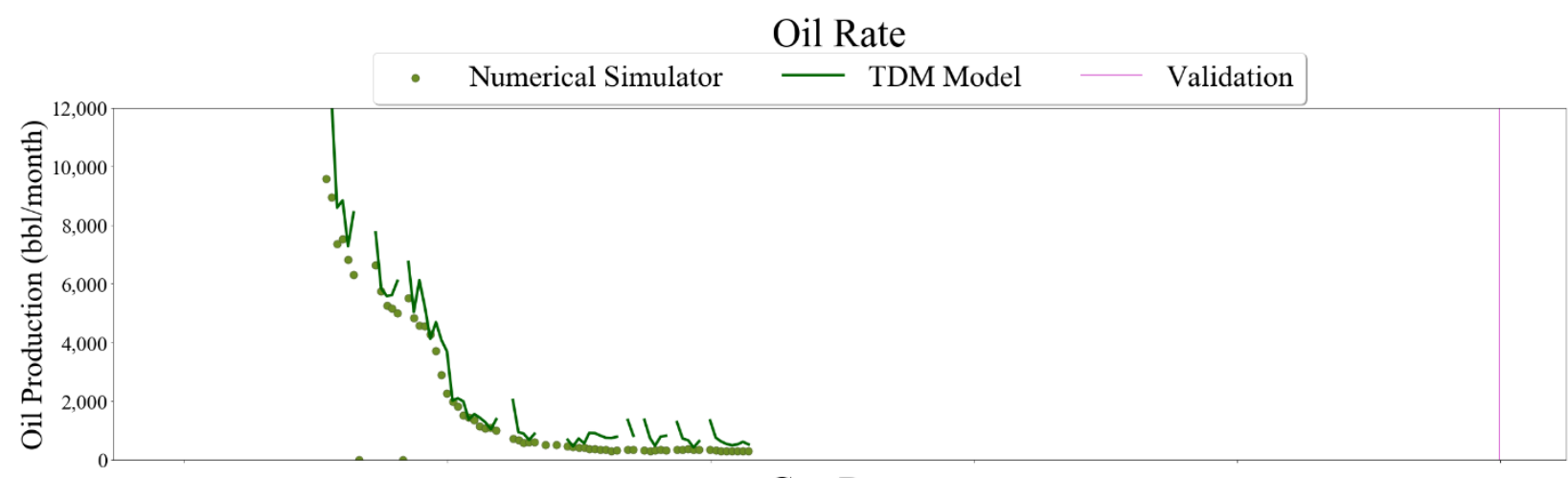

\section{Gas Rate}
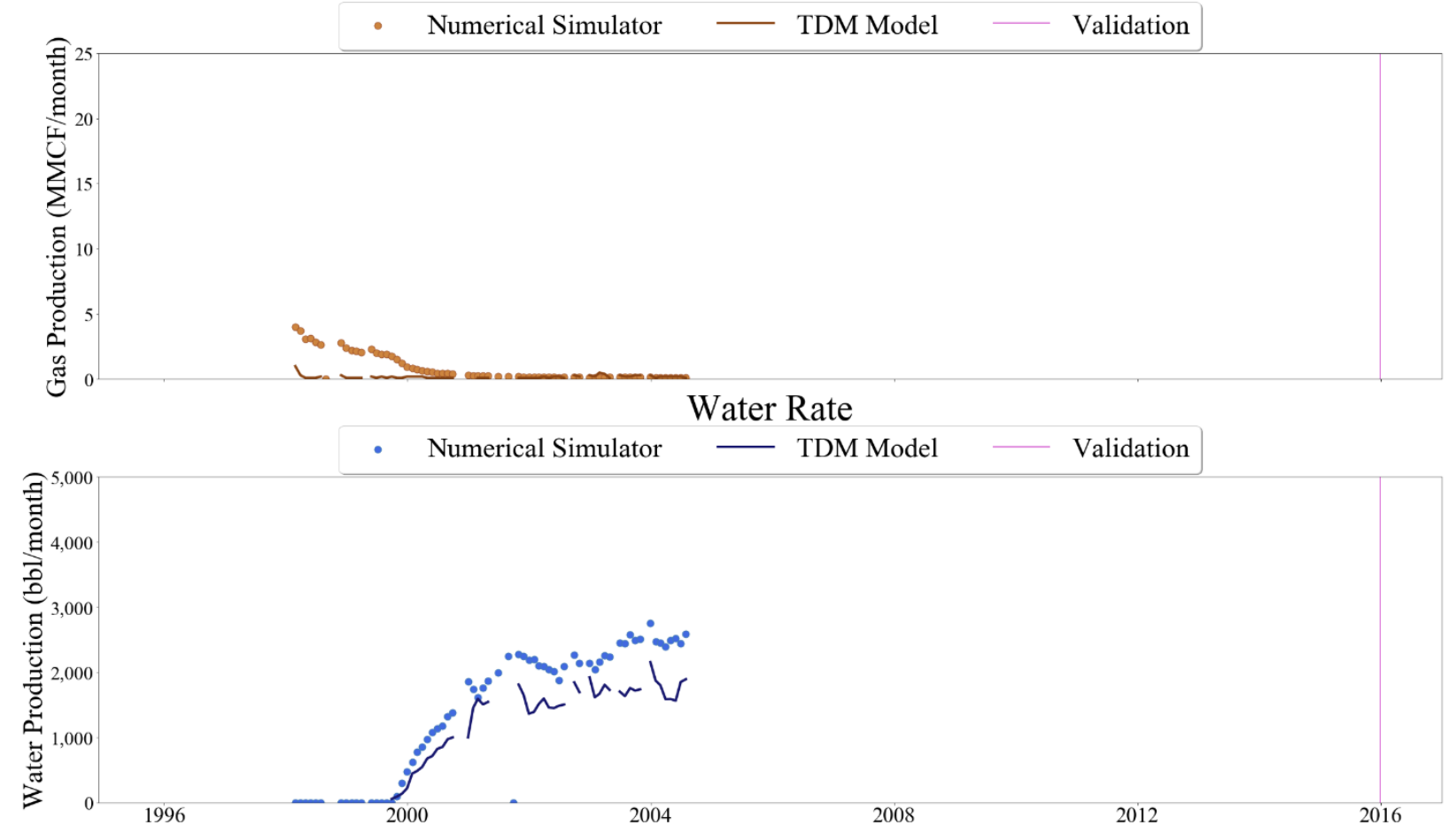

Figure 440 Well-014 oil, gas, and water rate TDM predictions vs actual simulation data plots for 1 Year Blind Validation TDM 

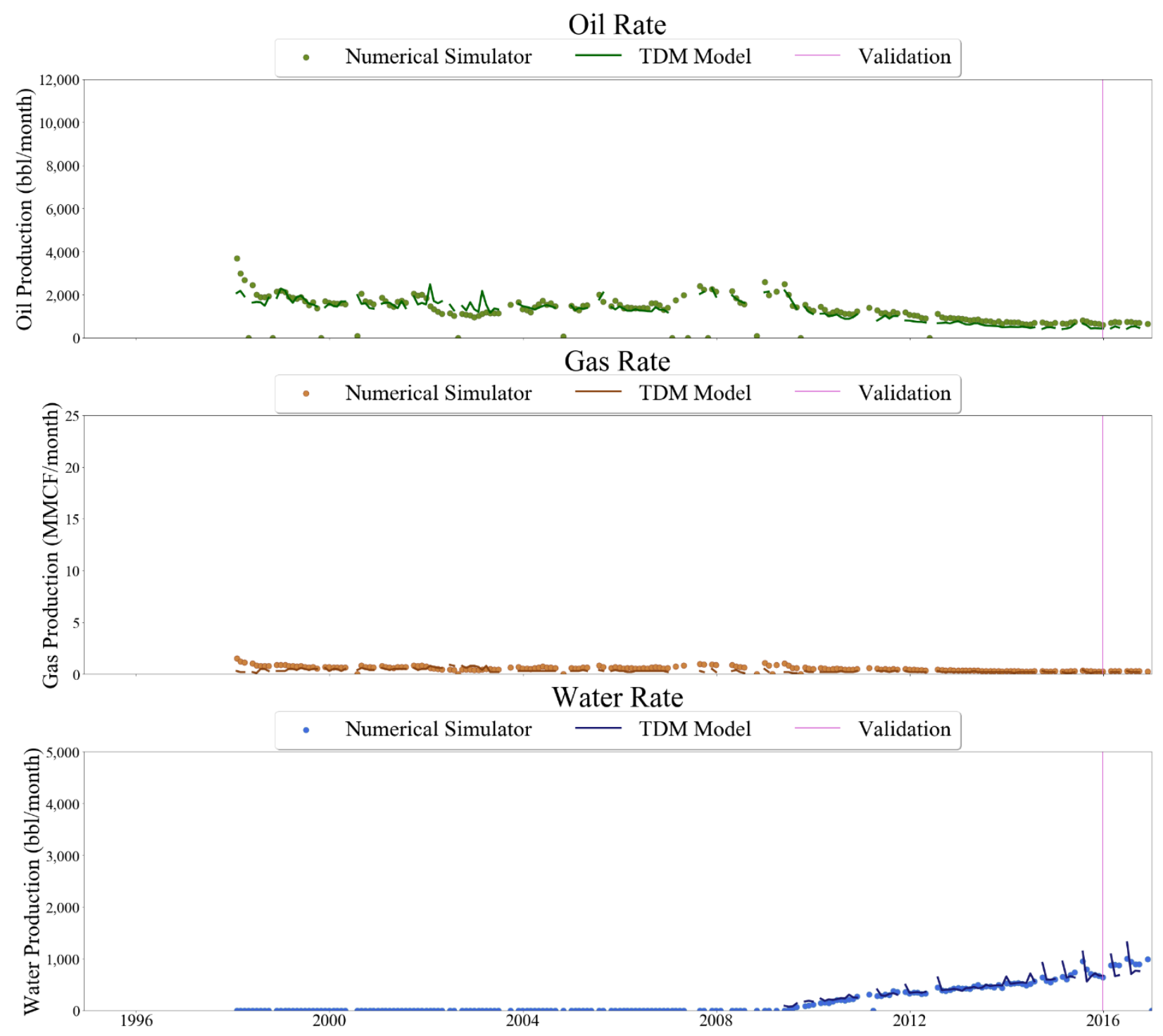

Figure 441 Well-015 oil, gas, and water rate TDM predictions vs actual simulation data plots for 1 Year Blind Validation TDM 

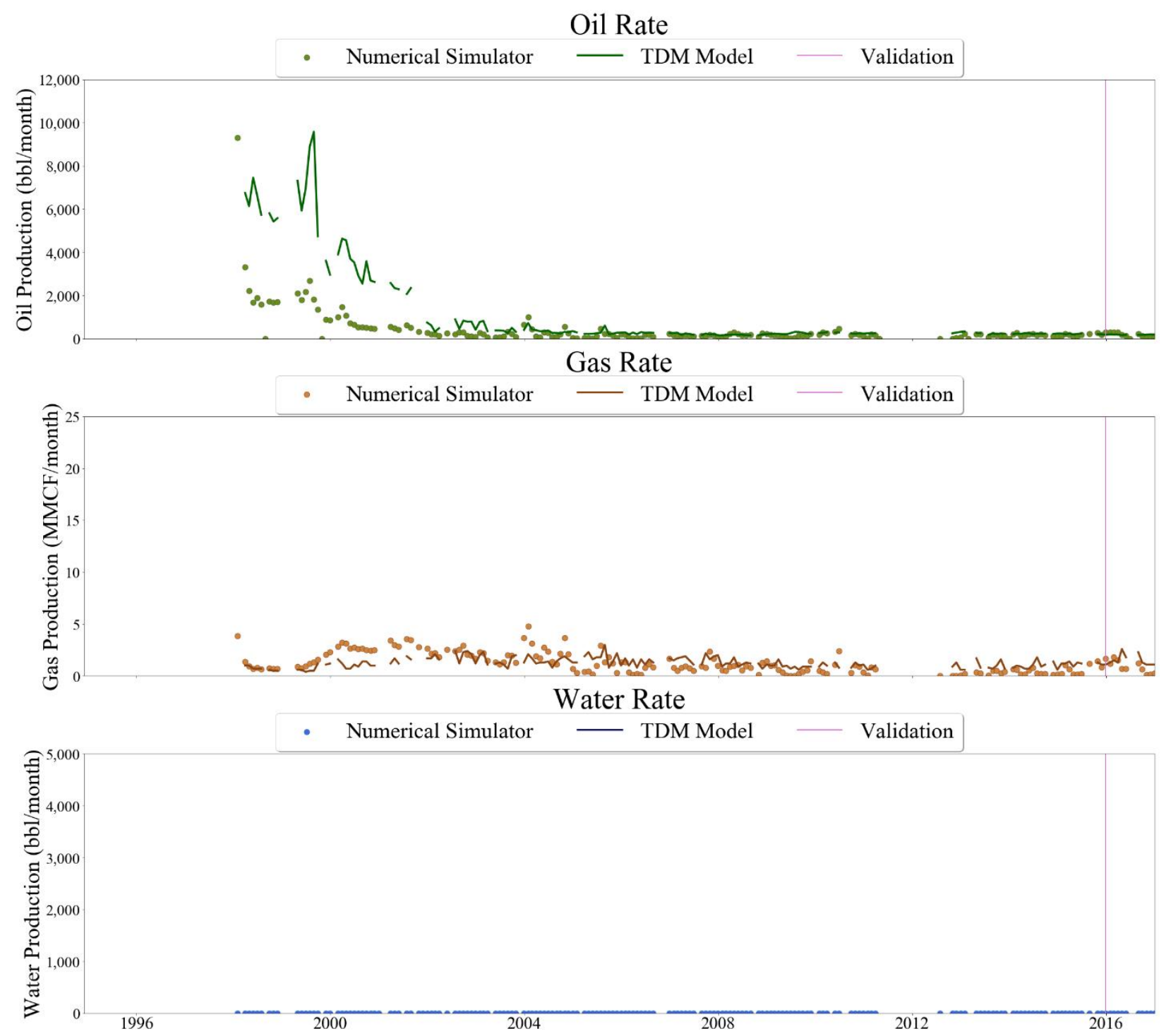

Figure 442 Well-016 oil, gas, and water rate TDM predictions vs actual simulation data plots for 1 Year Blind Validation TDM 

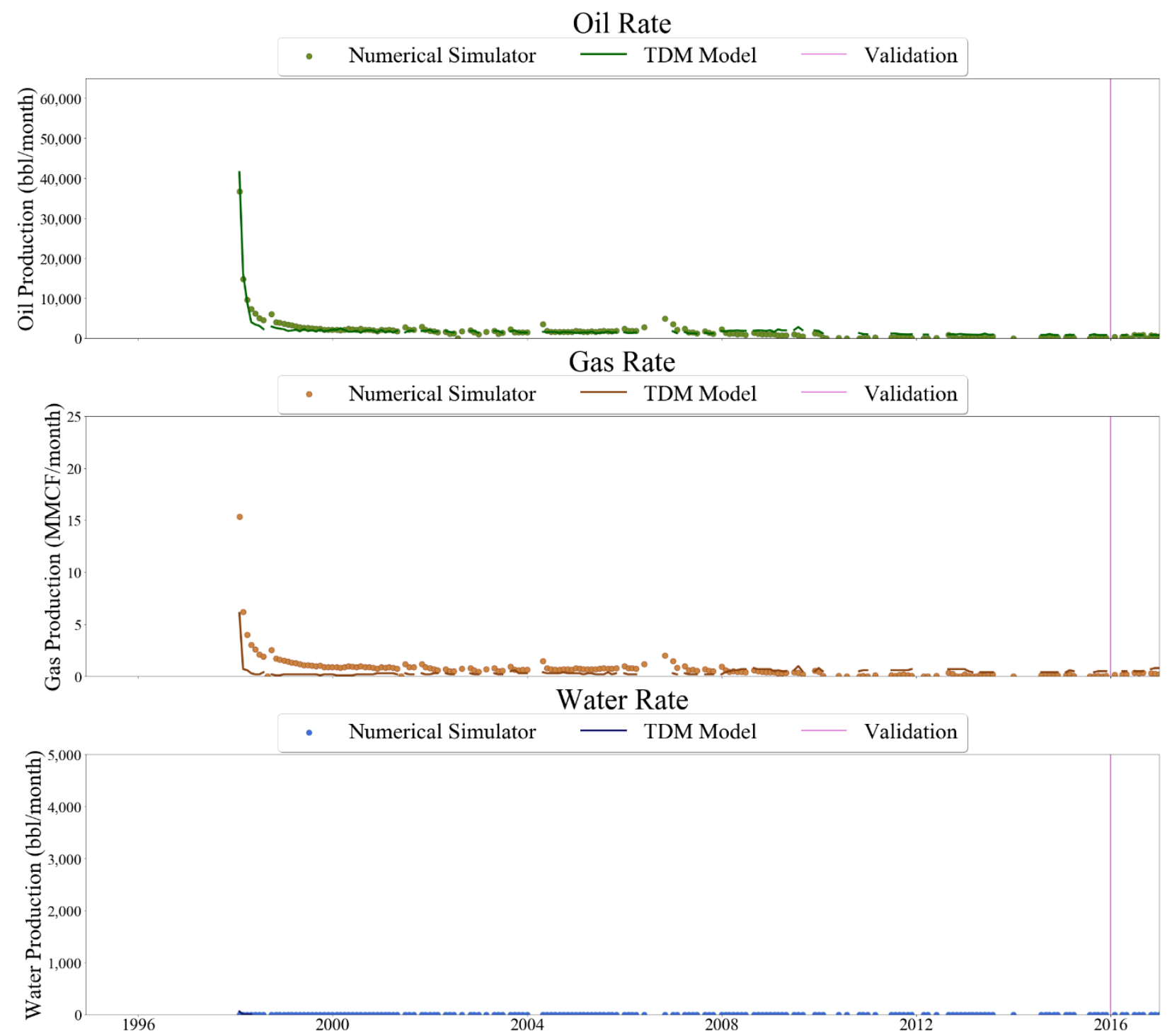

Figure 443 Well-017 oil, gas, and water rate TDM predictions vs actual simulation data plots for 1 Year Blind Validation TDM 

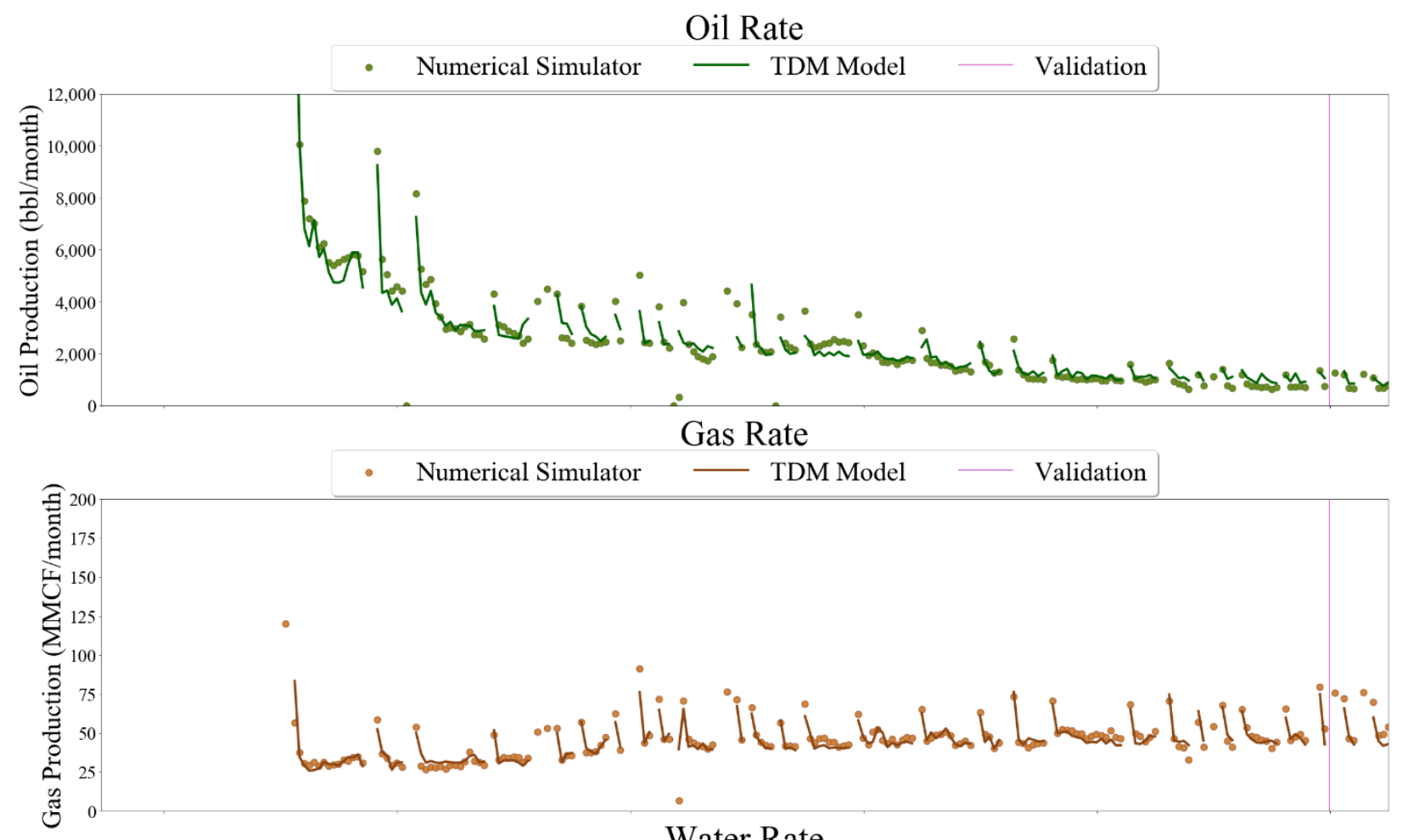

Water Rate

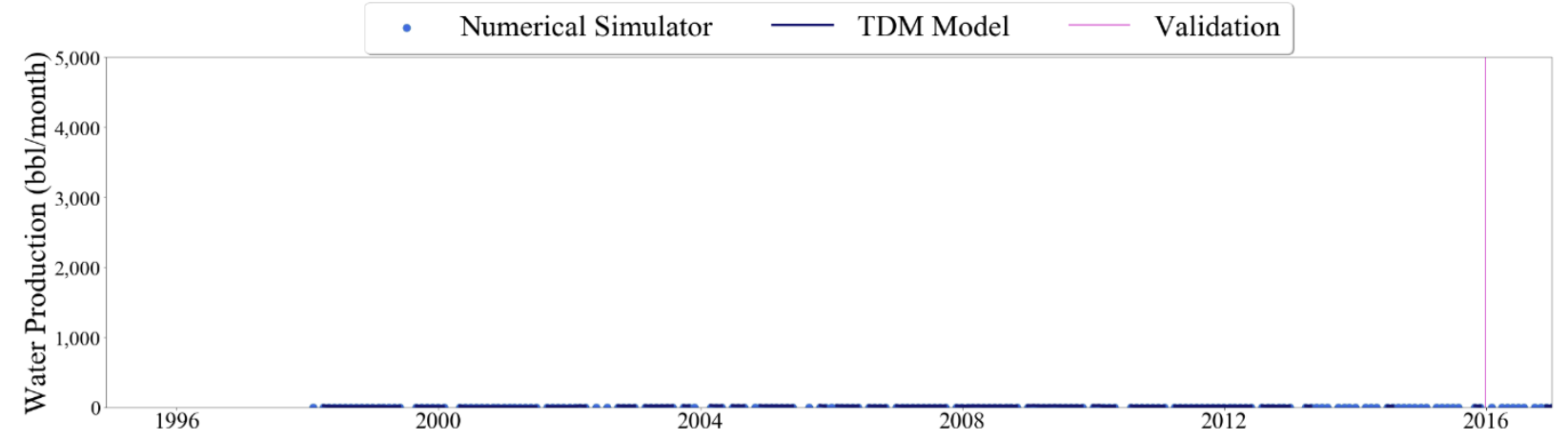

Figure 444 Well-018 oil, gas, and water rate TDM predictions vs actual simulation data plots for 1 Year Blind Validation TDM 

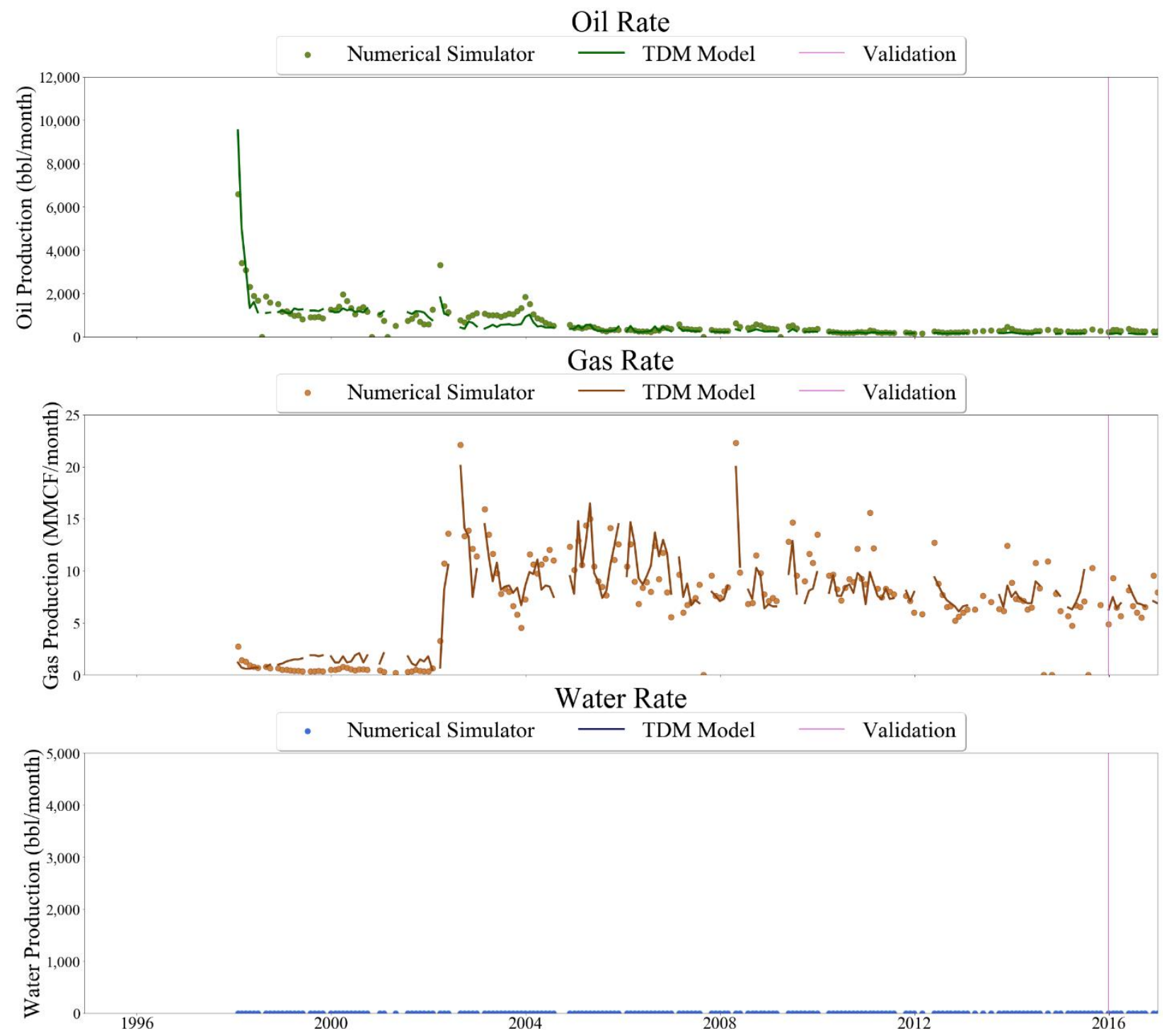

Figure 445 Well-019 oil, gas, and water rate TDM predictions vs actual simulation data plots for 1 Year Blind Validation TDM 

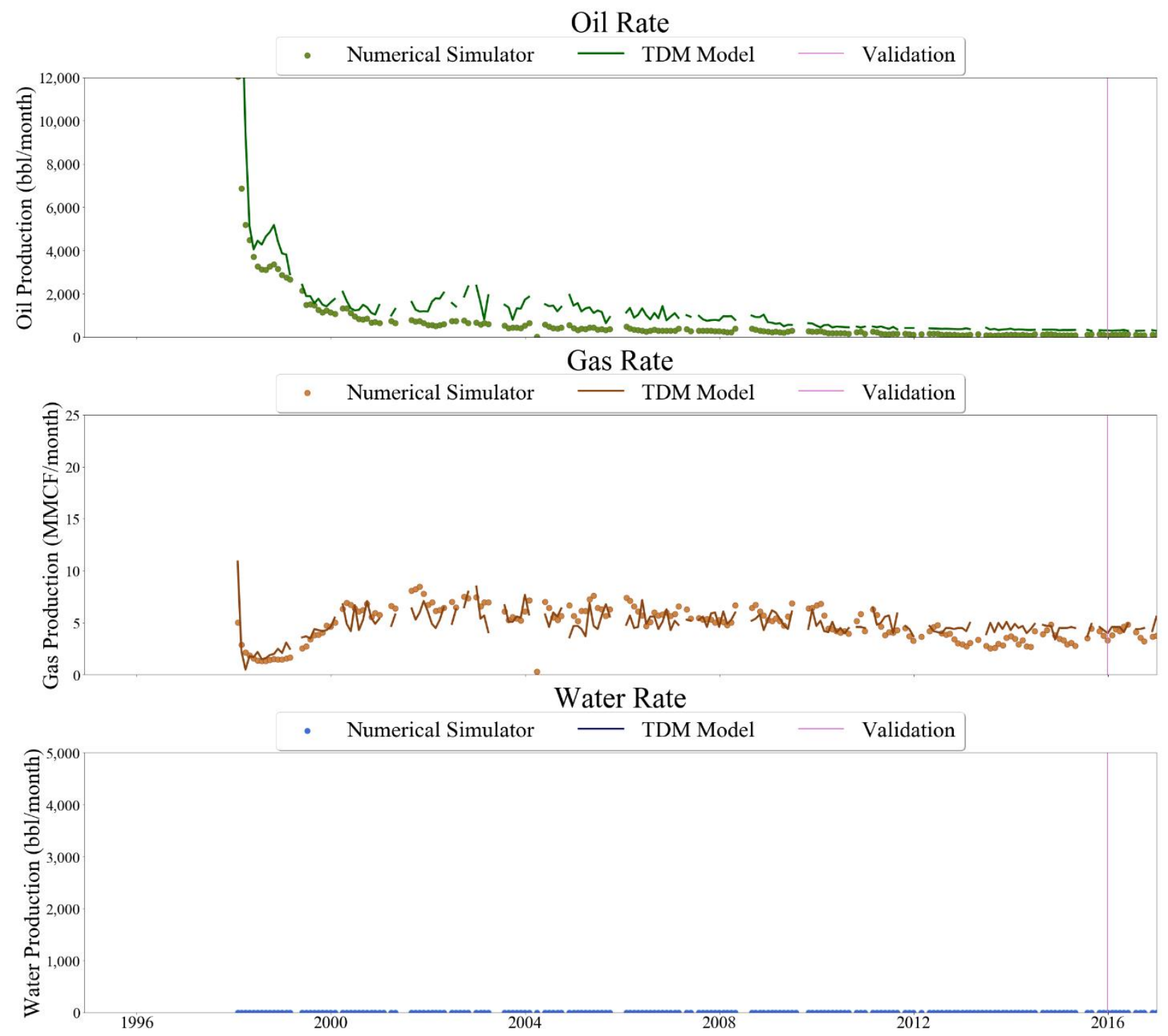

Figure 446 Well-020 oil, gas, and water rate TDM predictions vs actual simulation data plots for 1 Year Blind Validation TDM 

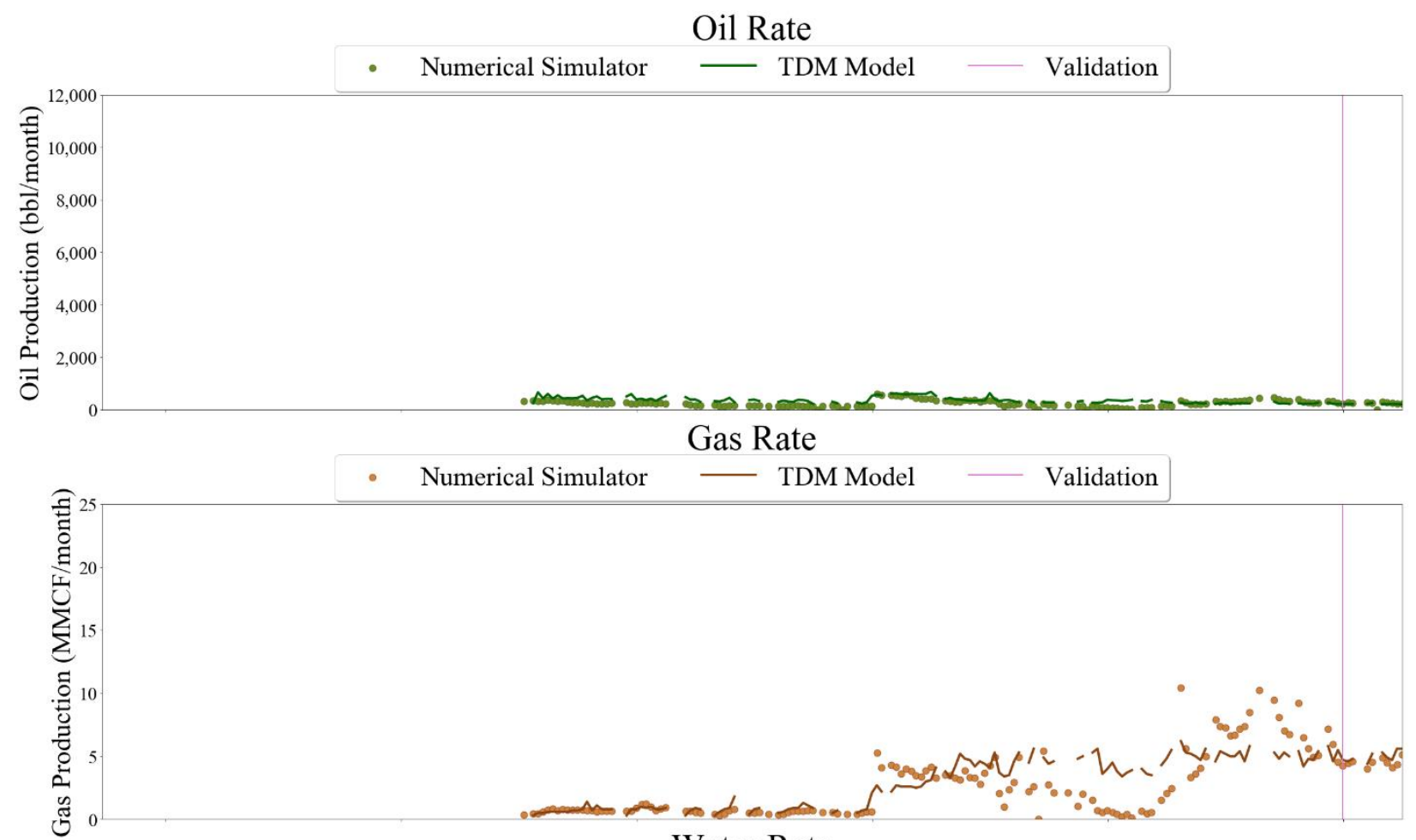

Water Rate

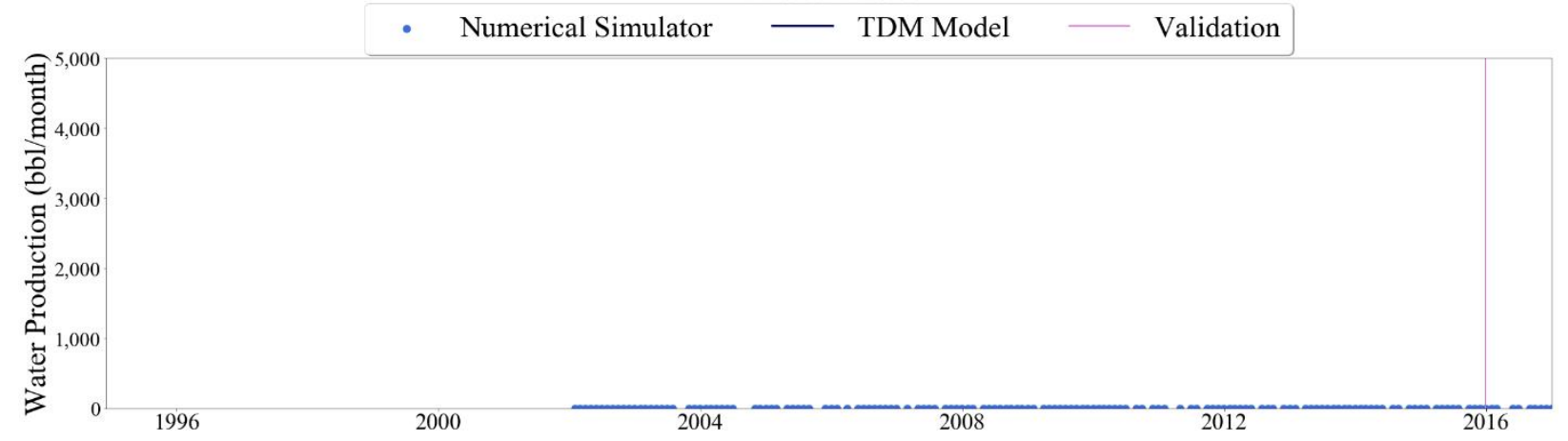

Figure 447 Well-021 oil, gas, and water rate TDM predictions vs actual simulation data plots for 1 Year Blind Validation TDM 

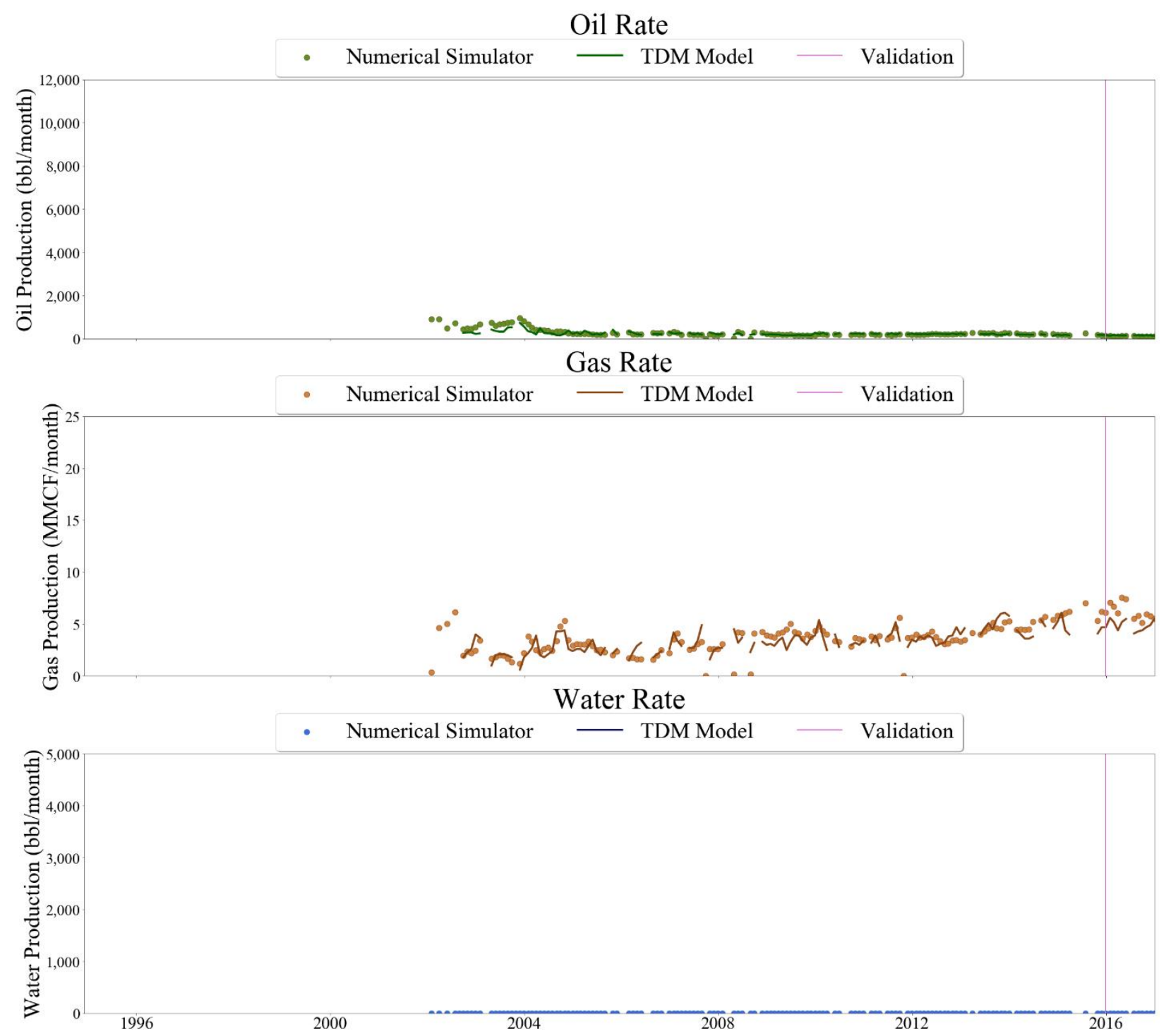

Figure 448 Well-022 oil, gas, and water rate TDM predictions vs actual simulation data plots for 1 Year Blind Validation TDM 

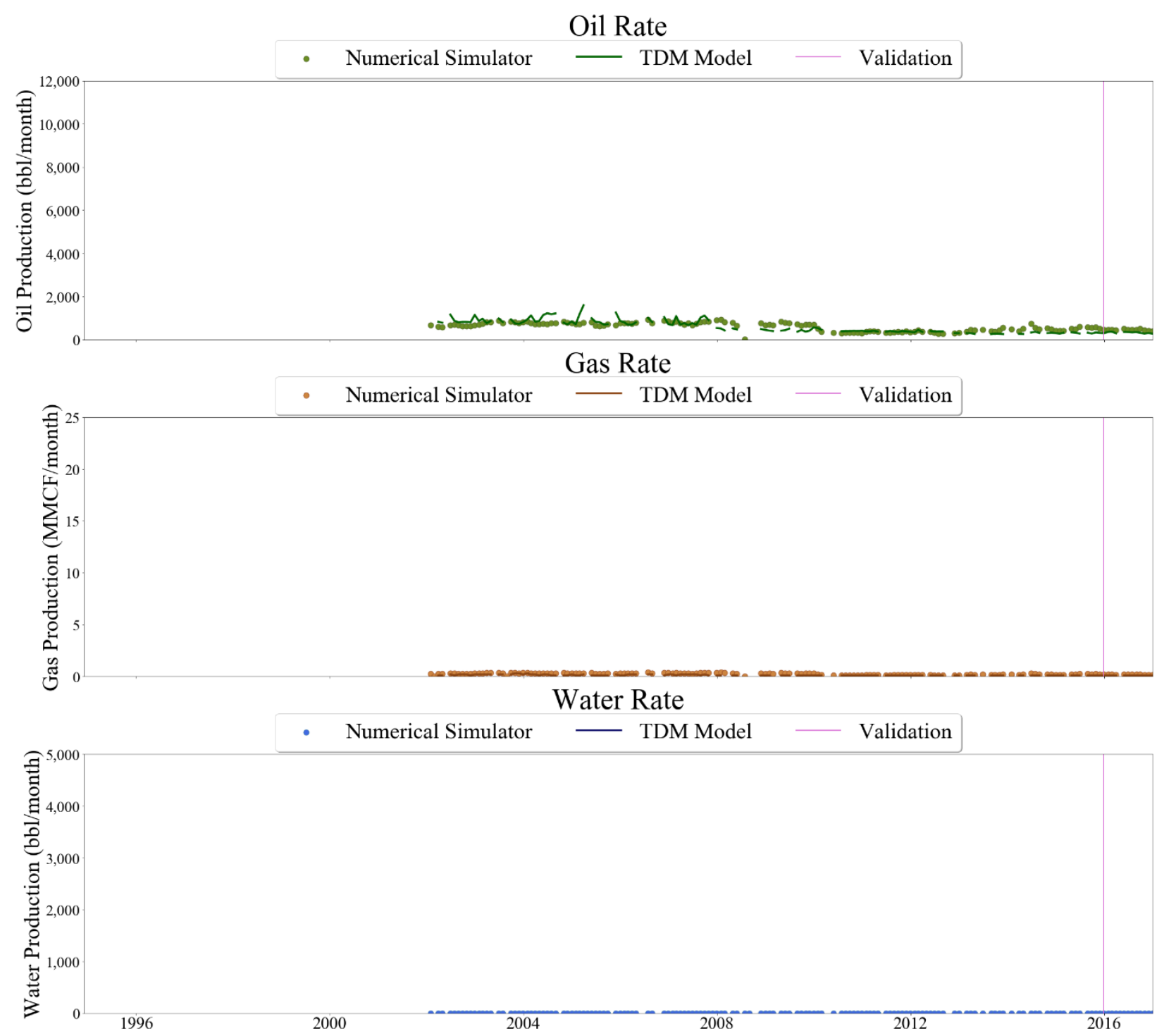

Figure 449 Well-023 oil, gas, and water rate TDM predictions vs actual simulation data plots for 1 Year Blind Validation TDM 

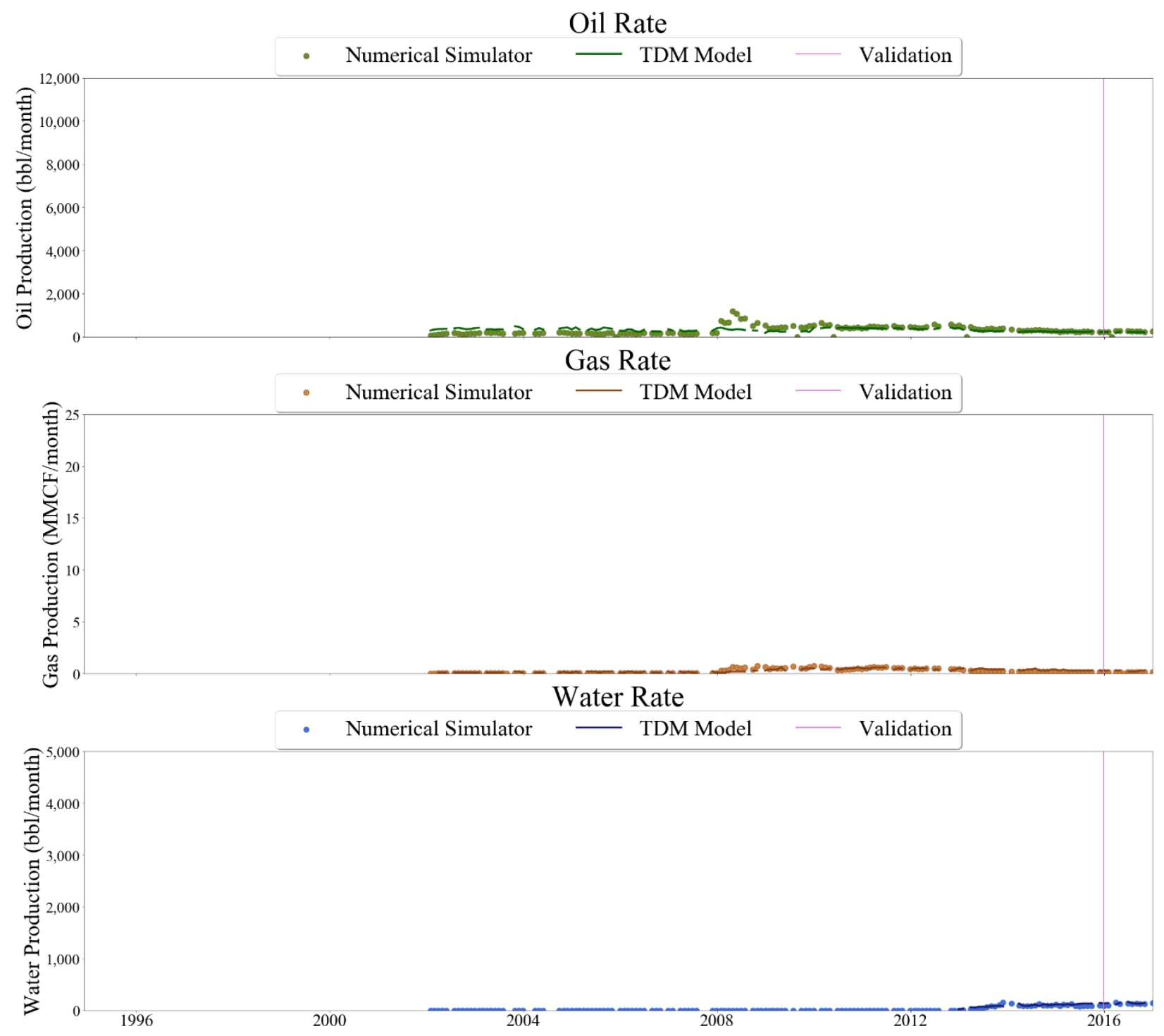

Figure 450 Well-024 oil, gas, and water rate TDM predictions vs actual simulation data plots for 1 Year Blind Validation TDM 

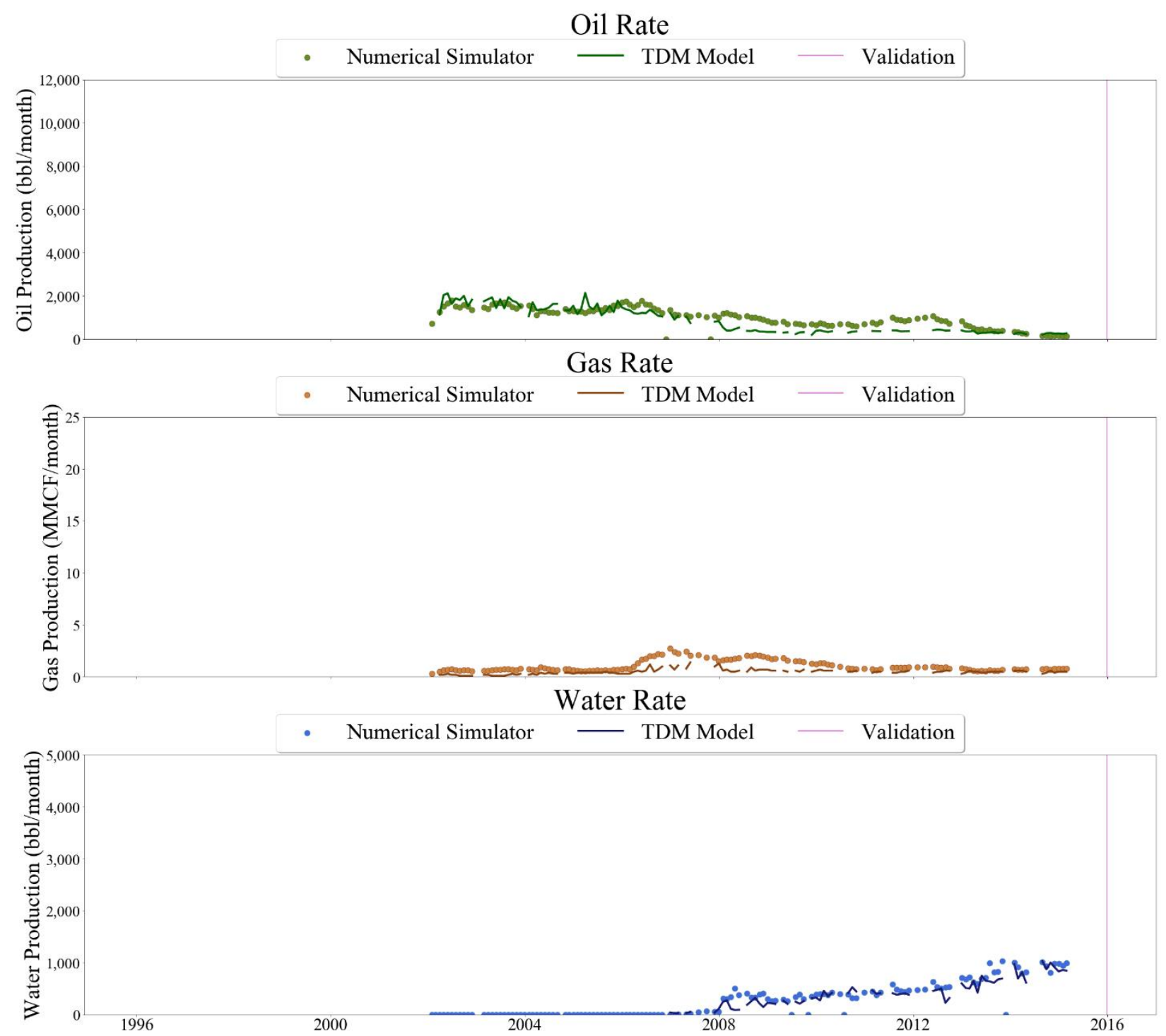

Figure 451 Well-025 oil, gas, and water rate TDM predictions vs actual simulation data plots for 1 Year Blind Validation TDM 


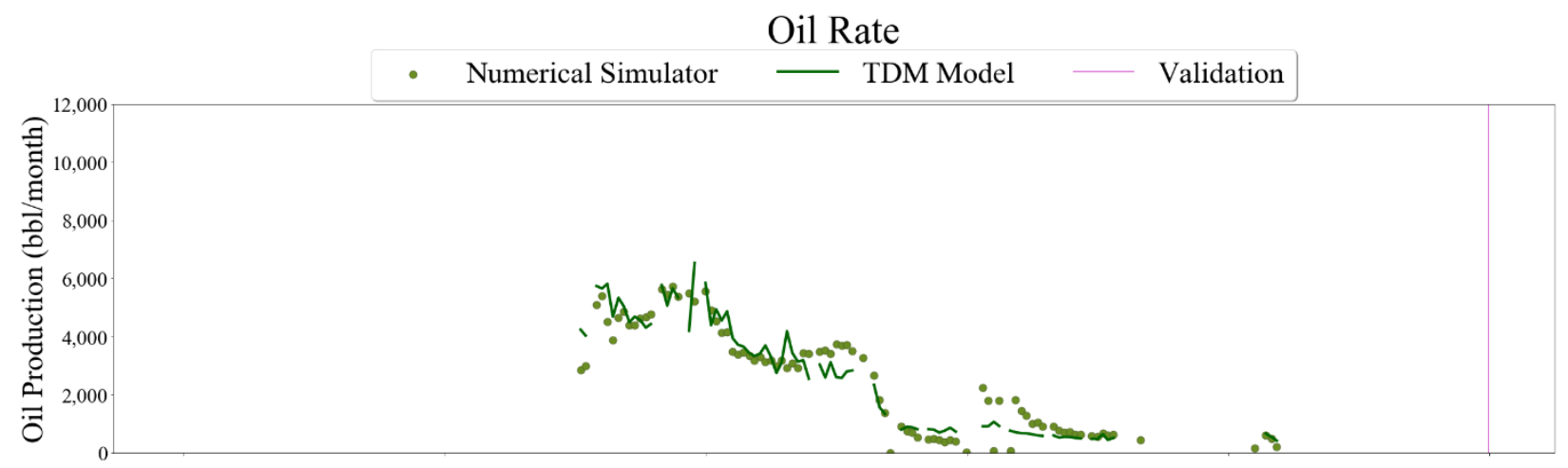

\section{Gas Rate}
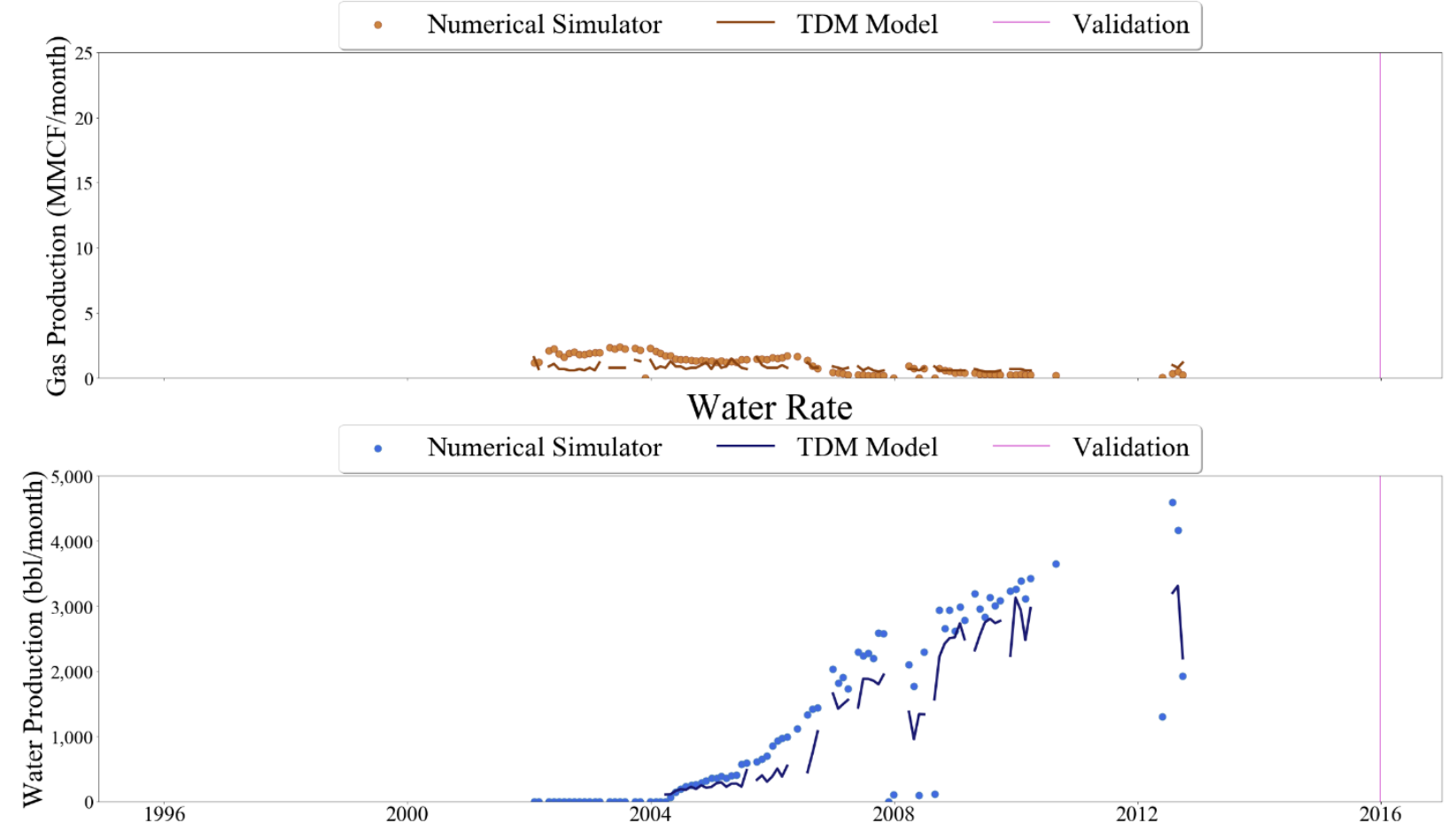

Figure 452 Well-026 oil, gas, and water rate TDM predictions vs actual simulation data plots for 1 Year Blind Validation TDM 


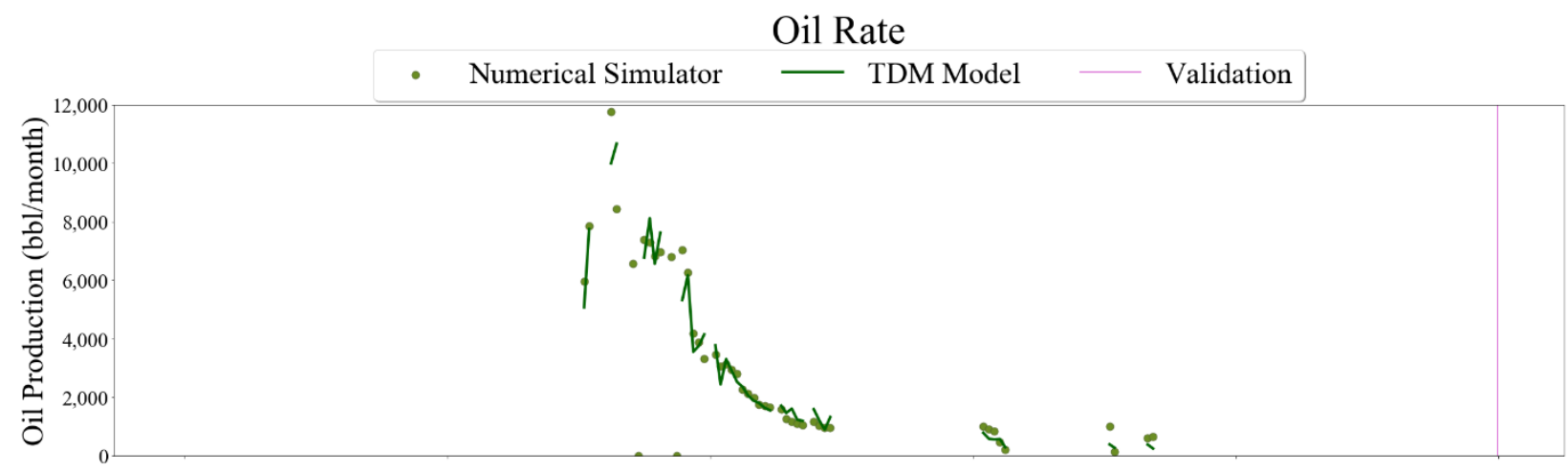

Gas Rate

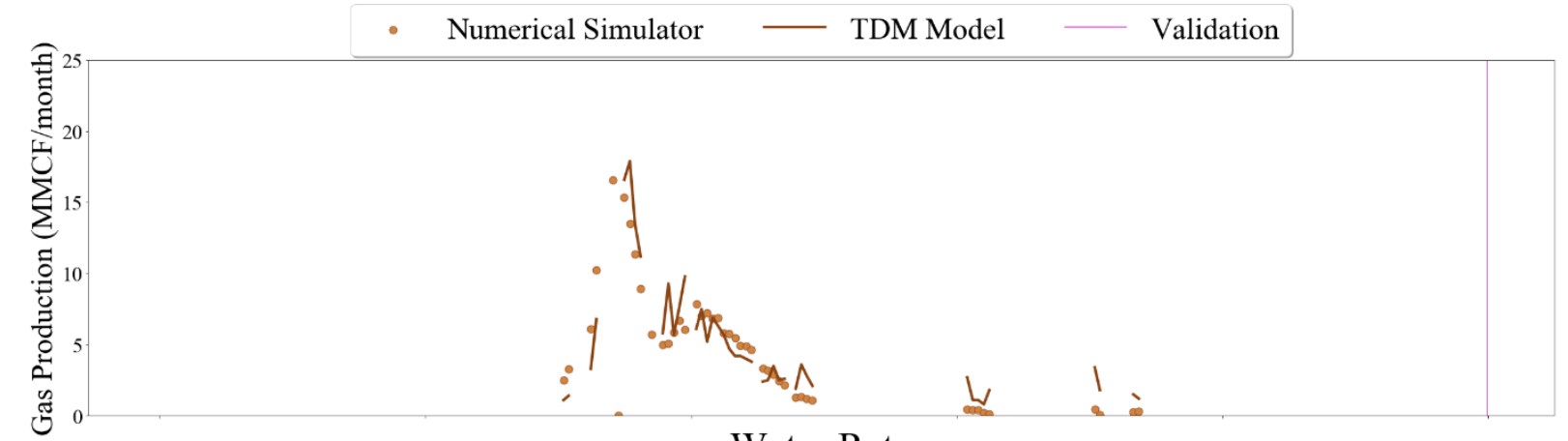

Water Rate

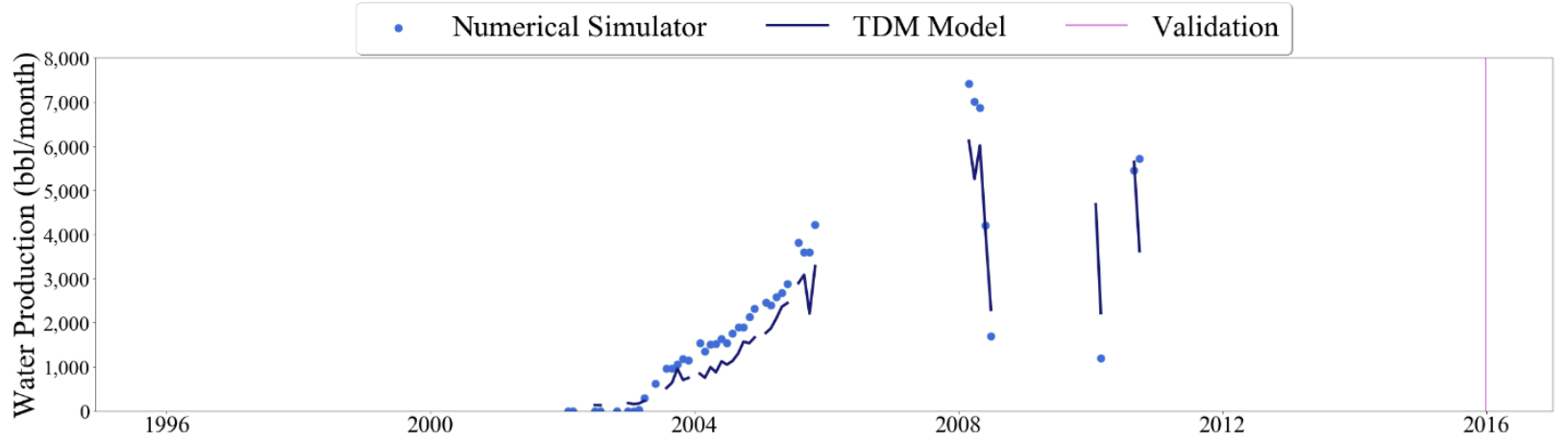

Figure 453 Well-027 oil, gas, and water rate TDM predictions vs actual simulation data plots for 1 Year Blind Validation TDM 


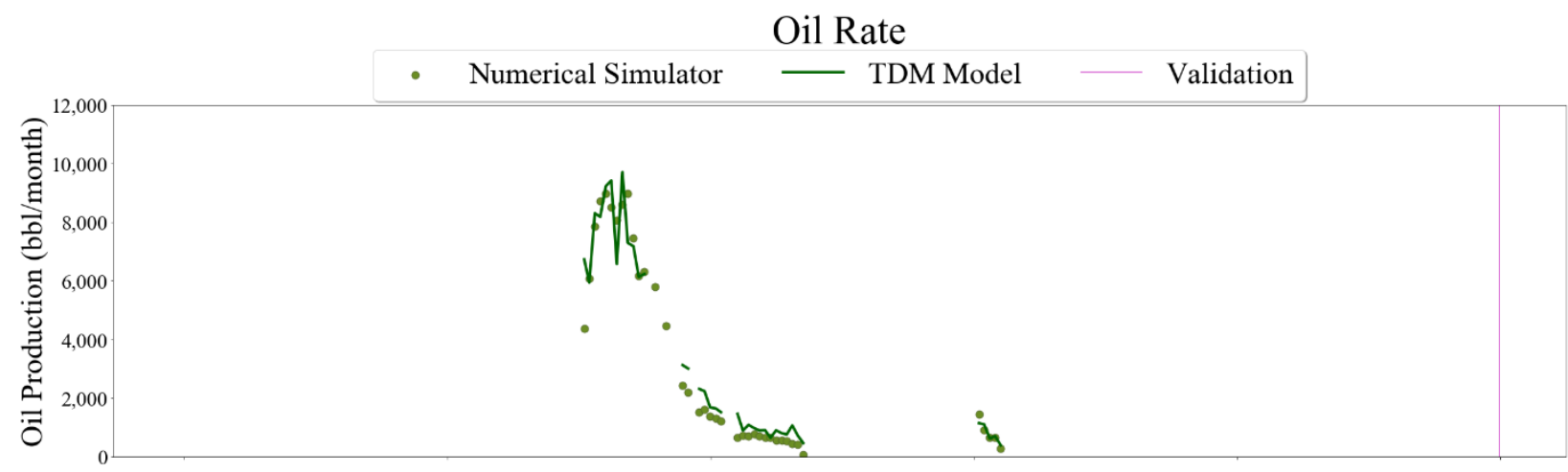

Gas Rate
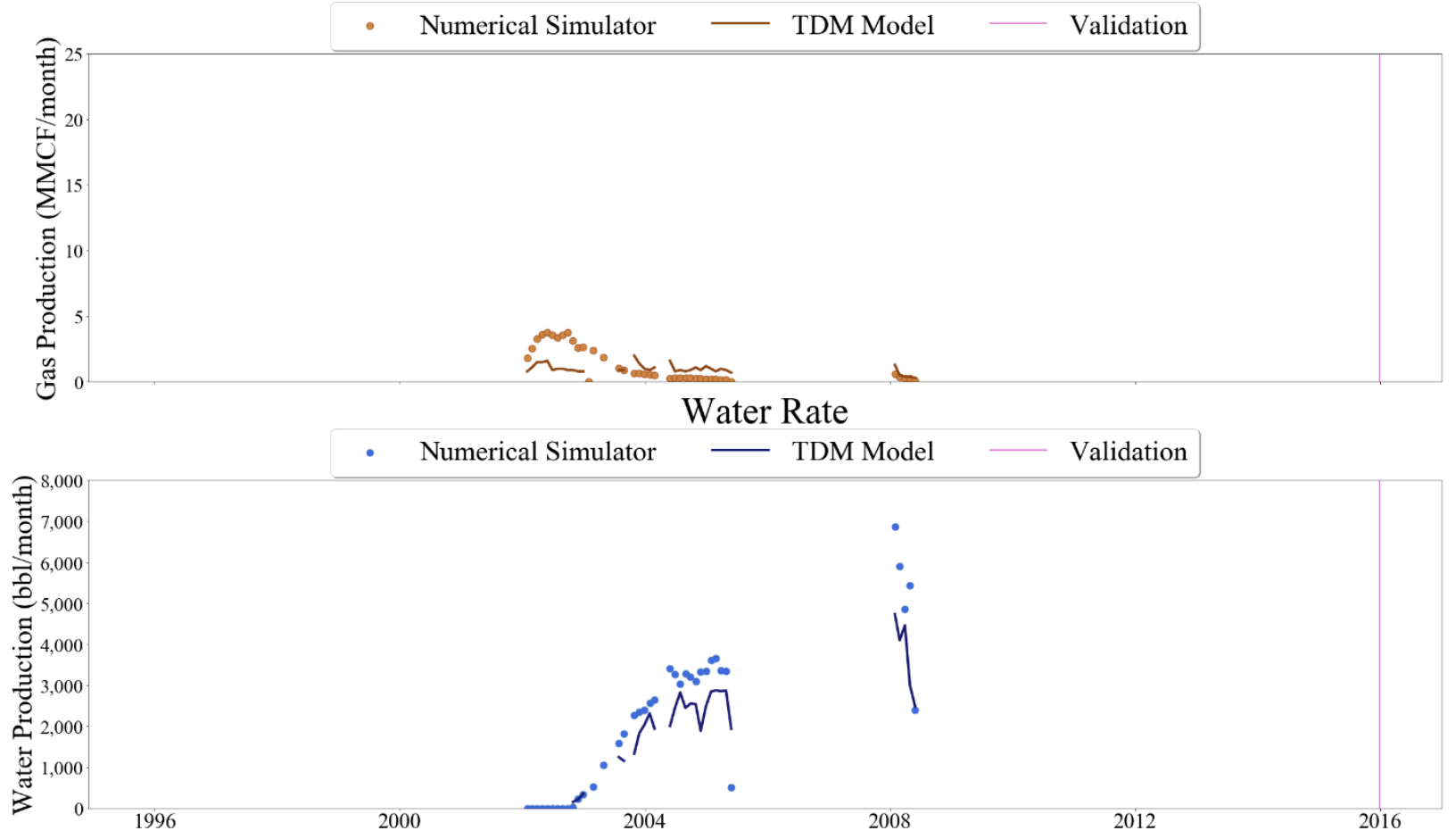

Figure 454 Well-028 oil, gas, and water rate TDM predictions vs actual simulation data plots for 1 Year Blind Validation TDM 

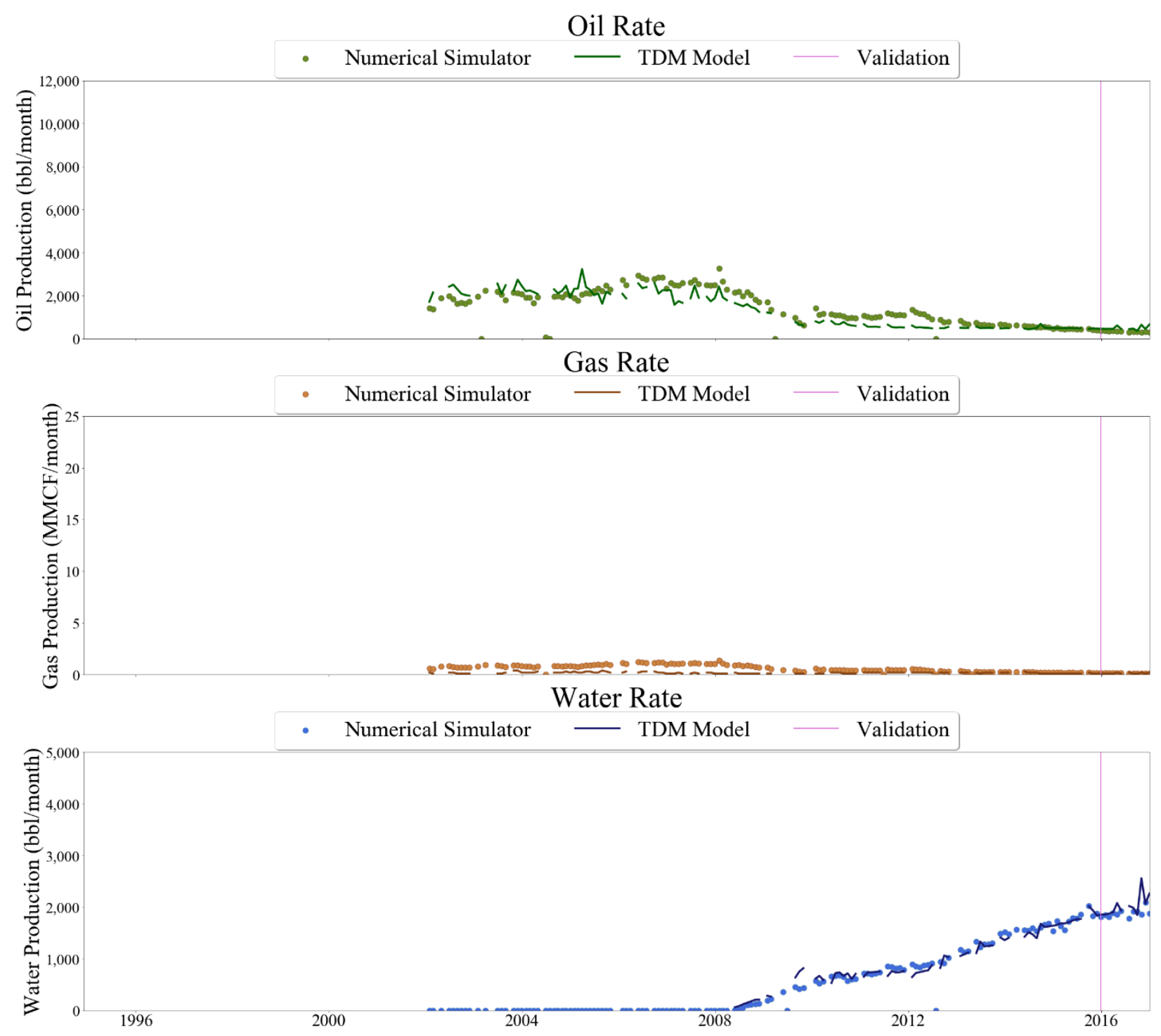

Figure 455 Well-029 oil, gas, and water rate TDM predictions vs actual simulation data plots for 1 Year Blind Validation TDM 


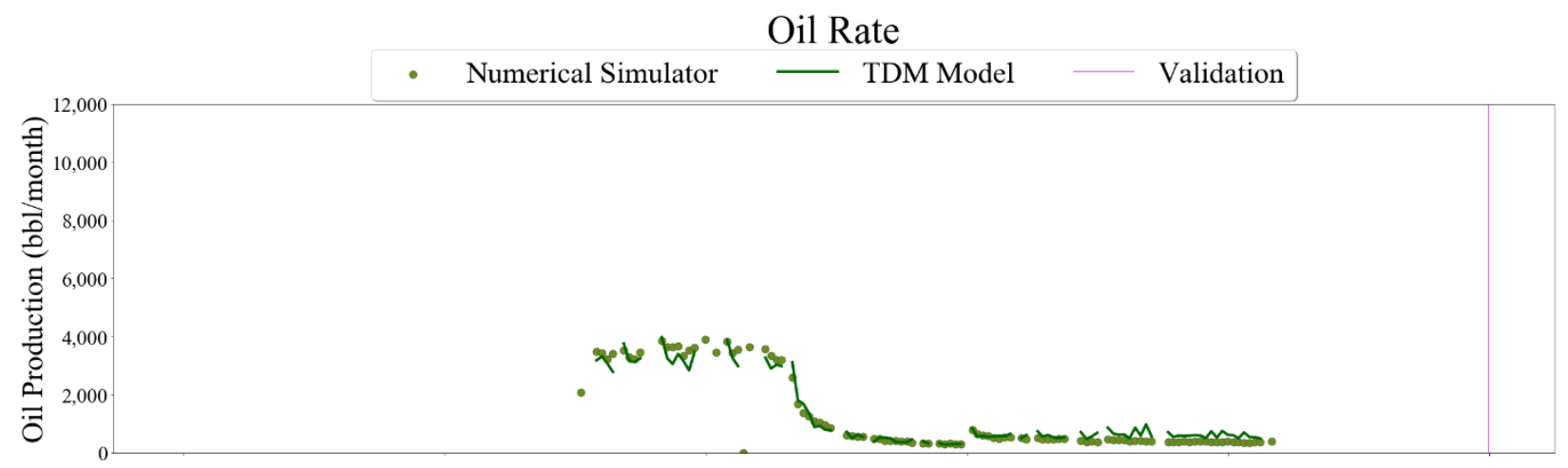

Gas Rate
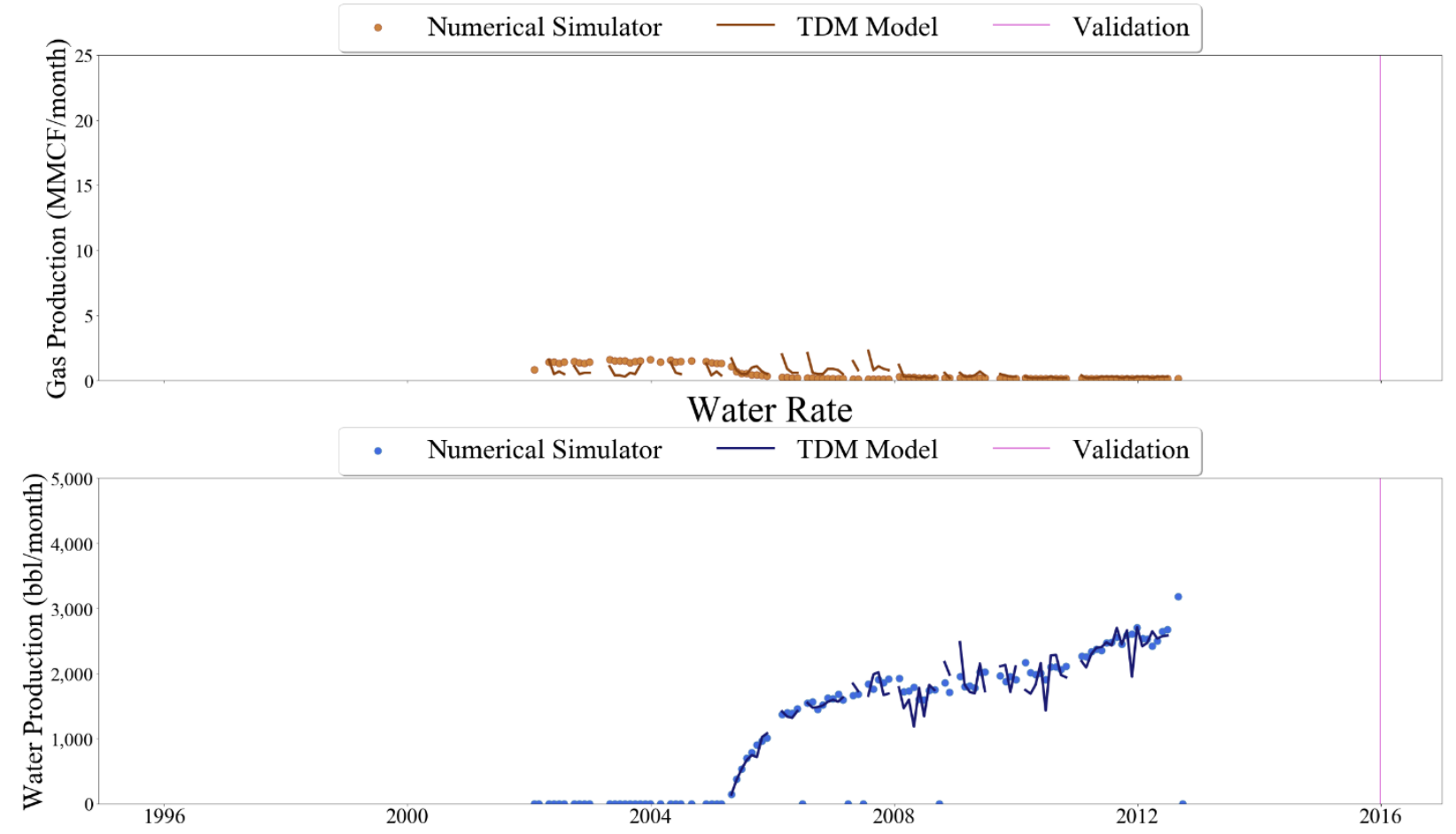

Figure 456 Well-030 oil, gas, and water rate TDM predictions vs actual simulation data plots for 1 Year Blind Validation TDM 

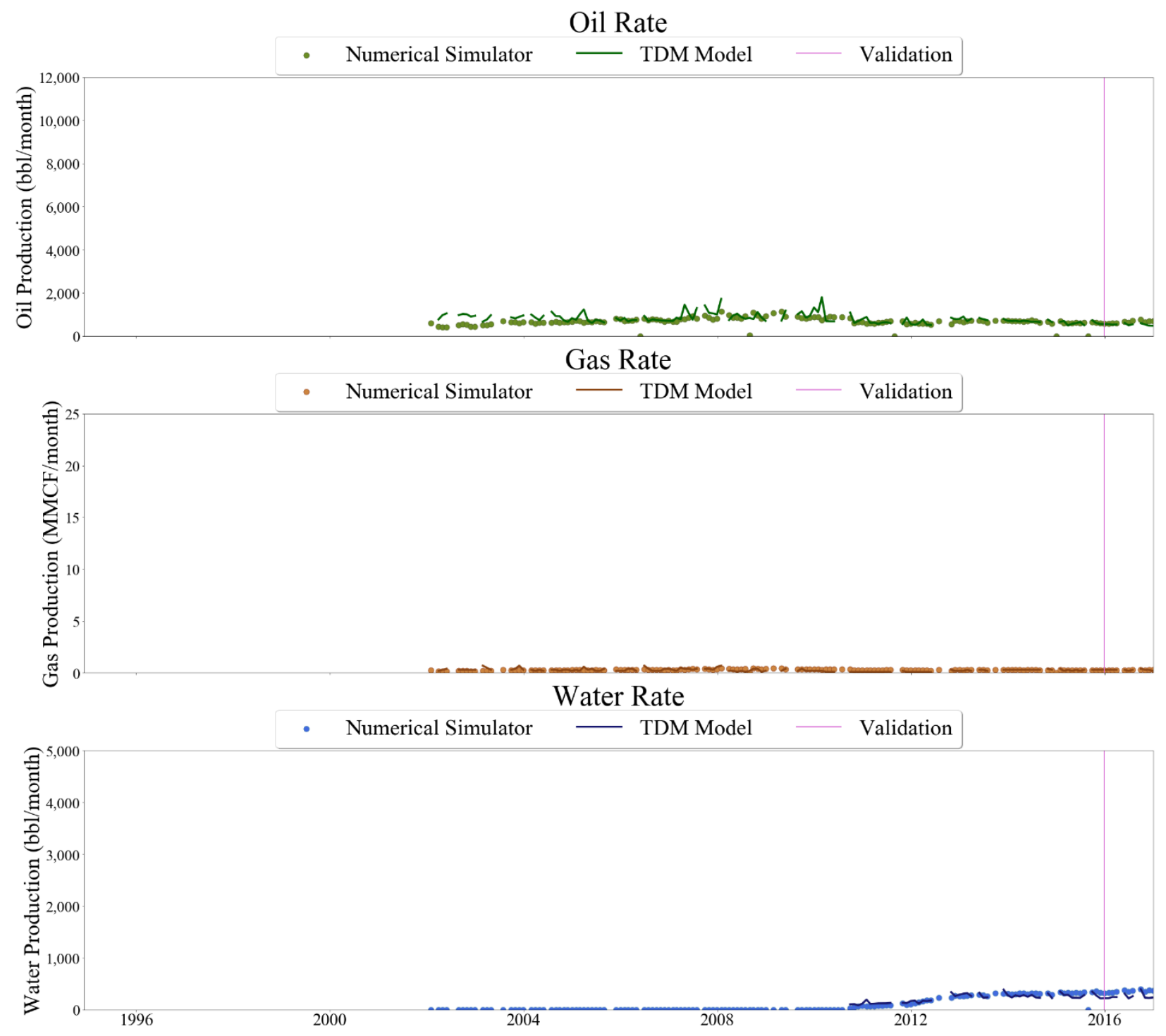

Figure 457 Well-031 oil, gas, and water rate TDM predictions vs actual simulation data plots for 1 Year Blind Validation TDM 


\section{Oil Rate}

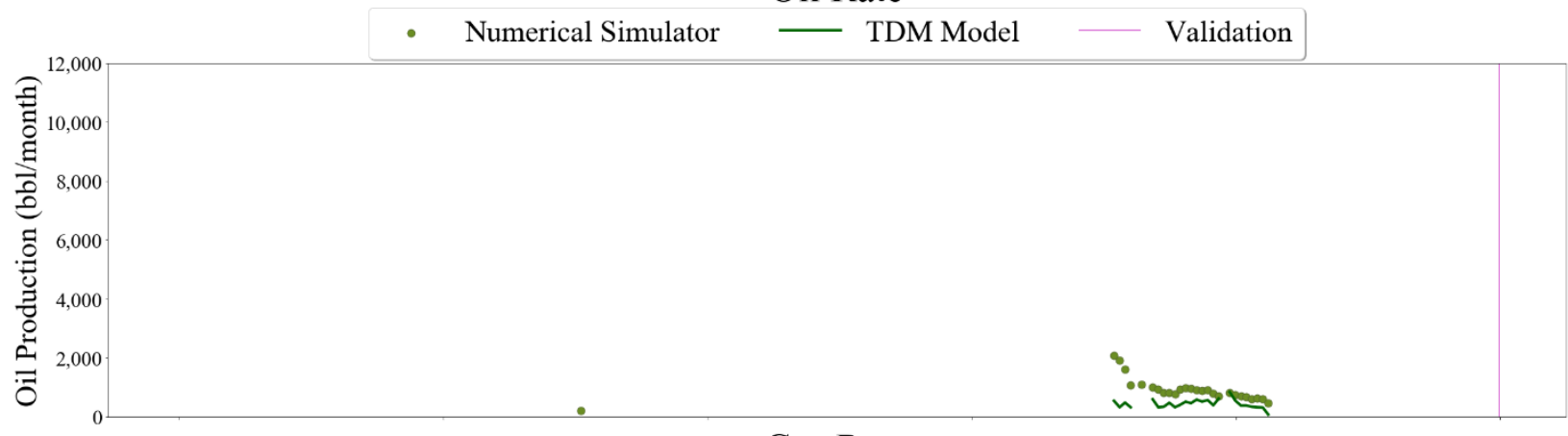

\section{Gas Rate}
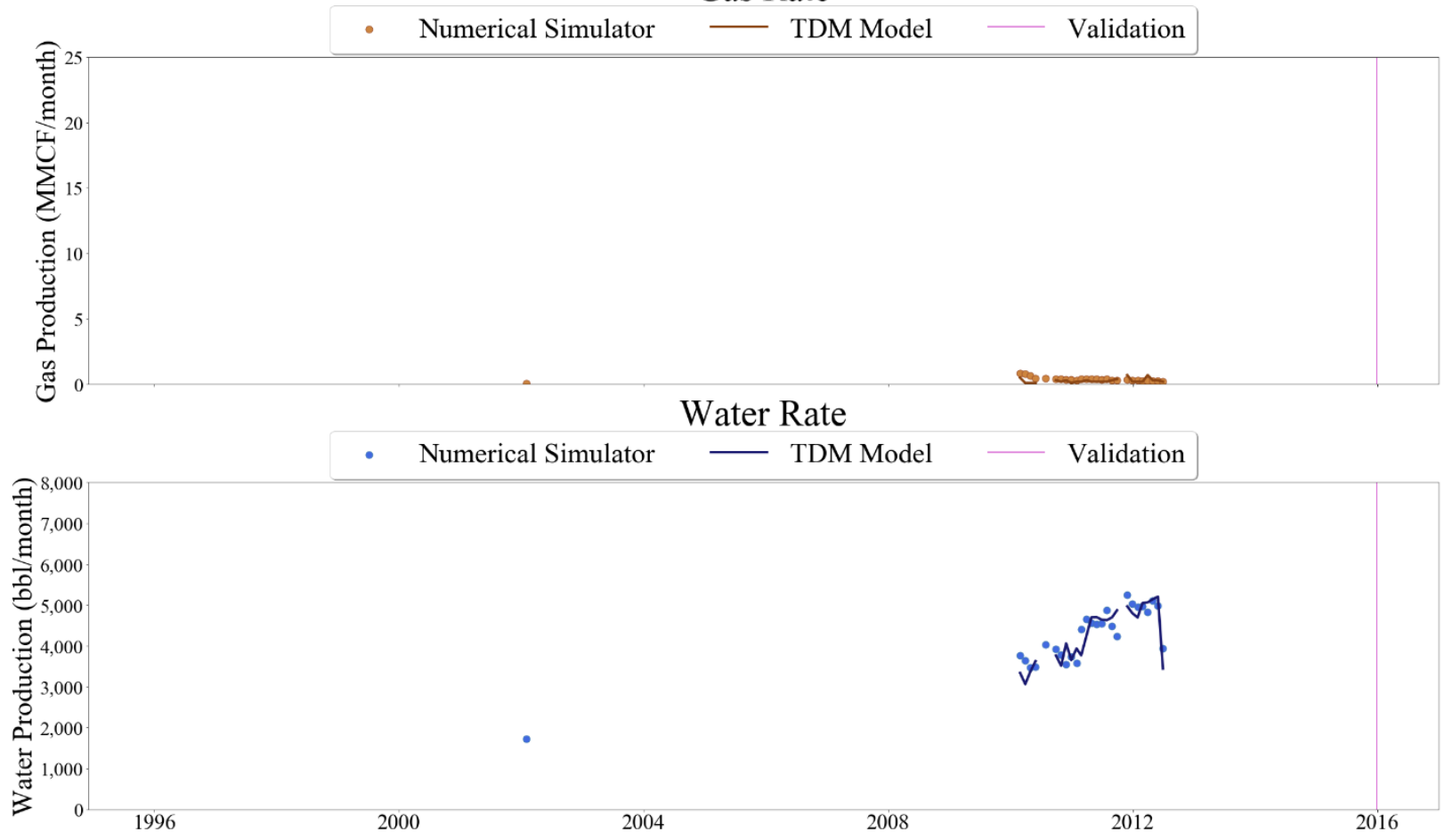

Figure 458 Well-032 oil, gas, and water rate TDM predictions vs actual simulation data plots for 1 Year Blind Validation TDM 


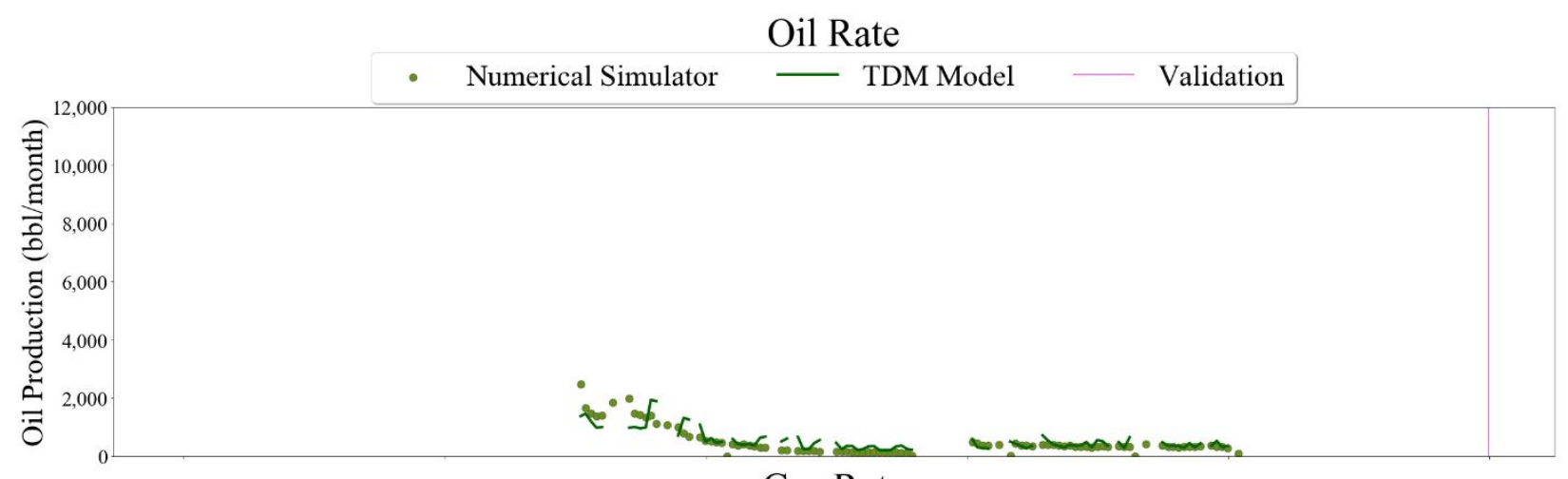

Gas Rate
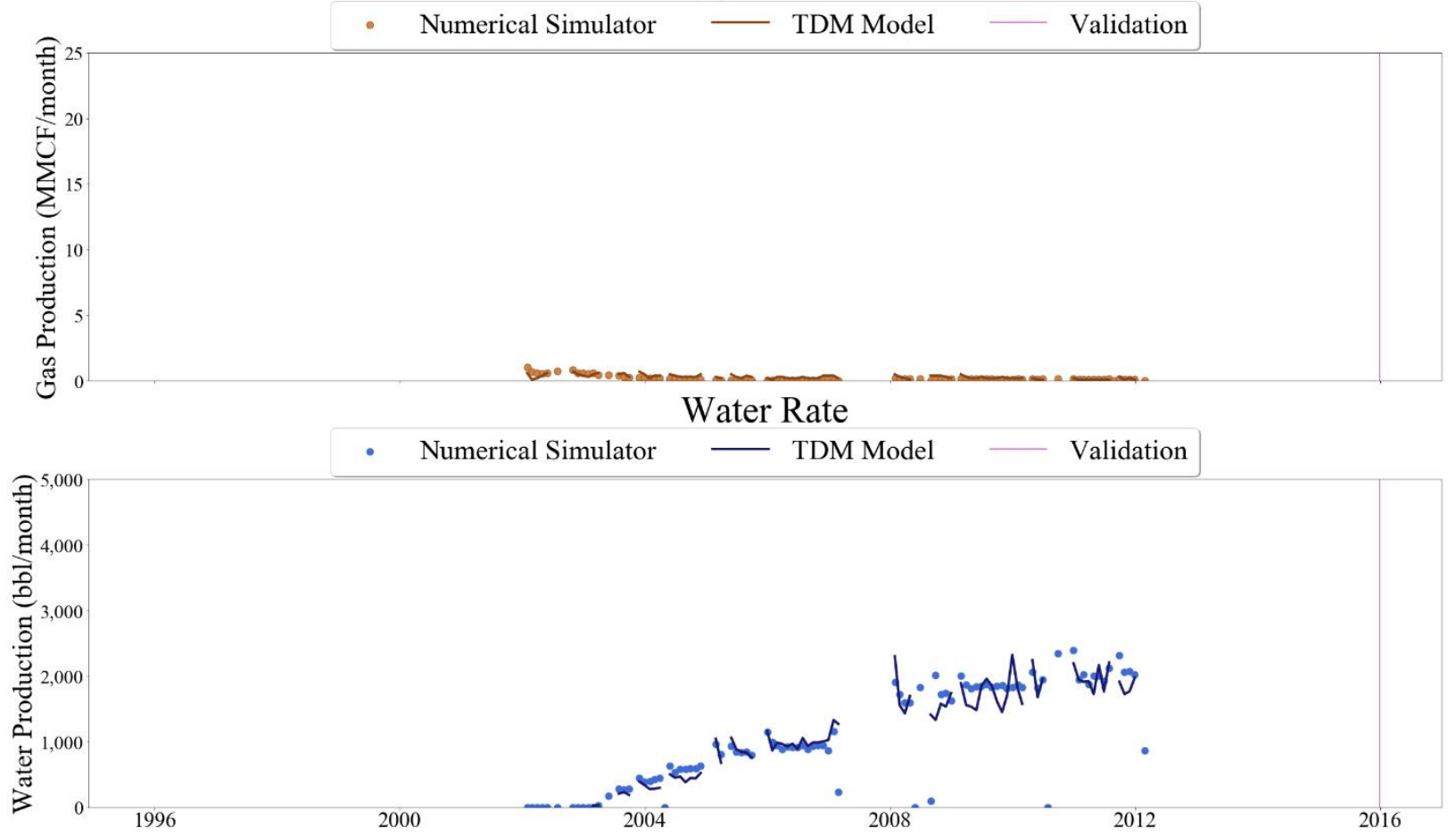

Figure 459 Well-033 oil, gas, and water rate TDM predictions vs actual simulation data plots for 1 Year Blind Validation TDM 

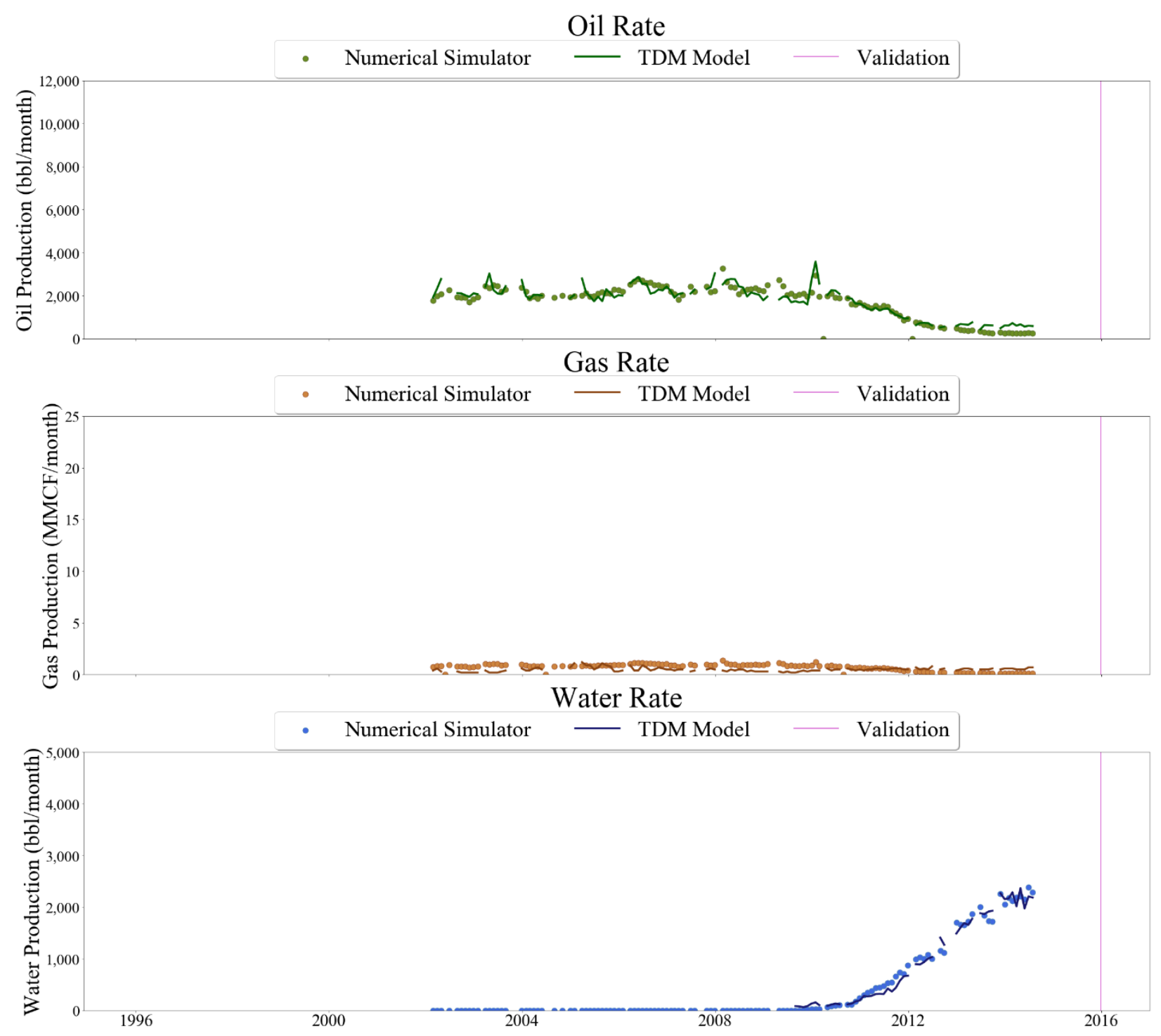

Figure 460 Well-034 oil, gas, and water rate TDM predictions vs actual simulation data plots for 1 Year Blind Validation TDM 


\section{Oil Rate}

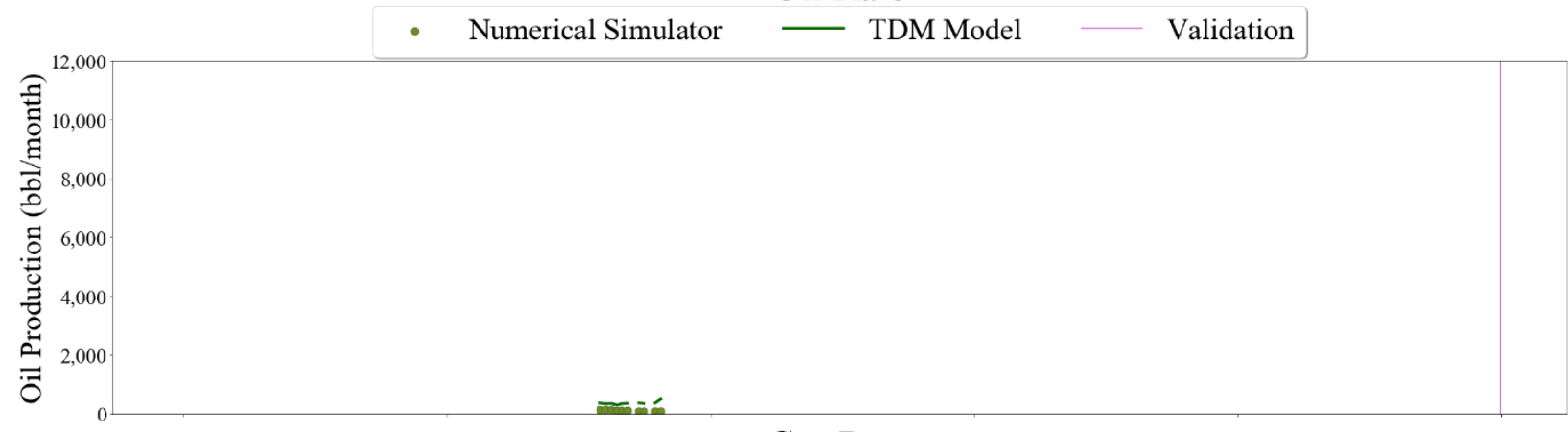

\section{Gas Rate}
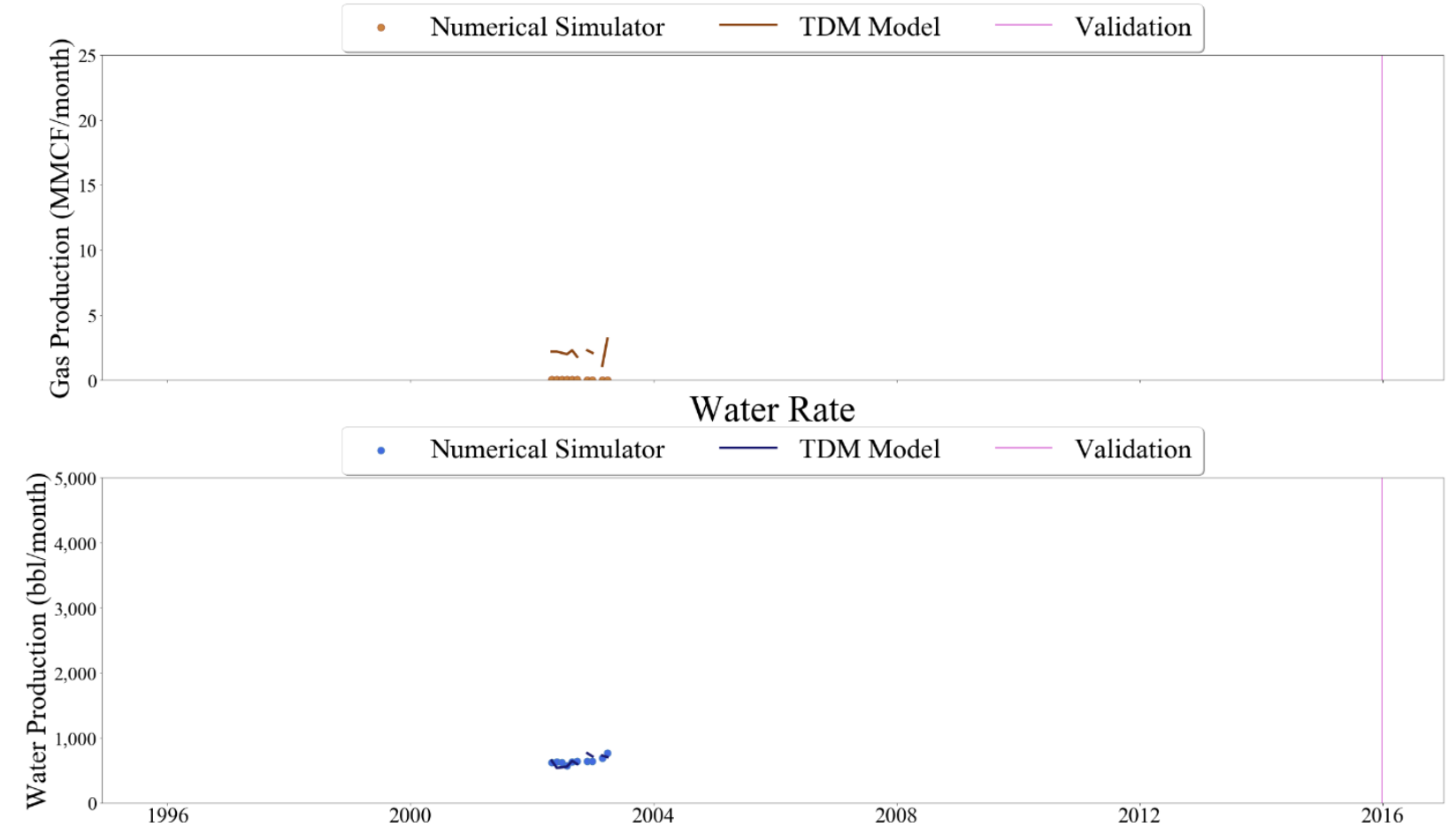

Figure 461 Well-035 oil, gas, and water rate TDM predictions vs actual simulation data plots for 1 Year Blind Validation TDM 

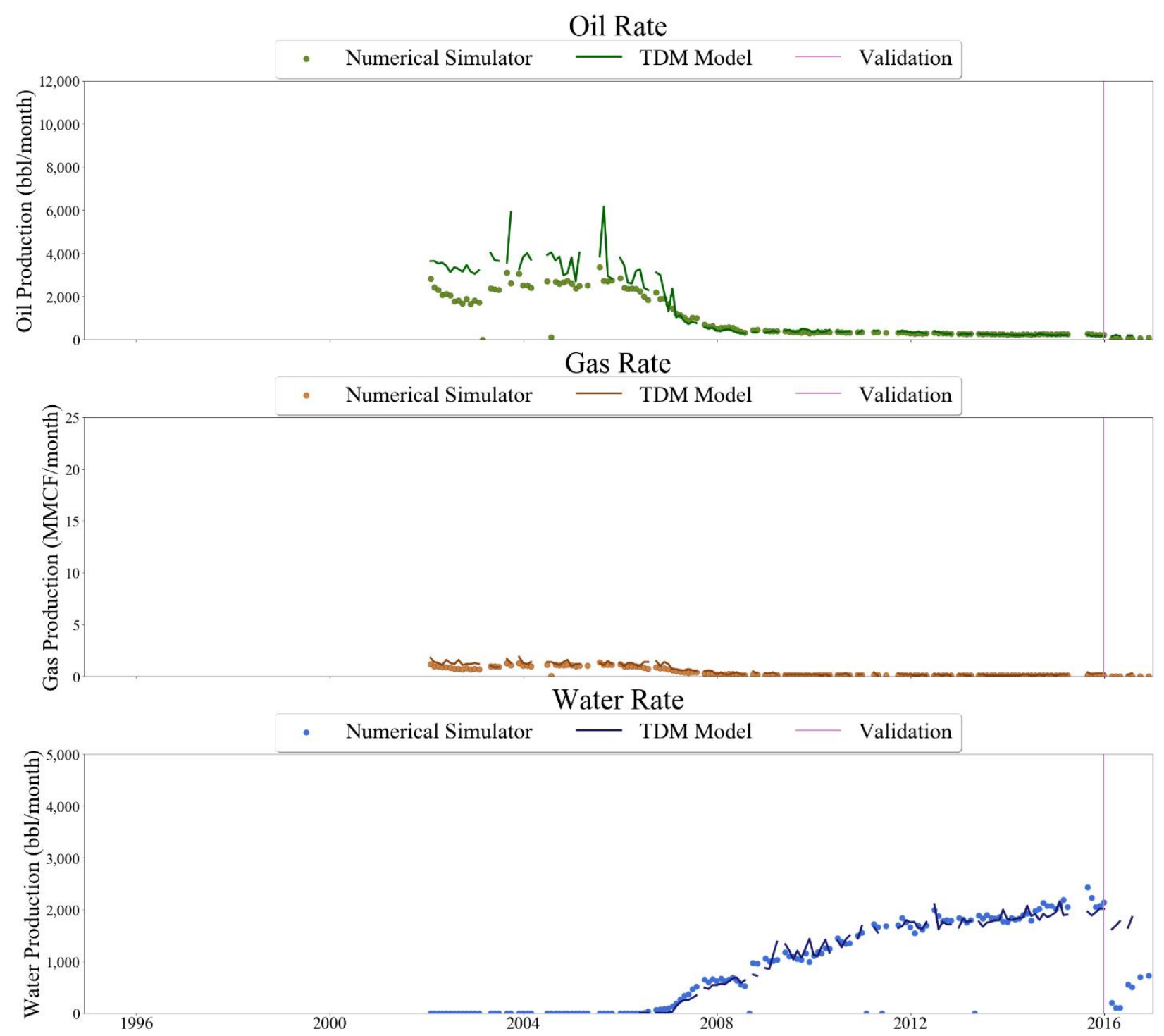

Figure 462 Well-036 oil, gas, and water rate TDM predictions vs actual simulation data plots for 1 Year Blind Validation TDM 

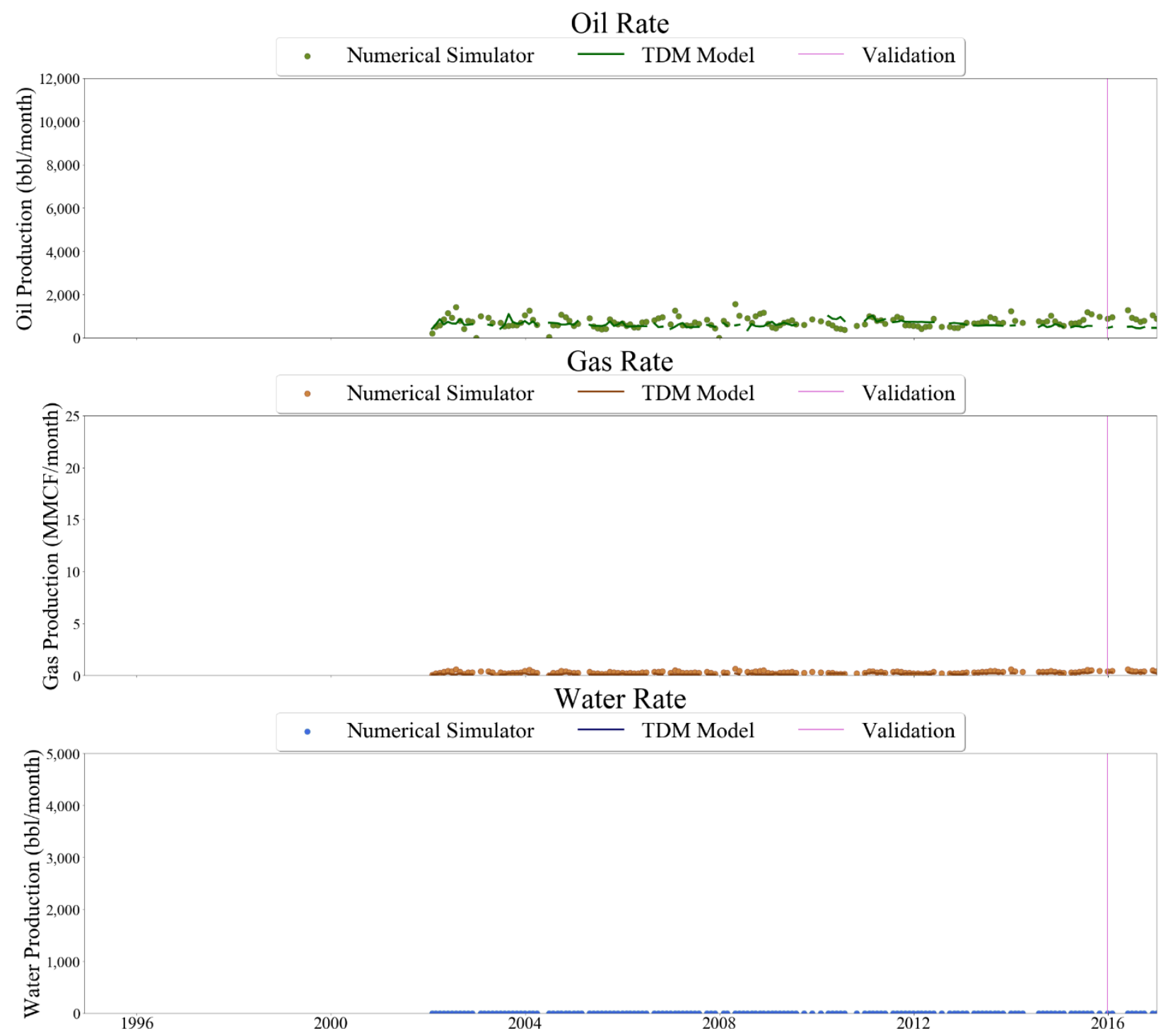

Figure 463 Well-037 oil, gas, and water rate TDM predictions vs actual simulation data plots for 1 Year Blind Validation TDM 


\section{Oil Rate}

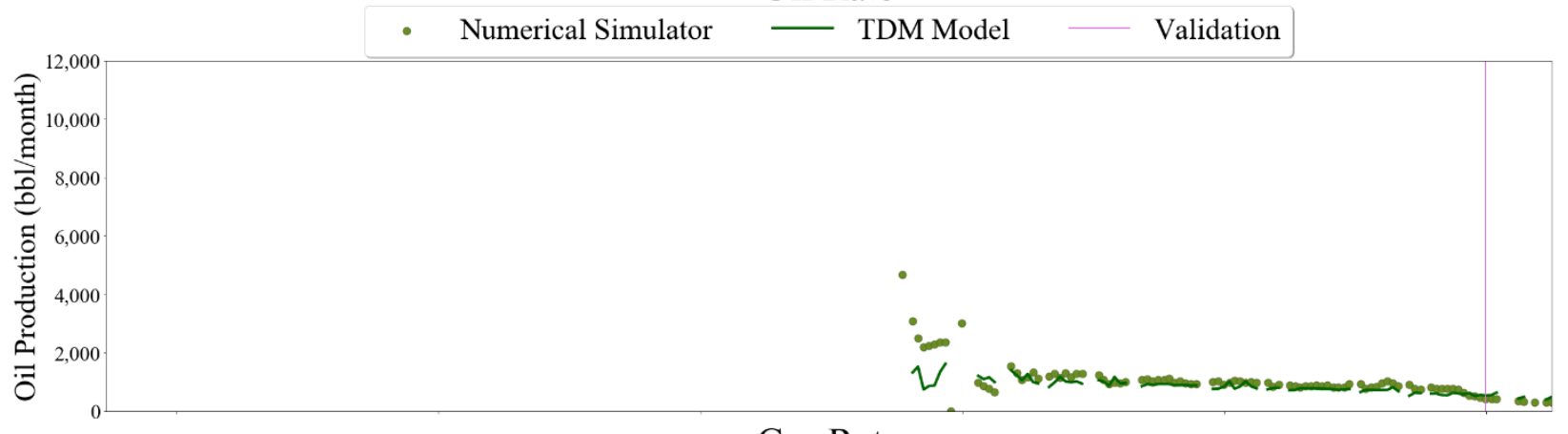

\section{Gas Rate}
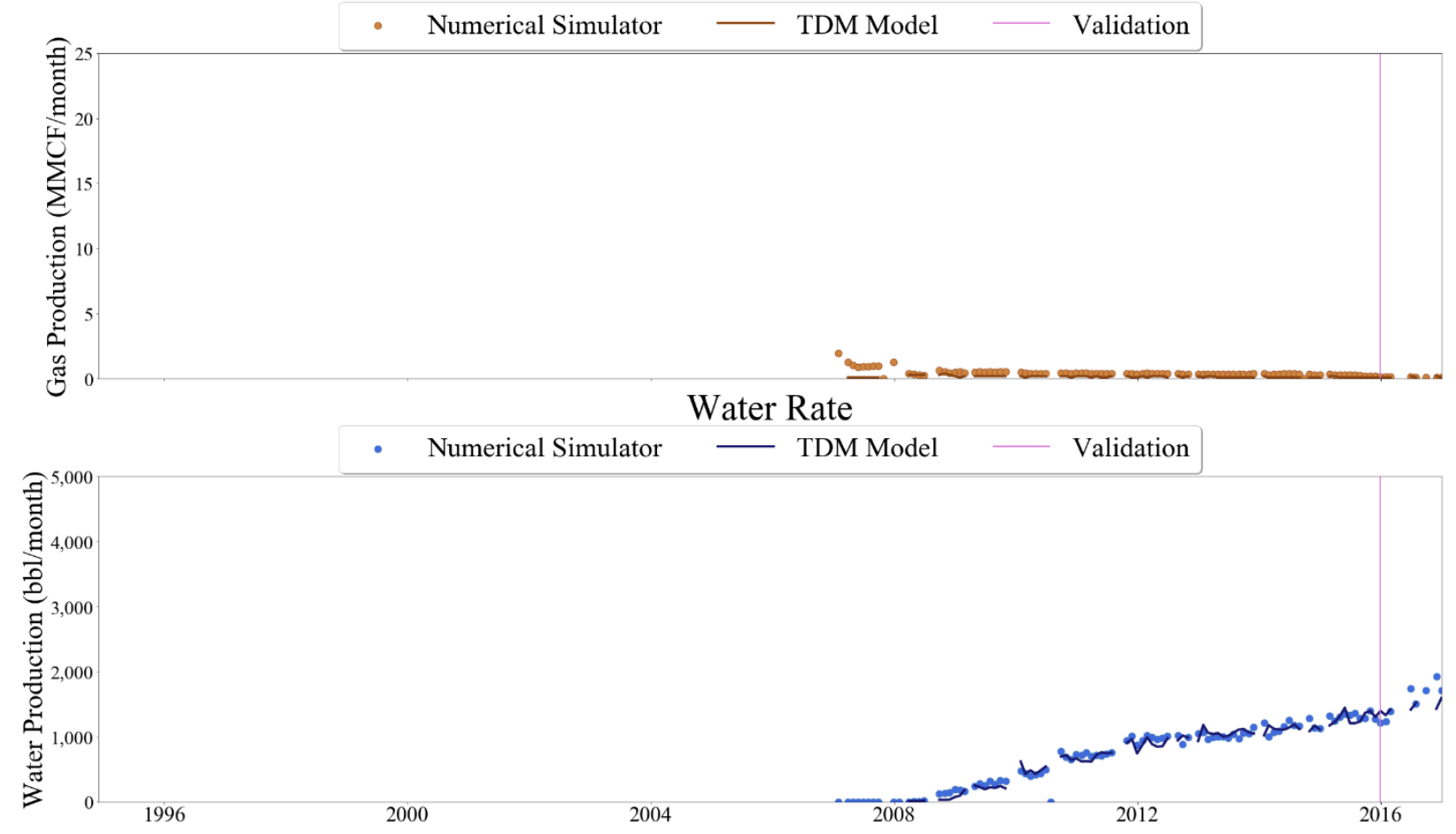

Figure 464 Well-038 oil, gas, and water rate TDM predictions vs actual simulation data plots for 1 Year Blind Validation TDM 


\section{Oil Rate}

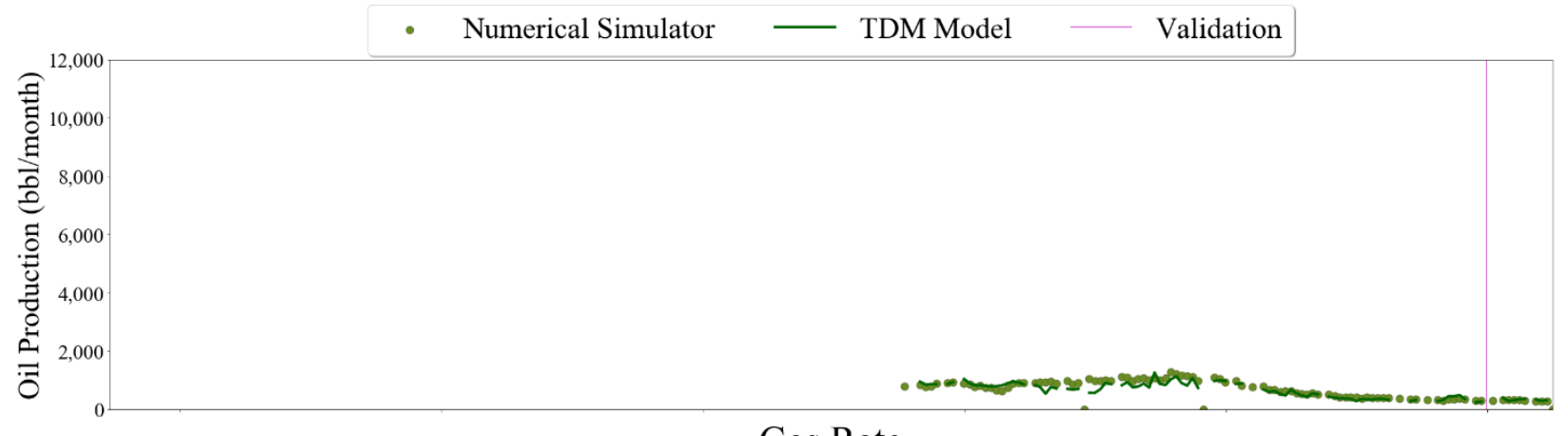

\section{Gas Rate}
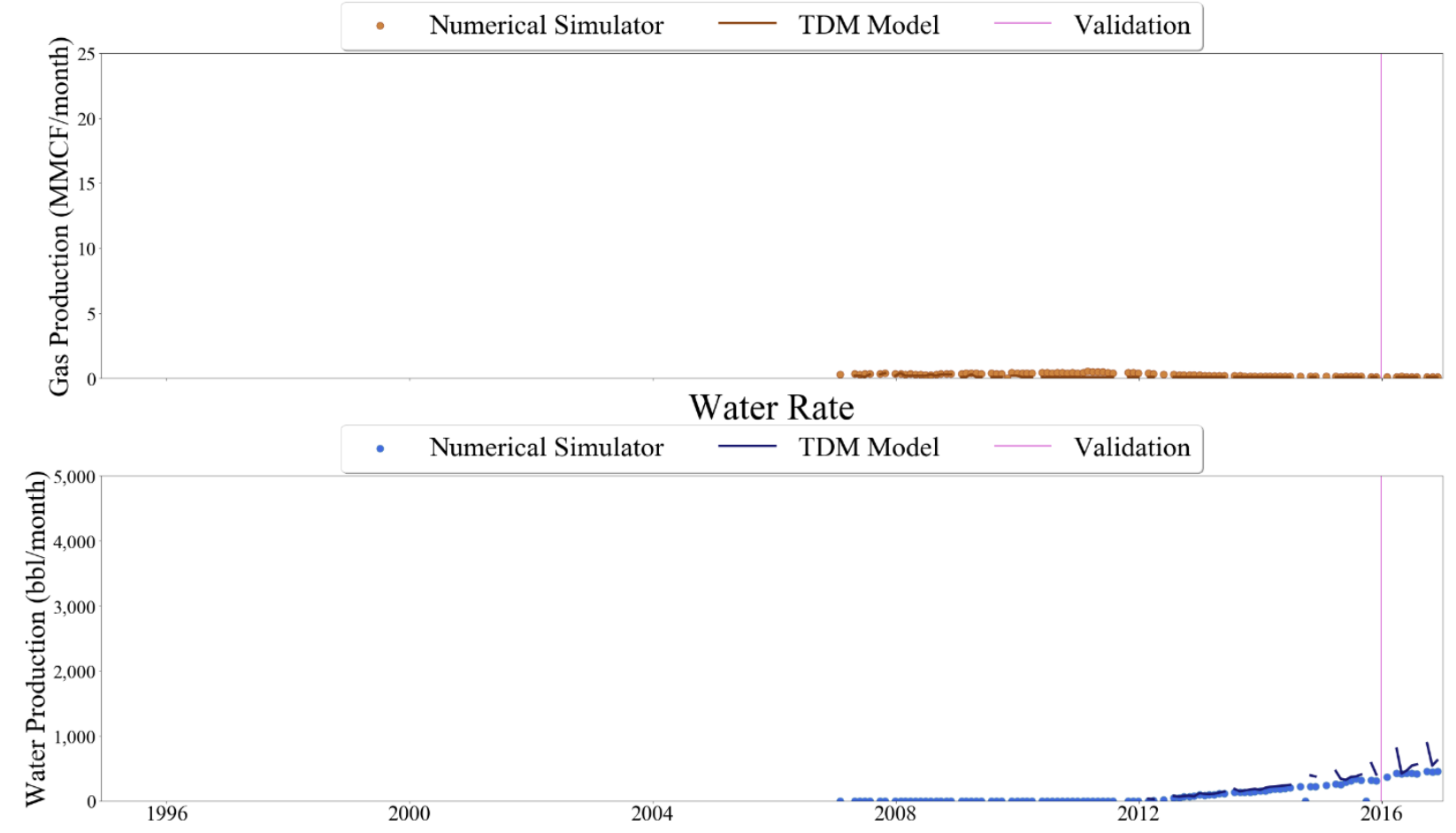

Figure 465 Well-039 oil, gas, and water rate TDM predictions vs actual simulation data plots for 1 Year Blind Validation TDM 


\section{Oil Rate}

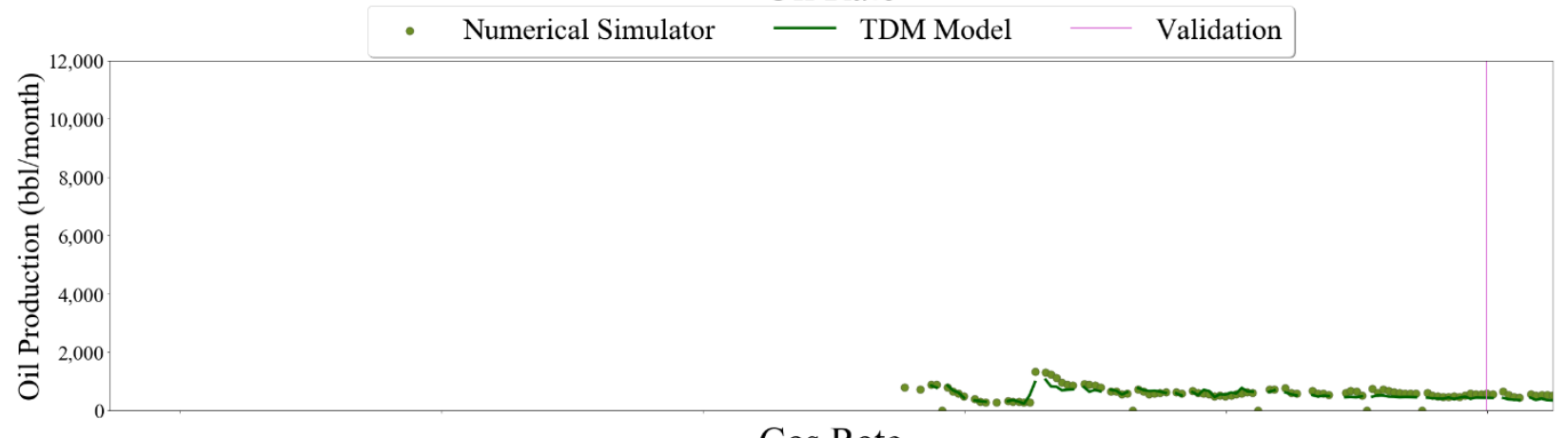

\section{Gas Rate}
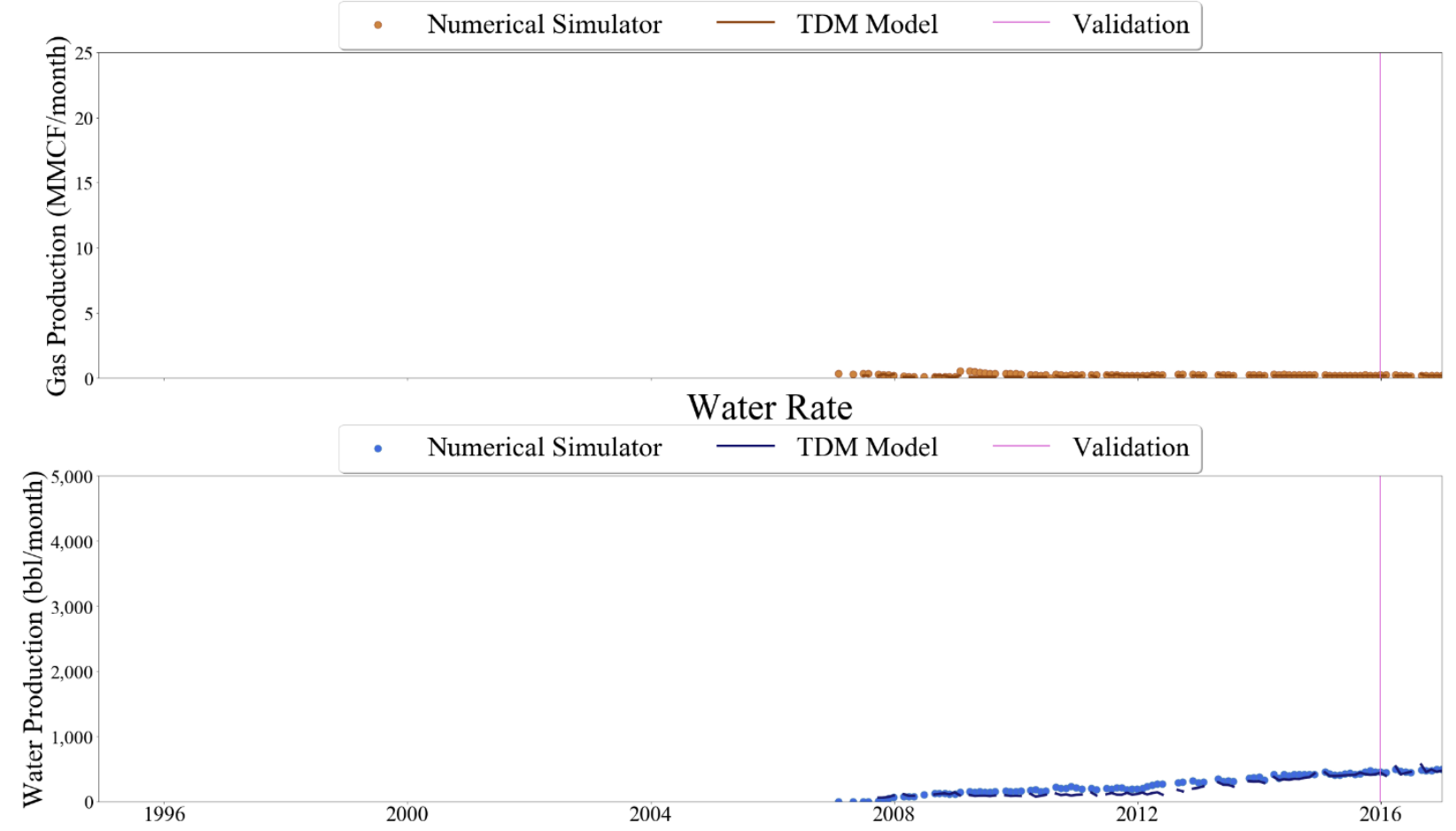

Figure 466 Well-040 oil, gas, and water rate TDM predictions vs actual simulation data plots for 1 Year Blind Validation TDM 


\section{Oil Rate}

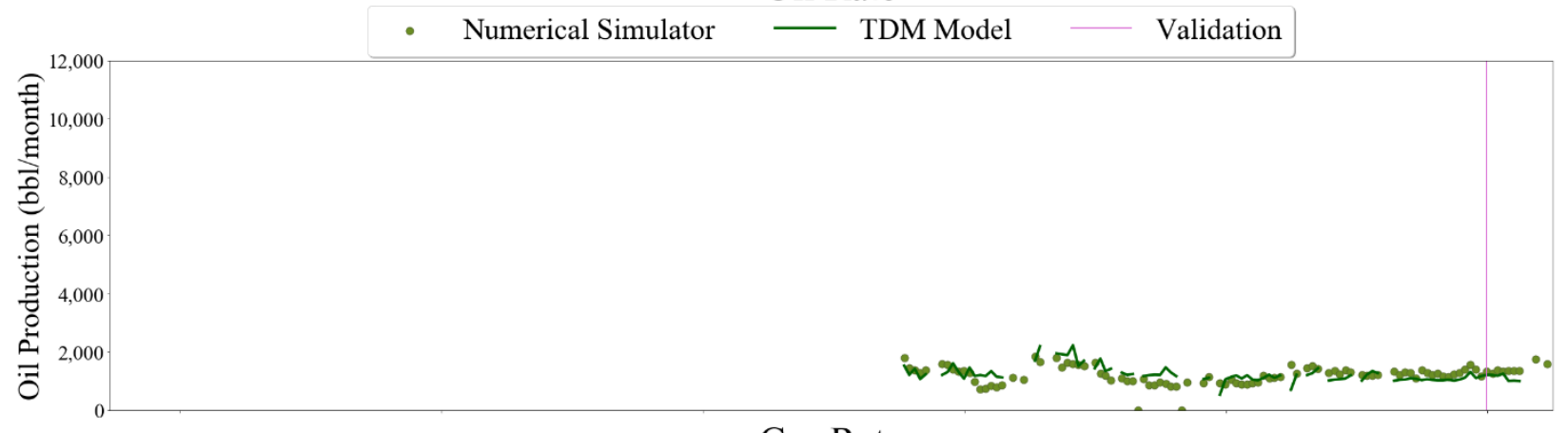

\section{Gas Rate}
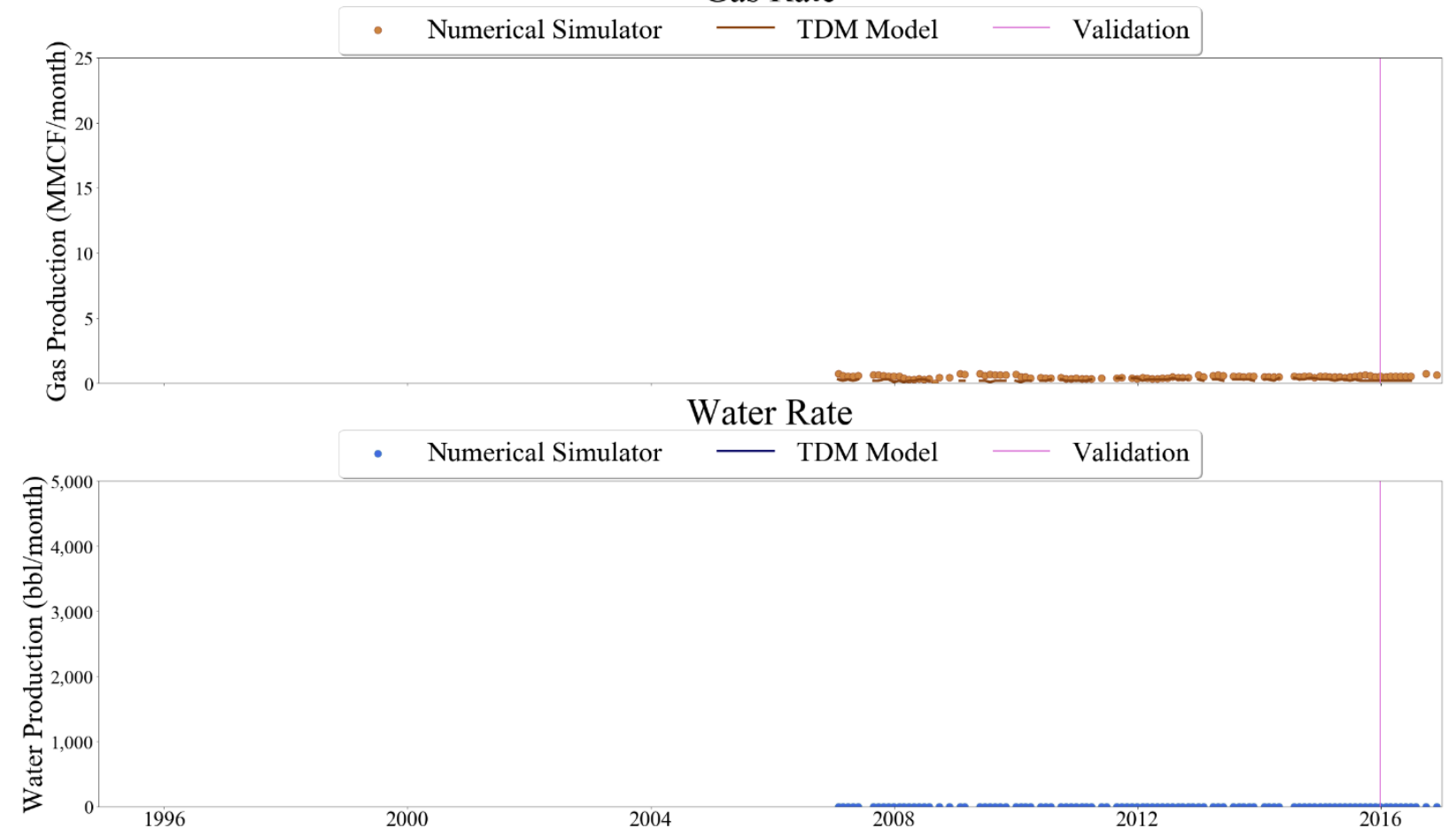

Figure 467 Well-041 oil, gas, and water rate TDM predictions vs actual simulation data plots for 1 Year Blind Validation TDM 


\section{Oil Rate}

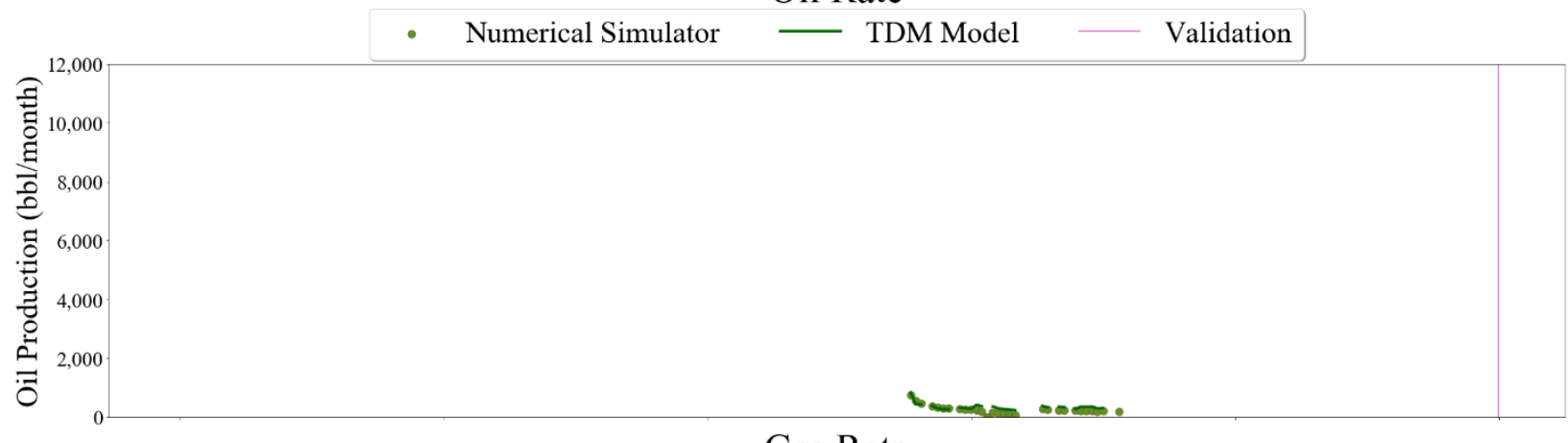

\section{Gas Rate}
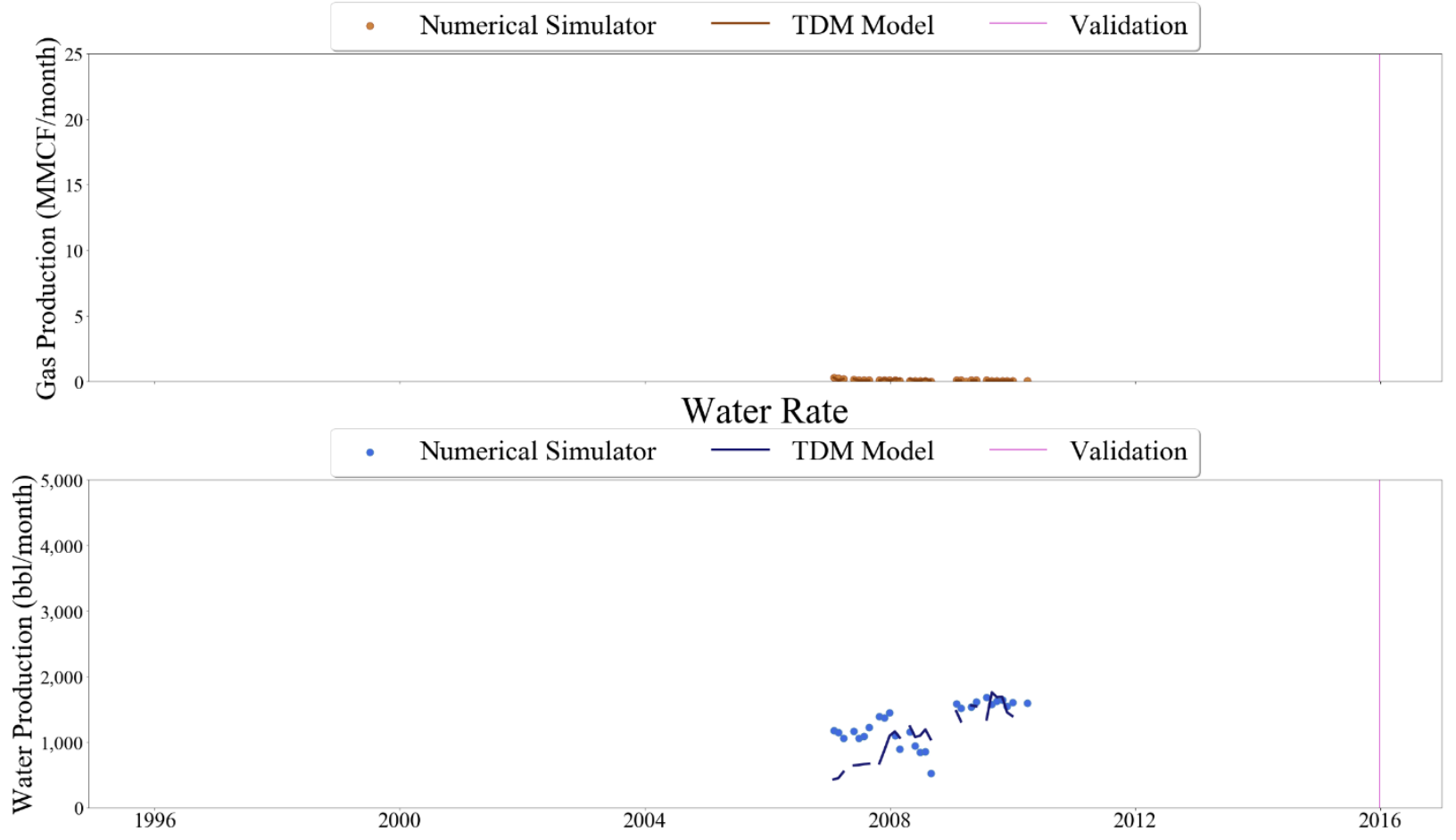

Figure 468 Well-042 oil, gas, and water rate TDM predictions vs actual simulation data plots for 1 Year Blind Validation TDM 


\section{Oil Rate}

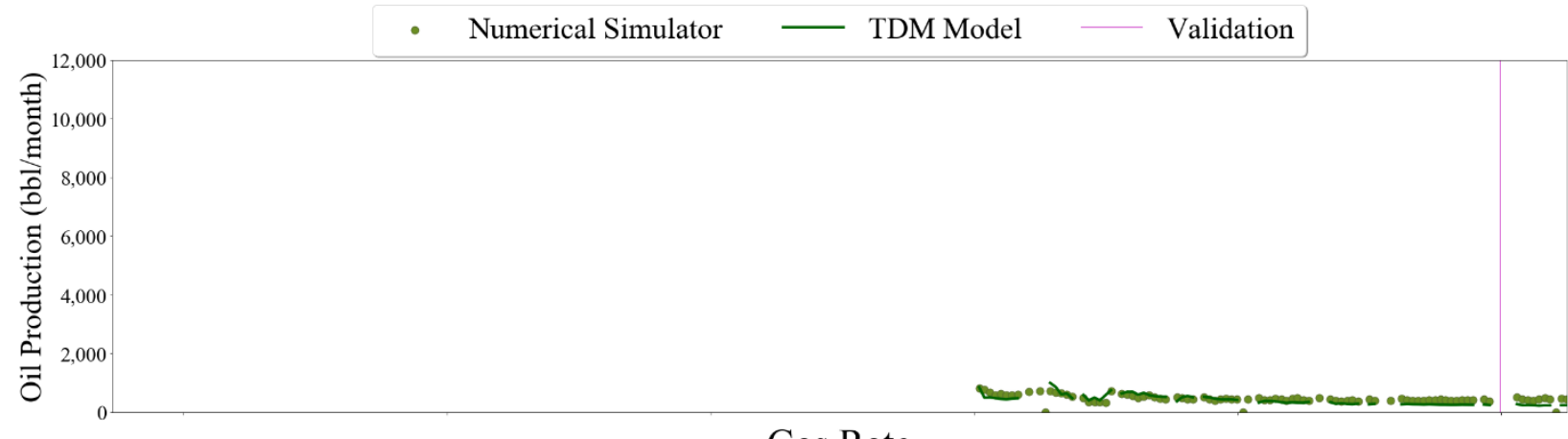

\section{Gas Rate}
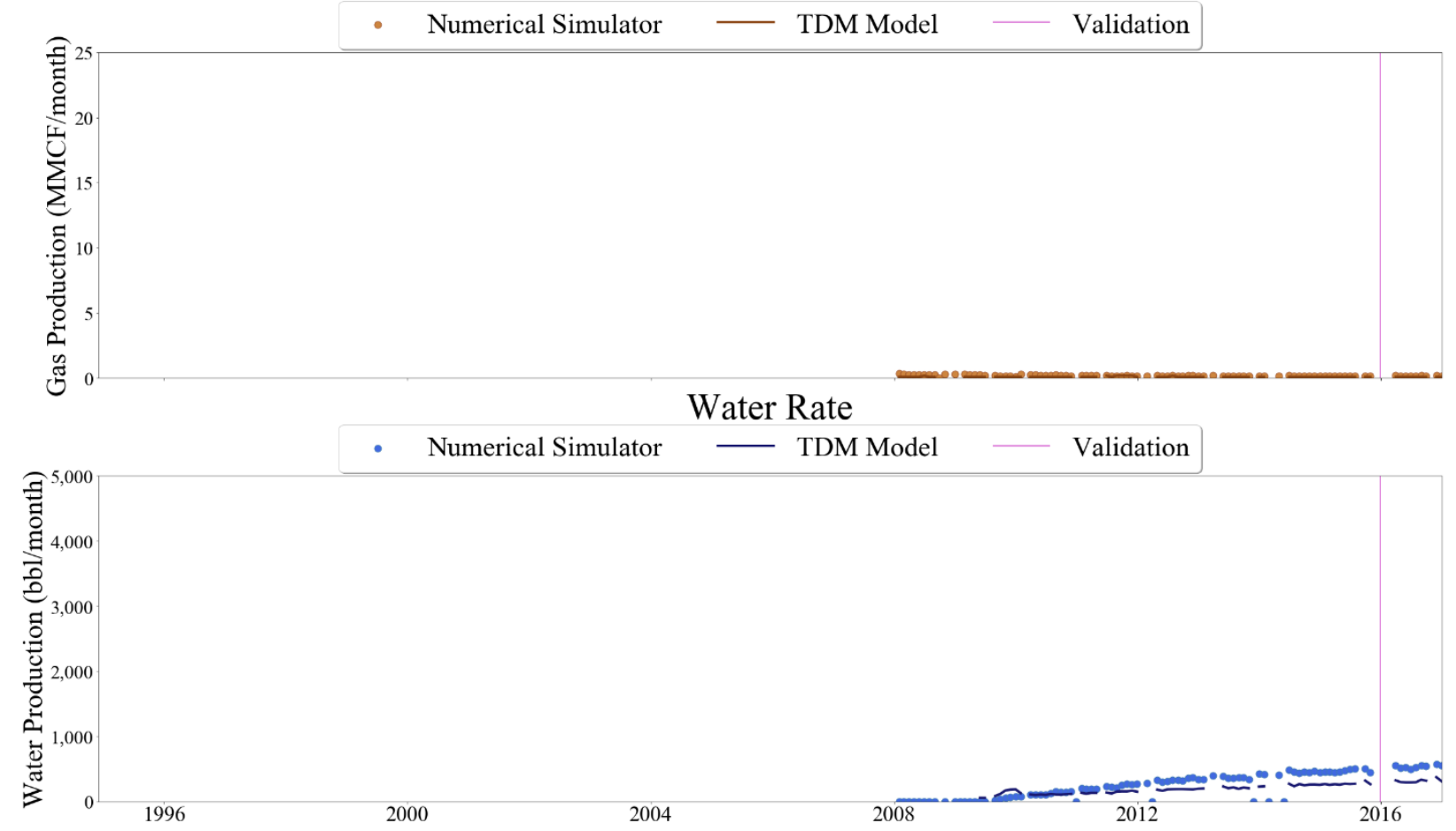

Figure 469 Well-043 oil, gas, and water rate TDM predictions vs actual simulation data plots for 1 Year Blind Validation TDM 


\section{Oil Rate}
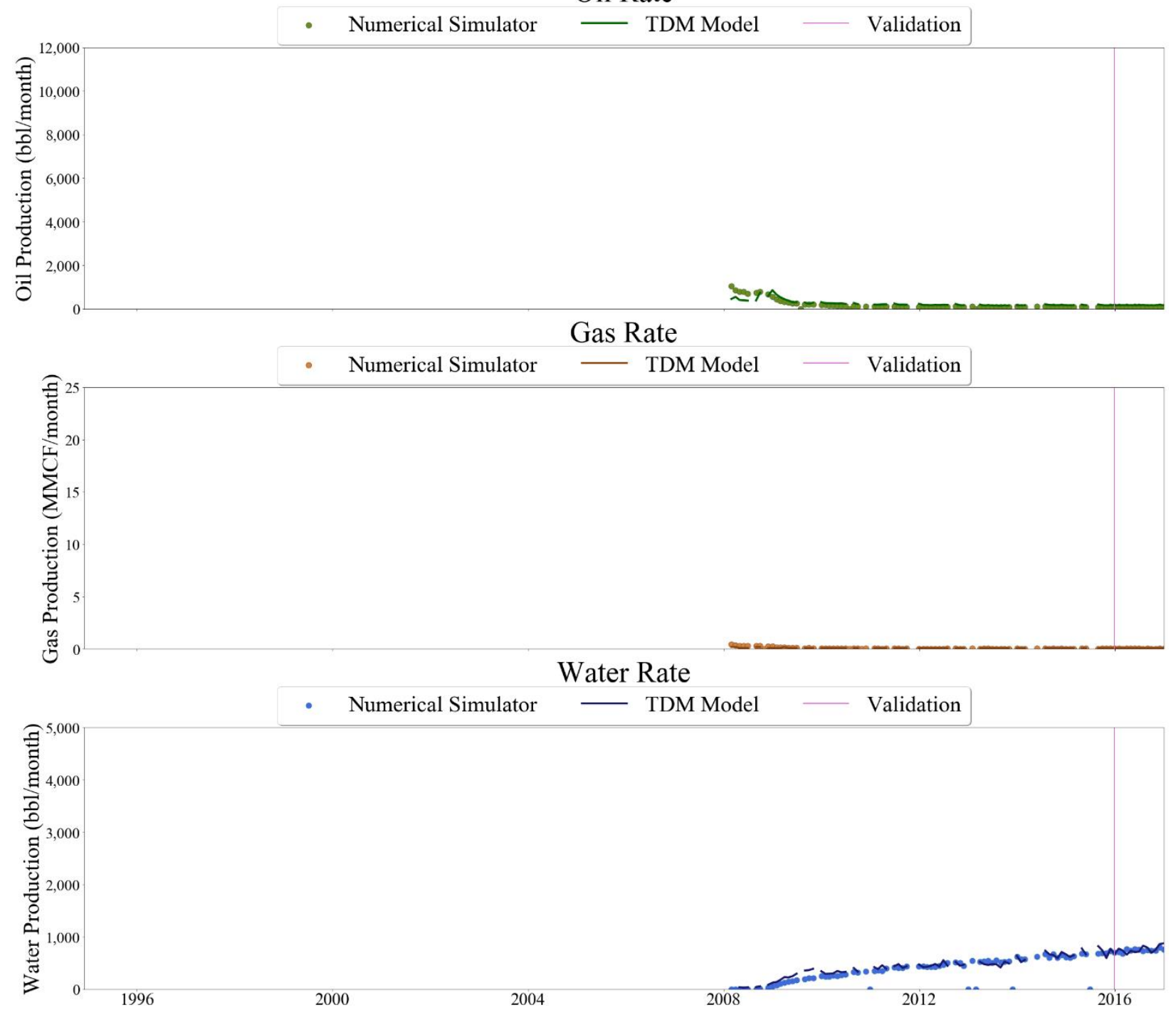

Figure 470 Well-044 oil, gas, and water rate TDM predictions vs actual simulation data plots for 1 Year Blind Validation TDM 

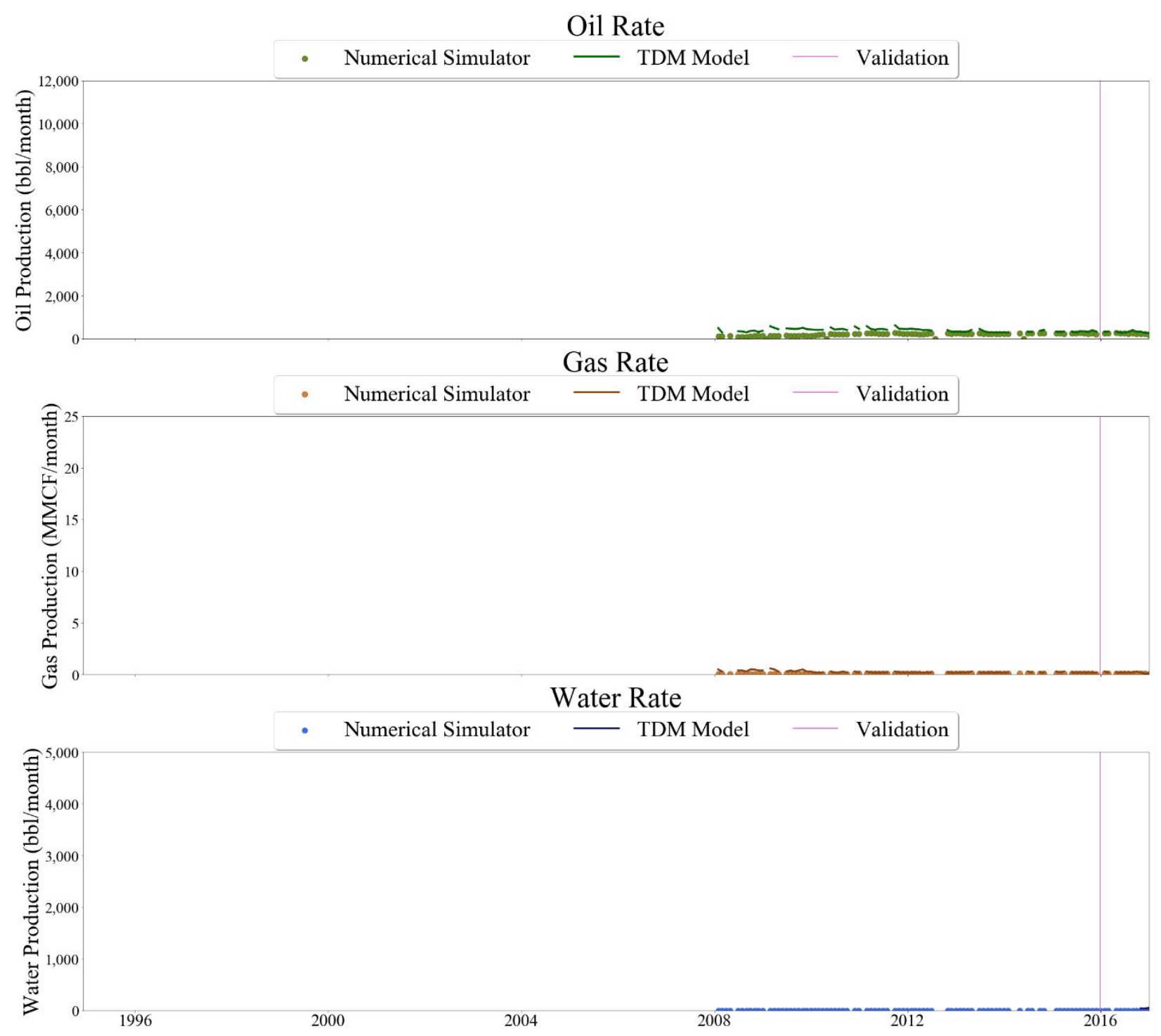

Figure 471 Well-045 oil, gas, and water rate TDM predictions vs actual simulation data plots for 1 Year Blind Validation 

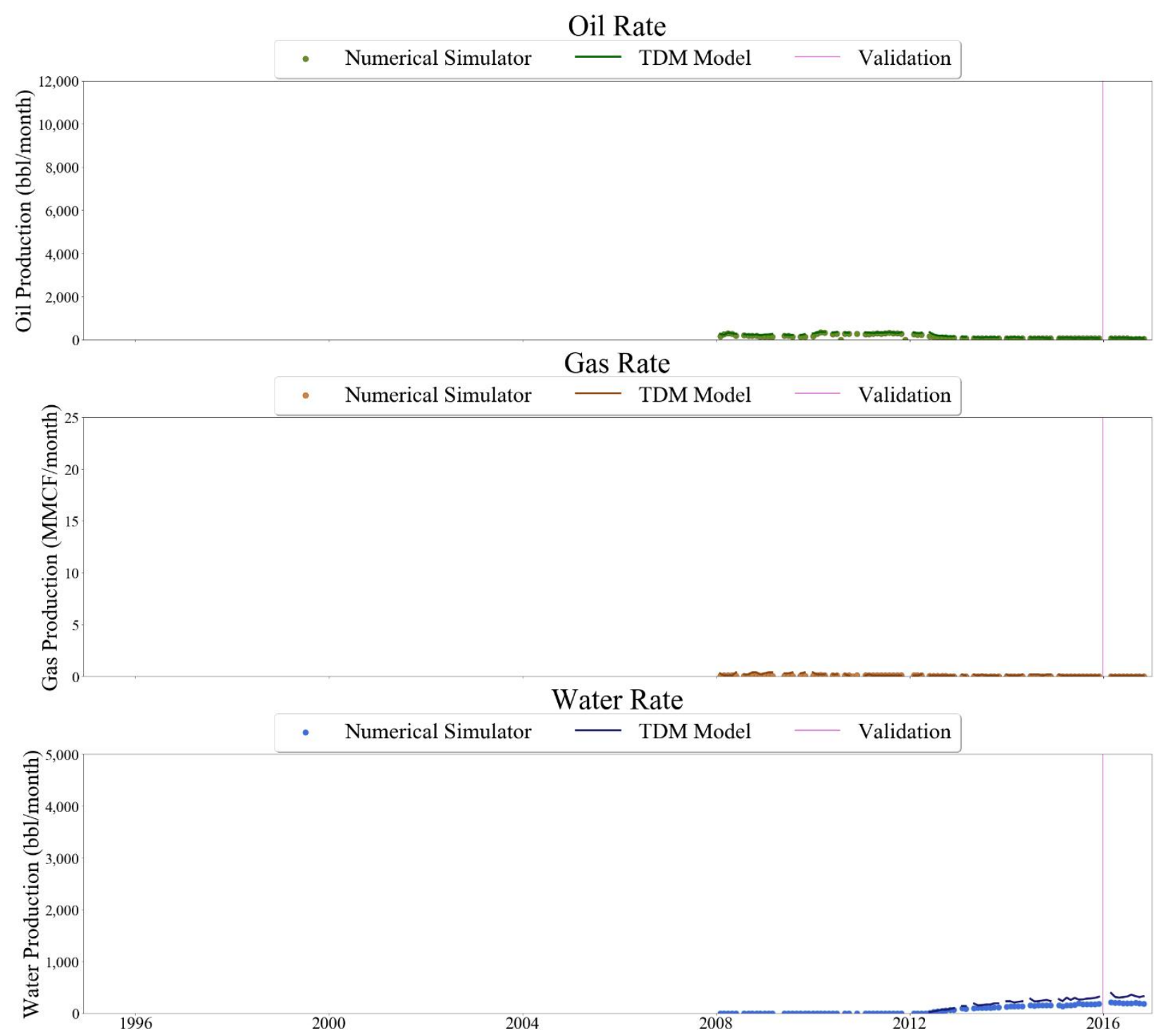

Figure 472 Well-046 oil, gas, and water rate TDM predictions vs actual simulation data plots for 1 Year Blind Validation TDM 


\section{Oil Rate}

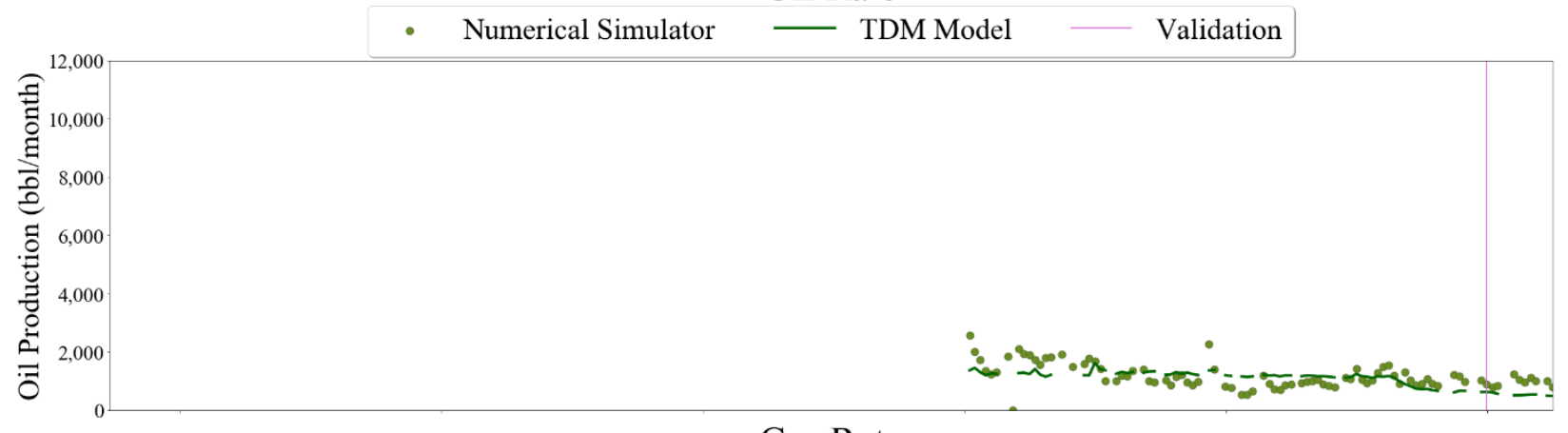

\section{Gas Rate}
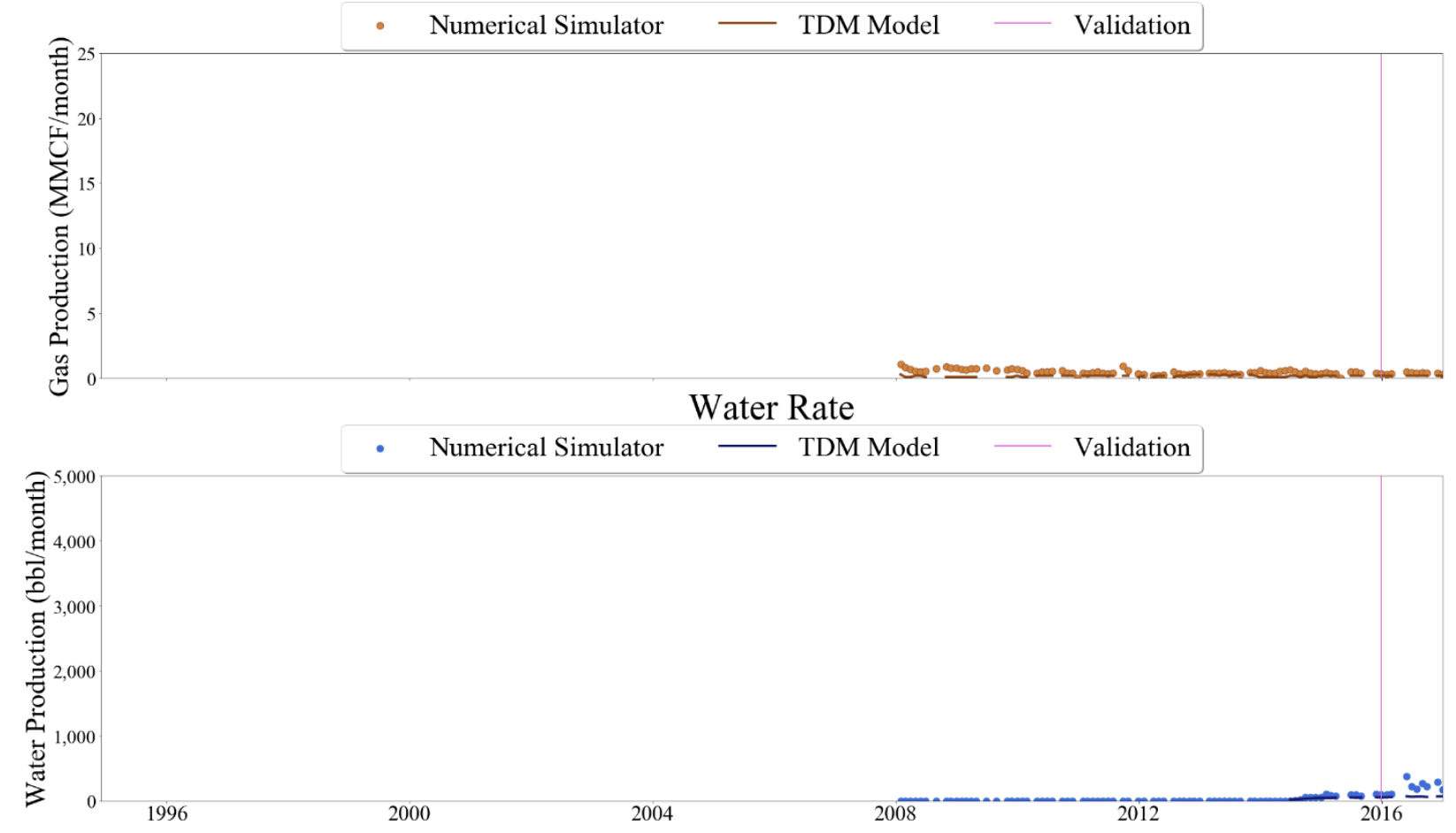

Figure 473 Well-047 oil, gas, and water rate TDM predictions vs actual simulation data plots for 1 Year Blind Validation TDM 


\section{Oil Rate}

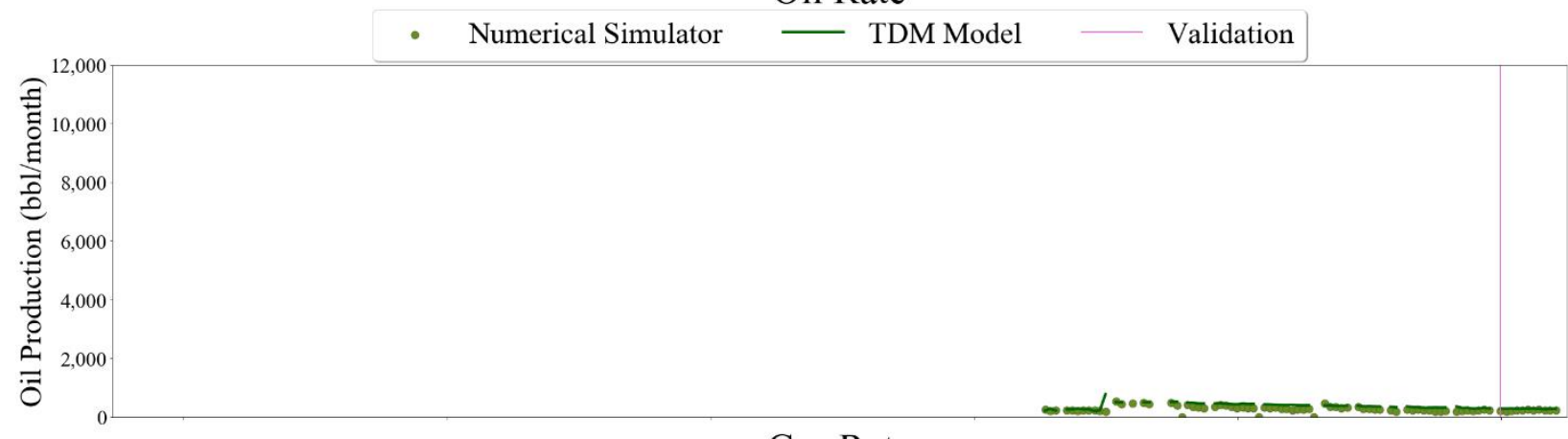

\section{Gas Rate}
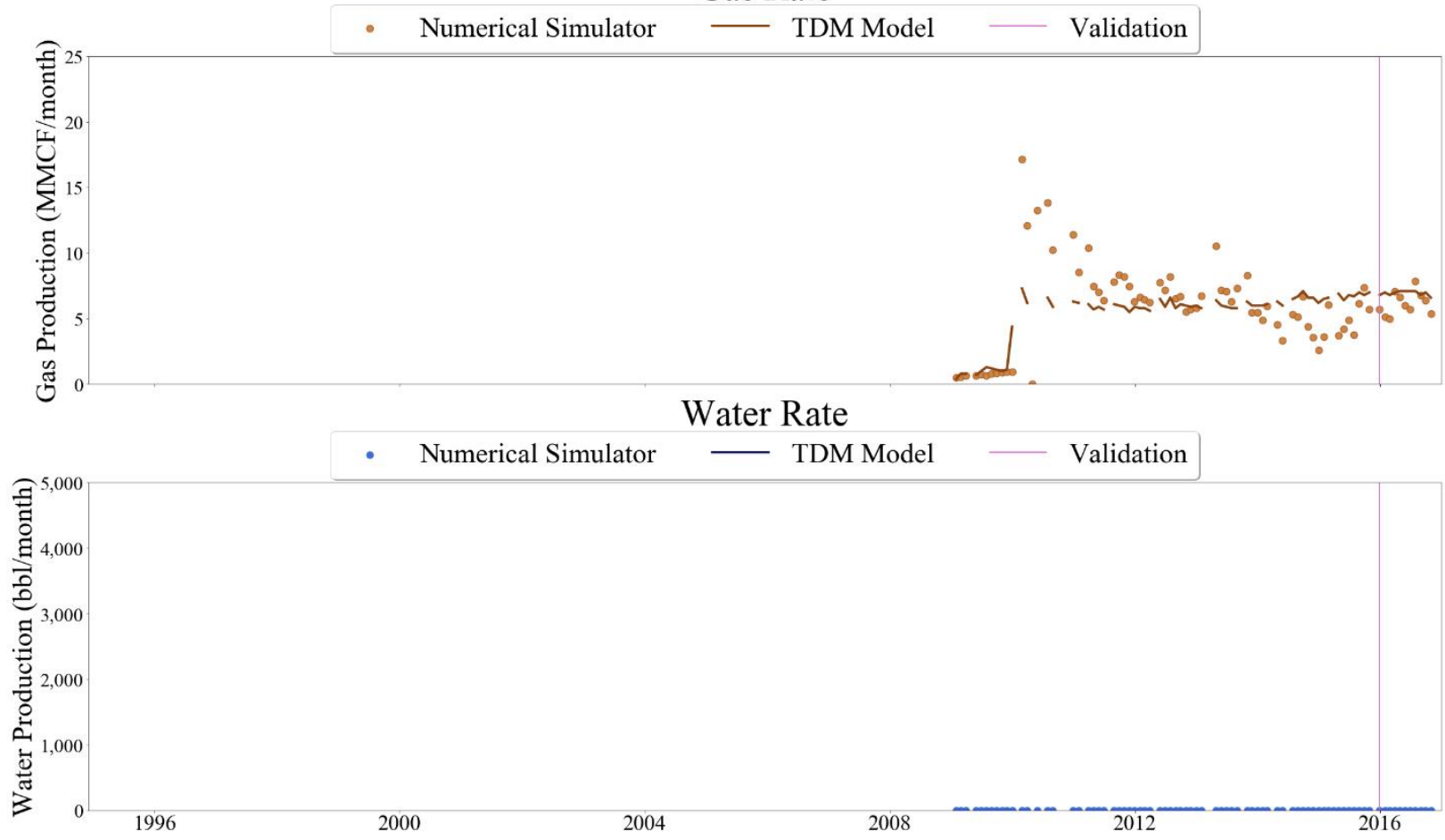

Figure 474 Well-048 oil, gas, and water rate TDM predictions vs actual simulation data plots for 1 Year Blind Validation TDM 

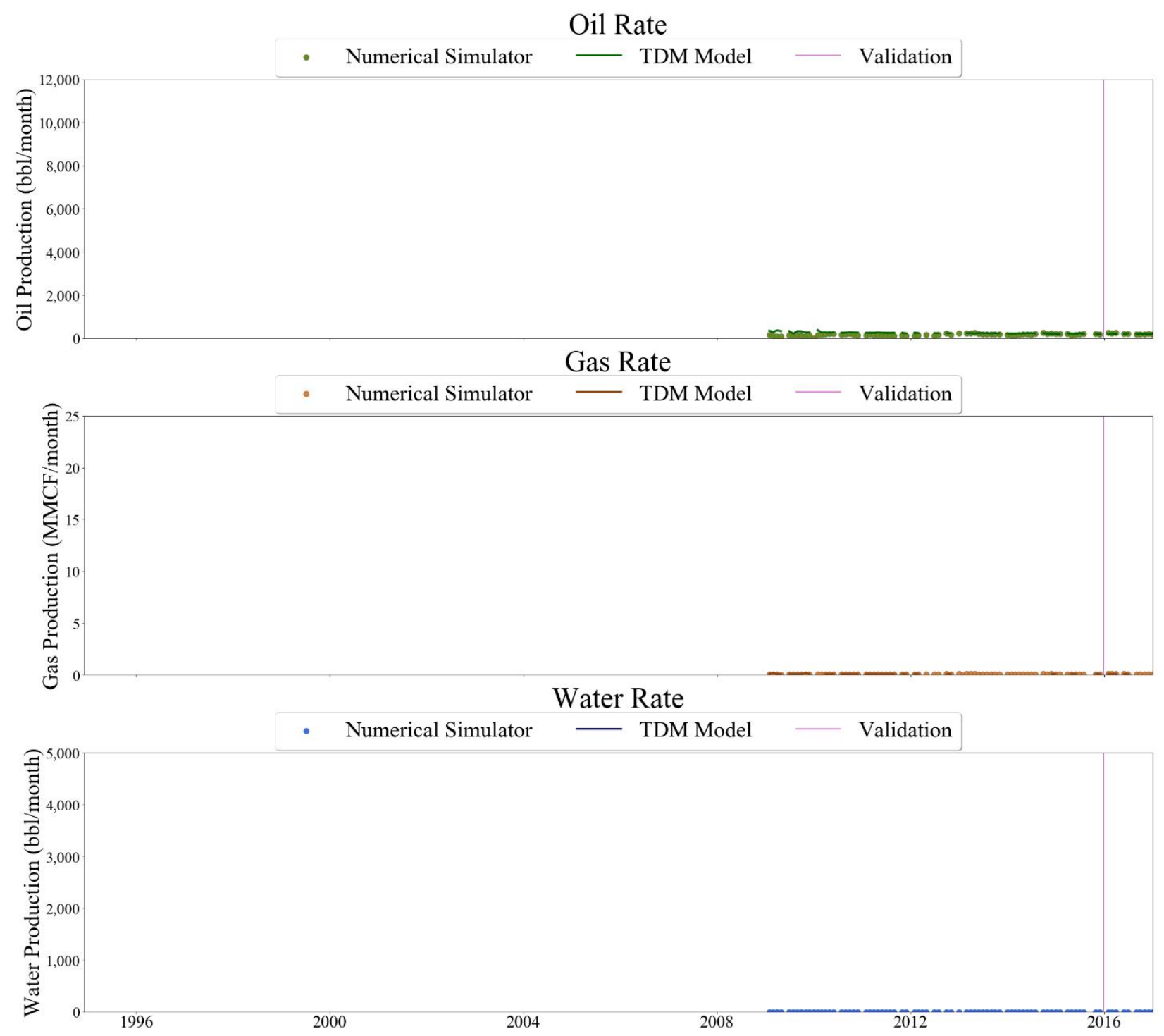

Figure 475 Well-049 oil, gas, and water rate TDM predictions vs actual simulation data plots for 1 Year Blind Validation TDM 


\section{Oil Rate}

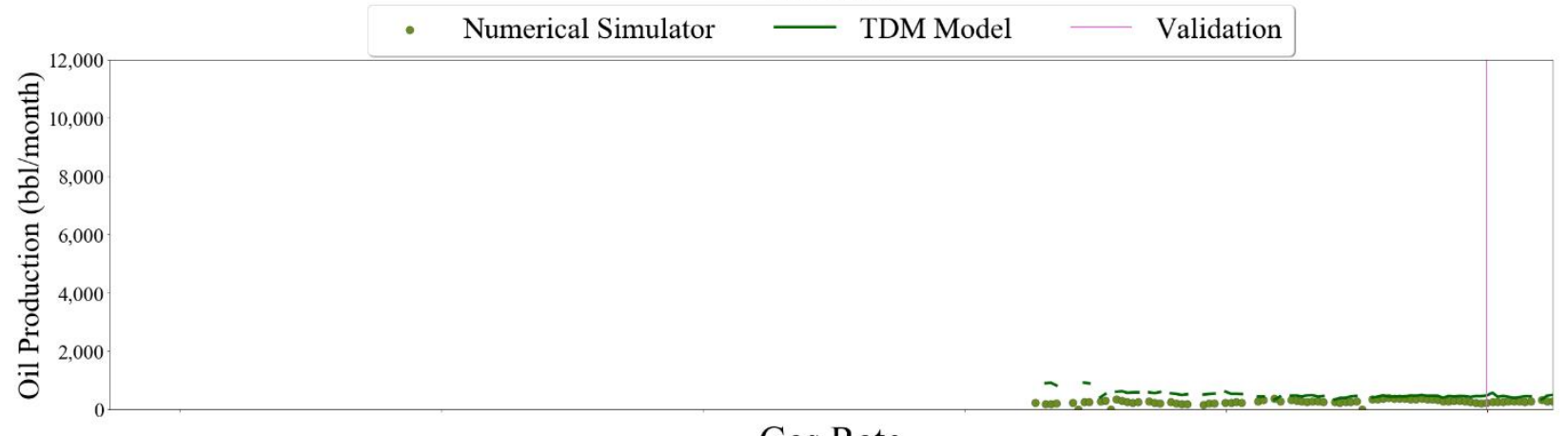

\section{Gas Rate}
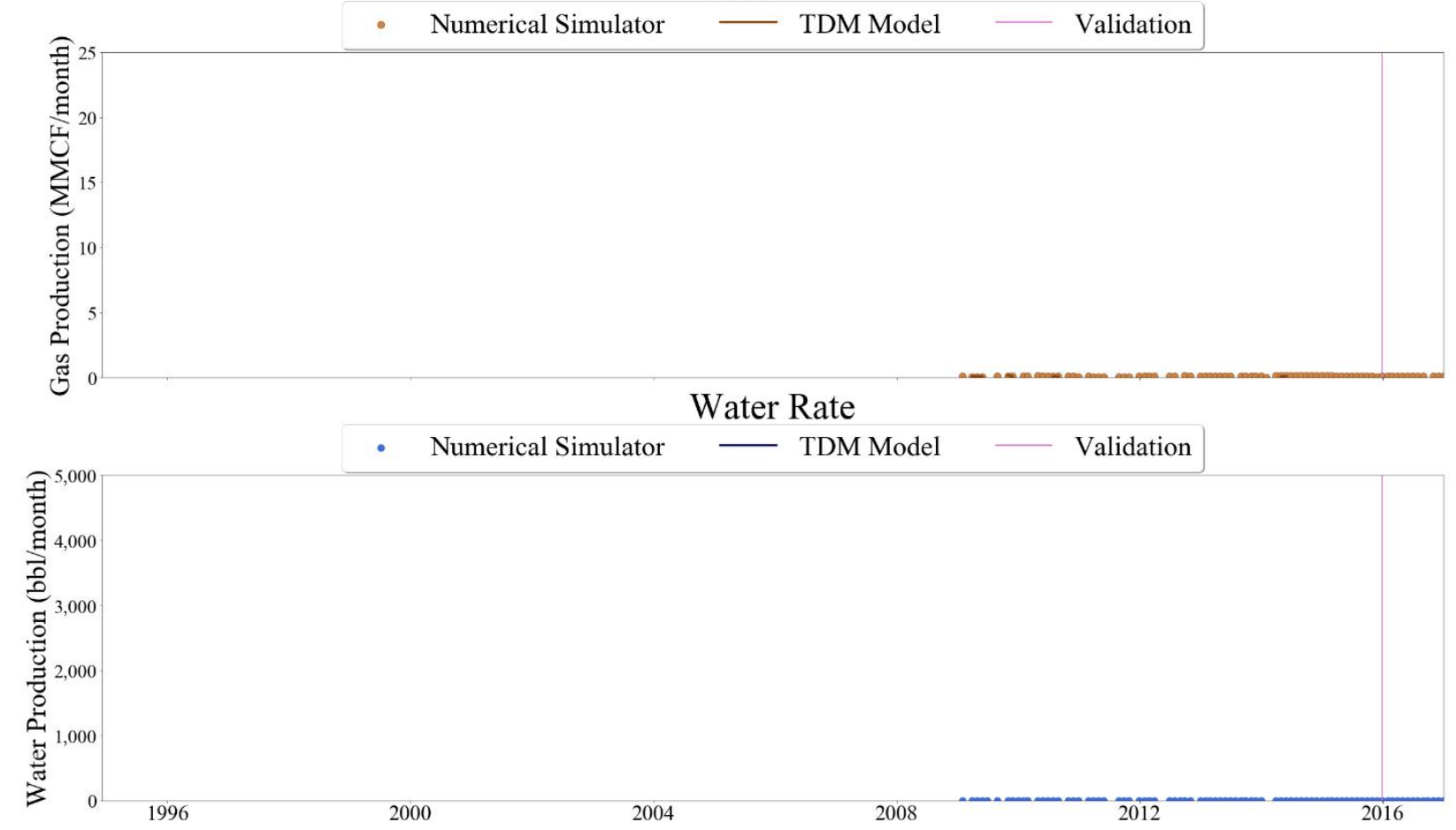

Figure 476 Well-050 oil, gas, and water rate TDM predictions vs actual simulation data plots for 1 Year Blind Validation TDM 

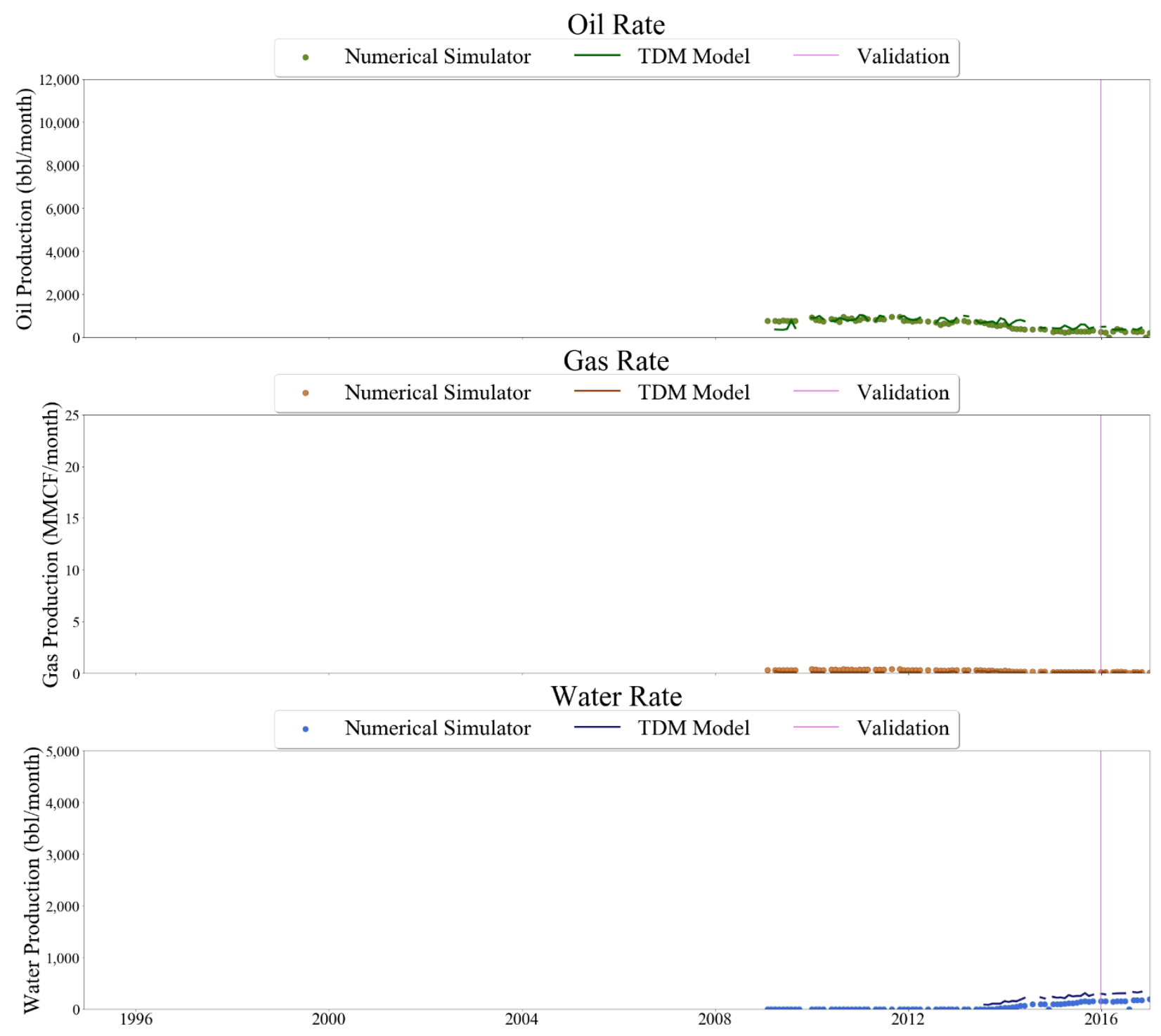

Figure 477 Well-051 oil, gas, and water rate TDM predictions vs actual simulation data plots for 1 Year Blind Validation TDM 


\section{Oil Rate}
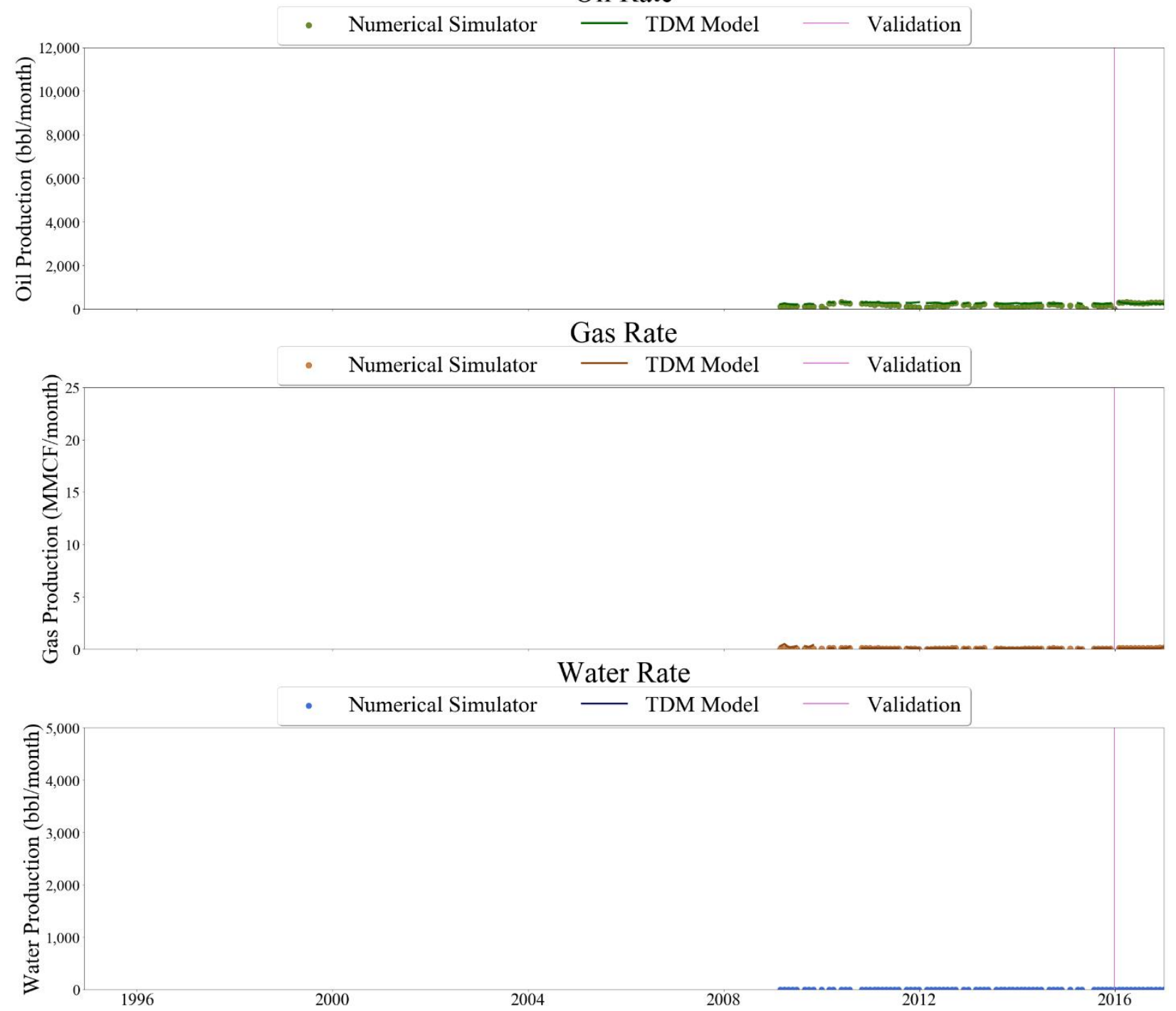

Figure 478 Well-052 oil, gas, and water rate TDM predictions vs actual simulation data plots for 1 Year Blind Validation TDM 


\section{Oil Rate}
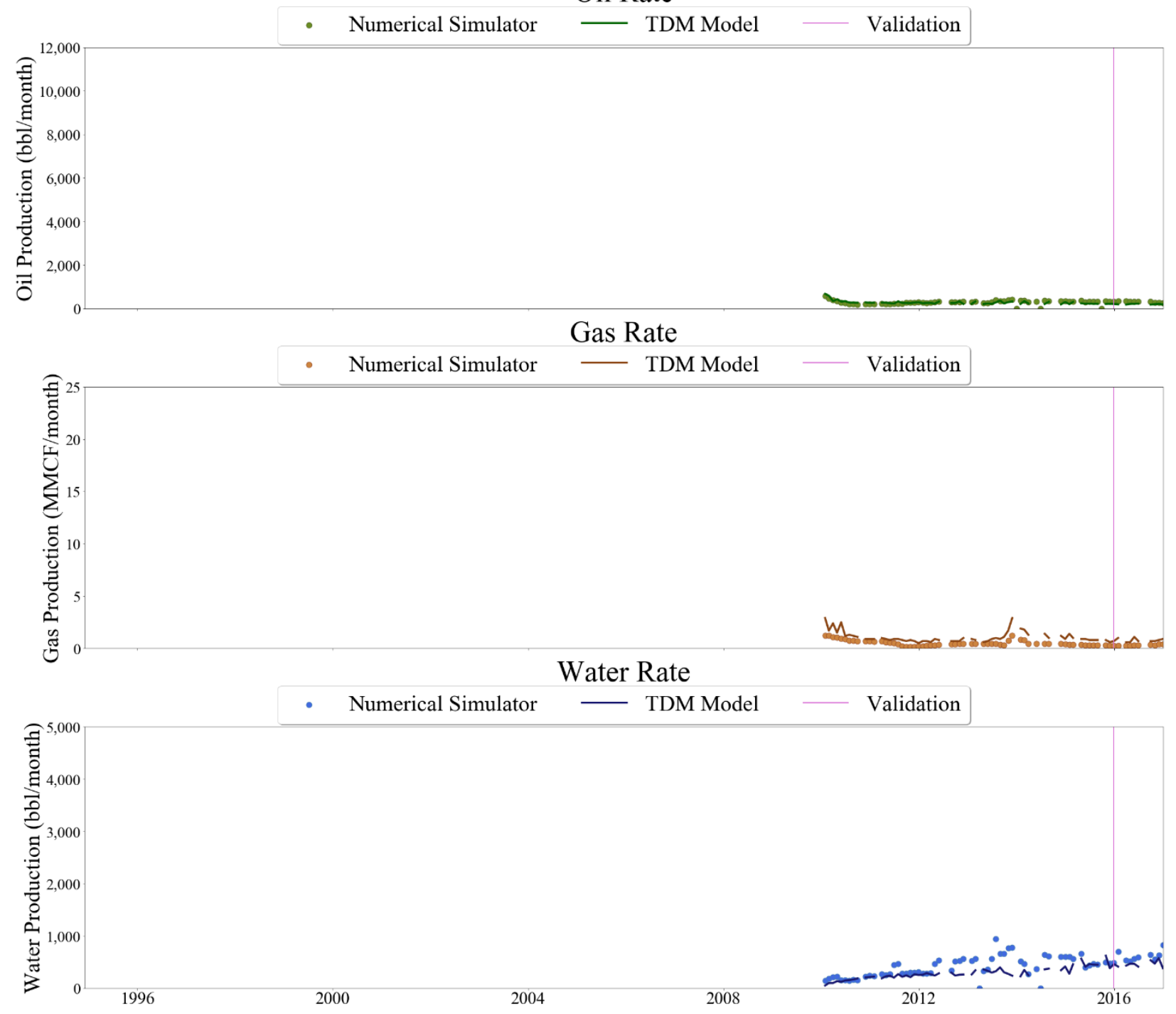

Figure 479 Well-053 oil, gas, and water rate TDM predictions vs actual simulation data plots for 1 Year Blind Validation TDM 


\section{Oil Rate}
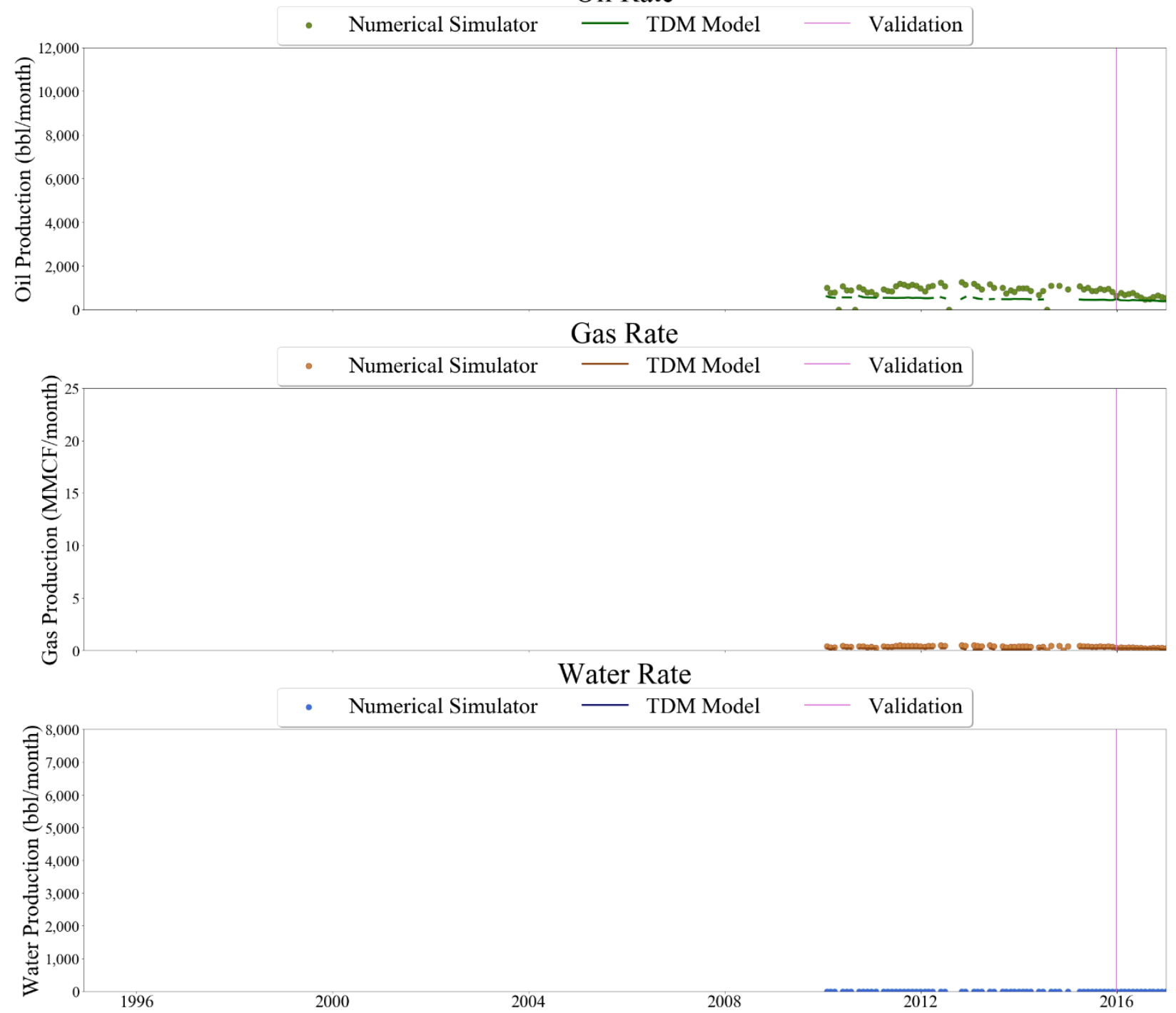

Figure 480 Well-054 oil, gas, and water rate TDM predictions vs actual simulation data plots for 1 Year Blind Validation TDM 


\section{Oil Rate}

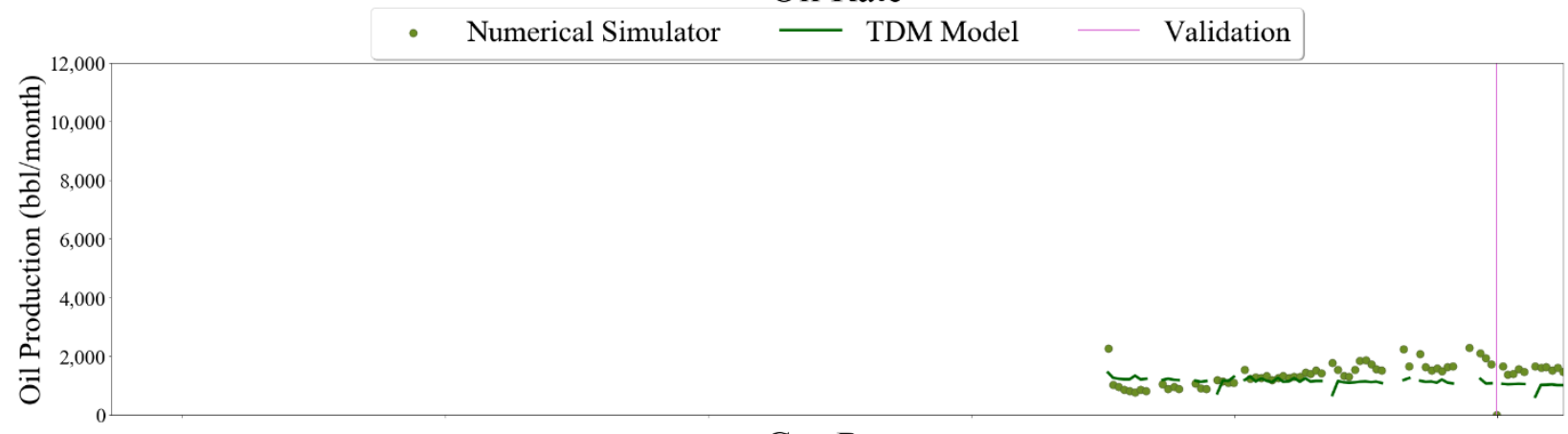

\section{Gas Rate}
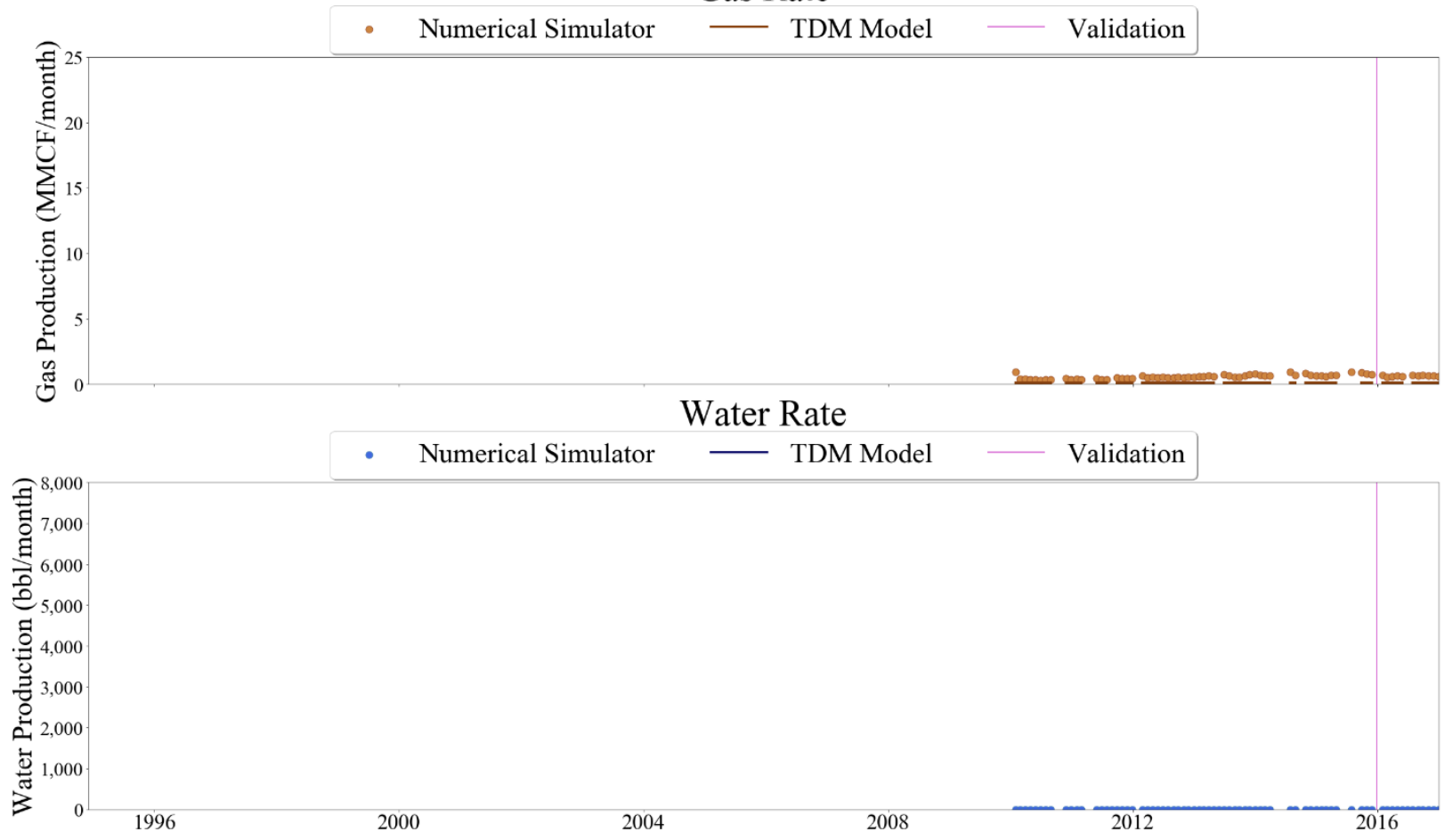

Figure 481 Well-055 oil, gas, and water rate TDM predictions vs actual simulation data plots for 1 Year Blind Validation TDM 


\section{Oil Rate}

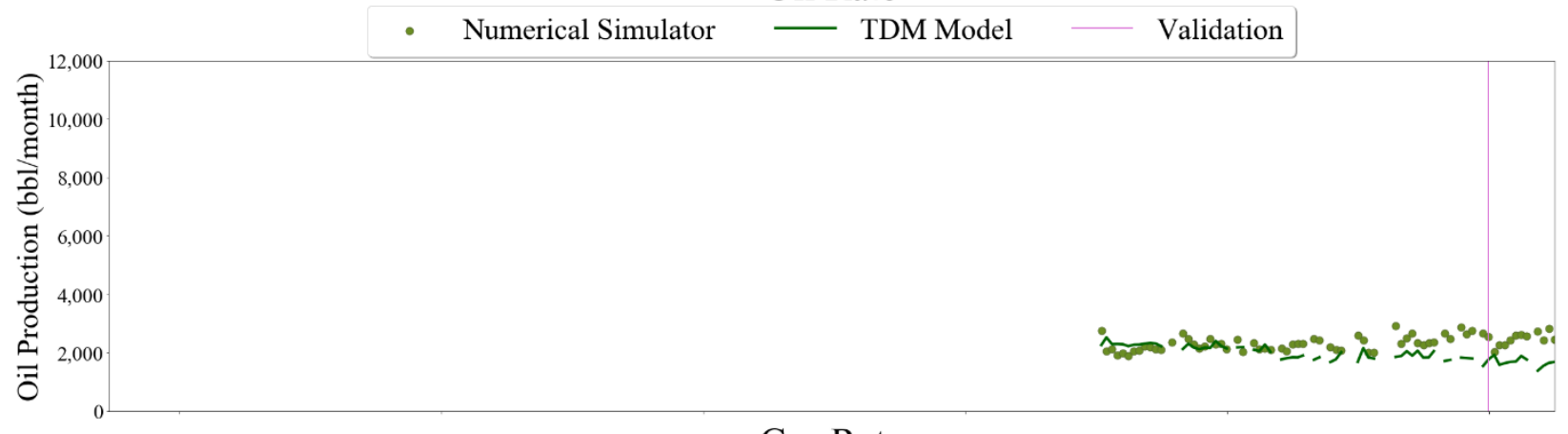

\section{Gas Rate}
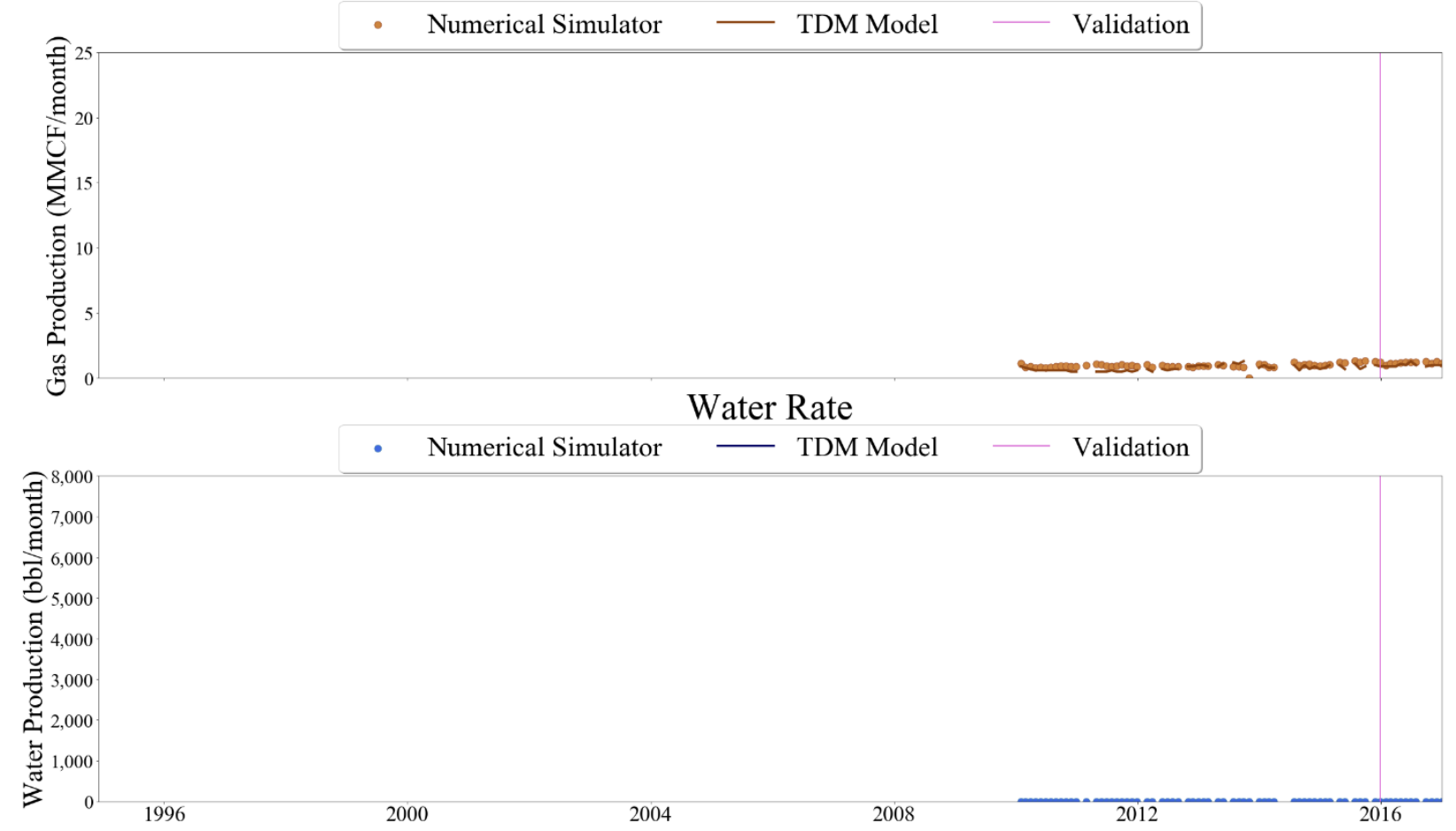

Figure 482 Well-056 oil, gas, and water rate TDM predictions vs actual simulation data plots for 1 Year Blind Validation TDM 


\section{Oil Rate}
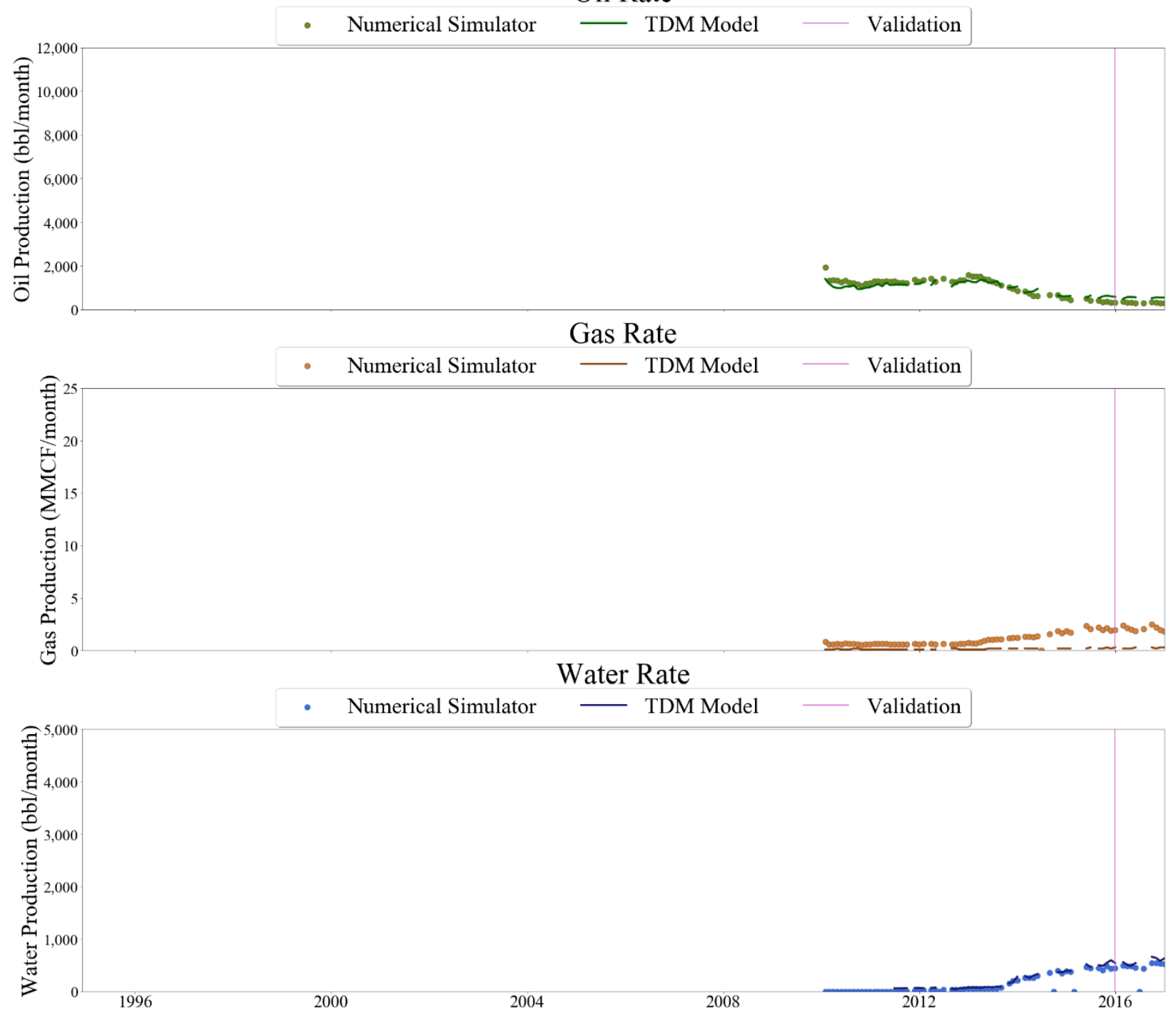

Figure 483 Well-057 oil, gas, and water rate TDM predictions vs actual simulation data plots for 1 Year Blind Validation TDM

\subsubsection{Heat Maps}

Reservoir Pressure (psi) at 2015-12-31
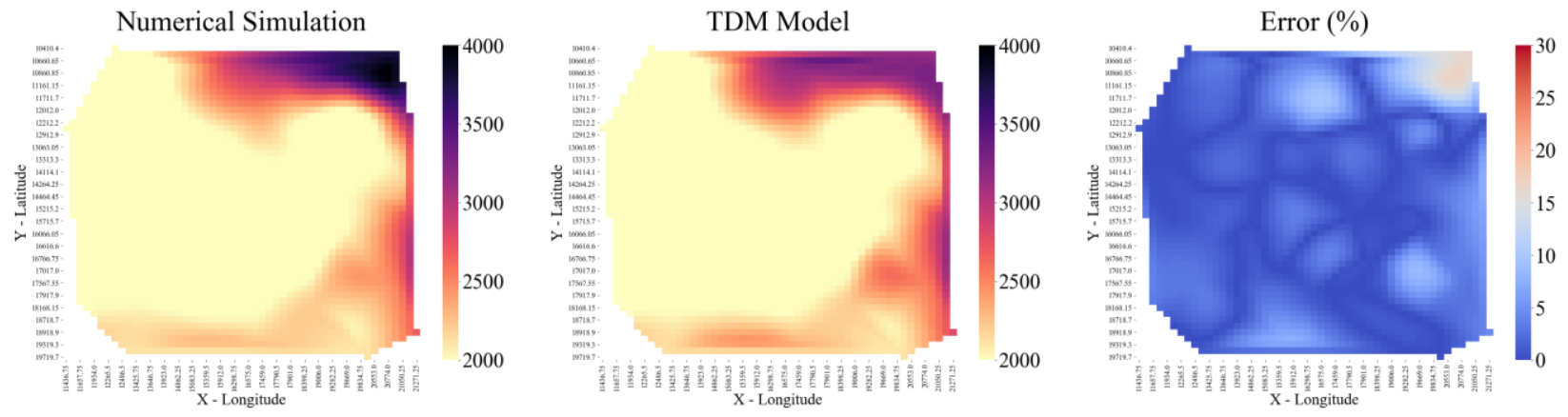

Figure 484 Entire reservoir heat map for reservoir pressure at December 31st, 2015 for 1 Year Blind Validation TDM 
Reservoir Pressure (psi) at 2016-02-29
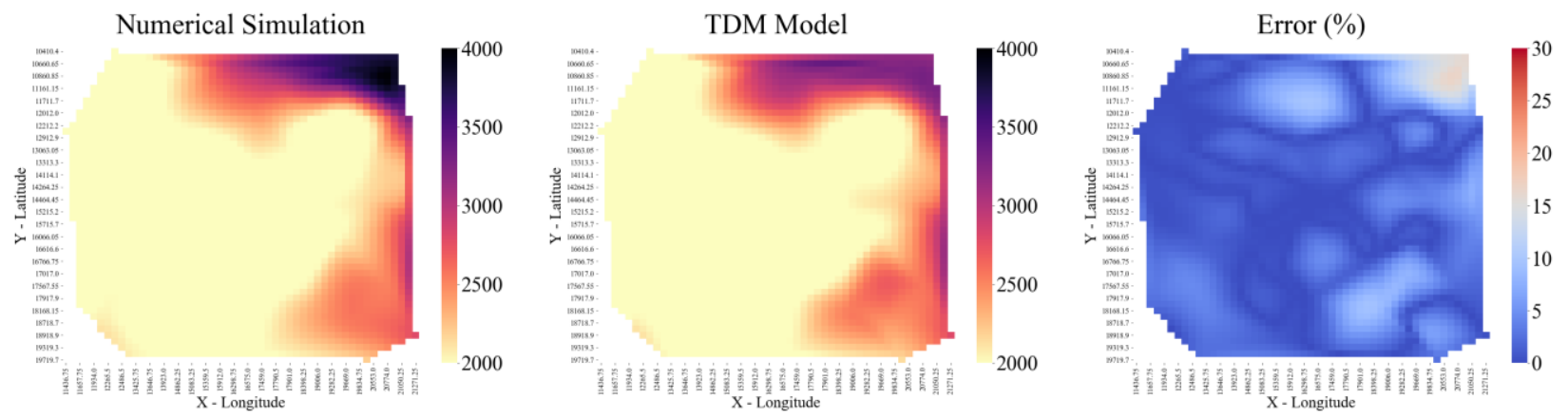

Figure 485 Entire reservoir heat map of reservoir pressure at February 29th, 2016 for 1 Year Blind Validation TDM

Reservoir Pressure (psi) at 2016-04-30
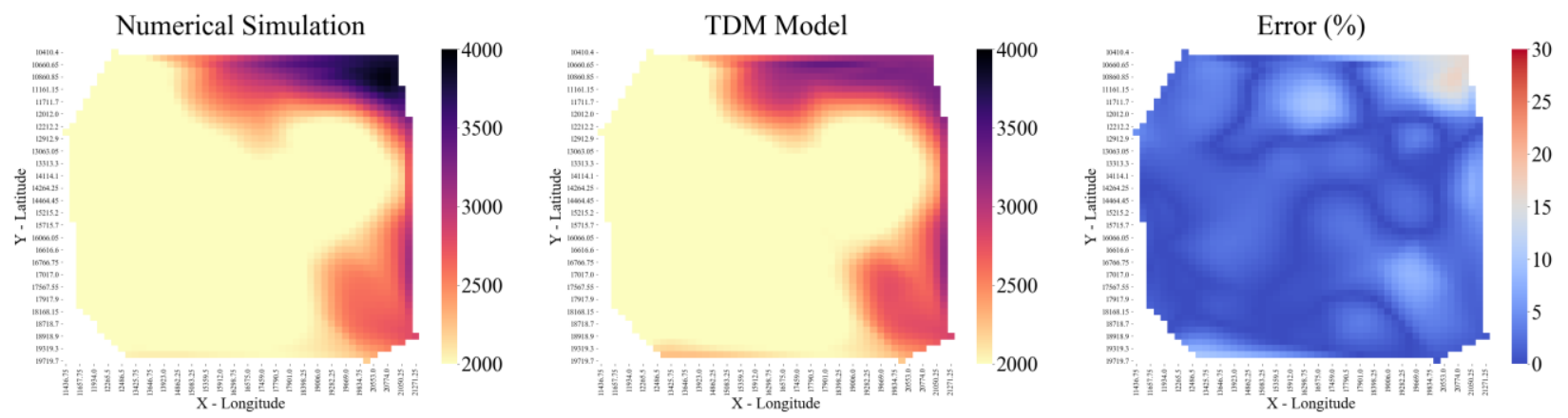

Figure 486 Entire reservoir heat map of reservoir pressure at April 30th, 2016 for 1 Year Blind Validation TDM

Reservoir Pressure (psi) at 2016-06-30
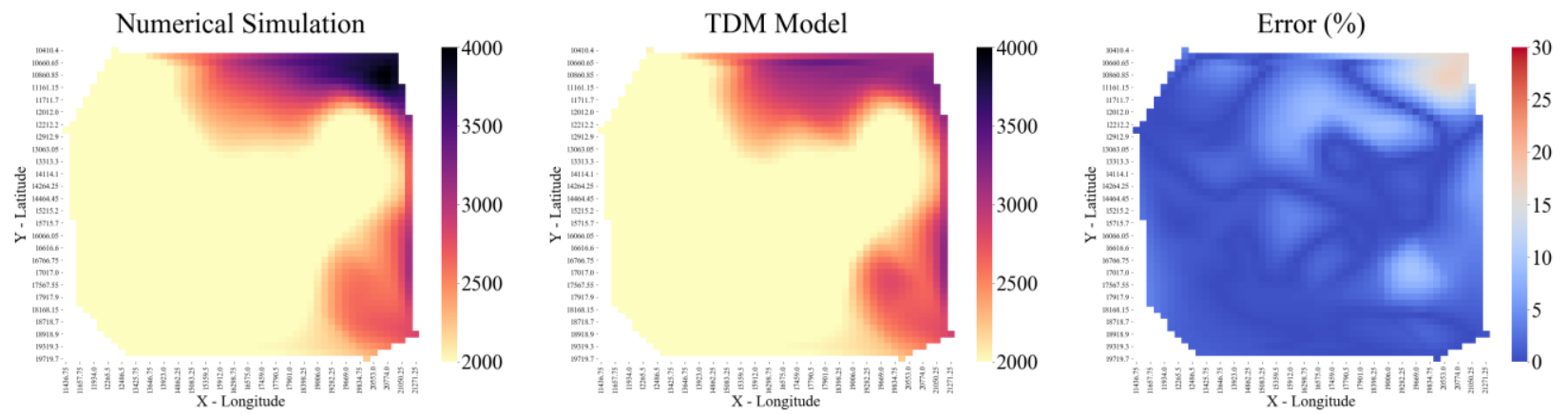

Figure 487 Entire reservoir heat map of reservoir pressure at June 30th, 2016 for 1 Year Blind Validation TDM 
Reservoir Pressure (psi) at 2016-08-31
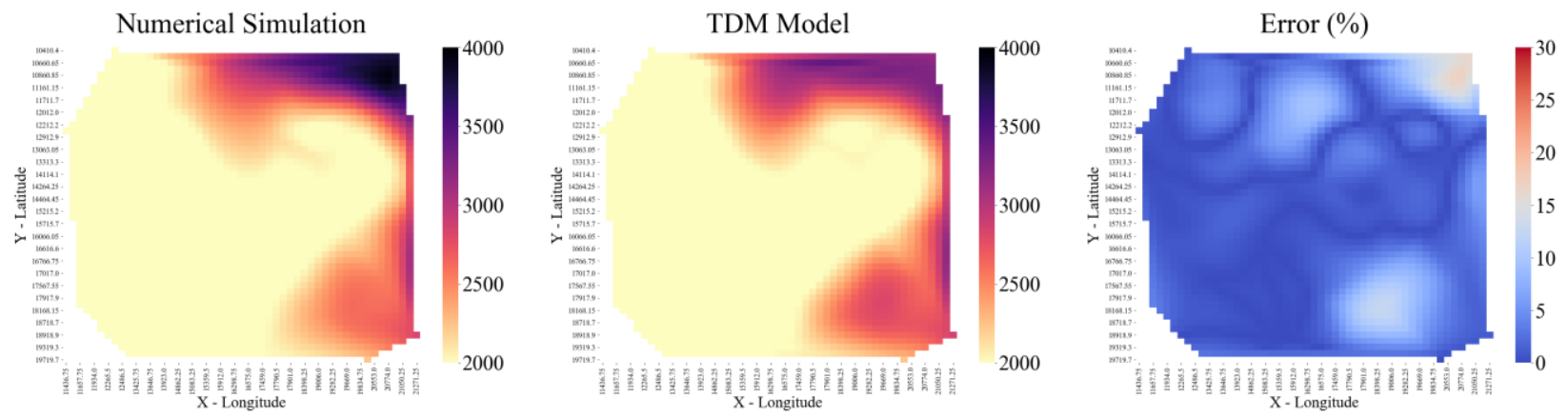

Figure 488 Entire reservoir heat map of reservoir pressure at August 31st, 2016 for 1 Year Blind Validation TDM

Reservoir Pressure (psi) at 2016-10-31
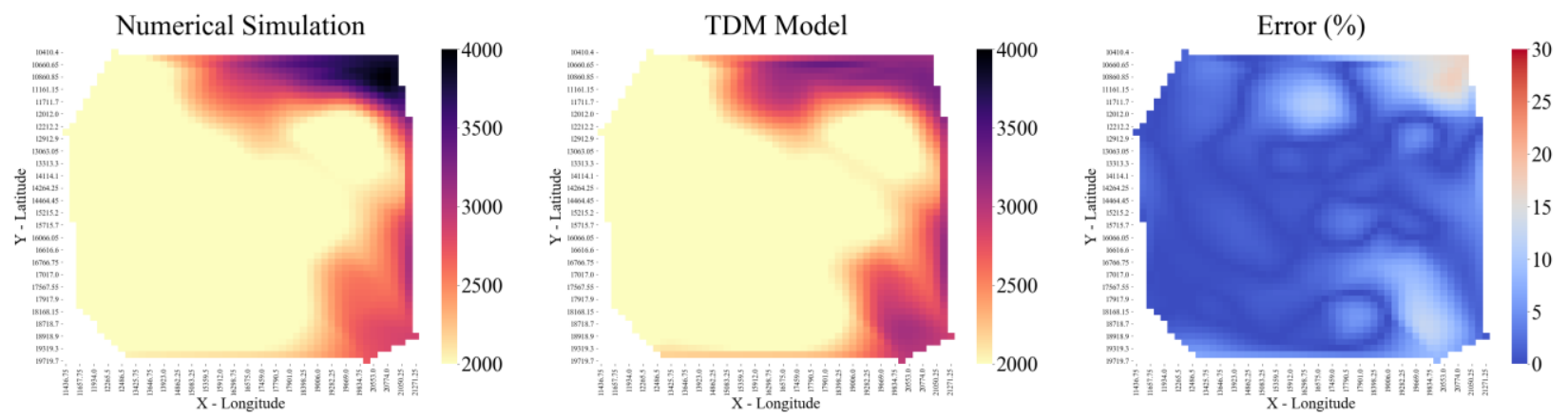

Figure 489 Entire reservoir heat map of reservoir pressure at October 31st, 2016 for 1 Year Blind Validation TDM

Reservoir Pressure (psi) at 2016-12-31
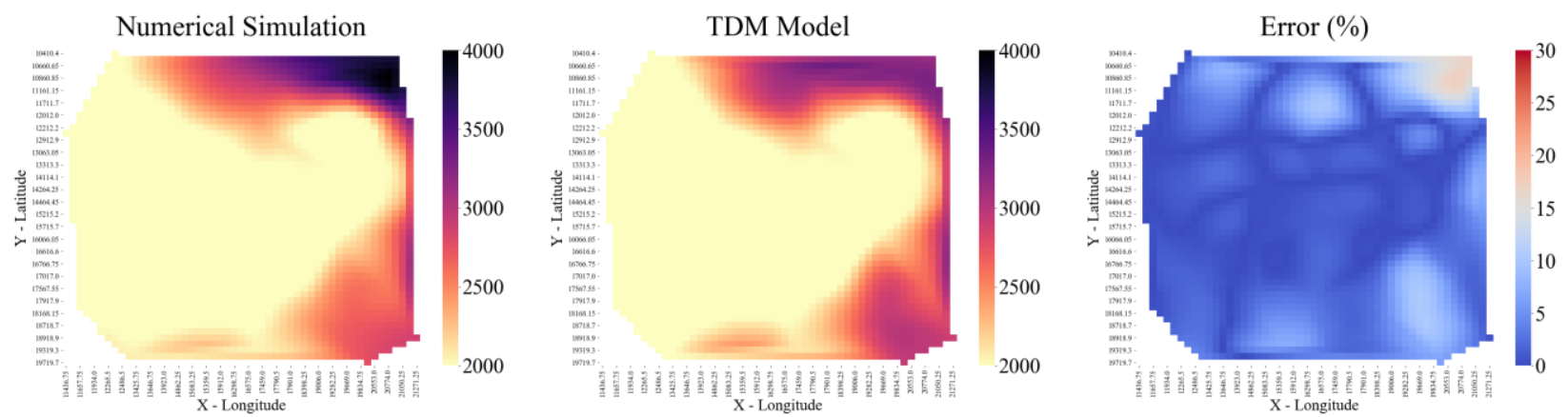

Figure 490 Entire reservoir heat map of reservoir pressure at December 31st, 2016 for 1 Year Blind Validation TDM 
Water Saturation (\%) at 2015-12-31
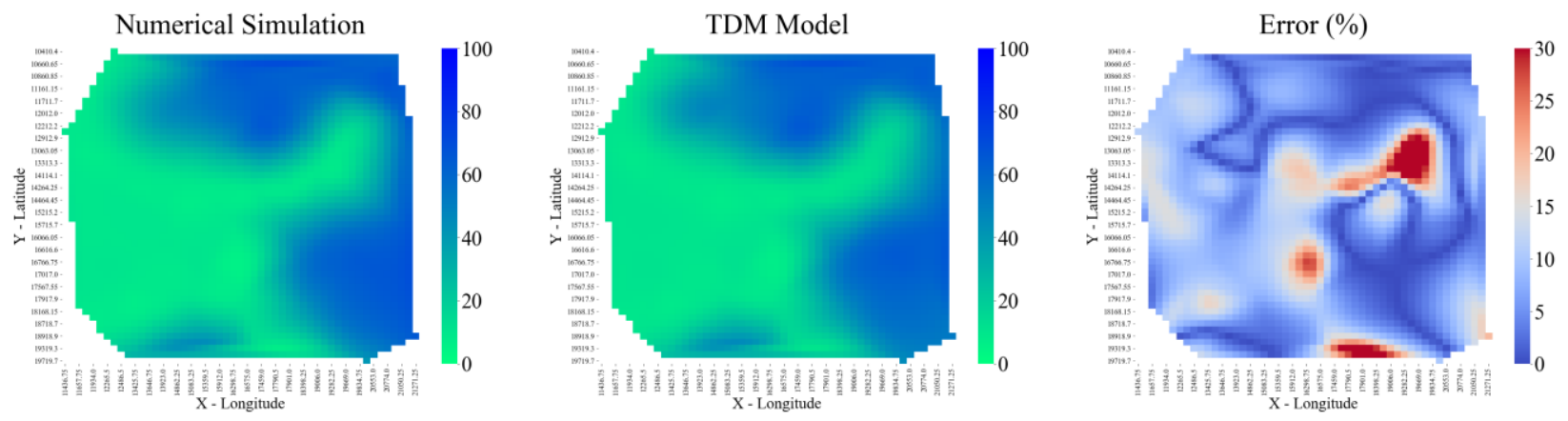

Figure 491 Entire reservoir heat map for water saturation at December 31st, 2015 for 1 Year Blind Validation TDM

Water Saturation (\%) at 2016-02-29
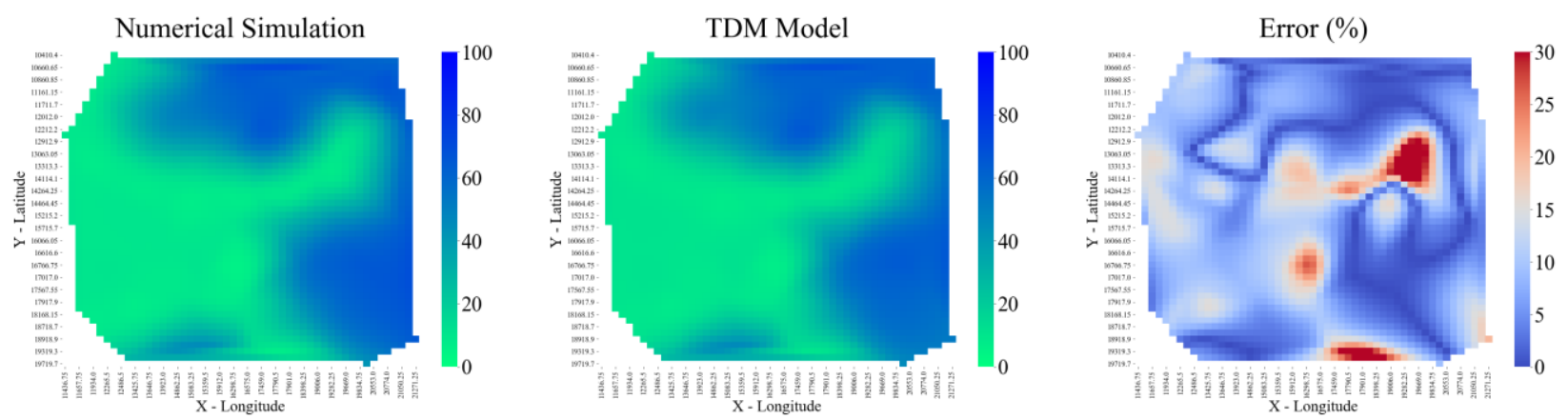

Figure 492 Entire reservoir heat map for water saturation February 29th, 2016 for 1 Year Blind Validation TDM

Water Saturation (\%) at 2016-04-30
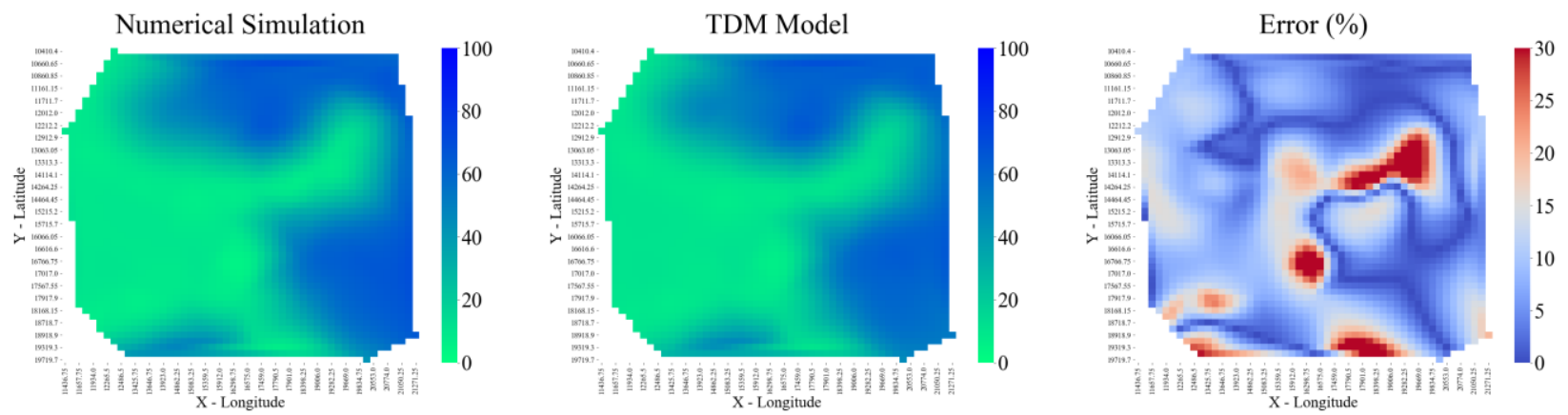

Figure 493 Entire reservoir heat map for water saturation April 30th, 2016 for 1 Year Blind Validation TDM 
Water Saturation (\%) at 2016-06-30
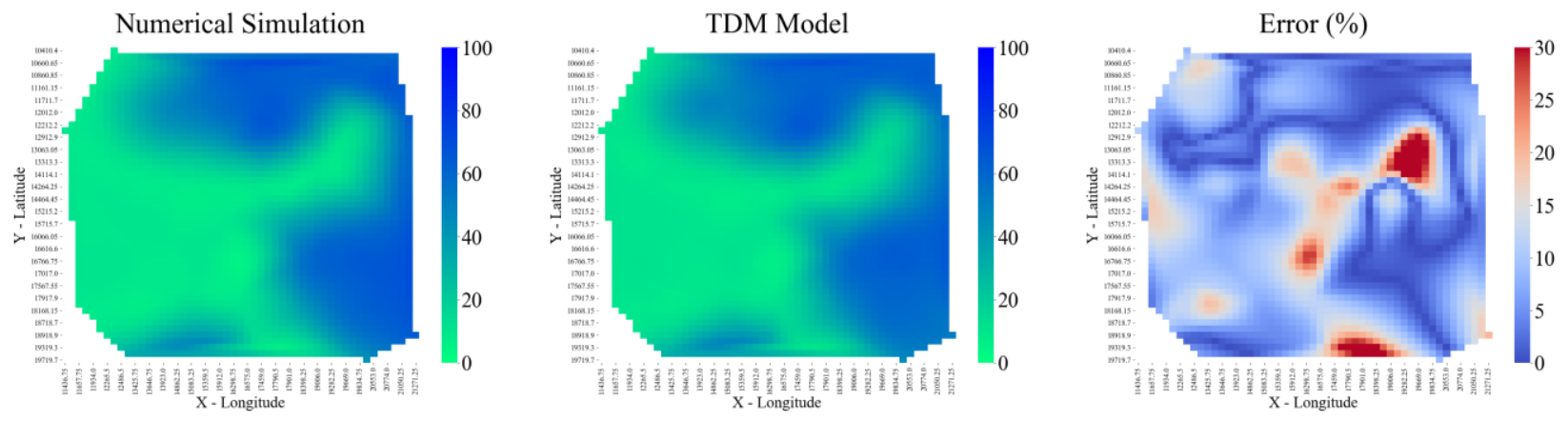

Figure 494 Entire reservoir heat map for water saturation at June 30th, 2016 for 1 Year Blind Validation TDM

Water Saturation (\%) at 2016-08-31
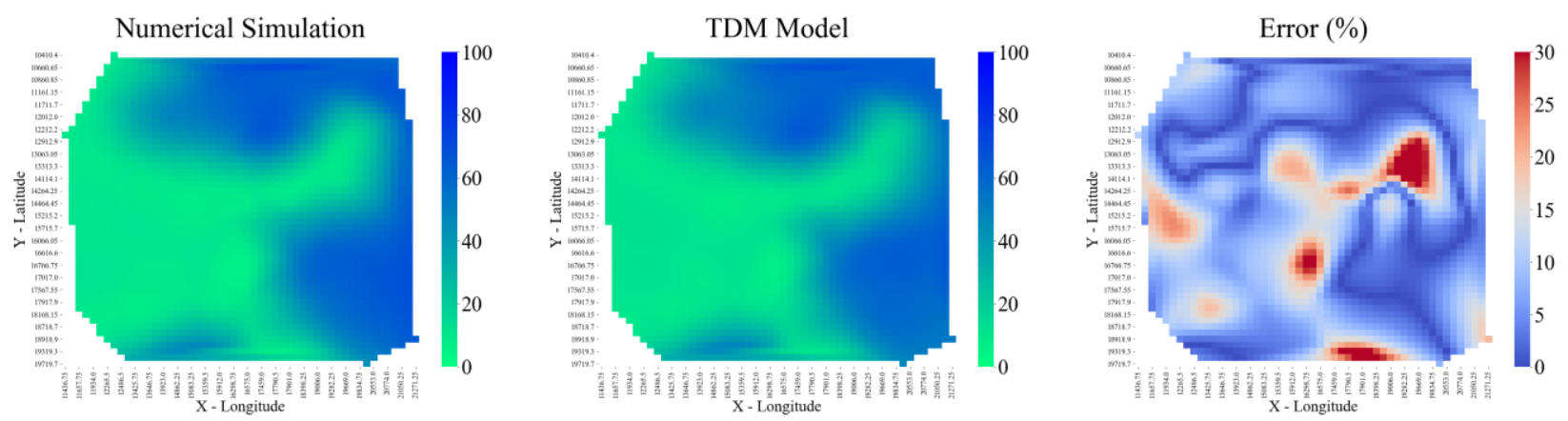

Figure 495 Entire reservoir heat map for water saturation at August 31st, 2016 for 1 Year Blind Validation TDM Water Saturation (\%) at 2016-10-31
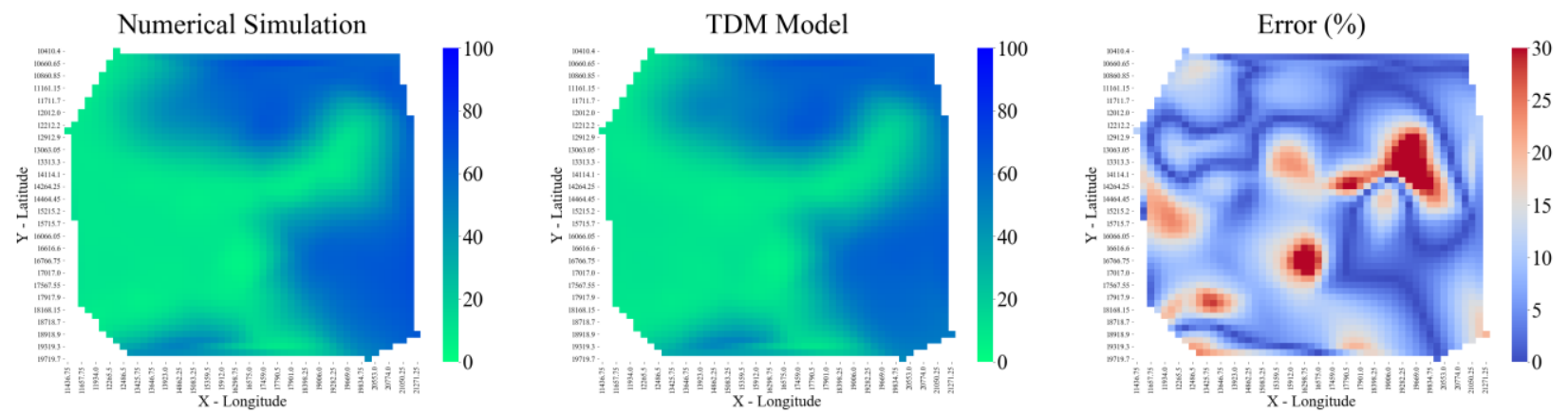

Figure 496 Entire reservoir heat map for water saturation at October 31st, 2016 for 1 Year Blind Validation TDM 
Water Saturation (\%) at 2016-12-31
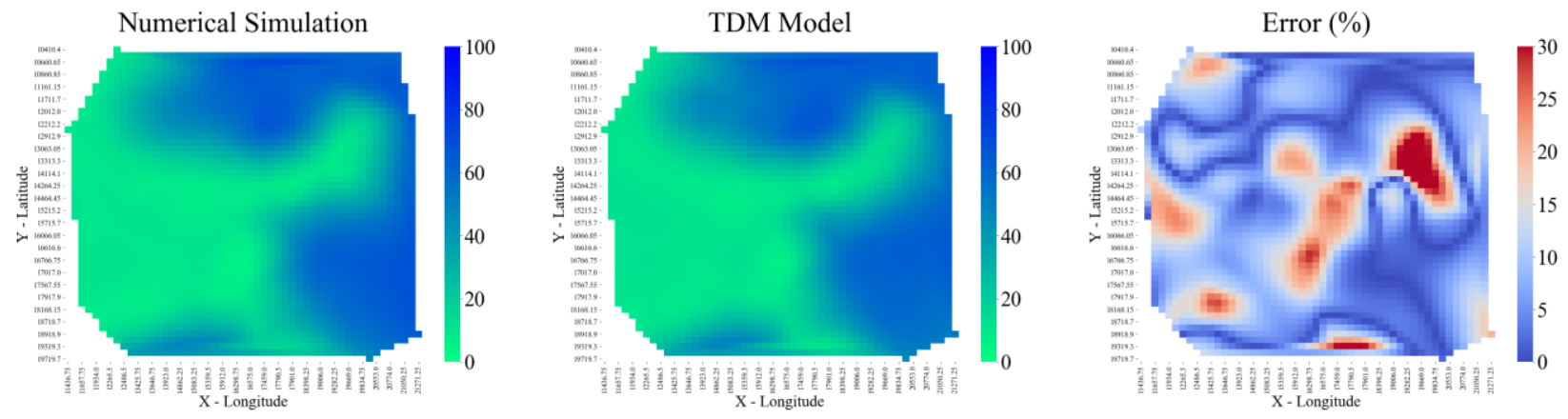

Figure 497 Entire reservoir heat map for water saturation at December 31st, 2016 for 1 Year Blind Validation TDM

\subsubsection{Multi-Random Partitioning-2 Year Blind Validation}

\subsubsection{Individual Well Production Plots}

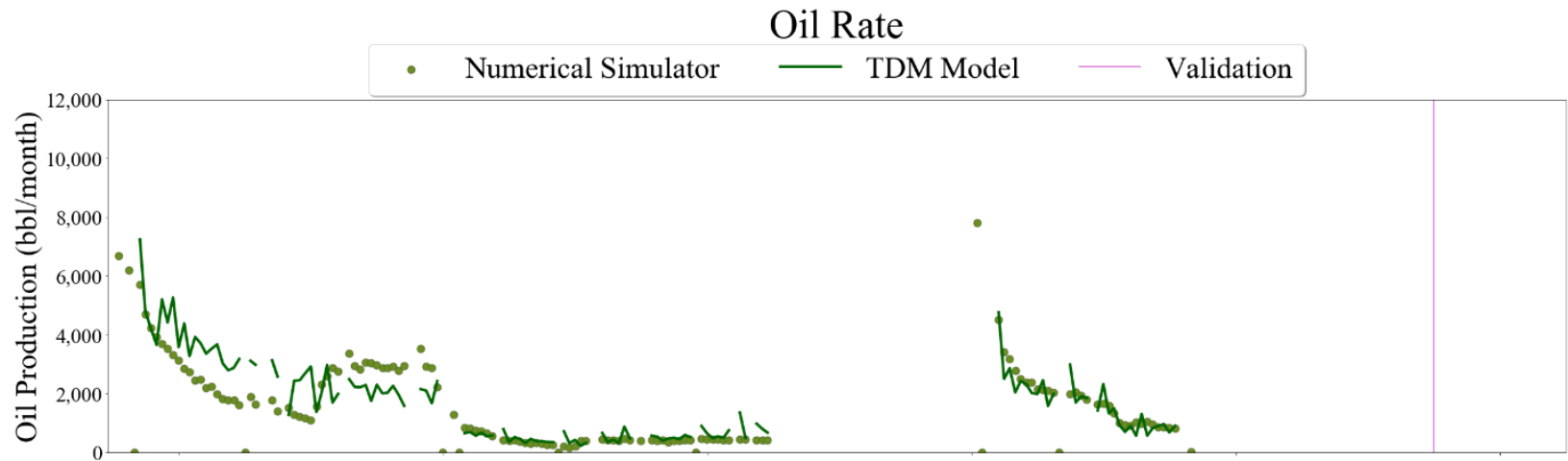

Gas Rate
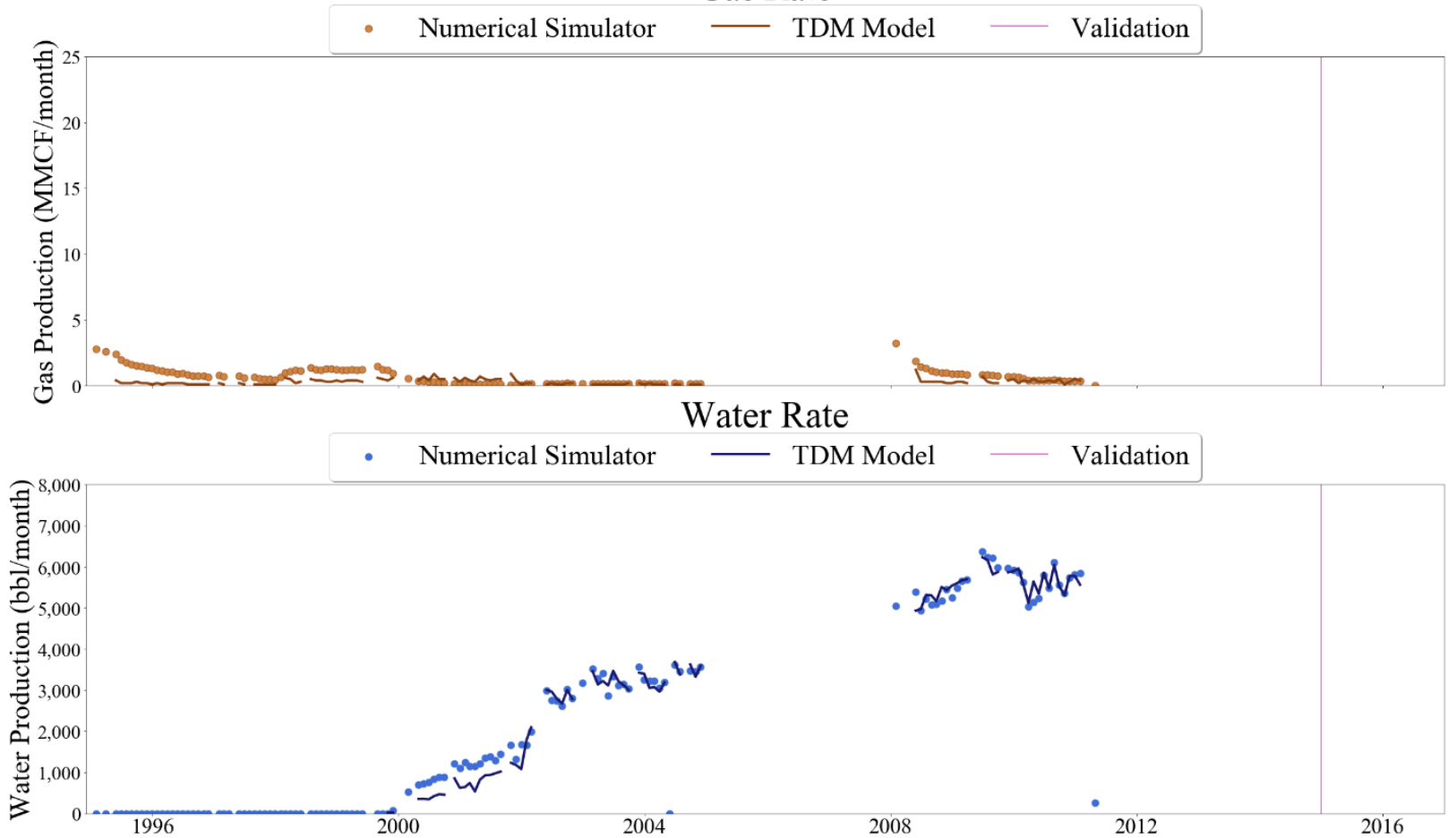

Figure 498 Well-001 oil, gas, and water rate TDM predictions vs actual simulation data plots for 2 Year Blind Validation TDM 

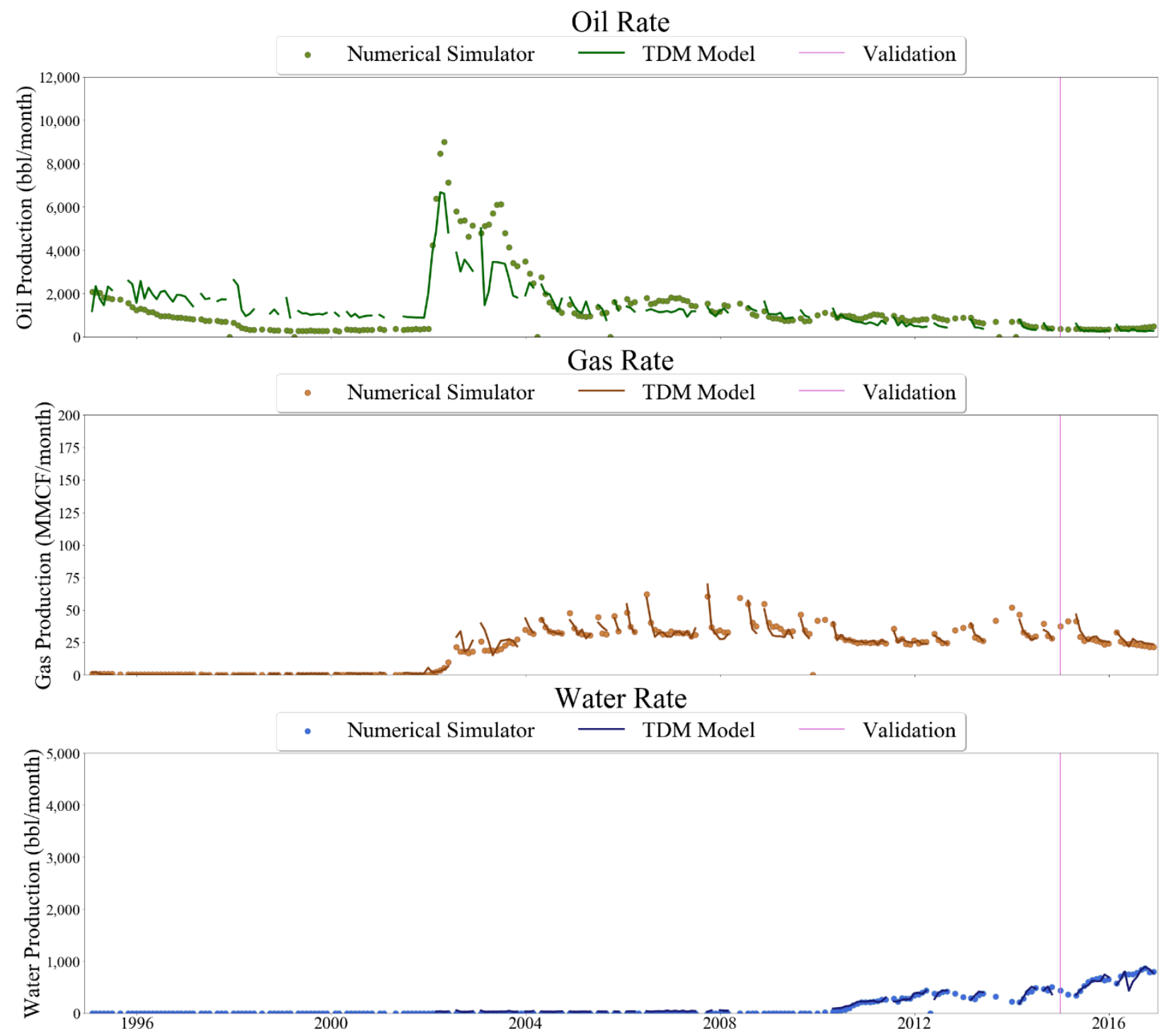

Figure 499 Well-002 oil, gas, and water rate TDM predictions vs actual simulation data plots for 2 Year Blind Validation TDM 


\section{Oil Rate}

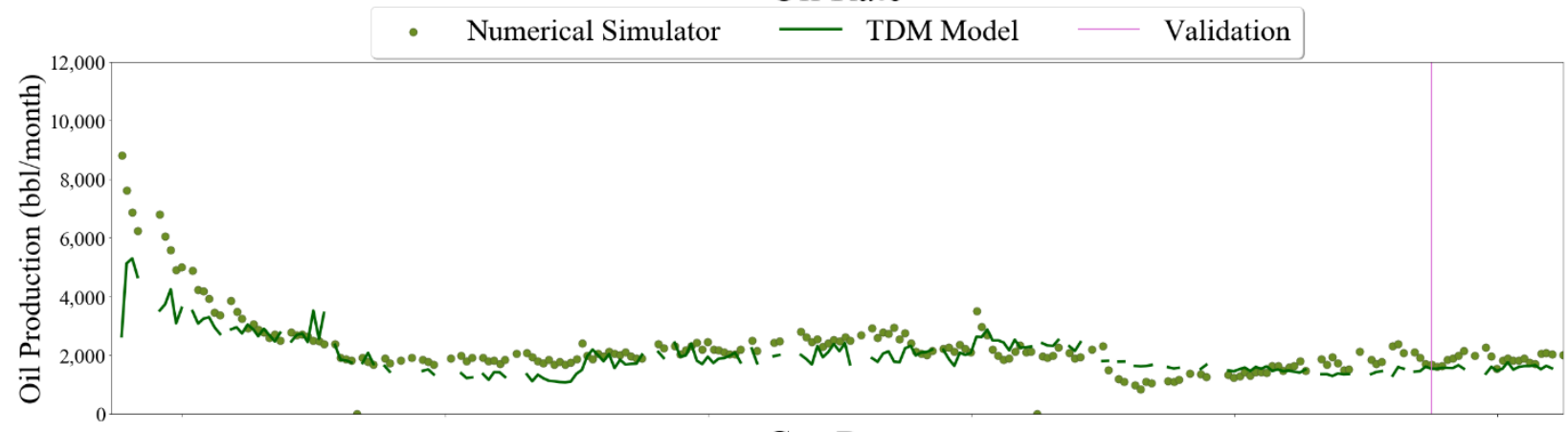

\section{Gas Rate}

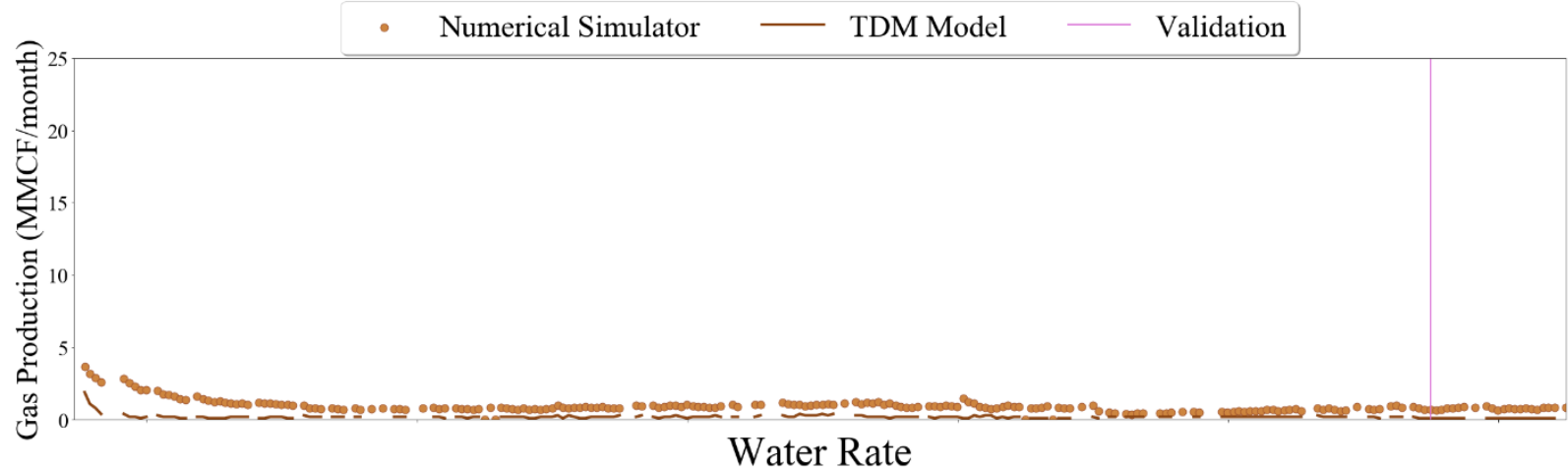

Water Rate

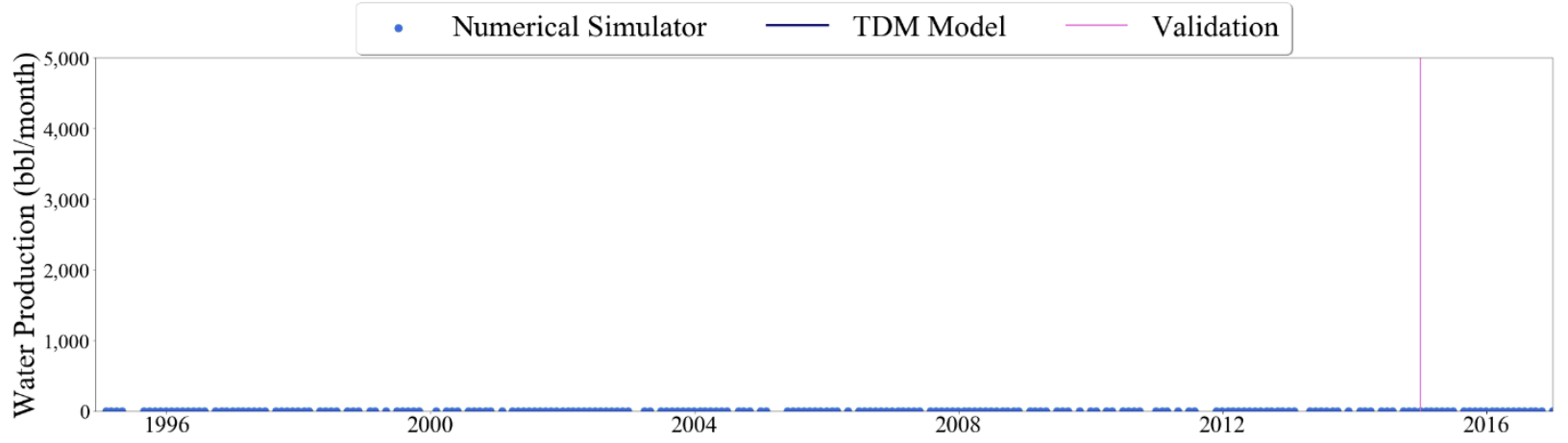

Figure 500 Well-003 oil, gas, and water rate TDM predictions vs actual simulation data plots for 2 Year Blind Validation TDM 


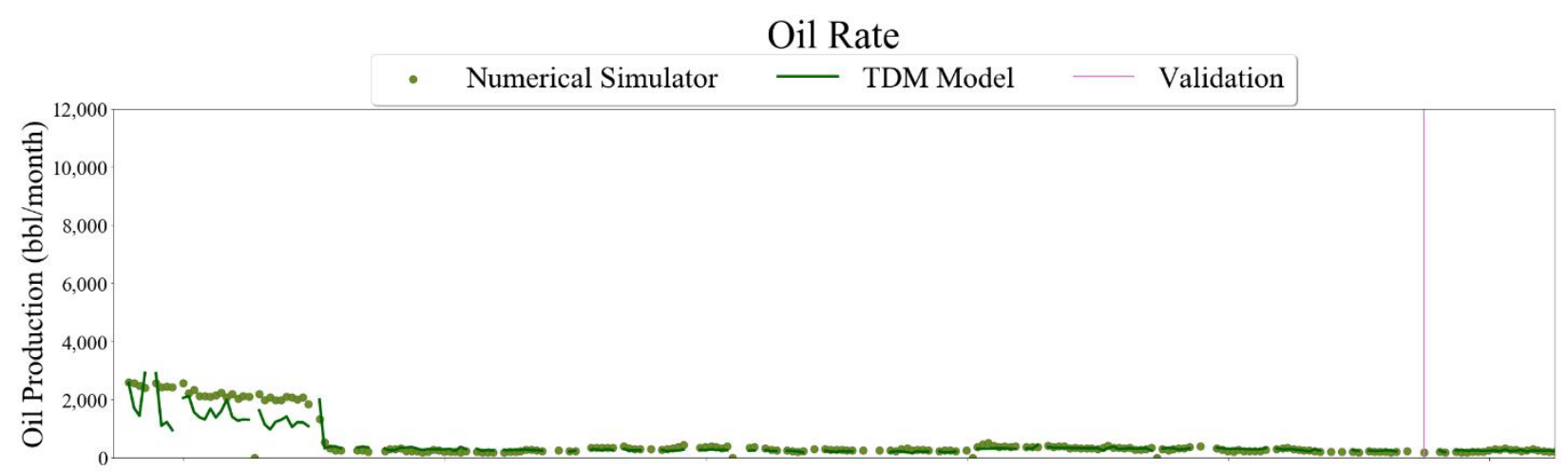

Gas Rate
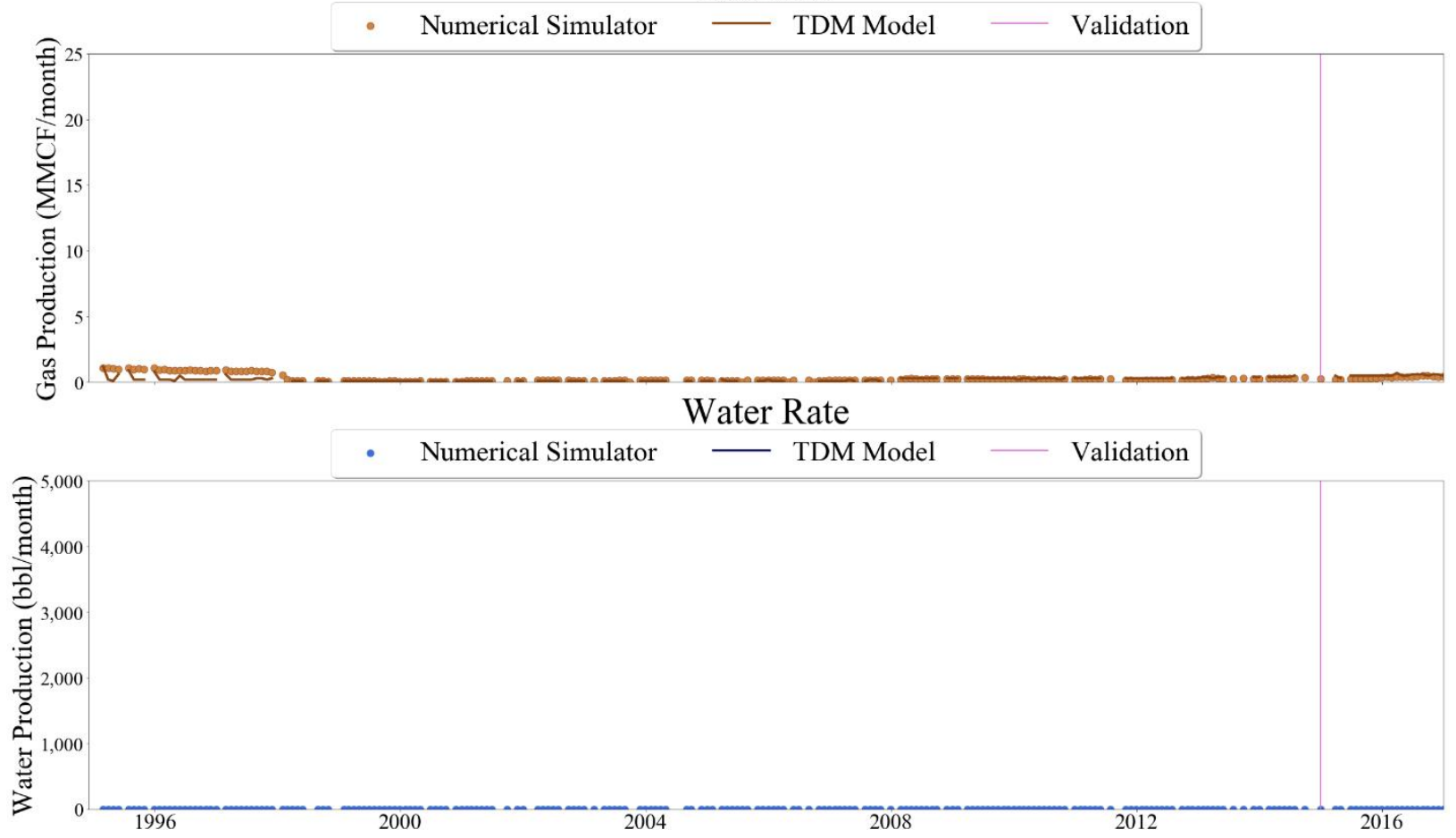

Figure 501 Well-004 oil, gas, and water rate TDM predictions vs actual simulation data plots for 2 Year Blind Validation TDM 


\section{Oil Rate}

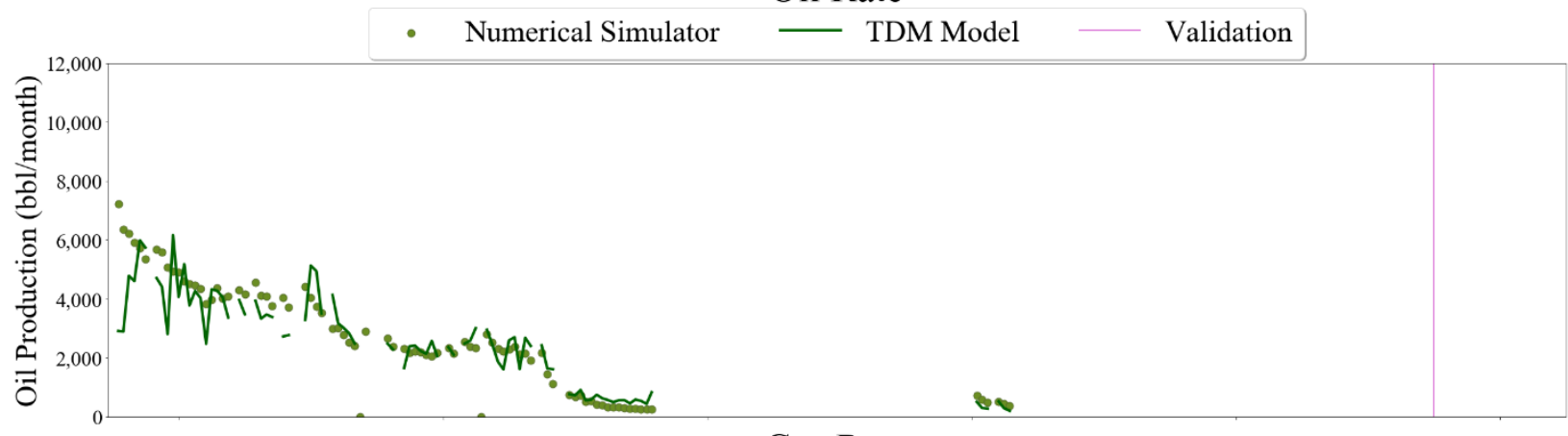

Gas Rate
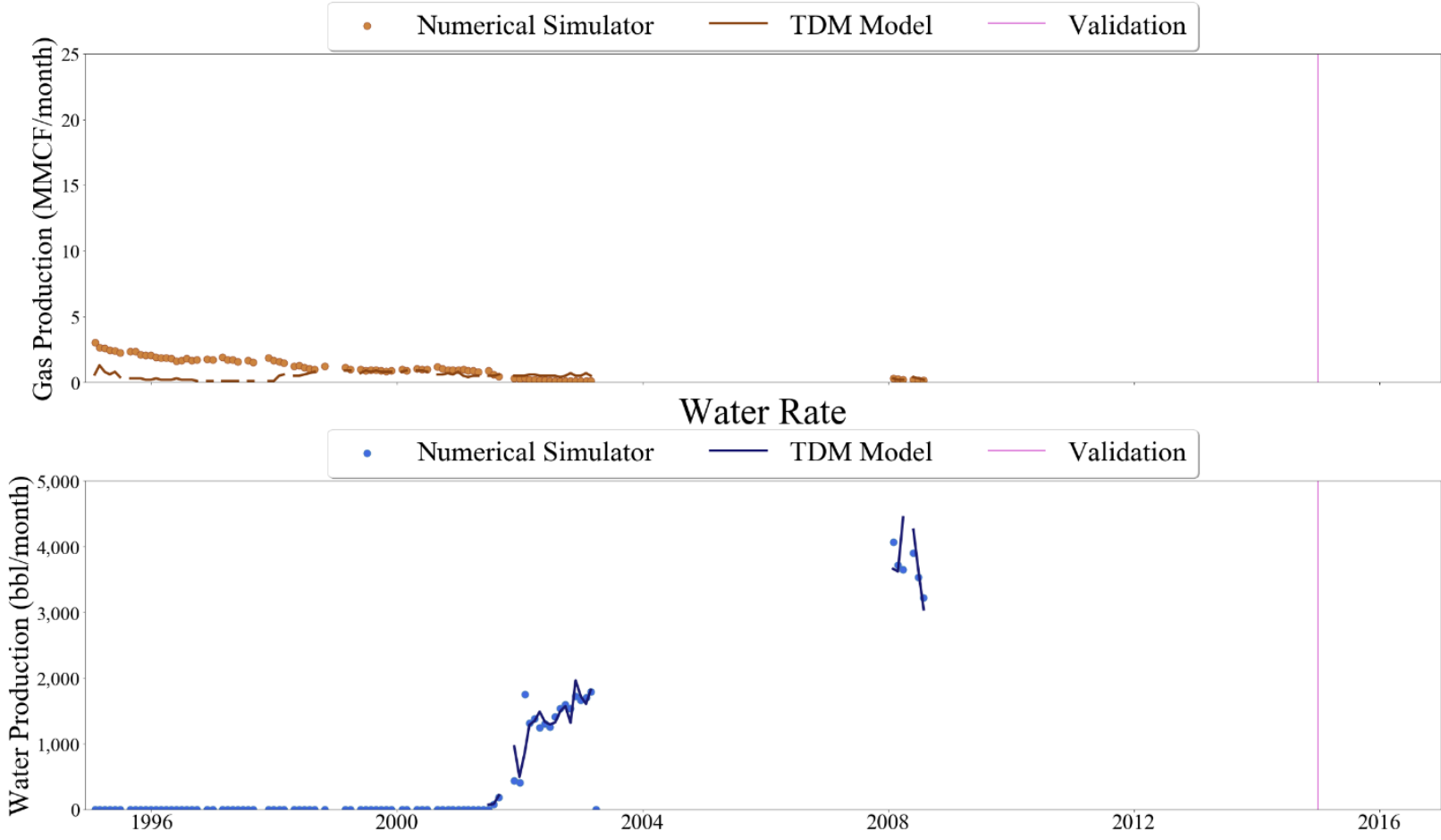

Figure 502 Well-005 oil, gas, and water rate TDM predictions vs actual simulation data plots for 2 Year Blind Validation TDM 

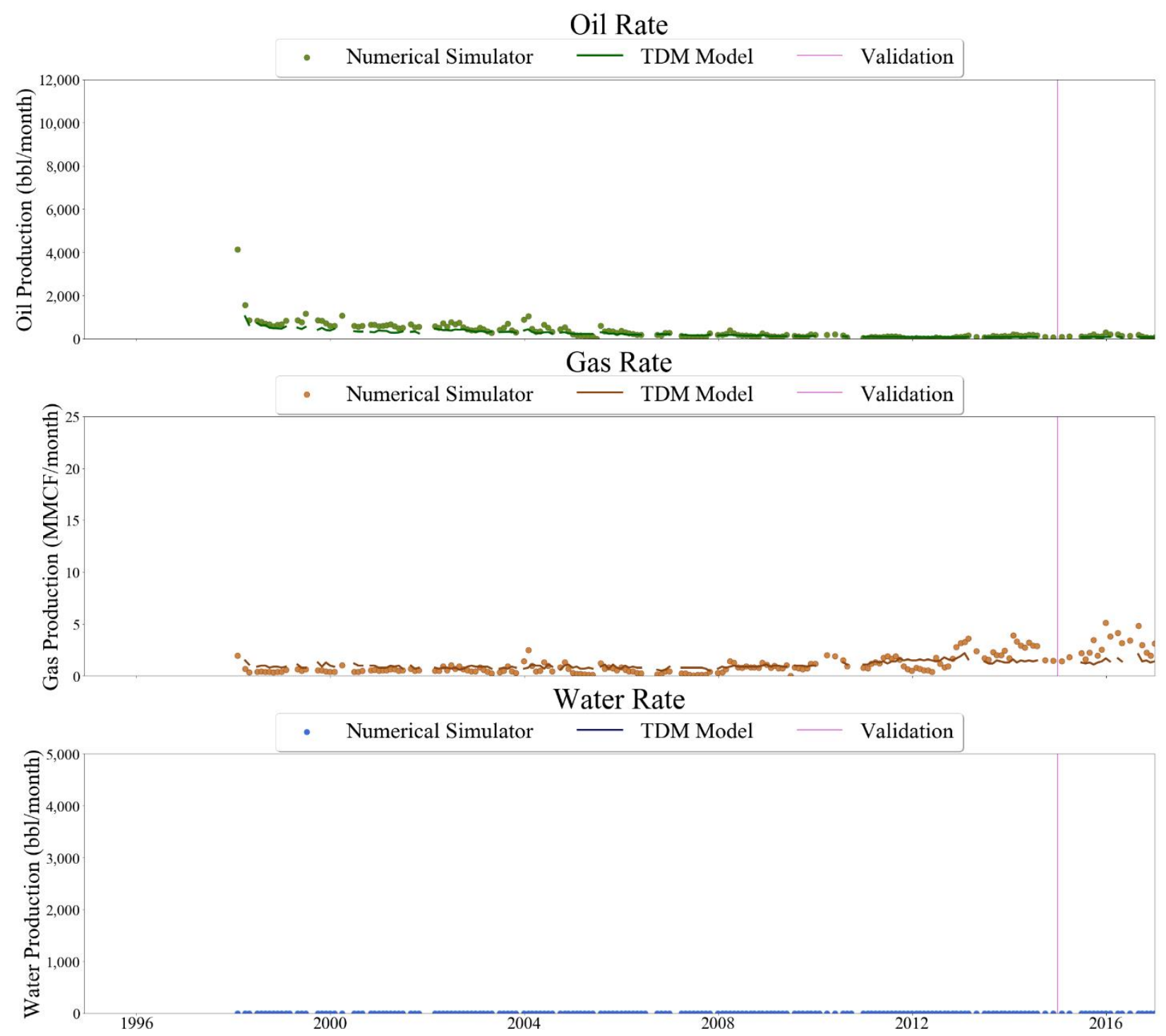

Figure 503 Well-006 oil, gas, and water rate TDM predictions vs actual simulation data plots for 2 Year Blind Validation TDM 


\section{Oil Rate}
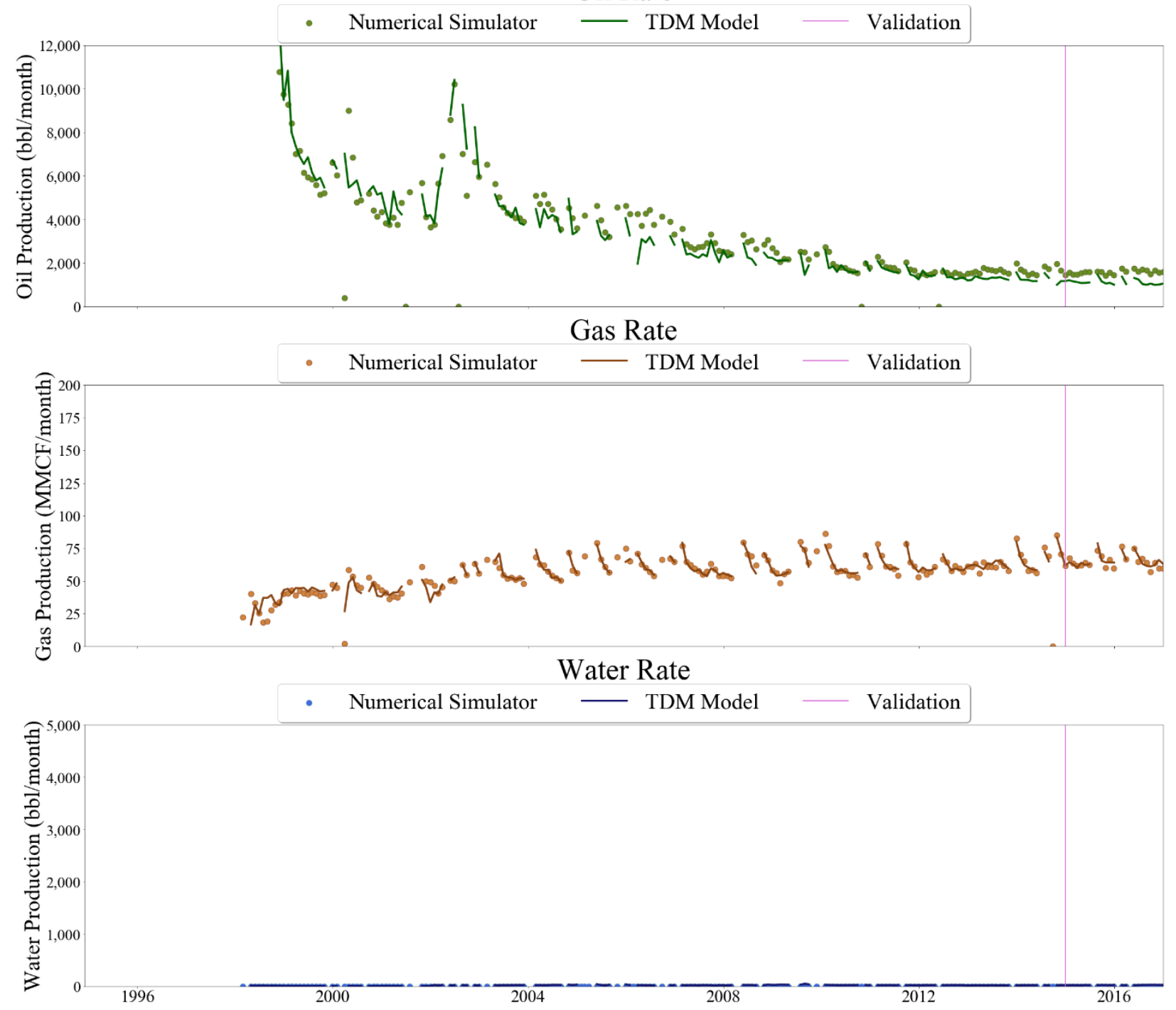

Figure 504 Well-007 oil, gas, and water rate TDM predictions vs actual simulation data plots for 2 Year Blind Validation TDM 


\section{Oil Rate}
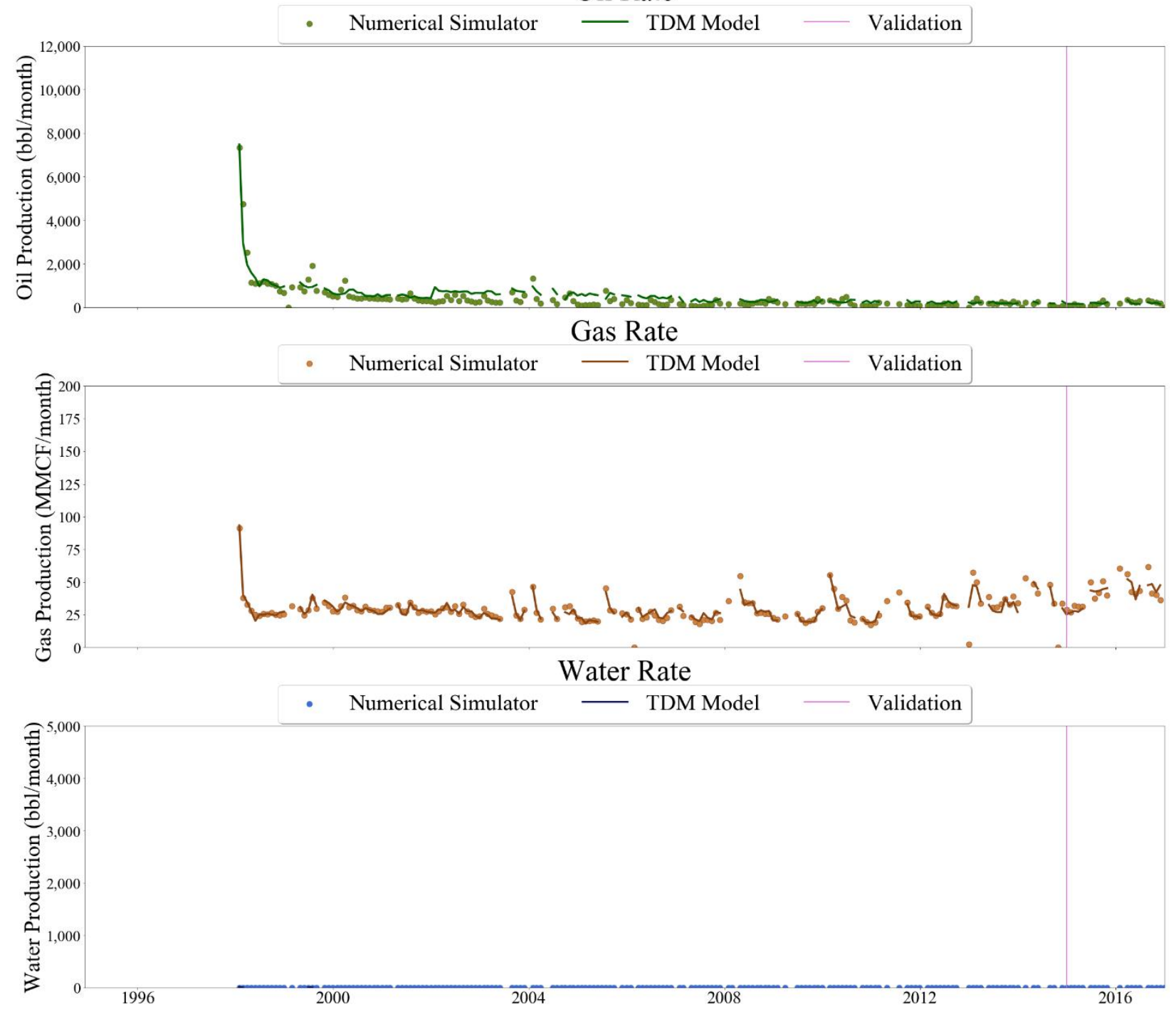

Figure 505 Well-008 oil, gas, and water rate TDM predictions vs actual simulation data plots for 2 Year Blind Validation TDM 

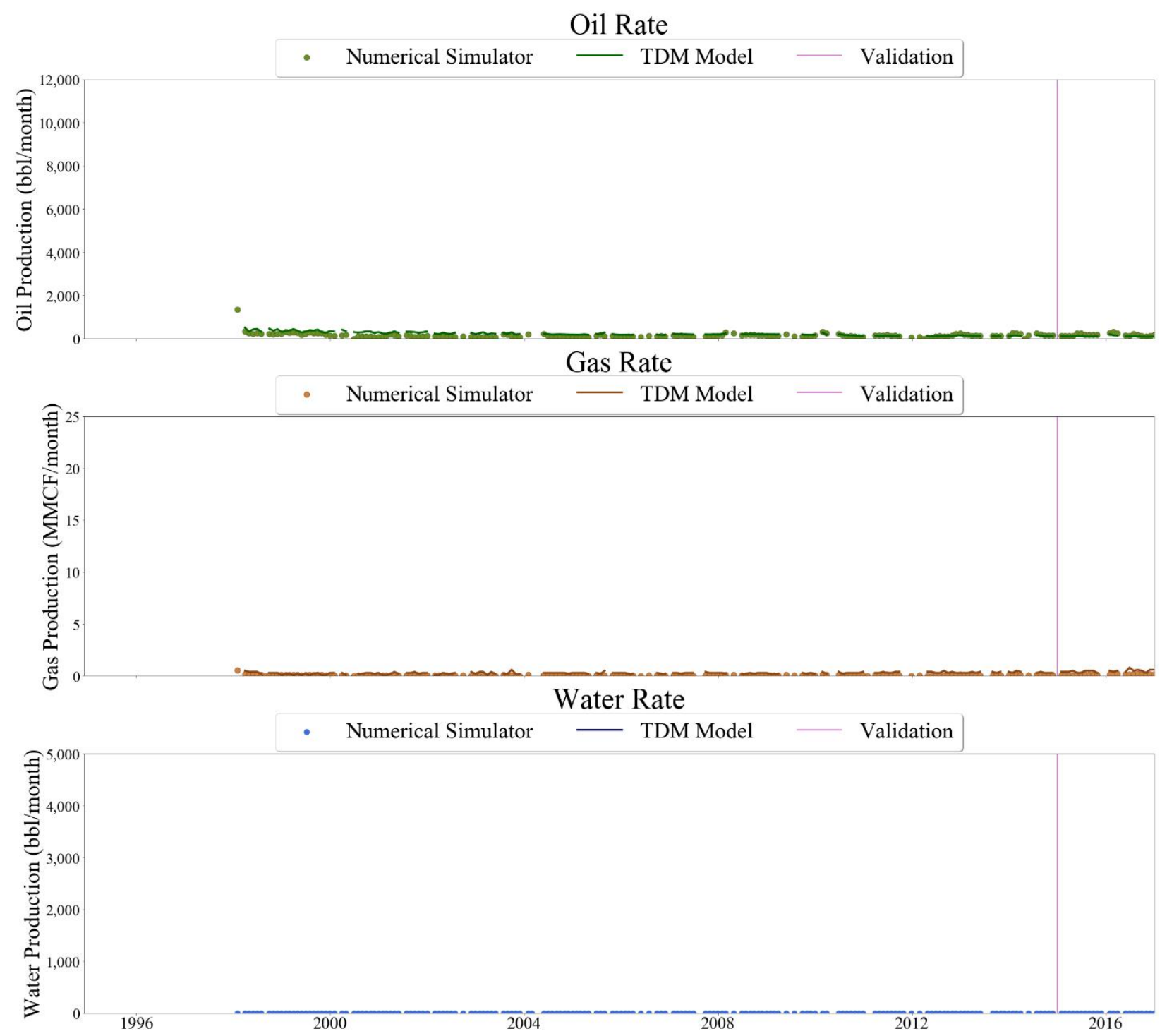

Figure 506 Well-009 oil, gas, and water rate TDM predictions vs actual simulation data plots for 2 Year Blind Validation TDM 


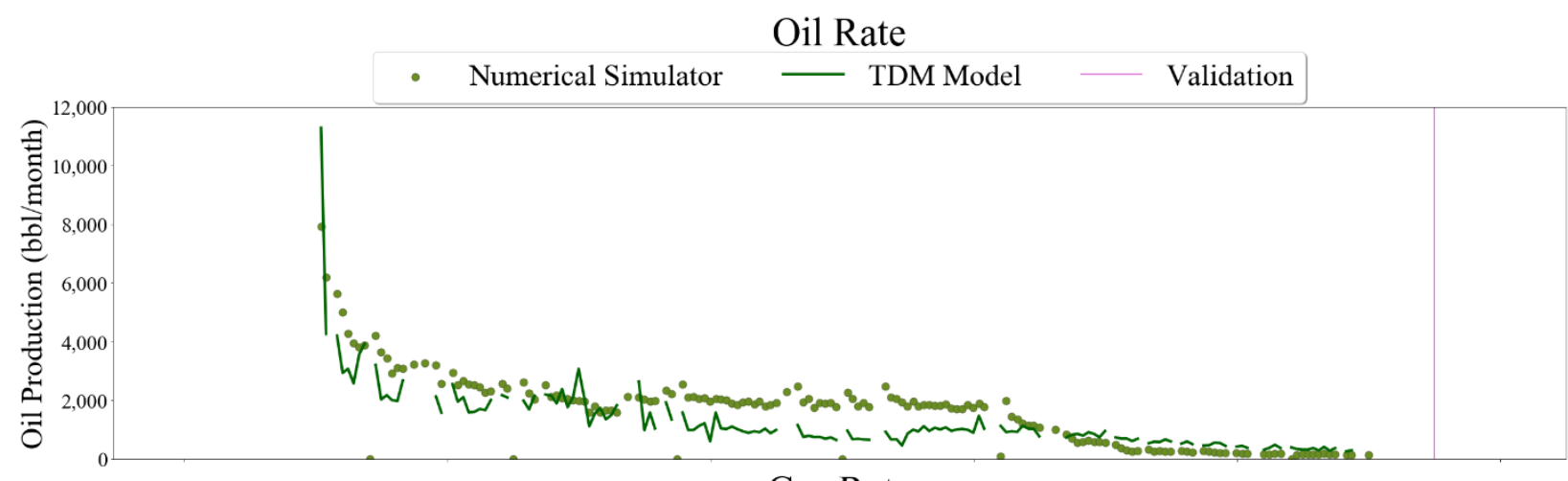

Gas Rate
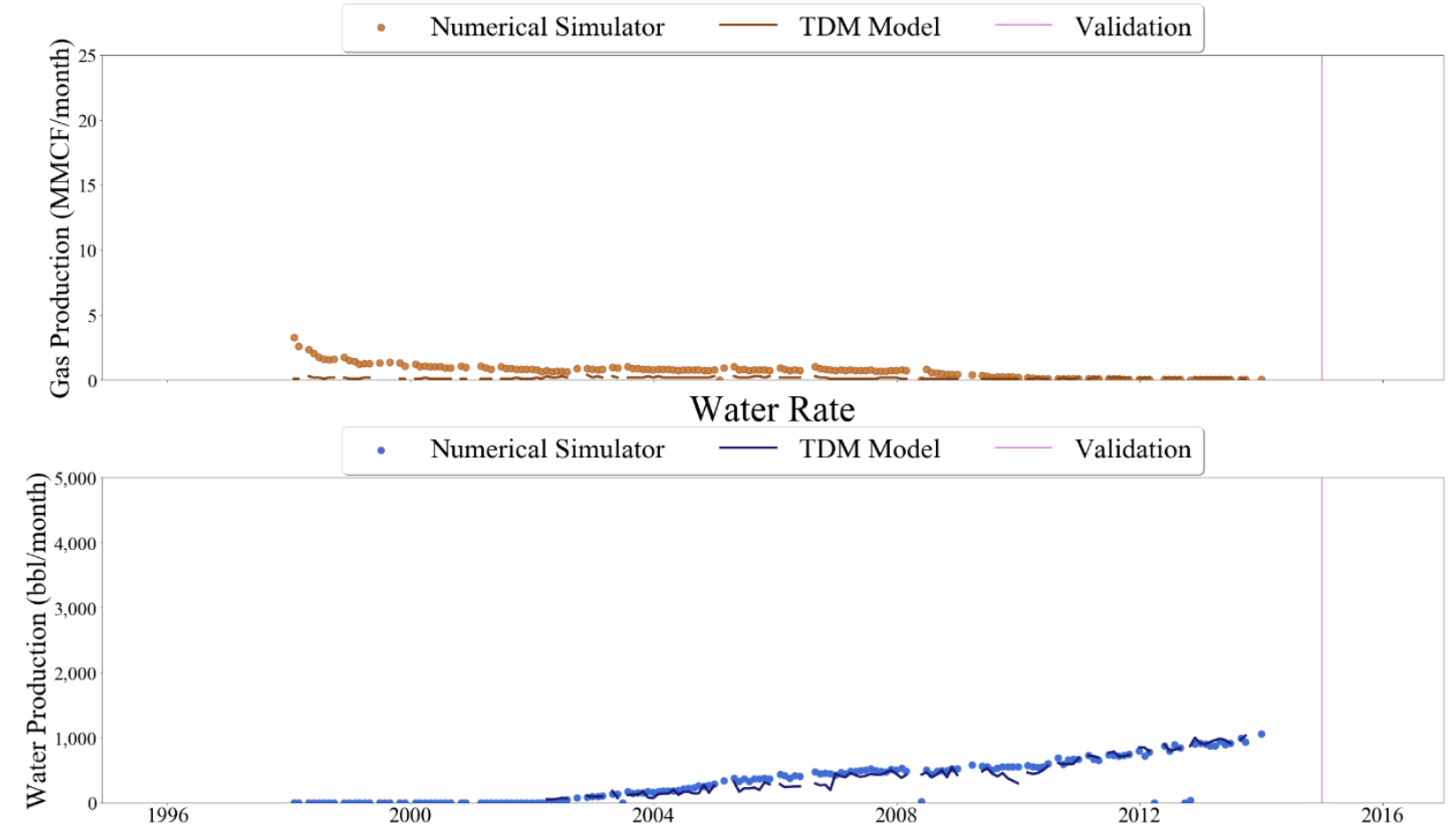

Figure 507 Well-010 oil, gas, and water rate TDM predictions vs actual simulation data plots for 2 Year Blind Validation 

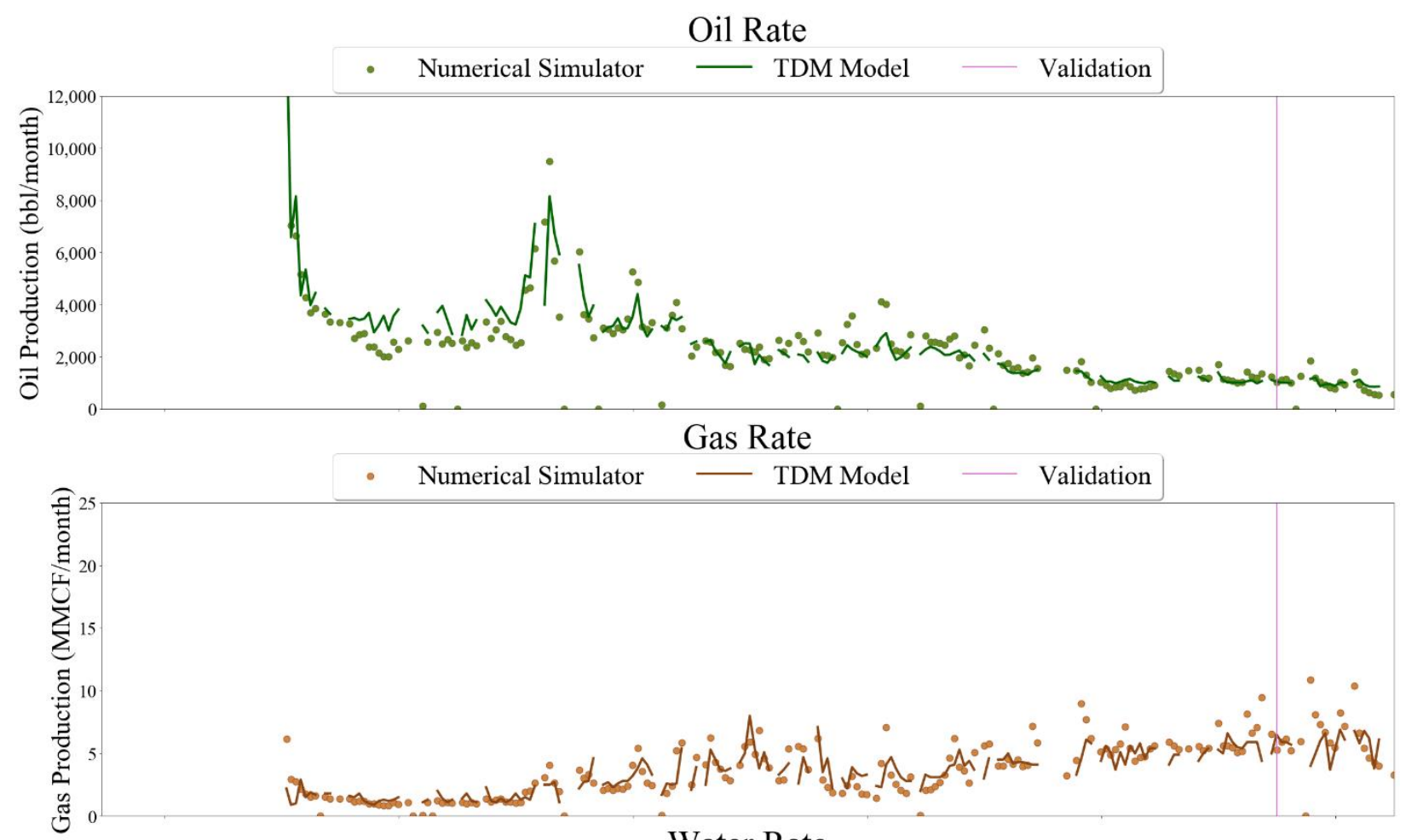

\section{Water Rate}

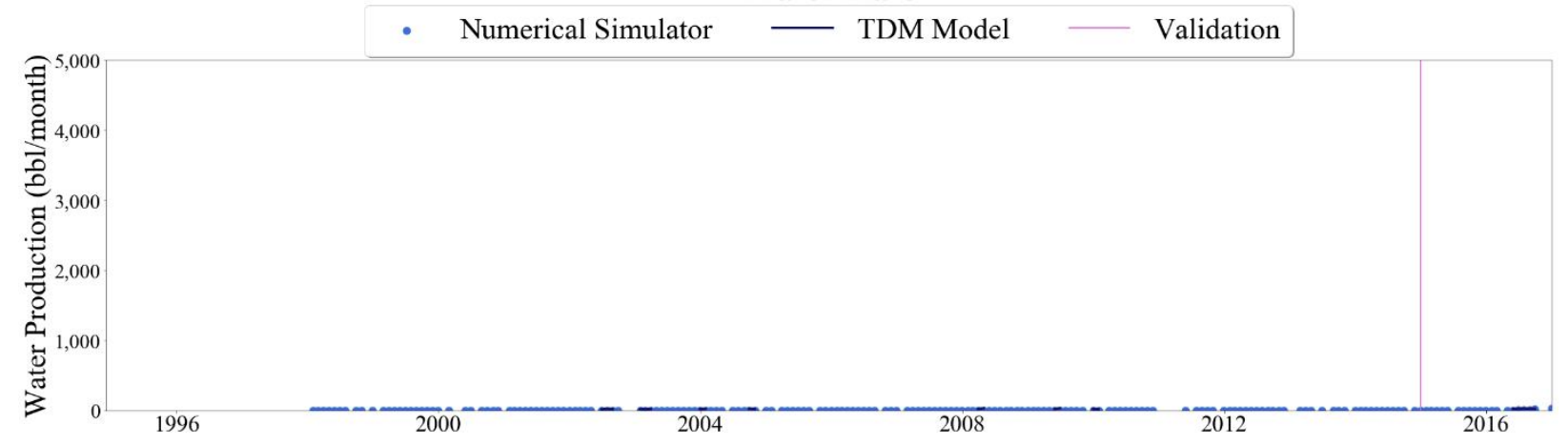

Figure 508 Well-011 oil, gas, and water rate TDM predictions vs actual simulation data plots for 2 Year Blind Validation TDM 

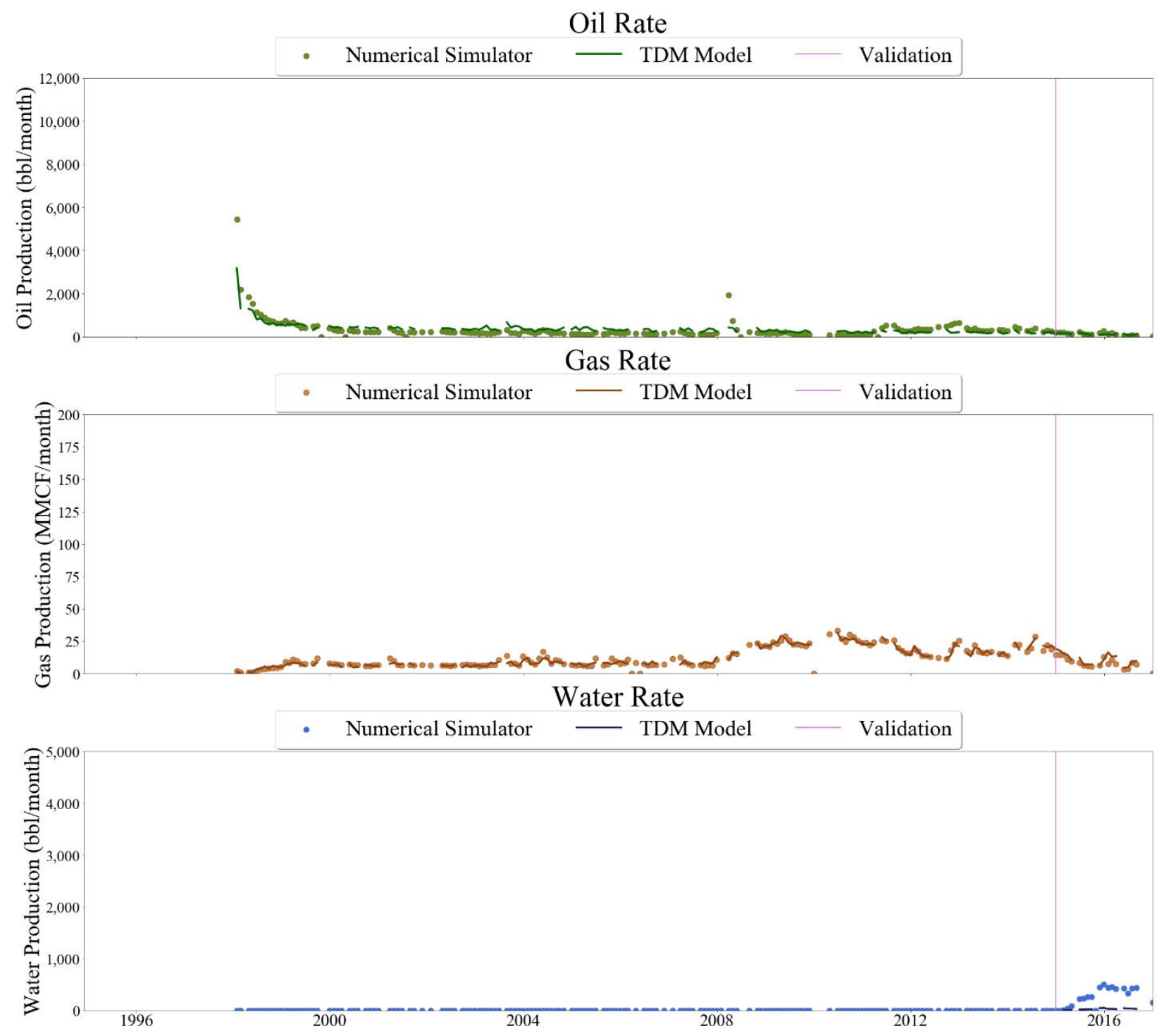

Figure 509 Well-012 oil, gas, and water rate TDM predictions vs actual simulation data plots for 2 Year Blind Validation TDM 

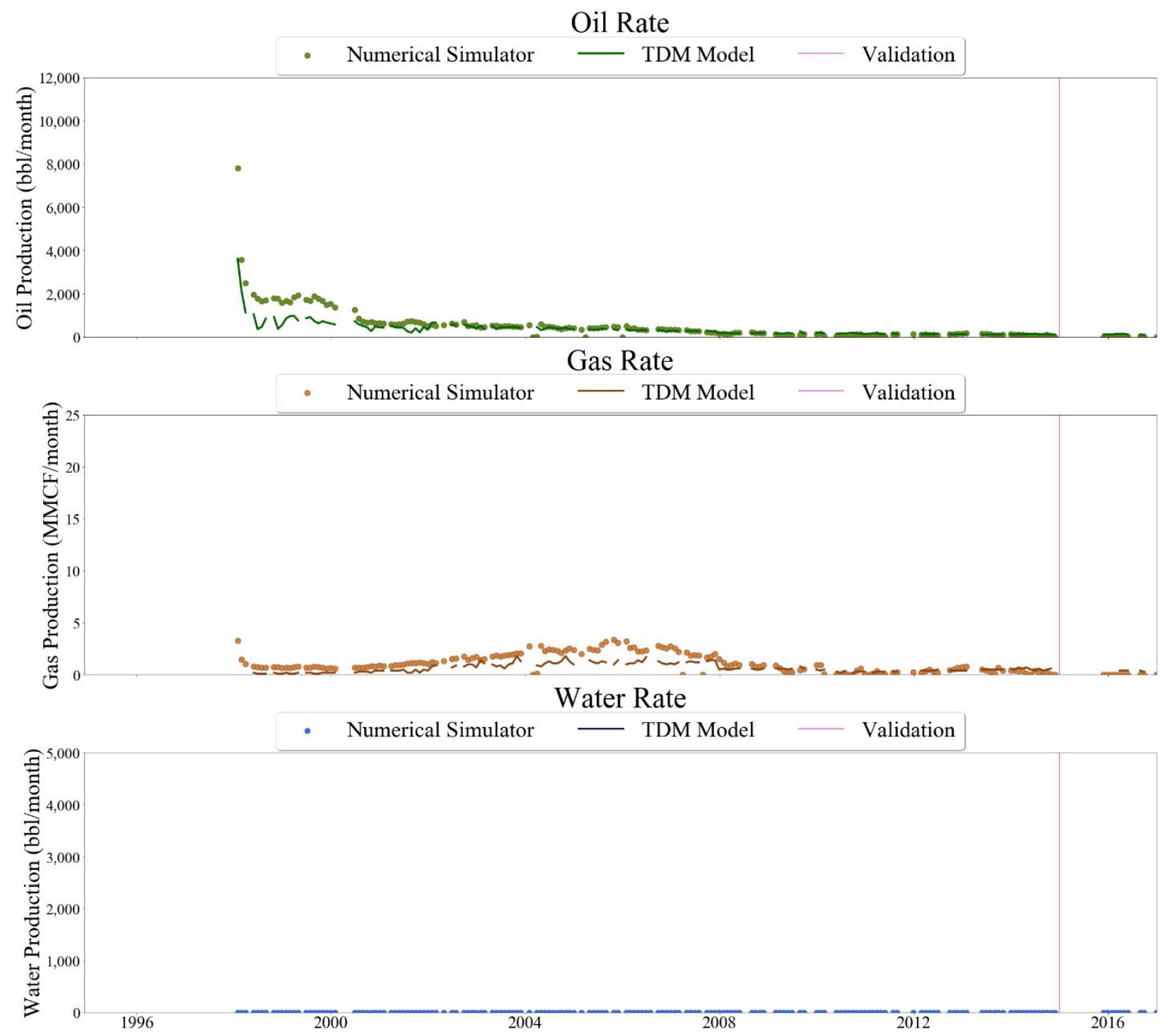

Figure 510 Well-013 oil, gas, and water rate TDM predictions vs actual simulation data plots for 2 Year Blind Validation TDM 


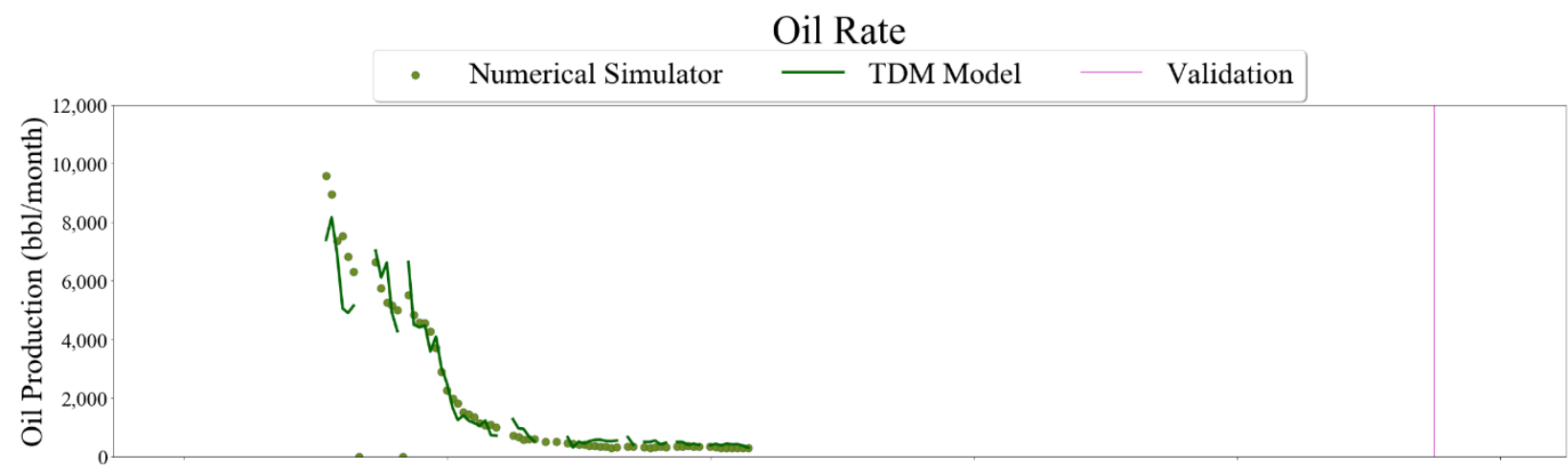

\section{Gas Rate}
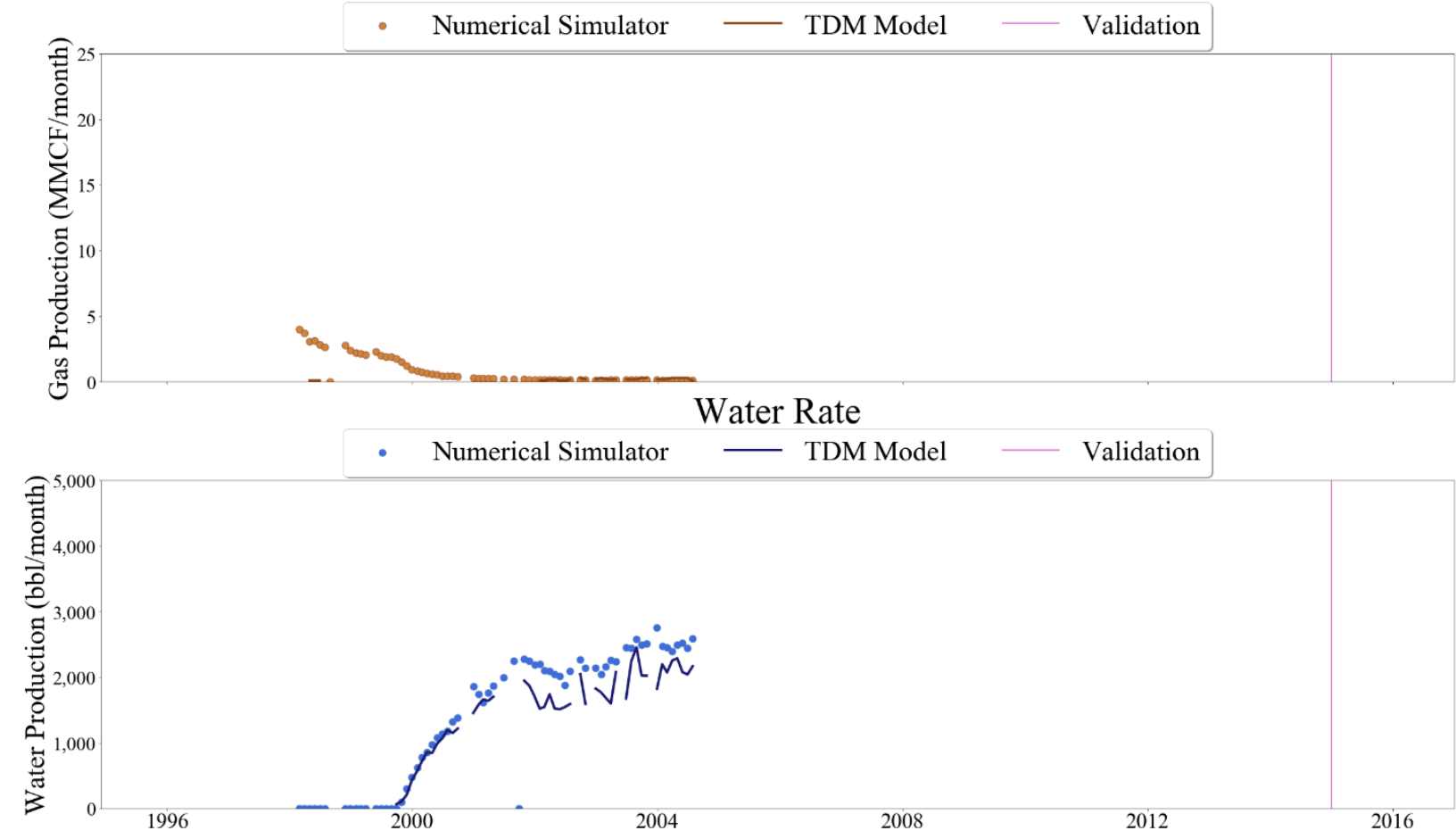

Figure 511 Well-014 oil, gas, and water rate TDM predictions vs actual simulation data plots for 2 Year Blind Validation TDM 

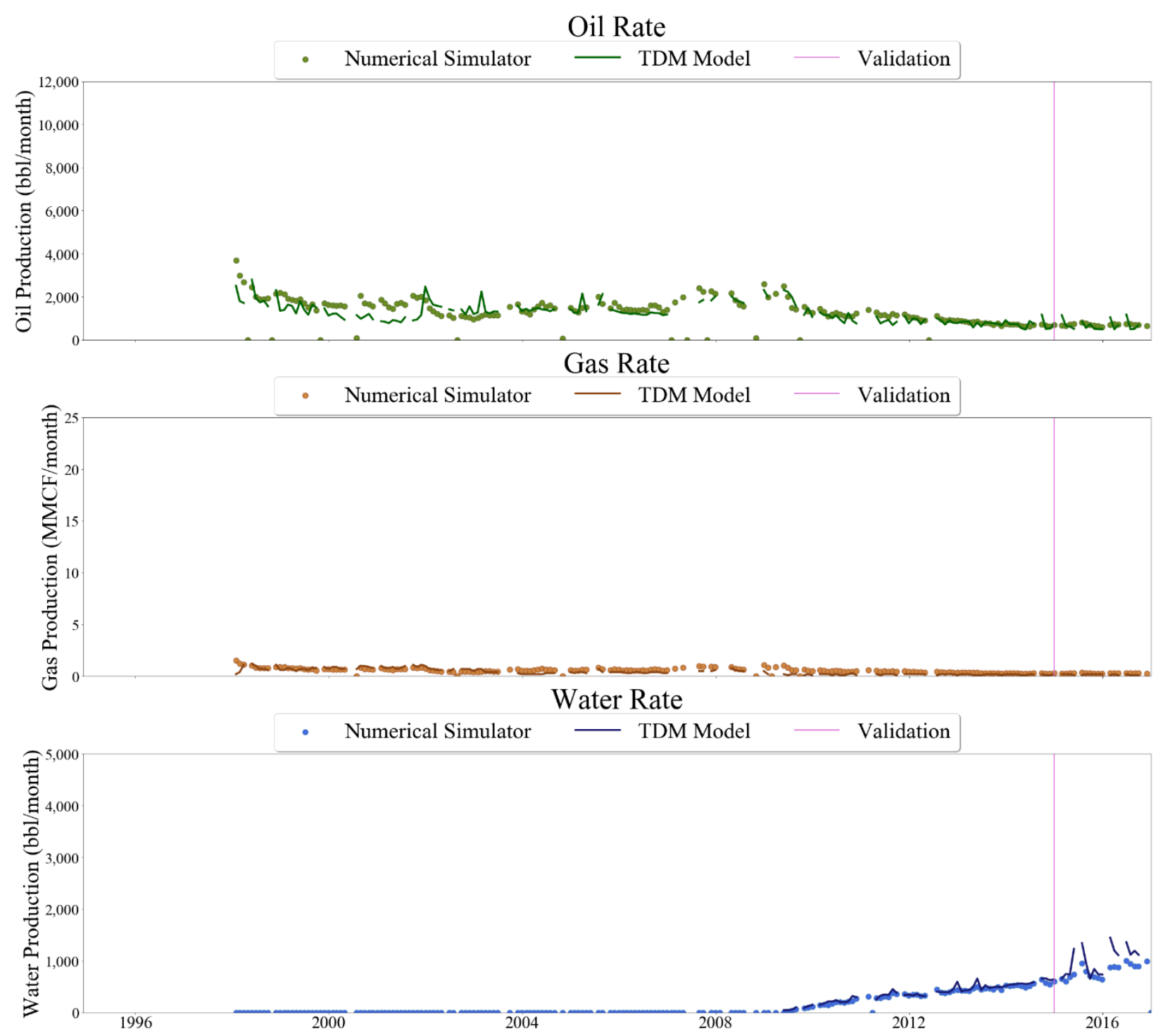

Figure 512 Well-015 oil, gas, and water rate TDM predictions vs actual simulation data plots for 2 Year Blind Validation TDM 

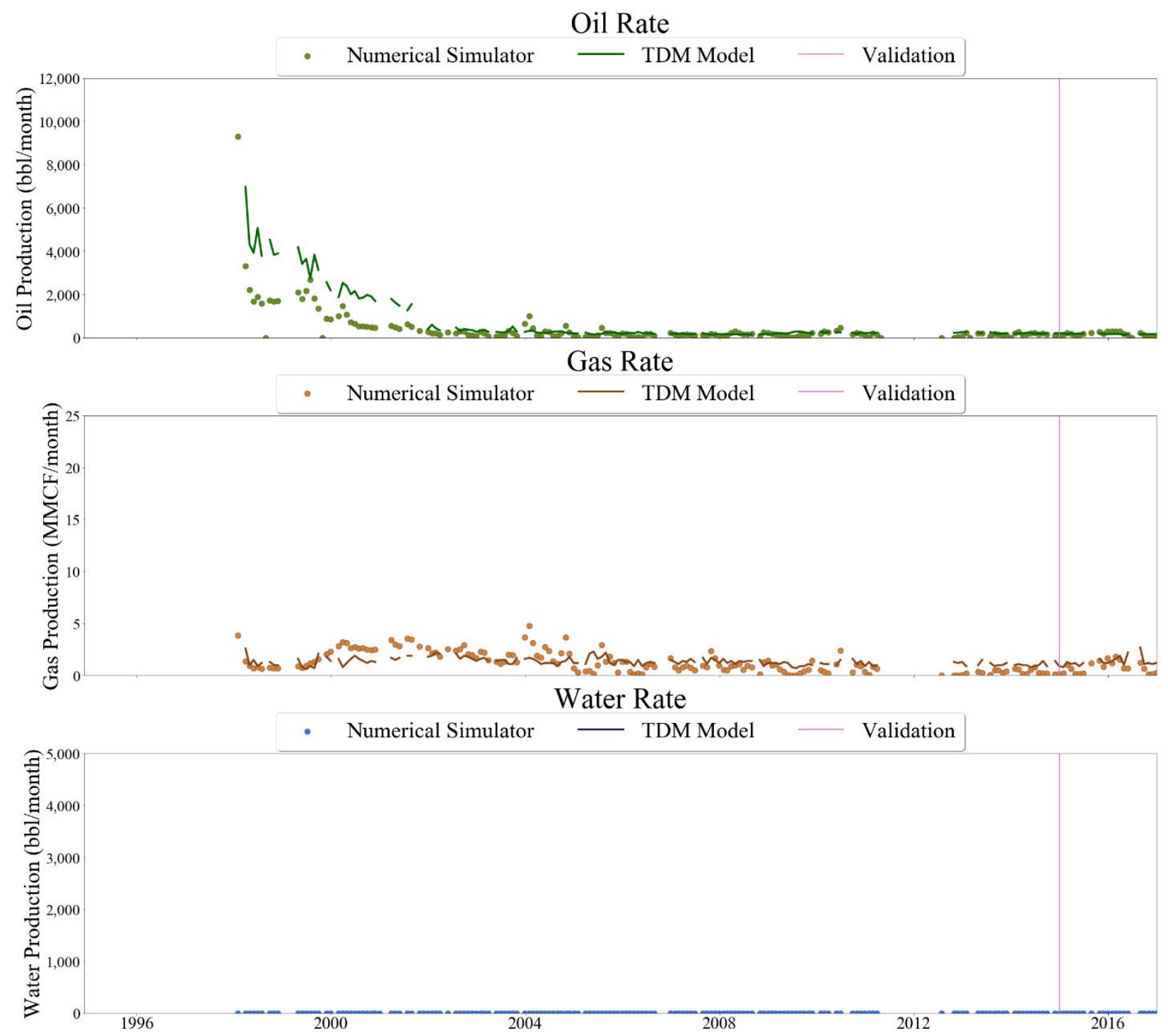

Figure 513 Well-016 oil, gas, and water rate TDM predictions vs actual simulation data plots for 2 Year Blind Validation TDM 

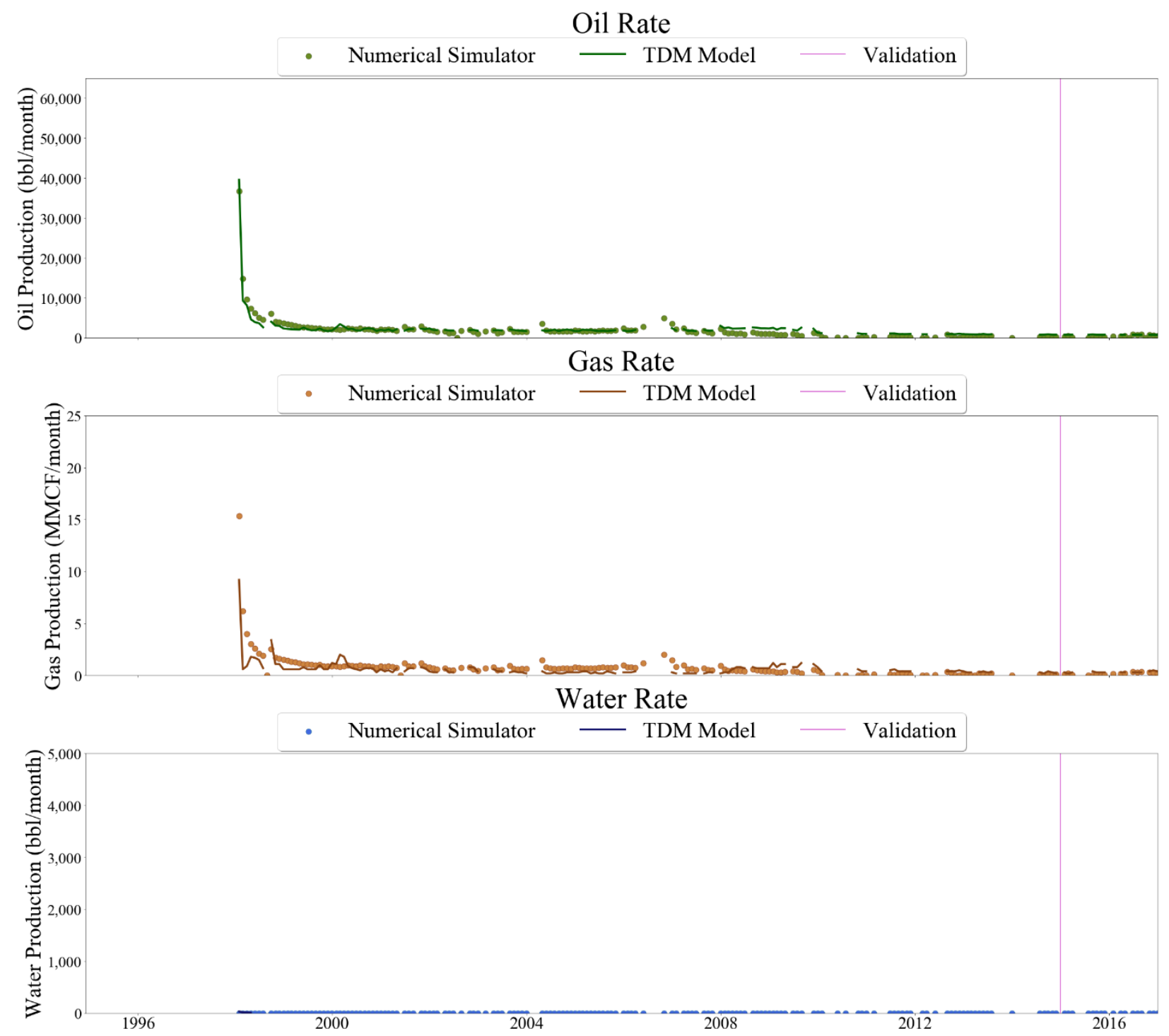

Figure 514 Well-017 oil, gas, and water rate TDM predictions vs actual simulation data plots for 2 Year Blind Validation TDM 

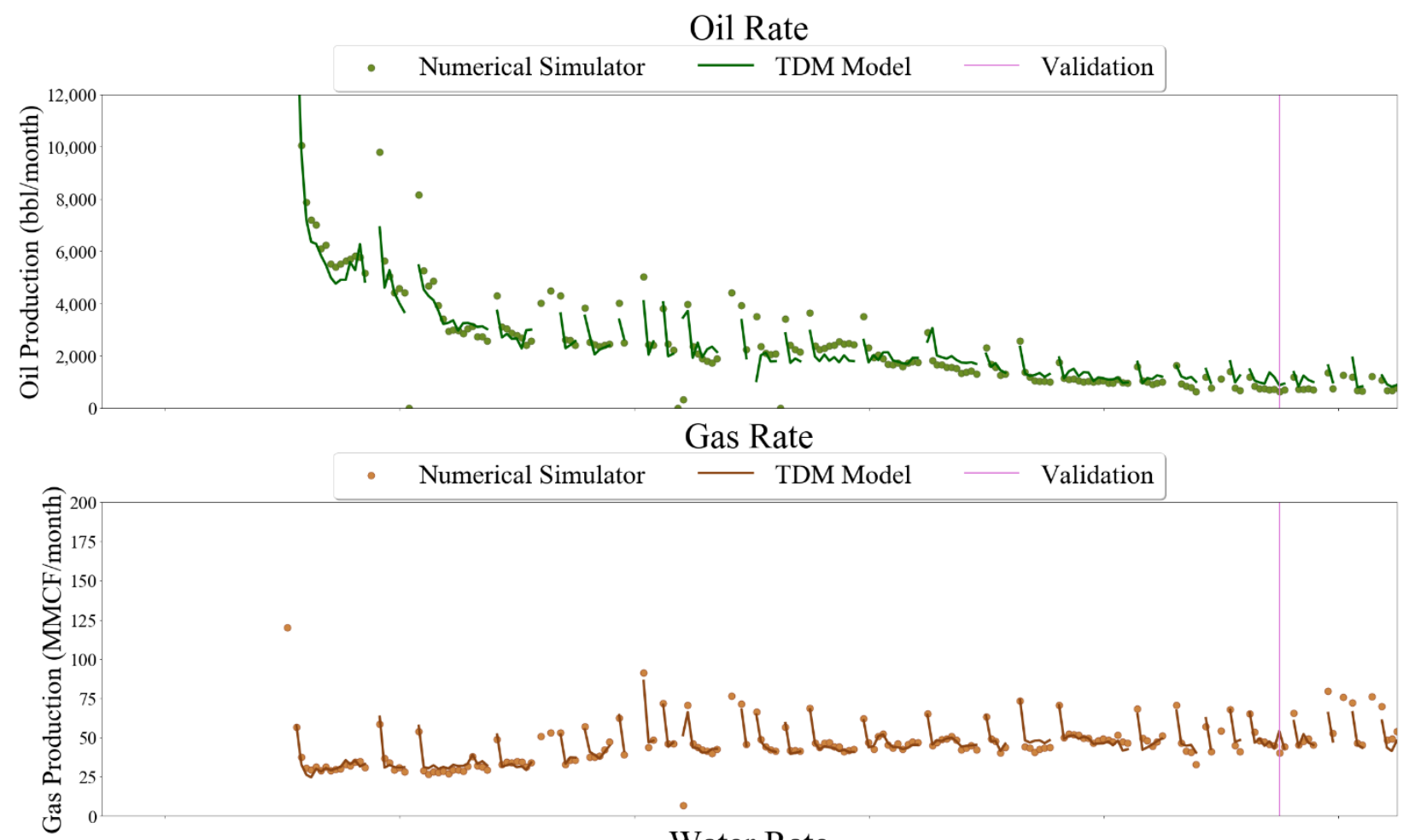

\section{Water Rate}

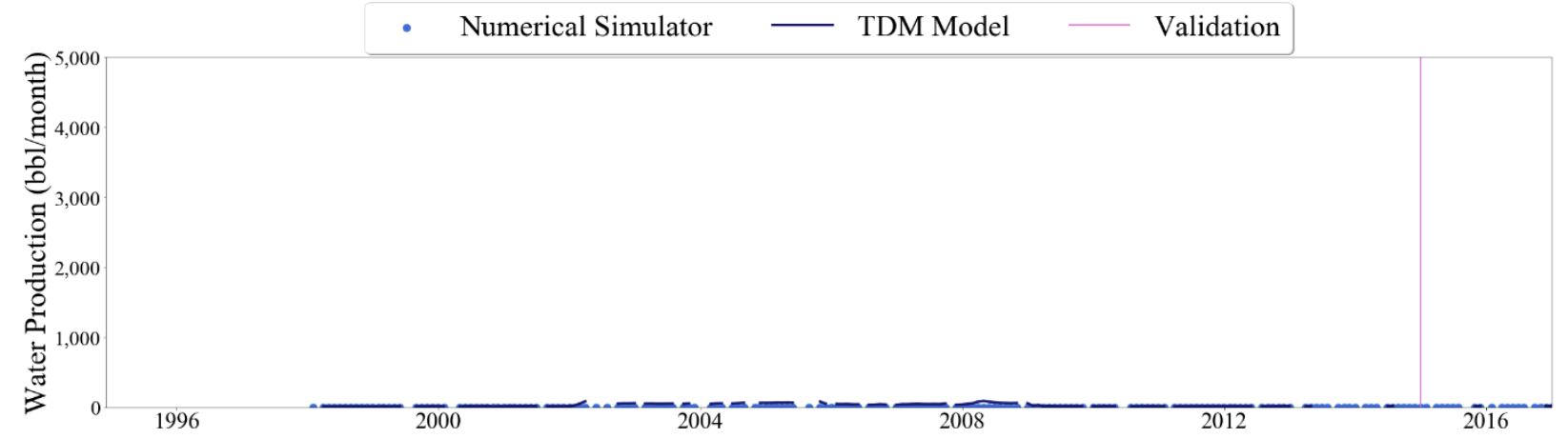

Figure 515 Well-018 oil, gas, and water rate TDM predictions vs actual simulation data plots for 2 Year Blind Validation TDM 

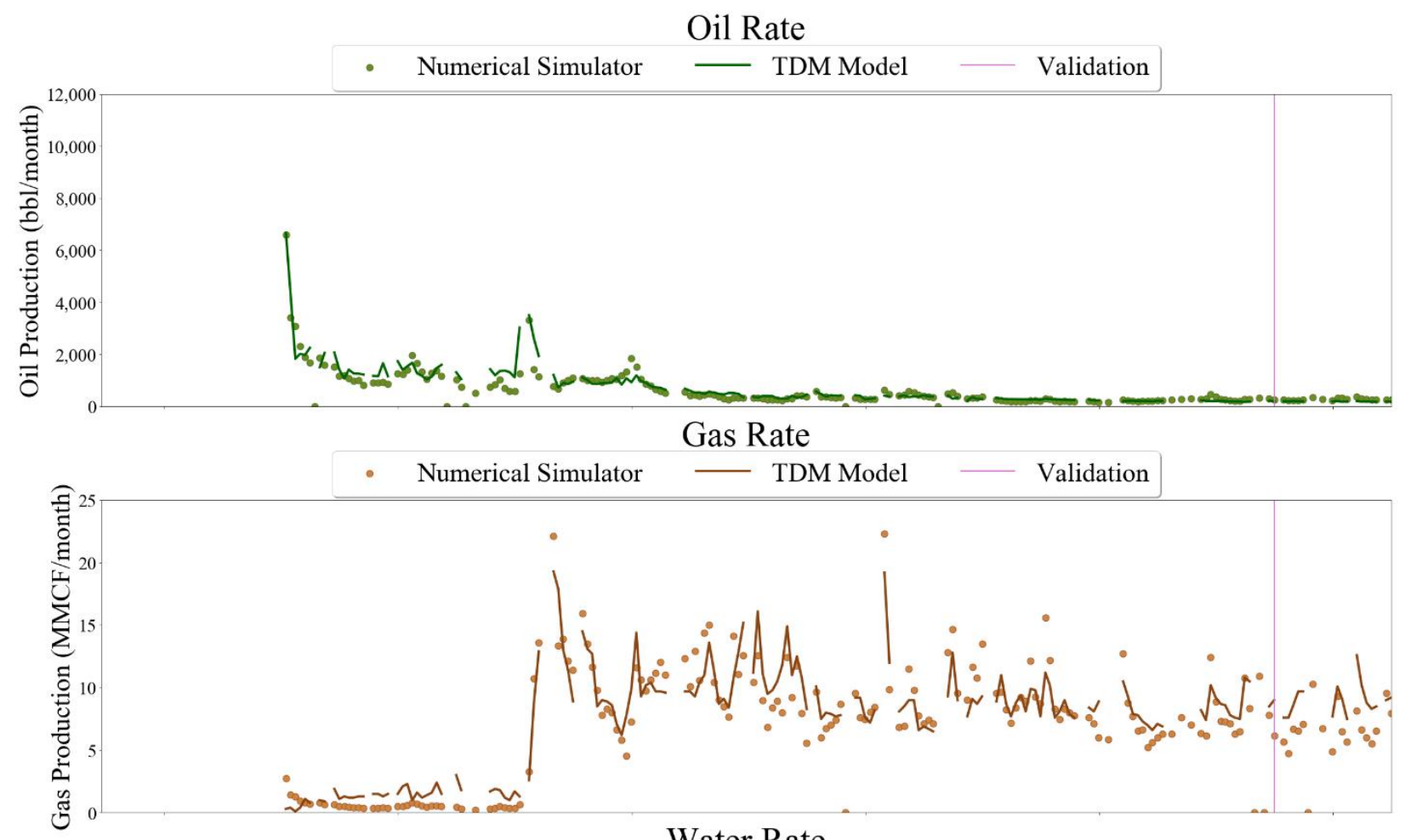

Water Rate

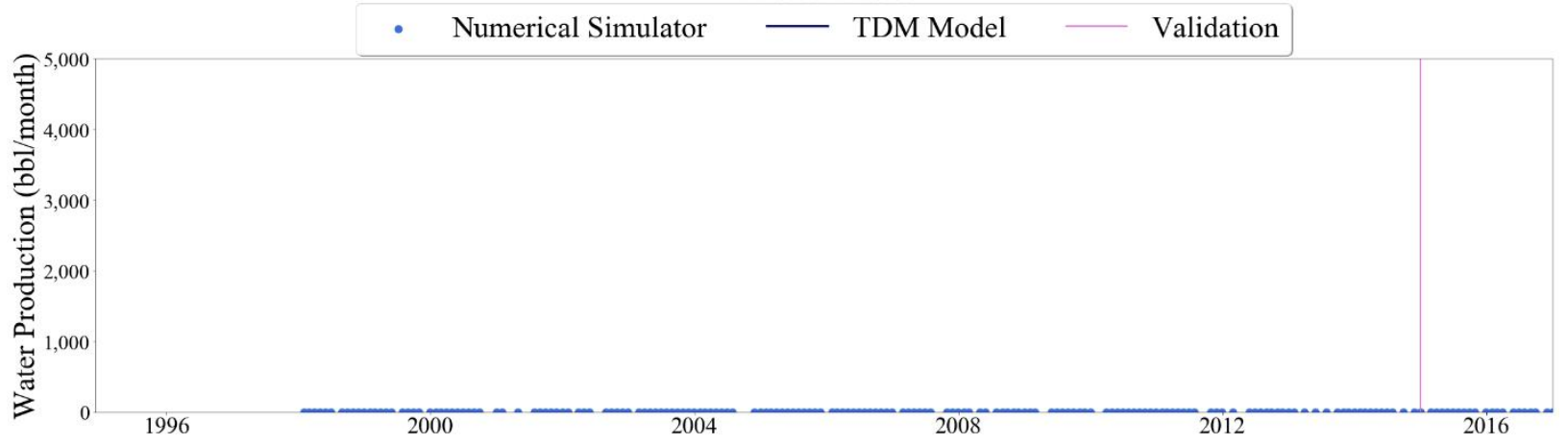

Figure 516 Well-019 oil, gas, and water rate TDM predictions vs actual simulation data plots for 2 Year Blind Validation TDM 

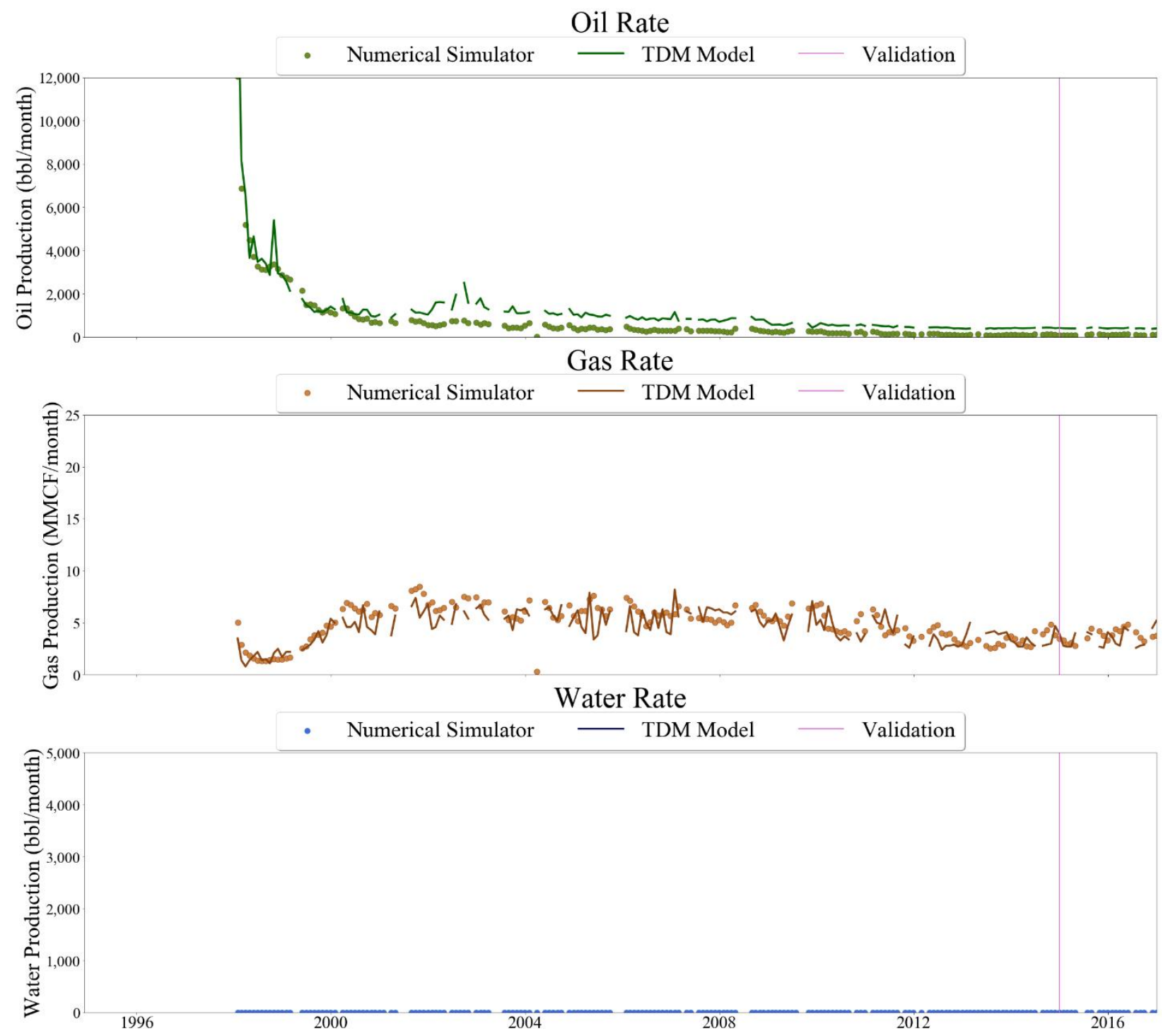

Figure 517 Well-020 oil, gas, and water rate TDM predictions vs actual simulation data plots for 2 Year Blind Validation TDM 

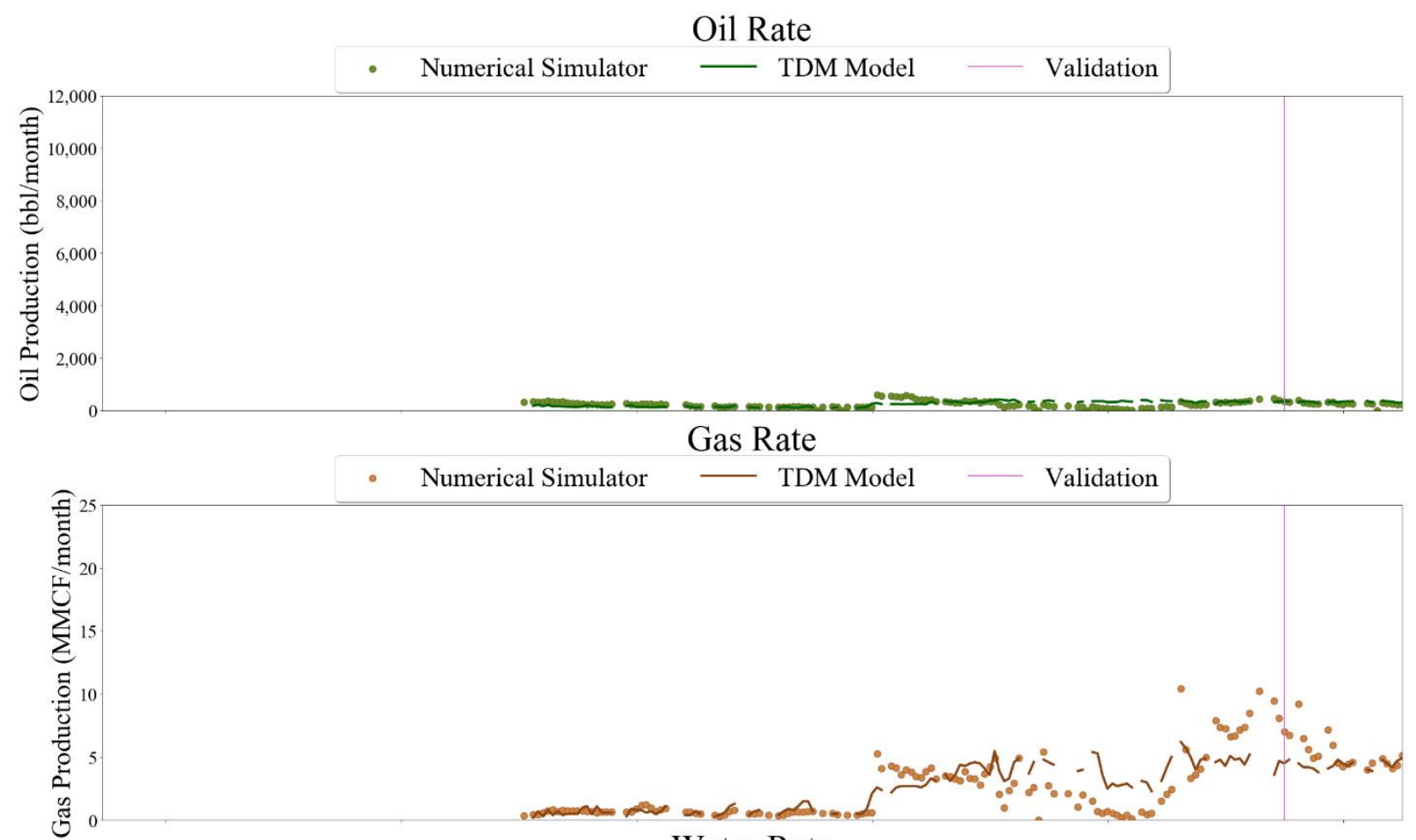

Water Rate

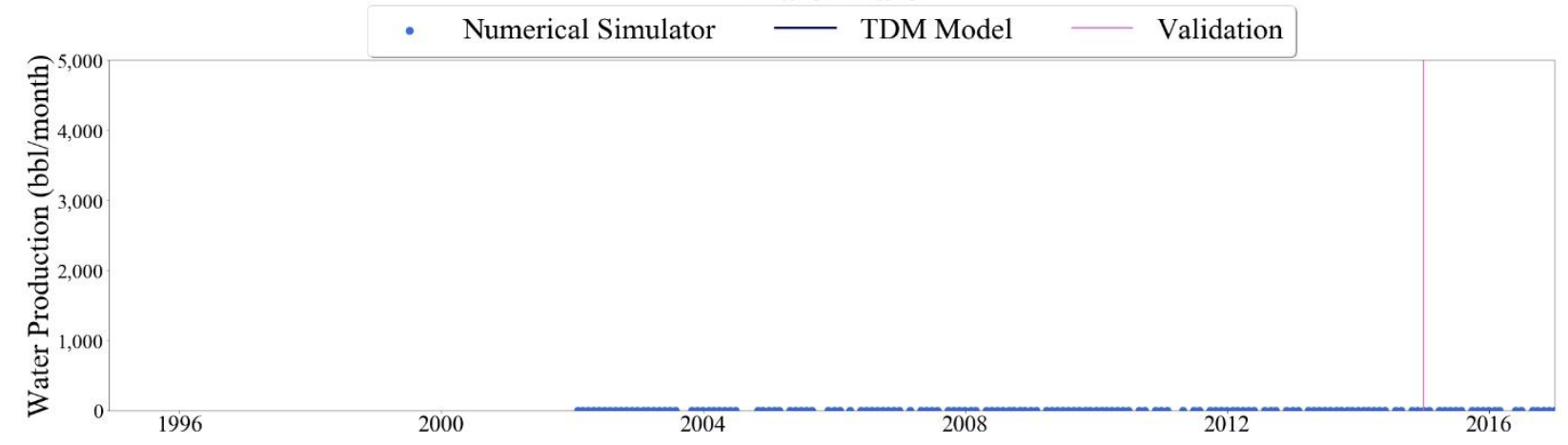

Figure 518 Well-021 oil, gas, and water rate TDM predictions vs actual simulation data plots for 2 Year Blind Validation TDM 


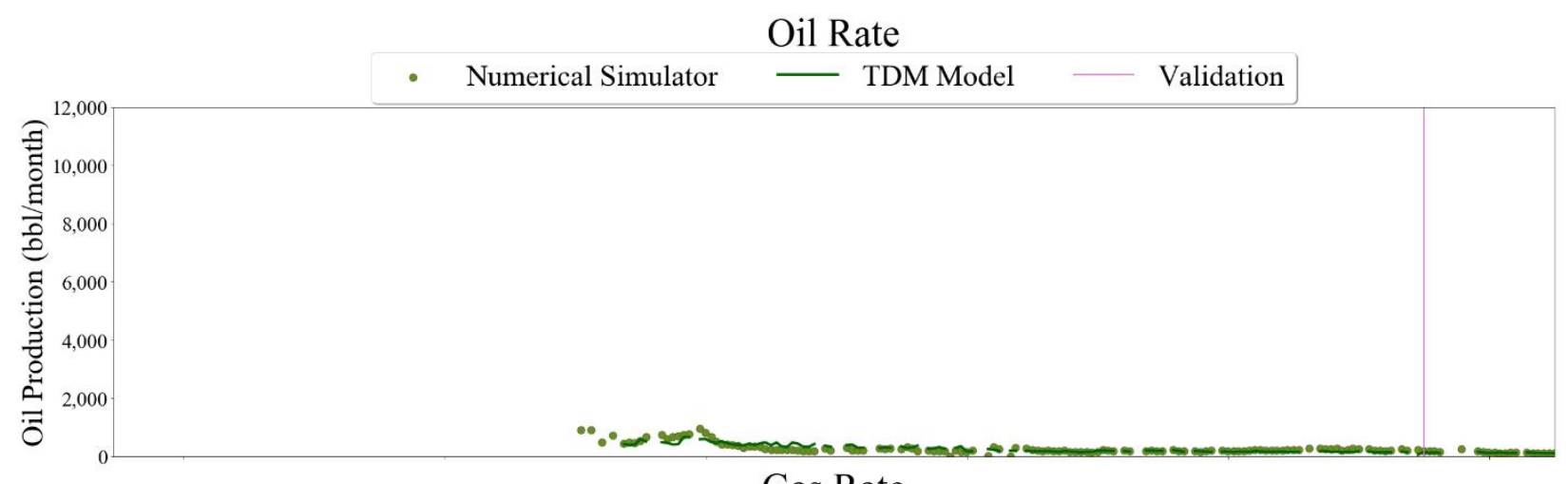

Gas Rate

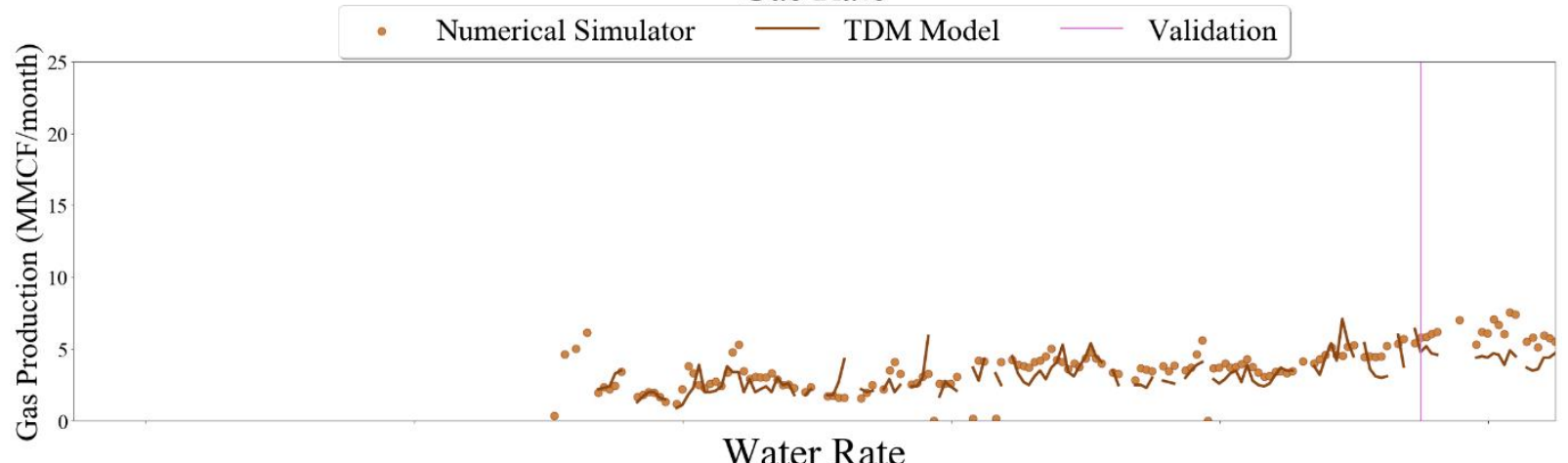

Water Rate

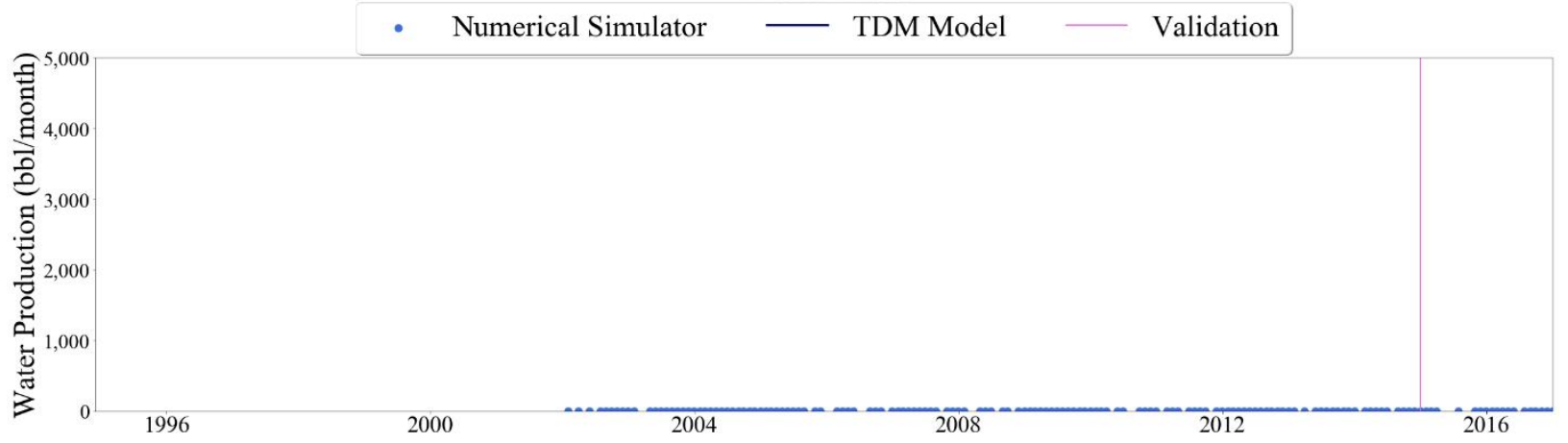

Figure 519 Well-022 oil, gas, and water rate TDM predictions vs actual simulation data plots for 2 Year Blind Validation TDM 

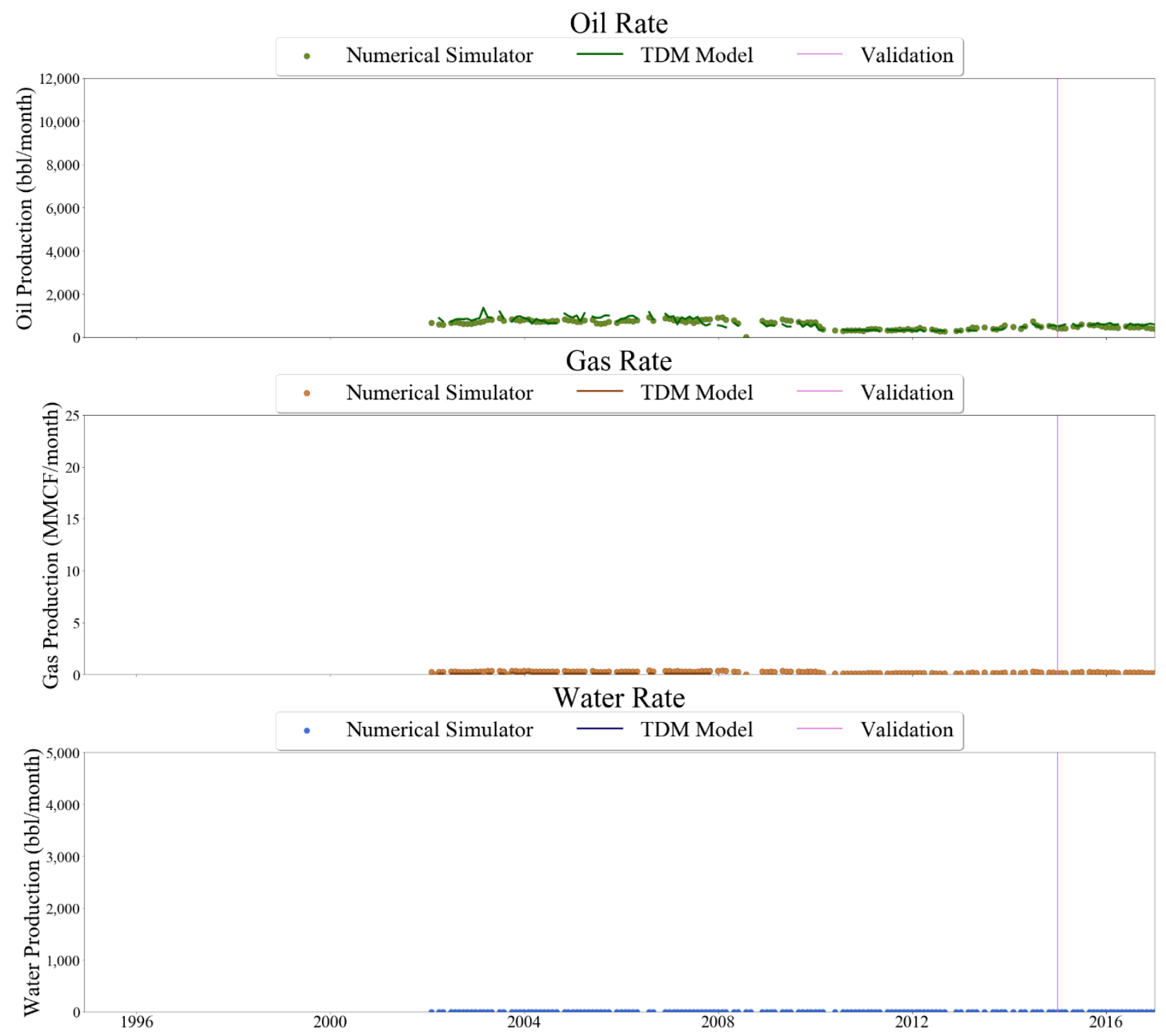

Figure 520 Well-023 oil, gas, and water rate TDM predictions vs actual simulation data plots for 2 Year Blind Validation TDM 

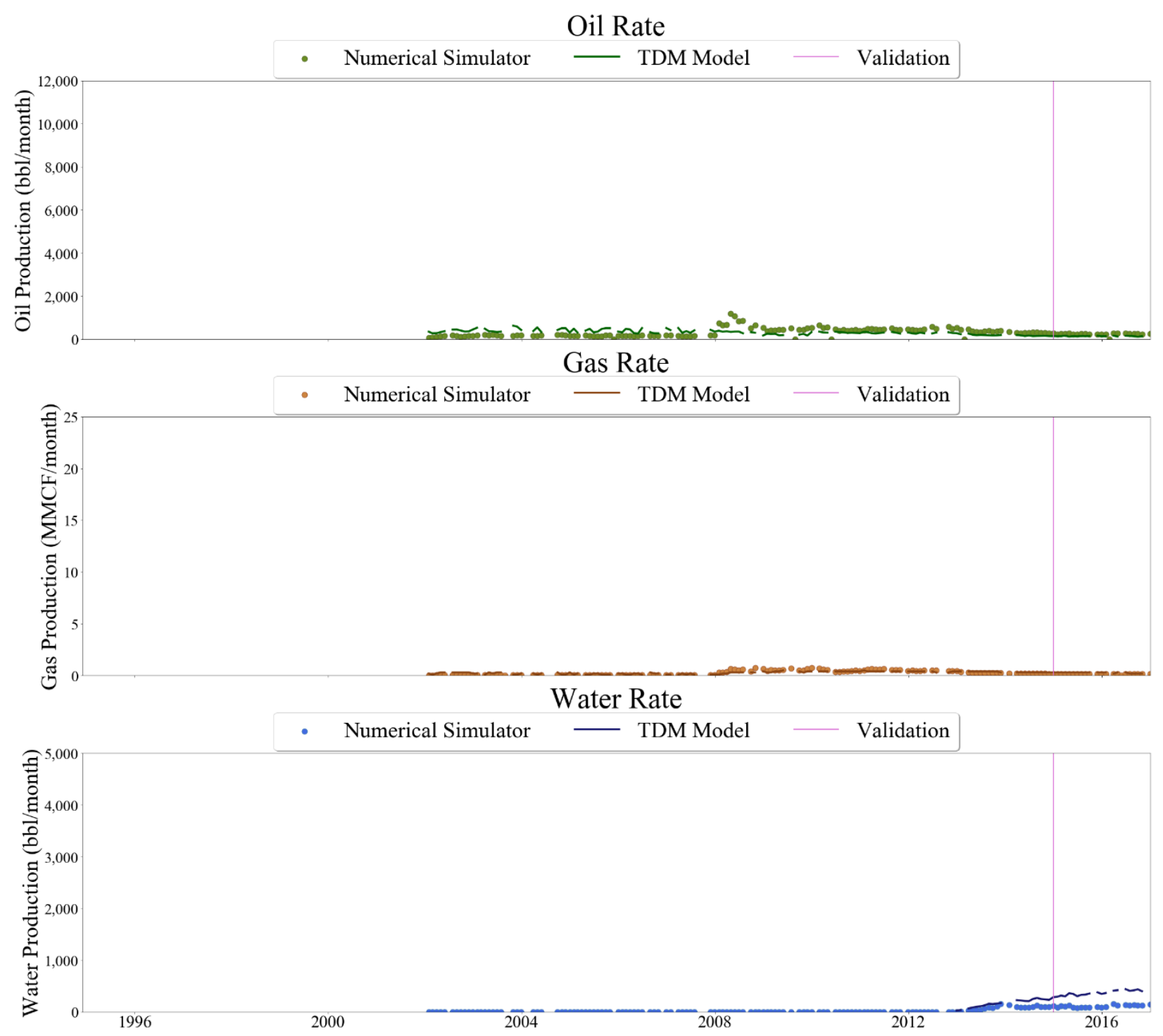

Figure 521 Well-024 oil, gas, and water rate TDM predictions vs actual simulation data plots for 2 Year Blind Validation TDM 


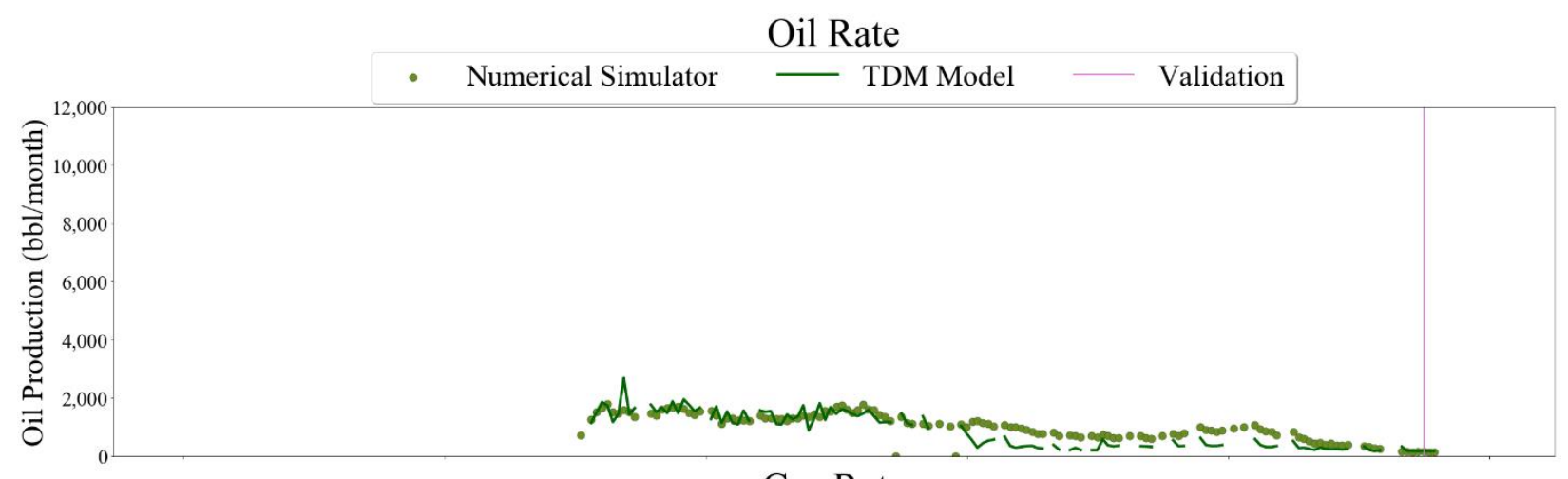

\section{Gas Rate}
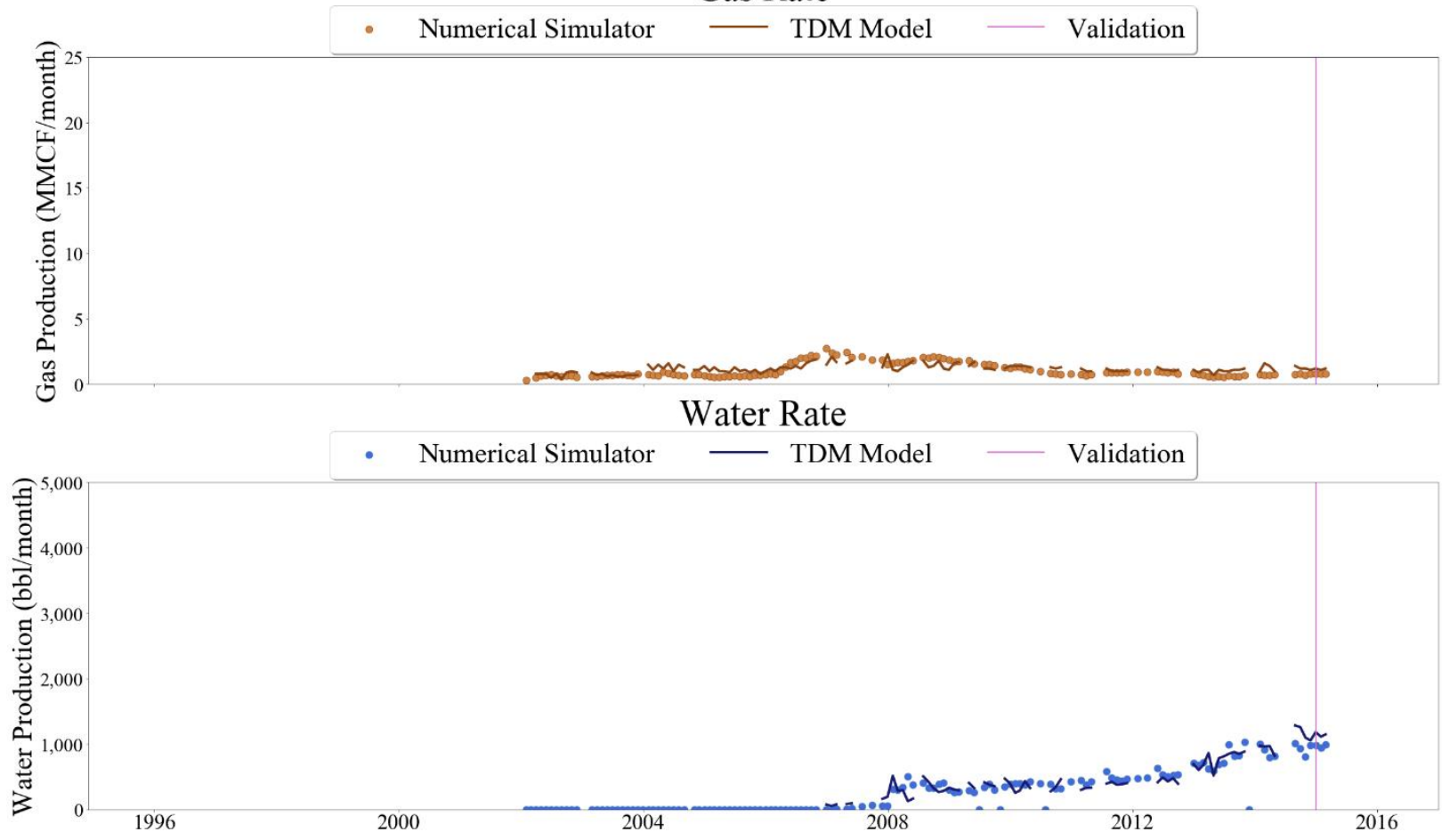

Figure 522 Well-025 oil, gas, and water rate TDM predictions vs actual simulation data plots for 2 Year Blind Validation TDM 


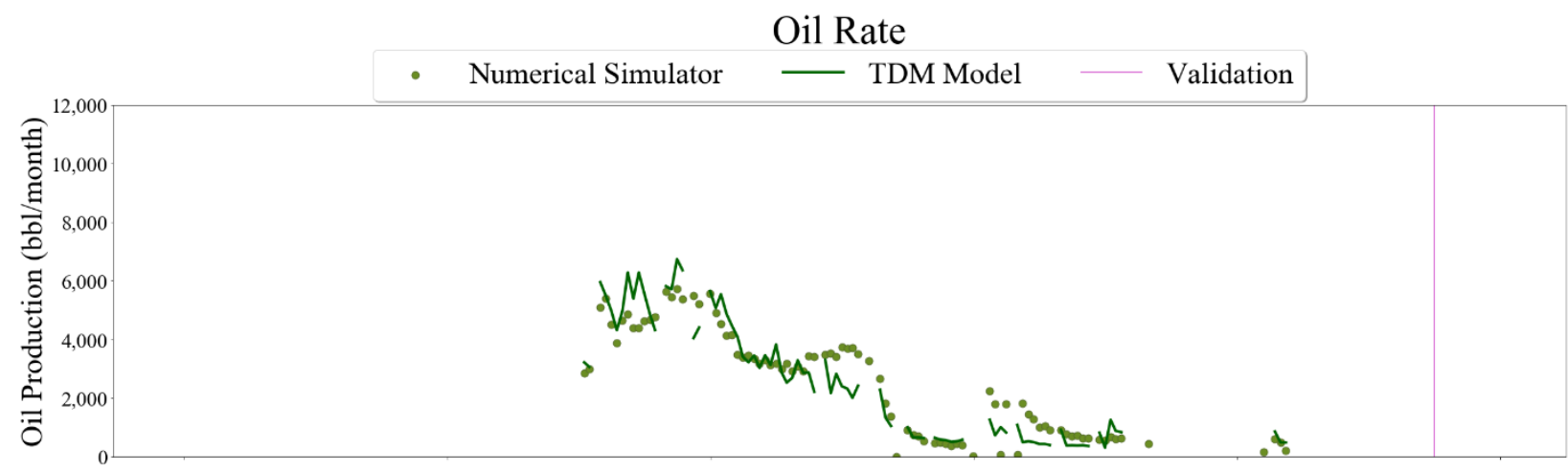

\section{Gas Rate}
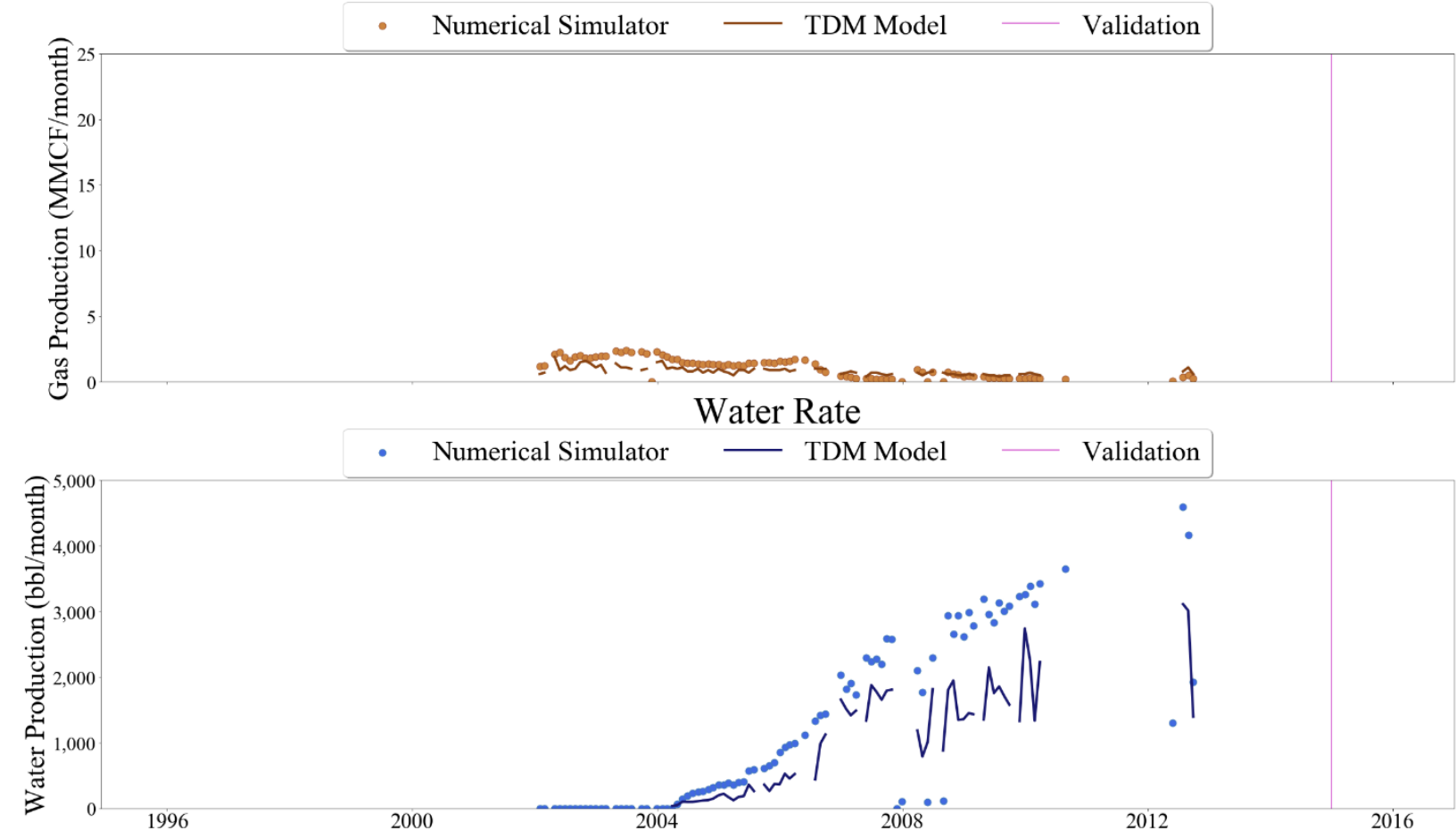

Figure 523 Well-026 oil, gas, and water rate TDM predictions vs actual simulation data plots for 2 Year Blind Validation TDM 


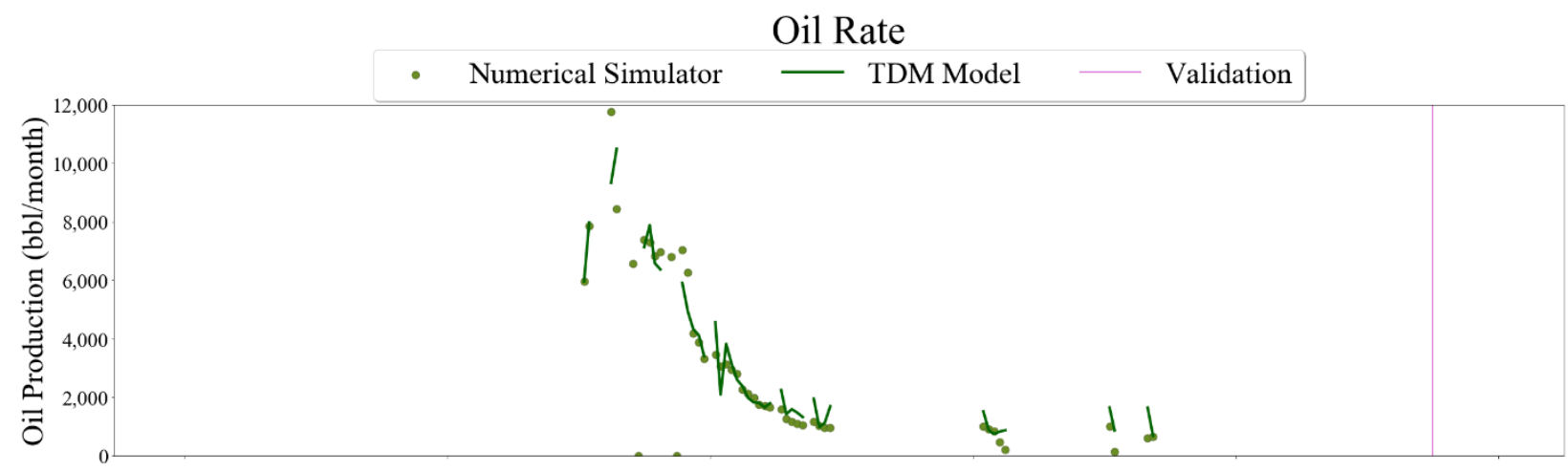

Gas Rate
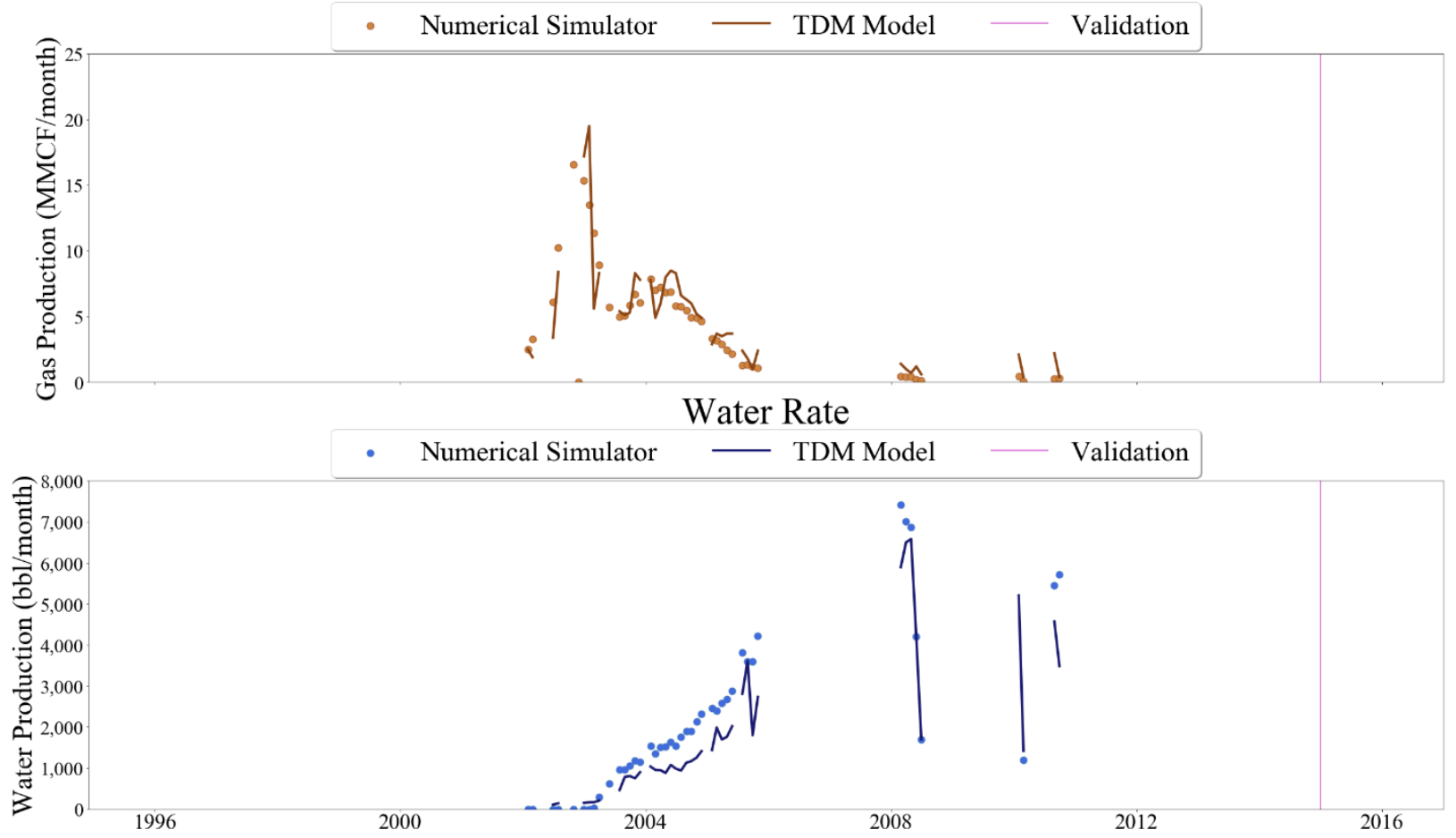

Figure 524 Well-027 oil, gas, and water rate TDM predictions vs actual simulation data plots for 2 Year Blind Validation TDM 


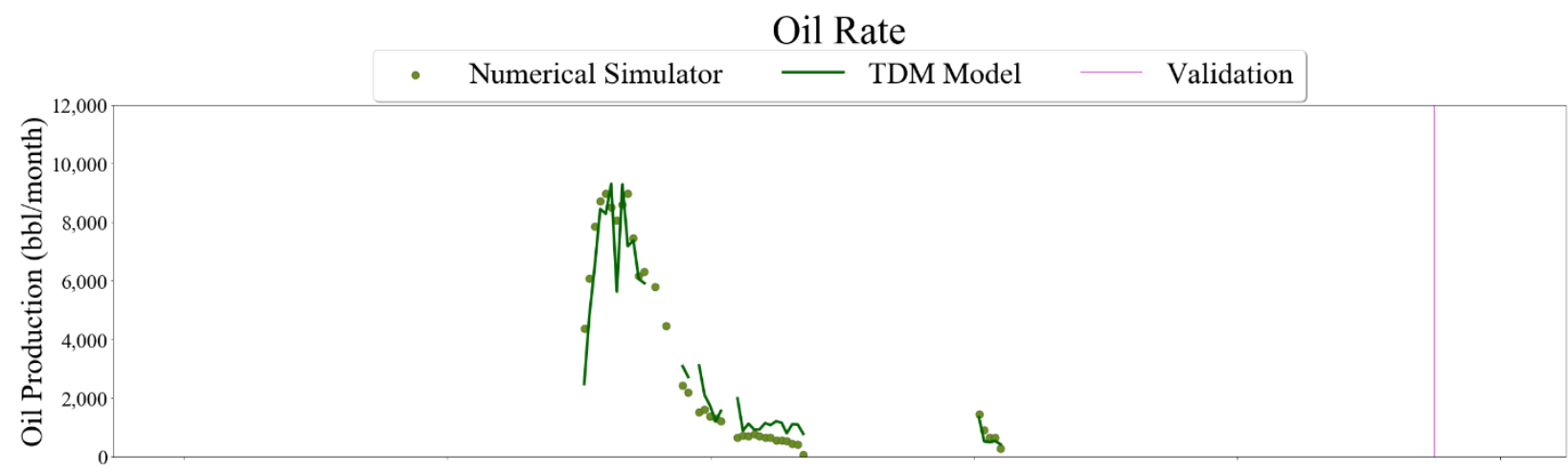

Gas Rate
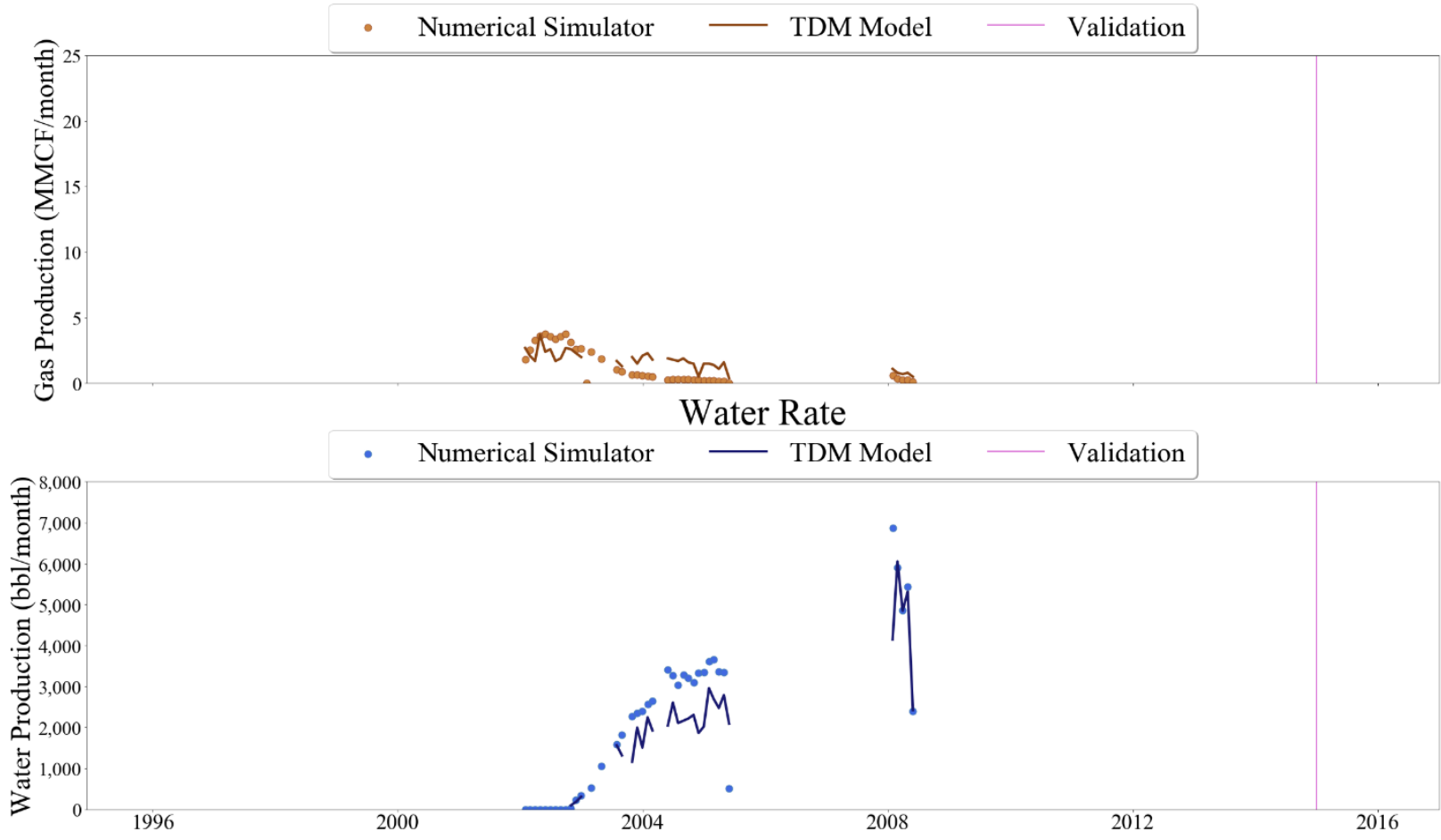

Figure 525 Well-028 oil, gas, and water rate TDM predictions vs actual simulation data plots for 2 Year Blind Validation TDM 

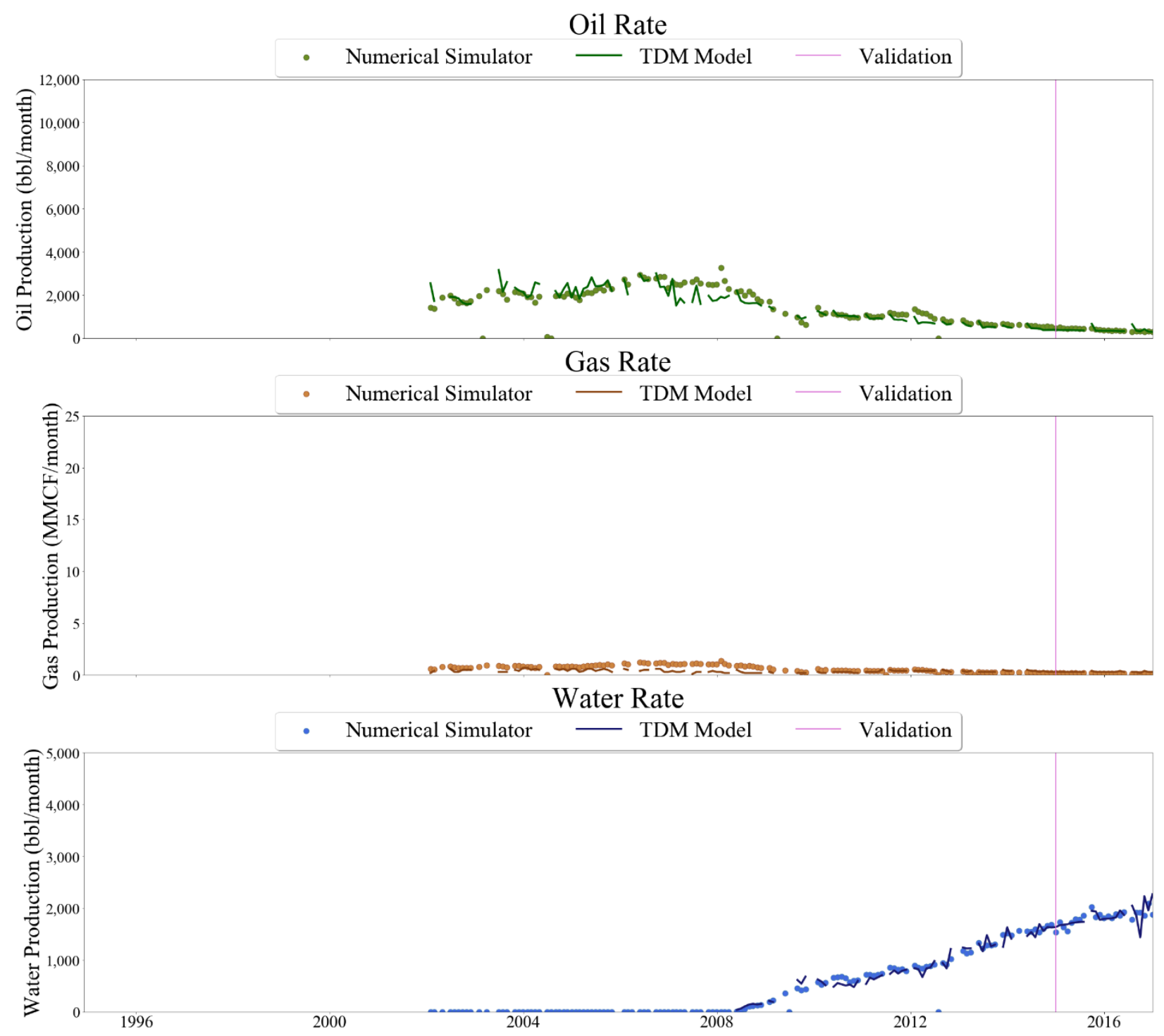

Figure 526 Well-029 oil, gas, and water rate TDM predictions vs actual simulation data plots for 2 Year Blind Validation TDM 


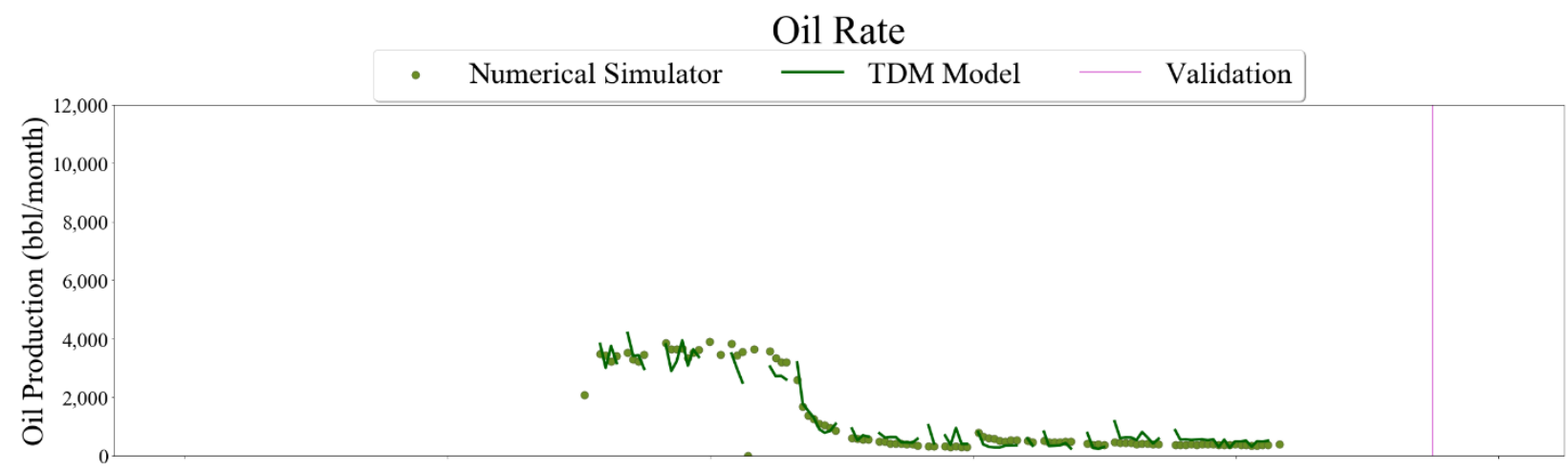

\section{Gas Rate}
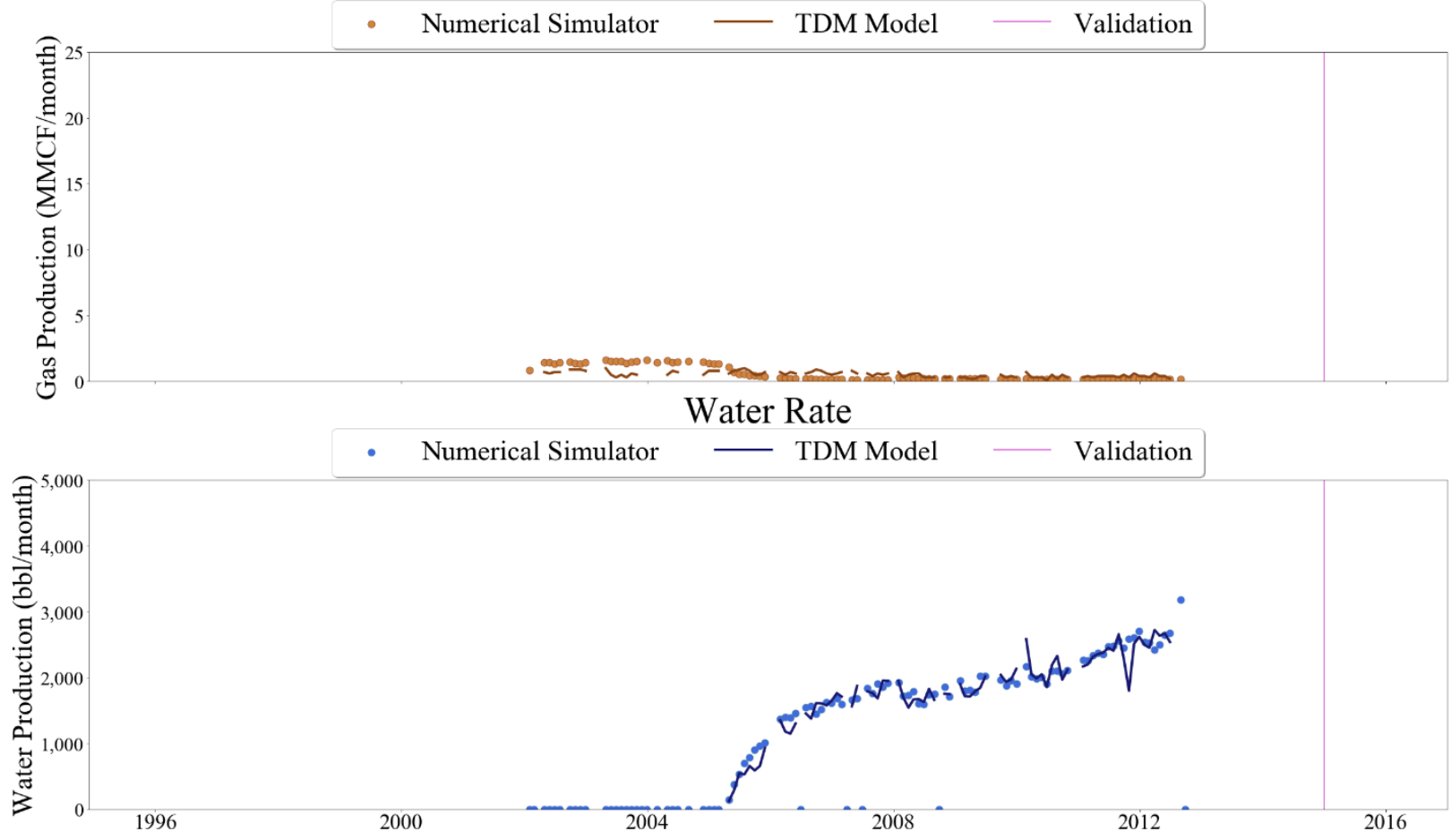

Figure 527 Well-030 oil, gas, and water rate TDM predictions vs actual simulation data plots for 2 Year Blind Validation TDM 

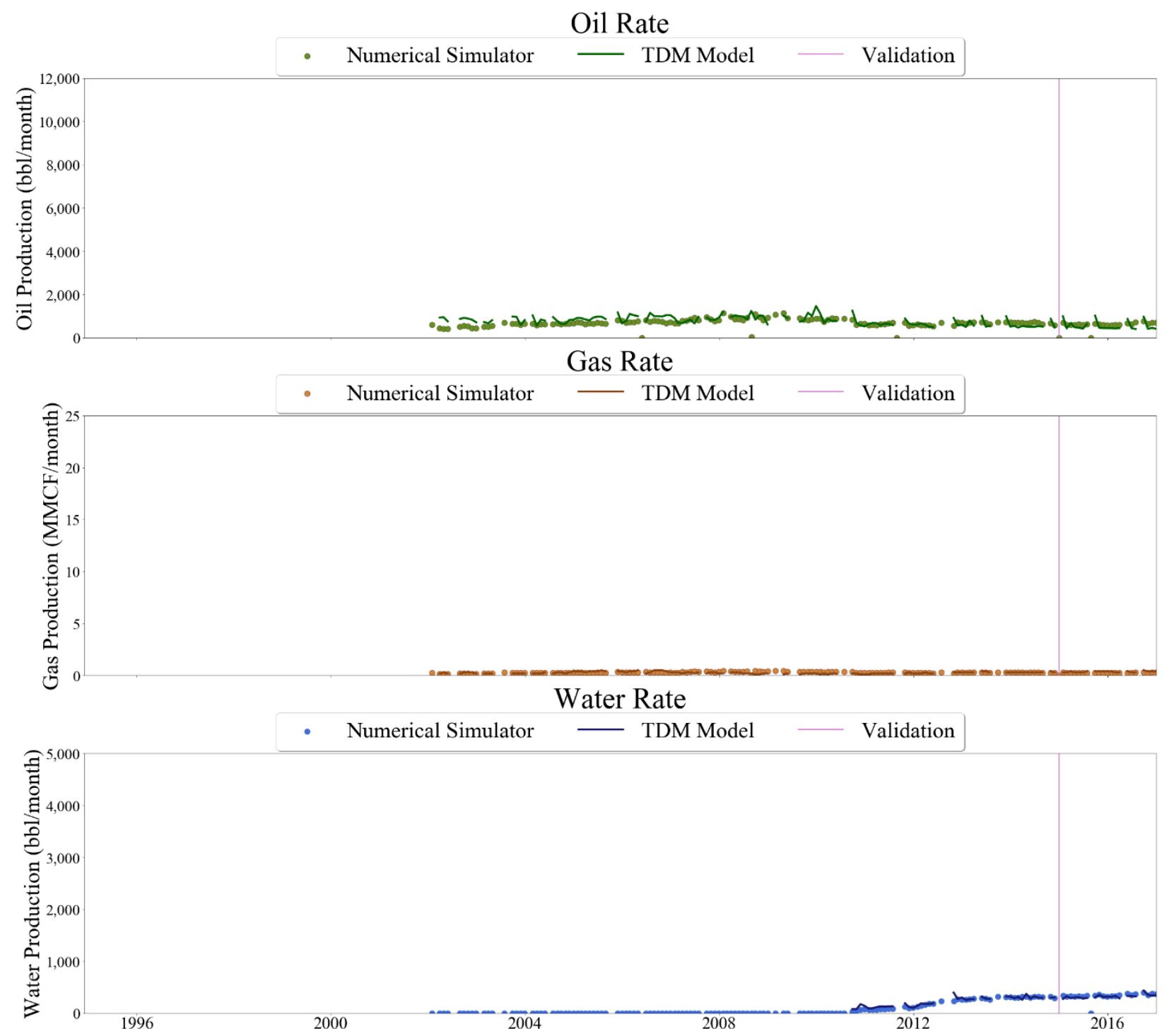

Figure 528 Well-031 oil, gas, and water rate TDM predictions vs actual simulation data plots for 2 Year Blind Validation TDM 


\section{Oil Rate}

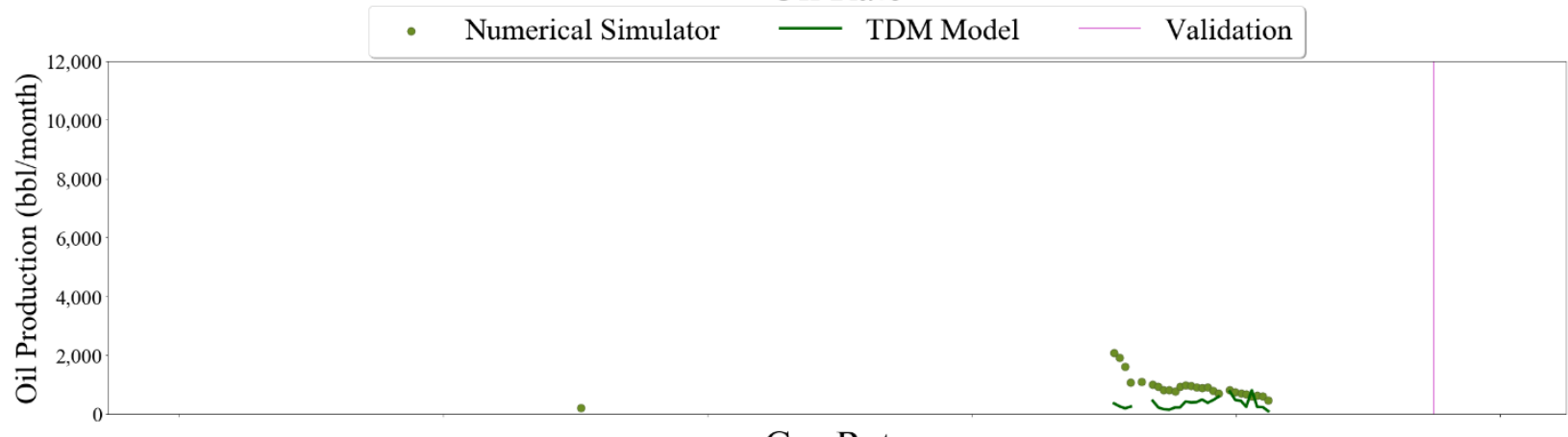

\section{Gas Rate}
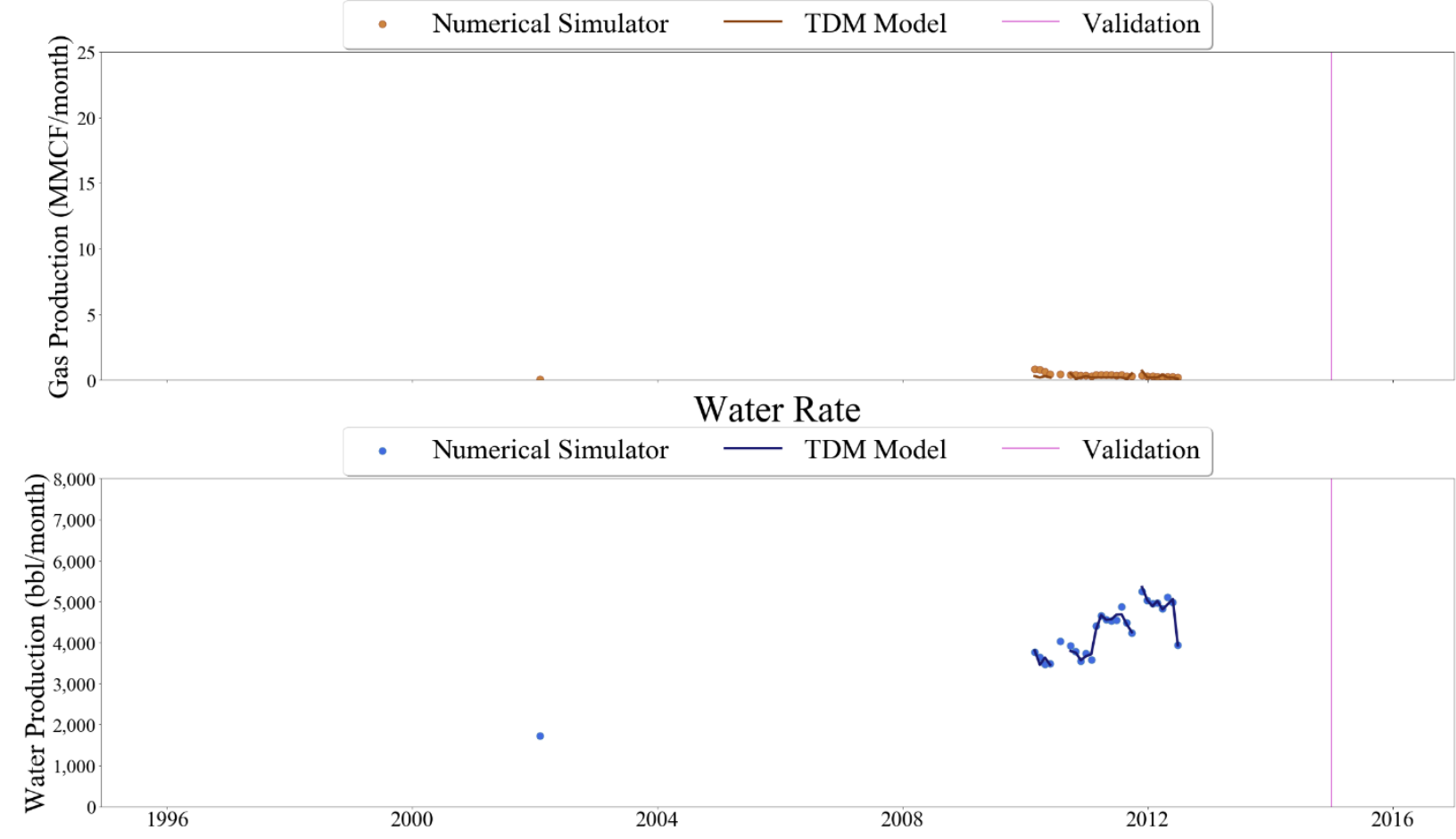

Figure 529 Well-032 oil, gas, and water rate TDM predictions vs actual simulation data plots for 2 Year Blind Validation TDM 


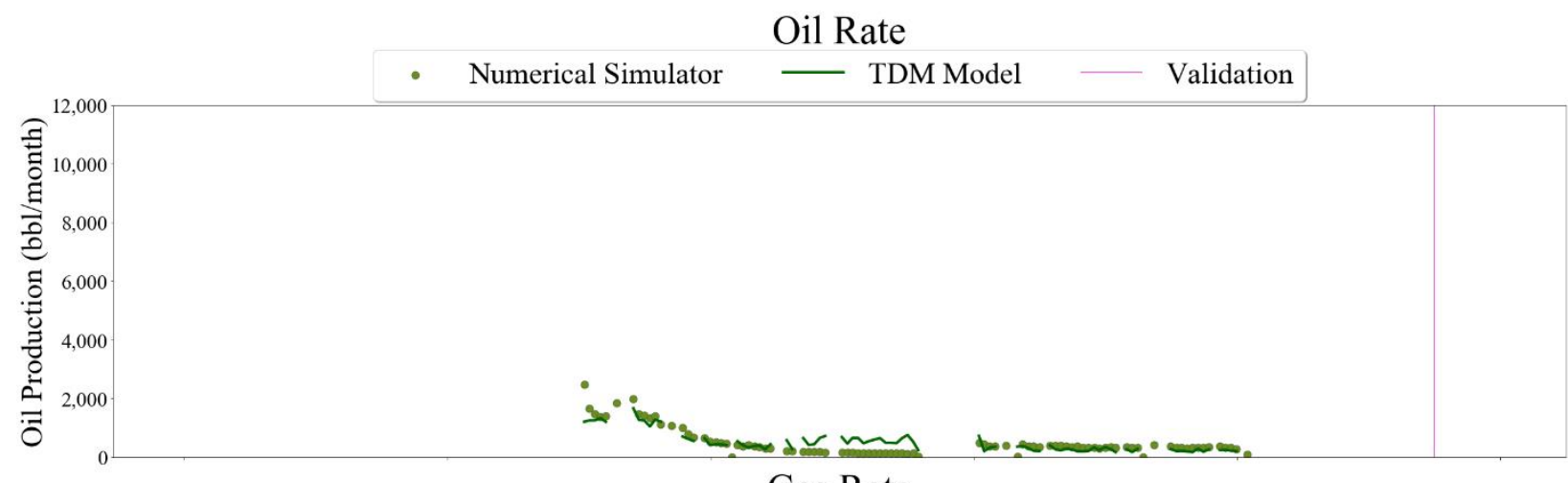

Gas Rate
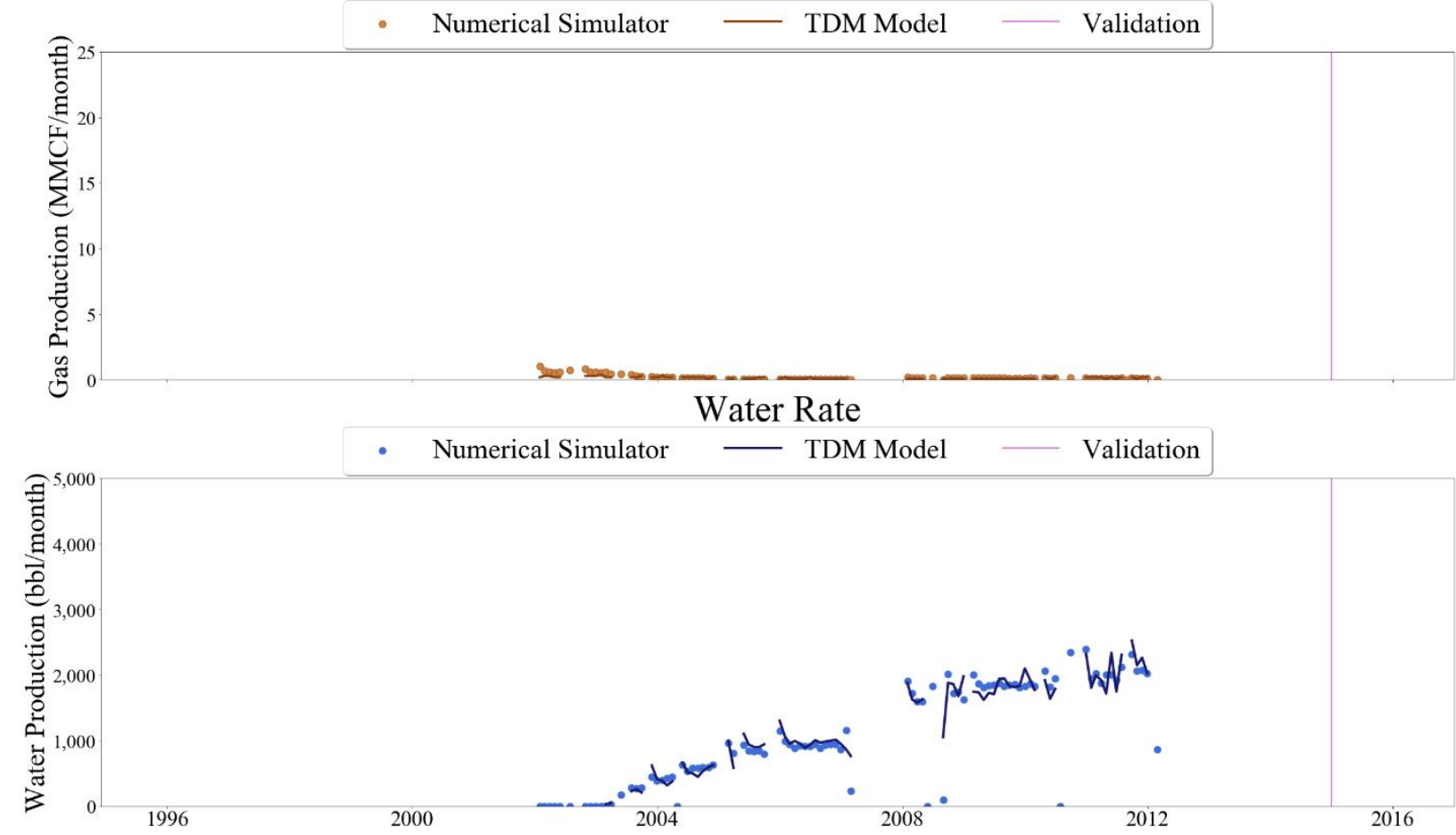

Figure 530 Well-033 oil, gas, and water rate TDM predictions vs actual simulation data plots for 2 Year Blind Validation TDM 

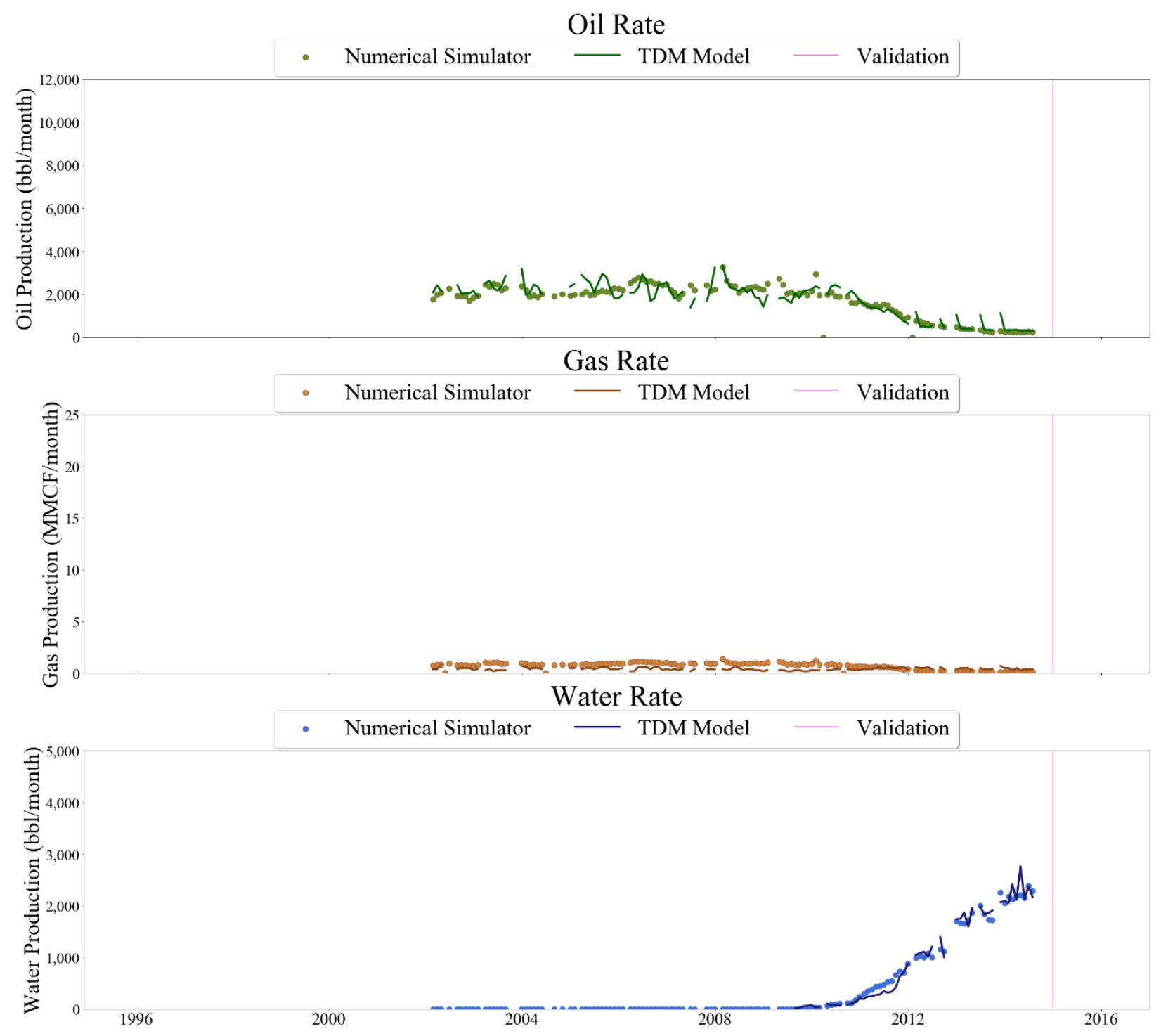

Figure 531 Well-034 oil, gas, and water rate TDM predictions vs actual simulation data plots for 2 Year Blind Validation TDM 


\section{Oil Rate}

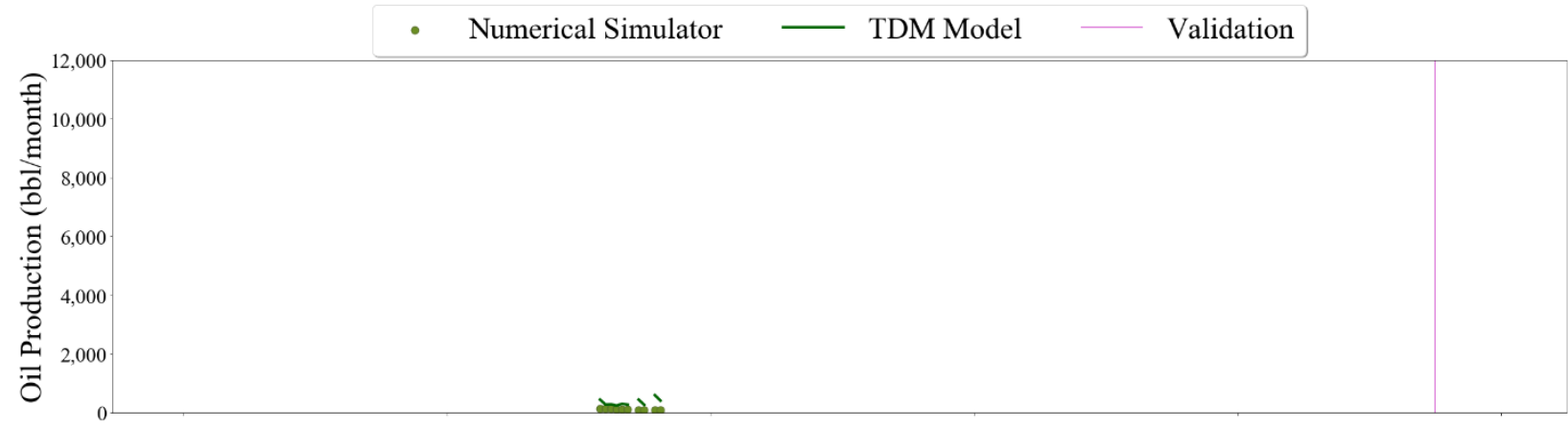

\section{Gas Rate}
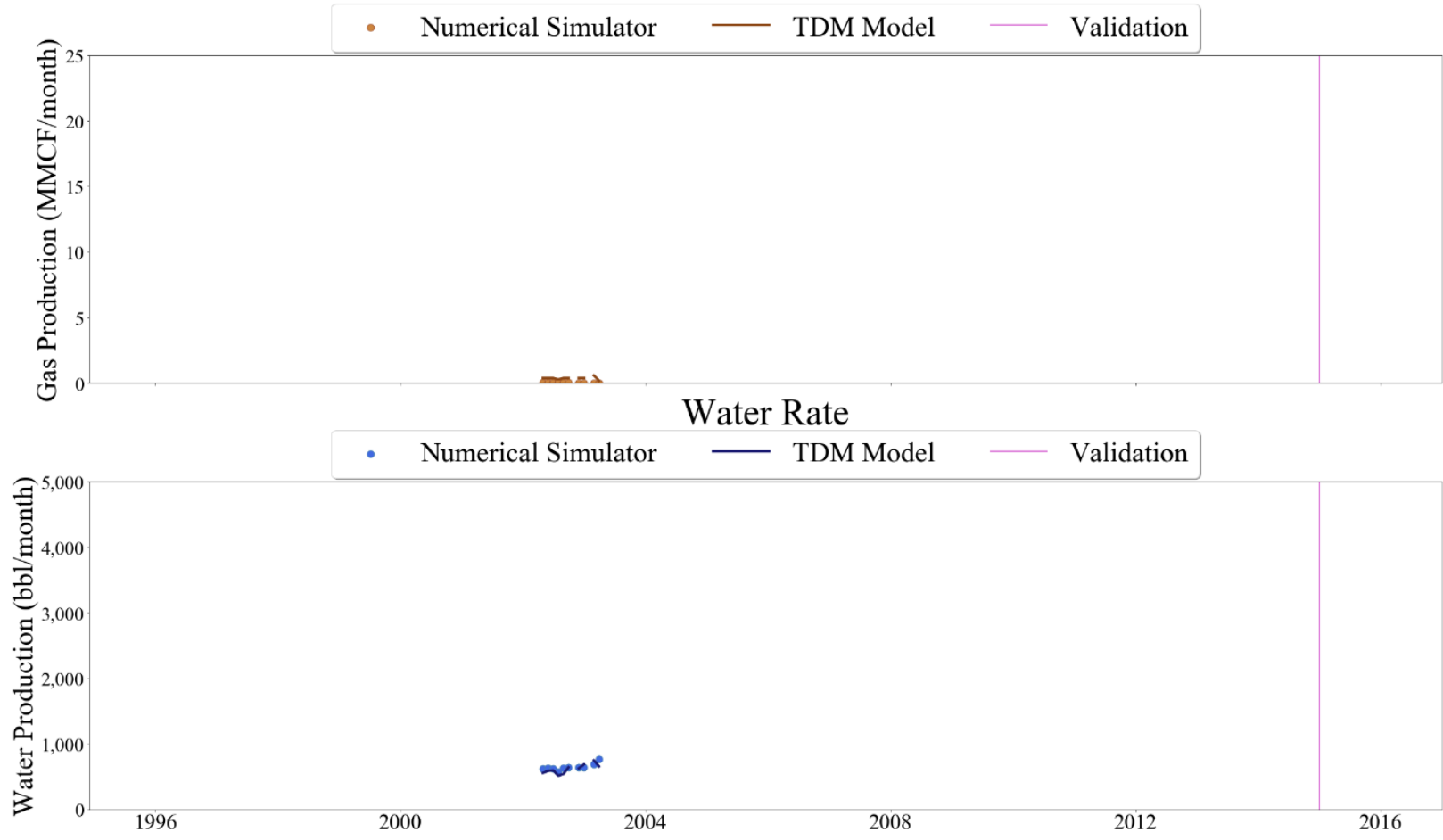

Figure 532 Well-035 oil, gas, and water rate TDM predictions vs actual simulation data plots for 2 Year Blind Validation TDM 

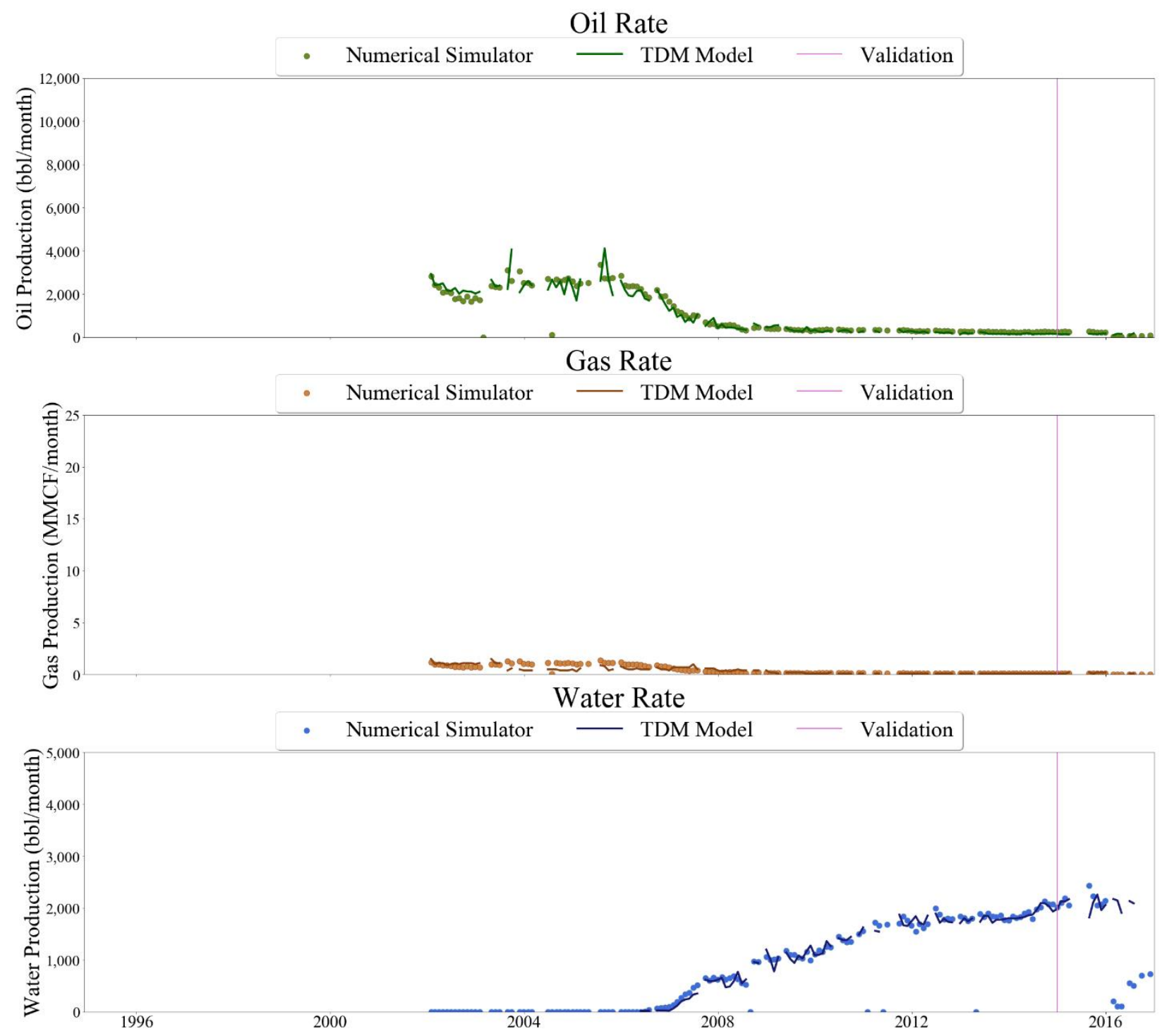

Figure 533 Well-036 oil, gas, and water rate TDM predictions vs actual simulation data plots for 2 Year Blind Validation TDM 


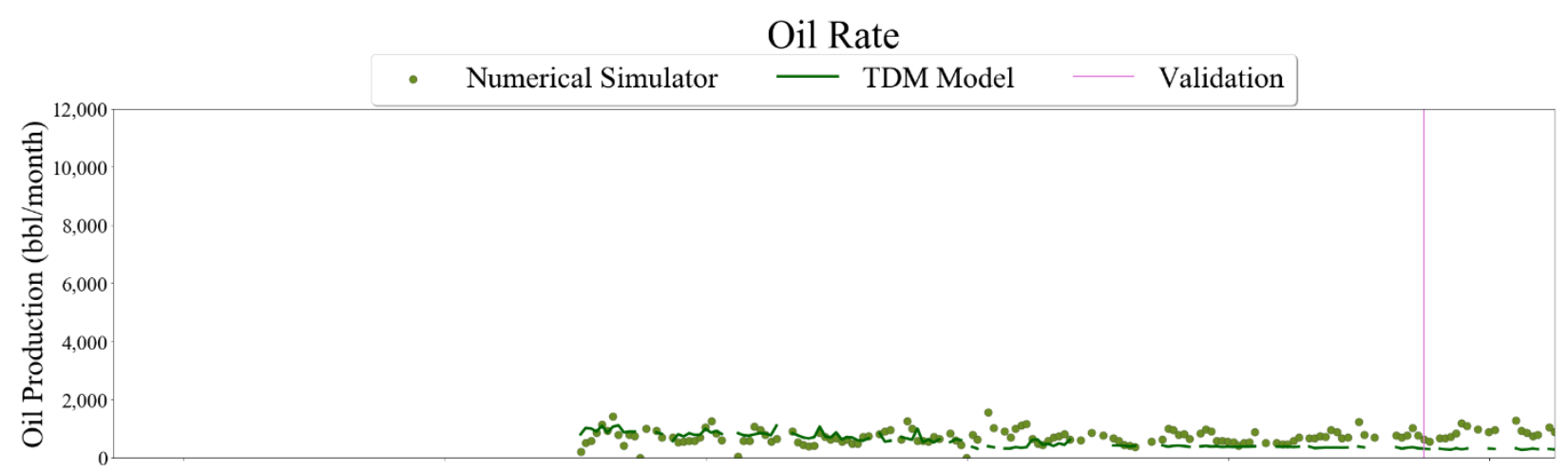

Gas Rate
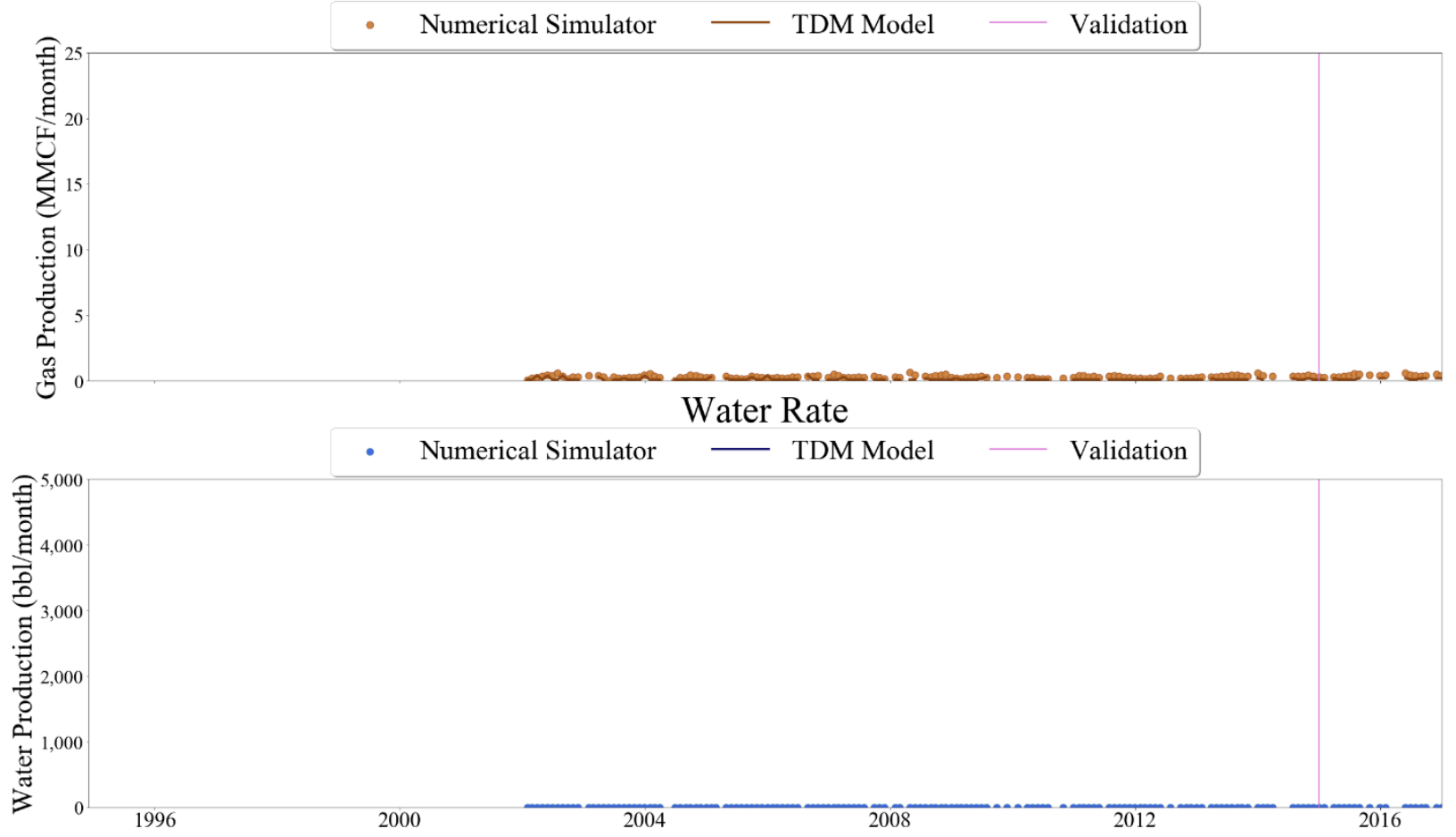

Figure 534 Well-037 oil, gas, and water rate TDM predictions vs actual simulation data plots for 2 Year Blind Validation TDM 


\section{Oil Rate}

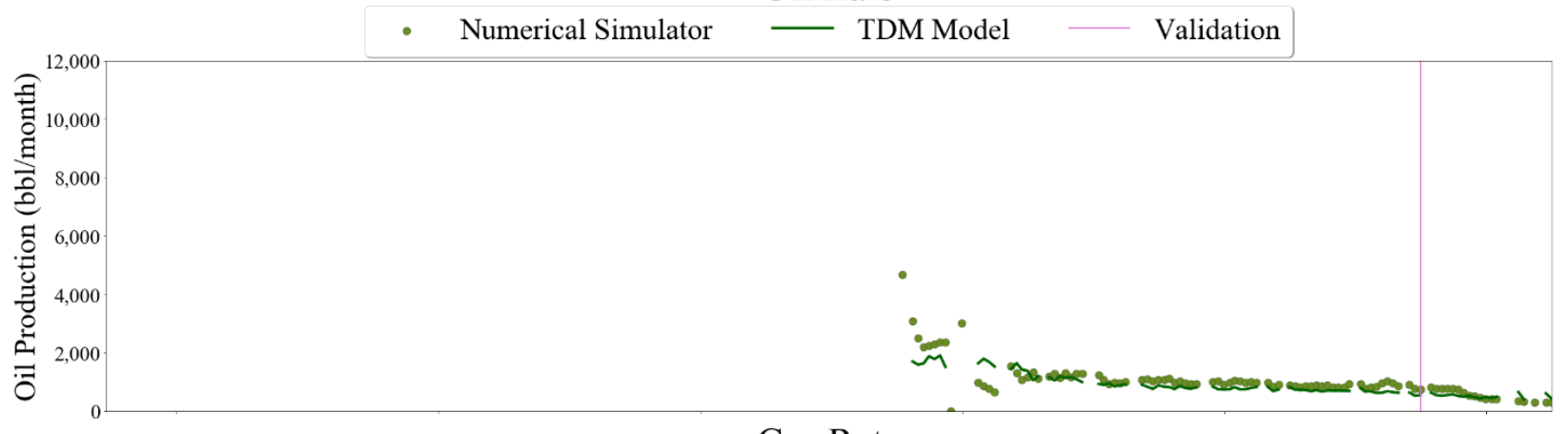

\section{Gas Rate}
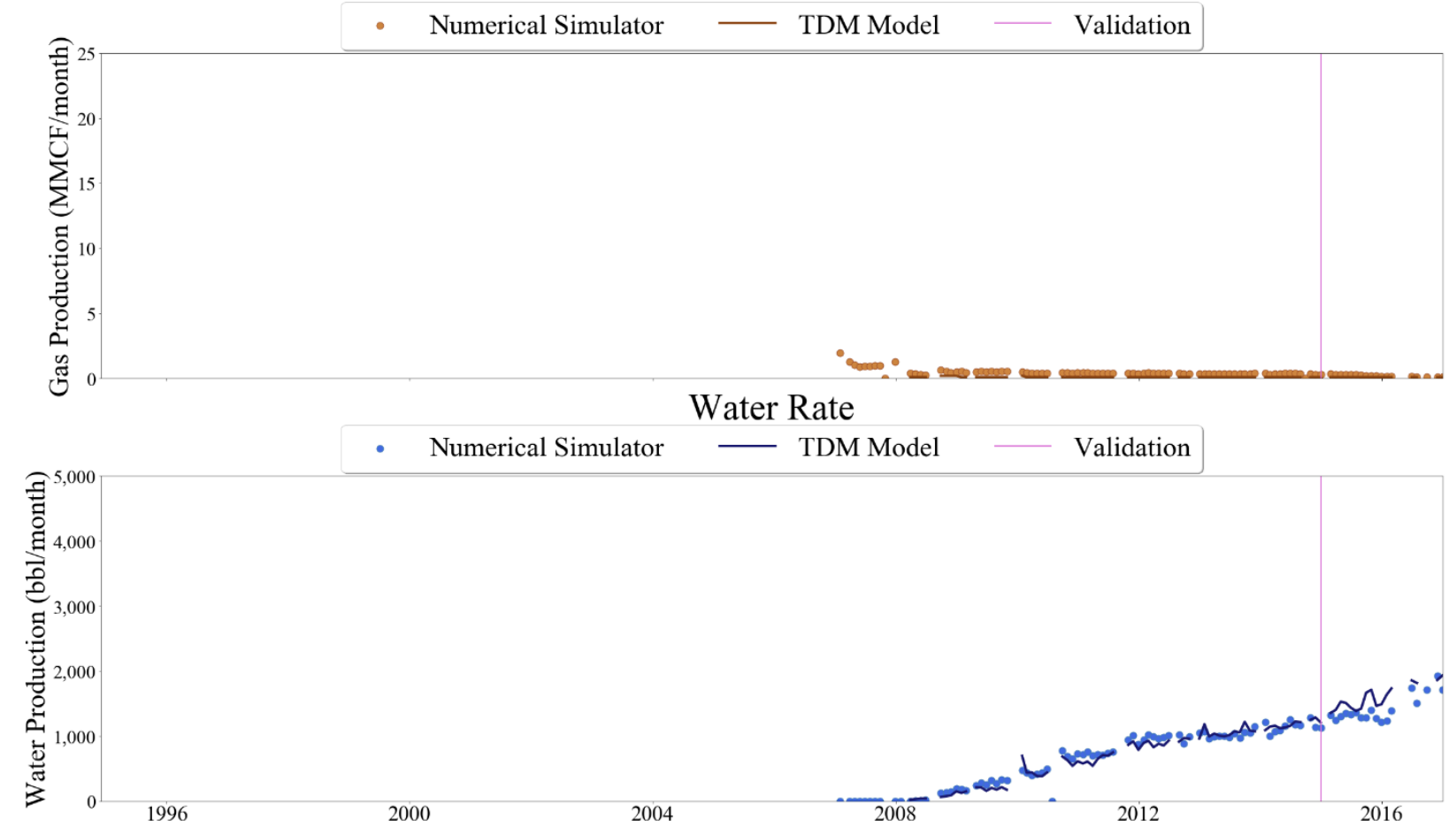

Figure 535 Well-038 oil, gas, and water rate TDM predictions vs actual simulation data plots for 2 Year Blind Validation TDM 


\section{Oil Rate}

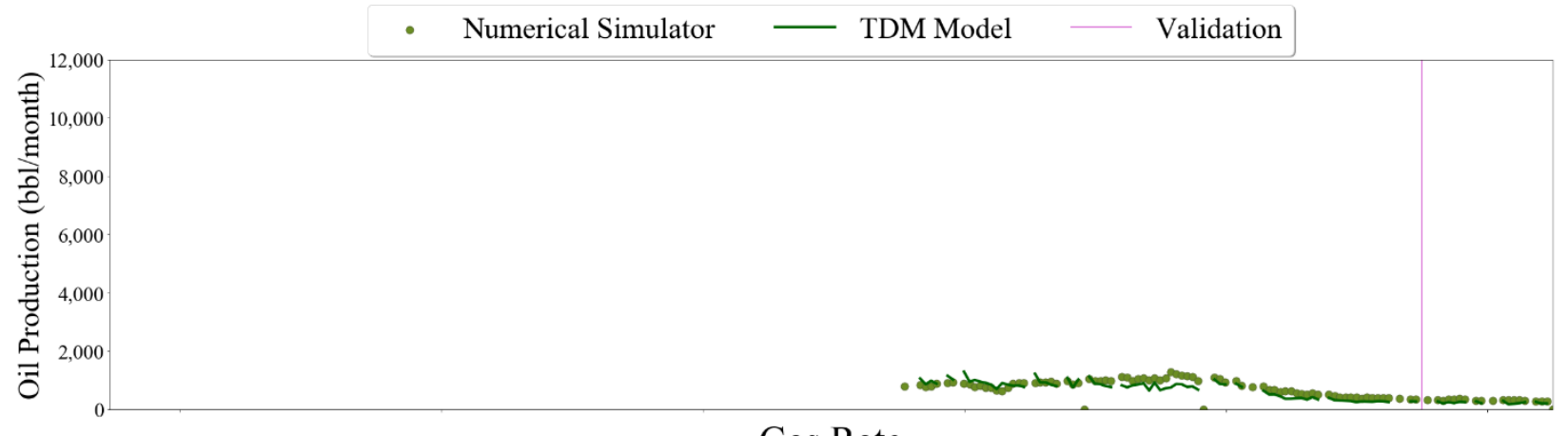

\section{Gas Rate}
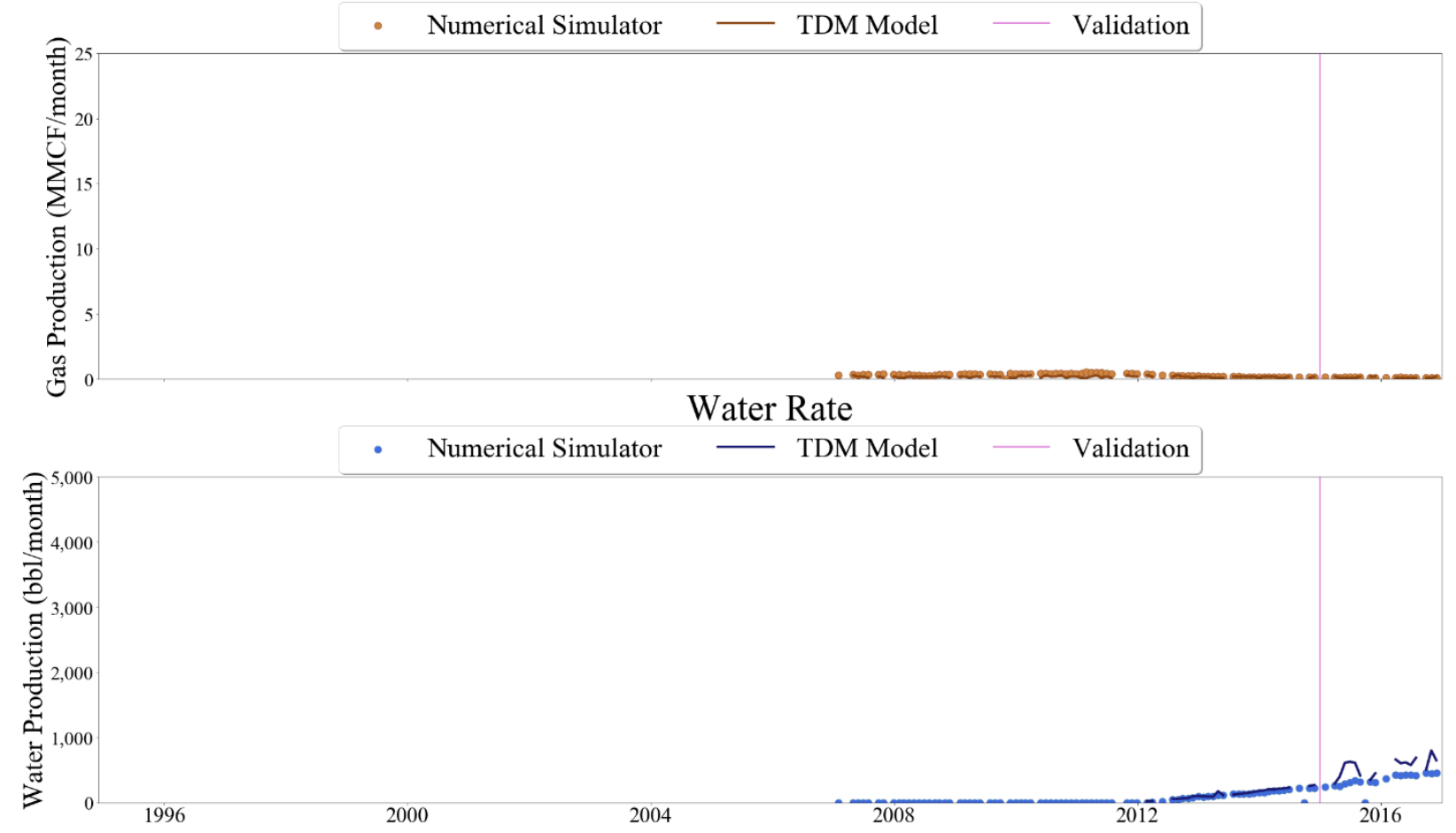

Figure 536 Well-039 oil, gas, and water rate TDM predictions vs actual simulation data plots for 2 Year Blind Validation TDM 


\section{Oil Rate}

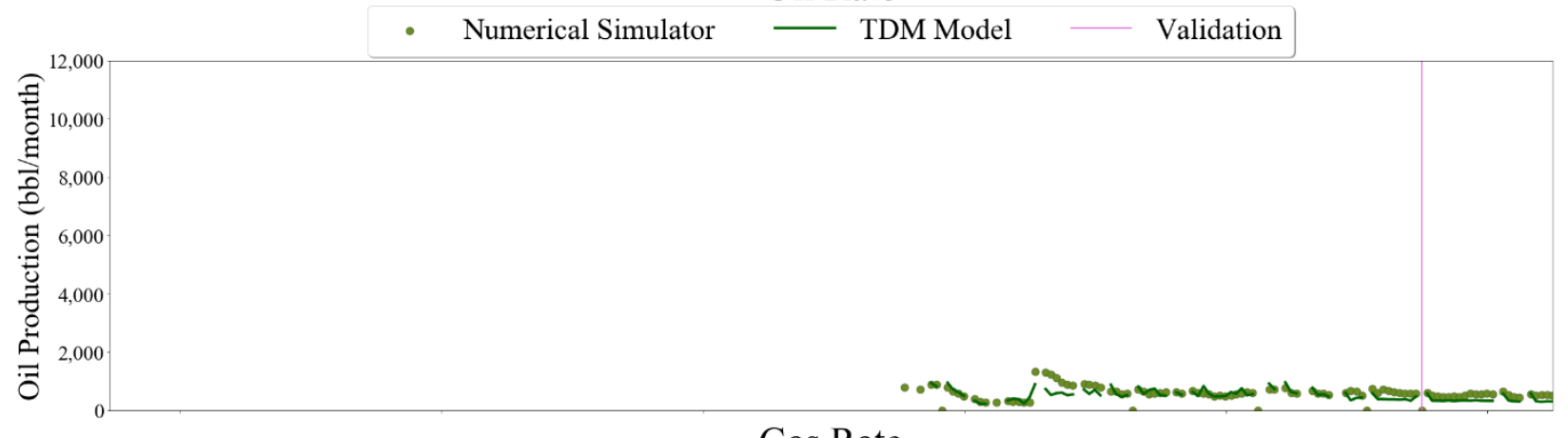

\section{Gas Rate}
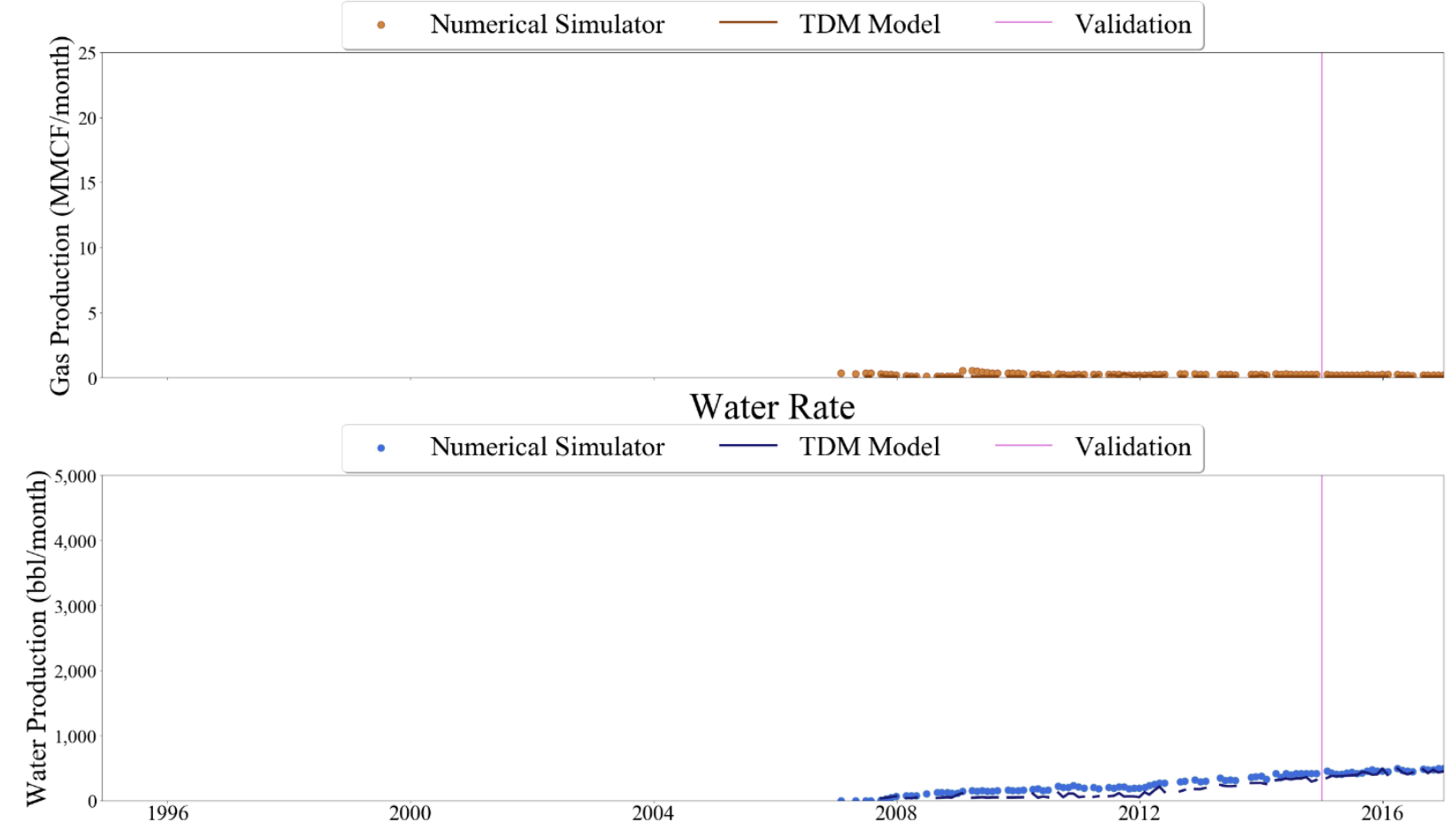

Figure 537 Well-040 oil, gas, and water rate TDM predictions vs actual simulation data plots for 2 Year Blind Validation TDM 


\section{Oil Rate}

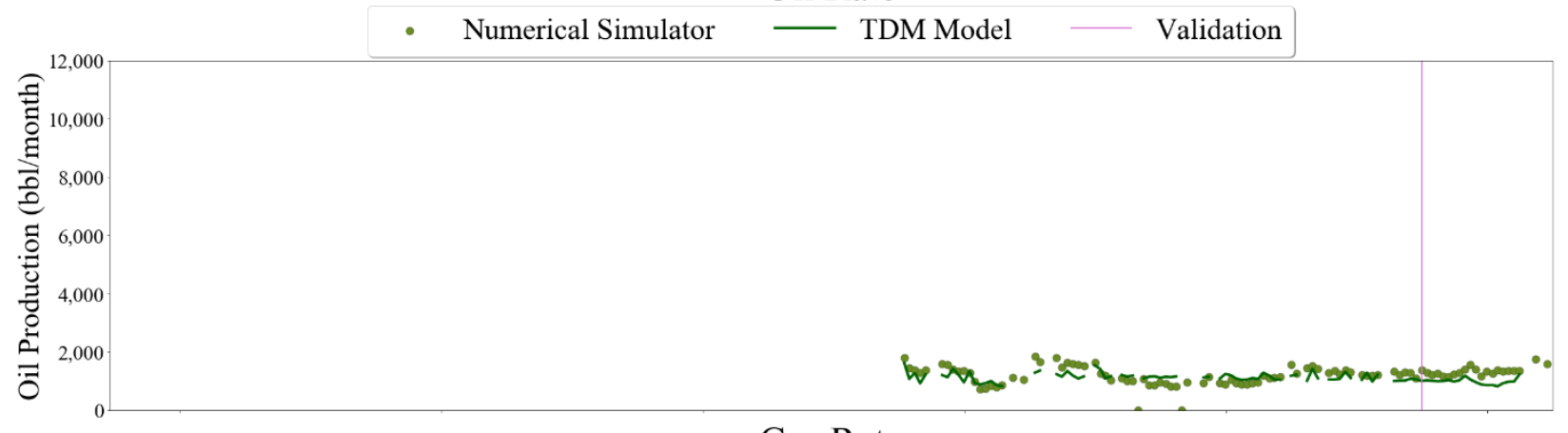

\section{Gas Rate}
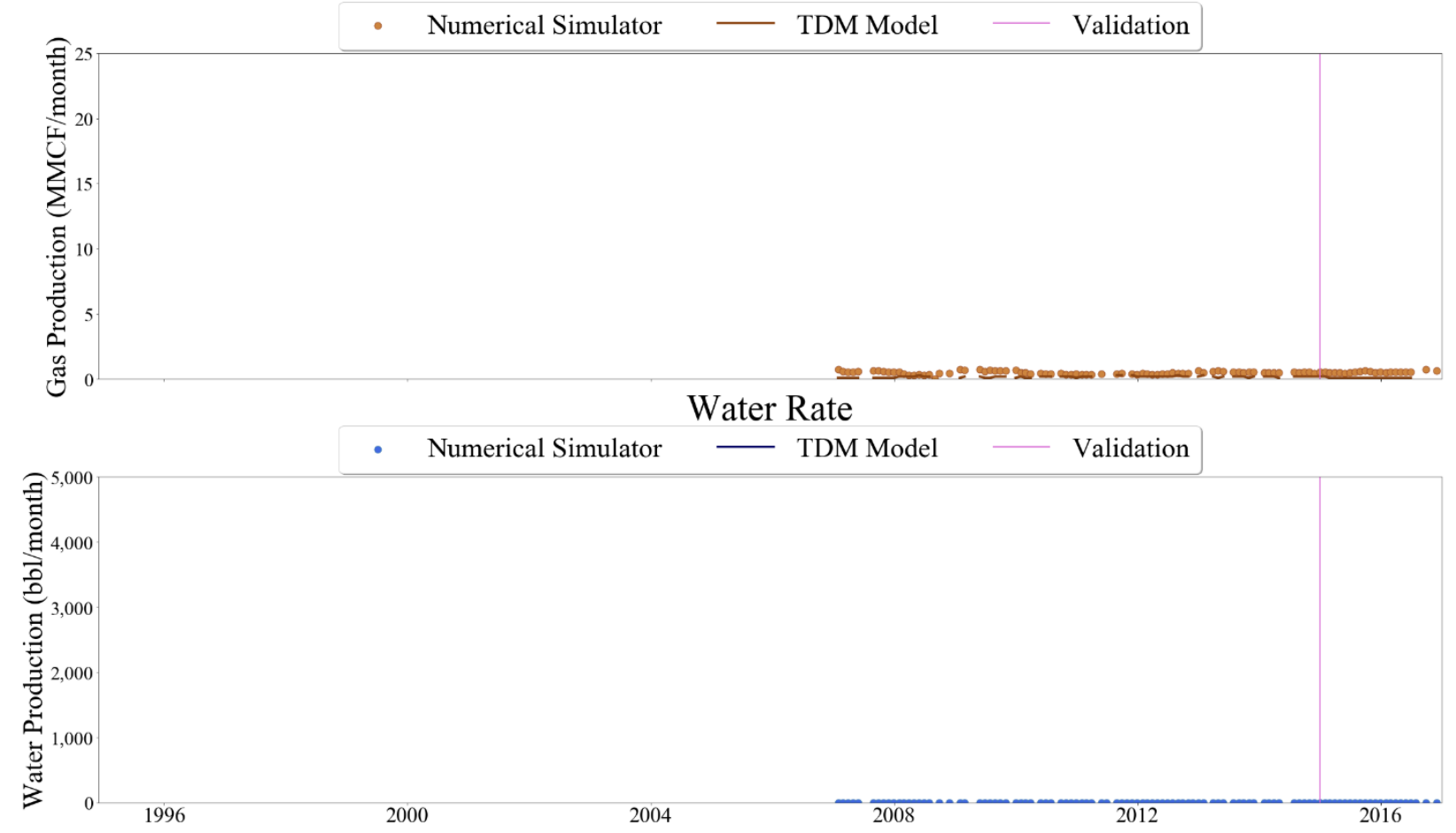

Figure 538 Well-041 oil, gas, and water rate TDM predictions vs actual simulation data plots for 2 Year Blind Validation TDM 


\section{Oil Rate}

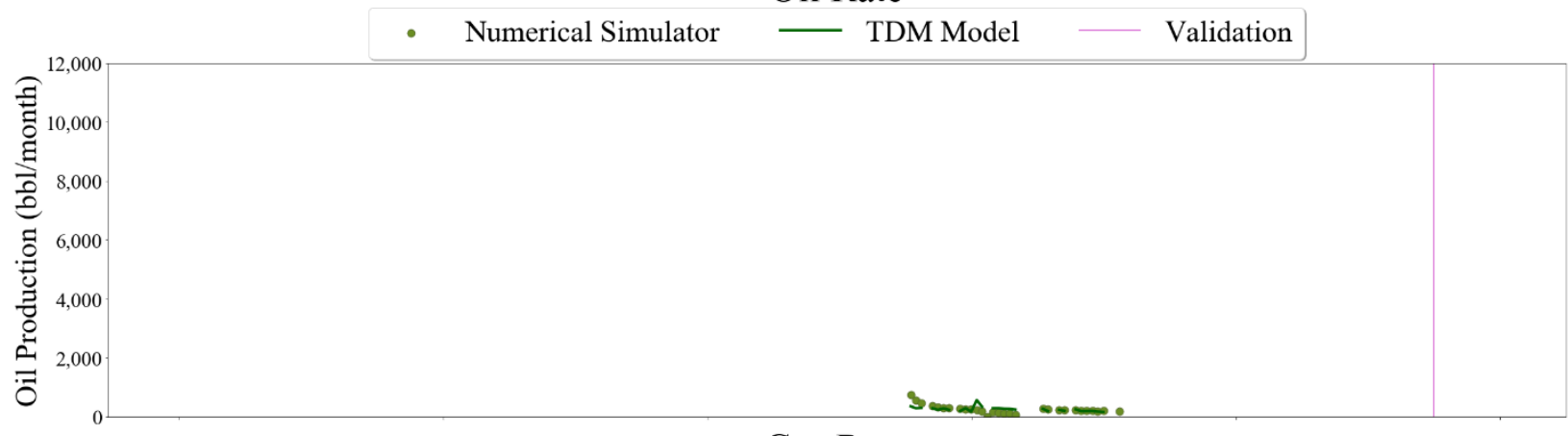

\section{Gas Rate}
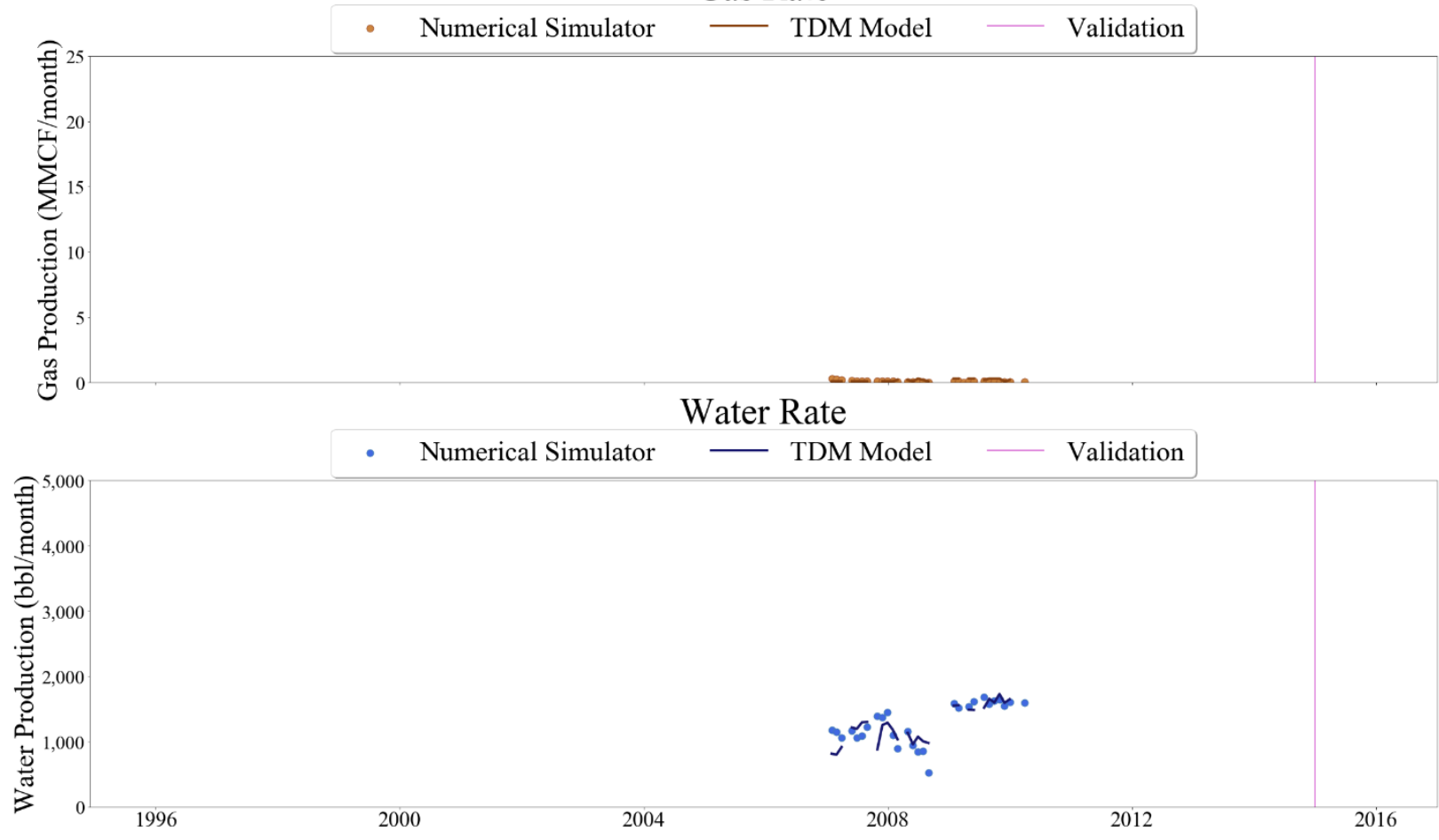

Figure 539 Well-042 oil, gas, and water rate TDM predictions vs actual simulation data plots for 2 Year Blind Validation TDM 


\section{Oil Rate}

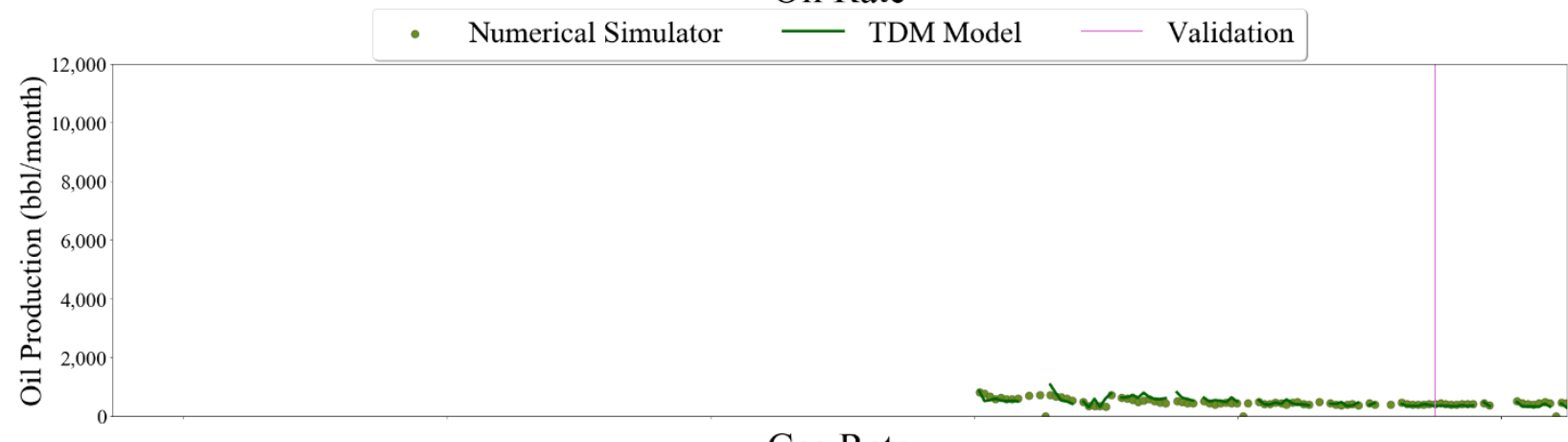

\section{Gas Rate}
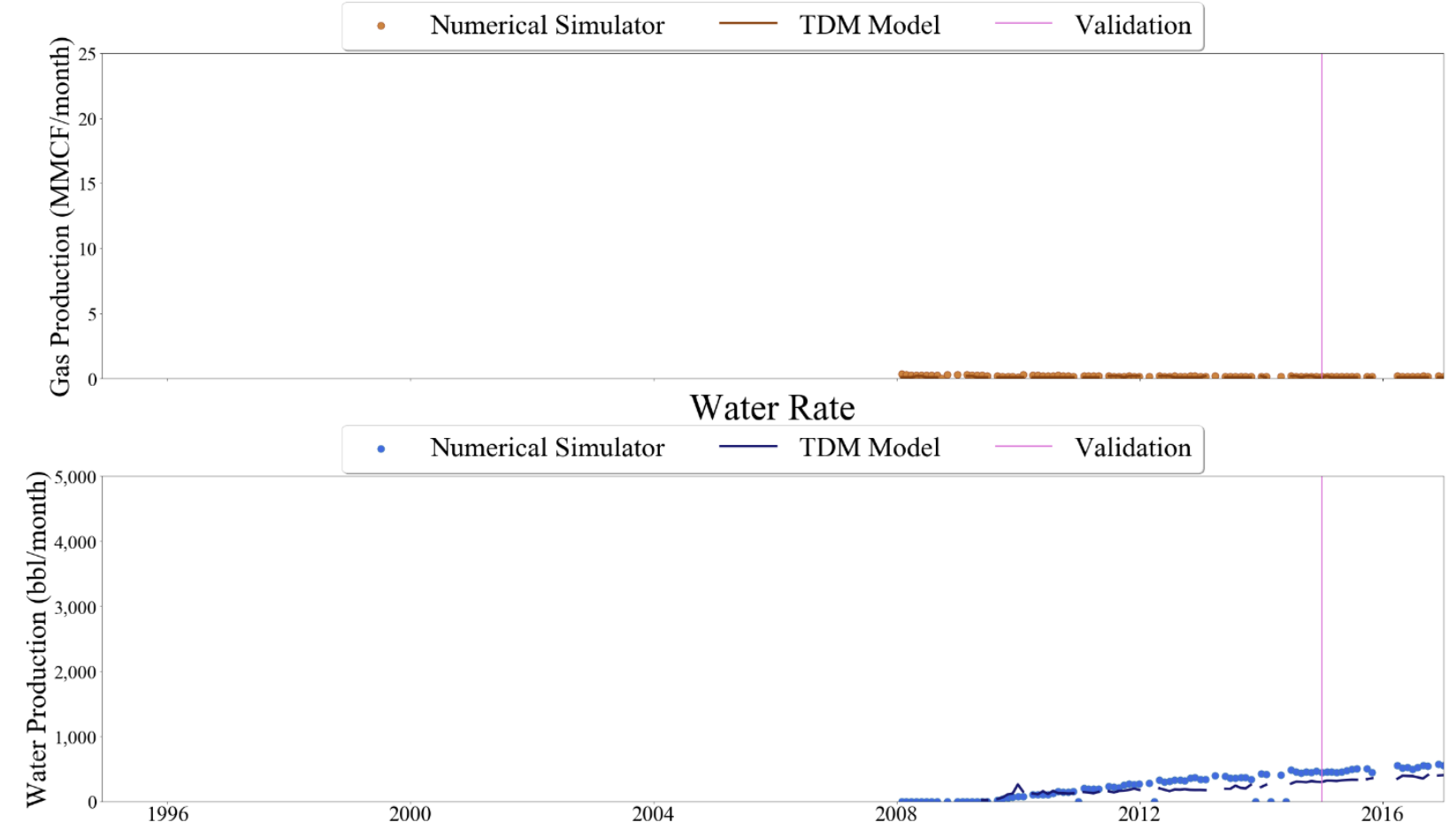

Figure 540 Well-043 oil, gas, and water rate TDM predictions vs actual simulation data plots for 2 Year Blind Validation TDM 


\section{Oil Rate}
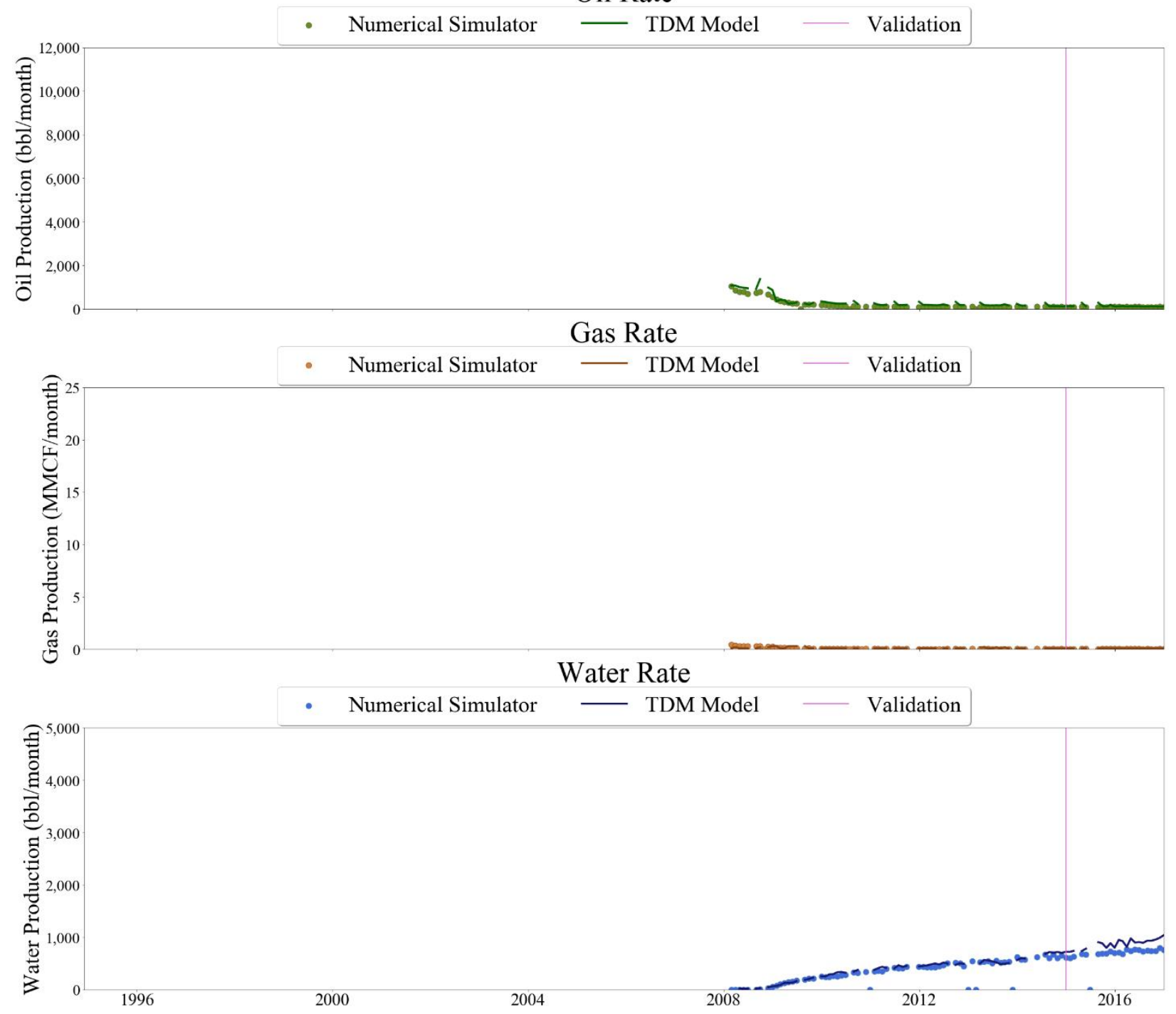

Figure 541 Well-044 oil, gas, and water rate TDM predictions vs actual simulation data plots for 2 Year Blind Validation TDM 

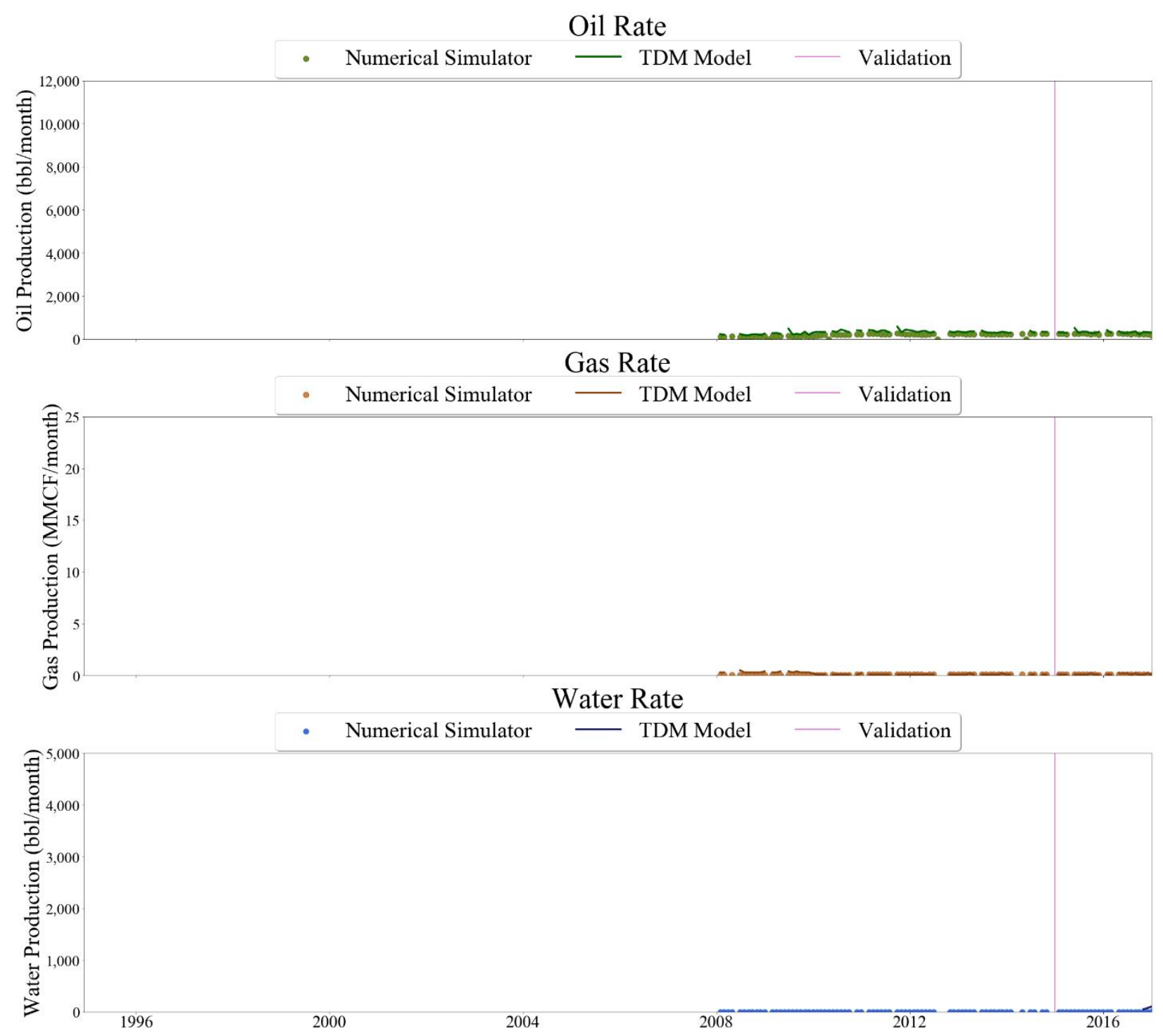

Figure 542 Well-045 oil, gas, and water rate TDM predictions vs actual simulation data plots for 2 Year Blind Validation TDM 

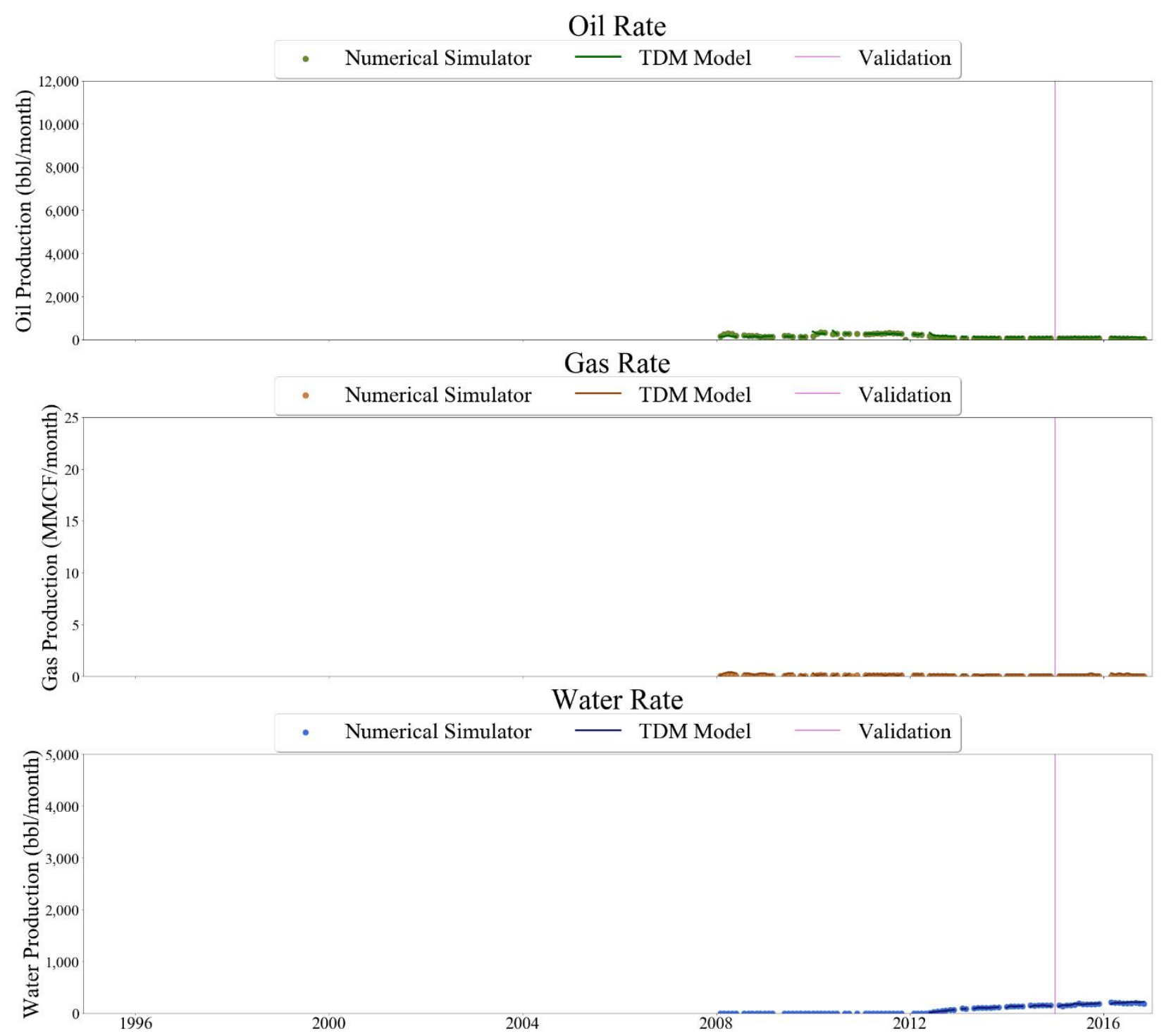

Figure 543 Well-046 oil, gas, and water rate TDM predictions vs actual simulation data plots for 2 Year Blind Validation TDM 


\section{Oil Rate}

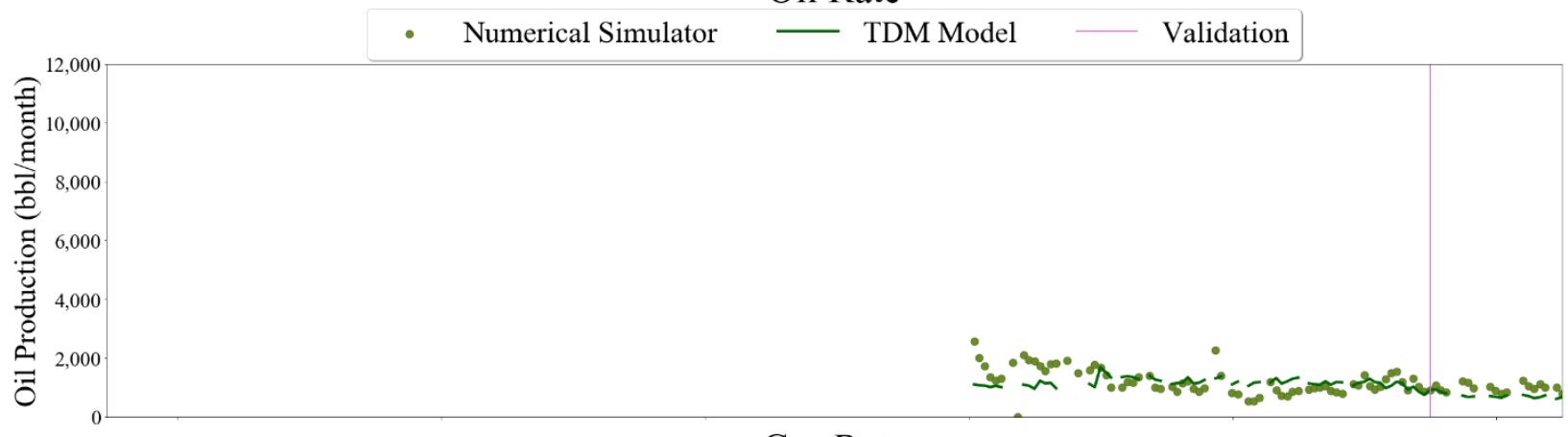

\section{Gas Rate}
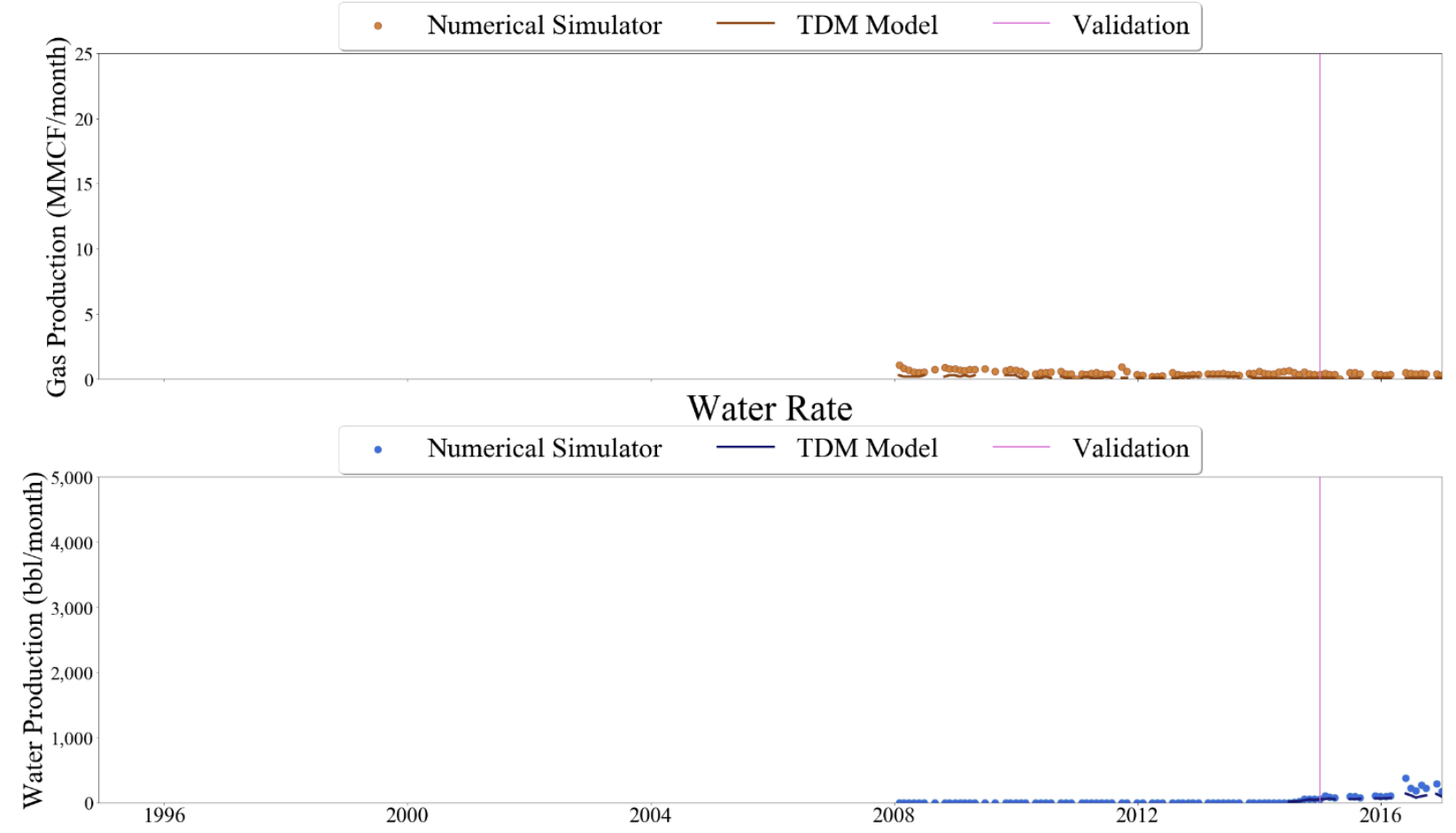

Figure 544 Well-047 oil, gas, and water rate TDM predictions vs actual simulation data plots for 2 Year Blind Validation TDM 


\section{Oil Rate}

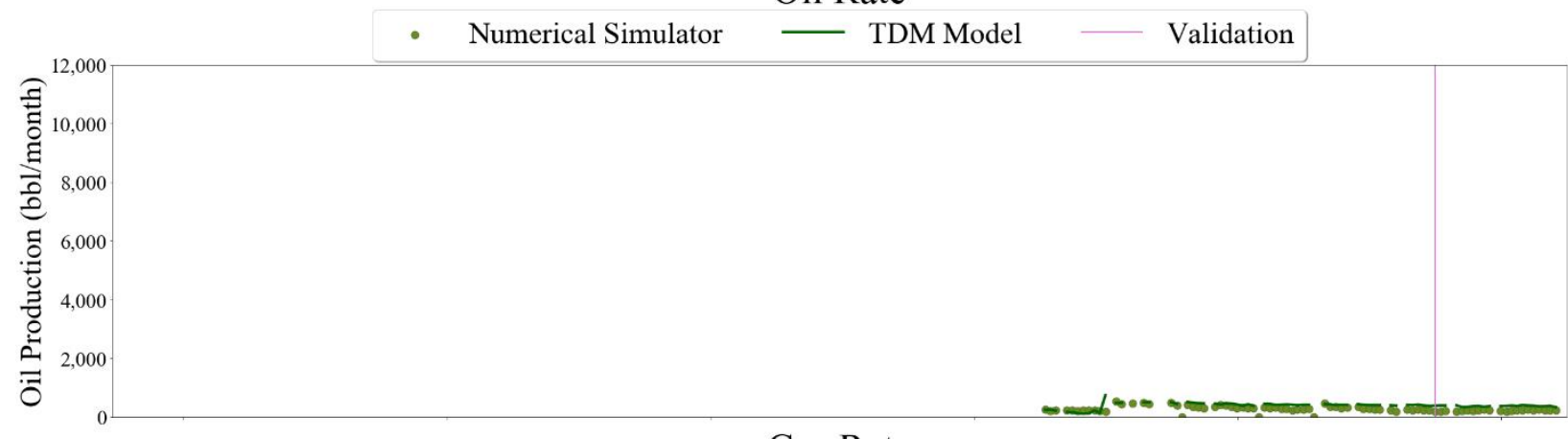

\section{Gas Rate}

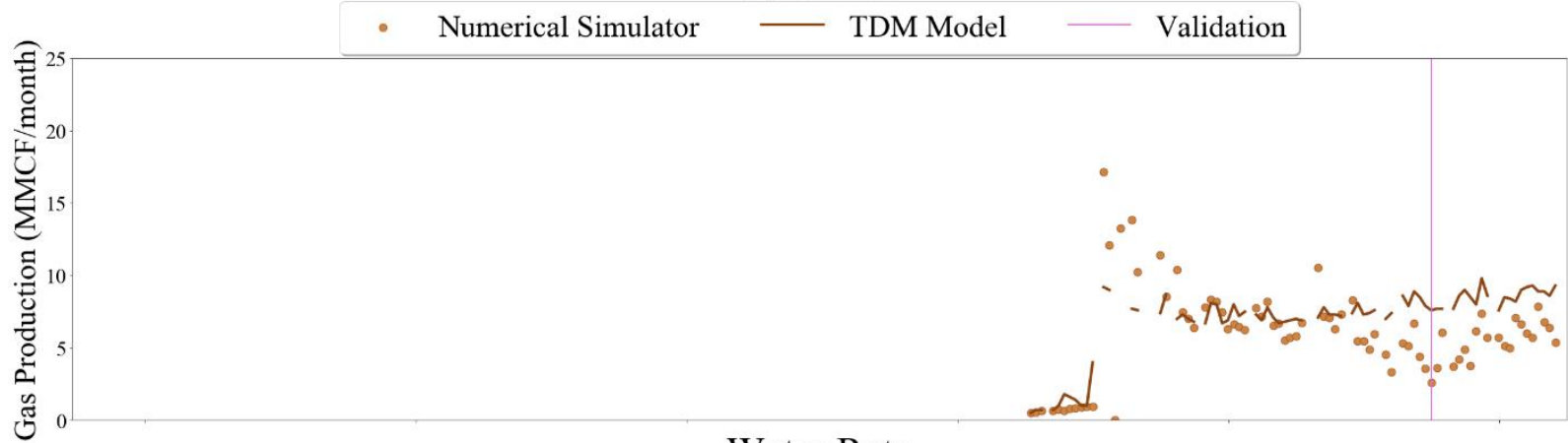

Water Rate

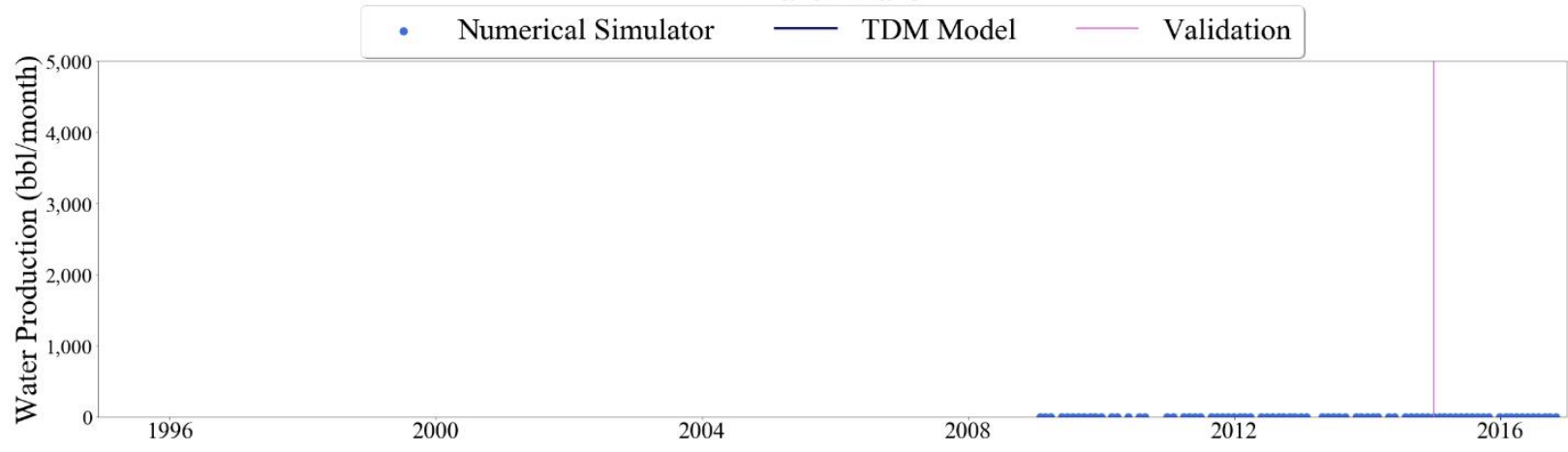

Figure 545 Well-048 oil, gas, and water rate TDM predictions vs actual simulation data plots for 2 Year Blind Validation TDM 


\section{Oil Rate}
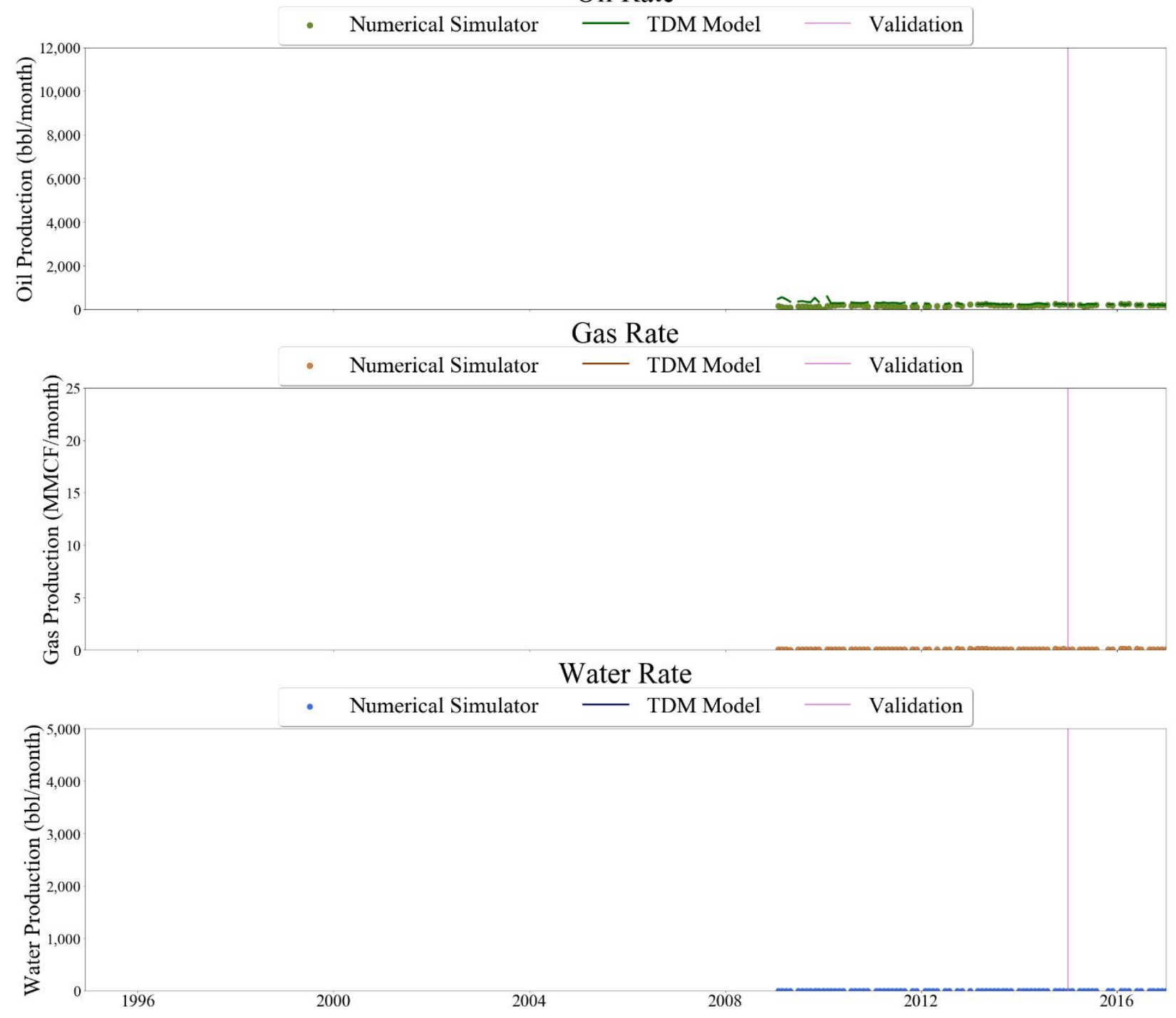

Figure 546 Well-049 oil, gas, and water rate TDM predictions vs actual simulation data plots for 2 Year Blind Validation TDM 


\section{Oil Rate}

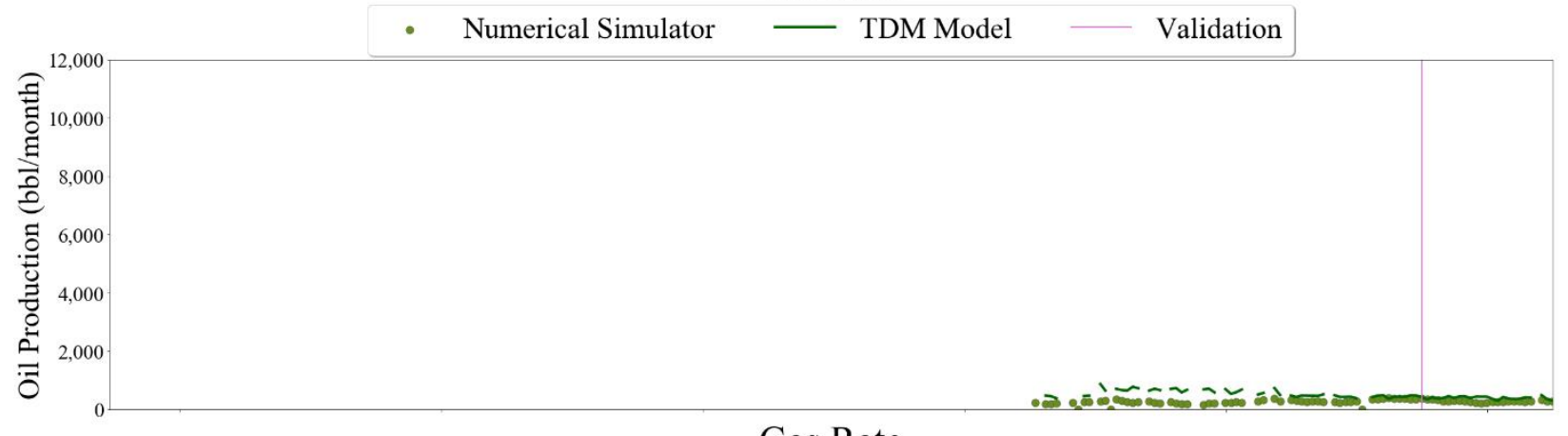

Gas Rate
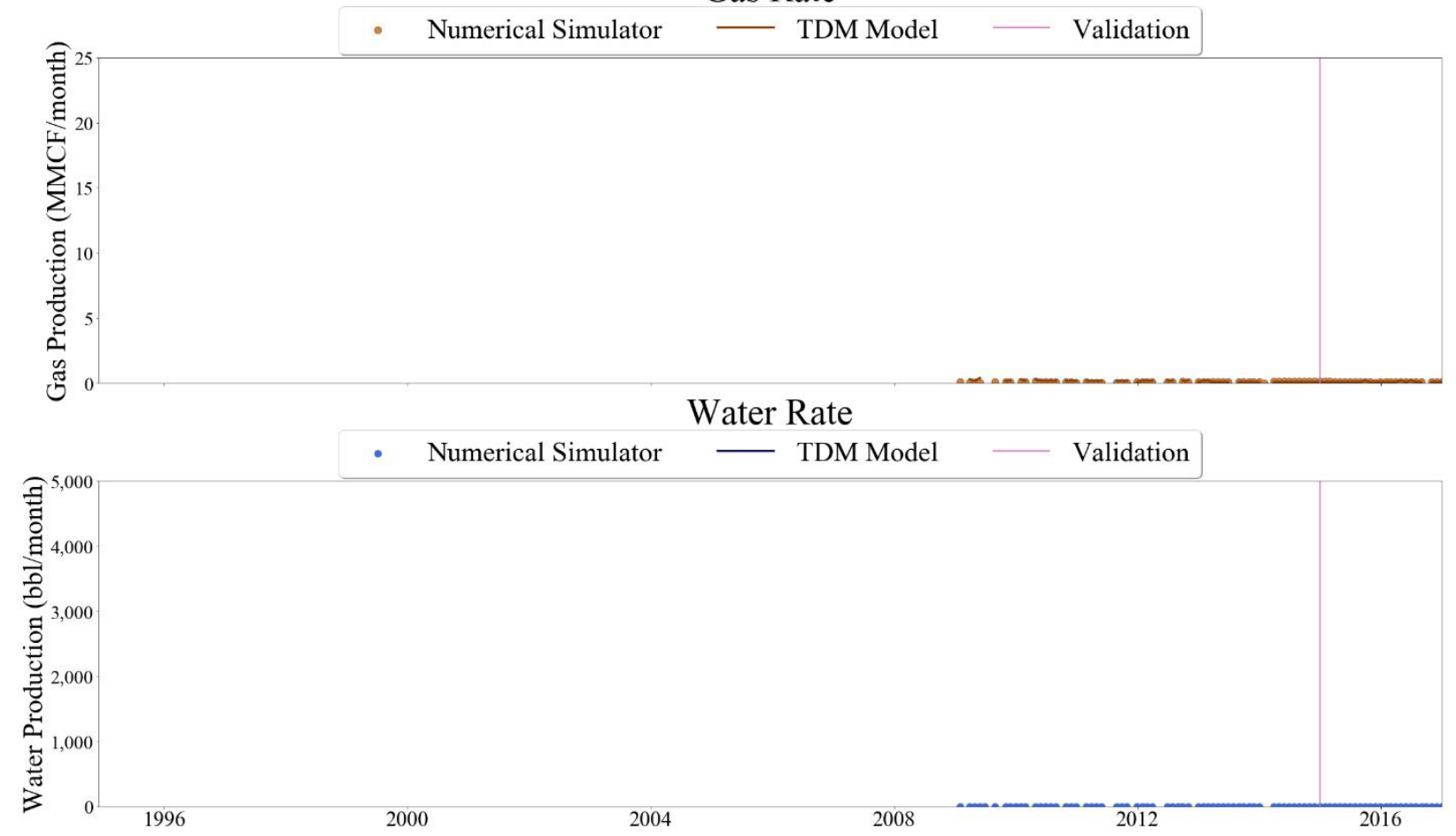

Figure 547 Well-050 oil, gas, and water rate TDM predictions vs actual simulation data plots for 2 Year Blind Validation TDM 


\section{Oil Rate}

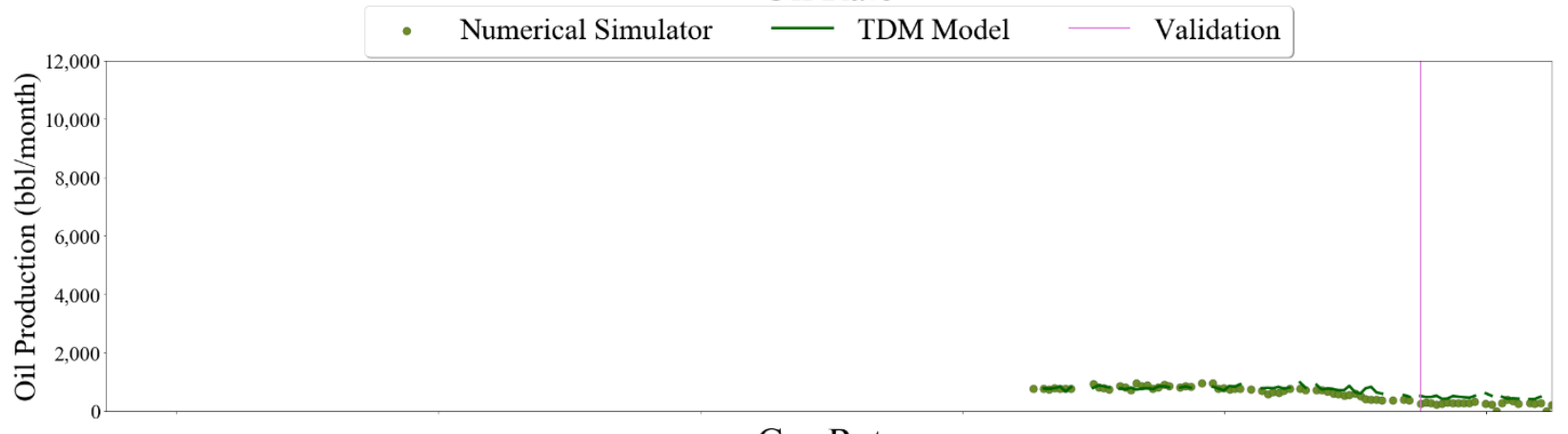

\section{Gas Rate}
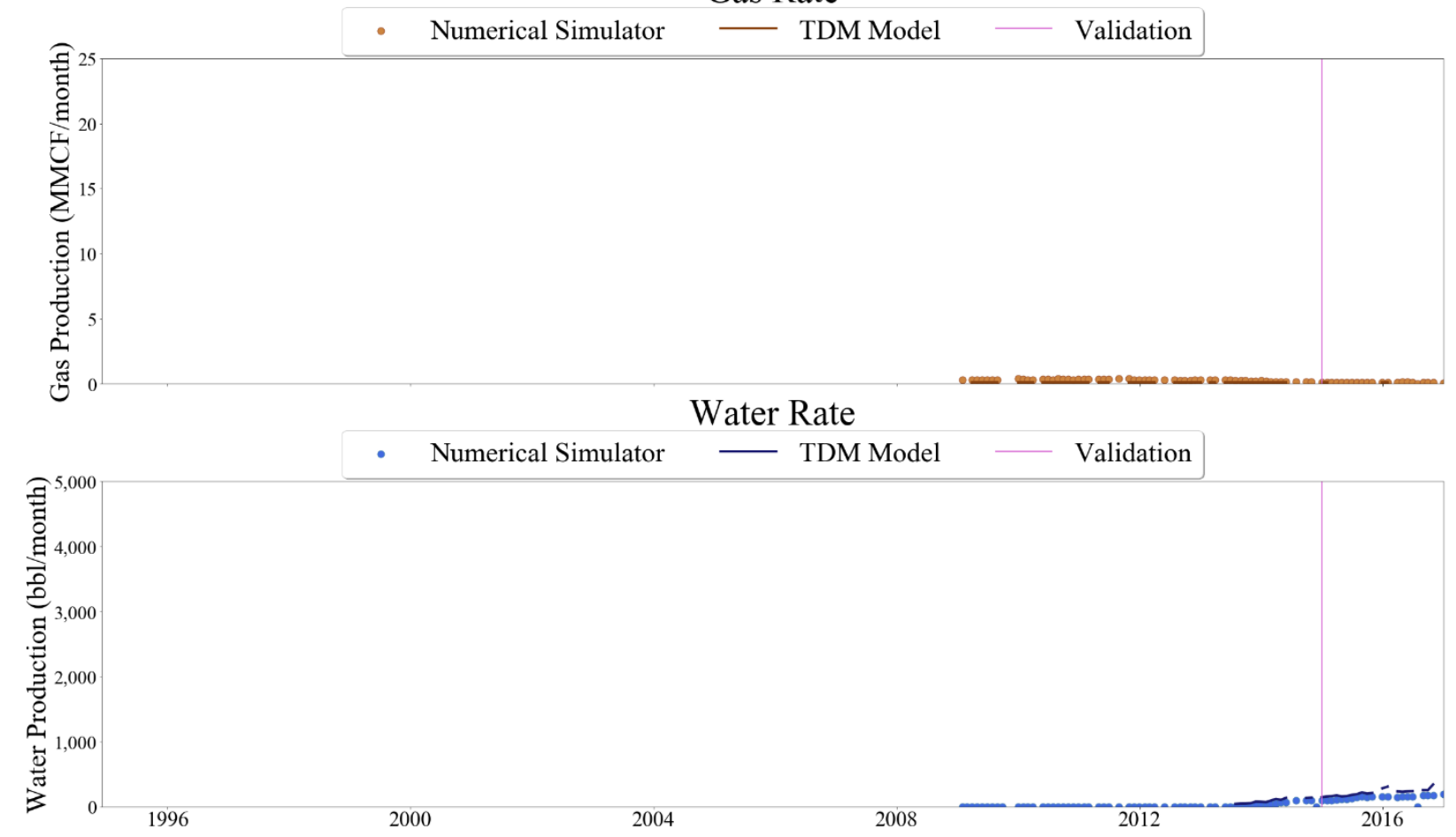

Figure 548 Well-051 oil, gas, and water rate TDM predictions vs actual simulation data plots for 2 Year Blind Validation TDM 


\section{Oil Rate}
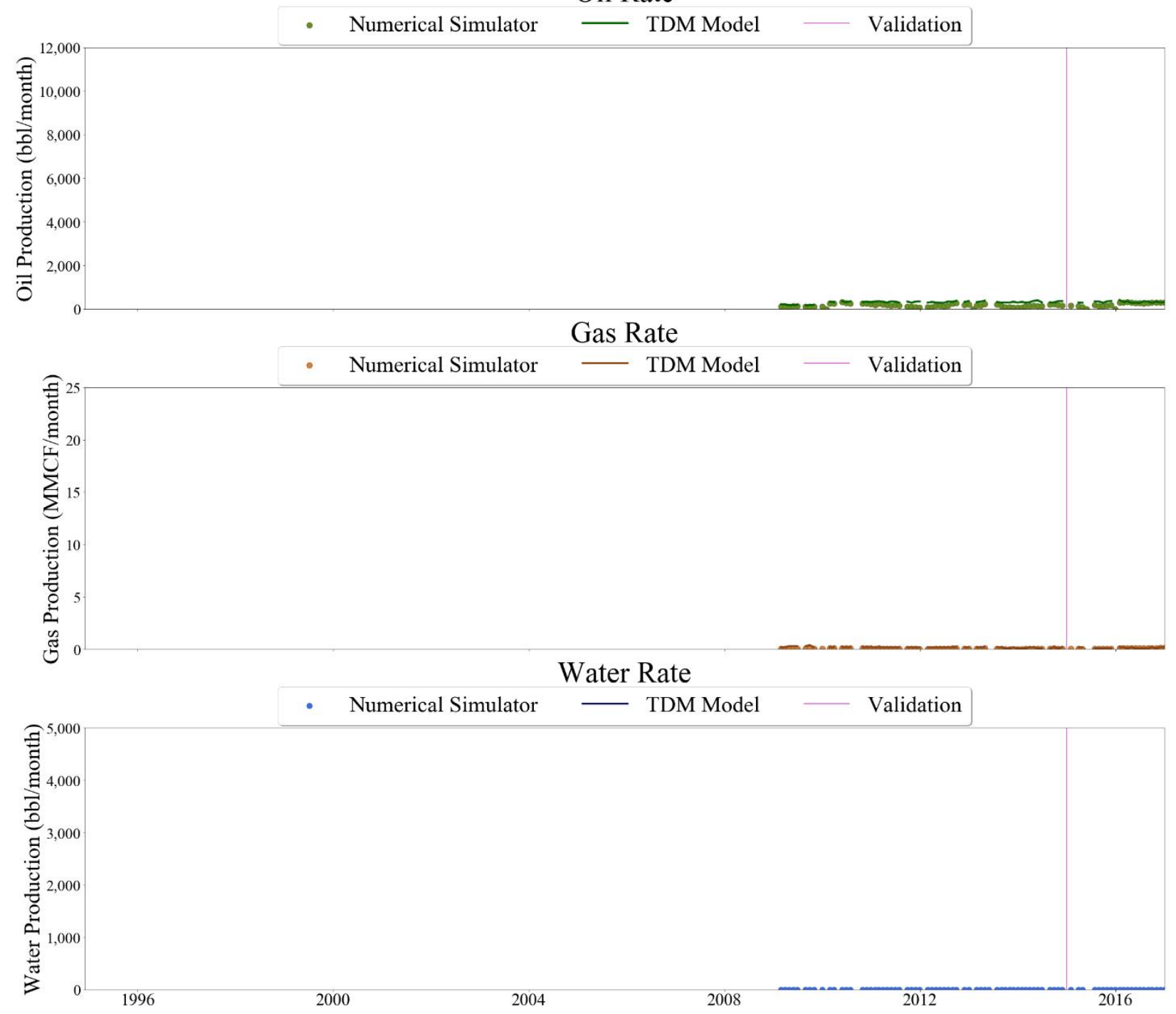

Figure 549 Well-052 oil, gas, and water rate TDM predictions vs actual simulation data plots for 2 Year Blind Validation TDM 


\section{Oil Rate}
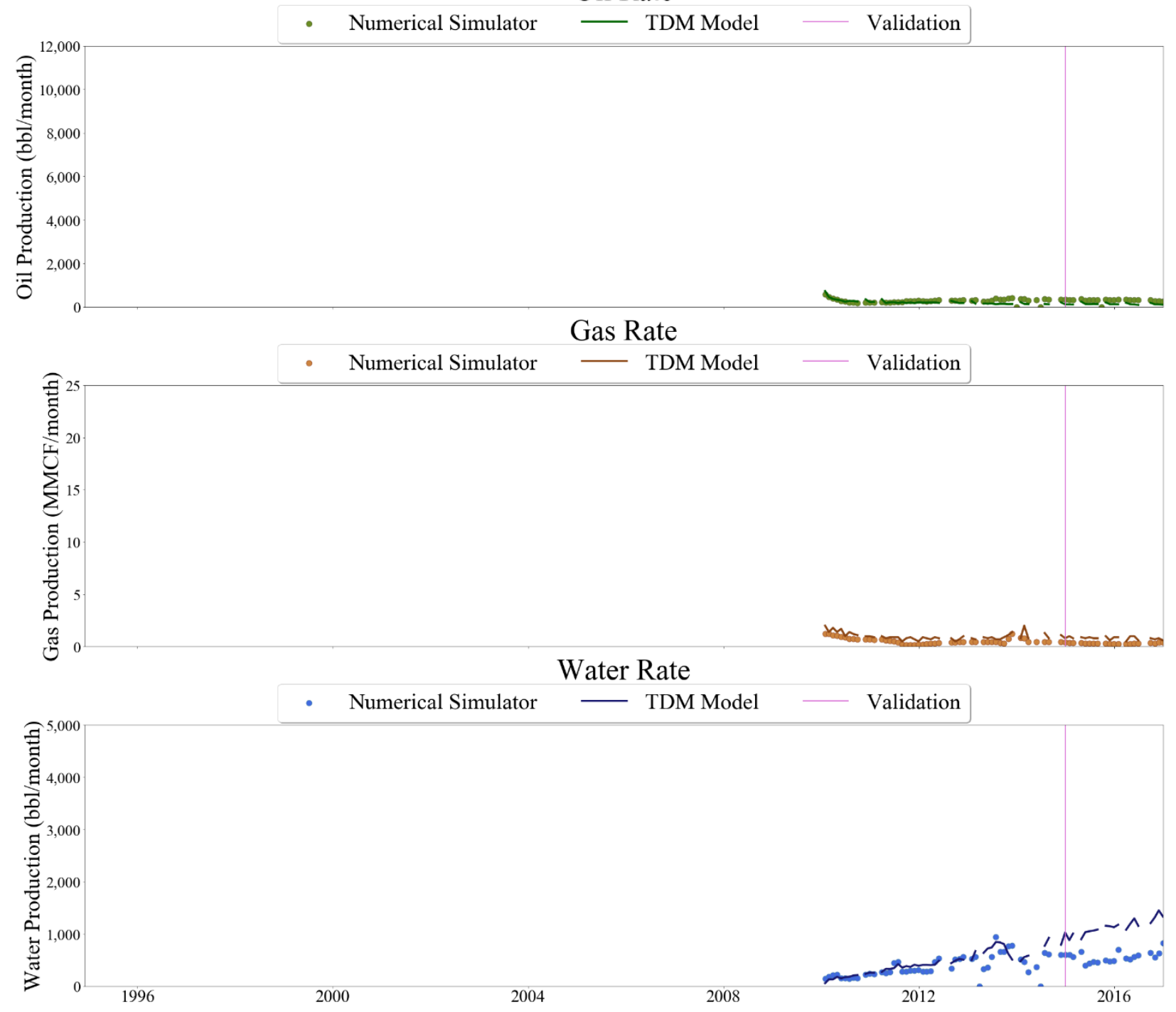

Figure 550 Well-053 oil. gas, and water rate TDM predictions vs actual simulation data plots for 2 Year Blind Validation TDM 


\section{Oil Rate}
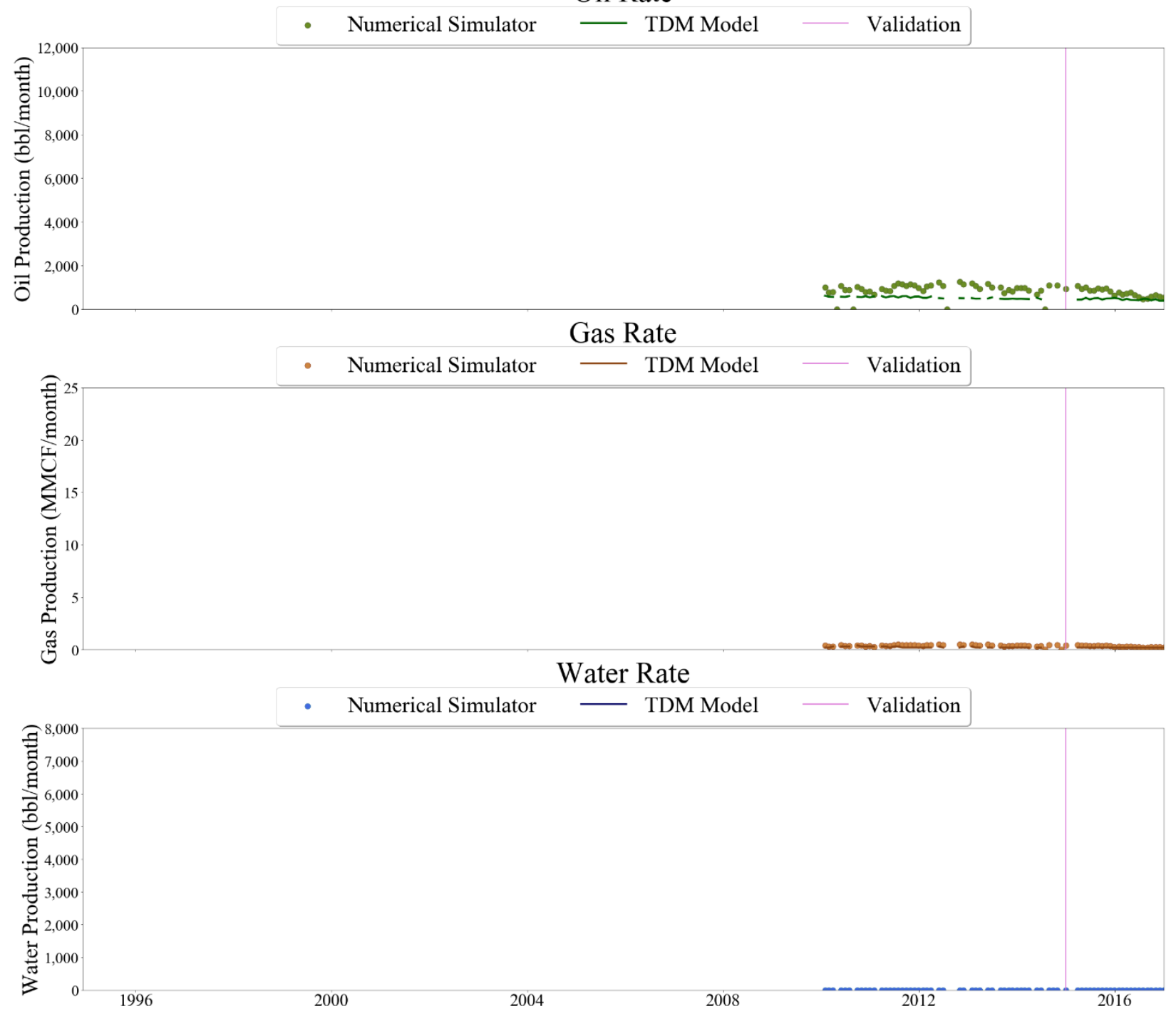

Figure 551 Well-054 oil, gas, and water rate TDM predictions vs actual simulation data plots for 2 Year Blind Validation TDM 


\section{Oil Rate}

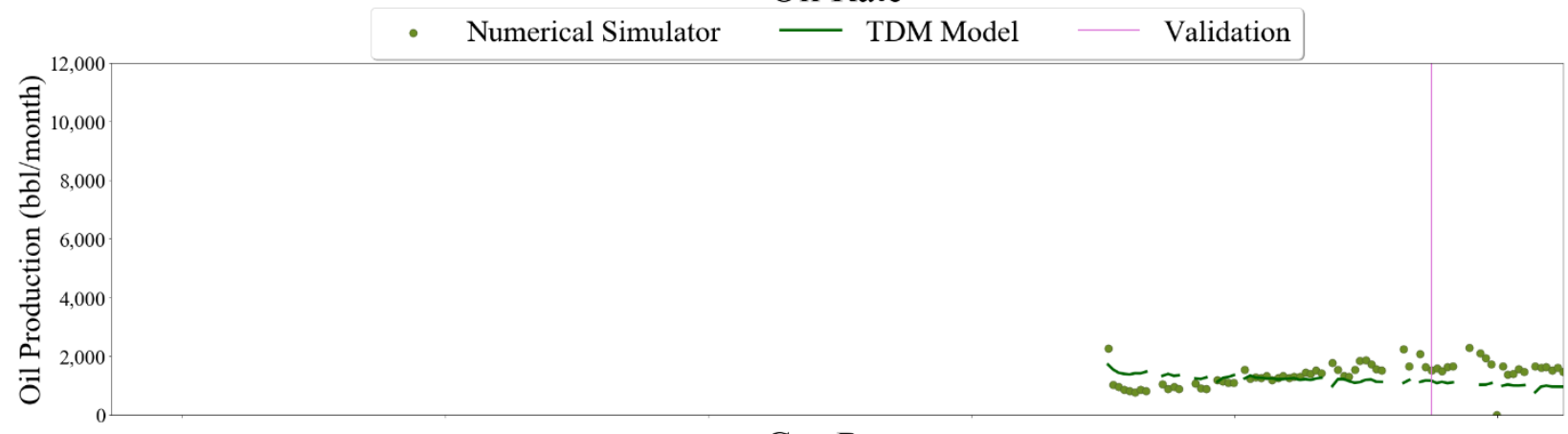

\section{Gas Rate}
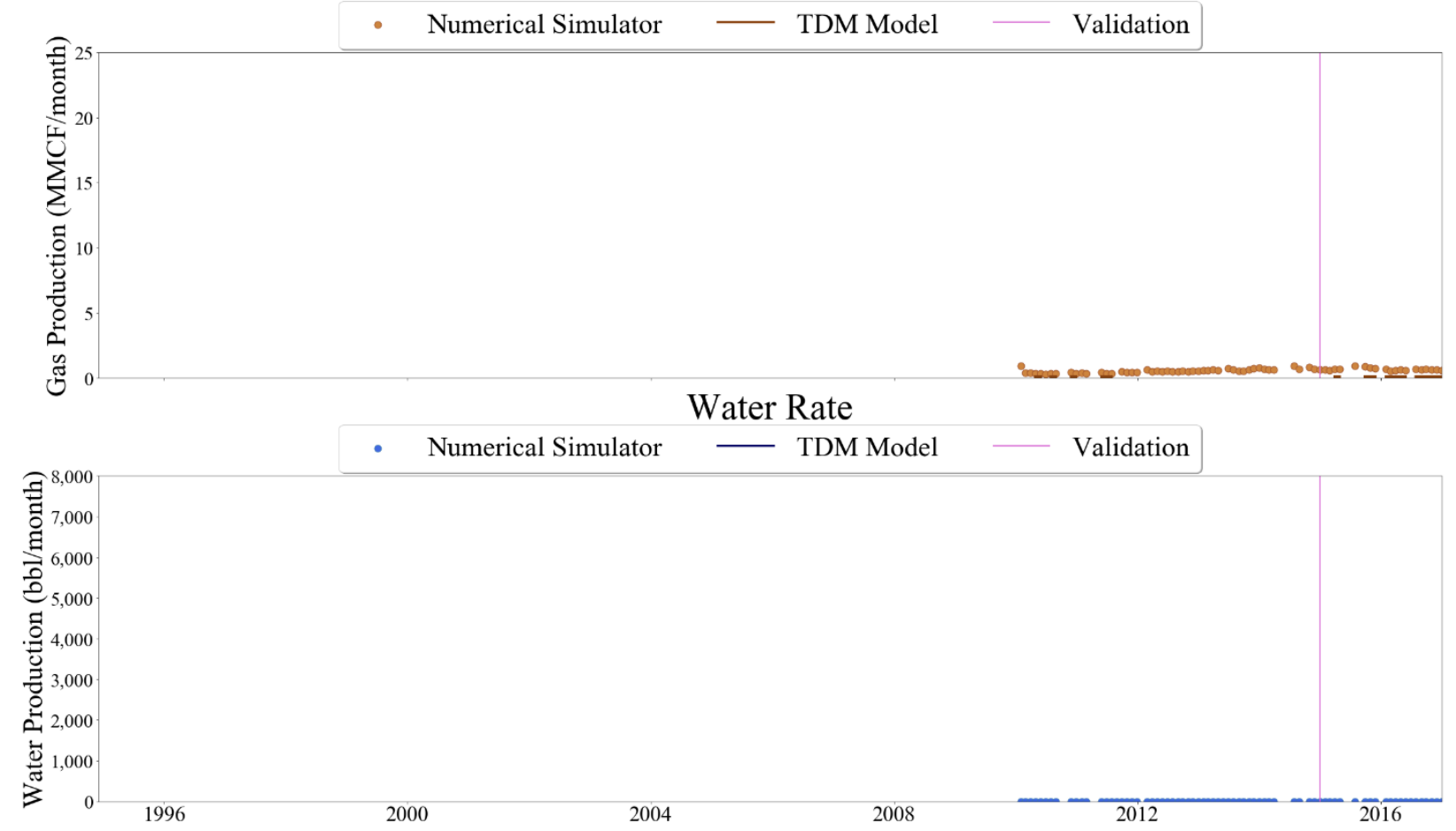

Figure 552 Well-055 oil, gas, and water rate TDM predictions vs actual simulation data plots for 2 Year Blind Validation TDM 


\section{Oil Rate}

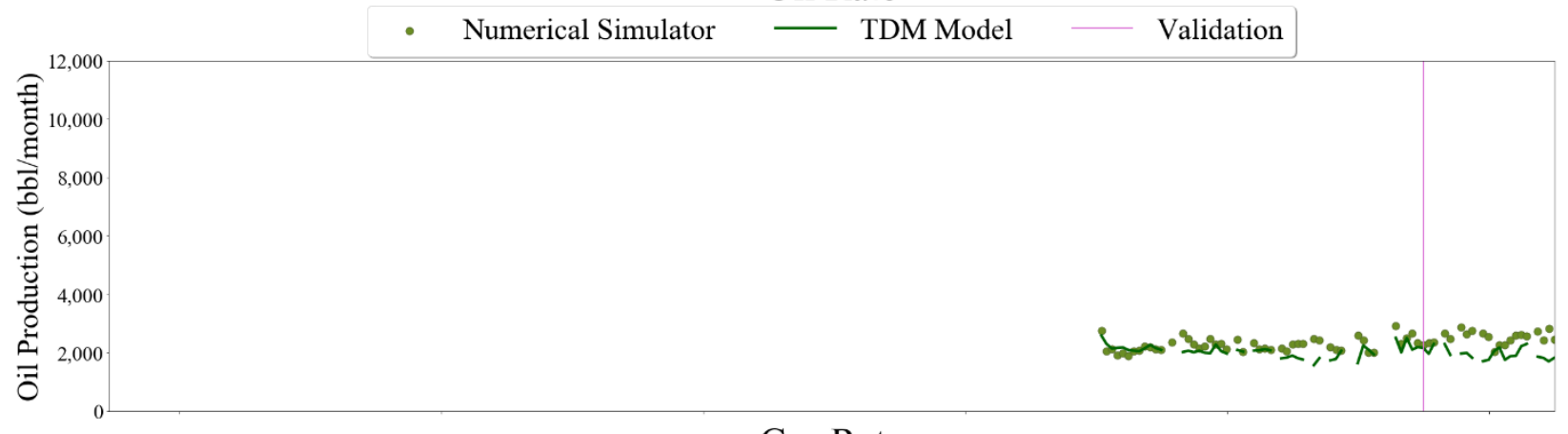

\section{Gas Rate}
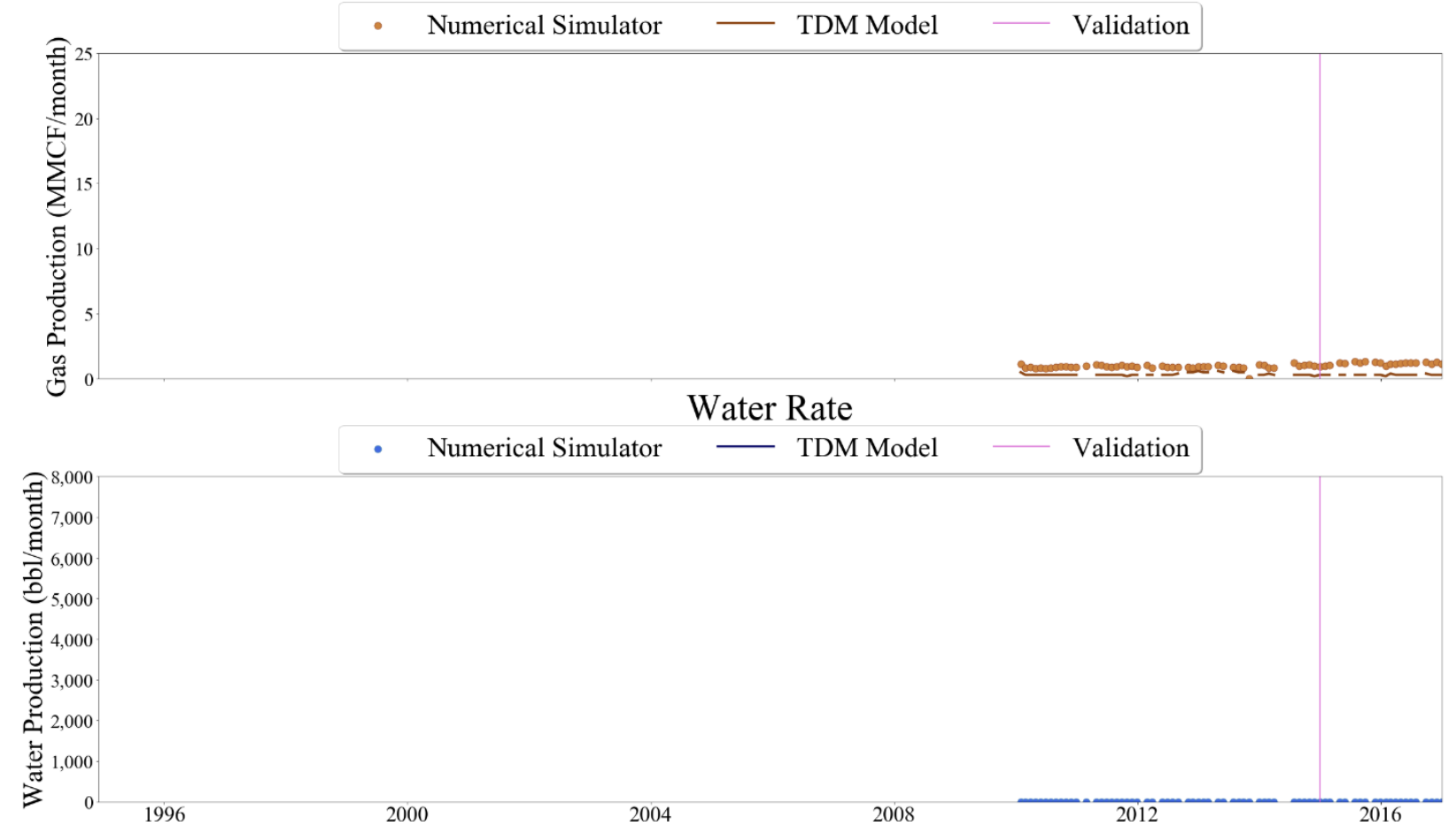

Figure 553 Well-056 oil, gas, and water rate TDM predictions vs actual simulation data plots for 2 Year Blind Validation TDM 


\section{Oil Rate}
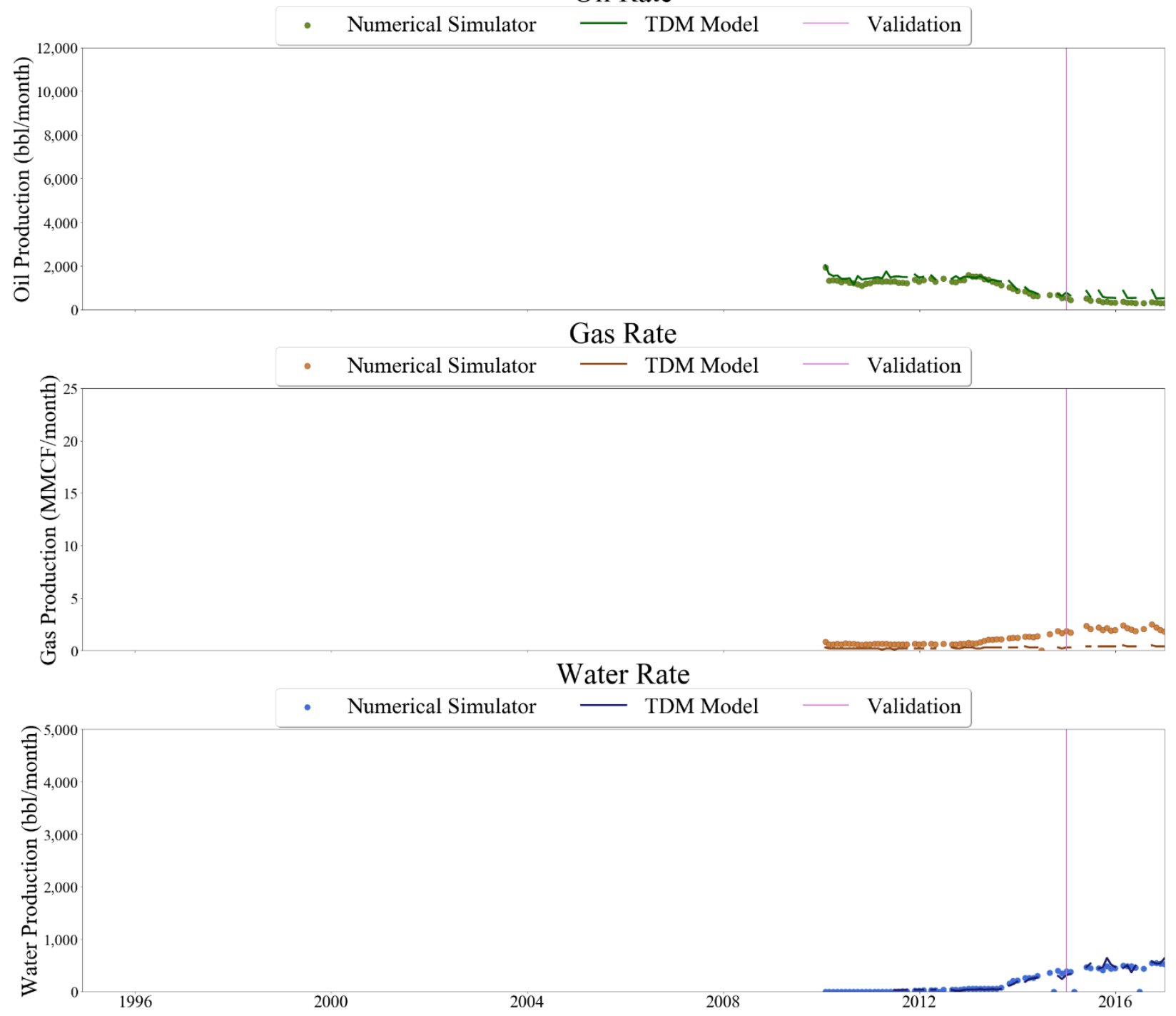

Figure 554 Well-057 oil, gas, and water rate TDM predictions vs actual simulation data plots for 2 Year Blind Validation TDM

\subsubsection{Heat Maps}

Reservoir Pressure (psi) at 2014-12-31
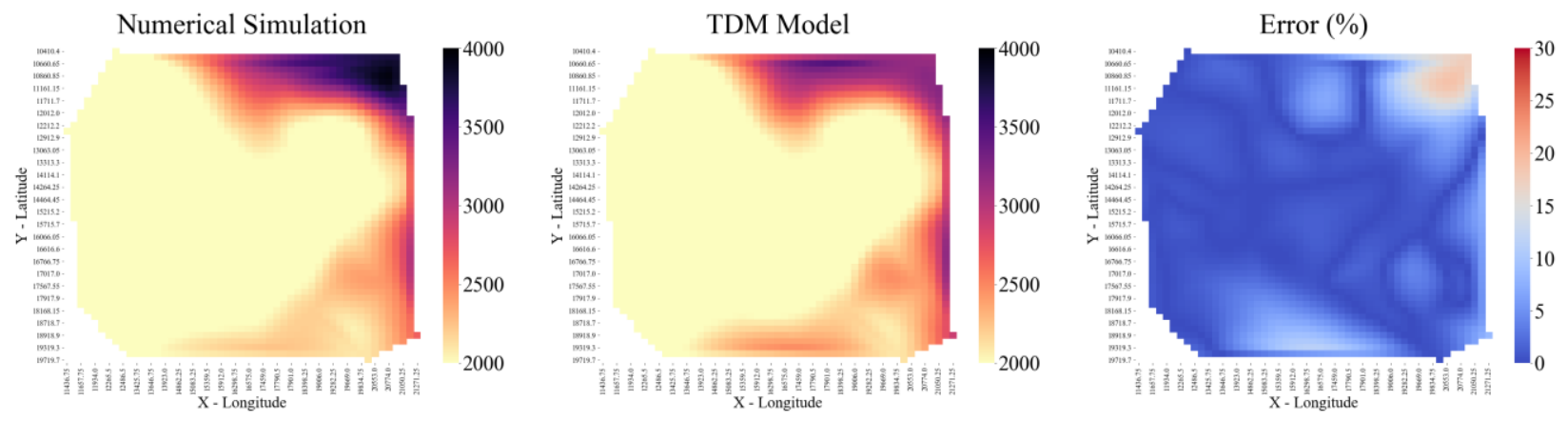

Figure 555 Entire reservoir heat map for reservoir pressure at December 31st, 2014 for 2 Year Blind Validation TDM 
Reservoir Pressure (psi) at 2015-03-31
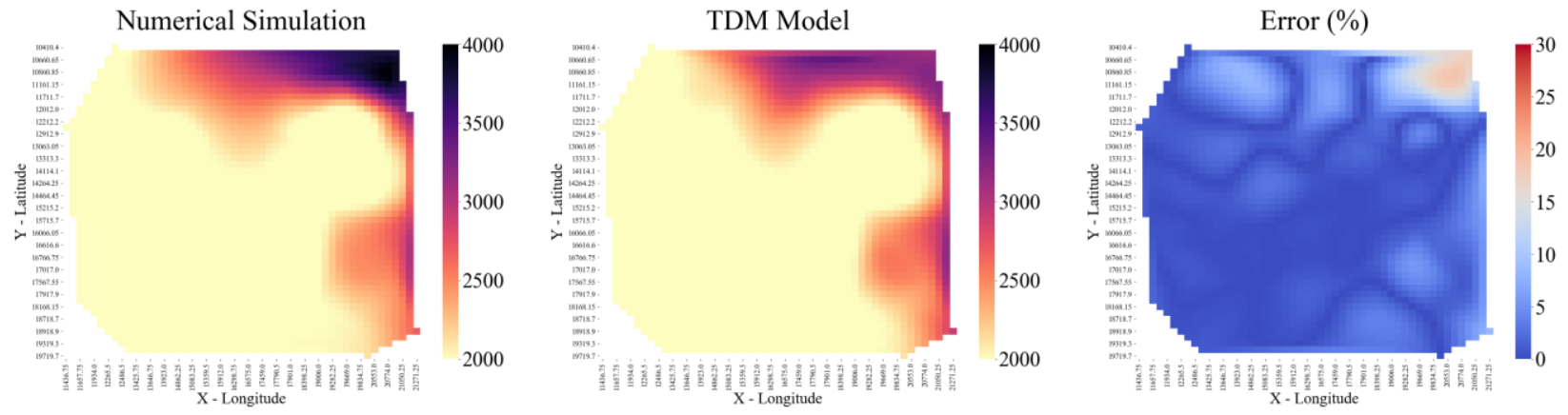

Figure 556 Entire reservoir heat map for reservoir pressure at March 31st, 2015 for 2 Year Blind Validation TDM

\section{Reservoir Pressure (psi) at 2015-06-30}
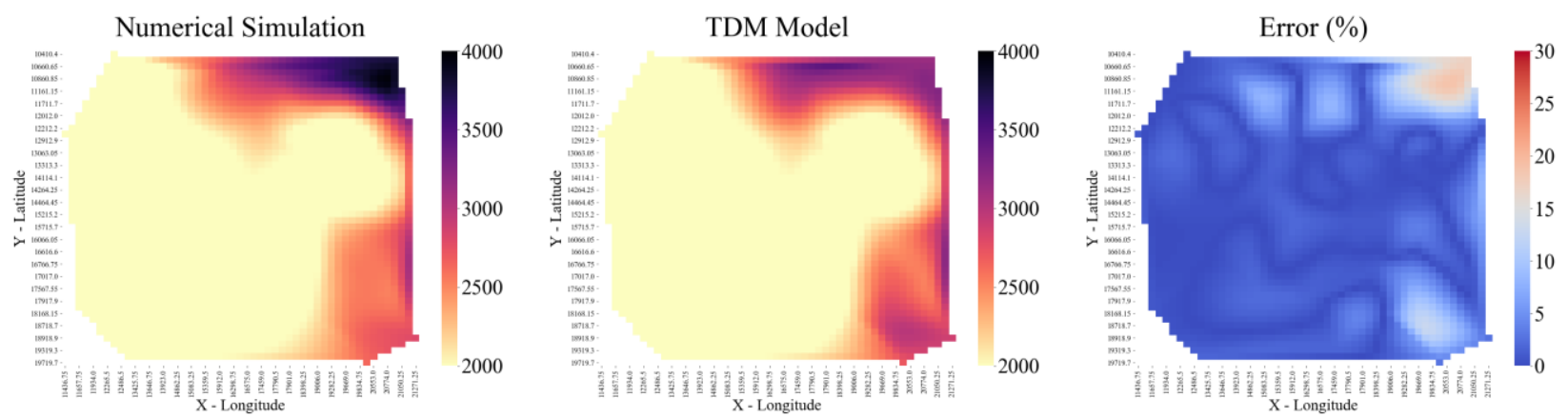

Figure 557 Entire reservoir heat map for reservoir pressure at June 30th, 2015 for 2 Year Blind Validation TDM

Reservoir Pressure (psi) at 2015-09-30
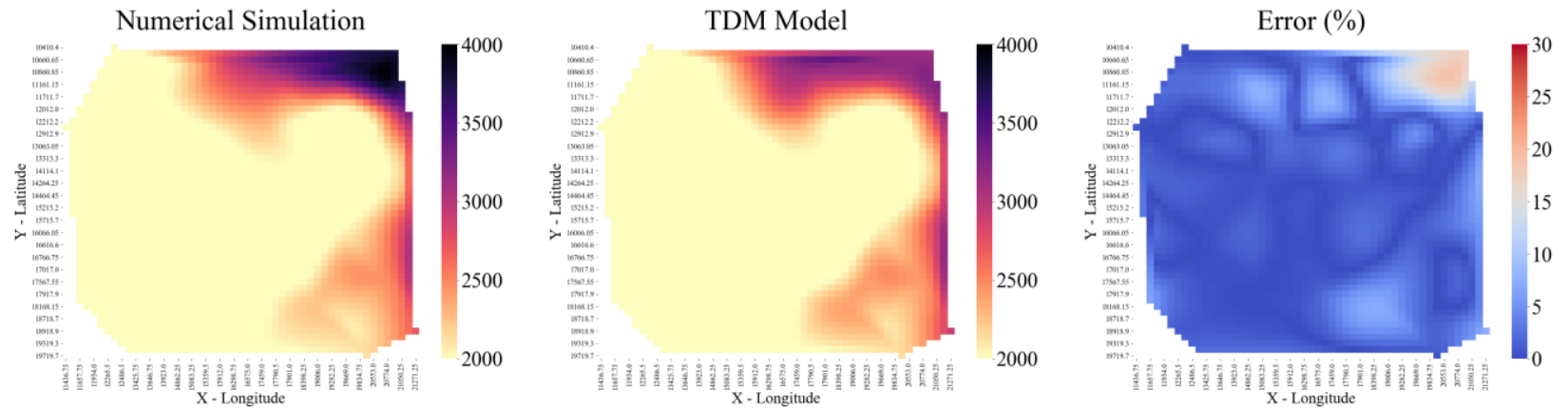

Figure 558 Entire reservoir heat map for reservoir pressure at September 30th, 2015 for 2 Year Blind Validation TDM 
Reservoir Pressure (psi) at 2015-12-31
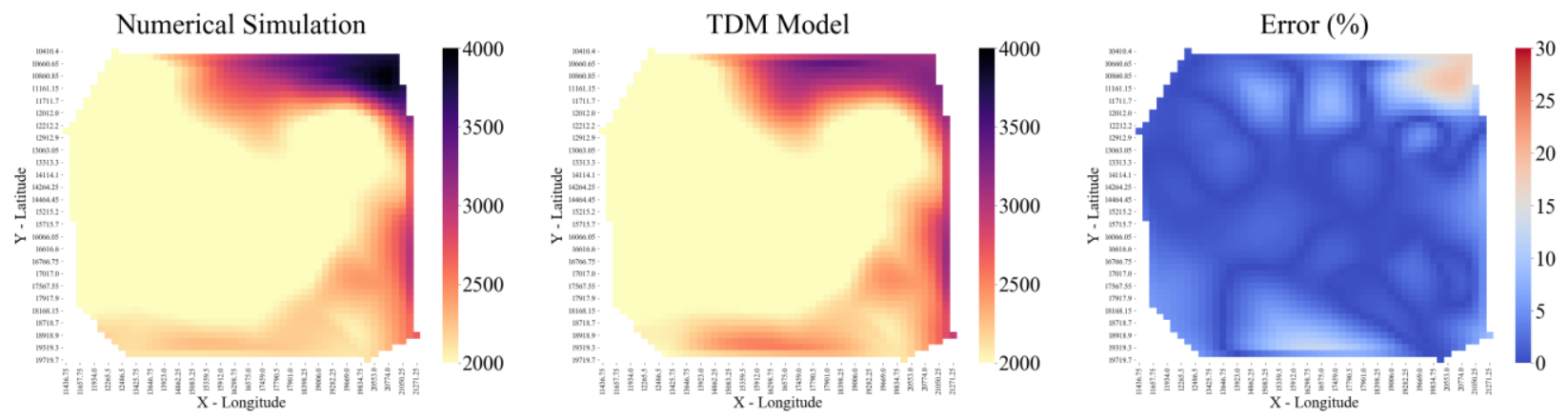

Figure 559 Entire reservoir heat map for reservoir pressure at December 31st, 2015 for 2 Year Blind Validation TDM

Reservoir Pressure (psi) at 2016-03-31
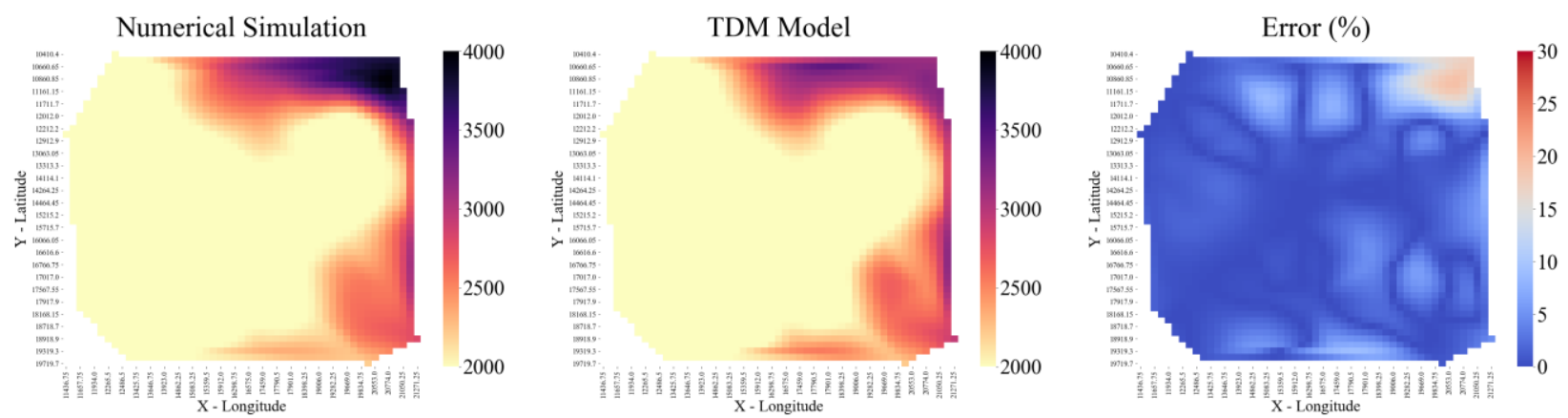

Figure 560 Entire reservoir heat map for reservoir pressure at March 31st, 2016 for 2 Year Blind Validation TDM

Reservoir Pressure (psi) at 2016-06-30
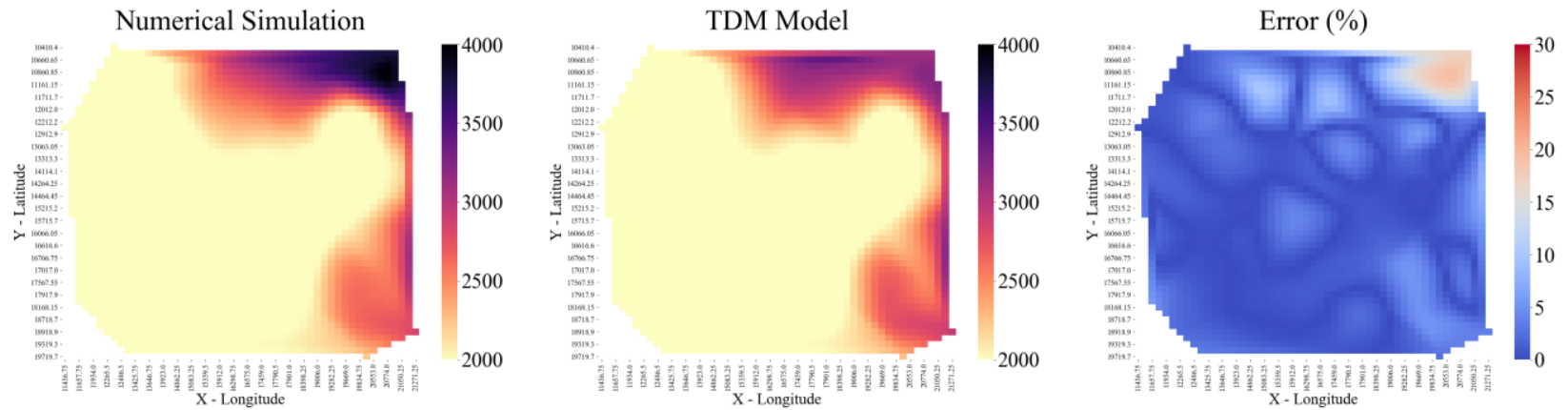

Figure 561 Entire reservoir heat map for reservoir pressure at June 30th, 2016 for 2 Year Blind Validation TDM 
Reservoir Pressure (psi) at 2016-09-30
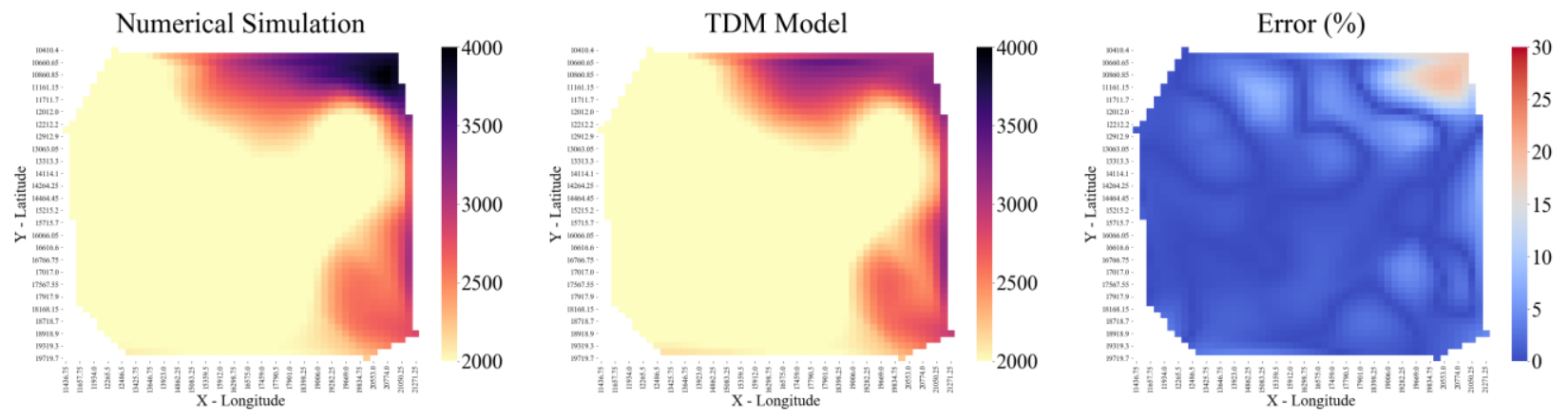

Figure 562 Entire reservoir heat map for reservoir pressure at September 30th, 2016 for 2 Year Blind Validation TDM

Reservoir Pressure (psi) at 2016-12-31
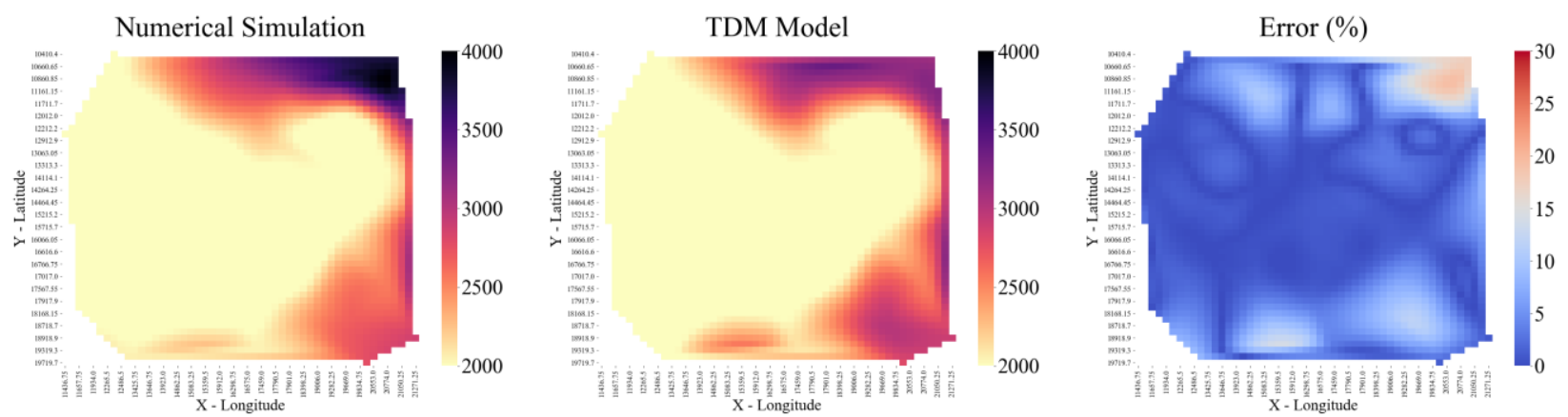

Figure 563 Entire reservoir heat map for reservoir pressure at December 31st, 2016 for 2 Year Blind Validation TDM

Water Saturation (\%) at 2014-12-31
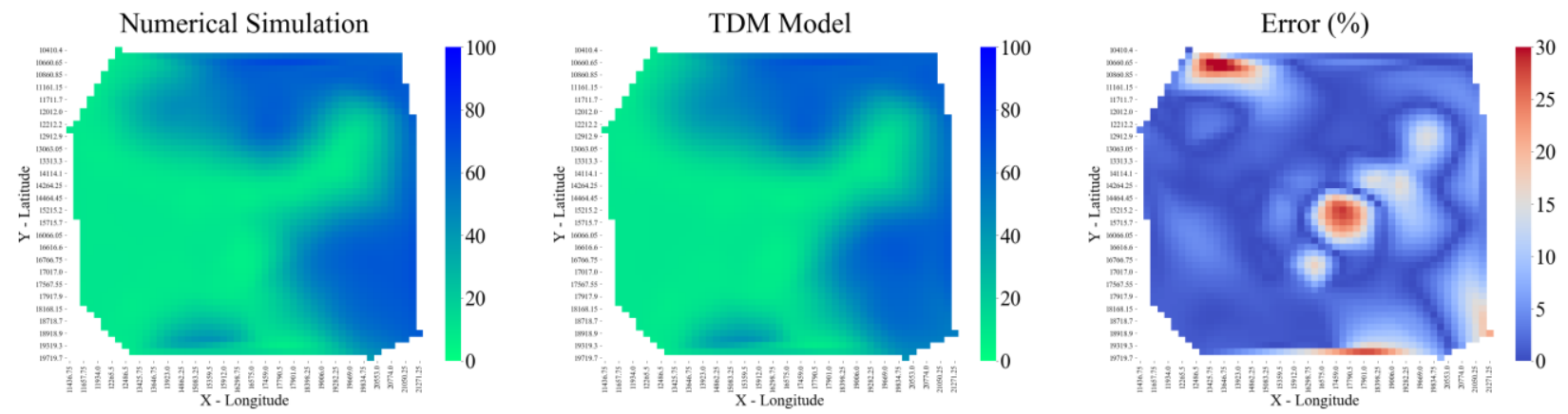

Figure 564 Entire reservoir heat map for water saturation at December 31st, 2014 for 2 Year Blind Validation TDM 


\section{Water Saturation (\%) at 2015-03-31}
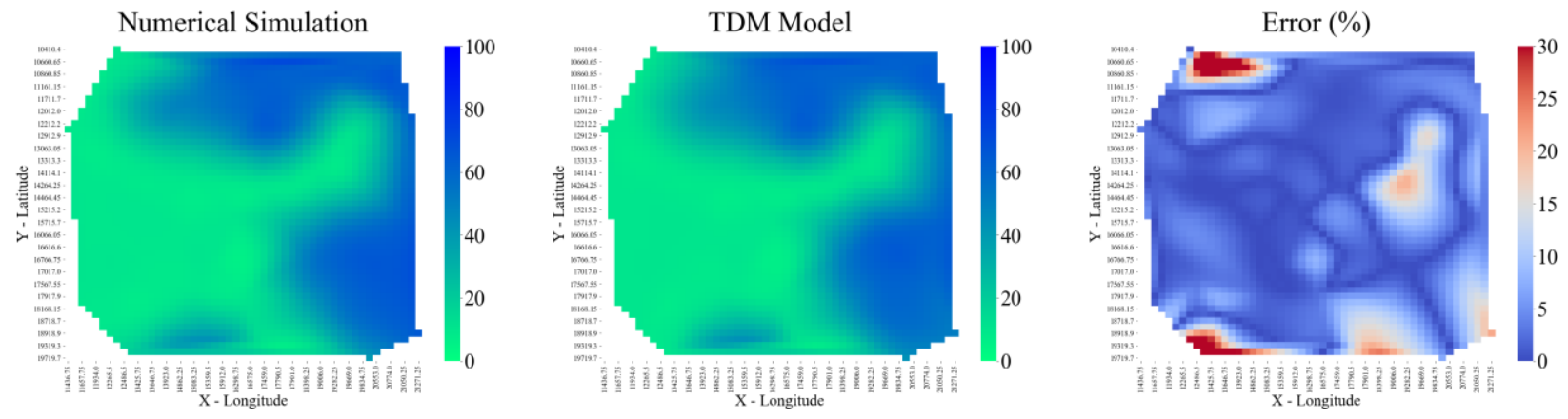

Figure 565 Entire reservoir heat map for water saturation at March 31st, 2015 for 2 Year Blind Validation TDM

\section{Water Saturation (\%) at 2015-06-30}
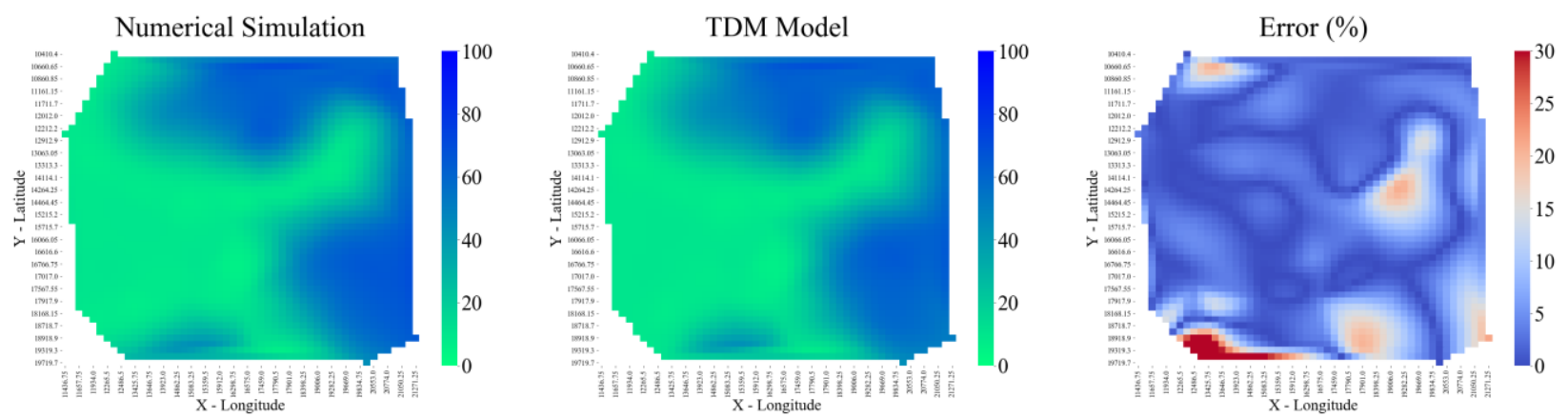

Figure 566 Entire reservoir heat map for water saturation at June 30th, 2015 for 2 Year Blind Validation TDM

Water Saturation (\%) at 2015-09-30
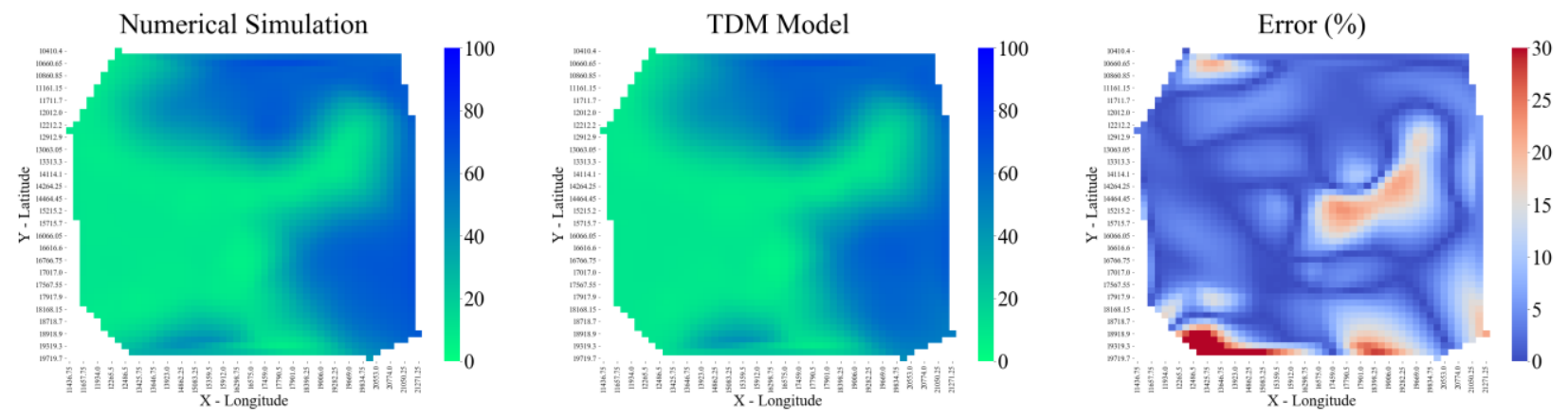

Figure 567 Entire reservoir heat map for water saturation at September 30th, 2015 for 2 Year Blind Validation TDM 
Water Saturation (\%) at 2015-12-31
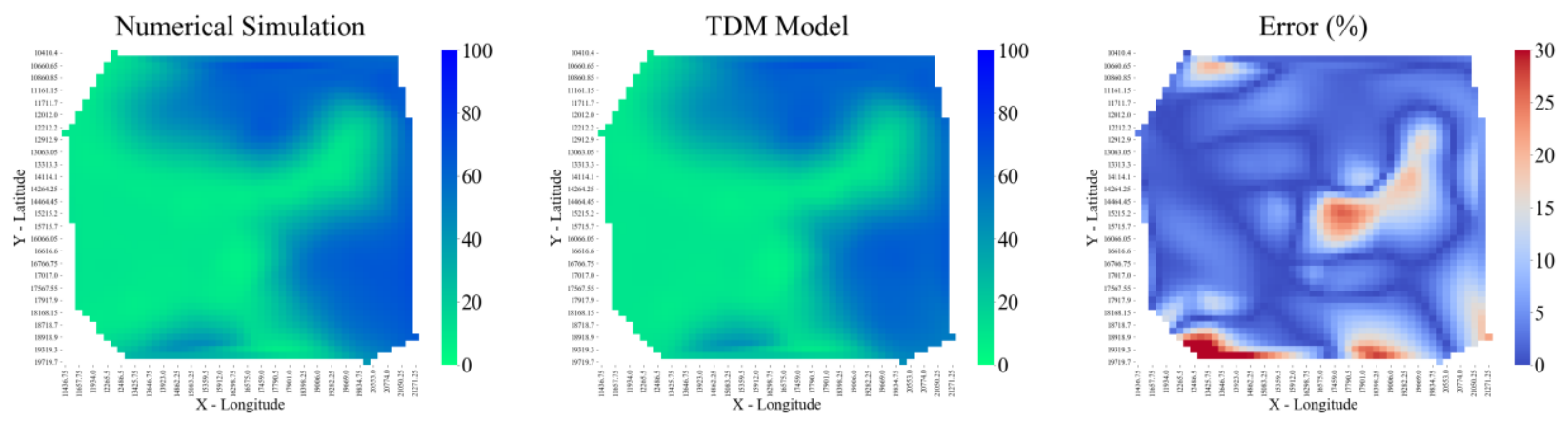

Figure 568 Entire reservoir heat map for water saturation at December 31st, 2015 for 2 Year Blind Validation TDM

\section{Water Saturation (\%) at 2016-03-31}
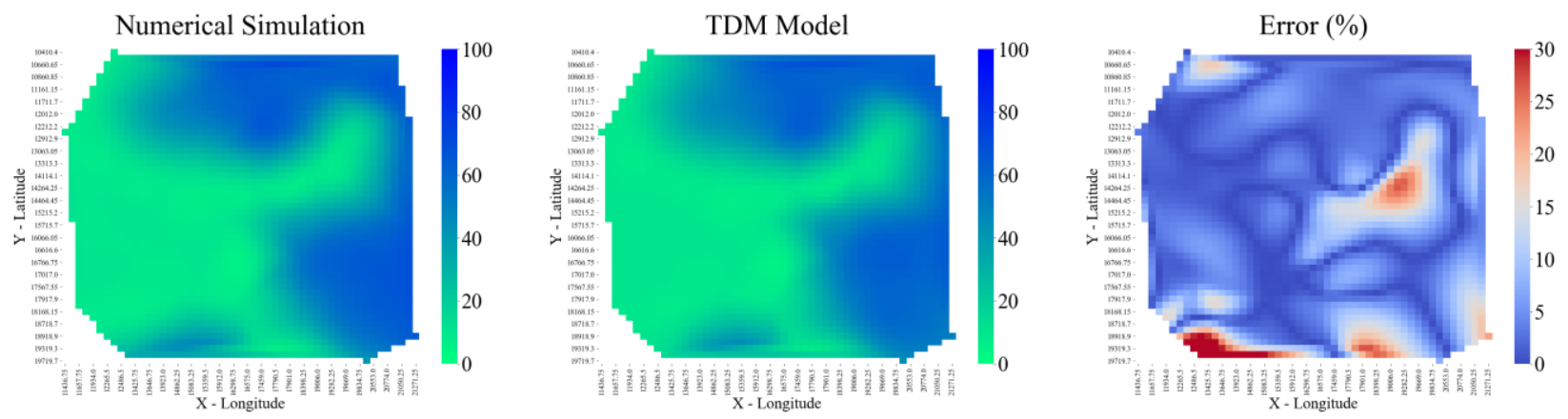

Figure 569 Entire reservoir heat map for water saturation at March 31st, 2016 for 2 Year Blind Validation TDM

Water Saturation (\%) at 2016-06-30
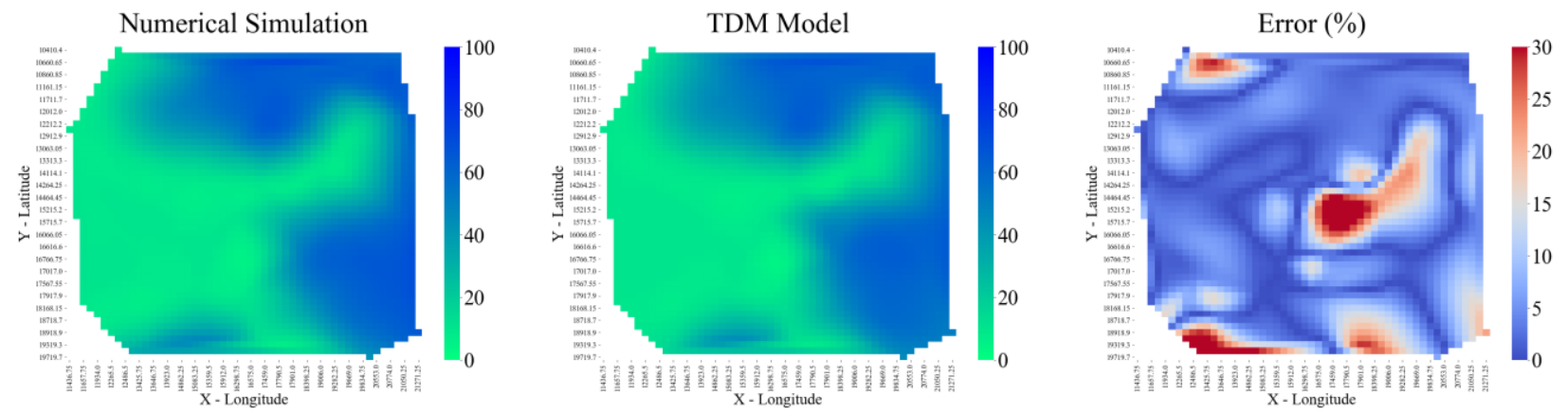

Figure 570 Entire reservoir heat map for water saturation at June 30th, 2016 for 2 Year Blind Validation TDM 
Water Saturation (\%) at 2016-09-30
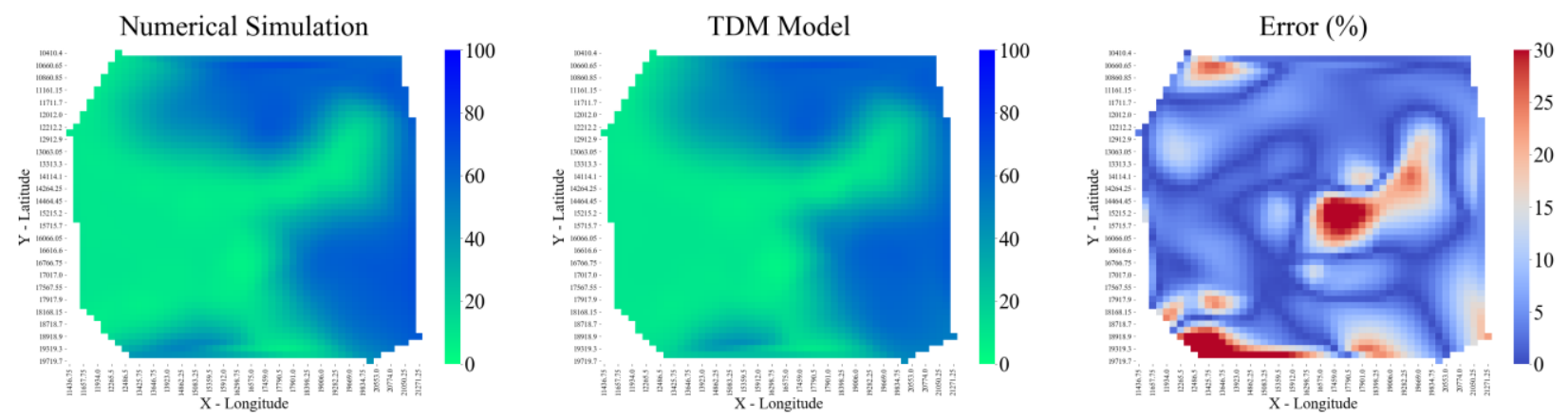

Figure 571 Entire reservoir heat map for water saturation at September 30th, 2016 for 2 Year Blind Validation TDM

\section{Water Saturation (\%) at 2016-12-31}
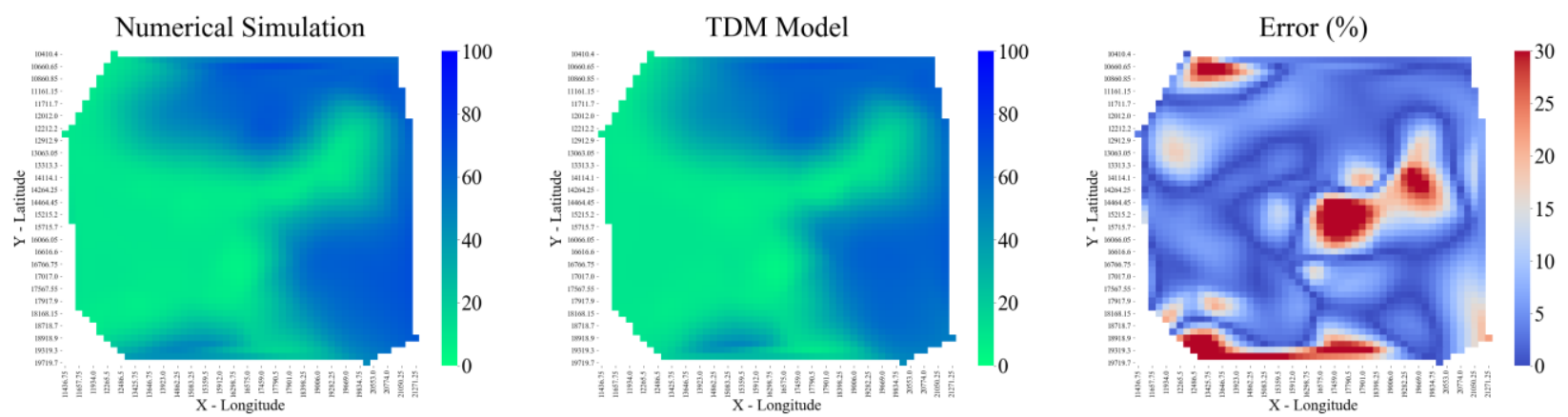

Figure 572 Entire reservoir heat map for water saturation at December 31st, 2016 for 2 Year Blind Validation TDM 
8.2.4 Multi-Random Partitioning- 3 Year Blind Validation

8.2.4.1 Individual Well Production Plots

Oil Rate

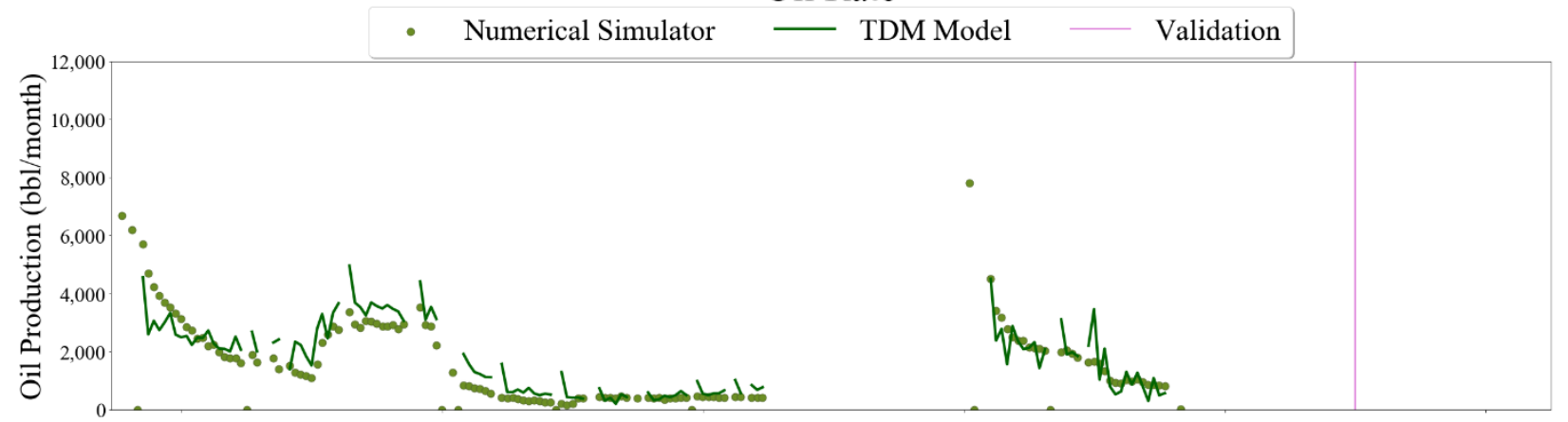

Gas Rate

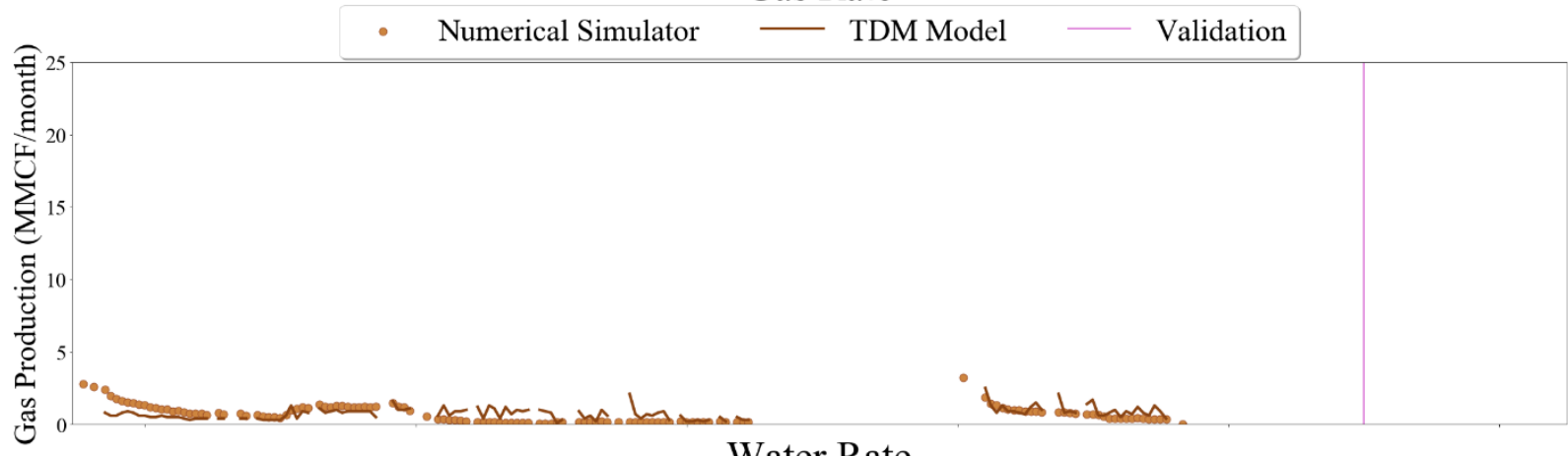

Water Rate

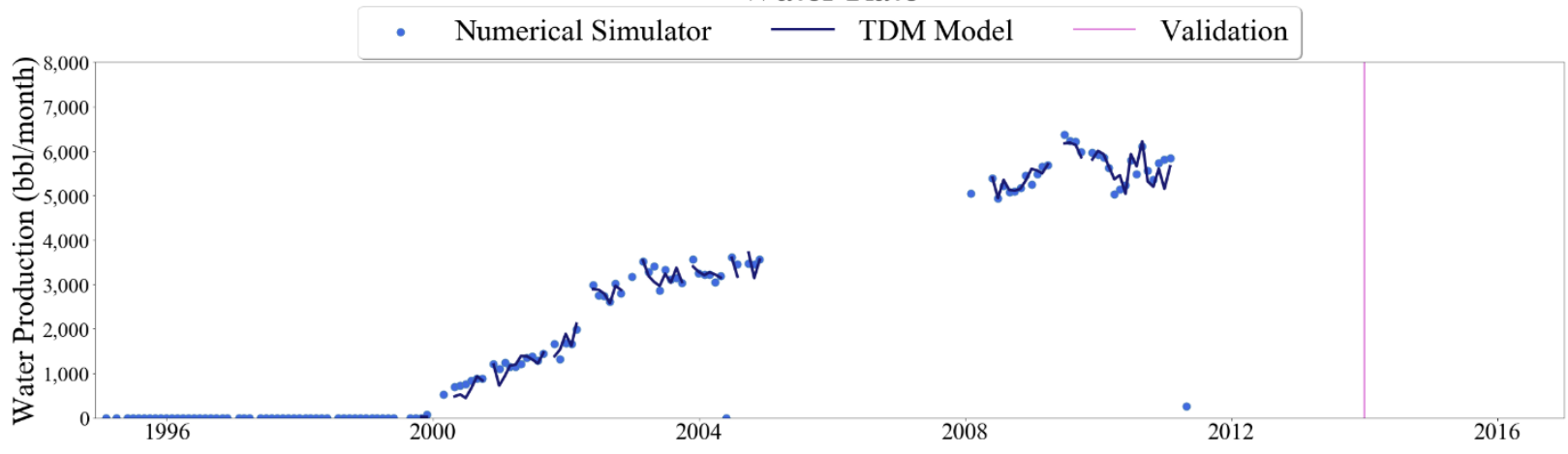

Figure 573 Well-001 oil, gas, and water rate TDM predictions vs actual simulation data plots for 4 Year Blind Validation TDM 

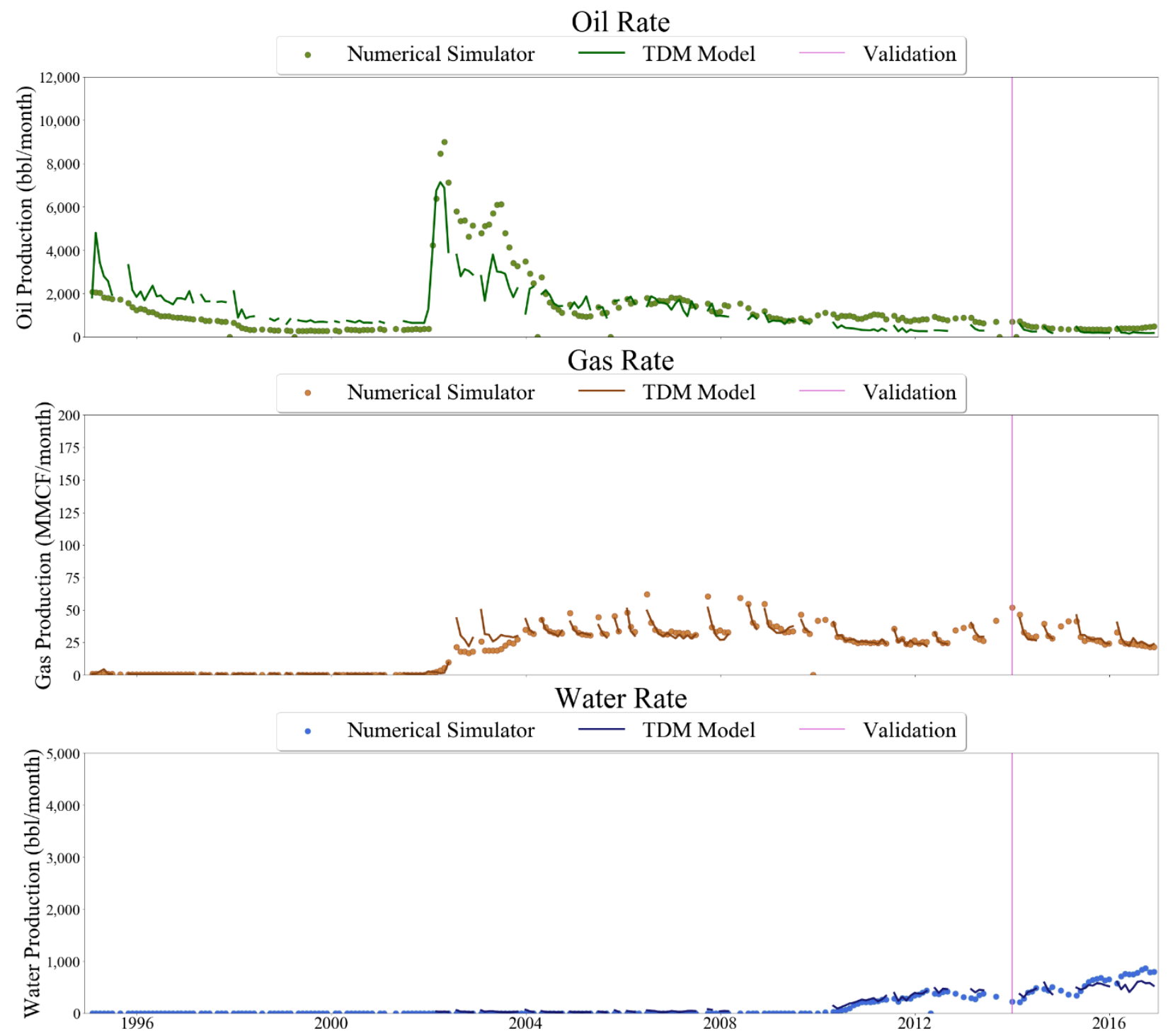

Figure 574 Well-002 oil, gas, and water rate TDM prediction vs actual simulation data plots for 3 Year Blind Validation TDM 


\section{Oil Rate}

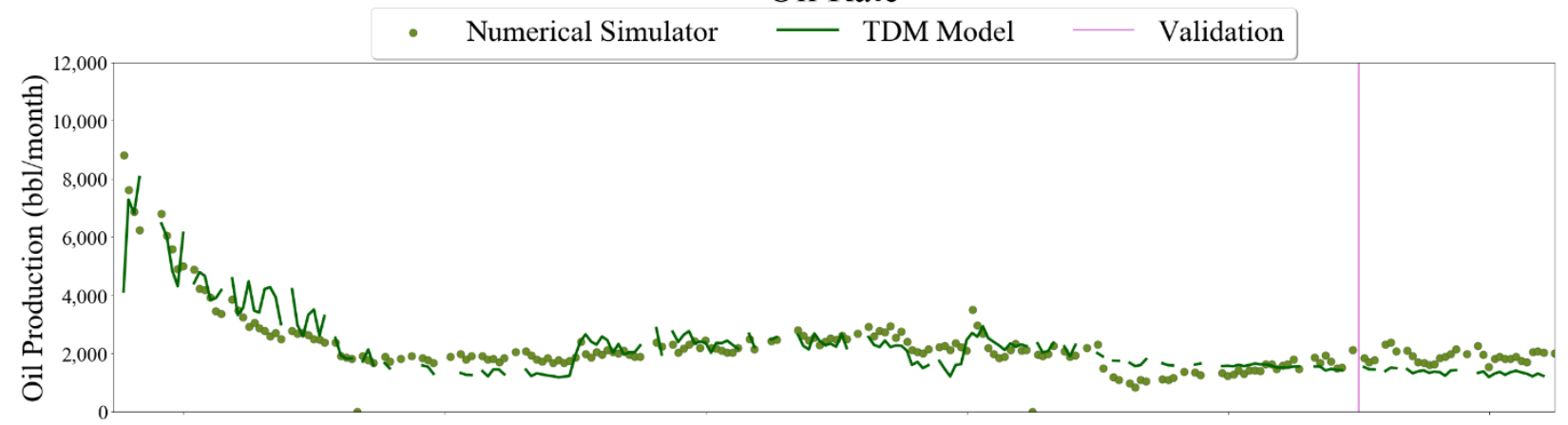

\section{Gas Rate}
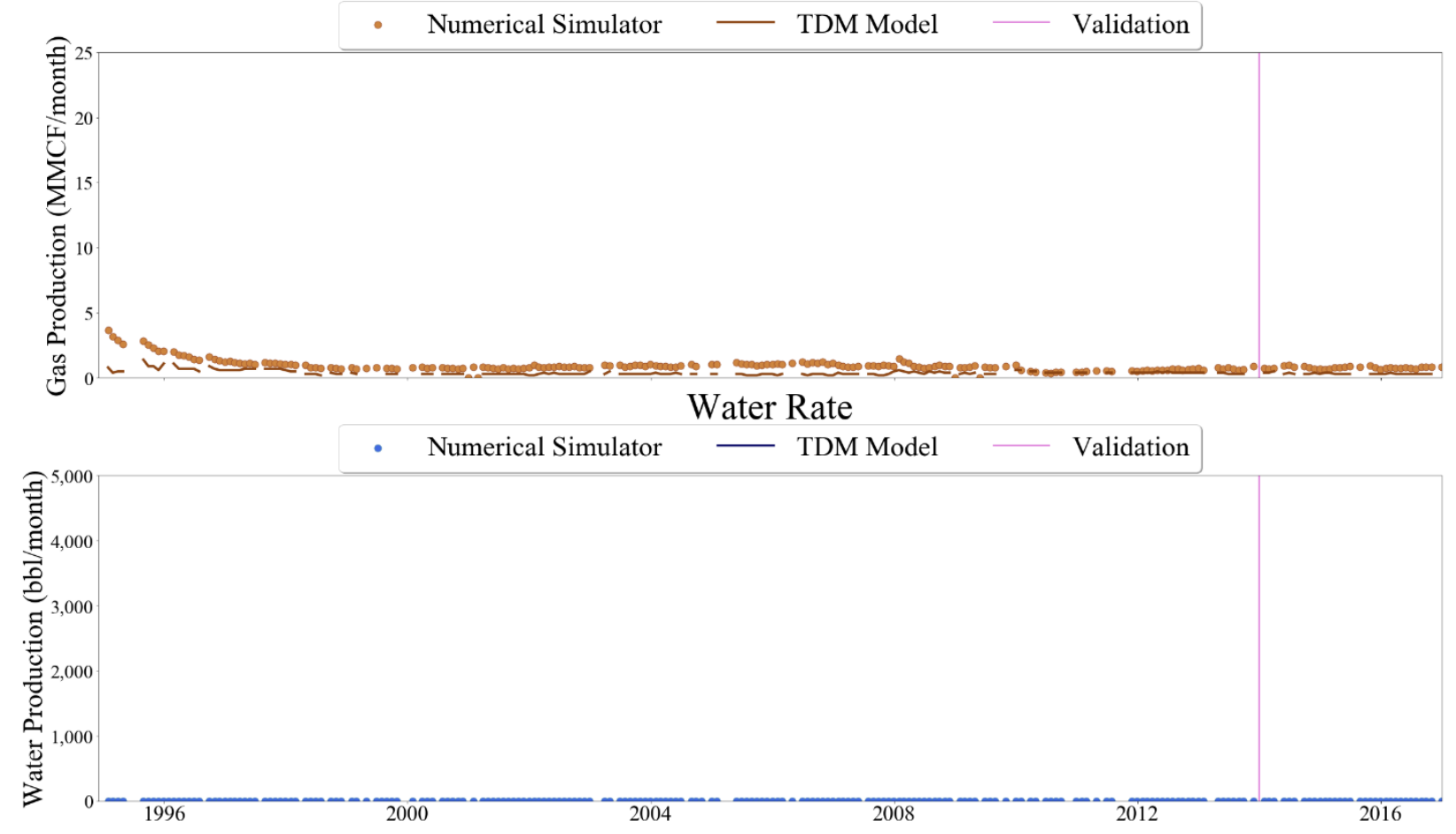

Figure 575 Well-003 oil, gas, and water rate TDM predictions vs actual simulation data plots for 3 Year Blind Validation TDM 

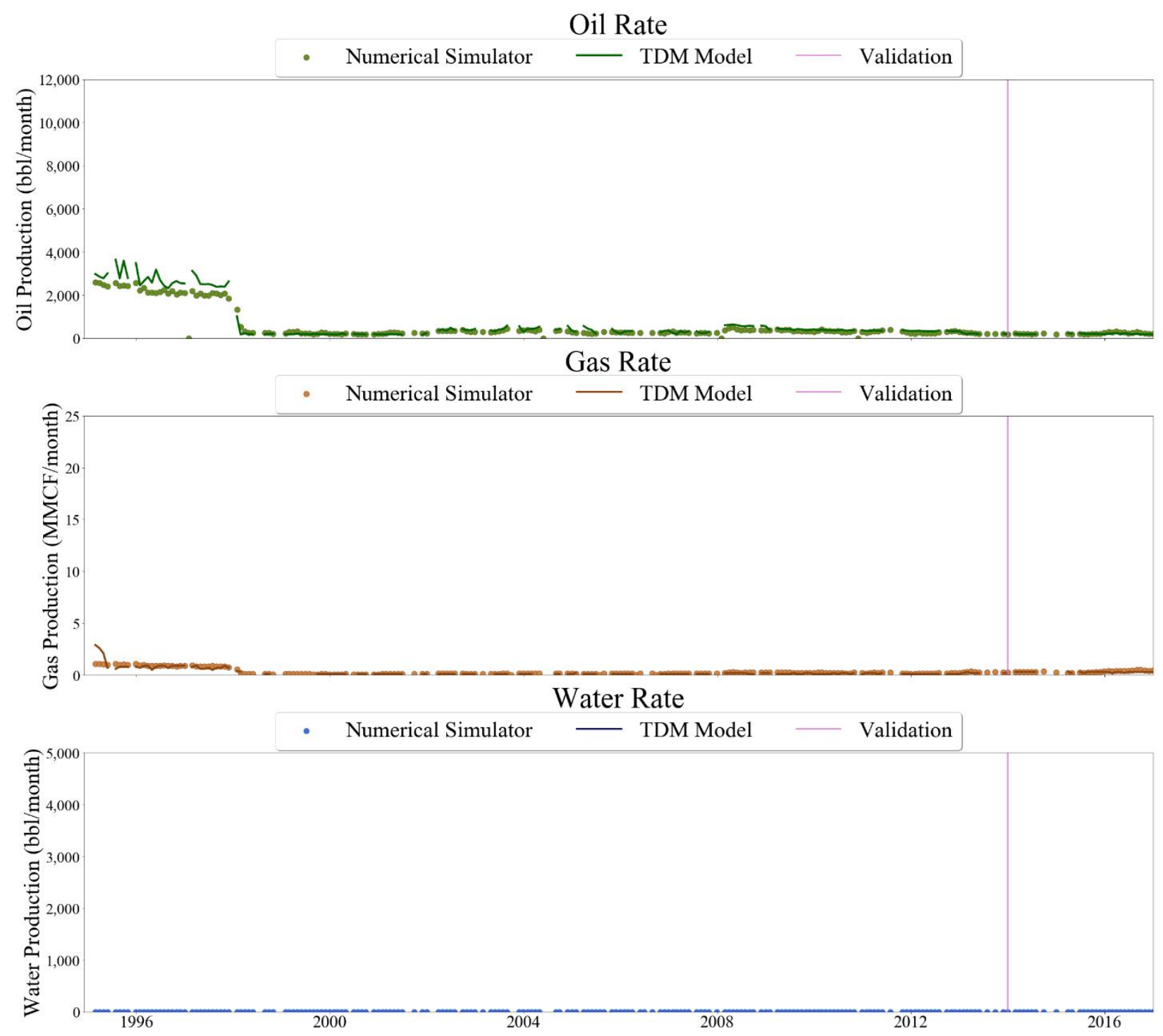

Figure 576 Well-004 oil, gas, and water rate TDM prediction vs actual simulation data plots for 3 Year Blind Validation TDM 


\section{Oil Rate}

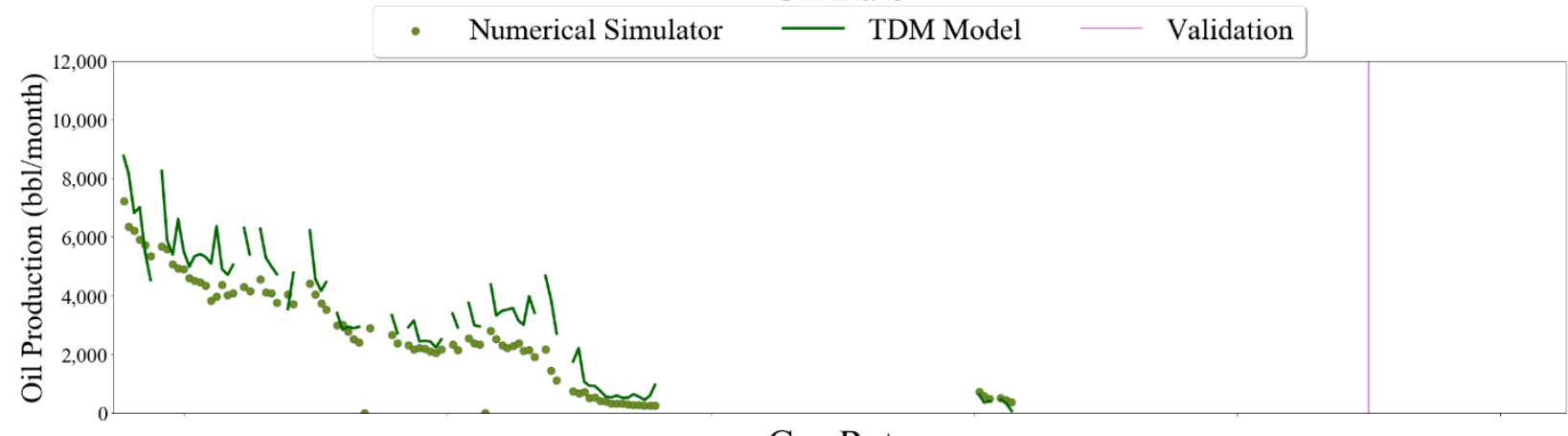

Gas Rate
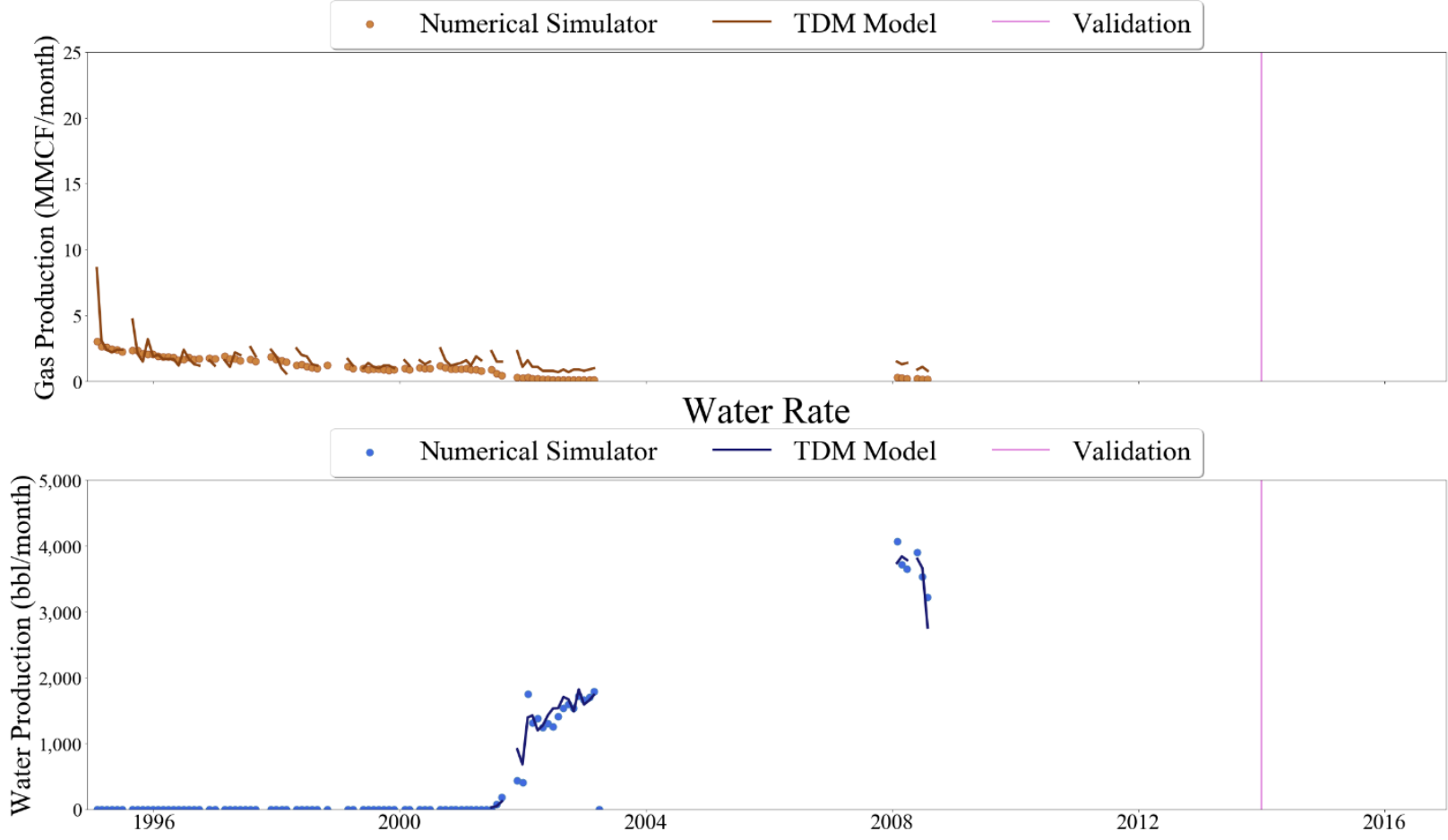

Figure 577 Well-005 oil, gas, and water rate TDM prediction vs actual simulation data plots for 3 Year Blind Validation TDM 

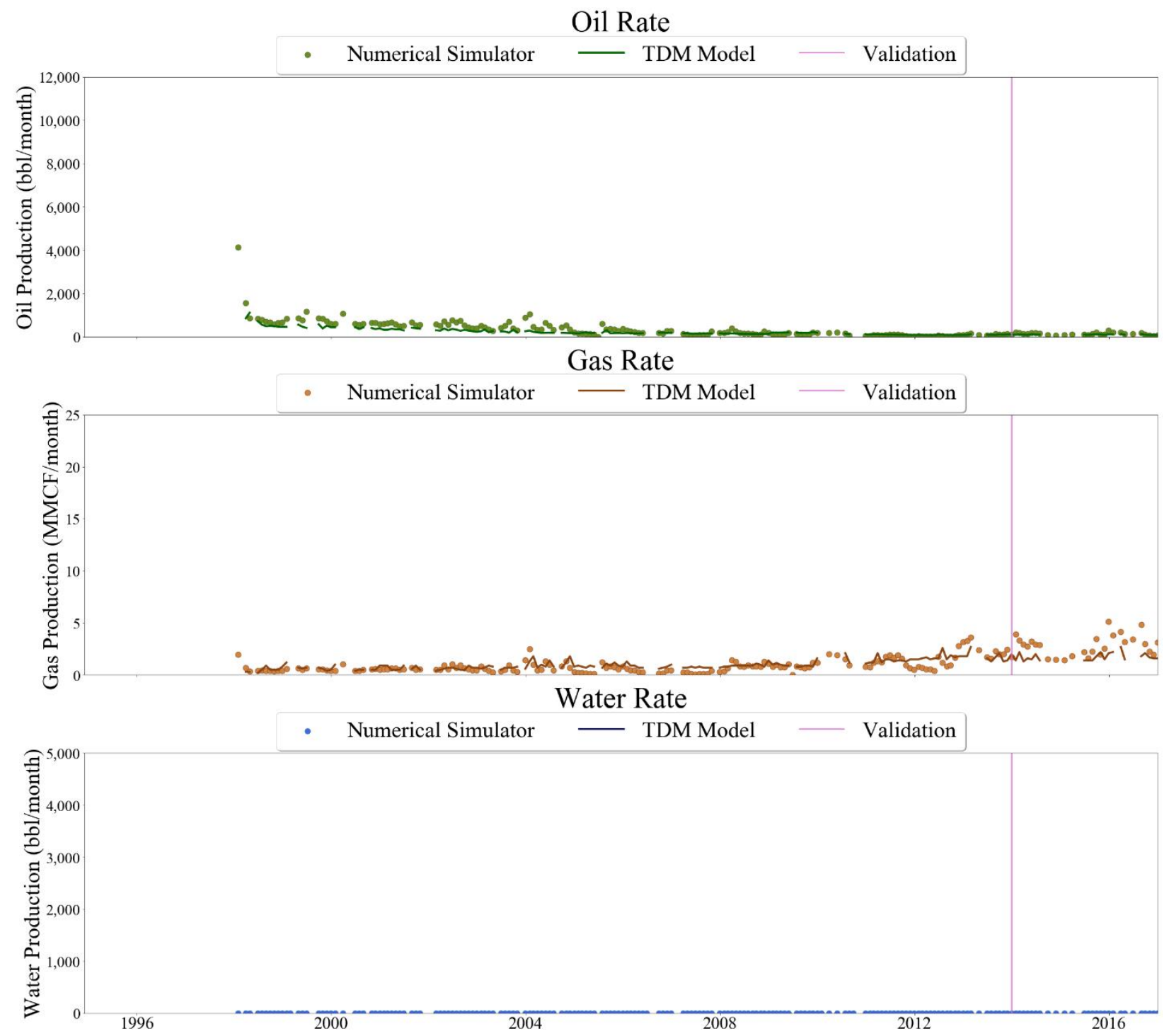

Figure 578 Well-006 oil, gas, and water rate TDM prediction vs actual simulation data plots for 3 Year Blind Validation TDM 

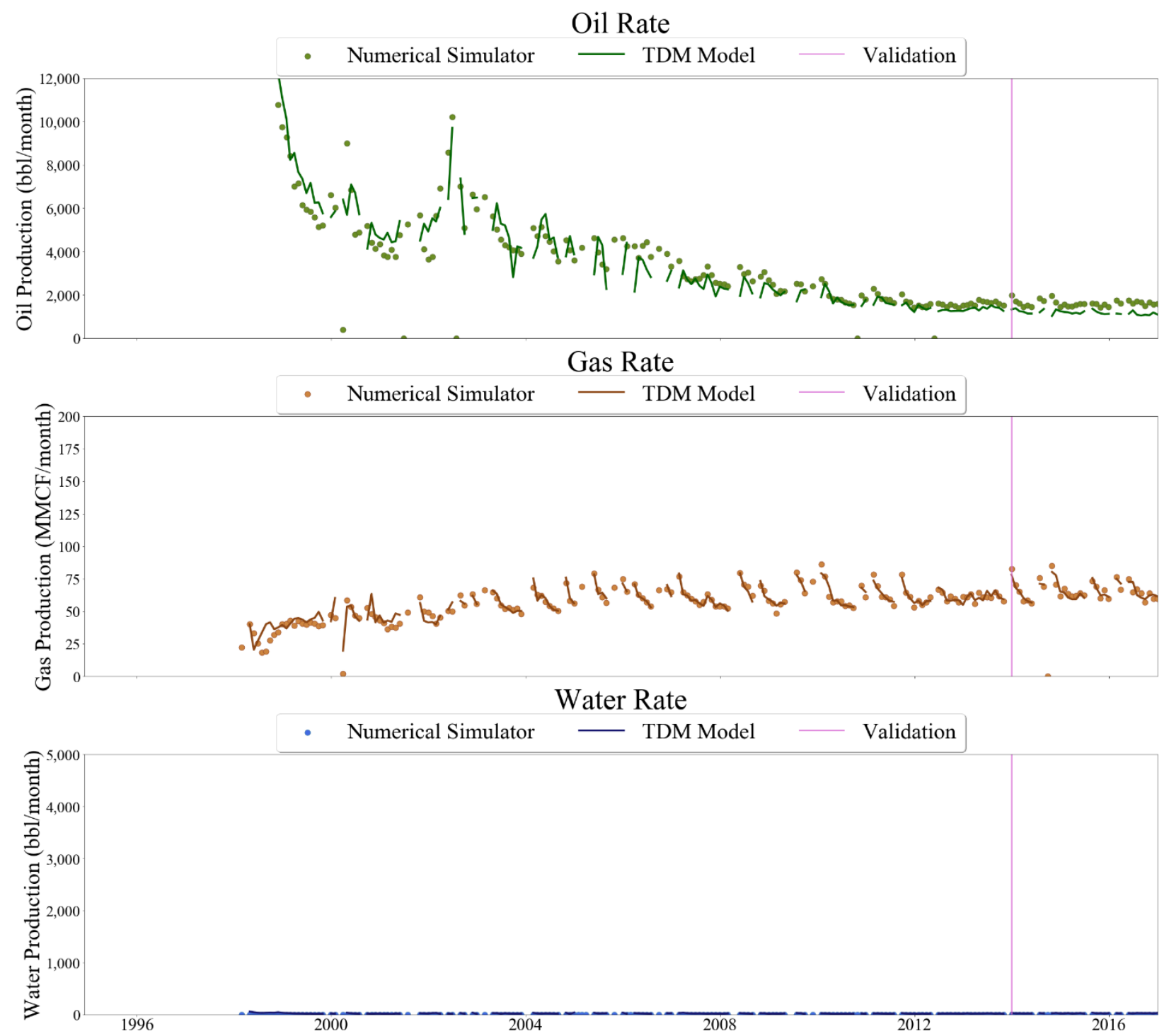

Figure 579 Well-007 oil, gas, and water rate TDM predictions vs actual simulation data plots for 3 Year Blind Validation TDM 


\section{Oil Rate}
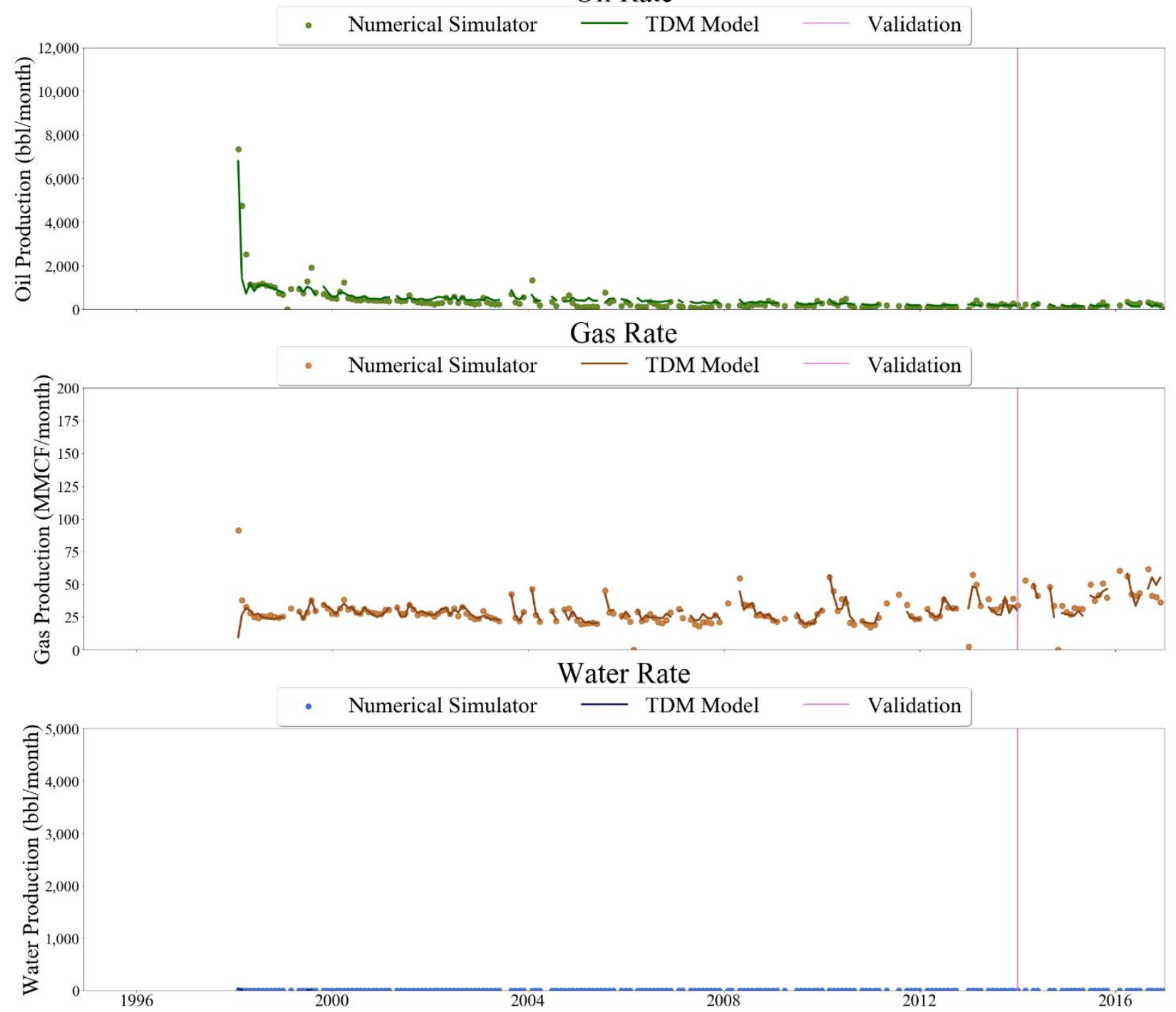

Figure 580 Well-008 oil, gas, and water rate TDM predictions vs actual simulation data plots for 3 Year Blind Validation TDM 

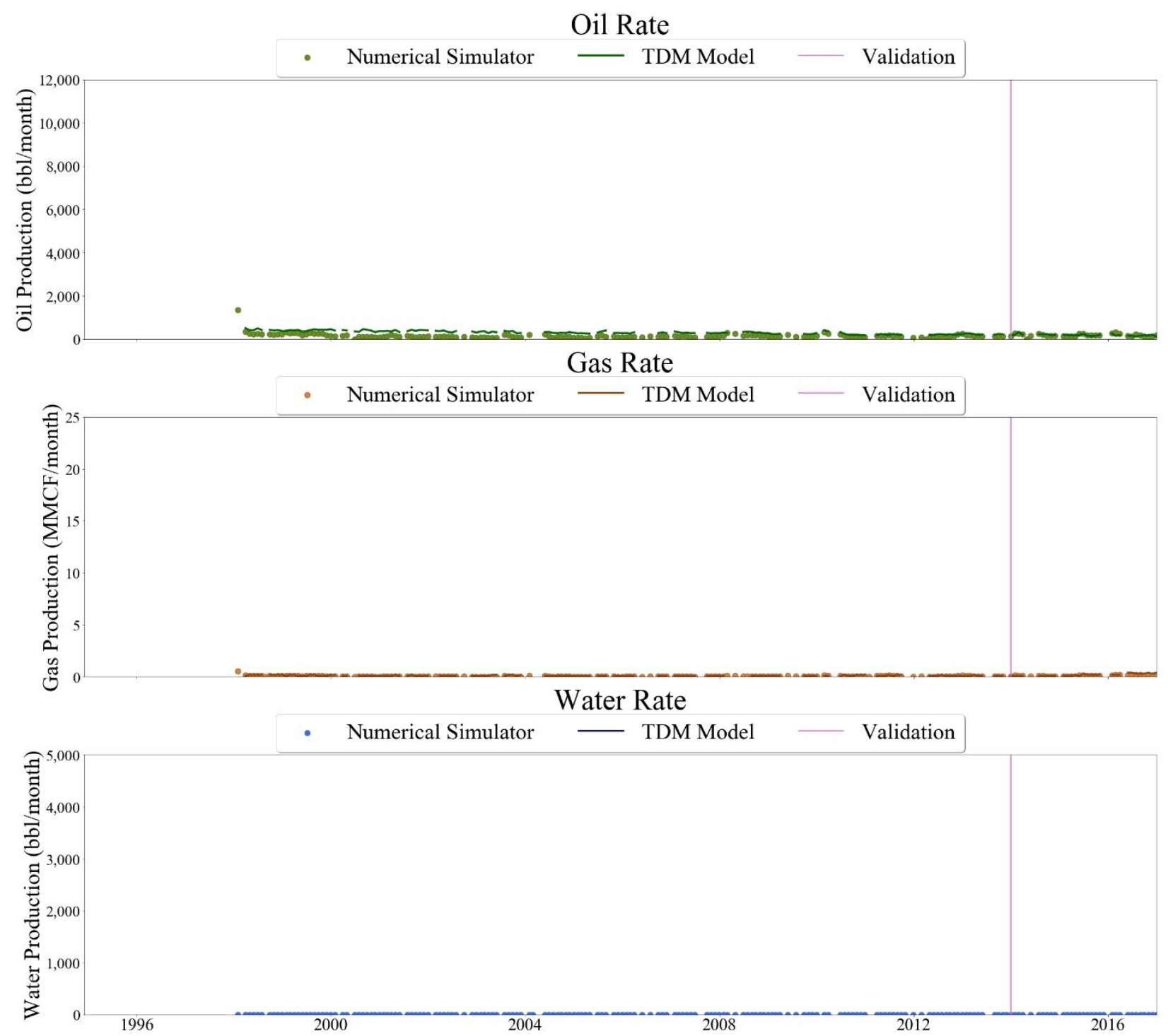

Figure 581 Well-009 oil, gas, and water rate TDM predictions vs actual simulation data plots for 3 Year Blind Validation TDM 


\section{Oil Rate}

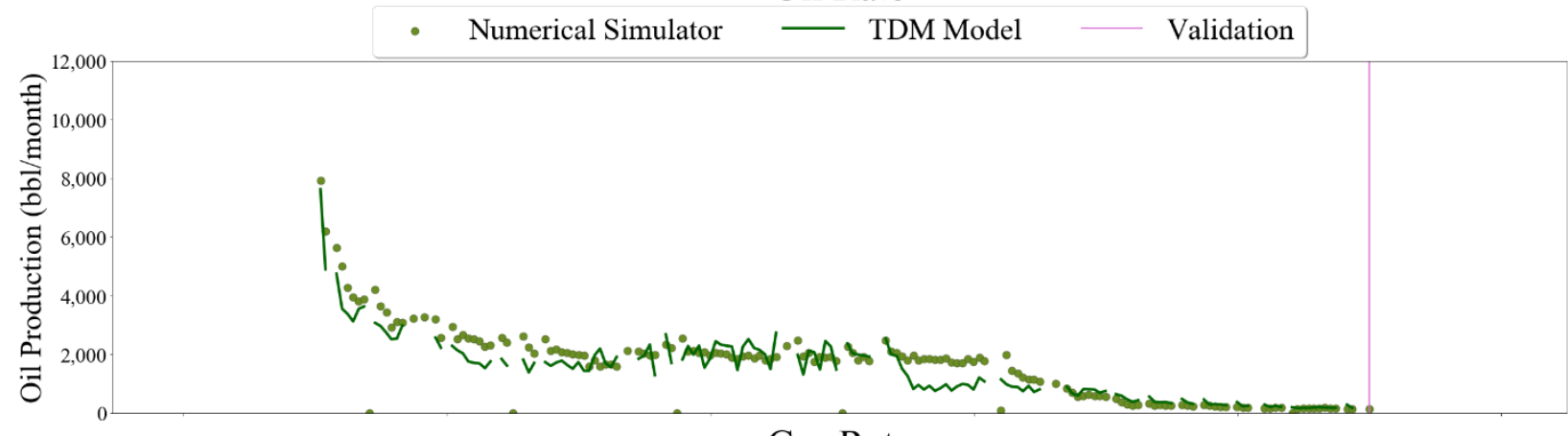

\section{Gas Rate}
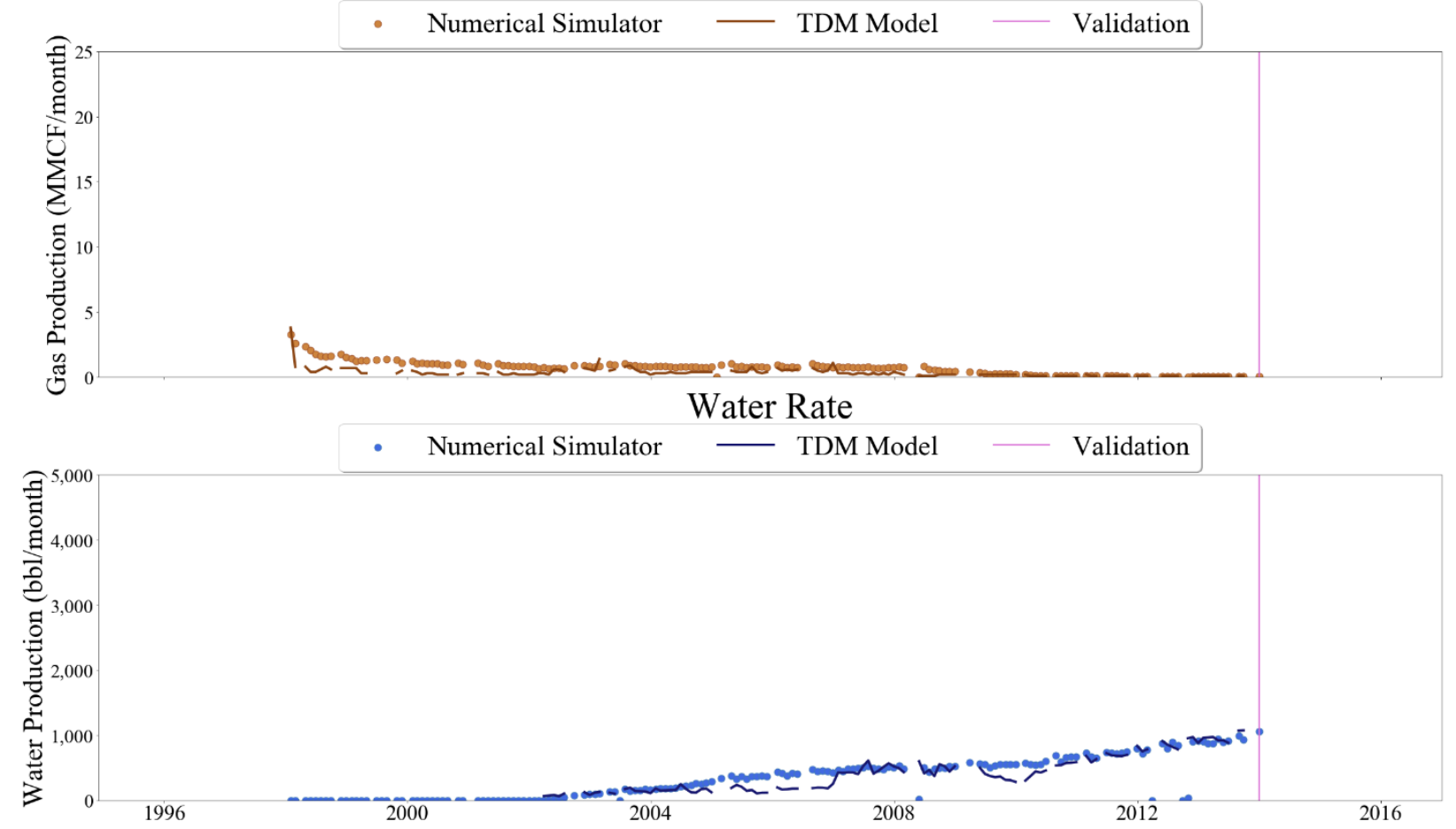

Figure 582 Well-010 oil, gas, and water rate TDM predictions vs actual simulation data plots for 3 Year Blind Validation TDM 

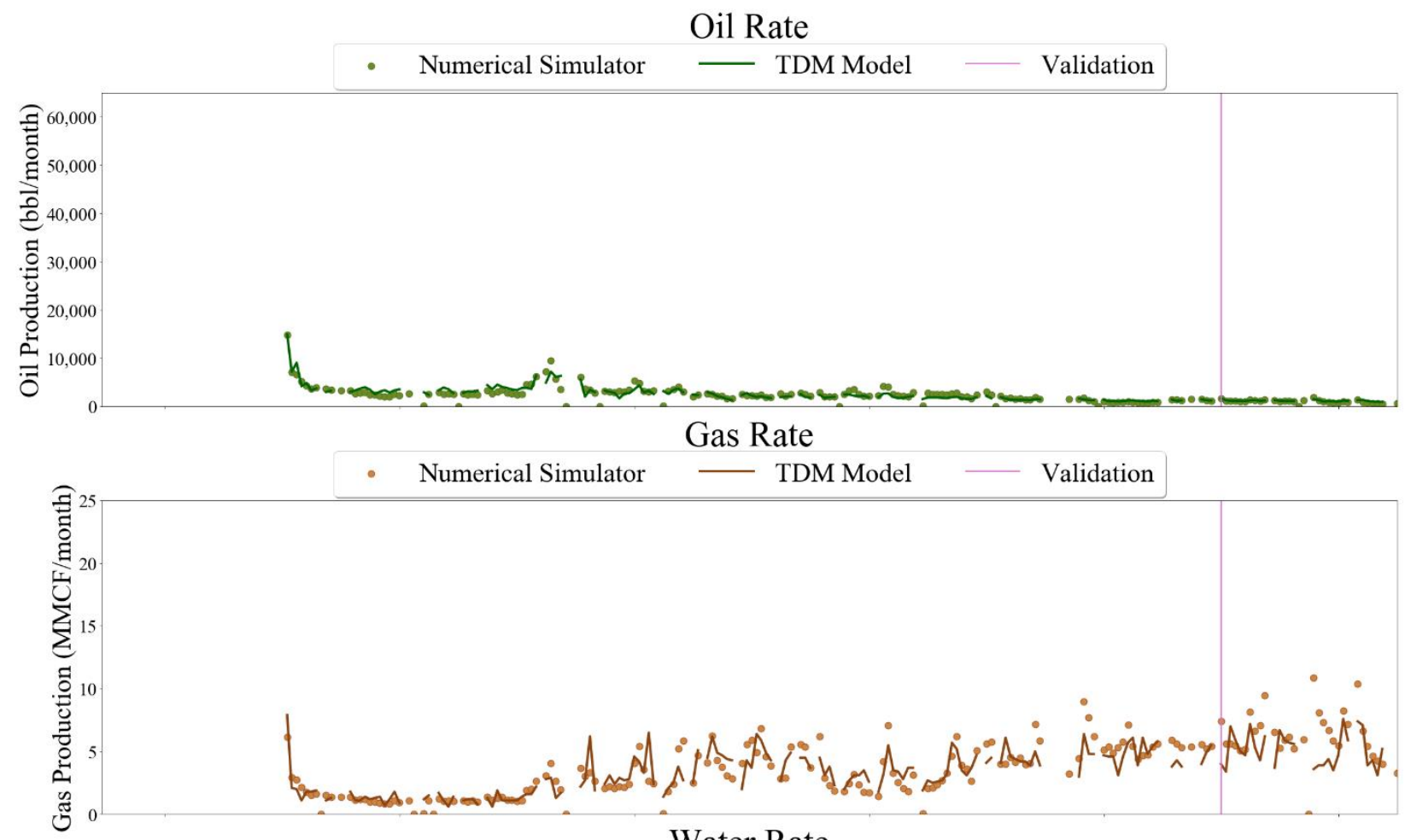

Water Rate

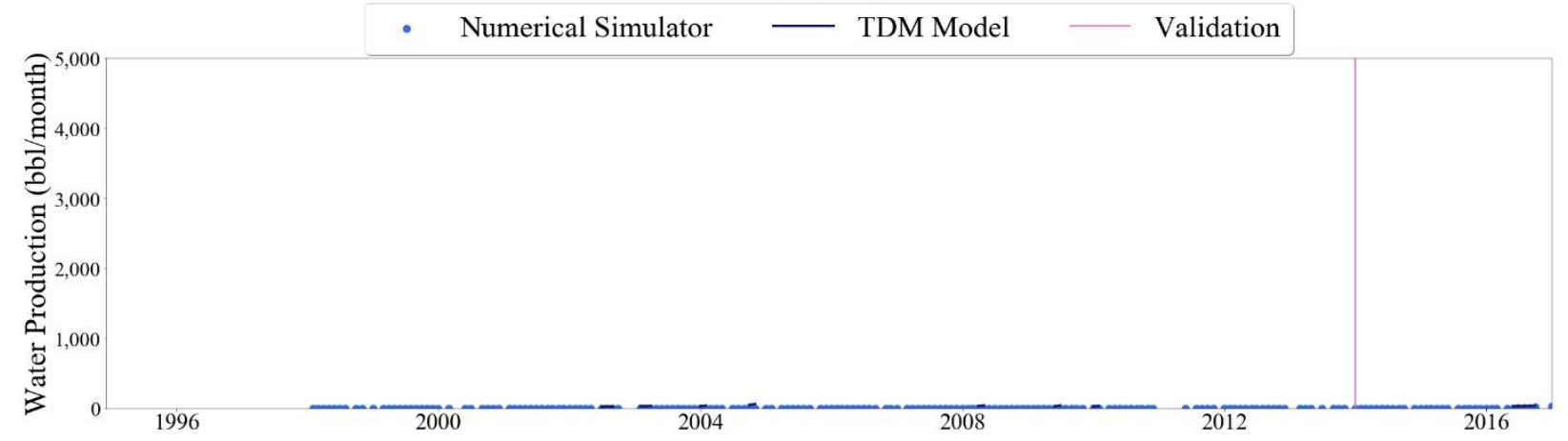

Figure 583 Well-011 oil, gas, and water rate TDM predictions vs actual simulation data plots for 3 Year Blind Validation TDM 

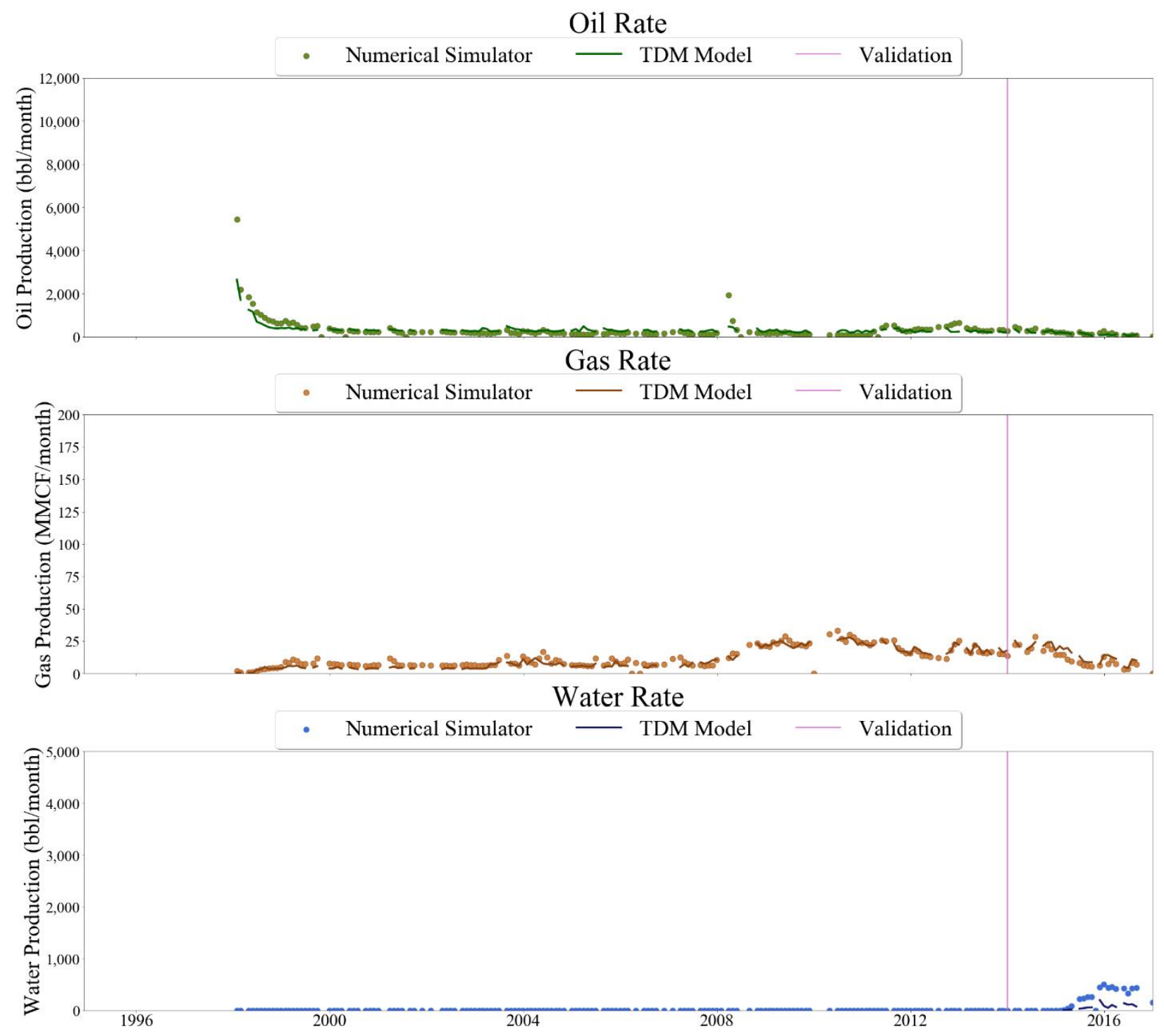

Figure 584 Well-012 oil, gas, and water rate TDM predictions vs actual simulation data plots for 3 Year Blind Validation TDM 

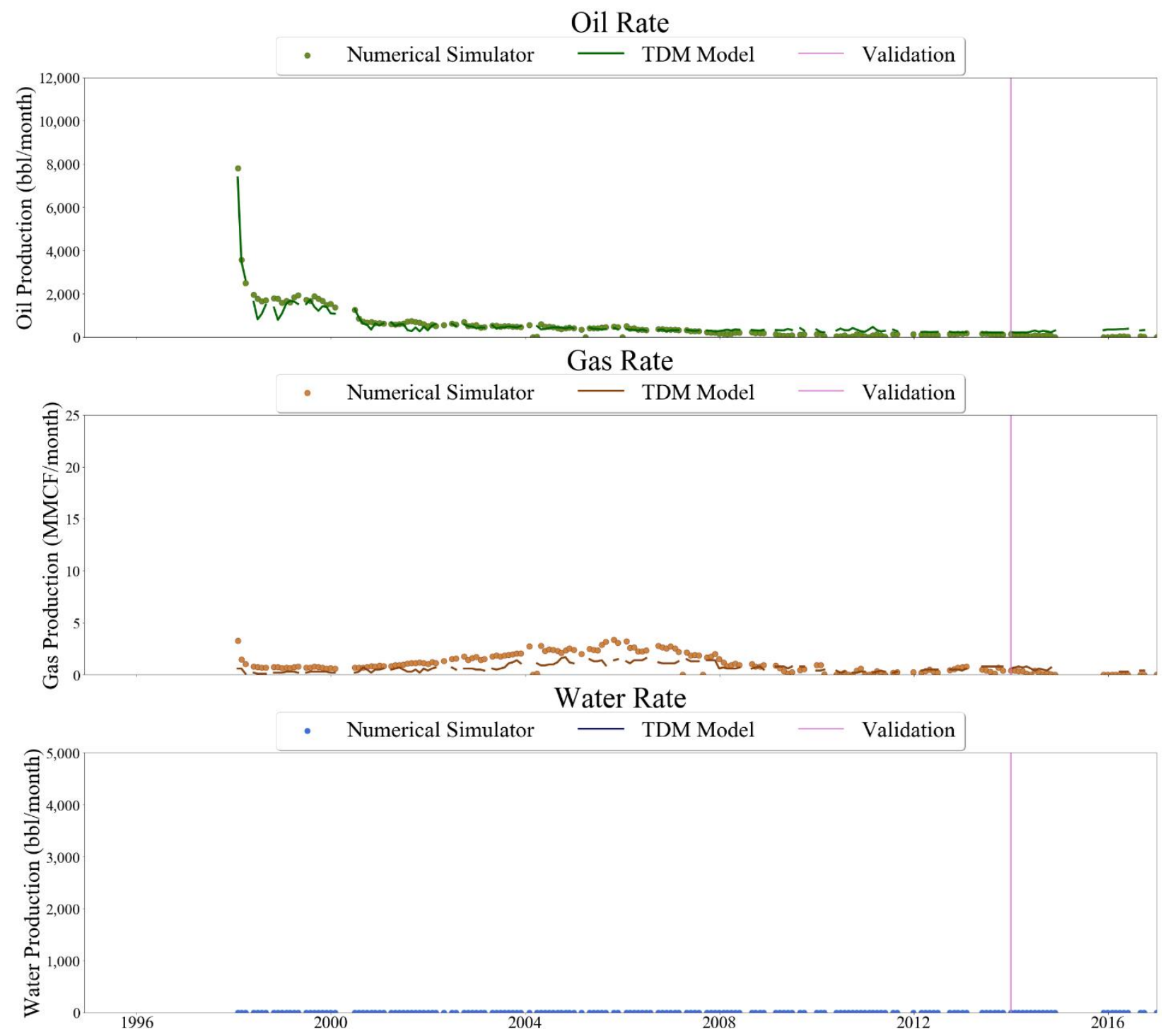

Figure 585 Well-013 oil, gas, and water rate TDM predictions vs actual simulation data plots for 3 Year Blind Validation TDM 


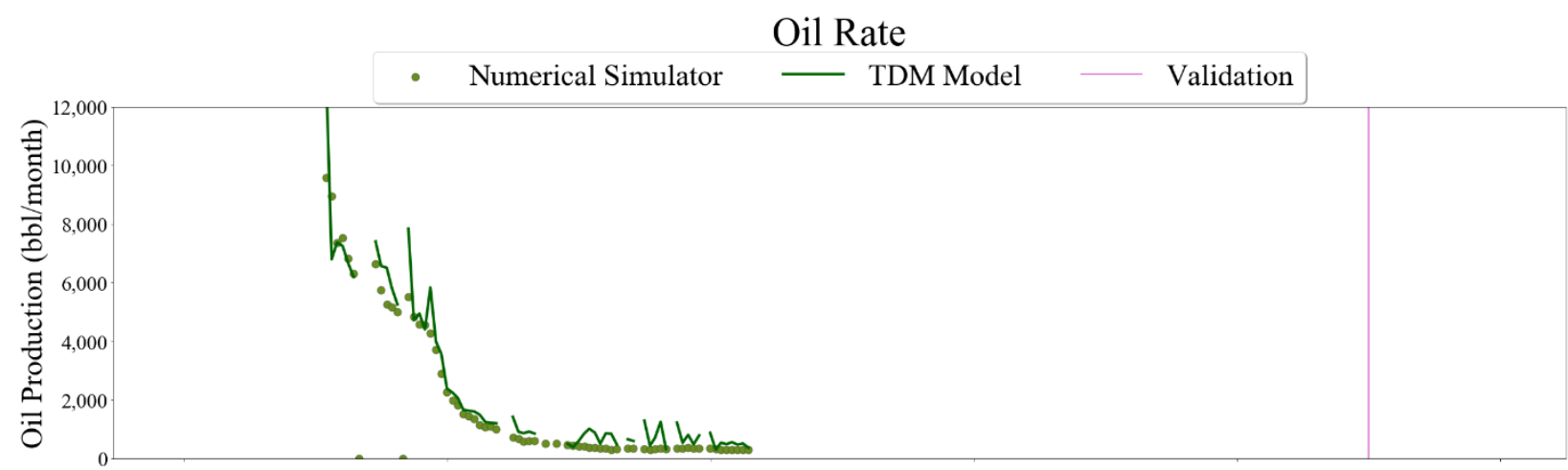

\section{Gas Rate}

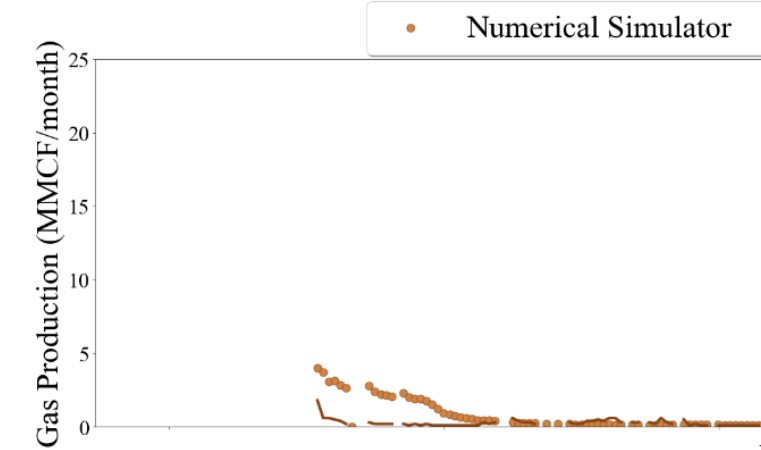

Water Rate

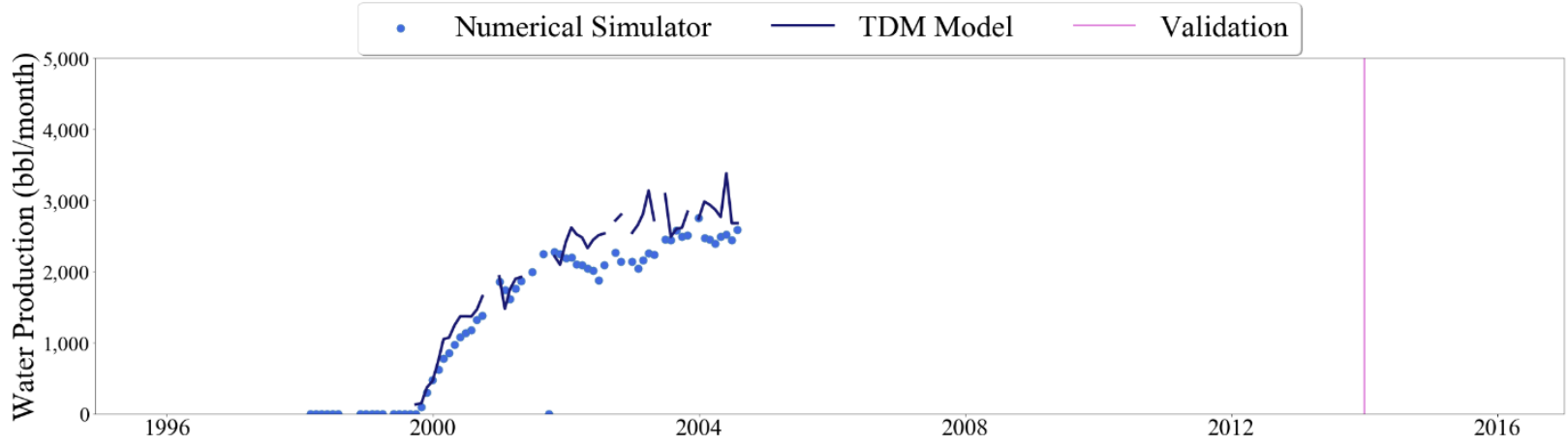

Figure 586 Well-014 oil, gas, and water rate TDM predictions vs actual simulation data plots for 3 Year Blind Validation TDM 

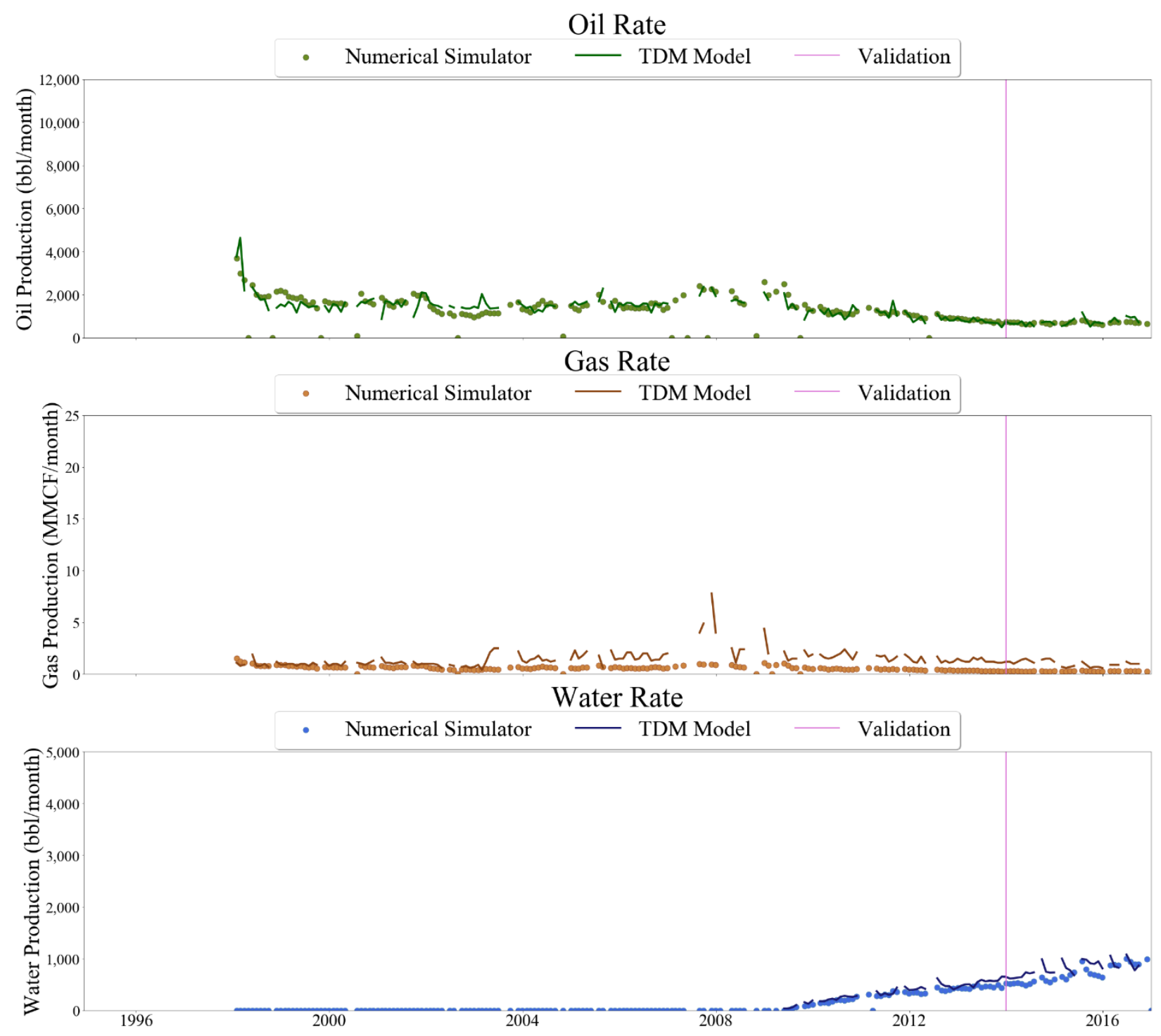

Figure 587 Well-015 oil, gas, and water rate TDM predictions vs actual simulation data plots for 3 Year Blind Validation TDM 

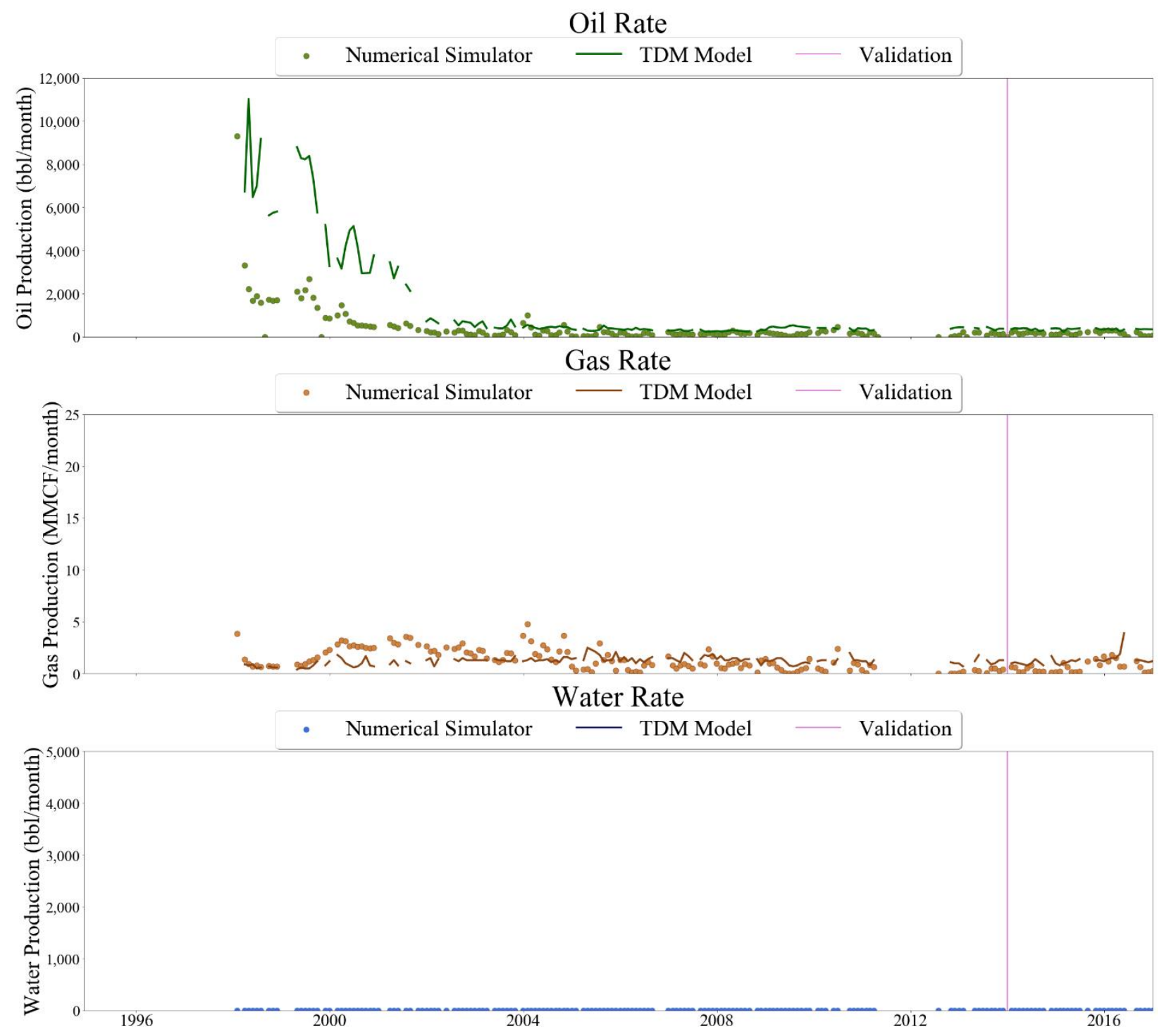

Figure 588 Well-016 oil, gas, and water rate TDM predictions vs actual simulation data plots for 3 Year Blind Validation TDM 

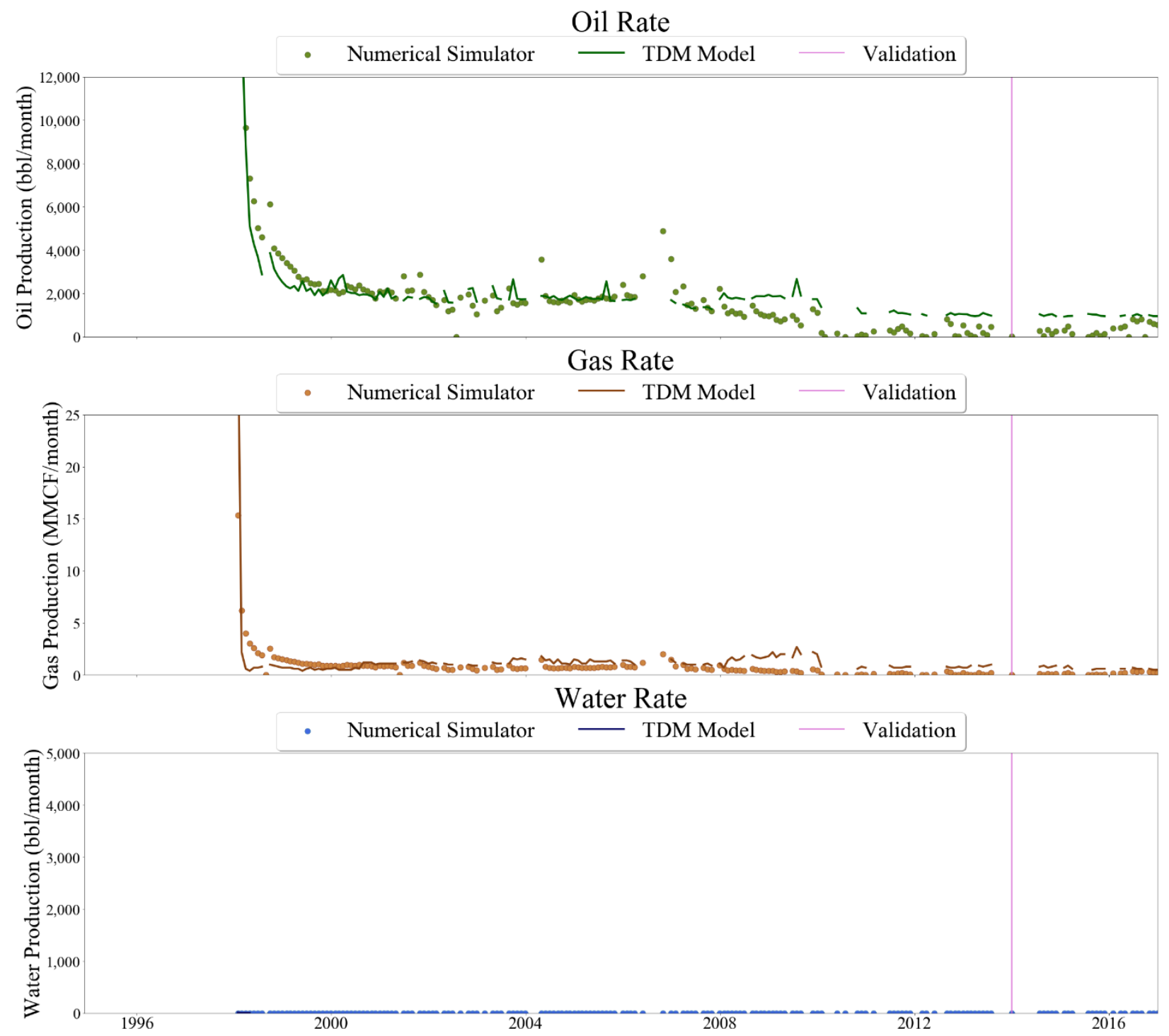

Figure 589 Well-017 oil, gas, and water rate TDM predictions vs actual simulation data plots for 3 Year Blind Validation TDM 

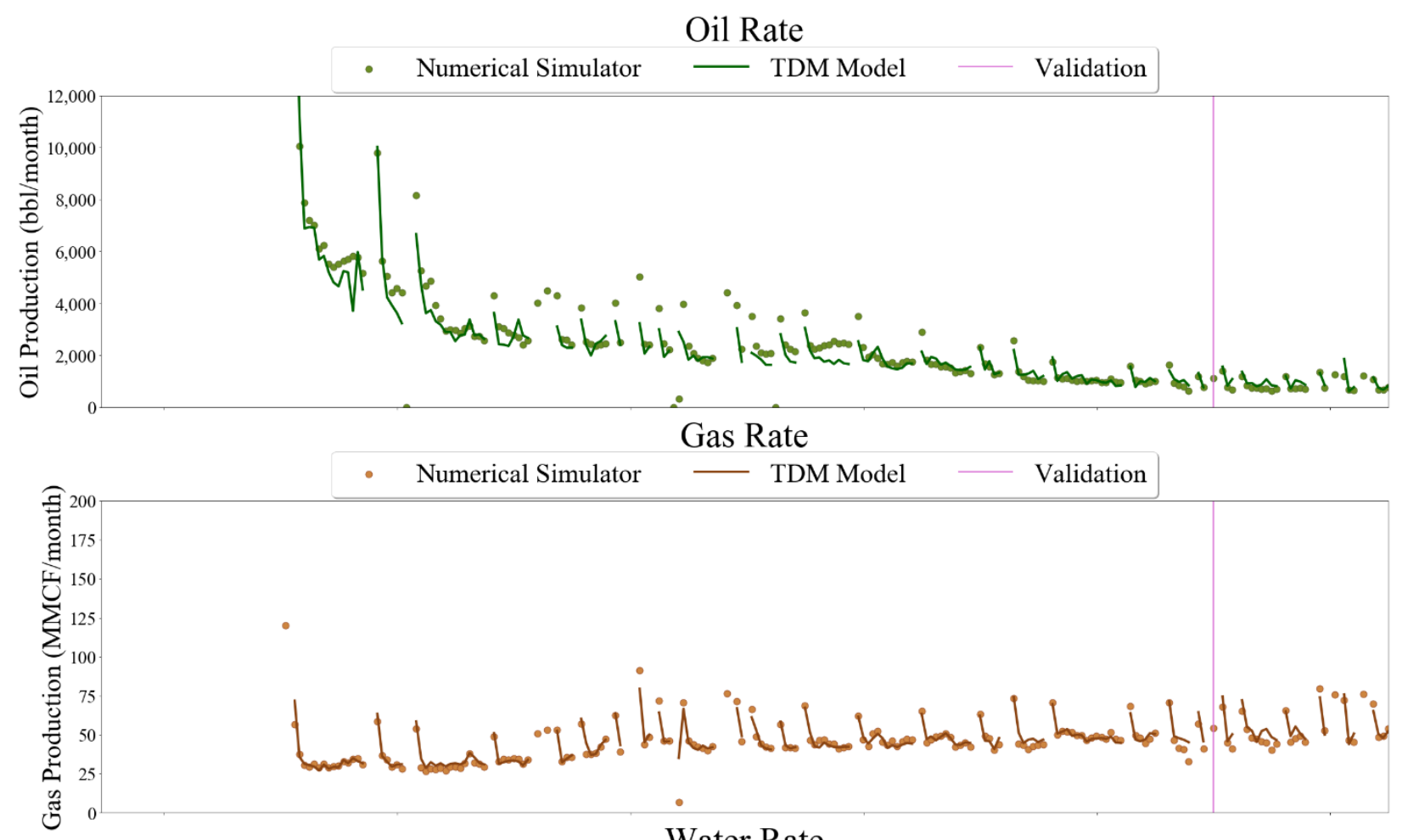

Water Rate

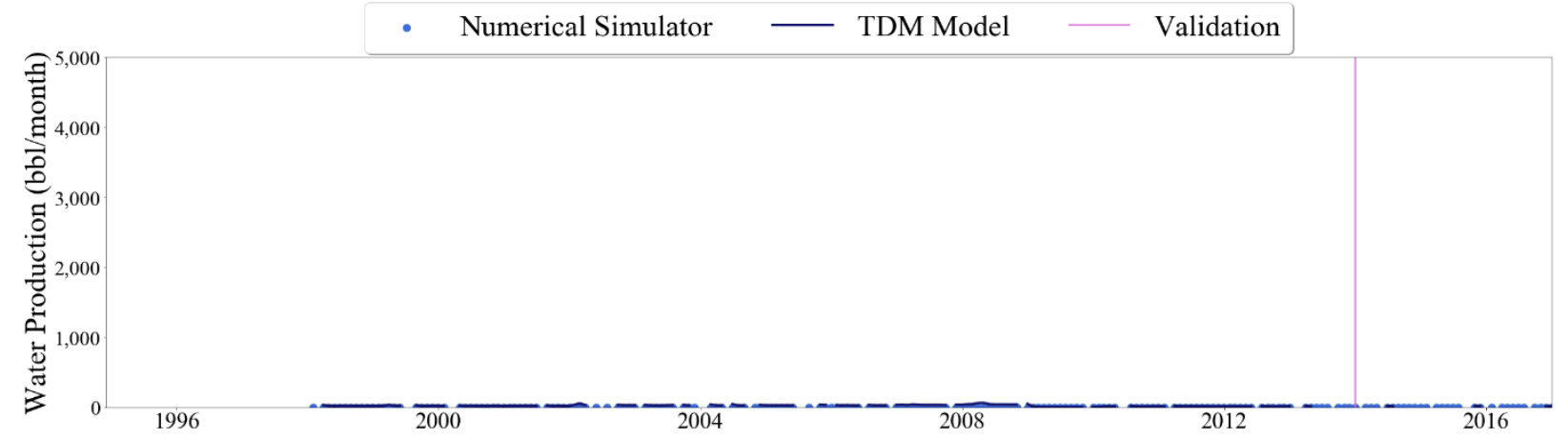

Figure 590 Well-018 oil, gas, and water rate TDM predictions vs actual simulation data plots for 3 Year Blind Validation TDM 

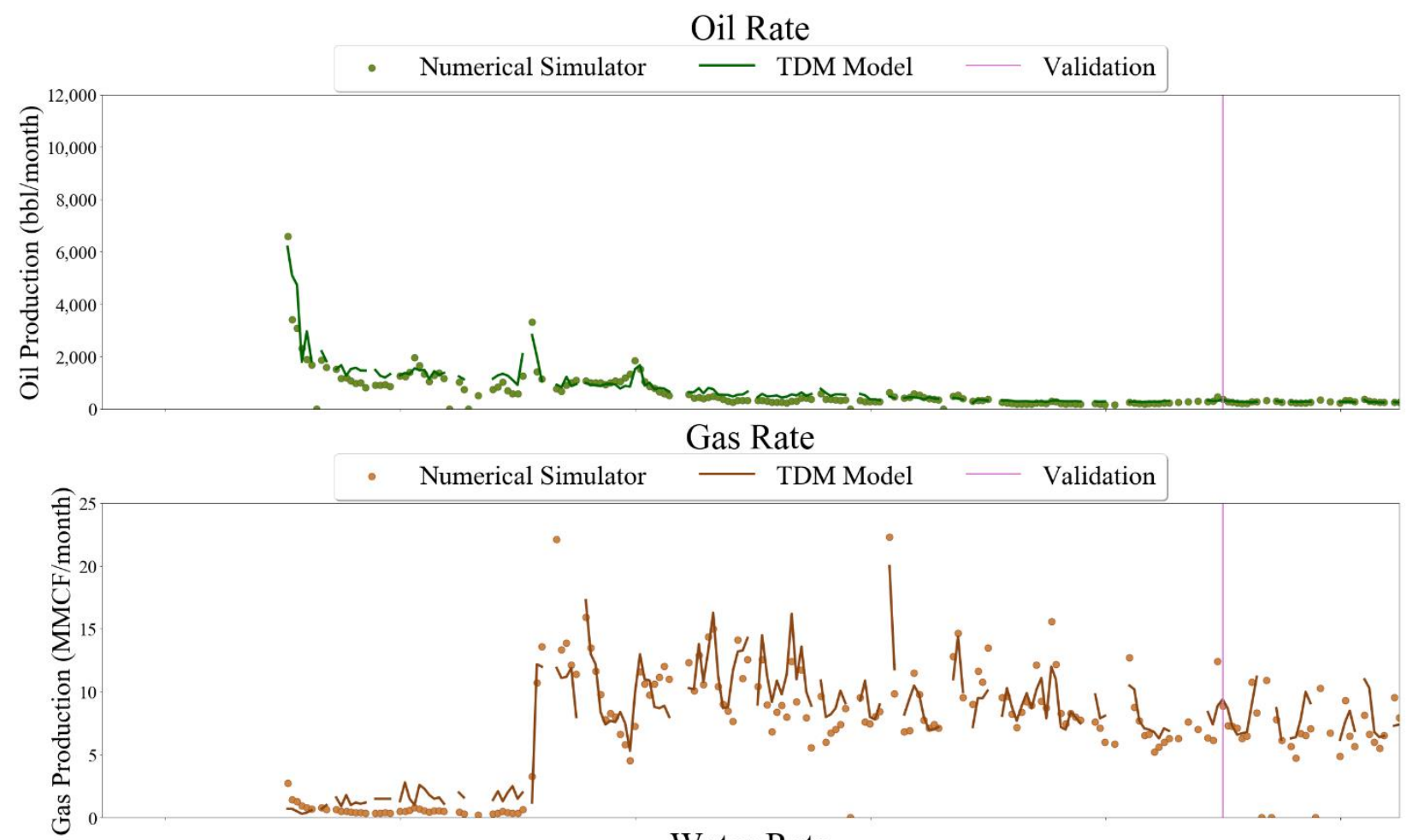

Water Rate

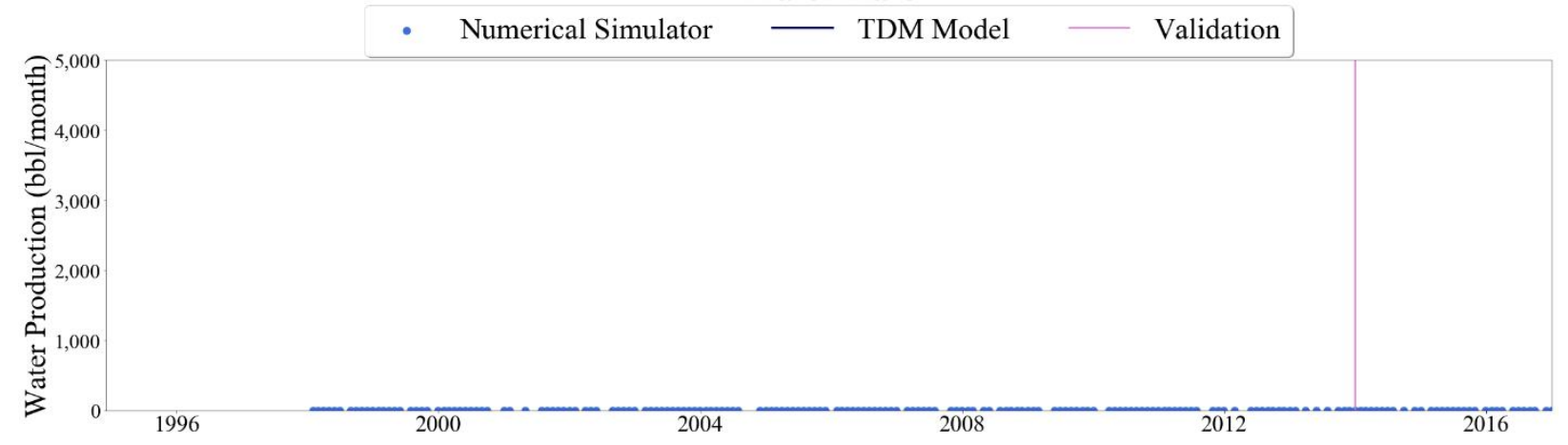

Figure 591 Well-019 oil, gas, and water rate TDM predictions vs actual simulation data plots for 3 Year Blind Validation TDM 


\section{Oil Rate}
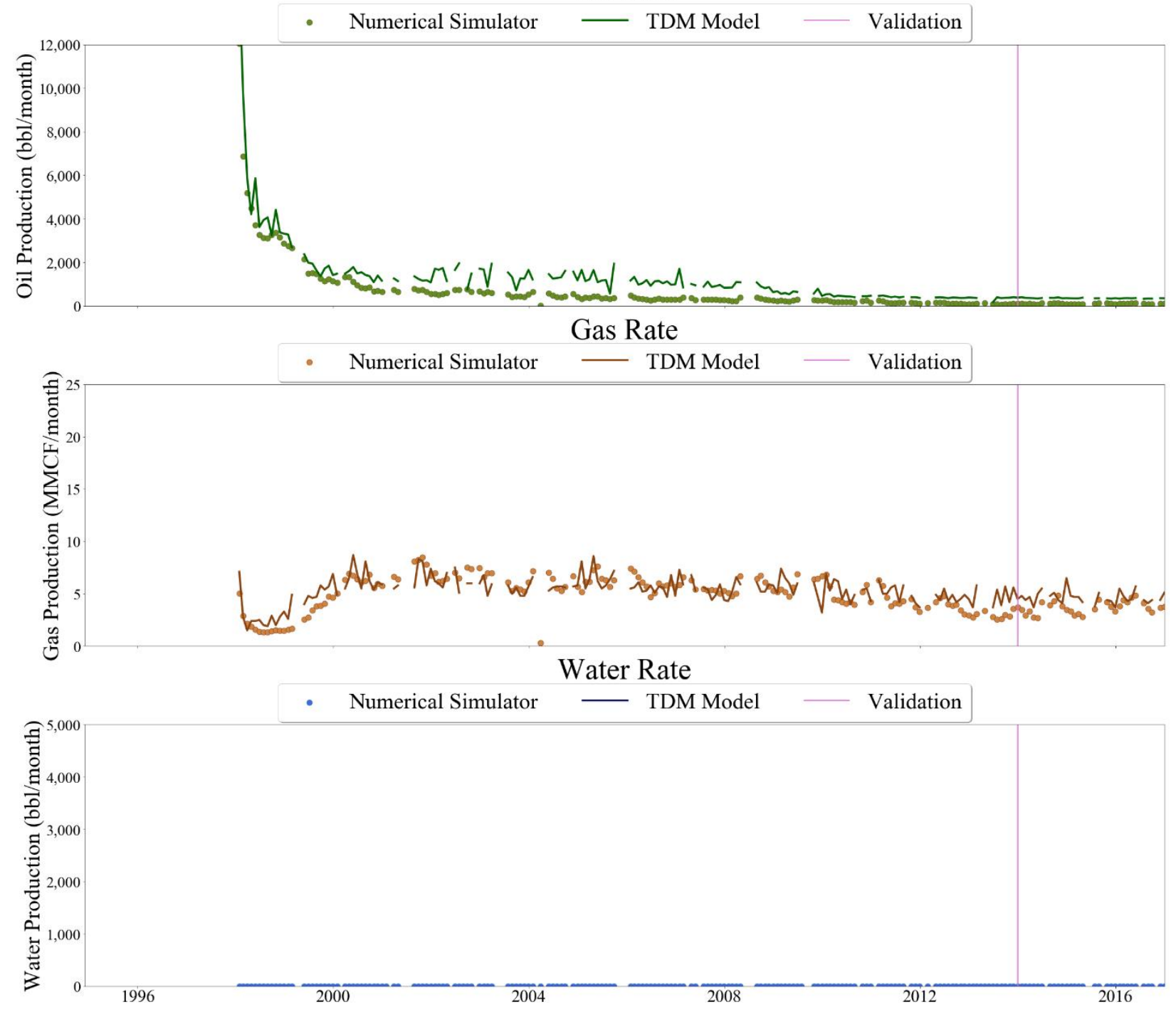

Figure 592 Well-020 oil, gas, and water rate TDM predictions vs actual simulation data plots for 3 Year Blind Validation TDM 

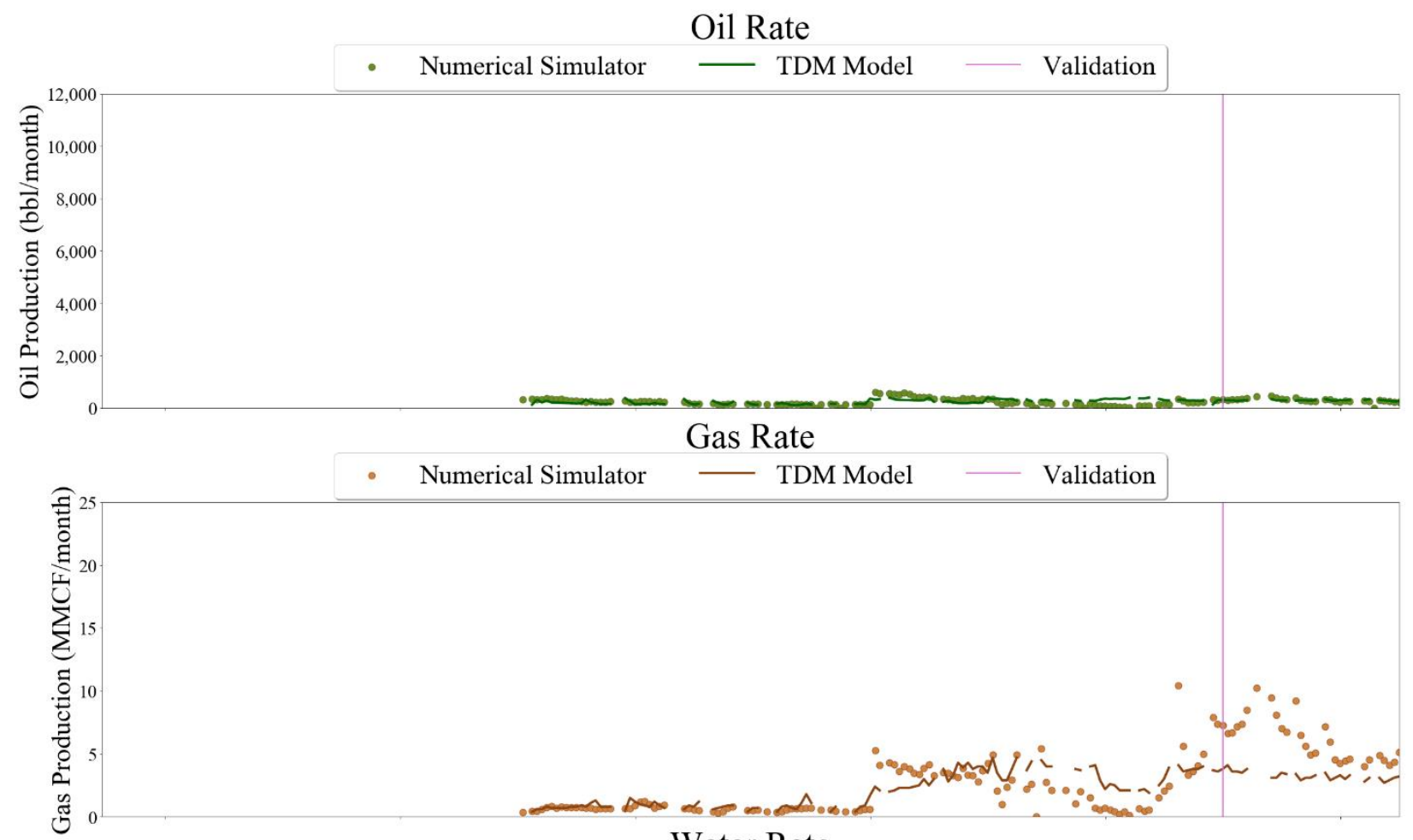

Water Rate

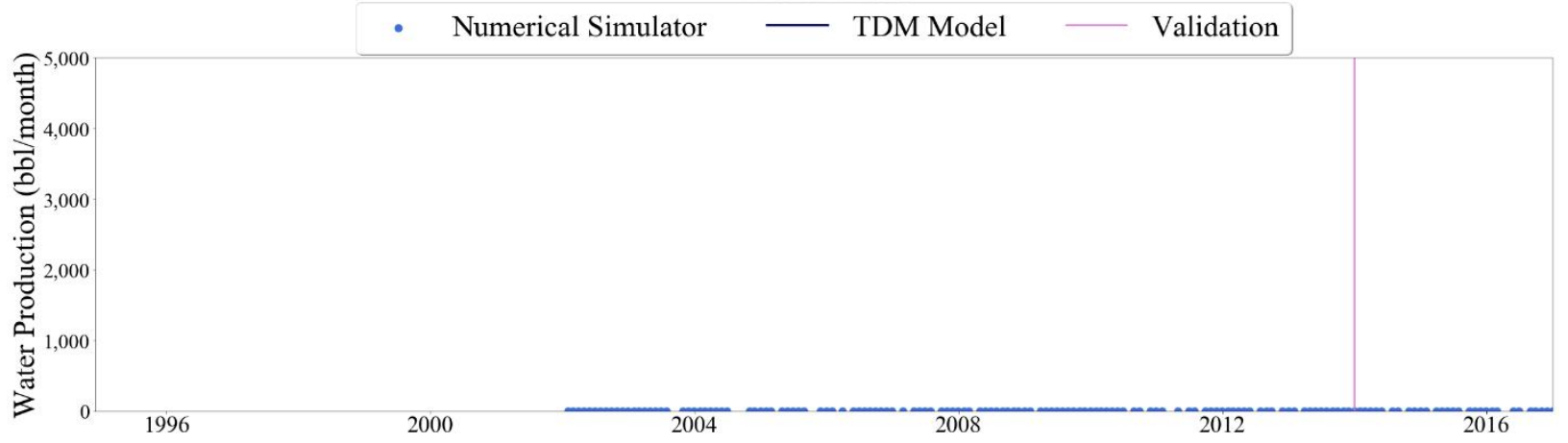

Figure 593 Well-021 oil, gas, and water rate TDM predictions vs actual simulation data plots for 3 Year Blind Validation TDM 


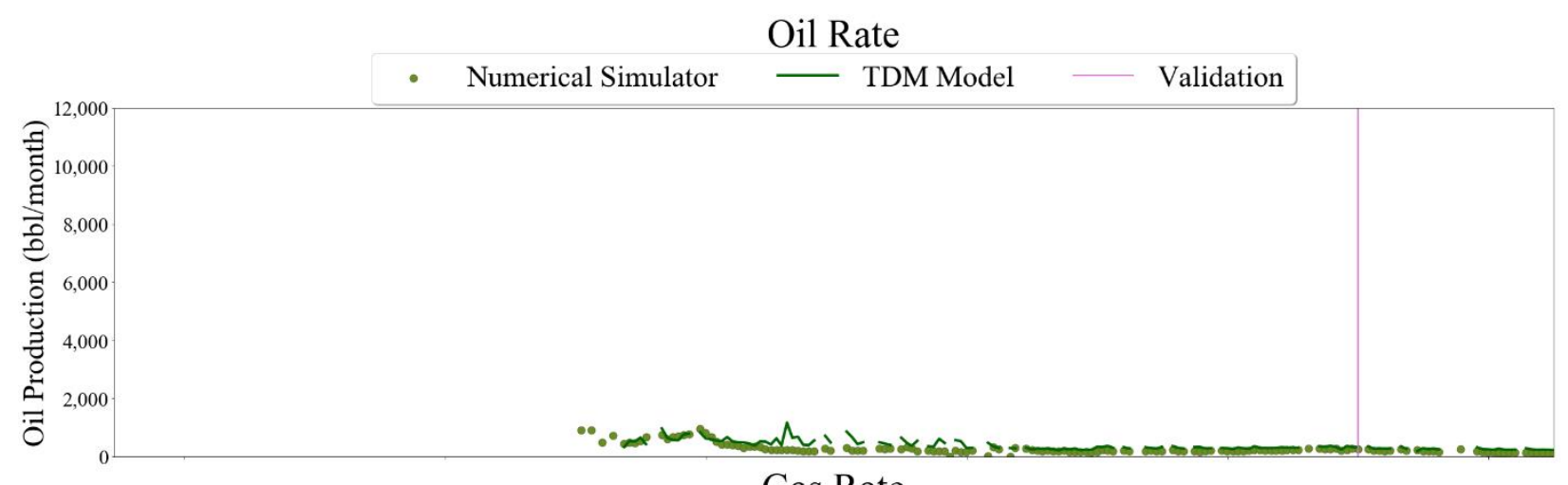

Gas Rate

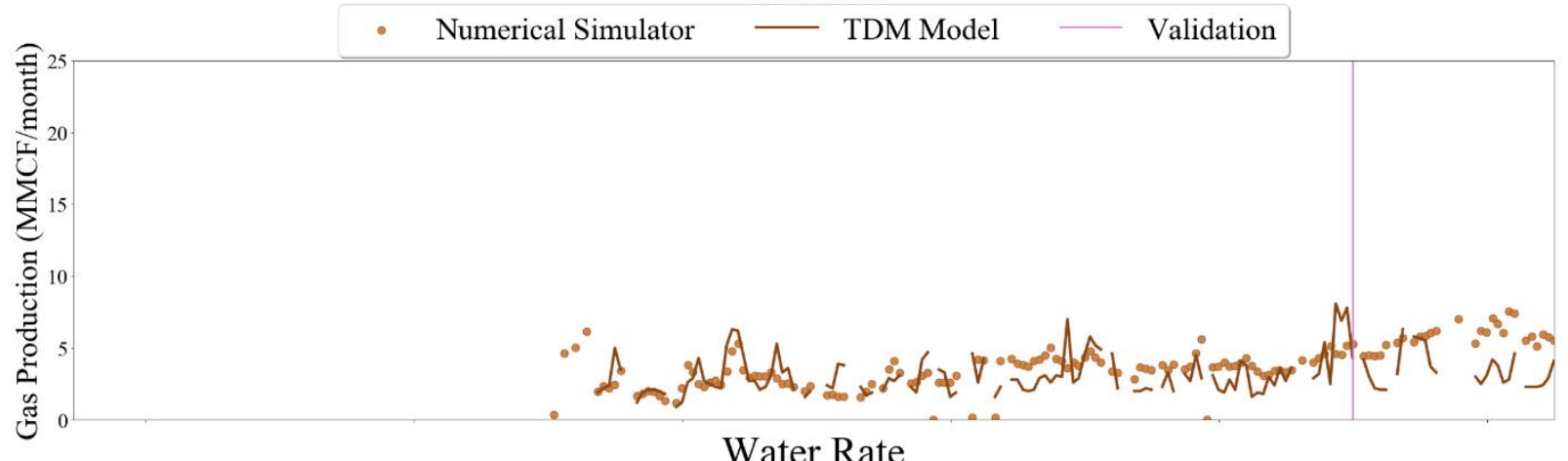

Water Rate

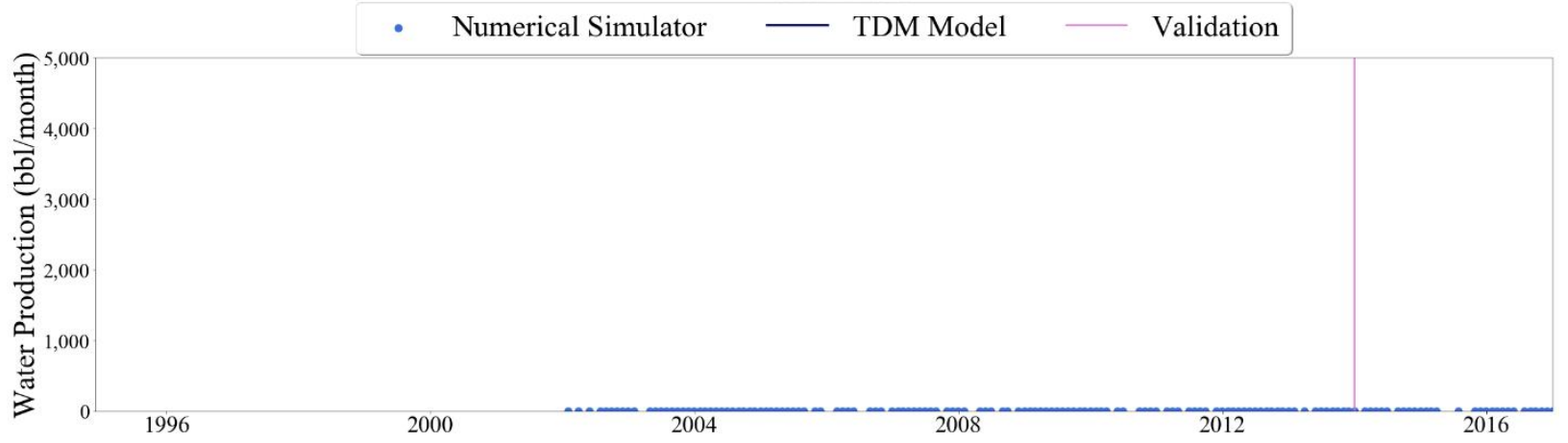

Figure 594 Well-022 oil, gas, and water rate TDM predictions vs actual simulation data plots for 3 Year Blind Validation TDM 

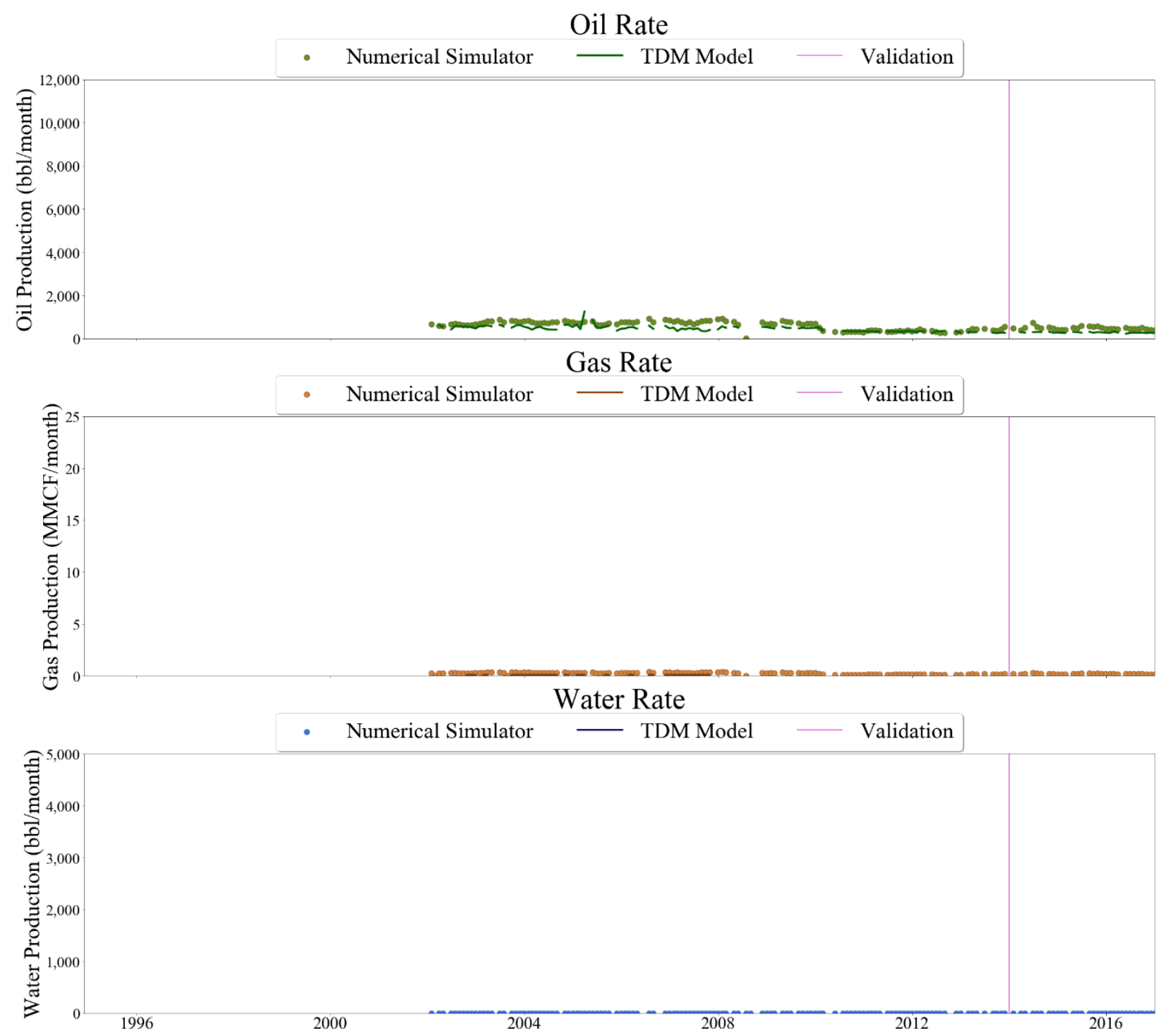

Figure 595 Well-023 oil, gas, and water rate TDM predictions vs actual simulation data plots for 3 Year Blind Validation TDM 

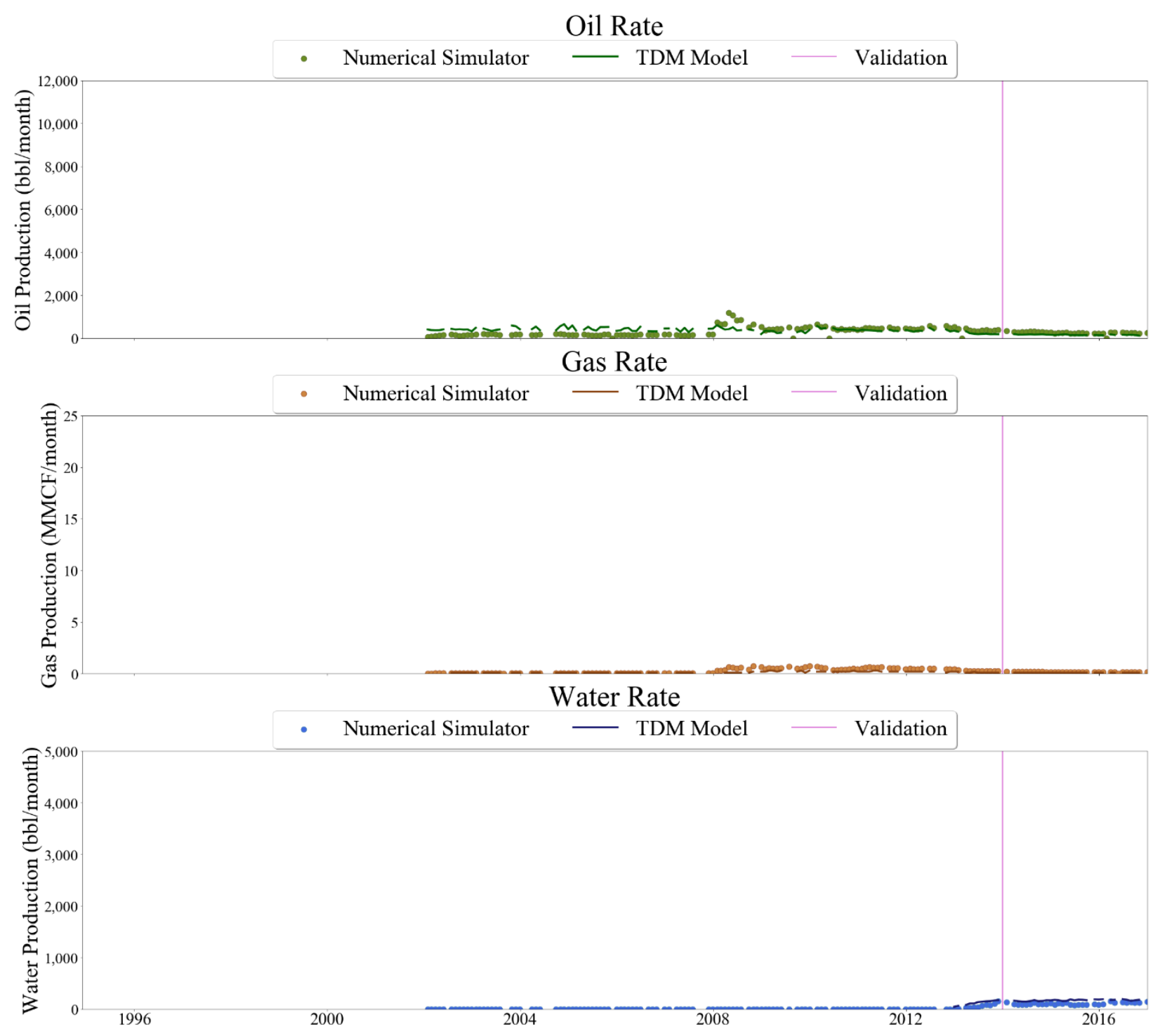

Figure 596 Well-024 oil, gas, and water rate TDM predictions vs actual simulation data plots for 3 Year Blind Validation TDM 


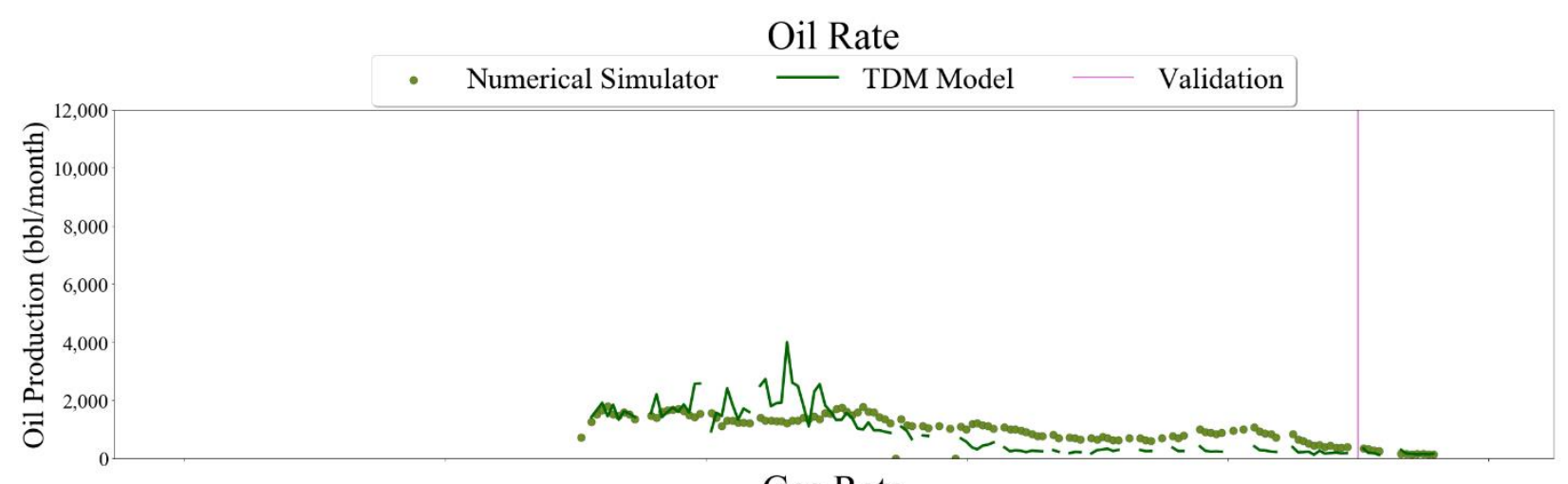

\section{Gas Rate}
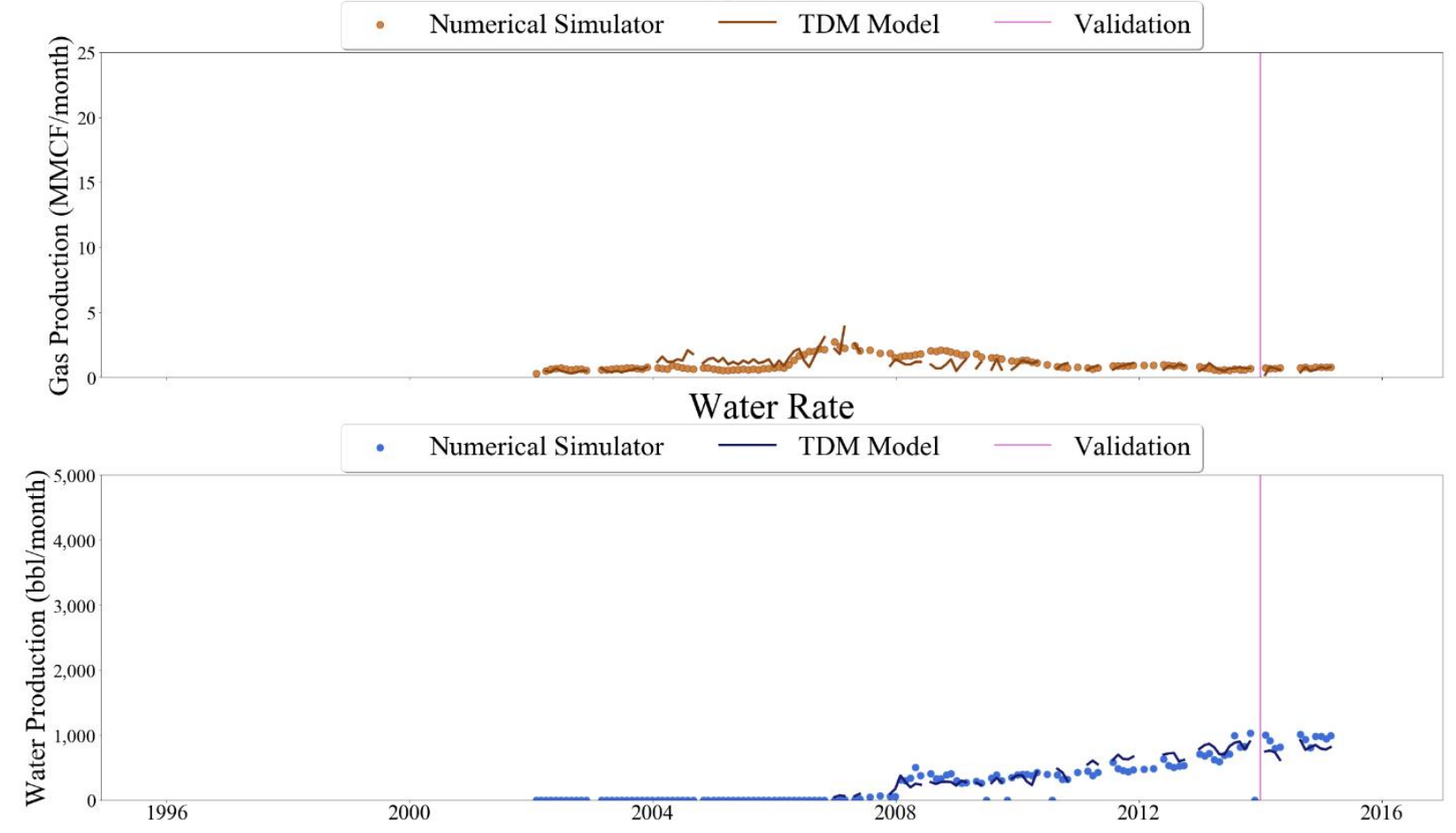

Figure 597 Well-025 oil, gas, and water rate TDM predictions vs actual simulation data plots for 3 Year Blind Validation TDM 


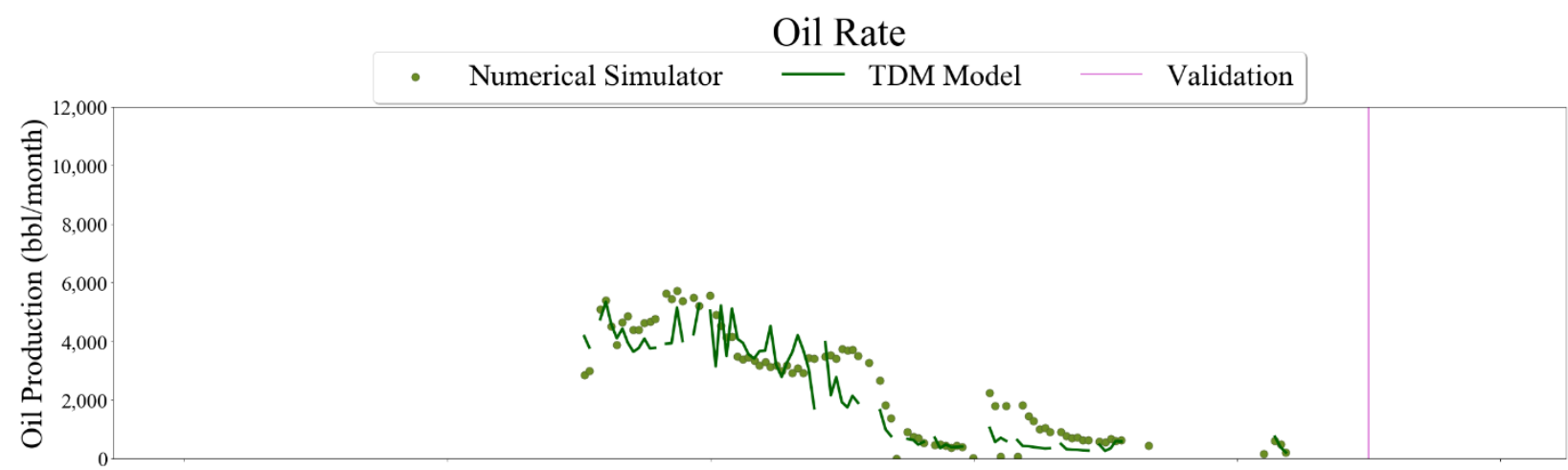

\section{Gas Rate}
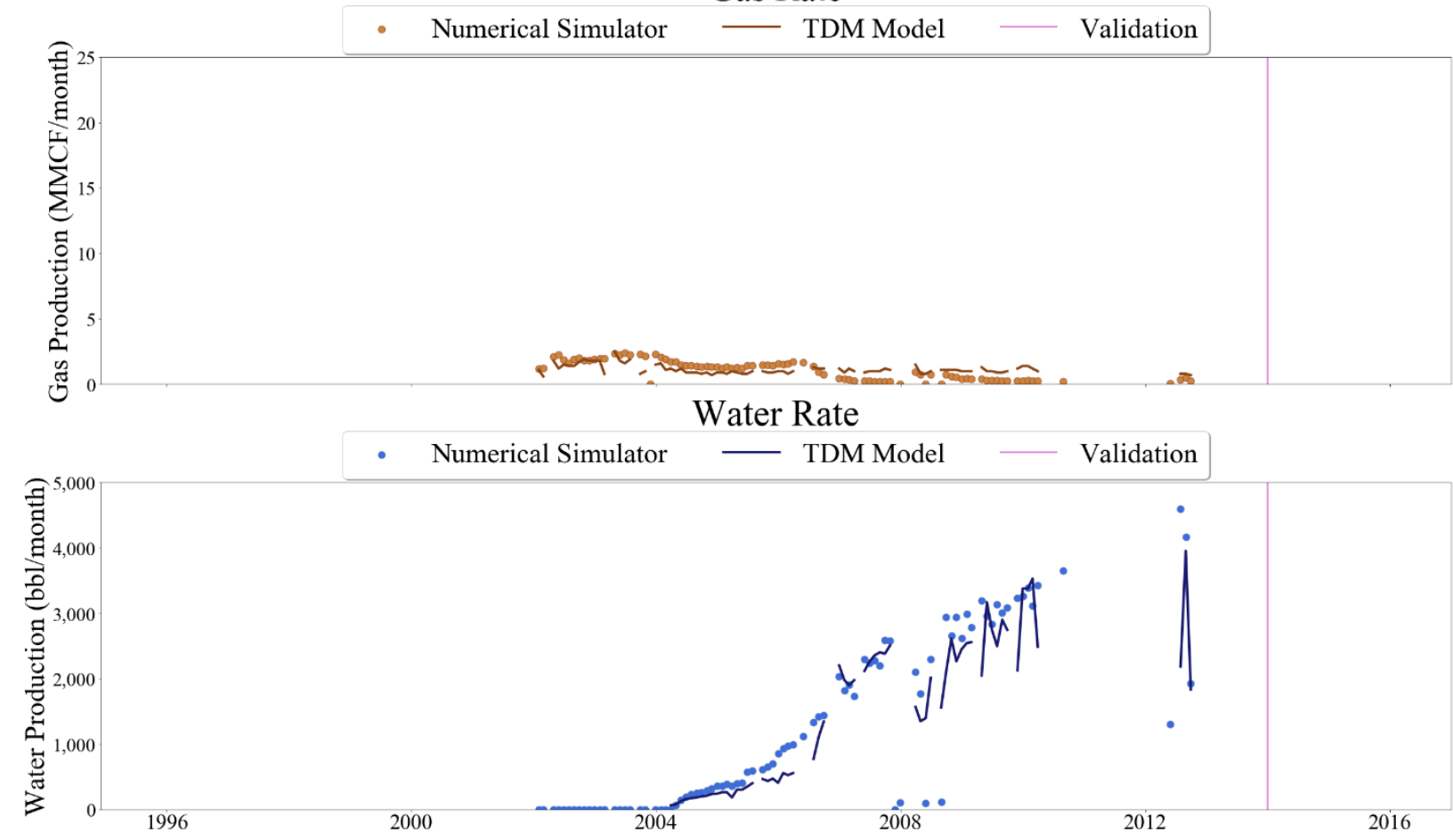

Figure 598 Well-026 oil, gas, and water rate TDM predictions vs actual simulation data plots for 3 Year Blind Validation TDM 


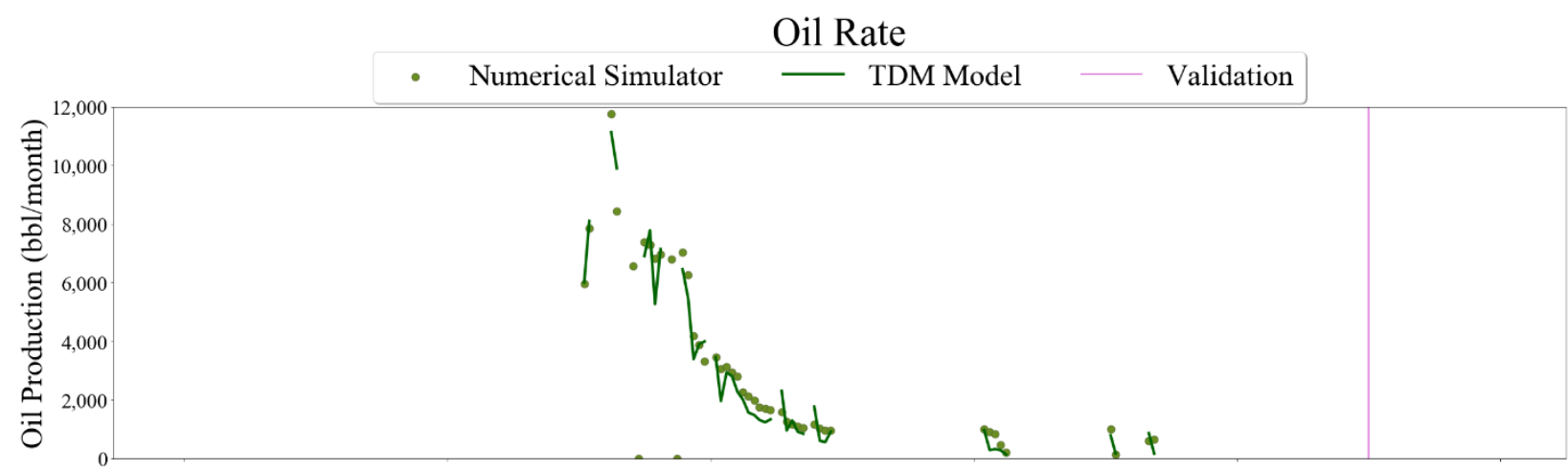

\section{Gas Rate}

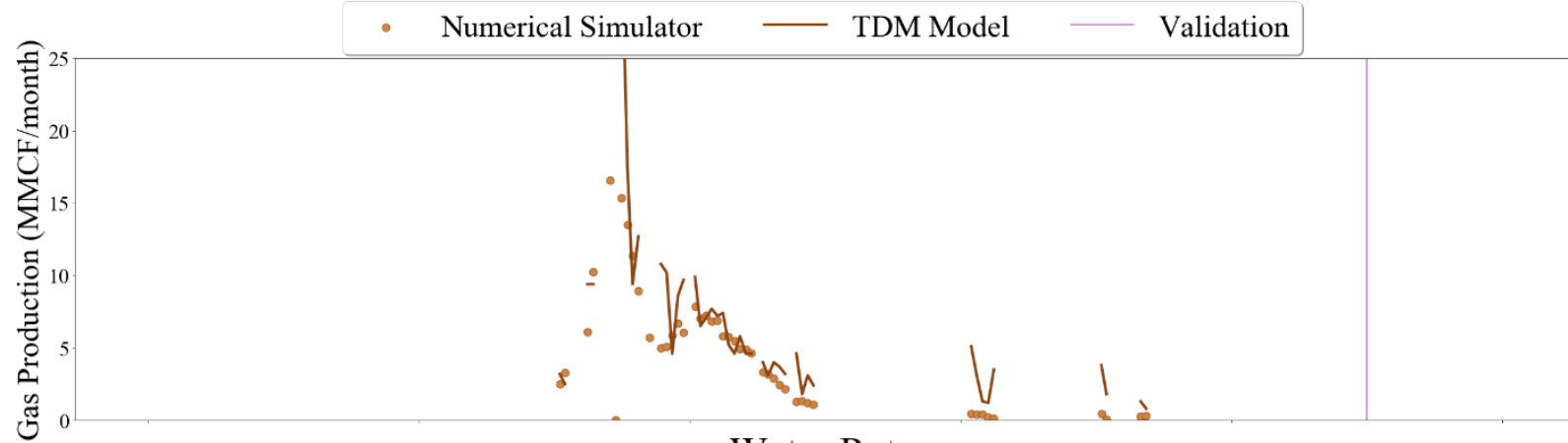

\section{Water Rate}

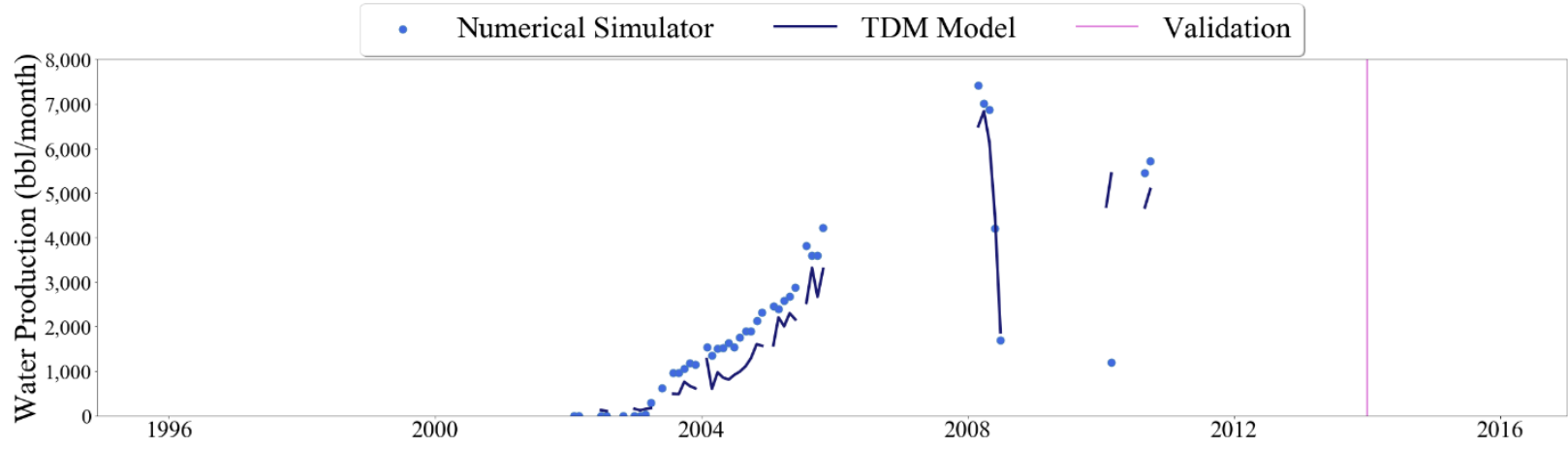

Figure 599 Well-027 oil, gas, and water rate TDM predictions vs actual simulation data plots for 3 Year Blind Validation TDM 


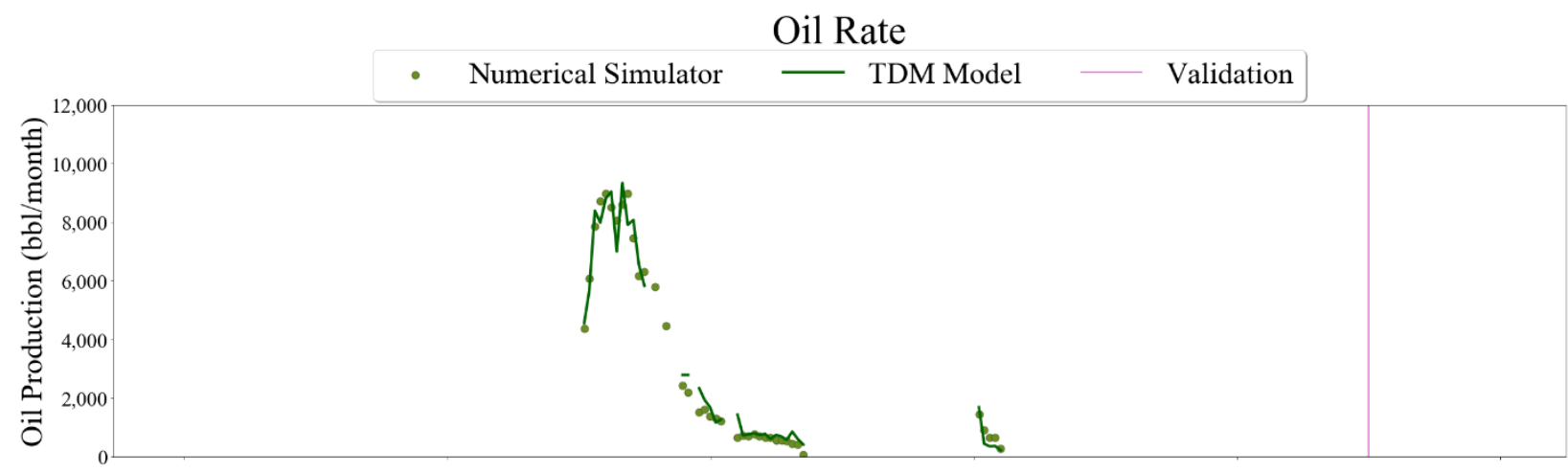

Gas Rate
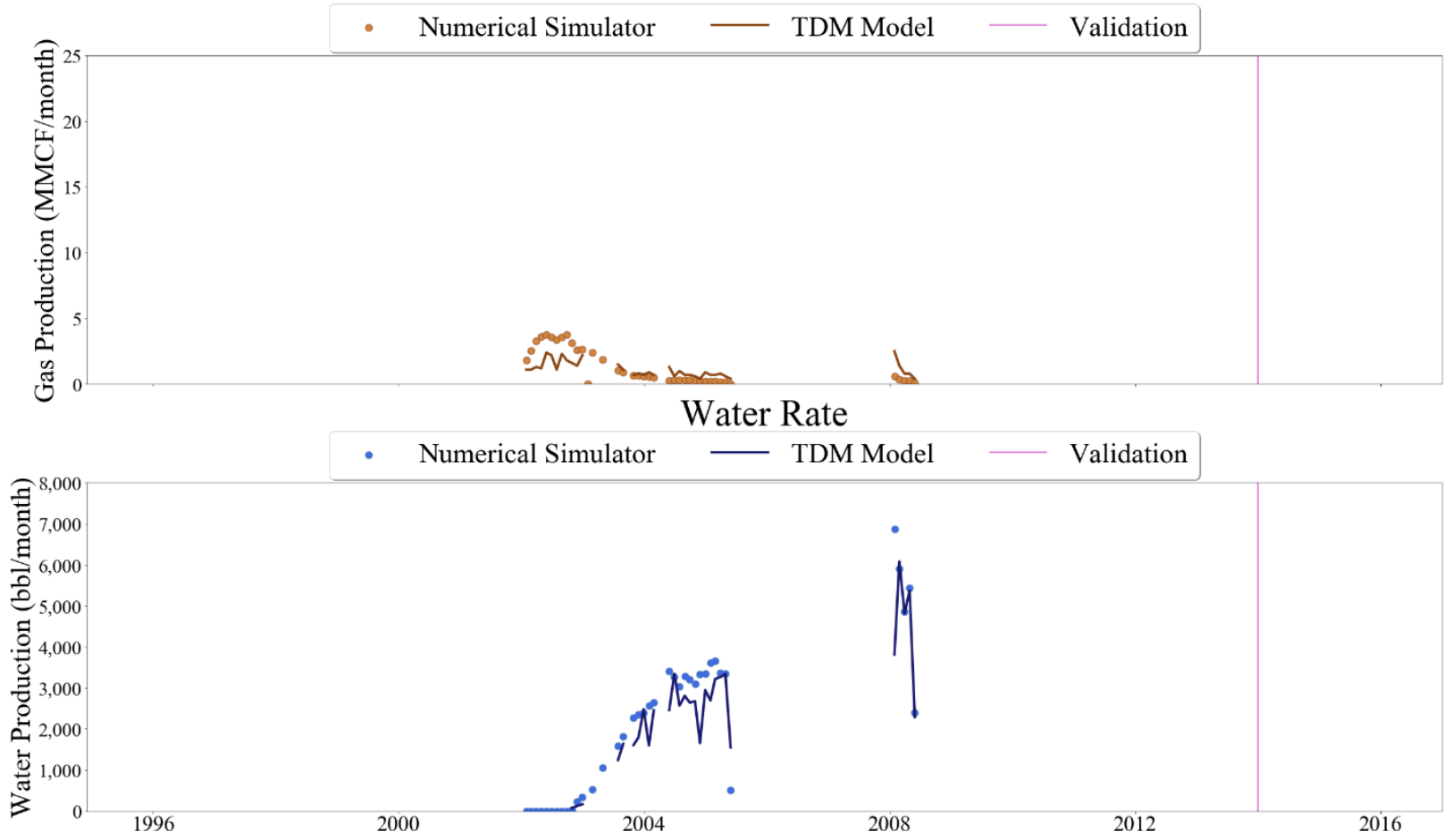

Figure 600 Well-028 oil, gas, and water rate TDM predictions vs actual simulation data plots for 3 Year Blind Validation TDM 

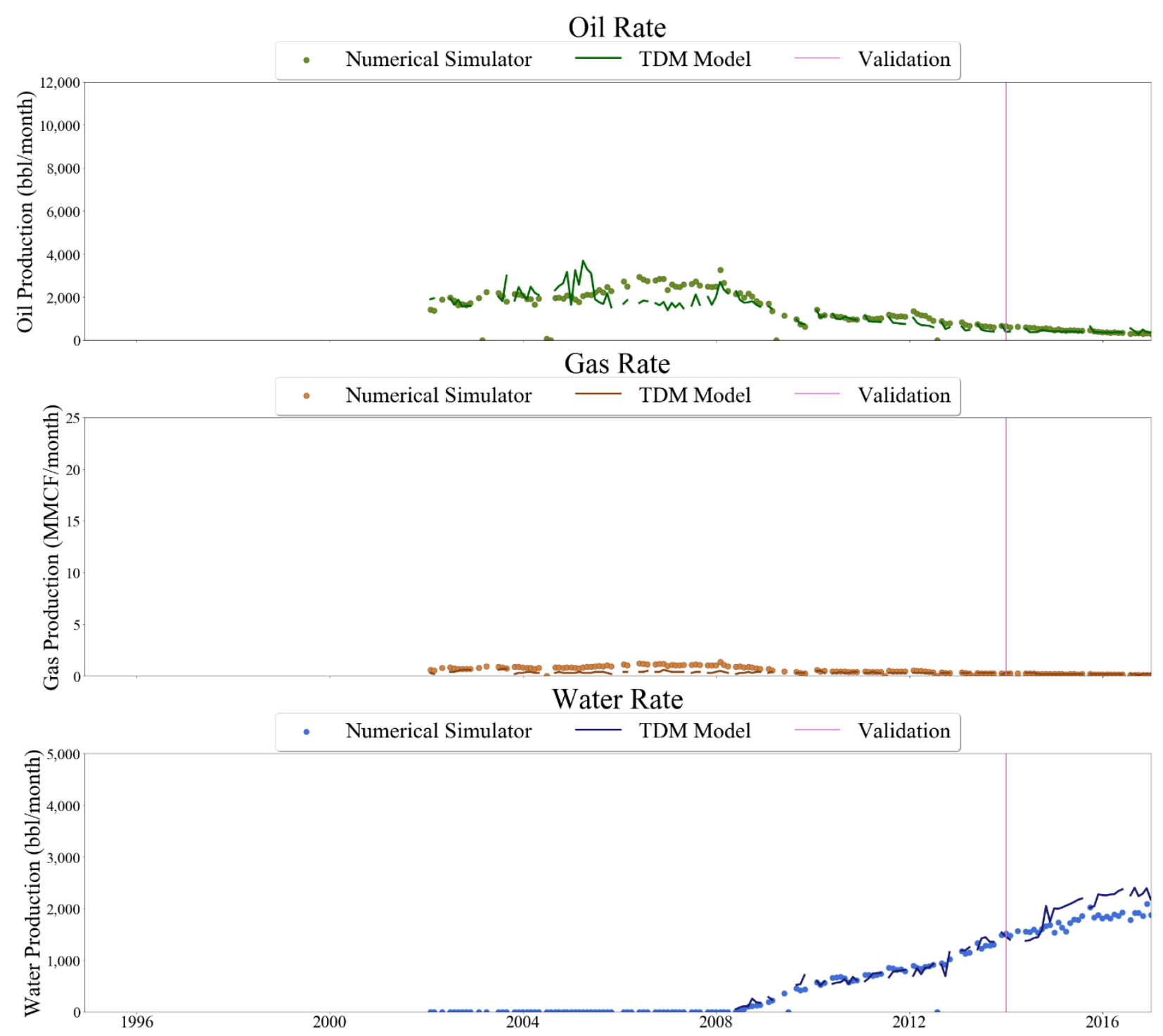

Figure 601 Well-029 oil, gas, and water rate TDM predictions vs actual simulation data plots for 3 Year Blind Validation TDM 

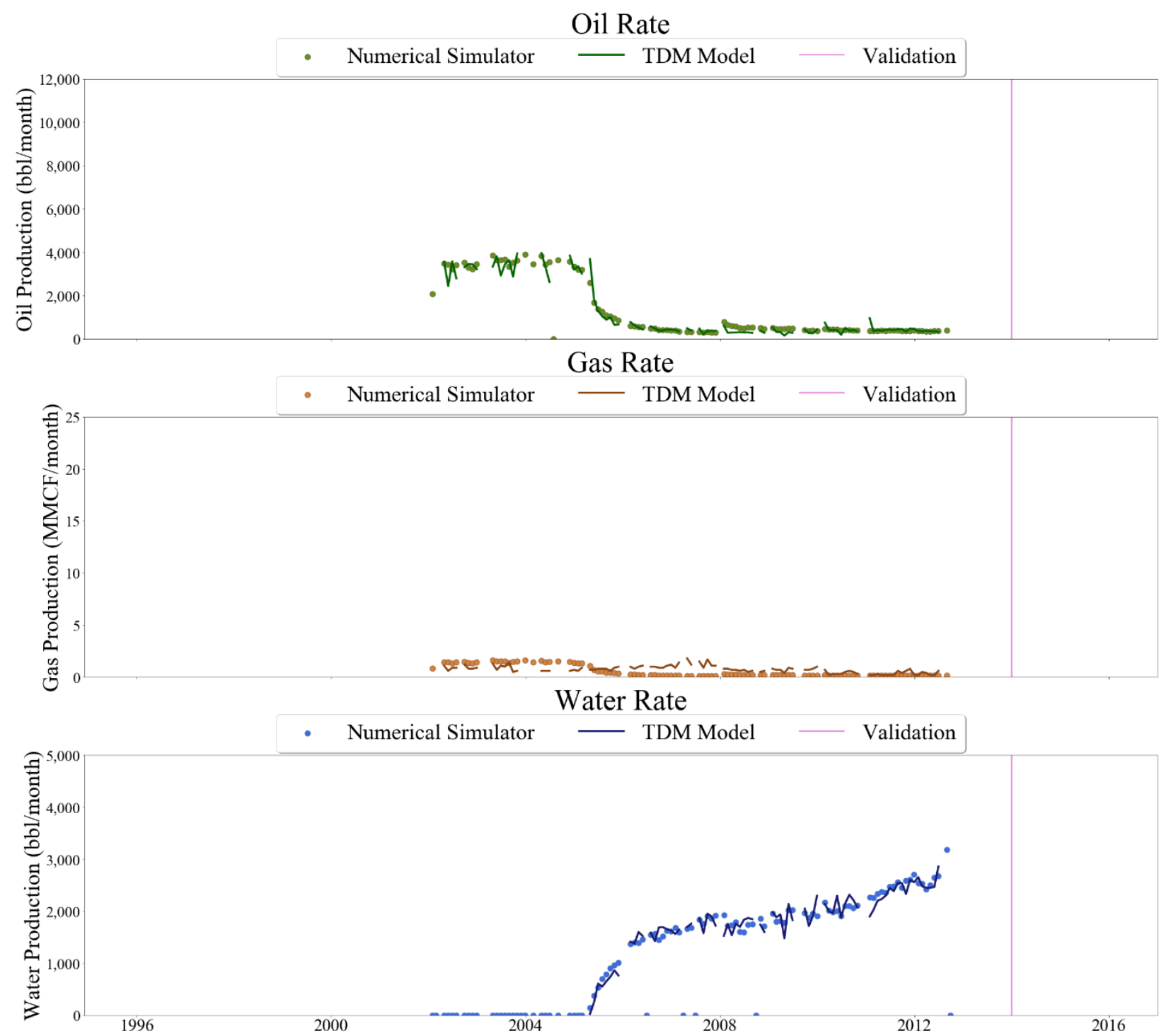

Figure 602 Well-030 oil, gas, and water rate TDM predictions vs actual simulation data plots for 3 Year Blind Validation TDM 

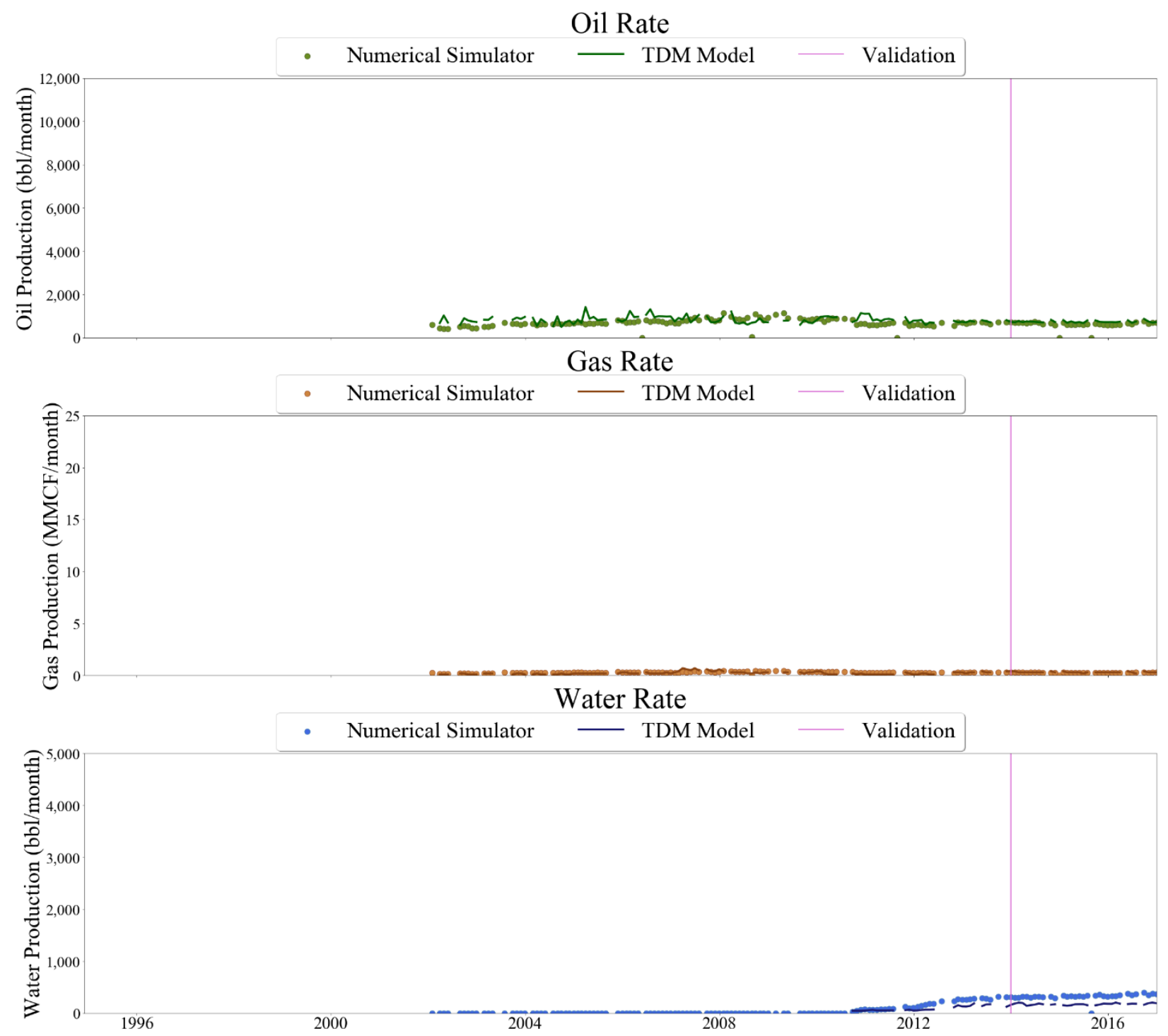

Figure 603 Well-031 oil, gas, and water rate TDM predictions vs actual simulation data plots for 3 Year Blind Validation TDM 


\section{Oil Rate}

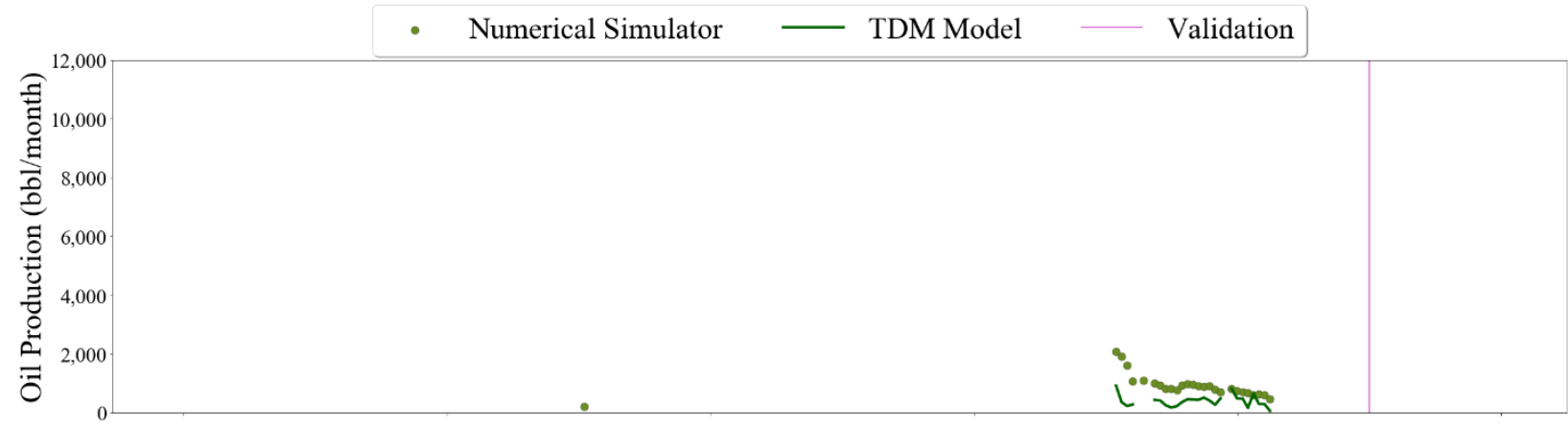

\section{Gas Rate}
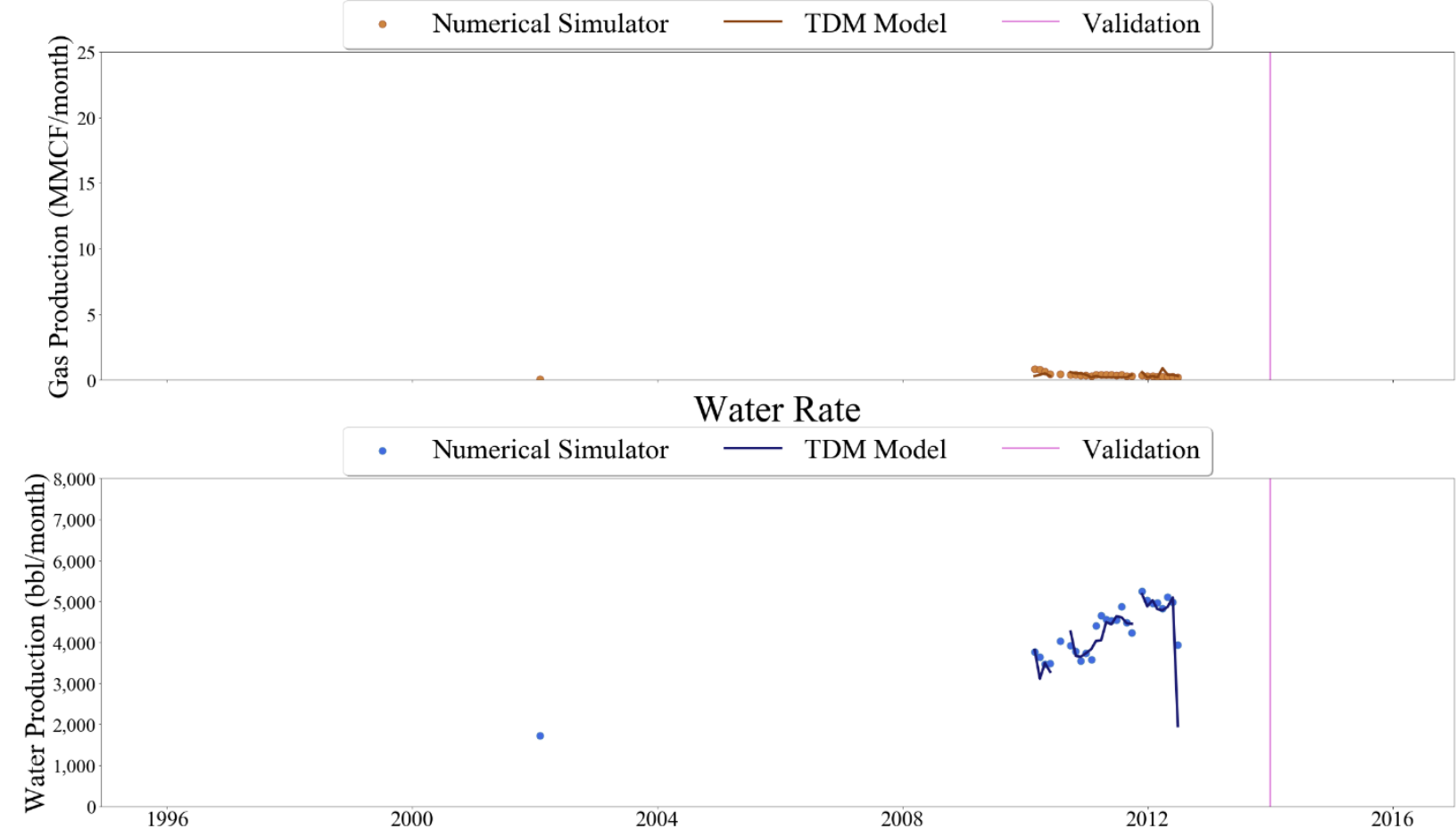

Figure 604 Well-032 oil, gas, and water rate TDM predictions vs actual simulation data plots for 3 Year Blind Validation TDM 


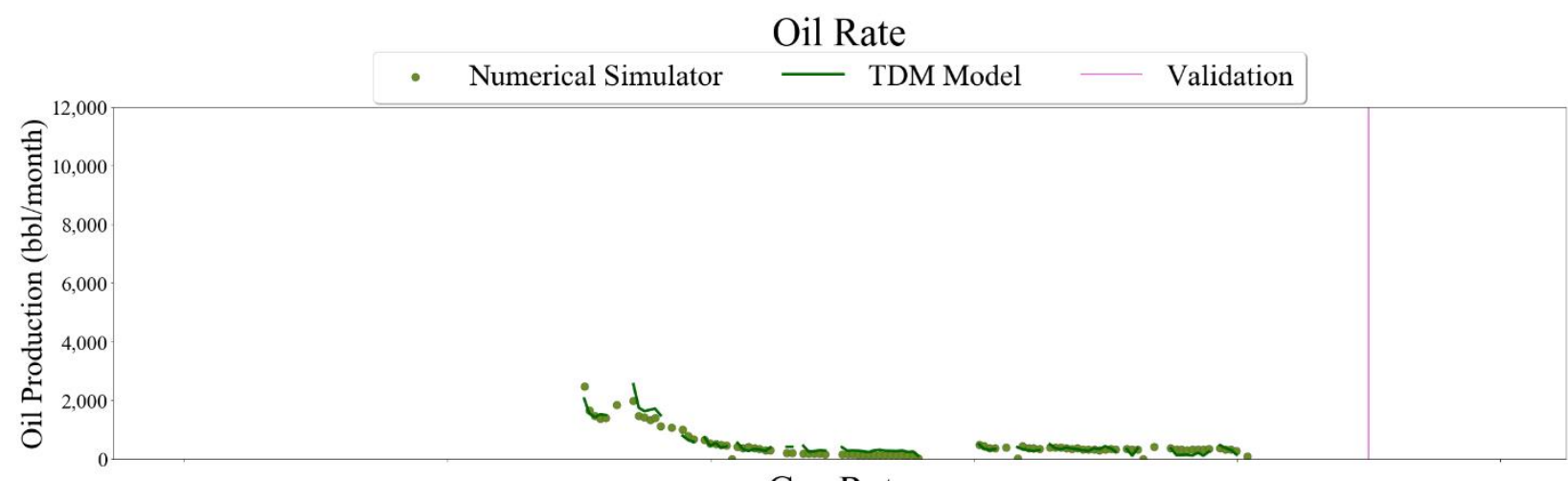

Gas Rate
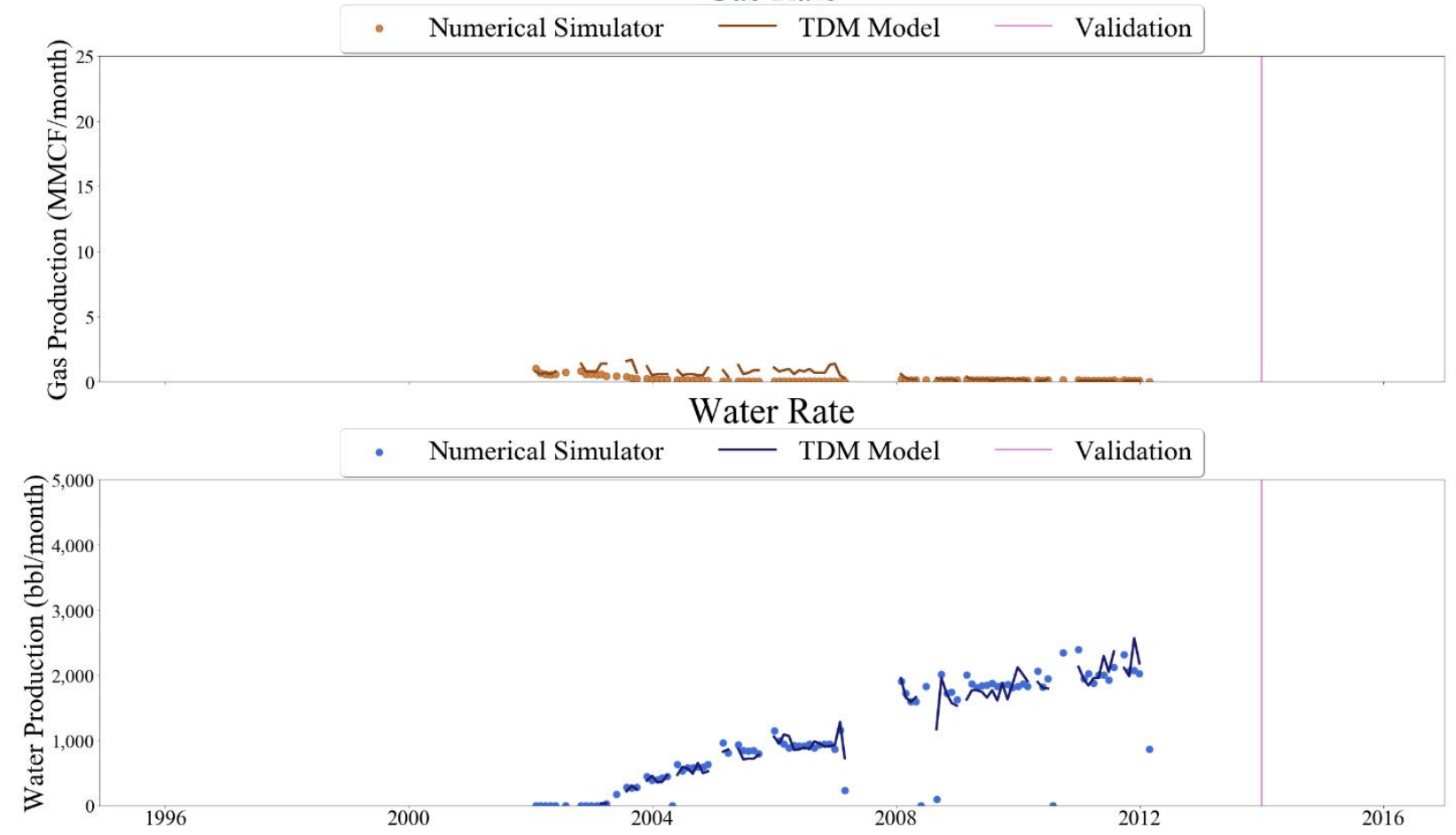

Figure 605 Well-033 oil, gas, and water rate TDM predictions vs actual simulation data plots for 3 Year Blind Validation TDM 

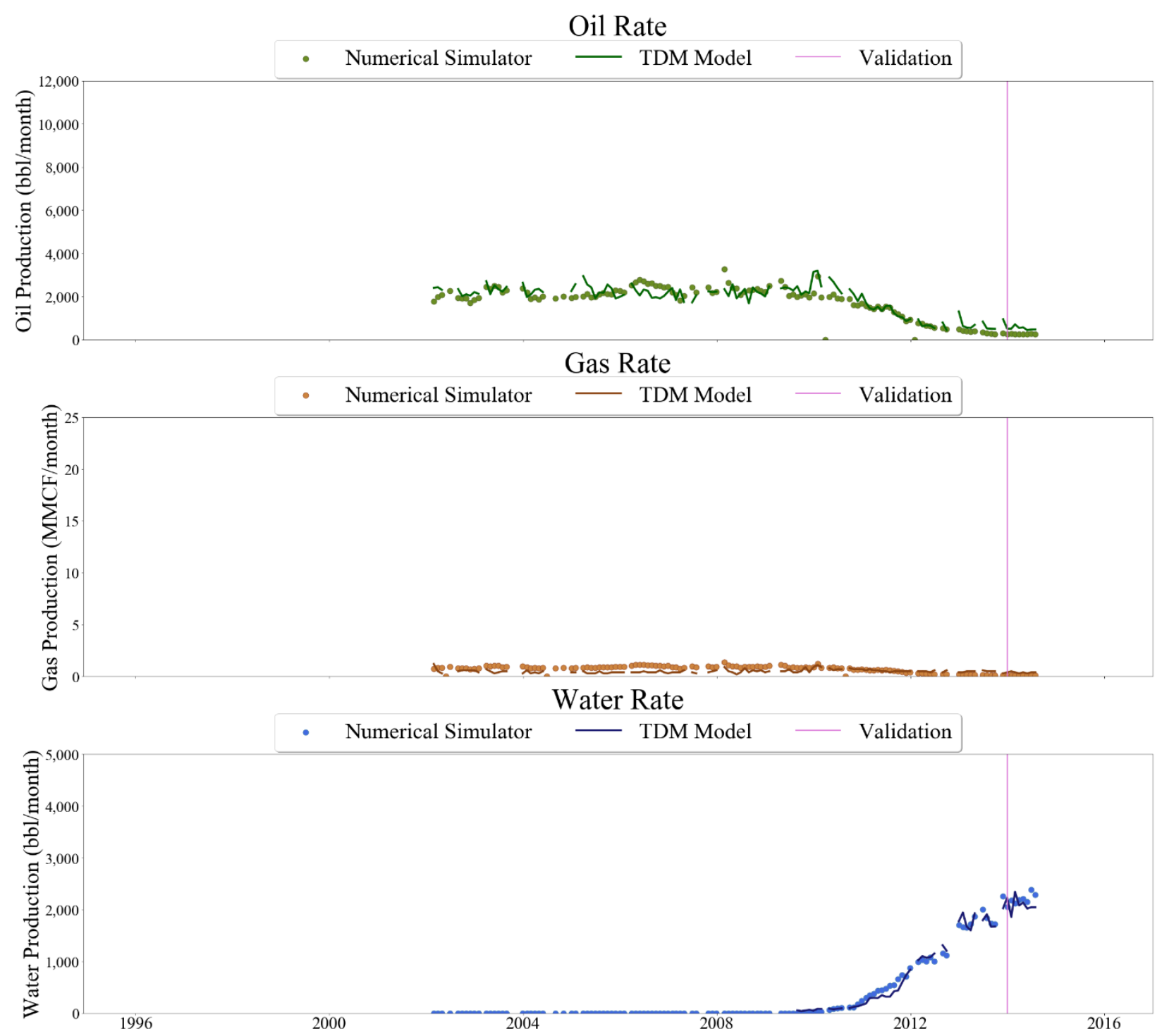

Figure 606 Well-034 oil, gas, and water rate TDM predictions vs actual simulation data plots for 3 Year Blind Validation TDM 


\section{Oil Rate}

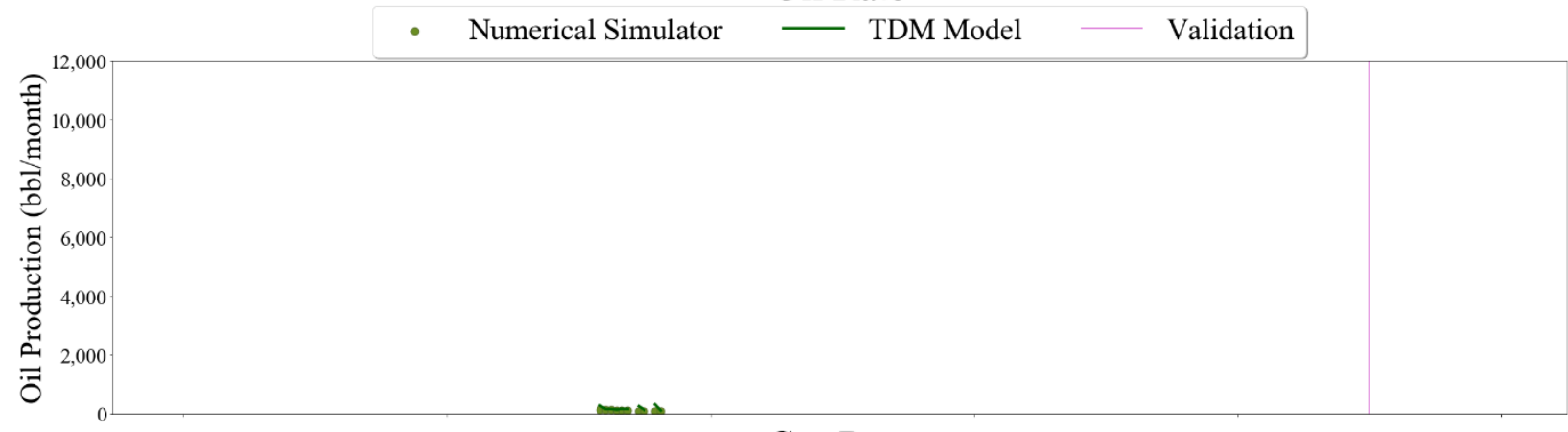

\section{Gas Rate}

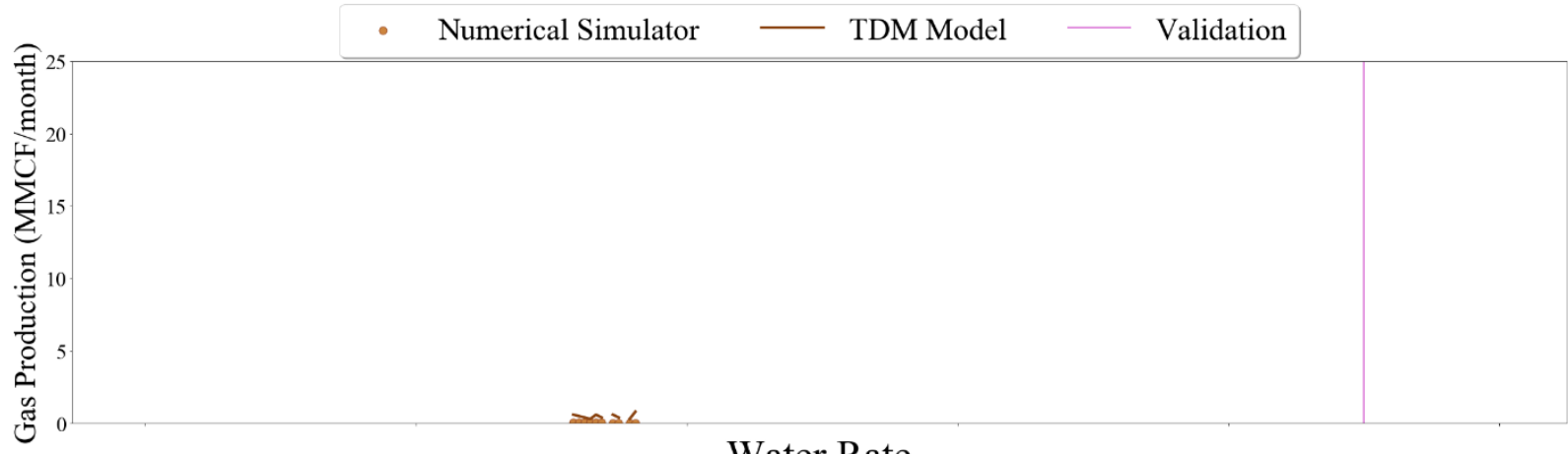

Water Rate

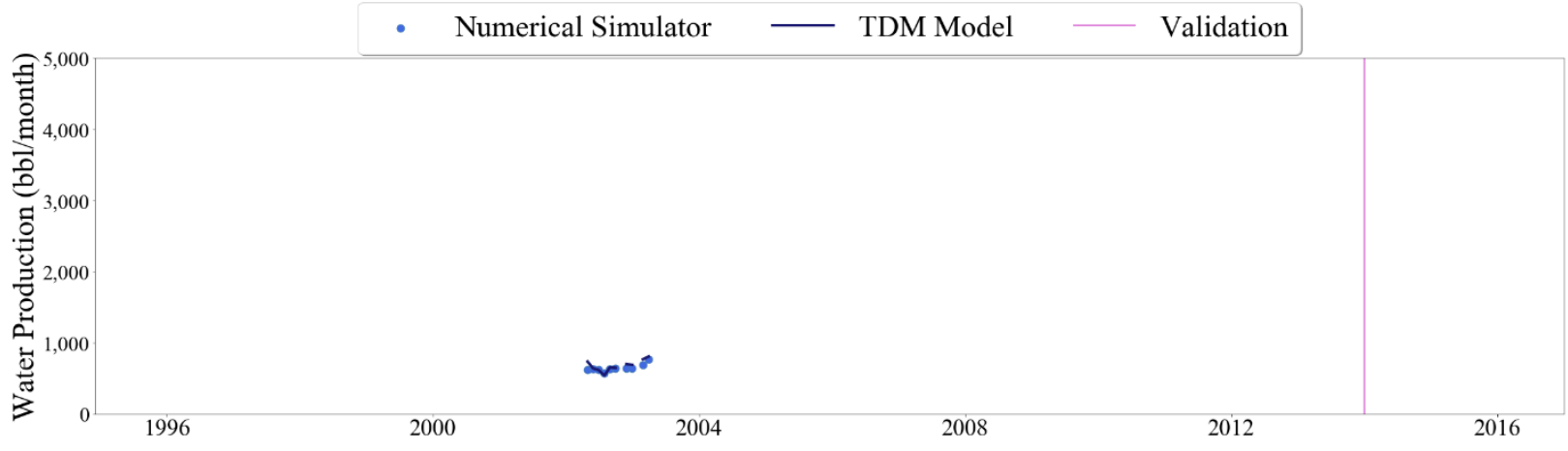

Figure 607 Well-035 oil, gas, ad water rate TDM predictions vs actual simulation data plots for 3 Year Blind Validation TDM 

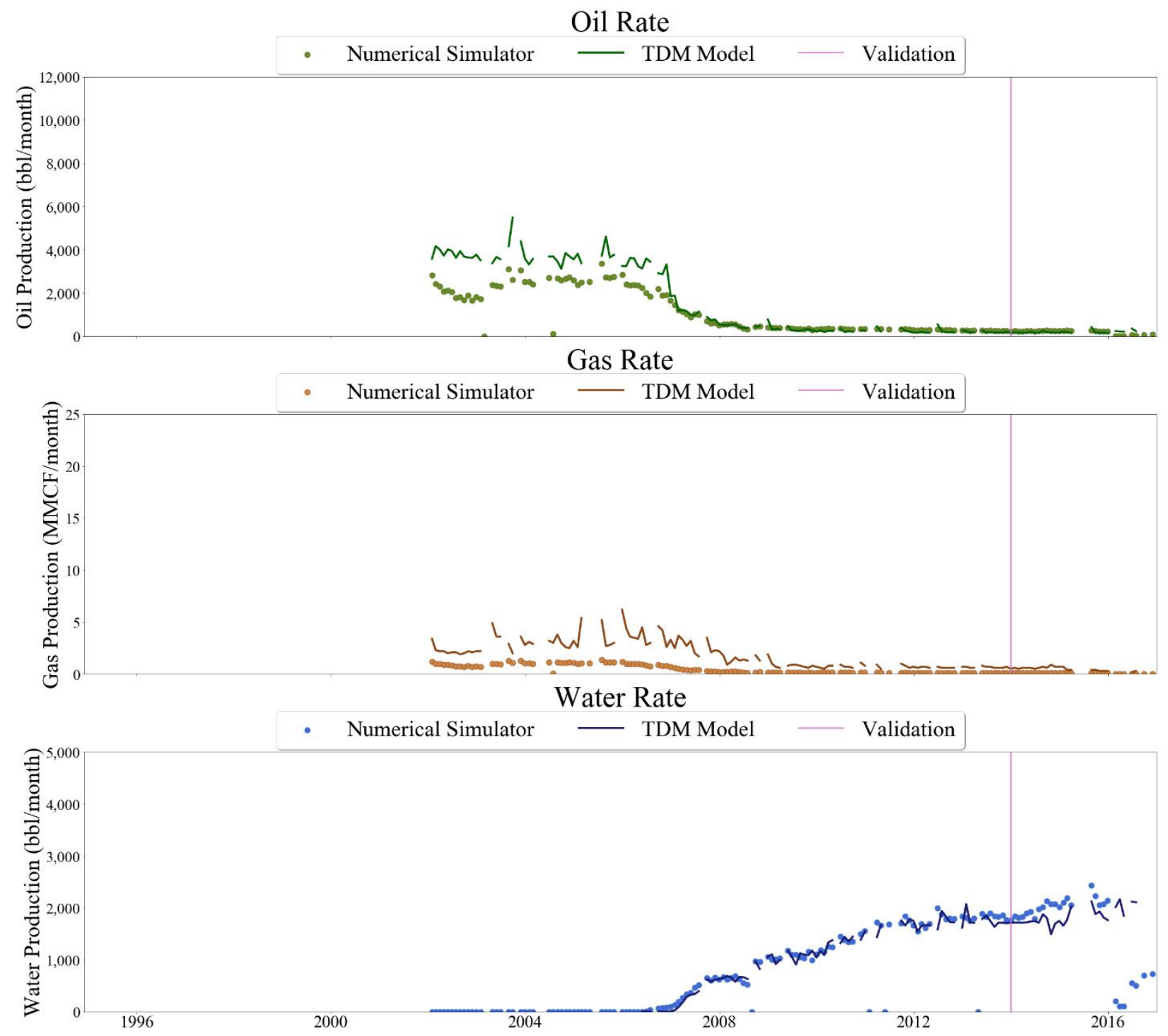

Figure 608 Well-036 oil, gas, and water rate TDM predictions vs actual simulation data plots for 3 Year Blind Validation TDM 

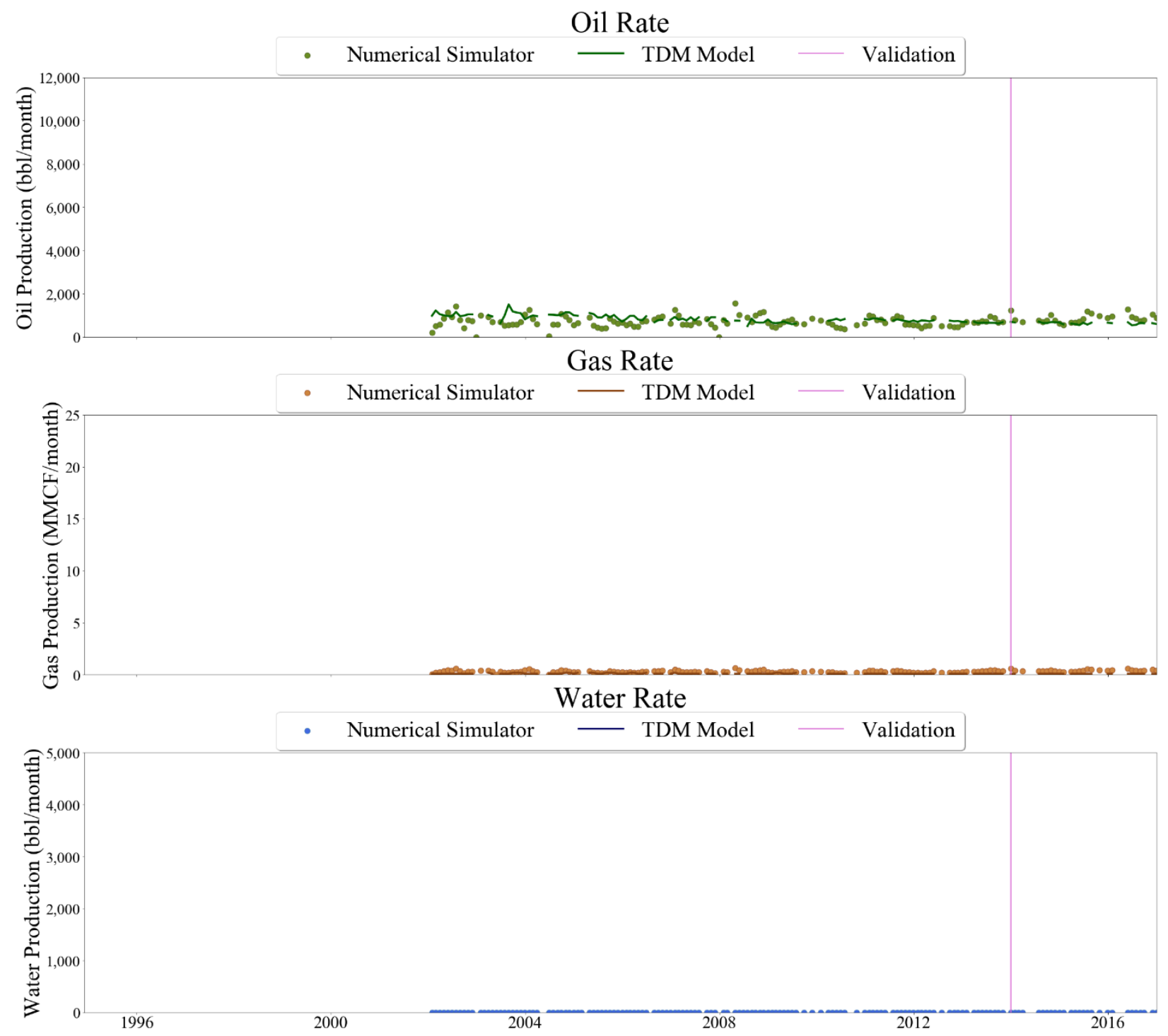

Figure 609 Well-037 oil, gas, ad water rate TDM predictions vs actual simulation data plots for 3 Year Blind Validation TDM 


\section{Oil Rate}

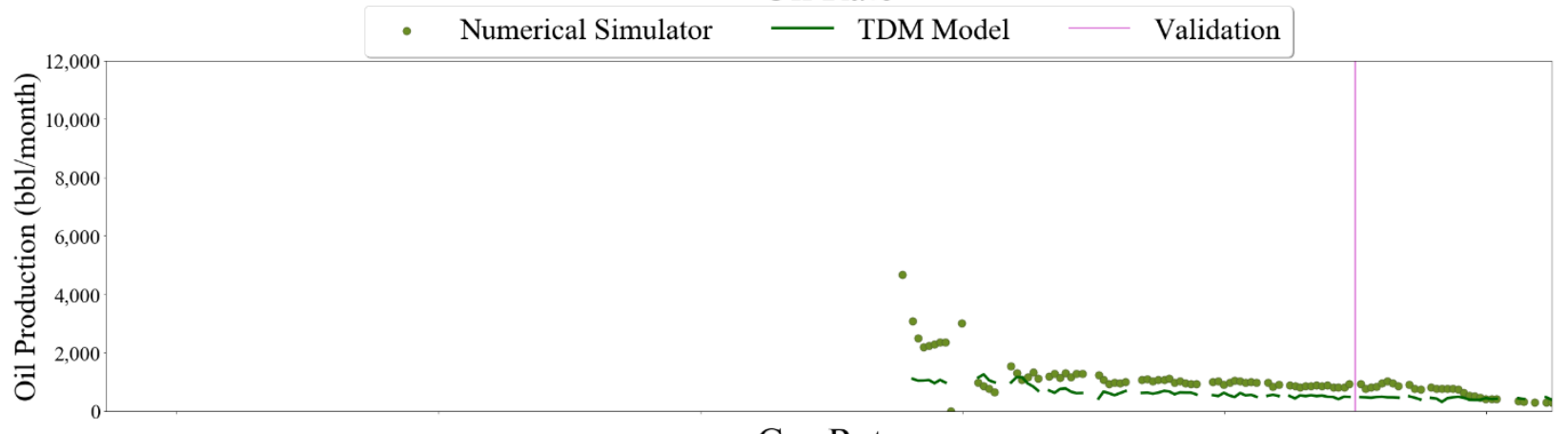

\section{Gas Rate}
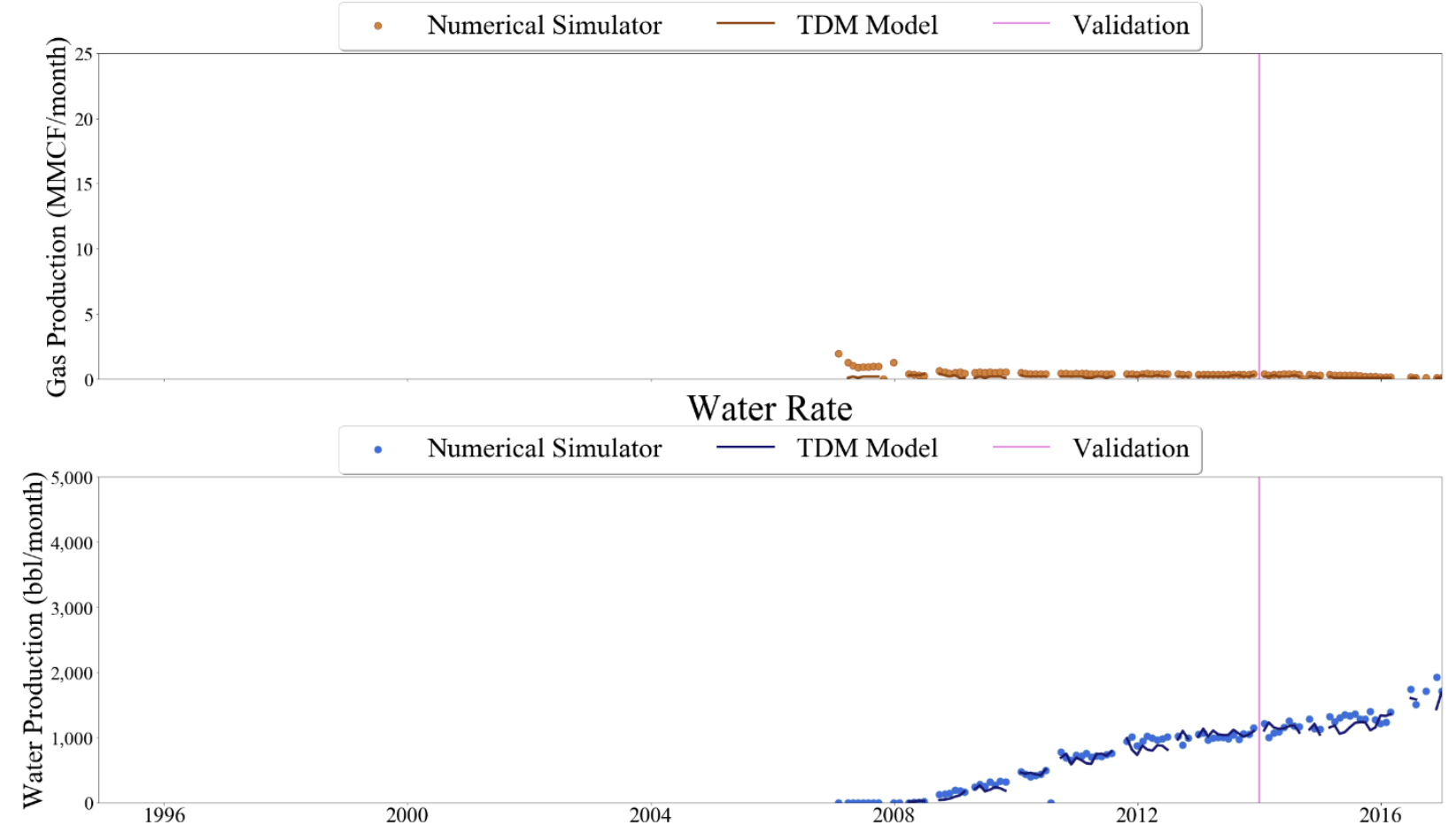

Figure 610 Well-038 oil, gas, and water rate TDM predictions vs actual simulation data plots for 3 Year Blind Validation TDM 


\section{Oil Rate}

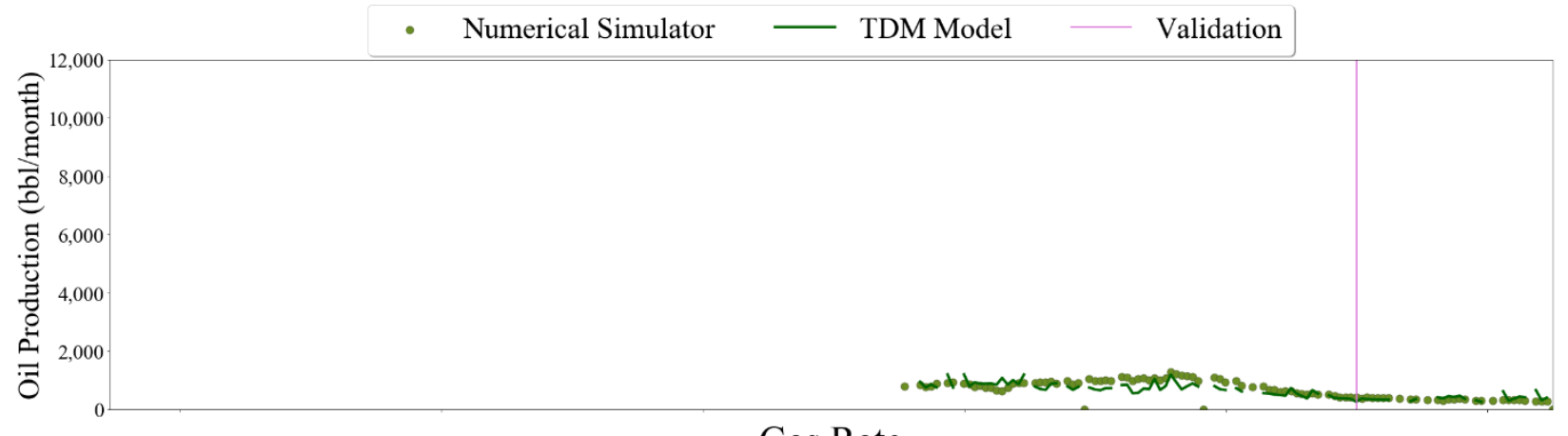

\section{Gas Rate}
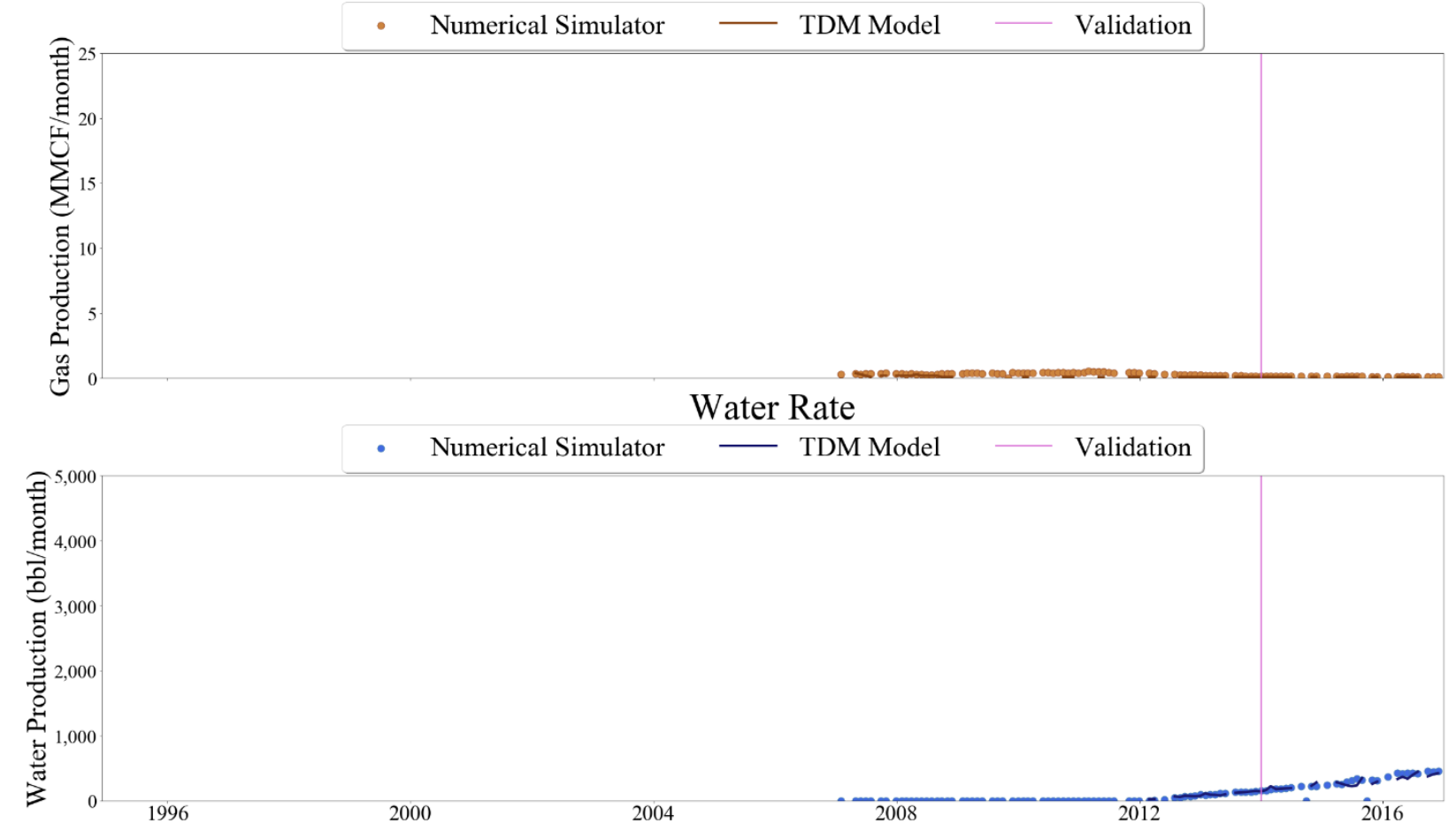

Figure 611 Well-039 oil, gas, and water rate TDM predictions vs actual simulation data plots for 3 Year Blind Validation TDM 


\section{Oil Rate}

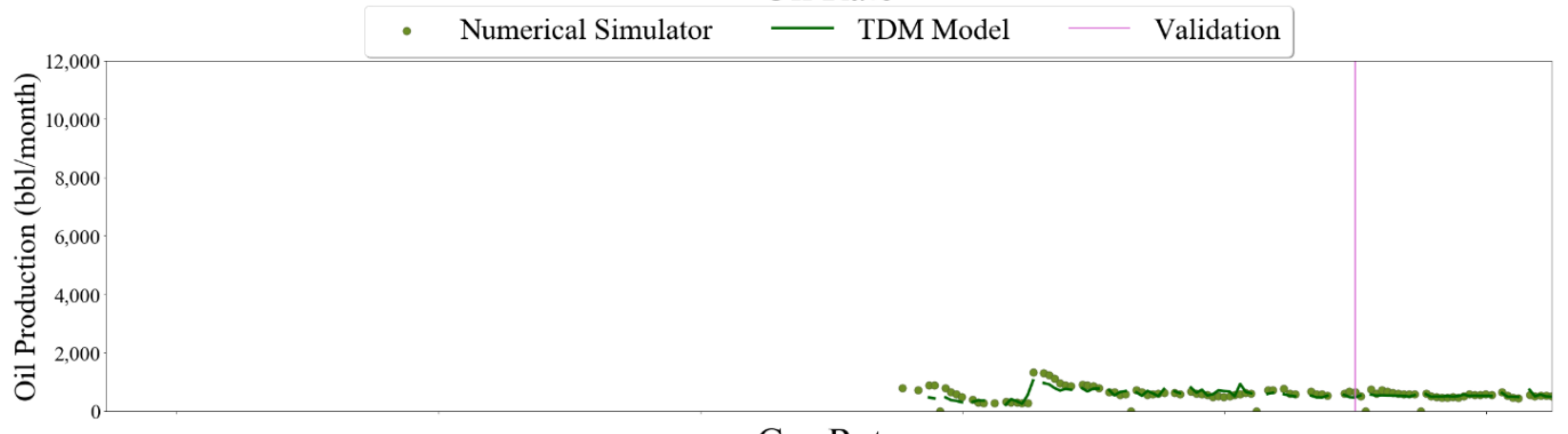

\section{Gas Rate}
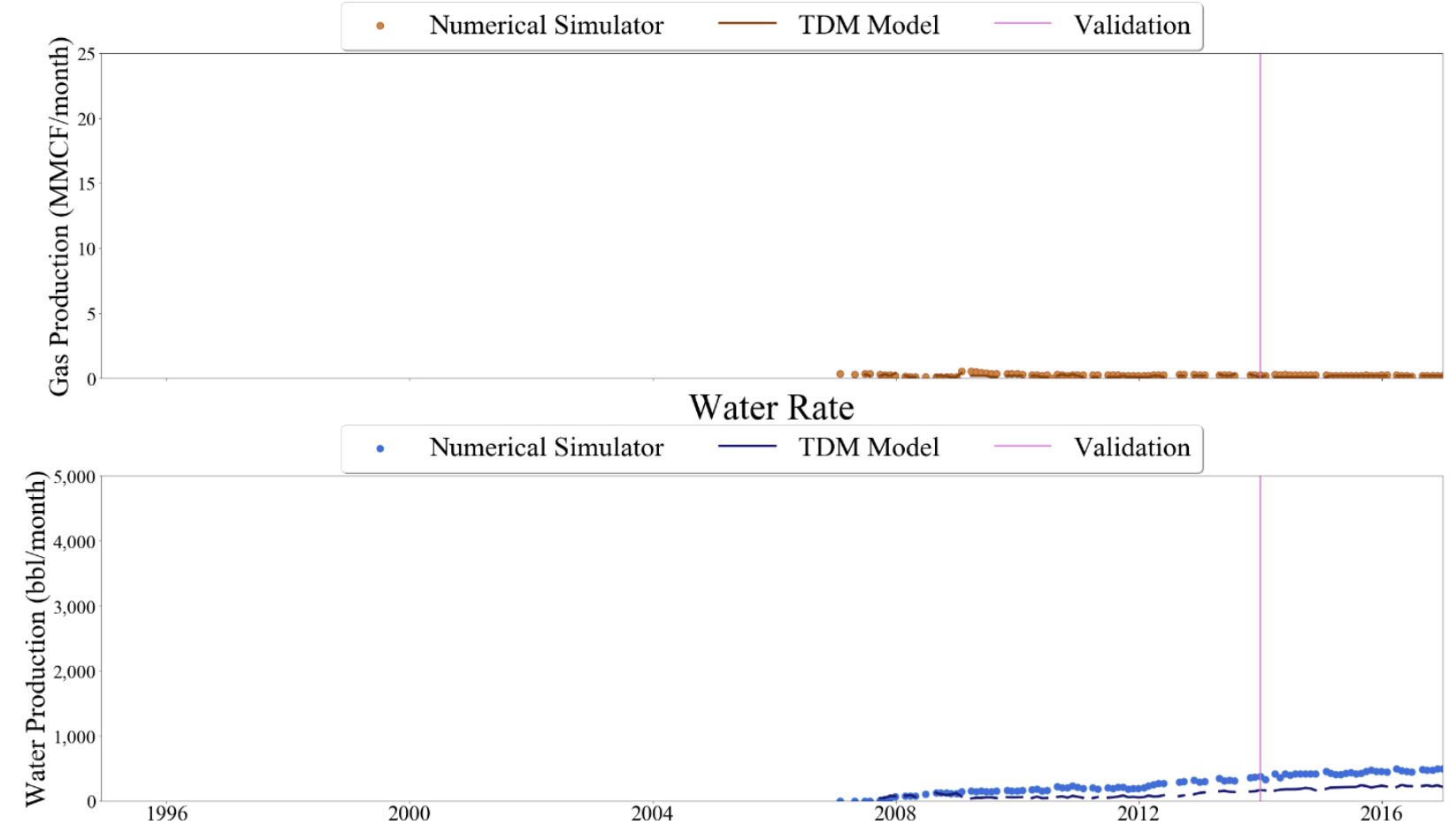

Figure 612 Well-040 oil, gas, and water rate TDM predictions vs actual simulation data plots for 3 Year Blind Validation TDM 


\section{Oil Rate}

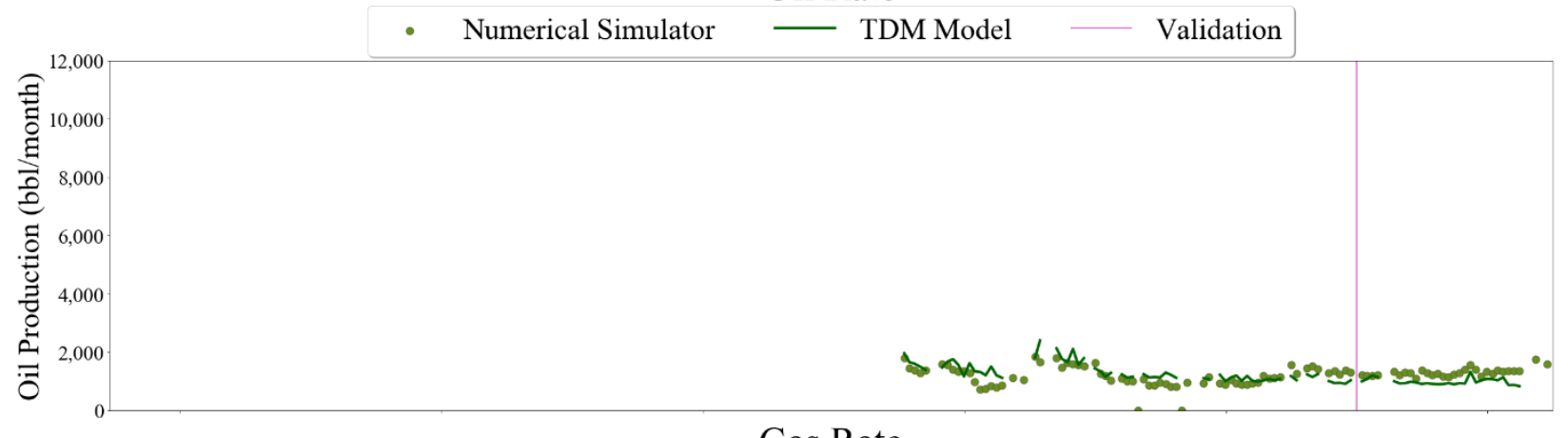

\section{Gas Rate}
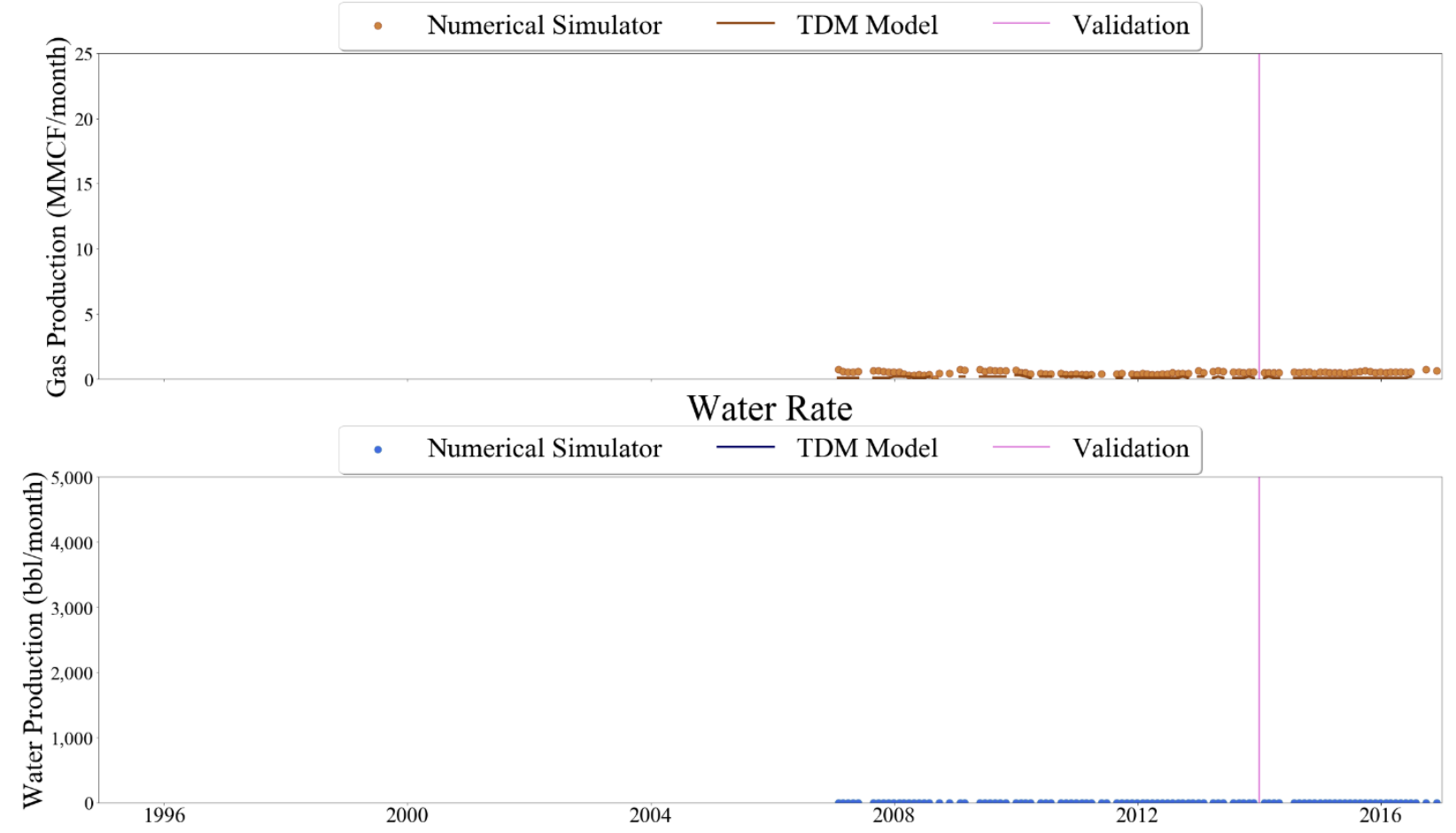

Figure 613 Well-041 oil, gas, and water rate TDM predictions vs actual simulation data plots for 3 Year Blind Validation TDM 


\section{Oil Rate}

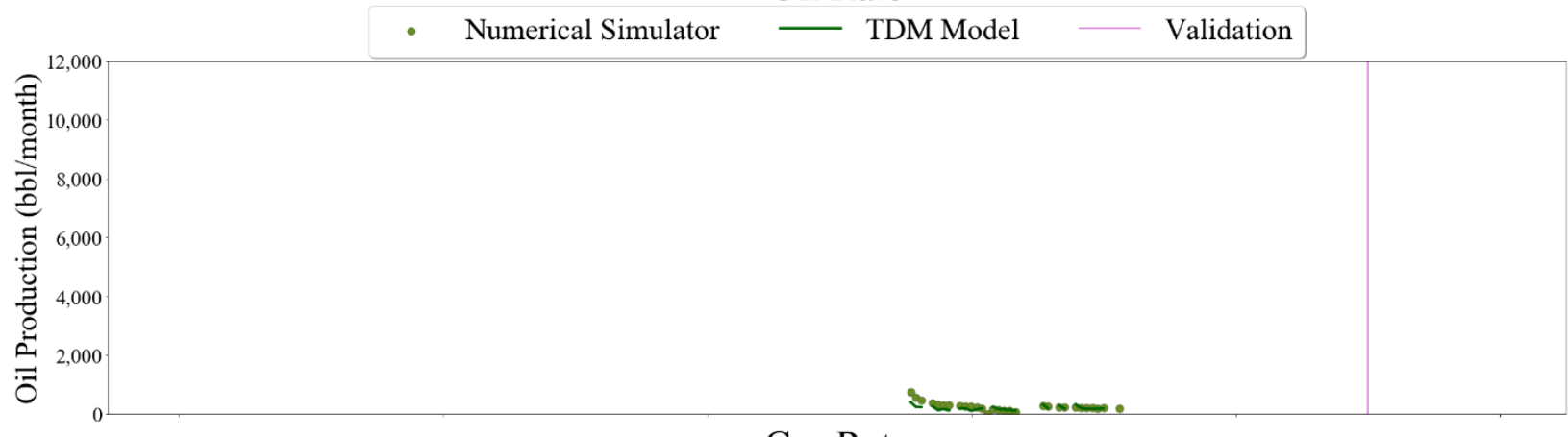

\section{Gas Rate}

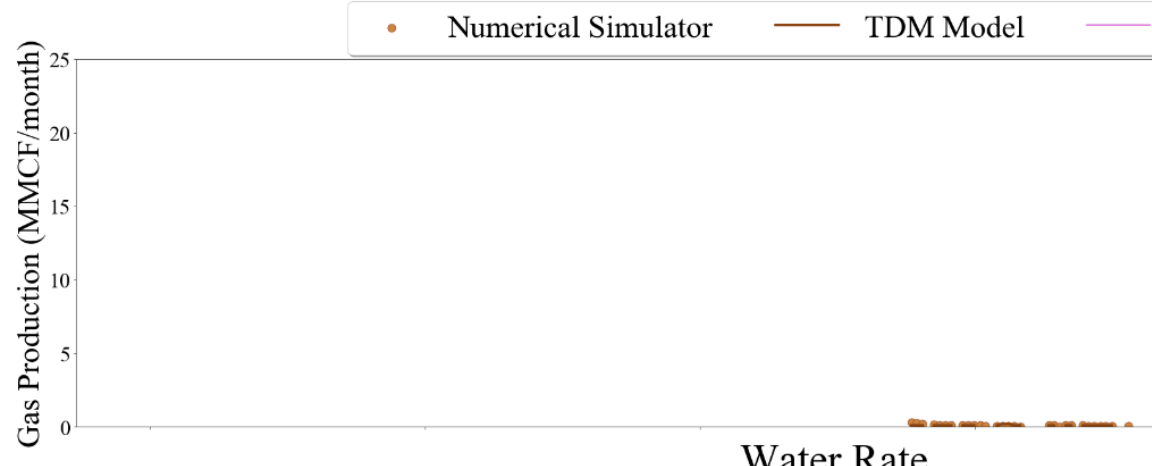

Water Rate

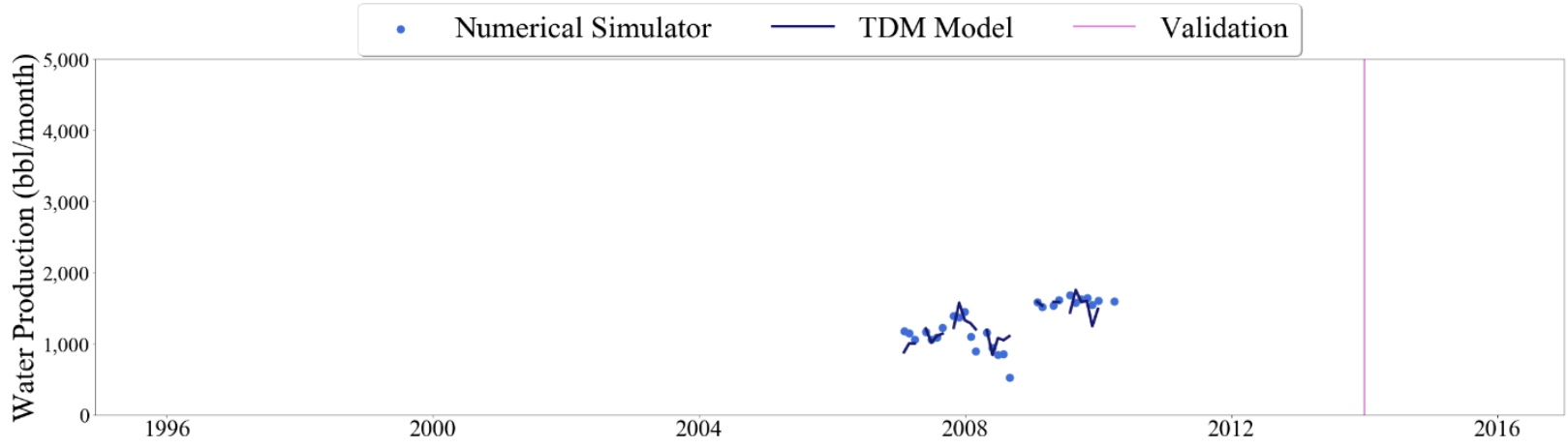

Figure 614 Well-042 oil, gas, and water rate TDM predictions vs actual simulation data plots for 3 Year Blind Validation TDM 


\section{Oil Rate}

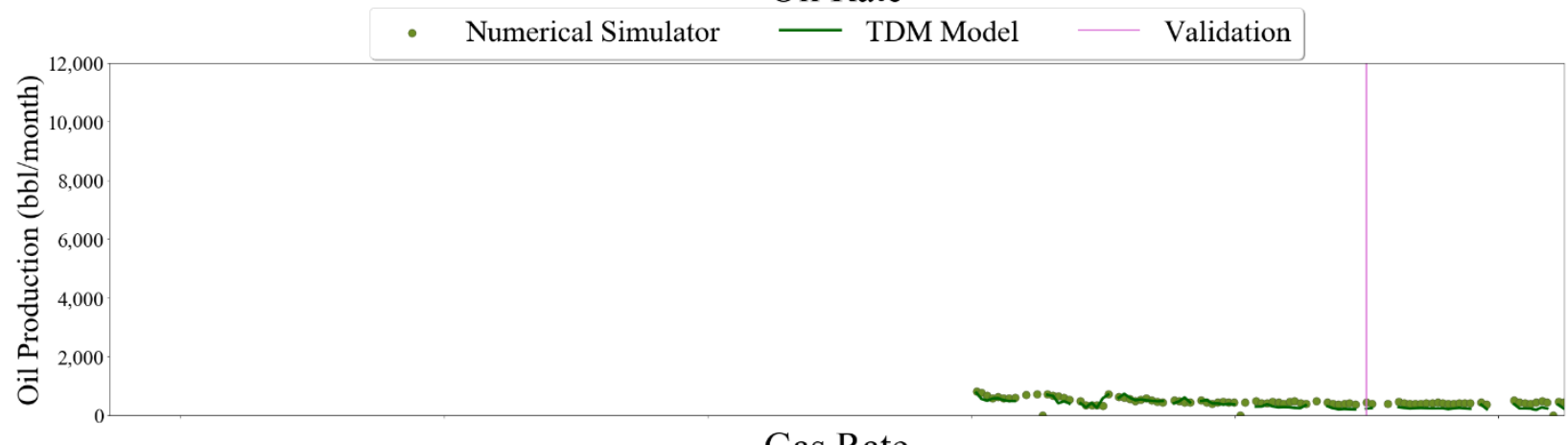

\section{Gas Rate}
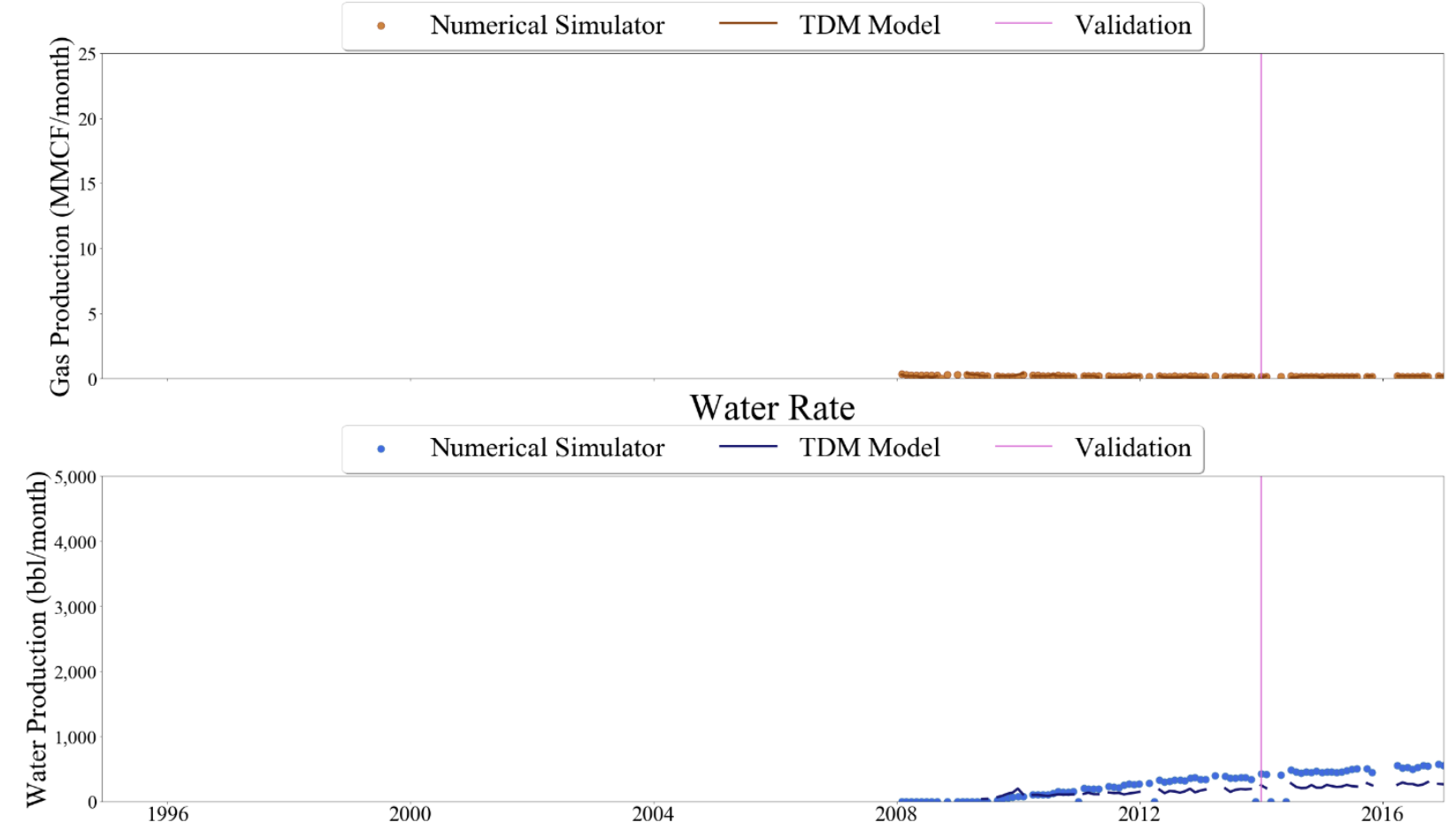

Figure 615 Well-043 oil, gas, and water rate TDM predictions vs actual simulation data plots for 3 Year Blind Validation TDM 


\section{Oil Rate}
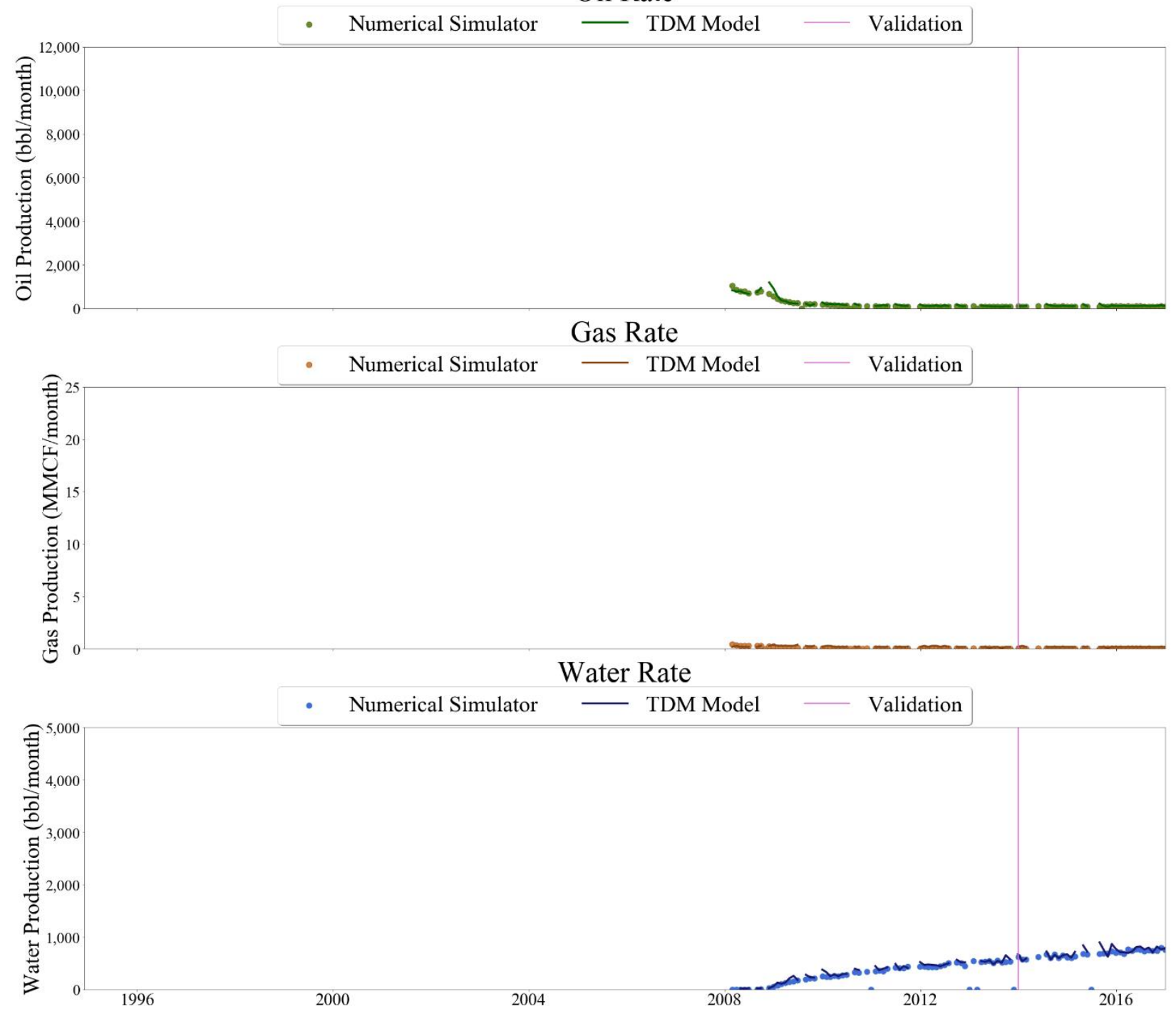

Figure 616 Well-044 oil. gas, and water rate TDM predictions vs actual simulation data plots for 3 Year Blind Validation TDM 


\section{Oil Rate}
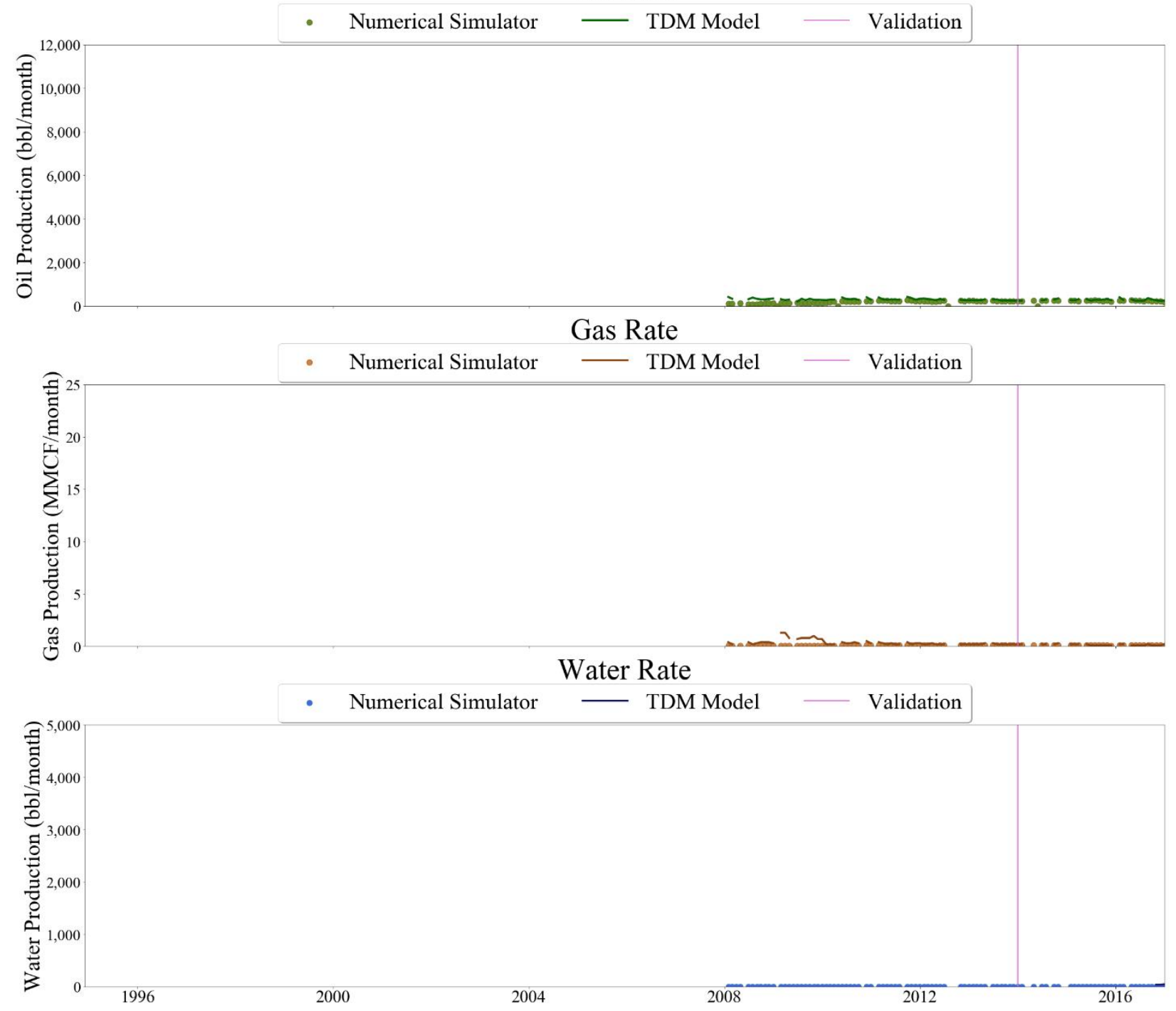

Figure 617 Well-045 oil, gas, and water rate TDM predictions vs actual simulation data plots for 3 Year Blind Validation TDM 


\section{Oil Rate}
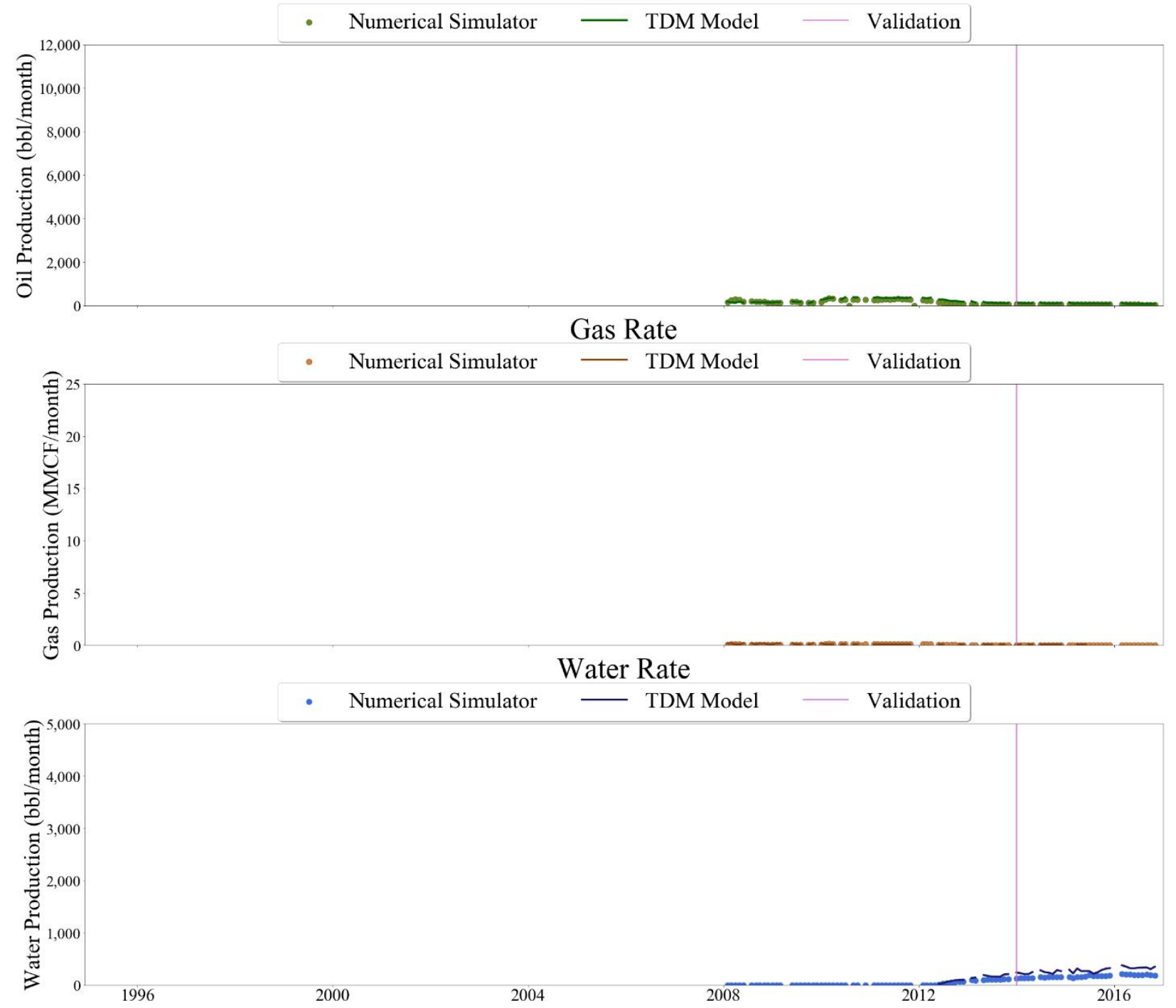

Figure 618 Well-046 oil, gas, and water rate TDM predictions vs actual simulation data plots for 3 Year Blind Validation TDM 


\section{Oil Rate}

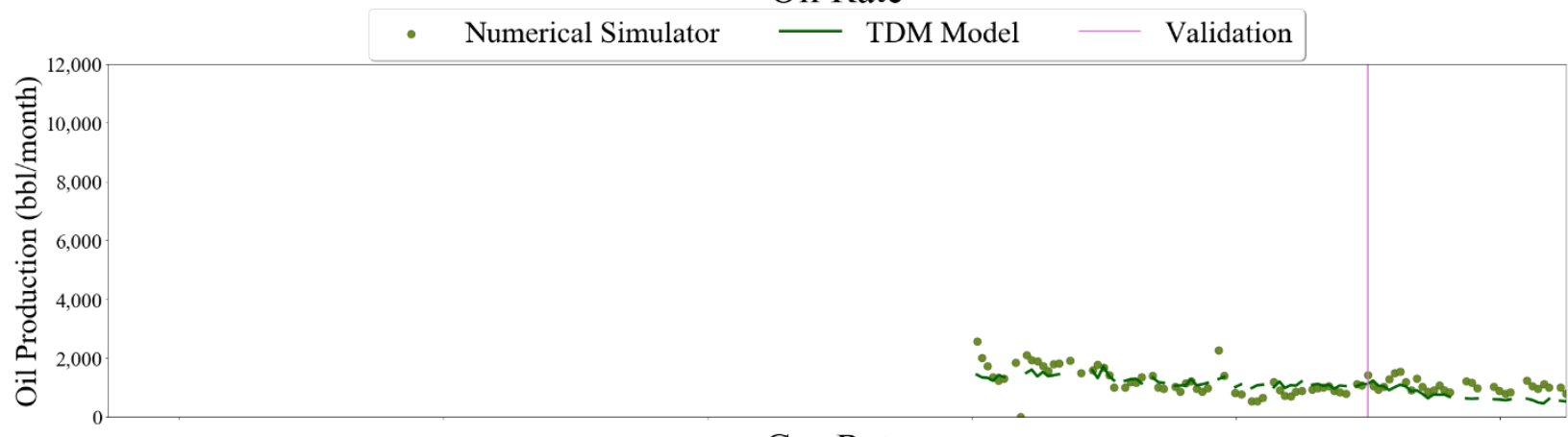

\section{Gas Rate}
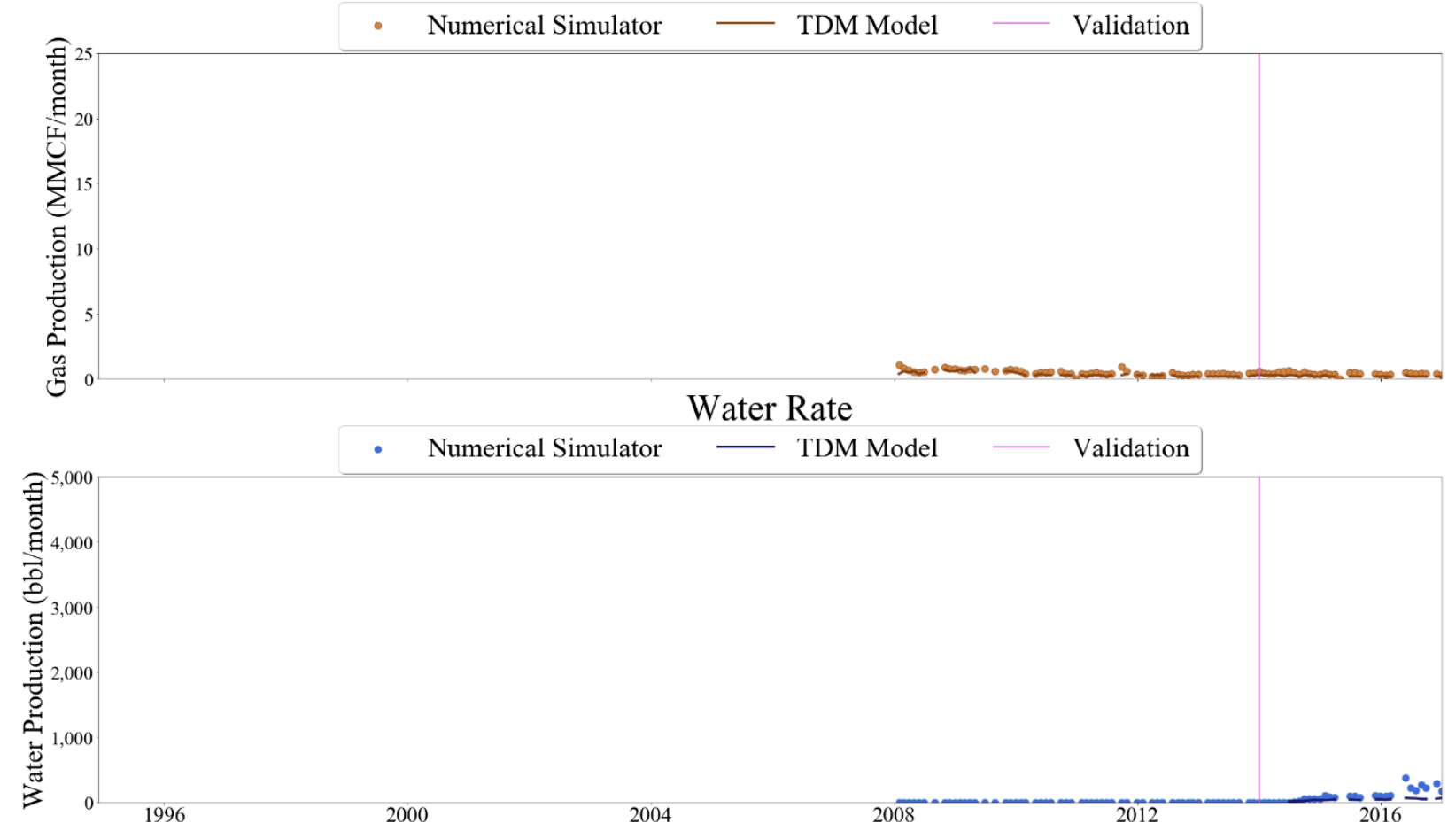

Figure 619 Well-047 oil, gas, and water rate TDM predictions vs actual simulation data plots for 3 Year Blind Validation TDM 


\section{Oil Rate}

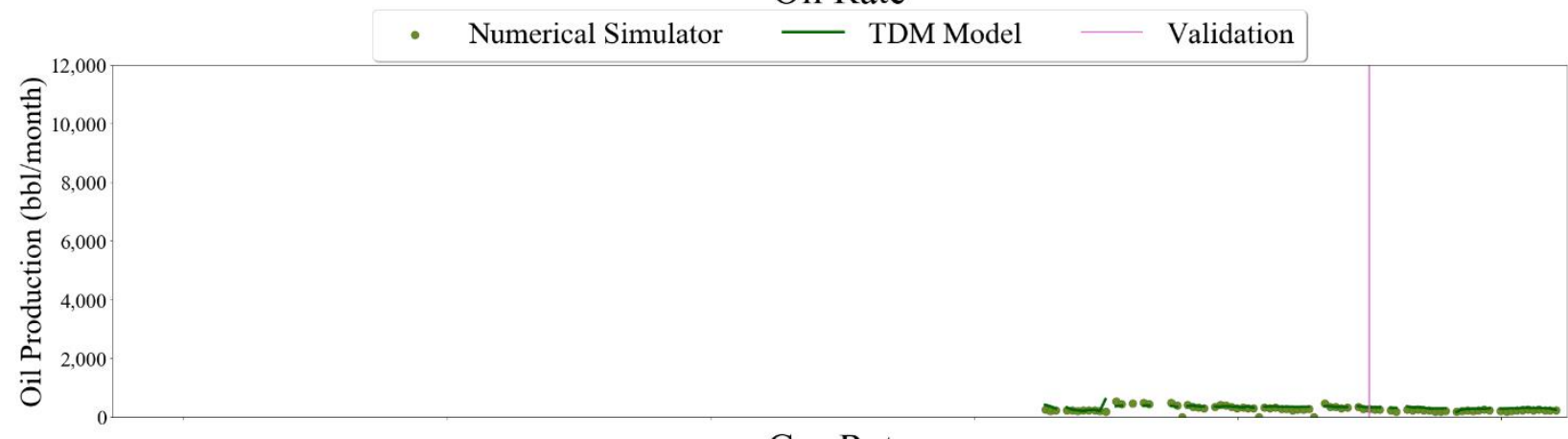

\section{Gas Rate}

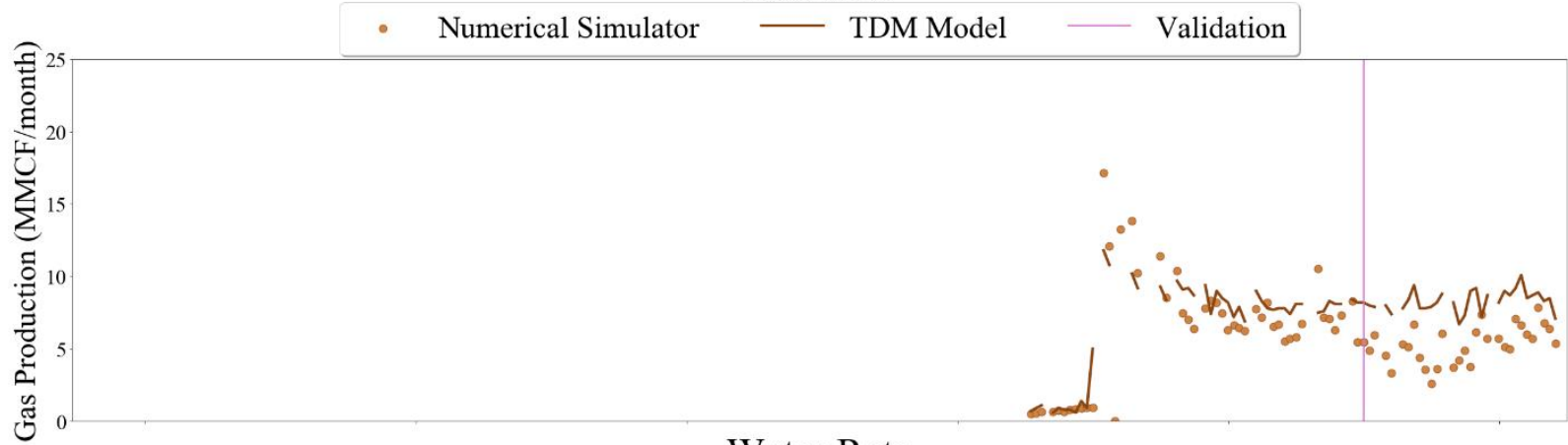

Water Rate

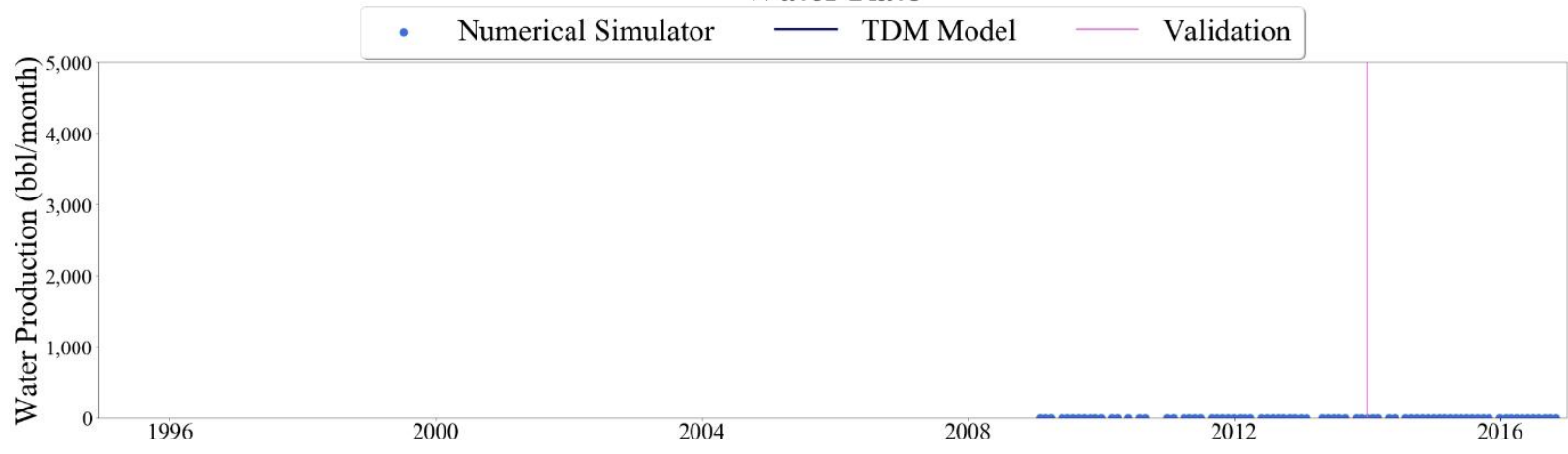

Figure 620 Well-048 oil, gas, and water rate TDM predictions vs actual simulation data plots for 3 Year Blind Validation TDM 


\section{Oil Rate}

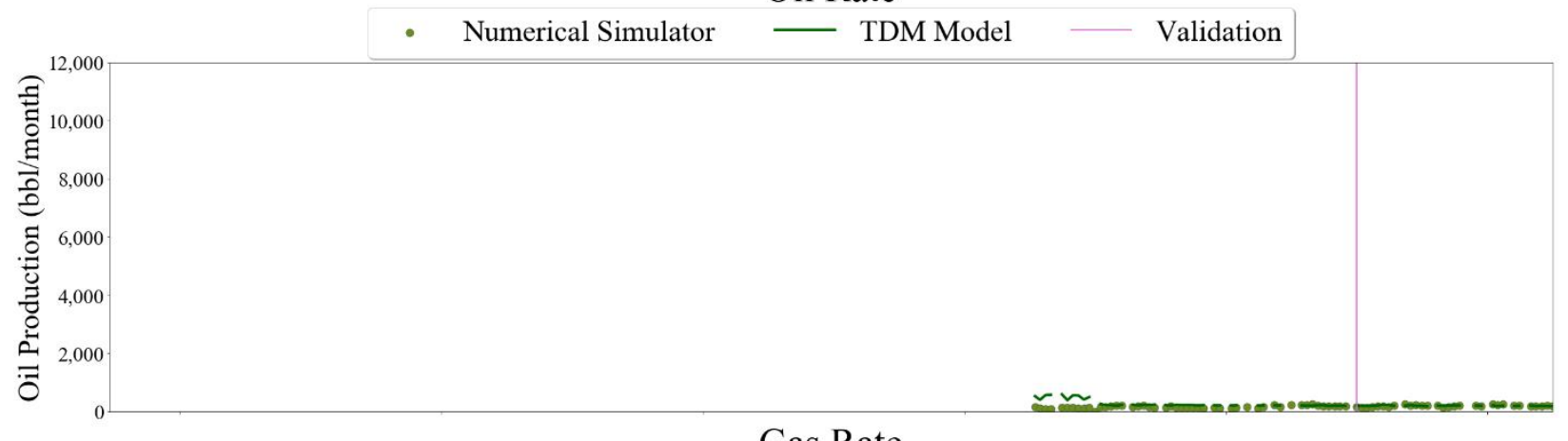

Gas Rate
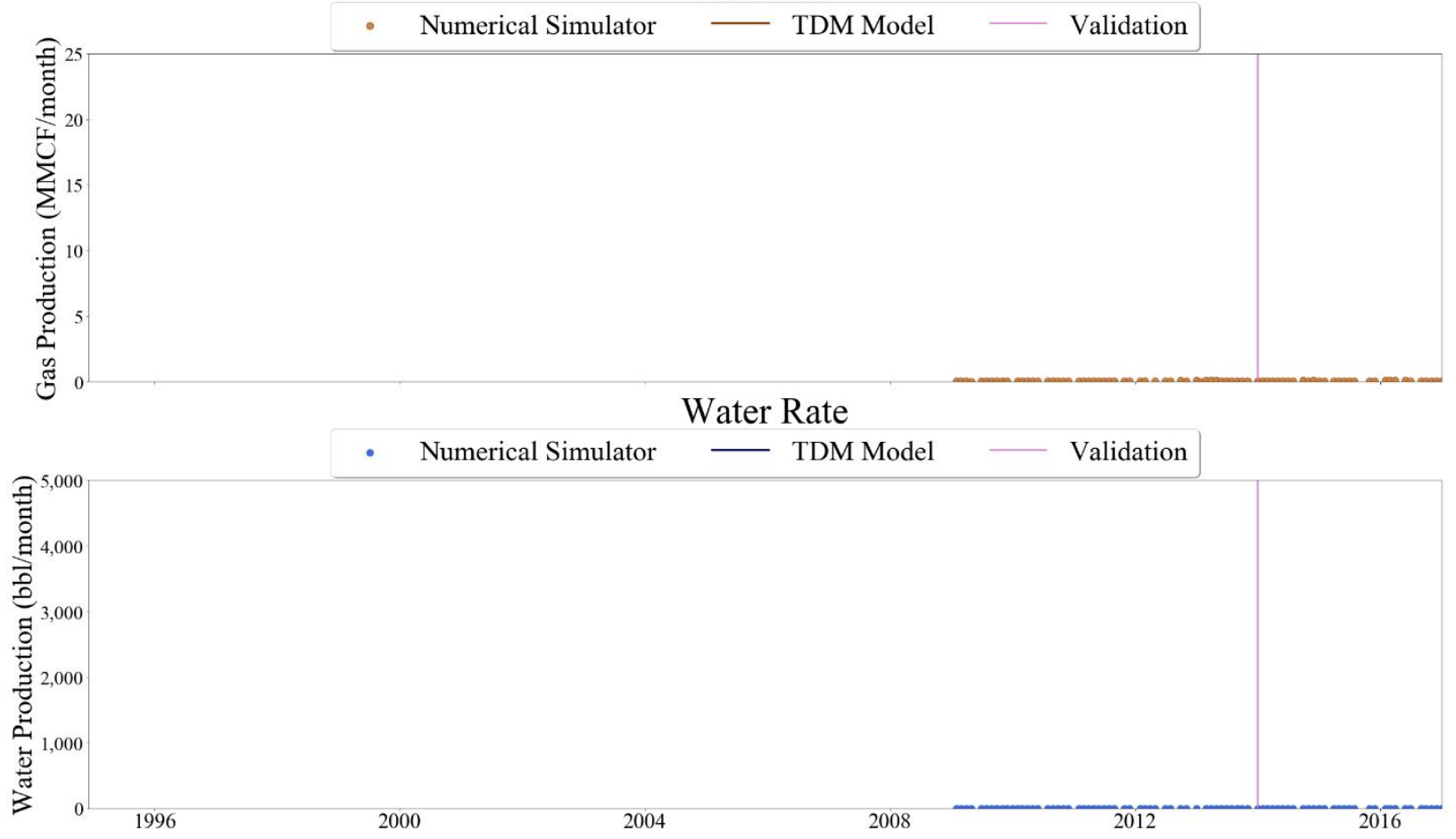

Figure 621 Well-049 oil, gas, and water rate TDM predictions vs actual simulation data plots for 3 Year Blind Validation TDM 


\section{Oil Rate}
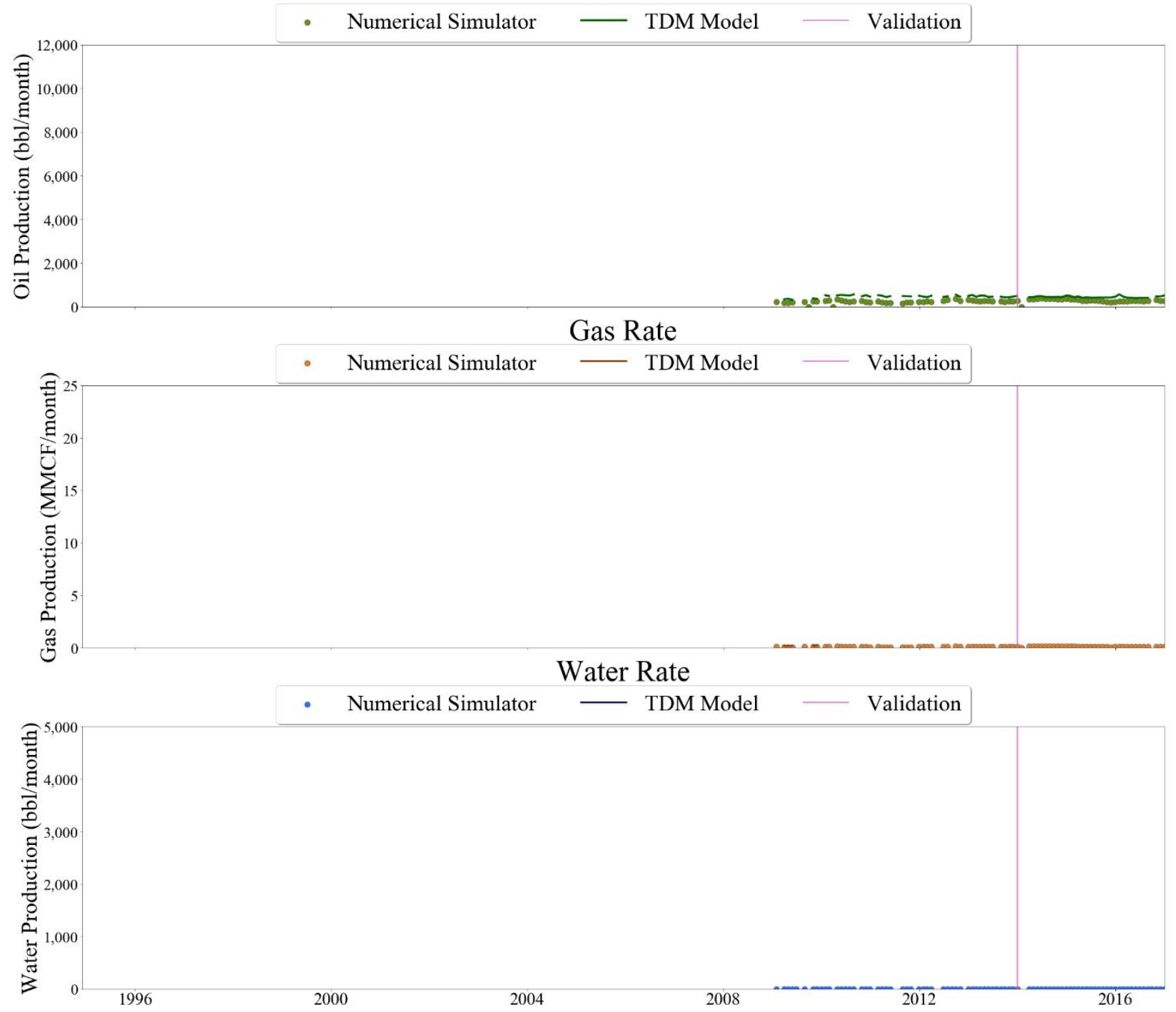

Figure 622 Well-050 oil, gas, and water rate TDM predictions vs actual simulation data plots for 3 Year Blind Validation TDM 


\section{Oil Rate}

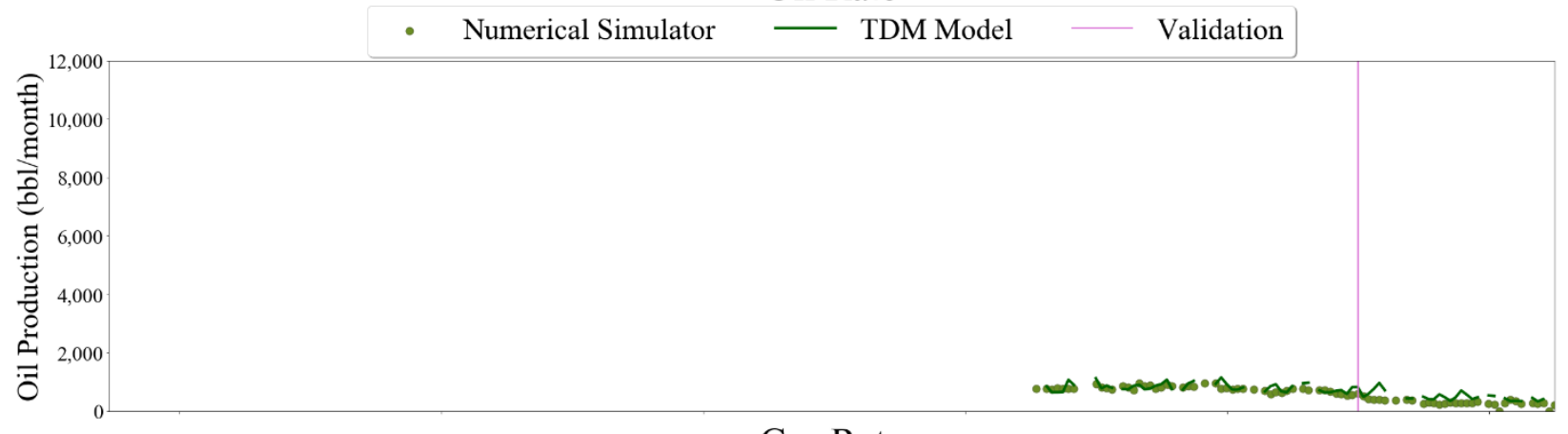

\section{Gas Rate}
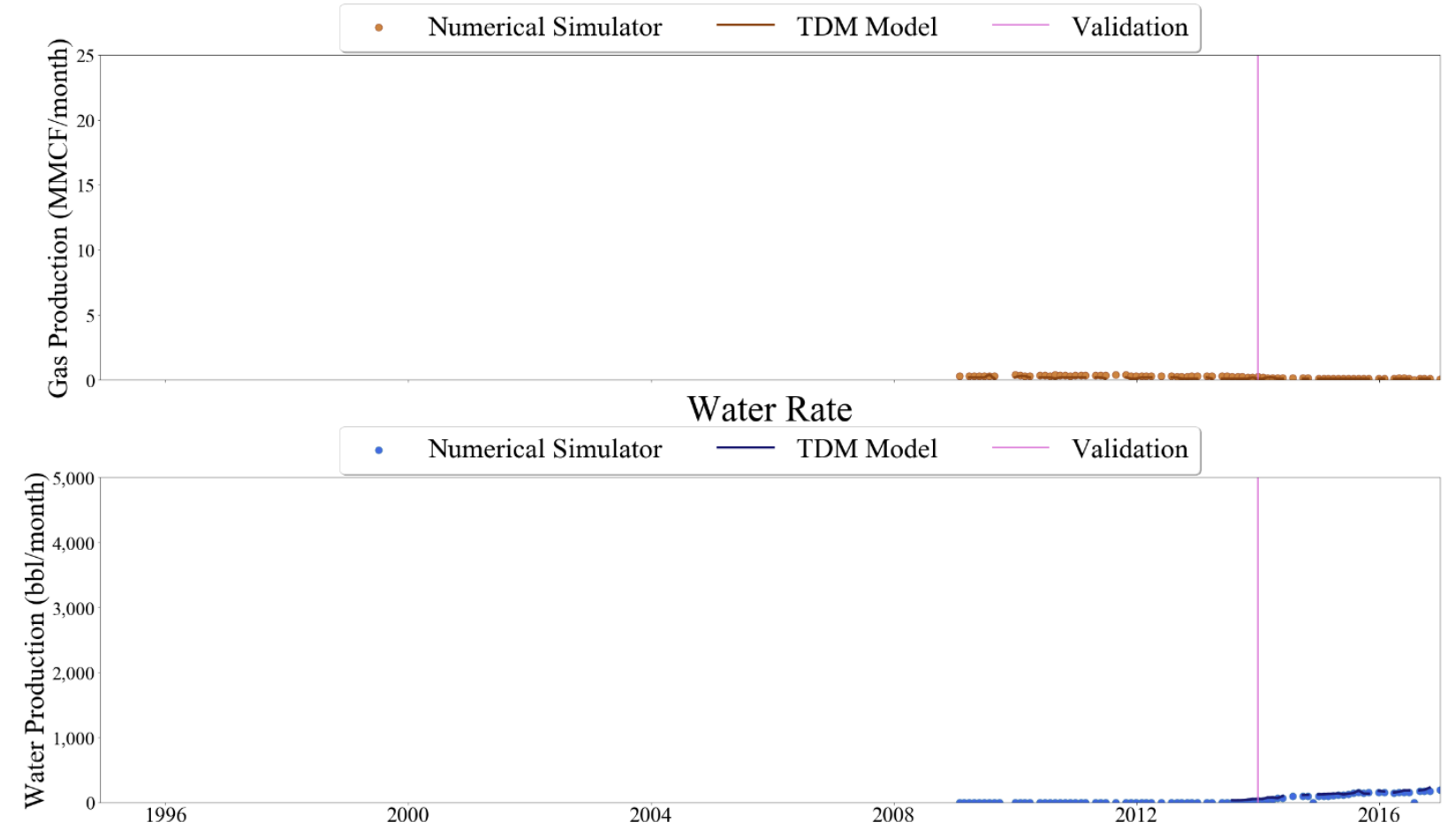

Figure 623 Well-051 oil, gas, and water rate TDM predictions vs actual simulation data plots for 3 Year Blind Validation TDM 


\section{Oil Rate}
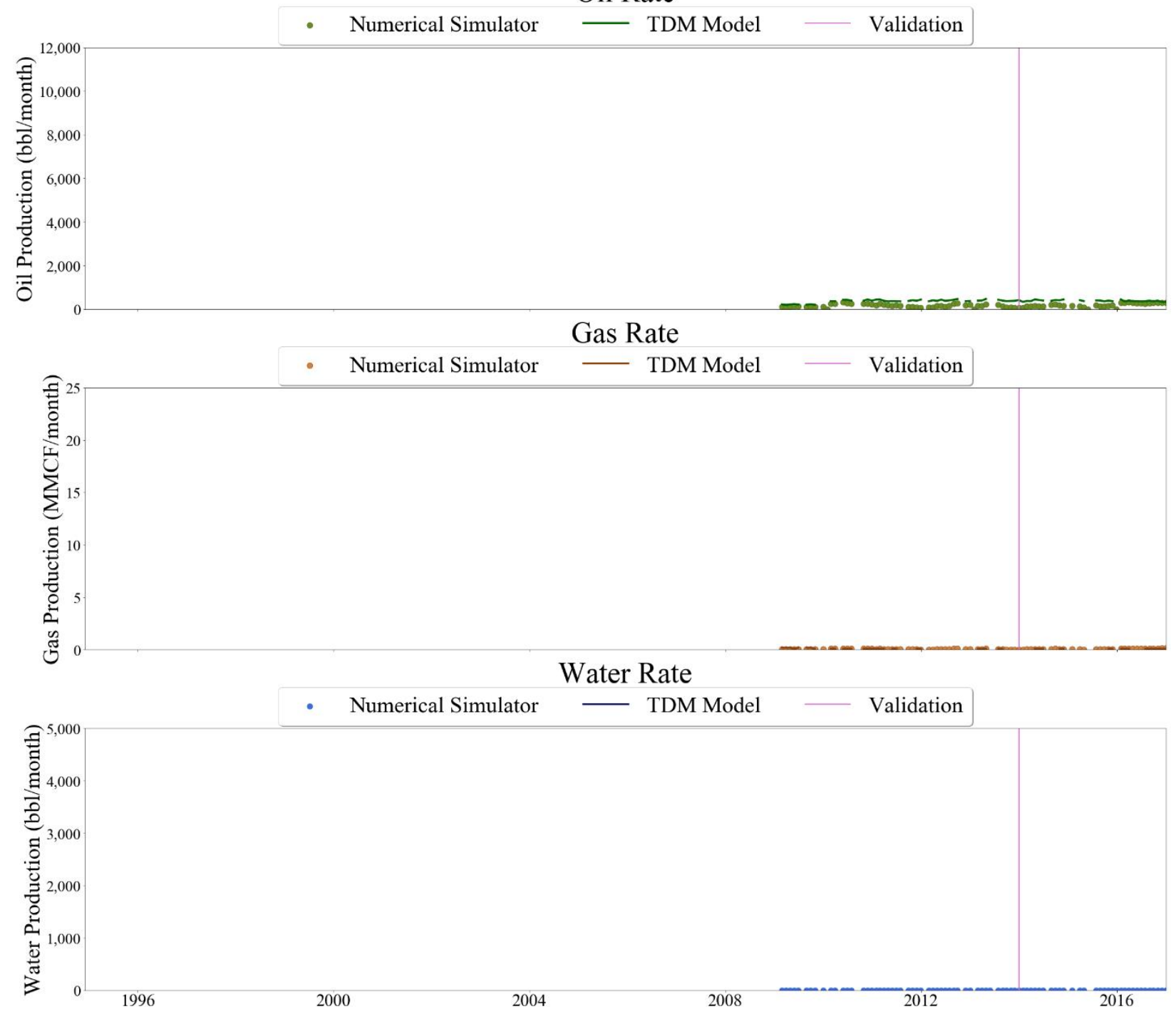

Figure 624 Well-052 oil, gas, and water rate TDM predictions vs actual simulation data plots for 3 Year Blind Validation TDM 


\section{Oil Rate}
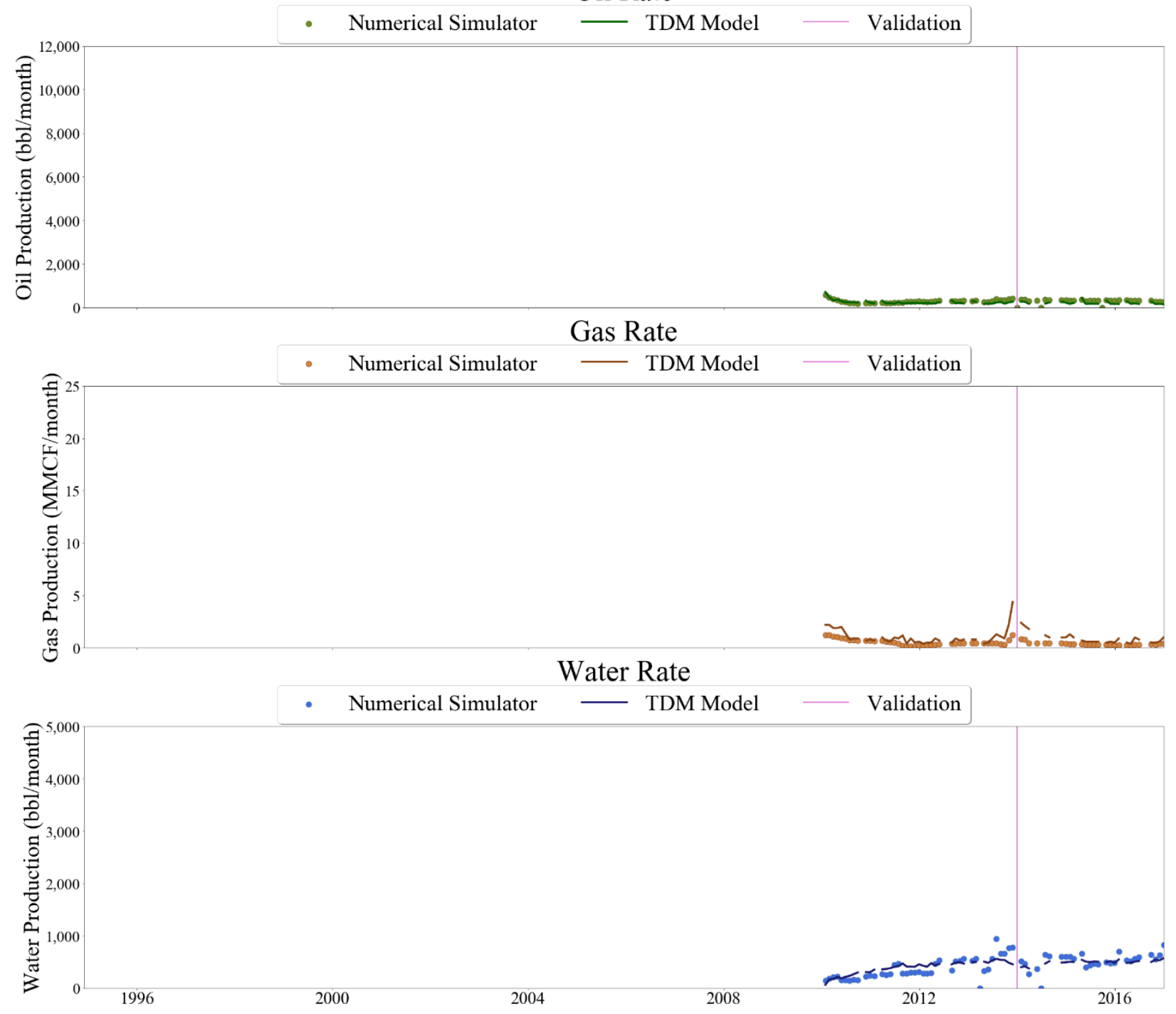

Figure 625 Well-053 oil, gas, and water rate TDM predictions vs actual simulation data plots for 3 Year Blind Validation TDM 


\section{Oil Rate}

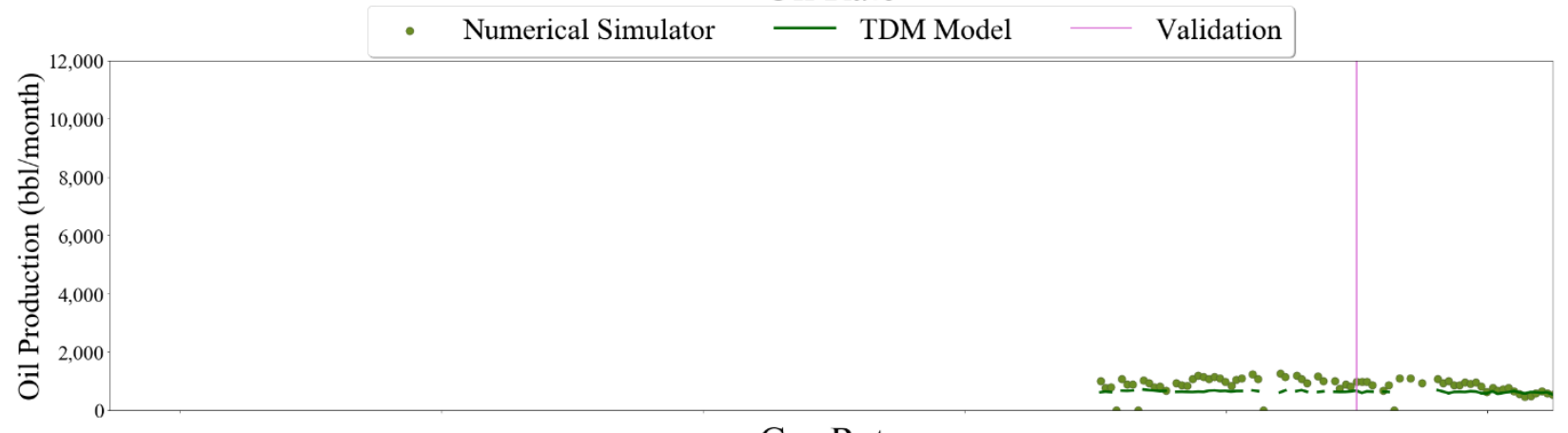

\section{Gas Rate}
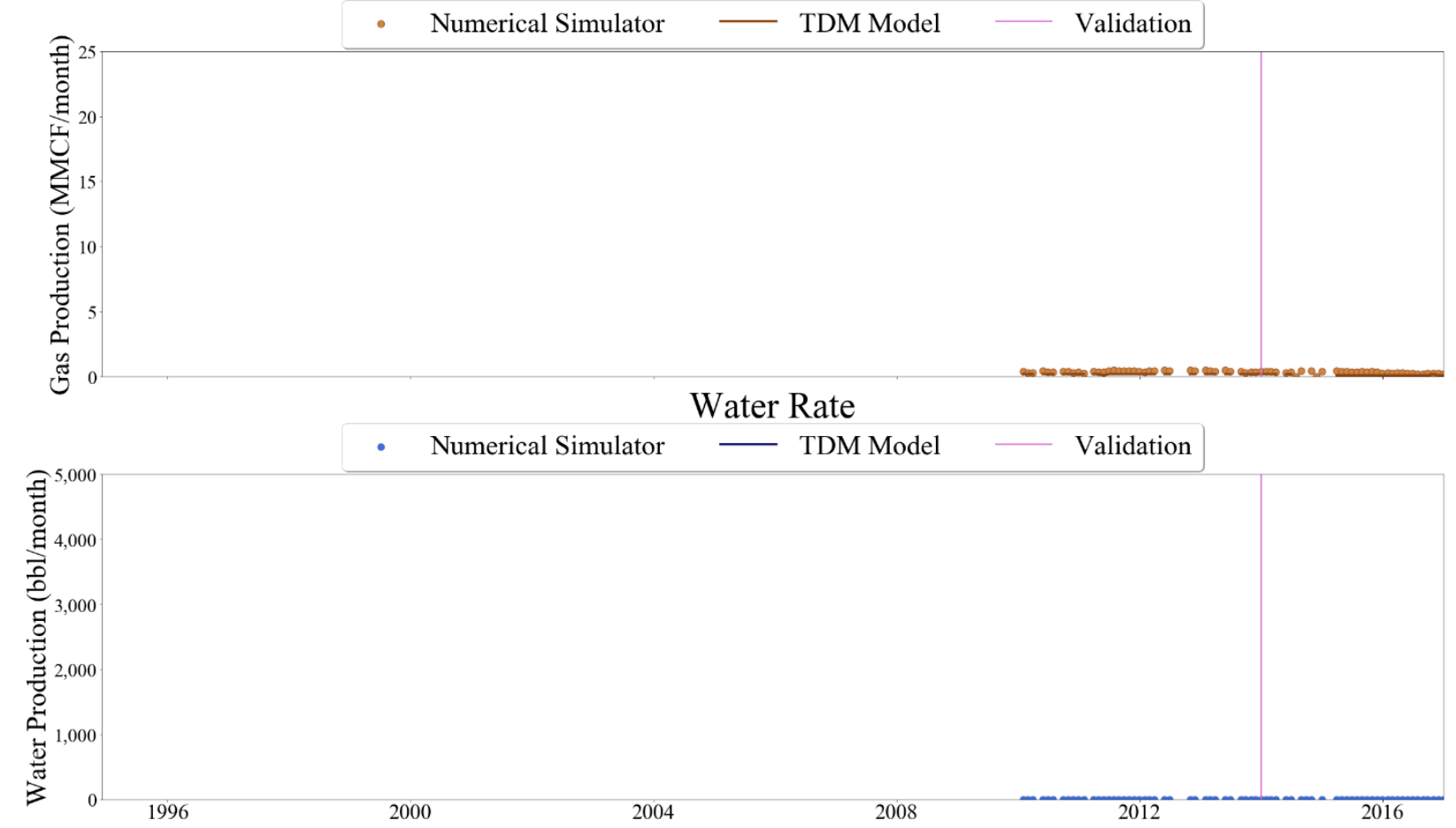

Figure 626 Well-054 oil, gas, and water rate TDM predictions vs actual simulation data plots for 3 Year Blind Validation TDM 


\section{Oil Rate}

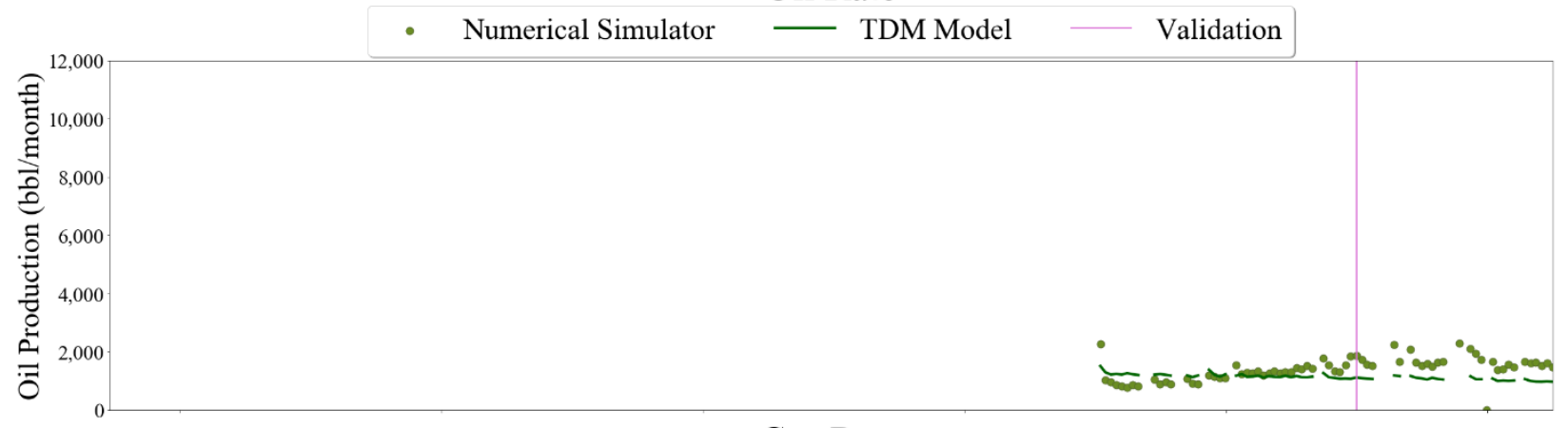

\section{Gas Rate}
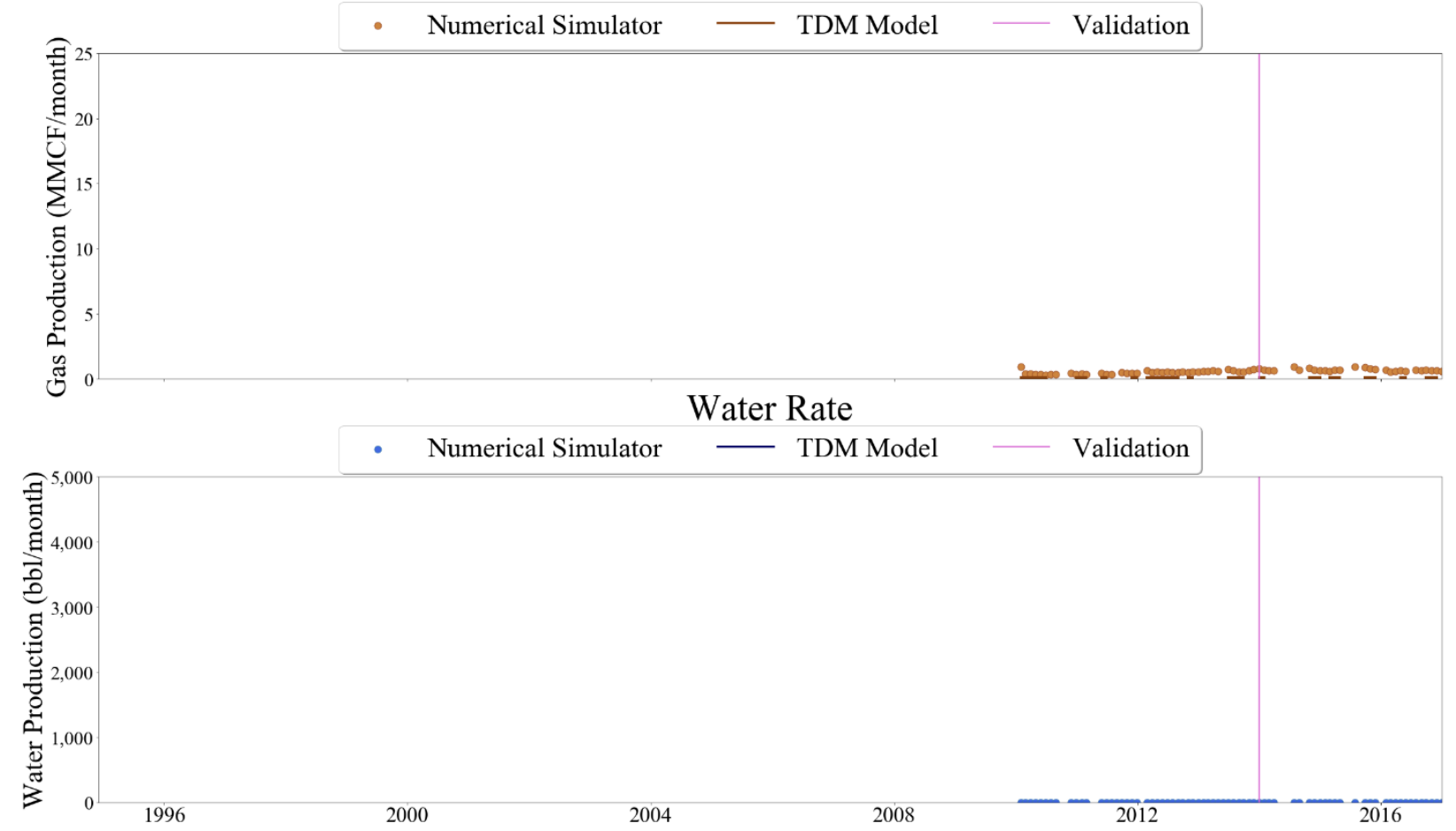

Figure 627 Well-055 oil, gas, and water rate TDM predictions vs actual simulation data plots for 3 Year Blind Validation TDM 


\section{Oil Rate}

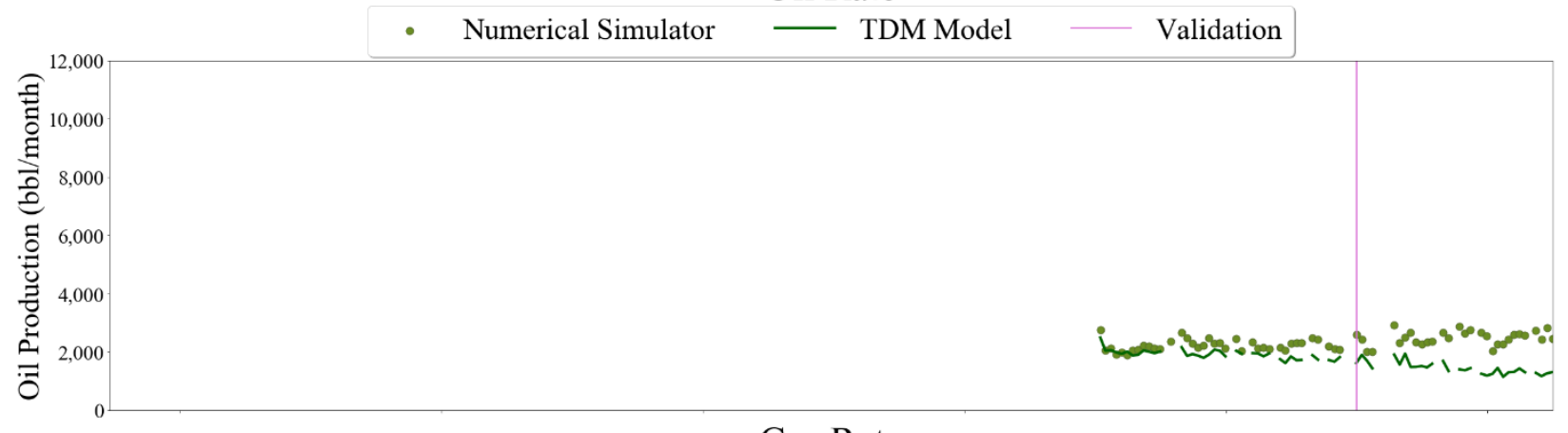

\section{Gas Rate}
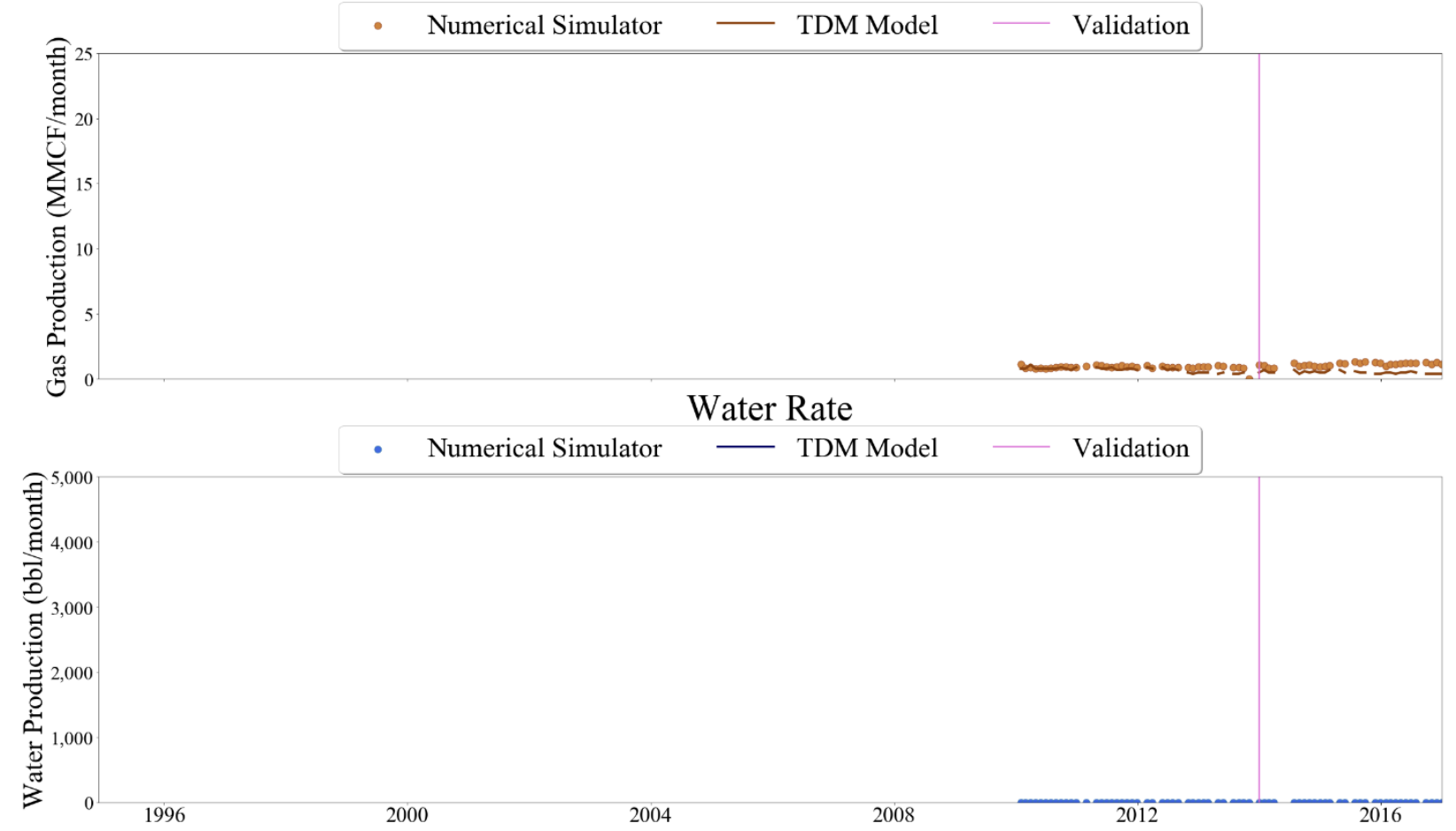

Figure 628 Well-056 oil, gas, and water rate TDM predictions vs actual simulation data plots for 3 Year Blind Validation TDM 


\section{Oil Rate}
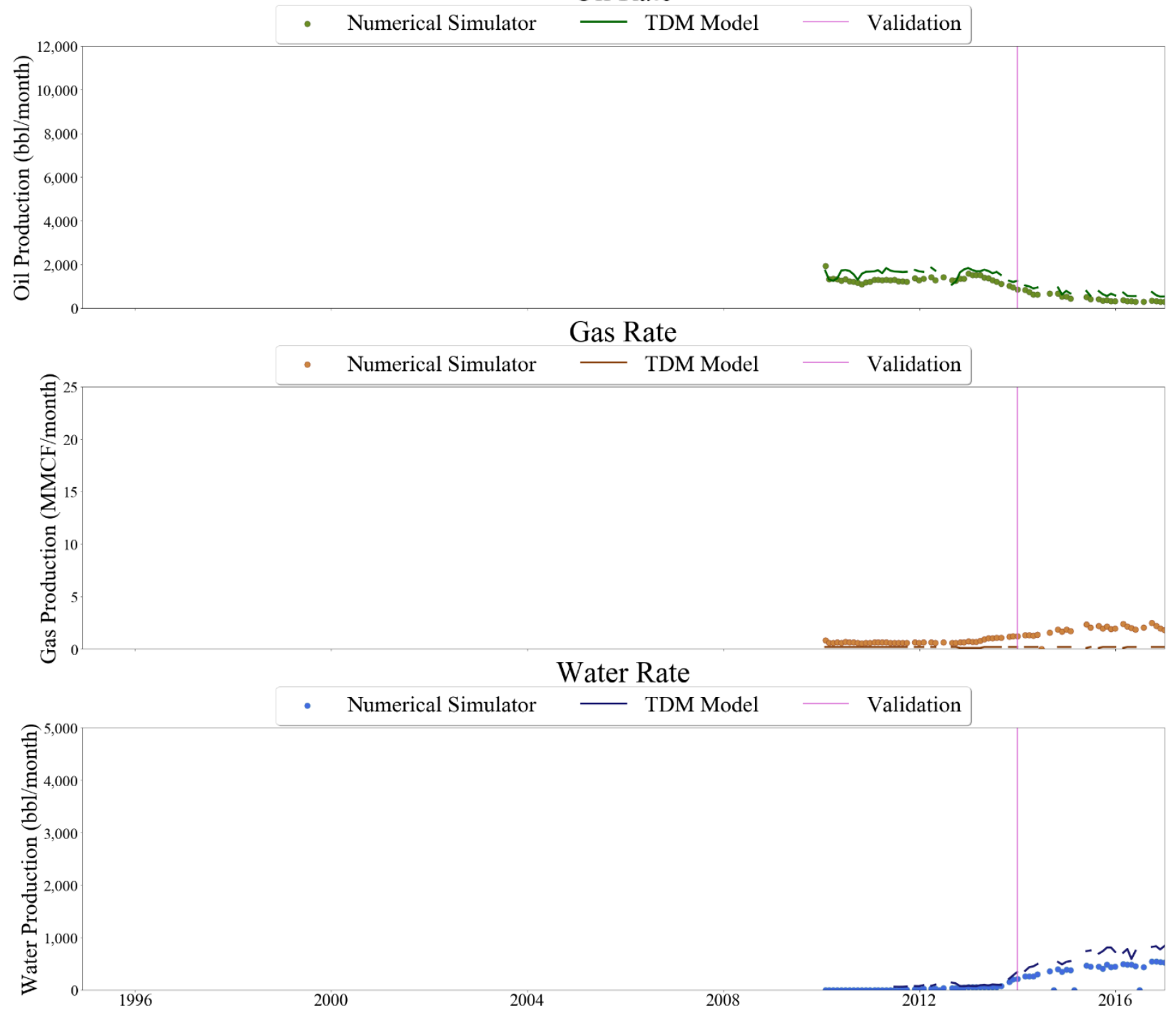

Figure 629 Well-057 oil, gas, and water rate TDM predictions vs actual simulation data plots for 3 Year Blind Validation TDM

\subsubsection{Heat Maps}

Reservoir Pressure (psi) at 2013-12-31
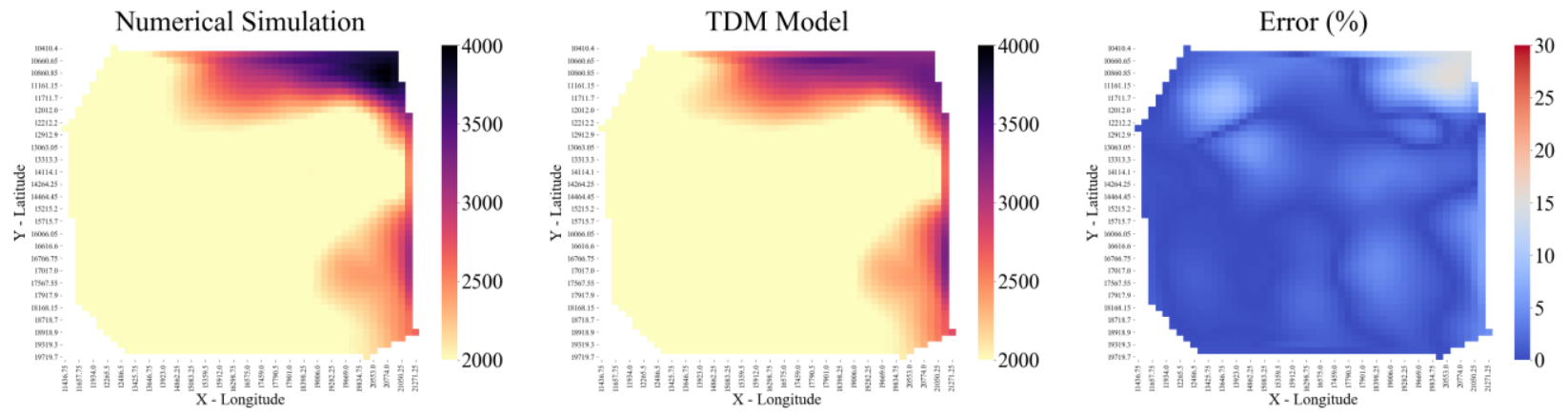

Figure 630 Entire reservoir heat map for reservoir pressure at December 31st, 2013 for 3 Year Blind Validation TDM 
Reservoir Pressure (psi) at 2014-03-31
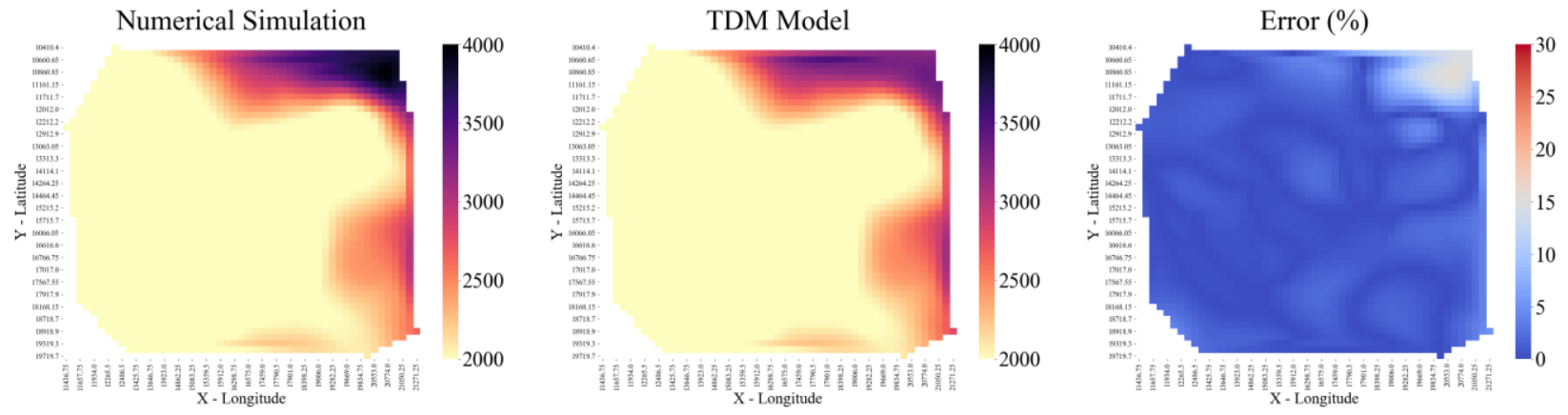

Figure 631 Entire reservoir heat map for reservoir pressure at March 31st, 2014 for 3 Year Blind Validation TDM

Reservoir Pressure (psi) at 2014-06-30
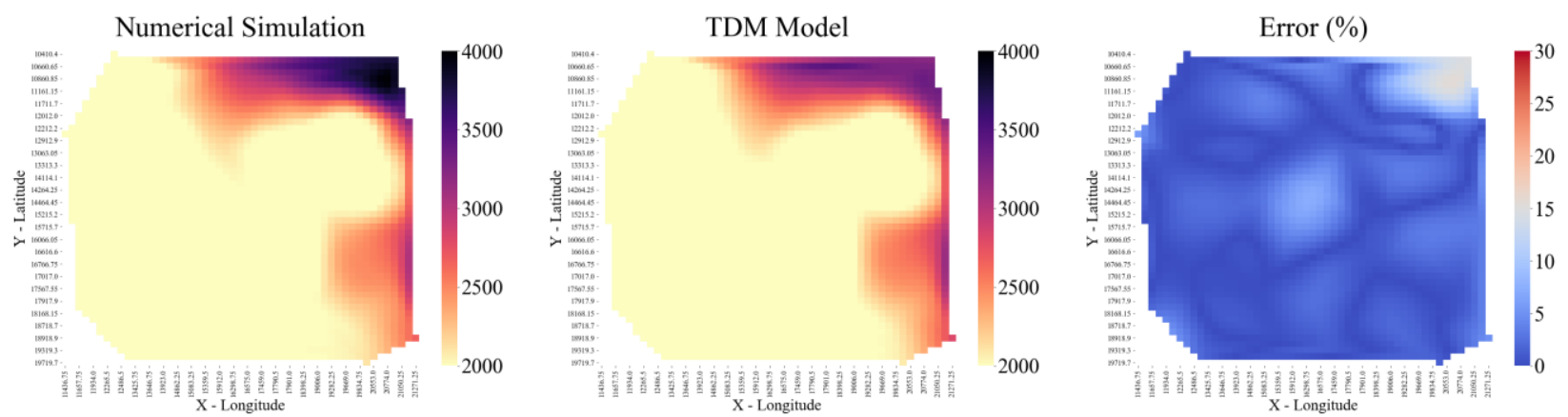

Figure 632 Entire reservoir heat map for reservoir pressure at June 30th, 2014 for 3 Year Blind Validation TDM

Reservoir Pressure (psi) at 2014-09-30
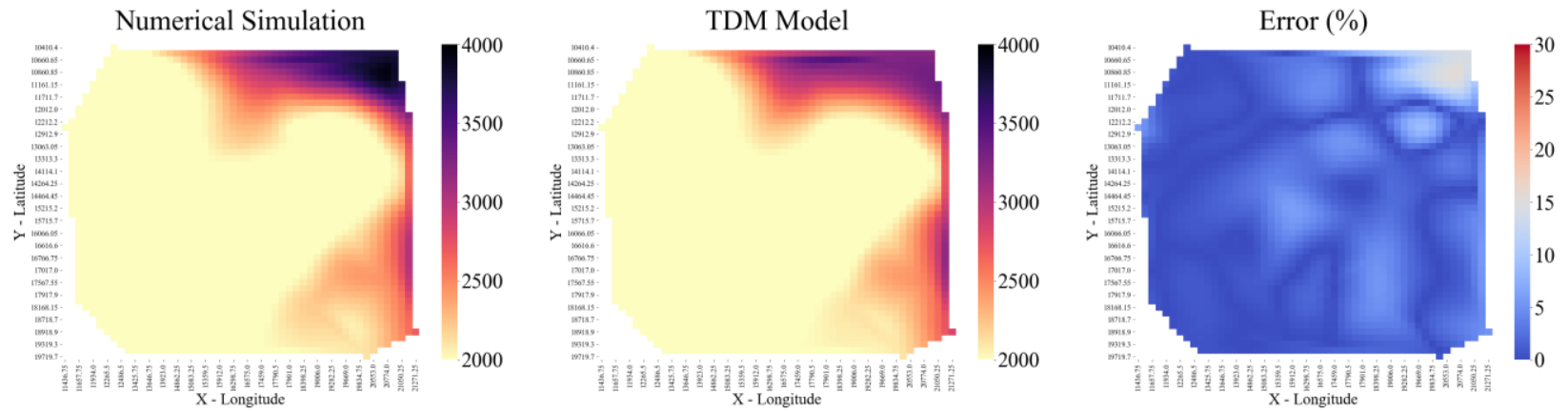

Figure 633 Entire reservoir heat map for September 30th, 2014 for 3 Year Blind Validation TDM 
Reservoir Pressure (psi) at 2014-12-31
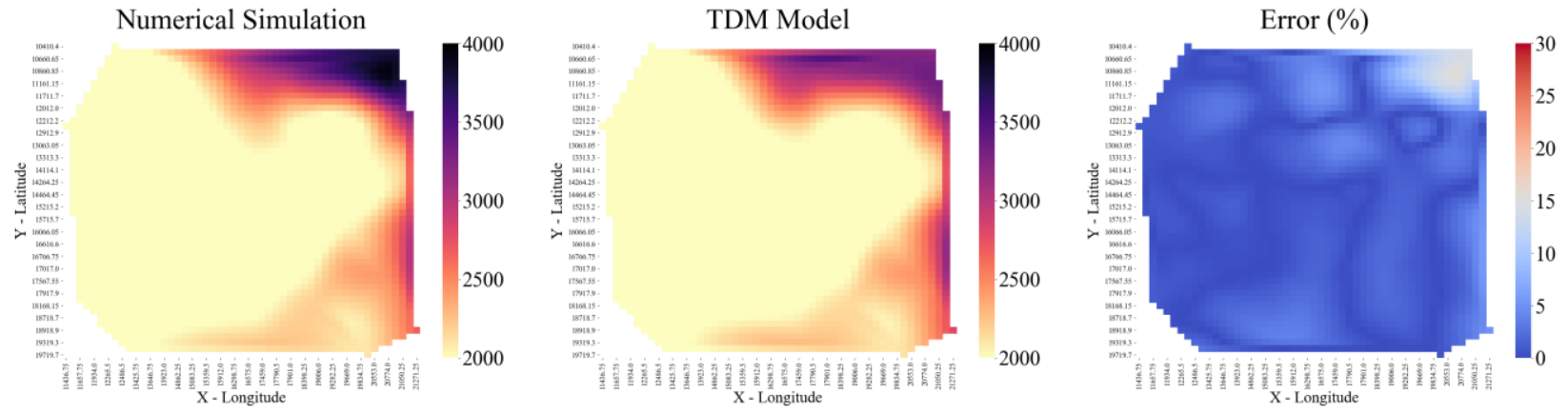

Figure 634 Entire reservoir heat map for reservoir pressure at December 31st, 2014 for 3 Year Blind Validation TDM

Reservoir Pressure (psi) at 2015-03-31
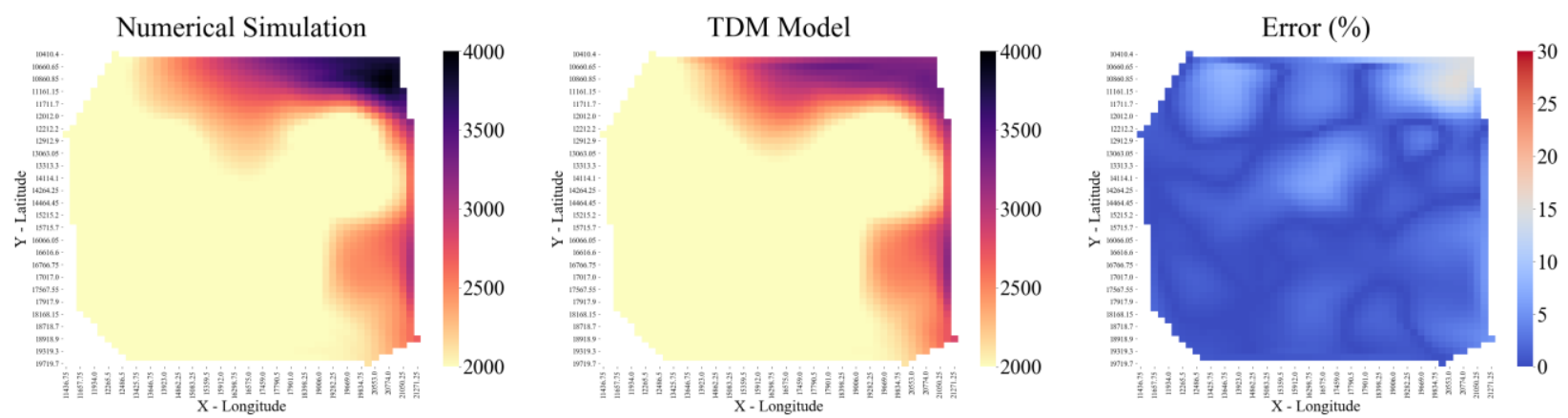

Figure 635 Entire reservoir heat map for reservoir pressure at March 31st, 2015 for 3 Year Blind Validation TDM

Reservoir Pressure (psi) at 2015-06-30
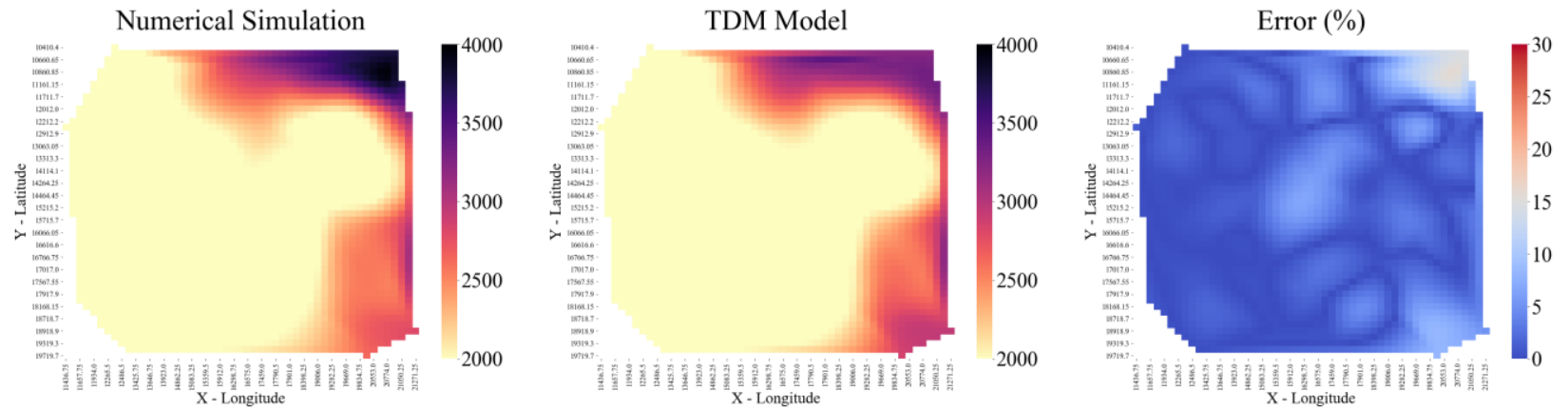

Figure 636 Entire reservoir heat map for reservoir pressure at June 30th, 2015 for 3 Year Blind Validation TDM 
Reservoir Pressure (psi) at 2015-09-30
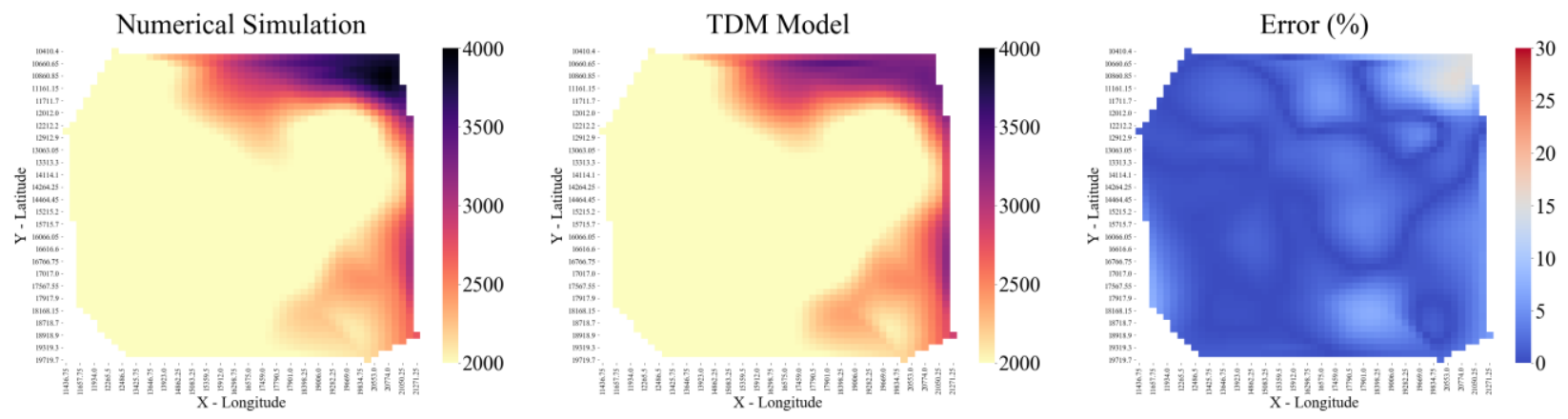

Figure 637 Entire reservoir heat map for reservoir pressure at September 30th, 2015 for 3 Year Blind Validation TDM

Reservoir Pressure (psi) at 2015-12-31
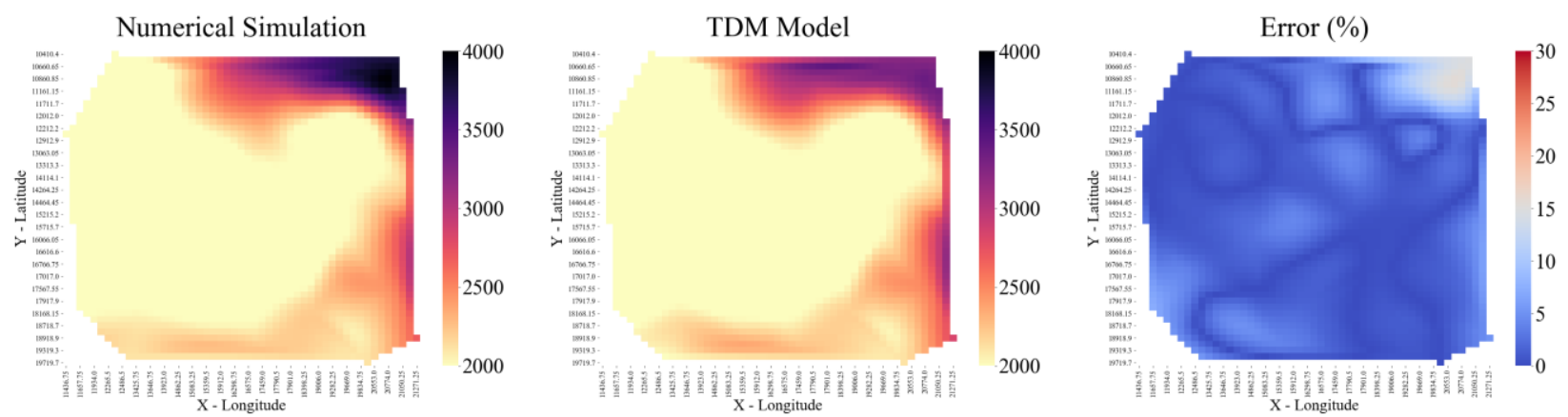

Figure 638 Entire reservoir heat map for reservoir pressure at December 31st, 2015 for 3 Year Blind Validation TDM

Reservoir Pressure (psi) at 2016-03-31
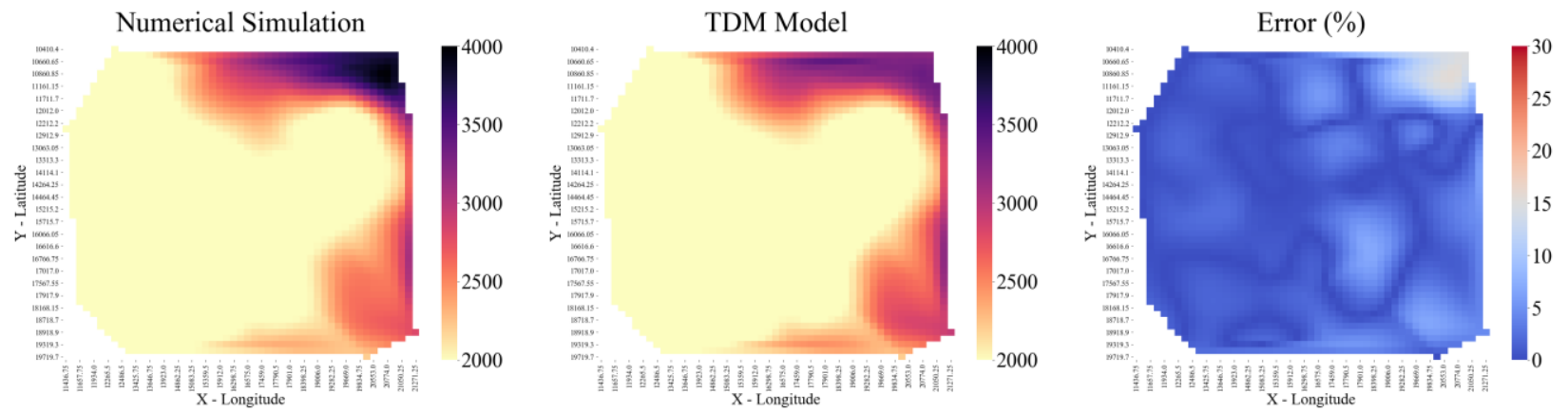

Figure 639 Entire reservoir heat map for reservoir pressure at March 31st, 2016 for 3 Year Blind Validation TDM 
Reservoir Pressure (psi) at 2016-06-30
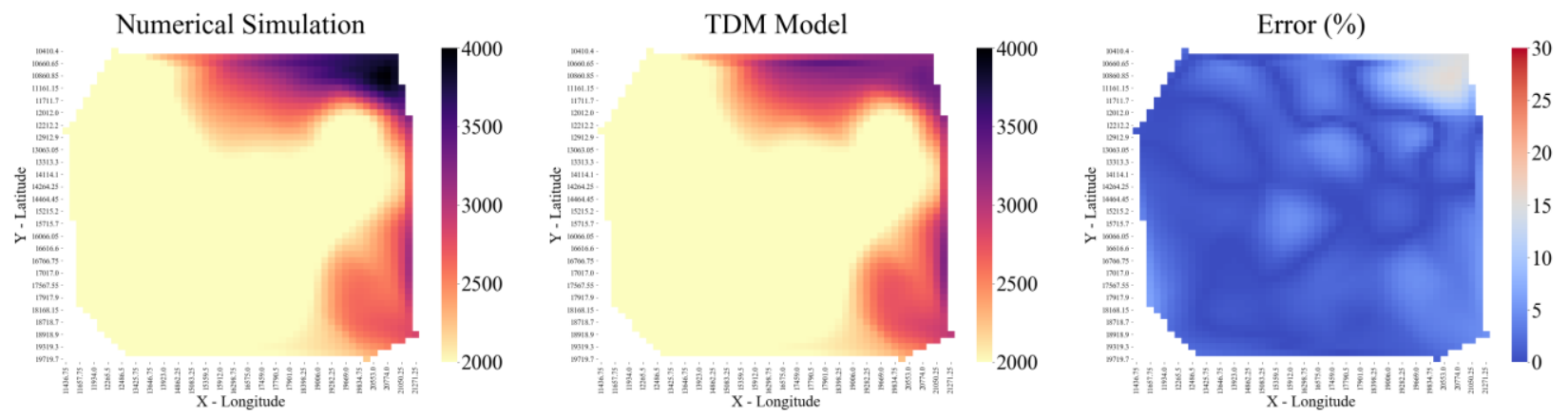

Figure 640 Entire reservoir heat map for reservoir pressure at June 30th, 2016 for 3 Year Blind Validation TDM

Reservoir Pressure (psi) at 2016-09-30
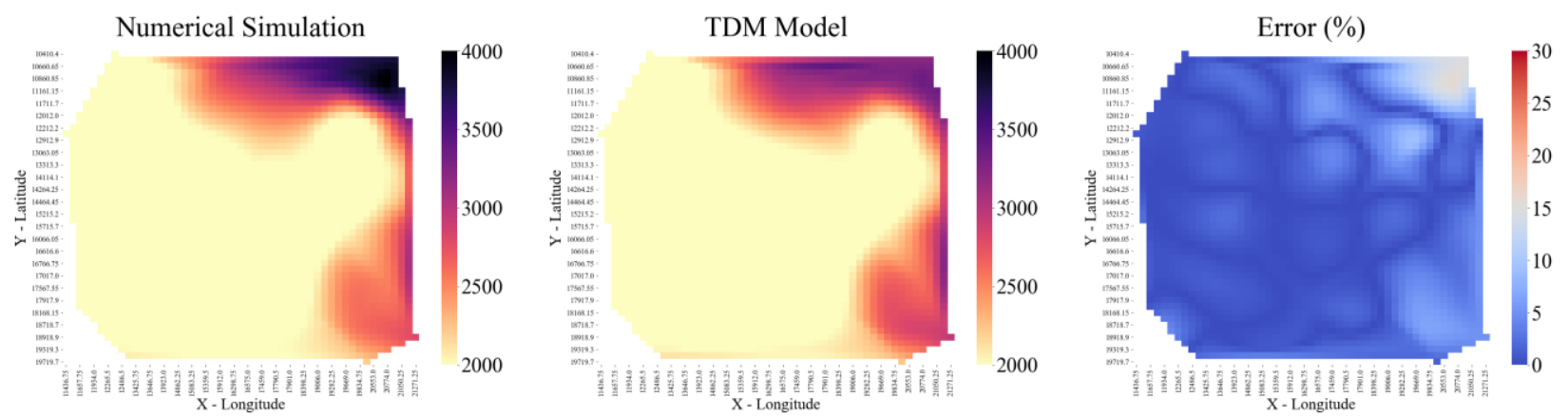

Figure 641 Entire reservoir heat map for reservoir pressure at September 30th, 2016 for 3 Year Blind Validation TDM

Reservoir Pressure (psi) at 2016-12-31
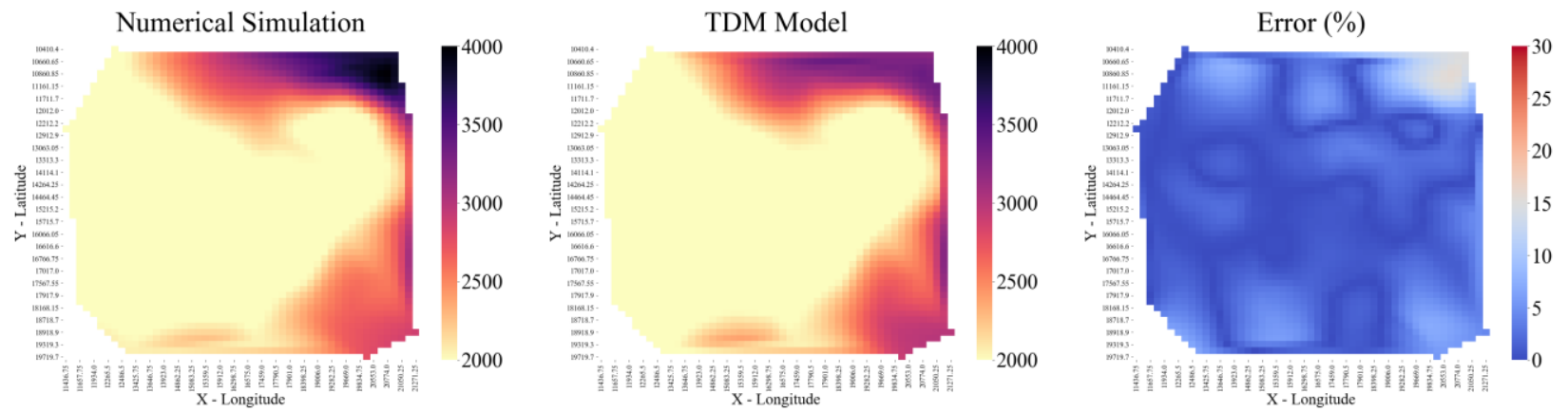

Figure 642 Entire reservoir heat map for reservoir pressure at December 31st, 2016 for 3 Year Blind Validation TDM 
Water Saturation (\%) at 2013-12-31
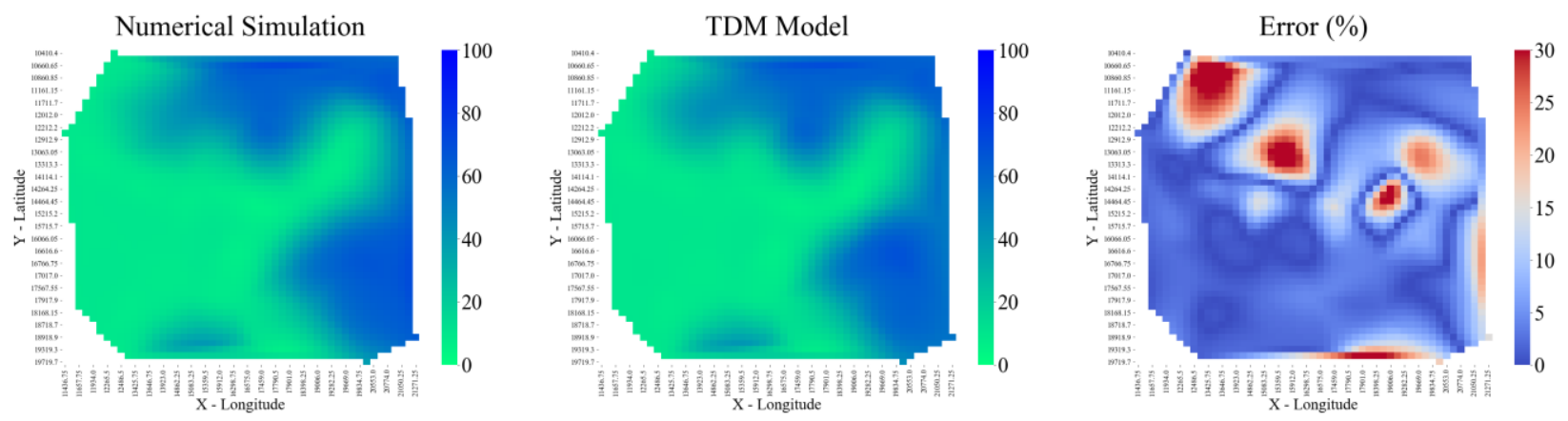

Figure 643 Entire reservoir heat map for water saturation at December 31st, 2013 for 3 Year Blind Validation TDM

\section{Water Saturation (\%) at 2014-03-31}
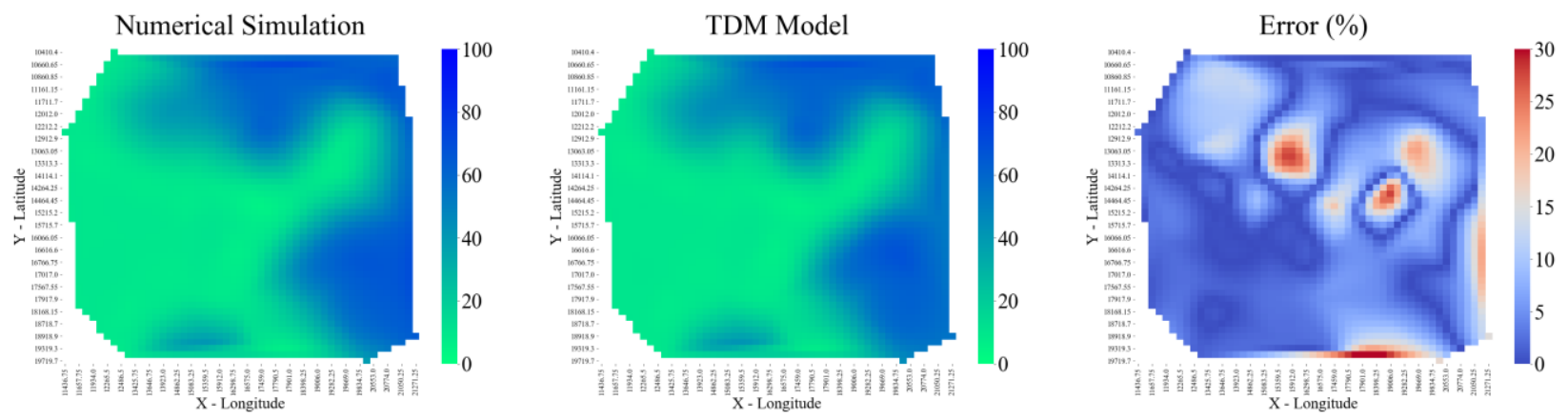

Figure 644 Entire reservoir heat map for water saturation at March 31st, 2014 for 3 Year Blind Validation TDM

Water Saturation (\%) at 2014-06-30
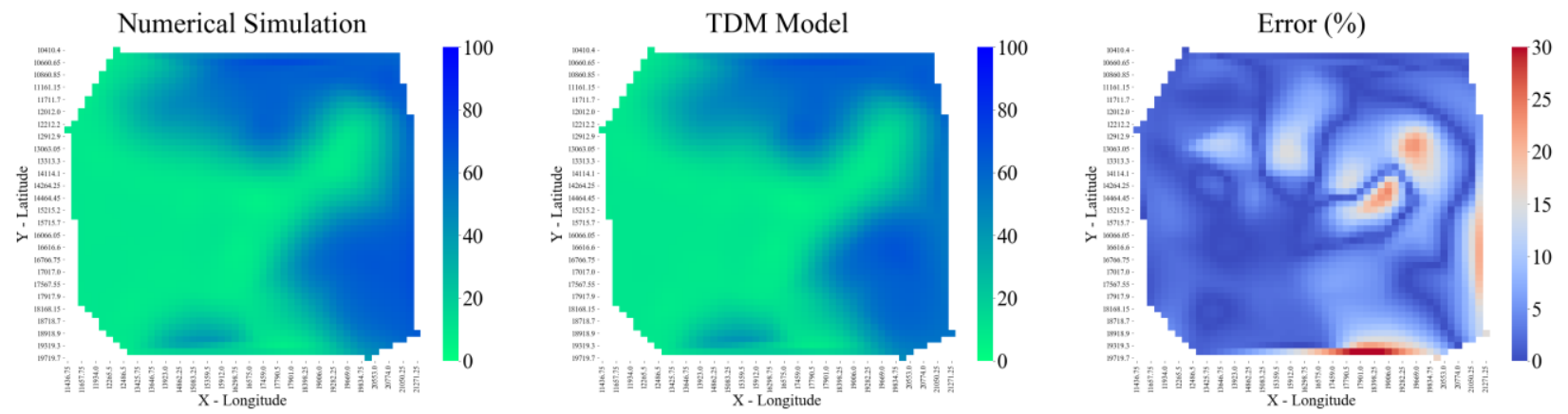

Figure 645 Entire reservoir heat map for water saturation at June 30th, 2014 for 3 Year Blind Validation TDM 
Water Saturation (\%) at 2014-09-30
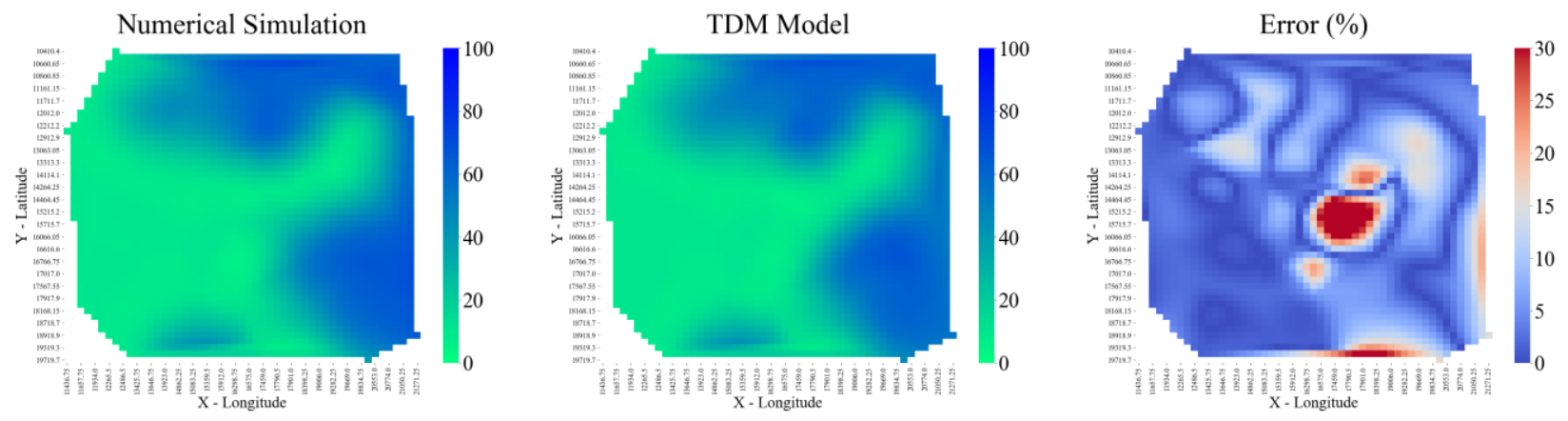

Figure 646 Entire reservoir heat map for water saturation at September 30th, 2014 for 3 Year Blind Validation TDM

Water Saturation (\%) at 2014-12-31
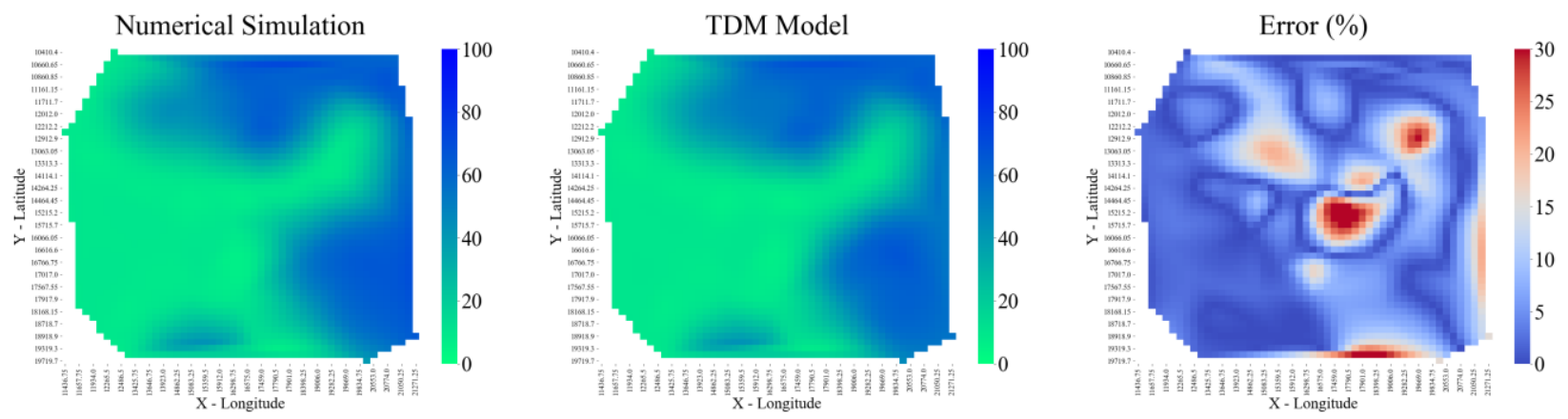

Figure 647 Entire reservoir heat map for water saturation at December 31st, 2014 for 3 Year Blind Validation TDM

Water Saturation (\%) at 2015-03-31
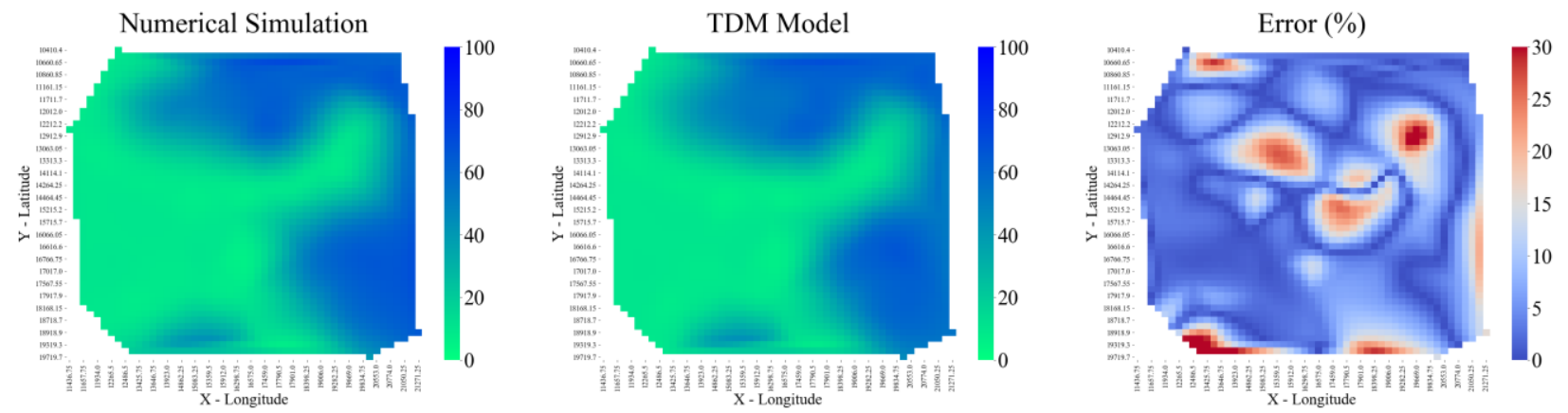

Figure 648 Entire reservoir heat map for water saturation at March 31st, 2015 for 3 Year Blind Validation TDM 
Water Saturation (\%) at 2015-06-30
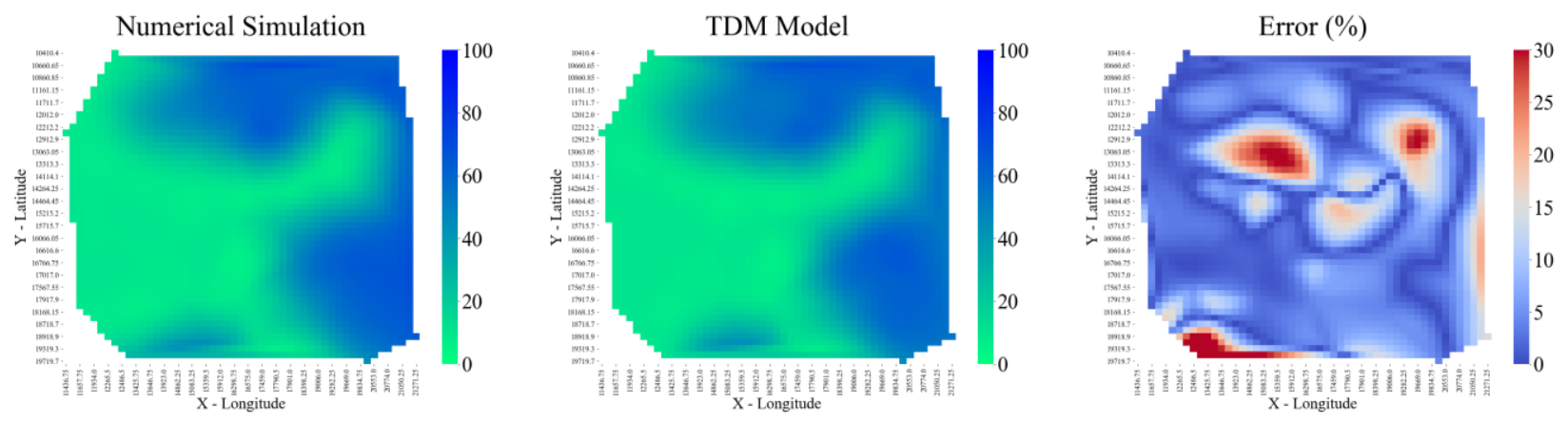

Figure 649 Entire reservoir heat map for water saturation at June 30th, 2015 for 3 Year Blind Validation TDM

Water Saturation (\%) at 2015-09-30
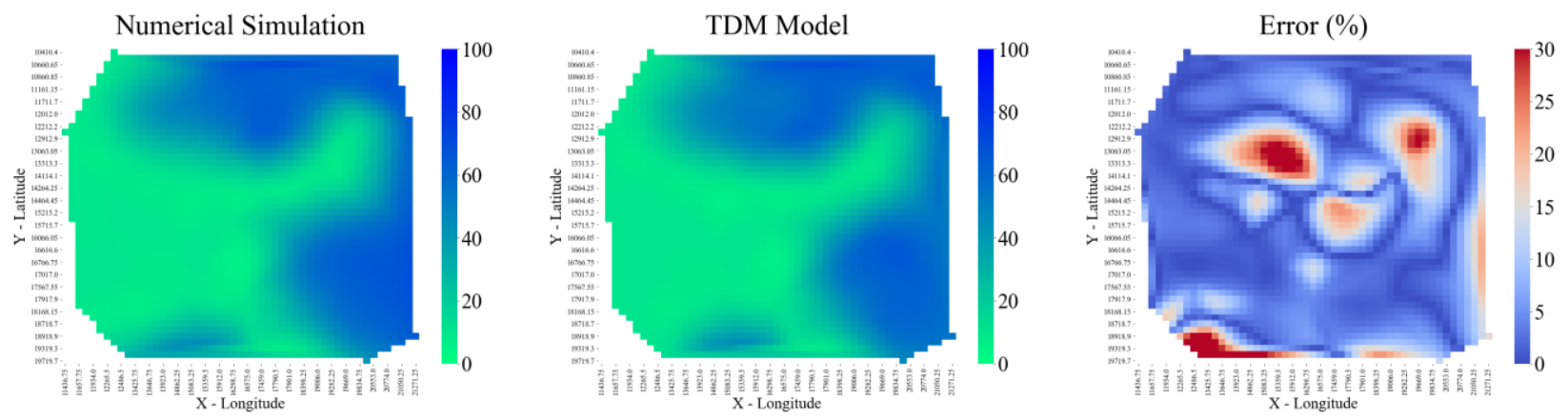

Figure 650 Entire reservoir heat map for water saturation at September 30th, 2015 for 3 Year Blind Validation TDM

Water Saturation (\%) at 2015-12-31
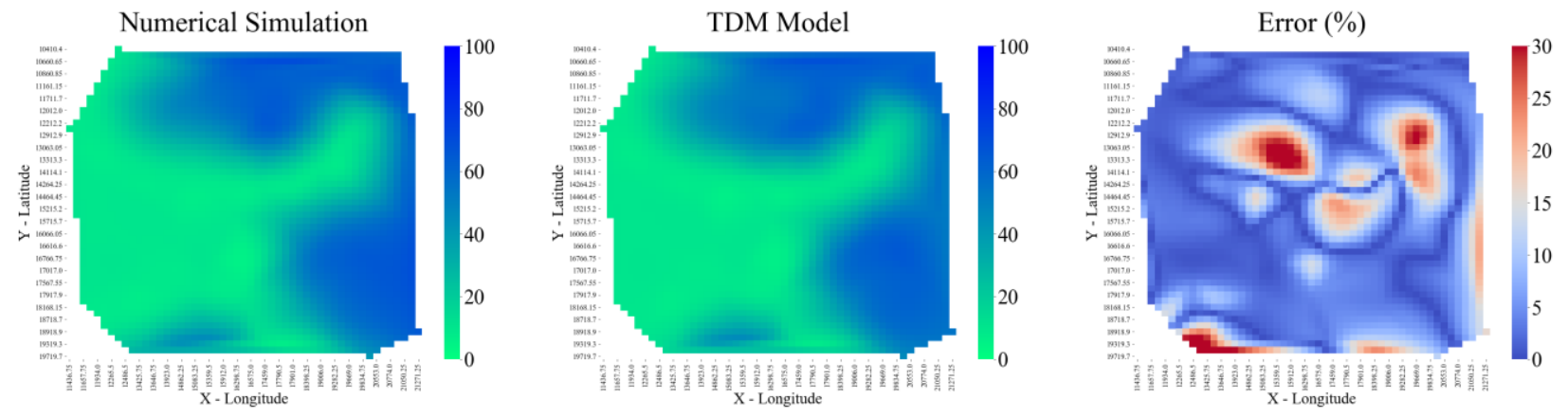

Figure 651 Entire reservoir heat map for water saturation at December 31st, 2015 for 3 Year Blind Validation TDM 
Water Saturation (\%) at 2016-03-31
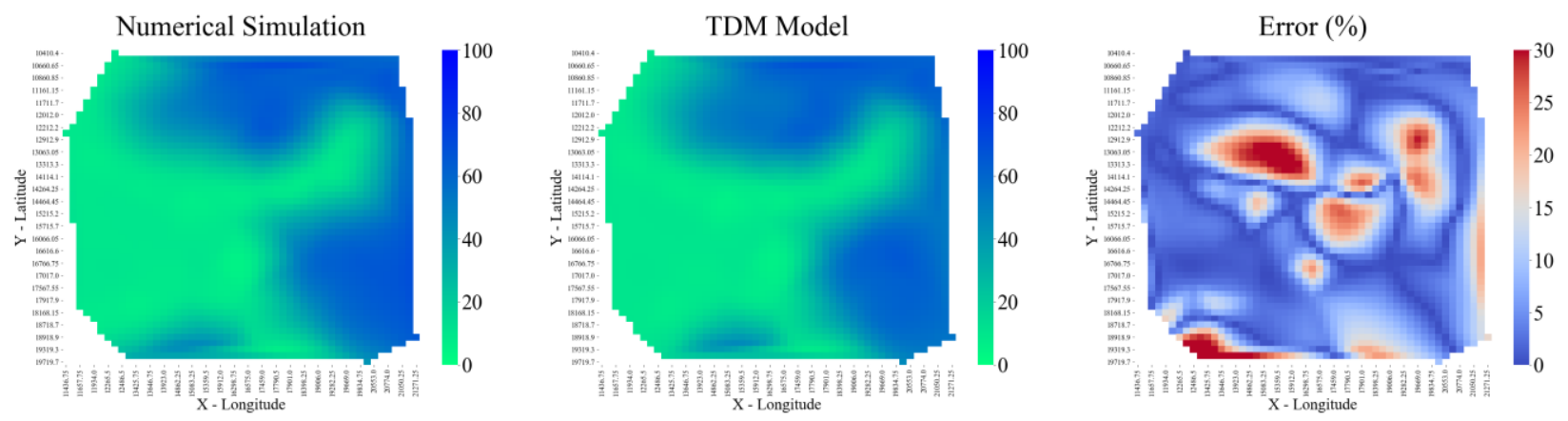

Figure 652 Entire reservoir heat map for water saturation at March 31st, 2016 for 3 Year Blind Validation TDM

\section{Water Saturation (\%) at 2016-06-30}
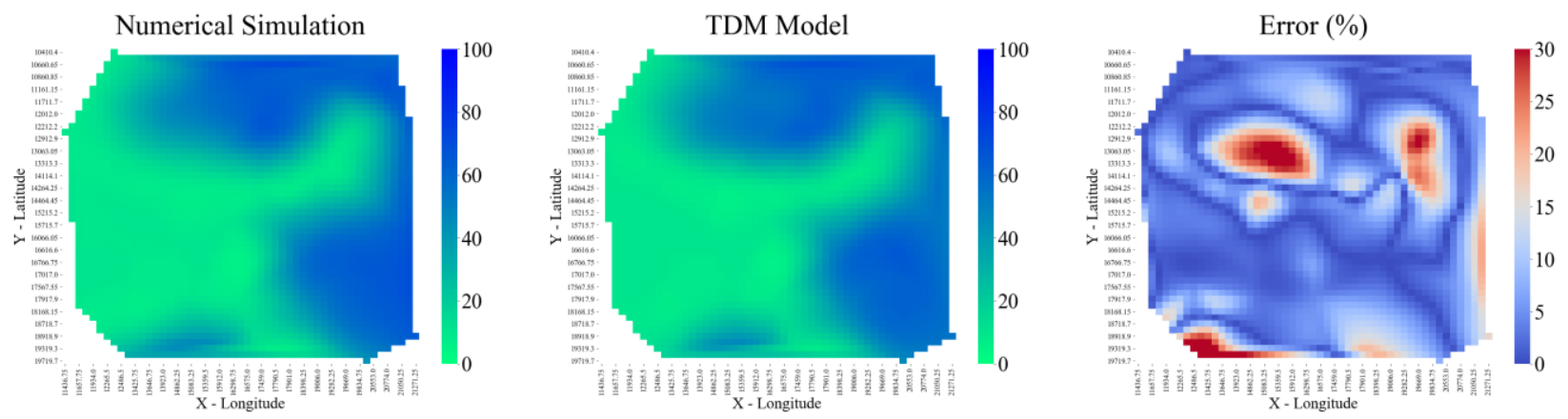

Figure 653 Entire reservoir heat map for water saturation at June 30th, 2016 for 3 Year Blind Validation TDM

Water Saturation (\%) at 2016-09-30
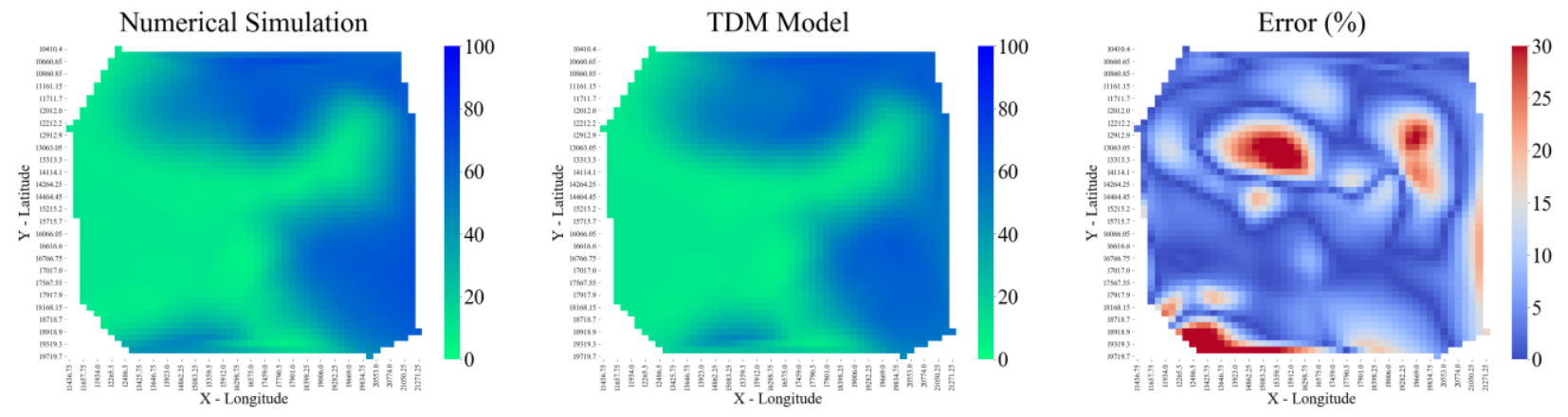

Figure 654 Entire reservoir heat map for water saturation at September 30th, 2016 for 3 Year Blind Validation TDM 
Water Saturation (\%) at 2016-12-31
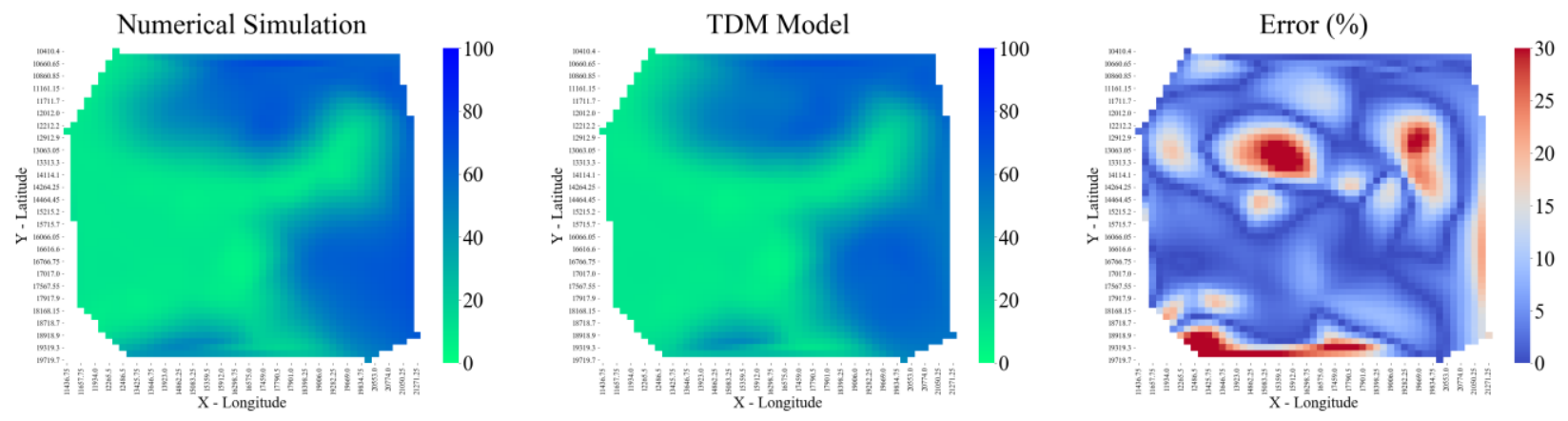

Figure 655 Entire reservoir heat map for water saturation at December 31st, 2016 for 3 Year Blind Validation TDM

\subsubsection{Multi-Random Partitioning- 4 Year Blind Validation}

\subsubsection{Individual Well Production Plots}

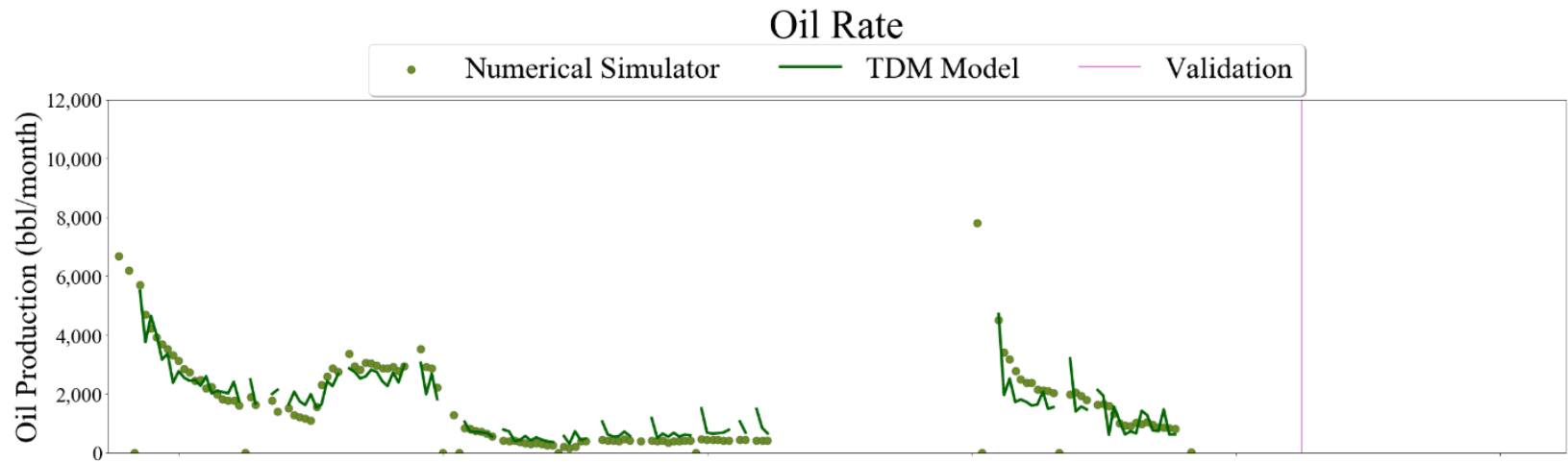

Gas Rate
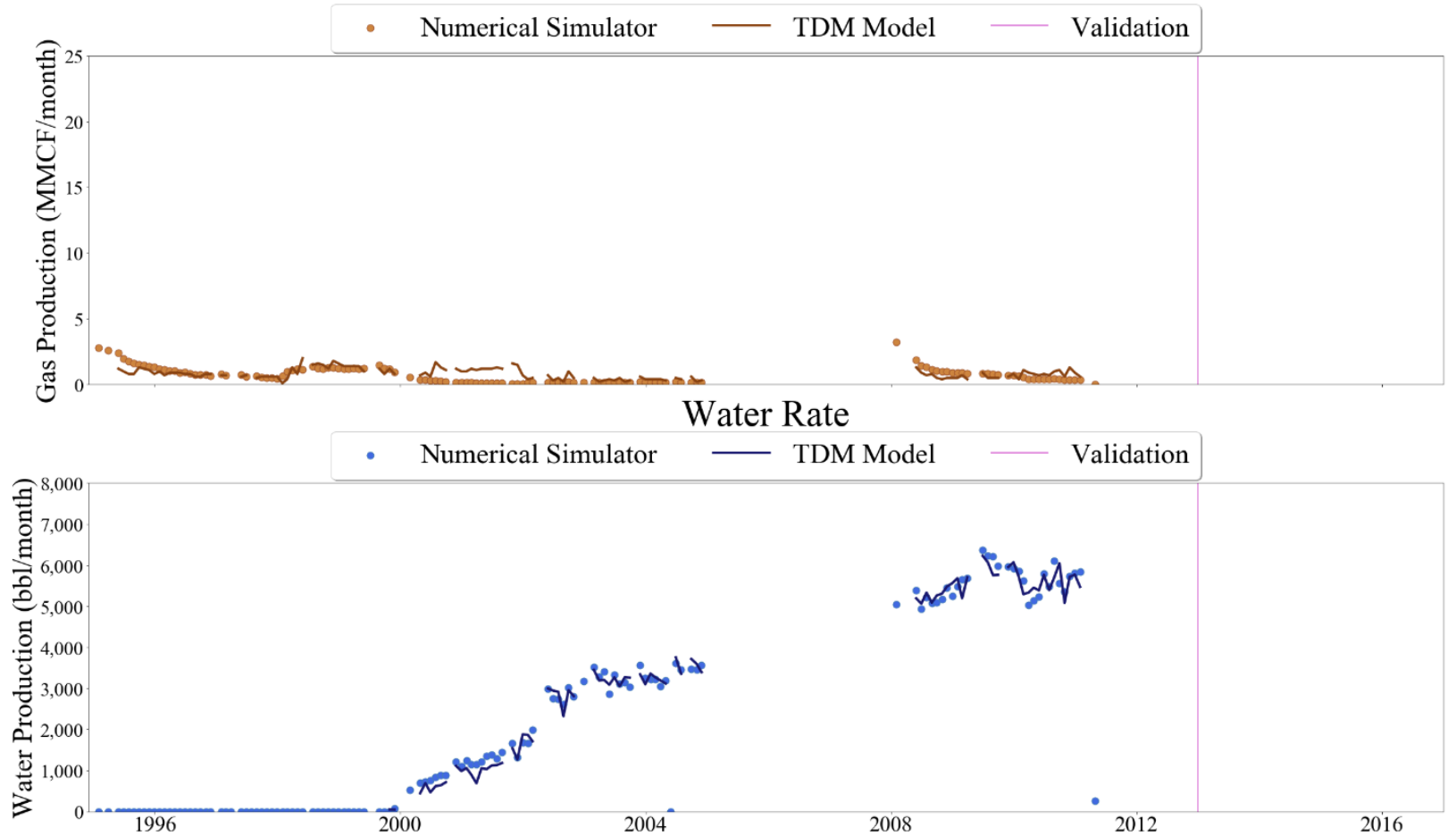

Figure 656 Well-001 oil, gas, and water rate TDM predictions vs actual simulation data plots for 4 Year Blind Validation TDM 

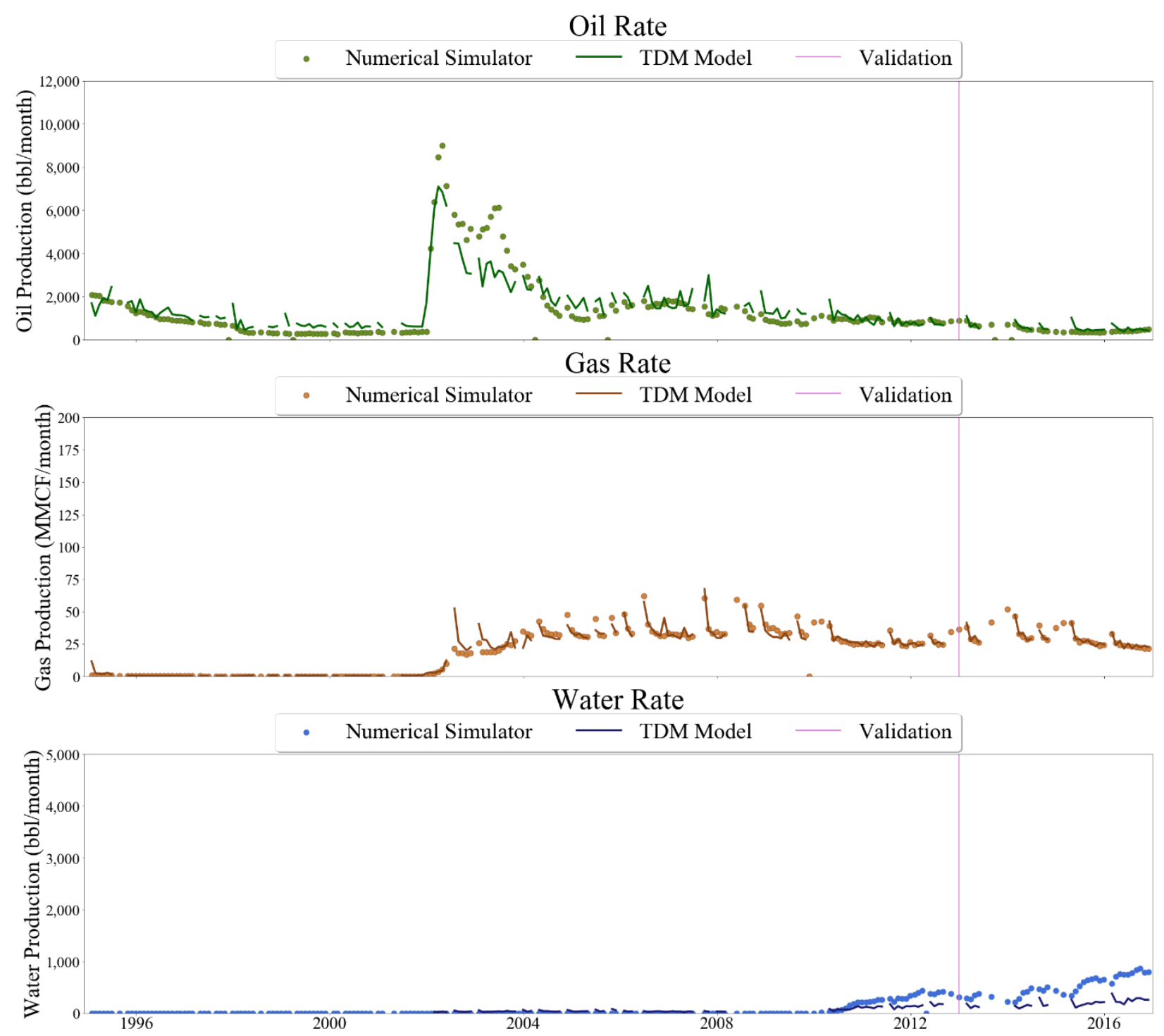

Figure 657 Well-002 oil, gas, and water rate TDM predictions vs actual simulation data plots for 4 Year Blind Validation TDM 


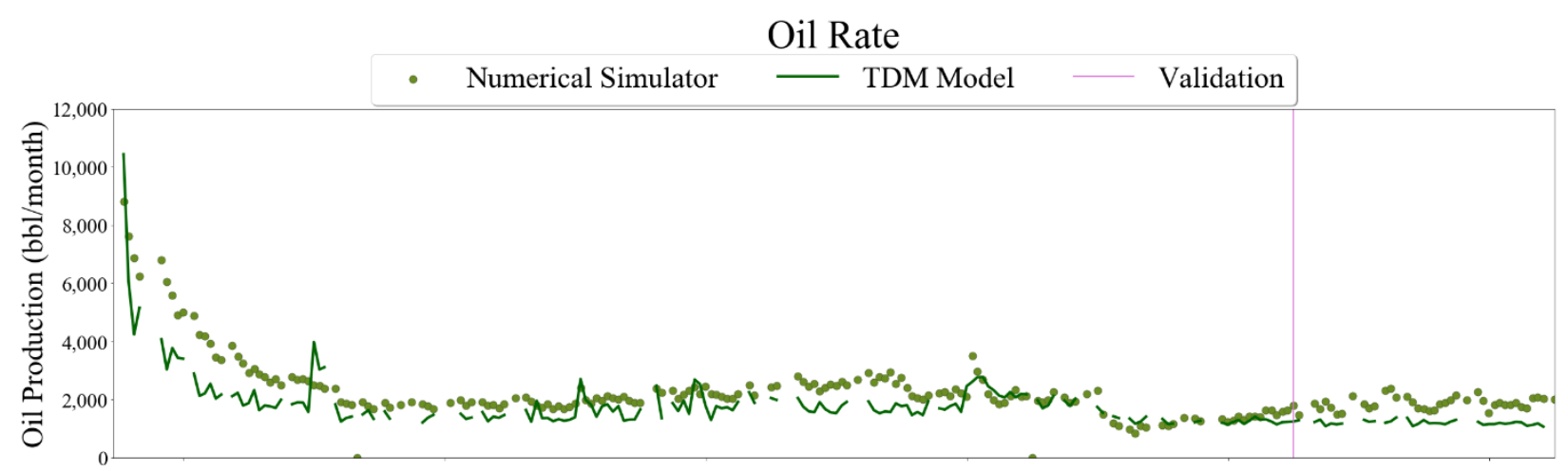

\section{Gas Rate}
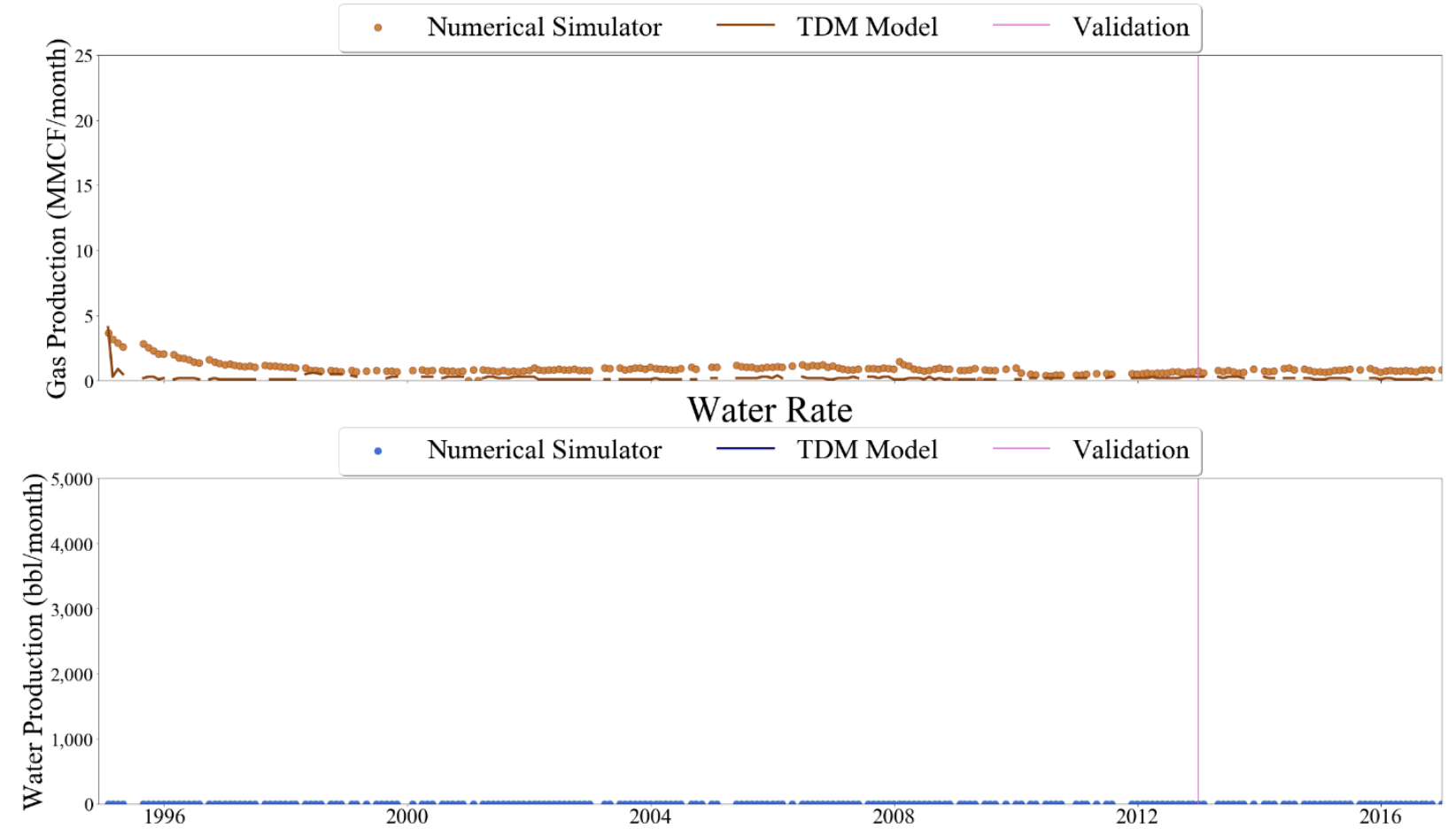

Figure 658 Well-003 oil, gas, and water rate TDM predictions vs actual simulation data plots for 4 Year Blind Validation TDM 


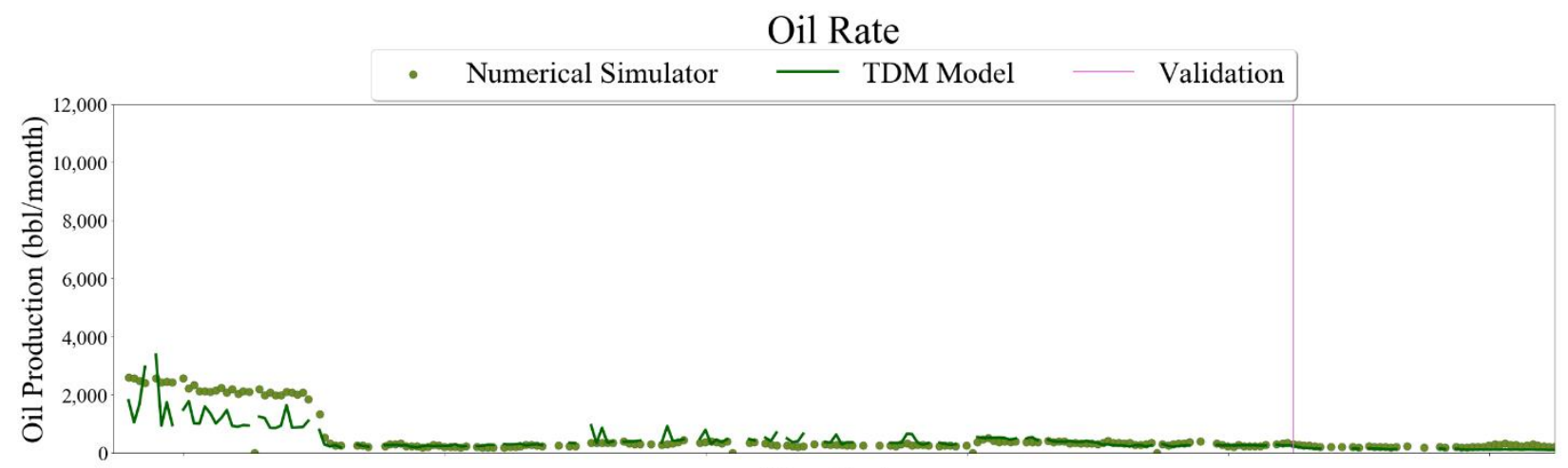

Gas Rate
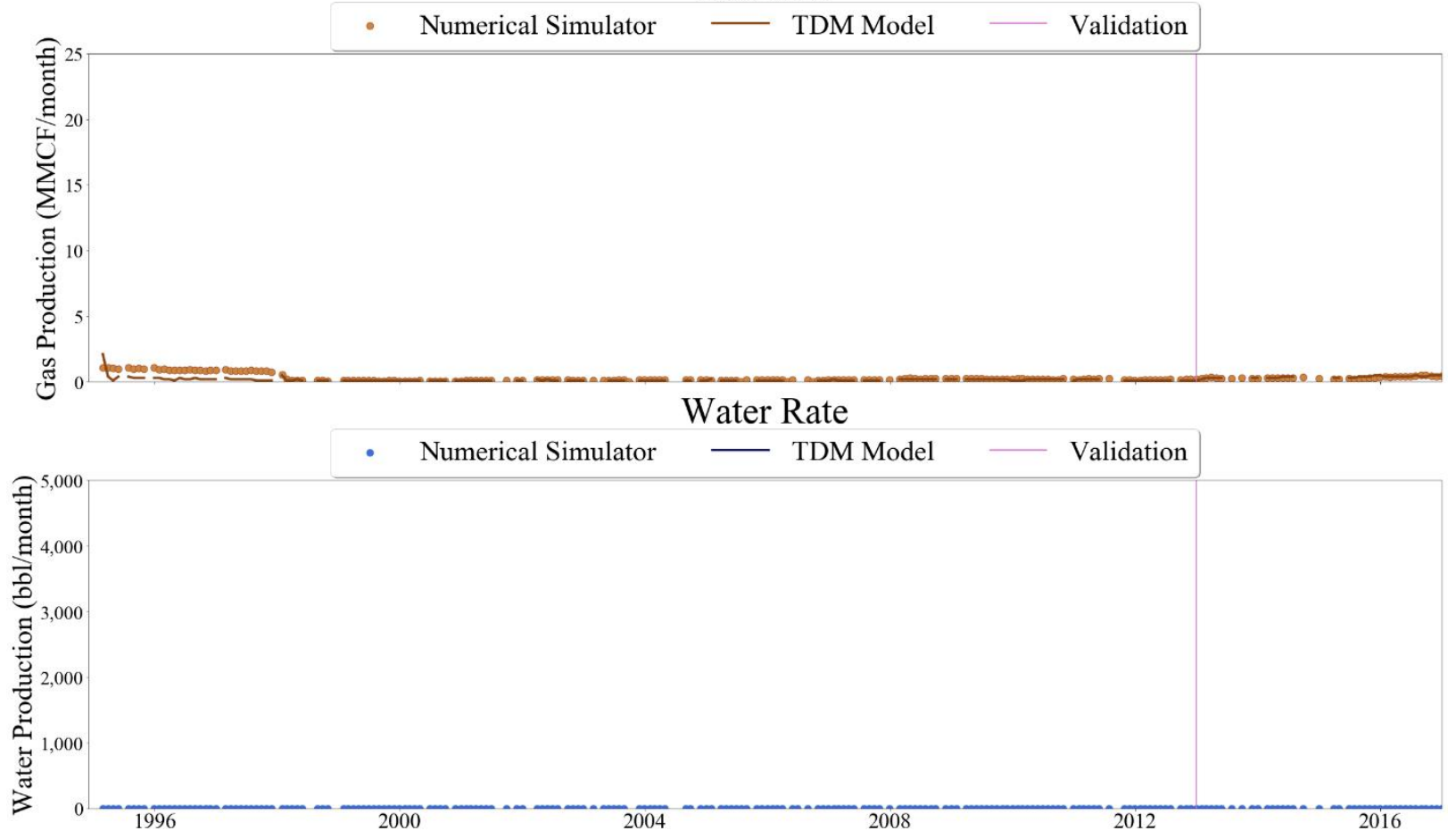

Figure 659 Well-004 oil, gas, and water rate TDM predictions vs actual simulation data plots for 4 Year Blind Validation TDM 


\section{Oil Rate}

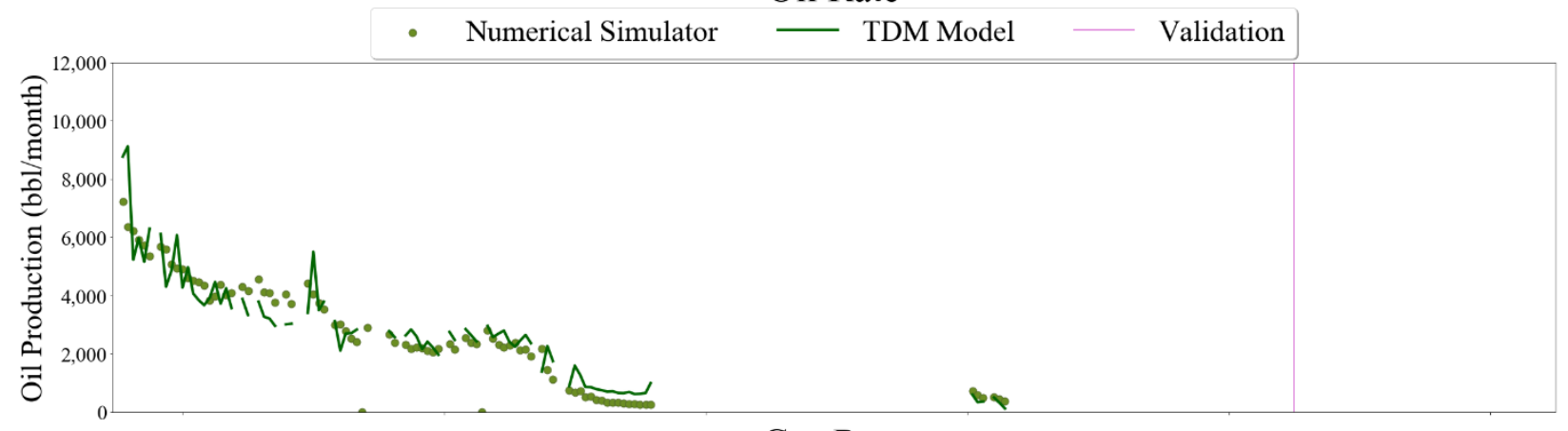

Gas Rate
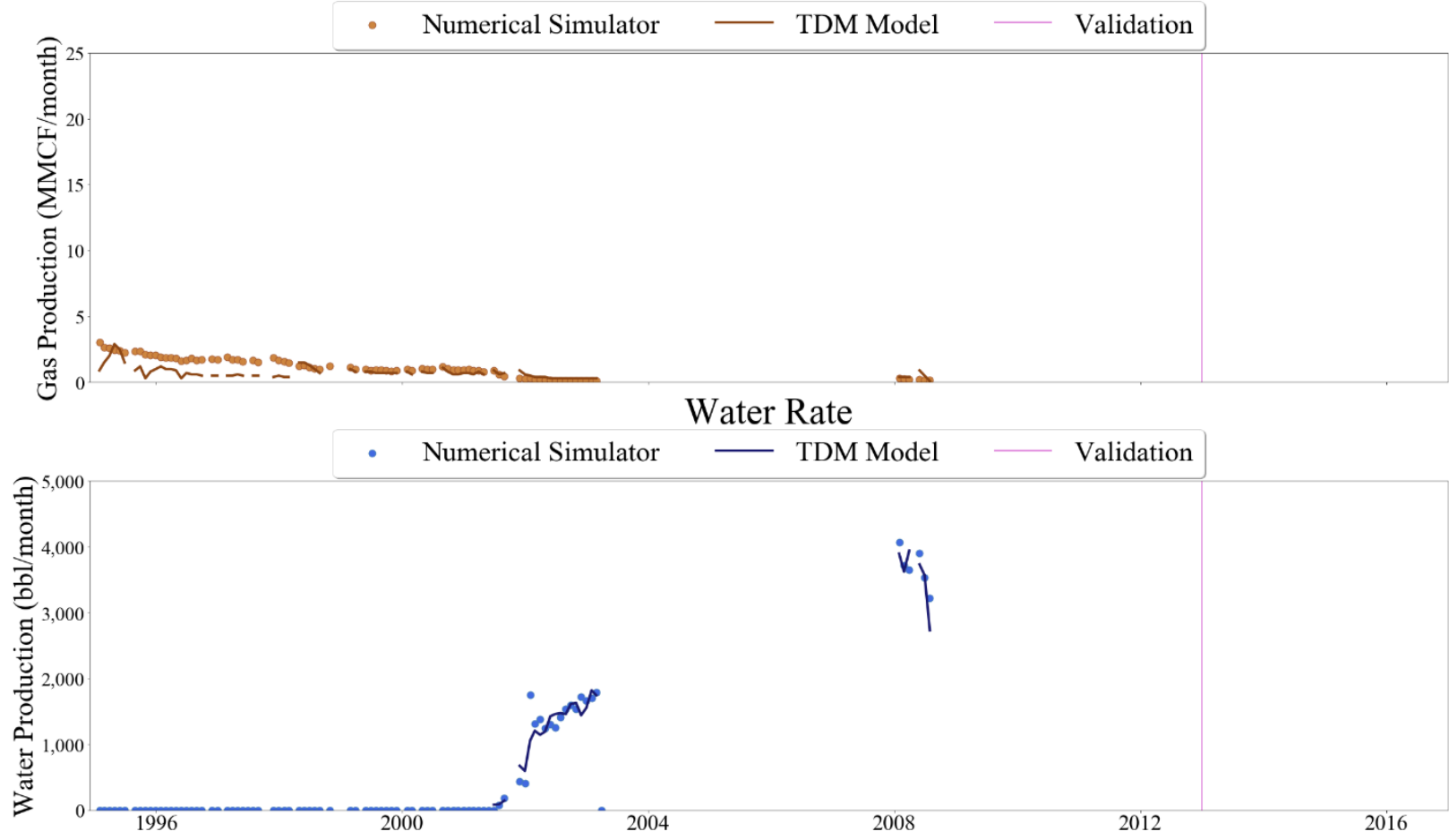

Figure 660 Well-005 oil, gas, and water rate TDM predictions vs actual simulation data plots for 4 Year Blind Validation TDM 


\section{Oil Rate}

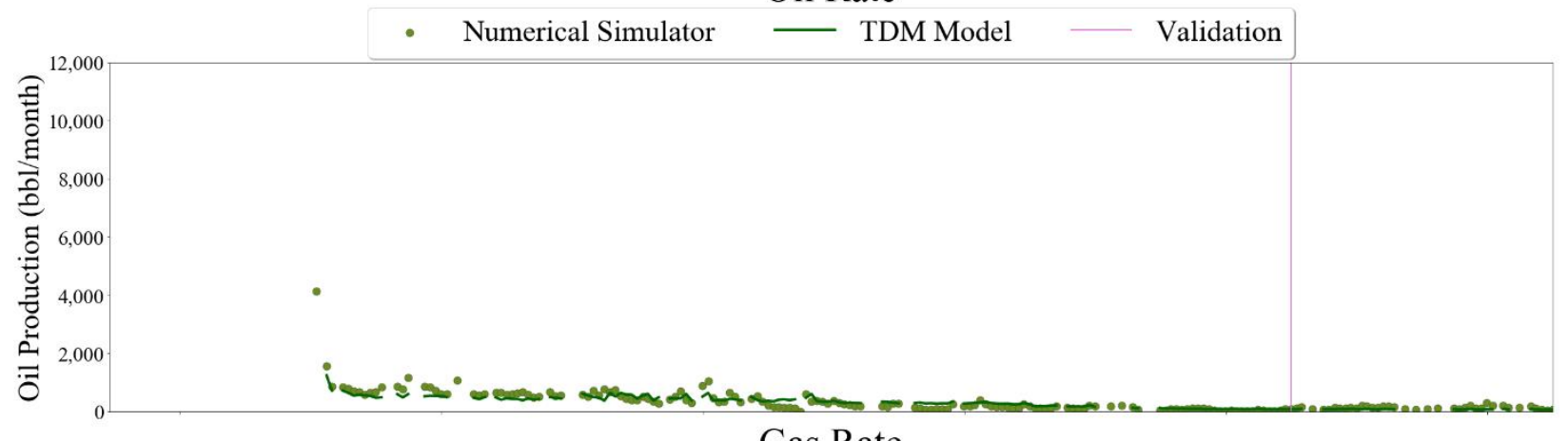

Gas Rate
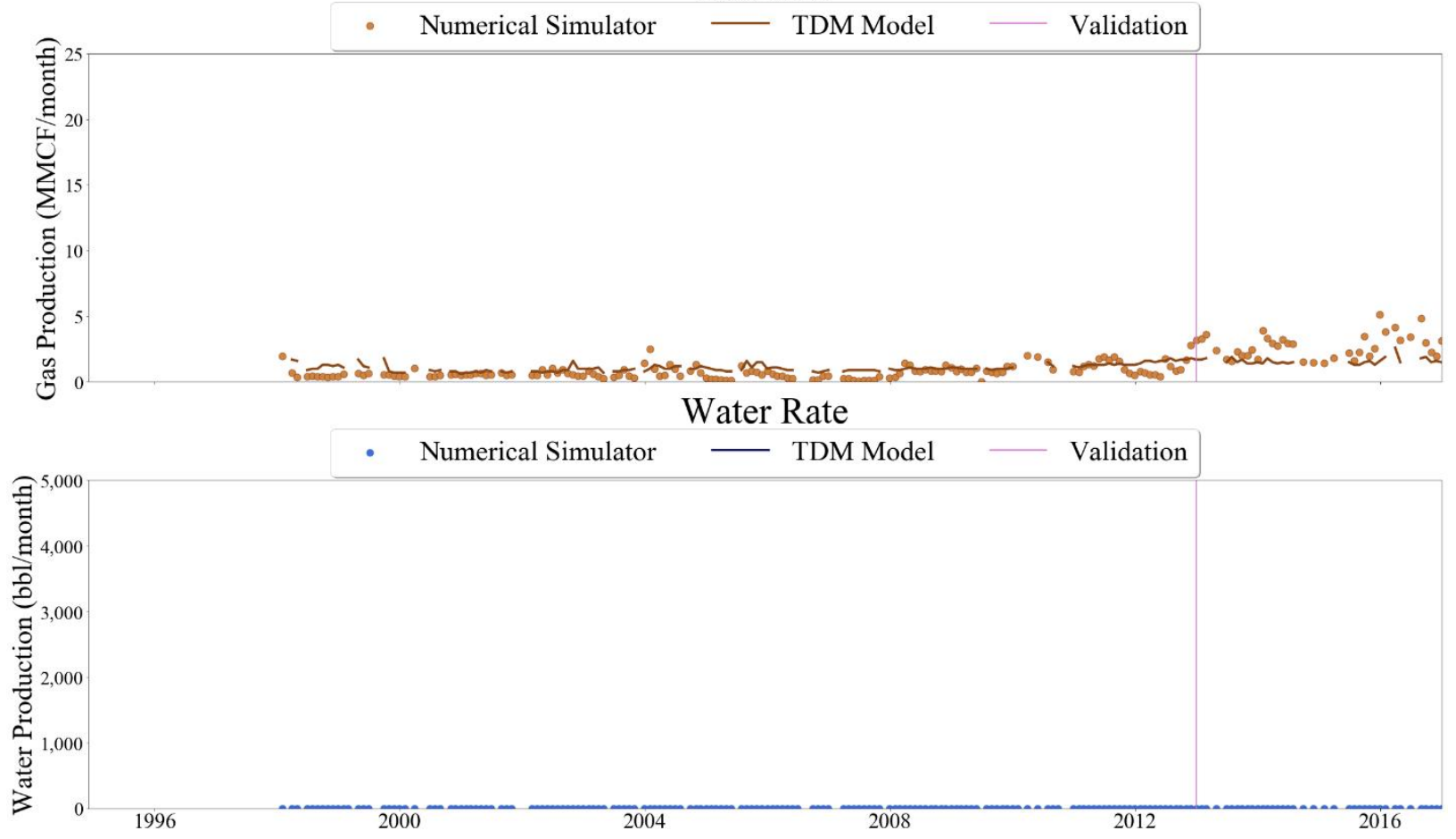

Figure 661 Well-006 oil, gas, and water rate TDM predictions vs actual simulation data plots for 4 Year Blind Validation TDM 

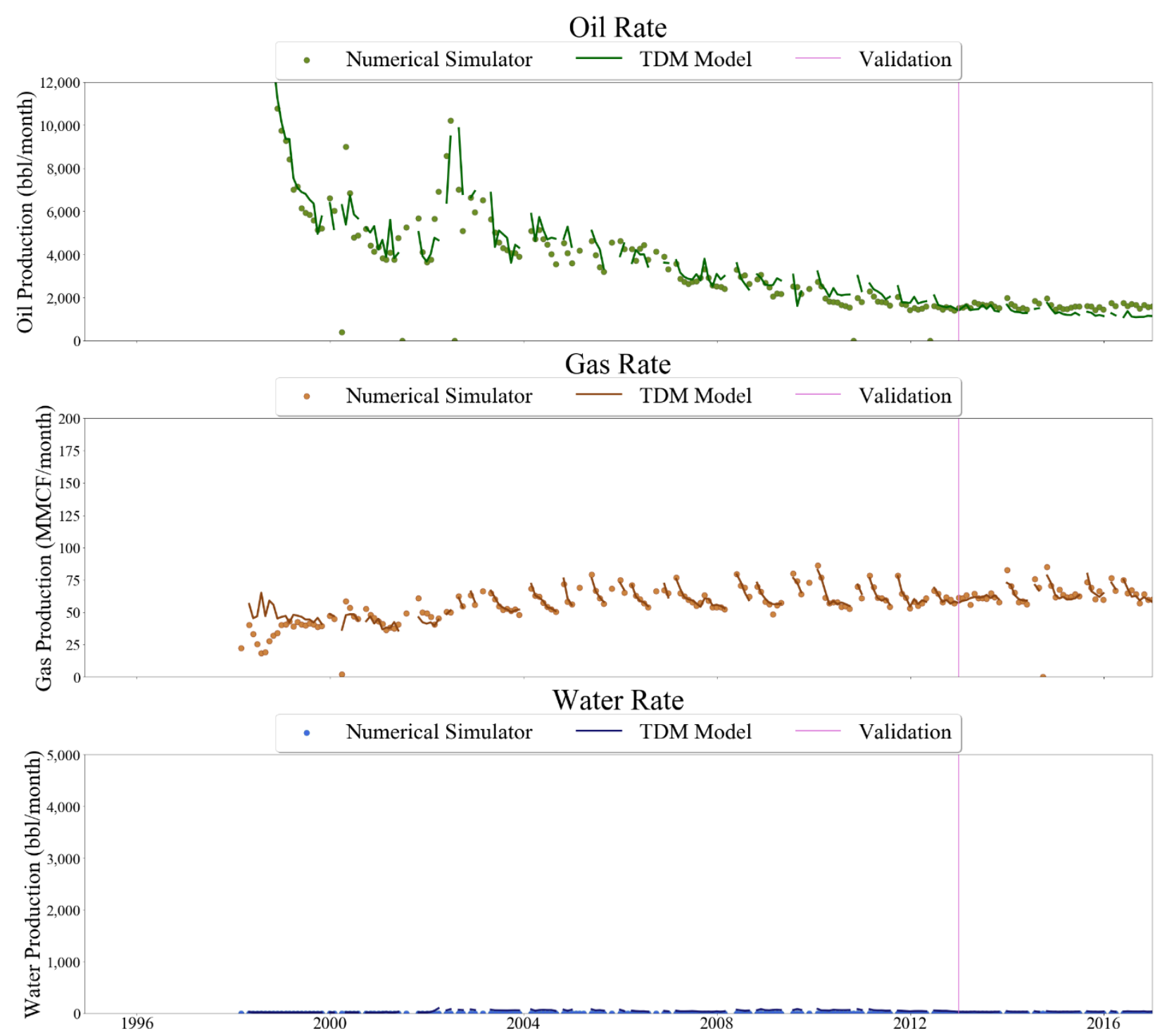

Figure 662 Well-007 oil, gas, and water rate TDM predictions vs actual simulation data plots for 4 Year Blind Validation TDM 


\section{Oil Rate}
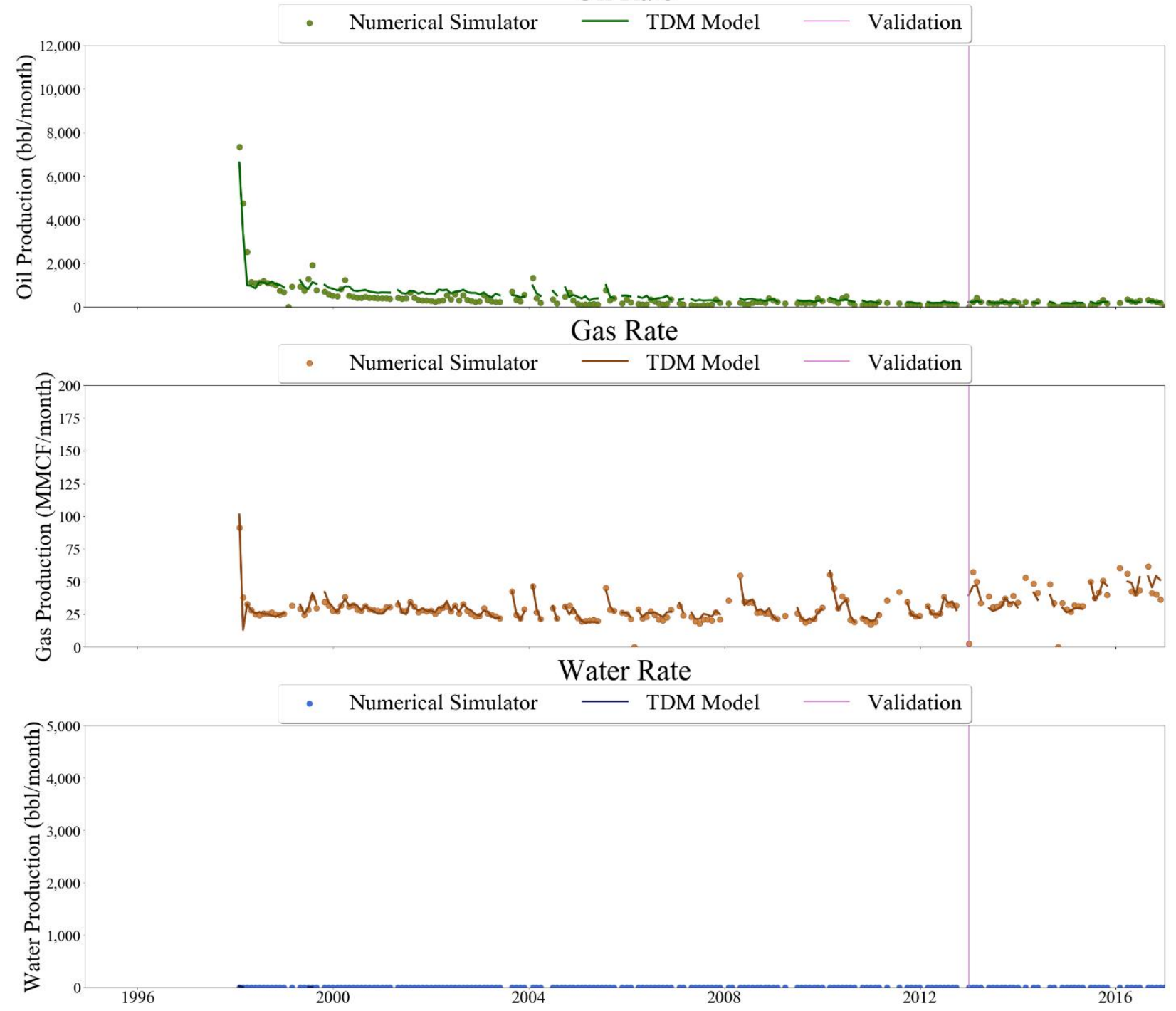

Figure 663 Well-008 oil, gas, and water rate TDM predictions vs actual simulation data plots for 4 Year Blind Validation TDM 

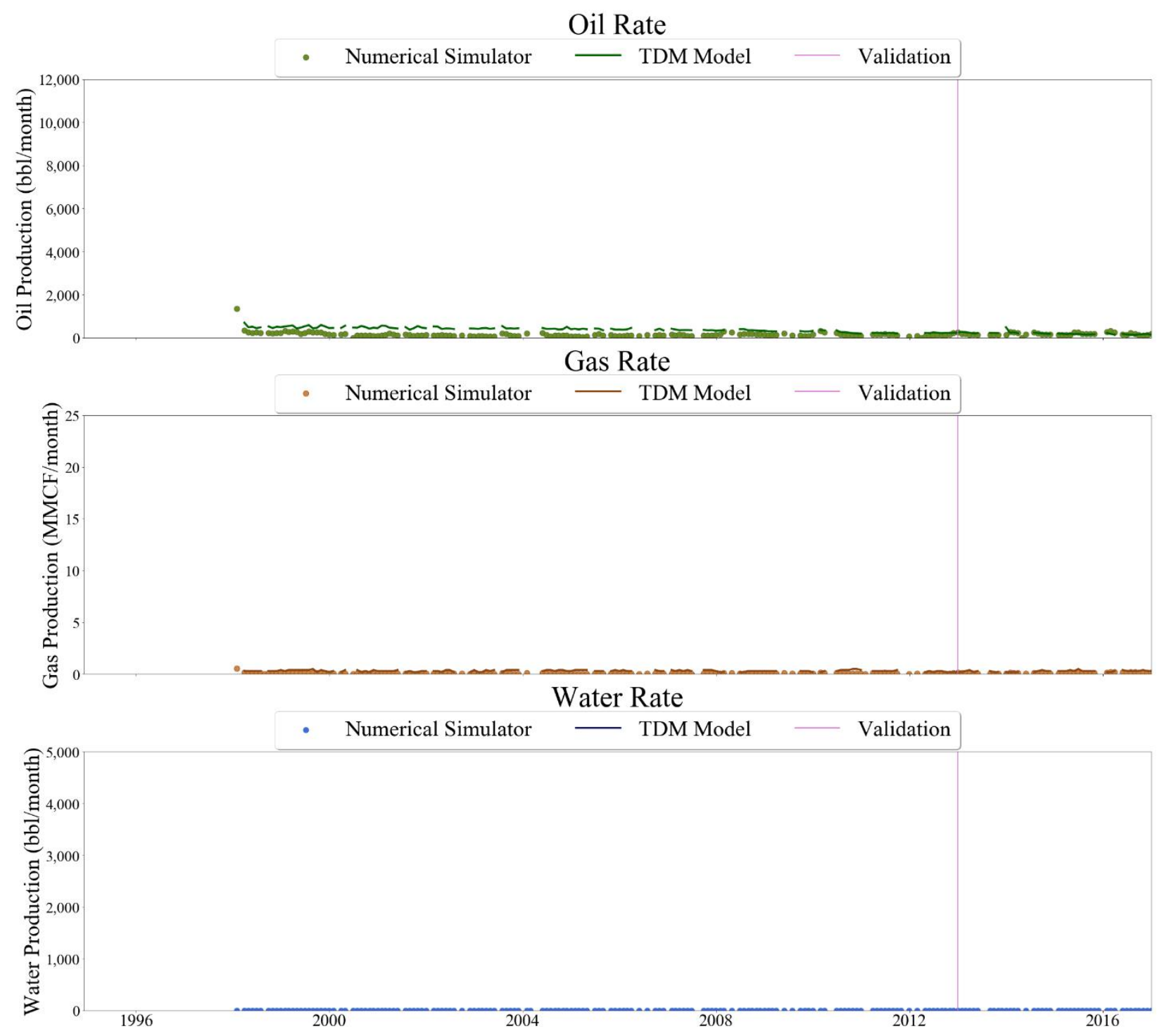

Figure 664 Well-009 oil, gas, and water rate TDM predictions vs actual simulation data plots for 4 Year Blind Validation TDM 


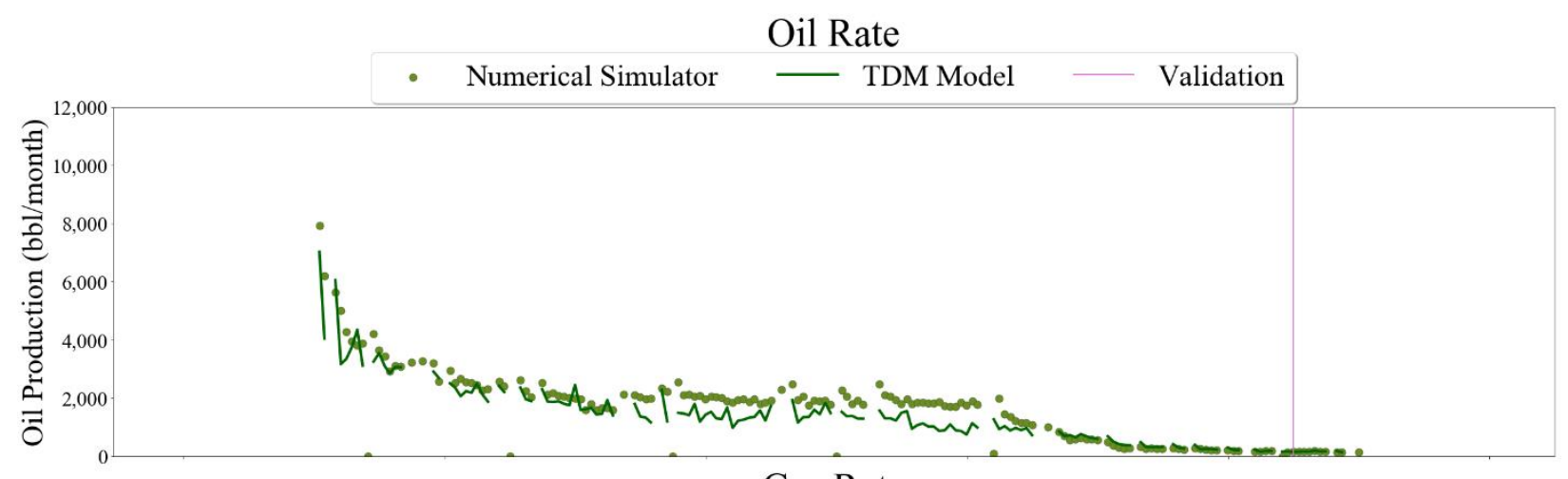

\section{Gas Rate}

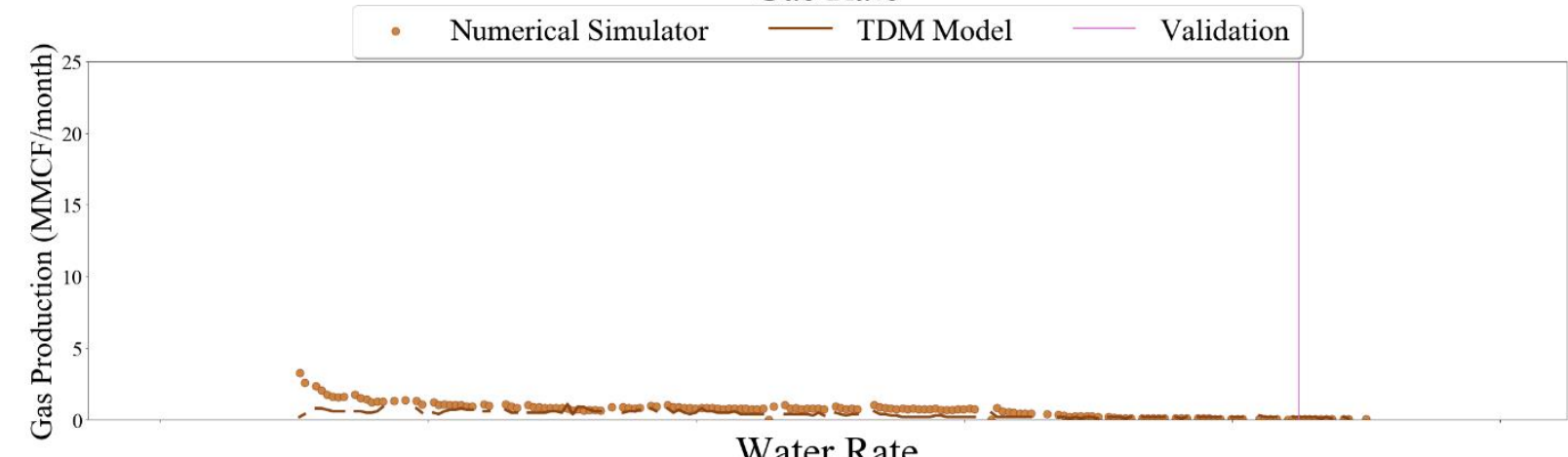

Water Rate

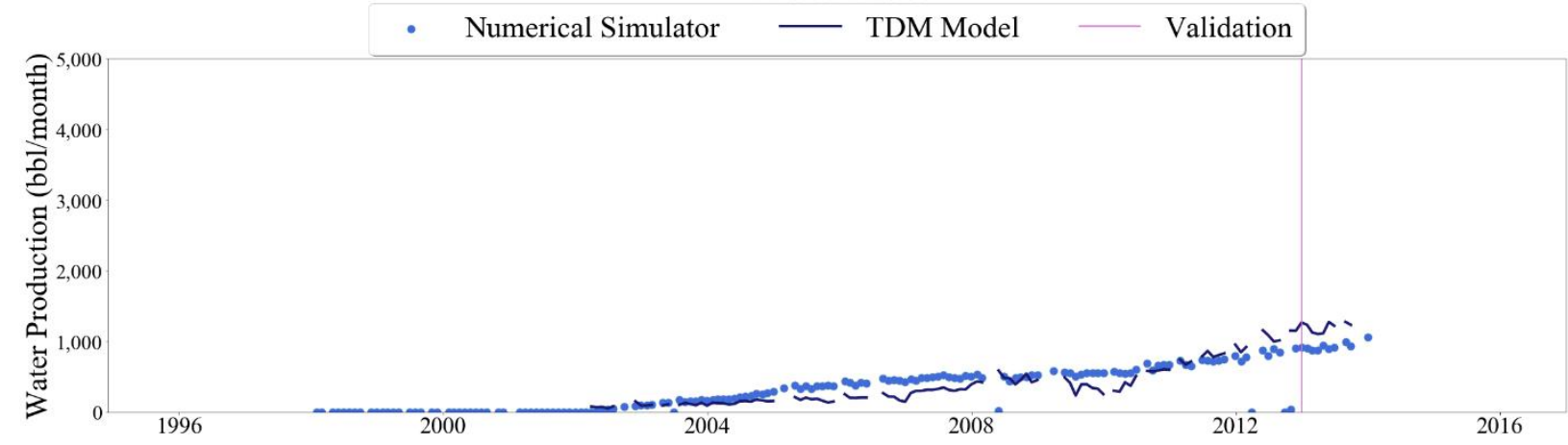

Figure 665 Well-010 oil, gas, and water rate TDM predictions vs actual simulation data plots for 4 Year Blind Validation TDM 


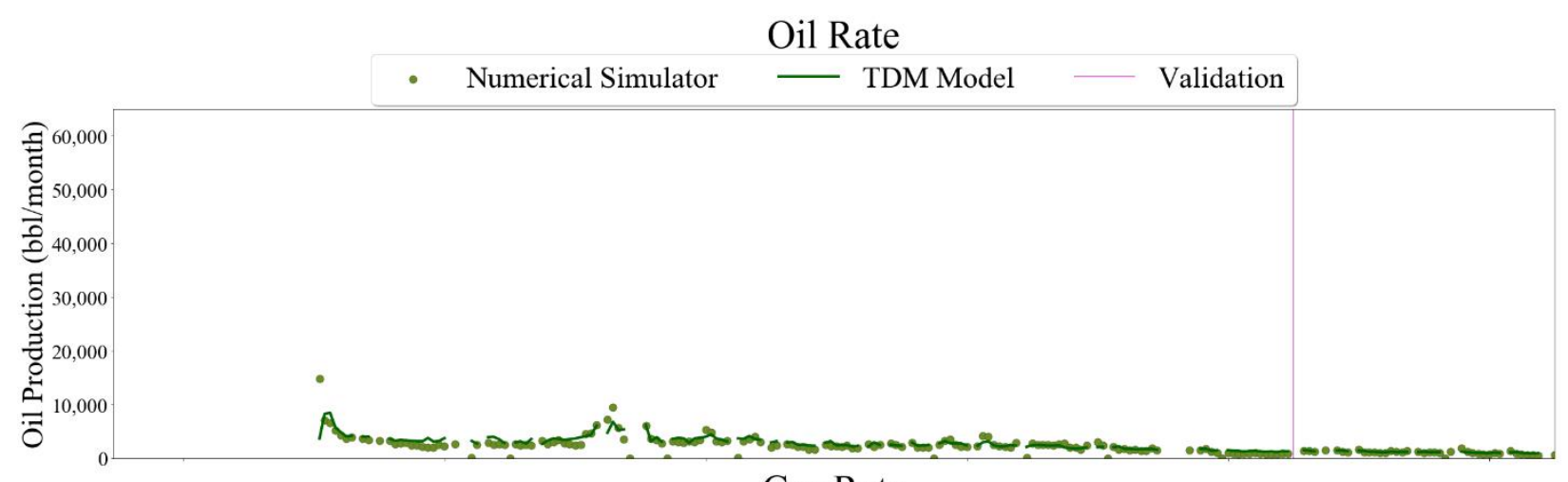

Gas Rate

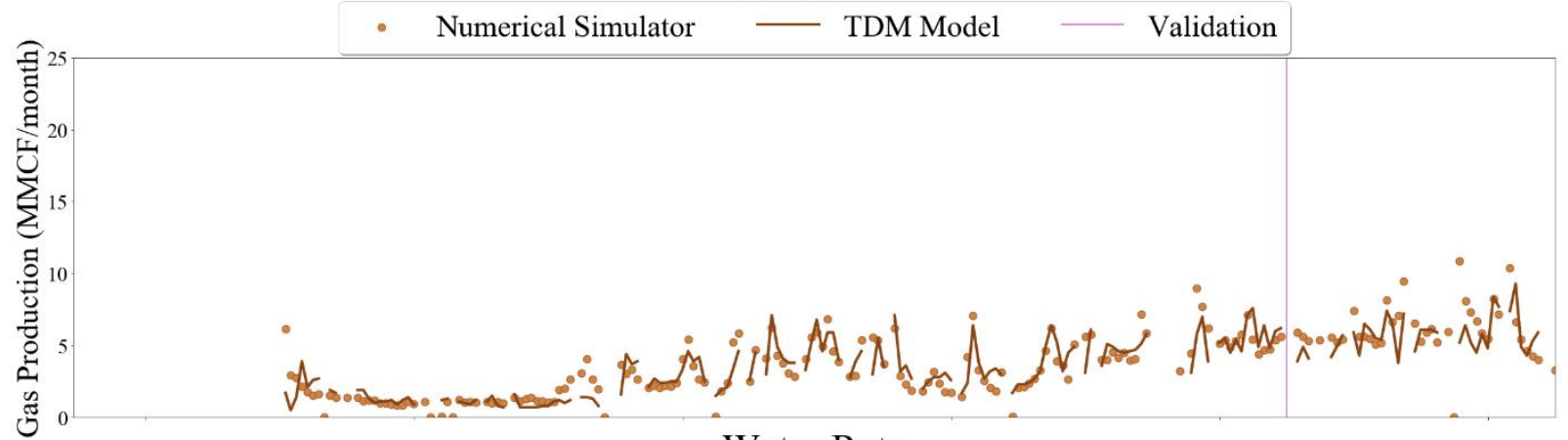

Water Rate

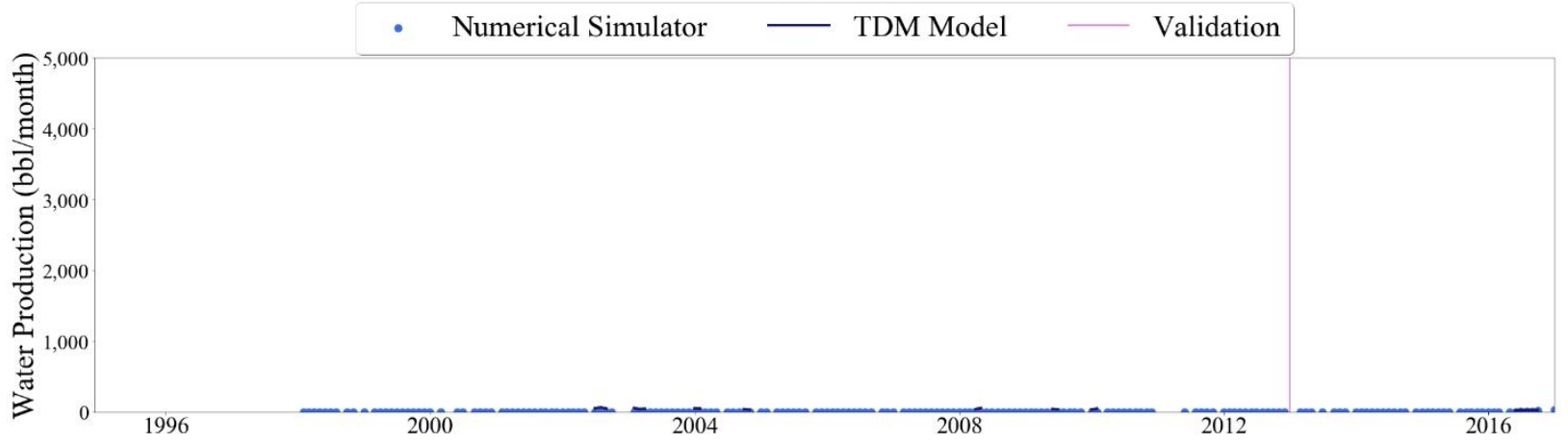

Figure 666 Well-011 oil, gas, and water rate TDM predictions vs actual simulation data plots for 4 Year Blind Validation TDM 


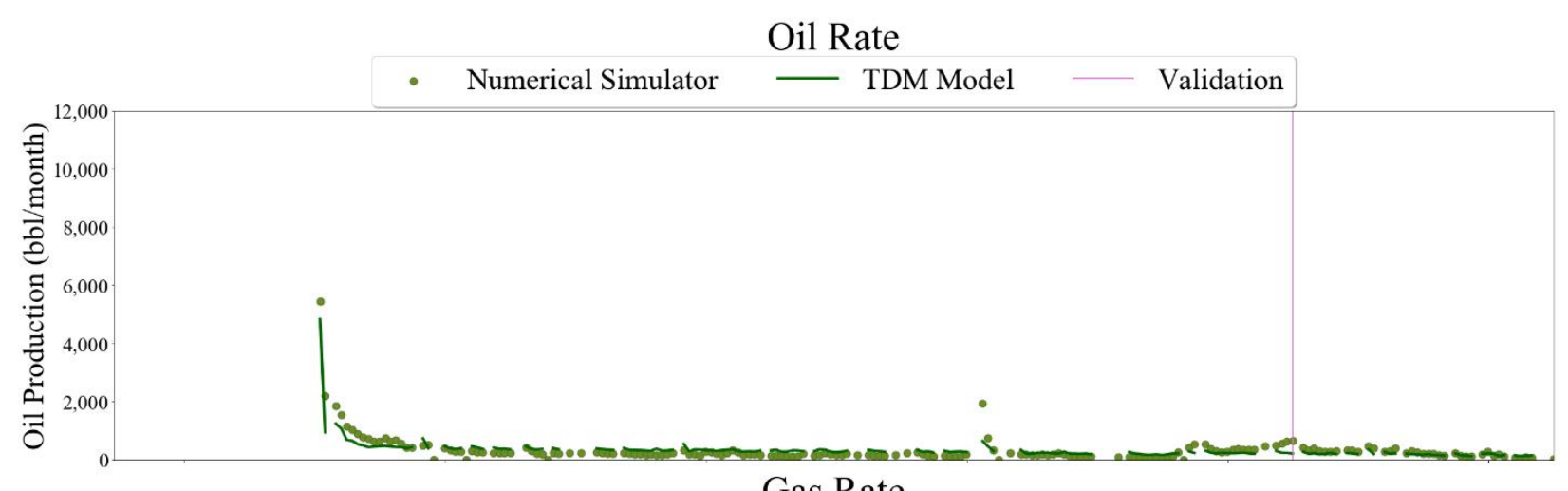

Gas Rate

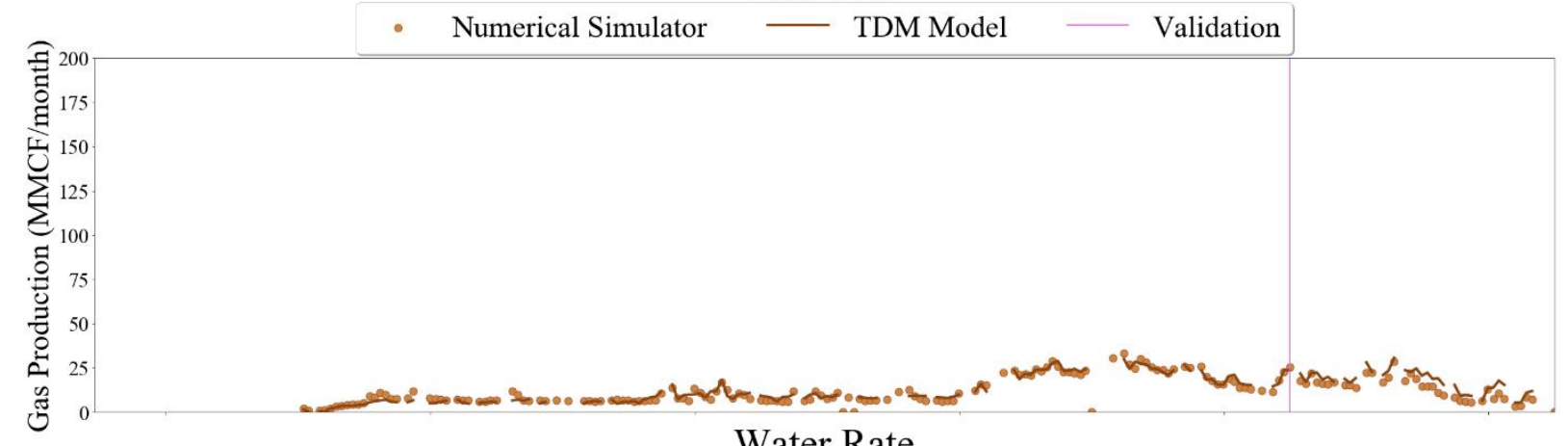

Water Rate

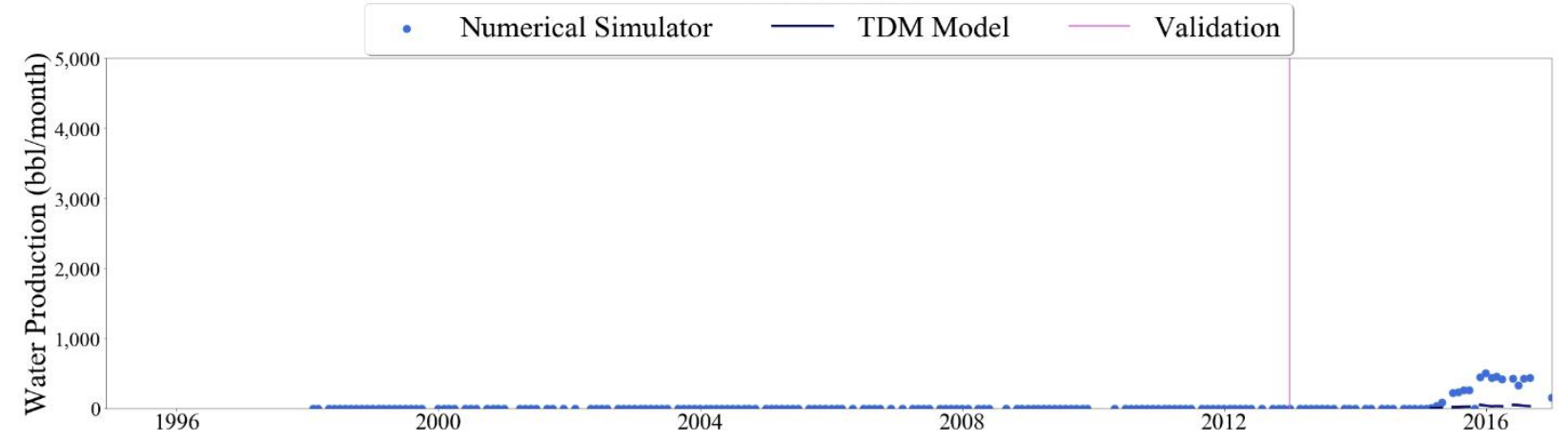

Figure 667 Well-012 oil, gas, and water rate TDM predictions vs actual simulation data plots for 4 Year Blind Validation TDM 

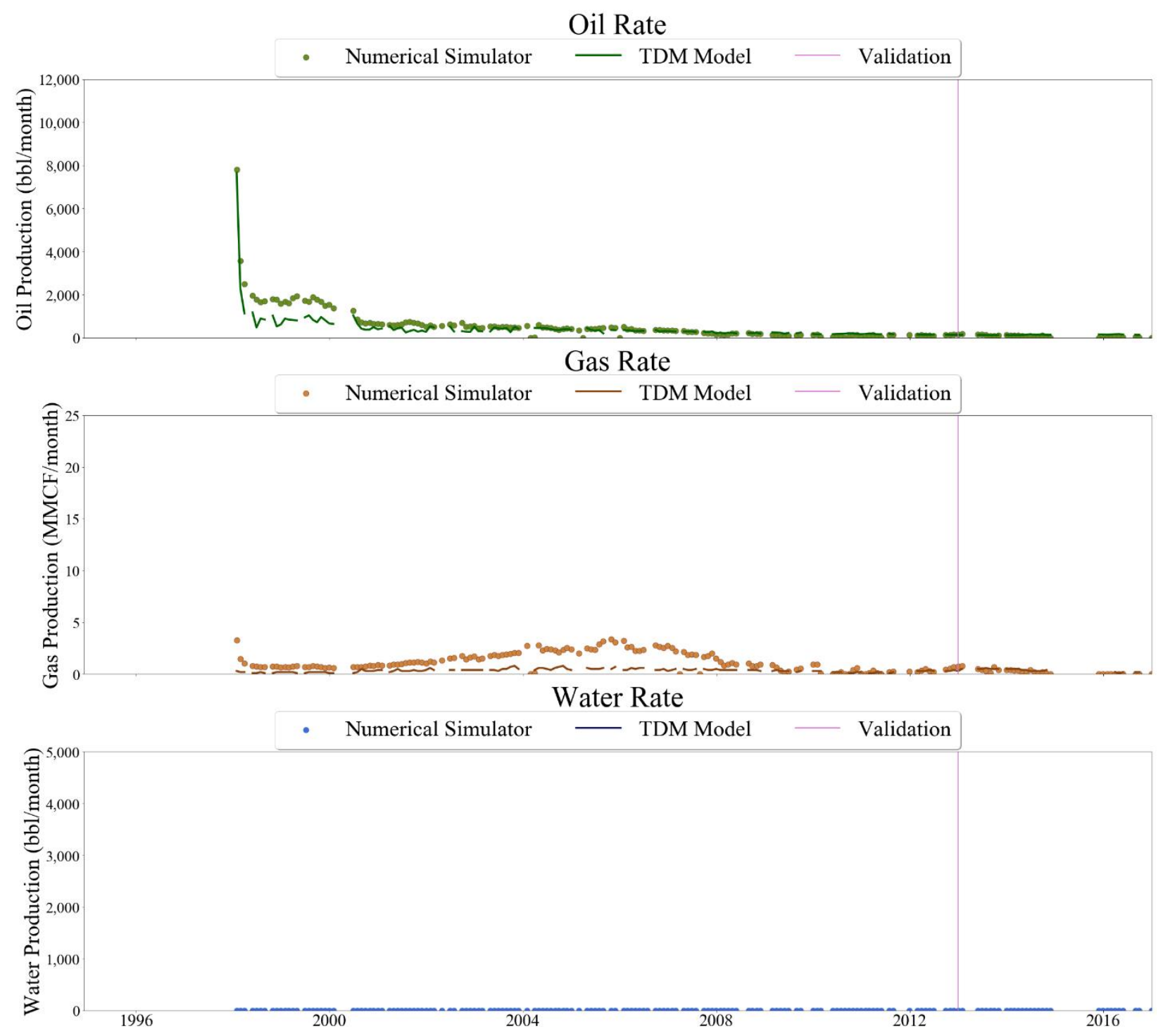

Figure 668 Well-013 oil, gas, and water rate TDM predictions vs actual simulation data plots for 4 Year Blind Validation TDM 


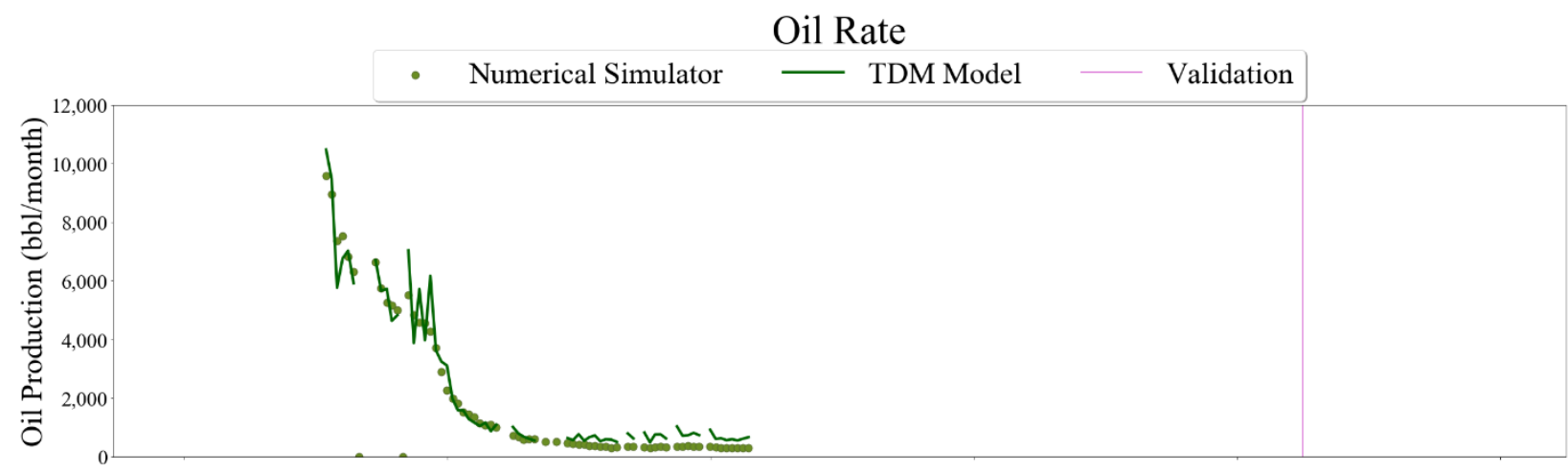

\section{Gas Rate}
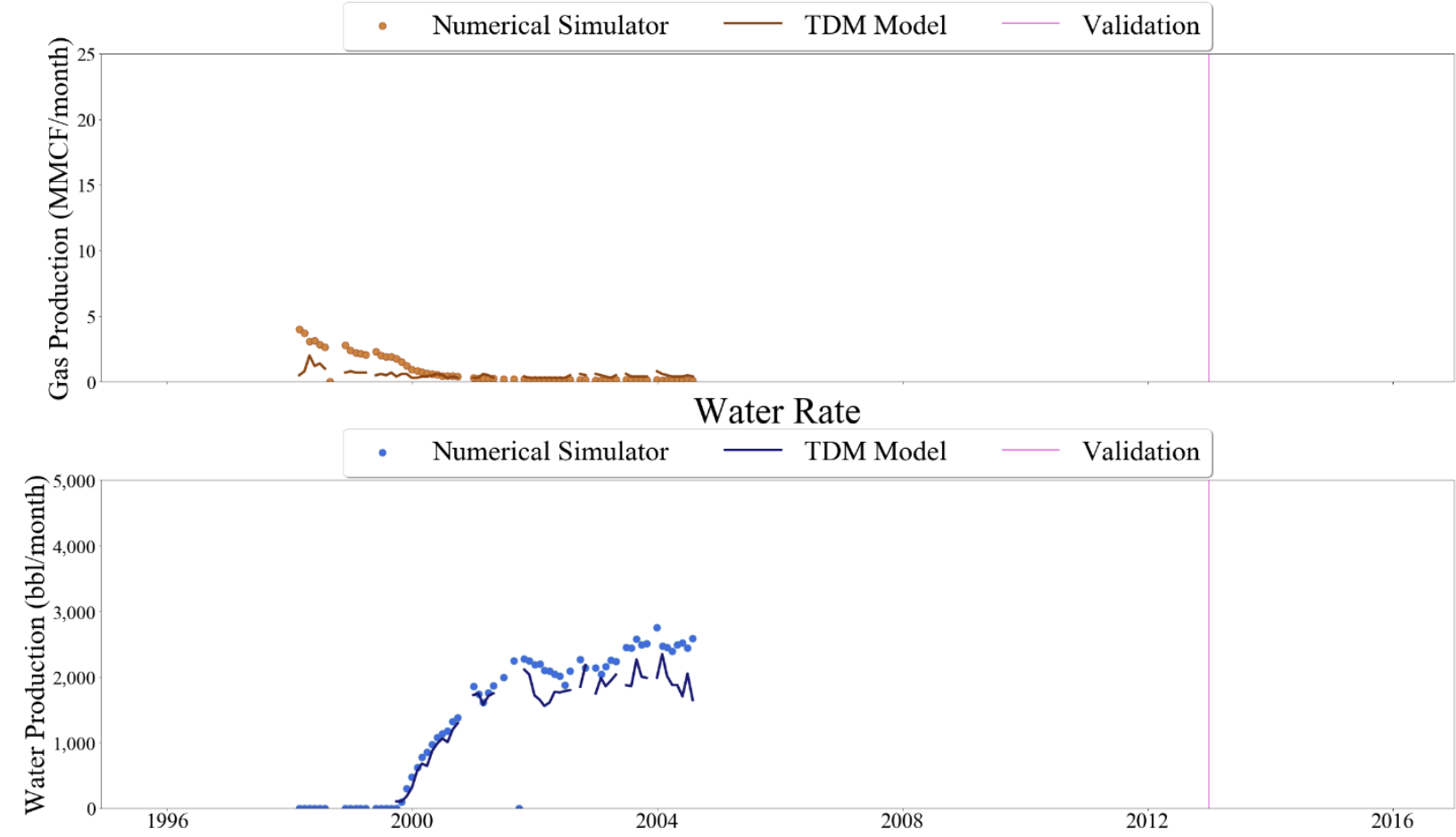

Figure 669 Well-014 oil, gas, and water rate TDM predictions vs actual simulation data plots for 4 Year Blind Validation TDM 

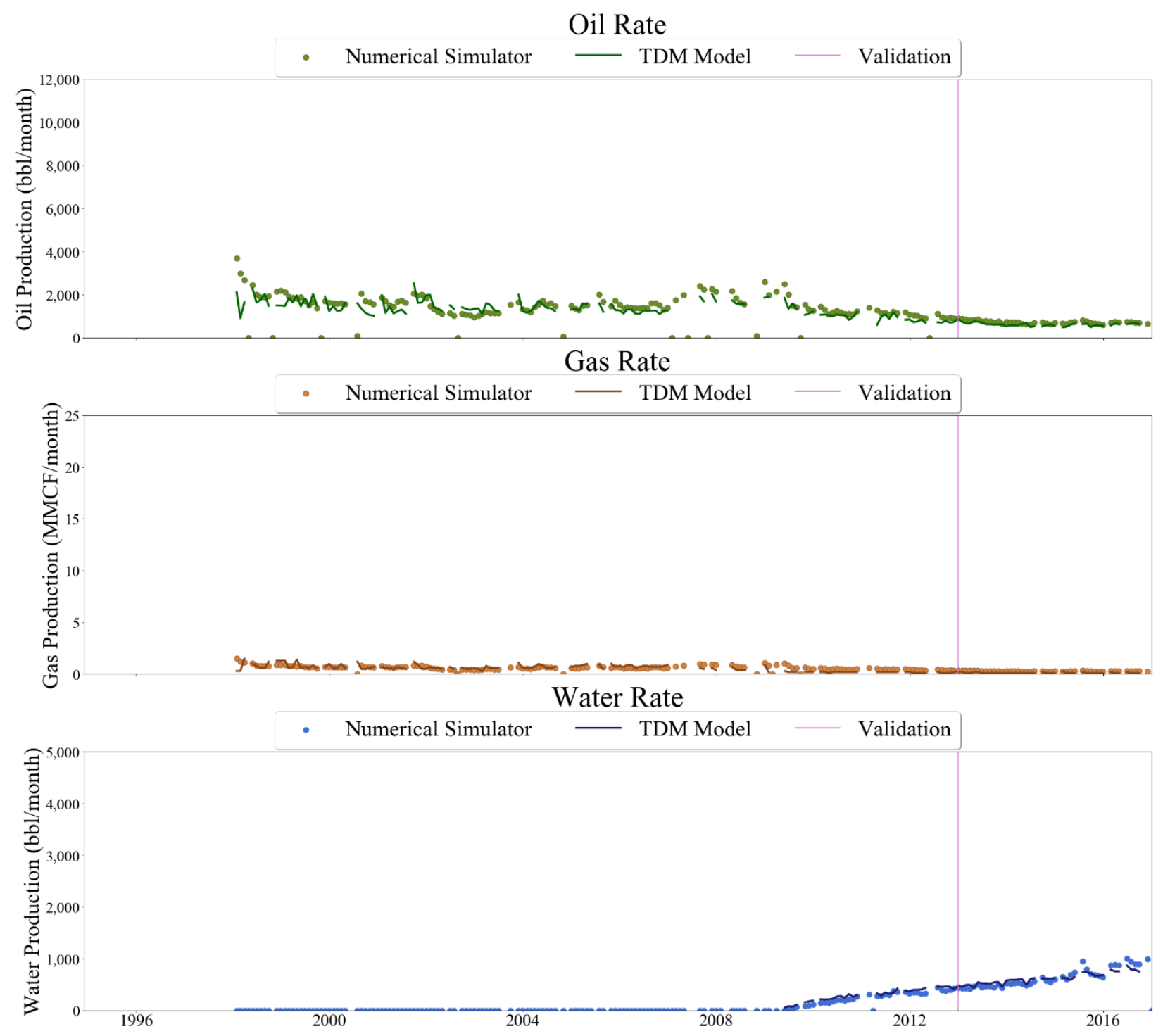

Figure 670 Well-015 oil, gas, and water rate TDM predictions vs actual simulation data plots for 4 Year Blind Validation TDM 

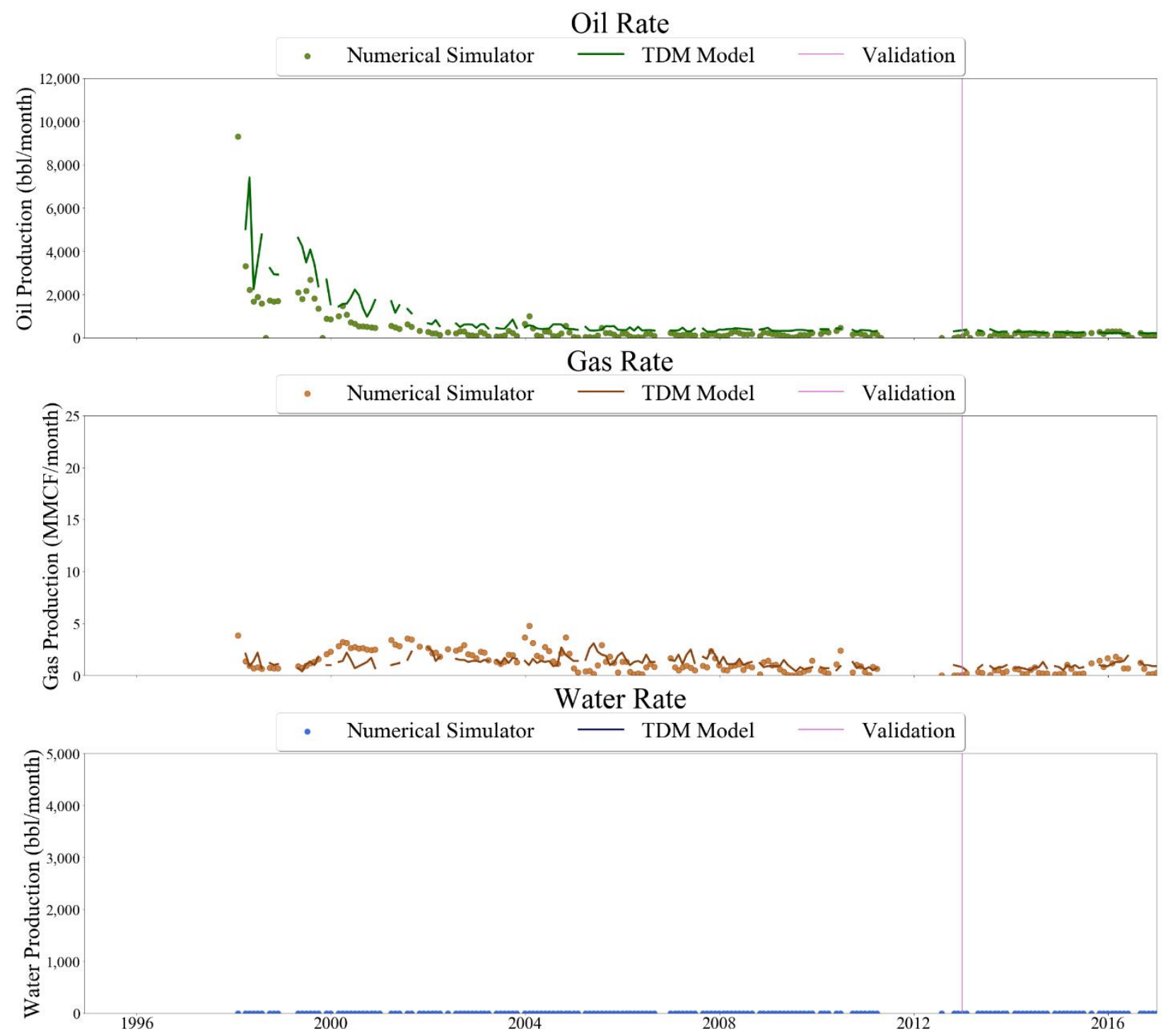

Figure 671 Well-016 oil, gas, and water rate TDM predictions vs actual simulation data plots for 4 Year Blind Validation TDM 

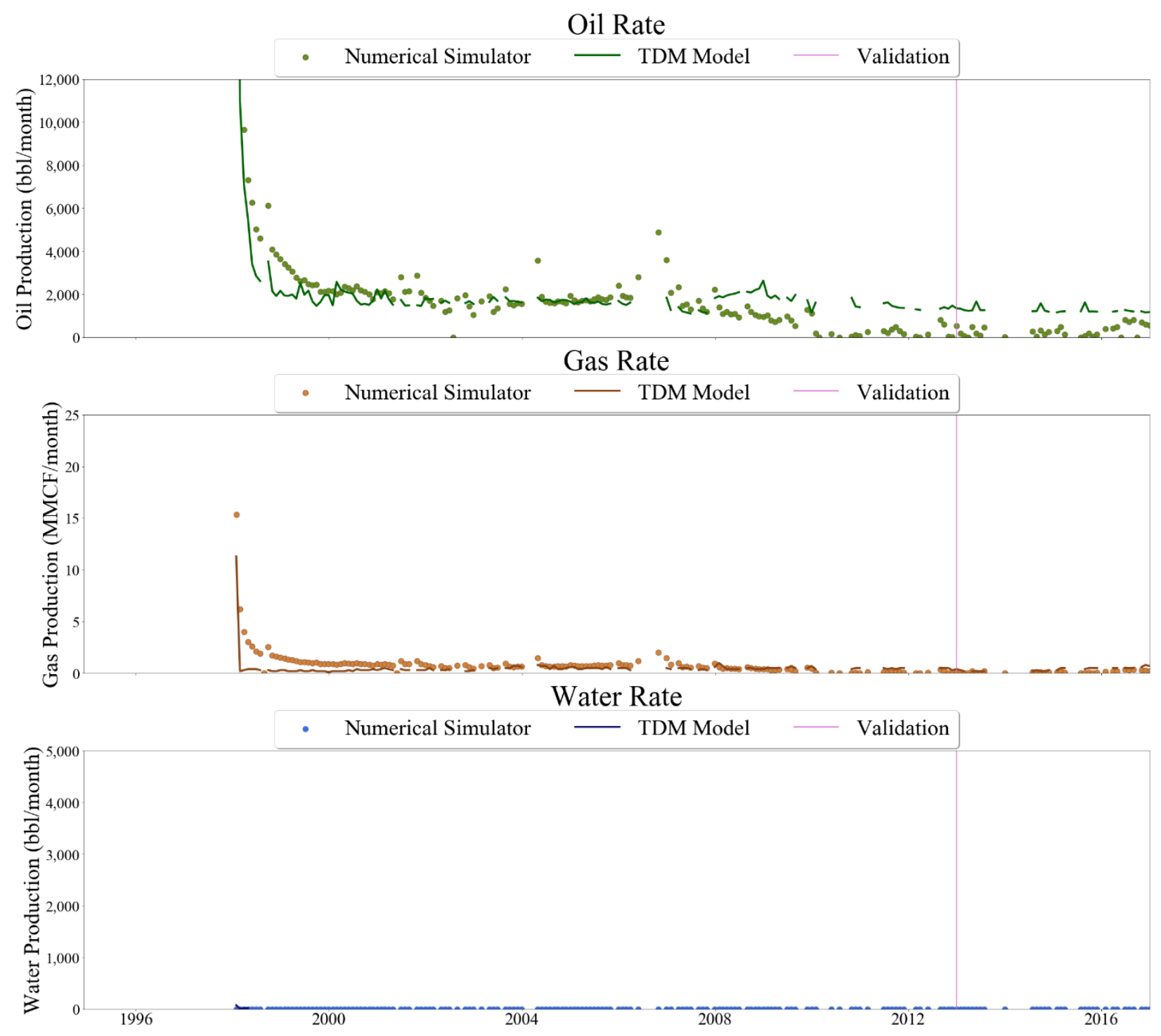

Figure 672 Well-017 oil, gas, and water rate TDM predictions vs actual simulation data plots for 4 Year Blind Validation TDM 

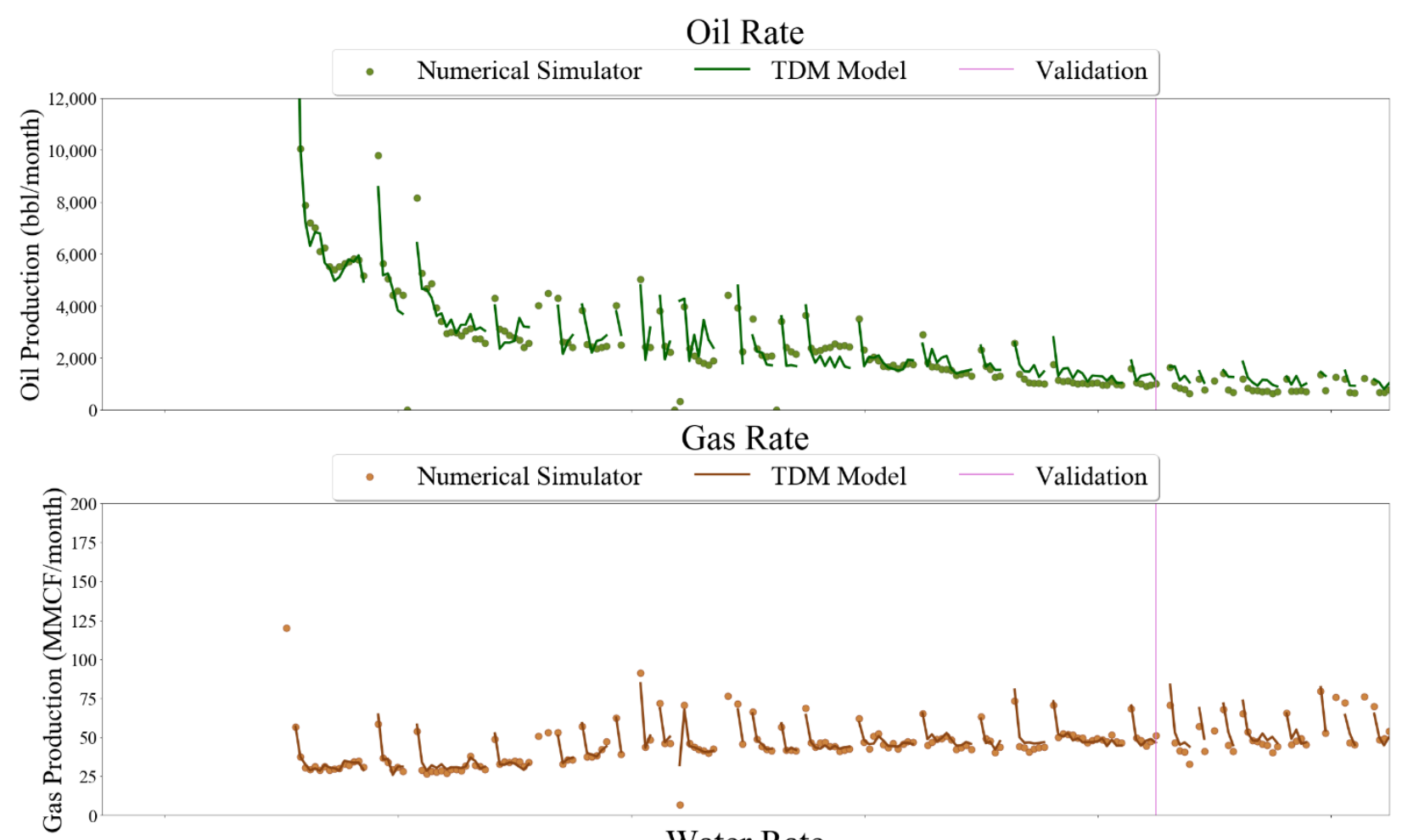

Water Rate

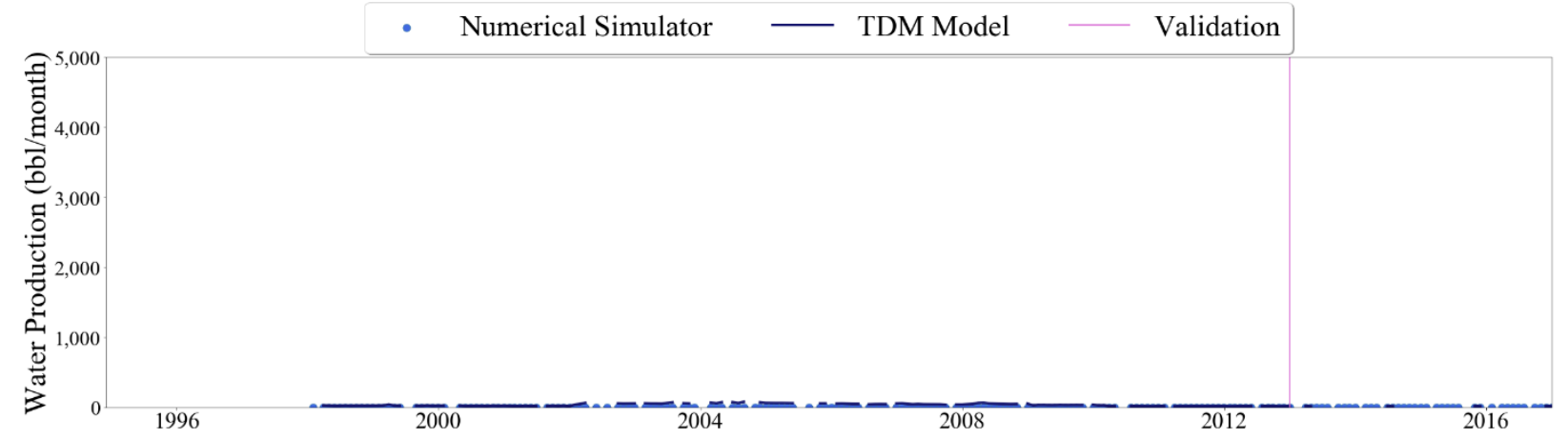

Figure 673 Well-018 oil, gas, and water rate TDM predictions vs actual simulation data plots for 4 Year Blind Validation TDM 

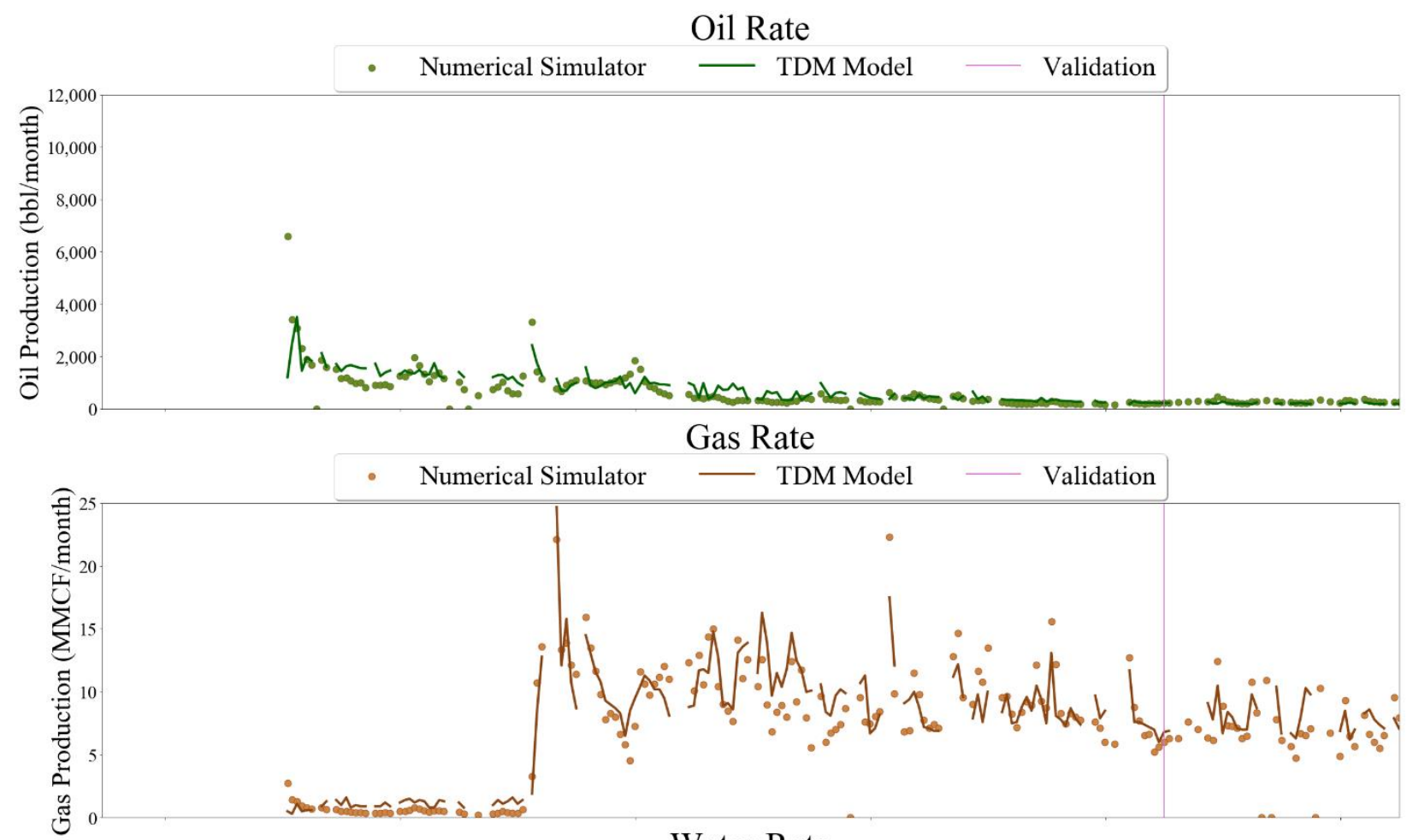

Water Rate

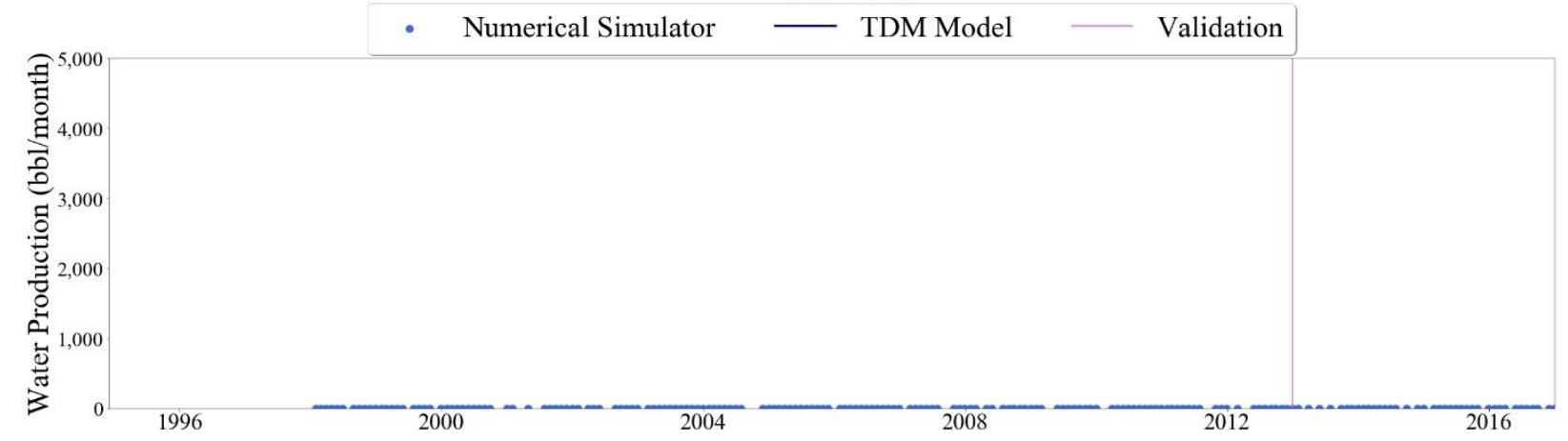

Figure 674 Well-019 oil, gas, and water rate TDM predictions vs actual simulation data plots for 4 Year Blind Validation TDM 


\section{Oil Rate}

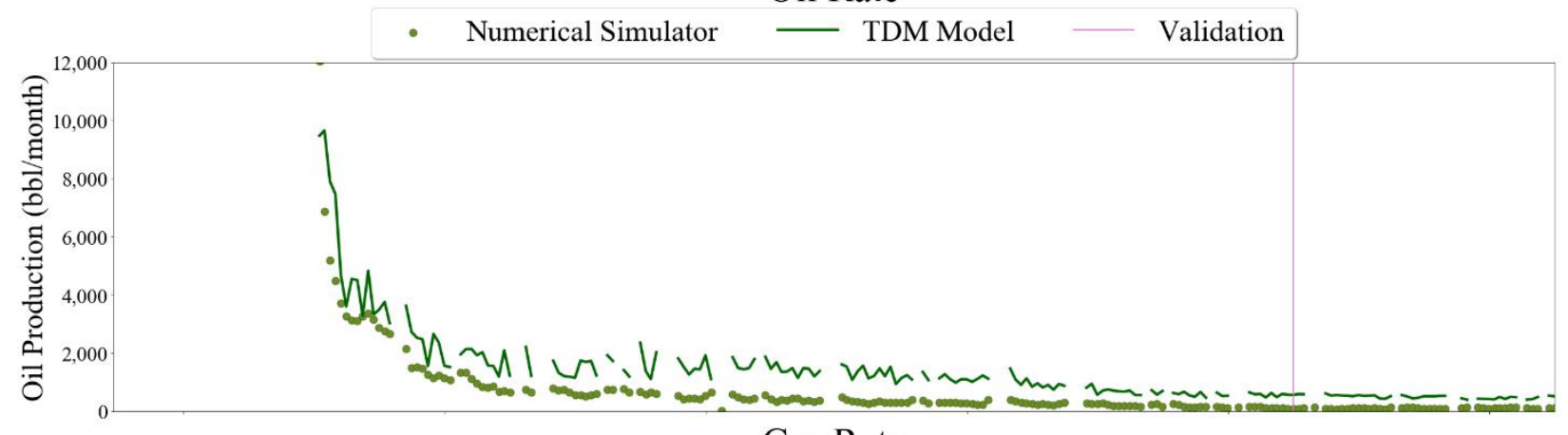

Gas Rate
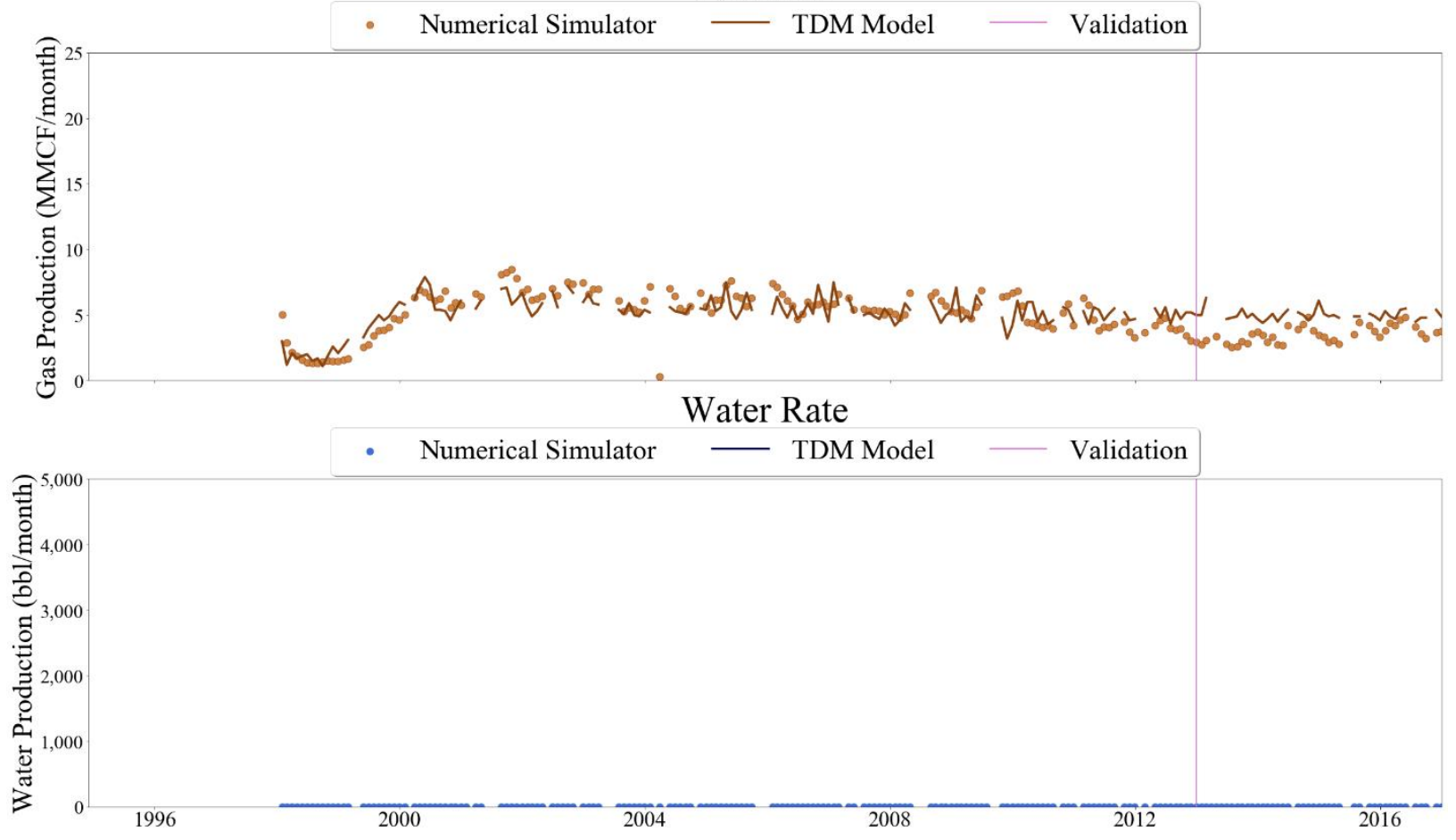

Figure 675 Well-020 oil, gas, and water rate TDM predictions vs actual simulation data plots for 4 Year Blind Validation TDM 

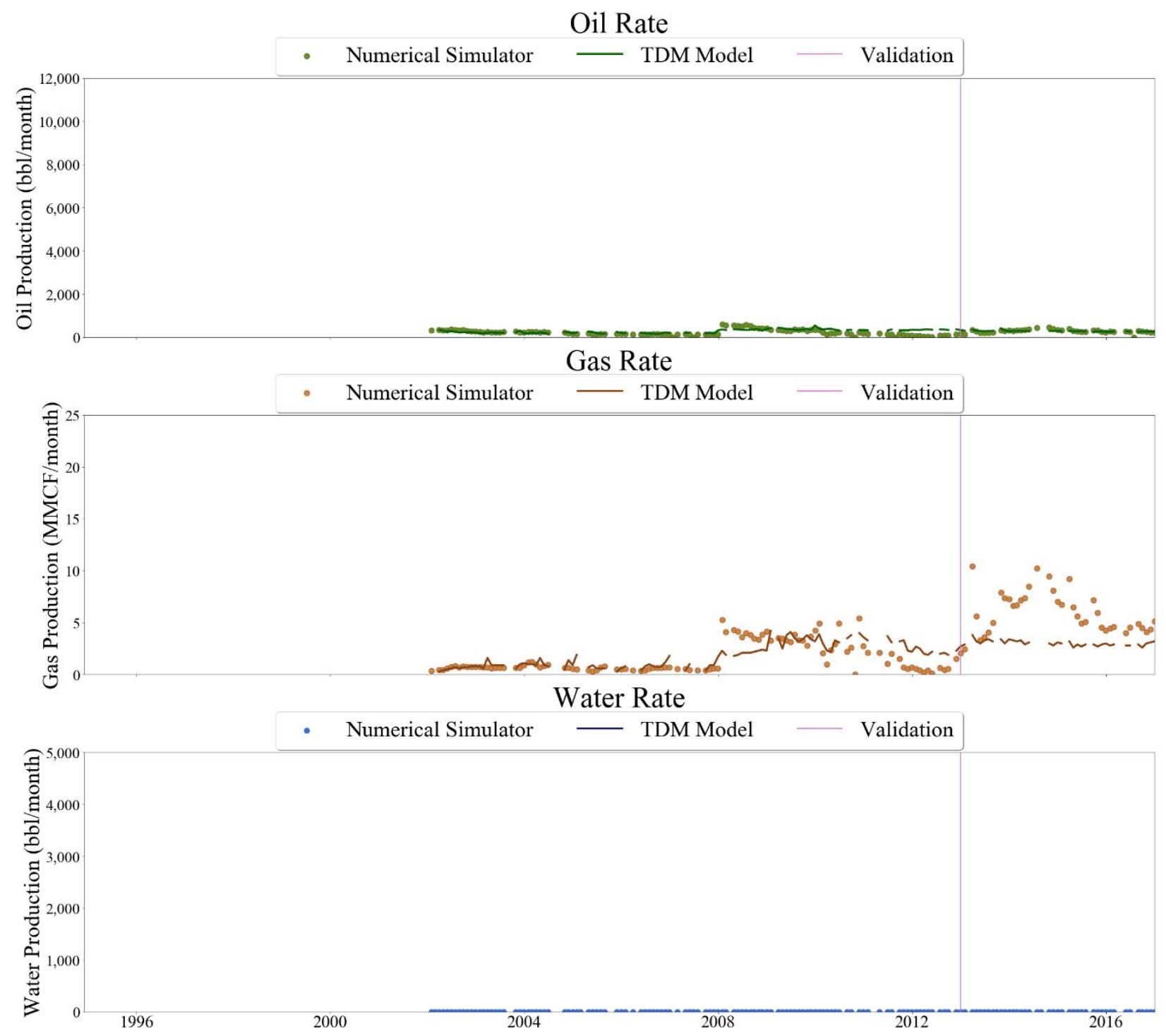

Figure 676 Well-021 oil, gas, and water rate TDM predictions vs actual simulation data plots for 4 Year Blind Validation TDM 


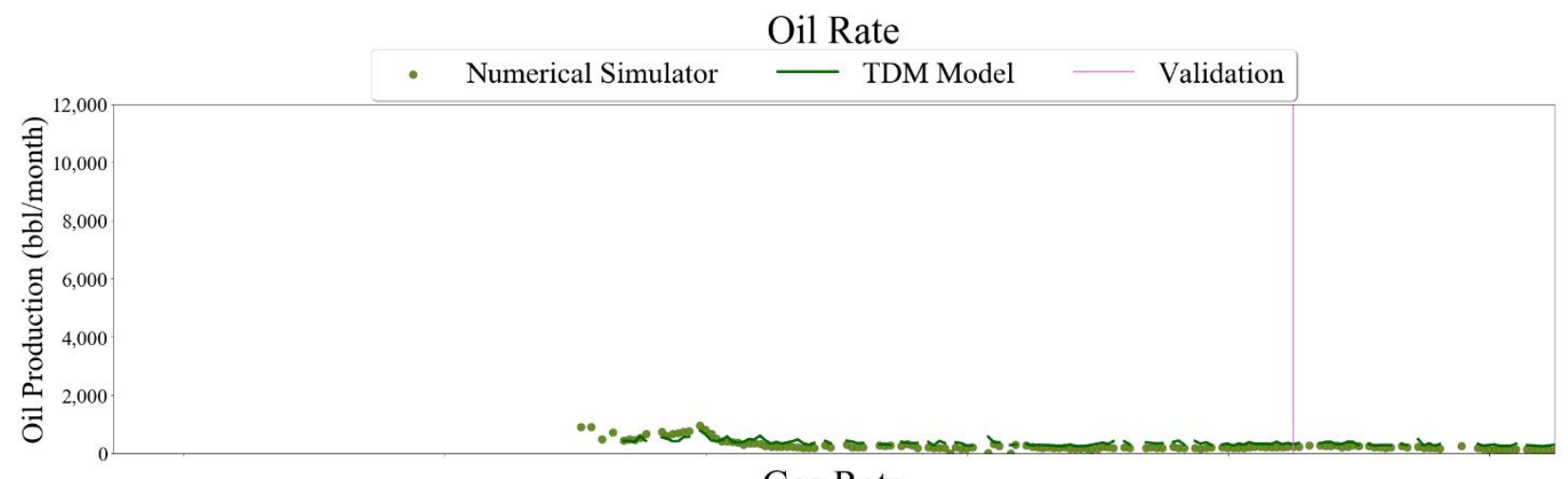

Gas Rate

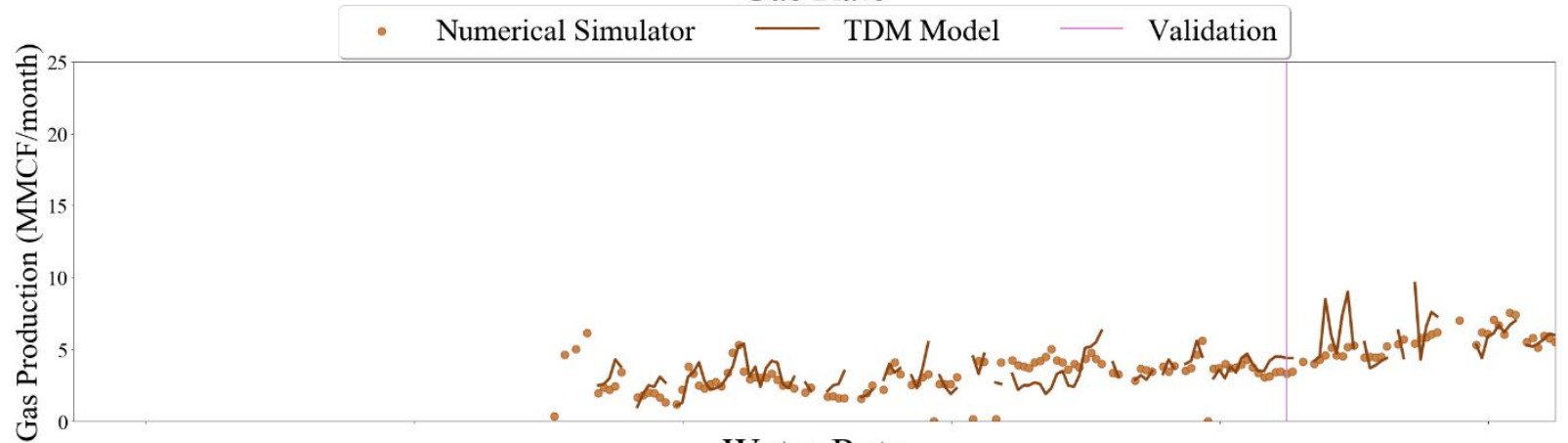

Water Rate

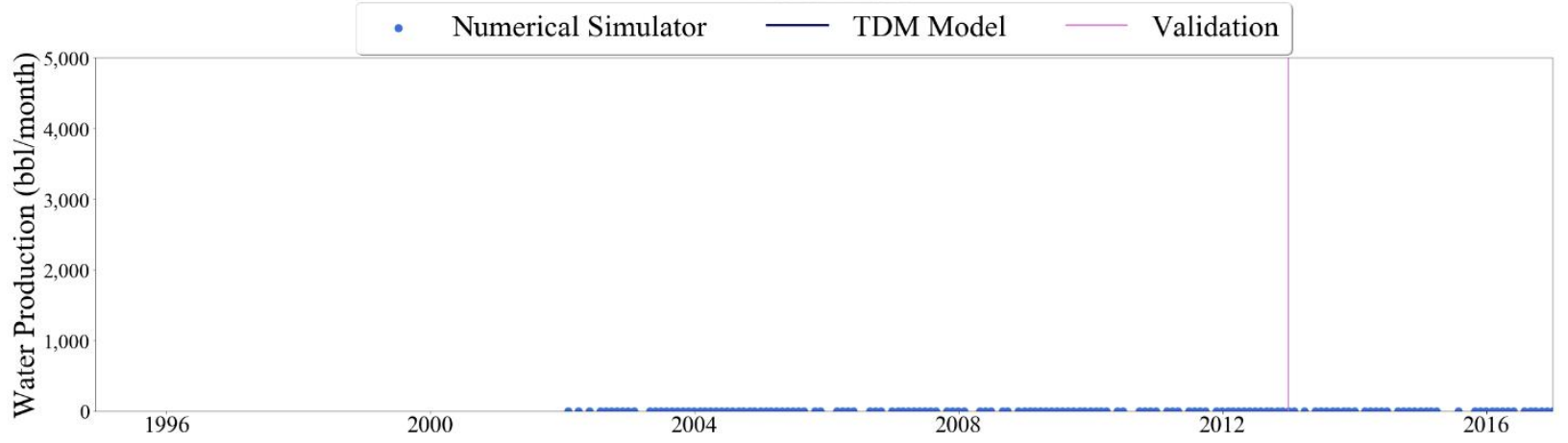

Figure 677 Well-022 oil, gas, and water rate TDM predictions vs actual simulation data plots for 4 Year Blind Validation TDM 


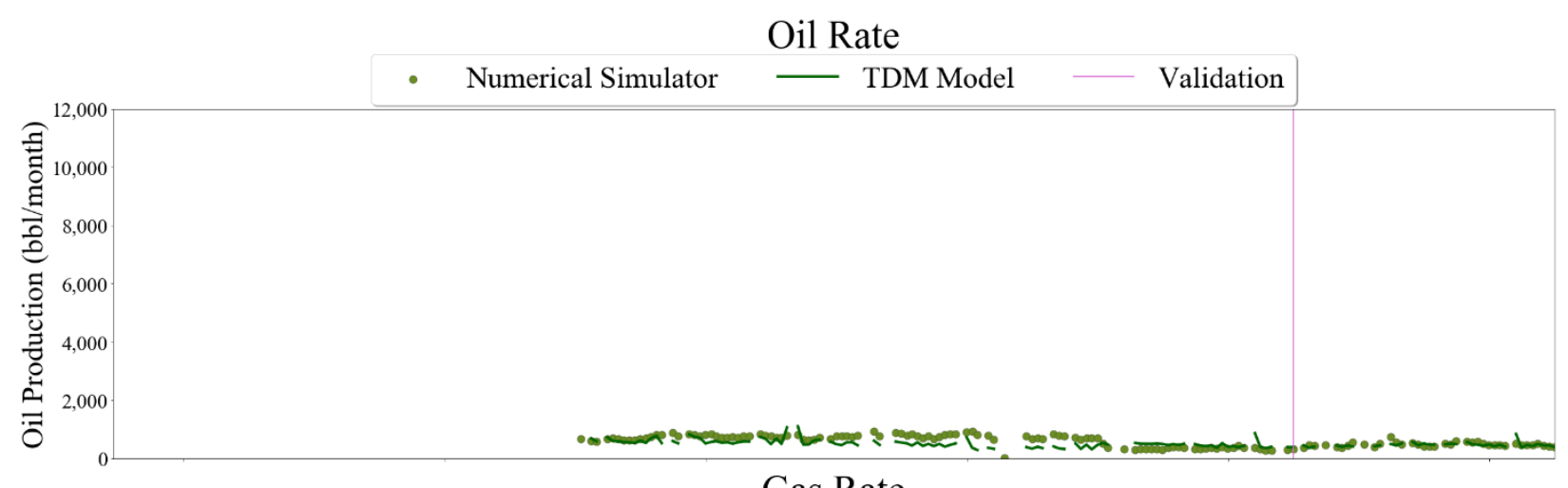

Gas Rate
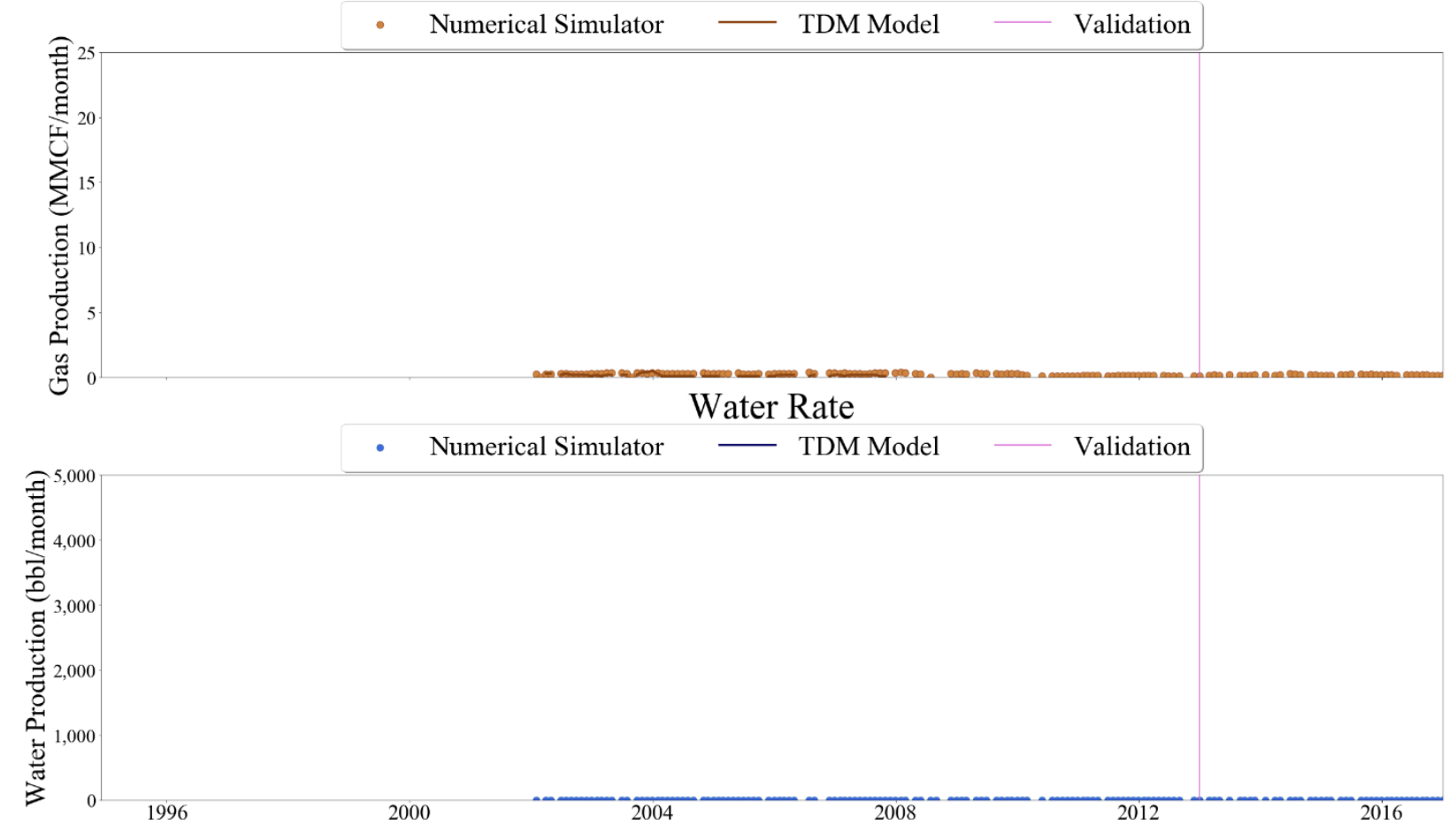

Figure 678 Well-023 oil, gas, aand water rate TDM predictions vs actual simulation data plots for 4 Year Blind Validation TDM 


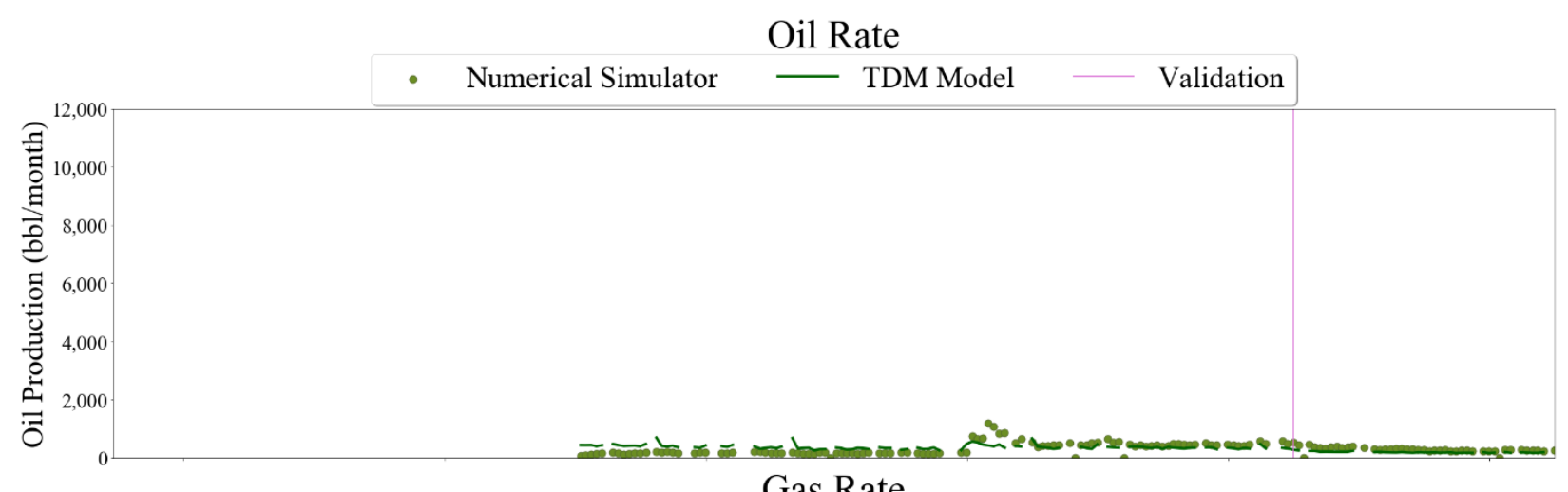

Gas Rate
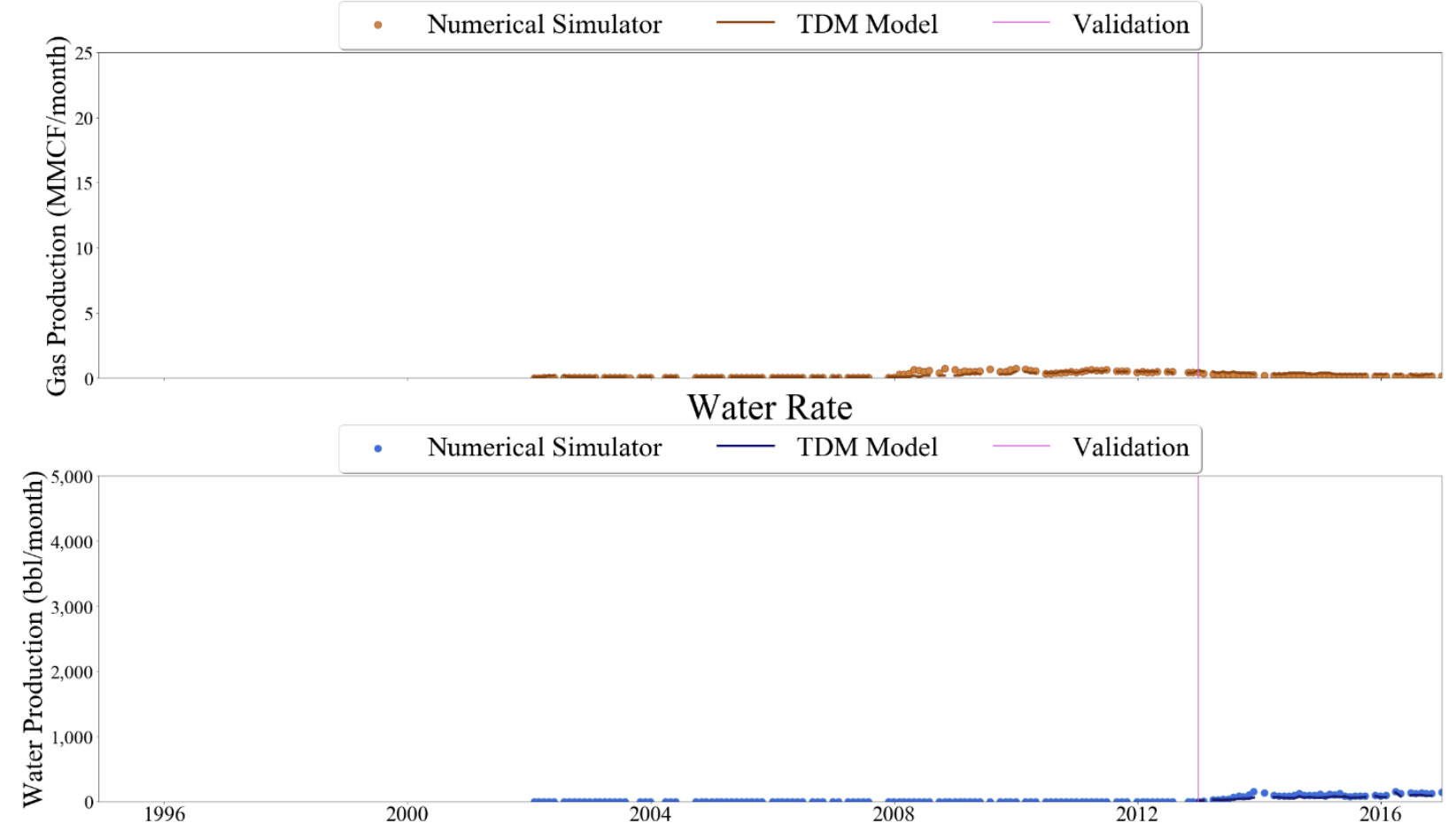

Figure 679 Well-024 oil, gas, and water rate TDM predictions vs actual simulation data plots for 4 Year Blind Validation TDM 

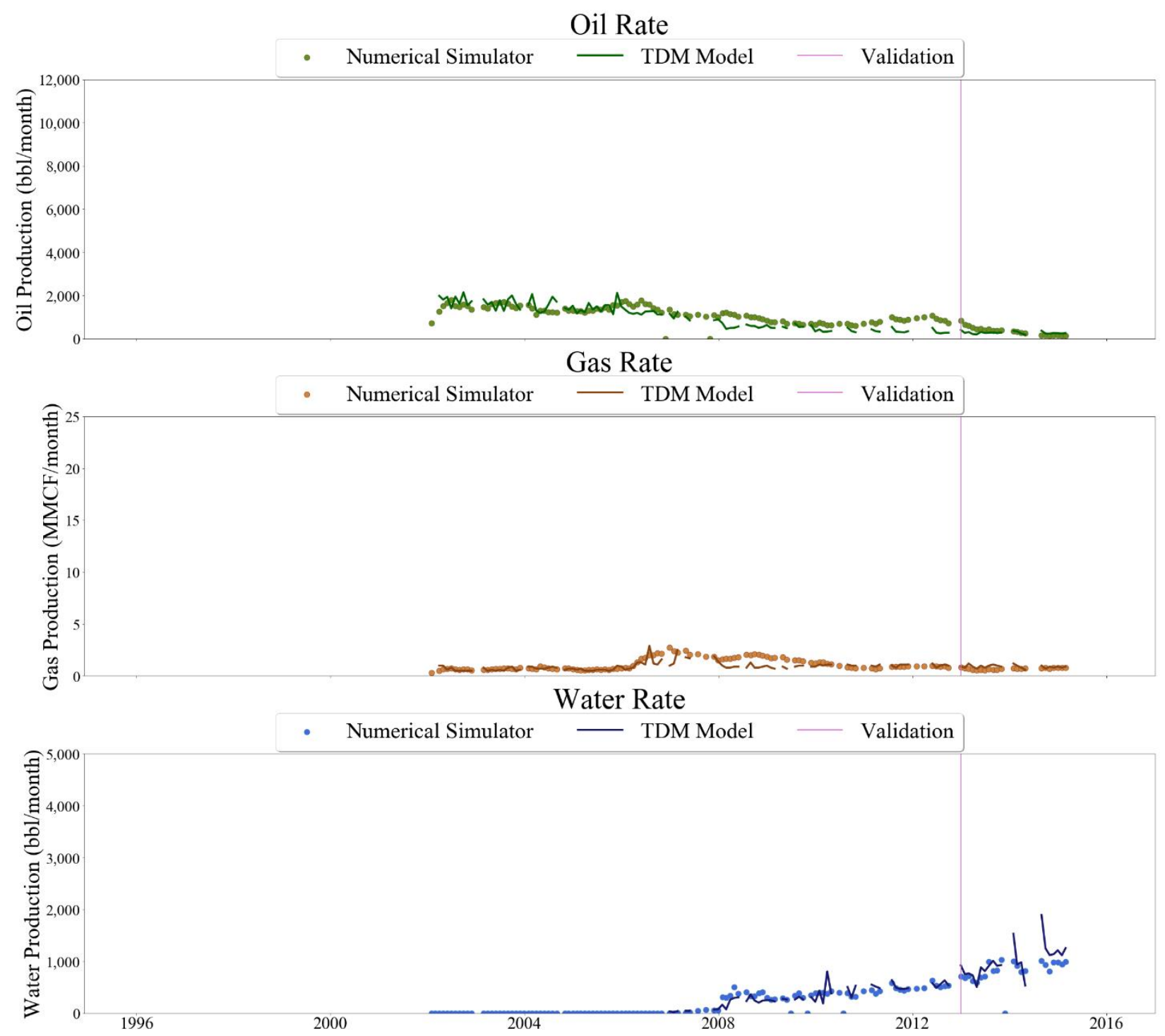

Figure 680 Well-025 oil, gas, and water rate TDM predictions vs actual simulation data plots for 4 Year Blind Validation TDM 


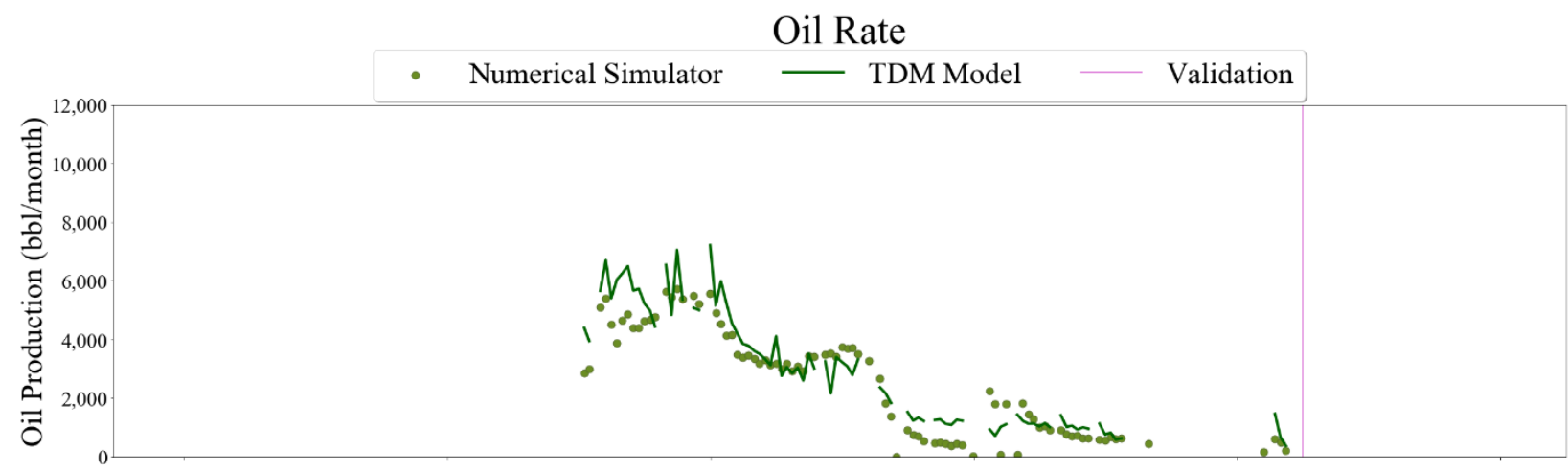

\section{Gas Rate}
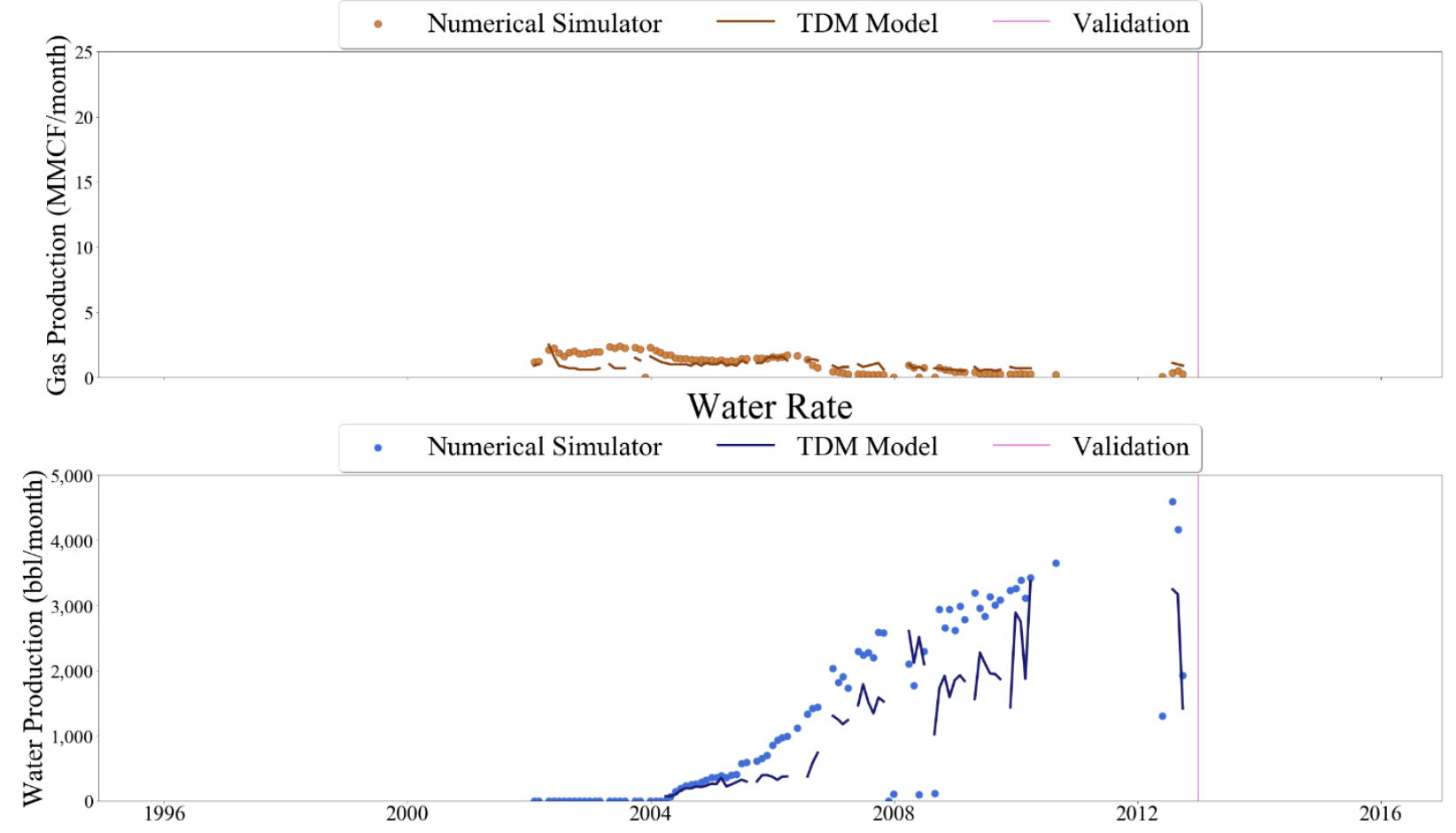

Figure 681 Well-026 oil, gas, and water rate TDM predictions vs actual simulation data plots for 4 Year Blind validation TDM 


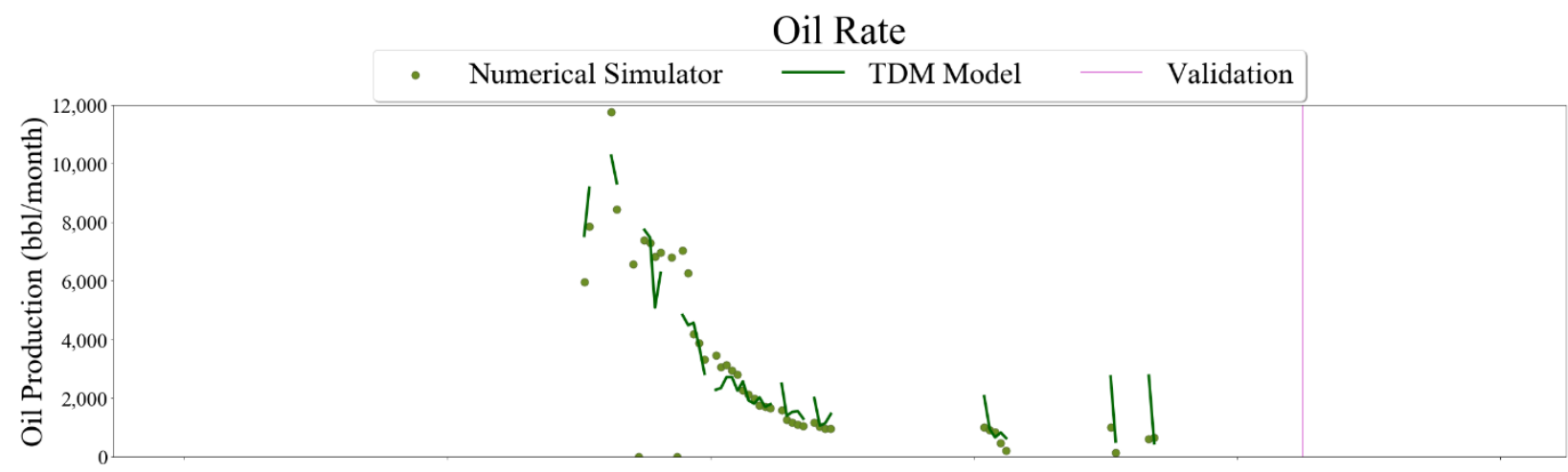

Gas Rate

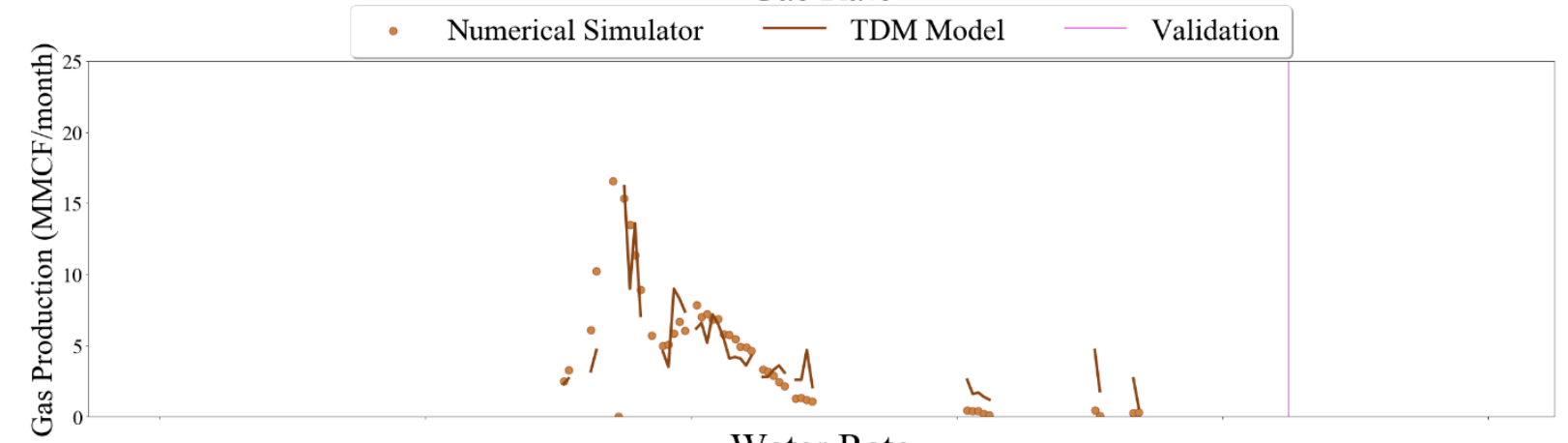

Water Rate

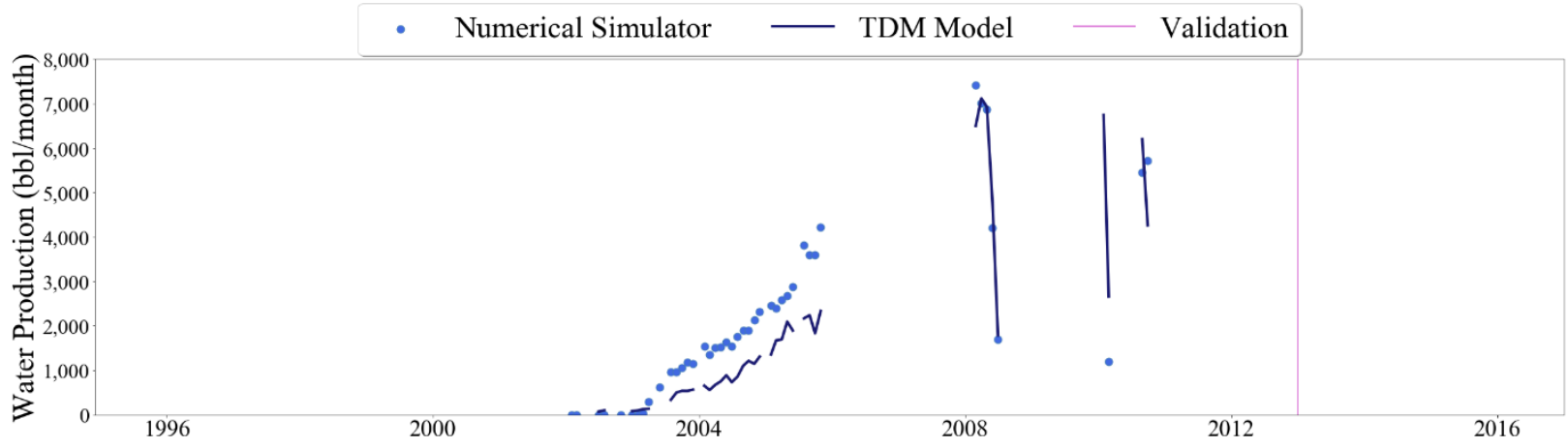

Figure 682 Well-027 oil, gas, and water rate TDM predictions vs actual simulation data plots for 4 Year Blind Validation TDM 


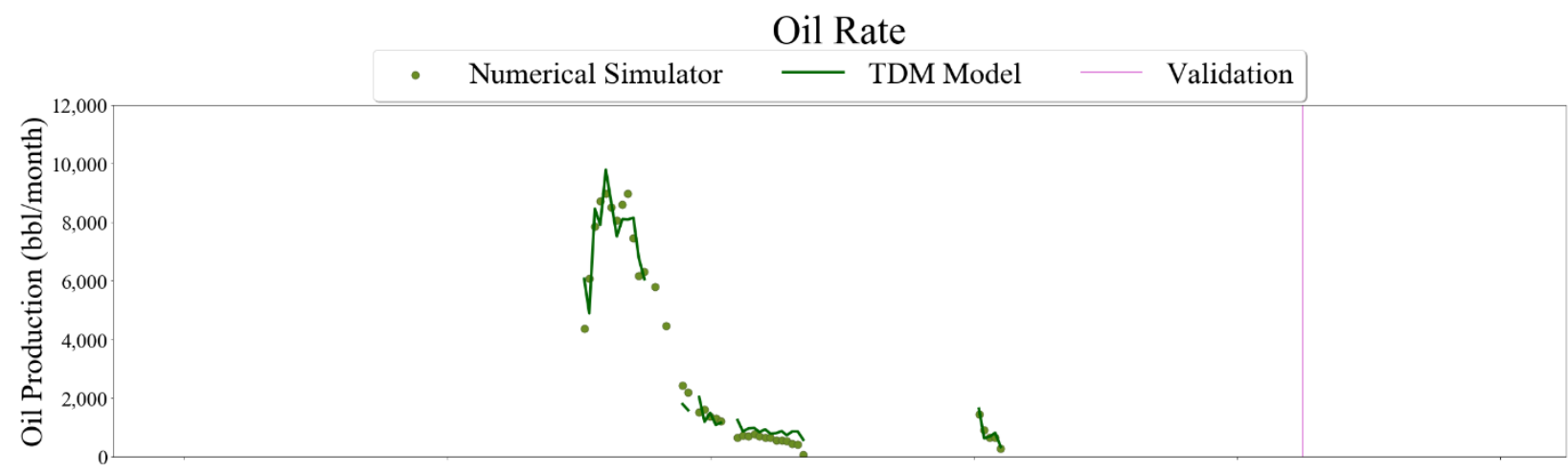

Gas Rate
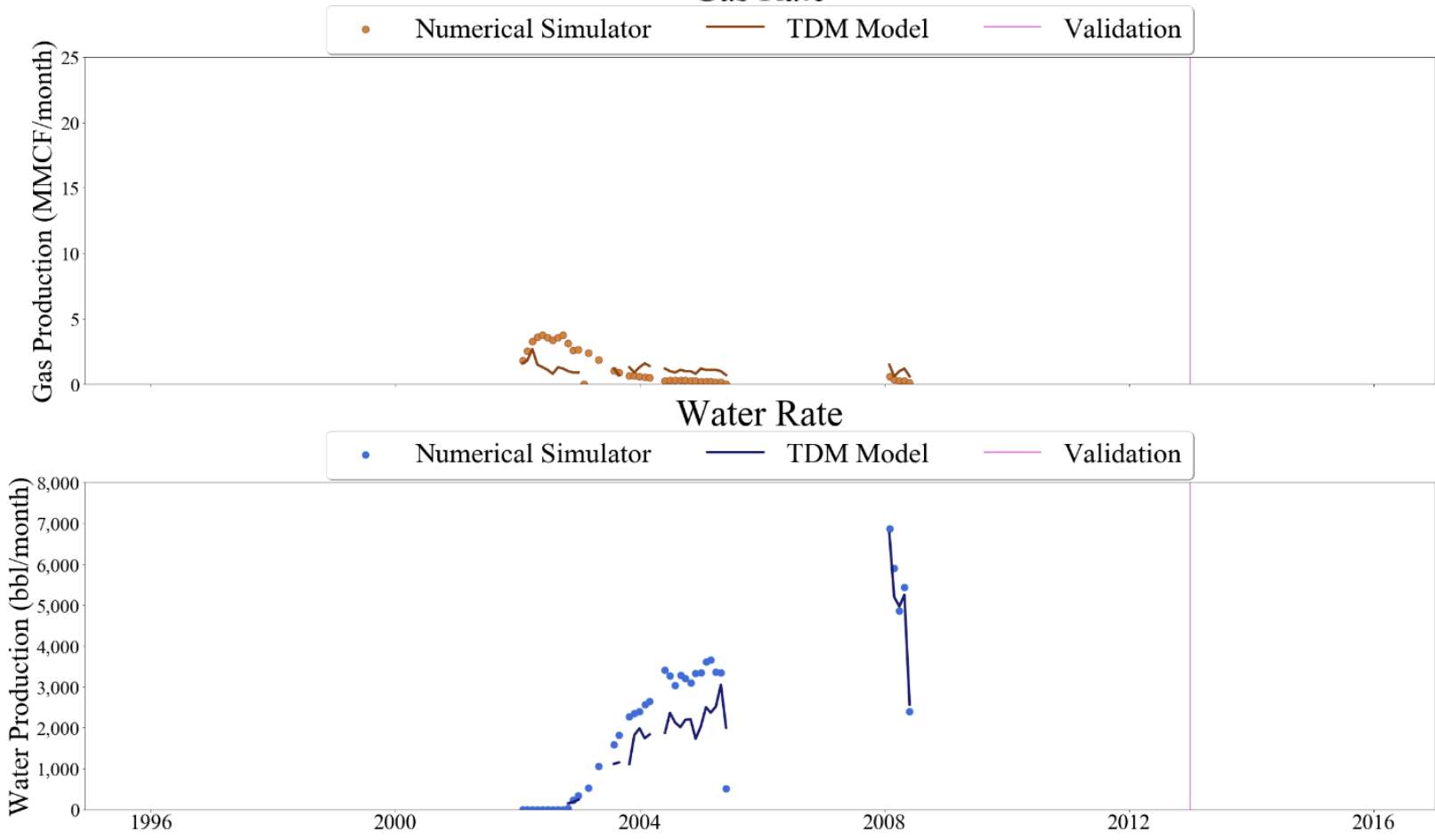

Figure 683 Well-028 oil, gas, and water rate TDM predictions vs actual simulation data plots for 4 Year Blind Validation TDM 

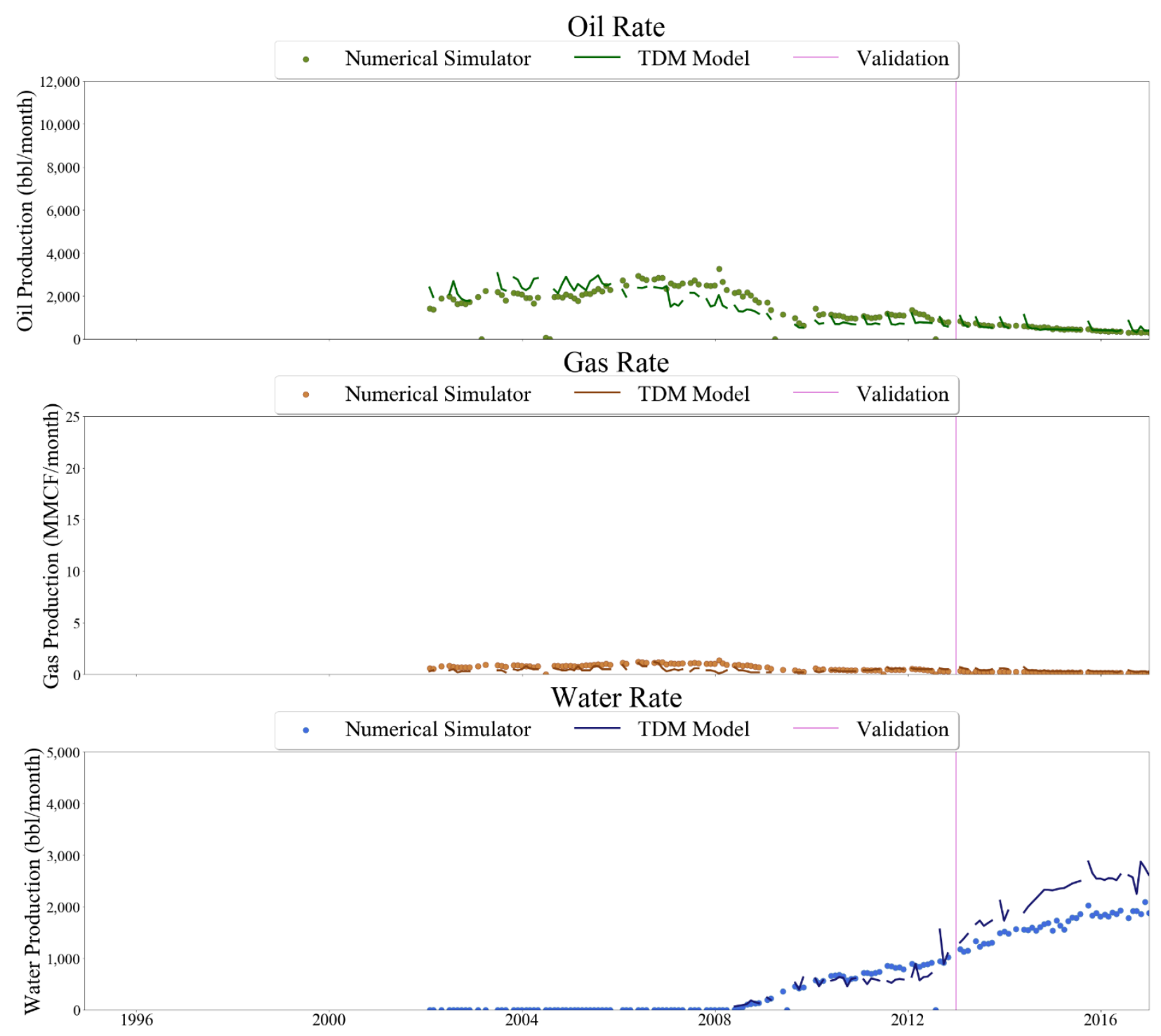

Figure 684 Well-029 oil, gas, and water rate TDM predictions vs actual simulation data plots for 4 Year Blind Validation TDM 


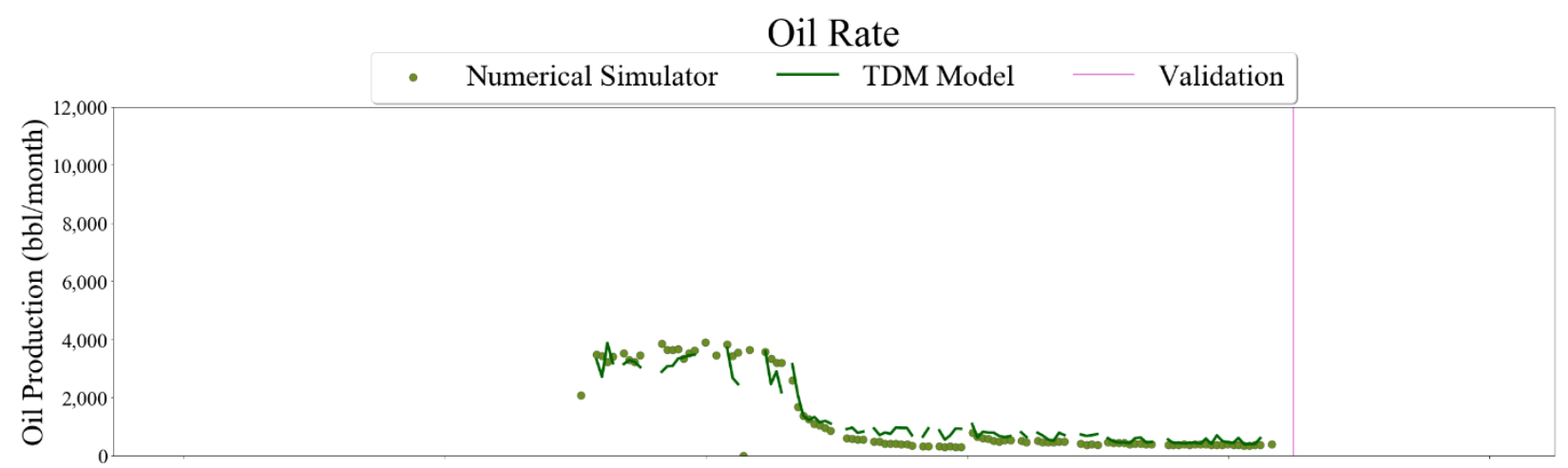

\section{Gas Rate}
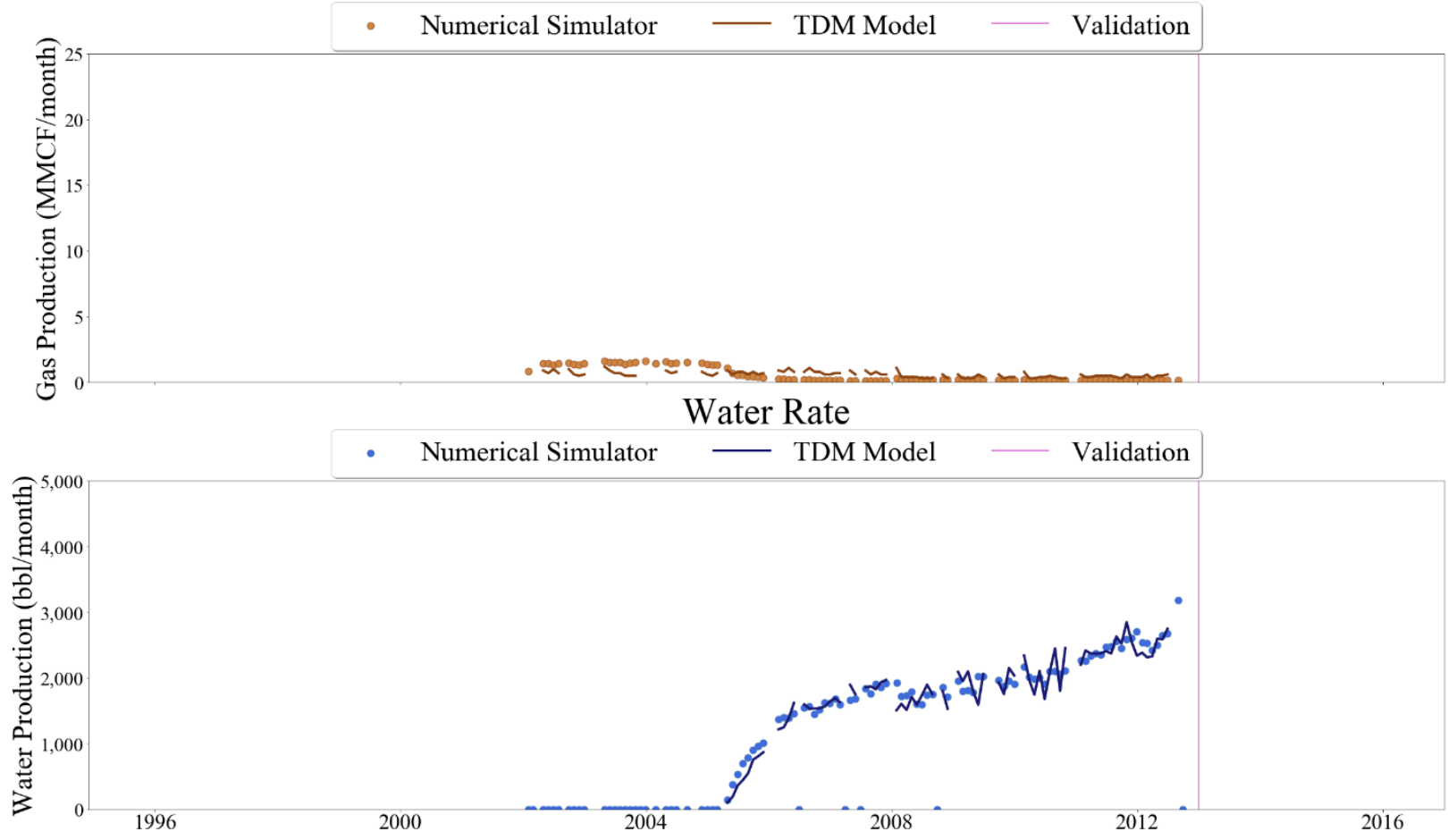

Figure 685 Well-030 oil, gas, and water rate TDM predictions vs actual simulation data plots for 4 Year Blind Validation TDM 


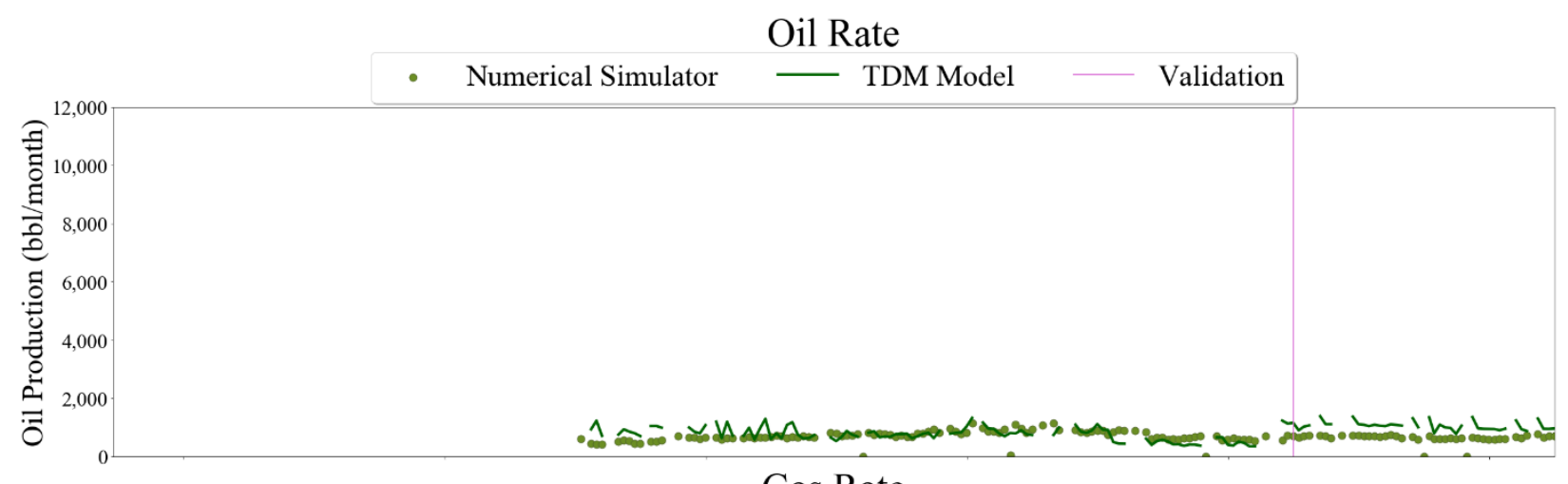

Gas Rate
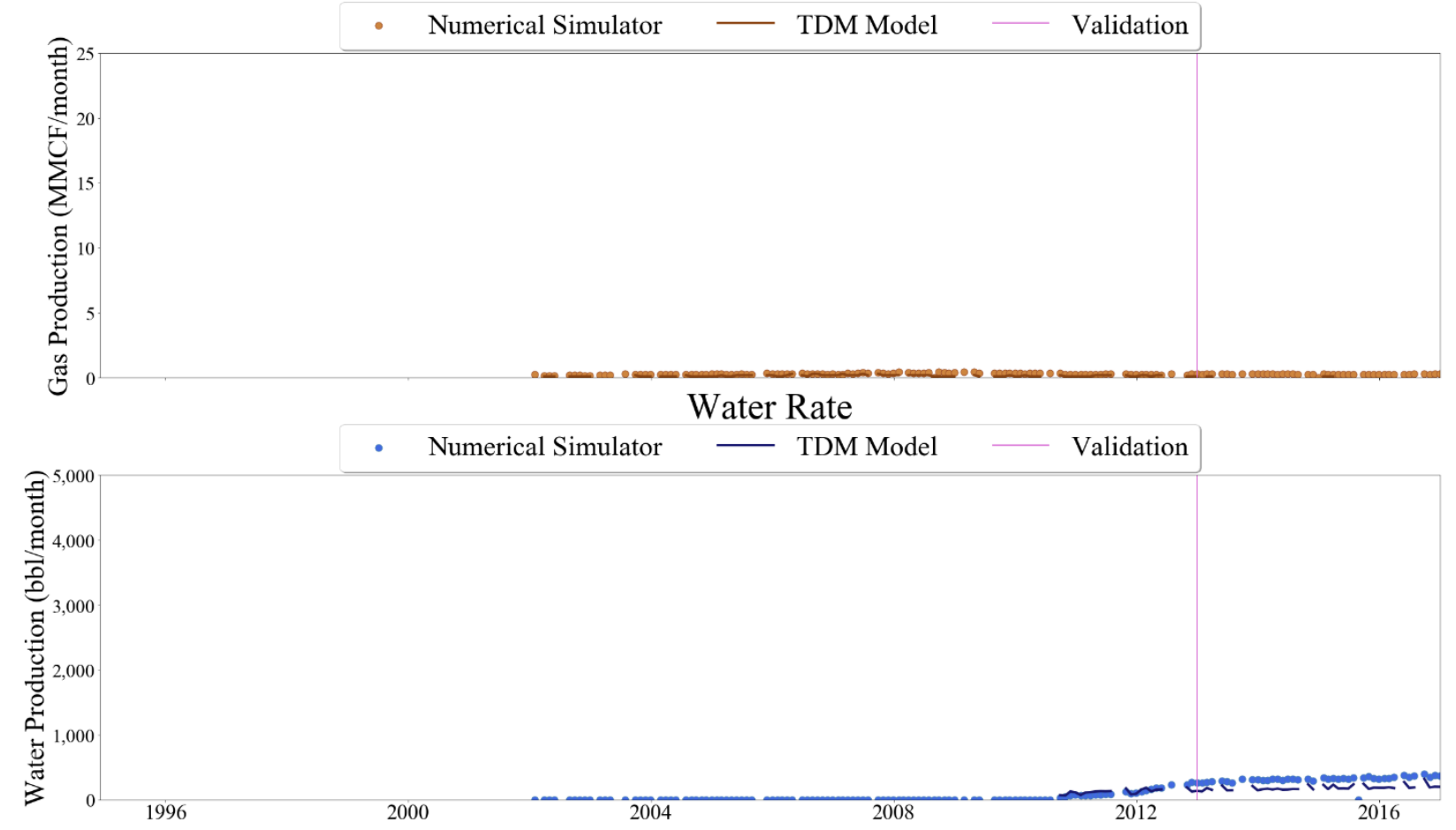

Figure 686 Well-031 oil, gas, and water rate TDM predictions vs actual simulation data plots for 4 Year Blind Validation TDM 


\section{Oil Rate}

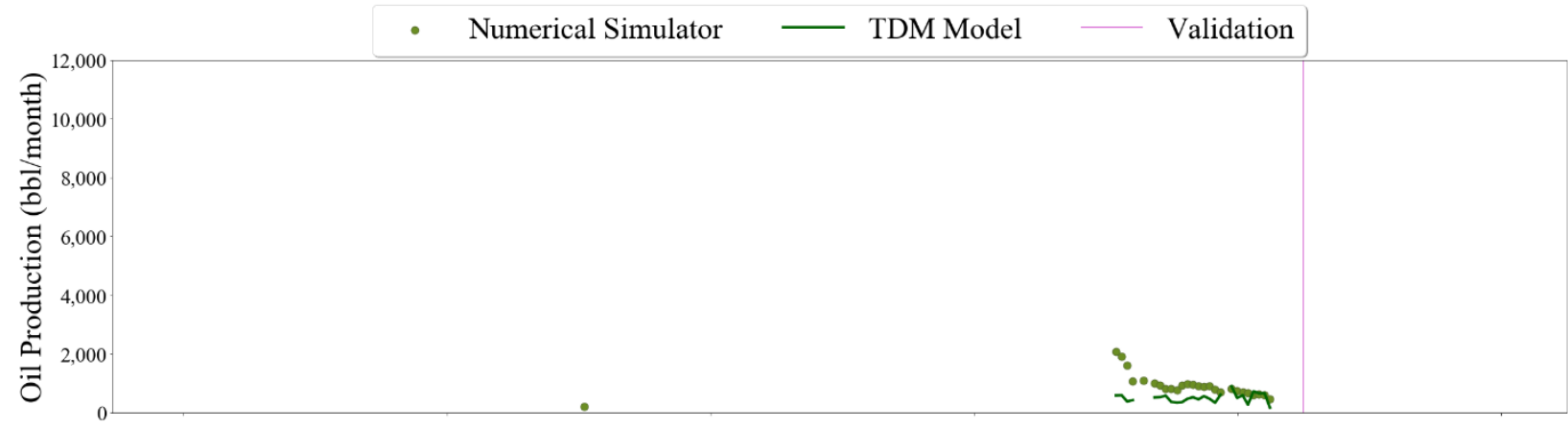

\section{Gas Rate}
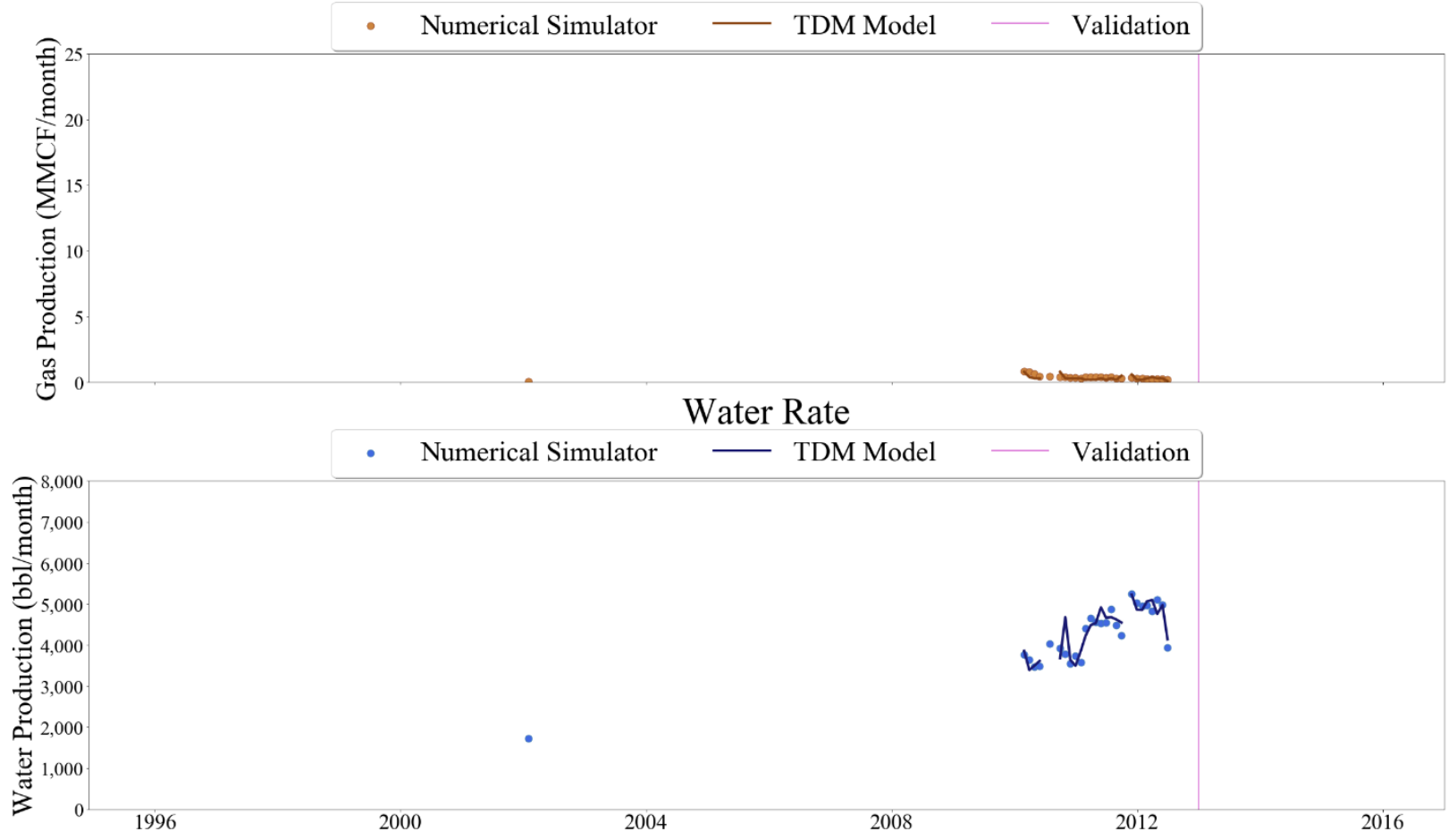

Figure 687 Well-032 oil, gas, and water rate TDM predictions vs actual simulation data plots for 4 Year Blind Validation TDM 


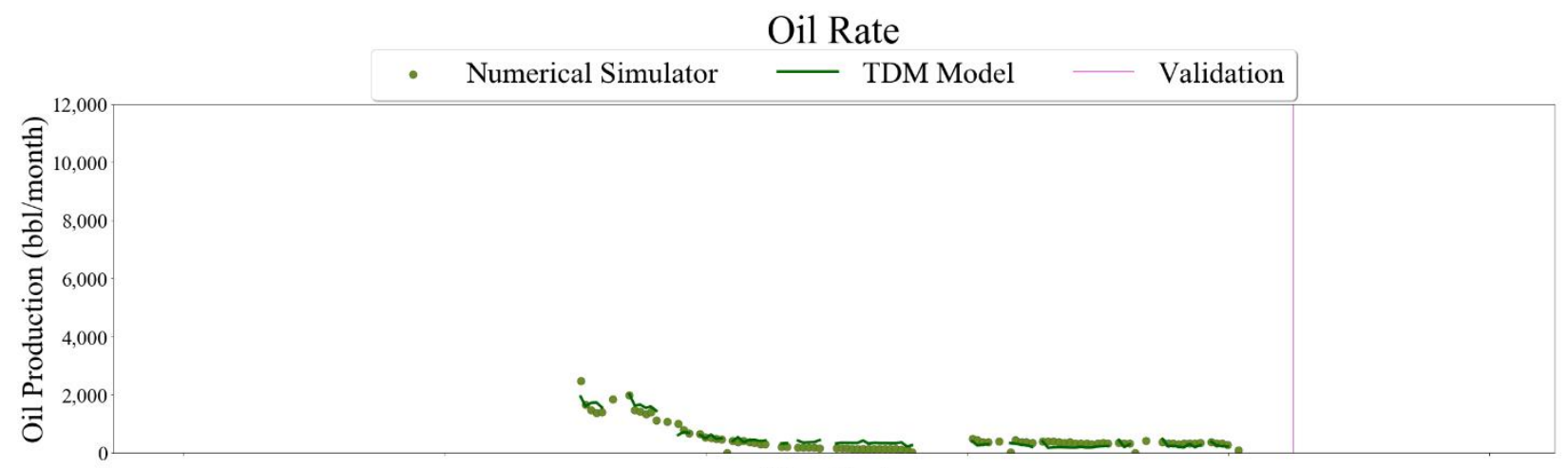

Gas Rate
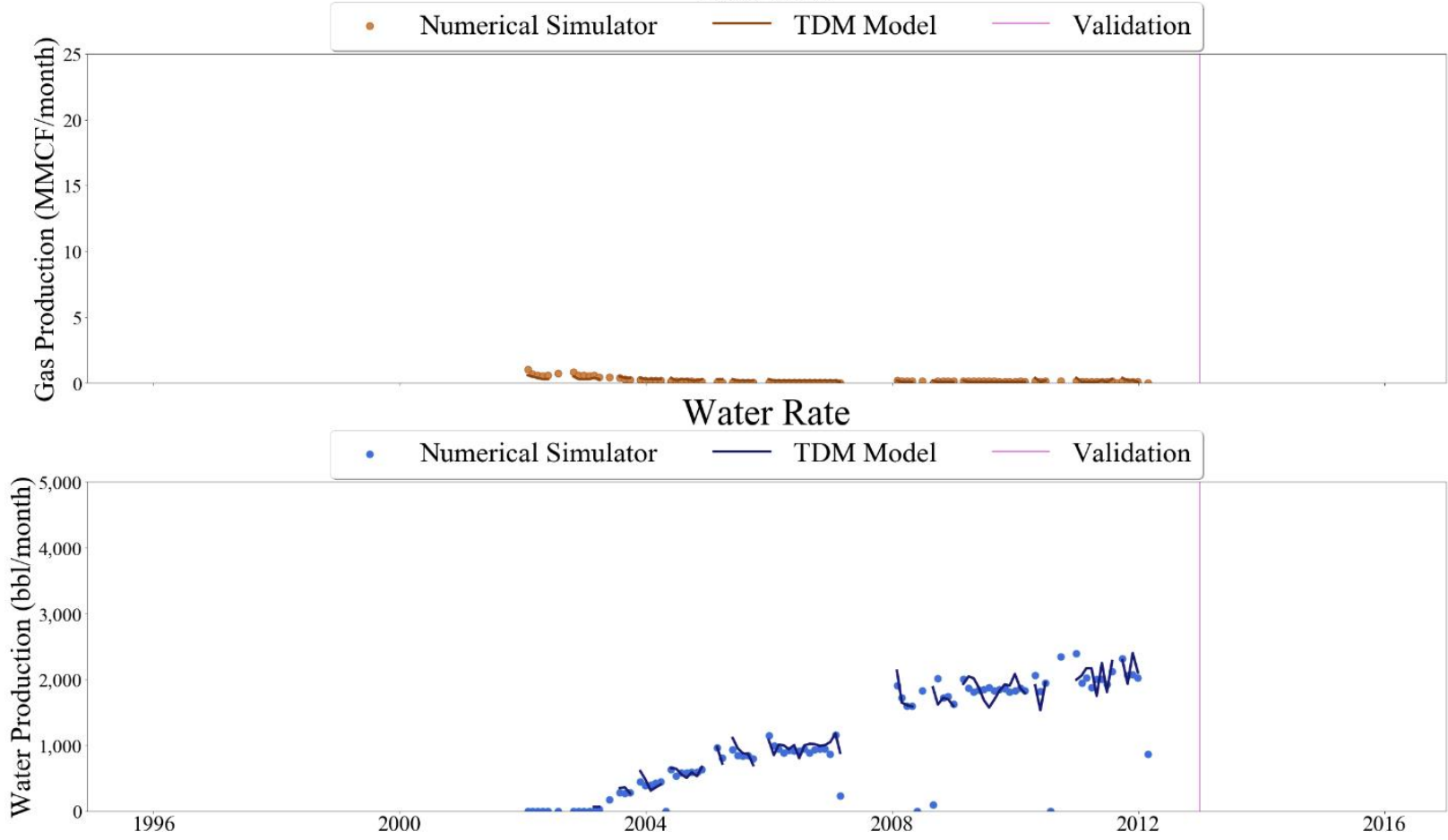

Figure 688 Well-033 oil, gas, and water rate TDM predictions vs actual simulation data plots for 4 Year Blind Validation TDM 

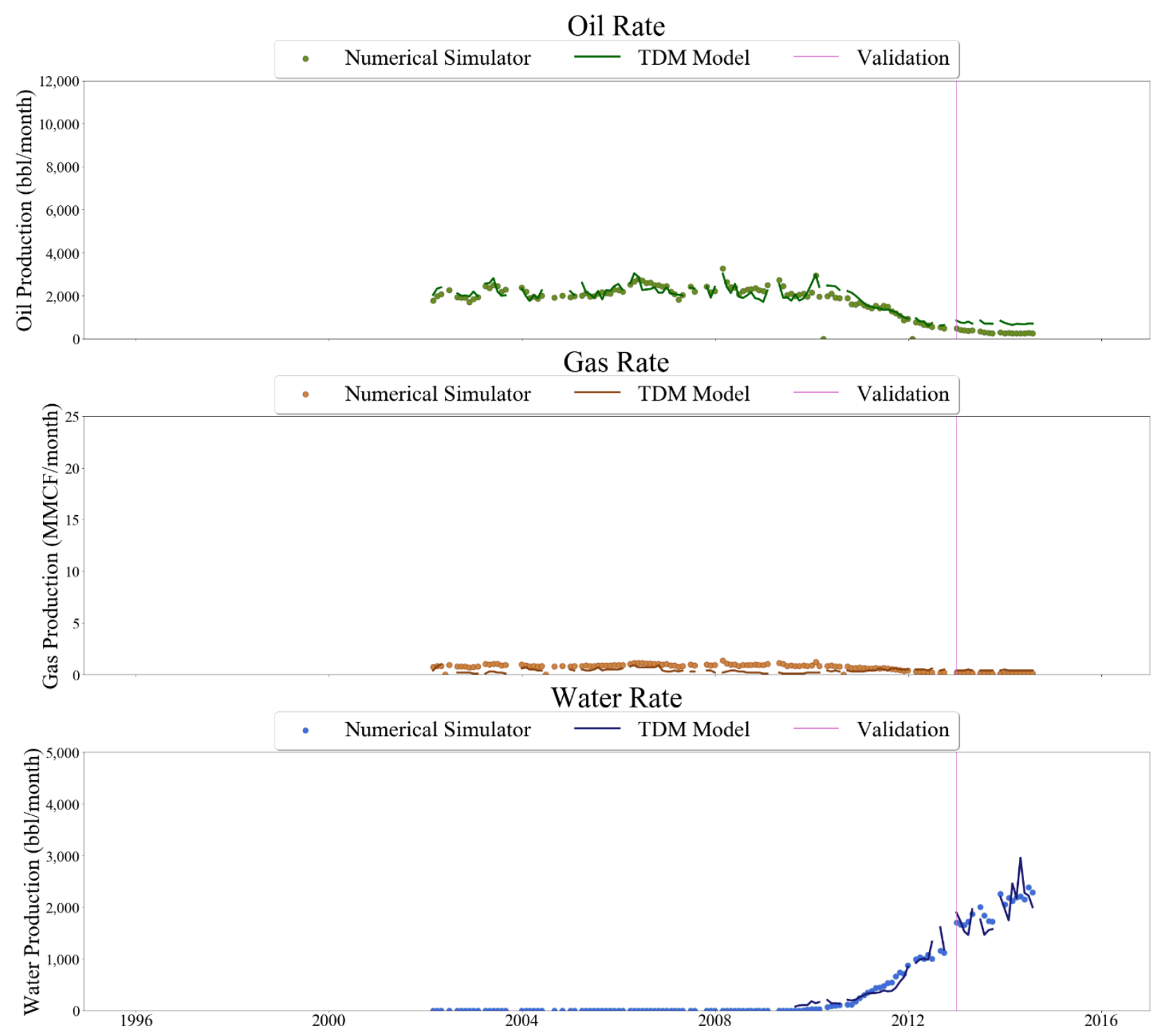

Figure 689 Well-034 oil, gas, and water rate TDM predictions vs actual simulation data plots for 4 Year Blind Validation TDM 


\section{Oil Rate}

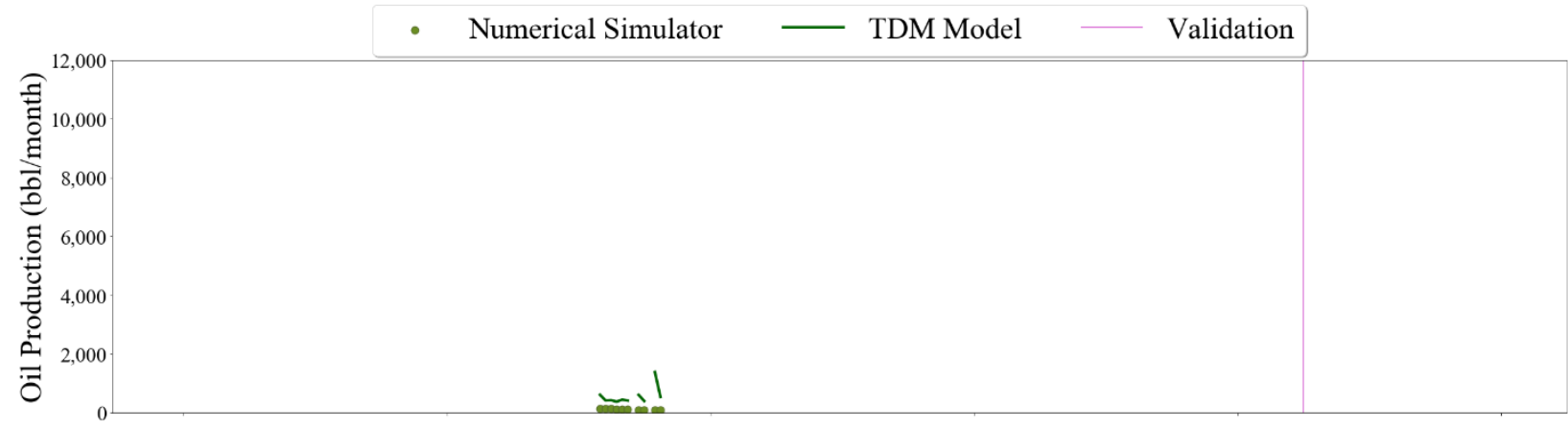

\section{Gas Rate}

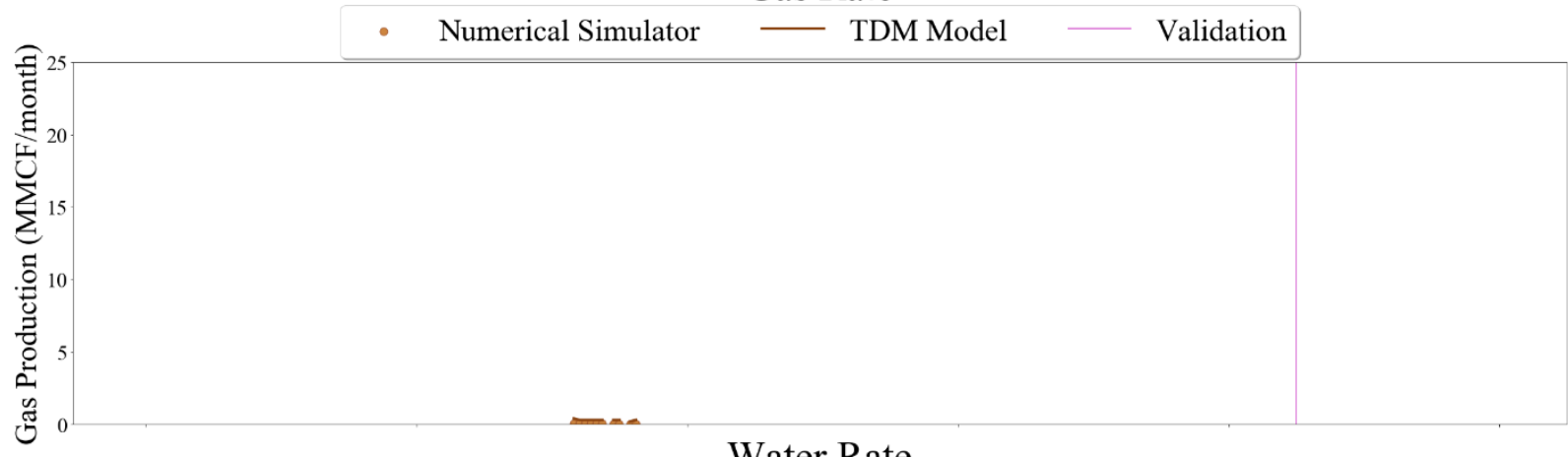

\section{Water Rate}

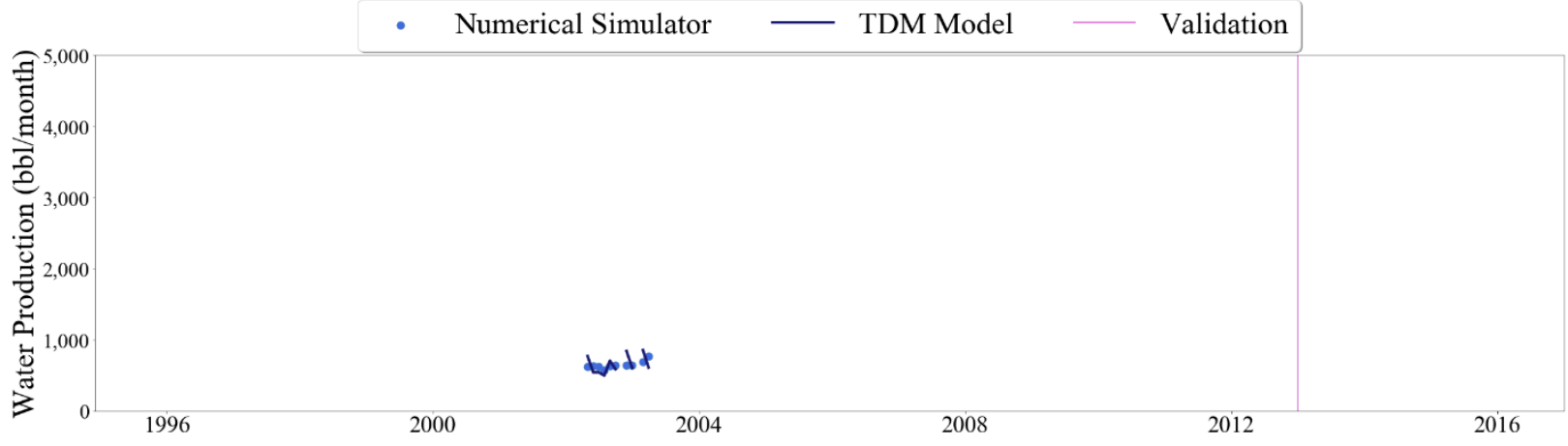

Figure 690 Well-035 oil, gas, and water rate TDM predictions vs actual simulation data plots for 4 Year Blind Validation TDM 

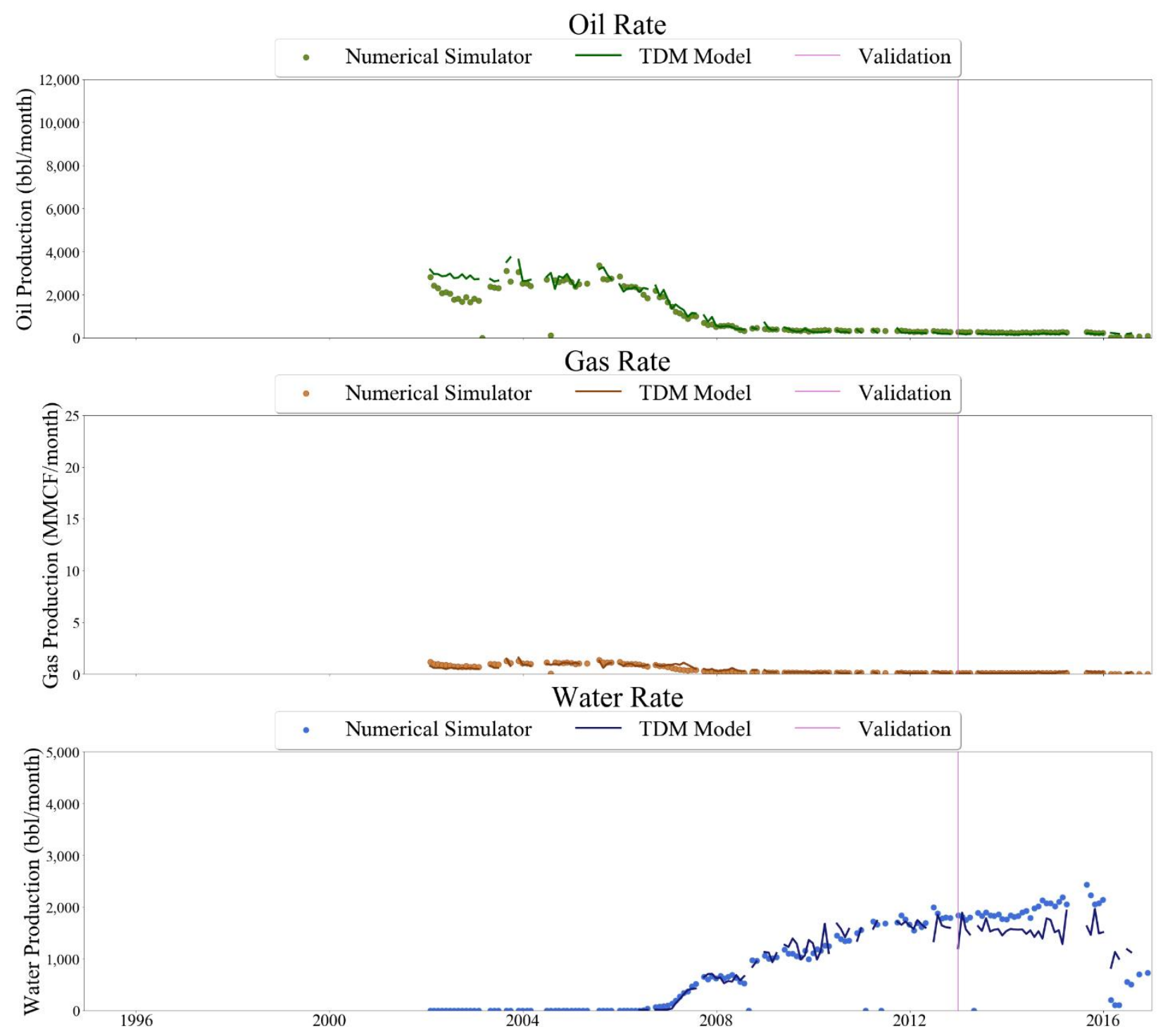

Figure 691 Well-036 oil, gas, and water rate TDM predictions vs actual simulation data plots for 4 Year Blind Validation TDM 


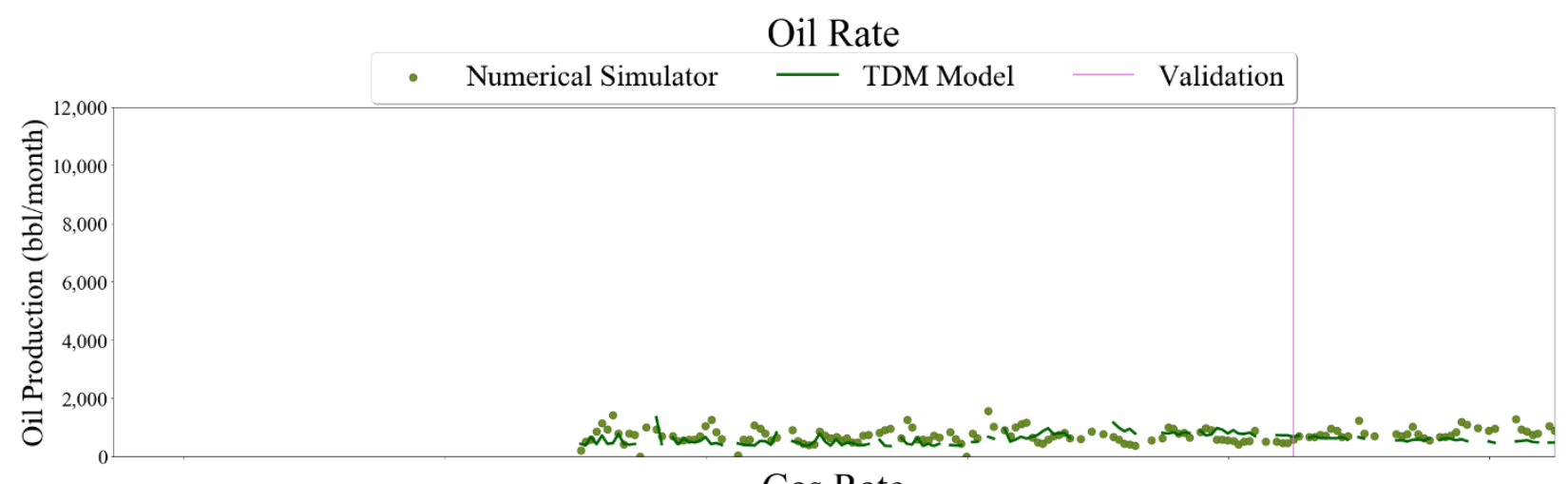

Gas Rate
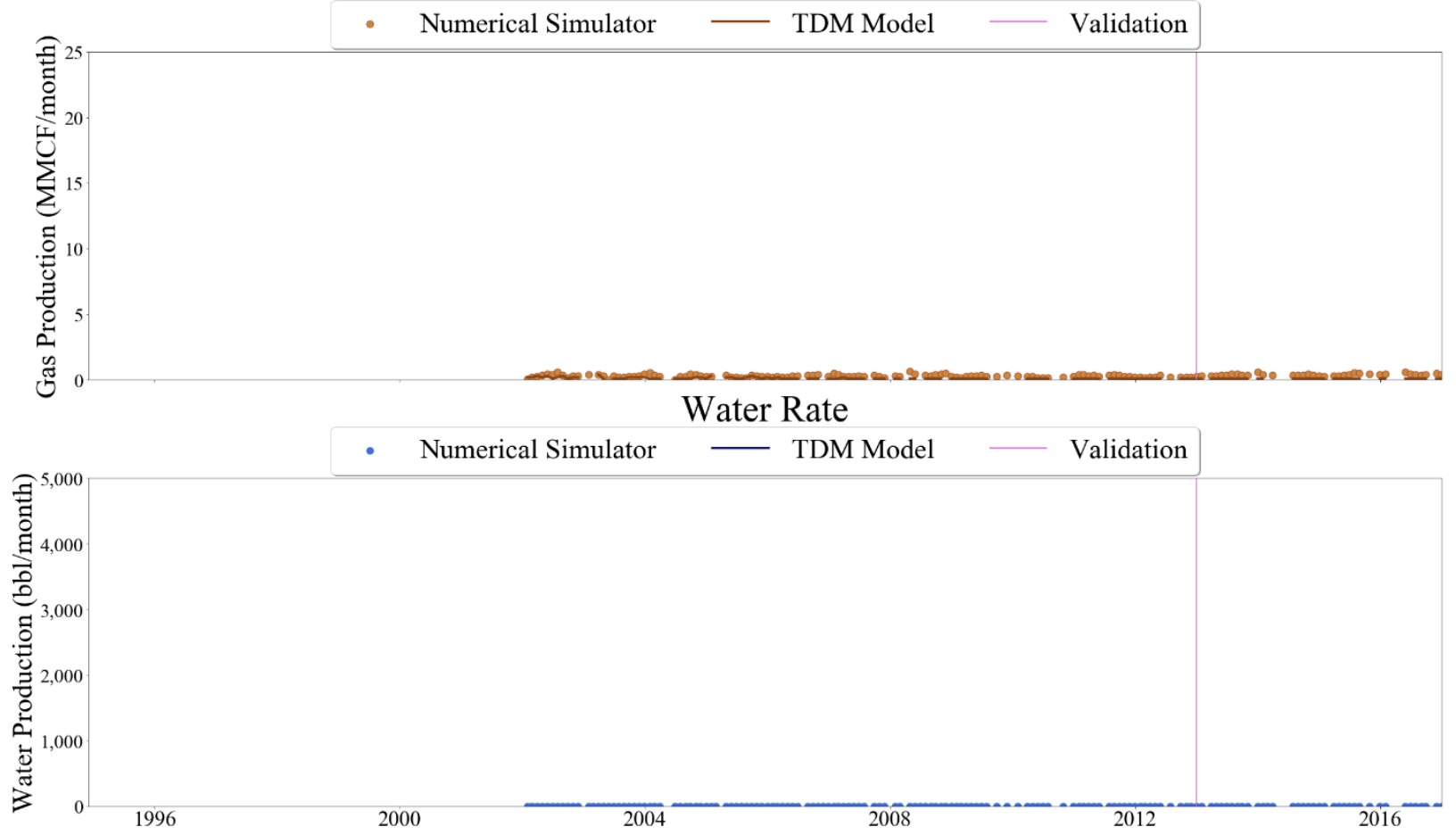

Figure 692 Well-037 oil, gas, and water rate TDM predictions vs actual simulation data plots for 4 Year Blind Validation TDM 


\section{Oil Rate}

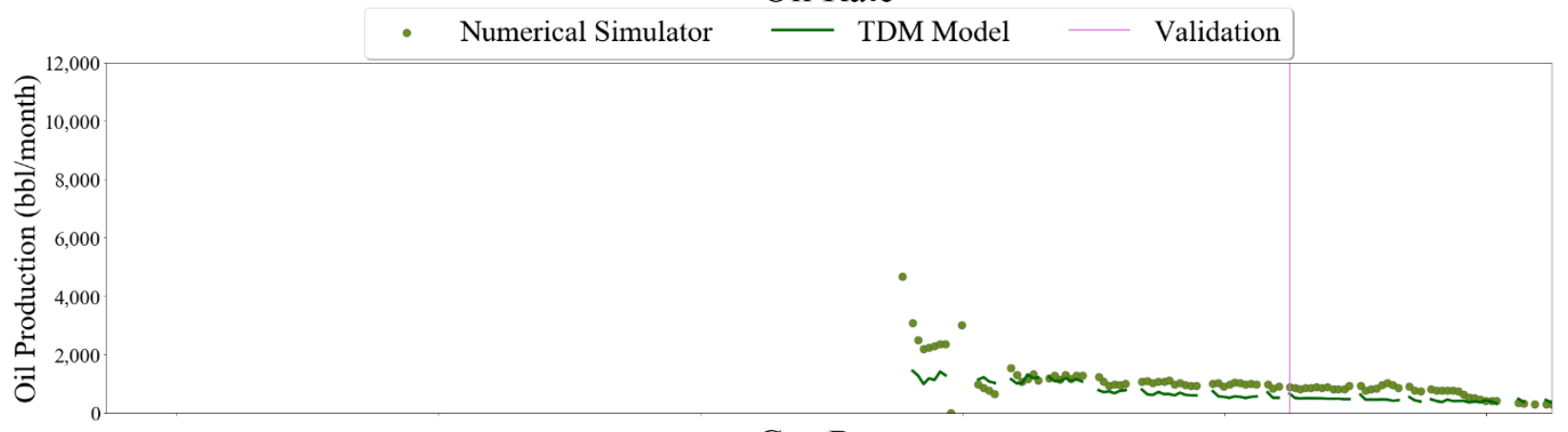

\section{Gas Rate}
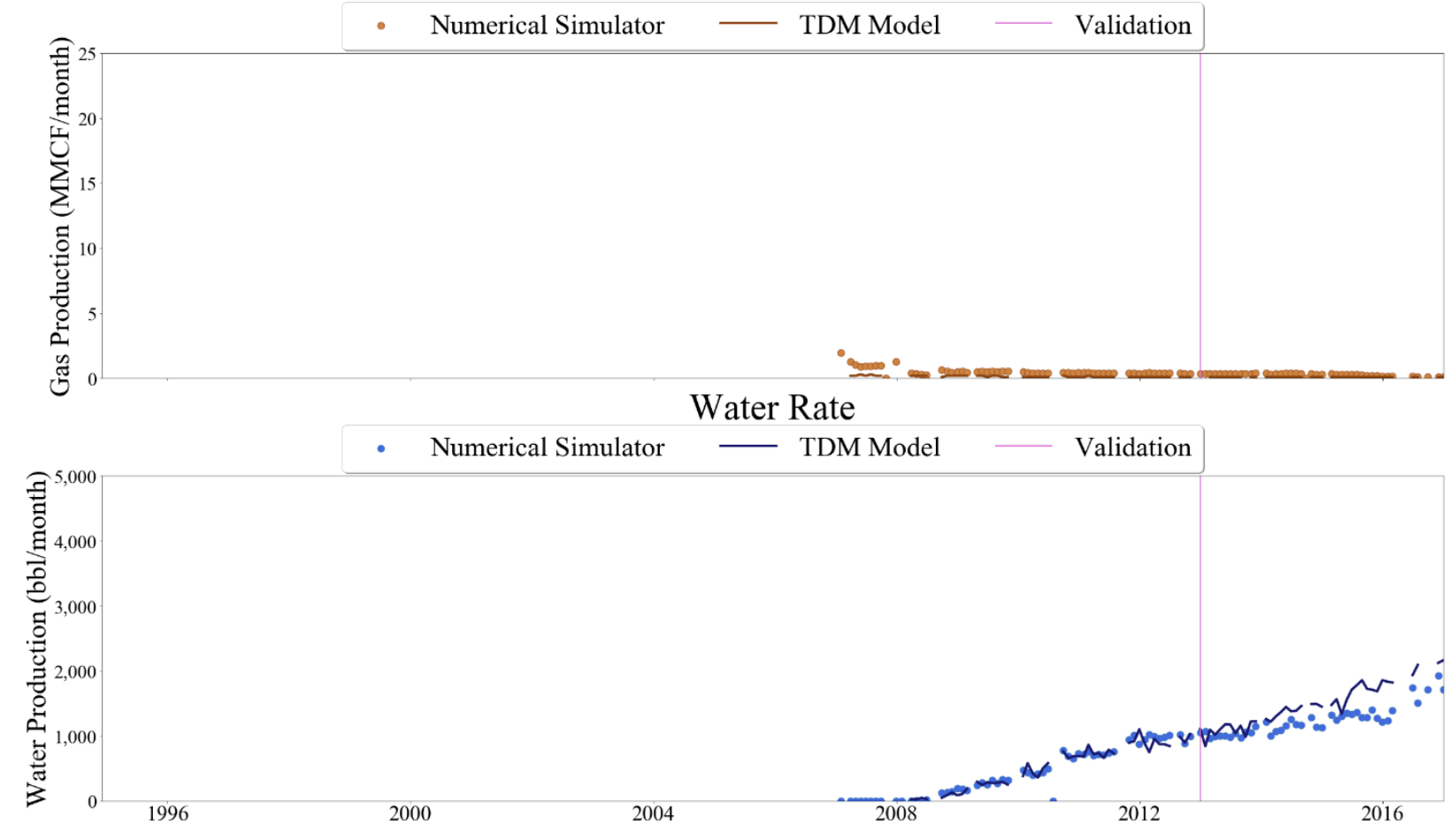

Figure 693 Well-038 oil, gas, and water rate TDM predictions vs actual simulation data plots for 4 Year Blind Validation TDM 


\section{Oil Rate}

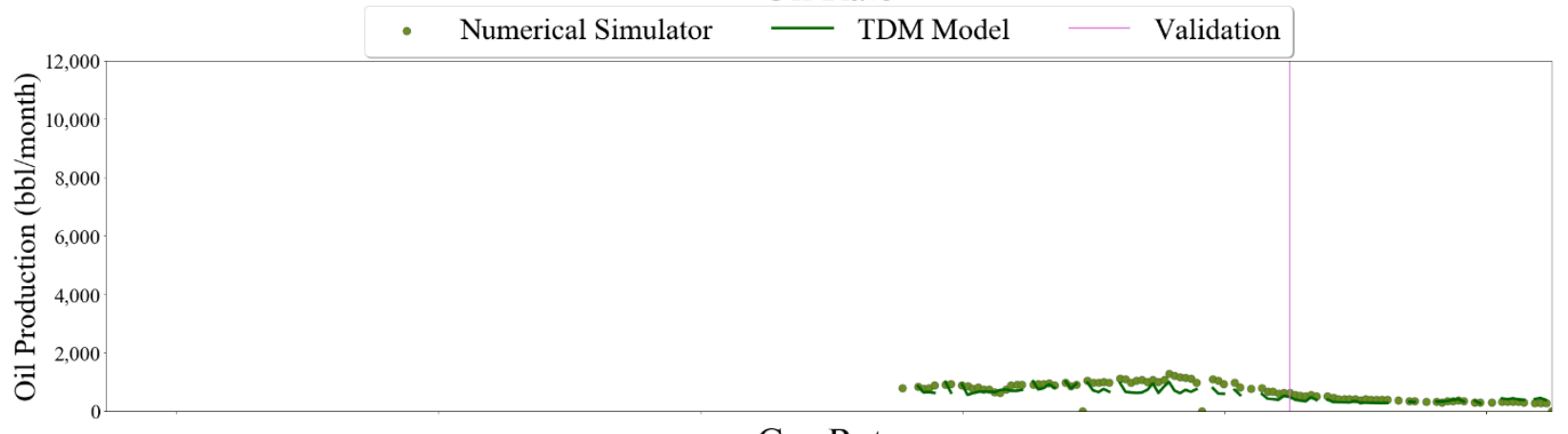

\section{Gas Rate}
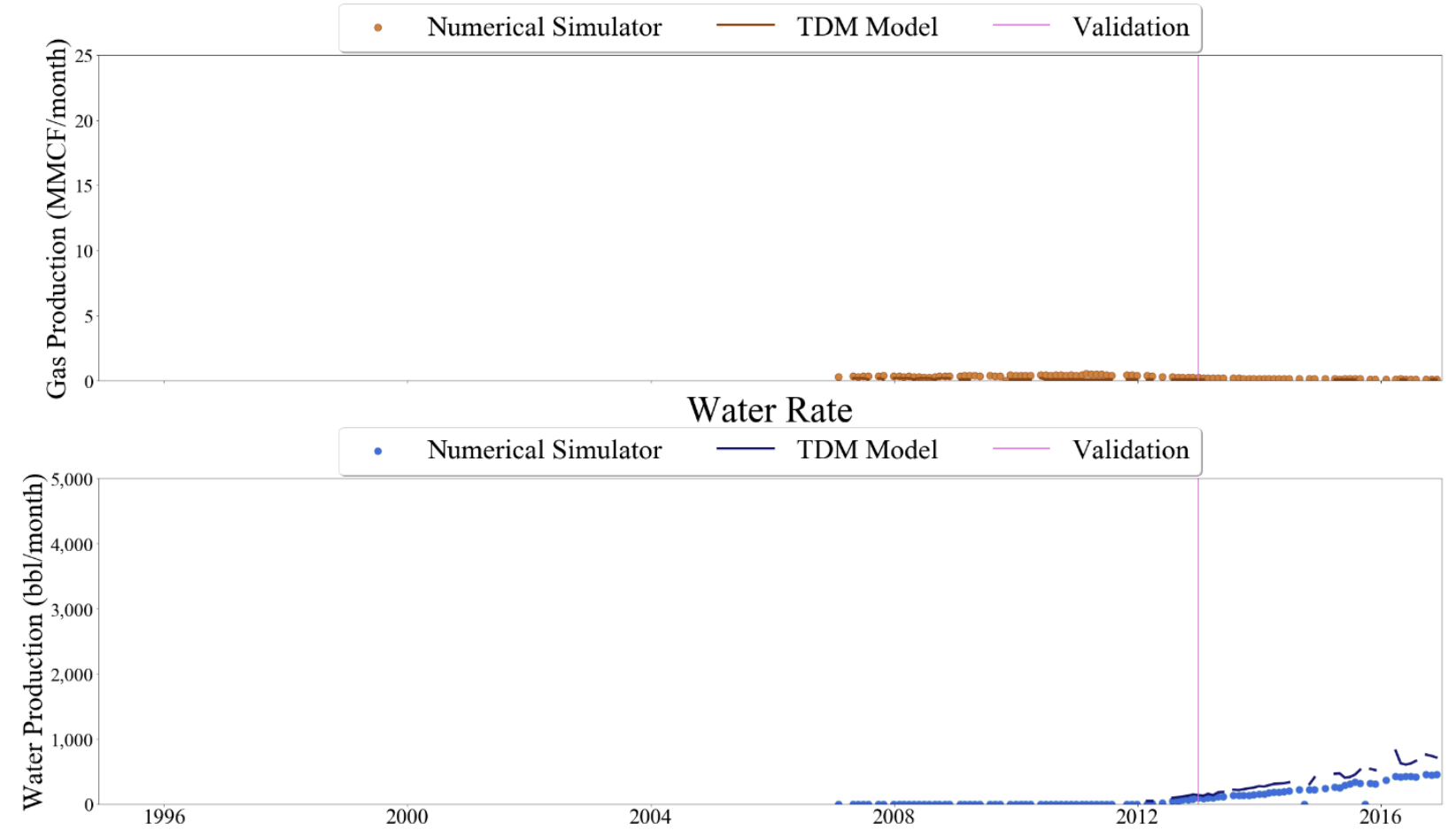

Figure 694 Well-039 oil, gas, and water rate TDM predictions vs actual simulation data plots for 4 Year Blind Validation TDM 


\section{Oil Rate}

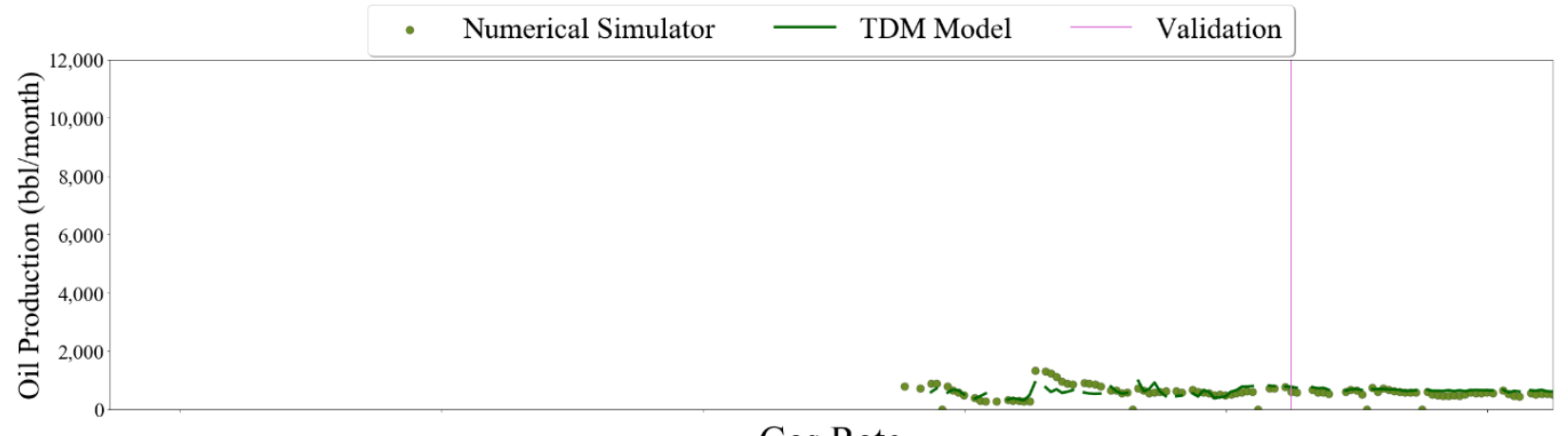

\section{Gas Rate}
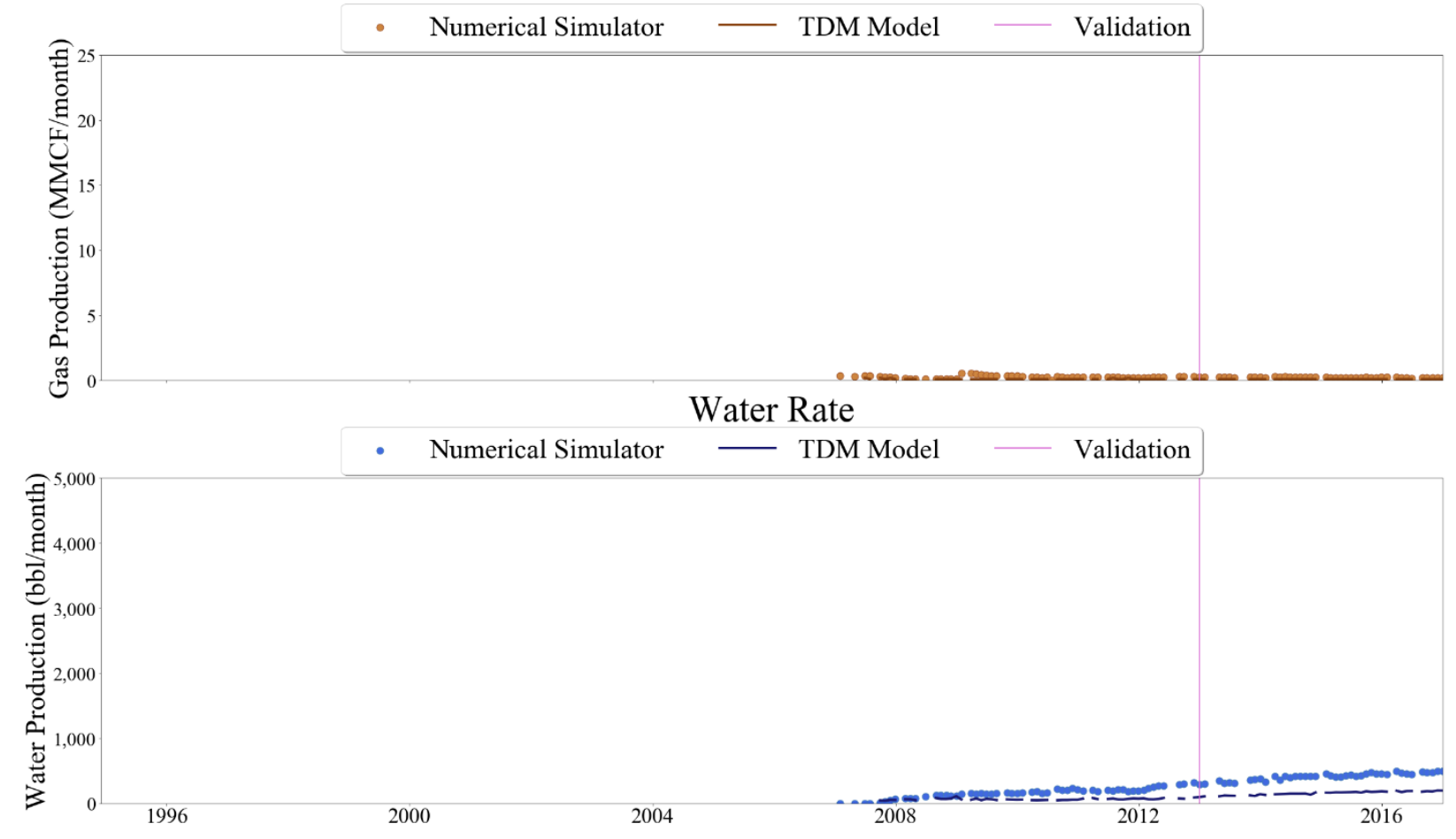

Figure 695 Well-040 oil, gas, and water rate TDM predictions vs actual simulation data plots for 4 Year Blind Validation TDM 


\section{Oil Rate}

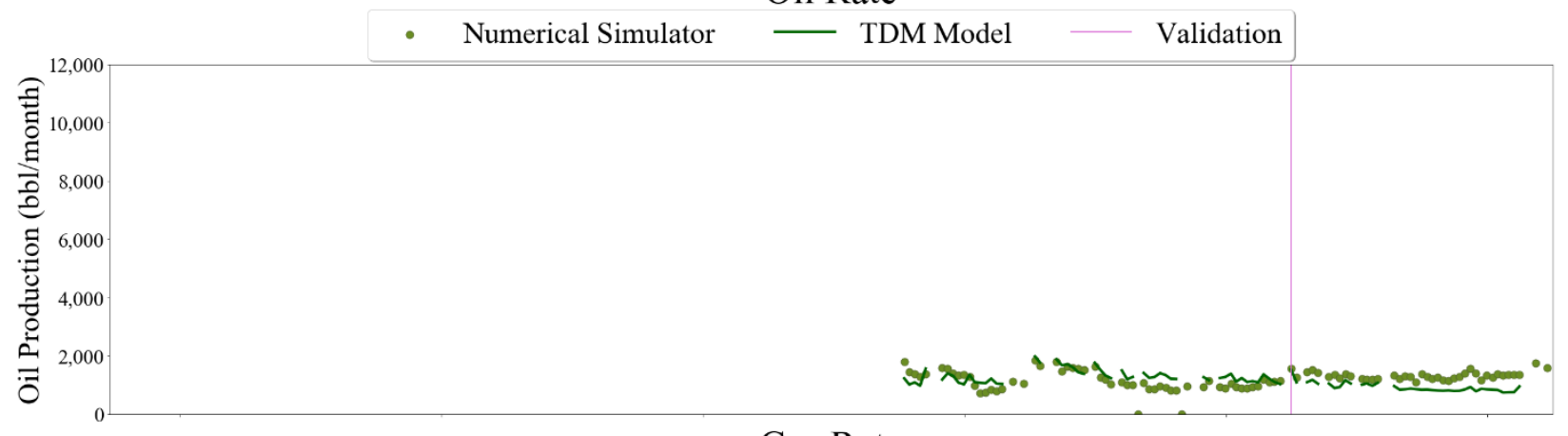

\section{Gas Rate}
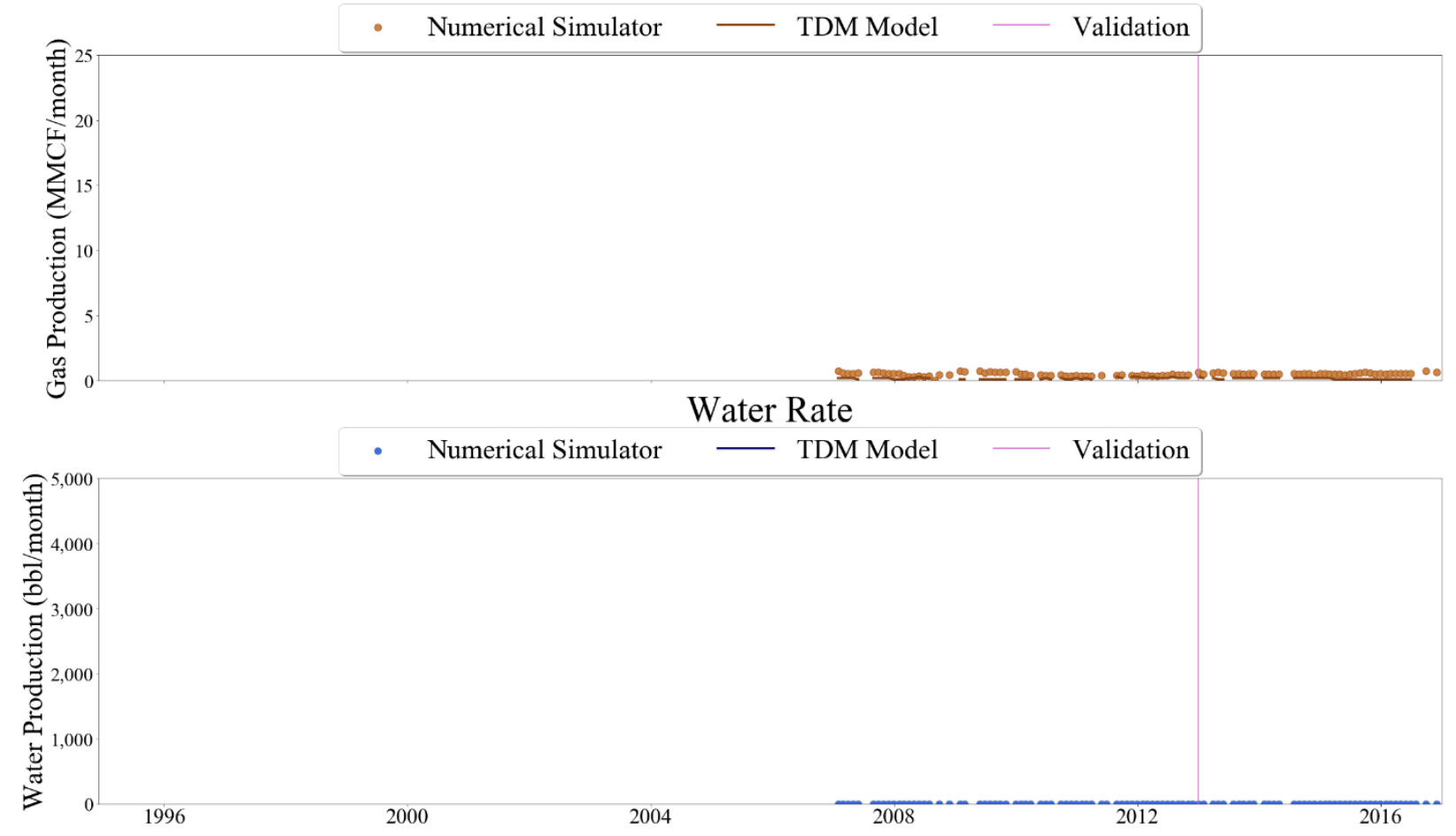

Figure 696 Well-041 oil, gas, and water rate TDM predictions vs actual simulation data plots for 4 Year Blind Validation TDM 


\section{Oil Rate}

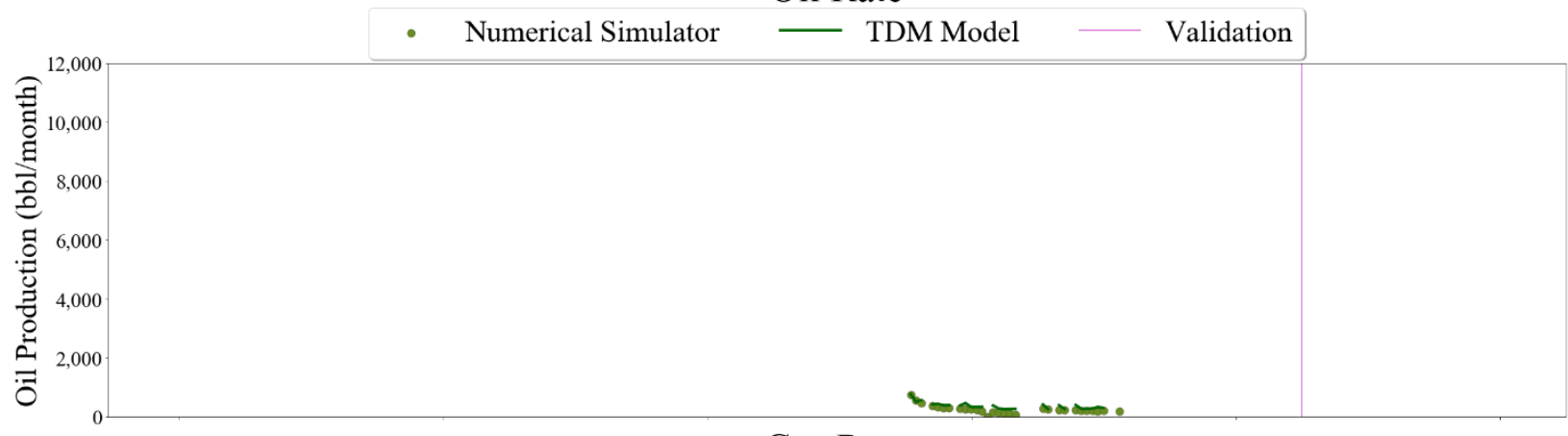

\section{Gas Rate}
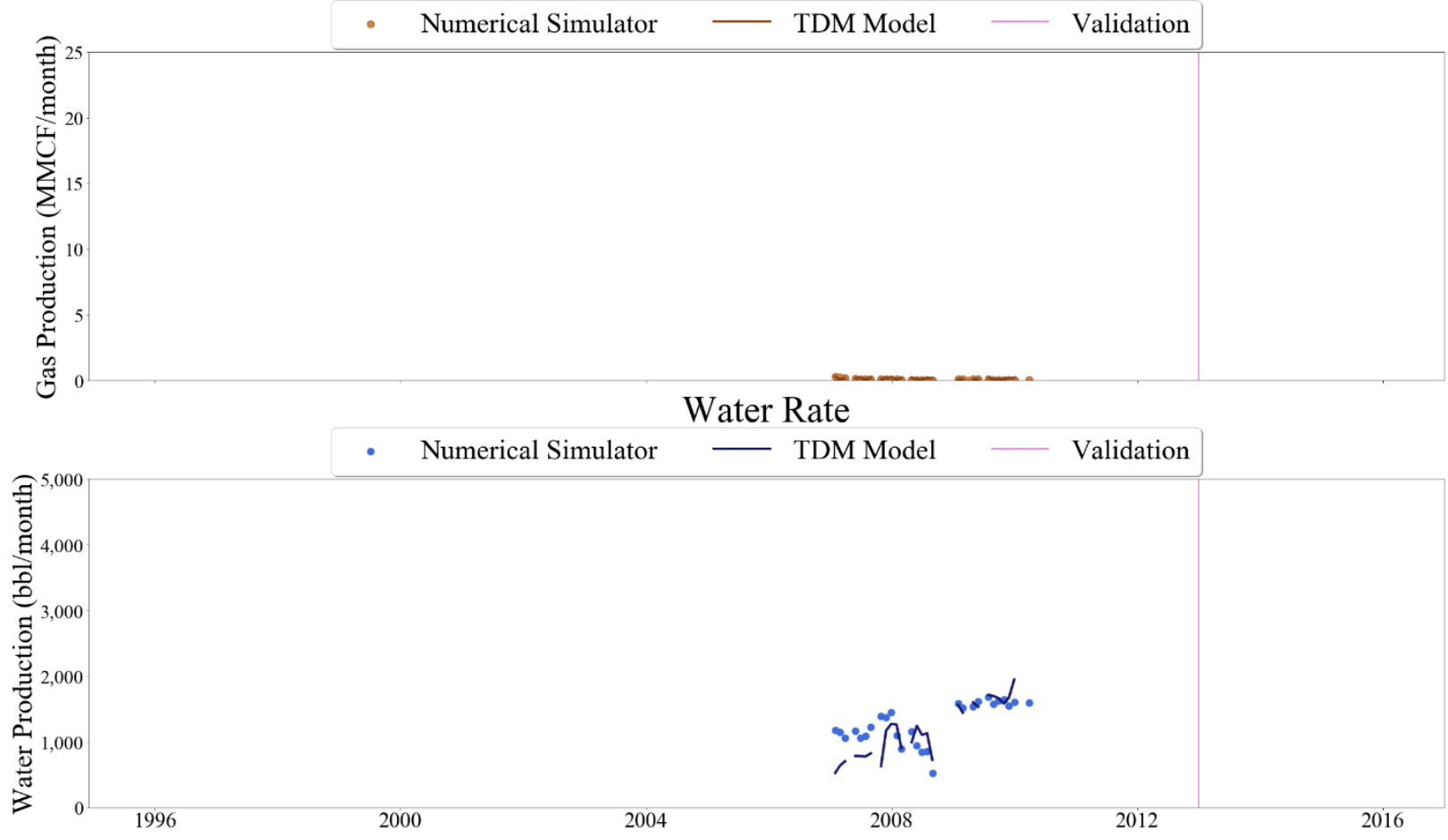

Figure 697 Well-042 oil, gas, and water rate TDM predictions vs actual simulation data plots for 4 Year Blind Validation TDM 


\section{Oil Rate}

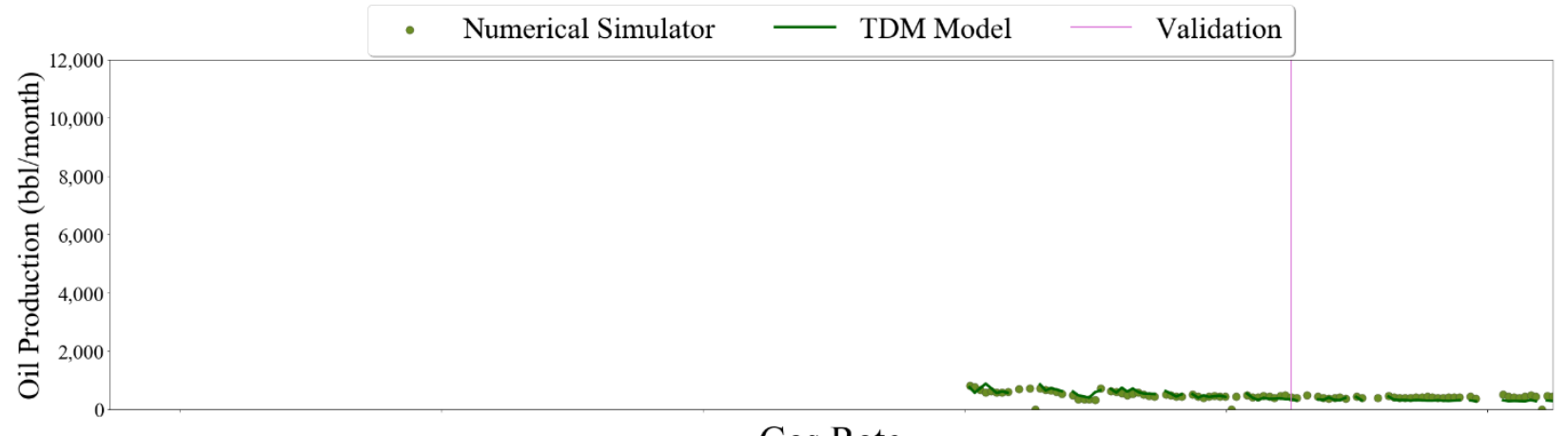

\section{Gas Rate}
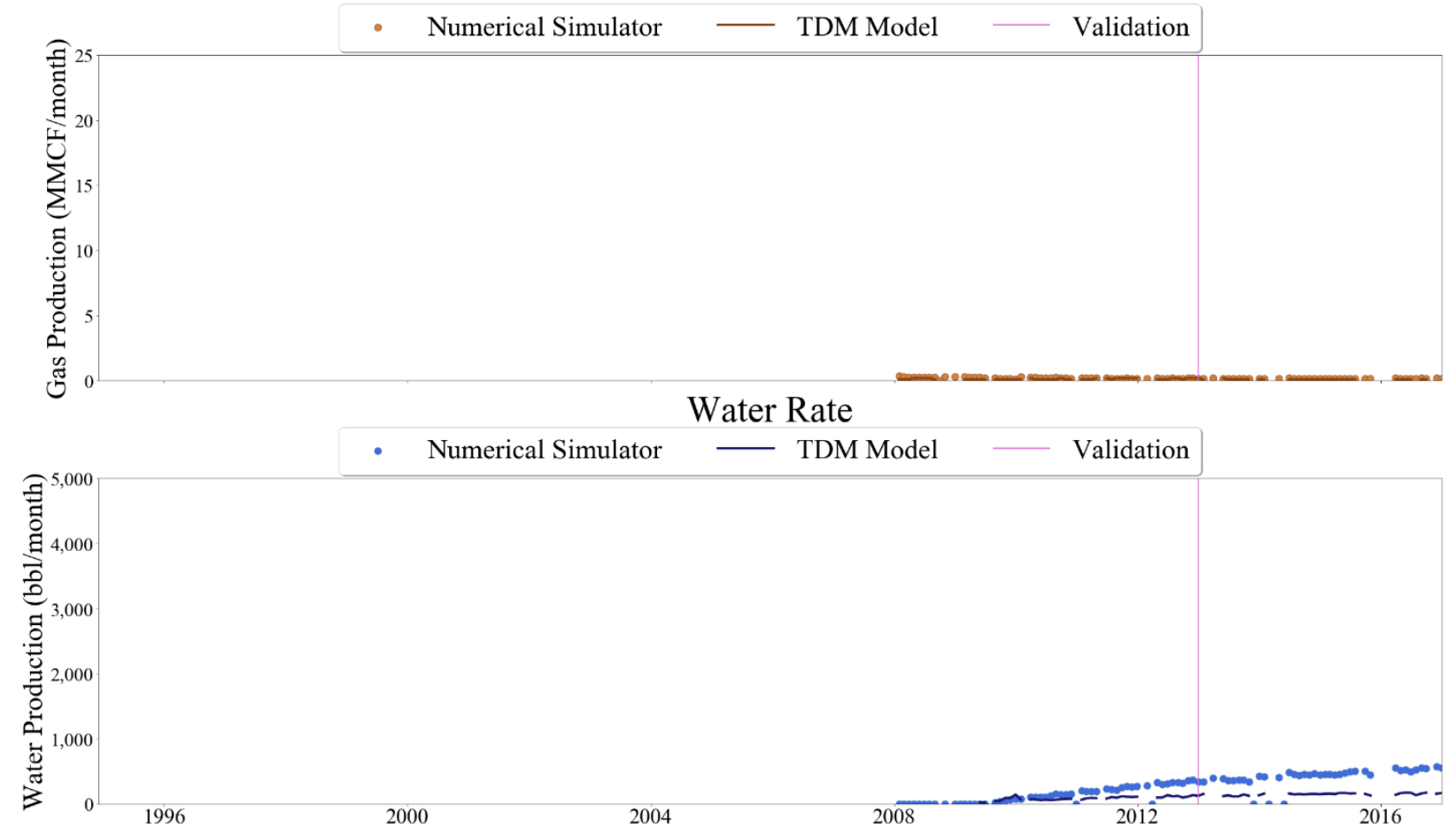

Figure 698 Well-043 oil, gas, annd water rate TDM predictions vs actual simulation data plots for 4 Year Blind Validation TDM 


\section{Oil Rate}
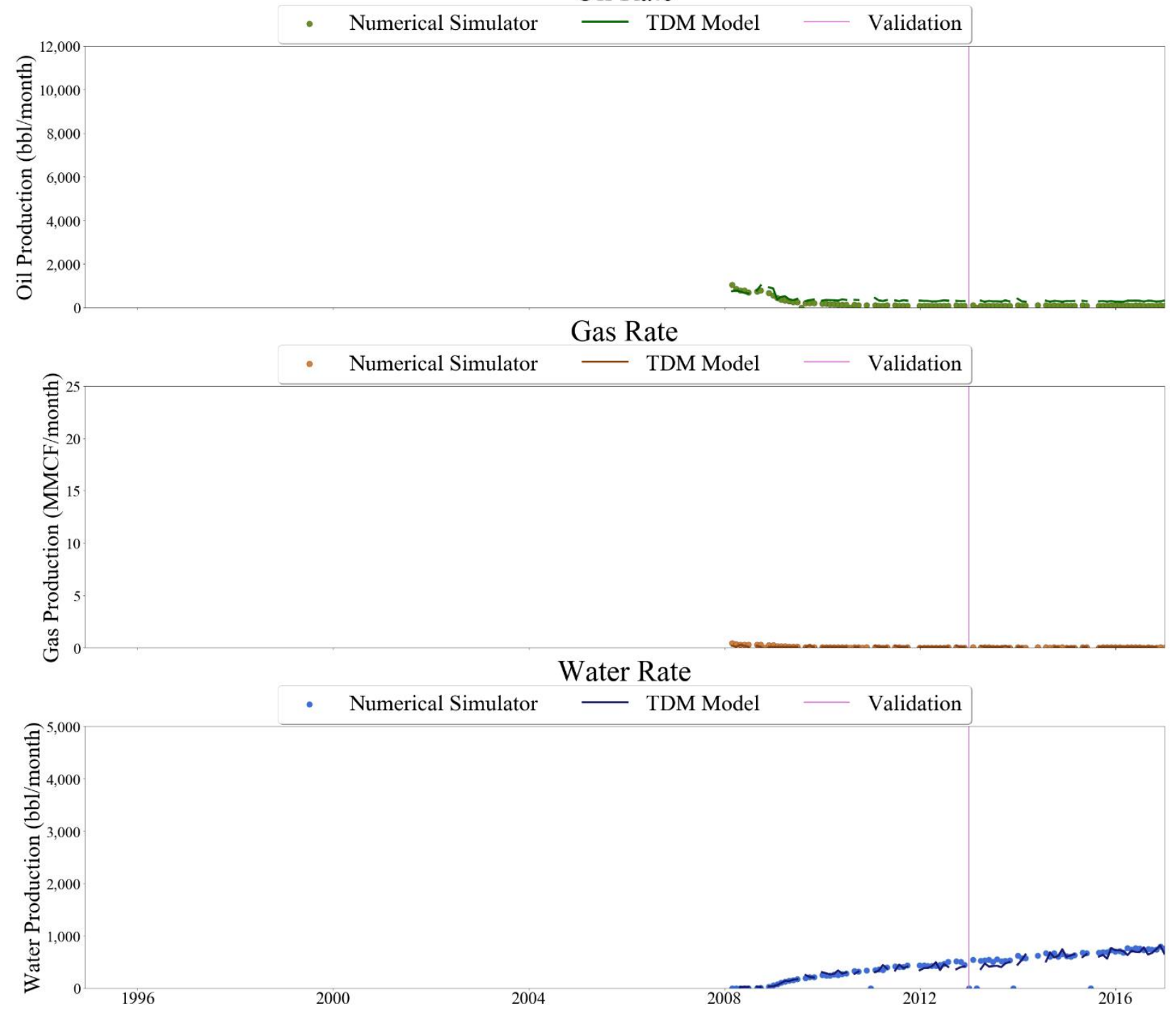

Figure 699 Well-044 oil, gas, and water rate TDM predictions vs actual simulation data plots for 4 Year Blind Validation TDM 

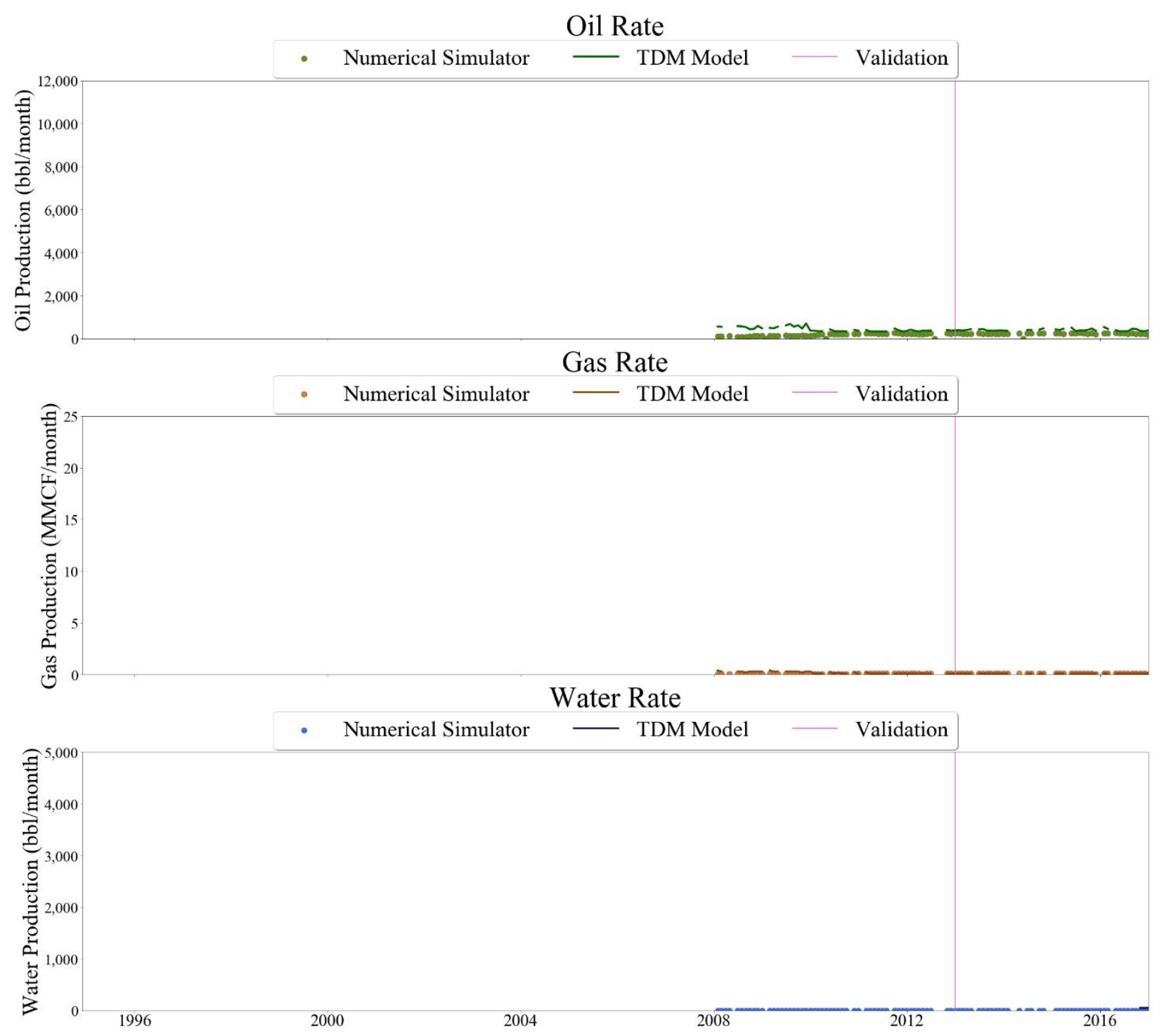

Figure 700 Well-045 oil, gas, and water rate TDM predictions vs actual simulation data plots for 4 Year Blind Validation TDM 


\section{Oil Rate}
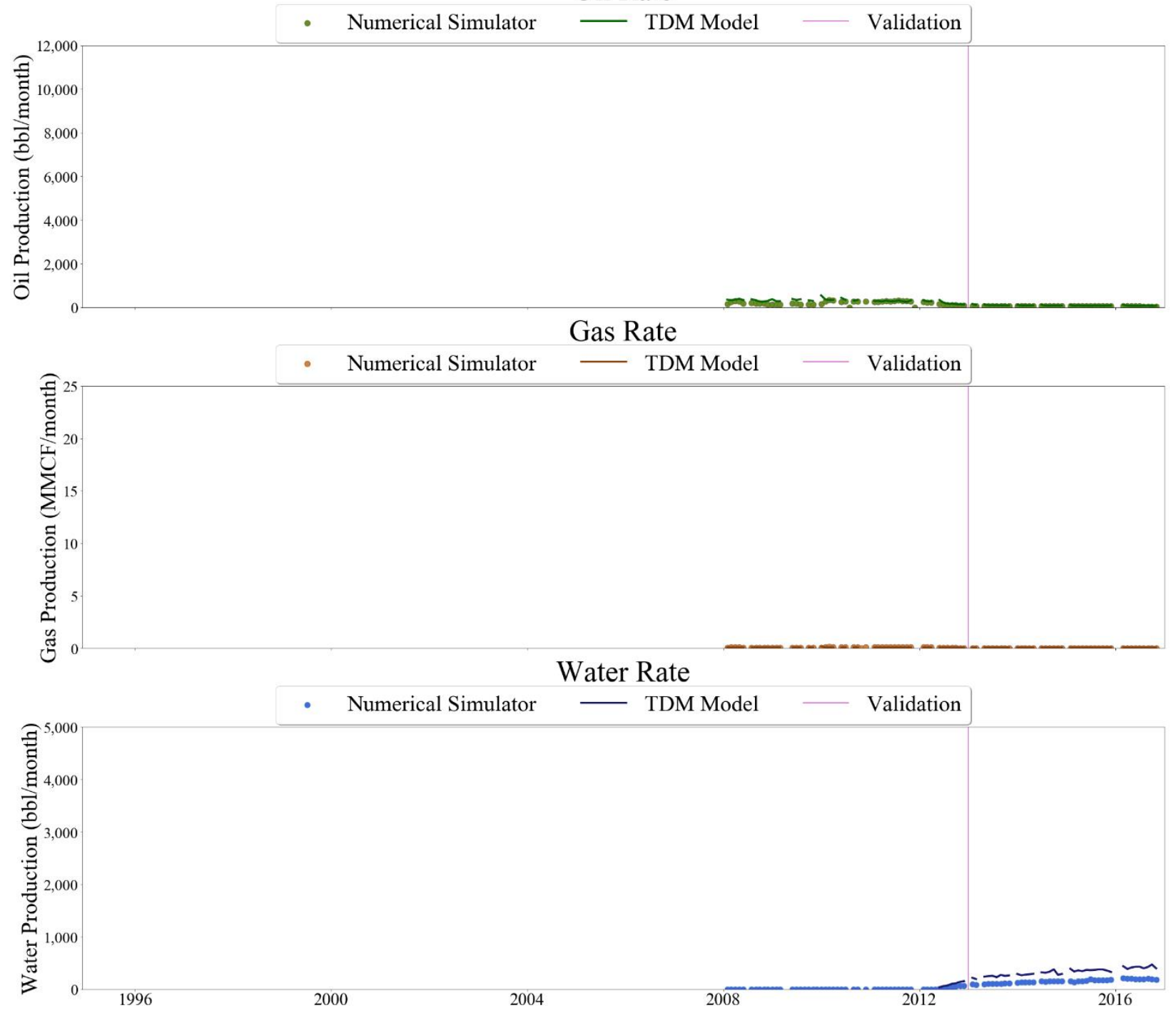

Figure 701 Well-046 oil, gas, and water rate TDM predictions vs actual simulation data plots for 4 Year Blind Validation TDM 


\section{Oil Rate}

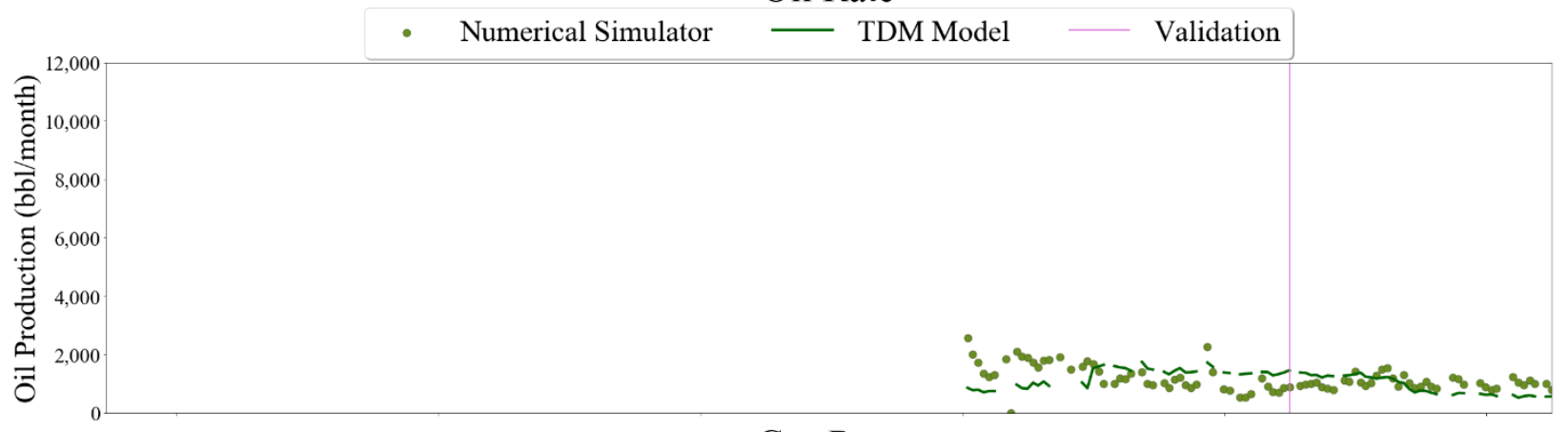

\section{Gas Rate}
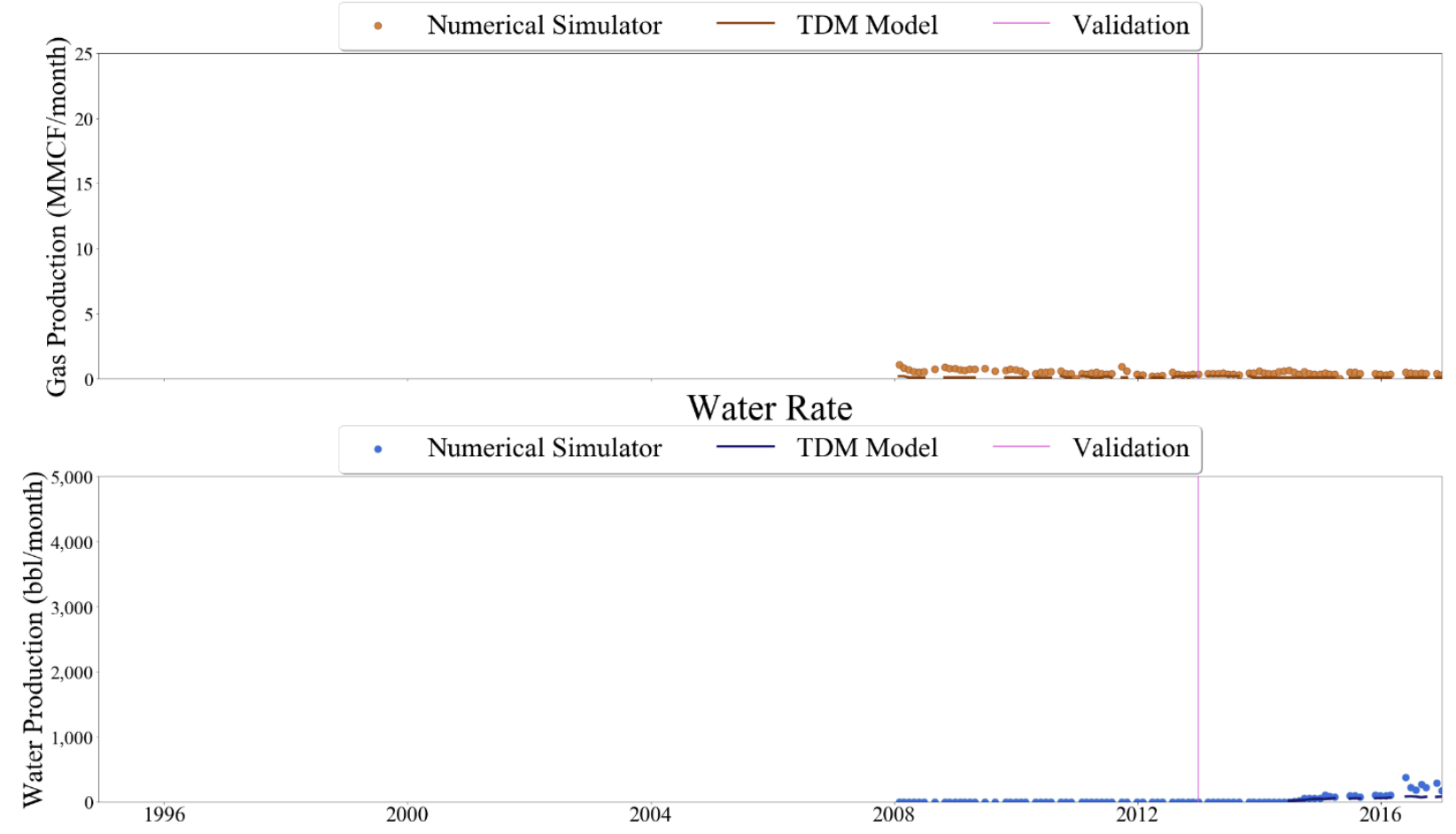

Figure 702 Well-047 oil, gas, and water rate TDM predictions vs actual simulation data plots for 4 Year Blind Validation TDM 


\section{Oil Rate}

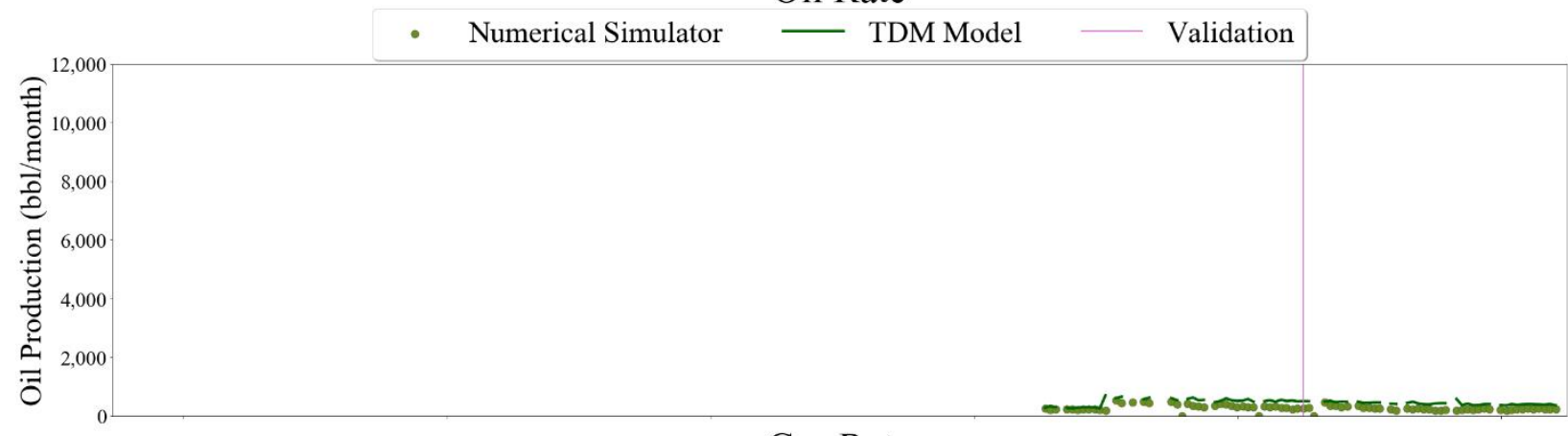

\section{Gas Rate}

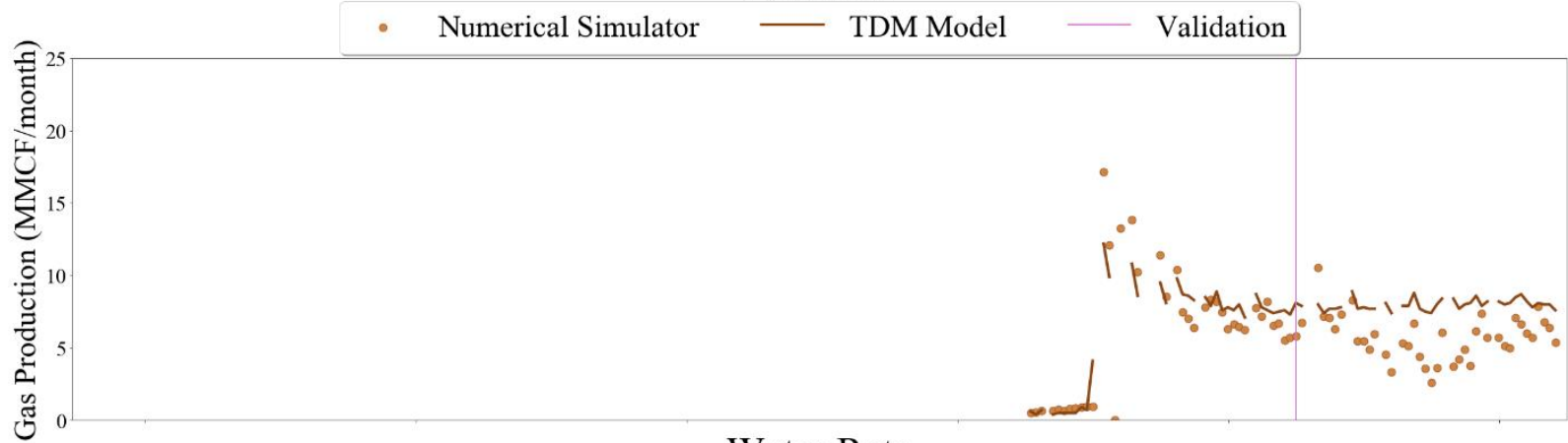

Water Rate

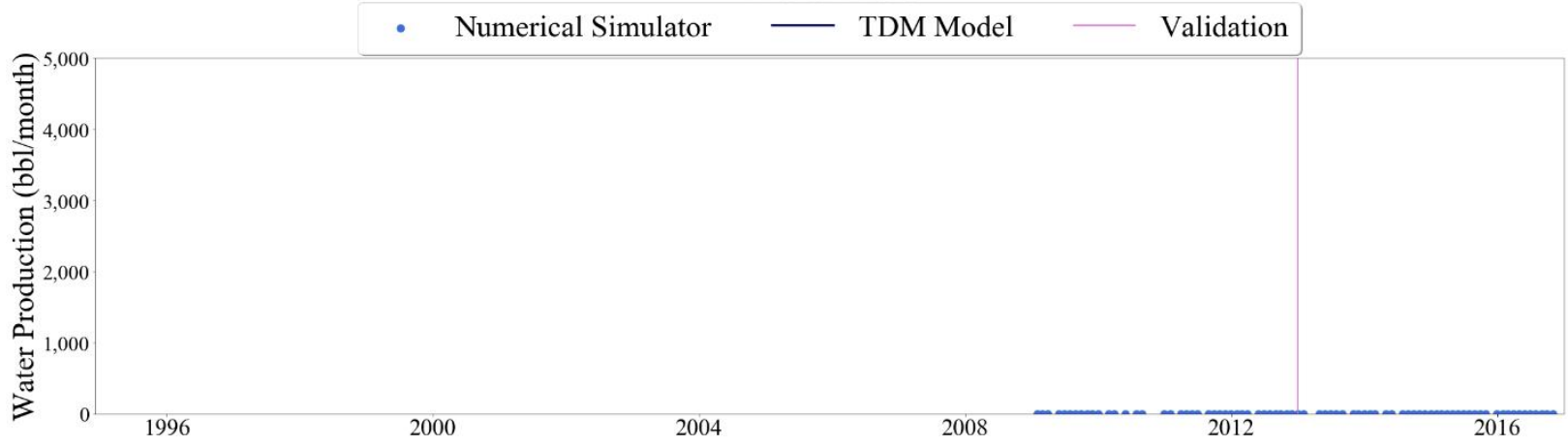

Figure 703 Well-048 oil, gas, and water rate TDM predictions vs actual simulation data plots for 4 Year Blind Validation TDM 


\section{Oil Rate}
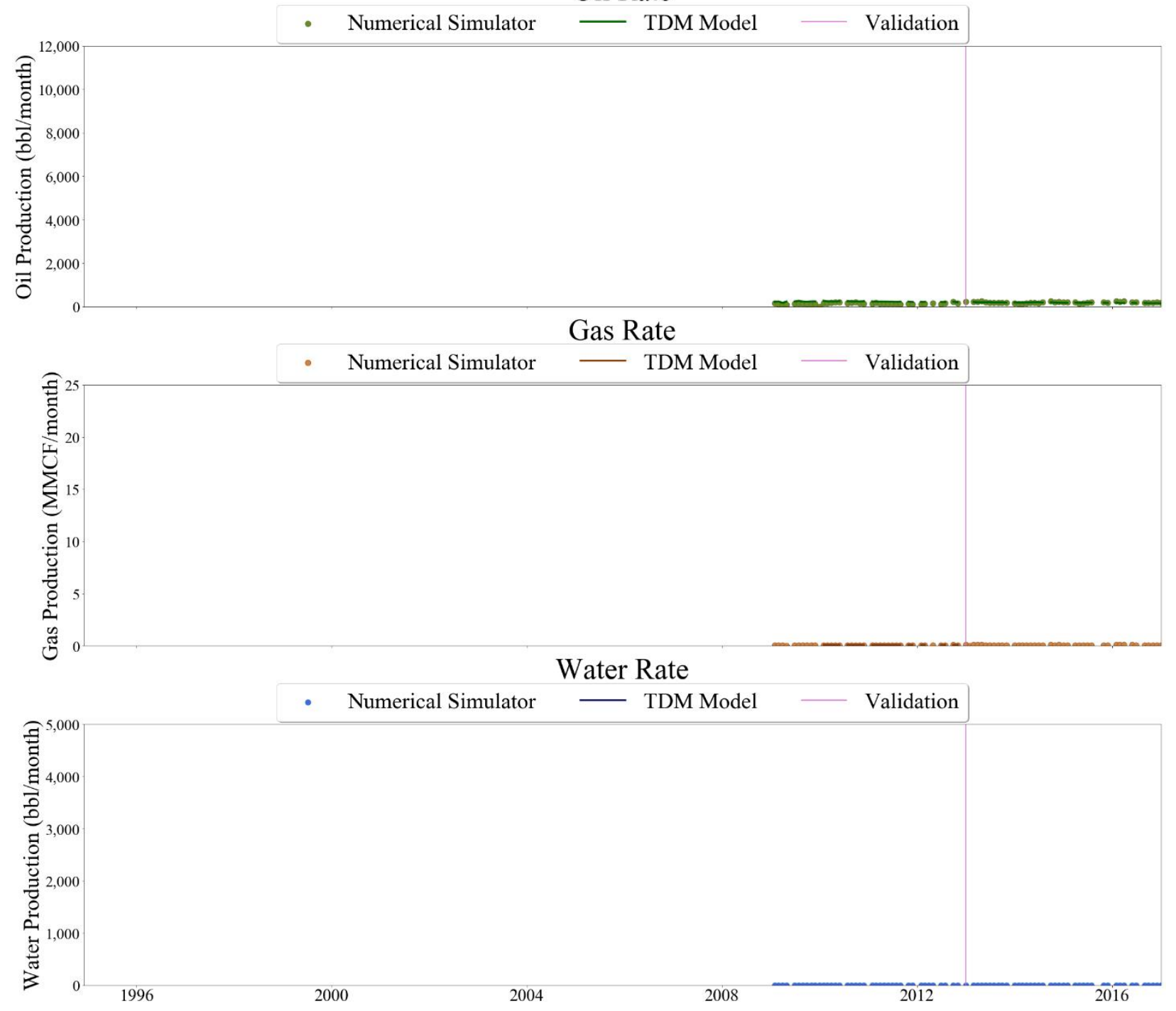

Figure 704 Well-049 oil, gas, and water rate TDM predictions vs actual simulation data plots for 4 Year Blind Validation TDM 


\section{Oil Rate}
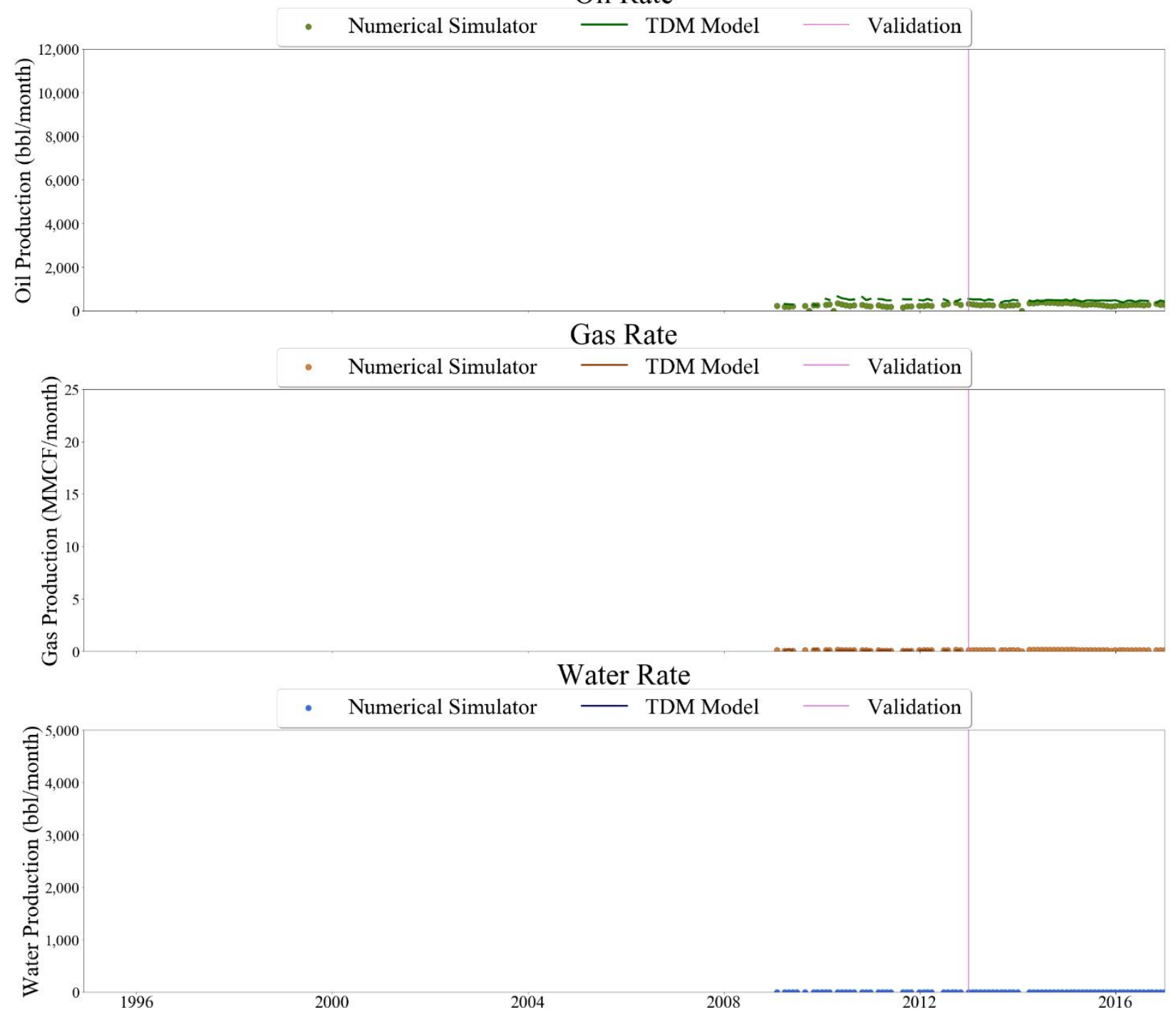

Figure 705 Well-050 oil, gas, and water rate TDM predictions vs actual simulation data plots for 4 Year Blind Validation TDM 


\section{Oil Rate}
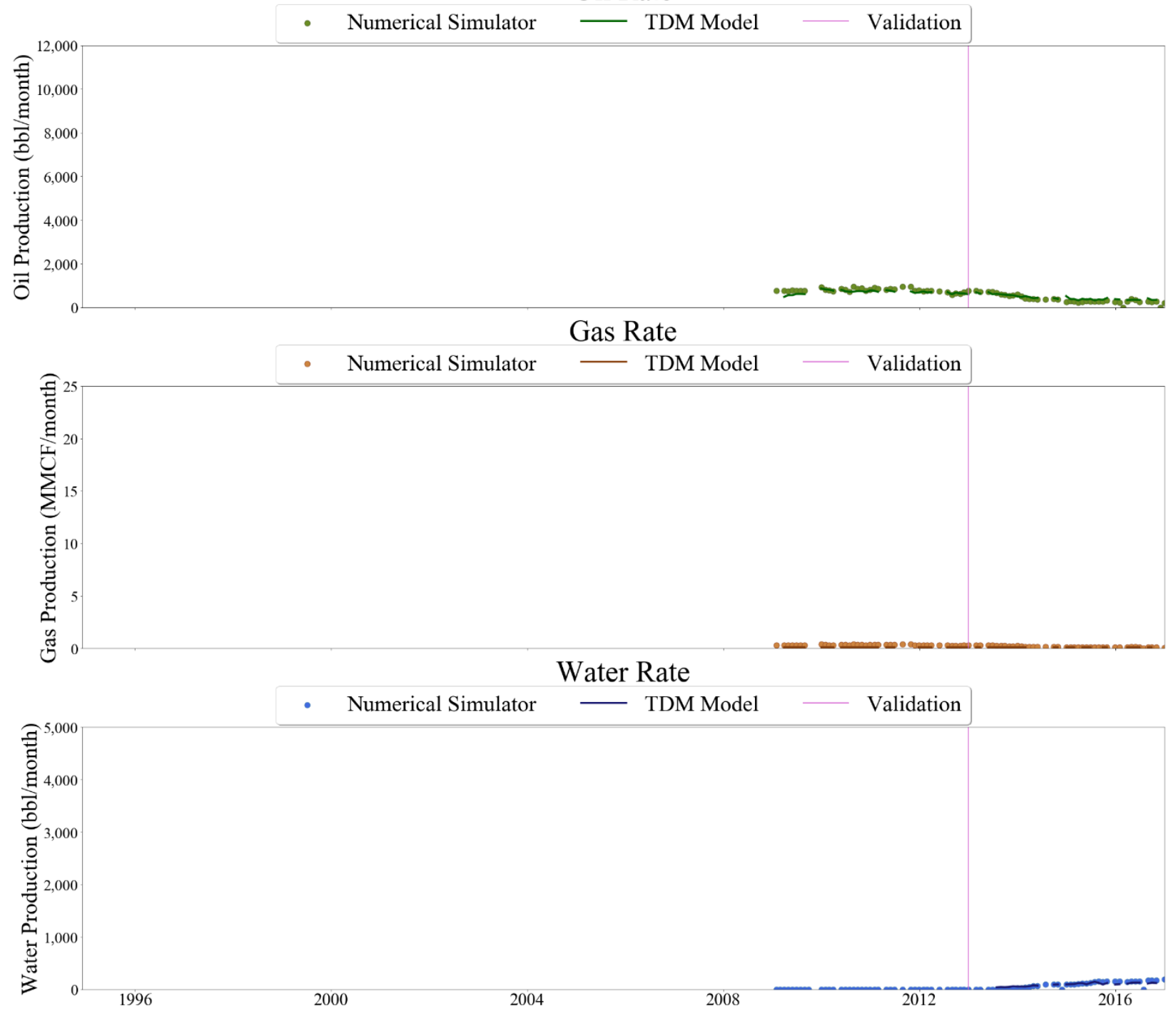

Figure 706 Well-051 oil, gas, and water rate TDM predictions vs actual simulation data plots for 4 Year Blind Validation TDM 


\section{Oil Rate}

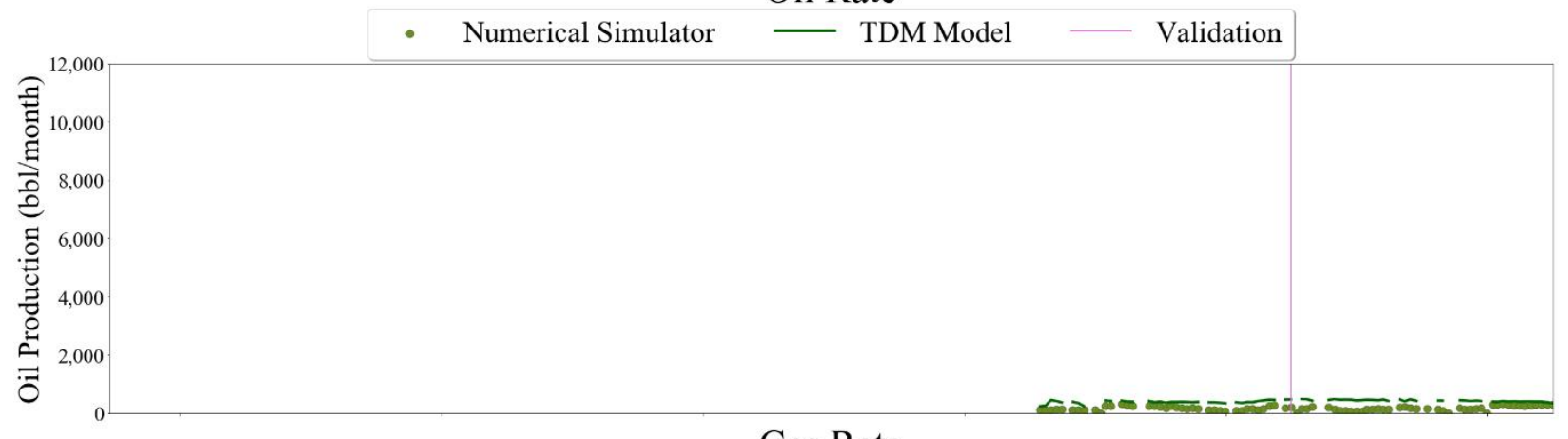

\section{Gas Rate}
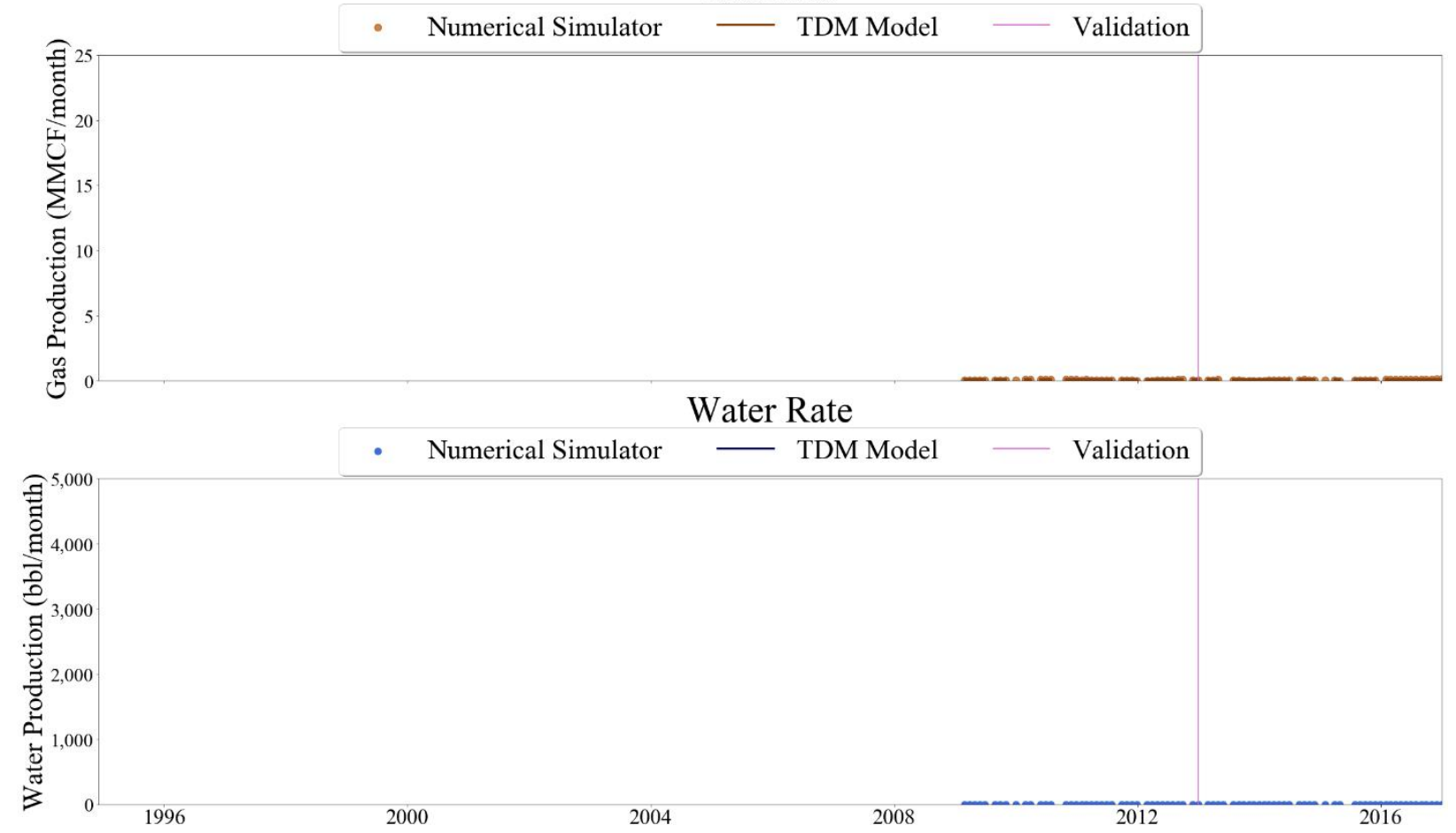

Figure 707 Well-052 oil, gas, and water rate TDM predictions vs actual simulation data plots for 4 Year Blind Validation TDM 


\section{Oil Rate}
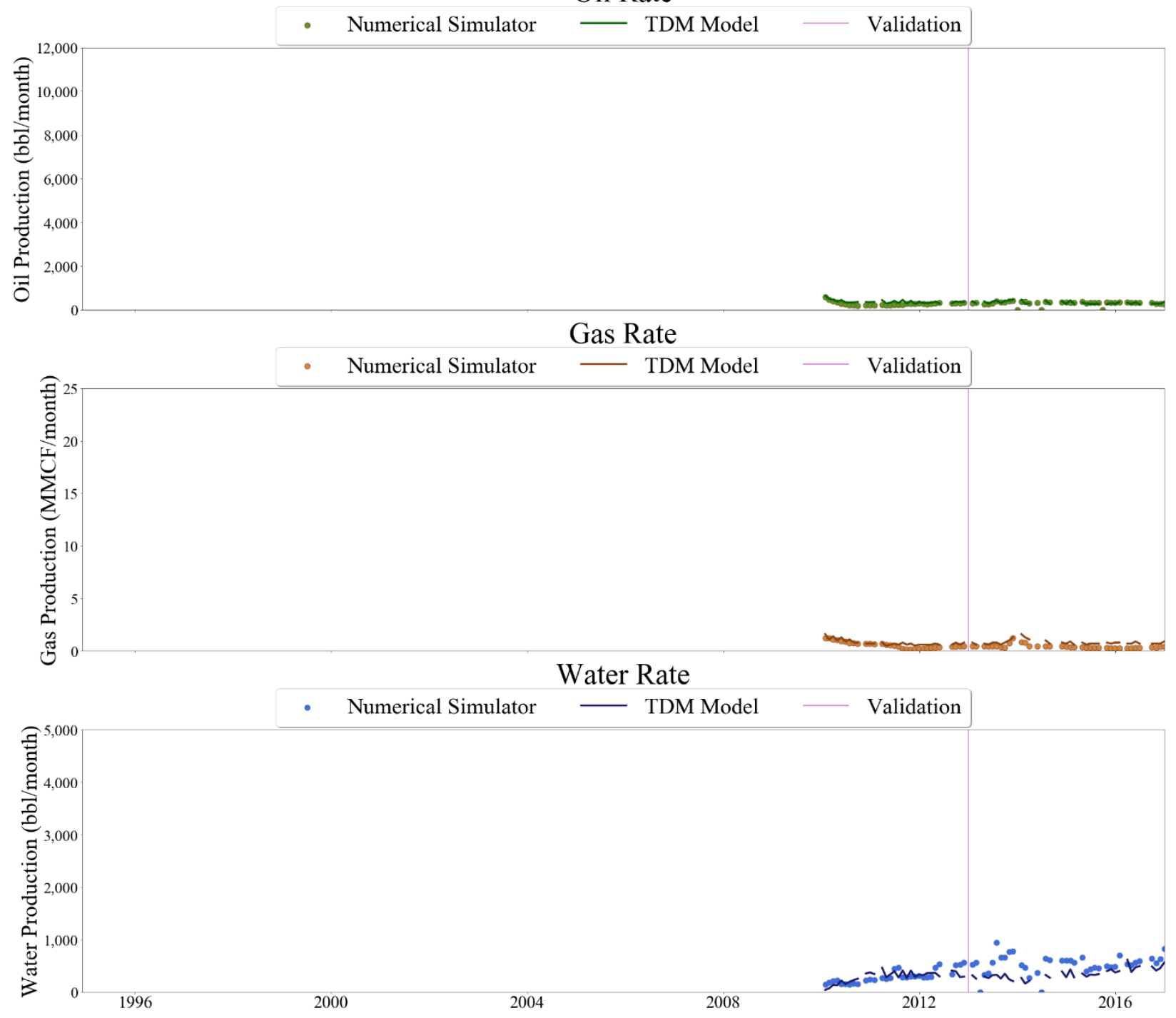

Figure 708 Well-053 oil, gas, and water rate TDM predictions vs actual simulation data plots for 4 Year Blind Validation TDM 


\section{Oil Rate}
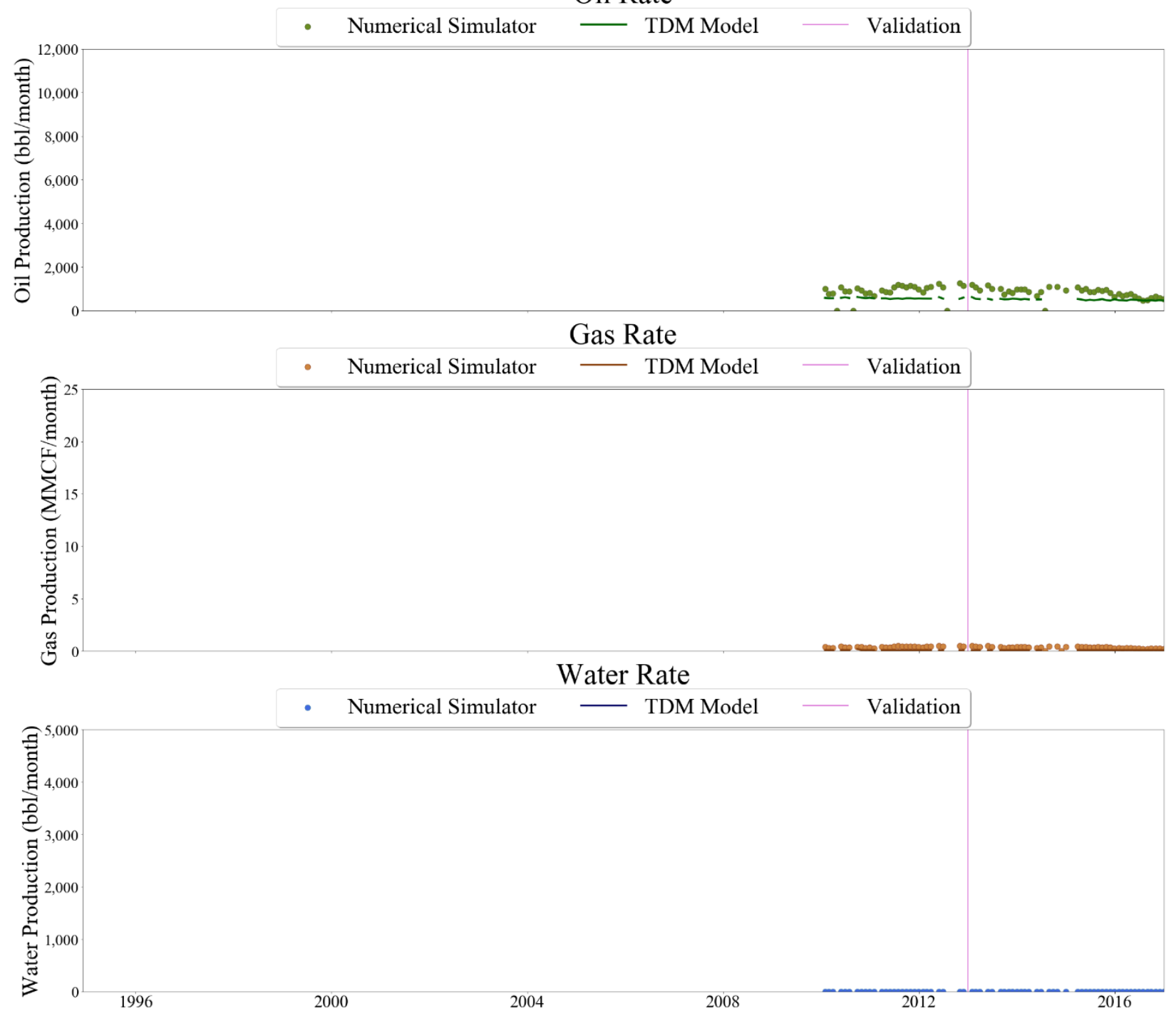

Figure 709 Well-054 oil, gas, and water rate TDM predictions vs actual simulation data plots for 4 Year Blind Validation TDM 


\section{Oil Rate}

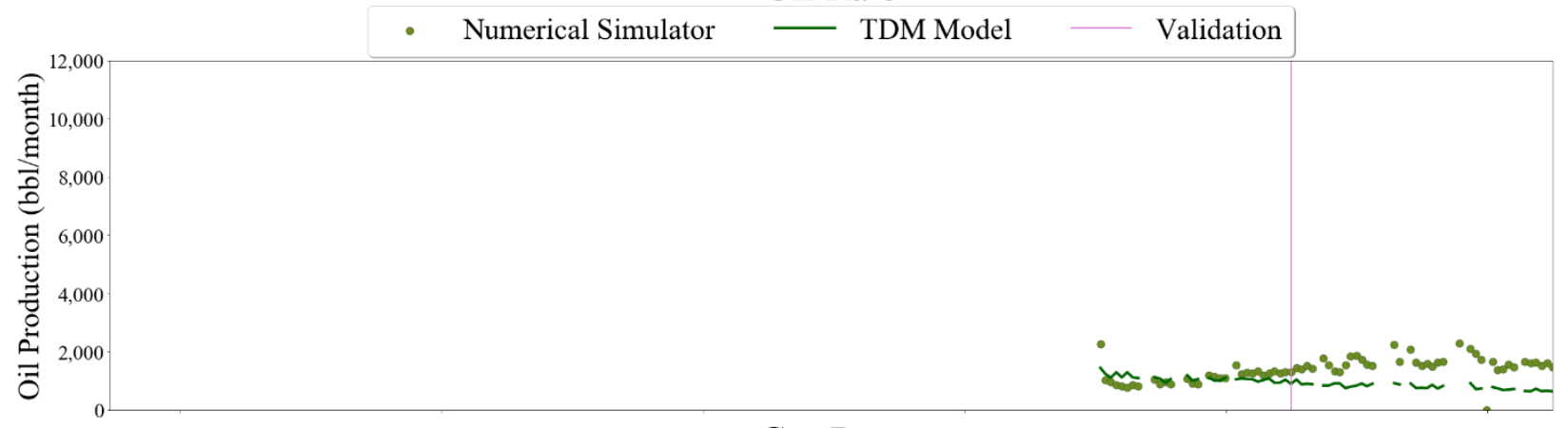

\section{Gas Rate}
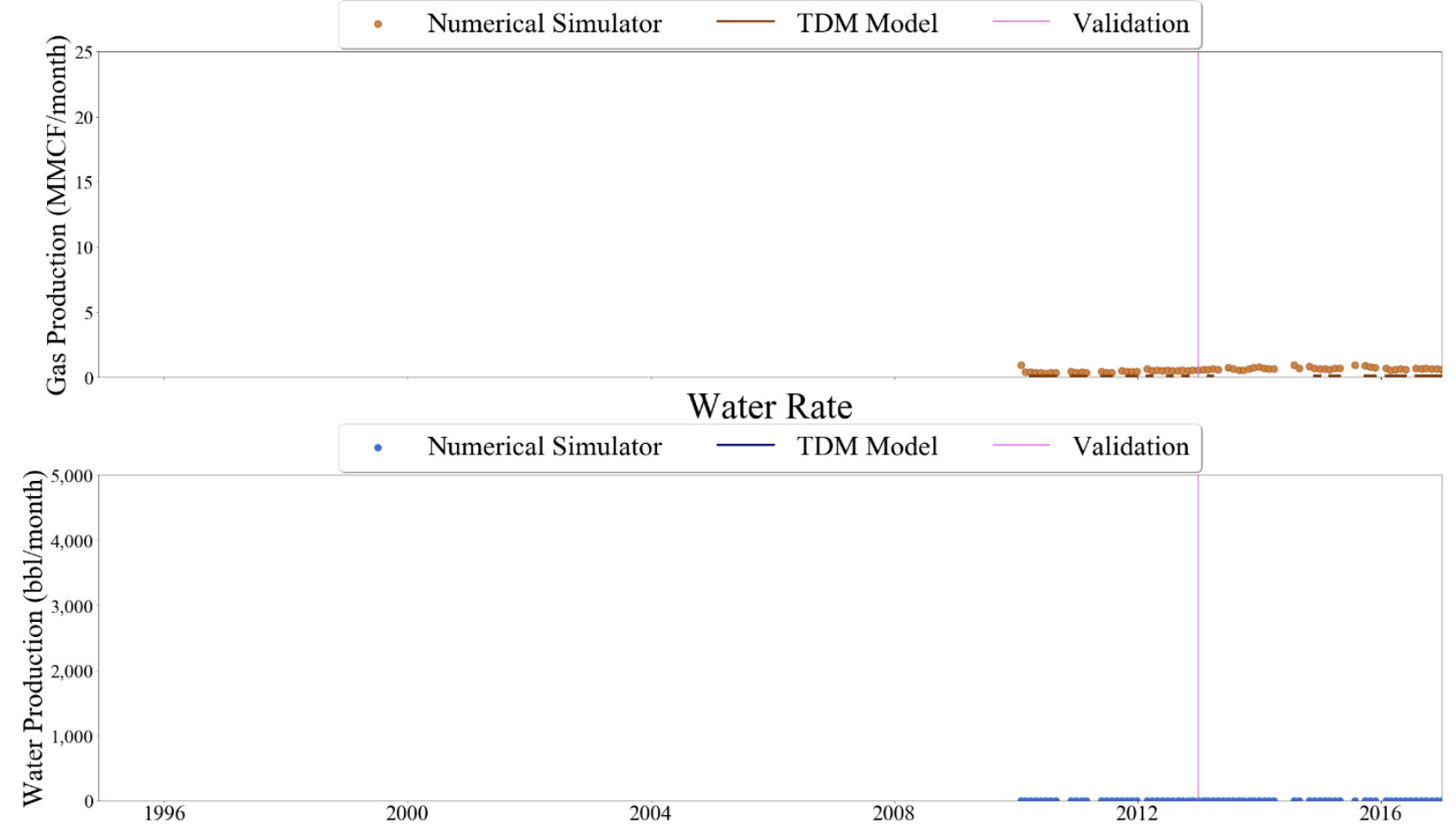

Figure 710 Well-055 oil, gas, and water rate TDM predictions vs actual simulation data plots for 4 Year Blind Validation TDM 


\section{Oil Rate}

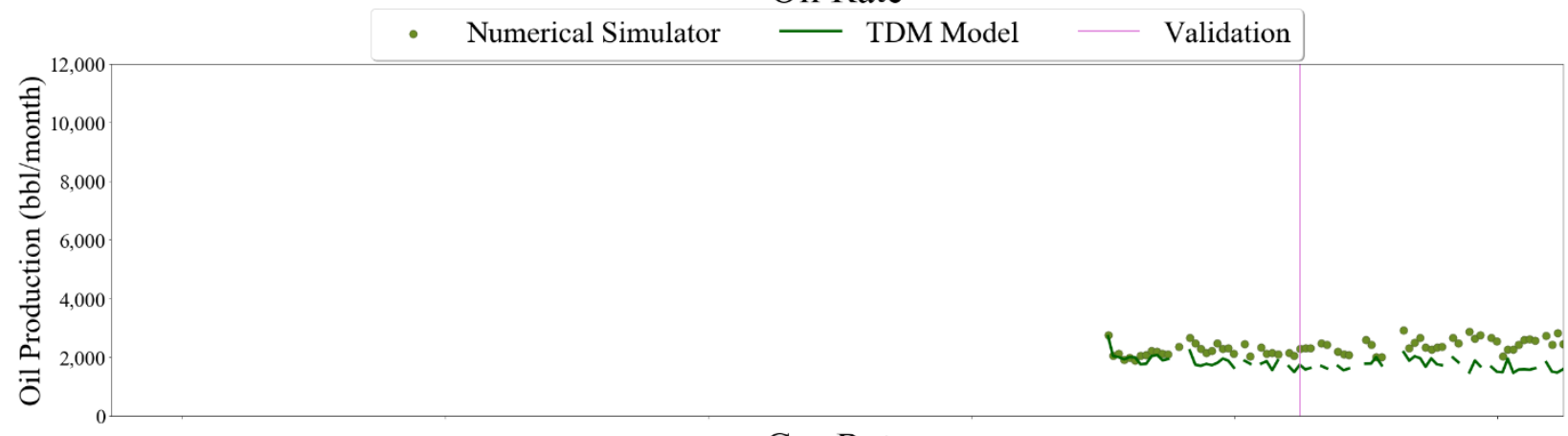

\section{Gas Rate}
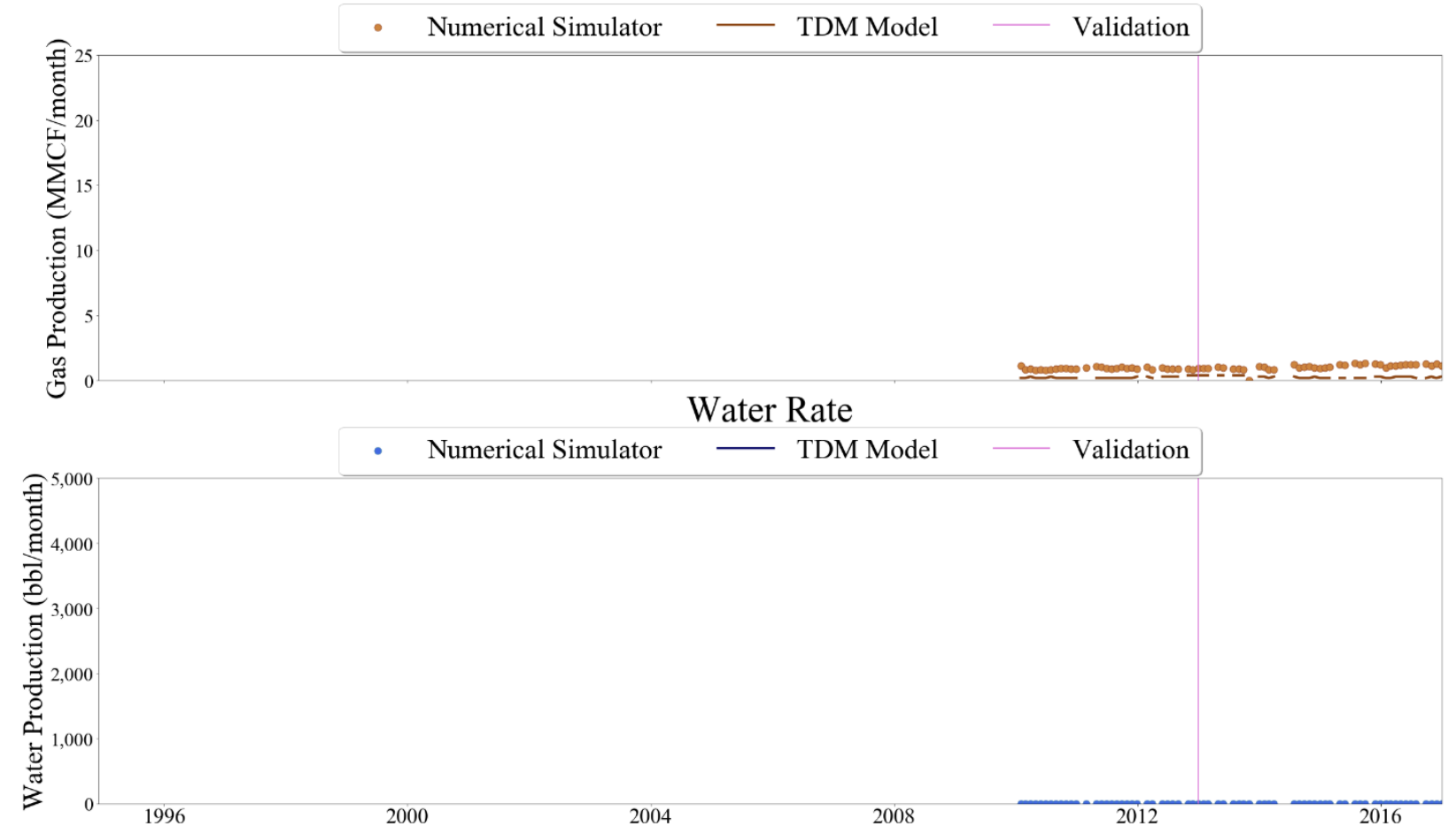

Figure 711 Well-056 oil, gas, and water rate TDM predictions vs actual simulation data plots for 4 Year Blind Validation TDM 


\section{Oil Rate}
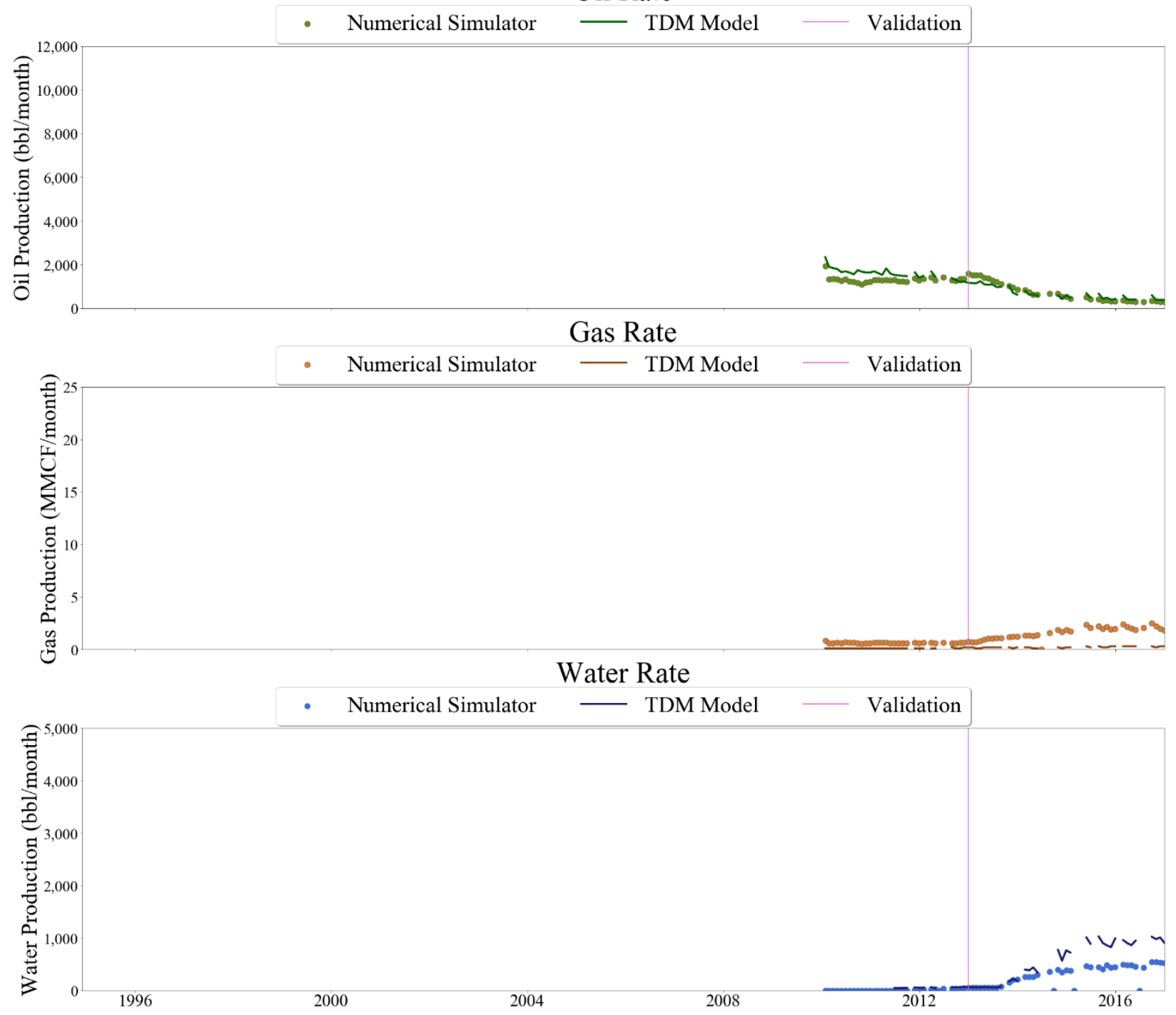

Figure 712 Well-057 oil, gas, and water rate TDM predictions vs actual simulation data plots for 4 Year Blind Validation TDM

\subsubsection{Heat Maps}

Reservoir Pressure (psi) at 2012-12-31
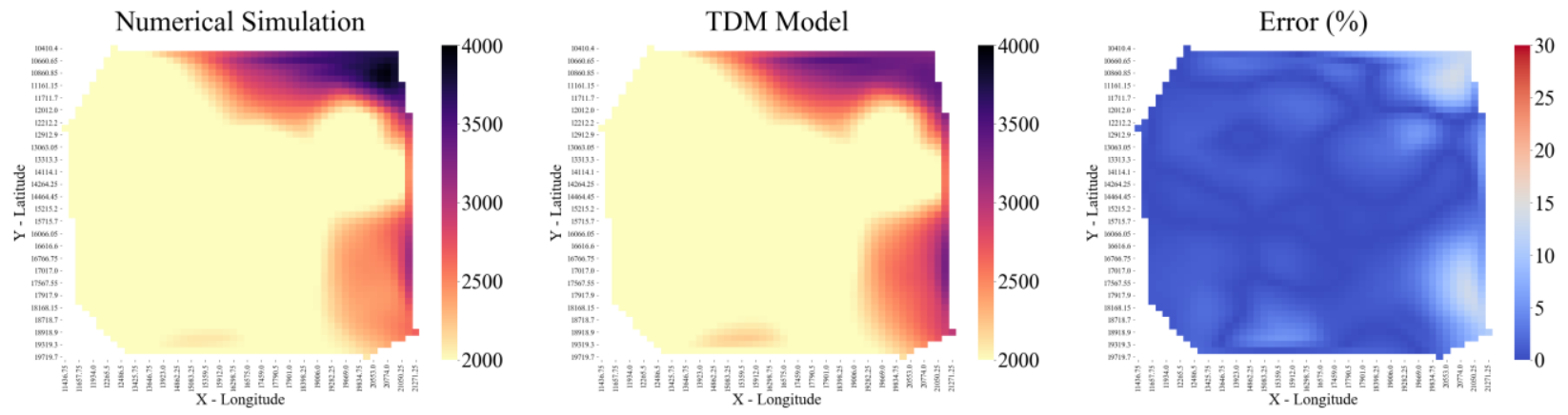

Figure 713 Entire reservoir heat map for reservoir pressure at December 31st, 2012 for 4 Year Blind Validation TDM 
Reservoir Pressure (psi) at 2013-03-31
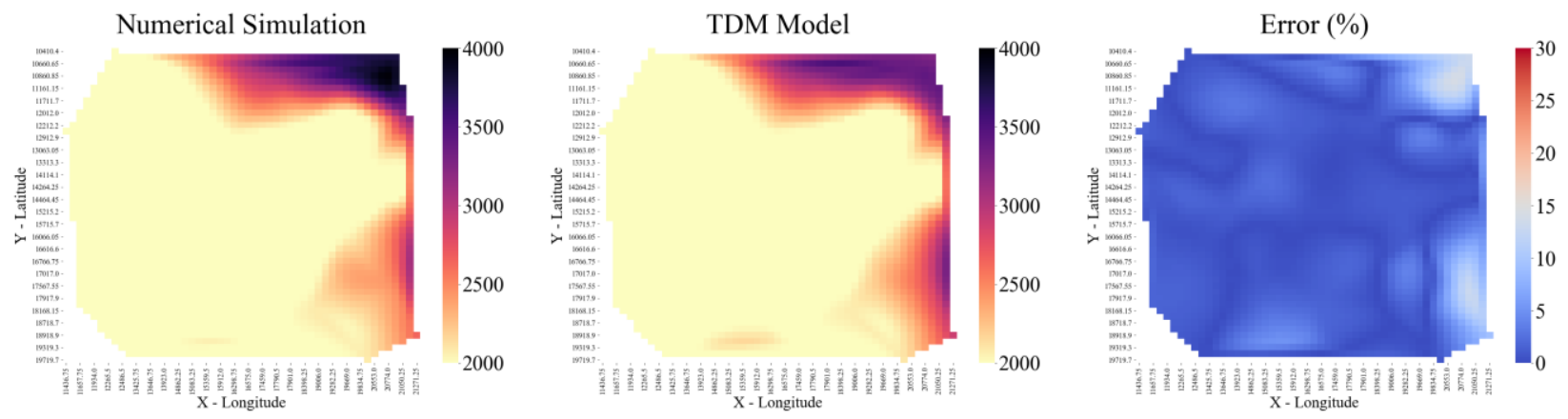

Figure 714 Entire reservoir heat map for reservoir pressure at March 31st, 2013 for 4 Year Blind Validation TDM

Reservoir Pressure (psi) at 2013-06-30
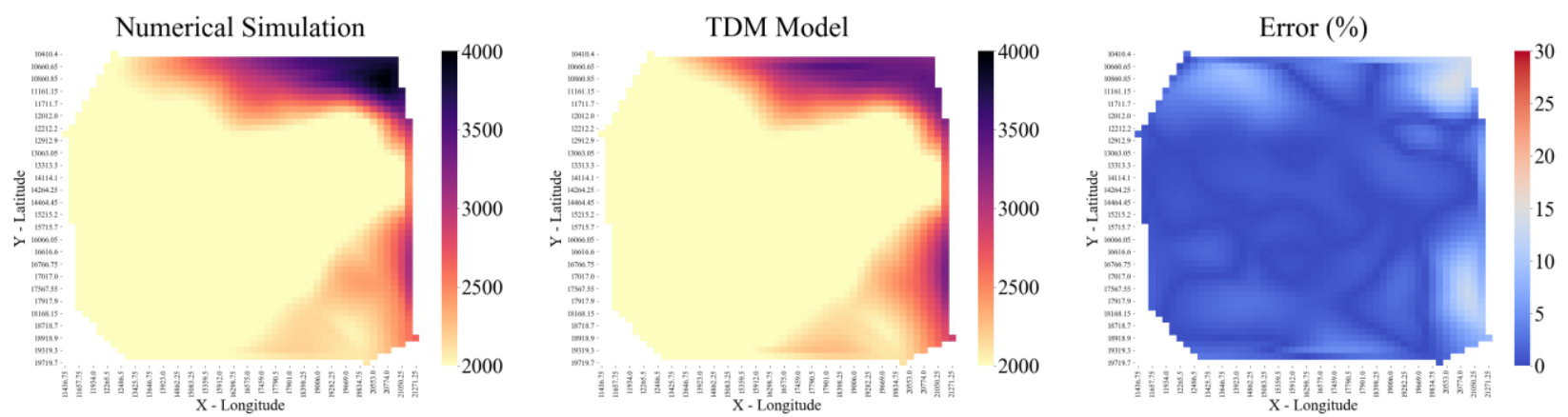

Figure 715 Entire reservoir heat map for reservoir pressure at June 30th, 2013 for 4 Year Blind Validation TDM

Reservoir Pressure (psi) at 2013-09-30
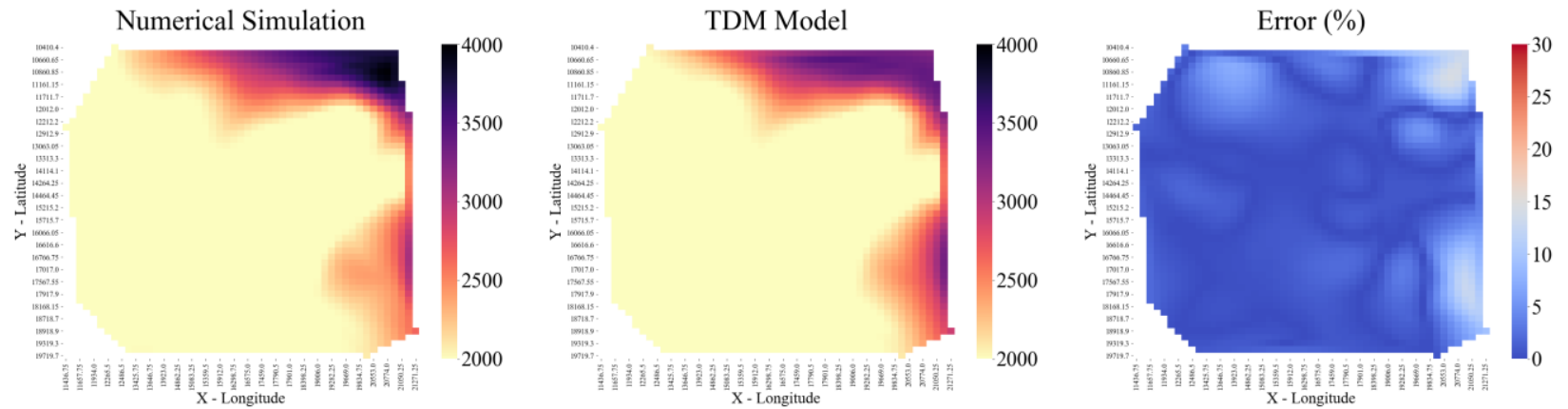

Figure 716 Entire reservoir heat map for reservoir pressure at September 30th, 2013 for 4 Year Blind Validation TDM 
Reservoir Pressure (psi) at 2013-12-31
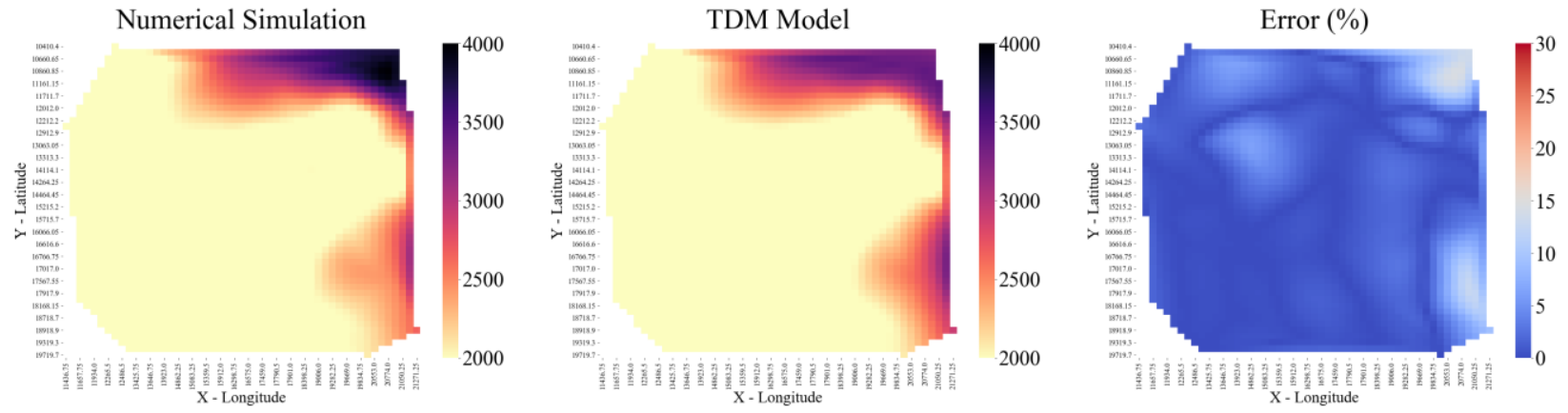

Figure 717 Entire reservoir heat map for reservoir pressure at December 31st, 2013 for 4 Year Blind Validation TDM

Reservoir Pressure (psi) at 2014-03-31
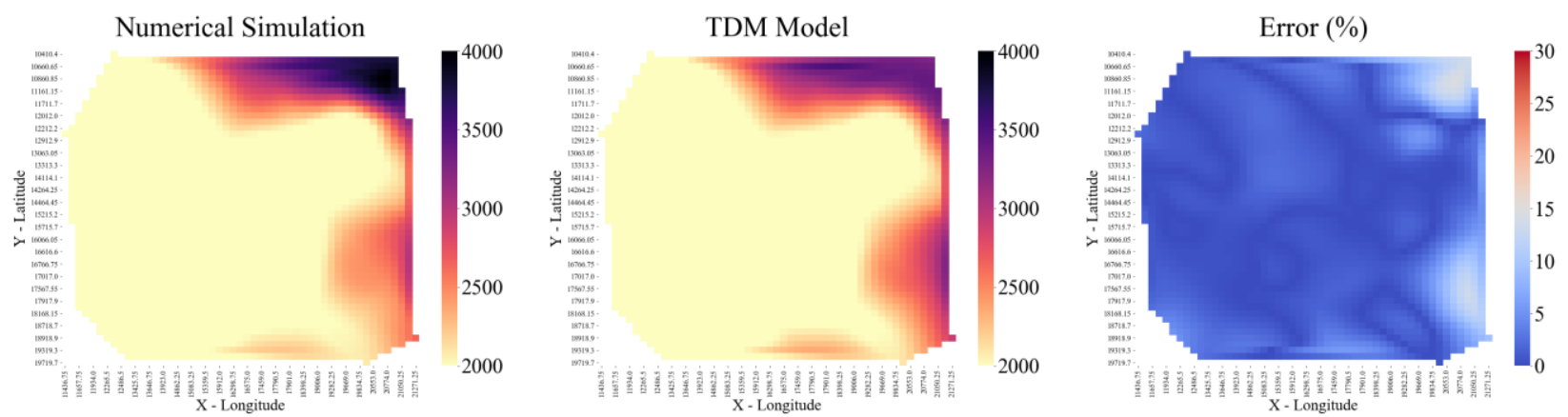

Figure 718 Entire reservoir heat map for reservoir pressure at March 31st, 2014 for 4 Year Blind Validation TDM

Reservoir Pressure (psi) at 2014-06-30
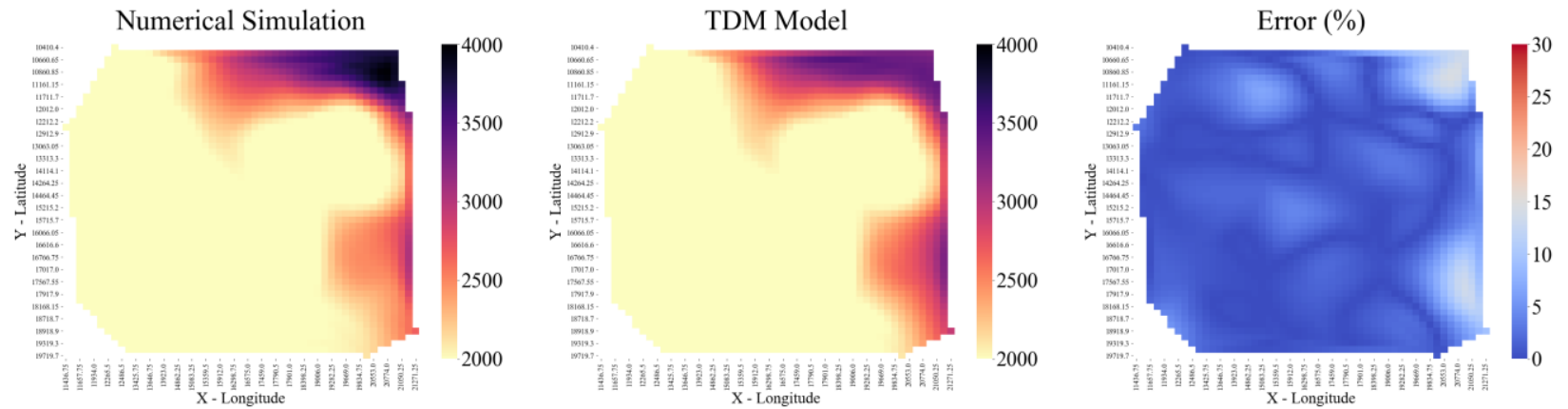

Figure 719 Entire reservoir heat map for reservoir pressure at June 30th, 2014 for 4 Year Blind Validation TDM 
Reservoir Pressure (psi) at 2014-09-30
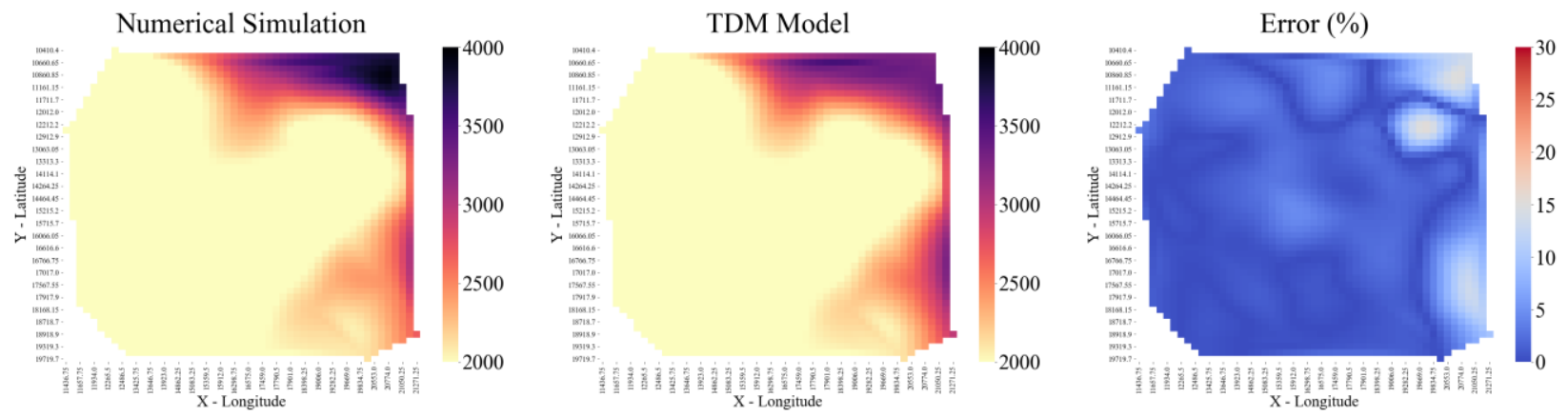

Figure 720 Entire reservoir heat map for reservoir pressure at September 30th, 2014 for 4 Year Blind Validation TDM

Reservoir Pressure (psi) at 2014-12-31
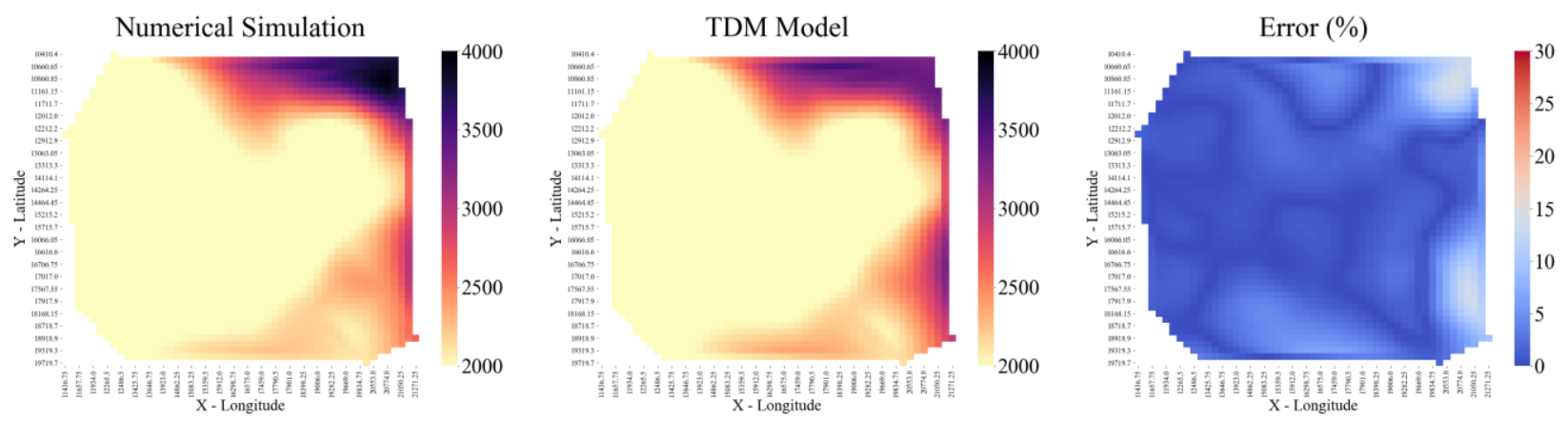

Figure 721 Entire reservoir heat map for reservoir pressure at December 31st, 2014 for 4 Year Blind Validation TDM

Reservoir Pressure (psi) at 2015-03-31
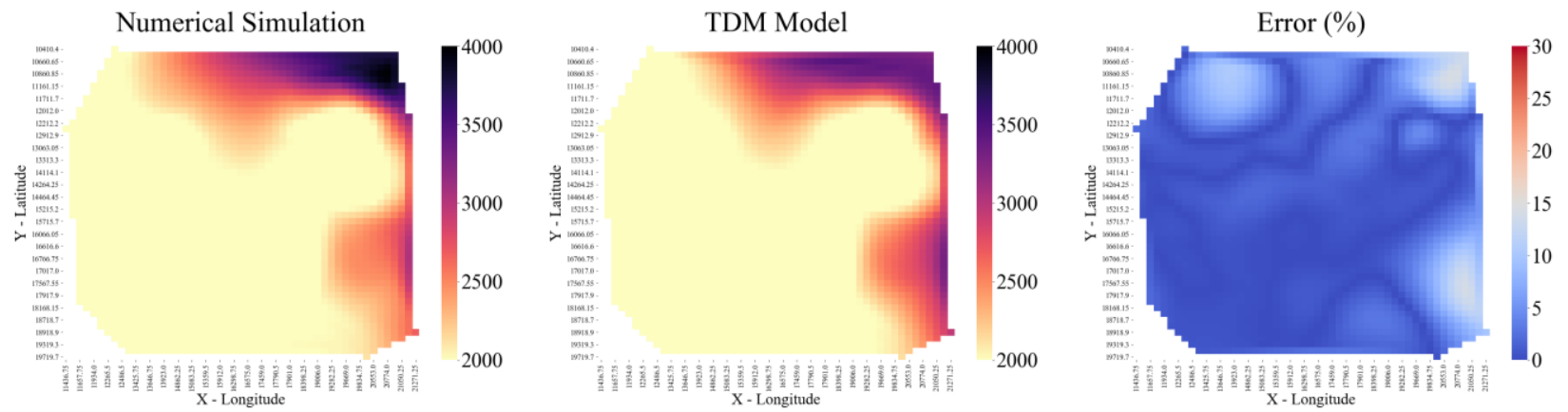

Figure 722 Entire reservoir heat map for reservoir pressure at March 31st, 2015 for 4 Year Blind Validation TDM 
Reservoir Pressure (psi) at 2015-06-30
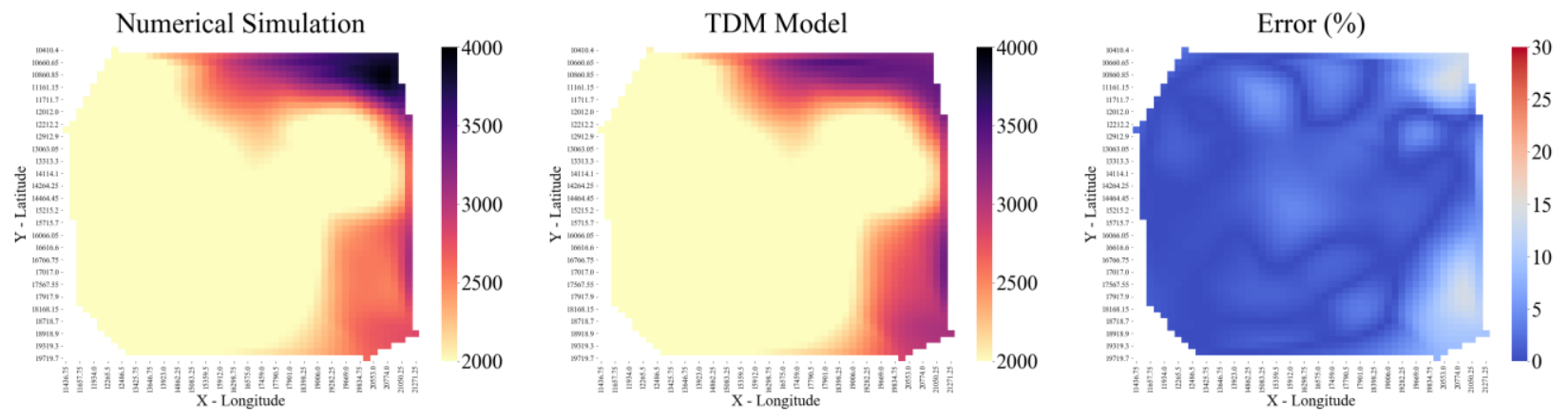

Figure 723 Entire reservoir heat map for reservoir pressure at June 30th, 2015 for 4 Year Blind Validation TDM

Reservoir Pressure (psi) at 2015-09-30
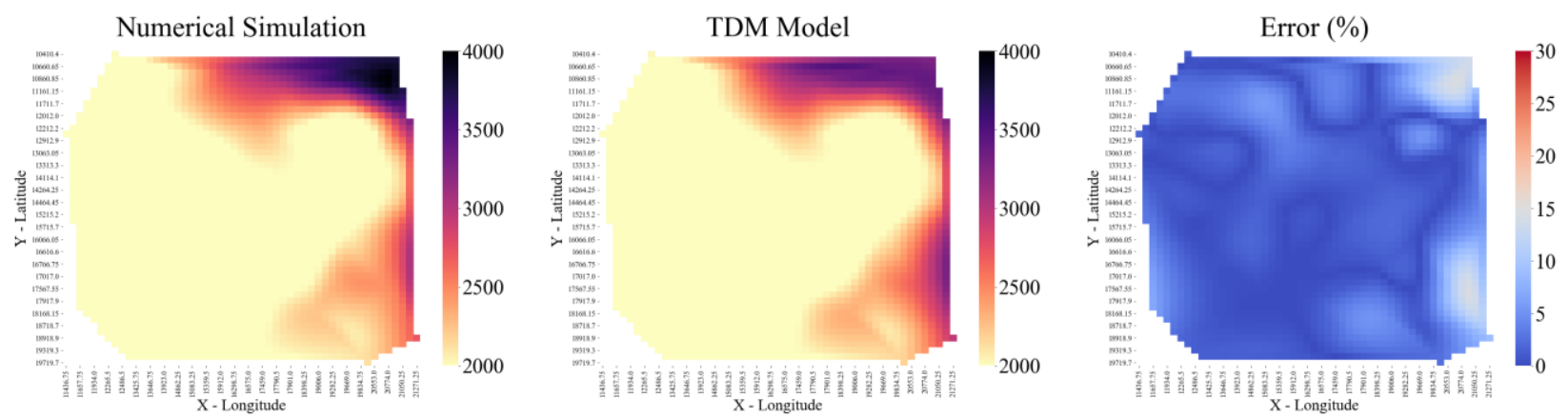

Figure 724 Entire reservoir heat map for reservoir pressure at September 30th, 2015 for 4 year Blind Validation TDM

Reservoir Pressure (psi) at 2015-12-31
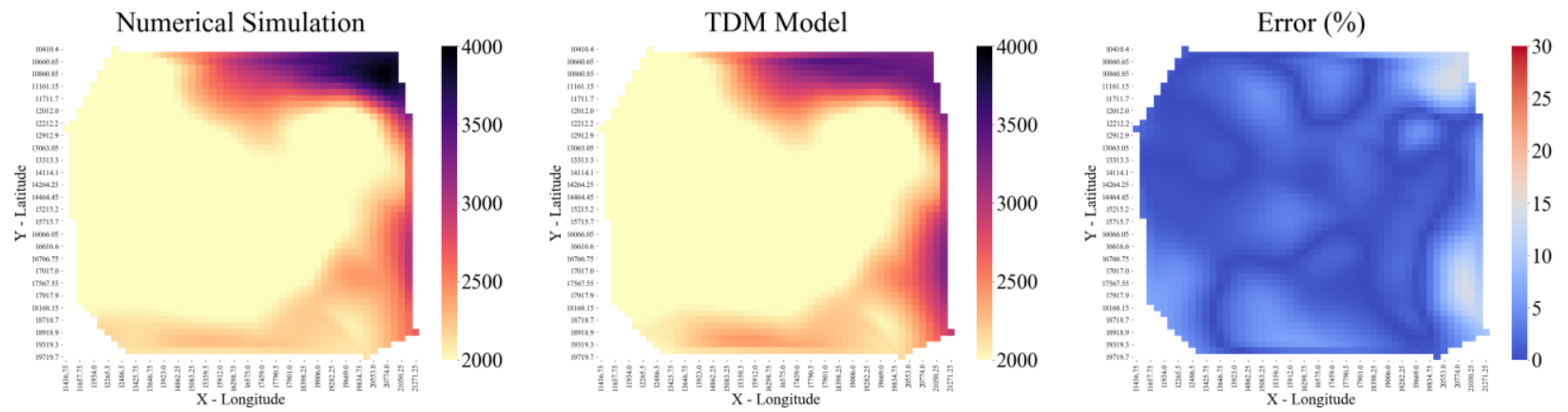

Figure 725 Entire reservoir heat map for reservoir pressure at December 31st, 2015 for 4 Year Blind Validation TDM 
Reservoir Pressure (psi) at 2016-03-31
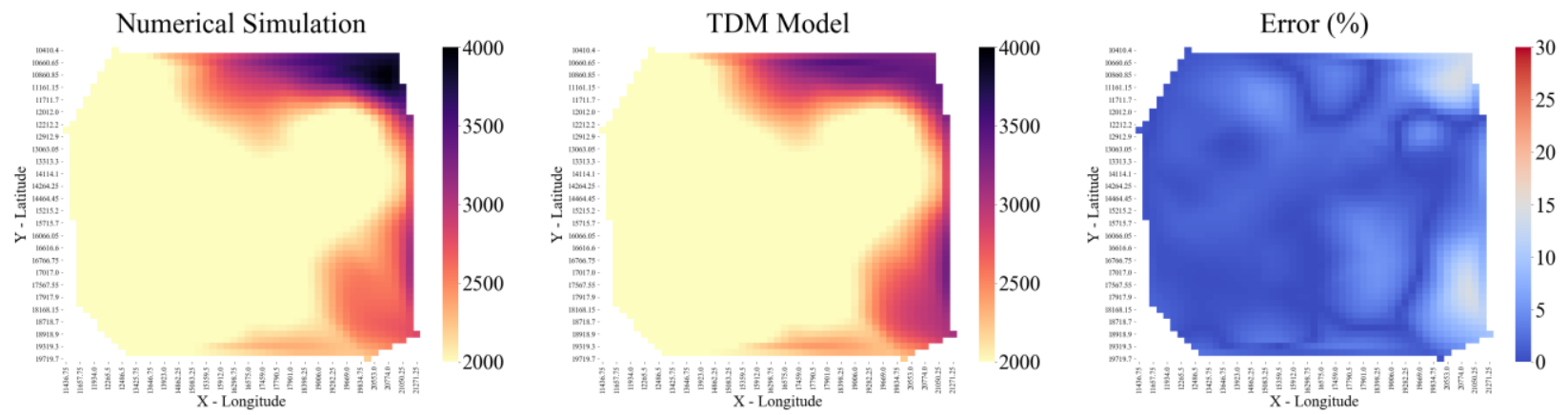

Figure 726 Entire reservoir heat map for reservoir pressure at March 31st, 2016 for 4 Year Blind Validation TDM

\section{Reservoir Pressure (psi) at 2016-06-30}
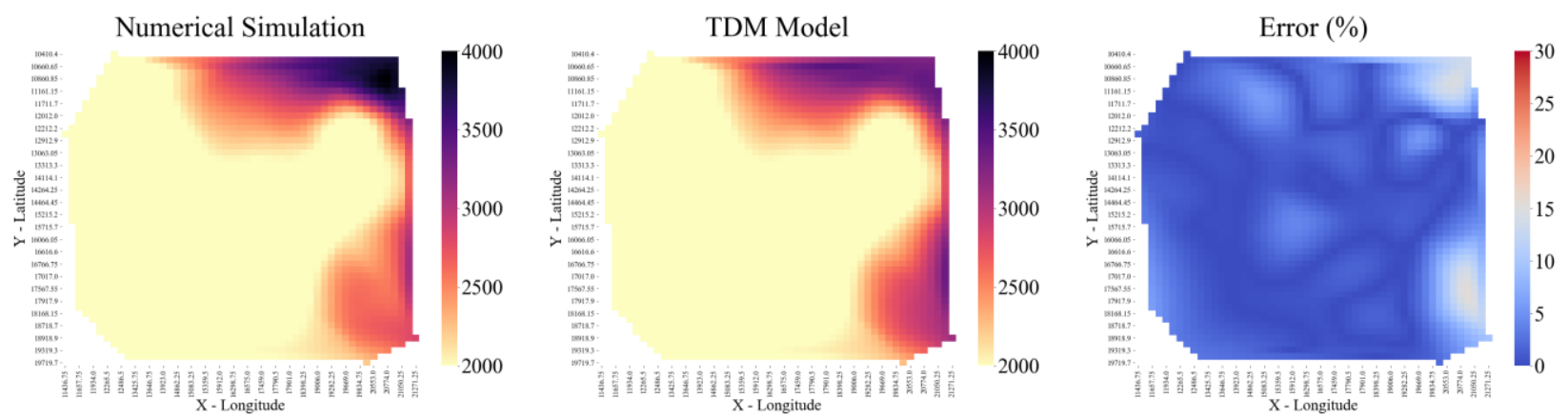

Figure 727 Entire reservoir heat map for reservoir pressure at June 30th, 2016 for 4 Year Blind Validation TDM

Reservoir Pressure (psi) at 2016-09-30
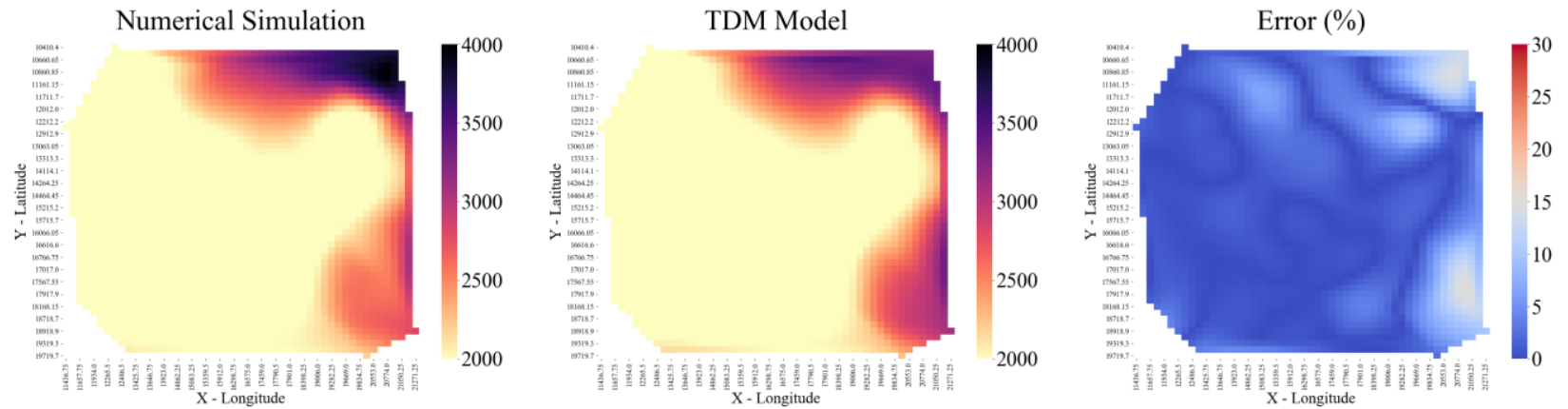

Figure 728 Entire reservoir heat map for reservoir pressure at September 30th, 2016 for 4 Year Blind Validation TDM 
Reservoir Pressure (psi) at 2016-12-31
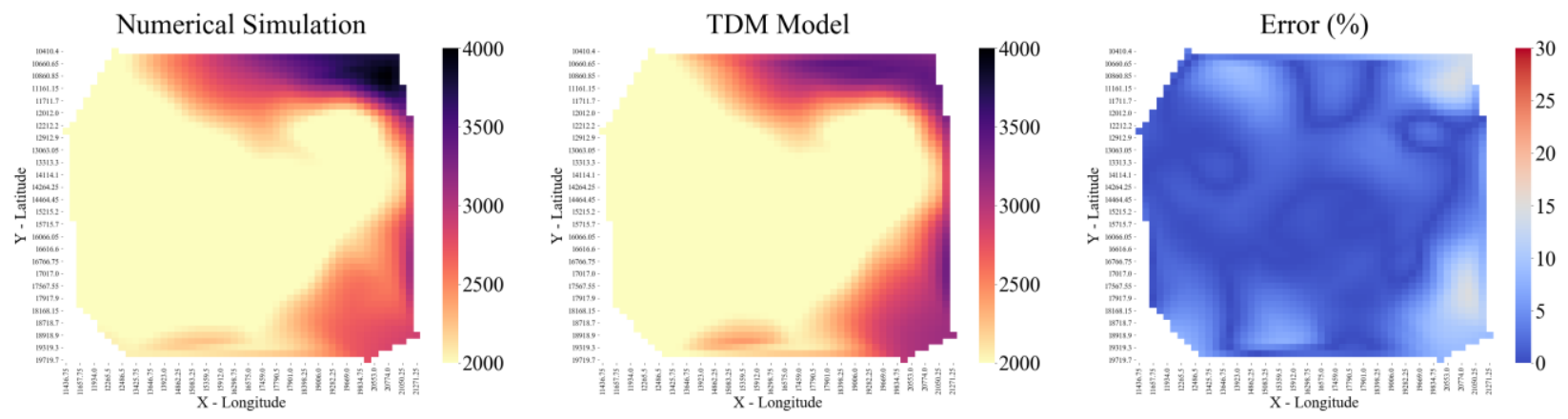

Figure 729 Entire reservoir heat map for reservoir pressure at December 31st, 2016 for 4 Year Blind Validation TDM

Water Saturation (\%) at 2012-12-31
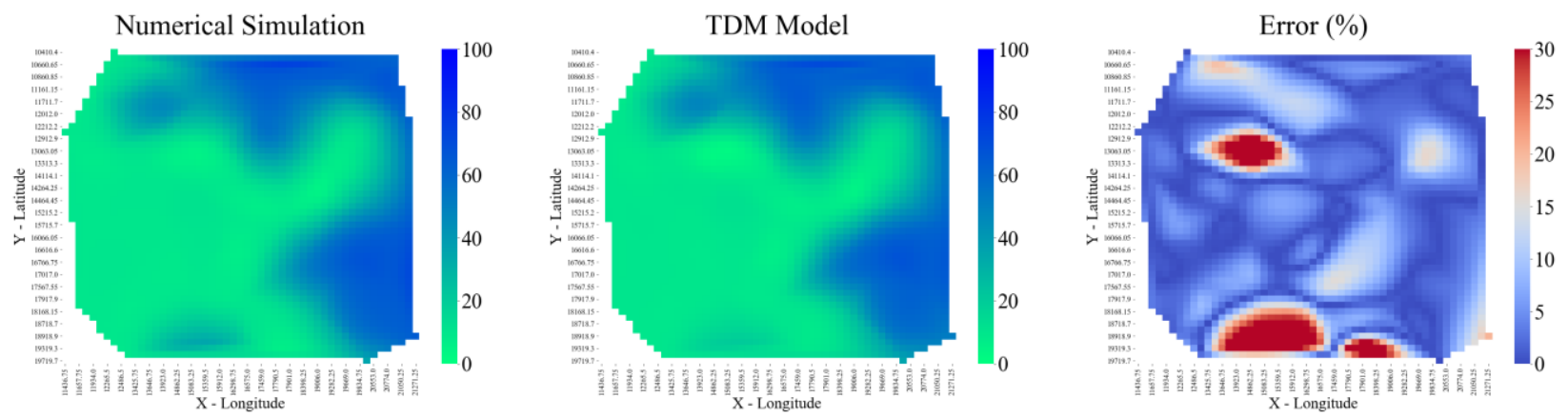

Figure 730 Entire reservoir heat map for water saturation at December 31 st, 2012 for 4 Year Blind Validation TDM

Water Saturation (\%) at 2013-03-31
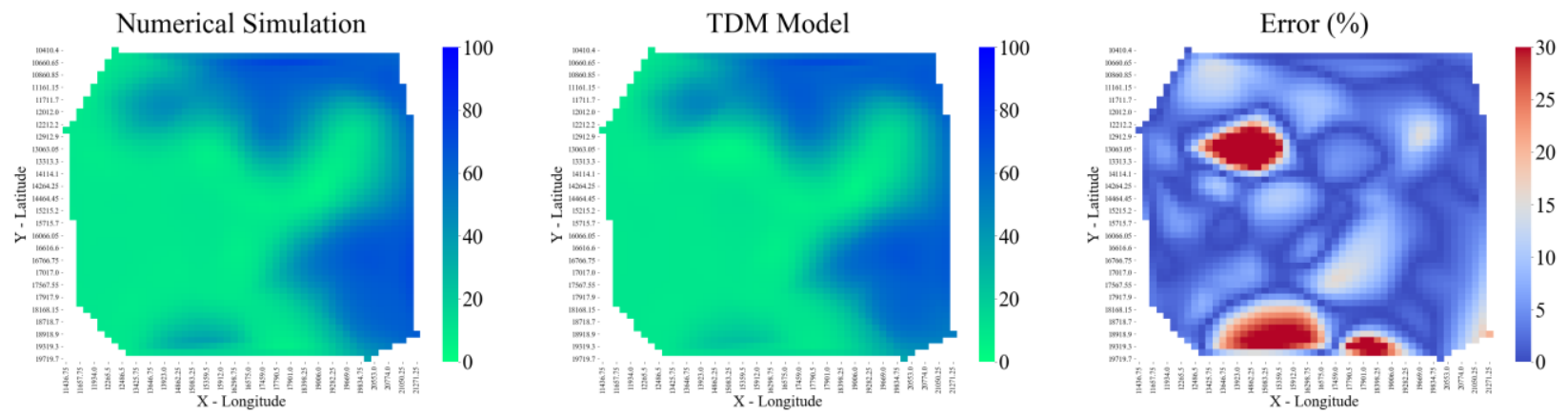

Figure 731 Entire reservoir heat map for water saturation at March 31st, 2013 for 4 Year Blind Validation TDM 
Water Saturation (\%) at 2013-06-30
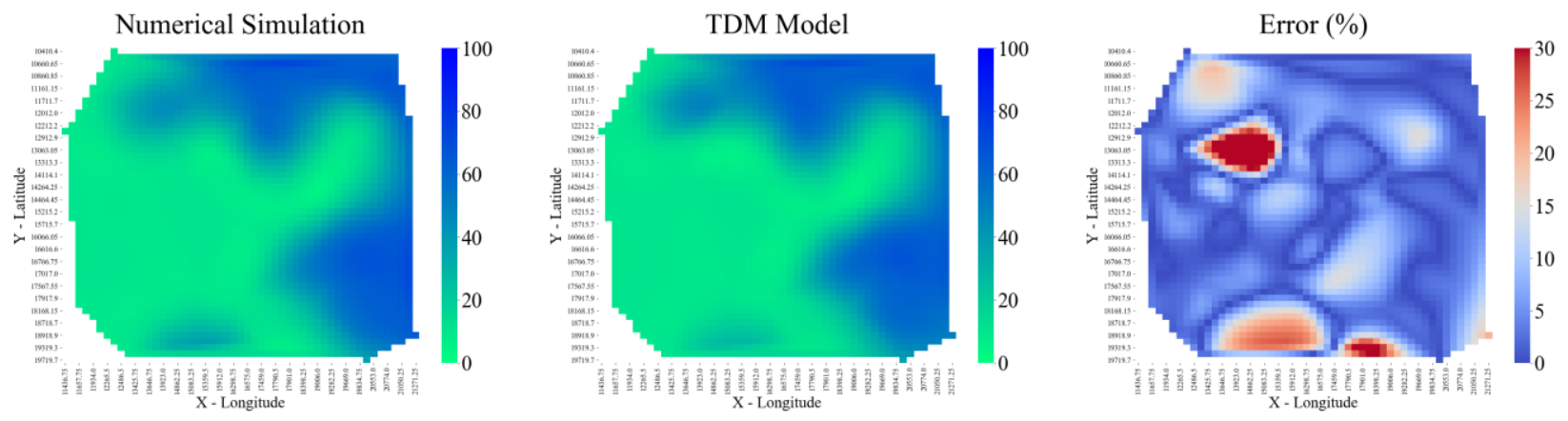

Figure 732 Entire reservoir heat map for water saturation at June 30th, 2013 for 4 Year Blind Validation TDM

Water Saturation (\%) at 2013-09-30
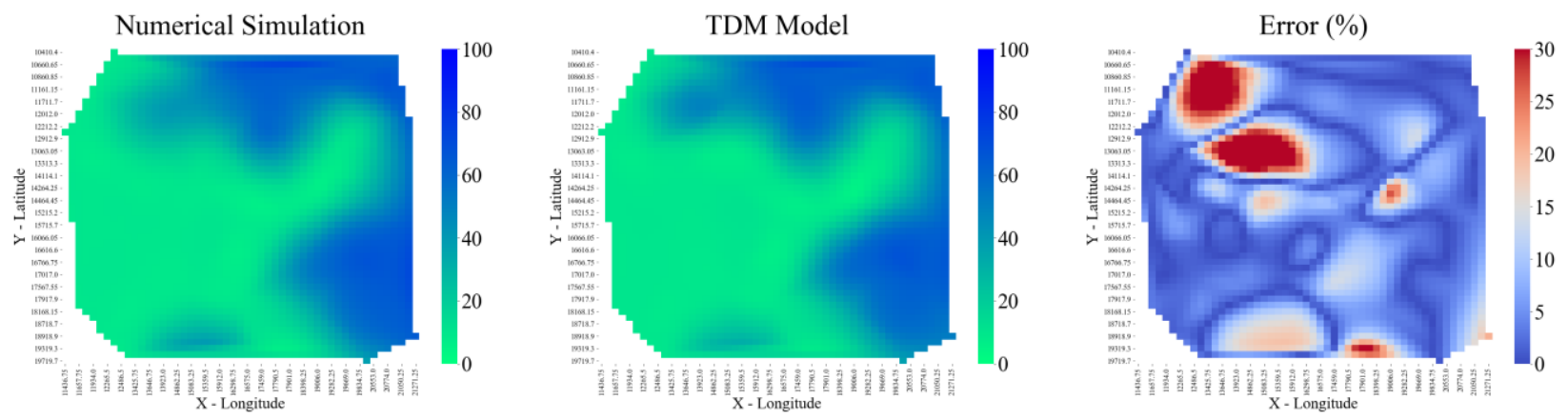

Figure 733 Entire reservoir heat map for water saturation at September 30th, 2013 for 4 Year Blind Validation TDM

Water Saturation (\%) at 2013-12-31
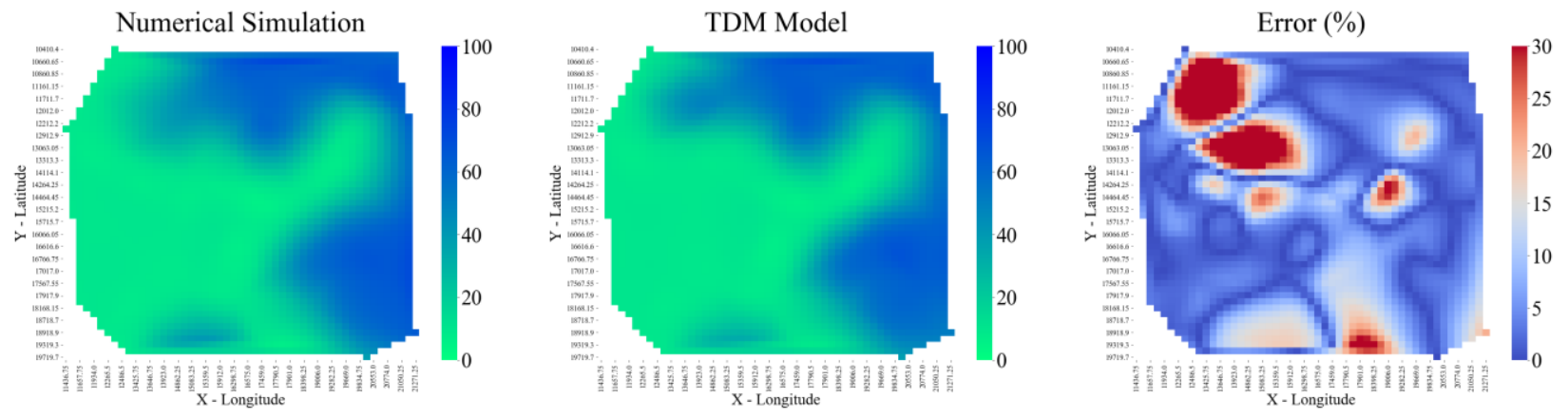

Figure 734 Entire reservoir heat map for water saturation at December 31st, 2013 for 4 Year Blind Validation TDM 
Water Saturation (\%) at 2014-03-31
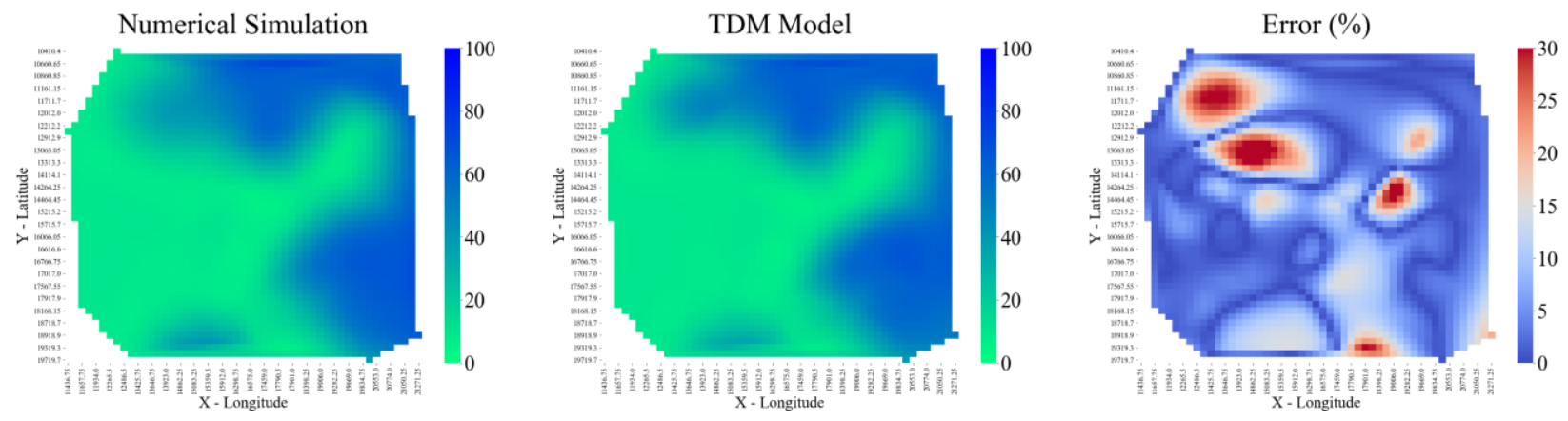

Figure 735 Entire reservoir heat map for water saturation at March 31st, 2014 for 4 Year Blind Validation TDM

\section{Water Saturation (\%) at 2014-06-30}
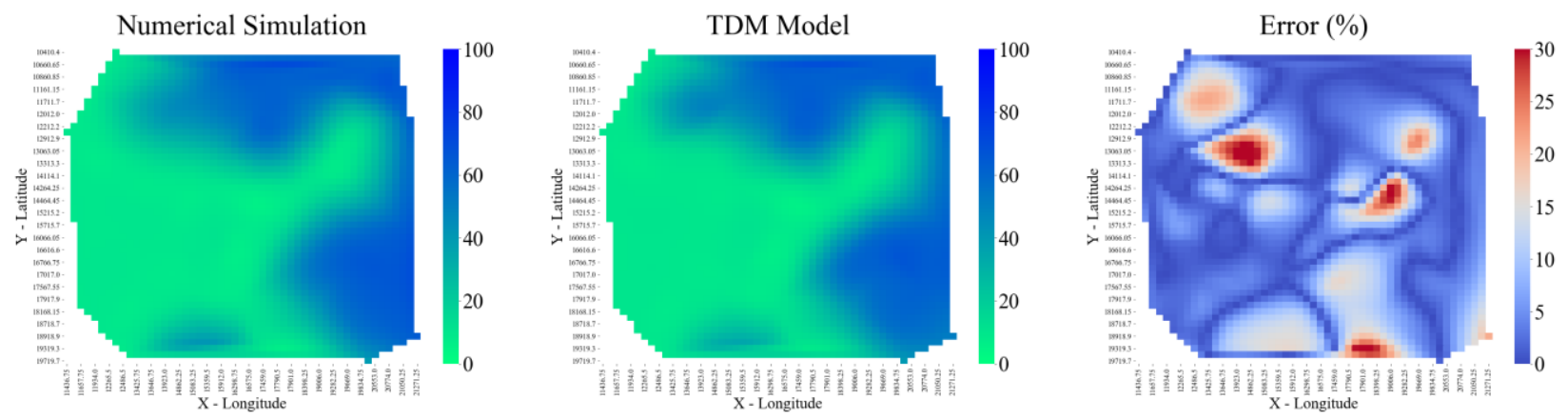

Figure 736 Entire reservoir heat map for water saturation at June 30th, 2014 for 4 Year Blind Validation TDM

Water Saturation (\%) at 2014-09-30
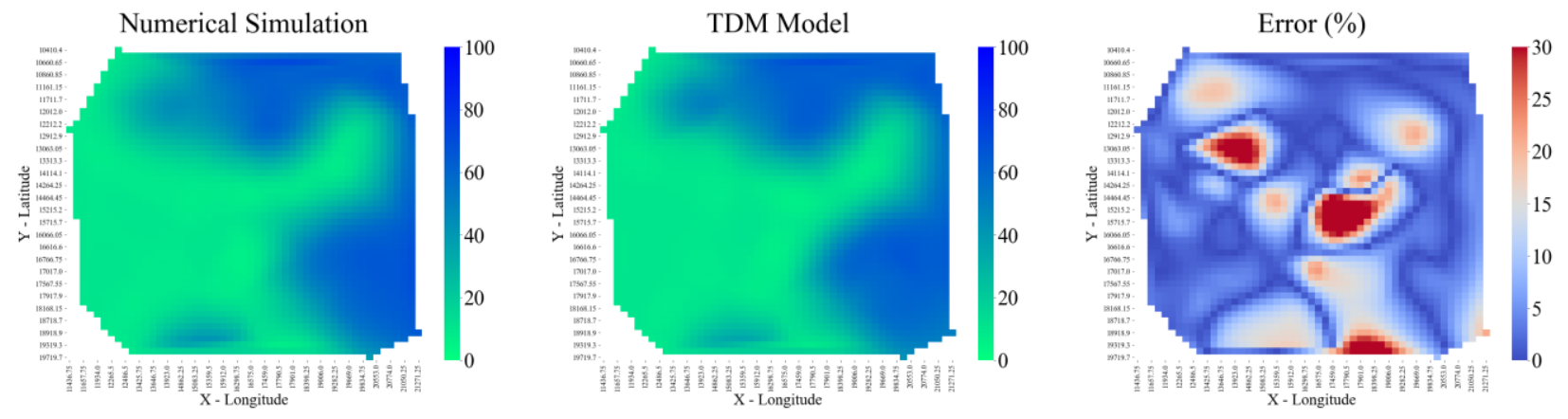

Figure 737 Entire reservoir heat map for water saturation at September 30th, 2014 for 4 Year Blind Validation TDM 
Water Saturation (\%) at 2014-12-31
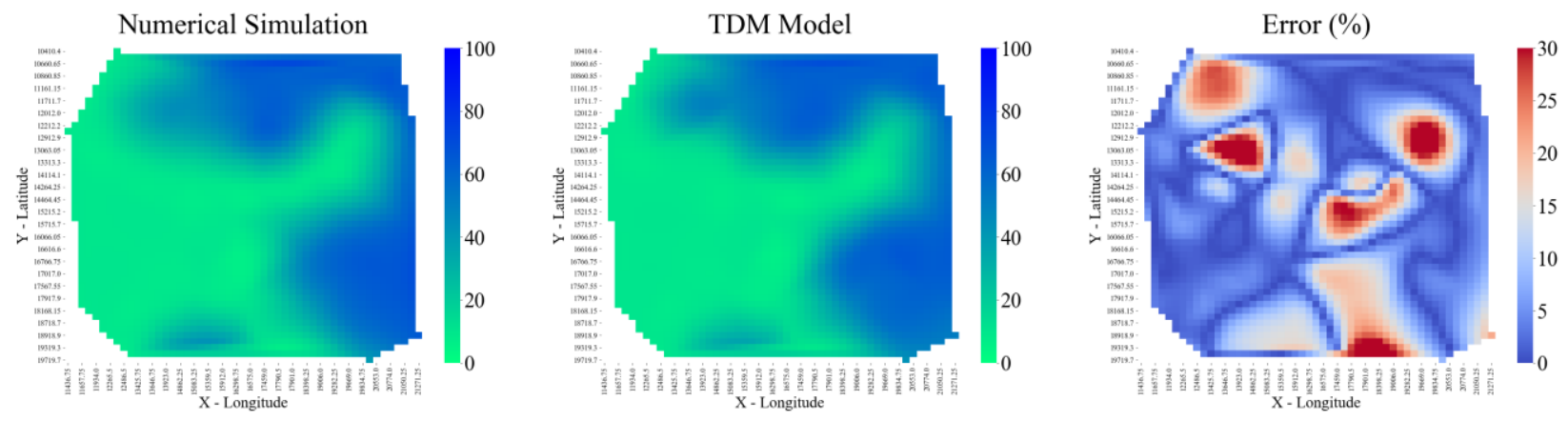

Figure 738 Entire reservoir heat map for water saturation at December 31st, 2014 for 4 Year Blind Validation TDM

Water Saturation (\%) at 2015-03-31
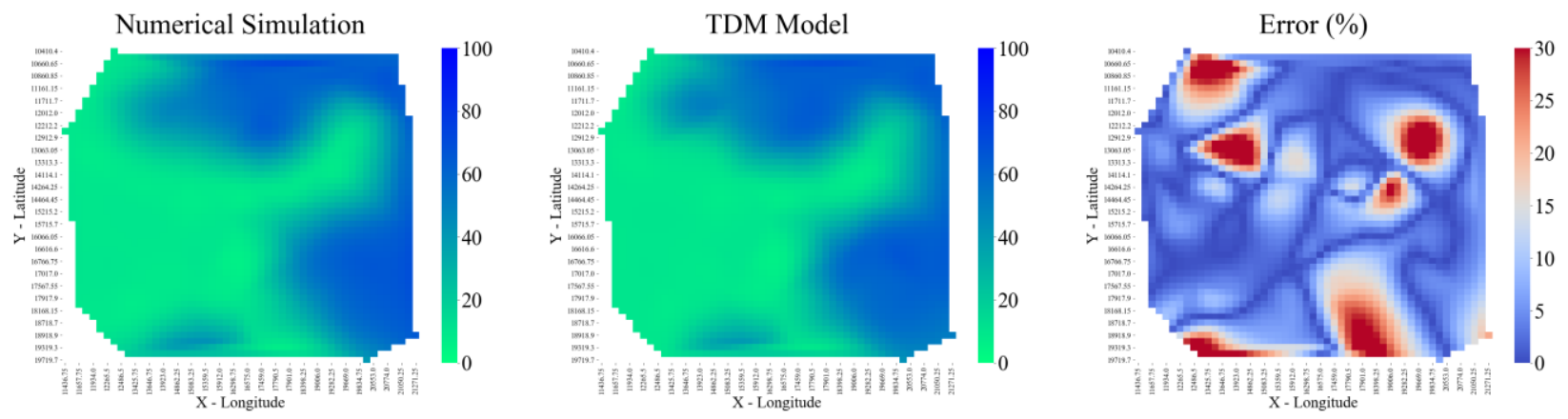

Figure 739 Entire reservoir heat map for water saturation at March 31st, 2015 for 4 Year Blind Validation TDM Water Saturation (\%) at 2015-06-30
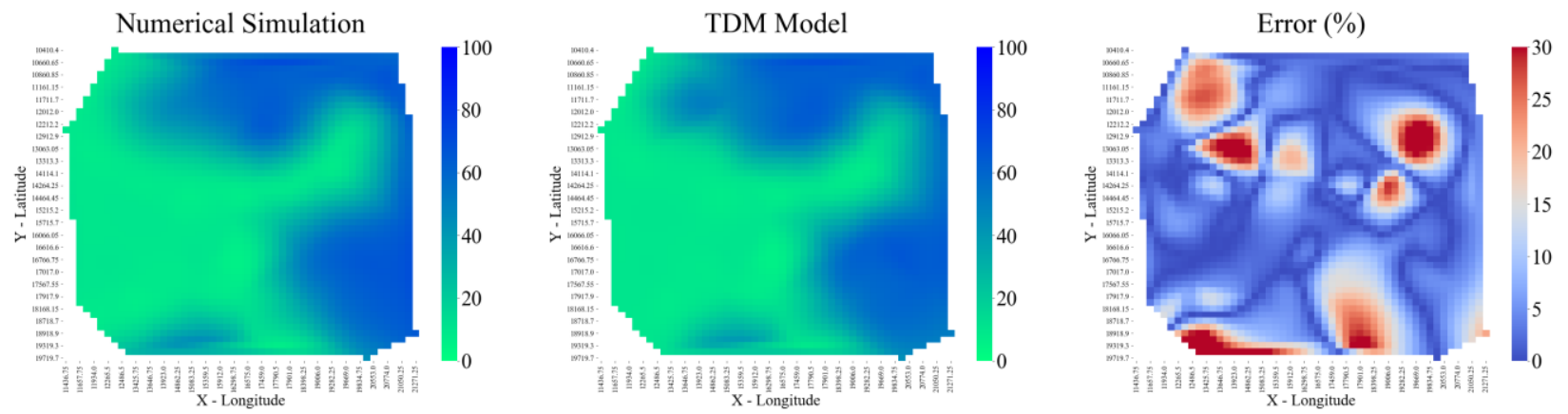

Figure 740 Entire reservoir heat map for water saturation at June 30th, 2015 for 4 Year Blind Validation 
Water Saturation (\%) at 2015-09-30
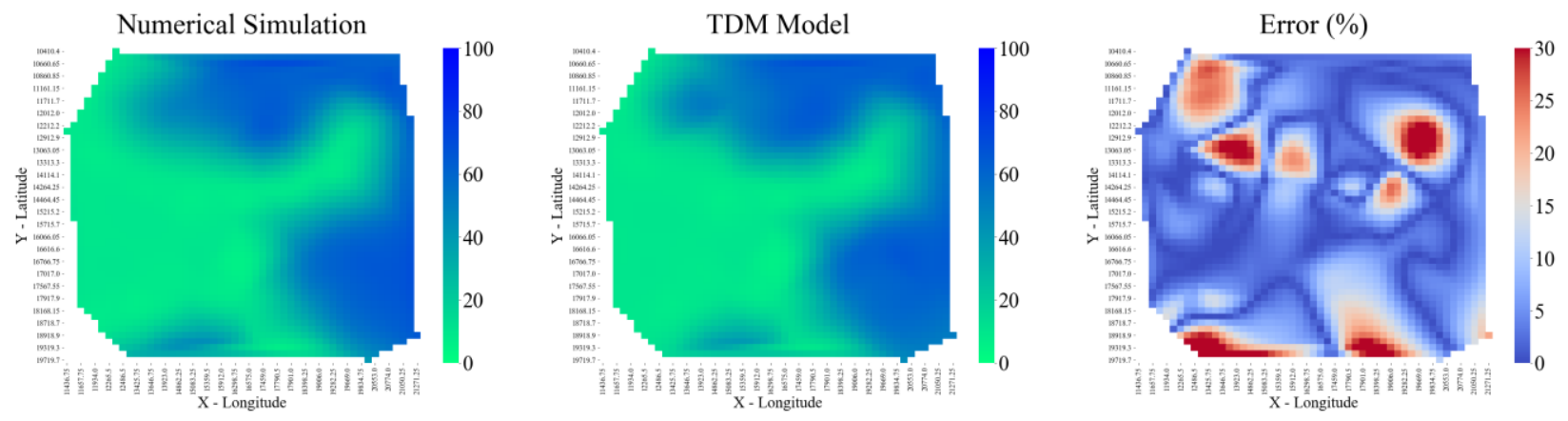

Figure 741 Entire reservoir heat map for water saturation at September 30th, 2015 for 4 Year Blind Validation TDM

Water Saturation (\%) at 2015-12-31
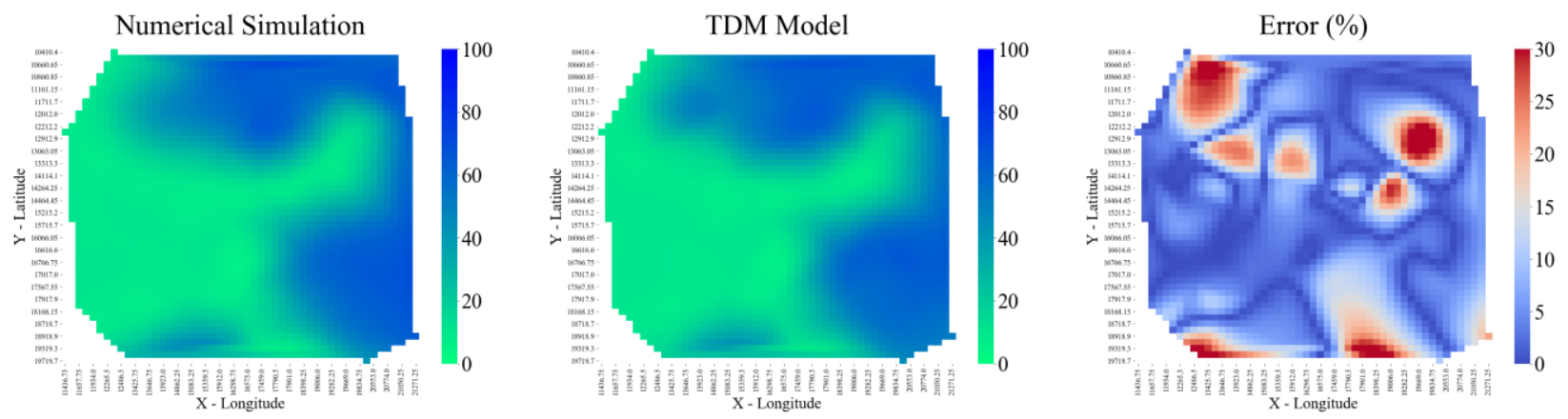

Figure 742 Entire reservoir heat map for water saturation at December 31st, 2015 for 4 Year Blind Validation TDM

Water Saturation (\%) at 2016-03-31
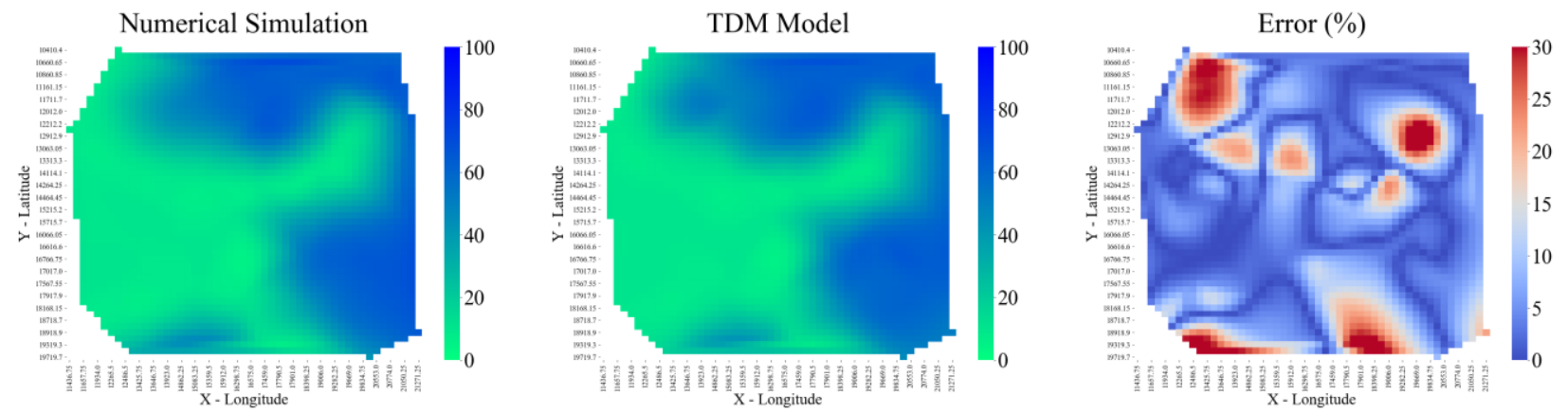

Figure 743 Entire reservoir heat map for water saturation at March 31st, 2016 for 4 Year Blind Validation TDM 
Water Saturation (\%) at 2016-06-30
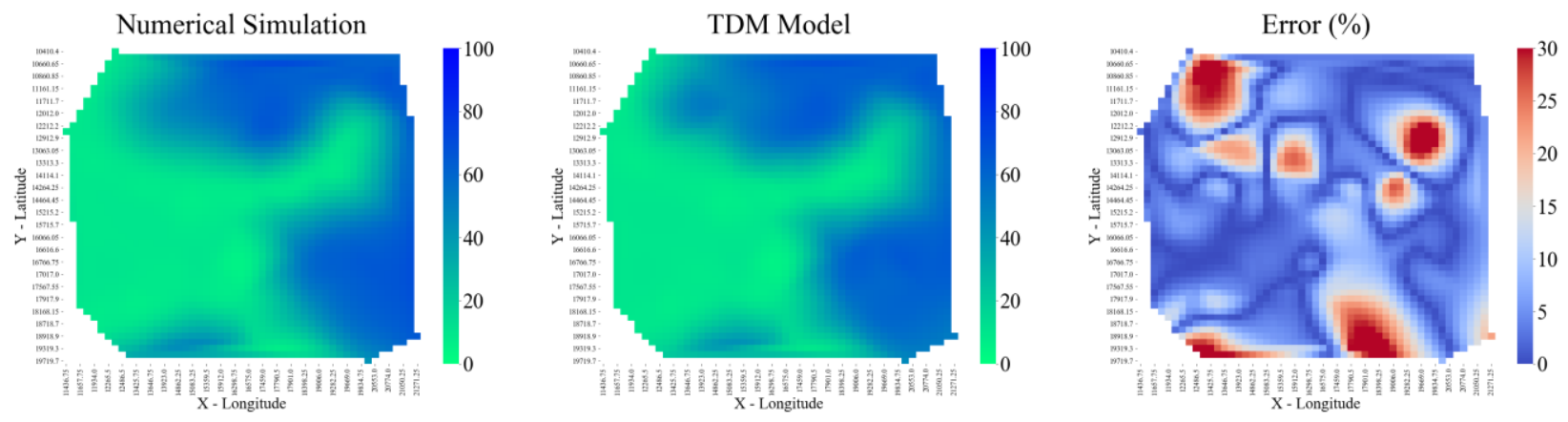

Figure 744 Entire reservoir heat map for water saturation at June 30th, 2016 for 4 Year Blind Validation TDM

Water Saturation (\%) at 2016-09-30
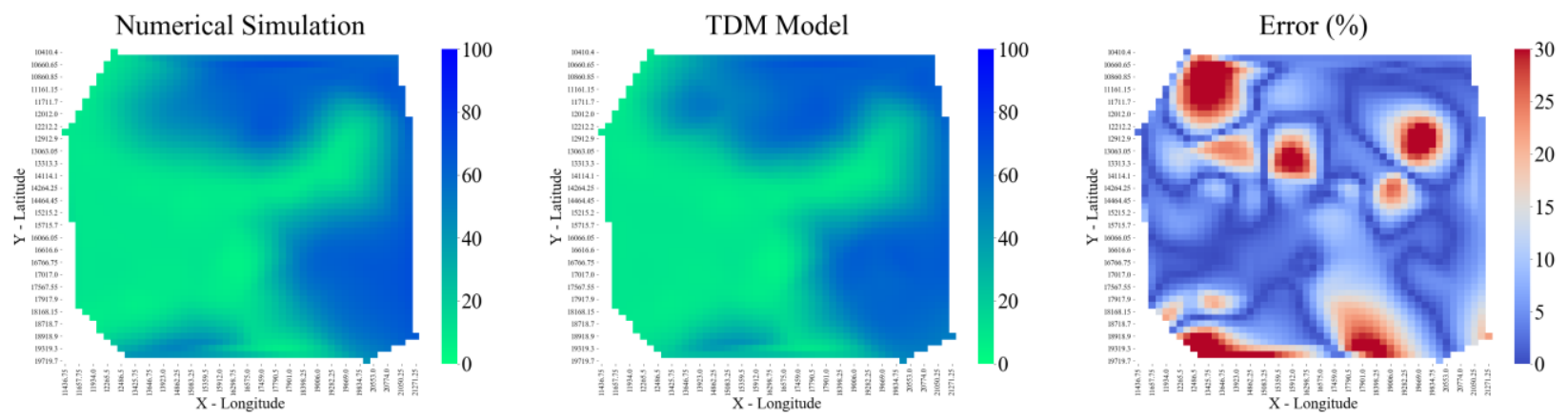

Figure 745 Entire reservoir heat map for water saturation at September 30th, 2016 for 4 Year Blind Validation TDM

Water Saturation (\%) at 2016-12-31
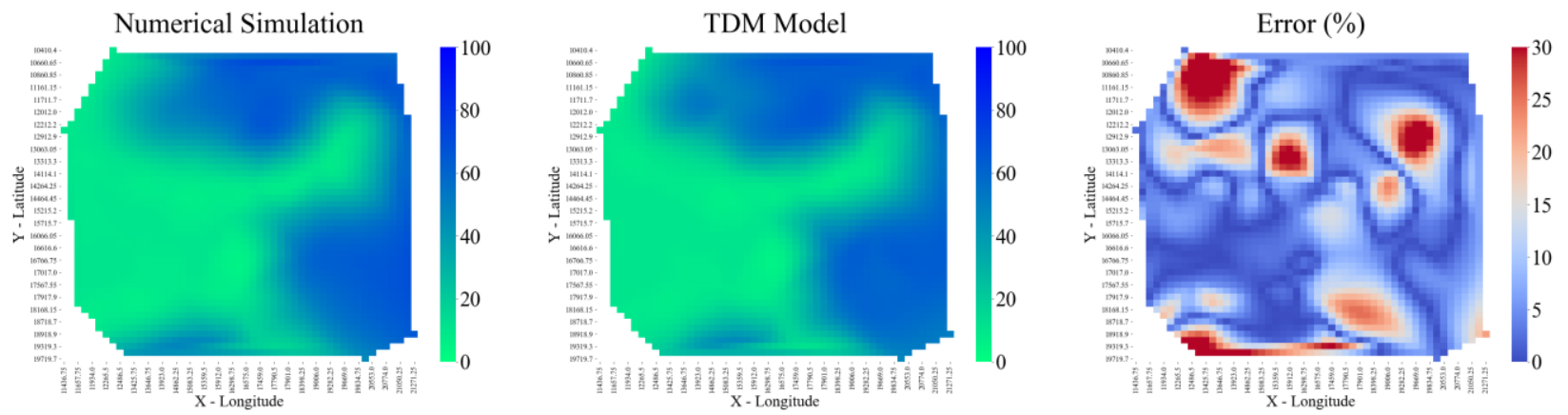

Figure 746 Entire reservoir heat map for water saturation at December 31st, 2016 for 4 Year Blind Validation TDM 
8.2.6 Multi-Random Partitioning- 5 Year Blind Validation

8.2.6.1 Individual Well Production Plots

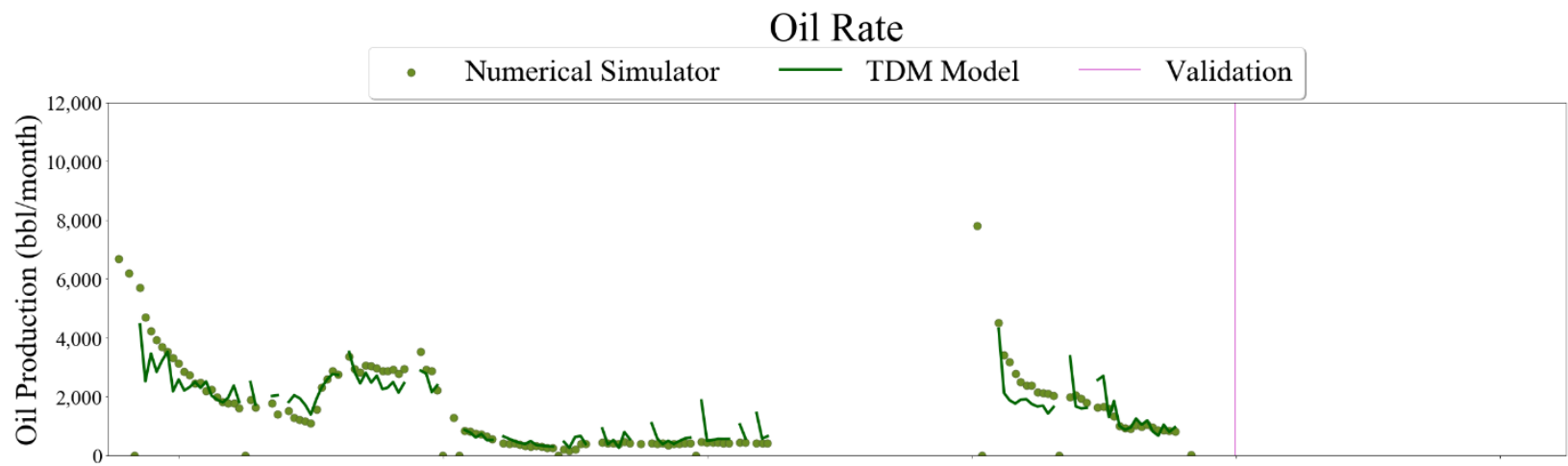

Gas Rate

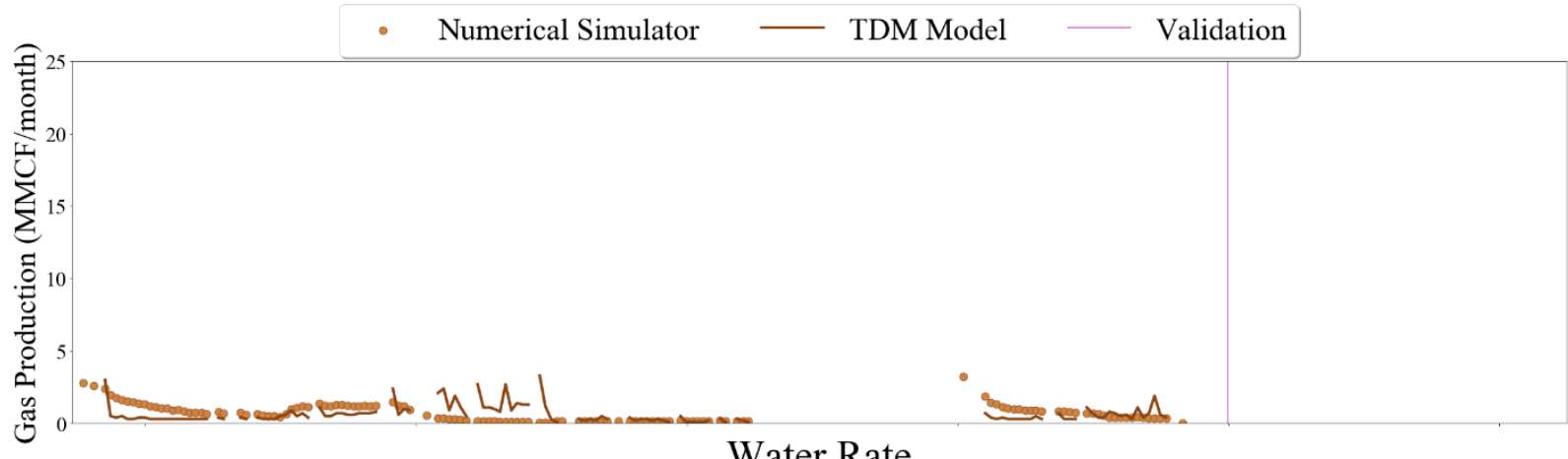

Water Rate

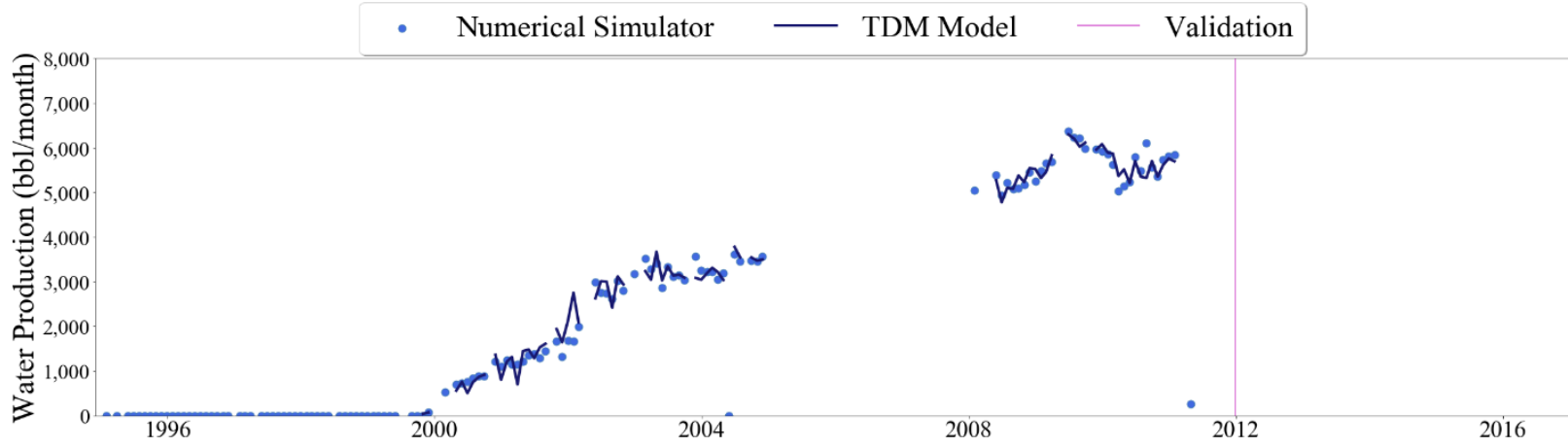

Figure 747 Well-001 oil, gas, and water rate TDM predictions vs actual simulation data plots for 5 Year Blind Validation TDM 

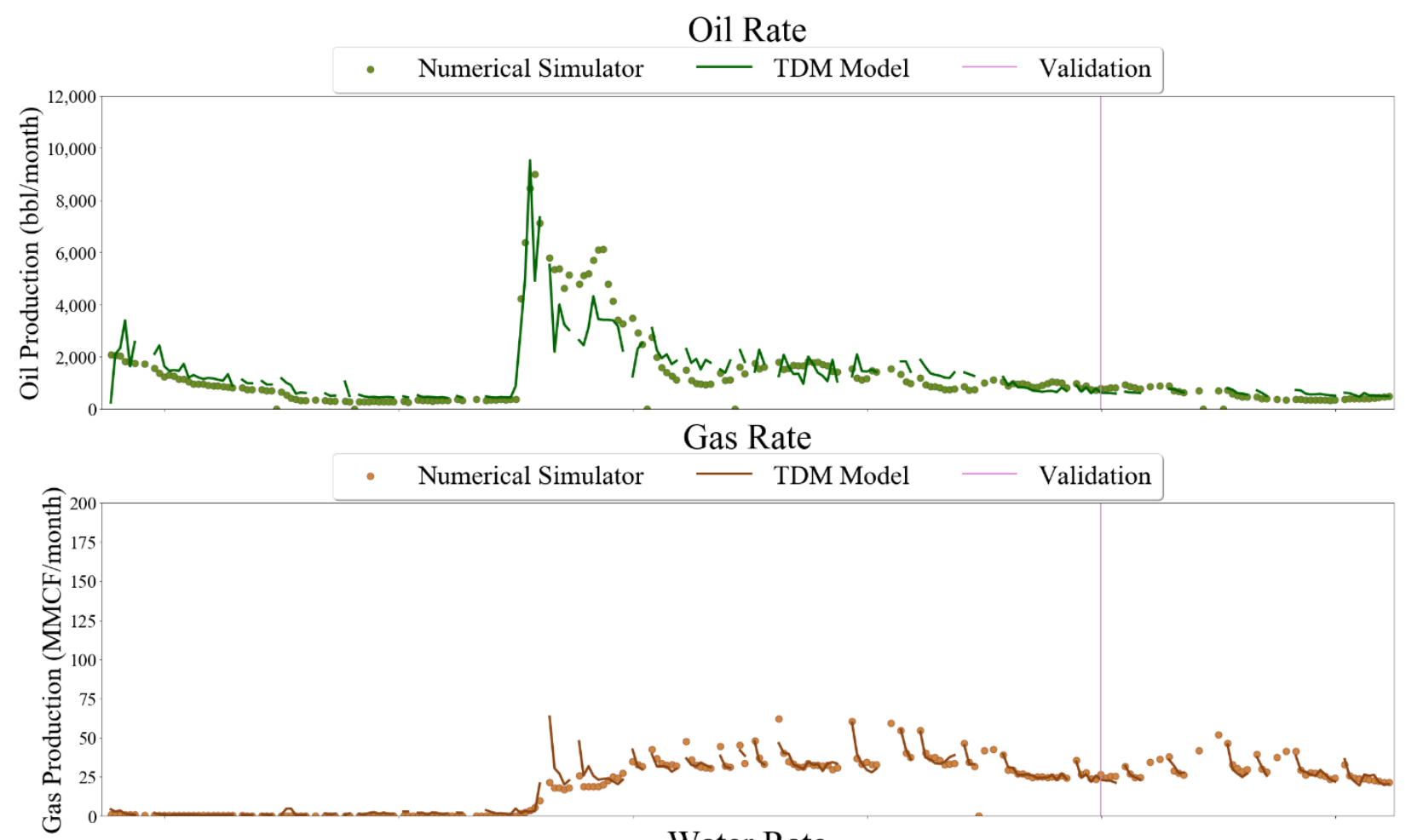

Water Rate

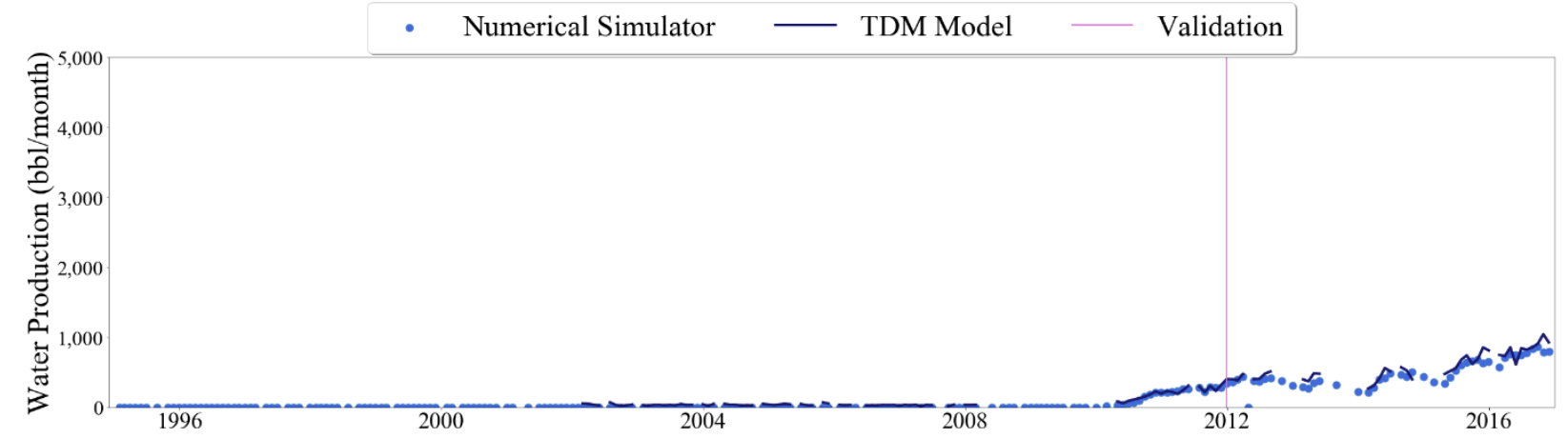

Figure 748 Well-002 oil, gas, and water rate TDM predictions vs actual simulation data plots for 5 Year Blind Validation TDM 


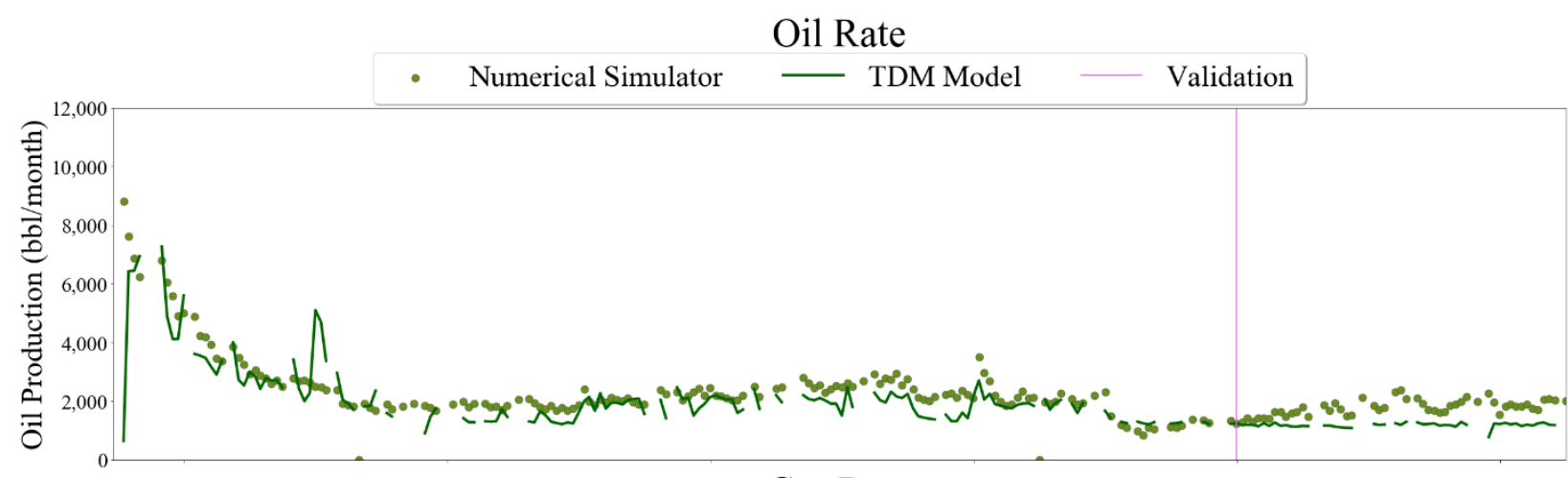

\section{Gas Rate}
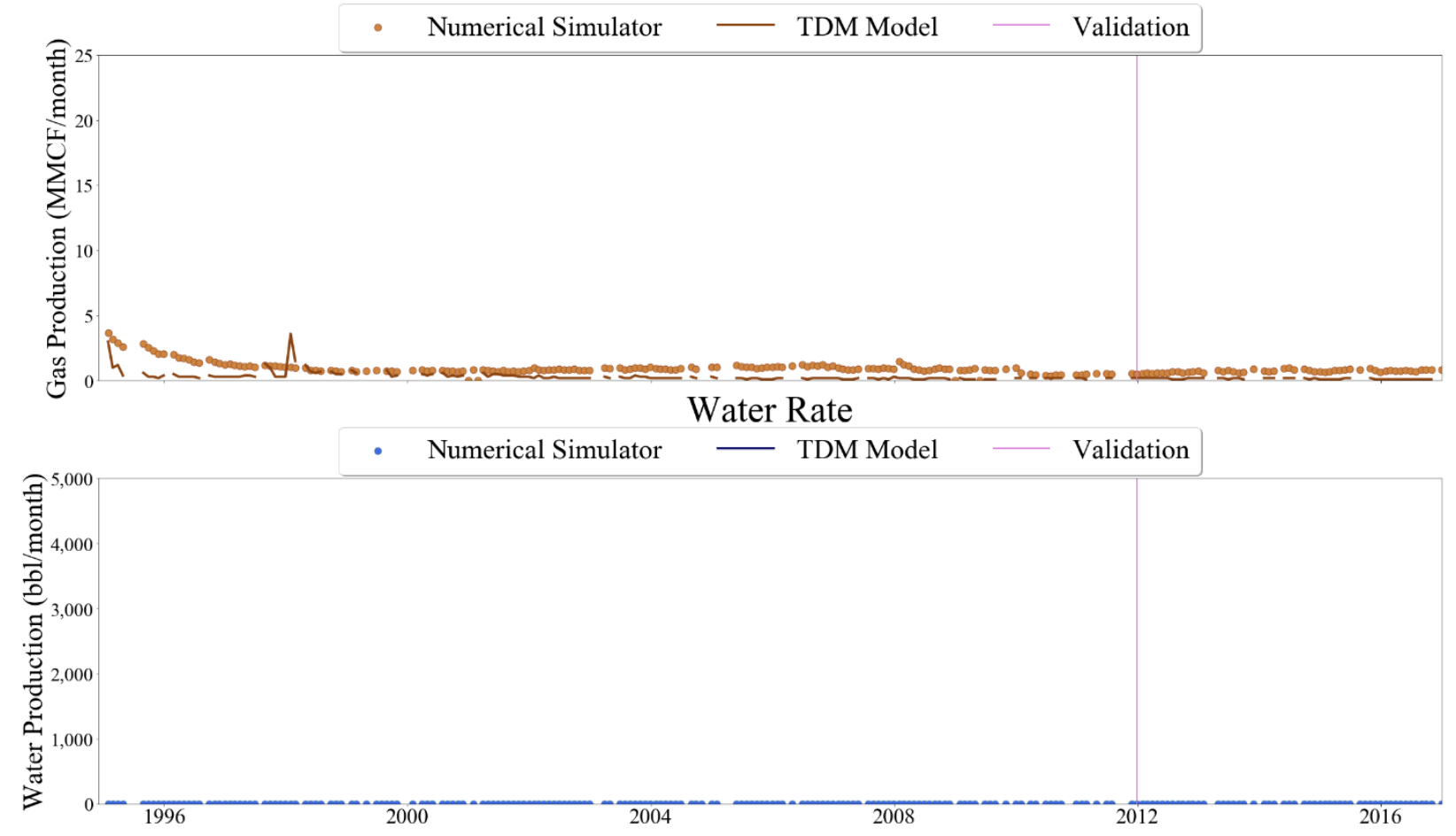

Figure 749 Well-003 oil, gas, and water rate TDM predictions vs actual simulation data plots for 5 Year Blind Validation TDM 


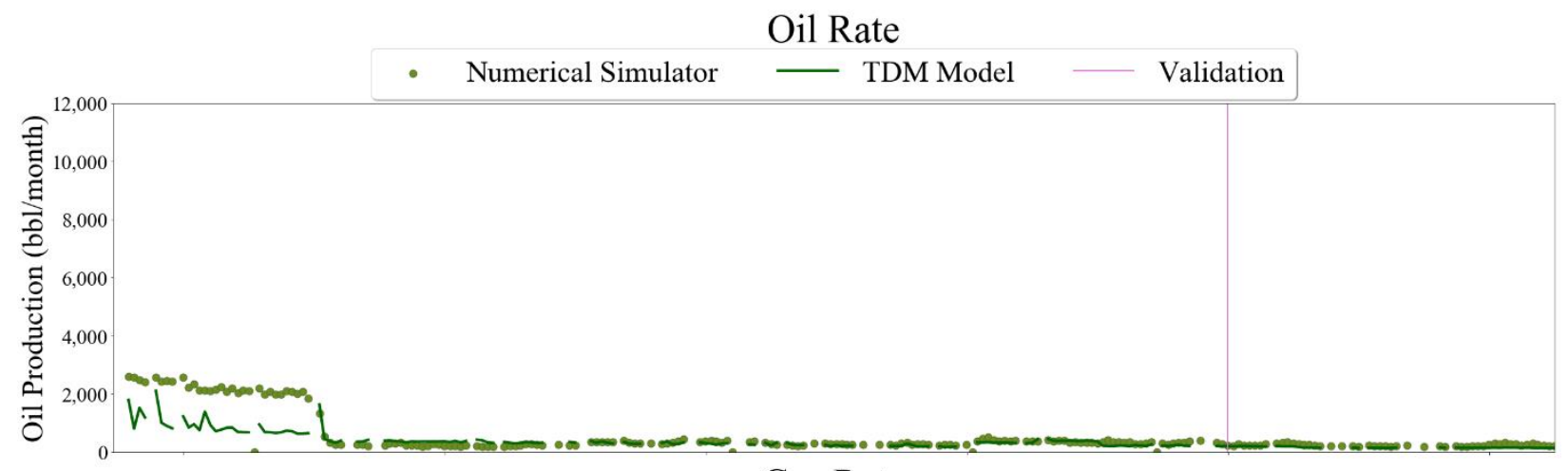

Gas Rate
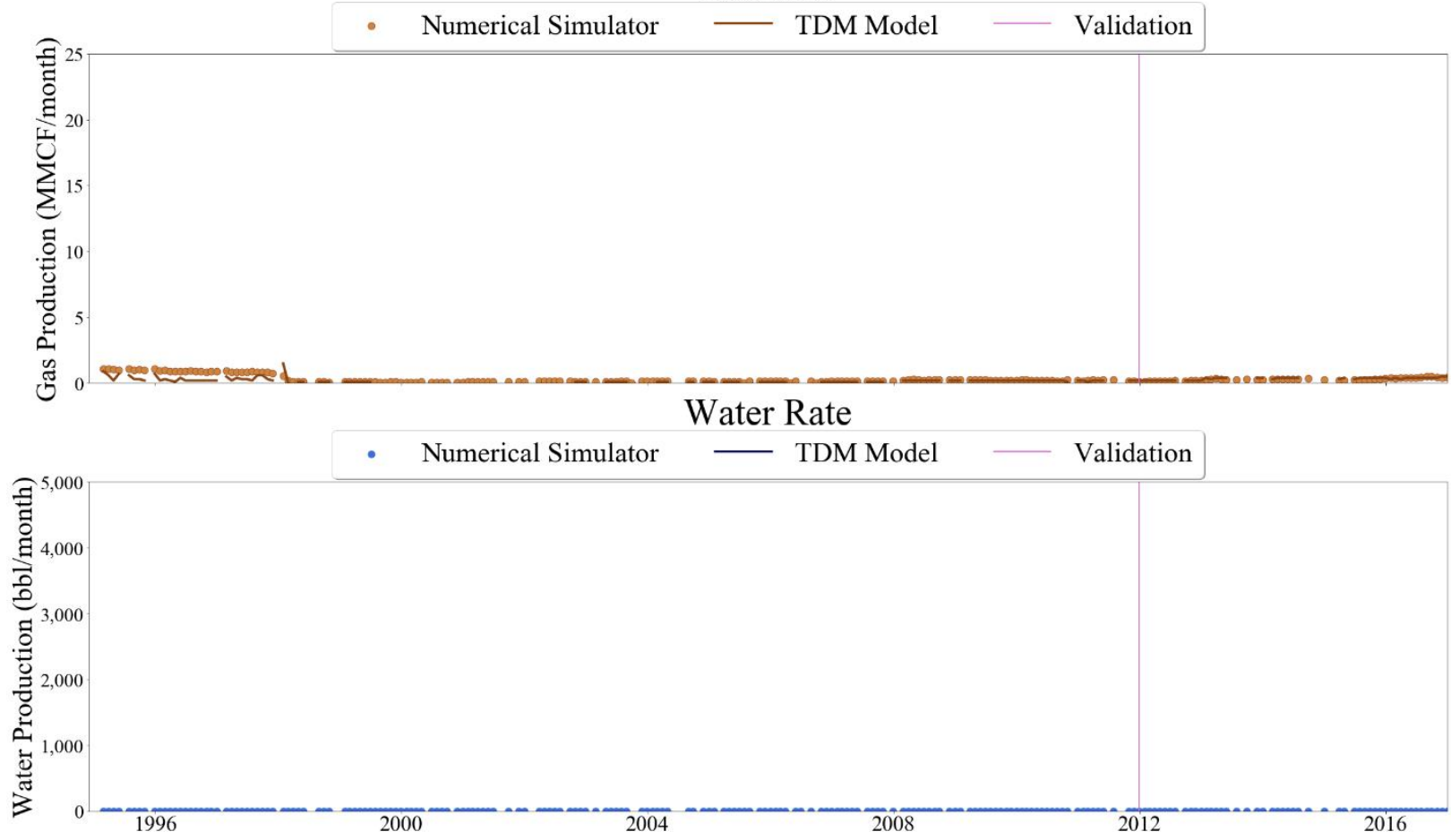

Figure 750 Well-004 oil, gas, and water rate TDM predictions vs actual simulation data plots for 5 Year Blind Validation TDM 


\section{Oil Rate}

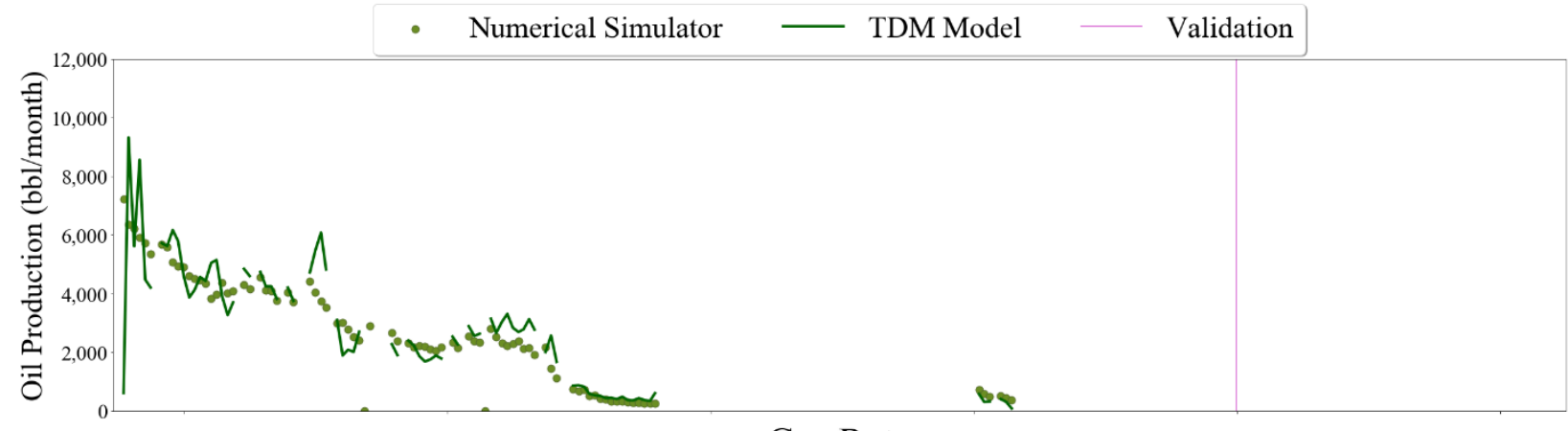

Gas Rate
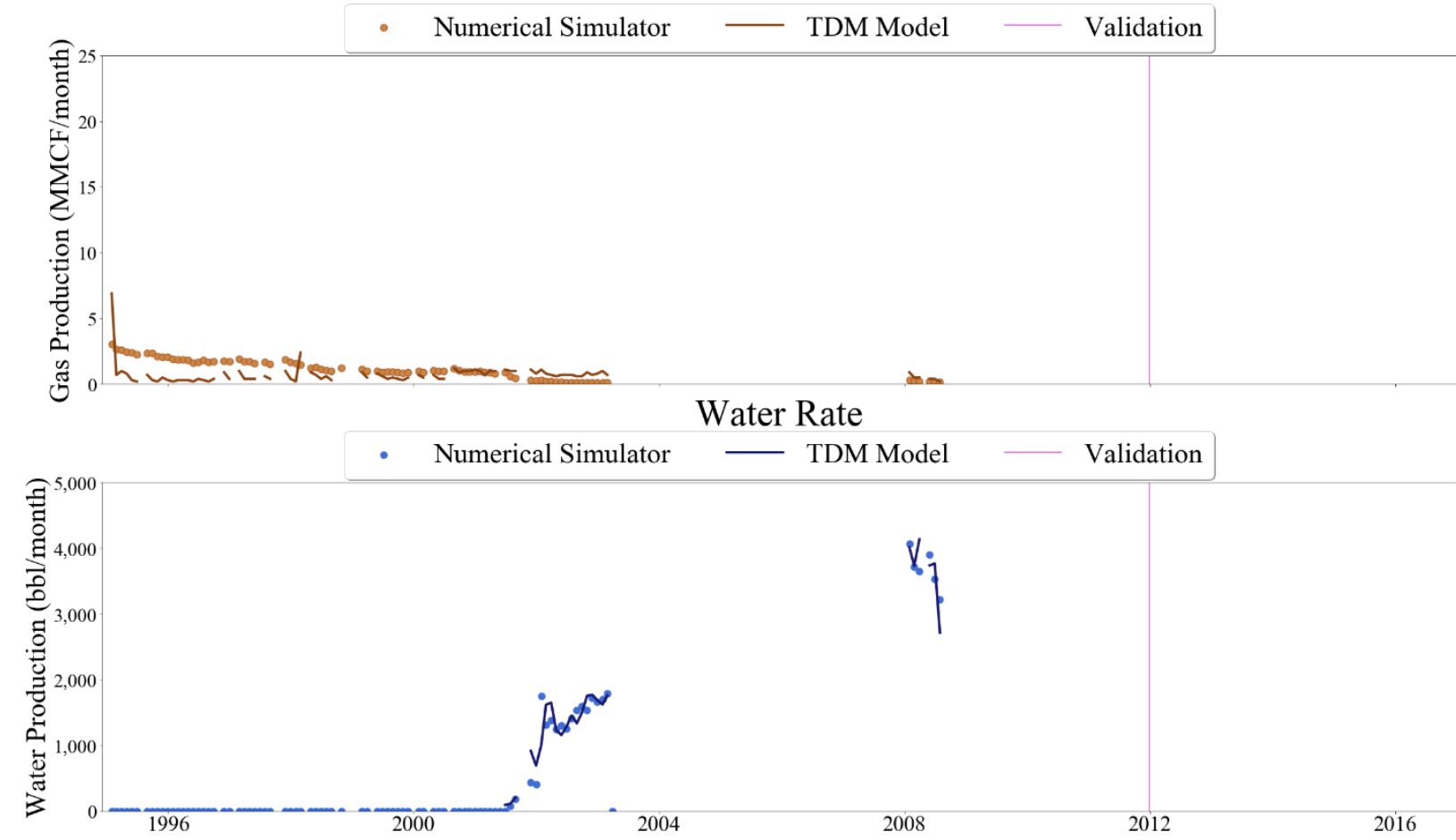

Figure 751 Well-005 oil, gas, and water rate TDM predictions vs actual simulation data plots for 5 Year Blind Validation TDM 

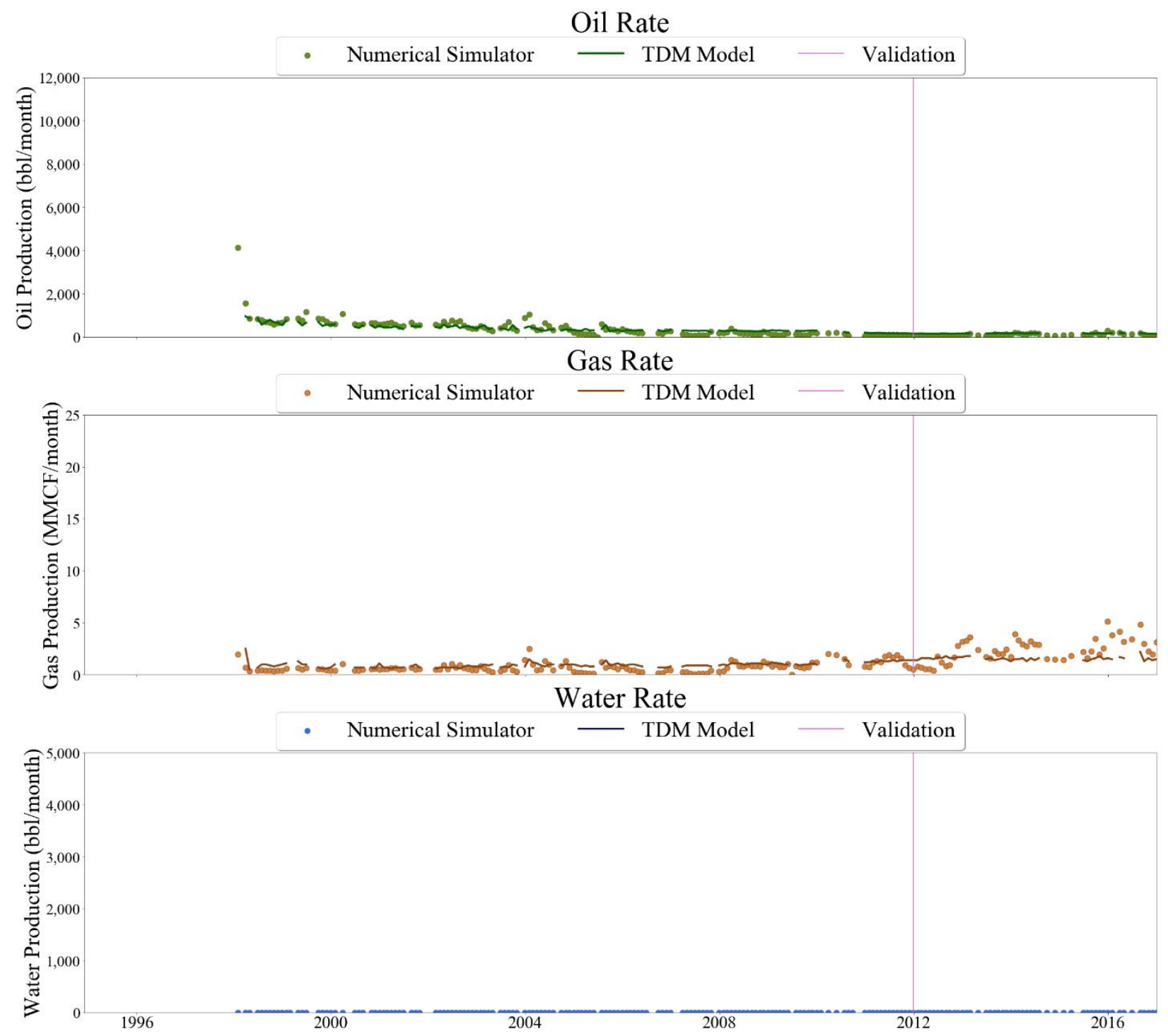

Figure 752 Well-006 oil, gas, and water rate TDM predictions vs actual simulation data plots for 5 Year Blind Validation TDM 

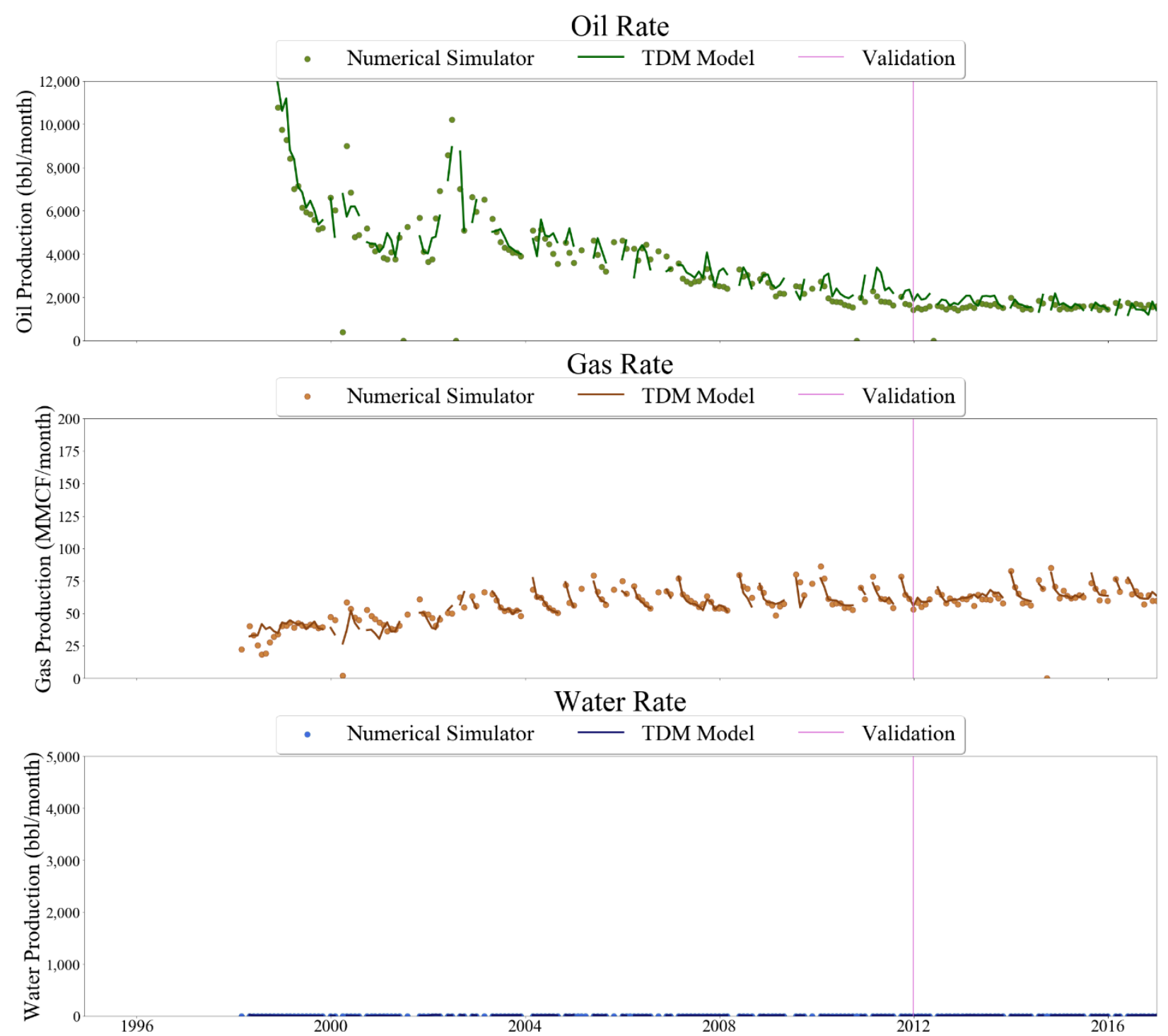

Figure 753 Well-007 oil, gas, and water rate TDM predictions vs actual simulation data plots for 5 Year Blind Validation TDM 


\section{Oil Rate}
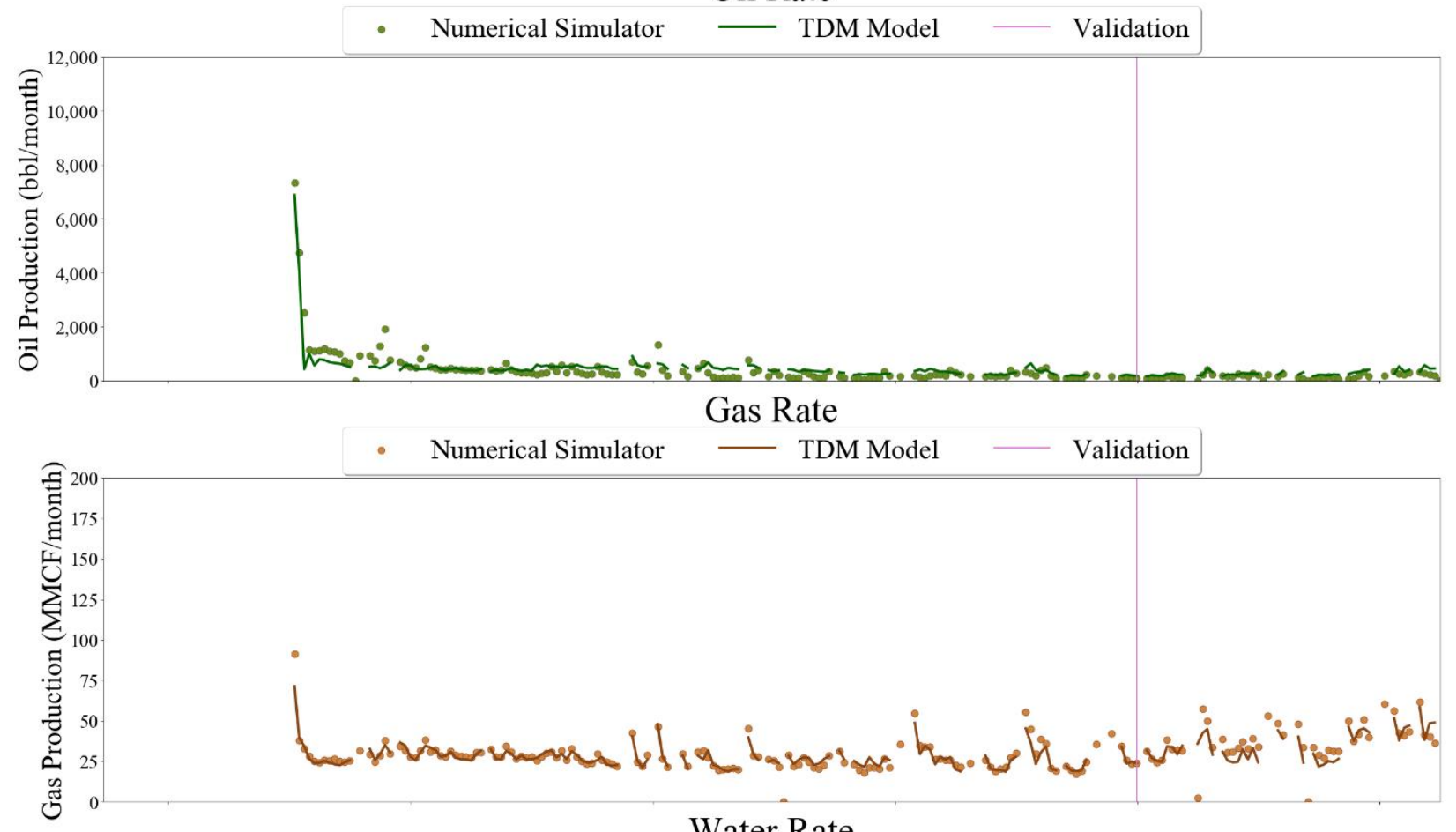

Water Rate

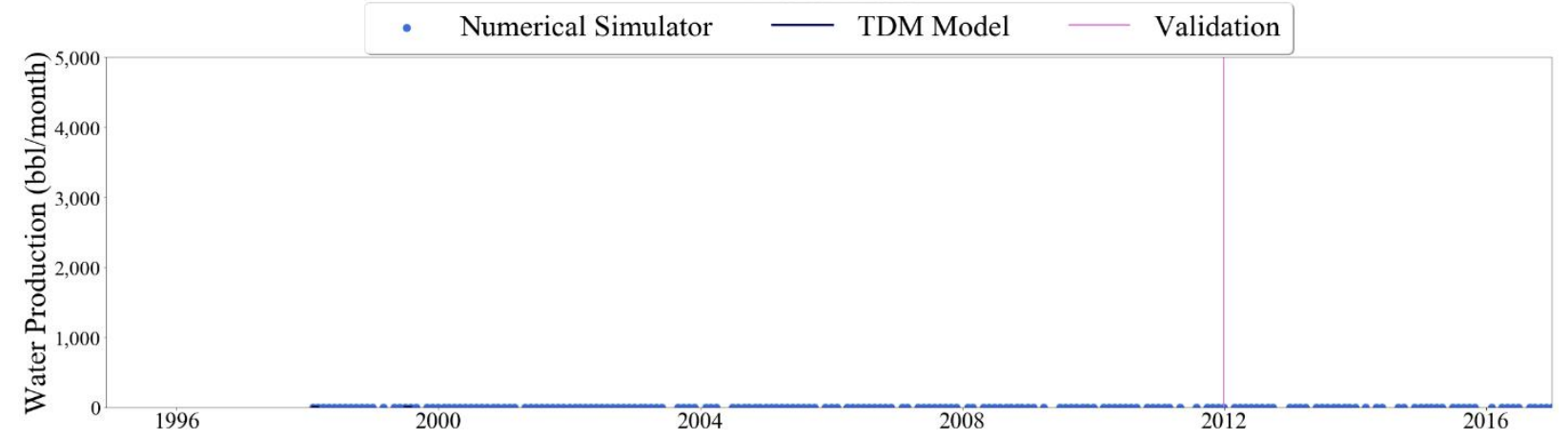

Figure 754 Well-008 oil, gas, and water rate TDM preditions vs actual simulation data plots for 5 Year Blind Validation TDM 

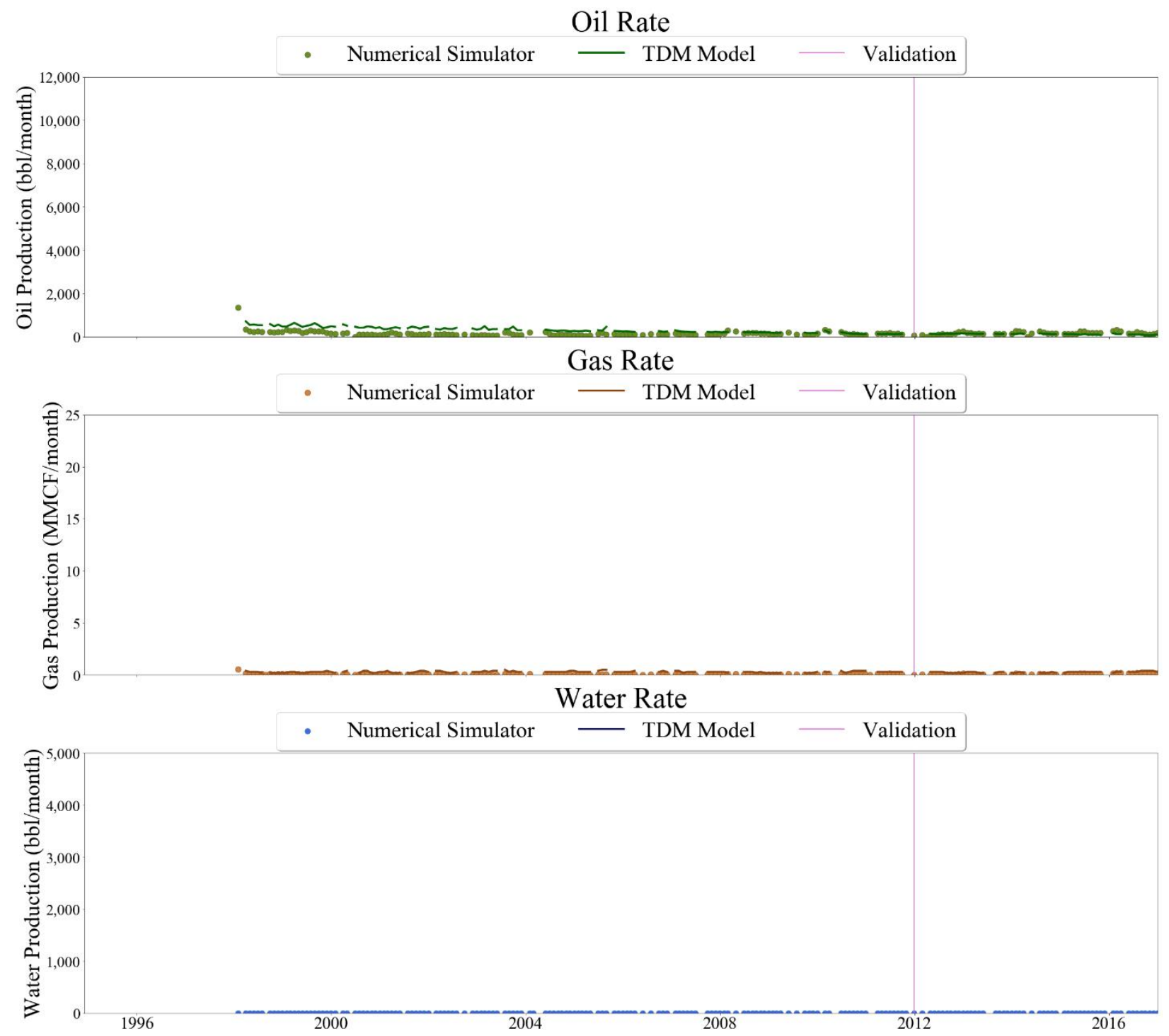

Figure 755 Well-009 oil, gas, and water rate TDM predictions vs actual simulation data plots for 5 Year Blind Validation TDM 


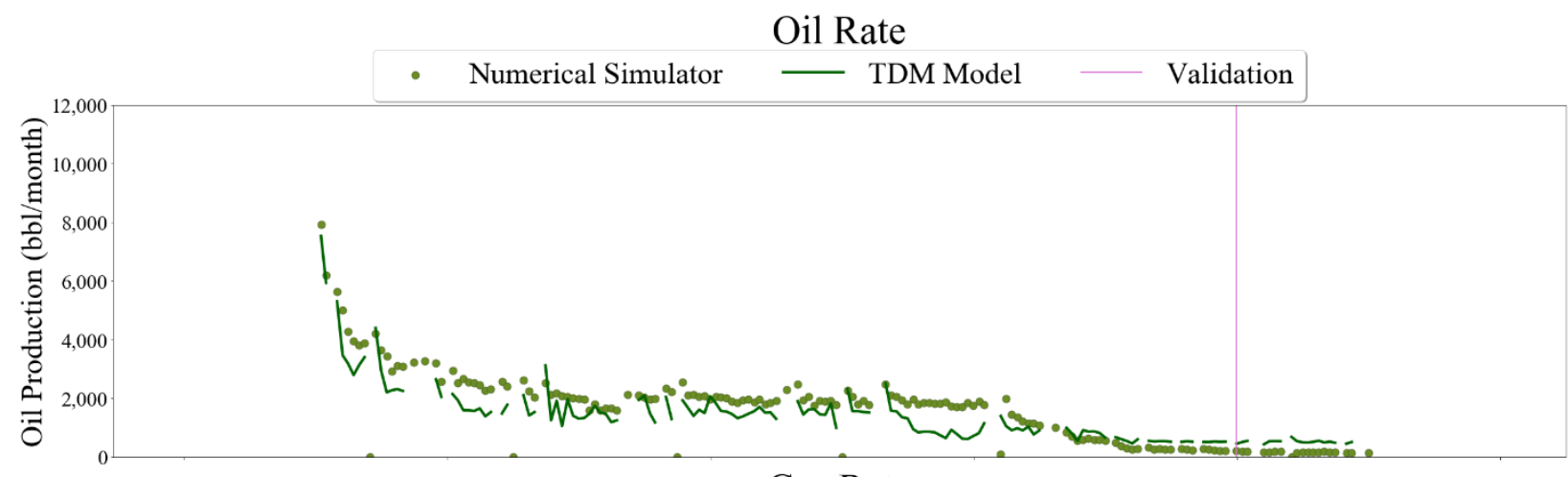

\section{Gas Rate}
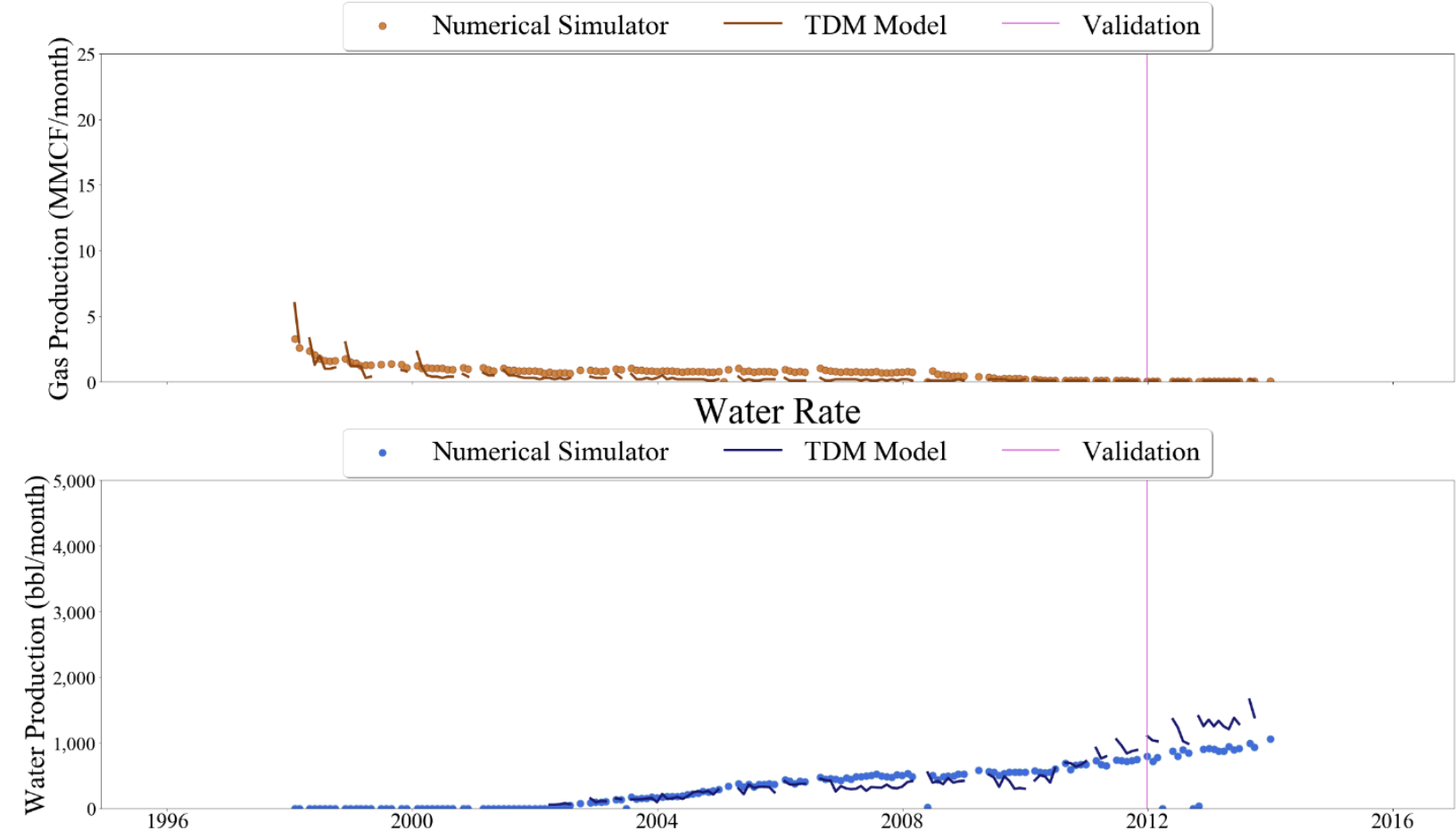

Figure 756 Well-010 oil, gas, and water rate TDM predictions vs actual simulation data plots for 5 Year Blind Validation TDM 


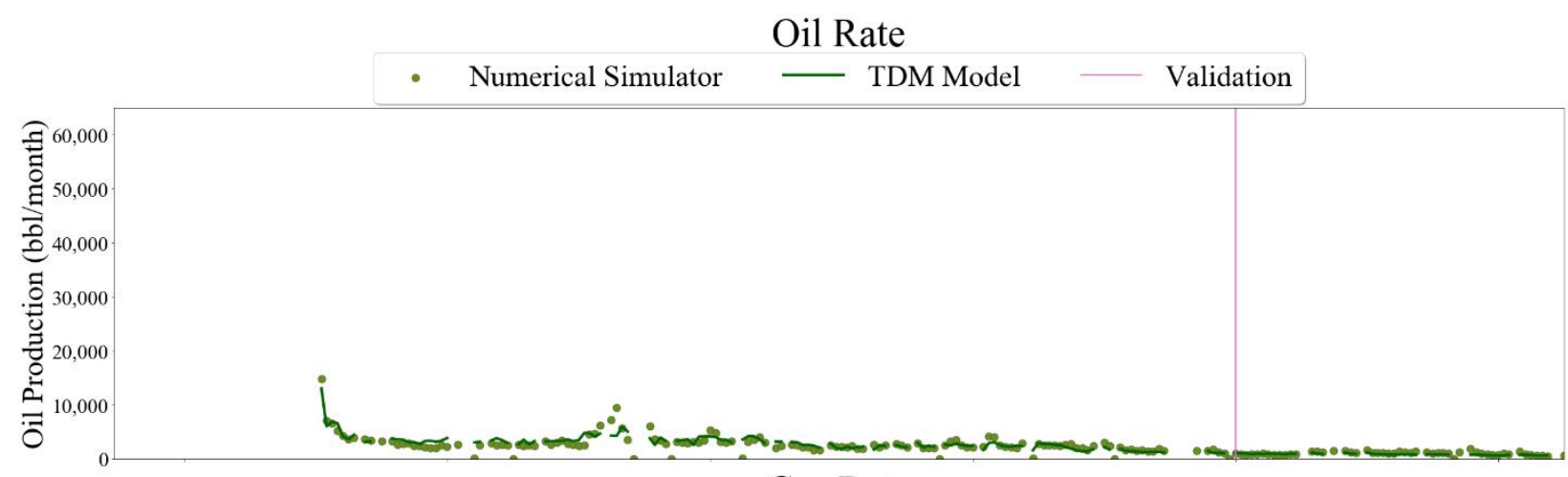

Gas Rate

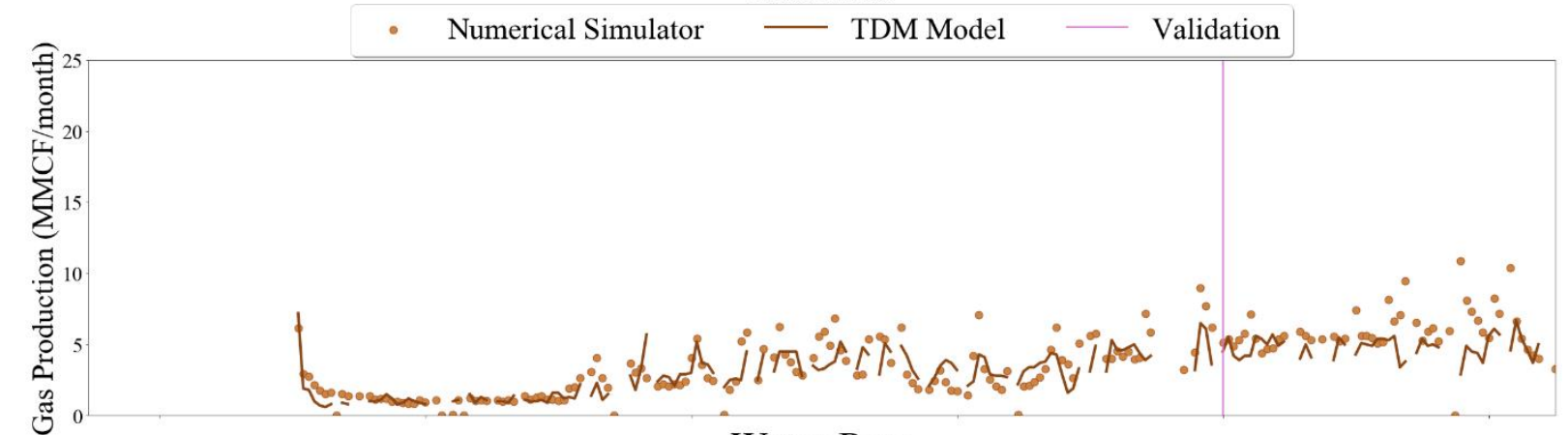

Water Rate

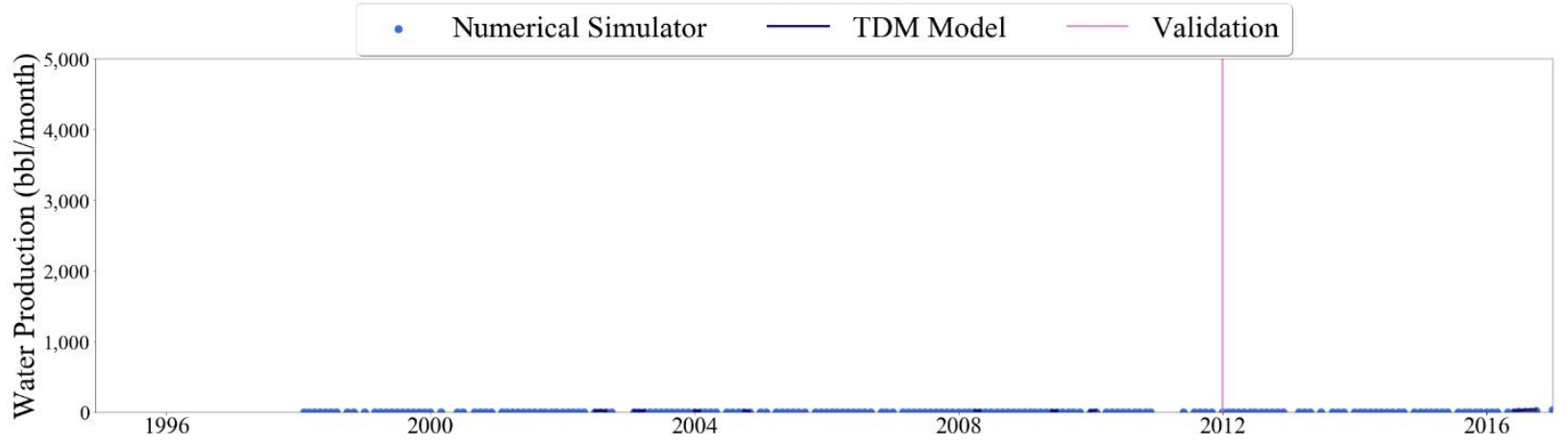

Figure 757 Well-011 oil, gas, and water rate TDM predictions vs actual simulation data plots for 5 Year Blind Validation TDM 

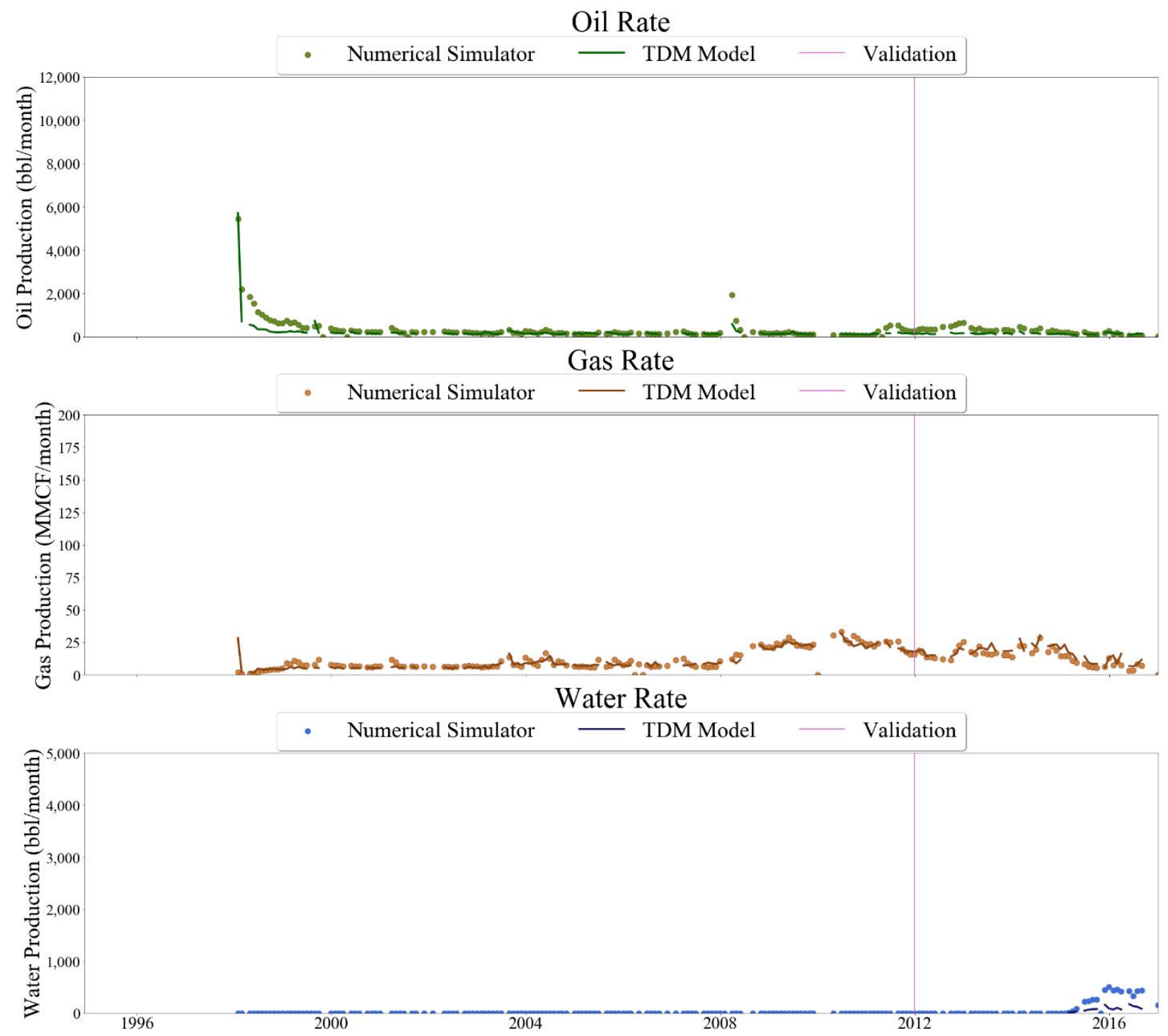

Figure 758 Well-012 oil, gas, and water rate TDM predictions vs actual simulation data plots for 5 Year Blind Validation TDM 

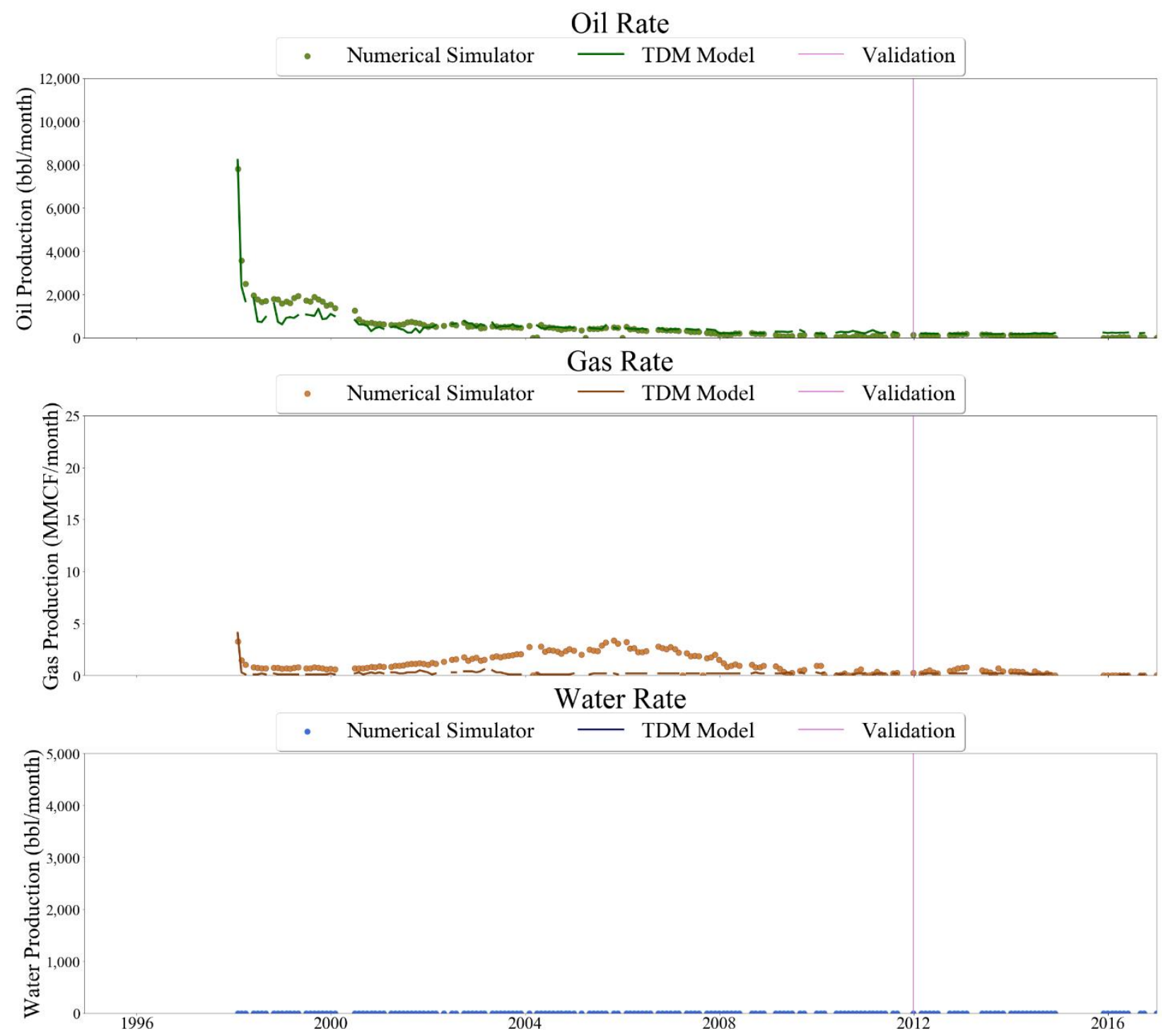

Figure 759 Well-013 oil, gas, and water rate TDM predictios vs actual simulation data plots for 5 Year Blind Validation TDM 


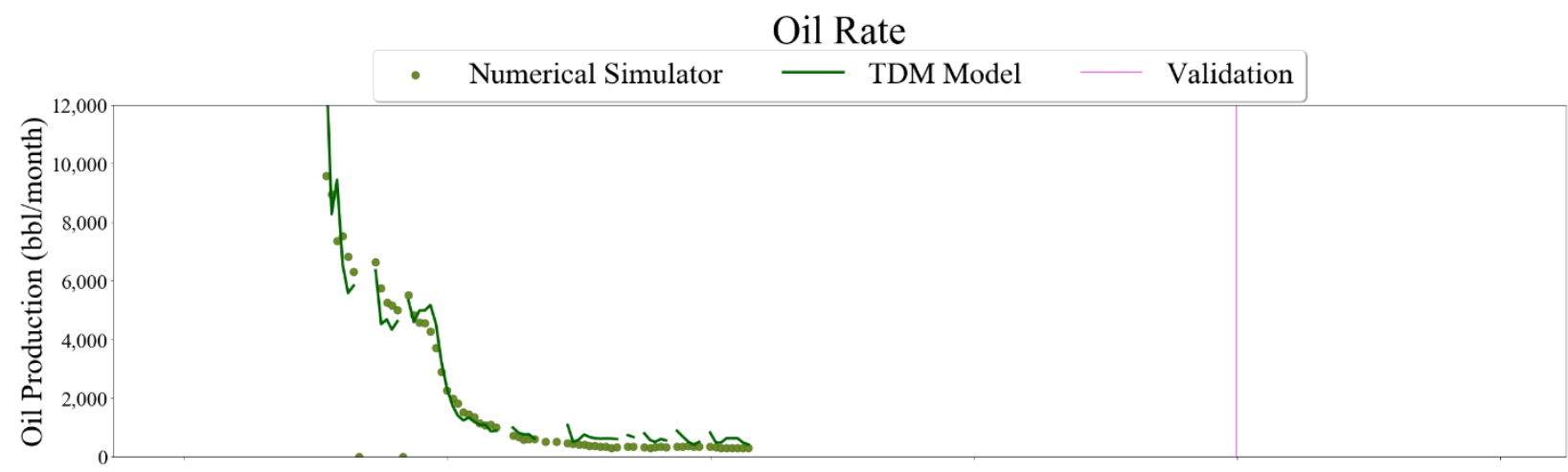

\section{Gas Rate}

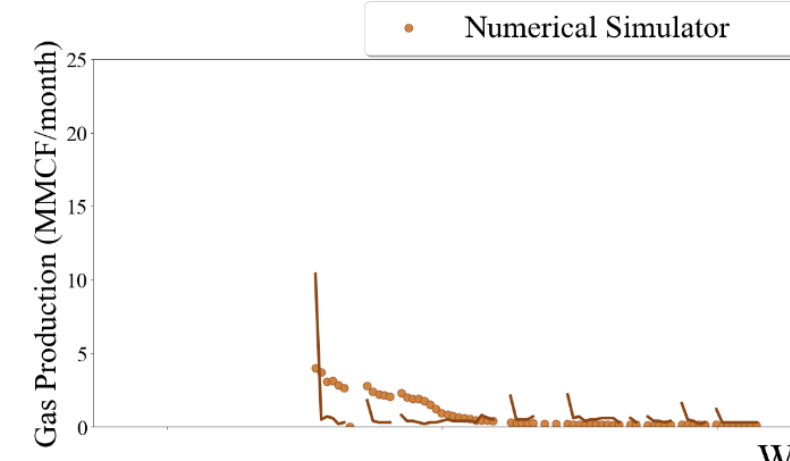

\section{Water Rate}

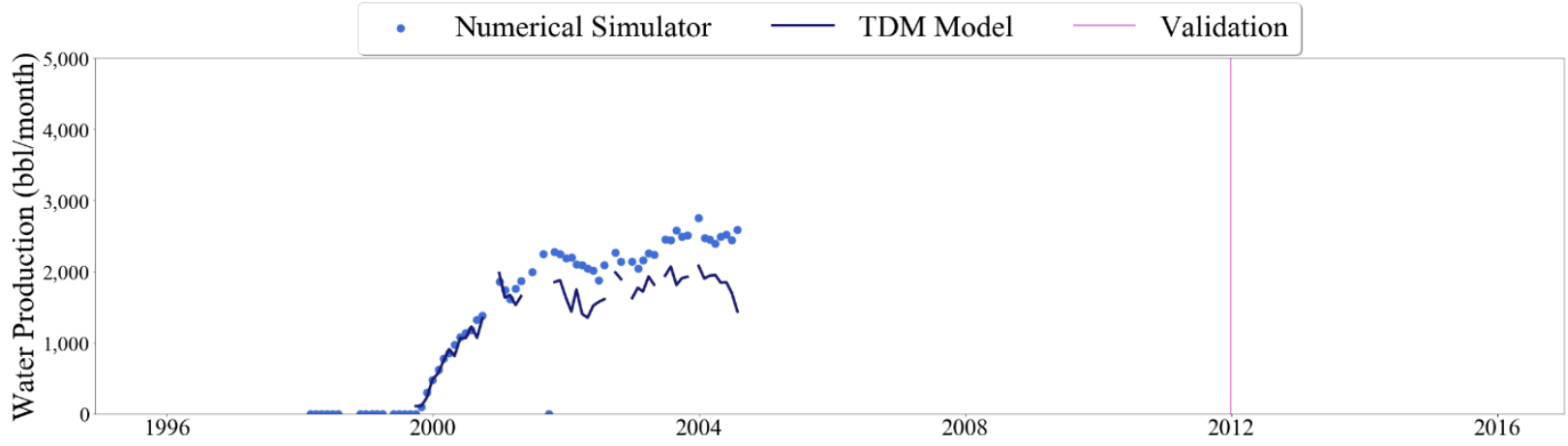

Figure 760 Well-014 oil, gas, and water rate TDM predictions vs actual simulation data plots for 5 Year Blind Validation TDM 

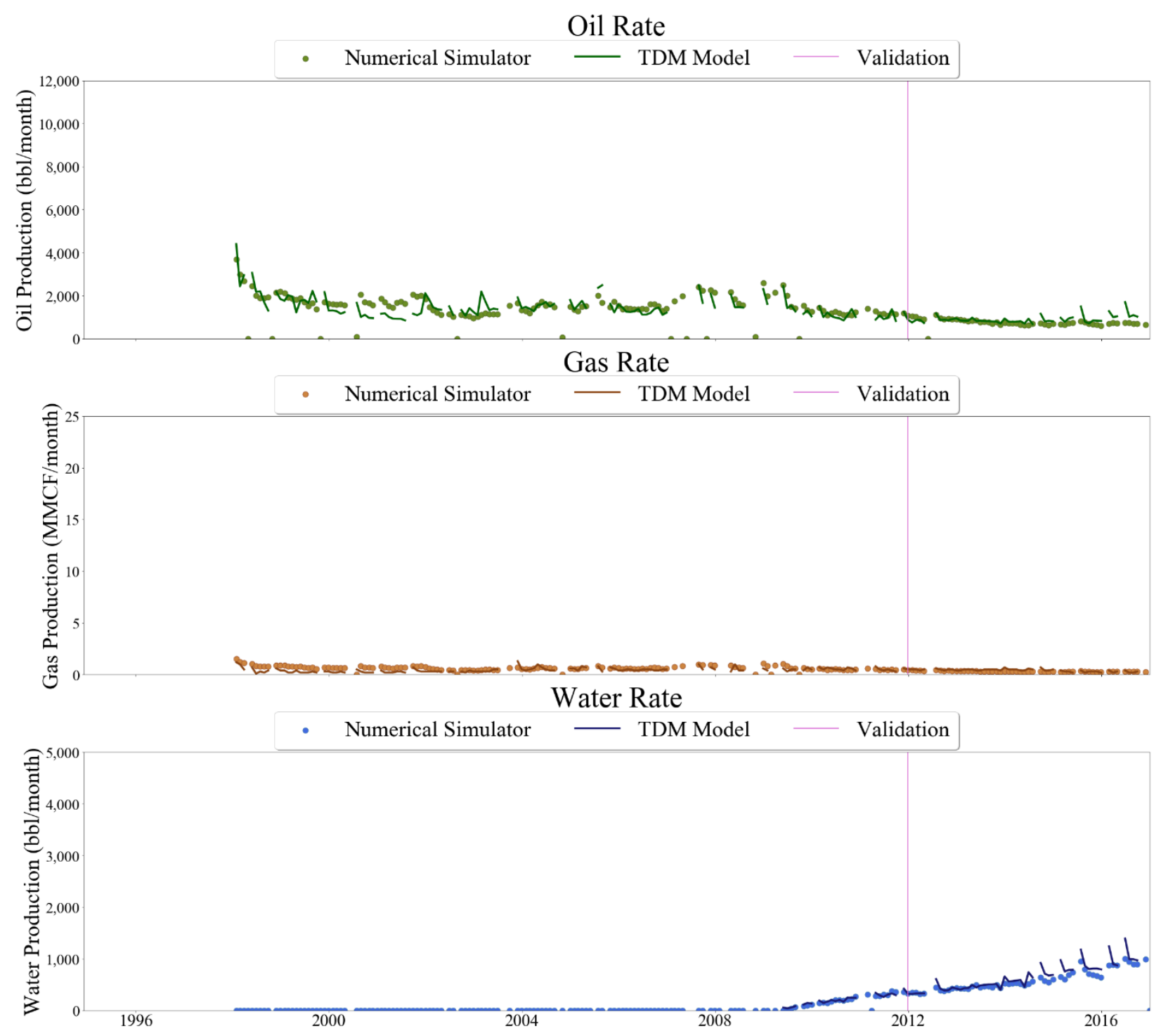

Figure 761 Well-015 oil, gas, and water rate TDM predictions vs actual simulation data plots for 5 Year Blind Validation TDM 

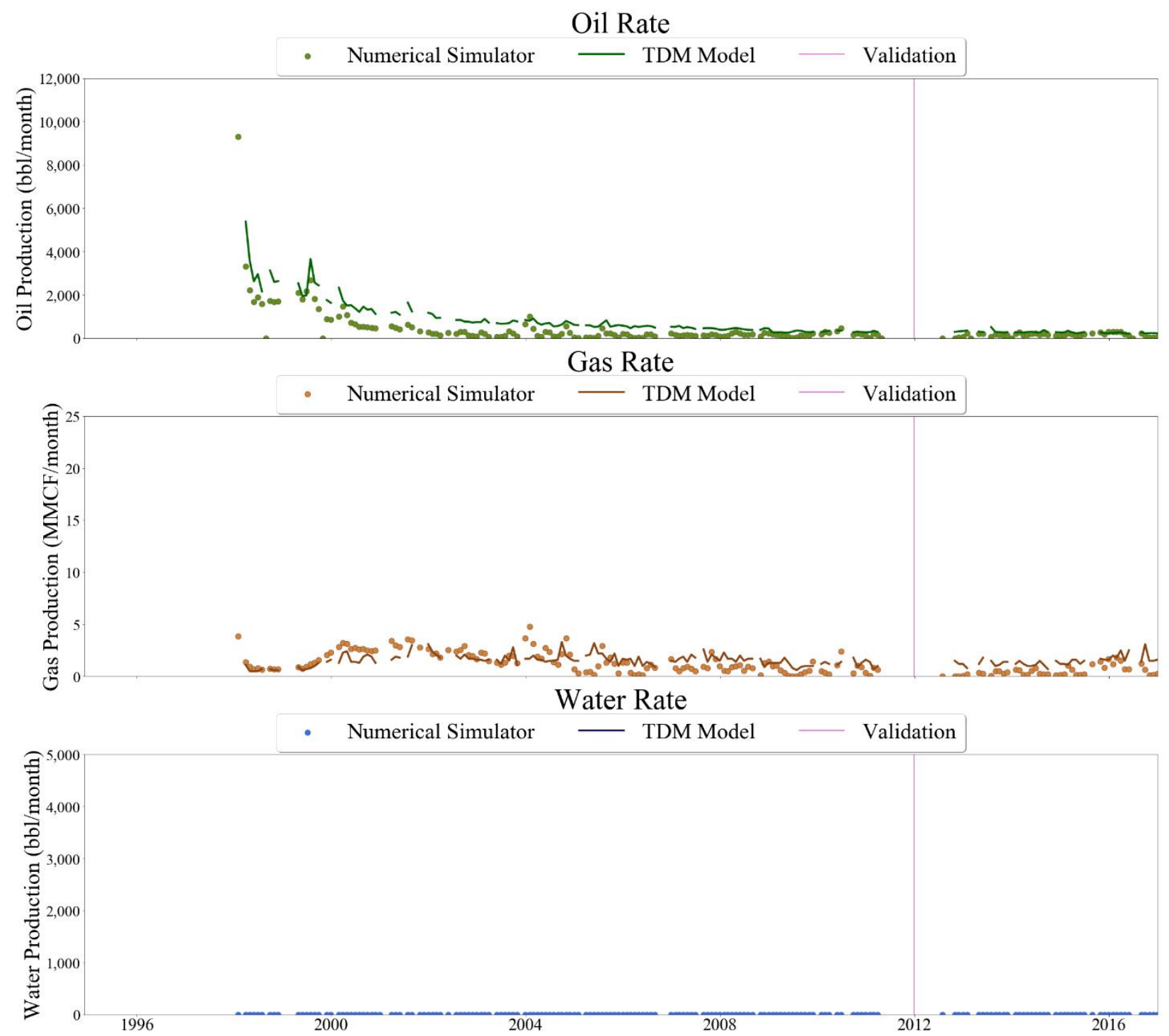

Figure 762 Well-016 oil, gas, and water rate TDM predictions vs actual simulation data plots for 5 Year Blind Validation TDM 

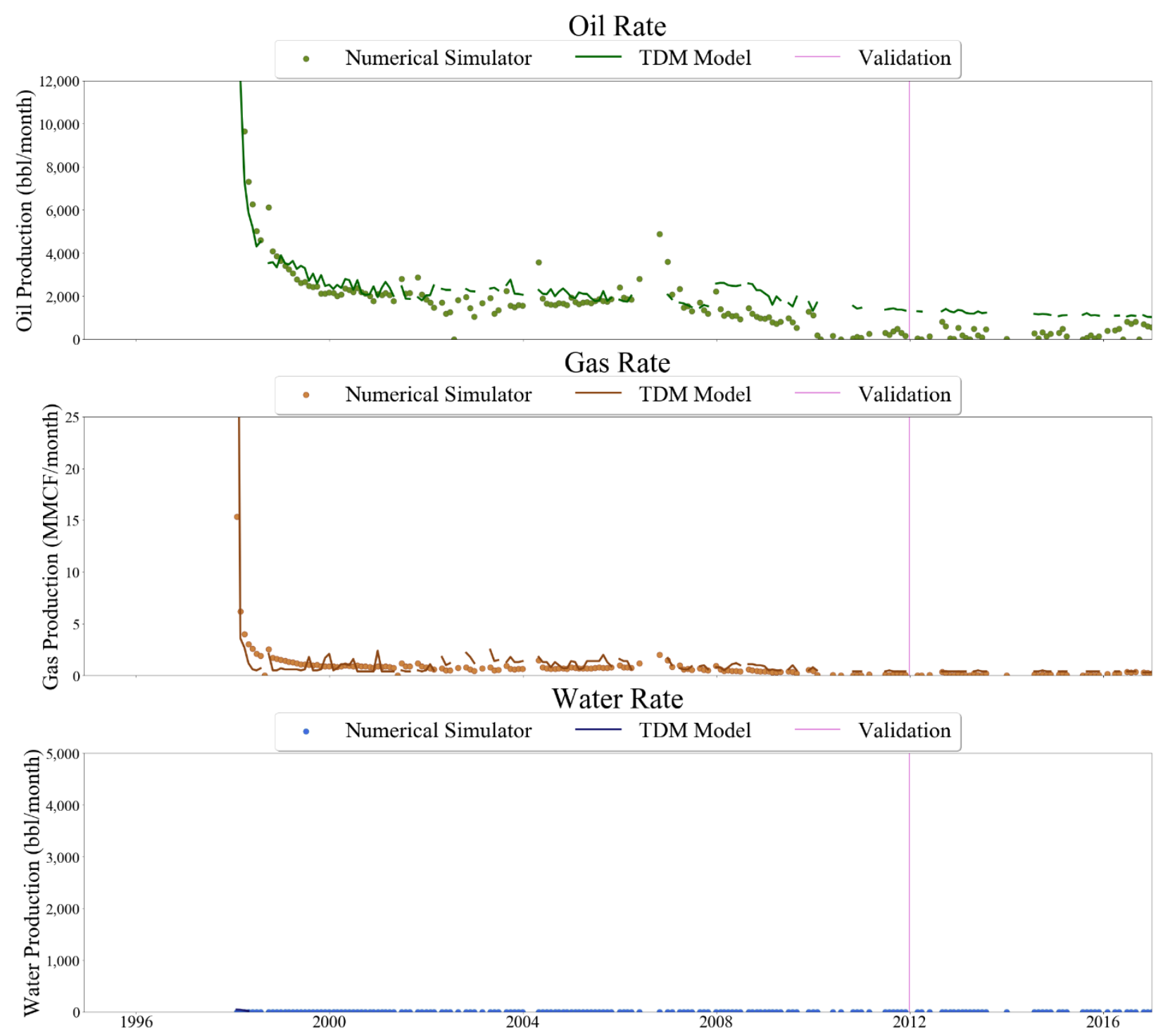

Figure 763 Well-017 oil, gas, and water rate TDM predictions vs actual simulation data plots for 5 Year Blind Validation TDM 

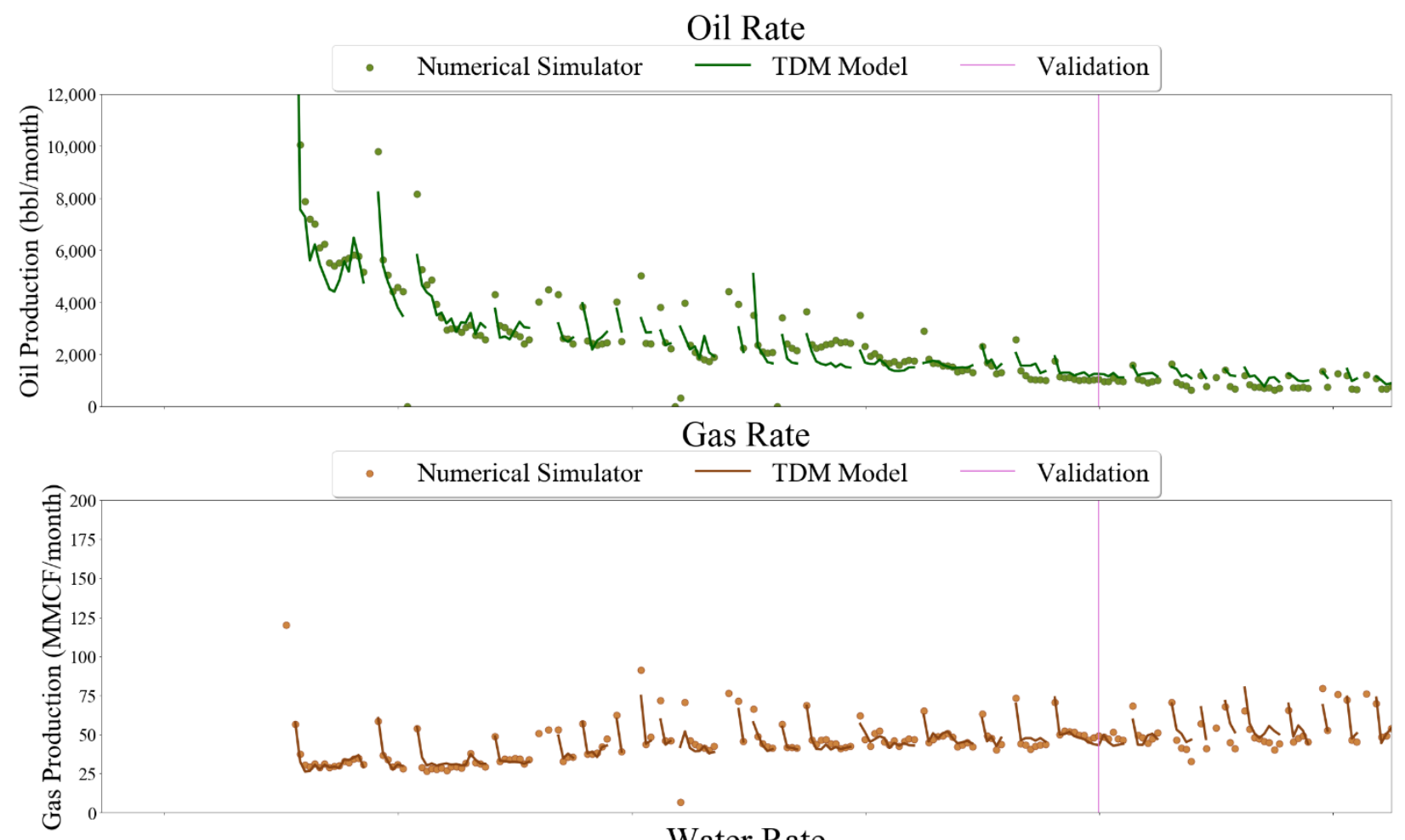

Water Rate

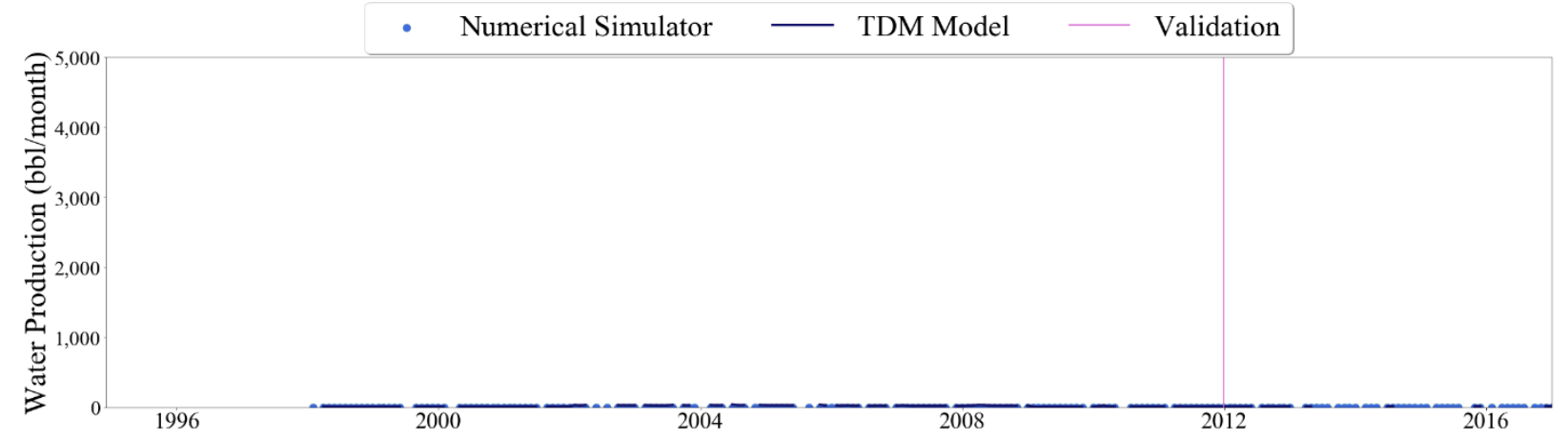

Figure 764 Well-018 oil, gas, and water rate TDM predictions vs actual simulation data plots for 5 Year Blind Validation TDM 

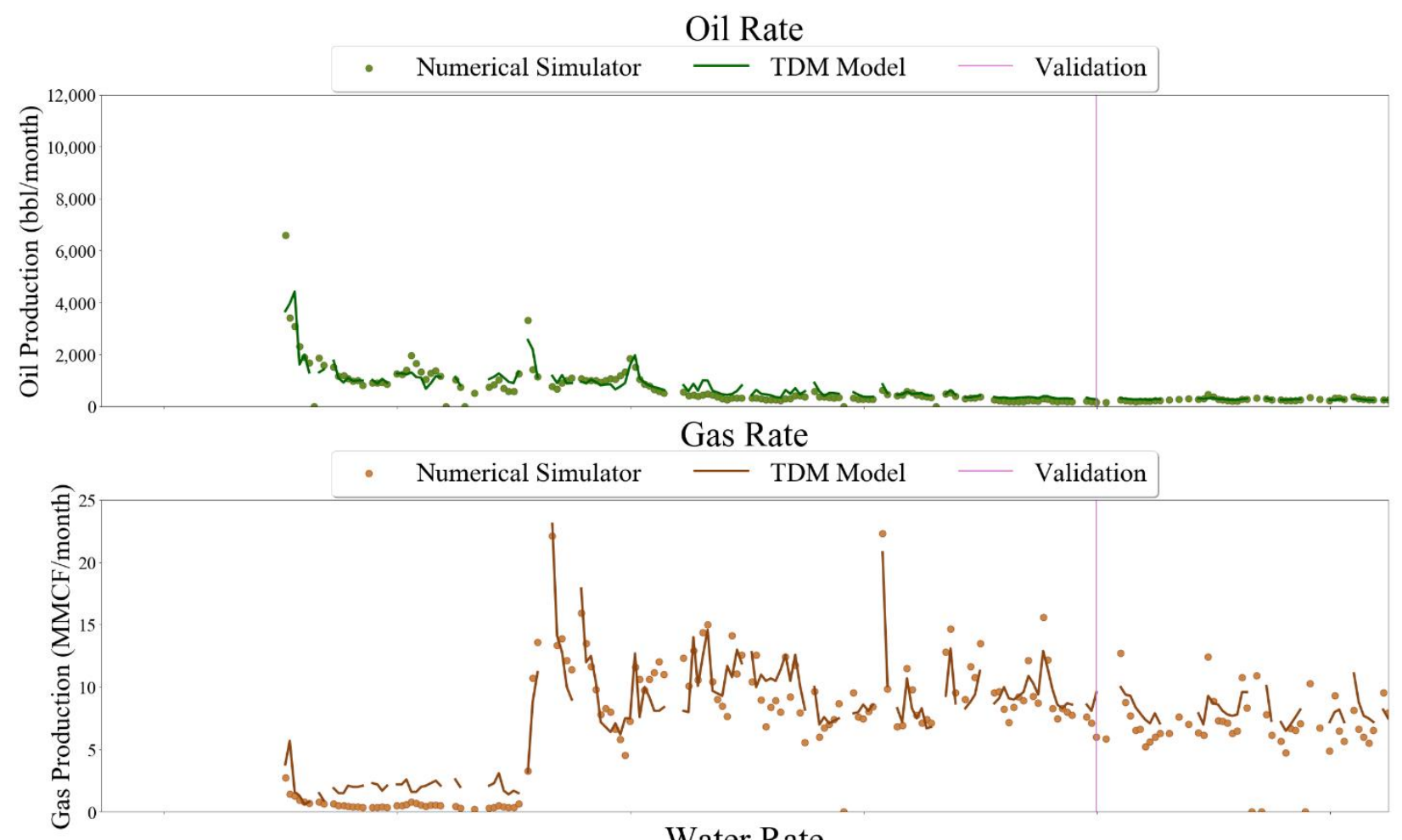

Water Rate

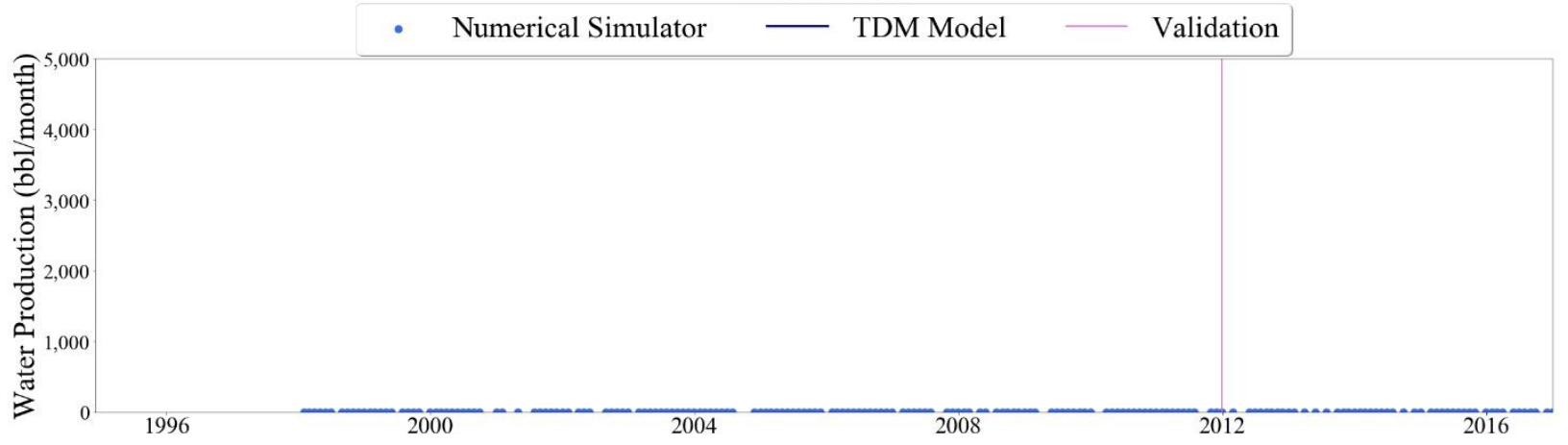

Figure 765 Well-019 oil, gas, and water rate TDM predictions vs actual simulation data plots for 5 Year Blind Validation TDM 


\section{Oil Rate}
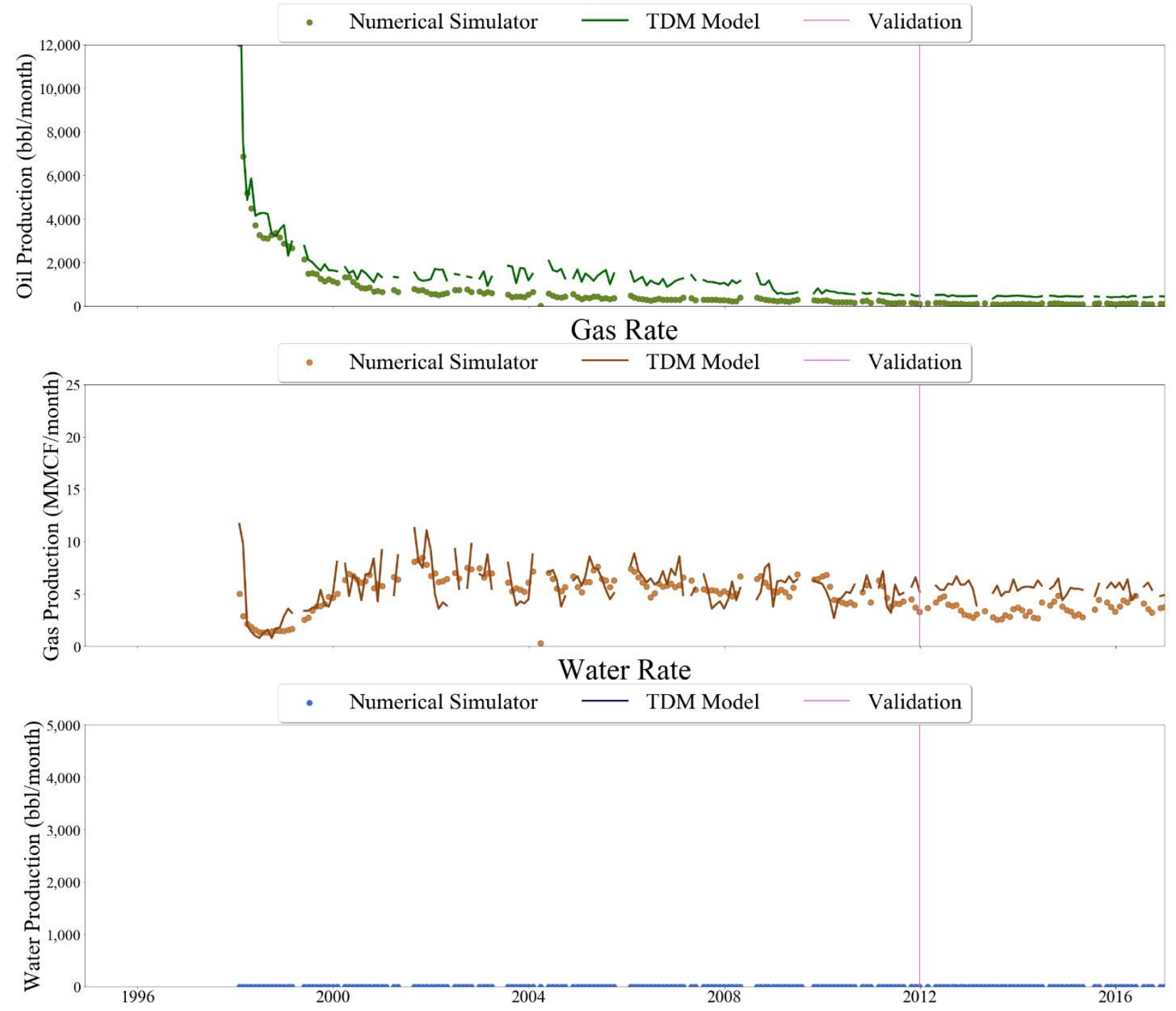

Figure 766 Well-020 oil, gas, and water rate TDM predictions vs actual simulation data plots for 5 Year Blind Validation TDM 

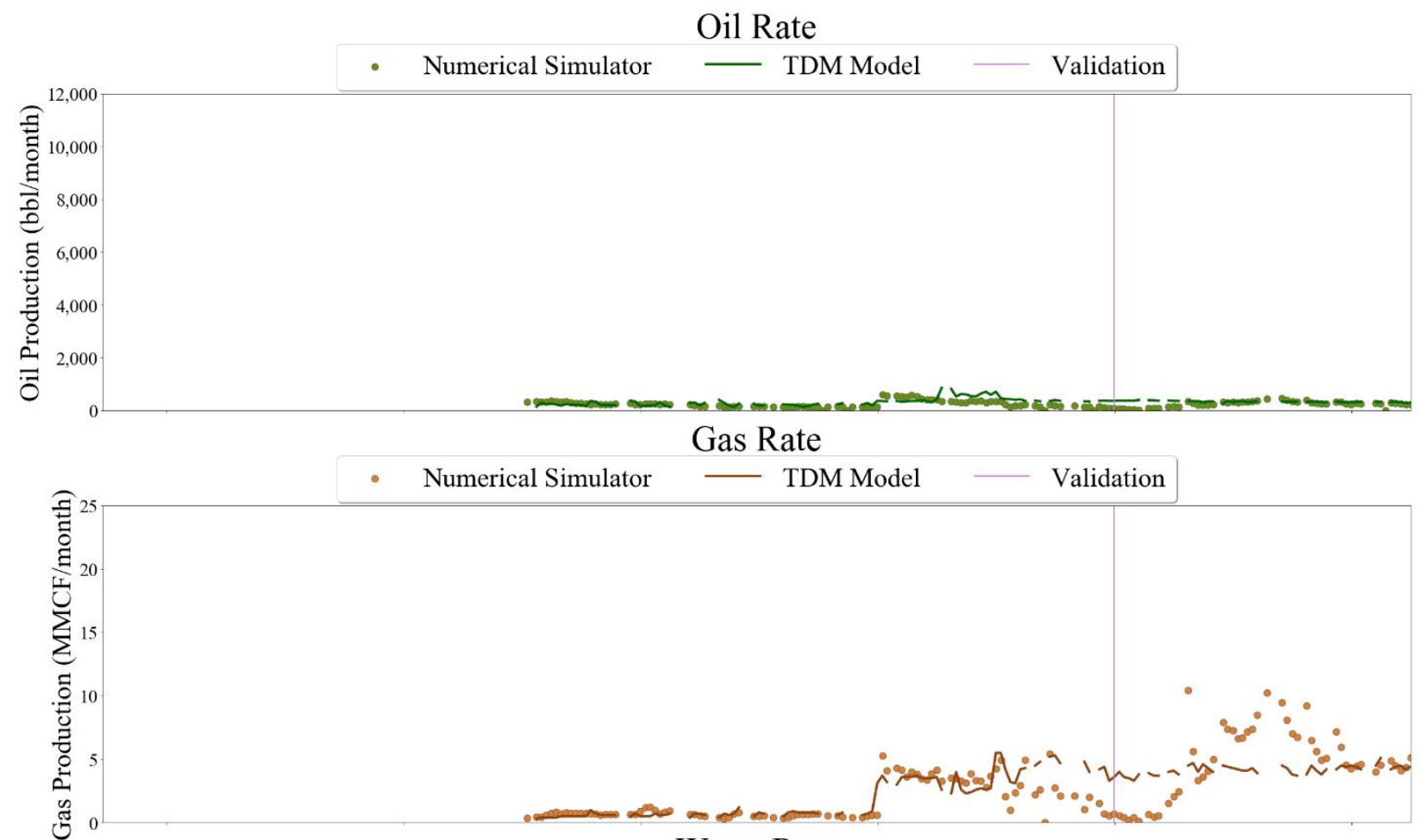

\section{Water Rate}

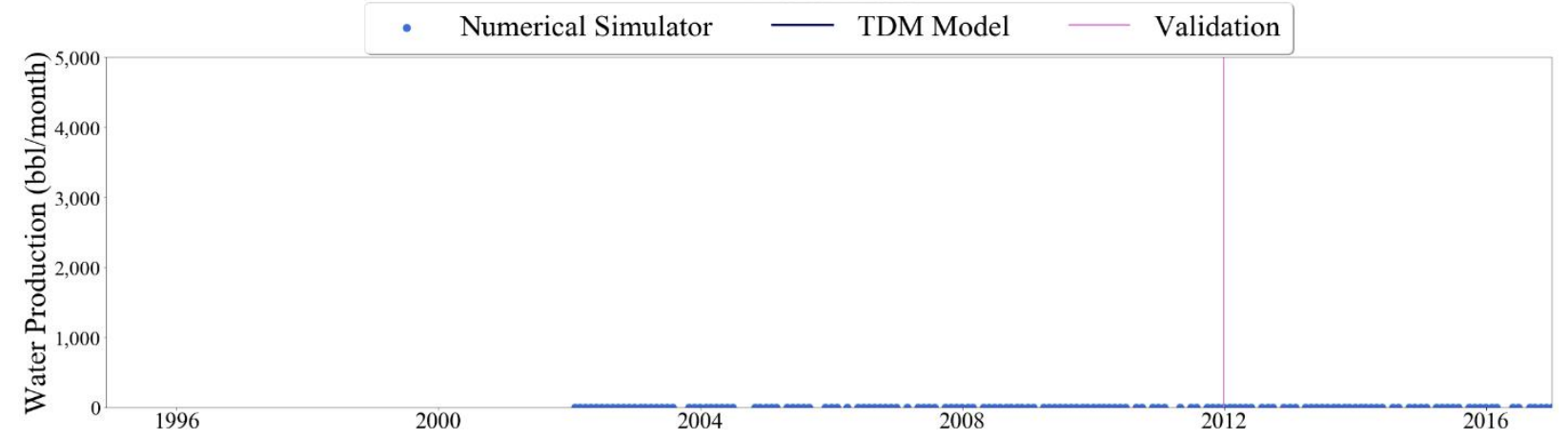

Figure 767 Well-021 oil, gas, and water rate TDM predictions vs actual simulation data plots for 5 Year Blind Validation TDM 

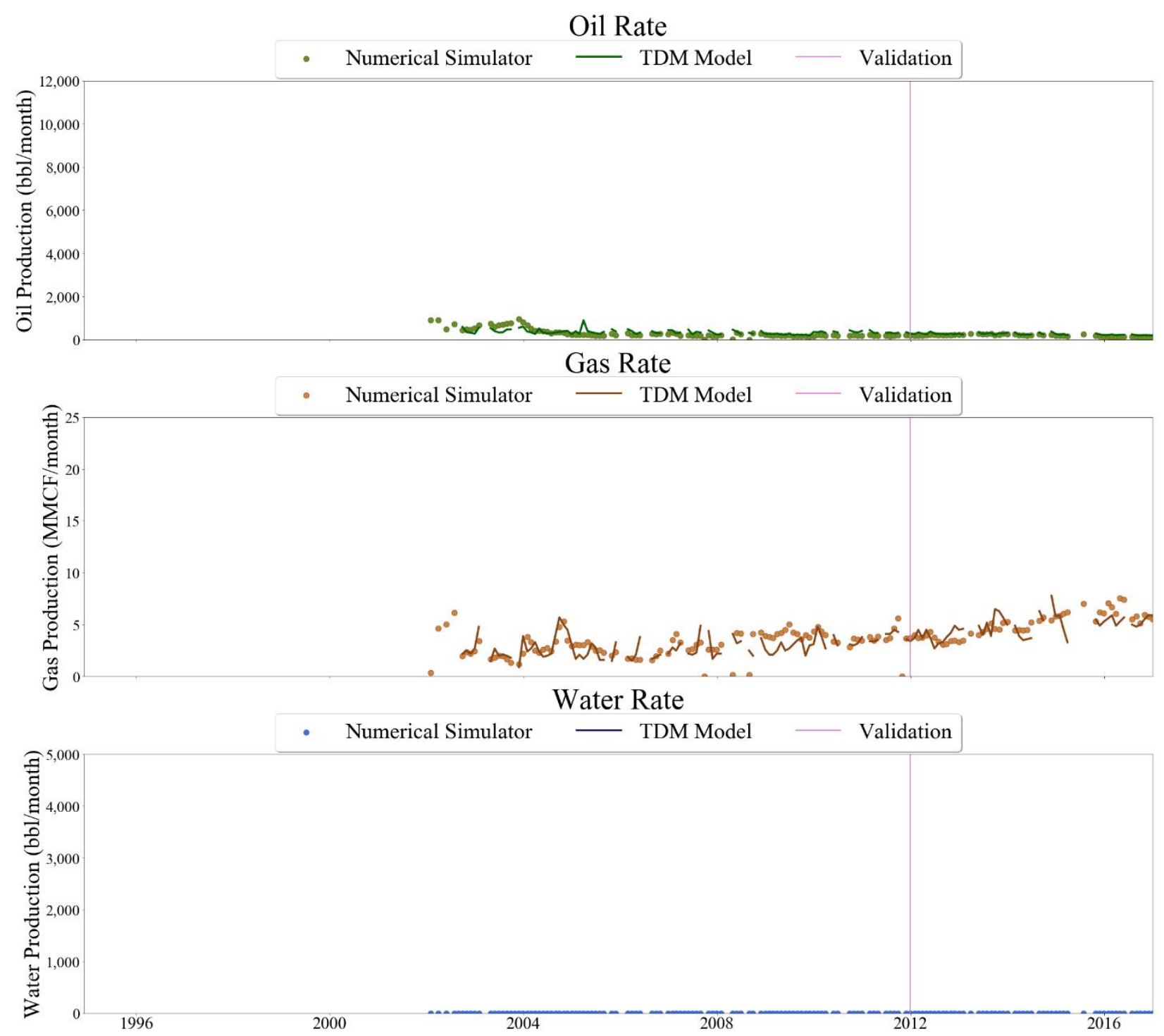

Figure 768 Well-022 oil, gas, and water rate TDM predictions vs actual simulation data plots for 5 Year Blind Validation TDM 


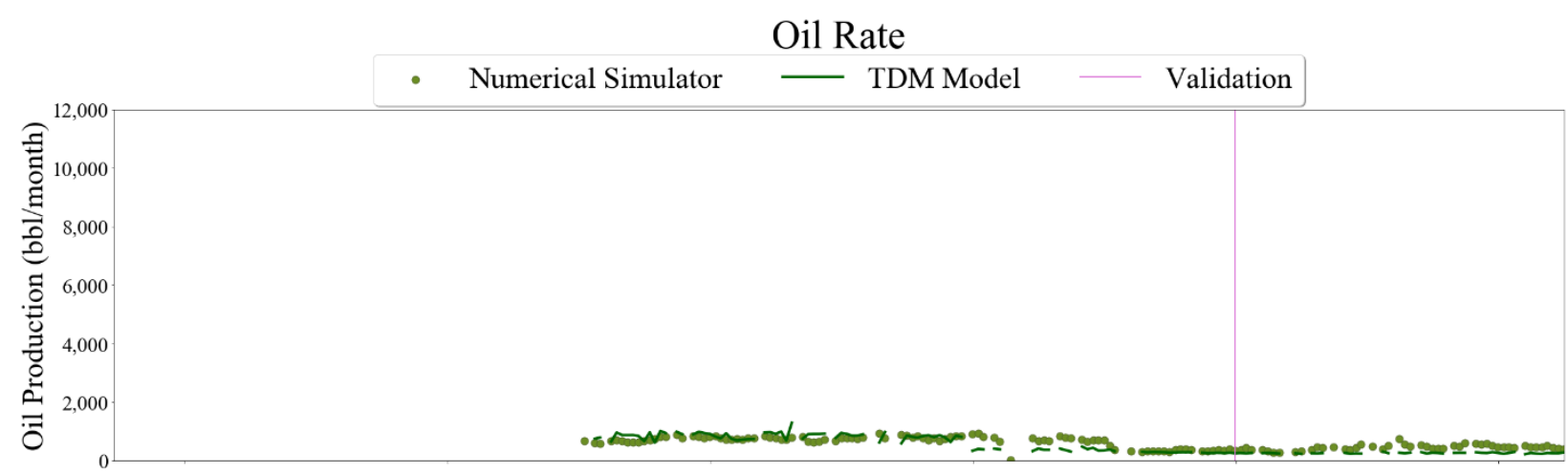

Gas Rate
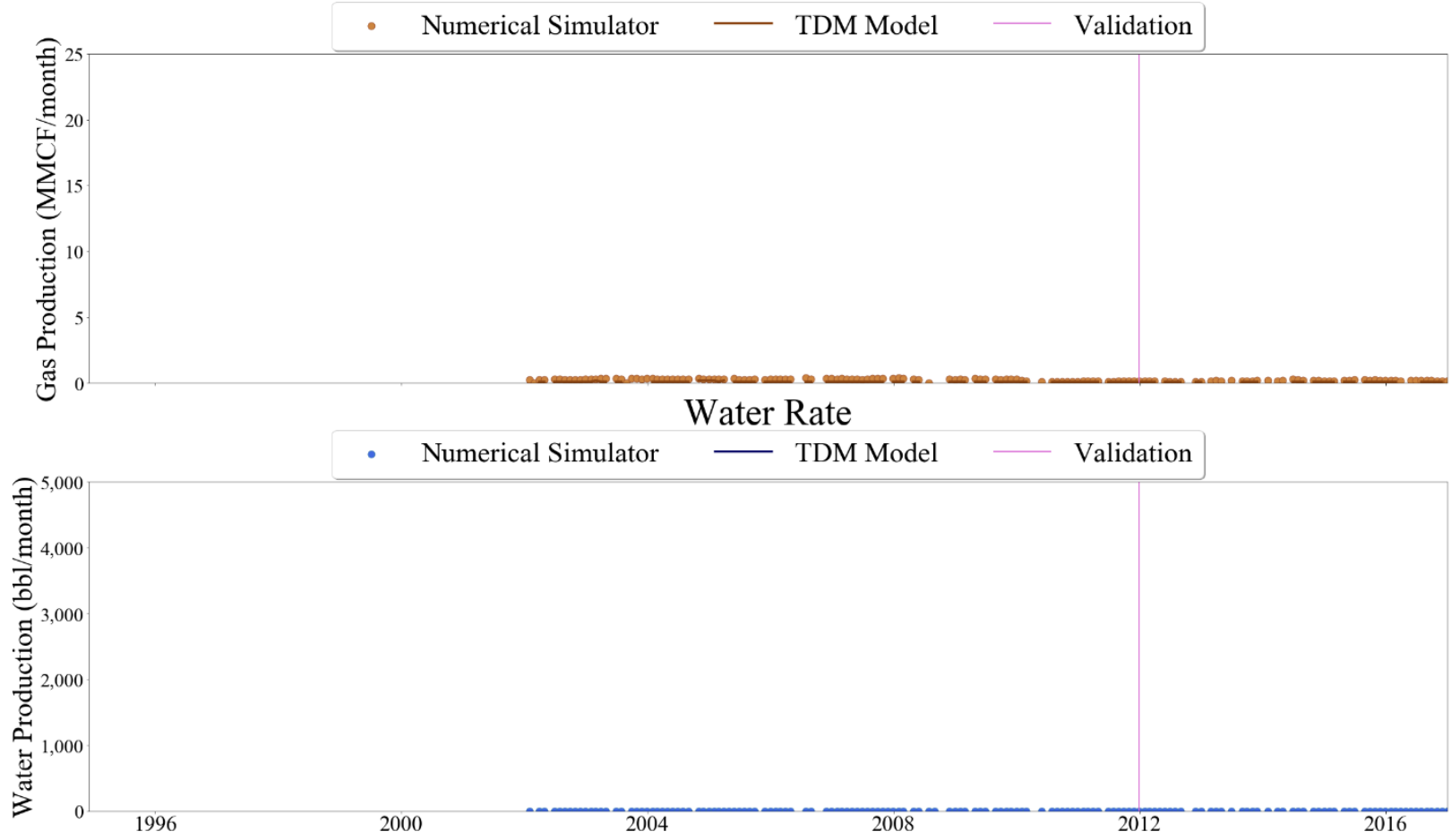

Figure 769 Well-023 oil, gas, and water rate TDM predictions vs actual simulation data plots for 5 Year Blind Validation TDM 

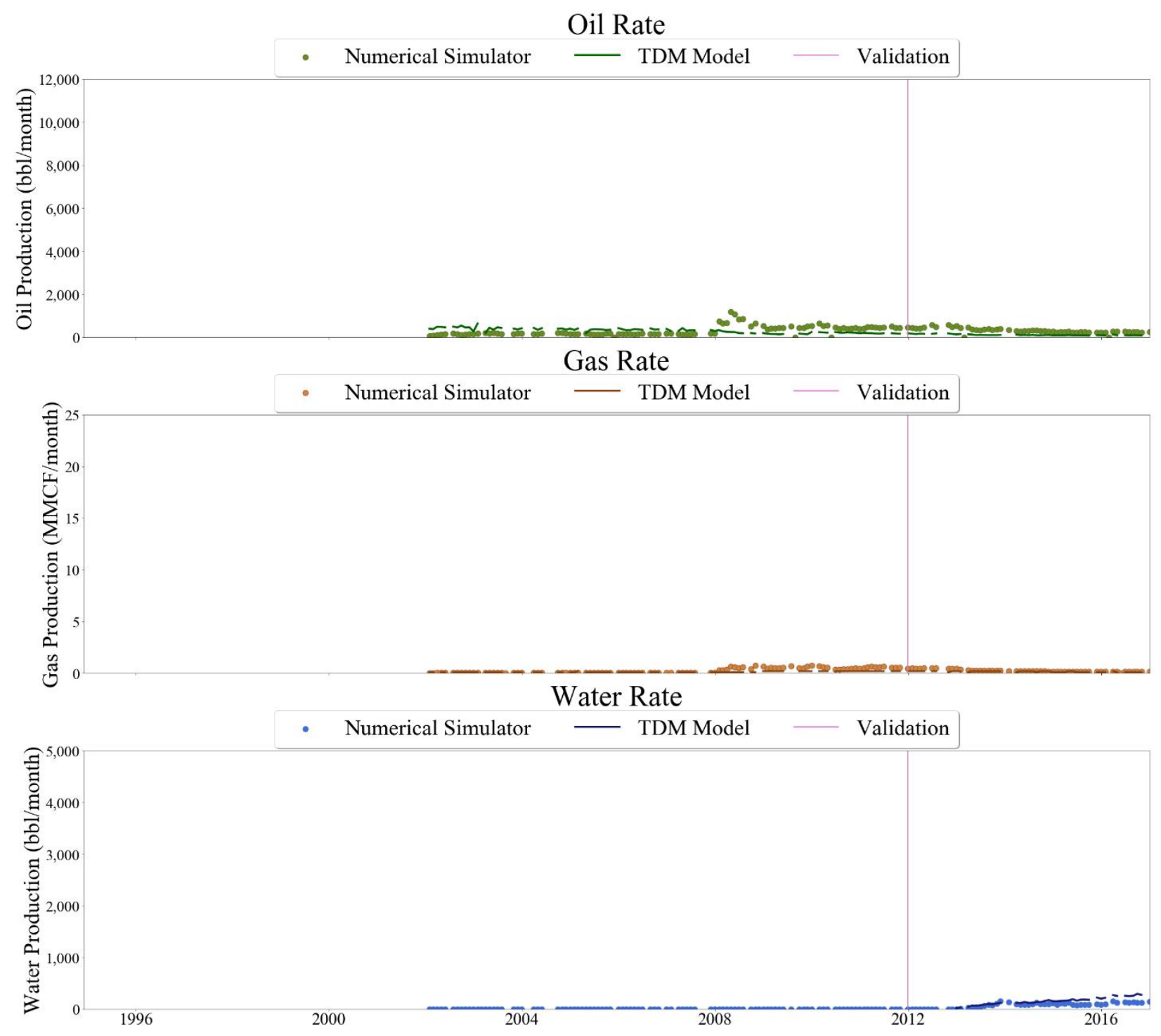

Figure 770 Well-024 oil, gas, and water rate TDM predictions vs actual simulation data plots for 5 Year Blind Validation TDM 


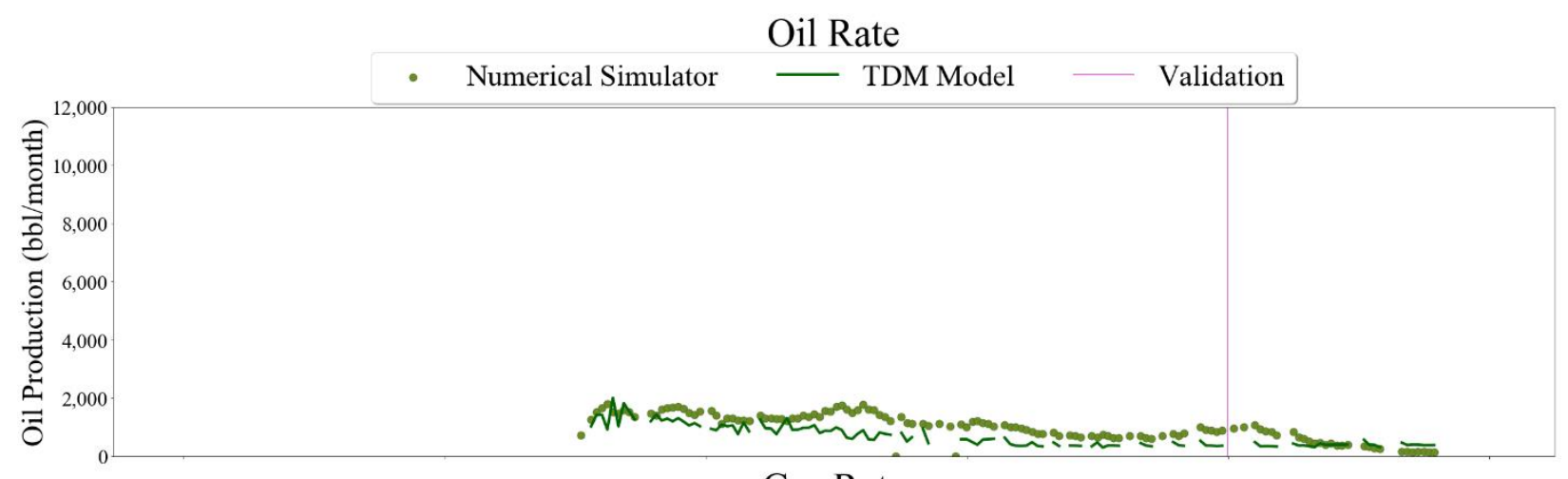

\section{Gas Rate}
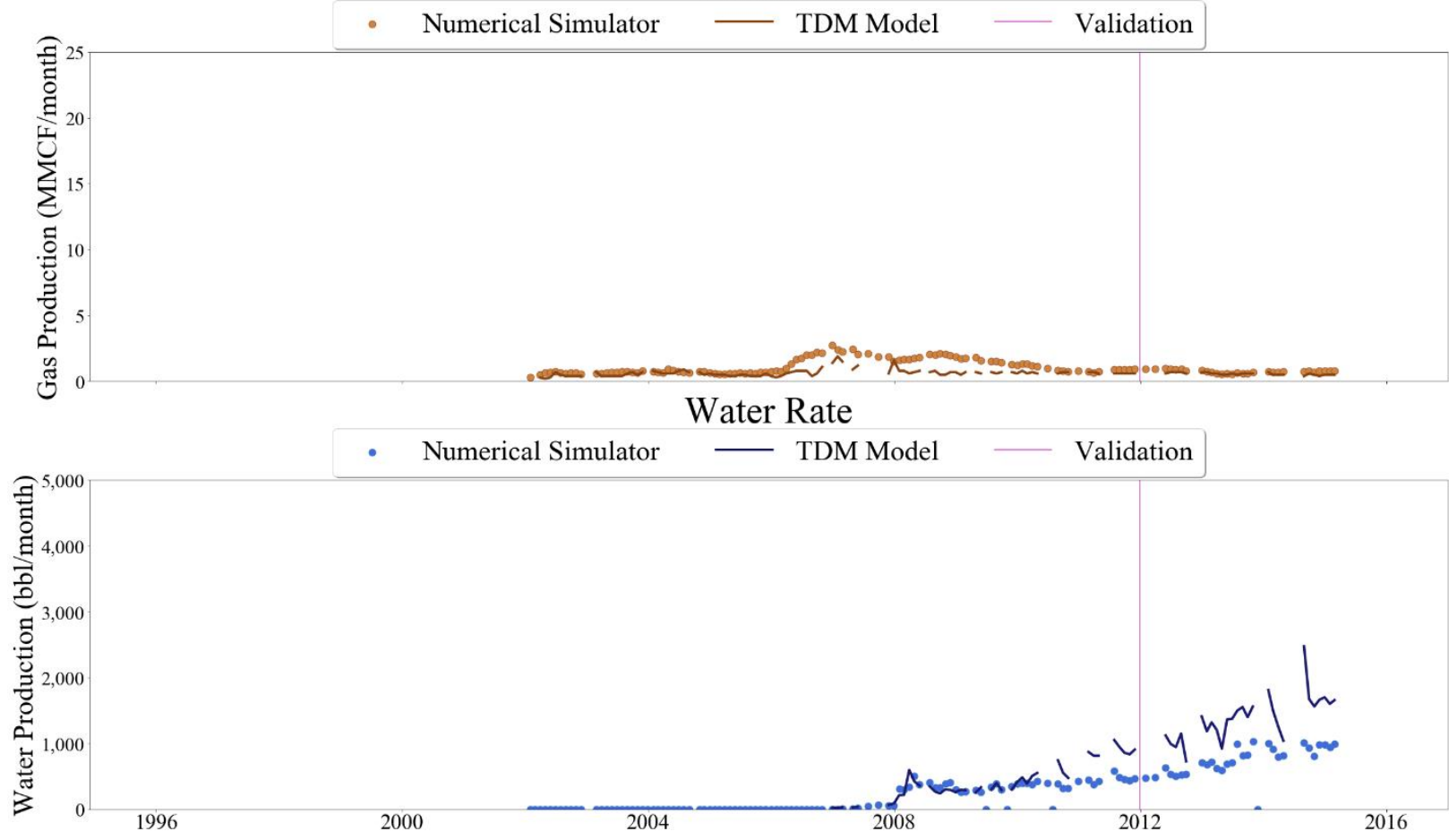

Figure 771 Well-025 oil, gas, and water rate TDM predictions vs actual simulation data plots for 5 Year Blind Validation TDM 


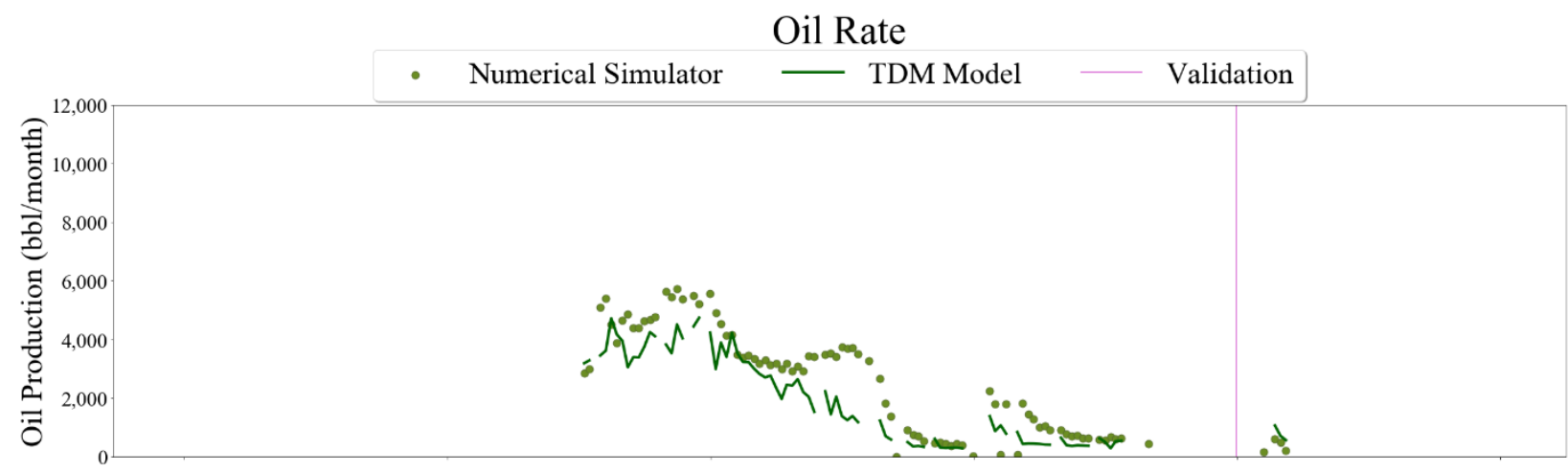

\section{Gas Rate}
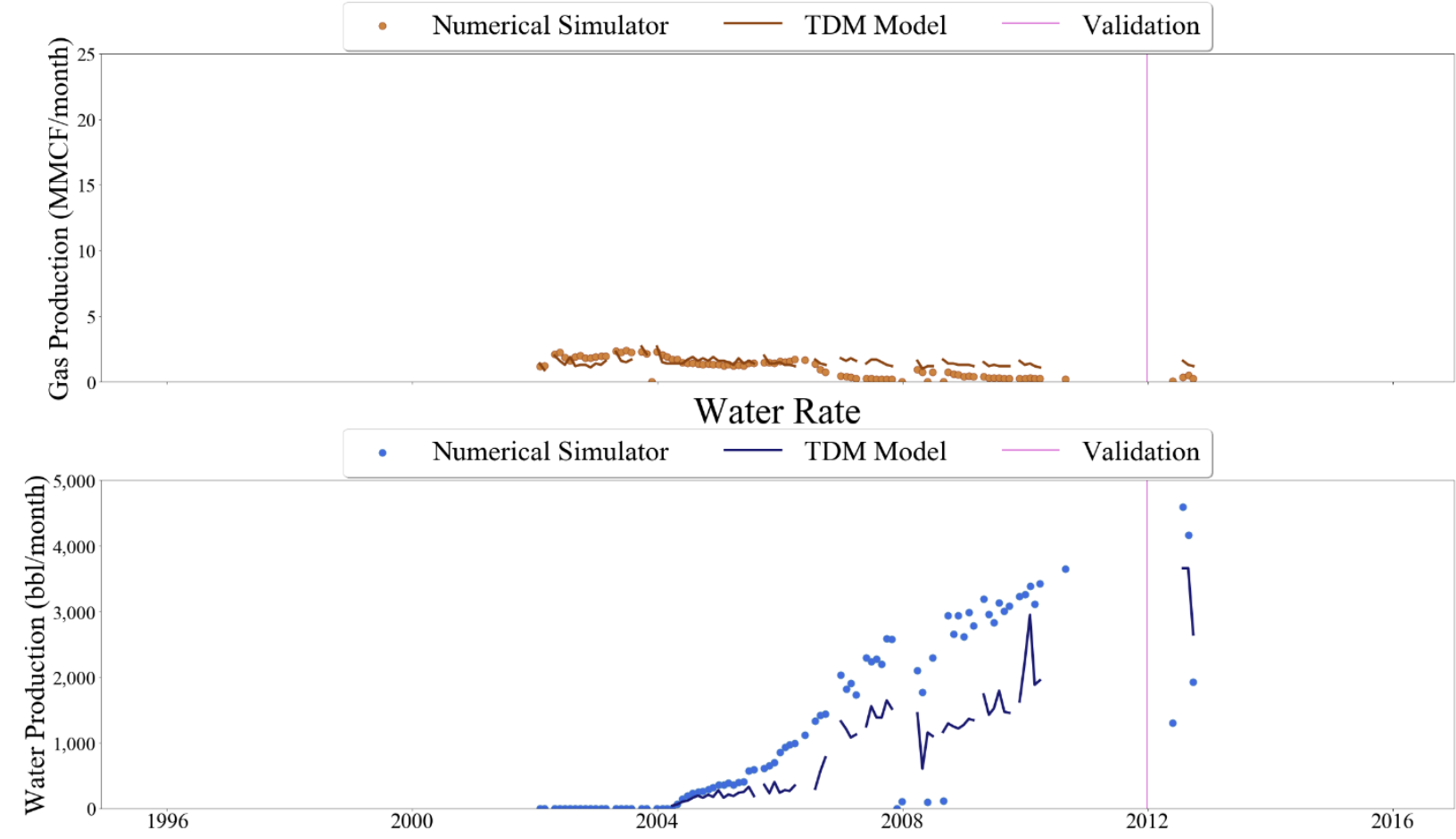

Figure 772 Well-026 oil, gas, and water rate TDM predictions vs actual simulation data plots for 5 Year Blind Validation TDM 


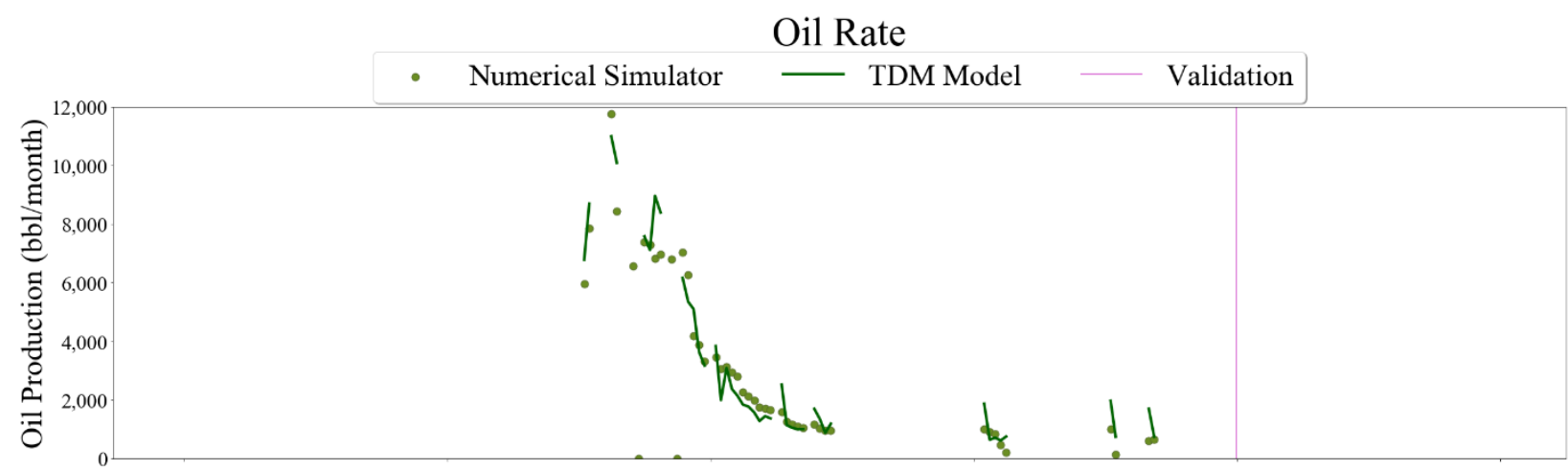

\section{Gas Rate}

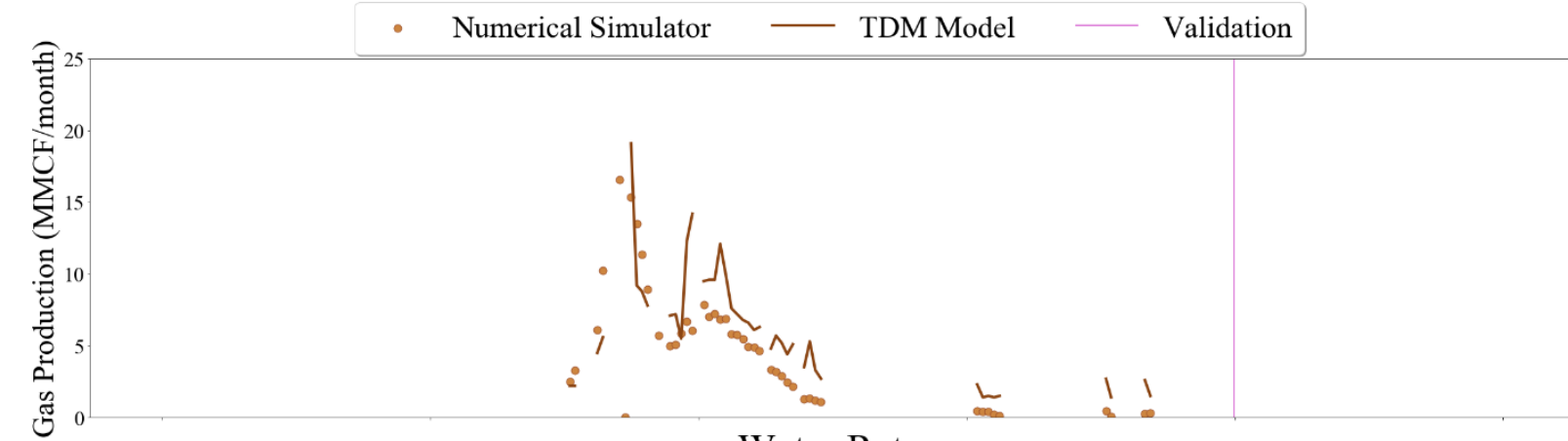

Water Rate

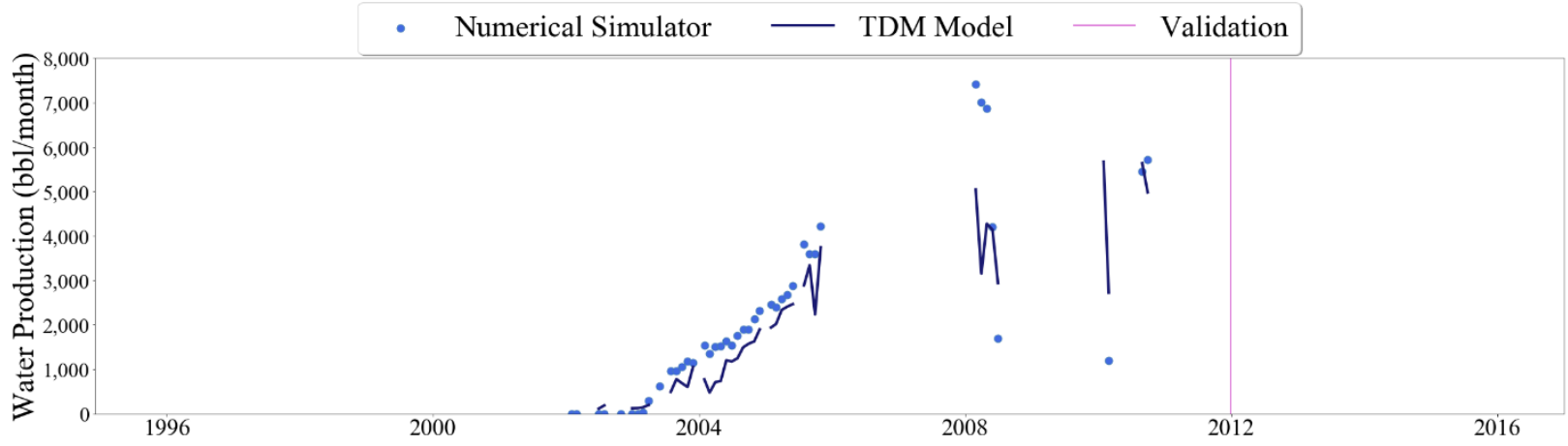

Figure 773 Well-027 oil, gas, and water rate TDM predictions vs actual simulation data plots for 5 Year Blind Validation TDM 


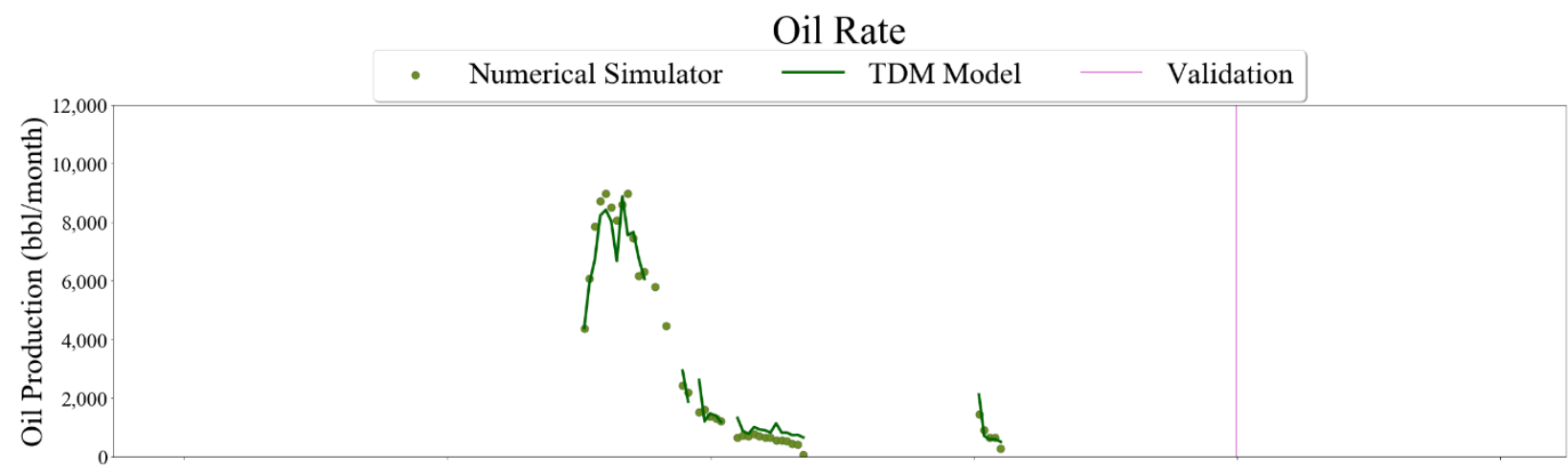

Gas Rate

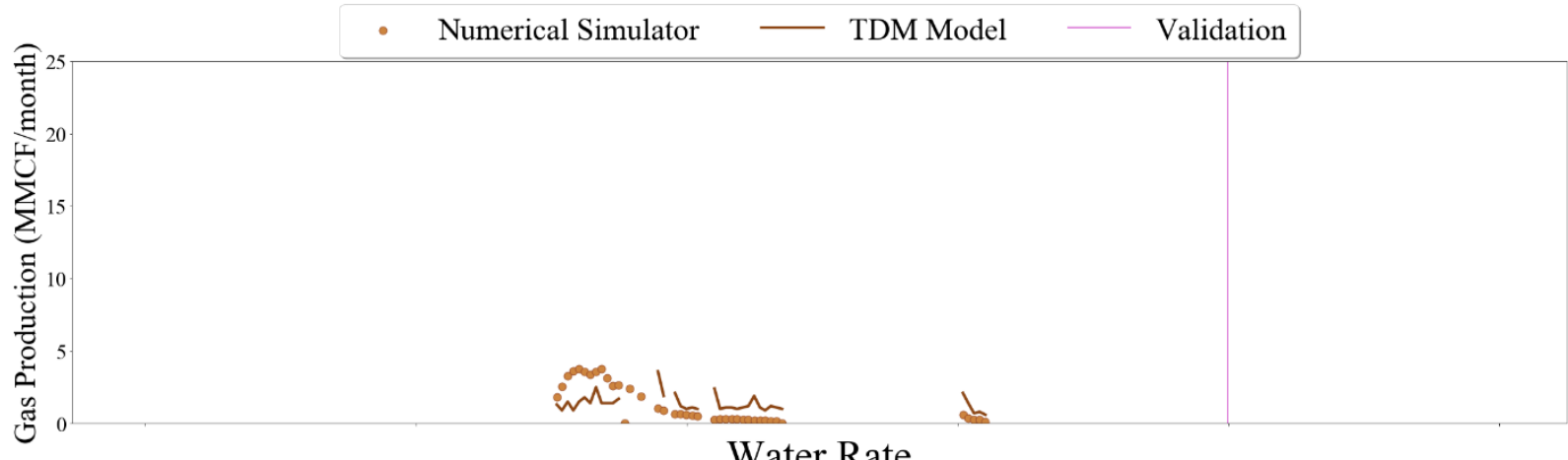

Water Rate

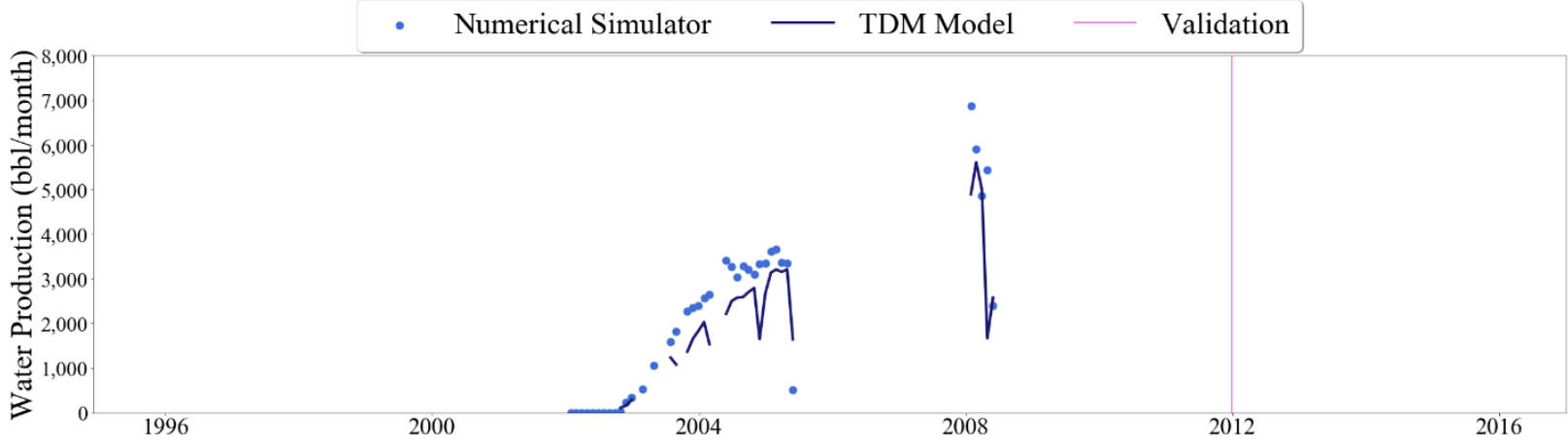

Figure 774 Well-028 oil, gas, and water rate TDM predictions vs actual simulation data plots for 5 Year Blind Validation TDM 

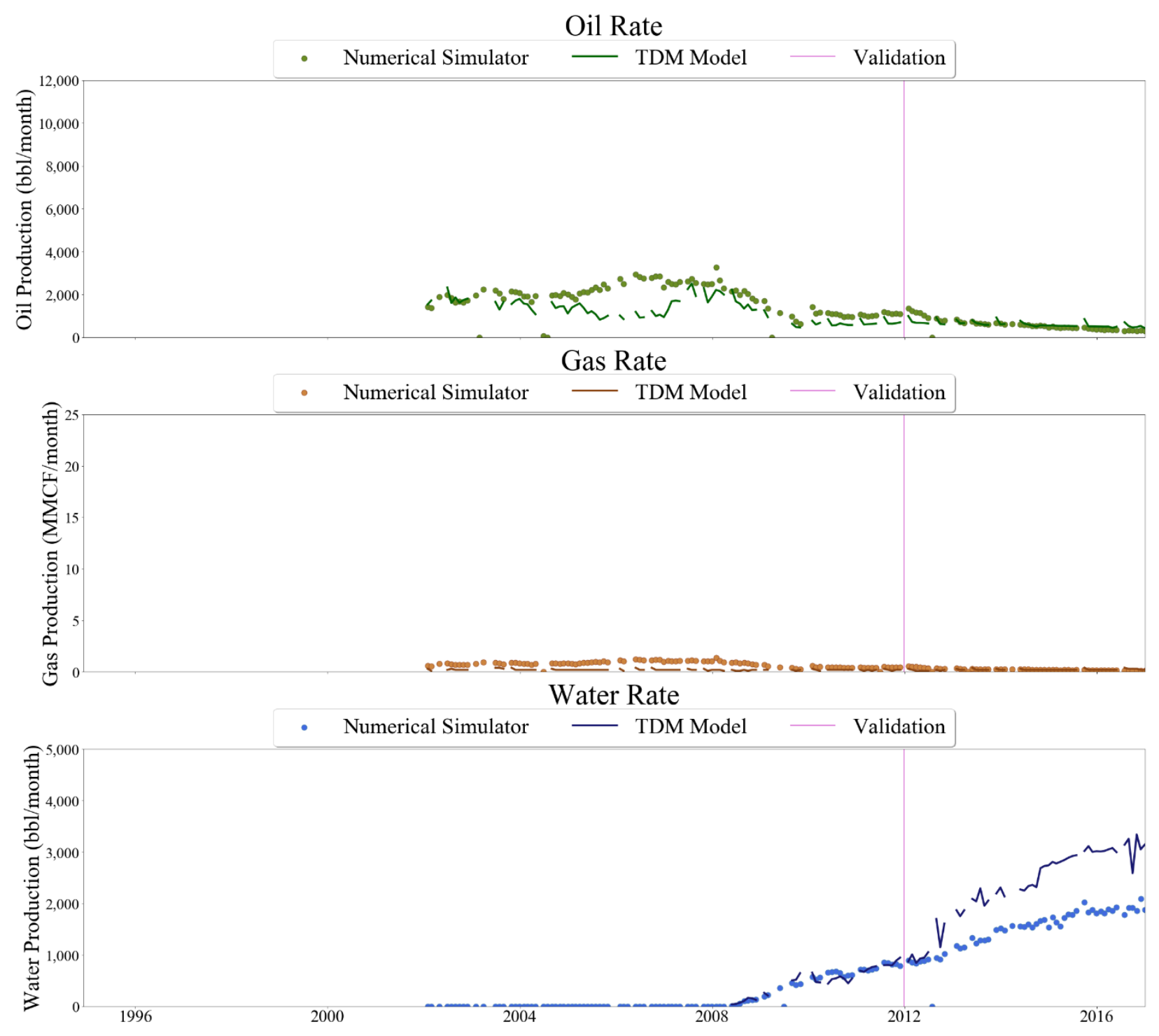

Figure 775 Well-029 oil, gas, and water rate TDM predictions vs actual simulation data plots for 5 Year Blind Validation TDM 


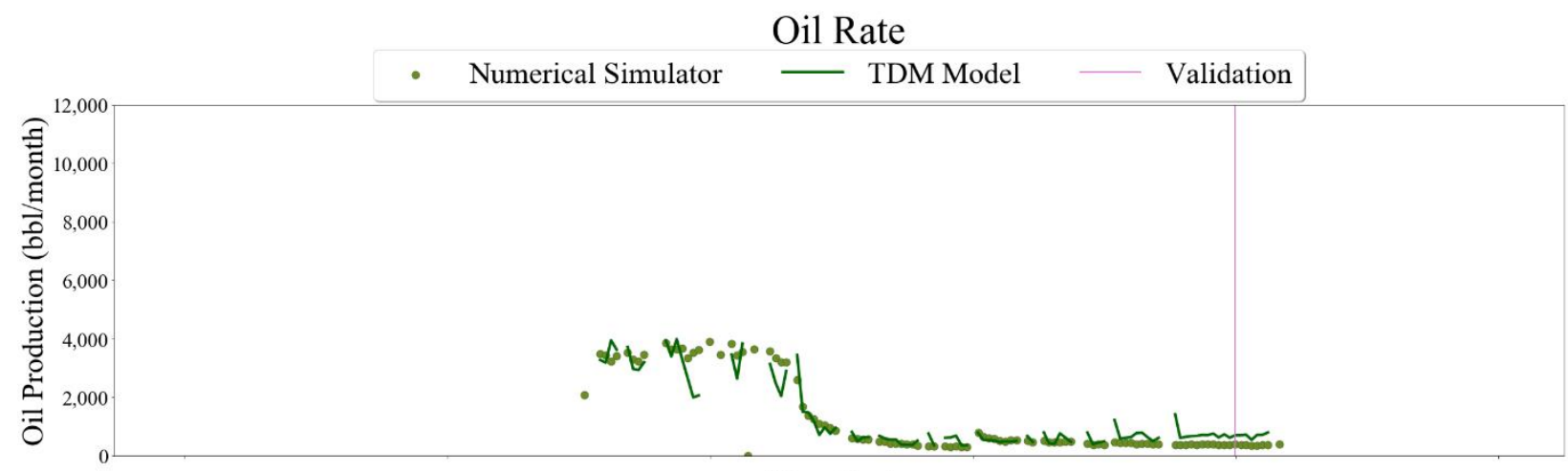

Gas Rate
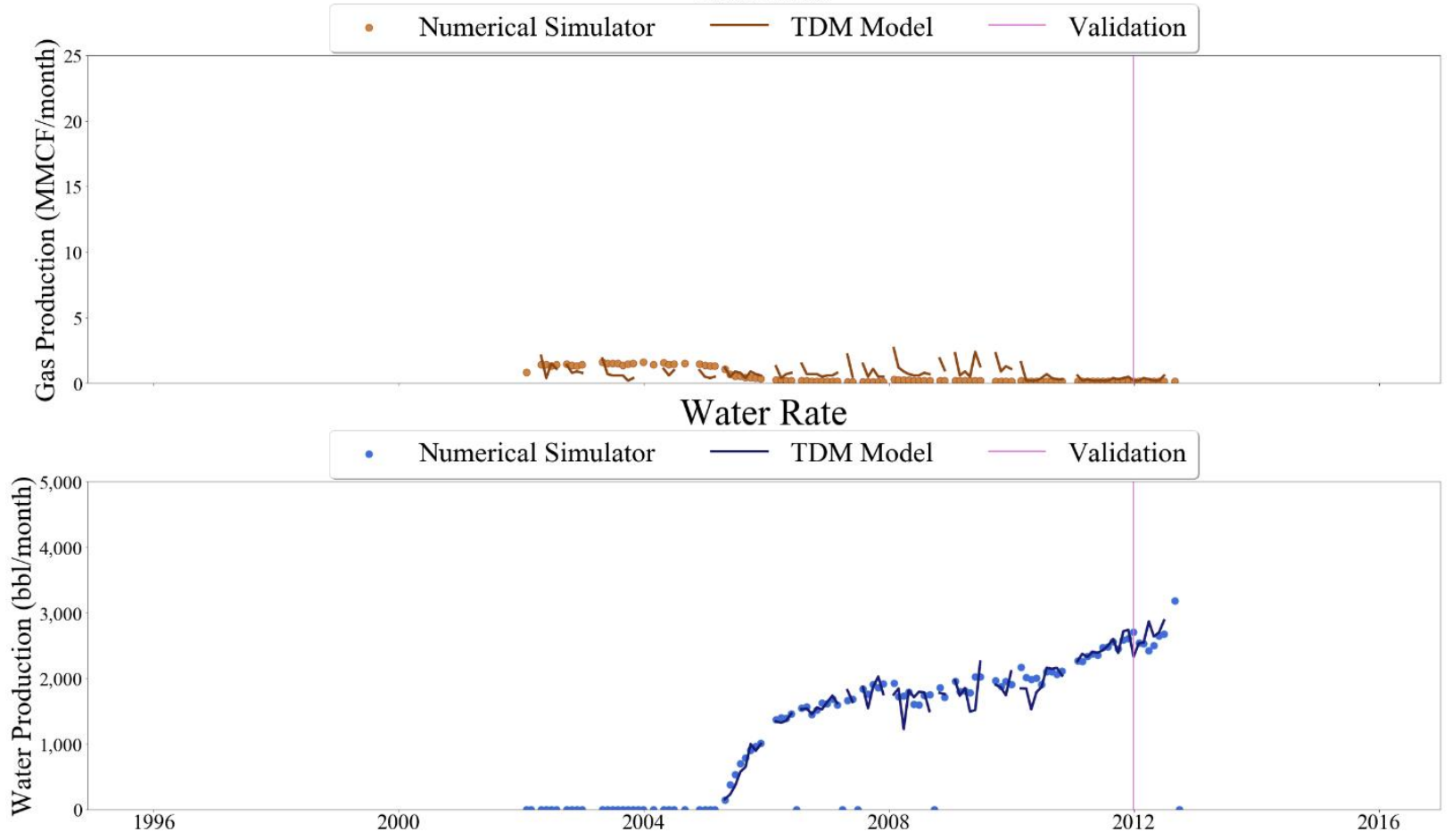

Figure 776 Well-030 oil, gas, and water rate TDM predictions vs actual simulation data plots for 5 Year Blind Validation TDM 


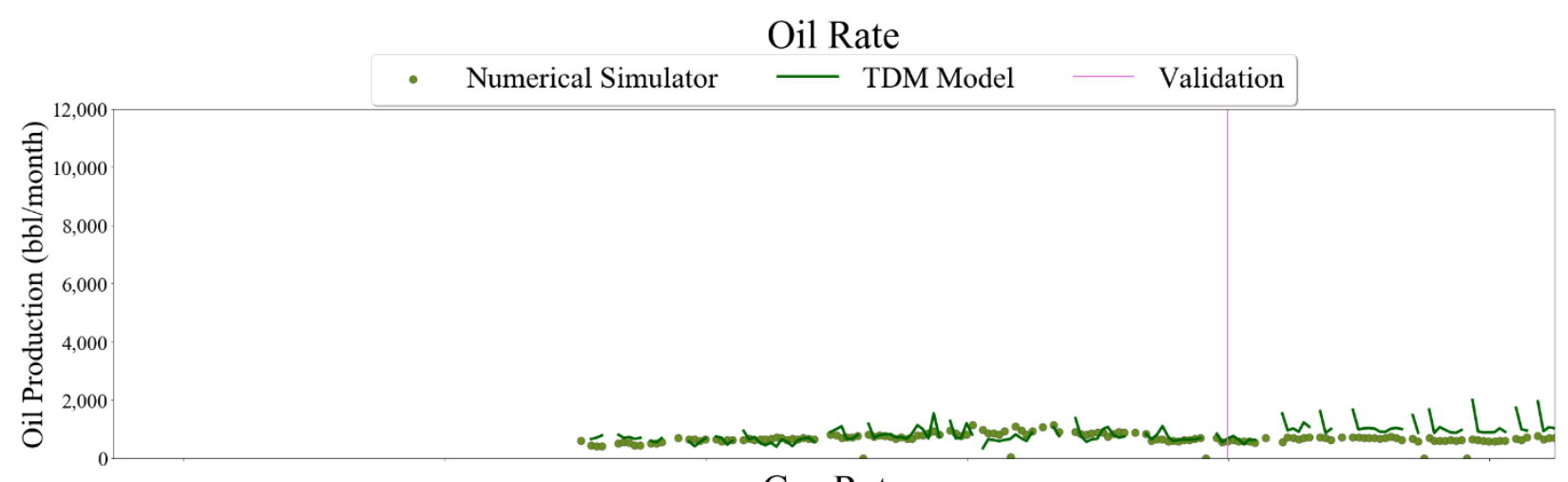

Gas Rate
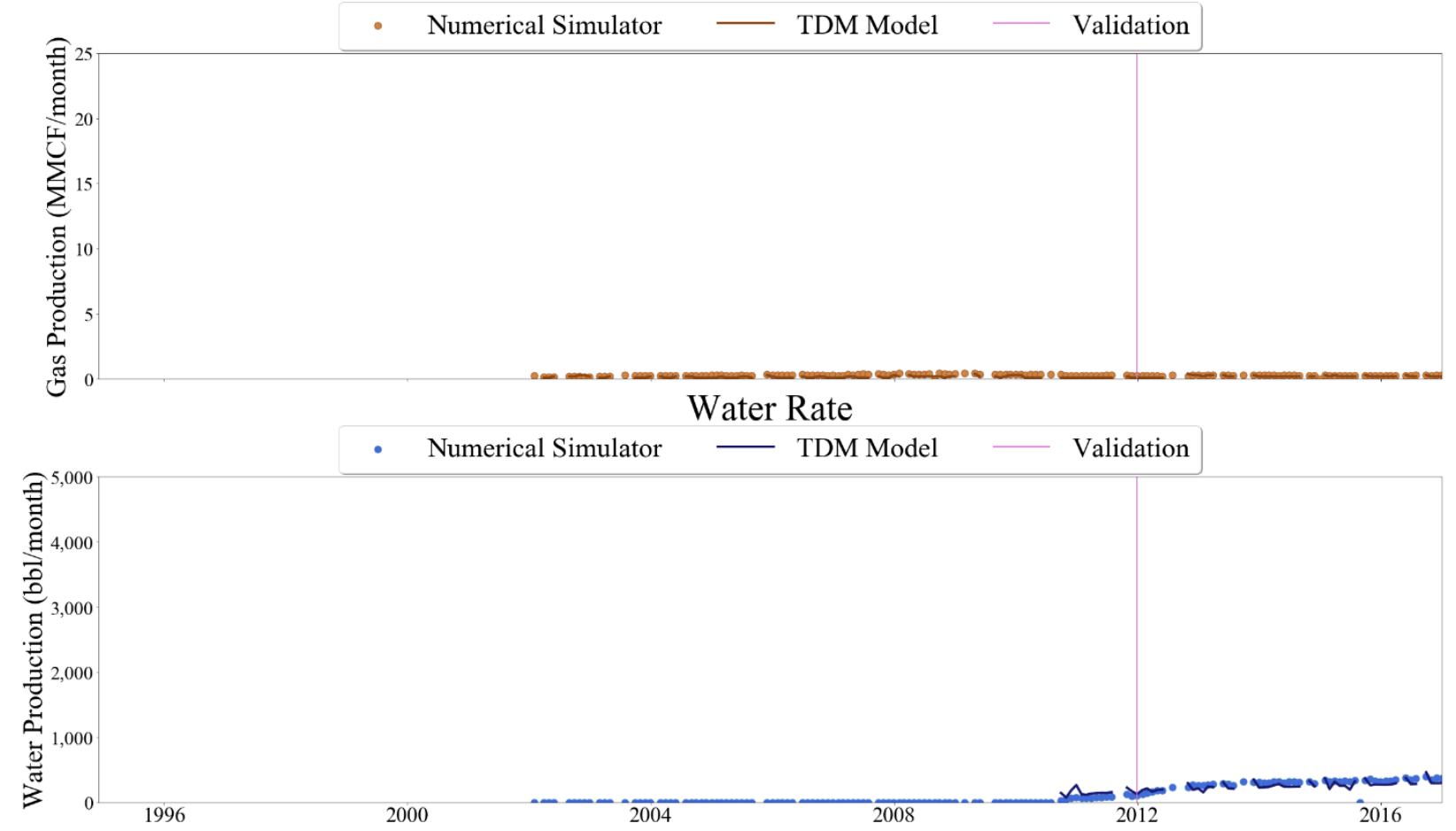

Figure 777 Well-031 oil, gas, and water rate TDM predictions vs actual simulation data plots for 5 Year Blind Validation TDM 


\section{Oil Rate}

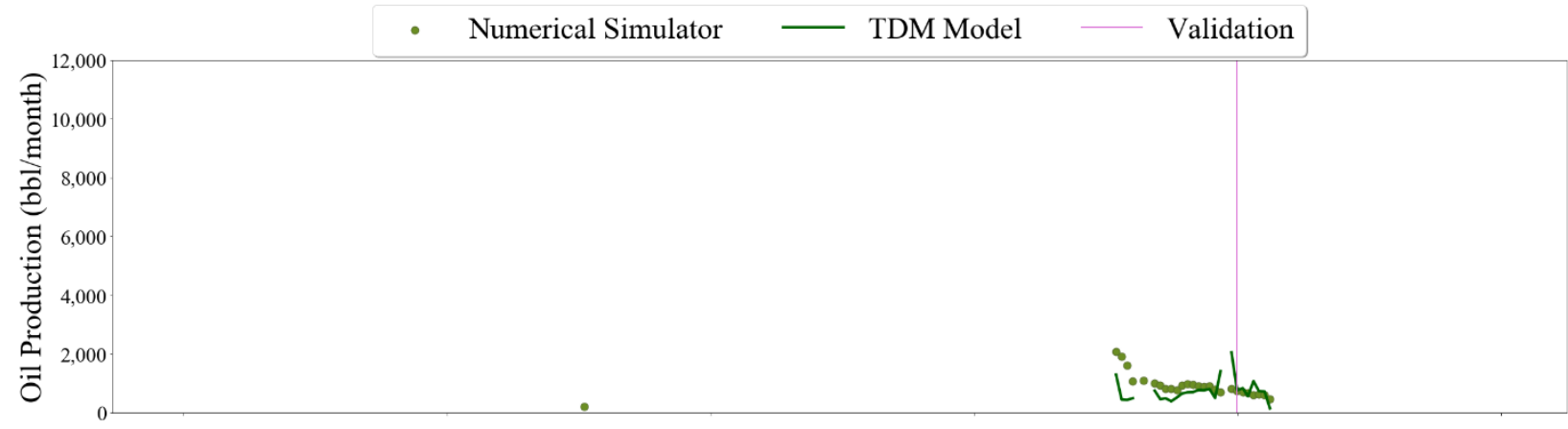

\section{Gas Rate}
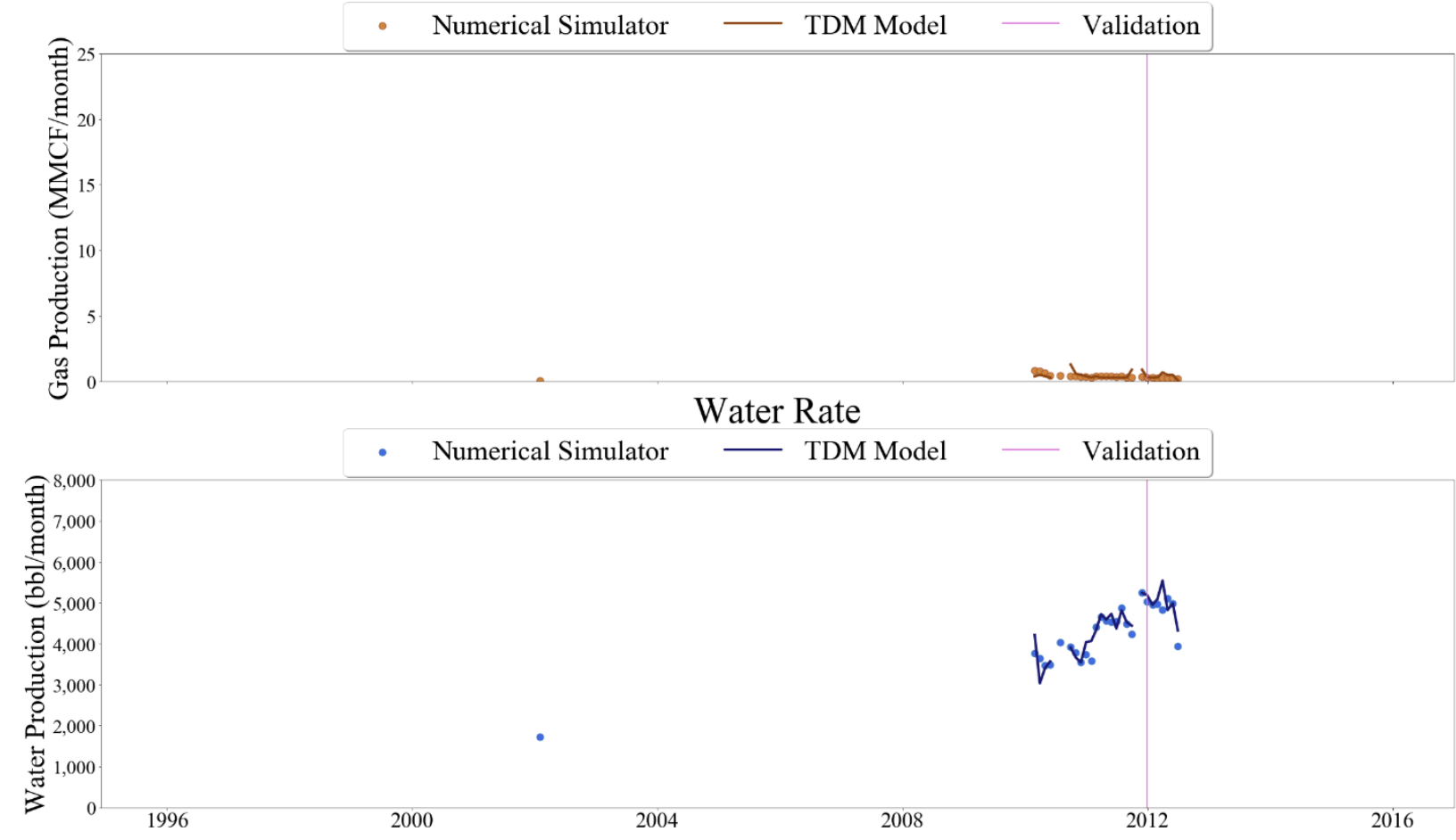

Figure 778 Well-032 oil, gas, and water rate TDM predictions vs actual simulation data plots for 5 Year Blind Validation TDM 


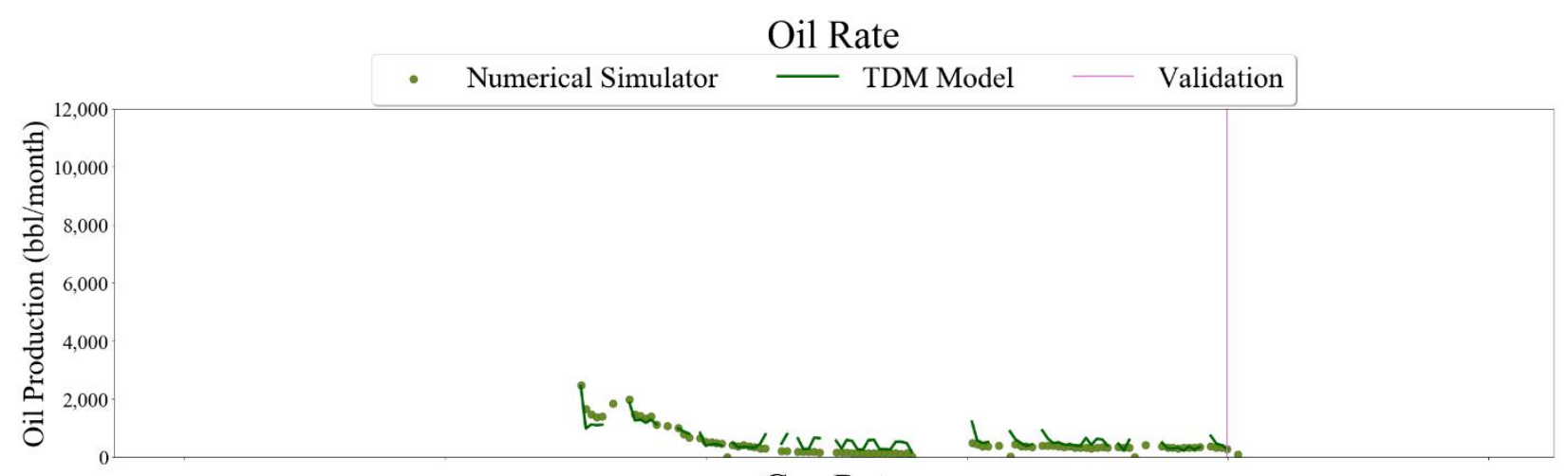

Gas Rate
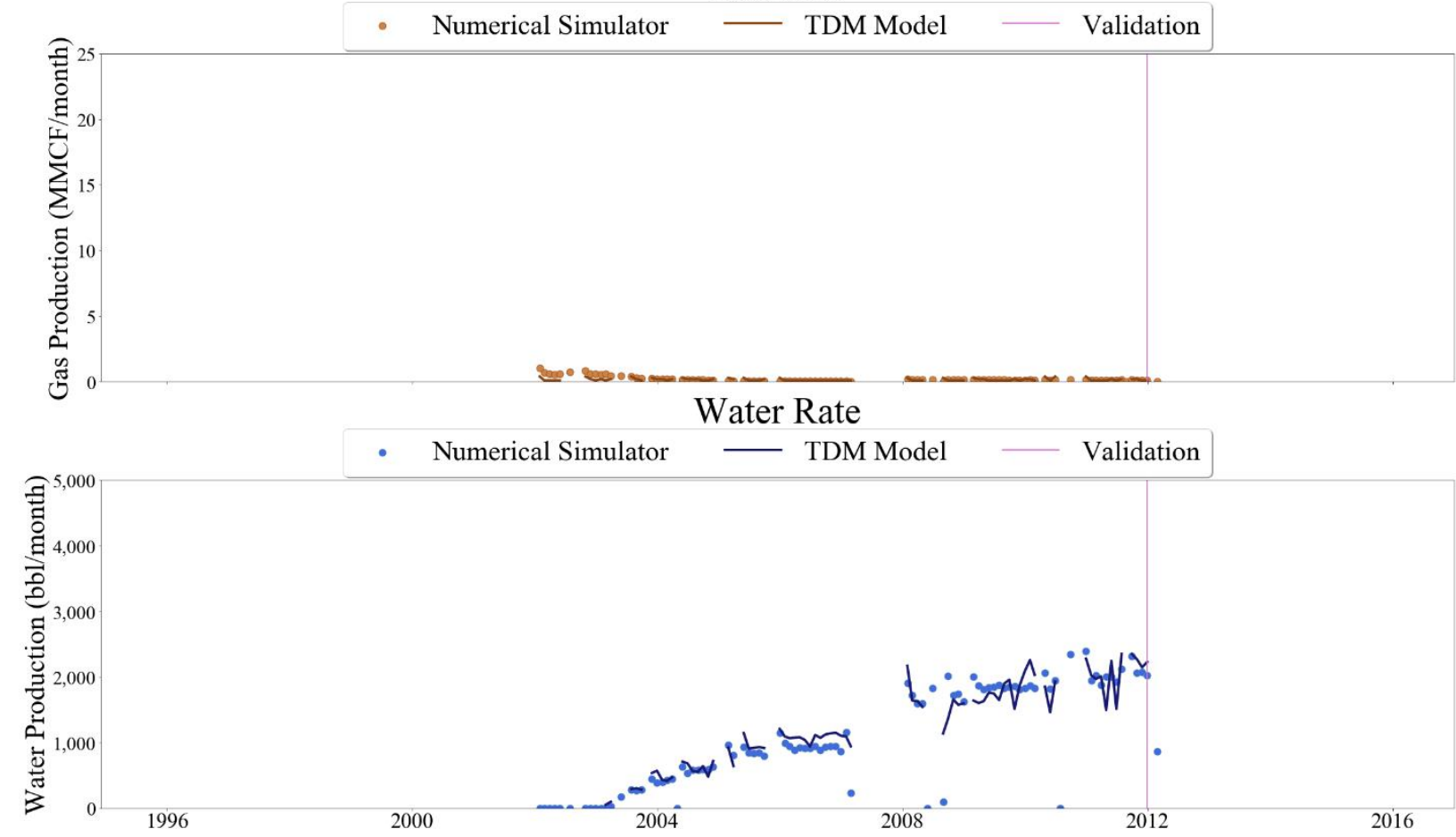

Figure 779 Well-033 oil, gas, and water rate TDM predictions vs actual simulation data plots for 5 Year Blind Validation TDM 

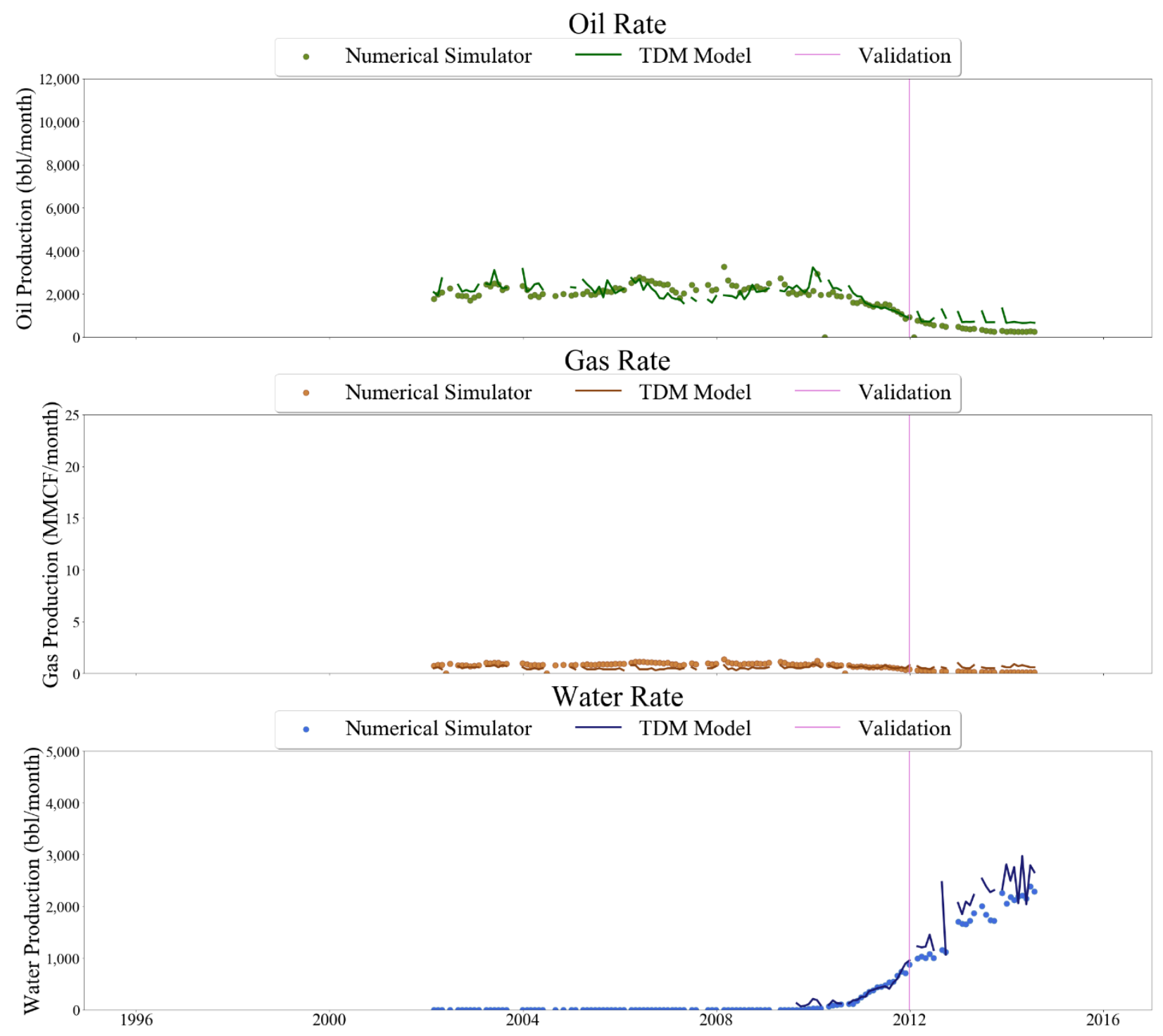

Figure 780 Well-034 oil, gas, and water rate TDM predictions vs actual simulation data plots for 5 Year Blind Validation TDM 


\section{Oil Rate}

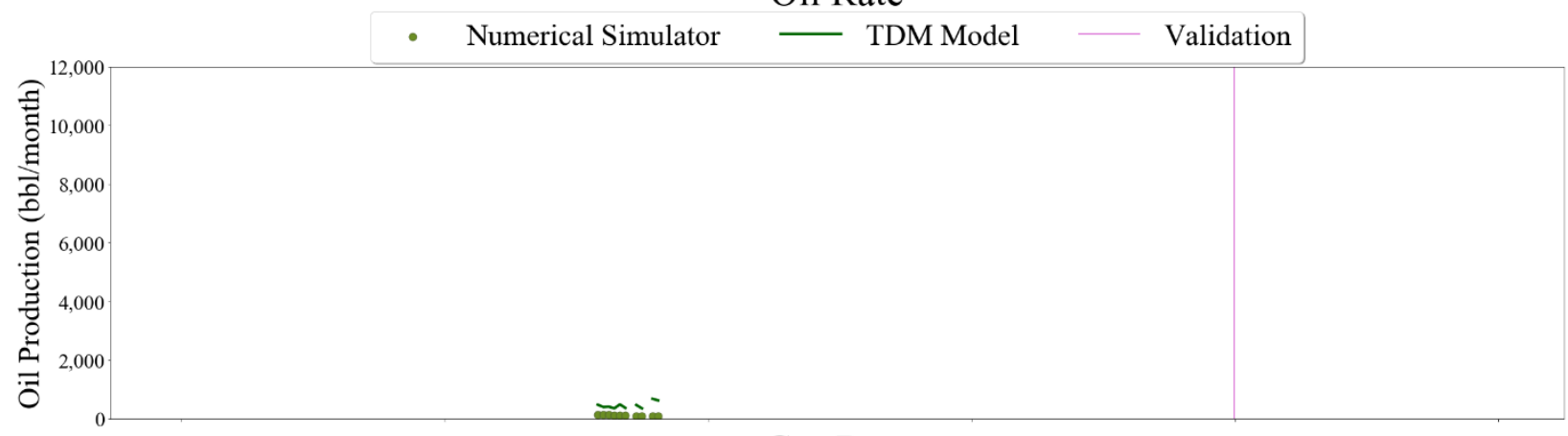

\section{Gas Rate}
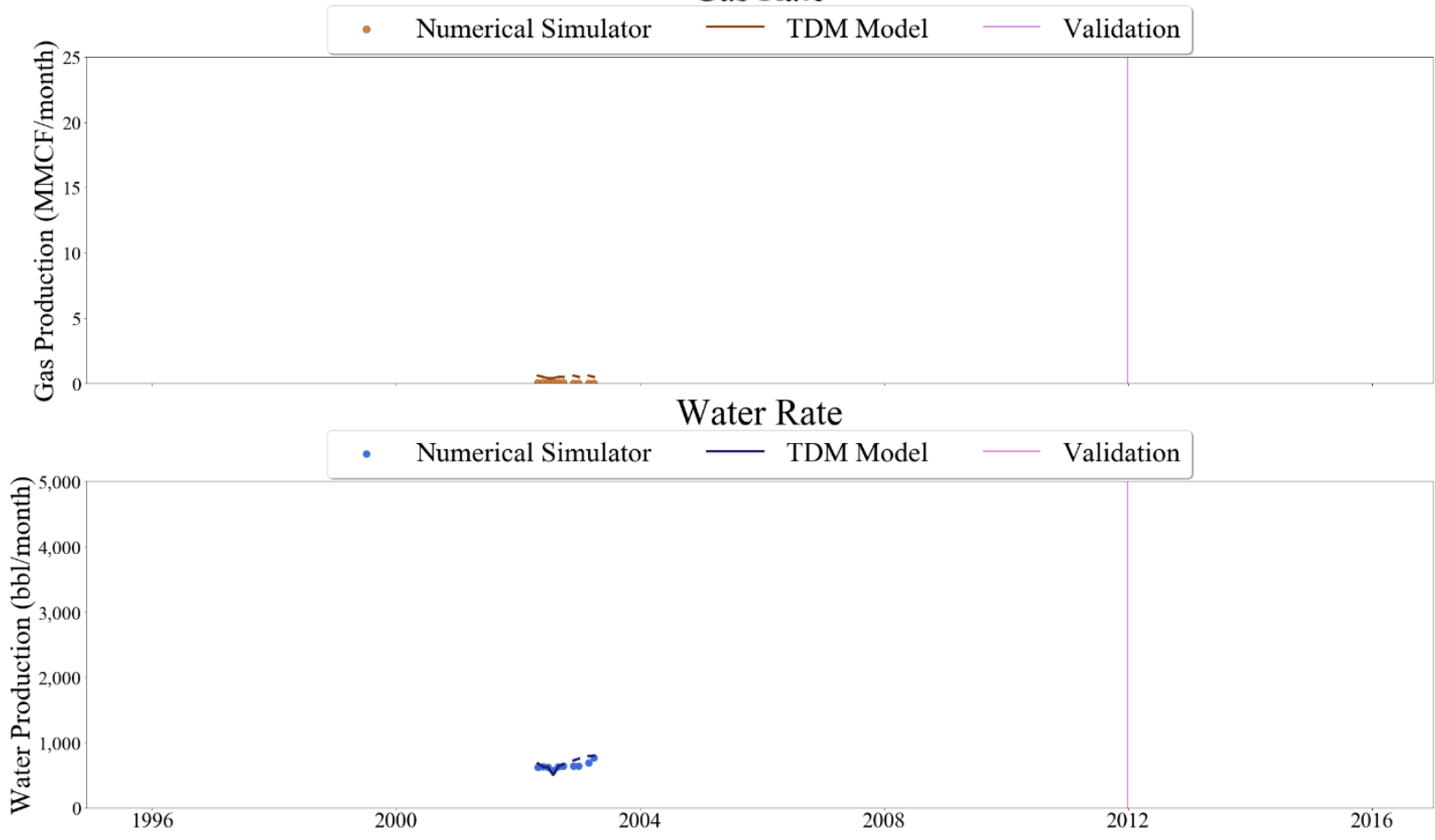

Figure 781 Well-035 oil, gas, and water rate TDM predictions vs actual simulation data plots for 5 Year Blind Validation TDM 

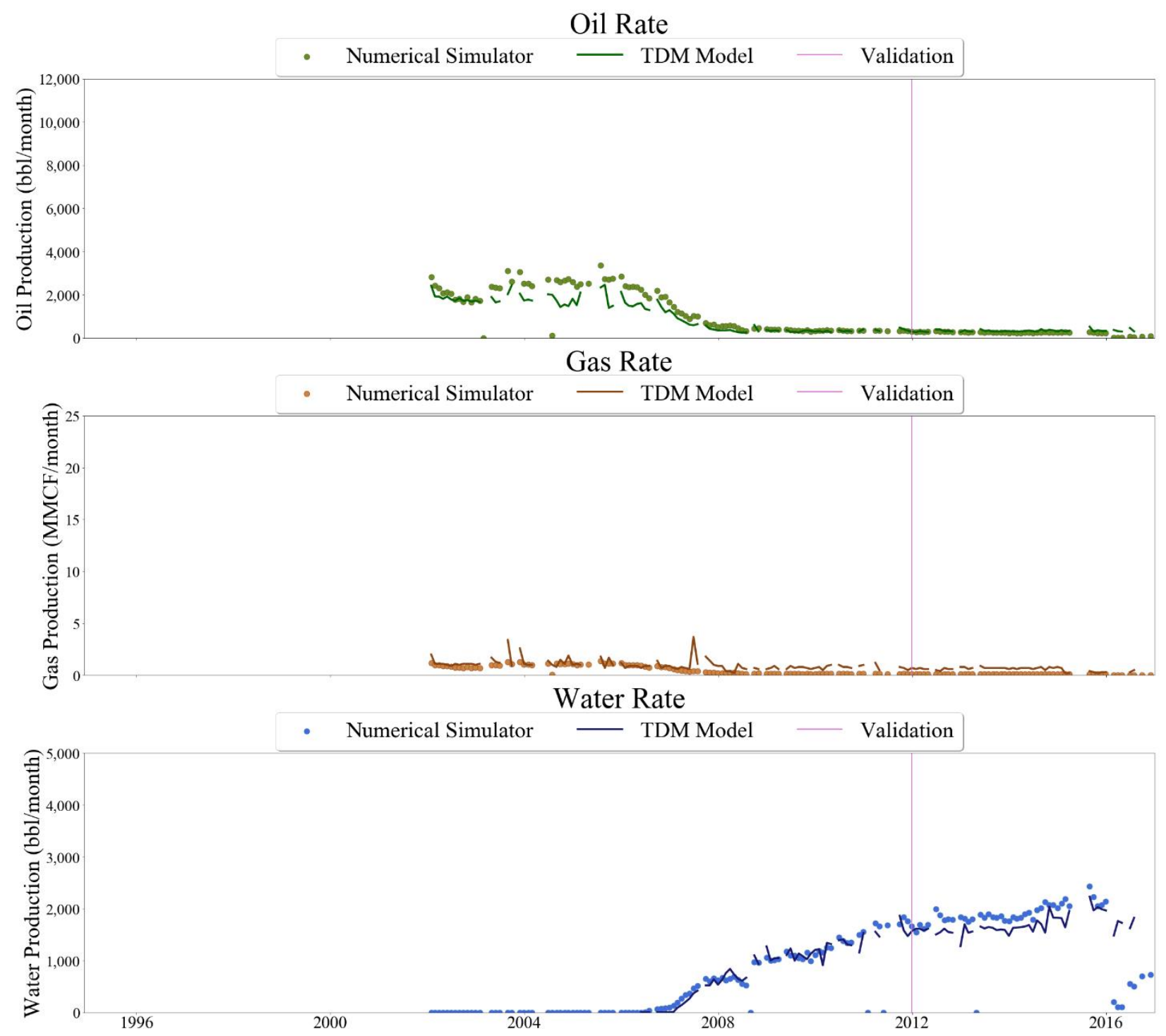

Figure 782 Well-036 oil, gas, and water rate TDM predictions vs actual simulation data plots for 5 Year Blind Validation TDM 


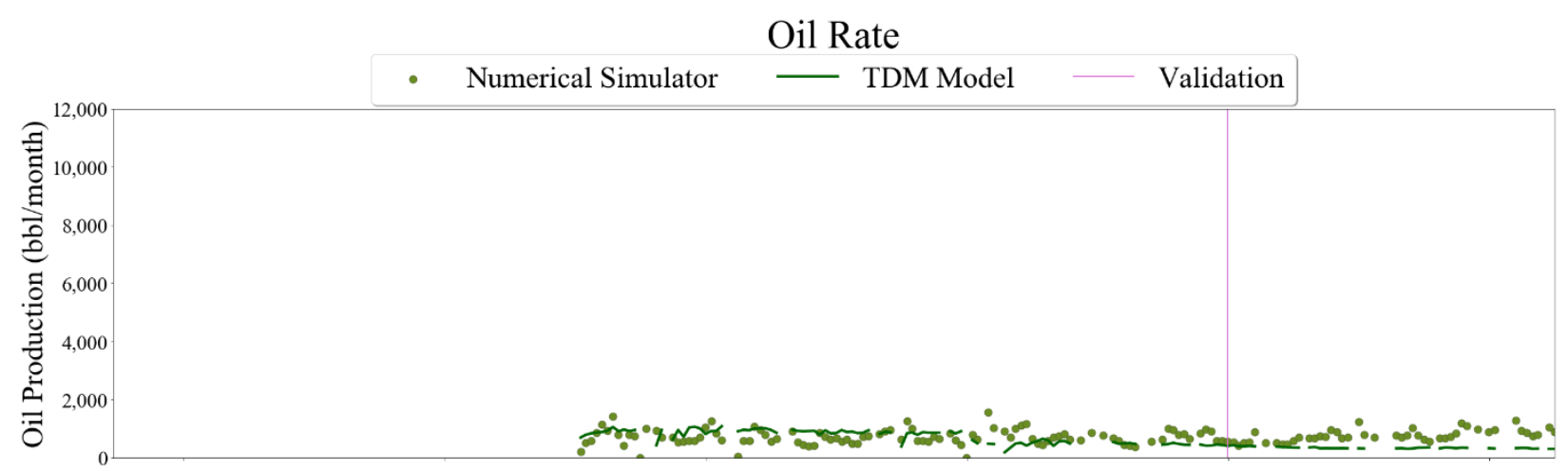

Gas Rate
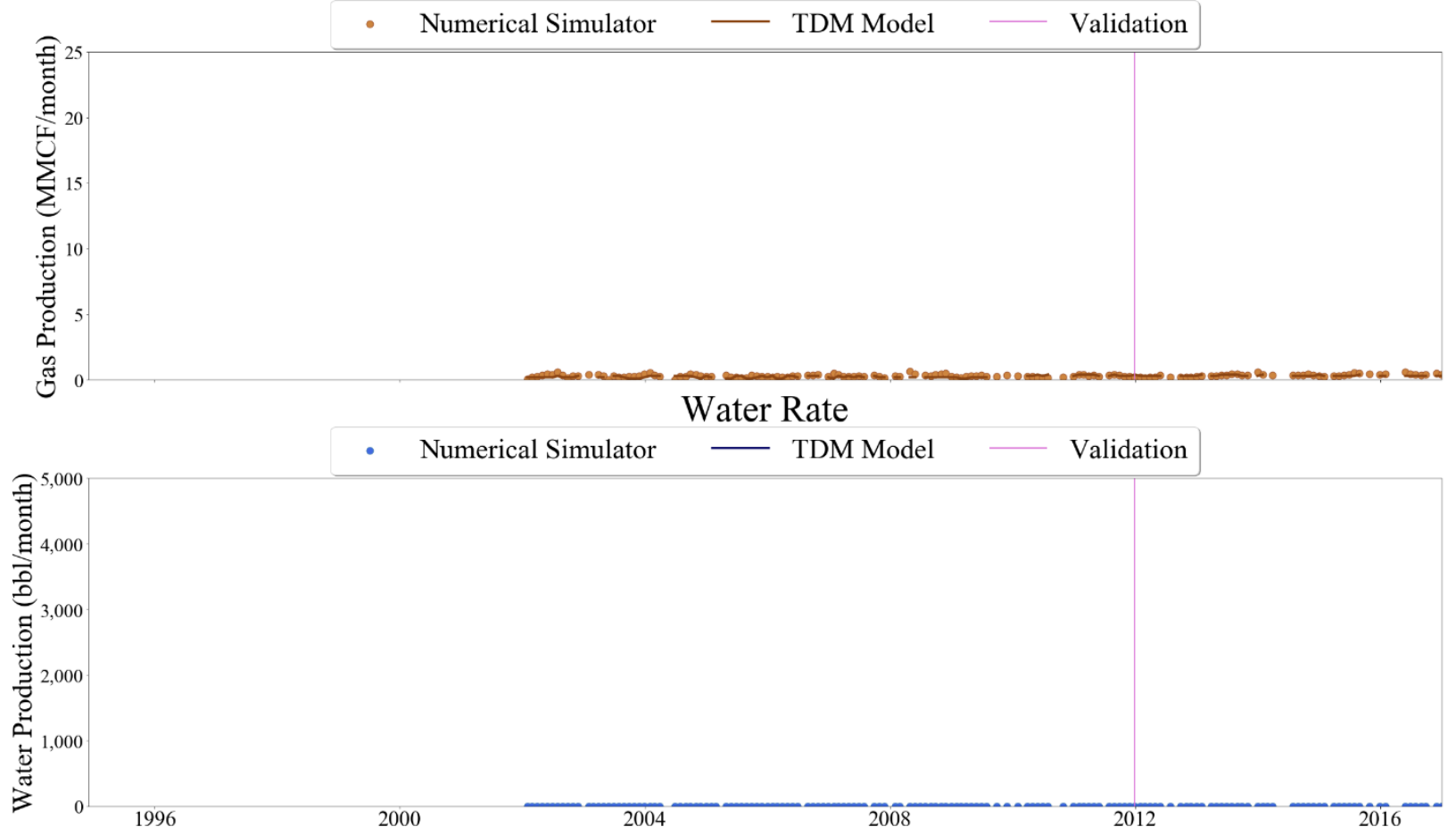

Figure 783 Well-037 oil, gas, and water rate TDM predictions vs actual simulation data plots for 5 Year Blind Validation TDM 


\section{Oil Rate}

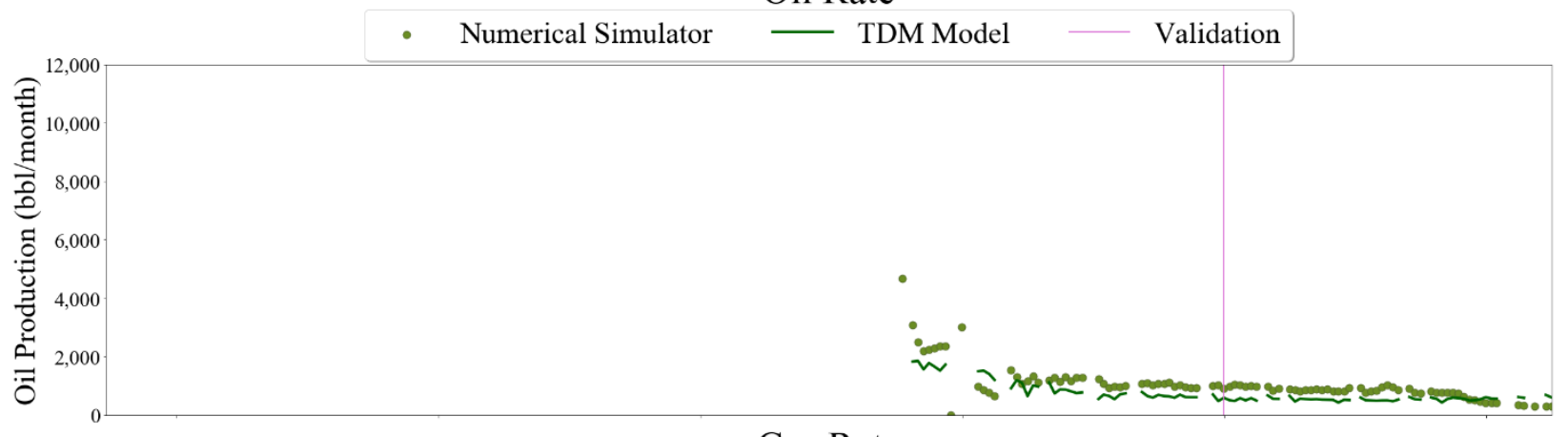

\section{Gas Rate}
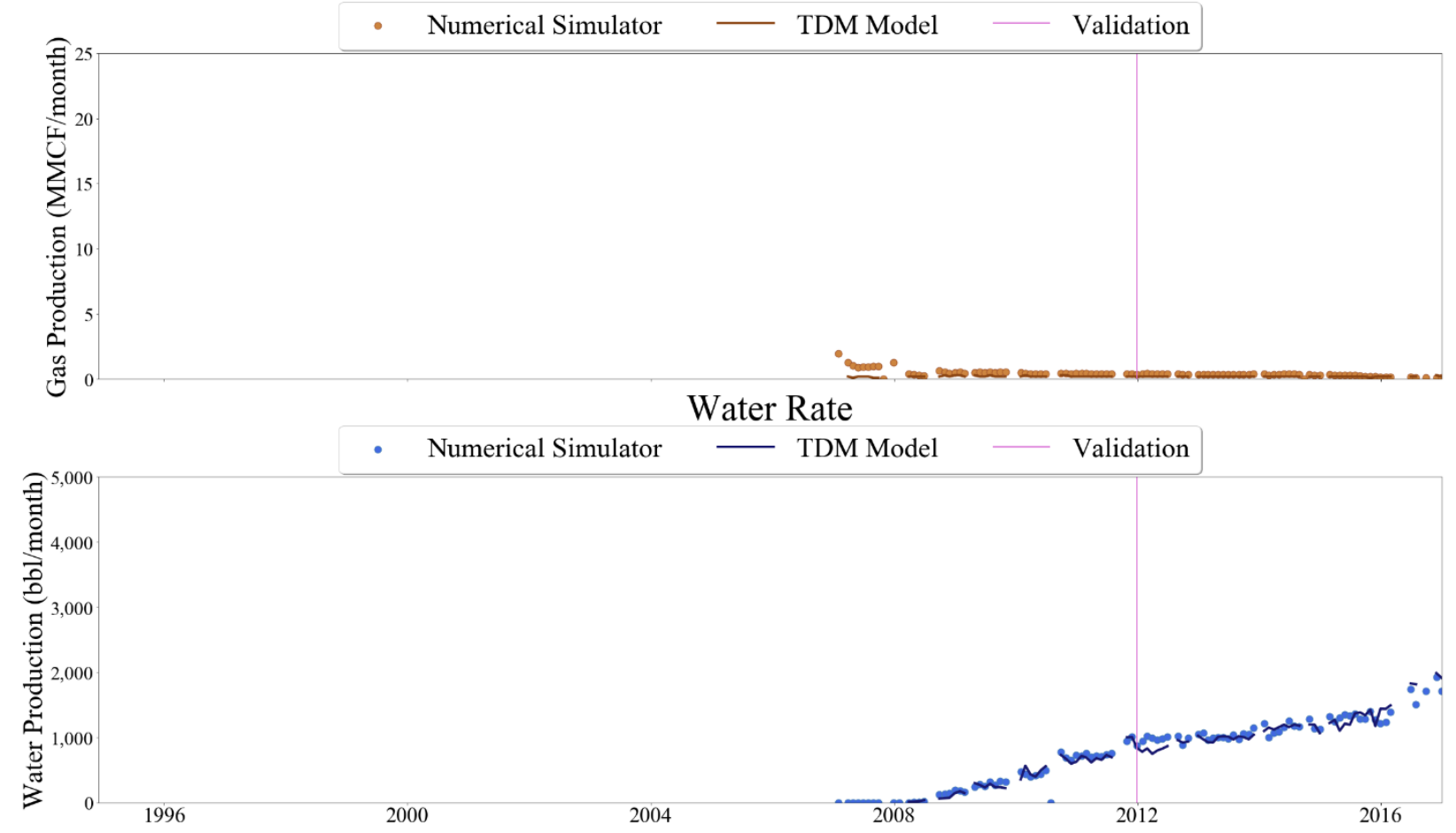

Figure 784 Well-038 oil, gas, and water rate TDM predictions vs actual simulation data plots for 5 Year Blind Validation TDM 


\section{Oil Rate}

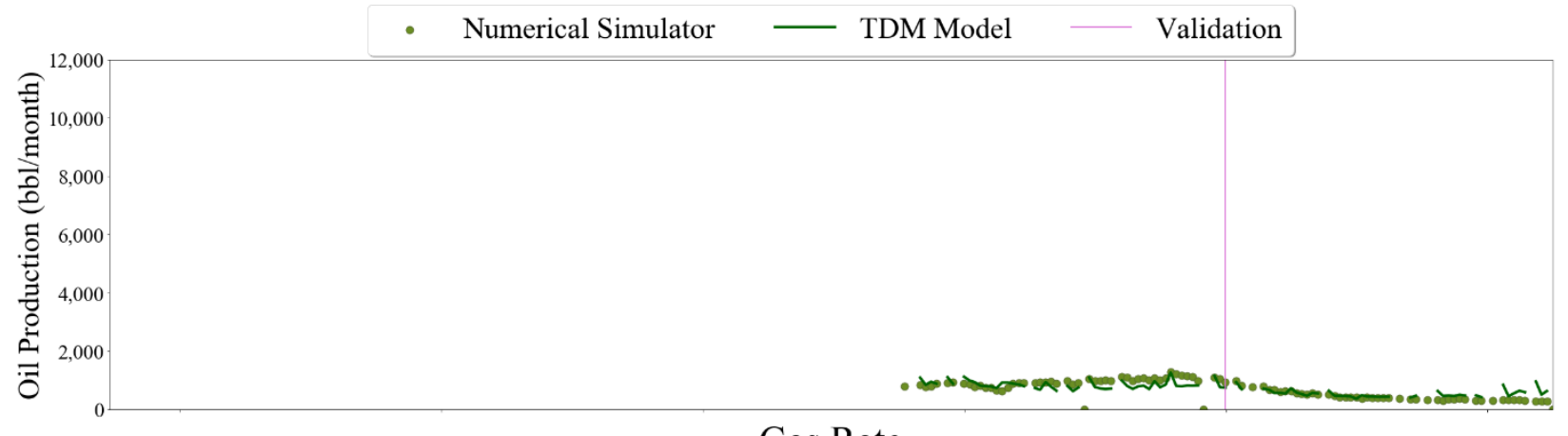

\section{Gas Rate}
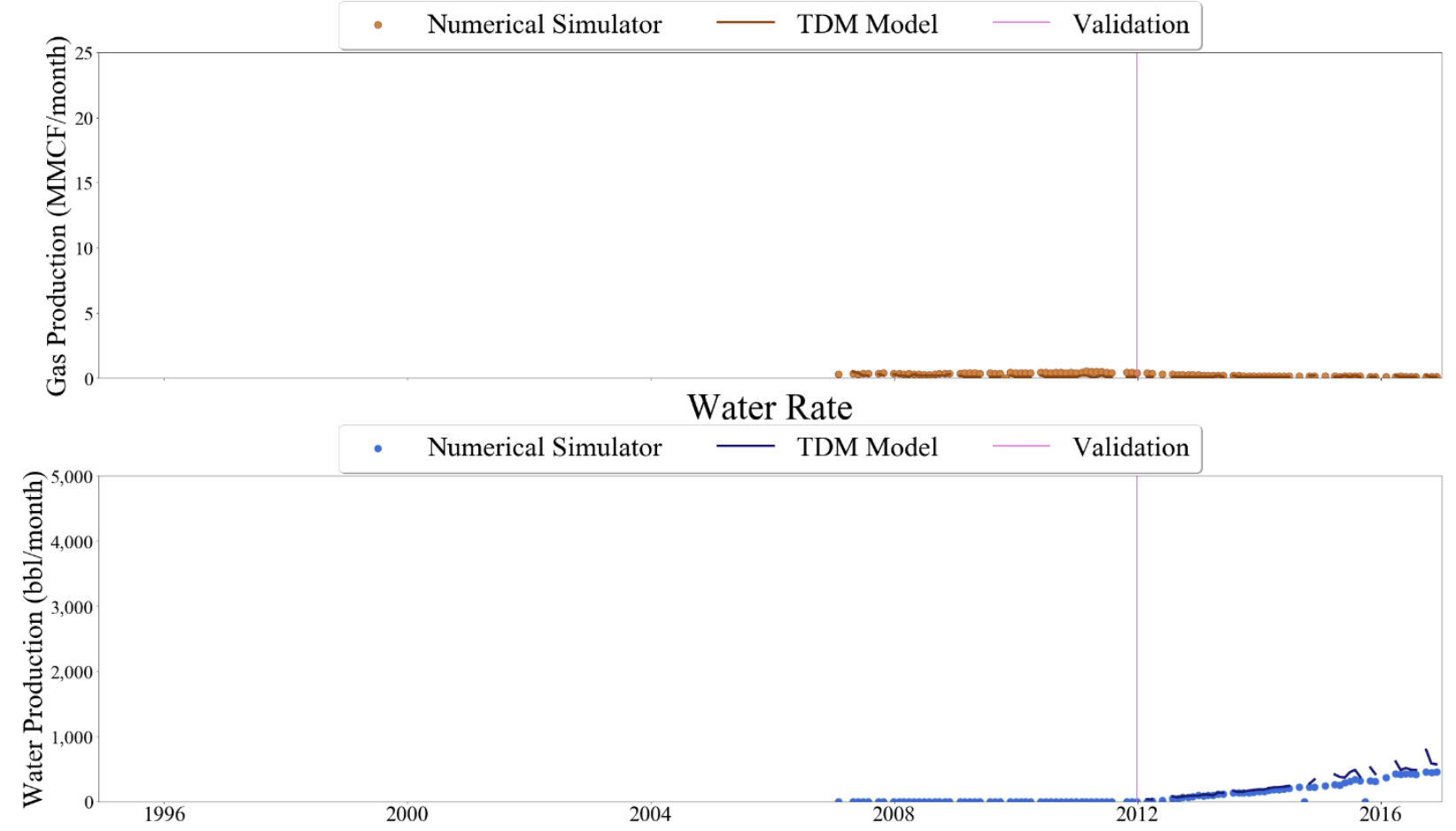

Figure 785 Well-039 oil, gas, and water rate TDM predictions vs actual simulation data plots for 5 Year Blind Validation TDM 


\section{Oil Rate}

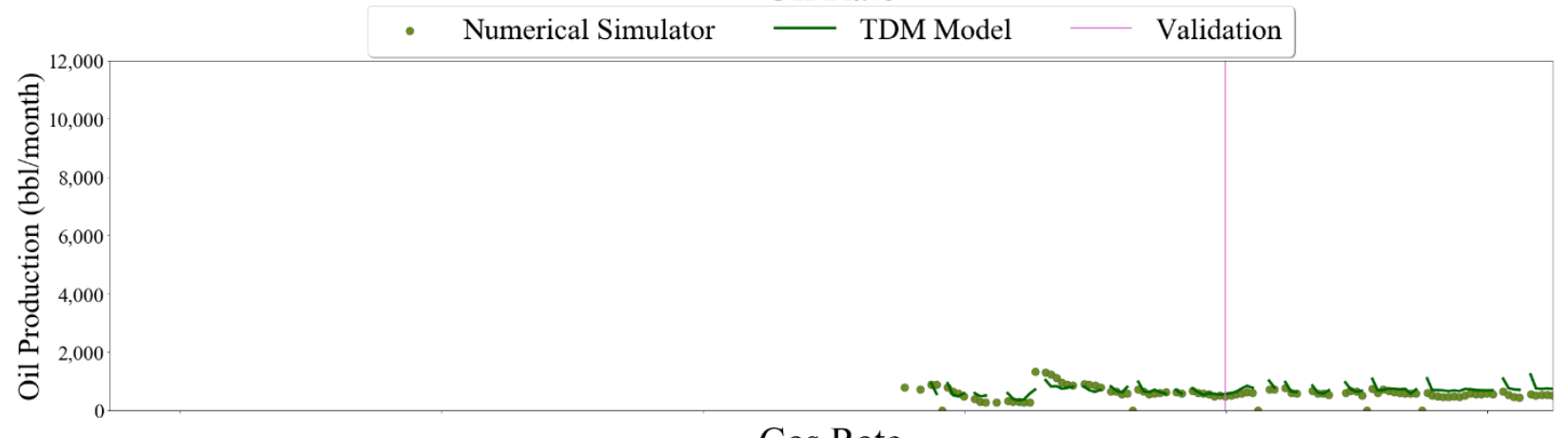

\section{Gas Rate}
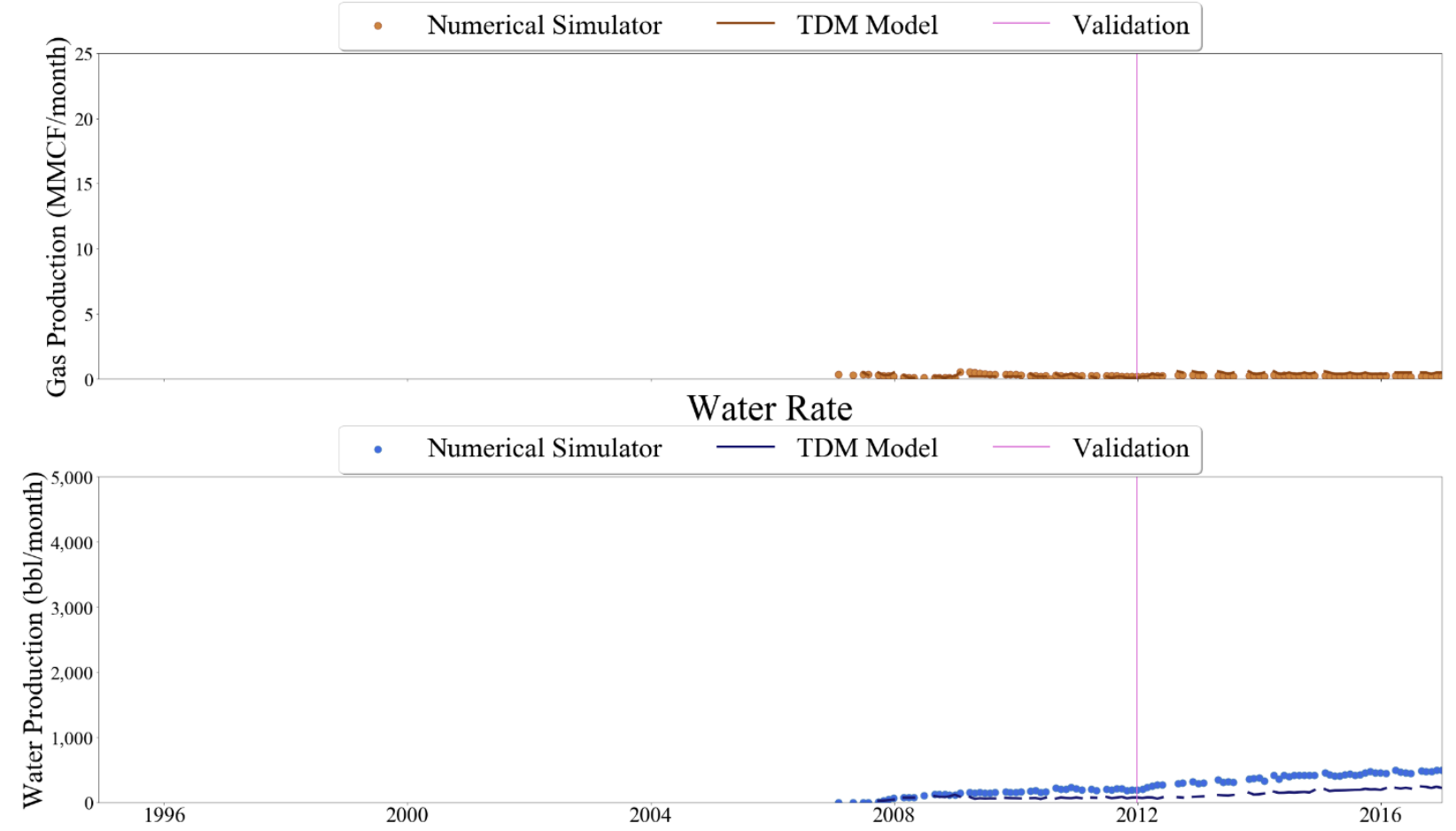

Figure 786 Well-040 oil, gas, and water rate TDM predictions vs actual simulation data plots for 5 Year Blind Validation TDM 


\section{Oil Rate}

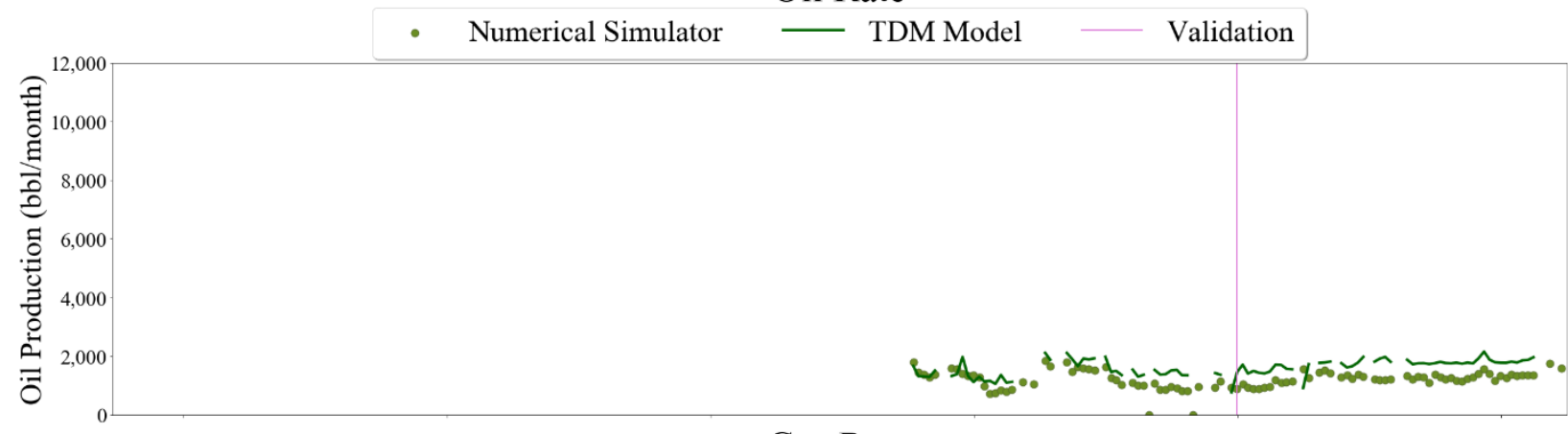

\section{Gas Rate}
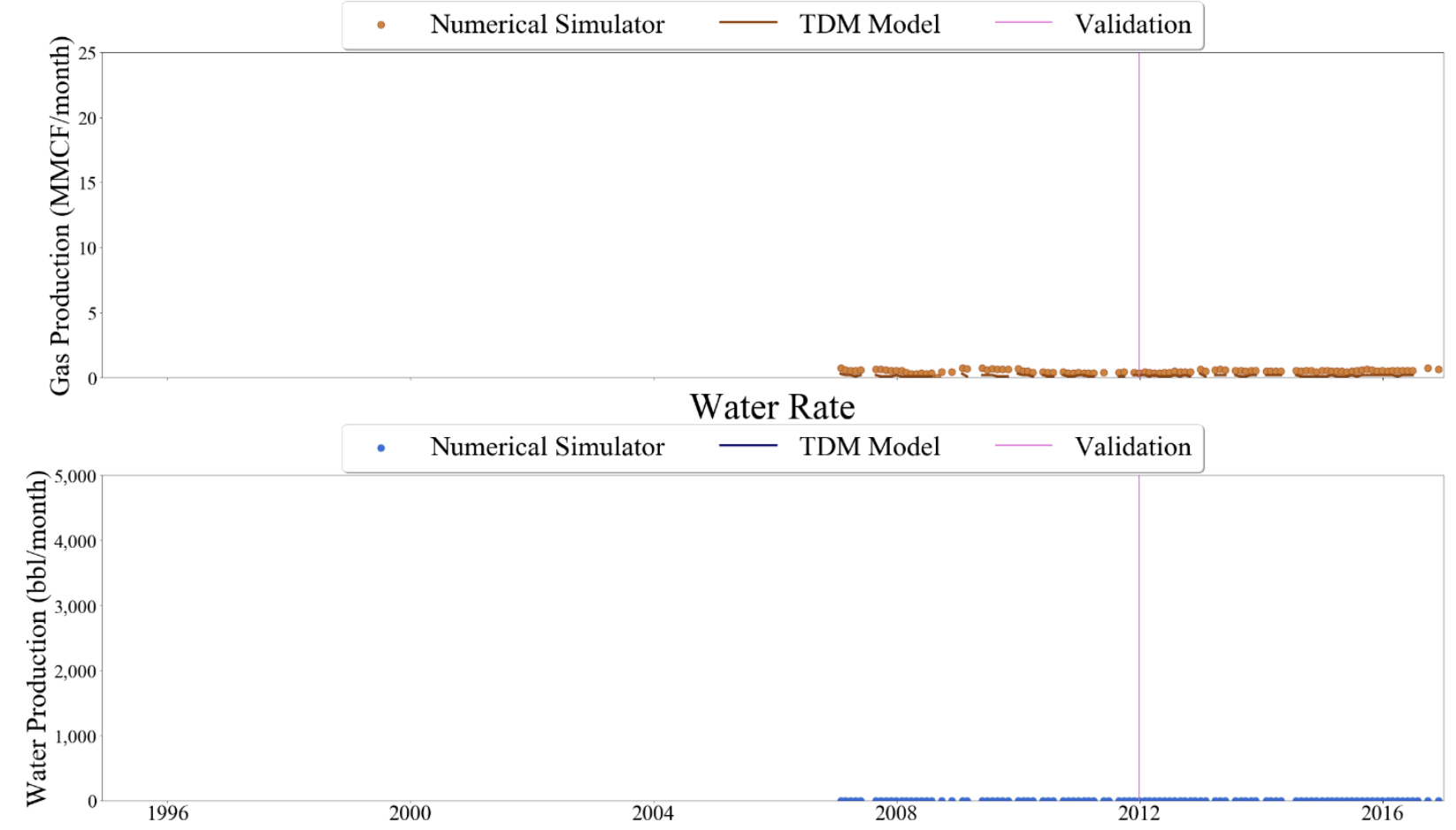

Figure 787 Well-041 oil, gas, and water rate TDM predictions vs actual simulation data plots for 5 Year Blind Validation TDM 


\section{Oil Rate}

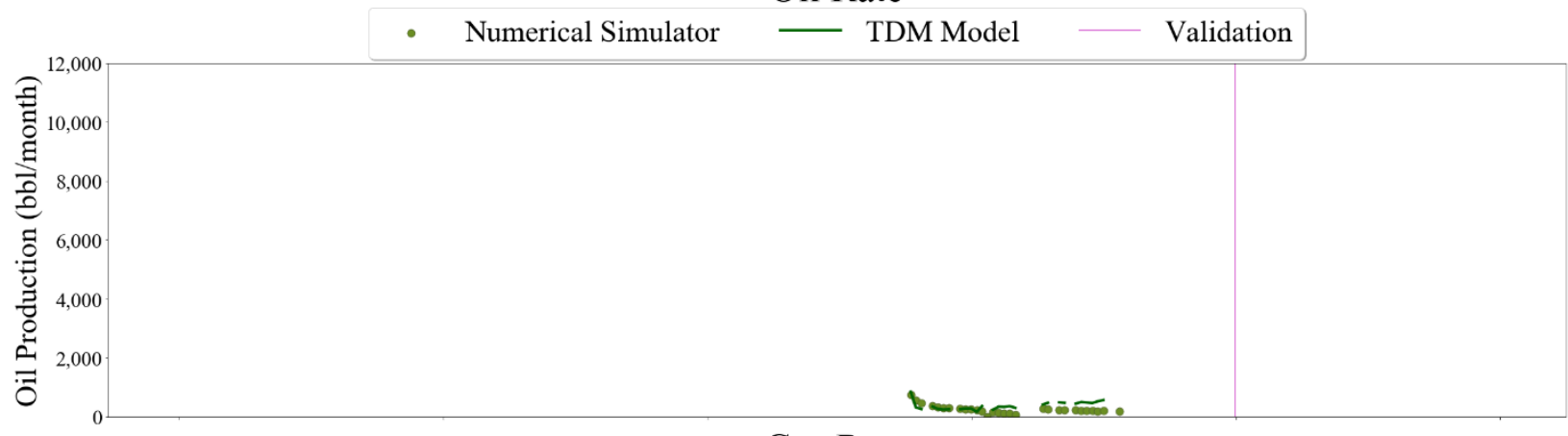

\section{Gas Rate}

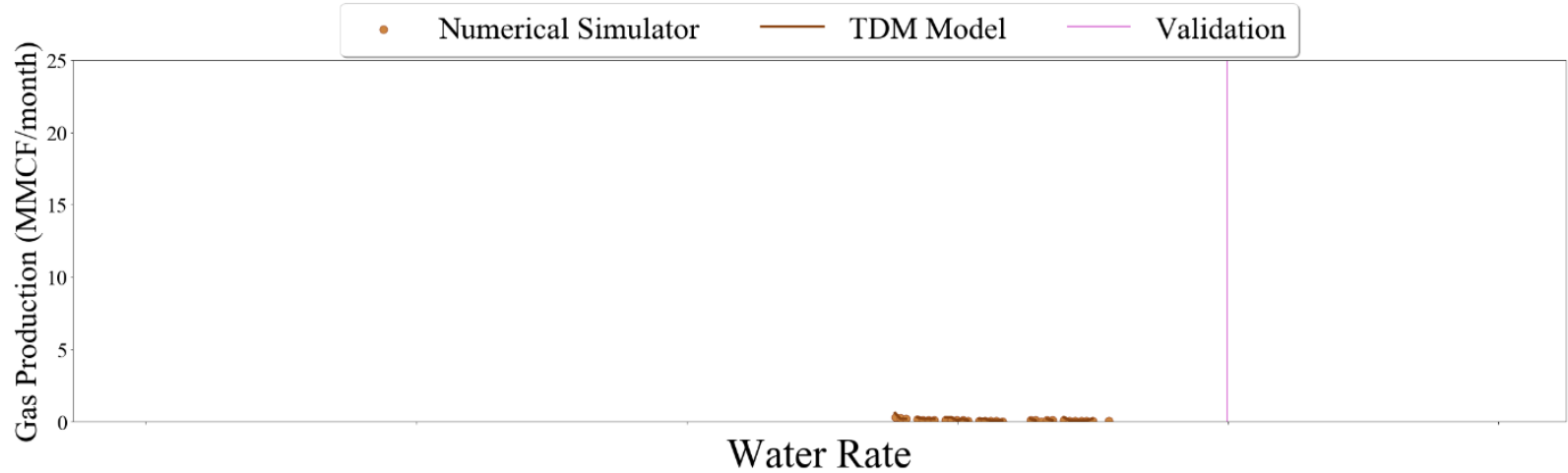

Water Rate

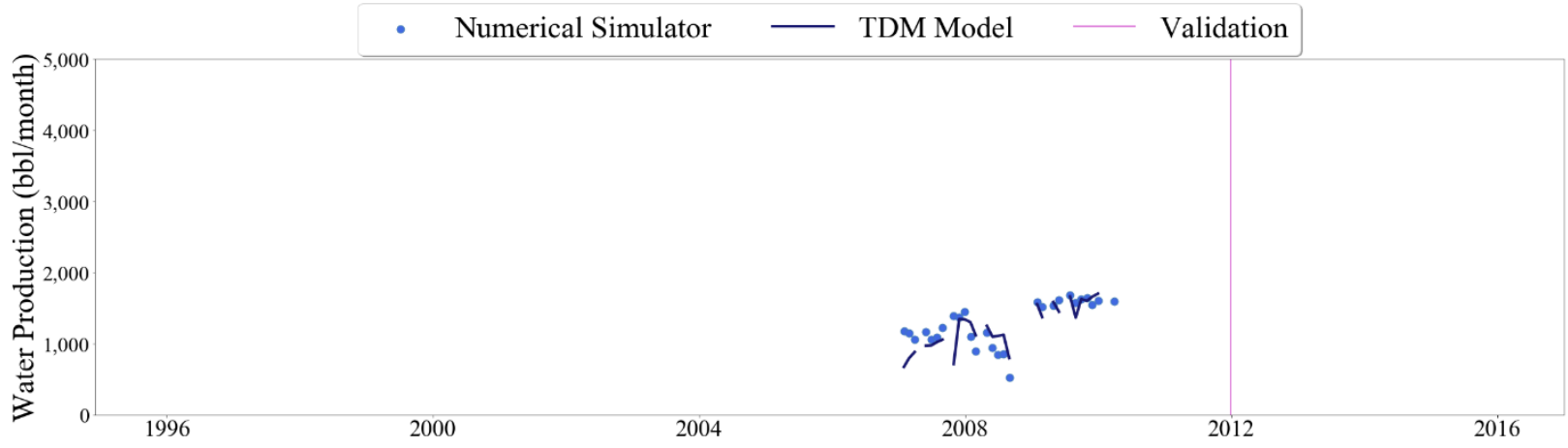

Figure 788 Well-042 oil, gas, and water rate TDM predictions vs actual simulation data plots for 5 Year Blind Validation TDM 


\section{Oil Rate}

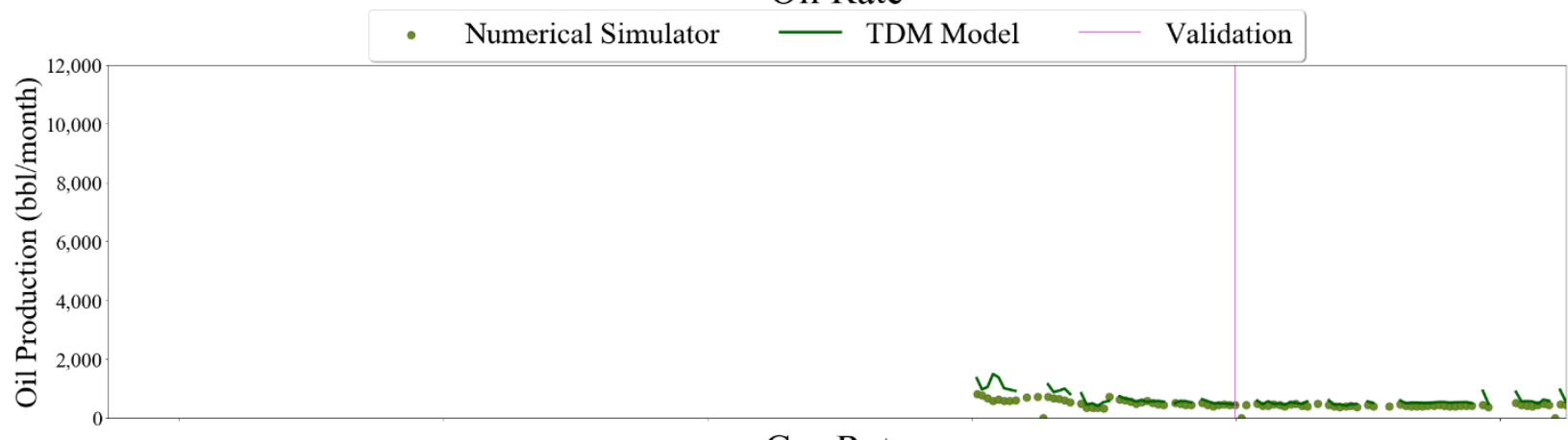

\section{Gas Rate}
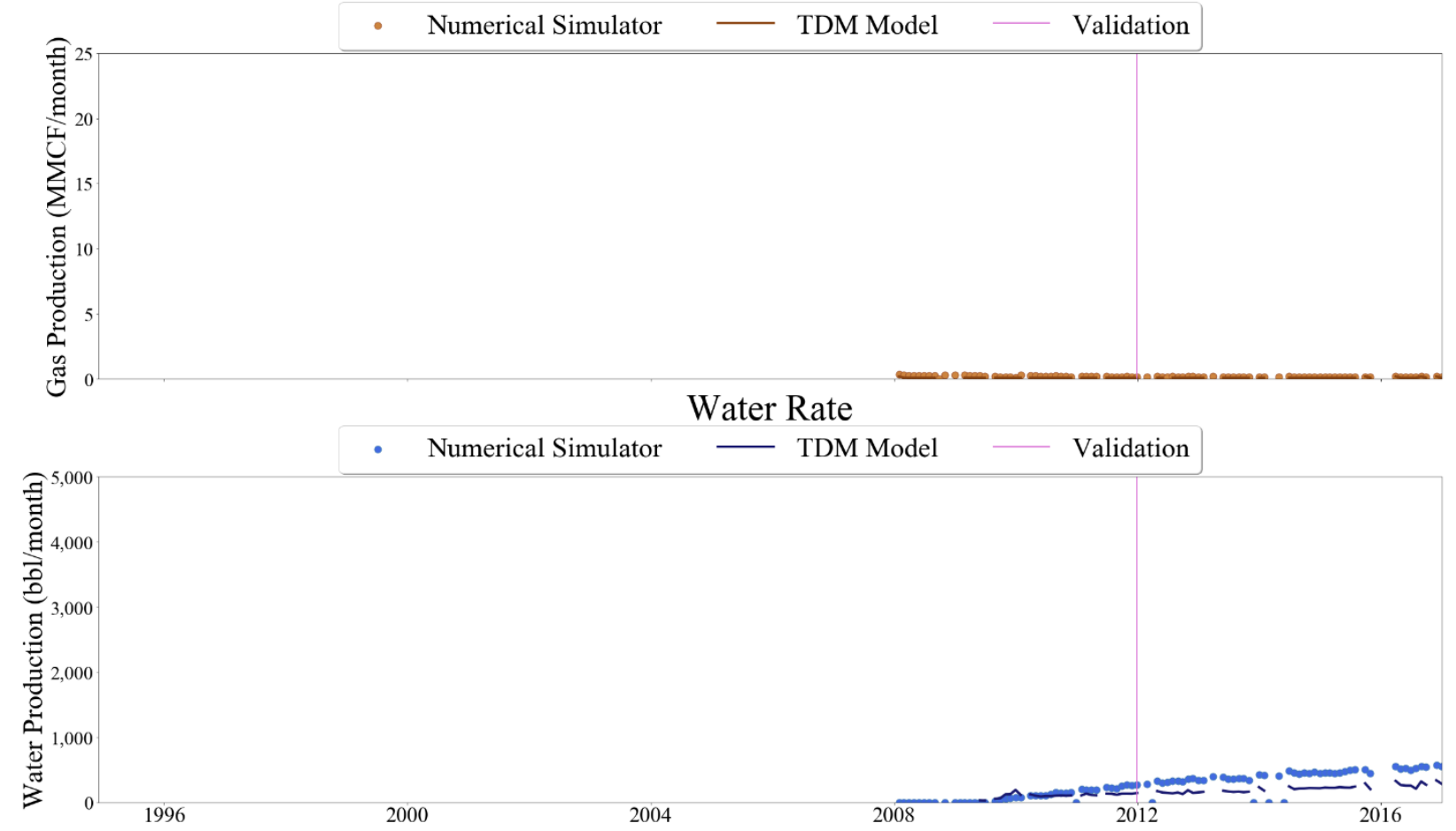

Figure 789 Well-043 oil, gas, and water rate TDM predictions vs actual simulation data plots for 5 Year Blind Validation TDM 


\section{Oil Rate}

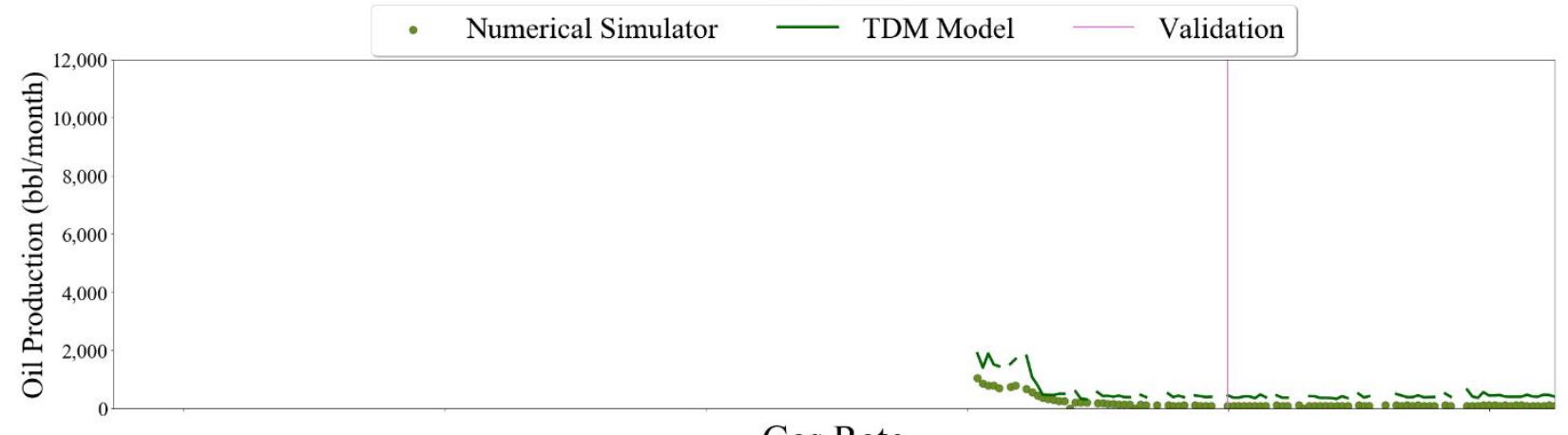

\section{Gas Rate}
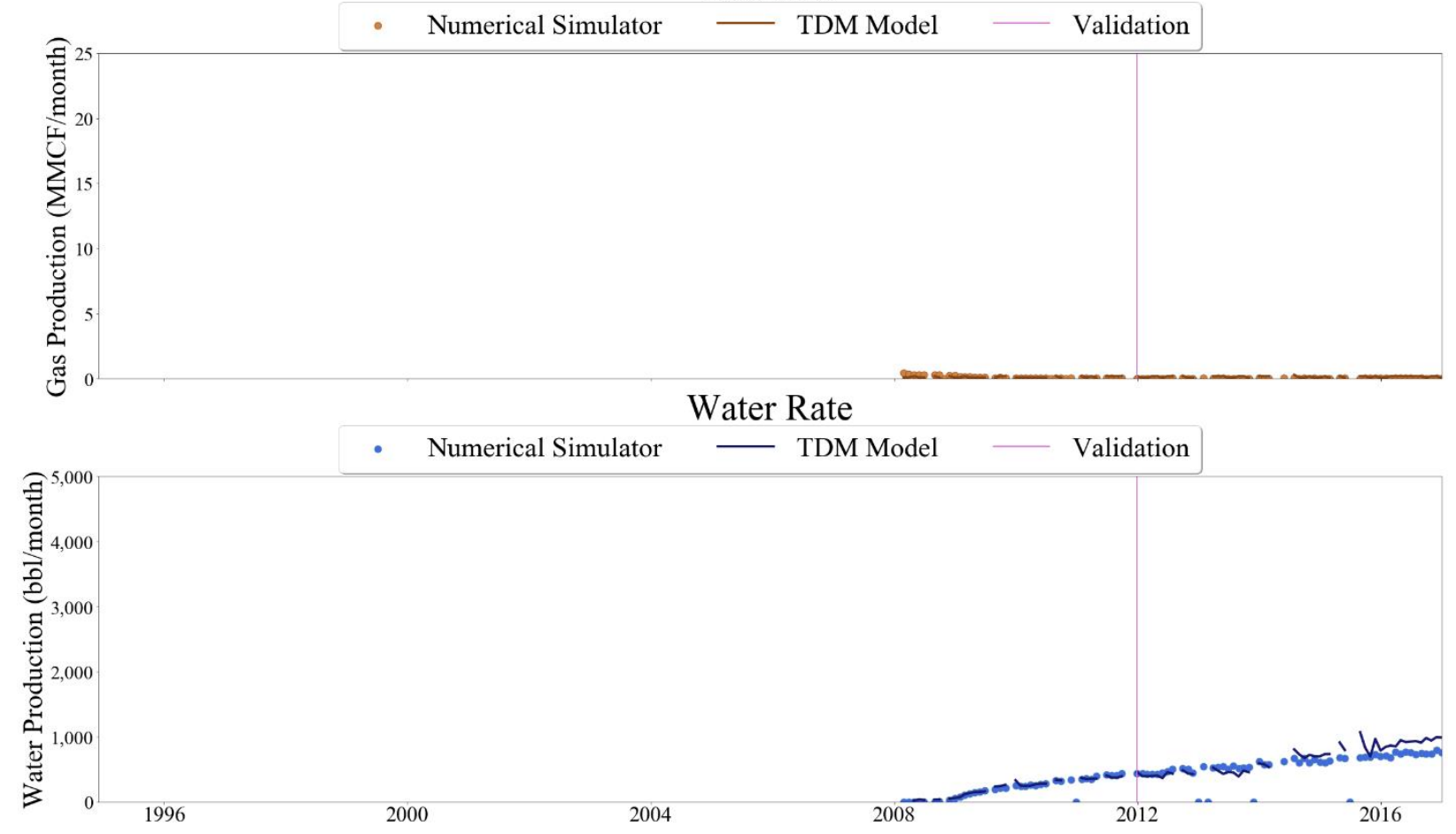

Figure 790 Well-044 oil, gas, and water rate TDM predictions vs actual simulation data plots for 5 Year Blind Validation TDM 


\section{Oil Rate}
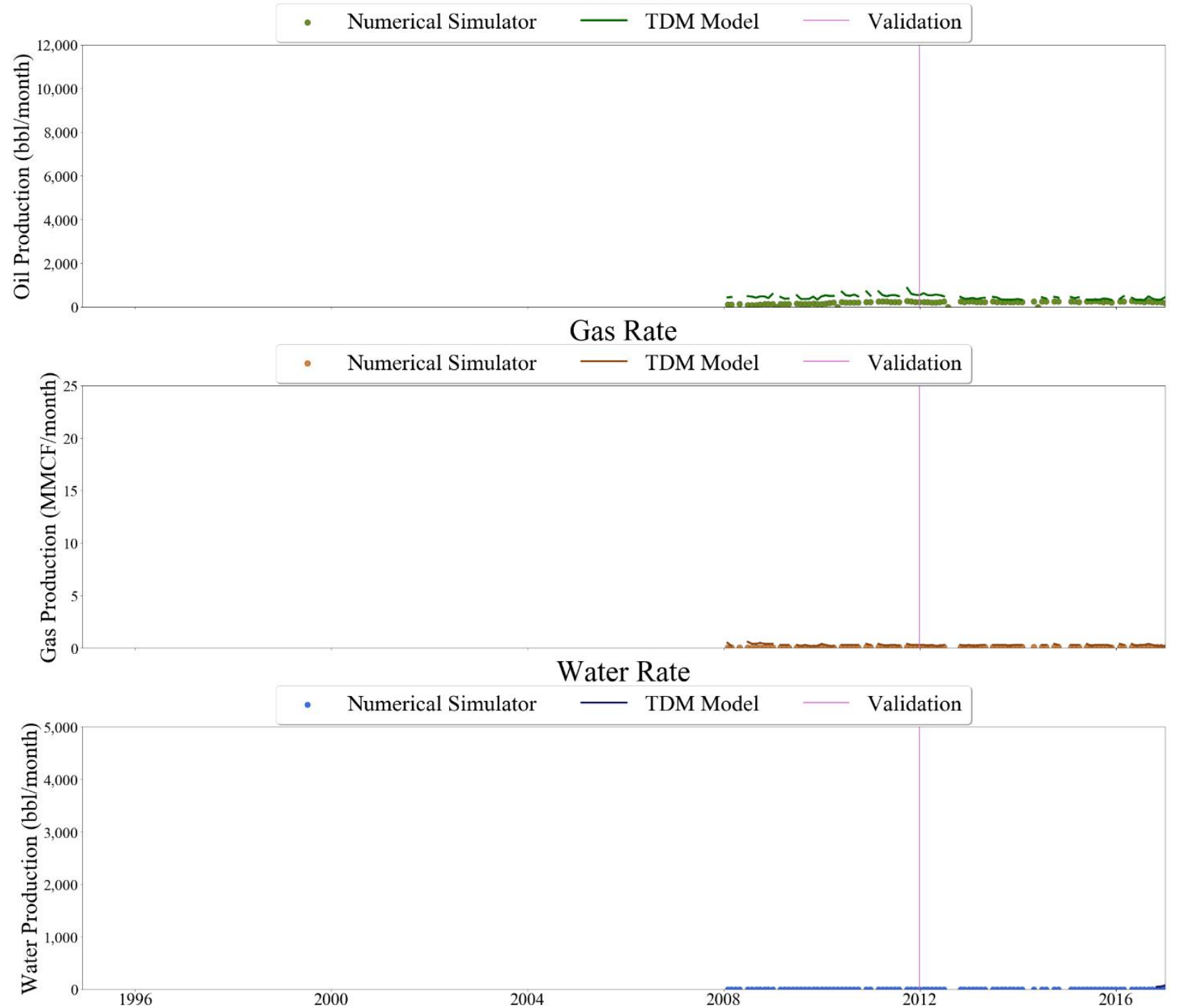

Figure 791 Well-045 oil, gas, and water rate TDM predictions vs actual simulation data plots for 5 Year Blind Validation TDM 


\section{Oil Rate}
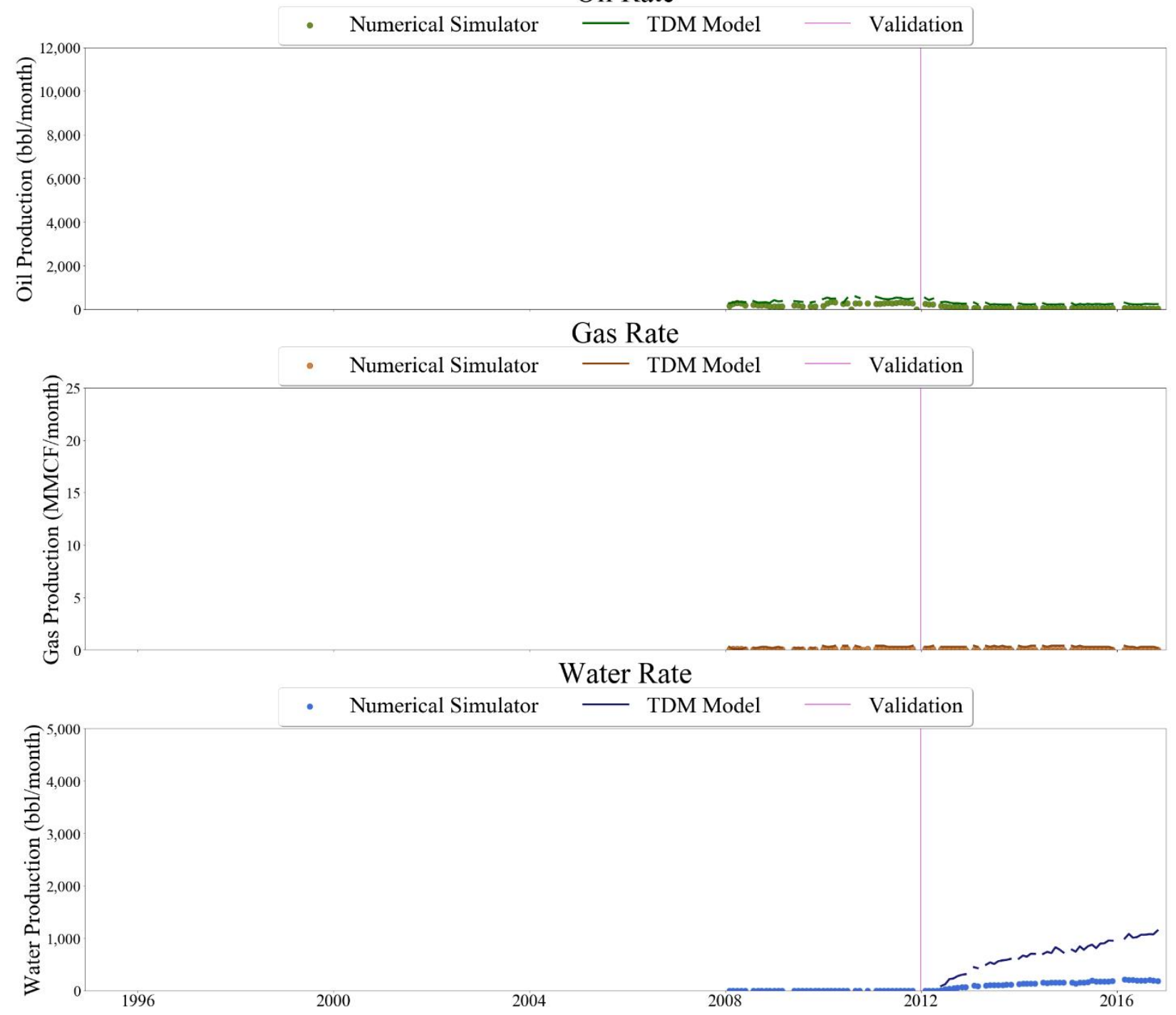

Figure 792 Well-046 oil, gas, and water rate TDM predictions vs actual simulation data plots for 5 Year Blind Validation TDM 


\section{Oil Rate}

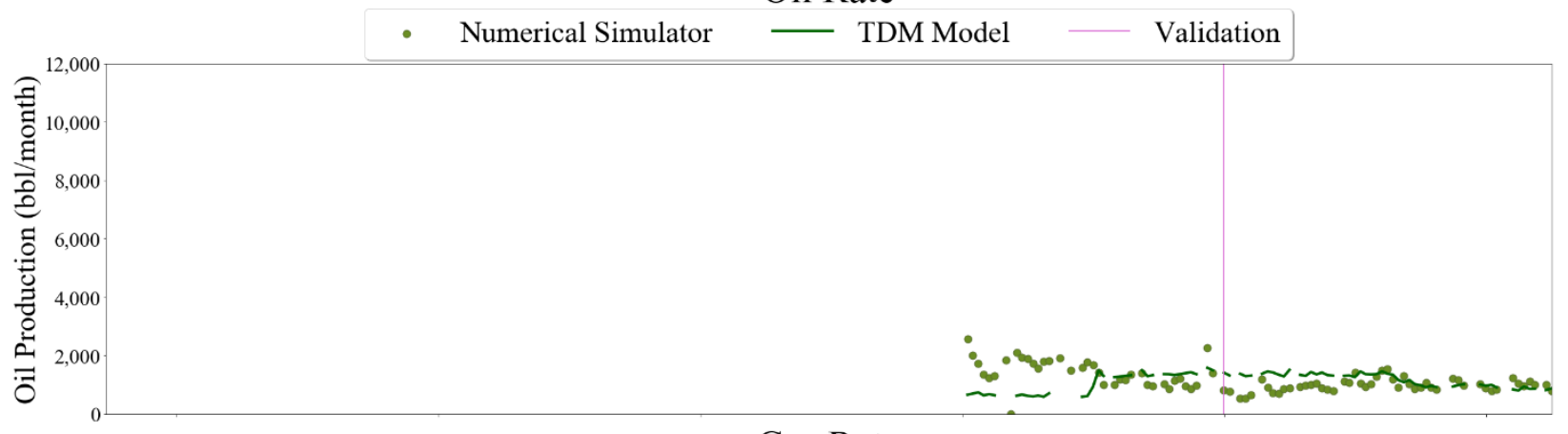

\section{Gas Rate}
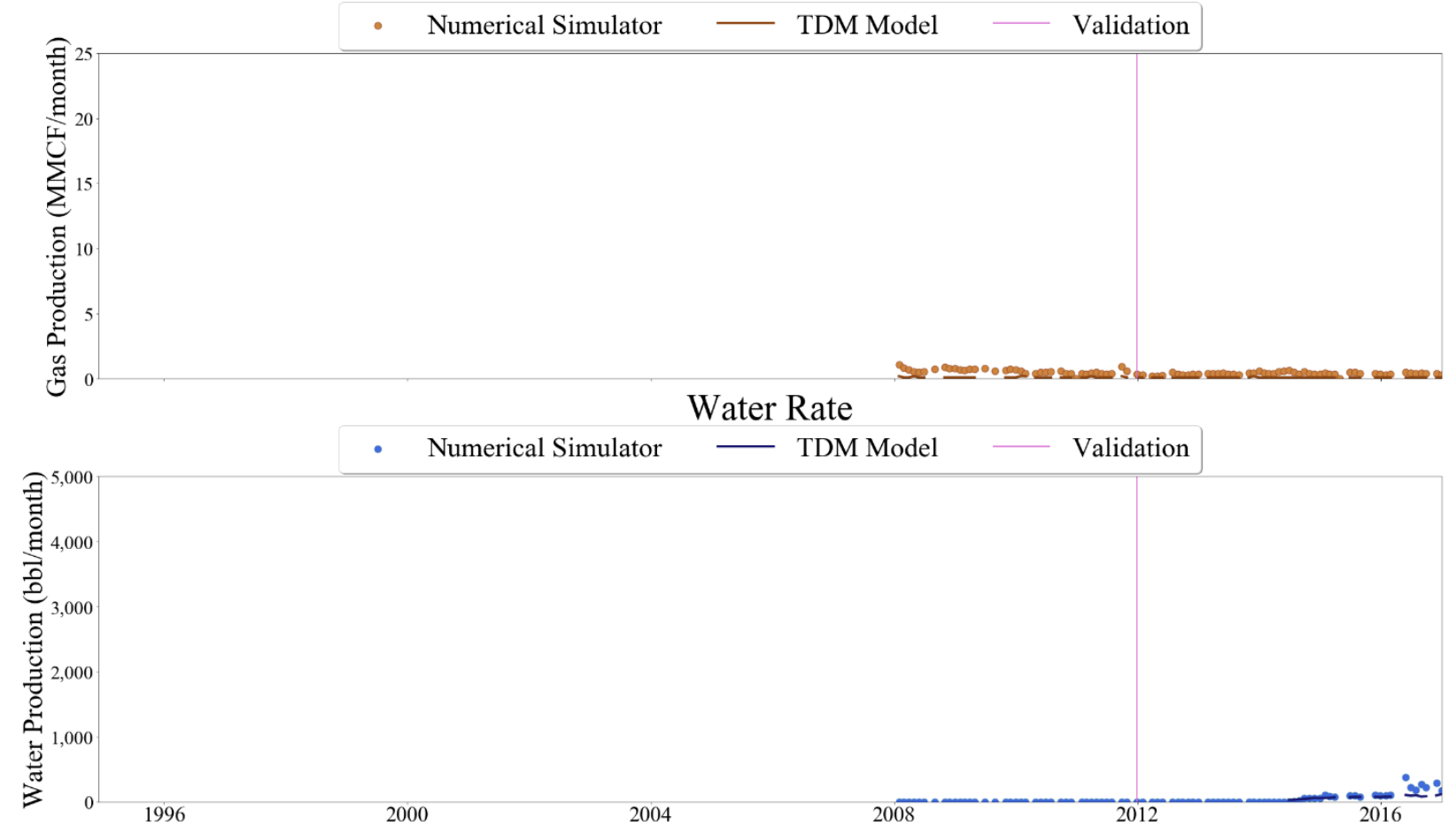

Figure 793 Well-047 oil, gas, and water rate TDM predictions vs actual simulation data plots for 5 Year Blind Validation TDM 


\section{Oil Rate}

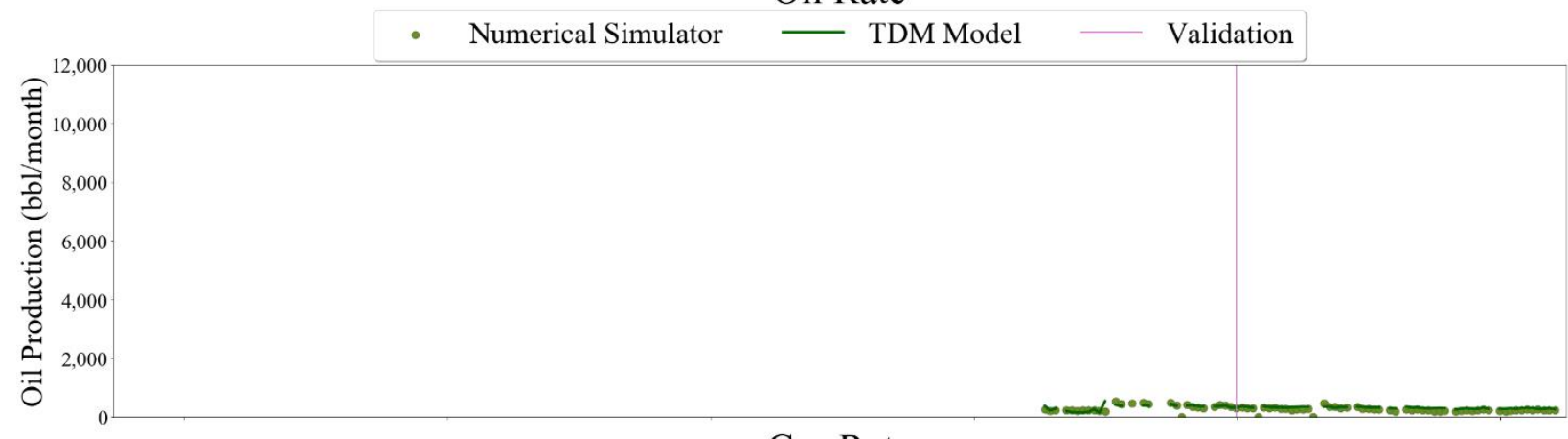

Gas Rate

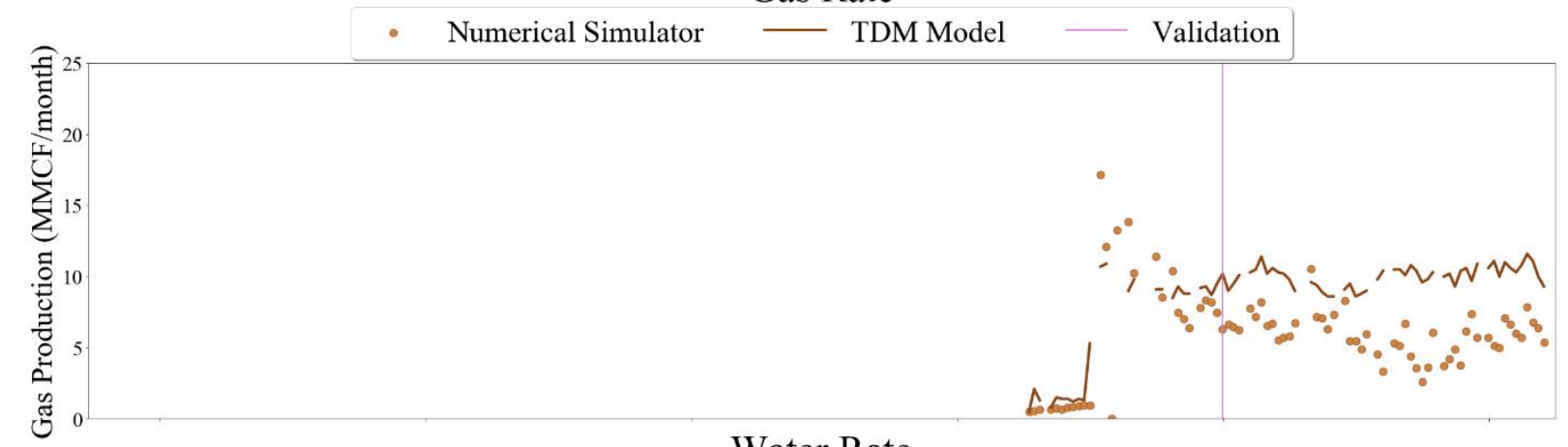

Water Rate

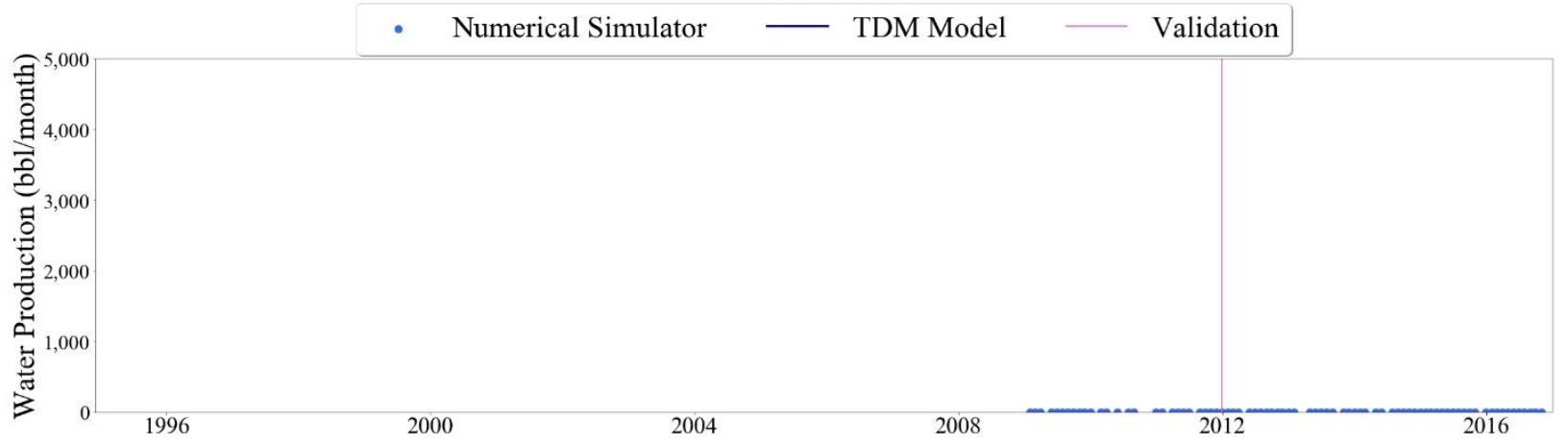

Figure 794 Well-048 oil, gas, and water rate TDM predictions vs actual simulation data plots for 5 Year Blind Validation TDM 


\section{Oil Rate}
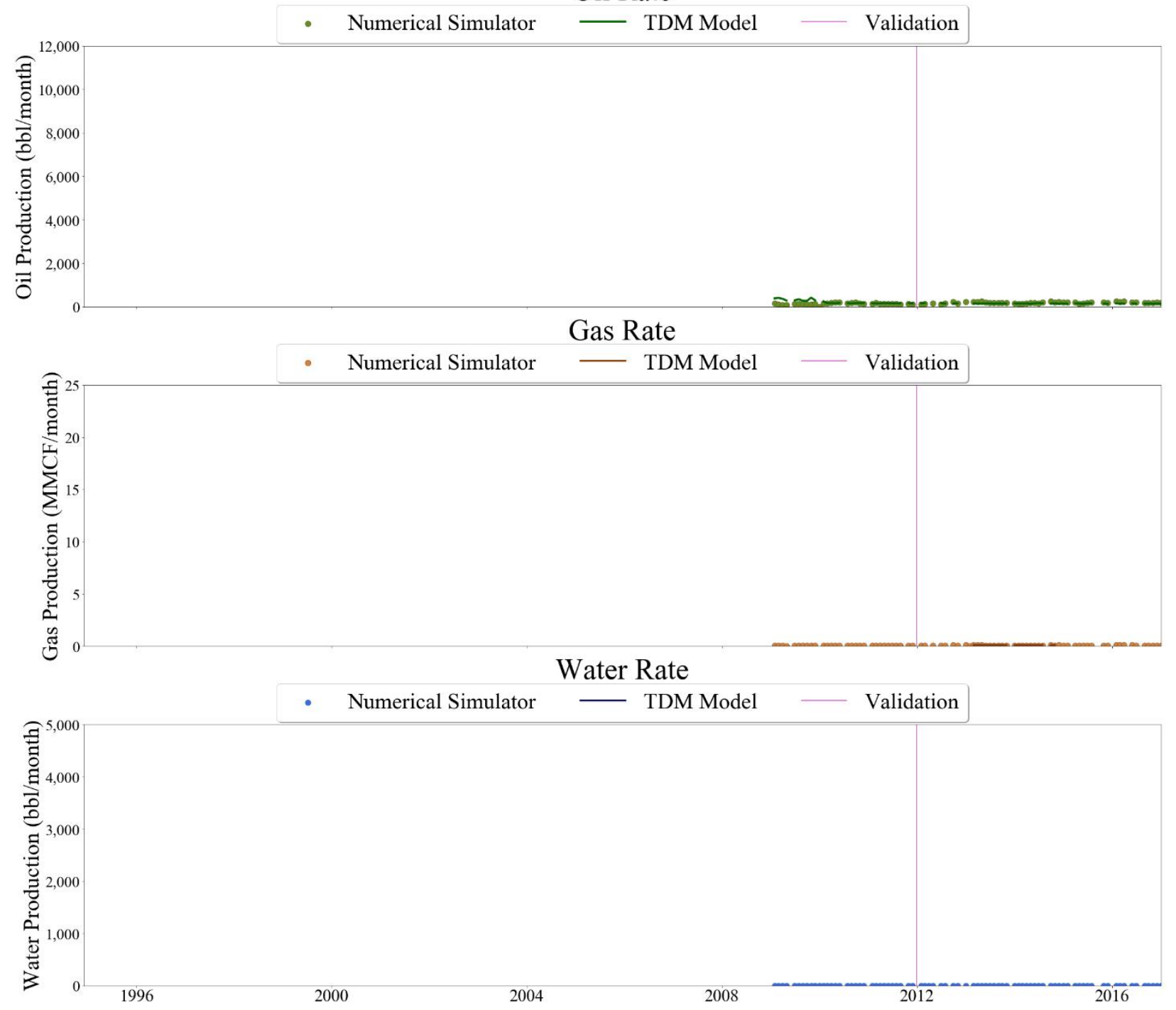

Figure 795 Well-049 oil, gas, and water rate TDM predictions vs actual simulation data plots for 5 Year Blind Validation TDM 


\section{Oil Rate}

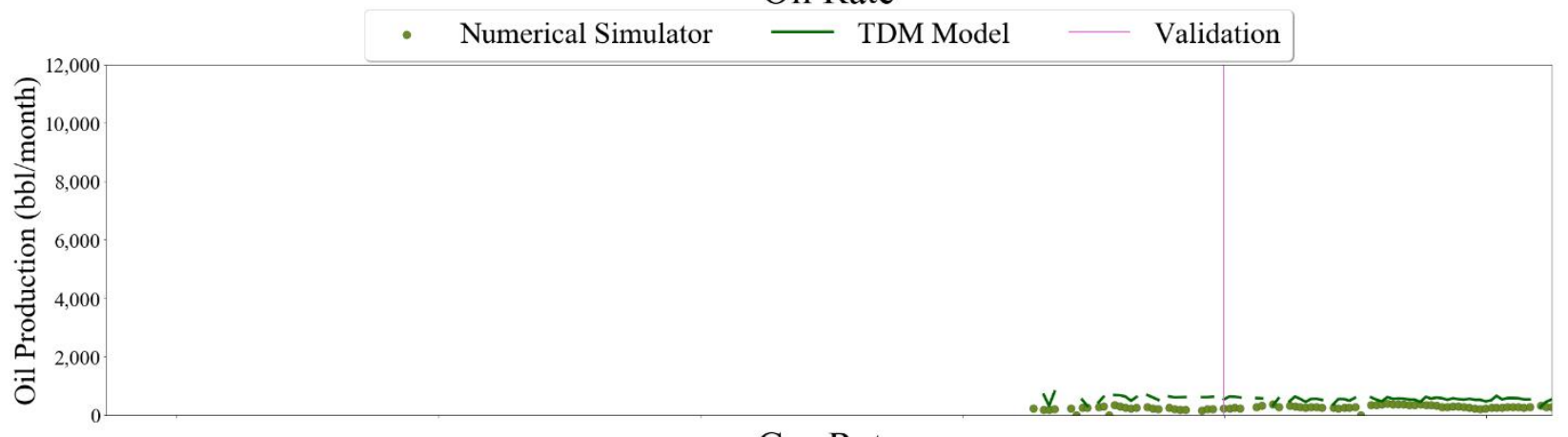

\section{Gas Rate}
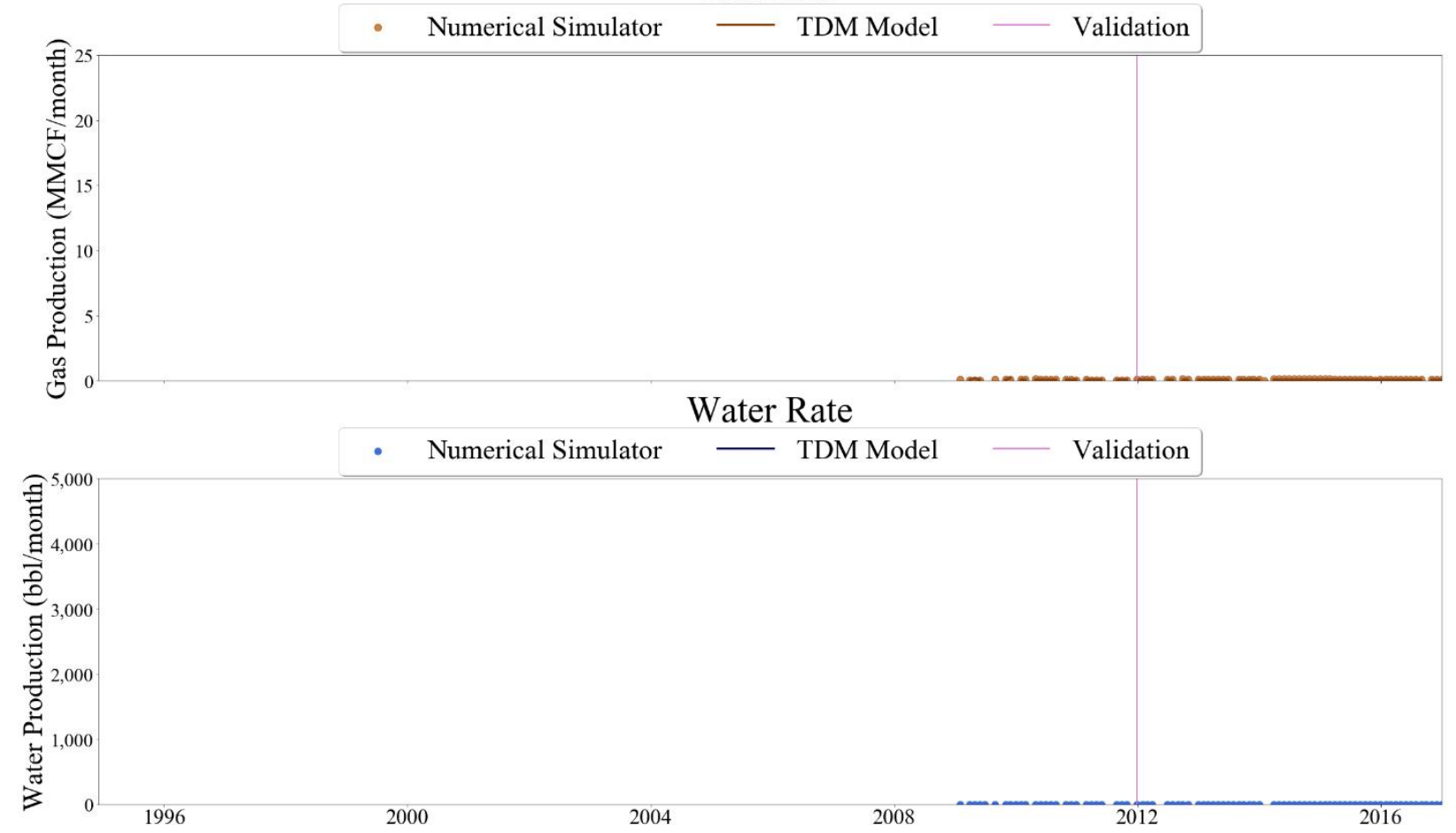

Figure 796 Well-050 oil, gas, and water rate TDM predictions vs actual simulation data plots for 5 Year Blind Validation TDM 


\section{Oil Rate}

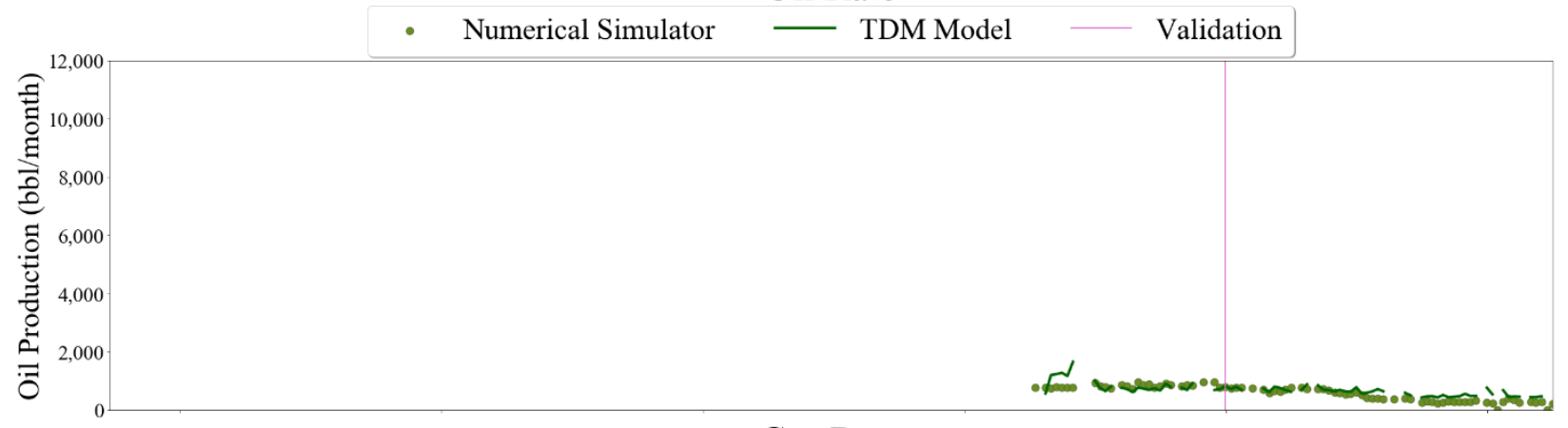

\section{Gas Rate}
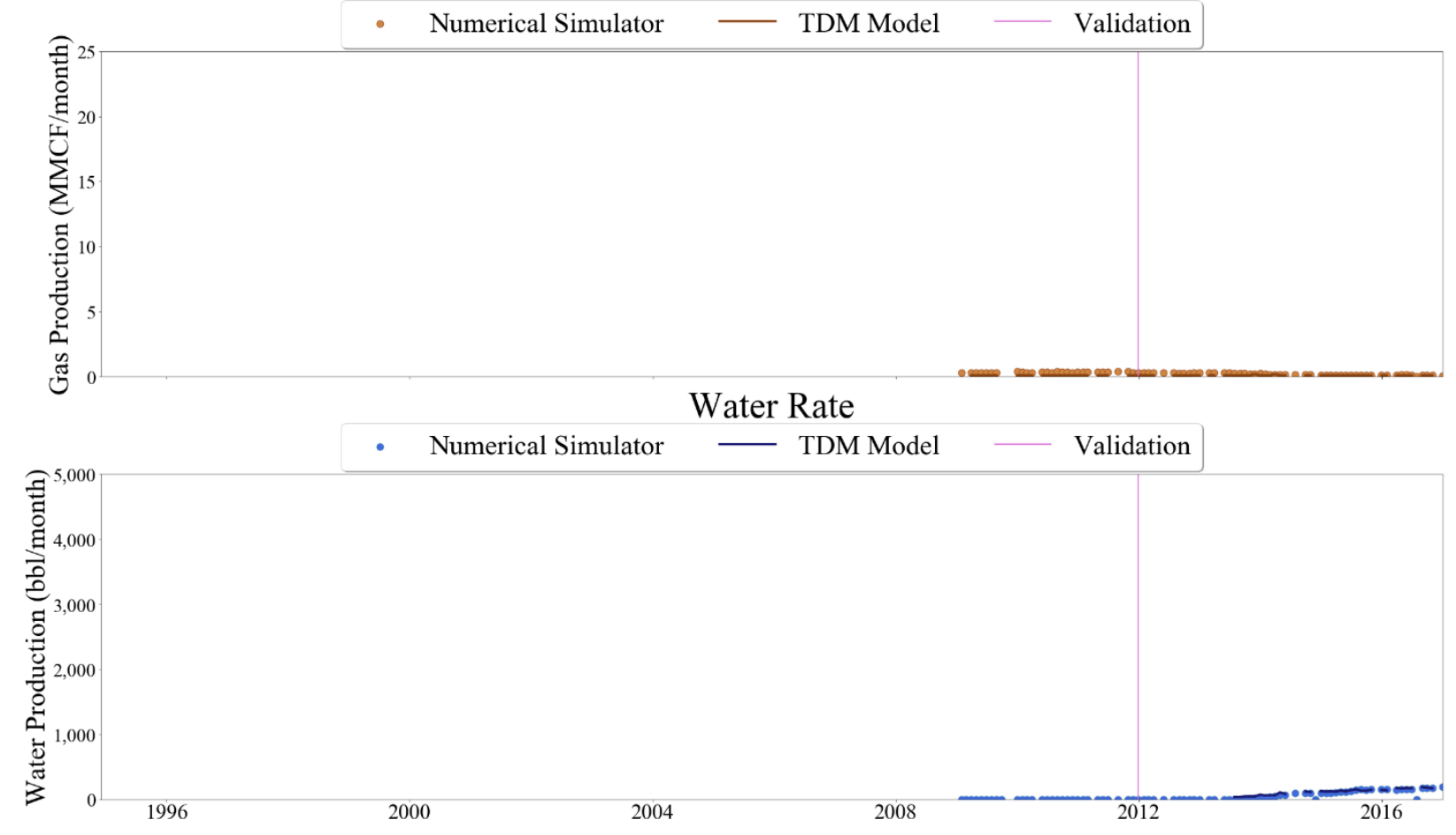

Figure 797 Well-051 oil, gas, and water rate TDM predictions vs actual simulation data plots for 5 Year Blind Validation TDM 


\section{Oil Rate}

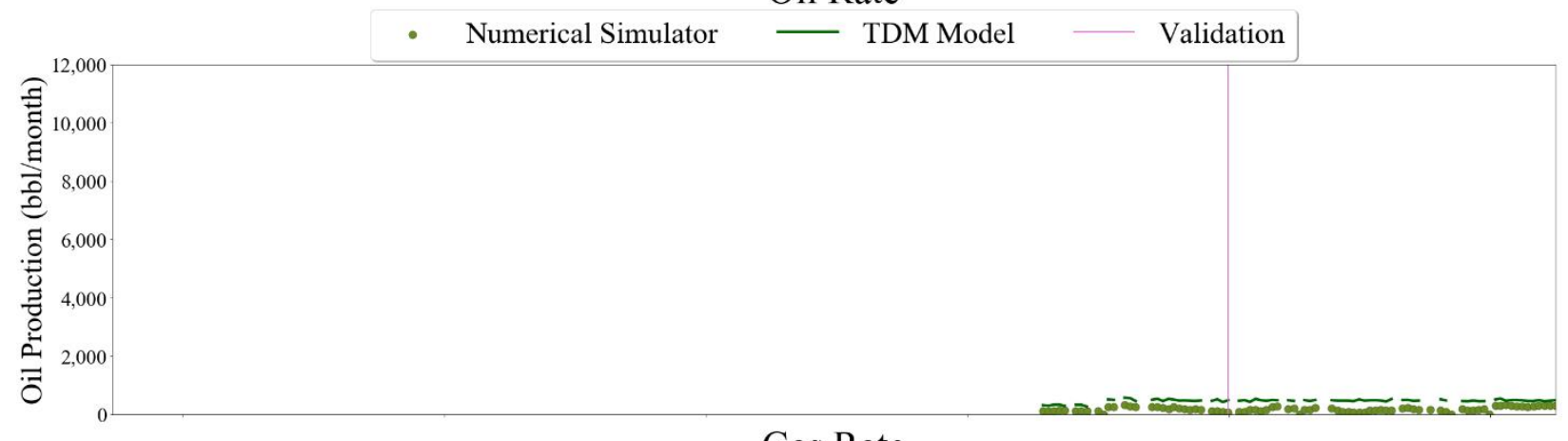

\section{Gas Rate}
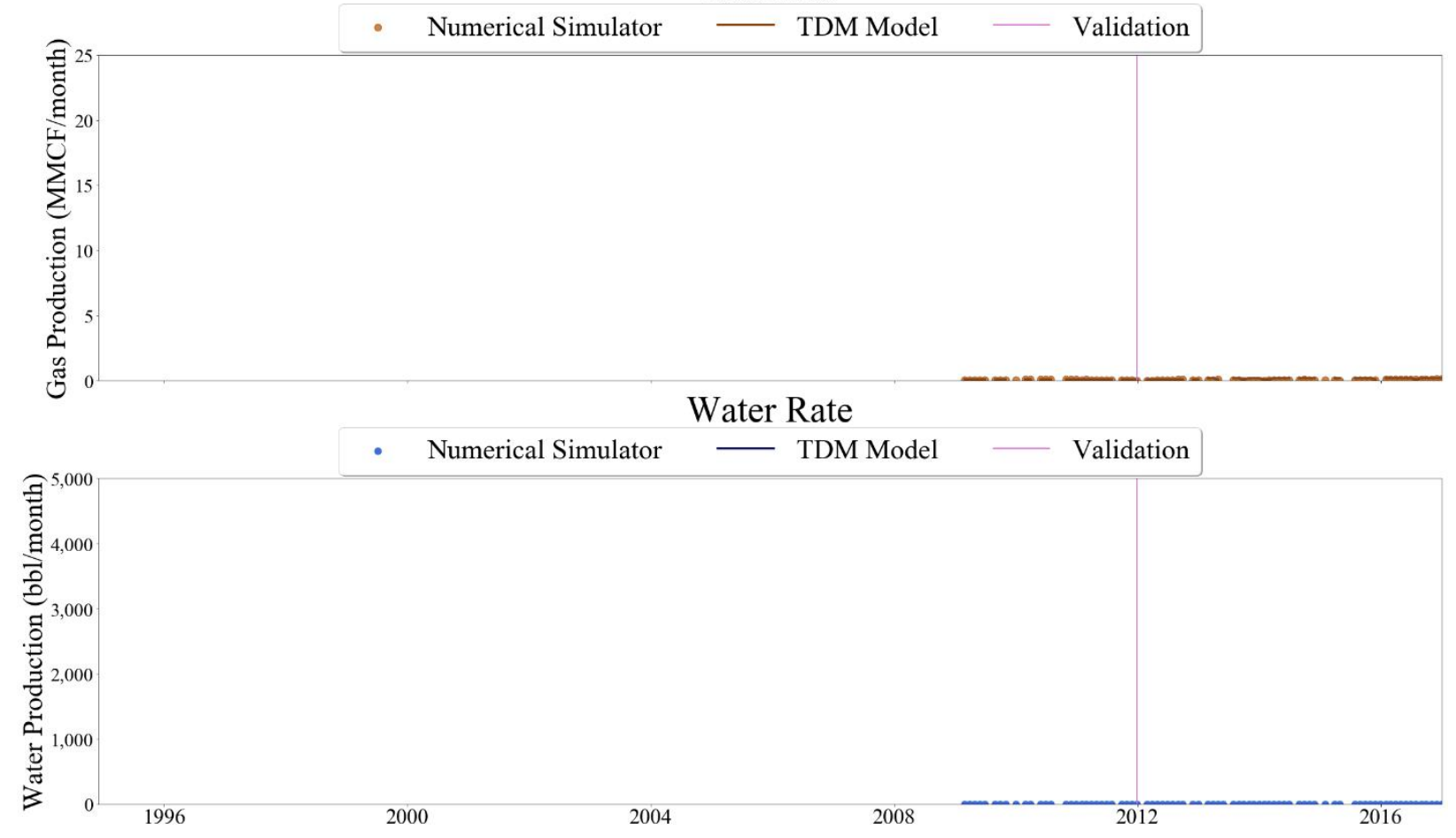

Figure 798 Well-051 oil, gas, and water rate TDM predictions vs actual simulation data plots for 5 Year Blind Validation TDM 


\section{Oil Rate}

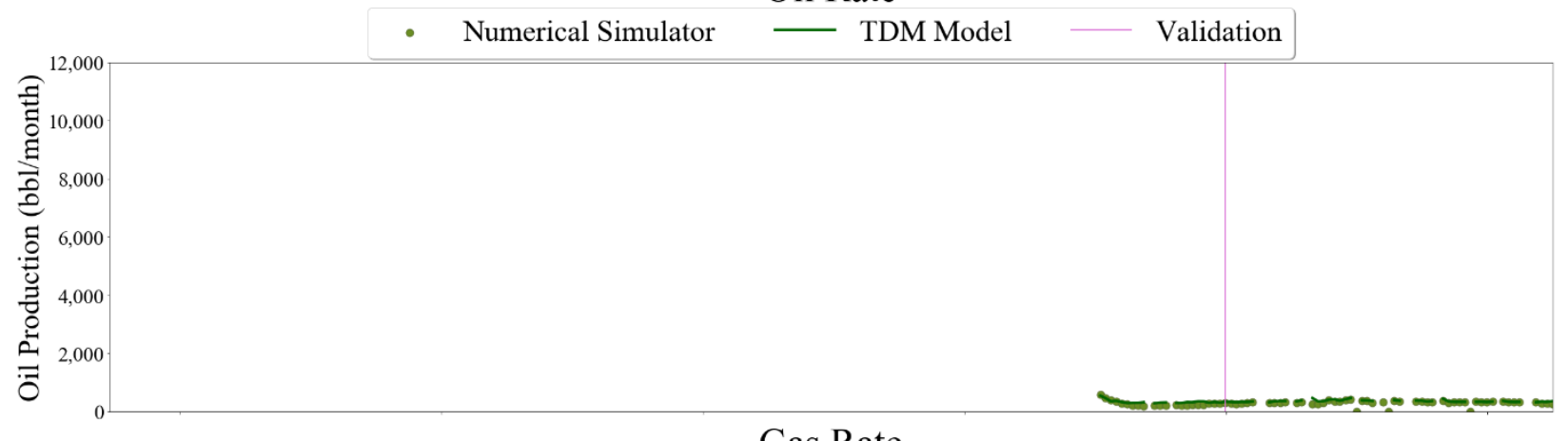

\section{Gas Rate}
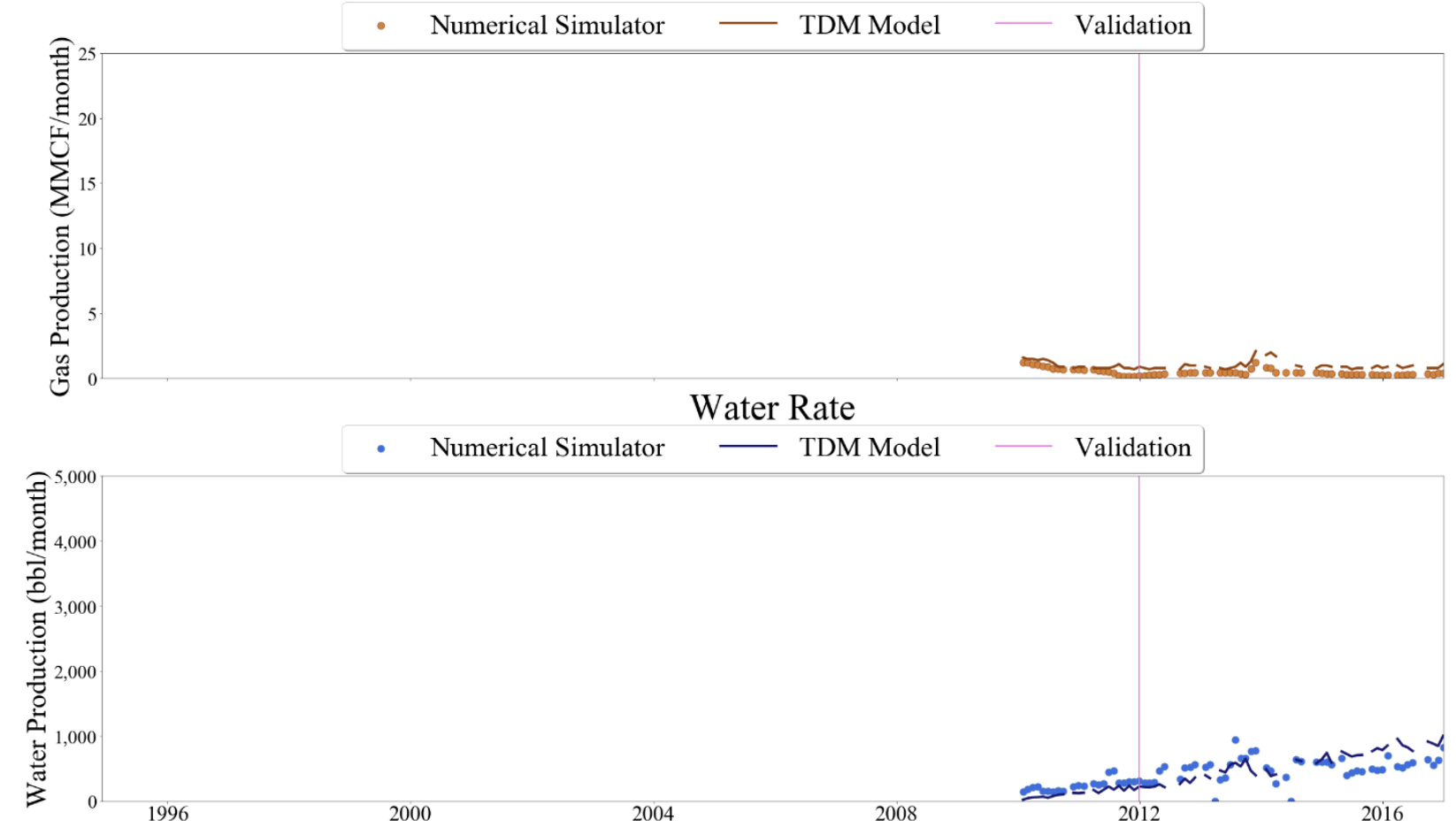

Figure 799 Well-053 oil, gas, and water rate TDM predictions vs actual simulation data plots for 5 Year Blind Validation TDM 


\section{Oil Rate}

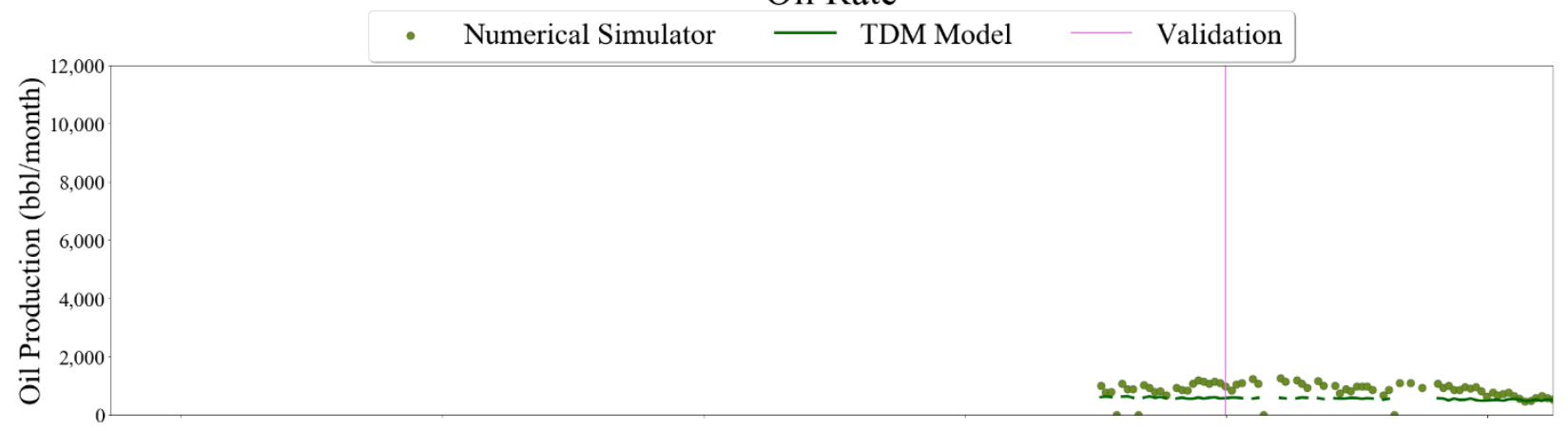

Gas Rate
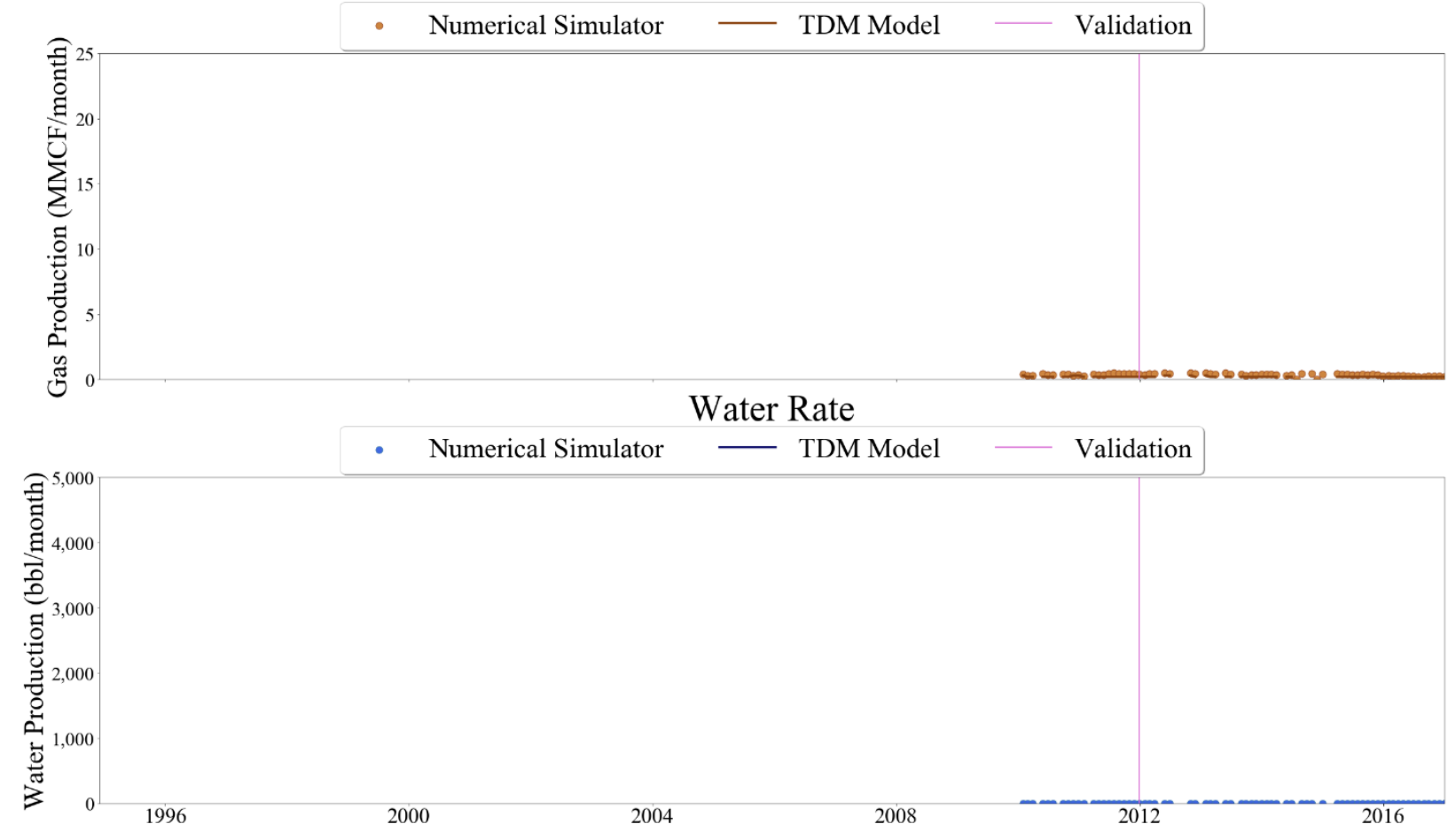

Figure 800 Well-054 oil, gas, and water rate TDM predictions vs actual simulation data plots for 5 Year Blind Validation TDM 


\section{Oil Rate}

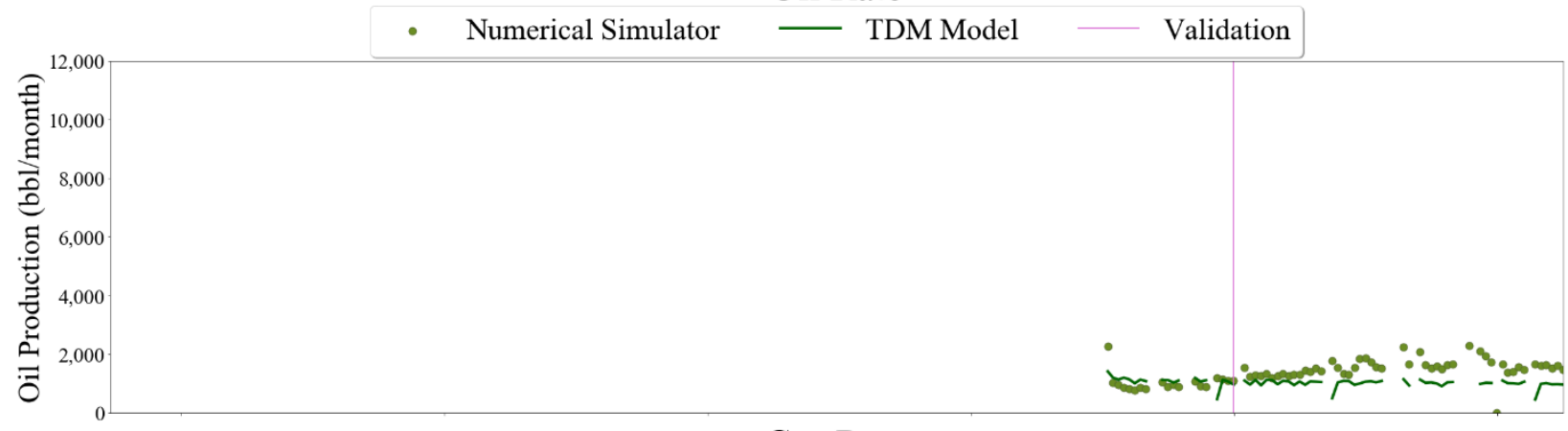

\section{Gas Rate}
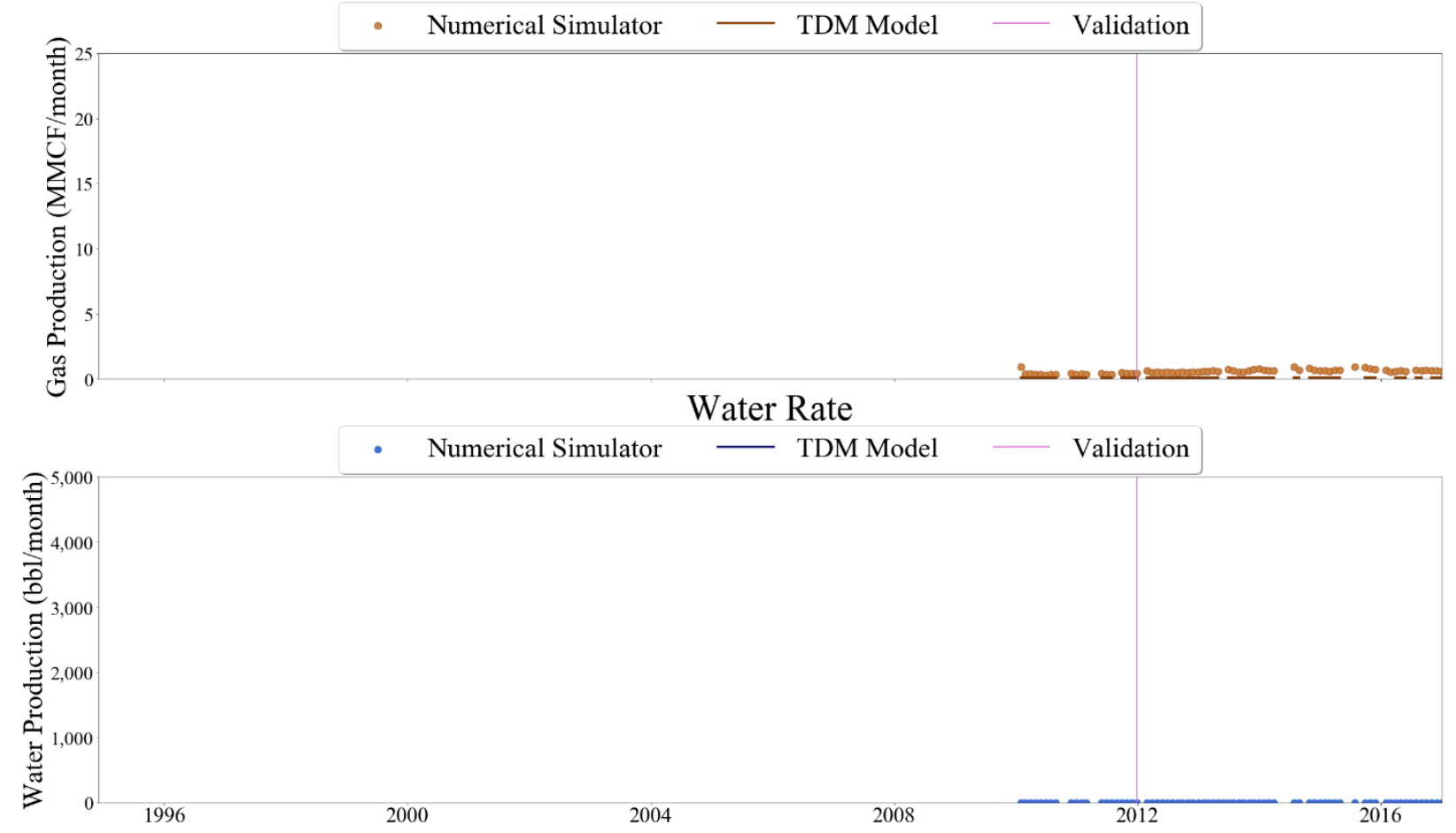

Figure 801 Well-055 oil, gas, and water rate TDM predictions vs actual simulation data plots for 5 Year Blind Validation TDM 


\section{Oil Rate}

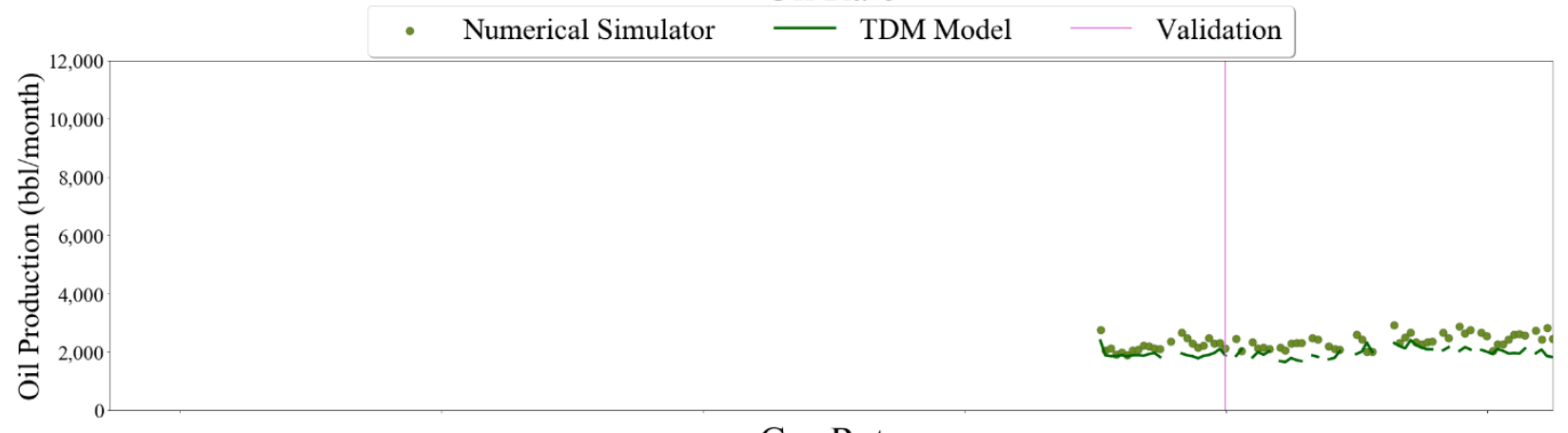

\section{Gas Rate}
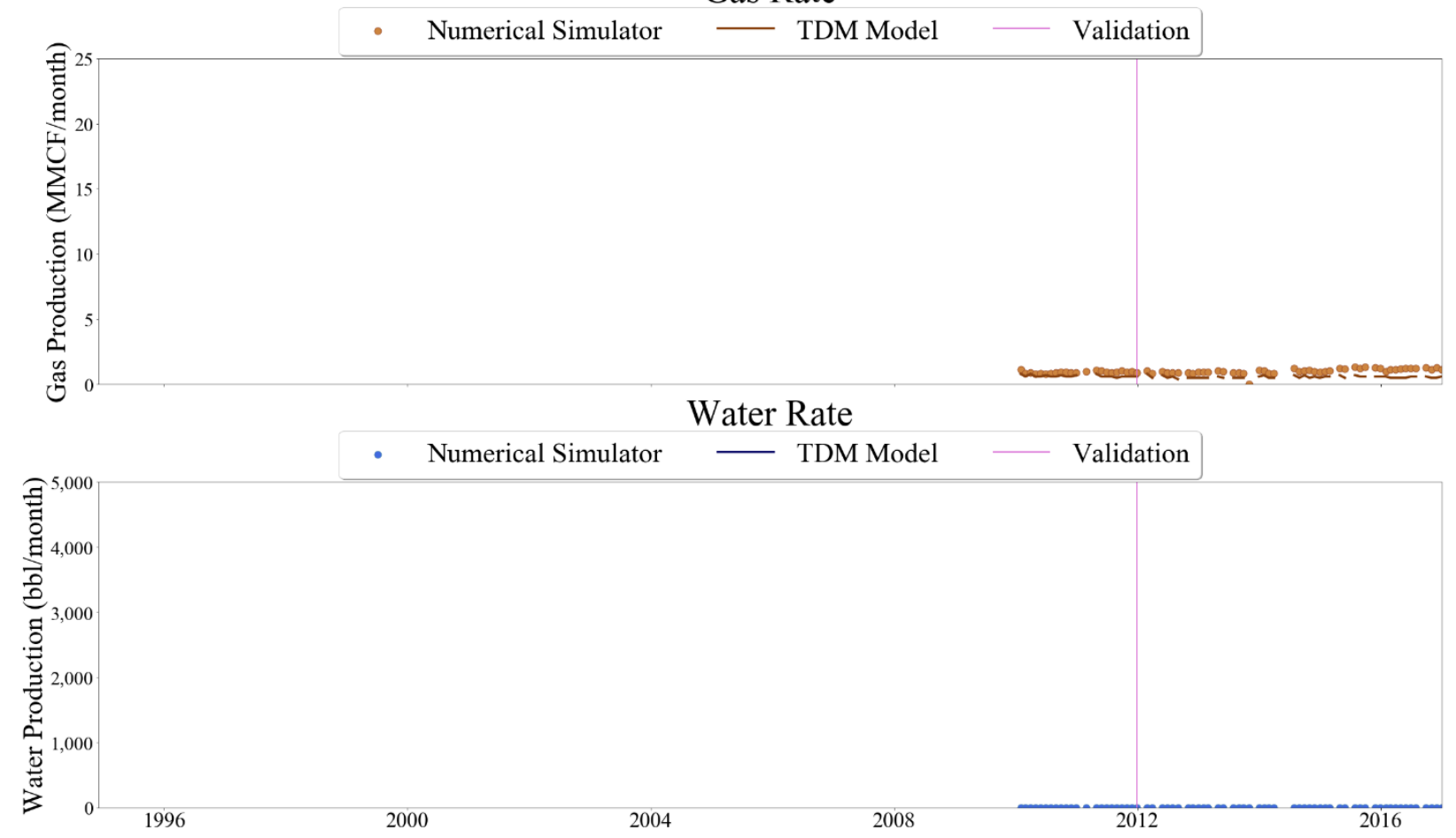

Figure 802 Well-056 oil, gas, and water rate TDM predictions vs actual simulation data plots for 5 Year Blind Validation TDM 


\section{Oil Rate}

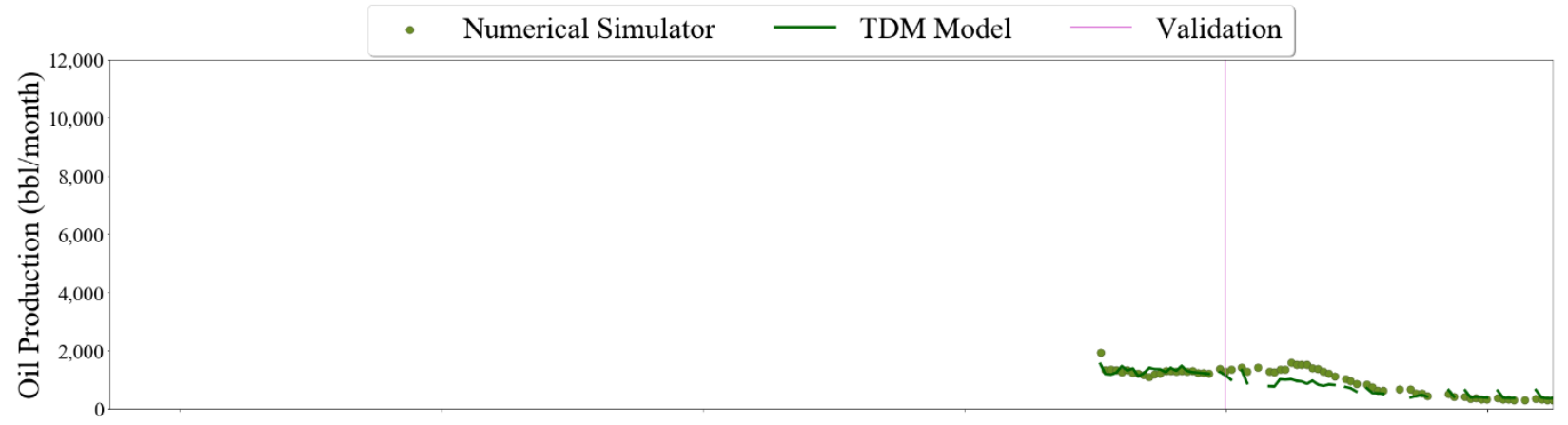

Gas Rate
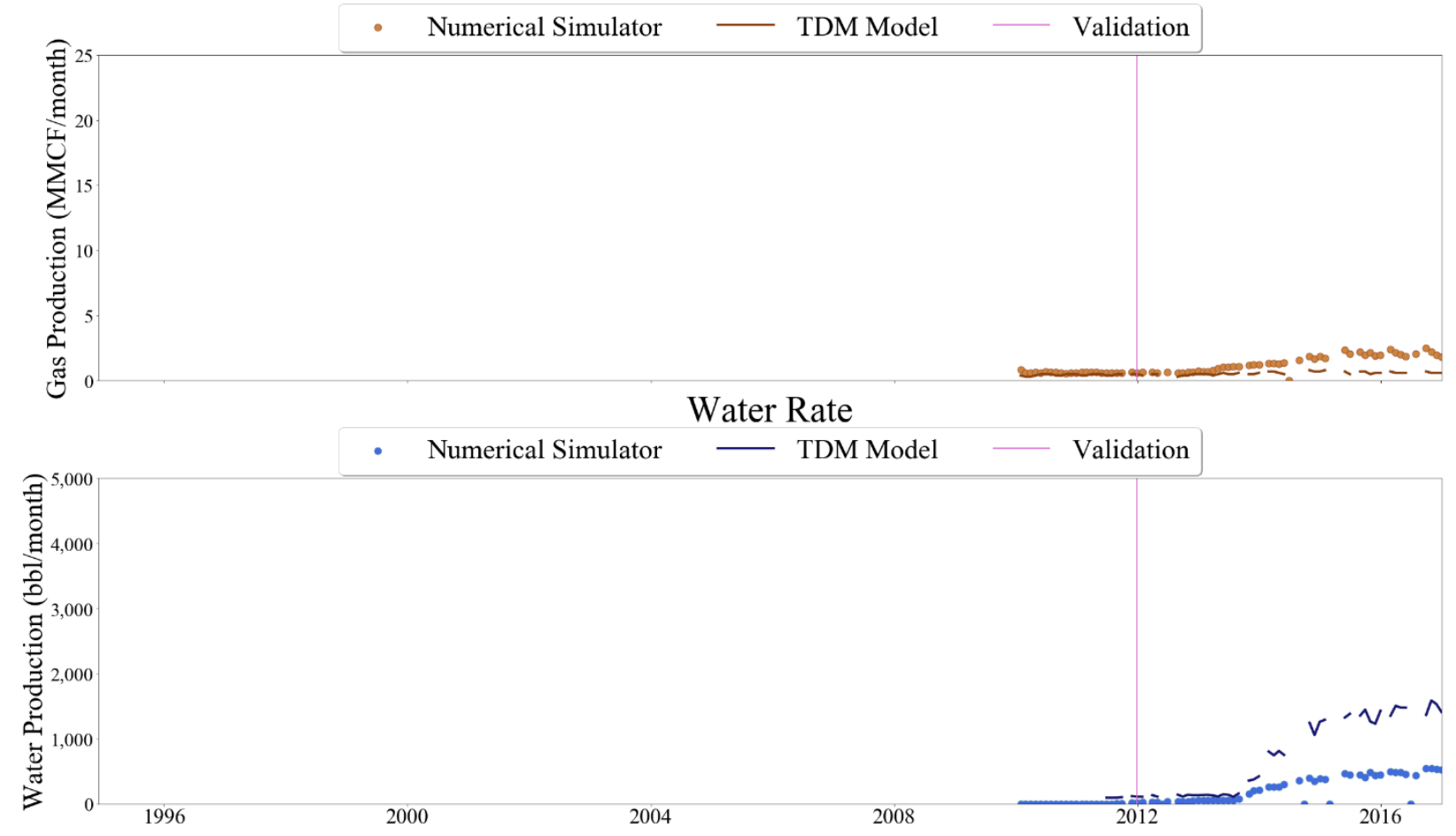

Figure 803 Well-057 oil, gas, and water rate TDM predictions vs actual simulation data plots for 5 Year Blind Validation TDM

\subsubsection{Heat Maps}

Reservoir Pressure (psi) at 2011-12-31
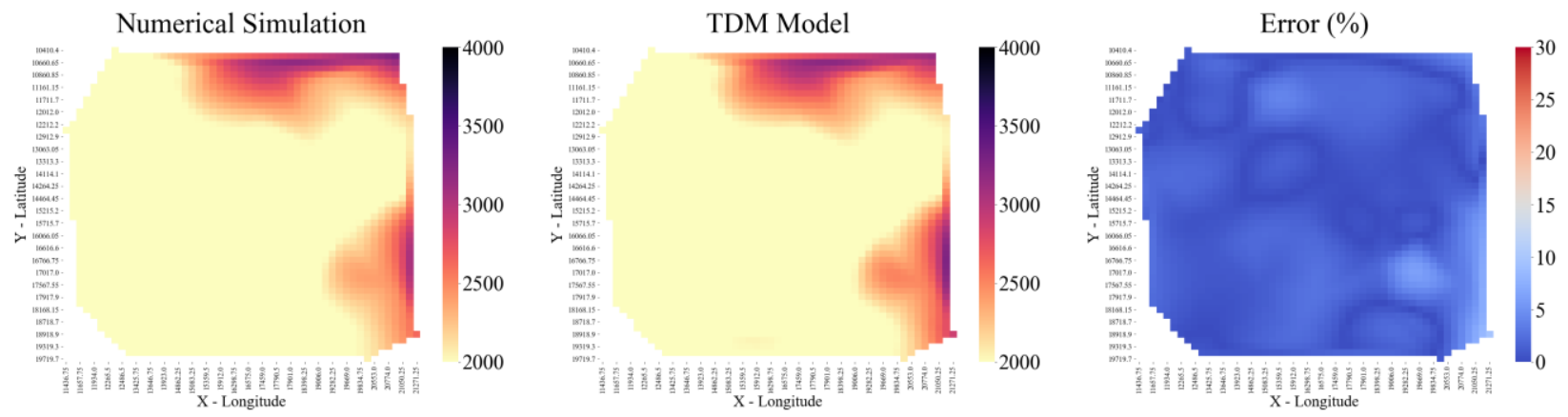

Figure 804 Entire reservoir heat map for reservoir pressure at December 31st, 2011 for 5 Year Blind Validation TDM 
Reservoir Pressure (psi) at 2012-06-30
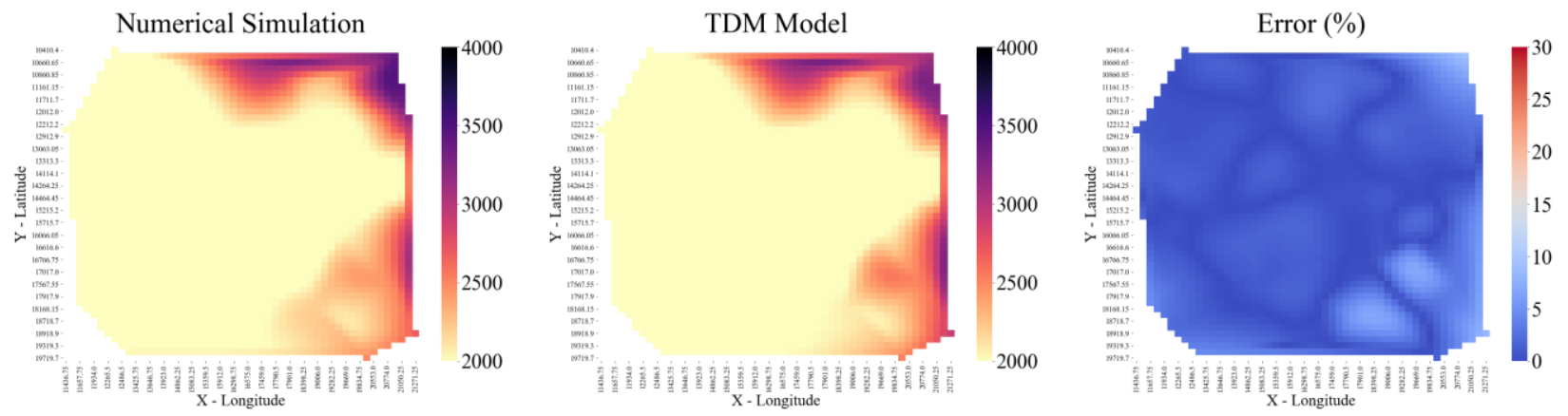

Figure 805 Entire reservoir heat map for reservoir pressure at June 30th, 2012 for 5 Year Blind Validation TDM

Reservoir Pressure (psi) at 2012-12-31
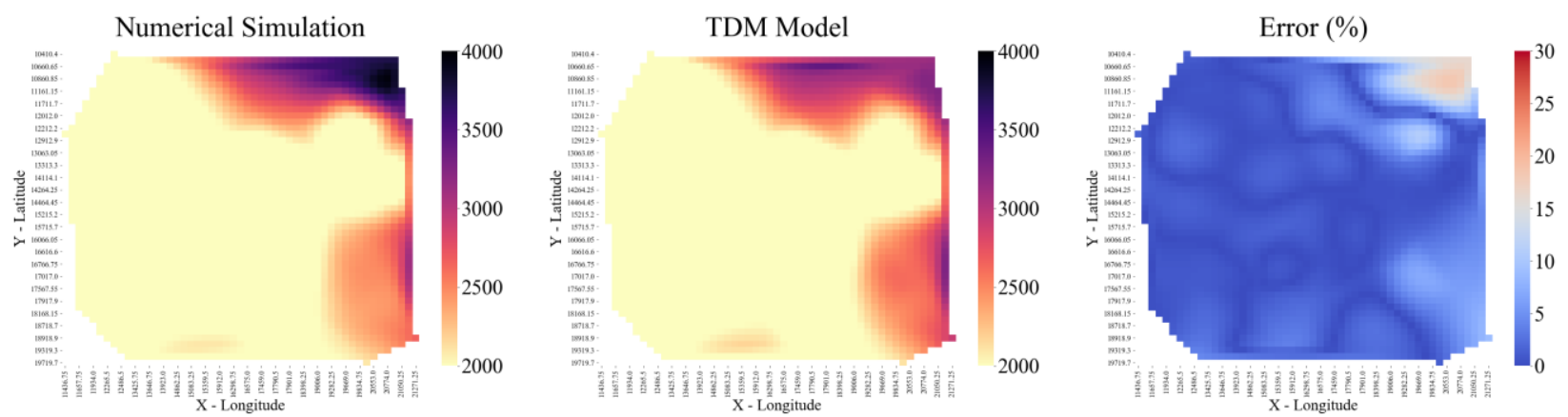

Figure 806 Entire reservoir heat map for reservoir pressure at December 31st, 2012 for 5 Year Blind Validation TDM

Reservoir Pressure (psi) at 2013-06-30
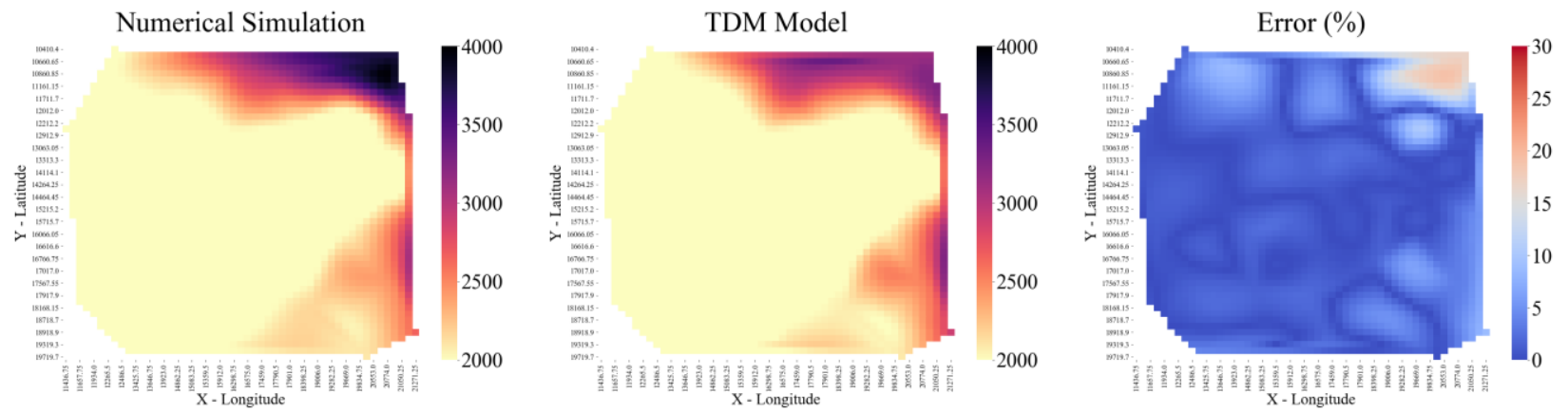

Figure 807 Entire reservoir heat map for reservoir pressure at June 30th, 2013 for 5 Year Blind Validation TDM 
Reservoir Pressure (psi) at 2013-12-31
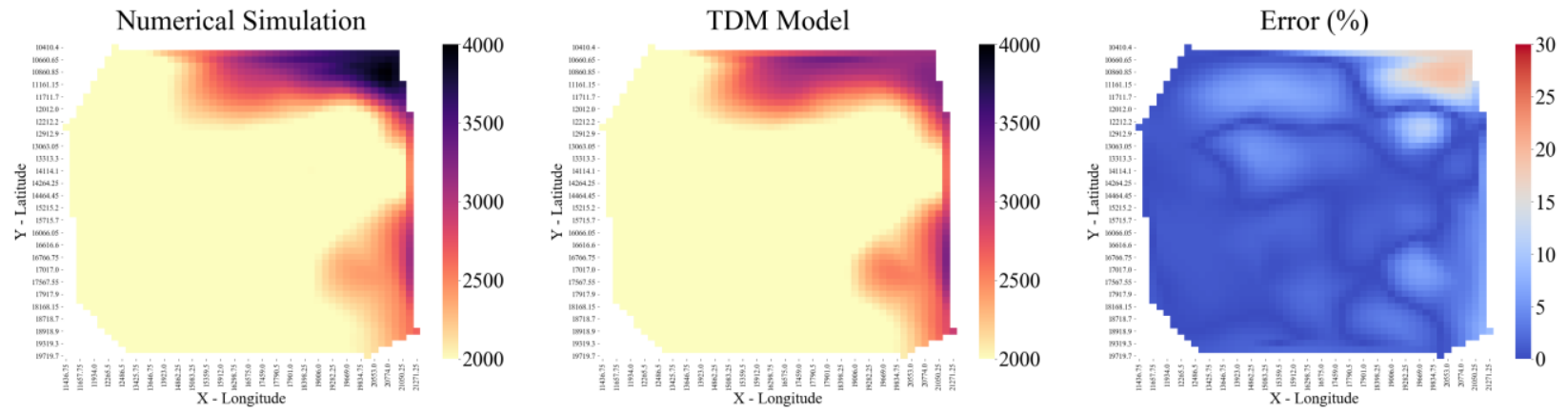

Figure 808 Entire reservoir heat map for reservoir pressure at December 31st, 2013 for 5 Year Blind Validation TDM

Reservoir Pressure (psi) at 2014-06-30
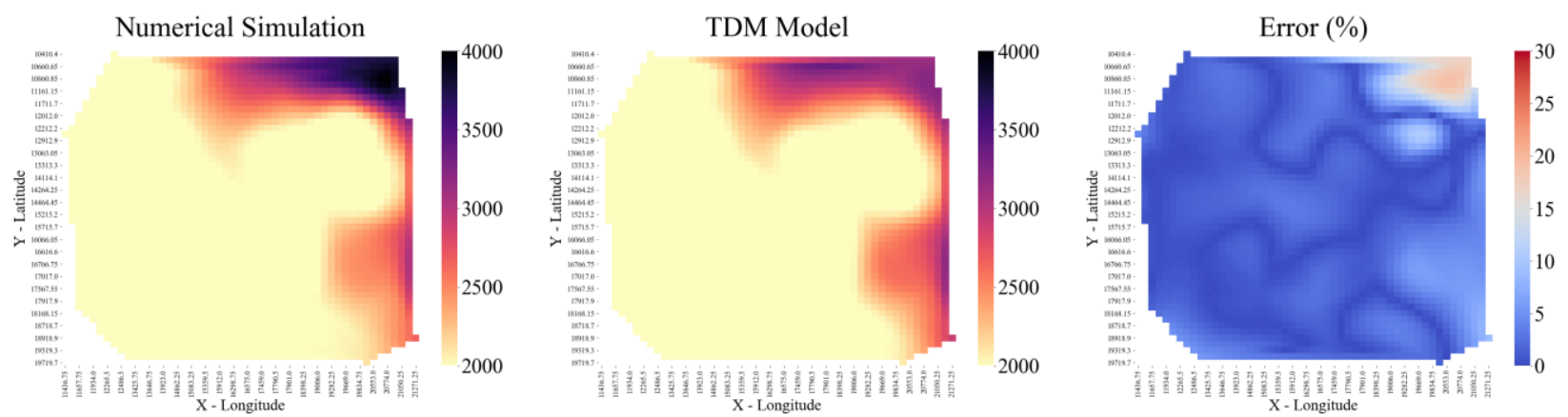

Figure 809 Entire reservoir heat map for reservoir heat map at June 30th, 2014 for 5 Year Blind Validation TDM

Reservoir Pressure (psi) at 2014-12-31
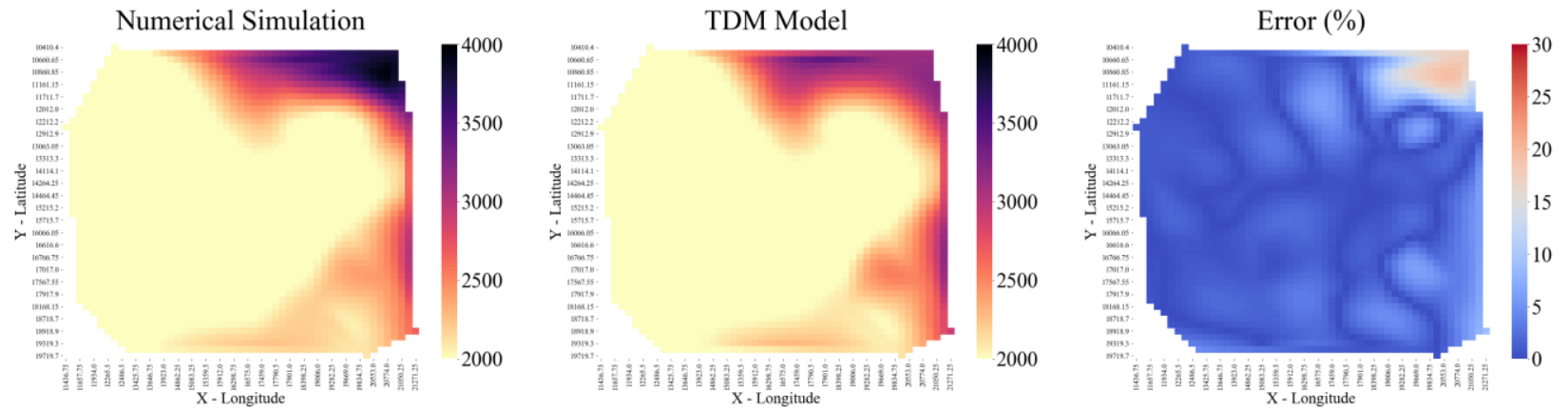

Figure 810 Entire reservoir heat map for reservoir pressure at December 31st, 2014 for 5 Year Blind Validation TDM 
Reservoir Pressure (psi) at 2015-06-30
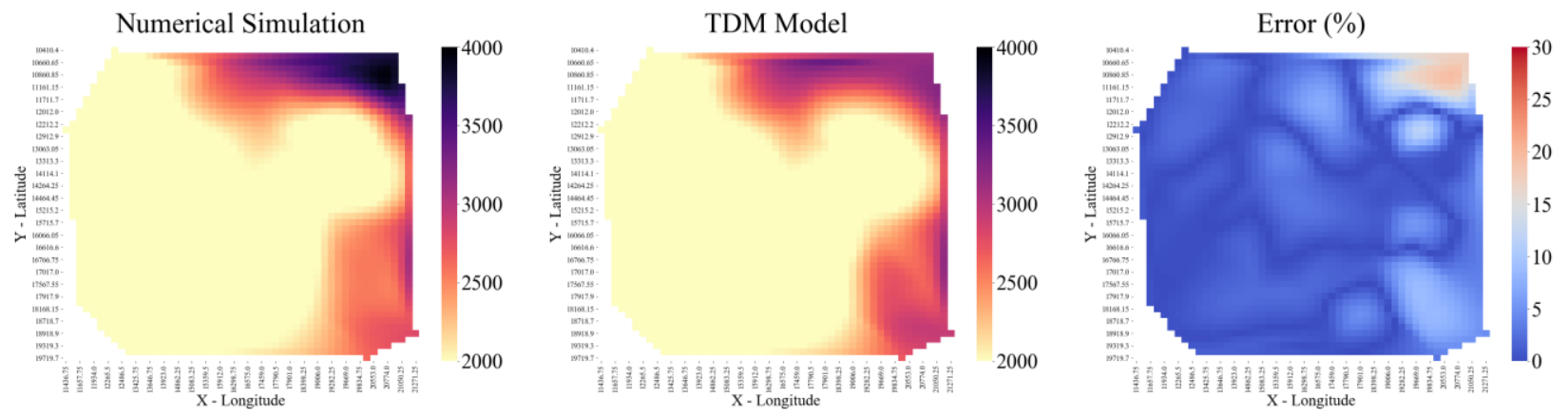

Figure 811 Entire reservoir heat map for reservoir pressure at June 30th, 2015 for 5 Year Blind Validation TDM

Reservoir Pressure (psi) at 2015-12-31
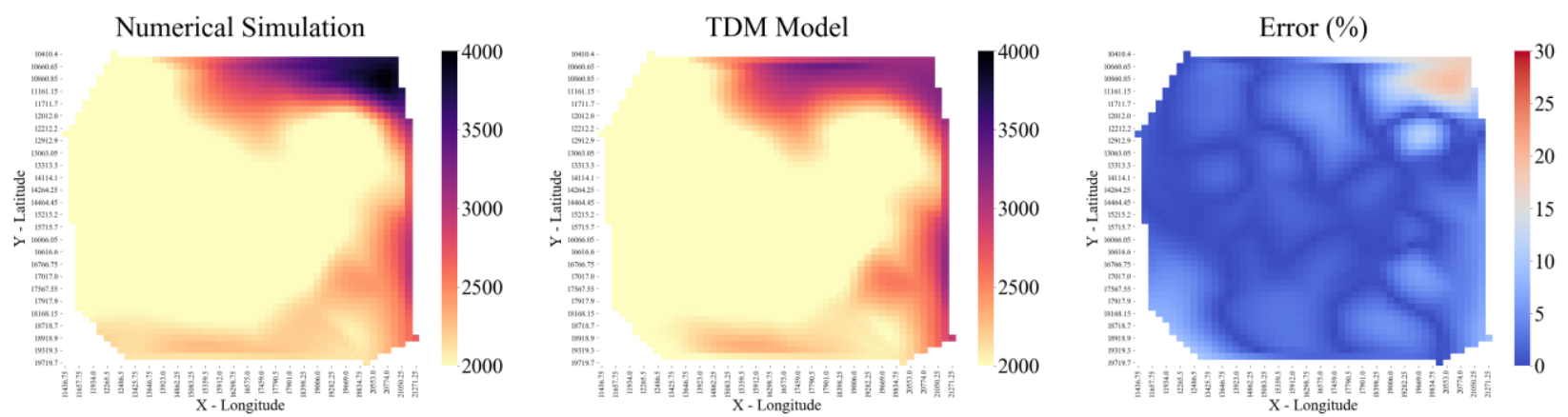

Figure 812 Entire reservoir heat map for reservoir pressure at December 31st, 2015 for 5 Year Blind Validation TDM

Reservoir Pressure (psi) at 2016-06-30
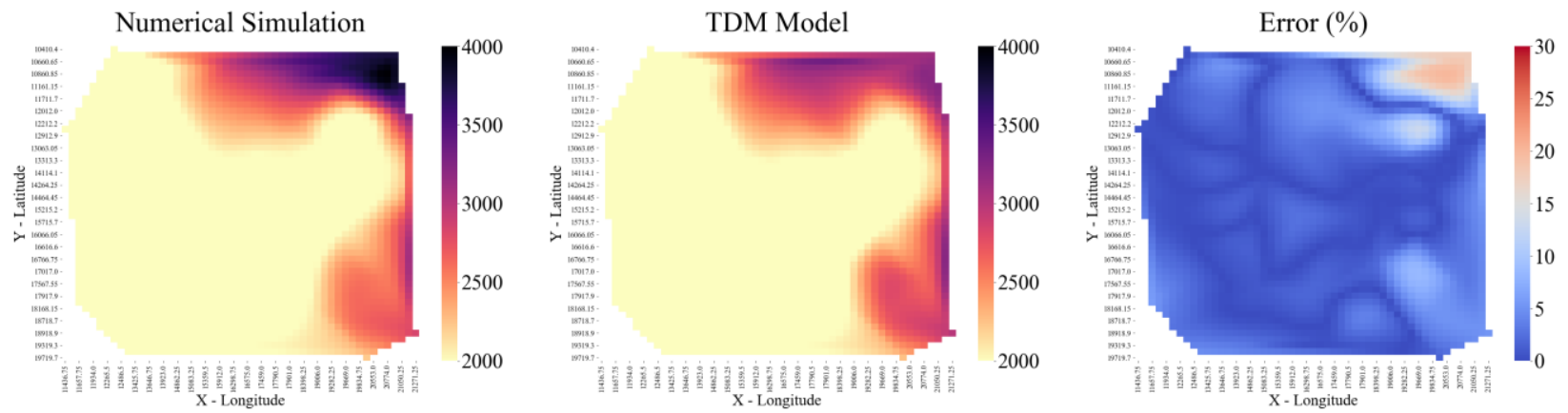

Figure 813 Entire reservoir heat map for reservoir pressure at June 30th, 2016 for 5 Year Blind Validation TDM 
Reservoir Pressure (psi) at 2016-12-31
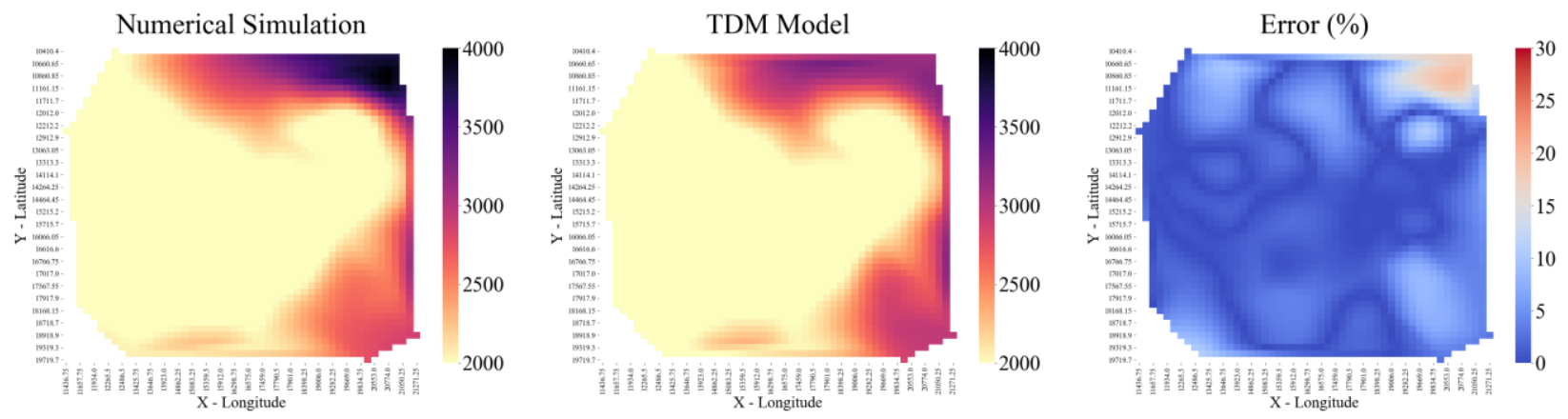

Figure 814 Entire reservoir heat map for reservoir pressure at December 31st, 2016 for 5 Year Blind Validation TDM

Water Saturation (\%) at 2011-12-31
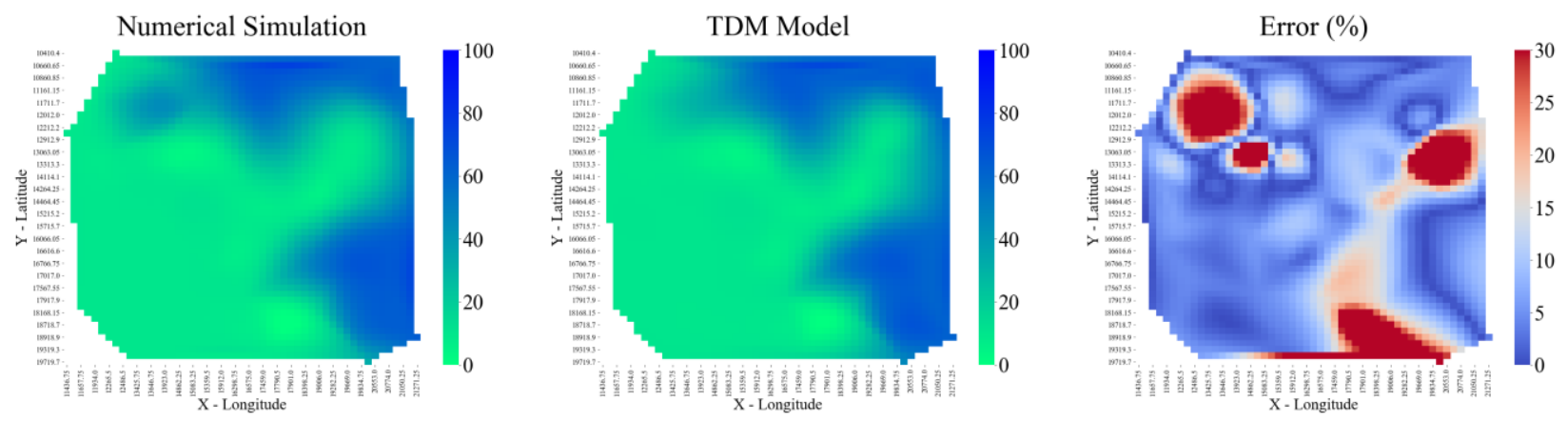

Figure 815 Entire reservoir heat map for water saturation at December 31st, 2011 for 5 Year Blind Validation TDM

Water Saturation (\%) at 2012-06-30
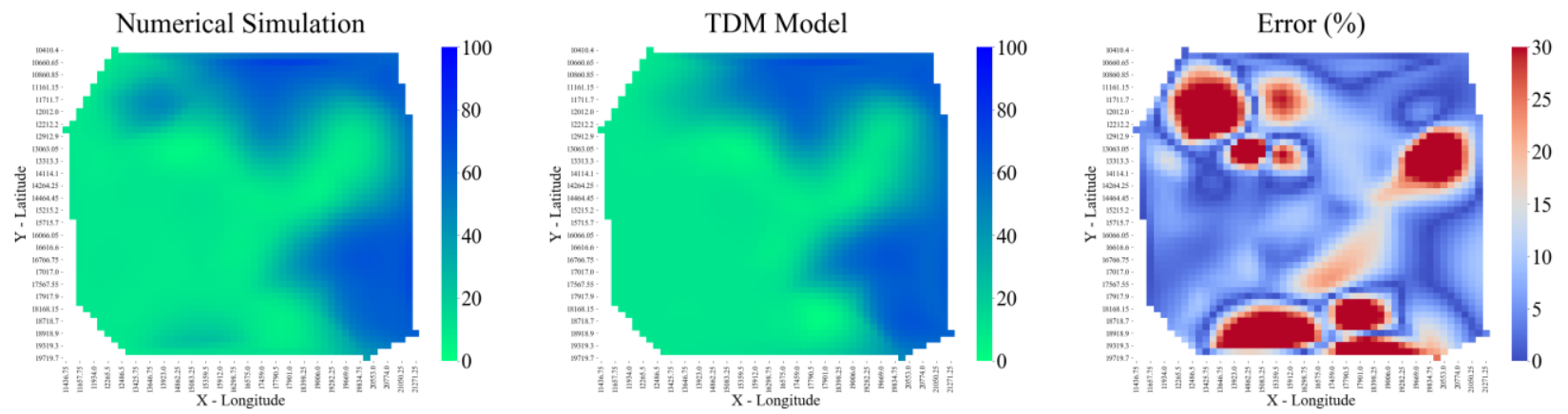

Figure 816 Entire reservoir heat map for water saturation at June 30th, 2012 for 5 Year Blind Validation TDM 
Water Saturation (\%) at 2012-12-31
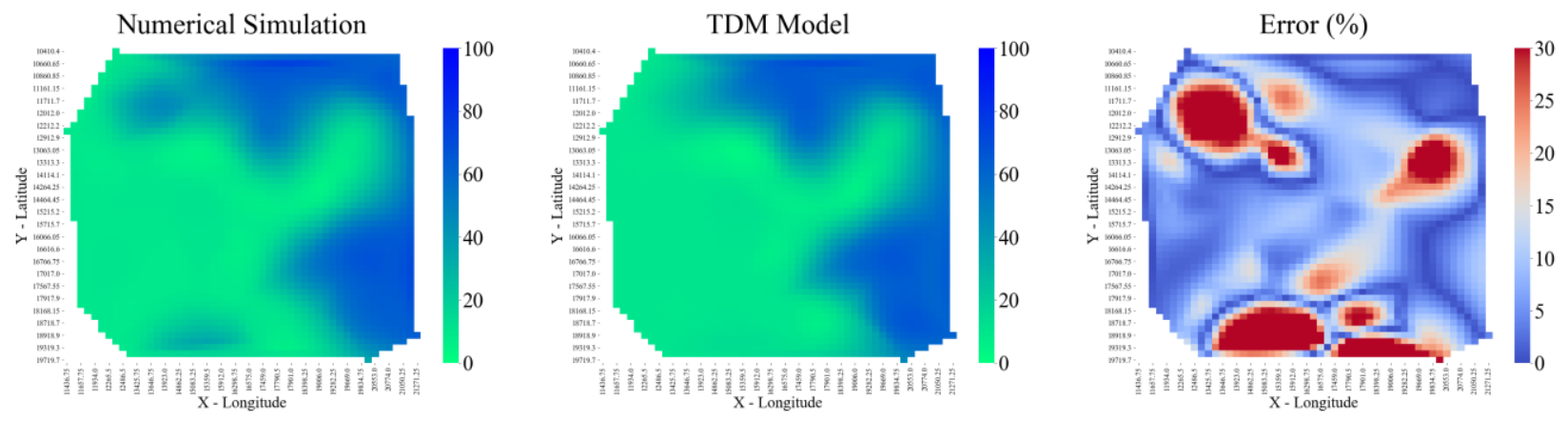

Figure 817 Entire reservoir heat map for water saturation at December 31st, 2012 for 5 Year Blind Validation TDM

Water Saturation (\%) at 2013-06-30
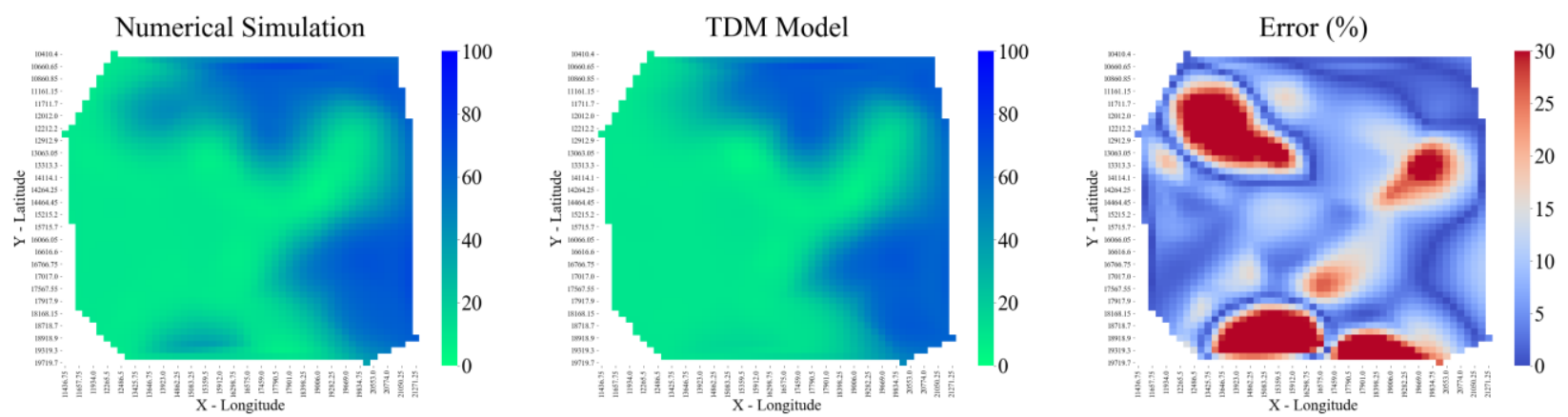

Figure 818 Entire reservoir heat map for water saturation at June 30th, 2013 for 5 Year Blind Validation TDM

Water Saturation (\%) at 2013-12-31
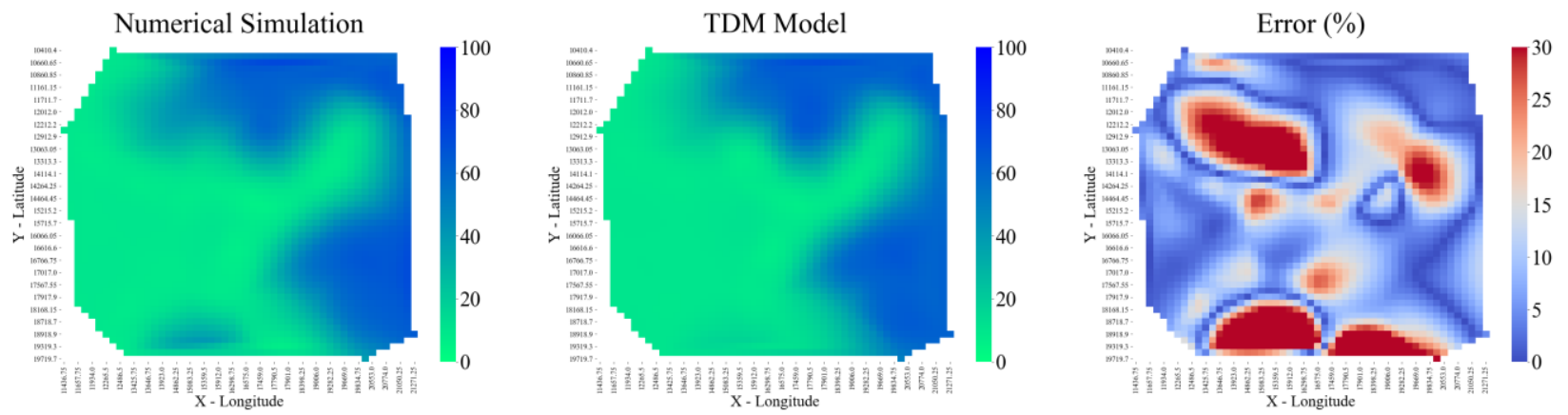

Figure 819 Entire reservoir heat map for water saturation at December 31st, 2013 for 5 Year Blind Validation TDM 
Water Saturation (\%) at 2014-06-30
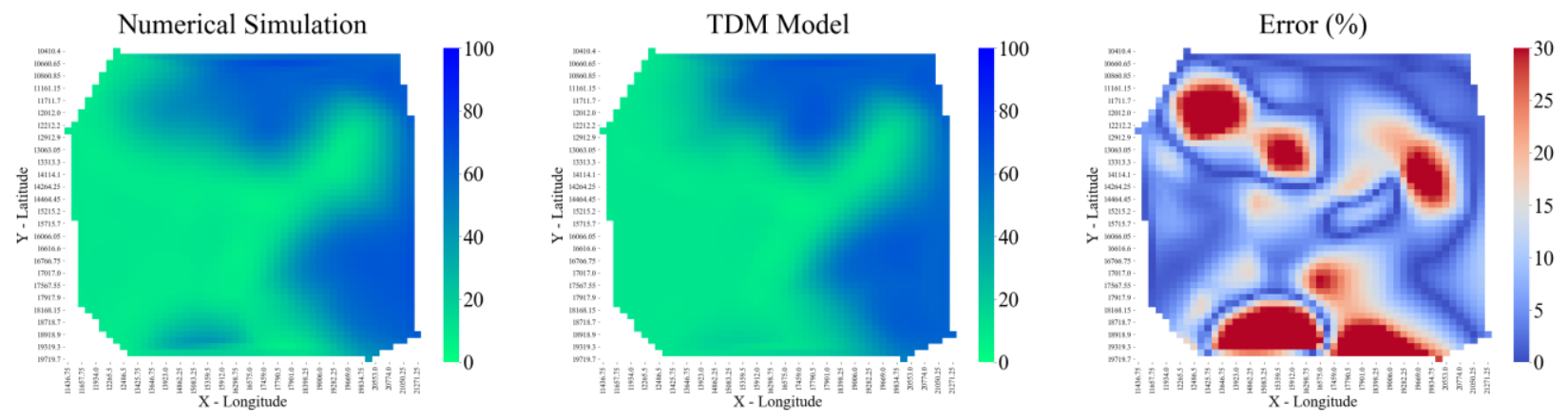

Figure 820 Entire reservoir heat map for water saturation at June 30th, 2014 for 5 Year Blind Validation TDM

Water Saturation (\%) at 2014-12-31
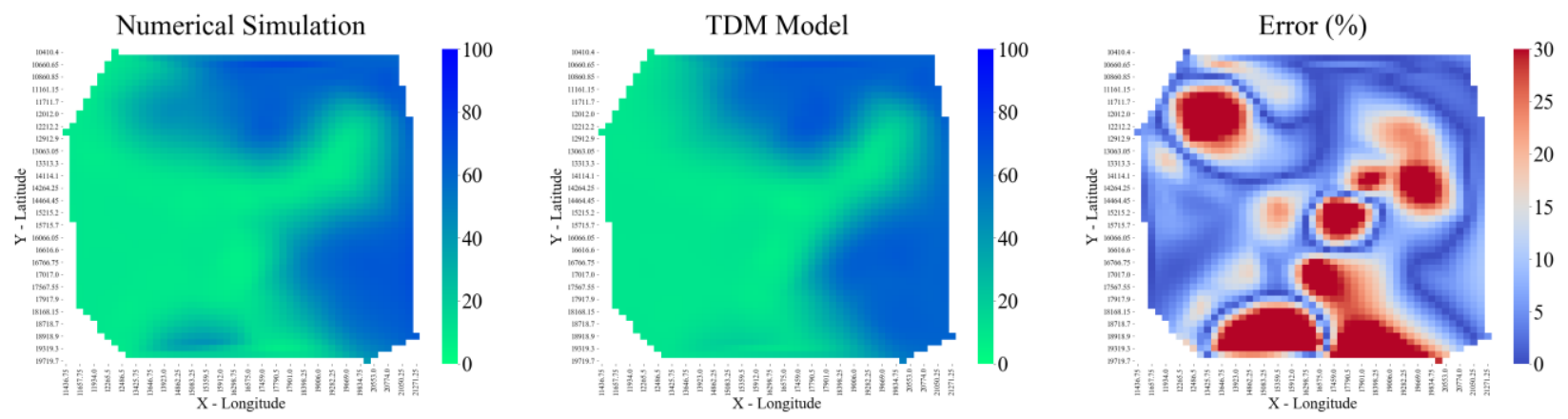

Figure 821 Entire reservoir heat map for water saturation at December 31st, 2014 for 5 Year Blind Validation TDM

Water Saturation (\%) at 2015-06-30
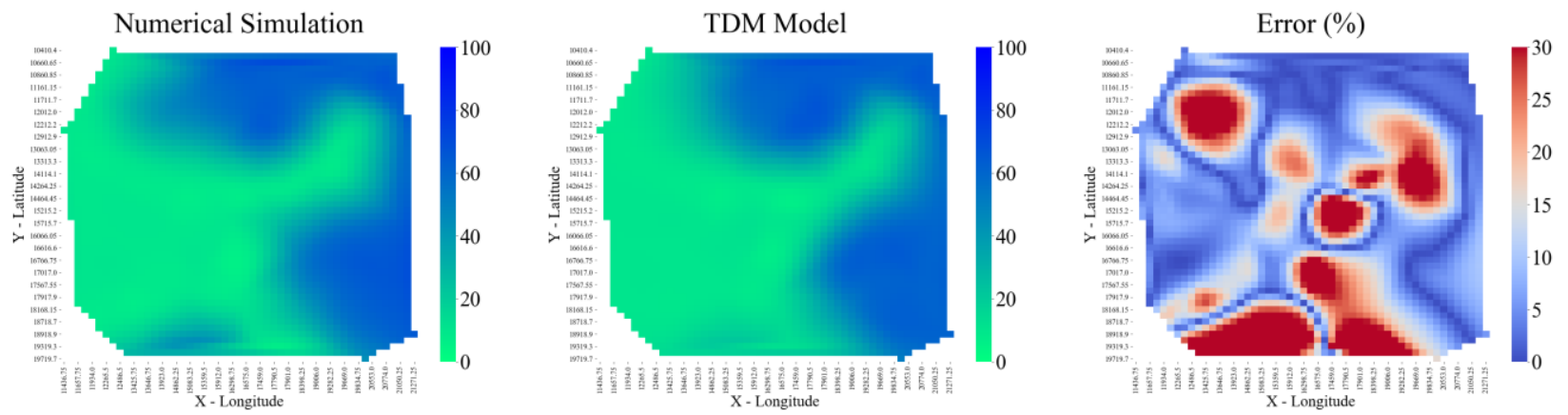

Figure 822 Entire reservoir heat map for water saturation at June 30th, 2015 for 5 Year Blind Validation TDM 
Water Saturation (\%) at 2015-12-31
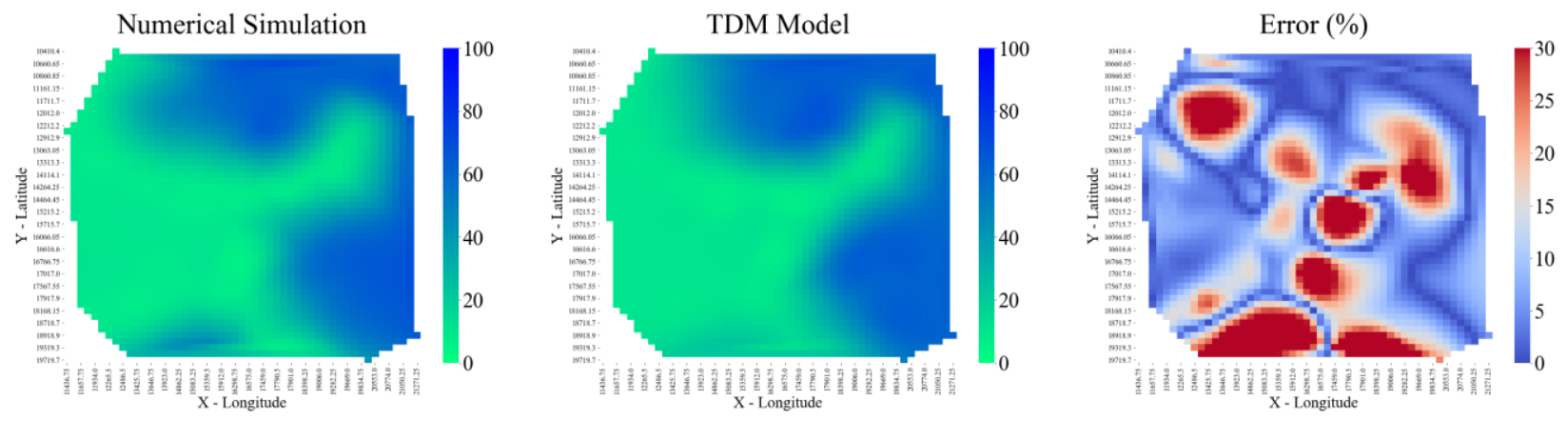

Figure 823 Entire reservoir heat map for water saturation at December 31st, 2015 for 5 Year Blind Validation TDM

Water Saturation (\%) at 2016-06-30
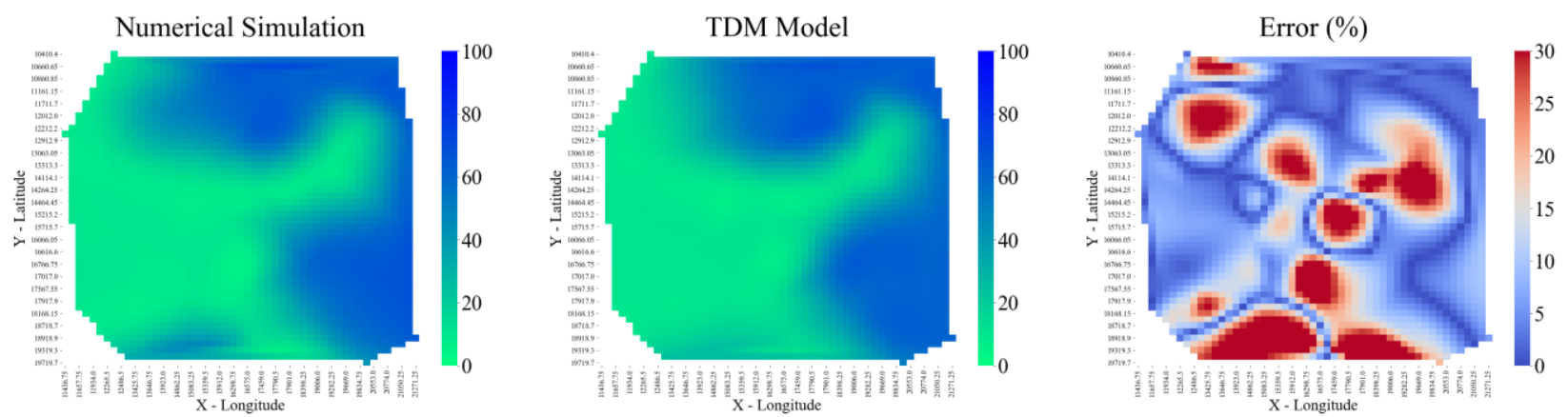

Figure 824 Entire reservoir heat map for water saturation at June 30th, 2016 for 5 Year Blind Validation TDM

Water Saturation (\%) at 2016-12-31
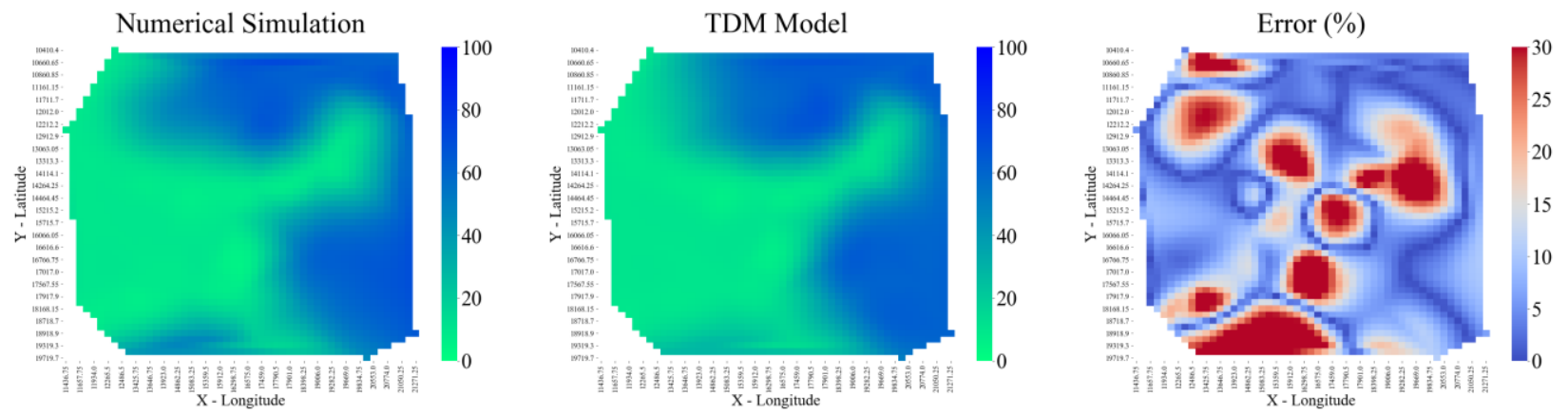

Figure 825 Entire reservoir heat map for water saturation at December 31st, 2016 for 5 Year Blind Validation TDM 
8.2.7 Multi-Random Partitioning- 6 Year Blind Validation

8.2.7.1 Individual Well Production Plots

Oil Rate

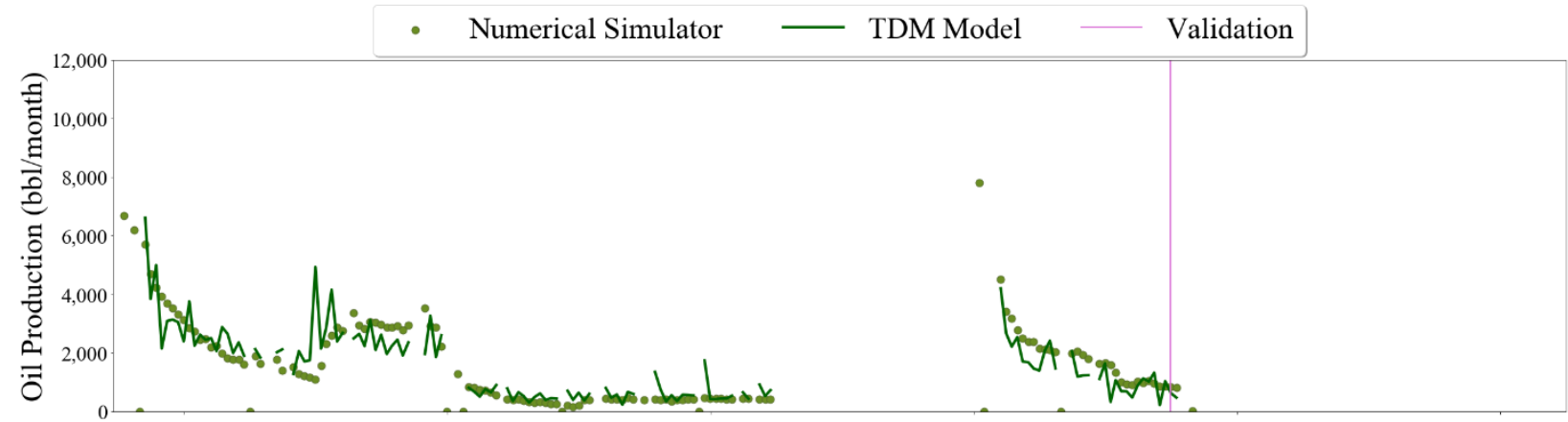

Gas Rate

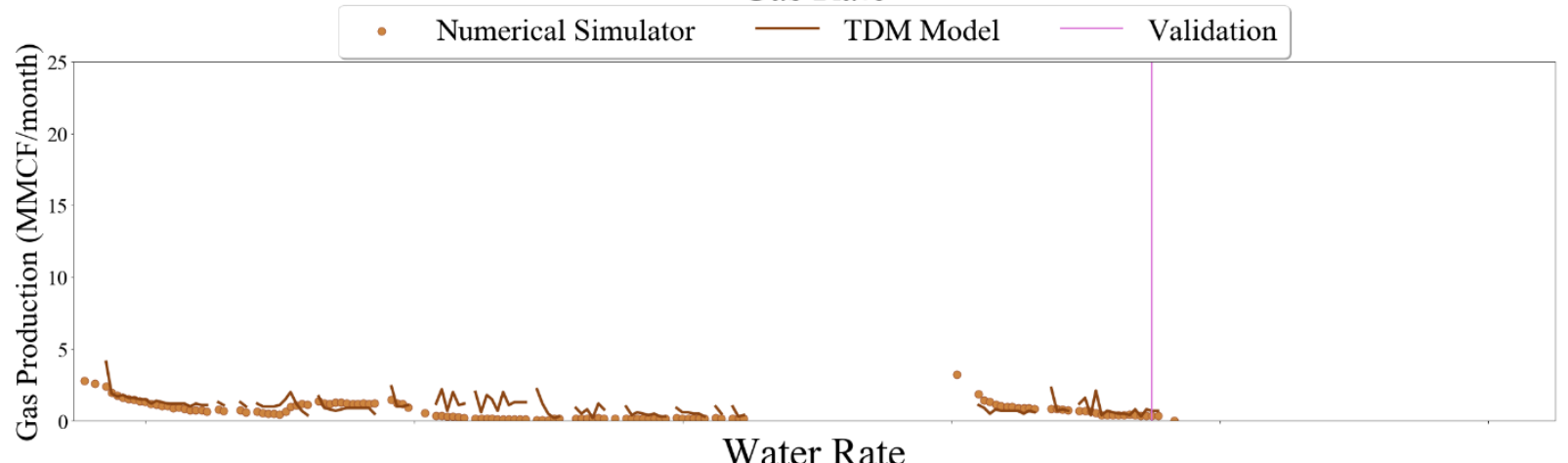

Water Rate

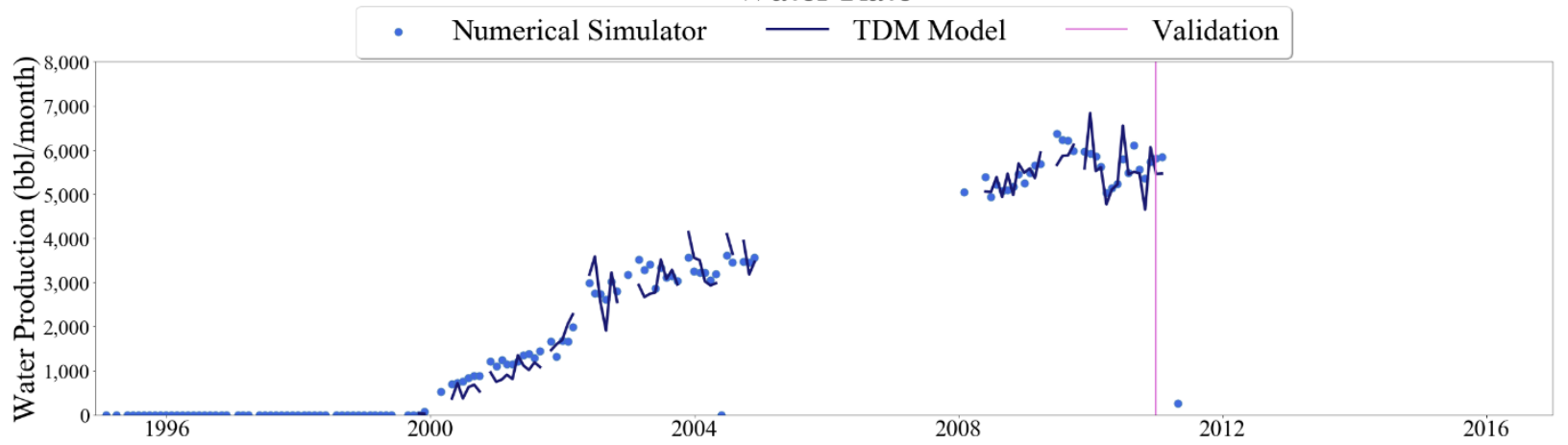

Figure 826 Well-001 oil, gas, and water rate TDM predictions vs actual simulation data plots for 6 Year Blind Validation TDM 

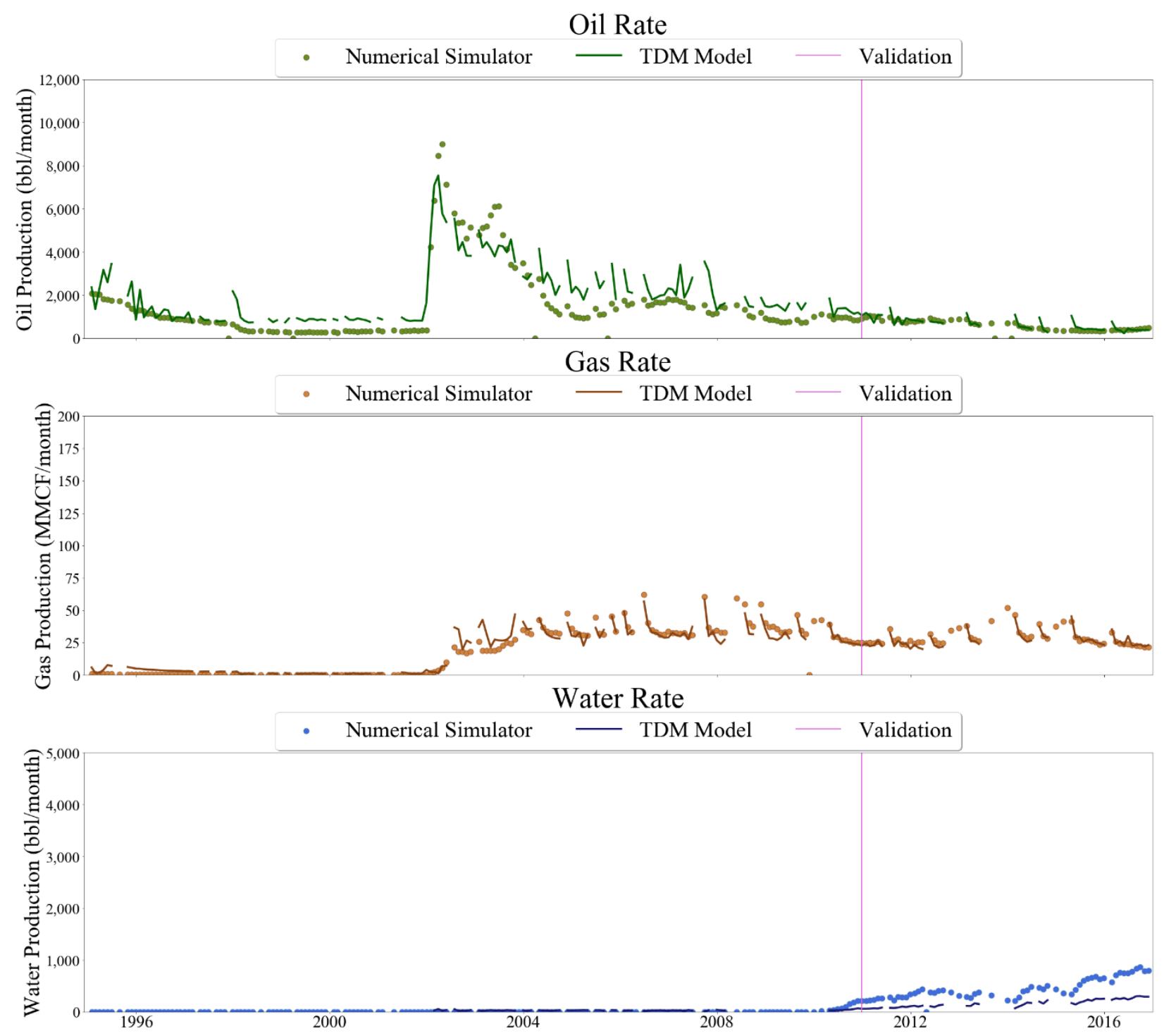

Figure 827 Well-002 oil, gas, and water rate TDM predictions vs actual simulation data plots for 6 Year Blind Validation TDM 


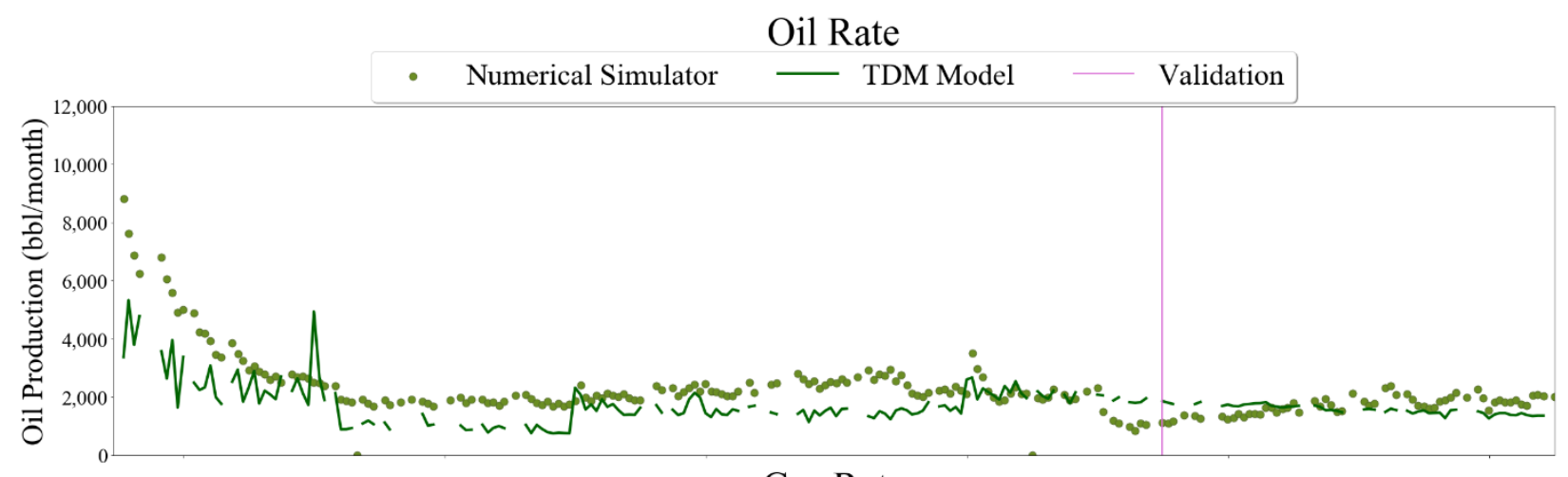

\section{Gas Rate}
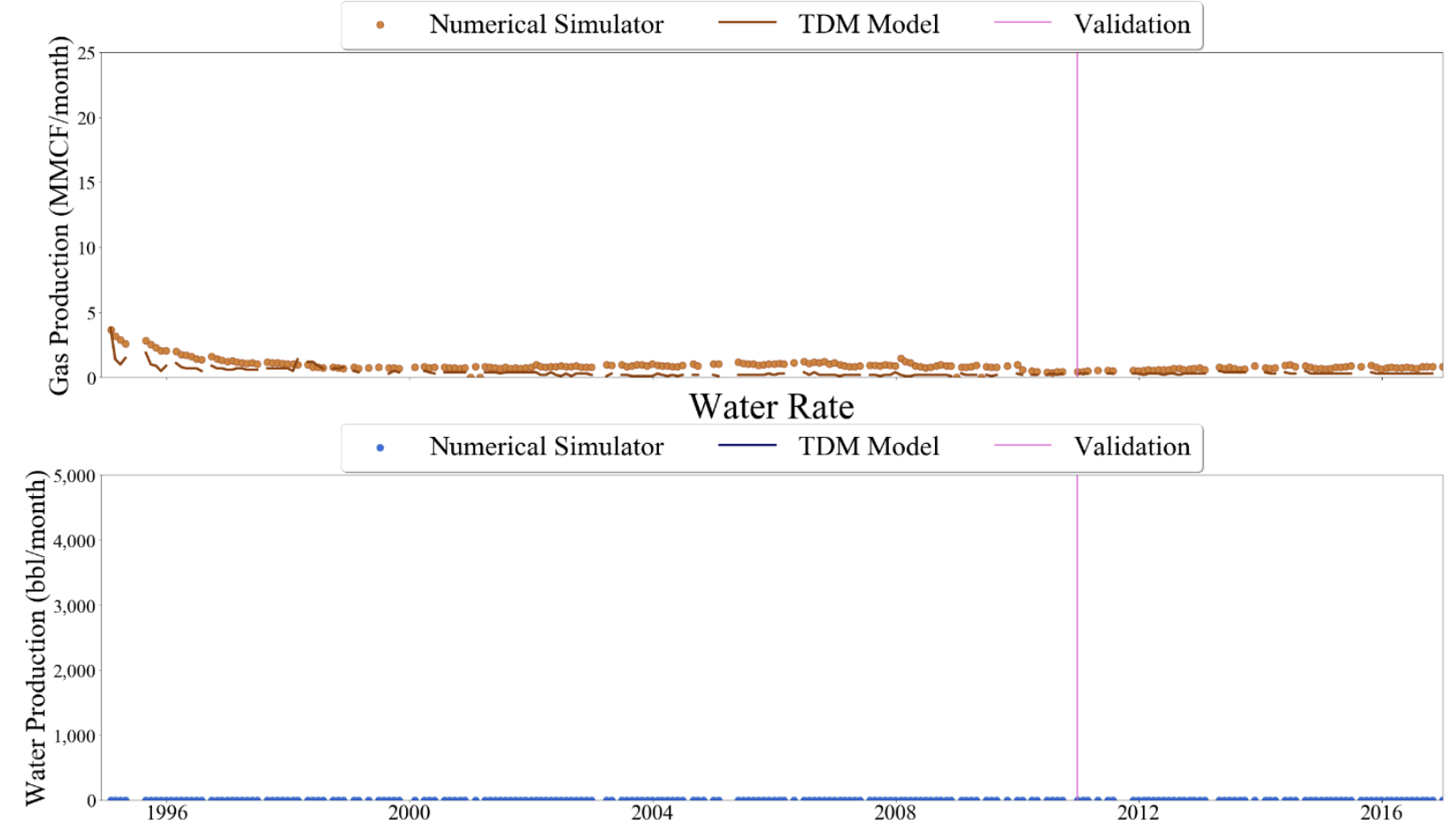

Figure 828 Well-003 oil, gas, and water rate TDM predictions vs actual simulation data plots for 6 Year Blind Validation TDM 


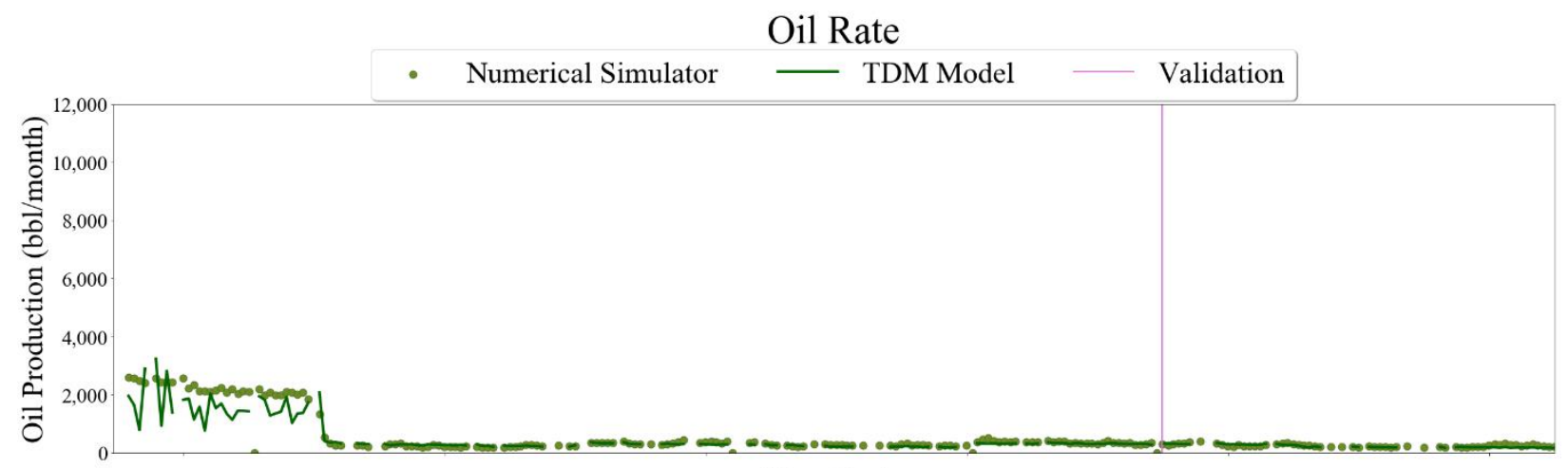

Gas Rate
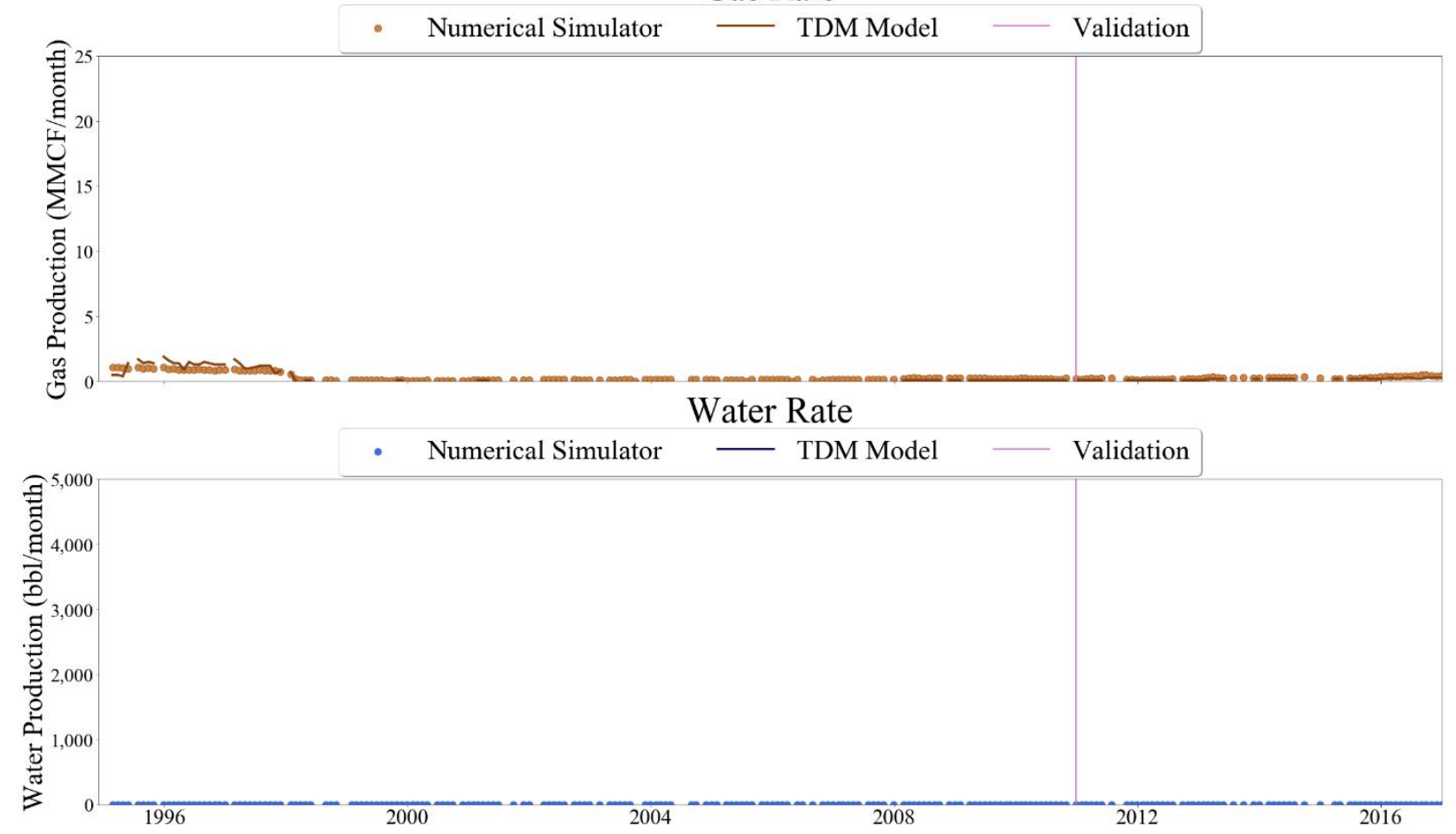

Figure 829 Well-004 oil, gas, and water rate TDM predictions vs actual simulation data plots for 6 Year Blind Validation TDM 


\section{Oil Rate}

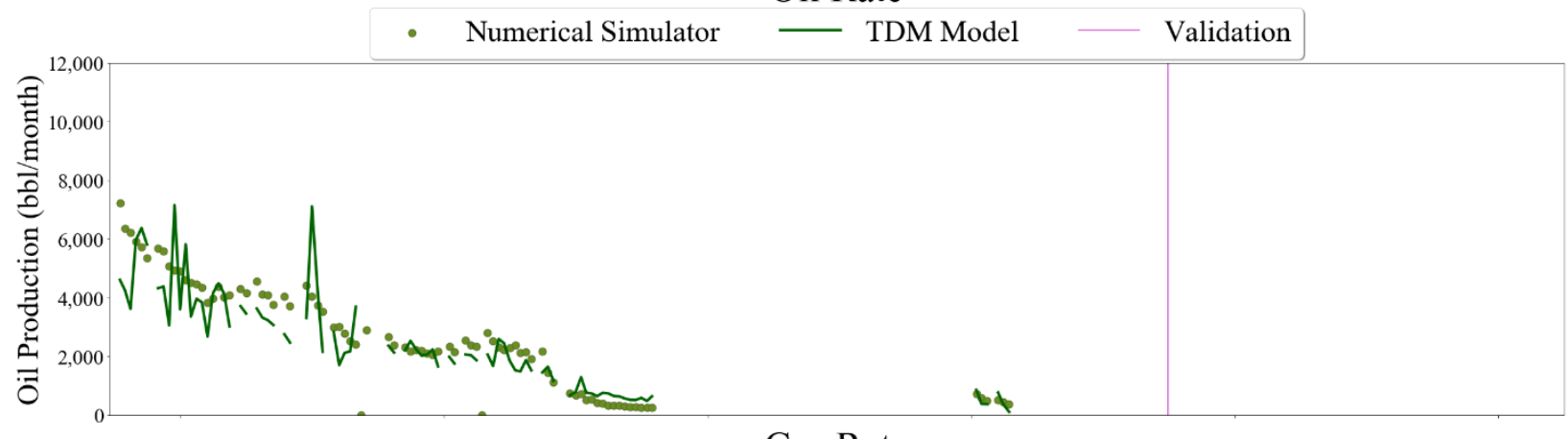

Gas Rate
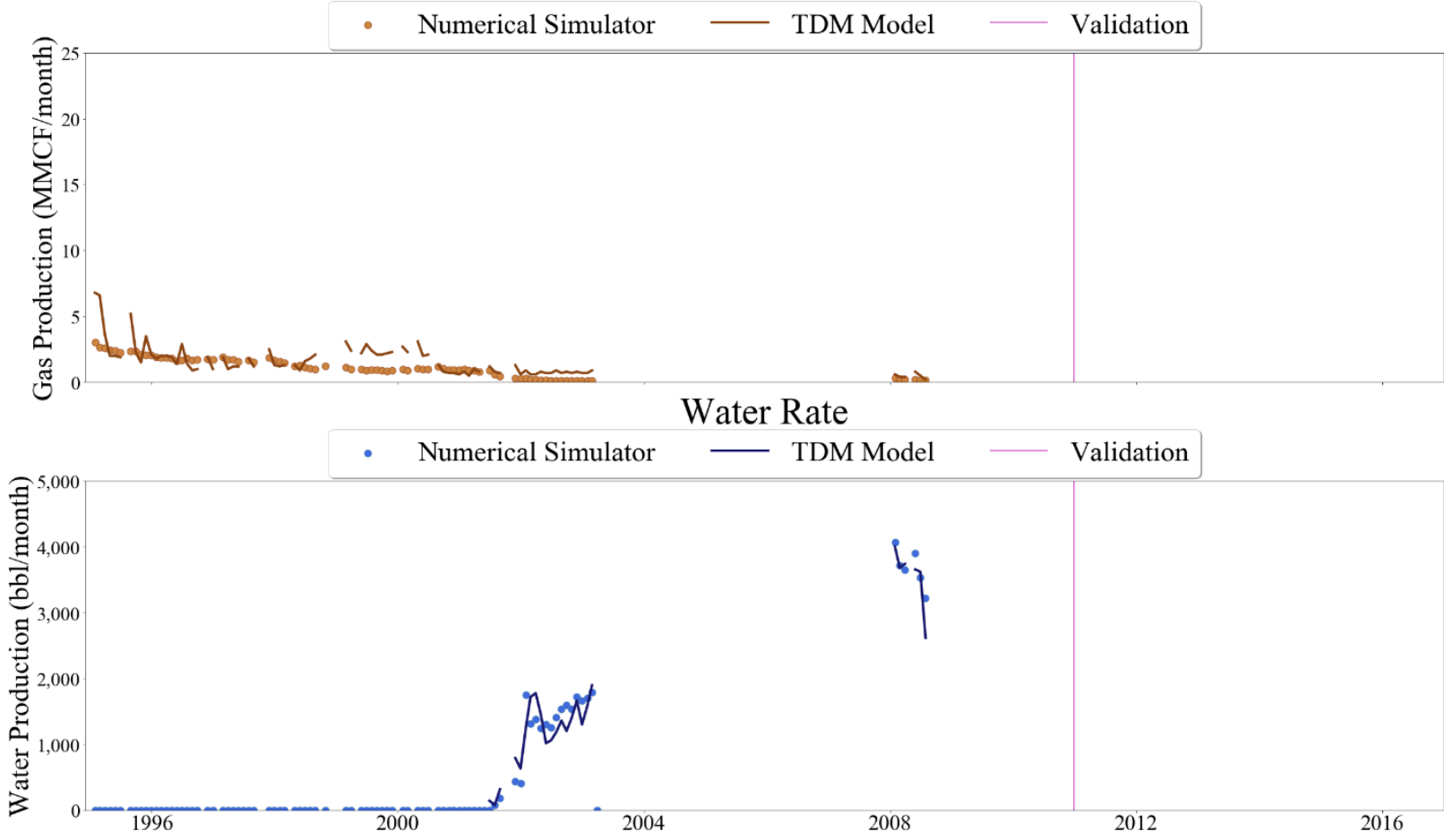

Figure 830 Well-005 oil, gas, and water rate TDM predictions vs actual simulation data plots for 6 Year Blind Validation TDM 

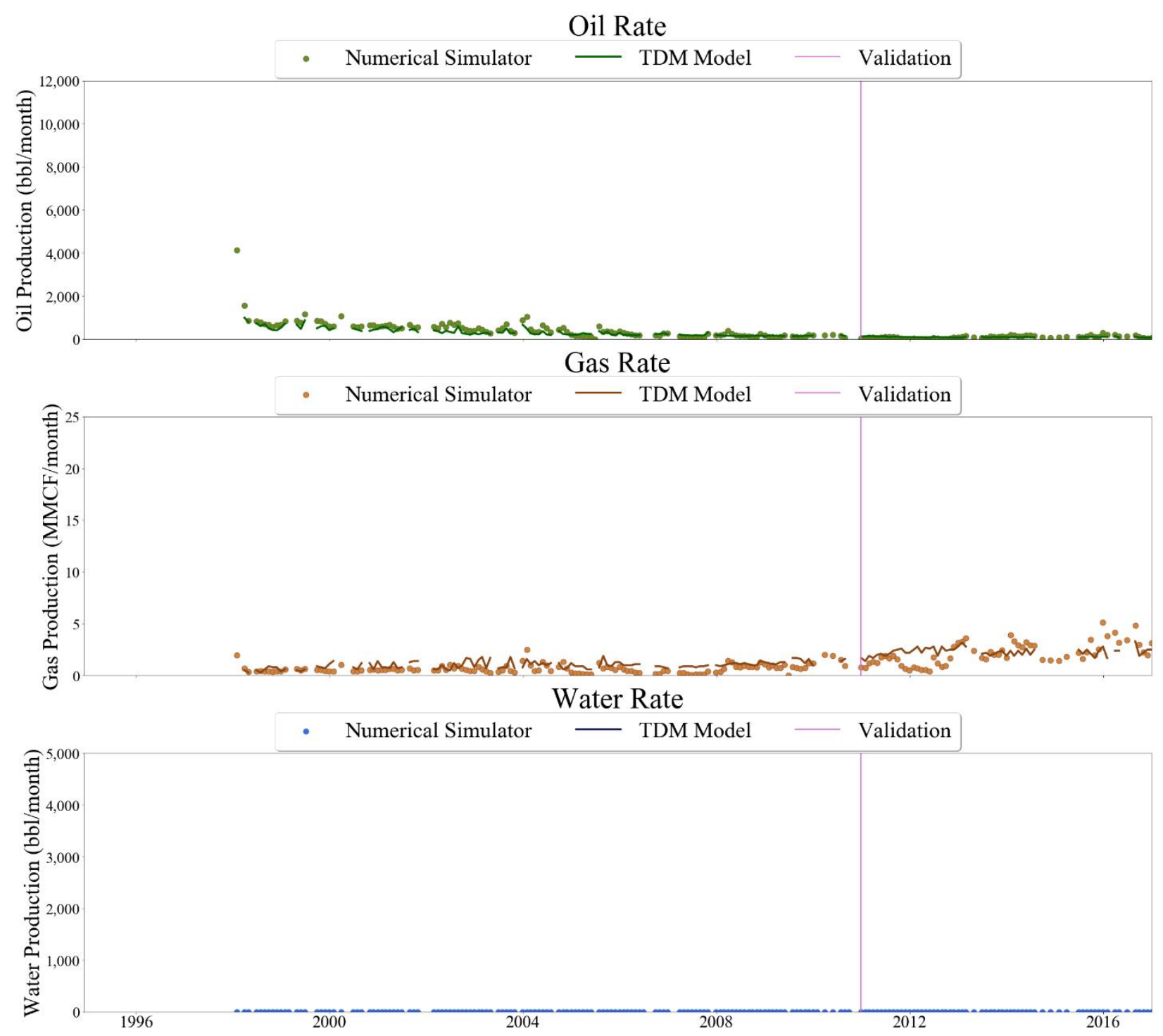

Figure 831 Well-006 oil, gas, and water rate TDM predictions vs actual simulation data plots for 6 Year Blind Validation TDM 

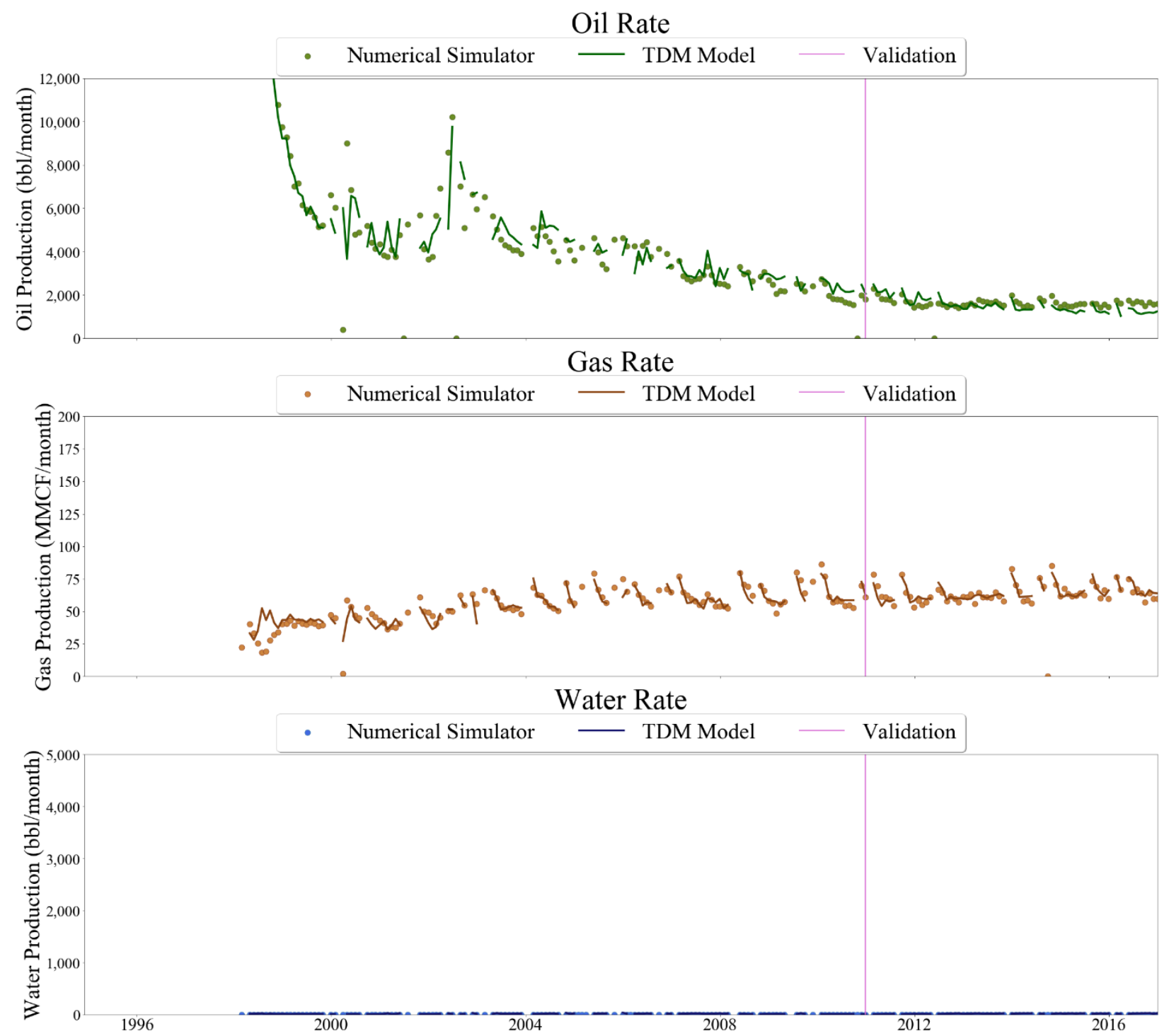

Figure 832 Well-007 oil, gas, and water rate TDM predictions vs actual simulation data plots for 6 Year Blind Validation TDM 


\section{Oil Rate}
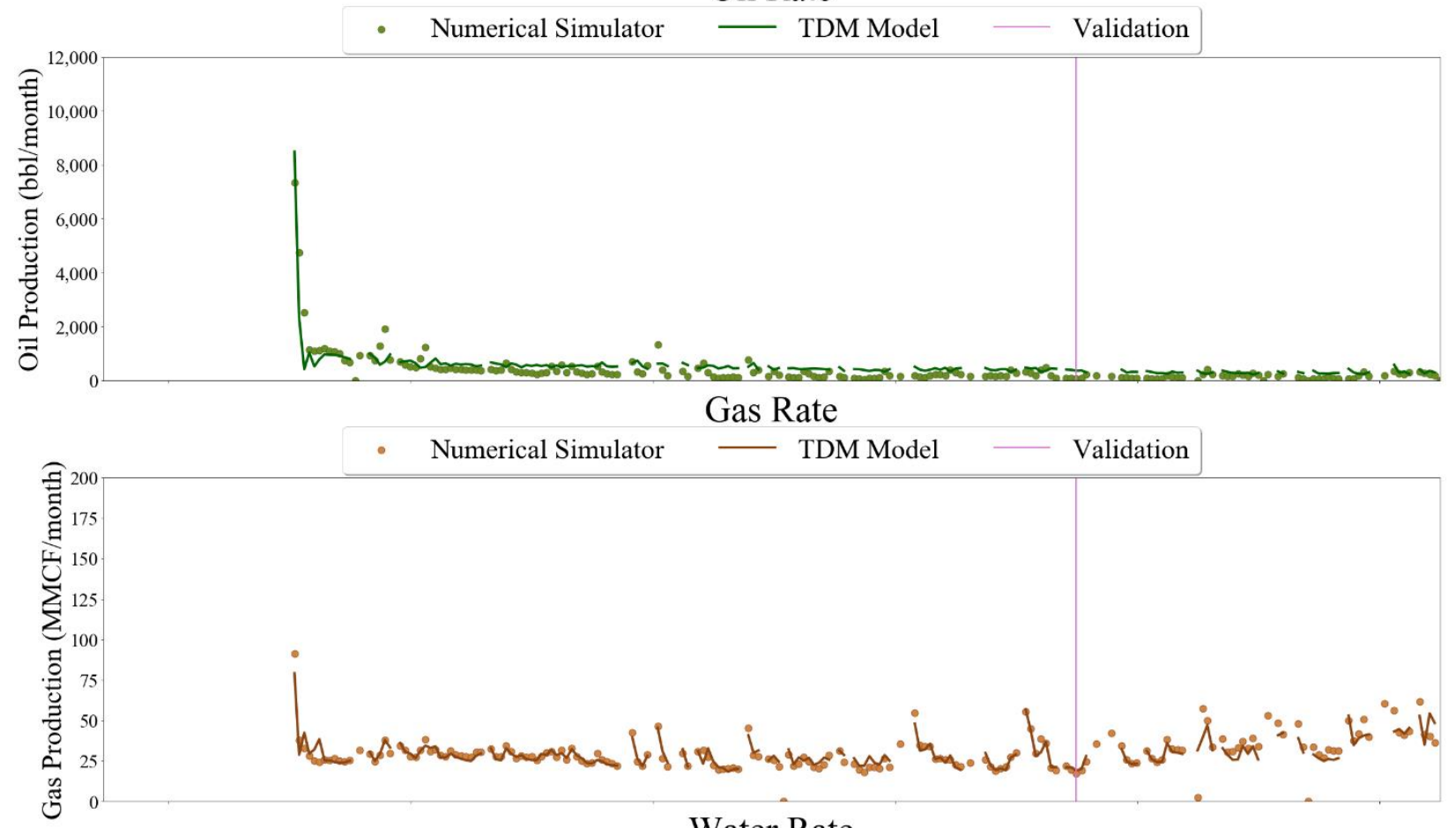

Water Rate

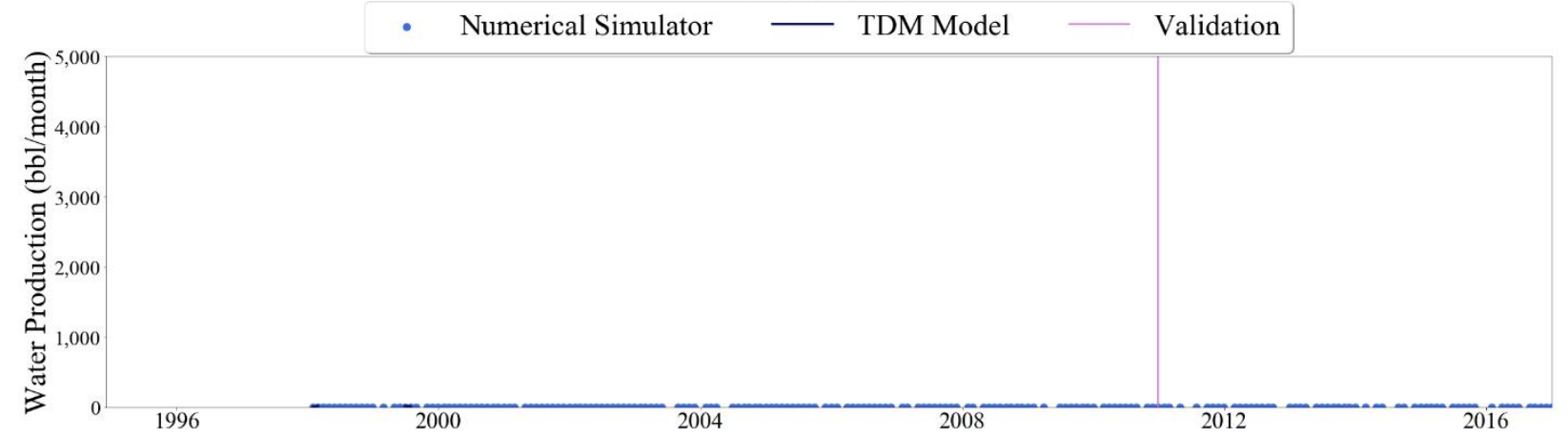

Figure 833 Well-008 oil, gas, and water rate TDM predictions vs actual simulation data plots for 6 Year Blind Validation TDM 

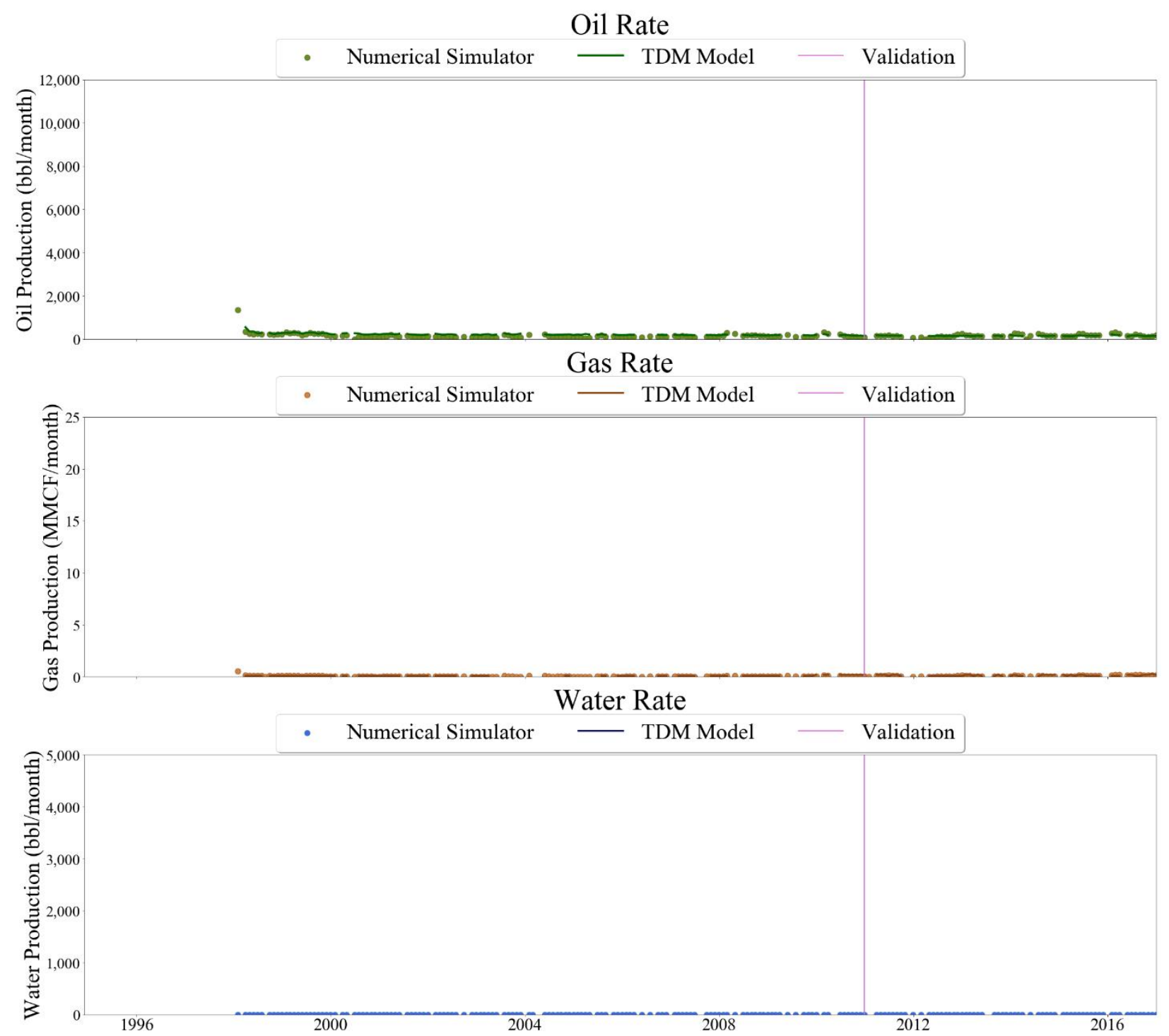

Figure 834 Well-009 oil, gas, and water rate TDM predictions vs actual simulation data plots for 6 Year Blind Validation TDM 


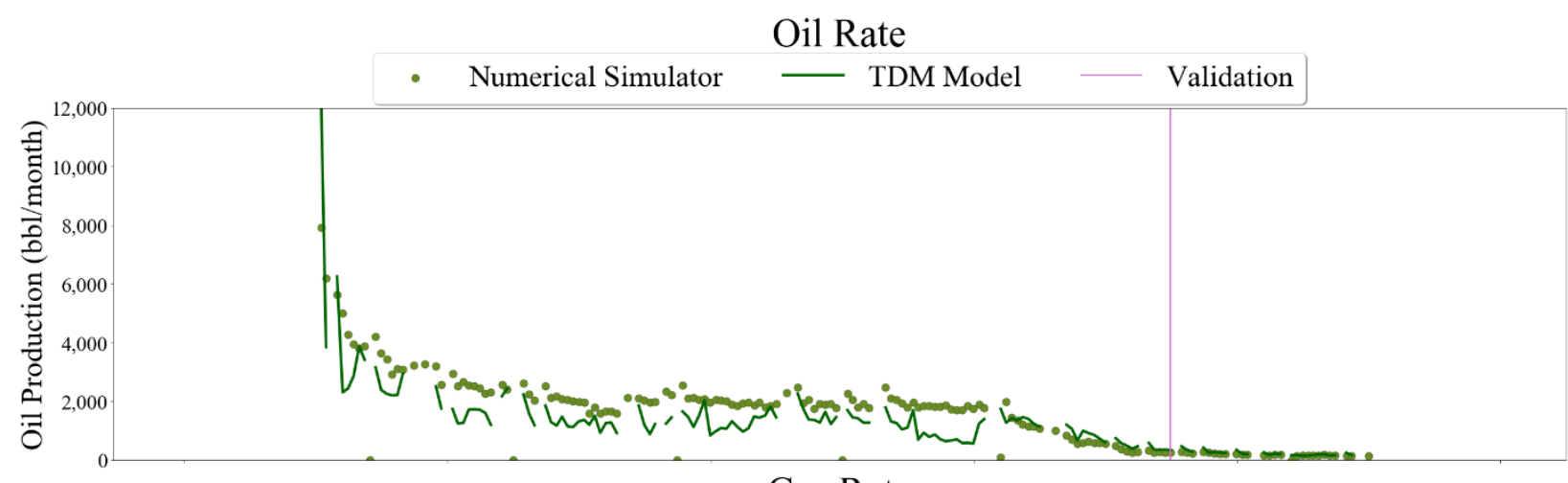

\section{Gas Rate}
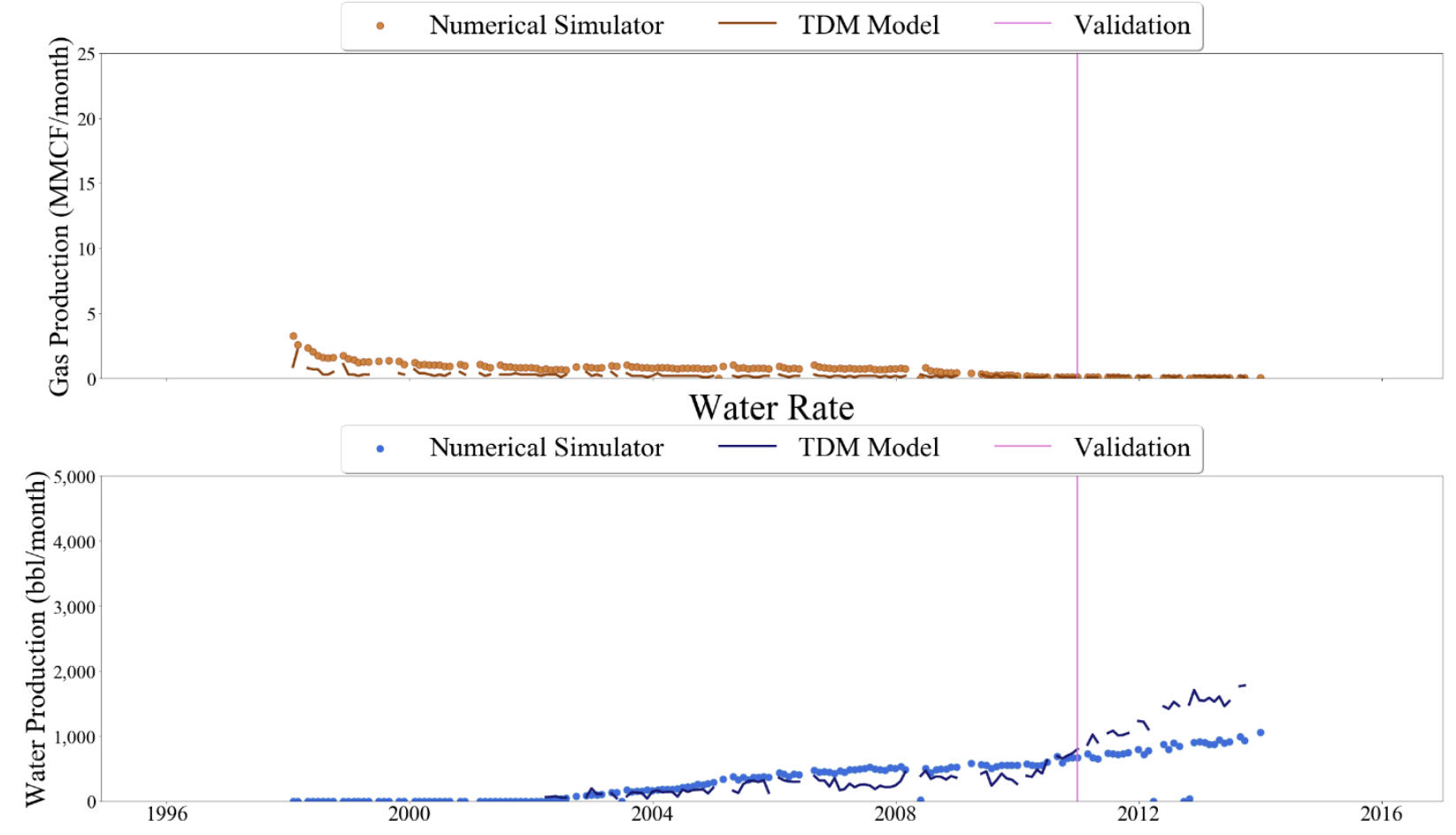

Figure 835 Well-010 oil, gas, and water rate TDM predictions vs actual simulation data plots for 6 Year Blind Validation TDM 


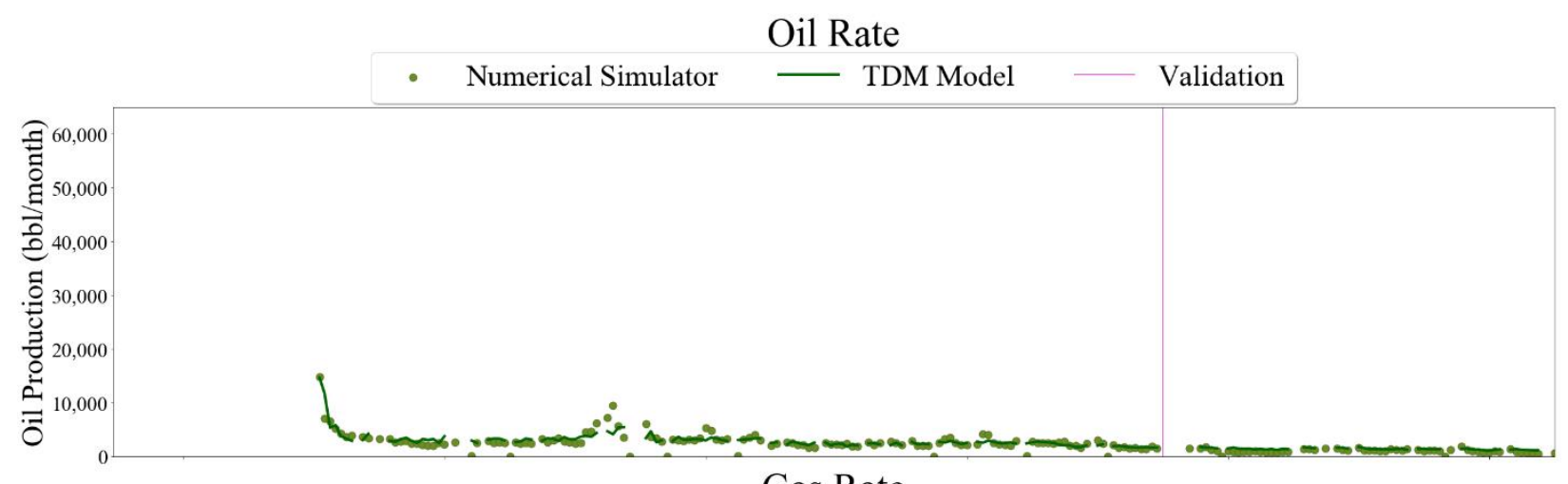

Gas Rate

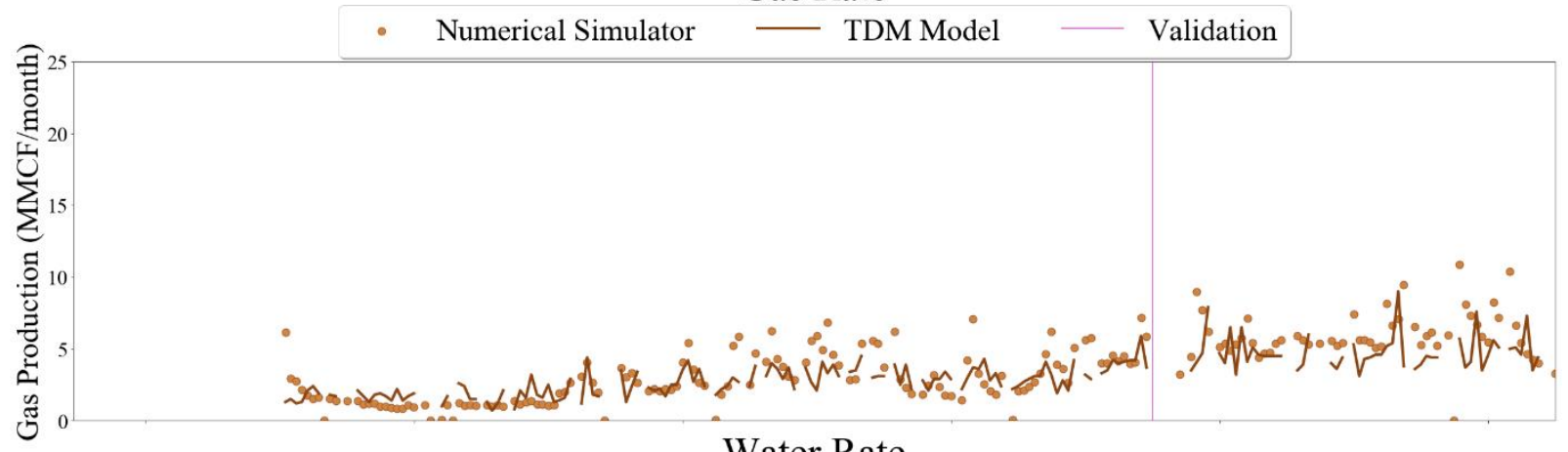

Water Rate

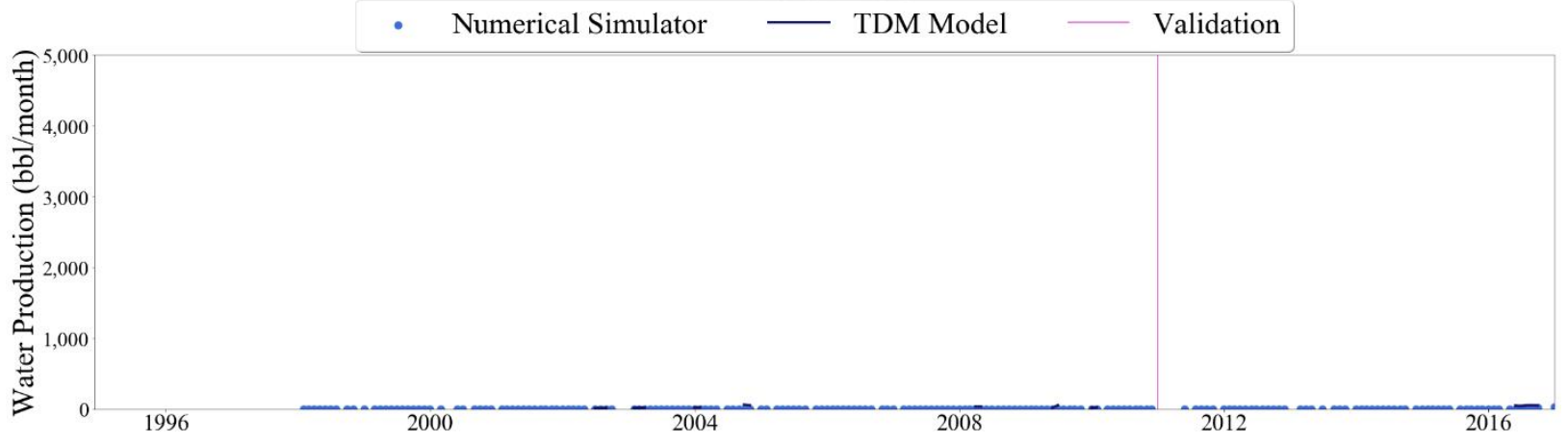

Figure 836 Well-011 oil, gas, and water rate TDM predictions vs actual simulation data plots for 6 Year Blind Validation TDM 

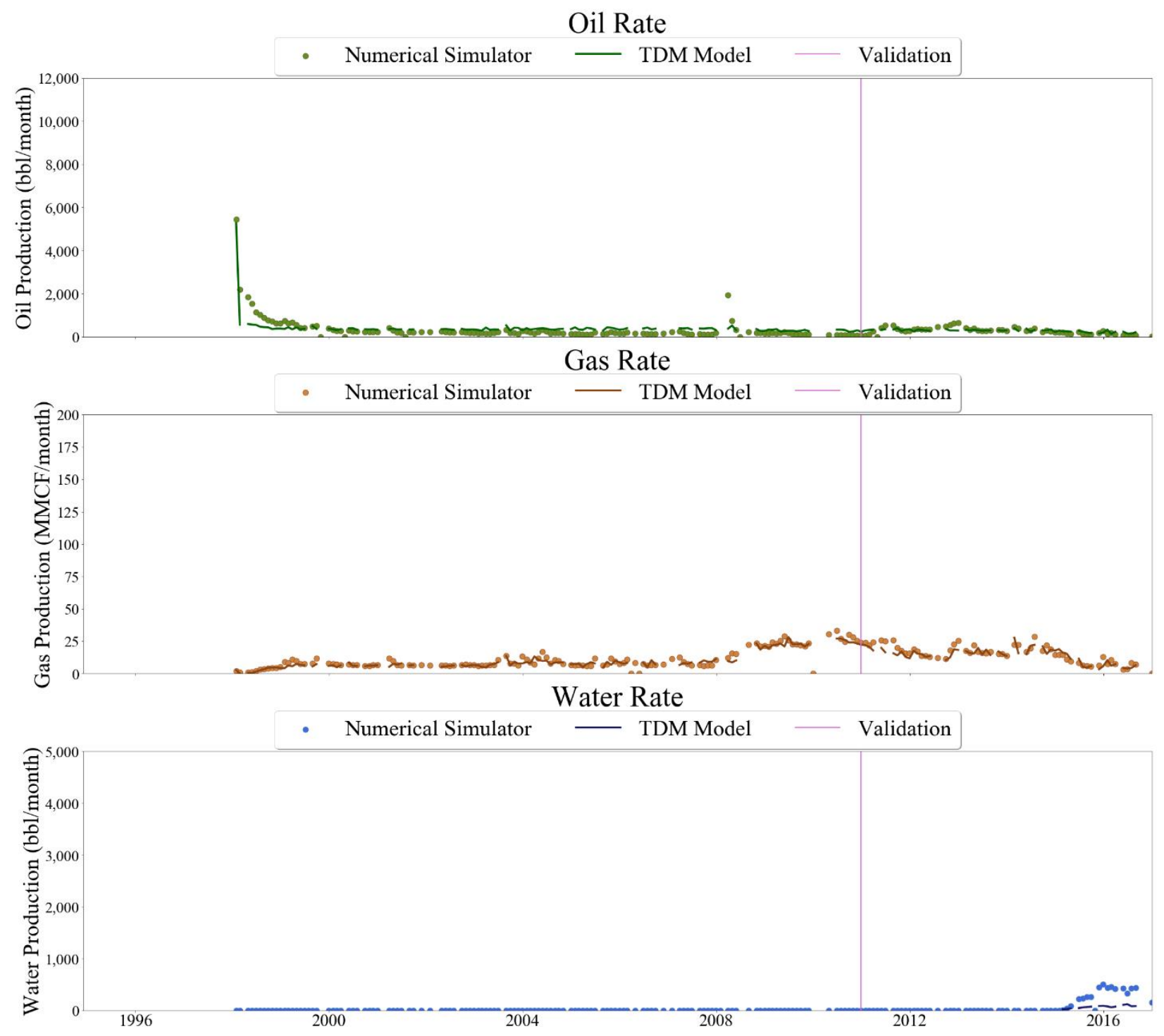

Figure 837 Well-012 oil, gas, and water rate TDM predictions vs actual simulation data plots for 6 Year Blind Validation TDM 

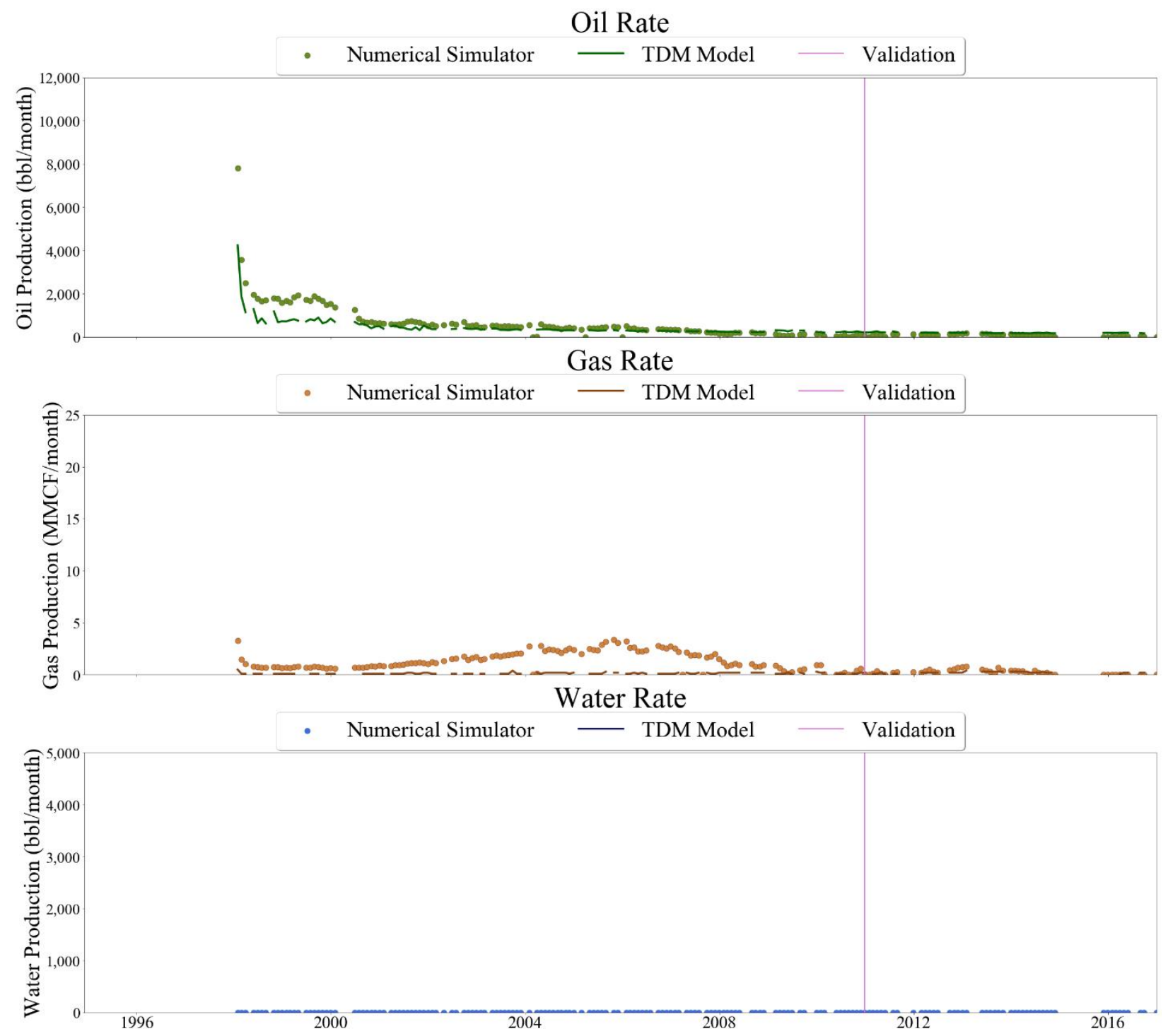

Figure 838 Well-013 oil, gas, and water rate TDM predictions vs actual simulation data plots for 6 Year Blind Validation TDM 


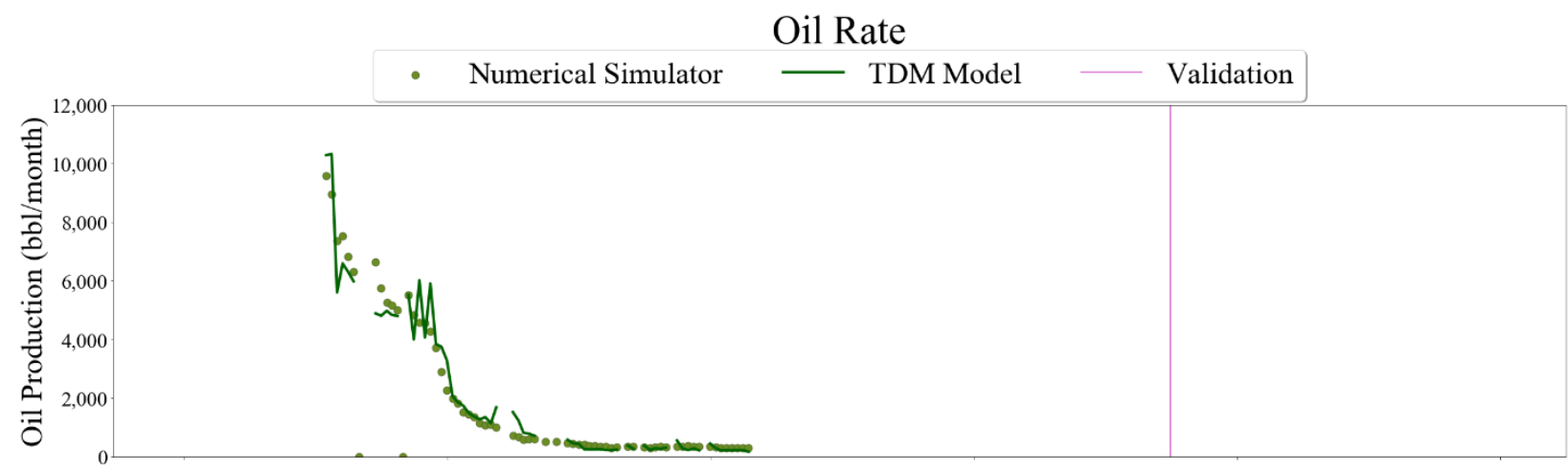

\section{Gas Rate}
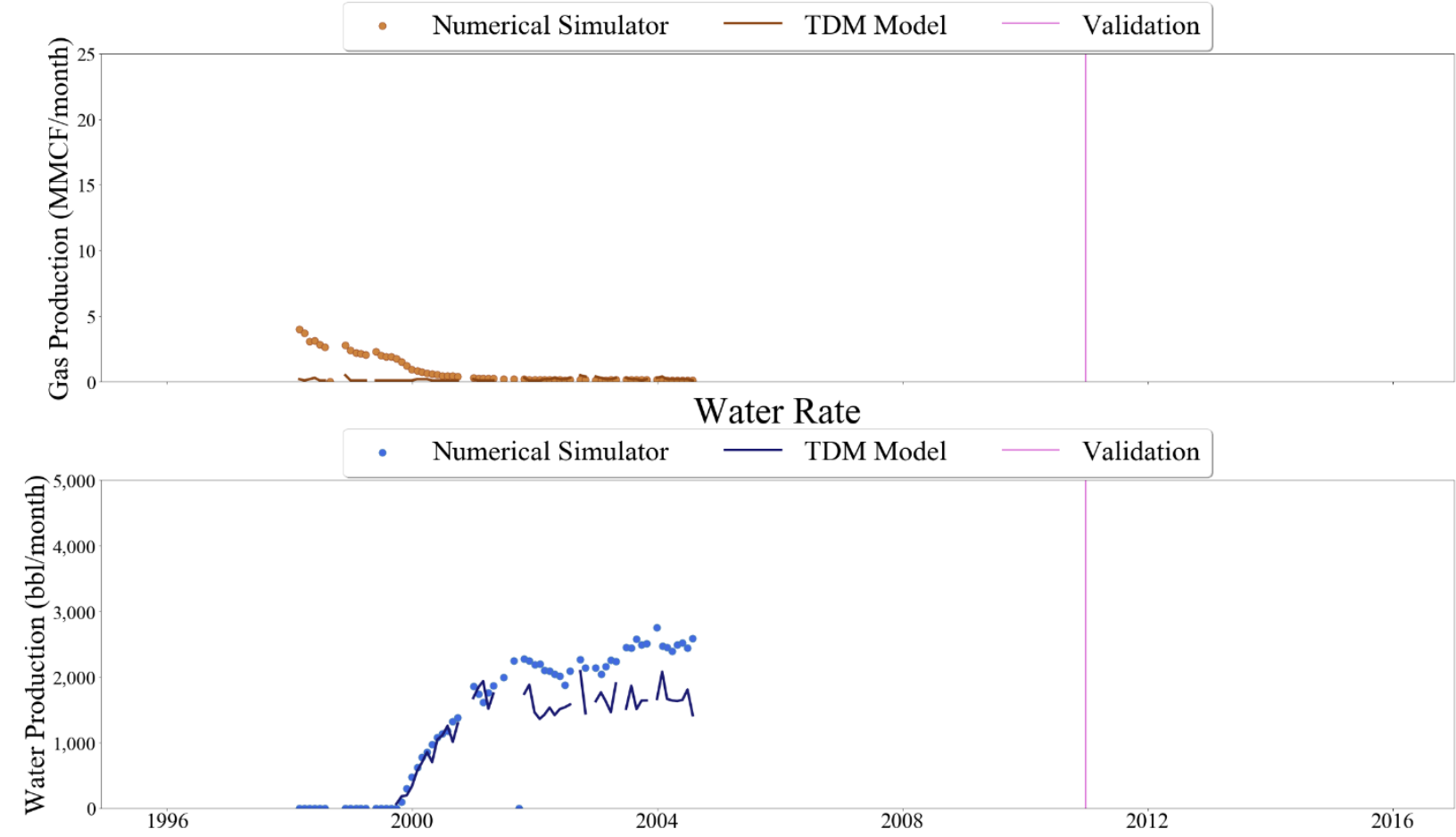

Figure 839 Well-014 oil, gas, and water rate TDM predictions vs actual simulation data plots for 6 Year Blind Validation TDM 

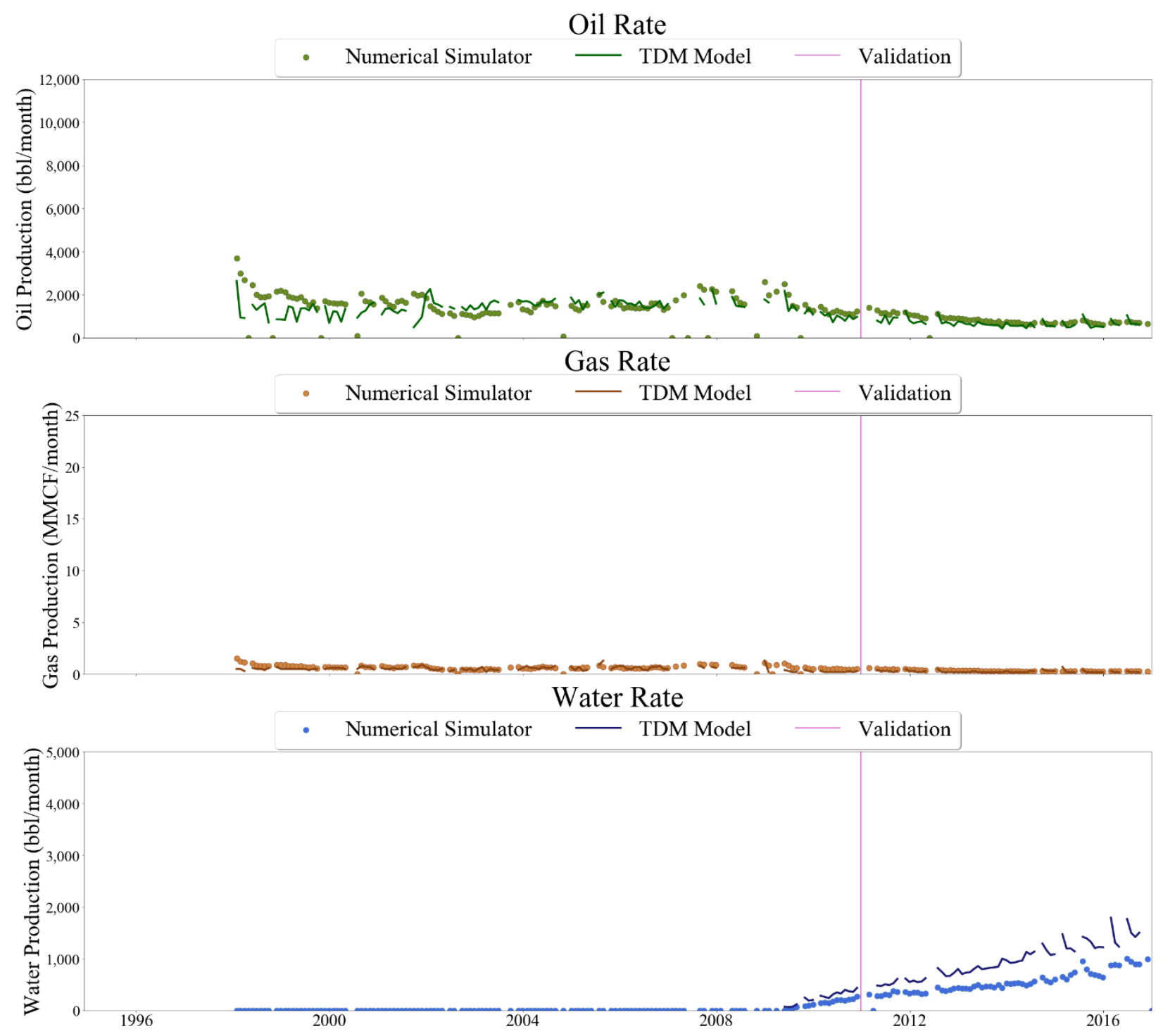

Figure 840 Well-015 oil, gas, and water rate TDM predictions vs actual simulation data plots for 6 Year Blind Validation TDM 

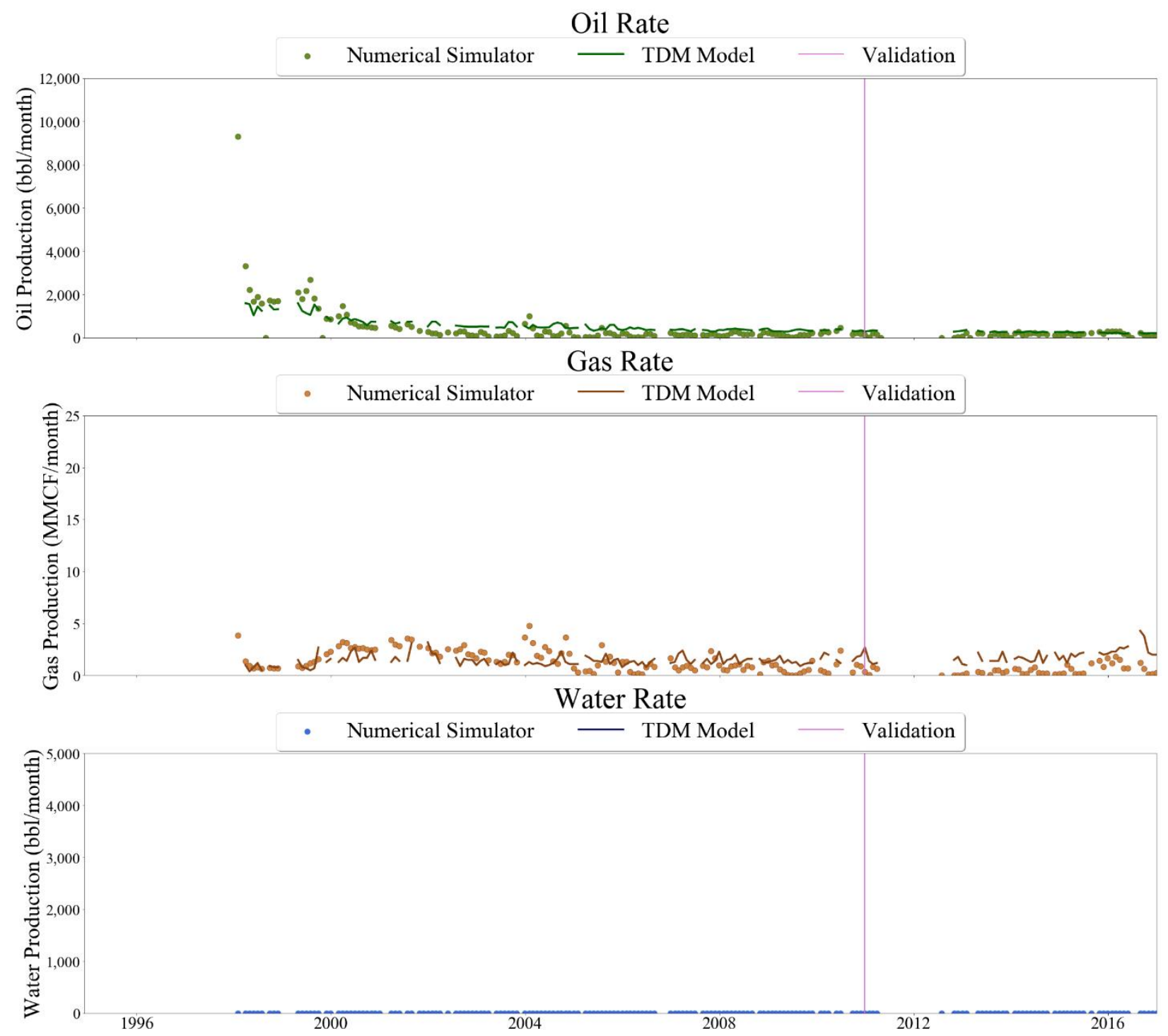

Figure 841 Well-016 oil, gas, and water rate TDM predictions vs actual simulation data plots for 6 Year Blind Validation TDM 

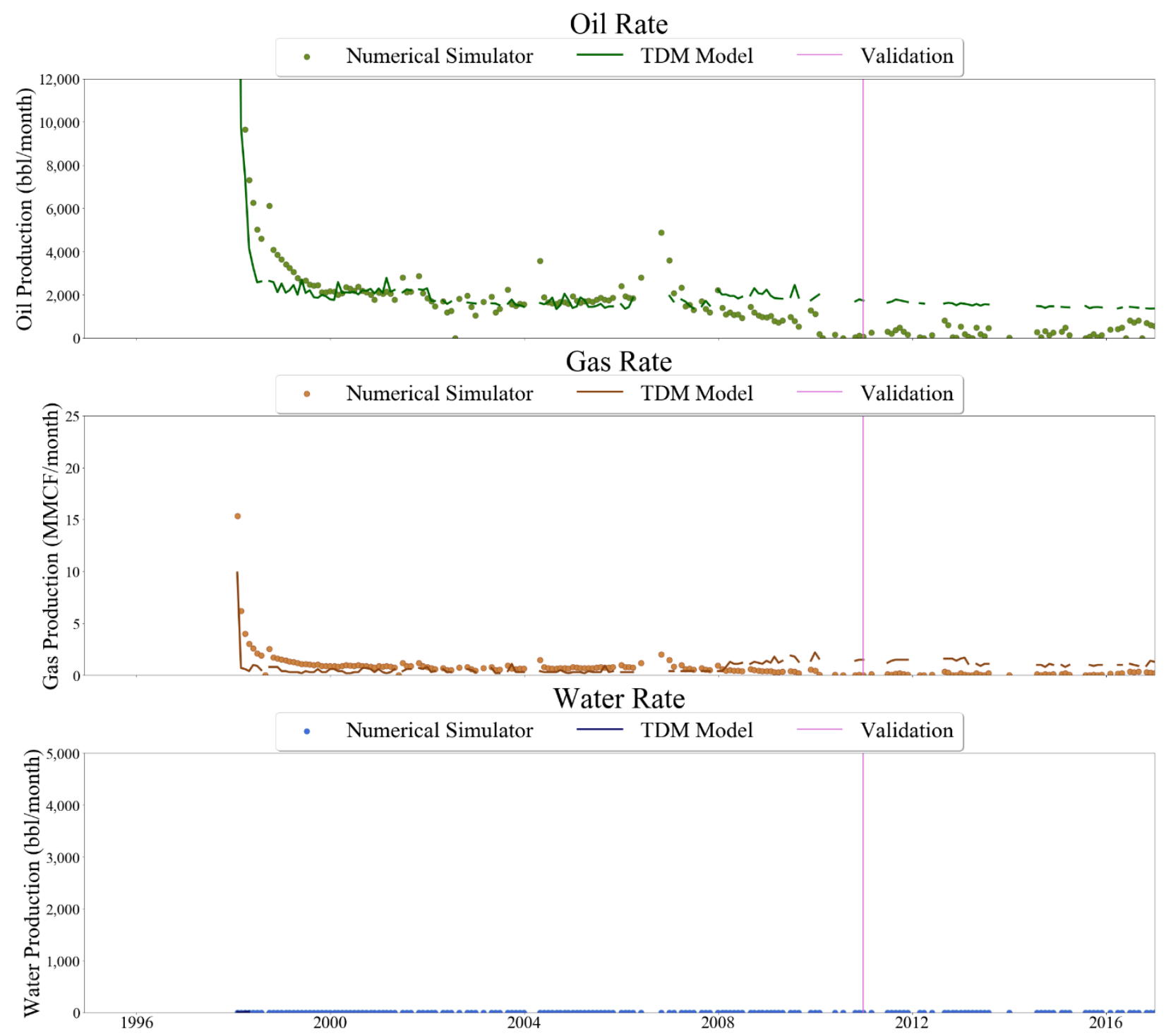

Figure 842 Well-017 oil, gas, and water rate TDM predictions vs actual simulation data plots for 6 Year Blind Validation TDM 

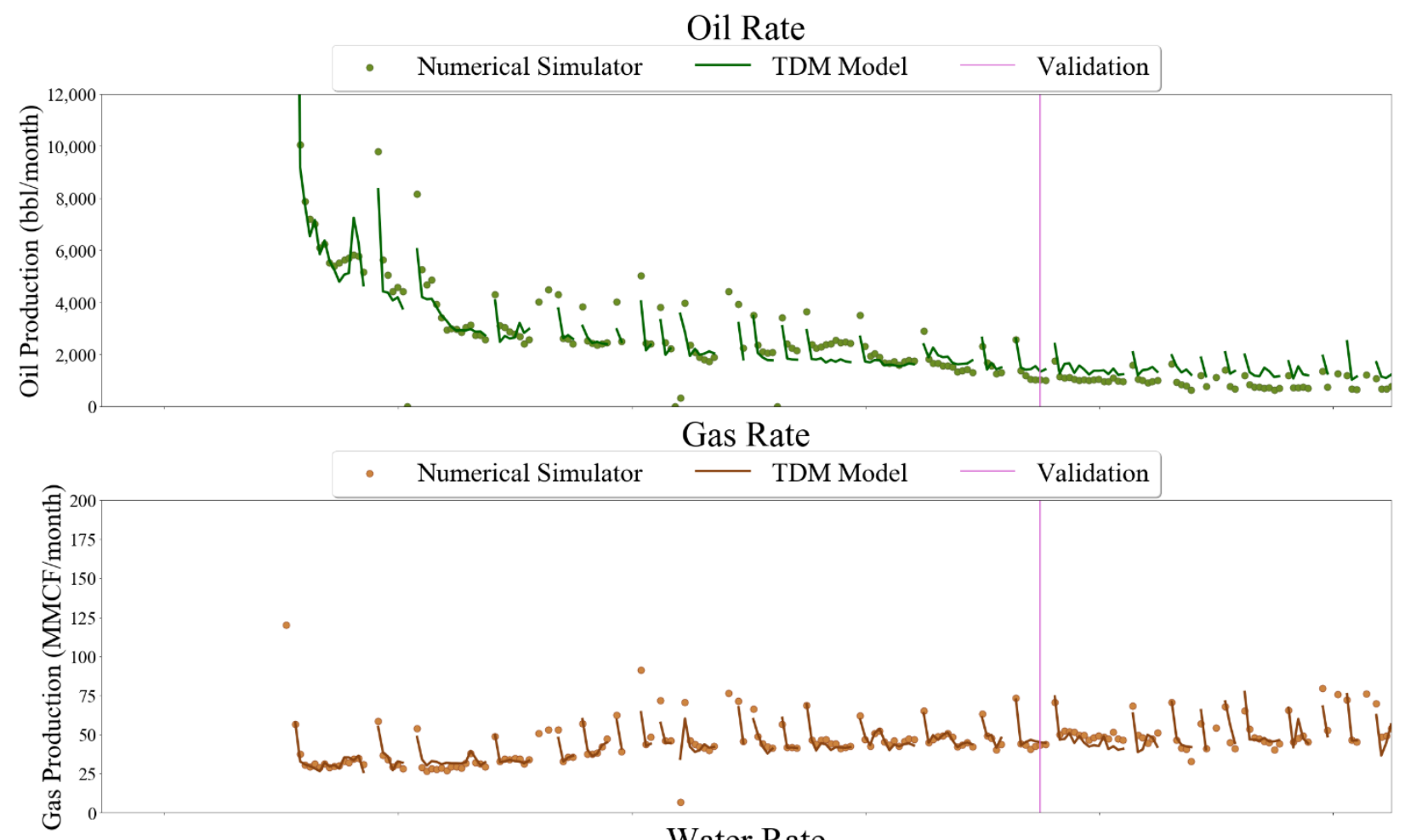

Water Rate

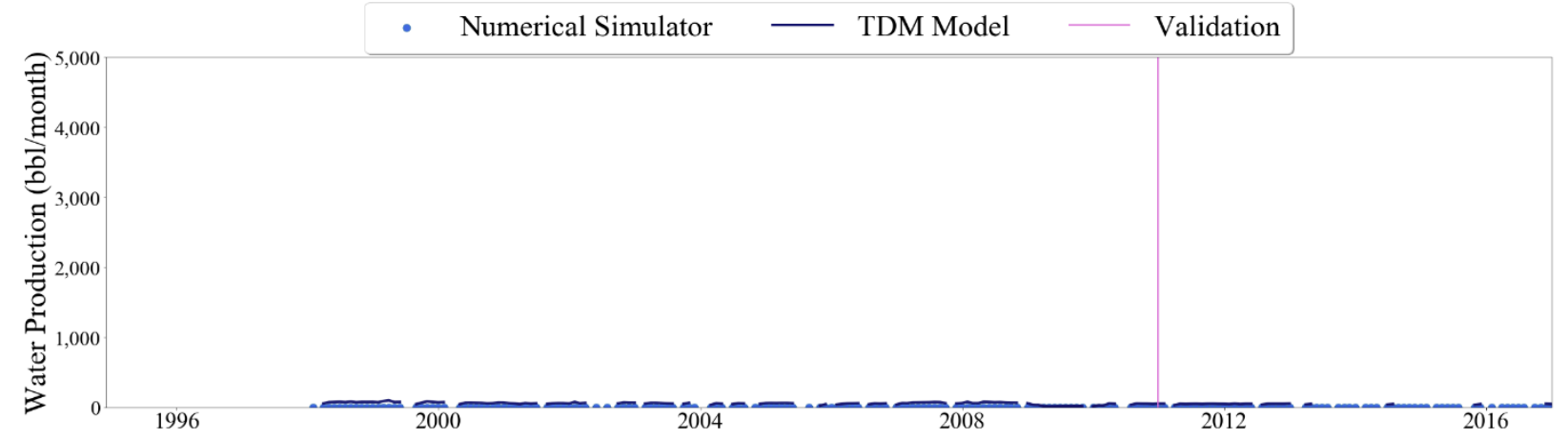

Figure 843 Well-018 oil, gas, and water rate TDM predictions vs actual simulation data plots for 6 Year Blind Validation TDM 

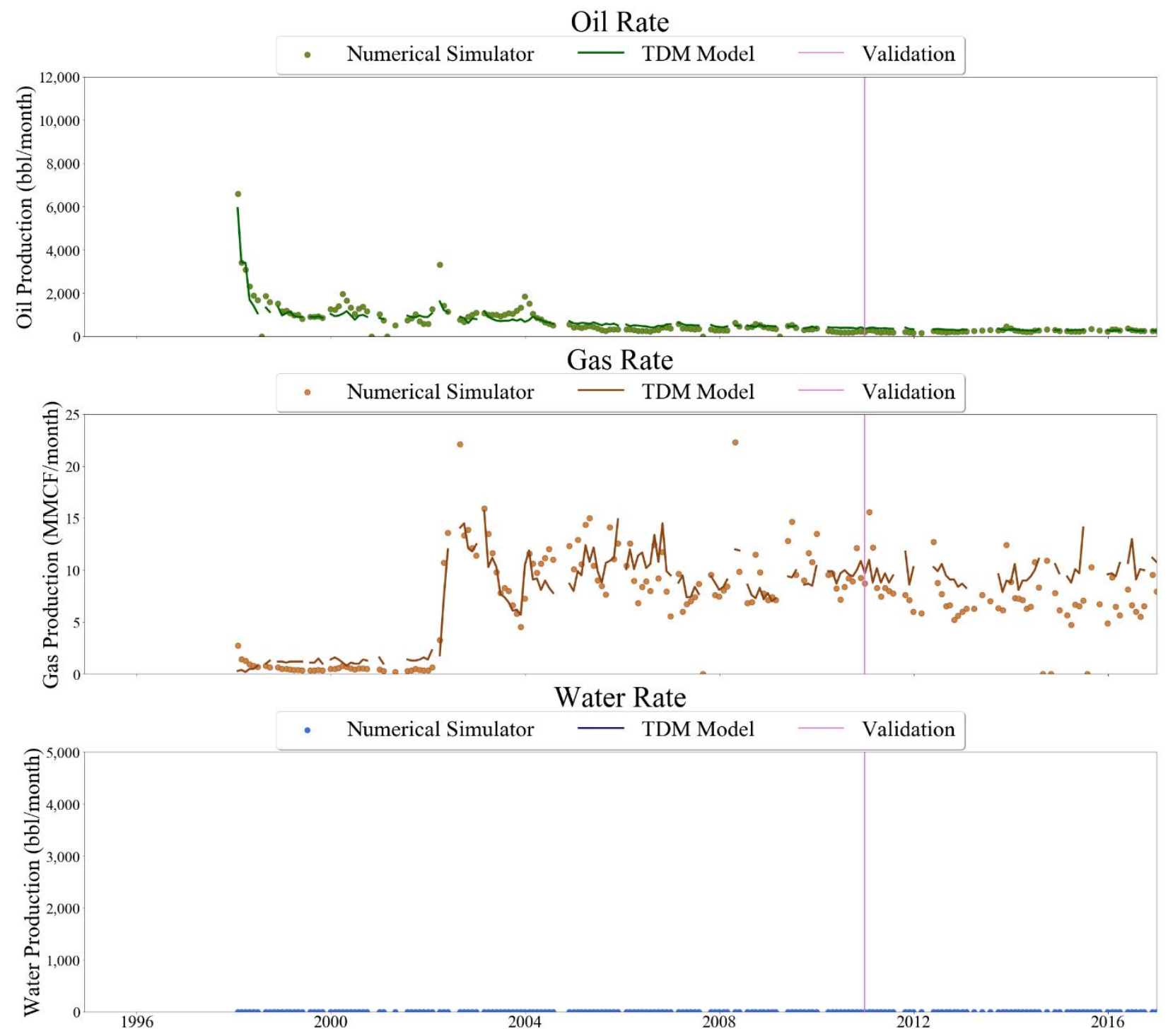

Figure 844 Well-019 oil, gas, and water rate TDM predictions vs actual simulation data plots for 6 Year Blind Validation TDM 

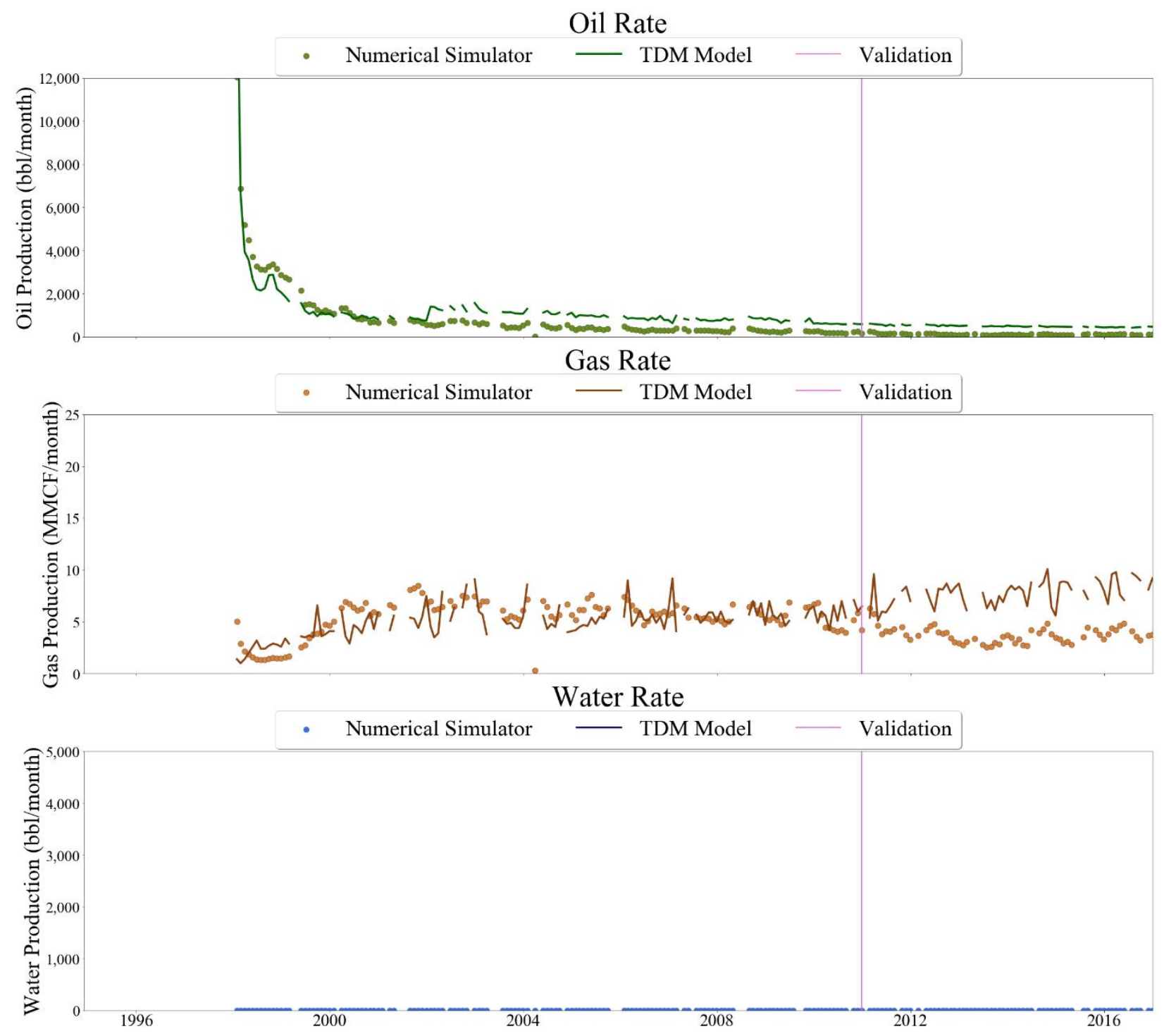

Figure 845 Well-020 oil, gas, and water rate TDM predictions vs actual simulation data plots for 6 Year Blind Validation TDM 

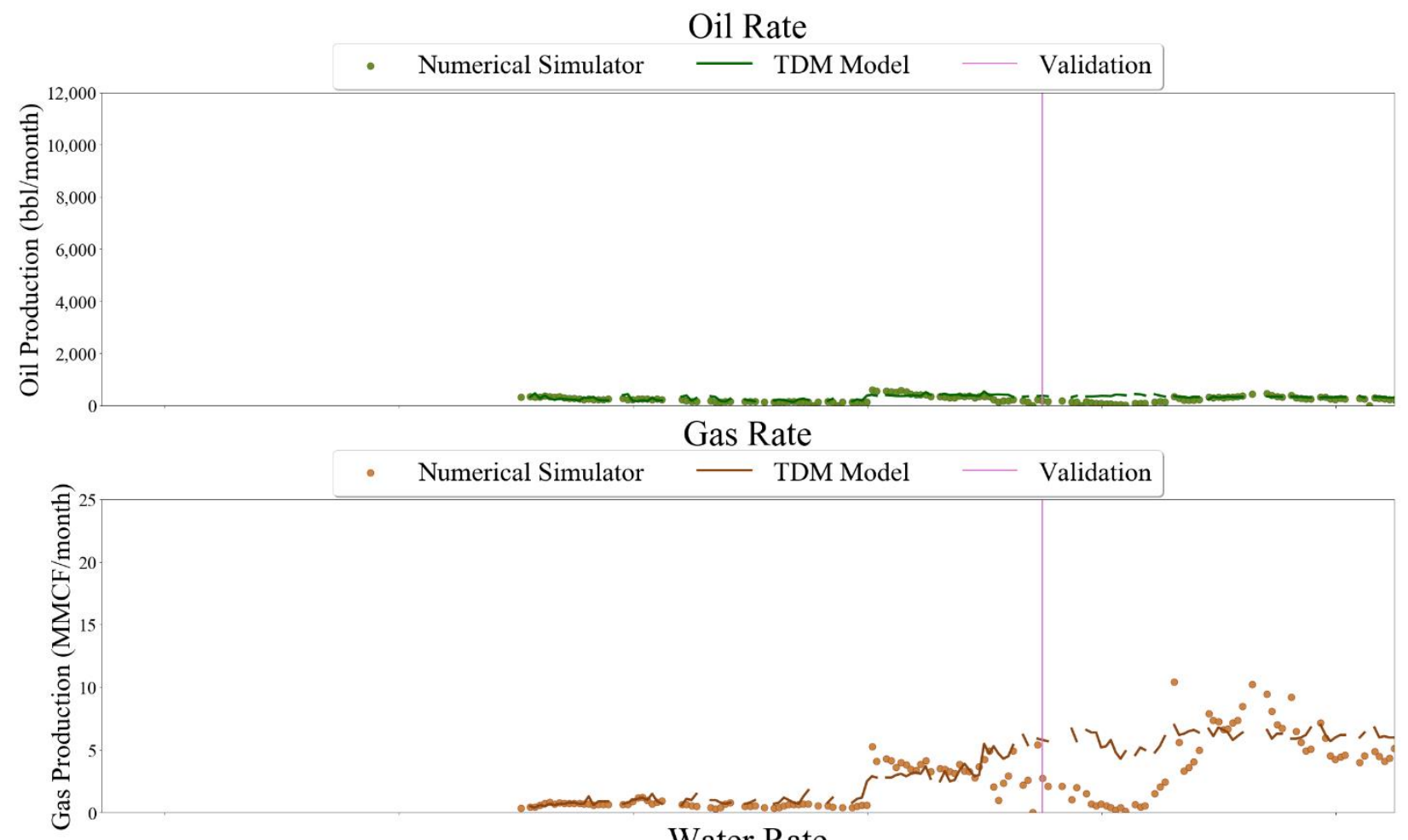

Water Rate

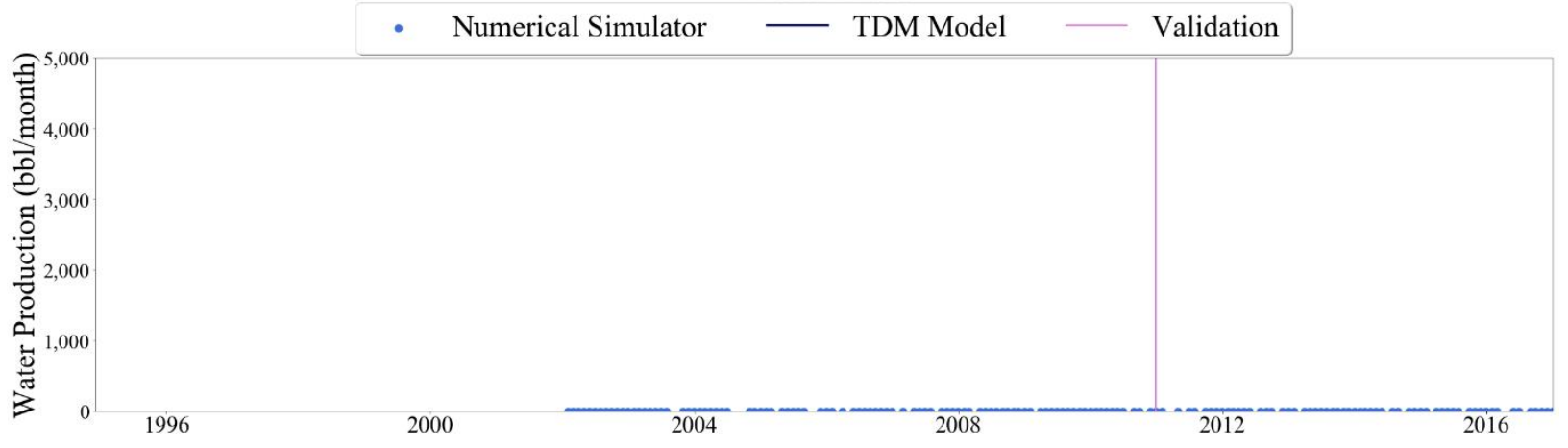

Figure 846 Well-021 oil, gas, and water rate TDM predictions vs actual simulation data plots for 6 Year Blind Validation TDM 

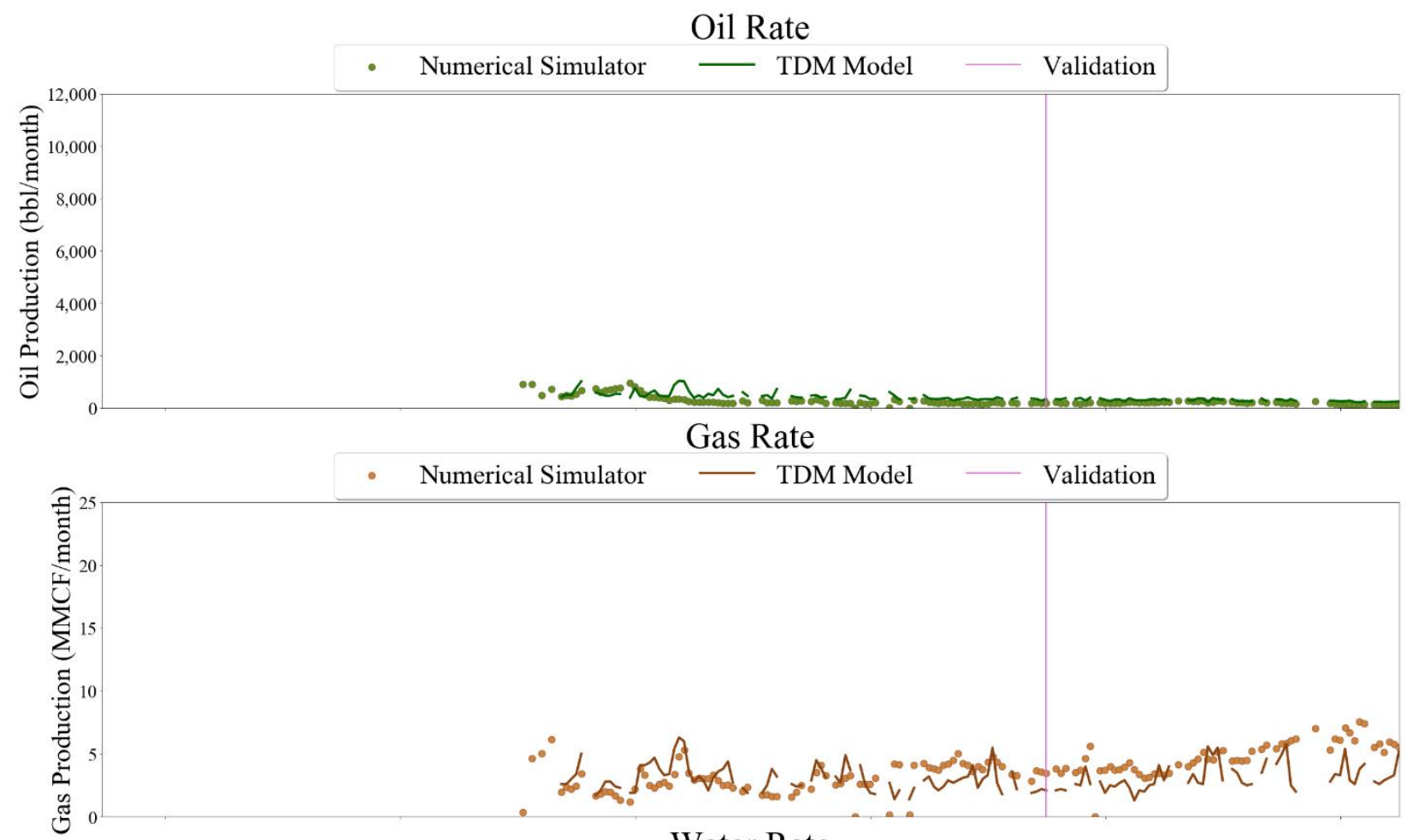

\section{Water Rate}

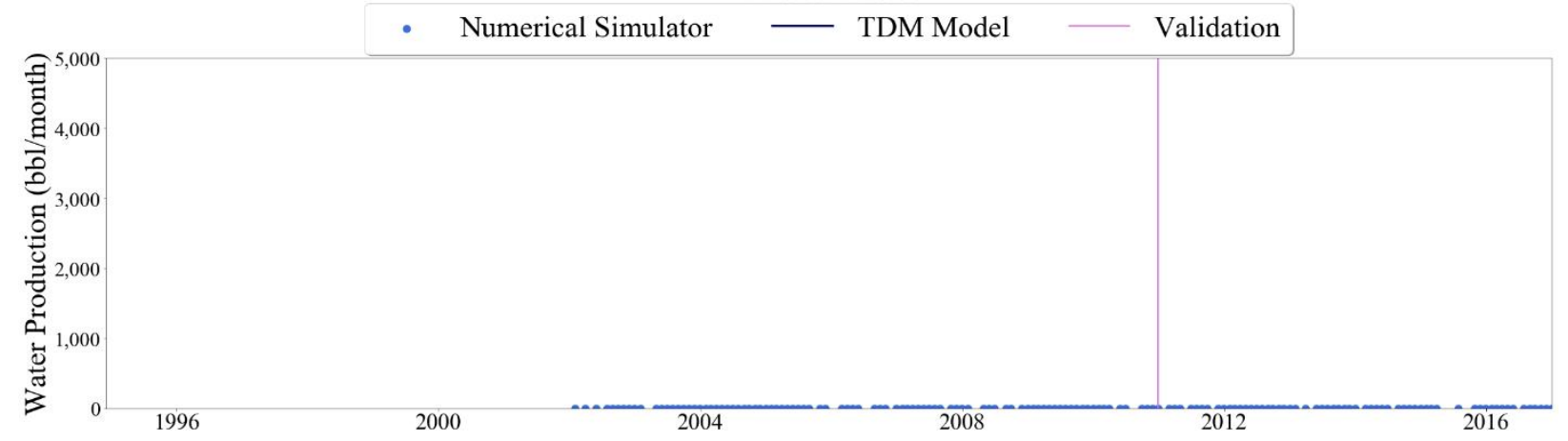

Figure 847 Well-002 oil, gas, and water rate TDM predictions vs actual simulation data plots for 6 Year Blind Validation TDM 

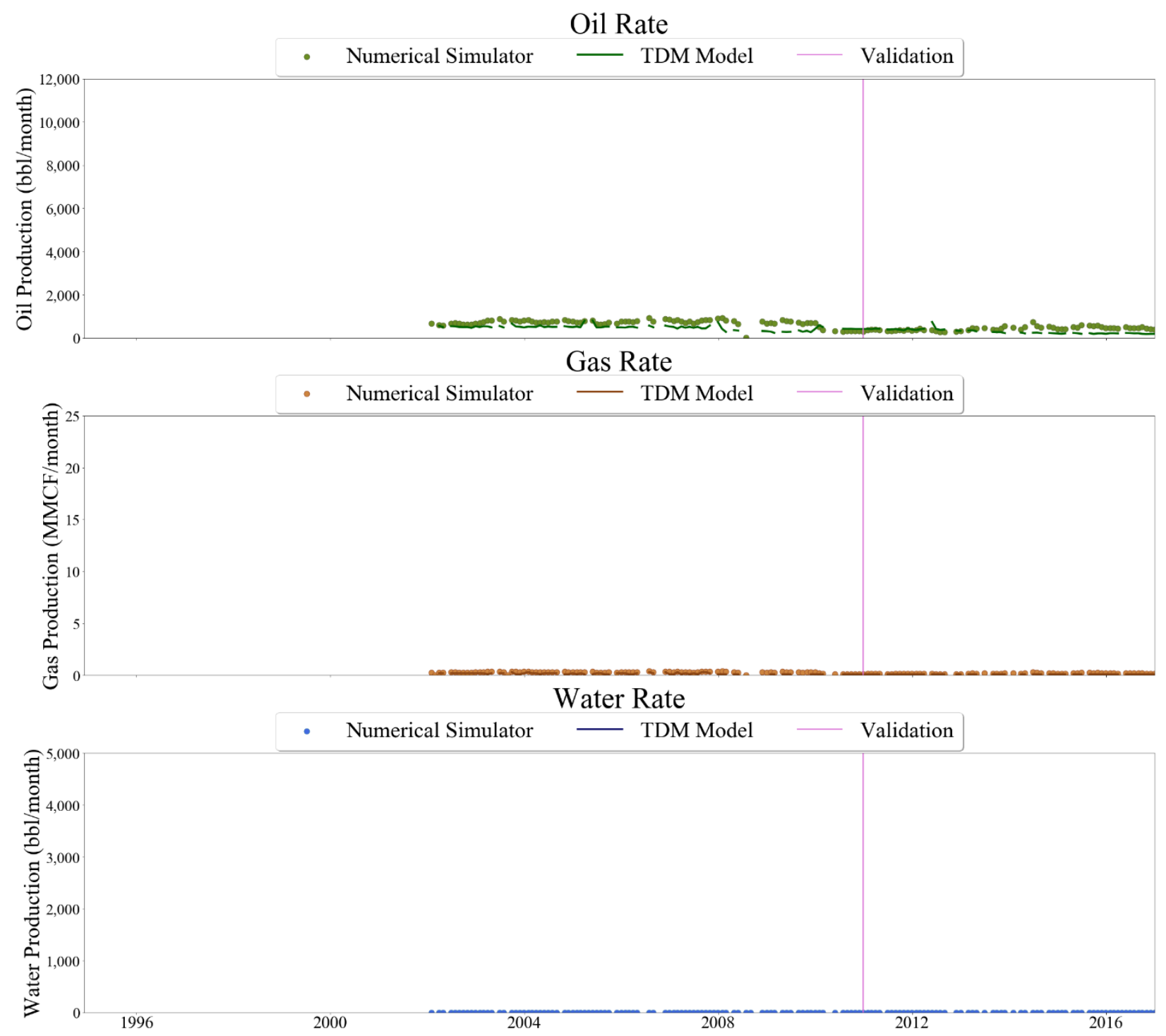

Figure 848 Well-023 oil, gas, and water rate TDM predictions vs actual simulation data plots for 6 Year Blind Validation TDM 

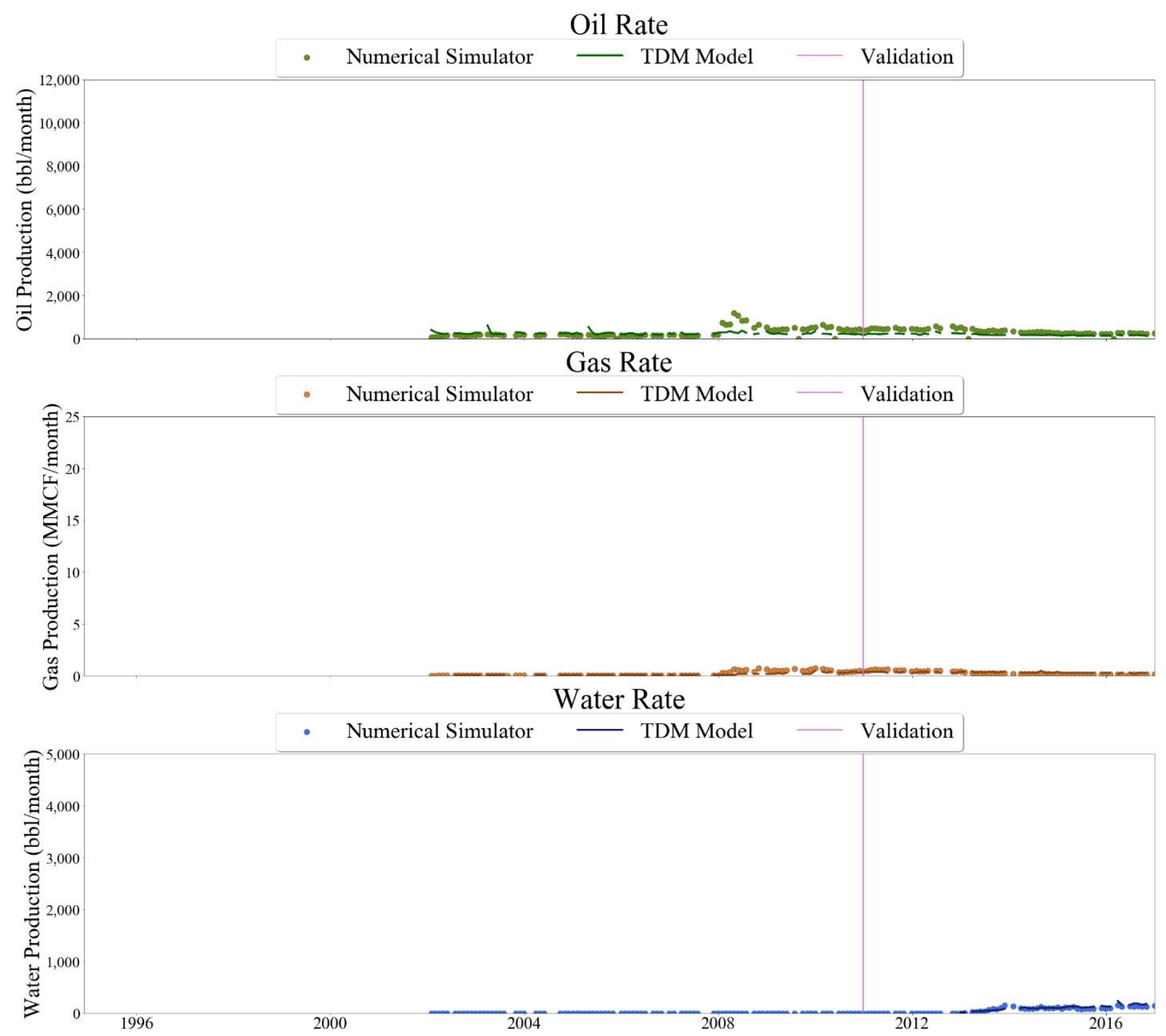

Figure 849 Well-024 oil, gas, and water rate TDM predictions vs actual simulation data plots for 6 Year Blind Validation TDM 


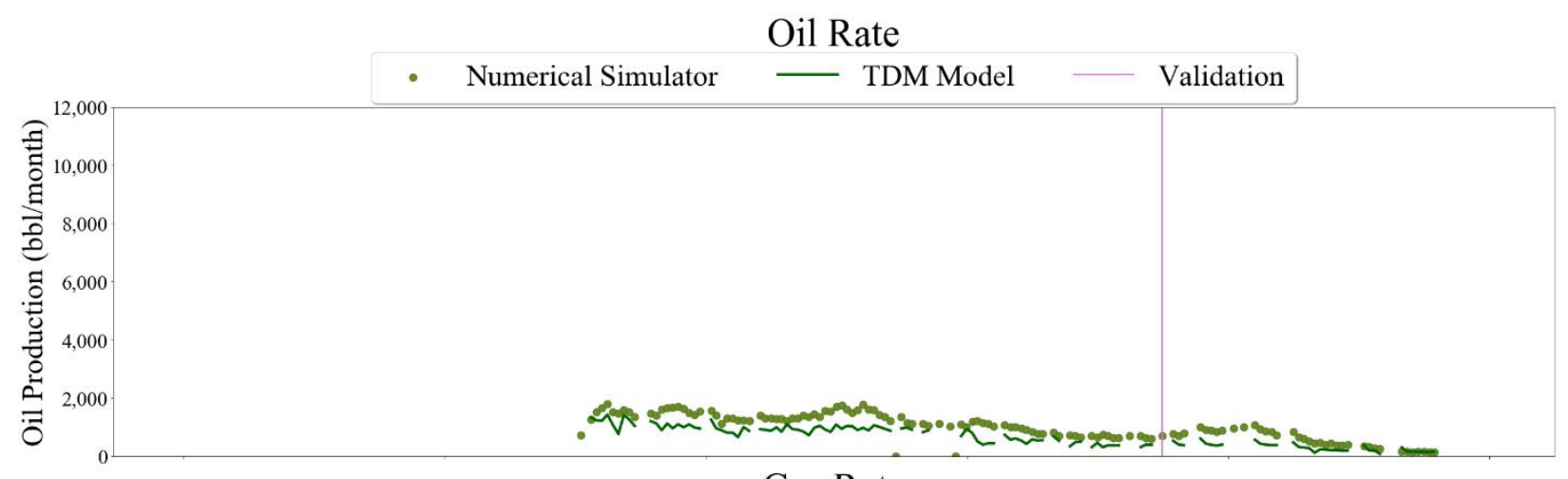

\section{Gas Rate}
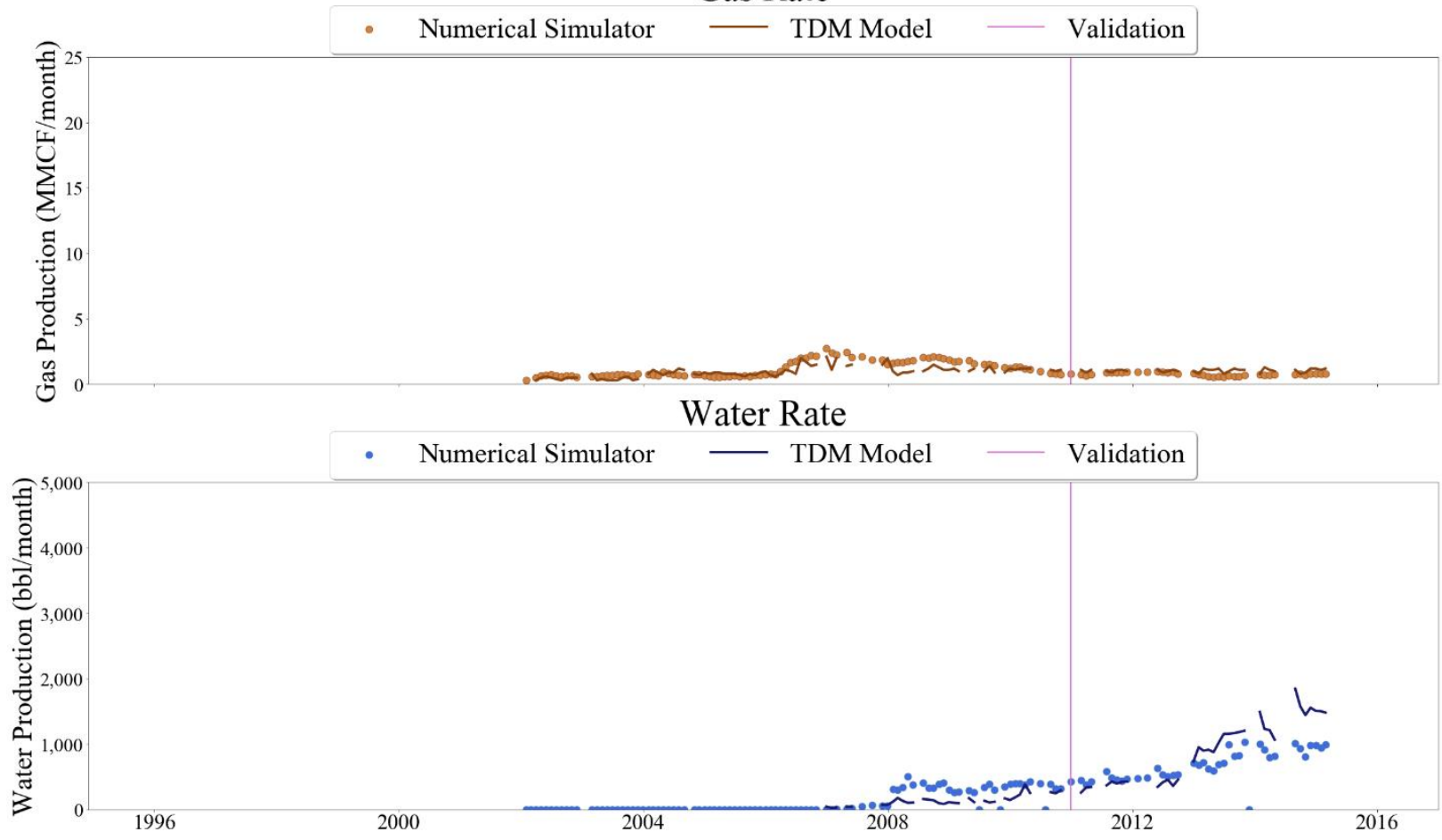

Figure 850 Well-025 oil, gas, and water rate TDM predictions vs actual simulation data plots for 6 Year Blind Validation TDM 


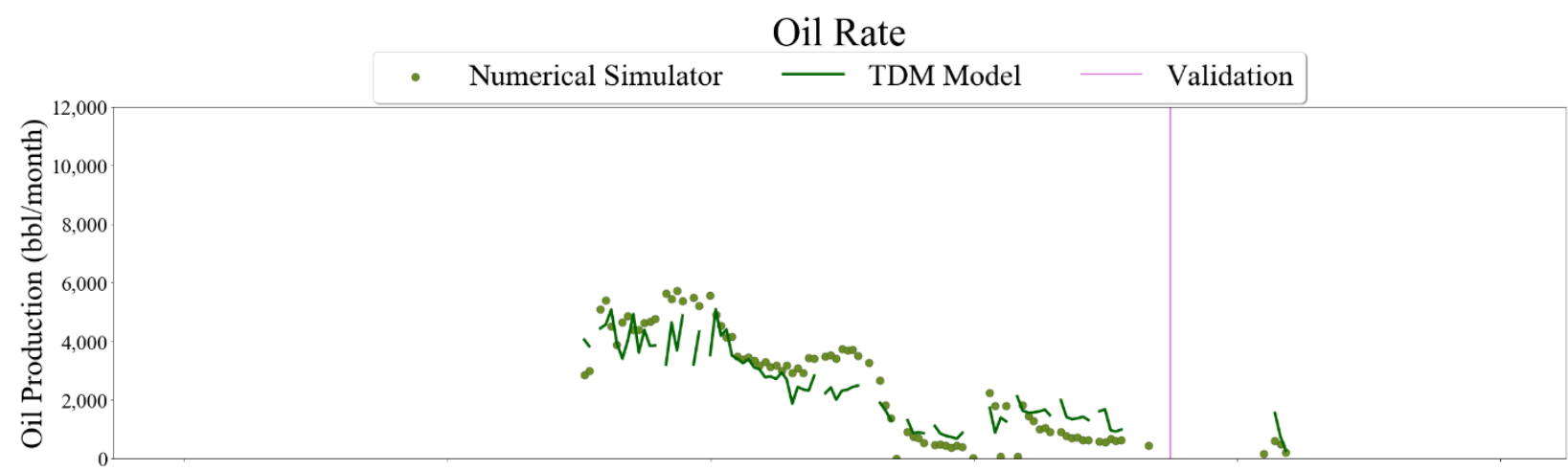

\section{Gas Rate}
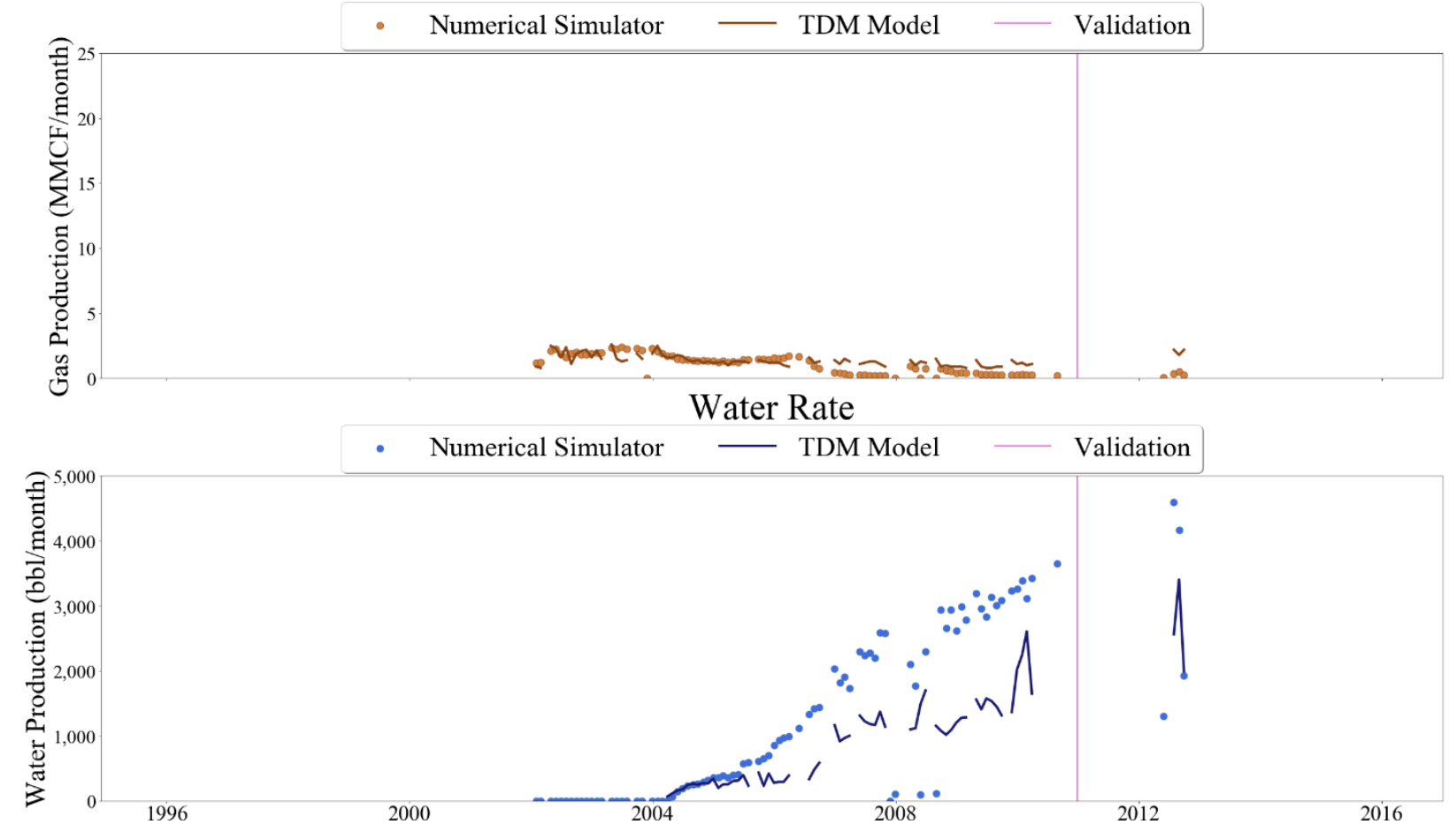

Figure 851 Well-026 oil, gas, and water rate TDM predictions vs actual simulation data plots for 6 Year Blind Validation TDM 

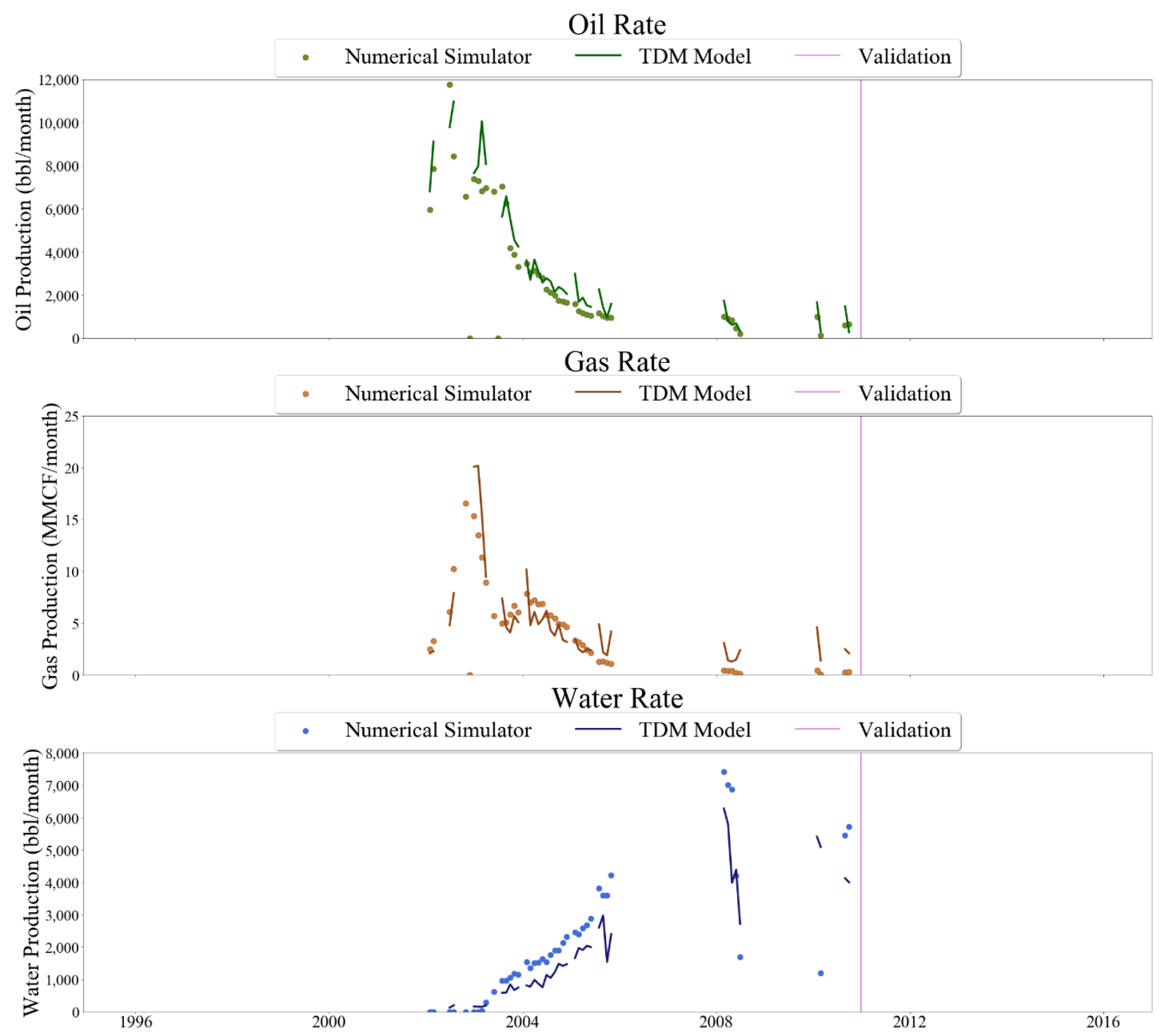

Figure 852 Well-027 oil, gas, and water rate TDM predictions vs actual simulaiton data plots for 6 Year Blind Validation TDM 


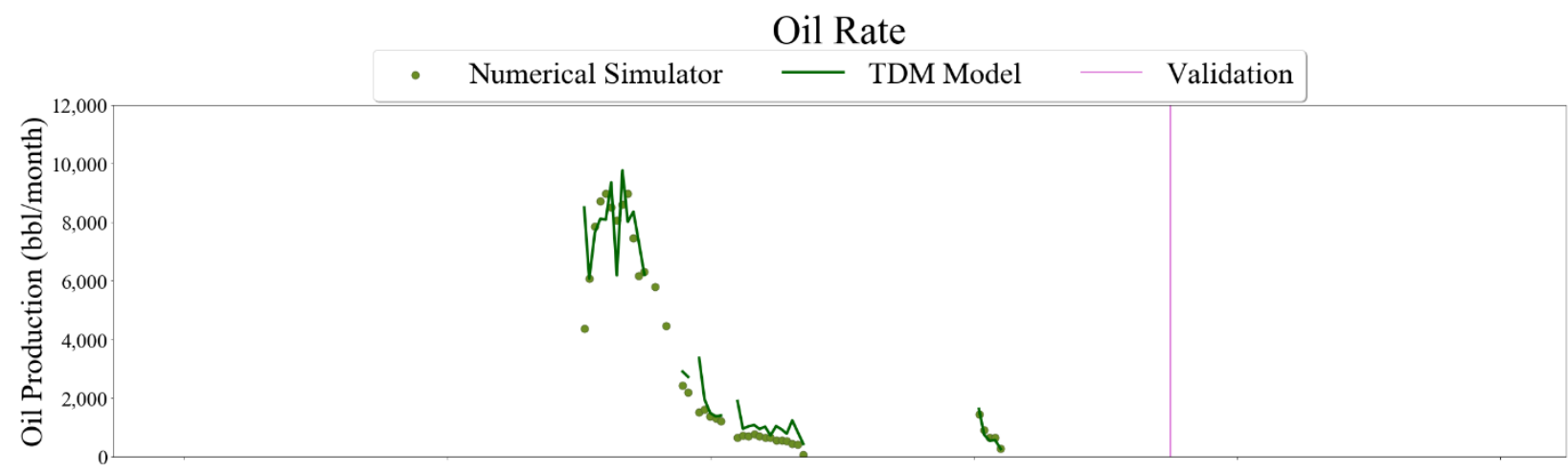

Gas Rate
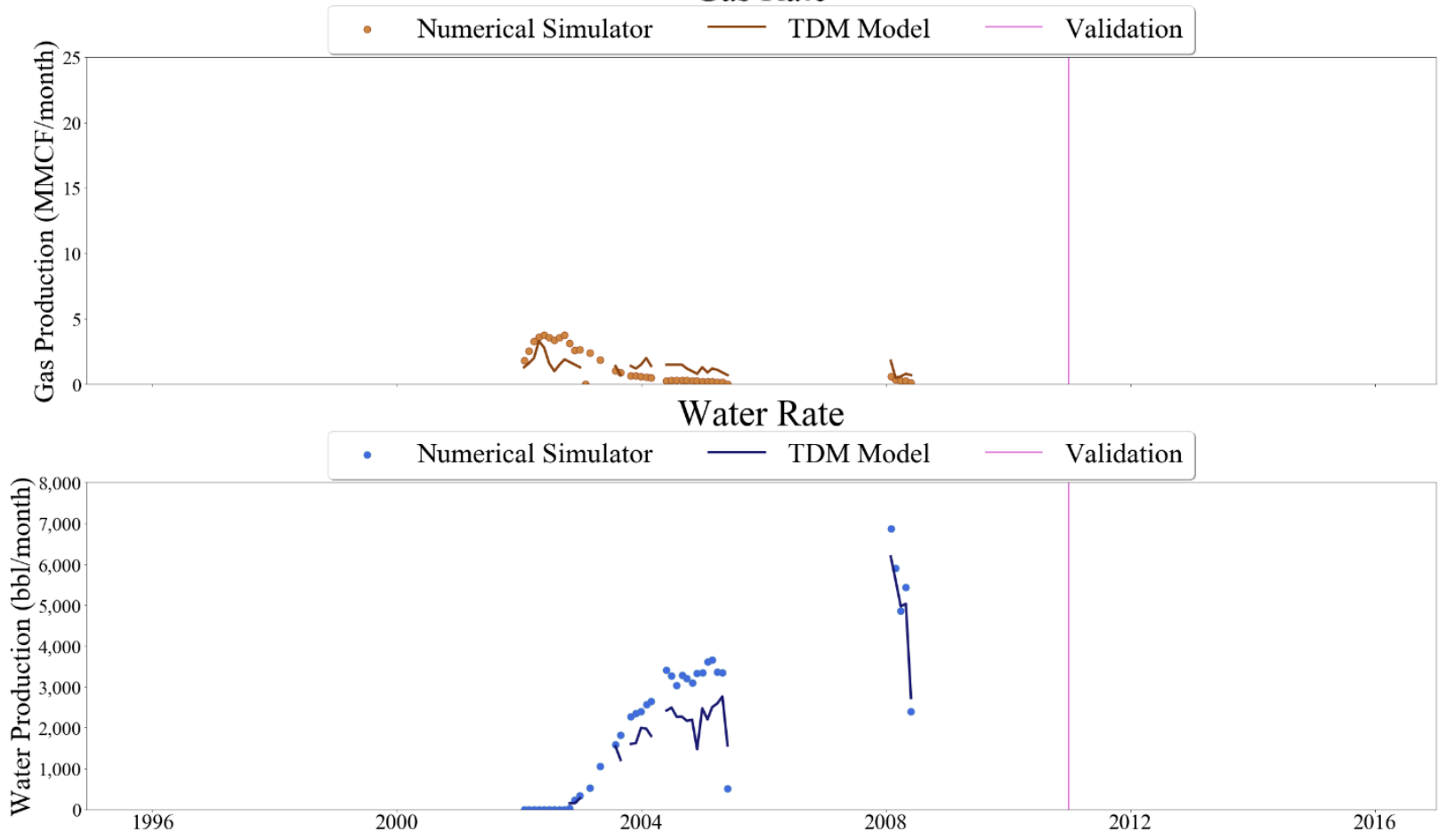

Figure 853 Well-028 oil, gas, and water rate TDM predictions vs actual simulation data plots for 6 Year Blind Validation TDM 

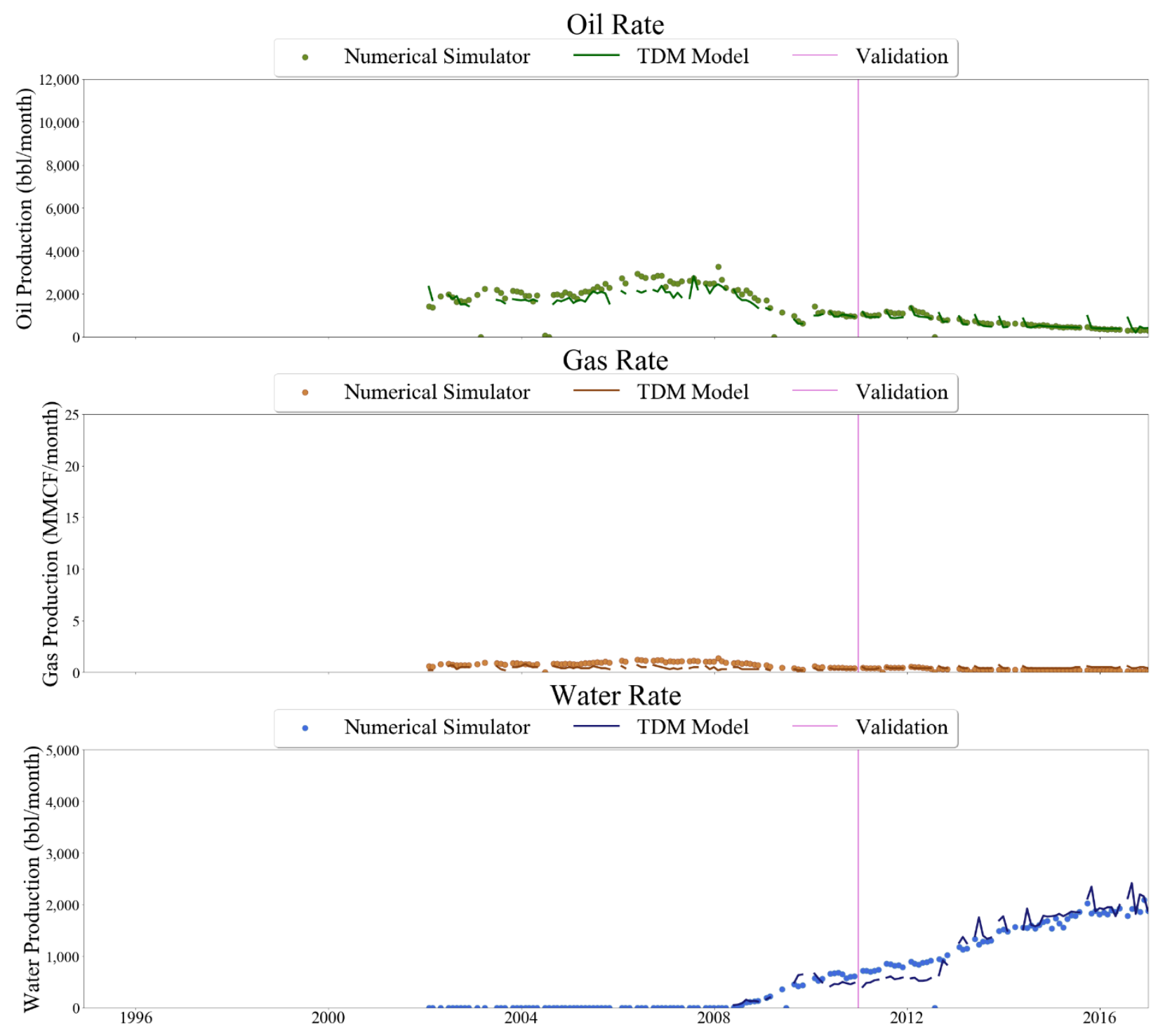

Figure 854 Well-029 oil, gas, and water rate TDM predictions vs actual simulation data plots for 6 Year Blind Validation TDM 


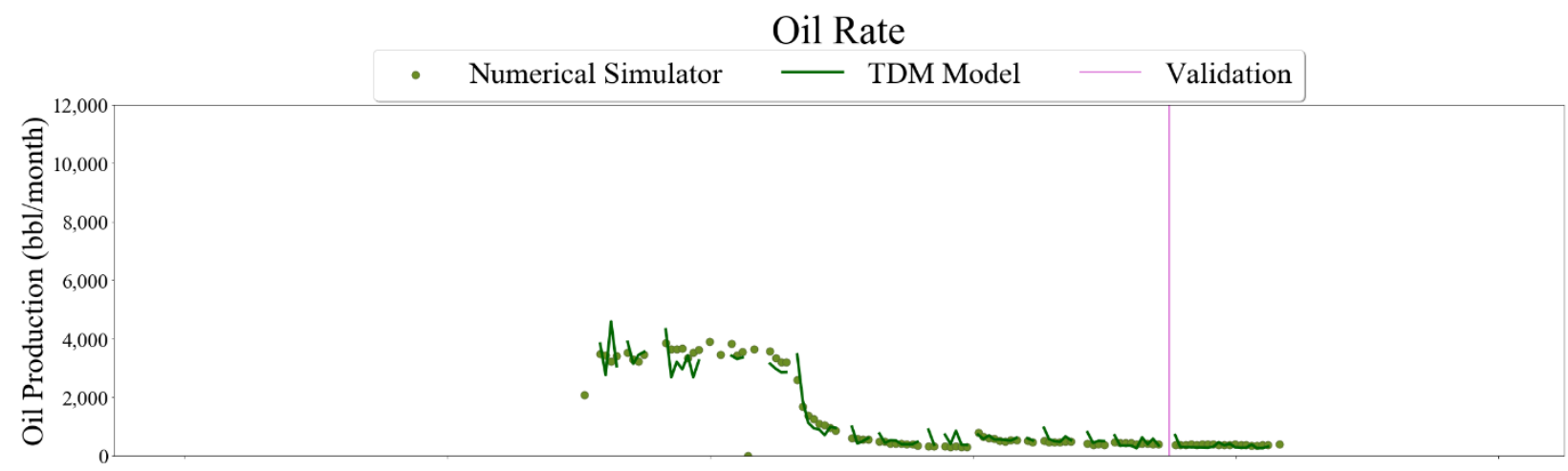

Gas Rate
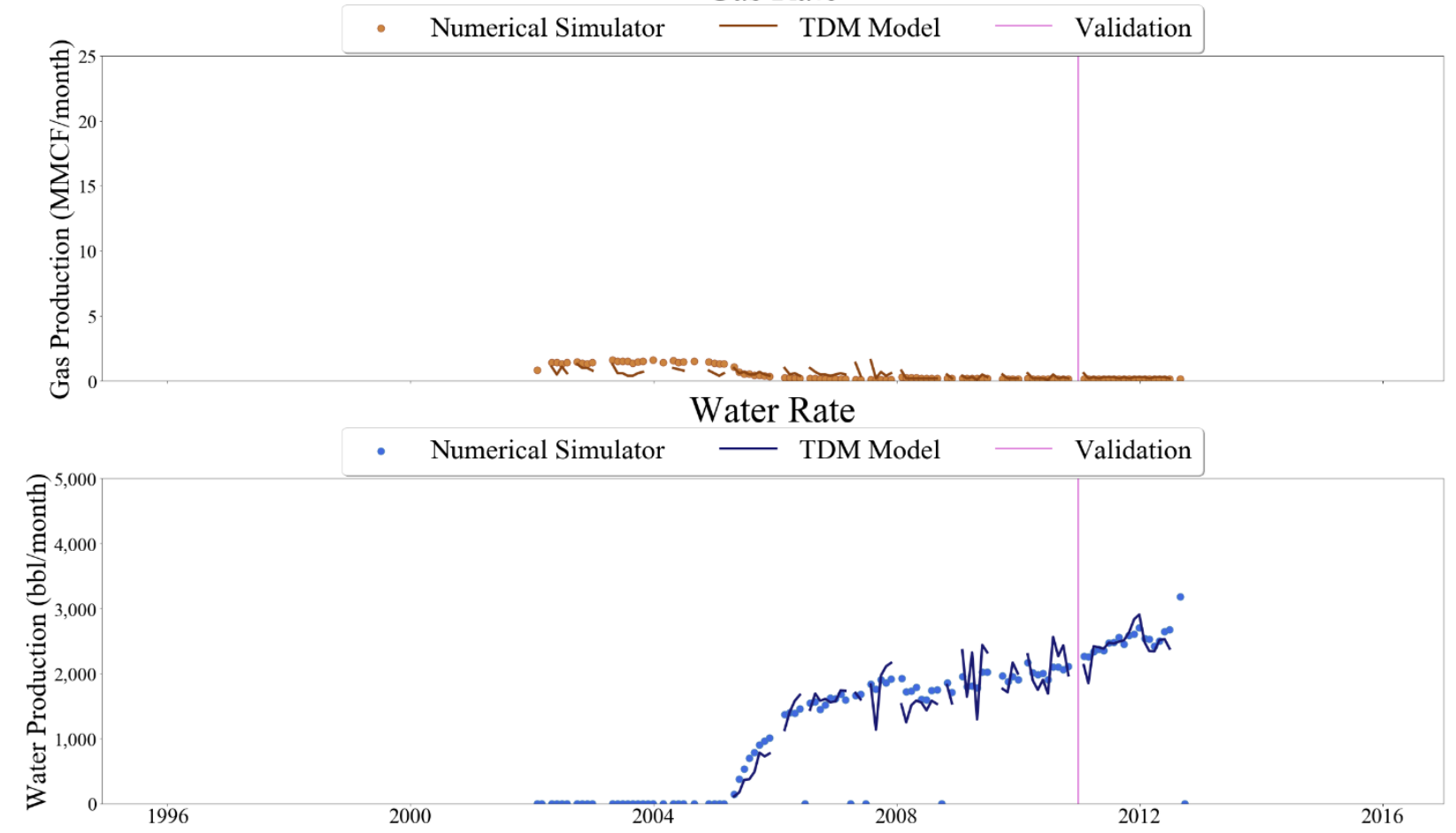

Figure 855 Well-030 oil, gas, and water rate TDM predictions vs actual simulation data plots for 6 Year Blind Validation TDM 


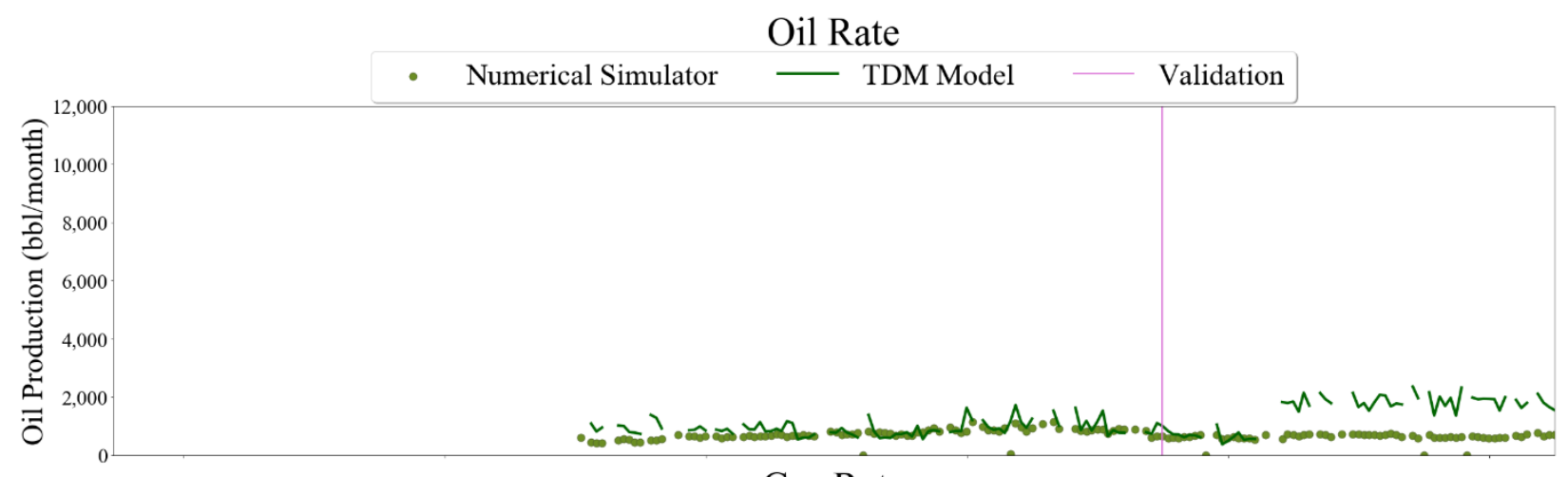

Gas Rate
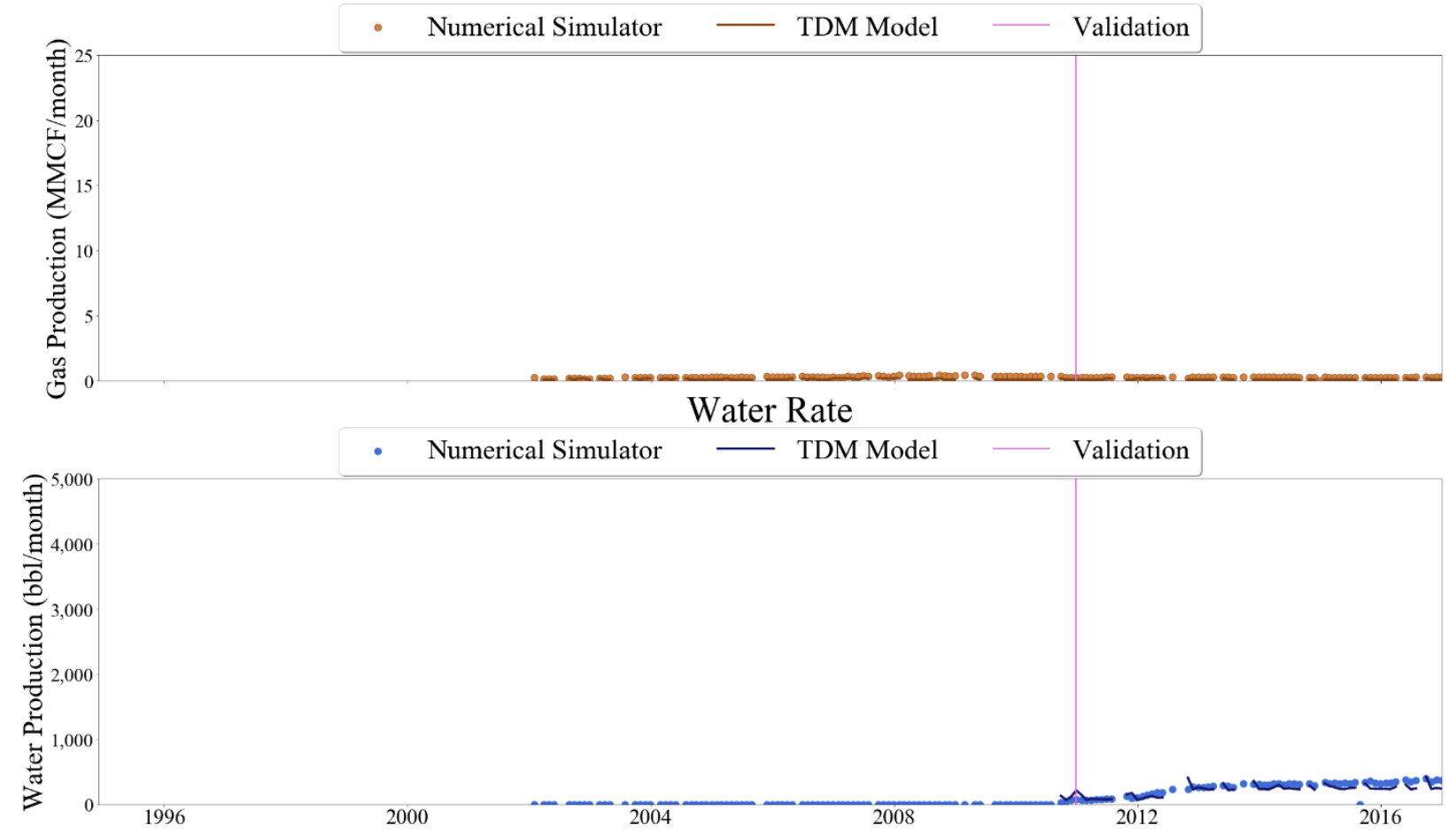

Figure 856 Well-031 oil, gas, and water rate TDM predictions vs actual simulation data plots for 6 Year Blind Validation TDM 


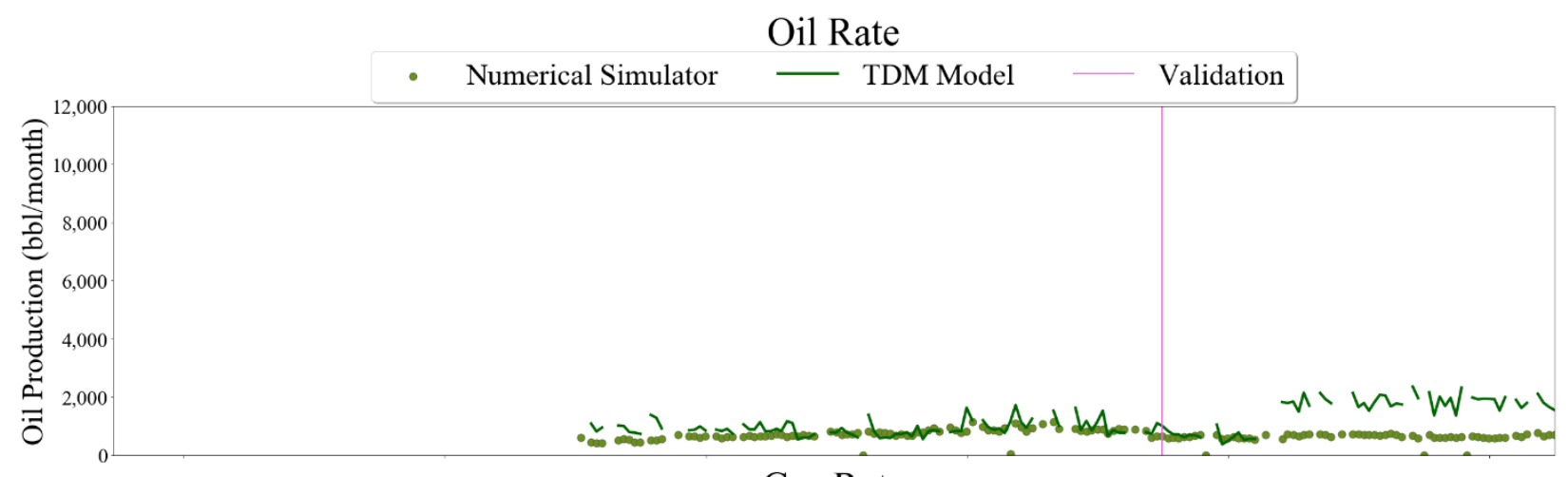

Gas Rate
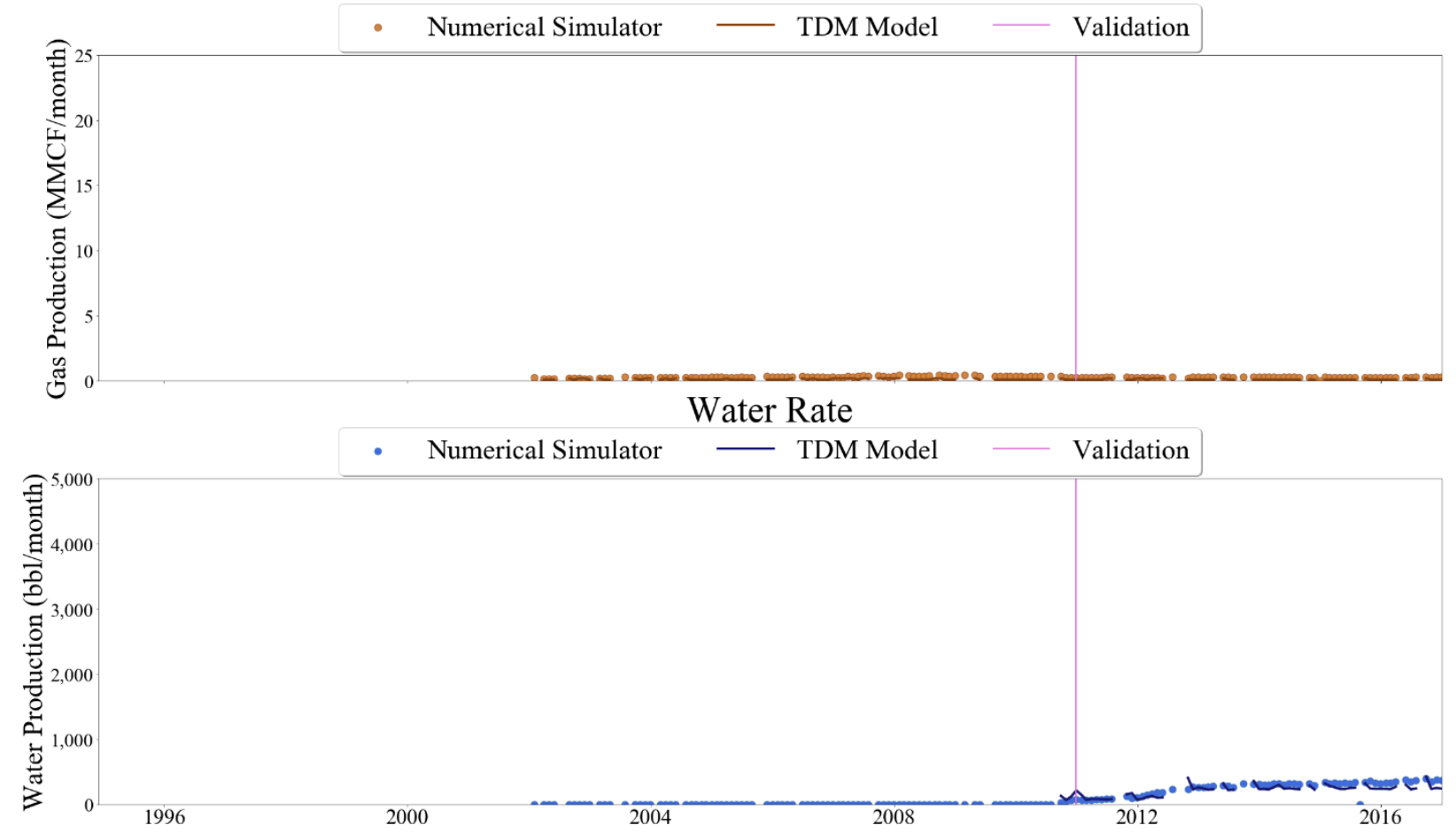

Figure 857 Well-032 oil, gas, and water rate TDM predictions vs actual simulation data plots for 6 Year Blind Validation TDM 


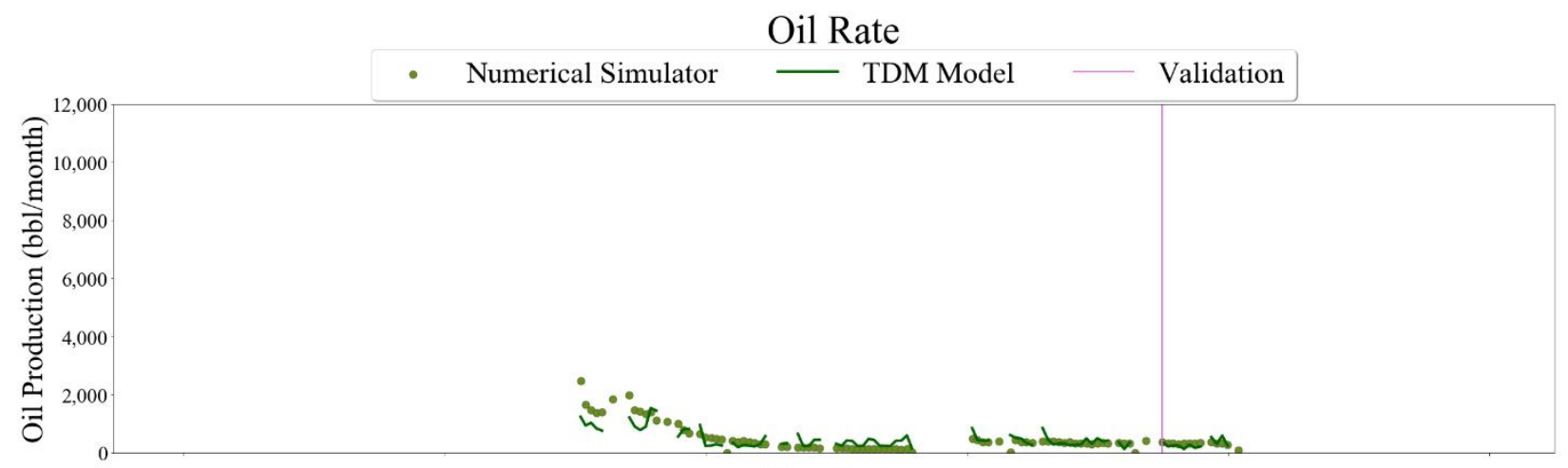

Gas Rate
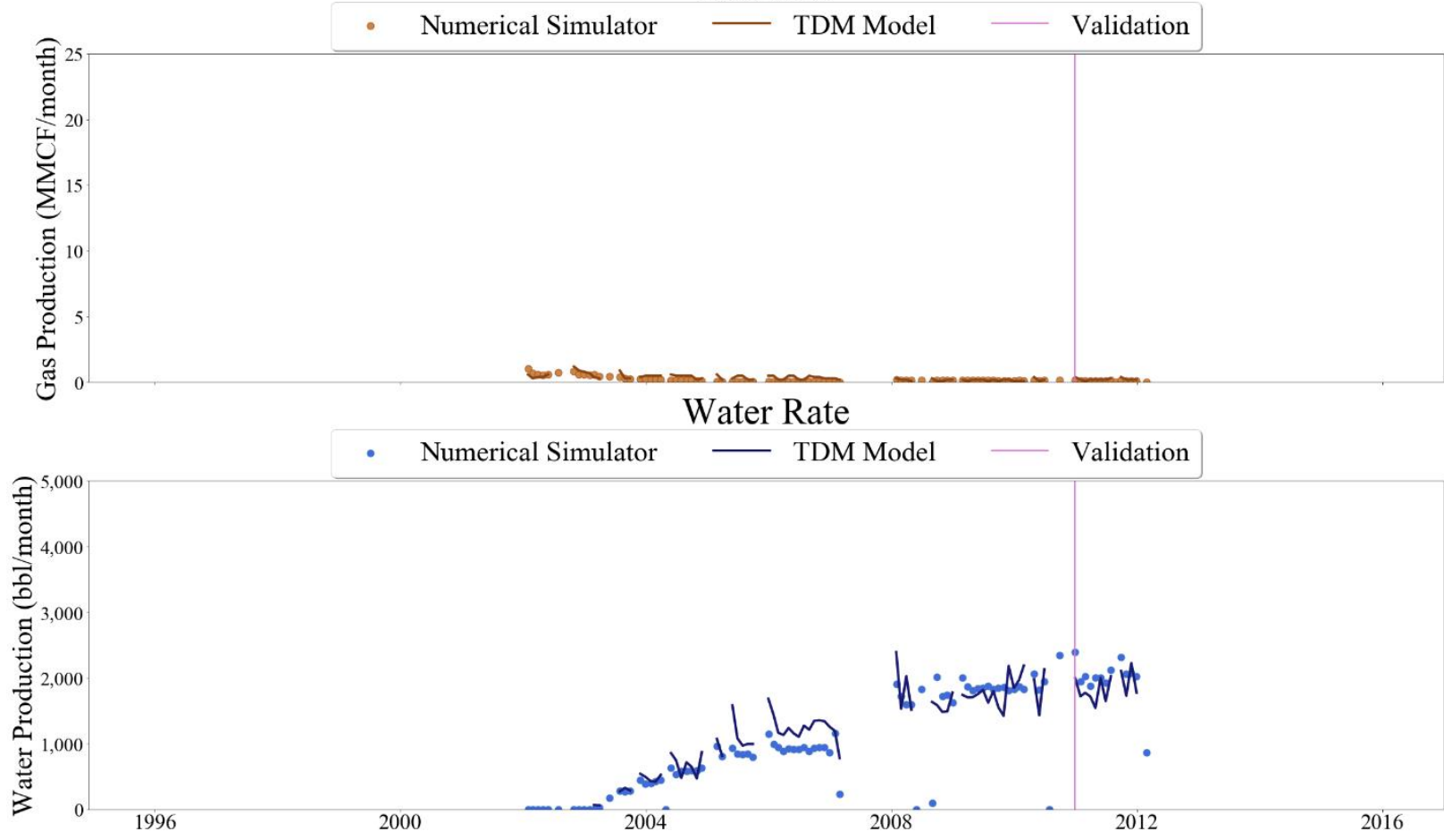

Figure 858 Well-033 oil, gas, and water rate TDM predictions vs actual simulation data plots for 6 Year Blind Validation TDM 


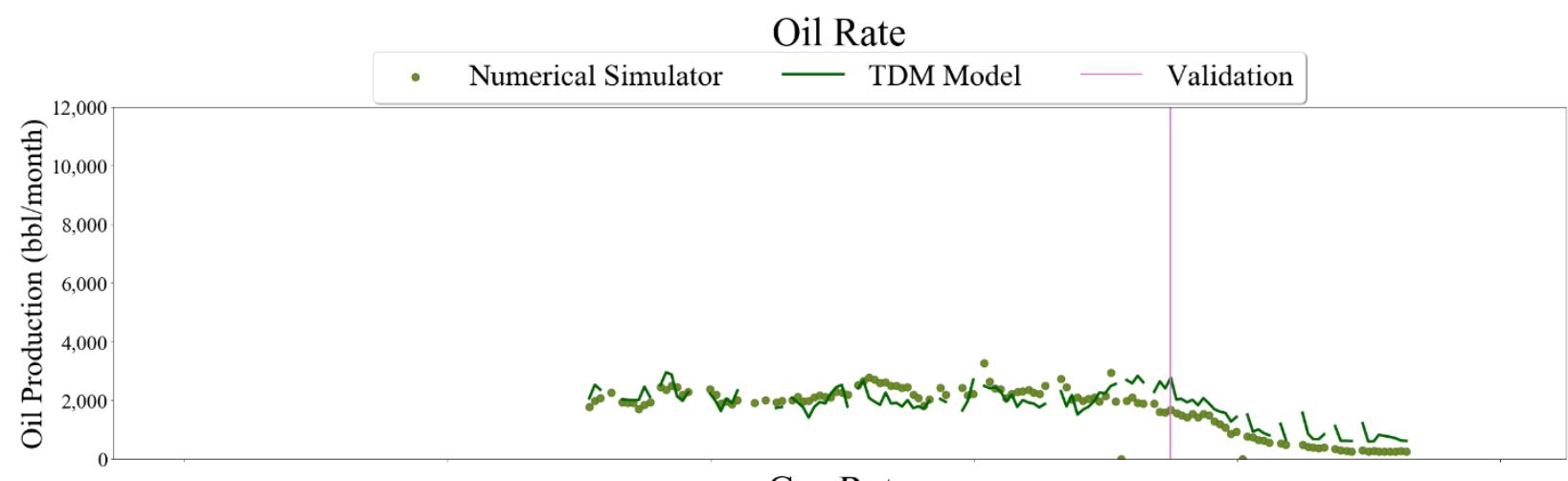

\section{Gas Rate}
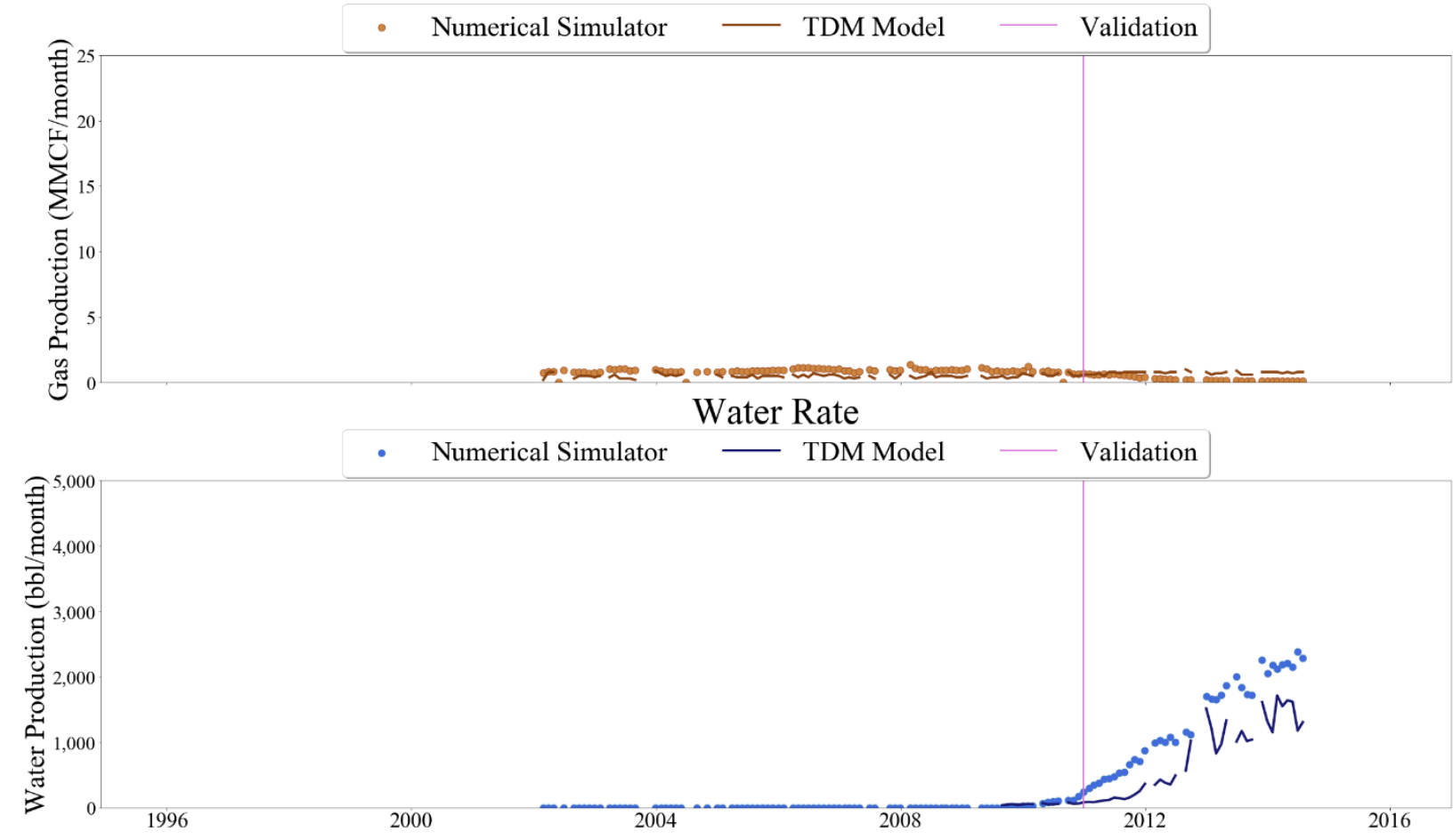

Figure 859 Well-034 oil, gas, and water rate TDM predictions vs actual simulation data plots for 6 Year Blind Validation TDM 


\section{Oil Rate}

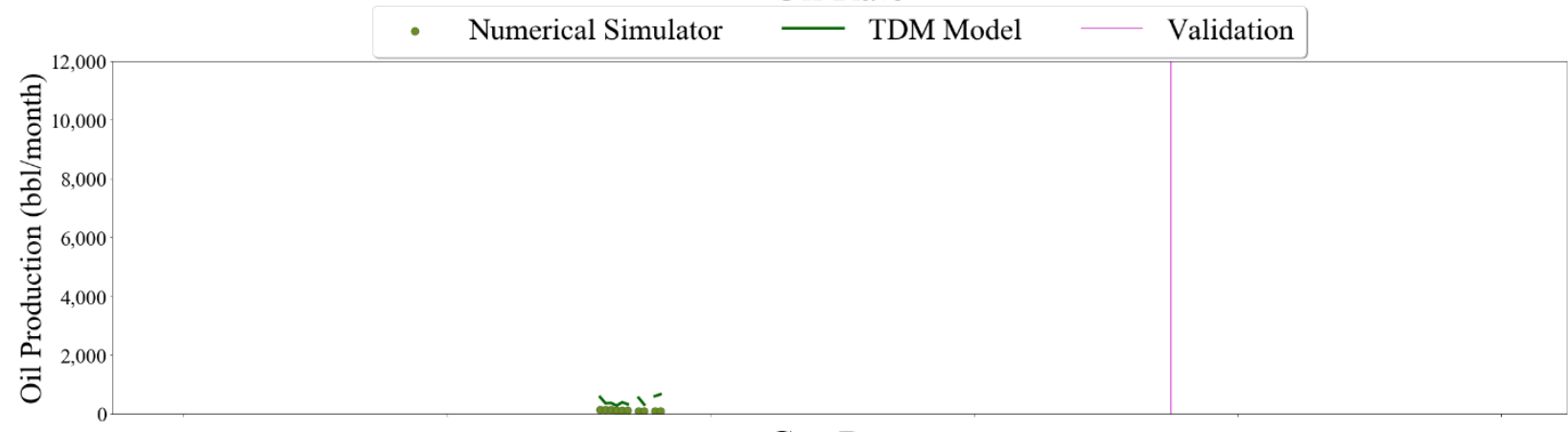

\section{Gas Rate}

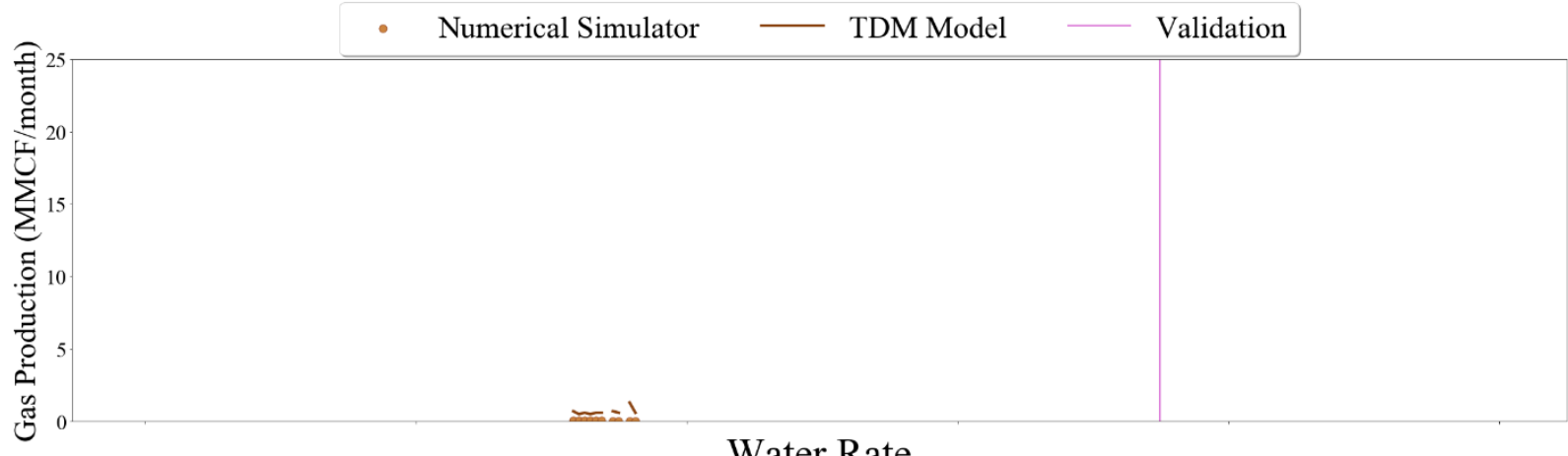

\section{Water Rate}

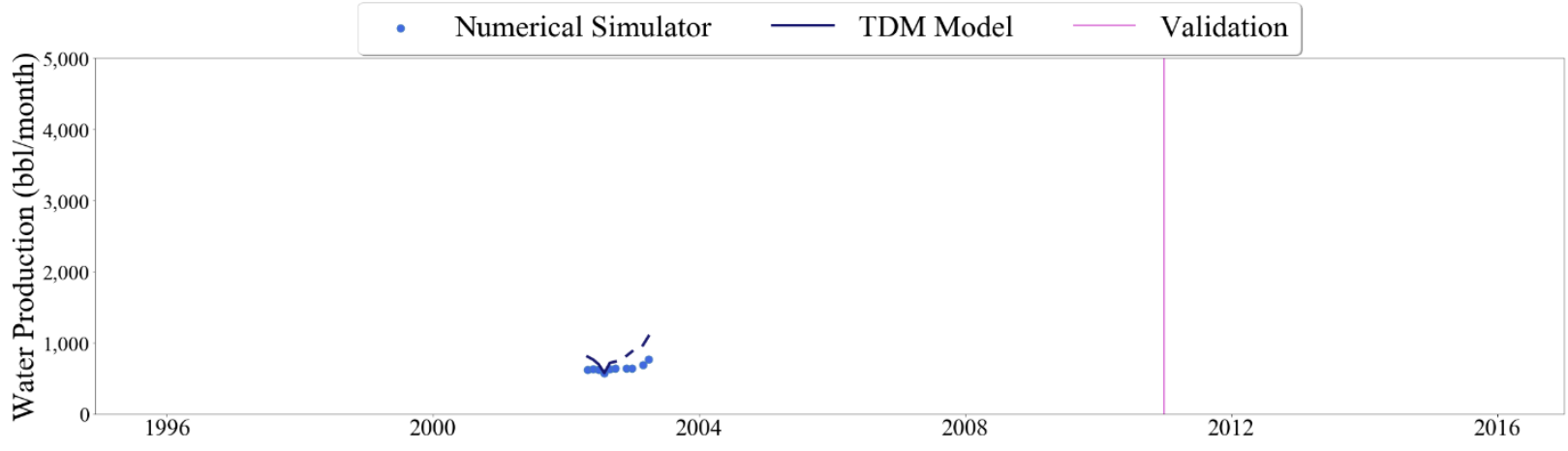

Figure 860 Well-035 oil, gas, and water rate TDM predictions vs actual simulation data plots for 6 Year blind Validation TDM 

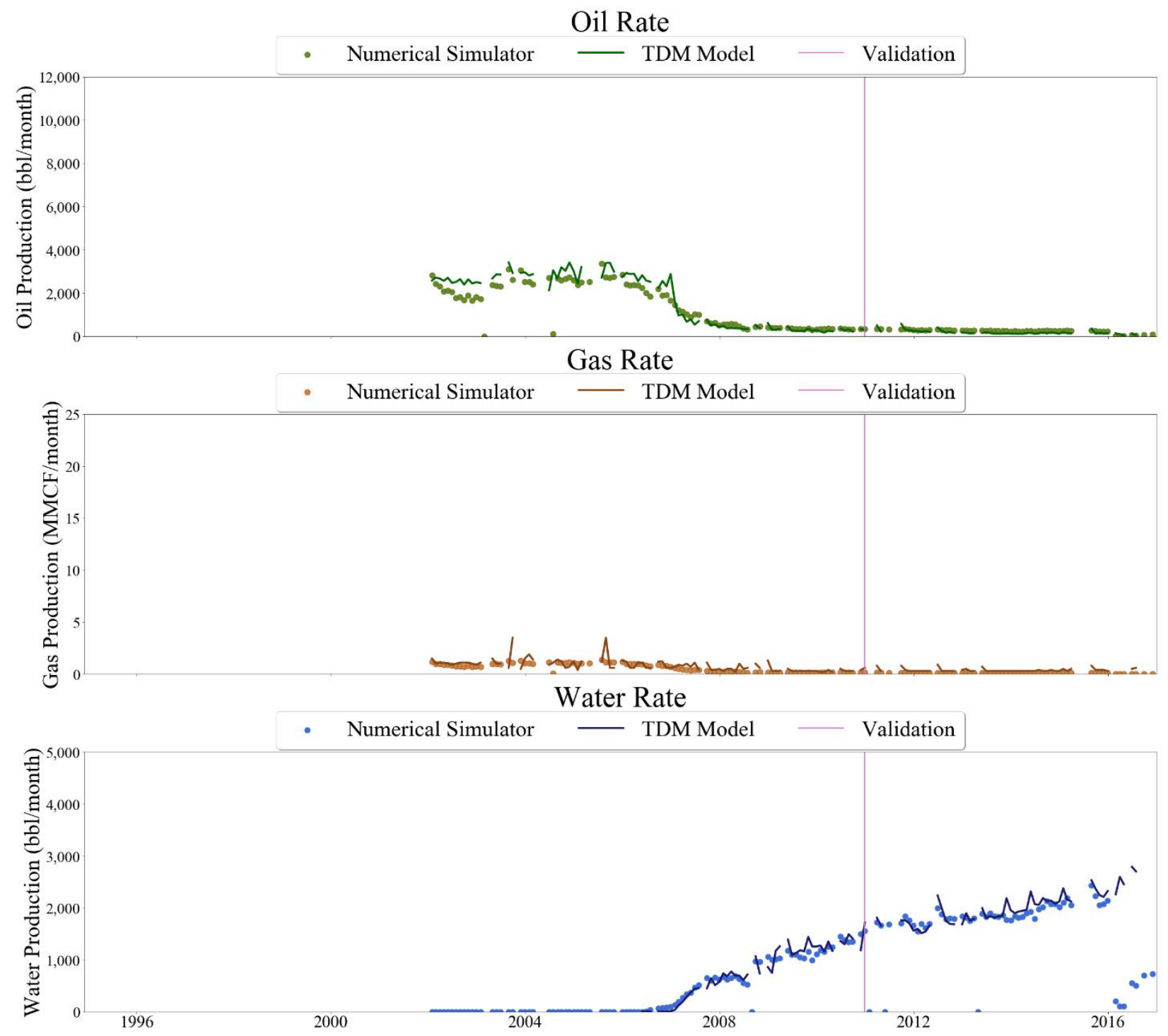

Figure 861 Well-036 oil, gas, and water rate TDM predictions vs actual simulation data plots for 6 Year Blind Validation TDM 

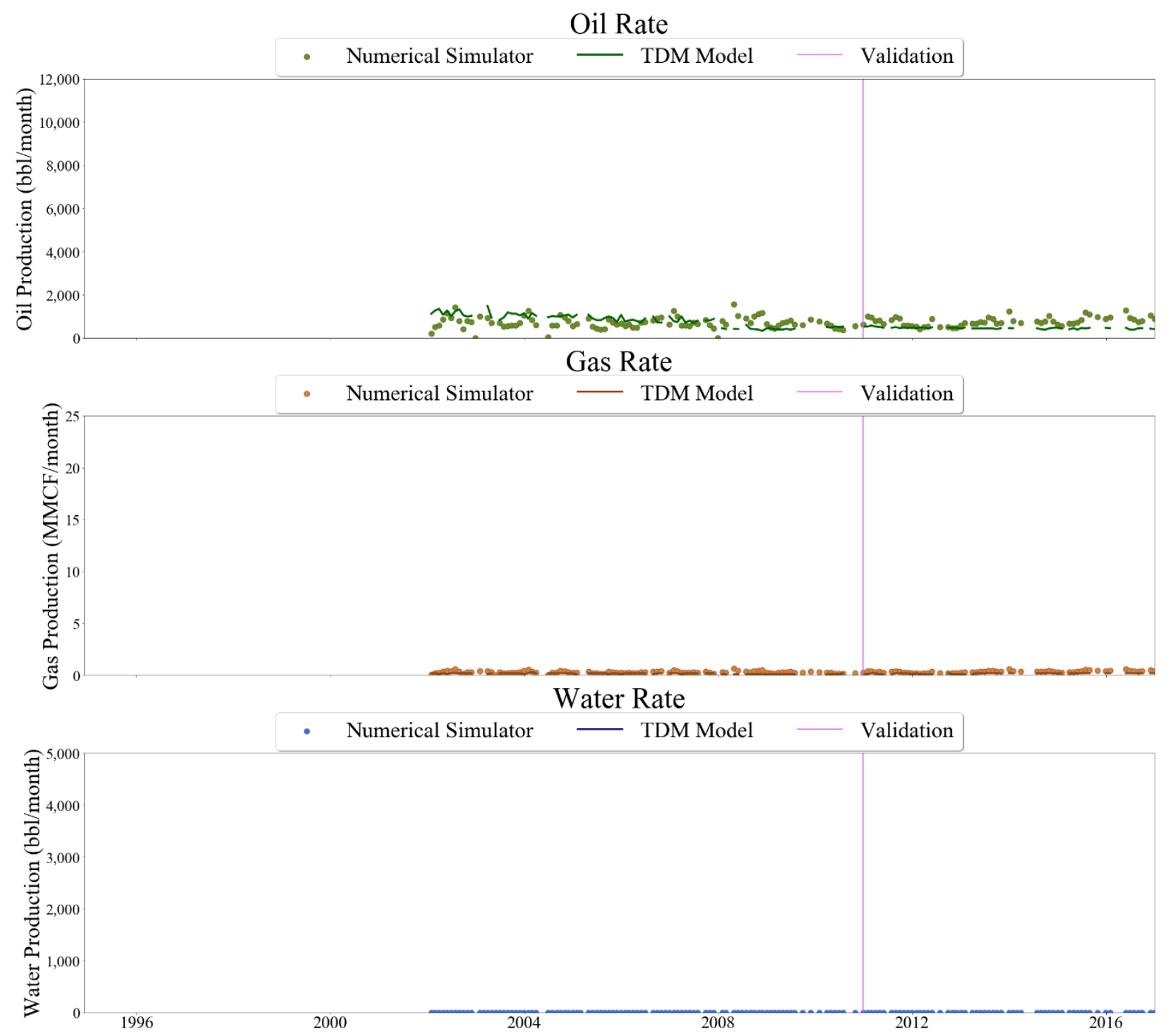

Figure 862 Well-037 oil, gas, and water rate TDM predictions vs actual simulation data plots for 6 Year Blind Validation TDM 


\section{Oil Rate}

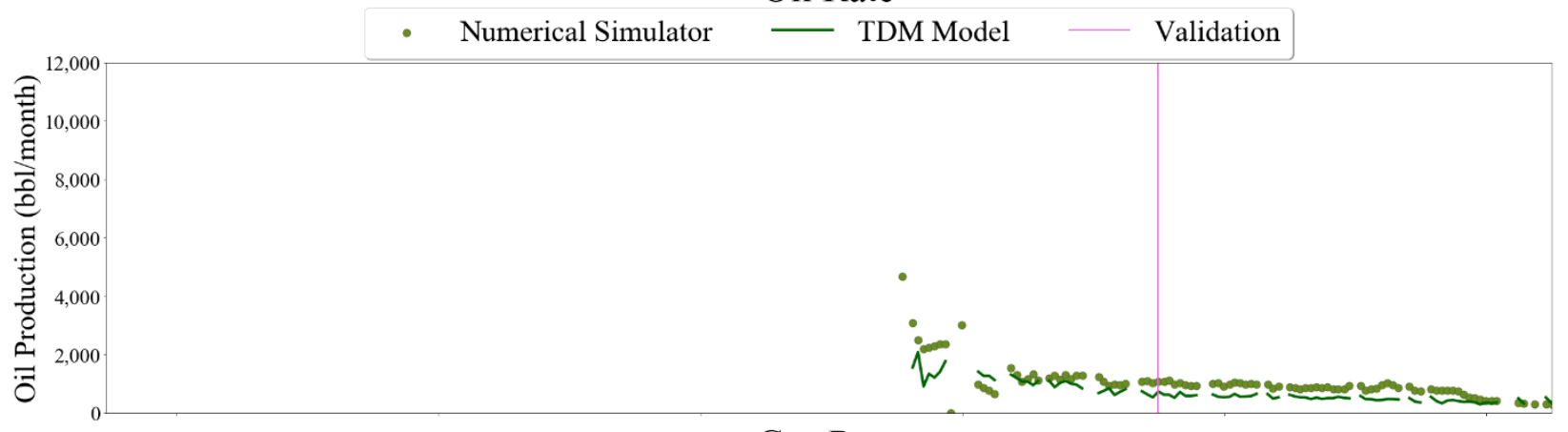

\section{Gas Rate}
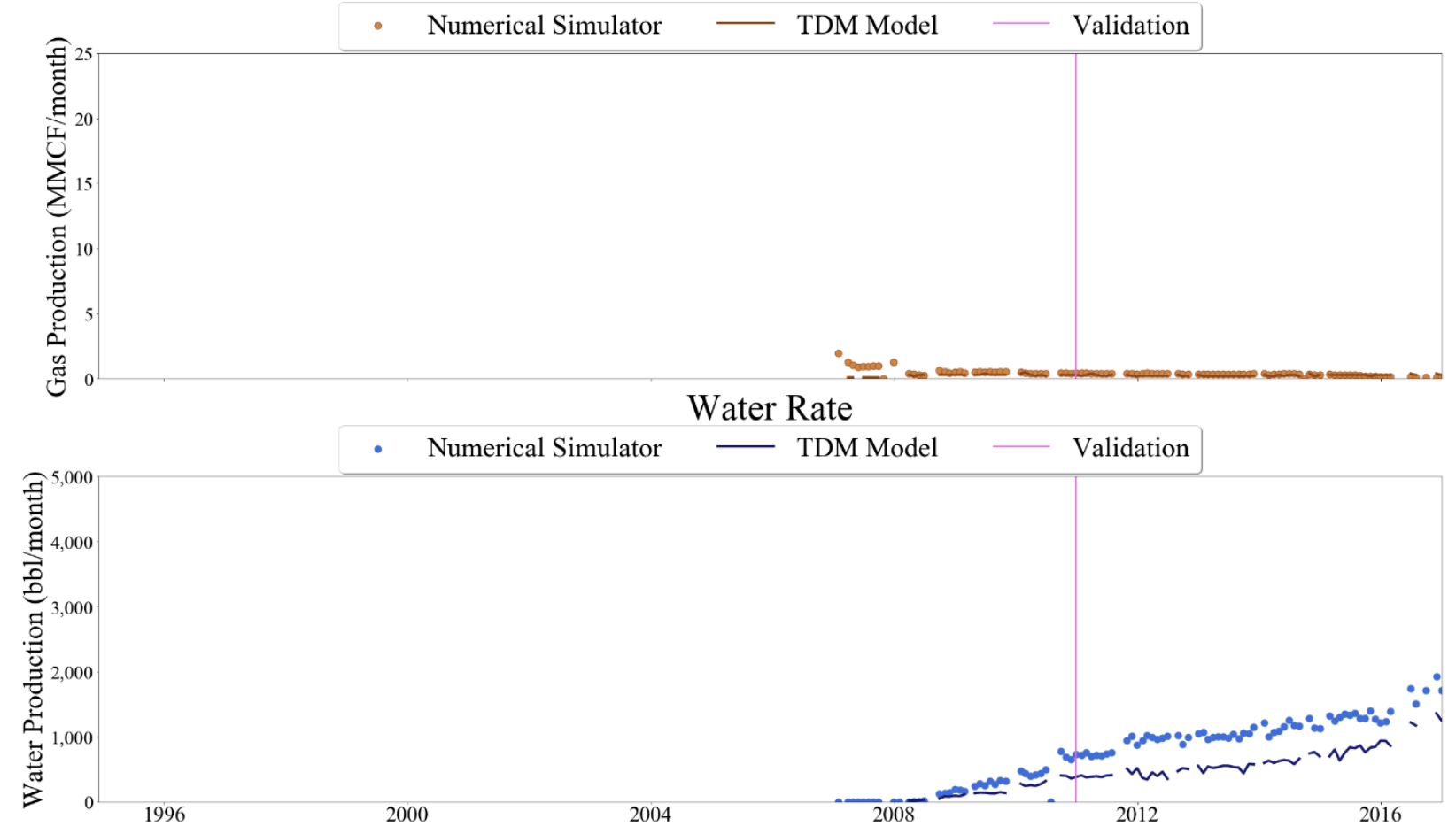

Figure 863 Well-038 oil, gas, and water rate TDM predictions vs actual simulation data plots for 6 Year Blind Validation TDM 


\section{Oil Rate}

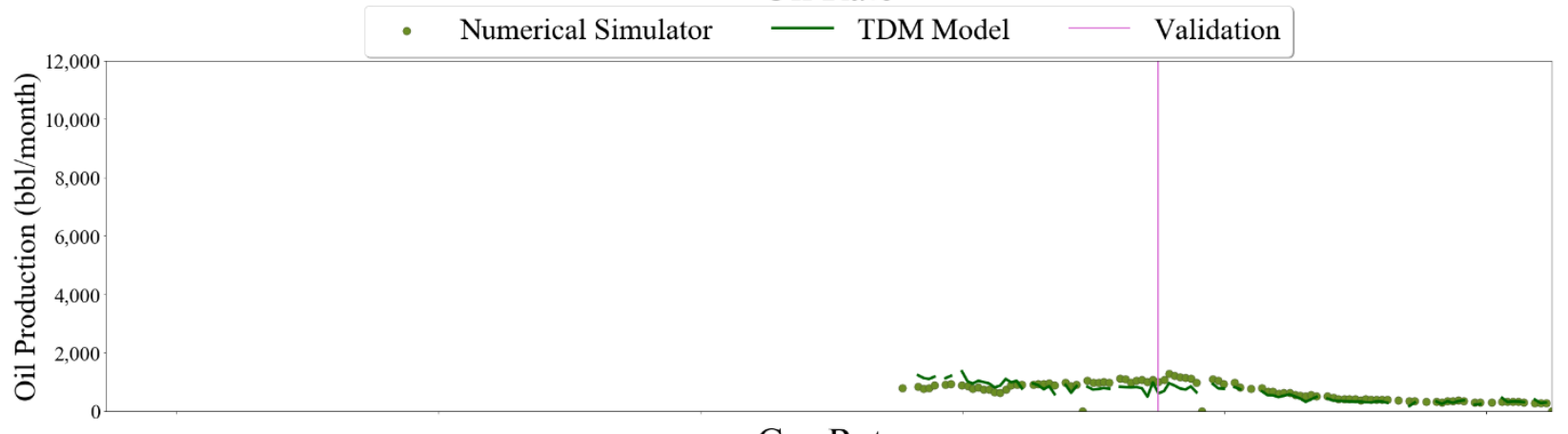

\section{Gas Rate}
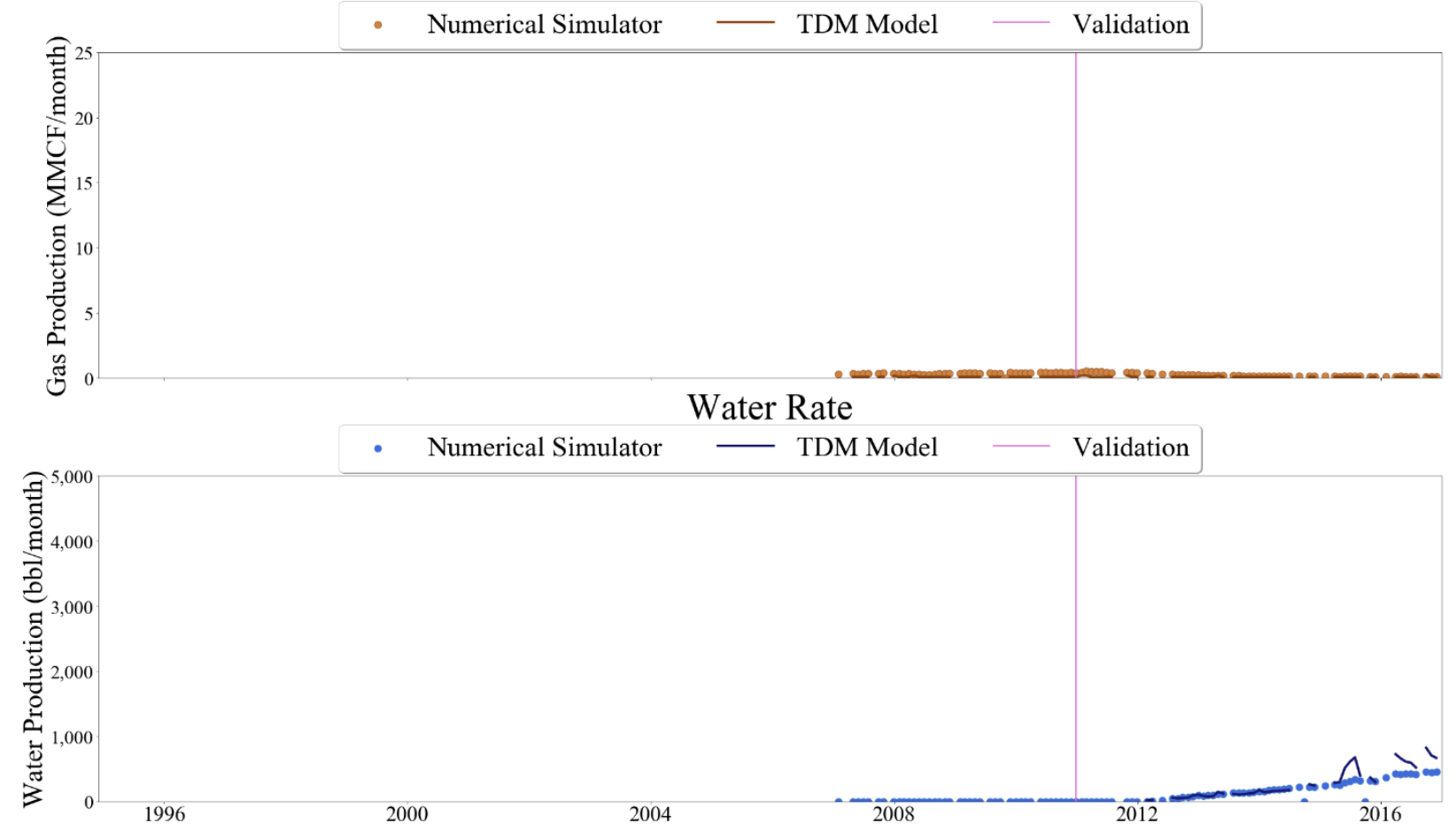

Figure 864 Well-039 oil, gas, and water rate TDM predictions vs actual simulation data plots for 6 Year Blind Validation TDM 


\section{Oil Rate}

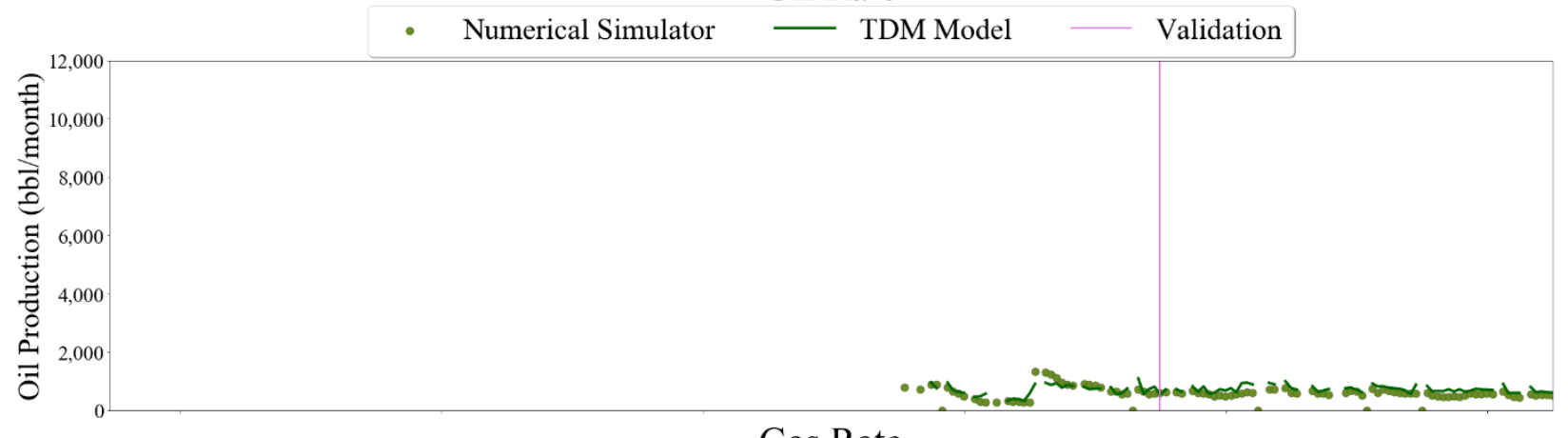

Gas Rate
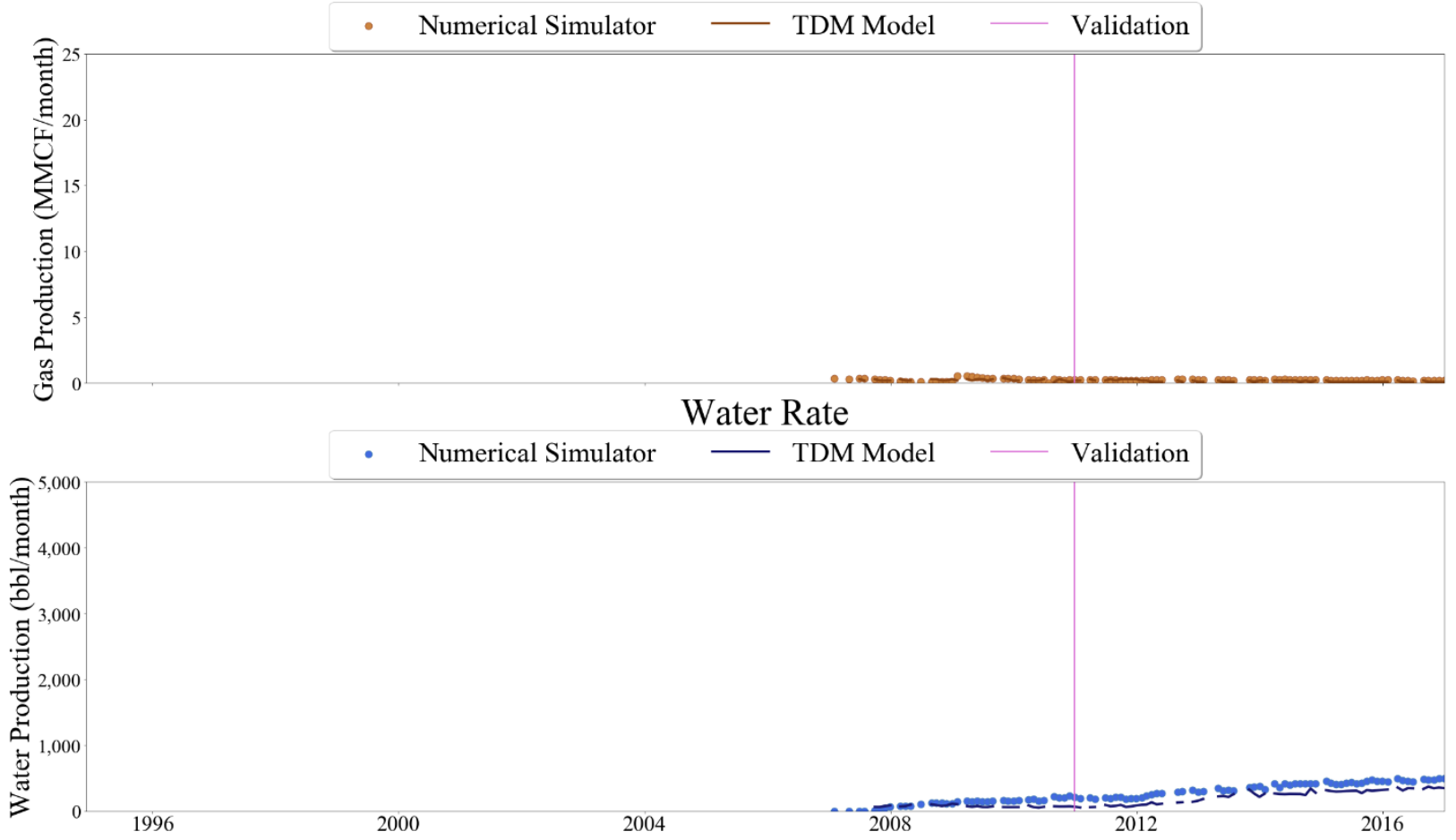

Figure 865 Well-040 oil, gas, and water rate TDM predictions vs actual simulation data plots for 6 Year Blind Validation TDM 


\section{Oil Rate}

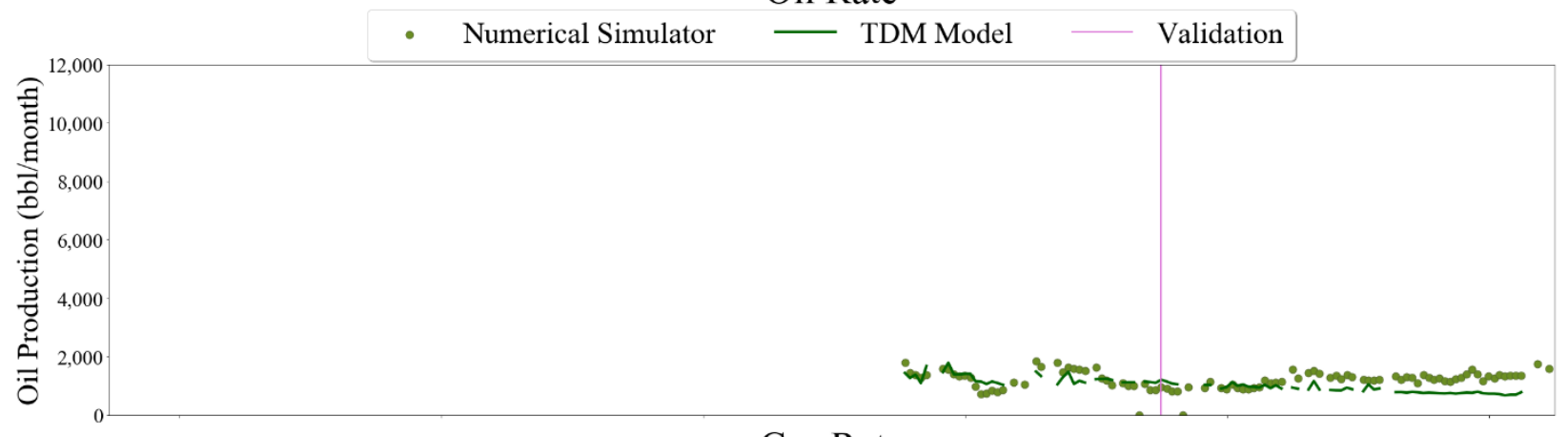

\section{Gas Rate}
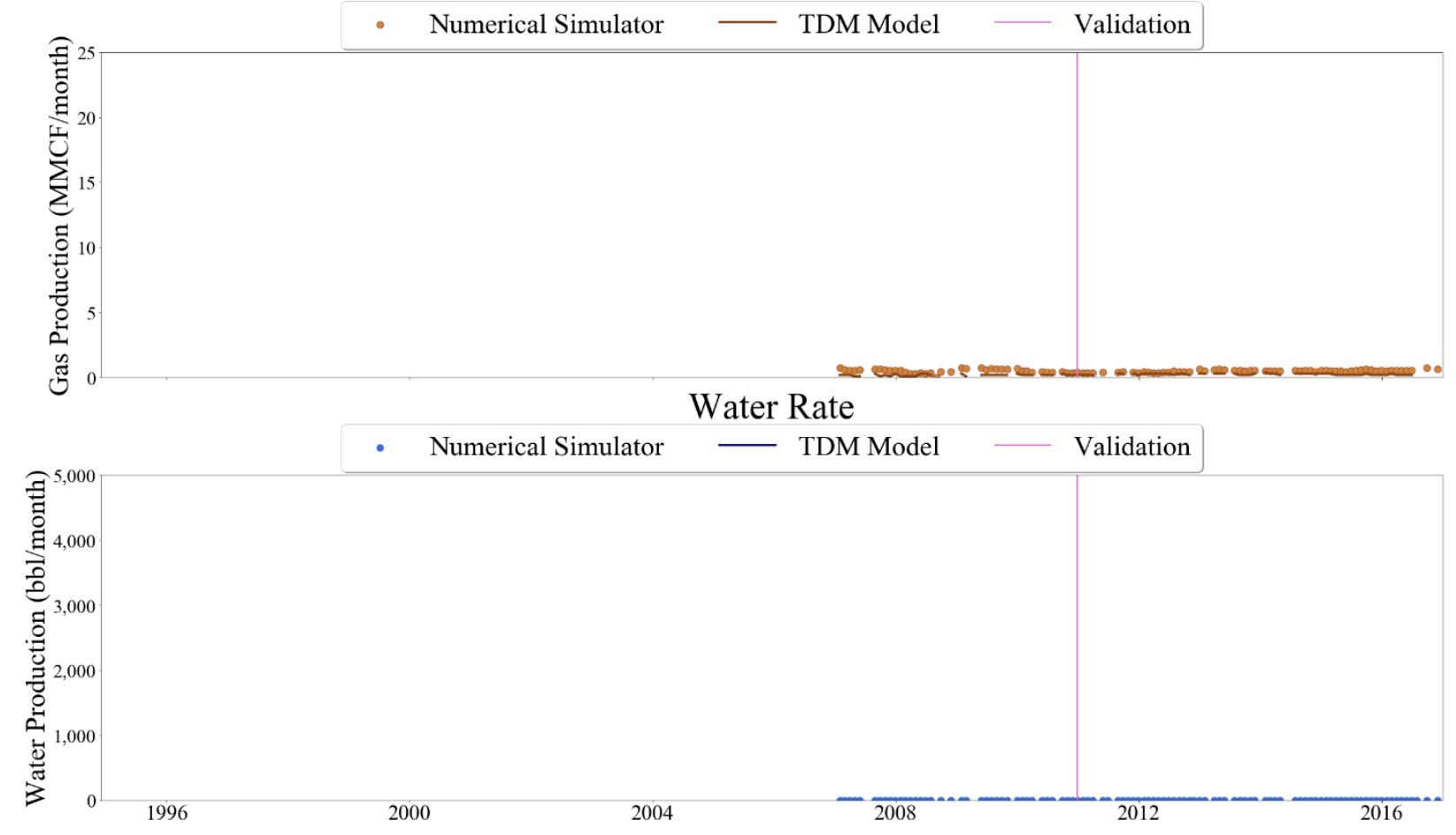

Figure 866 Well-041 oil, gas, and water rate TDM predictions vs actual simulation data plots for 6 Year Blind Validation TDM 


\section{Oil Rate}

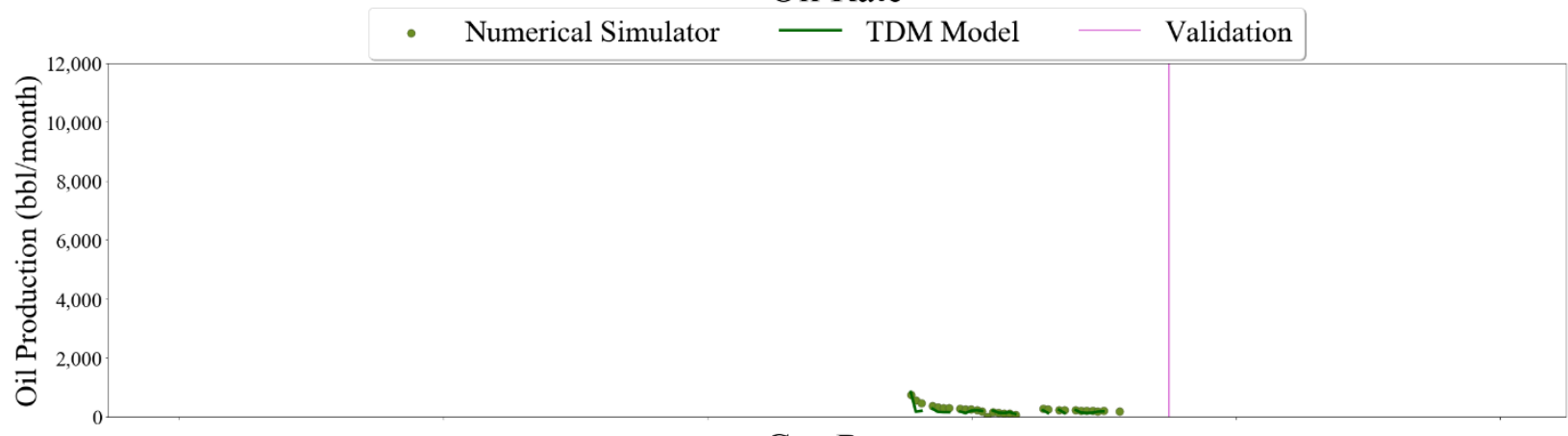

\section{Gas Rate}

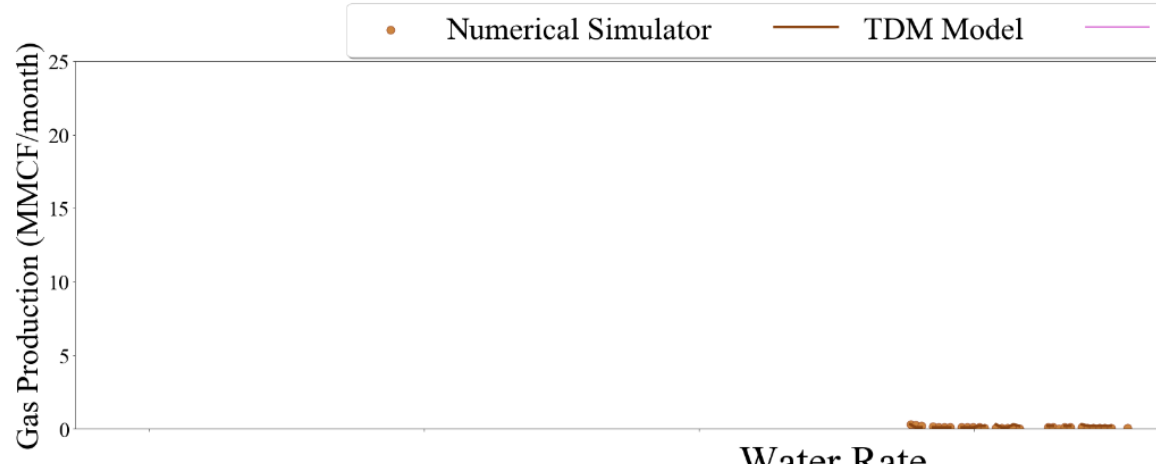

Water Rate

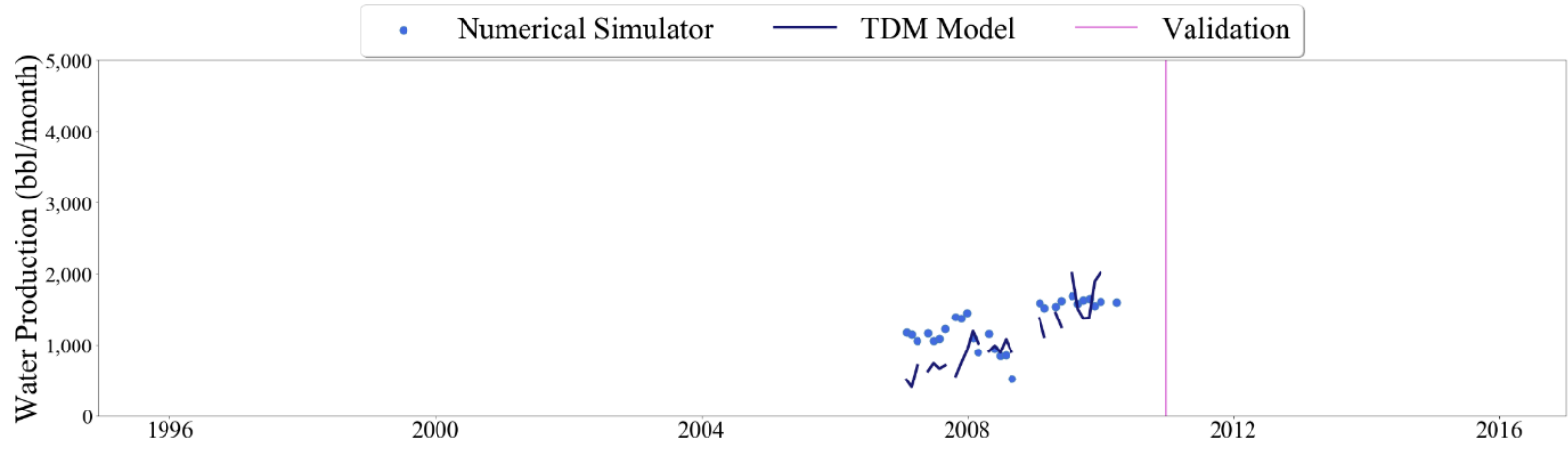

Figure 867 Well-042 oil, gas, and water rate TDM predictions vs actual simulation data plots for 6 Year Blind Validation TDM 


\section{Oil Rate}

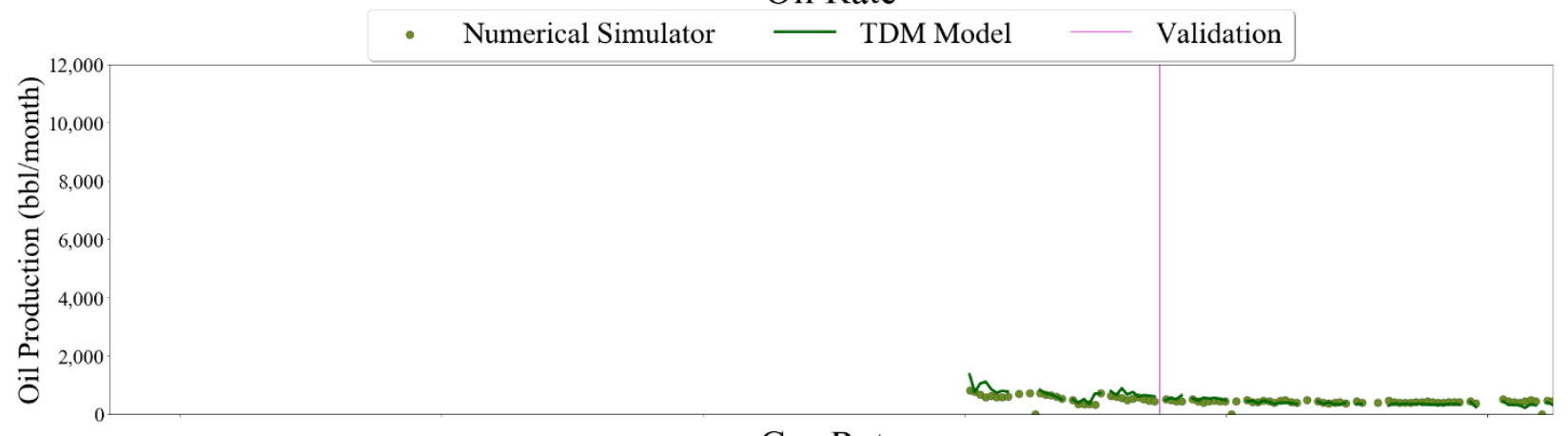

\section{Gas Rate}
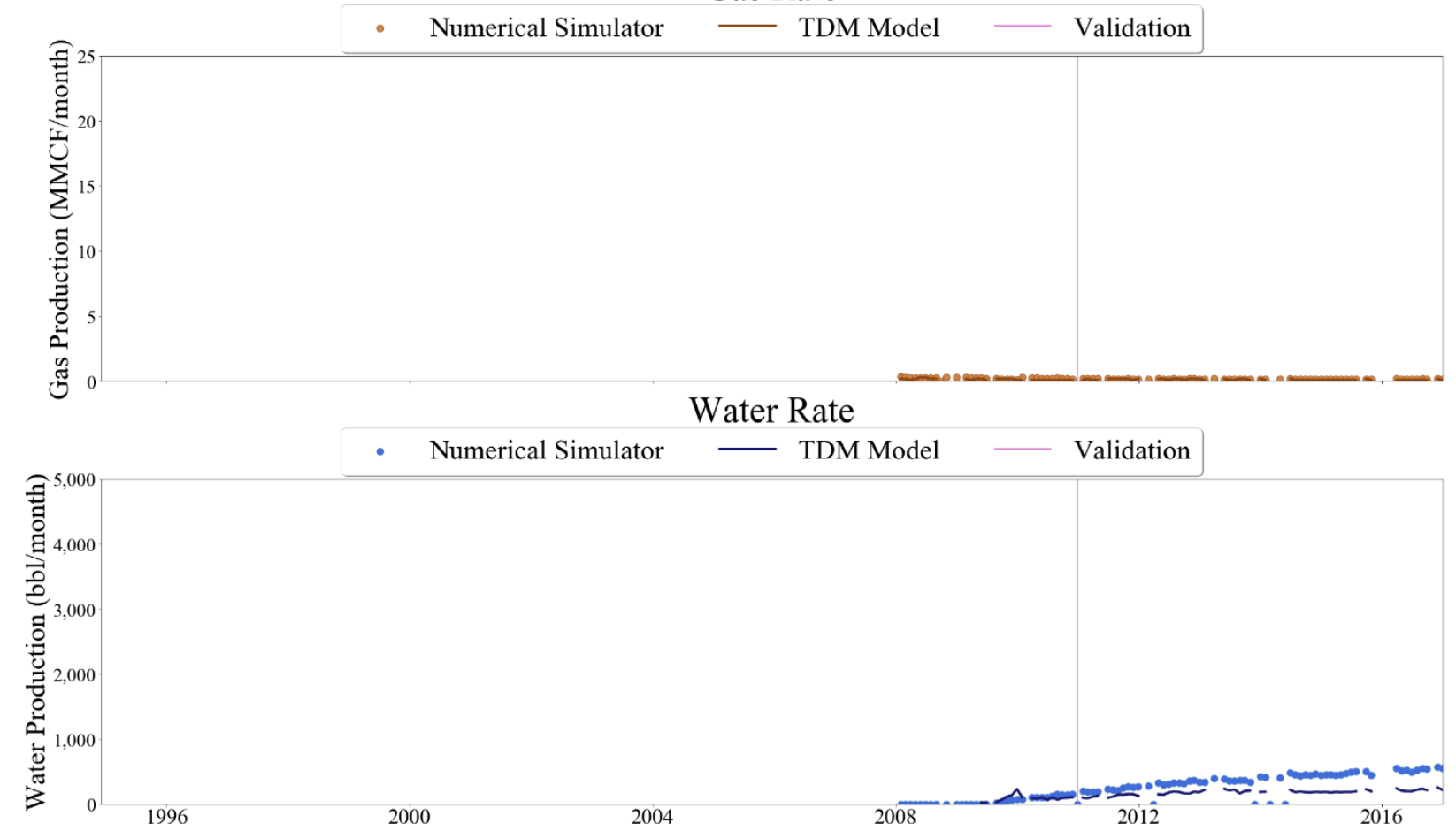

Figure 868 Well-043 oil, gas, and water rate TDM predictions vs actual simulation data plots for 6 Year Blind Validation TDM 


\section{Oil Rate}

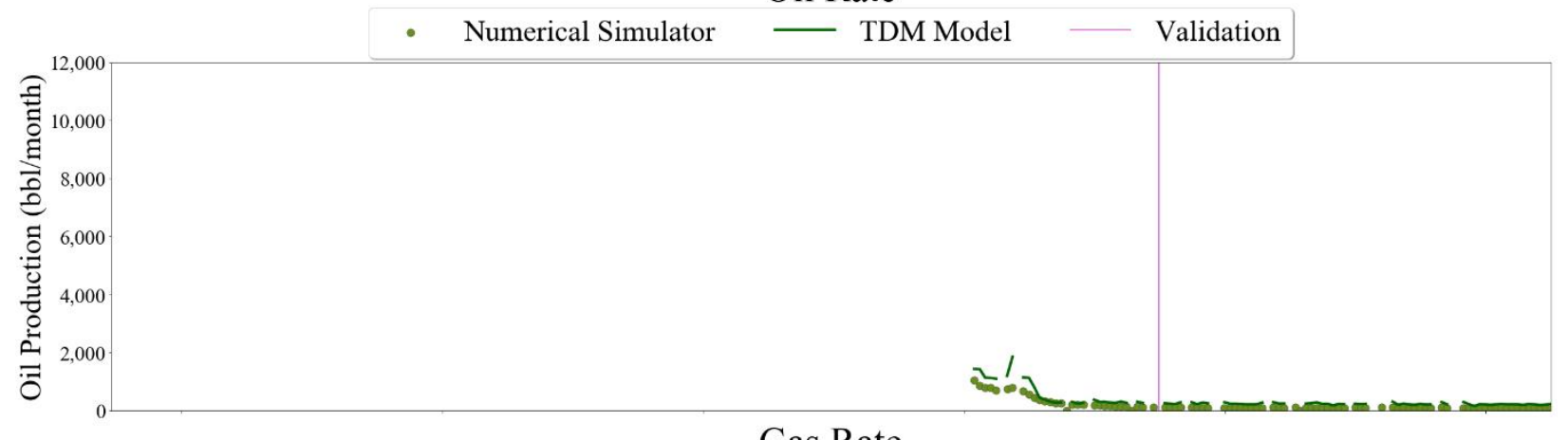

Gas Rate
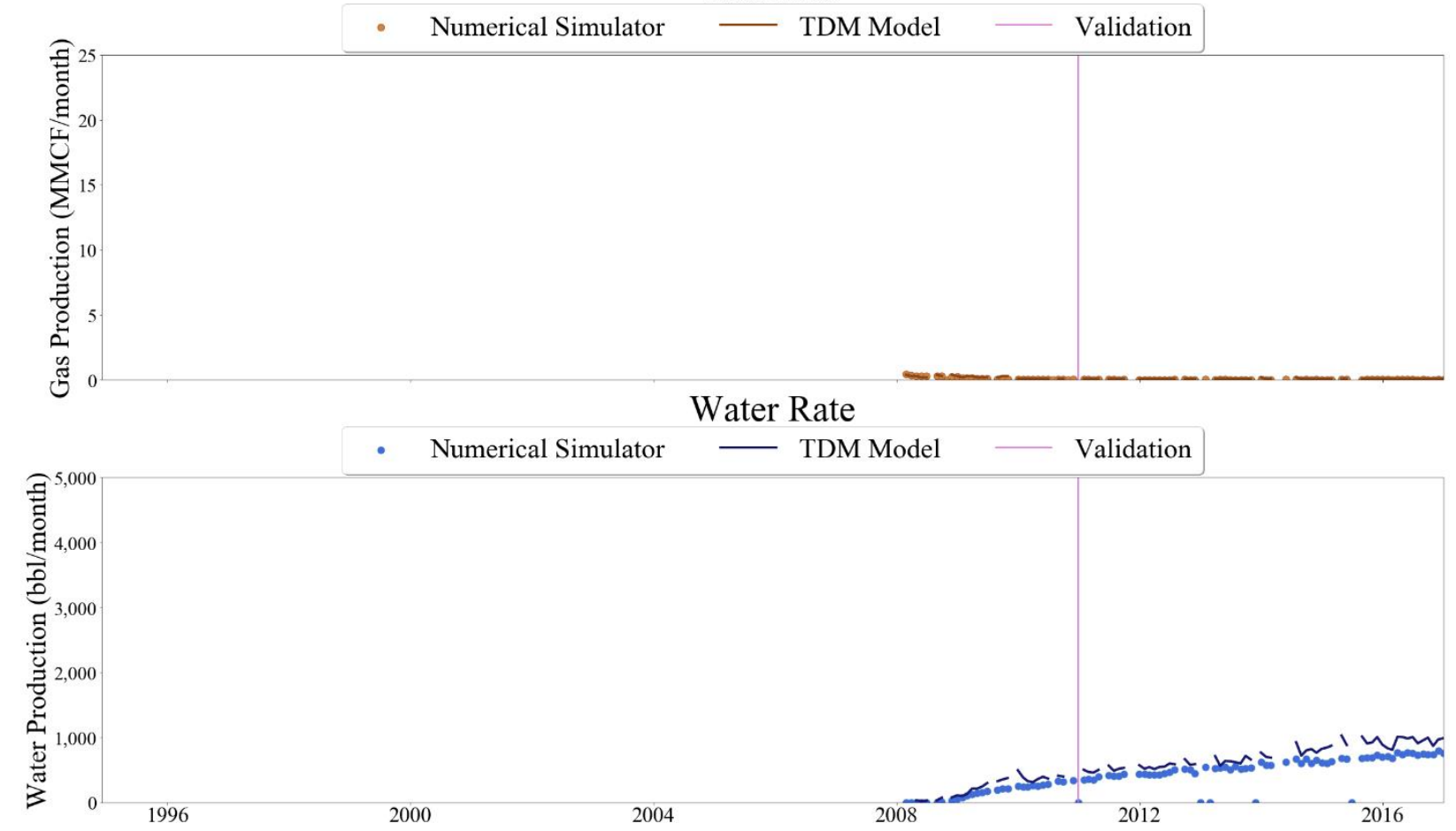

Figure 869 Well-044 oil, gas, and water rate TDM predictions vs actual simulation data plots for 6 Year Blind Validation TDM 


\section{Oil Rate}
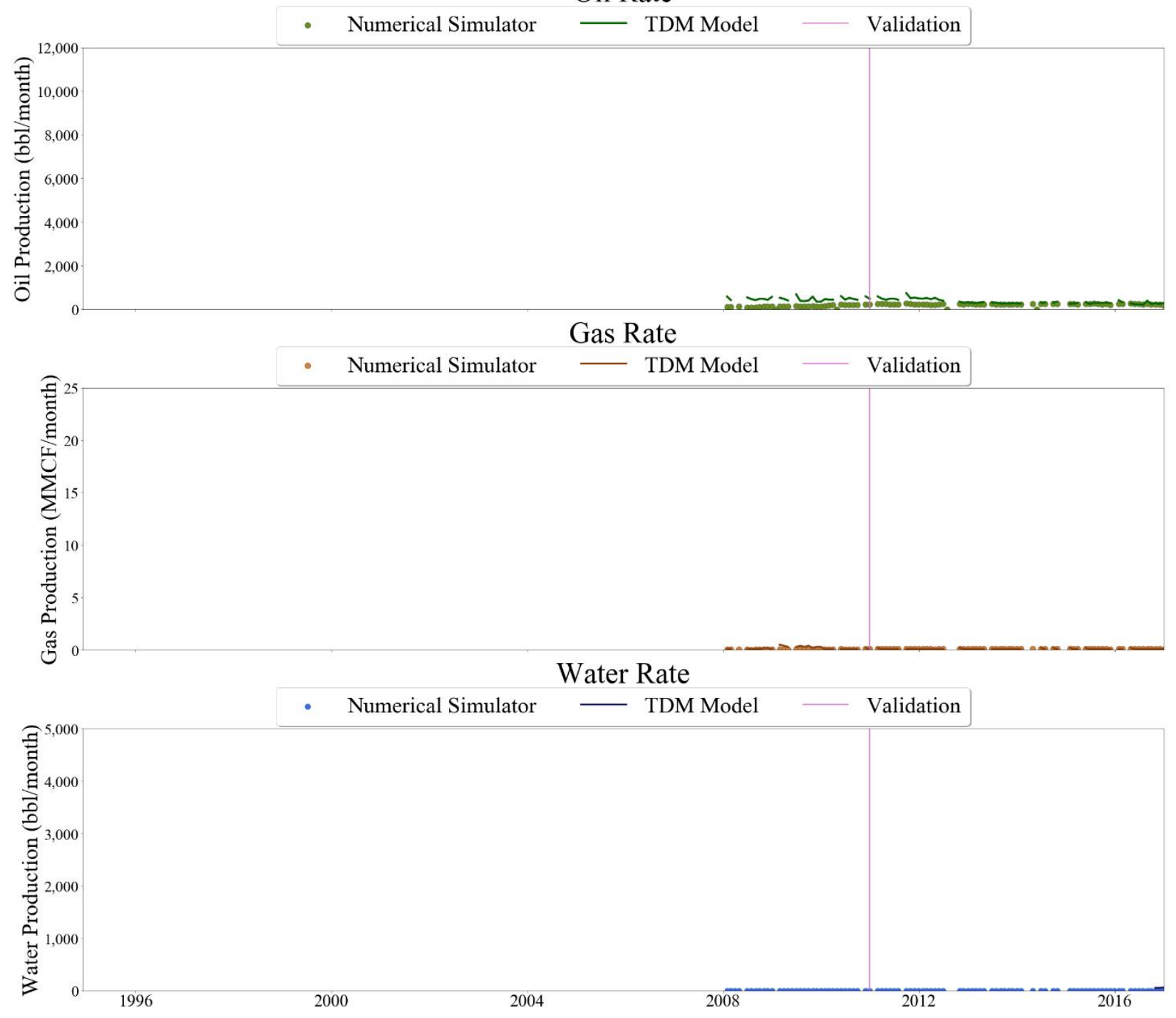

Figure 870 Well-045 oil, gas, and water rate TDM predictions vs actual simulation data plots for 6 Year Blind Validation TDM 


\section{Oil Rate}
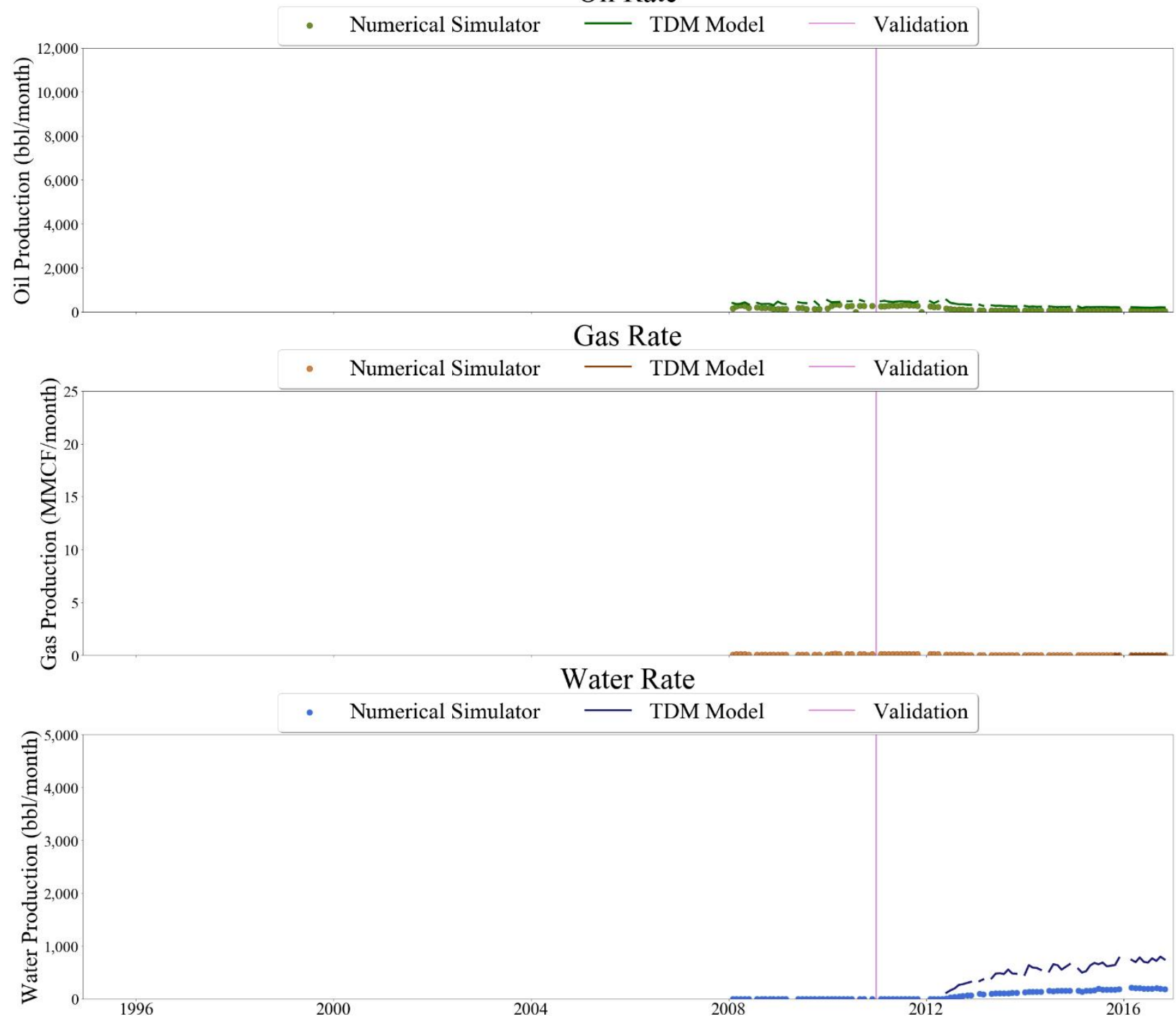

Figure 871 Well-046 oil, gas, and water rate TDM predictions vs actual simulation data plots for 6 Year Blind Validation TDM 


\section{Oil Rate}

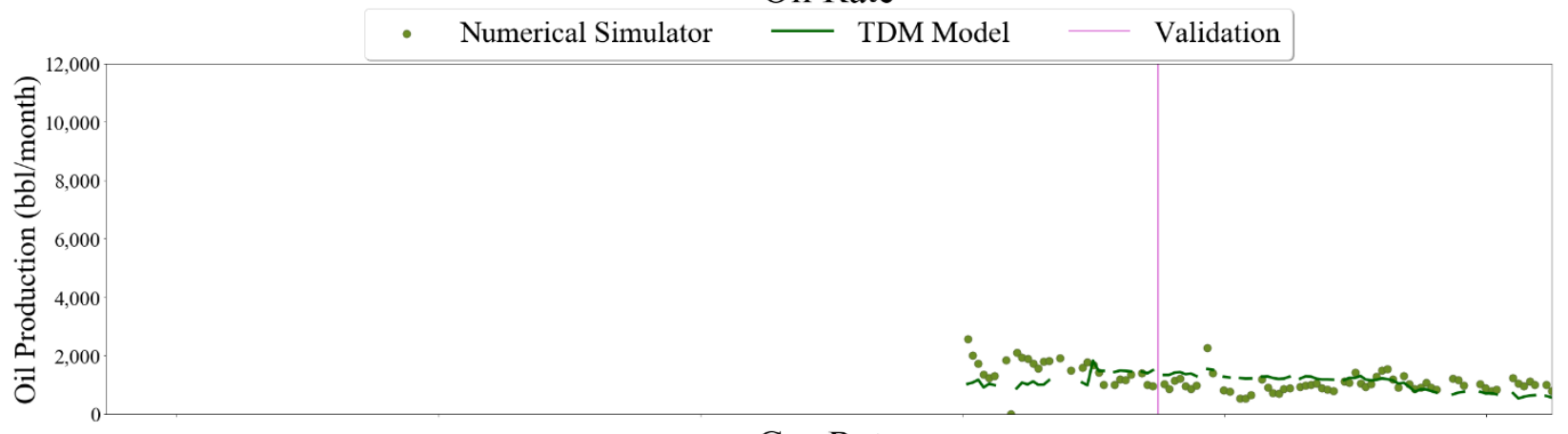

\section{Gas Rate}
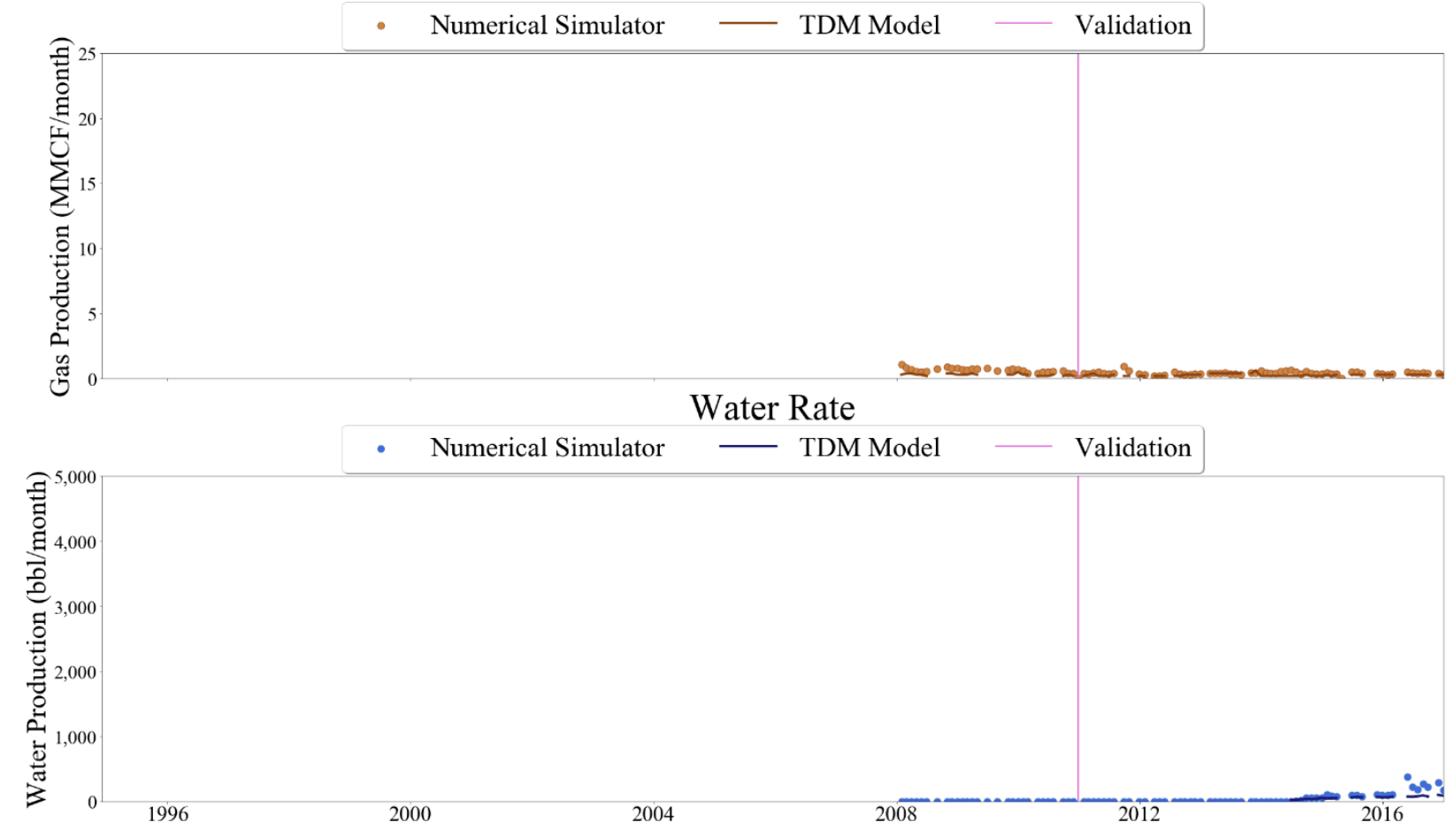

Figure 872 Well-047 oil, gas, and water rate TDM predicitons vs actual simulation data plots for 6 Year Blind Validation TDM 


\section{Oil Rate}

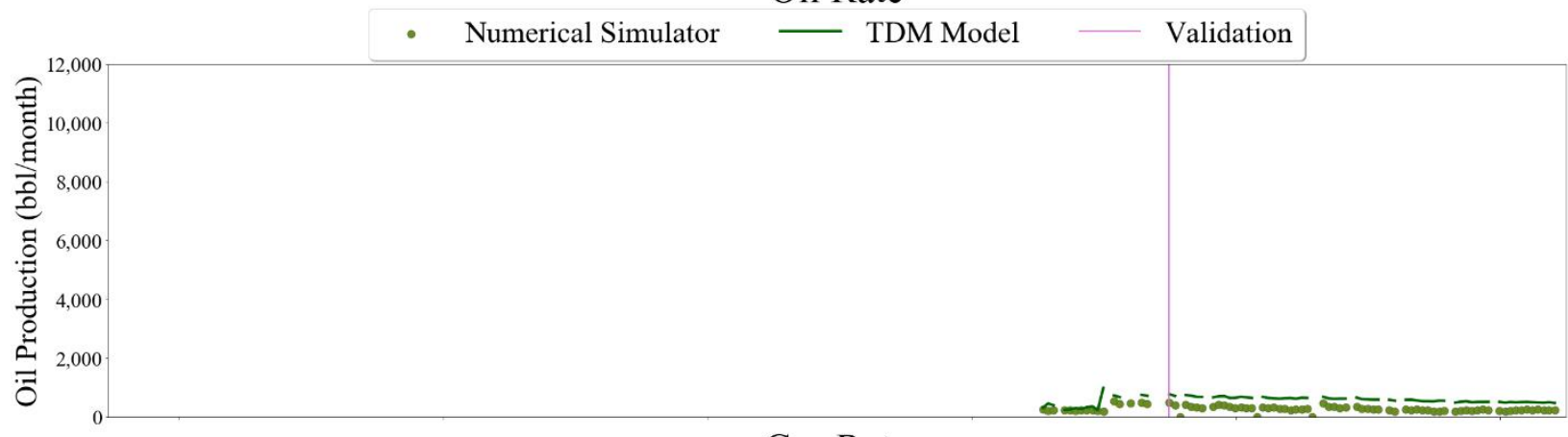

\section{Gas Rate}

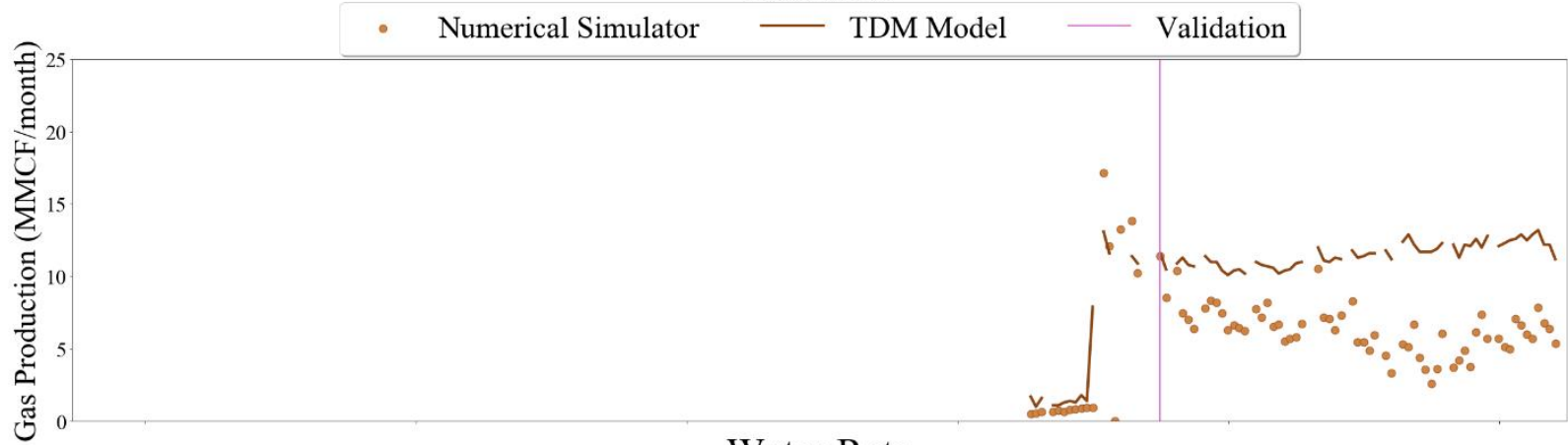

\section{Water Rate}

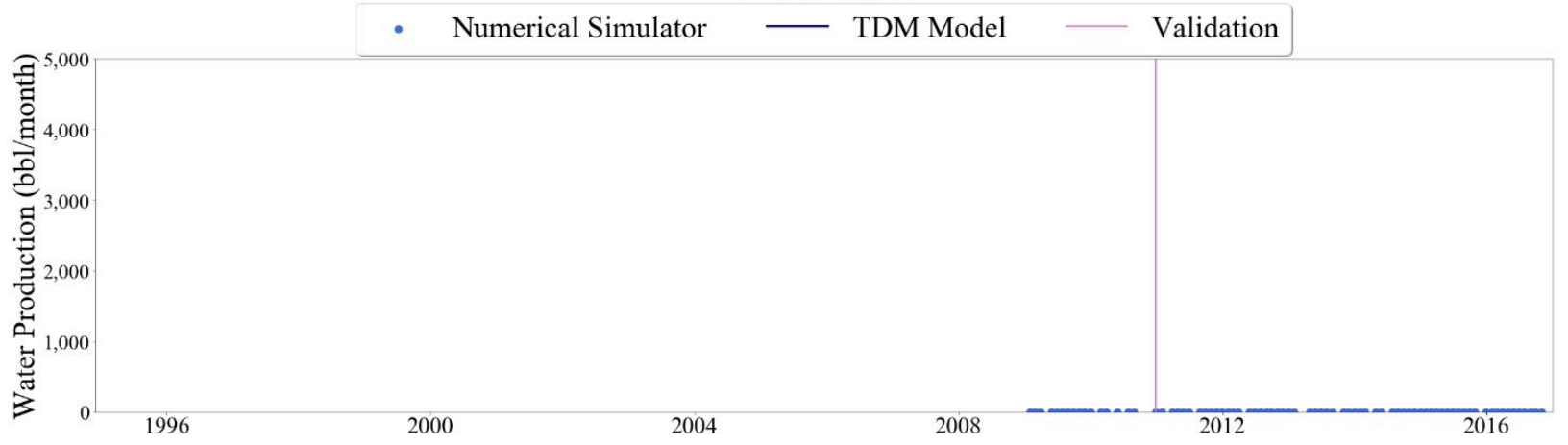

Figure 873 Well-048 oil, gas, and water rate TDM predictions vs actual simulation data plots for 6 Year Blind Validation TDM 


\section{Oil Rate}
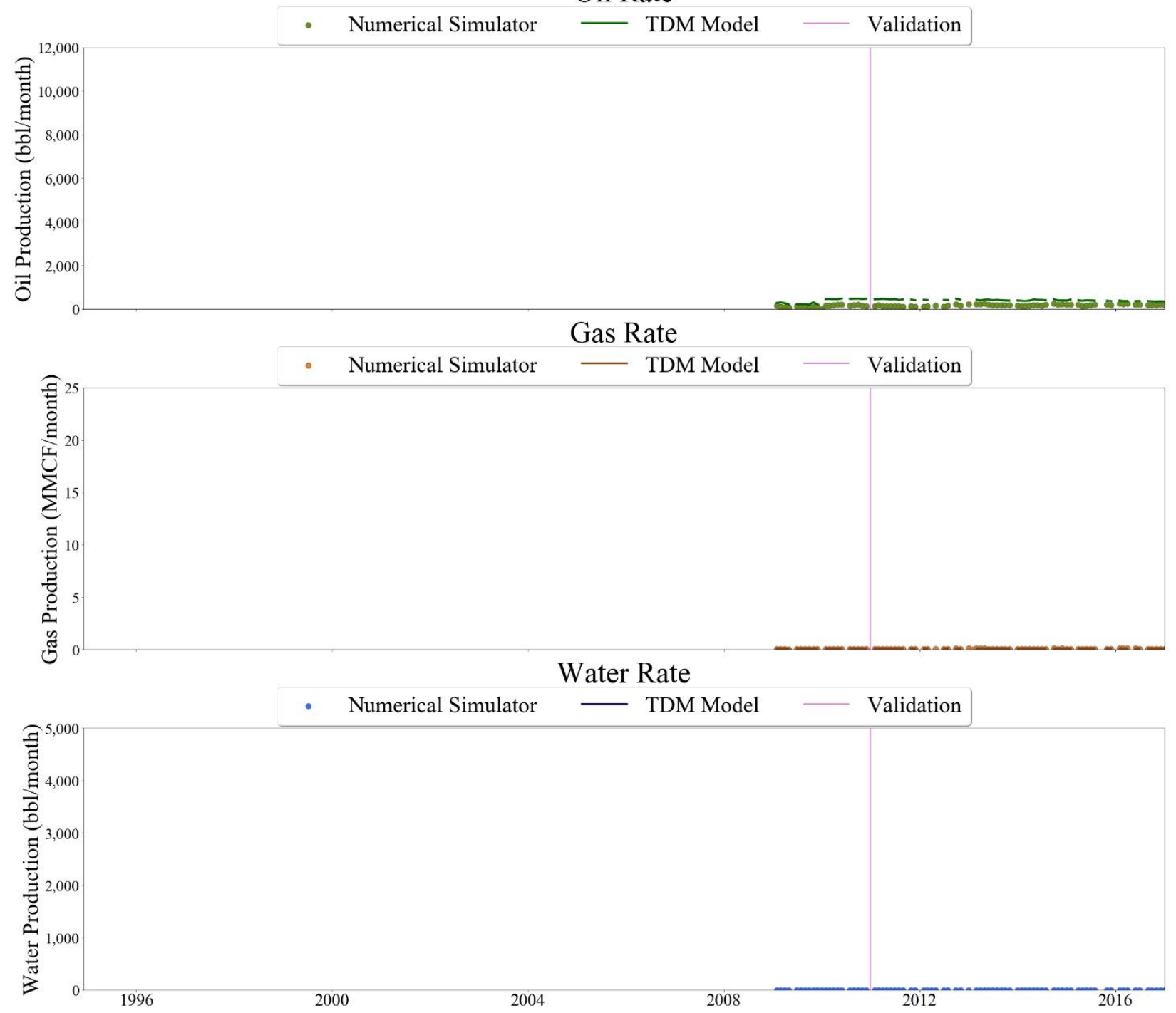

Figure 874 Well-049 oil, gas, and water rate TDM predictions vs actual simulation data plots for 6 Year Blind Validation TDM 


\section{Oil Rate}
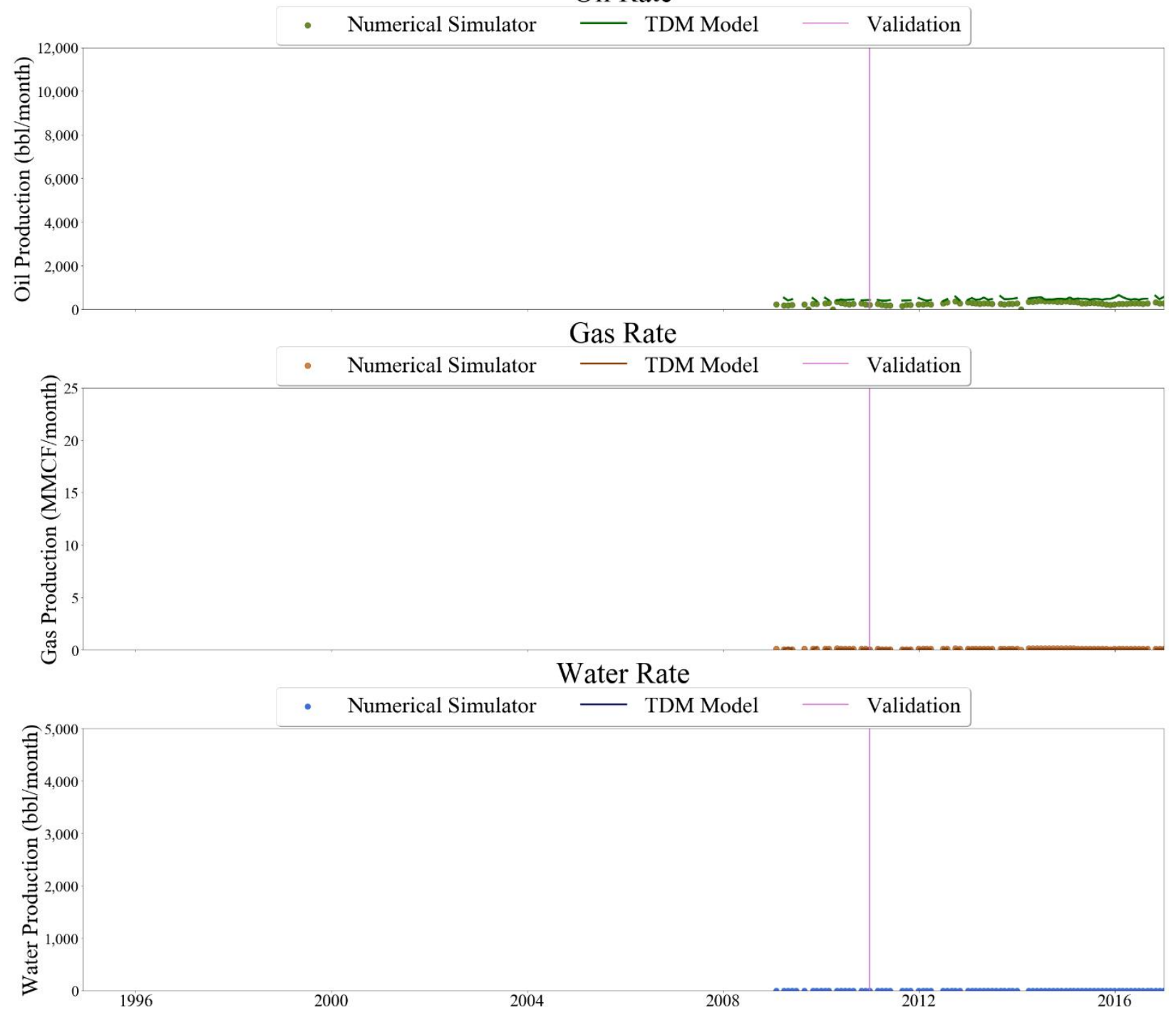

Figure 875 Well-050 oil, gas, and water rate TDM predictions vs actual simulation data plots for 6 Year Blind Validation TDM 


\section{Oil Rate}

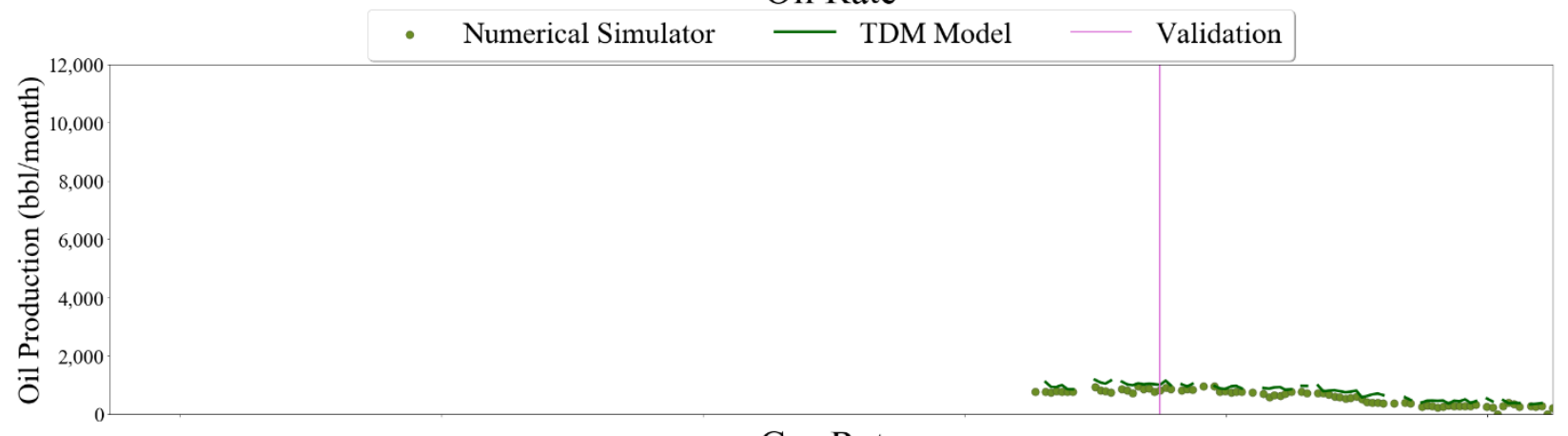

\section{Gas Rate}
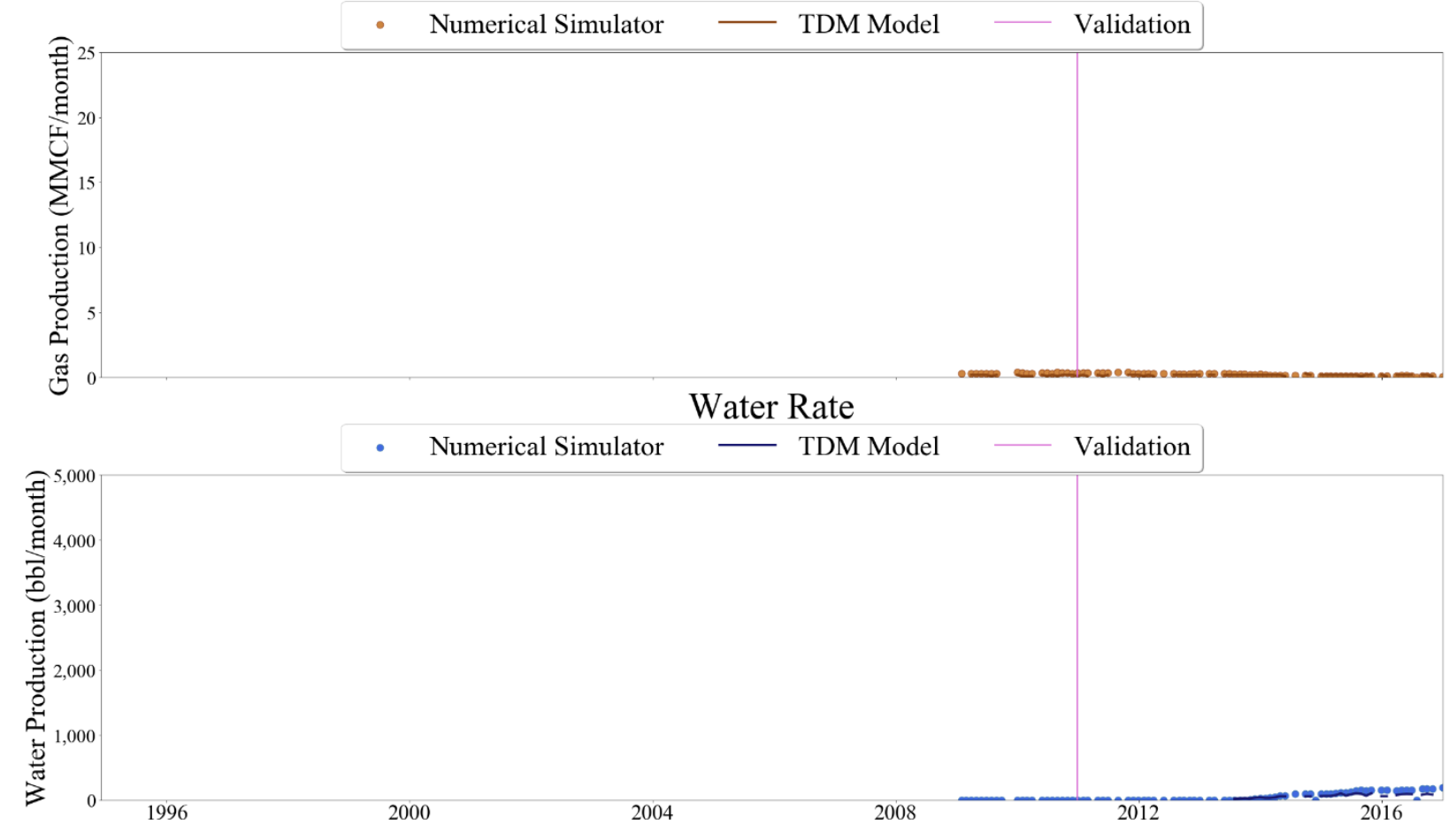

Figure 876 Well-051 oil, gas, and water rate TDM predictions vs actual simulation data plots for 6 Year Blind Validation TDM 


\section{Oil Rate}
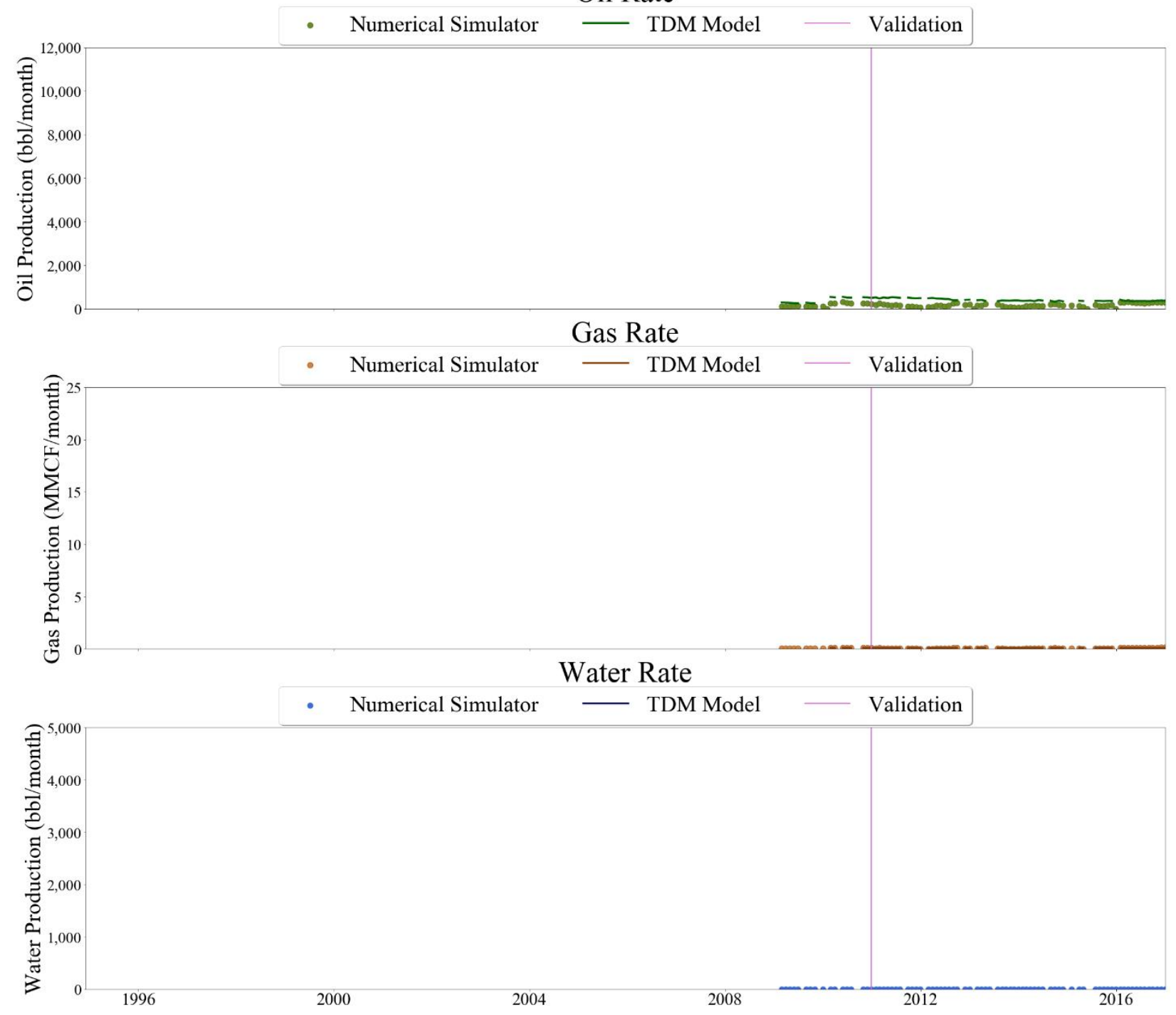

Figure 877 Well-052 oil, gas, and water rate TDM predictions vs actual simulation data plots for 6 Year Blind Validation TDM 


\section{Oil Rate}
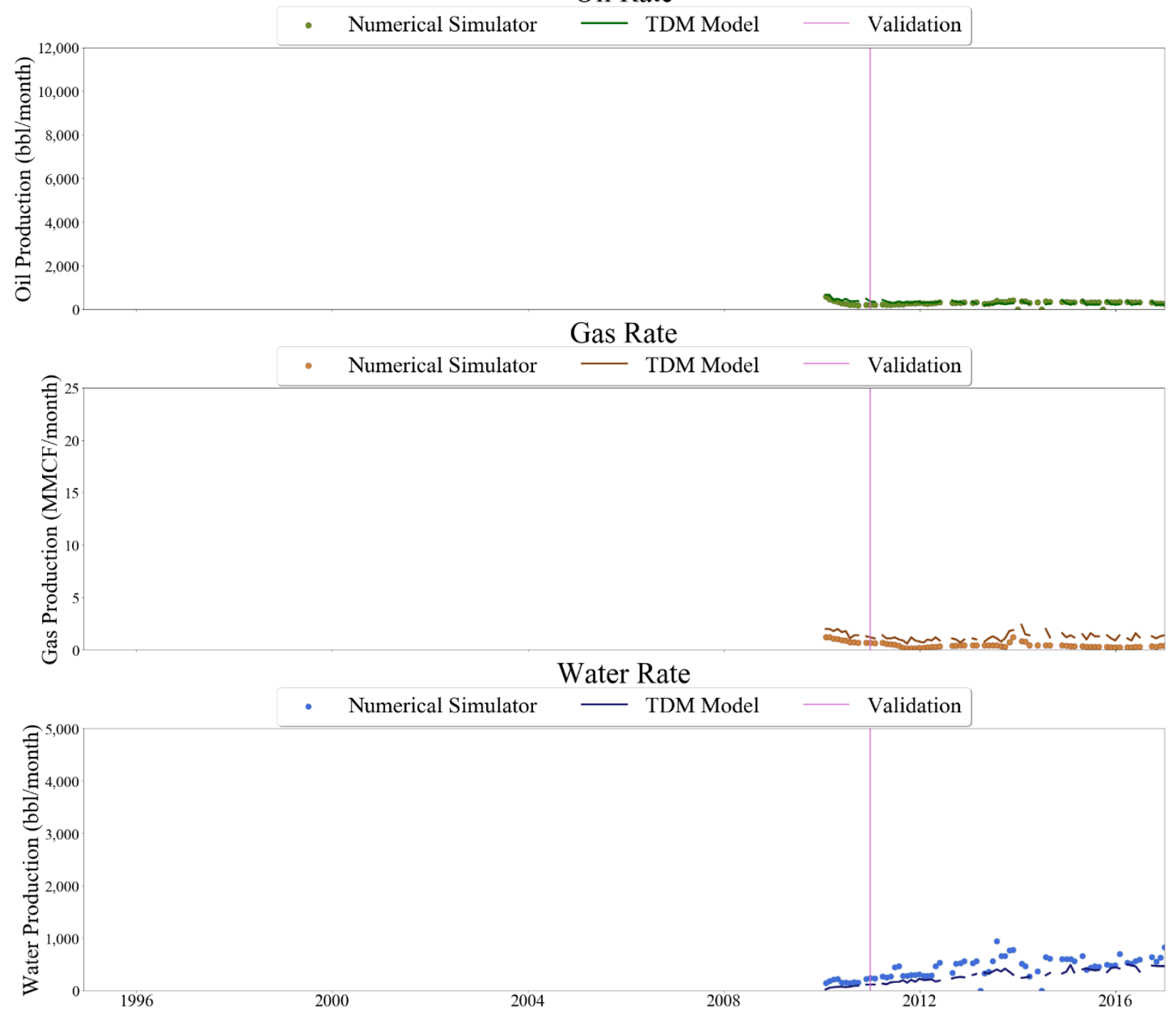

Figure 878 Well-053 oil, gas, and water rate TDM predictions vs actual simulation data plots for 6 Year Blind Validation TDM 


\section{Oil Rate}

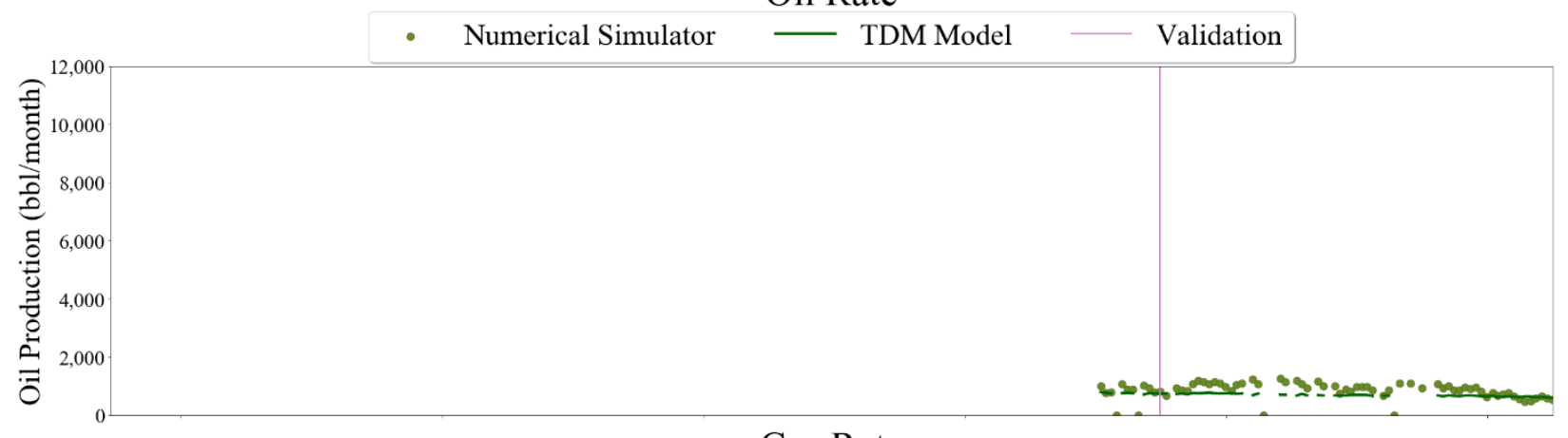

\section{Gas Rate}
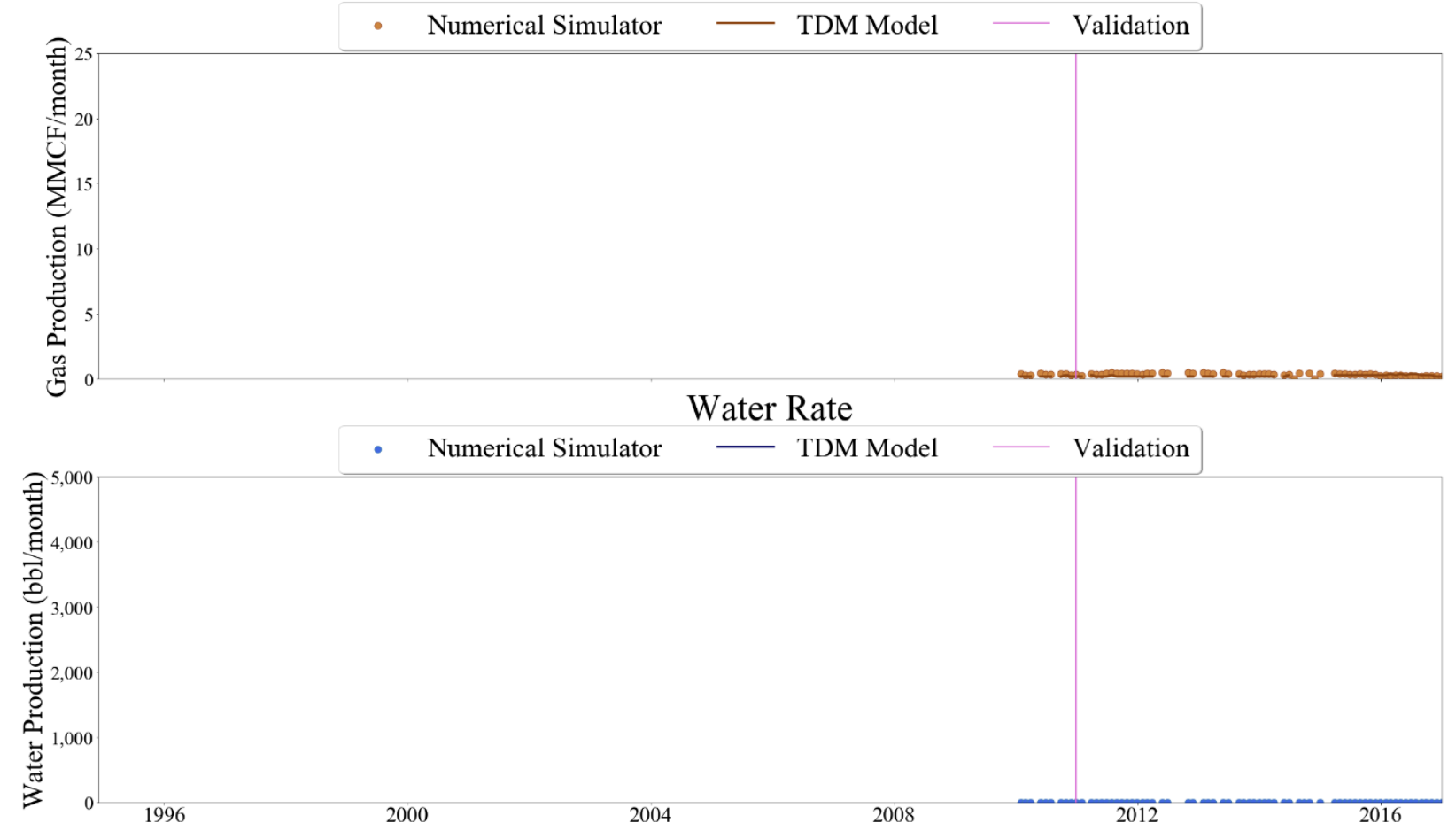

Figure 879 Well-054 oil, gas, and water rate TDM predictions vs actual simulation data plots for 6 Year Blind Validation TDM 


\section{Oil Rate}

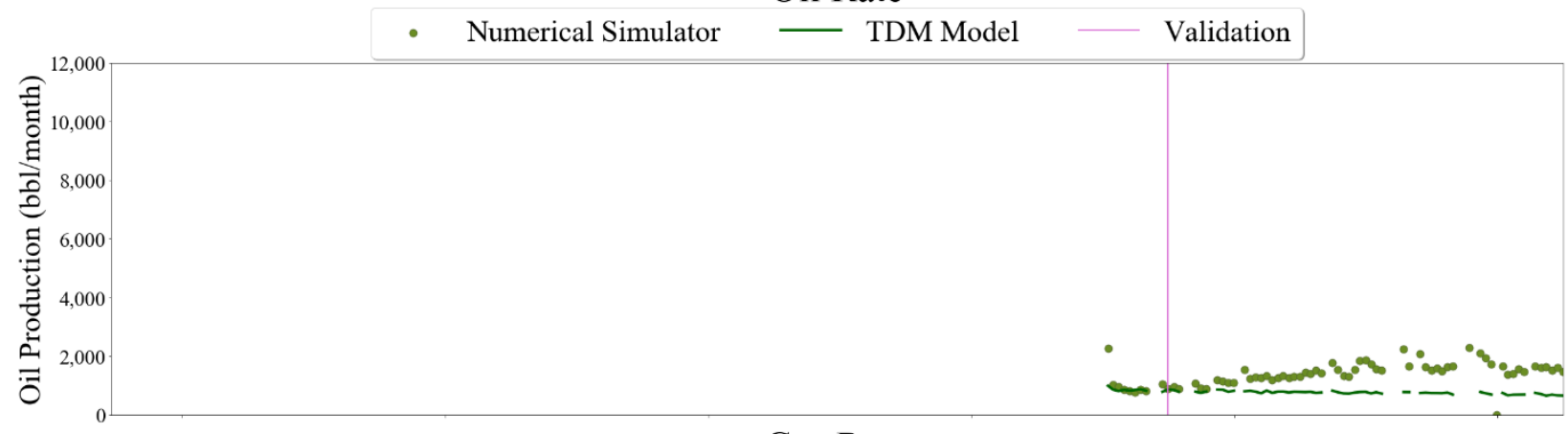

\section{Gas Rate}
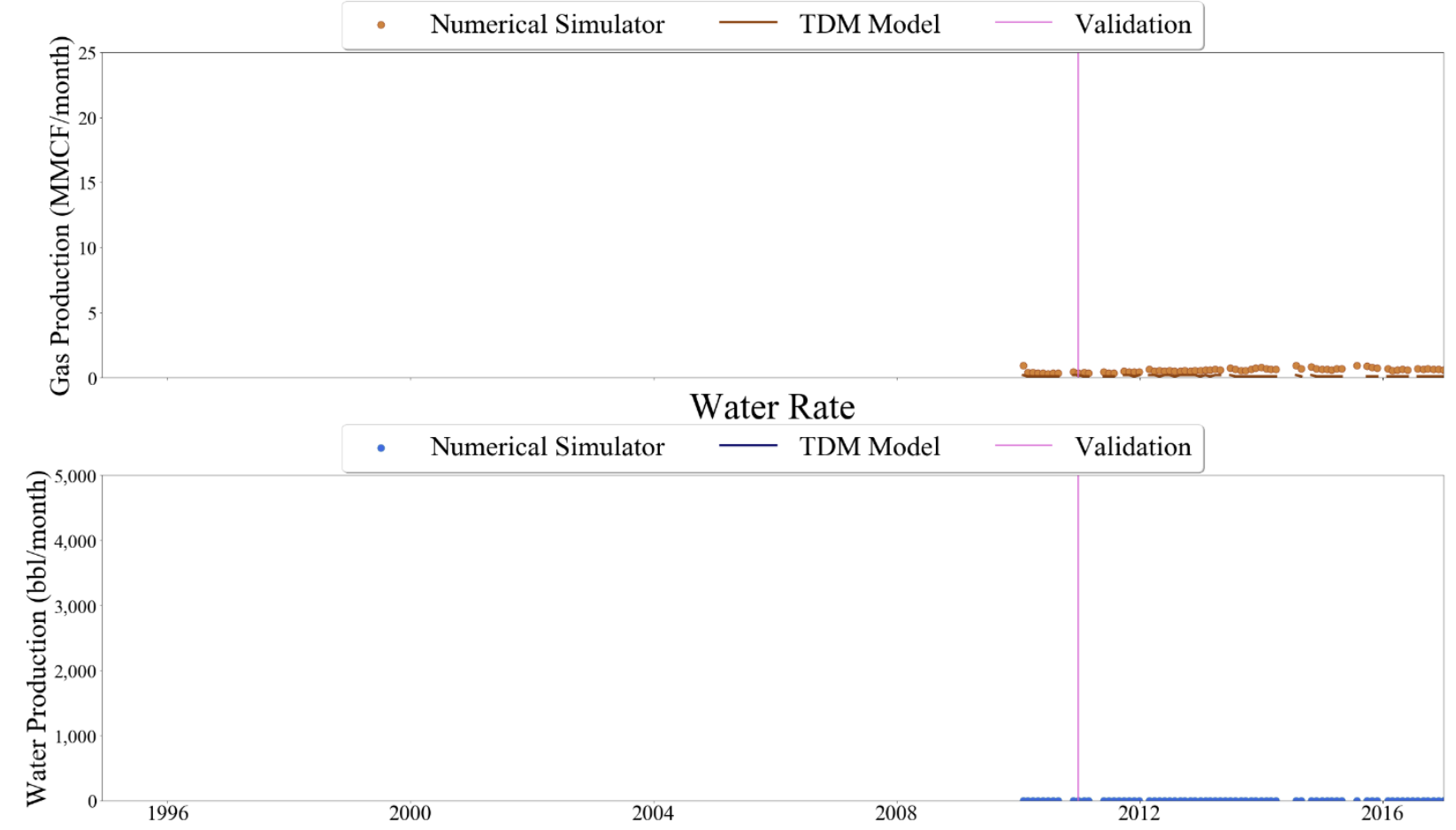

Figure 880 Well-055 oil, gas, and water rate TDM predictions vs actual simulation data plots for 6 Year Blind Validation TDM 


\section{Oil Rate}

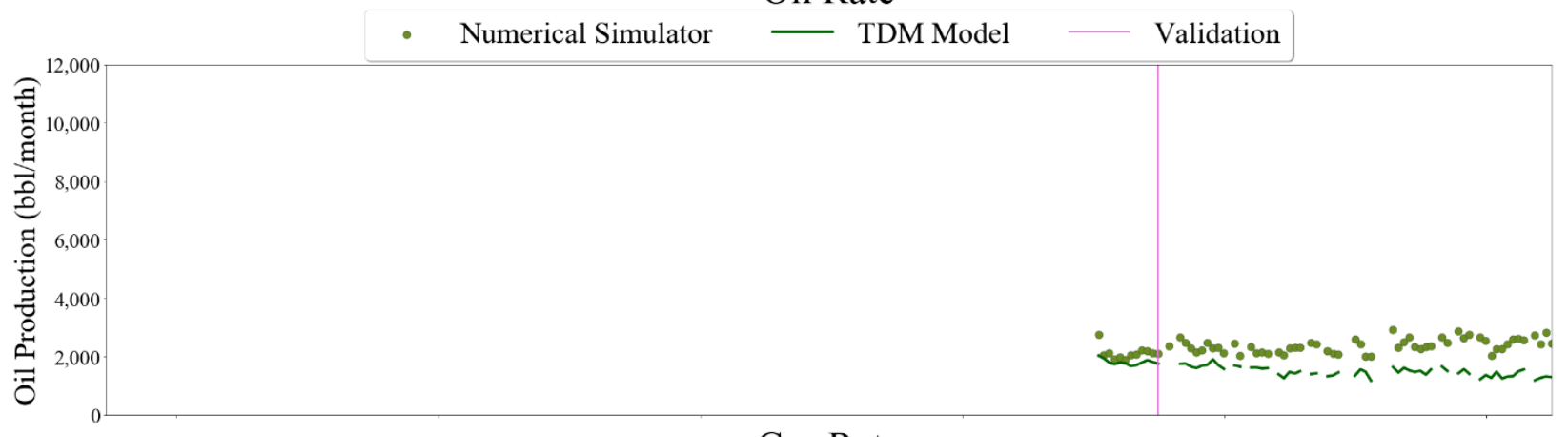

\section{Gas Rate}
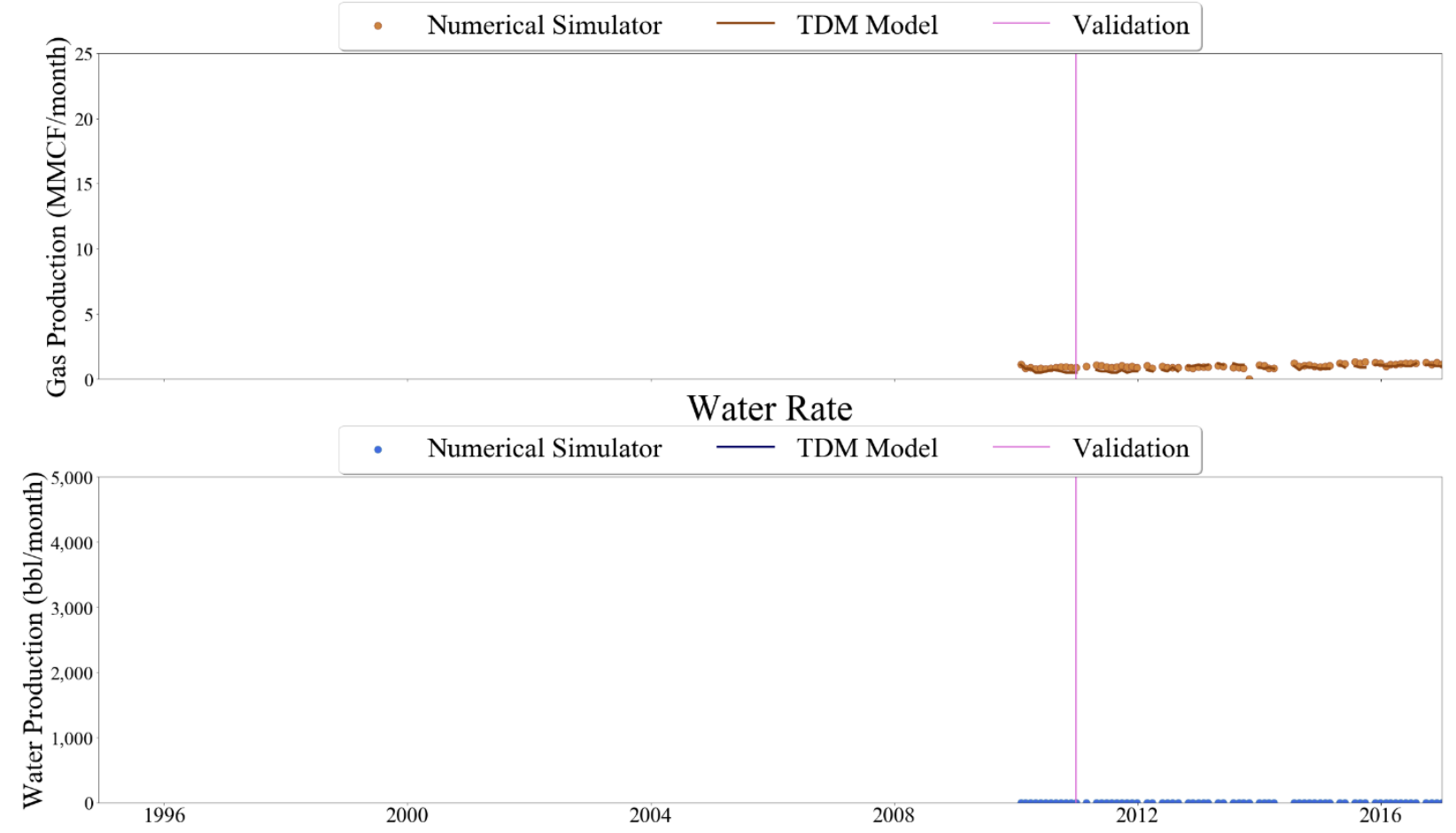

Figure 881 Well-056 oil, gas, and water rate TDM predictions vs actual simulation data plots for 6 Year Blind Validation TDM 


\section{Oil Rate}

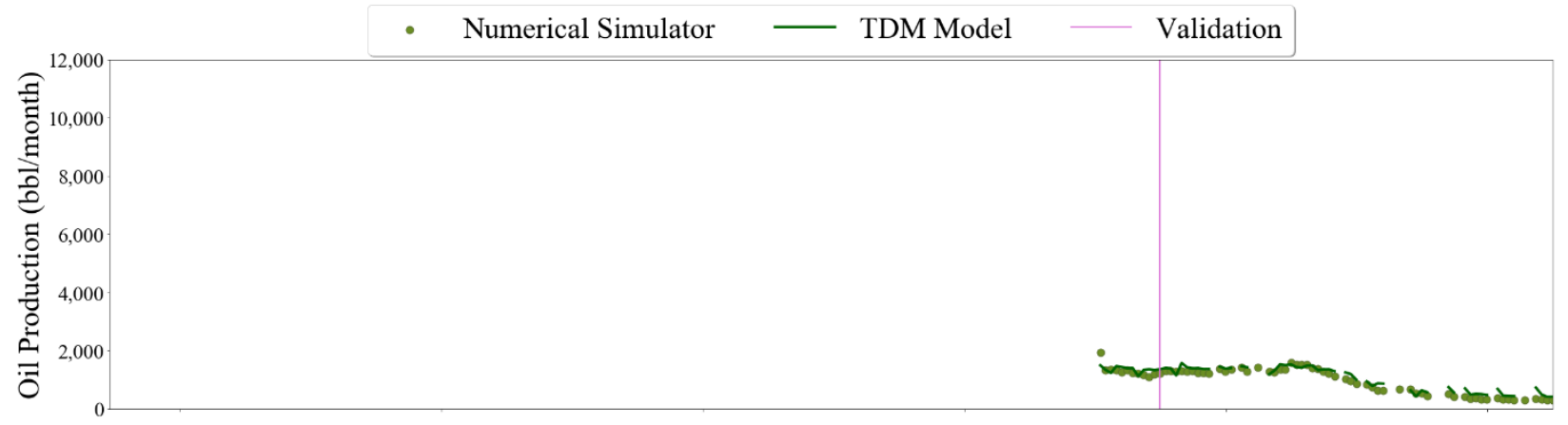

\section{Gas Rate}
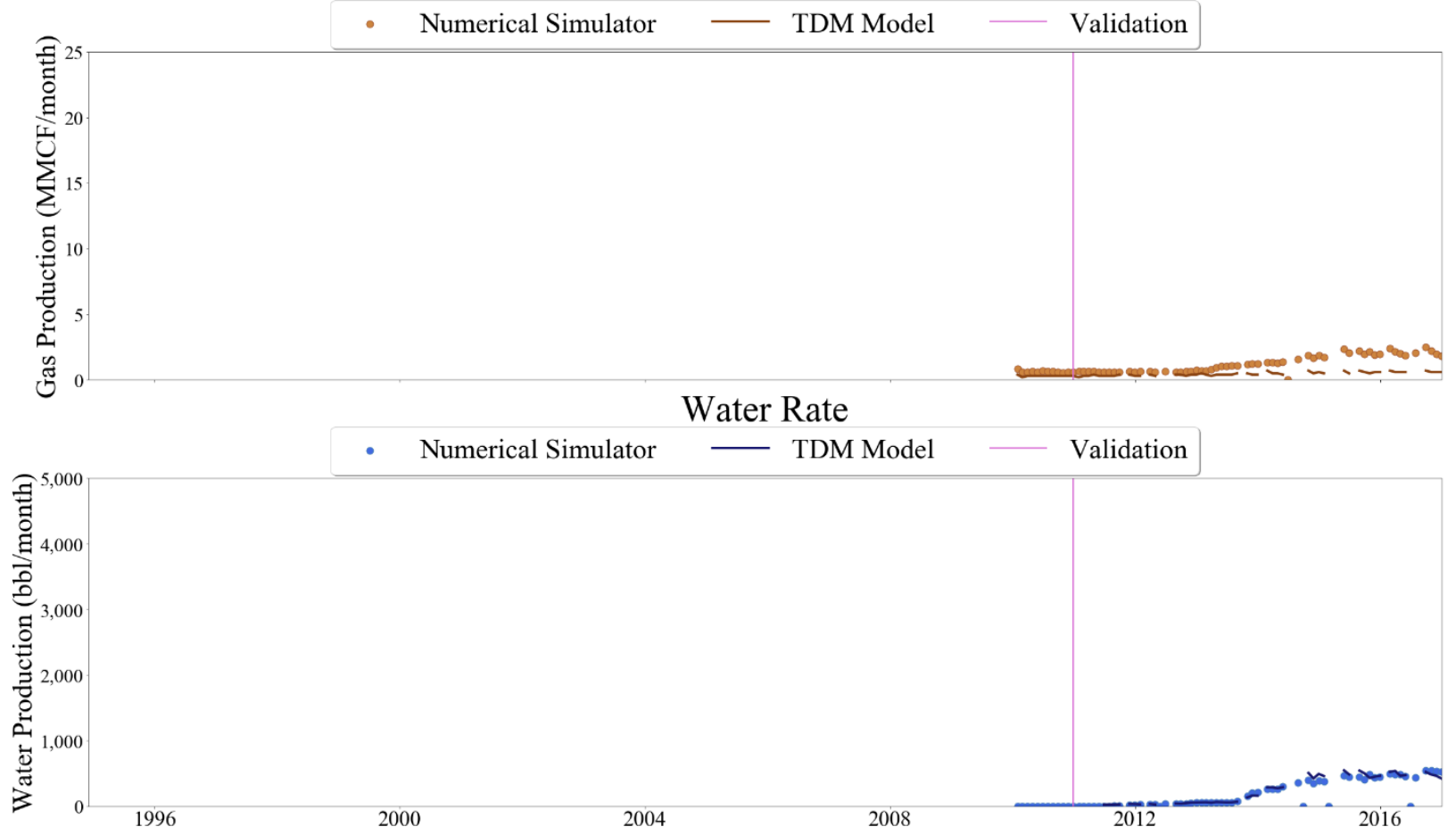

Figure 882 Well-057 oil, gas, and water rate TDM predictions vs actual simulation data plots for 6 Year Blind Validation TDM

\subsubsection{Heat Maps}

Reservoir Pressure (psi) at 2010-12-31
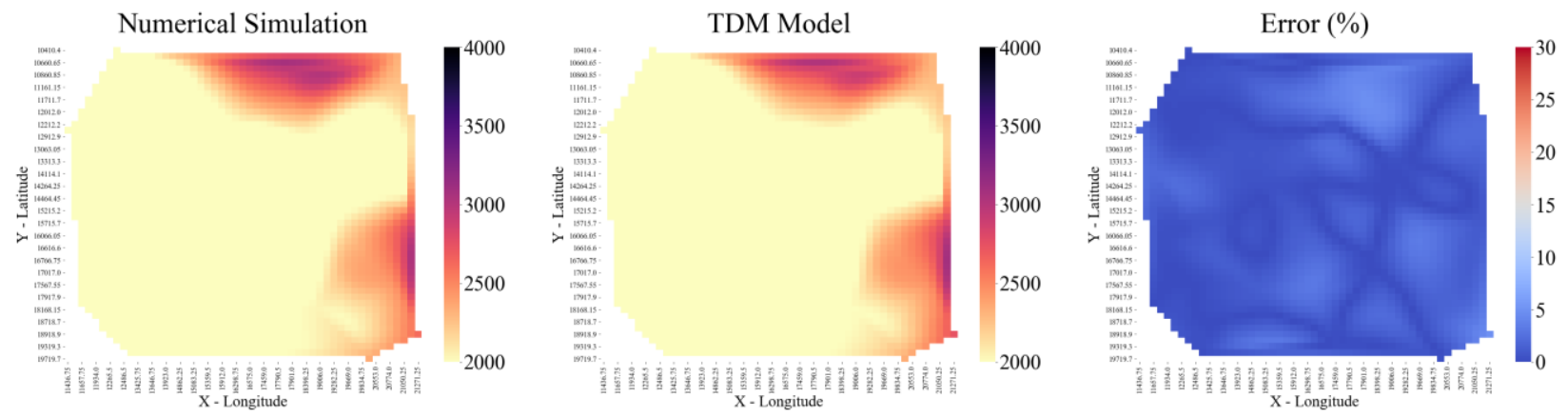

Figure 883 Entire reservoir heat map for reservoir pressure at December 31st, 2010 for 6 Year Blind Validation TDM 
Reservoir Pressure (psi) at 2011-06-30
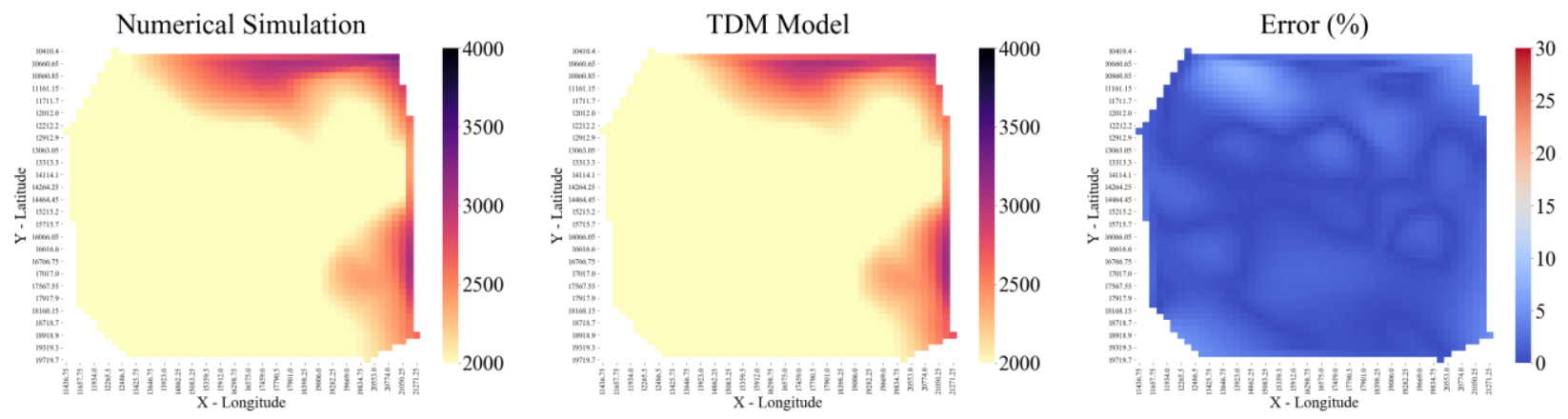

Figure 884 Entire reservoir heat map for reservoir pressure at June 30th, 2011 for 6 Year Blind Validation TDM

Reservoir Pressure (psi) at 2011-12-31
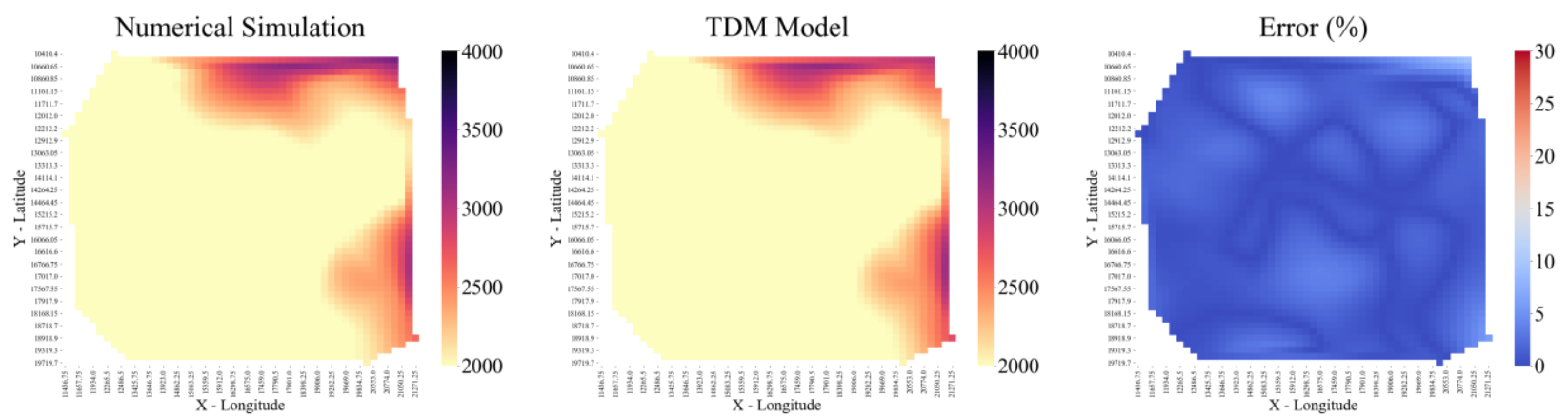

Figure 885 Entire reservoir heat map for reservoir pressure at December 31st, 2011 for 6 Year Blind Validation TDM

Reservoir Pressure (psi) at 2012-06-30
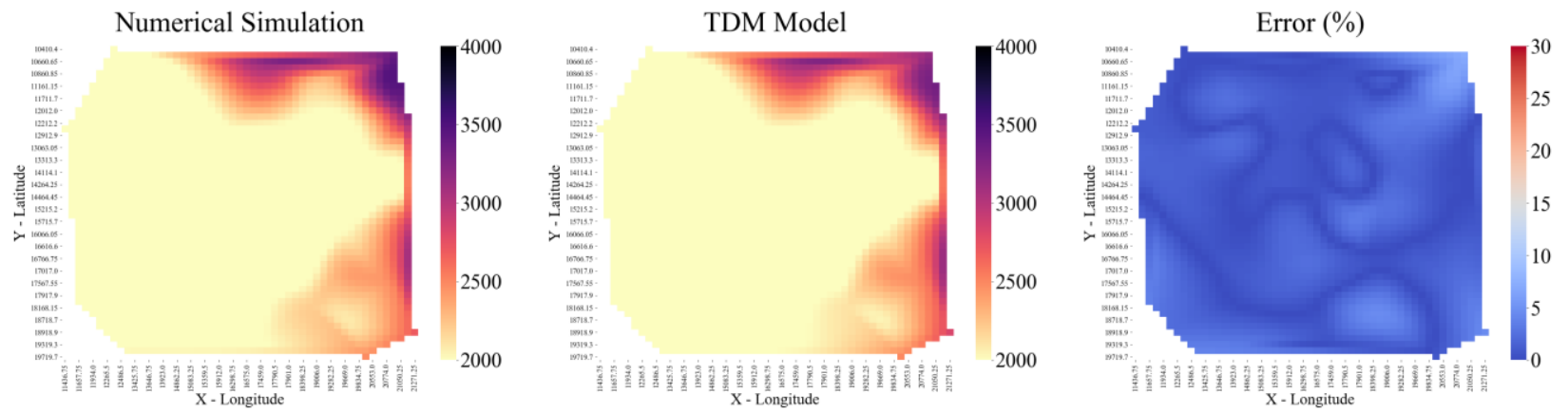

Figure 886 Entire reservoir heat map for reservoir pressure at June 30th, 2012 for 6 Year Blind Validation TDM 
Reservoir Pressure (psi) at 2012-12-31
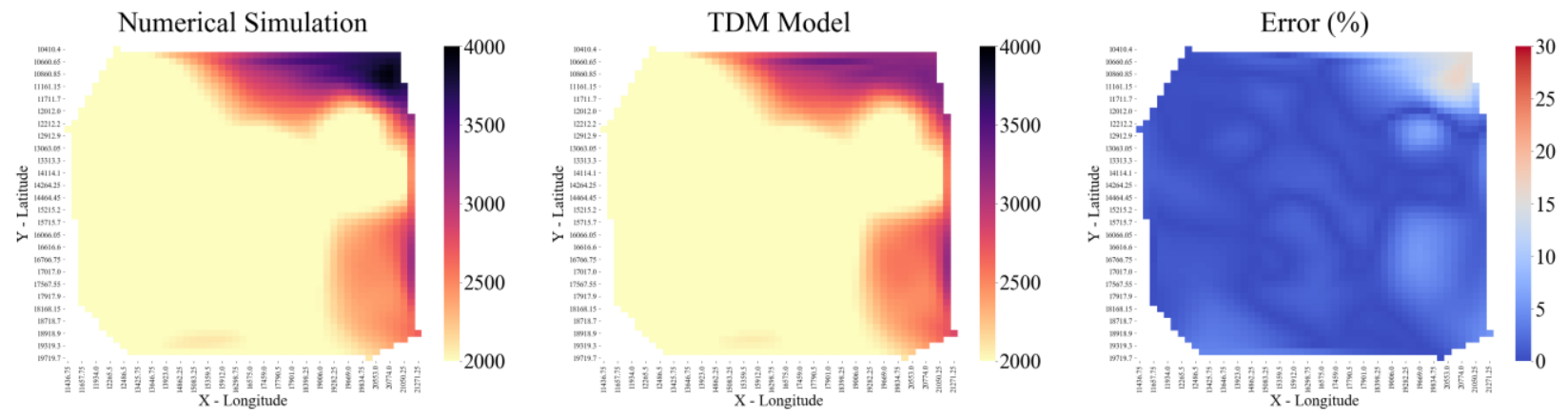

Figure 887 Entire reservoir heat map for reservoir pressure at December 31st, 2012 for 6 Year Blind Validation TDM

\section{Reservoir Pressure (psi) at 2013-06-30}
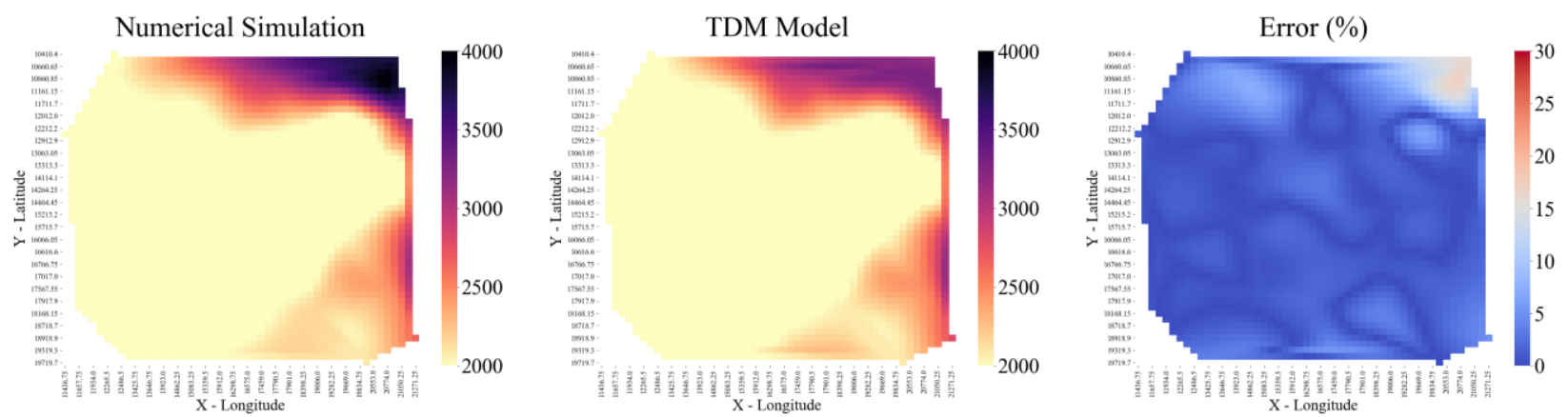

Figure 888 Entire reservoir heat map for reservoir pressure at June 30th, 2013 for 6 Year Blind Validation TDM

Reservoir Pressure (psi) at 2013-12-31
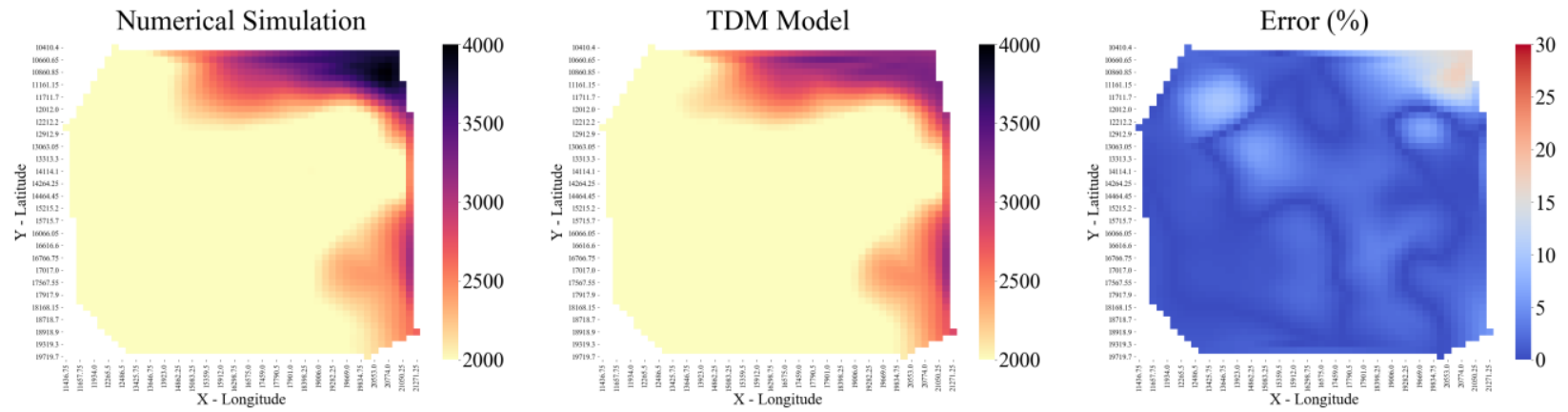

Figure 889 Entire reservoir heat map for reservoir pressure at December 31st, 2013 for 6 Year Blind Validation TDM 
Reservoir Pressure (psi) at 2014-06-30
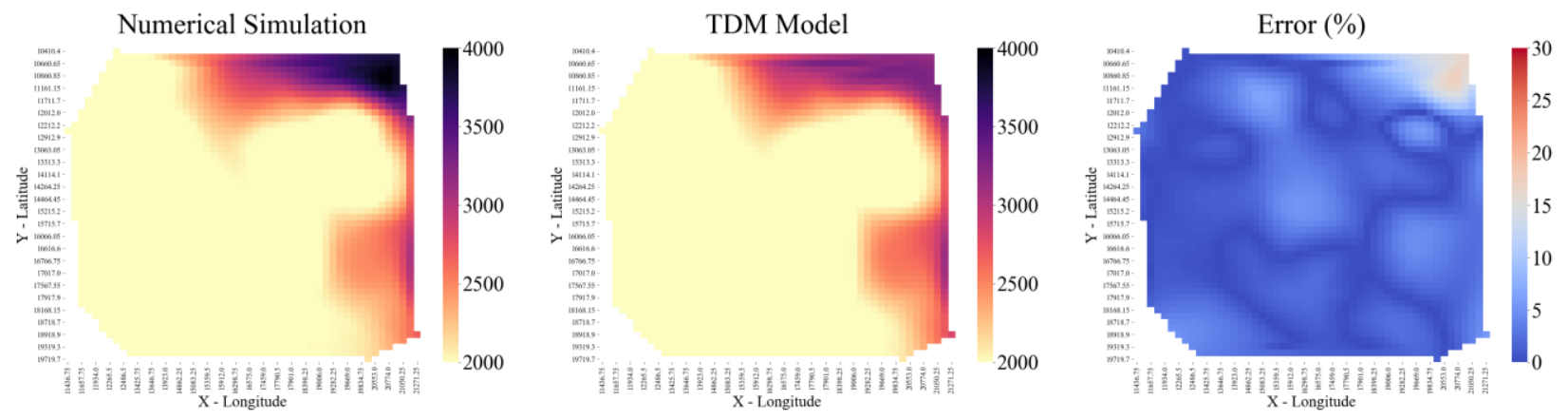

Figure 890 Entire reservoir heat map for reservoir pressure at June 30th, 2014 for 6 Year Blind Validation TDM

Reservoir Pressure (psi) at 2014-12-31
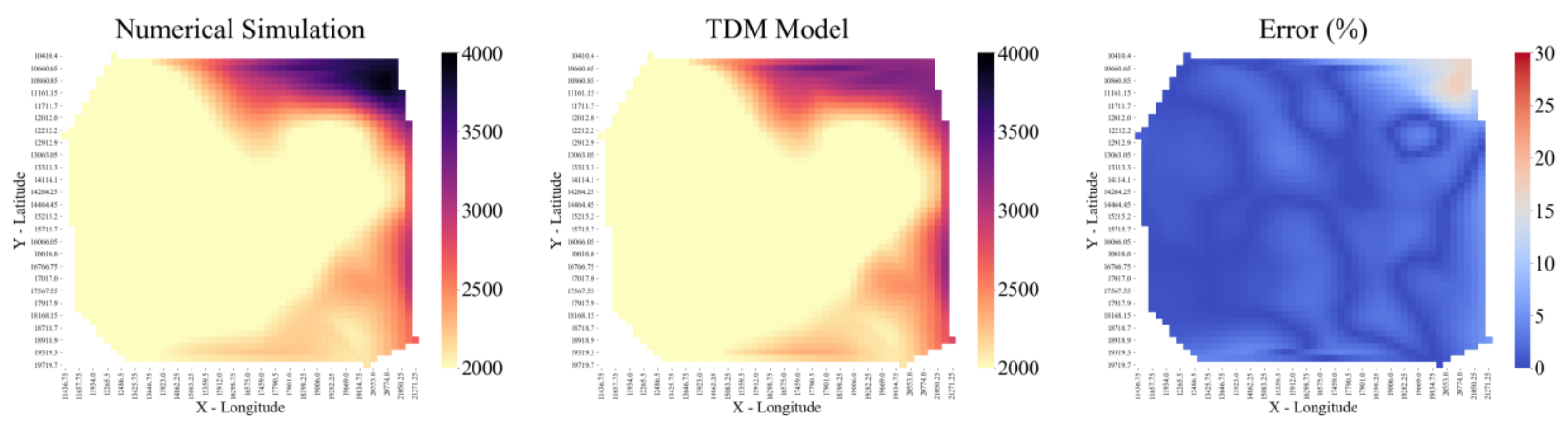

Figure 891 Entire reservoir heat map for reservoir pressure at December 31st, 2014 for 6 Year Blind Validation TDM

Reservoir Pressure (psi) at 2015-06-30
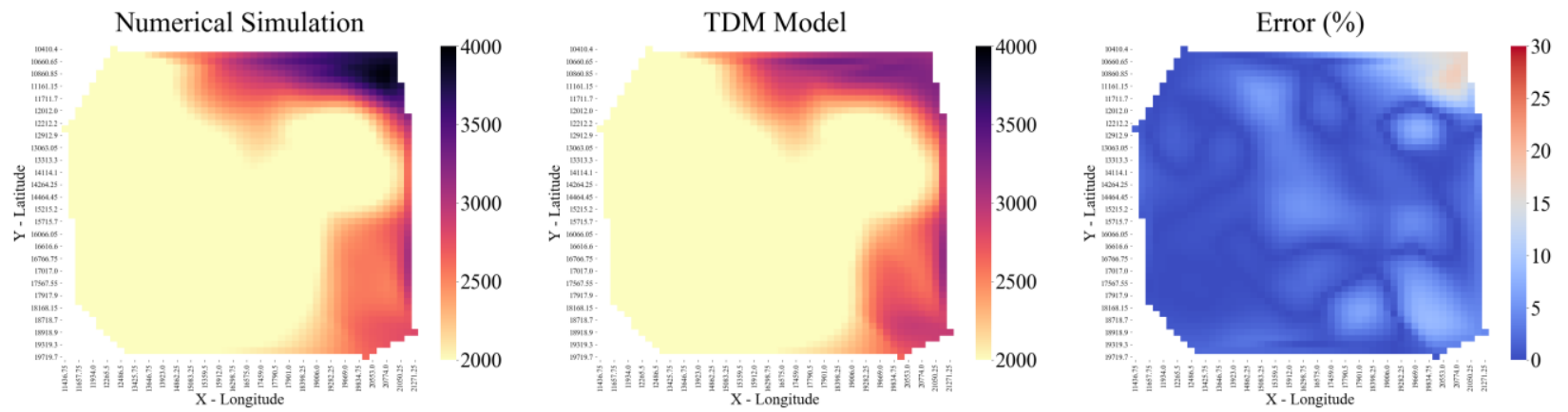

Figure 892 Entire reservoir heat map for reservoir pressure at June 30th, 2015 for 6 Year Blind Validation TDM 
Reservoir Pressure (psi) at 2015-12-31
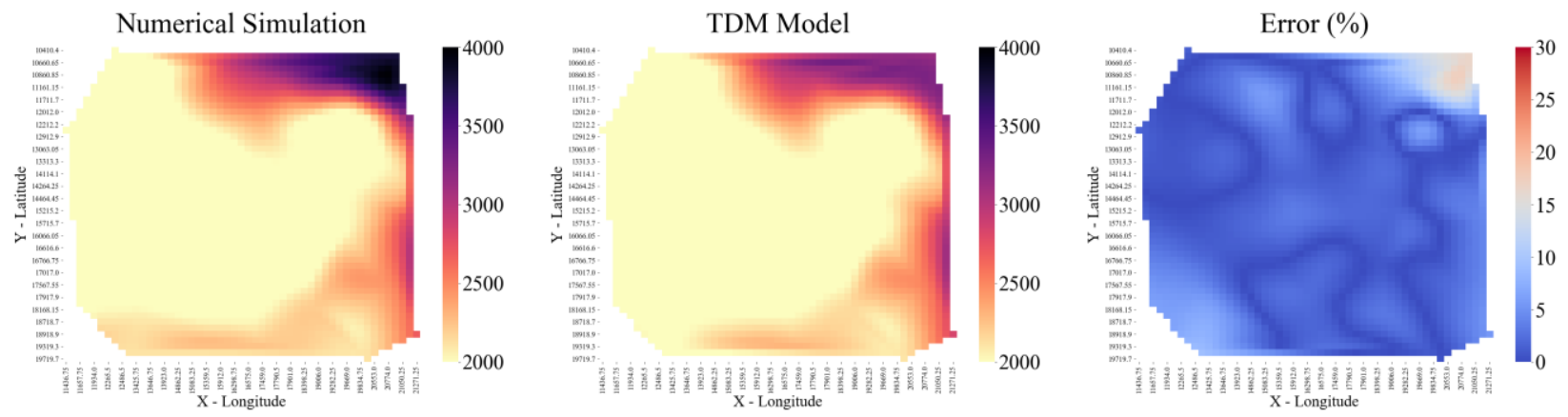

Figure 893 Entire reservoir heat map for reservoir pressure at December 31st, 2015 for 6 Year Blind Validation TDM

\section{Reservoir Pressure (psi) at 2016-06-30}
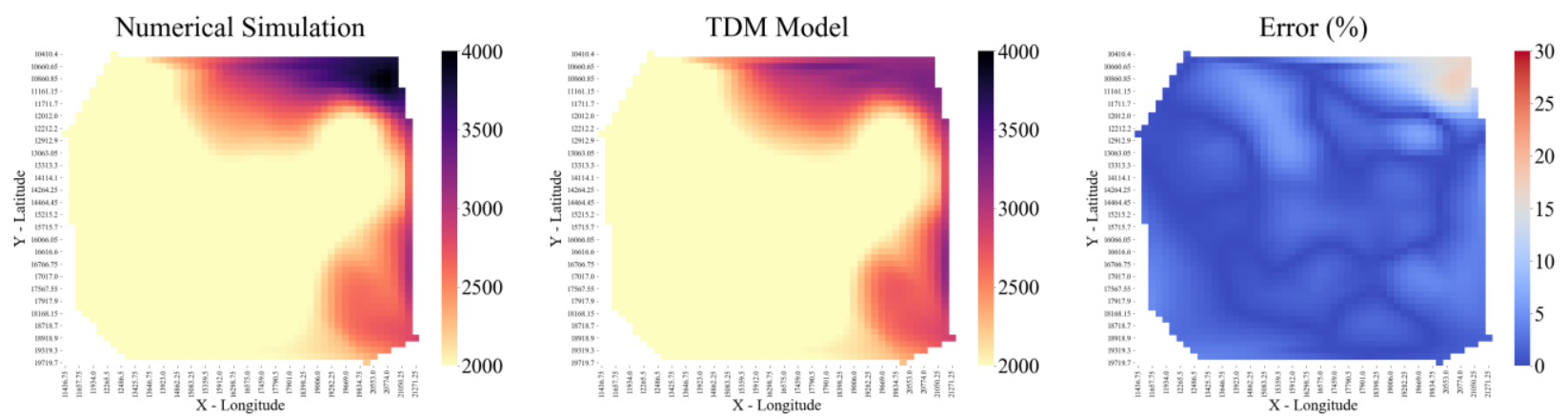

Figure 894 Entire reservoir heat map for reservoir pressure at June 30th, 2016 for 6 Year Blind Validation TDM

Reservoir Pressure (psi) at 2016-12-31
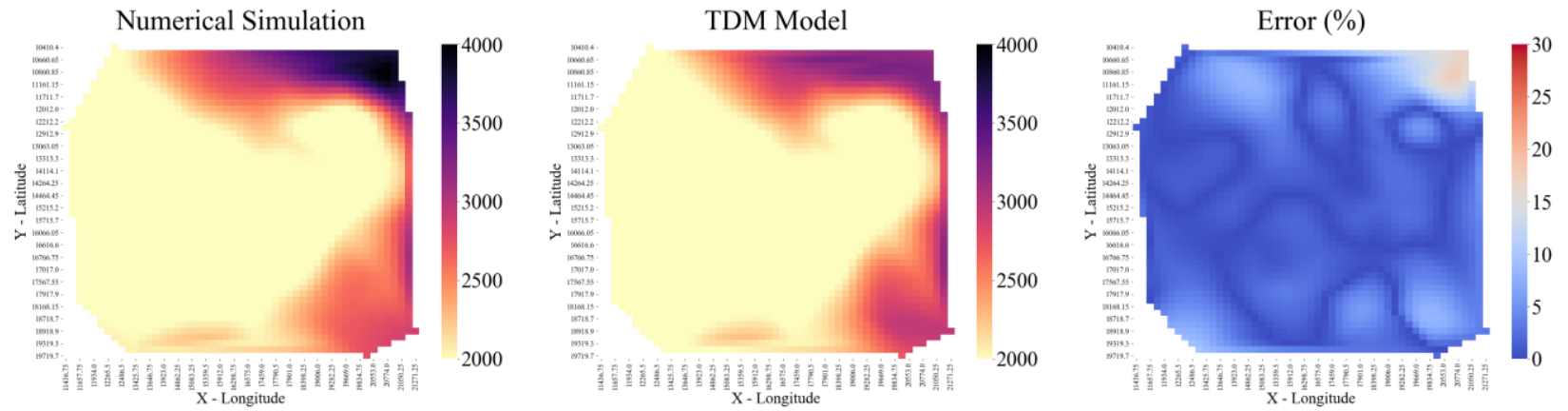

Figure 895 Entire reservoir heat map for reservoir pressure at December 31st, 2016 for 6 Year Blind Validation TDM 
Water Saturation (\%) at 2010-12-31
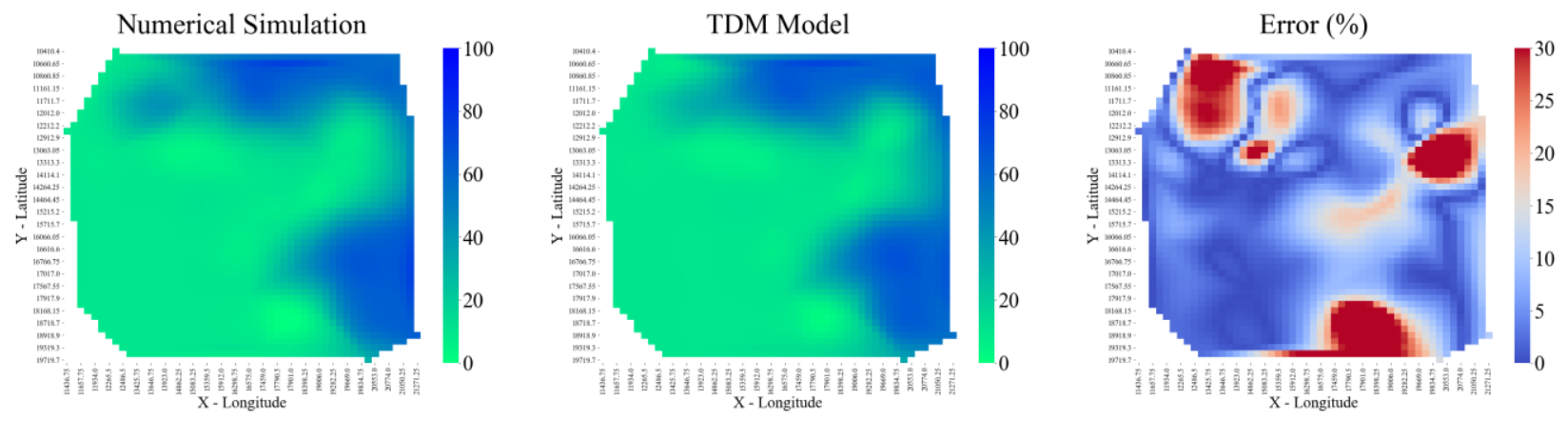

Figure 896 Entire reservoir heat map for water saturation at December 31st, 2010 for 6 Year Blind Validation TDM

Water Saturation (\%) at 2011-06-30
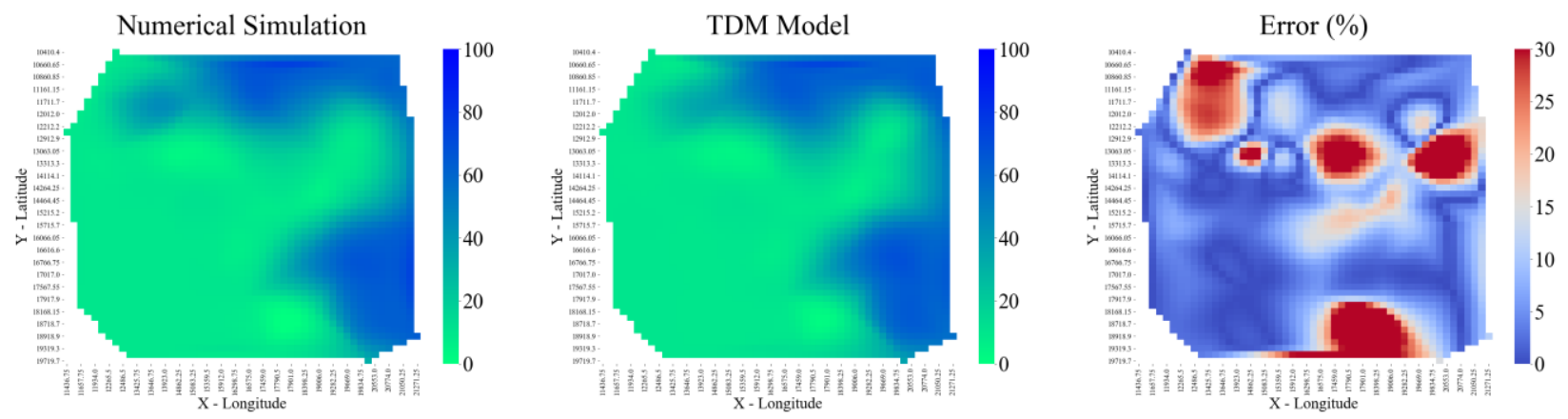

Figure 897 Entire reservoir heat map for water saturation at June 30th, 2011 for 6 Year Blind Validation TDM

Water Saturation (\%) at 2011-12-31
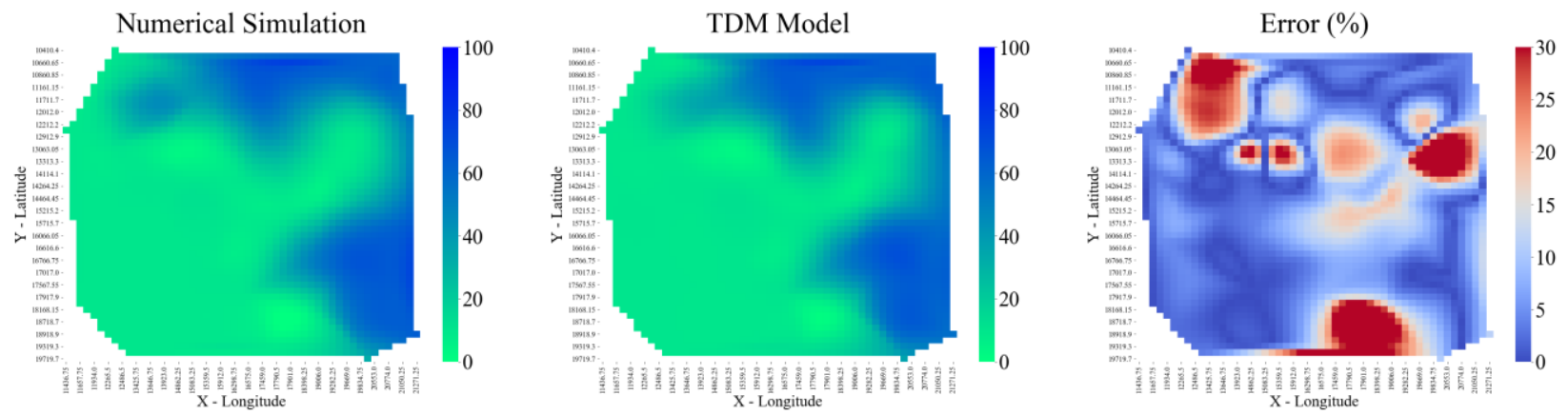

Figure 898 Entire reservoir heat map for water saturation at December 31st, 2011 for 6 Year Blind Validation TDM 
Water Saturation (\%) at 2012-06-30
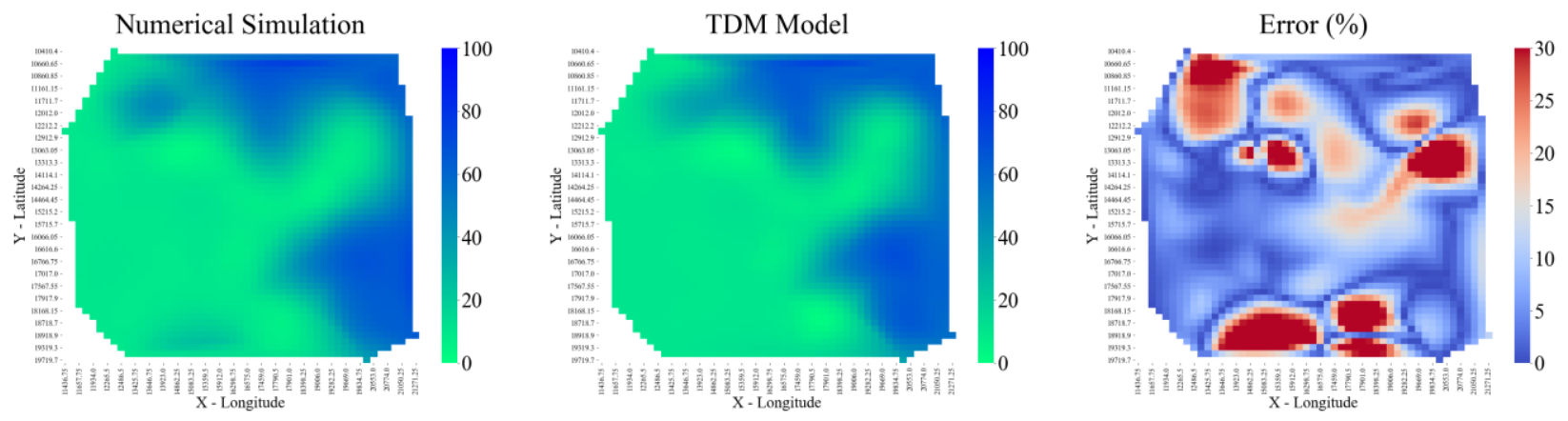

Figure 899 Entire reservoir heat map for water saturation at June 30th, 2012 for 6 Year Blind Validation TDM

Water Saturation (\%) at 2012-12-31
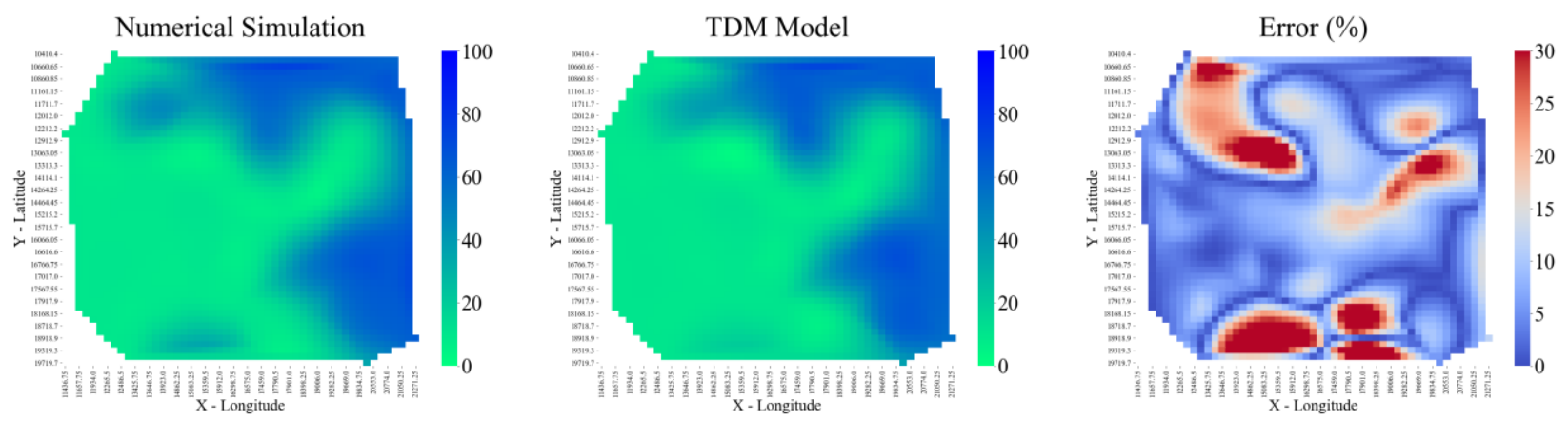

Figure 900 Entire reservoir heat map for water saturation at December 31st, 2012 for 6 Year Blind Validation TDM

Water Saturation (\%) at 2013-06-30
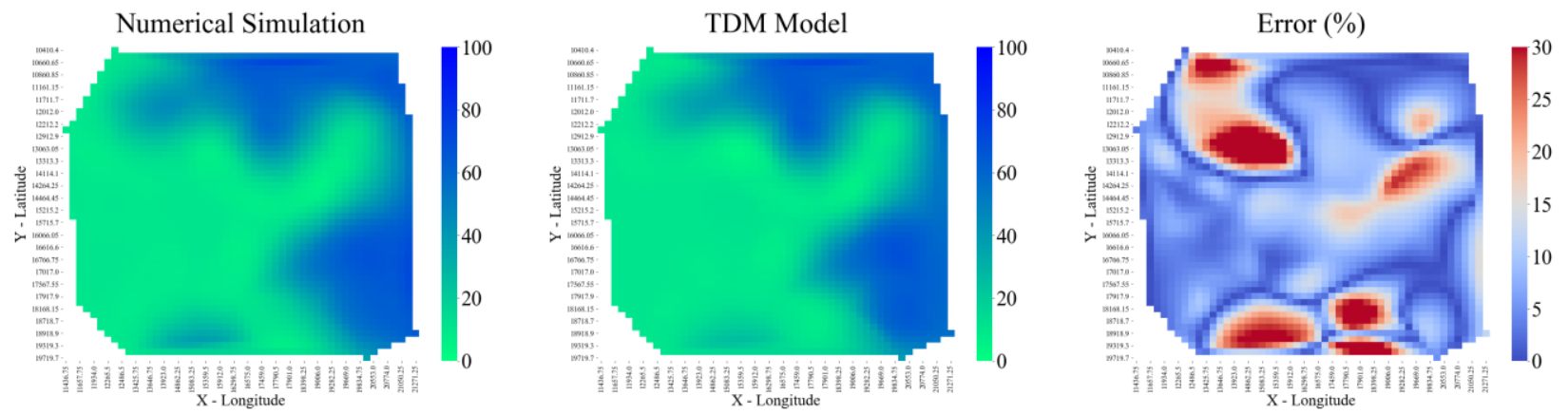

Figure 901 Entire reservoir heat map for water saturation at June 30th, 2013 for 6 Year Blind Validation TDM 
Water Saturation (\%) at 2013-12-31
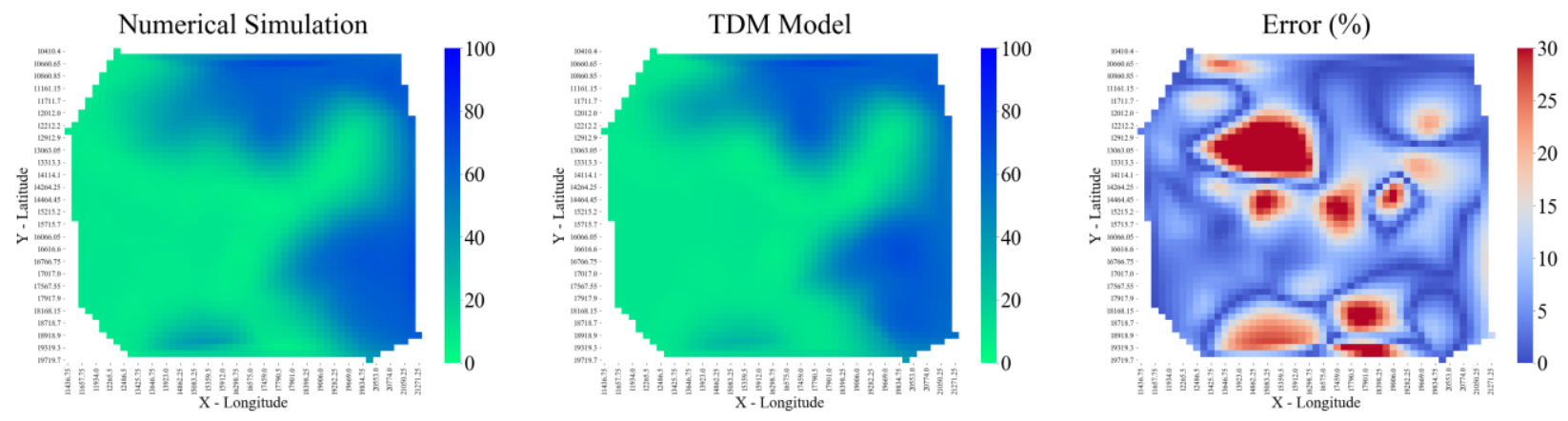

Figure 902 Entire reservoir heat map for water saturation at December 31st, 2013 for 6 Year Blind Validation TDM

Water Saturation (\%) at 2014-06-30
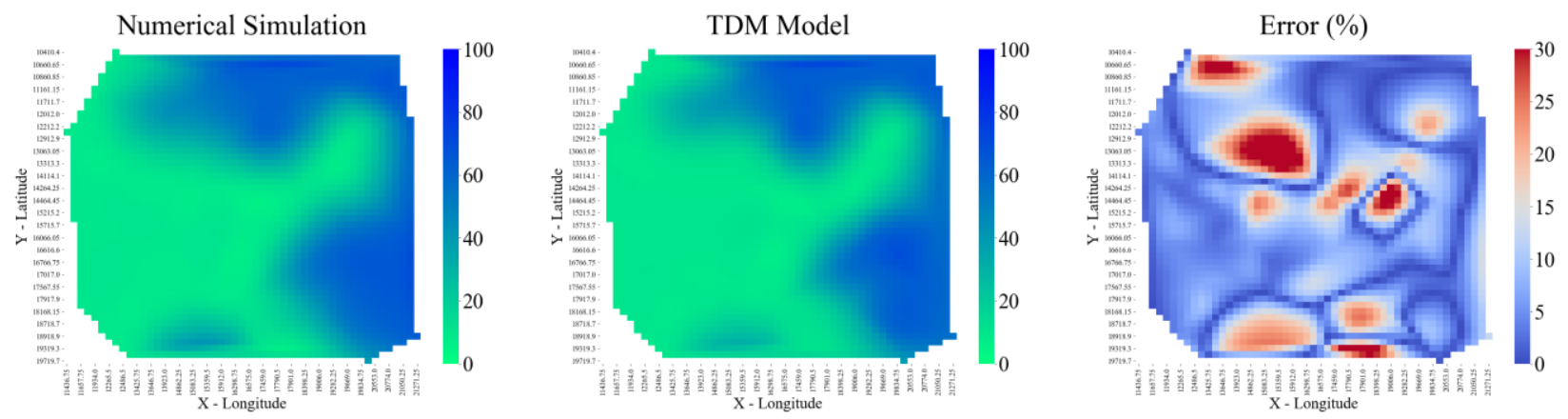

Figure 903 Entire reservoir heat map for water saturation at June 30th, 2014 for 6 Year Blind Validation TDM

Water Saturation (\%) at 2014-12-31
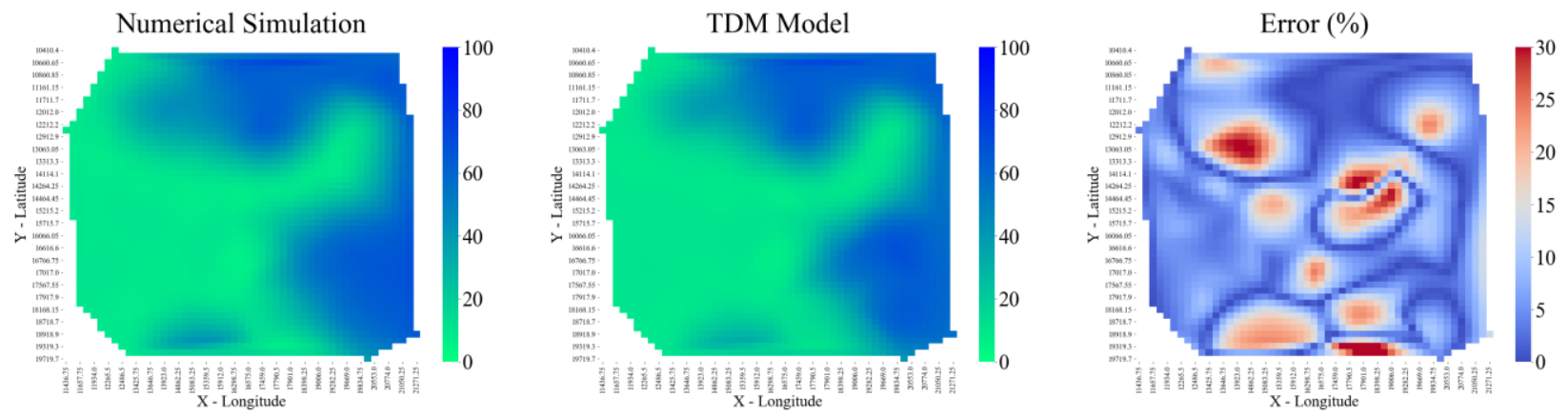

Figure 904 Entire reservoir heat map for water saturation at December 31st, 2014 for 6 Year Blind Validation TDM 
Water Saturation (\%) at 2015-06-30
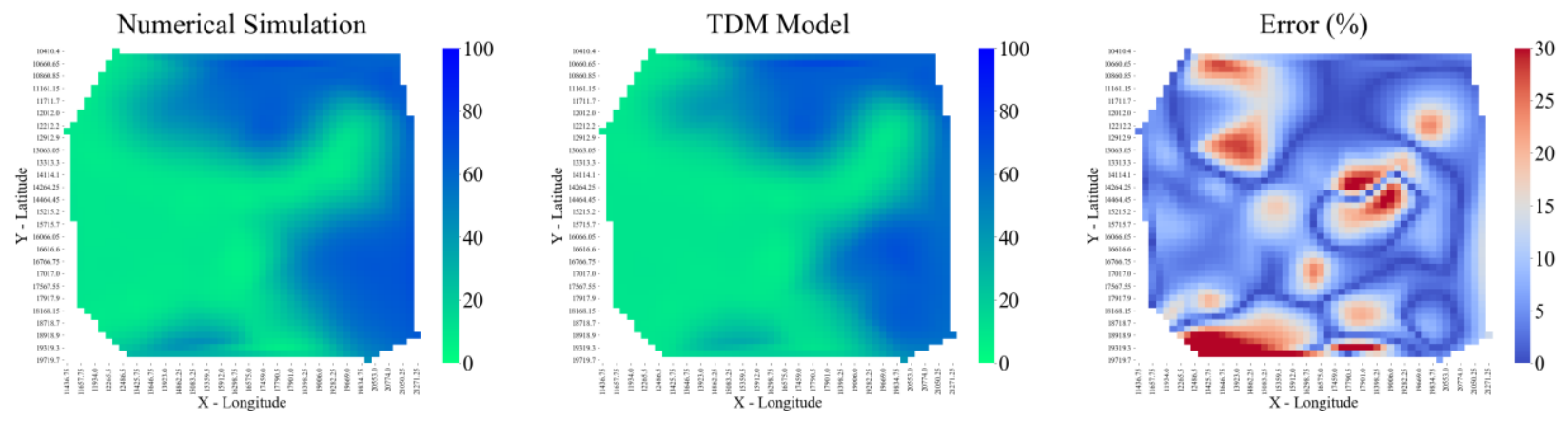

Figure 905 Entire reservoir heat map for water saturation at June 30th, 2015 for 6 Year Blind Validation TDM

Water Saturation (\%) at 2015-12-31
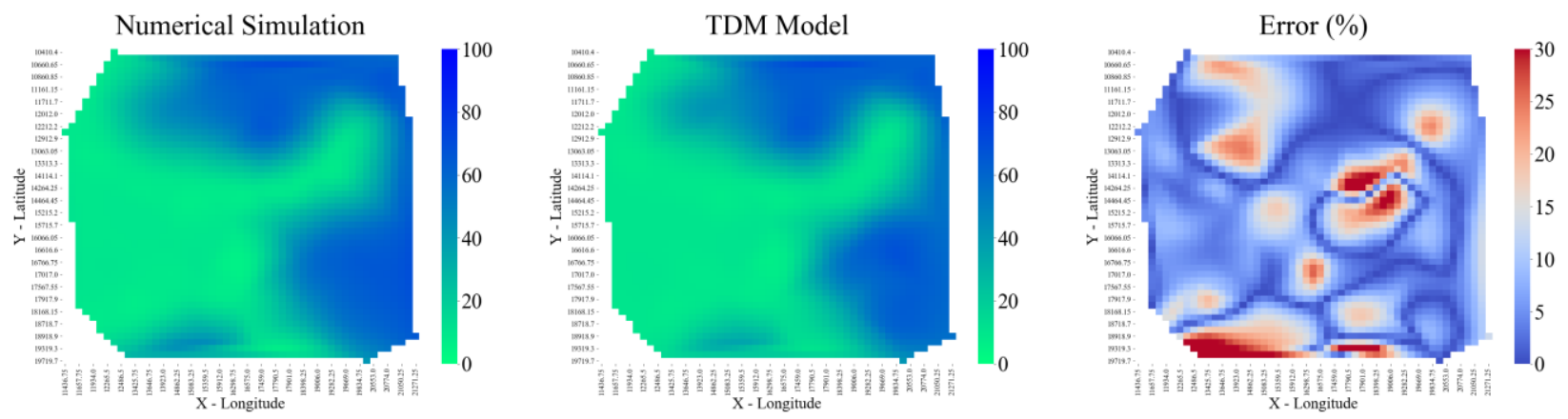

Figure 906 Entire reservoir heat map for water saturation at December 31st, 2015 for 6 Year Blind Validation TDM

Water Saturation (\%) at 2016-06-30
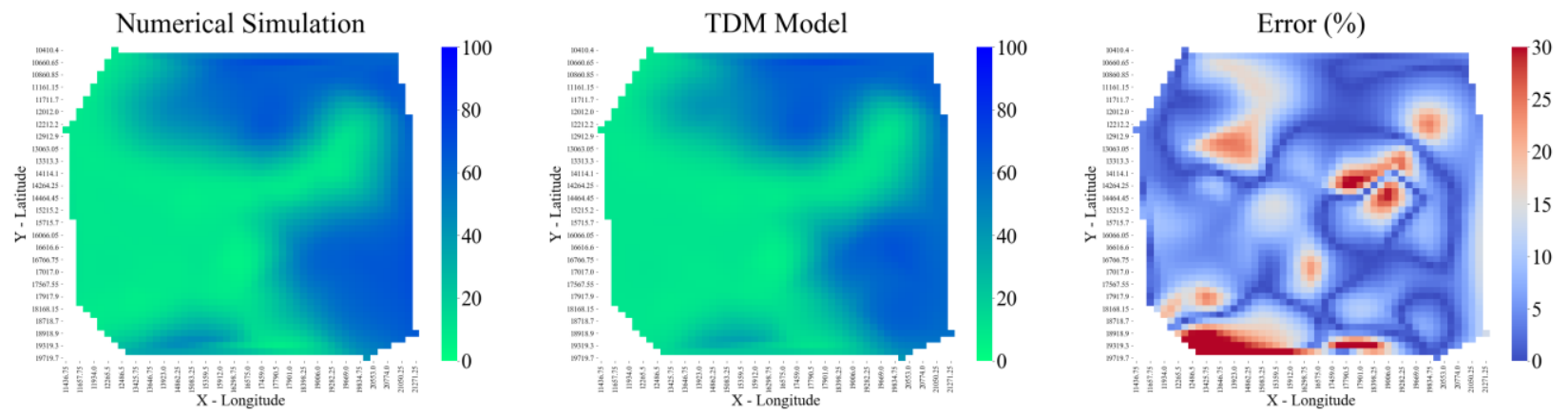

Figure 907 Entire reservoir heat map for water saturation at June 30th, 2016 for 6 Year Blind Validation TDM 
Water Saturation (\%) at 2016-12-31
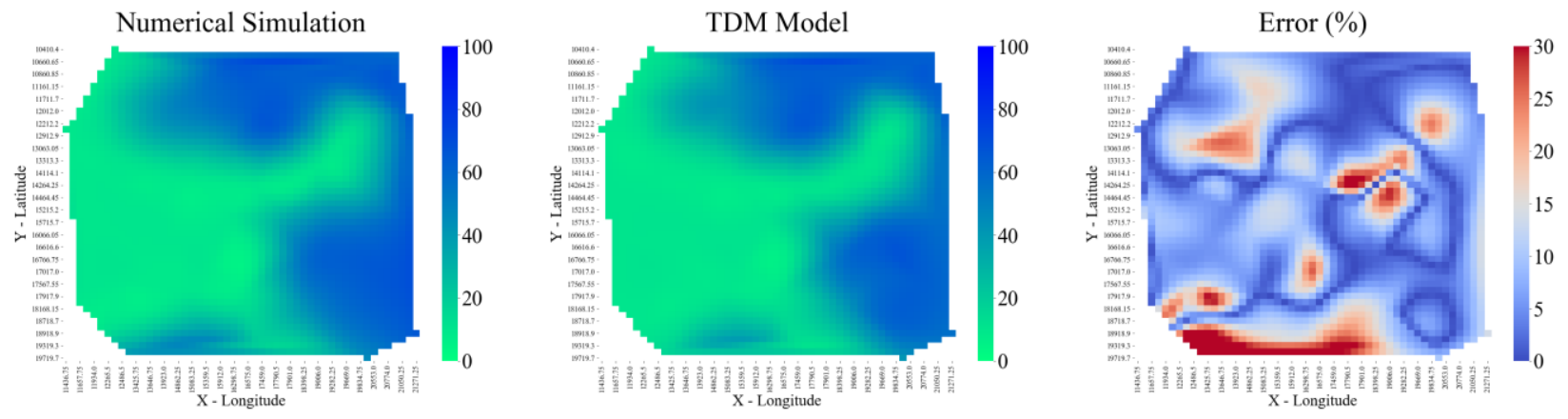

Figure 908 Entire reservoir heat map for water saturation at December 31st, 2016 for 6 Year Blind Validation TDM 